\title{
Phase Transition Enthalpy Measurements of Organic and Organometallic Compounds and Ionic Liquids. Sublimation, Vaporization, and Fusion Enthalpies from 1880 to 2015. Part 2. $C_{11}-C_{192}$
}

\author{
William Acree, Jr. \\ Department of Chemistry, University of North Texas, Denton, Texas 76203, USA
}

\author{
James S. Chickos \\ Department of Chemistry and Biochemistry, University of Missouri-St. Louis, One University Boulevard, St. Louis, Missouri 63121, USA
}

(Received 15 November 2016; accepted 15 November 2016; published online 8 March 2017)

\begin{abstract}
The second part of this compendium concludes with a collection of phase change enthalpies of organic molecules inclusive of $\mathrm{C}_{11}-\mathrm{C}_{192}$ reported over the period 1880-2015. Also included are phase change enthalpies including fusion, vaporization, and sublimation enthalpies for organometallic, ionic liquids, and a few inorganic compounds. Paper I of this compendium, published separately, includes organic compounds from $C_{1}$ to $\mathrm{C}_{10}$ and describes a group additivity method for evaluating solid, liquid, and gas phase heat capacities as well as temperature adjustments of phase changes. Paper II of this compendium also includes an updated version of a group additivity method for evaluating total phase change entropies which together with the fusion temperature can be useful in estimating total phase change enthalpies. Other uses include application in identifying potential substances that either form liquid or plastic crystals or exhibit additional phase changes such as undetected solid-solid transitions or behave anisotropically in the liquid state. () 2017 AIP Publishing LLC for the National Institute of Standards and Technology. [http://dx.doi.org/10.1063/1.4970519]
\end{abstract}

\section{CONTENTS}

1. Introduction

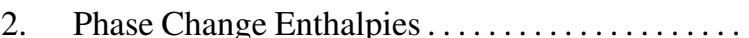

2.1. Total solid-liquid phase change entropies ...

2.2. The group additivity method $\ldots \ldots \ldots \ldots \ldots$

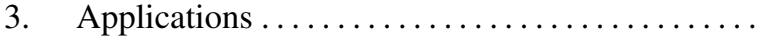

3.1. Estimations of acyclic and aromatic hydro-

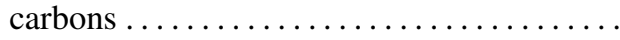

3.2. Estimations of polymers ............

3.3. Estimation of cyclic and polycyclic hydrocarbons ......................

3.4. Functionalized acyclic and aromatic hydro-

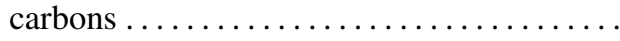

3.5. Estimations of functionalized cyclic and polycyclic hydrocarbons .............

4. Statistics in Evaluation of Group Values .........

5. The Phase Change Enthalpy Compendium, Paper

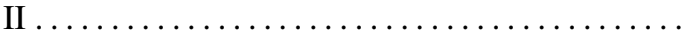

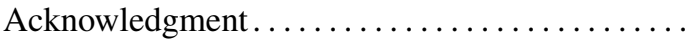

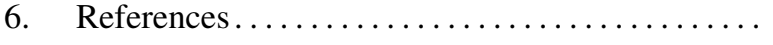

\section{List of Tables}

1. Contributions of the hydrocarbon portion of the molecule
2. Adjustments to contributions of the cyclic hydrocarbon portions of the molecule

3. Contributions of the functional group portion of the molecule; acyclic functional groups dependent on the substitution pattern ..............

4. Contributions of the functional group portion of the molecule; values for monosubstituted functional groups $\ldots \ldots \ldots \ldots \ldots \ldots \ldots \ldots \ldots \ldots \ldots$

5. Contributions of the functional group portion of the molecule; values for disubstituted functional

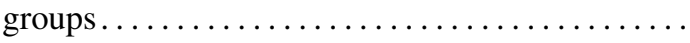

6. Contributions of the functional group portion of the molecule; values for trisubstituted functional

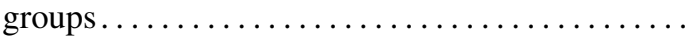

7. Contributions of the functional group portion of the molecule; values for tetrasubstituted functional groups .

8. Cyclic functional group adjustments to Eqs. (2) and $(3) \ldots \ldots \ldots \ldots \ldots \ldots \ldots \ldots \ldots \ldots \ldots \ldots \ldots \ldots \ldots \ldots \ldots \ldots$

9. List of acronyms and descriptions for methods ...

10. Phase change enthalpies of $\mathrm{C}_{11}$ organic com-

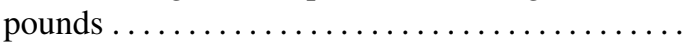

11. Phase change enthalpies of $\mathrm{C}_{12}$ to $\mathrm{C}_{13}$ organic

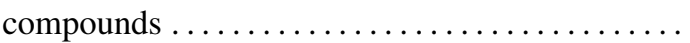

12. Phase change enthalpies of $\mathrm{C}_{14}$ to $\mathrm{C}_{18}$ organic compounds

13. Phase Change enthalpies of $\mathrm{C}_{17}$ to $\mathrm{C}_{18}$ organic compounds... 
14. Phase Change enthalpies of $\mathrm{C}_{19}-\mathrm{C}_{29}$ organic com-

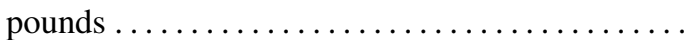

15. Phase change enthalpies of $\mathrm{C}_{30}$ to $\mathrm{C}_{49}$ organic

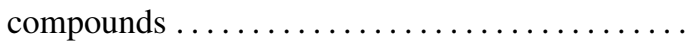

16. Phase change enthalpies of $\mathrm{C}_{50}$ to $\mathrm{C}_{192}$ organic

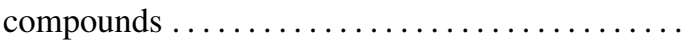

17. Phase change enthalpies of organometallic and inorganic compounds

18. Phase change enthalpies of organic salts and ionic

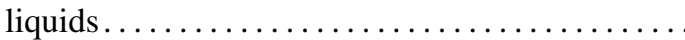

\section{List of Figures}

1. Estimations of acyclic and aromatic hydrocar-

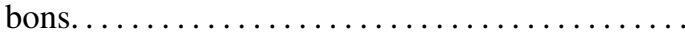

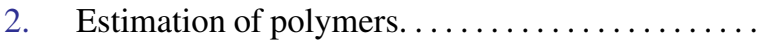

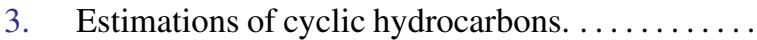

4. Estimation of aliphatic and aromatic derivatives. .

5. Estimations of complex cyclic and polycyclic

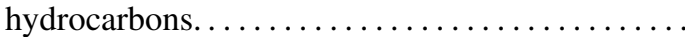

6. Distribution of differences between $\Delta S_{\text {tpce }}(\exp )$ $\Delta S_{\text {tpce }}$ (calc) for 3151 compounds. . . . . . . .

7. Distribution of differences between $\Delta H_{\text {tpce }}(\exp )$ $\Delta H_{\text {tpce }}$ (calc) for 3151 compounds. . . . . . . .

8. A comparison of $\Delta S_{\text {tpce }}(\exp )$ and $\Delta S_{\text {tpce }}$ (calc) for

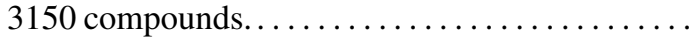

9. A comparison of $\Delta H_{\mathrm{tpce}}(\exp )$ and $\Delta H_{\mathrm{tpce}}$ (calc) for 3151 compounds. ................

\section{Introduction}

This compendium completes a previous report published in 2016 [2016ACR/CHI] on phase change enthalpies. Paper I of this compendium includes over 6600 organic compounds containing from one to ten carbon atoms published over the period of 1880-2015. [2016ACR/CHI] Paper II of this series contains over 7400 entries covering phase change enthalpies of organic compounds varying from eleven to one hundred ninety two carbon atoms, organometallic, and some inorganic compounds and ionic liquids. Many of these properties are freely available online [http://webbook.nist. gov/chemistry/ and http://trc.nist.gov/thermolit/]. As noted previously, a goal of this publication is to provide direct access to an entire collection of experimental phase change enthalpies that can be searched electronically. Additionally, some estimation methods and the use of various simple relationships for temperature adjustments of phase change enthalpies have been described and updated in Paper I. Paper II summarizes and updates a group method for estimating total phase change entropies. Uses include estimation of approximate fusion enthalpies and identification of potential substances exhibiting atypical behavior in either the solid or liquid state. Both the relationships and the estimation methods have proven useful to the authors.

\section{Phase Change Enthalpies}

Sublimation, vaporization, and fusion enthalpies are related to each other by Eq. (1) provided temperature $T$ for all three terms is the same. Estimation methods for each of these three terms have been developed but vary significantly in their accuracy. Numerous general methods for estimating vaporization enthalpies, some quite simple, have been developed and their degree of accuracy for many substances has been approaching the experimental uncertainty of the measurements.

$$
\Delta_{\text {sub }} H_{\mathrm{m}}(T)=\Delta_{\mathrm{v}} H_{\mathrm{m}}(T)+\Delta_{\text {fus }} H_{\mathrm{m}}(T)
$$

Estimations of sublimation and fusion enthalpies are more problematic, primarily because of the difficulties in estimating fusion enthalpies accurately. Group additivity methods, widely successful in estimations of many other thermochemical properties, frequently falter in estimation of fusion enthalpy because of dynamics, the structural complexity of the solid state, or nonisotropic behavior of the liquid phase. The occurrence of polymorphism, solid-solid phase transitions, plastic and liquid crystalline behavior, and formation of anisotropic liquids while each of relatively low probability are unpredictable, and combine together to produce significant difficulties in developing a successful estimation method for both fusion and sublimation enthalpy.

\subsection{Total solid-liquid phase change entropies}

An approach that has been taken to address the estimation of fusion enthalpy has focused initially on estimating entropy rather than enthalpy since the occurrence of solid-solid transitions are among the most common and these generally occur isothermally; many have also been measured. The thermodynamic function that was subjected to a group additivity approach was estimation of the total molar phase change entropy, $\Delta S_{\text {tpce }}$, the total entropy associated with isothermal events occurring from $T / \mathrm{K}=\left(0\right.$ to $\left.T_{\text {fus }}\right)$. For most substances with no additional phase transitions other than fusion, and isotropic behavior of the liquid, the product of $T_{\text {fus }} \cdot \Delta S_{\text {tpce }}$ can provide a reasonable estimate of the fusion enthalpy.

If the fusion enthalpy of the compound in question is known, the method described below offers a relatively simple manner of identifying potential substances that exhibit atypical behavior. For example, in an application of an earlier version of this estimation method applied to over 600 compounds known to form liquid crystals, this method greatly overestimated the total phase change entropies in comparison to solids with no additional phase transitions other than fusion. Even in cases where the total entropies of transition in the liquid crystals were known, the estimated values still exceeded the total values calculated. Only when the entropy associated with the heat capacity was also taken into account did the total entropy of the molecule correlate with homologous molecules that did not form liquid crystals as shown by Sorai et al. for the benzene hexa- $n$-alkanoates. [2003ASA/SOR, 2001ASA/SOR, 2006ACR/CHI] 
Atypical behavior in the liquid state, though less common, is also well known. Molecules containing segregated portions of fluorocarbons and hydrocarbons frequently do not behave isotropically in the liquid state and their fusion entropies are also overestimated by this approach. The overestimation is approximately $40 \%$ of the value estimated. [2003CHI/ACR]

While the fusion entropies of compounds exhibiting polymorphism can differ, their values are often quite similar. For such substances, the estimated total phase change entropy described below appears to correlate best with the thermodynamically most stable form. [2003CHI/ACR]

\subsection{The group additivity method}

The group method that has evolved mirrors both the complexity and diversity of the solid state. The protocol was last updated in 2009. [2009CHI/ACR] The group method described below in Secs. 3-5 is based mainly on structure. The focus of this update is to provide additional data to bolster the statistics of a number of group values previously assigned based on very limited data and to increase the scope of the method to include additional functional groups. Most group values reported previously have not been tampered with; exceptions include a few tentative values that have either been modified on the basis of the availability of new data or eliminated because of their unreliability. Group values are defined in Tables 1-8. Table 3 defines a few common groups whose values depend on the number of other functional groups present on the target molecule as defined in Table 3. Tables 4-7 segregate functional groups according to the number of additional attachments necessary to satisfy the valence requirements of the functional group; one, two, three, or four groups. Table 8 compliments Table 2 by providing contributions for cyclic functional groups. Groups in the tables are described in column 1 and defined in the second column; group values are provided in the third column. Group values in brackets have been evaluated by fewer than ten entries and are considered tentative assignments. Their value is based on the number of entries provided in parenthesis in column 1 of

TABLE 1. Contributions of the hydrocarbon portion of the molecule

\begin{tabular}{|c|c|c|c|}
\hline \multirow{2}{*}{$\frac{\text { Aliphatic and aromatic carbon groups }}{\text { Primary } \mathrm{sp}^{3} \mathrm{C}}$} & \multicolumn{2}{|c|}{$\begin{array}{l}\text { Group value }\left(\mathrm{G}_{\mathrm{i}}\right)^{\mathrm{a}} \\
\mathrm{J} \cdot \mathrm{mol}^{-1} \cdot \mathrm{K}^{-1}\end{array}$} & \multirow[t]{2}{*}{$\begin{array}{c}\text { Group } \\
\text { coefficient }(\mathrm{C})^{\mathrm{a}}\end{array}$} \\
\hline & $\mathrm{CH}_{3}-\mathrm{R}$ & 17.6 & \\
\hline Secondary $\mathrm{sp}^{3} \mathrm{C}$ & $\mathrm{R}_{2}>\mathrm{CH}_{2}$ & 7.1 & $1.31^{\mathrm{b}}$ \\
\hline Tertiary $\mathrm{sp}^{3} \mathrm{C}$ & $\mathrm{R}_{2}>\mathrm{CH}-\mathrm{R}$ & -16.4 & $0.60^{c}$ \\
\hline Quaternary $\mathrm{sp}^{3} \mathrm{C}$ & $\mathrm{R}_{2}>\mathbf{C}<\mathrm{R}_{2}$ & -34.8 & $0.66^{\mathrm{c}}$ \\
\hline Secondary $\mathrm{sp}^{2} \mathrm{C}$ & $=\mathrm{CH}_{2}$ & 17.3 & \\
\hline Tertiary $\mathrm{sp}^{2} \mathrm{C}$ & $=\mathbf{C H}-$ & 5.3 & $0.75^{\mathrm{c}}$ \\
\hline Quaternary $\mathrm{sp}^{2} \mathrm{C}$ & $=\mathbf{C}(\mathrm{R})-$ & -10.7 & \\
\hline Tertiary sp C & $\mathbf{H}-\mathbf{C} \equiv$ & 17.5 & \\
\hline Quaternary sp C & $\mathrm{R}-\mathrm{C} \equiv$ & -4.3 & \\
\hline Tertiary benzenoid $\mathrm{sp}^{2} \mathrm{C}$ & $=\mathbf{C}_{\mathbf{a}} \mathbf{H}-$ & 7.4 & \\
\hline Quaternary benzenoid $\mathrm{sp}^{2} \mathrm{C}$ adjacent to an $\mathrm{sp}^{3}$ atom ${ }^{\mathrm{d}}$ & $=\mathbf{C}_{\mathrm{a}}(\mathrm{R})-$ & -9.6 & \\
\hline Quaternary benzenoid $\mathrm{sp}^{2} \mathrm{C}$ adjacent to an $\mathrm{sp}^{2}$ atom ${ }^{\mathrm{e}}$ & $=\mathrm{C}_{\mathrm{a}}(\mathrm{R})-$ & -7.5 & \\
\hline Internal benzenoid quaternary adjacent to $3 \mathrm{sp}^{2}$ atoms ${ }^{\mathrm{f}}$ & $=\mathbf{C}_{\mathrm{a}}(\mathrm{R})-$ & -0.7 & \\
\hline
\end{tabular}

${ }^{\mathrm{a}} \mathrm{R}$ refers to any group or heteroatom; group coefficients are assumed to be 1 unless noted otherwise.

${ }^{b}$ The group coefficient of 1.31 for $\mathrm{C}_{\mathrm{CH}_{2}}$ is applied only when the number of consecutive methylene groups exceeds the sum of the remaining groups (both carbon and other functional groups); see text for additional details.

${ }^{\mathrm{c}}$ Applied only when a functional group is attached at this position.

${ }^{\mathrm{d}}$ This group applies to any quaternary benzenoid carbon adjacent to an $\mathrm{sp}^{3}$ hybridized atom with no lone pair of electrons.

${ }^{\mathrm{e}}$ This group applies to any quaternary benzenoid carbon adjacent to any $\mathrm{sp}^{2}$ hybridized atom and to $\mathrm{sp}^{3}$ hybridized atoms with a lone pair electrons with the exception of internal quaternary carbon atoms (see footnote $\mathrm{f}$ ).

${ }^{\mathrm{f}}$ Any internal quaternary benzenoid carbon that is not at the periphery of a molecule; for example the six internal quaternary benzenoid carbon atoms of coronene.

TABLE 2. Adjustments to contributions of the cyclic hydrocarbon portions of the molecule

\begin{tabular}{|c|c|c|c|}
\hline \multicolumn{2}{|c|}{ Contributions of cyclic carbons $\left(\mathrm{C}_{\mathrm{c}}\right)$} & \multicolumn{2}{|c|}{$\begin{array}{l}\text { Group value }\left(\mathrm{G}_{\mathrm{i}}\right)^{\mathrm{a}} \\
\mathrm{J} \cdot \mathrm{mol}^{-1} \cdot \mathrm{K}^{-1}\end{array}$} \\
\hline Cyclic tertiary $\mathrm{sp}^{3}$ carbon & $-\mathbf{C}_{\mathbf{c}} \mathbf{H}(\mathrm{R})-$ & -14.7 & \\
\hline Cyclic quaternary $\mathrm{sp}^{3}$ carbon & $-\mathbf{C}_{\mathbf{c}}<(\mathrm{R})_{2}-$ & -34.6 & \\
\hline Cyclic tertiary $\mathrm{sp}^{2}$ carbon & $=\mathrm{C}_{\mathrm{c}} \mathrm{H}-$ & -1.6 & $1.92^{\mathrm{b}}$ \\
\hline Cyclic quaternary $\mathrm{sp}^{2}$ carbon & $=\mathbf{C}_{\mathbf{c}}(\mathrm{R})-$ & -12.3 & \\
\hline Cyclic quaternary sp carbon & $=\mathbf{C}_{\mathbf{c}}=; \mathrm{R}-\mathbf{C}_{\mathbf{c}} \equiv$ & -4.7 & \\
\hline
\end{tabular}

${ }^{\mathrm{a}} \mathrm{R}$ refers to any organic fragment including other functional groups or heteroatom; group coefficients are assumed to be 1 unless noted otherwise.

${ }^{\mathrm{b}}$ Applied only when a cyclic functional group is attached at this position. 
TABLE 3. Contributions of the functional group portion of the molecule; acyclic functional groups dependent on the substitution pattern

\begin{tabular}{|c|c|c|c|c|c|}
\hline \multirow[b]{3}{*}{ Functional groups $^{\mathrm{a}}$} & & \multirow{3}{*}{$\begin{array}{l}\text { Group value }\left(\mathrm{G}_{\mathrm{k}}\right)^{\mathrm{a}} \\
\mathrm{J} \cdot \mathrm{mol}^{-1} \cdot \mathrm{K}^{-1}\end{array}$} & \multicolumn{3}{|c|}{ Group coefficient $\left(\mathrm{C}_{\mathrm{k}}\right)^{\mathrm{a}}$} \\
\hline & & & \multicolumn{3}{|c|}{$\mathrm{k}$} \\
\hline & & & 2 & 3 & $\mathbf{k}_{n}{ }^{\mathrm{b}}$ \\
\hline Chlorine & & 10.8 & 1.5 & 1.5 & 1.5 \\
\hline 2-fluorines on an acyclic $\mathrm{sp}^{3} \mathrm{C}$ & $\mathrm{R}_{2}>\mathbf{C F}_{\mathbf{2}}$ & 13.2 & 1.06 & 1.06 & 1.15 \\
\hline Alcohol & $\mathrm{R}-\mathrm{OH}$ & 1.7 & 10.4 & 9.7 & 13.1 \\
\hline Carboxylic acid & $\mathrm{R}-\mathrm{C}(=\mathbf{O}) \mathbf{O H}$ & 13.4 & 1.21 & 1.21 & 2.25 \\
\hline
\end{tabular}

${ }^{\mathrm{a}}$ Group coefficient refers to the total number of functional groups present; $\mathrm{R}$ refers to any organic fragment including other functional groups.

${ }^{\mathrm{b}}$ To be used in cases with 4 or more functional groups.

${ }^{\mathrm{c}}$ To be used in acyclic perfluorinated hydrocarbons.

TABLE 4. Contributions of the functional group portion of the molecule; values for monosubstituted functional groups

\begin{tabular}{|c|c|c|}
\hline Acyclic groups & & $\begin{array}{l}\text { Group value }\left(\mathrm{G}_{\mathrm{k}}\right)^{\mathrm{b}} \\
\mathrm{J} \cdot \mathrm{mol}^{-1} \cdot \mathrm{K}^{-1}\end{array}$ \\
\hline Bromine & $\mathrm{R}-\mathbf{B r}$ & 17.5 \\
\hline Fluorine on an $\mathrm{sp}^{2}$ carbon & $=\mathrm{CHF}$ & 19.5 \\
\hline Fluorine on an aromatic carbon & $=\mathrm{C}_{\mathrm{a}} \mathbf{F}-$ & 16.6 \\
\hline 3 -fluorines on an $\mathrm{sp}^{3}$ carbon & $\mathrm{CF}_{3}-\mathrm{R}$ & 13.2 \\
\hline 1-fluorine on an $\mathrm{sp}^{3}$ carbon & $\mathrm{R}_{2}>\mathrm{CF}-\mathrm{R}$ & 12.7 \\
\hline 1 or 2 Fluorines on a cyclic $\mathrm{sp}^{3}$ carbon & $>C_{\mathrm{c}} \mathrm{HF}->C_{\mathrm{c}} \mathbf{F}_{2}-$ & {$[17.5] / F$} \\
\hline Iodine & $\mathrm{R}-\mathbf{I}$ & 19.4 \\
\hline Phenol & $=\mathrm{C}-(\mathbf{O H})-$ & 20.3 \\
\hline Hydroperoxide (2) & $\mathrm{R}-\mathrm{OOH}$ & {$[31.8]$} \\
\hline Aldehyde & $\mathrm{R}-\mathbf{C H}(=\mathbf{O})$ & 21.5 \\
\hline Formate ester (4) & $\mathrm{R}-\mathbf{O}(\mathbf{C}=\mathbf{O}) \mathbf{H}$ & {$[22.3]$} \\
\hline Acyl chloride (1) & $\mathrm{R}-(\mathbf{C}=\mathbf{O}) \mathrm{Cl}$ & {$[25.8]$} \\
\hline Primary amine & $\mathrm{R}-\mathbf{N H}_{2}$ & 21.4 \\
\hline Azide (2) & $\mathrm{R}-\mathbf{N}_{3}$ & {$[36.3]$} \\
\hline Nitro group & $\mathrm{R}-\mathbf{N O}_{2}$ & 17.7 \\
\hline Nitrate ester & $\mathrm{R}-\mathrm{ONO}_{2}$ & 24.4 \\
\hline Nitrile & $\mathrm{R}-\mathrm{C} \equiv \mathbf{N}$ & 17.7 \\
\hline Isocyanide (1) & $\mathrm{R}-\mathbf{N C}$ & {$[17.5]$} \\
\hline Isocyanate (4) & $\mathrm{R}-\mathbf{N}=\mathbf{C}=\mathbf{O}$ & {$[23.1]$} \\
\hline Primary amide & $\mathrm{R}-\mathrm{CONH}_{2}$ & 27.9 \\
\hline $\mathrm{N}$-nitroso (3) & $>\mathrm{N}-\mathbf{N}=\mathbf{O}$ & {$[33.1]$} \\
\hline Oxime & $=\mathrm{N}-\mathrm{OH}$ & 13.6 \\
\hline Hydrazide (5) & $\mathrm{R}-\mathrm{C}(=\mathrm{O}) \mathbf{N H N H}_{2}$ & {$[26.0]$} \\
\hline Monosubstituted urea & $\mathrm{R}-\mathbf{N H C}(=\mathbf{O}) \mathbf{N H}_{2}$ & 14.1 \\
\hline Monosubstituted thiourea (4) & $\mathrm{R}-\mathbf{N H C}(=\mathbf{S}) \mathbf{N H}_{2}$ & {$[30.1]$} \\
\hline Phosphonic acid (2) & $\mathrm{R}-(\mathbf{P}=\mathbf{O})(\mathbf{O H})_{2}$ & {$[13]$} \\
\hline Thiols & $\mathrm{R}-\mathrm{SH}$ & 23.0 \\
\hline Thioamide (1) & $\mathrm{R}-\mathrm{C}(=\mathbf{S}) \mathbf{N H}_{2}$ & {$[30.0]$} \\
\hline Sulfonic acid (2) & $\mathrm{R}-\mathbf{S}(=\mathbf{O})_{2} \mathbf{O H}$ & {$[1.8]$} \\
\hline Sulfonamide (8) & $\mathrm{R}-\mathbf{S}(=\mathbf{O})_{2} \mathbf{N H}_{2}$ & 25.2 \\
\hline Sulfonyl chloride (2) & $\mathrm{R}-\mathbf{S}(=\mathbf{O})_{2} \mathrm{Cl}$ & {$[23.4]$} \\
\hline Alkyl arsonic acid (3) & $\mathrm{R}-(\mathbf{A s}=\mathbf{O})(\mathbf{O H})_{2}$ & {$[-2.9]$} \\
\hline
\end{tabular}

the table. Details of the application of the estimation method are illustrated by several specific examples.

\section{Applications}

Application of the group values in Tables 1-8 used to estimate total phase change entropies is illustrated below. The estimations are arranged in terms of increasing complexity.

3.1 Estimations of acyclic and aromatic hydrocarbons.
3.2 Estimation of polymers.

3.3 Estimations of cyclic and polycyclic hydrocarbons.

3.4 Estimations of functionalized acyclic and aromatic hydrocarbons.

3.5 Estimations of functionalized cyclic and polycyclic hydrocarbons.

3.6 Estimations of complex cyclic and polycyclic compounds.

Group values for carbon and for a number of other elements are defined on the basis of their substitution 
TABLE 5. Contributions of the functional group portion of the molecule; values for disubstituted functional groups

\begin{tabular}{|c|c|c|}
\hline Acyclic groups & & $\begin{array}{c}\text { Group value }\left(\mathrm{G}_{\mathrm{k}}\right)^{\mathrm{b}} \\
\mathrm{J} \cdot \mathrm{mol}^{-1} \cdot \mathrm{K}^{-1}\end{array}$ \\
\hline Ether & $\mathrm{R}-\mathrm{O}-\mathrm{R}$ & 4.71 \\
\hline Peroxide (1) & $\mathrm{R}-\mathrm{O}-\mathrm{O}-\mathrm{R}$ & {$[10.6]$} \\
\hline Ketone & $\mathrm{R}-\mathrm{C}(=\mathbf{O})-\mathrm{R}$ & 4.6 \\
\hline Ester & $\mathrm{R}-\mathbf{C}(=\mathbf{O}) \mathbf{O}-\mathrm{R}$ & 7.7 \\
\hline Carbonate (4) & $\mathrm{R}-\mathbf{O C}(=\mathbf{O}) \mathbf{O}-\mathrm{R}$ & {$[7.1]$} \\
\hline Anhydride (2) & $\mathrm{R}-(\mathbf{C}=\mathbf{O}) \mathbf{O}(\mathbf{C}=\mathbf{O})-\mathrm{R}$ & {$[10.0]$} \\
\hline Aromatic heterocyclic nitrogen & $=\mathbf{N}_{\mathrm{a}}-$ & 10.9 \\
\hline Acyclic $\mathrm{sp}^{2}$ nitrogen & $=\mathrm{N}-$ & -1.8 \\
\hline Secondary amine & $\mathrm{R}-\mathbf{N H}-\mathrm{R}$ & 0.2 \\
\hline Azoxy nitrogen (6) & $\mathrm{R}-\mathrm{N}=\mathbf{N}(\rightarrow \mathbf{O})-\mathrm{R}$ & [3.7] \\
\hline Secondary amide & $\mathrm{R}-\mathbf{C}(=\mathbf{O}) \mathbf{N H}-\mathrm{R}$ & 1.5 \\
\hline Iminohydrazide (4) & $\mathrm{R}-\mathbf{C}(=\mathbf{O}) \mathbf{N H N}=\mathrm{CHR}$ & [18.6] \\
\hline 1,1-disubstituted urea (2) & $\mathrm{R}_{2}>\mathbf{N C}(=\mathbf{O}) \mathbf{N H}_{2}$ & {$[19.5]$} \\
\hline 1,3-disubstituted urea & $\mathrm{RNHC}(=\mathbf{O}) \mathbf{N H}-\mathrm{R}$ & -8.1 \\
\hline 1,3-Diacyl substituted thiourea (3) & $\mathrm{R}[\mathbf{C}(=\mathbf{O}) \mathbf{N H}(\mathbf{C}=\mathrm{S}) \mathbf{N H C}(=\mathbf{O})] \mathrm{R}$ & {$[-76]$} \\
\hline Imide (3) & $\mathrm{R}-[\mathrm{C}(=\mathbf{O}) \mathbf{N H C}(=\mathbf{O})]-\mathrm{R}$ & [10.4] \\
\hline Phosphinic acid (3) & $\mathrm{R}_{2}>[(\mathbf{P}=\mathbf{O}) \mathbf{O H}]$ & [17] \\
\hline Phosphoroamidodithioate ester (1) & {$\left[\mathbf{N H}_{2} \mathbf{P}(=\mathbf{S})(\mathbf{S}-\mathbf{R})(\mathbf{O}]-\mathbf{R}\right)$} & [6.9] \\
\hline Sulfides & $\mathrm{R}-\mathrm{S}-\mathrm{R}$ & 2.1 \\
\hline Disulfides (6) & $\mathrm{R}-\mathbf{S S}-\mathrm{R}$ & [9.6] \\
\hline Sulfoxide (3) & $\mathrm{R}-\mathbf{S}(=\mathbf{O})-\mathrm{R}$ & [8.0] \\
\hline Sulfones & $\mathrm{R}-\mathbf{S}(=\mathbf{O})_{2}-\mathrm{R}$ & 0.6 \\
\hline Sulfonate ester (3) & $\mathrm{R}-\mathbf{S}(=\mathbf{O})_{2} \mathbf{O}-\mathrm{R}$ & [7.3] \\
\hline 1,3-disubstituted thiourea (6) & $\mathrm{R}-\mathbf{N H C}(=\mathbf{S}) \mathbf{N H}-\mathrm{R}$ & {$[7.8]$} \\
\hline Isothiourea(1) & $\mathrm{R}-\mathbf{S}-(\mathbf{C}=\mathbf{N H}) \mathbf{N H R}$ & {$[23.8]$} \\
\hline Thioamide (1) & $\mathrm{R}-\mathbf{C}(=\mathbf{S}) \mathbf{N H}_{2}$ & {$[15.0]$} \\
\hline $\mathrm{N}$-substituted sulfonamide & $\mathrm{R}-\mathbf{S}(\rightarrow \mathbf{O})_{2} \mathbf{N H}-\mathrm{R}$ & 4.5 \\
\hline Disubstituted selenium (9) & $\mathrm{R}_{2}-\mathrm{Se}$ & {$[-23]$} \\
\hline Disubstituted zinc (3) & $\mathrm{R}_{2} \mathbf{Z n}$ & [11.1] \\
\hline Disubstituted telluride (5) & $\mathrm{R}_{2} \mathrm{Te}$ & {$[5.1]$} \\
\hline Disubstituted arsenic acid & $\mathrm{R}_{2}(\mathbf{A s}=\mathbf{O}) \mathbf{O H}$ & -24 \\
\hline
\end{tabular}

TABLE 6. Contributions of the functional group portion of the molecule; values for trisubstituted functional groups

\begin{tabular}{|c|c|c|}
\hline Groups & & $\begin{array}{c}\text { Group value }\left(\mathrm{G}_{\mathrm{k}}\right)^{\mathrm{b}} \\
\mathrm{J} \cdot \mathrm{mol}^{-1} \cdot \mathrm{K}^{-1}\end{array}$ \\
\hline Tertiary amine & $\mathrm{R}_{3} \mathbf{N}$ & -22.2 \\
\hline Tertiary amide & $\mathrm{R}-(\mathbf{C}=\mathbf{O}) \mathbf{N}<\mathrm{R}_{2}$ & -11.2 \\
\hline 1,1,3-trisubstituted urea & $\mathrm{R}_{2}>\mathbf{N C}(=\mathbf{O}) \mathbf{N H}-\mathrm{R}$ & 0 \\
\hline Phosphine oxide (3) & $\mathrm{R}_{3} \mathbf{P}=\mathbf{O}$ & {$[-21.1]$} \\
\hline Phosphonate ester (1) & $\mathrm{R}-\mathbf{P}(=\mathbf{O})(\mathbf{O}-\mathrm{R})_{2}$ & {$[-3.4]$} \\
\hline Phosphoramidate ester (5) & $(\mathrm{R}-\mathbf{O})_{2} \mathbf{P}(=\mathbf{O}) \mathbf{N H}-\mathrm{R}$ & {$[-9.2]$} \\
\hline Phosphorothioate ester & $(\mathrm{R}-\mathbf{O})_{3} \mathbf{P}(=\mathbf{S})$ & 1.1 \\
\hline Phosphorodithioate ester (8) & $\mathrm{R}-\mathbf{S}-\mathbf{P}(=\mathbf{S})(\mathbf{O}-\mathbf{R})_{2}$ & -9.6 \\
\hline Phosphonothioate ester (2) & $\mathrm{R}-\mathbf{P}(=\mathbf{S})(\mathbf{O}-\mathrm{R})_{2}$ & {$[5.2]$} \\
\hline Phosphoroamidothioate ester (1) & $\mathrm{R}-\mathbf{N H P}(=\mathbf{S})(\mathbf{O}-\mathrm{R})_{2}$ & {$[16.0]$} \\
\hline Diacylsubstituted isoselenourea & $\mathrm{RC}(=\mathbf{O}) \mathbf{N}=\mathbf{C}(\mathrm{SeR}) \mathbf{N H C}(=\mathbf{O}) \mathrm{R}$ & -51 \\
\hline $\mathrm{N}, \mathrm{N}$-disubstitued thioamide (2) & $\mathrm{R}-\mathbf{C}(=\mathbf{S}) \mathbf{N R}_{2}$ & {$[-13.5]$} \\
\hline $\mathrm{N}, \mathrm{N}$ disubstituted thiocarbamate (1) & $\mathrm{R}-\mathbf{S C}(=\mathbf{O}) \mathbf{N}<\mathrm{R}_{2}$ & {$[5.5]$} \\
\hline N,N-disubstituted sulfonamide (4) & $\mathrm{R}-\mathbf{S}(=\mathbf{O})_{2} \mathbf{N}-\mathrm{R}_{2}$ & {$[-11.3]$} \\
\hline Trisubstituted aluminum (2) & $\mathrm{R}_{3} \mathrm{Al}$ & {$[-24.7]$} \\
\hline Trisubstituted arsenic (5) & $\mathrm{R}_{3} \mathrm{As}$ & {$[3.1]$} \\
\hline Trisubstituted boron (2) & $\mathrm{R}_{3} \mathrm{~B}$ & {$[-17.2]$} \\
\hline Trisubstituted gallium (2) & $\mathrm{R}_{3} \mathbf{G a}$ & {$[-11.3]$} \\
\hline Trisubstituted indium (2) & $\mathrm{R}_{3}$-In & {$[-19.3]$} \\
\hline
\end{tabular}

pattern and hybridization. The terms primary, secondary, tertiary, and quaternary are defined in terms of the number of attached hydrogens, 3, 2, 1, 0, respectively. Tables 1 and 2 define the typical carbon groups found in organic chemistry.

\subsection{Estimations of acyclic and aromatic hydrocarbons}

Examples of the estimations of simple hydrocarbons are illustrated in Fig. 1. These estimations follow a standard 
TABLE 7. Contributions of the functional group portion of the molecule; values for tetrasubstituted functional groups

\begin{tabular}{lcc}
\hline \hline Groups & & $\begin{array}{c}\text { Group value }\left(\mathrm{G}_{\mathrm{k}}\right)^{\mathrm{b}} \\
\mathrm{J} \cdot \mathrm{mol}^{-1} \cdot \mathrm{K}^{-1}\end{array}$ \\
\hline Tetrasubstituted thiourea (1) & $\mathrm{R}_{2}>\mathbf{N}(\mathbf{C}=\mathbf{S}) \mathbf{N}<\mathrm{R}_{2}$ & {$[-7.2]$} \\
Quaternary silicon & $\mathrm{R}_{4} \mathbf{S i}$ & -27.1 \\
Quaternary tin (6) & $\mathrm{R}_{4} \mathbf{S n}$ & {$[-24.2]$} \\
\hline
\end{tabular}

group additivity protocol with few exceptions. If the number of consecutive methylene groups $\left(\mathrm{C}_{\mathrm{CH} 2}\right.$, secondary $\left.\mathrm{sp}^{3} \mathrm{C}\right)$ equals or exceeds the sum total of carbon groups as defined in Tables 1 and 2 and other functional groups as defined in Tables 3-8, the value is incremented from 7.1 to 9.3 $\mathrm{J} \cdot \mathrm{mol}^{-1} \cdot \mathrm{K}^{-1}(7.1 \times 1.31$, Table 1$)$. Please note that only the groups in Tables 3-8 are considered functional groups in the discussion that follows. The remaining group coefficients identified in the last column of Table 1 with the exception of the value of 1.31 are only used with attached functional groups at those locations and their use is illustrated below. In both tridecane and diphenylmethane, estimation of the total phase change entropy is quite simple. Tridecane contains only two carbon groups, a methyl and methylene group. The groups can easily be identified in Table 1. Diphenylmethane contains two different types of aromatic carbon groups, a tertiary and quaternary carbon (no available hydrogens). Since the carbon of the methylene group is $\mathrm{sp}^{3}$ hybridized and has no available $p$ electrons, the value -9.6 is chosen for the quaternary benzenoid carbon. Heterocyclic aromatic compounds are estimated in a similar manner using the group value for an aromatic $\mathrm{sp}^{2}$ hybridized nitrogen reported in Table 5. Once the total phase change entropy is calculated, the product of $\Delta S_{\text {tpce }}$ and the melting point $T_{\text {fus }}$ can provide an estimate of the total phase change enthalpy, $\Delta H_{\text {tpce. }}$. Tridecane

TABLE 8. Cyclic functional group adjustments to Eqs. (2) and (3)

\begin{tabular}{|c|c|c|}
\hline $\begin{array}{l}\text { Heteroatoms and functional groups } \\
\text { comprising a portion of a ring }\end{array}$ & & $\begin{array}{l}\text { Group value }\left(\mathrm{G}_{\mathrm{k}}\right)^{\mathrm{b}} \\
\mathrm{J} \cdot \mathrm{mol}^{-1} \cdot \mathrm{K}^{-1}\end{array}$ \\
\hline Cyclic ether & $R-\boldsymbol{O}_{\mathrm{c}}-R$ & 1.2 \\
\hline Cyclic peroxide (4) & $R-[\boldsymbol{O O O}]_{\mathrm{c}}-R$ & [27.7] \\
\hline Cyclic ketone & $R-[\boldsymbol{C}(=\mathrm{O})]_{\mathrm{c}}-R$ & -1.4 \\
\hline Cyclic ester & $R-[\boldsymbol{C}(=\mathbf{O}) O]_{\mathrm{c}}-R$ & 3.1 \\
\hline Cyclic carbonate (6) & $R-[\boldsymbol{O C}(=\mathbf{O}) O]_{\mathrm{c}}-R$ & {$[1.3]$} \\
\hline Cyclic anhydride & $R-[\boldsymbol{C}(=\mathbf{O})-\boldsymbol{O}-\boldsymbol{C}(=O)]_{\mathfrak{c}}-R$ & 2.3 \\
\hline Cyclic $\mathrm{sp}^{2}$ nitrogen & $\mathrm{R} R C=\left[\mathrm{N}_{c}\right]-R$ & 0.5 \\
\hline Cyclic tertiary amine & $R_{2}>N_{c}-\mathrm{R}$ & -19.3 \\
\hline Cyclic hydrazine (2) & $R_{2}>N_{c} \mathbf{N H}_{2}$ & [21.7] \\
\hline Cyclic secondary amine & $R-N_{c} \mathbf{H}-R$ & 2.2 \\
\hline Cyclic tertiary amine-N-oxide (1) & $R-\boldsymbol{N}_{c}(\rightarrow \mathbf{O})-R \mathrm{R}$ & {$[-22.2]$} \\
\hline Cyclic azoxy group (2) & $R-N_{c}=N_{c}(\rightarrow \mathbf{O})-R$ & {$[2.9]$} \\
\hline Cyclic secondary amide & $R-[\boldsymbol{C}(=\mathbf{O}) N \mathrm{H}]_{\mathrm{c}}-R$ & 2.7 \\
\hline Cyclic tertiary amide & $R-[\boldsymbol{C}(=\mathbf{O}) N]_{\mathbf{c}}<R, \mathrm{R}$ & -21.7 \\
\hline Cyclic tertiary amide (4) & $\mathrm{R}-\boldsymbol{C}(=\mathbf{O})[N]_{\mathrm{c}}<R_{2}$ & {$[-7.0]$} \\
\hline Cyclic carbamate (2) & $R-[O C(=\mathbf{O}) N-\mathbf{H}]_{\mathrm{c}} R$ & {$[15.3]$} \\
\hline N substituted cyclic carbamate (3) & $R-[\boldsymbol{O C}(=\mathbf{O}) N]_{\mathrm{c}}<R \mathrm{R}$ & {$[-5.2]$} \\
\hline $\mathrm{N}, \mathrm{N}$ disubstituted cyclic carbamate & $\mathrm{R}-[O C(=\mathrm{O}) N]_{\mathrm{c}}<R_{2}$ & -22.2 \\
\hline $\mathrm{N}$-substituted cyclic imide (17) & $R-[\boldsymbol{C}(=\mathrm{O}) \boldsymbol{N}(\mathrm{R}) \boldsymbol{C}(=\mathbf{O})]_{\mathrm{c}}-R$ & -13.6 \\
\hline Cyclic imide (9) & $R-[C(=\mathbf{O}) N(\mathbf{H}) C(=\mathbf{O})]_{\mathrm{c}}-R$ & {$[2.8]$} \\
\hline Cyclic phosphorothioate (1) & $R-[\boldsymbol{O}-\boldsymbol{P}(=\mathbf{S})<(O R)]_{\mathrm{c}}(\mathrm{OR})$ & {$[-15.6]$} \\
\hline Cyclic phosphate ester (2) & {$[R-O-P(=\mathbf{O}) O R] \mathbf{O R}$} & {$[-17]$} \\
\hline Cyclic sulfide & $R-[S]_{c}-R$ & 2.9 \\
\hline Cyclic disulfide (5) & $R-[S S]_{c}-R$ & {$[-6.4]$} \\
\hline Cyclic disulfide S-oxide (2) & $R-\mathbf{S} \mathbf{S}(=\mathbf{O})_{\mathrm{c}}-R$ & {$[4.0]$} \\
\hline Cyclic sulfoxide (1) & $R-[\mathbf{S}(=\mathbf{O})]_{\mathrm{c}}-R$ & {$[-2.2]$} \\
\hline Cyclic sulfone (7) & $R-\left[\mathrm{S}(=\mathbf{O})_{2}\right]_{\mathrm{c}}-R$ & {$[0]$} \\
\hline Cyclic thiocarbonate (1) & $R-[\boldsymbol{O C}(=\mathbf{O}) S]_{\mathrm{c}}-R$ & [14.3] \\
\hline Cyclic sulfite (1) & $R-[\boldsymbol{O S}(=\mathbf{O}) O]_{\mathrm{c}}-R$ & {$[-5.8]$} \\
\hline Cyclic dithioester (2) & $R-[(C=\mathbf{S}) S]_{\mathrm{c}}-R$ & [11.0] \\
\hline Cyclic sulfate (1) & $R-\left[\mathrm{OS}(=\mathbf{O})_{2} \mathrm{O}\right]_{\mathrm{c}}-R$ & {$[0.9]$} \\
\hline Cyclic sulphonamide (1) & $R-\left[\mathrm{S}(=\mathbf{O})_{2} \mathrm{NH}\right]_{\mathrm{c}}-R$ & {$[-0.4]$} \\
\hline Cyclic N substituted sulfonamide (4) & $R-\left[\mathrm{SO}_{2} N\right]_{\mathrm{c}}-R \mathrm{R}$ & {$[-17.6]$} \\
\hline Cyclic carboxyl sulfimide (1) & $R-\left[\mathrm{SO}_{2} \mathrm{NH}-(\mathrm{C}=\mathbf{O})\right]_{\mathrm{c}}-R$ & [13.9] \\
\hline Cyclic thiocarbamate (1) & $R-[\mathbf{S}-(\boldsymbol{C}=\mathbf{O}) \boldsymbol{N H}]_{\mathrm{c}}-R$ & [13.9] \\
\hline Cyclic isothiocarbamate (3) & $R-[\boldsymbol{O}-(\mathrm{C}=\mathbf{S}) \mathbf{N H}]_{\mathrm{c}}-R$ & {$[2.6]$} \\
\hline Cyclic dithiocarbamate (1) & $R-[\mathrm{S}-(\mathrm{C}=\mathbf{S}) \boldsymbol{N H}]_{\mathrm{c}}-R$ & {$[3.8]$} \\
\hline Cyclic quaternary silicon & $\mathrm{R}_{2}>[S i]_{\mathrm{c}}<R_{2}$ & -34.7 \\
\hline
\end{tabular}

${ }^{\mathrm{a}} \mathrm{R}$ refers to any alkyl or aryl group unless specified otherwise; $\mathrm{X}$ refers to any halogen; values in brackets are tentative assignments; atoms in bold in column 2 define the atoms included in the functional group; all group values in this table are to be used with the ring Eqs. (2) or (3); values in brackets are tentative assignments and are based on the number of entries in parentheses reported in column 1; the $\mathrm{R}$ groups that comprise a portion of the ring structure are designated by italics; all group coefficients; Ck can be assumed to be 1 . 
Tridecane

$$
\begin{array}{cc}
\mathrm{CH}_{3} \mathrm{CH}_{2}\left(\mathrm{CH}_{2}\right)_{9} \mathrm{CH}_{2} \mathrm{CH}_{3} & \Delta H_{\text {trs }}=7900 \mathrm{~J} \mathrm{~mol}^{-1} \\
{[2 *(17.6)+11 *(7.1) *(1.31)]=137.5 \mathrm{~J} \mathrm{~mol}^{-1} \mathrm{~K}^{-1}} & T_{\text {trs }}=254.4 \mathrm{~K} \\
\Delta H_{\text {fus }}=137.5 * 267.3=36800 \mathrm{~J} \mathrm{~mol}^{-1} & \Delta H_{\text {fus }}=29620 \mathrm{~J} \mathrm{~mol}^{-1} \\
& T_{\text {fus }}=267.3 \mathrm{~K}^{-1} \\
\Delta S_{\text {tpce }}=141.9 \mathrm{~J} \mathrm{~mol}^{-1} \mathrm{~K}^{-1} \\
{[2005 \mathrm{HUA} / \mathrm{SIM}]}
\end{array}
$$

Diphenylmethane<smiles>c1ccc(Cc2ccccc2)cc1</smiles>

$$
\begin{aligned}
& {[10 *(7.4)+2 *(-9.6)+(7.1)]=61.9 \mathrm{~J} \mathrm{~mol}^{-1} \mathrm{~K}^{-1}} \\
& \Delta H_{\text {fus }}=61.9 * 298.4=18500 \mathrm{~J} \mathrm{~mol}^{-1}
\end{aligned}
$$

$$
\begin{gathered}
\Delta H_{\text {fus }}=19010 \mathrm{~J} \mathrm{~mol}^{-1} \\
T_{\text {fus }}=298.4 \mathrm{~K} \\
\Delta S_{\text {tpce }}=63.7 \mathrm{~J} \mathrm{~mol}^{-1} \mathrm{~K}^{-1}
\end{gathered}
$$

[2005CHI/STE2]

FIG. 1. Estimations of acyclic and aromatic hydrocarbons.

illustrates an example where the crystalline phase undergoes a solid-solid phase transition. The solid-solid phase transition enthalpy and transition temperature are denoted as $\Delta H_{\text {trs }}$ and $T_{\text {trs }}$, respectively, in the following illustrations. If the phase transition occurs close in temperature to the melting temperature, as in the case for tridecane, the predicted total phase change enthalpy is quite close to the experimental value: $(36.8-37.5) \mathrm{kJ} \cdot \mathrm{mol}^{-1}$. However, if the phase transition occurs at a much lower temperature, then the predicted total phase change enthalpy can be quite different from the fusion enthalpy. Based on the results for diphenylmethane, the estimation suggests that diphenylmethane does not have any substantial phase changes occurring at temperatures below ambient.

\subsection{Estimations of polymers}

Among the simplest class of molecules to estimate are microcrystalline linear polymers. Fusion enthalpies for these materials are usually reported per repeat unit. Poly- ethylene, poly(ethylene terephthalate) $\left(-\mathrm{CH}_{2}-,=\mathrm{C}_{\mathrm{a}} \mathrm{H}_{\mathrm{s}}-\right.$, $-\mathrm{C}(=\mathrm{O}) \mathrm{O}-$ ) and nylon 6,10 (poly(hexamethylene decanediamide) $\left(-\mathrm{CH}_{2}-,-\mathrm{C}(=\mathrm{O}) \mathrm{NH}-\right)$ in Fig. 2 are a few examples. Experimental values for all polymers are from [1990WUN, 1992VAN].

\subsection{Estimation of cyclic and polycyclic hydrocarbons}

The total phase change entropy of a cyclic or heterocyclic hydrocarbon is evaluated by first calculating the total phase change entropy of the parent cycloalkane containing only methylene groups. This is accomplished using Eq. (2) where $\mathrm{N}$ refers to the number of ring atoms. The resulting value is then adjusted for any changes in either the substitution pattern or hybridization from the parent cycloalkane using the group values in Table 2. Any additional acyclic hydrocarbon substituents are then added in normal group fashion to the ring using the values provided in Table 1. For polycyclic hydrocarbons, Eq. (3) is used followed by adjustments for the

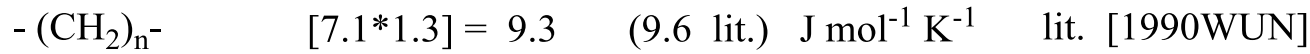

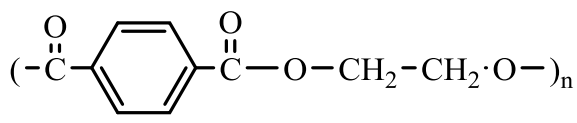

$$
\begin{aligned}
& {[2 * 7.1+4 * 7.4+2 *(-7.5)+2 * 7.7]=44.2 \text { (48.6 lit.) } \mathrm{J} \mathrm{mol}^{-1} \mathrm{~K}^{-1}} \\
& \left(-\left(\mathrm{CH}_{2}\right)_{6}-\mathrm{NH}-\stackrel{\mathrm{O}}{\mathrm{C}}-\left(\mathrm{CH}_{2}\right)_{8}-\stackrel{\mathrm{O}}{\mathrm{C}}-\mathrm{NH}-\right)_{\mathrm{n}} \\
& {[14 * 7.1 * 1.3+2 * 1.5]=132.2 \text { (141.6 lit.) } \mathrm{J} \mathrm{mol}^{-1} \mathrm{~K}^{-1}}
\end{aligned}
$$


1.5-Cyclooctadiene

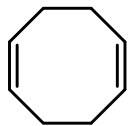

$$
\begin{aligned}
& {[33.4+3.7(8-3)+4(-1.6)]=45.5 \mathrm{~J} \mathrm{~mol}^{-1} \mathrm{~K}^{-1}} \\
& \Delta H_{\text {fus }}=45.5 * 204=9280 \mathrm{~J} \mathrm{~mol}^{-1}
\end{aligned}
$$

$$
\begin{gathered}
\Delta H_{\text {trs }}=380 \mathrm{~J} \mathrm{~mol}^{-1} \\
T_{\text {trs }}=194.4 \mathrm{~K} \\
\Delta H_{\text {fus }}=9830 \mathrm{~J} \mathrm{~mol}^{-1} \\
T_{\text {fus }}=204 \mathrm{~K} \\
\Delta S_{\text {tpce }}=50.1 \mathrm{~J} \mathrm{~mol}^{-1} \mathrm{~K}^{-1}
\end{gathered}
$$

[1996DOM/HEA]

endo Tetrahydrodicyclopentadiene

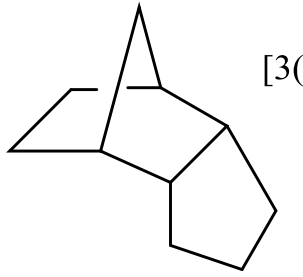

$[3(33.4)$

$$
\Delta H_{\text {fus }}=45.1 * 356.8=16100 \mathrm{~J} \mathrm{~mol}^{-1}
$$

$$
\begin{aligned}
\Delta H_{\text {trs }} & =10700 \mathrm{~J} \mathrm{~mol}^{-1} \\
T_{\text {trs }} & =194.4 \mathrm{~K} \\
\Delta H_{\text {fus }} & =3480 \mathrm{~J} \mathrm{~mol}^{-1} \\
T_{\text {fus }} & =356.8 \mathrm{~K} \\
\Delta \mathrm{S}_{\text {tpce }} & =50.1 \mathrm{~J} \mathrm{~mol}^{-1} \mathrm{~K}^{-1}
\end{aligned}
$$

[2002CHI/HIL]

1,3-Dithiane

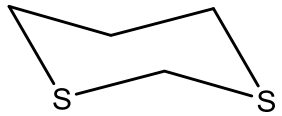

$$
\begin{gathered}
\left.[33.4)+3(3.7)+2(2.9)_{\mathrm{c}}\right]=50.3 \mathrm{~J} \mathrm{~mol}^{-1} \mathrm{~K}^{-1} \\
\Delta H_{\text {fus }}=50.3 * 327.2=16500 \mathrm{~J} \mathrm{~mol}^{-1}
\end{gathered}
$$$$
\Delta H_{\mathrm{trs}}=800 \mathrm{~J} \mathrm{~mol}^{-1}
$$$$
T_{\text {trs }}=316.4 \mathrm{~K}
$$$$
\Delta H_{\text {fus }}=14400 \mathrm{~J} \mathrm{~mol}^{-1}
$$$$
T_{\text {fus }}=327.2 \mathrm{~K}
$$$$
\Delta S_{\text {tpce }}=46.5 \mathrm{~J} \mathrm{~mol}^{-1} \mathrm{~K}^{-1}
$$

[1996DOM/HEA]

FIG. 3. Estimations of cyclic hydrocarbons.

bridgehead atoms, depending on their substitution pattern. The term R in Eq. (3) refers to the number of rings. In a polycyclic system, the value of $\mathrm{R}$ is determined by the minimum number of carbon-carbon bonds that must be broken to make the molecule completely acyclic. Additional substituents are added in normal fashion followed by adjustments for any changes in the substitution pattern and/or hybridization from the parent cycloalkane containing only cyclic secondary $\mathrm{sp}^{3}$ hybridized carbon. The most common carbon modifications to the cyclic carbon atoms are listed in Table 2. As described below, substitutions of cyclic heteroatoms for cyclic carbon in nonaromatic systems are handled in much the same manner. For ease of identity, functional groups selected from Table 8 are identified in the examples below with a subscript c. The $R$ groups in italics in Table 8 identify the remaining cyclic portion of the molecule,

Monocyclic ring systems:

$$
\Delta_{0}^{T_{\text {fus }}} S_{\text {tpce }} \Delta(\text { ring })=[33.4]+[3.7][N-3] \text {. }
$$

Polycyclic ring systems :

$$
\Delta_{0}^{T_{\text {fus }}} S_{\text {tpce }}(\text { ring })=[(33.4) R+3.7(N-3 R)],
$$

where $N=$ number of ring atoms and $R$ refers to the number of rings.

Both 1,5-cyclooctadiene (ring equation $+=C_{\mathrm{c}} \mathrm{H}-$ ) and endo tetrahydrodicyclopentadiene (ring equation $+C_{\mathrm{c}} \mathrm{H}<$ ) shown in Fig. 3 have solid-solid phase transitions. In the case of the former compound, the transition is small and occurs near the fusion temperature. In the latter case the solid-solid phase transition is large and quite separate from the fusion temperature. Although the total phase change enthalpy is reasonably reproduced, estimation of the fusion enthalpy for endo tetrahydrodicyclopentadiene is quite poor in this case because of the large difference in temperature between the transition and fusion temperatures.

1,3-dithiane (ring equation, $-[S]_{\mathrm{c}}-$ ) illustrates a simple example of an estimation of a nonaromatic heterocycle. Since the transition temperature is very close to the fusion 
temperature, the total phase change enthalpy is reasonably well reproduced, 15200 compared to $16500 \mathrm{~J} \cdot \mathrm{mol}^{-1}$.

\subsection{Functionalized acyclic and aromatic hydrocarbons}

Estimations of aromatic and aliphatic hydrocarbons derivatives also follow a standard group additivity protocol. Group values for acyclic functional groups are provided in Tables 3-7. The functional groups are segregated into groups according to the number of attached $\mathrm{R}$ groups necessary to complete the valence requirements of the functional group. As examples, an ether oxygen, an aromatic cyclic nitrogen, and a secondary amide (RCONHR) are grouped together for ease of location. Values for a few functional groups, those in Table 3, are segregated from the rest because their value is dependent on the total number of other functional groups attached to the molecule. The functional group itself is also included in this count but groups from Tables 1 and 2 are not. The group value to be used is simply the product of group coefficient and the group value. The group coefficients listed in the last column of Table 3 should be used for substances containing four or more functional groups. Fluorine substitution is the only exception and is considered a single substituent regardless of the number of fluorine atoms attached. Group values for the remaining acyclic functional groups are arranged according to the substitution pattern of the functional group. Terminal functional group values are reported in Table 4 while disubstituted, trisubstituted, and tetrasubstituted groups are arranged in Tables 5-7, respectively. Estimation of substances that fall in these two categories follows standard group additivity protocol with the exception just described for the functional groups listed in Table 3. The following examples in Fig. 4 illustrate typical estimations. None of the compounds listed as examples were included in evaluating any group values.

The top three estimations, derivatives of carboxylic acids, illustrate the application of the group coefficients in Table 3. The group value for carboxylic acids depends upon the total number of functional groups present as does the chlorine in the first estimation. The total number of functional groups in 2-benzoyl-3-chlorobenzoic acid $\left(=\mathrm{C}_{\mathrm{a}} \mathrm{H}-\right.$, $\left.=\mathrm{C}_{\mathrm{a}}\left(\mathrm{R}_{\mathrm{a}}\right)-,-\mathbf{C l},-\mathbf{C O}_{2} \mathbf{H},-\mathbf{C O}_{\mathbf{2}} \mathbf{R}\right)$ is three and two for 3,5-di-tert-butylsalicyclic acid as indicated in bold $\left(\mathrm{CH}_{3}\right.$-, $\left.\left.>\mathrm{C}<,=\mathrm{Ca}-(\mathrm{OH})-,=\mathrm{C}_{\mathrm{a}}\left(\mathrm{R}_{\mathrm{s}}\right)\right)-,=\mathrm{C}_{\mathrm{a}}\left(\mathrm{R}_{\mathrm{a}}\right)-,-\mathbf{C O}_{2} \mathbf{H}\right)$. The group coefficients are selected appropriately. The remaining groups are treated in normal fashion.

N-tetradecanoyl-(L)-alanine $\left(\mathrm{CH}_{3}-,-\mathrm{CH}_{2}->\mathrm{CH}-\right.$, $\left.-\mathrm{CO}_{2} \mathrm{H},-\mathrm{C}(=\mathrm{O}) \mathrm{NH}-\right)$ is an example of a compound that illustrates the application of two of the group coefficients in Table 1. The compound contains 10 consecutive methylene groups compared to a total of five other groups present, two functional groups and three carbon groups. Since the number of consecutive methylene groups is in excess, the contribution of each methylene group is the product of the group value and the group coefficient in Table 1. This estimation also illustrates the use of the group coefficient for a tertiary $\mathrm{sp}^{3}$ carbon. If a functional group is attached to any carbon with a group coefficient listed in these two tables, other than for a $\mathrm{CH}_{2}$ group, the contribution of the carbon group is the product of the group value and the group coefficient. Thus in $\mathrm{N}$-tetradecanoyl-(L)-alanine, the group value for the tertiary $\mathrm{sp}^{3}$ carbon is $(-16.4)(0.6)$.

The estimation of benz[a]acridine $\left(=\mathrm{C}_{\mathrm{a}} \mathrm{H}-,=\mathrm{C}_{\mathrm{a}}\left(\mathrm{R}_{\mathrm{a}}\right)\right.$-, $=\mathrm{N}_{\mathrm{a}}-$ ) is straightforward. Since the molecule is a totally aromatic compound, the estimation is simply the sum of the groups.

\subsection{Estimations of functionalized cyclic and polycyclic hydrocarbons}

Estimations of functionalized cyclic and polycyclic compounds use Eq. (2) or (3) to first evaluate the contribution of the ring or rings. The presence of substituents, double bonds, or heteroatoms on the ring is treated as adjustments to the parent cycloalkane as a result of hybridization and substitution changes, similar to the estimations provided above in Section 3.3.

Figure 5 summarizes adjustments to the ring equations resulting from structural modifications to the original cycloalkane and how they affect the total phase change entropy of the ring.

The estimation of phenothiazine (ring equation, $=\boldsymbol{C}_{\mathrm{c}}(R)$-, $\left.=C_{\mathrm{c}}(R)-,-\boldsymbol{N}_{\boldsymbol{c}} \mathrm{H}-,-\left[\boldsymbol{S}_{\boldsymbol{c}}\right]-,=\mathrm{C}_{\mathrm{a}} \mathrm{H}-\right)$ illustrates a slightly more complex situation. Since both the sulfur and nitrogen are cyclic but not aromatic, they both require the use of the ring equation and the group values listed in Table 8 . Thus the six membered heterocyclic ring must be estimated as cycloalkane. This brings into question how to treat the four $\mathrm{sp}^{2}$ hybridized carbons bound to the heteroatoms. The protocol developed requires these four atoms to be analyzed as cyclic quaternary $\mathrm{sp}^{2}$ hybridized carbons and not as quaternary aromatic carbons. The remaining portion of the molecule is treated normally as 8 tertiary aromatic $\mathrm{sp}^{2}$ hybridized carbons. In summary, estimations of nonaromatic rings take priority over the aromatic portion of the molecule.

The estimation of phenobarbitol illustrates a slightly different estimation. The heterocyclic six membered ring also necessitates the use of the ring equation for a six membered ring. The ring can be thought of being composed of a cyclic imide $\left.\left(R_{\mathrm{c}}(\mathbf{C}-\mathbf{O N H}) \mathbf{C}=\mathbf{O}\right) R_{\mathrm{c}}\right)$, a cyclic secondary amide $(R(\boldsymbol{C}=\boldsymbol{O}) \boldsymbol{N H} R)$, and a cyclic quaternary $\mathrm{sp}^{3}$ hybridized carbon $\left(-\boldsymbol{C}_{\mathbf{c}}<(\mathrm{R})_{2}-\right)$. The remaining ethyl and phenyl groups are evaluated normally. An alternative calculation substitutes group values for two cyclic secondary amides and a ketone together with the remaining portion of the molecule for which there is no ambiguity. The result in this case is essentially the same. Estimations of this sort for which the functional groups must be synthesized from existing components are usually associated with larger uncertainties.

\section{Statistics in Evaluation of Group Values}

The original 18 carbon groups and Eqs. (1) and (2) were derived from 192 hydrocarbons resulting in a standard deviation of $\pm 11.2 \mathrm{~J} \cdot \mathrm{mol}^{-1} \cdot \mathrm{K}^{-1}$ back in 1990. [1990CHI/HES] 
2-Benzoyl-3-chlorobenzoic acid

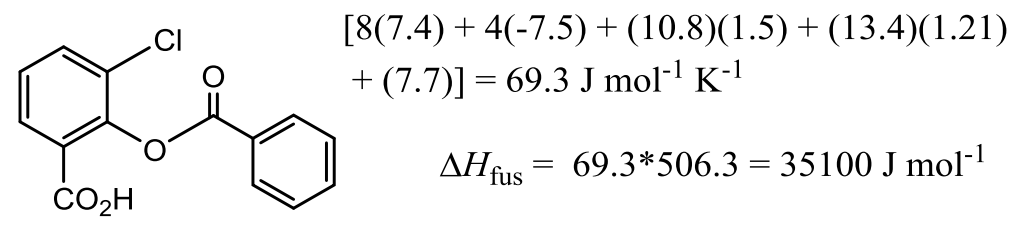

$$
\begin{gathered}
\Delta H_{\text {fus }}=35500 \mathrm{~J} \mathrm{~mol}^{-1} \\
T_{\text {fus }}=506.3 \mathrm{~K} \\
\Delta \mathrm{S}_{\text {tpce }}=70.1 \mathrm{~J} \mathrm{~mol}^{-1} \mathrm{~K}^{-1} \\
{[2013 \mathrm{YOU} / \mathrm{GAO}]}
\end{gathered}
$$

\section{3,5-Di-tert-butylsalicyclic acid}

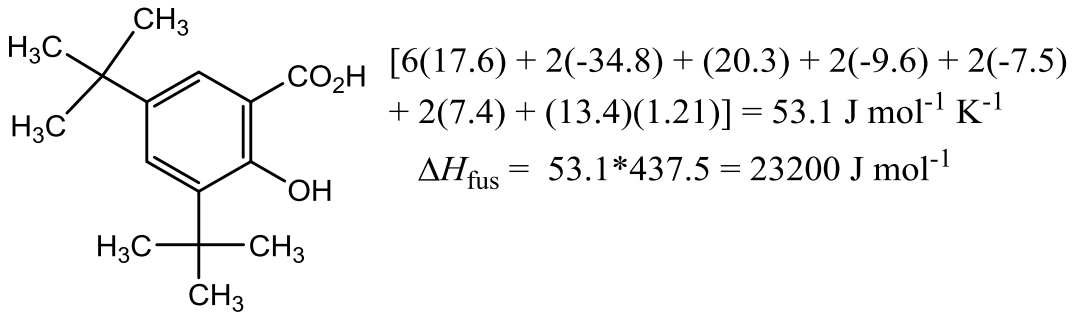

$$
\begin{gathered}
\Delta H_{\text {fus }}=22920 \mathrm{~J} \mathrm{~mol}^{-1} \\
T_{\text {fus }}=437.5 \mathrm{~K} \\
\Delta \mathrm{S}_{\text {tpce }}=52.4 \mathrm{~J} \mathrm{~mol}^{-1} \mathrm{~K}^{-1} \\
{[2003 \mathrm{YU} / \mathrm{TAN}]}
\end{gathered}
$$

N-Tetradecanoyl-(L)-alanine<smiles>CCCCCCCCCCCCCCCCCC(=O)O</smiles>

$$
\begin{gathered}
{[2(17.6)+12(7.1)(1.31)+} \\
\quad(-16.4)(0.6)+(13.4)(1.21) \\
+(1.5)]=154.7 \mathrm{~J} \mathrm{~mol}^{-1} \mathrm{~K}^{-1} \\
\Delta H_{\text {fus }}=154.7^{*} 367.1=56800 \mathrm{~J} \mathrm{~mol}^{-1}
\end{gathered}
$$

$$
\begin{gathered}
\Delta H_{\text {fus }}=52300 \mathrm{~J} \mathrm{~mol}^{-1} \\
T_{\text {fus }}=367.13 \mathrm{~K} \\
\Delta S_{\text {tpce }}=142.5 \mathrm{~J} \mathrm{~mol}^{-1} \mathrm{~K}^{-1} \\
{[1986 \mathrm{MIY} / \mathrm{MAT}]}
\end{gathered}
$$

Benz[a]acridine<smiles>c1ccc2nc3ccc4ccccc4c3cc2c1</smiles>

$$
\begin{aligned}
& {[11(7.4)+6(-7.5)+10.9]=47.3 \mathrm{~J} \mathrm{~mol}^{-1} \mathrm{~K}^{-1}} \\
& \Delta H_{\text {fus }}=47.3 * 402.8=19100 \mathrm{~J} \mathrm{~mol}^{-1}
\end{aligned}
$$

$\Delta H_{\text {fus }}=21900 \mathrm{~J} \mathrm{~mol}^{-1}$

$T_{\text {fus }}=36713 \mathrm{~K}$

$\Delta \mathrm{S}_{\text {tpce }}=54.4 \mathrm{~J} \mathrm{~mol}^{-1} \mathrm{~K}^{-1}$

[2010KES/AUC]

FIG. 4. Estimation of aliphatic and aromatic derivatives.

Their values have not changed much if any since. The update published in 1991 provided initial group values for 37 functional groups on the basis of 458 entries and in 1999 the total number of function groups was increased to 128 evaluated on the basis of a total of 1858 entries. [1991CHI/BRA] This resulted in a standard deviation of $\pm 13 \mathrm{~J} \cdot \mathrm{mol}^{-1} \cdot \mathrm{K}^{-1}$. In 2003 , an additional 547 entries resulted in changes to 18 of the 128 functional groups and resulted in a standard deviation of $\pm 18.6 \mathrm{~J} \cdot \mathrm{mol}^{-1} \cdot \mathrm{K}^{-1}$. [2003CHI/ACR]
The previous update in 2009 added approximately a thousand additional compounds of increasing complexity, increasing the total number of functional groups to 160 but retained a standard deviation of $\pm 18.6 \mathrm{~J} \cdot \mathrm{mol}^{-1} \cdot \mathrm{K}^{-1}$.

Most of the functional groups have not varied much since 1999 because it either has not been necessary or there has been a lack of additional data. Errors detected subsequently in some estimations have led to some changes. In a few cases newer experimental data have supplanted older data 


\section{Phenothiazine}<smiles>c1ccc2c(c1)Nc1ccccc1S2</smiles>

$$
\begin{aligned}
& {[33.4+3(3.7)+2.2+4(-12.3)} \\
& 2.9+8(7.4)]=59.6 \mathrm{~J} \mathrm{~mol}^{-1} \mathrm{~K}^{-1} \\
& \Delta H_{\text {fus }}=59.6 * 457.2=27200 \mathrm{~J} \mathrm{~mol}^{-1}
\end{aligned}
$$

$$
\begin{gathered}
\Delta H_{\text {fus }}=28400 \mathrm{~J} \mathrm{~mol}^{-1} \\
T_{\text {fus }}=457.2 \mathrm{~K} \\
\Delta \mathrm{S}_{\text {tpce }}=62.1 \mathrm{~J} \mathrm{~mol}^{-1} \mathrm{~K}^{-1} \\
{[2007 \mathrm{GUP} / \mathrm{SIN}]}
\end{gathered}
$$

Phenobarbitol

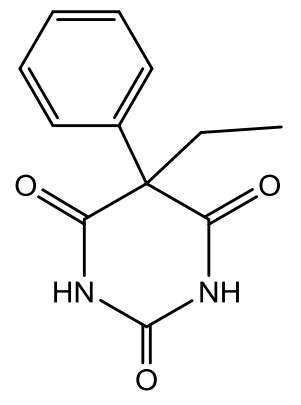

$$
\begin{aligned}
& {\left[33.4+3(3.7)+(2.8)_{\mathrm{c}}+(2.7)_{\mathrm{c}}+(-34.6)+\right.} \\
& 17.6+7.1+5(7.4)+(-9.6)]=67.5 \mathrm{~J} \mathrm{~mol}^{-1} \mathrm{~K}^{-1} \\
& \Delta H_{\text {fus }}=67.5 * 449=30300 \mathrm{~J} \mathrm{~mol}^{-1} \\
& {\left[33.4+3(3.7)+2(2.7)_{\mathrm{c}}+(-1.4)_{\mathrm{c}}+(-34.6)_{\mathrm{c}}+\right.} \\
& 17.6+7.1+5(7.4)+(-9.6)]=66.0 \mathrm{~J} \mathrm{~mol}^{-1} \mathrm{~K}^{-1} \\
& \Delta H_{\text {fus }}=66 * 449=29600 \mathrm{~J} \mathrm{~mol}^{-1}
\end{aligned}
$$$$
\Delta H_{\text {fus }}=28000 \mathrm{~J} \mathrm{~mol}^{-1}
$$$$
T_{\text {fus }}=449 \mathrm{~K}
$$$$
\Delta \mathrm{S}_{\text {tpce }}=62.4 \mathrm{~J} \mathrm{~mol}^{-1} \mathrm{~K}^{-1}
$$$$
\text { [2010ZEN/GEL] }
$$

Fig. 5. Estimations of complex cyclic and polycyclic hydrocarbons.

usually in better agreement with estimation. In this update, a few functional groups have also been eliminated on the basis of their variability and potential unreliability. This included a number of polynitrated compounds and some organometallics. A few new groups have also been added to the tables; these are identified in bold. Group values that have been changed from previous versions are reported in italics. Parameters for new values and values that were upgraded due to new data were evaluated by minimizing $R$ in equation (4),

$$
R=\sum_{1}^{n}\left(\frac{\Delta S_{\text {tpce }}(\text { exp })-\Delta S_{\text {tpce }}(\text { est })}{\Delta S_{\text {tpce }}(\exp )}\right)^{2} .
$$

The experimental total phase change entropies and enthalpies were calculated as a sum of the known experimental entropies and enthalpies for those compounds known to exhibit isothermal solid-solid phase transitions. The estimated total phase change entropy was calculated by the group method just discussed. The total phase change enthalpy was calculated as the product of the calculated total phase change entropy and the experimental fusion temperature. A total of 3299 compounds have been estimated on the basis of 128 functional groups. The original 192 hydrocarbons are not included in this total since most hydrocarbon groups have remained unchanged; neither are the 667 liquid crystals [2006ACR/CHI] and the 58 fluorinated compounds that are known to behave anisotropically as liquids. [2003CHI/ACR] Included are several new hydrocarbons in the 3299 compounds that comprise the current database and an additional 112 new compounds that have been added in this update. Of the 128 functional groups, 21 of them are based on only one entry. These entries were not included in generating the statistics. On the basis of the standard deviations obtained previously, $\pm 11.2, \pm 13, \pm 18.6$, and $\pm 18 \mathrm{~J} \cdot \mathrm{mol}^{-1} \cdot \mathrm{K}^{-1}$, estimations varying by more than 3 standard deviations were excluded from the database. An approximate value of $\pm 50 \mathrm{~J} \cdot \mathrm{mol}^{-1} \cdot \mathrm{K}^{-1}$ was chosen as $3 \sigma$. Of the 3299 compounds, 131 of them exhibited deviations in excess of $\pm 3 \sigma$ and all but four were eliminated from the database. The three materials in question exhibited deviations in excess of $\pm 3 \sigma$ but fractional errors less than 0.06 .

The standard deviation in the total phase change entropy and enthalpy associated with the 3151 compounds in the database is $\pm 14.6 \mathrm{~J} \cdot \mathrm{mol}^{-1} \cdot \mathrm{K}^{-1}$ and $\pm 5.7 \mathrm{~kJ} \cdot \mathrm{mol}^{-1}$, respectively. Figures 6 and 7 illustrate the error distributions in both $\Delta S_{\text {tpce }}$ and $\Delta H_{\text {tpce. }}$. While the uncertainty in $\Delta S_{\text {tpce }}$ has been constrained to $\pm 50 \mathrm{~J} \cdot \mathrm{mol}^{-1} \cdot \mathrm{K}^{-1}$, the uncertainty in $\Delta H_{\text {tpce }}$ is also dependent on the presence of additional unreported solid-solid phase transitions. Figures 8 and 9 compare experimental and estimation values for both entropy and

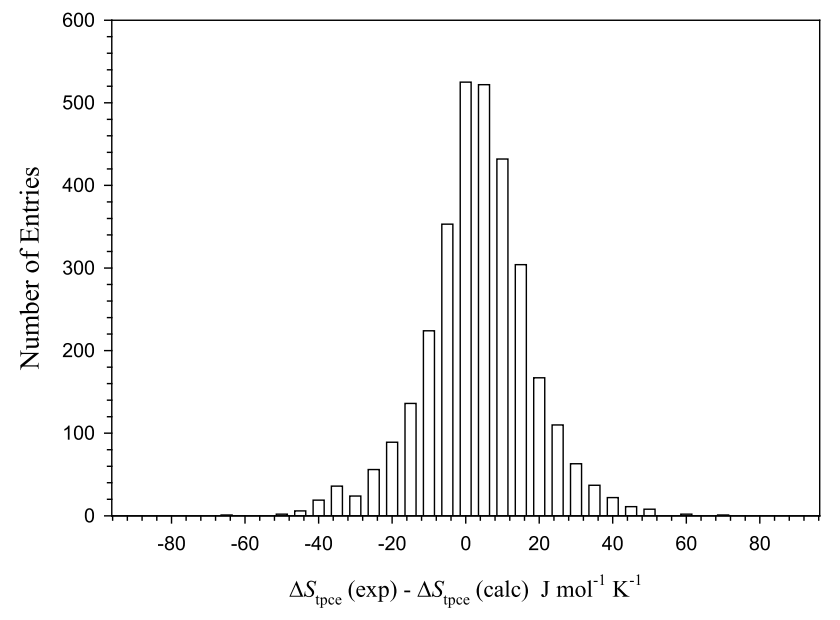

FIG. 6. Distribution of differences between $\Delta S_{\text {tpce }}$ (exp) $-\Delta S_{\text {tpce }}$ (calc) for 3151 compounds. The standard deviation is $\pm 14.6 \mathrm{~J} \cdot \mathrm{mol}^{-1} \cdot \mathrm{K}^{-1}$. 


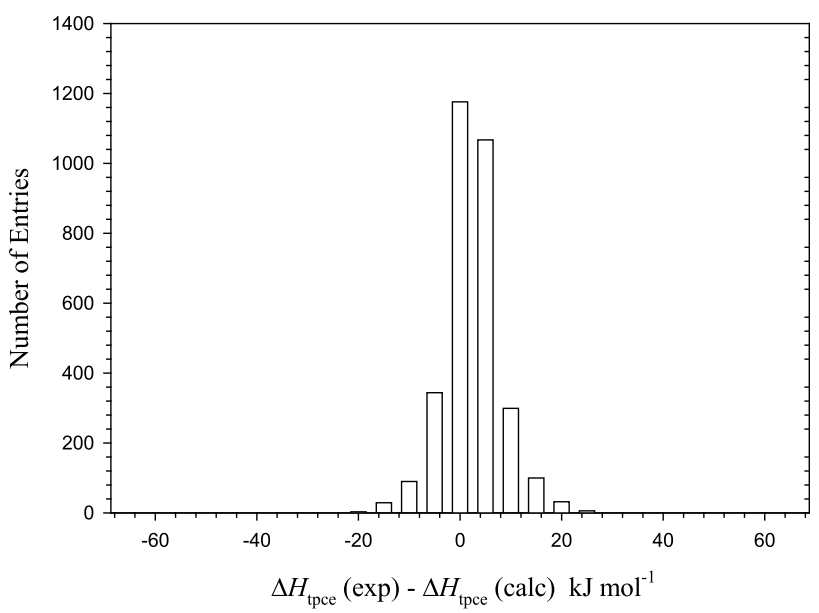

Fig. 7. Distribution of differences between $\Delta H_{\text {tpce }}(\exp )-\Delta H_{\text {tpce }}$ (calc) for 3151 compounds. Experimental enthalpies include enthalpies associated with all solid-solid phase changes. Calculated enthalpies are obtained as the product of $\Delta S_{\text {tpce }}$ (calc) ${ }^{*} T_{\text {fus }}$. The standard deviation is $\pm 5.7 \mathrm{~kJ} \cdot \mathrm{mol}^{-1}$.

enthalpy. The equations of the line are given below each caption. While most entropies in the database range from 10 to $200 \mathrm{~J} \cdot \mathrm{mol}^{-1} \cdot \mathrm{K}^{-1}$, a few long chain n-alkanes have significantly large entropies and enthalpies as illustrated in Figures 8 and 9 . Of the 3151 compounds in the database, $16 \%$ exhibited solid-solid phase changes. Analyzed separately, the 499 compounds with additional solid-solid phase transitions were reproduced with a standard error of $\pm 14.9 \mathrm{~J} \cdot \mathrm{mol}^{-1} \cdot \mathrm{K}^{-1}$ and $\pm 6.3 \mathrm{~kJ} \cdot \mathrm{mol}^{-1}$, compared to standard deviations of $\pm 14.4 \mathrm{~J} \cdot \mathrm{mol}^{-1} \cdot \mathrm{K}^{-1}$ and $\pm 5.5 \mathrm{~kJ} \cdot \mathrm{mol}^{-1}$ for those compounds with no known solid-solid transitions.

\section{The Phase Change Enthalpy Compendium, Paper II}

This portion of the compendium completes a summary of literature reports of experimental phase changes of $\mathrm{C}_{11}-\mathrm{C}_{192}$

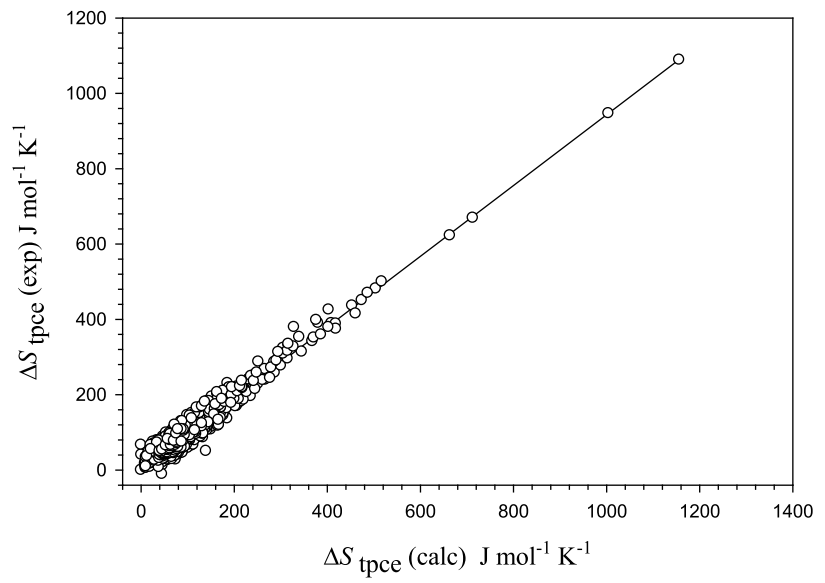

Fig. 8. A comparison of $\Delta S_{\text {tpce }}$ (exp) and $\Delta S_{\text {tpce }}$ (calc) for 3150 compounds. One n-alkane was omitted from this graph because of its large value, 1751.2 (exp), 1802.4 (calc) $\mathrm{J} \cdot \mathrm{mol}^{-1} \cdot \mathrm{K}^{-1}$ but is included in the statistics. The equation of the line is given by $\Delta S_{\text {tpce }}(\exp ) / \mathrm{J} \mathrm{mol}^{-1} \mathrm{~K}^{-1}=0.959^{*} \Delta S_{\text {tpce }}($ calc $)+2.13$, $r^{2}=0.949$.

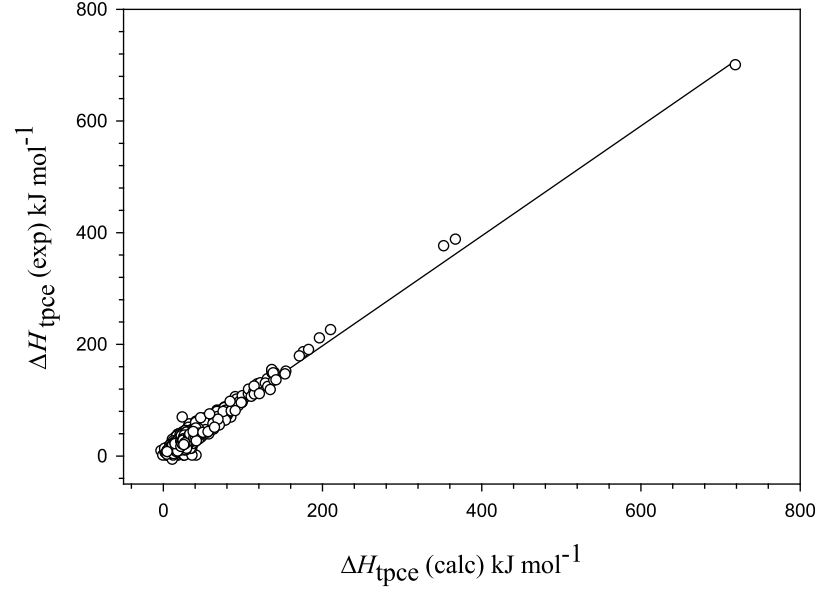

FIG. 9. A comparison of $\Delta H_{\text {tpce }}$ (exp) and $\Delta H_{\text {tpce }}$ (calc) for 3151 compounds. $\Delta H_{\text {tpce }}$ (calc) evaluated as the product of $\Delta S_{\text {tpce }}$ (calc)* $T_{\text {fus }}$. The equation of the line is given by $\Delta \boldsymbol{H}_{\text {tpce }}(\exp ) / \mathrm{kJ} \mathrm{mol}^{-1}=0.984 * \Delta \boldsymbol{H}_{\text {tpce }}($ calc $)+0.470 ; \mathrm{r}^{2}$ $=0.945$.

organic, organo-metallic, and a few inorganic compounds and ionic liquids. As described previously, organic compounds and ionic liquids are arranged according to the Hill system. This also applies to ionic liquids. Organometallic compounds are segregated alphabetically according to the symbol of the metal but also arranged according to the Hill system. Inorganic compounds containing the same element follow and are arranged alphabetically and incrementally. Formulas for inorganic salts are generally arranged by the cation followed by the anion.

The molecular formula is provided in the first column of the first row of each individual material. This is usually followed by the Chemical Abstracts reference number in the second column and the chemical name. The chemical name is usually the name provided by the authors of the article. The remaining information in subsequent columns includes the phase transition (column 2), the temperature, or temperature range if any associated with the transition (column 3), the enthalpy associated with the transition (column 4), the mean temperature associated with the transition if measured over a temperature range (column 5), the acronym associated with the method of measurement (column 6), and reference to the source of information (column 7), usually to the original work. The acronyms used are defined in Table 9 . If an entry is not provided in one of these columns, it is likely that the information was not available in the source consulted. For liquids, the vaporization enthalpy $\left(\Delta_{v} H\right)$ in $\mathrm{kJ} \cdot \mathrm{mol}^{-1}$ follows the name in subsequent rows. For solids, depending on the nature of the material, the order of entry includes any solid-solid transitions available $\left(\Delta_{t r s} H\right)$, followed in subsequent rows by each available solid-to-liquid transition $\left(\Delta_{f u s} H\right)$, the sublimation enthalpies available $\left(\Delta_{\text {sub }} H\right)$, and the vaporization enthalpies, if available. The enthalpies are identified in the data tables according to the type of transition, where TRS, FUS, SUB, and V denote solid-solid transitions, fusion, sublimation, and vaporization, respectively. Some enthalpy values are cited without an accompanying reference on the same line. In these cases the reference cited directly 
TABLE 9. List of acronyms and descriptions for methods

\begin{tabular}{|c|c|}
\hline A & $\begin{array}{l}\text { Calculated from the vapor pressure data reported by the } \\
\text { method of least squares }\end{array}$ \\
\hline $\mathrm{AC}$ & Adiabatic calorimeter \\
\hline B & $\begin{array}{l}\text { Calculated from the difference of the enthalpies of } \\
\text { sublimation at temperature } \mathrm{T} \text { and fusion at the melting point. }\end{array}$ \\
\hline BG & Bourdon gauge \\
\hline $\mathrm{BP}$ & Boiling point temperature at different pressures \\
\hline $\mathrm{C}$ & Calorimetric determination \\
\hline CATH & Cathetometer \\
\hline $\mathrm{CC}$ & Conduction calorimeter \\
\hline $\mathrm{CCM}$ & Cooling curve method \\
\hline CDG & Capacitance diaphragm gauge \\
\hline $\mathrm{CE}$ & Critical evaluated value \\
\hline CGC & Correlation-gas chromatography \\
\hline CGC-DSC & $\begin{array}{l}\text { Correlation gas chromatography combined with differential } \\
\text { scanning calorimeter }\end{array}$ \\
\hline $\mathrm{CGC}+\mathrm{Fus}$ & Correlation gas chromatography and fusion \\
\hline $\mathrm{CR}$ & Cryoscopy \\
\hline CRT & Chromatographic retention time \\
\hline CRYST & Crystallization \\
\hline CVC & Calvet calorimeter \\
\hline DBM & Dibutyl phthalate manometer \\
\hline DC & Dynamic calorimeter \\
\hline DFC & Differential calorimetry \\
\hline DFM & Differential manometer \\
\hline DFSC & Differential fast scanning calorimetry \\
\hline $\mathrm{DM}$ & Diaphragm manometer \\
\hline DP-LPD & Dew point low pressure distillation method \\
\hline DRC & Drop calorimetry \\
\hline DSC & Differential scanning calorimeter \\
\hline DTA & Differential thermal analysis \\
\hline $\mathrm{E}$ & Estimated value \\
\hline EB & Ebulliometry \\
\hline EM & Electronic manometer \\
\hline EST & Estimated value \\
\hline $\mathrm{EV}$ & Evaporation rates \\
\hline $\mathrm{F}$ & Fluorescence \\
\hline FPD & Freezing point depression \\
\hline FPM & Freezing point method \\
\hline FTIR & Fourier transform infrared spectroscopy \\
\hline GC & Gas chromatography \\
\hline GCC & Gas chromatography-calorimetry \\
\hline GCRT & Gas chromatographic retention time \\
\hline GC-RT & Gas chromatography retention time \\
\hline GS & Gas saturation, transpiration \\
\hline GSM & Glass spring manometer \\
\hline HBG & Heise-Bourdon gauge \\
\hline $\mathrm{HFC}$ & Heat flux calorimetry \\
\hline HG & Heise gauge \\
\hline HSA & Head space analysis \\
\hline I & Isoteniscope \\
\hline IP & Inclined piston manometry \\
\hline IPM & Inclined piston manometry \\
\hline IR & Infrared spectroscopy \\
\hline ITG & Isothermal thermogravimetry \\
\hline ITGA & Isothermogravimetric analysis \\
\hline $\mathrm{KG}$ & Knudsen gauge \\
\hline LE & Langmuir evaporation \\
\hline MCV & Method of calibrated volume \\
\hline MDSC & Modulated differential scanning calorimetry \\
\hline $\mathrm{ME}$ & Mass effusion-Knudsen effusion \\
\hline ME-MS & Mass effusion-mass spectrometry \\
\hline MEM & Modified entrainment method \\
\hline MG & McLeod gauge \\
\hline MM & Mercury manometer \\
\hline
\end{tabular}

TABLE 9. List of acronyms and descriptions for methods-Continued

\begin{tabular}{|c|c|}
\hline MS & Mass spectrometry \\
\hline $\mathrm{OM}$ & Oil manometer \\
\hline $\mathrm{OP}$ & Optical \\
\hline PG & Pressure gauge \\
\hline PGSM & Pressure gauge static method \\
\hline QCM & Quartz crystal microbalance \\
\hline QF & Quartz fiber \\
\hline QM & Quartz manometer \\
\hline QR & Quartz resonator \\
\hline $\mathrm{RC}$ & Radiation calorimeter \\
\hline RG & Rodebush gauge \\
\hline RS & Recirculating still \\
\hline $\mathrm{SC}$ & Solution calorimetry \\
\hline S-F & Sublimation-fusion \\
\hline SG & Spoon gauge \\
\hline SMZG & Silicon membrane zero gauge \\
\hline SRFG & Spinning rotor friction gauge \\
\hline SRM & Spinning rotor manometer \\
\hline Static & Static method \\
\hline STG & Strain gauge \\
\hline S-V & Sublimation-vaporization \\
\hline $\mathrm{T}$ & Tensiometer \\
\hline TA & Thermal analysis \\
\hline $\mathrm{TCC}$ & Tin Calvet calorimeter \\
\hline TCM & Thermal conductivity manometer \\
\hline $\mathrm{TE}$ & Torsion effusion \\
\hline TG & Thermal gravimetric \\
\hline TGA & Thermal gravimetric analysis \\
\hline TG-GS & Thermogravimetric based gas saturation method \\
\hline TG-TS & Thermogravimetric transpiration method \\
\hline THBC & Triple heat bridge calorimeter \\
\hline TPD & Temperature programmed desorption \\
\hline TPD-MS & $\begin{array}{l}\text { Temperature program desorption combined with mass } \\
\text { spectrometry }\end{array}$ \\
\hline TPD-UV & $\begin{array}{l}\text { Temperature program desorption combined with ultraviolet } \\
\text { spectroscopy }\end{array}$ \\
\hline TPTD & Temperature programmed thermal desorption \\
\hline TRM & Thermoradiometric method \\
\hline TSGC & Temperature scanning gas chromatography \\
\hline $\mathrm{U}$ & Unreliable \\
\hline UV & Ultraviolet spectroscopy \\
\hline UV/VIS & Ultraviolet-visible spectroscopy \\
\hline V & Visible spectroscopy \\
\hline $\mathrm{V}+\mathrm{F}$ & Vaporization+fusion \\
\hline VG & Viscosity gauge \\
\hline VP & Vapor pressure as a function of temperature \\
\hline $\mathrm{ZG}$ & Zimmerli gauge \\
\hline
\end{tabular}

below should be consulted since multiple enthalpy values may have been taken from the same source, particularly for compounds with multiple solid-solid phase transitions. A brief note summarizing some peculiarity in the data is also provided for some compounds, particularly if the value reported appears to be unreliable, U (e.g., "U $66 \pm 22.1$ ").

The phase change enthalpies are divided by elemental composition. The first set of tables contain substituted-organic compounds. Tables 10-16 contain phase change enthalpies of $\mathrm{C}_{11}, \mathrm{C}_{12}-\mathrm{C}_{13}, \mathrm{C}_{14}-\mathrm{C}_{16}, \mathrm{C}_{17}-\mathrm{C}_{18}, \mathrm{C}_{19}-\mathrm{C}_{29}, \mathrm{C}_{30}-\mathrm{C}_{49}$, and $\mathrm{C}_{50}-\mathrm{C}_{192}$ organic compounds. Table 17 contains phase change enthalpies of organometallic and some inorganic compounds. Table 18 contains phase change enthalpies of ionic liquids. 
TABLE 10. Phase change enthalpies of $\mathrm{C}_{11}$ organic compounds

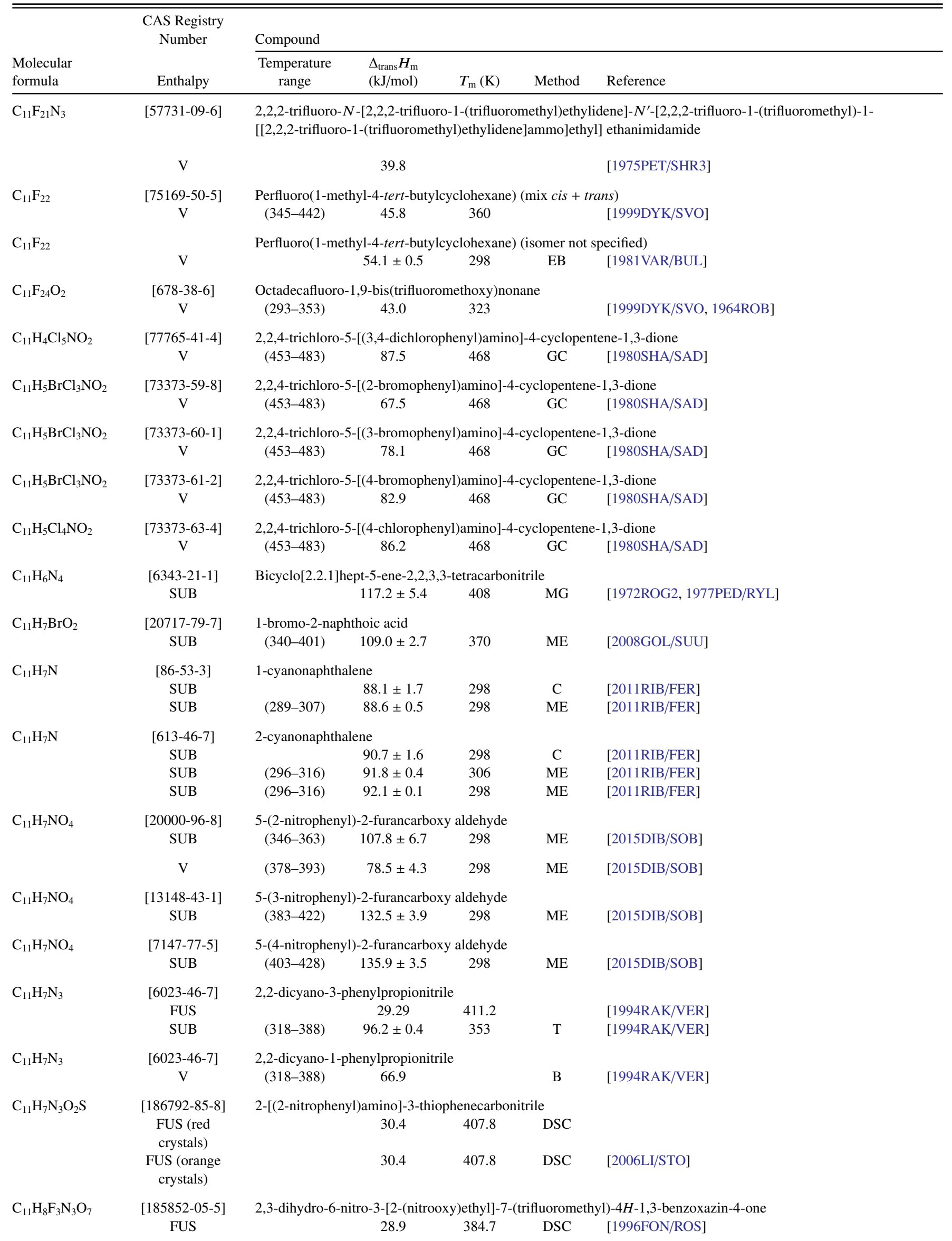


TABLE 10. Phase change enthalpies of $\mathrm{C}_{11}$ organic compounds-Continued

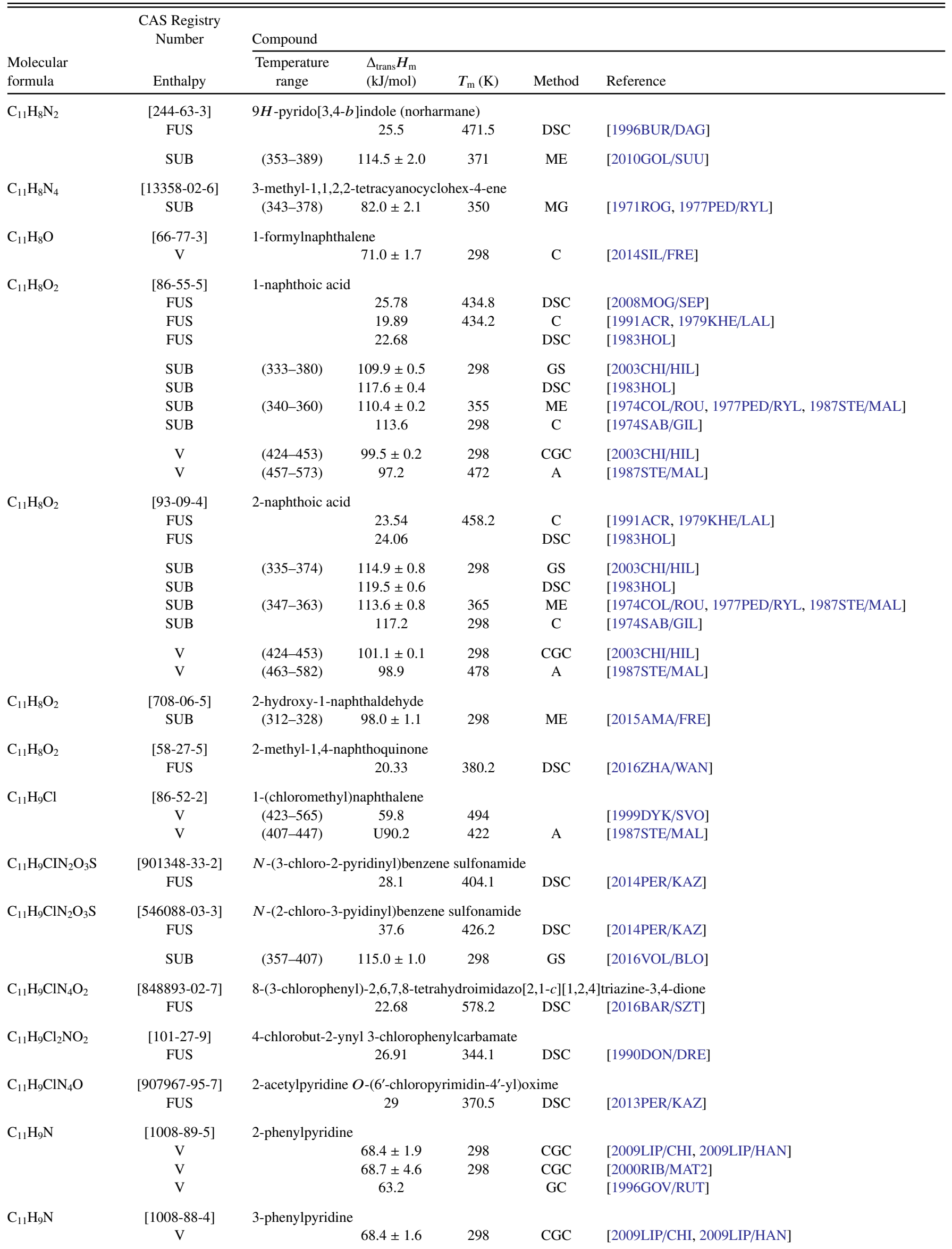


TABLE 10. Phase change enthalpies of $\mathrm{C}_{11}$ organic compounds-Continued

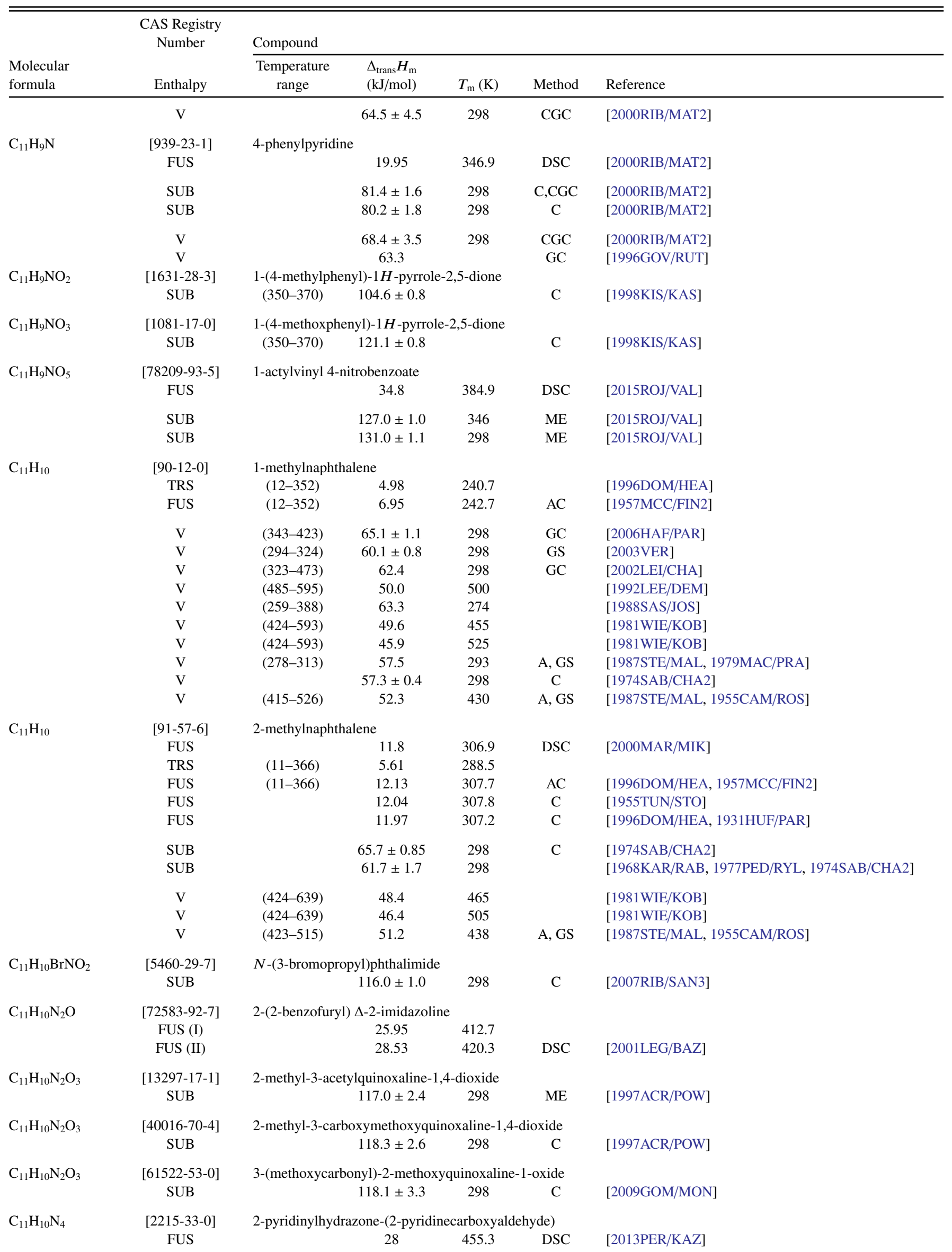


TABLE 10. Phase change enthalpies of $\mathrm{C}_{11}$ organic compounds-Continued

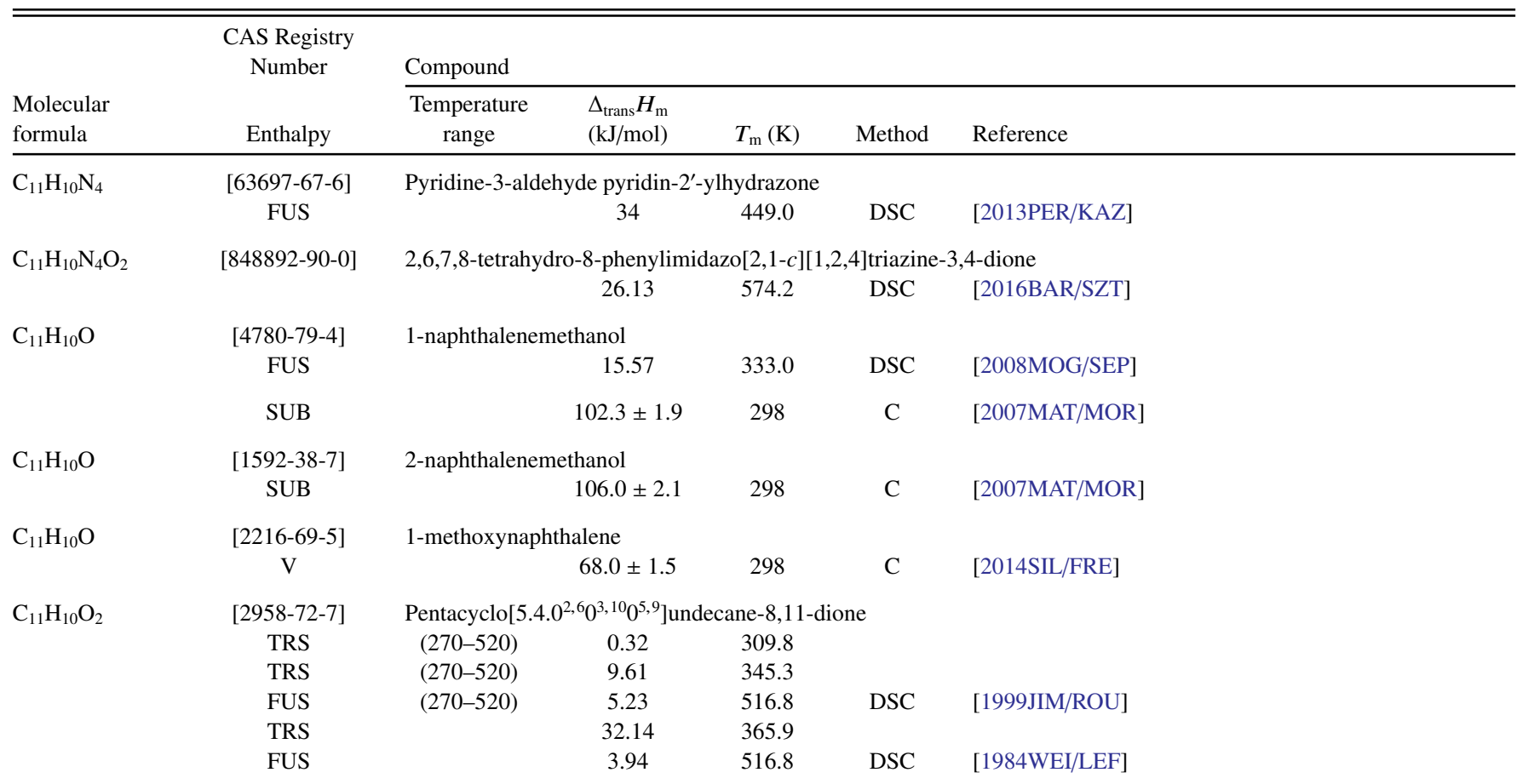

[Note: There is a large difference in the experimental enthalpies and transition temperature reported by the two research groups for the solid/solid transition around $355 \mathrm{~K}$.]

\begin{tabular}{|c|c|c|c|c|c|c|}
\hline & $\begin{array}{l}\text { SUB } \\
\text { SUB }\end{array}$ & $\begin{array}{l}(326-341) \\
(326-341)\end{array}$ & $\begin{array}{l}92.3 \pm 1.0 \\
92.6 \pm 1.0\end{array}$ & $\begin{array}{l}333 \\
298\end{array}$ & $\begin{array}{l}\text { ME } \\
\text { ME }\end{array}$ & $\begin{array}{l}\text { [1999JIM/ROU] } \\
\text { [1999JIM/ROU] }\end{array}$ \\
\hline $\mathrm{C}_{11} \mathrm{H}_{10} \mathrm{O}_{2}$ & $\begin{array}{l}\text { [711-79-5] } \\
\text { FUS }\end{array}$ & 2-acetyl-1-na & hol 22.52 & 371.8 & DSC & [1991ACR, 1990DOM] \\
\hline $\mathrm{C}_{11} \mathrm{H}_{10} \mathrm{O}_{2}$ & $\begin{array}{l}\text { [574-19-6] } \\
\text { FUS }\end{array}$ & \multicolumn{5}{|c|}{ 1-acetyl-2-naphthol } \\
\hline $\mathrm{C}_{11} \mathrm{H}_{10} \mathrm{O}_{3}$ & $\begin{array}{c}{[6295-35-8]} \\
\text { FUS }\end{array}$ & \multicolumn{5}{|c|}{ 6-methoxy-4-methylcoumarin } \\
\hline $\mathrm{C}_{11} \mathrm{H}_{10} \mathrm{O}_{4}$ & $\begin{array}{c}\text { [15721-10-5] } \\
\text { FUS }\end{array}$ & \multicolumn{5}{|c|}{$p$-methacryloyloxybenzoic acid } \\
\hline $\mathrm{C}_{11} \mathrm{H}_{11} \mathrm{Cl}_{3} \mathrm{O}_{3}$ & $\begin{array}{c}{[1928-40-1]} \\
\mathrm{V}\end{array}$ & \multicolumn{5}{|c|}{ 2,4,5-trichlorophenoxyacetic acid, propyl ester } \\
\hline $\mathrm{C}_{11} \mathrm{H}_{11} \mathrm{Cl}_{3} \mathrm{O}_{3}$ & $\begin{array}{c}\text { [25333-21-5] } \\
\text { FUS }\end{array}$ & \multicolumn{5}{|c|}{ Methyl 2-(2,4,5-trichlorophenoxy)butyrate } \\
\hline $\mathrm{C}_{11} \mathrm{H}_{11} \mathrm{~F}_{3} \mathrm{~N}_{2} \mathrm{O}_{3}$ & $\begin{array}{c}\text { [13311-84-7] } \\
\text { FUS }\end{array}$ & 2-methyl- $N$ - & $\begin{array}{l}\text { itro-3-(trifluorc } \\
\quad 28.4\end{array}$ & $\begin{array}{l}\text { ethyl)pl } \\
383.1\end{array}$ & $\begin{array}{l}\text { 1]propanar } \\
\text { DSC }\end{array}$ & $\begin{array}{l}\text { de (flutamide) } \\
\text { [2015NUR/BOO] }\end{array}$ \\
\hline $\mathrm{C}_{11} \mathrm{H}_{11} \mathrm{~N}$ & $\begin{array}{c}{[1198-37-4]} \\
\mathrm{V}\end{array}$ & $\begin{array}{l}\text { 2,4-dimethyl } \\
(458-543)\end{array}$ & $\begin{array}{l}\text { roline } \\
56.3\end{array}$ & 473 & A & [1987STE/MAL, 1964MAL/WEC] \\
\hline $\mathrm{C}_{11} \mathrm{H}_{11} \mathrm{~N}$ & $\begin{array}{l}\text { [877-43-0] } \\
\text { FUS }\end{array}$ & 2,6-dimethyl & $\begin{array}{l}\text { roline } \\
20.4\end{array}$ & 330.8 & AC,DSC & {$[2007 \mathrm{CHI} / \mathrm{JOH}]$} \\
\hline & SUB & & $84.5 \pm 1.5$ & 298 & $\mathrm{C}$ & [1995RIB/MAT3] \\
\hline & V & $(337-591)$ & $64.0 \pm 0.1$ & 340 & IPM,EB & [2007CHI/JOH] \\
\hline & $\mathrm{V}$ & $(337-591)$ & $61.1 \pm 0.1$ & 380 & IPM,EB & [2007CHI/JOH] \\
\hline & V & $(337-591)$ & $58.4 \pm 0.1$ & 420 & IPM,EB & {$[2007 \mathrm{CHI} / \mathrm{JOH}]$} \\
\hline & $\mathrm{V}$ & $(337-591)$ & $55.7 \pm 0.1$ & 460 & IPM,EB & [2007CHI/JOH] \\
\hline & $\begin{array}{l}\text { V } \\
\text { V }\end{array}$ & $\begin{array}{l}(337-591) \\
(337-591)\end{array}$ & $\begin{array}{l}53.0 \pm 0.1 \\
50.0+0.2\end{array}$ & $\begin{array}{l}500 \\
540\end{array}$ & $\begin{array}{l}\text { IPM,EB } \\
\text { IPM,EB }\end{array}$ & $\begin{array}{l}{[2007 \mathrm{CHI} / \mathrm{JOH}]} \\
{[2007 \mathrm{CHI} / \mathrm{JOH}]}\end{array}$ \\
\hline & $\mathrm{V}$ & $(337-591)$ & $46.8 \pm 0.4$ & 580 & IPM,EB & {$[2007 \mathrm{CHI} / \mathrm{JOH}]$} \\
\hline & $\mathrm{V}$ & $(337-591)$ & $67.1 \pm 0.2$ & 298 & IPM,EB & {$[2007 \mathrm{CHI} / \mathrm{JOH}]$} \\
\hline & V & $(461-541)$ & 55.7 & 476 & A & [1987STE/MAL, 1964MAL/WEC] \\
\hline
\end{tabular}

$\mathrm{C}_{11} \mathrm{H}_{11} \mathrm{~N} \quad$ [93-37-8] 2,7-dimethylquinoline 
TABLE 10. Phase change enthalpies of $\mathrm{C}_{11}$ organic compounds-Continued

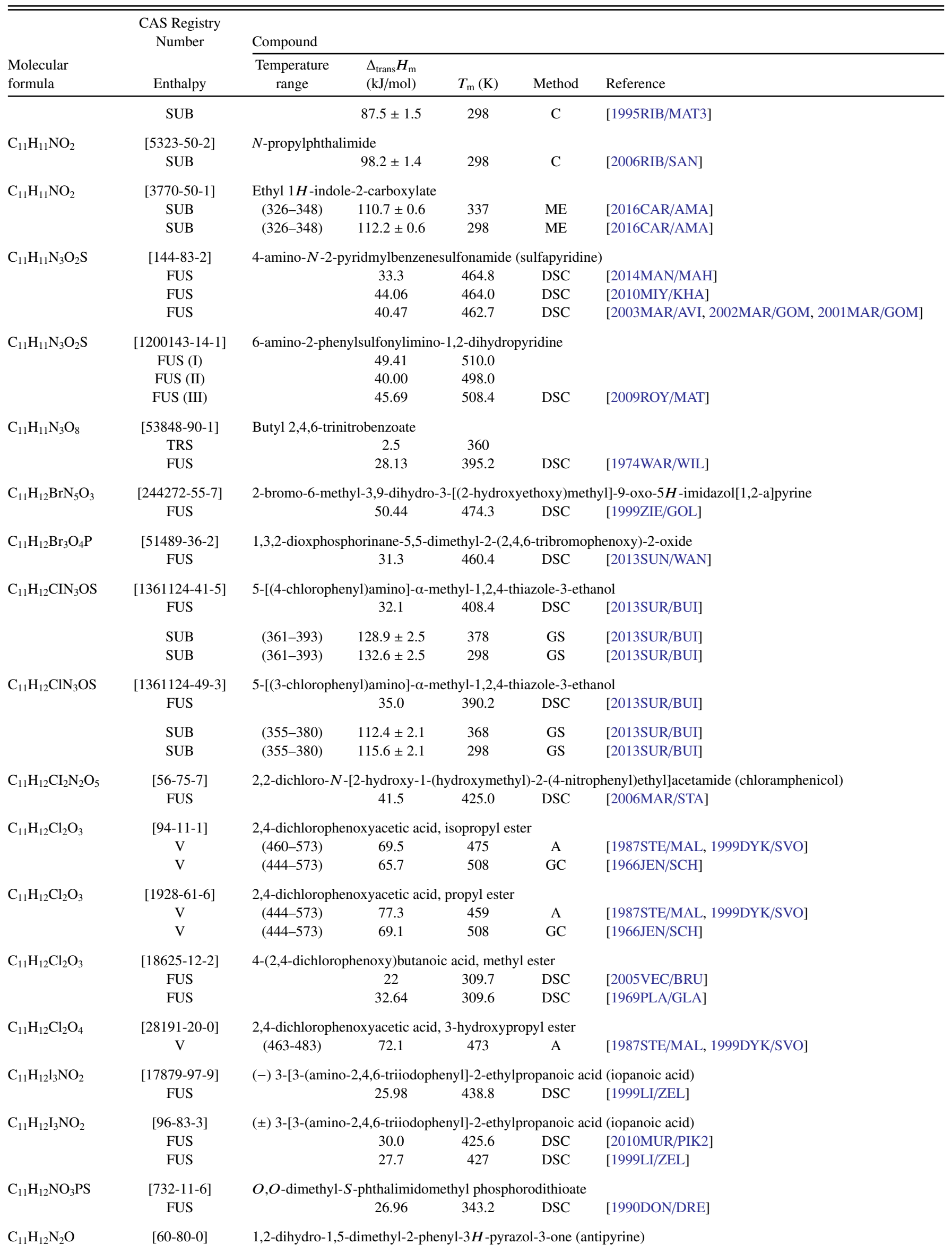


TABLE 10. Phase change enthalpies of $\mathrm{C}_{11}$ organic compounds-Continued

\begin{tabular}{|c|c|c|c|c|c|c|}
\hline \multirow[b]{2}{*}{$\begin{array}{l}\text { Molecular } \\
\text { formula }\end{array}$} & \multirow{2}{*}{$\begin{array}{c}\text { CAS Registry } \\
\text { Number } \\
\text { Enthalpy }\end{array}$} & \multicolumn{5}{|l|}{ Compound } \\
\hline & & $\begin{array}{l}\text { Temperature } \\
\text { range }\end{array}$ & $\begin{array}{l}\Delta_{\text {trans }} H_{\mathrm{m}} \\
(\mathrm{kJ} / \mathrm{mol})\end{array}$ & $T_{\mathrm{m}}(\mathrm{K})$ & Method & Reference \\
\hline & FUS & & 25.18 & 384.0 & DSC & [2010BAI/VAN] \\
\hline & FUS & & 24.5 & 383.7 & DSC & [2002SCH/LEN] \\
\hline & FUS & & 24.52 & 385.8 & & [1985OHM/LIP] \\
\hline \multirow[t]{3}{*}{$\mathrm{C}_{11} \mathrm{H}_{12} \mathrm{~N}_{2} \mathrm{OS}_{2}$} & [102-77-2] & 2-(4-morpholin & thio)benzothi & & & \\
\hline & FUS (I) & & 12.97 & 345 & & \\
\hline & FUS (II) & & 17.99 & 357 & DSC & [1978GUZ/LAR] \\
\hline \multirow[t]{4}{*}{$\mathrm{C}_{11} \mathrm{H}_{12} \mathrm{~N}_{2} \mathrm{O}_{2}$} & {$[73-22-3]$} & (l)-tryptophane & & & & \\
\hline & SUB & & $184.4 \pm 2.3$ & 450 & ME,MS & [2014TYU/KRA] \\
\hline & SUB & & $186.6 \pm 4.1$ & 298 & ME,MS & [2014TYU/KRA] \\
\hline & SUB & $(340-440)$ & $\mathrm{U} 87.9 \pm 8$ & 390 & LE & [1977GAF/PIE] \\
\hline \multirow{2}{*}{$\mathrm{C}_{11} \mathrm{H}_{12} \mathrm{~N}_{2} \mathrm{O}_{3}$} & [20771-72-6] & 4-[(4-nitropheny & 1)amino]pent- & he-2-one & & \\
\hline & SUB & & $121.9 \pm 3.9$ & 298 & $\mathrm{C}$ & [1993RIB/RIB] \\
\hline \multirow{2}{*}{$\mathrm{C}_{11} \mathrm{H}_{12} \mathrm{~N}_{2} \mathrm{O}_{5}$} & {$[143248-64-0]$} & 2,3-dihydro-6-m & ethyl-3-[2-(ni & xy)ethyl] & $I$-1,3-benz & xazin-4-one \\
\hline & FUS & & 27.1 & 351.2 & DSC & [1996FON/ROS] \\
\hline \multirow[t]{5}{*}{$\mathrm{C}_{11} \mathrm{H}_{12} \mathrm{~N}_{4} \mathrm{O}_{2} \mathrm{~S}$} & {$[127-79-7]$} & 2-(4-aminobenz & nesulfonamic & 4-methyl & midine (su & amerazine) \\
\hline & FUS & & 41.3 & 508.5 & DSC & [2003MAR/AVI, 2002MAR/GOM, 2001MAR/GOM] \\
\hline & FUS & & 41.1 & 509.7 & DTA & {$[1986 \mathrm{MAU} / \mathrm{RAM}]$} \\
\hline & FUS & & 45.8 & 506.4 & DSC & [1983KHA] \\
\hline & FUS & & 31.6 & 515.2 & DTA & [1971SUN/EIS] \\
\hline \multirow[t]{2}{*}{$\mathrm{C}_{11} \mathrm{H}_{12} \mathrm{~N}_{4} \mathrm{O}_{3} \mathrm{~S}$} & {$[80-35-3]$} & 4-amino- $N-(6-1$ & nethoxy-3-pyr & inyl)ben & esulfonami & e (sulphamethoxypyridazine) \\
\hline & FUS & & 22.3 & 453.4 & DSC & [1995BUS/ESC] \\
\hline \multicolumn{7}{|c|}{$\begin{array}{l}\text { [Note: Authors of [1995BUS/ESC] state that the solute was tested for purity in a differential scanning calorimeter (DSC) and the experimental heat } \\
\text { and temperature of fusion were } 22.3 \mathrm{~kJ} \mathrm{~mol}^{-1} \text { and } 453.4 \mathrm{~K} \text {, respectively. Later in the paper refer to the same numerical value as the heat of fusion at } \\
298 \mathrm{~K} \text {.] }\end{array}$} \\
\hline & FUS & & 33.9 & 453.6 & DSC & [1994ESC/BUS] \\
\hline & FUS & & 32.47 & 453.2 & $\mathrm{DSC}$ & [1985MAU/RAM] \\
\hline \multirow[t]{3}{*}{$\mathrm{C}_{11} \mathrm{H}_{12} \mathrm{NO}_{3} \mathrm{~S}$} & {$[152-47-6]$} & 4-amino- $N-(3-1$ & lethoxy-2-pyr & yl)benze & alfonamide & sulfamethoxypyrazine) \\
\hline & FUS & & 32.4 & 448.4 & DSC & [2013PER/RYZ] \\
\hline & SUB & & $130.4 \pm 1.3$ & 298 & GS & [2013PER/RYZ] \\
\hline \multirow[t]{2}{*}{$\mathrm{C}_{11} \mathrm{H}_{12} \mathrm{O}$} & & 2-ethylidiene-3- & henylpropan & & & \\
\hline & $\mathrm{V}$ & $(333-374)$ & 73.6 & 348 & A & [1987STE/MAL] \\
\hline \multirow[t]{2}{*}{$\mathrm{C}_{11} \mathrm{H}_{12} \mathrm{O}$} & {$[826-73-3]$} & 1-benzosuberon & & & & \\
\hline & V & & $69.0 \pm 1.7$ & 298 & $\mathrm{C}$ & [2010MIR/MOR] \\
\hline \multirow[t]{2}{*}{$\mathrm{C}_{11} \mathrm{H}_{12} \mathrm{O}_{2}$} & {$[2495-37-6]$} & Benzyl methacr & late & & & \\
\hline & V & $(347-431)$ & 70.5 & 362 & A & [1987STE/MAL] \\
\hline \multirow[t]{3}{*}{$\mathrm{C}_{11} \mathrm{H}_{12} \mathrm{O}_{2}$} & {$[103-36-6]$} & Ethyl cinnamate & & & & \\
\hline & $\mathrm{V}$ & & $70.4 \pm 1.4$ & 298 & CGC & [2015KOZ/GOB] \\
\hline & $\mathrm{V}$ & $(453-544)$ & 57.8 & 468 & A & [1987STE/MAL] \\
\hline \multirow[t]{2}{*}{$\mathrm{C}_{11} \mathrm{H}_{12} \mathrm{O}_{2}$} & [5331-64-6] & 1-phenyl-1,3-pe & tanedione & & & \\
\hline & $\mathrm{V}$ & $(371-550)$ & 64.6 & 386 & A & [1987STE/MAL] \\
\hline \multirow[t]{5}{*}{$\mathrm{C}_{11} \mathrm{H}_{12} \mathrm{O}_{2}$} & {$[39522-76-4]$} & 1-phenyl-4,7-di & xaspiro[2.4]h & & & \\
\hline & FUS & & 22.6 & 303.1 & DSC & [1998VER/PEN] \\
\hline & SUB & & $91.8 \pm 0.8$ & 298 & & [1998VER/PEN] \\
\hline & V & $(307-333)$ & $71.3 \pm 0.7$ & 298 & GS & [2002VER] \\
\hline & $\mathrm{V}$ & $(288-302)$ & $69.6 \pm 0.7$ & & GS & [1998VER/PEN] \\
\hline \multirow[t]{2}{*}{$\mathrm{C}_{11} \mathrm{H}_{12} \mathrm{O}_{2}$} & [40317-63-3] & 4-carboxymethy & Ipentacyclo [4 & $0 . .^{2,5} 0^{4,7}$ & lane & \\
\hline & $\mathrm{V}$ & (303-343) & $80.0 \pm 1.7$ & 333 & & [1984BEC/RUC] \\
\hline \multirow[t]{3}{*}{$\mathrm{C}_{11} \mathrm{H}_{12} \mathrm{O}_{2}$} & {$[33892-75-0]$} & 3,4-dihyro-5-me & thoxy-1(2H)- & hthalenor & & \\
\hline & FUS & & 22.2 & 362.5 & DSC & [2009MAT/SOU2] \\
\hline & SUB & & $97.9 \pm 0.4$ & 298 & $\mathrm{C}$ & [2009MAT/SOU2] \\
\hline
\end{tabular}


TABLE 10. Phase change enthalpies of $\mathrm{C}_{11}$ organic compounds-Continued

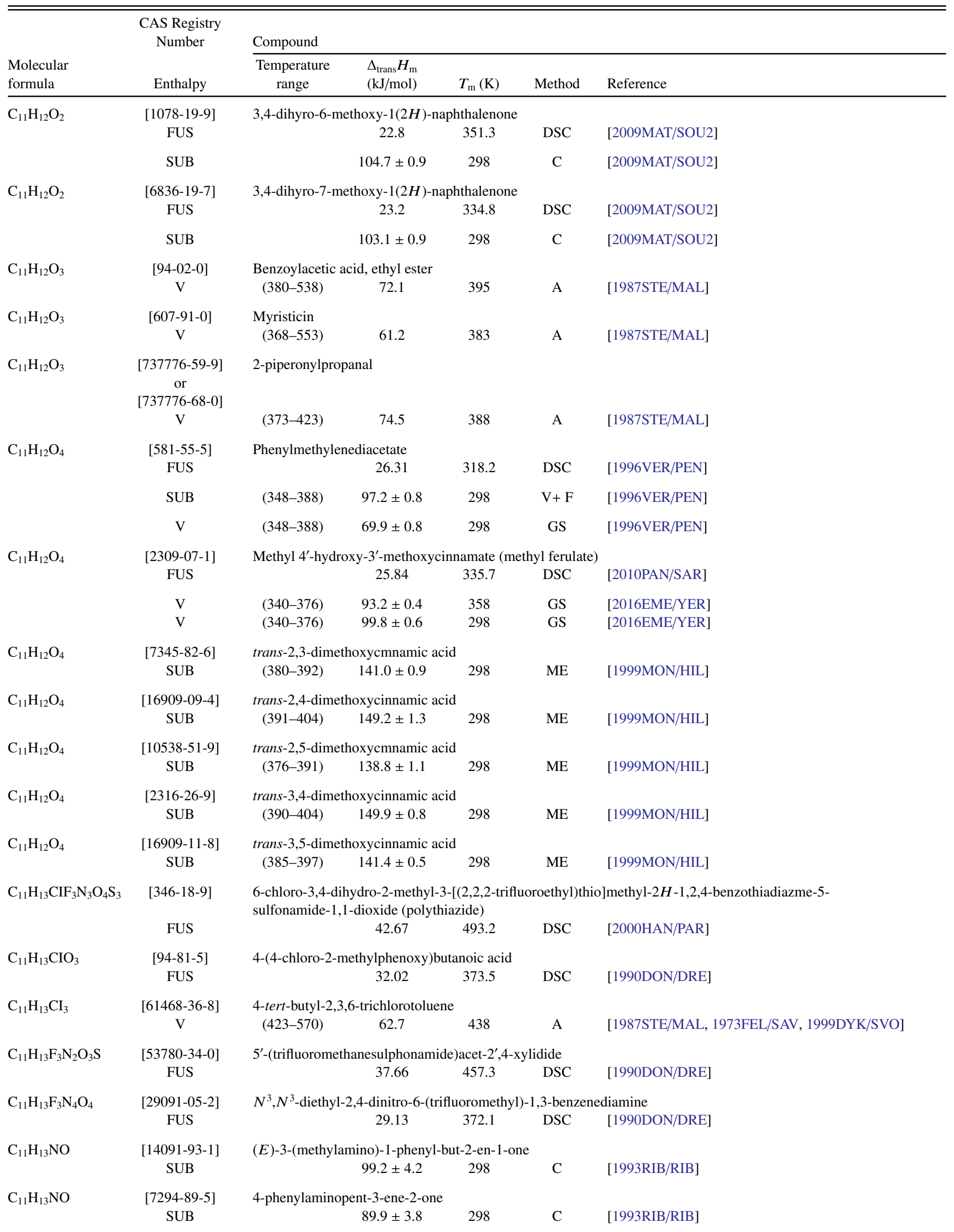


TABLE 10. Phase change enthalpies of $\mathrm{C}_{11}$ organic compounds-Continued

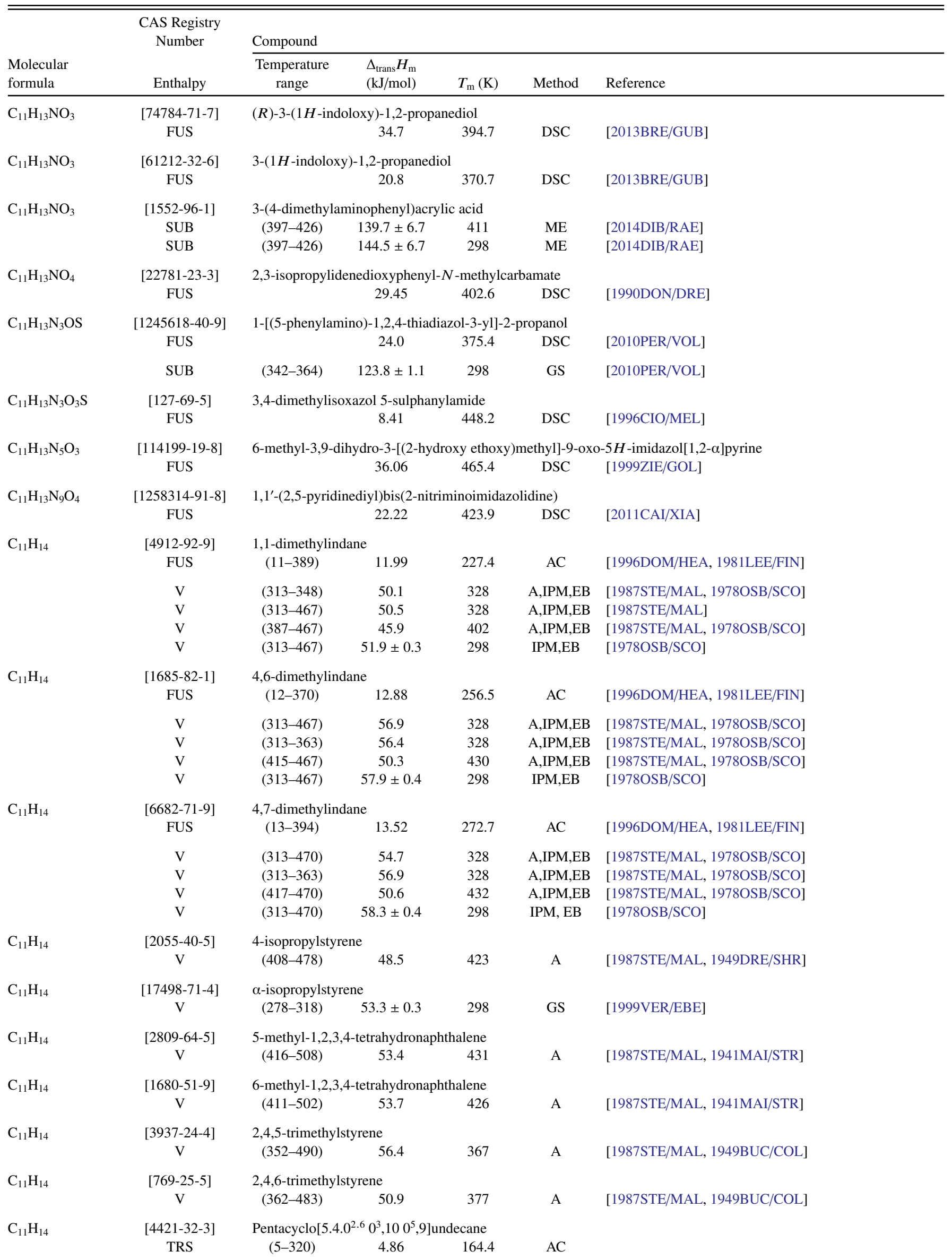


TABLE 10. Phase change enthalpies of $\mathrm{C}_{11}$ organic compounds-Continued

\begin{tabular}{|c|c|c|c|c|c|c|}
\hline \multirow[b]{2}{*}{$\begin{array}{l}\text { Molecular } \\
\text { formula }\end{array}$} & \multirow{2}{*}{$\begin{array}{c}\text { CAS Registry } \\
\text { Number } \\
\text { Enthalpy }\end{array}$} & \multicolumn{5}{|l|}{ Compound } \\
\hline & & $\begin{array}{c}\text { Temperature } \\
\text { range }\end{array}$ & $\begin{array}{l}\Delta_{\text {trans }} H_{\mathrm{m}} \\
(\mathrm{kJ} / \mathrm{mol})\end{array}$ & $T_{\mathrm{m}}(\mathrm{K})$ & Method & Reference \\
\hline & FUS & $(300-480)$ & 6.38 & 475.8 & $\mathrm{C}$ & {$[1995 \mathrm{KAB} / \mathrm{KOZ}]$} \\
\hline & SUB & & $54.7 \pm 0.9$ & 337 & $\mathrm{C}$ & [1995KAB/KOZ] \\
\hline & SUB & $(273-323)$ & $54.9 \pm 1.1$ & 298 & ME & [1995KAB/KOZ] \\
\hline \multirow[t]{2}{*}{$\mathrm{C}_{11} \mathrm{H}_{14} \mathrm{ClNO}$} & [1918-16-7] & \multicolumn{3}{|c|}{ 2-chloro- $N$-isopropyl- $N$-phenylacetamide } & & \\
\hline & FUS & & 26.05 & 351.4 & DSC & {$[1990 \mathrm{DON} / \mathrm{DRE}]$} \\
\hline \multirow[t]{2}{*}{$\mathrm{C}_{11} \mathrm{H}_{14} \mathrm{C}_{12}$} & {$[61468-35-7]$} & \multicolumn{3}{|c|}{ 4-tert-butyl-2,5-dichlorotoluene } & & \\
\hline & V & $(395-538)$ & 57.0 & 410 & A & [1987STE/MAL, 1973FEL/SAV, 1999DYK/SVO] \\
\hline \multirow[t]{2}{*}{$\mathrm{C}_{11} \mathrm{H}_{14} \mathrm{~N}_{2}$} & {$[4886-30-0]$} & \multicolumn{3}{|c|}{ 1-butylbenzimidazole } & & \\
\hline & V & $(328-375)$ & $83.4 \pm 0.3$ & 298 & GS & [2012GAR/VER] \\
\hline \multirow[t]{2}{*}{$\mathrm{C}_{11} \mathrm{H}_{14} \mathrm{~N}_{2} \mathrm{O}_{2}$} & {$[718-36-5]$} & \multicolumn{3}{|c|}{ 4-nitrobenzylidene tert-butylamine } & & \\
\hline & SUB & & $91.1 \pm 3.1$ & 298 & $\mathrm{C}$ & [1989ACR/KIR] \\
\hline $\mathrm{C}_{11} \mathrm{H}_{14} \mathrm{~N}_{2} \mathrm{O}_{2}$ & $\begin{array}{c}\text { [128478-71-7] } \\
\text { FUS }\end{array}$ & \multicolumn{3}{|c|}{ 2-cyano-2-nitroadamantane } & DSC & [1990FRI/DOG] \\
\hline \multicolumn{7}{|c|}{ [Note: Entropy seems low. Compound may have lower temperature phase transitions.] } \\
\hline & SUB & $(307-368)$ & $70.0 \pm 1.9$ & 338 & $\mathrm{~T}$ & [1990FRI/DOG] \\
\hline \multirow[t]{2}{*}{$\mathrm{C}_{11} \mathrm{H}_{14} \mathrm{~N}_{2} \mathrm{O}_{3}$} & {$[3585-88-4]$} & \multicolumn{3}{|c|}{ 4-nitrobenzylidene tert-butylamine $N$-oxide } & & \\
\hline & SUB & & $116.5 \pm 3.1$ & 298 & $\mathrm{C}$ & [1989ACR/KIR] \\
\hline \multirow[t]{4}{*}{$\mathrm{C}_{11} \mathrm{H}_{14} \mathrm{~N}_{2} \mathrm{O}_{4}$} & $\begin{array}{l}\text { [204189-06-0] } \\
\text { FUS }\end{array}$ & \multicolumn{3}{|c|}{$\begin{array}{c}\text { 3-nitro-3-(4-nitrophenyl)pentane } \\
20.29\end{array}$} & DSC & [1997VER3] \\
\hline & SUB & & $110.8 \pm 0.8$ & 298 & $\mathrm{~F}+\mathrm{V}$ & [1997VER3] \\
\hline & $\mathrm{V}$ & $(321-358)$ & $88.0 \pm 0.8$ & 340 & GS & [1997VER3] \\
\hline & $\mathrm{V}$ & $(321-358)$ & $90.5 \pm 0.8$ & 298 & GS & [1997VER3] \\
\hline \multirow[t]{2}{*}{$\mathrm{C}_{11} \mathrm{H}_{14} \mathrm{~N}_{4} \mathrm{O}_{9}$} & {$[165262-94-2]$} & \multicolumn{3}{|c|}{ 1-(trinitromethyl)-3-nitrateadamantane } & & \\
\hline & SUB & & $125.0 \pm 2.0$ & 298 & $\mathrm{C}$ & [2001MAT/LEB] \\
\hline \multirow[t]{2}{*}{$\mathrm{C}_{11} \mathrm{H}_{14} \mathrm{O}$} & {$[938-16-9]$} & \multicolumn{3}{|c|}{ tert-butyl phenyl ketone } & & \\
\hline & V & $(330-493)$ & 55.5 & 345 & A & [1987STE/MAL, 1947STU] \\
\hline \multirow[t]{2}{*}{$\mathrm{C}_{11} \mathrm{H}_{14} \mathrm{O}$} & {$[24569-60-6]$} & \multicolumn{3}{|c|}{ 2-ethyl-3-phenylpropanal } & & \\
\hline & V & $(343-388)$ & 64.6 & 358 & A & [1987STE/MAL] \\
\hline \multirow[t]{2}{*}{$\mathrm{C}_{11} \mathrm{H}_{14} \mathrm{O}$} & {$[582-62-7]$} & \multicolumn{3}{|c|}{ Isobutyl phenyl ketone } & & \\
\hline & V & $(331-501)$ & 55.7 & 346 & A & [1987STE/MAL, 1947STU] \\
\hline \multirow[t]{2}{*}{$\mathrm{C}_{11} \mathrm{H}_{14} \mathrm{O}$} & & 2,3,5-trimethy & etophenone & & & \\
\hline & $\mathrm{V}$ & $(352-557)$ & 57.9 & 367 & A & [1987STE/MAL, 1947STU] \\
\hline $\mathrm{C}_{11} \mathrm{H}_{14} \mathrm{O}_{2}$ & {$[18523-34-7]$} & 1,1-dimethoxy & phenylcyclop & ane & & \\
\hline & V & (278-313) & $63.9 \pm 0.6$ & & GS & [1998VER/PEN] \\
\hline $\mathrm{C}_{11} \mathrm{H}_{14} \mathrm{O}_{2}$ & {$[122-72-5]$} & 3-acetoxy-1-pl & ylpropane & & & \\
\hline & $\mathrm{V}$ & $(293-333)$ & 74.3 & 306 & A & [1987STE/MAL] \\
\hline & V & $(392-516)$ & 56.8 & 402 & & [1986CIH/VOJ] \\
\hline $\mathrm{C}_{11} \mathrm{H}_{14} \mathrm{O}_{2}$ & $\begin{array}{c}{[21009-92-7]} \\
\text { FUS }\end{array}$ & 2-acetyl-3,5-di & $\begin{array}{c}\text { thylanisole } \\
0.99\end{array}$ & 323.2 & DTA & [1989SAL/ABA] \\
\hline
\end{tabular}

[Note: Reported enthalpy of fusion is too small, and the published enthalpy and entropy of fusion data are internally inconsistent.]

$\begin{array}{ccccccc}\mathrm{C}_{11} \mathrm{H}_{14} \mathrm{O}_{2} & {[120-50-3]} & \text { Butyl benzoate } & & & \\ & \mathrm{V} & (374-474) & 63.2 & 394 & \text { BG } & {[1988 \mathrm{KAT} 2]} \\ & \mathrm{V} & (374-474) & 55.7 & 452 & \text { BG } & {[1988 \mathrm{KAT} 2]} \\ \mathrm{C}_{11} \mathrm{H}_{14} \mathrm{O}_{2} & \mathrm{~V} & (343-405) & 59.1 & 358 & \text { A } & {[1987 \mathrm{STE} / \mathrm{MAL}]} \\ & {[93-16-3]} & 1,2-\text { dimethoxy-4-(1-propenyl)benzne } & & \text { A } & {[1987 \mathrm{STE} / \mathrm{MAL}, 1947 \mathrm{STU}]} \\ \mathrm{C}_{11} \mathrm{H}_{14} \mathrm{O}_{2} & \mathrm{~V} & (358-521) & 61.9 & 373 & & \end{array}$


TABLE 10. Phase change enthalpies of $\mathrm{C}_{11}$ organic compounds-Continued

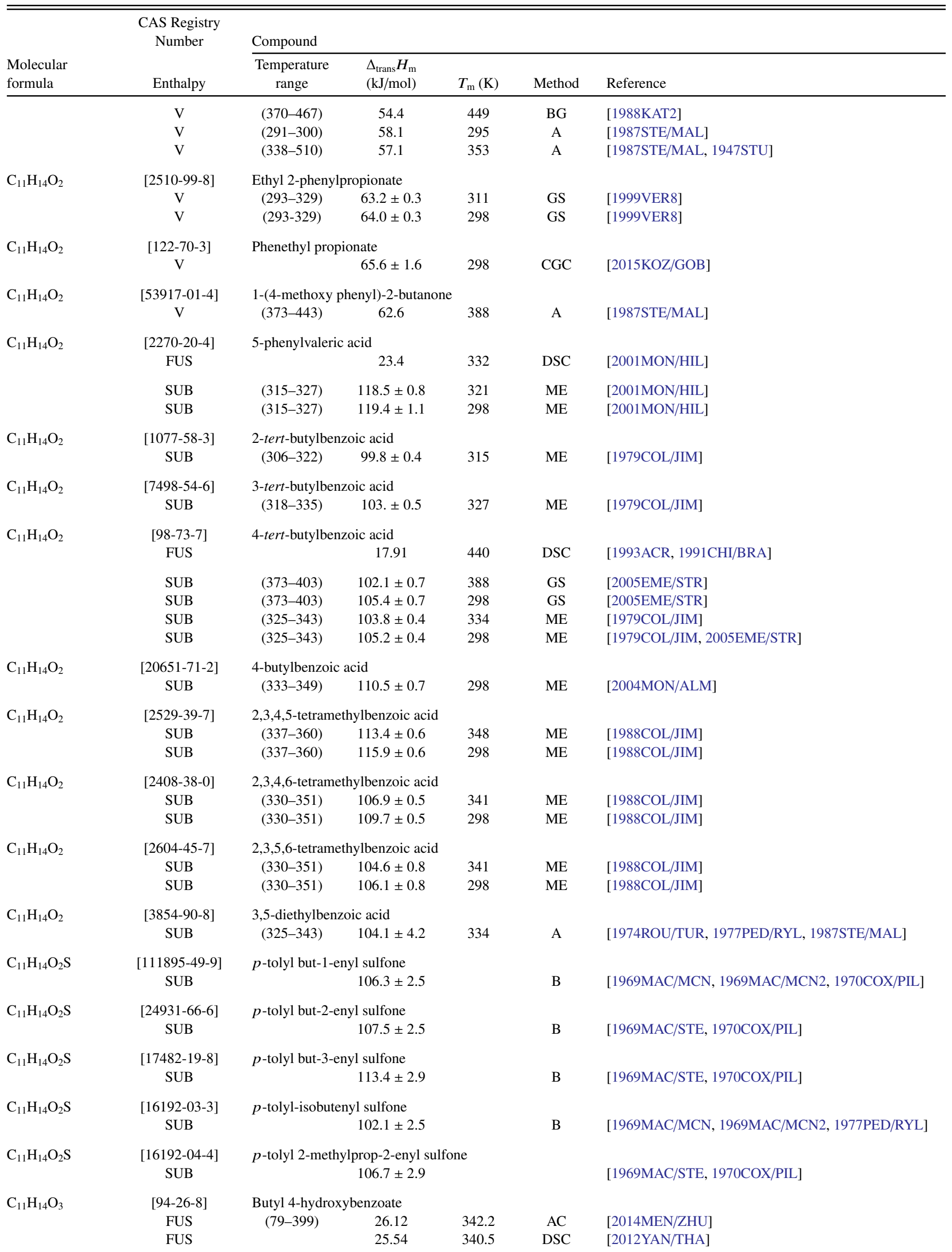


TABLE 10. Phase change enthalpies of $\mathrm{C}_{11}$ organic compounds-Continued

\begin{tabular}{|c|c|c|c|c|c|c|}
\hline \multirow[b]{2}{*}{$\begin{array}{l}\text { Molecular } \\
\text { formula }\end{array}$} & \multirow{2}{*}{$\begin{array}{c}\text { CAS Registry } \\
\text { Number } \\
\text { Enthalpy }\end{array}$} & \multicolumn{5}{|l|}{ Compound } \\
\hline & & $\begin{array}{l}\text { Temperature } \\
\text { range }\end{array}$ & $\begin{array}{l}\Delta_{\text {trans }} H_{\mathrm{m}} \\
(\mathrm{kJ} / \mathrm{mol})\end{array}$ & $T_{\mathrm{m}}(\mathrm{K})$ & Method & Reference \\
\hline & FUS & & 25.9 & 340.7 & DSC & [2011UMN/CHI] \\
\hline & FUS & & 29.3 & 342.2 & DSC & [2004STU/WIT] \\
\hline & FUS & & 26.6 & 341.8 & $\mathrm{DSC}$ & {$[1999 \mathrm{GIO} / \mathrm{BET}]$} \\
\hline & SUB & $(320-333)$ & $108.4 \pm 0.8$ & 298 & GS & [2005PER/ROD] \\
\hline & $\mathrm{V}$ & & $95.8 \pm 0.6$ & 298 & CGC & [2011UMN/CHI] \\
\hline & $\mathrm{V}$ & & 76.9 & & TGA & [2002CHA/DOL] \\
\hline & $\mathrm{V}$ & & 72.2 & & TGA & [2001CHA/DOL] \\
\hline \multirow[t]{2}{*}{$\mathrm{C}_{11} \mathrm{H}_{14} \mathrm{O}_{3}$} & & \multicolumn{5}{|c|}{$(d l)$-3-phenyl-3-hydroxy-2,2-dimethylpropanoic acid } \\
\hline & FUS & & 37.24 & 407 & & [1991CHI/BRA] \\
\hline \multirow[t]{2}{*}{$\mathrm{C}_{11} \mathrm{H}_{14} \mathrm{O}_{3}$} & & \multicolumn{5}{|c|}{ (d)-3-phenyl-3-hydroxy-2,2-dimethylpropanoic acid } \\
\hline & FUS & & 39.75 & 431 & & [1991CHI/BRA] \\
\hline \multirow[t]{2}{*}{$\mathrm{C}_{11} \mathrm{H}_{14} \mathrm{O}_{3}$} & {$[4521-28-2]$} & \multicolumn{5}{|c|}{ (4-methoxyphenyl)-4-butyric acid } \\
\hline & FUS & & 25.3 & 330.9 & & [1979ARM/JAM] \\
\hline \multirow[t]{2}{*}{$\mathrm{C}_{11} \mathrm{H}_{14} \mathrm{O}_{3}$} & & \multicolumn{5}{|c|}{$(d l)$-3-hydroxy-3-phenylvaleric acid } \\
\hline & FUS & & 35.15 & 394 & & [1991CHI/BRA] \\
\hline \multirow{2}{*}{$\mathrm{C}_{11} \mathrm{H}_{14} \mathrm{O}_{3}$} & & \multicolumn{5}{|c|}{ (d)-3-hydroxy-3-phenylvaleric acid } \\
\hline & FUS & & 30.96 & 379 & & [1991CHI/BRA] \\
\hline \multirow[t]{2}{*}{$\mathrm{C}_{11} \mathrm{H}_{14} \mathrm{O}_{3}$} & & \multicolumn{4}{|c|}{ 2-piperonylpropanol } & \\
\hline & $\mathrm{V}$ & $(373-443)$ & 84.8 & 388 & A & [1987STE/MAL] \\
\hline \multirow[t]{7}{*}{$\mathrm{C}_{11} \mathrm{H}_{14} \mathrm{O}_{3}$} & [1498-96-0] & \multicolumn{5}{|c|}{ 4-butoxybenzoic acid } \\
\hline & TRS (liq cryst) & & 18.83 & 420.7 & & \\
\hline & $\begin{array}{c}\text { TRS (liq } \\
\text { cryst-to-liq) }\end{array}$ & & 2.93 & 432.2 & DSC & [1967HER] \\
\hline & SUB & $(378-419)$ & $123.2 \pm 1.0$ & 298 & GS & [2015JAK/SPO] \\
\hline & SUB & $(351-373)$ & $125.2 \pm 0.4$ & 362 & $\mathrm{ME}$ & [2010FON/SAN] \\
\hline & SUB & $(351-373)$ & $127.7 \pm 0.8$ & 298 & ME & [2010FON/SAN] \\
\hline & SUB & & $129.0 \pm 0.8$ & 298 & & [2010RIB/FER3] \\
\hline \multirow[t]{2}{*}{$\mathrm{C}_{11} \mathrm{H}_{14} \mathrm{O}_{3}$} & [147578-43-6] & \multicolumn{5}{|c|}{ 2-isobutoxybenzoic acid } \\
\hline & V & $(344-398)$ & $95.6 \pm 0.6$ & 298 & GS & [2015JAK/SPO] \\
\hline \multirow[t]{2}{*}{$\mathrm{C}_{11} \mathrm{H}_{14} \mathrm{O}_{3}$} & {$[350997-58-9]$} & 3-isobutoxybe & oic acid & & & \\
\hline & $\mathrm{V}$ & $(355-382)$ & $99.2 \pm 0.9$ & 298 & GS & [2015JAK/SPO] \\
\hline $\mathrm{C}_{11} \mathrm{H}_{14} \mathrm{O}_{3}$ & {$[30762-00-6]$} & 4-isobutoxybe & oic acid & & & \\
\hline & SUB & (363-403) & $119.0 \pm 0.6$ & 298 & GS & [2015JAK/SPO] \\
\hline $\mathrm{C}_{11} \mathrm{H}_{14} \mathrm{O}_{3}$ & {$[6627-89-0]$} & tert-butyl phen & carbonate & & & \\
\hline & V & $(294-348)$ & $67.6 \pm 0.6$ & 298 & GS & [2008VER/EME2] \\
\hline $\mathrm{C}_{11} \mathrm{H}_{14} \mathrm{O}_{4}$ & [2107-70-2] & 3-(3,4-dimetho & phenyl)propi & acid & & \\
\hline & FUS & & 32.38 & 370.9 & DSC & [2001MON/HIL4] \\
\hline & SUB & $(352-366)$ & $140.3 \pm 0.8$ & 359 & ME & [2001MON/HIL4] \\
\hline & SUB & $(352-366)$ & $143.6 \pm 2.2$ & 298 & $\mathrm{ME}$ & [2001MON/HIL4] \\
\hline $\mathrm{C}_{11} \mathrm{H}_{15} \mathrm{BrO}$ & [99857-52-0] & 4-methyl-2-brc & ophenyl isobu & ether & & \\
\hline & V & $(293-328)$ & $71.0 \pm 0.3$ & 298 & GS & [2005STR/SPO] \\
\hline $\mathrm{C}_{11} \mathrm{H}_{15} \mathrm{BrO}_{3}$ & [929259-36-9] & 1-bromo-2-[2- & -methoxyethor & thoxy]b & ne & \\
\hline & V & $(310-373)$ & $83.1 \pm 0.3$ & 298 & GS & [2006DAB/SPO] \\
\hline $\mathrm{C}_{11} \mathrm{H}_{15} \mathrm{Cl}$ & [42597-10-4] & 4-tert-butyl-2- & lorotoluene & & & \\
\hline & V & $(372-503)$ & 54.0 & 387 & A & [1987STE/MAL, 1973FEL/SAV, 1999DYK/SVO] \\
\hline $\mathrm{C}_{11} \mathrm{H}_{15} \mathrm{~F}_{9} \mathrm{O}$ & [1193009-96-9] & $1,1,1,2,2,3,3,4$ & -nonafluoro-6 & ntyloxy) & ane & \\
\hline & FUS & & 18.4 & 203.0 & DSC & {$[2010 \mathrm{ZAG} / \mathrm{CON}]$} \\
\hline $\mathrm{C}_{11} \mathrm{H}_{15} \mathrm{~N}$ & {$[42525-65-5]$} & 2-phenylethyla & tidine & & & \\
\hline & V & $(302-333)$ & 62.2 & 317 & A & [1987STE/MAL, 1976KIP/TSV] \\
\hline $\mathrm{C}_{11} \mathrm{H}_{15} \mathrm{~N}$ & {$[4096-20-2]$} & $N$-phenylpipe & ine & & & \\
\hline
\end{tabular}


TABLE 10. Phase change enthalpies of $\mathrm{C}_{11}$ organic compounds-Continued

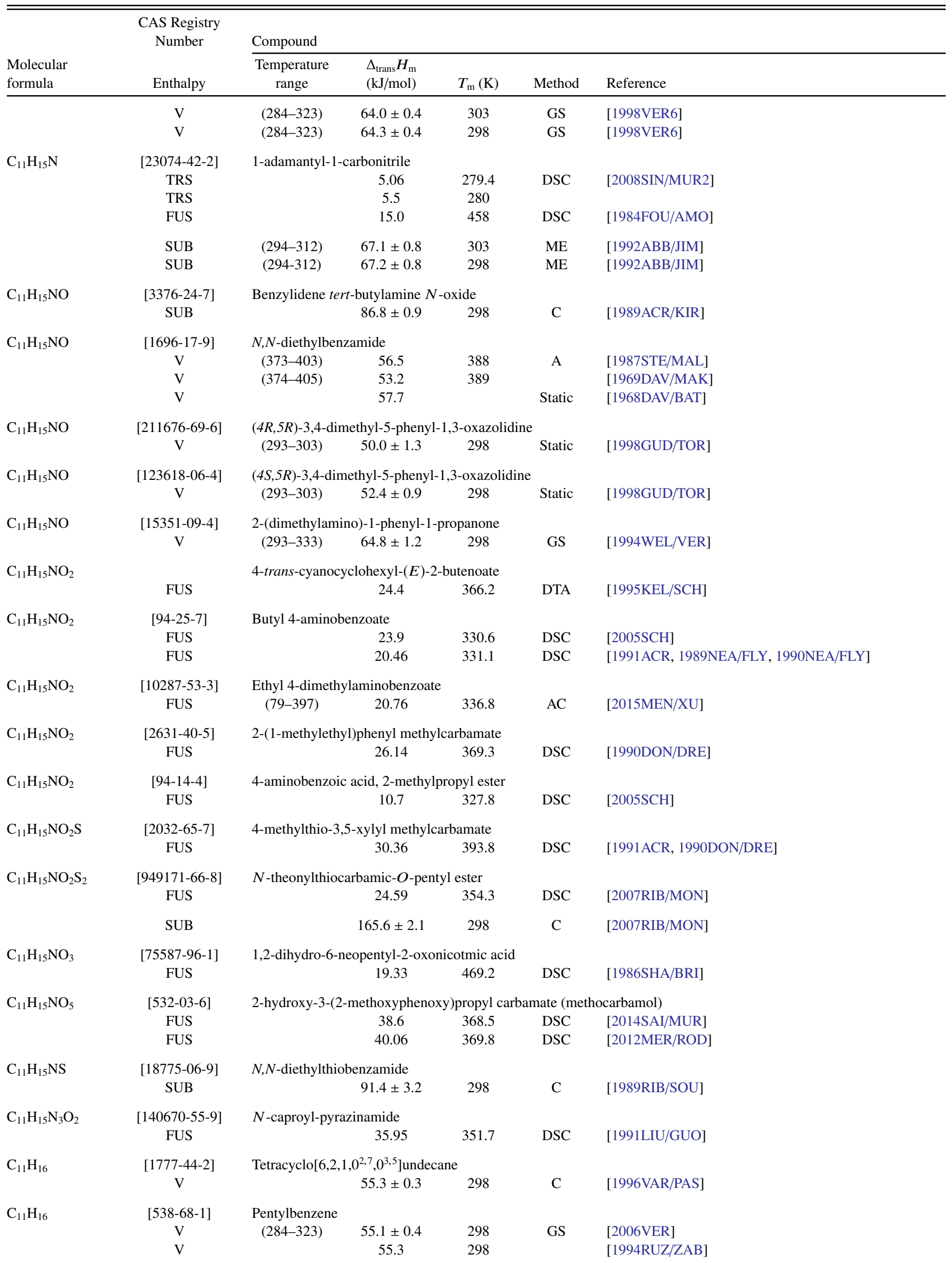


TABLE 10. Phase change enthalpies of $\mathrm{C}_{11}$ organic compounds-Continued

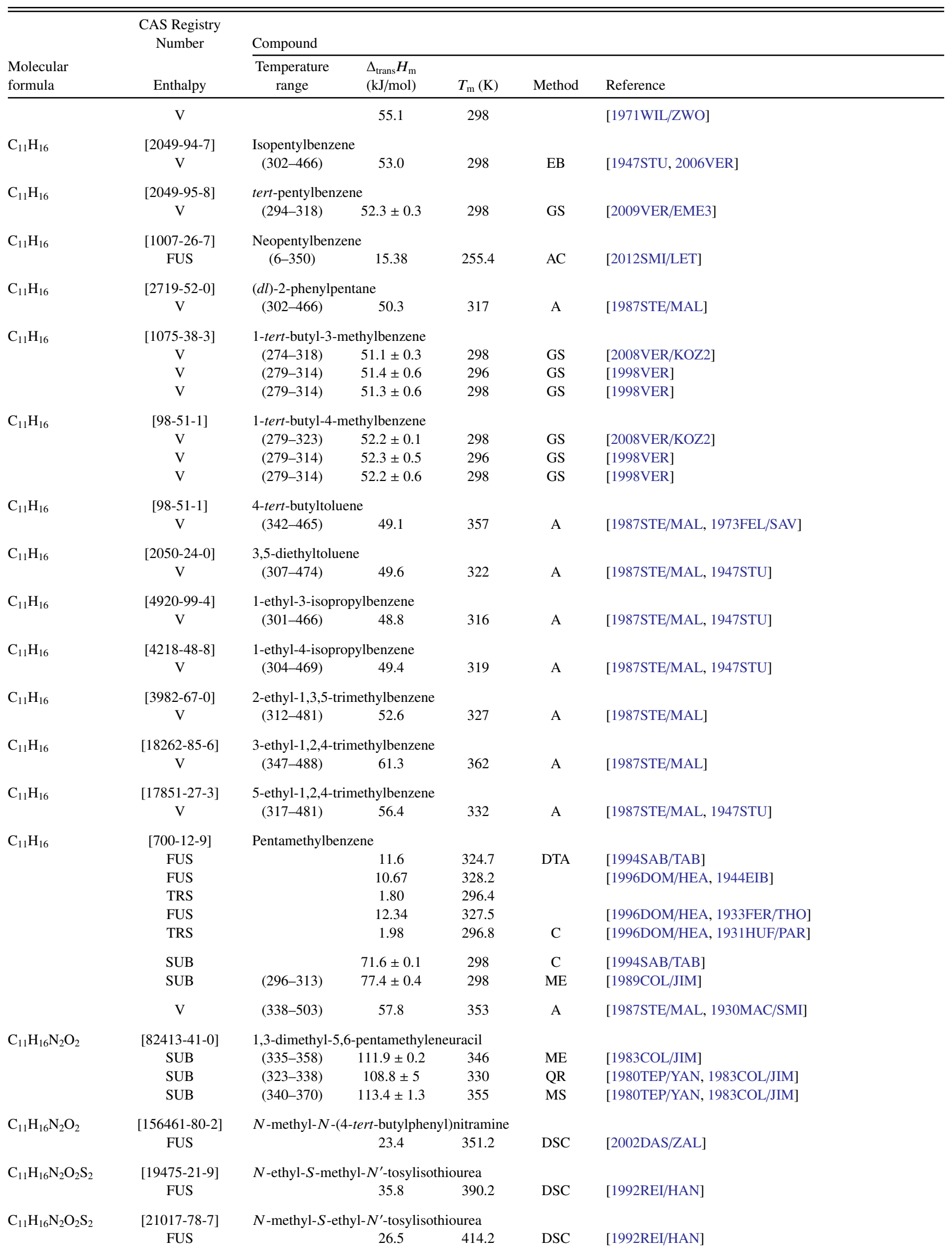


TABLE 10. Phase change enthalpies of $\mathrm{C}_{11}$ organic compounds-Continued

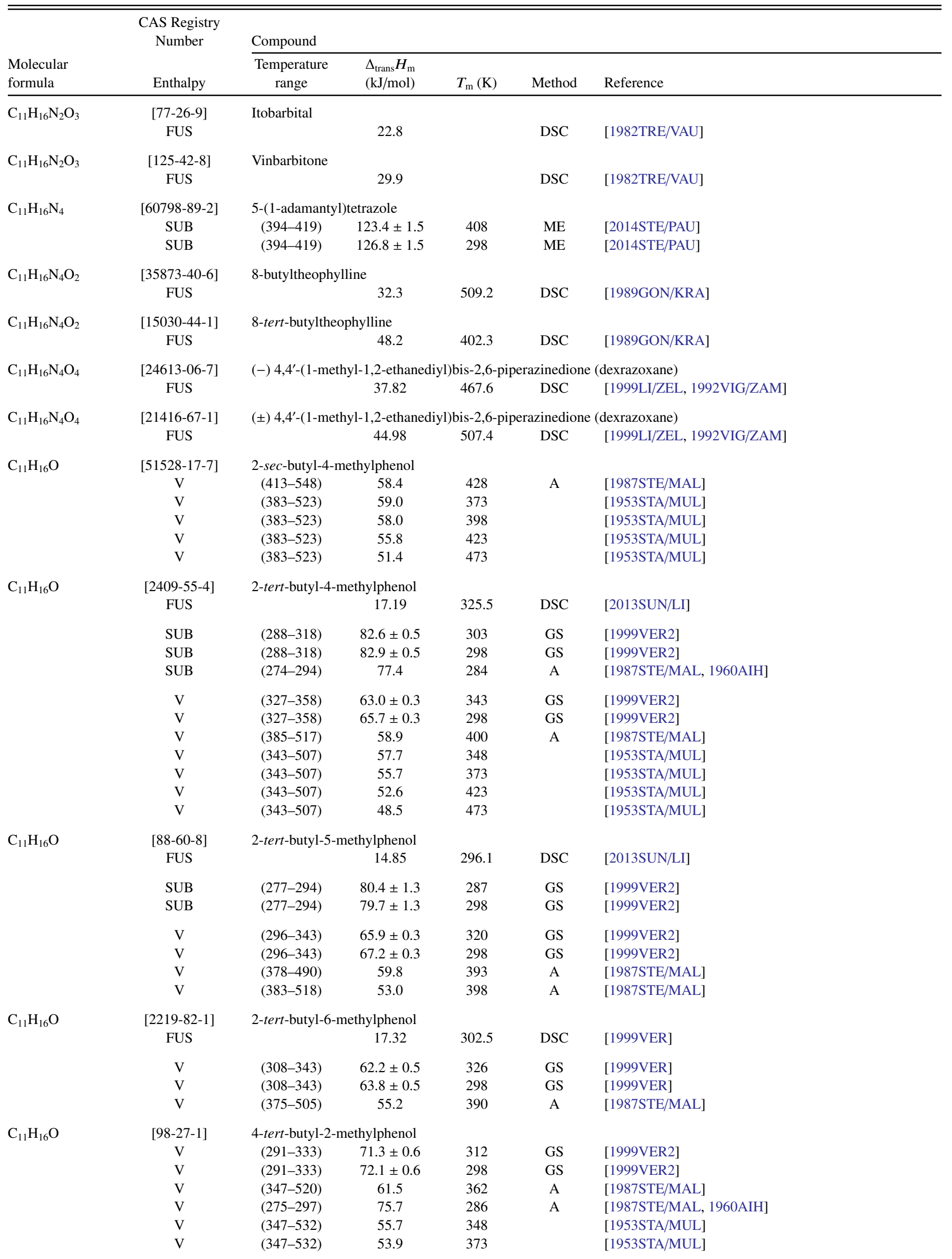


TABLE 10. Phase change enthalpies of $\mathrm{C}_{11}$ organic compounds-Continued

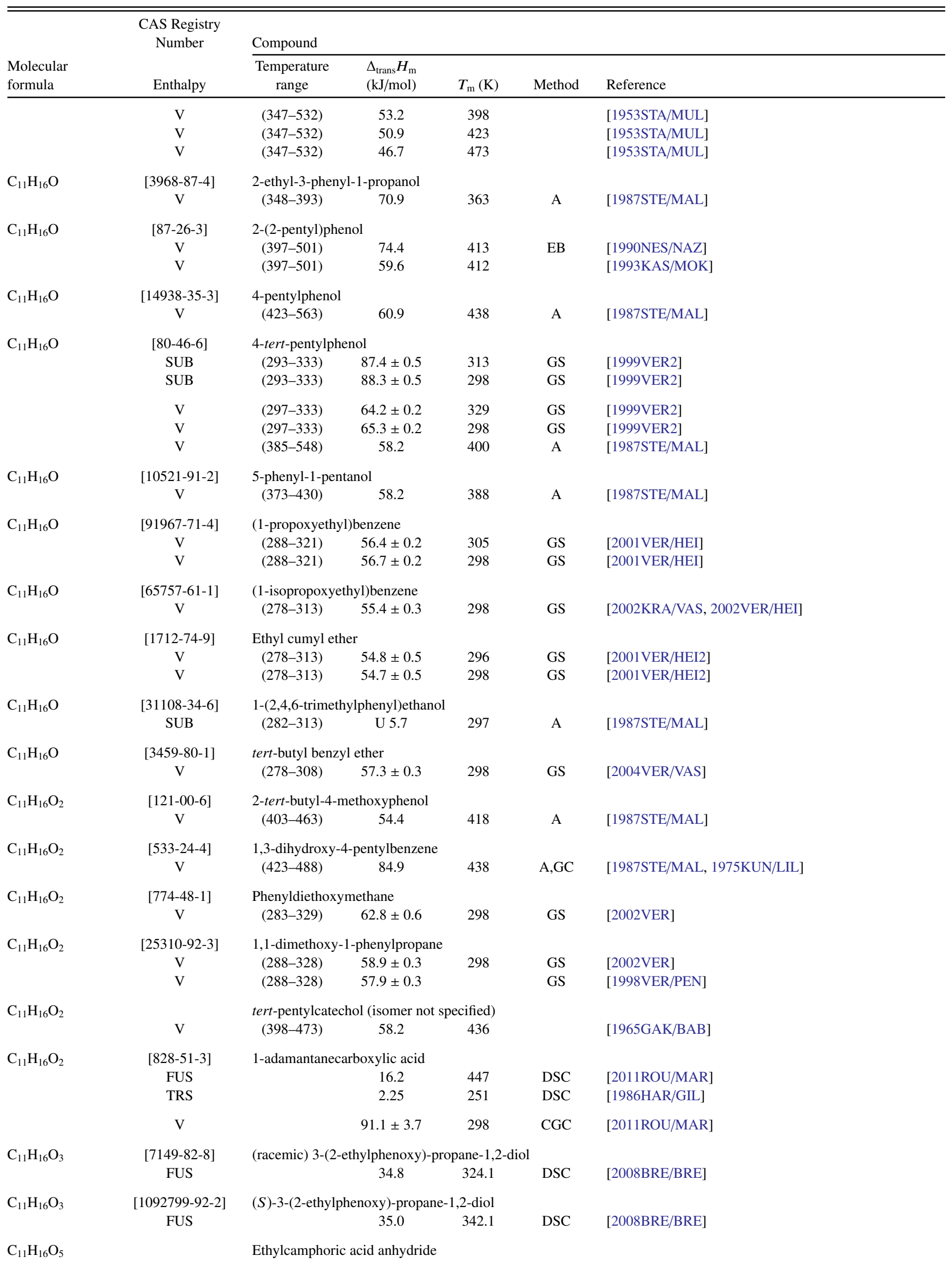


TABLE 10. Phase change enthalpies of $\mathrm{C}_{11}$ organic compounds-Continued

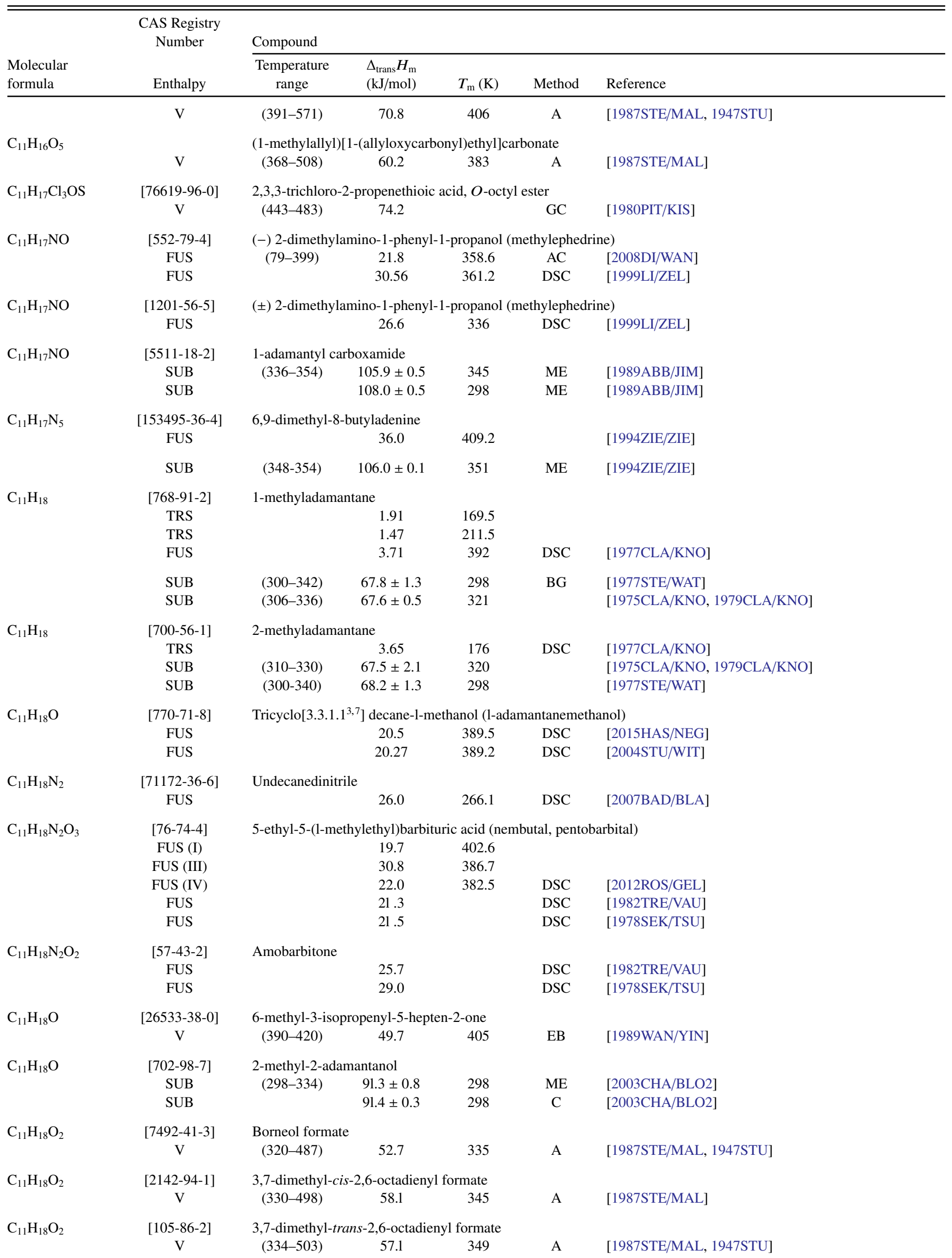


TABLE 10. Phase change enthalpies of $\mathrm{C}_{11}$ organic compounds-Continued

\begin{tabular}{|c|c|c|c|c|c|c|}
\hline \multirow[b]{2}{*}{$\begin{array}{l}\text { Molecular } \\
\text { formula }\end{array}$} & \multirow{2}{*}{$\begin{array}{l}\text { CAS Registry } \\
\text { Number } \\
\text { Enthalpy }\end{array}$} & \multicolumn{5}{|l|}{ Compound } \\
\hline & & $\begin{array}{l}\text { Temperature } \\
\text { range }\end{array}$ & $\begin{array}{l}\Delta_{\text {trans }} H_{\mathrm{m}} \\
(\mathrm{kJ} / \mathrm{mol})\end{array}$ & $T_{\mathrm{m}}(\mathrm{K})$ & Method & Reference \\
\hline $\mathrm{C}_{11} \mathrm{H}_{18} \mathrm{O}_{2}$ & $\begin{array}{c}{[1200-67-5]} \\
\mathrm{V}\end{array}$ & \multicolumn{2}{|c|}{$\begin{array}{l}\text { Isobomeol formate } \\
\quad(383-441)\end{array}$} & 398 & A & [1987STE/MAL, 1937RUD/KOR] \\
\hline $\mathrm{C}_{11} \mathrm{H}_{18} \mathrm{O}_{4}$ & $\begin{array}{c}{[4167-77-5]} \\
\mathrm{V}\end{array}$ & \multicolumn{4}{|c|}{ 1,1-cyclopentanedicarboxylic acid diethyl ester } & [1998VER/KUM] \\
\hline $\mathrm{C}_{11} \mathrm{H}_{18} \mathrm{O}_{5}$ & $\begin{array}{c}{[85710-88-9]} \\
\mathrm{V}\end{array}$ & \multicolumn{4}{|c|}{ 4-oxononanedioic acid, dimethyl ester } & [1987STE/MAL] \\
\hline $\mathrm{C}_{11} \mathrm{H}_{18} \mathrm{O}_{6}$ & $\begin{array}{l}{[16515-90-5]} \\
\mathrm{V}\end{array}$ & \multicolumn{2}{|c|}{ 1,1,1-tris(ethoxycarbonyl)methane } & $74.1 \pm 0.4$ & GS & [1995RAK/VER] \\
\hline $\mathrm{C}_{11} \mathrm{H}_{18} \mathrm{O}_{6}$ & $\begin{array}{c}{[170464-50-3]} \\
\mathrm{V}\end{array}$ & \multicolumn{3}{|c|}{$\begin{array}{l}\text { 1,1,1-tris(methoxy carbonyl)pentane } \\
(298-338) \quad 81.0 \pm 0.4\end{array}$} & GS & [1995RAK/VER] \\
\hline $\mathrm{C}_{11} \mathrm{H}_{19} \mathrm{NO}_{2}$ & $\begin{array}{c}{[62391-95-1]} \\
\mathrm{V}\end{array}$ & \multicolumn{3}{|c|}{ Ethyl bis(isopropyl)cyanoacetate } & GS & [1995VER/BEC] \\
\hline $\mathrm{C}_{11} \mathrm{H}_{19} \mathrm{NO}_{3}$ & $\begin{array}{c}{[114-26-1]} \\
\text { FUS }\end{array}$ & \multicolumn{3}{|c|}{ 2-isopropoxyphenyl $N$-methylcarbamate } & DSC & [1991ACR, 1990DON/DRE] \\
\hline $\mathrm{C}_{11} \mathrm{H}_{19} \mathrm{NO}_{5}$ & $\begin{array}{c}{[1446-19-1]} \\
\mathrm{V}\end{array}$ & \multicolumn{2}{|c|}{$N$-acetyl- $(l)$-glutamic acid, diethyl ester } & $\begin{array}{c}y l \text { ester } \\
418\end{array}$ & $\mathrm{~A}, \mathrm{~EB}$ & [1987STE/MAL, 1953MEL/VIO] \\
\hline $\mathrm{C}_{11} \mathrm{H}_{19} \mathrm{NS}$ & $\begin{array}{l}{[4175-69-3]} \\
\text { FUS }\end{array}$ & \multicolumn{3}{|c|}{ 2,4-di-tert-butylthiazole } & $\mathrm{C}$ & [1966MEY/MET] \\
\hline $\mathrm{C}_{11} \mathrm{H}_{19} \mathrm{~N}_{3} \mathrm{O}$ & $\begin{array}{l}{[23947-60-6]} \\
\text { FUS }\end{array}$ & \multicolumn{4}{|c|}{ 5-butyl-2-ethylamino-6-methylpyrimidin-4-ol } & [1991ACR, 1990DON/DRE] \\
\hline $\mathrm{C}_{11} \mathrm{H}_{19} \mathrm{~N}_{5} \mathrm{~S}$ & $\begin{array}{l}{[4147-51-7]} \\
\text { FUS }\end{array}$ & \multicolumn{5}{|c|}{ 6-ethylthio- $N, N^{\prime}$-bis(1-methylethyl)-1,3,5-triazine-2,4-diamine } \\
\hline $\mathrm{C}_{11} \mathrm{H}_{2} \mathrm{O}$ & $\begin{array}{c}{[2243-98-3]} \\
\mathrm{V}\end{array}$ & $\begin{array}{l}\text { 1-undecyne } \\
(422-468)\end{array}$ & 46.7 & 437 & EB & [1986ELV/KUD] \\
\hline $\mathrm{C}_{11} \mathrm{H}_{2} \mathrm{O}$ & $\begin{array}{c}{[2294-72-6]} \\
V\end{array}$ & $\begin{array}{l}\text { 5-undecyne } \\
(423-471)\end{array}$ & 47.3 & 438 & EB & [1986ELV/KUD] \\
\hline $\mathrm{C}_{11} \mathrm{H}_{2} \mathrm{O}$ & $\begin{array}{c}{[180-43-8]} \\
\mathrm{V}\end{array}$ & \multicolumn{2}{|c|}{ Spiro[5.5]undecane } & 298 & $\mathrm{C}$ & {$[1975 \mathrm{SUB} / \mathrm{ZWO}]$} \\
\hline $\mathrm{C}_{11} \mathrm{H}_{2} \mathrm{O}$ & $\begin{array}{c}{[1606-08-2]} \\
\mathrm{V}\end{array}$ & $\begin{array}{l}\text { Cyclopentylcy } \\
(383-488)\end{array}$ & $\begin{array}{r}\text { hexane } \\
47.9\end{array}$ & 398 & A & {$[1987 \mathrm{STE} / \mathrm{MAL}]$} \\
\hline $\mathrm{C}_{11} \mathrm{H}_{2} \mathrm{O}$ & $\begin{array}{c}{[180-43-8]} \\
\text { SUB }\end{array}$ & Bicyclo[3.3.3] & $\begin{array}{l}\text { decane } \\
63.6 \pm 0.8\end{array}$ & 298 & $\mathrm{C}$ & [1975PAR/STE, 1977PED/RYL] \\
\hline $\mathrm{C}_{11} \mathrm{H}_{20} \mathrm{Cl}_{4}$ & $\begin{array}{c}{[3922-34-7]} \\
\mathrm{V}\end{array}$ & $\begin{array}{c}1,1,1,11-\text { tetrac } \\
(303-353)\end{array}$ & $\begin{array}{c}\text { roundecane } \\
92.5\end{array}$ & 318 & A & [1987STE/MAL, 1999DYK/SVO, 1960MAL/MAL] \\
\hline $\mathrm{C}_{11} \mathrm{H}_{20} \mathrm{Cl}_{4}$ & $\begin{array}{c}{[210049-49-3]} \\
\mathrm{V}\end{array}$ & 1,2,10,11-tetr & $\begin{array}{c}\text { loroundecane } \\
78.7\end{array}$ & & & [1998DRO/TOM] \\
\hline $\mathrm{C}_{11} \mathrm{H}_{20} \mathrm{~N}_{2}$ & $\begin{array}{c}{[97142-71-7]} \\
\mathrm{V}\end{array}$ & $\begin{array}{l}\text { 1-heptyl-2-me } \\
(312-368)\end{array}$ & $\begin{array}{l}\text { limidazole } \\
79.4 \pm 0.2\end{array}$ & 298 & GS & [2011EME/POR2] \\
\hline $\mathrm{C}_{11} \mathrm{H}_{20} \mathrm{~N}_{2}$ & $\begin{array}{c}{[21252-69-7]} \\
\text { V }\end{array}$ & 1-octylimidaz & $81.0 \pm 1.2$ & 298 & $\mathrm{C}$ & [2015VIT/AGA] \\
\hline $\mathrm{C}_{11} \mathrm{H}_{20} \mathrm{~N}_{2} \mathrm{~S}$ & $\begin{array}{c}\text { [1394816-48-8] } \\
\text { FUS }\end{array}$ & Ethyl-(1-thia- & $\begin{array}{l}\text { aspiro }[5.5] \mathrm{u} \\
24.5\end{array}$ & $\begin{array}{c}\text { c-2-en- } 2 \\
378.4\end{array}$ & $\begin{array}{l}\operatorname{mine} \\
\text { DSC }\end{array}$ & [2013PER/BLO2] \\
\hline & SUB & & $100.8 \pm 1.1$ & 298 & GS & [2013PER/BLO2] \\
\hline $\mathrm{C}_{11} \mathrm{H}_{20} \mathrm{~N}_{6}$ & $\begin{array}{c}{[13452-85-2]} \\
\text { FUS }\end{array}$ & 1-pyrrolidinyl & $\begin{array}{l}\text {-bis(dimethy } \\
25.61\end{array}$ & $\begin{array}{c}\text { nino })-(s) \\
403.1\end{array}$ & DSC & [1991ACR, 1989BRA/RYT] \\
\hline $\mathrm{C}_{11} \mathrm{H}_{20} \mathrm{~N}_{6} \mathrm{O}$ & $\begin{array}{c}{[16269-02-6]} \\
\text { FUS }\end{array}$ & 1-morpholiny & $\begin{array}{c}\text { 5-bis(dimeth } \\
24.69\end{array}$ & $\begin{array}{c}\operatorname{nino})-(s) \\
397.4\end{array}$ & $\begin{array}{l}\text { zine } \\
\text { DSC }\end{array}$ & [1991ACR, 1989BRA/RYT] \\
\hline $\mathrm{C}_{11} \mathrm{H}_{20} \mathrm{~N}_{6} \mathrm{~S}$ & {$[41492-69-7]$} & 1-(thiomorpho & yl-3,5-bis(dir & hylamino & -triazine & \\
\hline
\end{tabular}


TABLE 10. Phase change enthalpies of $\mathrm{C}_{11}$ organic compounds-Continued

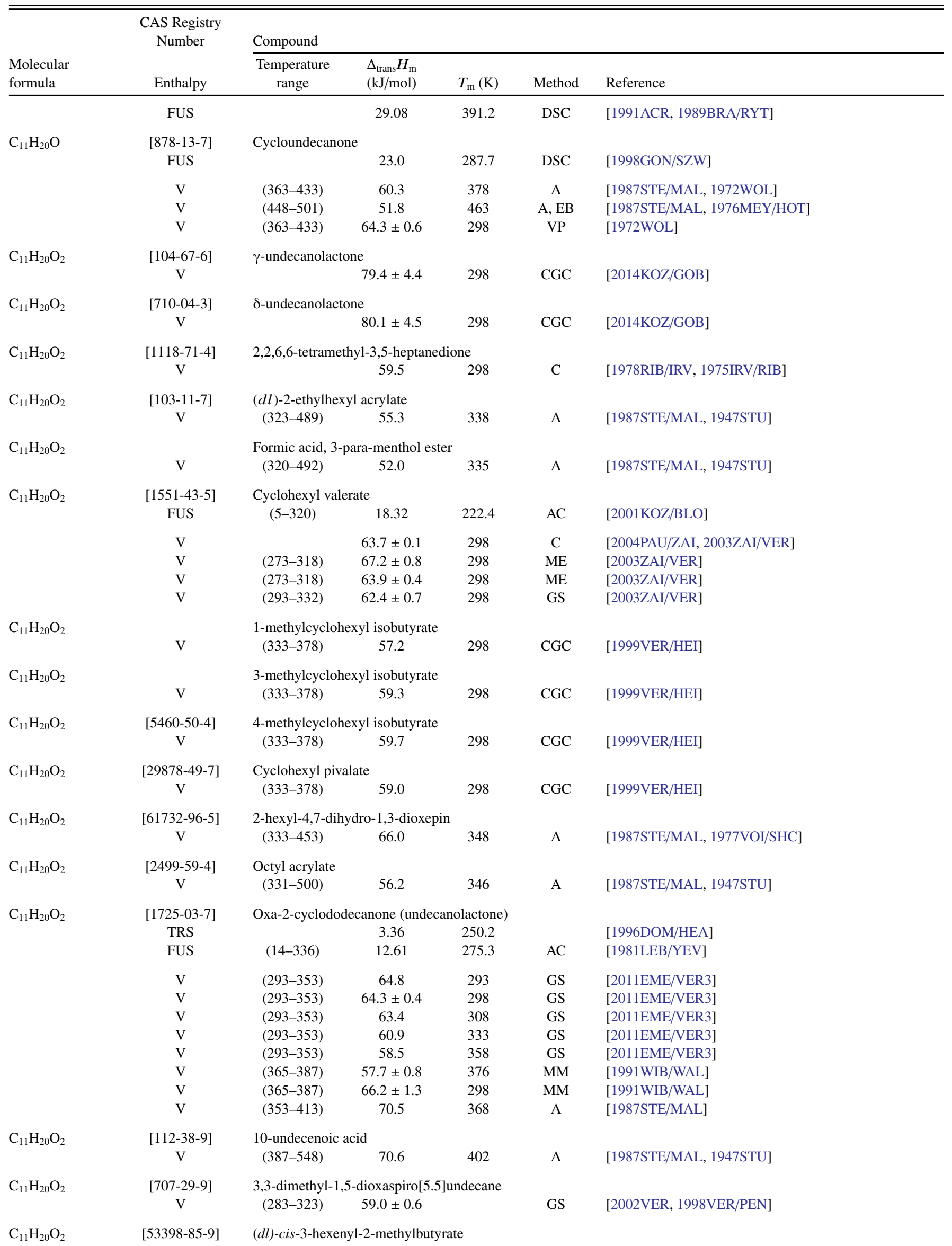


TABLE 10. Phase change enthalpies of $\mathrm{C}_{11}$ organic compounds-Continued

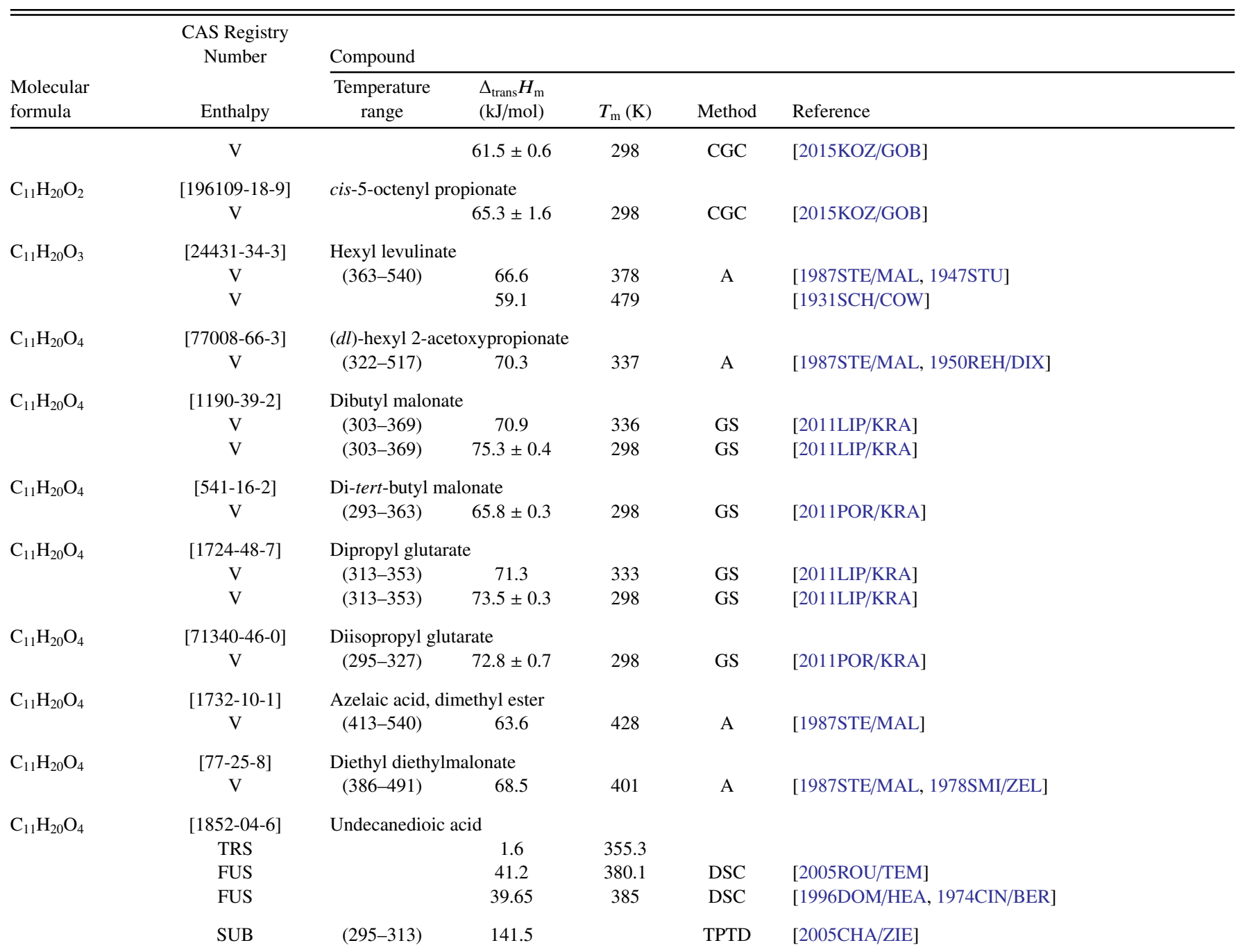

[Note: Values based on TPTD method are not consistent with values determined by other experimental methods.]

\begin{tabular}{|c|c|c|c|c|c|c|}
\hline & SUB & $(371-381)$ & $158.6 \pm 1.9$ & 376 & ME & [1999RIB/MON] \\
\hline & SUB & $(371-381)$ & $162.5 \pm 1.9$ & 298 & $\mathrm{ME}$ & [1999RIB/MON] \\
\hline & V & $(424-503)$ & $128.2 \pm 2.3$ & 298 & CGC & [2005ROU/TEM] \\
\hline $\mathrm{C}_{11} \mathrm{H}_{20} \mathrm{O}_{4}$ & $\begin{array}{c}{[1732-10-1]} \\
\mathrm{V}\end{array}$ & $\begin{array}{l}\text { Dimethyl azelate } \\
(298-373)\end{array}$ & $82.3 \pm 0.4$ & 298 & GS & [2006VER/KOZ] \\
\hline $\mathrm{C}_{11} \mathrm{H}_{20} \mathrm{O}_{5}$ & $\mathrm{~V}$ & $\begin{array}{l}\text { Hexyl[1-(methox } \\
\quad(371-538)\end{array}$ & $\begin{array}{c}\text { ycarbonyl)eth } \\
65.9\end{array}$ & $\begin{array}{l}\text { arbonate } \\
386\end{array}$ & A & [1987STE/MAL, 1950REH/DIX2] \\
\hline $\mathrm{C}_{11} \mathrm{H}_{20} \mathrm{O}_{5}$ & $\begin{array}{c}{[902261-26-1]} \\
\mathrm{V}\end{array}$ & $\begin{array}{l}\text { Propyl[1-(butoxy } \\
\quad(330-463)\end{array}$ & $\begin{array}{c}\text { carbonyl)ethy } \\
66.4\end{array}$ & $\begin{array}{l}\text { bonate } \\
345\end{array}$ & A & [1987STE/MAL, 1950REH/DIX2] \\
\hline $\mathrm{C}_{11} \mathrm{H}_{21} \mathrm{~N}$ & $\begin{array}{c}{[80606-32-2]} \\
\mathrm{V}\end{array}$ & $\begin{array}{l}\text { 2-butyl-2-methyll } \\
\text { (298-388) }\end{array}$ & $\begin{array}{c}\text { hexanenitrile } \\
59.8 \pm 0.4\end{array}$ & & GS & [1994RAK/VER] \\
\hline $\mathrm{C}_{11} \mathrm{H}_{21} \mathrm{~N}$ & $\begin{array}{c}\text { [2244-07-7] } \\
\text { V } \\
\text { V } \\
\text { V }\end{array}$ & $\begin{array}{l}\text { Undecanonitrile } \\
(290-340) \\
(355-534)\end{array}$ & $\begin{array}{c}71.8 \pm 0.3 \\
63.7 \\
71.1 \pm 0.1\end{array}$ & $\begin{array}{l}298 \\
370 \\
298\end{array}$ & $\begin{array}{l}\text { GS } \\
\text { A } \\
\text { C }\end{array}$ & $\begin{array}{l}\text { [2005EME/VER] } \\
\text { [1987STE/MAL] } \\
\text { [1977STRI/SUN] }\end{array}$ \\
\hline $\mathrm{C}_{11} \mathrm{H}_{21} \mathrm{~N}$ & $\begin{array}{c}\text { [3319-01-5] } \\
\mathrm{V} \\
\mathrm{V}\end{array}$ & $\begin{array}{l}N \text {-cyclohexylpip } \\
(288-328) \\
(288-328)\end{array}$ & $\begin{array}{l}59.9 \pm 0.6 \\
60.5 \pm 0.6\end{array}$ & $\begin{array}{l}308 \\
298\end{array}$ & $\begin{array}{l}\text { GS } \\
\text { GS }\end{array}$ & $\begin{array}{l}\text { [1998VER6] } \\
\text { [1998VER6] }\end{array}$ \\
\hline $\mathrm{C}_{11} \mathrm{H}_{21} \mathrm{NO}$ & $\begin{array}{c}{[15770-38-4]} \\
\text { V } \\
\text { V }\end{array}$ & $\begin{array}{l}N \text {-hexanoylpiper } \\
(383-433)\end{array}$ & $\begin{array}{l}\text { ridine } \\
66.3 \\
81.8\end{array}$ & 398 & $\begin{array}{c}\text { A } \\
\text { Static }\end{array}$ & $\begin{array}{l}\text { [1987STE/MAL] } \\
{[1968 \mathrm{DAV} / \mathrm{BAT}]}\end{array}$ \\
\hline
\end{tabular}


TABLE 10. Phase change enthalpies of $\mathrm{C}_{11}$ organic compounds-Continued

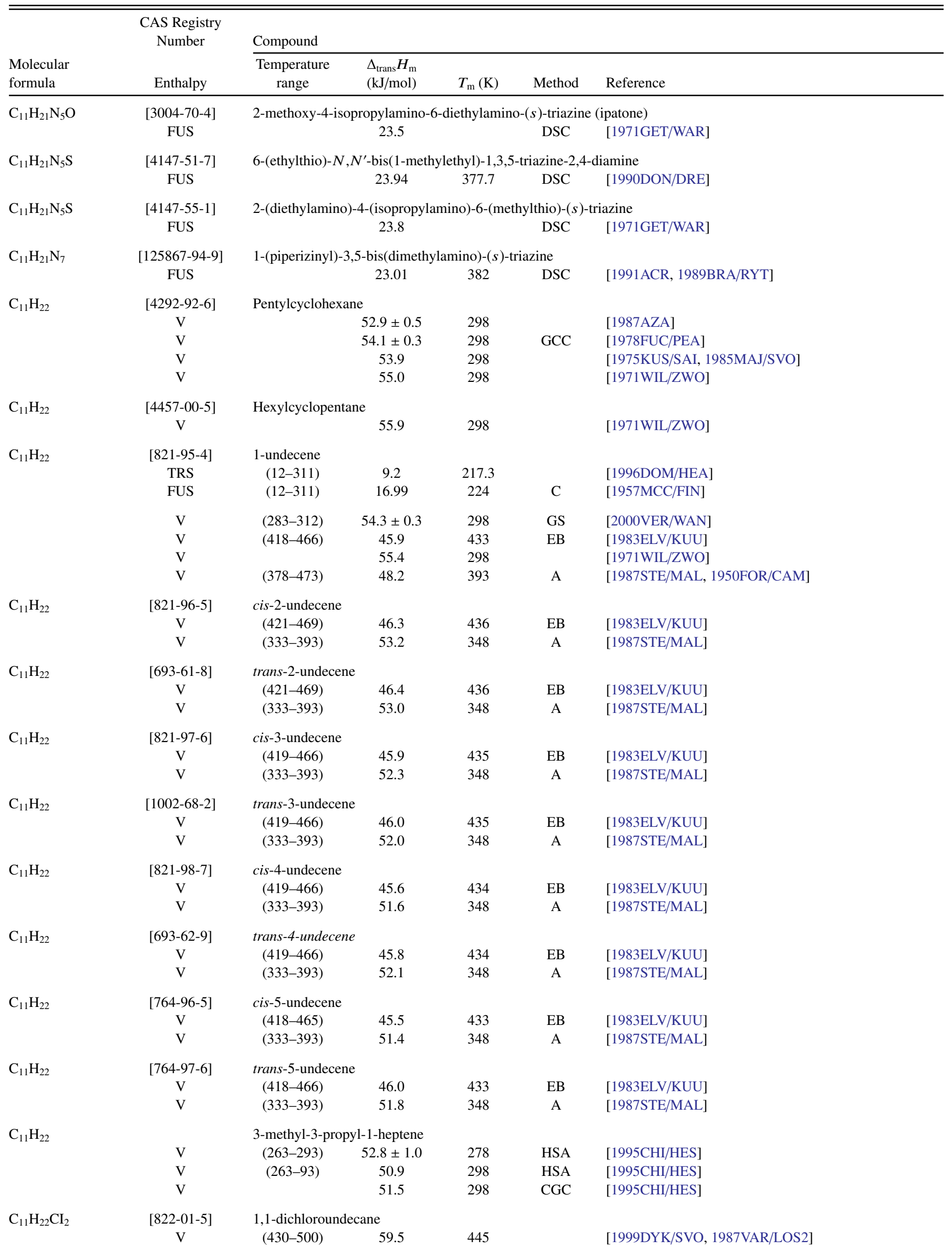


TABLE 10. Phase change enthalpies of $\mathrm{C}_{11}$ organic compounds-Continued

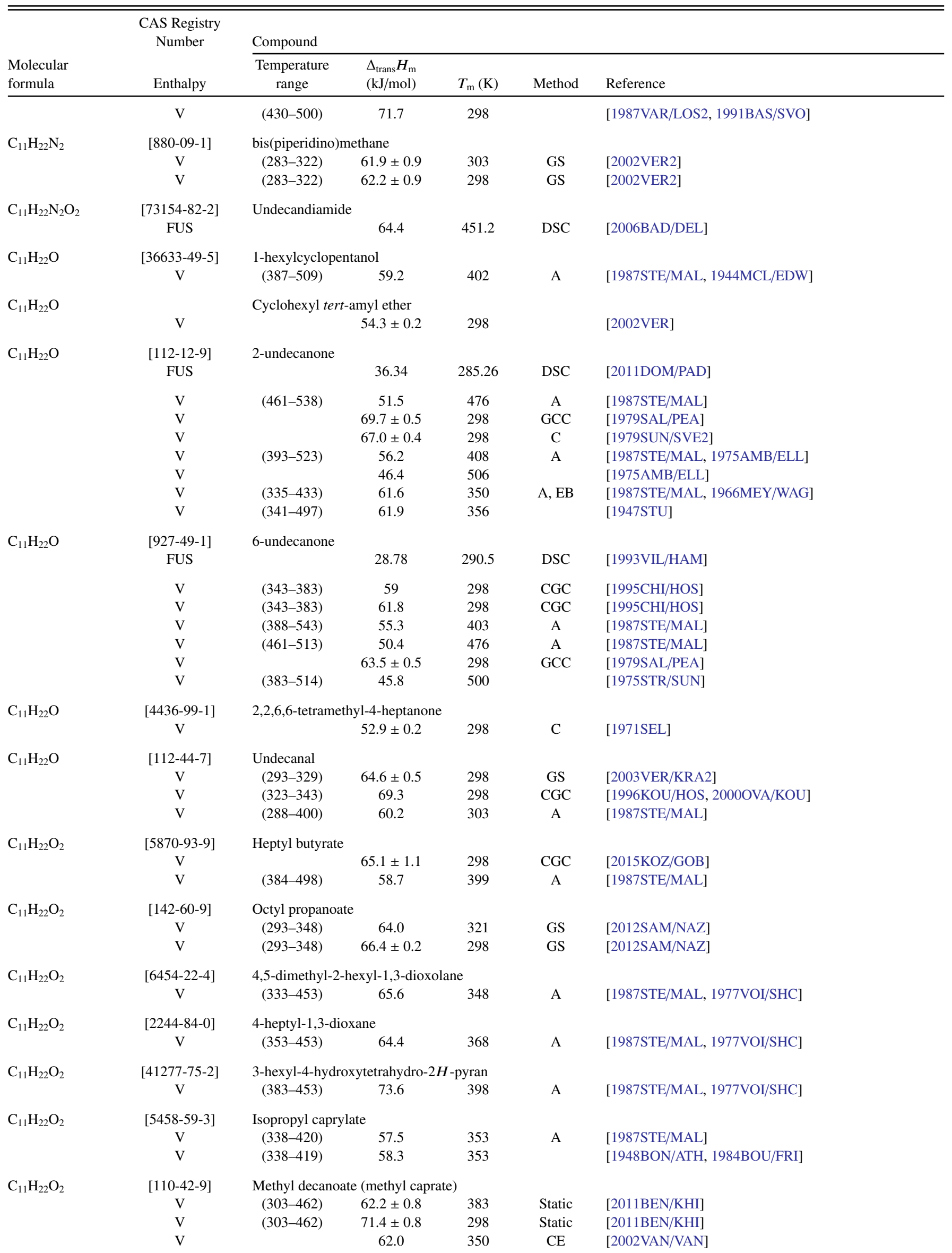


TABLE 10. Phase change enthalpies of $\mathrm{C}_{11}$ organic compounds-Continued

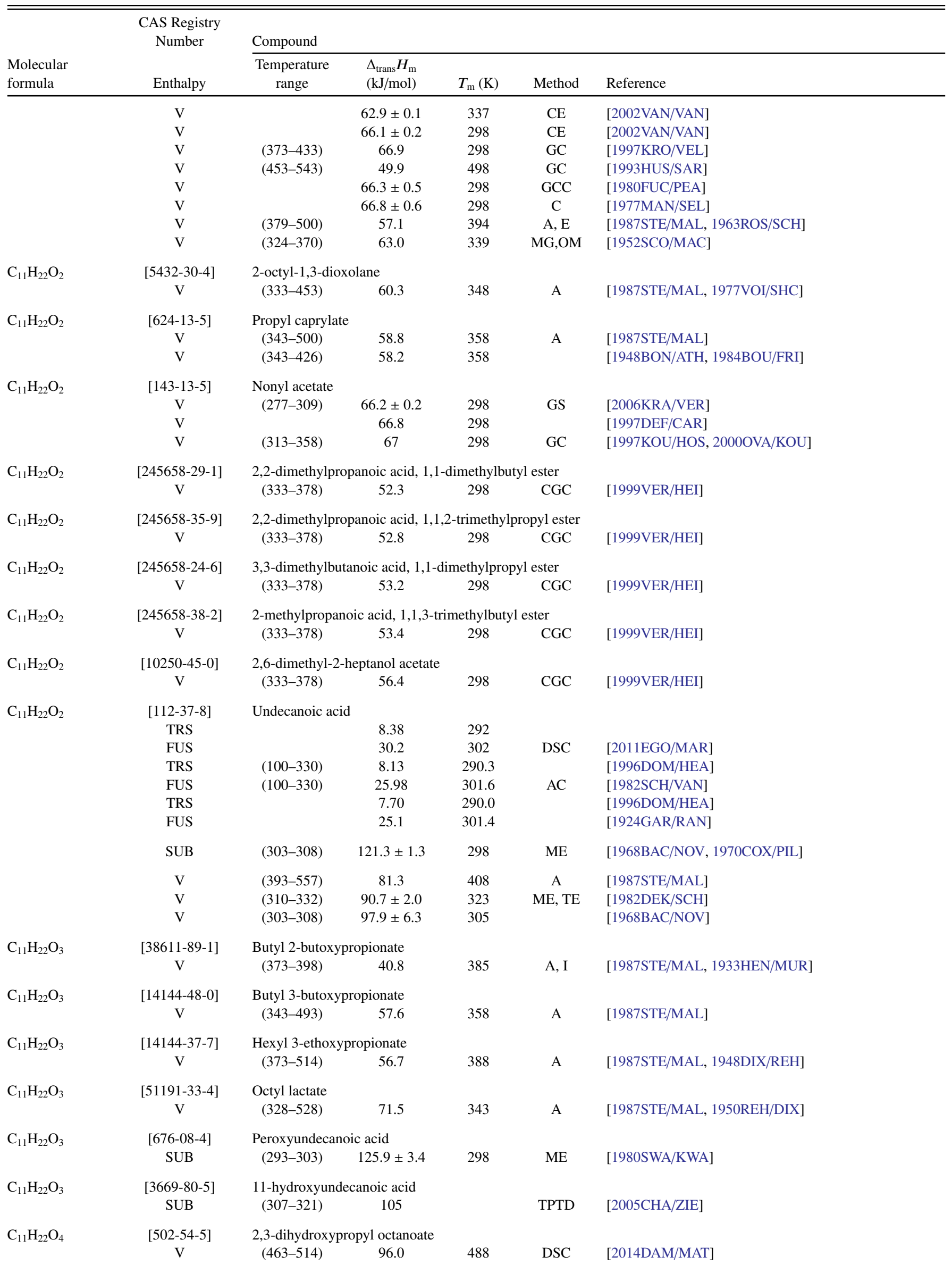


TABLE 10. Phase change enthalpies of $\mathrm{C}_{11}$ organic compounds-Continued

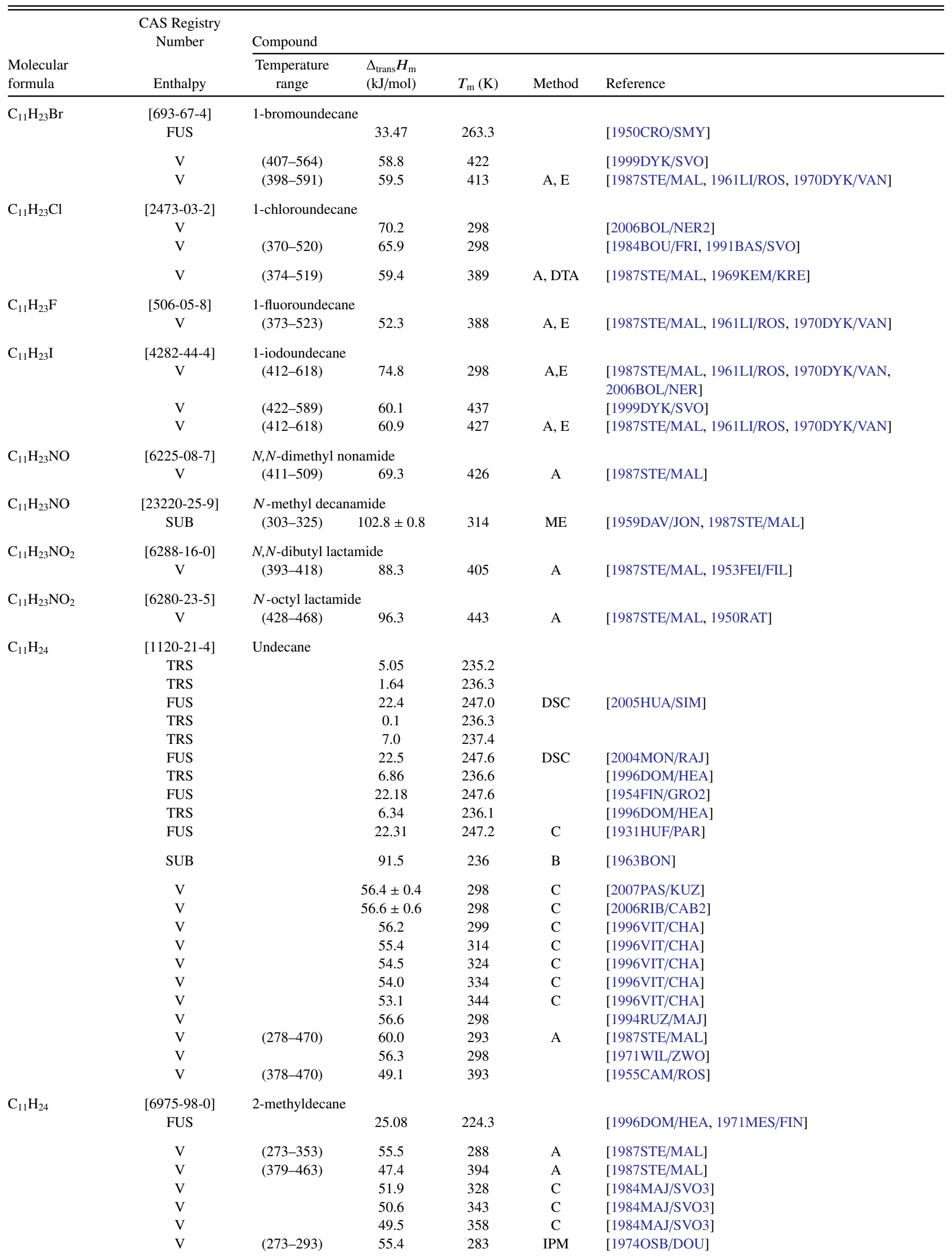


TABLE 10. Phase change enthalpies of $\mathrm{C}_{11}$ organic compounds-Continued

\begin{tabular}{|c|c|c|c|c|c|c|}
\hline \multirow[b]{2}{*}{$\begin{array}{l}\text { Molecular } \\
\text { formula }\end{array}$} & \multirow{2}{*}{$\begin{array}{l}\text { CAS Registry } \\
\text { Number } \\
\text { Enthalpy }\end{array}$} & \multicolumn{5}{|l|}{ Compound } \\
\hline & & $\begin{array}{l}\text { Temperature } \\
\text { range }\end{array}$ & $\begin{array}{l}\Delta_{\text {trans }} H_{\mathrm{m}} \\
(\mathrm{kJ} / \mathrm{mol})\end{array}$ & $T_{\mathrm{m}}(\mathrm{K})$ & Method & Reference \\
\hline $\mathrm{C}_{11} \mathrm{H}_{24}$ & $\begin{array}{c}\text { [13151-34-3] } \\
\mathrm{V}\end{array}$ & $\begin{array}{l}\text { 3-methyldecane } \\
(340-464)\end{array}$ & 46.5 & 355 & A & [1987STE/MAL] \\
\hline $\mathrm{C}_{11} \mathrm{H}_{24}$ & $\begin{array}{c}{[2847-72-5]} \\
\mathrm{V} \\
\mathrm{V} \\
\mathrm{V} \\
\mathrm{V}\end{array}$ & $\begin{array}{l}\text { 4-methyldecane } \\
\text { (339-460) }\end{array}$ & $\begin{array}{l}46.6 \\
50.4 \\
49.2 \\
48.5\end{array}$ & $\begin{array}{l}354 \\
343 \\
358 \\
368\end{array}$ & $\begin{array}{l}\mathrm{A} \\
\mathrm{C} \\
\mathrm{C} \\
\mathrm{C}\end{array}$ & $\begin{array}{l}\text { [1987STE/MAL] } \\
\text { [1984MAJ/SVO3] } \\
\text { [1984MAJ/SVO3] } \\
\text { [1984MAJ/SVO3] }\end{array}$ \\
\hline $\mathrm{C}_{11} \mathrm{H}_{24}$ & $\begin{array}{c}{[13151-35-4]} \\
\mathrm{V}\end{array}$ & $\begin{array}{l}\text { 5-methyldecane } \\
(334-452)\end{array}$ & 46.0 & 349 & A & [1987STE/MAL] \\
\hline $\mathrm{C}_{11} \mathrm{H}_{24}$ & $\begin{array}{c}\text { [2884-06-2] } \\
\mathrm{V}\end{array}$ & $\begin{array}{l}\text { 2,3-dimethylnona } \\
\quad(336-460)\end{array}$ & 45.1 & 351 & A & [1987STE/MAL] \\
\hline $\mathrm{C}_{11} \mathrm{H}_{24}$ & $\begin{array}{c}{[17302-24-8]} \\
\mathrm{V}\end{array}$ & $\begin{array}{l}\text { 2,4-dimethylnona } \\
\quad(334-452)\end{array}$ & 46.8 & 349 & A & [1987STE/MAL] \\
\hline $\mathrm{C}_{11} \mathrm{H}_{24}$ & $\begin{array}{c}{[62016-37-9]} \\
V\end{array}$ & $\begin{array}{l}\text { 2,4,6-trimethyloc } \\
(325-442)\end{array}$ & $\begin{array}{l}\text { tane } \\
44.9\end{array}$ & 340 & A & [1987STE/MAL] \\
\hline $\mathrm{C}_{11} \mathrm{H}_{24}$ & $\begin{array}{c}{[62016-38-0]} \\
\text { V } \\
\text { V } \\
\text { V }\end{array}$ & 2,4,7-trimethyloc & $\begin{array}{l}\text { tane } \\
\qquad 7.6 \\
46.4 \\
45.3\end{array}$ & $\begin{array}{l}328 \\
343 \\
358\end{array}$ & $\begin{array}{l}\mathrm{C} \\
\mathrm{C} \\
\mathrm{C}\end{array}$ & $\begin{array}{l}\text { [1984MAJ/SVO3] } \\
{[1984 \mathrm{MAJ} / \mathrm{SVO} 3]} \\
{[1984 \mathrm{MAJ} / \mathrm{SVO} 3]}\end{array}$ \\
\hline $\mathrm{C}_{11} \mathrm{H}_{24} \mathrm{~N}_{2} \mathrm{O}$ & $\begin{array}{c}\text { [17450-44-1] } \\
\text { TRS } \\
\text { FUS }\end{array}$ & 1-decyl urea & $\begin{array}{c}1.3 \\
38.3\end{array}$ & $\begin{array}{l}294.4 \\
385.3\end{array}$ & $\mathrm{DSC}$ & [2005HAS/TAJ] \\
\hline $\mathrm{C}_{11} \mathrm{H}_{24} \mathrm{O}$ & $\begin{array}{c}{[7289-52-3]} \\
\text { FUS } \\
\text { V } \\
\text { V } \\
\text { V } \\
\text { V } \\
\text { V }\end{array}$ & $\begin{array}{l}\text { Decyl methyl eth } \\
(12-349) \\
(341-429) \\
(341-471) \\
(341-471) \\
(341-471)\end{array}$ & $\begin{array}{c}31.71 \\
56.9 \\
57.0 \\
62.6 \\
45.5 \\
62.3 \pm 0.3\end{array}$ & $\begin{array}{l}243.5 \\
356 \\
356 \\
298 \\
489 \\
298\end{array}$ & $\begin{array}{l}\mathrm{AC} \\
\mathrm{A} \\
\mathrm{A}\end{array}$ & $\begin{array}{l}\text { [1996DOM/HEA, 1975AND/MAR] } \\
\text { [1987STE/MAL] } \\
\text { [1987STE/MAL, 1976AMB/ELL] } \\
\text { [1976AMB/ELL] } \\
\text { [1976AMB/ELL] } \\
\text { [1975FEN/HAR] }\end{array}$ \\
\hline $\mathrm{C}_{11} \mathrm{H}_{24} \mathrm{O}$ & $\begin{array}{c}{[16979-32-1]} \\
\mathrm{V}\end{array}$ & Ethyl nonyl ether & $60.3 \pm 0.1$ & 298 & $\mathrm{C}$ & [1985KUS] \\
\hline $\mathrm{C}_{11} \mathrm{H}_{24} \mathrm{O}$ & $\begin{array}{c}{[29379-41-7]} \\
\mathrm{V}\end{array}$ & Propyl octyl ethe & $58.8 \pm 0.1$ & 298 & $\mathrm{C}$ & [1985KUS] \\
\hline $\mathrm{C}_{11} \mathrm{H}_{24} \mathrm{O}$ & $\begin{array}{c}\text { [71112-90-8] } \\
\mathrm{V}\end{array}$ & Butyl heptyl ethe & $58.2 \pm 0.1$ & 298 & $\mathrm{C}$ & [1985KUS] \\
\hline $\mathrm{C}_{11} \mathrm{H}_{24} \mathrm{O}$ & $\begin{array}{c}{[78972-97-1]} \\
\mathrm{V}\end{array}$ & Heptyl tert-butyl & $\begin{array}{l}\text { ether } \\
56.6\end{array}$ & 298 & CGC & [UR/VER, 2002VER, 2003VER/KRA] \\
\hline $\mathrm{C}_{11} \mathrm{H}_{24} \mathrm{O}$ & V & Hexyl tert-amyl & ther & 298 & CGC & [UR/VER, 2002VER, 2003VER/KRA] \\
\hline $\mathrm{C}_{11} \mathrm{H}_{24} \mathrm{O}$ & $\begin{array}{c}\text { [405506-46-9] } \\
\mathrm{V}\end{array}$ & Propyl tert-octyl & $\begin{array}{l}\text { ether } \\
50.1 \pm 0.3\end{array}$ & 298 & CGC & [UR/VER, 2002VER, 2003VER/KRA] \\
\hline $\mathrm{C}_{11} \mathrm{H}_{24} \mathrm{O}$ & $\begin{array}{c}{[112-42-5]} \\
\text { FUS } \\
\text { FUS }\end{array}$ & 1-undecanol & $\begin{array}{l}37.52 \\
30.59\end{array}$ & $\begin{array}{c}306.2 \\
289.63\end{array}$ & $\begin{array}{l}\text { DSC } \\
\text { DSC }\end{array}$ & $\begin{array}{l}\text { [2008EGO/MAR] } \\
\text { [2005DOM/MAR] }\end{array}$ \\
\hline & $\begin{array}{l}\text { V } \\
\text { V } \\
\text { V } \\
\text { V } \\
\text { V } \\
\text { V } \\
\text { V } \\
\text { V }\end{array}$ & $\begin{array}{l}(313-354) \\
(313-354) \\
(373-423) \\
(353-393) \\
(293-342) \\
(283-393) \\
(393-523)\end{array}$ & $\begin{array}{c}85.8 \pm 2.1 \\
79.5 \\
84.7 \\
86.8 \\
85.6 \\
83.6 \\
83.5 \\
68.7\end{array}$ & $\begin{array}{l}298 \\
336 \\
298 \\
298 \\
298 \\
318 \\
298 \\
408\end{array}$ & $\begin{array}{c}\text { CGC } \\
\text { GS } \\
\text { GS } \\
\text { CGC } \\
\text { CGC }\end{array}$ & $\begin{array}{l}\text { [2006NIC/KWE] } \\
\text { [2001KUL/VER2] } \\
{[2001 \mathrm{KUL} / \mathrm{VER} 2]} \\
{[1995 \mathrm{CHI} / \mathrm{HOS}]} \\
{[1994 \mathrm{KOU} / \mathrm{HOS}, 2000 \mathrm{OVA} / \mathrm{KOU}]} \\
{[1992 \mathrm{NGU} / \mathrm{KAS}]} \\
{[1999 \mathrm{NGU} / \mathrm{BER}]} \\
{[1987 \mathrm{STE} / \mathrm{MAL}]}\end{array}$ \\
\hline
\end{tabular}


TABLE 10. Phase change enthalpies of $\mathrm{C}_{11}$ organic compounds-Continued

\begin{tabular}{|c|c|c|c|c|c|c|}
\hline \multirow[b]{2}{*}{$\begin{array}{l}\text { Molecular } \\
\text { formula }\end{array}$} & \multirow{2}{*}{$\begin{array}{l}\text { CAS Registry } \\
\text { Number } \\
\text { Enthalpy }\end{array}$} & \multicolumn{5}{|l|}{ Compound } \\
\hline & & $\begin{array}{l}\text { Temperature } \\
\text { range }\end{array}$ & $\begin{array}{l}\Delta_{\text {trans }} H_{\mathrm{m}} \\
(\mathrm{kJ} / \mathrm{mol})\end{array}$ & $T_{\mathrm{m}}(\mathrm{K})$ & Method & Reference \\
\hline & $\mathrm{V}$ & $(393-534)$ & 68.5 & 408 & A & [1987STE/MAL] \\
\hline & $\mathrm{V}$ & $(393-516)$ & 72.3 & 408 & & [1973WIL/ZWO] \\
\hline $\mathrm{C}_{11} \mathrm{H}_{24} \mathrm{O}$ & $\begin{array}{c}{[1653-30-1]} \\
\mathrm{V}\end{array}$ & $\begin{array}{c}\text { 2-undecanol } \\
(344-505)\end{array}$ & 61.4 & 359 & & [1947STU] \\
\hline $\mathrm{C}_{11} \mathrm{H}_{24} \mathrm{O}$ & $\begin{array}{c}{[57233-26-8]} \\
\mathrm{V}\end{array}$ & $\begin{array}{l}\text { 2,5-dimethyl- } \\
\text { (321-458) }\end{array}$ & $\begin{array}{l}\text { opropyl-3-he } \\
57.2\end{array}$ & ol 336 & & [1973WIL/ZWO] \\
\hline $\mathrm{C}_{11} \mathrm{H}_{24} \mathrm{O}$ & $\begin{array}{c}{[5457-41-0]} \\
\mathrm{V}\end{array}$ & $\begin{array}{l}\text { 2,2,4-trimethy } \\
(321-458)\end{array}$ & $\begin{array}{c}\text {-isopropyl-3- } \\
67.1\end{array}$ & $\begin{array}{c}\operatorname{tanol} \\
336\end{array}$ & & [1973WIL/ZWO] \\
\hline $\mathrm{C}_{11} \mathrm{H}_{24} \mathrm{OS}$ & $\begin{array}{c}\text { [3079-28-5] } \\
\text { FUS }\end{array}$ & Methyl decyl & $\begin{array}{l}\text { foxide } \\
\qquad 41.4\end{array}$ & NA & $\mathrm{C}$ & [1969COR/GOO] \\
\hline $\mathrm{C}_{11} \mathrm{H}_{24} \mathrm{O}_{2}$ & $\begin{array}{c}\text { [765-04-8] } \\
\text { FUS }\end{array}$ & 1,11-undecane & 45.9 & 334.1 & DSC & [2014BAD/NOW] \\
\hline & $\begin{array}{l}\text { V } \\
\text { V }\end{array}$ & & $\begin{array}{l}123.0 \pm 1.9 \\
131.0 \pm 3.0\end{array}$ & $\begin{array}{l}365 \\
298\end{array}$ & $\begin{array}{l}\mathrm{TE} \\
\mathrm{TE}\end{array}$ & $\begin{array}{l}\text { [1994PIA/FER] } \\
\text { [1994PIA/FER] }\end{array}$ \\
\hline $\mathrm{C}_{11} \mathrm{H}_{24} \mathrm{O}_{2} \mathrm{~S}$ & $\begin{array}{c}{[54581-75-8]} \\
\text { FUS }\end{array}$ & 3-(octylthio)-1 & $\begin{array}{c}\text { propanediol } \\
39.8\end{array}$ & 306.5 & DSC & [1993ACR, 1990VAN/VAN] \\
\hline $\mathrm{C}_{11} \mathrm{H}_{24} \mathrm{O}_{3}$ & $\begin{array}{c}\text { [10438-94-5] } \\
\text { FUS }\end{array}$ & 3-(octyloxy)-1 & $\begin{array}{c}\text { propanediol } \\
33.4\end{array}$ & 296.1 & DSC & [1993ACR, 1990VAN/VAN] \\
\hline $\mathrm{C}_{11} \mathrm{H}_{24} \mathrm{O}_{4}$ & $\begin{array}{c}{[75899-69-3]} \\
\mathrm{V}\end{array}$ & $\begin{array}{c}\text { Tripropylene } \\
(317-521)\end{array}$ & $\begin{array}{c}\text { col, monoeth } \\
60.0\end{array}$ & $\begin{array}{l}\text { her } \\
.332\end{array}$ & A & [1987STE/MAL] \\
\hline $\mathrm{C}_{11} \mathrm{H}_{24} \mathrm{~S}$ & $\begin{array}{c}{[5332-52-5]} \\
\mathrm{V}\end{array}$ & $\begin{array}{l}\text { 1-undecanethi } \\
(405-563)\end{array}$ & 59.3 & 420 & & [1999DYK/SVO] \\
\hline $\mathrm{C}_{11} \mathrm{H}_{24} \mathrm{~S}_{2}$ & $\begin{array}{c}{[63476-06-2]} \\
\mathrm{V}\end{array}$ & $\begin{array}{l}\text { 1,11-undecane } \\
(444-582)\end{array}$ & hiol & 459 & A & [1987STE/MAL, 1943HAL/REI, 1999DYK/SVO] \\
\hline $\mathrm{C}_{11} \mathrm{H}_{25} \mathrm{~N}$ & $\begin{array}{c}{[7307-55-3]} \\
\mathrm{V}\end{array}$ & $\begin{array}{l}\text { Undecylamine } \\
(428-527)\end{array}$ & 55.1 & 443 & $\mathrm{~A}, \mathrm{E}$ & [1987STE/MAL, 1956MAN2] \\
\hline $\mathrm{C}_{11} \mathrm{H}_{25} \mathrm{NO}_{2}$ & $\begin{array}{c}\text { [929-31-7] } \\
\text { FUS }\end{array}$ & 3-(octylamino & $\begin{array}{c}\text { 2-propanedi } \\
45.1\end{array}$ & 335.9 & DSC & [1993ACR, 1990VAN/VAN] \\
\hline $\mathrm{C}_{11} \mathrm{H}_{26} \mathrm{NO}_{2} \mathrm{PS}$ & $\begin{array}{c}{[50782-69-9]} \\
\text { V } \\
\text { V } \\
\text { V } \\
\text { V } \\
\text { V } \\
\text { V } \\
\text { V } \\
\text { V } \\
\text { V }\end{array}$ & Methylthioph & $\begin{array}{l}\text { honic acid, } C \\
86.0 \\
81.0 \\
77.3 \\
74.4 \\
71.3 \\
69.0 \\
67.2 \\
77.9 \\
101\end{array}$ & $\begin{array}{l}\text { yl- } S-[2- \\
263 \\
293 \\
323 \\
353 \\
393 \\
433 \\
473 \\
323 \\
295\end{array}$ & $\begin{array}{l}\text { V-diisopro } \\
\text { GC } \\
\text { A }\end{array}$ & $\begin{array}{l}\text { lamino)ethyl] ester } \\
\text { [2012TEV/BRO] } \\
\text { [2012TEV/BRO] } \\
\text { [2012TEV/BRO] } \\
\text { [2012TEV/BRO] } \\
\text { [2012TEV/BRO] } \\
\text { [2012TEV/BRO] } \\
\text { [2012TEV/BRO] } \\
\text { [2001RIT] } \\
\text { [1987STE/MAL, 1999DYK/SVO, 1974FRO] }\end{array}$ \\
\hline $\mathrm{C}_{11} \mathrm{H}_{26} \mathrm{NO}_{2} \mathrm{PS}$ & $\begin{array}{c}{[159939-87-4]} \\
\text { V } \\
\text { V } \\
\text { V } \\
\text { V } \\
\text { V } \\
\text { V } \\
\text { V } \\
\text { V }\end{array}$ & $\begin{array}{c}P \text {-methylphos } \\
(263-385)\end{array}$ & $\begin{array}{c}\text { onothioic aci } \\
86.1 \\
81.6 \\
78.2 \\
75.5 \\
72.8 \\
70.6 \\
68.9 \\
76.6\end{array}$ & $\begin{array}{c}-[2 \text { (dieth } \\
263 \\
293 \\
323 \\
353 \\
393 \\
433 \\
473 \\
324\end{array}$ & $\begin{array}{l}\text { nino)ethyl } \\
\text { GC }\end{array}$ & $\begin{array}{l}\text { D-(2-methylpropyl) ester } \\
\text { [2012TEV/BRO] } \\
\text { [2012TEV/BRO] } \\
{[2012 \mathrm{TEV} / \mathrm{BRO}]} \\
{[2012 \mathrm{TEV} / \mathrm{BRO}]} \\
{[2012 \mathrm{TEV} / \mathrm{BRO}]} \\
{[2012 \mathrm{TEV} / \mathrm{BRO}]} \\
{[2012 \mathrm{TEV} / \mathrm{BRO}]} \\
{[2001 \mathrm{RIT}]}\end{array}$ \\
\hline $\mathrm{C}_{11} \mathrm{H}_{26} \mathrm{~N}_{2}$ & $\begin{array}{c}\text { [822-08-2] } \\
\text { FUS }\end{array}$ & $\begin{array}{c}\text { Undecane- } 1,1 \\
48.08\end{array}$ & liamine & 313.6 & DSC & [2002DAL/DEL] \\
\hline & $\begin{array}{l}\mathrm{V} \\
\mathrm{V}\end{array}$ & $\begin{array}{l}(326-353) \\
(326-353)\end{array}$ & $\begin{array}{c}79.4 \\
84.7 \pm 0.4\end{array}$ & $\begin{array}{l}339 \\
298\end{array}$ & $\begin{array}{l}\text { GS } \\
\text { GS }\end{array}$ & $\begin{array}{l}\text { [2011POZ/VER] } \\
\text { [2011POZ/VER] }\end{array}$ \\
\hline
\end{tabular}


TABLE 11. Phase change enthalpies of $\mathrm{C}_{12}$ to $\mathrm{C}_{13}$ organic compounds

\begin{tabular}{|c|c|c|c|c|c|c|}
\hline \multirow[t]{2}{*}{$\begin{array}{l}\text { Molecular } \\
\text { formula }\end{array}$} & \multirow{2}{*}{$\begin{array}{c}\text { CAS Registry } \\
\text { Number } \\
\text { Enthalpy }\end{array}$} & \multicolumn{5}{|l|}{ Compound } \\
\hline & & $\begin{array}{c}\text { Temperature } \\
\text { range }\end{array}$ & $\begin{array}{c}\Delta_{\text {trans }} H_{\mathrm{m}} \\
(\mathrm{kJ} / \mathrm{mol})\end{array}$ & $\begin{array}{l}T_{\mathrm{m}} \\
(\mathrm{K})\end{array}$ & Method & References \\
\hline \multirow[t]{3}{*}{$\mathrm{C}_{12} \mathrm{Br}_{10} \mathrm{O}$} & [1163-19-5] & \multicolumn{5}{|c|}{ Decabromodiphenyl ether } \\
\hline & FUS & & 38.7 & 580.4 & DSC & [2011FU/SUU2] \\
\hline & SUB & $(444-505)$ & $157.1 \pm 3.5$ & & $\mathrm{ME}$ & [2011FU/SUU2] \\
\hline \multirow[t]{5}{*}{$\mathrm{C}_{12} \mathrm{Cl}_{8} \mathrm{O}$} & {$[39001-02-0]$} & \multicolumn{5}{|c|}{ Octachlorodibenzofuran } \\
\hline & FUS & & 28.0 & 526.3 & DSC & [2001SHI/YAM] \\
\hline & SUB & $(298-523)$ & 145.9 & 411 & ME & [2005RYA/GUL] \\
\hline & SUB & $(438-473)$ & $141.7 \pm 1.8$ & 455 & ME & [2004LI/SHI, 2002LI/SHI] \\
\hline & SUB & $(373-474)$ & 149.4 & 423 & $\mathrm{~T}$ & [1989ROR, 1986ROR] \\
\hline \multirow[t]{4}{*}{$\mathrm{C}_{12} \mathrm{Cl}_{8} \mathrm{O}_{2}$} & {$[3268-87-9]$} & \multicolumn{5}{|c|}{ Octachlorodibenzo[b,e][1,4] dioxin } \\
\hline & FUS & & 40.3 & 596.7 & DSC & [2001SHI/YAM] \\
\hline & SUB & $(298-523)$ & 147.4 & 411 & ME & [2005RYA/GUL] \\
\hline & SUB & $(463-493)$ & $145.7 \pm 4.0$ & 478 & $\mathrm{ME}$ & [2004LI/SHI] \\
\hline
\end{tabular}

[Note: The authors of [2004LI/SHI] attribute the difference in the enthalpy of sublimation from their earlier value of $131.1 \mathrm{~kJ} / \mathrm{mol}[2002 \mathrm{LI} / \mathrm{SHI}]$ to a temperature calibration.]

$\begin{array}{llll}\text { SUB } & (448-493) & 131.1 \pm 0.6 & 460 \quad \mathrm{ME} \quad[2002 \mathrm{LI} / \mathrm{SHI}]\end{array}$

$\begin{array}{llllll}\text { SUB } & (393-573) & 149.8 & 483 & \mathrm{~T} & \text { [1989ROR, 1986ROR] }\end{array}$

$\mathrm{C}_{12} \mathrm{Cl}_{10} \quad$ [2051-24-3] Decachlorobiphenyl

FUS $41.2 \quad 580.3 \quad$ DSC [1990DON/DRE]

FUS $28.7 \quad 578.9 \quad$ DSC $\quad$ [1984MIL/GHO]

FUS $39.34 \quad 577.7 \quad$ DSC [1991ACR, 1974RYB/MAR]

$\begin{array}{lllll}\text { SUB } & (338-358) & \text { U93.6 } & 348 & \text { ME }\end{array}$

$\begin{array}{lllll}\text { SUB } & (324-363) & 121.8 & 343 & \text { GS }\end{array}$

$\begin{array}{lllll}\mathrm{V} & (343-393) & 103.4 & 368 & \mathrm{GC}\end{array}$

$\begin{array}{lllll}\mathrm{V} & (343-453) & 103.4 & 398 & \text { GC }\end{array}$

$\mathrm{C}_{12} \mathrm{D}_{10}$

[1486-01-7] Biphenyl-d $d_{10}$

$\begin{array}{llll}\text { V } & 64.9 & 298 & \text { CGC }\end{array}$

$\mathrm{C}_{12} \mathrm{D}_{10} \quad[15067-26-2] \quad$ Acenaphthene- $\mathrm{d}_{10}$

$\begin{array}{llll}\mathrm{V} & 67.2 & 298 & \mathrm{CGC}\end{array}$

$\mathrm{C}_{12} \mathrm{D}_{18}$

[4342-40-9] Hexamethylbenzene-d ${ }_{18}$

$\mathrm{V}$

68.2

$298 \quad$ CGC $\quad$ [2008ZHA/UNH]

$\mathrm{C}_{12} \mathrm{D}_{10}$

[434-90-2] Decafluorobiphenyl

FUS $20.5 \quad 339.6 \quad$ DSC [2012HAS/DRA]

$\begin{array}{lcccc}\text { SUB } & (297-323) & 87.8 & 310 & \text { A }\end{array}$

$\begin{array}{lll}\text { SUB } & 85.3 \pm 2.3 & \text { [1974RAD/KAT] }\end{array}$

V $\quad 49.9 \quad 468 \quad 608) \quad$ DSC $\quad$ [1996BAC/GRZ]

$\mathrm{C}_{12} \mathrm{~F}_{18} \quad$ [23174-55-2] Hexakis(trifluoromethyl)bicyclo[2.2.0]hexa-2,5-diene

$\begin{array}{llll}\mathrm{V} & (293-343) & 41.4 & 308\end{array}$

$\mathrm{C}_{12} \mathrm{~F}_{18} \quad$ [22736-20-5] Hexakis(trifluoromethyl)tetracyclo[2.2.0.0 $\left.2,60^{3,5}\right]$ hexane

$\begin{array}{lllll}\text { SUB } & (293-306) & 49.2 & 299.5 & \text { A }\end{array}$

$\begin{array}{lllll}\text { V } & 313-353) & 33.1 & 328 & \text { A }\end{array}$

$\mathrm{C}_{12} \mathrm{~F}_{18} \quad$ [22186-64-7] Hexakis(trifluoromethyl)tricyclo[3.1.0.0 $\left.{ }^{2,6}\right]$ hex-3-ene

$\begin{array}{lllll}\mathrm{V} & (293-353) & 38.6 & 308 & \text { A }\end{array}$

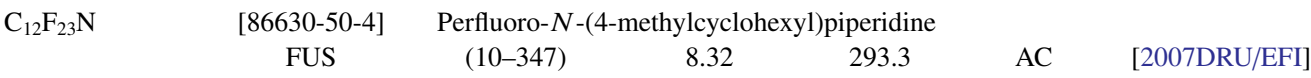

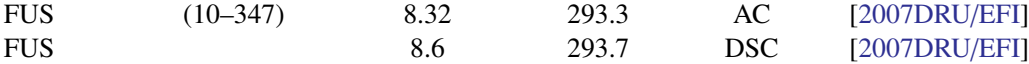

$\begin{array}{lllll}\text { V } & 56.6 & 298 & \mathrm{C} & \text { [2007DRU/EFI] }\end{array}$

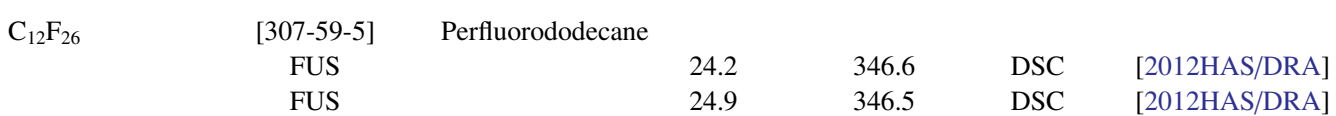


TABLE 11. Phase change enthalpies of $\mathrm{C}_{12}$ to $\mathrm{C}_{13}$ organic compounds-Continued

\begin{tabular}{|c|c|c|c|c|c|c|}
\hline \multirow[b]{2}{*}{ Molecular formula } & \multirow{2}{*}{$\begin{array}{l}\text { CAS Registry } \\
\text { Number } \\
\text { Enthalpy }\end{array}$} & \multicolumn{5}{|l|}{ Compound } \\
\hline & & $\begin{array}{l}\text { Temperature } \\
\text { range }\end{array}$ & $\begin{array}{l}\Delta_{\text {trans }} H_{\mathrm{m}} \\
(\mathrm{kJ} / \mathrm{mol})\end{array}$ & $\begin{array}{l}T_{\mathrm{m}} \\
(\mathrm{K})\end{array}$ & Method & References \\
\hline & FUS & & 21.6 & 349.3 & DSC & [1999VIS/TER] \\
\hline & TRS & $(5-317)$ & 5.07 & 177.5 & $\mathrm{AC}$ & [1994LEB/BYK] \\
\hline & TRS & & 7.2 & 177.7 & & \\
\hline & FUS & & 23.8 & 347.3 & DSC & [1994JIN/BOL] \\
\hline & TRS & & 6.9 & 170.2 & & \\
\hline & FUS & & U 38.16 & 348.5 & $\mathrm{DSC}$ & {$[1986 \mathrm{STA}]$} \\
\hline & SUB & $(313-348)$ & $85.8 \pm 0.3$ & 298 & GS & [2012HAS/DRA] \\
\hline & $\mathrm{V}$ & & $64.2 \pm 2.2$ & 298 & CGC & [2012HAS/DRA] \\
\hline \multirow[t]{2}{*}{$\mathrm{C}_{12} \mathrm{~F}_{26} \mathrm{O}_{10}$} & {$[927699-30-7]$} & \multicolumn{5}{|c|}{ Perfluoro-2,4,6,8,10,13,15.17,19,21-decaoxy-n-docosane } \\
\hline & $\mathrm{V}$ & $(397-468)$ & $74.6 \pm 2.9$ & 298 & $\mathrm{~EB}$ & [2006DRU/KRO] \\
\hline \multirow[t]{5}{*}{$\mathrm{C}_{12} \mathrm{~F}_{27} \mathrm{~N}$} & [311-89-7] & \multicolumn{5}{|c|}{ Perfluorotributylamine } \\
\hline & $\mathrm{V}$ & & $60.3 \pm 0.1$ & 298 & $\mathrm{C}$ & [1995VAR/DRO] \\
\hline & $\mathrm{V}$ & $(298-450)$ & 57.4 & 313 & A & [1987STE/MAL] \\
\hline & $\mathrm{V}$ & $(371-544)$ & 51.1 & 386 & A & [1987STE/MAL] \\
\hline & $\mathrm{V}$ & & $60.4 \pm 1.2$ & 298 & & [1977VAR/AMM2, 1977VAR/AMM] \\
\hline \multirow[t]{2}{*}{$\mathrm{C}_{12} \mathrm{HCl}_{7} \mathrm{O}_{2}$} & {$[58200-70-7]$} & \multicolumn{5}{|c|}{ 1,2,3,4,6,7,9-heptachlorodibenzo[b,e][1,4]dioxin } \\
\hline & SUB & $(418-453)$ & $144.2 \pm 0.3$ & 435 & $\mathrm{ME}$ & {$[2004 \mathrm{LI} / \mathrm{SHI}]$} \\
\hline \multirow[t]{2}{*}{$\mathrm{C}_{12} \mathrm{HCl}_{9}$} & {$[52663-77-1]$} & \multicolumn{5}{|c|}{$2,2^{\prime}, 3,3^{\prime}, 4,5,5^{\prime}, 6,6^{\prime}$-nonachlorobiphenyl } \\
\hline & FUS & & 22.6 & 455.8 & $\mathrm{DSC}$ & [1991ACR, 1984MIL/GHO] \\
\hline \multirow[t]{3}{*}{$\mathrm{C}_{12} \mathrm{HF}_{25}$} & {$[66563-68-6]$} & \multicolumn{5}{|c|}{$1,1,1,2,2,3,3,4,4,5,5,6,6,7,7,8,8,9,9,10,10,11,11,12,12$-pentacosofluorododecane } \\
\hline & FUS & & 21.0 & 345 & DSC & [1988HOP/PUG] \\
\hline & FUS & & 23.0 & 344.5 & $\mathrm{DSC}$ & [1986RUS/RAB] \\
\hline \multirow[t]{2}{*}{$\mathrm{C}_{12} \mathrm{H}_{2} \mathrm{Cl}_{6} \mathrm{O}_{2}$} & {$[58200-68-3]$} & \multicolumn{5}{|c|}{ 1,2,3,4,6,9-hexachlorodibenzo[b,e][1,4]dioxin } \\
\hline & SUB & $(418-438)$ & $128.5 \pm 1.5$ & 428 & $\mathrm{ME}$ & [2004LI/SHI] \\
\hline \multirow[t]{5}{*}{$\mathrm{C}_{12} \mathrm{H}_{2} \mathrm{C}_{18}$} & {$[2136-99-4]$} & \multicolumn{5}{|c|}{$2,2^{\prime}, 3,3^{\prime}, 5,5^{\prime}, 6,6^{\prime}$-octachlorobiphenyl } \\
\hline & FUS & & 22.8 & 433.8 & $\mathrm{DSC}$ & [1991ACR, 1984MIL/GHO] \\
\hline & SUB & $(302-334)$ & 101.7 & 318 & GS & [1984BUR/ARM] \\
\hline & $\mathrm{V}$ & $(343-393)$ & 92.9 & 368 & GC & [1994FAL/BID] \\
\hline & V & $(343-453)$ & 92.9 & 398 & GC & [1990HIN/BID2] \\
\hline \multirow[t]{3}{*}{$\mathrm{C}_{12} \mathrm{H}_{3} \mathrm{Br}_{7} \mathrm{O}$} & {$[327185-13-7]$} & \multicolumn{5}{|c|}{$2^{\prime}, 3,3^{\prime}, 4,4^{\prime}, 5,6$-heptabromodiphenyl ether } \\
\hline & $\mathrm{V}$ & $(363-473)$ & 115.8 & 418 & GC & [2001WON/LEI] \\
\hline & $\mathrm{V}$ & $(403-475)$ & 121.2 & & CGC & [2001TIT/TOM] \\
\hline \multirow[t]{2}{*}{$\mathrm{C}_{12} \mathrm{H}_{3} \mathrm{Cl}_{5} \mathrm{O}_{2}$} & {$[58802-08-7]$} & $1,2,4,7,8$-penta & lorodibenzo[b & {$[1,4]$ dio } & & \\
\hline & SUB & $(403-428)$ & $125.3 \pm 2.3$ & 415 & $\mathrm{ME}$ & [2004LI/SHI] \\
\hline $\mathrm{C}_{12} \mathrm{H}_{3} \mathrm{Cl}_{7}$ & {$[52663-68-0]$} & $2,2^{\prime}, 3,4^{\prime}, 5,5^{\prime}, 6-1$ & ptachlorobiph & & & \\
\hline & $\mathrm{V}$ & (343-393) & 94.0 & 368 & GC & [1994FAL/BID] \\
\hline $\mathrm{C}_{12} \mathrm{H}_{3} \mathrm{Cl}_{7}$ & {$[35065-29-3]$} & $2,2^{\prime}, 3,4,4^{\prime}, 5,5^{\prime}-1$ & ptachlorobiph & & & \\
\hline & FUS & & 24.8 & 383.4 & $\mathrm{DSC}$ & [2006NAK/SHI] \\
\hline & $\mathrm{V}$ & $(343-393)$ & 96.5 & 268 & GC & [1994FAL/BID] \\
\hline $\mathrm{C}_{12} \mathrm{H}_{3} \mathrm{Cl}_{7}$ & {$[52663-71-5]$} & $2,2^{\prime}, 3,3^{\prime}, 4,4^{\prime}, 6-1$ & ptachlorobiph & & & \\
\hline & FUS & & 20.3 & 395.4 & $\mathrm{DSC}$ & [1991ACR, 1984MIL/GHO] \\
\hline & $\mathrm{V}$ & & 109.1 & 298 & CGC & [2001PUR/CHI] \\
\hline & $\mathrm{V}$ & $(343-393)$ & 95.9 & 368 & $\mathrm{GC}$ & [1994FAL/BID] \\
\hline $\mathrm{C}_{12} \mathrm{H}_{3} \mathrm{Cl}_{7}$ & {$[35065-30-6]$} & $2,2^{\prime}, 3,3^{\prime}, 4,4^{\prime}, 5-1$ & ptachlorobiph & & & \\
\hline & FUS & & 26.9 & 412.2 & DSC & [2006NAK/SHI] \\
\hline & $\mathrm{V}$ & $(343-393)$ & 98.4 & 368 & GC & [1994FAL/BID] \\
\hline $\mathrm{C}_{12} \mathrm{H}_{3} \mathrm{Cl}_{7}$ & [38411-25-5] & $2,2^{\prime}, 3,3^{\prime}, 4,5,6^{\prime}-1$ & ptachlorobiph & & & \\
\hline & FUS & & 24.4 & 400.5 & DSC & [2006NAK/SHI] \\
\hline $\mathrm{C}_{12} \mathrm{H}_{3} \mathrm{Cl}_{7} \mathrm{O}$ & [67517-48-0] & $1,2,3,4,8$-penta & lorodibenzofu & & & \\
\hline & SUB & $(388-413)$ & $125.2 \pm 2.0$ & 400 & $\mathrm{ME}$ & [2004LI/SHI] \\
\hline
\end{tabular}


TABLE 11. Phase change enthalpies of $\mathrm{C}_{12}$ to $\mathrm{C}_{13}$ organic compounds-Continued

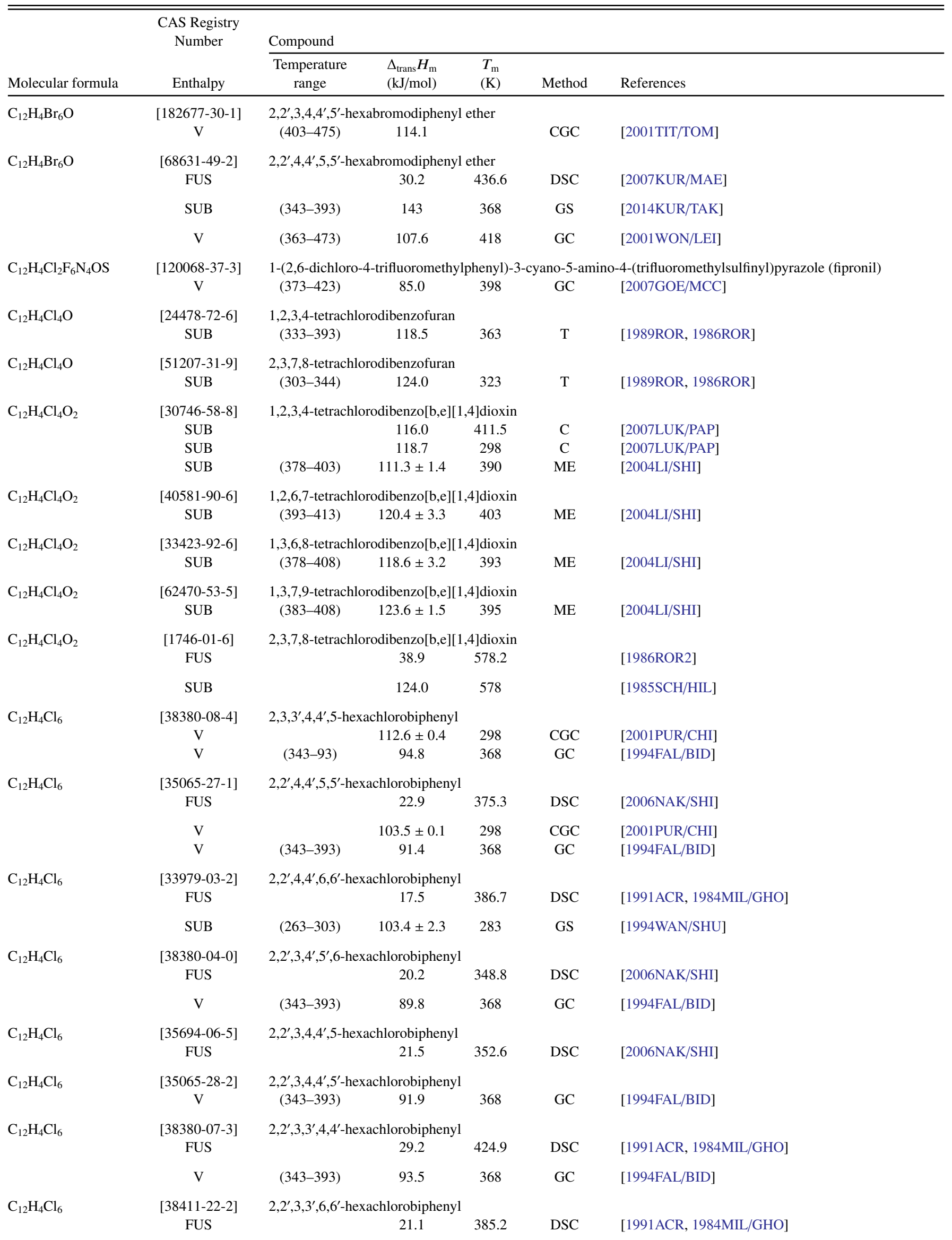


TABLE 11. Phase change enthalpies of $\mathrm{C}_{12}$ to $\mathrm{C}_{13}$ organic compounds-Continued

\begin{tabular}{|c|c|c|c|c|c|c|}
\hline \multirow[b]{2}{*}{ Molecular formula } & \multirow{2}{*}{$\begin{array}{l}\text { CAS Registry } \\
\text { Number } \\
\text { Enthalpy }\end{array}$} & \multicolumn{5}{|l|}{ Compound } \\
\hline & & $\begin{array}{l}\text { Temperature } \\
\text { range }\end{array}$ & $\begin{array}{l}\Delta_{\text {trans }} H_{\mathrm{m}} \\
(\mathrm{kJ} / \mathrm{mol})\end{array}$ & $\begin{array}{l}T_{\mathrm{m}} \\
(\mathrm{K})\end{array}$ & Method & References \\
\hline \multirow[t]{7}{*}{$\mathrm{C}_{12} \mathrm{H}_{4} \mathrm{~N}_{4}$} & [1518-16-7] & \multicolumn{5}{|c|}{ 7,7,8,8-tetracyanoquinodimethane } \\
\hline & SUB & \multicolumn{3}{|c|}{79.0} & TGA & [1995YAS/TAK] \\
\hline & SUB & $(452-553)$ & $108 \pm 2$ & 500 & $\mathrm{~T}$ & [1984KER/OPP] \\
\hline & SUB & $(382-464)$ & $122 \pm 2$ & 423 & ME & [1984KER/OPP] \\
\hline & SUB & & $126.1 \pm 1$ & 413 & ME,TE & [1980DEK/GOV] \\
\hline & SUB & $(433-499)$ & $104.8 \pm 10$ & 448 & A & [1980SWA/KWA, 1987STE/MAL] \\
\hline & SUB & & $105 \pm 9.2$ & 465 & MG & [1963BOY, 1970COX/PIL] \\
\hline \multirow[t]{2}{*}{$\mathrm{C}_{12} \mathrm{H}_{5} \mathrm{Br}_{5} \mathrm{O}$} & {$[182346-21-0]$} & \multicolumn{5}{|c|}{$2,2^{\prime}, 3,4,4^{\prime}$-pentabromodiphenyl ether } \\
\hline & $\mathrm{V}$ & $(403-475)$ & & 111 & $\mathrm{CGC}$ & [2001TIT/TOM] \\
\hline \multirow[t]{2}{*}{$\mathrm{C}_{12} \mathrm{H}_{5} \mathrm{Br}_{5} \mathrm{O}$} & [327185-11-5] & \multicolumn{5}{|c|}{ 2,2',3,3',4-pentabromodiphenyl ether } \\
\hline & $\mathrm{V}$ & $(363-473)$ & 99.1 & 418 & $\mathrm{GC}$ & [2001WON/LEI] \\
\hline \multirow[t]{5}{*}{$\mathrm{C}_{12} \mathrm{H}_{5} \mathrm{Br}_{5} \mathrm{O}$} & {$[60348-60-9]$} & \multicolumn{5}{|c|}{$2,2^{\prime}, 4,4^{\prime}, 5$-pentabromodiphenyl ether } \\
\hline & FUS & & 27.5 & 355.0 & $\mathrm{DSC}$ & [2007KUR/MAE] \\
\hline & SUB & $(323-373)$ & 115 & 348 & GS & [2014KUR/TAK] \\
\hline & $\mathrm{V}$ & $(363-473)$ & 100.3 & 418 & $\mathrm{GC}$ & [2001WON/LEI] \\
\hline & $\mathrm{V}$ & $(405-475)$ & 104.8 & & CGC & [2001TIT/TOM] \\
\hline \multirow[t]{2}{*}{$\mathrm{C}_{12} \mathrm{H}_{5} \mathrm{Br}_{5} \mathrm{O}$} & [189084-66-0] & \multicolumn{5}{|c|}{$2,2^{\prime}, 4,4^{\prime}, 6$-pentabromodiphenyl ether } \\
\hline & $\mathrm{V}$ & $(363-473)$ & 101.8 & 418 & GC & [2001WON/LEI] \\
\hline \multirow[t]{4}{*}{$\mathrm{C}_{12} \mathrm{H}_{5} \mathrm{Cl}_{3} \mathrm{O}$} & {$[58802-14-5]$} & \multicolumn{5}{|c|}{ 2,4,6-dibenzofuran } \\
\hline & SUB & $(298-418)$ & 121.6 & 358 & $\mathrm{ME}$ & [2005RYA/GUL] \\
\hline & SUB & $(338-373)$ & $108.8 \pm 2.2$ & 355 & $\mathrm{ME}$ & [2004LI/SHI] \\
\hline & $\mathrm{V}$ & $(448-548)$ & 84.8 & 498 & $\mathrm{ME}$ & [2005RYA/GUL] \\
\hline \multirow[t]{2}{*}{$\mathrm{C}_{12} \mathrm{H}_{5} \mathrm{Cl}_{3} \mathrm{O}_{2}$} & {$[54536-17-3]$} & \multicolumn{5}{|c|}{ 1,2,3-trichlorodibenzo[b,e][1,4]dioxin } \\
\hline & SUB & $(363-388)$ & $117.1 \pm 3.7$ & 375 & $\mathrm{ME}$ & [2004LI/SHI] \\
\hline \multirow[t]{3}{*}{$\mathrm{C}_{12} \mathrm{H}_{5} \mathrm{Cl}_{3} \mathrm{O}_{2}$} & {$[39227-58-2]$} & \multicolumn{5}{|c|}{ 1,2,4-trichlorodibenzo[b,e][1,4]dioxin } \\
\hline & SUB & (348-383) & $121.0 \pm 1.8$ & 365 & ME & [2004LI/SHI] \\
\hline & SUB & (310-374) & 118.8 & 342 & $\mathrm{~T}$ & [1989ROR, 1986ROR] \\
\hline \multirow[t]{3}{*}{$\mathrm{C}_{12} \mathrm{H}_{5} \mathrm{Cl}_{3} \mathrm{O}_{2}$} & {$[67028-17-5]$} & 1,3,7-trichlorod & enzo $[b, e][1,4]$ & oxin & & \\
\hline & FUS & & 30.8 & 421.7 & & [1986ROR2] \\
\hline & SUB & $(310-373)$ & 116.2 & 342 & $\mathrm{~T}$ & [1989ROR, 1986ROR] \\
\hline $\mathrm{C}_{12} \mathrm{H}_{5} \mathrm{Cl}_{3} \mathrm{O}_{2}$ & {$[82306-65-8]$} & 1,7,8-trichlorod & enzo $[\mathrm{b}, \mathrm{e}][1,4]$ & oxin & & \\
\hline & SUB & (358-388) & $113.5 \pm 3.3$ & 373 & $\mathrm{ME}$ & [2004LI/SHI] \\
\hline $\mathrm{C}_{12} \mathrm{H}_{5} \mathrm{Cl}_{3} \mathrm{O}_{2}$ & {$[33857-28-2]$} & 2,3,7-trichlorod & enzo $[b, e][1,4]$ & oxin & & \\
\hline & SUB & $(298-423)$ & 123.6 & 360 & $\mathrm{ME}$ & [2005RYA/GUL] \\
\hline & $\mathrm{V}$ & $(448-548)$ & 95.1 & 498 & $\mathrm{ME}$ & [2005RYA/GUL] \\
\hline $\mathrm{C}_{12} \mathrm{H}_{5} \mathrm{Cl}_{5}$ & {$[31508-00-6]$} & $2,3^{\prime}, 4,4^{\prime}, 5$-pent & hlorobiphenyl & & & \\
\hline & FUS & & 26.6 & 382.3 & $\mathrm{DSC}$ & [2006NAK/SHI] \\
\hline & $\mathrm{V}$ & $(343-393)$ & 89.3 & 368 & GC & [1994FAL/BID] \\
\hline $\mathrm{C}_{12} \mathrm{H}_{5} \mathrm{Cl}_{5}$ & {$[32598-14-4]$} & $2,3,3^{\prime}, 4,4^{\prime}$-pent & hlorobiphenyl & & & \\
\hline & $\mathrm{V}$ & (343-393) & 91.1 & 368 & GC & [1994FAL/BID] \\
\hline $\mathrm{C}_{12} \mathrm{H}_{5} \mathrm{Cl}_{5}$ & {$[37680-73-2]$} & $2,2^{\prime}, 4,5,5^{\prime}$-pent & hlorobiphenyl & & & \\
\hline & FUS & & 18.6 & 381.1 & DSC & [2006NAK/SHI] \\
\hline & FUS & & 18.8 & 350.1 & $\mathrm{DSC}$ & [1991ACR, 1984MIL/GHO] \\
\hline & SUB & $(303-313)$ & 92.7 & 308 & GS & [1981WES/SIM] \\
\hline & $\mathrm{V}$ & $(343-393)$ & 86.4 & 368 & GC & [1994FAL/BID] \\
\hline & $\mathrm{V}$ & $(343-453)$ & 83.7 & 398 & GC & [1990HIN/BID2] \\
\hline $\mathrm{C}_{12} \mathrm{H}_{5} \mathrm{Cl}_{5}$ & {$[38379-99-6]$} & $2,2^{\prime}, 3,5^{\prime}, 6$-pent & hlorobiphenyl & & & \\
\hline & FUS & & 23.3 & 367.7 & DSC & [2006NAK/SHI] \\
\hline
\end{tabular}


TABLE 11. Phase change enthalpies of $\mathrm{C}_{12}$ to $\mathrm{C}_{13}$ organic compounds-Continued

\begin{tabular}{|c|c|c|c|c|c|c|}
\hline \multirow[b]{2}{*}{ Molecular formula } & \multirow{2}{*}{$\begin{array}{c}\text { CAS Registry } \\
\text { Number } \\
\text { Enthalpy }\end{array}$} & \multicolumn{5}{|l|}{ Compound } \\
\hline & & $\begin{array}{c}\text { Temperature } \\
\text { range }\end{array}$ & $\begin{array}{l}\Delta_{\text {trans }} H_{\mathrm{m}} \\
(\mathrm{kJ} / \mathrm{mol})\end{array}$ & $\begin{array}{l}T_{\mathrm{m}} \\
(\mathrm{K})\end{array}$ & Method & References \\
\hline & $\mathrm{V}$ & & $92.3 \pm 0.6$ & 298 & CGC & [2001PUR/CHI] \\
\hline $\mathrm{C}_{12} \mathrm{H}_{5} \mathrm{Cl}_{5}$ & $\begin{array}{c}{[73575-54-9]} \\
\mathrm{V}\end{array}$ & $2,2^{\prime}, 3,6,6^{\prime}$-pen & $\begin{array}{l}\text { lorobiphenyl } \\
89.6 \pm 0.2\end{array}$ & 298 & CGC & [2001PUR/CHI] \\
\hline $\mathrm{C}_{12} \mathrm{H}_{5} \mathrm{Cl}_{5}$ & $\begin{array}{c}\text { [60233-25-2] } \\
\text { FUS }\end{array}$ & \multicolumn{4}{|c|}{$2,2^{\prime}, 3^{\prime}, 4,6$-pentachlorobiphenyl } & [2006NAK/SHI] \\
\hline $\mathrm{C}_{12} \mathrm{H}_{5} \mathrm{Cl}_{5}$ & $\begin{array}{c}{[60145-21-3]} \\
\mathrm{V}\end{array}$ & \multicolumn{5}{|c|}{$2,2^{\prime}, 4,5^{\prime}, 6$-pentachlorobiphenyl } \\
\hline $\mathrm{C}_{12} \mathrm{H}_{5} \mathrm{Cl}_{5}$ & $\begin{array}{c}{[38380-02-8]} \\
\mathrm{V}\end{array}$ & \multicolumn{5}{|c|}{$2,2^{\prime}, 3,4,5^{\prime}$-pentachlorobiphenyl } \\
\hline $\mathrm{C}_{12} \mathrm{H}_{5} \mathrm{Cl}_{5}$ & $\begin{array}{c}{[38380-01-7]} \\
\mathrm{V}\end{array}$ & \multicolumn{4}{|c|}{$2,2^{\prime}, 4,4^{\prime}, 5$-pentachlorobiphenyl } & [1994FAL/BID] \\
\hline $\mathrm{C}_{12} \mathrm{H}_{5} \mathrm{Cl}_{5}$ & $\begin{array}{c}\text { [18259-05-7] } \\
\text { FUS }\end{array}$ & \multicolumn{4}{|c|}{ 2,3,4,5,6-pentachlorobiphenyl } & [1991ACR, 1984MIL/GHO] \\
\hline $\mathrm{C}_{12} \mathrm{H}_{5} \mathrm{Cl}_{5}$ & $\begin{array}{c}\text { [38380-03-9] } \\
\text { FUS }\end{array}$ & \multicolumn{4}{|c|}{$2,3,3^{\prime}, 4^{\prime}, 6$-pentachlorobiphenyl } & [2006NAK/SHI] \\
\hline $\mathrm{C}_{12} \mathrm{H}_{5} \mathrm{Cl}_{5}$ & $\begin{array}{c}{[57465-28-8]} \\
\text { FUS }\end{array}$ & \multicolumn{4}{|c|}{$3,3^{\prime}, 4,4^{\prime}, 5$-pentachlorobiphenyl } & {$[2006 \mathrm{NAK} / \mathrm{SHI}]$} \\
\hline \multirow[t]{3}{*}{$\mathrm{C}_{12} \mathrm{H}_{6} \mathrm{Br}_{4} \mathrm{O}$} & $\begin{array}{c}{[5436-43-1]} \\
\text { FUS }\end{array}$ & \multicolumn{5}{|c|}{$2,2^{\prime}, 4,4^{\prime}$-tetrabromodiphenyl ether } \\
\hline & SUB & $(313-363)$ & 105 & 338 & GS & [2014KUR/TAK] \\
\hline & $\begin{array}{l}\mathrm{V} \\
\mathrm{V}\end{array}$ & $\begin{array}{l}(363-473) \\
(403-475)\end{array}$ & $\begin{array}{c}92.0 \\
103.1\end{array}$ & 418 & $\begin{array}{c}\mathrm{GC} \\
\mathrm{CGC}\end{array}$ & $\begin{array}{l}\text { [2001WON/LEI] } \\
\text { [2001PUR/CHI] }\end{array}$ \\
\hline $\mathrm{C}_{12} \mathrm{H}_{6} \mathrm{Br}_{4} \mathrm{O}$ & $\begin{array}{c}\text { [189084-61-5] } \\
\text { V }\end{array}$ & \multicolumn{5}{|c|}{$2,3^{\prime}, 4,4^{\prime}$-tetrabromodiphenyl ether } \\
\hline $\mathrm{C}_{12} \mathrm{H}_{6} \mathrm{Br}_{4} \mathrm{O}$ & $\begin{array}{c}{[327185-09-1]} \\
\mathrm{V}\end{array}$ & \multicolumn{5}{|c|}{ 2,3',4,6-tetrabromodiphenyl ether } \\
\hline $\mathrm{C}_{12} \mathrm{H}_{6} \mathrm{Br}_{4} \mathrm{O}$ & $\begin{array}{c}\text { [189084-63-7] } \\
\mathrm{V}\end{array}$ & \multicolumn{4}{|c|}{ 2,4,4',6-tetrabromodiphenyl ether } & [2001WON/LEI] \\
\hline $\mathrm{C}_{12} \mathrm{H}_{6} \mathrm{Br}_{4} \mathrm{O}$ & $\begin{array}{c}{[93703-48-1]} \\
\mathrm{V}\end{array}$ & \multicolumn{4}{|c|}{$3,3^{\prime}, 4,4^{\prime}$-tetrabromodiphenyl ether } & [2001WON/LEI] \\
\hline \multirow[t]{2}{*}{$\mathrm{C}_{12} \mathrm{H}_{6} \mathrm{Cl}_{2} \mathrm{O}$} & $\begin{array}{c}\text { [5409-83-6] } \\
\text { SUB } \\
\text { SUB }\end{array}$ & $\begin{array}{l}\text { 2,8-dichlorodi } \\
\quad(298-448) \\
(348-383)\end{array}$ & $\begin{array}{l}\text { zofuran } \\
\qquad \begin{array}{l}102.2 \\
110.3 \pm 1.2\end{array}\end{array}$ & $\begin{array}{l}373 \\
360\end{array}$ & $\begin{array}{l}\text { ME } \\
\text { ME }\end{array}$ & $\begin{array}{l}\text { [2005RYA/GUL] } \\
{[2004 \mathrm{LI} / \mathrm{SHI}]}\end{array}$ \\
\hline & $\mathrm{V}$ & $(473-523)$ & 87.9 & 498 & ME & [2005RYA/GUL] \\
\hline \multirow[t]{2}{*}{$\mathrm{C}_{12} \mathrm{H}_{6} \mathrm{Cl}_{2} \mathrm{O}$} & $\begin{array}{c}\text { [74918-40-4] } \\
\text { FUS }\end{array}$ & \multicolumn{5}{|c|}{ 3,6-dichlorodibenzofuran } \\
\hline & SUB & $(305-374)$ & 110.9 & 340 & $\mathrm{~T}$ & [1989ROR, 1986ROR] \\
\hline $\mathrm{C}_{12} \mathrm{H}_{6} \mathrm{Cl}_{2} \mathrm{O}_{2}$ & $\begin{array}{c}\text { [38178-38-0] } \\
\text { SUB }\end{array}$ & $\begin{array}{l}\text { 1,6-dichlorodi } \\
(348-383)\end{array}$ & $\begin{array}{c}\text { zo }[\mathrm{b}, \mathrm{e}][1,4] \mathrm{d} \\
113.6 \pm 2.3\end{array}$ & xin 365 & $\mathrm{ME}$ & [2004LI/SHI] \\
\hline $\mathrm{C}_{12} \mathrm{H}_{6} \mathrm{Cl}_{2} \mathrm{O}_{2}$ & $\begin{array}{c}\text { [29446-15-9] } \\
\text { FUS }\end{array}$ & 2,3-dichlorodi & $\begin{array}{c}\mathrm{zo}[\mathrm{b}, \mathrm{e}][1,4] \mathrm{di} \\
27.1\end{array}$ & in 431.6 & & [1999KOL/DOR] \\
\hline & $\begin{array}{l}\text { SUB } \\
\text { SUB } \\
\text { SUB } \\
\text { SUB } \\
\text { SUB }\end{array}$ & (306-374) & $\begin{array}{c}106.2 \pm 1.1 \\
108.6 \pm 1.0 \\
107.2 \pm 0.8 \\
108.6 \pm 1.0 \\
106.2\end{array}$ & $\begin{array}{l}358 \\
298 \\
358 \\
298 \\
340\end{array}$ & $\begin{array}{c}\mathrm{ME} \\
\mathrm{C} \\
\mathrm{C} \\
\mathrm{T}\end{array}$ & $\begin{array}{l}\text { [2004LI/SHI] } \\
{[1999 \mathrm{KOL} / \mathrm{DOR}]} \\
{[1998 \mathrm{PAP} / \mathrm{KOL}]} \\
{[1998 \mathrm{PAP} / \mathrm{KOL}]} \\
{[1989 \mathrm{ROR}, 1986 \mathrm{ROR}]}\end{array}$ \\
\hline $\mathrm{C}_{12} \mathrm{H}_{6} \mathrm{Cl}_{2} \mathrm{O}_{2}$ & $\begin{array}{c}\text { [33857-26-0] } \\
\text { SUB }\end{array}$ & $\begin{array}{l}\text { 2,7-dichlorodi } \\
\quad(298-483)\end{array}$ & $\begin{array}{c}\mathrm{zo}[\mathrm{b}, \mathrm{e}][1,4] \mathrm{di} \\
108.8\end{array}$ & in 390 & $\mathrm{ME}$ & [2005RYA/GUL] \\
\hline
\end{tabular}


TABLE 11. Phase change enthalpies of $\mathrm{C}_{12}$ to $\mathrm{C}_{13}$ organic compounds-Continued

\begin{tabular}{|c|c|c|c|c|c|c|}
\hline \multirow[b]{2}{*}{ Molecular formula } & \multirow{2}{*}{$\begin{array}{c}\text { CAS Registry } \\
\text { Number } \\
\text { Enthalpy }\end{array}$} & \multicolumn{5}{|l|}{ Compound } \\
\hline & & $\begin{array}{l}\text { Temperature } \\
\text { range }\end{array}$ & $\begin{array}{l}\Delta_{\text {trans }} H_{\mathrm{m}} \\
(\mathrm{kJ} / \mathrm{mol})\end{array}$ & $\begin{array}{l}T_{\mathrm{m}} \\
(\mathrm{K})\end{array}$ & Method & References \\
\hline & SUB & $(358-393)$ & $113.8 \pm 2.0$ & 375 & $\mathrm{ME}$ & [2004LI/SHI] \\
\hline & SUB & $(314-374)$ & 105.5 & 344 & $\mathrm{~T}$ & [1989ROR, 1986ROR] \\
\hline & $\mathrm{V}$ & $(503-523)$ & 64.3 & 513 & $\mathrm{ME}$ & [2005RYA/GUL] \\
\hline \multirow[t]{2}{*}{$\mathrm{C}_{12} \mathrm{H}_{6} \mathrm{Cl}_{2} \mathrm{O}_{2}$} & [38964-22-6] & \multicolumn{5}{|c|}{ 2,8-dichlorodibenzo[b,e][1,4]dioxin } \\
\hline & SUB & $(305-363)$ & 109 & 334 & $\mathrm{~T}$ & [1989ROR, 1986ROR] \\
\hline \multirow[t]{2}{*}{$\mathrm{C}_{12} \mathrm{H}_{6} \mathrm{Cl}_{4}$} & {$[38444-93-8]$} & \multicolumn{5}{|c|}{$2,2^{\prime}, 3,3^{\prime}$-tetrachlorobiphenyl } \\
\hline & $\mathrm{V}$ & $(343-398)$ & 81.8 & 368 & GC & [1994FAL/BID] \\
\hline \multirow[t]{6}{*}{$\mathrm{C}_{12} \mathrm{H}_{6} \mathrm{Cl}_{4}$} & [35693-99-3] & \multicolumn{5}{|c|}{ 2,2',5,5'-tetrachlorobiphenyl } \\
\hline & FUS & & 20.8 & 357.1 & DSC & [2006NAK/SHI] \\
\hline & SUB & $(323-353)$ & $102.0 \pm 0.5$ & 338 & $\mathrm{ME}$ & [2005NAK/SHI] \\
\hline & SUB & $(303-312)$ & 94.6 & 308 & GS & [1981WES/SIM] \\
\hline & V & $(343-398)$ & 80.8 & 368 & GC & [1994FAL/BID] \\
\hline & $\mathrm{V}$ & $(343-453)$ & 79 & 398 & GC & [1990HIN/BID2] \\
\hline \multirow[t]{2}{*}{$\mathrm{C}_{12} \mathrm{H}_{6} \mathrm{Cl}_{4}$} & {$[41464-39-5]$} & \multicolumn{5}{|c|}{ 2,2',3,5'-tetrachlorobiphenyl } \\
\hline & FUS & & 18.3 & 320.4 & DSC & [2006NAK/SHI] \\
\hline \multirow[t]{3}{*}{$\mathrm{C}_{12} \mathrm{H}_{6} \mathrm{Cl}_{4}$} & [33284-53-6] & \multicolumn{5}{|c|}{ 2,3,4,5-tetrachlorobiphenyl } \\
\hline & FUS & & 25.2 & 363.9 & DSC & [1991ACR, 1984MIL/GHO] \\
\hline & SUB & $(253-393)$ & $88.7 \pm 1.2$ & 273 & GS & [1994WAN/SHU] \\
\hline \multirow[t]{4}{*}{$\mathrm{C}_{12} \mathrm{H}_{6} \mathrm{Cl}_{4}$} & [32598-10-0] & \multicolumn{5}{|c|}{$2,3^{\prime}, 4,4^{\prime}$-tetrachlorobiphenyl } \\
\hline & FUS & & 27.9 & 398.4 & DSC & [2006NAK/SHI] \\
\hline & SUB & $(348-373)$ & $105.9 \pm 2.5$ & 353 & ME & [2005NAK/SHI] \\
\hline & $\mathrm{V}$ & $(343-398)$ & 83.3 & 368 & GC & [1994FAL/BID] \\
\hline \multirow[t]{3}{*}{$\mathrm{C}_{12} \mathrm{H}_{6} \mathrm{Cl}_{4}$} & {$[32598-11-1]$} & \multicolumn{5}{|c|}{ 2,3', $4^{\prime}, 5$-tetrachlorobiphenyl } \\
\hline & FUS & & 27.5 & 376.7 & DSC & [2006NAK/SHI] \\
\hline & $\mathrm{V}$ & $(343-398)$ & 84.8 & 368 & GC & [1994FAL/BID] \\
\hline \multirow[t]{3}{*}{$\mathrm{C}_{12} \mathrm{H}_{6} \mathrm{Cl}_{4}$} & {$[41464-40-8]$} & \multicolumn{5}{|c|}{$2,2^{\prime}, 4,5^{\prime}$-tetrachlorobiphenyl } \\
\hline & FUS & & 23.4 & 339.1 & DSC & [1991ACR, 1984MIL/GHO] \\
\hline & $\mathrm{V}$ & & $87.4 \pm 0.8$ & 298 & CGC & [2001PUR/CHI] \\
\hline \multirow[t]{3}{*}{$\mathrm{C}_{12} \mathrm{H}_{6} \mathrm{Cl}_{4}$} & {$[41464-41-9]$} & \multicolumn{5}{|c|}{ 2,2',5,6'-tetrachlorobiphenyl } \\
\hline & $\mathrm{V}$ & & $84.9 \pm 0.6$ & 298 & CGC & [2001PUR/CHI] \\
\hline & $\mathrm{V}$ & $(343-398)$ & 78.8 & 368 & GC & [1994FAL/BID] \\
\hline $\mathrm{C}_{12} \mathrm{H}_{6} \mathrm{Cl}_{4}$ & [32598-13-3] & $3,3^{\prime}, 4,4^{\prime}$-tetrac & robiphenyl & & & \\
\hline & FUS & & 34.5 & 451.2 & DSC & [2006NAK/SHI] \\
\hline & SUB & $(383-403)$ & $121.6 \pm 1.3$ & 393 & ME & [2005NAK/SHI] \\
\hline & $\mathrm{V}$ & $(343-393)$ & 87.2 & 368 & $\mathrm{GC}$ & [1994FAL/BID] \\
\hline $\mathrm{C}_{12} \mathrm{H}_{6} \mathrm{Cl}_{4} \mathrm{O}_{2} \mathrm{~S}$ & [116-29-0] & 1,2,4-trichloro & ((4-chlorophe & )sulfony & enzene & \\
\hline & FUS & & 28.94 & 419.9 & DSC & [1991ACR, 1990DON/DRE] \\
\hline $\mathrm{C}_{12} \mathrm{H}_{6} \mathrm{O}_{3}$ & [81-84-5] & 1-8-naphthalic & hydride (prote & & & \\
\hline & FUS & & 23.32 & 542.3 & DSC & [1990DON/DRE] \\
\hline $\mathrm{C}_{12} \mathrm{H}_{7} \mathrm{Br}_{3} \mathrm{O}$ & [41318-75-6] & $2,4,4^{\prime}$-tribrom & phenyl ether & & & \\
\hline & $\mathrm{V}$ & $(403-475)$ & 94.1 & & CGC & [2001TIT/TOM] \\
\hline $\mathrm{C}_{12} \mathrm{H}_{7} \mathrm{Br}_{3} \mathrm{O}$ & [147217-81-0] & 3,4,4'-tribrom & phenyl ether & & & \\
\hline & $\mathrm{V}$ & (363-473) & 86.7 & 418 & GC & [2001WON/LEI] \\
\hline $\mathrm{C}_{12} \mathrm{H}_{7} \mathrm{Br}_{3} \mathrm{O}$ & [155999-95-4] & 2,4,6-tribromo & henyl ether & & & \\
\hline & $\mathrm{V}$ & (363-473) & 85.1 & 418 & GC & [2001WON/LEI] \\
\hline $\mathrm{C}_{12} \mathrm{H}_{7} \mathrm{Br}_{3} \mathrm{O}$ & [189084-60-4] & 2,4',6-tribrom & ohenyl ether & & & \\
\hline & V & $(363-473)$ & 83.3 & 418 & GC & [2001WON/LEI] \\
\hline
\end{tabular}


TABLE 11. Phase change enthalpies of $\mathrm{C}_{12}$ to $\mathrm{C}_{13}$ organic compounds-Continued

\begin{tabular}{|c|c|c|c|c|c|c|}
\hline \multirow[b]{2}{*}{ Molecular formula } & \multirow{2}{*}{$\begin{array}{l}\text { CAS Registry } \\
\text { Number } \\
\text { Enthalpy }\end{array}$} & \multicolumn{5}{|l|}{ Compound } \\
\hline & & $\begin{array}{l}\text { Temperature } \\
\text { range }\end{array}$ & $\begin{array}{l}\Delta_{\text {trans }} H_{\mathrm{m}} \\
(\mathrm{kJ} / \mathrm{mol})\end{array}$ & $\begin{array}{l}T_{\mathrm{m}} \\
(\mathrm{K})\end{array}$ & Method & References \\
\hline \multirow[t]{2}{*}{$\mathrm{C}_{12} \mathrm{H}_{7} \mathrm{Br}_{3} \mathrm{O}$} & [147217-78-5] & \multicolumn{5}{|c|}{$2^{\prime}, 3,4$-tribromodiphenyl ether } \\
\hline & $\mathrm{V}$ & $(363-473)$ & 81.0 & 418 & GC & [2001WON/LEI] \\
\hline \multirow[t]{2}{*}{$\mathrm{C}_{12} \mathrm{H}_{7} \mathrm{Br}_{3} \mathrm{O}$} & [147217-80-9] & \multicolumn{5}{|c|}{ 3,3',4-tribromodiphenyl ether } \\
\hline & $\mathrm{V}$ & $(363-473)$ & 86.4 & 418 & GC & [2001WON/LEI] \\
\hline \multirow[t]{3}{*}{$\mathrm{C}_{12} \mathrm{H}_{7} \mathrm{ClO}$} & {$[51230-49-0]$} & \multicolumn{5}{|c|}{ 2-chlorodibenzofuran } \\
\hline & SUB & $(298-373)$ & 95.1 & 335 & ME & [2005RYA/GUL] \\
\hline & V & $(378-548)$ & 75.3 & 463 & $\mathrm{ME}$ & [2005RYA/GUL] \\
\hline \multirow[t]{8}{*}{$\mathrm{C}_{12} \mathrm{H}_{7} \mathrm{ClO}_{2}$} & {$[39227-53-7]$} & \multicolumn{5}{|c|}{ 1-chlorodibenzo[b,e][1,4]dioxin } \\
\hline & FUS & & 23.2 & 378.2 & & [1986ROR2] \\
\hline & SUB & $(298-373)$ & 96.3 & 335 & ME & [2005RYA/GUL] \\
\hline & SUB & $(308-343)$ & $100.5 \pm 0.8$ & 325 & $\mathrm{ME}$ & [2004LI/SHI] \\
\hline & SUB & & $95.2 \pm 1.1$ & 298 & $\mathrm{C}$ & [1999KOL/DOR] \\
\hline & SUB & & 95.2 & & & [1998PAP/LUK] \\
\hline & SUB & $(303-338)$ & 98.6 & 321 & $\mathrm{~T}$ & [1989ROR, 1986ROR] \\
\hline & $\mathrm{V}$ & $(398-523)$ & 79.9 & 460 & $\mathrm{ME}$ & [2005RYA/GUL] \\
\hline \multirow[t]{6}{*}{$\mathrm{C}_{12} \mathrm{H}_{7} \mathrm{ClO}_{2}$} & {$[39227-54-8]$} & \multicolumn{5}{|c|}{ 2-chlorodibenzo[b,e][1,4]dioxin } \\
\hline & FUS & & 23.1 & 362.2 & & [1986ROR2] \\
\hline & SUB & $(308-343)$ & $98.1 \pm 1.1$ & 298 & $\mathrm{ME}$ & [2004LI/SHI] \\
\hline & SUB & & 97.2 & 298 & $\mathrm{C}$ & [1999KOL/DOR] \\
\hline & SUB & & $97.2 \pm 0.6$ & 298 & $\mathrm{C}$ & [1996PAP/KOL] \\
\hline & SUB & $(305-348)$ & 97.2 & 327 & $\mathrm{~T}$ & [1989ROR, 1986ROR] \\
\hline \multirow[t]{3}{*}{$\mathrm{C}_{12} \mathrm{H}_{7} \mathrm{Cl}_{2} \mathrm{NO}_{3}$} & {$[1836-75-5]$} & \multicolumn{5}{|c|}{ 2,4-dichlorophenyl 4-nitrophenyl ether } \\
\hline & FUS & & 22.96 & 342 & $\mathrm{DSC}$ & [1990DON/DRE] \\
\hline & $\mathrm{V}$ & $(328-403)$ & 90.4 & 343 & A & [1987STE/MAL] \\
\hline \multirow[t]{2}{*}{$\mathrm{C}_{12} \mathrm{H}_{7} \mathrm{Cl}_{3}$} & {$[38444-85-8]$} & \multicolumn{5}{|c|}{ 2,3,4'-trichlorobiphenyl } \\
\hline & FUS & & 20.2 & 344.0 & $\mathrm{DSC}$ & [2006NAK/SHI] \\
\hline \multirow[t]{3}{*}{$\mathrm{C}_{12} \mathrm{H}_{7} \mathrm{Cl}_{3}$} & {$[15862-07-4]$} & \multicolumn{5}{|c|}{ 2,4,5-trichlorobiphenyl } \\
\hline & FUS & & 22.8 & 349.5 & DSC & [1991ACR, 1984MIL/GHO] \\
\hline & $\mathrm{V}$ & $(343-393)$ & 76.6 & 368 & GC & [1994FAL/BID] \\
\hline \multirow[t]{3}{*}{$\mathrm{C}_{12} \mathrm{H}_{7} \mathrm{Cl}_{3}$} & {$[35693-92-6]$} & \multicolumn{5}{|c|}{ 2,4,6-trichlorobiphenyl } \\
\hline & FUS & & 16.5 & 334.3 & $\mathrm{DSC}$ & [1991ACR, 1984MIL/GHO] \\
\hline & $\mathrm{V}$ & $(343-393)$ & 74.4 & 368 & GC & [1994FAL/BID] \\
\hline $\mathrm{C}_{12} \mathrm{H}_{7} \mathrm{Cl}_{3}$ & {$[16606-02-3]$} & $2,4^{\prime}, 5$-trichlor & henyl & & & \\
\hline & FUS & & 19.8 & 336.5 & $\mathrm{DSC}$ & [2006NAK/SHI] \\
\hline & V & $(343-398)$ & 77.7 & 368 & GC & [1994FAL/BID] \\
\hline $\mathrm{C}_{12} \mathrm{H}_{7} \mathrm{Cl}_{3}$ & {$[37680-65-2]$} & 2,2',5-trichlor & henyl & & & \\
\hline & FUS & & 18.9 & 316.6 & DSC & [2006NAK/SHI] \\
\hline & SUB & $(303-313)$ & $93.7 \pm 6.2$ & 308 & ME & [2005NAK/SHI] \\
\hline & V & & $80.2 \pm 0.9$ & 298 & CGC & [2001PUR/CHI] \\
\hline $\mathrm{C}_{12} \mathrm{H}_{7} \mathrm{Cl}_{3}$ & {$[7012-37-5]$} & 2,4,4'-trichlor & henyl & & & \\
\hline & FUS & & 19.7 & 329.3 & $\mathrm{DSC}$ & [2006NAK/SHI] \\
\hline & SUB & $(313-328)$ & $96.7 \pm 3.4$ & 320 & $\mathrm{ME}$ & [2005NAK/SHI] \\
\hline $\mathrm{C}_{12} \mathrm{H}_{7} \mathrm{Cl}_{3}$ & [38444-86-9] & $2^{\prime}, 3,4$-trichlor & henyl & & & \\
\hline & FUS & & 19.5 & 332.3 & DSC & [2006NAK/SHI] \\
\hline & SUB & $(313-328)$ & $98.2 \pm 5.5$ & 320 & $\mathrm{ME}$ & [2005NAK/SHI] \\
\hline $\mathrm{C}_{12} \mathrm{H}_{7} \mathrm{Cl}_{3} \mathrm{O}_{2}$ & [3380-34-5] & 5-chloro-2-(2, & ichloropheno & phenol & losan) & \\
\hline & FUS & & 17.75 & 331.1 & DSC & [2012LIM/JAN] \\
\hline
\end{tabular}


TABLE 11. Phase change enthalpies of $\mathrm{C}_{12}$ to $\mathrm{C}_{13}$ organic compounds-Continued

\begin{tabular}{|c|c|c|c|c|c|c|}
\hline \multirow[b]{2}{*}{ Molecular formula } & \multirow{2}{*}{$\begin{array}{l}\text { CAS Registry } \\
\text { Number } \\
\text { Enthalpy }\end{array}$} & \multicolumn{5}{|l|}{ Compound } \\
\hline & & $\begin{array}{l}\text { Temperature } \\
\text { range }\end{array}$ & $\begin{array}{l}\Delta_{\text {trans }} H_{\mathrm{m}} \\
(\mathrm{kJ} / \mathrm{mol})\end{array}$ & $\begin{array}{l}T_{\mathrm{m}} \\
(\mathrm{K})\end{array}$ & Method & References \\
\hline $\mathrm{C}_{12} \mathrm{H}_{8}$ & $\begin{array}{l}\text { [208-96-8] } \\
\text { TRS }\end{array}$ & $\begin{array}{l}\text { Acenaphthylene } \\
\quad(5-330)\end{array}$ & 1.4 & 116.6 & $\mathrm{AC}$ & [1994CHE/WES] \\
\hline \multicolumn{7}{|c|}{ [Note: Heat capacity versus temperature curve showed two peaks — the total transition enthalpy is $1.4 \mathrm{~kJ} / \mathrm{mol}$.] } \\
\hline & FUS & & 10.96 & 362 & DSC & [1990DON/DRE] \\
\hline \multicolumn{7}{|c|}{$\begin{array}{l}\text { [Note: The authors of [1990DON/DRE] gave the chemical name of the compound as acenaphthylene; however, they gave the CAS Registry Number } \\
\text { of acenaphthene.] }\end{array}$} \\
\hline & FUS & & 6.95 & 362.6 & $\mathrm{C}$ & {$[1969 \mathrm{SAD} / \mathrm{STE}]$} \\
\hline & SUB & $(297-318)$ & $74.2 \pm 8.2$ & 307 & $\mathrm{ME}$ & [2008GOL/SUU3] \\
\hline & SUB & & 70.0 & 298 & CGC-DSC & [1998CHI/HES] \\
\hline & SUB & $(313-453)$ & 77.2 & 383 & GS & [1995NAS/LEN] \\
\hline & SUB & $(238-323)$ & $73.2 \pm 0.5$ & 303 & GS & [1983SON/ZOL] \\
\hline & SUB & & $73.0 \pm 0.4$ & 298 & $\mathrm{C}$ & [1972MOR] \\
\hline & SUB & $(286-318)$ & $71.1 \pm 1.3$ & & A & [1970COX/PIL, 1987STE/MAL, 1965BOY/CHR] \\
\hline & $\mathrm{V}$ & & $64.6 \pm 5.8$ & 298 & $\mathrm{CGC}$ & [2008ROU/TEM] \\
\hline & $\mathrm{V}$ & & $69.1 \pm 2.2$ & 298 & GC & [2006HAF/PAR] \\
\hline \multirow[t]{6}{*}{$\mathrm{C}_{12} \mathrm{H}_{8}$} & {$[259-79-0]$} & Biphenylene & & & & \\
\hline & SUB & $(313-453)$ & 82.7 & 383 & GS & [1995NAS/LEN] \\
\hline & SUB & (309-336) & U 104.5 & 319 & & [1989ROR/RUT] \\
\hline & SUB & & $87.3 \pm 0.3$ & 298 & B & [1980OSB/SCO] \\
\hline & SUB & & $83.8 \pm 0.3$ & & $\mathrm{C}$ & {$[1972 \mathrm{MOR}]$} \\
\hline & SUB & $(371-381)$ & $\mathrm{U} 128.9 \pm 2$ & 376 & & [1955CAS/SPR, 1970COX/PIL, 1987STE/MAL] \\
\hline \multirow[t]{2}{*}{$\mathrm{C}_{12} \mathrm{H}_{8} \mathrm{Br}_{2}$} & & $(d l)$-1,2-dibromo & acenaphthene & & & \\
\hline & FUS & & 25.1 & 397 & DSC & {$[1976 \mathrm{LEC} / \mathrm{COL}]$} \\
\hline \multirow[t]{2}{*}{$\mathrm{C}_{12} \mathrm{H}_{8} \mathrm{Br}_{2}$} & & $(d)$-1,2-dibromoa & acenaphthene & & & \\
\hline & FUS & & 26.36 & 416 & DSC & {$[1976 \mathrm{LEC} / \mathrm{COL}]$} \\
\hline \multirow[t]{3}{*}{$\mathrm{C}_{12} \mathrm{H}_{8} \mathrm{Br}_{2}$} & {$[92-86-4]$} & $4,4^{\prime}$-dibromobiph & enyl & & & \\
\hline & FUS & & 28.38 & 440.7 & DSC & [2009RAI/RED] \\
\hline & SUB & $(358-404)$ & $105.5 \pm 0.4$ & 298 & GS & [2015SOL/VAR] \\
\hline \multirow[t]{2}{*}{$\mathrm{C}_{12} \mathrm{H}_{8} \mathrm{Br}_{2} \mathrm{O}$} & [171977-44-9] & 2,4-dibromodiph & enyl ether & & & \\
\hline & $\mathrm{V}$ & $(363-473)$ & 75.4 & 418 & GC & [2001WON/LEI] \\
\hline \multirow[t]{2}{*}{$\mathrm{C}_{12} \mathrm{H}_{8} \mathrm{Br}_{2} \mathrm{O}$} & [189084-59-1] & 3,4-dibromodiph & enyl ether & & & \\
\hline & $\mathrm{V}$ & $(363-473)$ & 77.4 & 418 & GC & [2001WON/LEI] \\
\hline \multirow[t]{2}{*}{$\mathrm{C}_{12} \mathrm{H}_{8} \mathrm{Br}_{2} \mathrm{O}$} & [83694-71-7] & 3,4'-dibromodiph & enyl ether & & & \\
\hline & V & $(363-473)$ & 77.4 & 418 & GC & [2001WON/LEI] \\
\hline \multirow[t]{5}{*}{$\mathrm{C}_{12} \mathrm{H}_{8} \mathrm{Br}_{2} \mathrm{O}$} & [2050-47-7] & 4,4'-dibromodiph & enyl ether & & & \\
\hline & FUS & & 18.4 & 331.9 & DSC & [2011FU/SUU2] \\
\hline & FUS & & 19.6 & 331.7 & $\mathrm{DSC}$ & [2007KUR/MAE] \\
\hline & SUB & $(300-328)$ & $102.0 \pm 3.5$ & & $\mathrm{ME}$ & [2011FU/SUU2] \\
\hline & $\mathrm{V}$ & $(363-473)$ & 78.0 & 418 & GC & [2001WON/LEI] \\
\hline \multirow[t]{2}{*}{$\mathrm{C}_{12} \mathrm{H}_{8} \mathrm{Br}_{2} \mathrm{O}$} & [147217-71-8] & 2,4'-dibromodiph & enyl ether & & & \\
\hline & $\mathrm{V}$ & $(363-473)$ & 76.4 & 418 & $\mathrm{GC}$ & [2001WON/LEI] \\
\hline \multirow[t]{2}{*}{$\mathrm{C}_{12} \mathrm{H}_{8} \mathrm{Br}_{2} \mathrm{O}$} & [51930-04-2] & 2,6-dibromodiph & enyl ether & & & \\
\hline & V & $(363-473)$ & 73.1 & 418 & GC & [2001PUR/CHI] \\
\hline \multirow[t]{5}{*}{$\mathrm{C}_{12} \mathrm{H}_{8} \mathrm{Cl}_{2}$} & [13029-08-8] & 2,2'-dichlorobiph & enyl & & & \\
\hline & FUS & & 16.6 & 330.9 & DSC & [2006NAK/SHI] \\
\hline & SUB & $(303-323)$ & $87.8 \pm 1.2$ & 313 & $\mathrm{ME}$ & [2005NAK/SHI] \\
\hline & SUB & $(310-328)$ & 96.1 & 314 & $\mathrm{ME}$ & {$[1964 \mathrm{SMI} / \mathrm{GOR}]$} \\
\hline & SUB & $(310-328)$ & $96.2 \pm 4.2$ & 298 & ME & [1964SMI/GOR, 1970COX/PIL, 1987STE/MAL] \\
\hline \multirow[t]{3}{*}{$\mathrm{C}_{12} \mathrm{H}_{8} \mathrm{Cl}_{2}$} & {$[33284-50-3]$} & 2,4-dichlorobiphe & enyl & & & \\
\hline & V & & $75.3 \pm 1.5$ & 298 & CGC & [2001PUR/CHI] \\
\hline & $\mathrm{V}$ & $(343-393)$ & 73.5 & 368 & GC & [1994FAL/BID] \\
\hline
\end{tabular}


TABLE 11. Phase change enthalpies of $\mathrm{C}_{12}$ to $\mathrm{C}_{13}$ organic compounds-Continued

\begin{tabular}{|c|c|c|c|c|c|c|}
\hline \multirow[b]{2}{*}{ Molecular formula } & \multirow{2}{*}{$\begin{array}{l}\text { CAS Registry } \\
\text { Number } \\
\text { Enthalpy }\end{array}$} & \multicolumn{5}{|l|}{ Compound } \\
\hline & & $\begin{array}{l}\text { Temperature } \\
\text { range }\end{array}$ & $\begin{array}{l}\Delta_{\text {trans }} H_{\mathrm{m}} \\
(\mathrm{kJ} / \mathrm{mol})\end{array}$ & $\begin{array}{l}T_{\mathrm{m}} \\
(\mathrm{K})\end{array}$ & Method & References \\
\hline \multirow[t]{3}{*}{$\mathrm{C}_{12} \mathrm{H}_{8} \mathrm{Cl}_{2}$} & {$[34883-43-7]$} & \multicolumn{5}{|c|}{ 2,4'-dichlorobiphenyl } \\
\hline & FUS & & 18.0 & 316.7 & DSC & [2006NAK/SHI] \\
\hline & SUB & $(303-313)$ & $94.3 \pm 5.6$ & & ME & [2006NAK/SHI] \\
\hline \multirow[t]{3}{*}{$\mathrm{C}_{12} \mathrm{H}_{8} \mathrm{Cl}_{2}$} & {$[34883-39-1]$} & \multicolumn{5}{|c|}{ 2,5-dichlorobiphenyl } \\
\hline & $\mathrm{V}$ & & $76.8 \pm 0.4$ & 298 & CGC & [2001PUR/CHI] \\
\hline & $\mathrm{V}$ & $(343-393)$ & 73.9 & 368 & $\mathrm{GC}$ & [1994FAL/BID] \\
\hline \multirow[t]{2}{*}{$\mathrm{C}_{12} \mathrm{H}_{8} \mathrm{Cl}_{2}$} & {$[33146-45-1]$} & \multicolumn{5}{|c|}{ 2,6-dichlorobiphenyl } \\
\hline & FUS & & 12.6 & 307.9 & DSC & [1991ACR, 1984MIL/GHO] \\
\hline \multirow[t]{3}{*}{$\mathrm{C}_{12} \mathrm{H}_{8} \mathrm{Cl}_{2}$} & {$[2050-67-1]$} & \multicolumn{5}{|c|}{ 3,3'-dichlorobiphenyl } \\
\hline & $\mathrm{V}$ & & $81.0 \pm 0.2$ & 298 & CGC & [2001PUR/CHI] \\
\hline & V & $(343-393)$ & 75.4 & 368 & $\mathrm{GC}$ & [1994FAL/BID] \\
\hline \multirow{7}{*}{$\mathrm{C}_{12} \mathrm{H}_{8} \mathrm{Cl}_{2}$} & {$[2050-68-2]$} & \multicolumn{5}{|c|}{ 4,4'-dichlorobiphenyl } \\
\hline & FUS & & 22.8 & 421.1 & DSC & [2006NAK/SHI] \\
\hline & SUB & $(263-303)$ & $95.3 \pm 1.3$ & 283 & GS & [1994WAN/SHU] \\
\hline & SUB & $(303-360)$ & 103.7 & 331 & $\mathrm{ME}$ & [1964SMI/GOR, 1987STE/MAL] \\
\hline & SUB & $(303-360)$ & $103.8 \pm 4.2$ & 298 & $\mathrm{ME}$ & [1964SMI/GOR, 1970COX/PIL] \\
\hline & $\mathrm{V}$ & & $81.4 \pm 0.3$ & 298 & CGC & [2001PUR/CHI] \\
\hline & $\mathrm{V}$ & $(343-393)$ & 76.0 & 368 & GC & [1994FAL/BID] \\
\hline \multirow[t]{2}{*}{$\mathrm{C}_{12} \mathrm{H}_{8} \mathrm{Cl}_{2}$} & & \multicolumn{5}{|c|}{$(d l)$-1,2-dichloroacenaphthene } \\
\hline & FUS & & 20.5 & 339 & DSC & [1976LEC/COL] \\
\hline \multirow[t]{2}{*}{$\mathrm{C}_{12} \mathrm{H}_{8} \mathrm{Cl}_{2}$} & & \multicolumn{5}{|c|}{ (d)-1,2-dichloroacenaphthene } \\
\hline & FUS & & 21.34 & 375 & DSC & [1976LEC/COL] \\
\hline \multirow[t]{3}{*}{$\mathrm{C}_{12} \mathrm{H}_{8} \mathrm{Cl}_{2} \mathrm{O}_{2} \mathrm{~S}$} & {$[80-07-9]$} & \multicolumn{5}{|c|}{ 4,4'-dichlorodiphenylsulfone } \\
\hline & FUS & $(14-480)$ & 24.4 & 422 & $\mathrm{AC}$ & [1996DOM/HEA, 1985NOV/TSV] \\
\hline & $\mathrm{V}$ & $(463-573)$ & 59.7 & 478 & & [1999DYK/SVO, 1979MIZ/DAN] \\
\hline \multirow[t]{2}{*}{$\mathrm{C}_{12} \mathrm{H}_{8} \mathrm{Cl}_{2} \mathrm{O}_{3} \mathrm{~S}$} & {$[80-33-1]$} & \multicolumn{5}{|c|}{ 4-chlorophenyl 4-chlorobenzenesulfonate } \\
\hline & FUS & & 23.63 & 360 & DSC & [1991ACR, 1990DON/DRE] \\
\hline $\mathrm{C}_{12} \mathrm{H}_{8} \mathrm{Cl}_{3} \mathrm{NO}_{2}$ & {$[77765-39-0]$} & 2,2,4-trichloro- & (2-methylphe & 1)amino & cyclopent & -1,3-dione \\
\hline & V & $(453-483)$ & 85 & 468 & GC & [1980SHA/SAD] \\
\hline $\mathrm{C}_{12} \mathrm{H}_{8} \mathrm{Cl}_{3} \mathrm{NO}_{2}$ & {$[77765-40-3]$} & 2,2,4-trichloro- & (2-methoxypl & lyl)amin & 4-cycloper & ne-1,3-dione \\
\hline & V & $(453-483)$ & 84.6 & 468 & $\mathrm{GC}$ & [1980SHA/SAD] \\
\hline $\mathrm{C}_{12} \mathrm{H}_{8} \mathrm{Cl}_{3} \mathrm{NO}_{2}$ & {$[73373-64-5]$} & 2,2,4-trichloro- & (3-methoxypl & yl)amin & 4-cycloper & e-1,3-dione \\
\hline & $\mathrm{V}$ & $(453-483)$ & 63.1 & 468 & GC & [1980SHA/SAD] \\
\hline $\mathrm{C}_{12} \mathrm{H}_{8} \mathrm{Cl}_{6}$ & [309-00-2] & $1,2,3,4,10,10-\mathrm{h}$ & chloro- $1,4,4$ & $8,8 \mathrm{a}-\mathrm{he}$ & ydro-endo & $o$-1,4:5,8-dimethylnaphthalene (aldrin) \\
\hline & TRS & & 16.59 & 383.7 & & \\
\hline & FUS & & 4.15 & 562.4 & DSC & [1995KSI/NAG] \\
\hline & SUB & $(309-343)$ & 91.8 & 326 & GS & [1982GRA/FOS] \\
\hline & $\mathrm{V}$ & $(343-453)$ & 75.1 & 398 & GC & [1990HIN/BID2] \\
\hline $\mathrm{C}_{12} \mathrm{H}_{8} \mathrm{Cl}_{6} \mathrm{O}$ & {$[60-57-1]$} & $\begin{array}{l}1,2,3,4,10,10-\mathrm{h} \\
\text { (dieldrin) }\end{array}$ & chloro-6,7-ep & $y-1,4,4$ & $6,7,8,8 \mathrm{a}-\mathrm{o}$ & hydro-1,4-endo-exo-5,8-dimethanonaphthalene \\
\hline & TRS & & 19.33 & 405.6 & & \\
\hline & FUS & & 3.04 & 452.9 & DSC & [1995KSI/NAG] \\
\hline & SUB & $(308-348)$ & 93.8 & 328 & GS & [1982GRA/FOS] \\
\hline & SUB & $(293-313)$ & 98.7 & 303 & GS & [1969SPE/CLI] \\
\hline & $\mathrm{V}$ & $(343-453)$ & 82.5 & 398 & GC & [1990HIN/BID2] \\
\hline $\mathrm{C}_{12} \mathrm{H}_{8} \mathrm{~F}_{2}$ & {$[388-82-9]$} & $2,2^{\prime}$-difluorobip & nyl & & & \\
\hline & SUB & (301-319) & 95.1 & 310 & A & [1987STE/MAL, 1964SMI/GOR] \\
\hline & SUB & $(301-318)$ & $95 \pm 4.2$ & 298 & $\mathrm{ME}$ & [1964SMI/GOR, 1970COX/PIL] \\
\hline
\end{tabular}


TABLE 11. Phase change enthalpies of $\mathrm{C}_{12}$ to $\mathrm{C}_{13}$ organic compounds-Continued

\begin{tabular}{|c|c|c|c|c|c|c|}
\hline \multirow[b]{2}{*}{ Molecular formula } & \multirow{2}{*}{$\begin{array}{l}\text { CAS Registry } \\
\text { Number } \\
\text { Enthalpy }\end{array}$} & \multicolumn{5}{|l|}{ Compound } \\
\hline & & $\begin{array}{c}\text { Temperature } \\
\text { range }\end{array}$ & $\begin{array}{l}\Delta_{\text {trans }} H_{\mathrm{m}} \\
(\mathrm{kJ} / \mathrm{mol})\end{array}$ & $\begin{array}{l}T_{\mathrm{m}} \\
(\mathrm{K})\end{array}$ & Method & References \\
\hline \multirow[t]{3}{*}{$\mathrm{C}_{12} \mathrm{H}_{8} \mathrm{~F}_{2}$} & [2050-68-2] & 4,4'-difluorobi & nyl & & & \\
\hline & SUB & $(294-318)$ & 91.4 & 306 & $\mathrm{ME}$ & [1964SMI/GOR] \\
\hline & SUB & $(294-318)$ & $91.2 \pm 4.2$ & 298 & $\mathrm{ME}$ & [1964SMI/GOR, 1970COX/PIL] \\
\hline \multirow[t]{6}{*}{$\mathrm{C}_{12} \mathrm{H}_{8} \mathrm{~N}_{2}$} & [230-46-6] & 1,7-phenanthrc & & & & \\
\hline & FUS & & 25.6 & 351.7 & DSC & [2016BRU/LAP] \\
\hline & SUB & $(3154-340)$ & $113.2 \pm 0.9$ & 329 & $\mathrm{TE}$ & [2016BRU/LAP] \\
\hline & SUB & $(315-340)$ & $114.2 \pm 1.0$ & 298 & $\mathrm{TE}$ & [2016BRU/LAP] \\
\hline & $\mathrm{V}$ & $(380-428)$ & $72.1 \pm 0.7$ & 401 & ITG & [2016BRU/LAP] \\
\hline & $\mathrm{V}$ & & $79.4 \pm 4.7$ & 298 & CGC & [2009LIP/CHI, 2009LIP/HAN] \\
\hline \multirow[t]{14}{*}{$\mathrm{C}_{12} \mathrm{H}_{8} \mathrm{~N}_{2}$} & {$[66-71-7]$} & 1,10-phenanth & & & & \\
\hline & FUS & & 13.4 & 390.9 & DSC & [2016BRU/LAP] \\
\hline & FUS & & 15.5 & 391.7 & $\mathrm{AC}$ & [2010CHI/KAZ] \\
\hline & FUS & & 11.8 & 391.1 & DSC & [2007BON/CAT] \\
\hline & FUS & & 15.0 & 398.0 & DSC & [1986AIR/SIL] \\
\hline & SUB & $(359-387)$ & $105.1 \pm 0.2$ & 373 & $\mathrm{ME}$ & [2016BRU/LAP] \\
\hline & SUB & $(321-367)$ & $104.3 \pm 0.5$ & 339 & $\mathrm{ME}$ & [2016BRU/LAP] \\
\hline & SUB & & $106.5 \pm 1.1$ & 298 & $\mathrm{ME}$ & [2016BRU/LAP] \\
\hline & SUB & & 98.3 & & ME & {$[1972 \mathrm{MIL}]$} \\
\hline & $\mathrm{V}$ & $(393-420)$ & $74.9 \pm 0.8$ & 407 & ITG & [2016BRU/LAP] \\
\hline & $\mathrm{V}$ & & $86.2 \pm 1.2$ & 298 & CGC & [2010LIP/CHI] \\
\hline & $\mathrm{V}$ & & $77.7 \pm 0.1$ & 520 & EB & [2010CHI/KAZ] \\
\hline & $\mathrm{V}$ & & $74.9 \pm 0.2$ & 560 & $\mathrm{~EB}$ & [2010CHI/KAZ] \\
\hline & $\mathrm{V}$ & & $72.1 \pm 0.2$ & 600 & EB & [2010CHI/KAZ] \\
\hline \multirow[t]{6}{*}{$\mathrm{C}_{12} \mathrm{H}_{8} \mathrm{~N}_{2}$} & {$[230-07-9]$} & 4,7-phenanthrc & & & & \\
\hline & FUS & & 22.4 & 444.1 & DSC & [2016BRU/LAP] \\
\hline & SUB & $(340-369)$ & $116.7 \pm 0.8$ & 353 & $\mathrm{TE}$ & [2016BRU/LAP] \\
\hline & SUB & $(340-369)$ & $118.4 \pm 1.2$ & 298 & $\mathrm{TE}$ & [2016BRU/LAP] \\
\hline & $\mathrm{V}$ & $(463-496)$ & $81.5 \pm 0.7$ & 480 & ITG & [2016BRU/LAP] \\
\hline & $\mathrm{V}$ & & $80.8 \pm 4.7$ & 298 & CGC & [2009LIP/CHI, 2009LIP/HAN] \\
\hline \multirow[t]{25}{*}{$\mathrm{C}_{12} \mathrm{H}_{8} \mathrm{~N}_{2}$} & {$[92-82-0]$} & Phenazine & & & & \\
\hline & FUS & & 19.3 & 446.6 & DSC & [2016BRU/LAP] \\
\hline & FUS $(\alpha)$ & & 23.5 & 447.2 & & \\
\hline & FUS $(\beta)$ & & 23.6 & 448.2 & DSC & [2010BRA/GRE] \\
\hline & FUS & $(6-522)$ & 24.92 & 447.9 & $\mathrm{AC}$ & [2010CHI/KAZ2] \\
\hline & FUS & & 20.92 & 450.2 & DSC & [1975MCE/SAN] \\
\hline & SUB & $(316-343)$ & $101.7 \pm 1.2$ & 330 & $\mathrm{ME}$ & [2016BRU/LAP] \\
\hline & SUB & $(292-324)$ & $103.4 \pm 1.7$ & 307 & $\mathrm{ME}$ & [2016BRU/LAP] \\
\hline & SUB & & $103.3 \pm 1.7$ & 298 & ME & [2016BRU/LAP] \\
\hline & SUB & & $95.9 \pm 0.4$ & 298 & & [2010CHI/KAZ2] \\
\hline & SUB & & $94.3 \pm 0.4$ & 354 & & [2010CHI/KAZ2] \\
\hline & SUB & & $92.7 \pm 0.4$ & 354 & & [1991SAB/WAT] \\
\hline & SUB & & $97.0 \pm 0.4$ & 298 & & [1991SAB/WAT] \\
\hline & SUB & & $91.8 \pm 2.1$ & 298 & $\mathrm{C}$ & [1990LEI/PIL] \\
\hline & SUB & $(280-318)$ & 92.4 & 295 & A & [1987STE/MAL] \\
\hline & SUB & & $99.9 \pm 2.5$ & & ME,GS & [1980ARS] \\
\hline & SUB & $(303-328)$ & $90.4 \pm 2.5$ & 298 & TE & [1975DEK/VAN] \\
\hline & SUB & $(303-323)$ & $90.0 \pm 1.5$ & 298 & TCM & [UR/DEK, 1975DEK/VAN] \\
\hline & SUB & $(281-293)$ & $90.4 \pm 1.7$ & & LE & [1975MCE/SAN] \\
\hline & SUB & & U 81.5 & & & [1946ALB/WIL] \\
\hline & $\mathrm{V}$ & $(450-469)$ & $71.4 \pm 0.6$ & 459 & ITG & [2016BRU/LAP] \\
\hline & $\mathrm{V}$ & & $76.6 \pm 1.1$ & 298 & CGC & [2010LIP/CHI] \\
\hline & $\mathrm{V}$ & $(450-470)$ & $66.1 \pm 0.1$ & 450 & IPM & [2010CHI/KAZ2] \\
\hline & $\mathrm{V}$ & $(450-470)$ & $65.5 \pm 0.1$ & 460 & IPM & [2010CHI/KAZ2] \\
\hline & $\mathrm{V}$ & $(450-470)$ & $65.0 \pm 0.1$ & 470 & IPM & [2010CHI/KAZ2] \\
\hline
\end{tabular}


TABLE 11. Phase change enthalpies of $\mathrm{C}_{12}$ to $\mathrm{C}_{13}$ organic compounds-Continued

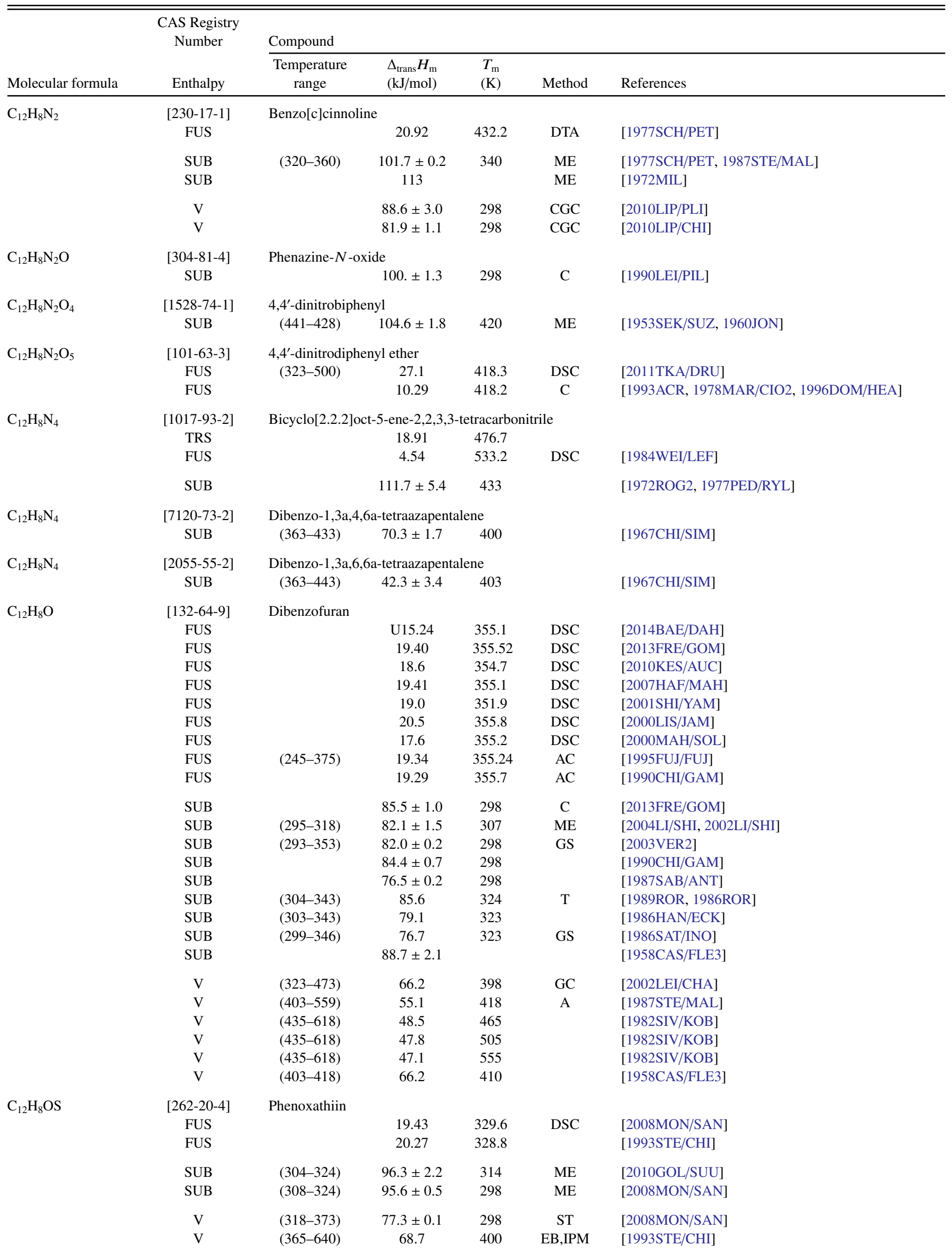


TABLE 11. Phase change enthalpies of $\mathrm{C}_{12}$ to $\mathrm{C}_{13}$ organic compounds-Continued

\begin{tabular}{|c|c|c|c|c|c|c|}
\hline \multirow[b]{2}{*}{ Molecular formula } & \multirow{2}{*}{$\begin{array}{l}\text { CAS Registry } \\
\text { Number } \\
\text { Enthalpy }\end{array}$} & \multicolumn{5}{|l|}{ Compound } \\
\hline & & $\begin{array}{l}\text { Temperature } \\
\text { range }\end{array}$ & $\begin{array}{l}\Delta_{\text {trans }} H_{\mathrm{m}} \\
(\mathrm{kJ} / \mathrm{mol})\end{array}$ & $\begin{array}{l}T_{\mathrm{m}} \\
(\mathrm{K})\end{array}$ & Method & References \\
\hline & $\mathrm{V}$ & $(365-640)$ & 66.0 & 440 & EB,IPM & [1993STE/CHI] \\
\hline & $\mathrm{V}$ & $(365-640)$ & 63.4 & 480 & EB,IPM & [1993STE/CHI] \\
\hline & $\mathrm{V}$ & $(365-640)$ & 60.8 & 520 & EB,IPM & [1993STE/CHI] \\
\hline & $\mathrm{V}$ & $(365-640)$ & 58.0 & 560 & EB,IPM & [1993STE/CHI] \\
\hline & $\mathrm{V}$ & $(365-640)$ & 55.1 & 600 & EB,IPM & [1993STE/CHI] \\
\hline \multirow[t]{2}{*}{$\mathrm{C}_{12} \mathrm{H}_{8} \mathrm{OS}_{2}$} & [49833-13-8] & \multicolumn{5}{|c|}{ Diphenylene-2,2'-disulfide $S$-oxide } \\
\hline & FUS & & 17.99 & 407 & DSC & [1996DOM/HEA, 1975CUC] \\
\hline \multirow[t]{8}{*}{$\mathrm{C}_{12} \mathrm{H}_{8} \mathrm{O}_{2}$} & {$[262-12-4]$} & \multicolumn{5}{|c|}{ Dibenzo[b,e][1,4]dioxin } \\
\hline & FUS & & 23.9 & 389.9 & DSC & [2001SHI/YAM] \\
\hline & FUS & & 23.2 & 395.7 & & [1986ROR2] \\
\hline & SUB & $(303-333)$ & $93.6 \pm 1.2$ & 318 & ME & [2002LI/SHI, 2004LI/SHI] \\
\hline & SUB & & $91.5 \pm 0.8$ & 298 & $\mathrm{C}$ & [2002PIM/MEL] \\
\hline & SUB & & $89.6 \pm 0.7$ & 298 & $\mathrm{C}$ & [1999KOL/DOR] \\
\hline & SUB & & $89.6 \pm 0.7$ & 318 & $\mathrm{C}$ & [1997LUK/KOL] \\
\hline & SUB & $(303-333)$ & 92.3 & 318 & $\mathrm{~T}$ & [1989ROR, 1986ROR] \\
\hline \multirow[t]{3}{*}{$\mathrm{C}_{12} \mathrm{H}_{8} \mathrm{O}_{2} \mathrm{~S}$} & {$[1016-05-3]$} & \multicolumn{5}{|c|}{ Dibenzothiophene sulfone } \\
\hline & FUS & & 27.17 & 509.2 & DSC & [2007RAM/ROJ] \\
\hline & FUS & & 23.72 & 507.8 & DSC & [UR/MAC] \\
\hline \multirow[t]{34}{*}{$\mathrm{C}_{12} \mathrm{H}_{8} \mathrm{~S}$} & {$[132-65-0]$} & \multicolumn{5}{|c|}{ Dibenzothiophene } \\
\hline & FUS & & 21.6 & 371.8 & DSC & [2000LIS/JAM] \\
\hline & FUS & $(5-515)$ & 21.7 & 371.8 & AC,DSC & [1991CHI/KNI] \\
\hline & FUS & $(220-560)$ & 21.6 & 371.0 & DSC & [1983ORO/MRA, 1996DOM/HEA] \\
\hline & FUS & & 22.35 & 372.4 & DSC & {$[1980 \mathrm{KRA} / \mathrm{PIG}]$} \\
\hline & SUB & & $93.2 \pm 0.5$ & 298 & $\mathrm{C}$ & [2009FRE/GOM] \\
\hline & SUB & & $85.1 \pm 0.4$ & 298 & $\mathrm{C}$ & [1987SAB/ANT, 1979SAB] \\
\hline & SUB & $(303-348)$ & 91.2 & 325 & $\mathrm{~T}$ & [1986HAN/ECK] \\
\hline & SUB & $(333-363)$ & 90.7 & 348 & GS & [1981EDW/PRA] \\
\hline & SUB & & 97.5 & 298 & & [1975AUB/MAY, 2009FRE/GOM] \\
\hline & $\mathrm{V}$ & $(413-473)$ & $78.3 \pm 1.1$ & 298 & GC & [2006HAF/PAR] \\
\hline & $\mathrm{V}$ & $(373-424)$ & 65.6 & 388 & & [1999DYK/SVO] \\
\hline & $\mathrm{V}$ & $(424-608)$ & 63.4 & 439 & & [1999DYK/SVO] \\
\hline & $\mathrm{V}$ & & $69.5 \pm 0.3$ & 380 & EB,IPM & [1995STE/CHI] \\
\hline & $\mathrm{V}$ & & $66.8 \pm 0.3$ & 420 & EB,IPM & [1995STE/CHI] \\
\hline & $\mathrm{V}$ & & $64.3 \pm 0.3$ & 460 & EB,IPM & [1995STE/CHI] \\
\hline & $\mathrm{V}$ & & $61.8 \pm 0.3$ & 500 & EB,IPM & [1995STE/CHI] \\
\hline & $\mathrm{V}$ & & $59.3 \pm 0.3$ & 540 & EB,IPM & [1995STE/CHI] \\
\hline & $\mathrm{V}$ & & $56.8 \pm 0.3$ & 580 & EB,IPM & [1995STE/CHI] \\
\hline & $\mathrm{V}$ & & $54.0 \pm 0.3$ & 620 & EB,IPM & [1995STE/CHI] \\
\hline & $\mathrm{V}$ & $(375-662)$ & $68.0 \pm 0.1$ & 400 & EB,IPM & [1991CHI/KNI] \\
\hline & $\mathrm{V}$ & $(375-662)$ & $64.9 \pm 0.1$ & 450 & EB,IPM & [1991CHI/KNI] \\
\hline & $\mathrm{V}$ & $(375-662)$ & $61.8 \pm 0.1$ & 500 & EB,IPM & [1991CHI/KNI] \\
\hline & $\mathrm{V}$ & $(375-662)$ & $58.7 \pm 0.1$ & 550 & EB,IPM & [1991CHI/KNI] \\
\hline & $\mathrm{V}$ & $(375-662)$ & $55.4 \pm 0.3$ & 600 & EB,IPM & [1991CHI/KNI] \\
\hline & $\mathrm{V}$ & $(375-662)$ & $51.8 \pm 0.4$ & 650 & EB,IPM & [1991CHI/KNI] \\
\hline & $\mathrm{V}$ & $(385-574)$ & 60.1 & 400 & A & [1987STE/MAL] \\
\hline & $\mathrm{V}$ & & 56.9 & 590 & $\mathrm{C}$ & [1984MRA/KEW] \\
\hline & $\mathrm{V}$ & & 55.3 & 610 & $\mathrm{C}$ & [1984MRA/KEW] \\
\hline & $\mathrm{V}$ & & 53.6 & 630 & $\mathrm{C}$ & [1984MRA/KEW] \\
\hline & $\mathrm{V}$ & $(425-607)$ & 63.1 & 465 & & [1982SIV/KOB] \\
\hline & $\mathrm{V}$ & $(425-607)$ & 62.6 & 505 & & [1982SIV/KOB] \\
\hline & $\mathrm{V}$ & $(425-607)$ & 62.3 & 555 & & [1982SIV/KOB] \\
\hline & $\mathrm{V}$ & $(373-403)$ & 69.4 & 385 & GS & [1981EDW/PRA] \\
\hline $\mathrm{C}_{12} \mathrm{H}_{8} \mathrm{~S}_{2}$ & {$[92-85-3]$} & Thianthrene & & & & \\
\hline & FUS & $(5-518)$ & 27.55 & 429.6 & AC,DSC & [1993STE/CHI] \\
\hline & FUS & & 25.44 & 428.4 & & [1989SAB/ELW] \\
\hline & SUB & $(344-364)$ & $105.1 \pm 0.6$ & 354 & $\mathrm{ME}$ & [2010MON/SOU] \\
\hline & SUB & (344-364) & $106.4 \pm 0.6$ & 298 & $\mathrm{ME}$ & [2010MON/SOU] \\
\hline
\end{tabular}


TABLE 11. Phase change enthalpies of $\mathrm{C}_{12}$ to $\mathrm{C}_{13}$ organic compounds-Continued

\begin{tabular}{|c|c|c|c|c|c|c|}
\hline \multirow[b]{2}{*}{ Molecular formula } & \multirow{2}{*}{$\begin{array}{c}\text { CAS Registry } \\
\text { Number } \\
\text { Enthalpy }\end{array}$} & \multicolumn{5}{|l|}{ Compound } \\
\hline & & $\begin{array}{c}\text { Temperature } \\
\text { range }\end{array}$ & $\begin{array}{l}\Delta_{\text {trans }} H_{\mathrm{m}} \\
(\mathrm{kJ} / \mathrm{mol})\end{array}$ & $\begin{array}{l}T_{\mathrm{m}} \\
(\mathrm{K})\end{array}$ & Method & References \\
\hline & SUB & & $103.6 \pm 0.4$ & 350 & IP & [1993STE/CHI] \\
\hline & SUB & $(338-368)$ & $98.6 \pm 0.5$ & 353 & & [1989SAB/ELW] \\
\hline & SUB & & $99.4 \pm 0.6$ & 298 & & [1989SAB/ELW] \\
\hline & SUB & $(358-428)$ & 98.0 & 393 & GS & [1981EDW/PRA] \\
\hline & SUB & $(338-368)$ & $97.5 \pm 6.3$ & 353 & HSA & [1979SAN/EPS] \\
\hline & $\mathrm{V}$ & $(429-460)$ & 71.2 & 444 & & [1999DYK/SVO] \\
\hline & $\mathrm{V}$ & $(460-539)$ & 68.4 & 475 & & [1999DYK/SVO] \\
\hline & V & $(395-639)$ & 72.7 & 440 & EB,IPM & [1993STE/CHI] \\
\hline & $\mathrm{V}$ & $(395-639)$ & 69.9 & 480 & EB,IPM & [1993STE/CHI] \\
\hline & V & $(395-639)$ & 67.2 & 520 & EB,IPM & [1993STE/CHI] \\
\hline & V & $(395-639)$ & 64.5 & 560 & EB,IPM & [1993STE/CHI] \\
\hline & $\mathrm{V}$ & $(395-639)$ & 61.7 & 600 & EB,IPM & [1993STE/CHI] \\
\hline & $\mathrm{V}$ & $(430-593)$ & 69.1 & 465 & & [1983SIV/KOB] \\
\hline & $\mathrm{V}$ & $(430-593)$ & 68.7 & 515 & & [1983SIV/KOB] \\
\hline & $\mathrm{V}$ & $(428-448)$ & 71.1 & 438 & GS & [1981EDW/PRA] \\
\hline \multirow[t]{2}{*}{$\mathrm{C}_{12} \mathrm{H}_{8} \mathrm{~S}_{2}$} & [230-26-2] & \multicolumn{5}{|c|}{ Dibenzo[c,e][1,2]dithiin } \\
\hline & FUS & & 19.3 & 386.2 & DSC & [1975CUC2] \\
\hline \multirow[t]{4}{*}{$\mathrm{C}_{12} \mathrm{H}_{8} \mathrm{~S}_{3}$} & [1081-34-1] & \multicolumn{5}{|c|}{$2,2^{\prime}: 5^{\prime}, 2^{\prime \prime}$-terthiophene } \\
\hline & FUS & & 20.7 & 365.3 & DSC & [2011COS/LIM] \\
\hline & SUB & & $112.5 \pm 0.3$ & 351 & $\mathrm{ME}$ & [2011COS/LIM] \\
\hline & SUB & & $114.7 \pm 0.4$ & 298 & ME & [2011COS/LIM] \\
\hline \multirow[t]{4}{*}{$\mathrm{C}_{12} \mathrm{H}_{8} \mathrm{~S}_{3}$} & {$[81294-16-8]$} & \multicolumn{5}{|c|}{$3,2^{\prime}: 5^{\prime}, 3^{\prime \prime}$-terthiophene } \\
\hline & FUS & & 29.9 & 461.0 & DSC & [2011COS/LIM] \\
\hline & SUB & & $119.0 \pm 0.7$ & 382 & $\mathrm{ME}$ & [2011COS/LIM] \\
\hline & SUB & & $122.6 \pm 0.8$ & 298 & $\mathrm{ME}$ & [2011COS/LIM] \\
\hline \multirow[t]{5}{*}{$\mathrm{C}_{12} \mathrm{H}_{9} \mathrm{Br}$} & {$[92-66-0]$} & \multicolumn{5}{|c|}{ 4-bromobiphenyl } \\
\hline & TRS & & 4.3 & 337.1 & & \\
\hline & FUS & & 16.3 & 362.1 & DSC & [2015SOL/VAR] \\
\hline & SUB & $(303-360)$ & $91.0 \pm 0.3$ & 298 & GS & [2015SOL/VAR] \\
\hline & $\mathrm{V}$ & $(371-583)$ & 62.2 & 386 & A & [1987STE/MAL, 1947STU] \\
\hline \multirow[t]{2}{*}{$\mathrm{C}_{12} \mathrm{H}_{9} \mathrm{Br}$} & {$[2051-98-1]$} & \multicolumn{5}{|c|}{ 5-bromoacenaphthene } \\
\hline & SUB & $(295-321)$ & $87.4 \pm 2.6$ & & $\mathrm{ME}$ & [2008GOL/SUU2] \\
\hline \multirow[t]{2}{*}{$\mathrm{C}_{12} \mathrm{H}_{9} \mathrm{BrO}$} & {$[7025-06-1]$} & \multicolumn{5}{|c|}{ 5-bromoacenaphthene } \\
\hline & V & $(363-473)$ & 63.7 & 418 & $\mathrm{GC}$ & [2001WON/LEI] \\
\hline \multirow[t]{2}{*}{$\mathrm{C}_{12} \mathrm{H}_{9} \mathrm{BrO}$} & {$[6876-00-2]$} & \multicolumn{5}{|c|}{ 3-bromodiphenyl ether } \\
\hline & V & $(363-473)$ & 65.4 & 418 & GC & [2001WON/LEI] \\
\hline \multirow[t]{2}{*}{$\mathrm{C}_{12} \mathrm{H}_{9} \mathrm{BrO}$} & {$[101-55-3]$} & \multicolumn{5}{|c|}{ 4-bromodiphenyl ether } \\
\hline & V & $(463-673)$ & 64.6 & 478 & A & [1987STE/MAL] \\
\hline $\mathrm{C}_{12} \mathrm{H}_{9} \mathrm{BrO}$ & {$[92-03-5]$} & 2-bromo-4-phe & Iphenol & & & \\
\hline & V & $(373-584)$ & 57.8 & 388 & A & [1987STE/MAL, 1947STU] \\
\hline $\mathrm{C}_{12} \mathrm{H}_{9} \mathrm{Br}_{2} \mathrm{NO}_{2} \mathrm{~S}$ & {$[901442-58-8]$} & $N$-(2,4-dibrom & enyl)benzene & Ifonami & & \\
\hline & FUS & & 31.9 & 411.8 & DSC & [2014PER/KAZ] \\
\hline $\mathrm{C}_{12} \mathrm{H}_{9} \mathrm{Cl}$ & {$[2051-60-7]$} & 2-chlorobiphen & & & & \\
\hline & FUS & $(12-327)$ & 14.54 & 304.9 & & [1991ACR, 1974GEI/DZH2] \\
\hline & FUS & & 15.3 & 305.3 & DSC & [1984MIL/GHO] \\
\hline & $\mathrm{V}$ & & $72.1 \pm 2.0$ & 298 & $\mathrm{CGC}$ & [2001PUR/CHI] \\
\hline & $\mathrm{V}$ & $(343-393)$ & 64.4 & 368 & $\mathrm{GC}$ & [1994FAL/BID] \\
\hline & V & $(409-540)$ & 57.8 & 424 & A & [1987STE/MAL, 1975GEI/DZH2] \\
\hline & $\mathrm{V}$ & $(306-350)$ & 74.5 & 328 & $\mathrm{ME}$ & [1983FER/PIA] \\
\hline & $\mathrm{V}$ & $(410-540)$ & 55.8 & 424 & QM & [1975GEI/DZH] \\
\hline & $\mathrm{V}$ & $(362-541)$ & 61.1 & 377 & A & [1987STE/MAL, 1947STU] \\
\hline
\end{tabular}


TABLE 11. Phase change enthalpies of $\mathrm{C}_{12}$ to $\mathrm{C}_{13}$ organic compounds-Continued

\begin{tabular}{|c|c|c|c|c|c|c|}
\hline \multirow[b]{2}{*}{ Molecular formula } & \multirow{2}{*}{$\begin{array}{l}\text { CAS Registry } \\
\text { Number } \\
\text { Enthalpy }\end{array}$} & \multicolumn{5}{|l|}{ Compound } \\
\hline & & $\begin{array}{l}\text { Temperature } \\
\text { range }\end{array}$ & $\begin{array}{l}\Delta_{\text {trans }} H_{\mathrm{m}} \\
(\mathrm{kJ} / \mathrm{mol})\end{array}$ & $\begin{array}{l}T_{\mathrm{m}} \\
(\mathrm{K})\end{array}$ & Method & References \\
\hline \multirow[t]{6}{*}{$\mathrm{C}_{12} \mathrm{H}_{9} \mathrm{Cl}$} & {$[2051-61-8]$} & \multicolumn{5}{|c|}{ 3-chlorobiphenyl } \\
\hline & $\mathrm{V}$ & & $74.3 \pm 1.1$ & 298 & CGC & [2001PUR/CHI] \\
\hline & $\mathrm{V}$ & $(343-393)$ & 66.6 & 368 & GC & [1994FAL/BID] \\
\hline & $\mathrm{V}$ & $(310-359)$ & 66.2 & 335 & ME & [1983FER/PIA] \\
\hline & $\mathrm{V}$ & $(341-402)$ & 69.2 & 372 & TE & [1983FER/PIA] \\
\hline & $\mathrm{V}$ & $(452-536)$ & 66.0 & 494 & QM & {$[1975 \mathrm{GEI} / \mathrm{DZH}]$} \\
\hline \multirow[t]{8}{*}{$\mathrm{C}_{12} \mathrm{H}_{9} \mathrm{Cl}$} & $\begin{array}{c}\text { [2051-62-9] } \\
\text { FUS }\end{array}$ & $\begin{array}{l}\text { 4-chlorobiphenyl } \\
\quad(12-370)\end{array}$ & 13.32 & 348.6 & $\mathrm{AC}$ & [1991ACR, 1975GEI/DZH2] \\
\hline & SUB & $(253-303)$ & $86.0 \pm 0.9$ & 278 & GS & [1994WAN/SHU] \\
\hline & SUB & $(306-346)$ & $73.7 \pm 0.7$ & 326 & TE,ME & [1983FER/PIA] \\
\hline & $\mathrm{V}$ & & $71.6 \pm 0.7$ & 298 & CGC & [2001PUR/CHI] \\
\hline & $\mathrm{V}$ & $(343-393)$ & 66.8 & 368 & GC & {$[1994 \mathrm{FAL} / \mathrm{BID}]$} \\
\hline & $\mathrm{V}$ & $(451-536)$ & 65.9 & 466 & A & [1987STE/MAL, 1975GEI/DZH2] \\
\hline & $\mathrm{V}$ & $(348-409)$ & 67.8 & 378 & $\mathrm{TE}$ & [1983FER/PIA] \\
\hline & $\mathrm{V}$ & $(369-566)$ & 59.0 & 384 & A & [1987STE/MAL, 1947STU] \\
\hline \multirow[t]{2}{*}{$\mathrm{C}_{12} \mathrm{H}_{9} \mathrm{ClN}_{2}$} & {$[4340-77-6]$} & \multicolumn{5}{|c|}{ 4-chloroazobenzene } \\
\hline & FUS & & 27.2 & 361.2 & & [1988BAU/PER] \\
\hline \multirow[t]{2}{*}{$\mathrm{C}_{12} \mathrm{H}_{9} \mathrm{ClO}$} & {$[666747-18-8]$} & \multicolumn{5}{|c|}{ 2-chloro-3-phenylphenol } \\
\hline & $\mathrm{V}$ & $(391-591)$ & 65.0 & 406 & A & [1987STE/MAL, 1947STU] \\
\hline \multirow[t]{2}{*}{$\mathrm{C}_{12} \mathrm{H}_{9} \mathrm{ClO}$} & {$[85-97-2]$} & \multicolumn{5}{|c|}{ 2-chloro-6-phenylphenol } \\
\hline & $\mathrm{V}$ & $(393-590)$ & 67.6 & 408 & A & [1987STE/MAL, 1947STU] \\
\hline \multirow[t]{2}{*}{$\mathrm{C}_{12} \mathrm{H}_{9} \mathrm{ClO}_{2} \mathrm{~S}$} & {$[80-38-6]$} & \multicolumn{5}{|c|}{ 4-chlorophenylbenzenesulfonate } \\
\hline & FUS & & 21.44 & 332.2 & DSC & {$[1990 \mathrm{DON} / \mathrm{DRE}]$} \\
\hline \multirow[t]{2}{*}{$\mathrm{C}_{12} \mathrm{H}_{9} \mathrm{Cl}_{2} \mathrm{NO}_{2} \mathrm{~S}$} & {$[92589-22-5]$} & \multicolumn{5}{|c|}{$N$-(2,3-dichlorophenyl)benzene sulfonamide } \\
\hline & FUS & & 27.2 & 387.2 & DSC & [2007PER/STR, 2014PER/KAZ] \\
\hline \multirow[t]{2}{*}{$\mathrm{C}_{12} \mathrm{H}_{9} \mathrm{Cl}_{3} \mathrm{~N}_{2} \mathrm{O}_{2} \mathrm{~S}$} & {$[1040012-16-5]$} & \multicolumn{5}{|c|}{ 4-amino- $N$-(2,4,5-trichlorophenyl)benzene sulfonamide } \\
\hline & FUS & & 39.7 & 461.2 & DSC & [2014PER/KAZ] \\
\hline $\mathrm{C}_{12} \mathrm{H}_{9} \mathrm{~F}_{3} \mathrm{~N}_{2} \mathrm{O}_{2}$ & {$[75706-12-6]$} & 5-methyl- $N$-[4-(tr & rifluoromethyl & henyl]- & xazolcarb & amide (leflunomide) \\
\hline & FUS & & 31.3 & 440.1 & DSC & [2016SHA/GAN] \\
\hline & FUS & & 30.9 & 444.4 & DSC & [2012BAN/MAH] \\
\hline & FUS & & 32.43 & 438.2 & DSC & [2006VEG/PET] \\
\hline $\mathrm{C}_{12} \mathrm{H}_{9} \mathrm{~N}$ & {$[86-74-8]$} & Carbazole & & & & \\
\hline & FUS & & 27.70 & 517.1 & DSC & [2016STA/KEI] \\
\hline & FUS & & 26.9 & 518.7 & DSC & [2000LIS/JAM] \\
\hline & FUS & & 27.08 & 518.3 & DTA & [1992SAB/ELW3] \\
\hline & FUS & & 21.17 & 519.5 & DSC & [1980KRA/PIG] \\
\hline & FUS & & 27.2 & 521.1 & DSC & [1996DOM/HEA, 1980RAD/RAD] \\
\hline & TRS & & 0.27 & 420 & & \\
\hline & FUS & & 26.9 & 519.3 & $\mathrm{DSC}$ & {$[1969 \mathrm{ROB} / \mathrm{SCO}]$} \\
\hline & SUB & $(348-383)$ & $104.3 \pm 0.5$ & 298 & GS & [2011VER/EME] \\
\hline & SUB & $(346-364)$ & $101.2 \pm 1.1$ & 355 & ME & [1990JIM/ROU] \\
\hline & SUB & & $103.3 \pm 1.1$ & 298 & ME & [1990JIM/ROU] \\
\hline & SUB & & $97.7 \pm 0.3$ & 298 & $\mathrm{C}$ & {$[1987 \mathrm{SAB} / \mathrm{ANT}]$} \\
\hline & SUB & & 108.8 & & & [1961ZIM/GEI, 1990JIM/ROU] \\
\hline & SUB & & $84.5 \pm 0.8$ & & & [1970COX/PIL, 1955AIH3] \\
\hline & $\mathrm{V}$ & & 76.2 & & GC & [1996GOV/RUT] \\
\hline & $\mathrm{V}$ & $(523-642)$ & 63.3 & 525 & Static & [1983SIV/MAR] \\
\hline & $\mathrm{V}$ & $(523-642)$ & 61.8 & 565 & Static & [1983SIV/MAR] \\
\hline & $\mathrm{V}$ & $(523-642)$ & 60.8 & 605 & Static & [1983SIV/MAR] \\
\hline & $\mathrm{V}$ & $(523-642)$ & $87.3 \pm 0.1$ & 298 & Static & [1983SIV/MAR, 2011VER/EME] \\
\hline & $\mathrm{V}$ & $(525-631)$ & 65.7 & 540 & A & [1987STE/MAL, 1923SEN/NEL, 1984BOU/FRI] \\
\hline & $\mathrm{V}$ & $(517-624)$ & 66.0 & 532 & & [1923MOR/MUR, 1984BOU/FRI] \\
\hline $\mathrm{C}_{12} \mathrm{H}_{9} \mathrm{NO}$ & {$[91-02-1]$} & 2-benzoylpyridine & & & & \\
\hline
\end{tabular}


TABLE 11. Phase change enthalpies of $\mathrm{C}_{12}$ to $\mathrm{C}_{13}$ organic compounds-Continued

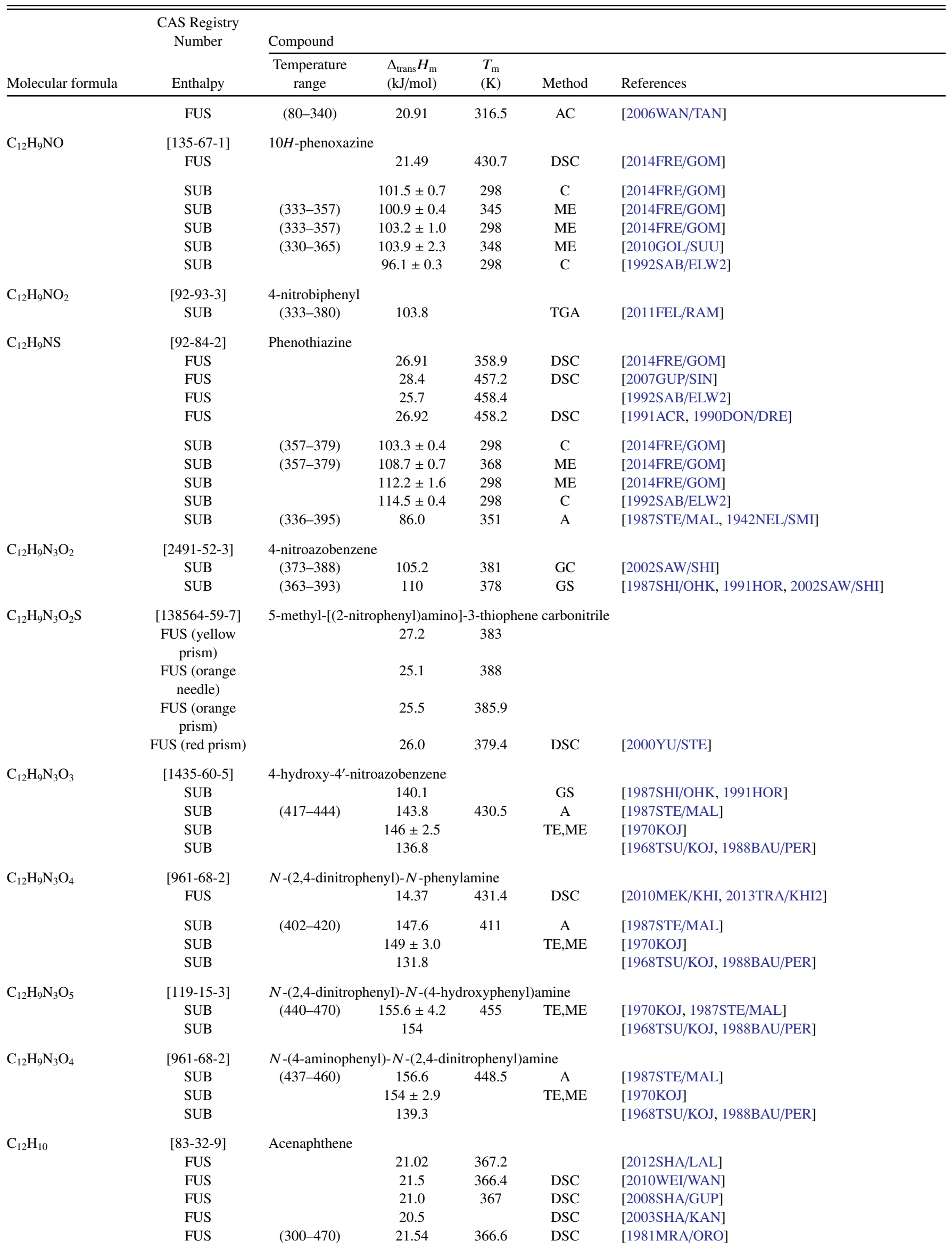


TABLE 11. Phase change enthalpies of $\mathrm{C}_{12}$ to $\mathrm{C}_{13}$ organic compounds-Continued

\begin{tabular}{|c|c|c|c|c|c|c|}
\hline \multirow[b]{2}{*}{ Molecular formula } & \multirow{2}{*}{$\begin{array}{l}\text { CAS Registry } \\
\text { Number } \\
\text { Enthalpy }\end{array}$} & \multicolumn{5}{|l|}{ Compound } \\
\hline & & $\begin{array}{c}\text { Temperature } \\
\text { range }\end{array}$ & $\begin{array}{l}\Delta_{\text {trans }} H_{\mathrm{m}} \\
(\mathrm{kJ} / \mathrm{mol})\end{array}$ & $\begin{array}{c}T_{\mathrm{m}} \\
(\mathrm{K})\end{array}$ & Method & References \\
\hline & FUS & $(11-436)$ & 21.46 & 366.6 & & [1996DOM/HEA, 1977FIN/MES] \\
\hline & FUS & & 21.8 & 363.1 & DSC & {$[1973 \mathrm{CAS} / \mathrm{VEC}]$} \\
\hline & FUS & & 21.9 & & DSC & [1972WAU/GET] \\
\hline & FUS & & 20.4 & 366.5 & & [1969SAD/STE] \\
\hline & FUS & & 20.7 & 366.5 & & {$[1935 \mathrm{SKA}]$} \\
\hline & SUB & $(299-320)$ & $78.7 \pm 2.2$ & 310 & ME & [2008GOL/SUU3] \\
\hline & SUB & & 84.6 & 298 & CGC-DSC & [1998CHI/HES] \\
\hline & SUB & $(313-453)$ & 83.2 & 383 & GS & [1995NAS/LEN] \\
\hline & SUB & $(293-342)$ & 77.0 & 318 & GS & [1986SAT/INO] \\
\hline & SUB & $(283-323)$ & $86.8 \pm 0.9$ & 303 & GS & [1983SON/ZOL] \\
\hline & SUB & & $83.4 \pm 1.0$ & 298 & & [1975OSB/DOU, 1977FIN/MES] \\
\hline & SUB & & 82.4 & 366 & $\mathrm{~B}$ & {$[1975 \mathrm{OSB} / \mathrm{DOU}]$} \\
\hline & SUB & & $84.7 \pm 2.7$ & & ME & [1974RAD/KAT] \\
\hline & SUB & $(290-340)$ & $86.2 \pm 0.8$ & & $\mathrm{ME}$ & [1965BOY/CHR, 1970COX/PIL] \\
\hline & SUB & $(291-310)$ & $82.1 \pm 0.4$ & 300 & $\mathrm{~V}$ & [1959AIH, 1987STE/MAL] \\
\hline & SUB & $(258-308)$ & 81.6 & & & {$[1958 \mathrm{HOY} / \mathrm{PEP}]$} \\
\hline & $\mathrm{V}$ & & 68.0 & 298 & CGC & [2008ZHA/UNH] \\
\hline & $\mathrm{V}$ & $(363-423)$ & $70.5 \pm 1.1$ & 298 & GC & [2006HAF/PAR] \\
\hline & $\mathrm{V}$ & & 61.1 & 366 & & [1998RUU/MOK, 2008HAN/NUT] \\
\hline & $\mathrm{V}$ & & 66.2 & 298 & & [1998RUU/MOK, 2008HAN/NUT] \\
\hline & V & $(323-473)$ & 63.9 & 398 & GC & [2002LEI/CHA] \\
\hline & $\mathrm{V}$ & & 66.2 & 298 & CGC & [1998CHI/HES] \\
\hline & $\mathrm{V}$ & & 60.6 & 378 & & [1995MOK/GUE, 2008HAN/NUT] \\
\hline & $\mathrm{V}$ & & $66.5 \pm 1.3$ & 298 & & [1995MOK/GUE, 2008HAN/NUT] \\
\hline & $\mathrm{V}$ & $(368-552)$ & 54 & 403 & A & {$[1987 \mathrm{STE} / \mathrm{MAL}]$} \\
\hline & $\mathrm{V}$ & $(368-413)$ & 60.3 & 383 & A & [1987STE/MAL, 1975OSB/DOU, 1984BOU/FRI] \\
\hline & $\mathrm{V}$ & & 61.3 & 395 & I & {$[1943 \mathrm{CRA}]$} \\
\hline & $\mathrm{V}$ & $(413-561)$ & 54.3 & 466 & $\mathrm{I}$ & [1923MOR/MUR] \\
\hline & $\mathrm{V}$ & $(420-561)$ & 55.4 & 435 & & [1923MOR/MUR, 1984BOU/FRI] \\
\hline \multirow[t]{30}{*}{$\mathrm{C}_{12} \mathrm{H}_{10}$} & {$[92-52-4]$} & Biphenyl & & & & \\
\hline & FUS & & 19.03 & 342.7 & DSC & [2016BOU/HAF] \\
\hline & FUS & & 18.78 & 341.4 & $\mathrm{DSC}$ & [2016BOU/DJE] \\
\hline & FUS & & 18.34 & 341.5 & DSC & [2014BAE/DAH] \\
\hline & FUS & & 18.54 & 342.1 & DSC & [2014CAB/GRA] \\
\hline & FUS & & 18.89 & 342.1 & $\mathrm{AC}$ & [2014LEY/LOS] \\
\hline & FUS & & 18.6 & 343.4 & & [2012SHA/LAL] \\
\hline & FUS & & 18.51 & 342.1 & DSC & [2012CHA/LAY] \\
\hline & FUS & & 19.7 & 342.3 & DSC & [2006KHI/DAH] \\
\hline & FUS & & 19.27 & 344.34 & DSC & [2004BEN/KHI] \\
\hline & FUS & $(5-440)$ & 18.58 & 342.1 & $\mathrm{AC}$ & [1989CHI/KNI] \\
\hline & FUS & $(220-480)$ & 18.58 & 342.2 & DSC & [1983ORO/MRA] \\
\hline & FUS & & 19.9 & 343.3 & DSC & [1982WAS/RAD] \\
\hline & FUS & & 18.80 & 344.1 & DSC & [1979SMI2] \\
\hline & FUS & & 18.90 & & DSC & [1972WAU/GET] \\
\hline & FUS & & 18.58 & 343.0 & & [1950UEB/ORT] \\
\hline & FUS & & 18.59 & 342.0 & & [1941SCH] \\
\hline & FUS & & 18.66 & 341.5 & Rad. Calor. & [1996DOM/HEA, 1932SPA/THO] \\
\hline & FUS & & 18.95 & 314.3 & & {$[1889 \mathrm{EYK}]$} \\
\hline & SUB & & 82.9 & 298 & CGC-DSC & [1998CHI/HES] \\
\hline & SUB & $(313-453)$ & 81.8 & 383 & GS & [1995NAS/LEN] \\
\hline & SUB & $(283-338)$ & 83.4 & 311 & EM & [1989SAS/NGU] \\
\hline & SUB & $(303-333)$ & U 113.3 & 318 & & [1989ROR/RUT] \\
\hline & SUB & & $81.5 \pm 0.2$ & 298 & & [1989CHI/KNI] \\
\hline & SUB & & $77.9 \pm 0.3$ & 298 & $\mathrm{C}$ & {$[1979 \mathrm{SAB} 2]$} \\
\hline & SUB & & $81.8 \pm 0.2$ & 298 & $\mathrm{C}$ & [1978MON/ROS] \\
\hline & SUB & $(306-332)$ & $80.4 \pm 1.6$ & 319 & TSGC & [1975CLA/KNO] \\
\hline & SUB & $(273-313)$ & $76.0 \pm 4.0$ & & HSA & {$[1975 \mathrm{CHI}]$} \\
\hline & SUB & & $83.6 \pm 2.5$ & & & [1974RAD/KAT] \\
\hline & SUB & $(298-318)$ & 75.2 & & $\mathrm{ME}$ & [1974PRI/POU] \\
\hline
\end{tabular}


TABLE 11. Phase change enthalpies of $\mathrm{C}_{12}$ to $\mathrm{C}_{13}$ organic compounds-Continued

\begin{tabular}{|c|c|c|c|c|c|c|}
\hline \multirow[b]{2}{*}{ Molecular formula } & \multirow{2}{*}{$\begin{array}{l}\text { CAS Registry } \\
\text { Number } \\
\text { Enthalpy }\end{array}$} & \multicolumn{5}{|l|}{ Compound } \\
\hline & & $\begin{array}{c}\text { Temperature } \\
\text { range }\end{array}$ & $\begin{array}{l}\Delta_{\text {trans }} H_{\mathrm{m}} \\
(\mathrm{kJ} / \mathrm{mol})\end{array}$ & $\begin{array}{l}T_{\mathrm{m}} \\
(\mathrm{K})\end{array}$ & Method & References \\
\hline & SUB & & $81.8 \pm 0.4$ & 298 & $\mathrm{C}$ & [1972MOR] \\
\hline & SUB & $(279-299)$ & $75.8 \pm 0.6$ & 289 & & [1955AIH3] \\
\hline & SUB & & $81.6 \pm 2.0$ & & & [1953BRA/CLE2, 1970COX/PIL, 1960JON] \\
\hline & SUB & $(287-307)$ & $75.1 \pm 1.7$ & 297 & & {$[1953 \mathrm{SEK} / \mathrm{SUZ}]$} \\
\hline & SUB & $(288-314)$ & $81.6 \pm 1.7$ & 301 & & [1953BRA/CLE] \\
\hline & SUB & $(278-307)$ & $72.8 \pm 3$ & 302 & $\mathrm{ME}$ & [1951BRI] \\
\hline & SUB & & $68.6 \pm 0.8$ & 292 & QF & [1938WOL/WEG] \\
\hline & $\mathrm{V}$ & & 65.0 & 298 & CGC & [2008ZHA/UNH] \\
\hline & $\mathrm{V}$ & $(323-473)$ & 62.5 & 298 & GC & [2002LEI/CHA] \\
\hline & $\mathrm{V}$ & & $64.5 \pm 2.2$ & 298 & GS & [2001PUR/CHI] \\
\hline & $\mathrm{V}$ & & 66.2 & 298 & $\mathrm{CGC}$ & [1998CHI/HES] \\
\hline & $\mathrm{V}$ & $(495-688)$ & 51.2 & 510 & DSC & [1996BAC/GRZ] \\
\hline & $\mathrm{V}$ & $(403-453)$ & 66.0 & 298 & CGC & [1995CHI/HOS] \\
\hline & $\mathrm{V}$ & $(348-453)$ & 59.6 & 363 & GS & [1989SAK/IWA] \\
\hline & $\mathrm{V}$ & $(350-578)$ & 64.9 & 298 & $\mathrm{~EB}$ & [1989CHI/KNI] \\
\hline & $\mathrm{V}$ & $(350-578)$ & 57.4 & 400 & $\mathrm{~EB}$ & {$[1989 \mathrm{CHI} / \mathrm{KNI}]$} \\
\hline & $\mathrm{V}$ & $(350-578)$ & 60.3 & 360 & $\mathrm{~EB}$ & [1989CHI/KNI] \\
\hline & $\mathrm{V}$ & $(350-578)$ & 50.4 & 500 & $\mathrm{~EB}$ & [1989CHI/KNI] \\
\hline & $\mathrm{V}$ & $(333-393)$ & 60.4 & 363 & & [1989SAS/NGU] \\
\hline & $\mathrm{V}$ & $(390-563)$ & 57.3 & 405 & A & [1987STE/MAL] \\
\hline & $\mathrm{V}$ & $(396-437)$ & 54.9 & 417 & GS & [1980NAS/HWA] \\
\hline & $\mathrm{V}$ & $(528-766)$ & 48.0 & 647 & & [1957GLA/RUL] \\
\hline & $\mathrm{V}$ & $(342-544)$ & 59.4 & 357 & & [1930CUN, 1984BOU/FRI] \\
\hline \multirow[t]{3}{*}{$\mathrm{C}_{12} \mathrm{H}_{10} \mathrm{BrN}_{3} \mathrm{O}_{4} \mathrm{~S}$} & {$[34392-67-1]$} & \multicolumn{5}{|c|}{ 4-amino- $N$-(2-bromo-4-nitrophenyl)benzenesulfonamide } \\
\hline & FUS & & 39.6 & 461 & DSC & [2013PER/RYZ, 2014PER/KAZ] \\
\hline & SUB & & $142.8 \pm 1.9$ & 298 & GS & [2013PER/RYZ] \\
\hline \multirow[t]{2}{*}{$\mathrm{C}_{12} \mathrm{H}_{10} \mathrm{ClNO}_{2} \mathrm{~S}$} & {$[21226-30-2]$} & \multicolumn{5}{|c|}{$N$-(2-chlorophenyl)benzene sulfonamide } \\
\hline & FUS & & 33.5 & 398.2 & DSC & [2007PER/STR, 2014PER/KAZ] \\
\hline \multirow[t]{2}{*}{$\mathrm{C}_{12} \mathrm{H}_{10} \mathrm{ClNO}_{2} \mathrm{~S}$} & {$[4750-28-1]$} & \multicolumn{5}{|c|}{$N$-(4-chlorophenyl)benzene sulfonamide } \\
\hline & FUS & & 25.8 & 394.6 & DSC & [2007PER/STR, 2014PER/KAZ] \\
\hline \multirow[t]{2}{*}{$\mathrm{C}_{12} \mathrm{H}_{10} \mathrm{ClN}_{3} \mathrm{~S}$} & {$[27429-35-2]$} & \multicolumn{5}{|c|}{$N$-2-pyridyl- $N^{\prime}$-(2-chlorophenyl) thiourea } \\
\hline & FUS & & 28.3 & 429.7 & DSC & {$[2002 \mathrm{KEL} / \mathrm{SZC}]$} \\
\hline \multirow[t]{2}{*}{$\mathrm{C}_{12} \mathrm{H}_{10} \mathrm{ClN}_{3} \mathrm{~S}$} & {$[53385-84-5]$} & \multicolumn{5}{|c|}{$N$-2-pyridyl- $N^{\prime}$-(4-chlorophenyl) thiourea } \\
\hline & FUS & & 34.3 & 462.2 & DSC & {$[2002 \mathrm{SZC} / \mathrm{KEL}]$} \\
\hline \multirow[t]{5}{*}{$\mathrm{C}_{12} \mathrm{H}_{10} \mathrm{Cl}_{2} \mathrm{~N}_{2} \mathrm{O}_{2} \mathrm{~S}$} & {$[943757-10-6]$} & \multicolumn{5}{|c|}{ 4-amino- $N$-(2,3-chlorophenyl)benzene sulfonamide } \\
\hline & FUS & & 40.9 & 454.3 & DSC & [2008PER/STR, 2014PER/KAZ] \\
\hline & SUB & $(345-391)$ & $137.5 \pm 0.7$ & 368 & GS & [2008PER/STR] \\
\hline & SUB & $(345-391)$ & $141.1 \pm 0.7$ & 298 & GS & [2008PER/STR] \\
\hline & $\mathrm{V}$ & 114.3 & & 298 & S-F & [2008PER/STR] \\
\hline \multirow[t]{5}{*}{$\mathrm{C}_{12} \mathrm{H}_{10} \mathrm{Cl}_{2} \mathrm{~N}_{2} \mathrm{O}_{2} \mathrm{~S}$} & {$[439118-58-8]$} & \multicolumn{5}{|c|}{ 4-amino- $N$-(2,5-chlorophenyl)benzene sulfonamide } \\
\hline & FUS & & 41.3 & 445.9 & DSC & [2008PER/STR, 2014PER/KAZ] \\
\hline & SUB & $(379-417)$ & $151.3 \pm 1.6$ & 398 & GS & [2008PER/STR] \\
\hline & SUB & $(379-417)$ & $155.4 \pm 1.6$ & 298 & GS & [2008PER/STR] \\
\hline & $\mathrm{V}$ & & 127.8 & 298 & $\mathrm{~S}-\mathrm{F}$ & [2008PER/STR] \\
\hline \multirow[t]{5}{*}{$\mathrm{C}_{12} \mathrm{H}_{10} \mathrm{Cl}_{2} \mathrm{~N}_{2} \mathrm{O}_{2} \mathrm{~S}$} & {$[34392-63-7]$} & \multicolumn{5}{|c|}{ 4-amino- $N$-(3,4-chlorophenyl)benzene sulfonamide } \\
\hline & FUS & & 51.5 & 497.9 & DSC & [2008PER/STR, 2014PER/KAZ] \\
\hline & SUB & $(418-448)$ & $161.4 \pm 3.6$ & 433 & GS & [2008PER/STR] \\
\hline & SUB & $(418-448)$ & $167.5 \pm 3.6$ & 298 & GS & [2008PER/STR] \\
\hline & V & & 136.7 & 298 & $\mathrm{~S}-\mathrm{F}$ & [2008PER/STR] \\
\hline $\mathrm{C}_{12} \mathrm{H}_{10} \mathrm{Cl}_{2} \mathrm{~N}_{2} \mathrm{O}_{2} \mathrm{~S}$ & [1036593-23-3] & 4-amino-2-chl & o- $N$-(2-chloro & enyl)ben & he sulfonar & \\
\hline & FUS & & 51.4 & 505.0 & DSC & [2014PER/KAZ] \\
\hline
\end{tabular}


TABLE 11. Phase change enthalpies of $\mathrm{C}_{12}$ to $\mathrm{C}_{13}$ organic compounds-Continued

\begin{tabular}{|c|c|c|c|c|c|c|}
\hline \multirow[b]{2}{*}{ Molecular formula } & \multirow{2}{*}{$\begin{array}{l}\text { CAS Registry } \\
\text { Number } \\
\text { Enthalpy }\end{array}$} & \multicolumn{5}{|l|}{ Compound } \\
\hline & & $\begin{array}{l}\text { Temperature } \\
\text { range }\end{array}$ & $\begin{array}{l}\Delta_{\text {trans }} H_{\mathrm{m}} \\
(\mathrm{kJ} / \mathrm{mol})\end{array}$ & $\begin{array}{l}T_{\mathrm{m}} \\
(\mathrm{K})\end{array}$ & Method & References \\
\hline & FUS & & 34.9 & 468.7 & DSC & [2014PER/KAZ] \\
\hline $\mathrm{C}_{12} \mathrm{H}_{10} \mathrm{~F}_{3} \mathrm{NO}_{2}$ & $\begin{array}{c}{[52840-38-7]} \\
\text { FUS }\end{array}$ & 4-(trifluoromet & $\begin{array}{l}\text { 1)-7-( } N \text {-ethyl } \\
30.47\end{array}$ & $\begin{array}{l}\text { ino)cou } \\
432.5\end{array}$ & in & [1991ZHA/HUA] \\
\hline $\mathrm{C}_{12} \mathrm{H}_{10} \mathrm{~F}_{3} \mathrm{NO}_{2}$ & $\begin{array}{l}{[53518-14-2]} \\
\text { FUS }\end{array}$ & 4-(trifluoromet & $\begin{array}{c}\text { 1)-7- }(N, N-\operatorname{din} \\
26.25\end{array}$ & $\begin{array}{l}\text { nylamin } \\
420.5\end{array}$ & $\begin{array}{r}\text { oumarin } \\
\text { DSC }\end{array}$ & [1991ZHA/HUA] \\
\hline $\mathrm{C}_{12} \mathrm{H}_{10} \mathrm{~F}_{3} \mathrm{~N}_{3} \mathrm{O}_{4}$ & $\begin{array}{l}{[63612-50-0]} \\
\text { FUS }\end{array}$ & 5,5-dimethyl-3 & $\begin{array}{l}\text {-nitro-3-(trifl } \\
31.03\end{array}$ & $\begin{array}{c}\text { omethy } \\
428\end{array}$ & $\begin{array}{l}\text { enyl]imid } \\
\text { DSC }\end{array}$ & $\begin{array}{l}\text { lidine-2,4-dione (nilutamide) } \\
\text { [2010BAI/VAN] }\end{array}$ \\
\hline $\mathrm{C}_{12} \mathrm{H}_{10} \mathrm{~N}_{2}$ & $\begin{array}{c}\text { [1080-16-6] } \\
\text { SUB } \\
\text { SUB } \\
\text { SUB }\end{array}$ & $\begin{array}{c}\text { cis-azobenzen } \\
(273-323) \\
(298-357) \\
(303-333)\end{array}$ & $\begin{array}{c}92.9 \\
92.9 \pm 0.12 \\
\text { U } 74.9\end{array}$ & $\begin{array}{l}288 \\
328 \\
318\end{array}$ & $\begin{array}{c}\text { A } \\
\mathrm{ME} \\
\mathrm{ME}\end{array}$ & $\begin{array}{l}\text { [1987STE/MAL] } \\
{[1977 \mathrm{SCH} / \mathrm{PET}]} \\
{[1950 \mathrm{BRI} / \mathrm{CAR}, 1960 \mathrm{JON}]}\end{array}$ \\
\hline $\mathrm{C}_{12} \mathrm{H}_{10} \mathrm{~N}_{2}$ & $\begin{array}{c}\text { [17082-12-1] } \\
\text { FUS } \\
\text { FUS } \\
\text { FUS } \\
\text { FUS } \\
\text { FUS } \\
\text { FUS } \\
\text { FUS } \\
\text { FUS } \\
\text { FUS } \\
\text { FUS }\end{array}$ & $\begin{array}{c}(299-408) \\
(83-356)\end{array}$ & $\begin{array}{c}23.0 \\
22.53 \\
22.53 \\
22.65 \\
22.1 \\
22.4 \\
24.7 \\
20.3 \\
21.3 \\
22.1\end{array}$ & $\begin{array}{l}341.0 \\
341.1 \\
341.1 \\
341.9 \\
340.5 \\
341.7\end{array}$ & $\begin{array}{c}\text { AC } \\
\text { AC } \\
\text { DTA } \\
\text { CR } \\
\text { DSC }\end{array}$ & $\begin{array}{l}\text { [1996STE/CHI2] } \\
\text { [1985BOU/DEL] } \\
\text { [1996DOM/HEA, 1984VAN/BOU] } \\
\text { [1977SCH/PET] } \\
\text { [1977SCH/PET] } \\
\text { [1974CIN/BER, 1985BOU/DEL] } \\
\text { [1919PAD, 1985BOU/DEL] } \\
\text { [1908BOG/WIN, 1985BOU/DEL] } \\
\text { [1894BRU, 1985BOU/DEL] } \\
\text { [1889EYK, 1985BOU/DEL] }\end{array}$ \\
\hline & $\begin{array}{l}\text { SUB } \\
\text { SUB } \\
\text { SUB } \\
\text { SUB } \\
\text { SUB } \\
\text { SUB } \\
\text { SUB }\end{array}$ & $\begin{array}{l}(298-302) \\
(298-341) \\
(299-317) \\
(299-317) \\
(298-347) \\
(303-333)\end{array}$ & $\begin{array}{l}94.1 \pm 0.8 \\
93.6 \pm 1.9 \\
92.1 \pm 0.9 \\
96.9 \pm 0.8 \\
94.9 \pm 0.8 \\
93.8 \pm 1.2 \\
\mathrm{U} 74.9\end{array}$ & $\begin{array}{l}298 \\
298 \\
319 \\
308 \\
308 \\
323 \\
318\end{array}$ & $\begin{array}{l}\text { B } \\
\text { ME } \\
\text { TE,ME } \\
\text { TE } \\
\text { ME } \\
\text { ME }\end{array}$ & $\begin{array}{l}\text { [1996STE/CHI2] } \\
\text { [1992DIA/MIN] } \\
{[1984 \mathrm{BOU} / \mathrm{OON}]} \\
\text { [1977DEK/VAN] } \\
\text { [1977DEK/VAN] } \\
\text { [1977SCH/PET] } \\
{[1950 \mathrm{BRI} / \mathrm{CAR}, 1960 J O N, 1987 \mathrm{STE} / \mathrm{MAL}]}\end{array}$ \\
\hline & $\begin{array}{l}\text { V } \\
\text { V } \\
\text { V } \\
\text { V } \\
\text { V } \\
\text { V } \\
\text { V }\end{array}$ & $\begin{array}{l}(436-626) \\
(436-626) \\
(436-626) \\
(436-626) \\
(436-626) \\
(436-626) \\
(376-566)\end{array}$ & $\begin{array}{l}72.8 \pm 0.7 \\
62.4 \pm 0.4 \\
59.9 \pm 0.4 \\
57.1 \pm 0.4 \\
54.2 \pm 0.4 \\
51.2 \pm 0.4 \\
62.3\end{array}$ & $\begin{array}{l}298 \\
440 \\
480 \\
520 \\
560 \\
600 \\
391\end{array}$ & $\begin{array}{l}\text { EB } \\
\text { EB } \\
\text { EB } \\
\text { EB } \\
\text { EB } \\
\text { EB } \\
\text { A }\end{array}$ & $\begin{array}{l}\text { [1996STE/CHI2] } \\
{[1996 \mathrm{STE} / \mathrm{CHI} 2]} \\
{[1996 \mathrm{STE} / \mathrm{CHI} 2]} \\
{[1996 \mathrm{STE} / \mathrm{CHI} 2]} \\
{[1996 \mathrm{STE} / \mathrm{CHI} 2]} \\
{[1996 \mathrm{STE} / \mathrm{CHI} 2]} \\
{[1987 \mathrm{STE} / \mathrm{MAL}, 1947 \mathrm{STU}]}\end{array}$ \\
\hline $\mathrm{C}_{12} \mathrm{H}_{10} \mathrm{~N}_{2}$ & $\begin{array}{l}\text { [486-84-0] } \\
\text { FUS }\end{array}$ & 1-methyl-9H- & $\begin{array}{c}\text { ido }[3,4-b] \text { inc } \\
27.2\end{array}$ & $\begin{array}{l}\text { (harma } \\
509.9\end{array}$ & DSC & [1996BUR/DAG] \\
\hline $\mathrm{C}_{12} \mathrm{H}_{10} \mathrm{~N}_{2} \mathrm{O}$ & $\begin{array}{c}{[20972-43-4]} \\
\text { FUS }\end{array}$ & trans-diphenyl & $\begin{array}{c}\text { zene } N \text {-oxid } \\
17.93\end{array}$ & 309.2 & & [1991ACR, 1983WEA] \\
\hline & SUB & & $98.6 \pm 0.9$ & 298 & $\mathrm{C}$ & [1986KIR/ACR] \\
\hline $\mathrm{C}_{12} \mathrm{H}_{10} \mathrm{~N}_{2} \mathrm{O}$ & $\begin{array}{c}{[1689-82-3]} \\
\text { FUS }\end{array}$ & 4-hydroxyazot & $\begin{array}{l}\text { zene } \\
\quad 32.99\end{array}$ & 425.2 & & [1988BAU/PER] \\
\hline $\mathrm{C}_{12} \mathrm{H}_{10} \mathrm{~N}_{2} \mathrm{O}$ & $\begin{array}{c}{[86-30-6]} \\
\text { FUS }\end{array}$ & $N$-nitroso- $N-1$ & $\begin{array}{l}\text { nyl-benzenar } \\
11.06\end{array}$ & e 340.0 & DSC & [2010MEK/KHI] \\
\hline $\mathrm{C}_{12} \mathrm{H}_{10} \mathrm{~N}_{2} \mathrm{O}_{2}$ & $\begin{array}{l}{[119-75-5]} \\
\text { FUS }\end{array}$ & $N$-(2-nitrophe & $\begin{array}{c}N \text {-phenyla } \\
26.14\end{array}$ & 347.9 & DSC & [2010MEK/KHI, 2013TRA/KHI] \\
\hline & $\begin{array}{l}\text { SUB } \\
\text { SUB } \\
\text { SUB }\end{array}$ & $(335-346)$ & $\begin{array}{c}100.9 \\
101.9 \pm 1.7 \\
108.4\end{array}$ & 340.5 & $\begin{array}{c}\text { A } \\
\text { TE,ME }\end{array}$ & $\begin{array}{l}\text { [1987STE/MAL] } \\
\text { [1970KOJ] } \\
\text { [1968TSU/KOJ, 1988BAU/PER] }\end{array}$ \\
\hline $\mathrm{C}_{12} \mathrm{H}_{10} \mathrm{~N}_{2} \mathrm{O}_{2}$ & $\begin{array}{c}\text { [836-30-6] } \\
\text { SUB } \\
\text { SUB }\end{array}$ & $\begin{array}{l}N \text {-(4-nitrophe } \\
\quad(382-403)\end{array}$ & $\begin{array}{c}-N \text {-phenyla } \\
130.6 \\
126.2 \pm 1.6\end{array}$ & 392.5 & $\begin{array}{c}\text { A } \\
\text { TE,ME }\end{array}$ & $\begin{array}{l}\text { [1987STE/MAL] } \\
{[1970 \mathrm{KOJ}]}\end{array}$ \\
\hline
\end{tabular}


TABLE 11. Phase change enthalpies of $\mathrm{C}_{12}$ to $\mathrm{C}_{13}$ organic compounds-Continued

\begin{tabular}{|c|c|c|c|c|c|c|}
\hline \multirow[b]{2}{*}{ Molecular formula } & \multirow{2}{*}{$\begin{array}{l}\text { CAS Registry } \\
\text { Number } \\
\text { Enthalpy }\end{array}$} & \multicolumn{5}{|l|}{ Compound } \\
\hline & & $\begin{array}{c}\text { Temperature } \\
\text { range }\end{array}$ & $\begin{array}{l}\Delta_{\text {trans }} H_{\mathrm{m}} \\
(\mathrm{kJ} / \mathrm{mol})\end{array}$ & $\begin{array}{l}T_{\mathrm{m}} \\
(\mathrm{K})\end{array}$ & Method & References \\
\hline & SUB & & 120.9 & & & [1968TSU/KOJ, 1988BAU/PER] \\
\hline \multirow[t]{3}{*}{$\mathrm{C}_{12} \mathrm{H}_{10} \mathrm{~N}_{2} \mathrm{O}_{4} \mathrm{~S}$} & {$[6933-51-3]$} & \multicolumn{5}{|c|}{$N$-(2-nitrophenyl)benzenesulfonamide } \\
\hline & FUS & & 22.3 & 371.8 & DSC & [2013PER/RYZ, 2014PER/KAZ] \\
\hline & SUB & & $126.8 \pm 0.9$ & 298 & GS & [2013PER/RYZ] \\
\hline \multirow[t]{5}{*}{$\mathrm{C}_{12} \mathrm{H}_{10} \mathrm{~N}_{2} \mathrm{O}_{4} \mathrm{~S}$} & {$[1829-81-8]$} & \multicolumn{5}{|c|}{$N$-(4-nitrophenyl)benzenesulfonamide } \\
\hline & FUS & & 28.7 & 411.3 & $\mathrm{DSC}$ & [2011PER/RYZ, 2014PER/KAZ] \\
\hline & SUB & $(380-401)$ & $127.9 \pm 1.6$ & 390 & GS & [2011PER/RYZ] \\
\hline & SUB & $(380-401)$ & $132.5 \pm 1.6$ & 298 & GS & [2011PER/RYZ] \\
\hline & $\mathrm{V}$ & & 111.7 & 298 & Sub-Fus & [2011PER/RYZ] \\
\hline \multirow[t]{2}{*}{$\mathrm{C}_{12} \mathrm{H}_{10} \mathrm{~N}_{4}$} & {$[69155-29-9]$} & \multicolumn{5}{|c|}{ 4,5-dimethy 1-1,1,2,2-tetracyanocyclohex-4-ene } \\
\hline & SUB & & $107.9 \pm 4.2$ & 378 & & [1972ROG2, 1977PED/RYL] \\
\hline \multirow[t]{10}{*}{$\mathrm{C}_{12} \mathrm{H}_{10} \mathrm{~N}_{4} \mathrm{O}_{2}$} & {$[730-40-5]$} & \multicolumn{5}{|c|}{ 4-amino-4'-nitroazobenzene } \\
\hline & FUS & & 31.88 & 488.2 & & [1988BAU/PER] \\
\hline & SUB & $(403-465)$ & 123 & 434 & GS & [1989NIS/AND] \\
\hline & SUB & & 140.1 & & GS & [1987SHI/OHK, 1991HOR] \\
\hline & SUB & & 127.6 & & UV & [1984KAR/ROD, 1984KAR/KRU] \\
\hline & SUB & & 136.4 & & $\mathrm{ME}$ & [1980NIG/DEP, 1991HOR] \\
\hline & SUB & & $140.4 \pm 1.2$ & & TE,ME & {$[1970 \mathrm{KOJ}]$} \\
\hline & SUB & & 134.3 & & ME & [1968TSU/KOJ, 1988BAU/PER] \\
\hline & SUB & $(404-424)$ & $137.7 \pm 0.8$ & 414 & $\mathrm{TE}$ & [1967GRE/JON, 1987STE/MAL] \\
\hline & SUB & $(404-423)$ & $136.4 \pm 5.0$ & 413 & ME & [1967GRE/JON, 1966JON/KRA] \\
\hline \multirow[t]{2}{*}{$\mathrm{C}_{12} \mathrm{H}_{10} \mathrm{~N}_{4} \mathrm{O}_{2}$} & {$[848892-92-2]$} & \multicolumn{5}{|c|}{ 2,6,7,8-tetrahydro-8-(2-methylphenyl)imidazo[2,1-c][1,2,4]triazme-3,4-dione } \\
\hline & FUS & & 25.64 & 506.2 & DSC & [2016BAR/SZT] \\
\hline \multirow[t]{2}{*}{$\mathrm{C}_{12} \mathrm{H}_{10} \mathrm{~N}_{4} \mathrm{O}_{2}$} & {$[848892-95-5]$} & \multicolumn{5}{|c|}{ 2,6,7,8-tetrahydro-8-(4-methylphenyl)imidazo[2,1-c][1,2,4]triazine-3,4-dione } \\
\hline & FUS & & 28.28 & 600.2 & DSC & [2016BAR/SZT] \\
\hline \multirow[t]{2}{*}{$\mathrm{C}_{12} \mathrm{H}_{10} \mathrm{~N}_{4} \mathrm{O}_{3}$} & {$[848892-99-9]$} & \multicolumn{5}{|c|}{ 2,6,7,8-tetrahydro-8-(2-methoxyphenyl)imidazo[2,1-c][1,2,4]triazine-3,4-dione } \\
\hline & FUS & & 23.70 & 509.2 & DSC & {$[2016 \mathrm{BAR} / \mathrm{SZT}]$} \\
\hline \multirow[t]{2}{*}{$\mathrm{C}_{12} \mathrm{H}_{10} \mathrm{~N}_{4} \mathrm{O}_{3}$} & {$[848893-00-5]$} & \multicolumn{5}{|c|}{ 2,6,7,8-tetrahydro-8-(4-methoxyphenyl)imidazo[2,1-c][1,2,4]triazine-3,4-dione } \\
\hline & FUS & & 20.80 & 567.2 & DSD & {$[2016 \mathrm{BAR} / \mathrm{SZT}]$} \\
\hline $\mathrm{C}_{12} \mathrm{H}_{10} \mathrm{O}$ & $\begin{array}{c}{[941-98-0]} \\
\mathrm{V}\end{array}$ & $\begin{array}{c}\text { 1-acetylnaphth } \\
(388-569)\end{array}$ & 65.4 & 403 & A & [1987STE/MAL] \\
\hline $\mathrm{C}_{12} \mathrm{H}_{10} \mathrm{O}$ & [93-08-3] & 2-acetylnaphth & & & & \\
\hline & SUB & $(295-316)$ & $87.9 \pm 0.4$ & 305 & $\mathrm{~V}$ & [1959AIH, 1987STE/MAL] \\
\hline & $\mathrm{V}$ & $(393-574)$ & 74.1 & 408 & A & {$[1987 \mathrm{STE} / \mathrm{MAL}]$} \\
\hline $\mathrm{C}_{12} \mathrm{H}_{10} \mathrm{O}$ & [101-84-8] & Diphenyl ethe & & & & \\
\hline & FUS & & 17.05 & 300.1 & DSC & [2014CAB/GRA] \\
\hline & FUS & & 16.51 & 300.4 & DSC & [1992BAB/HWA, 1994BAB/BEN] \\
\hline & FUS & & 17.23 & 301 & $\mathrm{C}$ & {$[1964 \mathrm{ARN}]$} \\
\hline & FUS & $(18-360)$ & 17.21 & 300 & $\mathrm{AC}$ & [1996DOM/HEA, 1953GIN/FUR, 1951FUR/GIN] \\
\hline & SUB & & $82 \pm 2.1$ & & $\mathrm{E}$ & [1958CAS/FLE3, 1970COX/PIL] \\
\hline & $\mathrm{V}$ & $(353-393)$ & 67.1 & 298 & CGC & [1995CHI/HOS] \\
\hline & $\mathrm{V}$ & $(477-544)$ & 65.0 & 298 & & [1976AMB/ELL] \\
\hline & $\mathrm{V}$ & $(477-544)$ & 48.2 & 531 & & [1976AMB/ELL] \\
\hline & $\mathrm{V}$ & $(477-544)$ & 53.0 & 492 & GS,EB & [1987STE/MAL, 1976AMB/ELL] \\
\hline & $\mathrm{V}$ & & $66.1 \pm 0.4$ & 298 & $\mathrm{C}$ & [1972MOR, 1965COL/COU] \\
\hline & $\mathrm{V}$ & $(313-333)$ & 64.2 & 323 & A & [1987STE/MAL, 1948BEN/FRA] \\
\hline $\mathrm{C}_{12} \mathrm{H}_{10} \mathrm{O}$ & [90-43-7] & 2-hydroxybiph & & & & \\
\hline & FUS & & 15.48 & 333.7 & DSC & [1998VER5] \\
\hline & FUS & $(12-350)$ & 16.21 & 330.6 & $\mathrm{AC}$ & {$[1973 \mathrm{GEI} / \mathrm{DZH}]$} \\
\hline & SUB & $(301-328)$ & $87.6 \pm 0.9$ & 314 & GS & [1998VER5] \\
\hline & SUB & $(301-328)$ & $88.5 \pm 0.9$ & 298 & GS & [1998VER5] \\
\hline
\end{tabular}


TABLE 11. Phase change enthalpies of $\mathrm{C}_{12}$ to $\mathrm{C}_{13}$ organic compounds-Continued

\begin{tabular}{|c|c|c|c|c|c|c|}
\hline \multirow[b]{2}{*}{ Molecular formula } & \multirow{2}{*}{$\begin{array}{c}\text { CAS Registry } \\
\text { Number } \\
\text { Enthalpy }\end{array}$} & \multicolumn{5}{|l|}{ Compound } \\
\hline & & $\begin{array}{c}\text { Temperature } \\
\text { range }\end{array}$ & $\begin{array}{l}\Delta_{\text {trans }} H_{\mathrm{m}} \\
(\mathrm{kJ} / \mathrm{mol})\end{array}$ & $\begin{array}{l}T_{\mathrm{m}} \\
(\mathrm{K})\end{array}$ & Method & References \\
\hline & SUB & $(292-314)$ & 82.9 & 303 & A & [1987STE/MAL, 1960AIH] \\
\hline & V & $(355-373)$ & $72.6 \pm 1.1$ & 298 & GS & [1998VER5] \\
\hline & $\mathrm{V}$ & $(434-547)$ & 94.2 & 449 & A & [1987STE/MAL] \\
\hline \multirow[t]{7}{*}{$\mathrm{C}_{12} \mathrm{H}_{10} \mathrm{O}$} & {$[92-69-3]$} & \multicolumn{5}{|c|}{ 4-hydroxybiphenyl } \\
\hline & FUS & & 31.5 & 439 & DSC & [2010BAI/VAN] \\
\hline & FUS & & 31.59 & 443.1 & DSC & [1998VER5] \\
\hline & SUB & $(333-368)$ & $106.6 \pm 1.0$ & 351 & GS & [1998VER5] \\
\hline & SUB & $(333-368)$ & $109.8 \pm 1.0$ & 298 & GS & [1998VER5] \\
\hline & SUB & $(327-348)$ & 97.0 & 337.5 & A & [1987STE/MAL, 1960AIH] \\
\hline & $\mathrm{V}$ & $(450-581)$ & 72.3 & 465 & A & [1987STE/MAL] \\
\hline \multirow[t]{5}{*}{$\mathrm{C}_{12} \mathrm{H}_{10} \mathrm{O}_{2}$} & [1806-29-7] & \multicolumn{5}{|c|}{ 2,2'-dihydroxybiphenyl } \\
\hline & FUS & & 25.36 & 386.7 & DSC & [1998VER5] \\
\hline & SUB & $(334-363)$ & $111.4 \pm 1.2$ & 349 & GS & [1998VER5] \\
\hline & SUB & $(334-363)$ & $114.4 \pm 1.2$ & 298 & GS & [1998VER5] \\
\hline & $\mathrm{V}$ & $(444-598)$ & 61.7 & 459 & A & [1987STE/MAL] \\
\hline \multirow[t]{4}{*}{$\mathrm{C}_{12} \mathrm{H}_{10} \mathrm{O}_{2}$} & {$[92-88-6]$} & \multicolumn{5}{|c|}{ 4,4'-dihydroxybiphenyl } \\
\hline & FUS & & 43.05 & 560.7 & DSC & [1998VER5] \\
\hline & SUB & $(354-388)$ & $138.6 \pm 2.0$ & 371 & GS & [1998VER5] \\
\hline & SUB & $(354-388)$ & $143.0 \pm 2.0$ & 298 & GS & [1998VER5] \\
\hline \multirow[t]{3}{*}{$\mathrm{C}_{12} \mathrm{H}_{10} \mathrm{O}_{2}$} & [713-68-8] & \multicolumn{5}{|c|}{ 3-phenoxyphenol } \\
\hline & V & & $90.4 \pm 2.3$ & 298 & $\mathrm{C}$ & [2011RIB/FER2] \\
\hline & $\mathrm{V}$ & $(416-494)$ & 69.5 & 431 & A & [1987STE/MAL] \\
\hline \multirow[t]{3}{*}{$\mathrm{C}_{12} \mathrm{H}_{10} \mathrm{O}_{2}$} & {$[831-82-3]$} & \multicolumn{5}{|c|}{ 4-phenoxyphenol } \\
\hline & SUB & $(325-343)$ & $117.3 \pm 0.7$ & 334 & $\mathrm{ME}$ & [2011RIB/FER2] \\
\hline & SUB & $(325-343)$ & $112.8 \pm 0.4$ & 298 & ME & [2011RIB/FER2] \\
\hline \multirow[t]{3}{*}{$\mathrm{C}_{12} \mathrm{H}_{10} \mathrm{O}_{2}$} & [830-81-9] & \multicolumn{5}{|c|}{$\alpha$-naphthyl acetate } \\
\hline & FUS & & 20.21 & 319.2 & & [1981BYS] \\
\hline & SUB & $(286-317)$ & $95.1 \pm 0.6$ & 298 & GS & [2003VER/ROU] \\
\hline \multirow[t]{4}{*}{$\mathrm{C}_{12} \mathrm{H}_{10} \mathrm{O}_{2}$} & [1523-11-1] & \multicolumn{5}{|c|}{$\beta$-naphthyl acetate } \\
\hline & FUS & & 22.86 & 342.0 & DSC & [2008MOG/SEP] \\
\hline & FUS & & 20.05 & 342.2 & & [1981BYS] \\
\hline & SUB & $(313-341)$ & $96.3 \pm 0.6$ & 298 & GS & [2003VER/ROU] \\
\hline \multirow[t]{5}{*}{$\mathrm{C}_{12} \mathrm{H}_{10} \mathrm{O}_{2}$} & {$[86-87-3]$} & \multicolumn{5}{|c|}{ 1-naphthaleneacetic acid } \\
\hline & TRS & & 1.77 & 368.9 & & \\
\hline & FUS & & 41.73 & 401.22 & DSC & [2008MOG/SEP] \\
\hline & FUS & & 22.26 & 405.3 & DSC & [1991ACR, 1990DON/DRE] \\
\hline & SUB & $(343-372)$ & $112.3 \pm 0.9$ & 298 & GS & [2004ROU/TEM] \\
\hline \multirow[t]{2}{*}{$\mathrm{C}_{12} \mathrm{H}_{10} \mathrm{O}_{2}$} & [581-96-4] & 2-naphthalenes & tic acid & & & \\
\hline & SUB & (343-372) & $124.6 \pm 1.0$ & 298 & GS & [2004ROU/TEM] \\
\hline $\mathrm{C}_{12} \mathrm{H}_{10} \mathrm{O}_{2}$ & {$[2459-25-8]$} & 2-carbomethox & aphthalene & & & \\
\hline & FUS & & 27.1 & 350.2 & DSC & [1978DOZ/FUJ] \\
\hline $\mathrm{C}_{12} \mathrm{H}_{10} \mathrm{O}_{2}$ & {$[5392-12-1]$} & 2-methoxy-1-n & hthaldehyde & & & \\
\hline & SUB & $(332-348)$ & $107.4 \pm 1.1$ & 298 & ME & [2015AMA/FRE] \\
\hline $\mathrm{C}_{12} \mathrm{H}_{10} \mathrm{O}_{2} \mathrm{~S}$ & [127-63-9] & Diphenyl sulfo & & & & \\
\hline & FUS & & 21.78 & 398.2 & DSC & [UR/MAC, 2000DEF/VAN] \\
\hline & SUB & & $106.3 \pm 2.9$ & & & [UR/MAC, 1970COX/PIL] \\
\hline $\mathrm{C}_{12} \mathrm{H}_{10} \mathrm{O}_{2} \mathrm{~S}$ & {$[2664-63-3]$} & 4,4'-thiodiphen & & & & \\
\hline & FUS & & 31.04 & 432.9 & DSC & [2001LI/HE] \\
\hline $\mathrm{C}_{12} \mathrm{H}_{10} \mathrm{O}_{4}$ & [106-34-3] & Quinhydrone & & & & \\
\hline
\end{tabular}


TABLE 11. Phase change enthalpies of $\mathrm{C}_{12}$ to $\mathrm{C}_{13}$ organic compounds-Continued

\begin{tabular}{|c|c|c|c|c|c|c|}
\hline \multirow[b]{2}{*}{ Molecular formula } & \multirow{2}{*}{$\begin{array}{l}\text { CAS Registry } \\
\text { Number } \\
\text { Enthalpy }\end{array}$} & \multicolumn{5}{|l|}{ Compound } \\
\hline & & $\begin{array}{l}\text { Temperature } \\
\text { range }\end{array}$ & $\begin{array}{l}\Delta_{\text {trans }} H_{\mathrm{m}} \\
(\mathrm{kJ} / \mathrm{mol})\end{array}$ & $\begin{array}{l}T_{\mathrm{m}} \\
(\mathrm{K})\end{array}$ & Method & References \\
\hline & SUB & $(317-334)$ & 89.1 & 325.5 & A & [1987STE/MAL] \\
\hline \multirow[t]{3}{*}{$\mathrm{C}_{12} \mathrm{H}_{10} \mathrm{O}_{4} \mathrm{~S}$} & {$[80-09-1]$} & \multicolumn{5}{|c|}{$4,4^{\prime}$-sulfonyldiphenol } \\
\hline & FUS & & 41.4 & 519.9 & DSC & {$[2014 \mathrm{COS} / \mathrm{DAV}]$} \\
\hline & SUB & $(465-490)$ & $162.0 \pm 2.1$ & 298 & ME & {$[2014 \mathrm{COS} / \mathrm{DAV}]$} \\
\hline \multirow[t]{3}{*}{$\mathrm{C}_{12} \mathrm{H}_{10} \mathrm{O}_{4} \mathrm{~S}_{2}$} & {$[10409-06-0]$} & \multicolumn{5}{|c|}{ Diphenyl disulfone } \\
\hline & SUB & & $161.9 \pm 3.3$ & & $\mathrm{E}$ & [1964MAC/OHA] \\
\hline & $\mathrm{V}$ & & $149.0 \pm 2.9$ & 298 & $\mathrm{E}$ & [1964MAC/OHA] \\
\hline \multirow[t]{10}{*}{$\mathrm{C}_{12} \mathrm{H}_{10} \mathrm{~S}$} & $\begin{array}{c}\text { [139-66-2] } \\
\text { FUS }\end{array}$ & \multicolumn{5}{|l|}{ Diphenyl sulfide } \\
\hline & SUB & & $95 . \pm 3.0$ & & $\mathrm{E}$ & [1962MAC/MAY3, 1970COX/PIL] \\
\hline & $\mathrm{V}$ & $(369-566)$ & 60.5 & 384 & & [1999DYK/SVO] \\
\hline & $\mathrm{V}$ & $(345-611)$ & $67.3 \pm 0.3$ & 360 & EB,IPM & [1995STE/CHI] \\
\hline & $\mathrm{V}$ & $(345-611)$ & $64.3 \pm 0.3$ & 400 & EB,IPM & [1995STE/CHI] \\
\hline & $\mathrm{V}$ & $(345-611)$ & $61.3 \pm 0.3$ & 440 & EB,IPM & [1995STE/CHI] \\
\hline & $\mathrm{V}$ & $(345-611)$ & $58.3 \pm 0.3$ & 480 & EB,IPM & [1995STE/CHI] \\
\hline & $\mathrm{V}$ & $(345-611)$ & $55.3 \pm 0.3$ & 520 & EB,IPM & [1995STE/CHI] \\
\hline & $\mathrm{V}$ & $(345-611)$ & $52.0 \pm 0.3$ & 560 & EB,IPM & [1995STE/CHI] \\
\hline & $\mathrm{V}$ & $(369-566)$ & 58.2 & 384 & A & [1987STE/MAL, 1949KRE/WIE] \\
\hline \multirow[t]{3}{*}{$\mathrm{C}_{12} \mathrm{H}_{10} \mathrm{~S}_{2}$} & $\begin{array}{c}{[882-33-7]} \\
\mathrm{V}\end{array}$ & \multicolumn{5}{|l|}{ Diphenyl disulfide } \\
\hline & $\mathrm{V}$ & & $78.7 \pm 2.9$ & 298 & & [1962MAC/MAY3] \\
\hline & $\mathrm{V}$ & $(404-583)$ & 74.4 & 419 & A & [1987STE/MAL, 1947STU] \\
\hline \multirow[t]{5}{*}{$\mathrm{C}_{12} \mathrm{H}_{11} \mathrm{ClN}_{2} \mathrm{O}_{2} \mathrm{~S}$} & [16803-92-2] & \multicolumn{5}{|c|}{ 4-amino- $N$-(4-chlorophenyl)benzene sulfonamide } \\
\hline & FUS & & 37.3 & 467.9 & DSC & [2008PER/STR, 2014PER/KAZ] \\
\hline & SUB & $(400-432)$ & $129.2 \pm 1.2$ & 416 & GS & [2008PER/STR] \\
\hline & SUB & $(400-432)$ & $134.1 \pm 1.2$ & 298 & GS & [2008PER/STR] \\
\hline & $\mathrm{V}$ & & 110.3 & 298 & $\mathrm{~S}-\mathrm{F}$ & [2008PER/STR] \\
\hline \multirow[t]{2}{*}{$\mathrm{C}_{12} \mathrm{H}_{11} \mathrm{ClN}_{2} \mathrm{O}_{5} \mathrm{~S}$} & {$[54-31-9]$} & \multicolumn{5}{|c|}{ 4-chloro-2-[(furan-2-ylmethyl)amino]-5-sulfamoylbenzoic acid (furosemide) } \\
\hline & FUS & & 21.07 & 508.2 & DSC & [2015GAU/VAN] \\
\hline \multirow[t]{3}{*}{$\mathrm{C}_{12} \mathrm{H}_{11} \mathrm{Cl}_{2} \mathrm{NO}$} & [175205-50-2] & \multicolumn{5}{|c|}{ 1-(3,5-dichlorophenyl)-2,5-dimethylpyrrole } \\
\hline & SUB & $(302-324)$ & $96.7 \pm 0.4$ & 313 & ME & [2013SAN/RIB3] \\
\hline & SUB & $(302-324)$ & $97.5 \pm 0.4$ & 298 & ME & [2013SAN/RIB3] \\
\hline \multirow[t]{2}{*}{$\mathrm{C}_{12} \mathrm{H}_{11} \mathrm{Cl}_{2} \mathrm{NO}$} & {$[23950-58-5]$} & \multicolumn{5}{|c|}{ 3,5-dichloro- $N$-(1,1-dimethyl-2-propynyl)benzamide } \\
\hline & FUS & & 28.68 & 428.4 & DSC & [1990DON/DRE] \\
\hline \multirow[t]{3}{*}{$\mathrm{C}_{12} \mathrm{H}_{11} \mathrm{FN}_{2} \mathrm{O}_{3}$} & [102916-95-0] & $(2 R, 4 S)$-6-fluoro- & -2-methyl-spi & chromar & $4^{\prime}$-imidazo & ine $]-2^{\prime}, 5^{\prime}$-dione \\
\hline & FUS (I) & & 26.3 & 517.5 & & \\
\hline & FUS (II) & & 31.3 & 501.0 & DSC & [1988ASH/UCH] \\
\hline $\mathrm{C}_{12} \mathrm{H}_{11} \mathrm{IN}_{2} \mathrm{O}_{2} \mathrm{~S}$ & {$[6965-75-9]$} & 4-amino- $N$-(4-iod & dophenyl)ben & le sulfor & iide & \\
\hline & FUS & & 36.4 & 468.7 & DSC & [2014PER/KAZ] \\
\hline $\mathrm{C}_{12} \mathrm{H}_{11} \mathrm{~N}$ & {$[90-41-5]$} & 2-aminobiphenyl & & & & \\
\hline & FUS & & 13.99 & 322.3 & & [1996DOM/HEA, 1991STE/CHI] \\
\hline & $\mathrm{V}$ & $(340-623)$ & 68.6 & 400 & EB,IPM & [1991STE/CHI] \\
\hline & $\mathrm{V}$ & $(340-623)$ & 65.1 & 440 & EB,IPM & [1991STE/CHI] \\
\hline & $\mathrm{V}$ & $(340-623)$ & 61.8 & 480 & EB,IPM & [1991STE/CHI] \\
\hline & $\mathrm{V}$ & $(340-623)$ & 58.5 & 520 & EB,IPM & [1991STE/CHI] \\
\hline & $\mathrm{V}$ & $(340-623)$ & 55.2 & 560 & EB,IPM & [1991STE/CHI] \\
\hline & $\mathrm{V}$ & $(340-623)$ & 51.7 & 600 & EB,IPM & [1991STE/CHI] \\
\hline $\mathrm{C}_{12} \mathrm{H}_{11} \mathrm{~N}$ & {$[122-39-4]$} & Diphenylamine & & & & \\
\hline & FUS & & 18.51 & 326.7 & & [2012SHA/LAL] \\
\hline & FUS & & 19.68 & 326.7 & DSC & [2010MEK/KHI, 2013TRA/KHI, 2013TRA/KHI2] \\
\hline & FUS & & 19.9 & 326.1 & DSC & [2009SUR/TER, 2010SUR/PER] \\
\hline
\end{tabular}


TABLE 11. Phase change enthalpies of $\mathrm{C}_{12}$ to $\mathrm{C}_{13}$ organic compounds-Continued

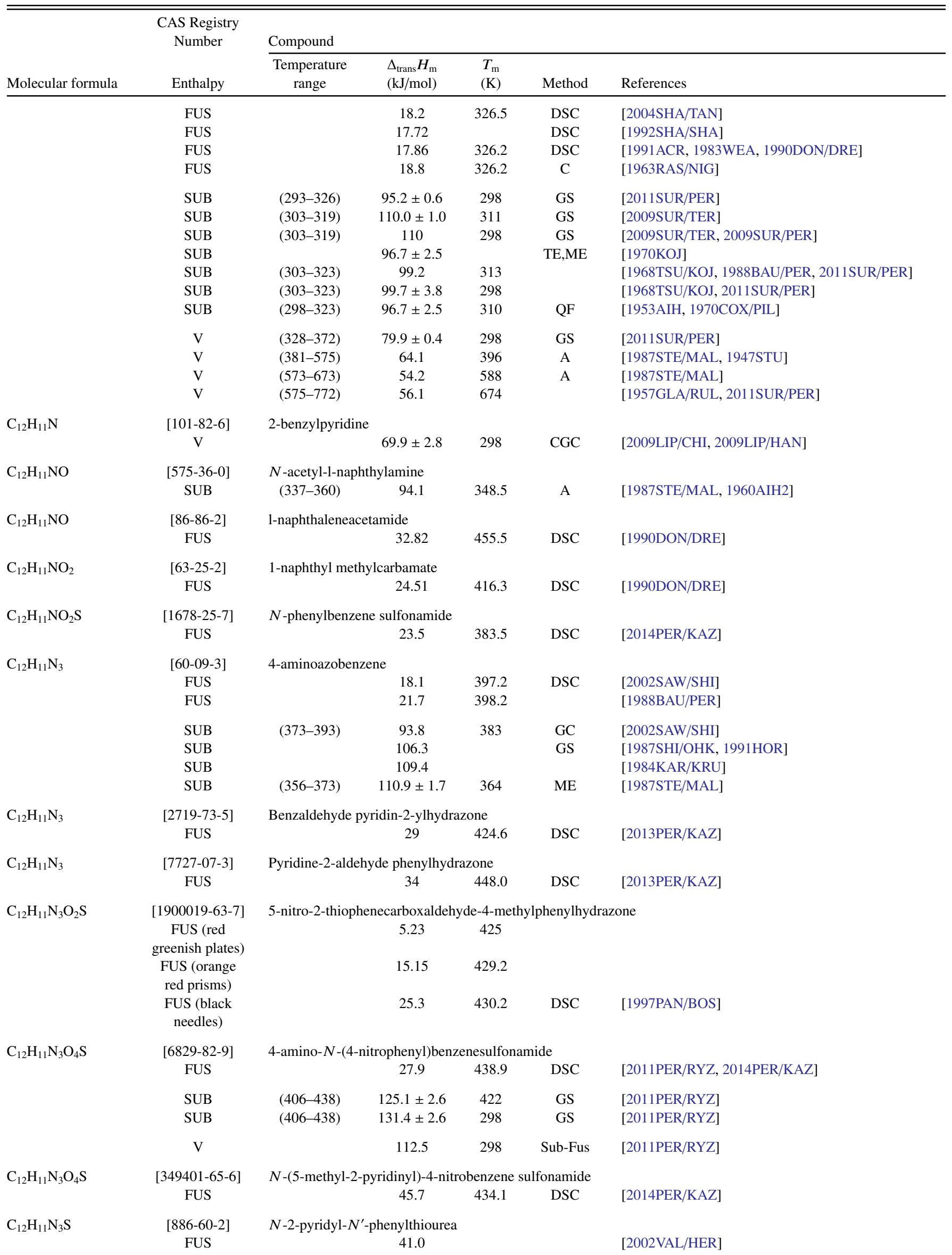


TABLE 11. Phase change enthalpies of $\mathrm{C}_{12}$ to $\mathrm{C}_{13}$ organic compounds-Continued

\begin{tabular}{|c|c|c|c|c|c|c|}
\hline \multirow[b]{2}{*}{ Molecular formula } & \multirow{2}{*}{$\begin{array}{c}\text { CAS Registry } \\
\text { Number } \\
\text { Enthalpy }\end{array}$} & \multicolumn{5}{|l|}{ Compound } \\
\hline & & $\begin{array}{c}\text { Temperature } \\
\text { range }\end{array}$ & $\begin{array}{l}\Delta_{\text {trans }} H_{\mathrm{m}} \\
(\mathrm{kJ} / \mathrm{mol})\end{array}$ & $\begin{array}{l}T_{\mathrm{m}} \\
(\mathrm{K})\end{array}$ & Method & References \\
\hline \multirow[t]{2}{*}{$\mathrm{C}_{12} \mathrm{H}_{11} \mathrm{~N}_{7}$} & {$[396-01-0]$} & \multicolumn{5}{|c|}{ 6-phenyl-2,4,7-pteridinetriamine (triamterene) } \\
\hline & FUS & & 73.4 & 602.2 & DSC & [2011DOM/POB] \\
\hline \multirow[t]{2}{*}{$\mathrm{C}_{12} \mathrm{H}_{11} \mathrm{O}_{2} \mathrm{P}$} & {$[1707-03-5]$} & \multicolumn{5}{|c|}{$P, P$-diphenylphosphinic acid } \\
\hline & FUS & & 21.91 & 466.1 & DSC & [2008ZHA/WAN] \\
\hline \multirow[t]{3}{*}{$\mathrm{C}_{12} \mathrm{H}_{12}$} & {$[571-58-4]$} & \multicolumn{5}{|c|}{ 1,4-dimethylnaphthalene } \\
\hline & FUS & & 10.6 & 279.2 & DSC & [2007CHE/KIM] \\
\hline & FUS & & 10.6 & 279.9 & & [1991ACR, 1980SMI] \\
\hline \multirow[t]{3}{*}{$\mathrm{C}_{12} \mathrm{H}_{12}$} & [571-61-9] & \multicolumn{5}{|c|}{ 1,5-dimethylnaphthalene } \\
\hline & FUS & & 20.0 & 355.2 & DSC & [2007CHE/KIM] \\
\hline & $\mathrm{V}$ & $(323-473)$ & 64.1 & 398 & GC & [2002LEI/CHA] \\
\hline \multirow[t]{3}{*}{$\mathrm{C}_{12} \mathrm{H}_{12}$} & {$[575-43-9]$} & \multicolumn{5}{|c|}{ 1,6-dimethylnaphthalene } \\
\hline & FUS & & 8.5 & 257 & DSC & [2007CHE/KIM] \\
\hline & $\mathrm{V}$ & $(323-473)$ & 63.6 & 398 & GC & [2002LEI/CHA] \\
\hline \multirow[t]{11}{*}{$\mathrm{C}_{12} \mathrm{H}_{12}$} & {$[569-41-5]$} & \multicolumn{5}{|c|}{ 1,8-dimethylnaphthalene } \\
\hline & FUS & & 18.53 & 338.2 & DSC & [2007CHE/KIM] \\
\hline & FUS & $(11-432)$ & 15.77 & 336.3 & & [1991ACR, 1977FIN/MES] \\
\hline & SUB & $(328-336)$ & 77.9 & 332 & A & [1987STE/MAL] \\
\hline & SUB & & 79.6 & 336 & B & [1975OSB/DOU, 1979COL/JIM2] \\
\hline & SUB & & $82.7 \pm 0.3$ & 298 & $\mathrm{C}$ & [1974MAN3, 1977PED/RYL] \\
\hline & $\mathrm{V}$ & $(338-413)$ & 62.8 & 353 & A & [1987STE/MAL, 1975OSB/DOU, 1984BOU/FRI] \\
\hline & $\mathrm{V}$ & $(338-413)$ & 64.8 & 336 & IP & {$[1977 \mathrm{FIN} / \mathrm{MES}]$} \\
\hline & $\mathrm{V}$ & $(338-413)$ & 62.2 & 360 & IP & [1977FIN/MES] \\
\hline & $\mathrm{V}$ & $(338-413)$ & 60.7 & 380 & IP & [1977FIN/MES] \\
\hline & $\mathrm{V}$ & $(338-413)$ & 59.7 & 400 & IP & [1977FIN/MES] \\
\hline \multirow[t]{10}{*}{$\mathrm{C}_{12} \mathrm{H}_{12}$} & {$[581-40-8]$} & \multicolumn{5}{|c|}{ 2,3-dimethylnaphthalene } \\
\hline & FUS & & 23.97 & 377.2 & DSC & [2007CHE/KIM] \\
\hline & FUS & & 15.9 & 378 & & [1991ACR, 1980SMI] \\
\hline & FUS & & 18.87 & 377.6 & DSC & [1971MAS/CHE] \\
\hline & SUB & $(333-373)$ & 82.8 & 348 & A & [1987STE/MAL] \\
\hline & SUB & $(287-300)$ & $82.2 \pm 0.4$ & 294 & $\mathrm{ME}$ & [1979COL/JIM2] \\
\hline & SUB & & 81.0 & & B & {$[1978 \mathrm{ARO} / \mathrm{STE}]$} \\
\hline & SUB & $(278-301)$ & $79.9 \pm 0.4$ & 290 & $\mathrm{~V}$ & [1959AIH, 1987STE/MAL] \\
\hline & $\mathrm{V}$ & & $60.9 \pm 0.7$ & 380 & & [1988MES/FIN] \\
\hline & $\mathrm{V}$ & $(378-408)$ & 60.0 & 393 & A & [1987STE/MAL] \\
\hline \multirow[t]{10}{*}{$\mathrm{C}_{12} \mathrm{H}_{12}$} & {$[581-42-0]$} & \multicolumn{5}{|c|}{ 2,6-dimethylnaphthalene } \\
\hline & FUS & & 25.3 & 385.2 & DSC & [2007CHE/KIM] \\
\hline & FUS & $(12-438)$ & 25.06 & 383.3 & & [1991ACR, 1977FIN/MES] \\
\hline & SUB & $(350-383)$ & $84.4 \pm 1.9$ & 366 & & [1977FIN/MES, 1975OSB/DOU, 1987STE/MAL] \\
\hline & SUB & & 82.5 & 383 & $\mathrm{~B}$ & {$[1975 \mathrm{OSB} / \mathrm{DOU}]$} \\
\hline & SUB & $(279-304)$ & 84.1 & 291 & $\mathrm{~V}$ & [1959AIH, 1987STE/MAL] \\
\hline & V & $(384-418)$ & 57.4 & 383 & IP & [1977FIN/MES] \\
\hline & $\mathrm{V}$ & $(384-418)$ & 56.6 & 400 & IP & [1977FIN/MES] \\
\hline & $\mathrm{V}$ & $(384-418)$ & 55.7 & 420 & IP & {$[1977 \mathrm{FIN} / \mathrm{MES}]$} \\
\hline & $\mathrm{V}$ & $(384-418)$ & 57.3 & 399 & A & [1987STE/MAL, 1975OSB/DOU, 1984BOU/FRI] \\
\hline $\mathrm{C}_{12} \mathrm{H}_{12}$ & {$[582-16-1]$} & 2,7-dimethyln & thalene & & & \\
\hline & FUS & & 22.2 & 370.2 & DSC & [2007CHE/KIM] \\
\hline & FUS & $(11-391)$ & 23.35 & 368.8 & & [1991ACR, 1977FIN/MES] \\
\hline & SUB & $(340-369)$ & $83.8 \pm 1.0$ & 345 & & [1977FIN/MES, 1975OSB/DOU] \\
\hline & SUB & & 83.2 & 369 & $\mathrm{~B}$ & {$[1975 O S B / D O U]$} \\
\hline & SUB & $(333-368)$ & 84.6 & 348 & & {$[1987 \mathrm{STE} / \mathrm{MAL}]$} \\
\hline & $\mathrm{V}$ & & 57.3 & 400 & & [1993CHI/KNI] \\
\hline & V & & 54.8 & 440 & & [1993CHI/KNI] \\
\hline
\end{tabular}


TABLE 11. Phase change enthalpies of $\mathrm{C}_{12}$ to $\mathrm{C}_{13}$ organic compounds-Continued

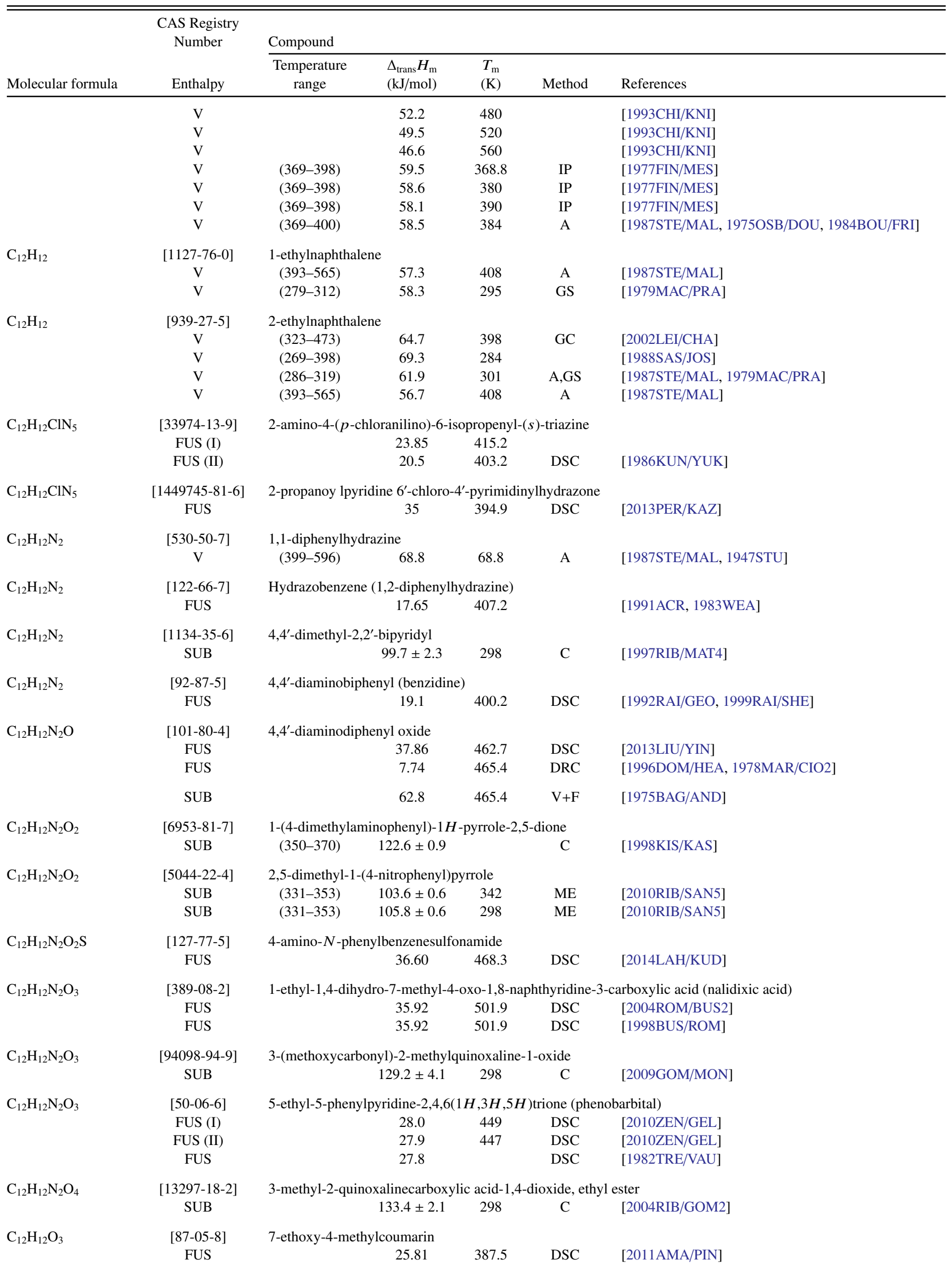


TABLE 11. Phase change enthalpies of $\mathrm{C}_{12}$ to $\mathrm{C}_{13}$ organic compounds-Continued

\begin{tabular}{|c|c|c|c|c|c|c|}
\hline \multirow[b]{2}{*}{ Molecular formula } & \multirow{2}{*}{$\begin{array}{l}\text { CAS Registry } \\
\text { Number } \\
\text { Enthalpy }\end{array}$} & \multicolumn{5}{|l|}{ Compound } \\
\hline & & $\begin{array}{l}\text { Temperature } \\
\text { range }\end{array}$ & $\begin{array}{l}\Delta_{\text {trans }} H_{\mathrm{m}} \\
(\mathrm{kJ} / \mathrm{mol})\end{array}$ & $\begin{array}{l}T_{\mathrm{m}} \\
(\mathrm{K})\end{array}$ & Method & References \\
\hline \multirow[t]{5}{*}{$\mathrm{C}_{12} \mathrm{H}_{12} \mathrm{O}_{4}$} & [29412-62-2] & \multicolumn{5}{|c|}{ 1,4-dimethylcubane dicarboxylate } \\
\hline & FUS & & 38.1 & 438.2 & DSC & [2005ROU/DAV] \\
\hline & FUS & & 41.0 & 437.8 & & [1996DOM/HEA, 1989KIR/CHU] \\
\hline & SUB & & $117.2 \pm 3.9$ & 298 & $\mathrm{~V}+\mathrm{F}$ & [2005ROU/DAV] \\
\hline & $\mathrm{V}$ & & $88.5 \pm 2.2$ & 298 & CGC & [2005ROU/DAV] \\
\hline \multirow[t]{4}{*}{$\mathrm{C}_{12} \mathrm{H}_{12} \mathrm{O}_{4}$} & {$[30296-80-1]$} & \multicolumn{5}{|c|}{ Dimethyl 2,6-cuneanedicarboxylate } \\
\hline & FUS & & 23.4 & 392.7 & DSC & [2005ROU/DAV] \\
\hline & SUB & & $106.8 \pm 3.0$ & 298 & $\mathrm{~V}+\mathrm{F}$ & [2005ROU/DAV] \\
\hline & $\mathrm{V}$ & & $89.7 \pm 2.1$ & 298 & CGC & [2005ROU/DAV] \\
\hline \multirow[t]{3}{*}{$\mathrm{C}_{12} \mathrm{H}_{12} \mathrm{O}_{6}$} & {$[2672-57-3]$} & \multicolumn{5}{|c|}{ 1,2,3-benzenetricarboxylic acid, trimethyl ester } \\
\hline & FUS & & 32.7 & 375.7 & DSC & [1993ACR, 1978DOZ/FUJ] \\
\hline & $\mathrm{V}$ & $(453-513)$ & 72.5 & 468 & A,GS & [1987STE/MAL, 1962KRA/BER] \\
\hline \multirow[t]{3}{*}{$\mathrm{C}_{12} \mathrm{H}_{12} \mathrm{O}_{6}$} & {$[28904-30-5]$} & \multicolumn{5}{|c|}{ 1,2,4-benzenetricarboxylic acid, trimethyl ester } \\
\hline & $\mathrm{V}$ & & $78.5 \pm 0.4$ & 399 & $\mathrm{C}$ & [1998MAK/KAB] \\
\hline & $\mathrm{V}$ & $(443-493)$ & 61.1 & 458 & A,GS & [1987STE/MAL, 1962KRA/BER] \\
\hline \multirow{8}{*}{$\mathrm{C}_{12} \mathrm{H}_{12} \mathrm{O}_{6}$} & {$[2672-58-4]$} & \multicolumn{5}{|c|}{ 1,3,5-benzenetricarboxylic acid, trimethyl ester } \\
\hline & TRS & & 4.6 & 408.2 & DSC & \\
\hline & FUS & & 17.6 & 419.4 & DSC & [1978DOZ/FUJ] \\
\hline & \multicolumn{6}{|c|}{ Fusion enthalpy may not be reliable_-authors reported a mass loss as the sample melted } \\
\hline & SUB & $(350-368)$ & $115.9 \pm 0.4$ & 359 & $\mathrm{ME}$ & [1995JIM/MEN] \\
\hline & SUB & & $118.9 \pm 0.4$ & 298 & & [1995JIM/MEN] \\
\hline & SUB & & $117.5 \pm 0.8$ & 298 & & [1967TUR2, 1995JIM/MEN] \\
\hline & $\mathrm{V}$ & $(443-513)$ & 75.4 & 458 & A & [1987STE/MAL] \\
\hline \multirow[t]{9}{*}{$\mathrm{C}_{12} \mathrm{H}_{12} \mathrm{~S}$} & {$[16587-33-0]$} & \multicolumn{5}{|c|}{ 1,2,3,4-tetrahydrodibenzothiophene } \\
\hline & FUS & & 32.03 & 275 & & [2004STE/CHI2] \\
\hline & $\mathrm{V}$ & $(360-600)$ & $70.3 \pm 0.3$ & 360 & IPM,EB & [2004STE/CHI2] \\
\hline & $\mathrm{V}$ & $(360-600)$ & $67.3 \pm 0.3$ & 400 & IPM,EB & [2004STE/CHI2] \\
\hline & $\mathrm{V}$ & $(360-600)$ & $64.5 \pm 0.2$ & 440 & IPM,EB & [2004STE/CHI2] \\
\hline & $\mathrm{V}$ & $(360-600)$ & $61.8 \pm 0.2$ & 480 & IPM,EB & [2004STE/CHI2] \\
\hline & $\mathrm{V}$ & $(360-600)$ & $59.2 \pm 0.3$ & 520 & IPM,EB & [2004STE/CHI2] \\
\hline & $\mathrm{V}$ & $(360-600)$ & $56.5 \pm 0.4$ & 560 & IPM,EB & [2004STE/CHI2] \\
\hline & $\mathrm{V}$ & $(360-600)$ & $75.3 \pm 0.7$ & 298 & IPM,EB & [2004STE/CHI2] \\
\hline \multirow[t]{2}{*}{$\mathrm{C}_{12} \mathrm{H}_{13} \mathrm{ClF}_{3} \mathrm{~N}_{3} \mathrm{O}_{4}$} & {$[33245-39-5]$} & \multicolumn{5}{|c|}{$N$-(2-chloroethyl)-2,6-dinitro- $N$-propyl-4-(trifluoromethyl)benzeneamine } \\
\hline & FUS & & 23.08 & 318.4 & DSC & {$[1990 \mathrm{DON} / \mathrm{DRE}]$} \\
\hline \multirow[t]{2}{*}{$\mathrm{C}_{12} \mathrm{H}_{13} \mathrm{Cl}_{3} \mathrm{O}_{3}$} & {$[93-79-8]$} & \multicolumn{5}{|c|}{ 2,4,5-trichlorophenoxyacetic acid, butyl ester } \\
\hline & $\mathrm{V}$ & $(460-573)$ & 87.3 & 475 & A & [1987STE/MAL] \\
\hline $\mathrm{C}_{12} \mathrm{H}_{13} \mathrm{~F}_{13}$ & [69125-80-0] & $1,1,1,2,2,3,3,4$ & ,5,6,6-trideca & orodode & & \\
\hline & $\mathrm{V}$ & (288-328) & $54.9 \pm 0.3$ & 298 & Static & [2015MOR/DAS] \\
\hline $\mathrm{C}_{12} \mathrm{H}_{13} \mathrm{~N}$ & {$[86-56-6]$} & $N, N$-dimethyl- & minonaphtha & & & \\
\hline & V & $(283-334)$ & $66.9 \pm 0.2$ & 298 & GS & [2007VER/GEO] \\
\hline $\mathrm{C}_{12} \mathrm{H}_{13} \mathrm{~N}$ & [83-24-9] & 2,5-dimethyl-1 & nenylpyrrole & & & \\
\hline & SUB & $(275-293)$ & $84.2 \pm 0.5$ & 284 & ME & [2010RIB/SAN5] \\
\hline & SUB & $(275-293)$ & $83.5 \pm 0.5$ & 298 & $\mathrm{ME}$ & [2010RIB/SAN5] \\
\hline $\mathrm{C}_{12} \mathrm{H}_{13} \mathrm{~N}$ & [942-01-8] & 1,2,3,4-tetrahy & carbazole & & & \\
\hline & FUS & & 17.85 & 391.3 & DSC & [2016STA/KEI] \\
\hline & SUB & $(336-366)$ & $97.2 \pm 0.9$ & 298 & GS & [2015STA/EME] \\
\hline $\mathrm{C}_{12} \mathrm{H}_{13} \mathrm{NO}_{2}$ & {$[87-01-4]$} & 4-methyl-7-din & hylaminocour & & & \\
\hline & FUS & & 23.92 & 416.1 & DSC & [1996DOM/HEA, 1989ZHA/HUA] \\
\hline $\mathrm{C}_{12} \mathrm{H}_{13} \mathrm{NO}_{2} \mathrm{~S}$ & {$[5234-68-4]$} & 5,6-dihydro-2- & thyl- $N$-pheny & ,4-oxatl & 3-carboxa & de (carboxin) \\
\hline
\end{tabular}


TABLE 11. Phase change enthalpies of $\mathrm{C}_{12}$ to $\mathrm{C}_{13}$ organic compounds-Continued

\begin{tabular}{|c|c|c|c|c|c|c|}
\hline \multirow[b]{2}{*}{ Molecular formula } & \multirow{2}{*}{$\begin{array}{l}\text { CAS Registry } \\
\text { Number } \\
\text { Enthalpy }\end{array}$} & \multicolumn{5}{|l|}{ Compound } \\
\hline & & $\begin{array}{l}\text { Temperature } \\
\text { range }\end{array}$ & $\begin{array}{l}\Delta_{\text {trans }} H_{\mathrm{m}} \\
(\mathrm{kJ} / \mathrm{mol})\end{array}$ & $\begin{array}{l}T_{\mathrm{m}} \\
(\mathrm{K})\end{array}$ & Method & References \\
\hline & FUS & $(79-380)$ & 28.19 & 365.3 & $\mathrm{AC}$ & [2004WAN/TAN] \\
\hline $\mathrm{C}_{12} \mathrm{H}_{13} \mathrm{NO}_{4} \mathrm{~S}$ & $\begin{array}{c}{[5259-88-1]} \\
\text { FUS }\end{array}$ & 2,3-dihydro-6- & $\begin{array}{l}\text { thyl-5-phenyl } \\
26.66\end{array}$ & $\begin{array}{l}\text { bamoyl } \\
401.5\end{array}$ & $\begin{array}{l}\text {-oxathiin- } \\
\text { DSC }\end{array}$ & $\begin{array}{l}\text {-dioxide } \\
\text { [1990DON/DRE] }\end{array}$ \\
\hline $\mathrm{C}_{12} \mathrm{H}_{13} \mathrm{~N}_{3}$ & $\begin{array}{c}\text { [53112-28-0] } \\
\text { FUS }\end{array}$ & $\begin{array}{l}\text { 4,6-dimethyl-1 } \\
\text { (78-391) }\end{array}$ & $\begin{array}{c}\text { henyl-2-pyrin } \\
21.23\end{array}$ & $\begin{array}{l}\text { linamine } \\
370.8\end{array}$ & $\mathrm{AC}$ & [2004SUN/SON] \\
\hline $\mathrm{C}_{12} \mathrm{H}_{14} \mathrm{ClN}_{3} \mathrm{OS}$ & $\begin{array}{c}\text { [1361124-59-5] } \\
\text { FUS }\end{array}$ & 5-[(5-chloro- 2 & $\begin{array}{l}\text { thylphenyl)an } \\
31.3\end{array}$ & $\begin{array}{l}10]-\alpha-m \\
374.5\end{array}$ & $\begin{array}{l}\text { 1-1,2,4-th } \\
\text { DSC }\end{array}$ & $\begin{array}{l}\text { azole-3-ethanol } \\
\text { [2013SUR/BUI] }\end{array}$ \\
\hline & $\begin{array}{l}\text { SUB } \\
\text { SUB }\end{array}$ & $\begin{array}{l}(347-367) \\
(347-367)\end{array}$ & $\begin{array}{c}98.9 \pm 1.5 \\
101.9 \pm 1.5\end{array}$ & $\begin{array}{l}357 \\
298\end{array}$ & $\begin{array}{l}\text { GS } \\
\text { GS }\end{array}$ & $\begin{array}{l}\text { [2013SUR/BUI] } \\
\text { [2013SUR/BUI] }\end{array}$ \\
\hline $\mathrm{C}_{12} \mathrm{H}_{14} \mathrm{ClN}_{3} \mathrm{OS}$ & $\begin{array}{l}\text { [1361124-42-6] } \\
\text { FUS } \\
\text { SUB } \\
\text { SUB }\end{array}$ & $\begin{array}{l}5-[(3-\text { chloro-4 } \\
\begin{aligned}(370-393) \\
(370-393)\end{aligned}\end{array}$ & $\begin{array}{l}\text { thylphenyl)an } \\
\qquad \begin{array}{l}29.6 \\
116.3 \pm 2.5 \\
120.7 \pm 2.5\end{array}\end{array}$ & $\begin{array}{l}10]-\alpha-m \\
402.3 \\
382 \\
298\end{array}$ & $\begin{array}{l}\text { 1-1,2,4-th } \\
\text { DSC } \\
\text { GS } \\
\text { GS }\end{array}$ & $\begin{array}{l}\text { azole-3-ethanol } \\
\text { [2013SUR/BUI] } \\
\text { [2013SUR/BUI] } \\
\text { [2013SUR/BUI] }\end{array}$ \\
\hline $\mathrm{C}_{12} \mathrm{H}_{14} \mathrm{Cl}_{2}$ & $\begin{array}{c}{[79995-39-4]} \\
\mathrm{V}\end{array}$ & $\begin{array}{c}\text { Cyclohexyl-3, } \\
(383-488)\end{array}$ & $\begin{array}{c}\text { chlorobenzen } \\
64.7\end{array}$ & 398 & & [1981GUS/KAS] \\
\hline $\mathrm{C}_{12} \mathrm{H}_{14} \mathrm{Cl}_{2} \mathrm{FNO}_{4} \mathrm{~S}$ & $\begin{array}{l}\text { FUS } \\
\text { FUS }\end{array}$ & $\begin{array}{l}\text { 2,2-dichloro- } \Lambda \\
((-) \text {-florfenico }\end{array}$ & $\begin{array}{l}\text {-(fluoromethy } \\
17.82 \\
83.36\end{array}$ & $\begin{array}{l}\text {-2-hydro } \\
427.25 \\
429.1\end{array}$ & $\begin{array}{l}\text { 2-[4-(met } \\
\text { DSC } \\
\text { DSC }\end{array}$ & $\begin{array}{l}\text { sulfonyl)phenyl]ethyl]acetamide } \\
\text { [2014SUN/HAO] } \\
\text { [2008MAR/STA] }\end{array}$ \\
\hline $\mathrm{C}_{12} \mathrm{H}_{14} \mathrm{Cl}_{2} \mathrm{O}_{3}$ & $\begin{array}{c}{[94-80-4]} \\
\mathrm{V} \\
\mathrm{V}\end{array}$ & $\begin{array}{l}\text { 2,4-dichloroph } \\
\quad(444-573) \\
(444-573)\end{array}$ & $\begin{array}{c}\text { xyacetic acid } \\
76.3 \\
70.6\end{array}$ & $\begin{array}{l}\text { utyl este } \\
459 \\
508\end{array}$ & $\begin{array}{c}\mathrm{A} \\
\mathrm{GC}\end{array}$ & $\begin{array}{l}\text { [1987STE/MAL, 1999DYK/SVO] } \\
\text { [1966JEN/SCH] }\end{array}$ \\
\hline $\mathrm{C}_{12} \mathrm{H}_{14} \mathrm{Cl}_{2} \mathrm{O}_{3}$ & $\begin{array}{c}{[94-79-1]} \\
\mathrm{V} \\
\mathrm{V}\end{array}$ & $\begin{array}{l}\text { 2,4-dichloroph } \\
\quad(444-573) \\
(444-573)\end{array}$ & $\begin{array}{c}\text { xyacetic acid } \\
74.2 \\
69.1\end{array}$ & $\begin{array}{c}\text { ec-butyl } \\
459 \\
508\end{array}$ & $\begin{array}{c}\text { A } \\
\text { GC }\end{array}$ & $\begin{array}{l}\text { [1987STE/MAL, 1999DYK/SVO] } \\
\text { [1966JEN/SCH] }\end{array}$ \\
\hline $\mathrm{C}_{12} \mathrm{H}_{14} \mathrm{Cl}_{2} \mathrm{O}_{4}$ & $\begin{array}{c}{[74944-83-5]} \\
V\end{array}$ & $\begin{array}{l}\text { 2,4-dichloroph } \\
\quad(443-503)\end{array}$ & $\begin{array}{l}\text { xyacetic acid } \\
63.5\end{array}$ & $\begin{array}{l}\text {-ethoxye } \\
458\end{array}$ & $\begin{array}{c}1 \text { ester } \\
\text { A }\end{array}$ & [1987STE/MAL] \\
\hline $\mathrm{C}_{12} \mathrm{H}_{14} \mathrm{Cl}_{2} \mathrm{O}_{4}$ & $\begin{array}{c}{[36227-43-7]} \\
V\end{array}$ & $\begin{array}{l}\text { 2,4-dichloroph } \\
\quad(443-503)\end{array}$ & $\begin{array}{l}\text { xyacetic acid } \\
72.1\end{array}$ & $\begin{array}{l}\text {-hydrox } \\
458\end{array}$ & $\begin{array}{l}\text { tyl ester } \\
\text { A }\end{array}$ & [1987STE/MAL] \\
\hline $\mathrm{C}_{12} \mathrm{H}_{14} \mathrm{FlN}_{2} \mathrm{O}_{5}$ & $\begin{array}{l}{[61787-10-8]} \\
\text { FUS }\end{array}$ & $5^{\prime}$-deoxy-5'-io & $\begin{array}{c}2^{\prime}, 3^{\prime}-O \text {-isopro } \\
13.7\end{array}$ & $\begin{array}{l}\text { idene-5- } \\
453.8\end{array}$ & $\begin{array}{l}\text { rouridine } \\
\text { DSC }\end{array}$ & [2014ZHA/ZHO] \\
\hline $\mathrm{C}_{12} \mathrm{H}_{14} \mathrm{~N}_{2} \mathrm{O}_{4} \mathrm{~S}$ & $\begin{array}{l}{[58168-20-0]} \\
\text { FUS }\end{array}$ & Ethyl 5-amino & $\begin{array}{l}\text { yano-3-(2-eth } \\
40.88\end{array}$ & $\begin{array}{l}\text { xy-2-oxo } \\
410.1\end{array}$ & $\begin{array}{l}\text { yl)-2-thior } \\
\text { DSC }\end{array}$ & $\begin{array}{l}\text { necarboxylate } \\
{[2016 \mathrm{HAN} / \mathrm{MEN}]}\end{array}$ \\
\hline $\mathrm{C}_{12} \mathrm{H}_{14} \mathrm{~N}_{2} \mathrm{O}_{5}$ & $\begin{array}{c}{[131-89-5]} \\
\text { FUS }\end{array}$ & 2-cyclohexyl-c & $\begin{array}{c}\text { dinitrophenol } \\
28.03\end{array}$ & 378.7 & DSC & [1969PLA/GLA] \\
\hline & $\mathrm{V}$ & $(405-565)$ & 88.6 & 420 & A & [1987STE/MAL, 1947STU] \\
\hline $\mathrm{C}_{12} \mathrm{H}_{14} \mathrm{~N}_{4} \mathrm{O}$ & $\begin{array}{c}\text { [2676-59-7] } \\
\text { FUS }\end{array}$ & $3,3^{\prime}, 4,4^{\prime}$-tetraa & $\begin{array}{l}\text { odiphenyl eth } \\
25.3\end{array}$ & 402.6 & & [1990DOM/HEA, 1977KAR/RAB] \\
\hline $\mathrm{C}_{12} \mathrm{H}_{14} \mathrm{~N}_{4} \mathrm{O}_{2} \mathrm{~S}$ & $\begin{array}{c}\text { [515-64-0] } \\
\text { FUS } \\
\text { FUS }\end{array}$ & 2,4-dimethyl-6 & $\begin{array}{c}\text { Ifamilamidop } \\
42.7 \\
45.11\end{array}$ & $\begin{array}{l}\text { midine } \\
523.6 \\
515.6\end{array}$ & $\begin{array}{l}\text { fisomidine } \\
\text { DTA } \\
\text { DSC }\end{array}$ & $\begin{array}{l}\text { [1971SUN/EIS] } \\
\text { [1982MAR/MIR] }\end{array}$ \\
\hline $\mathrm{C}_{12} \mathrm{H}_{14} \mathrm{~N}_{4} \mathrm{O}_{2} \mathrm{~S}$ & $\begin{array}{l}{[57-68-1]} \\
\text { FUS } \\
\text { FUS } \\
\text { FUS } \\
\text { FUS }\end{array}$ & 2-(4-aminoben & $\begin{array}{l}\text { lesulfonamido } \\
\begin{array}{c}37.7 \\
39.2 \\
44.80 \\
31.1\end{array}\end{array}$ & $\begin{array}{l}\text { 4,6-dime } \\
469.2 \\
469 \\
468.6 \\
471.6\end{array}$ & $\begin{array}{l}\text { lpyrimidir } \\
\text { DSC } \\
\text { DSC } \\
\text { DSC } \\
\text { DTA }\end{array}$ & $\begin{array}{l}\text { sulfamethazine) } \\
\text { [2016DEL/ALM] } \\
\text { [2003MAR/AVI, 2002MAR/GOM, 2001MAR/GOM] } \\
\text { [1983KHA] } \\
\text { [1971SUN/EIS] }\end{array}$ \\
\hline $\mathrm{C}_{12} \mathrm{H}_{14} \mathrm{~N}_{4} \mathrm{O}_{4} \mathrm{~S}$ & $\begin{array}{c}{[122-11-2]} \\
\text { FUS }\end{array}$ & 4-amino- $N-(2$ & $\begin{array}{l}\text { limethoxy-4-p } \\
45.6\end{array}$ & $\begin{array}{l}\text { imidinyl } \\
476.7\end{array}$ & $\begin{array}{l}\text { nzenesulf } \\
\text { DTA }\end{array}$ & $\begin{array}{c}\text { mide (sulfisomidine) } \\
\text { [1986MAU/RAM] }\end{array}$ \\
\hline
\end{tabular}

[Note: The molecular formula given in [1986MAU/RAM] was not consistent with the molecular structure.]

$\mathrm{C}_{12} \mathrm{H}_{14} \mathrm{O}_{2}$

[946-38-3] Ethyl cis-2-phenylcyclopropanecarboxylate 
TABLE 11. Phase change enthalpies of $\mathrm{C}_{12}$ to $\mathrm{C}_{13}$ organic compounds-Continued

\begin{tabular}{|c|c|c|c|c|c|c|}
\hline \multirow[b]{2}{*}{ Molecular formula } & \multirow{2}{*}{$\begin{array}{l}\text { CAS Registry } \\
\text { Number } \\
\text { Enthalpy }\end{array}$} & \multicolumn{5}{|l|}{ Compound } \\
\hline & & $\begin{array}{c}\text { Temperature } \\
\text { range }\end{array}$ & $\begin{array}{l}\Delta_{\text {trans }} H_{\mathrm{m}} \\
(\mathrm{kJ} / \mathrm{mol})\end{array}$ & $\begin{array}{l}T_{\mathrm{m}} \\
(\mathrm{K})\end{array}$ & Method & References \\
\hline & $\mathrm{V}$ & & $70.7 \pm 0.6$ & 298 & $\mathrm{C}$ & [1998KOL/PIM] \\
\hline \multirow[t]{2}{*}{$\mathrm{C}_{12} \mathrm{H}_{14} \mathrm{O}_{2}$} & [946-39-4] & \multicolumn{5}{|c|}{ Ethyl trans-2-phenylcyclopropanecarboxylate } \\
\hline & SUB & & $96.9 \pm 0.4$ & 298 & $\mathrm{C}$ & [1998KOL/PIM] \\
\hline \multirow[t]{2}{*}{$\mathrm{C}_{12} \mathrm{H}_{14} \mathrm{O}_{3}$} & {$[93-28-7]$} & \multicolumn{5}{|c|}{ 1-acetoxy-2-methoxy-4-allylbenzene (eugenol acetate) } \\
\hline & V & $(374-555)$ & 63.1 & 389 & A & [1987STE/MAL, 1947STU] \\
\hline \multirow[t]{2}{*}{$\mathrm{C}_{12} \mathrm{H}_{14} \mathrm{O}_{3}$} & {$[70637-00-2]$} & \multicolumn{5}{|c|}{ 4-methyl-1-phenyl-2,6,7-trioxabicyclo[2.2.2]octane } \\
\hline & FUS & & 20.9 & 410.2 & & [1995RAK/VER2] \\
\hline \multirow{2}{*}{$\mathrm{C}_{12} \mathrm{H}_{14} \mathrm{O}_{4}$} & {$[523-80-8]$} & \multicolumn{5}{|c|}{ 4,7-dimethoxy-5-(2-propen-1-yl)-1,3-benzodioxole (apiol) } \\
\hline & V & $(389-558)$ & 70.6 & 404 & A & {$[1987 \mathrm{STE} / \mathrm{MAL}]$} \\
\hline \multirow[t]{18}{*}{$\mathrm{C}_{12} \mathrm{H}_{14} \mathrm{O}_{4}$} & {$[84-66-2]$} & \multicolumn{5}{|c|}{ Diethyl phthalate } \\
\hline & FUS & & 17.99 & 269.9 & & [1996DOM/HEA, 1967CHA/HOR] \\
\hline & $\mathrm{V}$ & & $88.6 \pm 3.4$ & 298 & CRT & [2015GOB/CHI] \\
\hline & $\mathrm{V}$ & & $73.9 \pm 10$ & 298 & CGC & [2015GOB/CHI] \\
\hline & $\mathrm{V}$ & & $82.1 \pm 1.6$ & 298 & CGC & [2014GOB/CHI] \\
\hline & $\mathrm{V}$ & & $82.1 \pm 0.5$ & 298 & $\mathrm{~EB}, \mathrm{ME}$ & [2004ROH/RUZ] \\
\hline & $\mathrm{V}$ & & 87.4 & 298 & $\mathrm{~EB}, \mathrm{ME}$ & [2004ROH/RUZ] \\
\hline & $\mathrm{V}$ & $(404-520)$ & 74.6 & 426 & BG & [1988KAT] \\
\hline & $\mathrm{V}$ & $(404-520)$ & 70.0 & 441 & BG & [1988KAT] \\
\hline & $\mathrm{V}$ & $(404-520)$ & 66.5 & 459 & BG & [1988KAT] \\
\hline & $\mathrm{V}$ & $(404-520)$ & 64.2 & 478 & BG & [1988KAT] \\
\hline & $\mathrm{V}$ & $(404-520)$ & 63.3 & 497 & BG & [1988KAT] \\
\hline & $\mathrm{V}$ & $(345-453)$ & 77.9 & 360 & A & [1987STE/MAL] \\
\hline & $\mathrm{V}$ & $(421-570)$ & 59.1 & 436 & A & [1987STE/MAL] \\
\hline & $\mathrm{V}$ & $(307-333)$ & 86.8 & 310 & GS & [1982GRA/FOS] \\
\hline & $\mathrm{V}$ & & $81.1 \pm 0.8$ & 298 & GCC & [1980FUC/PEA] \\
\hline & $\mathrm{V}$ & & 82.4 & & & [1948SMA/SMA] \\
\hline & $\mathrm{V}$ & $(381-567)$ & 65.9 & 396 & & [1947STU] \\
\hline \multirow[t]{2}{*}{$\mathrm{C}_{12} \mathrm{H}_{14} \mathrm{O}_{4}$} & {$[636-09-9]$} & \multicolumn{5}{|c|}{ Diethyl terephthalate } \\
\hline & FUS & & 24.60 & 317.2 & & [1996DOM/HEA, 1956SMI/DOL] \\
\hline \multirow[t]{3}{*}{$\mathrm{C}_{12} \mathrm{H}_{14} \mathrm{O}_{4}$} & {$[28153-24-4]$} & \multicolumn{5}{|c|}{ 1,1-diacetoxy-1-phenylethane } \\
\hline & FUS & & 29.37 & 355.4 & DSC & [1996VER/PEN] \\
\hline & SUB & $(308-338)$ & $94.4 \pm 2.2$ & 318 & GS & [1996VER/PEN] \\
\hline \multirow[t]{2}{*}{$\mathrm{C}_{12} \mathrm{H}_{14} \mathrm{O}_{5}$} & {$[20733-94-2]$} & \multicolumn{5}{|c|}{ Methyl 4-hydroxy-3,5-dimethoxycinnamate (methyl sinapate) } \\
\hline & FUS & & 29.85 & 361.8 & DSC & [2010PAN/SAR] \\
\hline $\mathrm{C}_{12} \mathrm{H}_{15} \mathrm{ClNO}_{4} \mathrm{PS}_{2}$ & {$[2310-17-0]$} & $S$-6-chloro-2, & hydro-2-oxol & zoxazol & lmethyl $C$ & -diethylphosphorodithioate \\
\hline & FUS & & 30.03 & 320 & DSC & [1990DON/DRE] \\
\hline $\mathrm{C}_{12} \mathrm{H}_{15} \mathrm{~N}$ & {$[6247-00-3]$} & $N, N$-diallyl an & & & & \\
\hline & V & $(421-513)$ & 54.8 & 436 & A & [1987STE/MAL] \\
\hline $\mathrm{C}_{12} \mathrm{H}_{15} \mathrm{NO}$ & {$[4783-65-7]$} & 1-benzyl-2-pir & done & & & \\
\hline & V & & $91.3 \pm 1.0$ & 298 & $\mathrm{C}$ & [2006RIB/CAB] \\
\hline $\mathrm{C}_{12} \mathrm{H}_{15} \mathrm{NO}$ & [3612-20-2] & 1-benzy 1-4-pi & done & & & \\
\hline & V & & $78.0 \pm 0.8$ & 298 & $\mathrm{C}$ & [2006RIB/CAB] \\
\hline $\mathrm{C}_{12} \mathrm{H}_{15} \mathrm{NO}_{2}$ & {$[19288-59-6]$} & Phenylaminoe & methacrylat & & & \\
\hline & FUS & & 25.47 & 297.5 & $\mathrm{AC}$ & [1996DOM/HEA, 1985KAR/ABD] \\
\hline $\mathrm{C}_{12} \mathrm{H}_{15} \mathrm{NO}_{2} \mathrm{~S}$ & [21406-29-1] & $N$-benzoylthi & bamic $O$-but & ster & & \\
\hline & SUB & & $120.7 \pm 1.8$ & 298 & $\mathrm{C}$ & [2004RIB/SAN2] \\
\hline $\mathrm{C}_{12} \mathrm{H}_{15} \mathrm{NO}_{3}$ & {$[1563-66-2]$} & 2,3-dihydro-2, & imethylbenzc & an-7-yl & hylcarbar & \\
\hline & FUS & & 30.33 & 426.2 & DSC & [1990DON/DRE] \\
\hline $\mathrm{C}_{12} \mathrm{H}_{15} \mathrm{NO}_{3}$ & [1665-48-1] & 5 -[(3,5-dimeth & henoxy)meth & $1,3-\mathrm{oxa}$ & din-2-one & etaxalone) \\
\hline & FUS (triclinic) & & 30.3 & 395.1 & & \\
\hline & $\begin{array}{c}\text { FUS } \\
\text { (monoclinic) }\end{array}$ & & 26.4 & 395.4 & DSC & [2011AIT/CHO] \\
\hline
\end{tabular}


TABLE 11. Phase change enthalpies of $\mathrm{C}_{12}$ to $\mathrm{C}_{13}$ organic compounds-Continued

\begin{tabular}{|c|c|c|c|c|c|c|}
\hline \multirow[b]{2}{*}{ Molecular formula } & \multirow{2}{*}{$\begin{array}{l}\text { CAS Registry } \\
\text { Number } \\
\text { Enthalpy }\end{array}$} & \multicolumn{5}{|l|}{ Compound } \\
\hline & & $\begin{array}{c}\text { Temperature } \\
\text { range }\end{array}$ & $\begin{array}{l}\Delta_{\text {trans }} H_{\mathrm{m}} \\
(\mathrm{kJ} / \mathrm{mol})\end{array}$ & $\begin{array}{l}T_{\mathrm{m}} \\
(\mathrm{K})\end{array}$ & Method & References \\
\hline \multirow[t]{2}{*}{$\mathrm{C}_{12} \mathrm{H}_{15} \mathrm{~N}_{2} \mathrm{O}_{3} \mathrm{PS}$} & {$[13593-03-8]$} & \multicolumn{5}{|c|}{$O, O$-diethyl $O$-quinoxalin-2-yl phosphothioate } \\
\hline & FUS & & 25.4 & 304.1 & DSC & [1990DON/DRE] \\
\hline \multirow[t]{3}{*}{$\mathrm{C}_{12} \mathrm{H}_{15} \mathrm{~N}_{3} \mathrm{OS}$} & {$[1245618-41-0]$} & \multicolumn{5}{|c|}{ 1-[(5-p-tolylamino)-1,2,4-thiadiazol-3-yl]-2-propanol } \\
\hline & FUS & & 22.0 & 390.1 & DSC & [2010PER/VOL] \\
\hline & SUB & $(344-364)$ & $142.0 \pm 1.1$ & 298 & GS & [2010PER/VOL] \\
\hline \multirow[t]{4}{*}{$\mathrm{C}_{12} \mathrm{H}_{15} \mathrm{~N}_{3} \mathrm{OS}$} & {$[1275515-23-5]$} & \multicolumn{5}{|c|}{$\alpha$-methyl-5-[(3-methylphenyl)amino]-1,2,4-thiadiazole-3-ethanol } \\
\hline & FUS & & 26.8 & 361.5 & DSC & [2013SUR/BUI] \\
\hline & SUB & $(341-359)$ & $134.8 \pm 1.9$ & 350 & GS & [2013SUR/BUI] \\
\hline & SUB & $(341-359)$ & $137.3 \pm 1.9$ & 298 & GS & [2013SUR/BUI] \\
\hline \multirow{3}{*}{$\mathrm{C}_{12} \mathrm{H}_{15} \mathrm{~N}_{3} \mathrm{O}_{2}$} & {$[5972-07-6]$} & \multicolumn{5}{|c|}{ 3,6-bis(dimethylamino)phthalimide } \\
\hline & SUB & $(400-457)$ & 105 & 415 & A & [1987STE/MAL] \\
\hline & SUB & & 135.3 & & RG & [1958KLO] \\
\hline \multirow[t]{4}{*}{$\mathrm{C}_{12} \mathrm{H}_{15} \mathrm{~N}_{3} \mathrm{O}_{2} \mathrm{~S}$} & [1361124-43-7] & \multicolumn{5}{|c|}{$\alpha$-methyl-5-[(4-methoxyphenyl)amino]-1,2,4-thiadiazole-3-ethanol } \\
\hline & FUS & & 28.8 & 363.4 & DSC & [2013SUR/BUI] \\
\hline & SUB & $(343-356)$ & $126.1 \pm 2.1$ & 350 & GS & [2013SUR/BUI] \\
\hline & SUB & $(343-356)$ & $129.0 \pm 2.1$ & 298 & GS & [2013SUR/BUI] \\
\hline \multirow[t]{2}{*}{$\mathrm{C}_{12} \mathrm{H}_{15} \mathrm{~N}_{3} \mathrm{O}_{2} \mathrm{~S}$} & {$[54965-21-8]$} & \multicolumn{5}{|c|}{ Methyl [5-(propylthio)-1H-benzoimidazol-2-yl]carbamate (albendazole) } \\
\hline & FUS & & 44.71 & 483.2 & DSC & [2015GAU/VAN] \\
\hline \multirow[t]{3}{*}{$\mathrm{C}_{12} \mathrm{H}_{15} \mathrm{~N}_{3} \mathrm{O}_{6}$} & {$[81-15-2]$} & \multicolumn{5}{|c|}{ 2,4,6-trmitro-1,3-dimethyl-5-tert-butylbenzene } \\
\hline & FUS & & 20.79 & 386.7 & DSC & {$[2004 \mathrm{QU} / \mathrm{BAI}]$} \\
\hline & SUB & $(312-348)$ & 100.4 & 327 & A & [1987STE/MAL, 1956SER/VOI] \\
\hline \multirow{2}{*}{$\mathrm{C}_{12} \mathrm{H}_{15} \mathrm{~N}_{5} \mathrm{O}_{4}$} & & \multicolumn{5}{|c|}{ 9-[(2-acetoxyethoxy)methyl]-2-acetylamino-9H-purme } \\
\hline & FUS & & 42.33 & 407.2 & DSC & [1995KRI/VES] \\
\hline \multirow[t]{2}{*}{$\mathrm{C}_{12} \mathrm{H}_{15} \mathrm{~N}_{5} \mathrm{O}_{5}$} & {$[75128-73-3]$} & \multicolumn{5}{|c|}{ 9-[(2-acetoxyethoxy)methyl]-2-acetylamino-1,9-dihydro-6H-purm-6-one } \\
\hline & FUS & & 47.37 & 477.2 & DSC & [1995KRI/VES] \\
\hline $\mathrm{C}_{12} \mathrm{H}_{16}$ & $\begin{array}{c}\text { [827-52-1] } \\
\text { FUS }\end{array}$ & $\begin{array}{l}\text { Cyclohexylben } \\
(220-470)\end{array}$ & 15.3 & 280.5 & DSC & [1996DOM/HEA, 1983ORO/MRA] \\
\hline & $\mathrm{V}$ & $(344-462)$ & $60.8 \pm 0.2$ & 298 & MM & [1998MOK/RAU, 2006VER] \\
\hline & $\mathrm{V}$ & $(283-462)$ & 60.4 & 298 & & [1993KAS/MOK] \\
\hline & $\mathrm{V}$ & $(333-343)$ & 56.4 & 348 & & [1990SOH/OKA] \\
\hline & $\mathrm{V}$ & $(421-513)$ & 51.3 & 436 & A & [1987STE/MAL] \\
\hline & $\mathrm{V}$ & & $59.9 \pm 0.3$ & 298 & $\mathrm{C}$ & [1978MON/ROS] \\
\hline $\mathrm{C}_{12} \mathrm{H}_{16}$ & & Dicyclohexadi & & & & \\
\hline & $\mathrm{V}$ & $(377-505)$ & 77.9 & 329 & A & [1987STE/MAL] \\
\hline $\mathrm{C}_{12} \mathrm{H}_{16}$ & {$[2715-29-9]$} & 2,5-diethylstyr & & & & \\
\hline & $\mathrm{V}$ & $(322-496)$ & 52.2 & 337 & A & [1987STE/MAL, 1947STU] \\
\hline $\mathrm{C}_{12} \mathrm{H}_{16}$ & {$[5676-29-9]$} & $\alpha$-tert-butylsty & & & & \\
\hline & V & $(298-318)$ & $53.2 \pm 0.1$ & 298 & GS & [1999VER/EBE] \\
\hline $\mathrm{C}_{12} \mathrm{H}_{16}$ & {$[2388-14-9]$} & 1-isopropenyl- & sopropylbenz & & & \\
\hline & $\mathrm{V}$ & (403-479) & 50.9 & 418 & A & [1987STE/MAL] \\
\hline $\mathrm{C}_{12} \mathrm{H}_{16}$ & {$[24375-17-5]$} & Tetraspiro[2.0. & 2.0.2.0]dode & e $([4] \mathrm{r}$ & & \\
\hline & FUS & & 21 & 394.9 & DSC & [1995BEC/RUC] \\
\hline & SUB & $(298-338)$ & $74.9 \pm 0.5$ & & GS & [1995BEC/RUC] \\
\hline $\mathrm{C}_{12} \mathrm{H}_{16} \mathrm{Cl}_{2} \mathrm{~N}_{2} \mathrm{O}$ & [555-37-3] & $N$-butyl- $N^{\prime}-(3$ & dichlorophen & $N$-met & rea & \\
\hline & FUS & & 27.23 & 374.3 & DSC & [1990DON/DRE] \\
\hline $\mathrm{C}_{12} \mathrm{H}_{16} \mathrm{~N}_{2}$ & [126401-68-1] & 1-pentylbenzin & azole & & & \\
\hline & $\mathrm{V}$ & $(328-375)$ & $77.0 \pm 0.5$ & 298 & GS & [2012GAR/VER] \\
\hline $\mathrm{C}_{12} \mathrm{H}_{16} \mathrm{~N}_{2}$ & {$[61-50-7]$} & 2-(1H-mdol-3 & $-N, N$-dimet & ethanan & $(N, N-\mathrm{d}$ & thyltryptamine) \\
\hline
\end{tabular}


TABLE 11. Phase change enthalpies of $\mathrm{C}_{12}$ to $\mathrm{C}_{13}$ organic compounds-Continued

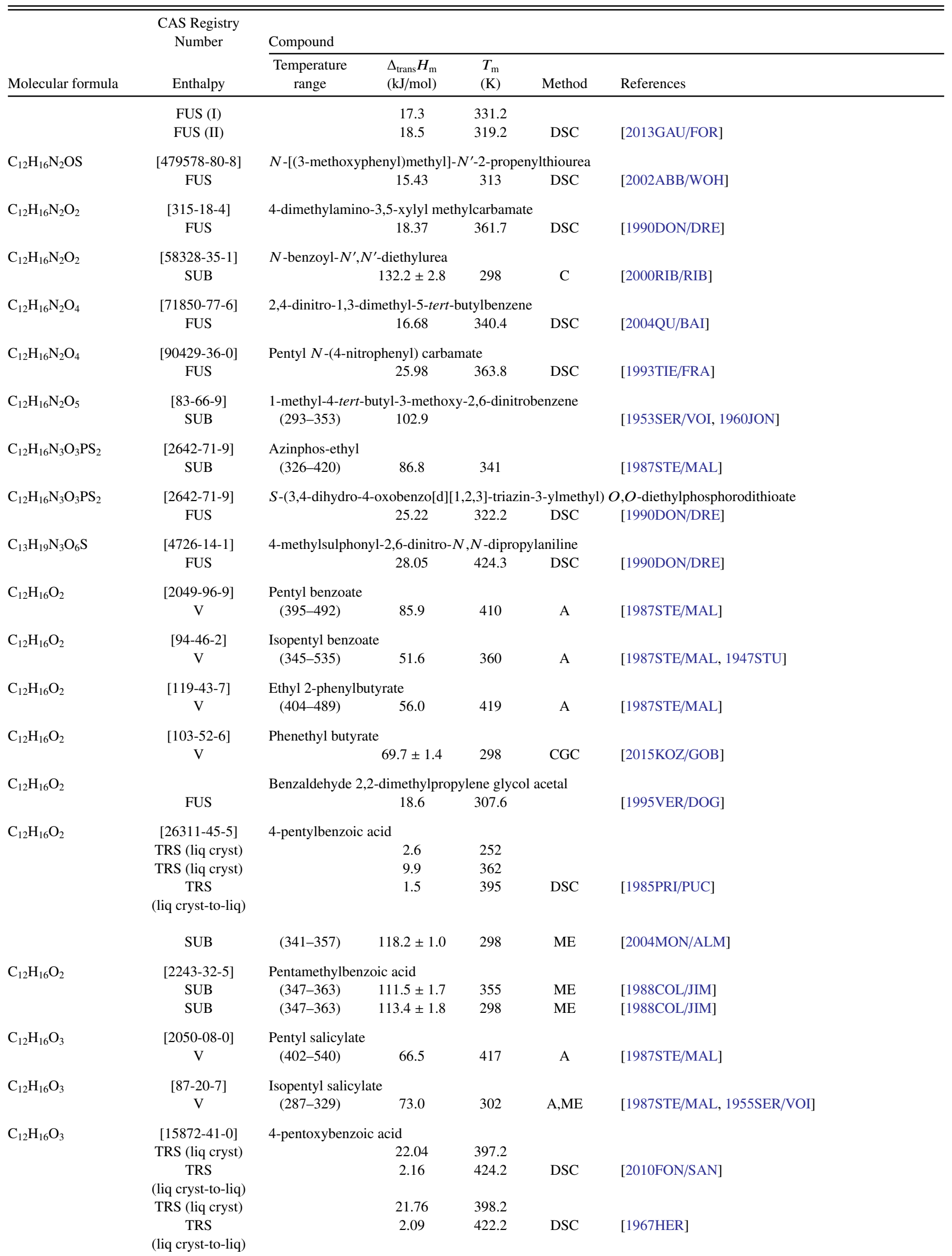


TABLE 11. Phase change enthalpies of $\mathrm{C}_{12}$ to $\mathrm{C}_{13}$ organic compounds-Continued

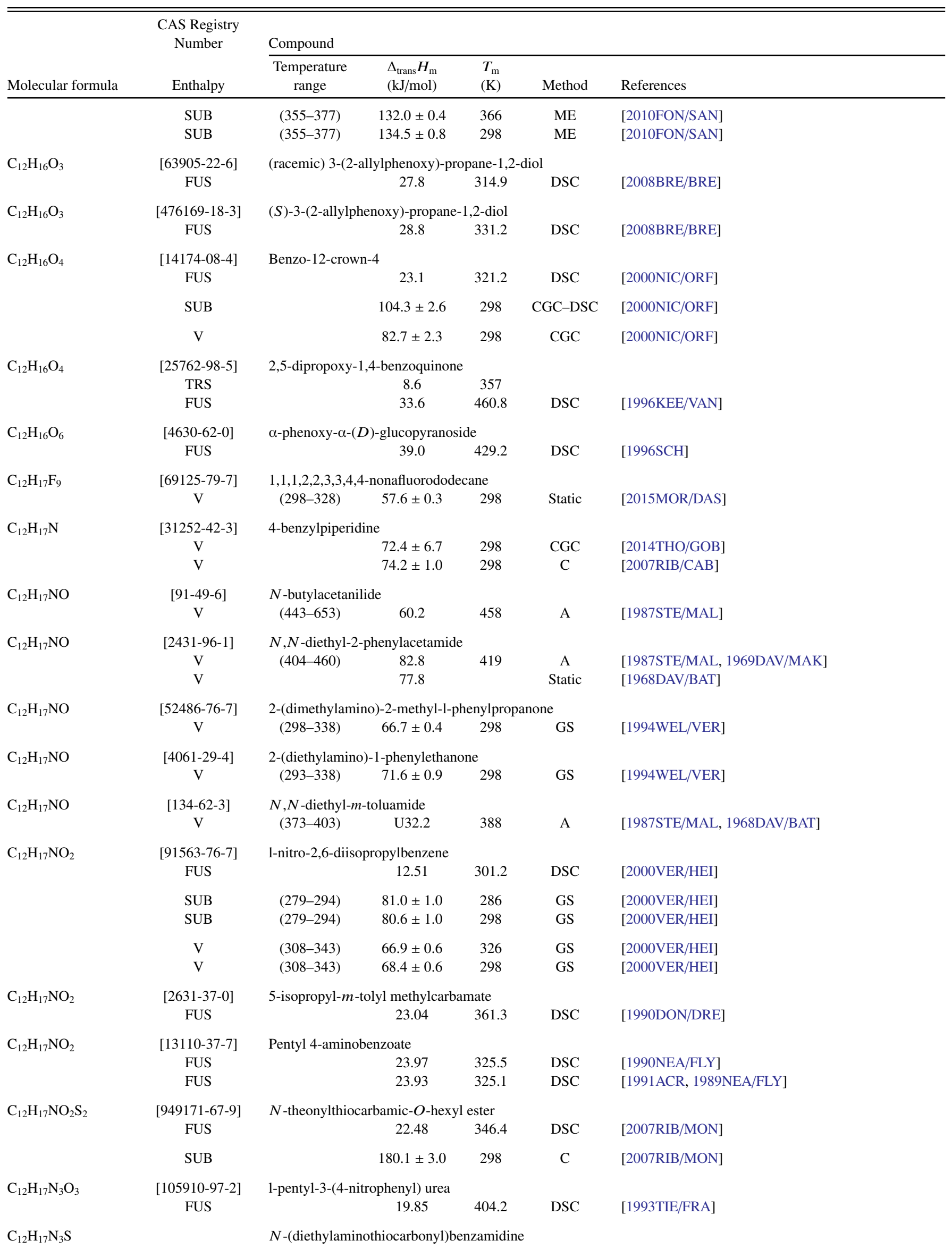


TABLE 11. Phase change enthalpies of $\mathrm{C}_{12}$ to $\mathrm{C}_{13}$ organic compounds-Continued

\begin{tabular}{|c|c|c|c|c|c|c|}
\hline \multirow[b]{2}{*}{ Molecular formula } & \multirow{2}{*}{$\begin{array}{c}\text { CAS Registry } \\
\text { Number } \\
\text { Enthalpy }\end{array}$} & \multicolumn{5}{|l|}{ Compound } \\
\hline & & $\begin{array}{c}\text { Temperature } \\
\text { range }\end{array}$ & $\begin{array}{l}\Delta_{\text {trans }} H_{\mathrm{m}} \\
(\mathrm{kJ} / \mathrm{mol})\end{array}$ & $\begin{array}{l}T_{\mathrm{m}} \\
(\mathrm{K})\end{array}$ & Method & References \\
\hline & SUB & & $126.0 \pm 1.5$ & 298 & $\mathrm{C}$ & [2004RIB/SAN] \\
\hline \multirow[t]{4}{*}{$\mathrm{C}_{12} \mathrm{H}_{18}$} & [4904-61-4] & \multicolumn{5}{|c|}{ 1-cis-5-trans-9-trans-cyclododecatriene } \\
\hline & $\mathrm{V}$ & $(344-387)$ & 49.9 & 359 & A & [1987STE/MAL] \\
\hline & $\mathrm{V}$ & $(400-423)$ & 60.0 & 411 & A & [1987STE/MAL] \\
\hline & $\mathrm{V}$ & $(426-503)$ & 47.8 & 441 & A & [1987STE/MAL] \\
\hline \multirow[t]{2}{*}{$\mathrm{C}_{12} \mathrm{H}_{18}$} & [706-31-0] & \multicolumn{5}{|c|}{ 1-trans-5-trans-9-cis-cyclododecatriene } \\
\hline & V & $(286-373)$ & 68.0 & 301 & A & [1987STE/MAL] \\
\hline \multirow[t]{4}{*}{$\mathrm{C}_{12} \mathrm{H}_{18}$} & {$[1077-16-3]$} & \multicolumn{5}{|l|}{ Hexylbenzene } \\
\hline & $\mathrm{V}$ & & 60.2 & 298 & & [1994RUZ/ZAB] \\
\hline & $\mathrm{V}$ & $(274-463)$ & 61.6 & 289 & & [1993KAS/MOK] \\
\hline & $\mathrm{V}$ & & 60.0 & 298 & & [1971WIL/ZWO] \\
\hline \multirow[t]{2}{*}{$\mathrm{C}_{12} \mathrm{H}_{18}$} & {$[577-55-9]$} & \multicolumn{5}{|c|}{ 1,2-diisopropylbenzene } \\
\hline & V & $(388-476)$ & 48.9 & 403 & A & [1987STE/MAL] \\
\hline \multirow[t]{4}{*}{$\mathrm{C}_{12} \mathrm{H}_{18}$} & [99-62-7] & \multicolumn{5}{|c|}{ 1,3-diisopropylbenzene } \\
\hline & V & $(283-318)$ & $56.0 \pm 0.8$ & 301 & GS & [1998VER7] \\
\hline & $\mathrm{V}$ & (283-318) & $56.2 \pm 0.8$ & 298 & GS & [1998VER7] \\
\hline & $\mathrm{V}$ & $(387-477)$ & 48.9 & 402 & A & [1987STE/MAL] \\
\hline \multirow[t]{9}{*}{$\mathrm{C}_{12} \mathrm{H}_{18}$} & {$[100-18-5]$} & \multicolumn{5}{|c|}{ 1,4-diisopropylbenzene } \\
\hline & $\mathrm{V}$ & $(366-530)$ & $50.7 \pm 0.2$ & 400 & EB & [2002STE/CHI6] \\
\hline & $\mathrm{V}$ & $(366-530)$ & $46.3 \pm 0.3$ & 440 & EB & [2002STE/CHI6] \\
\hline & $\mathrm{V}$ & $(366-530)$ & $43.0 \pm 0.5$ & 480 & EB & [2002STE/CHI6] \\
\hline & $\mathrm{V}$ & $(366-530)$ & $39.3 \pm 0.9$ & 520 & EB & [2002STE/CHI6] \\
\hline & $\mathrm{V}$ & $(283-318)$ & $56.3 \pm 0.3$ & 301 & GS & [1998VER7] \\
\hline & $\mathrm{V}$ & $(283-318)$ & $56.5 \pm 0.3$ & 298 & GS & [1998VER7] \\
\hline & $\mathrm{V}$ & $(393-485)$ & 47.6 & 408 & A & {$[1987 \mathrm{STE} / \mathrm{MAL}]$} \\
\hline & $\mathrm{V}$ & $(393-485)$ & 48.9 & 408 & & [1959MCD/SHR, 1984BOU/FRI] \\
\hline \multirow[t]{4}{*}{$\mathrm{C}_{12} \mathrm{H}_{18}$} & {$[98-19-1]$} & \multicolumn{5}{|c|}{ 1,3-dimethyl-5-tert-butylbenzene } \\
\hline & $\mathrm{V}$ & (284-318) & $56.5 \pm 0.6$ & 301 & GS & [1998VER] \\
\hline & $\mathrm{V}$ & (284-318) & $56.6 \pm 0.6$ & 298 & GS & [1998VER] \\
\hline & $\mathrm{V}$ & $(253-443)$ & 59.8 & 268 & & {$[1993 \mathrm{KAS} / \mathrm{MOK}]$} \\
\hline $\mathrm{C}_{12} \mathrm{H}_{18}$ & {$[87-85-4]$} & Hexamethylbe & & & & \\
\hline & FUS & & 23.27 & 438.7 & DSC & [2008MOG/SEP] \\
\hline & FUS & & 21.1 & 439.5 & DTA & {$[1994 \mathrm{SAB} / \mathrm{TAB}]$} \\
\hline & TRS & & 1.5 & 384.0 & DSC & [1996DOM/HEA, 1988PET/TSY] \\
\hline & TRS & & 1.1 & 115.5 & $\mathrm{AC}$ & [1996DOM/HEA, 1985YOS/FUJ] \\
\hline & TRS & & 0.98 & 117.5 & $\mathrm{AC}$ & [1982ATA/GYO] \\
\hline & TRS & & 1.1 & 116.5 & $\mathrm{AC}$ & [1965FRA/AST] \\
\hline & TRS & & 1.84 & 383.8 & & \\
\hline & FUS & & 20.59 & 438.4 & & [1996DOM/HEA, 1956MOM/SUG] \\
\hline & TRS & & 1.76 & 383.7 & & \\
\hline & FUS & & 20.63 & 438.7 & $\mathrm{RC}$. & [1996DOM/HEA, 1932SPA/THO] \\
\hline & TRS & & 1.0 & 108 & $\mathrm{C}$ & [1930HUF/PAR2] \\
\hline & SUB & & 80 & & TGA & [1997GIL/BOT] \\
\hline & SUB & & $81.4 \pm 0.1$ & 298 & $\mathrm{C}$ & [1994SAB/TAB] \\
\hline & SUB & $(288-304)$ & $85.0 \pm 0.2$ & 298 & $\mathrm{ME}$ & [1989COL/JIM] \\
\hline & SUB & & $74.9 \pm 0.6$ & & DSC & [1984HOL] \\
\hline & SUB & $(303-338)$ & 85.2 & 320 & A & [1976AMB/LAW] \\
\hline & SUB & & 86.1 & 298 & $\mathrm{H}$ & [1976AMB/LAW, 1993CHI/HOS] \\
\hline & SUB & $(314-364)$ & 83.2 & 329 & A & [1969OVE/STE] \\
\hline & SUB & & $74.7 \pm 2$ & & $\mathrm{ME}$ & [1965FRA/AST, 1970COX/PIL] \\
\hline & SUB & & 80.8 & & & [1957VAN, 1960JON] \\
\hline & SUB & & 80.8 & & & [1949NIT/SEK] \\
\hline & $\mathrm{V}$ & & 68.6 & 298 & CGC & {$[2008 \mathrm{ZHA} / \mathrm{UNH}]$} \\
\hline & $\mathrm{V}$ & $(443-537)$ & 56.8 & 458 & A & [1987STE/MAL, 1930MAC/SMI] \\
\hline$\left(\mathrm{C}_{12} \mathrm{H}_{18}\right)-\left(\mathrm{C}_{6} \mathrm{H}_{3} \mathrm{~N}_{2} \mathrm{ClO}_{4}\right)$ & {$[57230-36-1]$} & (hexamethylb & ene)-(picryl cl & ride) & & \\
\hline & SUB & & 93.7 & & & [1949NIT/SEK] \\
\hline
\end{tabular}


TABLE 11. Phase change enthalpies of $\mathrm{C}_{12}$ to $\mathrm{C}_{13}$ organic compounds-Continued

\begin{tabular}{|c|c|c|c|c|c|c|}
\hline \multirow[b]{2}{*}{ Molecular formula } & \multirow{2}{*}{$\begin{array}{l}\text { CAS Registry } \\
\text { Number } \\
\text { Enthalpy }\end{array}$} & \multicolumn{5}{|l|}{ Compound } \\
\hline & & $\begin{array}{l}\text { Temperature } \\
\text { range }\end{array}$ & $\begin{array}{l}\Delta_{\text {trans }} H_{\mathrm{m}} \\
(\mathrm{kJ} / \mathrm{mol})\end{array}$ & $\begin{array}{l}T_{\mathrm{m}} \\
(\mathrm{K})\end{array}$ & Method & References \\
\hline $\mathrm{C}_{12} \mathrm{H}_{18}$ & $\begin{array}{c}{[877-44-1]} \\
\mathrm{V}\end{array}$ & $\begin{array}{l}\text { 1,2,4-triethylb } \\
\text { (319-491) }\end{array}$ & ene 51.2 & 334 & A & [1987STE/MAL, 1947STU] \\
\hline $\mathrm{C}_{12} \mathrm{H}_{18}$ & $\begin{array}{c}{[102-25-0]} \\
\mathrm{V}\end{array}$ & $\begin{array}{l}\text { 1,3,5-triethylb } \\
(371-534)\end{array}$ & $59.2 \pm 0.3$ & 298 & EB & [1997STE/CHI2] \\
\hline $\mathrm{C}_{12} \mathrm{H}_{18}$ & $\begin{array}{c}{[10222-95-4]} \\
\mathrm{V}\end{array}$ & 1,2,4-trimethy & $\begin{array}{l}\text { isopropylben } \\
64.9\end{array}$ & 298 & & [1975VIL/PER] \\
\hline $\mathrm{C}_{12} \mathrm{H}_{18}$ & $\begin{array}{c}{[6902-73-4]} \\
\mathrm{V}\end{array}$ & $\begin{array}{l}\text { 2-isopropenyl- } \\
(348-404)\end{array}$ & $\begin{array}{l}\text { hethyl-1-viny } \\
47.8\end{array}$ & $\begin{array}{l}\text {-cycloh } \\
363\end{array}$ & A & [1987STE/MAL] \\
\hline $\mathrm{C}_{12} \mathrm{H}_{18}$ & $\begin{array}{l}{[676-22-2]} \\
\text { SUB } \\
\text { SUB }\end{array}$ & $\begin{array}{l}E, E, E-1,5,9-\mathrm{cy} \\
\quad(273-307)\end{array}$ & $\begin{array}{c}\text { dodecatriene } \\
75.2 \\
74.7 \pm 0.8\end{array}$ & 288 & A & $\begin{array}{l}\text { [1987STE/MAL] } \\
\text { [1973RAU/GEY, 1977PED/RYL] }\end{array}$ \\
\hline $\mathrm{C}_{12} \mathrm{H}_{18} \mathrm{ClNO}$ & $\begin{array}{l}\text { [41570-61-0] } \\
\text { FUS (I) } \\
\text { FUS (II) }\end{array}$ & 2-chloro- $\alpha-[[($ & $\begin{array}{c}\text { dimethylethy } \\
27.1 \\
25.4\end{array}$ & $\begin{array}{c}\operatorname{mino}] \mathrm{m} \\
364 \\
354\end{array}$ & $\begin{array}{l}\text { l]benzen } \\
\text { DSC }\end{array}$ & $\begin{array}{l}\text { thanol (tulobuterol) } \\
\text { [2004CAI/BOU] }\end{array}$ \\
\hline $\mathrm{C}_{12} \mathrm{H}_{18} \mathrm{Cl}_{2} \mathrm{NOPS}$ & $\begin{array}{c}{[42585-08-0]} \\
\mathrm{V}\end{array}$ & $\begin{array}{l}\text { (2-chloro-4-m } \\
(309-363)\end{array}$ & $\begin{array}{l}\text { lphenyl) } N- \\
62.6\end{array}$ & $\begin{array}{l}\text {-butyla } \\
324\end{array}$ & $\begin{array}{l}\text { o)(chloro } \\
\text { A }\end{array}$ & $\begin{array}{l}\text { hyl)thiophosphonate } \\
\text { [1987STE/MAL] }\end{array}$ \\
\hline $\mathrm{C}_{12} \mathrm{H}_{18} \mathrm{~N}_{2} \mathrm{O}$ & $\begin{array}{l}\text { [34123-59-6] } \\
\text { FUS }\end{array}$ & $N, N$-dimethy & $\begin{array}{c}\text { '-[4-(1-meth } \\
33.87\end{array}$ & $\begin{array}{l}\text { hyl)phe } \\
430.4\end{array}$ & $\begin{array}{l}\text { urea } \\
\text { DSC }\end{array}$ & [1991ACR, 1990DON/DRE] \\
\hline $\mathrm{C}_{12} \mathrm{H}_{18} \mathrm{~N}_{2} \mathrm{O}$ & $\begin{array}{c}\text { [34123-59-6] } \\
\text { FUS }\end{array}$ & $\begin{array}{c}N^{\prime}-(p \text {-cumeny } \\
\quad(78-346)\end{array}$ & $\begin{array}{c}N, N \text {-dimeth } \\
21.33\end{array}$ & $\begin{array}{l}\text { rea (iso } \\
427.4\end{array}$ & $\begin{array}{l}\text { uron) } \\
\mathrm{AC}\end{array}$ & [2003YU/TAN2] \\
\hline $\mathrm{C}_{12} \mathrm{H}_{18} \mathrm{~N}_{2} \mathrm{O}_{2}$ & $\begin{array}{c}\text { [315-18-4] } \\
\text { FUS }\end{array}$ & 3,5-dimethyl-4 & $\begin{array}{c}\text { imethylamin } \\
18.37\end{array}$ & $\begin{array}{l}\text { henyl m } \\
361.7\end{array}$ & $\begin{array}{l}\text { lcarbama } \\
\text { DSC }\end{array}$ & [1991ACR, 1990DON/DRE] \\
\hline $\mathrm{C}_{12} \mathrm{H}_{18} \mathrm{~N}_{2} \mathrm{O}_{2} \mathrm{~S}_{2}$ & $\begin{array}{c}\text { [120563-92-0] } \\
\text { FUS }\end{array}$ & $N$-isopropyl-S & $\begin{array}{l}\text { thyl- } N^{\prime} \text {-tosy } \\
32.7\end{array}$ & $\begin{array}{l}\text { thioure } \\
392.2\end{array}$ & DSC & [1992REI/HAN] \\
\hline $\mathrm{C}_{12} \mathrm{H}_{18} \mathrm{~N}_{2} \mathrm{O}_{2} \mathrm{~S}_{2}$ & $\begin{array}{c}\text { [145198-68-1] } \\
\text { FUS }\end{array}$ & $N$-ethyl-S-eth & $\begin{array}{l}V^{\prime} \text {-tosylisothi } \\
30.3\end{array}$ & $\begin{array}{l}\text { ea } \\
390.2\end{array}$ & DSC & [1992REI/HAN] \\
\hline $\mathrm{C}_{12} \mathrm{H}_{18} \mathrm{~N}_{2} \mathrm{O}_{3}$ & $\begin{array}{l}{[76-73-3]} \\
\text { FUS } \\
\text { FUS }\end{array}$ & 5-(1-methylbu & $\begin{array}{c}\text {-5-(2-propen } \\
22.9 \\
17.4\end{array}$ & $\begin{array}{l}\text { 1) }-2,4,6 \\
371.8\end{array}$ & $\begin{array}{c}\text { DH, } 3 H \\
\text { DSC } \\
\text { DSC }\end{array}$ & $\begin{array}{l}\text { [2008Wimidinetrione (secobarbital) } \\
\text { [1982TRE/HOL] } \\
\text { [19U] }\end{array}$ \\
\hline $\mathrm{C}_{12} \mathrm{H}_{18} \mathrm{~N}_{2} \mathrm{O}_{3} \mathrm{~S}$ & $\begin{array}{c}\text { [64-77-7] } \\
\text { TRS (I) } \\
\text { FUS (I) } \\
\text { FUS } \\
\text { FUS } \\
\text { FUS }\end{array}$ & $3-(p$-tolyl-4-st & $\begin{array}{c}\text { nyl)-1-butyl } \\
1.9 \\
23.8 \\
26.24 \\
27.2 \\
25.61\end{array}$ & $\begin{array}{c}\text { a (tolbu } \\
313.2 \\
401.2 \\
402 \\
400.2 \\
404.8\end{array}$ & $\begin{array}{l}\text { de) } \\
\text { DSC } \\
\text { DSC } \\
\text { DSC } \\
\text { DSC }\end{array}$ & $\begin{array}{l}\text { [2010THI/AIT] } \\
\text { [2010BAI/VAN] } \\
{[1999 \mathrm{KIM} / \mathrm{HIR}]} \\
{[1982 \mathrm{MAR} / \mathrm{MIR}]}\end{array}$ \\
\hline $\mathrm{C}_{12} \mathrm{H}_{18} \mathrm{~N}_{4} \mathrm{O}_{2}$ & $\begin{array}{c}\text { [35873-41-7] } \\
\text { FUS }\end{array}$ & 8-pentyltheopl & ine 35.1 & 498.4 & DSC & [1991ACR, 1989GON/KRA] \\
\hline $\mathrm{C}_{12} \mathrm{H}_{18} \mathrm{~N}_{4} \mathrm{O}_{6} \mathrm{~S}$ & $\begin{array}{c}\text { [19044-88-3] } \\
\text { FUS }\end{array}$ & 4-( $N, N$-dipro & $\begin{array}{c}\text { ammo) }-3,5-\mathrm{d} \\
38.48\end{array}$ & $\begin{array}{l}\text { robenze } \\
414.8\end{array}$ & $\begin{array}{l}\text { alphomm } \\
\text { DSC }\end{array}$ & [1990DON/DRE] \\
\hline $\mathrm{C}_{12} \mathrm{H}_{18} \mathrm{O}$ & $\begin{array}{c}{[5331-28-2]} \\
\text { FUS }\end{array}$ & $\begin{array}{l}\text { 4-tert-butyldip } \\
\quad(8-371)\end{array}$ & $\begin{array}{r}\text { iyl oxide } \\
21.99\end{array}$ & 327.8 & $\mathrm{AC}$ & [2015DRU/PIM] \\
\hline & SUB & $(299-324)$ & $100.1 \pm 1.0$ & 298 & GS & [2015DRU/PIM] \\
\hline $\mathrm{C}_{12} \mathrm{H}_{18} \mathrm{O}$ & $\begin{array}{c}{[4157-77-1]} \\
\mathrm{V}\end{array}$ & $\begin{array}{l}\text { (1-butoxyethyl } \\
(278-318)\end{array}$ & $\begin{array}{l}\text { nzene } \\
59.8 \pm 0.3\end{array}$ & 298 & GS & [2001VER/HEI] \\
\hline $\mathrm{C}_{12} \mathrm{H}_{18} \mathrm{O}$ & $\begin{array}{c}{[445251-36-5]} \\
\mathrm{V}\end{array}$ & $\begin{array}{l}(R S)-(1-\mathrm{sec}-\mathrm{bu} \\
\quad(296-332)\end{array}$ & $\begin{array}{c}\text { yethyl)benze } \\
58.7 \pm 0.5\end{array}$ & 298 & GS & [2002KRA/VAS, 2002VER/HEI] \\
\hline $\mathrm{C}_{12} \mathrm{H}_{18} \mathrm{O}$ & $\begin{array}{c}{[445251-38-7]} \\
\mathrm{V}\end{array}$ & $\begin{array}{l}(S S)-(1-\mathrm{sec}-\mathrm{bu} \\
\quad(297-332)\end{array}$ & $\begin{array}{l}\text { yethyl)benze } \\
59.1 \pm 0.5\end{array}$ & 298 & GS & [2002KRA/VAS, 2002BAE/SHI2] \\
\hline $\mathrm{C}_{12} \mathrm{H}_{18} \mathrm{O}$ & $\begin{array}{c}{[24142-77-6]} \\
V\end{array}$ & $\begin{array}{l}\text { Propyl cumyl } \\
(278-325)\end{array}$ & $59.1 \pm 0.2$ & 302 & GS & [2001VER/HEI2] \\
\hline
\end{tabular}


TABLE 11. Phase change enthalpies of $\mathrm{C}_{12}$ to $\mathrm{C}_{13}$ organic compounds-Continued

\begin{tabular}{|c|c|c|c|c|c|c|}
\hline \multirow[b]{2}{*}{ Molecular formula } & \multirow{2}{*}{$\begin{array}{l}\text { CAS Registry } \\
\text { Number } \\
\text { Enthalpy }\end{array}$} & \multicolumn{5}{|l|}{ Compound } \\
\hline & & $\begin{array}{l}\text { Temperature } \\
\text { range }\end{array}$ & $\begin{array}{l}\Delta_{\text {trans }} H_{\mathrm{m}} \\
(\mathrm{kJ} / \mathrm{mol})\end{array}$ & $\begin{array}{l}T_{\mathrm{m}} \\
(\mathrm{K})\end{array}$ & Method & References \\
\hline & V & $(278-325)$ & $59.3 \pm 0.2$ & 298 & GS & [2001VER/HEI2] \\
\hline $\mathrm{C}_{12} \mathrm{H}_{18} \mathrm{O}$ & $\begin{array}{c}{[6382-14-5]} \\
\mathrm{V}\end{array}$ & $\begin{array}{l}\text { Benzyl pentyl } \\
(363-512)\end{array}$ & 50.8 & 378 & A & [1987STE/MAL, 1969KRO] \\
\hline $\mathrm{C}_{12} \mathrm{H}_{18} \mathrm{O}$ & $\begin{array}{c}{[2934-05-6]} \\
\mathrm{V}\end{array}$ & $\begin{array}{l}\text { 2,4-diisopropy } \\
\quad(395-528)\end{array}$ & enol 58.4 & 410 & A & [1987STE/MAL] \\
\hline $\mathrm{C}_{12} \mathrm{H}_{18} \mathrm{O}$ & $\begin{array}{c}\text { [2078-54-8] } \\
\text { FUS }\end{array}$ & 2,6-diisopropy & $\begin{array}{l}\text { enol } \\
\qquad 14.64\end{array}$ & 292.8 & & [1975BER/PER] \\
\hline & $\begin{array}{l}\mathrm{V} \\
\mathrm{V}\end{array}$ & $\begin{array}{l}(293-328) \\
(293-328)\end{array}$ & $\begin{array}{l}67.9 \pm 0.3 \\
68.7 \pm 0.3\end{array}$ & $\begin{array}{l}310 \\
298\end{array}$ & $\begin{array}{l}\text { GS } \\
\text { GS }\end{array}$ & $\begin{array}{l}\text { [1999VER] } \\
\text { [1999VER] }\end{array}$ \\
\hline $\mathrm{C}_{12} \mathrm{H}_{18} \mathrm{O}$ & $\begin{array}{c}\text { [26886-05-5] } \\
\text { FUS }\end{array}$ & 3,5-diisopropy & $\begin{array}{l}\text { enol } \\
\qquad 12.13\end{array}$ & 326.3 & & [1975BER/PER] \\
\hline $\mathrm{C}_{12} \mathrm{H}_{18} \mathrm{O}$ & $\begin{array}{c}{[68189-19-5]} \\
\mathrm{V}\end{array}$ & $\begin{array}{l}\text { 2,3-dimethyl-4 } \\
\quad(418-523)\end{array}$ & $\begin{array}{l}r t \text {-butylphenol } \\
60.2\end{array}$ & 433 & A & [1987STE/MAL] \\
\hline $\mathrm{C}_{12} \mathrm{H}_{18} \mathrm{O}$ & $\begin{array}{c}{[46170-85-8]} \\
\mathrm{V}\end{array}$ & $\begin{array}{c}\text { 2,3-dimethyl-6 } \\
(412-525)\end{array}$ & $\begin{array}{l}r t \text {-butylphenol } \\
60.0\end{array}$ & 427 & A & [1987STE/MAL] \\
\hline $\mathrm{C}_{12} \mathrm{H}_{18} \mathrm{O}$ & $\begin{array}{c}{[1879-09-0]} \\
\mathrm{V} \\
\mathrm{V} \\
\mathrm{V} \\
\mathrm{V} \\
\mathrm{V} \\
\mathrm{V} \\
\mathrm{V} \\
\mathrm{V}\end{array}$ & $\begin{array}{l}\text { 2,4-dimethyl-6 } \\
(304-333) \\
(304-333) \\
(388-522) \\
(344-535) \\
(344-535) \\
(344-535) \\
(344-535) \\
(344-535)\end{array}$ & $\begin{array}{c}r t \text {-butylphenol } \\
67.2 \pm 0.8 \\
68.4 \pm 0.8 \\
58.4 \\
54.4 \\
52.7 \\
51.7 \\
49.7 \\
45.4\end{array}$ & $\begin{array}{l}318 \\
298 \\
403 \\
348 \\
373 \\
398 \\
423 \\
473\end{array}$ & $\begin{array}{c}\text { GS } \\
\text { GS } \\
\text { A }\end{array}$ & $\begin{array}{l}\text { [1999VER] } \\
{[1999 \mathrm{VER}]} \\
{[1987 \mathrm{STE} / \mathrm{MAL}]} \\
{[1953 \mathrm{STA} / \mathrm{MUL}]} \\
{[1953 \mathrm{STA} / \mathrm{MUL}]} \\
{[1953 \mathrm{STA} / \mathrm{MUL}]} \\
{[1953 \mathrm{STA} / \mathrm{MUL}]} \\
{[1953 \mathrm{STA} / \mathrm{MUL}]}\end{array}$ \\
\hline $\mathrm{C}_{12} \mathrm{H}_{18} \mathrm{O}$ & $\begin{array}{c}\text { [17696-37-6] } \\
\text { V } \\
\text { V } \\
\text { V } \\
\text { V } \\
\text { V }\end{array}$ & $\begin{array}{l}\text { 2,5-dimethyl-4 } \\
\begin{array}{l}(408-538) \\
(361-548) \\
(361-548) \\
(361-548) \\
(361-548)\end{array}\end{array}$ & $\begin{array}{c}r t \text {-butylphenol } \\
61.7 \\
62.0 \\
59.4 \\
57.1 \\
52.8\end{array}$ & $\begin{array}{l}423 \\
373 \\
398 \\
423 \\
473\end{array}$ & A & $\begin{array}{l}\text { [1987STE/MAL] } \\
{[1953 \mathrm{STA} / \mathrm{MUL}]} \\
{[1953 \mathrm{STA} / \mathrm{MUL}]} \\
\text { [1953STA/MUL] } \\
\text { [1953STA/MUL] }\end{array}$ \\
\hline $\mathrm{C}_{12} \mathrm{H}_{18} \mathrm{O}$ & $\begin{array}{c}\text { [879-97-0] } \\
\text { V } \\
\text { V } \\
\text { V } \\
\text { V } \\
\text { V } \\
\text { V }\end{array}$ & 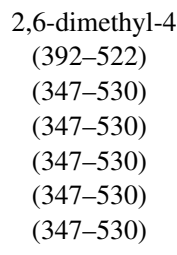 & $\begin{array}{c}r t \text {-butylphenol } \\
59.7 \\
58.4 \\
57.0 \\
55.4 \\
54.2 \\
49.3\end{array}$ & $\begin{array}{l}407 \\
348 \\
373 \\
398 \\
423 \\
473\end{array}$ & A & $\begin{array}{l}\text { [1987STE/MAL] } \\
\text { [1953STA/MUL] } \\
\text { [1953STA/MUL] } \\
\text { [1953STA/MUL] } \\
\text { [1953STA/MUL] } \\
\text { [1953STA/MUL] }\end{array}$ \\
\hline $\mathrm{C}_{12} \mathrm{H}_{18} \mathrm{O}$ & $\begin{array}{c}{[1445-23-4]} \\
\mathrm{V}\end{array}$ & $\begin{array}{c}\text { 3,4-dimethyl-6 } \\
(413-532)\end{array}$ & $\begin{array}{c}r t \text {-butylphenol } \\
62.7\end{array}$ & 428 & A & [1987STE/MAL] \\
\hline $\mathrm{C}_{12} \mathrm{H}_{18} \mathrm{O}$ & $\begin{array}{c}{[63452-61-9]} \\
\text { V } \\
\text { V } \\
\text { V } \\
\text { V }\end{array}$ & $\begin{array}{l}\text { 2-ethyl-4-tert- } \\
(428-623) \\
(397-543) \\
(397-543) \\
(397-543)\end{array}$ & $\begin{array}{r}\text { ylphenol } \\
61.6 \\
55.4 \\
54.2 \\
49.3\end{array}$ & $\begin{array}{l}443 \\
398 \\
423 \\
473\end{array}$ & A & $\begin{array}{l}\text { [1987STE/MAL] } \\
\text { [1953STA/MUL] } \\
\text { [1953STA/MUL] } \\
\text { [1953STA/MUL] }\end{array}$ \\
\hline $\mathrm{C}_{12} \mathrm{H}_{18} \mathrm{O}$ & $\begin{array}{c}{[63551-41-7]} \\
\mathrm{V}\end{array}$ & $\begin{array}{l}\text { 2-ethyl-6-tert-b } \\
\quad(393-443)\end{array}$ & $\begin{array}{r}\text { ylphenol } \\
58.2\end{array}$ & 408 & A & [1987STE/MAL] \\
\hline $\mathrm{C}_{12} \mathrm{H}_{18} \mathrm{O}$ & $\begin{array}{c}{[4237-25-6]} \\
\mathrm{V}\end{array}$ & $\begin{array}{l}\text { 3-ethyl-6-tert-b } \\
\quad(415-530)\end{array}$ & $\begin{array}{r}\text { ylphenol } \\
59.5\end{array}$ & 430 & A & [1987STE/MAL] \\
\hline $\mathrm{C}_{12} \mathrm{H}_{18} \mathrm{O}$ & $\begin{array}{c}\text { [96-70-8] } \\
\text { V } \\
\text { V } \\
\text { V } \\
\text { V }\end{array}$ & $\begin{array}{l}\text { 4-ethyl-2-tert-t } \\
(394-523) \\
(349-533) \\
(349-533) \\
(349-533)\end{array}$ & $\begin{array}{r}\text { ylphenol } \\
59.2 \\
57.0 \\
55.4 \\
54.2\end{array}$ & $\begin{array}{l}409 \\
373 \\
398 \\
423\end{array}$ & A & $\begin{array}{l}\text { [1987STE/MAL] } \\
\text { [1953STA/MUL] } \\
\text { [1953STA/MUL] } \\
\text { [1953STA/MUL] }\end{array}$ \\
\hline
\end{tabular}


TABLE 11. Phase change enthalpies of $\mathrm{C}_{12}$ to $\mathrm{C}_{13}$ organic compounds-Continued

\begin{tabular}{|c|c|c|c|c|c|c|}
\hline \multirow[b]{2}{*}{ Molecular formula } & \multirow{2}{*}{$\begin{array}{l}\text { CAS Registry } \\
\text { Number } \\
\text { Enthalpy }\end{array}$} & \multicolumn{5}{|l|}{ Compound } \\
\hline & & $\begin{array}{l}\text { Temperature } \\
\text { range }\end{array}$ & $\begin{array}{l}\Delta_{\text {trans }} H_{\mathrm{m}} \\
(\mathrm{kJ} / \mathrm{mol})\end{array}$ & $\begin{array}{l}T_{\mathrm{m}} \\
(\mathrm{K})\end{array}$ & Method & References \\
\hline & $\mathrm{V}$ & $(349-533)$ & 49.3 & 473 & & [1953STA/MUL] \\
\hline $\mathrm{C}_{12} \mathrm{H}_{18} \mathrm{O}$ & $\begin{array}{c}{[71745-63-6]} \\
\text { V } \\
\text { V } \\
\text { V }\end{array}$ & $\begin{array}{l}\text { 2-methyl-4-ter } \\
\begin{array}{c}(443-653) \\
(409-561) \\
(409-561)\end{array}\end{array}$ & $\begin{array}{l}\text { entylphenol } \\
65.6 \\
55.3 \\
50.7\end{array}$ & $\begin{array}{l}458 \\
423 \\
473\end{array}$ & $\mathrm{~A}$ & $\begin{array}{l}\text { [1987STE/MAL] } \\
\text { [1953STA/MUL] } \\
\text { [1953STA/MUL] }\end{array}$ \\
\hline $\mathrm{C}_{12} \mathrm{H}_{18} \mathrm{O}$ & $\begin{array}{l}\mathrm{V} \\
\mathrm{V} \\
\mathrm{V}\end{array}$ & $\begin{array}{c}\text { 3-methyl-4-ter } \\
(443-683) \\
(409-561) \\
(409-561)\end{array}$ & $\begin{array}{l}\text { entylphenol } \\
65.1 \\
55.3 \\
50.7\end{array}$ & $\begin{array}{l}458 \\
423 \\
473\end{array}$ & $\mathrm{~A}$ & $\begin{array}{l}\text { [1987STE/MAL] } \\
\text { [1953STA/MUL] } \\
\text { [1953STA/MUL] }\end{array}$ \\
\hline $\mathrm{C}_{12} \mathrm{H}_{18} \mathrm{O}$ & $\begin{array}{c}{[34072-71-4]} \\
\text { V } \\
\text { V } \\
\text { V } \\
\text { V }\end{array}$ & $\begin{array}{l}\text { 4-methyl-2-ter } \\
(423-653) \\
(394-538) \\
(394-538) \\
(394-538)\end{array}$ & $\begin{array}{c}\text { entylphenol } \\
61.4 \\
58.1 \\
55.3 \\
50.7\end{array}$ & $\begin{array}{l}438 \\
398 \\
423 \\
473\end{array}$ & A & $\begin{array}{l}\text { [1987STE/MAL] } \\
\text { [1953STA/MUL] } \\
\text { [1953STA/MUL] } \\
\text { [1953STA/MUL] }\end{array}$ \\
\hline $\mathrm{C}_{12} \mathrm{H}_{18} \mathrm{O}$ & $\begin{array}{c}{[1660-04-4]} \\
\text { SUB }\end{array}$ & $\begin{array}{c}\text { 1-adamantyl m } \\
(287-305)\end{array}$ & $\begin{array}{l}\text { yl ketone } \\
84.2 \pm 0.6\end{array}$ & 298 & ME & [1992ABB/JIM2] \\
\hline $\mathrm{C}_{12} \mathrm{H}_{18} \mathrm{O}$ & $\begin{array}{c}\text { [7273-98-5] } \\
\text { SUB } \\
\text { SUB }\end{array}$ & $\begin{array}{l}\text { exo-4-hydroxy- } \\
(303-343) \\
(303-343)\end{array}$ & $\begin{array}{c}\text { do-endo-tetro } \\
77.1 \pm 2.2 \\
79.0 \pm 2.5\end{array}$ & $\begin{array}{c}\text { clo[6.2. } \\
323 \\
298\end{array}$ & $\begin{array}{l}\left.{ }^{6} .0^{2,7}\right] \mathrm{doc} \\
\text { TSGC } \\
\text { TSGC }\end{array}$ & $\begin{array}{l}\text { [1980STE] } \\
\text { [1980STE] }\end{array}$ \\
\hline $\mathrm{C}_{12} \mathrm{H}_{18} \mathrm{O}$ & $\begin{array}{c}\text { [107133-43-7] } \\
\text { SUB } \\
\text { SUB }\end{array}$ & $\begin{array}{l}\text { exo-4-hydroxy- } \\
(323-353) \\
(323-353)\end{array}$ & $\begin{array}{c}\text {-endo-tetrac } \\
74.3 \pm 1.8 \\
76.3 \pm 2.0\end{array}$ & $\begin{array}{c}\mathrm{o}[6.2 .1 \\
338 \\
298\end{array}$ & $\begin{array}{c}\left.0^{2,7}\right] \text { dode } \\
\text { TSGC } \\
\text { TSGC }\end{array}$ & $\begin{array}{l}\text { [1980STE] } \\
\text { [1980STE] }\end{array}$ \\
\hline $\mathrm{C}_{12} \mathrm{H}_{18} \mathrm{O}$ & $\begin{array}{c}\text { [74007-11-7] } \\
\text { SUB } \\
\text { SUB }\end{array}$ & $\begin{array}{l}\text { exo-4-hydroxy- } \\
\quad(313-353) \\
(313-353)\end{array}$ & $\begin{array}{c}\text {-exo-tetracy } \\
73.9 \pm 2.0 \\
75.9 \pm 2.2\end{array}$ & 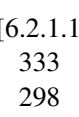 & $\begin{array}{c}2,7 \text { dodec } \\
\text { TSGC } \\
\text { TSGC }\end{array}$ & $\begin{array}{l}\text { [1980STE] } \\
\text { [1980STE] }\end{array}$ \\
\hline $\mathrm{C}_{12} \mathrm{H}_{18} \mathrm{O}$ & $\begin{array}{c}{[1011-12-7]} \\
\text { FUS }\end{array}$ & 2-(1'-cyclohexe & $\begin{array}{c}\text { 1)cyclohexan } \\
17.26\end{array}$ & 278.8 & $\mathrm{AC}$ & [1992MAR/KOZ] \\
\hline & $\mathrm{V}$ & $(298-358)$ & $72.8 \pm 0.8$ & 328 & ME & [1992MAR/KOZ] \\
\hline $\mathrm{C}_{12} \mathrm{H}_{18} \mathrm{O}_{2}$ & $\begin{array}{c}{[5673-09-6]} \\
\mathrm{V}\end{array}$ & $\begin{array}{l}\text { 1,3-dihydroxy- } \\
(433-494)\end{array}$ & $\begin{array}{c}\text { exylbenzene } \\
76.8\end{array}$ & 448 & $\mathrm{~A}, \mathrm{GC}$ & [1987STE/MAL, 1975KUN/LIL] \\
\hline $\mathrm{C}_{12} \mathrm{H}_{18} \mathrm{O}_{2}$ & $\begin{array}{c}\text { [136-77-6] } \\
\text { FUS }\end{array}$ & 1,3-dihydroxy- & $\begin{array}{c}\text { exylbenzene } \\
19.04\end{array}$ & 341.5 & DSC & [1991ACR, 1985OCH] \\
\hline & $\mathrm{V}$ & $(434-494)$ & 88.1 & 449 & $\mathrm{~A}, \mathrm{GC}$ & [1987STE/MAL, 1975KUN/LIL] \\
\hline $\mathrm{C}_{12} \mathrm{H}_{18} \mathrm{O}_{2}$ & $\begin{array}{c}\text { [711-01-3] } \\
\text { SUB } \\
\text { SUB }\end{array}$ & $\begin{array}{l}\text { 1-adamantyl-1- } \\
(267-283) \\
(267-283)\end{array}$ & $\begin{array}{c}\text { boxylic acid } \\
84.3 \pm 0.6 \\
82.4 \pm 0.6\end{array}$ & $\begin{array}{l}\text { thyl est } \\
275 \\
298\end{array}$ & $\begin{array}{l}\mathrm{ME} \\
\mathrm{ME}\end{array}$ & $\begin{array}{l}\text { [1992ABB/JIM] } \\
\text { [1992ABB/JIM] }\end{array}$ \\
\hline $\mathrm{C}_{12} \mathrm{H}_{18} \mathrm{O}_{2}$ & SUB & $\begin{array}{l}\text { trans-syn-trans } \\
\quad(240-310)\end{array}$ & $\begin{array}{l}\text { cahydro-3-h } \\
\text { NA }\end{array}$ & oxy-2-n & $\begin{array}{l}\text { thalene ac } \\
\text { ME }\end{array}$ & $\begin{array}{l}\gamma \text {-lactone } \\
{[1957 \mathrm{SPI}]}\end{array}$ \\
\hline $\mathrm{C}_{12} \mathrm{H}_{18} \mathrm{O}_{2}$ & SUB & $\begin{array}{l}\text { trans-anti-tran } \\
\quad(240-310)\end{array}$ & $\begin{array}{l}\text { ecahydro-3-h } \\
\text { NA }\end{array}$ & roxy-2- & $\begin{array}{l}\text { thalene a } \\
\text { ME }\end{array}$ & $\begin{array}{c}\text { c } \gamma \text {-lactone } \\
{[1957 \mathrm{SPI}]}\end{array}$ \\
\hline $\mathrm{C}_{12} \mathrm{H}_{18} \mathrm{O}_{3}$ & $\begin{array}{c}\text { [63991-78-6] } \\
\text { FUS }\end{array}$ & (racemic) 3-(2- & $\begin{array}{l}\text { pylphenoxy) } \\
29.5\end{array}$ & $\begin{array}{l}\text { opane- } \\
326.5\end{array}$ & $\begin{array}{l}\text { liol } \\
\text { DSC }\end{array}$ & [2008BRE/BRE] \\
\hline $\mathrm{C}_{12} \mathrm{H}_{18} \mathrm{O}_{3}$ & $\begin{array}{c}\text { [1092799-99-9] } \\
\text { FUS }\end{array}$ & (R)-3-(2-propy & $\begin{array}{c}\text { enoxy)-prop } \\
31.9\end{array}$ & $\begin{array}{l}-1,2-\mathrm{di} \\
340.5\end{array}$ & $\mathrm{DSC}$ & [2008BRE/BRE] \\
\hline $\mathrm{C}_{12} \mathrm{H}_{18} \mathrm{O}_{3}$ & $\begin{array}{c}\text { [204583-98-2] } \\
\text { FUS }\end{array}$ & (racemic) 3-(2- & $\begin{array}{c}\text { propylpheno } \\
31.5\end{array}$ & $\begin{array}{l}\text {-propan } \\
353.7\end{array}$ & $\begin{array}{l}\text { 2-diol } \\
\text { DSC }\end{array}$ & [2008BRE/BRE] \\
\hline $\mathrm{C}_{12} \mathrm{H}_{18} \mathrm{O}_{3}$ & $\begin{array}{c}\text { [204584-38-3] } \\
\text { FUS }\end{array}$ & $(R)$-3-(2-isopr & $\begin{array}{c}\text { lphenoxy)-p } \\
30.6\end{array}$ & $\begin{array}{c}\text { ane- } 1,2 \\
345\end{array}$ & DSC & [2008BRE/BRE] \\
\hline $\mathrm{C}_{12} \mathrm{H}_{18} \mathrm{O}_{4}$ & $\begin{array}{c}{[532-34-3]} \\
\mathrm{V}\end{array}$ & $\begin{array}{l}\text { 3,4-dihydro-2, } \\
(357-435)\end{array}$ & $\begin{array}{l}\text { imethyl-4-ox } \\
64.7\end{array}$ & $\begin{array}{c}H \text {-pyra } \\
372\end{array}$ & $\begin{array}{c}\text { carboxyli } \\
\text { A }\end{array}$ & $\begin{array}{l}\text { id, butyl ester } \\
\text { [1987STE/MAL] }\end{array}$ \\
\hline
\end{tabular}


TABLE 11. Phase change enthalpies of $\mathrm{C}_{12}$ to $\mathrm{C}_{13}$ organic compounds-Continued

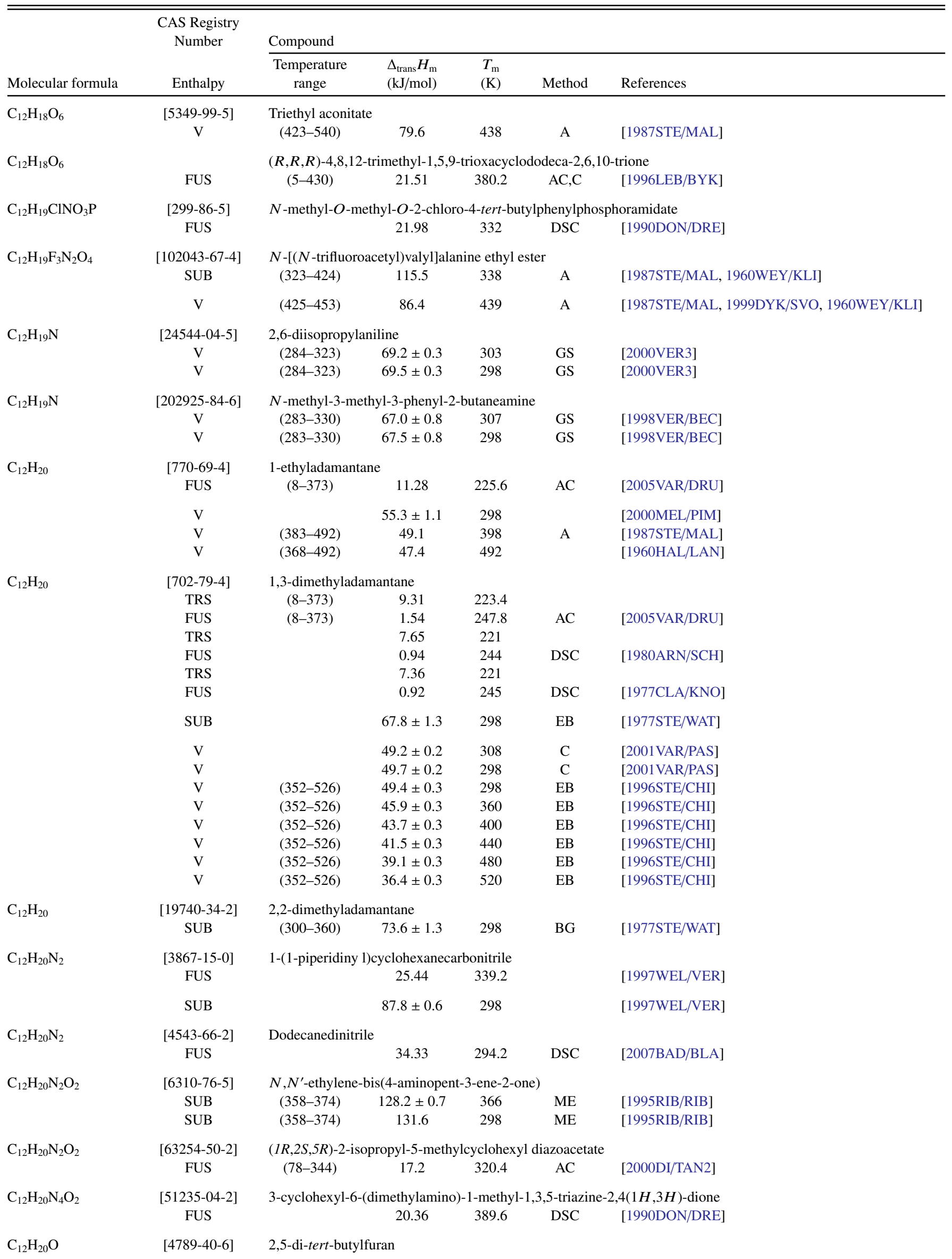


TABLE 11. Phase change enthalpies of $\mathrm{C}_{12}$ to $\mathrm{C}_{13}$ organic compounds-Continued

\begin{tabular}{|c|c|c|c|c|c|c|}
\hline \multirow[b]{2}{*}{ Molecular formula } & \multirow{2}{*}{$\begin{array}{c}\text { CAS Registry } \\
\text { Number } \\
\text { Enthalpy }\end{array}$} & \multicolumn{5}{|l|}{ Compound } \\
\hline & & $\begin{array}{c}\text { Temperature } \\
\text { range }\end{array}$ & $\begin{array}{l}\Delta_{\text {trans }} H_{\mathrm{m}} \\
(\mathrm{kJ} / \mathrm{mol})\end{array}$ & $\begin{array}{l}T_{\mathrm{m}} \\
(\mathrm{K})\end{array}$ & Method & References \\
\hline & V & $(274-323)$ & $56.1 \pm 1.1$ & 298 & GS & [1998VER/WEL] \\
\hline \multirow[t]{2}{*}{$\mathrm{C}_{12} \mathrm{H}_{20} \mathrm{O}$} & $\begin{array}{c}{[90-42-6]} \\
\text { FUS }\end{array}$ & \multicolumn{5}{|c|}{ 2-cyclohexylcyclohexanone } \\
\hline & V & $(370-481)$ & $54.0 \pm 0.6$ & 425 & GS & [1992MAR/KOZ] \\
\hline $\mathrm{C}_{12} \mathrm{H}_{20} \mathrm{O}_{2}$ & $\begin{array}{c}{[76-49-3]} \\
\mathrm{V}\end{array}$ & $\begin{array}{c}\text { Bornyl acetate } \\
(319-496)\end{array}$ & 50.8 & 334 & A & [1987STE/MAL, 1947STU] \\
\hline $\mathrm{C}_{12} \mathrm{H}_{20} \mathrm{O}_{2}$ & $\begin{array}{c}{[105-87-3]} \\
\mathrm{V}\end{array}$ & $\begin{array}{l}\text { Geranyl acetate } \\
\quad(346-516)\end{array}$ & 58.1 & 361 & A & [1987STE/MAL, 1947STU] \\
\hline $\mathrm{C}_{12} \mathrm{H}_{20} \mathrm{O}_{2}$ & $\begin{array}{c}{[125-12-2]} \\
\mathrm{V} \\
\mathrm{V} \\
\mathrm{V}\end{array}$ & $\begin{array}{l}\text { Isobornyl acetate } \\
(381-472) \\
(404-450)\end{array}$ & $\begin{array}{l}52.1 \\
56.1 \\
53.6\end{array}$ & $\begin{array}{l}400 \\
419\end{array}$ & $\begin{array}{c}\text { Boiling Pt } \\
\text { A }\end{array}$ & $\begin{array}{l}\text { [2014WAN/HUA] } \\
\text { [1987STE/MAL] } \\
\text { [1937RUD/KOR] }\end{array}$ \\
\hline $\mathrm{C}_{12} \mathrm{H}_{20} \mathrm{O}_{2}$ & V & $\begin{array}{l}\text { Bicyclo[2,2,1]hep } \\
\quad(293-323)\end{array}$ & $\begin{array}{c}\text { tane-7-one } 2 \\
60.5 \pm 0.9\end{array}$ & limethy & $\begin{array}{c}\text { opylene aceta } \\
\text { GS }\end{array}$ & [2002VER] \\
\hline $\mathrm{C}_{12} \mathrm{H}_{20} \mathrm{O}_{2}$ & $\begin{array}{c}{[115-95-7]} \\
\text { V } \\
\text { V } \\
\text { V }\end{array}$ & $\begin{array}{l}\text { 3,7-dimethyl-1,6-c } \\
(281-490) \\
(328-493)\end{array}$ & $\begin{array}{c}\text { octadien-3-ol } \\
62.5 \pm 0.6 \\
57.8 \\
56.8\end{array}$ & $\begin{array}{l}\text { etate (li } \\
298 \\
296 \\
343\end{array}$ & $\begin{array}{c}\text { yl acetate) } \\
\text { CGC } \\
\text { A }\end{array}$ & $\begin{array}{l}\text { [2015KOZ/GOB] } \\
{[1987 \mathrm{STE} / \mathrm{MAL}]} \\
{[1947 \mathrm{STU}]}\end{array}$ \\
\hline $\mathrm{C}_{12} \mathrm{H}_{20} \mathrm{O}_{2}$ & $\begin{array}{c}{[80-26-2]} \\
\mathrm{V}\end{array}$ & $\begin{array}{l}\text { Terpineol acetate } \\
(310-424)\end{array}$ & 68.1 & 325 & A & [1987STE/MAL] \\
\hline $\mathrm{C}_{12} \mathrm{H}_{20} \mathrm{O}_{2}$ & $\begin{array}{c}\text { [217467-40-8] } \\
\text { FUS }\end{array}$ & Bicyclo[2.2.1]hep & $\begin{array}{c}\text { tane-7-one } 2 \\
23.9\end{array}$ & $\begin{array}{l}\text { limethy } \\
346.7\end{array}$ & opylene ketal & [1998VER/PEN] \\
\hline & SUB & & $84.0 \pm 0.9$ & 298 & & [1998VER/PEN] \\
\hline $\mathrm{C}_{12} \mathrm{H}_{20} \mathrm{O}_{2}$ & $\begin{array}{c}\text { [10329-90-5] } \\
\text { FUS }\end{array}$ & 1,7-cyclododecane & $\begin{array}{l}\text { edione } \\
15.77\end{array}$ & 405.2 & & [1972ALV/BOR] \\
\hline $\mathrm{C}_{12} \mathrm{H}_{20} \mathrm{O}_{2}$ & $\begin{array}{c}\text { [28746-99-8] } \\
\text { FUS }\end{array}$ & $\begin{array}{l}2-\left(1^{\prime} \text {-hydroxycycl }\right. \\
\quad(5-310)\end{array}$ & $\begin{array}{c}\text { ohexyl)cyclo } \\
20.81\end{array}$ & $\begin{array}{l}\text { anone } \\
306.8\end{array}$ & DSC & [2006SHE/KAB] \\
\hline $\mathrm{C}_{12} \mathrm{H}_{20} \mathrm{O}_{3}$ & $\begin{array}{c}\text { [49540-29-6] } \\
\text { FUS }\end{array}$ & 3,3,6,6-tetramethy & $\begin{array}{c}\text { octanedioic } \\
18.83\end{array}$ & $\begin{array}{l}\text { ydride } \\
344.2\end{array}$ & & {$[1974 B O R]$} \\
\hline $\mathrm{C}_{12} \mathrm{H}_{20} \mathrm{O}_{4}$ & $\begin{array}{c}\text { [27198-40-9] } \\
\text { FUS }\end{array}$ & 1,5-cyclooctanedi & $\begin{array}{c}\text { one bis(ethyl } \\
18.03\end{array}$ & $\begin{array}{l}\text { ketal) } \\
296.2\end{array}$ & & [1972ALV/BOR] \\
\hline $\mathrm{C}_{12} \mathrm{H}_{20} \mathrm{O}_{4}$ & $\begin{array}{c}{[105-76-0]} \\
\mathrm{V}\end{array}$ & $\begin{array}{l}\text { Dibutyl maleate } \\
(255-550)\end{array}$ & 41.1 & 270 & A & [1987STE/MAL] \\
\hline $\mathrm{C}_{12} \mathrm{H}_{20} \mathrm{O}_{4}$ & $\begin{array}{c}\text { [2370-71-0] } \\
\text { FUS }\end{array}$ & Octyl maleate & 33.2 & 304.3 & $\mathrm{DSC}$ & [2016RIC/DEL] \\
\hline $\mathrm{C}_{12} \mathrm{H}_{20} \mathrm{O}_{5}$ & $\mathrm{~V}$ & $\begin{array}{l}\text { 2-ethoxycarbonylp } \\
(388-523)\end{array}$ & $\begin{array}{c}\text { propionic aci } \\
67.6\end{array}$ & $\begin{array}{l}\text { yclohex } \\
403\end{array}$ & $\begin{array}{l}\text { ester } \\
\text { A }\end{array}$ & [1987STE/MAL] \\
\hline $\mathrm{C}_{12} \mathrm{H}_{20} \mathrm{O}_{6}$ & $\begin{array}{c}{[139-45-7]} \\
\mathrm{V} \\
\mathrm{V}\end{array}$ & $\begin{array}{c}\text { Tripropionin } \\
(304-337)\end{array}$ & $\begin{array}{l}90.7 \pm 0.4 \\
91.4 \pm 0.4\end{array}$ & $\begin{array}{l}298 \\
298\end{array}$ & $\begin{array}{c}\mathrm{GS} \\
\mathrm{C}\end{array}$ & $\begin{array}{l}\text { [2010MAS/KRA] } \\
\text { [1986NIL/WAD] }\end{array}$ \\
\hline $\mathrm{C}_{12} \mathrm{H}_{20} \mathrm{O}_{7}$ & $\begin{array}{c}{[77-93-0]} \\
\mathrm{V}\end{array}$ & $\begin{array}{l}\text { Triethyl citrate } \\
(380-567)\end{array}$ & 68.2 & 395 & A & [1987STE/MAL] \\
\hline $\mathrm{C}_{12} \mathrm{H}_{20} \mathrm{~S}$ & $\begin{array}{c}\text { [880-36-4] } \\
\mathrm{V}\end{array}$ & 2-octylthiophene & $65.4 \pm 1.4$ & 298 & $\mathrm{C}$ & [2007RIB/SAN] \\
\hline $\mathrm{C}_{12} \mathrm{H}_{20} \mathrm{~S}$ & $\begin{array}{c}{[65016-62-8]} \\
\mathrm{V}\end{array}$ & 3-octylthiophene & $67.6 \pm 1.5$ & 298 & $\mathrm{C}$ & [2007RIB/SAN] \\
\hline $\mathrm{C}_{12} \mathrm{H}_{21} \mathrm{~N}$ & $\begin{array}{c}{[13392-28-4]} \\
\mathrm{V}\end{array}$ & 1-(1-adamantyl)et & $\begin{array}{l}\text { hylamine } \\
68.7 \pm 3.7\end{array}$ & 298 & CGC & [2013GOB/RAT] \\
\hline $\mathrm{C}_{12} \mathrm{H}_{21} \mathrm{~N}$ & {$[6326-88-1]$} & Dodecahydrocarbs & azole & & & \\
\hline
\end{tabular}


TABLE 11. Phase change enthalpies of $\mathrm{C}_{12}$ to $\mathrm{C}_{13}$ organic compounds-Continued

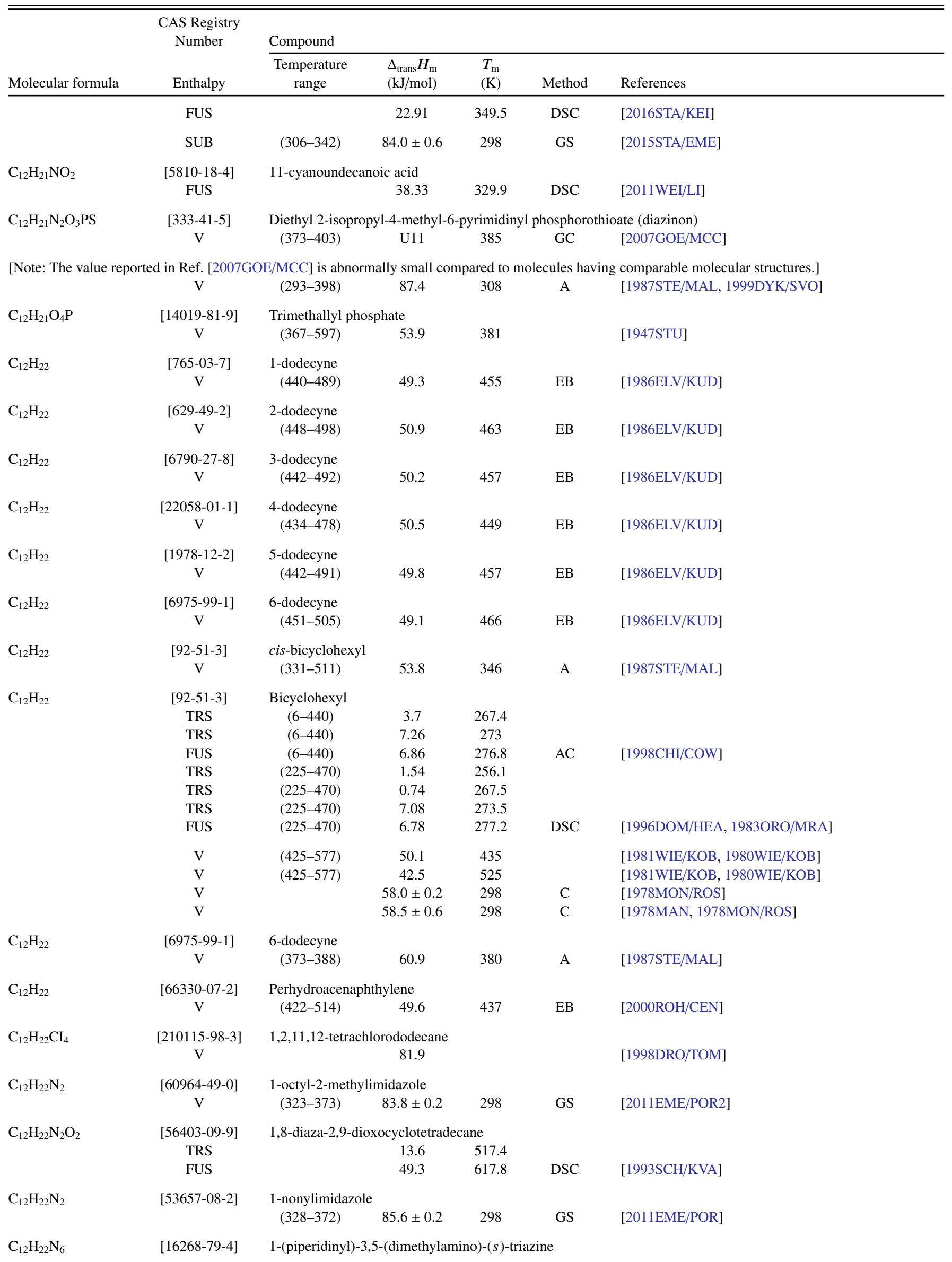


TABLE 11. Phase change enthalpies of $\mathrm{C}_{12}$ to $\mathrm{C}_{13}$ organic compounds-Continued

\begin{tabular}{|c|c|c|c|c|c|c|}
\hline \multirow[b]{2}{*}{ Molecular formula } & \multirow{2}{*}{$\begin{array}{l}\text { CAS Registry } \\
\text { Number } \\
\text { Enthalpy }\end{array}$} & \multicolumn{5}{|l|}{ Compound } \\
\hline & & $\begin{array}{l}\text { Temperature } \\
\text { range }\end{array}$ & $\begin{array}{l}\Delta_{\text {trans }} H_{\mathrm{m}} \\
(\mathrm{kJ} / \mathrm{mol})\end{array}$ & $\begin{array}{l}T_{\mathrm{m}} \\
(\mathrm{K})\end{array}$ & Method & References \\
\hline & FUS & & 23.22 & 361.5 & DSC & [1991ACR, 1989BRA/RYT] \\
\hline \multirow{6}{*}{$\mathrm{C}_{12} \mathrm{H}_{22} \mathrm{O}$} & [58879-21-3] & \multicolumn{2}{|c|}{ trans-2-cyclohexylcyclohexanol } & & & \\
\hline & FUS & $(5-337)$ & 14.52 & 325.8 & $\mathrm{AC}$ & [1997MAK/KAB] \\
\hline & SUB & $(293-325)$ & $96.6 \pm 0.6$ & 309 & ME & [1997MAK/KAB] \\
\hline & SUB & & $98.6 \pm 0.5$ & 320 & $\mathrm{C}$ & [1997MAK/KAB] \\
\hline & $\mathrm{V}$ & $(328-364)$ & $83.2 \pm 1.2$ & 346 & $\mathrm{ME}$ & [1997MAK/KAB] \\
\hline & $\mathrm{V}$ & & $80.3 \pm 0.3$ & 346 & $\mathrm{C}$ & [1997MAK/KAB] \\
\hline \multirow{9}{*}{$\mathrm{C}_{12} \mathrm{H}_{22} \mathrm{O}$} & [830-13-7] & \multirow[t]{3}{*}{ Cyclododecanone } & & & & \\
\hline & FUS & & 16.85 & 335.6 & DSC & [1996ROU/JIM, 1998GON/SZW] \\
\hline & FUS & & 16.75 & & DSC & {$[1972 \mathrm{WOL}]$} \\
\hline & SUB & $(282-300)$ & $83.2 \pm 0.3$ & 298 & $\mathrm{ME}$ & [1996ROU/JIM] \\
\hline & SUB & $(282-300)$ & $83.3 \pm 0.3$ & 291 & $\mathrm{ME}$ & [1996ROU/JIM] \\
\hline & $\mathrm{V}$ & $(373-443)$ & 61 & 388 & A & [1987STE/MAL, 1972WOL] \\
\hline & $\mathrm{V}$ & $(408-450)$ & 57.9 & 423 & $\mathrm{~A}, \mathrm{~EB}$ & [1987STE/MAL, 1976MEY/HOT] \\
\hline & $\mathrm{V}$ & $(458-556)$ & 54.7 & 473 & $\mathrm{~A}, \mathrm{~EB}$ & [1987STE/MAL, 1976MEY/HOT] \\
\hline & $\mathrm{V}$ & $(373-443)$ & $65.5 \pm 0.6$ & 298 & VP & {$[1972 \mathrm{WOL}]$} \\
\hline \multirow[t]{2}{*}{$\mathrm{C}_{12} \mathrm{H}_{22} \mathrm{O}$} & [33956-49-9] & \multicolumn{3}{|c|}{ trans,trans-8,10-dodecadien-1-ol } & & \\
\hline & $\mathrm{V}$ & & $92.3 \pm 2.6$ & 298 & CGC & [2015SCH/HAR] \\
\hline \multirow[t]{2}{*}{$\mathrm{C}_{12} \mathrm{H}_{22} \mathrm{O}$} & {$[81149-96-4]$} & \multirow{2}{*}{$\begin{array}{l}(Z) \text {-2-dodecenal } \\
\quad(323-363)\end{array}$} & & & & \\
\hline & $\mathrm{V}$ & & 72.5 & 298 & $\mathrm{CGC}$ & [1996KOU/HOS, 2000OVA/KOU] \\
\hline \multirow[t]{2}{*}{$\mathrm{C}_{12} \mathrm{H}_{22} \mathrm{O}$} & {$[20407-84-5]$} & \multicolumn{2}{|l|}{$(E)$-2-dodeceml } & & & \\
\hline & $\mathrm{V}$ & $(323-363)$ & 72.6 & 298 & CGC & [1996KOU/HOS, 2000OVA/KOU] \\
\hline \multirow[t]{2}{*}{$\mathrm{C}_{12} \mathrm{H}_{22} \mathrm{O}$} & {$[68141-15-1]$} & \multirow{2}{*}{$\begin{array}{l}(Z)-3 \text {-dodecenal } \\
\quad(323-363)\end{array}$} & & & & \\
\hline & V & & 69.6 & 298 & CGC & [1996KOU/HOS, 2000OVA/KOU] \\
\hline \multirow[t]{2}{*}{$\mathrm{C}_{12} \mathrm{H}_{22} \mathrm{O}$} & {$[76595-72-7]$} & \multirow{2}{*}{$\begin{array}{l}(E)-3 \text {-dodecenal } \\
\quad(323-363)\end{array}$} & & & & \\
\hline & V & & 70.2 & 298 & $\mathrm{CGC}$ & [1996KOU/HOS, 2000OVA/KOU] \\
\hline \multirow[t]{2}{*}{$\mathrm{C}_{12} \mathrm{H}_{22} \mathrm{O}$} & [21944-98-9] & \multirow{2}{*}{$\begin{array}{l}(Z)-4-d o d e c e n a l \\
\quad(323-363)\end{array}$} & & & & \\
\hline & $\mathrm{V}$ & & 69.4 & 298 & CGC & [1996KOU/HOS, 2000OVA/KOU] \\
\hline \multirow[t]{2}{*}{$\mathrm{C}_{12} \mathrm{H}_{22} \mathrm{O}$} & {$[174155-48-7]$} & $(E)$-4-dodecenal & & & & \\
\hline & V & $(323-363)$ & 69.9 & 298 & CGC & [1996KOU/HOS, 2000OVA/KOU] \\
\hline $\mathrm{C}_{12} \mathrm{H}_{22} \mathrm{O}$ & {$[68820-33-7]$} & (Z)-5-dodecenal & & & & \\
\hline & $\mathrm{V}$ & $(323-363)$ & 69.1 & 298 & CGC & [1996KOU/HOS, 2000OVA/KOU] \\
\hline $\mathrm{C}_{12} \mathrm{H}_{22} \mathrm{O}$ & {$[68820-34-8]$} & (E)-5-dodecenal & & & & \\
\hline & $\mathrm{V}$ & (323-363) & 69.6 & 298 & $\mathrm{CGC}$ & [1996KOU/HOS, 2000OVA/KOU] \\
\hline $\mathrm{C}_{12} \mathrm{H}_{22} \mathrm{O}$ & {$[126745-61-7]$} & (Z)-6-dodecenal & & & & \\
\hline & V & $(323-363)$ & 69.2 & 298 & CGC & [1996KOU/HOS, 2000OVA/KOU] \\
\hline $\mathrm{C}_{12} \mathrm{H}_{22} \mathrm{O}$ & {$[174155-49-8]$} & $(E)$-6-dodecenal & & & & \\
\hline & V & $(323-363)$ & 67.7 & 298 & CGC & [1996KOU/HOS, 2000OVA/KOU] \\
\hline $\mathrm{C}_{12} \mathrm{H}_{22} \mathrm{O}$ & {$[63851-40-1]$} & (Z)-7-dodecenal & & & & \\
\hline & $\mathrm{V}$ & $(323-363)$ & 69.4 & 298 & CGC & [1996KOU/HOS, 2000OVA/KOU] \\
\hline $\mathrm{C}_{12} \mathrm{H}_{22} \mathrm{O}$ & {$[82944-76-1]$} & $(E)$-7-dodecenal & & & & \\
\hline & V & $(323-363)$ & 69.6 & 298 & $\mathrm{CGC}$ & [1996KOU/HOS, 2000OVA/KOU] \\
\hline $\mathrm{C}_{12} \mathrm{H}_{22} \mathrm{O}$ & {$[139909-65-2]$} & (Z)-8-dodecenal & & & & \\
\hline & V & $(323-363)$ & 70.0 & 298 & CGC & [1996KOU/HOS, 2000OVA/KOU] \\
\hline $\mathrm{C}_{12} \mathrm{H}_{22} \mathrm{O}$ & [144298-64-6] & (E)-8-dodecenal & & & & \\
\hline & $\mathrm{V}$ & $(323-363)$ & 69.8 & 298 & CGC & [1996KOU/HOS, 2000OVA/KOU] \\
\hline $\mathrm{C}_{12} \mathrm{H}_{22} \mathrm{O}$ & {$[56219-03-5]$} & (Z)-9-dodecenal & & & & \\
\hline & V & $(323-363)$ & 70.1 & 298 & CGC & [1996KOU/HOS, 2000OVA/KOU] \\
\hline $\mathrm{C}_{12} \mathrm{H}_{22} \mathrm{O}$ & [155235-07-7] & $(E)$-9-dodecenal & & & & \\
\hline
\end{tabular}


TABLE 11. Phase change enthalpies of $\mathrm{C}_{12}$ to $\mathrm{C}_{13}$ organic compounds-Continued

\begin{tabular}{|c|c|c|c|c|c|c|}
\hline \multirow[b]{2}{*}{ Molecular formula } & \multirow{2}{*}{$\begin{array}{l}\text { CAS Registry } \\
\text { Number } \\
\text { Enthalpy }\end{array}$} & \multicolumn{5}{|l|}{ Compound } \\
\hline & & $\begin{array}{l}\text { Temperature } \\
\text { range }\end{array}$ & $\begin{array}{l}\Delta_{\text {trans }} H_{\mathrm{m}} \\
(\mathrm{kJ} / \mathrm{mol})\end{array}$ & $\begin{array}{l}T_{\mathrm{m}} \\
(\mathrm{K})\end{array}$ & Method & References \\
\hline & V & $(323-363)$ & 70.4 & 298 & $\mathrm{CGC}$ & [1996KOU/HOS, 2000OVA/KOU] \\
\hline $\mathrm{C}_{12} \mathrm{H}_{22} \mathrm{O}$ & $\begin{array}{c}{[81892-61-7]} \\
\mathrm{V}\end{array}$ & $\begin{array}{l}\text { (Z)-10-dodecenal } \\
\quad(323-363)\end{array}$ & 71.0 & 298 & CGC & [1996KOU/HOS, 2000OVA/KOU] \\
\hline $\mathrm{C}_{12} \mathrm{H}_{22} \mathrm{O}$ & $\begin{array}{c}{[81892-62-8]} \\
\mathrm{V}\end{array}$ & $\begin{array}{l}(E)-10 \text {-dodecenal } \\
(323-363)\end{array}$ & 70.9 & 298 & $\mathrm{CGC}$ & [1996KOU/HOS, 2000OVA/KOU] \\
\hline $\mathrm{C}_{12} \mathrm{H}_{22} \mathrm{O}_{2}$ & $\begin{array}{c}{[947-05-7]} \\
\text { V } \\
\text { V }\end{array}$ & $\begin{array}{l}\text { Dodecanolactone } \\
(377-403) \\
(377-403)\end{array}$ & $\begin{array}{l}64.2 \pm 1.1 \\
70.5 \pm 1.7\end{array}$ & $\begin{array}{l}390 \\
298\end{array}$ & $\begin{array}{l}\text { MM } \\
\text { MM }\end{array}$ & $\begin{array}{l}\text { [1991WIB/WAL] } \\
\text { [1991WIB/WAL] }\end{array}$ \\
\hline $\mathrm{C}_{12} \mathrm{H}_{22} \mathrm{O}_{2}$ & $\begin{array}{c}{[2305-05-7]} \\
\mathrm{V}\end{array}$ & $\gamma$-dodecanolactone & $83.9 \pm 4.6$ & 298 & CGC & [2014KOZ/GOB] \\
\hline $\mathrm{C}_{12} \mathrm{H}_{22} \mathrm{O}_{2}$ & $\begin{array}{c}{[713-95-1]} \\
\mathrm{V}\end{array}$ & 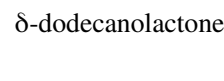 & $84.6 \pm 4.7$ & 298 & CGC & {$[2014 \mathrm{KOZ} / \mathrm{GOB}]$} \\
\hline $\mathrm{C}_{12} \mathrm{H}_{22} \mathrm{O}_{2}$ & $\begin{array}{c}{[32210-23-4]} \\
\mathrm{V}\end{array}$ & $\begin{array}{l}\text { Acetic acid, 4-tert- } \\
\quad(285-318)\end{array}$ & $\begin{array}{c}\text {-butylcycloh } \\
63.8\end{array}$ & $\begin{array}{c}1 \text { ester } \\
300\end{array}$ & $\mathrm{~A}, \mathrm{ME}$ & [1987STE/MAL, 1958SER/VOI, 1957SER/VOI] \\
\hline $\mathrm{C}_{12} \mathrm{H}_{22} \mathrm{O}_{2}$ & $\mathrm{~V}$ & $\begin{array}{l}\text { 3,3-dimethylbutanc } \\
\quad(333-378)\end{array}$ & $\begin{array}{l}\text { loic acid, cyc } \\
62.1\end{array}$ & $\begin{array}{l}\text { exyl est } \\
298\end{array}$ & CGC & [1999VER/HEI] \\
\hline $\mathrm{C}_{12} \mathrm{H}_{22} \mathrm{O}_{2}$ & $\mathrm{~V}$ & $\begin{array}{l}\text { 1-methylcyclohexy } \\
\text { (333-378) }\end{array}$ & $\begin{array}{c}\text { 1 pivalate } \\
57.9\end{array}$ & 298 & CGC & [1999VER/HEI] \\
\hline $\mathrm{C}_{12} \mathrm{H}_{22} \mathrm{O}_{2}$ & $\mathrm{~V}$ & $\begin{array}{l}\text { 3-methylcyclohexy } \\
\text { (333-378) }\end{array}$ & $\begin{array}{c}\text { yl pivalate } \\
60.5\end{array}$ & 298 & CGC & [1999VER/HEI] \\
\hline $\mathrm{C}_{12} \mathrm{H}_{22} \mathrm{O}_{2}$ & $\mathrm{~V}$ & $\begin{array}{l}\text { 4-methylcyclohexy } \\
\quad(333-378)\end{array}$ & $\begin{array}{c}\text { yl pivalate } \\
60.9\end{array}$ & 298 & CGC & [1999VER/HEI] \\
\hline $\mathrm{C}_{12} \mathrm{H}_{22} \mathrm{O}_{2}$ & $\begin{array}{c}{[16409-45-3]} \\
V\end{array}$ & $\begin{array}{l}\text { (d)-menthyl acetat } \\
(330-500)\end{array}$ & 55.3 & 345 & A & [1987STE/MAL, 1947STU] \\
\hline $\mathrm{C}_{12} \mathrm{H}_{22} \mathrm{O}_{2}$ & $\begin{array}{c}{[150-84-5]} \\
\mathrm{V} \\
\mathrm{V}\end{array}$ & $\begin{array}{l}\text { Citronellyl acetate } \\
(347-490)\end{array}$ & $\begin{array}{c}67.8 \pm 1.8 \\
68.7\end{array}$ & $\begin{array}{l}298 \\
362\end{array}$ & $\begin{array}{c}\mathrm{CGC} \\
\mathrm{A}\end{array}$ & $\begin{array}{l}\text { [2015KOZ/GOB] } \\
\text { [1987STE/MAL, 1947STU] }\end{array}$ \\
\hline $\mathrm{C}_{12} \mathrm{H}_{22} \mathrm{O}_{2}$ & $\begin{array}{c}{[61732-97-6]} \\
\mathrm{V}\end{array}$ & $\begin{array}{l}\text { 2-(1-ethylpentyl)-4 } \\
\quad(333-453)\end{array}$ & $\begin{array}{l}\text { 4,7-dihydro- } \\
66.3\end{array}$ & $\begin{array}{c}\text { dioxepi } \\
348\end{array}$ & A & [1987STE/MAL, 1977VOI/SHC] \\
\hline $\mathrm{C}_{12} \mathrm{H}_{22} \mathrm{O}_{2}$ & $\begin{array}{l}{[2664-55-3]} \\
\text { FUS }\end{array}$ & Nonyl acrylate & 23.36 & 236.5 & $\mathrm{AC}$ & [1990DOM/HEA, 1985KAR/ABD] \\
\hline $\mathrm{C}_{12} \mathrm{H}_{22} \mathrm{O}_{2}$ & $\begin{array}{l}\text { [2157-01-9] } \\
\text { FUS }\end{array}$ & Octyl methacrylate & 24.09 & 230.3 & $\mathrm{AC}$ & [1990DOM/HEA, 1985KAR/ABD] \\
\hline & $\mathrm{V}$ & $(384-513)$ & 55.6 & 399 & A & [1987STE/MAL] \\
\hline $\mathrm{C}_{12} \mathrm{H}_{22} \mathrm{O}_{2}$ & $\begin{array}{c}{[111-81-9]} \\
\mathrm{V}\end{array}$ & $\begin{array}{l}\text { Methyl 10-undecer } \\
(397-524)\end{array}$ & $\begin{array}{l}\text { noate } \\
59.2\end{array}$ & 412 & A & [1987STE/MAL] \\
\hline $\mathrm{C}_{12} \mathrm{H}_{22} \mathrm{O}_{2}$ & $\begin{array}{c}\text { [81634-99-3] } \\
\mathrm{V} \\
\mathrm{V}\end{array}$ & $\begin{array}{l}\text { (Z)-3-decenyl acet } \\
(313-358) \\
(299-313)\end{array}$ & $\begin{array}{l}\text { tate } \\
\begin{array}{r}69.5 \\
72.0\end{array}\end{array}$ & $\begin{array}{l}298 \\
306\end{array}$ & $\begin{array}{l}\text { GC } \\
\text { GC }\end{array}$ & $\begin{array}{l}\text { [1997KOU/HOS, 2000OVA/KOU] } \\
\text { [1983OLS/JON] }\end{array}$ \\
\hline $\mathrm{C}_{12} \mathrm{H}_{22} \mathrm{O}_{2}$ & $\begin{array}{c}{[81634-98-2]} \\
\mathrm{V}\end{array}$ & $\begin{array}{l}(E) \text {-3-decenyl acet } \\
(313-358)\end{array}$ & 70.1 & 298 & GC & [1997KOU/HOS, 2000OVA/KOU] \\
\hline $\mathrm{C}_{12} \mathrm{H}_{22} \mathrm{O}_{2}$ & $\begin{array}{c}{[67452-27-1]} \\
\mathrm{V}\end{array}$ & $\begin{array}{l}\text { (Z)-4-decenyl acet } \\
(313-358)\end{array}$ & tate 69.0 & 298 & GC & [1997KOU/HOS, 2000OVA/KOU] \\
\hline $\mathrm{C}_{12} \mathrm{H}_{22} \mathrm{O}_{2}$ & $\begin{array}{c}{[69222-16-8]} \\
\mathrm{V}\end{array}$ & $\begin{array}{l}(E) \text {-4-decenyl acet } \\
(313-358)\end{array}$ & 70.1 & 298 & GC & [1997KOU/HOS, 2000OVA/KOU] \\
\hline $\mathrm{C}_{12} \mathrm{H}_{22} \mathrm{O}_{2}$ & $\begin{array}{c}{[67446-07-5]} \\
\text { V } \\
V\end{array}$ & $\begin{array}{l}\text { (Z)-5-decenyl acet } \\
(313-358) \\
(299-313)\end{array}$ & $\begin{array}{l}\text { tate } \\
\begin{array}{r}69.7 \\
72.0\end{array}\end{array}$ & $\begin{array}{l}298 \\
306\end{array}$ & $\begin{array}{l}\mathrm{GC} \\
\mathrm{GC}\end{array}$ & $\begin{array}{l}\text { [1997KOU/HOS, 2000OVA/KOU] } \\
\text { [1983OLS/JON] }\end{array}$ \\
\hline
\end{tabular}


TABLE 11. Phase change enthalpies of $\mathrm{C}_{12}$ to $\mathrm{C}_{13}$ organic compounds-Continued

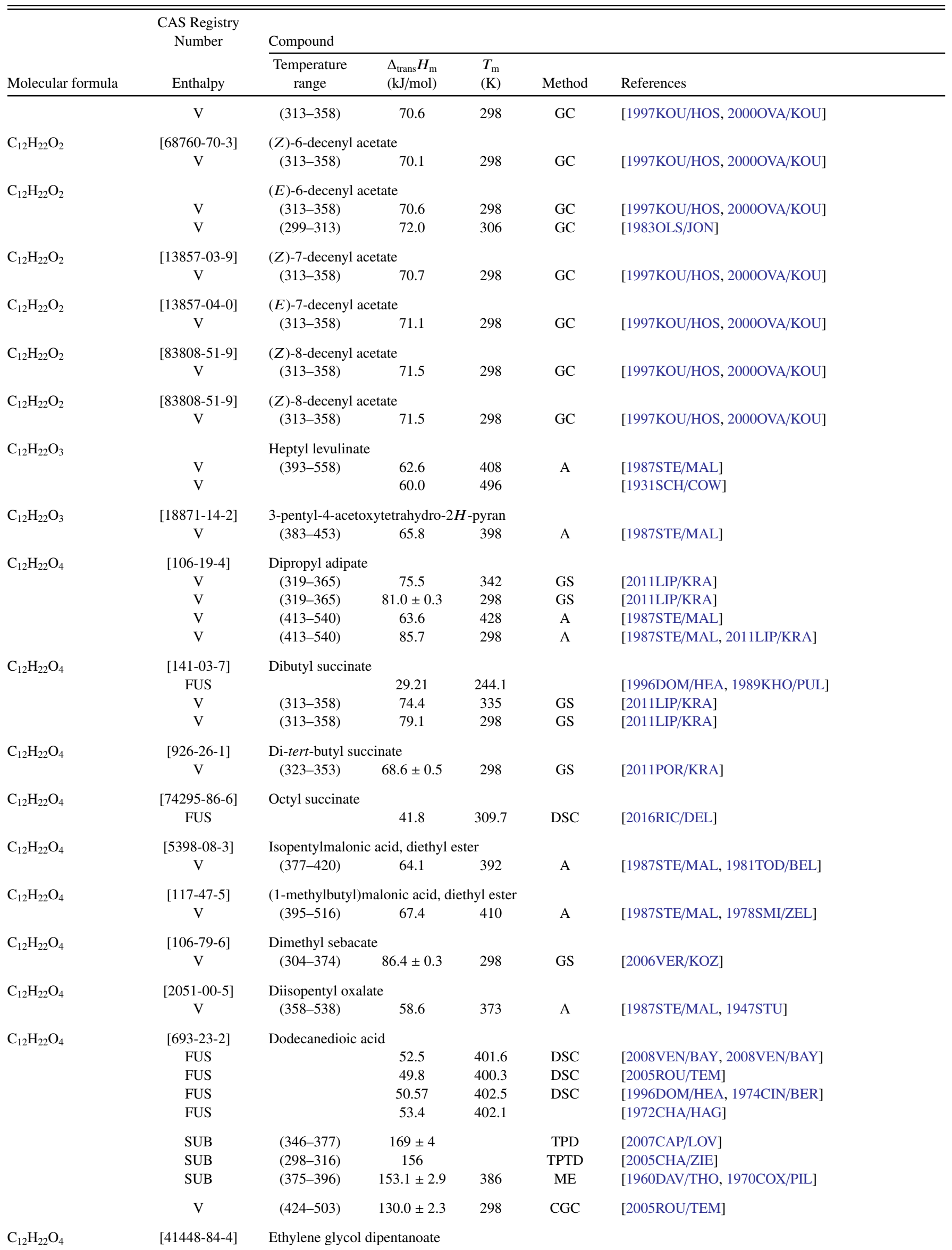


TABle 11. Phase change enthalpies of $\mathrm{C}_{12}$ to $\mathrm{C}_{13}$ organic compounds-Continued

\begin{tabular}{|c|c|c|c|c|c|c|}
\hline \multirow[b]{2}{*}{ Molecular formula } & \multirow{2}{*}{$\begin{array}{l}\text { CAS Registry } \\
\text { Number } \\
\text { Enthalpy }\end{array}$} & \multicolumn{5}{|l|}{ Compound } \\
\hline & & $\begin{array}{l}\text { Temperature } \\
\text { range }\end{array}$ & $\begin{array}{l}\Delta_{\text {trans }} H_{\mathrm{m}} \\
(\mathrm{kJ} / \mathrm{mol})\end{array}$ & $\begin{array}{l}T_{\mathrm{m}} \\
(\mathrm{K})\end{array}$ & Method & References \\
\hline & $\mathrm{V}$ & $(297-325)$ & \multicolumn{2}{|l|}{$80.2 \pm 1.0$} & GS & [2011MAS/KRA] \\
\hline $\mathrm{C}_{12} \mathrm{H}_{22} \mathrm{O}_{4}$ & $\begin{array}{c}{[20267-20-3]} \\
V\end{array}$ & $\begin{array}{l}\text { Ethylene glyco } \\
\quad(295-325)\end{array}$ & $\begin{array}{l}\text { (2,2-dimeth } \\
69.3 \pm 1.1\end{array}$ & opanoa & GS & [2011MAS/KRA] \\
\hline $\mathrm{C}_{12} \mathrm{H}_{22} \mathrm{O}_{4}$ & $\begin{array}{c}\text { [155514-29-7] } \\
\mathrm{V}\end{array}$ & $\begin{array}{c}\text { Ethylene glyco } \\
(297-327)\end{array}$ & $\begin{array}{c}\text { (2-methylbu } \\
71.5 \pm 1.1\end{array}$ & oate) & GS & [2011MAS/KRA] \\
\hline $\mathrm{C}_{12} \mathrm{H}_{22} \mathrm{O}_{4} \mathrm{~S}$ & $\begin{array}{c}{[4121-12-4]} \\
\mathrm{V}\end{array}$ & $\begin{array}{c}\text { Thiodiglycolic } \\
(298-383)\end{array}$ & $\begin{array}{c}\text { d, diethyl es } \\
75.7\end{array}$ & 313 & A & [1987STE/MAL, 1999DYK/SVO] \\
\hline $\mathrm{C}_{12} \mathrm{H}_{22} \mathrm{O}_{5}$ & $\begin{array}{c}{[902261-31-8]} \\
\mathrm{V}\end{array}$ & $\begin{array}{l}\text { Butyl[1-(butox } \\
\quad(338-513)\end{array}$ & \multicolumn{2}{|c|}{ Butyl[1-(butoxy carbonyl)ethyl] carbonate } & A & [1987STE/MAL, 1950REH/DIX2] \\
\hline $\mathrm{C}_{12} \mathrm{H}_{22} \mathrm{O}_{5}$ & $\mathrm{~V}$ & $\begin{array}{l}\text { Pentyl[1-(ethox } \\
\quad(368-513)\end{array}$ & $\begin{array}{c}\text { carbonyl)iso } \\
63.8\end{array}$ & $\begin{array}{l}383 \\
383 \text { carl }\end{array}$ & A & [1987STE/MAL] \\
\hline $\mathrm{C}_{12} \mathrm{H}_{22} \mathrm{O}_{6}$ & $\begin{array}{c}{[856371-29-4]} \\
\mathrm{V}\end{array}$ & $\begin{array}{l}\text { Lactic acid, } O \\
\quad(383-521)\end{array}$ & \multicolumn{2}{|c|}{ Lactic acid, $O$-ethoxycarbonyl, 2-butoxyethyl ester } & $\begin{array}{r}\text { ester } \\
\text { A }\end{array}$ & [1987STE/MAL] \\
\hline $\mathrm{C}_{12} \mathrm{H}_{22} \mathrm{O}_{6}$ & $\begin{array}{c}{[87-92-3]} \\
\mathrm{V}\end{array}$ & $\begin{array}{l}\text { Dibutyl tartrate } \\
\quad(428-511)\end{array}$ & 79.8 & 443 & A & [1987STE/MAL] \\
\hline $\mathrm{C}_{12} \mathrm{H}_{22} \mathrm{O}_{6}$ & $\begin{array}{c}{[4054-82-4]} \\
\mathrm{V}\end{array}$ & $\begin{array}{l}\text { (d)-diisobutyl } \\
(390-597)\end{array}$ & $\begin{array}{l}\text { rate } \\
64.6\end{array}$ & 405 & A & [1987STE/MAL] \\
\hline $\mathrm{C}_{12} \mathrm{H}_{22} \mathrm{O}_{11}$ & $\begin{array}{c}{[528-50-7]} \\
\text { SUB }\end{array}$ & $\begin{array}{c}(d) \text {-cellobiose } \\
(474-488)\end{array}$ & $302 \pm 44.0$ & 481 & $\mathrm{ME}$ & [1999OJA/SUU] \\
\hline $\mathrm{C}_{12} \mathrm{H}_{22} \mathrm{O}_{11}$ & $\begin{array}{c}{[14641-93-1]} \\
\text { FUS }\end{array}$ & $\alpha$-lactose & 75.2 & 496.2 & & [2000MAC/COU, 1983RAE/SCH] \\
\hline $\mathrm{C}_{12} \mathrm{H}_{22} \mathrm{O}_{11}$ & $\begin{array}{c}\text { [57-50-1] } \\
\text { FUS } \\
\text { TRS } \\
\text { FUS }\end{array}$ & Sucrose & $\begin{array}{c}45.21 \\
3.34 \\
39.39\end{array}$ & $\begin{array}{l}457.2 \\
419.2 \\
453.3\end{array}$ & $\begin{array}{l}\text { DSC } \\
\text { DSC }\end{array}$ & $\begin{array}{l}\text { [2014MAG/WUR] } \\
\text { [2011LEE/THO] }\end{array}$ \\
\hline
\end{tabular}

[Note: The authors performed measurements as a function of heating rate, and the above values correspond to a heating rate of $2^{\circ} \mathrm{C} / \mathrm{min}$.] FUS $46.2 \quad 459 \quad$ DSC [1988SOP/KEA]

$\mathrm{C}_{12} \mathrm{H}_{22} \mathrm{~S}$

[7133-46-2] TRS

FUS

V
V
V
V
V
V

$\mathrm{C}_{12} \mathrm{H}_{22} \mathrm{~N}$

[101-83-7]

V
V
V

$\mathrm{C}_{12} \mathrm{H}_{22} \mathrm{~N}$

$\mathrm{C}_{12} \mathrm{H}_{23} \mathrm{NO}$

[2437-25-4] FUS

V
V
V
V

[947-04-6]
Dicyclohexyl sulfide

e $5.68 \quad 284.2$

\section{(335-523)}

(335-523)

$(335-523)$

(335-523)

(335-523)

(421-523)

$65.4 \pm 0.2$

$62.5 \pm 0.1$

$59.5 \pm 0.1$

$56.6 \pm 0.1$

$53.7 \pm 0.1$

$69.0 \pm 0.7$

$(295-333)$
$(295-333)$
$(408-529)$

$62.8 \pm 0.2 \quad 314$

$64.0 \pm 0.3 \quad 298$

$54.0 \quad 423$

TRS

FUS

SUB
Dicyclohexylamine
Lauronitrile

(298-367)

(393-462)

(440-556)

$\begin{array}{cc}30.7 & 274.6 \\ 74.9 \pm 0.2 & 298 \\ 76.1 \pm 0.1 & 298 \\ 65.2 & 408 \\ 60.7 & 455\end{array}$

Azacyclotridecan-2-one

$$
1.1
$$$$
15.9
$$

$(370-420)$

$110.6 \pm 0.8 \quad 298$
284.2

380

420

440

480

298

298

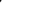

$\begin{array}{cl}\text { IPM,EB } & {[2004 \mathrm{STE} / \mathrm{CHI}]} \\ \text { IPM,EB } & {[2004 \mathrm{STE} / \mathrm{CHI}]} \\ \text { IPM,EB } & {[2004 \mathrm{STE} / \mathrm{CHI}]} \\ \text { IPM,EB } & {[2004 \mathrm{STE} / \mathrm{CHI}]} \\ \text { IPM,EB } & {[2004 \mathrm{STE} / \mathrm{CHI}]} \\ \text { EB } & {[1997 \mathrm{STE} / \mathrm{CHI} 4]}\end{array}$

$\begin{array}{cl}\text { EB } & \text { [2015VER/EME3] } \\ \text { EB } & \text { [2015VER/EME3] } \\ \text { A } & \text { [1987STE/MAL] }\end{array}$

DSC

[2013MEK/BEN]

GS [2005EME/VER]

C [1977STRI/SUN]

EB [1971MEY/REN]

A,EB [1987STE/MAL, 1971MEY/REN, 1973MEY/HOT]
DSC

[2012EME/VER]

GS

[2012EME/VER] 
TABLE 11. Phase change enthalpies of $\mathrm{C}_{12}$ to $\mathrm{C}_{13}$ organic compounds-Continued

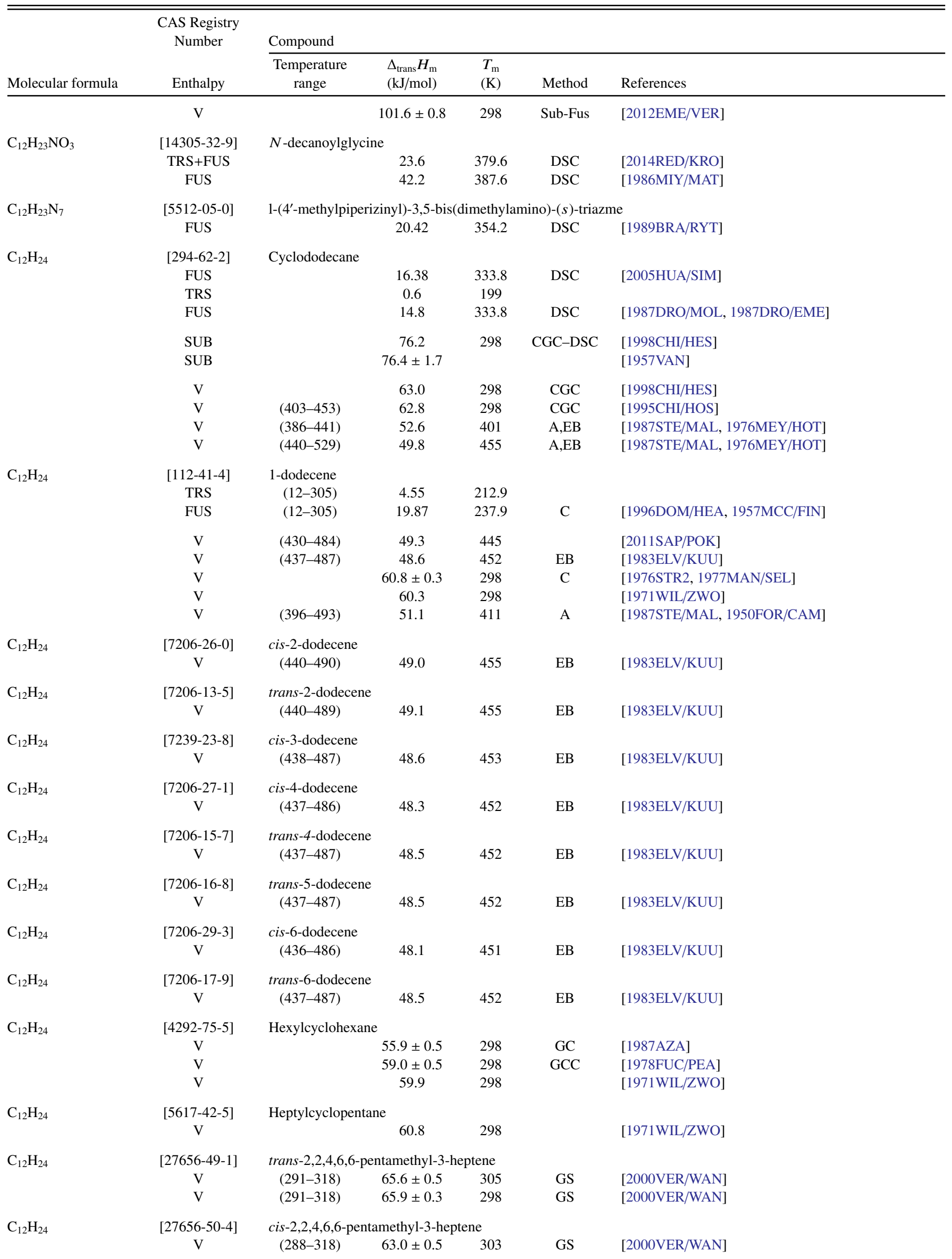


TABLE 11. Phase change enthalpies of $\mathrm{C}_{12}$ to $\mathrm{C}_{13}$ organic compounds-Continued

\begin{tabular}{|c|c|c|c|c|c|c|}
\hline \multirow[b]{2}{*}{ Molecular formula } & \multirow{2}{*}{$\begin{array}{l}\text { CAS Registry } \\
\text { Number } \\
\text { Enthalpy }\end{array}$} & \multicolumn{5}{|l|}{ Compound } \\
\hline & & $\begin{array}{l}\text { Temperature } \\
\text { range }\end{array}$ & $\begin{array}{l}\Delta_{\text {trans }} H_{\mathrm{m}} \\
(\mathrm{kJ} / \mathrm{mol})\end{array}$ & $\begin{array}{l}T_{\mathrm{m}} \\
(\mathrm{K})\end{array}$ & Method & References \\
\hline & $\mathrm{V}$ & $(288-318)$ & $53.2 \pm 0.5$ & 298 & GS & [2000VER/WAN] \\
\hline $\mathrm{C}_{12} \mathrm{H}_{24} \mathrm{Cl}_{2}$ & $\begin{array}{c}{[3922-28-9]} \\
V\end{array}$ & 1,12-dichlorododeca & $\begin{array}{l}\text { ane } \\
73.1\end{array}$ & & & [1998DRO/TOM] \\
\hline $\mathrm{C}_{12} \mathrm{H}_{24} \mathrm{~N}_{2} \mathrm{O}_{2}$ & $\begin{array}{c}\text { [10263-96-4] } \\
\text { FUS }\end{array}$ & $N, N^{\prime}$-di- $n$-propylad & $\begin{array}{l}\text { dipamide } \\
36.11\end{array}$ & 452 & & [1984DOM/EVA] \\
\hline $\mathrm{C}_{12} \mathrm{H}_{24} \mathrm{~N}_{2} \mathrm{O}_{2}$ & $\begin{array}{c}\text { [6224-99-3] } \\
\text { TRS } \\
\text { FUS }\end{array}$ & Dodecandiamide & $\begin{array}{l}5.09 \\
73.7\end{array}$ & $\begin{array}{l}422.8 \\
466.1\end{array}$ & DSC & [2006BAD/DEL] \\
\hline $\mathrm{C}_{12} \mathrm{H}_{24} \mathrm{~N}_{2} \mathrm{O}_{2}$ & $\begin{array}{l}{[3129-91-7]} \\
\text { SUB } \\
\text { SUB } \\
\text { SUB }\end{array}$ & $\begin{array}{l}\text { Dicyclohexyl ammor } \\
\quad(290-298) \\
(308-339)\end{array}$ & $\begin{array}{l}\text { nium nitrite } \\
99.1 \\
\text { U161.8 } \\
105.9\end{array}$ & 324 & $\mathrm{TE}$ & $\begin{array}{l}\text { [1987STE/MAL, 1965MAR] } \\
\text { [1985TRU/KRA] } \\
\text { [1961ROZ/POL] }\end{array}$ \\
\hline $\mathrm{C}_{12} \mathrm{H}_{24} \mathrm{O}$ & $\begin{array}{c}{[1724-39-6]} \\
\mathrm{V} \\
\mathrm{V}\end{array}$ & $\begin{array}{l}\text { Cyclododecanol } \\
(405-468) \\
(467-557)\end{array}$ & $\begin{array}{l}68.8 \\
57.1\end{array}$ & $\begin{array}{l}420 \\
482\end{array}$ & $\begin{array}{l}\mathrm{A} \\
\mathrm{A}\end{array}$ & $\begin{array}{l}\text { [1987STE/MAL] } \\
\text { [1987STE/MAL] }\end{array}$ \\
\hline $\mathrm{C}_{12} \mathrm{H}_{24} \mathrm{O}$ & $\begin{array}{c}\text { [112-54-9] } \\
\text { FUS } \\
\text { V } \\
\text { V } \\
\text { V }\end{array}$ & $\begin{array}{l}\text { Dodecanal } \\
\begin{array}{r}(314-347) \\
(308-353) \\
(350-530)\end{array}\end{array}$ & $\begin{array}{c}43.38 \\
68.3 \pm 0.9 \\
70.2 \\
56.5\end{array}$ & $\begin{array}{c}286.5 \\
298 \\
298 \\
365\end{array}$ & $\begin{array}{l}\text { DSC } \\
\text { GS } \\
\text { CGC } \\
\text { A }\end{array}$ & $\begin{array}{l}\text { [2012BEI/RUE] } \\
\text { [2003VER/KRA2] } \\
\text { [1996KOU/HOS, 2000OVA/KOU] } \\
\text { [1987STE/MAL, 1947STU] }\end{array}$ \\
\hline $\mathrm{C}_{12} \mathrm{H}_{24} \mathrm{O}$ & $\begin{array}{c}{[6175-49-1]} \\
\text { FUS }\end{array}$ & 2-dodecanone & 39.48 & 294.3 & DSC & [2011DOM/PAD] \\
\hline & $\begin{array}{l}\mathrm{V} \\
\mathrm{V} \\
\mathrm{V} \\
\mathrm{V}\end{array}$ & $\begin{array}{l}(350-520) \\
(386-609) \\
(386-609)\end{array}$ & $\begin{array}{l}61.1 \\
71.8 \pm 0.6 \\
60.8 \\
48.1\end{array}$ & $\begin{array}{l}365 \\
298 \\
401 \\
524\end{array}$ & $\begin{array}{l}\mathrm{A} \\
\mathrm{C} \\
\mathrm{A}\end{array}$ & $\begin{array}{l}\text { [1987STE/MAL, 1947STU] } \\
\text { [1977SEL] } \\
\text { [1987STE/MAL, 1975AMB/ELL] } \\
\text { [1975AMB/ELL] }\end{array}$ \\
\hline $\mathrm{C}_{12} \mathrm{H}_{24} \mathrm{O}$ & $\begin{array}{c}\text { [19321-39-2] } \\
\mathrm{V}\end{array}$ & $\begin{array}{l}\text { Ethyl } p \text {-methyl ether } \\
\quad(366-414)\end{array}$ & 50.9 & 381 & A & [1987STE/MAL] \\
\hline $\mathrm{C}_{12} \mathrm{H}_{24} \mathrm{O}$ & $\begin{array}{c}{[20999-39-7]} \\
\text { V }\end{array}$ & $\begin{array}{l}\text { 1-heptylcyclopentan } \\
(395-524)\end{array}$ & 58.6 & 410 & A & [1987STE/MAL, 1944MCL/EDW] \\
\hline $\mathrm{C}_{12} \mathrm{H}_{24} \mathrm{O}$ & $\begin{array}{c}{[3964-63-4]} \\
\mathrm{V}\end{array}$ & $\begin{array}{l}\text { 1-hexylcyclohexanol } \\
\text { (380-491) }\end{array}$ & 53.5 & 395 & A & [1987STE/MAL, 1947WIL/EDW] \\
\hline $\mathrm{C}_{12} \mathrm{H}_{24} \mathrm{O}$ & $\begin{array}{c}{[69064-36-4]} \\
\mathrm{V}\end{array}$ & $\begin{array}{l}\text { (Z)-2-dodecen-1-ol } \\
\quad(333-373)\end{array}$ & 90.7 & 298 & CGC & [2000OVA/KOU, 1994KOU/HOS] \\
\hline $\mathrm{C}_{12} \mathrm{H}_{24} \mathrm{O}$ & $\begin{array}{c}{[69064-37-5]} \\
\mathrm{V}\end{array}$ & $\begin{array}{l}(E) \text {-2-dodecen-1-ol } \\
\quad(333-373)\end{array}$ & 91.0 & 298 & CGC & [2000OVA/KOU, 1994KOU/HOS] \\
\hline $\mathrm{C}_{12} \mathrm{H}_{24} \mathrm{O}$ & $\begin{array}{c}{[32451-95-9]} \\
\mathrm{V}\end{array}$ & $\begin{array}{l}\text { (Z)-3-dodecen-1-ol } \\
\quad(333-373)\end{array}$ & 89.3 & 298 & CGC & [2000OVA/KOU, 1994KOU/HOS] \\
\hline $\mathrm{C}_{12} \mathrm{H}_{24} \mathrm{O}$ & $\begin{array}{c}{[68900-87-8]} \\
\mathrm{V}\end{array}$ & $\begin{array}{l}(E) \text {-3-dodecen-1-ol } \\
\quad(333-373)\end{array}$ & 89.2 & 298 & CGC & [2000OVA/KOU, 1994KOU/HOS] \\
\hline $\mathrm{C}_{12} \mathrm{H}_{24} \mathrm{O}$ & $\begin{array}{c}{[40642-37-3]} \\
\mathrm{V}\end{array}$ & $\begin{array}{l}\text { (Z)-4-dodecen-1-ol } \\
\quad(333-373)\end{array}$ & 89.9 & 298 & CGC & [2000OVA/KOU, 1994KOU/HOS] \\
\hline $\mathrm{C}_{12} \mathrm{H}_{24} \mathrm{O}$ & $\begin{array}{c}{[81745-38-2]} \\
\mathrm{V}\end{array}$ & $\begin{array}{l}(E)-4 \text {-dodecen-1-ol } \\
\quad(333-373)\end{array}$ & 90.6 & 298 & CGC & [2000OVA/KOU, 1994KOU/HOS] \\
\hline $\mathrm{C}_{12} \mathrm{H}_{24} \mathrm{O}$ & $\begin{array}{c}{[40642-38-4]} \\
\mathrm{V}\end{array}$ & $\begin{array}{l}(Z)-5 \text {-dodecen-1-ol } \\
\quad(333-373)\end{array}$ & 90.2 & 298 & CGC & [2000OVA/KOU, 1994KOU/HOS] \\
\hline $\mathrm{C}_{12} \mathrm{H}_{24} \mathrm{O}$ & $\begin{array}{c}{[62936-12-3]} \\
\mathrm{V}\end{array}$ & $\begin{array}{l}(E)-5 \text {-dodecen-1-ol } \\
\quad(333-373)\end{array}$ & 90.7 & 298 & CGC & [2000OVA/KOU, 1994KOU/HOS] \\
\hline $\mathrm{C}_{12} \mathrm{H}_{24} \mathrm{O}$ & $\begin{array}{c}{[40642-39-5]} \\
\mathrm{V}\end{array}$ & $\begin{array}{l}\text { (Z)-6-dodecen-1-ol } \\
\quad(333-373)\end{array}$ & 90.2 & 298 & $\mathrm{CGC}$ & [2000OVA/KOU, 1994KOU/HOS] \\
\hline
\end{tabular}


TABLE 11. Phase change enthalpies of $C_{12}$ to $C_{13}$ organic compounds-Continued

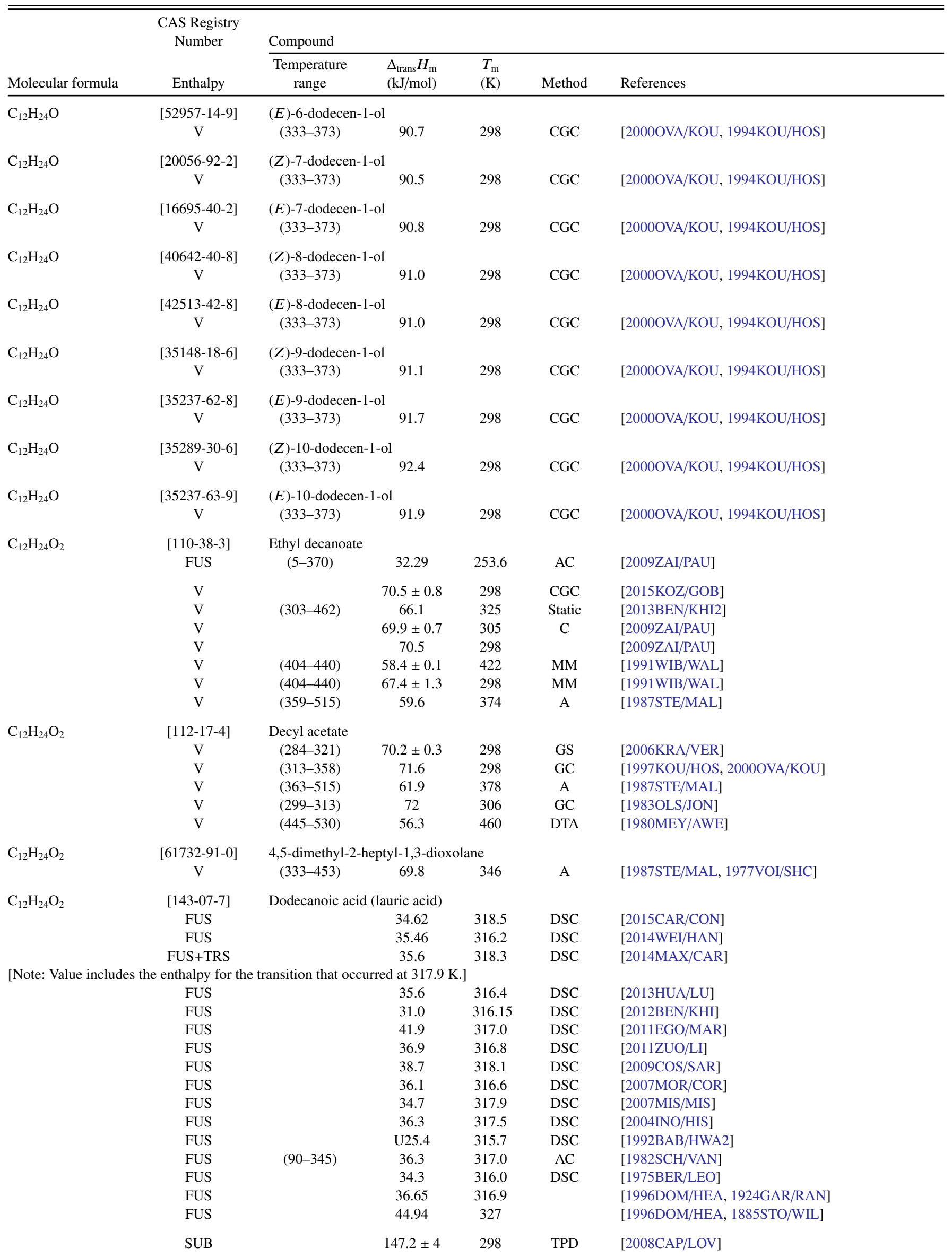


TABLE 11. Phase change enthalpies of $\mathrm{C}_{12}$ to $\mathrm{C}_{13}$ organic compounds-Continued

\begin{tabular}{|c|c|c|c|c|c|c|}
\hline \multirow[b]{2}{*}{ Molecular formula } & \multirow{2}{*}{$\begin{array}{l}\text { CAS Registry } \\
\text { Number } \\
\text { Enthalpy }\end{array}$} & \multicolumn{5}{|l|}{ Compound } \\
\hline & & $\begin{array}{c}\text { Temperature } \\
\text { range }\end{array}$ & $\begin{array}{l}\Delta_{\text {trans }} H_{\mathrm{m}} \\
(\mathrm{kJ} / \mathrm{mol})\end{array}$ & $\begin{array}{l}T_{\mathrm{m}} \\
(\mathrm{K})\end{array}$ & Method & References \\
\hline & SUB & $(293-303)$ & 127.9 & 298 & A & [1987STE/MAL] \\
\hline & SUB & $(293-308)$ & 132.6 & 300 & $\mathrm{ME}$ & [1968BAC/NOV] \\
\hline & SUB & $(296-314)$ & $140.2 \pm 3.3$ & 304 & $\mathrm{ME}$ & [1961DAV/MAL] \\
\hline & SUB & $(293-313)$ & $117.2 \pm 2.9$ & 303 & $\mathrm{ME}$ & {$[1957 \mathrm{LIT}]$} \\
\hline & $\mathrm{V}$ & $(393-573)$ & 88.8 & 408 & A & [1987STE/MAL] \\
\hline & $\mathrm{V}$ & $(321-341)$ & 95.8 & 332 & ME,TE & [1982FUC/HAL] \\
\hline & $\mathrm{V}$ & & 81.3 & 437 & I & [1943CRA] \\
\hline \multirow[t]{2}{*}{$\mathrm{C}_{12} \mathrm{H}_{24} \mathrm{O}_{2}$} & {$[61732-93-2]$} & \multicolumn{5}{|c|}{ 2-(1-ethylpentyl)-1,3-dioxepane } \\
\hline & V & $(333-373)$ & 68.1 & 348 & A & [1987STE/MAL, 1977VOI/SHC2] \\
\hline \multirow{2}{*}{$\mathrm{C}_{12} \mathrm{H}_{24} \mathrm{O}_{2}$} & {$[61732-92-1]$} & \multicolumn{5}{|c|}{ 2-heptyl-1,3-dioxepane } \\
\hline & $\mathrm{V}$ & $(328-373)$ & 70.3 & 343 & A & [1987STE/MAL, 1977VOI/SHC2] \\
\hline \multirow{2}{*}{$\mathrm{C}_{12} \mathrm{H}_{24} \mathrm{O}_{2}$} & [62159-06-2] & \multicolumn{5}{|c|}{ 3-heptyl-4-hydroxytetrahydro-2H-pyran } \\
\hline & V & $(383-453)$ & 77.6 & 398 & A & [1987STE/MAL, 1977VOI/SHC] \\
\hline $\mathrm{C}_{12} \mathrm{H}_{24} \mathrm{O}_{2}$ & $\begin{array}{c}{[23433-02-5]} \\
\mathrm{V}\end{array}$ & \multicolumn{4}{|c|}{ 4-octyl-1,3-dioxane } & [1987STE/MAL, 1977VOI/SHC] \\
\hline \multirow[t]{7}{*}{$\mathrm{C}_{12} \mathrm{H}_{24} \mathrm{O}_{2}$} & {$[1731-86-8]$} & \multicolumn{5}{|c|}{ Methyl undecanoate } \\
\hline & $\mathrm{V}$ & & 66.1 & 350 & $\mathrm{CE}$ & [2002VAN/VAN] \\
\hline & $\mathrm{V}$ & & $67.0 \pm 0.1$ & 340 & $\mathrm{CE}$ & [2002VAN/VAN] \\
\hline & $\mathrm{V}$ & & $70.8 \pm 0.4$ & 298 & $\mathrm{CE}$ & [2002VAN/VAN] \\
\hline & $\mathrm{V}$ & $(433-473)$ & 70.6 & 298 & CGC & [1995CHI/HOS] \\
\hline & $\mathrm{V}$ & & $71.4 \pm 0.3$ & 298 & $\mathrm{C}$ & {$[1977 \mathrm{MAN} / \mathrm{SEL}]$} \\
\hline & $\mathrm{V}$ & $(393-473)$ & 60.9 & 408 & $\mathrm{~A}, \mathrm{E}$ & [1987STE/MAL, 1963ROS/SCH] \\
\hline \multirow[t]{2}{*}{$\mathrm{C}_{12} \mathrm{H}_{24} \mathrm{O}_{2}$} & {$[245658-36-0]$} & \multicolumn{5}{|c|}{ 3,3-dimethylbutanoic acid, 1,1,2-trimethylpropyl ester } \\
\hline & & $(333-378)$ & 57.1 & 298 & CGC & [1999VER/HEI] \\
\hline \multirow[t]{2}{*}{$\mathrm{C}_{12} \mathrm{H}_{24} \mathrm{O}_{2}$} & {$[245658-40-6]$} & \multicolumn{5}{|c|}{ 2,2-dimethylpropanoic acid, 1,1,3-trimethylbutyl ester } \\
\hline & V & $(333-378)$ & 54.2 & 298 & CGC & [1999VER/HEI] \\
\hline \multirow[t]{2}{*}{$\mathrm{C}_{12} \mathrm{H}_{24} \mathrm{O}_{2}$} & {$[245658-43-9]$} & \multicolumn{5}{|c|}{ 2,6-dimethyl-2-heptanol propanoate } \\
\hline & V & $(333-378)$ & 59.4 & 298 & CGC & [1999VER/HEI] \\
\hline $\mathrm{C}_{12} \mathrm{H}_{24} \mathrm{O}_{3}$ & {$[2388-12-7]$} & Peroxydodecan & c acid & & & \\
\hline & SUB & $(293-303)$ & $131.4 \pm 1.7$ & 298 & ME & [1980SWA/KWA] \\
\hline $\mathrm{C}_{12} \mathrm{H}_{24} \mathrm{O}_{3}$ & & Pentyl 2-butox & ropionate & & & \\
\hline & $\mathrm{V}$ & $(373-398)$ & 47.3 & 385 & $\mathrm{~A}, \mathrm{I}$ & [1987STE/MAL, 1933HEN/MUR] \\
\hline $\mathrm{C}_{12} \mathrm{H}_{24} \mathrm{O}_{3}$ & {$[7419-98-9]$} & Methyl 3-octyl & ypropionate & & & \\
\hline & V & $(373-513)$ & 59.8 & 388 & A & [1987STE/MAL] \\
\hline $\mathrm{C}_{12} \mathrm{H}_{24} \mathrm{O}_{4}$ & {$[53759-20-9]$} & 2,2,8,8-tetrame & yl-1,3,7,9-tetr & ycycloc & decane & \\
\hline & FUS & & 23.4 & 383 & & [1975BOR] \\
\hline $\mathrm{C}_{12} \mathrm{H}_{24} \mathrm{O}_{4}$ & {$[43091-26-5]$} & $1,3,9,11$-tetrao & cy clohexadec & & & \\
\hline & FUS & & 35.56 & 332 & & {$[1973 \mathrm{DAL} / \mathrm{EKE}]$} \\
\hline $\mathrm{C}_{12} \mathrm{H}_{24} \mathrm{O}_{4}$ & {$[20732-35-8]$} & 3,6-dimethyl-3 & -di-tert-butyl- & 4,5-tetr & xyacyclohexa & \\
\hline & $\mathrm{V}$ & $(403-473)$ & 53.7 & 298 & CGC & [2007CAN/EYL] \\
\hline $\mathrm{C}_{12} \mathrm{H}_{24} \mathrm{O}_{6}$ & {$[24748-23-0]$} & 3,6,9-triethyl-3 & ,9-trimethyl-1 & $, 5,7,8-1$ & xaoxacyclono & ane \\
\hline & $\mathrm{V}$ & $(403-473)$ & 59.2 & 298 & CGC & [2007CAN/EYL] \\
\hline $\mathrm{C}_{12} \mathrm{H}_{24} \mathrm{O}_{6}$ & [17455-13-9] & $1,4,7,10,13,16$ & xaoxacyclooc & ecane ( & -crown-6) & \\
\hline & FUS & & 40.0 & 312.3 & DSC & [2016SAN/CRU] \\
\hline & FUS & & 35.5 & 312.3 & DSC & [2013KOV/PUS] \\
\hline & FUS & & 35.5 & 312.6 & DSC & [2011KOV/GOL] \\
\hline & FUS & & 40.9 & 312.2 & DSC & {$[2000 N I C / O R F]$} \\
\hline & FUS & & 35.66 & 312.4 & DSC & [1998DOM, 1998DOM/VEN] \\
\hline & FUS & & 34.0 & 312.2 & & {$[1972 \mathrm{DAL} / \mathrm{KRI}]$} \\
\hline & SUB & & $139.7 \pm 3.6$ & 298 & $\mathrm{~V}+\mathrm{F}$ & [2016SAN/CRU] \\
\hline & SUB & & $119.1 \pm 6.7$ & 298 & CGC-DSC & [2000NIC/ORF] \\
\hline & SUB & & $133.2 \pm 0.3$ & 298 & $\mathrm{C}$ & [1990BRI/WAD] \\
\hline
\end{tabular}


TABLE 11. Phase change enthalpies of $\mathrm{C}_{12}$ to $\mathrm{C}_{13}$ organic compounds-Continued

\begin{tabular}{|c|c|c|c|c|c|c|}
\hline \multirow[b]{2}{*}{ Molecular formula } & \multirow{2}{*}{$\begin{array}{l}\text { CAS Registry } \\
\text { Number } \\
\text { Enthalpy }\end{array}$} & \multicolumn{5}{|l|}{ Compound } \\
\hline & & $\begin{array}{l}\text { Temperature } \\
\text { range }\end{array}$ & $\begin{array}{l}\Delta_{\text {trans }} H_{\mathrm{m}} \\
(\mathrm{kJ} / \mathrm{mol})\end{array}$ & $\begin{array}{l}T_{\mathrm{m}} \\
(\mathrm{K})\end{array}$ & Method & References \\
\hline & $\mathrm{V}$ & $(430-480)$ & $73.6 \pm 0.2$ & 455 & DTA & [2016SAN/CRU] \\
\hline & $\mathrm{V}$ & $(430-480)$ & $98.2 \pm 3.0$ & 298 & DTA & [2016SAN/CRU] \\
\hline & $\mathrm{V}$ & & $86.1 \pm 6.7$ & 298 & CGC & {$[2000 \mathrm{NIC} / \mathrm{ORF}]$} \\
\hline \multirow[t]{2}{*}{$\mathrm{C}_{12} \mathrm{H}_{24} \mathrm{O}_{11}$} & {$[585-88-6]$} & \multicolumn{5}{|c|}{ 1,4-O- $\alpha$-D-glucopyranosyl-D-glucitol (maltitol) } \\
\hline & FUS & & 55.07 & 420 & DSC & [2001LEB/VAN, 2003LEB/VAN] \\
\hline \multirow{2}{*}{$\mathrm{C}_{12} \mathrm{H}_{24} \mathrm{O}_{11}$} & {$[534-73-6]$} & \multicolumn{5}{|c|}{$\alpha$ - $(d)$-glucopyranosyl-1,6-sorbitol } \\
\hline & FUS & & 56.4 & 439 & DSC & [1996CAM/FIG] \\
\hline \multirow[t]{2}{*}{$\mathrm{C}_{12} \mathrm{H}_{24} \mathrm{O}_{11}$} & [20942-99-8] & \multicolumn{5}{|c|}{$\alpha$ - $(D)$-glucopyranosyl-1,6-mannitol } \\
\hline & FUS & & 55.0 & 440.8 & DSC & [1996CAM/FIG] \\
\hline \multirow[t]{2}{*}{$\mathrm{C}_{12} \mathrm{H}_{24} \mathrm{O}_{11}$} & {$[64519-82-0]$} & \multicolumn{5}{|c|}{ 6-O- $\mathrm{O}$-D-glucopyranosyl-D-arabino-hexitol (isomalt) } \\
\hline & FUS & & 44.3 & & DSC & [2002BOR/CES] \\
\hline \multirow[t]{5}{*}{$\mathrm{C}_{12} \mathrm{H}_{24} \mathrm{~S}_{4}$} & [297181-32-9] & \multicolumn{5}{|c|}{ 1,4,8,11-tetrathiacyclohexadecane } \\
\hline & TRS (needles) & & 32.0 & 328.2 & & \\
\hline & FUS & & 5.2 & 333.2 & & \\
\hline & TRS (plates) & & 27.0 & 328.2 & & \\
\hline & FUS & & 5.2 & 333.2 & DSC & [2002ROC/GRI] \\
\hline \multirow[t]{3}{*}{$\mathrm{C}_{12} \mathrm{H}_{25} \mathrm{Br}$} & {$[143-15-7]$} & \multicolumn{5}{|l|}{ 1-bromododecane } \\
\hline & $\mathrm{V}$ & & $74.8 \pm 0.4$ & 298 & $\mathrm{C}$ & [1976STR3, 1977MAN/SEL] \\
\hline & $\mathrm{V}$ & $(411-610)$ & 62.2 & 426 & $\mathrm{~A}, \mathrm{E}$ & [1987STE/MAL, 1961LI/ROS, 1970DYK/VAN] \\
\hline \multirow[t]{7}{*}{$\mathrm{C}_{12} \mathrm{H}_{25} \mathrm{Cl}$} & {$[112-52-7]$} & \multicolumn{5}{|l|}{ 1-chlorododecane } \\
\hline & $\mathrm{V}$ & & 75.8 & 298 & & [2006BOL/NER2] \\
\hline & $\mathrm{V}$ & & $73.9 \pm 1.4$ & 298 & GS & [2001PUR/CHI] \\
\hline & $\mathrm{V}$ & $(390-520)$ & 70.5 & 298 & & [1984BOU/FRI, 1991BAS/SVO] \\
\hline & $\mathrm{V}$ & & $71.9 \pm 0.3$ & 298 & $\mathrm{C}$ & [1977MAN/SEL] \\
\hline & $\mathrm{V}$ & & $70.3 \pm 0.5$ & 298 & $\mathrm{C}$ & [1975STR/SUN] \\
\hline & $\mathrm{V}$ & $(389-519)$ & 62.4 & 404 & A,DTA & [1987STE/MAL, 1969KEM/KRE] \\
\hline \multirow[t]{2}{*}{$\mathrm{C}_{12} \mathrm{H}_{25} \mathrm{Cl}$} & {$[2350-12-1]$} & \multicolumn{5}{|c|}{$(d l)$-2-chlorododecane } \\
\hline & $\mathrm{V}$ & $(283-328)$ & 65.3 & 298 & A & [1987STE/MAL, 1970DYK/VAN, 1962GEI/QUI] \\
\hline $\mathrm{C}_{12} \mathrm{H}_{25} \mathrm{Cl}$ & {$[2350-12-1]$} & $(d l)$-3-chlorododec & cane & & & \\
\hline & $\mathrm{V}$ & $(283-328)$ & 65.9 & 298 & A & [1987STE/MAL, 1970DYK/VAN, 1962GEI/QUI] \\
\hline $\mathrm{C}_{12} \mathrm{H}_{25} \mathrm{Cl}$ & {$[2350-13-2]$} & $(d l)$-4-chlorododec & cane & & & \\
\hline & $\mathrm{V}$ & $(283-328)$ & 64.1 & 298 & A & [1987STE/MAL, 1970DYK/VAN, 1962GEI/QUI] \\
\hline $\mathrm{C}_{12} \mathrm{H}_{25} \mathrm{Cl}$ & {$[2350-14-3]$} & $(d l)$-5-chlorododec & cane & & & \\
\hline & $\mathrm{V}$ & $(283-328)$ & 65.9 & 298 & A & [1987STE/MAL, 1970DYK/VAN, 1962GEI/QUI] \\
\hline $\mathrm{C}_{12} \mathrm{H}_{25} \mathrm{Cl}$ & {$[26535-66-0]$} & 6-chlorododecane & & & & \\
\hline & V & $(283-328)$ & 65.5 & 298 & A & [1987STE/MAL, 1970DYK/VAN, 1962GEI/QUI] \\
\hline $\mathrm{C}_{12} \mathrm{H}_{25} \mathrm{~F}$ & {$[334-68-9]$} & 1-fluorododecane & & & & \\
\hline & $\mathrm{V}$ & $(288-328)$ & $64.0 \pm 0.2$ & 298 & GS & {$[1997 \mathrm{SCH} / \mathrm{VER}]$} \\
\hline & $\mathrm{V}$ & $(374-533)$ & 56.2 & 389 & $\mathrm{~A}, \mathrm{E}$ & [1987STE/MAL, 1961LI/ROS, 1970DYK/VAN] \\
\hline $\mathrm{C}_{12} \mathrm{H}_{25} \mathrm{I}$ & {$[4292-19-7]$} & 1-iodododecane & & & & \\
\hline & $\mathrm{V}$ & $(426-636)$ & 79.9 & 298 & $\mathrm{~A}, \mathrm{E}$ & $\begin{array}{l}\text { [1987STE/MAL, 1961LI/ROS, 1970DYK/VAN, } \\
\text { 2006BOL/NER] }\end{array}$ \\
\hline & $\mathrm{V}$ & $(426-636)$ & 63.5 & 441 & $\mathrm{~A}, \mathrm{E}$ & [1987STE/MAL, 1961LI/ROS, 1970DYK/VAN] \\
\hline $\mathrm{C}_{12} \mathrm{H}_{25} \mathrm{NO}$ & {$[996-97-4]$} & $N, N$-diethylcapryla & amide & & & \\
\hline & $\mathrm{V}$ & $(373-510)$ & 71.2 & 388 & A & [1987STE/MAL, 1968DAV/BAT] \\
\hline $\mathrm{C}_{12} \mathrm{H}_{25} \mathrm{NO}$ & {$[1120-16-7]$} & Dodecanamide & & & & \\
\hline & TRS & & 9.7 & 321.1 & & \\
\hline & FUS & & 36.3 & 373.3 & DSC & {$[2008 \mathrm{ABA} / \mathrm{BAD}]$} \\
\hline & SUB & $(349-368)$ & $152.7 \pm 0.8$ & 358.5 & $\mathrm{ME}$ & [1959DAV/JON2, 1987STE/MAL] \\
\hline $\mathrm{C}_{12} \mathrm{H}_{26}$ & {$[112-40-3]$} & Dodecane & & & & \\
\hline & FUS & & 34.63 & 263.2 & DSC & [2005HUA/SIM] \\
\hline & FUS & & 35.7 & 263.1 & DSC & [2004MON/RAJ] \\
\hline
\end{tabular}


TABLE 11. Phase change enthalpies of $\mathrm{C}_{12}$ to $\mathrm{C}_{13}$ organic compounds-Continued

\begin{tabular}{|c|c|c|c|c|c|c|}
\hline \multirow[b]{2}{*}{ Molecular formula } & \multirow{2}{*}{$\begin{array}{l}\text { CAS Registry } \\
\text { Number } \\
\text { Enthalpy }\end{array}$} & \multicolumn{5}{|l|}{ Compound } \\
\hline & & $\begin{array}{c}\text { Temperature } \\
\text { range }\end{array}$ & $\begin{array}{l}\Delta_{\text {trans }} H_{\mathrm{m}} \\
(\mathrm{kJ} / \mathrm{mol})\end{array}$ & $\begin{array}{l}T_{\mathrm{m}} \\
(\mathrm{K})\end{array}$ & Method & References \\
\hline & FUS & & 37.1 & 263.6 & $\mathrm{C}$ & [1955TUN/STO] \\
\hline & FUS & & 36.84 & 263.6 & & [1996DOM/HEA, 1954FIN/GRO2] \\
\hline & FUS & & 36.58 & 263.5 & $\mathrm{C}$ & [1996DOM/HEA, 1931HUF/PAR] \\
\hline & SUB & & 100.2 & 298 & $\mathrm{~B}$ & [1972MOR3] \\
\hline & SUB & & 101.7 & 263 & $\mathrm{~B}$ & {$[1963 \mathrm{BON}]$} \\
\hline & $\mathrm{V}$ & & $62.1 \pm 0.2$ & 298 & GS & [2001PUR/CHI] \\
\hline & $\mathrm{V}$ & & $60.3 \pm 0.8$ & 298 & CGC & [2000NIC/ORF] \\
\hline & $\mathrm{V}$ & & 61.4 & 299 & $\mathrm{C}$ & [1996VIT/CHA] \\
\hline & $\mathrm{V}$ & & 58.1 & 334 & $\mathrm{C}$ & [1996VIT/CHA] \\
\hline & $\mathrm{V}$ & & 57.4 & 344 & $\mathrm{C}$ & [1996VIT/CHA] \\
\hline & $\mathrm{V}$ & $(373-423)$ & 60.7 & 298 & CGC & [1995CHI/HOS] \\
\hline & $\mathrm{V}$ & $(363-413)$ & 61.2 & 298 & CGC & [1995CHI/HOS] \\
\hline & $\mathrm{V}$ & $(423-473)$ & 61.2 & 298 & CGC & [1995CHI/HOS] \\
\hline & $\mathrm{V}$ & & 61.5 & 298 & & [1994RUZ/MAJ] \\
\hline & $\mathrm{V}$ & $(263-371)$ & 65.7 & 278 & & [1988SAS/JOS] \\
\hline & V & $(278-400)$ & 61.8 & 293 & A & [1987STE/MAL] \\
\hline & $\mathrm{V}$ & $(298-389)$ & 61.1 & 313 & GS & [1986ALL/JOS] \\
\hline & $\mathrm{V}$ & & $61.8 \pm 0.5$ & 298 & $\mathrm{C}$ & [1976MEL/MAN] \\
\hline & $\mathrm{V}$ & & $61.2 \pm 0.2$ & 298 & $\mathrm{C}$ & [1974MAN4] \\
\hline & $\mathrm{V}$ & & $60.4 \pm 0.3$ & 298 & $\mathrm{C}$ & [1972MOR2] \\
\hline & $\mathrm{V}$ & & 61.3 & 298 & & [1971WIL/ZWO] \\
\hline & $\mathrm{V}$ & & $59.5 \pm 0.2$ & 298 & $\mathrm{C}$ & [1963MOR/SUN] \\
\hline & $\mathrm{V}$ & $(400-492)$ & 51.6 & 415 & $\mathrm{~A}, \mathrm{MM}$ & [1987STE/MAL, 1945WIL/TAY] \\
\hline \multirow[t]{2}{*}{$\mathrm{C}_{12} \mathrm{H}_{26}$} & {$[7045-71-8]$} & \multicolumn{2}{|l|}{ 2-methylundecane } & & & \\
\hline & V & $(356-484)$ & 49.5 & 371 & A & [1987STE/MAL] \\
\hline \multirow[t]{2}{*}{$\mathrm{C}_{12} \mathrm{H}_{26}$} & {$[1002-43-3]$} & \multicolumn{2}{|c|}{$(d l)$-3-methylundecane } & & & \\
\hline & V & $(357-485)$ & 48.8 & 372 & A & [1987STE/MAL] \\
\hline \multirow[t]{2}{*}{$\mathrm{C}_{12} \mathrm{H}_{26}$} & {$[2980-69-0]$} & \multicolumn{2}{|l|}{ 4-methylundecane } & & & \\
\hline & $\mathrm{V}$ & $(359-481)$ & 51.6 & 374 & A & [1987STE/MAL] \\
\hline \multirow[t]{2}{*}{$\mathrm{C}_{12} \mathrm{H}_{26}$} & [1632-70-8] & \multicolumn{2}{|l|}{ 5-methylundecane } & & & \\
\hline & V & $(357-480)$ & 50.3 & 372 & A & [1987STE/MAL] \\
\hline \multirow[t]{2}{*}{$\mathrm{C}_{12} \mathrm{H}_{26}$} & [17312-44-6] & \multicolumn{2}{|l|}{ 2,3-dimethyldecane } & & & \\
\hline & $\mathrm{V}$ & $(369-480)$ & 50.0 & 384 & A & [1987STE/MAL] \\
\hline \multirow[t]{2}{*}{$\mathrm{C}_{12} \mathrm{H}_{26}$} & [2801-84-5] & \multicolumn{2}{|l|}{ 2,4-dimethyldecane } & & & \\
\hline & $\mathrm{V}$ & $(348-472)$ & 47.5 & 363 & A & [1987STE/MAL] \\
\hline \multirow[t]{2}{*}{$\mathrm{C}_{12} \mathrm{H}_{26}$} & [62184-10-5] & \multicolumn{2}{|c|}{ 2,4,6-trimethylnonane } & & & \\
\hline & $\mathrm{V}$ & $(339-459)$ & 46.4 & 354 & A & [1987STE/MAL] \\
\hline \multirow[t]{2}{*}{$\mathrm{C}_{12} \mathrm{H}_{26}$} & [62199-46-6] & 3,3,6,6-tetramethyl & loctane & & & \\
\hline & $\mathrm{V}$ & $(347-463)$ & 52.9 & 362 & A & [1987STE/MAL] \\
\hline $\mathrm{C}_{12} \mathrm{H}_{26}$ & [13475-82-6] & 2,2,4,6,6-pentametl & thylheptane & & & \\
\hline & $\mathrm{V}$ & & $49.0 \pm 0.2$ & 298 & $\mathrm{C}$ & [1976MEL/MAN] \\
\hline $\mathrm{C}_{12} \mathrm{H}_{26} \mathrm{~N}_{2} \mathrm{O}$ & [4128-38-5] & 1-undecyl urea & & & & \\
\hline & FUS & & 38.4 & 385.6 & DSC & [2005HAS/TAJ] \\
\hline $\mathrm{C}_{12} \mathrm{H}_{26} \mathrm{~N}_{2} \mathrm{O}_{6} \mathrm{P}_{2}$ & [256374-76-2] & 1,2-bis(2-oxo-5,5-d & dimethyl-1,3 & dioxyph & hacycloh & -2-imino)ethane \\
\hline & FUS & & 20.6 & 564.7 & DSC & [2013QI/WAN] \\
\hline $\mathrm{C}_{12} \mathrm{H}_{26} \mathrm{O}$ & [55962-01-1] & Ethyl decyl ether & & & & \\
\hline & V & & $65.9 \pm 0.1$ & 298 & $\mathrm{C}$ & [1985KUS] \\
\hline $\mathrm{C}_{12} \mathrm{H}_{26} \mathrm{O}$ & [112-58-3] & Dihexyl ether & & & & \\
\hline & V & & $63.6 \pm 0.8$ & 298 & $\mathrm{CGC}$ & [2000NIC/ORF] \\
\hline & $\mathrm{V}$ & $(353-393)$ & 63.5 & 298 & CGC & [1995CHI/HOS] \\
\hline & $\mathrm{V}$ & $(372-510)$ & 52.9 & 387 & A & [1987STE/MAL] \\
\hline & $\mathrm{V}$ & & $64.1 \pm 0.1$ & 298 & $\mathrm{C}$ & [1985KUS] \\
\hline $\mathrm{C}_{12} \mathrm{H}_{26} \mathrm{O}$ & [51323-70-7] & Octyl tert-butyl eth & her & & & \\
\hline
\end{tabular}


TABLE 11. Phase change enthalpies of $\mathrm{C}_{12}$ to $\mathrm{C}_{13}$ organic compounds-Continued

\begin{tabular}{|c|c|c|c|c|c|c|}
\hline \multirow[b]{2}{*}{ Molecular formula } & \multirow{2}{*}{$\begin{array}{l}\text { CAS Registry } \\
\text { Number } \\
\text { Enthalpy }\end{array}$} & \multicolumn{5}{|l|}{ Compound } \\
\hline & & $\begin{array}{c}\text { Temperature } \\
\text { range }\end{array}$ & $\begin{array}{l}\Delta_{\text {trans }} H_{\mathrm{m}} \\
(\mathrm{kJ} / \mathrm{mol})\end{array}$ & $\begin{array}{l}T_{\mathrm{m}} \\
(\mathrm{K})\end{array}$ & Method & References \\
\hline & $\mathrm{V}$ & & 61.4 & 298 & CGC & [UR/VER, 2002VER, 2003VER/KRA] \\
\hline \multirow[t]{2}{*}{$\mathrm{C}_{12} \mathrm{H}_{26} \mathrm{O}$} & \multicolumn{6}{|c|}{ Isobutyl tert-octyl ether } \\
\hline & $\mathrm{V}$ & & 51.6 & 298 & CGC & [UR/VER, 2002VER, 2003VER/KRA] \\
\hline \multirow[t]{2}{*}{$\mathrm{C}_{12} \mathrm{H}_{26} \mathrm{O}$} & {$[153296-45-8]$} & butyl tert-octy & & & & \\
\hline & $\mathrm{V}$ & & $52.9 \pm 0.4$ & 298 & $\mathrm{CGC}$ & [UR/VER, 2002VER, 2003VER/KRA] \\
\hline \multirow[t]{5}{*}{$\mathrm{C}_{12} \mathrm{H}_{26} \mathrm{O}$} & {$[112-53-8]$} & 1-dodecanol ( & l alcohol) & & & \\
\hline & FUS & & 38.46 & 297.53 & DSC & [2015CAR/CON] \\
\hline & FUS & & 38.4 & 297.8 & DSC & [2014CAR/DOS] \\
\hline & FUS & & 39.05 & 297.15 & DSC & [2013WEI/ZHA] \\
\hline & FUS & & 42.7 & 312 & DSC & [2008EGO/MAR] \\
\hline
\end{tabular}

[Note: The melting point reported in [2008EGO/MAR] is considerably higher than melting points reported in synthetic papers and other thermodynamic papers.]

\begin{tabular}{|c|c|c|c|c|c|c|}
\hline & FUS & $(5-390)$ & 40.31 & 297.3 & & [2003VAN/VAN] \\
\hline & FUS & & U 34.3 & 297.0 & DSC & [1992BAB/HWA, 1994BAB/BEN] \\
\hline & FUS & & 40.17 & 300.2 & & [1993ACR, 1991CHI/BRA] \\
\hline & FUS & & 38.42 & 297.0 & DSC & [1978ECK/MUL] \\
\hline & SUB & $(285-294)$ & $130.1 \pm 1.2$ & 290 & ME & [1965DAV/KYB, 1987STE/MAL] \\
\hline & SUB & & 129.3 & 298 & & {$[1965 \mathrm{DAV} / \mathrm{KYB}]$} \\
\hline & $\mathrm{V}$ & & $90.8 \pm 1.2$ & 298 & $\mathrm{CGC}$ & [2006NIC/KWE] \\
\hline & $\mathrm{V}$ & $(303-348)$ & 85.8 & 327 & GS & [2001KUL/VER2] \\
\hline & $\mathrm{V}$ & $(303-348)$ & 90 & 298 & GS & [2001KUL/VER2] \\
\hline & $\mathrm{V}$ & $(373-423)$ & 91.7 & 298 & CGC & [1995CHI/HOS] \\
\hline & $\mathrm{V}$ & $(353-393)$ & 91.7 & 298 & $\mathrm{CGC}$ & [1994KOU/HOS, 2000OVA/KOU] \\
\hline & $\mathrm{V}$ & $(303-413)$ & 80.5 & 358 & & {$[1992 \mathrm{NGU} / \mathrm{KAS}]$} \\
\hline & $\mathrm{V}$ & $(383-438)$ & 73.8 & 398 & A & [1987STE/MAL] \\
\hline & $\mathrm{V}$ & $(505-550)$ & 57.1 & 520 & A & [1987STE/MAL] \\
\hline & V & & $84.7 \pm 0.5$ & 343 & $\mathrm{C}$ & [1979SEV] \\
\hline & $\mathrm{V}$ & & $91.8 \pm 0.6$ & 298 & $\mathrm{C}$ & [1979SEV] \\
\hline & V & & $92.0 \pm 0.6$ & 298 & $\mathrm{C}$ & [1977MAN/SEL] \\
\hline & $\mathrm{V}$ & $(297-363)$ & 92.5 & 312 & & [1973WIL/ZWO] \\
\hline & $\mathrm{V}$ & $(411-487)$ & 67.6 & 426 & & [1973WIL/ZWO] \\
\hline & $\mathrm{V}$ & $(425-550)$ & 66.7 & 440 & $\mathrm{~A}, \mathrm{~EB}$ & [1987STE/MAL, 1970AMB/SPR] \\
\hline & V & $(400-538)$ & 71.5 & 415 & DTA & [1969KEM/KRE] \\
\hline & $\mathrm{V}$ & $(297-313)$ & 95.4 & 305 & ME & {$[1965 \mathrm{DAV} / \mathrm{KYB}]$} \\
\hline & V & $(303-363)$ & 83.3 & 333 & A,ME & [1987STE/MAL, 1962GEI/QUI2] \\
\hline & $\mathrm{V}$ & $(411-487)$ & 67.6 & 426 & & {$[1958 \mathrm{ROS} / \mathrm{PAP}]$} \\
\hline $\mathrm{C}_{12} \mathrm{H}_{26} \mathrm{O}$ & {$[10203-28-8]$} & 2-dodecanol & & & & \\
\hline & $\mathrm{V}$ & $(293-393)$ & 87.0 & 308 & & [1999NGU/BER] \\
\hline & $\mathrm{V}$ & $(293-343)$ & 85.0 & 318 & A,ME & [1987STE/MAL, 1962GEI/QUI2] \\
\hline $\mathrm{C}_{12} \mathrm{H}_{26} \mathrm{O}$ & {$[10203-30-2]$} & $(d l)$-3-dodecanol & & & & \\
\hline & $\mathrm{V}$ & $(293-343)$ & 78.3 & 318 & A,ME & [1987STE/MAL, 1962GEI/QUI2] \\
\hline $\mathrm{C}_{12} \mathrm{H}_{26} \mathrm{O}$ & {$[10203-32-4]$} & 4-dodecanol & & & & \\
\hline & $\mathrm{V}$ & (293-343) & 80.6 & 318 & A,ME & [1987STE/MAL, 1962GEI/QUI2] \\
\hline $\mathrm{C}_{12} \mathrm{H}_{26} \mathrm{O}$ & [10203-33-5] & 5-dodecanol & & & & 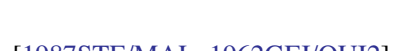 \\
\hline & V & (293-343) & 79.4 & 318 & $\mathrm{~A}, \mathrm{ME}$ & [1987STE/MAL, 1962GEI/QUI2] \\
\hline $\mathrm{C}_{12} \mathrm{H}_{26} \mathrm{O}$ & {$[6836-38-0]$} & 6-dodecanol & & & & \\
\hline & $\mathrm{V}$ & $(293-343)$ & 81.5 & 318 & $\mathrm{~A}, \mathrm{ME}$ & [1987STE/MAL, 1962GEI/QUI2] \\
\hline $\mathrm{C}_{12} \mathrm{H}_{26} \mathrm{O}$ & {$[5457-42-1]$} & di-tert-butyl-isop & opylmethanol & & & \\
\hline & FUS & & 2.09 & 314 & DSC & [1998VER3] \\
\hline [Note: $\mathrm{Tl}$ & rely has an unm & ured solid phase tr & ansition.] & & & \\
\hline & SUB & (274-308) & $59.3 \pm 0.8$ & 298 & GS & [1998VER3] \\
\hline & SUB & $(274-308)$ & $59.7 \pm 0.8$ & 291 & GS & [1998VER3] \\
\hline & $\mathrm{V}$ & $(317-348)$ & $54.9 \pm 0.8$ & 333 & GS & [1998VER5] \\
\hline & $\mathrm{V}$ & $(317-348)$ & $57.0 \pm 0.8$ & 298 & GS & [1998VER5] \\
\hline
\end{tabular}


TABLE 11. Phase change enthalpies of $\mathrm{C}_{12}$ to $\mathrm{C}_{13}$ organic compounds-Continued

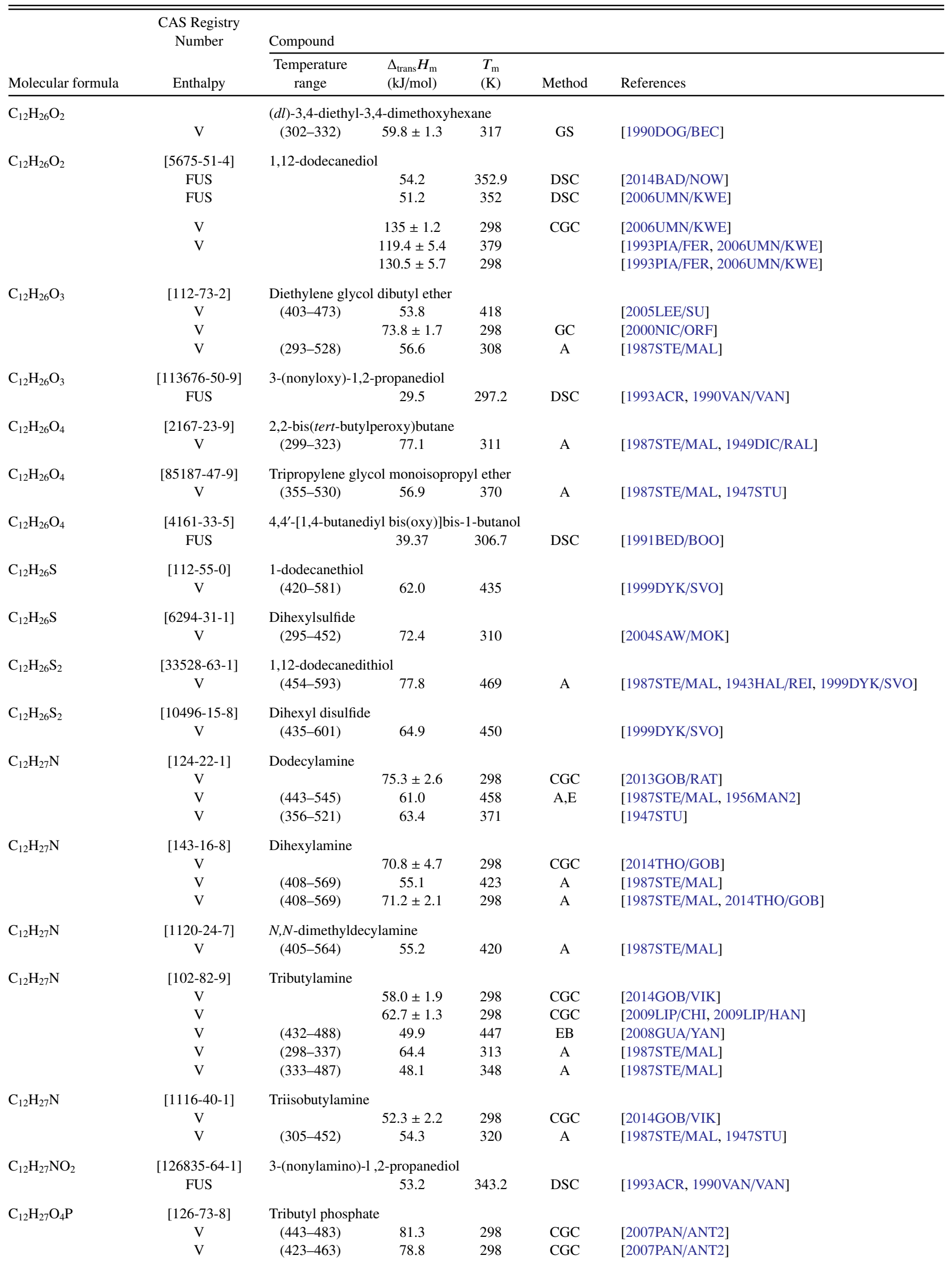


TABLE 11. Phase change enthalpies of $\mathrm{C}_{12}$ to $\mathrm{C}_{13}$ organic compounds-Continued

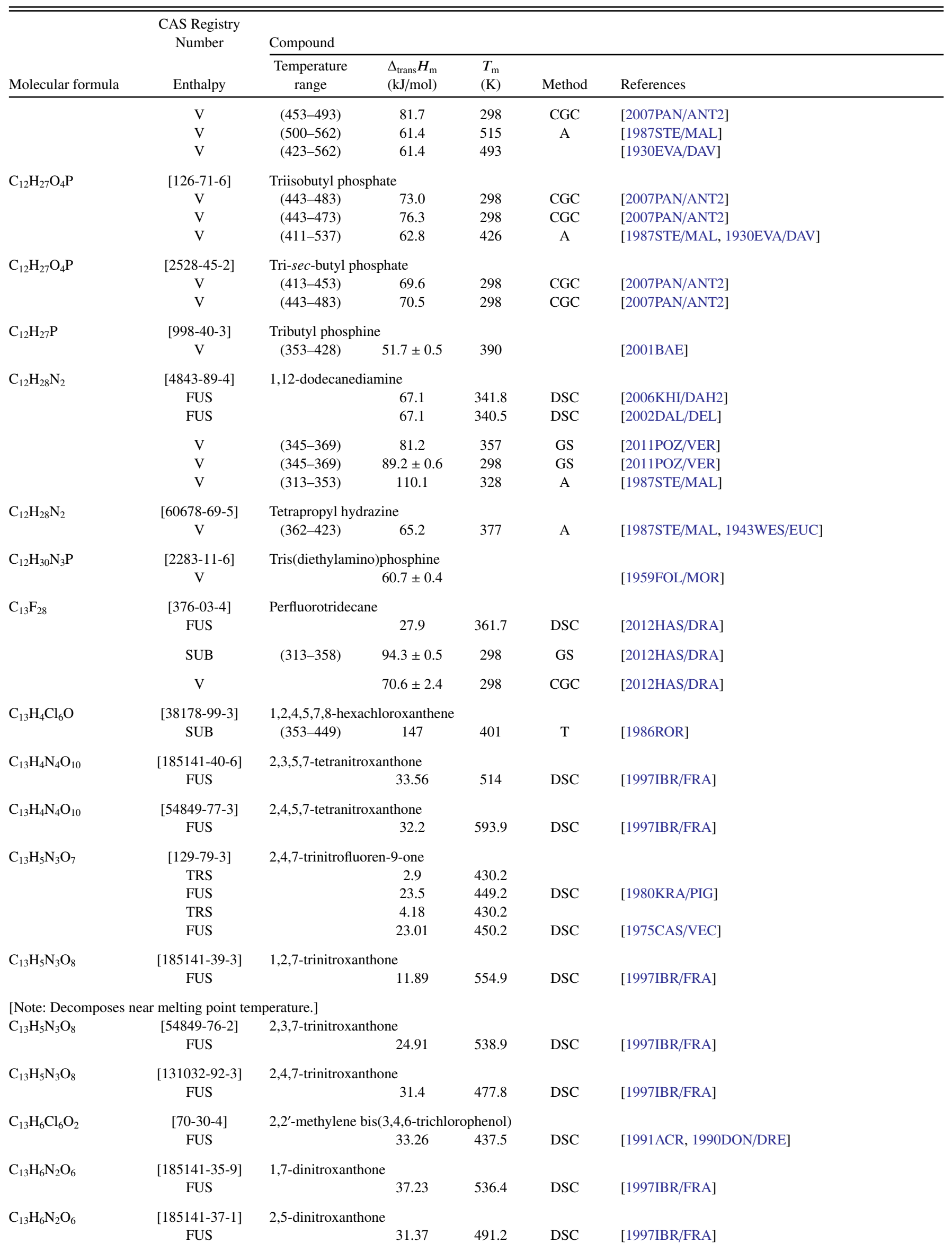


TABLE 11. Phase change enthalpies of $\mathrm{C}_{12}$ to $\mathrm{C}_{13}$ organic compounds-Continued

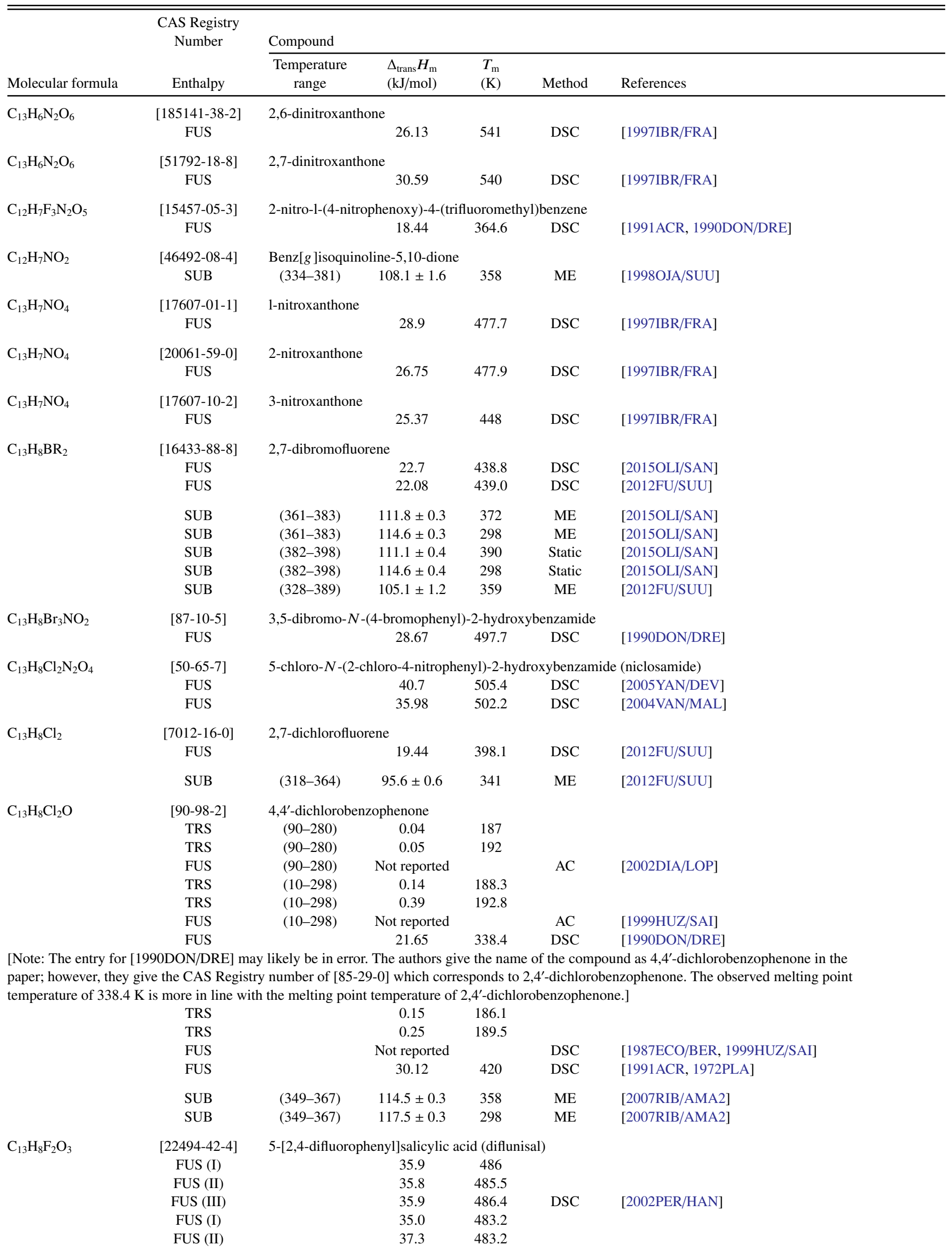


TABLE 11. Phase change enthalpies of $\mathrm{C}_{12}$ to $\mathrm{C}_{13}$ organic compounds-Continued

\begin{tabular}{|c|c|c|c|c|c|c|}
\hline \multirow[b]{2}{*}{ Molecular formula } & \multirow{2}{*}{$\begin{array}{l}\text { CAS Registry } \\
\text { Number } \\
\text { Enthalpy }\end{array}$} & \multicolumn{5}{|l|}{ Compound } \\
\hline & & $\begin{array}{l}\text { Temperature } \\
\text { range }\end{array}$ & $\begin{array}{l}\Delta_{\text {trans }} H_{\mathrm{m}} \\
(\mathrm{kJ} / \mathrm{mol})\end{array}$ & $\begin{array}{c}T_{\mathrm{m}} \\
(\mathrm{K})\end{array}$ & Method & References \\
\hline & FUS (III) & & 36.0 & 483.2 & DSC & [1994MAR/MAR] \\
\hline & SUB & $(349-414)$ & $119.3 \pm 0.6$ & 382 & GS & [2003PER/KUR] \\
\hline $\mathrm{C}_{13} \mathrm{H}_{8} \mathrm{~N}_{2} \mathrm{O}_{2}$ & $\begin{array}{c}\text { [2538-68-3] } \\
\text { FUS }\end{array}$ & 1-phenazinecar & $\begin{array}{c}\text { xylic acid } \\
35.44\end{array}$ & 514.5 & DSC & [1997CIO/MEL] \\
\hline $\mathrm{C}_{13} \mathrm{H}_{8} \mathrm{~N}_{2} \mathrm{O}_{5}$ & $\begin{array}{c}\text { [21222-05-9] } \\
\text { SUB }\end{array}$ & 3,3'-dinitroben & $\begin{array}{l}\text { phenone } \\
147.4 \pm 3.9\end{array}$ & 298 & $\mathrm{C}$ & [2011RIB/AMA3] \\
\hline $\mathrm{C}_{13} \mathrm{H}_{8} \mathrm{~N}_{4}$ & $\begin{array}{c}\text { [19139-24-3] } \\
\text { TRS } \\
\text { TRS } \\
\text { FUS }\end{array}$ & $8,8,9,9$-tetracy & $\begin{array}{c}\text { quadracyclo[2 } \\
4.14 \\
0.37 \\
14.47\end{array}$ & $\begin{array}{l}1.0^{3,5} \cdot 2 \\
425.8 \\
462.1 \\
467.9\end{array}$ & $\begin{array}{l}\text { nane } \\
\text { DSC }\end{array}$ & [1984WEI/LEF] \\
\hline $\mathrm{C}_{13} \mathrm{H}_{8} \mathrm{O}$ & $\begin{array}{c}{[548-39-0]} \\
\text { SUB }\end{array}$ & $\begin{array}{l}\text { Perinaphthenor } \\
\quad(326-348)\end{array}$ & $97.2 \pm 2.5$ & 337 & ME & [1998OJA/SUU] \\
\hline $\mathrm{C}_{13} \mathrm{H}_{8} \mathrm{O}$ & $\begin{array}{l}\text { [486-25-9] } \\
\text { FUS } \\
\text { FUS } \\
\text { FUS } \\
\text { FUS }\end{array}$ & $\begin{array}{l}\text { 9-fluorenone } \\
\qquad(5-520)\end{array}$ & $\begin{array}{c}17.6 \\
16.36 \\
14.85 \\
18.12\end{array}$ & $\begin{array}{l}356.2 \\
356.5 \\
353.3 \\
356.4\end{array}$ & $\begin{array}{l}\mathrm{DSC} \\
\mathrm{AC} \\
\mathrm{DSC}\end{array}$ & $\begin{array}{l}\text { [2012MON/NOT2] } \\
\text { [2012CHI/KAZ] } \\
\text { [1998VER4] } \\
\text { [1991ACR, 1988SAB/ELW2] }\end{array}$ \\
\hline & $\begin{array}{l}\text { SUB } \\
\text { SUB } \\
\text { SUB } \\
\text { SUB } \\
\text { SUB } \\
\text { SUB } \\
\text { SUB } \\
\text { SUB }\end{array}$ & $\begin{array}{l}(312-334) \\
(327-352) \\
(301-343) \\
(324-348) \\
(324-348)\end{array}$ & $\begin{array}{l}94.7 \pm 0.5 \\
95.1 \pm 0.5 \\
92.2 \pm 1.7 \\
88.5 \pm 3.7 \\
91.6 \pm 1.8 \\
93.9 \pm 1.8 \\
87.6 \pm 0.3 \\
88.4 \pm 0.4\end{array}$ & $\begin{array}{l}298 \\
298 \\
298 \\
322 \\
336 \\
298 \\
319 \\
298\end{array}$ & $\begin{array}{c}\text { ME } \\
\text { Static } \\
\text { C } \\
\text { ME } \\
\text { GS } \\
\text { GS } \\
\text { C } \\
\text { C }\end{array}$ & $\begin{array}{l}\text { [2012MON/NOT2] } \\
\text { [2012MON/NOT2] } \\
\text { [2012MON/NOT2] } \\
\text { [2010GOL/SUU] } \\
\text { [1998VER4] } \\
\text { [1998VER4] } \\
\text { [1988SAB/ELW2] } \\
\text { [1988SAB/ELW2] }\end{array}$ \\
\hline & $\begin{array}{l}\text { V } \\
\text { V } \\
\text { V } \\
\text { V } \\
\text { V } \\
\text { V } \\
\text { V } \\
\text { V } \\
\text { V } \\
\text { V } \\
\text { V } \\
\text { V } \\
\text { V }\end{array}$ & $\begin{array}{l}(328-441) \\
(368-668) \\
(368-668) \\
(368-668) \\
(368-668) \\
(368-668) \\
(368-668) \\
(368-668)\end{array}$ & $\begin{array}{c}80.6 \pm 0.2 \\
79.1 \pm 0.6 \\
77.3 \pm 0.5 \\
74.3 \pm 0.3 \\
71.3 \pm 0.3 \\
68.5 \pm 0.3 \\
65.8 \pm 0.2 \\
63.1 \pm 0.2 \\
60.9 \\
59.8 \\
59.1 \\
58.6 \\
57.9\end{array}$ & $\begin{array}{l}298 \\
298 \\
320 \\
360 \\
400 \\
440 \\
480 \\
520 \\
435 \\
475 \\
525 \\
565 \\
595\end{array}$ & $\begin{array}{c}\text { Static } \\
\text { IPM,EB } \\
\text { IPM,EB } \\
\text { IPM,EB } \\
\text { IPM,EB } \\
\text { IPM,EB } \\
\text { IPM,EB } \\
\text { IPM,EB } \\
\text { Static } \\
\text { Static } \\
\text { Static } \\
\text { Static } \\
\text { Static }\end{array}$ & $\begin{array}{l}\text { [2012MON/NOT2] } \\
\text { [2012CHI/KAZ] } \\
\text { [2012CHI/KAZ] } \\
\text { [2012CHI/KAZ] } \\
\text { [2012CHI/KAZ] } \\
\text { [2012CHI/KAZ] } \\
\text { [2012CHI/KAZ] } \\
\text { [2012CHI/KAZ] } \\
\text { [1983SIV/MAR] } \\
\text { [1983SIV/MAR] } \\
\text { [1983SIV/MAR] } \\
\text { [1983SIV/MAR] } \\
\text { [1983SIV/MAR] }\end{array}$ \\
\hline $\mathrm{C}_{18} \mathrm{H}_{8} \mathrm{OS}$ & $\begin{array}{l}{[492-22-8]} \\
\text { FUS } \\
\text { FUS }\end{array}$ & Thioxanthone & $\begin{array}{l}28.4 \\
35.5\end{array}$ & $\begin{array}{l}486.6 \\
487.9\end{array}$ & $\mathrm{DSC}$ & $\begin{array}{l}\text { [2010MON/SOU] } \\
\text { [1992SAB/ELW] }\end{array}$ \\
\hline & $\begin{array}{l}\text { SUB } \\
\text { SUB } \\
\text { SUB } \\
\text { SUB }\end{array}$ & $\begin{array}{l}(369-387) \\
(369-387)\end{array}$ & $\begin{array}{l}110.9 \pm 1.2 \\
113.8 \pm 1.2 \\
106.5 \pm 1.2 \\
114.8 \pm 0.4\end{array}$ & $\begin{array}{l}378 \\
298 \\
298 \\
298\end{array}$ & $\begin{array}{c}\mathrm{ME} \\
\mathrm{ME} \\
\mathrm{C} \\
\mathrm{C}\end{array}$ & $\begin{array}{l}{[2010 \mathrm{MON} / \mathrm{SOU}]} \\
{[2010 \mathrm{MON} / \mathrm{SOU}]} \\
{[2010 \mathrm{FRE} / \mathrm{GOM} 3]} \\
{[1992 \mathrm{SAB} / \mathrm{ELW}]}\end{array}$ \\
\hline $\mathrm{C}_{13} \mathrm{H}_{8} \mathrm{O}_{2}$ & $\begin{array}{l}{[90-47-1]} \\
\text { FUS } \\
\text { FUS } \\
\text { FUS }\end{array}$ & $\begin{array}{l}\text { Xanthone } \\
\quad(5-521)\end{array}$ & $\begin{array}{c}27.48 \\
26.6 \\
26.12\end{array}$ & $\begin{array}{l}448.8 \\
448.3 \\
449.7\end{array}$ & $\begin{array}{c}\mathrm{AC} \\
\mathrm{DSC}\end{array}$ & $\begin{array}{l}\text { [2015CHI/KAZ] } \\
\text { [2010MON/SOU] } \\
\text { [1996DOM/HEA, 1988SAB/ELW] }\end{array}$ \\
\hline & $\begin{array}{l}\text { SUB } \\
\text { SUB } \\
\text { SUB } \\
\text { SUB }\end{array}$ & $\begin{array}{l}(342-362) \\
(342-362)\end{array}$ & $\begin{array}{l}103.9 \pm 0.6 \\
105.8 \pm 0.6 \\
102.7 \pm 2.3 \\
98.57 \pm 0.4\end{array}$ & $\begin{array}{l}352 \\
298 \\
298 \\
298\end{array}$ & $\begin{array}{c}\mathrm{ME} \\
\mathrm{ME} \\
\mathrm{C} \\
\mathrm{C}\end{array}$ & $\begin{array}{l}\text { [2010MON/SOU] } \\
{[2010 \mathrm{MON} / \mathrm{SOU}]} \\
{[2009 \mathrm{FRE} / \mathrm{GOM} 2]} \\
{[1988 \mathrm{SAB} / \mathrm{ELW}]}\end{array}$ \\
\hline $\mathrm{C}_{13} \mathrm{H}_{8} \mathrm{O}_{2}$ & $\begin{array}{c}{[5472-84-4]} \\
\text { SUB }\end{array}$ & $\begin{array}{c}\text { 3-hydroxy-1H } \\
(402-432)\end{array}$ & $\begin{array}{c}\text { henalen-1-one } \\
151.5 \pm 4.7\end{array}$ & 417 & $\mathrm{ME}$ & [19980JA/SUU] \\
\hline
\end{tabular}


TABLE 11. Phase change enthalpies of $\mathrm{C}_{12}$ to $\mathrm{C}_{13}$ organic compounds-Continued

\begin{tabular}{|c|c|c|c|c|c|c|}
\hline \multirow[b]{2}{*}{ Molecular formula } & \multirow{2}{*}{$\begin{array}{l}\text { CAS Registry } \\
\text { Number } \\
\text { Enthalpy }\end{array}$} & \multicolumn{5}{|l|}{ Compound } \\
\hline & & $\begin{array}{l}\text { Temperature } \\
\text { range }\end{array}$ & $\begin{array}{l}\Delta_{\text {trans }} H_{\mathrm{m}} \\
(\mathrm{kJ} / \mathrm{mol})\end{array}$ & $\begin{array}{l}T_{\mathrm{m}} \\
(\mathrm{K})\end{array}$ & Method & References \\
\hline \multirow[t]{10}{*}{$\mathrm{C}_{13} \mathrm{H}_{9} \mathrm{Br}$} & [1133-80-8] & 2-bromofluorene & & & & \\
\hline & FUS & & 17.02 & 387.4 & DSC & [2015OLI/SAN] \\
\hline & FUS & & 16.02 & 382.3 & $\mathrm{DSC}$ & [2012FU/SUU] \\
\hline & SUB & $(327-349)$ & $96.7 \pm 0.4$ & 338 & $\mathrm{ME}$ & [2015OLI/SAN] \\
\hline & SUB & $(327-349)$ & $98.1 \pm 0.4$ & 298 & ME & [2015OLI/SAN] \\
\hline & SUB & $(351-380)$ & $94.1 \pm 0.1$ & 366 & Static & [2015OLI/SAN] \\
\hline & SUB & $(351-380)$ & $96.4 \pm 0.1$ & 298 & Static & [2015OLI/SAN] \\
\hline & SUB & $(303-354)$ & $93.1 \pm 1.6$ & 328 & $\mathrm{ME}$ & [2012FU/SUU] \\
\hline & $\mathrm{V}$ & $(374-420)$ & $73.6 \pm 0.1$ & 397 & Static & [2015OLI/SAN] \\
\hline & $\mathrm{V}$ & $(374-420)$ & $81.7 \pm 0.1$ & 298 & Static & [2015OLI/SAN] \\
\hline \multirow[t]{3}{*}{$\mathrm{C}_{13} \mathrm{H}_{9} \mathrm{Br}$} & [1940-57-4] & 9-bromofluorene & & & & \\
\hline & FUS & & 13.36 & 373.7 & DSC & [2012FU/SUU] \\
\hline & SUB & $(303-344)$ & $98.4 \pm 2.5$ & 324 & ME & [2012FU/SUU] \\
\hline \multirow[t]{3}{*}{$\mathrm{C}_{13} \mathrm{H}_{9} \mathrm{Cl}$} & {$[6630-65-5]$} & 9-chlorofluorene & & & & \\
\hline & FUS & & 14.21 & 361.2 & $\mathrm{DSC}$ & [2012FU/SUU] \\
\hline & SUB & $(303-330)$ & $94.0 \pm 3.0$ & 317 & $\mathrm{ME}$ & [2012FU/SUU] \\
\hline \multirow[t]{2}{*}{$\mathrm{C}_{13} \mathrm{H}_{9} \mathrm{ClO}$} & {$[5162-03-8]$} & 2-chlorobenzophe & none & & & \\
\hline & SUB & & $100.2 \pm 0.4$ & 298 & $\mathrm{C}$ & [2007RIB/AMA2] \\
\hline \multirow[t]{3}{*}{$\mathrm{C}_{13} \mathrm{H}_{9} \mathrm{ClO}$} & [1016-78-0] & 3-chlorobenzophe & none & & & \\
\hline & SUB & (321-339) & $108.8 \pm 0.4$ & 330 & $\mathrm{ME}$ & [2007RIB/AMA2] \\
\hline & SUB & (321-339) & $110.4 \pm 0.4$ & 298 & $\mathrm{ME}$ & [2007RIB/AMA2] \\
\hline \multirow[t]{3}{*}{$\mathrm{C}_{13} \mathrm{H}_{9} \mathrm{ClO}$} & {$[134-85-0]$} & 4-chlorobenzophe & none & & & \\
\hline & SUB & $(320-338)$ & $105.4 \pm 0.3$ & 329 & $\mathrm{ME}$ & [2007RIB/AMA2] \\
\hline & SUB & $(320-338)$ & $108.2 \pm 0.3$ & 298 & $\mathrm{ME}$ & [2007RIB/AMA2] \\
\hline \multirow[t]{3}{*}{$\mathrm{C}_{13} \mathrm{H}_{9} \mathrm{ClO}_{2}$} & {$[85-19-8]$} & 5-chloro-2-hydro & xybenzophenc & & & \\
\hline & SUB & $(293-367)$ & 91.9 & 308 & A & [1987STE/MAL, 1960SCH/HIR] \\
\hline & $\mathrm{V}$ & $(367-493)$ & 73.3 & 382 & A,UV & [1987STE/MAL, 1960SCH/HIR] \\
\hline \multirow[t]{2}{*}{$\mathrm{C}_{13} \mathrm{H}_{9} \mathrm{Cl}_{3} \mathrm{~N}_{2} \mathrm{O}$} & {$[33422-33-2]$} & \multicolumn{5}{|c|}{ Benzoic acid, 2,4,6-trichlorophenyl hydrazide } \\
\hline & FUS & & 32.71 & 439.7 & DSC & [1990DON/DRE] \\
\hline \multirow[t]{5}{*}{$\mathrm{C}_{13} \mathrm{H}_{9} \mathrm{Cl}_{3} \mathrm{~N}_{2} \mathrm{O}$} & {$[101-20-2]$} & $3,4,4^{\prime}$-trichloroca & banilide & & & \\
\hline & FUS & & 41.94 & 528.2 & DSC & [2013LIM/JAN2] \\
\hline & TRS & & 6.1 & 428 & DSC & [2010RIB/RIB2] \\
\hline & FUS & & 41.94 & 528.2 & DSC & [2009ARA/SOS, 2010CHI/DEG] \\
\hline & SUB & & $182.2 \pm 1.7$ & 298 & $\mathrm{C}$ & [2010RIB/RIB2] \\
\hline \multirow[t]{11}{*}{$\mathrm{C}_{13} \mathrm{H}_{9} \mathrm{~F}_{3} \mathrm{~N}_{2} \mathrm{O}_{2}$} & {$[4394-00-7]$} & \multicolumn{5}{|c|}{ 2-[3-(trifluoromethyl)anilino)nicotinic acid (niflumic acid) } \\
\hline & FUS (I) & & 33.1 & 477.9 & & \\
\hline & FUS (II) & & 37.0 & 476.8 & DSC & [2012BAG/RED] \\
\hline & FUS & & 30.54 & 478 & DSC & [2012KAC/RUS] \\
\hline & FUS & & 36.5 & 478 & $\mathrm{DSC}$ & $\begin{array}{l}\text { [2007PER/SUR2, 2009SUR/TER, 2008SUR/SUR, } \\
\text { 2010SUR/PER, 2015SUR/SIM] }\end{array}$ \\
\hline & FUS & & 35.7 & 476.4 & DSC & [2004ROM/BUS] \\
\hline & FUS & & 32.73 & 477.2 & DSC & [1998BUS/PEN] \\
\hline & FUS & & 38.0 & 476 & DSC & {$[1989 \mathrm{PIN} / \mathrm{GON}]$} \\
\hline & SUB & $(355-396)$ & $127.8 \pm 0.8$ & 376 & GS & [2007PER/SUR2, 2009SUR/TER] \\
\hline & SUB & $(355-396)$ & $130.2 \pm 0.8$ & 298 & GS & $\begin{array}{l}\text { [2007PER/SUR2, 2009SUR/TER, 2009SUR/PER, } \\
\text { 2008SUR/SUR] }\end{array}$ \\
\hline & $\mathrm{V}$ & & 107.5 & 298 & $\mathrm{~S}-\mathrm{F}$ & [2007PER/SUR2] \\
\hline \multirow[t]{3}{*}{$\mathrm{C}_{13} \mathrm{H}_{9} \mathrm{~N}$} & {$[260-94-6]$} & Acridine & & & & \\
\hline & FUS & & 20.5 & 383 & DSC & [2016BRU/LAP] \\
\hline & FUS (VII) & & 17.0 & 382.2 & & \\
\hline
\end{tabular}


TABLE 11. Phase change enthalpies of $\mathrm{C}_{12}$ to $\mathrm{C}_{13}$ organic compounds-Continued

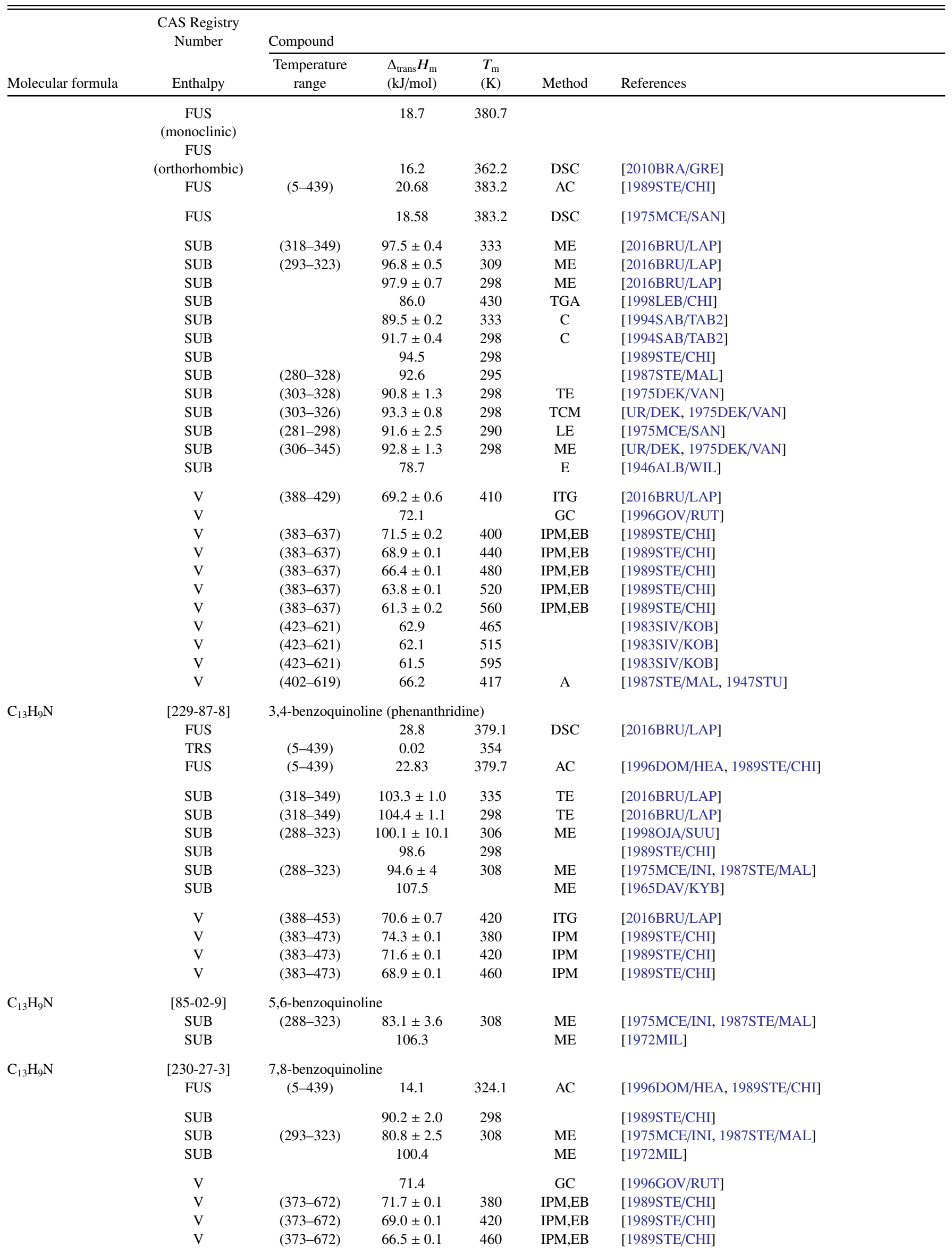


TABLE 11. Phase change enthalpies of $\mathrm{C}_{12}$ to $\mathrm{C}_{13}$ organic compounds-Continued

\begin{tabular}{|c|c|c|c|c|c|c|}
\hline \multirow[b]{2}{*}{ Molecular formula } & \multirow{2}{*}{$\begin{array}{l}\text { CAS Registry } \\
\text { Number } \\
\text { Enthalpy }\end{array}$} & \multicolumn{5}{|l|}{ Compound } \\
\hline & & $\begin{array}{c}\text { Temperature } \\
\text { range }\end{array}$ & $\begin{array}{l}\Delta_{\text {trans }} H_{\mathrm{m}} \\
(\mathrm{kJ} / \mathrm{mol})\end{array}$ & $\begin{array}{l}T_{\mathrm{m}} \\
(\mathrm{K})\end{array}$ & Method & References \\
\hline & $\mathrm{V}$ & $(373-672)$ & $64.0 \pm 0.1$ & 500 & IPM,EB & [1989STE/CHI] \\
\hline & $\mathrm{V}$ & $(373-672)$ & $61.5 \pm 0.3$ & 540 & IPM,EB & [1989STE/CHI] \\
\hline & $\mathrm{V}$ & $(373-672)$ & $59.0 \pm 0.3$ & 580 & IPM,EB & [1989STE/CHI] \\
\hline \multirow[t]{4}{*}{$\mathrm{C}_{13} \mathrm{H}_{9} \mathrm{NO}$} & {$[578-95-0]$} & Acridone & & & & \\
\hline & FUS & & 32.5 & 640 & DSC & [2003STO/KRZ] \\
\hline & SUB & & 133 & & DSC & [2003STO/KRZ] \\
\hline & SUB & & $136.2 \pm 0.5$ & 298 & $\mathrm{C}$ & [1992SAB/ELW] \\
\hline \multirow[t]{2}{*}{$\mathrm{C}_{13} \mathrm{H}_{9} \mathrm{NO}$} & [2157-52-0] & 9-fluorenoneox & & & & \\
\hline & SUB & $(375-399)$ & $79.6 \pm 1.2$ & 387 & $\mathrm{ME}$ & [2010GOL/SUU] \\
\hline \multirow[t]{3}{*}{$\mathrm{C}_{13} \mathrm{H}_{9} \mathrm{NO}_{2}$} & [2382-08-3] & $N$-methyl-1,8- & phthalimide & & & \\
\hline & SUB & (379-398) & $107.4 \pm 0.8$ & 389 & $\mathrm{ME}$ & [2000ROU/JIM] \\
\hline & SUB & (379-398) & $109.7 \pm 0.8$ & 298 & $\mathrm{ME}$ & [2000ROU/JIM] \\
\hline \multirow[t]{7}{*}{$\mathrm{C}_{13} \mathrm{H}_{9} \mathrm{NO}_{22}$} & {$[607-57-8]$} & 2-nitrofluorene & & & & \\
\hline & FUS & & 24.75 & 429.9 & DSC & [2014OLI/MON] \\
\hline & SUB & $(357-379)$ & $113.6 \pm 0.3$ & 368 & ME & [2014OLI/MON] \\
\hline & SUB & $(357-379)$ & $116.2 \pm 0.3$ & 298 & $\mathrm{ME}$ & [2014OLI/MON] \\
\hline & SUB & & $114.2 \pm 2.5$ & 298 & $\mathrm{C}$ & [2014OLI/MON] \\
\hline & SUB & $(349-384)$ & $114.2 \pm 3.0$ & 366 & $\mathrm{ME}$ & {$[2008 \mathrm{GOL} / \mathrm{SUU}]$} \\
\hline & SUB & $(349-384)$ & $116.8 \pm 1.4$ & 298 & $\mathrm{ME}$ & [2008GOL/SUU, 2014OLI/MON] \\
\hline \multirow[t]{4}{*}{$\mathrm{C}_{13} \mathrm{H}_{9} \mathrm{NO}_{3}$} & {$[2243-80-3]$} & 3-nitrobenzoph & one & & & \\
\hline & SUB & & $123.3 \pm 2.4$ & 298 & $\mathrm{C}$ & [2011RIB/AMA3] \\
\hline & SUB & $(349-365)$ & $119.3 \pm 0.6$ & 357 & $\mathrm{ME}$ & [2011RIB/AMA3] \\
\hline & SUB & $(349-365)$ & $121.6 \pm 0.6$ & 298 & $\mathrm{ME}$ & [2011RIB/AMA3] \\
\hline \multirow[t]{4}{*}{$\mathrm{C}_{13} \mathrm{H}_{9} \mathrm{NO}_{3}$} & {$[1144-74-7]$} & 4-nitrobenzoph & one & & & \\
\hline & SUB & & $122.3 \pm 2.7$ & 298 & $\mathrm{C}$ & [2011RIB/AMA3] \\
\hline & SUB & $(360-375)$ & $120.2 \pm 0.7$ & 367 & ME & [2011RIB/AMA3] \\
\hline & SUB & $(360-375)$ & $122.9 \pm 0.7$ & 298 & $\mathrm{ME}$ & [2011RIB/AMA3] \\
\hline \multirow[t]{2}{*}{$\mathrm{C}_{13} \mathrm{H}_{9} \mathrm{NO}_{4}$} & [75965-74-1] & \multicolumn{5}{|c|}{ 2-nitro-7-methoxynaphtho[2,1b]furan } \\
\hline & FUS & & 28.7 & 460.4 & DSC & [2010KES/AUC] \\
\hline \multirow[t]{27}{*}{$\mathrm{C}_{13} \mathrm{H}_{10}$} & {$[86-73-7]$} & Fluorene & & & & \\
\hline & FUS & & 20.3 & 388 & DSC & [2012MON/PIN] \\
\hline & FUS & & 19.4 & 386.7 & DSC & [2011RIC/FU] \\
\hline & FUS & & 18.55 & 387.7 & DSC & [2008MOG/SEP] \\
\hline & FUS & & 19.1 & 387.7 & DSC & [2000LIS/JAM] \\
\hline & FUS & $(196-419)$ & 19.40 & 387.78 & $\mathrm{AC}$ & {$[1995 \mathrm{FUJ} / \mathrm{FUJ}]$} \\
\hline & FUS & $(12-426)$ & 19.58 & 387.9 & & [1996DOM/HEA, 1977FIN/MES] \\
\hline & FUS & & 19.65 & 389.0 & DSC & [1980KRA/PIG] \\
\hline & FUS & & 19.5 & & DSC & [1972WAU/GET] \\
\hline & FUS & & 19.87 & 387.0 & & [1944EIB] \\
\hline & SUB & $(292-320)$ & $88.3 \pm 0.2$ & 306 & ME & [2012MON/PIN] \\
\hline & SUB & $(292-320)$ & $88.6 \pm 0.2$ & 298 & $\mathrm{ME}$ & [2012MON/PIN] \\
\hline & SUB & $(313-378)$ & $85.8 \pm 0.1$ & 346 & PGSM & [2012MON/PIN] \\
\hline & SUB & $(313-378)$ & $87.3 \pm 0.1$ & 298 & PGSM & [2012MON/PIN] \\
\hline & SUB & & $87.7 \pm 1.1$ & 298 & $\mathrm{C}$ & [2012MON/PIN] \\
\hline & SUB & $(296-317)$ & $82.6 \pm 2.5$ & & $\mathrm{ME}$ & [2011RIC/FU] \\
\hline & SUB & $(298-324)$ & $88.1 \pm 2.3$ & 311 & $\mathrm{ME}$ & [2010GOL/SUU] \\
\hline & SUB & $(298-324)$ & $87.1 \pm 1.9$ & 311 & ME & [2008GOL/SUU3] \\
\hline & SUB & $(289-359)$ & $86.1 \pm 0.1$ & 298 & GS & [2004VER] \\
\hline & SUB & & 87.6 & 298 & CGC-DSC & [1998CHI/HES] \\
\hline & SUB & $(313-453)$ & 84.9 & 383 & GS & [1995NAS/LEN] \\
\hline & SUB & $(323-363)$ & $84.9 \pm 0.4$ & 343 & GS & [1994RAK/VER2] \\
\hline & SUB & & $85.1 \pm 0.4$ & 298 & & [1994RAK/VER2] \\
\hline & SUB & $(318-333)$ & $87.0 \pm 1.0$ & 318 & PG & [1988SAS/JOS] \\
\hline & SUB & & $80.2 \pm 0.2$ & 298 & $\mathrm{C}$ & [1987SAB/ANT] \\
\hline & SUB & $(348-388)$ & 78.9 & 363 & A & [1987STE/MAL] \\
\hline & SUB & $(308-347)$ & 83.2 & 328 & GS & [1986SAT/INO] \\
\hline
\end{tabular}


TABLE 11. Phase change enthalpies of $\mathrm{C}_{12}$ to $\mathrm{C}_{13}$ organic compounds-Continued

\begin{tabular}{|c|c|c|c|c|c|c|}
\hline \multirow[b]{2}{*}{ Molecular formula } & \multirow{2}{*}{$\begin{array}{l}\text { CAS Registry } \\
\text { Number } \\
\text { Enthalpy }\end{array}$} & \multicolumn{5}{|l|}{ Compound } \\
\hline & & $\begin{array}{l}\text { Temperature } \\
\text { range }\end{array}$ & $\begin{array}{l}\Delta_{\text {trans }} H_{\mathrm{m}} \\
(\mathrm{kJ} / \mathrm{mol})\end{array}$ & $\begin{array}{l}T_{\mathrm{m}} \\
(\mathrm{K})\end{array}$ & Method & References \\
\hline & SUB & $(298-343)$ & 92.2 & 320 & $\mathrm{~T}$ & [1986HAN/ECK] \\
\hline & SUB & $(283-323)$ & $88.4 \pm 0.6$ & 303 & GS & {$[1983 \mathrm{SON} / \mathrm{ZOL}]$} \\
\hline & SUB & $(350-388)$ & $83.1 \pm 1.3$ & & & [1977FIN/MES, 1975OSB/DOU] \\
\hline & SUB & & 81.8 & 388 & $\mathrm{~B}$ & {$[19750 S B / D O U]$} \\
\hline & SUB & $(286-300)$ & $80.3 \pm 0.8$ & 293 & $\mathrm{TE}$ & [1960BUD] \\
\hline & SUB & $(306-323)$ & 82.8 & 315 & & [1953BRA/CLE, 1987STE/MAL] \\
\hline & SUB & $(306-322)$ & 82.8 & & & [1953BRA/CLE2, 1960JON] \\
\hline & V & $(387-412)$ & $63.1 \pm 0.2$ & 399 & PGSM & [2012MON/PIN] \\
\hline & $\mathrm{V}$ & $(387-412)$ & $72.1 \pm 0.2$ & 298 & PGSM & [2012MON/PIN] \\
\hline & V & & $72.4 \pm 1.7$ & 298 & CGC & [2008HAN/NUT] \\
\hline & $\mathrm{V}$ & $(373-423)$ & $74.4 \pm 1.2$ & 298 & GC & [2006HAF/PAR] \\
\hline & $\mathrm{V}$ & $(323-473)$ & 66.9 & 398 & $\mathrm{GC}$ & [2002LEI/CHA] \\
\hline & $\mathrm{V}$ & & 72.3 & 298 & CGC & [1998CHI/HES] \\
\hline & $\mathrm{V}$ & $(403-453)$ & 72.2 & 298 & CGC & [1995CHI/HOS] \\
\hline & $\mathrm{V}$ & $(323-363)$ & 65.7 & 298 & B & [1994RAK/VER2] \\
\hline & $\mathrm{V}$ & $(383-427)$ & 63.3 & 398 & & [1988SAS/JOS] \\
\hline & $\mathrm{V}$ & $(402-568)$ & 54.2 & 417 & A & [1987STE/MAL] \\
\hline & $\mathrm{V}$ & $(425-639)$ & 56.5 & 465 & & [1982SIV/KOB] \\
\hline & $\mathrm{V}$ & $(425-639)$ & 55.5 & 505 & & [1982SIV/KOB] \\
\hline & $\mathrm{V}$ & $(425-639)$ & 54.3 & 555 & & [1982SIV/KOB] \\
\hline & $\mathrm{V}$ & $(423-573)$ & 56.6 & 498 & I & [1923MOR/MUR] \\
\hline \multirow[t]{2}{*}{$\mathrm{C}_{13} \mathrm{H}_{10} \mathrm{BrCl}_{2} \mathrm{O}_{2} \mathrm{PS}$} & {$[21609-90-5]$} & \multicolumn{5}{|c|}{$O$-(4-bromo-2,5-dichlorophenyl) $O$-methyl phenylphosphonothioate } \\
\hline & FUS & & 31.35 & 345.6 & DSC & {$[1990 \mathrm{DON} / \mathrm{DRE}]$} \\
\hline \multirow[t]{2}{*}{$\mathrm{C}_{13} \mathrm{H}_{10} \mathrm{BrN}_{3} \mathrm{O}_{4}$} & {$[192219-62-8]$} & \multicolumn{5}{|c|}{ 2-cyano-6-mtro-1(2H)-quinolinecarboxylic acid, 2-bromoethyl ester } \\
\hline & FUS & & 19.79 & 419.2 & DSC & {$[2005 \mathrm{LIZ} / \mathrm{ZAB}]$} \\
\hline \multirow[t]{2}{*}{$\mathrm{C}_{13} \mathrm{H}_{10} \mathrm{CIN}_{3} \mathrm{O}_{4}$} & {$[850836-65-6]$} & \multicolumn{5}{|c|}{ 2-cyano-6-mtro-1(2H)-quinolinecarboxylic acid, 2-chloroethyl ester } \\
\hline & FUS & & 17.17 & 418.9 & DSC & {$[2005 \mathrm{LIZ} / \mathrm{ZAB}]$} \\
\hline \multirow[t]{3}{*}{$\mathrm{C}_{13} \mathrm{H}_{10} \mathrm{CIN}_{3} \mathrm{O}_{4} \mathrm{~S}_{2}$} & {$[70374-39-9]$} & \multicolumn{5}{|c|}{$\begin{array}{l}\text { (3E)-6-chloro-3-[hydroxy(pyridin-2-ylamino)methylene]-2-methyl-2,3-dihydro- } 4 H \text {-thieno[2,3- } \\
e][1,2] \text { thiazin-4-one 1,1-dioxide }\end{array}$} \\
\hline & FUS & & 54.29 & 479.8 & DSC & [2013KHA/SUB] \\
\hline & FUS & & 54.2 & 481.7 & DSC & {$[2012 \mathrm{KHA} / \mathrm{ACH}]$} \\
\hline \multirow[t]{2}{*}{$\mathrm{C}_{13} \mathrm{H}_{10} \mathrm{Cl}_{2} \mathrm{~S}$} & {$[103-17-3]$} & \multicolumn{5}{|c|}{$p$-chlorobenzyl $p$-chlorophenyl sulfide } \\
\hline & FUS & & 32.22 & 343.8 & $\mathrm{DSC}$ & [1969PLA/GLA] \\
\hline \multirow[t]{3}{*}{$\mathrm{C}_{13} \mathrm{H}_{10} \mathrm{~N}_{2}$} & {$[622-16-2]$} & \multicolumn{5}{|c|}{$N, N^{\prime}$-diphenylcarbondiimide } \\
\hline & FUS & $(5-330)$ & 18.55 & 287.4 & & [1990DOM/HEA, 1984LEB/BYK] \\
\hline & $\mathrm{V}$ & $(500-599)$ & 65.6 & 515 & $\mathrm{~A}, \mathrm{I}$ & [1987STE/MAL, 1962JOH/MCE] \\
\hline \multirow[t]{2}{*}{$\mathrm{C}_{13} \mathrm{H}_{10} \mathrm{~N}_{2}$} & {$[90-45-9]$} & \multicolumn{5}{|c|}{ 9-aminoacridine } \\
\hline & SUB & & 115 & 520 & TGA & [1998STO/KRZ] \\
\hline \multirow[t]{3}{*}{$\mathrm{C}_{13} \mathrm{H}_{10} \mathrm{~N}_{2}$} & {$[716-79-0]$} & \multicolumn{5}{|c|}{ 2-phenylbenzimidazole } \\
\hline & FUS & & 22.18 & 572.2 & $\mathrm{DSC}$ & [1971KAM/MIT] \\
\hline & SUB & & $123.0 \pm 1.7$ & 298 & $\mathrm{C}$ & [2005RIB/RIB] \\
\hline $\mathrm{C}_{13} \mathrm{H}_{10} \mathrm{~N}_{2} \mathrm{O}_{2}$ & {$[785-80-8]$} & $N$-phenyl 4-n & benzaldehyde & & & \\
\hline & FUS & & 24.56 & 347.2 & DSC & [1997VER/MOR] \\
\hline & SUB & & $126.0 \pm 1.3$ & 298 & $\mathrm{~V}+\mathrm{F}$ & [1997VER/MOR] \\
\hline & $\mathrm{V}$ & $(359-387)$ & $96.9 \pm 1.3$ & 373 & GS & [1997VER/MOR] \\
\hline & $\mathrm{V}$ & $(359-387)$ & $101.4 \pm 1.3$ & 298 & GS & [1997VER/MOR] \\
\hline $\mathrm{C}_{13} \mathrm{H}_{10} \mathrm{~N}_{2} \mathrm{O}_{3}$ & [1775-95-7] & 2-amino-5-nit & enzophenone & & & \\
\hline & FUS & & 37.9 & 440 & DSC & [2007DRA/JAN] \\
\hline $\mathrm{C}_{13} \mathrm{H}_{10} \mathrm{~N}_{2} \mathrm{O}_{4}$ & {$[50-35-1]$} & Thalidomide & & & & \\
\hline & FUS (I) & & 39.97 & 546.7 & $\mathrm{DSC}$ & [2007LAR/PER] \\
\hline & FUS (II) & & 37.91 & 550.8 & DSC & [2007LAR/PER] \\
\hline & FUS & & 36.02 & 548.2 & DSC & [2002GOO/LAI] \\
\hline
\end{tabular}


TABLE 11. Phase change enthalpies of $\mathrm{C}_{12}$ to $\mathrm{C}_{13}$ organic compounds-Continued

\begin{tabular}{|c|c|c|c|c|c|c|}
\hline \multirow[b]{2}{*}{ Molecular formula } & \multirow{2}{*}{$\begin{array}{l}\text { CAS Registry } \\
\text { Number } \\
\text { Enthalpy }\end{array}$} & \multicolumn{5}{|l|}{ Compound } \\
\hline & & $\begin{array}{l}\text { Temperature } \\
\text { range }\end{array}$ & $\begin{array}{l}\Delta_{\text {trans }} H_{\mathrm{m}} \\
(\mathrm{kJ} / \mathrm{mol})\end{array}$ & $\begin{array}{c}T_{\mathrm{m}} \\
(\mathrm{K})\end{array}$ & Method & References \\
\hline \multirow[t]{2}{*}{$\mathrm{C}_{13} \mathrm{H}_{10} \mathrm{~N}_{4}$} & {$[7477-73-8]$} & \multicolumn{5}{|c|}{ 1,5-diphenyltetrazole } \\
\hline & SUB & $(348-363)$ & $121.5 \pm 4.2$ & 355 & $\mathrm{ME}$ & [1951MCE/RIG, 1970COX/PIL] \\
\hline \multirow[t]{2}{*}{$\mathrm{C}_{13} \mathrm{H}_{10} \mathrm{~N}_{4}$} & {$[18039-45-7]$} & \multicolumn{5}{|c|}{ 2,5-diphenyltetrazole } \\
\hline & SUB & $(333-353)$ & $119.7 \pm 4.2$ & 343 & ME & [1951MCE/RIG, 1970COX/PIL] \\
\hline \multirow[t]{2}{*}{$\mathrm{C}_{13} \mathrm{H}_{10} \mathrm{~N}_{4} \mathrm{O}$} & [14031-13-1] & \multicolumn{5}{|c|}{ 1-phenazinecarboxylic acid hydrazide } \\
\hline & FUS & & 27.62 & 505 & $\mathrm{DSC}$ & [1997CIO/MEL] \\
\hline \multirow[t]{2}{*}{$\mathrm{C}_{13} \mathrm{H}_{10} \mathrm{O}$} & {$[3218-36-8]$} & \multicolumn{5}{|c|}{ 4-biphenylcarboxaldehyde } \\
\hline & FUS & & 21.09 & 332 & DSC & [2010BAI/VAN] \\
\hline \multirow[t]{31}{*}{$\mathrm{C}_{13} \mathrm{H}_{10} \mathrm{O}$} & {$[119-61-9]$} & \multicolumn{5}{|l|}{ Benzophenone } \\
\hline & FUS & $(6-351)$ & 18.47 & 321.3 & $\mathrm{AC}$ & [2002HAN/HIK] \\
\hline & FUS & $(5-440)$ & 18.81 & 321.2 & $\mathrm{AC}$ & [2002CHI/KNI2] \\
\hline & FUS & $(81-330)$ & 18.19 & 324.2 & & [1996DOM/HEA, 1983DEK/VAN] \\
\hline & FUS & & 16.9 & 321.4 & $\mathrm{C}$ & [1963RAS/NIG] \\
\hline & FUS & & 19.2 & & $\mathrm{RC}$. & {$[1927 \mathrm{STE} / \mathrm{JOH}]$} \\
\hline & FUS & & 16.5 & & $\mathrm{AC}$ & [1922STR/PAR, 1927STE/JOH] \\
\hline & FUS & & 18.2 & & & [1915BRI, 1927STE/JOH] \\
\hline & FUS & & 17.8 & & $\mathrm{C}$ & [1899TAM, 1927STE/JOH] \\
\hline & FUS & & 18.0 & & $\mathrm{C}$ & [1894BRU, 1927STE/JOH] \\
\hline & FUS & & 17.67 & 321.2 & & {$[1889 \mathrm{EYK}]$} \\
\hline & SUB & & $93.1 \pm 2.1$ & 298 & GS & [1998VER4] \\
\hline & SUB & & $94.7 \pm 1.0$ & 321 & DM & [1983DEK/VAN] \\
\hline & SUB & & $92 \pm 0.83$ & 298 & $\mathrm{C}$ & [1974SAB, 1983DEK/VAN] \\
\hline & SUB & $(295-313)$ & $95.0 \pm 0.2$ & 304 & ME & [1980COL/JIM2] \\
\hline & SUB & & $84.4 \pm 1.13$ & 298 & $\mathrm{C}$ & [1978SAB/LAF2] \\
\hline & SUB & $(297-317)$ & $93.9 \pm 0.5$ & 307 & TE,ME & [1977DEK/VAN] \\
\hline & SUB & $(293-318)$ & $95.0 \pm 1.5$ & 305 & TE & [1975DEK/VAN] \\
\hline & SUB & $(294-318)$ & $92.9 \pm 0.8$ & 306 & ME & [1975VAN/DEK] \\
\hline & SUB & $(278-311)$ & $77.0 \pm 2.5$ & 298 & ME & [1974ARS] \\
\hline & SUB & $(298-318)$ & 89.96 & 308 & ME & [1987STE/MAL, 1974PRI/POU] \\
\hline & SUB & $(295-304)$ & $94.6 \pm 0.8$ & 298 & TCM & {$[1973 \mathrm{DEK} / \mathrm{OON}]$} \\
\hline & SUB & & $93.4 \pm 0.3$ & 298 & $\mathrm{C}$ & [1972MOR3] \\
\hline & SUB & $(293-319)$ & 96.1 & 306 & & [1956SER/VOI] \\
\hline & SUB & & 91.2 & & & {$[1950 \mathrm{NIT} / \mathrm{SEK}]$} \\
\hline & SUB & $(290-315)$ & $78.2 \pm 1.2$ & 303 & & [1938WOL/WEG, 1934WOL/TRI] \\
\hline & SUB & & $95 \pm 2.5$ & 298 & $\mathrm{TE}$ & [1932NEU/VOL, 1970COX/PIL, 1960JON] \\
\hline & SUB & $(273-320)$ & $91.2 \pm 1.6$ & 298 & ME & {$[1925 \mathrm{VOL} / \mathrm{KIR}]$} \\
\hline & $\mathrm{V}$ & $(433-673)$ & 65.1 & 448 & A & {$[1987 \mathrm{STE} / \mathrm{MAL}]$} \\
\hline & $\mathrm{V}$ & $(473-579)$ & 62.2 & 488 & & [1949DRE/SHR, 1949DRE/MAR, 1984BOU/FRI] \\
\hline & $\mathrm{V}$ & $(530-575)$ & 59.0 & 545 & & [1904JAQ/WAS, 1984BOU/FRI] \\
\hline \multirow[t]{9}{*}{$\mathrm{C}_{13} \mathrm{H}_{10} \mathrm{O}$} & {$[92-83-1]$} & \multicolumn{5}{|l|}{ Xanthene } \\
\hline & FUS & & 20.67 & 374.6 & DSC & {$[2008 \mathrm{MON} / \mathrm{SAN}]$} \\
\hline & FUS & & 15.87 & 374.3 & DSC & [2000MAH/SOL] \\
\hline & FUS & & 19.2 & 373.7 & & [1991ACR, 1988COO/SED] \\
\hline & SUB & & $92 \pm 1.1$ & 298 & $\mathrm{C}$ & [2010FRE/GOM2] \\
\hline & SUB & $(308-326)$ & $93.2 \pm 0.9$ & 298 & ME & [2008MON/SAN] \\
\hline & SUB & $(318-371)$ & $92.6 \pm 1.2$ & 298 & Static & [2008MON/SAN] \\
\hline & SUB & $(305-353)$ & 92.5 & 329 & $\mathrm{~T}$ & [1986ROR] \\
\hline & SUB & & $112.1 \pm 2.1$ & & & [1958CAS/FLE3, 1970COX/PIL] \\
\hline
\end{tabular}

[Note: The authors refer to the compound as dibenzopyran; however, the melting point temperature corresponds to xanthene.]

$\begin{array}{lccccl}\text { V } & (358-382) & 73.4 \pm 0.1 & 298 & \text { Static } & \text { [2008MON/SAN] } \\ \text { V } & (424-589) & 64.5 & 435 & \text { Static } & {[1984 \mathrm{SIV} / \text { KOB] }} \\ \text { V } & (424-589) & 61.1 & 475 & \text { Static } & {[1984 \mathrm{SIV} / \text { KOB] }} \\ \text { V } & (424-589) & 59.2 & 515 & \text { Static } & {[1984 \mathrm{SIV} / \mathrm{KOB}]} \\ \text { V } & (424-589) & 56.7 & 555 & \text { Static } & {[1984 \mathrm{SIV} / \mathrm{KOB}]} \\ \text { V } & (424-589) & 54.4 & 585 & \text { Static } & {[1984 \mathrm{SIV} / \mathrm{KOB}]} \\ \text { V } & (413-433) & 88.7 & 423 & \mathrm{~A} & {[1987 \mathrm{STE} / \mathrm{MAL}, 1958 \mathrm{CAS} / \mathrm{FLE} 3]}\end{array}$


TABLE 11. Phase change enthalpies of $\mathrm{C}_{12}$ to $\mathrm{C}_{13}$ organic compounds-Continued

\begin{tabular}{|c|c|c|c|c|c|c|}
\hline \multirow[b]{2}{*}{ Molecular formula } & \multirow{2}{*}{$\begin{array}{c}\text { CAS Registry } \\
\text { Number } \\
\text { Enthalpy }\end{array}$} & \multicolumn{5}{|l|}{ Compound } \\
\hline & & $\begin{array}{c}\text { Temperature } \\
\text { range }\end{array}$ & $\begin{array}{l}\Delta_{\text {trans }} H_{\mathrm{m}} \\
(\mathrm{kJ} / \mathrm{mol})\end{array}$ & $\begin{array}{l}T_{\mathrm{m}} \\
(\mathrm{K})\end{array}$ & Method & References \\
\hline \multirow[t]{9}{*}{$\mathrm{C}_{13} \mathrm{H}_{10} \mathrm{O}$} & [1689-64-1] & \multicolumn{5}{|c|}{ 9-hydroxyfluorene } \\
\hline & FUS & & 24.2 & 428.7 & DSC & [2012MON/NOT2] \\
\hline & SUB & $(337-359)$ & $109.0 \pm 0.8$ & 298 & $\mathrm{ME}$ & [2012MON/NOT2] \\
\hline & SUB & $(351-424)$ & $108.3 \pm 0.5$ & 298 & Static & [2012MON/NOT2] \\
\hline & SUB & $(326-366)$ & $97.4 \pm 2.3$ & 346 & $\mathrm{ME}$ & [2010GOL/SUU] \\
\hline & V & $(410-441)$ & $91.6 \pm 0.4$ & 298 & Static & [2012MON/NOT2] \\
\hline & $\mathrm{V}$ & & 50.4 & 435 & Static & [1983SIV/MAR] \\
\hline & $\mathrm{V}$ & & 49.7 & 465 & Static & [1983SIV/MAR] \\
\hline & $\mathrm{V}$ & & 48.9 & 505 & Static & [1983SIV/MAR] \\
\hline \multirow[t]{2}{*}{$\mathrm{C}_{13} \mathrm{H}_{10} \mathrm{O}_{2}$} & [947-84-2] & \multicolumn{5}{|c|}{ 2-biphenylcarboxylic acid } \\
\hline & SUB & & $121.3 \pm 4.3$ & 298 & $\mathrm{C}$ & [2004MAT/MIR2] \\
\hline \multirow[t]{4}{*}{$\mathrm{C}_{13} \mathrm{H}_{10} \mathrm{O}_{2}$} & [92-92-2] & \multicolumn{5}{|c|}{ 4-biphenylcarboxylic acid } \\
\hline & FUS & & 32.1 & 499.5 & DSC & [2012UMN/HAS] \\
\hline & FUS & & 32.26 & 500 & DSC & [2010BAI/VAN] \\
\hline & SUB & & $127.5 \pm 4.1$ & 298 & $\mathrm{C}$ & [2004MAT/MIR2] \\
\hline \multirow[t]{5}{*}{$\mathrm{C}_{13} \mathrm{H}_{10} \mathrm{O}_{2}$} & [93-99-2] & \multicolumn{5}{|c|}{ Phenylbenzoate } \\
\hline & SUB & & $99.0 \pm 0.4$ & 298 & & {$[1971 \mathrm{CAR} / \mathrm{FIN}]$} \\
\hline & SUB & & $89.5 \pm 4.2$ & & & [1971KIP/RAB, 1977PED/RYL] \\
\hline & SUB & & $96.2 \pm 1.7$ & & & [1947STU, 1970COX/PIL] \\
\hline & $\mathrm{V}$ & $(379-587)$ & 62.4 & 394 & A & [1987STE/MAL, 1947STU] \\
\hline \multirow[t]{3}{*}{$\mathrm{C}_{13} \mathrm{H}_{10} \mathrm{O}_{2}$} & [117-99-7] & \multicolumn{5}{|c|}{ (2-hydroxyphenyl)phenylmethanone } \\
\hline & FUS & & 18.7 & 312.3 & DSC & [2010DAV/GUE] \\
\hline & FUS & & 0.67 & 308.2 & DTA & [1989SAL/ABA] \\
\hline
\end{tabular}

[Note: Reported enthalpy of fusion is too small, and the published enthalpy and entropy of fusion data are internally inconsistent.]

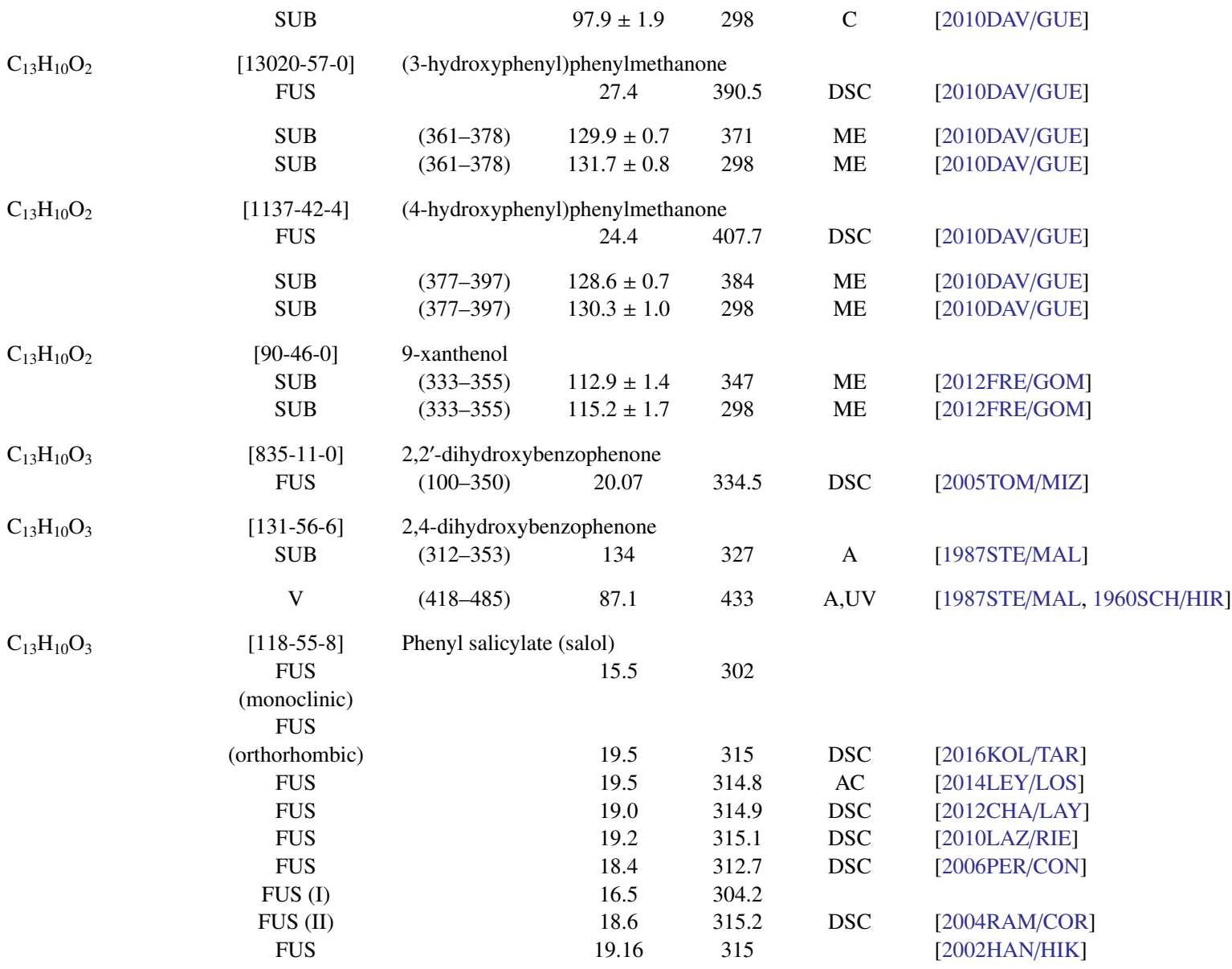


TABLE 11. Phase change enthalpies of $\mathrm{C}_{12}$ to $\mathrm{C}_{13}$ organic compounds-Continued

\begin{tabular}{|c|c|c|c|c|c|c|}
\hline \multirow[b]{2}{*}{ Molecular formula } & \multirow{2}{*}{$\begin{array}{l}\text { CAS Registry } \\
\text { Number } \\
\text { Enthalpy }\end{array}$} & \multicolumn{5}{|l|}{ Compound } \\
\hline & & $\begin{array}{c}\text { Temperature } \\
\text { range }\end{array}$ & $\begin{array}{l}\Delta_{\text {trans }} H_{\mathrm{m}} \\
(\mathrm{kJ} / \mathrm{mol})\end{array}$ & $\begin{array}{l}T_{\mathrm{m}} \\
(\mathrm{K})\end{array}$ & Method & References \\
\hline & FUS & & 18.98 & 314.2 & DSC & [1995MUR/PAI] \\
\hline & SUB & $(279-315)$ & 109.1 & 294 & $\mathrm{~A}$ & [1987STE/MAL] \\
\hline & SUB & & $92.0 \pm 4.2$ & & & [1947STU, 1970COX/PIL] \\
\hline & $\mathrm{V}$ & $(423-587)$ & 69.9 & 438 & $\mathrm{~A}, \mathrm{UV}$ & [1987STE/MAL, 1960SCH/HIR] \\
\hline \multirow[t]{6}{*}{$\mathrm{C}_{13} \mathrm{H}_{10} \mathrm{O}_{3}$} & {$[102-09-0]$} & \multicolumn{5}{|c|}{ Diphenyl carbonate } \\
\hline & FUS & & 24.30 & 355.95 & DSC & [2008WEI/PEI] \\
\hline & FUS & & 23.43 & 355 & DSC & [1971CAR/FIN] \\
\hline & SUB & & $107.6 \pm 0.5$ & 298 & $\mathrm{ME}$ & {$[2014 \mathrm{FON} / \mathrm{GUS}]$} \\
\hline & SUB & & $90 \pm 8.4$ & 298 & $\mathrm{E}$ & [1971CAR/FIN, 1977PED/RYL] \\
\hline & $\mathrm{V}$ & $(355-381)$ & $80.9 \pm 0.6$ & 298 & GS & [2008VER/EME2] \\
\hline \multirow[t]{3}{*}{$\mathrm{C}_{13} \mathrm{H}_{10} \mathrm{O}_{3}$} & {$[58110-42-2]$} & \multicolumn{5}{|c|}{ 3-(5-phenylfur-2-yl)acrylic acid } \\
\hline & SUB & $(389-421)$ & $148.7 \pm 7.6$ & 405 & ME & [2014DIB/RAE] \\
\hline & SUB & $(389-421)$ & $152.6 \pm 7.6$ & 298 & ME & [2014DIB/RAE] \\
\hline \multirow[t]{4}{*}{$\mathrm{C}_{13} \mathrm{H}_{10} \mathrm{O}_{4}$} & {$[1470-79-7]$} & \multicolumn{5}{|c|}{ 2,4,4'-trihydroxybenzophenone } \\
\hline & FUS & & 31.3 & 482.6 & DSC & [1999PRI/HAW] \\
\hline & SUB & & 139 & & TGA & [1999PRI/HAW] \\
\hline & $\mathrm{V}$ & & 107.6 & & TGA & [1999PRI/HAW] \\
\hline \multirow[t]{5}{*}{$\mathrm{C}_{13} \mathrm{H}_{10} \mathrm{O}_{5}$} & {$[131-55-5]$} & \multicolumn{5}{|c|}{$2,2^{\prime}, 4,4^{\prime}$-tetrahydroxybenzophenone } \\
\hline & FUS & & 28.0 & 472 & DSC & [1999PRI/HAW] \\
\hline & SUB & & 178.5 & & $\mathrm{~B}$ & [1999PRI/HAW] \\
\hline & SUB & $(363-471)$ & 143.4 & 378 & A & [1987STE/MAL] \\
\hline & $\mathrm{V}$ & & 150.5 & & TGA & [1999PRI/HAW] \\
\hline \multirow[t]{8}{*}{$\mathrm{C}_{13} \mathrm{H}_{10} \mathrm{~S}$} & [261-31-4] & \multicolumn{5}{|l|}{ Thioxanthene } \\
\hline & FUS & & 26.8 & 402.7 & $\mathrm{~S}-\mathrm{V}$ & [2009FRE/MON] \\
\hline & FUS & & 26.1 & 401.8 & & [1991ACR, 1988COO/SED] \\
\hline & SUB & & $101.7 \pm 1.6$ & 298 & $\mathrm{C}$ & [2009FRE/MON] \\
\hline & SUB & $(339-402)$ & $98.4 \pm 0.2$ & 370 & Static & [2009FRE/MON] \\
\hline & SUB & $(339-402)$ & $100.9 \pm 0.2$ & 298 & Static & [2009FRE/MON] \\
\hline & $\mathrm{V}$ & $(383-447)$ & $69.5 \pm 0.2$ & 415 & Static & [2009FRE/MON] \\
\hline & $\mathrm{V}$ & $(383-447)$ & $77.8 \pm 2.6$ & 298 & Static & [2009FRE/MON] \\
\hline \multirow[t]{2}{*}{$\mathrm{C}_{13} \mathrm{H}_{10} \mathrm{~S}$} & {$[7372-88-5]$} & \multicolumn{5}{|c|}{ 4-methyldibenzothiophene } \\
\hline & SUB & & $90.3 \pm 0.7$ & 298 & $\mathrm{C}$ & [2010FRE/GOM] \\
\hline \multirow[t]{2}{*}{$\mathrm{C}_{13} \mathrm{H}_{11} \mathrm{BrO}_{5}$} & [111171-29-0] & \multicolumn{5}{|c|}{ 8-(hydroxymethyl)-6-bromo-2-oxo-2H-1-benzopyran-3-carboxylic acid, ethyl ester } \\
\hline & FUS & & 28.19 & 434.1 & DSC & [1992HUA/ZHO2] \\
\hline \multirow[t]{2}{*}{$\mathrm{C}_{13} \mathrm{H}_{11} \mathrm{CI}$} & {$[90-99-3]$} & \multicolumn{5}{|c|}{ Chlorodiphenylmethane } \\
\hline & $\mathrm{V}$ & $(381-450)$ & 70.4 & 396 & A & {$[1987 \mathrm{STE} / \mathrm{MAL}]$} \\
\hline \multirow[t]{2}{*}{$\mathrm{C}_{13} \mathrm{H}_{11} \mathrm{CIN}_{2} \mathrm{O}_{2}$} & {$[556836-77-2]$} & \multicolumn{5}{|c|}{ 4-chloro-2'-hydroxy-4'-methoxyazobenzene } \\
\hline & FUS & & 33.7 & 390 & DSC & [2003PAJ/ROS] \\
\hline \multirow[t]{3}{*}{$\mathrm{C}_{13} \mathrm{H}_{11} \mathrm{CIO}_{5}$} & [111171-28-9] & \multicolumn{5}{|c|}{ 8-(hydroxymethyl)-6-chloro-2-oxo-2H-1-benzopyran-3-carboxylic acid, ethyl ester } \\
\hline & TRS & & 15.53 & 418.2 & & \\
\hline & FUS & & 22.47 & 424 & DSC & [1992HUA/ZHO2] \\
\hline $\mathrm{C}_{13} \mathrm{H}_{11} \mathrm{CI}_{2} \mathrm{NO}_{2} \mathrm{~S}$ & [1245836-03-6] & $N$-(2,6-dichlo & henyl)-3-methy & lbenzenes & onamide & \\
\hline & FUS (I) & & U115.4 & 441.4 & DSC & \\
\hline & FUS (II) & & U107.1 & 440.5 & DSC & [2010SAN/SAR] \\
\hline
\end{tabular}

[Note: Enthalpies of fusion of both crystalline forms are out of line with other substituted benzenesulfonamides determined by the authors.]

$\mathrm{C}_{13} \mathrm{H}_{11} \mathrm{~F}$

$\mathrm{C}_{13} \mathrm{H}_{11} \mathrm{~N}$ [579-55-5] Fluorodiphenylmethane
$\mathrm{V}$
(288-333)
$69.8 \pm 0.4 \quad 298$
GS
[1997SCH/VER]

[1013-88-3]

Benzophenone imine

(308-338)

$74.2 \pm 1.0$

323

GS

[1997VER/MOR] 
TABLE 11. Phase change enthalpies of $\mathrm{C}_{12}$ to $\mathrm{C}_{13}$ organic compounds-Continued

\begin{tabular}{|c|c|c|c|c|c|c|}
\hline \multirow[b]{2}{*}{ Molecular formula } & \multirow{2}{*}{$\begin{array}{l}\text { CAS Registry } \\
\text { Number } \\
\text { Enthalpy }\end{array}$} & \multicolumn{5}{|l|}{ Compound } \\
\hline & & $\begin{array}{l}\text { Temperature } \\
\text { range }\end{array}$ & $\begin{array}{l}\Delta_{\text {trans }} H_{\mathrm{m}} \\
(\mathrm{kJ} / \mathrm{mol})\end{array}$ & $\begin{array}{l}T_{\mathrm{m}} \\
(\mathrm{K})\end{array}$ & Method & References \\
\hline & $\mathrm{V}$ & $(308-338)$ & $75.7 \pm 1.0$ & 298 & GS & [1997VER/MOR] \\
\hline & $\mathrm{V}$ & $(373-422)$ & 62.3 & 388 & A & [1987STE/MAL] \\
\hline \multirow[t]{5}{*}{$\mathrm{C}_{13} \mathrm{H}_{11} \mathrm{~N}$} & $\begin{array}{c}\text { [538-51-2] } \\
\text { FUS }\end{array}$ & \multicolumn{5}{|c|}{$N$-phenyl-benzaldehyde imine } \\
\hline & SUB & $(294-326)$ & $97.4 \pm 1.2$ & 309 & GS & [1997VER/MOR] \\
\hline & SUB & $(294-326)$ & $98.1 \pm 1.2$ & 298 & GS & [1997VER/MOR] \\
\hline & SUB & & $93.7 \pm 0.9$ & 298 & $\mathrm{C}$ & [1986KIR/ACR] \\
\hline & SUB & & $85.5 \pm 2.1$ & 293 & $\mathrm{E}$ & {$[1948 \mathrm{COA} / \mathrm{SUT}]$} \\
\hline \multirow{13}{*}{$\mathrm{C}_{13} \mathrm{H}_{11} \mathrm{~N}$} & [1484-12-4] & \multicolumn{5}{|c|}{ 9-methylcarbazole } \\
\hline & FUS & & 16.43 & 361.0 & DSC & [2016STA/KEI] \\
\hline & FUS & $(13-388)$ & 17.15 & 362.5 & $\mathrm{AC}$ & [1996DOM/HEA, 1988MES/TOD] \\
\hline & SUB & $(313-332)$ & 95.0 & 322 & $\mathrm{ME}$ & [1990JIM/ROU] \\
\hline & SUB & $(313-332)$ & 95.5 & 298 & ME & [1990JIM/ROU] \\
\hline & $\mathrm{V}$ & & $79.5 \pm 3.2$ & 298 & CGC & [2009LIP/CHI, 2009LIP/HAN] \\
\hline & $\mathrm{V}$ & $(373-673)$ & 73.4 & 400 & EB,IPM & {$[1992 \mathrm{STE} / \mathrm{CHI}]$} \\
\hline & $\mathrm{V}$ & $(373-673)$ & 70.5 & 440 & EB,IPM & [1992STE/CHI] \\
\hline & $\mathrm{V}$ & $(373-673)$ & 67.7 & 480 & EB,IPM & [1992STE/CHI] \\
\hline & $\mathrm{V}$ & $(373-673)$ & 65.0 & 520 & EB,IPM & [1992STE/CHI] \\
\hline & $\mathrm{V}$ & $(373-673)$ & 62.1 & 560 & EB,IPM & [1992STE/CHI] \\
\hline & $\mathrm{V}$ & $(373-673)$ & 59.1 & 600 & EB,IPM & [1992STE/CHI] \\
\hline & V & $(373-673)$ & 55.9 & 640 & $\mathrm{~EB}, \mathrm{IPM}$ & {$[1992 \mathrm{STE} / \mathrm{CHI}]$} \\
\hline \multirow[t]{5}{*}{$\mathrm{C}_{13} \mathrm{H}_{11} \mathrm{~N}$} & [153-78-6] & \multicolumn{5}{|c|}{ 2-aminofluorene } \\
\hline & FUS & & 23.81 & 400.9 & DSC & {$[2014 \mathrm{OLI} / \mathrm{MON}]$} \\
\hline & SUB & $(345-365)$ & $110.3 \pm 0.4$ & 355 & ME & [2014OLI/MON] \\
\hline & SUB & $(345-365)$ & $112.3 \pm 0.4$ & 298 & ME & [2014OLI/MON] \\
\hline & SUB & & $110.4 \pm 1.7$ & 298 & $\mathrm{C}$ & {$[2014 \mathrm{OLI} / \mathrm{MON}]$} \\
\hline \multirow[t]{2}{*}{$\mathrm{C}_{13} \mathrm{H}_{11} \mathrm{~N}$} & {$[5097-92-7]$} & \multicolumn{5}{|c|}{ cis-4-(2-phenylethyenyl)pyridine } \\
\hline & FUS & & 9.14 & 400.3 & $\mathrm{DSC}$ & [2007LIU/LIA] \\
\hline \multirow[t]{2}{*}{$\mathrm{C}_{13} \mathrm{H}_{11} \mathrm{NO}$} & {$[1137-96-8]$} & \multicolumn{5}{|c|}{$N$-phenylmethylene benzenamine $N$-oxide } \\
\hline & SUB & & $115.0 \pm 0.8$ & 298 & $\mathrm{C}$ & [1986KIR/ACR] \\
\hline \multirow[t]{3}{*}{$\mathrm{C}_{13} \mathrm{H}_{11} \mathrm{NO}$} & {$[779-84-0]$} & \multicolumn{5}{|c|}{ 2-hydroxybenzaldehyde $N$-phenylimine } \\
\hline & SUB & $(288-325)$ & 115.9 & 303 & A & [1987STE/MAL] \\
\hline & SUB & $(348-408)$ & 129.9 & 378 & & {$[1958 \mathrm{HOY} / \mathrm{PEP}]$} \\
\hline \multirow[t]{3}{*}{$\mathrm{C}_{13} \mathrm{H}_{11} \mathrm{NO}$} & {$[1689-73-2]$} & \multicolumn{5}{|c|}{ 4-hydroxybenzaldehyde $N$-phenylimine } \\
\hline & SUB & $(348-408)$ & 127.9 & 363 & A & [1987STE/MAL] \\
\hline & SUB & $(288-338)$ & 116 & 313 & & {$[1958 \mathrm{HOY} / \mathrm{PEP}]$} \\
\hline \multirow[t]{6}{*}{$\mathrm{C}_{13} \mathrm{H}_{11} \mathrm{NO}$} & {$[93-98-1]$} & Benzanilide & & & & \\
\hline & FUS & & 31.2 & 435.0 & DSC & [2012UMN/CHI] \\
\hline & FUS & & 32.4 & 436.3 & DSC & [2006MAT/MIR2] \\
\hline & FUS & & 29.61 & 436.5 & DTA & [1996DOM/HEA, 1992SAB/ELW3] \\
\hline & SUB & & $125.4 \pm 2.3$ & 298 & $\mathrm{C}$ & [2006MAT/MIR2] \\
\hline & SUB & $(352-369)$ & 99.2 & 360.5 & A & [1987STE/MAL, 1960AIH2] \\
\hline \multirow[t]{3}{*}{$\mathrm{C}_{13} \mathrm{H}_{11} \mathrm{NO}$} & {$[1137-41-3]$} & 4-aminobenzo & none & & & \\
\hline & FUS & & 21.2 & 395.2 & DTA & [2015NAT/USH] \\
\hline & V & & 56.4 & 578.2 & DTA,TGA & [2015NAT/USH] \\
\hline $\mathrm{C}_{13} \mathrm{H}_{11} \mathrm{NO}$ & {$[1207-72-3]$} & $N$-methylphen & liazine & & & \\
\hline & SUB & $(337-359)$ & $108.9 \pm 0.8$ & 298 & ME & [2015OLI/FRE] \\
\hline & SUB & & $103.6 \pm 2.3$ & 298 & $\mathrm{C}$ & [2015OLI/FRE] \\
\hline $\mathrm{C}_{13} \mathrm{H}_{11} \mathrm{NO}_{2}$ & {$[20357-59-9]$} & $N$-(2-hydroxy & nylmethylene & enzenan & $N$-oxide & \\
\hline & SUB & & $116.5 \pm 1.4$ & 298 & $\mathrm{C}$ & [1986KIR/ACR] \\
\hline $\mathrm{C}_{13} \mathrm{H}_{11} \mathrm{NO}_{2}$ & [91-40-7] & $N$-phenylanth & ilic acid & & & \\
\hline & FUS & & 39.7 & 458.2 & DSC & [2009SUR/TER, 2010SUR/PER, 2015SUR/SIM] \\
\hline
\end{tabular}


TABLE 11. Phase change enthalpies of $\mathrm{C}_{12}$ to $\mathrm{C}_{13}$ organic compounds-Continued

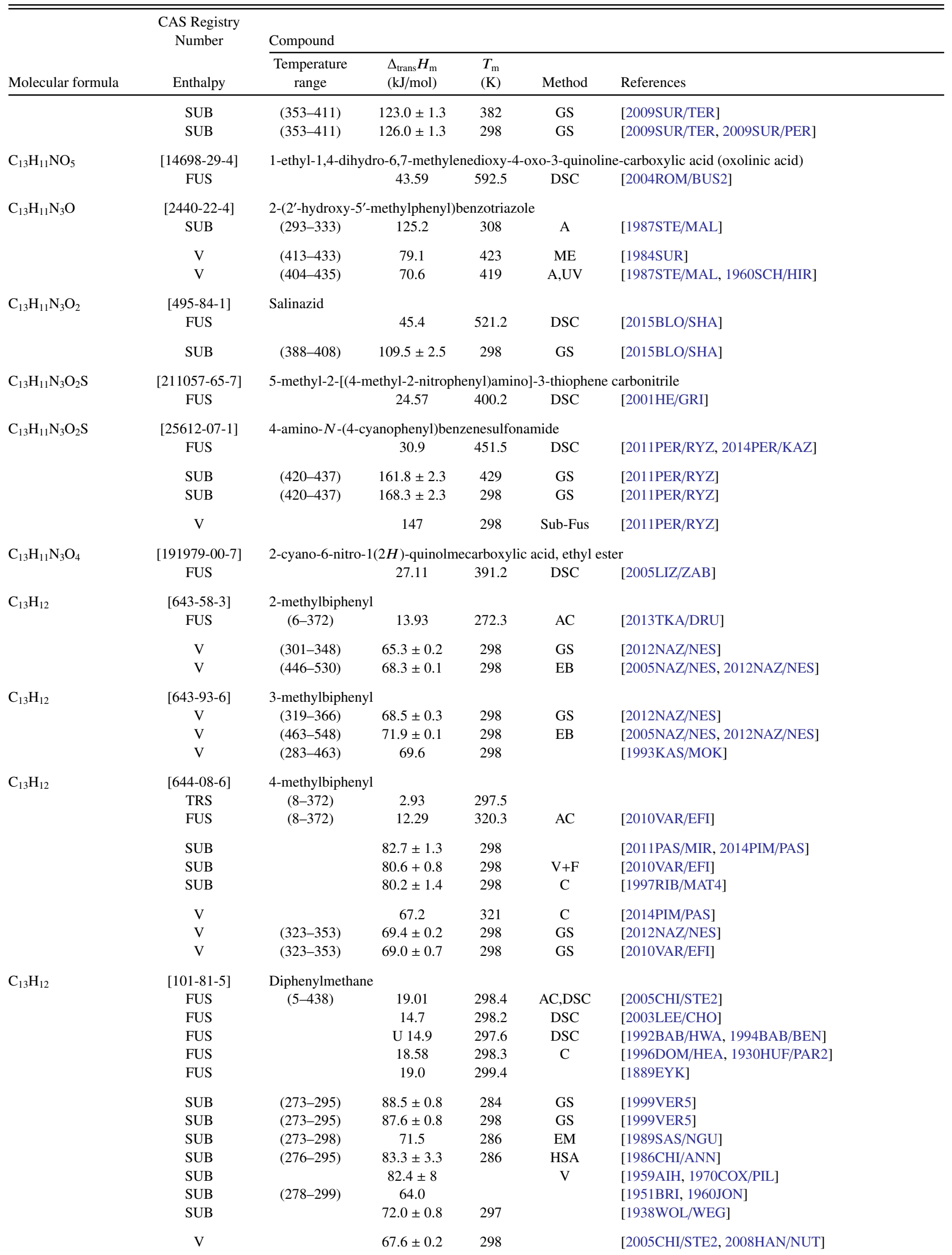


TABLE 11. Phase change enthalpies of $\mathrm{C}_{12}$ to $\mathrm{C}_{13}$ organic compounds-Continued

\begin{tabular}{|c|c|c|c|c|c|c|}
\hline \multirow[b]{2}{*}{ Molecular formula } & \multirow{2}{*}{$\begin{array}{l}\text { CAS Registry } \\
\text { Number } \\
\text { Enthalpy }\end{array}$} & \multicolumn{5}{|l|}{ Compound } \\
\hline & & $\begin{array}{c}\text { Temperature } \\
\text { range }\end{array}$ & $\begin{array}{l}\Delta_{\text {trans }} H_{\mathrm{m}} \\
(\mathrm{kJ} / \mathrm{mol})\end{array}$ & $\begin{array}{l}T_{\mathrm{m}} \\
(\mathrm{K})\end{array}$ & Method & References \\
\hline & $\mathrm{V}$ & $(343-393)$ & $64.7 \pm 0.2$ & 298 & & [2006HAF/PAR] \\
\hline & $\mathrm{V}$ & $(330-588)$ & $64.1 \pm 0.1$ & 340 & IPM,EB & [2005CHI/STE2] \\
\hline & $\mathrm{V}$ & $(330-588)$ & $61.0 \pm 0.1$ & 380 & IPM,EB & [2005CHI/STE2] \\
\hline & $\mathrm{V}$ & $(330-588)$ & $57.9 \pm 0.1$ & 420 & IPM,EB & [2005CHI/STE2] \\
\hline & $\mathrm{V}$ & $(330-588)$ & $55.0 \pm 0.1$ & 460 & IPM,EB & [2005CHI/STE2] \\
\hline & $\mathrm{V}$ & $(330-588)$ & $52.0 \pm 0.2$ & 500 & IPM,EB & [2005CHI/STE2] \\
\hline & $\mathrm{V}$ & $(330-588)$ & $48.9 \pm 0.3$ & 540 & IPM,EB & [2005CHI/STE2] \\
\hline & $\mathrm{V}$ & & 65.7 & 298 & GC & [2002VAN/PAR] \\
\hline & $\mathrm{V}$ & $(303-343)$ & $66.4 \pm 0.5$ & 323 & GS & [1999VER5] \\
\hline & V & $(303-343)$ & $67.9 \pm 0.5$ & 298 & GS & [1999VER5] \\
\hline & $\mathrm{V}$ & $(353-433)$ & 61.8 & 368 & & [1990SOH/OKA] \\
\hline & $\mathrm{V}$ & $(303-402)$ & 63.7 & 363 & & [1989SAS/NGU] \\
\hline & $\mathrm{V}$ & $(295-383)$ & 72.2 & 310 & A & [1987STE/MAL] \\
\hline & $\mathrm{V}$ & $(423-583)$ & 56.7 & 438 & A & [1987STE/MAL] \\
\hline & $\mathrm{V}$ & $(425-648)$ & 55.8 & 445 & & [1981WIE/KOB, 1980WIE/KOB] \\
\hline & $\mathrm{V}$ & $(425-648)$ & 49.0 & 535 & & [1981WIE/KOB, 1980WIE/KOB] \\
\hline & $\mathrm{V}$ & & $66.6 \pm 0.1$ & 298 & $\mathrm{C}$ & {$[1972 \mathrm{MOR}]$} \\
\hline & $\mathrm{V}$ & $(490-555)$ & 54.2 & 505 & & [1915CRA, 1984BOU/FRI] \\
\hline \multirow[t]{3}{*}{$\mathrm{C}_{13} \mathrm{H}_{12} \mathrm{ClNO}_{2} \mathrm{~S}$} & {$[16964-20-8]$} & \multicolumn{5}{|c|}{$N$-(4-methylphenyl) 3-chlorobenzenesulfonamide } \\
\hline & FUS (I) & & 26.44 & 366.8 & & \\
\hline & FUS (II) & & 18.16 & 364.8 & DSC & {$[2010 \mathrm{SAN} / \mathrm{SAR}]$} \\
\hline \multirow[t]{2}{*}{$\mathrm{C}_{13} \mathrm{H}_{12} \mathrm{ClN}_{3} \mathrm{~S}$} & [281212-47-3] & \multicolumn{5}{|c|}{$N$-2-(3-picolyl)- $N^{\prime}$-(2-chlorophenyl) thiourea } \\
\hline & FUS & & 11.2 & 400.2 & DSC & {$[2002 \mathrm{KEL} / \mathrm{SZC}]$} \\
\hline \multirow[t]{2}{*}{$\mathrm{C}_{13} \mathrm{H}_{12} \mathrm{ClN}_{3} \mathrm{~S}$} & {$[457886-93-0]$} & \multicolumn{5}{|c|}{$N$-2-(4-picolyl)- $N^{\prime}$-(2-chlorophenyl) thiourea } \\
\hline & FUS & & 44.5 & 441.2 & DSC & {$[2002 \mathrm{KEL} / \mathrm{SZC}]$} \\
\hline \multirow[t]{2}{*}{$\mathrm{C}_{13} \mathrm{H}_{12} \mathrm{ClN}_{3} \mathrm{~S}$} & {$[457886-96-3]$} & \multicolumn{5}{|c|}{$N$-2-(5-picolyl)- $N^{\prime}$-(2-chlorophenyl) thiourea } \\
\hline & FUS & & 24.2 & 460.2 & DSC & {$[2002 \mathrm{KEL} / \mathrm{SZC}]$} \\
\hline \multirow[t]{2}{*}{$\mathrm{C}_{13} \mathrm{H}_{12} \mathrm{ClN}_{3} \mathrm{~S}$} & {$[457886-94-1]$} & \multicolumn{5}{|c|}{$N$-2-(6-picolyl)- $N^{\prime}$-(2-chlorophenyl) thiourea } \\
\hline & FUS & & 27.3 & 449.7 & DSC & {$[2002 \mathrm{KEL} / \mathrm{SZC}]$} \\
\hline \multirow[t]{2}{*}{$\mathrm{C}_{13} \mathrm{H}_{12} \mathrm{ClN}_{3} \mathrm{~S}$} & {$[53385-87-8]$} & \multicolumn{5}{|c|}{$N$-2-(3-picolyl)- $N^{\prime}$-(4-chlorophenyl) thiourea } \\
\hline & FUS & & 16.8 & 391.2 & DSC & {$[2002 \mathrm{SZC} / \mathrm{KEL}]$} \\
\hline \multirow[t]{2}{*}{$\mathrm{C}_{13} \mathrm{H}_{12} \mathrm{ClN}_{3} \mathrm{~S}$} & {$[53385-88-9]$} & \multicolumn{5}{|c|}{$N$-2-(4-picolyl)- $N^{\prime}$-(4-chlorophenyl) thiourea } \\
\hline & FUS & & 35.2 & 460.2 & DSC & {$[2002 \mathrm{SZC} / \mathrm{KEL}]$} \\
\hline \multirow[t]{2}{*}{$\mathrm{C}_{13} \mathrm{H}_{12} \mathrm{ClN}_{3} \mathrm{~S}$} & {$[53385-89-0]$} & \multicolumn{5}{|c|}{$N$-2-(5-picolyl)- $N^{\prime}$-(4-chlorophenyl) thiourea } \\
\hline & FUS & & 51.1 & 473.7 & DSC & {$[2002 \mathrm{SZC} / \mathrm{KEL}]$} \\
\hline $\mathrm{C}_{13} \mathrm{H}_{12} \mathrm{ClN}_{3} \mathrm{~S}$ & {$[53385-90-3]$} & $N-2-(6-$ picoly & $N^{\prime}$-(4-chlorop & hyl) thio & & \\
\hline & FUS & & 40.1 & 464.2 & DSC & [2002SZC/KEL] \\
\hline $\mathrm{C}_{13} \mathrm{H}_{12} \mathrm{FNO}_{2} \mathrm{~S}$ & $\begin{array}{c}\text { [13198-87-3] } \\
\text { FUS }\end{array}$ & $N$-(3-fluoropl & l) 4-methylb & enesulfo & mide & \\
\hline & $\begin{array}{l}\text { FUS (I) } \\
\text { FUS (II) }\end{array}$ & & $\begin{array}{l}18.61 \\
18.83\end{array}$ & $\begin{array}{l}385.1 \\
384.4\end{array}$ & DSC & [2010SAN/SAR] \\
\hline $\mathrm{C}_{13} \mathrm{H}_{12} \mathrm{~F}_{2} \mathrm{~N}_{6} \mathrm{O}$ & {$[86386-73-4]$} & 2-(2,4-difluor & enyl)-1,3-bis( & $-1,2,4-\mathrm{t}$ & zol-1-yl)pro & in-2-ol (fluconazole) \\
\hline & FUS & & 37.76 & 413.25 & DSC & [2015PAT/PAT] \\
\hline $\mathrm{C}_{13} \mathrm{H}_{12} \mathrm{~N}_{2} \mathrm{O}$ & {$[102-07-8]$} & 1,3-diphenylu & & & & \\
\hline & FUS & & 37.7 & & DSC & {$[1995 \mathrm{STR} / \mathrm{ARG}]$} \\
\hline & FUS & & 34.62 & 512.1 & DSC & [1996DOM/HEA, 1991ACR, 1987FER/DEL] \\
\hline & SUB & $(445-484)$ & $152 \pm 6$ & & $\mathrm{TE}$ & {$[1987 \mathrm{FER} / \mathrm{DEL}]$} \\
\hline $\mathrm{C}_{13} \mathrm{H}_{12} \mathrm{~N}_{2} \mathrm{O}$ & {$[442-51-3]$} & 7-methoxy-1- & hyl-9H-pyric & $3,4-b]$ in & e (harmine) & \\
\hline & FUS & & 48.8 & 536.6 & DSC & [1996BUR/DAG] \\
\hline $\mathrm{C}_{13} \mathrm{H}_{12} \mathrm{~N}_{2} \mathrm{O}_{2}$ & [23042-34-4] & $N$-methyl- $N$ & biphenylyl)ni & nine & & \\
\hline & FUS & & 24.0 & 415.1 & $\mathrm{DSC}$ & [2002DAS/ZAL] \\
\hline $\mathrm{C}_{13} \mathrm{H}_{12} \mathrm{~N}_{2} \mathrm{O}_{2}$ & [17954-23-3] & 2-cyano-1(2H & uinolinecarbc & lic acid, & yl ester & \\
\hline & FUS & & 19.88 & 337 & DSC & [2005LIZ/ZAB] \\
\hline
\end{tabular}


TABLE 11. Phase change enthalpies of $\mathrm{C}_{12}$ to $\mathrm{C}_{13}$ organic compounds-Continued

\begin{tabular}{|c|c|c|c|c|c|c|}
\hline \multirow[b]{2}{*}{ Molecular formula } & \multirow{2}{*}{$\begin{array}{l}\text { CAS Registry } \\
\text { Number } \\
\text { Enthalpy }\end{array}$} & \multicolumn{5}{|l|}{ Compound } \\
\hline & & $\begin{array}{l}\text { Temperature } \\
\text { range }\end{array}$ & $\begin{array}{l}\Delta_{\text {trans }} H_{\mathrm{m}} \\
(\mathrm{kJ} / \mathrm{mol})\end{array}$ & $\begin{array}{l}T_{\mathrm{m}} \\
(\mathrm{K})\end{array}$ & Method & References \\
\hline \multirow{5}{*}{$\mathrm{C}_{13} \mathrm{H}_{12} \mathrm{~N}_{2} \mathrm{O}_{5} \mathrm{~S}$} & {$[51803-78-2]$} & \multicolumn{5}{|c|}{$N$-(4-nitro-2-phenoxyphenyl)methanesulfonamide (nimesulide) } \\
\hline & FUS & & 32.7 & 418.7 & DSC & [2011SAN/SAR] \\
\hline & FUS & & 47.4 & 422.2 & DSC & [2010DOM/POB] \\
\hline & FUS & & 33.38 & 423 & DSC & [2010BAI/VAN] \\
\hline & FUS & & 37.3 & 422.5 & $\mathrm{DSC}$ & [2007MON/PER] \\
\hline \multirow[t]{2}{*}{$\mathrm{C}_{13} \mathrm{H}_{12} \mathrm{~N}_{4} \mathrm{O}_{2}$} & & \multicolumn{5}{|c|}{ 4'-nitro-2-methylaminoazobenzene } \\
\hline & SUB & & 134.7 & & GS & [1987SHI/OHK, 1991HOR] \\
\hline \multirow[t]{2}{*}{$\mathrm{C}_{13} \mathrm{H}_{12} \mathrm{~N}_{4} \mathrm{O}_{5} \mathrm{~S}$} & {$[1169390-40-2]$} & \multicolumn{5}{|c|}{$N$-[3-[[(4-nitrophenyl)sulfonyl]amino]-4-pyridinyl]aceamide } \\
\hline & FUS & & 41.4 & 511.1 & DSC & {$[2014 \mathrm{PER} / \mathrm{KAZ}]$} \\
\hline $\mathrm{C}_{13} \mathrm{H}_{12} \mathrm{O}$ & $\begin{array}{c}\text { [946-80-5] } \\
\mathrm{V}\end{array}$ & \multicolumn{4}{|c|}{ Benzyl phenyl ether } & [1987STE/MAL, 1947STU] \\
\hline \multirow[t]{9}{*}{$\mathrm{C}_{13} \mathrm{H}_{12} \mathrm{O}$} & {$[91-01-0]$} & \multicolumn{5}{|c|}{ Diphenylmethanol } \\
\hline & FUS & & 23.7 & 339.4 & DSC & [2016DAV/GUE] \\
\hline & FUS & & 23.0 & 338.5 & DSC & [1996DOM/HEA, 1974CIN/BER] \\
\hline & SUB & $(302-317)$ & $103.4 \pm 1.0$ & 298 & ME & [2016DAV/GUE] \\
\hline & SUB & $(301-335)$ & $105.7 \pm 0.7$ & 298 & GS & [1998VER3] \\
\hline & SUB & $(301-335)$ & $104.5 \pm 0.7$ & 318 & GS & [1998VER3] \\
\hline & $\mathrm{V}$ & $(342-373)$ & $79.4 \pm 0.7$ & 358 & GS & [1998VER5] \\
\hline & $\mathrm{V}$ & $(342-373)$ & $83.0 \pm 0.7$ & 298 & GS & [1998VER5] \\
\hline & $\mathrm{V}$ & $(438-574)$ & 65.4 & 453 & A & [1987STE/MAL] \\
\hline \multirow[t]{2}{*}{$\mathrm{C}_{13} \mathrm{H}_{12} \mathrm{O}$} & {$[2876-63-3]$} & \multicolumn{5}{|c|}{ Ethyl 1-naphthyl ketone } \\
\hline & $\mathrm{V}$ & $(397-579)$ & 74.1 & 412 & A & [1987STE/MAL, 1947STU] \\
\hline \multirow[t]{4}{*}{$\mathrm{C}_{13} \mathrm{H}_{12} \mathrm{O}$} & {$[28994-41-4]$} & \multicolumn{5}{|c|}{ 2-(phenylmethyl)phenol (2-benzylphenol) } \\
\hline & FUS (I) & & 21.8 & 326.2 & & \\
\hline & FUS (II) & & 17.0 & 288.2 & DSC & [2004ROM/ROC] \\
\hline & FUS & & 23.4 & 325.7 & DSC & [1995MUR/PAI] \\
\hline \multirow[t]{2}{*}{$\mathrm{C}_{13} \mathrm{H}_{12} \mathrm{O}$} & {$[101-53-1]$} & \multicolumn{5}{|c|}{ 4-benzylphenol } \\
\hline & SUB & $(313-335)$ & 97.4 & 324 & A & [1987STE/MAL, 1960AIH] \\
\hline \multirow[t]{6}{*}{$\mathrm{C}_{13} \mathrm{H}_{12} \mathrm{O}$} & {$[2928-43-0]$} & 2-biphenylmet & 10l & & & \\
\hline & FUS & & 19.7 & 324 & DSC & [2007PIN/BER, 2006DIO/PIN] \\
\hline & FUS & & 18.5 & 326.8 & DSC & {$[2006 \mathrm{BAR} / \mathrm{DAV}]$} \\
\hline & SUB & & $106.0 \pm 1.1$ & 315 & $\mathrm{ME}$ & [2007PIN/BER] \\
\hline & SUB & & $107.1 \pm 1.1$ & 298 & $\mathrm{ME}$ & [2007PIN/BER] \\
\hline & $\mathrm{V}$ & & $85.6 \pm 0.6$ & 326 & $\mathrm{C}$ & [2007PIN/BER] \\
\hline $\mathrm{C}_{13} \mathrm{H}_{12} \mathrm{O}$ & {$[3597-91-9]$} & 4-biphenylmet & & & & \\
\hline & FUS & & 25.06 & 373 & DSC & {$[2010 \mathrm{BAI} / \mathrm{VAN}]$} \\
\hline & FUS & & 27.0 & 375.5 & DSC & [2007PIN/BER, 2006DIO/PIN] \\
\hline & SUB & & $105.7 \pm 1.8$ & 349 & $\mathrm{C}$ & [2007PIN/BER] \\
\hline & SUB & & $107.3 \pm 1.8$ & 298 & $\mathrm{C}$ & [2007PIN/BER] \\
\hline $\mathrm{C}_{13} \mathrm{H}_{12} \mathrm{OS}$ & {$[40932-63-6]$} & 3-acetyl-2-met & 1-5-phenylthio & ene & & \\
\hline & SUB & $(321-339)$ & $107.3 \pm 0.4$ & 330 & $\mathrm{ME}$ & [2010RIB/SAN] \\
\hline & SUB & (321-339) & $108.9 \pm 0.4$ & 298 & $\mathrm{ME}$ & [2010RIB/SAN] \\
\hline $\mathrm{C}_{13} \mathrm{H}_{12} \mathrm{O}_{2}$ & {$[103-16-2]$} & 4-benzyloxyph & & & & \\
\hline & SUB & $(347-369)$ & $128.9 \pm 0.6$ & 358 & ME & [2011RIB/FER3] \\
\hline & SUB & $(347-369)$ & $131.0 \pm 0.9$ & 298 & ME & [2011RIB/FER3] \\
\hline $\mathrm{C}_{13} \mathrm{H}_{12} \mathrm{O}_{2}$ & {$[620-92-8]$} & $4,4^{\prime}$-methylene & s(phenol) & & & \\
\hline & FUS & & 36.8 & 436.2 & DSC & {$[2014 \mathrm{COS} / \mathrm{DAV}]$} \\
\hline & SUB & $(375-399)$ & $140.8 \pm 0.6$ & 298 & $\mathrm{ME}$ & [2014DAV/HER, 2014COS/DAV] \\
\hline $\mathrm{C}_{13} \mathrm{H}_{12} \mathrm{~S}$ & {$[831-91-4]$} & Phenyl benzyl & fide & & & \\
\hline & SUB & & $98.4 \pm 1.4$ & 298 & $\mathrm{C}$ & [2006MUL/MOZ] \\
\hline
\end{tabular}


TABLE 11. Phase change enthalpies of $\mathrm{C}_{12}$ to $\mathrm{C}_{13}$ organic compounds-Continued

\begin{tabular}{|c|c|c|c|c|c|c|}
\hline \multirow[b]{2}{*}{ Molecular formula } & \multirow{2}{*}{$\begin{array}{l}\text { CAS Registry } \\
\text { Number } \\
\text { Enthalpy }\end{array}$} & \multicolumn{5}{|l|}{ Compound } \\
\hline & & $\begin{array}{l}\text { Temperature } \\
\text { range }\end{array}$ & $\begin{array}{l}\Delta_{\text {trans }} H_{\mathrm{m}} \\
(\mathrm{kJ} / \mathrm{mol})\end{array}$ & $\begin{array}{l}T_{\mathrm{m}} \\
(\mathrm{K})\end{array}$ & Method & References \\
\hline \multirow[t]{2}{*}{$\mathrm{C}_{13} \mathrm{H}_{13} \mathrm{BrS}$} & [148681-80-5] & \multicolumn{5}{|c|}{ 2-propyl-5-(4-bromophenyl)thiophene } \\
\hline & FUS & & 15.7 & 360.4 & DSC & [1993BRE/DUN] \\
\hline \multirow[t]{4}{*}{$\mathrm{C}_{13} \mathrm{H}_{13} \mathrm{ClN}_{2} \mathrm{O}_{2} \mathrm{~S}$} & {$[34392-72-8]$} & \multicolumn{5}{|c|}{ 4-amino- $N$-(5-chloro-2-methylphenyl)benzenesulfonamide } \\
\hline & FUS & & 36.8 & 422.7 & DSC & [2009PER/TKA, 2014PER/KAZ] \\
\hline & SUB & & $130 \pm 1$ & 298 & GS & [2009PER/TKA] \\
\hline & $\mathrm{V}$ & & 104 & 298 & $\mathrm{~S}-\mathrm{F}$ & [2009PER/TKA] \\
\hline \multirow[t]{3}{*}{$\mathrm{C}_{13} \mathrm{H}_{13} \mathrm{ClN}_{2} \mathrm{O}_{2} \mathrm{~S}$} & [952915-63-8] & \multicolumn{5}{|c|}{ 4-amino- $N$-(3-chloro-4-methylphenyl)benzenesulfonamide } \\
\hline & FUS & & 45.6 & 477.6 & DSC & [2013PER/RYZ, 2014PER/KAZ] \\
\hline & SUB & & $144.6 \pm 1.8$ & 298 & GS & [2013PER/RYZ] \\
\hline \multirow[t]{2}{*}{$\mathrm{C}_{13} \mathrm{H}_{13} \mathrm{ClN}_{2} \mathrm{O}_{2} \mathrm{~S}$} & {$[1039862-63-9]$} & \multicolumn{5}{|c|}{ 4-amino-(2-chloro-4-methylphenyl)benzene sulfonamide } \\
\hline & FUS & & 31.7 & 428.8 & DSC & [2014PER/KAZ] \\
\hline \multirow{2}{*}{$\mathrm{C}_{13} \mathrm{H}_{13} \mathrm{ClN}_{2} \mathrm{O}_{2} \mathrm{~S}$} & {$[1016780-94-1]$} & \multicolumn{5}{|c|}{ 4-amino-(2-chloro-4-methylphenyl)benzene sulfonamide } \\
\hline & FUS & & 36.8 & 422.7 & DSC & [2014PER/KAZ] \\
\hline \multirow[t]{2}{*}{$\mathrm{C}_{13} \mathrm{H}_{13} \mathrm{ClN}_{2} \mathrm{O}_{2} \mathrm{~S}$} & [1094869-61-0] & \multicolumn{5}{|c|}{ 4-amino-(4-chlorophenyl)-2-methylbenzene sulfonamide } \\
\hline & FUS & & 35.7 & 446.5 & DSC & [2014PER/KAZ] \\
\hline \multirow[t]{2}{*}{$\mathrm{C}_{13} \mathrm{H}_{13} \mathrm{ClN}_{2} \mathrm{O}_{2} \mathrm{~S}$} & {$[1094869-83-6]$} & \multicolumn{5}{|c|}{ 4-amino-(2-chlorophenyl)-2-methylbenzene sulfonamide } \\
\hline & FUS & & 47.3 & 479.5 & DSC & [2014PER/KAZ] \\
\hline \multirow[t]{3}{*}{$\mathrm{C}_{13} \mathrm{H}_{13} \mathrm{ClN}_{2} \mathrm{O}_{3} \mathrm{~S}$} & [1169390-41-3] & \multicolumn{5}{|c|}{ 4-amino- $N$-(3-chloro-2-methoxyphenyl)benzenesulfonamide } \\
\hline & FUS & & 30.7 & 403.9 & DSC & [2013PER/RYZ, 2014PER/KAZ] \\
\hline & SUB & & $147.2 \pm 1.5$ & 298 & GS & [2013PER/RYZ] \\
\hline \multirow[t]{2}{*}{$\mathrm{C}_{13} \mathrm{H}_{13} \mathrm{~N}$} & [552-82-9] & \multicolumn{5}{|c|}{$N$-methyldiphenylamine } \\
\hline & $\mathrm{V}$ & $(376-555)$ & 65.2 & 391 & A & [1987STE/MAL, 1947STU] \\
\hline \multirow[t]{7}{*}{$\mathrm{C}_{13} \mathrm{H}_{13} \mathrm{~N}$} & [103-32-2] & \multicolumn{5}{|c|}{$N$-benzylaniline } \\
\hline & FUS & & 20.08 & 313.9 & DSC & [1997VER] \\
\hline & FUS & & 16.76 & 305.6 & & [1991ACR, 1983WEA] \\
\hline & SUB & $(293-312)$ & $103.6 \pm 1.6$ & 303 & GS & [1997VER] \\
\hline & SUB & & 51.3 & & & [1980AIH, 1997VER] \\
\hline & V & $(316-343)$ & $79.6 \pm 1.1$ & 330 & GS & [1997VER] \\
\hline & $\mathrm{V}$ & & 79.5 & & & [1980AIH] \\
\hline
\end{tabular}

[Note: The value reported in [1980AIH] for the enthalpy of sublimation is smaller than the value given for the enthalpy of vaporization. The author of [1980AIH] noted the anomalous behavior.]

\begin{tabular}{|c|c|c|c|c|c|}
\hline \multirow[t]{2}{*}{$\mathrm{C}_{13} \mathrm{H}_{13} \mathrm{NO}$} & {$[3449-48-7]$} & \multicolumn{2}{|c|}{ 1-keto-1,2,3,4-tetrahydro-6-methylcarbazole } & \multirow[b]{2}{*}{ DSC } & \multirow[b]{2}{*}[2006\mathrm{COR}/\mathrm{LOP}]{} \\
\hline & FUS & 26.9 & 468.5 & & \\
\hline \multirow[t]{2}{*}{$\mathrm{C}_{13} \mathrm{H}_{13} \mathrm{NO}$} & {$[25363-55-7]$} & \multicolumn{2}{|c|}{ 2-(4-methoxyphenyl)-5-methylpyridine } & & \\
\hline & FUS & 20.0 & 328 & DSC & [2000MOR/HAR] \\
\hline \multirow[t]{3}{*}{$\mathrm{C}_{13} \mathrm{H}_{13} \mathrm{NO}$} & {$[83-18-1]$} & \multicolumn{3}{|c|}{ 2,5-dimethyl-1-phenyl-3-pyrrolecarboxaldehyde } & \\
\hline & SUB & $\begin{array}{ll}(326-348) & 106.0 \pm 0.4\end{array}$ & 337 & $\mathrm{ME}$ & [2013SAN/RIB3] \\
\hline & SUB & $(326-348)$ & 298 & ME & [2013SAN/RIB3] \\
\hline \multirow[t]{2}{*}{$\mathrm{C}_{13} \mathrm{H}_{13} \mathrm{NO}_{2}$} & & \multicolumn{3}{|c|}{$(d l)$-2-(1-naphthoxy)propionamide } & \\
\hline & FUS & 37.66 & 445 & DSC & {$[1976 \mathrm{LEC} / \mathrm{COL}]$} \\
\hline \multirow[t]{2}{*}{$\mathrm{C}_{13} \mathrm{H}_{13} \mathrm{NO}_{2}$} & & \multicolumn{3}{|c|}{ (d)-2-(1-naphthoxy)propionamide } & \\
\hline & FUS & 38.07 & 475 & DSC & {$[1976 \mathrm{LEC} / \mathrm{COL}]$} \\
\hline \multirow[t]{2}{*}{$\mathrm{C}_{13} \mathrm{H}_{13} \mathrm{~N}_{3}$} & {$[102-06-7]$} & 1,3-diphenylguanidine & & & \\
\hline & FUS & 22.52 & 421.15 & DSC & [2016XU/WAN2] \\
\hline \multirow{2}{*}{$\mathrm{C}_{13} \mathrm{H}_{13} \mathrm{~N}_{3} \mathrm{O}$} & {$[13256-75-2]$} & \multicolumn{3}{|c|}{$N$-(4-methylphenyl)- $N^{\prime}$-(2-pyridyl) urea } & \\
\hline & FUS & 204.45 & 447 & DSC & [2002LU/SON, 2004SON/TAN] \\
\hline
\end{tabular}

[Note: The value is too large. The compound may have decomposed upon melting, or there is a decimal place error in the numerical value.]

$\mathrm{C}_{13} \mathrm{H}_{13} \mathrm{~N}_{3} \mathrm{OS} \quad$ [1187083-48-2] 1-(3-methylsulfanylphenyl)-3-pyridin-2-ylurea

FUS (I) $\quad 11.1 \quad 413.2$ 
TABle 11. Phase change enthalpies of $\mathrm{C}_{12}$ to $\mathrm{C}_{13}$ organic compounds-Continued

\begin{tabular}{|c|c|c|c|c|c|c|}
\hline \multirow[b]{2}{*}{ Molecular formula } & \multirow{2}{*}{$\begin{array}{l}\text { CAS Registry } \\
\text { Number } \\
\text { Enthalpy }\end{array}$} & \multicolumn{5}{|l|}{ Compound } \\
\hline & & $\begin{array}{c}\text { Temperature } \\
\text { range }\end{array}$ & $\begin{array}{l}\Delta_{\text {trans }} H_{\mathrm{m}} \\
(\mathrm{kJ} / \mathrm{mol})\end{array}$ & $\begin{array}{l}T_{\mathrm{m}} \\
(\mathrm{K})\end{array}$ & Method & References \\
\hline & FUS (II) & & 26.3 & 417.2 & & \\
\hline & FUS (III) & & 23.0 & 413.2 & & \\
\hline & FUS (IV) & & 34.5 & 426.2 & $\mathrm{DSC}$ & [2010FUC/QUR] \\
\hline \multirow[t]{2}{*}{$\mathrm{C}_{13} \mathrm{H}_{13} \mathrm{~N}_{3} \mathrm{O}_{4} \mathrm{~S}$} & {$[1169390-42-4]$} & \multicolumn{5}{|c|}{ 4-amino-2-methyl- $N$-(2-nitrophenyl)benzene sulfonamide } \\
\hline & FUS & & 40.0 & 451.4 & DSC & [2014PER/KAZ] \\
\hline \multirow[t]{2}{*}{$\mathrm{C}_{13} \mathrm{H}_{13} \mathrm{~N}_{3} \mathrm{~S}$} & {$[53385-83-4]$} & \multicolumn{5}{|c|}{$N$-2-(6-picolyl)- $N^{\prime}$-phenylthiourea } \\
\hline & FUS & & 43.5 & 460.7 & DSC & [2002VAL/HER] \\
\hline \multirow[t]{2}{*}{$\mathrm{C}_{13} \mathrm{H}_{13} \mathrm{OP}$} & [2129-89-7] & \multicolumn{5}{|c|}{ Methyldiphenylphosphine oxide } \\
\hline & FUS & & 20.37 & 385.4 & DSC & [2010HU/WAN] \\
\hline \multirow[t]{2}{*}{$\mathrm{C}_{13} \mathrm{H}_{14}$} & {$[2245-38-7]$} & \multicolumn{5}{|c|}{ 1,6,7-trimethylnaphthalene } \\
\hline & $\mathrm{V}$ & $(323-473)$ & 68.6 & 398 & GC & [2002LEI/CHA] \\
\hline \multirow[t]{2}{*}{$\mathrm{C}_{13} \mathrm{H}_{14}$} & {$[6158-45-8]$} & \multicolumn{5}{|c|}{ 1-isopropylnaphthalene } \\
\hline & $\mathrm{V}$ & $(402-541)$ & 50.4 & 417 & A & [1987STE/MAL] \\
\hline \multirow[t]{2}{*}{$\mathrm{C}_{13} \mathrm{H}_{14}$} & {$[2027-17-0]$} & \multicolumn{5}{|c|}{ 2-isopropylnaphthalene } \\
\hline & $\mathrm{V}$ & $(402-541)$ & 60.3 & 417 & A & [1987STE/MAL] \\
\hline \multirow[t]{2}{*}{$\mathrm{C}_{13} \mathrm{H}_{14}$} & & \multicolumn{5}{|c|}{ Isopropylnaphthalene } \\
\hline & $\mathrm{V}$ & $(426-454)$ & 52.8 & 440 & EB & [1974MAN/LOG] \\
\hline
\end{tabular}

[Note: The authors do not specify which isomer; however they report a boiling point temperature of $268.2^{\circ} \mathrm{C}$ for the compound.]

\begin{tabular}{|c|c|c|c|c|c|c|}
\hline \multirow[t]{2}{*}{$\mathrm{C}_{13} \mathrm{H}_{14} \mathrm{ClN}_{5}$} & \multirow{2}{*}{$\begin{array}{c}\text { [1449745-82-7] } \\
\text { FUS }\end{array}$} & \multicolumn{4}{|c|}{ 2-butanolypyridine 6'-chloro-4'-pyrimidinylhydrazone } & \multirow[b]{2}{*}{ [2013PER/KAZ] } \\
\hline & & & 37 & 384.4 & DSC & \\
\hline \multirow[t]{4}{*}{$\mathrm{C}_{13} \mathrm{H}_{14} \mathrm{Cl}_{2} \mathrm{~N}_{2} \mathrm{~S}$} & [1343440-37-8] & \multicolumn{5}{|c|}{ (3,4-dichlorophenyl)-[3-thia-1-azabicyclo[3.3.1]non-2-ylidene]amine } \\
\hline & FUS & & 23.5 & 370.9 & DSC & [2014BLO/OLK] \\
\hline & SUB & $(352-363)$ & $122.2 \pm 1.1$ & 357 & GS & [2014BLO/OLK] \\
\hline & SUB & $(352-363)$ & $125.0 \pm 1.1$ & 298 & GS & [2014BLO/OLK] \\
\hline \multirow[t]{2}{*}{$\mathrm{C}_{13} \mathrm{H}_{14} \mathrm{~N}_{2}$} & [1208-52-2] & \multicolumn{2}{|c|}{ 2,4'-diaminodiphenylmethane } & & & \\
\hline & $\mathrm{V}$ & $(353-403)$ & 111.5 & 368 & A & [1987STE/MAL] \\
\hline \multirow[t]{6}{*}{$\mathrm{C}_{13} \mathrm{H}_{14} \mathrm{~N}_{2}$} & {$[101-77-9]$} & \multicolumn{5}{|c|}{ 4,4'-diaminodiphenylmethane } \\
\hline & FUS & & 19.69 & 362.7 & DSC & [2006KHI/DAH] \\
\hline & FUS & & 9.23 & 363.7 & & [1996DOM/HEA, 1978MAR/CIO2] \\
\hline & $\mathrm{V}$ & $(343-393)$ & 109.3 & 358 & A & [1987STE/MAL] \\
\hline & V & $(486-545)$ & 98.0 & 501 & A & [1987STE/MAL] \\
\hline & $\mathrm{V}$ & $(471-545)$ & 100.6 & 502 & A & [1966ZAL/STR] \\
\hline \multirow[t]{2}{*}{$\mathrm{C}_{13} \mathrm{H}_{14} \mathrm{~N}_{2}$} & {$[6582-52-1]$} & \multicolumn{2}{|c|}{ 2,2'-diaminodiphenylmethane } & & & \\
\hline & SUB & $(343-403)$ & 111.3 & 358 & A & [1987STE/MAL] \\
\hline \multirow[t]{4}{*}{$\mathrm{C}_{13} \mathrm{H}_{14} \mathrm{~N}_{2} \mathrm{O}_{3} \mathrm{~S}$} & [19837-74-2] & \multicolumn{5}{|c|}{ 4-amino- $N$-(4-methoxyphenyl)benzenesulfonamide } \\
\hline & FUS & & 38.6 & 467.4 & DSC & [2009PER/TKA, 2014PER/KAZ] \\
\hline & SUB & & $124 \pm 1$ & 298 & GS & [2009PER/TKA] \\
\hline & $\mathrm{V}$ & & 99.4 & 298 & $\mathrm{~S}-\mathrm{F}$ & [2009PER/TKA] \\
\hline \multirow[t]{2}{*}{$\mathrm{C}_{13} \mathrm{H}_{14} \mathrm{O}_{3}$} & {$[36112-95-5]$} & \multicolumn{4}{|c|}{ 3-(1-naphthaleneyloxy)-1,2-propanediol } & \\
\hline & FUS & & 32.2 & 370.1 & DSC & [2013BRE/GUB] \\
\hline \multirow[t]{2}{*}{$\mathrm{C}_{13} \mathrm{H}_{14} \mathrm{O}_{3}$} & {$[56715-19-6]$} & \multicolumn{4}{|c|}{ (S)-3-(1-naphthaleneyloxy)-1,2-propanediol } & \\
\hline & FUS & & 39.0 & 383.9 & DSC & [2013BRE/GUB] \\
\hline \multirow[t]{2}{*}{$\mathrm{C}_{13} \mathrm{H}_{15} \mathrm{BrN}_{4} \mathrm{O}_{2}$} & {$[56518-41-3]$} & \multicolumn{5}{|c|}{ 5-[(4-bromo-3,5-dimethoxyphenyl)methyl]-2,4-pyrimidinediamine } \\
\hline & FUS & & 49.9 & 505.4 & DSC & [2007CAI/BET] \\
\hline \multirow[t]{4}{*}{$\mathrm{C}_{13} \mathrm{H}_{15} \mathrm{CIN}_{2} \mathrm{~S}$} & [1583299-21-1] & \multicolumn{5}{|c|}{ (4-chlorophenyl)-[3-thia-1-azabicyclo[3.3.1]non-2-ylidene]amine } \\
\hline & FUS & & 11.8 & 411.8 & DSC & [2014BLO/OLK] \\
\hline & SUB & $(368-391)$ & $133.1 \pm 1.2$ & 379 & GS & [2014BLO/OLK] \\
\hline & SUB & $(368-391)$ & $136.7 \pm 1.2$ & 298 & GS & [2014BLO/OLK] \\
\hline $\mathrm{C}_{13} \mathrm{H}_{15} \mathrm{CI}_{2} \mathrm{~N}_{3}$ & {$[66246-88-6]$} & \multicolumn{5}{|c|}{ 1-[2-(2,4-dichlorophenyl)pentyl]-1H-1,2,4-triazole (penconazole) } \\
\hline
\end{tabular}


TABLE 11. Phase change enthalpies of $\mathrm{C}_{12}$ to $\mathrm{C}_{13}$ organic compounds-Continued

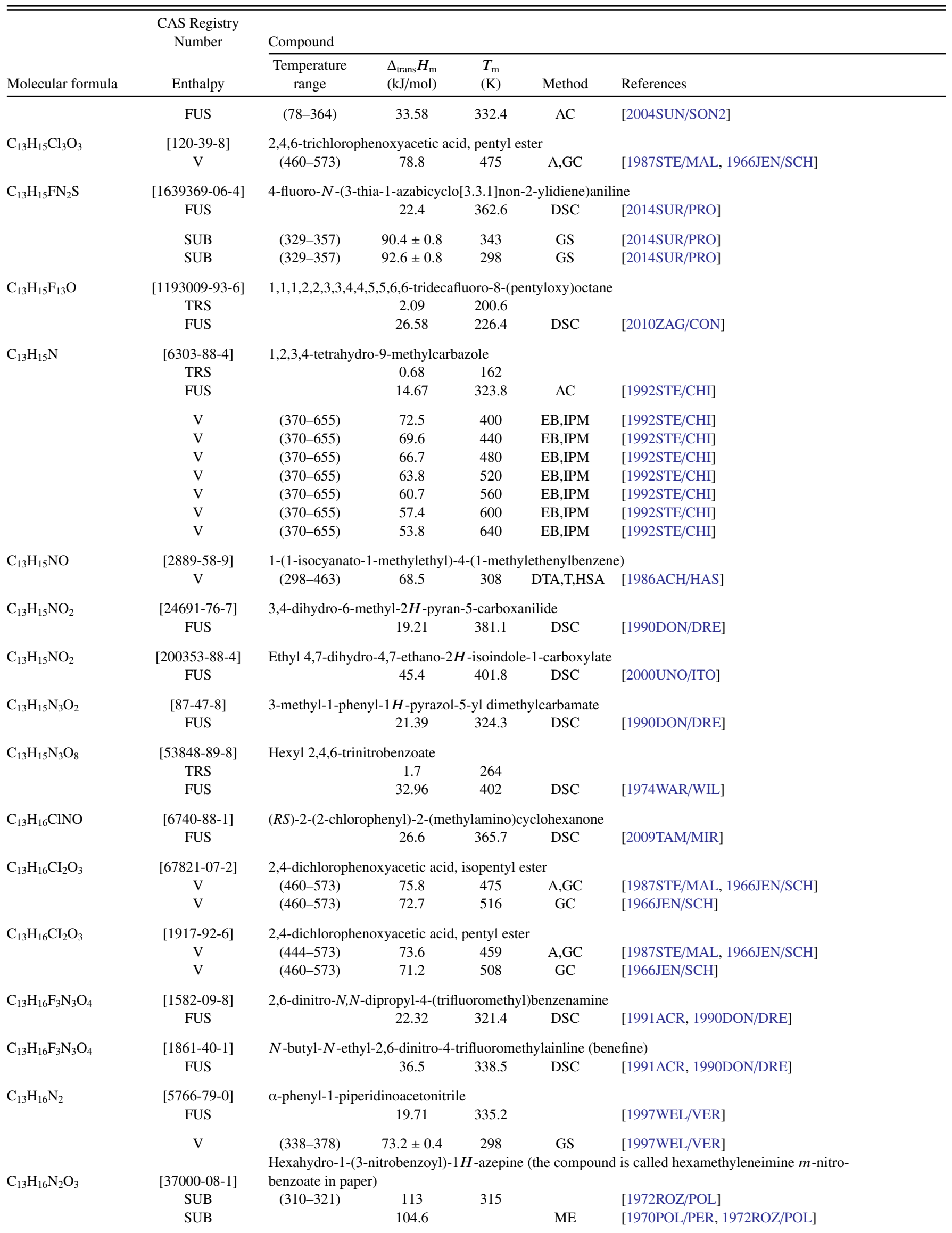


TABLE 11. Phase change enthalpies of $\mathrm{C}_{12}$ to $\mathrm{C}_{13}$ organic compounds-Continued

\begin{tabular}{|c|c|c|c|c|c|c|}
\hline \multirow[b]{2}{*}{ Molecular formula } & \multirow{2}{*}{$\begin{array}{l}\text { CAS Registry } \\
\text { Number } \\
\text { Enthalpy }\end{array}$} & \multicolumn{5}{|l|}{ Compound } \\
\hline & & $\begin{array}{c}\text { Temperature } \\
\text { range }\end{array}$ & $\begin{array}{l}\Delta_{\text {trans }} H_{\mathrm{m}} \\
(\mathrm{kJ} / \mathrm{mol})\end{array}$ & $\begin{array}{l}T_{\mathrm{m}} \\
(\mathrm{K})\end{array}$ & Method & References \\
\hline \multirow[t]{2}{*}{$\mathrm{C}_{13} \mathrm{H}_{16} \mathrm{~N}_{2} \mathrm{O}_{6}$} & [159432-36-7] & \multicolumn{5}{|c|}{ 2,3-dihydro-7-(1-methylethoxy)-3-[2-(nitrooxy)ethyl]-4H-1,3-benzoxazin-4-one } \\
\hline & FUS & & 32.1 & 344.7 & DSC & [1996FON/ROS] \\
\hline \multirow[t]{2}{*}{$\mathrm{C}_{13} \mathrm{H}_{16} \mathrm{O}_{4}$} & {$[7479-28-9]$} & \multicolumn{5}{|c|}{ 2,3,5-trimethyl-1,4-diacetoxybenzene } \\
\hline & FUS & & 22.32 & 381.6 & DSC & [2013YAN/YIN] \\
\hline \multirow[t]{2}{*}{$\mathrm{C}_{13} \mathrm{H}_{17} \mathrm{ClN}_{2} \mathrm{O}_{4}$} & {$[138517-06-3]$} & \multicolumn{5}{|c|}{ (4-nitrophenyl)-6-chlorohexyl carbamate } \\
\hline & FUS & & 30.31 & 360.8 & DSC & [1993TIE/FRA] \\
\hline \multirow[t]{2}{*}{$\mathrm{C}_{13} \mathrm{H}_{17} \mathrm{~N}$} & {$[14611-51-9]$} & \multicolumn{5}{|c|}{$(R)-N$-methyl- $N$-(1-phenylpropan-2-yl)prop-1-yn-3-amine $((R)$-deprenyl) } \\
\hline & V & & $64.3 \pm 2.2$ & 298 & CGC & {$[2014 \mathrm{GOB} / \mathrm{VIK}]$} \\
\hline \multirow[t]{3}{*}{$\mathrm{C}_{13} \mathrm{H}_{17} \mathrm{NO}$} & {$[3626-62-8]$} & \multicolumn{5}{|c|}{ 1-(phenacyl)piperidine } \\
\hline & V & $(381-446)$ & 51.4 & 396 & A & [1987STE/MAL, 1969DAV/MAK, 1968DAV/BAT] \\
\hline & $\mathrm{V}$ & $(382-450)$ & 47.2 & 416 & Static & {$[1969 \mathrm{DAV} / \mathrm{MAK}]$} \\
\hline \multirow{2}{*}{$\mathrm{C}_{13} \mathrm{H}_{17} \mathrm{NO}$} & {$[13290-48-7]$} & \multicolumn{5}{|c|}{ 1-(m-toluoyl)piperidine } \\
\hline & $\mathrm{V}$ & $(373-403)$ & 53.8 & 388 & A & [1987STE/MAL, 1969DAV/MAK, 1968DAV/BAT] \\
\hline \multirow{2}{*}{$\mathrm{C}_{13} \mathrm{H}_{17} \mathrm{NO}_{3}$} & [4134-09-2] & \multicolumn{5}{|c|}{$(d l)-N$-acetylphenylalanine, ether ester } \\
\hline & V & $(438-528)$ & 82.4 & 453 & A & [1987STE/MAL] \\
\hline \multirow[t]{2}{*}{$\mathrm{C}_{13} \mathrm{H}_{17} \mathrm{NO}_{3}$} & & \multicolumn{5}{|c|}{ Morpholine cinnamate } \\
\hline & SUB & $(298-349)$ & 118.8 & 313 & A & [1987STE/MAL] \\
\hline \multirow[t]{3}{*}{$\mathrm{C}_{13} \mathrm{H}_{17} \mathrm{~N}_{3} \mathrm{O}_{2} \mathrm{~S}$} & {$[1245618-42-1]$} & 1-[5-(4-ethoxy & nylamino)-1 & -thiadia & 1-3-yl]-2-pro & anol \\
\hline & FUS & & 27.5 & 385.1 & DSC & [2010PER/VOL] \\
\hline & SUB & $(343-361)$ & $152.8 \pm 2.2$ & 298 & GS & [2010PER/VOL] \\
\hline $\mathrm{C}_{13} \mathrm{H}_{18}$ & [941-60-6] & 1,1,4,6-tetrame & lindane & & & \\
\hline & FUS & $(12-376)$ & 15.74 & 273.6 & $\mathrm{AC}$ & [1991ACR, 1981LEE/FIN] \\
\hline & V & $(313-383)$ & 59.4 & 328 & $\mathrm{~A}, \mathrm{~EB}, \mathrm{IPM}$ & [1987STE/MAL, 1978OSB/SCO] \\
\hline & $\mathrm{V}$ & $(313-469)$ & 60.2 & 328 & $\mathrm{~A}, \mathrm{~EB}, \mathrm{IPM}$ & [1987STE/MAL, 1978OSB/SCO] \\
\hline & $\mathrm{V}$ & $(423-469)$ & 51.9 & 439 & A,EB,IPM & [1987STE/MAL, 1978OSB/SCO] \\
\hline & $\mathrm{V}$ & $(313-469)$ & $61.4 \pm 0.5$ & 298 & EB,IP & {$[1978 \mathrm{OSB} / \mathrm{SCO}]$} \\
\hline $\mathrm{C}_{13} \mathrm{H}_{18}$ & [1078-04-2] & 1,1,4,7-tetrame & lindane & & & \\
\hline & FUS & & 11.28 & 245.6 & $\mathrm{AC}$ & [1991ACR, 1981LEE/FIN] \\
\hline & $\mathrm{V}$ & $(313-388)$ & 59.6 & 328 & A,EB,IPM & [1987STE/MAL, 1978OSB/SCO] \\
\hline & $\mathrm{V}$ & $(313-469)$ & 60.4 & 328 & A,EB,IPM & [1987STE/MAL, 1978OSB/SCO] \\
\hline & V & $(431-469)$ & 52.0 & 446 & A,EB,IPM & [1987STE/MAL, 1978OSB/SCO] \\
\hline & $\mathrm{V}$ & $(313-469)$ & $61.4 \pm 0.6$ & 298 & EB,IP & {$[1978 \mathrm{OSB} / \mathrm{SCO}]$} \\
\hline $\mathrm{C}_{13} \mathrm{H}_{18} \mathrm{Br}_{2} \mathrm{~N}_{2} \mathrm{O}$ & [18683-91-5] & trans-4-[[(2-an & -3,5-dibrom & nenyl)m & tyl]amino]cy & ohexanol (ambroxol) \\
\hline & FUS (I) & & 31.46 & 372.7 & & \\
\hline & FUS (II) & & 36.52 & 365.6 & DSC & [2004CAI/FOP] \\
\hline $\mathrm{C}_{13} \mathrm{H}_{18} \mathrm{ClNO}$ & {$[7287-36-7]$} & $N$-(4-chloroph & 1)-2,2-dimet & pentana & & \\
\hline & FUS & & 23.31 & 360.2 & DSC & [1990DON/DRE] \\
\hline $\mathrm{C}_{13} \mathrm{H}_{18} \mathrm{ClNO}$ & {$[2307-68-8]$} & $N$-(3-chloro-4 & thylphenyl)- & nethylpe & anamide & \\
\hline & FUS & & 16.35 & 353.2 & DSC & [1990DON/DRE] \\
\hline $\mathrm{C}_{13} \mathrm{H}_{18} \mathrm{~N}_{2} \mathrm{O}_{2}$ & [2164-08-1] & 3-cyclohexyl-6 & lihydro-1 $H$ - & lopenta & rimidine-2,4 & $3 H, 5 H$ )-dione \\
\hline & FUS & & 42.31 & 584.3 & DSC & [1990DON/DRE] \\
\hline $\mathrm{C}_{13} \mathrm{H}_{18} \mathrm{~N}_{2} \mathrm{O}_{2} \mathrm{~S}_{2}$ & & $N$-allyl-S-ethy & $I^{\prime}$-tosylisothi & & & \\
\hline & FUS & & 28.0 & 335.2 & DSC & [1992REI/HAN] \\
\hline $\mathrm{C}_{13} \mathrm{H}_{18} \mathrm{~N}_{2} \mathrm{O}_{3}$ & [509-86-4] & Heptabarbiton & & & & \\
\hline & FUS & & 38.6 & & DSC & [1982TRE/VAU] \\
\hline $\mathrm{C}_{13} \mathrm{H}_{18} \mathrm{~N}_{2} \mathrm{O}_{4}$ & [87458-01-3] & hexyl $N$-(4-nit & henyl) carba & & & \\
\hline & FUS & & 32.74 & 376.7 & DSC & [1993TIE/FRA] \\
\hline $\mathrm{C}_{13} \mathrm{H}_{18} \mathrm{~N}_{4} \mathrm{O}_{3}$ & [6493-05-6] & 1-(5-oxohexyl) & 7-dimethylxa & ine (per & xifylline) & \\
\hline & FUS & & 36.6 & 376.8 & DSC & [2009DOM/POB] \\
\hline
\end{tabular}


TABLE 11. Phase change enthalpies of $\mathrm{C}_{12}$ to $\mathrm{C}_{13}$ organic compounds-Continued

\begin{tabular}{|c|c|c|c|c|c|c|}
\hline \multirow[b]{2}{*}{ Molecular formula } & \multirow{2}{*}{$\begin{array}{l}\text { CAS Registry } \\
\text { Number } \\
\text { Enthalpy }\end{array}$} & \multicolumn{5}{|l|}{ Compound } \\
\hline & & $\begin{array}{l}\text { Temperature } \\
\text { range }\end{array}$ & $\begin{array}{l}\Delta_{\text {trans }} H_{\mathrm{m}} \\
(\mathrm{kJ} / \mathrm{mol})\end{array}$ & $\begin{array}{l}T_{\mathrm{m}} \\
(\mathrm{K})\end{array}$ & Method & References \\
\hline \multirow[t]{2}{*}{$\mathrm{C}_{13} \mathrm{H}_{18} \mathrm{O}$} & [5195-24-4] & \multicolumn{5}{|c|}{ 4,4-dimethyl-1-phenyl-3-pentanone } \\
\hline & $\mathrm{V}$ & $(405-520)$ & 63.5 & 420 & A & [1987STE/MAL] \\
\hline \multirow[t]{2}{*}{$\mathrm{C}_{13} \mathrm{H}_{18} \mathrm{O}$} & [103-95-7] & \multicolumn{5}{|c|}{$p$-isopropyl- $\alpha$-methylhydrocinnamaldehyde } \\
\hline & $\mathrm{V}$ & $(283-499)$ & 72.6 & 298 & A & {$[1987 \mathrm{STE} / \mathrm{MAL}]$} \\
\hline \multirow[t]{2}{*}{$\mathrm{C}_{13} \mathrm{H}_{18} \mathrm{O}$} & {$[1671-75-6]$} & \multicolumn{5}{|c|}{ 1-phenyl-1-heptanone } \\
\hline & $\mathrm{V}$ & $(373-550)$ & 64.6 & 388 & A & [1987STE/MAL, 1947STU] \\
\hline \multirow[t]{5}{*}{$\mathrm{C}_{13} \mathrm{H}_{18} \mathrm{O}_{2}$} & {$[21643-38-9]$} & \multicolumn{5}{|c|}{ 4-hexylbenzoic acid } \\
\hline & TRS (liq cryst) & & 17.4 & 371 & & \\
\hline & TRS (liq & & 2.4 & 380 & DSC & [1985PRI/PUC] \\
\hline & \multicolumn{6}{|l|}{ cryst-to-liq) } \\
\hline & SUB & $(347-363)$ & $123.6 \pm 1.0$ & 298 & $\mathrm{ME}$ & [2004MON/ALM] \\
\hline \multirow[t]{2}{*}{$\mathrm{C}_{13} \mathrm{H}_{18} \mathrm{O}_{2}$} & {$[15687-27-1]$} & \multicolumn{5}{|c|}{ ( \pm ) $\alpha$-methyl-4-(isobutyl)phenylacetic acid (ibuprofen) } \\
\hline & FUS & & 29.77 & 353.3 & DSC & [2016ABI/ARM] \\
\hline \multicolumn{7}{|c|}{ [Note: The authors of [2016ABI/ARM] report an enthalpy of fusion of $29.77 \mathrm{~J} / \mathrm{g}$, which we have taken to $29.77 \mathrm{~kJ} / \mathrm{mol}$.] } \\
\hline & FUS & & 24.7 & 352.2 & DSC & [2015NOK/HOM] \\
\hline & FUS & & 26.2 & 348.3 & DSC & [2013YUA/CAP] \\
\hline & FUS & & 25.69 & 347.5 & DSC & [2013BOU/TEY] \\
\hline & FUS (I) & & 25.8 & 349 & & \\
\hline & FUS (II) & & 7.0 & 290 & DSC & [2013DUD/COR, 2008DUD/DAN] \\
\hline \multicolumn{7}{|c|}{$\begin{array}{l}\text { [Note: The authors of [2008DUD/DAN] report that phase II melts at } 290 \mathrm{~K} \text {, recrystallizes to phase I at } 303 \mathrm{~K} \text {, and then at } 348 \mathrm{~K} \text { phase I } \\
\text { melts.] }\end{array}$} \\
\hline & FUS & & 26.3 & 349.5 & DSC & [2010ELK/ESS] \\
\hline [Note: The authors & 2010ELK/ESS] re & ort that the entha & of fusion dec & ases sig & cantly witl & creasing crystallinity.] \\
\hline & FUS & & 25.3 & 347.6 & DSC & [2010YUA/CAP] \\
\hline & FUS & & 28.92 & 347.4 & DSC & [2010MIY/KHA] \\
\hline & FUS & & 26.6 & 352.2 & DSC & [2010NOK/AMI] \\
\hline & FUS & & 25.99 & 348.2 & $\mathrm{DSC}$ & [2010KRU/MAJ] \\
\hline & FUS & & 39.5 & 350.4 & DSC & [2010CIL/ALB] \\
\hline & FUS & & 26.42 & 348.3 & DSC & [2010HAH/GRA] \\
\hline & FUS & & 27.94 & 347.6 & DSC & [2010HON/HUA] \\
\hline & FUS & & 31.7 & 351.7 & DSC & [2010BAN/ARC] \\
\hline & FUS & & 26.48 & 350 & DSC & [2010BAI/VAN] \\
\hline & FUS & & 25.47 & 348.5 & DSC & [2009GAS/CEN] \\
\hline & FUS & & 25.0 & 348.2 & DSC & [2007VIP/WAN] \\
\hline & FUS & & 25.47 & 348.6 & & [2007TUR/UPP] \\
\hline & FUS & & 26.6 & 346.4 & DSC & [2006WAS/HOL] \\
\hline & FUS & $(78-400)$ & 26.65 & 348.0 & $\mathrm{AC}$ & [2004XU/SUN] \\
\hline & FUS & & 25.0 & 348.4 & DSC & [2004XU/SUN] \\
\hline & FUS & & 25.5 & 347.2 & DSC & [2002GRA/RAS] \\
\hline & FUS & & 25.7 & 350.9 & DSC & [1999LI/ZEL] \\
\hline & FUS & & 25.28 & & DSC & [1998REI/ZIM] \\
\hline & FUS & & 23.1 & 347.2 & & [1998MUR/BET2] \\
\hline & FUS & & 25.7 & 348.7 & DSC & [1996BUR/KOL] \\
\hline & FUS & & 26.9 & 344.0 & $\mathrm{DSC}$ & [1992DWI/SAT] \\
\hline & SUB & & 121 & 317 & & [1990ERT/HEA] \\
\hline $\mathrm{C}_{13} \mathrm{H}_{18} \mathrm{O}_{2}$ & [51146-56-6] & $(S)$ - $\alpha$-methyl- & 2-methylpropy & oenzene & ic acid $((S$ & buprofen) \\
\hline & FUS & & 18.3 & 324.5 & DSC & [2013YUA/CAP] \\
\hline & FUS & & 19.0 & 322.8 & DSC & [2012MAX/CHI] \\
\hline & FUS & & 28.3 & 326.9 & DSC & [2010CIL/ALB] \\
\hline & FUS & $(80-370)$ & 18.05 & 324.2 & $\mathrm{AC}$ & [2005XU/SUN] \\
\hline & FUS & & 15.4 & 323.5 & & [2001LI/GRA] \\
\hline & FUS & & 17.9 & 327.2 & DSC & [1999LI/ZEL] \\
\hline & FUS & & 18.7 & 325.5 & DSC & [1996BUR/KOL] \\
\hline & FUS & & 17.9 & 323.5 & DSC & [1993ROM/RHO] \\
\hline & FUS & & 19.9 & 319.0 & DSC & [1992DWI/SAT] \\
\hline & $\mathrm{V}$ & $(443-474)$ & $106.0 \pm 5.5$ & 298 & $\mathrm{CGC}$ & [2012MAX/CHI] \\
\hline $\mathrm{C}_{13} \mathrm{H}_{18} \mathrm{O}_{2}$ & [51146-57-7] & $(R)$ - $\alpha$-methyl- & 2-methylpropy & benzene & tic acid $((I$ & ibuprofen) \\
\hline & FUS & & 18.1 & 324.5 & DSC & [2013YUA/CAP] \\
\hline
\end{tabular}


TABLE 11. Phase change enthalpies of $\mathrm{C}_{12}$ to $\mathrm{C}_{13}$ organic compounds-Continued

\begin{tabular}{|c|c|c|c|c|c|c|}
\hline \multirow[b]{2}{*}{ Molecular formula } & \multirow{2}{*}{$\begin{array}{l}\text { CAS Registry } \\
\text { Number } \\
\text { Enthalpy }\end{array}$} & \multicolumn{5}{|l|}{ Compound } \\
\hline & & $\begin{array}{l}\text { Temperature } \\
\text { range }\end{array}$ & $\begin{array}{l}\Delta_{\text {trans }} H_{\mathrm{m}} \\
(\mathrm{kJ} / \mathrm{mol})\end{array}$ & $\begin{array}{l}T_{\mathrm{m}} \\
(\mathrm{K})\end{array}$ & Method & References \\
\hline $\mathrm{C}_{13} \mathrm{H}_{18} \mathrm{O}_{3}$ & $\begin{array}{c}\text { [200570-98-5] } \\
\text { FUS }\end{array}$ & 3-hexyloxyben & $\begin{array}{l}c \text { acid } \\
22.72\end{array}$ & 343 & $\mathrm{DSC}$ & [2001LAI/LEE] \\
\hline $\mathrm{C}_{13} \mathrm{H}_{18} \mathrm{O}_{3}$ & $\begin{array}{c}{[1142-39-8]} \\
\text { TRS }\end{array}$ & 4-hexyloxyben & $\begin{array}{l}\text { c acid } \\
5.95\end{array}$ & 342.2 & DSC & [2010FON/SAN] \\
\hline & $\begin{array}{c}\text { SUB } \\
\text { SUB (II) }\end{array}$ & $\begin{array}{l}(363-379) \\
(363-379)\end{array}$ & $\begin{array}{l}130.8 \pm 0.4 \\
139.4 \pm 0.9\end{array}$ & $\begin{array}{l}371 \\
298\end{array}$ & $\begin{array}{l}\mathrm{ME} \\
\mathrm{ME}\end{array}$ & $\begin{array}{l}{[2010 \mathrm{FON} / \mathrm{SAN}]} \\
{[2010 \mathrm{FON} / \mathrm{SAN}]}\end{array}$ \\
\hline $\mathrm{C}_{13} \mathrm{H}_{18} \mathrm{O}_{5} \mathrm{~S}$ & $\begin{array}{l}\text { [26225-79-6] } \\
\text { FUS }\end{array}$ & $(d l)$-2-ethoxy-2 & $\begin{array}{l}\text { dihydro-3,3- } \\
26.25\end{array}$ & $\begin{array}{l}\text { nethyl-5 } \\
344.1\end{array}$ & $\begin{array}{l}\text { Dofuranyl } \\
\text { DSC }\end{array}$ & $\begin{array}{l}\text { thanesulfonate } \\
\text { [1990DON/DRE] }\end{array}$ \\
\hline $\mathrm{C}_{13} \mathrm{H}_{18} \mathrm{O}_{7}$ & $\begin{array}{l}{[138-52-3]} \\
\text { FUS }\end{array}$ & 2-(hydroxymet & $\begin{array}{l}\text { phenyl- } \beta-(L \\
55.5\end{array}$ & $\begin{array}{l}\text { glucopy } \\
474.7\end{array}$ & $\begin{array}{l}\text { side, }(D) \\
\text { DSC }\end{array}$ & $\begin{array}{l}\text { icin } \\
\text { [2008PIN/DIO, 2008DIO/PIN] }\end{array}$ \\
\hline $\mathrm{C}_{13} \mathrm{H}_{19} \mathrm{BrO}_{4}$ & $\begin{array}{c}{[929259-37-0]} \\
\mathrm{V}\end{array}$ & $\begin{array}{c}\text { 1-bromo-2- }\{2- \\
(333-370)\end{array}$ & $\begin{array}{c}\text { (2-methoxyet } \\
96.1 \pm 0.4\end{array}$ & $\begin{array}{l}\text { xy)etho } \\
298\end{array}$ & $\begin{array}{l}\text { thoxy } \text { bee } \\
\text { GS }\end{array}$ & [2006DAB/SPO] \\
\hline $\mathrm{C}_{13} \mathrm{H}_{19} \mathrm{NO}$ & $\begin{array}{c}{[18859-19-3]} \\
\mathrm{V}\end{array}$ & $\begin{array}{l}\text { 3-phenylpropic } \\
(353-439)\end{array}$ & $\begin{array}{l}\text { acid, } N, N-\mathrm{d} \\
46.5\end{array}$ & $\begin{array}{l}\text { nylamid } \\
368\end{array}$ & A & [1987STE/MAL, 1968DAV/BAT] \\
\hline $\mathrm{C}_{13} \mathrm{H}_{19} \mathrm{NO}$ & $\begin{array}{c}{[141271-51-4]} \\
\mathrm{V}\end{array}$ & $\begin{array}{l}(4 R, 5 R)-2,2,3 \\
\quad(293-301)\end{array}$ & $\begin{array}{c}\text { etramethyl-5 } \\
61.6 \pm 1.8\end{array}$ & $\begin{array}{c}\text { ienyl-1, } \\
298\end{array}$ & azolidine & [1998GUD/TOR] \\
\hline $\mathrm{C}_{13} \mathrm{H}_{19} \mathrm{NO}_{2}$ & $\begin{array}{c}\text { [3129-92-8] } \\
\text { SUB }\end{array}$ & $\begin{array}{l}\text { Cyclohexyl am } \\
(289-298)\end{array}$ & $\begin{array}{l}\text { nium benzoa } \\
103.1\end{array}$ & 293.5 & A & [1987STE/MAL, 1965MAR] \\
\hline $\mathrm{C}_{13} \mathrm{H}_{19} \mathrm{NO}_{2}$ & $\begin{array}{c}\text { [13476-55-6] } \\
\text { FUS }\end{array}$ & Hexyl 4-amino & $\begin{array}{l}\text { nzoate } \\
33.1\end{array}$ & 334.2 & $\mathrm{DSC}$ & [1990NEA/FLY] \\
\hline $\mathrm{C}_{13} \mathrm{H}_{19} \mathrm{NO}_{2}$ & $\begin{array}{c}\text { [7461-26-9] } \\
\text { FUS }\end{array}$ & Hexyl $N$-phen & $\begin{array}{r}\text { arbamate } \\
32.76\end{array}$ & 328 & & [1971PRI] \\
\hline $\mathrm{C}_{13} \mathrm{H}_{19} \mathrm{NO}_{2}$ & $\begin{array}{c}\text { [147169-48-0] } \\
\text { FUS }\end{array}$ & $(S)$-tert-butyl- & $\begin{array}{c}\text { henylethylcar } \\
29.73\end{array}$ & $\begin{array}{l}\text { mate } \\
359.5\end{array}$ & DSC & [2011ZEN/YU] \\
\hline $\mathrm{C}_{13} \mathrm{H}_{19} \mathrm{NO}_{4}$ & $\begin{array}{c}\text { [73243-69-3] } \\
\text { FUS }\end{array}$ & $N$-phenylethyl & $\begin{array}{c}\text { amino- } 1,5-\mathrm{di} \\
39.9\end{array}$ & $\begin{array}{r}x y-(D) \\
455.8\end{array}$ & $\begin{array}{l}\text { copyrano } \\
\text { DSC }\end{array}$ & [1994BLU/PRA] \\
\hline $\mathrm{C}_{13} \mathrm{H}_{19} \mathrm{NO}_{4} \mathrm{~S}$ & $\begin{array}{c}\text { [57-66-9] } \\
\text { FUS } \\
\text { FUS }\end{array}$ & 4-[(dipropylam & $\begin{array}{c}\text { sulfonyl]ber } \\
33.57 \\
40.9\end{array}$ & $\begin{array}{c}\text { ic acid } \\
471 \\
472.1\end{array}$ & $\begin{array}{c}\text { benecid) } \\
\text { DSC } \\
\text { DSC }\end{array}$ & $\begin{array}{l}\text { [2009PEN/ESC] } \\
\text { [2006WAS/HOL] }\end{array}$ \\
\hline $\mathrm{C}_{13} \mathrm{H}_{19} \mathrm{~N}_{3} \mathrm{O}_{3}$ & $\begin{array}{c}\text { [16577-64-3] } \\
\text { FUS }\end{array}$ & 1-hexyl-3-(4-ni & $\begin{array}{c}\text { phenyl) urea } \\
25.47\end{array}$ & 384.4 & $\mathrm{DSC}$ & [1993TIE/FRA] \\
\hline $\mathrm{C}_{13} \mathrm{H}_{19} \mathrm{~N}_{3} \mathrm{O}_{4}$ & $\begin{array}{c}\text { [40487-42-1] } \\
\text { FUS }\end{array}$ & $N$-(1-ethylpro| & $\begin{array}{c}\text {-2,6-dinitro- } \\
25.19\end{array}$ & $\begin{array}{c}-x y l i d i n \\
327.5\end{array}$ & DSC & [1991ACR, 1990DON/DRE] \\
\hline $\mathrm{C}_{13} \mathrm{H}_{20}$ & $\begin{array}{c}{[1078-71-3]} \\
\mathrm{V} \\
\mathrm{V} \\
\mathrm{V}\end{array}$ & $\begin{array}{l}\text { Heptylbenzene } \\
(292-353) \\
(423-527)\end{array}$ & $\begin{array}{c}64.2 \pm 0.2 \\
54.0 \\
64.9\end{array}$ & $\begin{array}{l}298 \\
438 \\
298\end{array}$ & $\begin{array}{c}\text { GS } \\
\text { A }\end{array}$ & $\begin{array}{l}\text { [2006VER] } \\
{[1987 \mathrm{STE} / \mathrm{MAL}]} \\
{[1971 \mathrm{WIL} / \mathrm{ZWO}]}\end{array}$ \\
\hline $\mathrm{C}_{13} \mathrm{H}_{20} \mathrm{~N}_{2} \mathrm{O}_{2}$ & $\begin{array}{l}\text { [59-46-1] } \\
\text { FUS } \\
\text { FUS }\end{array}$ & 2-(diethylamin & $\begin{array}{c}\text { thyl 4-amino } \\
26.07 \\
26.2\end{array}$ & $\begin{array}{c}\text { tzoate }( \\
428.6 \\
335\end{array}$ & $\begin{array}{l}\text { aine) } \\
\text { DTA } \\
\text { DSC }\end{array}$ & $\begin{array}{l}\text { [2013FUL/VLA] } \\
\text { [2010BAI/VAN] }\end{array}$ \\
\hline
\end{tabular}

[Note: There is a significant difference noted in the melting point temperatures determined by the two research groups.]

\begin{tabular}{|c|c|c|c|c|c|}
\hline \multirow[t]{2}{*}{$\mathrm{C}_{13} \mathrm{H}_{20} \mathrm{~N}_{2} \mathrm{O}_{2} \mathrm{~S}_{2}$} & {$[81261-44-1]$} & \multicolumn{2}{|c|}{$N$-isobutyl- $S$-methyl- $N^{\prime}$-tosylisothiourea } & \multirow[b]{2}{*}{ DSC } & \multirow[b]{2}{*}{ [1992REI/HAN] } \\
\hline & FUS & 29.4 & 363.2 & & \\
\hline \multirow[t]{2}{*}{$\mathrm{C}_{13} \mathrm{H}_{20} \mathrm{~N}_{2} \mathrm{O}_{2} \mathrm{~S}_{2}$} & [120563-91-9] & \multicolumn{2}{|c|}{$N$-tert-butyl- $S$-methyl- $N^{\prime}$-tosylisothiourea } & & \\
\hline & FUS & 30.4 & 394.2 & DSC & [1992REI/HAN] \\
\hline \multirow[t]{2}{*}{$\mathrm{C}_{13} \mathrm{H}_{20} \mathrm{~N}_{2} \mathrm{O}_{2} \mathrm{~S}_{2}$} & [145198-70-5] & \multicolumn{2}{|c|}{$N$-isopropyl- $S$-ethyl- $N^{\prime}$-tosylisothiourea } & & \\
\hline & FUS & 29.1 & 392.2 & DSC & [1992REI/HAN] \\
\hline $\mathrm{C}_{13} \mathrm{H}_{20} \mathrm{O}$ & $\mathrm{V}$ & $\begin{array}{l}\text { Butyl cumyl ether } \\
(278-318) \quad 63.8 \pm 0.5\end{array}$ & 298 & GS & [2001VER/HEI2] \\
\hline
\end{tabular}


TABLE 11. Phase change enthalpies of $\mathrm{C}_{12}$ to $\mathrm{C}_{13}$ organic compounds-Continued

\begin{tabular}{|c|c|c|c|c|c|c|}
\hline \multirow[b]{2}{*}{ Molecular formula } & \multirow{2}{*}{$\begin{array}{l}\text { CAS Registry } \\
\text { Number } \\
\text { Enthalpy }\end{array}$} & \multicolumn{5}{|l|}{ Compound } \\
\hline & & $\begin{array}{l}\text { Temperature } \\
\text { range }\end{array}$ & $\begin{array}{l}\Delta_{\text {trans }} H_{\mathrm{m}} \\
(\mathrm{kJ} / \mathrm{mol})\end{array}$ & $\begin{array}{l}T_{\mathrm{m}} \\
(\mathrm{K})\end{array}$ & Method & References \\
\hline $\mathrm{C}_{13} \mathrm{H}_{20} \mathrm{O}$ & $\begin{array}{c}{[127-41-3]} \\
\mathrm{V} \\
\mathrm{V}\end{array}$ & $\begin{array}{l}\alpha \text {-ionone } \\
\quad(352-523) \\
(286-333)\end{array}$ & $\begin{array}{l}62.0 \\
67.5\end{array}$ & $\begin{array}{l}367 \\
301\end{array}$ & $\begin{array}{c}\text { A } \\
\mathrm{A}, \mathrm{ME}\end{array}$ & $\begin{array}{l}\text { [1987STE/MAL, 1947STU] } \\
\text { [1987STE/MAL, 1957SER/VOI] }\end{array}$ \\
\hline $\mathrm{C}_{13} \mathrm{H}_{20} \mathrm{O}$ & $\begin{array}{c}{[14901-07-6]} \\
\mathrm{V}\end{array}$ & $\begin{array}{l}\beta \text {-ionone } \\
\quad(291-334)\end{array}$ & 69.0 & 306 & $\mathrm{~A}, \mathrm{ME}$ & [1987STE/MAL, 1957SER/VOI] \\
\hline $\mathrm{C}_{13} \mathrm{H}_{20} \mathrm{O}$ & $\begin{array}{c}{[16647-05-5]} \\
\mathrm{V}\end{array}$ & $\begin{array}{l}\text { 6,10-dimethyl- } \\
(349-421)\end{array}$ & $\begin{array}{l}9 \text {-undecatrie } \\
63.6 \pm 1.4\end{array}$ & -one & Static & [1988BAG/GUR] \\
\hline $\mathrm{C}_{13} \mathrm{H}_{20} \mathrm{O}$ & $\begin{array}{c}{[141-10-6]} \\
\mathrm{V}\end{array}$ & $\begin{array}{l}\text { 6,10-dimethyl- } \\
(382-457)\end{array}$ & $\begin{array}{l}9 \text {-undecatrie } \\
67.6 \pm 1.1\end{array}$ & -one & Static & [1988BAG/GUR] \\
\hline $\mathrm{C}_{13} \mathrm{H}_{20} \mathrm{O}$ & $\begin{array}{c}{[79-77-6]} \\
\mathrm{V}\end{array}$ & $\begin{array}{l}\text { 4-(2,6,6-trimet } \\
\quad(373-442)\end{array}$ & $\begin{array}{c}-1-\text { cyclohexe } \\
49.6 \pm 1.1\end{array}$ & $\begin{array}{l}-y 1)-3-1 \\
408\end{array}$ & $\begin{array}{l}\text {-2-one } \\
\text { Static }\end{array}$ & [1988BAG/GUR] \\
\hline $\mathrm{C}_{13} \mathrm{H}_{20} \mathrm{O}_{2}$ & $\begin{array}{c}{[500-67-4]} \\
\mathrm{V}\end{array}$ & $\begin{array}{l}\text { 1,3-dihydroxy } \\
(443-504)\end{array}$ & $\begin{array}{l}\text { heptylbenzen } \\
91.6\end{array}$ & 458 & $\mathrm{~A}, \mathrm{GC}$ & [1987STE/MAL, 1975KUN/LIL] \\
\hline $\mathrm{C}_{13} \mathrm{H}_{20} \mathrm{O}_{2}$ & $\begin{array}{c}{[41395-27-1]} \\
\mathrm{V}\end{array}$ & $\begin{array}{l}\text { 1,3-dihydroxy } \\
(433-493)\end{array}$ & $\begin{array}{l}\text { nethyl-2-hexy } \\
\qquad 82.8\end{array}$ & $\begin{array}{c}\text { enzene } \\
448\end{array}$ & $\mathrm{~A}, \mathrm{GC}$ & [1987STE/MAL, 1975KUN/LIL] \\
\hline $\mathrm{C}_{13} \mathrm{H}_{21} \mathrm{CI}_{3} \mathrm{OS}$ & $\begin{array}{c}{[76619-97-1]} \\
\mathrm{V}\end{array}$ & $\begin{array}{l}\text { 2,3,3-trichloro } \\
\quad(483-503)\end{array}$ & $\begin{array}{l}\text { propenethioic } \\
79.9\end{array}$ & $\mathrm{~d}, O-\mathrm{d}$ & $\begin{array}{l}\text { ester } \\
\text { GC }\end{array}$ & [1980PIT/KIS] \\
\hline $\mathrm{C}_{13} \mathrm{H}_{21} \mathrm{~N}$ & $\begin{array}{c}\text { [29772-98-3] } \\
\text { V } \\
\text { V }\end{array}$ & $\begin{array}{c}N, N \text {-dimethyl- } \\
\quad(283-330) \\
(283-330)\end{array}$ & $\begin{array}{c}\text { hethyl-3-phen } \\
59.8 \pm 0.7 \\
60.3 \pm 0.7\end{array}$ & $\begin{array}{l}\text { 2-butar } \\
307 \\
298\end{array}$ & $\begin{array}{l}\text { line } \\
\text { GS } \\
\text { GS }\end{array}$ & $\begin{array}{l}{[1998 \mathrm{VER} / \mathrm{BEC}]} \\
{[1998 \mathrm{VER} / \mathrm{BEC}]}\end{array}$ \\
\hline $\mathrm{C}_{13} \mathrm{H}_{21} \mathrm{~N}$ & $\begin{array}{c}{[29772-82-5]} \\
\text { V } \\
\text { V }\end{array}$ & $\begin{array}{c}N \text {-methyl-2,3 } \\
\quad(285-332) \\
(285-332)\end{array}$ & $\begin{array}{c}\text { nethyl-3-pher } \\
71.9 \pm 1.1 \\
72.5 \pm 1.1\end{array}$ & $\begin{array}{l}\text { 2-butar } \\
309 \\
298\end{array}$ & $\begin{array}{l}\text { nine } \\
\text { GS } \\
\text { GS }\end{array}$ & $\begin{array}{l}\text { [1998VER/BEC] } \\
{[1998 \mathrm{VER} / \mathrm{BEC}]}\end{array}$ \\
\hline $\mathrm{C}_{13} \mathrm{H}_{21} \mathrm{~N}$ & $\begin{array}{c}{[585-48-8]} \\
\mathrm{V} \\
\mathrm{V}\end{array}$ & $\begin{array}{l}\text { 2,6-di-tert-but } \\
\quad(293-313)\end{array}$ & $\begin{array}{l}\text { yridine } \\
56.6 \pm 1.2 \\
57.3\end{array}$ & $\begin{array}{l}298 \\
298\end{array}$ & $\mathrm{C}$ & $\begin{array}{l}\text { [2008FRI/ACR] } \\
\text { [1979ARN/CHA] }\end{array}$ \\
\hline $\mathrm{C}_{13} \mathrm{H}_{21} \mathrm{NO}$ & $\begin{array}{c}{[90-84-6]} \\
\mathrm{V}\end{array}$ & $\begin{array}{l}\text { 2-(diethylamin } \\
\quad(293-333)\end{array}$ & $\begin{array}{l}1-\text { phenyl-1-p } \\
71.6 \pm 1.0\end{array}$ & $\begin{array}{c}\text { anone } \\
298\end{array}$ & GS & [1994WEL/VER] \\
\hline $\mathrm{C}_{13} \mathrm{H}_{21} \mathrm{NO}$ & $\begin{array}{c}{[1502-00-7]} \\
\text { SUB } \\
\text { SUB }\end{array}$ & $\begin{array}{c}N, N \text {-dimethyl- } \\
\quad(303-322) \\
(303-322)\end{array}$ & $\begin{array}{c}\text { damantylcarb } \\
96.9 \pm 0.3 \\
97.5 \pm 0.3\end{array}$ & $\begin{array}{c}\text { amide } \\
313 \\
298\end{array}$ & $\begin{array}{l}\mathrm{ME} \\
\mathrm{ME}\end{array}$ & $\begin{array}{l}\text { [1993ABB/JIM, 1995ABB/JIM] } \\
\text { [1995ABB/JIM] }\end{array}$ \\
\hline $\mathrm{C}_{13} \mathrm{H}_{21} \mathrm{NO}_{2}$ & $\begin{array}{l}\text { [3246-04-6] } \\
\text { SUB }\end{array}$ & $\begin{array}{l}N \text {-(3-phenoxy } \\
\quad(323-348)\end{array}$ & $\begin{array}{l}\text { 1ydroxypropy } \\
133.9\end{array}$ & $\begin{array}{l}335.5 \\
33 \text { utylam }\end{array}$ & A & [1987STE/MAL] \\
\hline $\mathrm{C}_{13} \mathrm{H}_{22}$ & $\begin{array}{c}{[886027-03-8]} \\
\mathrm{V}\end{array}$ & $\begin{array}{l}\text { 2-allyl-cis-dec } \\
\quad(296-320)\end{array}$ & $\begin{array}{c}\text { dronaphthale } \\
89.9\end{array}$ & 308 & A & [1987STE/MAL, 1940ZIL] \\
\hline $\mathrm{C}_{13} \mathrm{H}_{22}$ & $\begin{array}{c}{[886026-95-5]} \\
\mathrm{V}\end{array}$ & $\begin{array}{l}\text { 2-allyl-trans-d } \\
\quad(296-320)\end{array}$ & $\begin{array}{l}\text { hydronaphth } \\
91.7\end{array}$ & 308 & $\mathrm{~A}$ & [1987STE/MAL, 1940ZIL] \\
\hline $\mathrm{C}_{13} \mathrm{H}_{22}$ & $\begin{array}{c}{[5744-03-6]} \\
\mathrm{V}\end{array}$ & $\begin{array}{c}\text { Dodecahydrof } \\
(332-525)\end{array}$ & rene & 347 & A & [1987STE/MAL] \\
\hline $\mathrm{C}_{13} \mathrm{H}_{22}$ & $\begin{array}{c}\text { [707-35-7] } \\
\text { TRS } \\
\text { FUS } \\
\text { FUS } \\
\text { TRS } \\
\text { FUS }\end{array}$ & $\begin{array}{c}\text { 1,3,5-trimethy } \\
(8-356)\end{array}$ & $\begin{array}{c}\text { mantane } \\
8.19 \\
2.06 \\
1.92 \\
6.3 \\
1.73\end{array}$ & $\begin{array}{l}234.4 \\
255.6 \\
255.0 \\
228.2 \\
253.6\end{array}$ & $\begin{array}{c}\mathrm{AC} \\
\mathrm{DSC} \\
\mathrm{DSC}\end{array}$ & $\begin{array}{l}\text { [2000DRU/VAR2] } \\
\text { [1980ARN/SCH] } \\
\text { [1977CLA/KNO] }\end{array}$ \\
\hline & SUB & $(300-360)$ & $77.8 \pm 1.3$ & 298 & BG & [1977STE/WAT] \\
\hline & $\begin{array}{l}\mathrm{V} \\
\mathrm{V} \\
\mathrm{V}\end{array}$ & $(385-482)$ & $\begin{array}{c}51.7 \pm 0.2 \\
45.4 \\
51.0 \pm 0.2\end{array}$ & $\begin{array}{l}298 \\
400 \\
313\end{array}$ & $\mathrm{C}$ & $\begin{array}{l}\text { [2000DRU/VAR, 2000MEL/PIM, 2000PAS/KOR] } \\
\text { [2000PAS/KOR] } \\
\text { [2000PAS/KOR] }\end{array}$ \\
\hline $\mathrm{C}_{13} \mathrm{H}_{22} \mathrm{Cl}_{2} \mathrm{O}_{4}$ & [3843-83-2] & 2,2-bis(chloror & hyl)-1,3-prop & diol di & rate & \\
\hline
\end{tabular}


TABLE 11. Phase change enthalpies of $\mathrm{C}_{12}$ to $\mathrm{C}_{13}$ organic compounds-Continued

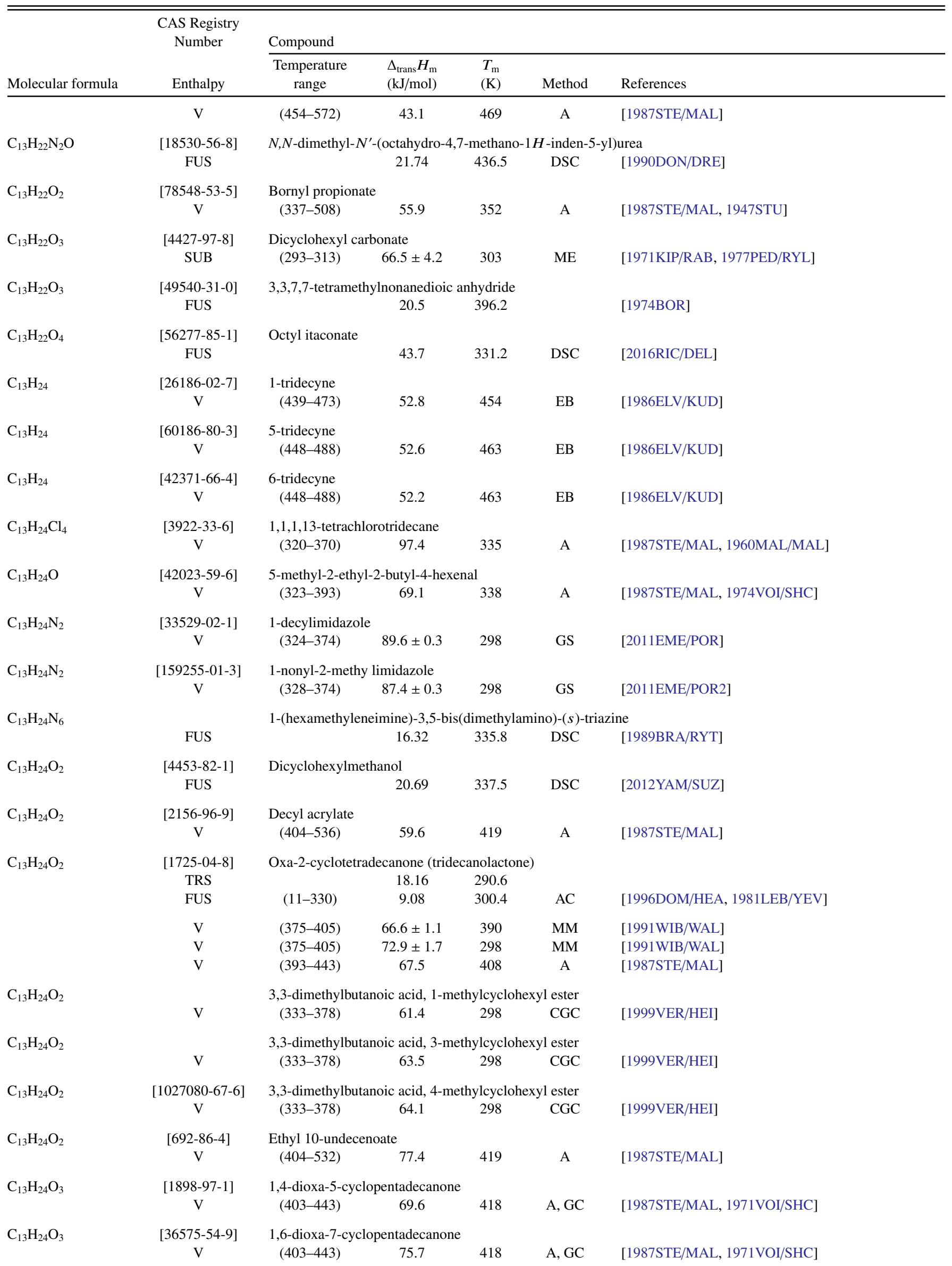


TABLE 11. Phase change enthalpies of $\mathrm{C}_{12}$ to $\mathrm{C}_{13}$ organic compounds-Continued

\begin{tabular}{|c|c|c|c|c|c|c|}
\hline \multirow[b]{2}{*}{ Molecular formula } & \multirow{2}{*}{$\begin{array}{c}\text { CAS Registry } \\
\text { Number } \\
\text { Enthalpy }\end{array}$} & \multicolumn{5}{|l|}{ Compound } \\
\hline & & $\begin{array}{l}\text { Temperature } \\
\text { range }\end{array}$ & $\begin{array}{l}\Delta_{\text {trans }} H_{\mathrm{m}} \\
(\mathrm{kJ} / \mathrm{mol})\end{array}$ & $\begin{array}{c}T_{\mathrm{m}} \\
(\mathrm{K})\end{array}$ & Method & References \\
\hline $\mathrm{C}_{13} \mathrm{H}_{24} \mathrm{O}_{3}$ & $\begin{array}{c}{[36575-53-8]} \\
\mathrm{V}\end{array}$ & $\begin{array}{l}\text { 1,8-dioxa-9-cyclop } \\
\quad(403-443)\end{array}$ & $\begin{array}{c}\text { pentadecano } \\
66.5\end{array}$ & 418 & $\mathrm{~A}, \mathrm{GC}$ & [1987STE/MAL, 1971VOI/SHC] \\
\hline $\mathrm{C}_{13} \mathrm{H}_{24} \mathrm{O}_{3}$ & $\begin{array}{c}\text { [18871-17-5] } \\
\mathrm{V}\end{array}$ & $\begin{array}{l}\text { 3-hexyl-4-acetoxyt } \\
\quad(383-453)\end{array}$ & $\begin{array}{c}\text { tetrahydro-2 } \\
72.1\end{array}$ & $\begin{array}{r}\text { pyran } \\
398\end{array}$ & A & [1987STE/MAL] \\
\hline $\mathrm{C}_{13} \mathrm{H}_{24} \mathrm{O}_{3}$ & $\begin{array}{c}{[41780-57-8]} \\
\mathrm{V} \\
\mathrm{V}\end{array}$ & $\begin{array}{l}\text { Octyl levulinate } \\
\quad(413-565)\end{array}$ & $\begin{array}{l}66.3 \\
65.1\end{array}$ & $\begin{array}{l}428 \\
507\end{array}$ & $\mathrm{~A}$ & $\begin{array}{l}\text { [1987STE/MAL] } \\
{[1933 \mathrm{COW} / \mathrm{SCH}]}\end{array}$ \\
\hline $\mathrm{C}_{13} \mathrm{H}_{24} \mathrm{O}_{4}$ & $\begin{array}{c}{[855376-33-9]} \\
\text { V }\end{array}$ & $\begin{array}{l}\text { Octyl 3-acetoxypro } \\
\quad(420-440)\end{array}$ & $\begin{array}{r}\text { opionate } \\
88.4\end{array}$ & 430 & A & [1987STE/MAL, 1948FEI/FIS] \\
\hline $\mathrm{C}_{13} \mathrm{H}_{24} \mathrm{O}_{4}$ & $\begin{array}{c}\text { [77478-67-2] } \\
\mathrm{V}\end{array}$ & $\begin{array}{l}\text { Ethylisopentylmald } \\
(392-501)\end{array}$ & $\begin{array}{c}\text { onic acid, et } \\
73.1\end{array}$ & $\begin{array}{c}\text { methyl } \\
407\end{array}$ & A & [1987STE/MAL, 1981TOD/BEL] \\
\hline $\mathrm{C}_{13} \mathrm{H}_{24} \mathrm{O}_{4}$ & $\begin{array}{c}\text { [505-52-2] } \\
\text { FUS } \\
\text { FUS } \\
\text { FUS }\end{array}$ & 1,13-tridecanedioic & $\begin{array}{c}\text { c acid (brass } \\
51.6 \\
49.4 \\
45.3\end{array}$ & $\begin{array}{l}\text { (acid) } \\
386.2 \\
386.3 \\
397.5\end{array}$ & $\begin{array}{l}\text { DSC } \\
\text { DSC } \\
\text { DSC }\end{array}$ & $\begin{array}{l}\text { [2015TAN/DAI] } \\
\text { [2005ROU/TEM] } \\
\text { [1991ACR, 1974CIN/BER] }\end{array}$ \\
\hline $\mathrm{C}_{13} \mathrm{H}_{24} \mathrm{O}_{4}$ & $\begin{array}{c}{[6624-57-3]} \\
\mathrm{V} \\
\mathrm{V}\end{array}$ & $\begin{array}{l}\text { Dibutyl glutarate } \\
\quad(318-370) \\
(318-370)\end{array}$ & $\begin{array}{c}77.2 \\
83.1 \pm 0.2\end{array}$ & $\begin{array}{l}344 \\
298\end{array}$ & $\begin{array}{l}\text { GS } \\
\text { GS }\end{array}$ & $\begin{array}{l}\text { [2011LIP/KRA] } \\
\text { [2011LIP/KRA] }\end{array}$ \\
\hline $\mathrm{C}_{13} \mathrm{H}_{24} \mathrm{O}_{4}$ & $\begin{array}{c}\text { [43052-39-7] } \\
\mathrm{V}\end{array}$ & $\begin{array}{l}\text { Di-tert-butyl glutar } \\
\quad(295-325)\end{array}$ & $\begin{array}{l}\text { Irate } \\
71.7 \pm 0.5\end{array}$ & 298 & GS & [2011POR/KRA] \\
\hline $\mathrm{C}_{13} \mathrm{H}_{24} \mathrm{O}_{5}$ & $\begin{array}{c}{[5456-15-5]} \\
\mathrm{V}\end{array}$ & $\begin{array}{l}\text { Octyl }[1-(\text { methoxyc } \\
\quad(391-566)\end{array}$ & $\begin{array}{c}\text { carbonyl)eth } \\
70.0\end{array}$ & $\begin{array}{c}\text { carbon } \\
406\end{array}$ & A & [1987STE/MAL, 1950REH/DIX2] \\
\hline $\mathrm{C}_{13} \mathrm{H}_{24} \mathrm{O}_{5}$ & $\begin{array}{c}{[902261-31-8]} \\
\mathrm{V}\end{array}$ & $\begin{array}{l}\text { Pentyl[1-(butoxyca } \\
\quad(348-513)\end{array}$ & $\begin{array}{c}\text { arbonyl)ethy } \\
70.1\end{array}$ & $\begin{array}{c}\text { arbona } \\
363\end{array}$ & A & [1987STE/MAL, 1950REH/DIX2] \\
\hline $\mathrm{C}_{13} \mathrm{H}_{25} \mathrm{~N}$ & $\begin{array}{c}{[629-60-7]} \\
\mathrm{V} \\
\mathrm{V}\end{array}$ & $\begin{array}{l}\text { Tridecanonitrile } \\
\qquad(301-363) \\
(380-566)\end{array}$ & $\begin{array}{c}80.3 \pm 0.4 \\
69.5\end{array}$ & $\begin{array}{l}298 \\
395\end{array}$ & $\begin{array}{c}\text { GS } \\
\text { A }\end{array}$ & $\begin{array}{l}\text { [2005EME/VER] } \\
\text { [1987STE/MAL] }\end{array}$ \\
\hline $\mathrm{C}_{13} \mathrm{H}_{25} \mathrm{NO}$ & $\begin{array}{c}\text { [20299-83-6] } \\
\mathrm{V}\end{array}$ & $\begin{array}{l}\text { 1-octanoyl piperidi } \\
(373-443)\end{array}$ & line 50.0 & 388 & A & [1987STE/MAL, 1968DAV/BAT] \\
\hline $\mathrm{C}_{13} \mathrm{H}_{25} \mathrm{NO}_{3}$ & $\begin{array}{c}\text { [83871-09-4] } \\
\text { TRS+FUS }\end{array}$ & $N$-(1-oxoundecyl) & $\begin{array}{r}\text { glycine } \\
26.3\end{array}$ & 383.3 & DSC & [2014RED/KRO] \\
\hline $\mathrm{C}_{13} \mathrm{H}_{26}$ & $\begin{array}{c}{[7367-38-6]} \\
\mathrm{V}\end{array}$ & $\begin{array}{l}\text { 5-butyl-4-nonene } \\
\quad(310-361)\end{array}$ & 55.8 & 325 & $\mathrm{~A}, \mathrm{MG}$ & [1987STE/MAL, 1955SCH/WHI] \\
\hline $\mathrm{C}_{13} \mathrm{H}_{26}$ & $\begin{array}{c}{[2437-56-1]} \\
\mathrm{V} \\
\mathrm{V}\end{array}$ & $\begin{array}{l}\text { 1-tridecene } \\
(413-509)\end{array}$ & $\begin{array}{l}65.3 \\
53.9\end{array}$ & $\begin{array}{l}298 \\
428\end{array}$ & A & $\begin{array}{l}\text { [1971WIL/ZWO] } \\
\text { [1987STE/MAL, 1955CAM/ROS] }\end{array}$ \\
\hline $\mathrm{C}_{13} \mathrm{H}_{26}$ & $\begin{array}{c}{[1795-20-6]} \\
\mathrm{V}\end{array}$ & $n$-octylcyclopentar & 65.8 & 298 & & [1971WIL/ZWO] \\
\hline $\mathrm{C}_{13} \mathrm{H}_{26}$ & $\begin{array}{c}{[5617-41-4]} \\
\text { FUS } \\
\text { V }\end{array}$ & $n$-heptylcyclohexa & $\begin{array}{l}\text { ane } \\
22.22 \\
64.9\end{array}$ & $\begin{array}{c}232.8 \\
298\end{array}$ & & $\begin{array}{l}\text { [1996DOM/HEA, 1949PAR/MOO2] } \\
\text { [1971WIL/ZWO] }\end{array}$ \\
\hline $\mathrm{C}_{13} \mathrm{H}_{26}$ & $\begin{array}{c}\text { [295-02-3] } \\
\text { TRS } \\
\text { FUS }\end{array}$ & Cyclotridecane & $\begin{array}{l}0.9 \\
7.4\end{array}$ & $\begin{array}{l}285.6 \\
297.6\end{array}$ & DSC & [1987DRO/MOL, 1987DRO/EME] \\
\hline $\mathrm{C}_{13} \mathrm{H}_{26} \mathrm{O}$ & $\begin{array}{c}{[53144-53-9]} \\
\mathrm{V}\end{array}$ & $\begin{array}{l}\text { 5-methyl-2-ethyl-2 } \\
\text { (333-393) }\end{array}$ & $\begin{array}{l}\text { 2-butyl-4-he } \\
76.9\end{array}$ & $\begin{array}{r}\text { e- } 1-\text { ol } \\
348\end{array}$ & A & [1987STE/MAL, 1974VOI/SHC] \\
\hline $\mathrm{C}_{13} \mathrm{H}_{26} \mathrm{O}$ & $\begin{array}{c}{[30089-09-9]} \\
\mathrm{V}\end{array}$ & $\begin{array}{l}\text { 1-octylcyclopentan } \\
(468-541)\end{array}$ & nol 60.9 & 483 & A & [1987STE/MAL] \\
\hline $\mathrm{C}_{13} \mathrm{H}_{26} \mathrm{O}$ & $\begin{array}{c}{[10486-19-8]} \\
\mathrm{V}\end{array}$ & $\begin{array}{l}\text { Tridecanal } \\
(325-349)\end{array}$ & $73.3 \pm 0.4$ & 298 & GS & [2003VER/KRA2] \\
\hline
\end{tabular}


TABLE 11. Phase change enthalpies of $\mathrm{C}_{12}$ to $\mathrm{C}_{13}$ organic compounds-Continued

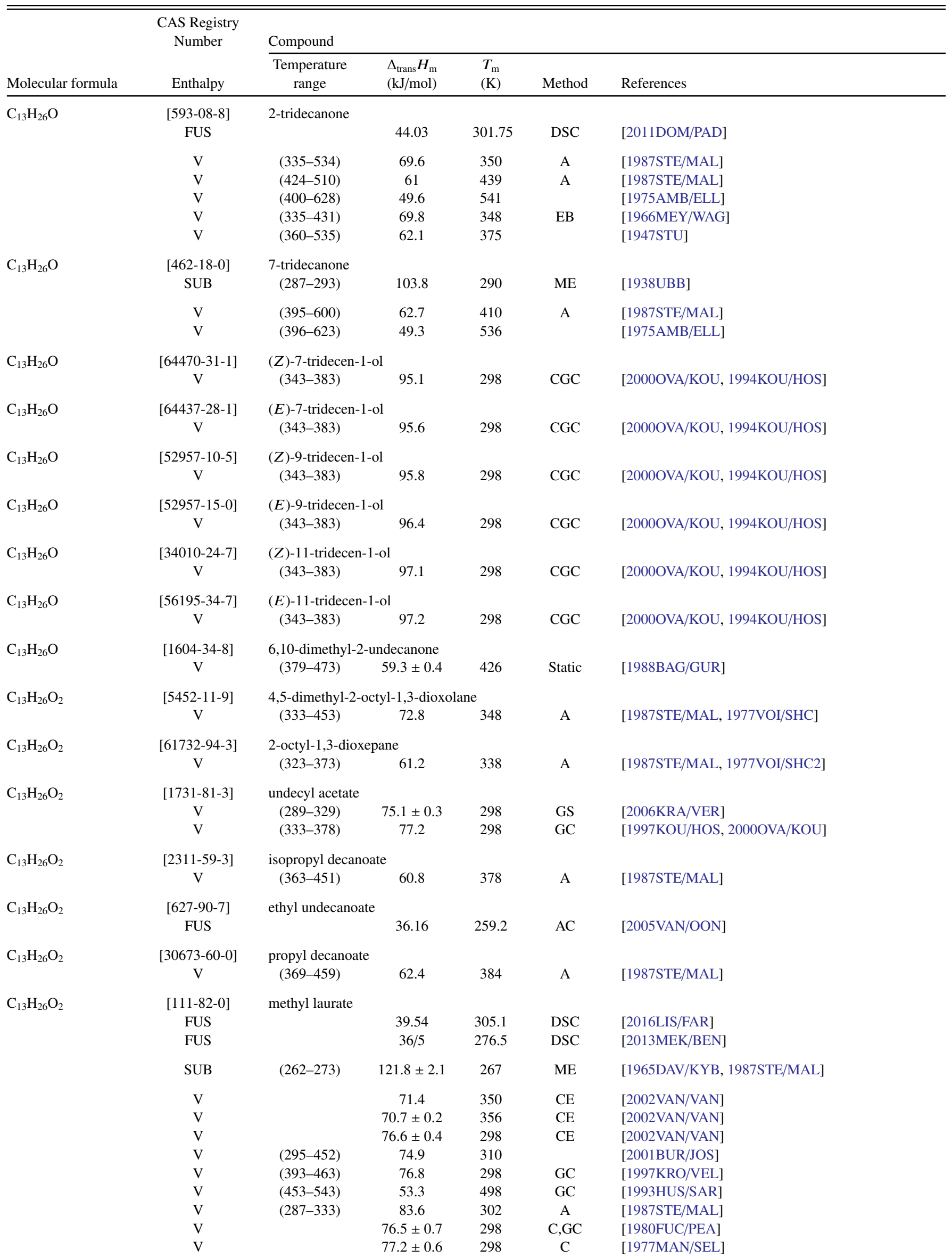


TABLE 11. Phase change enthalpies of $\mathrm{C}_{12}$ to $\mathrm{C}_{13}$ organic compounds-Continued

\begin{tabular}{|c|c|c|c|c|c|c|}
\hline \multirow[b]{2}{*}{ Molecular formula } & \multirow{2}{*}{$\begin{array}{c}\text { CAS Registry } \\
\text { Number } \\
\text { Enthalpy }\end{array}$} & \multicolumn{5}{|l|}{ Compound } \\
\hline & & $\begin{array}{c}\text { Temperature } \\
\text { range }\end{array}$ & $\begin{array}{l}\Delta_{\text {trans }} H_{\mathrm{m}} \\
(\mathrm{kJ} / \mathrm{mol})\end{array}$ & $\begin{array}{l}T_{\mathrm{m}} \\
(\mathrm{K})\end{array}$ & Method & References \\
\hline & $\mathrm{V}$ & $(407-540)$ & 63.6 & 422 & A & [1987STE/MAL, 1963ROS/SCH] \\
\hline & $\mathrm{V}$ & $(336-409)$ & 71.4 & 351 & MG,OM & {$[1952 \mathrm{SCO} / \mathrm{MAC}]$} \\
\hline & $\mathrm{V}$ & $(373-439)$ & 62.3 & 388 & & [1944ALT/TRI] \\
\hline \multirow[t]{2}{*}{$\mathrm{C}_{13} \mathrm{H}_{26} \mathrm{O}_{2}$} & {$[245658-41-7]$} & \multicolumn{4}{|c|}{ 3,3-dimethylbutanoic acid, 1,1,3-trimethylbutyl ester } & \\
\hline & $\mathrm{V}$ & $(333-378)$ & 58.4 & 298 & CGC & [1999VER/HEI] \\
\hline \multirow[t]{2}{*}{$\mathrm{C}_{13} \mathrm{H}_{26} \mathrm{O}_{2}$} & & \multicolumn{4}{|c|}{ 2,6-dimethyl-2-heptanol butanoate } & \\
\hline & $\mathrm{V}$ & $(333-378)$ & 62.5 & 298 & CGC & [1999VER/HEI] \\
\hline \multirow[t]{2}{*}{$\mathrm{C}_{13} \mathrm{H}_{26} \mathrm{O}_{2}$} & {$[245658-44-0]$} & \multicolumn{4}{|c|}{ 2-methylpropanoic acid, 1,1,5-trimethylhexyl ester } & \\
\hline & V & $(333-378)$ & 60.8 & 298 & CGC & [1999VER/HEI] \\
\hline \multirow[t]{11}{*}{$\mathrm{C}_{13} \mathrm{H}_{26} \mathrm{O}_{2}$} & [638-53-9] & \multicolumn{4}{|c|}{ tridecanoic acid } & \\
\hline & TRS & & 8.14 & 306.1 & & \\
\hline & FUS & & 38.2 & 314.6 & DSC & [2011EGO/MAR] \\
\hline & TRS & & 0.06 & 287.7 & & \\
\hline & TRS & & 8.5 & 309.1 & & \\
\hline & FUS & & 33.0 & 314.6 & DSC & [2007GBA/NEG] \\
\hline & TRS & $(90-340)$ & 8.72 & 307.1 & & \\
\hline & FUS & $(90-340)$ & 33.74 & 315 & $\mathrm{AC}$ & [1996DOM/HEA, 1982SCH/VAN2] \\
\hline & FUS & & 33.1 & 314.5 & & {$[1975 B E R / L E O]$} \\
\hline & SUB & $(271-282)$ & 112.5 & & TPTD & [2005CHA/ZIE] \\
\hline & SUB & $(282-299)$ & 170 & & TPTD & [2001CHA/TOB] \\
\hline \multicolumn{7}{|c|}{ [Note: Experimental values based on the TPTD method are often inconsistent with values determined using other experimental methods.] } \\
\hline & V & $(409-585)$ & 90.1 & 424 & A & [1987STE/MAL] \\
\hline & V & $(328-350)$ & $100.4 \pm 2.0$ & 340 & ME,TE & [1982DEK/SCH] \\
\hline \multirow[t]{2}{*}{$\mathrm{C}_{13} \mathrm{H}_{26} \mathrm{O}_{3}$} & {$[42175-34-8]$} & decyl lactate & & & & \\
\hline & V & $(349-556)$ & 76.6 & 364 & A & [1987STE/MAL, 1950REH/DIX] \\
\hline \multirow[t]{2}{*}{$\mathrm{C}_{13} \mathrm{H}_{26} \mathrm{O}_{3}$} & {$[500787-64-4]$} & \multicolumn{4}{|c|}{ octyl 3-ethoxypropionate } & \\
\hline & $\mathrm{V}$ & $(398-543)$ & 56.9 & 413 & A & [1987STE/MAL, 1948DIX/REH] \\
\hline \multirow[t]{2}{*}{$\mathrm{C}_{13} \mathrm{H}_{26} \mathrm{O}_{3}$} & {$[14144-56-0]$} & \multicolumn{4}{|c|}{ pentyl 3-pentyloxypropionate } & \\
\hline & V & $(378-498)$ & 62.3 & 393 & A & [1987STE/MAL, 1947REH/DIX] \\
\hline \multirow[t]{2}{*}{$\mathrm{C}_{13} \mathrm{H}_{26} \mathrm{O}_{3}$} & {$[40915-96-6]$} & \multicolumn{2}{|c|}{ peroxytridecanoic acid } & & & \\
\hline & SUB & $(293-303)$ & $142.7 \pm 5$ & 298 & $\mathrm{ME}$ & [1980SWA/KWA] \\
\hline \multirow[t]{2}{*}{$\mathrm{C}_{13} \mathrm{H}_{26} \mathrm{O}_{4}$} & {$[2277-23-8]$} & \multicolumn{2}{|c|}{ 2,3-dihydroxypropyl decanoate } & & & \\
\hline & $\mathrm{V}$ & $(479-524)$ & 111.7 & 502 & DSC & [2014DAM/MAT] \\
\hline
\end{tabular}

[Note: The authors of [2014DAM/MAT] gave a CAS Registry Number of [26402-22-2] which was not consistent with the IUPAC chemical name in the paper.]

\begin{tabular}{|c|c|c|c|c|c|c|}
\hline $\mathrm{C}_{13} \mathrm{H}_{27} \mathrm{Br}$ & $\begin{array}{c}{[765-09-3]} \\
\mathrm{V}\end{array}$ & $\begin{array}{l}\text { 1-bromotridecane } \\
(425-628)\end{array}$ & 64.6 & 440 & $\mathrm{~A}, \mathrm{E}$ & [1987STE/MAL, 1961LI/ROS, 1970DYK/VAN] \\
\hline $\mathrm{C}_{13} \mathrm{H}_{27} \mathrm{Cl}$ & $\begin{array}{c}{[822-13-9]} \\
\mathrm{V} \\
\mathrm{V}\end{array}$ & $\begin{array}{l}\text { 1-chlorotridecane } \\
\text { (414-611) }\end{array}$ & $\begin{array}{l}81.3 \\
63.0\end{array}$ & $\begin{array}{l}298 \\
429\end{array}$ & $\mathrm{~A}, \mathrm{E}$ & $\begin{array}{l}\text { [2006BOL/NER2] } \\
\text { [1987STE/MAL, 1961LI/ROS, 1970DYK/VAN] }\end{array}$ \\
\hline $\mathrm{C}_{13} \mathrm{H}_{27} \mathrm{~F}$ & $\begin{array}{c}{[1536-21-6]} \\
\mathrm{V}\end{array}$ & $\begin{array}{l}\text { 1-fluorotridecane } \\
\text { (387-558) }\end{array}$ & 58.9 & 402 & $\mathrm{~A}, \mathrm{E}$ & [1987STE/MAL, 1961LI/ROS, 1970DYK/VAN] \\
\hline $\mathrm{C}_{13} \mathrm{H}_{27} \mathrm{I}$ & $\begin{array}{c}{[35599-77-0]} \\
\text { V }\end{array}$ & $\begin{array}{l}\text { 1-iodotridecane } \\
(440-655) \\
(440-655)\end{array}$ & $\begin{array}{l}85.0 \\
66.1\end{array}$ & 455 & $\begin{array}{l}\mathrm{A}, \mathrm{E} \\
\mathrm{A}, \mathrm{E}\end{array}$ & $\begin{array}{l}\text { [1987STE/MAL, 1961LI/ROS, 1970DYK/VAN, } \\
\text { 2006BOL/NER] } \\
\text { [1987STE/MAL, 1961LI/ROS, 1970DYK/VAN] }\end{array}$ \\
\hline $\mathrm{C}_{13} \mathrm{H}_{27} \mathrm{NO}$ & $\begin{array}{c}{[27563-67-3]} \\
\text { SUB }\end{array}$ & $\begin{array}{l}N \text {-methyl dodecar } \\
\quad(323-337)\end{array}$ & $\begin{array}{l}\text { namide } \\
116.6 \pm 0.8\end{array}$ & 330 & ME & [1959DAV/JON, 1987STE/MAL] \\
\hline $\mathrm{C}_{13} \mathrm{H}_{27} \mathrm{NO}_{2}$ & $\begin{array}{c}{[6280-24-6]} \\
\mathrm{V}\end{array}$ & $\begin{array}{l}N \text {-decyl lactamide } \\
\quad(413-483)\end{array}$ & 97.9 & 428 & A & [1987STE/MAL, 1950RAT] \\
\hline $\mathrm{C}_{13} \mathrm{H}_{27} \mathrm{NO}_{2}$ & $\mathrm{~V}$ & $\begin{array}{l}O \text {-decyl lactamide } \\
\quad(413-483)\end{array}$ & 95.0 & 428 & A & [1987STE/MAL] \\
\hline
\end{tabular}


TABLE 11. Phase change enthalpies of $\mathrm{C}_{12}$ to $\mathrm{C}_{13}$ organic compounds-Continued

\begin{tabular}{|c|c|c|c|c|c|c|}
\hline \multirow[b]{2}{*}{ Molecular formula } & \multirow{2}{*}{$\begin{array}{l}\text { CAS Registry } \\
\text { Number } \\
\text { Enthalpy }\end{array}$} & \multicolumn{5}{|l|}{ Compound } \\
\hline & & $\begin{array}{l}\text { Temperature } \\
\text { range }\end{array}$ & $\begin{array}{l}\Delta_{\text {trans }} H_{\mathrm{m}} \\
(\mathrm{kJ} / \mathrm{mol})\end{array}$ & $\begin{array}{l}T_{\mathrm{m}} \\
(\mathrm{K})\end{array}$ & Method & References \\
\hline \multirow[t]{22}{*}{$\mathrm{C}_{13} \mathrm{H}_{28}$} & [629-50-5] & tridecane & & & & \\
\hline & TRS & & 7.9 & 254.4 & & \\
\hline & FUS & & 29.62 & 267.3 & DSC & [2005HUA/SIM] \\
\hline & TRS & & 7.7 & 255.2 & & \\
\hline & FUS & & 28.9 & 267.7 & DSC & [2004MON/RAJ] \\
\hline & TRS & & 7.66 & 255 & & \\
\hline & FUS & & 28.49 & 267.8 & & [1996DOM/HEA, 1954FIN/GRO2] \\
\hline & SUB & & 91.4 & 298 & $\mathrm{~B}$ & [1972MOR3] \\
\hline & $\mathrm{V}$ & & 65.3 & 309 & $\mathrm{C}$ & [1996VIT/CHA] \\
\hline & $\mathrm{V}$ & & 64.9 & 314 & $\mathrm{C}$ & [1996VIT/CHA] \\
\hline & $\mathrm{V}$ & & 64.2 & 324 & $\mathrm{C}$ & [1996VIT/CHA] \\
\hline & $\mathrm{V}$ & & 63.3 & 334 & $\mathrm{C}$ & [1996VIT/CHA] \\
\hline & $\mathrm{V}$ & & 62.4 & 344 & $\mathrm{C}$ & [1996VIT/CHA] \\
\hline & $\mathrm{V}$ & & 62.3 & 349 & $\mathrm{C}$ & [1996VIT/CHA] \\
\hline & $\mathrm{V}$ & & 66.7 & 298 & & [1994RUZ/MAJ] \\
\hline & $\mathrm{V}$ & & 65.6 & 308 & $\mathrm{C}$ & [1979SUN/SVE] \\
\hline & $\mathrm{V}$ & & 64.6 & 318 & $\mathrm{C}$ & [1979SUN/SVE] \\
\hline & $\mathrm{V}$ & & 61.7 & 348 & $\mathrm{C}$ & [1979SUN/SVE] \\
\hline & $\mathrm{V}$ & & $66.5 \pm 0.2$ & 298 & $\mathrm{C}$ & [1979SUN/SVE] \\
\hline & $\mathrm{V}$ & & $66.4 \pm 0.3$ & 298 & $\mathrm{C}$ & [1972MOR2] \\
\hline & $\mathrm{V}$ & & 66.2 & 298 & & [1971WIL/ZWO] \\
\hline & $\mathrm{V}$ & $(417-511)$ & 54.5 & 432 & A & [1987STE/MAL, 1955CAM/ROS] \\
\hline $\mathrm{C}_{13} \mathrm{H}_{28}$ & $\begin{array}{c}{[1560-97-0]} \\
\mathrm{V}\end{array}$ & $\begin{array}{l}\text { 2-methyldodecane } \\
\text { (373-503) }\end{array}$ & 52.5 & 388 & A & [1987STE/MAL] \\
\hline $\mathrm{C}_{13} \mathrm{H}_{28}$ & $\begin{array}{c}{[17312-57-1]} \\
\mathrm{V}\end{array}$ & $\begin{array}{l}\text { 3-methyldodecane } \\
\text { (372-504) }\end{array}$ & 51.4 & 387 & A & [1987STE/MAL] \\
\hline $\mathrm{C}_{13} \mathrm{H}_{28}$ & $\begin{array}{c}{[6117-97-1]} \\
\mathrm{V}\end{array}$ & $\begin{array}{l}\text { 4-methyldodecane } \\
\text { (372-501) }\end{array}$ & 52.0 & 387 & A & [1987STE/MAL] \\
\hline $\mathrm{C}_{13} \mathrm{H}_{28}$ & $\begin{array}{c}\text { [17453-93-9] } \\
\mathrm{V}\end{array}$ & $\begin{array}{l}\text { 5-methyldodecane } \\
\quad(368-500)\end{array}$ & 50.6 & 383 & A & [1987STE/MAL] \\
\hline $\mathrm{C}_{13} \mathrm{H}_{28}$ & $\begin{array}{c}{[17312-77-5]} \\
\mathrm{V}\end{array}$ & \multicolumn{2}{|c|}{ 2,3-dimethylundecane } & 398 & A & [1987STE/MAL] \\
\hline $\mathrm{C}_{13} \mathrm{H}_{28}$ & {$[17312-80-0]$} & \multicolumn{2}{|c|}{ 2,4-dimethylundecane } & & & \\
\hline & V & $(365-490)$ & 52.1 & 380 & A & [1987STE/MAL] \\
\hline $\mathrm{C}_{13} \mathrm{H}_{28}$ & $\begin{array}{c}\text { [62108-27-4] } \\
\mathrm{V}\end{array}$ & $\begin{array}{l}\text { 2,4,6-trimethyldeca } \\
\quad(352-478)\end{array}$ & 48.7 & 367 & A & [1987STE/MAL] \\
\hline $\mathrm{C}_{13} \mathrm{H}_{28}$ & {$[17312-74-2]$} & \multicolumn{2}{|c|}{ 5-ethyl-5-methyldecane } & & & \\
\hline & V & $(273-307)$ & $61.4 \pm 1.1$ & 290 & HSA & [1995CHI/HES] \\
\hline & $\mathrm{V}$ & & $60.5 \pm 1.1$ & 298 & & [1995CHI/HES] \\
\hline & $\mathrm{V}$ & & $61.4 \pm 1.8$ & 298 & $\mathrm{CGC}$ & [1995CHI/HES] \\
\hline $\mathrm{C}_{13} \mathrm{H}_{28}$ & $\begin{array}{c}{[17312-63-9]} \\
\mathrm{V}\end{array}$ & $\begin{array}{l}\text { 5-butylnonane } \\
\text { (298-365) }\end{array}$ & 52.6 & 313 & $\mathrm{~A}, \mathrm{MG}$ & [1987STE/MAL, 1955SCH/WHI] \\
\hline \multirow[t]{7}{*}{$\mathrm{C}_{13} \mathrm{H}_{28}$} & {$[35660-96-9]$} & \multicolumn{2}{|c|}{ Tri-tert-butylmethane } & & & \\
\hline & FUS & & 3.53 & 358.2 & $\mathrm{DSC}$ & [1986FLA/BEC] \\
\hline & SUB & & 55.4 & 298 & CGC-DSC & [1998CHI/HES] \\
\hline & SUB & $(265-319)$ & $57.0 \pm 0.4$ & 288 & $\mathrm{~T}$ & [1997VER/NOL] \\
\hline & SUB & $(273-306)$ & $57.7 \pm 2.8$ & 290 & HSA & [1995CHI/HES] \\
\hline & SUB & & $61.1 \pm 1.3$ & & & [1995CHI/HES] \\
\hline & SUB & $(295-330)$ & $7.7 \pm 0.1$ & 311 & & [1986FLA/BEC] \\
\hline \multirow[t]{3}{*}{$\mathrm{C}_{13} \mathrm{H}_{28} \mathrm{~N}_{2} \mathrm{O}$} & {$[2158-09-0]$} & \multicolumn{2}{|l|}{ 1-dodecyl urea } & & & \\
\hline & TRS & & 1.3 & 275.4 & & \\
\hline & FUS & & 46.6 & 379.2 & DSC & [2005HAS/TAJ] \\
\hline \multirow[t]{2}{*}{$\mathrm{C}_{13} \mathrm{H}_{28} \mathrm{O}$} & [508181-43-9] & \multicolumn{2}{|c|}{ Pentyl tert-octyl ether } & & & \\
\hline & $\mathrm{V}$ & $(278-303)$ & $55.9 \pm 0.3$ & 298 & GS & [UR/VER, 2002VER, 2003VER/KRA] \\
\hline
\end{tabular}


TABLE 11. Phase change enthalpies of $\mathrm{C}_{12}$ to $\mathrm{C}_{13}$ organic compounds-Continued

\begin{tabular}{|c|c|c|c|c|c|c|}
\hline \multirow[b]{2}{*}{ Molecular formula } & \multirow{2}{*}{$\begin{array}{l}\text { CAS Registry } \\
\text { Number } \\
\text { Enthalpy }\end{array}$} & \multicolumn{5}{|l|}{ Compound } \\
\hline & & $\begin{array}{l}\text { Temperature } \\
\text { range }\end{array}$ & $\begin{array}{l}\Delta_{\text {trans }} H_{\mathrm{m}} \\
(\mathrm{kJ} / \mathrm{mol})\end{array}$ & $\begin{array}{l}T_{\mathrm{m}} \\
(\mathrm{K})\end{array}$ & Method & References \\
\hline \multirow[t]{3}{*}{$\mathrm{C}_{13} \mathrm{H}_{28} \mathrm{O}$} & [112-70-9] & 1-tridecanol & & & & \\
\hline & TRS & & 20.8 & 317.4 & & \\
\hline & FUS & & 24.87 & 318.3 & DSC & [2008EGO/MAR] \\
\hline \multicolumn{7}{|c|}{$\begin{array}{l}\text { [Note: Transition temperatures reported in [2008EGO/MAR] are considerably higher than melting points reported in synthetic papers and othe } \\
\text { thermodynamic papers.] }\end{array}$} \\
\hline & FUS & $(5-370)$ & 44.78 & 304.7 & & [2003VAN/VAN] \\
\hline & FUS $(\beta)$ & & 45.1 & 304.6 & & \\
\hline & FUS $(\gamma)$ & & 41.42 & 304.5 & & \\
\hline & FUS $(\alpha)$ & & 23.3 & 303.5 & & \\
\hline & $\operatorname{TRS}(\beta$ to $\gamma)$ & & 3.6 & 301.6 & & \\
\hline & $\operatorname{TRS}(\beta$ to $\alpha)$ & & 22.09 & 305.8 & & \\
\hline & $\operatorname{TRS}(\gamma$ to $\alpha)$ & & 18.74 & 306.6 & $\mathrm{AC}$ & [1974MOS/MOU] \\
\hline & $\mathrm{V}$ & & $94.7 \pm 0.4$ & 298 & CGC & [2006NIC/KWE] \\
\hline & $\mathrm{V}$ & $(307-348)$ & 91.1 & 327 & GS & [2001KUL/VER2] \\
\hline & $\mathrm{V}$ & (307-348) & 95.8 & 298 & GS & [2001KUL/VER2] \\
\hline & $\mathrm{V}$ & $(313-373)$ & 87.4 & 343 & & [1992NGU/KAS] \\
\hline & $\mathrm{V}$ & $(431-568)$ & 69.2 & 446 & A & [1987STE/MAL] \\
\hline \multirow[t]{2}{*}{$\mathrm{C}_{13} \mathrm{H}_{28} \mathrm{O}$} & {$[42930-67-6]$} & \multicolumn{5}{|c|}{ 2,2-dimethyl-3-tert-butyl-3-heptanol } \\
\hline & $\mathrm{V}$ & $(379-513)$ & 58.3 & 394 & & [1973WIL/ZWO] \\
\hline \multirow[t]{2}{*}{$\mathrm{C}_{13} \mathrm{H}_{28} \mathrm{O}$} & & \multicolumn{4}{|c|}{ 3,3,5,5-tetramethyl-4-ethyl-4-heptanol } & \\
\hline & $\mathrm{V}$ & $(393-526)$ & 55.9 & 408 & & [1973WIL/ZWO] \\
\hline \multirow[t]{2}{*}{$\mathrm{C}_{13} \mathrm{H}_{28} \mathrm{O}$} & & \multicolumn{4}{|c|}{ 3,3,6-trimethyl-4-isopropyl-4-heptanol } & \\
\hline & $\mathrm{V}$ & $(381-512)$ & 59.1 & 396 & & [1973WIL/ZWO] \\
\hline \multirow[t]{2}{*}{$\mathrm{C}_{13} \mathrm{H}_{28} \mathrm{O}$} & & \multicolumn{4}{|c|}{ 3,3,6-trimethyl-4-propyl-4-heptanol } & \\
\hline & $\mathrm{V}$ & $(383-513)$ & 60.1 & 398 & & [1973WIL/ZWO] \\
\hline \multirow[t]{2}{*}{$\mathrm{C}_{12} \mathrm{H}_{28} \mathrm{O}$} & {$[32579-70-7]$} & \multicolumn{4}{|c|}{ 2,2,5-trimethyl-3-tert-butyl-3-hexanol } & \\
\hline & $\mathrm{V}$ & $(377-513)$ & 57.6 & 392 & & [1973WIL/ZWO] \\
\hline \multirow[t]{5}{*}{$\mathrm{C}_{13} \mathrm{H}_{28} \mathrm{O}$} & [41902-42-5] & \multicolumn{4}{|c|}{ Tri-tert-butylmethanol } & \\
\hline & TRS & & 7.2 & 302 & & \\
\hline & FUS & & 3.43 & 390 & DSC & [1996DOM/HEA, 1983MAS/STE] \\
\hline & SUB (plastic) & $(278-318)$ & $56.5 \pm 1.0$ & 298 & $\mathrm{TE}$ & [1983MAS/STE] \\
\hline & SUB (crys) & $(269-300)$ & $63.2 \pm 1.2$ & 298 & $\mathrm{TE}$ & [1983MAS/STE] \\
\hline \multirow[t]{6}{*}{$\mathrm{C}_{13} \mathrm{H}_{28} \mathrm{O}_{2}$} & {$[13362-52-2]$} & \multicolumn{3}{|c|}{ 1,13-tridecanediol } & & \\
\hline & FUS+TRS & & 54.4 & 350.3 & DSC & [2014BAD/NOW] \\
\hline & TRS & & 28.9 & 343 & & \\
\hline & FUS & & 17.8 & 351 & DSC & [1999OGA/NAK] \\
\hline & $\mathrm{V}$ & & $122.0 \pm 7.6$ & 372 & TE & [1994PIA/FER, 2006UMN/KWE] \\
\hline & $\mathrm{V}$ & & $132.8 \pm 7.8$ & 298 & $\mathrm{TE}$ & [1994PIA/FER, 2006UMN/KWE] \\
\hline $\mathrm{C}_{13} \mathrm{H}_{28} \mathrm{O}_{2} \mathrm{~S}$ & {$[24724-30-9]$} & 3-(decylthio)-1 & propanediol & & & \\
\hline & TRS & & 17.3 & 291.9 & & \\
\hline & FUS & & 17.3 & 311.9 & DSC & [1993ACR, 1990VAN/VAN] \\
\hline $\mathrm{C}_{13} \mathrm{H}_{28} \mathrm{O}_{3}$ & {$[10430-97-4]$} & 3-(decyloxy)-1 & propanediol & & & \\
\hline & FUS & & 38.9 & 311 & DSC & [1993ACR, 1990VAN/VAN] \\
\hline $\mathrm{C}_{13} \mathrm{H}_{28} \mathrm{O}_{4}$ & [57499-93-1] & Tripropylene g & ol, monobutyl & her & & \\
\hline & $\mathrm{V}$ & $(374-543)$ & 67.1 & 389 & A & [1987STE/MAL, 1947STU] \\
\hline $\mathrm{C}_{13} \mathrm{H}_{28} \mathrm{O}_{5} \mathrm{~S}_{2}$ & [123483-21-6] & $(L)$-arabinose & utyl dithioace & & & \\
\hline & FUS & & 41.5 & 380.4 & DSC & [1989VAN/VAN] \\
\hline $\mathrm{C}_{13} \mathrm{H}_{28} \mathrm{~S}$ & {$[19484-26-5]$} & 1-tridecanethic & & & & \\
\hline & $\mathrm{V}$ & $(433-598)$ & 64.7 & 448 & & [1999DYK/SVO] \\
\hline $\mathrm{C}_{13} \mathrm{H}_{29} \mathrm{~N}$ & {$[2869-34-3]$} & Tridecylamine & & & & \\
\hline & $\mathrm{V}$ & $(458-562)$ & 60.1 & 473 & $\mathrm{~A}, \mathrm{E}$ & [1987STE/MAL, 1956MAN2] \\
\hline $\mathrm{C}_{13} \mathrm{H}_{29} \mathrm{NO}_{2}$ & {$[1191-45-3]$} & 3-(decylamino & 2-propanedio & & & \\
\hline & FUS & & 54.8 & 346.6 & DSC & [1993ACR, 1990VAN/VAN] \\
\hline
\end{tabular}


TABle 12. Phase change enthalpies of $\mathrm{C}_{14}$ to $\mathrm{C}_{18}$ organic compounds

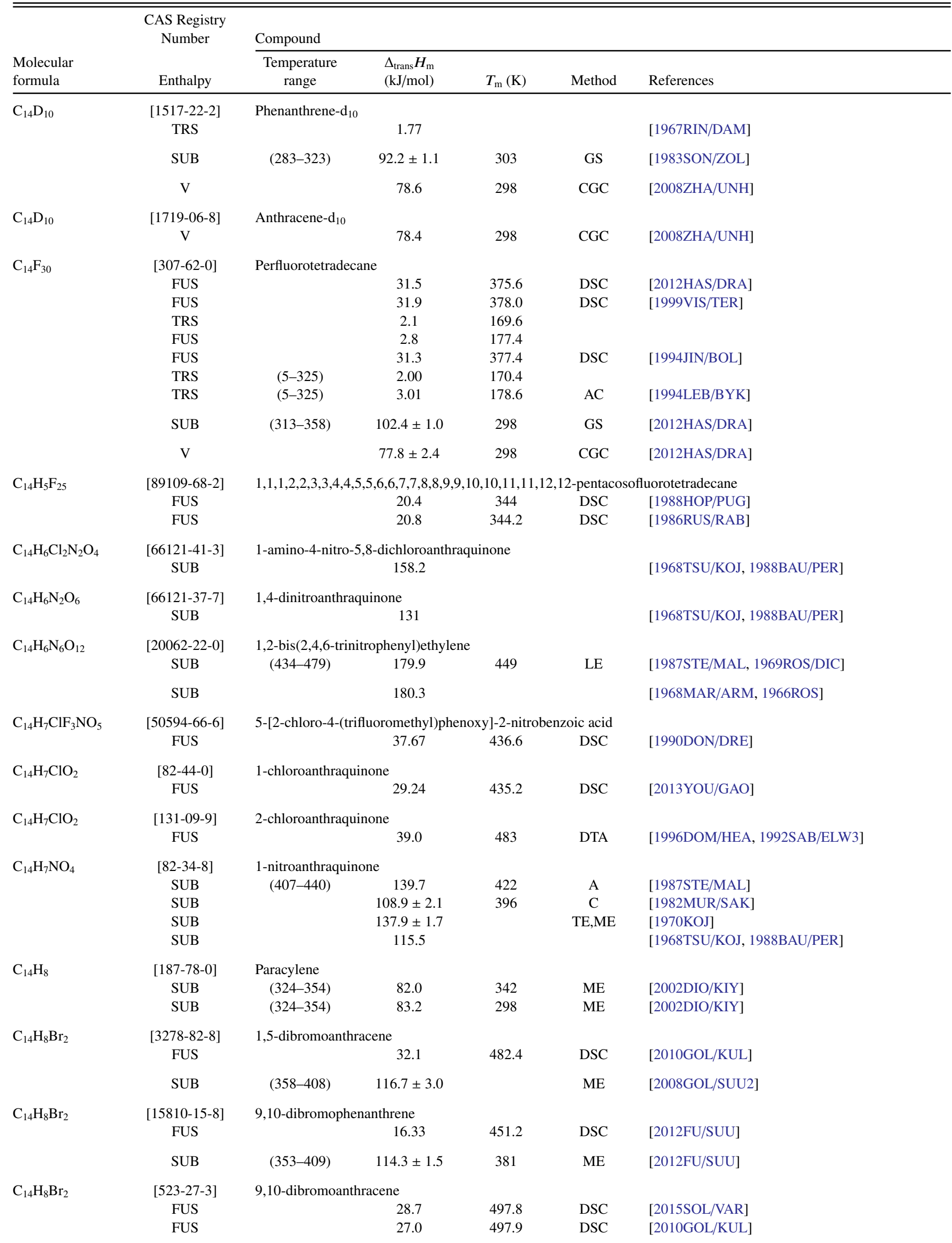


TABLE 12. Phase change enthalpies of $\mathrm{C}_{14}$ to $\mathrm{C}_{18}$ organic compounds-Continued

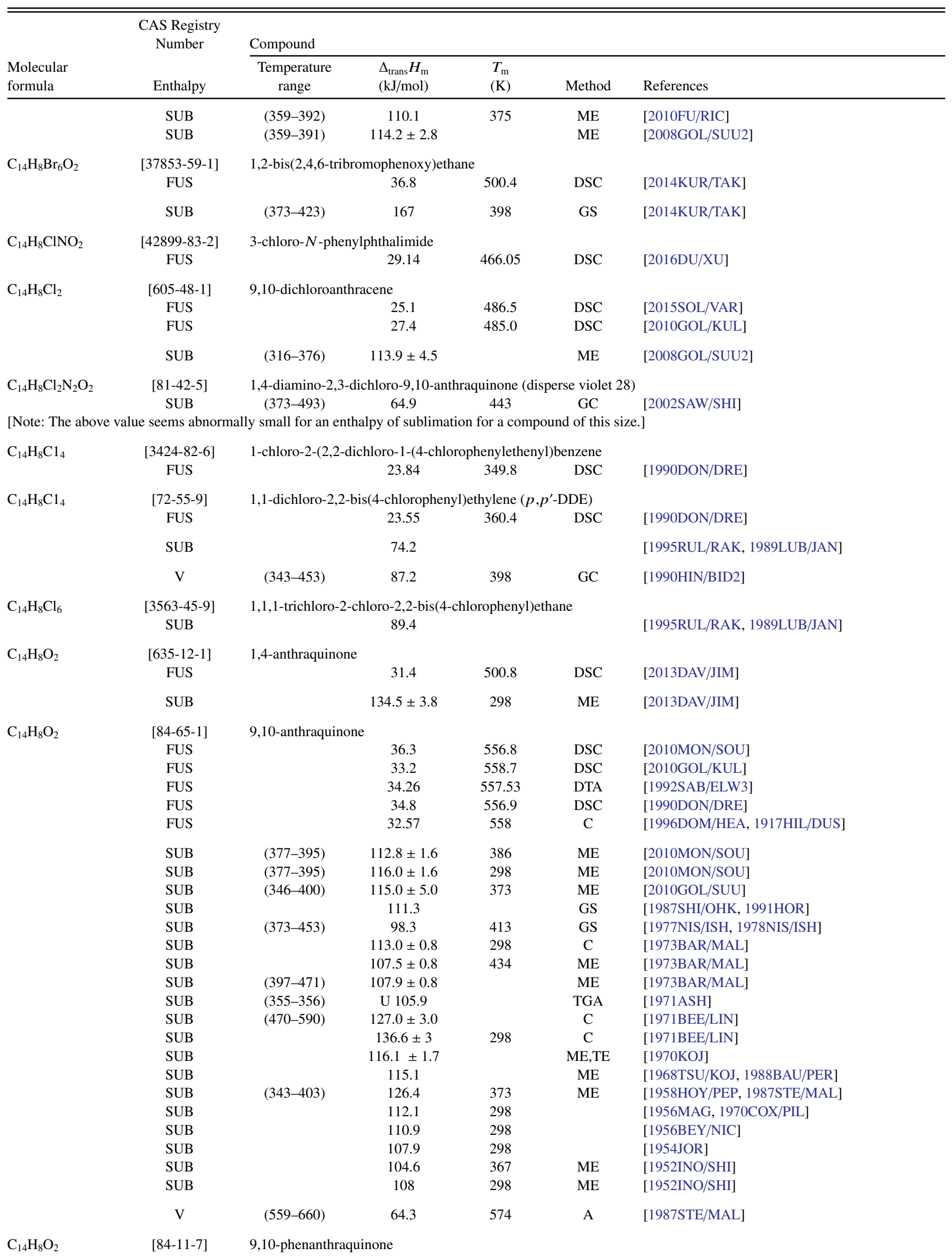


TABLE 12. Phase change enthalpies of $\mathrm{C}_{14}$ to $\mathrm{C}_{18}$ organic compounds-Continued

\begin{tabular}{|c|c|c|c|c|c|c|}
\hline \multirow[b]{2}{*}{$\begin{array}{l}\text { Molecular } \\
\text { formula }\end{array}$} & \multirow{2}{*}{$\begin{array}{c}\text { CAS Registry } \\
\text { Number } \\
\text { Enthalpy }\end{array}$} & \multicolumn{5}{|l|}{ Compound } \\
\hline & & $\begin{array}{c}\text { Temperature } \\
\text { range }\end{array}$ & $\begin{array}{l}\Delta_{\text {trans }} H_{\mathrm{m}} \\
(\mathrm{kJ} / \mathrm{mol})\end{array}$ & $\begin{array}{l}T_{\mathrm{m}} \\
(\mathrm{K})\end{array}$ & Method & References \\
\hline & SUB & & 108.1 & 289 & $\mathrm{C}$ & [1989RIB/RIB] \\
\hline & SUB & & 132 & 383 & & [1956MAG, 1970COX/PIL] \\
\hline \multirow[t]{4}{*}{$\mathrm{C}_{14} \mathrm{H}_{8} \mathrm{O}_{3}$} & {$[129-43-1]$} & 1-hydroxy-9,10 & hraquinone & & & \\
\hline & SUB & & 113.4 & & GS & [1987SHI/OHK, 1991HOR] \\
\hline & SUB & $(333-383)$ & 120.6 & 358 & & [1958HOY/PEP, 1987STE/MAL] \\
\hline & SUB & & $101.3 \pm 0.4$ & 407 & HSA & {$[1956 \mathrm{BEY} / \mathrm{NIC}]$} \\
\hline \multirow[t]{3}{*}{$\mathrm{C}_{14} \mathrm{H}_{8} \mathrm{O}_{3}$} & [605-32-3] & 2-hydroxy-9,10 & hraquinone & & & \\
\hline & SUB & & 136.8 & & GS & [1987SHI/OHK, 1991HOR] \\
\hline & SUB & $(393-453)$ & 153.1 & 408 & A & [1987STE/MAL] \\
\hline \multirow[t]{3}{*}{$\mathrm{C}_{14} \mathrm{H}_{8} \mathrm{O}_{3}$} & [74553-57-4] & 9-hydroxy-1,4- & raquinone & & & \\
\hline & SUB & (377-394) & $108.2 \pm 2.2$ & 386 & ME & [2002JIM/ROU] \\
\hline & SUB & (377-394) & $109.5 \pm 2.2$ & 298 & ME & [2002JIM/ROU] \\
\hline \multirow[t]{3}{*}{$\mathrm{C}_{14} \mathrm{H}_{8} \mathrm{O}_{3}$} & {$[6050-13-1]$} & 2,2'-biphenyldi & oxylic anhydr & & & \\
\hline & SUB & & $120.7 \pm 4.0$ & 298 & $\mathrm{C}$ & [2005MAT/MIR2] \\
\hline & SUB & $(433-490)$ & 91.4 & 448 & A & [1987STE/MAL] \\
\hline \multirow[t]{5}{*}{$\mathrm{C}_{14} \mathrm{H}_{8} \mathrm{O}_{4}$} & {$[72-48-0]$} & 1,2-dihydroxya & aquinone & & & \\
\hline & SUB & $(368-498)$ & 123.8 & 383 & A & [1987STE/MAL] \\
\hline & SUB & & $121.9 \pm 0.5$ & 469 & $\mathrm{C}$ & [1973MAL/BAR] \\
\hline & SUB & $(434-505)$ & $121.5 \pm 0.4$ & 469 & ME & [1973MAL/BAR] \\
\hline & SUB & & 123.9 & 403 & $\mathrm{ME}$ & [1958HOY/PEP] \\
\hline \multirow[t]{12}{*}{$\mathrm{C}_{14} \mathrm{H}_{8} \mathrm{O}_{4}$} & {$[81-64-1]$} & 1,4-dihydroxy- & -anthraquinon & inizarin) & & \\
\hline & FUS & & 19.41 & 473.2 & DSC & [2015CHE/SVA] \\
\hline & SUB & $(363-393)$ & 115.3 & 363 & TGA, GS & [2003HIN/RAF] \\
\hline & SUB & & 114.6 & & GS & [1987SHI/OHK, 1991HOR] \\
\hline & SUB & $(353-373)$ & $102.4 \pm 4.4$ & 363 & & {$[1984 \mathrm{KRI}]$} \\
\hline & SUB & $(473-553)$ & 89.1 & 513 & GS & [1977NIS/ISH, 1978NIS/ISH] \\
\hline & SUB & $(394-463)$ & $121.9 \pm 0.8$ & 429 & ME & [1973MAL/BAR] \\
\hline & SUB & & $121.1 \pm 4$ & 429 & $\mathrm{C}$ & [1973MAL/BAR] \\
\hline & SUB & $(324-351)$ & U 94.5 & 338 & TGA & {$[1971 \mathrm{ASH}]$} \\
\hline & SUB & & 123.5 & 376 & & [1958HOY/PEP, 1987STE/MAL] \\
\hline & SUB & & $103.5 \pm 1.3$ & 409 & HSA & {$[1956 \mathrm{BEY} / \mathrm{NIC}]$} \\
\hline & $\mathrm{V}$ & $(469-633)$ & 74.0 & 484 & A & [1987STE/MAL, 1947STU] \\
\hline \multirow[t]{5}{*}{$\mathrm{C}_{14} \mathrm{H}_{8} \mathrm{O}_{4}$} & {$[117-12-4]$} & 1,5-dihydroxya & aquinone & & & \\
\hline & SUB & & $123.2 \pm 7$ & & ME & [1973BAR/MAL] \\
\hline & SUB & $(363-433)$ & 126.8 & 398 & $\mathrm{ME}$ & [1958HOY/PEP, 1987STE/MAL] \\
\hline & SUB & & 111.3 & 456 & HSA & {$[1956 \mathrm{BEY} / \mathrm{NIC}]$} \\
\hline & SUB & & 117.6 & 298 & HSA & [1956BEY/NIC] \\
\hline \multirow[t]{8}{*}{$\mathrm{C}_{14} \mathrm{H}_{8} \mathrm{O}_{4}$} & {$[117-10-2]$} & 1,8-dihydroxya & aquinone & & & \\
\hline & TRS & & 4.96 & 437.16 & & \\
\hline & FUS & & 20.06 & 471.2 & DSC & [2015CHE/KHA] \\
\hline & SUB & & 116.8 & & $\mathrm{ME}$ & [1973BAR/MAL] \\
\hline & SUB & $(333-403)$ & 123 & 368 & $\mathrm{ME}$ & [1958HOY/PEP, 1987STE/MAL] \\
\hline & SUB & $(335-356)$ & U 96.5 & 345 & TGA & [1971ASH] \\
\hline & SUB & & $105.8 \pm 8$ & 404 & HSA & [1956BEY/NIC] \\
\hline & SUB & & $109.6 \pm 8$ & 298 & HSA & [1956BEY/NIC] \\
\hline \multirow[t]{2}{*}{$\mathrm{C}_{14} \mathrm{H}_{8} \mathrm{O}_{4}$} & [84-60-6] & 2,6-dihydroxya & aquinone & & & \\
\hline & SUB & $(463-533)$ & 173.8 & 498 & & [1958HOY/PEP, 1987STE/MAL] \\
\hline \multirow[t]{2}{*}{$\mathrm{C}_{14} \mathrm{H}_{8} \mathrm{O}_{6}$} & [81-60-7] & 1,4,5,8-tetrahy & yanthraquinor & & & \\
\hline & SUB & $(403-473)$ & 151.6 & 438 & & [1958HOY/PEP, 1987STE/MAL] \\
\hline \multirow[t]{3}{*}{$\mathrm{C}_{14} \mathrm{H}_{9} \mathrm{Br}$} & [7321-27-9] & 2-bromoanthra & & & & \\
\hline & FUS & & 26.23 & 493.6 & DSC & [2012FU/SUU] \\
\hline & SUB & $(334-390)$ & $101.3 \pm 0.6$ & 362 & $\mathrm{ME}$ & [2012FU/SUU] \\
\hline
\end{tabular}


TABLE 12. Phase change enthalpies of $\mathrm{C}_{14}$ to $\mathrm{C}_{18}$ organic compounds-Continued

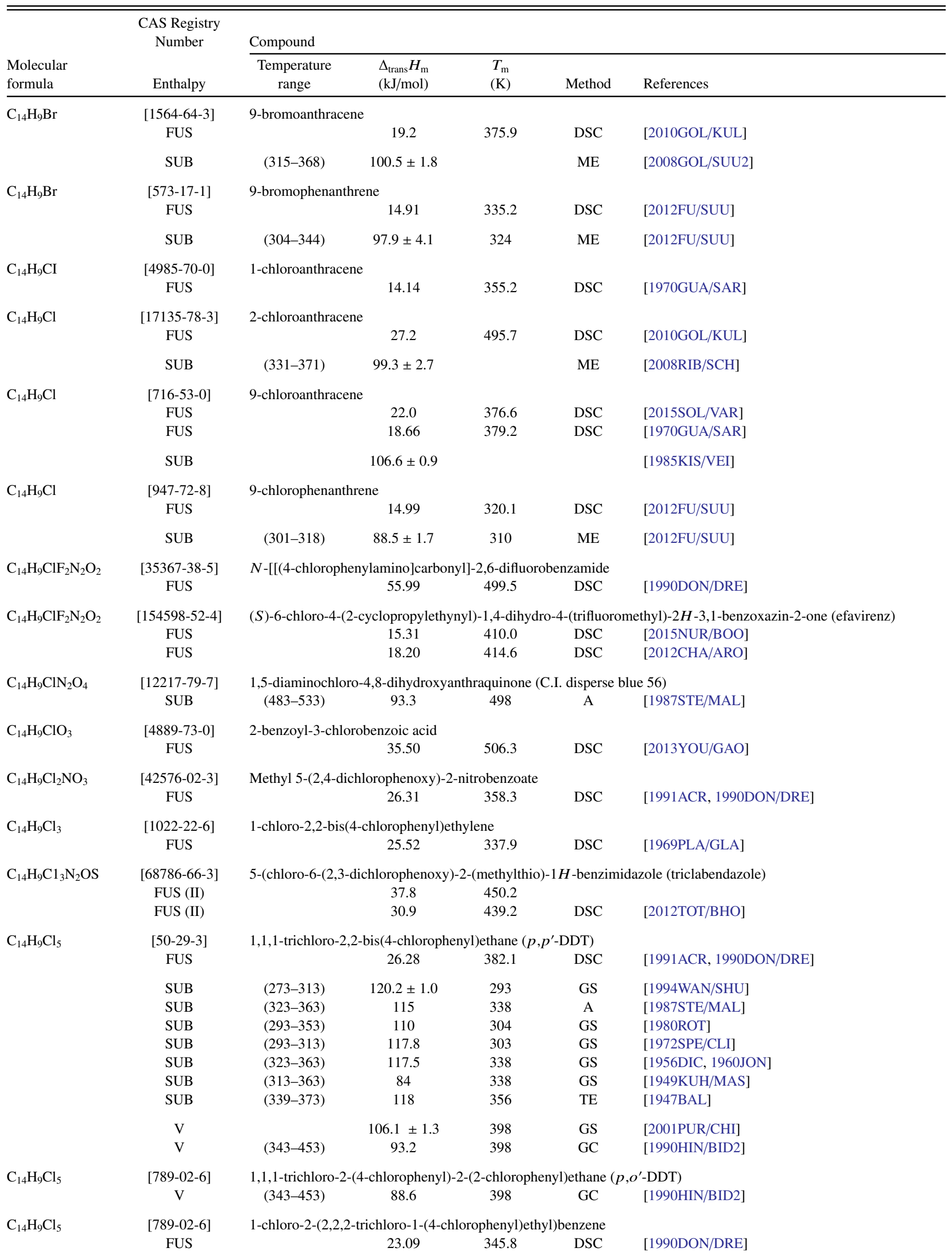


TABLE 12. Phase change enthalpies of $\mathrm{C}_{14}$ to $\mathrm{C}_{18}$ organic compounds-Continued

\begin{tabular}{|c|c|c|c|c|c|c|}
\hline \multirow[b]{2}{*}{$\begin{array}{l}\text { Molecular } \\
\text { formula }\end{array}$} & \multirow{2}{*}{$\begin{array}{l}\text { CAS Registry } \\
\text { Number } \\
\text { Enthalpy }\end{array}$} & \multicolumn{5}{|l|}{ Compound } \\
\hline & & $\begin{array}{l}\text { Temperature } \\
\text { range }\end{array}$ & $\begin{array}{l}\Delta_{\text {trans }} H_{\mathrm{m}} \\
(\mathrm{kJ} / \mathrm{mol})\end{array}$ & $\begin{array}{l}T_{\mathrm{m}} \\
(\mathrm{K})\end{array}$ & Method & References \\
\hline $\mathrm{C}_{14} \mathrm{H}_{9} \mathrm{Cl}_{5}$ & $\mathrm{~V}$ & $\begin{array}{l}\text { DDT } \\
\qquad(313-363)\end{array}$ & 83.7 & 338 & & [1949KUH/MAS] \\
\hline $\mathrm{C}_{14} \mathrm{H}_{9} \mathrm{Cl}_{5} \mathrm{O}$ & $\begin{array}{l}\text { [10606-46-9] } \\
\text { FUS }\end{array}$ & 2-chloro- $\alpha-(4-$ & $\begin{array}{l}\text { ophenyl })-\alpha-(\mathrm{t} \\
25.2\end{array}$ & $\begin{array}{l}\text { romethy } \\
396.3\end{array}$ & $\begin{array}{l}\text { zenemeth } \\
\text { DSC }\end{array}$ & [1991ACR, 1990DON/DRE] \\
\hline $\mathrm{C}_{14} \mathrm{H}_{9} \mathrm{Cl}_{5} \mathrm{O}$ & $\begin{array}{c}{[115-32-2]} \\
\text { FUS }\end{array}$ & 4-chloro- $\alpha-(4-$ & $\begin{array}{l}\text { ophenyl })-\alpha-(\mathrm{t} \\
19.56\end{array}$ & $\begin{array}{l}\text { romethy } \\
347.2\end{array}$ & $\begin{array}{l}\text { zenemeth } \\
\text { DSC }\end{array}$ & [1990DON/DRE] \\
\hline $\mathrm{C}_{14} \mathrm{H}_{9} \mathrm{~F}_{3} \mathrm{O}_{2}$ & $\begin{array}{c}{[893-33-4]} \\
\text { SUB }\end{array}$ & 4,4,4-trifluoro- & $\begin{array}{l}\text { naphthyl)-but } \\
108.7 \pm 0.6\end{array}$ & $\begin{array}{l}3 \text {-dione } \\
298\end{array}$ & ME & [1997RIB/GON] \\
\hline $\mathrm{C}_{14} \mathrm{H}_{9} \mathrm{~F}_{17} \mathrm{O}_{2}$ & $\begin{array}{l}\text { [1996-88-9] } \\
\text { TRS } \\
\text { FUS }\end{array}$ & Perfluorooctyle & $\begin{array}{l}\text { ene methacryl } \\
5.0 \\
9.0\end{array}$ & $\begin{array}{l}210 \\
253\end{array}$ & DSC & [1992HOP/FAU] \\
\hline $\mathrm{C}_{14} \mathrm{H}_{9} \mathrm{~F}_{21} \mathrm{O}$ & $\begin{array}{l}{[39239-81-1]} \\
\text { FUS }\end{array}$ & $\omega$-perfluorodec & $\begin{array}{r}\text { butanol } \\
21.3\end{array}$ & 360 & DSC & [1991HOP/MOL] \\
\hline $\mathrm{C}_{14} \mathrm{H}_{9} \mathrm{NO}_{2}$ & $\begin{array}{l}{[82-45-1]} \\
\text { FUS } \\
\text { FUS } \\
\text { SUB } \\
\text { SUB } \\
\text { SUB } \\
\text { SUB } \\
\text { SUB } \\
\text { SUB } \\
\text { SUB } \\
\text { SUB } \\
\text { SUB }\end{array}$ & $\begin{array}{l}(423-443) \\
(413-443) \\
(368-393) \\
(473-553) \\
(361-386)\end{array}$ & $\begin{array}{c}26.3 \\
28.78 \\
121.1 \\
121.8 \\
126.5 \\
116.3 \pm 3.9 \\
103.3 \\
U 90.9 \\
125.9 \pm 2.5 \\
131 \\
113 \pm 0.4\end{array}$ & $\begin{array}{l}524.7 \\
524.2 \\
433 \\
428 \\
380 \\
513 \\
374 \\
\\
463\end{array}$ & $\begin{array}{c}\text { GC } \\
\text { GS } \\
\text { A } \\
\text { GS } \\
\text { TGA } \\
\text { TE,ME } \\
\text { HSA }\end{array}$ & $\begin{array}{l}\text { [2002SAW/SHI] } \\
{[1988 \mathrm{BAU} / \mathrm{PER}]} \\
\text { [2002SAW/SHI] } \\
{[1987 \mathrm{SHI} / \mathrm{OHK}, 1991 \mathrm{HOR}]} \\
{[1987 \mathrm{STE} / \mathrm{MAL}]} \\
{[1984 \mathrm{KRI}]} \\
{[1977 \mathrm{NIS} / \mathrm{ISH}, 1978 \mathrm{NIS} / \mathrm{ISH}]} \\
{[1971 \mathrm{ASH}]} \\
{[1970 \mathrm{KOJ}]} \\
{[1968 \mathrm{TSU} / \mathrm{KOJ}, 1988 \mathrm{BAU} / \mathrm{PER}]} \\
\text { [1956BEY/NIC] }\end{array}$ \\
\hline $\mathrm{C}_{14} \mathrm{H}_{9} \mathrm{NO}_{2}$ & $\begin{array}{l}\text { [117-79-3] } \\
\text { SUB } \\
\text { SUB } \\
\text { SUB }\end{array}$ & 2-aminoanthra & $\begin{array}{l}136.8 \\
143.5 \pm 2.9 \\
162.3\end{array}$ & & $\begin{array}{c}\text { GS } \\
\text { TE,ME }\end{array}$ & $\begin{array}{l}\text { [1987SHI/OHK, 1991HOR] } \\
\text { [1970KOJ] } \\
\text { [1968TSU/KOJ, 1988BAU/PER] }\end{array}$ \\
\hline $\mathrm{C}_{14} \mathrm{H}_{9} \mathrm{NO}_{2}$ & $\begin{array}{l}\text { [602-60-8] } \\
\text { FUS } \\
\text { SUB } \\
\text { SUB }\end{array}$ & $\begin{array}{l}(361-377) \\
(361-377)\end{array}$ & $\begin{array}{c}20.1 \\
111.9 \pm 0.6 \\
115.4 \pm 0.6\end{array}$ & $\begin{array}{l}420.4 \\
369 \\
298\end{array}$ & $\begin{array}{l}\text { DSC } \\
\mathrm{ME} \\
\mathrm{ME}\end{array}$ & $\begin{array}{l}\text { [2010KES/AUC] } \\
\text { [2006RIB/AMA3] } \\
\text { [2006RIB/AMA3] }\end{array}$ \\
\hline $\mathrm{C}_{14} \mathrm{H}_{9} \mathrm{NO}_{3}$ & $\begin{array}{c}\text { [116-85-8] } \\
\text { SUB } \\
\text { SUB } \\
\text { SUB } \\
\text { SUB } \\
\text { SUB } \\
\text { SUB } \\
\text { SUB }\end{array}$ & $\begin{array}{c}\text { 1-hydroxy-4-ar } \\
\quad(433-453) \\
(418-438) \\
(444-473)\end{array}$ & $\begin{array}{c}\text { anthraquinone } \\
129.3 \\
127.2 \\
131.3 \\
144 \\
119.6 \\
133.5 \pm 2.1 \\
120.1\end{array}$ & $\begin{array}{c}443 \\
428 \\
458.5\end{array}$ & $\begin{array}{c}\text { GC } \\
\text { GS } \\
\text { A } \\
\text { A } \\
\text { TE,ME }\end{array}$ & $\begin{array}{l}\text { [2002SAW/SHI] } \\
\text { [1987SHI/OHK, 1991HOR] } \\
\text { [1987STE/MAL, 1980ROD/KRU] } \\
\text { [1987STE/MAL] } \\
\text { [1984KAR/KRU] } \\
\text { [1970KOJ] } \\
\text { [1968TSU/KOJ, 1988BAU/PER] }\end{array}$ \\
\hline $\mathrm{C}_{14} \mathrm{H}_{9} \mathrm{~N}_{3} \mathrm{O}_{4}$ & $\begin{array}{c}{[82-33-7]} \\
\text { SUB (not } \\
\text { crystalline) }\end{array}$ & $\begin{array}{l}\text { 1,4-diamino-5- } \\
(473-553)\end{array}$ & $\begin{array}{l}\text { anthraquinon } \\
\text { U50.2 }\end{array}$ & 513 & GS & [1977NIS/ISH, 1978NIS/ISH] \\
\hline $\mathrm{C}_{14} \mathrm{H}_{10}$ & $\begin{array}{c}\text { [120-12-7] } \\
\text { FUS } \\
\text { FUS } \\
\text { FUS } \\
\text { FUS } \\
\text { FUS } \\
\text { FUS } \\
\text { FUS } \\
\text { FUS } \\
\text { FUS } \\
\text { FUS }\end{array}$ & $(463-503)$ & $\begin{array}{l}27.4 \\
24.96 \\
27.8 \\
28.2 \\
27.8 \\
29.8 \\
31.5 \\
28.8 \\
29.0 \\
28.97\end{array}$ & $\begin{array}{c}490 \\
489.5 \\
487 \\
489.7 \\
490 \\
492 \\
491 \\
489.4 \\
490.6 \\
491.3\end{array}$ & $\begin{array}{l}\text { DSC } \\
\text { DSC } \\
\text { DSC } \\
\text { DSC } \\
\text { DSC } \\
\text { DSC } \\
\text { DSC } \\
\text { DSC } \\
\text { DSC } \\
\text { DSC }\end{array}$ & $\begin{array}{l}\text { [2015RIC/FU] } \\
\text { [2015DIN/YIN] } \\
\text { [2011RIC/FU] } \\
\text { [2010GOL/KUL] } \\
\text { [2010RIC/FU, 2010RIC/SUU, 2011RIC/FU] } \\
\text { [2003ROJ/ORO] } \\
{[2003 S T O / K R Z]} \\
\text { [2000LIS/JAM] } \\
\text { [1980RAD/RAD] } \\
\text { [1980KRA/PIG] }\end{array}$ \\
\hline
\end{tabular}


TABLE 12. Phase change enthalpies of $\mathrm{C}_{14}$ to $\mathrm{C}_{18}$ organic compounds-Continued

\begin{tabular}{|c|c|c|c|c|c|c|}
\hline \multirow[b]{2}{*}{$\begin{array}{l}\text { Molecular } \\
\text { formula }\end{array}$} & \multirow{2}{*}{$\begin{array}{c}\text { CAS Registry } \\
\text { Number } \\
\text { Enthalpy }\end{array}$} & \multicolumn{5}{|l|}{ Compound } \\
\hline & & $\begin{array}{l}\text { Temperature } \\
\text { range }\end{array}$ & $\begin{array}{l}\Delta_{\text {trans }} H_{\mathrm{m}} \\
(\mathrm{kJ} / \mathrm{mol})\end{array}$ & $\begin{array}{l}T_{\mathrm{m}} \\
(\mathrm{K})\end{array}$ & Method & References \\
\hline & FUS & & 29.0 & & DSC & [1972WAU/GET] \\
\hline & FUS & $(5-520)$ & 29.37 & 488.9 & $\mathrm{AC}$ & [1996DOM/HEA, 1970GOU/GIR] \\
\hline & FUS & & 27.9 & 490.0 & DSC & {$[1969 \mathrm{ROB} / \mathrm{SCO}]$} \\
\hline & FUS & & 28.83 & 490.0 & & [1950UEB/ORT] \\
\hline & FUS & & 28.87 & 489.7 & $\mathrm{C}$ & [1917HIL/DUS] \\
\hline & SUB & $(300-373)$ & 97.1 & 336 & ME & [2015RIC/FU] \\
\hline & SUB & $(321-360)$ & $99.8 \pm 0.5$ & 341 & $\mathrm{ME}$ & [2011FON/PFO] \\
\hline & SUB & $(321-360)$ & $100.8 \pm 0.5$ & 298 & $\mathrm{ME}$ & [2011FON/PFO] \\
\hline & SUB & $(322-367)$ & $100.5 \pm 0.3$ & 345 & $\mathrm{ME}$ & [2011SAN/LIM] \\
\hline & SUB & $(322-367)$ & $101.8 \pm 0.3$ & 298 & $\mathrm{ME}$ & [2011SAN/LIM] \\
\hline & SUB & $(331-375)$ & $99.2 \pm 0.9$ & 349 & ME & [2011SAN/LIM] \\
\hline & SUB & $(331-375)$ & $100.6 \pm 0.9$ & 298 & ME & [2011SAN/LIM] \\
\hline & SUB & $(300-373)$ & $97.1 \pm 1.1$ & 337 & $\mathrm{ME}$ & [2010RIC/FU, 2011RIC/FU] \\
\hline & SUB & $(322-348)$ & $98.5 \pm 3.3$ & 335 & ME & [2010GOL/SUU, 2008GOL/SUU3] \\
\hline & SUB & $(339-399)$ & $97.6 \pm 1.3$ & 369 & $\mathrm{ME}$ & [2009SID/SID] \\
\hline & SUB & $(339-399)$ & 98.2 & 298 & ME & [2009SID/SID] \\
\hline & SUB & $(320-355)$ & $97.9 \pm 0.6$ & & ME & [2009OJA/CHE] \\
\hline & SUB & $(320-350)$ & $98.4 \pm 0.7$ & & ME & [2009OJA/CHE] \\
\hline & SUB & $(320-354)$ & $95.6 \pm 1.2$ & 337 & $\mathrm{ME}$ & [2006CHE/OJA] \\
\hline & SUB & $(340-360)$ & $98.8 \pm 0.4$ & 350 & $\mathrm{ME}$ & [2006RIB/MON] \\
\hline & SUB & $(340-360)$ & $100.2 \pm 0.4$ & 298 & $\mathrm{ME}$ & [2006RIB/MON] \\
\hline & SUB & $(348-368)$ & $102.5 \pm 1.9$ & 358 & $\mathrm{ME}$ & [2004VER] \\
\hline & SUB & & $96.3 \pm 0.7$ & 298 & DSC & [2003ROJ/ORO] \\
\hline & SUB & & 106 & & DSC & [2003STO/KRZ] \\
\hline & SUB & $(348-368)$ & $102.5 \pm 1.9$ & 358 & ME & [2002LI/SHI] \\
\hline & SUB & $(423-488)$ & 94.5 & & MEM & [1999EMM/PIC] \\
\hline & SUB & $(338-353)$ & 102.5 & & ME & [1998KLO/LAU] \\
\hline & SUB & & 99.4 & 298 & CGC-DSC & [1998CHI/HES] \\
\hline & SUB & $(318-363)$ & $100.0 \pm 2.8$ & 341 & ME & [1998OJA/SUU] \\
\hline & SUB & $(343-448)$ & $84.0 \pm 3.0$ & 298 & TGA & [1997TES/PIK] \\
\hline & SUB & $(313-453)$ & 99.7 & 383 & GS & [1995NAS/LEN] \\
\hline & SUB & $(318-373)$ & 98.7 & 346 & GS & [1986ROR] \\
\hline & SUB & $(313-363)$ & 102.6 & 338 & GS & [1986HAN/ECK] \\
\hline & SUB & & $100 \pm 2$ & & & [1985KIS/VEI] \\
\hline & SUB & $(353-399)$ & 94.3 & & GS & [1983BEN/BIE] \\
\hline & SUB & $(283-323)$ & $91.8 \pm 0.9$ & 303 & GS & [1983SON/ZOL] \\
\hline & SUB & $(323-353)$ & 91.2 & 338 & GS & [1982GRA/FOS] \\
\hline & SUB & & $97.4 \pm 1.1$ & & GS,C & [1981BRO/MCE] \\
\hline & SUB & & $97.8 \pm 0.1$ & & HSA & [1980DYG/STE] \\
\hline & SUB & $(337-361)$ & $104.5 \pm 1.5$ & 298 & TE,ME & [1980DEK] \\
\hline & SUB & $(358-393)$ & 94.8 & 376 & GS & [1979MAC/PRA] \\
\hline & SUB & $(363-448)$ & $98.8 \pm 0.4$ & & HSA & [1977DYG/STE] \\
\hline & SUB & $(328-372)$ & 97.2 & & ME & [1976TAY/CRO] \\
\hline & SUB & & 97.1 & & $\mathrm{C}$ & [1975ADE/BRO] \\
\hline & SUB & $(323-353)$ & $102.9 \pm 4.8$ & 298 & $\mathrm{TE}$ & [1975DEK/VAN] \\
\hline & SUB & $(283-323)$ & $95.8 \pm 6$ & & LE & [1973MCE/SAN] \\
\hline & SUB & $(353-432)$ & $101.0 \pm 0.5$ & & $\mathrm{ME}$ & [1973MAL/GIG] \\
\hline & SUB & & 99.7 & 393 & $\mathrm{C}$ & [1973MAL/GIG] \\
\hline & SUB & $(290-358)$ & 84.1 & & ME & [1972WIE] \\
\hline & SUB & $(373-403)$ & 100.3 & 393 & MG & {$[1971 \mathrm{ROG}]$} \\
\hline & SUB & & $\mathrm{U} 126 \pm 4$ & & DSC & [1971BEE/LIN] \\
\hline & SUB & $(368-480)$ & 90.1 & 424 & & [1967BRA/SMI] \\
\hline & SUB & $(342-359)$ & $98.3 \pm 2.1$ & & & [1964KEL/RIC, 1970COX/PIL] \\
\hline & SUB & $(327-346)$ & $90 \pm 01.3$ & 337 & $\mathrm{TE}$ & {$[1960 \mathrm{BUD}]$} \\
\hline & SUB & & 100.8 & & & [1958HOY/PEP, 1970COX/PIL] \\
\hline & SUB & $(303-373)$ & $103.4 \pm 2.9$ & & & [1958HOY/PEP, 1970COX/PIL] \\
\hline & SUB & & $100.8 \pm 4.2$ & & & [1958HOY/PEP, 1970COX/PIL] \\
\hline & SUB & $(396-421)$ & $97.5 \pm 2$ & & HSA & [1953STE] \\
\hline & SUB & $(339-353)$ & 102.1 & 346 & & {$[1953 \mathrm{BRA} / \mathrm{CLE}]$} \\
\hline & SUB & $(338-353)$ & $102.1 \pm 2.1$ & & & [1953BRA/CLE2, 1970COX/PIL] \\
\hline & SUB & & $92.0 \pm 2.1$ & 364 & $\mathrm{ME}$ & {$[1952 \mathrm{INO} / \mathrm{SHI}]$} \\
\hline & SUB & & 90.4 & 353 & ME & [1951INO] \\
\hline
\end{tabular}


TABLE 12. Phase change enthalpies of $\mathrm{C}_{14}$ to $\mathrm{C}_{18}$ organic compounds-Continued

\begin{tabular}{|c|c|c|c|c|c|c|}
\hline \multirow[b]{2}{*}{$\begin{array}{l}\text { Molecular } \\
\text { formula }\end{array}$} & \multirow{2}{*}{$\begin{array}{l}\text { CAS Registry } \\
\text { Number } \\
\text { Enthalpy }\end{array}$} & \multicolumn{5}{|l|}{ Compound } \\
\hline & & $\begin{array}{c}\text { Temperature } \\
\text { range }\end{array}$ & $\begin{array}{l}\Delta_{\text {trans }} H_{\mathrm{m}} \\
(\mathrm{kJ} / \mathrm{mol})\end{array}$ & $\begin{array}{l}T_{\mathrm{m}} \\
(\mathrm{K})\end{array}$ & Method & References \\
\hline & SUB & & 95.4 & & & [1951NIT/SEK] \\
\hline & SUB & & 95.0 & & & [1950NIT/SEK3] \\
\hline & SUB & $(378-398)$ & $97.3 \pm 1.2$ & & RG & [1949SEA/HOP2] \\
\hline & SUB & & $104.6 \pm 4.2$ & & & [1949KLA, 1970COX/PIL] \\
\hline & SUB & & $93.3 \pm 4.2$ & 353 & & [1938WOL/WEG] \\
\hline & $\mathrm{V}$ & & 66.7 & 498 & DSC & [2003ROJ/ORO, 2008HAN/NUT] \\
\hline & $\mathrm{V}$ & & 78.5 & 298 & CGC & {$[2008 \mathrm{ZHA} / \mathrm{UNH}]$} \\
\hline & $\mathrm{V}$ & $(413-473)$ & $79.5 \pm 1.2$ & 298 & $\mathrm{GC}$ & [2006HAF/PAR] \\
\hline & $\mathrm{V}$ & $(323-473)$ & 72.4 & 398 & GC & [2002LEI/CHA] \\
\hline & $\mathrm{V}$ & & 79.1 & 298 & CGC & [2001PUR/CHI] \\
\hline & $\mathrm{V}$ & & 79.8 & 298 & CGC & [1998CHI/HES] \\
\hline & $\mathrm{V}$ & $(453-503)$ & 79.6 & 298 & CGC & [1995CHI/HOS] \\
\hline & $\mathrm{V}$ & $(343-453)$ & 69.7 & 398 & GC & [1990HIN/BID2] \\
\hline & $\mathrm{V}$ & $(504-615)$ & 58.6 & 519 & A & {$[1987 \mathrm{STE} / \mathrm{MAL}]$} \\
\hline & $\mathrm{V}$ & & 62.1 & 500 & & [1979KUD/KUD2, 2008HAN/NUT] \\
\hline & $\mathrm{V}$ & $(500-616)$ & 59.2 & 558 & $\mathrm{I}$ & {$[1923 \mathrm{MOR} / \mathrm{MUR}]$} \\
\hline & $\mathrm{V}$ & $(500-616)$ & 60.3 & 515 & $\mathrm{I}$ & [1923MOR/MUR, 1984BOU/FRI] \\
\hline & $\mathrm{V}$ & $(496-614)$ & 59.6 & 555 & $\mathrm{I}$ & {$[1922 N E L / S E N]$} \\
\hline & $\mathrm{V}$ & $(496-614)$ & 60.7 & 511 & I & [1922NEL/SEN, 1984BOU/FRI] \\
\hline \multirow[t]{42}{*}{$\mathrm{C}_{14} \mathrm{H}_{10}$} & {$[85-01-8]$} & Phenanthrene & & & & \\
\hline & FUS & & 15.2 & 372 & DSC & [2015RIC/FU] \\
\hline & FUS & & 16.1 & 371.1 & DSC & [2011RIC/FU] \\
\hline & FUS & & 16.69 & 372.1 & DSC & [2008WEI] \\
\hline & FUS & & 14.09 & 371.3 & DSC & [2008MOG/SEP] \\
\hline & FUS & & 18.1 & & DSC & [2003SHA/KAN] \\
\hline & FUS & $(353-383)$ & 16.6 & 367.6 & DSC & [2003ROJ/ORO] \\
\hline & TRS & & 0.22 & 347.5 & & \\
\hline & FUS & & 16.2 & 372.9 & DSC & [2000LIS/JAM] \\
\hline & TRS & & 0.22 & 347.5 & & \\
\hline & FUS & $(12-408)$ & 16.46 & 372.4 & & [1996DOM/HEA, 1977FIN/MES] \\
\hline & FUS & & 15.72 & 373.8 & DTA & [1992SAB/ELW3] \\
\hline & FUS & & 18.23 & & DSC & [1992SHA/SHA] \\
\hline & FUS & & 16.3 & & DSC & [1972WAU/GET] \\
\hline & TRS & & 1.1 & 341.9 & DTA & [1966MAT] \\
\hline & TRS & & 1.6 & 341.2 & & [1966ARN/DAM, 1967RIN/DAM] \\
\hline & FUS & & 18.0 & 373.2 & $\mathrm{C}$ & [1964RAS/BAS] \\
\hline & FUS & & 17.5 & 372.2 & DTA & [1958VAR] \\
\hline & TRS & & 2.6 & 342 & & \\
\hline & FUS & & 18.62 & 373 & & [1950UEB/ORT] \\
\hline & FUS & & 17.15 & 371.4 & & [1944EIB] \\
\hline & FUS & & 17.14 & 371.7 & & {$[1941 \mathrm{SCH}]$} \\
\hline & SUB & $(296-313)$ & 88.5 & 304 & $\mathrm{ME}$ & [2015RIC/FU] \\
\hline & SUB & $(303-372)$ & $89.9 \pm 0.8$ & 298 & GS & [2014ABO/MOK] \\
\hline & SUB & $(296-308)$ & $88.5 \pm 1.0$ & & $\mathrm{ME}$ & [2011RIC/FU] \\
\hline & SUB & $(296-333)$ & $92.1 \pm 0.2$ & 314 & $\mathrm{ME}$ & [2011FON/PFO] \\
\hline & SUB & $(296-333)$ & $92.7 \pm 0.2$ & 298 & ME & [2011FON/PFO] \\
\hline & SUB & $(323-363)$ & $88.0 \pm 1.0$ & 343 & TGA & [2007SID/ATA] \\
\hline & SUB & (313-333) & $91.6 \pm 0.4$ & 323 & $\mathrm{ME}$ & [2006RIB/MON] \\
\hline & SUB & $(313-333)$ & $92.5 \pm 0.4$ & 298 & $\mathrm{ME}$ & [2006RIB/MON] \\
\hline & SUB & & $89.6 \pm 0.8$ & 298 & DSC & [2003ROJ/ORO] \\
\hline & SUB & & $92 \pm 1$ & & LE & [1998PRI/HAW] \\
\hline & SUB & & 90.5 & 298 & CGC-DSC & [1998CHI/HES] \\
\hline & SUB & $(303-333)$ & $95.0 \pm 4.4$ & 318 & $\mathrm{ME}$ & [1998OJA/SUU] \\
\hline & SUB & $(313-453)$ & 88.9 & 383 & GS & [1995NAS/LEN] \\
\hline & SUB & & $87.2 \pm 1.1$ & 350 & DSC & [1988TOR/BAR] \\
\hline & SUB & & $90.9 \pm 1.7$ & 298 & DSC & [1988TOR/BAR] \\
\hline & SUB & $(323-348)$ & 96.2 & 335 & GS & [1986SAT/INO] \\
\hline & SUB & $(317-362)$ & $82 \pm 2$ & 340 & $\mathrm{TE}$ & [1983FER/IMP] \\
\hline & SUB & $(283-323)$ & $95.0 \pm 0.6$ & 303 & GS & [1983SON/ZOL] \\
\hline & SUB & $(315-335)$ & $92.5 \pm 2$ & 298 & TE,ME & [1980DEK] \\
\hline & SUB & $(325-364)$ & 87.2 & 345 & GS & [1979MAC/PRA] \\
\hline
\end{tabular}


TABLE 12. Phase change enthalpies of $\mathrm{C}_{14}$ to $\mathrm{C}_{18}$ organic compounds-Continued

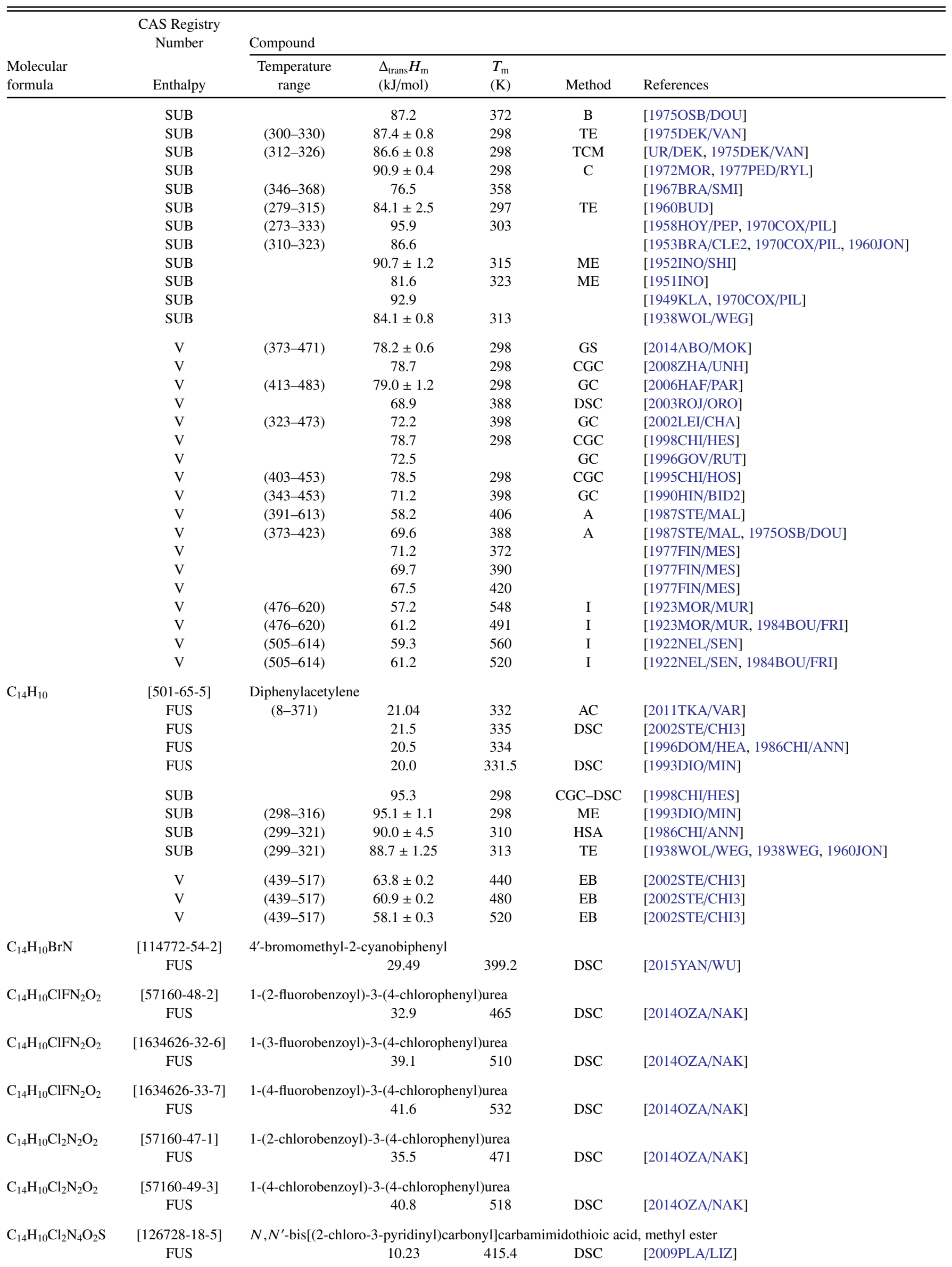


TABLE 12. Phase change enthalpies of $\mathrm{C}_{14}$ to $\mathrm{C}_{18}$ organic compounds-Continued

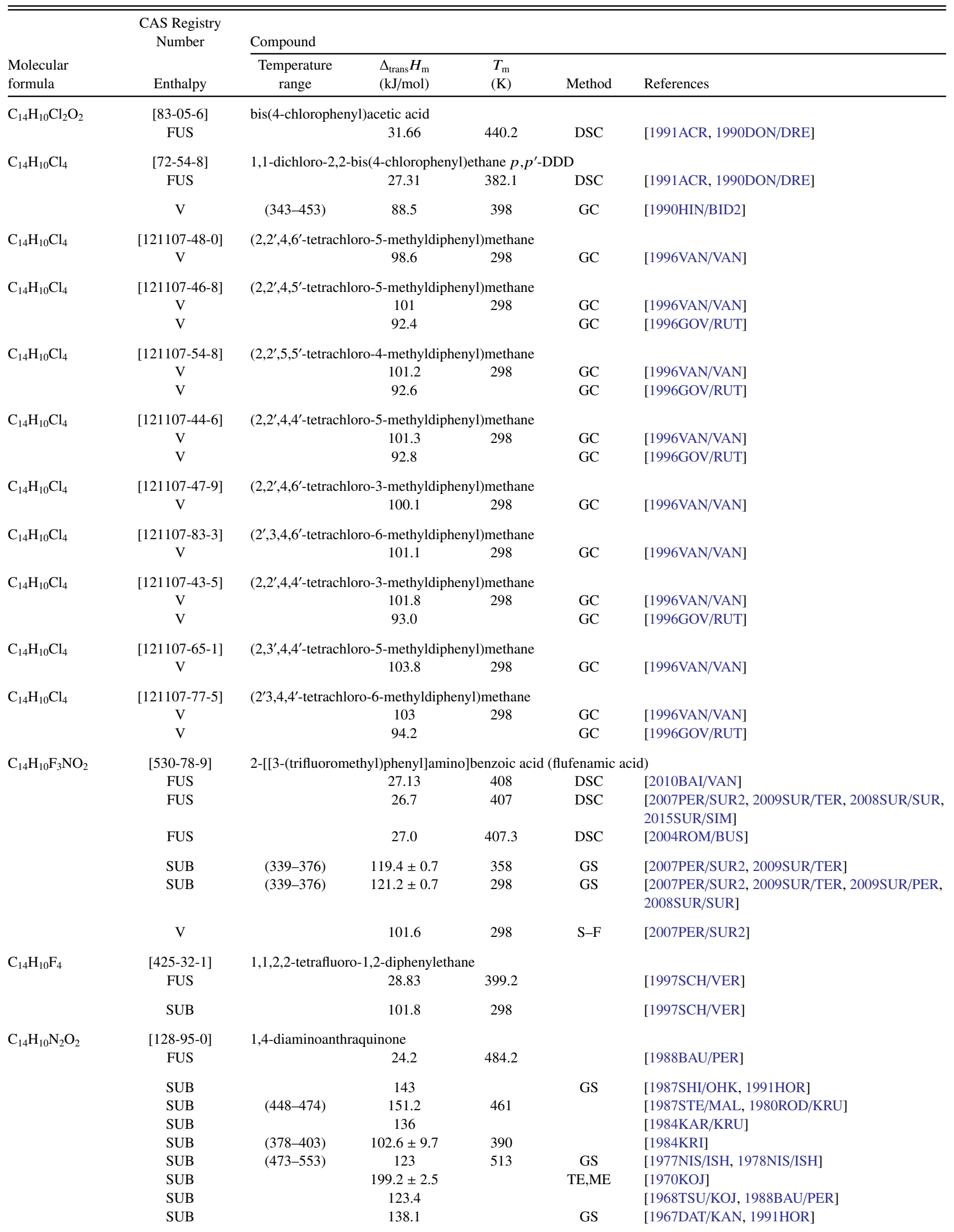

$\mathrm{C}_{14} \mathrm{H}_{10} \mathrm{~N}_{2} \mathrm{O}_{2} \quad$ [129-44-2] 1,5-diaminoanthraquinone 
TABLE 12. Phase change enthalpies of $\mathrm{C}_{14}$ to $\mathrm{C}_{18}$ organic compounds-Continued

\begin{tabular}{|c|c|c|c|c|c|c|}
\hline \multirow[b]{2}{*}{$\begin{array}{l}\text { Molecular } \\
\text { formula }\end{array}$} & \multirow{2}{*}{$\begin{array}{l}\text { CAS Registry } \\
\text { Number } \\
\text { Enthalpy }\end{array}$} & \multicolumn{5}{|l|}{ Compound } \\
\hline & & $\begin{array}{l}\text { Temperature } \\
\text { range }\end{array}$ & $\begin{array}{l}\Delta_{\text {trans }} H_{\mathrm{m}} \\
(\mathrm{kJ} / \mathrm{mol})\end{array}$ & $\begin{array}{l}T_{\mathrm{m}} \\
(\mathrm{K})\end{array}$ & Method & References \\
\hline & SUB & $(405-427)$ & $118.5 \pm 4.8$ & 416 & & [1984KRI] \\
\hline $\mathrm{C}_{14} \mathrm{H}_{10} \mathrm{~N}_{2} \mathrm{O}_{2}$ & $\begin{array}{l}\text { [4870-16-0] } \\
\text { TRS } \\
\text { FUS }\end{array}$ & $N$-anilinophth & $\begin{array}{l}1.62 \\
26.9\end{array}$ & $\begin{array}{l}401 \\
457\end{array}$ & DSC & [1998BOT/ELL] \\
\hline $\mathrm{C}_{14} \mathrm{H}_{10} \mathrm{~N}_{2} \mathrm{O}_{3}$ & $\begin{array}{l}{[58658-02-9]} \\
\text { FUS }\end{array}$ & 10-methyl-2-ni & $\begin{array}{l}\text { tridin-9 }(10 H) \\
37.6\end{array}$ & 561 & DSC & [2003STO/KRZ] \\
\hline $\mathrm{C}_{14} \mathrm{H}_{10} \mathrm{O}$ & $\begin{array}{l}\text { [90-44-8] } \\
\text { FUS } \\
\text { FUS }\end{array}$ & Anthrone & $\begin{array}{l}22.4 \\
26.8\end{array}$ & $\begin{array}{l}429.5 \\
429.0\end{array}$ & DSC & $\begin{array}{l}\text { [2010MON/SOU] } \\
\text { [1996DOM/HEA, 1991ELW/SAB] }\end{array}$ \\
\hline [Note: Some & $\begin{array}{l}\text { osition upon me } \\
\qquad \begin{array}{c}\text { SUB } \\
\text { SUB } \\
\text { SUB } \\
\text { SUB } \\
\text { SUB } \\
\text { SUB }\end{array}\end{array}$ & $\begin{array}{l}(346-365) \\
(346-365)\end{array}$ & $\begin{array}{c}105.6 \pm 0.8 \\
107.6 \pm 0.8 \\
106.7 \pm 1.8 \\
106.1 \pm 0.8 \\
103.3 \\
99.6\end{array}$ & $\begin{array}{l}356 \\
298 \\
298 \\
298 \\
298 \\
354\end{array}$ & $\begin{array}{c}\mathrm{ME} \\
\mathrm{ME} \\
\mathrm{C} \\
\mathrm{GS} \\
\mathrm{C}\end{array}$ & $\begin{array}{l}{[2010 \mathrm{MON} / \mathrm{SOU}]} \\
{[2010 \mathrm{MON} / \mathrm{SOU}]} \\
{[2010 \mathrm{FRE} / \mathrm{GOM} 2]} \\
{[1998 \mathrm{VER} 4]} \\
{[1991 \mathrm{ELW} / \mathrm{SAB}, 1992 \mathrm{SAB} / \mathrm{WAT}]} \\
{[1991 \mathrm{ELW} / \mathrm{SAB}]}\end{array}$ \\
\hline $\mathrm{C}_{14} \mathrm{H}_{10} \mathrm{O}$ & $\begin{array}{l}{[30084-90-3]} \\
\text { SUB }\end{array}$ & $\begin{array}{l}\text { 2-fluorenecarb } \\
\qquad(338-356)\end{array}$ & $\begin{array}{l}\text { lehyde } \\
100.0 \pm 3.4\end{array}$ & 347 & $\mathrm{ME}$ & [2008GOL/SUU] \\
\hline \multirow[t]{3}{*}{$\mathrm{C}_{14} \mathrm{H}_{10} \mathrm{O}_{2}$} & $\begin{array}{c}\text { [134-81-6] } \\
\text { FUS } \\
\text { FUS } \\
\text { FUS } \\
\text { FUS } \\
\text { FUS } \\
\text { FUS } \\
\text { FUS } \\
\text { TRS } \\
\text { FUS } \\
\text { TRS } \\
\text { FUS }\end{array}$ & $\begin{array}{l}\text { Benzil } \\
\begin{array}{r}(15-300) \\
(60-100)\end{array}\end{array}$ & $\begin{array}{c}22.76 \\
23.31 \\
23.19 \\
22.88 \\
23.2 \\
23.8 \\
23.8 \\
0.04 \\
23.56 \\
0.05 \\
22.6\end{array}$ & $\begin{array}{c}369.6 \\
368.0 \\
368.2 \\
368.1 \\
\\
369.2 \\
369.2 \\
84.0 \\
368 \\
84.1 \\
368.1\end{array}$ & $\begin{array}{c}\text { DSC } \\
\text { DSC } \\
\text { DSC } \\
\text { DSC } \\
\text { DSC } \\
\text { DSC } \\
\text { AC } \\
\text { AC } \\
\text { AC }\end{array}$ & $\begin{array}{l}\text { [2014TU/CHE] } \\
\text { [2012CHA/LAY] } \\
\text { [2012SHA/LAL] } \\
\text { [2005FAT/KAS] } \\
\text { [2003SHA/KAN] } \\
\text { [2001RAI/VAR] } \\
\text { [1998RAI/RAI] } \\
\text { [1996DOM/HEA, 1980AND/CON] } \\
\text { [1977DWO/FUC] } \\
\text { [1972BOO/HAU] }\end{array}$ \\
\hline & $\begin{array}{l}\text { SUB } \\
\text { SUB } \\
\text { SUB }\end{array}$ & $(319-340)$ & $\begin{array}{l}106.3 \pm 2.8 \\
98.4 \pm 1.1 \\
82.8\end{array}$ & $\begin{array}{l}298 \\
329\end{array}$ & $\mathrm{C}$ & $\begin{array}{l}\text { [2005FAT/KAS] } \\
\text { [1959AIH, 1970COX/PIL, 1987STE/MAL] } \\
\text { [1938WOL/WEG, 1938WEG, 1960JON] }\end{array}$ \\
\hline & $\mathrm{V}$ & $(401-620)$ & 69.2 & 416 & A & [1987STE/MAL, 1947STU] \\
\hline \multirow[t]{2}{*}{$\mathrm{C}_{14} \mathrm{H}_{10} \mathrm{O}_{2}$} & $\begin{array}{l}\text { [1989-33-9] } \\
\text { FUS }\end{array}$ & 9-fluorenecarb & $\begin{array}{l}c \text { acid } \\
\quad 30.24\end{array}$ & 503.8 & DSC & [2013OLI/CAL] \\
\hline & $\begin{array}{l}\text { SUB } \\
\text { SUB } \\
\text { SUB }\end{array}$ & $\begin{array}{l}(384-406) \\
(384-406) \\
(349-418)\end{array}$ & $\begin{array}{l}126.8 \pm 0.7 \\
130.4 \pm 0.8 \\
110.1 \pm 4.6\end{array}$ & $\begin{array}{l}395 \\
298 \\
383\end{array}$ & $\begin{array}{l}\mathrm{ME} \\
\mathrm{ME} \\
\mathrm{ME}\end{array}$ & $\begin{array}{l}{[2013 \mathrm{OLI} / \mathrm{CAL}]} \\
{[2013 \mathrm{OLI} / \mathrm{CAL}]} \\
{[2008 \mathrm{GOL} / \mathrm{SUU}]}\end{array}$ \\
\hline \multirow[t]{3}{*}{$\mathrm{C}_{14} \mathrm{H}_{10} \mathrm{O}_{3}$} & $\begin{array}{l}\text { [93-97-0] } \\
\text { FUS }\end{array}$ & Benzoic acid a & $\begin{array}{l}\text { ride } \\
17.15\end{array}$ & 313.2 & DSC & [1971CAR/FIN] \\
\hline & $\begin{array}{l}\text { SUB } \\
\text { SUB }\end{array}$ & & $\begin{array}{l}96.2 \pm 4.2 \\
96.7 \pm 4.2\end{array}$ & 298 & $\mathrm{~B}$ & $\begin{array}{l}\text { [1971CAR/FIN, 1977PED/RYL] } \\
\text { [1947STU, 1970COX/PIL] }\end{array}$ \\
\hline & $\mathrm{V}$ & $(416-633)$ & 69.1 & 431 & A & [1987STE/MAL, 1947STU] \\
\hline $\mathrm{C}_{14} \mathrm{H}_{10} \mathrm{O}_{3}$ & $\begin{array}{c}{[82-07-5]} \\
\text { SUB } \\
\text { SUB } \\
\text { SUB }\end{array}$ & $\begin{array}{r}9 \text {-xanthenecarl } \\
\quad(383-405) \\
(383-405)\end{array}$ & $\begin{array}{l}\text { lic acid } \\
125.5 \pm 3.0 \\
126.0 \pm 1.0 \\
130.8 \pm 2.2\end{array}$ & $\begin{array}{l}298 \\
394 \\
298\end{array}$ & $\begin{array}{c}\mathrm{C} \\
\mathrm{ME} \\
\mathrm{ME}\end{array}$ & $\begin{array}{l}{[2012 \text { FRE/GOM] }} \\
{[2012 \text { FRE/GOM] }} \\
{[2012 \text { FRE/GOM] }}\end{array}$ \\
\hline $\mathrm{C}_{14} \mathrm{H}_{10} \mathrm{O}_{4}$ & $\begin{array}{l}{[94-36-0]} \\
\quad \text { FUS }\end{array}$ & Benzoyl perox & 22.59 & 378 & FPD & [1967FIN/GRA] \\
\hline
\end{tabular}


TABLE 12. Phase change enthalpies of $\mathrm{C}_{14}$ to $\mathrm{C}_{18}$ organic compounds-Continued

\begin{tabular}{|c|c|c|c|c|c|c|}
\hline \multirow[b]{2}{*}{$\begin{array}{l}\text { Molecular } \\
\text { formula }\end{array}$} & \multirow{2}{*}{$\begin{array}{l}\text { CAS Registry } \\
\text { Number } \\
\text { Enthalpy }\end{array}$} & \multicolumn{5}{|l|}{ Compound } \\
\hline & & $\begin{array}{l}\text { Temperature } \\
\text { range }\end{array}$ & $\begin{array}{l}\Delta_{\text {trans }} H_{\mathrm{m}} \\
(\mathrm{kJ} / \mathrm{mol})\end{array}$ & $\begin{array}{l}T_{\mathrm{m}} \\
(\mathrm{K})\end{array}$ & Method & References \\
\hline \multirow[t]{3}{*}{$\mathrm{C}_{14} \mathrm{H}_{10} \mathrm{O}_{4}$} & $\begin{array}{c}\text { [3155-16-6] } \\
\text { FUS }\end{array}$ & \multicolumn{5}{|l|}{ Diphenyl oxalate } \\
\hline & SUB & & $102.5 \pm 8.4$ & & B & [1971CAR/FIN, 1977PED/RYL] \\
\hline & $\mathrm{V}$ & $(463-598)$ & 68.2 & & & [1971CAR/FIN] \\
\hline $\mathrm{C}_{14} \mathrm{H}_{10} \mathrm{O}_{4}$ & $\begin{array}{l}{[482-05-3]} \\
\text { SUB } \\
\text { SUB }\end{array}$ & $\begin{array}{l}\text { 2,2'-biphenyldicar } \\
\text { (433-493) }\end{array}$ & $\begin{array}{l}\text { oxylic acid } \\
151.9 \pm 3.5 \\
166.1\end{array}$ & $\begin{array}{l}298 \\
448\end{array}$ & $\begin{array}{l}\mathrm{C} \\
\mathrm{A}\end{array}$ & $\begin{array}{l}\text { [2004MAT/MIR2] } \\
\text { [1987STE/MAL] }\end{array}$ \\
\hline $\mathrm{C}_{14} \mathrm{H}_{10} \mathrm{O}_{4}$ & $\begin{array}{l}\text { [787-70-2] } \\
\text { SUB }\end{array}$ & \multicolumn{5}{|c|}{ 4,4'-biphenyldicarboxylic acid } \\
\hline $\mathrm{C}_{14} \mathrm{H}_{10} \mathrm{O}_{4}$ & $\begin{array}{l}{[40498-13-3]} \\
\text { SUB }\end{array}$ & \multicolumn{5}{|c|}{ 2,3-dihydro-1,4-dihydroxy-9,10-anthraquinone } \\
\hline $\mathrm{C}_{14} \mathrm{H}_{10} \mathrm{O}_{5}$ & $\begin{array}{l}{[962-16-3]} \\
\text { SUB } \\
\text { SUB }\end{array}$ & $O$-phenyl- $O, O$-be & $\begin{array}{l}\text { zoyl peroxyc } \\
97.9 \pm 2.5 \\
133.9 \pm 4.2\end{array}$ & & $\mathrm{E}$ & $\begin{array}{l}\text { [1975CAR/LAY, 1977PED/RYL] } \\
\text { [1971KIP/RAB, 1977PED/RYL] }\end{array}$ \\
\hline $\mathrm{C}_{14} \mathrm{H}_{10} \mathrm{O}_{5}$ & $\begin{array}{l}\text { [552-94-3] } \\
\text { FUS }\end{array}$ & \multicolumn{5}{|l|}{ Salicylsalicylic acid } \\
\hline $\mathrm{C}_{14} \mathrm{H}_{11} \mathrm{BrN}_{2} \mathrm{~S}$ & $\begin{array}{c}\text { [109768-69-6] } \\
\text { FUS }\end{array}$ & \multicolumn{4}{|c|}{$N$-(4-bromophenyl)-4H-3,1-benzothiazin-2-amine } & {$[2004 \mathrm{GON} / \mathrm{KOS}]$} \\
\hline $\mathrm{C}_{14} \mathrm{H}_{11} \mathrm{ClN}_{2} \mathrm{O}_{2}$ & $\begin{array}{l}{[57160-46-0]} \\
\text { FUS }\end{array}$ & 1-benzoyl-3-(4-chl & $\begin{array}{l}\text { rophenyl)ure } \\
39.1\end{array}$ & 510 & DSC & [2014OZA/NAK] \\
\hline $\mathrm{C}_{14} \mathrm{H}_{11} \mathrm{ClN}_{2} \mathrm{~S}$ & $\begin{array}{c}{[461662-90-8]} \\
\text { FUS }\end{array}$ & \multicolumn{5}{|c|}{$N$-(4-chlorophenyl)-4H-3,1-benzothiazin-2-amine } \\
\hline $\mathrm{C}_{14} \mathrm{H}_{11} \mathrm{Cl}_{2} \mathrm{NO}_{2}$ & $\begin{array}{l}\text { [32809-16-8] } \\
\text { FUS }\end{array}$ & \multicolumn{5}{|c|}{ 3-(3,5-dichlorophenyl)-1,5-dimethyl-3-azabicyclo[3.1.0]hexanedione } \\
\hline $\mathrm{C}_{14} \mathrm{H}_{11} \mathrm{Cl}_{2} \mathrm{NO}_{2}$ & $\begin{array}{c}\text { [15307-86-5] } \\
\text { FUS } \\
\text { FUS } \\
\text { FUS }\end{array}$ & 2-[(2,6-dichloroph & $\begin{array}{c}\text { nyl)amino]be } \\
40.4 \\
39.4 \\
38.4\end{array}$ & $\begin{array}{l}\operatorname{acid}(\mathrm{di} \\
452.6 \\
454.2 \\
453.7\end{array}$ & $\begin{array}{c}\text { nac acid) } \\
\text { DSC } \\
\text { DSC } \\
\text { DSC }\end{array}$ & $\begin{array}{l}\text { [2009SUR/TER, 2008SUR/SUR, 2010SUR/PER] } \\
\text { [2007PAS/BET] } \\
\text { [2003GIO/ROS] }\end{array}$ \\
\hline & $\begin{array}{l}\text { SUB } \\
\text { SUB }\end{array}$ & $\begin{array}{l}(323-355) \\
(323-355)\end{array}$ & $\begin{array}{l}114.7 \pm 1.3 \\
115.6 \pm 1.3\end{array}$ & $\begin{array}{l}339 \\
298\end{array}$ & $\begin{array}{l}\text { GS } \\
\text { GS }\end{array}$ & $\begin{array}{l}\text { [2007PER/SUR, 2009SUR/TER] } \\
\text { [2007PER/SUR, 2009SUR/TER, 2008SUR/SUR] }\end{array}$ \\
\hline $\mathrm{C}_{14} \mathrm{H}_{11} \mathrm{FO}_{3}$ & $\begin{array}{l}{[3119-88-8]} \\
\text { SUB }\end{array}$ & $\begin{array}{l}\text { 2'-fluoro-2-hydrox } \\
\quad(307-318)\end{array}$ & \multicolumn{3}{|c|}{ 2'-fluoro-2-hydroxy-4-methoxybenzophenone } & [1987STE/MAL, 1966GRA/BUR] \\
\hline $\mathrm{C}_{14} \mathrm{H}_{11} \mathrm{FO}_{3}$ & $\begin{array}{l}{[3506-35-2]} \\
\text { SUB }\end{array}$ & $\begin{array}{l}\text { 3'-fluoro-2-hydrox } \\
\quad(322-343)\end{array}$ & \multicolumn{3}{|c|}{ 3'-fluoro-2-hydroxy-4-methoxybenzophenone } & [1987STE/MAL, 1966GRA/BUR] \\
\hline $\mathrm{C}_{14} \mathrm{H}_{11} \mathrm{FO}_{3}$ & $\begin{array}{l}{[3602-47-9]} \\
\text { SUB }\end{array}$ & \multicolumn{5}{|c|}{ 4'-fluoro-2-hydroxy-4-methoxybenzophenone } \\
\hline $\mathrm{C}_{14} \mathrm{H}_{11} \mathrm{~F}_{3}$ & $\begin{array}{l}{[68936-77-6]} \\
\text { FUS }\end{array}$ & \multicolumn{4}{|c|}{ 1,1,2-trifluoro-1,2-diphenylethane } & [1997SCH/VER] \\
\hline & SUB & & 93.1 & 298 & & [1997SCH/VER] \\
\hline $\mathrm{C}_{14} \mathrm{H}_{11} \mathrm{~F}_{3}$ & $\begin{array}{c}{[384-94-1]} \\
\mathrm{V}\end{array}$ & $\begin{array}{l}\text { 1,1,1-trifluoro-2,2- } \\
\quad(286-328)\end{array}$ & $\begin{array}{l}\text { liphenylethan } \\
69.1 \pm 0.9\end{array}$ & 298 & GS & {$[1997 \mathrm{SCH} / \mathrm{VER}]$} \\
\hline $\mathrm{C}_{14} \mathrm{H}_{11} \mathrm{IO}_{3} \mathrm{~S}$ & $\begin{array}{l}{[313057-05-5]} \\
\text { FUS }\end{array}$ & 4-(2-propenyloxy) & $\begin{array}{l}\text { henyl 5-iodo- } \\
\quad 83.68\end{array}$ & $\begin{array}{l}\text { phene c } \\
383.2\end{array}$ & $\begin{array}{l}\text { xylate } \\
\text { DSC }\end{array}$ & [2000WU/WAN] \\
\hline $\mathrm{C}_{14} \mathrm{H}_{11} \mathrm{~N}$ & $\begin{array}{l}{[948-65-2]} \\
\text { SUB } \\
\text { SUB }\end{array}$ & $\begin{array}{l}\text { 2-phenylindole } \\
\text { (360-382) } \\
(360-382)\end{array}$ & $\begin{array}{l}111.7 \pm 0.8 \\
114.4 \pm 0.8\end{array}$ & $\begin{array}{l}371 \\
298\end{array}$ & $\begin{array}{l}\mathrm{ME} \\
\mathrm{ME}\end{array}$ & $\begin{array}{l}\text { [2015CAR/AMA] } \\
{[2015 \mathrm{CAR} / \mathrm{AMA}]}\end{array}$ \\
\hline $\mathrm{C}_{14} \mathrm{H}_{11} \mathrm{NO}$ & $\begin{array}{c}\text { [574-39-0] } \\
\text { FUS }\end{array}$ & $N$-acetylcarbazole & 15.1 & 349.9 & & [2001JAM/DOB] \\
\hline $\mathrm{C}_{14} \mathrm{H}_{11} \mathrm{NO}$ & [719-54-0] & 10-methylacridin- & $(10 H)$-one & & & \\
\hline
\end{tabular}


TABLE 12. Phase change enthalpies of $\mathrm{C}_{14}$ to $\mathrm{C}_{18}$ organic compounds-Continued

\begin{tabular}{|c|c|c|c|c|c|c|}
\hline \multirow[b]{2}{*}{$\begin{array}{l}\text { Molecular } \\
\text { formula }\end{array}$} & \multirow{2}{*}{$\begin{array}{c}\text { CAS Registry } \\
\text { Number } \\
\text { Enthalpy }\end{array}$} & \multicolumn{5}{|l|}{ Compound } \\
\hline & & $\begin{array}{c}\text { Temperature } \\
\text { range }\end{array}$ & $\begin{array}{l}\Delta_{\text {trans }} H_{\mathrm{m}} \\
(\mathrm{kJ} / \mathrm{mol})\end{array}$ & $\begin{array}{l}T_{\mathrm{m}} \\
(\mathrm{K})\end{array}$ & Method & References \\
\hline & FUS & & 29.7 & 479 & DSC & [2003STO/KRZ] \\
\hline & SUB & & 105 & & DSC & [2003STO/KRZ] \\
\hline $\mathrm{C}_{14} \mathrm{H}_{11} \mathrm{NO}_{2}$ & $\begin{array}{c}\text { [5813-90-1] } \\
\text { SUB } \\
\text { SUB } \\
\text { SUB }\end{array}$ & $\begin{array}{l}\text { 9-xanthenecarl } \\
(399-421) \\
(399-421)\end{array}$ & $\begin{array}{l}\text { mide } \\
\qquad \begin{array}{l}130.0 \pm 4.2 \\
130.5 \pm 1.1 \\
136.1 \pm 2.5\end{array}\end{array}$ & $\begin{array}{l}298 \\
410 \\
298\end{array}$ & $\begin{array}{c}\mathrm{C} \\
\mathrm{ME} \\
\mathrm{ME}\end{array}$ & $\begin{array}{l}{[2012 \text { FRE/GOM] }} \\
{[2012 \text { FRE/GOM] }} \\
{[2012 \text { FRE/GOM] }}\end{array}$ \\
\hline $\mathrm{C}_{14} \mathrm{H}_{11} \mathrm{NO}_{3}$ & $\begin{array}{c}\text { [841-12-3] } \\
\text { FUS }\end{array}$ & $N$-salicylidene & $\begin{array}{c}\text { aminobenzoic } \\
33.11\end{array}$ & 464 & DSC & [1996DOM/HEA, 1991WU/XIO] \\
\hline $\mathrm{C}_{14} \mathrm{H}_{11} \mathrm{NS}$ & $\begin{array}{c}{[150993-53-6]} \\
\text { SUB }\end{array}$ & 2-cyanophenyl & $\begin{array}{l}\text { zyl sulfide } \\
117.8 \pm 2.1\end{array}$ & 298 & $\mathrm{C}$ & [2006MUL/MOZ] \\
\hline $\mathrm{C}_{14} \mathrm{H}_{11} \mathrm{~N}_{3} \mathrm{O}_{2}$ & $\begin{array}{c}\text { [6407-69-8] } \\
\text { SUB }\end{array}$ & $\begin{array}{c}\text { 1,4,5-triamino } \\
\quad(473-553)\end{array}$ & $\begin{array}{r}\text { aquinone } \\
\text { U70.3 }\end{array}$ & 513 & GS & [1977NIS/ISH, 1978NIS/ISH] \\
\hline $\mathrm{C}_{14} \mathrm{H}_{11} \mathrm{~N}_{3} \mathrm{O}_{4}$ & $\begin{array}{c}\text { [191979-14-3] } \\
\text { FUS }\end{array}$ & 2-cyano-6-nitr & $\begin{array}{l}H \text { )-quinoline } \\
26.87\end{array}$ & $\begin{array}{l}\text { xylic ac } \\
383.7\end{array}$ & $\begin{array}{c}\text { propenyl e } \\
\text { DSC }\end{array}$ & [2005LIZ/ZAB] \\
\hline $\mathrm{C}_{14} \mathrm{H}_{11} \mathrm{~N}_{3} \mathrm{O}_{4}$ & $\begin{array}{c}\text { [191979-17-6] } \\
\text { FUS }\end{array}$ & 2-cyano-6-nitr & $\begin{array}{l}H \text { )-quinoline } \\
26.27\end{array}$ & $\begin{array}{l}\text { xylic ac } \\
389.4\end{array}$ & $\begin{array}{l}\text { propenyl e } \\
\text { DSC }\end{array}$ & [2005LIZ/ZAB] \\
\hline $\mathrm{C}_{14} \mathrm{H}_{12}$ & $\begin{array}{c}\text { [1730-37-6] } \\
\text { SUB }\end{array}$ & $\begin{array}{c}\text { 1-methylfluore } \\
\text { (285-317) }\end{array}$ & $91.2 \pm 0.4$ & 298 & GS & [2004VER] \\
\hline & $\begin{array}{l}\mathrm{V} \\
\mathrm{V} \\
\mathrm{V}\end{array}$ & $\begin{array}{l}(361-375) \\
(323-473)\end{array}$ & $\begin{array}{c}77.2 \pm 3.6 \\
78.7 \pm 0.7 \\
71.1\end{array}$ & $\begin{array}{l}298 \\
298 \\
398\end{array}$ & $\begin{array}{l}\text { CGC } \\
\text { GS } \\
\text { GC }\end{array}$ & $\begin{array}{l}\text { [2008HAN/NUT] } \\
{[2004 \mathrm{VER}]} \\
{[2002 \mathrm{LEI} / \mathrm{CHA}]}\end{array}$ \\
\hline $\mathrm{C}_{14} \mathrm{H}_{12}$ & $\begin{array}{c}\text { [2523-37-7] } \\
\text { FUS }\end{array}$ & 9-methylfluore & 16.32 & 319.2 & DSC & [1994RAK/VER2] \\
\hline & $\begin{array}{l}\text { SUB } \\
\text { SUB } \\
\text { SUB }\end{array}$ & $\begin{array}{l}(285-317) \\
(318-358)\end{array}$ & $\begin{array}{l}83.7 \pm 0.6 \\
82.8 \pm 0.3 \\
82.8 \pm 0.3\end{array}$ & $\begin{array}{l}298 \\
338 \\
298\end{array}$ & $\begin{array}{c}\text { GS } \\
\text { B }\end{array}$ & $\begin{array}{l}\text { [2004VER] } \\
\text { [1994RAK/VER2] } \\
\text { [1994RAK/VER2] }\end{array}$ \\
\hline & $\begin{array}{l}\mathrm{V} \\
\mathrm{V} \\
\mathrm{V}\end{array}$ & $\begin{array}{l}(320-353) \\
(318-358) \\
(318-358)\end{array}$ & $\begin{array}{c}70.6 \pm 0.3 \\
71.3 \pm 0.2 \\
66.5\end{array}$ & $\begin{array}{l}298 \\
298 \\
298\end{array}$ & $\begin{array}{c}\text { GS } \\
\text { GS } \\
\text { B }\end{array}$ & $\begin{array}{l}\text { [2004VER] } \\
\text { [2004VER] } \\
\text { [1994RAK/VER2] }\end{array}$ \\
\hline $\mathrm{C}_{14} \mathrm{H}_{12}$ & $\begin{array}{l}\text { [613-31-0] } \\
\text { SUB } \\
\text { SUB } \\
\text { SUB } \\
\text { SUB } \\
\text { SUB }\end{array}$ & $\begin{array}{l}\text { 9,10-dihydroar } \\
\quad(313-453) \\
(318-379) \\
(279-328)\end{array}$ & $\begin{array}{l}\text { cene } \\
\qquad \begin{array}{l}93.9 \\
92.4 \pm 4 \\
94.2 \pm 0.8 \\
93.3 \pm 4 \\
89.5\end{array}\end{array}$ & $\begin{array}{l}383 \\
298 \\
304 \\
388\end{array}$ & $\begin{array}{l}\text { GS } \\
\text { ME } \\
\text { ME }\end{array}$ & $\begin{array}{l}\text { [1995NAS/LEN] } \\
\text { [1975MAL/GIG, 1987STE/MAL] } \\
\text { [1975MAL/GIG] } \\
\text { [1958HOY/PEP, 1970COX/PIL] } \\
\text { [1951MAG/HAR, 1960JON] }\end{array}$ \\
\hline $\mathrm{C}_{14} \mathrm{H}_{12}$ & $\begin{array}{c}\text { [776-35-2] } \\
\text { FUS }\end{array}$ & $\begin{array}{l}\text { 9,10-dihydropl } \\
\quad(11-350)\end{array}$ & $\begin{array}{r}\text { nthrene } \\
12.8\end{array}$ & 306.5 & $\mathrm{AC}$ & [1996DOM/HEA, 1979LEE/HOS] \\
\hline & $\begin{array}{l}\mathrm{V} \\
\mathrm{V} \\
\mathrm{V}\end{array}$ & $\begin{array}{l}(417-453) \\
(353-418) \\
(353-418)\end{array}$ & $\begin{array}{c}64.0 \\
72.3 \pm 0.6 \\
76.6 \pm 0.1\end{array}$ & $\begin{array}{l}432 \\
340 \\
298\end{array}$ & $\begin{array}{c}\text { A } \\
\text { IPM } \\
\text { IPM }\end{array}$ & $\begin{array}{l}\text { [1987STE/MAL] } \\
{[1979 \mathrm{LEE} / \mathrm{HOS}]} \\
{[1979 \mathrm{LEE} / \mathrm{HOS}]}\end{array}$ \\
\hline $\mathrm{C}_{14} \mathrm{H}_{12}$ & $\begin{array}{c}{[530-48-3]} \\
\mathrm{V} \\
\mathrm{V} \\
\mathrm{V}\end{array}$ & $\begin{array}{l}\text { 1,1-diphenylet } \\
\quad(298-331) \\
(298-331) \\
(360-550)\end{array}$ & $\begin{array}{l}70.2 \pm 0.7 \\
71.2 \pm 0.7 \\
59.3\end{array}$ & $\begin{array}{l}314 \\
298 \\
375\end{array}$ & $\begin{array}{c}\text { GS } \\
\text { GS } \\
\text { A }\end{array}$ & $\begin{array}{l}\text { [1999VER/EBE] } \\
{[1999 \mathrm{VER} / \mathrm{EBE}]} \\
\text { [1987STE/MAL, 1947STU] }\end{array}$ \\
\hline $\mathrm{C}_{14} \mathrm{H}_{12}$ & $\begin{array}{c}{[645-49-8]} \\
\mathrm{V} \\
\mathrm{V} \\
\mathrm{V}\end{array}$ & $\begin{array}{l}\text { cis-1,2-diphen } \\
\quad(308-343) \\
(373-428) \\
(276-286)\end{array}$ & $\begin{array}{c}\text { ylene (cis-stil } \\
70.5 \pm 0.4 \\
66.5 \\
50.3 \pm 1.0\end{array}$ & $\begin{array}{l}298 \\
388 \\
298\end{array}$ & $\begin{array}{c}\text { GS } \\
\text { A }\end{array}$ & $\begin{array}{l}\text { [2009CAM/EME] } \\
\text { [1987STE/MAL] } \\
\text { [1952BRA/PLE, 2009CAM/EME] }\end{array}$ \\
\hline $\mathrm{C}_{14} \mathrm{H}_{12}$ & $\begin{array}{l}{[103-30-0]} \\
\text { FUS } \\
\text { FUS }\end{array}$ & $\begin{array}{l}\text { trans-1,2-diph } \\
\quad(317-411) \\
(8-450)\end{array}$ & $\begin{array}{l}\text { thylene (tran } \\
\begin{array}{l}27.7 \\
27.4\end{array}\end{array}$ & $\begin{array}{l}\text { ene) } \\
397.4 \\
398.2\end{array}$ & $\begin{array}{c}\mathrm{AC} \\
\mathrm{AC}, \mathrm{DSC}\end{array}$ & $\begin{array}{l}\text { [1985BOU/DEL] } \\
\text { [1991ACR, 1984VAN/BOU] }\end{array}$ \\
\hline
\end{tabular}


TABLE 12. Phase change enthalpies of $\mathrm{C}_{14}$ to $\mathrm{C}_{18}$ organic compounds-Continued

\begin{tabular}{|c|c|c|c|c|c|c|}
\hline \multirow[b]{2}{*}{$\begin{array}{l}\text { Molecular } \\
\text { formula }\end{array}$} & \multirow{2}{*}{$\begin{array}{l}\text { CAS Registry } \\
\text { Number } \\
\text { Enthalpy }\end{array}$} & \multicolumn{5}{|l|}{ Compound } \\
\hline & & $\begin{array}{c}\text { Temperature } \\
\text { range }\end{array}$ & $\begin{array}{l}\Delta_{\text {trans }} H_{\mathrm{m}} \\
(\mathrm{kJ} / \mathrm{mol})\end{array}$ & $\begin{array}{l}T_{\mathrm{m}} \\
(\mathrm{K})\end{array}$ & Method & References \\
\hline & FUS & & 27.7 & 397.4 & $\mathrm{AC}$ & [1985BOU/DEL] \\
\hline & SUB & $(324-367)$ & $102 \pm 0.4$ & 298 & GS & [2009CAM/EME] \\
\hline & SUB & & 102 & 298 & CGC-DSC & [1998CHI/HES] \\
\hline & SUB & $(298-343)$ & 99.6 & 313 & $\mathrm{~A}$ & [1987STE/MAL] \\
\hline & SUB & & U 61.1 & & MS & [1983MAJ/AZZ] \\
\hline & SUB & $(293-338)$ & $103.8 \pm 2.5$ & 315 & & [1983KRA/BEC] \\
\hline & SUB & & $100.7 \pm 0.4$ & 298 & SRFG & [1983VAN/JAC] \\
\hline & SUB & $(310-340)$ & $99.6 \pm 1.7$ & 298 & TE & [1975DEK/VAN] \\
\hline & SUB & & $102.1 \pm 0.6$ & & TCM & [1973DEK/OON] \\
\hline & SUB & & $99.2 \pm 0.4$ & & & [1972MOR3] \\
\hline & SUB & $(303-315)$ & $86.5 \pm 0.1$ & 309 & $\mathrm{~T}$ & [1955ENG] \\
\hline & V & & 79.7 & 298 & CGC & [1998CHI/HES] \\
\hline & V & $(453-503)$ & 79.8 & 298 & CGC & [1995CHI/HOS] \\
\hline & $\mathrm{V}$ & $(403-453)$ & 79.6 & 298 & CGC & [1995CHI/HOS] \\
\hline & $\mathrm{V}$ & $(419-580)$ & 65.5 & 434 & A & [1987STE/MAL] \\
\hline \multirow[t]{3}{*}{$\mathrm{C}_{14} \mathrm{H}_{12} \mathrm{BrNOS}$} & [127351-08-0] & \multicolumn{5}{|c|}{ 3-bromo- $N$-(4-methoxyphenyl)benzenecarbothioamide } \\
\hline & FUS (I) & & 28.5 & 376.7 & & \\
\hline & FUS (II) & & 22.6 & 373.2 & $\mathrm{DSC}$ & [2007BAS/AND] \\
\hline \multirow[t]{2}{*}{$\mathrm{C}_{14} \mathrm{H}_{12} \mathrm{ClN}$} & {$[33442-36-3]$} & \multicolumn{5}{|c|}{ 4-chlorobenzylidene-4'-methylaniline } \\
\hline & FUS & & 25.58 & 400.4 & DSC & [1999GAL/COL] \\
\hline \multirow[t]{10}{*}{$\mathrm{C}_{14} \mathrm{H}_{12} \mathrm{ClNO}_{2}$} & [13710-19-5] & \multicolumn{5}{|c|}{ 2-[(3-chloro-2-methylphenyl)amino]benzoic acid (tolfenamic acid) } \\
\hline & FUS & & 38.83 & 486 & DSC & [2010BAI/VAN] \\
\hline & FUS (white & & 41.0 & 484.2 & & \\
\hline & crys) & & & & & \\
\hline & FUS & & 49.0 & 485.8 & DSC & [2009SUR/SZT, 2015SUR/SIM] \\
\hline & (yellowcrys) & & & & & \\
\hline & FUS & & 38.6 & 484.3 & DSC & [2009SUR/TER] \\
\hline & FUS & & 41.2 & 485.3 & & [2007BER/WAS] \\
\hline & SUB & $(346-373)$ & $125.7 \pm 0.8$ & 360 & GS & [2009SUR/TER] \\
\hline & SUB & $(346-373)$ & $128.4 \pm 0.8$ & 298 & GS & [2009SUR/TER, 2009SUR/PER] \\
\hline \multirow[t]{3}{*}{$\mathrm{C}_{14} \mathrm{H}_{12} \mathrm{~F}_{2}$} & {$[350-62-9]$} & \multicolumn{5}{|c|}{ 1,1-difluoro-1,2-diphenylethane } \\
\hline & FUS & & 24.35 & 339.2 & & [1997SCH/VER] \\
\hline & SUB & & $94.7 \pm 0.9$ & 298 & & [1997SCH/VER] \\
\hline \multirow[t]{2}{*}{$\mathrm{C}_{14} \mathrm{H}_{12} \mathrm{~F}_{3} \mathrm{NO}_{4} \mathrm{~S}_{2}$} & [37924-13-3] & \multicolumn{5}{|c|}{ 1,1,1-trifluoro- $N$-[2-methyl-4-(phenylsulphonyl)phenyl]methanesulfonamide } \\
\hline & FUS & & 31.79 & 418.4 & DSC & {$[1990 \mathrm{DON} / \mathrm{DRE}]$} \\
\hline \multirow[t]{2}{*}{$\mathrm{C}_{14} \mathrm{H}_{12} \mathrm{~N}_{2}$} & {$[22739-29-3]$} & \multicolumn{5}{|c|}{$N$-methyl-9-acridinamine } \\
\hline & SUB & & 107 & 480 & TGA & [1998STO/KRZ] \\
\hline \multirow[t]{2}{*}{$\mathrm{C}_{14} \mathrm{H}_{12} \mathrm{~N}_{2}$} & {$[5291-44-1]$} & \multicolumn{5}{|c|}{ 10-methyl-9-acridinimine } \\
\hline & SUB & & 94 & 550 & TGA & [1998STO/KRZ] \\
\hline \multirow[t]{2}{*}{$\mathrm{C}_{14} \mathrm{H}_{12} \mathrm{~N}_{2}$} & {$[588-68-1]$} & \multicolumn{5}{|c|}{ Dibenzylideneazine } \\
\hline & SUB & & $93.3 \pm 2.1$ & 293 & $\mathrm{E}$ & [1948COA/SUT] \\
\hline $\mathrm{C}_{14} \mathrm{H}_{12} \mathrm{~N}_{2}$ & {$[484-11-7]$} & 2,9-dimethyl-1 & phenanthrolin & & & \\
\hline & FUS & & 17.6 & 435.9 & $\mathrm{DSC}$ & [2007BON/CAT] \\
\hline $\mathrm{C}_{14} \mathrm{H}_{12} \mathrm{~N}_{2}$ & {$[621-72-7]$} & 2-benzylbenzir & zole & & & \\
\hline & SUB & $(393-412)$ & $134.5 \pm 0.5$ & 403 & ME & [2005RIB/RIB] \\
\hline & SUB & $(393-412)$ & $136.2 \pm 0.5$ & 298 & ME & [2005RIB/RIB] \\
\hline $\mathrm{C}_{14} \mathrm{H}_{12} \mathrm{~N}_{2} \mathrm{O}_{2}$ & [192998-96-2] & $c i s-5 a, 6,11 a, 1$ & trahdro[1,4]be & iazino[3 & ]$[1,4]$-benzox & zine \\
\hline & SUB & (383-392) & 122 & 387 & ME & [1997GUD/TOR] \\
\hline & SUB & $(383-392)$ & $129.0 \pm 1.3$ & 298 & $\mathrm{ME}$ & [1997GUD/TOR] \\
\hline $\mathrm{C}_{14} \mathrm{H}_{12} \mathrm{~N}_{2} \mathrm{O}_{2}$ & [730-39-2] & 4-nitro-4'-meth & enzylidene ani & & & \\
\hline & FUS & & 27.3 & 402 & $\mathrm{DSC}$ & [1997KER/LOC] \\
\hline $\mathrm{C}_{14} \mathrm{H}_{12} \mathrm{~N}_{2} \mathrm{O}_{3}$ & [1821-33-6] & 1-benzoyl-3-pl & lurea & & & \\
\hline
\end{tabular}


TABLE 12. Phase change enthalpies of $\mathrm{C}_{14}$ to $\mathrm{C}_{18}$ organic compounds-Continued

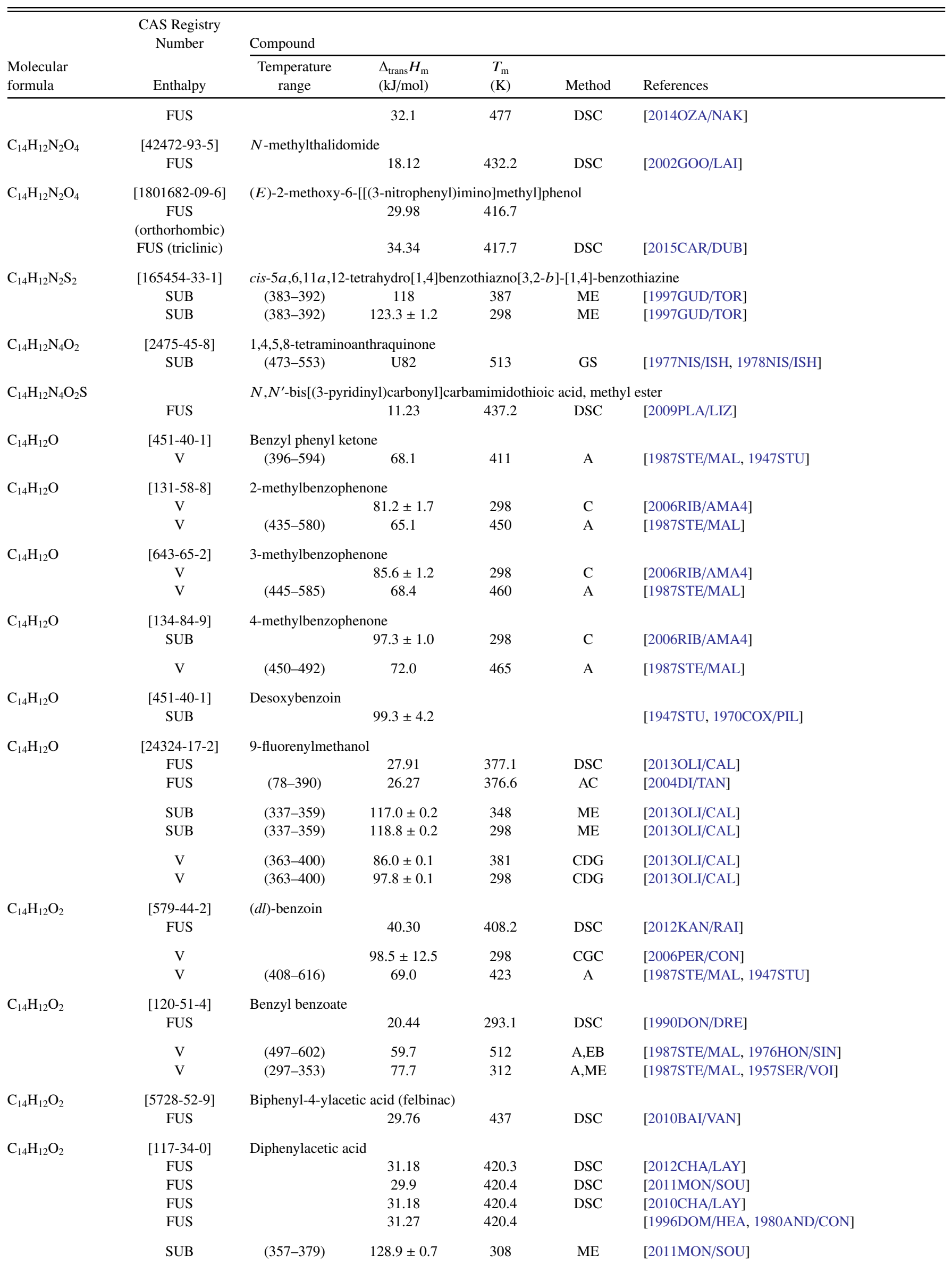


TABLE 12. Phase change enthalpies of $\mathrm{C}_{14}$ to $\mathrm{C}_{18}$ organic compounds-Continued

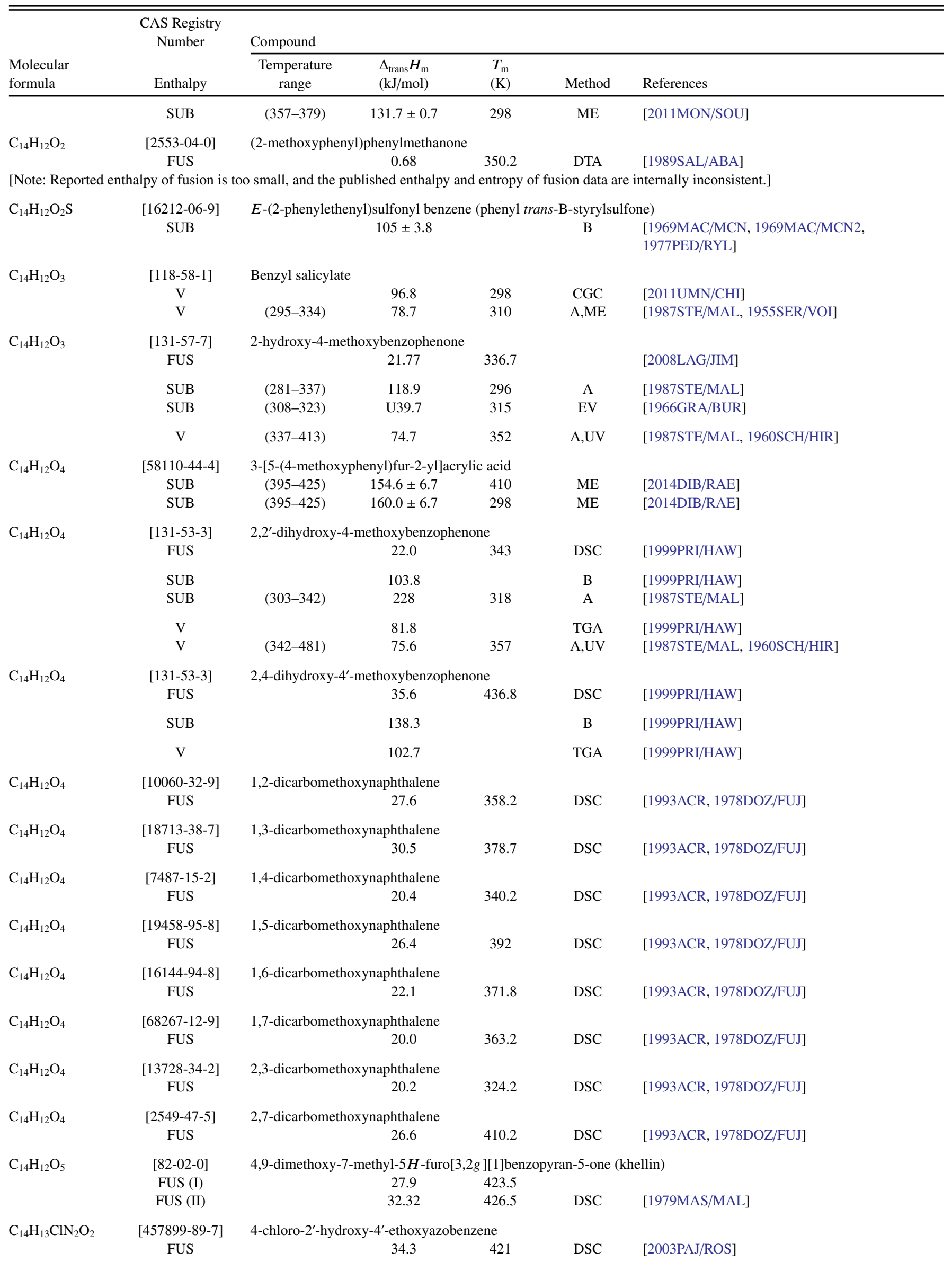


TABLE 12. Phase change enthalpies of $\mathrm{C}_{14}$ to $\mathrm{C}_{18}$ organic compounds-Continued

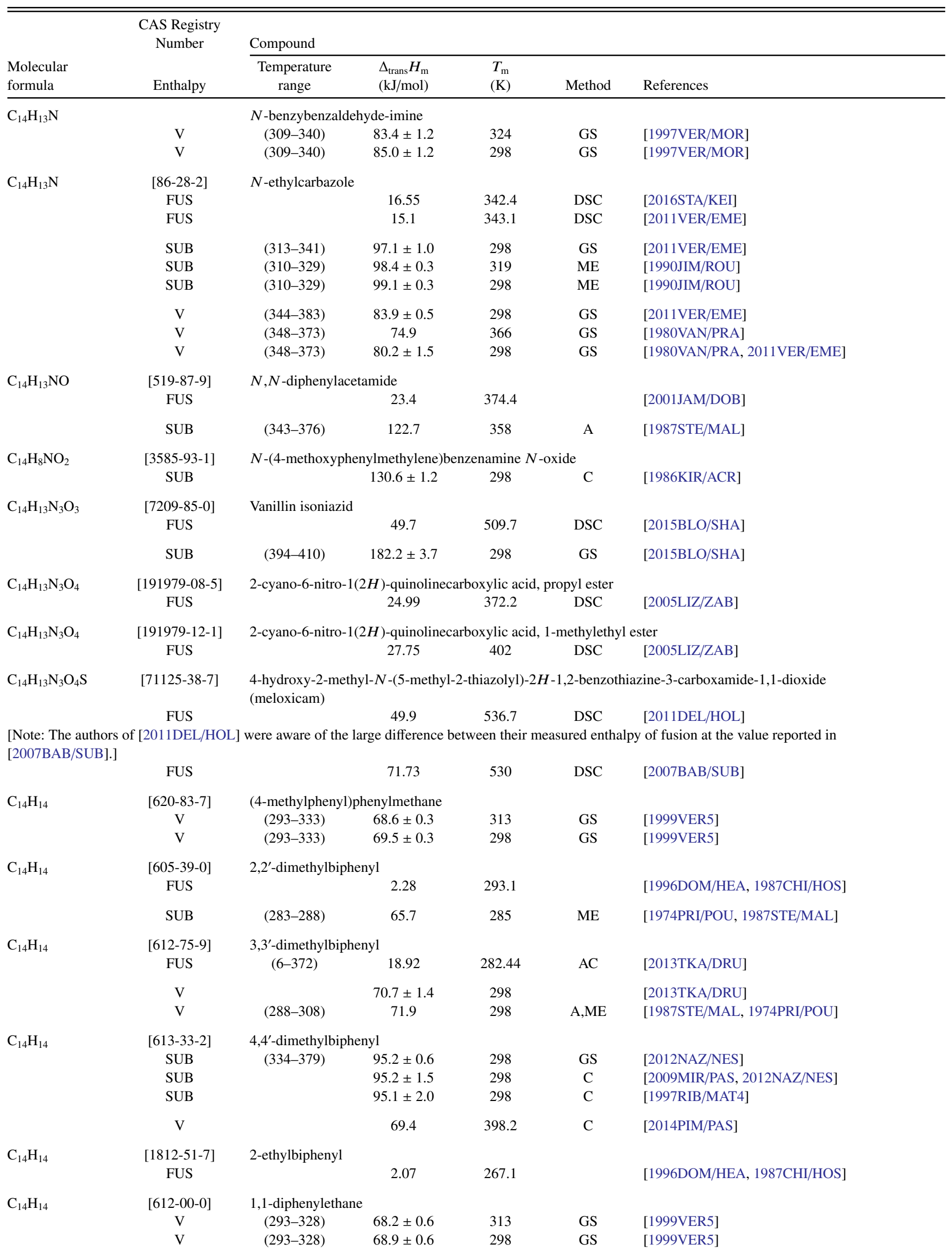


TABLE 12. Phase change enthalpies of $\mathrm{C}_{14}$ to $\mathrm{C}_{18}$ organic compounds-Continued

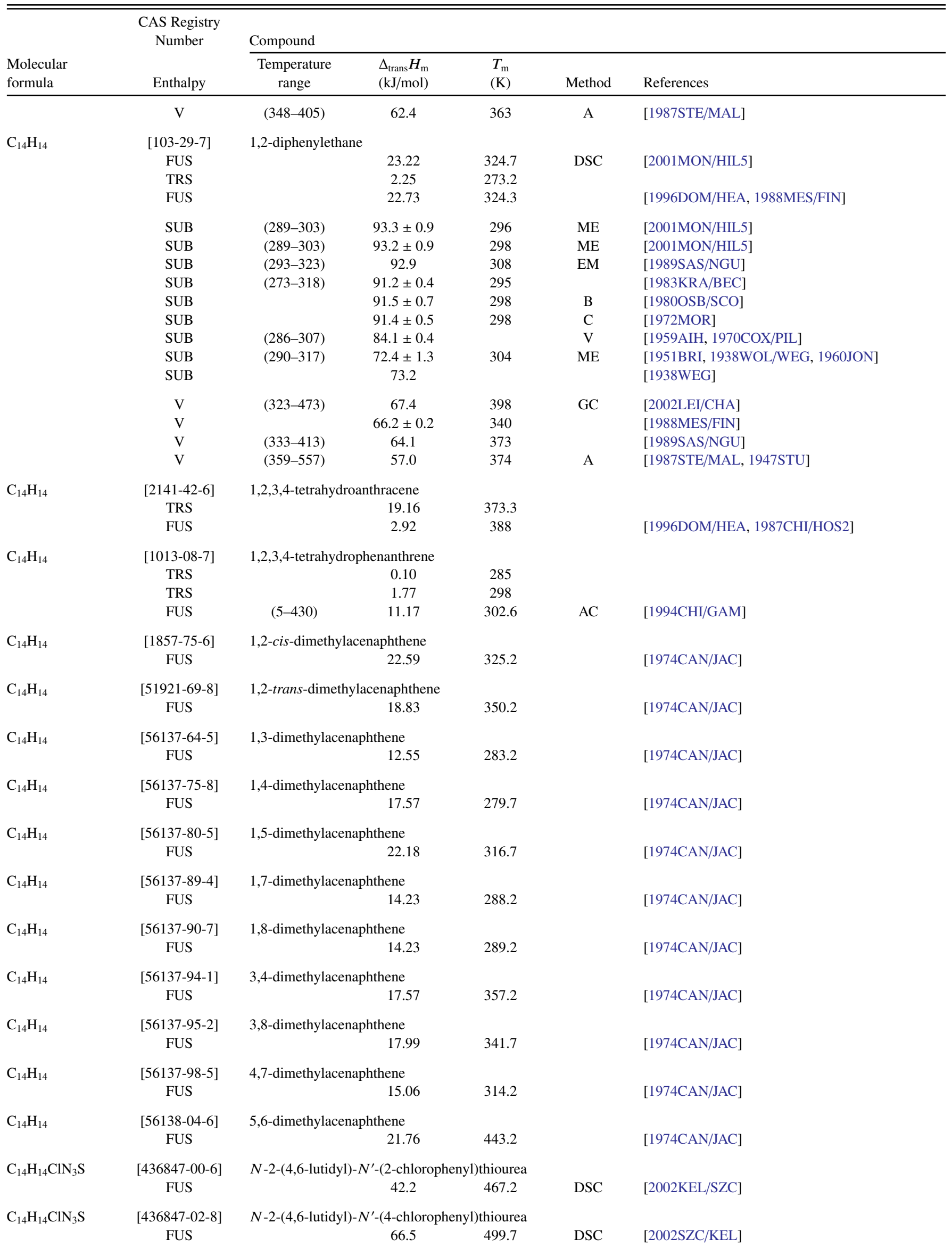


TABLE 12. Phase change enthalpies of $\mathrm{C}_{14}$ to $\mathrm{C}_{18}$ organic compounds-Continued

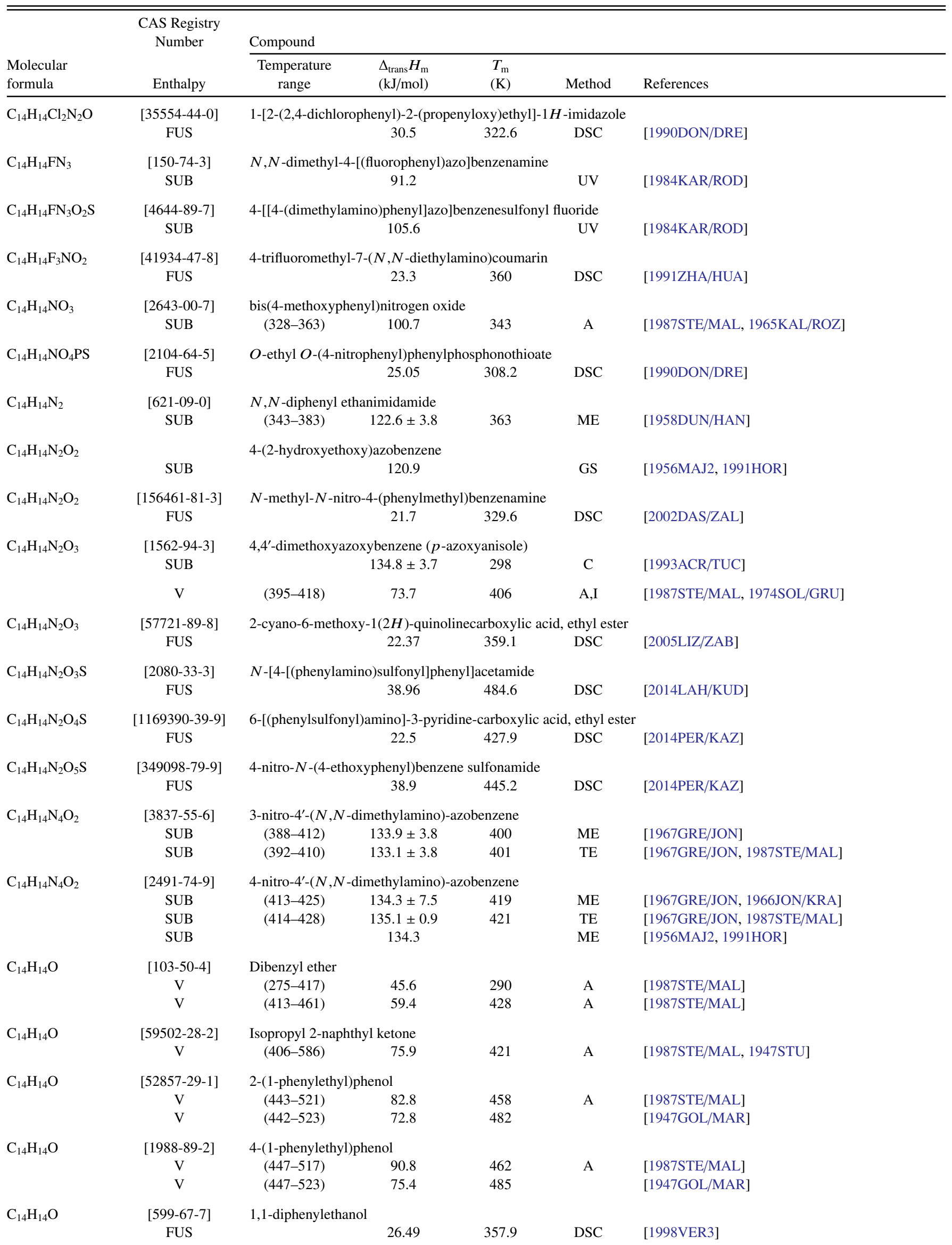


TABLE 12. Phase change enthalpies of $\mathrm{C}_{14}$ to $\mathrm{C}_{18}$ organic compounds-Continued

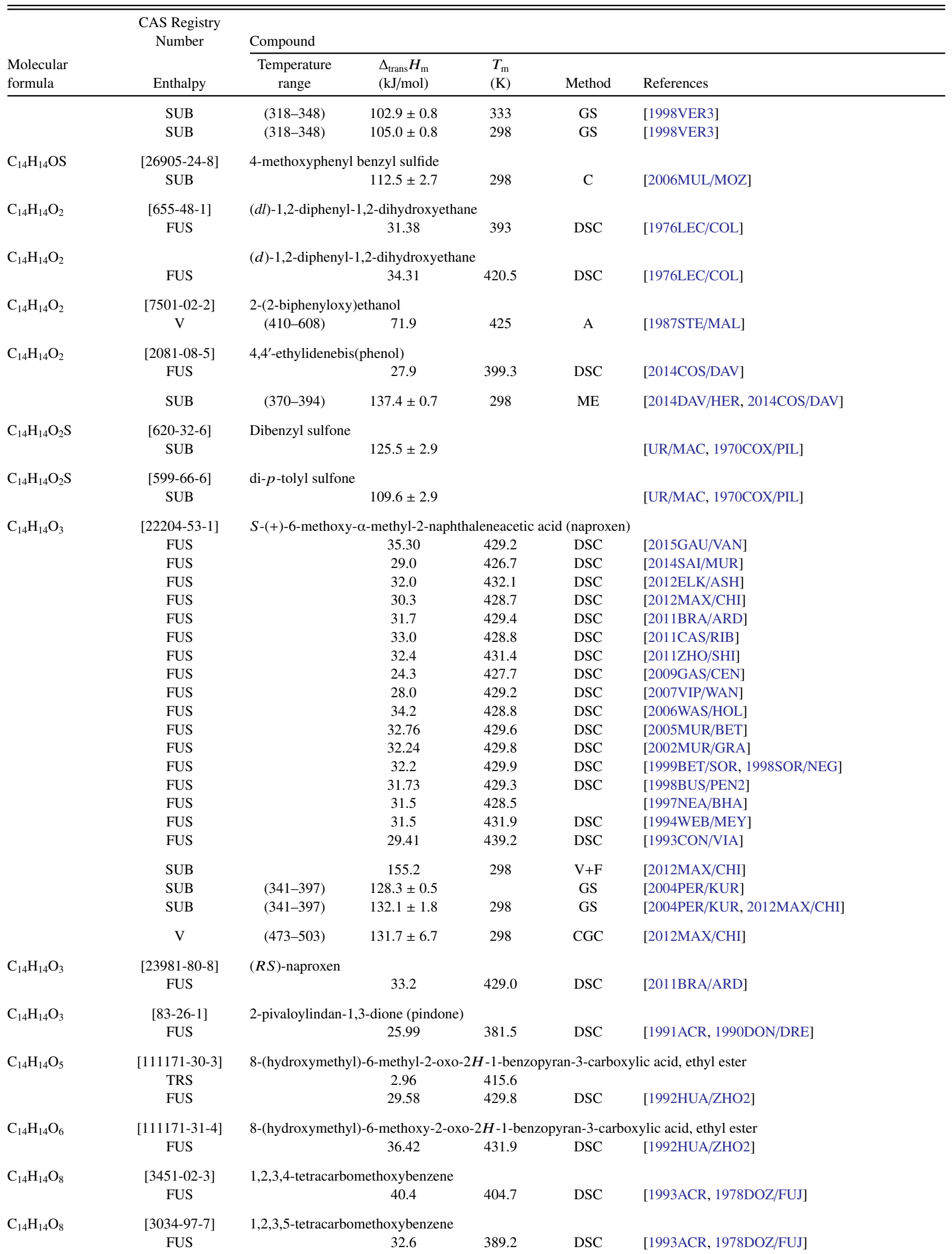


TABLE 12. Phase change enthalpies of $\mathrm{C}_{14}$ to $\mathrm{C}_{18}$ organic compounds-Continued

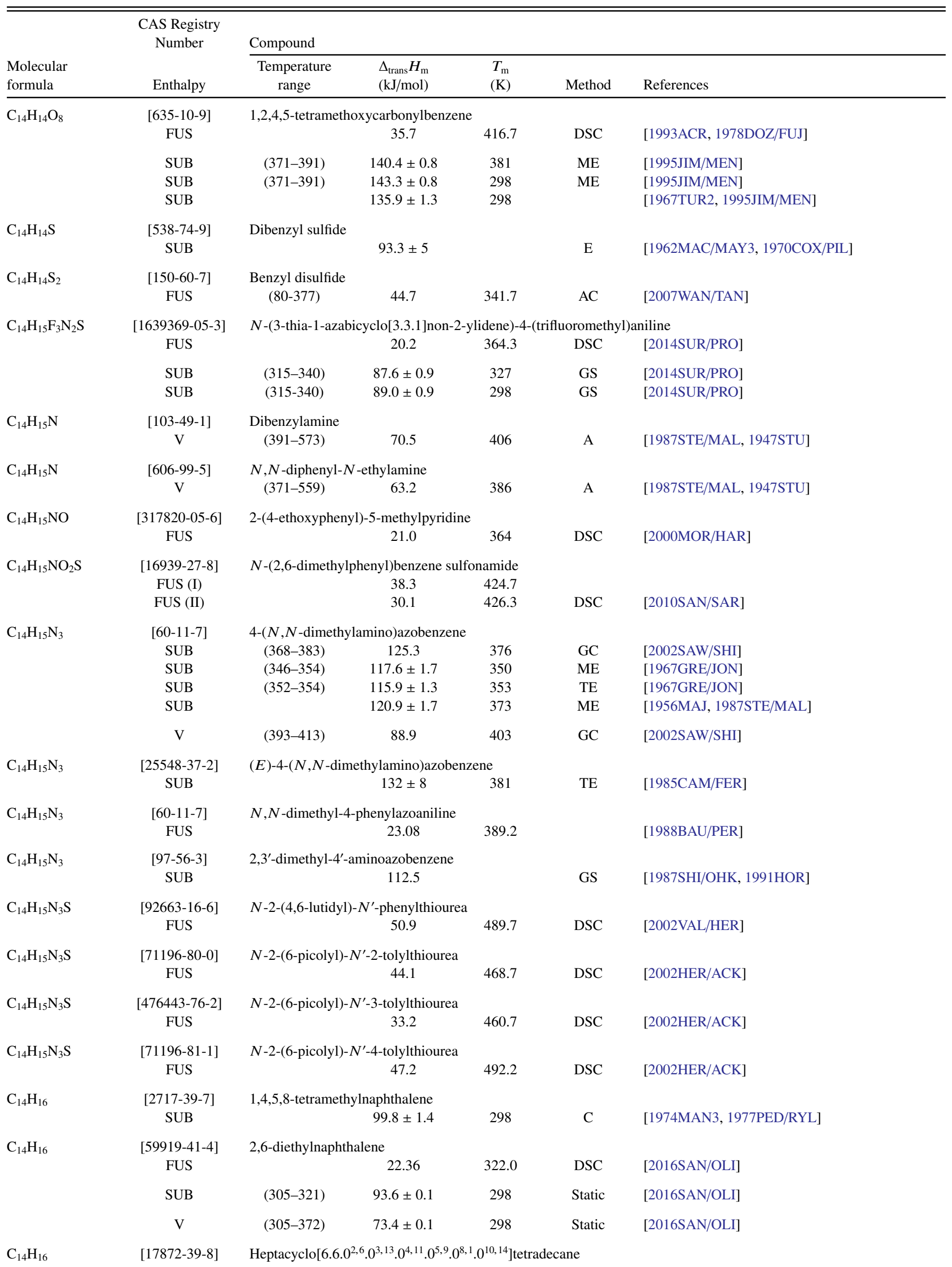


TABLE 12. Phase change enthalpies of $\mathrm{C}_{14}$ to $\mathrm{C}_{18}$ organic compounds-Continued

\begin{tabular}{|c|c|c|c|c|c|c|}
\hline \multirow[b]{2}{*}{$\begin{array}{l}\text { Molecular } \\
\text { formula }\end{array}$} & \multirow{2}{*}{$\begin{array}{l}\text { CAS Registry } \\
\text { Number } \\
\text { Enthalpy }\end{array}$} & \multicolumn{5}{|l|}{ Compound } \\
\hline & & $\begin{array}{l}\text { Temperature } \\
\text { range }\end{array}$ & $\begin{array}{l}\Delta_{\text {trans }} H_{\mathrm{m}} \\
(\mathrm{kJ} / \mathrm{mol})\end{array}$ & $\begin{array}{l}T_{\mathrm{m}} \\
(\mathrm{K})\end{array}$ & Method & References \\
\hline & TRS & & 14.67 & 355 & & \\
\hline & FUS & $(5-304)$ & 5.57 & 440 & $\mathrm{AC}$ & [1994KAB/KOZ] \\
\hline & SUB & & $79.3 \pm 0.4$ & 298 & $\mathrm{C}$ & [1994KAB/KOZ] \\
\hline & SUB & $(298-349)$ & $83.8 \pm 1.2$ & 321 & ME & [1994KAB/KOZ] \\
\hline & SUB & $(298-349)$ & $84.6 \pm 1.2$ & 298 & $\mathrm{ME}$ & [1994KAB/KOZ] \\
\hline \multirow{4}{*}{$\mathrm{C}_{14} \mathrm{H}_{16} \mathrm{ClN}_{3} \mathrm{O}$} & [67129-08-2] & \multicolumn{5}{|c|}{ 2-chloro- $N$-(2,6-dimethylphenyl)- $N$-(1H-pyrazol-1-ylmethyl)acetamide (metazachlor) } \\
\hline & FUS (I) & & 19.7 & 356.2 & & \\
\hline & FUS (II) & & 23 & 353.2 & & \\
\hline & FUS (III) & & 26.6 & 349.2 & DSC & [2004GRI/WEI] \\
\hline \multirow[t]{2}{*}{$\mathrm{C}_{14} \mathrm{H}_{16} \mathrm{ClN}_{3} \mathrm{O}_{2}$} & [43121-43-3] & \multicolumn{5}{|c|}{1 -(4-chlorophenoxy)-3,3-dimethyl-1-(1H-1,2,4-triazol-1-yl) } butanone (triadimefon) \\
\hline & SUB & $(298-343)$ & $111.1 \pm 2.2$ & 303 & GS & {$[1997 \mathrm{DAS} / \mathrm{DAS}]$} \\
\hline \multirow[t]{2}{*}{$\mathrm{C}_{14} \mathrm{H}_{16} \mathrm{ClN}_{3} \mathrm{O}_{2}$} & {$[43121-43-3]$} & \multicolumn{5}{|c|}{ 1-(4-chlorophenoxy)-3,3-dimethyl-(1H,1,2,4-triazol-1-yl)-2-butanone } \\
\hline & FUS & & 22.87 & 351.4 & DSC & {$[1990 \mathrm{DON} / \mathrm{DRE}]$} \\
\hline \multirow[t]{2}{*}{$\mathrm{C}_{14} \mathrm{H}_{16} \mathrm{~F}_{3} \mathrm{~N}_{3} \mathrm{O}_{4}$} & {$[26399-36-0]$} & \multicolumn{5}{|c|}{$N$-(cyclopropylmethyl)-2,6-dmitro- $N$-propyl-4-(trifluoromethyl)benzenamine } \\
\hline & FUS & & 22.51 & 305.8 & DSC & {$[1990 \mathrm{DON} / \mathrm{DRE}]$} \\
\hline \multirow[t]{2}{*}{$\mathrm{C}_{14} \mathrm{H}_{16} \mathrm{~N}_{2} \mathrm{O}_{2}$} & {$[2778-42-9]$} & \multicolumn{5}{|c|}{ 1,3-bis(1-isocyanato-1-methylethyl)benzene } \\
\hline & V & $(298-426)$ & 65.2 & 361 & HSA,T,DTA & [1986ACH/HAS] \\
\hline \multirow[t]{2}{*}{$\mathrm{C}_{14} \mathrm{H}_{16} \mathrm{~N}_{2} \mathrm{O}_{2}$} & {$[2778-41-8]$} & \multicolumn{5}{|c|}{ 1,4-bis(1-isocyanato-1-methylethyl)benzene } \\
\hline & V & $(373-428)$ & 74.0 & 400 & HSA,T,DTA & [1986ACH/HAS] \\
\hline \multirow[t]{4}{*}{$\mathrm{C}_{14} \mathrm{H}_{16} \mathrm{~N}_{2} \mathrm{O}_{2} \mathrm{~S}$} & {$[108929-67-5]$} & \multicolumn{5}{|c|}{ 4-amino- $N$-(4-ethylphenyl)benzenesulfonamide } \\
\hline & FUS & & 36.3 & 436.2 & DSC & [2009PER/TKA, 2014PER/KAZ] \\
\hline & SUB & & $143.6 \pm 0.9$ & 298 & GS & [2009PER/TKA] \\
\hline & $\mathrm{V}$ & & 118.8 & 298 & $\mathrm{~S}-\mathrm{F}$ & [2009PER/TKA] \\
\hline \multirow[t]{2}{*}{$\mathrm{C}_{14} \mathrm{H}_{16} \mathrm{~N}_{2} \mathrm{O}_{3}$} & {$[946568-73-6]$} & \multicolumn{5}{|c|}{ 2-tert-butyoxycarbonyl-3-methylquinoxaline $N$-oxide } \\
\hline & SUB & & $140.8 \pm 3.1$ & 298 & $\mathrm{C}$ & [2012VIV/FRE] \\
\hline \multirow[t]{2}{*}{$\mathrm{C}_{14} \mathrm{H}_{16} \mathrm{~N}_{2} \mathrm{O}_{4}$} & {$[793716-72-0]$} & \multicolumn{5}{|c|}{ 2-tert-butyoxycarbonyl-3-methylquinoxaline $N, N^{\prime}$-dioxide } \\
\hline & SUB & & $164.1 \pm 1.8$ & 298 & $\mathrm{C}$ & [2007GOM/SOU] \\
\hline $\mathrm{C}_{14} \mathrm{H}_{16} \mathrm{~N}_{2} \mathrm{O}_{7}$ & {$[152672-90-7]$} & $\begin{array}{l}\text { 2-methylpropar } \\
\text { yl ester }\end{array}$ & acid 2,3-dihyc & (1-meth & 1oxy)-3-[2-(ni & rooxy)-ethyl]-4-oxo-2H-1,3-benzoxazin-7- \\
\hline & FUS & & 26 & 345.7 & DSC & {$[1996 \mathrm{FON} / \mathrm{ROS}]$} \\
\hline $\mathrm{C}_{14} \mathrm{H}_{16} \mathrm{O}_{5}$ & {$[20666-86-8]$} & Benzoyl (3-cyc & xyloxy)carbor & roxide & & \\
\hline & SUB & $(293-313)$ & $96.2 \pm 4.2$ & 303 & $\mathrm{ME}$ & [1971KIP/RAB, 1977PED/RYL] \\
\hline $\mathrm{C}_{14} \mathrm{H}_{17} \mathrm{ClN}_{2} \mathrm{~S}$ & {$[1583299-22-2]$} & (3-chloro-4-me & phenyl)-[3-thi & rabicycl & .1]non-2-ylid & ene]amine \\
\hline & FUS & & 22.7 & 406.6 & DSC & [2014BLO/OLK] \\
\hline & SUB & $(376-396)$ & $146.2 \pm 1.8$ & 386 & GS & [2014BLO/OLK] \\
\hline & SUB & (376-396) & $150.5 \pm 1.8$ & 298 & GS & {$[2014 \mathrm{BLO} / \mathrm{OLK}]$} \\
\hline $\mathrm{C}_{14} \mathrm{H}_{17} \mathrm{ClNO}_{4} \mathrm{PS}_{2}$ & [10311-84-9] & $S$-[2-chloro-1-( & dihydro-1,3-d & $2 H$-isor & -2-yl)ethyl] $O$ & $O$-diethylphosphorodithioate \\
\hline & FUS & & 25.27 & 340 & DSC & [1990DON/DRE] \\
\hline $\mathrm{C}_{14} \mathrm{H}_{17} \mathrm{Cl}_{2} \mathrm{~N}_{3} \mathrm{O}$ & [79983-71-4] & $\alpha$-butyl- $\alpha-(2,4-$ & lorophenyl)-1 & 2,4-triaz & 1-ethanol $( \pm)-1$ & exaconazole \\
\hline & SUB & $(318-358)$ & 160.1 & 338 & ME & [1997GOO] \\
\hline $\mathrm{C}_{14} \mathrm{H}_{17} \mathrm{Cl}_{3} \mathrm{O}_{3}$ & [2630-13-9] & Hexyl 2,4,5-tric & rophenoxyace & & & \\
\hline & V & (460-573) & 85.3 & 475 & A & [1987STE/MAL] \\
\hline & $\mathrm{V}$ & $(460-573)$ & 81.1 & 516 & & [1966JEN/SCH] \\
\hline $\mathrm{C}_{14} \mathrm{H}_{17} \mathrm{~N}$ & & $N$-ethyl-1,2,3, & rahydrocarbaz & & & \\
\hline & FUS & & 12.41 & 282.4 & DSC & [2016STA/KEI] \\
\hline & $\mathrm{V}$ & $(308-371)$ & $82.8 \pm 0.4$ & 298 & GS & [2015STA/EME] \\
\hline $\mathrm{C}_{14} \mathrm{H}_{17} \mathrm{NO}_{2}$ & {$[91-44-1]$} & 4-methyl-7-die & aminocoumari & & & \\
\hline & FUS & & 17.88 & 343.8 & DSC & [1996DOM/HEA, 1989ZHA/HUA] \\
\hline $\mathrm{C}_{14} \mathrm{H}_{17} \mathrm{~N}_{5} \mathrm{O}_{3}$ & {$[51940-44-4]$} & 8-ethyl-5,8-dih & $-5-o x o-2-(1-p$ & cinyl)py & 2,3-d]-pyrimi & line-6-carboxylic acid (pipemidic acid) \\
\hline
\end{tabular}


TABLE 12. Phase change enthalpies of $\mathrm{C}_{14}$ to $\mathrm{C}_{18}$ organic compounds-Continued

\begin{tabular}{|c|c|c|c|c|c|c|}
\hline \multirow[b]{2}{*}{$\begin{array}{l}\text { Molecular } \\
\text { formula }\end{array}$} & \multirow{2}{*}{$\begin{array}{l}\text { CAS Registry } \\
\text { Number } \\
\text { Enthalpy }\end{array}$} & \multicolumn{5}{|l|}{ Compound } \\
\hline & & $\begin{array}{l}\text { Temperature } \\
\text { range }\end{array}$ & $\begin{array}{l}\Delta_{\text {trans }} H_{\mathrm{m}} \\
(\mathrm{kJ} / \mathrm{mol})\end{array}$ & $\begin{array}{l}T_{\mathrm{m}} \\
(\mathrm{K})\end{array}$ & Method & References \\
\hline & FUS & & 32.85 & 529.9 & & [2004ROM/BUS2] \\
\hline \multirow[t]{2}{*}{$\mathrm{C}_{14} \mathrm{H}_{18}$} & $\begin{array}{l}{[1079-71-6]} \\
\text { TRS } \\
\text { FUS }\end{array}$ & $1,2,3,4,5,6,7,8$ & $\begin{array}{c}\text { ydroanthrac } \\
2.51 \\
18.34\end{array}$ & $\begin{array}{l}331.4 \\
345.4\end{array}$ & & [1996DOM/HEA] \\
\hline & $\begin{array}{l}\mathrm{V} \\
\mathrm{V}\end{array}$ & $\begin{array}{l}(437-498) \\
(348-433)\end{array}$ & $\begin{array}{l}45.6 \\
\text { NA }\end{array}$ & 452 & $\begin{array}{c}\text { A } \\
\text { IPM }\end{array}$ & $\begin{array}{l}{[1987 \mathrm{STE} / \mathrm{MAL}]} \\
{[1982 \mathrm{GAM} / \mathrm{CAL}]}\end{array}$ \\
\hline $\mathrm{C}_{14} \mathrm{H}_{18}$ & $\begin{array}{c}{[5325-97-3]} \\
\mathrm{V}\end{array}$ & \multicolumn{5}{|c|}{$1,2,3,4,5,6,7,8$-octahydrophenanthrene } \\
\hline $\mathrm{C}_{14} \mathrm{H}_{18} \mathrm{ClN}_{3} \mathrm{O}_{2}$ & $\begin{array}{c}\text { [70585-35-2] } \\
\text { FUS (I) } \\
\text { FUS (II) } \\
\text { FUS (III) }\end{array}$ & $(1 \mathrm{RS}, 2 \mathrm{SR})-1-($ & $\begin{array}{c}\text { orophenoxy) } \\
32 \\
33.1 \\
25.1\end{array}$ & $\begin{array}{l}\text { imethyl } \\
411.2 \\
406.2 \\
385.2\end{array}$ & DSC & [2000BUR/VAN] \\
\hline $\mathrm{C}_{14} \mathrm{H}_{18} \mathrm{ClN}_{3} \mathrm{O}_{2}$ & $\begin{array}{l}{[70585-37-4]} \\
\text { FUS }\end{array}$ & \multicolumn{5}{|c|}{ (1RR,2SS)-1-(4-chlorophenoxy)-3,3-dimethyl-1-(1H-1,2,4-triazol-1-yl)-butan-2-ol (threo triadimenol) } \\
\hline $\mathrm{C}_{14} \mathrm{H}_{18} \mathrm{ClN}_{3} \mathrm{O}_{2}$ & $\begin{array}{l}{[55219-65-3]} \\
\text { FUS }\end{array}$ & \multicolumn{5}{|c|}{$\beta$-(4-chlorophenoxy)- $\alpha$-(1,1-dimethylethyl)-1H-1,2,4-triazole-1-ethanol } \\
\hline $\mathrm{C}_{14} \mathrm{H}_{18} \mathrm{Cl}_{2} \mathrm{O}_{3}$ & $\begin{array}{c}{[1917-95-9]} \\
\mathrm{V} \\
\mathrm{V}\end{array}$ & $\begin{array}{l}\text { Hexyl 2,4-dich } \\
\quad(444-573) \\
\quad(444-573)\end{array}$ & $\begin{array}{l}\text { henoxyaceta } \\
\begin{array}{l}81.3 \\
76.3\end{array}\end{array}$ & $\begin{array}{l}459 \\
508\end{array}$ & $\begin{array}{c}\mathrm{A}, \mathrm{GC} \\
\mathrm{GC}\end{array}$ & $\begin{array}{l}\text { [1987STE/MAL, 1966JEN/SCH] } \\
\text { [1966JEN/SCH] }\end{array}$ \\
\hline $\mathrm{C}_{14} \mathrm{H}_{18} \mathrm{Cl}_{2} \mathrm{O}_{3}$ & $\begin{array}{c}{[1917-93-7]} \\
\text { V } \\
\text { V }\end{array}$ & $\begin{array}{l}\text { Isohexyl 2,4-di } \\
\qquad(460-573) \\
\quad(460-573)\end{array}$ & $\begin{array}{c}\text { ophenoxyac } \\
69.1 \\
72.0\end{array}$ & $\begin{array}{l}475 \\
516\end{array}$ & $\begin{array}{c}\mathrm{A}, \mathrm{GC} \\
\mathrm{GC}\end{array}$ & $\begin{array}{l}\text { [1987STE/MAL, 1999DYK/SVO, 1966JEN/SCH] } \\
\text { [1966JEN/SCH] }\end{array}$ \\
\hline $\mathrm{C}_{14} \mathrm{H}_{18} \mathrm{~N}_{2}$ & $\begin{array}{l}{[10075-69-1]} \\
\text { SUB }\end{array}$ & $\begin{array}{l}1,5-N, N, N^{\prime}, N \\
\quad(318-356)\end{array}$ & $\begin{array}{l}\text { ramethyldian } \\
98.6 \pm 0.4\end{array}$ & $\begin{array}{l}\text { aphthale } \\
298\end{array}$ & GS & [2007VER/GEO] \\
\hline $\mathrm{C}_{14} \mathrm{H}_{18} \mathrm{~N}_{2}$ & $\begin{array}{l}{[20734-58-1]} \\
\text { SUB }\end{array}$ & $\begin{array}{l}1,8-N, N, N^{\prime}, N \\
\quad(324-364)\end{array}$ & $\begin{array}{l}\text { ramethyldian } \\
94.7 \pm 0.8\end{array}$ & $\begin{array}{l}\text { aphthale } \\
298\end{array}$ & GS & [2007VER/GEO] \\
\hline & $\mathrm{V}$ & $(324-364)$ & $76.7 \pm 0.4$ & 298 & GS & [2007VER/GEO] \\
\hline $\mathrm{C}_{14} \mathrm{H}_{18} \mathrm{~N}_{2} \mathrm{O}_{3}$ & $\begin{array}{l}{[3625-25-0]} \\
\text { FUS }\end{array}$ & 5-bicyclo[3.2.1 & $\begin{array}{l}\text { 2-en-3-yl-5-c } \\
22.7\end{array}$ & $2,4,6(1 H$ & $\begin{array}{l}5 H) \text {-pyril } \\
\text { DSC }\end{array}$ & $\begin{array}{r}\text { inetrione (reposal) } \\
\text { [1982TRE/VAU] }\end{array}$ \\
\hline $\mathrm{C}_{14} \mathrm{H}_{18} \mathrm{~N}_{2} \mathrm{O}_{5}$ & $\begin{array}{l}{[81-14-1]} \\
\text { FUS }\end{array}$ & 2,6-dimethyl-3 & $\begin{array}{l}\text { itro-4-tert-b } \\
\quad 23.81\end{array}$ & $\begin{array}{l}\text { etophen } \\
408.5\end{array}$ & $\mathrm{DSC}$ & [2004QU/BAI] \\
\hline & SUB & $(293-353)$ & 107.9 & 323 & $\mathrm{ME}$ & [1953SER/VOI, 1960JON] \\
\hline $\mathrm{C}_{14} \mathrm{H}_{18} \mathrm{~N}_{2} \mathrm{~S}$ & $\begin{array}{l}\text { [1583299-19-7] } \\
\text { FUS }\end{array}$ & (3-methylpheny & $\begin{array}{c}\text {-thia-1-azab } \\
26.5\end{array}$ & $\begin{array}{c}{[3.3 .1] \mathrm{n}} \\
398.5\end{array}$ & $\begin{array}{l}\text { lidene]an } \\
\text { DSC }\end{array}$ & [2014BLO/OLK] \\
\hline & $\begin{array}{l}\text { SUB } \\
\text { SUB }\end{array}$ & $\begin{array}{l}(353-376) \\
(353-376)\end{array}$ & $\begin{array}{l}128.2 \pm 1.0 \\
131.0 \pm 1.0\end{array}$ & $\begin{array}{l}365 \\
298\end{array}$ & $\begin{array}{l}\text { GS } \\
\text { GS }\end{array}$ & $\begin{array}{l}{[2014 \mathrm{BLO} / \mathrm{OLK}]} \\
{[2014 \mathrm{BLO} / \mathrm{OLK}]}\end{array}$ \\
\hline $\mathrm{C}_{14} \mathrm{H}_{18} \mathrm{~N}_{2} \mathrm{~S}$ & $\begin{array}{c}{[1639369-07-5]} \\
\text { FUS }\end{array}$ & 4-methyl- $N$-(3 & $\begin{array}{c}\text {-1-azabicycl } \\
20.5\end{array}$ & $\begin{array}{l}\text { ]non-2- } \\
347.6\end{array}$ & $\begin{array}{l}\text { e)aniline } \\
\text { DSC }\end{array}$ & [2014SUR/PRO] \\
\hline & $\begin{array}{l}\text { SUB } \\
\text { SUB }\end{array}$ & $\begin{array}{l}(327-347) \\
(327-347)\end{array}$ & $\begin{array}{c}99.2 \pm 0.6 \\
101.1 \pm 0.6\end{array}$ & $\begin{array}{l}337 \\
298\end{array}$ & $\begin{array}{l}\text { GS } \\
\text { GS }\end{array}$ & $\begin{array}{l}\text { [2014SUR/PRO] } \\
\text { [2014SUR/PRO] }\end{array}$ \\
\hline $\mathrm{C}_{14} \mathrm{H}_{18} \mathrm{~N}_{4} \mathrm{O}_{3}$ & $\begin{array}{l}\text { [738-70-5] } \\
\text { FUS } \\
\text { FUS } \\
\text { FUS } \\
\text { FUS } \\
\text { FUS } \\
\text { FUS }\end{array}$ & 5 -[(3,4,5-trime & $\begin{array}{c}\text { phenyl)metl } \\
47.44 \\
38.5 \\
48.4 \\
49.8 \\
53.65 \\
46.55\end{array}$ & $\begin{array}{c}\text { 4-pyrim } \\
475.0 \\
471.2 \\
477.4 \\
472.9 \\
474\end{array}$ & $\begin{array}{c}\text { iamine }(\mathrm{t} \\
\text { DSC } \\
\text { DSC } \\
\text { DSC } \\
\text { DSC } \\
\text { DSC }\end{array}$ & $\begin{array}{l}\text { ethoprim) } \\
\text { [2015MAD/SWA] } \\
\text { [2013AGA/MOS] } \\
\text { [2012ELS/HAN] } \\
\text { [2006WAS/HOL] } \\
\text { [1998ISS/ELA] } \\
\text { [1990TIM/CRA, 1985CHA] }\end{array}$ \\
\hline $\mathrm{C}_{14} \mathrm{H}_{18} \mathrm{O}$ & $\begin{array}{c}{[122-40-7]} \\
\mathrm{V}\end{array}$ & $\begin{array}{c}\alpha \text {-pentylcinnan } \\
\quad(282-333)\end{array}$ & $\begin{array}{l}\text { hyde } \\
75.3\end{array}$ & 297 & A,ME & [1987STE/MAL, 1955SER/VOI] \\
\hline $\mathrm{C}_{14} \mathrm{H}_{18} \mathrm{O}$ & [30545-23-4] & Diamantanone & & & & \\
\hline
\end{tabular}


TABLE 12. Phase change enthalpies of $\mathrm{C}_{14}$ to $\mathrm{C}_{18}$ organic compounds-Continued

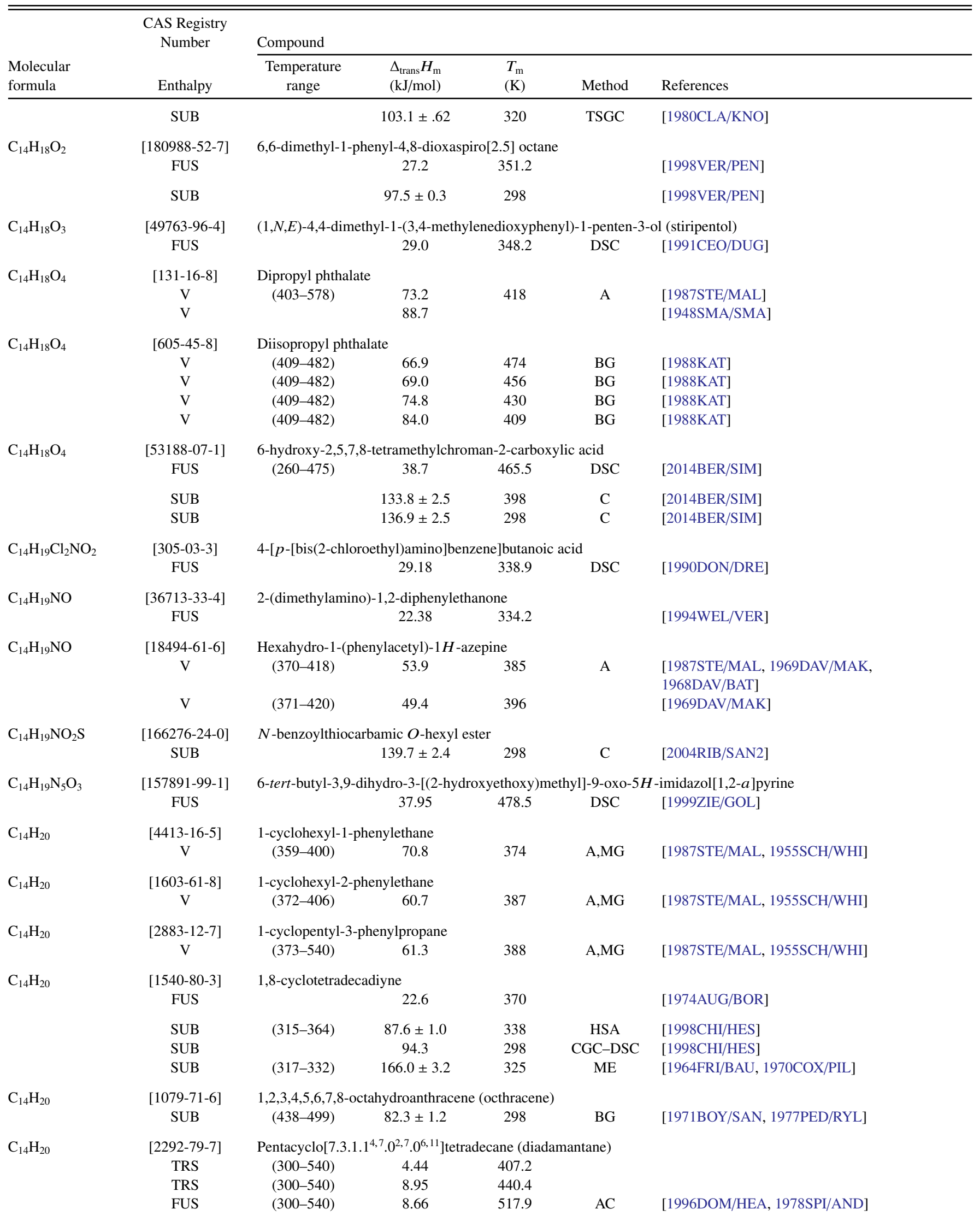

[Note: Westrum et al. [1978WES/MCK] report a very small thermal anomaly at $35.7 \mathrm{~K}$ as determined by adiabatic calorimetric measurements.]

$\begin{array}{lccccc}\text { SUB } & (305-333) & 96.0 \pm 0.8 & 319 & \text { TSGC } & \text { [1975CLA/KNO] } \\ \text { SUB } & & 117.2 \pm 8 & & B & \text { [1971CAR/LAY] }\end{array}$

$\mathrm{C}_{14} \mathrm{H}_{20} \mathrm{ClNO}_{2}$

[15972-60-8] 2-chloro- $N$-(2,6-diethylphenyl)- $N$-(methoxymethyl)acetamide 
TABLE 12. Phase change enthalpies of $\mathrm{C}_{14}$ to $\mathrm{C}_{18}$ organic compounds-Continued

\begin{tabular}{|c|c|c|c|c|c|c|}
\hline \multirow[b]{2}{*}{$\begin{array}{l}\text { Molecular } \\
\text { formula }\end{array}$} & \multirow{2}{*}{$\begin{array}{l}\text { CAS Registry } \\
\text { Number } \\
\text { Enthalpy }\end{array}$} & \multicolumn{5}{|l|}{ Compound } \\
\hline & & $\begin{array}{l}\text { Temperature } \\
\text { range }\end{array}$ & $\begin{array}{l}\Delta_{\text {trans }} H_{\mathrm{m}} \\
(\mathrm{kJ} / \mathrm{mol})\end{array}$ & $\begin{array}{l}T_{\mathrm{m}} \\
(\mathrm{K})\end{array}$ & Method & References \\
\hline & FUS & & 26.7 & 317.7 & DSC & [2005SBI/VEC] \\
\hline & FUS & & 25.31 & 315.9 & DSC & [1990DON/DRE] \\
\hline & $\mathrm{V}$ & & $85 \pm 1$ & 436 & TGA & {$[2007 \mathrm{VEC}]$} \\
\hline $\mathrm{C}_{14} \mathrm{H}_{20} \mathrm{Cl}_{2}$ & \multicolumn{6}{|c|}{ 1,2-dichloro-3,4,5,6-tetraethylbenzene } \\
\hline $\mathrm{C}_{14} \mathrm{H}_{20} \mathrm{C}_{12}$ & $\mathrm{~V}$ & \multicolumn{5}{|c|}{ 1,4-dichloro-2,3,5,6-tetraethylbenzene } \\
\hline $\mathrm{C}_{14} \mathrm{H}_{20} \mathrm{~N}_{2} \mathrm{O}$ & $\begin{array}{c}{[27262-40-4]} \\
\text { FUS }\end{array}$ & \multicolumn{5}{|c|}{$N$-(2,6-dimethylphenyl)-2-piperidinecarboxamide } \\
\hline $\mathrm{C}_{14} \mathrm{H}_{20} \mathrm{~N}_{2} \mathrm{OS}$ & $\begin{array}{c}\text { [90473-84-0] } \\
\text { SUB }\end{array}$ & \multicolumn{5}{|c|}{$N$-(diethylaminothiocarbonyl)benzimido ethyl ester } \\
\hline $\mathrm{C}_{14} \mathrm{H}_{20} \mathrm{~N}_{2} \mathrm{O}_{2}$ & $\begin{array}{c}\text { [26328-11-0] } \\
\text { FUS }\end{array}$ & \multicolumn{5}{|c|}{ (-)-1-(1H-indol-4-yloxy)-3-(isopropylamino)-2-propanol (pindolol) } \\
\hline \multirow[t]{2}{*}{$\mathrm{C}_{14} \mathrm{H}_{20} \mathrm{~N}_{2} \mathrm{O}_{2}$} & $\begin{array}{l}\text { [13523-86-9] } \\
\text { FUS } \\
\text { FUS } \\
\text { FUS } \\
\text { FUS }\end{array}$ & $( \pm)-1-(1 H$-ind & $\begin{array}{c}\text { yloxy)-3-(iso } \\
58.0 \\
60.6 \\
58.0 \\
57.9\end{array}$ & $\begin{array}{l}\text { lamino) } \\
442.9 \\
423.6 \\
443.8 \\
442.9\end{array}$ & $\begin{array}{l}\text { panol (pin } \\
\text { DSC } \\
\text { DSC } \\
\text { DSC }\end{array}$ & $\begin{array}{l}\text { ol) } \\
\text { [2010CAN/CAS] } \\
\text { [2007PER/VOL] } \\
\text { [2004NUN/EUS] } \\
\text { [1999LI/ZEL, 1993NEA/SHI] }\end{array}$ \\
\hline & SUB & $(355-427)$ & $146.0 \pm 1.2$ & 298 & GS & [2007PER/VOL] \\
\hline $\mathrm{C}_{14} \mathrm{H}_{20} \mathrm{~N}_{3} \mathrm{O}_{5} \mathrm{PS}$ & $\begin{array}{c}\text { [13457-18-6] } \\
\text { FUS }\end{array}$ & \multicolumn{5}{|c|}{$O$-6-ethoxycarbonyl-5-methylpyrazolo[1,5- $a$ ]pyrimidin-2-yl $O, O$-diethyl phosphorothioate } \\
\hline $\mathrm{C}_{14} \mathrm{H}_{20} \mathrm{O}$ & $\begin{array}{c}{[61812-55-3]} \\
\mathrm{V}\end{array}$ & $\begin{array}{c}\text { (1-cyclohexylo } \\
(286-338)\end{array}$ & $\begin{array}{l}\text { hyl)benzene } \\
69.8 \pm 0.5\end{array}$ & 298 & GS & [2002KRA/VAS, 2002VER/HEI] \\
\hline $\mathrm{C}_{14} \mathrm{H}_{20} \mathrm{O}$ & $\begin{array}{c}\text { [30545-14-3] } \\
\text { TRS } \\
\text { TRS } \\
\text { FUS }\end{array}$ & Diamantan-1-o & $\begin{array}{l}18.0 \\
4.9 \\
9.6\end{array}$ & $\begin{array}{l}395 \\
408 \\
573\end{array}$ & DSC & [1974CLA/MCK] \\
\hline & SUB & $(319-349)$ & 118. \pm 0.6 & 334 & & [1980CLA/KNO, 1975CLA/KNO] \\
\hline $\mathrm{C}_{14} \mathrm{H}_{20} \mathrm{O}$ & $\begin{array}{c}\text { [30545-24-5] } \\
\text { SUB }\end{array}$ & $\begin{array}{l}\text { Diamantan-3-o } \\
\quad(323-354)\end{array}$ & $116.1 \pm 4.4$ & 338 & & [1980CLA/KNO, 1975CLA/KNO] \\
\hline $\mathrm{C}_{14} \mathrm{H}_{20} \mathrm{O}$ & $\begin{array}{c}\text { [30651-03-7] } \\
\text { TRS } \\
\text { FUS }\end{array}$ & Diamantan-4-o & $\begin{array}{l}9.77 \\
16.4\end{array}$ & $\begin{array}{l}448 \\
484\end{array}$ & DSC & [1974CLA/MCK] \\
\hline & SUB & $(322-353)$ & $117.8 \pm 0.2$ & 337 & & [1980CLA/KNO, 1975CLA/KNO] \\
\hline $\mathrm{C}_{14} \mathrm{H}_{20} \mathrm{O}_{2}$ & $\begin{array}{c}\text { [3383-21-9] } \\
\text { FUS } \\
\text { FUS }\end{array}$ & 3,5-di-tert-buty & $\begin{array}{c}\text { benzoquinon } \\
26.21 \\
26.53\end{array}$ & $\begin{array}{l}388.5 \\
387.9\end{array}$ & $\begin{array}{l}\text { DSC } \\
\text { DSC }\end{array}$ & $\begin{array}{l}\text { [2016PAS/ABA] } \\
{[2005 \mathrm{FAT} / \mathrm{KAS}]}\end{array}$ \\
\hline & SUB & & $106.1 \pm 1.3$ & 298 & $\mathrm{C}$ & [2005FAT/KAS] \\
\hline $\mathrm{C}_{14} \mathrm{H}_{20} \mathrm{O}_{2}$ & $\begin{array}{c}\text { [34105-76-5] } \\
\text { FUS }\end{array}$ & 3,6-di-tert-buty & $\begin{array}{c}\text { benzoquinon } \\
27.98\end{array}$ & 475.3 & DSC & [2016PAS/ABA] \\
\hline $\mathrm{C}_{14} \mathrm{H}_{20} \mathrm{O}_{2}$ & $\begin{array}{c}{[38350-87-7]} \\
\text { SUB }\end{array}$ & $\begin{array}{l}\text { 4-heptylbenzoi } \\
\text { (353-369) }\end{array}$ & $\begin{array}{l}\mathrm{d} \\
130.0 \pm 0.9\end{array}$ & 298 & ME & [2004MON/ALM] \\
\hline $\mathrm{C}_{14} \mathrm{H}_{20} \mathrm{O}_{2}$ & $\begin{array}{c}\text { [950-99-2] } \\
\text { FUS }\end{array}$ & $\begin{array}{l}2,2,5,7,8 \text {-penta } \\
\quad(260-380)\end{array}$ & $\begin{array}{l}\text { ylchroman-6 } \\
27.0\end{array}$ & 367.5 & DSC & [2014BER/SIM] \\
\hline & $\begin{array}{l}\text { SUB } \\
\text { SUB } \\
\text { SUB } \\
\text { SUB }\end{array}$ & $\begin{array}{l}(331-352) \\
(331-352)\end{array}$ & $\begin{array}{l}105.8 \pm 1.6 \\
107.3 \pm 1.6 \\
105.9 \pm 0.9 \\
107.4 \pm 0.9\end{array}$ & $\begin{array}{l}341 \\
298 \\
341 \\
298\end{array}$ & $\begin{array}{c}\mathrm{ME} \\
\mathrm{ME} \\
\mathrm{C} \\
\mathrm{C}\end{array}$ & $\begin{array}{l}\text { [2014BER/SIM] } \\
{[2014 \mathrm{BER} / \mathrm{SIM}]} \\
{[2014 \mathrm{BER} / \mathrm{SIM}]} \\
{[2014 \mathrm{BER} / \mathrm{SIM}]}\end{array}$ \\
\hline $\mathrm{C}_{14} \mathrm{H}_{20} \mathrm{O}_{2}$ & $\begin{array}{c}{[6290-37-5]} \\
\mathrm{V}\end{array}$ & Phenethyl hexa & $78.8 \pm 1.5$ & 298 & CGC & [2015KOZ/GOB] \\
\hline
\end{tabular}


TABLE 12. Phase change enthalpies of $\mathrm{C}_{14}$ to $\mathrm{C}_{18}$ organic compounds-Continued

\begin{tabular}{|c|c|c|c|c|c|c|}
\hline \multirow[b]{2}{*}{$\begin{array}{l}\text { Molecular } \\
\text { formula }\end{array}$} & \multirow{2}{*}{$\begin{array}{l}\text { CAS Registry } \\
\text { Number } \\
\text { Enthalpy }\end{array}$} & \multicolumn{5}{|l|}{ Compound } \\
\hline & & $\begin{array}{c}\text { Temperature } \\
\text { range }\end{array}$ & $\begin{array}{l}\Delta_{\text {trans }} H_{\mathrm{m}} \\
(\mathrm{kJ} / \mathrm{mol})\end{array}$ & $\begin{array}{l}T_{\mathrm{m}} \\
(\mathrm{K})\end{array}$ & Method & References \\
\hline \multirow[t]{2}{*}{$\mathrm{C}_{14} \mathrm{H}_{20} \mathrm{O}_{3}$} & & \multicolumn{5}{|c|}{ 2-(4-tert-butylphenoxy)ethyl acetate } \\
\hline & $\mathrm{V}$ & $(391-578)$ & 78.8 & 406 & A & [1987STE/MAL, 1947STU] \\
\hline \multirow[t]{3}{*}{$\mathrm{C}_{14} \mathrm{H}_{20} \mathrm{O}_{3}$} & {$[15872-42-1]$} & \multicolumn{5}{|c|}{ 4-heptyloxybenzoic acid } \\
\hline & SUB & $(355-361)$ & $155.1 \pm 1.0$ & 358 & $\mathrm{ME}$ & [2010FON/SAN] \\
\hline & SUB & $(355-361)$ & $157.2 \pm 1.2$ & 298 & $\mathrm{ME}$ & [2010FON/SAN] \\
\hline \multirow{4}{*}{$\mathrm{C}_{14} \mathrm{H}_{20} \mathrm{O}_{4}$} & {$[58608-07-4]$} & \multicolumn{5}{|c|}{ 2,5-dibutoxy-1,4-benzoquinone } \\
\hline & TRS & & 4.7 & 328.3 & & \\
\hline & TRS & & 2.3 & 364.5 & & \\
\hline & FUS & & 31.5 & 473.3 & DSC & [1996KEE/VAN] \\
\hline \multirow[t]{4}{*}{$\mathrm{C}_{14} \mathrm{H}_{20} \mathrm{O}_{5}$} & [14098-44-3] & \multicolumn{5}{|c|}{ benzo-15-crown-5 } \\
\hline & FUS & & 28.3 & 351.2 & DSC & {$[2000 \mathrm{NIC} / \mathrm{ORF}]$} \\
\hline & SUB & & $128.1 \pm 10.8$ & 298 & CGC-DSC & {$[2000 \mathrm{NIC} / \mathrm{ORF}]$} \\
\hline & $\mathrm{V}$ & & $98.9 \pm 1.3$ & 298 & CGC & {$[2000 \mathrm{NIC} / \mathrm{ORF}]$} \\
\hline \multirow{3}{*}{$\mathrm{C}_{14} \mathrm{H}_{21} \mathrm{~F}_{3} \mathrm{~N}_{2} \mathrm{O}_{4}$} & [2768-49-2] & \multicolumn{5}{|c|}{ Proline, 1-[N-(trifluoroacetyl)-(1)-leucyl]methyl ester } \\
\hline & SUB & $(313-366)$ & 121.3 & 328 & A & [1987STE/MAL, 1960WEY/KLI] \\
\hline & $\mathrm{V}$ & $(366-453)$ & 105.8 & 381 & A & [1987STE/MAL, 1960WEY/KLI] \\
\hline \multirow{2}{*}{$\mathrm{C}_{14} \mathrm{H}_{21} \mathrm{~F}_{9} \mathrm{O}$} & {$[1240205-63-3]$} & \multicolumn{5}{|c|}{1 - $[(3,3,4,4,5,5,6,6,6$-nonafluorohexyl)oxy]octane } \\
\hline & FUS & & 19.65 & 237.8 & DSC & {$[2010 \mathrm{ZAG} / \mathrm{CON}]$} \\
\hline \multirow[t]{3}{*}{$\mathrm{C}_{14} \mathrm{H}_{21} \mathrm{~N}$} & {$[861622-66-4]$} & \multicolumn{5}{|c|}{$N$-ethyloctahydrocarbazole } \\
\hline & FUS & & 20.58 & 316.0 & DSC & [2016STA/KEI] \\
\hline & $\mathrm{V}$ & $(324-366)$ & $81.9 \pm 0.5$ & 298 & GS & [2015STA/EME] \\
\hline \multirow[t]{2}{*}{$\mathrm{C}_{14} \mathrm{H}_{21} \mathrm{NO}$} & {$[121678-88-4]$} & \multicolumn{5}{|c|}{ 4-isopropylbenzylidene tert-butylamine $N$-oxide } \\
\hline & SUB & & $101.8 \pm 4.1$ & 298 & $\mathrm{C}$ & [1989ACR/KIR] \\
\hline $\mathrm{C}_{14} \mathrm{H}_{21} \mathrm{~N}_{3} \mathrm{O}_{3} \mathrm{~S}$ & [1156-19-0] & $N$-[(azepan-1- & nino)carbonyl] & thylben & sulfonamide & olazamide) \\
\hline & FUS (I) & & 38.4 & 444 & & \\
\hline & FUS (II) & & 37.9 & 444 & DSC & [2015BOL/ARK] \\
\hline & FUS & & 43.44 & 445 & DSC & [2010BAI/VAN] \\
\hline $\mathrm{C}_{14} \mathrm{H}_{21} \mathrm{~N}_{3} \mathrm{O}_{4}$ & [33629-47-9] & 4-(1,1-dimethy & yl)-N-(1-meth & pyl)-2,6 & itrobenzenean & \\
\hline & FUS & & 20.84 & 338.8 & DSC & [1990DON/DRE] \\
\hline $\mathrm{C}_{14} \mathrm{H}_{21} \mathrm{~N}_{3} \mathrm{~S}$ & [90473-92-0] & $N$-(diethylami & iiocarbony)- $N$ - & oethylbe & mide & \\
\hline & SUB & & $141.2 \pm 1.2$ & 298 & $\mathrm{C}$ & [2006RIB/SAN3] \\
\hline $\mathrm{C}_{14} \mathrm{H}_{22}$ & {$[1012-72-2]$} & 1,4-di-tert-buty & nzene & & & \\
\hline & TRS & & 14.4 & 350.7 & $\mathrm{AC}, \mathrm{DSC}$ & [2009CHI/STE] \\
\hline & FUS & & 8.2 & 350.8 & $\mathrm{AC}, \mathrm{DSC}$ & [2009CHI/STE] \\
\hline & FUS & & 22.48 & 341.5 & & [1997STE/CHI3] \\
\hline & SUB & $(288-333)$ & $82.1 \pm 0.4$ & 310 & GS & [1998VER] \\
\hline & SUB & & $82.8 \pm 0.4$ & 298 & & [1998VER] \\
\hline & SUB & $(285-325)$ & 82.8 & 305 & ME & [1951HOP/SEA, 1987STE/MAL] \\
\hline & $\mathrm{V}$ & (319-559) & $63.0 \pm 0.1$ & 298 & EB,IPM & [2009CHI/STE] \\
\hline & $\mathrm{V}$ & $(319-559)$ & $55.8 \pm 0.1$ & 360 & EB,IPM & [2009CHI/STE] \\
\hline & $\mathrm{V}$ & $(319-559)$ & $54.6 \pm 0.1$ & 400 & EB,IPM & [2009CHI/STE] \\
\hline & $\mathrm{V}$ & $(319-559)$ & $61.4 \pm 0.1$ & 440 & EB,IPM & [2009CHI/STE] \\
\hline & $\mathrm{V}$ & $(319-559)$ & $46.4 \pm 0.2$ & 480 & EB,IPM & {$[2009 \mathrm{CHI} / \mathrm{STE}]$} \\
\hline & $\mathrm{V}$ & (319-559) & $44.6 \pm 0.3$ & 520 & EB,IPM & [2009CHI/STE] \\
\hline & $\mathrm{V}$ & $(354-382)$ & $61.4 \pm 0.3$ & 298 & GS & [2008VER/KOZ2] \\
\hline & $\mathrm{V}$ & $(387-559)$ & $63.0 \pm 0.6$ & 298 & $\mathrm{~EB}$ & [1997STE/CHI3] \\
\hline $\mathrm{C}_{14} \mathrm{H}_{22}$ & {$[1014-60-4]$} & 1,3-di-tert-buty & nzene & & & \\
\hline & V & $(288-333)$ & $58.9 \pm 0.5$ & 310 & GS & [1998VER] \\
\hline & $\mathrm{V}$ & & $59.6 \pm 0.5$ & 298 & & [1998VER] \\
\hline & $\mathrm{V}$ & $(346-374)$ & 58.0 & 360 & A & [1987STE/MAL] \\
\hline $\mathrm{C}_{14} \mathrm{H}_{22}$ & [2189-60-8] & Octylbenzene & & & & \\
\hline
\end{tabular}


TABLE 12. Phase change enthalpies of $\mathrm{C}_{14}$ to $\mathrm{C}_{18}$ organic compounds-Continued

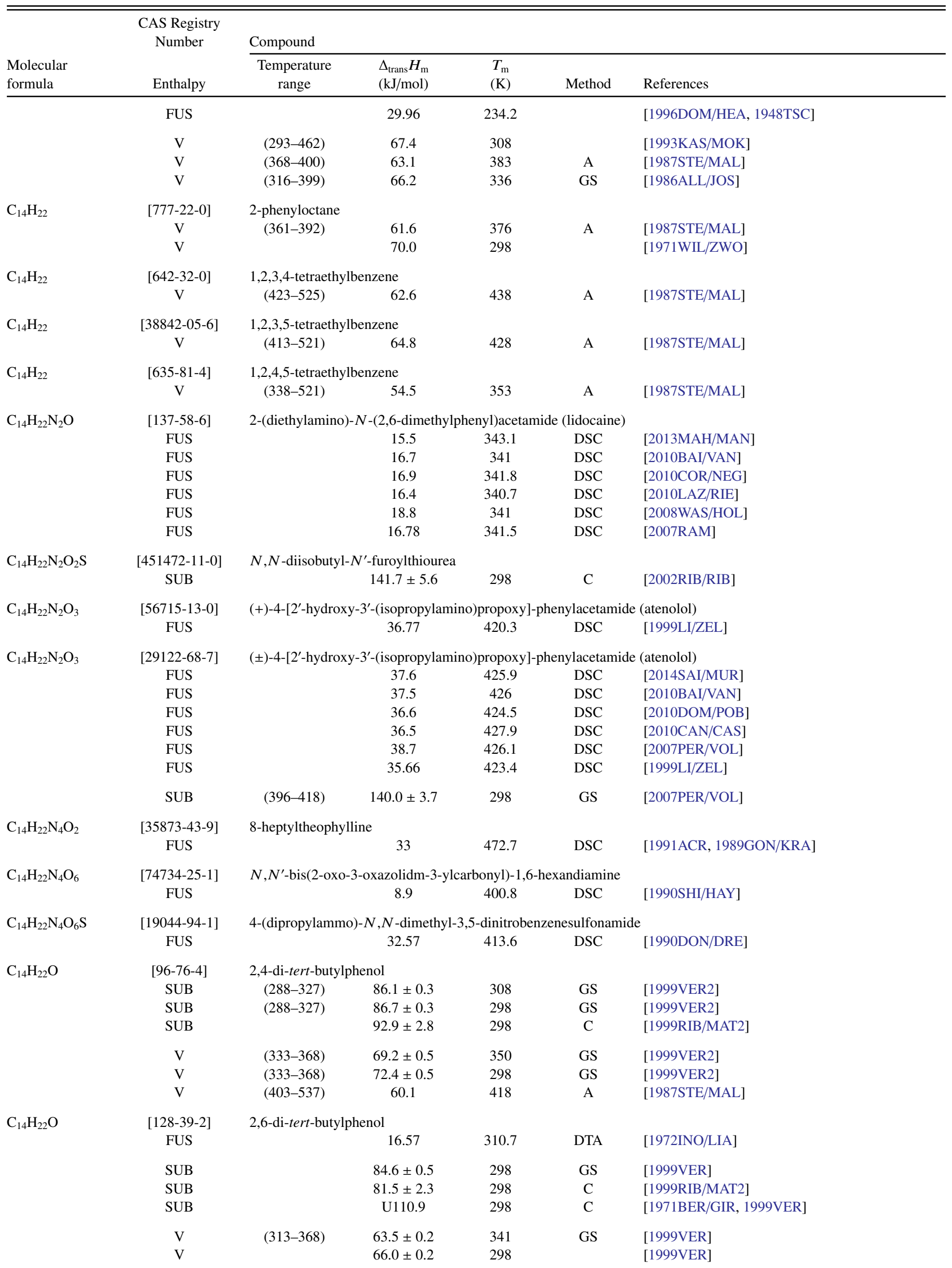


TABLE 12. Phase change enthalpies of $\mathrm{C}_{14}$ to $\mathrm{C}_{18}$ organic compounds-Continued

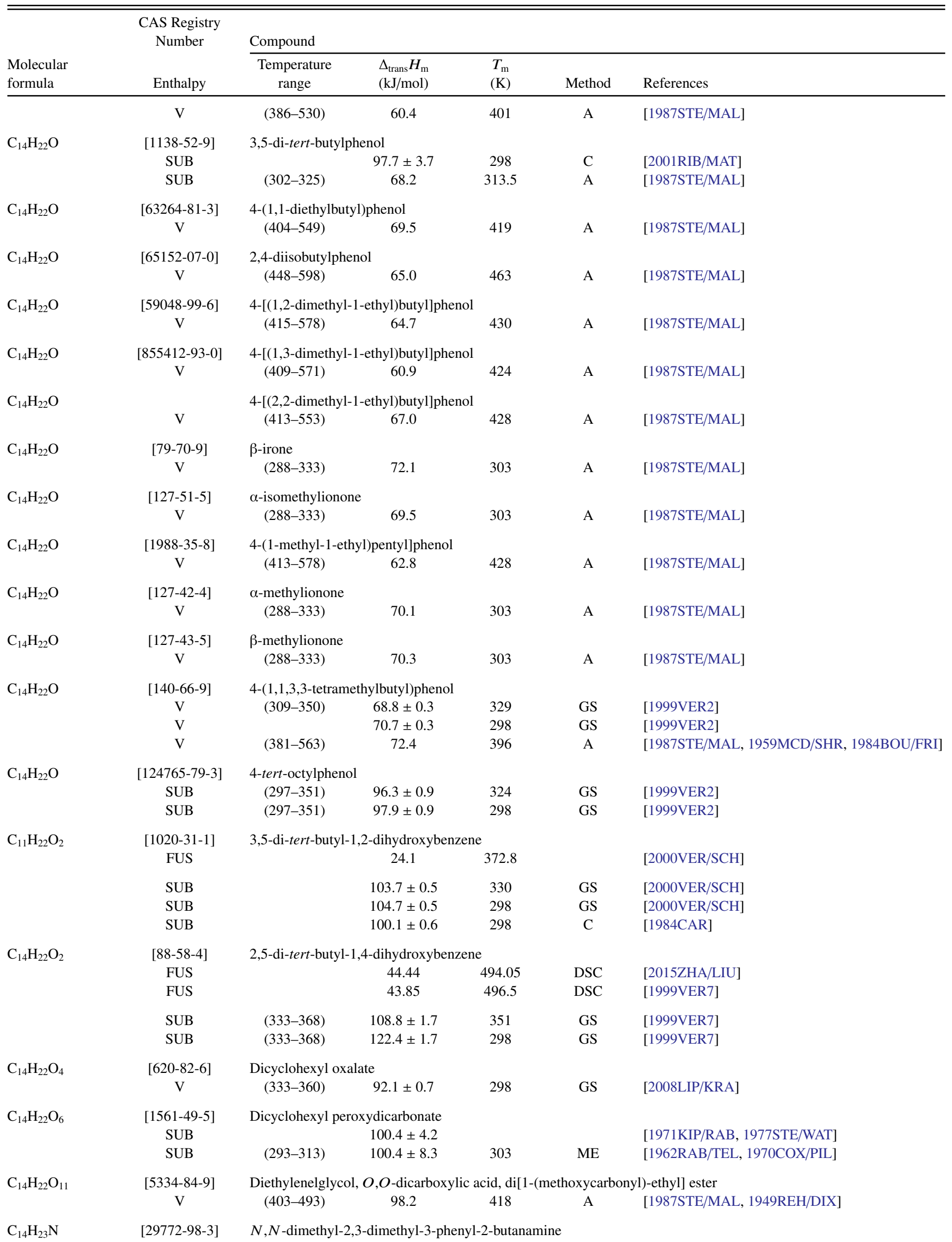


TABLE 12. Phase change enthalpies of $\mathrm{C}_{14}$ to $\mathrm{C}_{18}$ organic compounds-Continued

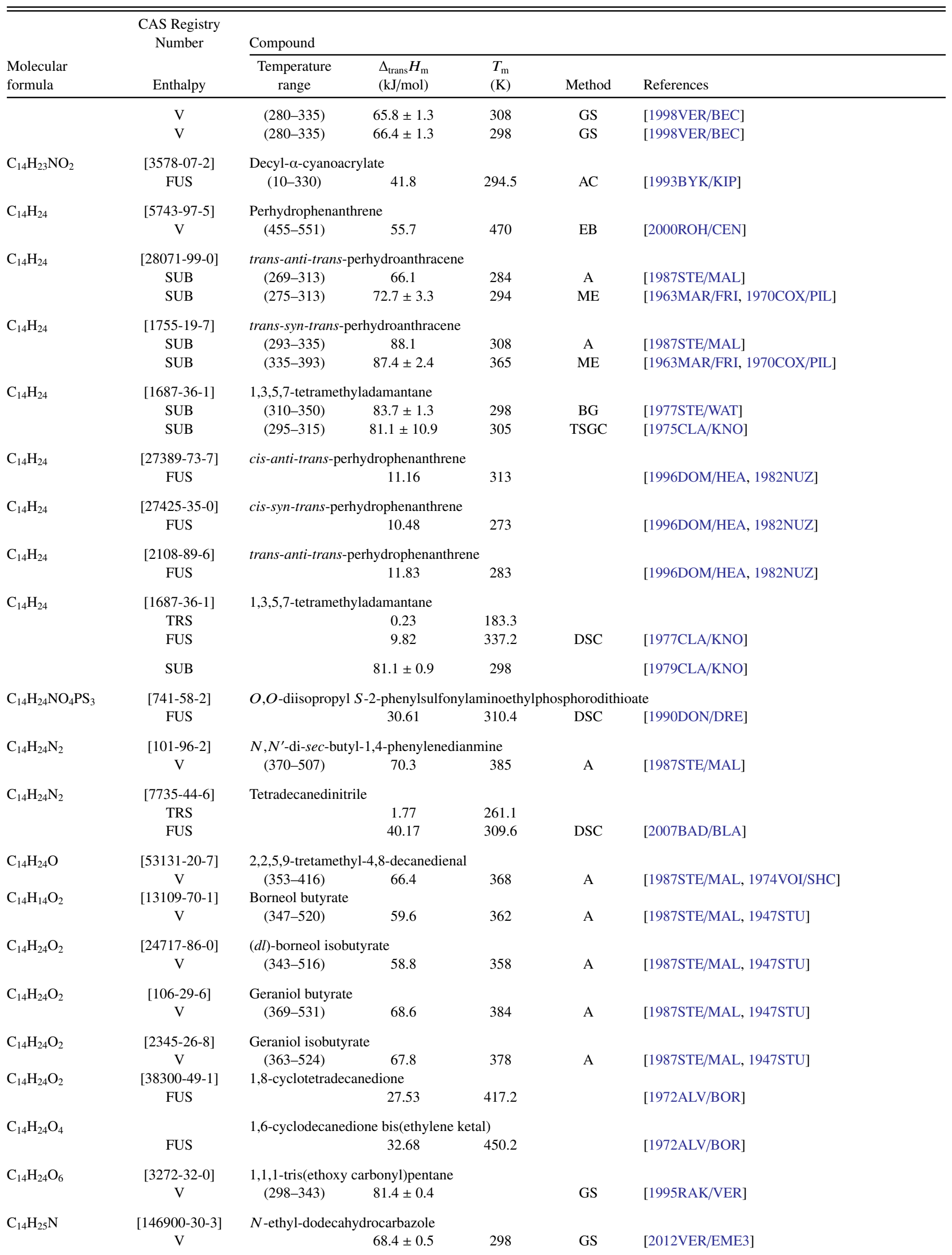


TABLE 12. Phase change enthalpies of $\mathrm{C}_{14}$ to $\mathrm{C}_{18}$ organic compounds-Continued

\begin{tabular}{|c|c|c|c|c|c|c|}
\hline \multirow[b]{2}{*}{$\begin{array}{l}\text { Molecular } \\
\text { formula }\end{array}$} & \multirow{2}{*}{$\begin{array}{l}\text { CAS Registry } \\
\text { Number } \\
\text { Enthalpy }\end{array}$} & \multicolumn{5}{|l|}{ Compound } \\
\hline & & $\begin{array}{l}\text { Temperature } \\
\text { range }\end{array}$ & $\begin{array}{l}\Delta_{\text {trans }} H_{\mathrm{m}} \\
(\mathrm{kJ} / \mathrm{mol})\end{array}$ & $\begin{array}{l}T_{\mathrm{m}} \\
(\mathrm{K})\end{array}$ & Method & References \\
\hline $\mathrm{C}_{14} \mathrm{H}_{26}$ & $\begin{array}{c}{[638-60-8]} \\
\mathrm{V}\end{array}$ & $\begin{array}{l}\text { 2-tetradecyne } \\
\quad(458-491)\end{array}$ & 57.1 & 473 & & [1986ELV/KUD] \\
\hline $\mathrm{C}_{14} \mathrm{H}_{26}$ & $\begin{array}{c}{[60212-32-0]} \\
\mathrm{V}\end{array}$ & $\begin{array}{c}\text { 3-tetradecyne } \\
(455-494)\end{array}$ & 56.3 & 470 & & [1986ELV/KUD] \\
\hline $\mathrm{C}_{14} \mathrm{H}_{26}$ & $\begin{array}{c}{[60212-32-0]} \\
\mathrm{V}\end{array}$ & $\begin{array}{l}\text { 5-tetradecyne } \\
\quad(453-490)\end{array}$ & 55.5 & 468 & & [1986ELV/KUD] \\
\hline $\mathrm{C}_{14} \mathrm{H}_{26}$ & $\begin{array}{c}{[3730-08-3]} \\
\mathrm{V}\end{array}$ & $\begin{array}{c}\text { 6-tetradecyne } \\
(461-484)\end{array}$ & 55.6 & 473 & & [1986ELV/KUD] \\
\hline $\mathrm{C}_{14} \mathrm{H}_{26}$ & $\begin{array}{c}\text { [35216-11-6] } \\
\mathrm{V}\end{array}$ & $\begin{array}{l}\text { 7-tetradecyne } \\
\quad(478-505)\end{array}$ & 53.6 & 491 & & [1986ELV/KUD] \\
\hline $\mathrm{C}_{14} \mathrm{H}_{26}$ & $\begin{array}{c}{[2319-61-1]} \\
\mathrm{V}\end{array}$ & $\begin{array}{l}\text { 1,1-dicyclohexyleth } \\
\quad(370-402)\end{array}$ & 62.1 & 385 & A & [1987STE/MAL] \\
\hline $\mathrm{C}_{14} \mathrm{H}_{26}$ & $\begin{array}{c}{[3321-50-4]} \\
\mathrm{V}\end{array}$ & $\begin{array}{l}\text { 1,2-dicyclohexylethe } \\
\quad(371-402)\end{array}$ & ine 65.4 & 386 & A & [1987STE/MAL] \\
\hline $\mathrm{C}_{14} \mathrm{H}_{26} \mathrm{~N}_{2}$ & $\begin{array}{c}{[42032-30-4]} \\
\mathrm{V}\end{array}$ & $\begin{array}{l}\text { 1-decyl-2-methylimi } \\
\text { (343-383) }\end{array}$ & $\begin{array}{l}\text { dazole } \\
91.8 \pm 0.4\end{array}$ & 298 & GS & [2011EME/POR2] \\
\hline $\mathrm{C}_{14} \mathrm{H}_{26} \mathrm{O}$ & $\begin{array}{c}{[53965-17-6]} \\
\mathrm{V}\end{array}$ & $\begin{array}{l}\text { cis-2,2,5,9-tetrameth } \\
\quad(363-393)\end{array}$ & $\begin{array}{l}\text { yl-4,8-deca } \\
94.0\end{array}$ & $\begin{array}{l}-\mathrm{ol} \\
378\end{array}$ & A & [1987STE/MAL, 1974VOI/SHC] \\
\hline $\mathrm{C}_{14} \mathrm{H}_{26} \mathrm{O}$ & $\begin{array}{c}{[53965-18-7]} \\
\mathrm{V}\end{array}$ & $\begin{array}{l}\text { trans-2,2,5,9-tetram } \\
\quad(363-393)\end{array}$ & $\begin{array}{l}\text { thyl- } 4,8 \text {-de } \\
86.3\end{array}$ & $\begin{array}{r}-1-\mathrm{ol} \\
378\end{array}$ & A & [1987STE/MAL, 1974VOI/SHC] \\
\hline $\mathrm{C}_{14} \mathrm{H}_{26} \mathrm{O}$ & $\begin{array}{c}{[174155-51-2]} \\
\mathrm{V}\end{array}$ & $\begin{array}{l}\text { (Z)-3-tetradecenal } \\
\quad(353-393)\end{array}$ & 79.4 & 298 & CGC & [1996KOU/HOS, 2000OVA/KOU] \\
\hline $\mathrm{C}_{14} \mathrm{H}_{26} \mathrm{O}$ & $\begin{array}{c}{[174155-50-1]} \\
\mathrm{V}\end{array}$ & $\begin{array}{l}\text { (E)-3-tetradecenal } \\
(353-393)\end{array}$ & 80.1 & 298 & CGC & [1996KOU/HOS, 2000OVA/KOU] \\
\hline $\mathrm{C}_{14} \mathrm{H}_{26} \mathrm{O}$ & $\begin{array}{c}{[115018-49-0]} \\
\mathrm{V}\end{array}$ & $\begin{array}{l}\text { (Z)-4-tetradecenal } \\
\quad(353-393)\end{array}$ & 79.2 & 298 & CGC & [1996KOU/HOS, 2000OVA/KOU] \\
\hline $\mathrm{C}_{14} \mathrm{H}_{26} \mathrm{O}$ & $\begin{array}{c}{[115018-39-8]} \\
\mathrm{V}\end{array}$ & $\begin{array}{l}\text { (E)-4-tetradecenal } \\
\quad(353-393)\end{array}$ & 79.9 & 298 & CGC & [1996KOU/HOS, 2000OVA/KOU] \\
\hline $\mathrm{C}_{14} \mathrm{H}_{26} \mathrm{O}$ & $\begin{array}{c}{[63851-42-3]} \\
\mathrm{V}\end{array}$ & $\begin{array}{l}\text { (Z)-5-tetradecenal } \\
\quad(353-393)\end{array}$ & 78.4 & 298 & CGC & [1996KOU/HOS, 2000OVA/KOU] \\
\hline $\mathrm{C}_{14} \mathrm{H}_{26} \mathrm{O}$ & $\begin{array}{c}{[174155-52-3]} \\
\mathrm{V}\end{array}$ & $\begin{array}{l}\text { (E)-5-tetradecenal } \\
(353-393)\end{array}$ & 79.1 & 298 & CGC & [1996KOU/HOS, 2000OVA/KOU] \\
\hline $\mathrm{C}_{14} \mathrm{H}_{26} \mathrm{O}$ & $\begin{array}{c}{[174155-53-4]} \\
V\end{array}$ & $\begin{array}{l}\text { (Z)-6-tetradecenal } \\
\quad(353-393)\end{array}$ & 78.5 & 298 & CGC & [1996KOU/HOS, 2000OVA/KOU] \\
\hline $\mathrm{C}_{14} \mathrm{H}_{26} \mathrm{O}$ & $\begin{array}{c}{[174155-54-5]} \\
\mathrm{V}\end{array}$ & $\begin{array}{l}\text { (E)-6-tetradecenal } \\
(353-393)\end{array}$ & 79.3 & 298 & CGC & [1996KOU/HOS, 2000OVA/KOU] \\
\hline $\mathrm{C}_{14} \mathrm{H}_{26} \mathrm{O}$ & $\begin{array}{c}{[65128-96-3]} \\
\mathrm{V}\end{array}$ & $\begin{array}{l}\text { (Z)-7-tetradecenal } \\
\quad(353-393)\end{array}$ & 78.7 & 298 & CGC & [1996KOU/HOS, 2000OVA/KOU] \\
\hline $\mathrm{C}_{14} \mathrm{H}_{26} \mathrm{O}$ & $\begin{array}{c}\text { [37011-96-4] } \\
\text { V }\end{array}$ & $\begin{array}{l}\text { (E)-7-tetradecenal } \\
(353-393)\end{array}$ & 79.2 & 298 & CGC & [1996KOU/HOS, 2000OVA/KOU] \\
\hline
\end{tabular}


TABLE 12. Phase change enthalpies of $\mathrm{C}_{14}$ to $\mathrm{C}_{18}$ organic compounds-Continued

\begin{tabular}{|c|c|c|c|c|c|c|}
\hline \multirow[b]{2}{*}{$\begin{array}{l}\text { Molecular } \\
\text { formula }\end{array}$} & \multirow{2}{*}{$\begin{array}{l}\text { CAS Registry } \\
\text { Number } \\
\text { Enthalpy }\end{array}$} & \multicolumn{5}{|l|}{ Compound } \\
\hline & & $\begin{array}{l}\text { Temperature } \\
\text { range }\end{array}$ & $\begin{array}{l}\Delta_{\text {trans }} H_{\mathrm{m}} \\
(\mathrm{kJ} / \mathrm{mol})\end{array}$ & $\begin{array}{l}T_{\mathrm{m}} \\
(\mathrm{K})\end{array}$ & Method & References \\
\hline & $\mathrm{V}$ & $(353-393)$ & 78.8 & 298 & CGC & [1996KOU/HOS, 2000OVA/KOU] \\
\hline $\mathrm{C}_{14} \mathrm{H}_{26} \mathrm{O}$ & $\begin{array}{c}{[174155-55-6]} \\
\mathrm{V}\end{array}$ & $\begin{array}{l}(E) \text {-8-tetradecenal } \\
\quad(353-393)\end{array}$ & 79.3 & 298 & CGC & [1996KOU/HOS, 2000OVA/KOU] \\
\hline $\mathrm{C}_{14} \mathrm{H}_{26} \mathrm{O}$ & $\begin{array}{c}{[53939-27-8]} \\
\mathrm{V}\end{array}$ & $\begin{array}{l}\text { (Z)-9-tetradecenal } \\
\quad(353-393)\end{array}$ & 79.1 & 298 & CGC & [1996KOU/HOS, 2000OVA/KOU] \\
\hline $\mathrm{C}_{14} \mathrm{H}_{26} \mathrm{O}$ & $\begin{array}{c}{[71377-13-4]} \\
\mathrm{V}\end{array}$ & $\begin{array}{l}(E)-9 \text {-tetradecenal } \\
(353-393)\end{array}$ & 79.5 & 298 & $\mathrm{CGC}$ & [1996KOU/HOS, 2000OVA/KOU] \\
\hline $\mathrm{C}_{14} \mathrm{H}_{26} \mathrm{O}$ & $\begin{array}{c}{[144525-16-6]} \\
\mathrm{V}\end{array}$ & $\begin{array}{l}\text { (Z)-10-tetradecenal } \\
\quad(353-393)\end{array}$ & 79.6 & 298 & CGC & [1996KOU/HOS, 2000OVA/KOU] \\
\hline $\mathrm{C}_{14} \mathrm{H}_{26} \mathrm{O}$ & $\begin{array}{c}{[148238-39-5]} \\
\mathrm{V}\end{array}$ & $\begin{array}{l}(E)-10 \text {-tetradecenal } \\
\quad(353-393)\end{array}$ & 79.8 & 298 & CGC & [1996KOU/HOS, 2000OVA/KOU] \\
\hline $\mathrm{C}_{14} \mathrm{H}_{26} \mathrm{O}$ & $\begin{array}{c}{[35237-64-0]} \\
\mathrm{V}\end{array}$ & $\begin{array}{l}\text { (Z)-11-tetradecenal } \\
\quad(353-393)\end{array}$ & 80.3 & 298 & CGC & [1996KOU/HOS, 2000OVA/KOU] \\
\hline $\mathrm{C}_{14} \mathrm{H}_{26} \mathrm{O}$ & $\begin{array}{c}{[35746-21-5]} \\
V\end{array}$ & $\begin{array}{l}\text { (E)-11-tetradecenal } \\
\quad(353-393)\end{array}$ & 80.5 & 298 & CGC & [1996KOU/HOS, 2000OVA/KOU] \\
\hline $\mathrm{C}_{14} \mathrm{H}_{26} \mathrm{O}$ & $\begin{array}{c}{[174155-56-7]} \\
\mathrm{V}\end{array}$ & $\begin{array}{l}\text { (Z)-12-tetradecenal } \\
\quad(353-393)\end{array}$ & 80.8 & 298 & CGC & [1996KOU/HOS, 2000OVA/KOU] \\
\hline $\mathrm{C}_{14} \mathrm{H}_{26} \mathrm{O}$ & $\begin{array}{c}{[124499-92-9]} \\
\mathrm{V}\end{array}$ & $\begin{array}{l}\text { (E)-12-tetradecenal } \\
\quad(353-393)\end{array}$ & 80.8 & 298 & CGC & [1996KOU/HOS, 2000OVA/KOU] \\
\hline $\mathrm{C}_{14} \mathrm{H}_{26} \mathrm{O}$ & $\begin{array}{c}{[99914-84-8]} \\
\mathrm{V}\end{array}$ & $\begin{array}{l}\text { 2-(1,2-dimethylpropy } \\
\quad(385-535)\end{array}$ & $\begin{array}{l}\text { 1)-5,6-dir } \\
60.0\end{array}$ & $\begin{array}{c}\text { ptenal } \\
400\end{array}$ & $\mathrm{~EB}$ & [1987MIL/FEN2] \\
\hline $\mathrm{C}_{14} \mathrm{H}_{26} \mathrm{O}$ & $\begin{array}{c}{[3021-89-4]} \\
\mathrm{V}\end{array}$ & $\begin{array}{l}\text { 2-pentyl-2-nonenal } \\
\quad(385-553)\end{array}$ & 65.0 & 409 & EB & [1987MIL/FEN2] \\
\hline $\mathrm{C}_{14} \mathrm{H}_{26} \mathrm{O}$ & $\begin{array}{c}\text { [295-17-0] } \\
\text { SUB }\end{array}$ & Cyclotetradecanone & 80.75 & & & [1938WOL/WEG, 1960JON] \\
\hline $\mathrm{C}_{14} \mathrm{H}_{26} \mathrm{O}$ & $\begin{array}{c}\text { [37608-02-9] } \\
\text { FUS }\end{array}$ & 4,4,8,8-tetramethylcy & $\begin{array}{c}\text { clodecan } \\
16.32\end{array}$ & 378.2 & & [1976BOR/DAL] \\
\hline $\mathrm{C}_{14} \mathrm{H}_{26} \mathrm{O}_{2}$ & $\begin{array}{c}\text { [3179-47-3] } \\
\text { FUS }\end{array}$ & Decyl methacrylate & 30.55 & 250.7 & $\mathrm{AC}$ & [1996DOM/HEA, 1985KAR/ABD] \\
\hline & V & $(350-541)$ & 62.7 & 365 & A & [1987STE/MAL] \\
\hline $\mathrm{C}_{14} \mathrm{H}_{26} \mathrm{O}_{2}$ & $\begin{array}{c}{[84801-15-0]} \\
\mathrm{V}\end{array}$ & $\begin{array}{l}\text { (Z)-2-dodecenyl acet } \\
\quad(333-378)\end{array}$ & $\begin{array}{l}\text { tate } \\
79.7\end{array}$ & 298 & GC & [1997KOU/HOS, 2000OVA/KOU] \\
\hline $\mathrm{C}_{14} \mathrm{H}_{26} \mathrm{O}_{2}$ & $\begin{array}{c}{[84801-16-1]} \\
\mathrm{V}\end{array}$ & $\begin{array}{l}\text { (E)-2-dodecenyl acet } \\
\quad(333-378)\end{array}$ & $\begin{array}{l}\text { tate } \\
\qquad 81.0\end{array}$ & 298 & GC & [1997KOU/HOS, 2000OVA/KOU] \\
\hline $\mathrm{C}_{14} \mathrm{H}_{26} \mathrm{O}_{2}$ & $\begin{array}{c}{[38363-24-5]} \\
V\end{array}$ & $\begin{array}{l}\text { (Z)-3-dodecenyl acet } \\
\quad(333-378)\end{array}$ & 79.3 & 298 & GC & [1997KOU/HOS, 2000OVA/KOU] \\
\hline $\mathrm{C}_{14} \mathrm{H}_{26} \mathrm{O}_{2}$ & $\begin{array}{c}{[56218-63-4]} \\
\mathrm{V}\end{array}$ & $\begin{array}{l}(E) \text {-3-dodecenyl acet } \\
\quad(333-378)\end{array}$ & $\begin{array}{l}\text { tate } \\
79.8\end{array}$ & 298 & GC & [1997KOU/HOS, 2000OVA/KOU] \\
\hline $\mathrm{C}_{14} \mathrm{H}_{26} \mathrm{O}_{2}$ & $\begin{array}{c}{[38363-25-6]} \\
V\end{array}$ & $\begin{array}{l}\text { (Z)-4-dodecenyl acet } \\
\quad(333-378)\end{array}$ & tate 78.6 & 298 & GC & [1997KOU/HOS, 2000OVA/KOU] \\
\hline $\mathrm{C}_{14} \mathrm{H}_{26} \mathrm{O}_{2}$ & $\begin{array}{c}{[38363-26-7]} \\
\mathrm{V}\end{array}$ & $\begin{array}{l}(E) \text {-4-dodecenyl acet } \\
\quad(333-378)\end{array}$ & $\begin{array}{l}\text { tate } \\
79.8\end{array}$ & 298 & GC & [1997KOU/HOS, 2000OVA/KOU] \\
\hline $\mathrm{C}_{14} \mathrm{H}_{26} \mathrm{O}_{2}$ & $\begin{array}{c}{[16676-96-3]} \\
V\end{array}$ & $\begin{array}{l}\text { (Z)-5-dodecenyl acet } \\
\quad(333-378)\end{array}$ & tate 79.2 & 298 & GC & [1997KOU/HOS, 2000OVA/KOU] \\
\hline $\mathrm{C}_{14} \mathrm{H}_{26} \mathrm{O}_{2}$ & $\begin{array}{c}{[16676-97-4]} \\
\mathrm{V}\end{array}$ & $\begin{array}{l}(E)-5 \text {-dodecenyl acet } \\
\quad(333-378)\end{array}$ & $\begin{array}{l}\text { tate } \\
\qquad 80.0\end{array}$ & 298 & GC & [1997KOU/HOS, 2000OVA/KOU] \\
\hline $\mathrm{C}_{14} \mathrm{H}_{26} \mathrm{O}_{2}$ & $\begin{array}{c}{[16974-12-2]} \\
\mathrm{V}\end{array}$ & $\begin{array}{l}\text { (Z)-6-dodecenyl acet } \\
\quad(333-378)\end{array}$ & tate 79.3 & 298 & GC & [1997KOU/HOS, 2000OVA/KOU] \\
\hline
\end{tabular}


TABLE 12. Phase change enthalpies of $\mathrm{C}_{14}$ to $\mathrm{C}_{18}$ organic compounds-Continued

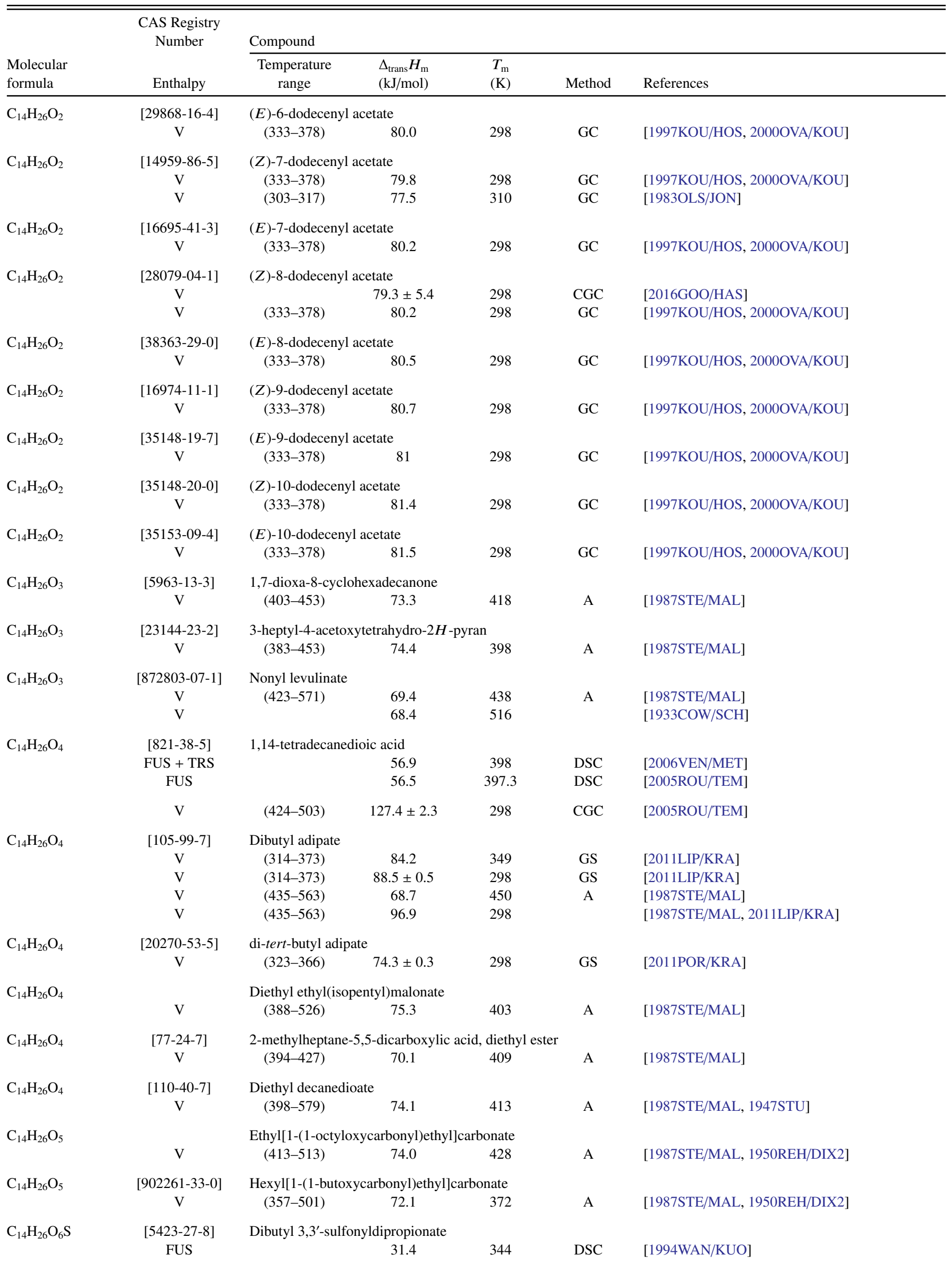


TABLE 12. Phase change enthalpies of $\mathrm{C}_{14}$ to $\mathrm{C}_{18}$ organic compounds-Continued

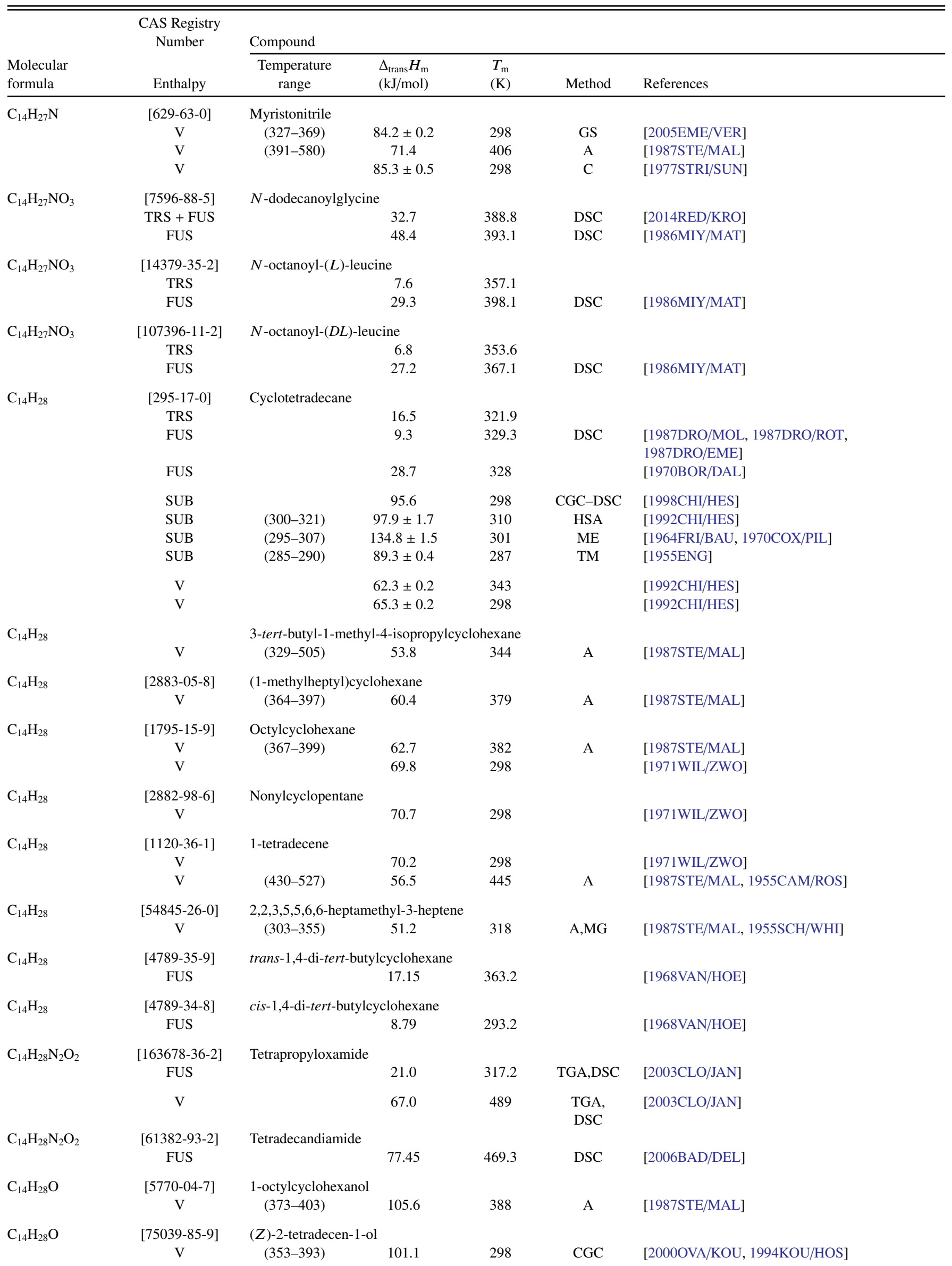


TABLE 12. Phase change enthalpies of $\mathrm{C}_{14}$ to $\mathrm{C}_{18}$ organic compounds-Continued

\begin{tabular}{|c|c|c|c|c|c|c|}
\hline \multirow[b]{2}{*}{$\begin{array}{l}\text { Molecular } \\
\text { formula }\end{array}$} & \multirow{2}{*}{$\begin{array}{l}\text { CAS Registry } \\
\text { Number } \\
\text { Enthalpy }\end{array}$} & \multicolumn{5}{|l|}{ Compound } \\
\hline & & $\begin{array}{l}\text { Temperature } \\
\text { range }\end{array}$ & $\begin{array}{l}\Delta_{\text {trans }} H_{\mathrm{m}} \\
(\mathrm{kJ} / \mathrm{mol})\end{array}$ & $\begin{array}{l}T_{\mathrm{m}} \\
(\mathrm{K})\end{array}$ & Method & References \\
\hline $\mathrm{C}_{14} \mathrm{H}_{28} \mathrm{O}$ & $\begin{array}{c}{[75039-86-0]} \\
\mathrm{V}\end{array}$ & $\begin{array}{l}\text { (E)-2-tetradecen-1-ol } \\
\quad(353-393)\end{array}$ & 101.5 & 298 & CGC & [2000OVA/KOU, 1994KOU/HOS] \\
\hline $\mathrm{C}_{14} \mathrm{H}_{28} \mathrm{O}$ & $\begin{array}{c}{[68892-27-3]} \\
\mathrm{V}\end{array}$ & $\begin{array}{l}\text { (Z)-3-tetradecen-1-ol } \\
\quad(353-393)\end{array}$ & 99.8 & 298 & CGC & [2000OVA/KOU, 1994KOU/HOS] \\
\hline $\mathrm{C}_{14} \mathrm{H}_{28} \mathrm{O}$ & $\begin{array}{c}{[68900-86-7]} \\
\mathrm{V}\end{array}$ & $\begin{array}{l}\text { (E)-3-tetradecen-1-ol } \\
\quad(353-393)\end{array}$ & 99.7 & 298 & CGC & [2000OVA/KOU, 1994KOU/HOS] \\
\hline $\mathrm{C}_{14} \mathrm{H}_{28} \mathrm{O}$ & $\begin{array}{c}{[40642-41-9]} \\
\mathrm{V}\end{array}$ & $\begin{array}{l}\text { (Z)-4-tetradecen-1-ol } \\
\quad(353-393)\end{array}$ & 100.0 & 298 & CGC & [2000OVA/KOU, 1994KOU/HOS] \\
\hline $\mathrm{C}_{14} \mathrm{H}_{28} \mathrm{O}$ & $\begin{array}{c}{[59101-24-5]} \\
\mathrm{V}\end{array}$ & $\begin{array}{l}\text { (E)-4-tetradecen-1-ol } \\
\quad(353-393)\end{array}$ & 100.7 & 298 & CGC & [2000OVA/KOU, 1994KOU/HOS] \\
\hline $\mathrm{C}_{14} \mathrm{H}_{28} \mathrm{O}$ & $\begin{array}{c}{[40642-42-0]} \\
\mathrm{V}\end{array}$ & $\begin{array}{l}\text { (Z)-5-tetradecen-1-ol } \\
\quad(353-393)\end{array}$ & 100.3 & 298 & CGC & [2000OVA/KOU, 1994KOU/HOS] \\
\hline $\mathrm{C}_{14} \mathrm{H}_{28} \mathrm{O}$ & $\begin{array}{c}{[62936-14-5]} \\
\mathrm{V}\end{array}$ & $\begin{array}{l}\text { (E)-5-tetradecen-1-ol } \\
\quad(353-393)\end{array}$ & 100.8 & 298 & CGC & [2000OVA/KOU, 1994KOU/HOS] \\
\hline $\mathrm{C}_{14} \mathrm{H}_{28} \mathrm{O}$ & $\begin{array}{c}{[68760-63-4]} \\
\mathrm{V}\end{array}$ & $\begin{array}{l}\text { (Z)-6-tetradecen-1-ol } \\
\quad(353-393)\end{array}$ & 100 & 298 & CGC & [2000OVA/KOU, 1994KOU/HOS] \\
\hline $\mathrm{C}_{14} \mathrm{H}_{28} \mathrm{O}$ & $\begin{array}{c}{[68760-62-3]} \\
\mathrm{V}\end{array}$ & $\begin{array}{l}\text { (E)-6-tetradecen-1-ol } \\
\quad(353-393)\end{array}$ & 100.5 & 298 & CGC & [2000OVA/KOU, 1994KOU/HOS] \\
\hline $\mathrm{C}_{14} \mathrm{H}_{28} \mathrm{O}$ & $\begin{array}{c}{[40642-43-1]} \\
\mathrm{V}\end{array}$ & $\begin{array}{l}\text { (Z)-7-tetradecen-1-ol } \\
\quad(353-393)\end{array}$ & 99.9 & 298 & CGC & [2000OVA/KOU, 1994KOU/HOS] \\
\hline $\mathrm{C}_{14} \mathrm{H}_{28} \mathrm{O}$ & $\begin{array}{c}{[37011-95-3]} \\
\mathrm{V}\end{array}$ & $\begin{array}{l}\text { (E)-7-tetradecen-1-ol } \\
\quad(353-393)\end{array}$ & 100.5 & 298 & CGC & [2000OVA/KOU, 1994KOU/HOS] \\
\hline $\mathrm{C}_{14} \mathrm{H}_{28} \mathrm{O}$ & $\begin{array}{c}{[64470-32-2]} \\
\mathrm{V}\end{array}$ & $\begin{array}{l}\text { (Z)-8-tetradecen-1-ol } \\
\quad(353-393)\end{array}$ & 100.3 & 298 & CGC & [2000OVA/KOU, 1994KOU/HOS] \\
\hline $\mathrm{C}_{14} \mathrm{H}_{28} \mathrm{O}$ & $\begin{array}{c}{[64437-34-9]} \\
\mathrm{V}\end{array}$ & $\begin{array}{l}\text { (E)-8-tetradecen-1-ol } \\
\quad(353-393)\end{array}$ & 101.4 & 298 & CGC & [2000OVA/KOU, 1994KOU/HOS] \\
\hline $\mathrm{C}_{14} \mathrm{H}_{28} \mathrm{O}$ & $\begin{array}{c}{[35153-15-2]} \\
\mathrm{V}\end{array}$ & $\begin{array}{l}\text { (Z)-9-tetradecen-1-ol } \\
\quad(353-393)\end{array}$ & 100.6 & 298 & CGC & [2000OVA/KOU, 1994KOU/HOS] \\
\hline $\mathrm{C}_{14} \mathrm{H}_{28} \mathrm{O}$ & $\begin{array}{c}{[52957-16-1]} \\
\mathrm{V}\end{array}$ & $\begin{array}{l}\text { (E)-9-tetradecen-1-ol } \\
\quad(353-393)\end{array}$ & 101 & 298 & $\mathrm{CGC}$ & [2000OVA/KOU, 1994KOU/HOS] \\
\hline $\mathrm{C}_{14} \mathrm{H}_{28} \mathrm{O}$ & $\begin{array}{c}{[57393-02-9]} \\
\mathrm{V}\end{array}$ & $\begin{array}{l}\text { (Z)-10-tetradecen-1-o } \\
\quad(353-393)\end{array}$ & 101.1 & 298 & CGC & [2000OVA/KOU, 1994KOU/HOS] \\
\hline $\mathrm{C}_{14} \mathrm{H}_{28} \mathrm{O}$ & $\begin{array}{c}{[64437-35-0]} \\
\mathrm{V}\end{array}$ & $\begin{array}{l}\text { (E)-10-tetradecen-1-o } \\
\quad(353-393)\end{array}$ & 101.5 & 298 & CGC & [2000OVA/KOU, 1994KOU/HOS] \\
\hline $\mathrm{C}_{14} \mathrm{H}_{28} \mathrm{O}$ & $\begin{array}{c}{[34010-15-6]} \\
\mathrm{V}\end{array}$ & $\begin{array}{l}\text { (Z)-11-tetradecen-1-o } \\
\quad(353-393)\end{array}$ & 101.7 & 298 & CGC & [2000OVA/KOU, 1994KOU/HOS] \\
\hline $\mathrm{C}_{14} \mathrm{H}_{28} \mathrm{O}$ & $\begin{array}{c}{[35153-18-5]} \\
\mathrm{V}\end{array}$ & $\begin{array}{l}(E) \text {-11-tetradecen-1-o } \\
\quad(353-393)\end{array}$ & 101.8 & 298 & CGC & [2000OVA/KOU, 1994KOU/HOS] \\
\hline $\mathrm{C}_{14} \mathrm{H}_{28} \mathrm{O}$ & $\begin{array}{c}{[70711-48-7]} \\
\mathrm{V}\end{array}$ & $\begin{array}{l}\text { (Z)-12-tetradecen-1-o } \\
\quad(353-393)\end{array}$ & 102.5 & 298 & CGC & [2000OVA/KOU, 1994KOU/HOS] \\
\hline $\mathrm{C}_{14} \mathrm{H}_{28} \mathrm{O}$ & $\begin{array}{c}{[70711-49-8]} \\
\mathrm{V}\end{array}$ & $\begin{array}{l}\text { (E)-12-tetradecen-1-o } \\
\quad(353-393)\end{array}$ & 102.5 & 298 & CGC & [2000OVA/KOU, 1994KOU/HOS] \\
\hline $\mathrm{C}_{14} \mathrm{H}_{28} \mathrm{O}$ & $\begin{array}{c}\text { [2345-27-9] } \\
\text { FUS }\end{array}$ & 2-tetradecanone & 49.12 & 306.7 & DSC & [1996DOM/HEA, 1979SUN/SVE2] \\
\hline & SUB & & $30.9 \pm 0.5$ & 298 & $\mathrm{C}$ & [1979SUN/SVE2] \\
\hline & $\begin{array}{l}\mathrm{V} \\
\mathrm{V} \\
\mathrm{V} \\
\mathrm{V}\end{array}$ & $\begin{array}{l}(411-560) \\
(549-643) \\
(412-643)\end{array}$ & $\begin{array}{c}65.6 \\
55.6 \\
82.1 \pm 0.6 \\
51.6\end{array}$ & $\begin{array}{l}426 \\
564 \\
298 \\
556\end{array}$ & $\begin{array}{c}\text { A } \\
\text { A } \\
\text { S-F }\end{array}$ & $\begin{array}{l}\text { [1987STE/MAL] } \\
\text { [1987STE/MAL] } \\
\text { [1979SUN/SVE2] } \\
\text { [1975AMB/ELL] }\end{array}$ \\
\hline
\end{tabular}


TABLE 12. Phase change enthalpies of $\mathrm{C}_{14}$ to $\mathrm{C}_{18}$ organic compounds-Continued

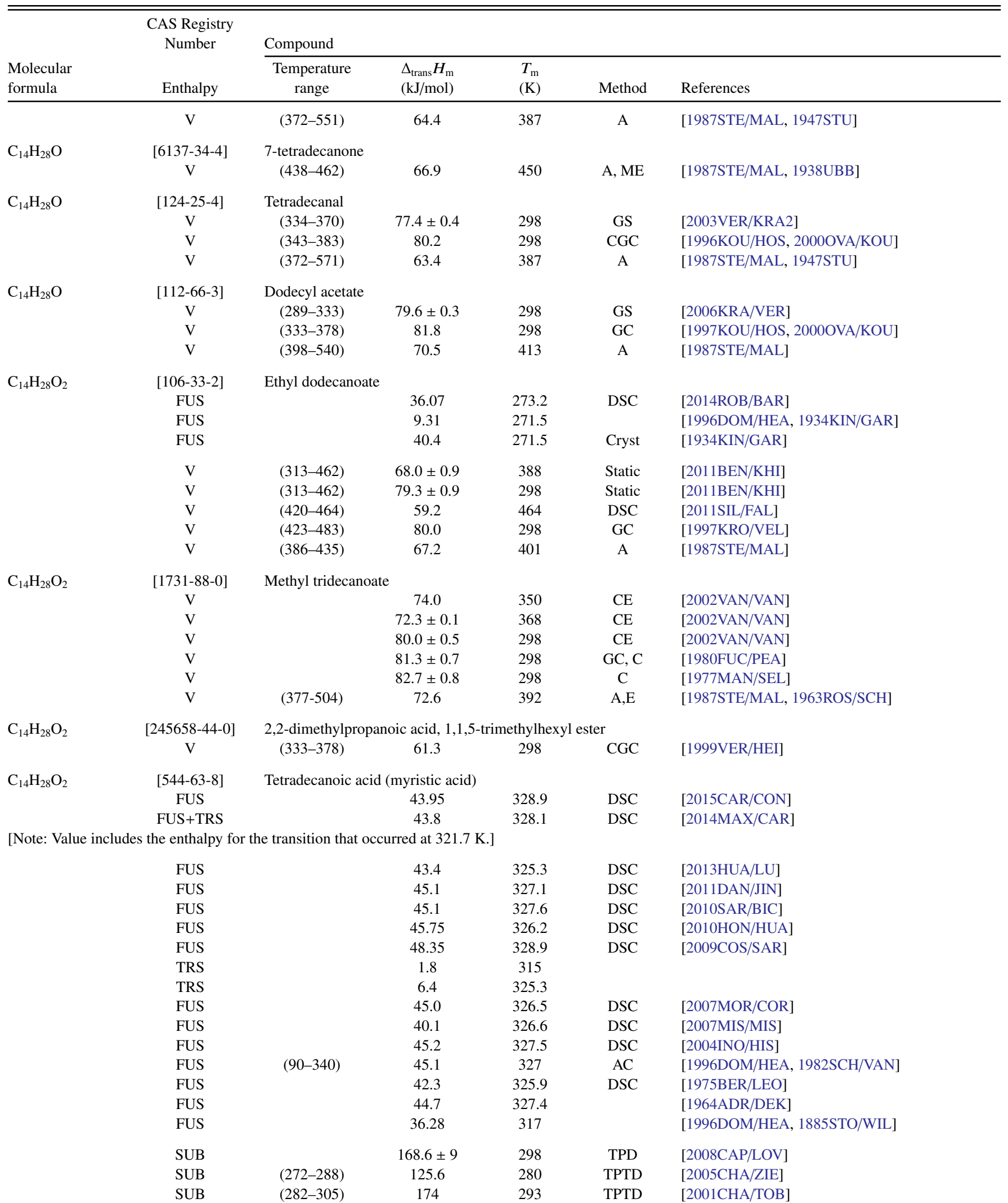

[Note: Experimental values based on the TPTD method are often inconsistent with values determined using other experimental methods.]

$\begin{array}{cccccl}\text { SUB } & (312-325) & 139.7 \pm 3.8 & 318 & \text { ME } & \text { [1961DAV/MAL, 1970COX/PIL] } \\ \text { V } & & 110.7 \pm 6.1 & 298 & \text { CGC } & {[2015 \mathrm{WIL} / \mathrm{GOB}]} \\ \text { V } & & 111.2 \pm 8.0 & 298 & \text { CGC } & \text { [2013WIL/CHI] } \\ \text { V } & (383-459) & 100.4 & 398 & \text { A } & {[1987 \mathrm{STE} / \mathrm{MAL}]}\end{array}$


TABLE 12. Phase change enthalpies of $\mathrm{C}_{14}$ to $\mathrm{C}_{18}$ organic compounds-Continued

\begin{tabular}{|c|c|c|c|c|c|c|}
\hline \multirow[b]{2}{*}{$\begin{array}{l}\text { Molecular } \\
\text { formula }\end{array}$} & \multirow{2}{*}{$\begin{array}{l}\text { CAS Registry } \\
\text { Number } \\
\text { Enthalpy }\end{array}$} & \multicolumn{5}{|l|}{ Compound } \\
\hline & & $\begin{array}{l}\text { Temperature } \\
\text { range }\end{array}$ & $\begin{array}{l}\Delta_{\text {trans }} H_{\mathrm{m}} \\
(\mathrm{kJ} / \mathrm{mol})\end{array}$ & $\begin{array}{l}T_{\mathrm{m}} \\
(\mathrm{K})\end{array}$ & Method & References \\
\hline & $\mathrm{V}$ & $(423-599)$ & 91.6 & 438 & A & [1987STE/MAL] \\
\hline & $\mathrm{V}$ & $(339-358)$ & $104.1 \pm 2.0$ & 349 & ME, TE & [1982DEK/SCH] \\
\hline & $\mathrm{V}$ & & 88.9 & 455 & I & [1943CRA] \\
\hline \multirow[t]{2}{*}{$\mathrm{C}_{14} \mathrm{H}_{28} \mathrm{O}_{3}$} & & \multicolumn{5}{|c|}{ Decyl 3-methoxypropionate } \\
\hline & V & $(403-513)$ & 68.9 & 418 & A & [1987STE/MAL] \\
\hline \multirow[t]{2}{*}{$\mathrm{C}_{14} \mathrm{H}_{28} \mathrm{O}_{3}$} & {$[19816-73-0]$} & \multicolumn{5}{|c|}{ Peroxytetradecanoic acid } \\
\hline & SUB & (293-303) & $156.0 \pm 4.1$ & & ME & [1980SWA/KWA] \\
\hline \multirow{2}{*}{$\mathrm{C}_{14} \mathrm{H}_{28} \mathrm{O}_{4}$} & {$[56444-61-2]$} & \multicolumn{5}{|c|}{ 2,2,9,9-tetramethyl-1,3,8,10-tetraoxacyclotetradecane } \\
\hline & FUS & & 30.5 & 409.4 & & {$[1975 \mathrm{BOR}]$} \\
\hline \multirow[t]{2}{*}{$\mathrm{C}_{14} \mathrm{H}_{28} \mathrm{O}_{4}$} & {$[55208-76-9]$} & \multicolumn{5}{|c|}{ 3,3,6,6-tetrapropyl-1,2,4,5-tetraoxacyclohexane } \\
\hline & $\mathrm{V}$ & $(403-473)$ & 65.1 & 298 & CGC & [2007CAN/EYL] \\
\hline \multirow[t]{3}{*}{$\mathrm{C}_{14} \mathrm{H}_{28} \mathrm{O}_{6}$} & {$[125590-73-0]$} & \multicolumn{5}{|c|}{ 2-ethylhexyl $\alpha$ - $D$-glucoside } \\
\hline & TRS & & 33.47 & 341.2 & & \\
\hline & FUS & & 3.56 & 387.2 & DSC & [1998NIL/SOE] \\
\hline \multirow[t]{2}{*}{$\mathrm{C}_{14} \mathrm{H}_{28} \mathrm{O}_{6}$} & [125590-74-1] & \multicolumn{5}{|c|}{ 2-ethylhexyl $\beta$ - $D$-glucoside } \\
\hline & FUS & & 10.88 & 330.2 & DSC & [1998NIL/SOE] \\
\hline \multirow[t]{2}{*}{$\mathrm{C}_{14} \mathrm{H}_{29} \mathrm{Br}$} & [112-71-0] & \multicolumn{5}{|l|}{ 1-bromotetradecane } \\
\hline & V & $(437-645)$ & 67.1 & 452 & $\mathrm{~A}, \mathrm{E}$ & [1987STE/MAL, 1961LI/ROS, 1970DYK/VAN] \\
\hline \multirow[t]{7}{*}{$\mathrm{C}_{14} \mathrm{H}_{29} \mathrm{Cl}$} & {$[2425-54-9]$} & 1-chlorotetradecane & & & & \\
\hline & V & & 86.6 & 298 & & [2006BOL/NER2] \\
\hline & $\mathrm{V}$ & $(313-373)$ & 80.2 & 313 & $\mathrm{GC}$ & [1980JON/MAT] \\
\hline & $\mathrm{V}$ & $(313-373)$ & 78.0 & 333 & GC & [1980JON/MAT] \\
\hline & $\mathrm{V}$ & $(313-373)$ & 74.4 & 353 & $\mathrm{GC}$ & [1980JON/MAT] \\
\hline & $\mathrm{V}$ & $(313-373)$ & 72.9 & 373 & GC & [1980JON/MAT] \\
\hline & V & $(414-570)$ & 68.7 & 429 & A, DTA & [1987STE/MAL, 1969KEM/KRE] \\
\hline $\mathrm{C}_{14} \mathrm{H}_{29} \mathrm{~F}$ & [593-33-9] & 1-fluorotetradecane & & & & \\
\hline & V & $(288-335)$ & $73.5 \pm 0.4$ & 298 & GS & {$[1997 \mathrm{SCH} / \mathrm{VER}]$} \\
\hline & $\mathrm{V}$ & $(400-593)$ & 61.4 & 415 & $\mathrm{~A}, \mathrm{E}$ & [1987STE/MAL, 1961LI/ROS, 1970DYK/VAN] \\
\hline $\mathrm{C}_{14} \mathrm{H}_{29} \mathrm{I}$ & {$[19218-94-1]$} & 1-iodotetradecane & & & & \\
\hline & $\mathrm{V}$ & $(452-672)$ & 90.0 & 298 & $\mathrm{~A}, \mathrm{E}$ & $\begin{array}{l}\text { [1987STE/MAL, 1961LI/ROS, 1970DYK/VAN, } \\
\text { 2006BOL/NER] }\end{array}$ \\
\hline & $\mathrm{V}$ & $(452-672)$ & 68.6 & 467 & $\mathrm{~A}, \mathrm{E}$ & [1987STE/MAL, 1961LI/ROS, 1970DYK/VAN] \\
\hline $\mathrm{C}_{14} \mathrm{H}_{29} \mathrm{NO}$ & {$[638-58-4]$} & Tetradecanamide & & & & \\
\hline & SUB & $(248-375)$ & $167.4 \pm 2.5$ & 352 & $\mathrm{ME}$ & [1959DAV/JON2, 1987STE/MAL] \\
\hline $\mathrm{C}_{14} \mathrm{H}_{30}$ & {$[629-59-4]$} & Tetradecane & & & & \\
\hline & FUS & & 43.3 & 279.1 & DSC & [2005HUA/SIM] \\
\hline & FUS & & 42.7 & 278.3 & DSC & [2004MON/RAJ] \\
\hline & FUS & & 42,8 & 278.3 & DSC & [1999MET/RAJ] \\
\hline & FUS & & 45.07 & 279.0 & & [1996DOM/HEA, 1954FIN/GRO2] \\
\hline & TRS & & 0.18 & 194.0 & & \\
\hline & FUS & & 44.27 & 288.7 & & [1996DOM/HEA, 1934PAR/LIG] \\
\hline & SUB & & 117.6 & 298 & $\mathrm{~B}$ & [1972MOR3] \\
\hline & $\mathrm{V}$ & $(303-462)$ & 68.5 & 325 & Static & [2013BEN/KHI2] \\
\hline & $\mathrm{V}$ & $(283-313)$ & $71.6 \pm 1.3$ & 298 & GS & [2009LEG/BAC] \\
\hline & $\mathrm{V}$ & & 72.1 & 298 & GS & [2001PUR/CHI] \\
\hline & $\mathrm{V}$ & & $72.0 \pm 2.4$ & 298 & CGC & [2000NIC/ORF] \\
\hline & V & & 69.0 & 324 & $\mathrm{C}$ & [1996VIT/CHA] \\
\hline & $\mathrm{V}$ & & 68.6 & 329 & $\mathrm{C}$ & [1996VIT/CHA] \\
\hline & V & & 67.9 & 334 & $\mathrm{C}$ & [1996VIT/CHA] \\
\hline & $\mathrm{V}$ & & 66.8 & 344 & $\mathrm{C}$ & [1996VIT/CHA] \\
\hline & V & & 65.7 & 359 & $\mathrm{C}$ & [1996VIT/CHA] \\
\hline & $\mathrm{V}$ & $(423-473)$ & 71.2 & 298 & CGC & [1995CHI/HOS] \\
\hline & $\mathrm{V}$ & $(363-413)$ & 71.4 & 298 & CGC & [1995CHI/HOS] \\
\hline & $\mathrm{V}$ & & 71.7 & 298 & & [1994RUZ/MAJ] \\
\hline
\end{tabular}


TABLE 12. Phase change enthalpies of $\mathrm{C}_{14}$ to $\mathrm{C}_{18}$ organic compounds-Continued

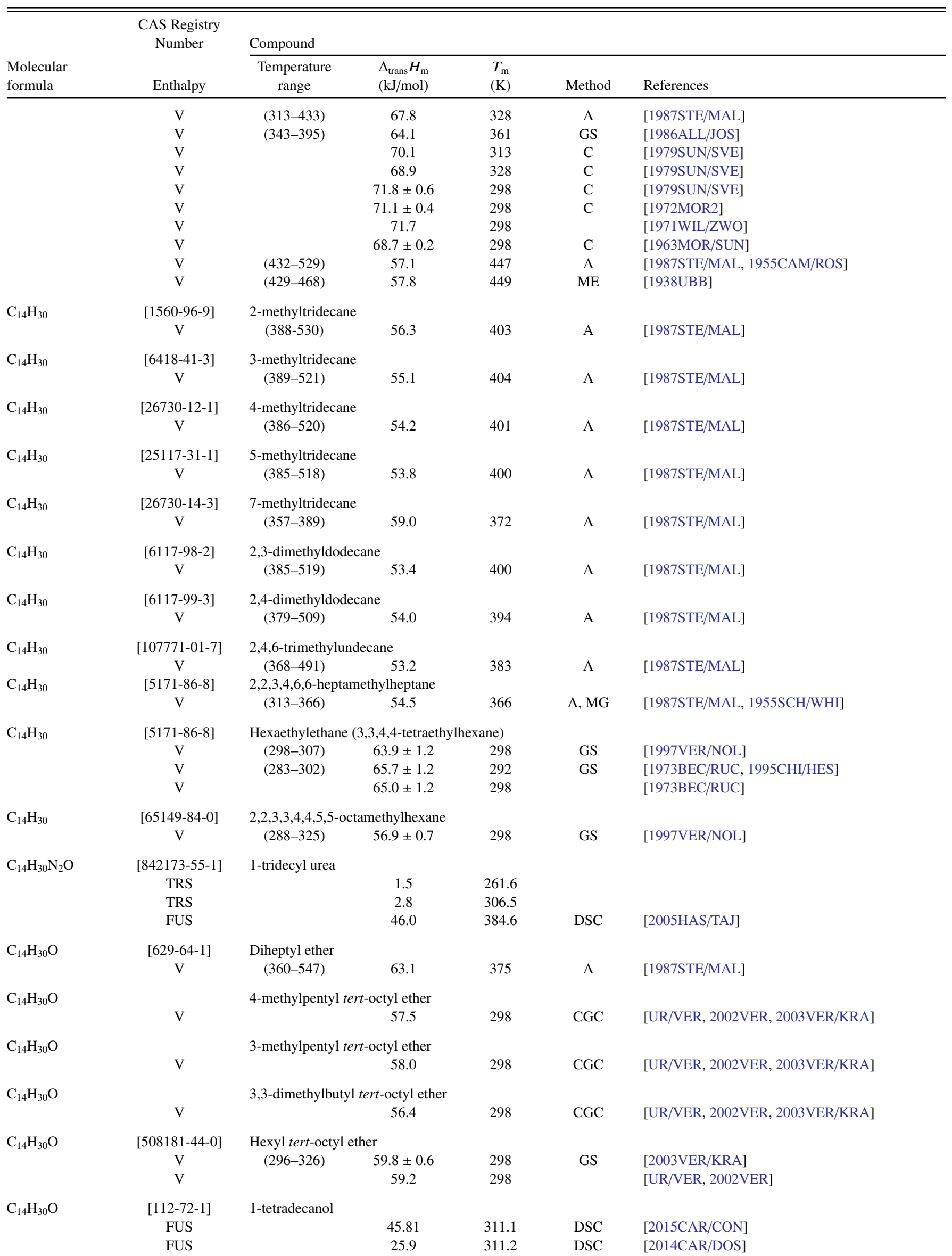


TABLE 12. Phase change enthalpies of $\mathrm{C}_{14}$ to $\mathrm{C}_{18}$ organic compounds-Continued

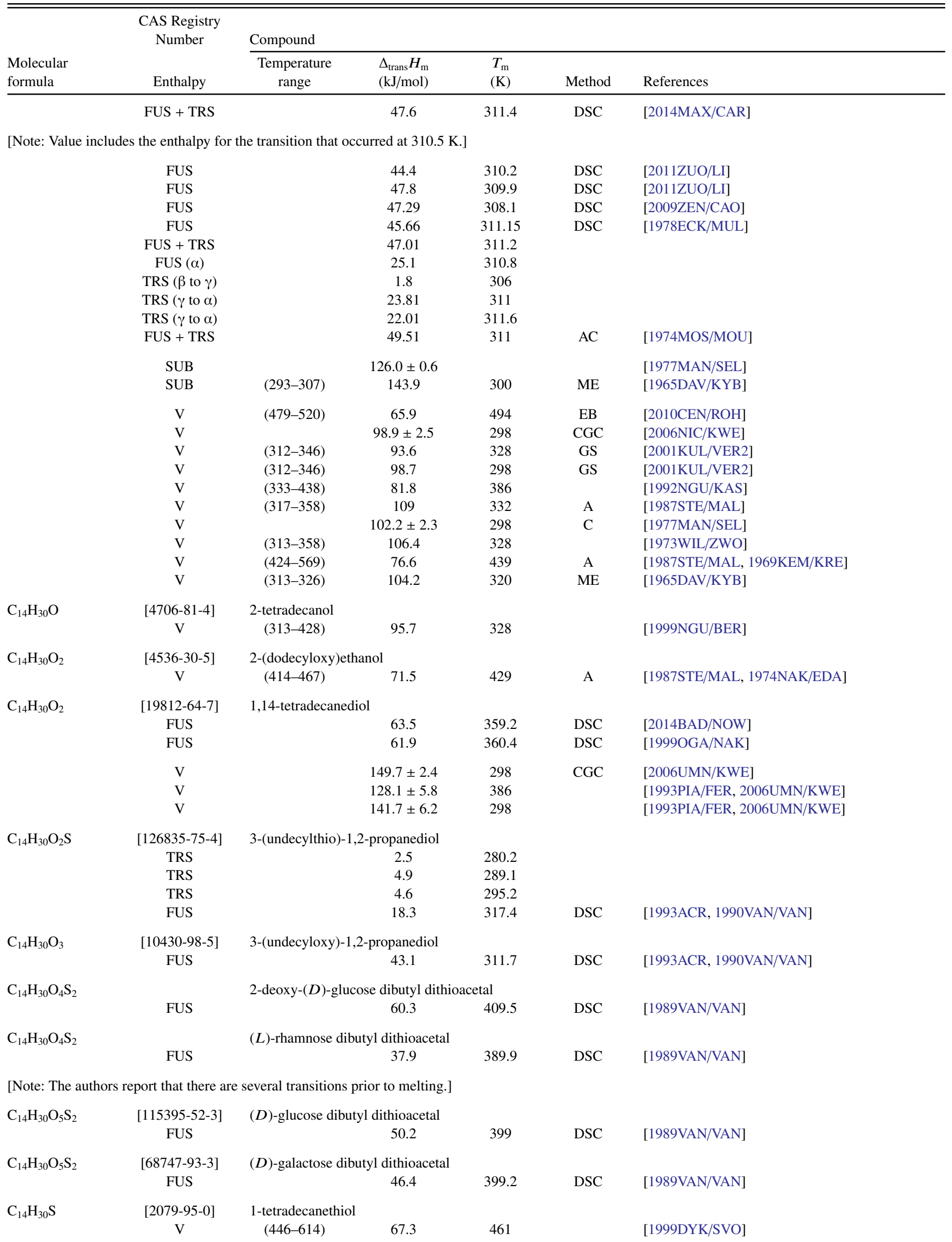


TABLE 12. Phase change enthalpies of $\mathrm{C}_{14}$ to $\mathrm{C}_{18}$ organic compounds-Continued

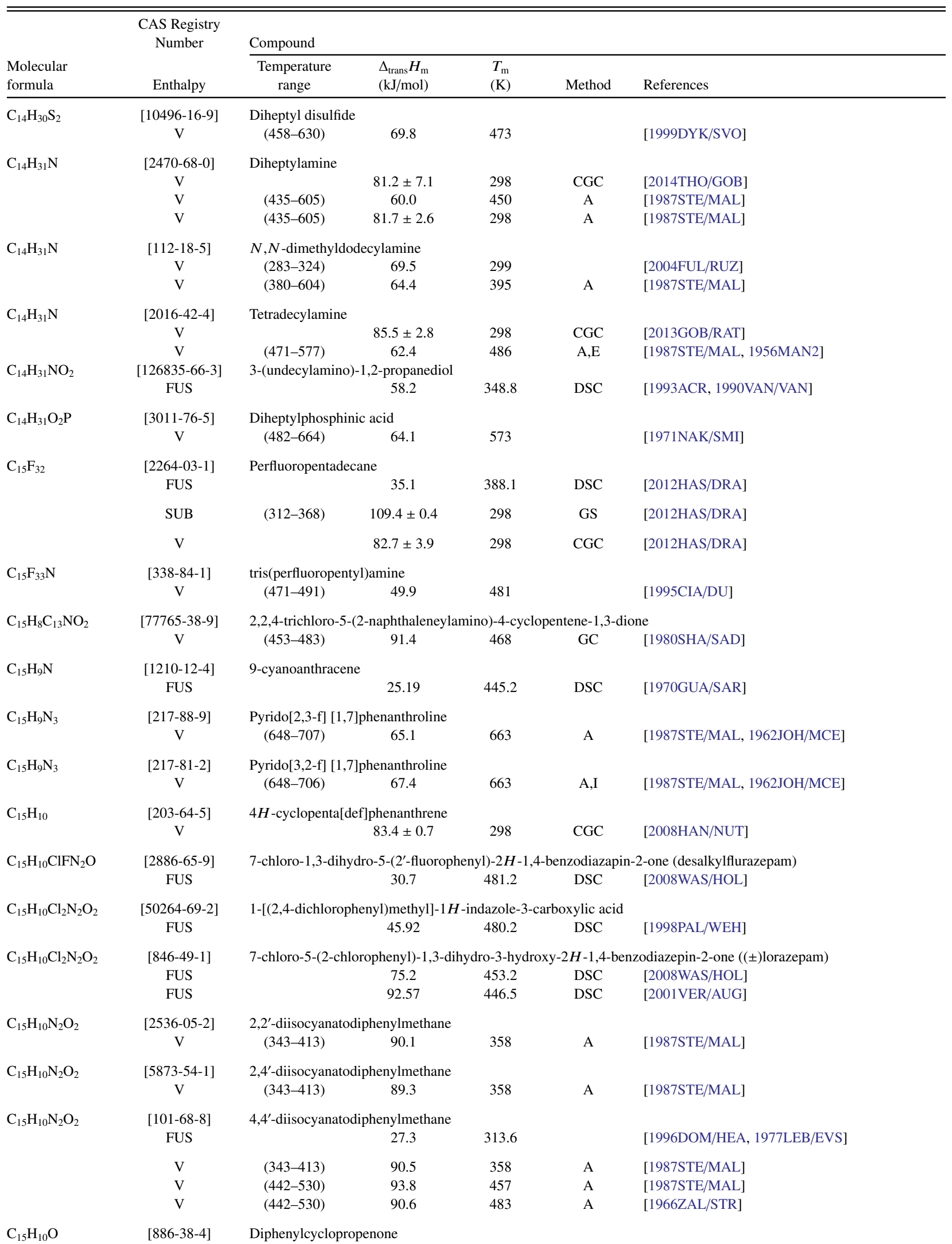


TABLE 12. Phase change enthalpies of $\mathrm{C}_{14}$ to $\mathrm{C}_{18}$ organic compounds-Continued

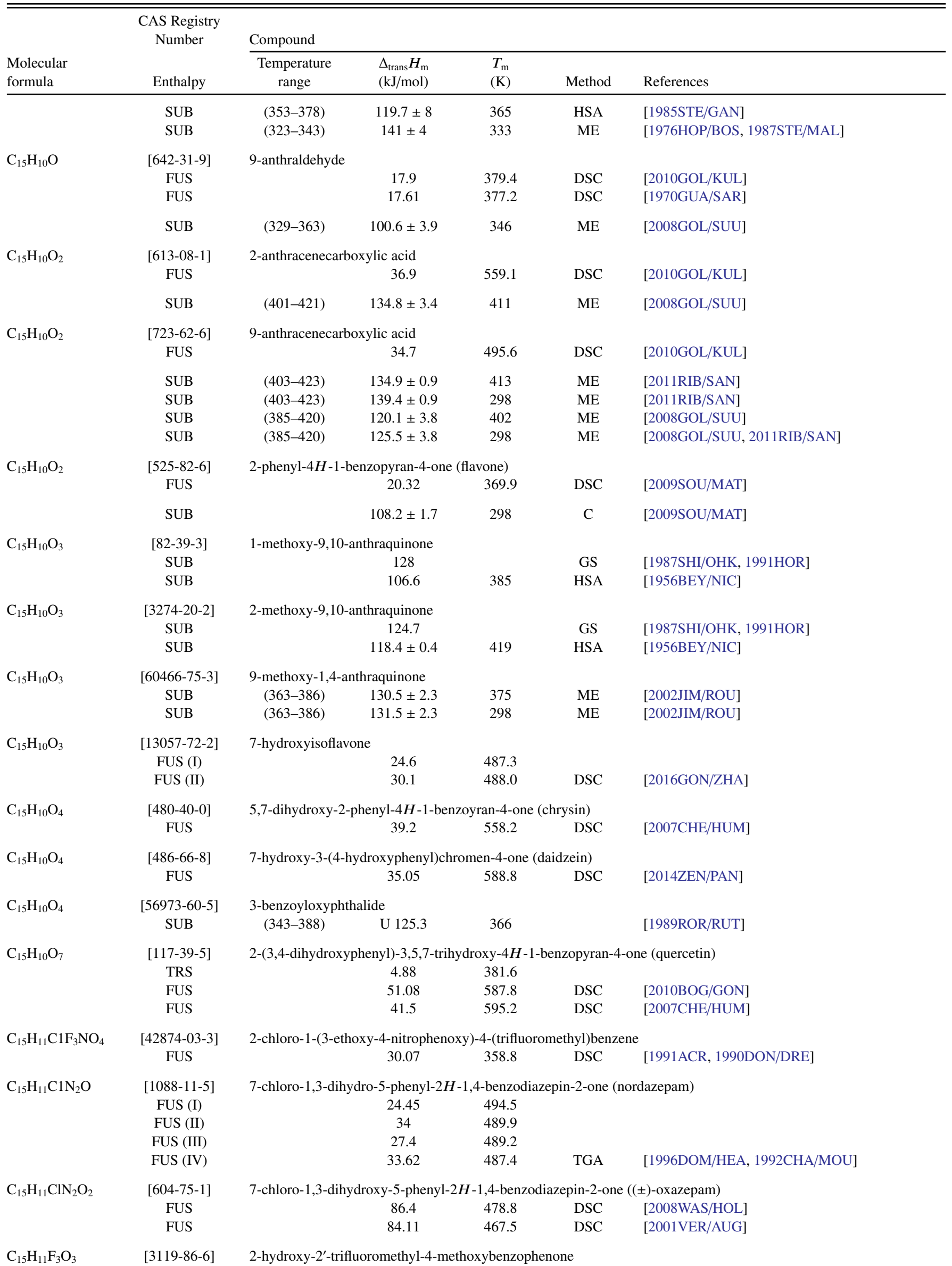


TABLE 12. Phase change enthalpies of $\mathrm{C}_{14}$ to $\mathrm{C}_{18}$ organic compounds-Continued

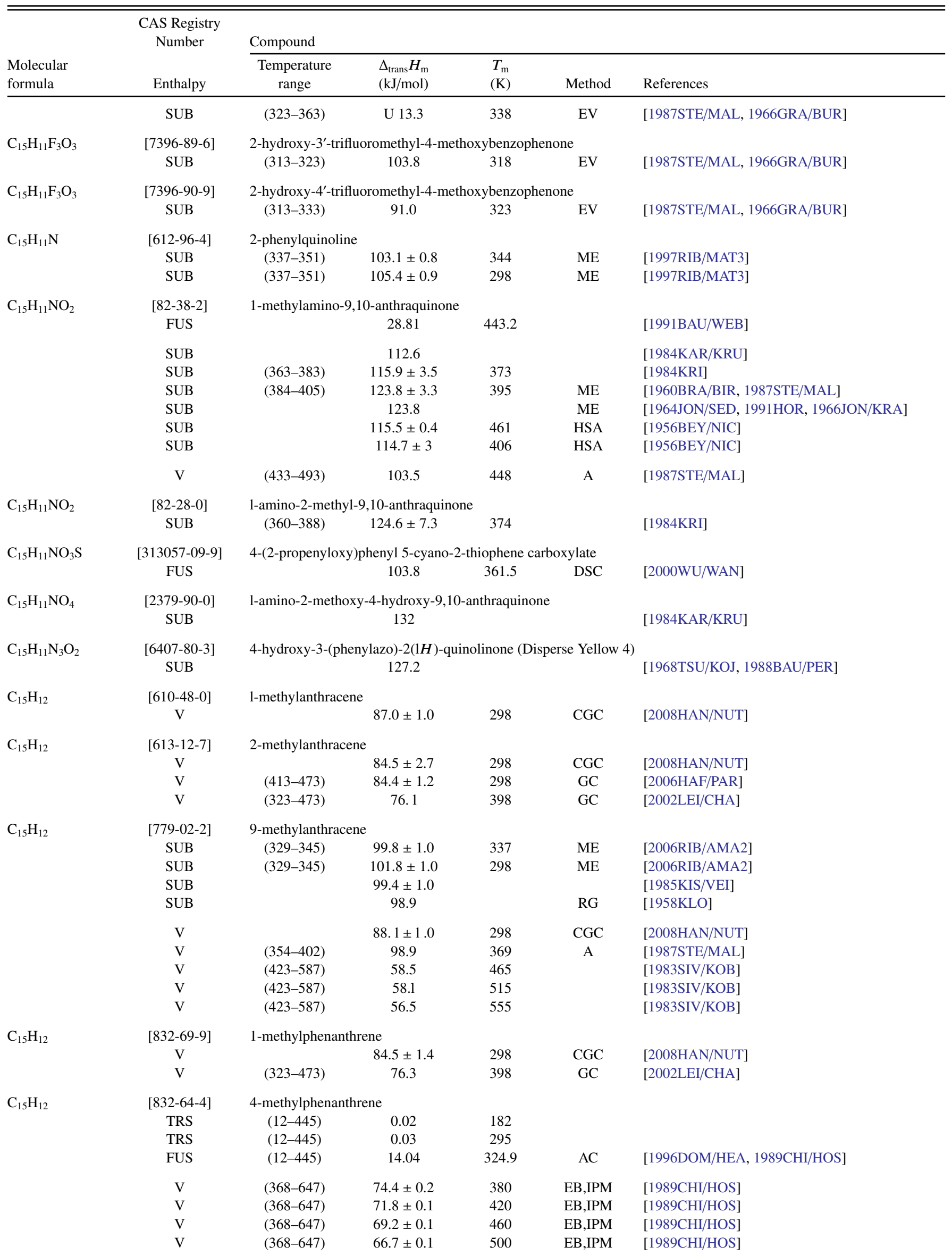


TABLE 12. Phase change enthalpies of $\mathrm{C}_{14}$ to $\mathrm{C}_{18}$ organic compounds-Continued

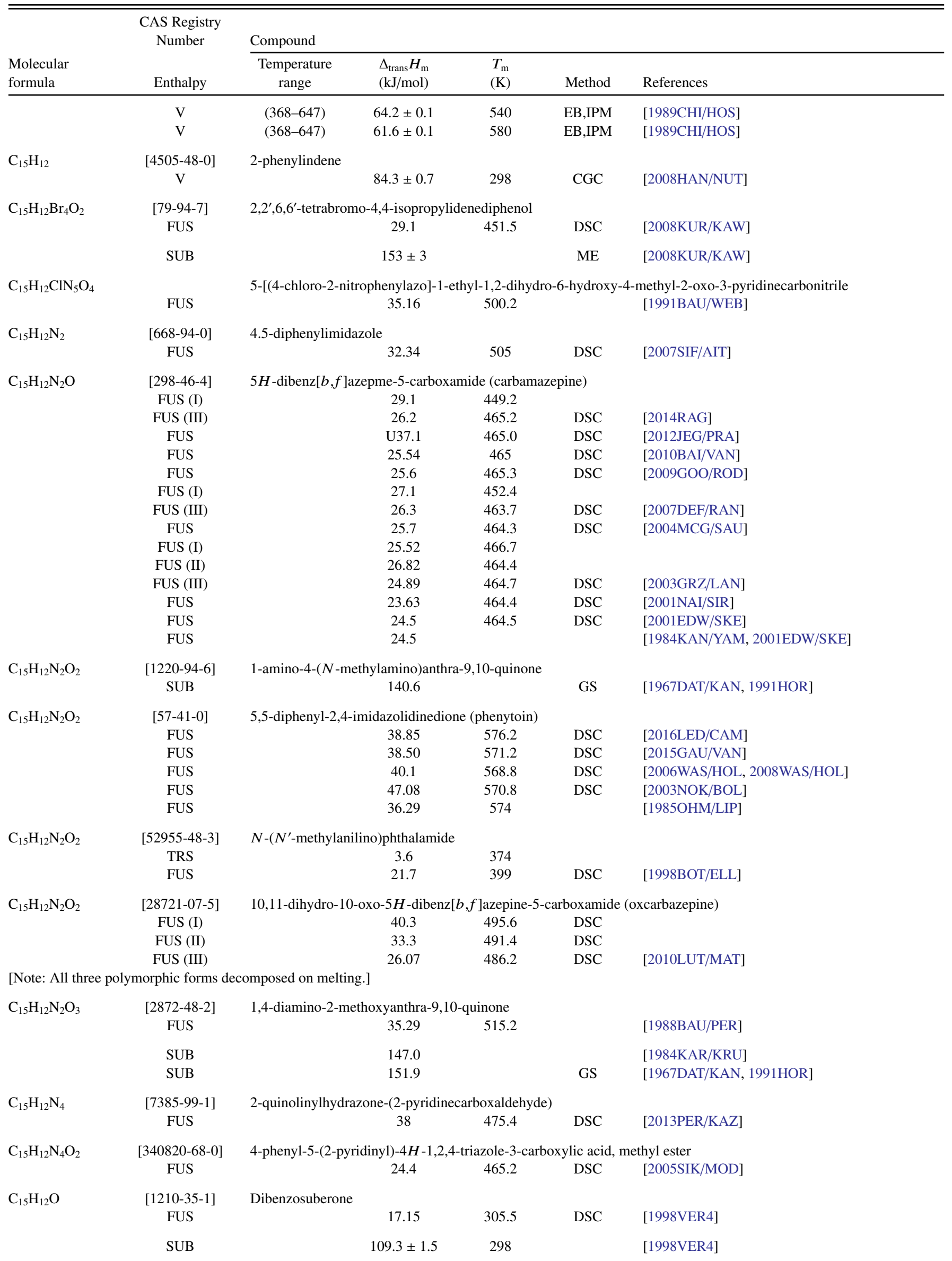


TABLE 12. Phase change enthalpies of $\mathrm{C}_{14}$ to $\mathrm{C}_{18}$ organic compounds-Continued

\begin{tabular}{|c|c|c|c|c|c|c|}
\hline \multirow[b]{2}{*}{$\begin{array}{l}\text { Molecular } \\
\text { formula }\end{array}$} & \multirow{2}{*}{$\begin{array}{l}\text { CAS Registry } \\
\text { Number } \\
\text { Enthalpy }\end{array}$} & \multicolumn{5}{|l|}{ Compound } \\
\hline & & $\begin{array}{l}\text { Temperature } \\
\text { range }\end{array}$ & $\begin{array}{l}\Delta_{\text {trans }} H_{\mathrm{m}} \\
(\mathrm{kJ} / \mathrm{mol})\end{array}$ & $\begin{array}{l}T_{\mathrm{m}} \\
(\mathrm{K})\end{array}$ & Method & References \\
\hline & $\mathrm{V}$ & $(314-338)$ & $90.0 \pm 1.5$ & 326 & GS & [1998VER4] \\
\hline $\mathrm{C}_{15} \mathrm{H}_{12} \mathrm{O}$ & $\begin{array}{l}{[2395-96-2]} \\
\text { SUB }\end{array}$ & \multicolumn{5}{|c|}{ 9-methoxyanthracene } \\
\hline \multirow[t]{2}{*}{$\mathrm{C}_{15} \mathrm{H}_{12} \mathrm{O}$} & $\begin{array}{c}{[1139-82-8]} \\
\text { FUS }\end{array}$ & \multicolumn{5}{|c|}{ 5,7-dihydro-6H-dibenzo[ $a, c]$ cyclohepten-6-one } \\
\hline & SUB & $(323-347)$ & $95.6 \pm 0.8$ & 298 & GS & [1998VER4] \\
\hline $\mathrm{C}_{15} \mathrm{H}_{12} \mathrm{OS}$ & $\begin{array}{l}{[1215-43-6]} \\
\text { SUB }\end{array}$ & \multicolumn{5}{|c|}{ monthiodibenzoylmethane } \\
\hline $\mathrm{C}_{15} \mathrm{H}_{12} \mathrm{O}_{2}$ & $\begin{array}{c}\text { [120-46-7] } \\
\text { SUB } \\
\text { SUB } \\
\text { SUB }\end{array}$ & $\begin{array}{l}\text { Dibenzoylmetl } \\
\qquad \begin{array}{c}(339-348) \\
(339-348)\end{array}\end{array}$ & $\begin{array}{l}113.3 \pm 4.8 \\
114.4 \pm 0.9 \\
115.7 \pm 0.9\end{array}$ & $\begin{array}{l}298 \\
343 \\
298\end{array}$ & $\begin{array}{c}\mathrm{C} \\
\mathrm{ME} \\
\mathrm{ME}\end{array}$ & $\begin{array}{l}\text { [2004RIB/SAN3] } \\
{[1992 \mathrm{RIB} / \mathrm{MON}]} \\
{[1992 \mathrm{RIB} / \mathrm{MON}]}\end{array}$ \\
\hline $\mathrm{C}_{15} \mathrm{H}_{12} \mathrm{O}_{2}$ & $\begin{array}{c}{[120-46-7]} \\
\mathrm{V}\end{array}$ & \multicolumn{4}{|c|}{ 1,3-diphenyl-1,3-propanedione } & [1987STE/MAL] \\
\hline \multirow[t]{2}{*}{$\mathrm{C}_{15} \mathrm{H}_{12} \mathrm{O}_{2}$} & $\begin{array}{c}{[487-26-3]} \\
\text { FUS }\end{array}$ & \multicolumn{5}{|c|}{ 2,3-dihydro-2-phenyl-4H-1-benzopyran-4-one (flavanone) } \\
\hline & SUB & & $107.2 \pm 2.3$ & 298 & $\mathrm{C}$ & [2009SOU/MAT] \\
\hline $\mathrm{C}_{15} \mathrm{H}_{12} \mathrm{O}_{5}$ & $\begin{array}{l}{[480-41-1]} \\
\text { FUS }\end{array}$ & \multicolumn{5}{|c|}{ 2,3-dihydro-5,7-dihydroxy-2-(4-hydroxyphenyl)-4H-1-benzopyran-4-one (naringenin) } \\
\hline $\mathrm{C}_{15} \mathrm{H}_{12} \mathrm{O}_{8}$ & $\begin{array}{l}{[27200-12-0]} \\
\text { FUS }\end{array}$ & \multicolumn{5}{|c|}{ Dihydromyricetin } \\
\hline $\mathrm{C}_{15} \mathrm{H}_{13} \mathrm{ClN}_{2} \mathrm{O}_{2}$ & $\begin{array}{c}{[107485-57-4]} \\
\text { FUS }\end{array}$ & \multicolumn{5}{|c|}{ 1-(2-methylbenzoyl)-3-(4-chlorophenyl)urea } \\
\hline $\mathrm{C}_{15} \mathrm{H}_{13} \mathrm{ClN}_{2} \mathrm{O}_{2}$ & $\begin{array}{c}{[1634626-34-8]} \\
\text { FUS }\end{array}$ & \multicolumn{4}{|c|}{ 1-(4-methylbenzoyl)-3-(4-chlorophenyl)urea } & [2014OZA/NAK] \\
\hline $\mathrm{C}_{15} \mathrm{H}_{13} \mathrm{ClN}_{2} \mathrm{O}_{3}$ & $\begin{array}{c}{[107485-58-5]} \\
\text { FUS }\end{array}$ & \multicolumn{4}{|c|}{ 1-(2-methoxybenzoyl)-3-(4-chlorophenyl)urea } & [2014OZA/NAK] \\
\hline $\mathrm{C}_{15} \mathrm{H}_{13} \mathrm{ClN}_{2} \mathrm{O}_{5}$ & $\begin{array}{l}{[1562-85-2]} \\
\text { SUB }\end{array}$ & \multicolumn{4}{|c|}{ Gallocyanine (C. I. Disperse Blue 95) } & {$[1987 \mathrm{STE} / \mathrm{MAL}]$} \\
\hline $\mathrm{C}_{15} \mathrm{H}_{13} \mathrm{ClN}_{2} \mathrm{~S}$ & $\begin{array}{c}{[688319-94-0]} \\
\text { FUS }\end{array}$ & \multicolumn{4}{|c|}{$N$-(2-methyl-4-chlorophenyl)-4H-3,1-benzothiazin-2-amine } & {$[2004 \mathrm{GON} / \mathrm{KOS}]$} \\
\hline $\mathrm{C}_{15} \mathrm{H}_{13} \mathrm{Cl}_{2} \mathrm{NO}_{2}$ & $\begin{array}{l}{[117-27-1]} \\
\text { FUS }\end{array}$ & \multicolumn{4}{|c|}{ 1,1-(di- $p$-chlorophenyl)-2-nitropropane } & [1990DON/DRE] \\
\hline \multirow[t]{3}{*}{$\mathrm{C}_{15} \mathrm{H}_{13} \mathrm{FO}_{2}$} & $\begin{array}{c}\text { [5104-49-4] } \\
\text { FUS } \\
\text { FUS } \\
\text { FUS } \\
\text { FUS } \\
\text { FUS }\end{array}$ & 2-fluoro- $\alpha$-me & $\begin{array}{c}\text { 1'-biphenyl] } \\
28.2 \\
27.41 \\
27.8 \\
28.0 \\
27.9\end{array}$ & $\begin{array}{c}\text { tic acid } \\
387.1 \\
388 \\
387.0 \\
387.9 \\
386.7\end{array}$ & $\begin{array}{c}\text { flurbipro } \\
\text { DSC } \\
\text { DSC } \\
\text { DSC } \\
\text { DSC } \\
\text { DAC }\end{array}$ & $\begin{array}{l}{[2012 \mathrm{UMN} / \mathrm{HAS}]} \\
{[2010 \mathrm{BAI} / \mathrm{VAN}]} \\
{[2009 \mathrm{GAS} / \mathrm{CEN}]} \\
{[2007 \mathrm{VIP} / \mathrm{WAN}]} \\
{[1999 \mathrm{HEN} / \mathrm{KUH}]}\end{array}$ \\
\hline & $\begin{array}{l}\text { SUB } \\
\text { SUB }\end{array}$ & $(342-378)$ & $\begin{array}{l}110.2 \pm 0.5 \\
108.4 \pm 0.5\end{array}$ & $\begin{array}{l}298 \\
360\end{array}$ & GS & $\begin{array}{l}\text { [2008KUR/PER, 2012UMN/HAS] } \\
\text { [2003PER/KUR] }\end{array}$ \\
\hline & $\mathrm{V}$ & & $127.5 \pm 5.5$ & 298 & CGC & [2012UMN/HAS] \\
\hline $\mathrm{C}_{15} \mathrm{H}_{13} \mathrm{FO}_{2}$ & $\begin{array}{l}{[51543-40-9]} \\
\text { FUS }\end{array}$ & 2-fluoro- $\alpha$-me & $\begin{array}{l}\text {, 1'-biphenyl] } \\
23.3\end{array}$ & $\begin{array}{l}\text { tic acid } \\
380.6\end{array}$ & $\begin{array}{l}\text { Irbiprofer } \\
\text { DSC }\end{array}$ & [2012UMN/HAS] \\
\hline
\end{tabular}

[Note: The above enthalpy of fusion includes two solid-solid phase transition enthalpies totaling $0.5 \mathrm{~kJ} / \mathrm{mol}$.]

\begin{tabular}{|c|c|c|c|c|c|}
\hline & $\mathrm{V}$ & $127.4 \pm 4.7$ & 298 & CGC & [2012UMN/HAS] \\
\hline \multirow[t]{3}{*}{$\mathrm{C}_{15} \mathrm{H}_{13} \mathrm{NO}$} & [3558-24-5] & 1-methyl-2-phenylindole & & & \\
\hline & SUB & $\begin{array}{ll}(331-353) & 109.5 \pm 0.7\end{array}$ & 342 & ME & [2015CAR/AMA] \\
\hline & SUB & $111.1 \pm 0.7$ & 298 & ME & [2015CAR/AMA] \\
\hline
\end{tabular}


TABLE 12. Phase change enthalpies of $\mathrm{C}_{14}$ to $\mathrm{C}_{18}$ organic compounds-Continued

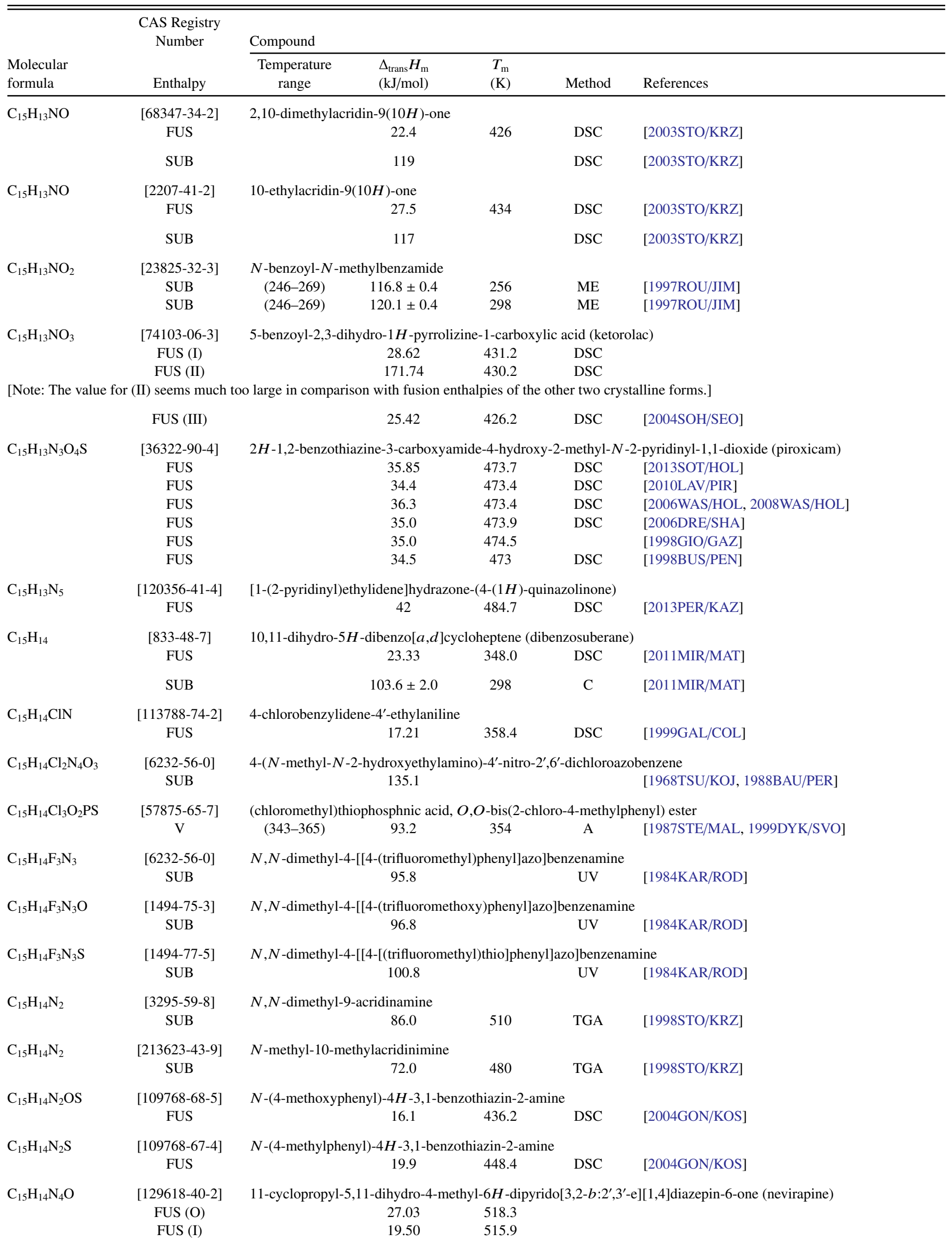


TABLE 12. Phase change enthalpies of $\mathrm{C}_{14}$ to $\mathrm{C}_{18}$ organic compounds-Continued

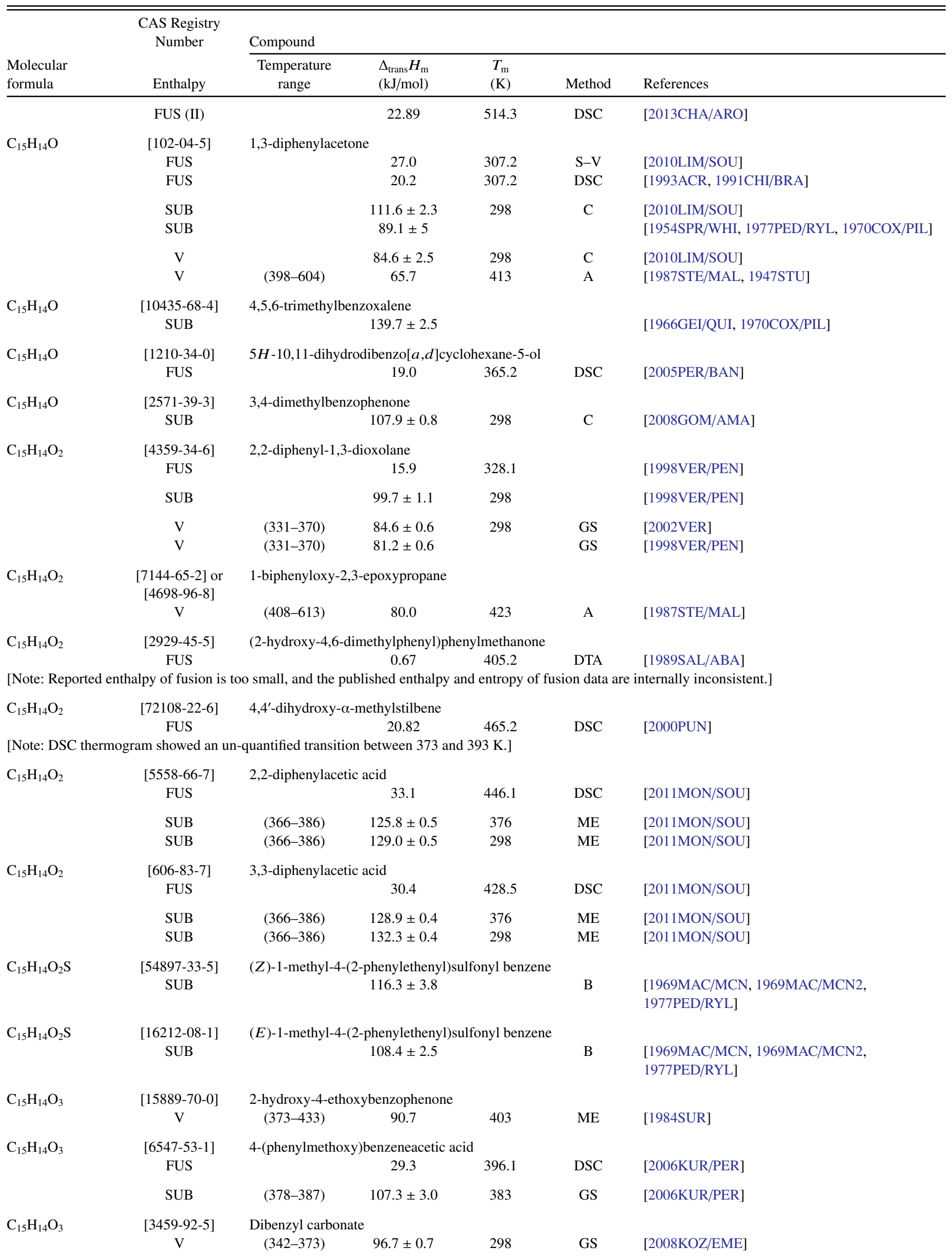


TABLE 12. Phase change enthalpies of $\mathrm{C}_{14}$ to $\mathrm{C}_{18}$ organic compounds-Continued

\begin{tabular}{|c|c|c|c|c|c|c|}
\hline \multirow[b]{2}{*}{$\begin{array}{l}\text { Molecular } \\
\text { formula }\end{array}$} & \multirow{2}{*}{$\begin{array}{l}\text { CAS Registry } \\
\text { Number } \\
\text { Enthalpy }\end{array}$} & \multicolumn{5}{|l|}{ Compound } \\
\hline & & $\begin{array}{c}\text { Temperature } \\
\text { range }\end{array}$ & $\begin{array}{l}\Delta_{\text {trans }} H_{\mathrm{m}} \\
(\mathrm{kJ} / \mathrm{mol})\end{array}$ & $\begin{array}{l}T_{\mathrm{m}} \\
(\mathrm{K})\end{array}$ & Method & References \\
\hline \multirow[t]{4}{*}{$\mathrm{C}_{15} \mathrm{H}_{14} \mathrm{O}_{4}$} & {$[6131-38-0]$} & \multicolumn{5}{|c|}{ 2-hydroxy-4,4'-dimethoxybenzophenone } \\
\hline & FUS & & 37.6 & 390.4 & DSC & {$[1999 \mathrm{PRI} / \mathrm{HAW}]$} \\
\hline & SUB & & 121.1 & & B & [1999PRI/HAW] \\
\hline & $\mathrm{V}$ & & 83.5 & & TGA & [1999PRI/HAW] \\
\hline \multirow[t]{2}{*}{$\mathrm{C}_{15} \mathrm{H}_{14} \mathrm{O}_{4} \mathrm{~S}$} & [313057-13-5] & \multicolumn{5}{|c|}{ 4-(2-propenyloxy)phenyl 5-methoxy-2-thiophene carboxylate } \\
\hline & FUS & & 66.94 & 336.9 & DSC & [2000WU/WAN] \\
\hline \multirow[t]{5}{*}{$\mathrm{C}_{15} \mathrm{H}_{14} \mathrm{O}_{5}$} & {$[131-54-4]$} & \multicolumn{5}{|c|}{ 2,2'-dihydroxy-4,4'-dimethoxybenzophenone } \\
\hline & FUS & & 33.2 & 412.3 & DSC & [1999PRI/HAW] \\
\hline & SUB & & 130.2 & & $\mathrm{~B}$ & [1999PRI/HAW] \\
\hline & $\mathrm{V}$ & & 96.9 & & TGA & [1999PRI/HAW] \\
\hline & $\mathrm{V}$ & $(406-497)$ & 77.4 & 423 & A,UV & [1987STE/MAL, 1960SCH/HIR] \\
\hline \multirow[t]{2}{*}{$\mathrm{C}_{15} \mathrm{H}_{14} \mathrm{O}_{6}$} & [490-46-0] & \multicolumn{5}{|c|}{ (2R-cis)-2-(3,4-dihydroxyphenyl)-3,4-dihydro-2 $H$-1-benzopyran-3,5,7-triol (epicatechin) } \\
\hline & FUS & & 56.72 & 525.0 & DSC & [2010PAR/LEE] \\
\hline $\mathrm{C}_{15} \mathrm{H}_{15} \mathrm{Cl}$ & [13389-70-3] & \multicolumn{5}{|c|}{ Chloro-di-4-tolylmethane } \\
\hline \multirow[t]{2}{*}{$\mathrm{C}_{15} \mathrm{H}_{15} \mathrm{ClN}_{2} \mathrm{O}_{2}$} & {$[1982-47-4]$} & \multicolumn{5}{|c|}{ 3-[4-[4-chlorophenoxy]phenyl]-1,1-dimethylurea } \\
\hline & FUS & & 34.87 & 425.8 & DSC & [1991ACR, 1990DON/DRE] \\
\hline \multirow[t]{2}{*}{$\mathrm{C}_{15} \mathrm{H}_{15} \mathrm{ClN}_{2} \mathrm{O}_{2}$} & {$[556836-79-4]$} & \multicolumn{5}{|c|}{ 4-chloro-2'-hydroxy-4'-propoxyazobenzene } \\
\hline & FUS & & 29.8 & 371 & DSC & [2003PAJ/ROS] \\
\hline \multirow[t]{2}{*}{$\mathrm{C}_{15} \mathrm{H}_{15} \mathrm{CIO}_{5}$} & $\begin{array}{c}\text { [111171-33-6] } \\
\text { TRS }\end{array}$ & \multicolumn{5}{|c|}{ 8-(hydroxymethyl)-6-chloro-5,7-dimethyl-2-oxo- $2 H$-1-benzopyran-3-carboxylic acid, ethyl ester } \\
\hline & FUS & & 25.08 & 456.4 & DSC & {$[1992 \mathrm{HUA} / \mathrm{ZHO} 2]$} \\
\hline \multirow[t]{2}{*}{$\mathrm{C}_{15} \mathrm{H}_{15} \mathrm{~F}_{17} \mathrm{O}$} & {$[1240205-61-1]$} & \multicolumn{5}{|c|}{$1,1,1,2,2,3,3,4,4,5,5,6,6,7,7,8,8$-heptadecafluoro-10-(pentyloxy)decane } \\
\hline & FUS & & 36.82 & 253.8 & DSC & [2010ZAG/CON] \\
\hline $\mathrm{C}_{15} \mathrm{H}_{15} \mathrm{~N}$ & {$[1484-10-2]$} & $N$-propylcarba & & & & \\
\hline & FUS & & 19.57 & 320.4 & DSC & [2016STA/KEI] \\
\hline & $\mathrm{V}$ & $(324-371)$ & $87.8 \pm 0.4$ & 298 & GS & [2015EME/VAR] \\
\hline $\mathrm{C}_{15} \mathrm{H}_{15} \mathrm{~N}$ & {$[1484-09-9]$} & $N$-isopropylca & & & & \\
\hline & FUS & & 18.26 & 393.9 & DSC & [2016STA/KEI] \\
\hline & TRS & & 0.64 & 137.5 & DSC & [1986BER/COL] \\
\hline & TRS & & 0.38 & 180 & & \\
\hline & FUS & & 17.73 & 395.2 & DSC & [1991ACR, 1990KAL/DRE] \\
\hline & SUB & $(340-375)$ & $97.7 \pm 1.0$ & 298 & GS & [2015EME/VAR] \\
\hline $\mathrm{C}_{15} \mathrm{H}_{15} \mathrm{NO}$ & {$[954-21-2]$} & $N$-methyldiph & cetamide & & & \\
\hline & FUS & & 30.23 & 439.8 & DSC & [1990DON/DRE] \\
\hline $\mathrm{C}_{15} \mathrm{H}_{15} \mathrm{NO}$ & {$[1404112-28-2]$} & $N$-(4'-methylb & tyl-3-yl)acet & & & \\
\hline & FUS & & 29.0 & 422.4 & DSC & [2015OWU/CHE] \\
\hline $\mathrm{C}_{15} \mathrm{H}_{15} \mathrm{NO}$ & {$[1215-21-0]$} & $N$-(4'-methylb & yyl-4-yl)acet & & & \\
\hline & FUS & & 28.9 & 498.3 & DSC & [2015OWU/CHE] \\
\hline $\mathrm{C}_{15} \mathrm{H}_{15} \mathrm{NO}_{2}$ & {$[61-68-7]$} & 2 -[(2,3-dimeth & nyl)amino]b & $\operatorname{acid}(\mathrm{m}$ & mic acid) & \\
\hline & FUS & & 41.5 & 503.2 & DSC & [2015GAU/VAN] \\
\hline & FUS & & $\mathrm{U} 71.2$ & 503.1 & DSC & [2010DOM/POB] \\
\hline & FUS & & 38.24 & 502.0 & DSC & {$[2010 A V U / A L E]$} \\
\hline & FUS & & 38.7 & 503.5 & DSC & [2009SUR/TER, 2010SUR/PER, 2015SUR/SIM] \\
\hline & TRS & & 18.1 & 463.2 & & \\
\hline & FUS & & 38.25 & 503.6 & DSC & [2004ROM/BUS] \\
\hline & FUS & & 38.2 & 503.6 & DSC & [1999ROM/ESC] \\
\hline & SUB & $(357-398)$ & $132.7 \pm 0.8$ & 377 & GS & [2009SUR/TER] \\
\hline & SUB & $(357-398)$ & $136.3 \pm 0.8$ & 298 & GS & [2009SUR/TER, 2009SUR/PER] \\
\hline
\end{tabular}


TABLE 12. Phase change enthalpies of $\mathrm{C}_{14}$ to $\mathrm{C}_{18}$ organic compounds-Continued

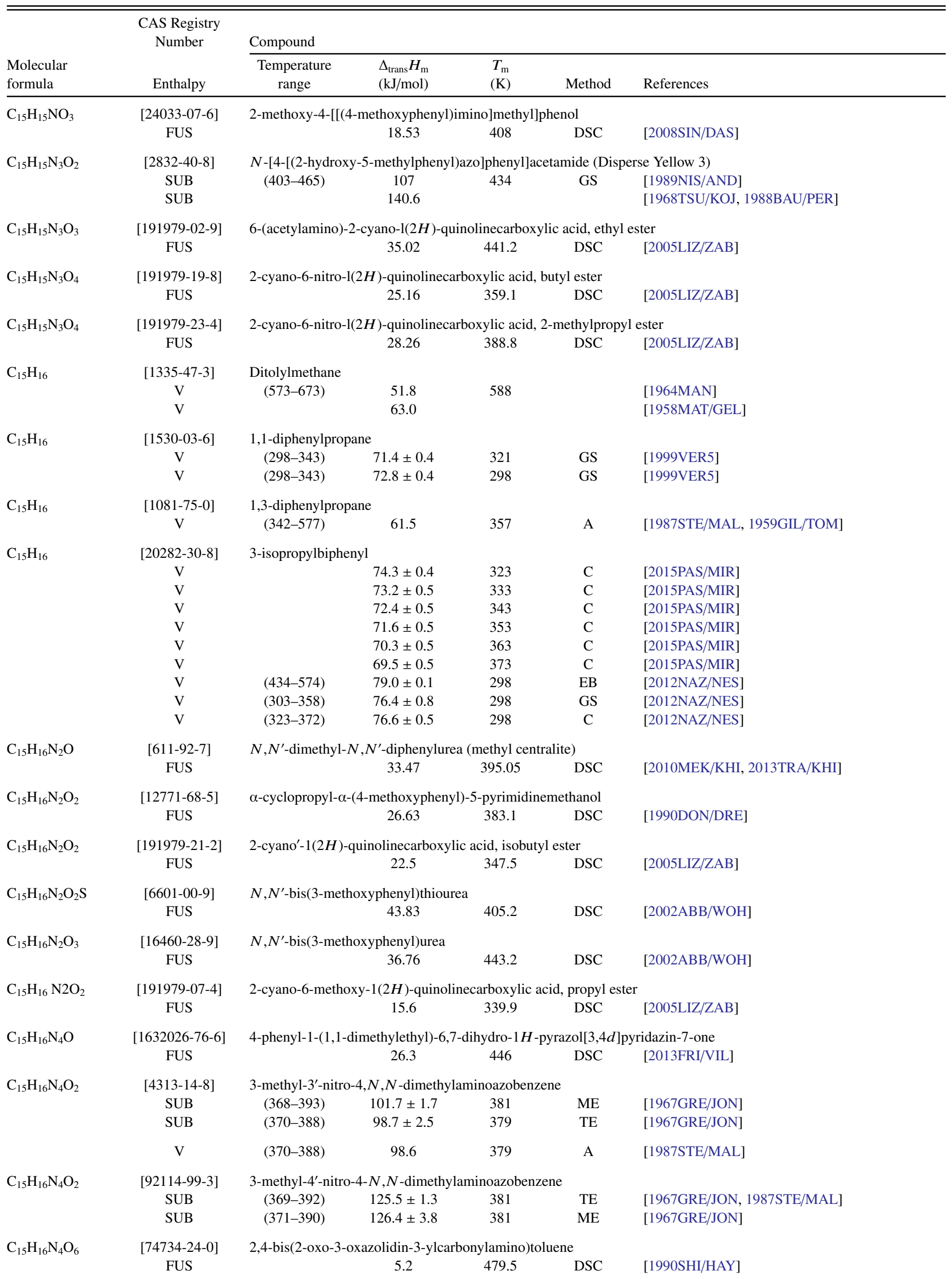


TABLE 12. Phase change enthalpies of $\mathrm{C}_{14}$ to $\mathrm{C}_{18}$ organic compounds-Continued

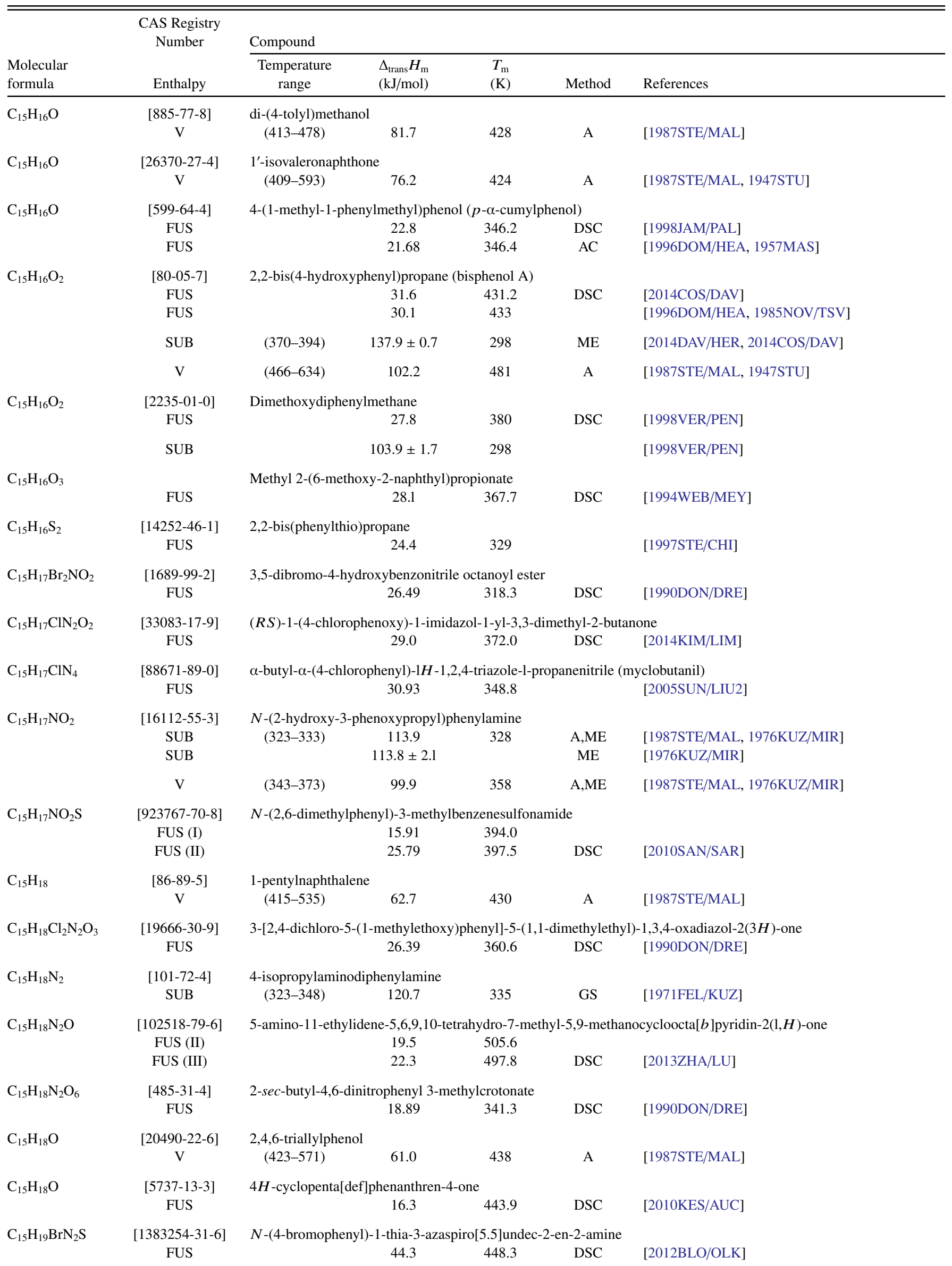


TABLE 12. Phase change enthalpies of $\mathrm{C}_{14}$ to $\mathrm{C}_{18}$ organic compounds-Continued

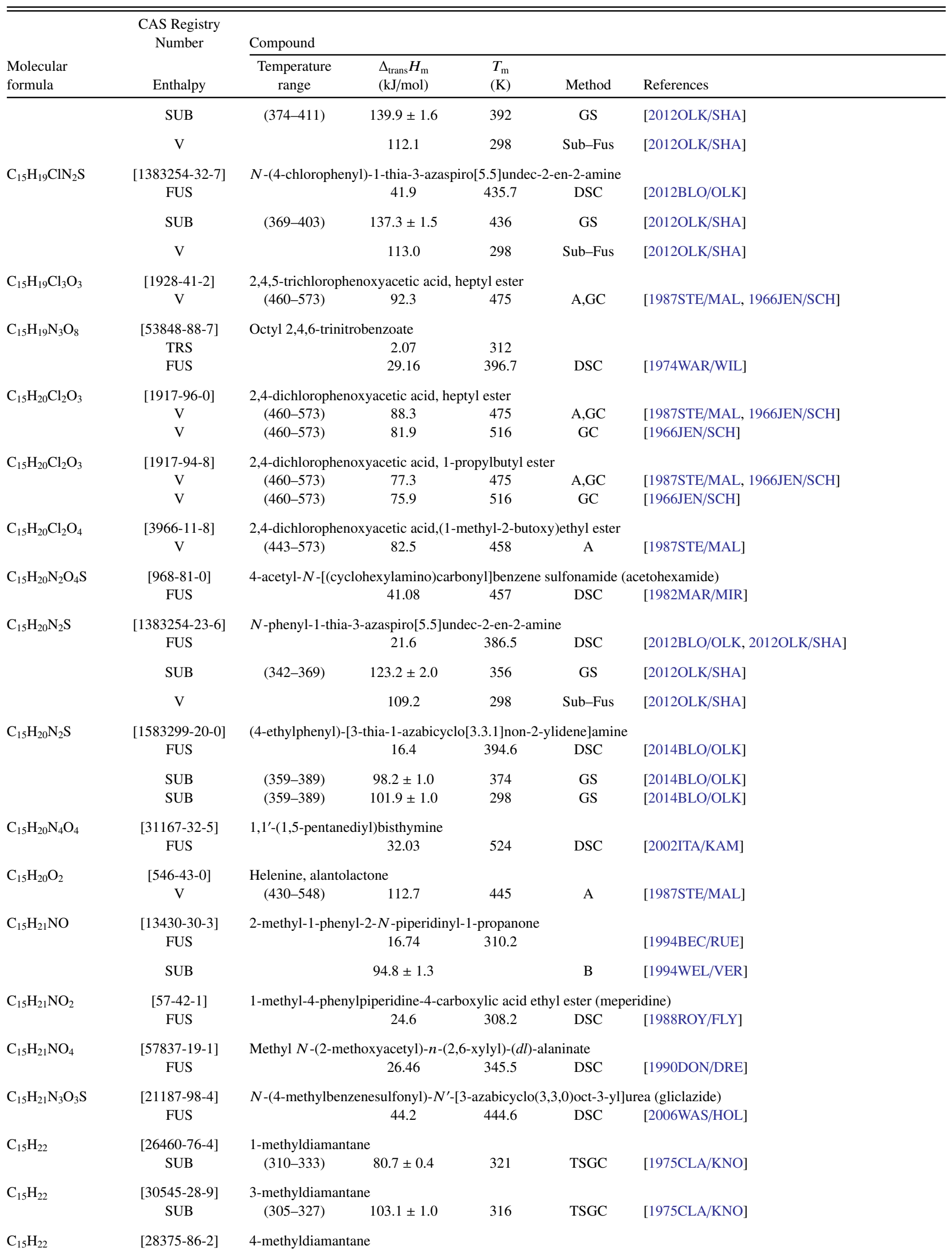


TABLE 12. Phase change enthalpies of $\mathrm{C}_{14}$ to $\mathrm{C}_{18}$ organic compounds-Continued

\begin{tabular}{|c|c|c|c|c|c|c|}
\hline \multirow[b]{2}{*}{$\begin{array}{l}\text { Molecular } \\
\text { formula }\end{array}$} & \multirow{2}{*}{$\begin{array}{l}\text { CAS Registry } \\
\text { Number } \\
\text { Enthalpy }\end{array}$} & \multicolumn{5}{|l|}{ Compound } \\
\hline & & $\begin{array}{l}\text { Temperature } \\
\text { range }\end{array}$ & $\begin{array}{l}\Delta_{\text {trans }} H_{\mathrm{m}} \\
(\mathrm{kJ} / \mathrm{mol})\end{array}$ & $\begin{array}{l}T_{\mathrm{m}} \\
(\mathrm{K})\end{array}$ & Method & References \\
\hline & SUB & $(310-333)$ & $79.4 \pm 1.25$ & 321 & TSGC & {$[1975 \mathrm{CLA} / \mathrm{KNO}]$} \\
\hline \multirow[t]{2}{*}{$\mathrm{C}_{15} \mathrm{H}_{22} \mathrm{ClNO}_{2}$} & $\begin{array}{l}{[51218-45-2]} \\
\text { FUS }\end{array}$ & \multicolumn{5}{|c|}{ 2-chloro- $N$-(2-ethyl-6-methylphenyl)- $N$-(2-methoxy-1-methylethyl)acetamide } \\
\hline & V & & $70 \pm 1$ & 436 & TGA & {$[2007 \mathrm{VEC}]$} \\
\hline $\mathrm{C}_{15} \mathrm{H}_{22} \mathrm{~N}_{2} \mathrm{O}$ & $\begin{array}{l}{[24358-84-7]} \\
\text { FUS }\end{array}$ & $N$-(2,6-dimeth & $\begin{array}{l}\text { enyl)-1-methy } \\
17.77\end{array}$ & peridin & $\begin{array}{l}\text { xamide } \\
\text { DSC }\end{array}$ & {$[1997 N E M / A C S]$} \\
\hline $\mathrm{C}_{15} \mathrm{H}_{22} \mathrm{~N}_{2} \mathrm{O}_{2}$ & $\begin{array}{c}{[5124-30-1]} \\
\mathrm{V}\end{array}$ & \multicolumn{5}{|c|}{$\begin{array}{l}\text { Dicyclohexylmethane-4,4'-diisocyanate } \\
\begin{array}{lll}(326-404) & 80.4 & 341\end{array}\end{array}$} \\
\hline $\mathrm{C}_{15} \mathrm{H}_{22} \mathrm{~N}_{2} \mathrm{O}_{4}$ & $\begin{array}{l}{[92700-71-5]} \\
\text { FUS }\end{array}$ & \multicolumn{5}{|c|}{$\begin{array}{c}\text { Octyl } N \text {-(4-nitrophenyl) carbamate } \\
38.85\end{array}$} \\
\hline $\mathrm{C}_{15} \mathrm{H}_{22} \mathrm{~N}_{2} \mathrm{O}_{5}$ & $\begin{array}{l}{[138517-11-0]} \\
\text { FUS }\end{array}$ & \multicolumn{4}{|c|}{ (4-nitrophenyl)-8-hydroxyoctyl carbamate } & [1993TIE/FRA] \\
\hline $\mathrm{C}_{15} \mathrm{H}_{22} \mathrm{O}_{2}$ & $\begin{array}{l}{[16225-26-6]} \\
\text { SUB }\end{array}$ & \multicolumn{4}{|c|}{ 3,5-di-tert-butylbenzoic acid } & [1974ROU/TUR, 1987STE/MAL, 1977PED/RYL] \\
\hline $\mathrm{C}_{15} \mathrm{H}_{22} \mathrm{O}_{2}$ & $\begin{array}{l}{[37942-07-7]} \\
\text { SUB } \\
\text { SUB }\end{array}$ & $\begin{array}{l}\text { 3,5-di-tert-buty } \\
\quad(296-312) \\
\quad(296-312)\end{array}$ & $\begin{array}{l}\text { hydroxybenza } \\
\begin{array}{l}95.7 \pm 0.5 \\
96.0 \pm 0.5\end{array}\end{array}$ & $\begin{array}{l}\text { de } \\
304 \\
298\end{array}$ & $\begin{array}{l}\mathrm{ME} \\
\mathrm{ME}\end{array}$ & $\begin{array}{l}{[2010 \mathrm{RIB} / \mathrm{GON}]} \\
{[2010 \mathrm{RIB} / \mathrm{GON}]}\end{array}$ \\
\hline $\mathrm{C}_{15} \mathrm{H}_{22} \mathrm{O}_{2}$ & $\begin{array}{c}\text { [3575-31-3] } \\
\text { SUB (I) } \\
\text { SUB (II) }\end{array}$ & $\begin{array}{l}\text { 4-octylbenzoic } \\
\qquad \begin{array}{c}(357-365) \\
(367-372)\end{array}\end{array}$ & $\begin{array}{l}134.7 \pm 1.5 \\
135.4 \pm 1.3\end{array}$ & $\begin{array}{l}298 \\
298\end{array}$ & $\begin{array}{l}\mathrm{ME} \\
\mathrm{ME}\end{array}$ & $\begin{array}{l}\text { [2004MON/ALM] } \\
\text { [2004MON/ALM] }\end{array}$ \\
\hline $\mathrm{C}_{15} \mathrm{H}_{22} \mathrm{O}_{3}$ & $\begin{array}{l}{[79785-45-8]} \\
\text { FUS }\end{array}$ & 3-octyloxyben & $\begin{array}{l}\text { acid } \\
\quad 33.12\end{array}$ & 347.1 & DSC & [2001LAI/LEE] \\
\hline $\mathrm{C}_{15} \mathrm{H}_{22} \mathrm{O}_{3}$ & $\begin{array}{c}\text { [2493-84-7] } \\
\text { SUB } \\
\text { SUB (II) } \\
\text { SUB }\end{array}$ & $\begin{array}{l}\text { 4-octyloxyben } \\
\qquad(363-372) \\
(363-372)\end{array}$ & $\begin{array}{l}\text { acid } \\
141.1 \pm 0.9 \\
161.4 \pm 1.2 \\
163.0 \pm 1.2\end{array}$ & $\begin{array}{l}368 \\
298 \\
298\end{array}$ & $\begin{array}{l}\mathrm{ME} \\
\mathrm{ME}\end{array}$ & $\begin{array}{l}\text { [2010FON/SAN] } \\
{[2010 \text { FON/SAN] }} \\
\text { [2010RIB/FER3] }\end{array}$ \\
\hline $\mathrm{C}_{15} \mathrm{H}_{22} \mathrm{O}_{3}$ & $\begin{array}{c}\text { [19715-19-6] } \\
\text { FUS } \\
\text { SUB }\end{array}$ & 3,5-di-tert-buty & $\begin{array}{c}\text { icylic acid } \\
22.92 \\
83.9 \pm 2.6\end{array}$ & 437.5 & $\begin{array}{l}\text { DSC } \\
\text { DSC }\end{array}$ & $\begin{array}{l}\text { [2003YU/TAN] } \\
\text { [2003YU/TAN] }\end{array}$ \\
\hline $\mathrm{C}_{15} \mathrm{H}_{22} \mathrm{O}_{5}$ & $\begin{array}{l}\text { [63968-64-9] } \\
\text { FUS } \\
\text { (orthorhombic) } \\
\text { FUS (triclinic) } \\
\text { FUS (I) } \\
\text { FUS (II) }\end{array}$ & Octahydro-3,6 & $\begin{array}{c}19.9 \\
22.8 \\
23.41\end{array}$ & $\begin{array}{l}427.1 \\
428.2 \\
428.1\end{array}$ & $\begin{array}{l}\text { DSC } \\
\text { DSC }\end{array}$ & $\begin{array}{l}\text { enzodioxepin-10(3H)-one (artemisinine) } \\
\text { [2014HOR/SEI] } \\
\text { [1997CHA/YUE] }\end{array}$ \\
\hline $\mathrm{C}_{15} \mathrm{H}_{23} \mathrm{NO}_{2}$ & $\begin{array}{l}{[23846-72-2]} \\
\text { FUS }\end{array}$ & \multicolumn{5}{|c|}{ (+)-1-(o-allylphenoxy)-3-(isopropylamino)-2-propanol (alprenolol) } \\
\hline $\mathrm{C}_{15} \mathrm{H}_{23} \mathrm{NO}_{2}$ & $\begin{array}{l}{[13655-52-2]} \\
\text { FUS }\end{array}$ & \multicolumn{5}{|c|}{$( \pm)-1-(o$-allylphenoxy)-3-(isopropylamino)-2-propanol (alprenolol) } \\
\hline $\mathrm{C}_{15} \mathrm{H}_{23} \mathrm{~N}_{3} \mathrm{O}_{2}$ & $\begin{array}{l}{[135742-55-1]} \\
\text { FUS }\end{array}$ & $N$-capryl-pyra & $\begin{array}{l}\text { mide } \\
\qquad 50.58\end{array}$ & 360.5 & DSC & {$[1991 \mathrm{LIU} / \mathrm{GUO}]$} \\
\hline $\mathrm{C}_{15} \mathrm{H}_{23} \mathrm{~N}_{3} \mathrm{O}_{4} \mathrm{~S}$ & $\begin{array}{l}{[23672-07-3]} \\
\text { FUS }\end{array}$ & \multicolumn{5}{|c|}{ (-)-N-1-(ethylpyrrolidm-2-ylmethyl)-2-methoxy-5-sulfamoylbenzamide (sulpiride) } \\
\hline $\mathrm{C}_{15} \mathrm{H}_{23} \mathrm{~N}_{3} \mathrm{O}_{4} \mathrm{~S}$ & $\begin{array}{l}{[15676-16-1]} \\
\text { FUS }\end{array}$ & $( \pm)-N-1-($ ethy & $\begin{array}{l}\text { olidin-2-ylme } \\
46.15\end{array}$ & 2-meth & $\begin{array}{l}\text { sulfamoyl } \\
\text { DSC }\end{array}$ & $\begin{array}{l}\text { nzamide (sulpiride) } \\
\text { [1999LI/ZEL, 1987PIT/VAL] }\end{array}$ \\
\hline $\mathrm{C}_{15} \mathrm{H}_{24}$ & $\begin{array}{c}\text { [1081-77-2] } \\
\mathrm{V} \\
\mathrm{V} \\
\mathrm{V}\end{array}$ & $\begin{array}{l}\text { Nonylbenzene } \\
\quad(304-466) \\
(316-415)\end{array}$ & $\begin{array}{c}74.1 \pm 0.5 \\
69.7 \\
74.8\end{array}$ & $\begin{array}{l}298 \\
331 \\
298\end{array}$ & $\begin{array}{c}\text { MM } \\
\text { GS }\end{array}$ & $\begin{array}{l}\text { [1998MOK/RAU, 2006VER/KOZ] } \\
\text { [1986ALL/JOS] } \\
\text { [1971WIL/ZWO] }\end{array}$ \\
\hline
\end{tabular}


TABLE 12. Phase change enthalpies of $\mathrm{C}_{14}$ to $\mathrm{C}_{18}$ organic compounds-Continued

\begin{tabular}{|c|c|c|c|c|c|c|}
\hline \multirow[b]{2}{*}{$\begin{array}{l}\text { Molecular } \\
\text { formula }\end{array}$} & \multirow{2}{*}{$\begin{array}{l}\text { CAS Registry } \\
\text { Number } \\
\text { Enthalpy }\end{array}$} & \multicolumn{5}{|l|}{ Compound } \\
\hline & & $\begin{array}{c}\text { Temperature } \\
\text { range }\end{array}$ & $\begin{array}{l}\Delta_{\text {trans }} H_{\mathrm{m}} \\
(\mathrm{kJ} / \mathrm{mol})\end{array}$ & $\begin{array}{l}T_{\mathrm{m}} \\
(\mathrm{K})\end{array}$ & Method & References \\
\hline \multirow[t]{4}{*}{$\mathrm{C}_{15} \mathrm{H}_{24}$} & {$[717-74-8]$} & \multicolumn{5}{|c|}{ 1,3,5-triisopropylbenzene } \\
\hline & V & (283-323) & $64.3 \pm 0.3$ & 303 & GS & [1998VER7] \\
\hline & $\mathrm{V}$ & $(283-323)$ & $64.6 \pm 0.6$ & 298 & GS & [1998VER7] \\
\hline & $\mathrm{V}$ & (282-388) & 67.4 & 297 & & [1993KAS/MOK] \\
\hline \multirow[t]{5}{*}{$\mathrm{C}_{15} \mathrm{H}_{24}$} & {$[15181-11-0]$} & \multicolumn{5}{|c|}{ 1,3-di-tert-butyl-5-methylbenzene } \\
\hline & SUB & $(275-301)$ & $82.4 \pm 0.5$ & 288 & $\mathrm{~T}$ & [1998VER] \\
\hline & SUB & $(275-301)$ & $81.8 \pm 0.5$ & 298 & $\mathrm{~T}$ & [1998VER] \\
\hline & V & $(309-338)$ & $61.8 \pm 0.9$ & 310 & GS & [1998VER] \\
\hline & $\mathrm{V}$ & $(309-338)$ & $63.3 \pm 0.9$ & 298 & GS & [1998VER] \\
\hline \multirow[t]{2}{*}{$\mathrm{C}_{15} \mathrm{H}_{24}$} & {$[18794-84-8]$} & \multicolumn{5}{|l|}{$(E)-\beta$-farnesene } \\
\hline & $\mathrm{V}$ & $(363-473)$ & 72.5 & 298 & GC & [2005HOS/GRY] \\
\hline \multirow[t]{2}{*}{$\mathrm{C}_{15} \mathrm{H}_{24}$} & {$[87-44-5]$} & \multicolumn{5}{|l|}{$\beta$-caryophyllene } \\
\hline & $\mathrm{V}$ & $(363-463)$ & 65.5 & 298 & GC & [2005HOS/GRY] \\
\hline \multirow[t]{2}{*}{$\mathrm{C}_{15} \mathrm{H}_{24} \mathrm{~N}_{2} \mathrm{O}_{3}$} & {$[490-98-2]$} & \multicolumn{5}{|c|}{ 4-(butylamino)-2-hydroxybenzoic acid, 2-(dimethylamino)ethyl ester (salicaine) } \\
\hline & FUS & & 26.8 & 319.4 & DSC & {$[2006 \mathrm{SCH} / \mathrm{SCH}]$} \\
\hline \multirow[t]{2}{*}{$\mathrm{C}_{15} \mathrm{H}_{24} \mathrm{O}$} & [497-39-2] & \multicolumn{5}{|c|}{ 2,4-di-tert-butyl-5-methylphenol } \\
\hline & V & $(376-555)$ & 67.0 & 391 & A & [1987STE/MAL, 1947STU] \\
\hline \multirow[t]{2}{*}{$\mathrm{C}_{15} \mathrm{H}_{24} \mathrm{O}$} & {$[616-55-7]$} & \multicolumn{5}{|c|}{ 2,4-di-tert-butyl-6-methylphenol } \\
\hline & V & (359-543) & 59.8 & 374 & A & [1987STE/MAL] \\
\hline \multirow[t]{11}{*}{$\mathrm{C}_{15} \mathrm{H}_{24} \mathrm{O}$} & {$[128-37-0]$} & \multicolumn{5}{|c|}{ 2,6-di-tert-butyl-4-methylphenol } \\
\hline & FUS & & 19.08 & 345.0 & DSC & [2010PAR/LEE] \\
\hline & FUS & & 19.85 & 341.7 & DSC & [1999VER] \\
\hline & FUS & & 23.85 & 343.7 & DTA & [1972INO/LIA] \\
\hline & SUB & & $91.9 \pm 3.2$ & 298 & $\mathrm{C}$ & [2001RIB/MAT] \\
\hline & SUB & $(298-338)$ & $86.8 \pm 0.8$ & 319 & GS & [1999VER] \\
\hline & SUB & $(298-338)$ & $88.0 \pm 0.8$ & 298 & GS & [1999VER] \\
\hline & SUB & $(303-343)$ & 87.8 & 318 & GS & [1987STE/MAL, 1971FEL/KUZ] \\
\hline & SUB & & U117.3 & 298 & $\mathrm{C}$ & [1971BER/GIR, 1999VER] \\
\hline & $\mathrm{V}$ & $(303-343)$ & 87.8 & 318 & A & [1987STE/MAL] \\
\hline & $\mathrm{V}$ & $(358-536)$ & 61.5 & 373 & A & [1987STE/MAL, 1947STU] \\
\hline \multirow[t]{2}{*}{$\mathrm{C}_{15} \mathrm{H}_{24} \mathrm{O}$} & {$[2219-84-3]$} & 2-methyl-4-(1,1 & -tetramethylb & henol & & \\
\hline & V & (447-683) & 67.1 & 462 & A & [1987STE/MAL] \\
\hline $\mathrm{C}_{15} \mathrm{H}_{24} \mathrm{O}$ & [2219-84-3] & 3-methyl-4-(1,1 & -tetramethylb & henol & & \\
\hline & V & (436-549) & 65.5 & 451 & A & [1987STE/MAL] \\
\hline $\mathrm{C}_{15} \mathrm{H}_{24} \mathrm{O}$ & {$[4979-46-8]$} & 4-methyl-2-(1,1 & -tetramethylb & henol & & \\
\hline & V & $(415-545)$ & 65.0 & 430 & A & [1987STE/MAL] \\
\hline $\mathrm{C}_{15} \mathrm{H}_{24} \mathrm{O}$ & {$[406944-31-8]$} & 4-(3,6-dimethyl & an-3-yl)phen & & & \\
\hline & V & & 89.4 & 298 & ME & {$[2001 \mathrm{LAL} / \mathrm{SCH}]$} \\
\hline $\mathrm{C}_{15} \mathrm{H}_{24} \mathrm{O}$ & {$[104-40-5]$} & 4-nonylphenol & & & & \\
\hline & V & $(487-595)$ & 65.0 & 502 & $\mathrm{~A}, \mathrm{~EB}$ & [1987STE/MAL, 1976HON/SIN] \\
\hline $\mathrm{C}_{15} \mathrm{H}_{24} \mathrm{O}$ & {$[115-71-9]$} & $\alpha$-santalol & & & & \\
\hline & V & $(293-450)$ & 58.3 & 308 & A & [1987STE/MAL] \\
\hline $\mathrm{C}_{15} \mathrm{H}_{24} \mathrm{O}_{2}$ & [1991-52-2] & 2,5-di-tert-buty & nethoxyphen & & & \\
\hline & FUS & & 26.9 & 374.4 & & [1972ALV/BOR] \\
\hline & $\mathrm{V}$ & $(423-453)$ & 64.4 & 438 & A & [1987STE/MAL] \\
\hline $\mathrm{C}_{15} \mathrm{H}_{24} \mathrm{O}_{2}$ & {$[6121-64-8]$} & 1,3-dimethoxy- & ptylbenzene & & & \\
\hline & V & $(419-488)$ & 75.5 & 434 & $\mathrm{~A}, \mathrm{GC}$ & [1987STE/MAL, 1975KUN/LIL] \\
\hline $\mathrm{C}_{15} \mathrm{H}_{24} \mathrm{O}_{2}$ & {$[41442-51-7]$} & 1,3-dimethoxy- & ethyl-2-hexy & & & \\
\hline & $\mathrm{V}$ & $(410-475)$ & 72.3 & 425 & A,GC & [1987STE/MAL, 1975KUN/LIL] \\
\hline $\mathrm{C}_{15} \mathrm{H}_{24} \mathrm{O}_{4}$ & {$[1152-57-4]$} & Dicyclohexyl m & nate & & & \\
\hline
\end{tabular}


TABle 12. Phase change enthalpies of $\mathrm{C}_{14}$ to $\mathrm{C}_{18}$ organic compounds-Continued

\begin{tabular}{|c|c|c|c|c|c|c|}
\hline \multirow[b]{2}{*}{$\begin{array}{l}\text { Molecular } \\
\text { formula }\end{array}$} & \multirow{2}{*}{$\begin{array}{l}\text { CAS Registry } \\
\text { Number } \\
\text { Enthalpy }\end{array}$} & \multicolumn{5}{|l|}{ Compound } \\
\hline & & $\begin{array}{c}\text { Temperature } \\
\text { range }\end{array}$ & $\begin{array}{l}\Delta_{\text {trans }} H_{\mathrm{m}} \\
(\mathrm{kJ} / \mathrm{mol})\end{array}$ & $\begin{array}{l}T_{\mathrm{m}} \\
(\mathrm{K})\end{array}$ & Method & References \\
\hline & $\mathrm{V}$ & $(324-353)$ & $93.7 \pm 1.1$ & 298 & GS & [2008LIP/KRA] \\
\hline $\mathrm{C}_{15} \mathrm{H}_{24} \mathrm{O}_{6}$ & $\begin{array}{c}{[64617-28-3]} \\
\mathrm{V}\end{array}$ & $\begin{array}{c}\text { Aconitic acid, } \\
\quad(359-500)\end{array}$ & $\begin{array}{c}\text { pyl ester } \\
72.3\end{array}$ & 374 & A & [1987STE/MAL, 1953MAG/MOD] \\
\hline $\mathrm{C}_{15} \mathrm{H}_{26} \mathrm{O}$ & $\begin{array}{c}{[489-86-1]} \\
\mathrm{V}\end{array}$ & $\begin{array}{l}\text { Guaiol } \\
\qquad(373-561)\end{array}$ & 62.2 & 388 & A & [1987STE/MAL] \\
\hline $\mathrm{C}_{15} \mathrm{H}_{26} \mathrm{O}_{6}$ & $\mathrm{~V}$ & $\begin{array}{c}\text { Camphorenic a } \\
\quad(423-574)\end{array}$ & $\begin{array}{l}\text { triethyl ester } \\
69.0\end{array}$ & 438 & A & [1987STE/MAL, 1947STU] \\
\hline $\mathrm{C}_{15} \mathrm{H}_{26} \mathrm{O}_{6}$ & $\begin{array}{c}{[5333-54-0]} \\
\mathrm{V}\end{array}$ & $\begin{array}{l}\text { Tripropyl 1,2,3 } \\
\quad(360-460)\end{array}$ & $\begin{array}{c}\text { panetricarboxy } \\
76.5\end{array}$ & 375 & A & [1987STE/MAL, 1953MAG/MOD] \\
\hline $\mathrm{C}_{15} \mathrm{H}_{26} \mathrm{O}_{6}$ & $\begin{array}{c}{[60-01-5]} \\
\text { V } \\
\text { V } \\
\text { V } \\
\text { V } \\
\text { V } \\
\text { V }\end{array}$ & $\begin{array}{l}\text { Tributyrin } \\
\qquad(324-354) \\
(318-364) \\
(318-364)\end{array}$ & $\begin{array}{l}98.5 \pm 0.4 \\
83.5 \\
84.9 \pm 2.5 \\
81.4 \\
107.1 \pm 1.0 \\
88.2\end{array}$ & $\begin{array}{l}298 \\
308 \\
298 \\
333 \\
298 \\
298\end{array}$ & $\begin{array}{l}\text { GS } \\
\text { TGA } \\
\text { TGA } \\
\text { A } \\
\text { C } \\
\text { T }\end{array}$ & $\begin{array}{l}\text { [2010MAS/KRA] } \\
\text { [1990KIS/SHO] } \\
\text { [1990KIS/SHO] } \\
\text { [1987STE/MAL, 1949PER/WEB2] } \\
\text { [1986NIL/WAD] } \\
\text { [2010MAS/KRA, 1949PER/WEB2] }\end{array}$ \\
\hline $\mathrm{C}_{15} \mathrm{H}_{26} \mathrm{O}_{6}$ & $\begin{array}{c}{[14295-64-8]} \\
\mathrm{V}\end{array}$ & $\begin{array}{l}\text { Glycerol tri(2-1 } \\
\quad(329-371)\end{array}$ & $\begin{array}{c}\text { ypropanoate) } \\
95.3 \pm 0.6\end{array}$ & 298 & GS & [2010MAS/KRA] \\
\hline $\mathrm{C}_{15} \mathrm{H}_{28} \mathrm{Cl}_{4}$ & $\begin{array}{c}{[3922-32-5]} \\
\mathrm{V}\end{array}$ & $\begin{array}{l}\text { 1,1,1,15-tetracl } \\
\quad(340-392)\end{array}$ & $\begin{array}{c}\text { pentadecane } \\
103.5\end{array}$ & 355 & A & [1987STE/MAL, 1960MAL/MAL] \\
\hline $\mathrm{C}_{15} \mathrm{H}_{28} \mathrm{O}$ & $\begin{array}{c}{[1604-35-9]} \\
\mathrm{V}\end{array}$ & $\begin{array}{l}\text { 3,7,11-trimethy } \\
\quad(401-524)\end{array}$ & $\begin{array}{c}\text { lodecyn-3-ol } \\
43.2 \pm 1.1\end{array}$ & 463 & Static & [1988BAG/GUR, 1986WHI] \\
\hline $\mathrm{C}_{15} \mathrm{H}_{28} \mathrm{O}$ & $\begin{array}{c}{[502-72-7]} \\
\text { FUS }\end{array}$ & Cyclopentadec & 8.8 & 338.4 & DSC & [1997JIM/ROU] \\
\hline & $\begin{array}{l}\text { SUB } \\
\text { SUB }\end{array}$ & $(296-315)$ & $\begin{array}{l}86.0 \pm 0.6 \\
77.4 \pm 0.8\end{array}$ & 305 & ME & $\begin{array}{l}\text { [1938WOL/WEG, 1960JON] [1997JIM/ROU] } \\
\text { [1970COX/PIL] }\end{array}$ \\
\hline $\mathrm{C}_{15} \mathrm{H}_{28} \mathrm{O}_{2}$ & $\begin{array}{c}{[2156-97-0]} \\
\mathrm{V}\end{array}$ & $\begin{array}{l}\text { Dodecyl acryla } \\
\quad(432-573)\end{array}$ & 64.6 & 447 & A & [1987STE/MAL] \\
\hline $\mathrm{C}_{15} \mathrm{H}_{28} \mathrm{O}_{2}$ & $\begin{array}{l}{[106-02-5]} \\
\text { FUS }\end{array}$ & 1,15-pentadeca & 7.0 & 309.5 & DSC & [2011EME/VER] \\
\hline & $\begin{array}{l}\text { SUB } \\
\text { SUB }\end{array}$ & $(290-310)$ & $\begin{array}{l}85.9 \pm 0.5 \\
81.3\end{array}$ & $\begin{array}{l}298 \\
300\end{array}$ & $\begin{array}{l}\mathrm{V}+\mathrm{F} \\
\mathrm{ME}\end{array}$ & $\begin{array}{l}\text { [2011EME/VER] } \\
\text { [1987STE/MAL, 1960JON, 1954SER/VOI] }\end{array}$ \\
\hline & $\begin{array}{l}\mathrm{V} \\
\mathrm{V} \\
\mathrm{V} \\
\mathrm{V} \\
\mathrm{V} \\
\mathrm{V} \\
\mathrm{V}\end{array}$ & $\begin{array}{l}(318-378) \\
(318-378) \\
(318-378) \\
(318-378) \\
(318-378) \\
(363-443) \\
(310-320)\end{array}$ & $\begin{array}{c}77.3 \\
74.6 \\
73.2 \\
71.8 \\
79.1 \pm 0.5 \\
78.2 \\
74.2\end{array}$ & $\begin{array}{l}318 \\
348 \\
363 \\
378 \\
298 \\
378 \\
315\end{array}$ & $\begin{array}{l}\text { GS } \\
\text { GS } \\
\text { GS } \\
\text { GS } \\
\text { GS } \\
\text { A } \\
\text { A,ME }\end{array}$ & $\begin{array}{l}\text { [2011EME/VER] } \\
\text { [2011EME/VER] } \\
\text { [2011EME/VER] } \\
\text { [2011EME/VER] } \\
\text { [2011EME/VER] } \\
\text { [1987STE/MAL] } \\
{[1987 \mathrm{STE} / \mathrm{MAL}, 1954 \mathrm{SER} / \mathrm{VOI}]}\end{array}$ \\
\hline $\mathrm{C}_{15} \mathrm{H}_{28} \mathrm{O}_{2}$ & $\begin{array}{c}{[65954-19-0]} \\
\mathrm{V}\end{array}$ & (Z)-4-trideceny & $\begin{array}{l}\text { etate } \\
82.7 \pm 5.5\end{array}$ & 298 & CGC & [2016GOO/HAS] \\
\hline $\mathrm{C}_{15} \mathrm{H}_{28} \mathrm{O}_{2}$ & $\begin{array}{c}{[34270-22-9]} \\
\mathrm{V}\end{array}$ & $\begin{array}{l}\text { (Z)-7-trideceny } \\
\quad(343-388)\end{array}$ & $\begin{array}{l}\text { etate } \\
\qquad 84.3\end{array}$ & 298 & GC & [1997KOU/HOS, 2000OVA/KOU] \\
\hline $\mathrm{C}_{15} \mathrm{H}_{28} \mathrm{O}_{2}$ & $\begin{array}{c}{[56577-30-1]} \\
\mathrm{V}\end{array}$ & $\begin{array}{l}\text { (E)-7-tridecen } \\
\quad(343-388)\end{array}$ & $\begin{array}{l}\text { etate } \\
\qquad 84.8\end{array}$ & 298 & GC & [1997KOU/HOS, 2000OVA/KOU] \\
\hline $\mathrm{C}_{15} \mathrm{H}_{28} \mathrm{O}_{2}$ & $\begin{array}{l}{[35835-78-0]} \\
\mathrm{V}\end{array}$ & $\begin{array}{l}\text { (Z)-9-trideceny } \\
\quad(343-388)\end{array}$ & $\begin{array}{l}\text { etate } \\
\qquad 85.1\end{array}$ & 298 & GC & [1997KOU/HOS, 2000OVA/KOU] \\
\hline $\mathrm{C}_{15} \mathrm{H}_{28} \mathrm{O}_{2}$ & $\begin{array}{c}{[52957-19-4]} \\
\mathrm{V}\end{array}$ & $\begin{array}{l}\text { (E)-9-tridecen } \\
\quad(343-388)\end{array}$ & $\begin{array}{l}\text { etate } \\
85.5\end{array}$ & 298 & GC & [1997KOU/HOS, 2000OVA/KOU] \\
\hline $\mathrm{C}_{15} \mathrm{H}_{28} \mathrm{O}_{2}$ & {$[33951-95-0]$} & (Z)-11-tridecer & cetate & & & \\
\hline
\end{tabular}


TABLE 12. Phase change enthalpies of $\mathrm{C}_{14}$ to $\mathrm{C}_{18}$ organic compounds-Continued

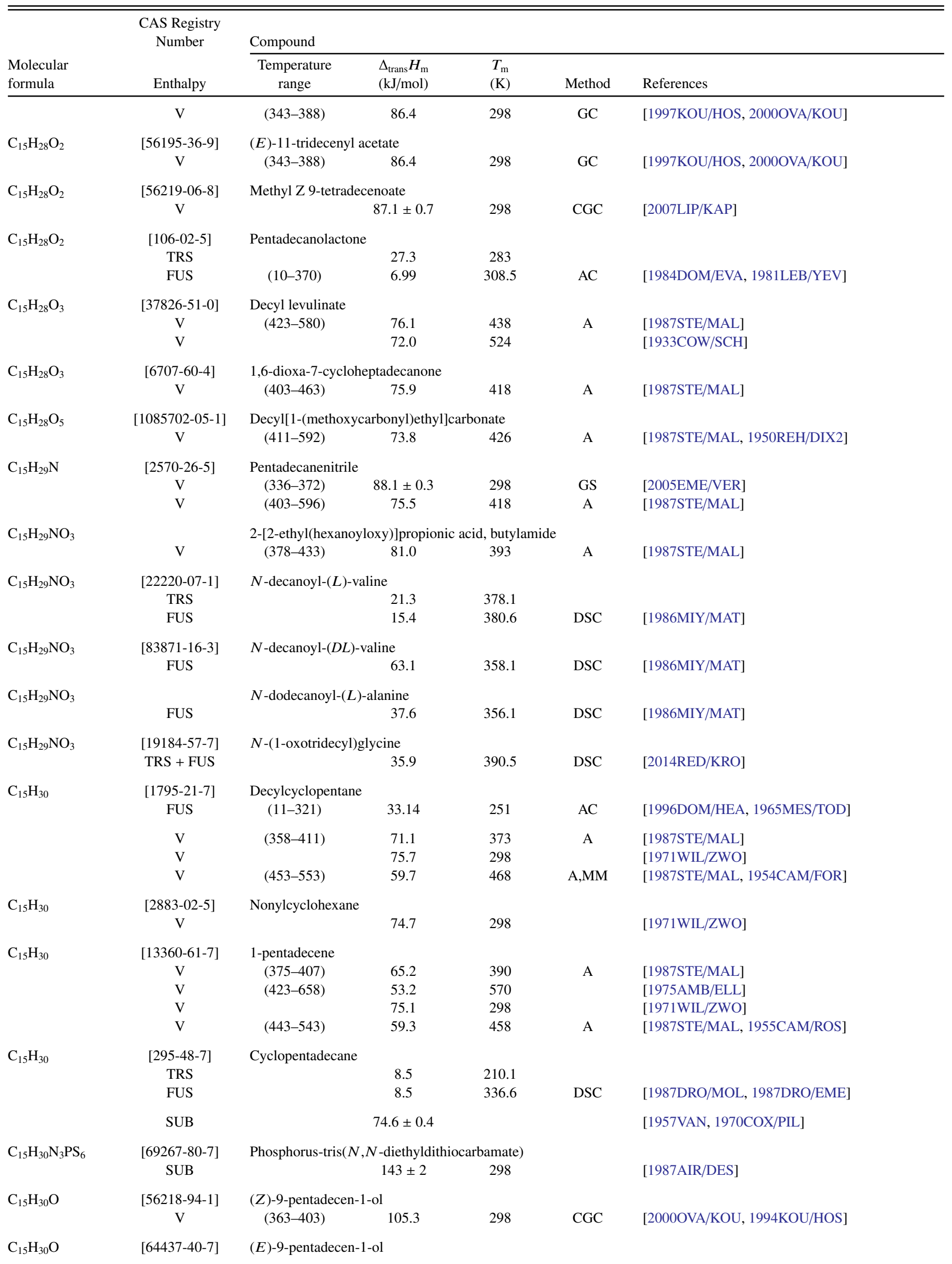


TABle 12. Phase change enthalpies of $\mathrm{C}_{14}$ to $\mathrm{C}_{18}$ organic compounds-Continued

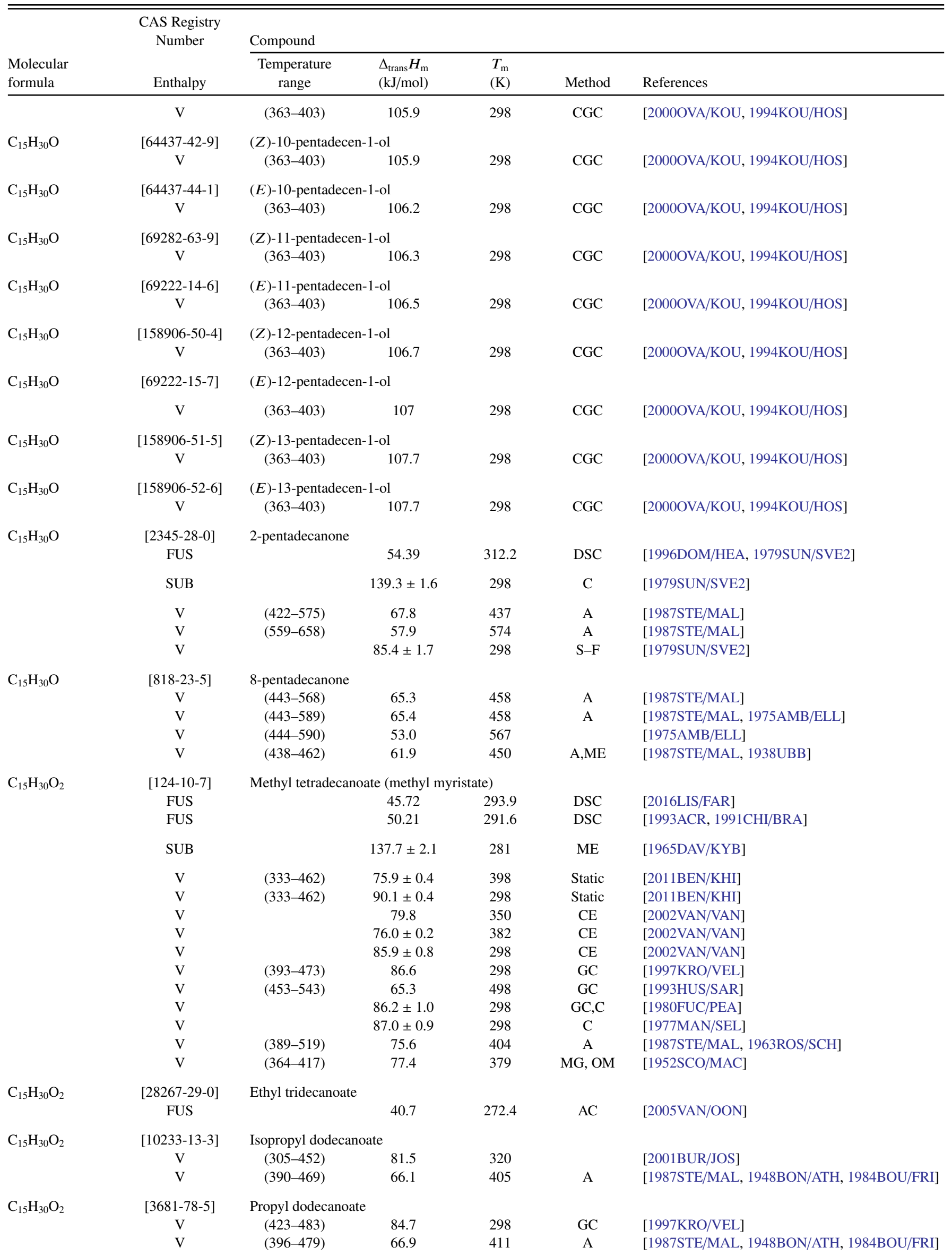


TABLE 12. Phase change enthalpies of $\mathrm{C}_{14}$ to $\mathrm{C}_{18}$ organic compounds-Continued

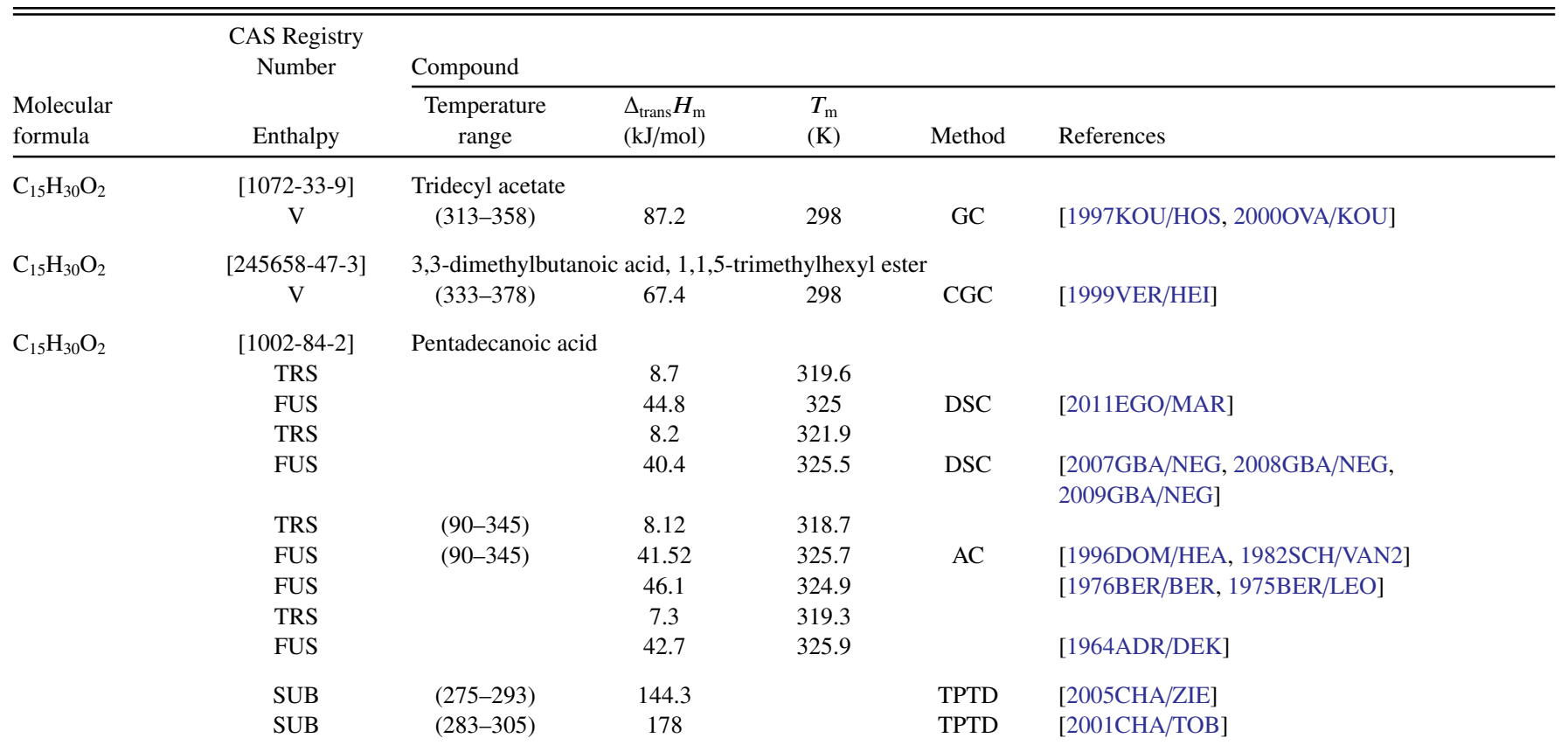

[Note: Experimental values based on the TPTD method are often inconsistent with values determined using other experimental methods.]

$\begin{array}{cccccc}\mathrm{V} & & 116.6 \pm 9.2 & 298 & \mathrm{CGC} & {[2013 \mathrm{WIL} / \mathrm{CHI}]} \\ \mathrm{V} & (431-613) & 94 & 446 & \mathrm{~A} & {[1987 \mathrm{STE} / \mathrm{MAL}]} \\ \mathrm{V} & (347-367) & 108.5 \pm 2.0 & 357 & \mathrm{ME}, \mathrm{TE} & {[1982 \mathrm{DEK} / \mathrm{SCH}]}\end{array}$

$\mathrm{C}_{15} \mathrm{H}_{30} \mathrm{O}_{3}$

[4617-33-8] 15-hydroxypentadecanoic acid
SUB
(294-316)
103
TPTD [2005CHA/ZIE]

[Note: Experimental values based on the TPTD method are often inconsistent with values determined using other experimental methods.]

\begin{tabular}{|c|c|c|c|c|c|c|}
\hline $\mathrm{C}_{15} \mathrm{H}_{30} \mathrm{O}_{3}$ & $\begin{array}{c}{[6283-92-7]} \\
V\end{array}$ & $\begin{array}{l}\text { Dodecyl lactate } \\
\quad(367-583)\end{array}$ & 80.5 & 382 & A & [1987STE/MAL, 1950REH/DIX] \\
\hline $\mathrm{C}_{15} \mathrm{H}_{30} \mathrm{O}_{3}$ & $\begin{array}{c}\text { [70160-09-7] } \\
\mathrm{V}\end{array}$ & $\begin{array}{l}\text { Decyl 2-ethoxypropio } \\
\quad(423-523)\end{array}$ & $\begin{array}{l}\text { nate } \\
69.8\end{array}$ & 438 & A & [1987STE/MAL, 1948DIX/REH] \\
\hline $\mathrm{C}_{15} \mathrm{H}_{30} \mathrm{O}_{6}$ & $\begin{array}{c}\text { [63364-38-5] } \\
\mathrm{V}\end{array}$ & $\begin{array}{l}\text { 3,3,6,6,9,9-tetraethyl- } \\
\quad(403-473)\end{array}$ & $\begin{array}{l}1,2,4,5,7,8 \\
63.6\end{array}$ & $\begin{array}{c}\text { xacycl } \\
298\end{array}$ & CGC & [2007CAN/EYL] \\
\hline $\mathrm{C}_{15} \mathrm{H}_{31} \mathrm{Br}$ & $\begin{array}{c}{[629-72-1]} \\
\mathrm{V}\end{array}$ & $\begin{array}{l}\text { 1-bromopentadecane } \\
\quad(450-661)\end{array}$ & 69.5 & 465 & $\mathrm{~A}, \mathrm{E}$ & [1987STE/MAL, 1961LI/ROS, 1970DYK/VAN] \\
\hline $\mathrm{C}_{15} \mathrm{H}_{31} \mathrm{Cl}$ & $\begin{array}{l}{[4862-03-7]} \\
\text { V } \\
\text { V }\end{array}$ & $\begin{array}{l}\text { 1-chloropentadecane } \\
\text { (439-645) }\end{array}$ & $\begin{array}{l}92.6 \\
55.4\end{array}$ & $\begin{array}{l}298 \\
454\end{array}$ & $\mathrm{~A}, \mathrm{E}$ & $\begin{array}{l}\text { [2006BOL/NER2] } \\
\text { [1987STE/MAL, 1961LI/ROS, 1970DYK/VAN] }\end{array}$ \\
\hline $\mathrm{C}_{15} \mathrm{H}_{31} \mathrm{~F}$ & $\begin{array}{l}{[1555-17-5]} \\
\mathrm{V}\end{array}$ & $\begin{array}{l}\text { 1-fluoropentadecane } \\
\quad(413-593)\end{array}$ & 63.8 & 428 & $\mathrm{~A}, \mathrm{E}$ & [1987STE/MAL, 1961LI/ROS, 1970DYK/VAN] \\
\hline $\mathrm{C}_{15} \mathrm{H}_{31} \mathrm{I}$ & $\begin{array}{l}{[35599-78-1]} \\
\mathrm{V} \\
\mathrm{V}\end{array}$ & $\begin{array}{l}\text { 1-iodopentadecane } \\
\quad(464-673) \\
(464-673)\end{array}$ & $\begin{array}{l}94.6 \\
70.6\end{array}$ & $\begin{array}{l}298 \\
479\end{array}$ & $\begin{array}{l}\mathrm{A}, \mathrm{E} \\
\mathrm{A}, \mathrm{E}\end{array}$ & $\begin{array}{l}\text { [1987STE/MAL, 1961LI/ROS, 1970DYK/VAN, } \\
\text { 2006BOL/NER] } \\
\text { [1987STE/MAL, 1961LI/ROS, 1970DYK/VAN] }\end{array}$ \\
\hline $\mathrm{C}_{15} \mathrm{H}_{31} \mathrm{NO}$ & $\begin{array}{l}\text { [7438-09-7] } \\
\text { SUB }\end{array}$ & $\begin{array}{c}N \text {-methyl tetradecana } \\
(332-347)\end{array}$ & $\begin{array}{l}\text { mide } \\
30.4 \pm 0.8\end{array}$ & 340 & ME & [1959DAV/JON, 1987STE/MAL] \\
\hline $\mathrm{C}_{15} \mathrm{H}_{31} \mathrm{NO}_{2}$ & $\begin{array}{c}{[5468-40-6]} \\
\mathrm{V}\end{array}$ & $\begin{array}{l}N, N \text {-dihexyl lactami } \\
\quad(418-453)\end{array}$ & 79.4 & 433 & A & [1987STE/MAL, 1953FEI/FIL] \\
\hline $\mathrm{C}_{15} \mathrm{H}_{31} \mathrm{NO}_{2}$ & $\begin{array}{c}{[5422-41-3]} \\
\mathrm{V}\end{array}$ & $\begin{array}{l}N \text {-dodecyl lactamide } \\
\quad(408-476)\end{array}$ & 103.9 & 423 & A & [1987STE/MAL, 1950RAT] \\
\hline $\mathrm{C}_{15} \mathrm{H}_{32}$ & $\begin{array}{c}\text { [629-62-9] } \\
\text { TRS } \\
\text { FUS } \\
\text { TRS } \\
\text { FUS } \\
\text { TRS }\end{array}$ & Pentadecane & $\begin{array}{c}8.88 \\
34.62 \\
9.37 \\
36.8 \\
8.7\end{array}$ & $\begin{array}{l}269.3 \\
282.3 \\
270.5 \\
282.8 \\
270.3\end{array}$ & $\begin{array}{l}\text { DSC } \\
\text { DSC }\end{array}$ & $\begin{array}{l}\text { [2015VEL/ORT] } \\
{[2005 \mathrm{HUA} / \mathrm{SIM}]}\end{array}$ \\
\hline
\end{tabular}


TABLE 12. Phase change enthalpies of $\mathrm{C}_{14}$ to $\mathrm{C}_{18}$ organic compounds-Continued

\begin{tabular}{|c|c|c|c|c|c|c|}
\hline \multirow[b]{2}{*}{$\begin{array}{l}\text { Molecular } \\
\text { formula }\end{array}$} & \multirow{2}{*}{$\begin{array}{l}\text { CAS Registry } \\
\text { Number } \\
\text { Enthalpy }\end{array}$} & \multicolumn{5}{|l|}{ Compound } \\
\hline & & $\begin{array}{l}\text { Temperature } \\
\text { range }\end{array}$ & $\begin{array}{l}\Delta_{\text {trans }} H_{\mathrm{m}} \\
(\mathrm{kJ} / \mathrm{mol})\end{array}$ & $\begin{array}{l}T_{\mathrm{m}} \\
(\mathrm{K})\end{array}$ & Method & References \\
\hline & FUS & & 34.2 & 282.7 & DSC & [2004MON/RAJ] \\
\hline & TRS & & 9.17 & 270.9 & & \\
\hline & FUS & & 34.2 & 282.7 & DSC & [1999MET/RAJ] \\
\hline & TRS & & 9.17 & 270.9 & & \\
\hline & FUS & & 34.6 & 283.1 & & [1996DOM/HEA, 1954FIN/GRO2] \\
\hline & SUB & & 107.8 & 298 & $\mathrm{~B}$ & [1972MOR3] \\
\hline & $\mathrm{V}$ & & 72.9 & 334 & $\mathrm{C}$ & [1996VIT/CHA] \\
\hline & $\mathrm{V}$ & & 71.8 & 344 & $\mathrm{C}$ & [1996VIT/CHA] \\
\hline & $\mathrm{V}$ & $(453-503)$ & 75.7 & 298 & CGC & [1995CHI/HOS] \\
\hline & $\mathrm{V}$ & $(423-473)$ & 76.2 & 298 & CGC & [1995CHI/HOS] \\
\hline & $\mathrm{V}$ & $(363-413)$ & 76.4 & 298 & CGC & [1995CHI/HOS] \\
\hline & $\mathrm{V}$ & & 76.8 & 298 & & [1994RUZ/MAJ] \\
\hline & $\mathrm{V}$ & $(366-409)$ & 67.5 & 381 & A & [1987STE/MAL] \\
\hline & $\mathrm{V}$ & $(333-409)$ & 66.4 & 350 & GS & [1986ALL/JOS] \\
\hline & $\mathrm{V}$ & & $75.4 \pm 1.2$ & 298 & $\mathrm{C}$ & [1979SUN/SVE] \\
\hline & $\mathrm{V}$ & & 70.8 & 353 & $\mathrm{C}$ & [1979SUN/SVE] \\
\hline & V & & 68.8 & 373 & $\mathrm{C}$ & [1979SUN/SVE] \\
\hline & $\mathrm{V}$ & & $72.2 \pm 1.2$ & 333 & $\mathrm{C}$ & [1979SUN/SVE] \\
\hline & $\mathrm{V}$ & & $76.2 \pm 0.4$ & 298 & $\mathrm{C}$ & [1972MOR2] \\
\hline & $\mathrm{V}$ & & 76.2 & 298 & & [1971WIL/ZWO] \\
\hline & $\mathrm{V}$ & $(447-546)$ & 59.6 & 462 & A & [1987STE/MAL, 1955CAM/ROS] \\
\hline & $\mathrm{V}$ & $(430-464)$ & 61.9 & 447 & ME & {$[1938 \mathrm{UBB}]$} \\
\hline \multirow[t]{2}{*}{$\mathrm{C}_{15} \mathrm{H}_{32}$} & {$[1560-95-8]$} & \multirow{2}{*}{$\begin{array}{l}\text { 2-methyltetradecane } \\
\quad(402-537)\end{array}$} & & & & \\
\hline & $\mathrm{V}$ & & 58.8 & 417 & A & [1987STE/MAL] \\
\hline \multirow[t]{2}{*}{$\mathrm{C}_{15} \mathrm{H}_{32}$} & {$[18435-22-8]$} & \multirow{2}{*}{$\begin{array}{l}\text { 3-methyltetradecane } \\
\quad(403-538)\end{array}$} & & & & \\
\hline & $\mathrm{V}$ & & 58.4 & 418 & A & [1987STE/MAL] \\
\hline \multirow[t]{2}{*}{$\mathrm{C}_{15} \mathrm{H}_{32}$} & {$[25117-24-2]$} & \multirow{2}{*}{$\begin{array}{l}\text { 4-methyltetradecane } \\
\text { (398-536) }\end{array}$} & & & & \\
\hline & $\mathrm{V}$ & & 55.9 & 413 & A & [1987STE/MAL] \\
\hline \multirow[t]{2}{*}{$\mathrm{C}_{15} \mathrm{H}_{32}$} & {$[25117-32-2]$} & \multirow{2}{*}{$\begin{array}{l}\text { 5-methyltetradecane } \\
\text { (398-535) }\end{array}$} & & & & \\
\hline & $\mathrm{V}$ & & 56.1 & 413 & A & [1987STE/MAL] \\
\hline \multirow[t]{2}{*}{$\mathrm{C}_{15} \mathrm{H}_{32}$} & {$[18435-20-6]$} & \multirow{2}{*}{$\begin{array}{l}\text { 2,3-dimethyltridecane } \\
\quad \text { (399-537) }\end{array}$} & & & & \\
\hline & $\mathrm{V}$ & & 56.3 & 414 & A & [1987STE/MAL] \\
\hline \multirow[t]{2}{*}{$\mathrm{C}_{15} \mathrm{H}_{32}$} & {$[61868-05-1]$} & \multirow{2}{*}{$\begin{array}{l}\text { 2,4-dimethyltridecane } \\
\quad(393-523)\end{array}$} & & & & \\
\hline & $\mathrm{V}$ & & 57.9 & 408 & A & [1987STE/MAL] \\
\hline \multirow[t]{2}{*}{$\mathrm{C}_{15} \mathrm{H}_{32}$} & {$[103387-11-7]$} & \multicolumn{2}{|c|}{ 2,4,6-trimethyldodecane } & & & \\
\hline & V & $(382-508)$ & 55.8 & 397 & A & [1987STE/MAL] \\
\hline \multirow[t]{4}{*}{$\mathrm{C}_{15} \mathrm{H}_{32} \mathrm{~N}_{2} \mathrm{O}$} & [32954-73-7] & 1-tetradecyl urea & & & & \\
\hline & TRS & & 1.0 & 227.1 & & \\
\hline & TRS & & 1.7 & 369.2 & & \\
\hline & FUS & & 50.9 & 387.4 & DSC & [2005HAS/TAJ] \\
\hline $\mathrm{C}_{15} \mathrm{H}_{32} \mathrm{O}$ & {$[629-76-5]$} & 1-pentadecanol & & & & \\
\hline & FUS & & 29.6 & 316.4 & DSC & [2004VEN/CAL] \\
\hline & FUS & $(298-380)$ & 53.62 & 316.9 & & [2003VAN/VAN] \\
\hline [Note: The v & 53.62 includes bo & the enthalpy of fusion & as well as $\mathrm{t}$ & halpy of & -to-solid t & sition that occurs at $315.4 \mathrm{~K}]$. \\
\hline & $\operatorname{TRS}(\beta$ to $\alpha)$ & & 23.64 & 316 & & \\
\hline & FUS $(\alpha)$ & & 30.35 & 316.9 & & \\
\hline & FUS $(\beta)$ & & 54.73 & 316.6 & $\mathrm{AC}$ & [1974MOS/MOU] \\
\hline & $\mathrm{V}$ & & $103.5 \pm 3.3$ & 298 & CGC & [2006NIC/KWE] \\
\hline & $\mathrm{V}$ & $(319-358)$ & 95.5 & 339 & GS & [2001KUL/VER2] \\
\hline & $\mathrm{V}$ & $(319-358)$ & 102.5 & 298 & GS & [2001KUL/VER2] \\
\hline & $\mathrm{V}$ & $(353-393)$ & 107.2 & 298 & CGC & [1994KOU/HOS, 2000OVA/KOU] \\
\hline & $\mathrm{V}$ & $(343-393)$ & 92.4 & 368 & & [1992NGU/KAS] \\
\hline & $\mathrm{V}$ & $(438-600)$ & 75.0 & 453 & A & [1987STE/MAL] \\
\hline & $\mathrm{V}$ & $(453-584)$ & 72.4 & 468 & A & [1987STE/MAL] \\
\hline
\end{tabular}


TABLE 12. Phase change enthalpies of $\mathrm{C}_{14}$ to $\mathrm{C}_{18}$ organic compounds-Continued

\begin{tabular}{|c|c|c|c|c|c|c|}
\hline \multirow[b]{2}{*}{$\begin{array}{l}\text { Molecular } \\
\text { formula }\end{array}$} & \multirow{2}{*}{$\begin{array}{l}\text { CAS Registry } \\
\text { Number } \\
\text { Enthalpy }\end{array}$} & \multicolumn{5}{|l|}{ Compound } \\
\hline & & $\begin{array}{c}\text { Temperature } \\
\text { range }\end{array}$ & $\begin{array}{l}\Delta_{\text {trans }} H_{\mathrm{m}} \\
(\mathrm{kJ} / \mathrm{mol})\end{array}$ & $\begin{array}{l}T_{\mathrm{m}} \\
(\mathrm{K})\end{array}$ & Method & References \\
\hline \multirow[t]{9}{*}{$\mathrm{C}_{15} \mathrm{H}_{32} \mathrm{O}_{2}$} & {$[14722-40-8]$} & \multicolumn{5}{|c|}{ 1,15-pentadecanediol } \\
\hline & TRS & & 38.3 & 349.0 & & \\
\hline & FUS & & 27.0 & 361.2 & DSC & [2014BAD/NOW] \\
\hline & TRS & & 33.0 & 346.2 & & \\
\hline & FUS & & 24.2 & 364.0 & DSC & [2009EGO/MAR] \\
\hline & TRS & & 35.1 & 349.4 & & \\
\hline & FUS & & 23.6 & 361.4 & DSC & [1999OGA/NAK] \\
\hline & V & & $124.3 \pm 3.8$ & 390 & $\mathrm{TE}$ & [1994PIA/FER, 2006UMN/KWE] \\
\hline & V & & $139.2 \pm 4.4$ & 298 & $\mathrm{TE}$ & [1994PIA/FER, 2006UMN/KWE] \\
\hline \multirow[t]{3}{*}{$\mathrm{C}_{15} \mathrm{H}_{32} \mathrm{O}_{2} \mathrm{~S}$} & {$[18023-86-4]$} & \multicolumn{5}{|c|}{ 3-(dodecylthio)-1,2-propanediol } \\
\hline & TRS & & 18.1 & 299 & & \\
\hline & FUS & & 20.3 & 325.5 & DSC & [1993ACR, 1990VAN/VAN] \\
\hline \multirow[t]{2}{*}{$\mathrm{C}_{15} \mathrm{H}_{32} \mathrm{O}_{3}$} & {$[1561-07-5]$} & \multicolumn{5}{|c|}{ 3-(dodecyloxy)-1,2-propanediol } \\
\hline & FUS & & 51.4 & 323 & $\mathrm{DSC}$ & [1993ACR, 1990VAN/VAN] \\
\hline \multirow[t]{2}{*}{$\mathrm{C}_{15} \mathrm{H}_{32} \mathrm{O}_{4}$} & {$[4161-34-6]$} & \multicolumn{5}{|c|}{ 5,5'-[1,5-pentanediylbis(oxy)]bis-1-pentanol } \\
\hline & FUS & & 35.66 & 302.7 & DSC & [1991BED/BOO] \\
\hline \multirow[t]{2}{*}{$\mathrm{C}_{15} \mathrm{H}_{32} \mathrm{O}_{5}$} & & \multicolumn{5}{|c|}{ Tetrapropylene glycol monoisopropyl ether } \\
\hline & $\mathrm{V}$ & $(389-566)$ & 71.5 & 404 & A & [1987STE/MAL, 1947STU] \\
\hline \multirow[t]{2}{*}{$\mathrm{C}_{15} \mathrm{H}_{32} \mathrm{O}_{5} \mathrm{~S}_{2}$} & & \multicolumn{5}{|c|}{$(L)$-arabinose dipentyl dithioacetal } \\
\hline & FUS & & 37.3 & 368 & DSC & [1989VAN/VAN] \\
\hline \multirow[t]{2}{*}{$\mathrm{C}_{15} \mathrm{H}_{32} \mathrm{~S}$} & {$[25276-70-4]$} & \multicolumn{5}{|c|}{ 1-pentadecanethiol } \\
\hline & V & $(459-629)$ & 69.8 & 474 & & [1999DYK/SVO] \\
\hline \multirow[t]{2}{*}{$\mathrm{C}_{15} \mathrm{H}_{33} \mathrm{~N}$} & {$[2570-26-5]$} & 1-aminopentad & & & & \\
\hline & V & $(400-594)$ & 71.2 & 415 & $\mathrm{~A}, \mathrm{E}$ & [1987STE/MAL, 1956MAN2] \\
\hline $\mathrm{C}_{15} \mathrm{H}_{33} \mathrm{NO}_{2}$ & {$[821-91-0]$} & 3-(dodecylami & ,2-propaned & & & \\
\hline & FUS & & 62.1 & 351.9 & DSC & [1993ACR, 1990VAN/VAN] \\
\hline $\mathrm{C}_{15} \mathrm{H}_{33} \mathrm{O}_{4} \mathrm{P}$ & {$[2528-38-3]$} & Tripentyl phos & & & & \\
\hline & $\mathrm{V}$ & $(443-473)$ & 92.3 & 298 & CGC & [2007PAN/ANT2] \\
\hline & $\mathrm{V}$ & $(443-483)$ & 90.7 & 298 & CGC & [2007PAN/ANT2] \\
\hline $\mathrm{C}_{15} \mathrm{H}_{33} \mathrm{O}_{4} \mathrm{P}$ & {$[919-62-0]$} & Triisopentyl pl & late & & & \\
\hline & $\mathrm{V}$ & $(453-493)$ & 86.6 & 298 & CGC & [2007PAN/ANT2] \\
\hline & $\mathrm{V}$ & $(483-513)$ & 86.5 & 298 & CGC & [2007PAN/ANT2] \\
\hline $\mathrm{C}_{15} \mathrm{H}_{33} \mathrm{O}_{4} \mathrm{P}$ & [646521-37-1] & Tri-sec-pentyl & phate & & & \\
\hline & $\mathrm{V}$ & $(463-493)$ & 80.7 & 298 & $\mathrm{CGC}$ & [2007PAN/ANT2] \\
\hline & $\mathrm{V}$ & $(453-493)$ & 81.5 & 298 & CGC & [2007PAN/ANT2] \\
\hline $\mathrm{C}_{15} \mathrm{H}_{33} \mathrm{O}_{4} \mathrm{P}$ & {$[45241-53-0]$} & Tri-(2-methylb & phosphate & & & \\
\hline & $\mathrm{V}$ & $(463-493)$ & 86.1 & 298 & CGC & [2007PAN/ANT2] \\
\hline & $\mathrm{V}$ & $(493-523)$ & 86.7 & 298 & CGC & [2007PAN/ANT2] \\
\hline $\mathrm{C}_{16} \mathrm{~F}_{34}$ & [355-49-7] & $n$-perfluorohex & ane & & & \\
\hline & FUS & & 38.7 & 399.7 & DSC & [2012HAS/DRA] \\
\hline & FUS & & 37 & 401.8 & DSC & [1999VIS/TER] \\
\hline & TRS & & 3.2 & 174.6 & & \\
\hline & TRS & & 1.5 & 183.9 & & \\
\hline & FUS & & 37.3 & 400.0 & DSC & [1994JIN/BOL] \\
\hline & TRS & $(5-320)$ & 0.87 & 175.5 & & \\
\hline & TRS & $(5-320)$ & 1.88 & 177.3 & & \\
\hline & TRS & $(5-320)$ & 1.16 & 186.9 & $\mathrm{AC}$ & [1994LEB/BYK] \\
\hline & TRS & $(5-320)$ & 0.87 & 175.5 & & \\
\hline & TRS & $(5-320)$ & 1.88 & 177.3 & $\mathrm{AC}$ & [1993LEB/BYK] \\
\hline & TRS & & 1.13 & 176.5 & & \\
\hline & TRS & & 3.01 & 177.7 & & \\
\hline & TRS & & 1.89 & 186.7 & & \\
\hline & FUS & & U 61.09 & 402.2 & DSC & [1986STA] \\
\hline
\end{tabular}


TABLE 12. Phase change enthalpies of $\mathrm{C}_{14}$ to $\mathrm{C}_{18}$ organic compounds-Continued

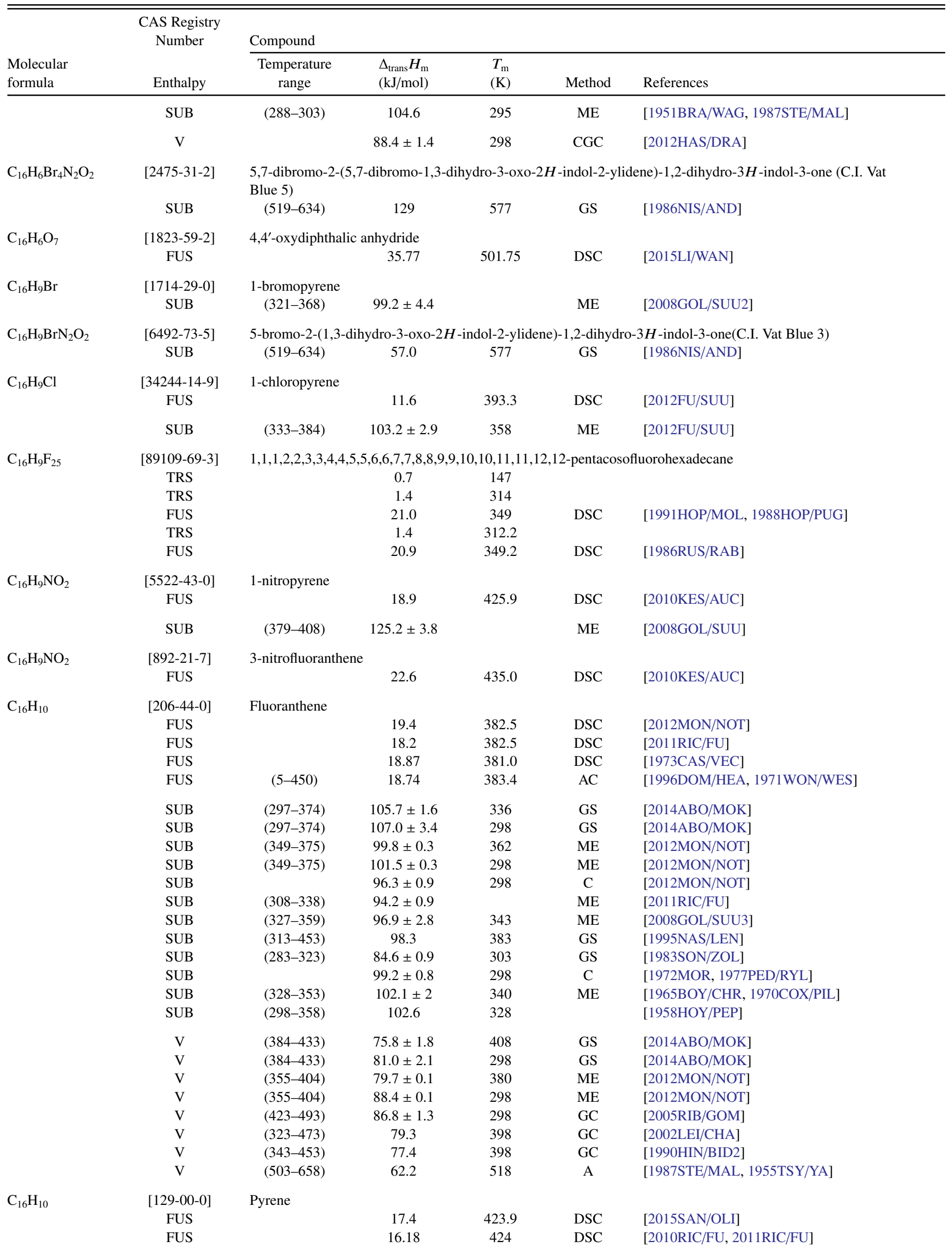


TABLE 12. Phase change enthalpies of $\mathrm{C}_{14}$ to $\mathrm{C}_{18}$ organic compounds-Continued

\begin{tabular}{|c|c|c|c|c|c|c|}
\hline \multirow[b]{2}{*}{$\begin{array}{l}\text { Molecular } \\
\text { formula }\end{array}$} & \multirow{2}{*}{$\begin{array}{l}\text { CAS Registry } \\
\text { Number } \\
\text { Enthalpy }\end{array}$} & \multicolumn{5}{|l|}{ Compound } \\
\hline & & $\begin{array}{c}\text { Temperature } \\
\text { range }\end{array}$ & $\begin{array}{l}\Delta_{\text {trans }} H_{\mathrm{m}} \\
(\mathrm{kJ} / \mathrm{mol})\end{array}$ & $\begin{array}{l}T_{\mathrm{m}} \\
(\mathrm{K})\end{array}$ & Method & References \\
\hline & FUS & & 17.4 & 422.2 & DSC & [2004GUP/SIN] \\
\hline & FUS & $(403-433)$ & 16.7 & 422.4 & DSC & [2003ROJ/ORO] \\
\hline & FUS & & 17.28 & 425.4 & DSC & [1980KRA/PIG] \\
\hline & FUS & & 15.3 & & DSC & [1972WAU/GET] \\
\hline & TRS & & 0.29 & 120.8 & & \\
\hline & FUS & $(5-479)$ & 17.36 & 423.8 & $\mathrm{AC}$ & [1996DOM/HEA, 1971WON/WES] \\
\hline & SUB & $(345-369)$ & $98.3 \pm 0.6$ & 356 & ME & [2015SAN/OLI] \\
\hline & SUB & $(345-369)$ & $100.1 \pm 0.6$ & 298 & ME & [2015SAN/OLI] \\
\hline & SUB & $(333-375)$ & $97.1 \pm 1.2$ & & ME & [2011RIC/FU] \\
\hline & SUB & $(324-359)$ & 93.1 & 341 & ME & [2010FU/RIC] \\
\hline & SUB & $(315-378)$ & 97.1 & 346 & ME & [2010RIC/FU] \\
\hline & SUB & $(341-418)$ & $103.3 \pm 2.1$ & 380 & ME & [2009SID/SID] \\
\hline & SUB & $(341-418)$ & 104.5 & 298 & ME & [2009SID/SID] \\
\hline & SUB & $(322-381)$ & $97.8 \pm 3.3$ & 352 & $\mathrm{ME}$ & [2008GOL/SUU3] \\
\hline & SUB & & $98.5 \pm 1.0$ & 298 & DSC & [2003ROJ/ORO] \\
\hline & SUB & $(308-398)$ & $103.1 \pm 6.5$ & 353 & $\mathrm{ME}$ & [1998OJA/SUU] \\
\hline & SUB & $(313-453)$ & 97.9 & 383 & GS & [1995NAS/LEN] \\
\hline & SUB & $(369-383)$ & $100.3 \pm 0.3$ & 353 & PG & [1988SAS/JOS] \\
\hline & SUB & $(283-323)$ & $91.2 \pm 0.5$ & 303 & GS & [1983SON/ZOL] \\
\hline & SUB & $(398-423)$ & $100.2 \pm 0.4$ & 410 & IPM & [1980SMI/STE] \\
\hline & SUB & & $101.0 \pm 0.5$ & & $\mathrm{C}$ & [1974MAL/BAR] \\
\hline & SUB & $(348-419)$ & $100.8 \pm 1.5$ & & $\mathrm{ME}$ & {$[1974 \mathrm{MAL} / \mathrm{BAR}]$} \\
\hline & SUB & & 95.7 & & ME & [1953BRA/CLE2, 1977PED/RYL, 1970COX/PIL] \\
\hline & SUB & $(298-363)$ & 100.5 & 330 & ME & {$[1958 \mathrm{HOY} / \mathrm{PEP}]$} \\
\hline & SUB & $(345-358)$ & $100.1 \pm 1.7$ & 351 & $\mathrm{ME}$ & [1952INO/SHI] \\
\hline & V & & $92.4 \pm 1.1$ & 298 & CGC & [2008HAN/NUT] \\
\hline & $\mathrm{V}$ & $(423-493)$ & $87.2 \pm 1.3$ & 298 & GC & [2006TEO/BAR] \\
\hline & $\mathrm{V}$ & & 66.1 & 443 & DSC & [2003ROJ/ORO] \\
\hline & $\mathrm{V}$ & $(343-453)$ & 78.6 & 398 & $\mathrm{GC}$ & [1990HIN/BID2] \\
\hline & $\mathrm{V}$ & $(413-467)$ & 76 & 428 & & [1988SAS/JOS] \\
\hline & $\mathrm{V}$ & $(398-458)$ & 76.4 & 440 & IPM & [1980SMI/STE] \\
\hline & V & $(513-668)$ & 73 & 528 & A & [1987STE/MAL, 1955TSY/YA] \\
\hline \multirow[t]{2}{*}{$\mathrm{C}_{16} \mathrm{H}_{10} \mathrm{ClN}_{3} \mathrm{O}$} & {$[191978-95-7]$} & \multicolumn{5}{|c|}{ 1-[(2-chloro-3-pyridinyl)carbonyl]-1,2-dihydro-2-quinolinecarbonitrile } \\
\hline & FUS & & 50.89 & 489.8 & DSC & {$[2005 \mathrm{LIZ} / \mathrm{ZAB}]$} \\
\hline \multirow[t]{3}{*}{$\mathrm{C}_{16} \mathrm{H}_{10} \mathrm{~N}_{2} \mathrm{O}_{2}$} & {$[482-89-3]$} & \multicolumn{5}{|c|}{ 2-(1,3-dihydro-3-oxo-2 $\boldsymbol{H}$-indol-2-ylidene)-1,2-dihydro-3H-indol-3-one (C.I. Vat Blue 1$)$} \\
\hline & SUB & (519-634) & 136 & 577 & GS & {$[1986 \mathrm{NIS} / \mathrm{AND}]$} \\
\hline & SUB & & 148.2 & 298 & & [1986NIS/AND, 2014MIR/CHI] \\
\hline \multirow[t]{2}{*}{$\mathrm{C}_{16} \mathrm{H}_{10} \mathrm{O}$} & {$[5315-79-7]$} & \multicolumn{5}{|c|}{ 1-hydroxypyrene } \\
\hline & SUB & $(369-394)$ & $129.0 \pm 3.2$ & 382 & ME & {$[19980 J A / S U U]$} \\
\hline \multirow[t]{3}{*}{$\mathrm{C}_{16} \mathrm{H}_{10} \mathrm{O}$} & {$[243-24-3]$} & \multicolumn{5}{|c|}{ 2,3,5,6-dibenzoxalene (benz $[b]$ indeno $[1,2-e]$ pyran) } \\
\hline & SUB & (375-388) & 125.9 & 381.5 & A & [1987STE/MAL] \\
\hline & SUB & & $129.4 \pm 1.3$ & & & [1966GEI/QUI, 1970COX/PIL] \\
\hline \multirow[t]{2}{*}{$\mathrm{C}_{16} \mathrm{H}_{10} \mathrm{O}$} & {$[955-83-9]$} & 2,5-diphenylfu & & & & \\
\hline & SUB & & 102 & 340 & HSA & {$[1989 \mathrm{SCH} / \mathrm{PEN}]$} \\
\hline \multirow[t]{2}{*}{$\mathrm{C}_{16} \mathrm{H}_{10} \mathrm{O}$} & {$[205-39-0]$} & Benzo $[b]$ naph & $1,2 d]$ furan & & & \\
\hline & FUS & & 13.7 & 315.9 & DSC & [2010KES/AUC] \\
\hline \multirow[t]{2}{*}{$\mathrm{C}_{16} \mathrm{H}_{10} \mathrm{O}$} & {$[239-30-5]$} & Benzo $[b]$ naph & $2,1 d]$ furan & & & \\
\hline & FUS & & 20.9 & 373.7 & DSC & [2010KES/AUC] \\
\hline \multirow[t]{2}{*}{$\mathrm{C}_{16} \mathrm{H}_{10} \mathrm{~S}$} & {$[239-35-0]$} & 1,2-benzodiph & ene sulfide & & & \\
\hline & SUB & $(325-373)$ & $111.9 \pm 1.2$ & 349 & ME & [1998OJA/SUU] \\
\hline \multirow[t]{2}{*}{$\mathrm{C}_{16} \mathrm{H}_{10} \mathrm{~S}$} & {$[205-43-6]$} & Dibenzo $[b] \mathrm{na}$ & o[ $[1,2 d]$ thiopn & & & \\
\hline & FUS & & 19.0 & 375.5 & $\mathrm{DSC}$ & [2010KES/AUC] \\
\hline \multirow[t]{2}{*}{$\mathrm{C}_{16} \mathrm{H}_{10} \mathrm{~S}_{4}$} & {$[5632-29-1]$} & \multicolumn{5}{|c|}{$2,2^{\prime}, 5^{\prime}, 2^{\prime \prime}, 5^{\prime \prime}, 2^{\prime \prime \prime}$-quaterthiophene } \\
\hline & SUB & $(383-413)$ & 132.6 & & $\mathrm{ME}$ & [1998KLO/LAU] \\
\hline
\end{tabular}


TABLE 12. Phase change enthalpies of $\mathrm{C}_{14}$ to $\mathrm{C}_{18}$ organic compounds-Continued

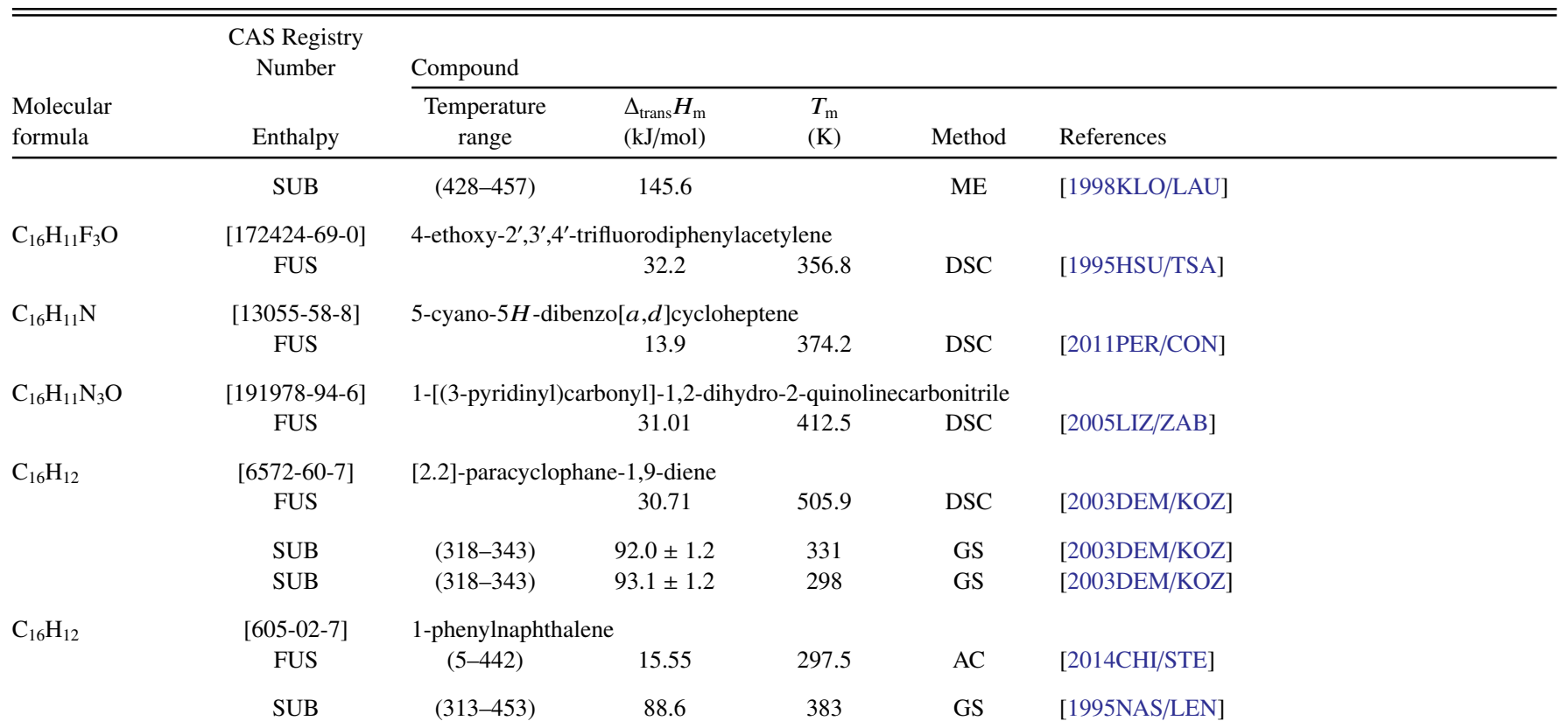

[Note: The authors of [1995NAS/LEN] label the value as an enthalpy of sublimation.]

$\begin{array}{lccccc}\mathrm{V} & (375-630) & 75.1 \pm 0.3 & 380 & \text { IPM,EB } & {[2014 \mathrm{CHI} / \mathrm{STE}]} \\ \mathrm{V} & (375-630) & 71.8 \pm 0.3 & 420 & \text { IPM,EB } & {[2014 \mathrm{CHI} / \mathrm{STE}]} \\ \mathrm{V} & (375-630) & 68.6 \pm 0.3 & 460 & \text { IPM,EB } & {[2014 \mathrm{CHI} / \mathrm{STE}]} \\ \mathrm{V} & (375-630) & 65.4 \pm 0.3 & 500 & \text { IPM,EB } & {[2014 \mathrm{CHI} / \mathrm{STE}]} \\ \mathrm{V} & (375-630) & 62.2 \pm 0.3 & 540 & \text { IPM,EB } & {[2014 \mathrm{CHI} / \mathrm{STE}]} \\ \mathrm{V} & (375-630) & 58.8 \pm 0.5 & 580 & \text { IPM,EB } & {[2014 \mathrm{CHI} / \mathrm{STE}]} \\ \mathrm{V} & (375-630) & 55.2 \pm 0.8 & 620 & \text { IPM,EB } & {[2014 \mathrm{CHI} / \mathrm{STE}]} \\ \mathrm{V} & & 81.1 \pm 1.8 & 298 & \mathrm{C} & {[2008 \mathrm{ROU} / \mathrm{LIM}]}\end{array}$

$\begin{array}{cccccc}\mathrm{C}_{16} \mathrm{H}_{12} & \text { [612-94-2] } & \text { 2-phenylnaphthalene } & & & \\ \text { FUS } & (5-442) & 22.62 & 374.8 & \text { AC } & \text { [2014CHI/STE] } \\ \text { FUS } & & 17.9 & 373.5 & \text { DSC } & {[2008 \text { ROU/LIM] }} \\ \text { SUB } & (333-353) & 106.6 \pm 0.4 & 343 & \text { ME } & {[2008 \text { ROU/LIM] }} \\ \text { SUB } & (333-353) & 107.6 \pm 0.6 & 298 & \text { ME } & {[2008 \text { ROU/LIM] }} \\ \text { V } & (483-604) & 69.3 \pm 0.3 & 500 & \text { EB } & {[2014 \mathrm{CHI} / \mathrm{STE}]} \\ \text { V } & (483-604) & 66.2 \pm 0.3 & 540 & \text { EB } & {[2014 \mathrm{CHI} / \mathrm{STE}]} \\ \text { V } & (483-604) & 63.0 \pm 0.4 & 580 & \text { EB } & {[2014 \mathrm{CHI} / \mathrm{STE}]}\end{array}$

\begin{tabular}{|c|c|c|c|c|c|}
\hline $\mathrm{C}_{16} \mathrm{H}_{12} \mathrm{ClN}_{5}$ & [142740-67-8] & 2-benzoylpyridine $6^{\prime}$-chloro- $3^{\prime}$-pyrida & inylhydrazone & & \\
\hline & FUS & 35 & 440.3 & DSC & [2013PER/KAZ] \\
\hline $\mathrm{C}_{16} \mathrm{H}_{12} \mathrm{ClN}_{5}$ & [907968-02-9] & 2-benzoylpyridine $6^{\prime}$-chloro-4'-pyrimi & linylhydrazone & & \\
\hline & FUS & 34 & 456.3 & DSC & [2013PER/KAZ] \\
\hline $\mathrm{C}_{16} \mathrm{H}_{12} \mathrm{Cl}_{2} \mathrm{~N}_{2} \mathrm{O}_{2} \mathrm{~S}$ & {$[20098-72-0]$} & $N, N^{\prime}$-bis(4-chlorobenzoyl)carbamimi & dothioic acid, $\mathrm{m}$ & nethyl ester & \\
\hline & FUS & 14.14 & 455.3 & DSC & [2009PLA/LIZ] \\
\hline $\mathrm{C}_{16} \mathrm{H}_{12} \mathrm{~F}_{2}$ & [145698-42-6] & 4-ethyl-3',4'-difluorodiphenylacetylen & & & \\
\hline & FUS & 16.6 & 301.2 & DSC & [1995HSU/TSA] \\
\hline $\mathrm{C}_{16} \mathrm{H}_{12} \mathrm{~F}_{2} \mathrm{O}$ & [172424-66-7] & 4-ethoxy-2',4'-difluorodiphenylacetyle & & & \\
\hline & FUS & 27.0 & 343.4 & DSC & [1995HSU/TSA] \\
\hline $\mathrm{C}_{16} \mathrm{H}_{12} \mathrm{~N}_{2}$ & {$[6672-73-7]$} & 5-cyano-7H-dibenzo $[a, c]$ cyclohepter & -6-amine & & \\
\hline & FUS & 14.86 & 374.1 & DSC & [2013PER/CON] \\
\hline $\mathrm{C}_{16} \mathrm{H}_{12} \mathrm{~N}_{2} \mathrm{O}$ & [842-07-9] & 2-hydroxy-1-phenylazonaphthalene & & & \\
\hline & SUB & $(350-374) \quad 116.7 \pm 5.4$ & 362 & & [1984KRI] \\
\hline $\mathrm{C}_{16} \mathrm{H}_{12} \mathrm{~N}_{2} \mathrm{O}_{3}$ & [19803-53-3] & (3-methyl-2-quinoxalinyl)phenylmeth & anone $N, N^{\prime}$-dic & xide & \\
\hline & SUB & $153.8 \pm 1.8$ & 298 & $\mathrm{C}$ & [2007GOM/SOU] \\
\hline $\mathrm{C}_{16} \mathrm{H}_{12} \mathrm{O}_{2}$ & [134852-10-1] & 5-hydroxymethylene-5H-6,7-dihydro & libenzo $[a, c]$-cy & clohepten- 6 & -one \\
\hline & FUS & 16.9 & 357.7 & DSC & [2006PER/CON] \\
\hline
\end{tabular}


TABLE 12. Phase change enthalpies of $\mathrm{C}_{14}$ to $\mathrm{C}_{18}$ organic compounds-Continued

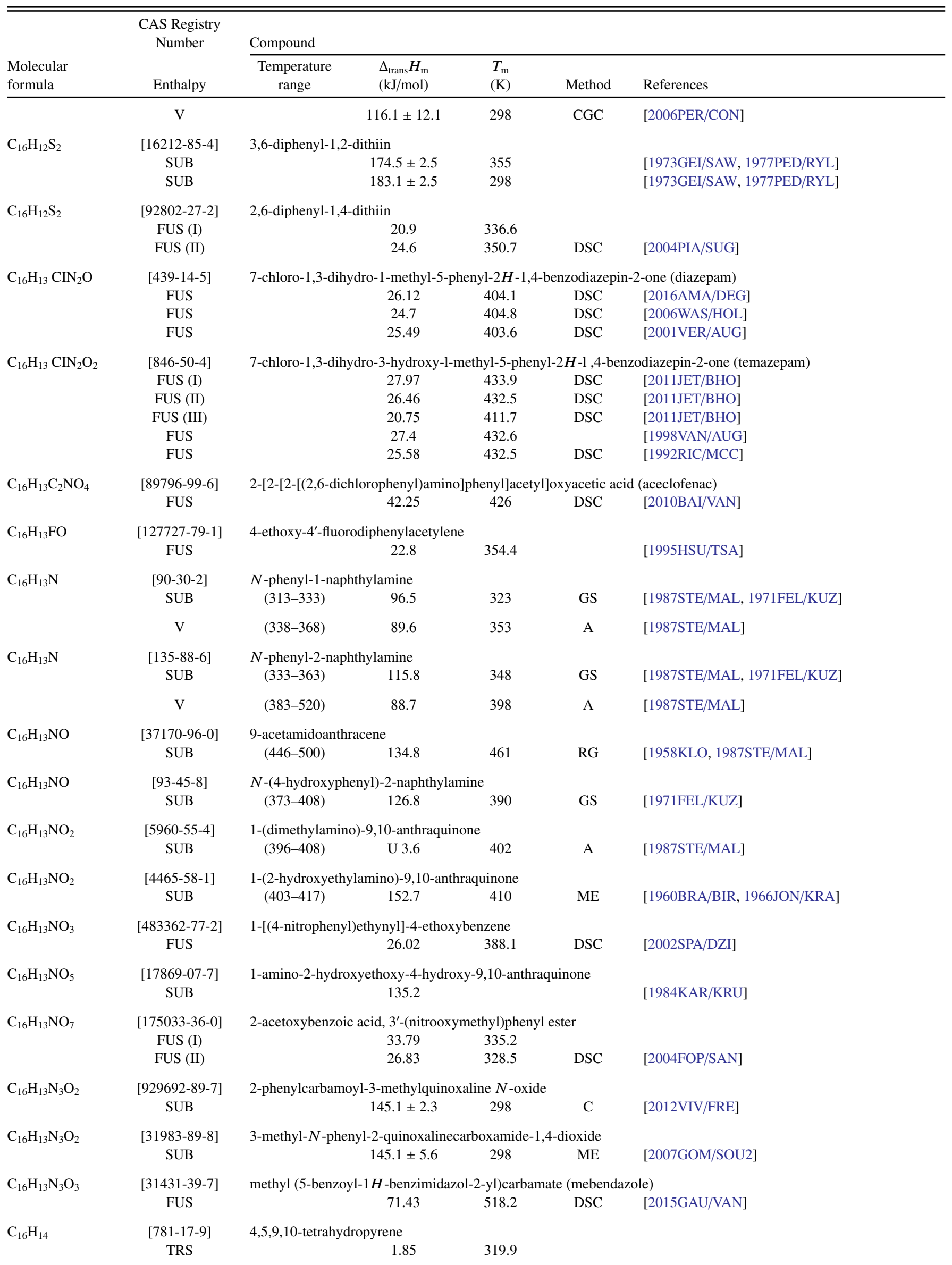


TABLE 12. Phase change enthalpies of $\mathrm{C}_{14}$ to $\mathrm{C}_{18}$ organic compounds-Continued

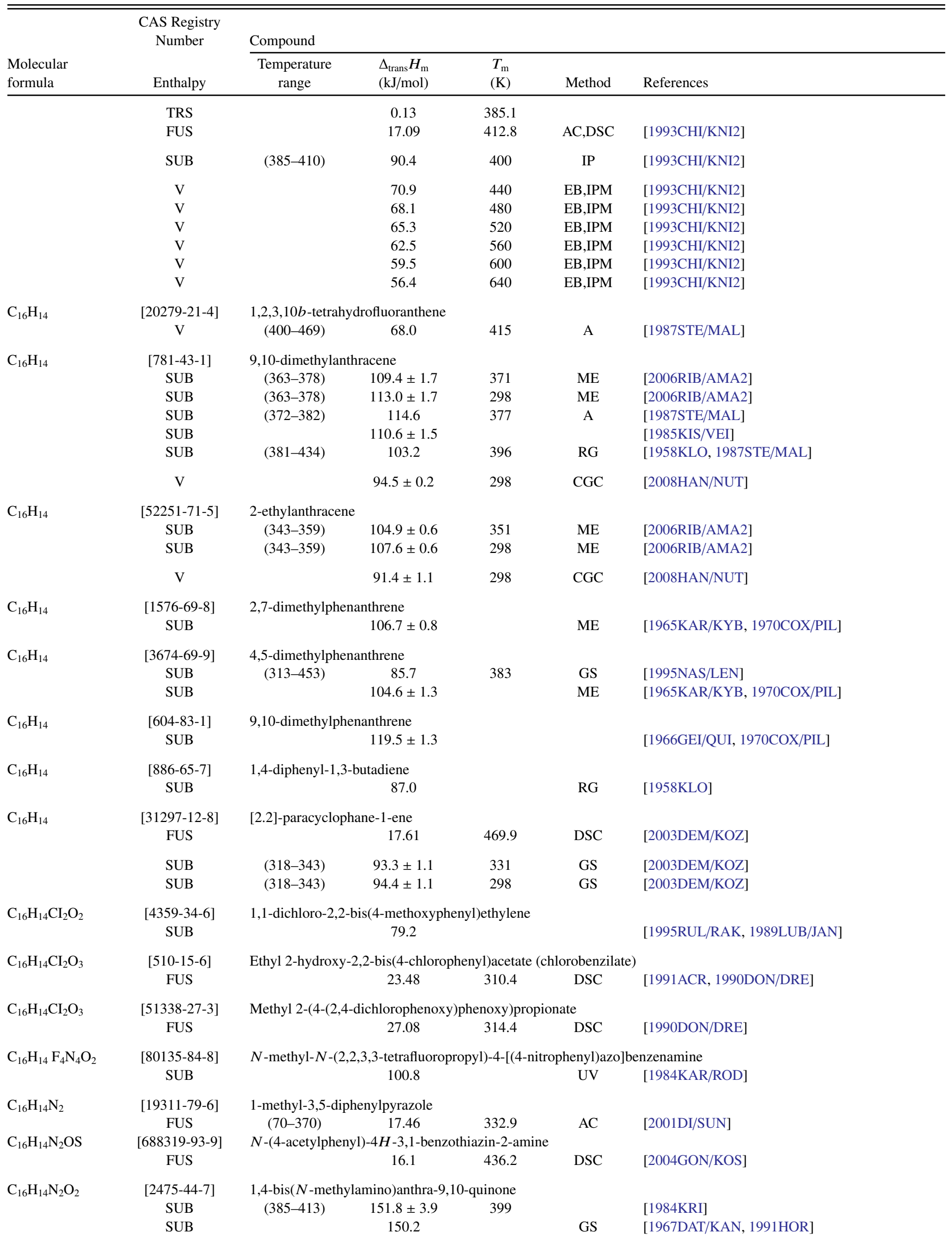


TABLE 12. Phase change enthalpies of $\mathrm{C}_{14}$ to $\mathrm{C}_{18}$ organic compounds-Continued

\begin{tabular}{|c|c|c|c|c|c|c|}
\hline \multirow[b]{2}{*}{$\begin{array}{l}\text { Molecular } \\
\text { formula }\end{array}$} & \multirow{2}{*}{$\begin{array}{l}\text { CAS Registry } \\
\text { Number } \\
\text { Enthalpy }\end{array}$} & \multicolumn{5}{|l|}{ Compound } \\
\hline & & $\begin{array}{l}\text { Temperature } \\
\text { range }\end{array}$ & $\begin{array}{l}\Delta_{\text {trans }} H_{\mathrm{m}} \\
(\mathrm{kJ} / \mathrm{mol})\end{array}$ & $\begin{array}{l}T_{\mathrm{m}} \\
(\mathrm{K})\end{array}$ & Method & References \\
\hline $\mathrm{C}_{16} \mathrm{H}_{14} \mathrm{~N}_{2} \mathrm{O}_{2}$ & $\begin{array}{c}\text { [65990-96-7] } \\
\text { SUB }\end{array}$ & \multicolumn{5}{|c|}{ 2-methyl-3-(phenylmethyl)quinoxaline-1,4-dioxide } \\
\hline $\mathrm{C}_{16} \mathrm{H}_{14} \mathrm{~N}_{2} \mathrm{O}_{2} \mathrm{~S}$ & $\begin{array}{c}\text { [19921-98-3] } \\
\text { FUS }\end{array}$ & \multicolumn{5}{|c|}{$N, N^{\prime}$-dibenzoylcarbamimidothioic acid, methyl ester } \\
\hline $\mathrm{C}_{16} \mathrm{H}_{14} \mathrm{~N}_{2} \mathrm{O}_{3} \mathrm{~S}$ & $\begin{array}{c}\text { [181695-72-7] } \\
\text { FUS }\end{array}$ & \multicolumn{5}{|c|}{ 4-(5-methyl-3-phenyl-4-isoxazolyl)benzenesulfonamide (valdecoxib) } \\
\hline $\mathrm{C}_{16} \mathrm{H}_{14} \mathrm{~N}_{4}$ & $\begin{array}{c}\text { [70845-34-0] } \\
\text { FUS }\end{array}$ & \multicolumn{5}{|c|}{ [1-(2-pyridinyl)ethylidene]hydrazone-(2-(1H)-quinolinone) } \\
\hline $\mathrm{C}_{16} \mathrm{H}_{14} \mathrm{~N}_{4} \mathrm{O}_{2}$ & $\begin{array}{c}\text { [340820-69-1] } \\
\text { FUS }\end{array}$ & 4-(4-methylphe & $\begin{array}{c}-5-(2-\text {-pyridin } y \\
38.2\end{array}$ & $\begin{array}{l}I-1,2,4- \\
423.4\end{array}$ & $\begin{array}{l}\text { e-3-carbo } \\
\text { DSC }\end{array}$ & $\begin{array}{l}\text { ic acid, methyl ester } \\
\text { [2005SIK/MOD] }\end{array}$ \\
\hline \multirow[t]{3}{*}{$\mathrm{C}_{16} \mathrm{H}_{14} \mathrm{O}$} & $\begin{array}{c}{[838-15-3]} \\
\text { FUS }\end{array}$ & \multicolumn{5}{|c|}{ 2,3:6,7-dibenzocycloocta-2,6-dien-1-one } \\
\hline & SUB & & $103.3 \pm 3.2$ & 298 & $\mathrm{~V}+\mathrm{F}$ & [2003PER/CON] \\
\hline & $\mathrm{V}$ & & $90.6 \pm 2.0$ & 298 & $\mathrm{CGC}$ & [2003PER/CON] \\
\hline \multirow[t]{3}{*}{$\mathrm{C}_{16} \mathrm{H}_{14} \mathrm{O}$} & $\begin{array}{c}{[6374-70-5]} \\
\text { FUS }\end{array}$ & \multicolumn{5}{|c|}{ 2,3: 7,8-dibenzocycloocta-2,7-dien-1-one } \\
\hline & SUB & & $112.8 \pm 4.1$ & 298 & $\mathrm{~V}+\mathrm{F}$ & [2003PER/CON] \\
\hline & $\mathrm{V}$ & & $92.0 \pm 2.9$ & 298 & $\mathrm{CGC}$ & {$[2003 \mathrm{PER} / \mathrm{CON}]$} \\
\hline $\mathrm{C}_{16} \mathrm{H}_{14} \mathrm{O}_{2}$ & $\begin{array}{c}{[103-41-3]} \\
\mathrm{V}\end{array}$ & $\begin{array}{l}\text { Benzyl cinnam } \\
\quad(446-623)\end{array}$ & 89.4 & 461 & A & [1987STE/MAL, 1947STU] \\
\hline $\mathrm{C}_{16} \mathrm{H}_{14} \mathrm{O}_{2}$ & $\begin{array}{c}\text { [495-71-6] } \\
\text { TRS } \\
\text { FUS }\end{array}$ & 1,2-dibenzoyle & $\begin{array}{c}0.22 \\
38.99\end{array}$ & $\begin{array}{c}187 \\
418.6\end{array}$ & $\mathrm{RC}$ & [1996DOM/HEA, 1932SPA/THO] \\
\hline $\mathrm{C}_{16} \mathrm{H}_{14} \mathrm{O}_{2}$ & $\begin{array}{c}\text { [2395-97-3] } \\
\text { SUB }\end{array}$ & 9,10-dimethox & $\begin{array}{l}\text { hracene } \\
123.0 \pm 2.0\end{array}$ & & & [1985KIS/VEI] \\
\hline $\mathrm{C}_{16} \mathrm{H}_{14} \mathrm{O}_{3}$ & $\begin{array}{c}\text { [22071-15-4] } \\
\text { FUS } \\
\text { FUS } \\
\text { FUS } \\
\text { FUS } \\
\text { FUS } \\
\text { FUS } \\
\text { FUS } \\
\text { FUS } \\
\text { FUS } \\
\text { FUS } \\
\text { FUS } \\
\text { FUS } \\
\text { FUS } \\
\text { FUS } \\
\text { FUS }\end{array}$ & $( \pm) \alpha-(3$-benzo $)$ & $\begin{array}{c}\text { nyl)propionic } \\
29.65 \\
44.5 \\
28.8 \\
45.5 \\
\mathrm{U} 87.3 \\
28.31 \\
20.3 \\
30.83 \\
26.0 \\
21.5 \\
28.4 \\
37.3 \\
27.38 \\
25.04 \\
28.23\end{array}$ & $\begin{array}{c}( \pm)-\mathrm{ke} \\
368.2 \\
369.8 \\
365.9 \\
369.4 \\
370 \\
368 \\
366.3 \\
369.2 \\
369.7 \\
366.2 \\
\\
368 \\
367.7 \\
369 \\
367.4\end{array}$ & $\begin{array}{l}\text { DSC } \\
\text { DSC } \\
\text { DSC } \\
\text { DSC } \\
\text { DSC } \\
\text { DSC } \\
\text { DSC } \\
\text { DSC } \\
\text { DSC } \\
\text { DSC } \\
\text { DSC } \\
\text { DSC } \\
\text { DSC }\end{array}$ & $\begin{array}{l}\text { [2015GAU/VAN] } \\
\text { [2014ARD/ELN] } \\
\text { [2013YAD/KUM] } \\
\text { [2013DIX/KUL] } \\
\text { [2011TIT/FUL] } \\
\text { [2010BAI/VAN] } \\
\text { [2010YUA/CAP] } \\
\text { [2010BAN/ARC] } \\
\text { [2009CIR/MAE] } \\
\text { [2009GAS/CEN] } \\
\text { [2007BLA/SCH] } \\
\text { [2006WAS/HOL, 2008WAS/HOL] } \\
\text { [2004LU/CHI] } \\
\text { [1998MUR/BET2, 1999MUR/FAU] } \\
\text { [1995ESP/BIS] }\end{array}$ \\
\hline & SUB & $(341-365)$ & $110.1 \pm 0.5$ & & GS & [2003PER/KUR2] \\
\hline $\mathrm{C}_{16} \mathrm{H}_{14} \mathrm{O}_{3}$ & $\begin{array}{c}{[22161-81-5]} \\
\text { FUS }\end{array}$ & $(+) \alpha-(3$-benzo $)$ & $\begin{array}{l}\text { nyl)propionic } \\
22.78\end{array}$ & $\begin{array}{l}((+)-\mathrm{ke} \\
348.6\end{array}$ & DSC & [2004LU/CHI] \\
\hline $\mathrm{C}_{16} \mathrm{H}_{14} \mathrm{O}_{3}$ & $\begin{array}{c}\text { [36330-85-5] } \\
\text { FUS } \\
\text { FUS } \\
\text { FUS } \\
\text { FUS }\end{array}$ & 3-(4-biphenyly & $\begin{array}{c}\text { onyl)propion } \\
73.84 \\
42.4 \\
41.1 \\
46.2\end{array}$ & $\begin{array}{l}\text { d fenbu } \\
459.1 \\
458.2 \\
462.9 \\
459.3\end{array}$ & $\begin{array}{l}\text { DSC } \\
\text { DSC } \\
\text { DSC } \\
\text { DSC }\end{array}$ & $\begin{array}{l}{[2011 \mathrm{DOM} / \mathrm{POB}]} \\
{[2009 \mathrm{GAS} / \mathrm{CEN}]} \\
{[2008 \mathrm{KUR} / \mathrm{PER}]} \\
{[2006 \mathrm{WAS} / \mathrm{HOL}]}\end{array}$ \\
\hline & SUB & $(378-420)$ & $154.9 \pm 0.8$ & 298 & GS & [2008KUR/PER] \\
\hline $\mathrm{C}_{16} \mathrm{H}_{14} \mathrm{O}_{4}$ & {$[5673-22-3]$} & 1,2-cis-dicarbo & loxyacenaphtl & & & \\
\hline
\end{tabular}


TABLE 12. Phase change enthalpies of $\mathrm{C}_{14}$ to $\mathrm{C}_{18}$ organic compounds-Continued

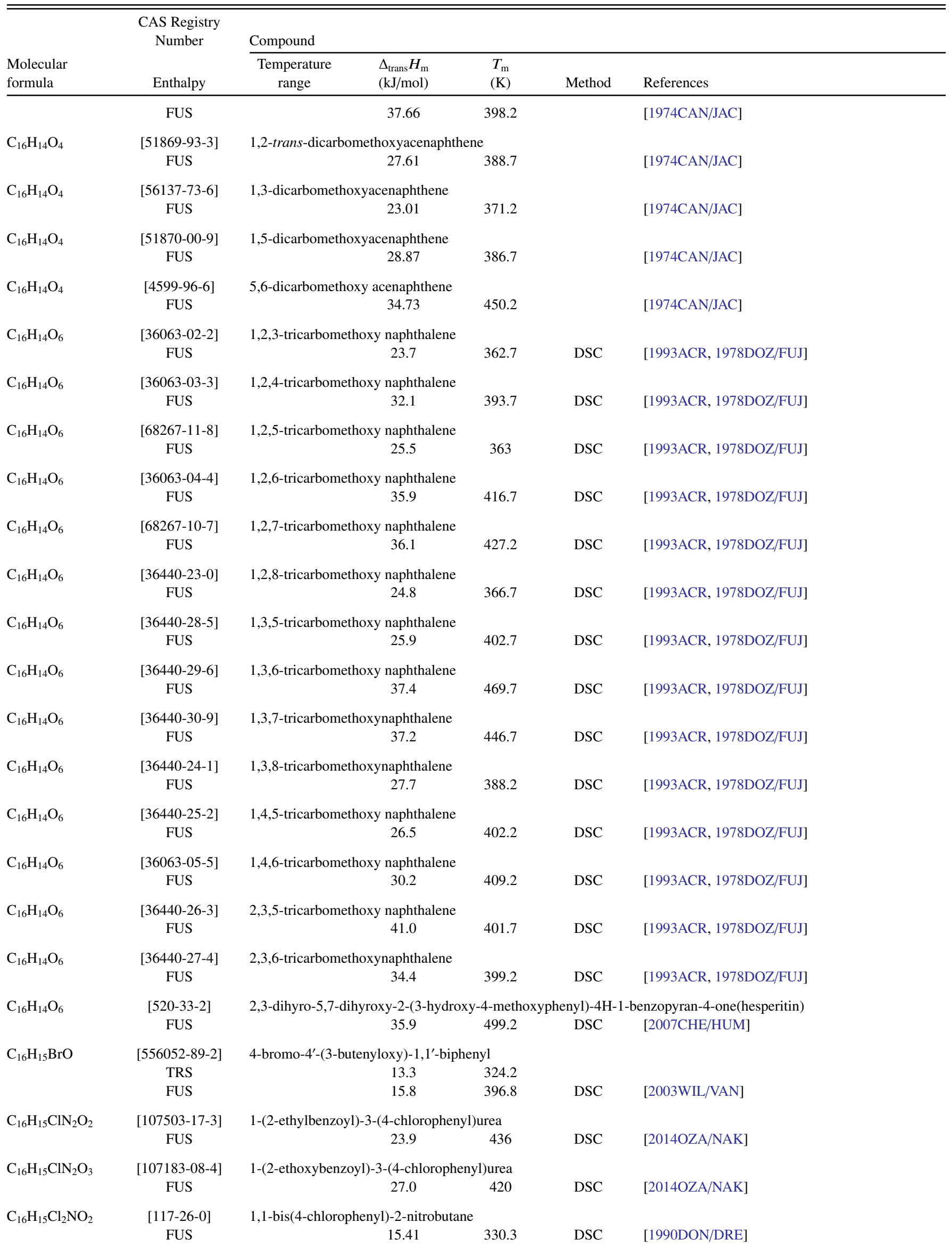


TABLE 12. Phase change enthalpies of $\mathrm{C}_{14}$ to $\mathrm{C}_{18}$ organic compounds-Continued

\begin{tabular}{|c|c|c|c|c|c|c|}
\hline \multirow[b]{2}{*}{$\begin{array}{l}\text { Molecular } \\
\text { formula }\end{array}$} & \multirow{2}{*}{$\begin{array}{l}\text { CAS Registry } \\
\text { Number } \\
\text { Enthalpy }\end{array}$} & \multicolumn{5}{|l|}{ Compound } \\
\hline & & $\begin{array}{c}\text { Temperature } \\
\text { range }\end{array}$ & $\begin{array}{l}\Delta_{\text {trans }} H_{\mathrm{m}} \\
(\mathrm{kJ} / \mathrm{mol})\end{array}$ & $\begin{array}{l}T_{\mathrm{m}} \\
(\mathrm{K})\end{array}$ & Method & References \\
\hline \multirow[t]{2}{*}{$\mathrm{C}_{16} \mathrm{H}_{15} \mathrm{Cl}_{3} \mathrm{O}_{2}$} & [30667-99-3] & \multicolumn{5}{|c|}{ 1-methoxy-2-(2,2,2-trichloro-1-(4-methoxyphenyl)ethyl)benzene } \\
\hline & FUS & & 22.45 & 347.6 & DSC & [1990DON/DRE] \\
\hline \multirow[t]{2}{*}{$\mathrm{C}_{16} \mathrm{H}_{15} \mathrm{CI}_{3} \mathrm{O}_{2}$} & {$[72-43-5]$} & \multicolumn{5}{|c|}{ 1,1'-(2,2,2-trichloroethylidene-bis(4-methoxy)benzene } \\
\hline & FUS & & 27.48 & 360.6 & DSC & [1990DON/DRE] \\
\hline \multirow{2}{*}{$\mathrm{C}_{16} \mathrm{H}_{15} \mathrm{IO}_{3} \mathrm{~S}$} & [313057-06-6] & \multicolumn{5}{|c|}{ 4-(4-pentenyloxy)phenyl 5-iodo-2-thiophene carboxylate } \\
\hline & FUS & & 68.2 & 332.7 & DSC & [2000WU/WAN] \\
\hline \multirow{4}{*}{$\mathrm{C}_{16} \mathrm{H}_{15} \mathrm{~N}$} & {$[58743-76-3]$} & \multicolumn{5}{|c|}{ 4'-propylbiphenyl-4-carbonitrile } \\
\hline & FUS & $(12-383)$ & 22.7 & 338.8 & $\mathrm{AC}$ & [1996DOM/HEA, 1991ASA/SOR] \\
\hline & FUS (I) & & 19.9 & 338.4 & & \\
\hline & FUS (II) & & 16.7 & 326.3 & $\mathrm{DSC}$ & [1983HAA/PAU] \\
\hline \multirow[t]{3}{*}{$\mathrm{C}_{16} \mathrm{H}_{15} \mathrm{~N}$} & [13228-39-2] & \multicolumn{5}{|c|}{ 1-ethyl-2-phenylindole } \\
\hline & SUB & $(327-349)$ & $113.4 \pm 0.6$ & 338 & $\mathrm{ME}$ & [2015CAR/AMA] \\
\hline & SUB & $(327-349)$ & $115.0 \pm 0.6$ & 298 & ME & [2015CAR/AMA] \\
\hline \multirow[t]{2}{*}{$\mathrm{C}_{16} \mathrm{H}_{15} \mathrm{NO}$} & [18594-93-9] & \multicolumn{5}{|c|}{ 3-anilino-1-phenylbut-2-enone } \\
\hline & SUB & & $126.8 \pm 3.0$ & 298 & $\mathrm{C}$ & [1993RIB/RIB] \\
\hline \multirow[t]{2}{*}{$\mathrm{C}_{16} \mathrm{H}_{15} \mathrm{NO}_{4}$} & {$[483362-66-9]$} & \multicolumn{5}{|c|}{ 2-(4-nitrophenyl)-1-(4-ethoxyphenyl)ethanone } \\
\hline & FUS & & 28.2 & 390.3 & DSC & [2002SPA/DZI] \\
\hline \multirow[t]{2}{*}{$\mathrm{C}_{16} \mathrm{H}_{15} \mathrm{~N}_{5}$} & [120356-36-7] & \multicolumn{5}{|c|}{ [1-(2-pyridinyl)propylidene]hydrazone-(1-(2H)phthalazinone) } \\
\hline & FUS & & 34 & 415.0 & DSC & [2013PER/KAZ] \\
\hline \multirow[t]{2}{*}{$\mathrm{C}_{16} \mathrm{H}_{15} \mathrm{~N}_{5} \mathrm{O}_{3}$} & [157892-00-7] & 6-phenyl-3,9-d & ro-3-[(2-hydr & hoxy)me & 9-охо-5H & nidazol[1,2-a]pyrine \\
\hline & FUS & & 63.78 & 485.8 & DSC & [1999ZIE/GOL] \\
\hline $\mathrm{C}_{16} \mathrm{H}_{16}$ & {$[1732-13-4]$} & $1,2,3,6,7,8-$ hex & Iropyrene & & & \\
\hline & TRS & & 5.02 & 377 & & \\
\hline & FUS & & 18.09 & 407.7 & $\mathrm{AC}, \mathrm{DSC}$ & [1993CHI/KNI2] \\
\hline & SUB & $(390-405)$ & 92.3 & 398 & IP & [1993CHI/KNI2] \\
\hline & $\mathrm{V}$ & & 72.0 & 440 & EB,IPM & [1993CHI/KNI2] \\
\hline & $\mathrm{V}$ & & 69.4 & 480 & EB,IPM & [1993CHI/KNI2] \\
\hline & $\mathrm{V}$ & & 66.8 & 520 & EB,IPM & [1993CHI/KNI2] \\
\hline & $\mathrm{V}$ & & 64.2 & 560 & EB,IPM & [1993CHI/KNI2] \\
\hline & $\mathrm{V}$ & & 61.5 & 600 & EB,IPM & [1993CHI/KNI2] \\
\hline $\mathrm{C}_{16} \mathrm{H}_{16}$ & {$[1633-22-3]$} & Tricyclo[8.2.2. & hexadeca-4,6, & $, 13,15-h$ & $\mathrm{e}([2.2]-p c$ & -cyclophane) \\
\hline & FUS & $(10-350)$ & 0.21 & 323.2 & $\mathrm{AC}$ & [1970AND/WES] \\
\hline & SUB & $(353-409)$ & $96.4 \pm 1.5$ & & TSGC & [1980NIS/SAK] \\
\hline & SUB & & $96.3 \pm 4.2$ & & & [1973ROD/WES, 1977PED/RYL] \\
\hline & SUB & $(343-383)$ & $92.9 \pm 0.8$ & 363 & ME & [1966BOY, 1987STE/MAL, 1970COX/PIL] \\
\hline $\mathrm{C}_{16} \mathrm{H}_{16}$ & [2319-97-3] & Tricyclo[9.3.1. & hexadeca-1(1 & $8(16), 1$ & lexaene ([2 & -meta-cyclophane) \\
\hline & FUS & & 21.42 & 404 & DSL & [1969SHI/MCN] \\
\hline & SUB & $(308-332)$ & $91.6 \pm 1.7$ & 320 & ME & [1969SHI/MCN, 1977PED/RYL, 1987STE/MAL] \\
\hline & SUB & & $92.0 \pm 2.0$ & 298 & ME & [1969SHI/MCN, 1977PED/RYL] \\
\hline $\mathrm{C}_{16} \mathrm{H}_{16}$ & {$[5385-36-4]$} & Tricyclo[9.2.2. & hexadeca-4,6, & $11,13,1$ & aene $([2.2]$ & eta-para-cyclophane) \\
\hline & TRS & & 0.98 & 315 & & \\
\hline & FUS & & 12.76 & 354 & $\mathrm{DSC}$ & {$[1969 \mathrm{SHI} / \mathrm{MCN}]$} \\
\hline & SUB & $(311-328)$ & 86.6 & 319 & ME & [1969SHI/MCN, 1977PED/RYL, 1987STE/MAL] \\
\hline & SUB & & $87.5 \pm 0.9$ & 298 & ME & [1969SHI/MCN, 1977PED/RYL] \\
\hline $\mathrm{C}_{16} \mathrm{H}_{16}$ & {$[2919-20-2]$} & 1,1-bis(4-meth & enyl)ethene & & & \\
\hline & FUS & & 23.31 & 334.1 & & [1999VER6] \\
\hline & SUB & $(309-332)$ & $100.3 \pm 1.4$ & 320 & GS & [1999VER6] \\
\hline & SUB & $(309-332)$ & $101.0 \pm 1.4$ & 298 & GS & {$[1999 \mathrm{VER} 6]$} \\
\hline $\mathrm{C}_{16} \mathrm{H}_{16} \mathrm{ClN}$ & $\begin{array}{c}{[113788-75-3]} \\
\text { FUS }\end{array}$ & 4-chlorobenzy & $\begin{array}{c}\text { e-4'-propylan } \\
24.61\end{array}$ & 343.7 & DSC & [1999GAL/COL] \\
\hline
\end{tabular}


TABLE 12. Phase change enthalpies of $\mathrm{C}_{14}$ to $\mathrm{C}_{18}$ organic compounds-Continued

\begin{tabular}{|c|c|c|c|c|c|c|}
\hline \multirow[b]{2}{*}{$\begin{array}{l}\text { Molecular } \\
\text { formula }\end{array}$} & \multirow{2}{*}{$\begin{array}{l}\text { CAS Registry } \\
\text { Number } \\
\text { Enthalpy }\end{array}$} & \multicolumn{5}{|l|}{ Compound } \\
\hline & & $\begin{array}{c}\text { Temperature } \\
\text { range }\end{array}$ & $\begin{array}{l}\Delta_{\text {trans }} H_{\mathrm{m}} \\
(\mathrm{kJ} / \mathrm{mol})\end{array}$ & $\begin{array}{l}T_{\mathrm{m}} \\
(\mathrm{K})\end{array}$ & Method & References \\
\hline \multirow[t]{3}{*}{$\mathrm{C}_{16} \mathrm{H}_{16} \mathrm{ClN}_{3} \mathrm{O}_{3} \mathrm{~S}$} & {$[26807-65-8]$} & \multicolumn{5}{|c|}{ 3-(aminosulfonyl)-4-chloro- $N$-(2,3-dihydro-2-methyl- $1 H$-indol-1-yl)-benzamide (indapamide) } \\
\hline & FUS & & 26.7 & 441.0 & DSC & [2015NUR/BOO] \\
\hline & FUS & & 57.18 & 462.2 & DSC & [2010GHU/DON] \\
\hline \multirow[t]{3}{*}{$\mathrm{C}_{16} \mathrm{H}_{16} \mathrm{~N}_{2} \mathrm{O}_{2}$} & {$[94-93-9]$} & \multicolumn{5}{|c|}{$N, N^{\prime}$-bis(salicylaldehydo)ethylenediimine } \\
\hline & FUS & & 34.09 & 397.9 & DSC & {$[2004 \mathrm{RIB} / \mathrm{GON}]$} \\
\hline & SUB & $(348-363)$ & $141.3 \pm 3.2$ & 298 & $\mathrm{ME}$ & [2004RIB/GON] \\
\hline \multirow[t]{2}{*}{$\mathrm{C}_{16} \mathrm{H}_{16} \mathrm{~N}_{2} \mathrm{O}_{2}$} & {$[2299-73-2]$} & \multicolumn{5}{|c|}{ 2-[(4-methoxyphenyl)methylene]hydrazone-4-methoxybenzaldehyde (anisaldazine) } \\
\hline & FUS & & 29.75 & 442 & DSC & [1996DOM/HEA, 1967BAR/POR] \\
\hline \multirow[t]{2}{*}{$\mathrm{C}_{16} \mathrm{H}_{16} \mathrm{~N}_{2} \mathrm{O}_{4}$} & {$[13684-56-5]$} & \multicolumn{5}{|c|}{ Ethyl [3-[[(phenylamino)carbonyl]oxy]phenylcarbamate] } \\
\hline & FUS & & 32.75 & 394.1 & DSC & [1990DON/DRE] \\
\hline \multirow[t]{5}{*}{$\mathrm{C}_{16} \mathrm{H}_{16} \mathrm{~N}_{2} \mathrm{O}_{3} \mathrm{~S}$} & {$[144060-53-7]$} & \multicolumn{5}{|c|}{ 2-(3-cyano-4-isobutoxyphenyl)-4-methyl-1,3-thiazole-5-carboxylic acid (febuxostat) } \\
\hline & FUS (I) & & 27.59 & 486.5 & & \\
\hline & FUS (II) & & 32.37 & 481.5 & & \\
\hline & FUS (III) & & 31.04 & 477.0 & DSC & {$[2015 \mathrm{PAT} / \mathrm{JAG}]$} \\
\hline & FUS & & 34.8 & 383.3 & DSC & {$[2015 \mathrm{PAN} / \mathrm{MEH}]$} \\
\hline \multirow[t]{2}{*}{$\mathrm{C}_{16} \mathrm{H}_{16} \mathrm{~N}_{2} \mathrm{O}_{4}$} & {$[13684-63-4]$} & \multicolumn{5}{|c|}{ Methyl 3-m-tolylcarbamoyloxyphenylcarbamate } \\
\hline & FUS & & 39.62 & 423.8 & $\mathrm{DSC}$ & [1990DON/DRE] \\
\hline \multirow[t]{2}{*}{$\mathrm{C}_{16} \mathrm{H}_{16} \mathrm{~N}_{2} \mathrm{O}_{4}$} & {$[54946-22-4]$} & \multicolumn{5}{|c|}{$N$-propylthalidomide } \\
\hline & FUS & & 27.28 & 409.2 & DSC & {$[2002 \mathrm{GOO} / \mathrm{LAI}]$} \\
\hline \multirow[t]{2}{*}{$\mathrm{C}_{16} \mathrm{H}_{16} \mathrm{O}$} & {$[130935-82-9]$} & \multicolumn{5}{|c|}{ 6-hydroxymethyl-5,6-dihydro-7H-dibenzo[ $a, c]$ cycloheptane } \\
\hline & FUS & & 25.9 & 405.7 & DSC & {$[2005 \mathrm{PER} / \mathrm{BAN}]$} \\
\hline \multirow[t]{2}{*}{$\mathrm{C}_{16} \mathrm{H}_{16} \mathrm{O}$} & {$[29817-04-7]$} & \multicolumn{5}{|c|}{ 5-hydroxymethyl-5,6-dihydro-7H-dibenzo $[a, c]$ cycloheptane } \\
\hline & FUS & & 16 & 352.5 & DSC & {$[2005 \mathrm{PER} / \mathrm{BAN}]$} \\
\hline \multirow[t]{2}{*}{$\mathrm{C}_{16} \mathrm{H}_{16} \mathrm{O}_{2}$} & {$[29783-24-2]$} & \multicolumn{5}{|c|}{ trans-9,10-bishydroxymethyl-9,10-dihydrophenanthrene } \\
\hline & FUS & & 30.3 & 450.8 & DSC & {$[2005 \mathrm{PER} / \mathrm{BAN}]$} \\
\hline \multirow[t]{2}{*}{$\mathrm{C}_{16} \mathrm{H}_{16} \mathrm{O}_{2}$} & {$[29790-58-7]$} & \multicolumn{5}{|c|}{ trans-5-hydroxymethyl-5,6-dihydro-7H-dibenzo[ $a, c]$ cycloheptan-6-ol } \\
\hline & FUS & & 31.8 & 460.2 & DSC & {$[2005 \mathrm{PER} / \mathrm{BAN}]$} \\
\hline \multirow[t]{2}{*}{$\mathrm{C}_{16} \mathrm{H}_{16} \mathrm{O}_{2}$} & & \multicolumn{5}{|c|}{ (d)-2-( $p$-methoxyphenyl)propiophenone } \\
\hline & FUS & & 21.76 & 326 & DSC & {$[1976 \mathrm{LEC} / \mathrm{COL}]$} \\
\hline \multirow[t]{2}{*}{$\mathrm{C}_{16} \mathrm{H}_{16} \mathrm{O}_{2}$} & & $(d l)-2-(p-$ meth & henyl)propio & & & \\
\hline & FUS & & 26.36 & 353 & $\mathrm{DSC}$ & [1976LEC/COL] \\
\hline $\mathrm{C}_{16} \mathrm{H}_{16} \mathrm{O}_{2}$ & [46863-20-1] & (2-hydroxyphe & 2,4,6-trimeth & nylmeth & & \\
\hline & FUS & & 0.49 & 353.2 & DTA & [1989SAL/ABA] \\
\hline
\end{tabular}

[Note: Reported enthalpy of fusion is too small, and the published enthalpy and entropy of fusion data are internally inconsistent.]

$$
\begin{aligned}
& \mathrm{C}_{16} \mathrm{H}_{16} \mathrm{O}_{3} \\
& \mathrm{C}_{16} \mathrm{H}_{16} \mathrm{O}_{3} \\
& \mathrm{C}_{16} \mathrm{H}_{16} \mathrm{O}_{10}
\end{aligned}
$$

$\mathrm{C}_{16} \mathrm{H}_{17} \mathrm{ClN}_{4} \mathrm{O}_{3}$

$\mathrm{C}_{16} \mathrm{H}_{17} \mathrm{ClN}_{4} \mathrm{O}_{4}$

$\mathrm{C}_{16} \mathrm{H}_{17} \mathrm{Cl}_{2} \mathrm{~N}_{5} \mathrm{O}_{4}$

$$
\begin{aligned}
& \text { [24650-42-8] } \\
& \text { FUS } \\
& \text { [7074-00-2] } \\
& \text { SUB } \\
& \text { [3327-06-8] } \\
& \text { FUS } \\
& \text { SUB } \\
& \text { SUB } \\
& \text { SUB } \\
& \text { [3180-81-2] } \\
& \text { SUB } \\
& \text { [4540-00-5] } \\
& \text { FUS }
\end{aligned}
$$

FUS 2,2-dimethoxy-1,2-diphenylethanone

$$
\begin{array}{lll}
20.86 & 338.5 & \text { DSC }
\end{array}
$$

2-phenylisopropoxybenzoate

$$
(293-313) \quad 43.1 \pm 4.2
$$$$
303 \quad \text { ME }
$$$$
\text { [1971KIP/RAB, 1977PED/RYL] }
$$

Pentamethoxycarbonylbenzene

$$
38.0
$$

$424.7 \quad$ DSC

[1978DOZ/FUJ]

$$
\begin{array}{ll}
(389-413) & 160.0 \pm 0.8 \\
(389-413) & 165.1 \pm 0.8
\end{array}
$$

$401 \quad \mathrm{ME}$

[1995JIM/MEN]

$165.1 \pm 0.8$

298

$\mathrm{ME}$

[1995JIM/MEN]

[1967TUR2, 1995JIM/MEN]

4-( $N$-ethyl- $N$-2-hydroxyethylamino)-4'-nitro-2'-chloroazobenzene

$$
142.7
$$

[1968TSU/KOJ, 1988BAU/PER]

2,2'-[[3-chloro-4-[(4-nitrophenyl)azo]phenyl]imino]bis-ethanol

$$
29.78 \quad 463.2 \quad \text { [1988BAU/PER] }
$$

1-[[2-chloro-4-[(2-chloro-4-nitrophenyl)azo]-5-(methylamino)phenyl]amino]-2-propanol $N$-oxide $30.62 \quad 371.2 \quad$ [1991BAU/WEB] 
TABLE 12. Phase change enthalpies of $\mathrm{C}_{14}$ to $\mathrm{C}_{18}$ organic compounds-Continued

\begin{tabular}{|c|c|c|c|c|c|c|}
\hline \multirow[b]{2}{*}{$\begin{array}{l}\text { Molecular } \\
\text { formula }\end{array}$} & \multirow{2}{*}{$\begin{array}{l}\text { CAS Registry } \\
\text { Number } \\
\text { Enthalpy }\end{array}$} & \multicolumn{5}{|l|}{ Compound } \\
\hline & & $\begin{array}{l}\text { Temperature } \\
\text { range }\end{array}$ & $\begin{array}{l}\Delta_{\text {trans }} H_{\mathrm{m}} \\
(\mathrm{kJ} / \mathrm{mol})\end{array}$ & $\begin{array}{l}T_{\mathrm{m}} \\
(\mathrm{K})\end{array}$ & Method & References \\
\hline \multirow[t]{3}{*}{$\mathrm{C}_{16} \mathrm{H}_{17} \mathrm{~F}$} & {$[193472-70-7]$} & \multicolumn{5}{|c|}{ 2-fluoro-2-methyl-1,3-diphenylpropane } \\
\hline & FUS & & 29.7 & 332.7 & & [1997SCH/VER] \\
\hline & SUB & & $102.2 \pm 1.1$ & 298 & & [1997SCH/VER] \\
\hline \multirow[t]{2}{*}{$\mathrm{C}_{16} \mathrm{H}_{17} \mathrm{~F}_{15} \mathrm{O}$} & {$[41049-15-4]$} & \multicolumn{5}{|c|}{$1,1,1,2,2,3,3,4,4,5,5,6,6,7,7$-pentadecafluoro-8-hexadecanone } \\
\hline & FUS & & 34.2 & 285.8 & DSC & [1992VIL/WEI] \\
\hline \multirow[t]{3}{*}{$\mathrm{C}_{16} \mathrm{H}_{17} \mathrm{~N}$} & {$[1484-08-8]$} & \multicolumn{5}{|c|}{$N$-butylcarbazole } \\
\hline & FUS & & 22.80 & 330.6 & DSC & [2016STA/KEI] \\
\hline & $\mathrm{V}$ & $(331-373)$ & $92.5 \pm 0.6$ & 298 & GS & [2015EME/VAR] \\
\hline \multirow[t]{2}{*}{$\mathrm{C}_{16} \mathrm{H}_{17} \mathrm{NO}$} & [36713-33-4] & \multicolumn{5}{|c|}{ 1,2-diphenyl-2- $N, N$-dimethylamino-1-ethanone } \\
\hline & SUB & & $140.1 \pm 1.9$ & & $\mathrm{~B}$ & [1994WEL/VER] \\
\hline \multirow{2}{*}{$\mathrm{C}_{16} \mathrm{H}_{17} \mathrm{NO}$} & [99081-88-6] & \multicolumn{5}{|c|}{$N$-(4-isopropylphenylmethylene)benzenamine $N$-oxide } \\
\hline & SUB & & $127.2 \pm 1.7$ & 298 & $\mathrm{C}$ & {$[1986 \mathrm{KIR} / \mathrm{ACR}]$} \\
\hline \multirow[t]{2}{*}{$\mathrm{C}_{16} \mathrm{H}_{17} \mathrm{NO}$} & {$[957-51-7]$} & \multicolumn{5}{|c|}{$N, N$-dimethyl-2,2-diphenylacetamide } \\
\hline & FUS & & 25.43 & 407.1 & DSC & [1991ACR, 1990DON/DRE] \\
\hline \multirow[t]{2}{*}{$\mathrm{C}_{16} \mathrm{H}_{17} \mathrm{~N}_{3} \mathrm{O}_{3}$} & [850836-66-7] & \multicolumn{5}{|c|}{ 6-(acetylamino)-2-cyano-1(2H)-quinolinecarboxylic acid, 1-methylethyl ester } \\
\hline & FUS & & 12.66 & 377.4 & DSC & {$[2005 \mathrm{LIZ} / \mathrm{ZAB}]$} \\
\hline \multirow[t]{2}{*}{$\mathrm{C}_{16} \mathrm{H}_{18}$} & {$[5080-10-4]$} & \multicolumn{5}{|c|}{ 1-(2-tolyl)-1-(4-tolyl)ethane } \\
\hline & V & $(298-473)$ & 85.6 & 313 & A & [1987STE/MAL, 1963BES] \\
\hline \multirow[t]{3}{*}{$\mathrm{C}_{16} \mathrm{H}_{18}$} & [719-79-9] & 1,1-diphenylbu & & & & \\
\hline & V & $(298-342)$ & $75.9 \pm 0.6$ & 320 & GS & [1999VER5] \\
\hline & $\mathrm{V}$ & $(298-342)$ & $77.2 \pm 0.6$ & 298 & GS & [1999VER5] \\
\hline $\mathrm{C}_{16} \mathrm{H}_{18}$ & {$[1520-44-1]$} & $(d l)$-1,3-diphe & tane & & & \\
\hline & SUB & (288-303) & 73.6 & 296 & $\mathrm{ME}$ & [1974PRI/POU, 1987STE/MAL] \\
\hline $\mathrm{C}_{16} \mathrm{H}_{18}$ & [5789-35-5] & 2,3-diphenylbu & & & & \\
\hline & SUB & (293-348) & 96.7 & 326 & & [1984BEC/RUC] \\
\hline $\mathrm{C}_{16} \mathrm{H}_{18}$ & [1634-11-3] & 2-methyl-1,1-c & nylpropane & & & \\
\hline & V & $(298-338)$ & $72.0 \pm 0.5$ & 318 & GS & [1999VER5] \\
\hline & V & (298-338) & $73.2 \pm 0.5$ & 298 & GS & [1999VER5] \\
\hline $\mathrm{C}_{16} \mathrm{H}_{18}$ & [530-45-0] & 1,1-bis(4-meth & enyl)ethane & & & \\
\hline & V & (298-338) & $75.3 \pm 0.6$ & 318 & GS & [1999VER5] \\
\hline & $\mathrm{V}$ & (298-338) & $76.5 \pm 0.6$ & 298 & GS & [1999VER5] \\
\hline $\mathrm{C}_{16} \mathrm{H}_{18}$ & [2113-60-2] & 3-tert-butylbip & & & & \\
\hline & V & $(323-361)$ & $77.1 \pm 0.2$ & 298 & GS & [2012NAZ/NES] \\
\hline & V & (486-537) & $82.3 \pm 0.3$ & 298 & $\mathrm{~EB}$ & [2012NAZ/NES] \\
\hline $\mathrm{C}_{16} \mathrm{H}_{18}$ & [1625-92-9] & 4-tert-butylbip & & & & \\
\hline & FUS & $(8-372)$ & 19.87 & 324.7 & $\mathrm{AC}$ & [2010VAR/EFI] \\
\hline & SUB & $(301-326)$ & $98.0 \pm 1.0$ & 298 & GS & [2010VAR/EFI] \\
\hline & SUB & & $98.1 \pm 2.1$ & 298 & $\mathrm{C}$ & [2009MEL/PIM] \\
\hline & $\mathrm{V}$ & $(448-593)$ & $84.0 \pm 0.4$ & 298 & GS & [2012NAZ/NES] \\
\hline & $\mathrm{V}$ & $(327-378)$ & $79.9 \pm 0.8$ & 298 & GS & [2010VAR/EFI] \\
\hline & $\mathrm{V}$ & & $80.0 \pm 1.9$ & 298 & $\mathrm{C}$ & [2009MEL/PIM] \\
\hline $\mathrm{C}_{16} \mathrm{H}_{18} \mathrm{Cl}_{4} \mathrm{O}_{4}$ & [3015-66-5] & Dibutyl tetracl & phthalate & & & \\
\hline & $\mathrm{V}$ & $(368-421)$ & 99.7 & 383 & $\mathrm{~A}, \mathrm{~T}$ & $\begin{array}{l}\text { [1987STE/MAL, 1949PER/WEB, } \\
\text { 1999DYK/SVO] }\end{array}$ \\
\hline $\mathrm{C}_{16} \mathrm{H}_{18} \mathrm{FN}_{3} \mathrm{O}_{3}$ & [70458-96-7] & 1-ethyl-6-fluor & 1-dihydro-4-o & 1-piper & -3-quinol & carboxylic acid (norfloxacin) \\
\hline & FUS & & 32.42 & 492.6 & DSC & [2009OLI/BER] \\
\hline & FUS (I) & & 39.1 & 492.2 & & \\
\hline & FUS (II) & & 41.5 & 480.2 & DSC & [2007BAR/PRO] \\
\hline & FUS & & 32.97 & 500.2 & DSC & [1994YU/ZIP] \\
\hline $\mathrm{C}_{16} \mathrm{H}_{18} \mathrm{NO}_{5}$ & [3788-15-6] & bis(2,4-dimeth & henyl)nitroge & & & \\
\hline
\end{tabular}


TABLE 12. Phase change enthalpies of $\mathrm{C}_{14}$ to $\mathrm{C}_{18}$ organic compounds-Continued

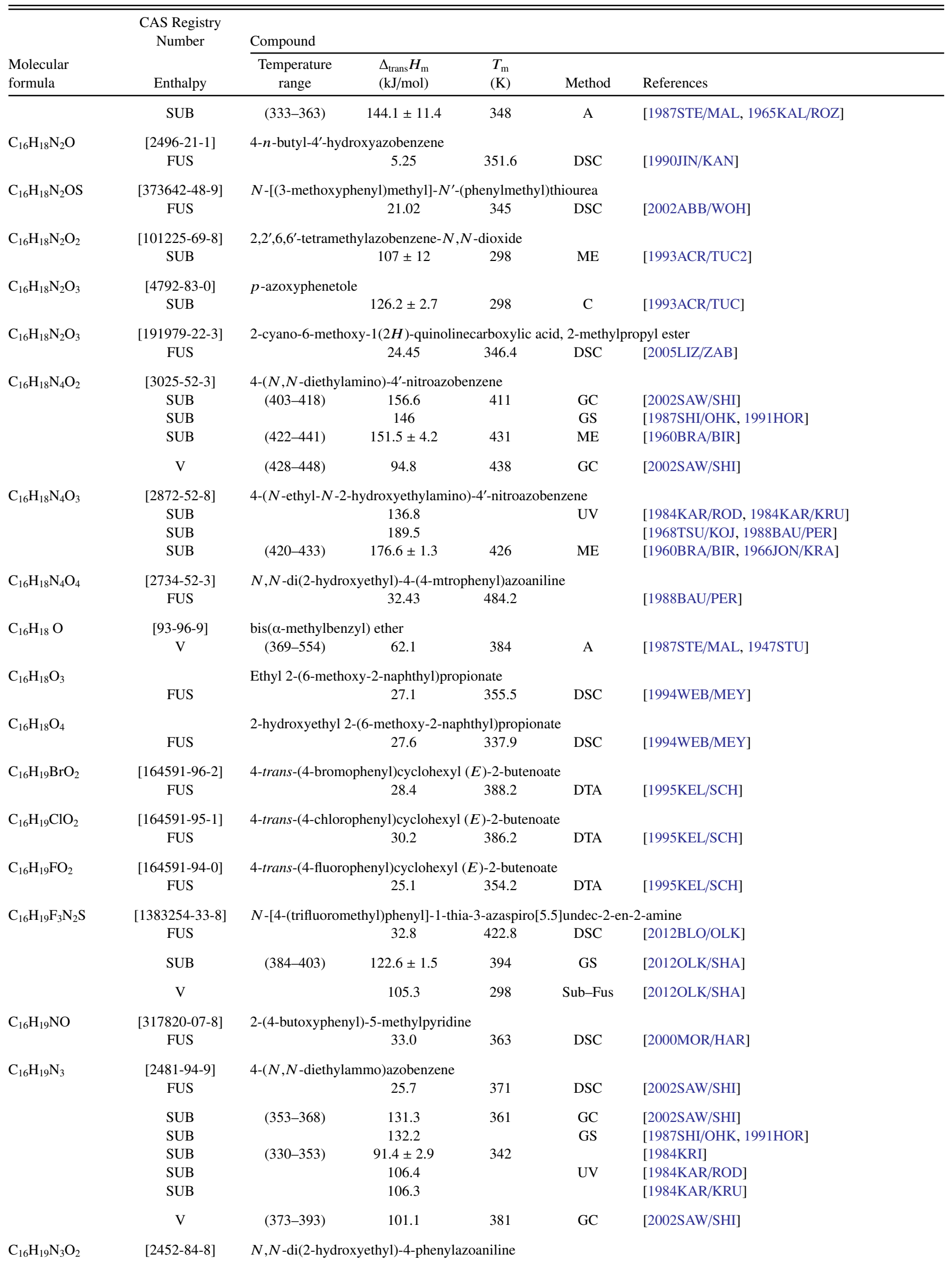


TABLE 12. Phase change enthalpies of $\mathrm{C}_{14}$ to $\mathrm{C}_{18}$ organic compounds-Continued

\begin{tabular}{|c|c|c|c|c|c|c|}
\hline \multirow[b]{2}{*}{$\begin{array}{l}\text { Molecular } \\
\text { formula }\end{array}$} & \multirow{2}{*}{$\begin{array}{l}\text { CAS Registry } \\
\text { Number } \\
\text { Enthalpy }\end{array}$} & \multicolumn{5}{|l|}{ Compound } \\
\hline & & $\begin{array}{c}\text { Temperature } \\
\text { range }\end{array}$ & $\begin{array}{l}\Delta_{\text {trans }} H_{\mathrm{m}} \\
(\mathrm{kJ} / \mathrm{mol})\end{array}$ & $\begin{array}{l}T_{\mathrm{m}} \\
(\mathrm{K})\end{array}$ & Method & References \\
\hline & FUS & & 29.96 & 407 & 298 & [1988BAU/PER] \\
\hline \multirow[t]{2}{*}{$\mathrm{C}_{16} \mathrm{H}_{20}$} & $\begin{array}{c}\text { [24157-81-1] } \\
\text { FUS }\end{array}$ & 2,6-diisopropy & $\begin{array}{l}\text { hthalene } \\
18.84\end{array}$ & 343.0 & DSC & [2016SAN/OLI] \\
\hline & SUB & $(301-323)$ & $98.0 \pm 0.2$ & 298 & ME & {$[2016 \mathrm{SAN} / \mathrm{OLI}]$} \\
\hline \multirow[t]{2}{*}{$\mathrm{C}_{16} \mathrm{H}_{20} \mathrm{ClNO}_{2} \mathrm{~S}$} & $\begin{array}{c}\text { [424810-52-6] } \\
\text { FUS }\end{array}$ & $N$-adamantan- & $\begin{array}{c}\text {-4-chlorobenz } \\
25.3\end{array}$ & $\begin{array}{c}\text { Ifonami } \\
459.9\end{array}$ & DSC & {$[2016 \mathrm{PER} / \mathrm{VOL}]$} \\
\hline & $\begin{array}{l}\text { SUB } \\
\text { SUB }\end{array}$ & $\begin{array}{l}(379-435) \\
(379-435)\end{array}$ & $\begin{array}{l}120.1 \pm 1.4 \\
126.2 \pm 1.4\end{array}$ & $\begin{array}{l}384 \\
298\end{array}$ & $\begin{array}{l}\text { GS } \\
\text { GS }\end{array}$ & $\begin{array}{l}\text { [2016PER/VOL] } \\
{[2016 \mathrm{PER} / \mathrm{VOL}]}\end{array}$ \\
\hline \multirow[t]{2}{*}{$\mathrm{C}_{16} \mathrm{H}_{20} \mathrm{FNO}_{2} \mathrm{~S}$} & $\begin{array}{c}\text { [398995-61-2] } \\
\text { FUS }\end{array}$ & $N$-adamantan- & $\begin{array}{c}\text {-4-fluorobenze } \\
18.8\end{array}$ & $\begin{array}{c}\text { Ifonamic } \\
419.3\end{array}$ & DSC & [2016PER/VOL] \\
\hline & $\begin{array}{l}\text { SUB } \\
\text { SUB }\end{array}$ & $\begin{array}{l}(363-406) \\
(363-406)\end{array}$ & $\begin{array}{l}116.5 \pm 1.2 \\
121.4 \pm 1.2\end{array}$ & $\begin{array}{l}384 \\
298\end{array}$ & $\begin{array}{l}\text { GS } \\
\text { GS }\end{array}$ & $\begin{array}{l}\text { [2016PER/VOL] } \\
{[2016 \mathrm{PER} / \mathrm{VOL}]}\end{array}$ \\
\hline \multirow[t]{2}{*}{$\mathrm{C}_{16} \mathrm{H}_{20} \mathrm{~N}_{2}$} & $\begin{array}{c}\text { [19219-01-3] } \\
\text { FUS }\end{array}$ & Tetracycloprop & $\begin{array}{c}\text { ccinonitrile } \\
22.3\end{array}$ & 390 & & [1996DOM/HEA, 1984BER/BEC2] \\
\hline & SUB & & $110.2 \pm 1.5$ & & & [1984BER/BEC2] \\
\hline \multirow[t]{2}{*}{$\mathrm{C}_{16} \mathrm{H}_{20} \mathrm{~N}_{2} \mathrm{OS}$} & $\begin{array}{c}\text { [1383254-28-1] } \\
\text { FUS }\end{array}$ & & \multicolumn{3}{|c|}{$N$-1-thia-3-azaspiro[5.5]undec-2-en-2-yl-benzamide } & [2012BLO/OLK, 2013OLK/BLO] \\
\hline & $\begin{array}{l}\text { SUB } \\
\text { SUB }\end{array}$ & $\begin{array}{l}(353-386) \\
(353-386)\end{array}$ & $\begin{array}{l}88.3 \pm 0.9 \\
92.0 \pm 0.9\end{array}$ & $\begin{array}{l}369 \\
298\end{array}$ & $\begin{array}{l}\text { GS } \\
\text { GS }\end{array}$ & $\begin{array}{l}{[20130 L K / B L O]} \\
{[20130 L K / B L O]}\end{array}$ \\
\hline $\mathrm{C}_{16} \mathrm{H}_{20} \mathrm{~N}_{4} \mathrm{O}_{3} \mathrm{~S}$ & $\begin{array}{l}{[56211-40-6]} \\
\text { FUS (I) } \\
\text { FUS (II) }\end{array}$ & $N$-[[(1-methyle & $\begin{array}{c}\text { amino]carbo } \\
37.2 \\
29.0\end{array}$ & $\begin{array}{c}-\left[\left(3^{\prime}-\mathrm{me}\right.\right. \\
434.7 \\
430\end{array}$ & $\begin{array}{l}\text { henyl)amir } \\
\text { DSC }\end{array}$ & $\begin{array}{l}\text {-3-pyridinesulfonamide (torasemide) } \\
\text { [2002ROL/GST] }\end{array}$ \\
\hline $\mathrm{C}_{16} \mathrm{H}_{20} \mathrm{O}_{2}$ & $\begin{array}{c}{[105443-43-4]} \\
\text { FUS }\end{array}$ & \multicolumn{5}{|c|}{ 2-isopropyl-6-(1-hydroperoxy-1-methyethyl)naphthalene } \\
\hline $\mathrm{C}_{16} \mathrm{H}_{20} \mathrm{O}_{3}$ & $\begin{array}{c}\text { [146683-17-2] } \\
\text { FUS }\end{array}$ & \multicolumn{5}{|c|}{ 3-benzoyl-1,2,2-trimethylcyclopentanecarboxylic acid } \\
\hline $\mathrm{C}_{16} \mathrm{H}_{20} \mathrm{O}_{4}$ & $\begin{array}{c}\text { [96783-79-8] } \\
\text { FUS }\end{array}$ & \multicolumn{5}{|c|}{ 2,6-bis(1-hydroperoxy-1-methylethyl)naphthalene } \\
\hline $\mathrm{C}_{16} \mathrm{H}_{20} \mathrm{O}_{6} \mathrm{P}_{2} \mathrm{~S}_{3}$ & $\begin{array}{c}\text { [3383-96-8] } \\
\text { FUS }\end{array}$ & \multicolumn{5}{|c|}{$O, O, O^{\prime}, O^{\prime}$-tetramethyl $O, O^{\prime}$-thiodi- $p$-phenylene bis(phosphorothioate) } \\
\hline \multirow[t]{3}{*}{$\mathrm{C}_{16} \mathrm{H}_{21} \mathrm{CIN}_{2} \mathrm{~S}$} & $\begin{array}{c}\text { [1383254-30-5] } \\
\text { FUS }\end{array}$ & \multicolumn{5}{|c|}{$N$-(3-chloro-4-methylphenyl)-1-thia-3-azaspiro[5.5]undec-2-en-2-amine } \\
\hline & SUB & $(385-407)$ & $114.5 \pm 1.9$ & 396 & GS & {$[2012 \mathrm{OLK} / \mathrm{SHA}]$} \\
\hline & $\mathrm{V}$ & & 96.6 & 298 & $\mathrm{~S}-\mathrm{F}$ & {$[2012 \mathrm{OLK} / \mathrm{SHA}]$} \\
\hline $\mathrm{C}_{16} \mathrm{H}_{21} \mathrm{CI}_{3} \mathrm{O}_{3}$ & $\begin{array}{c}{[1928-47-8]} \\
\mathrm{V}\end{array}$ & \multicolumn{5}{|c|}{ 2,4,5-trichlorophenoxyacetic acid, (2-ethylhexyl) ester } \\
\hline $\mathrm{C}_{16} \mathrm{H}_{21} \mathrm{CI}_{3} \mathrm{O}_{3}$ & $\begin{array}{c}{[2630-15-1]} \\
\mathrm{V}\end{array}$ & \multicolumn{4}{|c|}{ 2,4,5-trichlorophenoxyacetic acid, octyl ester } & [1987STE/MAL, 1966JEN/SCH] \\
\hline $\mathrm{C}_{16} \mathrm{H}_{21} \mathrm{~N}$ & $\begin{array}{c}\text { [61203-99-4] } \\
\text { TRS } \\
\text { FUS }\end{array}$ & $\begin{array}{l}\text { 4-(trans-4-pro } \\
\qquad \begin{array}{l}(15-385) \\
(15-385)\end{array}\end{array}$ & $\begin{array}{c}\text { clohexyl)benz } \\
20.4 \\
1.1\end{array}$ & $\begin{array}{l}\text { le } \\
316.3 \\
319\end{array}$ & $\mathrm{AC}$ & [1998ASA/SOR] \\
\hline $\mathrm{C}_{16} \mathrm{H}_{21} \mathrm{NO}_{2}$ & $\begin{array}{l}\text { [4199-09-1] } \\
\text { FUS } \\
\text { FUS }\end{array}$ & (-)-1-(isoprop & $\begin{array}{l}\text { ino)-3-(1-nap } \\
36.25 \\
34.2\end{array}$ & $\begin{array}{l}\text { oxy) }-2-p \\
344.7 \\
344.6\end{array}$ & $\begin{array}{l}\text { ol (proprai } \\
\text { DSC } \\
\text { DSC }\end{array}$ & $\begin{array}{l}\text { ol) } \\
{[1999 \mathrm{LI} / \mathrm{ZEL}]} \\
{[1993 \mathrm{NEA} / \mathrm{SHI}]}\end{array}$ \\
\hline $\mathrm{C}_{16} \mathrm{H}_{21} \mathrm{NO}_{2}$ & $\begin{array}{l}{[525-66-6]} \\
\text { FUS } \\
\text { FUS }\end{array}$ & $( \pm)$-1-(isopropy & $\begin{array}{l}\text { no })-3-(1-\text { naph } \\
43.45 \\
38.1\end{array}$ & $\begin{array}{c}\text { xy)-2-pr } \\
365.5 \\
365.2\end{array}$ & $\begin{array}{l}1 \text { (propran } \\
\text { DSC } \\
\text { DSC }\end{array}$ & $\begin{array}{l}\text { 1) } \\
\text { [1999LI/ZEL] } \\
{[1993 \mathrm{NEA} / \mathrm{SHI}]}\end{array}$ \\
\hline
\end{tabular}


TABLE 12. Phase change enthalpies of $\mathrm{C}_{14}$ to $\mathrm{C}_{18}$ organic compounds-Continued

\begin{tabular}{|c|c|c|c|c|c|c|}
\hline \multirow[b]{2}{*}{$\begin{array}{l}\text { Molecular } \\
\text { formula }\end{array}$} & \multirow{2}{*}{$\begin{array}{l}\text { CAS Registry } \\
\text { Number } \\
\text { Enthalpy }\end{array}$} & \multicolumn{5}{|l|}{ Compound } \\
\hline & & $\begin{array}{c}\text { Temperature } \\
\text { range }\end{array}$ & $\begin{array}{l}\Delta_{\text {trans }} H_{\mathrm{m}} \\
(\mathrm{kJ} / \mathrm{mol})\end{array}$ & $\begin{array}{l}T_{\mathrm{m}} \\
(\mathrm{K})\end{array}$ & Method & References \\
\hline & FUS & & 14.7 & 397.4 & $\mathrm{DSC}$ & [2016PER/VOL] \\
\hline & SUB & $(366-394)$ & $119.1 \pm 0.8$ & 380 & GS & [2016PER/VOL] \\
\hline & SUB & $(366-394)$ & $123.6 \pm 0.8$ & 298 & GS & [2016PER/VOL] \\
\hline \multirow[t]{2}{*}{$\mathrm{C}_{16} \mathrm{H}_{22} \mathrm{CINO}_{3}$} & {$[4199-10-4]$} & \multicolumn{5}{|c|}{ (-)-1-(isopropylamino)-3-(1-naphthyloxy)-2-propanol hydrochloride (propranolol hydrochloride) } \\
\hline & FUS & & 36.0 & 467.8 & DSC & {$[1999 \mathrm{LI} / \mathrm{ZEL}]$} \\
\hline \multirow[t]{2}{*}{$\mathrm{C}_{16} \mathrm{H}_{22} \mathrm{CINO}_{3}$} & {$[318-98-9]$} & \multicolumn{5}{|c|}{ ( \pm )-1-(isopropylamino)-3-(1-naphthyloxy)-2-propanol hydrochloride (propranolol hydrochloride) } \\
\hline & FUS & & 16.31 & 437.1 & DSC & [2016AMB/CER] \\
\hline
\end{tabular}

[Note: The authors of [2016AMB/CER] refer to the compound simply as propranolol. The purchased compound was the hydrochloride salt, and there is no mention in the paper that the compound was converted to the neutral base.]

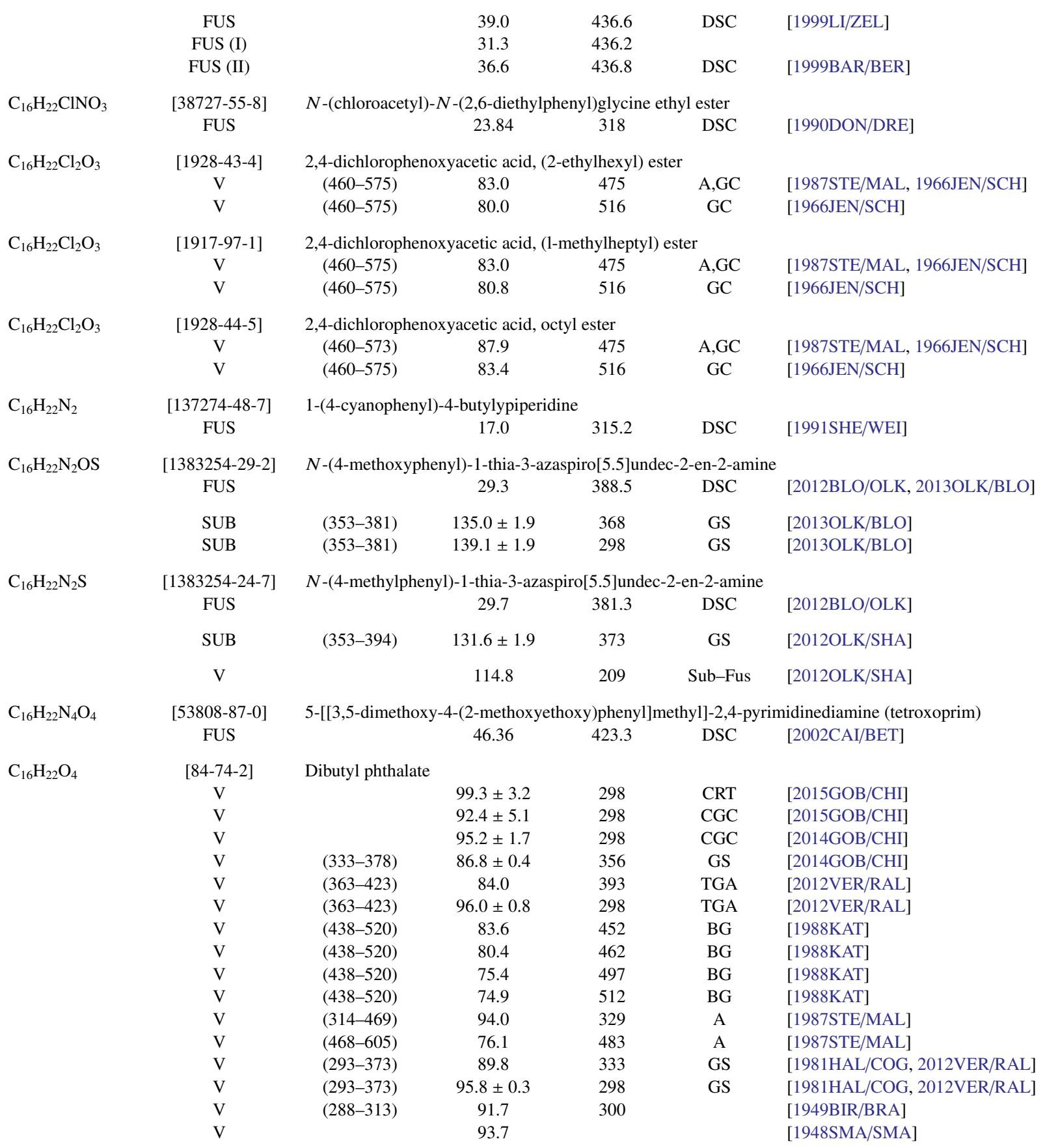


TABLE 12. Phase change enthalpies of $\mathrm{C}_{14}$ to $\mathrm{C}_{18}$ organic compounds-Continued

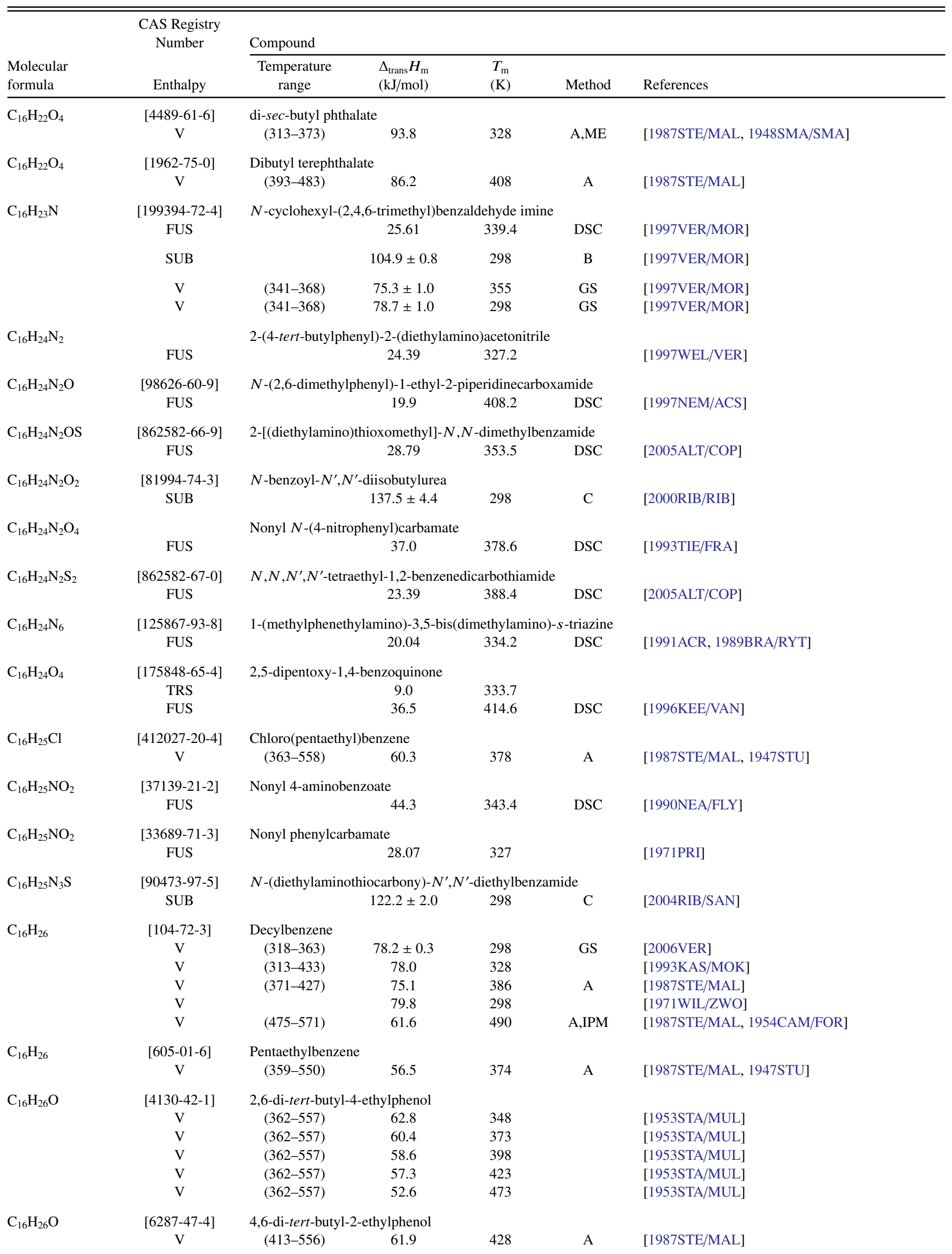


TABLE 12. Phase change enthalpies of $\mathrm{C}_{14}$ to $\mathrm{C}_{18}$ organic compounds-Continued

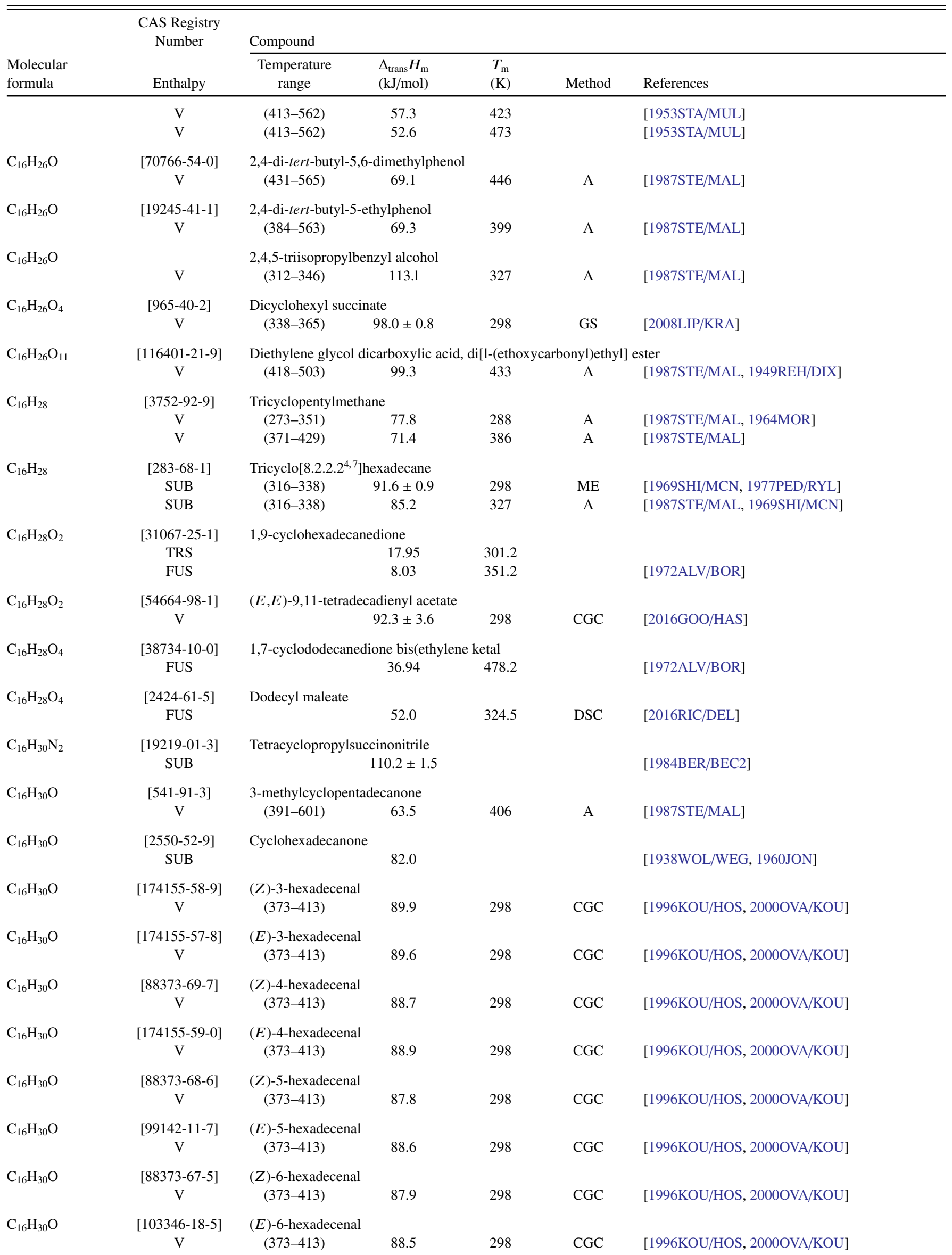


TABLE 12. Phase change enthalpies of $\mathrm{C}_{14}$ to $\mathrm{C}_{18}$ organic compounds-Continued

\begin{tabular}{|c|c|c|c|c|c|c|}
\hline \multirow[b]{2}{*}{$\begin{array}{l}\text { Molecular } \\
\text { formula }\end{array}$} & \multirow{2}{*}{$\begin{array}{c}\text { CAS Registry } \\
\text { Number } \\
\text { Enthalpy }\end{array}$} & \multicolumn{5}{|l|}{ Compound } \\
\hline & & $\begin{array}{l}\text { Temperature } \\
\text { range }\end{array}$ & $\begin{array}{l}\Delta_{\text {trans }} H_{\mathrm{m}} \\
(\mathrm{kJ} / \mathrm{mol})\end{array}$ & $\begin{array}{l}T_{\mathrm{m}} \\
(\mathrm{K})\end{array}$ & Method & References \\
\hline $\mathrm{C}_{16} \mathrm{H}_{30} \mathrm{O}$ & $\begin{array}{c}{[56797-40-1]} \\
\mathrm{V}\end{array}$ & $\begin{array}{l}\text { (Z)-7-hexadecenal } \\
\quad(373-413)\end{array}$ & 87.8 & 298 & CGC & [1996KOU/HOS, 2000OVA/KOU] \\
\hline $\mathrm{C}_{16} \mathrm{H}_{30} \mathrm{O}$ & $\begin{array}{c}{[72698-27-2]} \\
\mathrm{V}\end{array}$ & $\begin{array}{l}(E) \text {-7-hexadecenal } \\
\quad(373-413)\end{array}$ & 88.6 & 298 & CGC & [1996KOU/HOS, 2000OVA/KOU] \\
\hline $\mathrm{C}_{16} \mathrm{H}_{30} \mathrm{O}$ & $\begin{array}{c}{[66644-98-2]} \\
\mathrm{V}\end{array}$ & $\begin{array}{l}\text { (Z)-8-hexadecenal } \\
\quad(373-413)\end{array}$ & 87.7 & 298 & CGC & [1996KOU/HOS, 2000OVA/KOU] \\
\hline $\mathrm{C}_{16} \mathrm{H}_{30} \mathrm{O}$ & $\begin{array}{c}{[72698-28-3]} \\
V\end{array}$ & $\begin{array}{l}(E) \text {-8-hexadecenal } \\
\quad(373-413)\end{array}$ & 88.4 & 298 & CGC & [1996KOU/HOS, 2000OVA/KOU] \\
\hline $\mathrm{C}_{16} \mathrm{H}_{30} \mathrm{O}$ & $\begin{array}{c}{[56219-04-6]} \\
\mathrm{V}\end{array}$ & $\begin{array}{l}\text { (Z)-9-hexadecenal } \\
\quad(373-413)\end{array}$ & 88.0 & 298 & CGC & [1996KOU/HOS, 2000OVA/KOU] \\
\hline $\mathrm{C}_{16} \mathrm{H}_{30} \mathrm{O}$ & $\begin{array}{c}{[72698-29-4]} \\
\mathrm{V}\end{array}$ & $\begin{array}{l}(E)-9 \text {-hexadecenal } \\
\quad(373-413)\end{array}$ & 88.6 & 298 & CGC & [1996KOU/HOS, 2000OVA/KOU] \\
\hline $\mathrm{C}_{16} \mathrm{H}_{30} \mathrm{O}$ & $\begin{array}{c}{[68279-24-3]} \\
\mathrm{V}\end{array}$ & $\begin{array}{l}\text { (Z)-10-hexadecenal } \\
\quad(373-413)\end{array}$ & 88.2 & 298 & CGC & [1996KOU/HOS, 2000OVA/KOU] \\
\hline $\mathrm{C}_{16} \mathrm{H}_{30} \mathrm{O}$ & $\begin{array}{c}{[72698-30-7]} \\
\mathrm{V}\end{array}$ & $\begin{array}{l}(E)-10 \text {-hexadecenal } \\
\quad(373-413)\end{array}$ & 88.8 & 298 & CGC & [1996KOU/HOS, 2000OVA/KOU] \\
\hline $\mathrm{C}_{16} \mathrm{H}_{30} \mathrm{O}$ & $\begin{array}{c}{[53939-28-9]} \\
\mathrm{V}\end{array}$ & $\begin{array}{l}(Z)-11 \text {-hexadecenal } \\
\quad(373-413)\end{array}$ & 88.5 & 298 & CGC & [1996KOU/HOS, 2000OVA/KOU] \\
\hline $\mathrm{C}_{16} \mathrm{H}_{30} \mathrm{O}$ & $\begin{array}{c}{[57491-33-5]} \\
\mathrm{V}\end{array}$ & $\begin{array}{l}(E)-11 \text {-hexadecenal } \\
\quad(373-413)\end{array}$ & 89.2 & 298 & CGC & [1996KOU/HOS, 2000OVA/KOU] \\
\hline $\mathrm{C}_{16} \mathrm{H}_{30} \mathrm{O}$ & $\begin{array}{c}{[72698-31-8]} \\
\mathrm{V}\end{array}$ & $\begin{array}{l}\text { (Z)-12-hexadecenal } \\
\quad(373-413)\end{array}$ & 89.3 & 298 & CGC & [1996KOU/HOS, 2000OVA/KOU] \\
\hline $\mathrm{C}_{16} \mathrm{H}_{30} \mathrm{O}$ & $\begin{array}{c}{[72698-32-9]} \\
V\end{array}$ & $\begin{array}{l}(E) \text {-12-hexadecenal } \\
\quad(373-413)\end{array}$ & 89.3 & 298 & CGC & [1996KOU/HOS, 2000OVA/KOU] \\
\hline $\mathrm{C}_{16} \mathrm{H}_{30} \mathrm{O}$ & $\begin{array}{c}{[71545-96-5]} \\
\mathrm{V}\end{array}$ & $\begin{array}{l}\text { (Z)-13-hexadecenal } \\
\quad(373-413)\end{array}$ & 89.7 & 298 & CGC & [1996KOU/HOS, 2000OVA/KOU] \\
\hline $\mathrm{C}_{16} \mathrm{H}_{30} \mathrm{O}$ & $\begin{array}{c}{[72698-33-0]} \\
\mathrm{V}\end{array}$ & $\begin{array}{l}(E) \text {-13-hexadecenal } \\
\quad(373-413)\end{array}$ & 90.0 & 298 & CGC & [1996KOU/HOS, 2000OVA/KOU] \\
\hline $\mathrm{C}_{16} \mathrm{H}_{30} \mathrm{O}_{2}$ & $\begin{array}{c}{[142-90-5]} \\
\mathrm{V}\end{array}$ & $\begin{array}{l}\text { Dodecyl methacrylate } \\
\quad(438-580)\end{array}$ & 64.9 & 453 & A & [1987STE/MAL] \\
\hline $\mathrm{C}_{16} \mathrm{H}_{30} \mathrm{O}_{2}$ & $\begin{array}{c}{[109-29-5]} \\
\mathrm{V}\end{array}$ & $\begin{array}{l}\text { Oxa-2-cycloheptadec } \\
\quad(403-463)\end{array}$ & $\begin{array}{l}\text { anone } \\
71.6\end{array}$ & 418 & A & [1987STE/MAL] \\
\hline $\mathrm{C}_{16} \mathrm{H}_{30} \mathrm{O}_{2}$ & $\begin{array}{c}{[51309-20-7]} \\
\mathrm{V}\end{array}$ & $\begin{array}{l}\text { (Z)-2-tetradecenyl ac } \\
\quad(353-398)\end{array}$ & $\begin{array}{l}\text { etate } \\
89.1\end{array}$ & 298 & GC & [1997KOU/HOS, 2000OVA/KOU] \\
\hline $\mathrm{C}_{16} \mathrm{H}_{30} \mathrm{O}_{2}$ & $\begin{array}{c}{[51309-21-8]} \\
\mathrm{V}\end{array}$ & $\begin{array}{l}(E) \text {-2-tetradecenyl ac } \\
\quad(353-398)\end{array}$ & $\begin{array}{l}90.3 \\
90 t a t e\end{array}$ & 298 & GC & [1997KOU/HOS, 2000OVA/KOU] \\
\hline $\mathrm{C}_{16} \mathrm{H}_{30} \mathrm{O}_{2}$ & $\begin{array}{c}{[54897-65-3]} \\
\mathrm{V}\end{array}$ & $\begin{array}{l}\text { (Z)-3-tetradecenyl ac } \\
\quad(353-398)\end{array}$ & $\begin{array}{l}\text { etate } \\
88.5\end{array}$ & 298 & GC & [1997KOU/HOS, 2000OVA/KOU] \\
\hline $\mathrm{C}_{16} \mathrm{H}_{30} \mathrm{O}_{2}$ & $\begin{array}{c}{[56221-90-0]} \\
\mathrm{V}\end{array}$ & $\begin{array}{l}(E) \text {-3-tetradecenyl ac } \\
(353-398)\end{array}$ & $\begin{array}{l}89.2 \\
89.2\end{array}$ & 298 & GC & [1997KOU/HOS, 2000OVA/KOU] \\
\hline $\mathrm{C}_{16} \mathrm{H}_{30} \mathrm{O}_{2}$ & $\begin{array}{c}{[54897-66-4]} \\
\mathrm{V}\end{array}$ & $\begin{array}{l}\text { (Z)-4-tetradecenyl ac } \\
\quad(353-398)\end{array}$ & $\begin{array}{l}87.8 \\
8 \text { etate }\end{array}$ & 298 & GC & [1997KOU/HOS, 2000OVA/KOU] \\
\hline $\mathrm{C}_{16} \mathrm{H}_{30} \mathrm{O}_{2}$ & $\begin{array}{c}{[56209-67-7]} \\
\mathrm{V}\end{array}$ & $\begin{array}{l}(E) \text {-4-tetradecenyl ac } \\
\quad(353-398)\end{array}$ & $\begin{array}{l}89.0 \\
\quad \text { etate }\end{array}$ & 298 & GC & [1997KOU/HOS, 2000OVA/KOU] \\
\hline $\mathrm{C}_{16} \mathrm{H}_{30} \mathrm{O}_{2}$ & $\begin{array}{c}{[35153-13-0]} \\
\mathrm{V}\end{array}$ & $\begin{array}{l}\text { (Z)-5-tetradecenyl ac } \\
\quad(353-398)\end{array}$ & $\begin{array}{l}\text { etate } \\
88.3\end{array}$ & 298 & GC & [1997KOU/HOS, 2000OVA/KOU] \\
\hline $\mathrm{C}_{16} \mathrm{H}_{30} \mathrm{O}_{2}$ & $\begin{array}{c}{[34010-13-4]} \\
\mathrm{V}\end{array}$ & $\begin{array}{l}(E)-5 \text {-tetradecenyl ac } \\
(353-398)\end{array}$ & $\begin{array}{l}\text { etate } \\
89.1\end{array}$ & 298 & GC & [1997KOU/HOS, 2000OVA/KOU] \\
\hline
\end{tabular}


TABLE 12. Phase change enthalpies of $\mathrm{C}_{14}$ to $\mathrm{C}_{18}$ organic compounds-Continued

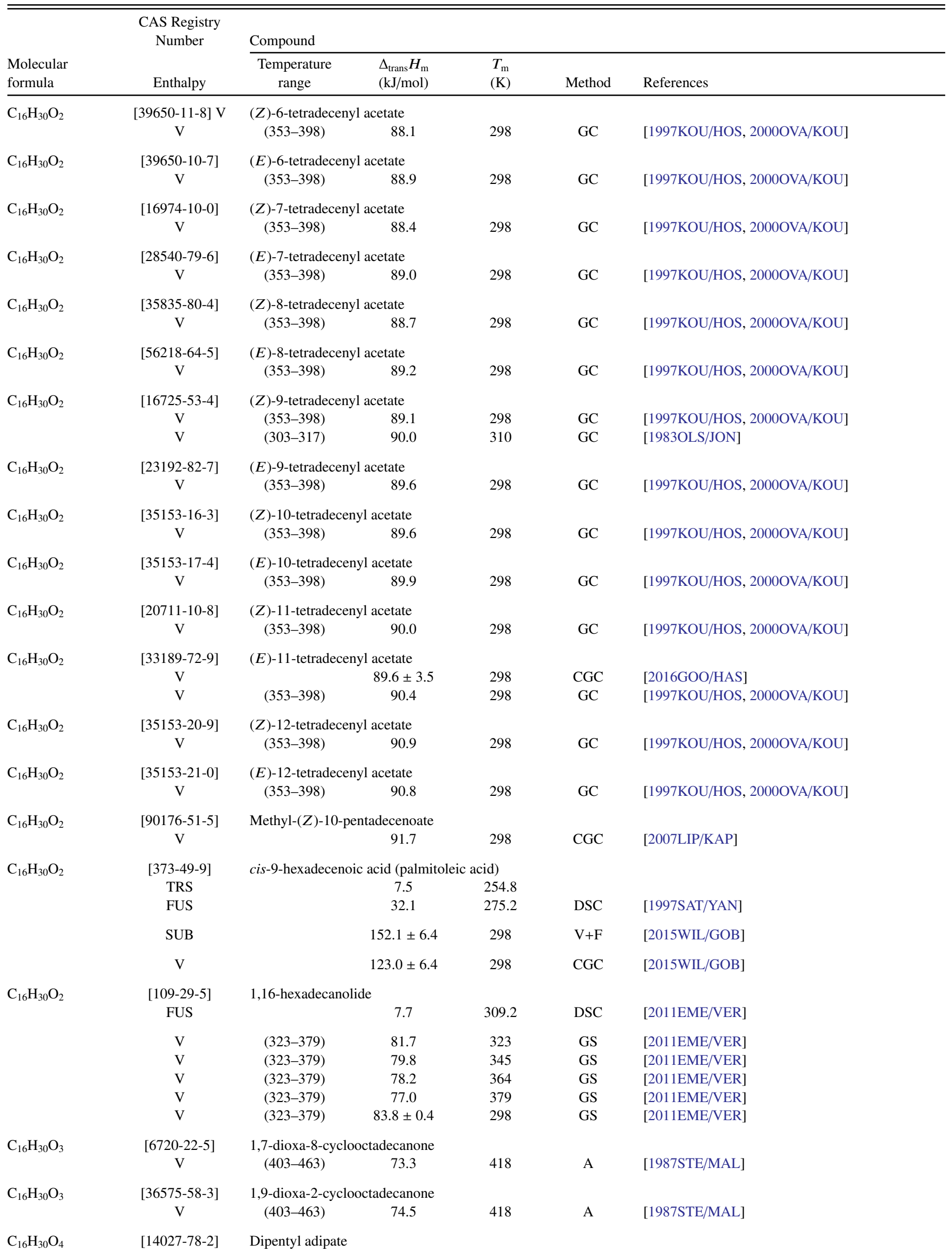


TABLE 12. Phase change enthalpies of $\mathrm{C}_{14}$ to $\mathrm{C}_{18}$ organic compounds-Continued

\begin{tabular}{|c|c|c|c|c|c|c|}
\hline \multirow[b]{2}{*}{$\begin{array}{l}\text { Molecular } \\
\text { formula }\end{array}$} & \multirow{2}{*}{$\begin{array}{l}\text { CAS Registry } \\
\text { Number } \\
\text { Enthalpy }\end{array}$} & \multicolumn{5}{|l|}{ Compound } \\
\hline & & $\begin{array}{l}\text { Temperature } \\
\text { range }\end{array}$ & $\begin{array}{l}\Delta_{\text {trans }} H_{\mathrm{m}} \\
(\mathrm{kJ} / \mathrm{mol})\end{array}$ & $\begin{array}{l}T_{\mathrm{m}} \\
(\mathrm{K})\end{array}$ & Method & References \\
\hline & $\mathrm{V}$ & $(449-575)$ & 74.7 & 464 & A & [1987STE/MAL] \\
\hline \multirow[t]{2}{*}{$\mathrm{C}_{16} \mathrm{H}_{30} \mathrm{O}_{4}$} & $\begin{array}{l}{[505-54-4]} \\
\text { FUS+TRS } \\
\quad \text { FUS }\end{array}$ & Hexadecanedioic acid & $\begin{array}{r}60.1 \\
52.2\end{array}$ & $\begin{array}{l}396.4 \\
395.4\end{array}$ & $\begin{array}{l}\text { DSC } \\
\text { DSC }\end{array}$ & $\begin{array}{l}{[2006 \mathrm{VEN} / \mathrm{MET}]} \\
{[2005 \mathrm{ROU} / \mathrm{TEM}]}\end{array}$ \\
\hline & $\begin{array}{l}\text { SUB } \\
\text { SUB }\end{array}$ & $\begin{array}{l}(377-398) \\
(377-398)\end{array}$ & $\begin{array}{l}151.0 \pm 3.3 \\
155.4 \pm 3.3\end{array}$ & $\begin{array}{l}388 \\
298\end{array}$ & $\begin{array}{l}\mathrm{ME} \\
\mathrm{ME}\end{array}$ & $\begin{array}{l}\text { [1960DAV/THO, 1987STE/MAL] } \\
\text { [1960DAV/THO, 1999RIB/MON] }\end{array}$ \\
\hline $\mathrm{C}_{16} \mathrm{H}_{30} \mathrm{O}_{4}$ & $\begin{array}{l}{[21668-03-1]} \\
\text { FUS }\end{array}$ & \multicolumn{5}{|l|}{ Dodecyl succinate } \\
\hline $\mathrm{C}_{16} \mathrm{H}_{30} \mathrm{O}_{5}$ & $\begin{array}{c}{[5420-72-4]} \\
\mathrm{V}\end{array}$ & $\begin{array}{l}\text { Ocyl[1-(butoxycarbon } \\
\quad(374-503)\end{array}$ & \multicolumn{3}{|c|}{ Ocyl[1-(butoxycarbonyl)ethyl]carbonate } & [1987STE/MAL, 1950REH/DIX2] \\
\hline $\mathrm{C}_{16} \mathrm{H}_{31} \mathrm{~N}$ & $\begin{array}{c}{[629-79-8]} \\
\text { V } \\
\text { V }\end{array}$ & $\begin{array}{l}\text { Hexadecanenitrile (pal } \\
\quad(345-382)\end{array}$ & $\begin{array}{l}\text { almitonitrile } \\
93.3 \pm 0.4 \\
70.1\end{array}$ & $\begin{array}{l}298 \\
518\end{array}$ & A & $\begin{array}{l}\text { [2005EME/VER] } \\
{[1987 \mathrm{STE} / \mathrm{MAL}]}\end{array}$ \\
\hline $\mathrm{C}_{16} \mathrm{H}_{31} \mathrm{NO}_{3}$ & $\begin{array}{l}\text { [14246-55-0] } \\
\text { TRS+FUS } \\
\text { TRS } \\
\text { FUS }\end{array}$ & $N$-tetradecanoylglycin & $\begin{array}{l}\text { ine } \\
38.7 \\
6.8 \\
47.4\end{array}$ & $\begin{array}{l}393.6 \\
379.6 \\
396.6\end{array}$ & DSC & $\begin{array}{l}\text { [2014RED/KRO] } \\
\text { [1986MIY/MAT] }\end{array}$ \\
\hline $\mathrm{C}_{16} \mathrm{H}_{31} \mathrm{NO}_{3}$ & $\begin{array}{l}\text { [14379-38-5] } \\
\text { TRS } \\
\text { FUS }\end{array}$ & $N$-decanoyl- $(L)$-leuci & $\begin{array}{l}\text { cine } \\
\qquad \begin{array}{c}1.2 \\
27.5\end{array}\end{array}$ & $\begin{array}{l}343.1 \\
383.1\end{array}$ & $\mathrm{DSC}$ & [1986MIY/MAT] \\
\hline $\mathrm{C}_{16} \mathrm{H}_{31} \mathrm{NO}_{3}$ & $\begin{array}{c}{[107396-12-3]} \\
\text { FUS }\end{array}$ & $N$-decanoyl- $(D L)$-leuc & $\begin{array}{l}\text { ucine } \\
28.9\end{array}$ & 357.1 & DSC & [1986MIY/MAT] \\
\hline $\mathrm{C}_{16} \mathrm{H}_{32}$ & $\begin{array}{c}{[15220-85-6]} \\
\mathrm{V}\end{array}$ & $\begin{array}{l}\text { Tetraisobutylene } \\
\quad(381-440)\end{array}$ & 54.5 & 397 & & [1943STE] \\
\hline $\mathrm{C}_{16} \mathrm{H}_{32}$ & $\begin{array}{l}{[1795-16-0]} \\
\text { FUS }\end{array}$ & $\begin{array}{l}\text { Decylcyclohexane } \\
\quad(12-300)\end{array}$ & 38.62 & 271.4 & $\mathrm{AC}$ & [1991ACR, 1965FIN/MES] \\
\hline & $\begin{array}{l}\mathrm{V} \\
\mathrm{V} \\
\mathrm{V}\end{array}$ & $\begin{array}{l}(371-425) \\
(469-571)\end{array}$ & $\begin{array}{l}76.7 \\
79.7 \\
61.6\end{array}$ & $\begin{array}{l}386 \\
298 \\
484\end{array}$ & $\begin{array}{c}\mathrm{A} \\
\mathrm{A}, \mathrm{MM}\end{array}$ & $\begin{array}{l}\text { [1987STE/MAL] } \\
\text { [1971WIL/ZWO] } \\
\text { [1987STE/MAL, 1954CAM/FOR] }\end{array}$ \\
\hline $\mathrm{C}_{16} \mathrm{H}_{32}$ & $\begin{array}{c}{[6785-23-5]} \\
\mathrm{V}\end{array}$ & Undecylcyclopentane & 80.6 & 298 & & [1971WIL/ZWO] \\
\hline $\mathrm{C}_{16} \mathrm{H}_{32}$ & $\begin{array}{l}\text { [629-73-2] } \\
\text { TRS } \\
\text { FUS } \\
\text { FUS }\end{array}$ & $\begin{array}{c}\text { 1-hexadecene } \\
(12-304)\end{array}$ & $\begin{array}{l}3.87 \\
30.21 \\
30.19\end{array}$ & $\begin{array}{l}249.2 \\
277.5 \\
277.5\end{array}$ & $\mathrm{C}$ & $\begin{array}{l}\text { [1990MES/TOD] } \\
{[1957 \mathrm{MCC} / \mathrm{FIN}]}\end{array}$ \\
\hline & $\begin{array}{l}\mathrm{V} \\
\mathrm{V} \\
\mathrm{V} \\
\mathrm{V}\end{array}$ & $(461-558)$ & $\begin{array}{c}80.3 \pm 0.4 \\
80.3 \pm 0.4 \\
80.1 \\
61.5\end{array}$ & $\begin{array}{l}298 \\
298 \\
298 \\
476\end{array}$ & $\begin{array}{l}\mathrm{C} \\
\mathrm{C} \\
\mathrm{A}\end{array}$ & $\begin{array}{l}\text { [1977MAN/SEL] } \\
\text { [1976STR2] } \\
\text { [1971WIL/ZWO] } \\
\text { [1987STE/MAL, 1954CAM/FOR] }\end{array}$ \\
\hline $\mathrm{C}_{16} \mathrm{H}_{32}$ & $\begin{array}{c}\text { [295-65-8] } \\
\text { TRS } \\
\text { FUS } \\
\text { TRS } \\
\text { TRS } \\
\text { FUS }\end{array}$ & Cyclohexadecane & $\begin{array}{c}19.6 \\
7.6 \\
18.83 \\
1.26 \\
4.18\end{array}$ & $\begin{array}{l}268.9 \\
333.7 \\
271.2 \\
283.2 \\
332.2\end{array}$ & DSC & [1975BJO/BOR2] \\
\hline & SUB & & $81.8 \pm 0.4$ & & & [1957VAN, 1970COX/PIL] \\
\hline $\mathrm{C}_{16} \mathrm{H}_{32} \mathrm{O}$ & $\begin{array}{c}\text { [141694-91-9] } \\
\mathrm{V}\end{array}$ & $\begin{array}{l}\text { (Z)-3-hexadecen-1-ol } \\
\quad(373-413)\end{array}$ & 110.7 & 298 & CGC & [2000OVA/KOU, 1994KOU/HOS] \\
\hline $\mathrm{C}_{16} \mathrm{H}_{32} \mathrm{O}$ & $\begin{array}{c}{[128999-42-8]} \\
\mathrm{V}\end{array}$ & $\begin{array}{l}(E) \text {-3-hexadecen-1-ol } \\
\quad(373-413)\end{array}$ & ol 110.8 & 298 & $\mathrm{CGC}$ & [2000OVA/KOU, 1994KOU/HOS] \\
\hline $\mathrm{C}_{16} \mathrm{H}_{32} \mathrm{O}$ & {$[145235-63-8]$} & (Z)-4-hexadecen-1-ol & & & & \\
\hline
\end{tabular}


TABle 12. Phase change enthalpies of $\mathrm{C}_{14}$ to $\mathrm{C}_{18}$ organic compounds-Continued

\begin{tabular}{|c|c|c|c|c|c|c|}
\hline \multirow[b]{2}{*}{$\begin{array}{l}\text { Molecular } \\
\text { formula }\end{array}$} & \multirow{2}{*}{$\begin{array}{l}\text { CAS Registry } \\
\text { Number } \\
\text { Enthalpy }\end{array}$} & \multicolumn{5}{|l|}{ Compound } \\
\hline & & $\begin{array}{l}\text { Temperature } \\
\text { range }\end{array}$ & $\begin{array}{l}\Delta_{\text {trans }} H_{\mathrm{m}} \\
(\mathrm{kJ} / \mathrm{mol})\end{array}$ & $\begin{array}{l}T_{\mathrm{m}} \\
(\mathrm{K})\end{array}$ & Method & References \\
\hline & $\mathrm{V}$ & $(373-413)$ & 110.6 & 298 & CGC & [2000OVA/KOU, 1994KOU/HOS] \\
\hline $\mathrm{C}_{16} \mathrm{H}_{32} \mathrm{O}$ & $\begin{array}{c}{[59101-23-4]} \\
\mathrm{V}\end{array}$ & $\begin{array}{l}(E) \text {-4-hexadecen-1-ol } \\
\quad(373-413)\end{array}$ & 111.5 & 298 & CGC & [2000OVA/KOU, 1994KOU/HOS] \\
\hline $\mathrm{C}_{16} \mathrm{H}_{32} \mathrm{O}$ & $\begin{array}{c}{[106463-48-3]} \\
V\end{array}$ & $\begin{array}{l}\text { (Z)-5-hexadecen-1-ol } \\
\quad(373-413)\end{array}$ & 110.9 & 298 & CGC & [2000OVA/KOU, 1994KOU/HOS] \\
\hline $\mathrm{C}_{16} \mathrm{H}_{32} \mathrm{O}$ & $\begin{array}{c}{[85388-16-5]} \\
\mathrm{V}\end{array}$ & $\begin{array}{l}(E)-5 \text {-hexadecen-1-ol } \\
\quad(373-413)\end{array}$ & 111.4 & 298 & CGC & [2000OVA/KOU, 1994KOU/HOS] \\
\hline $\mathrm{C}_{16} \mathrm{H}_{32} \mathrm{O}$ & $\begin{array}{c}{[40642-45-3]} \\
\mathrm{V}\end{array}$ & $\begin{array}{l}\text { (Z)-6-hexadecen-1-ol } \\
\quad(373-413)\end{array}$ & 110.5 & 298 & CGC & [2000OVA/KOU, 1994KOU/HOS] \\
\hline $\mathrm{C}_{16} \mathrm{H}_{32} \mathrm{O}$ & $\begin{array}{c}{[34500-33-9]} \\
\mathrm{V}\end{array}$ & $\begin{array}{l}\text { (E)-6-hexadecen-1-ol } \\
\quad(373-413)\end{array}$ & 111 & 298 & CGC & [2000OVA/KOU, 1994KOU/HOS] \\
\hline $\mathrm{C}_{16} \mathrm{H}_{32} \mathrm{O}$ & $\begin{array}{c}{[24880-48-6]} \\
V\end{array}$ & $\begin{array}{l}\text { (Z)-7-hexadecen-1-ol } \\
\quad(373-413)\end{array}$ & 110.2 & 298 & CGC & [2000OVA/KOU, 1994KOU/HOS] \\
\hline $\mathrm{C}_{16} \mathrm{H}_{32} \mathrm{O}$ & $\begin{array}{c}{[51824-10-3]} \\
\mathrm{V}\end{array}$ & $\begin{array}{l}\text { (E)-7-hexadecen-1-ol } \\
\quad(373-413)\end{array}$ & 111.4 & 298 & CGC & [2000OVA/KOU, 1994KOU/HOS] \\
\hline $\mathrm{C}_{16} \mathrm{H}_{32} \mathrm{O}$ & $\begin{array}{c}{[64437-46-3]} \\
\mathrm{V}\end{array}$ & $\begin{array}{l}\text { (Z)-8-hexadecen-1-ol } \\
\quad(373-413)\end{array}$ & 110.4 & 298 & CGC & [2000OVA/KOU, 1994KOU/HOS] \\
\hline $\mathrm{C}_{16} \mathrm{H}_{32} \mathrm{O}$ & $\begin{array}{c}{[64470-33-3]} \\
\mathrm{V}\end{array}$ & $\begin{array}{l}(E)-8 \text {-hexadecen-1-ol } \\
\quad(373-413)\end{array}$ & 111.1 & 298 & CGC & [2000OVA/KOU, 1994KOU/HOS] \\
\hline $\mathrm{C}_{16} \mathrm{H}_{32} \mathrm{O}$ & $\begin{array}{c}{[10378-01-5]} \\
V\end{array}$ & $\begin{array}{l}\text { (Z)-9-hexadecen-1-ol } \\
\quad(373-413)\end{array}$ & 110.6 & 298 & CGC & [2000OVA/KOU, 1994KOU/HOS] \\
\hline $\mathrm{C}_{16} \mathrm{H}_{32} \mathrm{O}$ & $\begin{array}{c}{[64437-47-4]} \\
\mathrm{V}\end{array}$ & $\begin{array}{l}\text { (E)-9-hexadecen-1-ol } \\
\quad(373-413)\end{array}$ & 111.3 & 298 & CGC & [2000OVA/KOU, 1994KOU/HOS] \\
\hline $\mathrm{C}_{16} \mathrm{H}_{32} \mathrm{O}$ & $\begin{array}{c}{[64437-48-5]} \\
\mathrm{V}\end{array}$ & $\begin{array}{l}\text { (Z)-10-hexadecen-1-ol } \\
\quad(373-413)\end{array}$ & 111 & 298 & CGC & [2000OVA/KOU, 1994KOU/HOS] \\
\hline $\mathrm{C}_{16} \mathrm{H}_{32} \mathrm{O}$ & $\begin{array}{c}{[54502-94-2]} \\
\mathrm{V}\end{array}$ & $\begin{array}{l}\text { (E)-10-hexadecen-1-ol } \\
\quad(373-413)\end{array}$ & ol 111.5 & 298 & CGC & [2000OVA/KOU, 1994KOU/HOS] \\
\hline $\mathrm{C}_{16} \mathrm{H}_{32} \mathrm{O}$ & $\begin{array}{c}{[56683-54-6]} \\
V\end{array}$ & $\begin{array}{l}\text { (Z)-11-hexadecen-1-ol } \\
\quad(373-413)\end{array}$ & 111.3 & 298 & CGC & [2000OVA/KOU, 1994KOU/HOS] \\
\hline $\mathrm{C}_{16} \mathrm{H}_{32} \mathrm{O}$ & $\begin{array}{c}{[61301-56-2]} \\
\mathrm{V}\end{array}$ & $\begin{array}{l}\text { (E)-11-hexadecen-1-ol } \\
\quad(373-413)\end{array}$ & ol 111.8 & 298 & CGC & [2000OVA/KOU, 1994KOU/HOS] \\
\hline $\mathrm{C}_{16} \mathrm{H}_{32} \mathrm{O}$ & $\begin{array}{c}{[72698-34-1]} \\
\mathrm{V}\end{array}$ & $\begin{array}{l}\text { (Z)-12-hexadecen-1-ol } \\
\quad(373-413)\end{array}$ & 111.8 & 298 & CGC & [2000OVA/KOU, 1994KOU/HOS] \\
\hline $\mathrm{C}_{16} \mathrm{H}_{32} \mathrm{O}$ & $\begin{array}{c}{[72698-35-2]} \\
\mathrm{V}\end{array}$ & $\begin{array}{l}(E) \text {-12-hexadecen-1-ol } \\
\quad(373-413)\end{array}$ & ol 112.1 & 298 & CGC & [2000OVA/KOU, 1994KOU/HOS] \\
\hline $\mathrm{C}_{16} \mathrm{H}_{32} \mathrm{O}$ & $\begin{array}{c}{[69282-65-1]} \\
\mathrm{V}\end{array}$ & $\begin{array}{l}\text { (Z)-13-hexadecen-1-ol } \\
\quad(373-413)\end{array}$ & 112.3 & 298 & CGC & [2000OVA/KOU, 1994KOU/HOS] \\
\hline $\mathrm{C}_{16} \mathrm{H}_{32} \mathrm{O}$ & $\begin{array}{c}{[69282-66-2]} \\
\mathrm{V}\end{array}$ & $\begin{array}{l}\text { (E)-13-hexadecen-1-ol } \\
\quad(373-413)\end{array}$ & 112.6 & 298 & CGC & [2000OVA/KOU, 1994KOU/HOS] \\
\hline $\mathrm{C}_{16} \mathrm{H}_{32} \mathrm{O}$ & $\begin{array}{c}{[18787-63-8]} \\
\mathrm{V}\end{array}$ & $\begin{array}{l}\text { 2-hexadecanone } \\
\qquad(382-580)\end{array}$ & 72.3 & 397 & A & [1987STE/MAL] \\
\hline $\mathrm{C}_{16} \mathrm{H}_{32} \mathrm{O}$ & $\begin{array}{c}{[629-80-1]} \\
\mathrm{V} \\
\mathrm{V}\end{array}$ & $\begin{array}{r}\text { Hexadecanal } \\
(343-383) \\
(394-594)\end{array}$ & $\begin{array}{l}89.7 \\
67.6\end{array}$ & $\begin{array}{l}298 \\
409\end{array}$ & $\begin{array}{c}\text { CGC } \\
\mathrm{A}\end{array}$ & $\begin{array}{l}\text { [1996KOU/HOS, 2000OVA/KOU] } \\
\text { [1987STE/MAL, 1947STU] }\end{array}$ \\
\hline $\mathrm{C}_{16} \mathrm{H}_{32} \mathrm{O}_{2}$ & $\begin{array}{c}\text { [7132-64-1] } \\
\text { V } \\
\text { V } \\
\text { V } \\
\text { V }\end{array}$ & $\begin{array}{c}\text { Methyl pentadecanoate } \\
79 \\
(433-473)\end{array}$ & $\begin{array}{l}82.1 \\
79.8 \pm 0.2 \\
39.3 \pm 0.8 \\
88.8\end{array}$ & $\begin{array}{l}350 \\
372 \\
298 \\
298\end{array}$ & $\begin{array}{c}\text { CE } \\
\mathrm{CE} \\
\mathrm{CE} \\
\mathrm{CGC}\end{array}$ & $\begin{array}{l}{[2002 \mathrm{VAN} / \mathrm{VAN}]} \\
{[2002 \mathrm{VAN} / \mathrm{VAN}]} \\
{[2002 \mathrm{VAN} / \mathrm{VAN}]} \\
{[1995 \mathrm{CHI} / \mathrm{HOS}]}\end{array}$ \\
\hline
\end{tabular}


TABLE 12. Phase change enthalpies of $\mathrm{C}_{14}$ to $\mathrm{C}_{18}$ organic compounds-Continued

\begin{tabular}{|c|c|c|c|c|c|c|}
\hline \multirow[b]{2}{*}{$\begin{array}{l}\text { Molecular } \\
\text { formula }\end{array}$} & \multirow{2}{*}{$\begin{array}{l}\text { CAS Registry } \\
\text { Number } \\
\text { Enthalpy }\end{array}$} & \multicolumn{5}{|l|}{ Compound } \\
\hline & & $\begin{array}{c}\text { Temperature } \\
\text { range }\end{array}$ & $\begin{array}{l}\Delta_{\text {trans }} H_{\mathrm{m}} \\
(\mathrm{kJ} / \mathrm{mol})\end{array}$ & $\begin{array}{l}T_{\mathrm{m}} \\
(\mathrm{K})\end{array}$ & Method & References \\
\hline & $\mathrm{V}$ & & $91.6 \pm 0.9$ & 298 & $\mathrm{GC}, \mathrm{C}$ & [1980FUC/PEA] \\
\hline & $\mathrm{V}$ & & $93.5 \pm 1.0$ & 298 & $\mathrm{C}$ & [1977MAN/SEL] \\
\hline & $\mathrm{V}$ & $(295-303)$ & $87.9 \pm 1.3$ & 299 & & [1968BAC/NOV] \\
\hline & $\mathrm{V}$ & $(400-527)$ & 78.3 & 415 & $\mathrm{~A}, \mathrm{E}$ & [1987STE/MAL, 1963ROS/SCH] \\
\hline \multirow[t]{4}{*}{$\mathrm{C}_{16} \mathrm{H}_{32} \mathrm{O}_{2}$} & [124-06-1] & \multicolumn{5}{|l|}{ Ethyl tetradecanoate } \\
\hline & $\mathrm{V}$ & $(333-462)$ & 79.1 & 358 & Static & [2013BEN/KHI2] \\
\hline & $\mathrm{V}$ & $(446-492)$ & 79.0 & 464 & DSC & [2011SIL/FAL] \\
\hline & $\mathrm{V}$ & $(407-568)$ & 71.8 & 422 & A & [1987STE/MAL] \\
\hline \multirow[t]{3}{*}{$\mathrm{C}_{16} \mathrm{H}_{32} \mathrm{O}_{2}$} & [106-18-3] & \multicolumn{5}{|l|}{ Butyl dodecanoate } \\
\hline & $\mathrm{V}$ & $(423-483)$ & 89.2 & 298 & $\mathrm{GC}$ & [1997KRO/VEL] \\
\hline & $\mathrm{V}$ & $(343-383)$ & 75.8 & 358 & A & [1987STE/MAL] \\
\hline $\mathrm{C}_{16} \mathrm{H}_{32} \mathrm{O}_{2}$ & $\begin{array}{c}\text { [30673-38-2] } \\
\mathrm{V}\end{array}$ & \multicolumn{5}{|l|}{ Isobutyl dodecanoate } \\
\hline \multirow[t]{4}{*}{$\mathrm{C}_{16} \mathrm{H}_{32} \mathrm{O}_{2}$} & {$[638-59-5]$} & \multicolumn{5}{|l|}{ Tetradecyl acetate } \\
\hline & $\mathrm{V}$ & $(303-340)$ & $89.9 \pm 0.2$ & 298 & GS & [2006KRA/VER] \\
\hline & $\mathrm{V}$ & $(353-398)$ & 91.7 & 298 & GC & [1997KOU/HOS, 2000OVA/KOU] \\
\hline & $\mathrm{V}$ & $(411-462)$ & 72.7 & 426 & A & [1987STE/MAL] \\
\hline \multirow[t]{23}{*}{$\mathrm{C}_{16} \mathrm{H}_{32} \mathrm{O}_{2}$} & {$[57-10-3]$} & \multicolumn{5}{|c|}{ Hexadecanoic acid (palmitic acid) } \\
\hline & FUS & & 53.02 & 336.4 & DSC & [2016CAR/CON] \\
\hline & FUS & & 53.3 & 336.3 & DSC & [2014MAX/CAR] \\
\hline & FUS & & 56.18 & 333.75 & DSC & [2012BEN/KHI] \\
\hline & FUS & & 55.0 & 335.9 & DSC & [2010SAR/BIC] \\
\hline & FUS & & 55.9 & 335.4 & DSC & [2009COS/SAR] \\
\hline & TRS & & 3.8 & 318.8 & & \\
\hline & FUS & & 53.9 & 335.4 & DSC & [2009GBA/NEG] \\
\hline & FUS & & 51.37 & 332.7 & DSC & [2009ZEN/CAO] \\
\hline & TRS & & 3.1 & 316.7 & & \\
\hline & TRS & & 4.9 & 317.5 & & \\
\hline & FUS & & 53.0 & 334.7 & DSC & [2007MOR/COR] \\
\hline & FUS & & 47.0 & 336.5 & DSC & [2007MIS/MIS] \\
\hline & FUS & & 52.3 & 335.8 & DSC & [2004INO/HIS] \\
\hline & FUS & & 53.4 & 337.7 & DSC & [2001CED/PRI] \\
\hline & FUS & $(100-345)$ & 53.7 & 335.7 & $\mathrm{AC}$ & [1996DOM/HEA, 1982SCH/VAN] \\
\hline & FUS & & 51.5 & 334.6 & DSC & {$[1975 \mathrm{BER} / \mathrm{LEO}]$} \\
\hline & FUS & & 54.9 & 336.0 & DTA & [1996DOM/HEA, 1967PAC] \\
\hline & FUS & & 54.89 & 335.7 & $\mathrm{C}$ & [1996DOM/HEA, 1952WAR/SIN] \\
\hline & FUS & & 61.3 & 335.8 & & [1924STR/PAR] \\
\hline & SUB & & $193.8 \pm 11$ & 298 & TPD & [2008CAP/LOV] \\
\hline & SUB & $(273-303)$ & 134 & & TPTD & [2005CHA/ZIE] \\
\hline & SUB & $(294-316)$ & 154 & & TPTD & [2001CHA/TOB] \\
\hline
\end{tabular}

[Note: Experimental values based on the TPTD method are often inconsistent with values determined using other experimental methods.]

\begin{tabular}{|c|c|c|c|c|c|c|}
\hline & SUB & $(320-333)$ & $154.4 \pm 4.2$ & 326 & $\mathrm{ME}$ & [1961DAV/MAL, 1970COX/PIL, 1987STE/MAL] \\
\hline & V & & $121.3 \pm 4.2$ & 298 & CGC & [2015WIL/GOB] \\
\hline & $\mathrm{V}$ & & $121.6 \pm 7.4$ & 298 & CGC & [2013WIL/CHI] \\
\hline & $\mathrm{V}$ & $(440-625)$ & 97.5 & 455 & A & [1987STE/MAL] \\
\hline & $\mathrm{V}$ & $(347-374)$ & $110.2 \pm 2.0$ & 364 & ME,TE & [1982DEK/SCH] \\
\hline & $\mathrm{V}$ & & 90.1 & 475 & I & [1943CRA] \\
\hline $\mathrm{C}_{16} \mathrm{H}_{32} \mathrm{O}_{3}$ & [764-67-0] & 2-hydroxyhex & noic acid & & & \\
\hline & SUB & (294-311) & 121 & & TPTD & [2005CHA/ZIE] \\
\hline
\end{tabular}

[Note: Experimental values based on the TPTD method are often inconsistent with values determined using other experimental methods.]

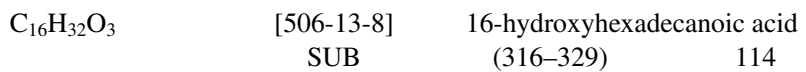

TPTD [2005CHA/ZIE]

[Note: Experimental values based on the TPTD method are often inconsistent with values determined using other experimental methods.] 
TABLE 12. Phase change enthalpies of $\mathrm{C}_{14}$ to $\mathrm{C}_{18}$ organic compounds-Continued

\begin{tabular}{|c|c|c|c|c|c|c|}
\hline \multirow[b]{2}{*}{$\begin{array}{l}\text { Molecular } \\
\text { formula }\end{array}$} & \multirow{2}{*}{$\begin{array}{l}\text { CAS Registry } \\
\text { Number } \\
\text { Enthalpy }\end{array}$} & \multicolumn{5}{|l|}{ Compound } \\
\hline & & $\begin{array}{l}\text { Temperature } \\
\text { range }\end{array}$ & $\begin{array}{l}\Delta_{\text {trans }} H_{\mathrm{m}} \\
(\mathrm{kJ} / \mathrm{mol})\end{array}$ & $\begin{array}{l}T_{\mathrm{m}} \\
(\mathrm{K})\end{array}$ & Method & References \\
\hline \multirow{2}{*}{$\mathrm{C}_{16} \mathrm{H}_{32} \mathrm{O}_{4}$} & {$[43091-27-6]$} & \multicolumn{5}{|c|}{ 6,6,14,14-tetramethyl-1,3,9,11-tetraoxacyclohexadecane } \\
\hline & FUS & & 29.71 & 358.6 & & [1973DAL/EKE] \\
\hline \multirow{2}{*}{$\mathrm{C}_{16} \mathrm{H}_{32} \mathrm{O}_{4}$} & [43091-28-7] & \multicolumn{5}{|c|}{ 2,2,10,10-tetramethyl-1,3,9,11-tetraoxacyclohexadecane } \\
\hline & FUS & & 25.94 & 371.3 & & {$[1973 \mathrm{DAL} / \mathrm{EKE}]$} \\
\hline \multirow[t]{2}{*}{$\mathrm{C}_{16} \mathrm{H}_{32} \mathrm{O}_{8}$} & {$[33089-37-1]$} & \multicolumn{5}{|c|}{$1,4,7,10,13,16,19,22$-octaoxacyclotetracosane } \\
\hline & FUS & & 34.5 & 292.2 & & [1972DAL/KRI] \\
\hline \multirow[t]{3}{*}{$\mathrm{C}_{16} \mathrm{H}_{33} \mathrm{Br}$} & [112-82-3] & \multicolumn{5}{|c|}{ 1-bromohexadecane } \\
\hline & $\mathrm{V}$ & & $94.4 \pm 1.5$ & 298 & $\mathrm{C}$ & [1996WEB/DEF2] \\
\hline & V & $(461-673)$ & 71.9 & 476 & $\mathrm{~A}, \mathrm{E}$ & [1987STE/MAL, 1961LI/ROS, 1970DYK/VAN] \\
\hline \multirow[t]{5}{*}{$\mathrm{C}_{16} \mathrm{H}_{33} \mathrm{Cl}$} & {$[4860-03-1]$} & \multicolumn{5}{|c|}{ 1-chlorohexadecane } \\
\hline & $\mathrm{V}$ & & 97.9 & 298 & & [2006BOL/NER2] \\
\hline & $\mathrm{V}$ & & $96.4 \pm 0.9$ & 298 & GS & [2001PUR/CHI] \\
\hline & $\mathrm{V}$ & & $91.8 \pm 1.1$ & 298 & $\mathrm{C}$ & [1977MAN/SEL] \\
\hline & $\mathrm{V}$ & $(439-600)$ & 73.3 & 454 & DTA & {$[1969 \mathrm{KEM} / \mathrm{KRE}]$} \\
\hline \multirow[t]{2}{*}{$\mathrm{C}_{16} \mathrm{H}_{33} \mathrm{~F}$} & {$[408-38-8]$} & \multicolumn{5}{|c|}{ 1-fluorohexadecane } \\
\hline & V & $(425-608)$ & 66.1 & 440 & $\mathrm{~A}, \mathrm{E}$ & [1970DYK/VAN] \\
\hline \multirow[t]{4}{*}{$\mathrm{C}_{16} \mathrm{H}_{33} \mathrm{I}$} & [544-77-4] & \multicolumn{5}{|c|}{ 1-iodohexadecane } \\
\hline & FUS & & 46.2 & 295.4 & DSC & [1992BAB/HWA, 1994BAB/BEN] \\
\hline & $\mathrm{V}$ & $(475-673)$ & 99.6 & 298 & $\mathrm{~A}, \mathrm{E}$ & $\begin{array}{l}\text { [1987STE/MAL, 1961LI/ROS, 1970DYK/VAN, } \\
\text { 2006BOL/NER] }\end{array}$ \\
\hline & $\mathrm{V}$ & $(475-673)$ & 73.0 & 490 & $\mathrm{~A}, \mathrm{E}$ & [1987STE/MAL, 1961LI/ROS, 1970DYK/VAN] \\
\hline \multirow[t]{4}{*}{$\mathrm{C}_{16} \mathrm{H}_{33} \mathrm{NO}$} & [629-54-9] & \multicolumn{5}{|c|}{ Hexadecanamide } \\
\hline & TRS & & 10.4 & 355.5 & & \\
\hline & FUS & & 45.4 & 376 & DSC & {$[2008 \mathrm{ABA} / \mathrm{BAD}]$} \\
\hline & SUB & $(364-378)$ & $181.6 \pm 1.3$ & 371 & ME & [1959DAV/JON2, 1987STE/MAL] \\
\hline $\mathrm{C}_{16} \mathrm{H}_{33} \mathrm{NO}$ & {$[74534-10-4]$} & $N$-hexyldecan & & & & \\
\hline & TRS & & 6.0 & 301 & & \\
\hline & FUS & & 31.0 & 311 & DSC & [1980CAR/BUS] \\
\hline $\mathrm{C}_{16} \mathrm{H}_{33} \mathrm{NO}$ & [6284-08-8] & $N$-butyldodec & & & & \\
\hline & FUS & & 39.0 & 322.1 & DSC & [1980CAR/BUS] \\
\hline $\mathrm{C}_{16} \mathrm{H}_{33} \mathrm{NO}$ & {$[57303-23-8]$} & $N, N$-dibutylo & mide & & & \\
\hline & V & $(463-513)$ & $75.6 \pm 0.7$ & 298 & CGC & [2009PAN/ANT] \\
\hline $\mathrm{C}_{16} \mathrm{H}_{34}$ & {$[544-76-3]$} & Hexadecane & & & & \\
\hline & FUS & & 53.2 & 290.9 & DSC & [2015VEL/KHA] \\
\hline & FUS & & 57.7 & 291.4 & DSC & [2013JEO/JEO] \\
\hline & FUS & & 53.0 & & DSC & [2005ESP/WHI] \\
\hline & FUS & & 53.25 & 290.6 & DSC & [2005HUA/SIM] \\
\hline & FUS & & 53.0 & 290.7 & DSC & [2004MON/RAJ] \\
\hline & FUS & & 53.0 & 290.7 & DSC & [1999MET/RAJ] \\
\hline & FUS & & $\mathrm{U} 48.9$ & 293.2 & DSC & [1992BAB/HWA, 1994BAB/BEN] \\
\hline & FUS & & 53.35 & 291.3 & & [1996DOM/HEA, 1954FIN/GRO2] \\
\hline & FUS & & 51.54 & 291.1 & & [1996DOM/HEA, 1949PAR/MOO2] \\
\hline & SUB & & 135.1 & 298 & $\mathrm{~B}$ & [1972MOR3] \\
\hline & SUB & & 134.9 & 291 & $\mathrm{~B}$ & [1963BON] \\
\hline & SUB & $(288-290)$ & $\mathrm{U} 83.4 \pm 8$ & & $\mathrm{ME}$ & [1949BRA/SHE] \\
\hline & $\mathrm{V}$ & $(303-364)$ & 74.9 & 333 & GS & [2012VER/RAL] \\
\hline & $\mathrm{V}$ & $(303-364)$ & $80.7 \pm 0.4$ & 298 & GS & [2012VER/RAL] \\
\hline & $\mathrm{V}$ & $(323-383)$ & 74.5 & 353 & TGA & [2012VER/RAL] \\
\hline & $\mathrm{V}$ & $(323-383)$ & $81.7 \pm 0.8$ & 298 & TGA & [2012VER/RAL] \\
\hline & $\mathrm{V}$ & & $81.8 \pm 1.3$ & 298 & CGC & [2000NIC/ORF] \\
\hline & $\mathrm{V}$ & $(453-503)$ & 81.4 & 298 & CGC & [1995CHI/HOS] \\
\hline & $\mathrm{V}$ & $(423-473)$ & 81.4 & 298 & CGC & [1995CHI/HOS] \\
\hline & $\mathrm{V}$ & $(363-413)$ & 81.2 & 298 & CGC & [1995CHI/HOS] \\
\hline
\end{tabular}


TABLE 12. Phase change enthalpies of $\mathrm{C}_{14}$ to $\mathrm{C}_{18}$ organic compounds-Continued

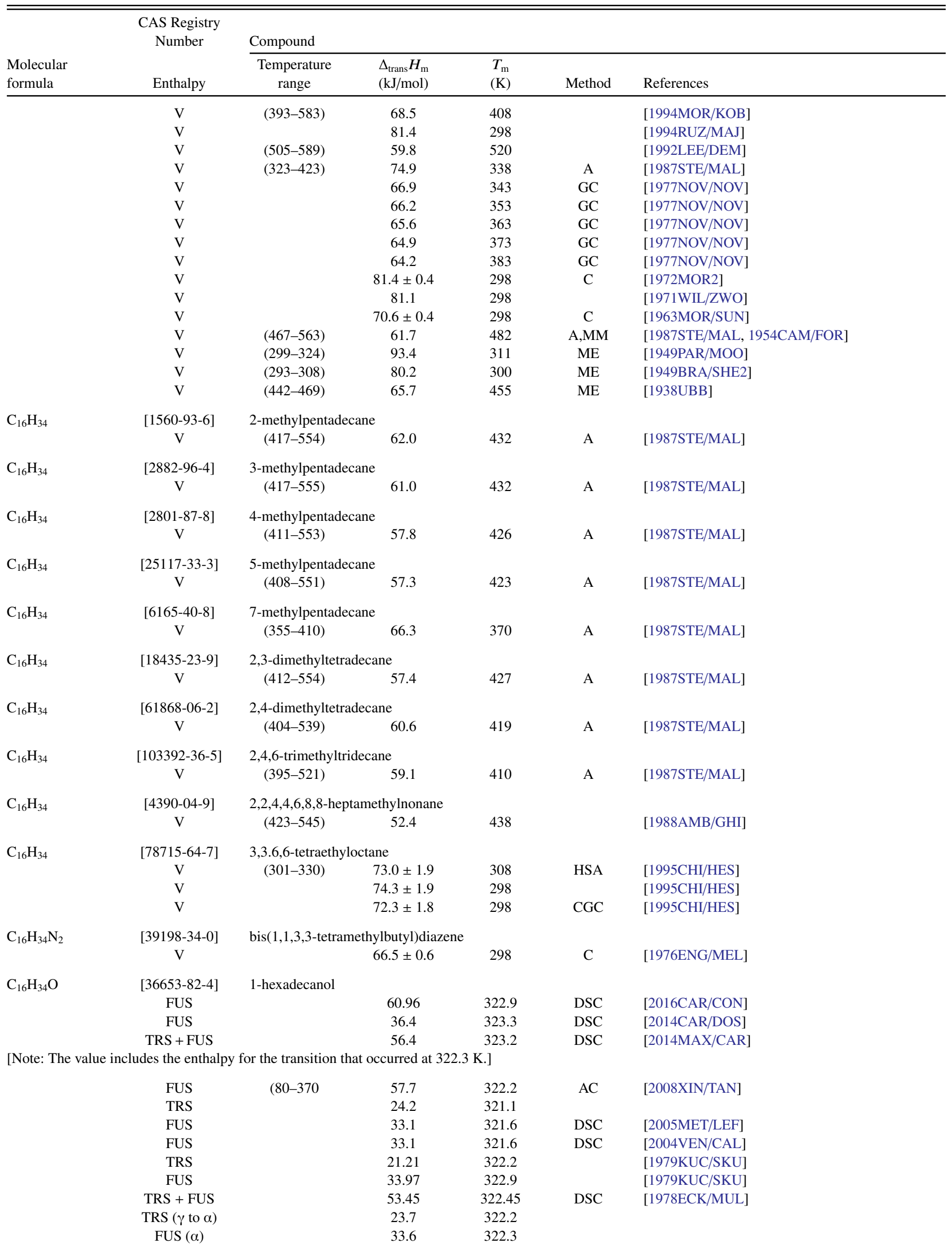


TABLE 12. Phase change enthalpies of $\mathrm{C}_{14}$ to $\mathrm{C}_{18}$ organic compounds-Continued

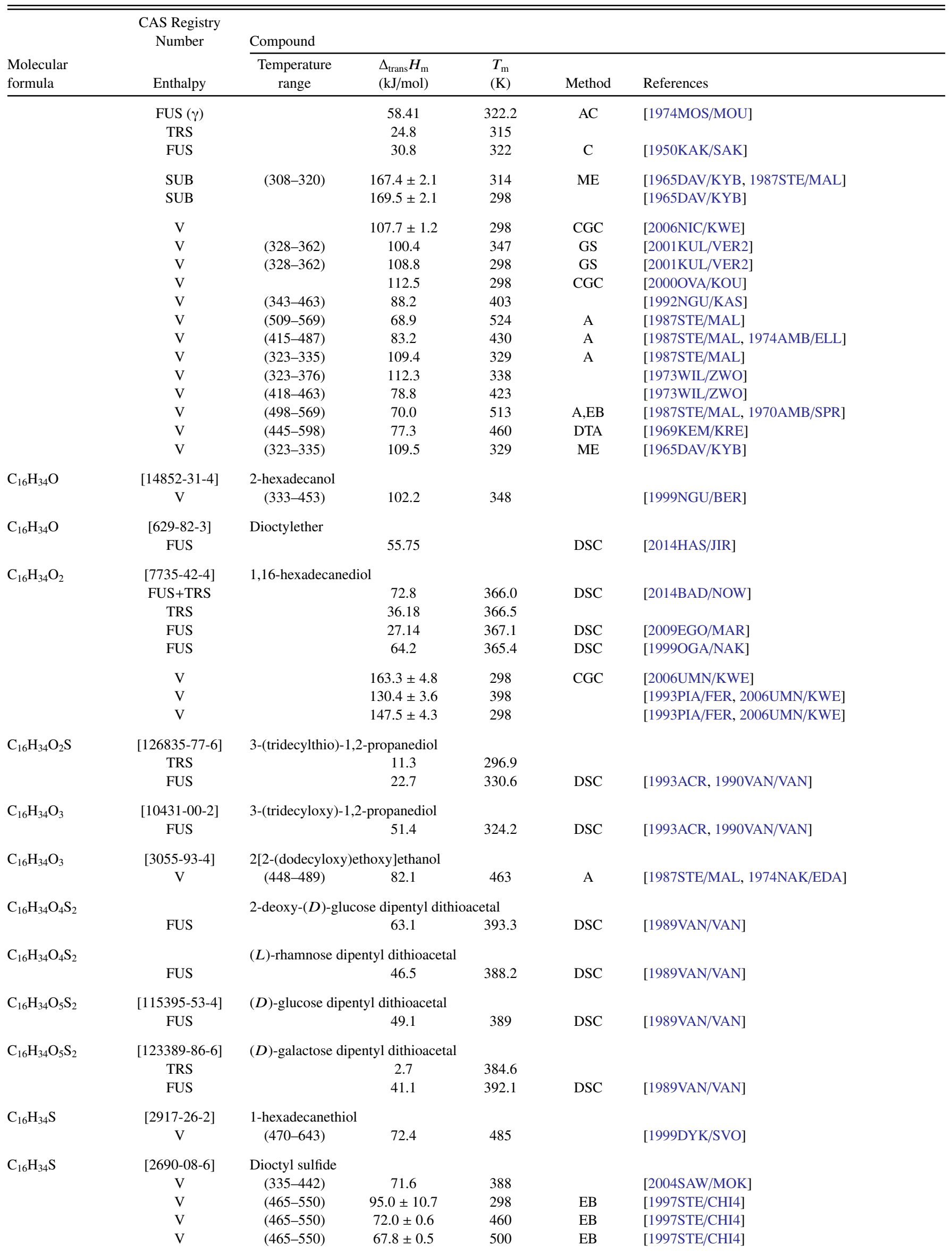


TABLE 12. Phase change enthalpies of $\mathrm{C}_{14}$ to $\mathrm{C}_{18}$ organic compounds-Continued

\begin{tabular}{|c|c|c|c|c|c|c|}
\hline \multirow[b]{2}{*}{$\begin{array}{l}\text { Molecular } \\
\text { formula }\end{array}$} & \multirow{2}{*}{$\begin{array}{l}\text { CAS Registry } \\
\text { Number } \\
\text { Enthalpy }\end{array}$} & \multicolumn{5}{|l|}{ Compound } \\
\hline & & $\begin{array}{l}\text { Temperature } \\
\text { range }\end{array}$ & $\begin{array}{l}\Delta_{\text {trans }} H_{\mathrm{m}} \\
(\mathrm{kJ} / \mathrm{mol})\end{array}$ & $\begin{array}{l}T_{\mathrm{m}} \\
(\mathrm{K})\end{array}$ & Method & References \\
\hline & $\mathrm{V}$ & $(465-550)$ & $63.7 \pm 0.6$ & 540 & EB & [1997STE/CHI4] \\
\hline $\mathrm{C}_{16} \mathrm{H}_{34} \mathrm{~S}_{2}$ & $\begin{array}{c}{[822-27-5]} \\
V\end{array}$ & $\begin{array}{l}\text { Diheptyl disulfide } \\
\quad(479-656)\end{array}$ & 73.9 & 494 & & [1999DYK/SVO] \\
\hline $\mathrm{C}_{16} \mathrm{H}_{35} \mathrm{~N}$ & $\begin{array}{c}{[112-75-4]} \\
V\end{array}$ & $\mathrm{~N}, \mathrm{~N}$-dimethyltetrac & $\begin{array}{l}\text { ecylamine } \\
77.3 \pm 1.9\end{array}$ & 298 & CGC & [2014GOB/VIK] \\
\hline $\mathrm{C}_{16} \mathrm{H}_{35} \mathrm{~N}$ & $\begin{array}{c}\text { [99916-30-0] } \\
\mathrm{V}\end{array}$ & $\begin{array}{l}N, N \text {-dimethyl-2-pe } \\
\quad(401-552)\end{array}$ & $\begin{array}{l}\text { tylnonylamine } \\
64.8\end{array}$ & 425 & EB & [1987MIL/FEN2] \\
\hline $\mathrm{C}_{16} \mathrm{H}_{35} \mathrm{NO}_{2}$ & $\begin{array}{c}{[126835-68-5]} \\
\text { FUS }\end{array}$ & 3-(tridecylamino)- & $\begin{array}{l}\text { 2-propanediol } \\
\quad 68.7\end{array}$ & 354.9 & DSC & [1993ACR, 1990VAN/VAN] \\
\hline $\mathrm{C}_{16} \mathrm{H}_{36} \mathrm{~N}_{2}$ & $\begin{array}{c}{[60678-70-8]} \\
V\end{array}$ & $\begin{array}{l}\text { Tetrabutyl hydrazir } \\
\quad(392-453)\end{array}$ & 51.1 & 407 & A & [1987STE/MAL, 1943WES/EUC] \\
\hline
\end{tabular}


TABle 13. Phase change enthalpies of $\mathrm{C}_{17}$ to $\mathrm{C}_{18}$ organic compounds

\begin{tabular}{|c|c|c|c|c|c|c|}
\hline \multirow[b]{2}{*}{$\begin{array}{l}\text { Molecular } \\
\text { formula }\end{array}$} & \multirow{2}{*}{$\begin{array}{c}\text { CAS Registry } \\
\text { Number } \\
\text { Enthalpy }\end{array}$} & \multicolumn{5}{|l|}{ Compound } \\
\hline & & $\begin{array}{c}\text { Temperature } \\
\text { Range }\end{array}$ & $\begin{array}{l}\Delta_{\text {trans }} H_{\mathrm{m}} \\
(\mathrm{kJ} \mathrm{mol})\end{array}$ & $\begin{array}{l}T_{\mathrm{m}} \\
(\mathrm{K})\end{array}$ & Method & Reference \\
\hline \multirow[t]{2}{*}{$\mathrm{C}_{17} \mathrm{H}_{10} \mathrm{CIN}_{3} \mathrm{O}_{3}$} & [191978-90-2] & \multicolumn{5}{|c|}{ 1-(4-chlorobenzoyl)-1,2-dihydro-6-nitro-1-quinolinecarbonitrile } \\
\hline & FUS & & 25.62 & 430.5 & DSC & {$[2005 \mathrm{LIZ} / \mathrm{ZAB}]$} \\
\hline \multirow[t]{20}{*}{$\mathrm{C}_{17} \mathrm{H}_{10} \mathrm{O}$} & {$[82-05-3]$} & \multicolumn{5}{|l|}{ Benzanthrone } \\
\hline & FUS & & 26.36 & 446.8 & $\mathrm{CVC}$ & {$[2014 \mathrm{FON} / \mathrm{GUS}]$} \\
\hline & SUB & & $127.3 \pm 0.6$ & 298 & $\mathrm{ME}$ & [2014FON/GUS] \\
\hline & SUB & $(388-402)$ & $122.5 \pm 0.6$ & 395 & $\mathrm{ME}$ & [2011SAN/LIM] \\
\hline & SUB & $(388-402)$ & $125.3 \pm 1.1$ & 298 & $\mathrm{ME}$ & [2011SAN/LIM] \\
\hline & SUB & (389-403) & $122.6 \pm 0.7$ & 396 & ME & [2011SAN/LIM] \\
\hline & SUB & $(389-403)$ & $125.4 \pm 1.1$ & 298 & $\mathrm{ME}$ & [2011SAN/LIM] \\
\hline & SUB & $(392-408)$ & $122.4 \pm 0.5$ & 400 & $\mathrm{ME}$ & [2011SAN/LIM] \\
\hline & SUB & $(392-408)$ & $125.4 \pm 1.0$ & 298 & $\mathrm{ME}$ & [2011SAN/LIM] \\
\hline & SUB & $(390-410)$ & $122.6 \pm 0.6$ & 400 & $\mathrm{ME}$ & [2006RIB/MON] \\
\hline & SUB & $(390-410)$ & $125.6 \pm 0.6$ & 298 & $\mathrm{ME}$ & [2006RIB/MON] \\
\hline & SUB & (389-409) & $121.6 \pm 0.6$ & 399 & ME & [1999RIB/FER] \\
\hline & SUB & $(389-409)$ & $126.6 \pm 0.6$ & 298 & $\mathrm{ME}$ & [1999RIB/FER] \\
\hline & SUB & $(373-393)$ & $129.7 \pm 2.1$ & 298 & QR & [1999RIB/FER] \\
\hline & SUB & $(373-393)$ & $125.5 \pm 2.1$ & 382 & QR & [1999RIB/FER] \\
\hline & SUB & $(353-388)$ & $119.7 \pm 5.4$ & 370 & $\mathrm{ME}$ & [1984BUR/MOR] \\
\hline & SUB & $(353-388)$ & $124.6 \pm 6.0$ & 298 & ME & [1984BUR/MOR] \\
\hline & SUB & & $114.2 \pm 0.8$ & & QR & [1979YAN/TEP] \\
\hline & SUB & & 115.5 & 398 & $\mathrm{ME}$ & [1952INO/SHI, 1960JON] \\
\hline & $\mathrm{V}$ & $(498-673)$ & 91.4 & 513 & A & [1987STE/MAL, 1947STU] \\
\hline \multirow[t]{2}{*}{$\mathrm{C}_{17} \mathrm{H}_{10} \mathrm{O}$} & [116232-62-3] & \multicolumn{5}{|c|}{ Benzo $[a]$ fluorenone } \\
\hline & FUS & & 15.6 & 406.9 & $\mathrm{DSC}$ & [2010KES/AUC] \\
\hline \multirow[t]{6}{*}{$\mathrm{C}_{17} \mathrm{H}_{10} \mathrm{O}$} & & \multicolumn{5}{|c|}{ 1-pyrenecarboxaldehy de } \\
\hline & TRS & & 2.8 & 340.4 & & [2015SAN/OLI] \\
\hline & FUS & & 11.0 & 399.9 & $\mathrm{DSC}$ & [2015SAN/OLI] \\
\hline & SUB & $(377-400)$ & $110.9 \pm 0.4$ & 388 & $\mathrm{ME}$ & [2015SAN/OLI] \\
\hline & SUB (I) & $(377-400)$ & $114.6 \pm 0.4$ & 298 & $\mathrm{ME}$ & [2015SAN/OLI] \\
\hline & SUB (II) & $(377-400)$ & $117.4 \pm 0.4$ & 298 & $\mathrm{ME}$ & {$[2015 \mathrm{SAN} / \mathrm{OLI}]$} \\
\hline \multirow[t]{2}{*}{$\mathrm{C}_{17} \mathrm{H}_{11} \mathrm{ClN}_{2} \mathrm{O}_{2}$} & {$[191979-25-6]$} & \multicolumn{5}{|c|}{ 2-cyano-1(2H)-quinolinecarboxylic acid, 4-chlorophenyl ester } \\
\hline & FUS & & 37.19 & 424.2 & DSC & [2005LIZ/ZAB] \\
\hline \multirow[t]{2}{*}{$\mathrm{C}_{17} \mathrm{H}_{11} \mathrm{~N}$} & {$[225-11-6]$} & \multicolumn{5}{|c|}{ Benz $[a]$ acridine } \\
\hline & FUS & & 21.9 & 402.8 & $\mathrm{DSC}$ & [2010KES/AUC] \\
\hline \multirow[t]{2}{*}{$\mathrm{C}_{17} \mathrm{H}_{11} \mathrm{~N}$} & [225-51-4] & \multicolumn{5}{|c|}{ Benz $[c]$ acridine } \\
\hline & FUS & & 20.3 & 381.4 & DSC & [2010KES/AUC] \\
\hline \multirow[t]{5}{*}{$\mathrm{C}_{17} \mathrm{H}_{12}$} & {$[238-84-6]$} & \multicolumn{5}{|c|}{ 1,2-benzofluorene } \\
\hline & TRS & & 3.8 & 399.9 & & \\
\hline & FUS & & 18.4 & 462.8 & DSC & [1996DOM/HEA, 1979FAR/SHA] \\
\hline & SUB & $(313-453)$ & 105.4 & 383 & GS & [1995NAS/LEN] \\
\hline & $\mathrm{V}$ & $(323-473)$ & 83.7 & 398 & GC & [2002LEI/CHA] \\
\hline $\mathrm{C}_{17} \mathrm{H}_{12}$ & [243-17-4] & 2,3-benzofluor & & & & \\
\hline & FUS & & 23.4 & 489.7 & $\mathrm{DSC}$ & [1996DOM/HEA, 1979FAR/SHA] \\
\hline & SUB & $(344-398)$ & $119.3 \pm 1.3$ & 371 & ME & [1998OJA/SUU] \\
\hline & SUB & $(313-453)$ & 111.2 & 383 & GS & [1995NAS/LEN] \\
\hline & V & & $97.5 \pm 3.9$ & 298 & CGC & [2008HAN/NUT] \\
\hline & $\mathrm{V}$ & $(323-473)$ & 84.7 & 398 & $\mathrm{GC}$ & [2002LEI/CHA] \\
\hline $\mathrm{C}_{17} \mathrm{H}_{12}$ & {$[2381-21-7]$} & 1-methylpyren & & & & \\
\hline & $\mathrm{V}$ & $(423-493)$ & $92.3 \pm 1.3$ & 298 & GC & [2006HAF/PAR] \\
\hline $\mathrm{C}_{17} \mathrm{H}_{12} \mathrm{Cl}_{2} \mathrm{~N}_{4}$ & {$[28911-01-5]$} & 8-chloro-6-(2-c & ophenyl)-1-m & $-4 H-[1$, & azolo[4,3-a] & 1,4]benzodiazepme (triazolam) \\
\hline & FUS & & 41.0 & 514.5 & DSC & [2008WAS/HOL] \\
\hline
\end{tabular}


TABLE 13. Phase change enthalpies of $\mathrm{C}_{17}$ to $\mathrm{C}_{18}$ organic compounds-Continued

\begin{tabular}{|c|c|c|c|c|c|c|}
\hline \multirow[b]{2}{*}{$\begin{array}{l}\text { Molecular } \\
\text { formula }\end{array}$} & \multirow{2}{*}{$\begin{array}{l}\text { CAS Registry } \\
\text { Number } \\
\text { Enthalpy }\end{array}$} & \multicolumn{5}{|l|}{ Compound } \\
\hline & & $\begin{array}{c}\text { Temperature } \\
\text { range }\end{array}$ & $\begin{array}{l}\Delta_{\text {trans }} H_{\mathrm{m}} \\
(\mathrm{kJ} / \mathrm{mol})\end{array}$ & $\begin{array}{l}T_{\mathrm{m}} \\
(\mathrm{K})\end{array}$ & Method & References \\
\hline $\mathrm{C}_{17} \mathrm{H}_{12} \mathrm{~N}_{2} \mathrm{O}_{2}$ & $\begin{array}{c}\text { [40448-93-9] } \\
\text { FUS }\end{array}$ & \multicolumn{5}{|c|}{ 2-cyano-1(2H)-quinolinecarboxylic acid, phenyl ester } \\
\hline $\mathrm{C}_{17} \mathrm{H}_{12} \mathrm{O}$ & $\begin{array}{c}\text { [152464-07-8] } \\
\text { FUS }\end{array}$ & \multicolumn{5}{|c|}{ 4-ethynyl-1-[(4-ethynylphenyl)methoxy]benzene } \\
\hline $\mathrm{C}_{17} \mathrm{H}_{12} \mathrm{O}_{2}$ & $\begin{array}{c}\text { [24776-44-1] } \\
\text { FUS }\end{array}$ & \multicolumn{4}{|c|}{ 4-benzoyl-1-naphthol } & [1991ACR, 1990DOM2] \\
\hline $\mathrm{C}_{17} \mathrm{H}_{12} \mathrm{O}_{2}$ & $\begin{array}{c}\text { [6333-07-9] } \\
\text { FUS }\end{array}$ & \multicolumn{4}{|c|}{ 1-benzoyl-2-naphthol } & [1991ACR, 1990DOM2] \\
\hline $\mathrm{C}_{17} \mathrm{H}_{12} \mathrm{O}_{2}$ & $\begin{array}{c}\text { [21009-99-4] } \\
\text { FUS }\end{array}$ & \multicolumn{4}{|c|}{ 2-benzoyl-1-naphthol } & [1991ACR, 1990DOM2] \\
\hline $\mathrm{C}_{17} \mathrm{H}_{12} \mathrm{O}_{2}$ & $\begin{array}{c}{[93-44-7]} \\
\text { FUS }\end{array}$ & \multicolumn{5}{|c|}{ 2-naphthyl benzoate } \\
\hline $\mathrm{C}_{17} \mathrm{H}_{13} \mathrm{CIN}_{4}$ & $\begin{array}{c}\text { [28981-97-7] } \\
\text { FUS } \\
\text { FUS }\end{array}$ & 8-chloro-1-me & $\begin{array}{c}\text { 5-phenyl-4H } \\
32.0 \\
30.68\end{array}$ & $\begin{array}{c}\text { 4]triazol } \\
501.8 \\
500.2\end{array}$ & $\begin{array}{l}\text { a][1,4]-b } \\
\text { DSC } \\
\text { DSC }\end{array}$ & $\begin{array}{l}\text { diazepine (alprazolam) } \\
\text { [2008WAS/HOL] } \\
\text { [2007NOV/PEE] }\end{array}$ \\
\hline $\mathrm{C}_{17} \mathrm{H}_{13} \mathrm{~F}_{3} \mathrm{O}$ & $\begin{array}{c}\text { [172424-70-3] } \\
\text { FUS }\end{array}$ & \multicolumn{4}{|c|}{ 4- $n$-propoxy-2', 3', 4'-trifluorodiphenylacetylene } & [1995HSU/TSA] \\
\hline \multirow[t]{2}{*}{$\mathrm{C}_{17} \mathrm{H}_{13} \mathrm{~N}$} & $\begin{array}{l}{[6626-64-8]} \\
\text { SUB }\end{array}$ & \multicolumn{5}{|c|}{ 5-methyl-5H-indeno[2,1-b] quinoline } \\
\hline & $\mathrm{V}$ & $(375-388)$ & 122.2 & 381 & A & [1966GEI/QUI, 1970COX/PIL] \\
\hline $\mathrm{C}_{17} \mathrm{H}_{14} \mathrm{~F}_{3} \mathrm{~N}_{3} \mathrm{O}_{2} \mathrm{~S}$ & $\begin{array}{c}\text { [169590-42-5] } \\
\text { FUS } \\
\text { FUS } \\
\text { FUS } \\
\text { FUS } \\
\text { FUS } \\
\text { FUS }\end{array}$ & 4-[5-(3-methy] & $\begin{array}{c}\text { yl)-3-(trifluo } \\
38.48 \\
32.07 \\
34.9 \\
37.42 \\
31.86 \\
34.35\end{array}$ & $\begin{array}{c}\text { hyl })-1 H \\
433.2 \\
433.8 \\
434.3 \\
436 \\
437 \\
436\end{array}$ & $\begin{array}{c}\text { ol-1-yl]- } \\
\text { DSC } \\
\text { DSC } \\
\text { DSC } \\
\text { DSC } \\
\text { DSC }\end{array}$ & $\begin{array}{l}\text { zenesulphonamide (celecoxib) } \\
\text { [2015GAU/VAN] } \\
{[2015 \mathrm{NUR} / \mathrm{BOO}]} \\
{[2013 \mathrm{MOD} / \mathrm{DAN}]} \\
{[2010 \mathrm{BAI} / \mathrm{VAN}]} \\
{[2009 \mathrm{THI} / \mathrm{SUB}]} \\
{[2003 \mathrm{CHA} / \mathrm{GUP}]}\end{array}$ \\
\hline $\mathrm{C}_{17} \mathrm{H}_{14} \mathrm{~N}_{2} \mathrm{O}_{2}$ & $\begin{array}{l}{[1229-55-6]} \\
\text { SUB }\end{array}$ & $\begin{array}{l}\text { 1-[(2-methoxy } \\
\text { (374-388) }\end{array}$ & $\begin{array}{l}\text { yl)azo]-2-hyr } \\
142.4 \pm 2.2\end{array}$ & $\begin{array}{l}\text { phthale } \\
381\end{array}$ & & [1984KRI] \\
\hline $\mathrm{C}_{17} \mathrm{H}_{14} \mathrm{~N}_{2} \mathrm{O}_{2}$ & $\begin{array}{l}\text { [1156-51-0] } \\
\text { FUS }\end{array}$ & \multicolumn{5}{|c|}{ 2,2-bis(4-cyanatophenyl)propane } \\
\hline $\mathrm{C}_{17} \mathrm{H}_{14} \mathrm{~N}_{4} \mathrm{O}_{3}$ & $\begin{array}{c}{[243445-12-7]} \\
\text { FUS }\end{array}$ & \multicolumn{5}{|c|}{ 2[4,5-dihydro-5-oxo-4-phenyl-3-(2-pyridyl)-1,2,4-triazine-6(1H)-ylidene]acetic acid, methyl ester } \\
\hline \multirow[t]{2}{*}{$\mathrm{C}_{17} \mathrm{H}_{14} \mathrm{O}$} & $\begin{array}{c}\text { [24330-03-8] } \\
\text { FUS }\end{array}$ & \multicolumn{5}{|c|}{ 2:3,6:7-dibenzobicyclo[3.2.2]nona-2,6-dien-4-one } \\
\hline & $\mathrm{V}$ & & $94.5 \pm 2.2$ & 298 & CGC & [2006PER/CON] \\
\hline $\mathrm{C}_{17} \mathrm{H}_{14} \mathrm{O}_{4} \mathrm{~S}$ & $\begin{array}{c}\text { [162011-90-7] } \\
\text { FUS }\end{array}$ & 3-phenyl-4-[4- & $\begin{array}{l}\text { ylsulfonyl)p } \\
11.98\end{array}$ & $\begin{array}{r}-2(5 H) \\
482.1\end{array}$ & $\begin{array}{l}\text { one (rofe } \\
\text { DSC }\end{array}$ & $\begin{array}{l}\text { ib) } \\
\text { [2008TUN/TAB] }\end{array}$ \\
\hline $\mathrm{C}_{17} \mathrm{H}_{14} \mathrm{O}_{5}$ & $\begin{array}{l}{[117-52-2]} \\
\text { FUS }\end{array}$ & 3-[1-(2-furany] & $\begin{array}{l}\text { xobutyl]-4-h } \\
33.88\end{array}$ & $\begin{array}{c}\mathrm{y}-2 H-1 \\
391.8\end{array}$ & $\begin{array}{l}\text { pyran-2- } \\
\text { DSC }\end{array}$ & [1990DON/DRE] \\
\hline
\end{tabular}


TABLE 13. Phase change enthalpies of $\mathrm{C}_{17}$ to $\mathrm{C}_{18}$ organic compounds-Continued

\begin{tabular}{|c|c|c|c|c|c|c|}
\hline \multirow[b]{2}{*}{$\begin{array}{l}\text { Molecular } \\
\text { formula }\end{array}$} & \multirow{2}{*}{$\begin{array}{l}\text { CAS Registry } \\
\text { Number } \\
\text { Enthalpy }\end{array}$} & \multicolumn{5}{|l|}{ Compound } \\
\hline & & $\begin{array}{l}\text { Temperature } \\
\text { range }\end{array}$ & $\begin{array}{l}\Delta_{\text {trans }} H_{\mathrm{m}} \\
(\mathrm{kJ} / \mathrm{mol})\end{array}$ & $\begin{array}{l}T_{\mathrm{m}} \\
(\mathrm{K})\end{array}$ & Method & References \\
\hline & FUS & & 24.1 & 324 & DSC & [1995HSU/TSA] \\
\hline $\mathrm{C}_{17} \mathrm{H}_{15} \mathrm{FO}$ & $\begin{array}{c}{[145532-20-3]} \\
\text { FUS }\end{array}$ & \multicolumn{4}{|c|}{ 4- $n$-propoxy-4'-fluorodiphenylacetylene } & [1995HSU/TSA] \\
\hline $\mathrm{C}_{17} \mathrm{H}_{15} \mathrm{NO}_{2}$ & $\begin{array}{l}\text { [154924-24-0] } \\
\text { FUS }\end{array}$ & \multicolumn{4}{|c|}{ 1-[(4-nitrophenyl)ethynyl]-4-propylbenzene } & [2002SPA/DZI] \\
\hline $\mathrm{C}_{17} \mathrm{H}_{15} \mathrm{NO}_{3}$ & $\begin{array}{c}{[483362-78-3]} \\
\text { FUS }\end{array}$ & \multicolumn{4}{|c|}{ 1-[(4-nitrophenyl)ethynyl]-4-propoxy benzene } & [2002SPA/DZI] \\
\hline $\mathrm{C}_{17} \mathrm{H}_{15} \mathrm{NO}_{3}$ & $\begin{array}{l}\text { [31842-01-0] } \\
\text { FUS } \\
\text { FUS }\end{array}$ & 4-(1,3-dihydro- & $\begin{array}{c}-2 H \text {-isoindo } \\
36.04 \\
40.3\end{array}$ & $\begin{array}{l}-\alpha \text {-met } \\
485 \\
484.6\end{array}$ & $\begin{array}{l}\text { DSCeneacet } \\
\text { DSC }\end{array}$ & $\begin{array}{l}\text { cid ( } \pm \text { )-indoprofen } \\
\text { [2010BAI/VAN] } \\
\text { [2006WAS/HOL, 2008WAS/HOL] }\end{array}$ \\
\hline $\mathrm{C}_{17} \mathrm{H}_{15} \mathrm{NO}_{3} \mathrm{~S}$ & $\begin{array}{c}{[313057-10-2]} \\
\text { FUS }\end{array}$ & \multicolumn{4}{|c|}{ 4-(4-pentenyloxy)phenyl 5-cyano-2-thiophene carboxylate } & [2000WU/WAN] \\
\hline $\mathrm{C}_{17} \mathrm{H}_{15} \mathrm{~N}_{3} \mathrm{O}_{3}$ & $\begin{array}{c}{[111888-46-1]} \\
\text { SUB }\end{array}$ & \multicolumn{5}{|c|}{ 3-methyl- $N$-(2-methylphenyl)-2-quinoxalinecarboxamide-1,4-dioxide } \\
\hline $\mathrm{C}_{17} \mathrm{H}_{16} \mathrm{Br}_{2} \mathrm{O}_{3}$ & $\begin{array}{l}{[18181-80-1]} \\
\text { FUS }\end{array}$ & isopropyl $4,4^{\prime}-\mathrm{c}$ & $\begin{array}{l}\text { mobenzilate } \\
24.55\end{array}$ & 348.1 & DSC & [1996DOM/HEA, 1990DON/DRE] \\
\hline \multirow[t]{3}{*}{$\mathrm{C}_{17} \mathrm{H}_{16} \mathrm{C}_{1 \mathrm{~N}_{5}} \mathrm{O}_{3}$} & $\begin{array}{l}{[40880-51-1]} \\
\text { FUS }\end{array}$ & \multicolumn{5}{|c|}{ 3-[[4-[(2-chloro-4-nitrophenyl)azo]phenyl](2-hydroxyethyl)amino]propanenitrile } \\
\hline & SUB & $(398-408)$ & 127.1 & 403 & GC & [2002SAW/SHI] \\
\hline & $\mathrm{V}$ & $(423-503)$ & 104.9 & 463 & GC & [2002SAW/SHI] \\
\hline $\mathrm{C}_{17} \mathrm{H}_{16} \mathrm{~F}_{4} \mathrm{~N}_{4} \mathrm{O}_{2}$ & $\begin{array}{l}{[91488-84-5]} \\
\text { SUB }\end{array}$ & \multicolumn{5}{|c|}{$N$-ethyl-N-(2,2,3,3-tetrafluoropropyl)-4-[4-nitrophenyl]azobenzenamine } \\
\hline $\mathrm{C}_{17} \mathrm{H}_{16} \mathrm{~F}_{4} \mathrm{~N}_{4} \mathrm{O}_{4}$ & $\begin{array}{c}{[1543-74-4]} \\
\text { SUB }\end{array}$ & \multicolumn{5}{|c|}{ 2-[[4-(4-nitrophenyl)azo]phenyl](2,2,3,3-tetrafluoropropyl)amino]ethanol } \\
\hline $\mathrm{C}_{17} \mathrm{H}_{16} \mathrm{~N}_{2} \mathrm{O}_{4}$ & $\begin{array}{c}{[129555-39-1]} \\
\text { FUS }\end{array}$ & \multicolumn{5}{|c|}{ 5-phenoyxmethyl-3-phenylcarbamoyl-2-oxazolidone } \\
\hline $\mathrm{C}_{17} \mathrm{H}_{16} \mathrm{OS}$ & $\begin{array}{l}\text { [37014-01-0] } \\
\text { SUB } \\
\text { SUB }\end{array}$ & tetrahydro-2,6-c & $\begin{array}{c}\text { nyl- } 4 H \text {-thio } \\
136 \\
144 \pm 3\end{array}$ & $\begin{array}{c}-4-\text { one } \\
375 \\
298\end{array}$ & $\begin{array}{l}\mathrm{ME} \\
\mathrm{ME}\end{array}$ & $\begin{array}{l}\text { [1972GEI/SAW] } \\
{[1972 \mathrm{GEI} / \mathrm{SAW}, 1977 \mathrm{PED} / \mathrm{RYL}]}\end{array}$ \\
\hline \multirow[t]{2}{*}{$\mathrm{C}_{17} \mathrm{H}_{16} \mathrm{O}_{4}$} & $\begin{array}{c}{[54334-63-3]} \\
\text { FUS }\end{array}$ & \multicolumn{5}{|c|}{ diphenylmethylene diacetate } \\
\hline & $\begin{array}{l}\text { SUB } \\
\text { SUB }\end{array}$ & $\begin{array}{l}(348-388) \\
(348-388)\end{array}$ & $\begin{array}{l}122.1 \pm 1.2 \\
123.6 \pm 1.2\end{array}$ & $\begin{array}{l}368 \\
298\end{array}$ & $\begin{array}{l}\text { GS } \\
\text { GS }\end{array}$ & $\begin{array}{l}{[1996 \mathrm{VER} / \mathrm{PEN}]} \\
{[1996 \mathrm{VER} / \mathrm{PEN}]}\end{array}$ \\
\hline $\mathrm{C}_{17} \mathrm{H}_{17} \mathrm{ClO}_{6}$ & $\begin{array}{c}\text { FUS } \\
\text { FUS (I) } \\
\text { FUS (II) } \\
\text { FUS (III) } \\
\text { FUS } \\
\text { FUS } \\
\text { FUS } \\
\text { FUS } \\
\text { FUS } \\
\text { FUS } \\
\text { FUS }\end{array}$ & $\begin{array}{l}([1 S] \text {-trans }-7-\mathrm{c} \\
\text { dione(griseoful }\end{array}$ & $\begin{array}{c}\text {-, } 2^{\prime}, 4,6 \text {-trim } \\
39.3 \\
42.3 \\
28.6 \\
35.6 \\
39.12 \\
37.8 \\
36.7 \\
41.0 \\
44.7 \\
41.2 \\
39.39\end{array}$ & $\begin{array}{c}y-6^{\prime}-\mathrm{met} \\
489.5 \\
493.2 \\
487.2 \\
478.2 \\
491 \\
494.2 \\
489.9 \\
493.2 \\
491.2 \\
493.0 \\
495.2\end{array}$ & $\begin{array}{l}\text { DSC } \\
\text { DSC } \\
\text { DSC } \\
\text { DSC } \\
\text { DSC } \\
\text { DSC } \\
\text { DSC }\end{array}$ & $\begin{array}{l}\text { [2013MAN/WIL] } \\
\text { [2010BAI/VAN] } \\
\text { [2010MUR/PIK2] } \\
\text { [2008ZHO/ZHA] } \\
\text { [2007VIP/WAN] } \\
\text { [2006WAS/HOL, 2008WAS/HOL] } \\
\text { [1995WUL/ALD] } \\
\text { [1983GRA/ABO] }\end{array}$ \\
\hline $\mathrm{C}_{17} \mathrm{H}_{17} \mathrm{Cl}_{2} \mathrm{~N}_{5} \mathrm{O}_{4}$ & \multicolumn{6}{|c|}{$N$-[4-chloro-2-[(2-chloro-4-nitrophenyl)azo]-5-[(2-hydroxypropyl)amino]phenyl] acetamide } \\
\hline $\mathrm{C}_{17} \mathrm{H}_{17} \mathrm{NO}$ & [1404112-27-1] & $N$-(4'-methylbi & yl-3-yl)cyclo & necarbo & & \\
\hline
\end{tabular}


TABLE 13. Phase change enthalpies of $\mathrm{C}_{17}$ to $\mathrm{C}_{18}$ organic compounds-Continued

\begin{tabular}{|c|c|c|c|c|c|c|}
\hline \multirow[b]{2}{*}{$\begin{array}{l}\text { Molecular } \\
\text { formula }\end{array}$} & \multirow{2}{*}{$\begin{array}{l}\text { CAS Registry } \\
\text { Number } \\
\text { Enthalpy }\end{array}$} & \multicolumn{5}{|l|}{ Compound } \\
\hline & & $\begin{array}{l}\text { Temperature } \\
\text { range }\end{array}$ & $\begin{array}{l}\Delta_{\text {trans }} H_{\mathrm{m}} \\
(\mathrm{kJ} / \mathrm{mol})\end{array}$ & $\begin{array}{l}T_{\mathrm{m}} \\
(\mathrm{K})\end{array}$ & Method & References \\
\hline & FUS & & 40.4 & 469.5 & $\mathrm{DSC}$ & [2015OWU/CHE] \\
\hline $\mathrm{C}_{17} \mathrm{H}_{17} \mathrm{NO}_{4}$ & $\begin{array}{c}{[483362-67-0]} \\
\text { FUS }\end{array}$ & 2-(4-nitropheny) & $\begin{array}{l}\text { 4-propoxy } \\
31.97\end{array}$ & $\begin{array}{l}\text { ethanon } \\
372.4\end{array}$ & DSC & [2002SPA/DZI] \\
\hline $\mathrm{C}_{17} \mathrm{H}_{17} \mathrm{NO}_{4}$ & $\begin{array}{c}\text { [1161-13-3] } \\
\text { FUS } \\
\text { FUS }\end{array}$ & $\begin{array}{l}N \text {-benzyloxyc } \\
\quad(79-395)\end{array}$ & $\begin{array}{c}\text { yl- } L-3 \text {-phe } \\
31.77 \\
27.3\end{array}$ & $\begin{array}{l}\text { ine } \\
358.8 \\
359.1\end{array}$ & $\begin{array}{c}\mathrm{AC} \\
\mathrm{DSC}\end{array}$ & $\begin{array}{l}{[2010 \mathrm{ZHA} / \mathrm{SUN}]} \\
{[2010 \mathrm{ZHA} / \mathrm{SUN}]}\end{array}$ \\
\hline $\mathrm{C}_{17} \mathrm{H}_{17} \mathrm{~N}_{5} \mathrm{O}_{2}$ & $\begin{array}{l}{[31482-56-1]} \\
\text { SUB }\end{array}$ & 4-nitro-4'-[N-2 & $\begin{array}{l}\text { loethyl- } N \text { - } \\
\quad 147.3\end{array}$ & ino]azo & & [1984KAR/KRU] \\
\hline $\mathrm{C}_{17} \mathrm{H}_{17} \mathrm{~N}_{5} \mathrm{O}_{4}$ & $\begin{array}{c}\text { [231629-80-4] } \\
\text { FUS }\end{array}$ & 6-(4-methoxyp & $\begin{array}{l}\text {-3,9-dihyd } \\
48.41\end{array}$ & $\begin{array}{l}\text { 2-hydro } \\
507.3\end{array}$ & $\begin{array}{l}\text { xy)methy } \\
\text { DSC }\end{array}$ & $\begin{array}{l}\text {-oxo-5 } \boldsymbol{H} \text {-imidazol[1,2-a]pyrine } \\
\text { [1999ZIE/GOL] }\end{array}$ \\
\hline $\mathrm{C}_{17} \mathrm{H}_{18} \mathrm{ClNO}_{2} \mathrm{~S}$ & $\begin{array}{c}{[178870-32-1]} \\
\text { FUS }\end{array}$ & $N$-[4-chloro-3 & $\begin{array}{l}\text { lethyl-2-bu } \\
\quad 36.94\end{array}$ & $\begin{array}{l}\text { xy]phen } \\
400.8\end{array}$ & $\begin{array}{l}\text { methyl-3- } \\
\text { DSC }\end{array}$ & $\begin{array}{l}\text { Incarbothiamide } \\
\text { [2001DAM/BLA] }\end{array}$ \\
\hline $\mathrm{C}_{17} \mathrm{H}_{18} \mathrm{FNO}_{2}$ & $\begin{array}{c}{[164591-98-4]} \\
\text { FUS }\end{array}$ & 4-trans-(3-fluo & $\begin{array}{l}\text { yanopheny } \\
21.1\end{array}$ & $\begin{array}{l}\text { hexyl- }(1 \\
393.2\end{array}$ & $\begin{array}{l}\text {-2-enoate } \\
\text { DTA }\end{array}$ & [1995KEL/SCH] \\
\hline $\mathrm{C}_{17} \mathrm{H}_{18} \mathrm{FN}_{3} \mathrm{O}_{3}$ & $\begin{array}{l}{[85721-33-1]} \\
\text { FUS }\end{array}$ & 1-cyclopropyl- & $\begin{array}{l}\text { ro-1,4-dihy } \\
\quad 64.48\end{array}$ & $\begin{array}{l}5 \times-7-(1 \\
541.5\end{array}$ & $\begin{array}{l}\text { azinyl)-3 } \\
\text { DSC }\end{array}$ & $\begin{array}{l}\text { nolinecarboxylic acid (ciprofloxacin) } \\
\text { [1994YU/ZIP] }\end{array}$ \\
\hline $\mathrm{C}_{17} \mathrm{H}_{18} \mathrm{~N}_{2} \mathrm{O}_{3} \mathrm{~S}$ & $\begin{array}{c}{[479578-81-9]} \\
\text { FUS }\end{array}$ & 4-methoxy- $N$ - & $\begin{array}{l}\text { methoxyph } \\
\quad 31.02\end{array}$ & $\begin{array}{l}\text { ethyl]an } \\
389.2\end{array}$ & $\begin{array}{l}\text { nioxometl } \\
\text { DSC }\end{array}$ & $\begin{array}{l}\text { benzamide } \\
\text { [2002ABB/WOH] }\end{array}$ \\
\hline $\mathrm{C}_{17} \mathrm{H}_{18} \mathrm{~N}_{2} \mathrm{O}_{6}$ & $\begin{array}{c}\text { [21829-25-4] } \\
\text { FUS } \\
\text { FUS } \\
\text { FUS } \\
\text { FUS (I) } \\
\text { FUS (II) } \\
\text { FUS } \\
\text { FUS } \\
\text { FUS }\end{array}$ & 1,4-dihydro-2, & $\begin{array}{c}\text { ethyl-4- }(2-1 \\
38.1 \\
37.6 \\
38.19 \\
36.0 \\
24.2 \\
39.9 \\
36.6 \\
38.0\end{array}$ & $\begin{array}{c}\text { enyl }-3 \\
446.1 \\
446.6 \\
446 \\
444.2 \\
435.2 \\
445.3 \\
445.3 \\
449.2\end{array}$ & $\begin{array}{l}\text { DSC } \\
\text { DSC } \\
\text { DSC } \\
\text { DSC }\end{array}$ & $\begin{array}{l}\text { lic acid dimethyl ester (nifedipine) } \\
\text { [2015NUR/BOO] } \\
{[2015 \mathrm{KUM} / \mathrm{THI}]} \\
{[2010 \mathrm{BAI} / \mathrm{VAN}]} \\
{[2007 \mathrm{GRO} / \mathrm{DEV}, 2011 \mathrm{GRO} / \mathrm{LIE}]} \\
{[2006 \mathrm{MAR} / \mathrm{KON}]} \\
{[2004 \mathrm{MAR} / \mathrm{KOZ}]} \\
{[1997 \mathrm{SQU} / \mathrm{NEE}]}\end{array}$ \\
\hline $\mathrm{C}_{17} \mathrm{H}_{18} \mathrm{~N}_{4} \mathrm{O}_{5}$ & $\begin{array}{l}\text { [3846-49-9] } \\
\text { FUS }\end{array}$ & $N, N^{\prime}$-diethyl- & $\begin{array}{l}\text { '-bis(4-nitr } \\
35.60\end{array}$ & $\begin{array}{l}\text { urea }(4 \\
420.5\end{array}$ & $\begin{array}{l}\text { iitroethyl } \\
\text { DSC }\end{array}$ & $\begin{array}{l}\text { ralite) } \\
{[2010 \mathrm{MEK} / \mathrm{KHI}]}\end{array}$ \\
\hline $\mathrm{C}_{17} \mathrm{H}_{18} \mathrm{O}_{3}$ & $\begin{array}{c}{[15131-43-8]} \\
\mathrm{V}\end{array}$ & $\begin{array}{l}\text { 2-hydroxy-4-bu } \\
\quad(393-443)\end{array}$ & $\begin{array}{l}\text { enzophen } \\
92.7\end{array}$ & 418 & ME & [1984SUR] \\
\hline $\mathrm{C}_{17} \mathrm{H}_{18} \mathrm{O}_{3}$ & $\begin{array}{c}{[87-18-3]} \\
\text { SUB }\end{array}$ & $\begin{array}{l}\text { 4-tert-butylphe } \\
\text { (293-336) }\end{array}$ & $\begin{array}{r}\text { licylate } \\
137.4\end{array}$ & 308 & A & [1987STE/MAL] \\
\hline & $\mathrm{V}$ & $(336-438)$ & 90.4 & 351 & A, UV & [1987STE/MAL, 1960SCH/HIR] \\
\hline $\mathrm{C}_{17} \mathrm{H}_{18} \mathrm{O}_{4}$ & $\begin{array}{c}\text { [101595-31-7] } \\
\text { FUS }\end{array}$ & 2-hydroxy-4,4' & $\begin{array}{c}\text { oxybenzop } \\
34.7\end{array}$ & 373.6 & DSC & [1999PRI/HAW] \\
\hline & SUB & & 134.9 & & $\mathrm{~B}$ & [1999PRI/HAW] \\
\hline & $\mathrm{V}$ & & 100.2 & & TGA & [1999PRI/HAW] \\
\hline $\mathrm{C}_{17} \mathrm{H}_{18} \mathrm{O}_{4}$ & FUS & 2-oxopropyl 2- & $\begin{array}{l}\text { thoxy-2-nc } \\
\quad 32.4\end{array}$ & $\begin{array}{l}\text { propion } \\
355.2\end{array}$ & $\mathrm{DSC}$ & [1994WEB/MEY] \\
\hline $\mathrm{C}_{17} \mathrm{H}_{18} \mathrm{O}_{4} \mathrm{~S}$ & $\begin{array}{c}\text { [313057-14-6] } \\
\text { FUS }\end{array}$ & 4-(4-pentenylo & $\begin{array}{l}\text { enyl 5-met } \\
74.48\end{array}$ & $\begin{array}{l}\text { thiophe } \\
333.7\end{array}$ & $\begin{array}{c}\text { boxylate } \\
\text { DSC }\end{array}$ & [2000WU/WAN] \\
\hline $\mathrm{C}_{17} \mathrm{H}_{18} \mathrm{O}_{8} \mathrm{P}_{2}$ & $\begin{array}{c}\text { [55120-33-7] } \\
\text { FUS }\end{array}$ & 3,9-diphenoxy- & $\begin{array}{l}\text { 10-tetraox } \\
36.46\end{array}$ & $\begin{array}{l}\text { phosph } \\
469.58\end{array}$ & $\begin{array}{l}\text { [5.5]unde } \\
\text { DSC }\end{array}$ & $\begin{array}{l}\text {-3,9-dioxide } \\
\text { [2010GUO/WAN2] }\end{array}$ \\
\hline $\mathrm{C}_{17} \mathrm{H}_{19} \mathrm{~F}_{3} \mathrm{O}_{3}$ & $\begin{array}{c}\text { [164591-97-3] } \\
\text { FUS }\end{array}$ & 4-trans-(trifluo & $\begin{array}{l}\text { hoxypheny } \\
21.6\end{array}$ & $\begin{array}{l}\text { hexyl- }(1 \\
340.2\end{array}$ & $\begin{array}{l}\text {-2-enoate } \\
\text { DTA }\end{array}$ & [1995KEL/SCH] \\
\hline $\mathrm{C}_{17} \mathrm{H}_{19} \mathrm{NO}_{3}$ & $\begin{array}{l}{[57-27-2]} \\
\text { FUS }\end{array}$ & 7,8-didehydro- & $\begin{array}{l}\text { oxy-17-m } \\
42.23\end{array}$ & $\begin{array}{l}\text { orphinan } \\
529 / 7\end{array}$ & $\begin{array}{l}\text { liol (morp } \\
\text { DSC }\end{array}$ & [2015MUS/MAT] \\
\hline & FUS & & 28.87 & 528.2 & DTA & [1988ROY/FLY] \\
\hline
\end{tabular}


TABLE 13. Phase change enthalpies of $\mathrm{C}_{17}$ to $\mathrm{C}_{18}$ organic compounds-Continued

\begin{tabular}{|c|c|c|c|c|c|c|}
\hline \multirow[b]{2}{*}{$\begin{array}{l}\text { Molecular } \\
\text { formula }\end{array}$} & \multirow{2}{*}{$\begin{array}{l}\text { CAS Registry } \\
\text { Number } \\
\text { Enthalpy }\end{array}$} & \multicolumn{5}{|l|}{ Compound } \\
\hline & & $\begin{array}{c}\text { Temperature } \\
\text { range }\end{array}$ & $\begin{array}{l}\Delta_{\text {trans }} H_{\mathrm{m}} \\
(\mathrm{kJ} / \mathrm{mol})\end{array}$ & $\begin{array}{l}T_{\mathrm{m}} \\
(\mathrm{K})\end{array}$ & Method & References \\
\hline $\mathrm{C}_{17} \mathrm{H}_{19} \mathrm{NO}_{3}$ & $\begin{array}{l}\text { [466-99-9] } \\
\text { FUS }\end{array}$ & hydromorphone & 38.53 & 531.8 & DSC & [2015MUS/MAT] \\
\hline $\mathrm{C}_{17} \mathrm{H}_{19} \mathrm{NO}_{3}$ & $\begin{array}{l}\text { [94-62-2] } \\
\text { FUS (I) } \\
\text { FUS (II) } \\
\text { FUS (III) }\end{array}$ & $(E, E)-1$-pipero & $\begin{array}{c}\text { iperidine (pip } \\
31.38 \\
25.10 \\
24.69\end{array}$ & $\begin{array}{l}405.7 \\
401.2 \\
389.7\end{array}$ & DSC & [2015PFU/CHA] \\
\hline $\mathrm{C}_{17} \mathrm{H}_{19} \mathrm{NO}_{4}$ & $\begin{array}{c}\text { [72490-01-8] } \\
\text { FUS } \\
\text { FUS }\end{array}$ & $\begin{array}{c}N \text {-[2-(4-phenox } \\
(79-360)\end{array}$ & $\begin{array}{l}\text { henoxy)ethyl] } \\
28.46 \\
26.98\end{array}$ & $\begin{array}{c}\text { mic acic } \\
326.7 \\
326.3\end{array}$ & $\begin{array}{l}1 \text { ester (fer } \\
\text { DSC } \\
\text { AC }\end{array}$ & $\begin{array}{l}\text { ycarb) } \\
\text { [2013KUH/SVA] } \\
\text { [2005SUN/LIU4] }\end{array}$ \\
\hline $\mathrm{C}_{17} \mathrm{H}_{19} \mathrm{NO}_{4}$ & $\begin{array}{l}\text { [76-41-5] } \\
\text { FUS }\end{array}$ & Oxymorphone & 25.01 & 518.3 & DSC & [2015MUS/MAT] \\
\hline $\mathrm{C}_{17} \mathrm{H}_{19} \mathrm{~N}_{3} \mathrm{O}_{3}$ & $\begin{array}{c}\text { [191979-20-1] } \\
\text { FUS }\end{array}$ & 6-(acetylamino) & $\begin{array}{c}\text { cyano- } 1(2 H)- \\
36.44\end{array}$ & $\begin{array}{l}\text { linecarb } \\
436.1\end{array}$ & $\begin{array}{l}\text { acid, buty } \\
\text { DSC }\end{array}$ & $\begin{array}{l}\text { ster } \\
{[2005 \text { LIZ/ZAB] }}\end{array}$ \\
\hline $\mathrm{C}_{17} \mathrm{H}_{20} \mathrm{~N}_{2} \mathrm{O}$ & $\begin{array}{l}\text { [85-98-3] } \\
\text { FUS }\end{array}$ & $N, N^{\prime}$-diethyl- $\Lambda$ & $\begin{array}{l}\mathrm{V}^{\prime} \text {-diphenylur } \\
33.54\end{array}$ & $\begin{array}{l}\text { hyl cent } \\
345.0\end{array}$ & DSC & [2010MEK/KHI, 2013TRA/KHI] \\
\hline $\mathrm{C}_{17} \mathrm{H}_{20} \mathrm{~N}_{2} \mathrm{O}_{2} \mathrm{~S}$ & $\begin{array}{c}{[373642-58-1]} \\
\text { FUS }\end{array}$ & $N, N^{\prime}$-bis $[(3-\mathrm{me}$ & $\begin{array}{l}\text { xyphenyl)me } \\
33.22\end{array}$ & $\begin{array}{r}\text { hiourea } \\
354.7\end{array}$ & DSC & [2002ABB/WOH] \\
\hline $\mathrm{C}_{17} \mathrm{H}_{20} \mathrm{O}_{2}$ & $\begin{array}{l}{[6397-77-9]} \\
\text { FUS }\end{array}$ & Diethoxydiphen & $\begin{array}{l}\text { hethane } \\
19.9\end{array}$ & 323.2 & & [1998VER/PEN] \\
\hline $\mathrm{C}_{17} \mathrm{H}_{20} \mathrm{O}_{3}$ & & Propyl 2-(6-met & $\begin{array}{c}97.1 \pm 1.1 \\
\text { cy-2-naphthyl }\end{array}$ & $\begin{array}{r}298 \\
\text { ionate }\end{array}$ & & [1998VER/PEN] \\
\hline $\mathrm{C}_{17} \mathrm{H}_{20} \mathrm{O}_{5}$ & FUS & 2,3-dihydroxypr & $\begin{array}{l}\text { yl 2-(6-metho } \\
29.0\end{array}$ & $\begin{array}{r}\text { naphthyl } \\
343.9\end{array}$ & $\begin{array}{l}\text { ionate } \\
\text { DSC }\end{array}$ & [1994WEB/MEY] \\
\hline $\mathrm{C}_{17} \mathrm{H}_{21} \mathrm{CIO}_{4}$ & $\begin{array}{c}{[104225-37-8]} \\
\text { FUS }\end{array}$ & 3-(3-chloro-4-m & $\begin{array}{l}\text { oxybenzoyl)- } \\
29.1\end{array}$ & $\begin{array}{l}\text { trimethy } \\
440.2\end{array}$ & $\begin{array}{l}\text { opentaneca } \\
\text { DSC }\end{array}$ & $\begin{array}{l}\text { oxylic acid } \\
\text { [1992TER/PAU] }\end{array}$ \\
\hline $\mathrm{C}_{17} \mathrm{H}_{21} \mathrm{~F}_{15}$ & $\begin{array}{c}\text { [139277-00-2] } \\
\text { TRS } \\
\text { FUS }\end{array}$ & $1,1,1,2,3,3,4,4,5$ & $\begin{array}{c}\text { 6,6-dodecaflu } \\
3.0 \\
18.0\end{array}$ & $\begin{array}{c}\text {-(trifluor } \\
220 \\
261\end{array}$ & $\begin{array}{l}\text { hyl)hexade } \\
\text { DSC }\end{array}$ & [1992HOP/MOL] \\
\hline $\mathrm{C}_{17} \mathrm{H}_{21} \mathrm{~N}$ & $\begin{array}{c}{[156-08-1]} \\
\mathrm{V}\end{array}$ & $(S)$-benzphetam & $77.2 \pm 0.7$ & 298 & CGC & [2014GOB/VIK] \\
\hline $\mathrm{C}_{17} \mathrm{H}_{21} \mathrm{NO}_{2}$ & $\begin{array}{c}\text { [15299-99-7] } \\
\text { FUS }\end{array}$ & $N, N$-diethyl-2 & $\begin{array}{c}\text { naphthyloxy) } \\
24.57\end{array}$ & $\begin{array}{c}\text { onamide } \\
345.3\end{array}$ & DSC & [1990DON/DRE] \\
\hline $\mathrm{C}_{17} \mathrm{H}_{21} \mathrm{NO}_{3}$ & $\begin{array}{l}\text { [509-60-4] } \\
\text { FUS }\end{array}$ & 4,5-epoxy-3-hy & $\begin{array}{l}\text { xy-17-methyl } \\
35.61\end{array}$ & $\begin{array}{r}\text { ninan-6- } \\
539.2\end{array}$ & $\begin{array}{l}\text { ydromorp } \\
\text { DSC }\end{array}$ & $\begin{array}{l}\text { e) } \\
\text { [1988ROY/FLY] }\end{array}$ \\
\hline $\mathrm{C}_{17} \mathrm{H}_{21} \mathrm{NO}_{3}$ & $\begin{array}{c}\text { [41340-25-4] } \\
\text { FUS }\end{array}$ & $2-[(1 R S)-1,8-\mathrm{d}$ & $\begin{array}{c}\text { yl-1,3,4,9-tet } \\
26.2\end{array}$ & $\begin{array}{c}\text { ropyranc } \\
426.9\end{array}$ & $\begin{array}{l}\text { ]-indol-1- } \\
\text { DSC }\end{array}$ & $\begin{array}{l}\text { acetic acid (etodolac) } \\
\text { [2014RAT/DES] }\end{array}$ \\
\hline $\mathrm{C}_{17} \mathrm{H}_{21} \mathrm{NO}_{4}$ & $\begin{array}{l}\text { [50-36-2] } \\
\text { SUB } \\
\text { SUB }\end{array}$ & (294-314) & $\begin{array}{c}127.2 \\
112.3 \pm 2.8\end{array}$ & 304 & $\begin{array}{l}\text { GS } \\
\text { GS }\end{array}$ & $\begin{array}{l}\text { [1996ZIE/EIK] } \\
\text { [1984LAW/ELI] }\end{array}$ \\
\hline
\end{tabular}


TABLE 13. Phase change enthalpies of $\mathrm{C}_{17}$ to $\mathrm{C}_{18}$ organic compounds-Continued

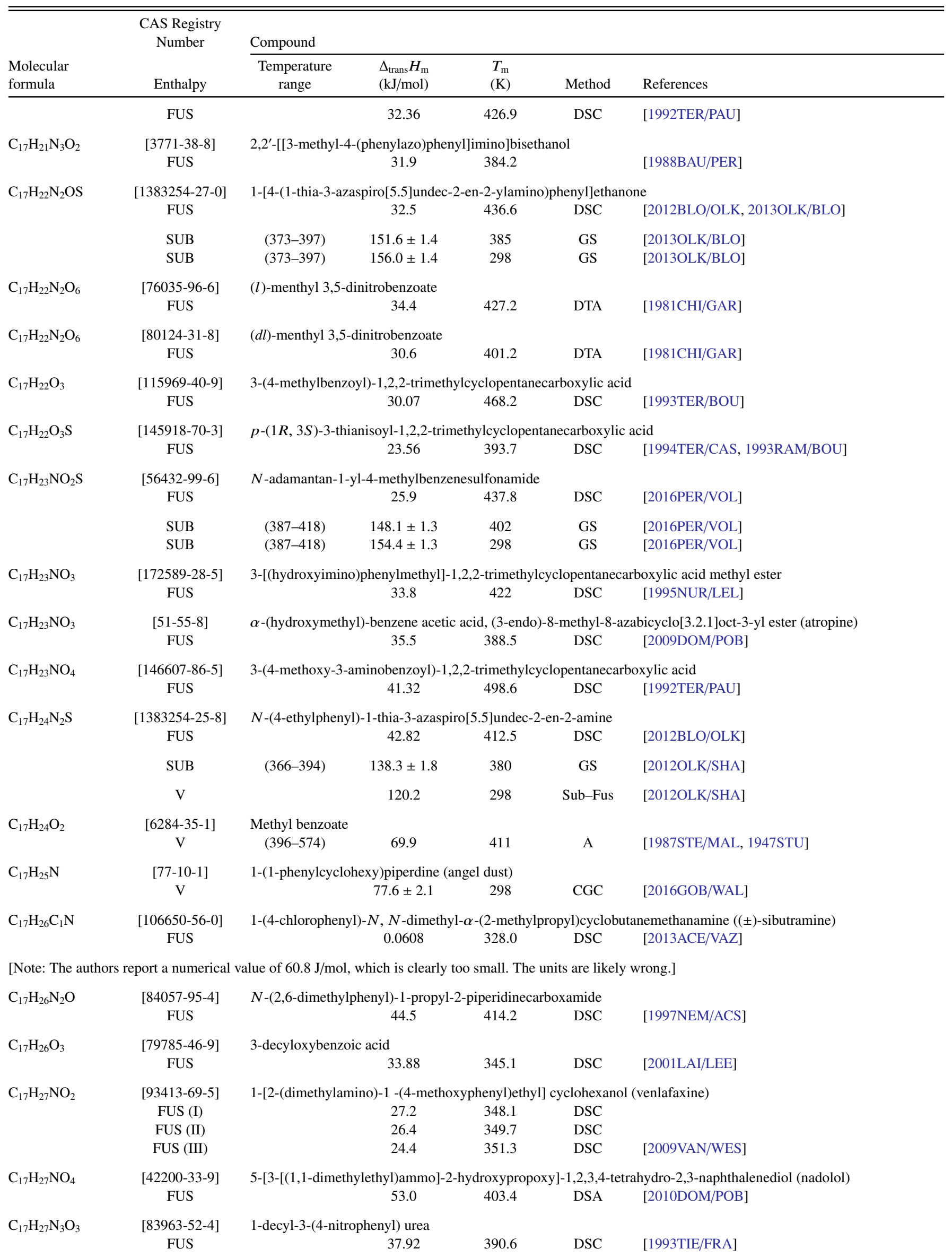


TABLE 13. Phase change enthalpies of $\mathrm{C}_{17}$ to $\mathrm{C}_{18}$ organic compounds-Continued

\begin{tabular}{|c|c|c|c|c|c|c|}
\hline \multirow[b]{2}{*}{$\begin{array}{l}\text { Molecular } \\
\text { formula }\end{array}$} & \multirow{2}{*}{$\begin{array}{l}\text { CAS Registry } \\
\text { Number } \\
\text { Enthalpy }\end{array}$} & \multicolumn{5}{|l|}{ Compound } \\
\hline & & $\begin{array}{c}\text { Temperature } \\
\text { range }\end{array}$ & $\begin{array}{l}\Delta_{\text {trans }} H_{\mathrm{m}} \\
(\mathrm{kJ} / \mathrm{mol})\end{array}$ & $\begin{array}{l}T_{\mathrm{m}} \\
(\mathrm{K})\end{array}$ & Method & References \\
\hline $\mathrm{C}_{17} \mathrm{H}_{28}$ & $\begin{array}{c}{[6742-54-7]} \\
\text { V } \\
\text { V } \\
\text { V }\end{array}$ & $\begin{array}{l}\text { Undecylbenzen } \\
(313-364) \\
(450-622)\end{array}$ & $\begin{array}{c}82.4 \pm 0.4 \\
66.7 \\
84.7\end{array}$ & $\begin{array}{l}298 \\
465 \\
298\end{array}$ & GS & $\begin{array}{l}\text { [2006VER] } \\
\text { [1999DYK/SVO] } \\
{[1971 \mathrm{WIL} / \mathrm{ZWO}]}\end{array}$ \\
\hline $\mathrm{C}_{17} \mathrm{H}_{28} \mathrm{~N}_{2} \mathrm{OS}$ & $\begin{array}{c}\text { [373642-33-2] } \\
\text { FUS }\end{array}$ & $N$-[(3-methoxy & $\begin{array}{l}\text { nyl)methyl]- } \Lambda \\
37.94\end{array}$ & $\begin{array}{l}\text { ylthiour } \\
350.7\end{array}$ & DSC & {$[2002 \mathrm{ABB} / \mathrm{WOH}]$} \\
\hline $\mathrm{C}_{17} \mathrm{H}_{28} \mathrm{O}$ & $\begin{array}{c}{[56103-67-4]} \\
\mathrm{V}\end{array}$ & $\begin{array}{c}\text { 4-methyl-2,6-di } \\
(438-556)\end{array}$ & $\begin{array}{c}t \text {-pentylpheno } \\
65.9\end{array}$ & 453 & A & {$[1987 \mathrm{STE} / \mathrm{MAL}]$} \\
\hline $\mathrm{C}_{17} \mathrm{H}_{28} \mathrm{O}_{2}$ & $\begin{array}{c}{[55095-35-7]} \\
\mathrm{V}\end{array}$ & $\begin{array}{l}\text { 1,3-dimethoxy- } \\
\text { (443-509) }\end{array}$ & $\begin{array}{c}\text { onylbenzene } \\
79.2\end{array}$ & 458 & $\mathrm{~A}, \mathrm{GC}$ & [1987STE/MAL, 1975KUN/LIL] \\
\hline $\mathrm{C}_{17} \mathrm{H}_{28} \mathrm{O}_{4}$ & $\begin{array}{c}{[3220-58-4]} \\
\mathrm{V}\end{array}$ & $\begin{array}{c}\text { Dicyclohexyl gl } \\
(341-370)\end{array}$ & $\begin{array}{l}\text { rate } \\
\qquad 101.1 \pm 0.8\end{array}$ & 298 & GS & [2008LIP/KRA] \\
\hline $\mathrm{C}_{17} \mathrm{H}_{30} \mathrm{O}_{2}$ & $\begin{array}{c}{[65733-18-8]} \\
\mathrm{V}\end{array}$ & $S$-(+)-ethyl-(2E & $\begin{array}{c}\text { E)-3,7,11-trim } \\
92.4 \pm 3.6\end{array}$ & $\begin{array}{l}2,4-\mathrm{doc} \\
298\end{array}$ & $\begin{array}{l}\text { enoate } \\
\text { CGC }\end{array}$ & {$[2016 \mathrm{GOO} / \mathrm{HAS}]$} \\
\hline $\mathrm{C}_{17} \mathrm{H}_{30} \mathrm{O}_{4}$ & $\begin{array}{c}{[23405-96-1]} \\
\text { FUS }\end{array}$ & Dodecyl itacona & 57.2 & 347.8 & DSC & [2016RIC/DEL] \\
\hline $\mathrm{C}_{17} \mathrm{H}_{32}$ & $\begin{array}{c}{[26186-00-5]} \\
V\end{array}$ & $\begin{array}{c}\text { 1-heptadecyne } \\
\text { (438-607) }\end{array}$ & 62.7 & 453 & & [1999DYK/SVO] \\
\hline $\mathrm{C}_{17} \mathrm{H}_{32}$ & $\begin{array}{c}{[61847-96-9]} \\
\mathrm{V}\end{array}$ & $\begin{array}{l}\text { 2-heptadecyne } \\
\text { (446-619) }\end{array}$ & 63.7 & 461 & & [1999DYK/SVO] \\
\hline $\mathrm{C}_{17} \mathrm{H}_{32}$ & $\begin{array}{c}{[61886-63-3]} \\
V\end{array}$ & $\begin{array}{c}\text { 3-heptadecyne } \\
\text { (438-607) }\end{array}$ & 62.5 & 453 & & [1999DYK/SVO] \\
\hline $\mathrm{C}_{17} \mathrm{H}_{32} \mathrm{Cl}_{4}$ & $\begin{array}{c}{[93479-16-4]} \\
\mathrm{V}\end{array}$ & $\begin{array}{l}\text { 1,1,1,17-tetrach } \\
\quad(351-418)\end{array}$ & $\begin{array}{l}\text { heptadecane } \\
108\end{array}$ & 366 & A & [1987STE/MAL, 1960MAL/MAL] \\
\hline $\mathrm{C}_{17} \mathrm{H}_{32} \mathrm{O}$ & $\begin{array}{c}\text { [3661-77-6] } \\
\text { SUB }\end{array}$ & Cycloheptadeca & 75.7 & & & [1938WOL/WEG, 1960JON, 1970COX/PIL] \\
\hline $\mathrm{C}_{17} \mathrm{H}_{32} \mathrm{O}_{2}$ & $\begin{array}{c}{[5637-97-8]} \\
\mathrm{V}\end{array}$ & $\begin{array}{l}\text { Oxa-2-cyclotetr } \\
\quad(403-463)\end{array}$ & $\begin{array}{l}\text { canone } \\
73.5\end{array}$ & 418 & A & [1987STE/MAL] \\
\hline $\mathrm{C}_{17} \mathrm{H}_{32} \mathrm{O}_{2}$ & $\begin{array}{c}{[21643-42-5]} \\
V\end{array}$ & $\begin{array}{l}\text { Tetradecyl acryl } \\
\quad(458-601)\end{array}$ & 69.4 & 473 & A & [1987STE/MAL] \\
\hline $\mathrm{C}_{17} \mathrm{H}_{32} \mathrm{O}_{2}$ & $\begin{array}{c}{[35835-77-9]} \\
\mathrm{V}\end{array}$ & $\begin{array}{l}\text { (Z)-9-pentadece } \\
\quad(363-408)\end{array}$ & $\begin{array}{r}1 \text { acetate } \\
93.6\end{array}$ & 298 & $\mathrm{GC}$ & [1997KOU/HOS, 2000OVA/KOU] \\
\hline $\mathrm{C}_{17} \mathrm{H}_{32} \mathrm{O}_{2}$ & $\begin{array}{c}{[64437-41-8]} \\
\mathrm{V}\end{array}$ & $\begin{array}{c}(E)-9 \text {-pentadece } \\
\quad(363-408)\end{array}$ & $\begin{array}{l}1 \text { acetate } \\
94.3\end{array}$ & 298 & GC & [1997KOU/HOS, 2000OVA/KOU] \\
\hline $\mathrm{C}_{17} \mathrm{H}_{32} \mathrm{O}_{2}$ & $\begin{array}{c}{[64437-43-0]} \\
\mathrm{V}\end{array}$ & $\begin{array}{l}(Z) \text {-10-pentadec } \\
\quad(363-408)\end{array}$ & $\begin{array}{c}\text { yl acetate } \\
94.1\end{array}$ & 298 & GC & [1997KOU/HOS, 2000OVA/KOU] \\
\hline $\mathrm{C}_{17} \mathrm{H}_{32} \mathrm{O}_{2}$ & $\begin{array}{c}{[64437-45-2]} \\
\mathrm{V}\end{array}$ & $\begin{array}{l}(E)-10 \text {-pentade } \\
\quad(363-408)\end{array}$ & $\begin{array}{r}y l \text { acetate } \\
94.6\end{array}$ & 298 & GC & [1997KOU/HOS, 2000OVA/KOU] \\
\hline $\mathrm{C}_{17} \mathrm{H}_{32} \mathrm{O}_{2}$ & $\begin{array}{c}{[35153-25-4]} \\
\mathrm{V}\end{array}$ & $\begin{array}{l}\text { (Z)-11-pentadec } \\
\quad(363-408)\end{array}$ & $\begin{array}{r}y l \text { acetate } \\
94.6\end{array}$ & 298 & GC & [1997KOU/HOS, 2000OVA/KOU] \\
\hline $\mathrm{C}_{17} \mathrm{H}_{32} \mathrm{O}_{2}$ & $\begin{array}{c}{[40535-40-8]} \\
\mathrm{V}\end{array}$ & $\begin{array}{l}(E)-11 \text {-pentade } \\
\quad(363-408)\end{array}$ & $\begin{array}{r}y l \text { acetate } \\
94.9\end{array}$ & 298 & GC & [1997KOU/HOS, 2000OVA/KOU] \\
\hline $\mathrm{C}_{17} \mathrm{H}_{32} \mathrm{O}_{2}$ & $\begin{array}{c}{[70711-45-4]} \\
\mathrm{V}\end{array}$ & $\begin{array}{l}(Z) \text {-12-pentade } \\
\quad(363-408)\end{array}$ & $\begin{array}{c}\text { yl acetate } \\
95.1\end{array}$ & 298 & GC & [1997KOU/HOS, 2000OVA/KOU] \\
\hline $\mathrm{C}_{17} \mathrm{H}_{32} \mathrm{O}_{2}$ & $\begin{array}{c}{[73304-17-3]} \\
\mathrm{V}\end{array}$ & $\begin{array}{c}(E) \text {-12-pentade } \\
\quad(363-408)\end{array}$ & $\begin{array}{r}\text { yl acetate } \\
94.5\end{array}$ & 298 & GC & [1997KOU/HOS, 2000OVA/KOU] \\
\hline $\mathrm{C}_{17} \mathrm{H}_{32} \mathrm{O}_{2}$ & {$[70711-46-5]$} & $(Z)$-13-pentadec & yl acetate & & & \\
\hline
\end{tabular}


TABLE 13. Phase change enthalpies of $\mathrm{C}_{17}$ to $\mathrm{C}_{18}$ organic compounds-Continued

\begin{tabular}{|c|c|c|c|c|c|c|}
\hline \multirow[b]{2}{*}{$\begin{array}{l}\text { Molecular } \\
\text { formula }\end{array}$} & \multirow{2}{*}{$\begin{array}{l}\text { CAS Registry } \\
\text { Number } \\
\text { Enthalpy }\end{array}$} & \multicolumn{5}{|l|}{ Compound } \\
\hline & & $\begin{array}{l}\text { Temperature } \\
\text { range }\end{array}$ & $\begin{array}{l}\Delta_{\text {trans }} H_{\mathrm{m}} \\
(\mathrm{kJ} / \mathrm{mol})\end{array}$ & $\begin{array}{l}T_{\mathrm{m}} \\
(\mathrm{K})\end{array}$ & Method & References \\
\hline & $\mathrm{V}$ & $(363-408)$ & 95.9 & 298 & GC & [1997KOU/HOS, 2000OVA/KOU] \\
\hline $\mathrm{C}_{17} \mathrm{H}_{32} \mathrm{O}_{2}$ & $\begin{array}{c}{[39639-20-8]} \\
\mathrm{V}\end{array}$ & $\begin{array}{l}(E) \text {-13-pentadecenyl } \\
\quad(363-408)\end{array}$ & $\begin{array}{r}\text { acetate } \\
95.9\end{array}$ & 298 & GC & [1997KOU/HOS, 2000OVA/KOU] \\
\hline $\mathrm{C}_{17} \mathrm{H}_{32} \mathrm{O}_{2}$ & $\begin{array}{c}{[1120-25-8]} \\
\mathrm{V}\end{array}$ & Methyl-(Z)-9-hexade & $\begin{array}{l}\text { ecenoate } \\
96.4 \pm 0.7\end{array}$ & 298 & CGC & [2007LIP/KAP] \\
\hline $\mathrm{C}_{17} \mathrm{H}_{32} \mathrm{O}_{2}$ & $\begin{array}{c}{[75190-82-8]} \\
\mathrm{V}\end{array}$ & Methyl-(Z)-10-hepta & $\begin{array}{l}\text { adecenoate } \\
100.8\end{array}$ & 298 & CGC & [2007LIP/KAP] \\
\hline $\mathrm{C}_{17} \mathrm{H}_{32} \mathrm{O}_{3}$ & $\begin{array}{c}{[1725-00-4]} \\
\mathrm{V}\end{array}$ & $\begin{array}{l}\text { 1,8-dioxa-9-cyclonon } \\
\quad(403-463)\end{array}$ & $\begin{array}{c}\text { nadecanone } \\
77.0\end{array}$ & 418 & A & [1987STE/MAL] \\
\hline $\mathrm{C}_{17} \mathrm{H}_{32} \mathrm{O}_{4}$ & $\begin{array}{c}{[2917-73-9]} \\
\mathrm{V}\end{array}$ & $\begin{array}{l}\text { Dibutyl nonadioate } \\
\quad(313-450)\end{array}$ & 88.4 & 328 & A & [1987STE/MAL] \\
\hline $\mathrm{C}_{17} \mathrm{H}_{32} \mathrm{O}_{5}$ & $\mathrm{~V}$ & $\begin{array}{l}\text { Nonyl[1-(butoxycarb } \\
\quad(420-534)\end{array}$ & $\begin{array}{c}\text { onyl)ethyl] } \\
73.8\end{array}$ & $\begin{array}{l}\text { nate } \\
435\end{array}$ & A & [1987STE/MAL, 1950REH/DIX2] \\
\hline $\mathrm{C}_{17} \mathrm{H}_{33} \mathrm{~N}$ & $\begin{array}{c}{[5399-02-0]} \\
\text { V } \\
\text { V }\end{array}$ & $\begin{array}{l}\text { Heptadecanonitrile } \\
\qquad(348-385) \\
(425-620)\end{array}$ & $\begin{array}{l}98.9 \pm 0.4 \\
\quad 81.2\end{array}$ & $\begin{array}{l}298 \\
440\end{array}$ & $\begin{array}{c}\text { GS } \\
\text { A }\end{array}$ & $\begin{array}{l}\text { [2005EME/VER] } \\
\text { [1987STE/MAL] }\end{array}$ \\
\hline $\mathrm{C}_{17} \mathrm{H}_{33} \mathrm{NO}_{2}$ & $\begin{array}{c}\text { [42373-46-6] } \\
\text { FUS }\end{array}$ & $N$-dodecyl- $(D L)$-vali & ine 64.4 & 364.6 & DSC & [1986MIY/MAT] \\
\hline $\mathrm{C}_{17} \mathrm{H}_{33} \mathrm{NO}_{3}$ & $\begin{array}{c}{[71448-29-8]} \\
\text { FUS }\end{array}$ & $N$-tetradecanoyl- $(L)$ & $\begin{array}{c}\text {-alanine } \\
52.3\end{array}$ & 367.1 & $\mathrm{DSC}$ & [1986MIY/MAT] \\
\hline $\mathrm{C}_{17} \mathrm{H}_{33} \mathrm{NO}_{3}$ & $\begin{array}{l}{[35054-70-7]} \\
\text { FUS }\end{array}$ & $N$-dodecanoyl- $(D L)-$ & $\begin{array}{r}\text {-valine } \\
33.1\end{array}$ & 380.1 & DSC & [1986MIY/MAT] \\
\hline $\mathrm{C}_{17} \mathrm{H}_{33} \mathrm{NO}_{3}$ & $\begin{array}{c}{[155300-71-3]} \\
\text { TRS + FUS }\end{array}$ & $N$-(1-oxopentadecyl) & $\begin{array}{c}\text { glycine } \\
42.6\end{array}$ & 394.8 & $\mathrm{DSC}$ & [2014RED/KRO] \\
\hline $\mathrm{C}_{17} \mathrm{H}_{34}$ & $\begin{array}{c}{[5634-30-0]} \\
\mathrm{V} \\
\mathrm{V}\end{array}$ & $\begin{array}{l}\text { Dodecylcyclopentane } \\
\quad(450-619)\end{array}$ & $\begin{array}{l}68.0 \\
85.5\end{array}$ & $\begin{array}{l}465 \\
298\end{array}$ & & $\begin{array}{l}\text { [1999DYK/SVO] } \\
\text { [1971WIL/ZWO] }\end{array}$ \\
\hline $\mathrm{C}_{17} \mathrm{H}_{34}$ & $\begin{array}{c}{[54105-66-7]} \\
\text { V } \\
\text { V }\end{array}$ & $\begin{array}{l}\text { Undecylcyclohexane } \\
\qquad(450-622)\end{array}$ & $\begin{array}{l}67.0 \\
84.6\end{array}$ & $\begin{array}{l}465 \\
298\end{array}$ & & $\begin{array}{l}\text { [1999DYK/SVO] } \\
\text { [1971WIL/ZWO] }\end{array}$ \\
\hline $\mathrm{C}_{17} \mathrm{H}_{34}$ & $\begin{array}{c}{[6765-39-5]} \\
\mathrm{V} \\
\mathrm{V} \\
\mathrm{V}\end{array}$ & $\begin{array}{l}\text { 1-heptadecene } \\
\quad(598-746) \\
(376-432)\end{array}$ & $\begin{array}{l}55.5 \\
72.3 \\
84.9\end{array}$ & $\begin{array}{l}613 \\
391 \\
298\end{array}$ & A & $\begin{array}{l}\text { [1999DYK/SVO] } \\
\text { [1987STE/MAL] } \\
\text { [1971WIL/ZWO] }\end{array}$ \\
\hline $\mathrm{C}_{17} \mathrm{H}_{34}$ & $\begin{array}{c}\text { [295-97-6] } \\
\text { SUB }\end{array}$ & Cycloheptadecane & $66.1 \pm 0.6$ & & & [1957VAN, 1970COX/PIL] \\
\hline $\mathrm{C}_{17} \mathrm{H}_{34} \mathrm{O}$ & $\begin{array}{c}{[2922-51-2]} \\
\mathrm{V}\end{array}$ & $\begin{array}{l}\text { 2-heptadecanone } \\
\quad(402-593)\end{array}$ & 77.0 & 417 & A & [1987STE/MAL, 1947STU] \\
\hline $\mathrm{C}_{17} \mathrm{H}_{34} \mathrm{O}$ & $\begin{array}{c}{[6064-42-2]} \\
\mathrm{V}\end{array}$ & 7-heptadecanone & $94.5 \pm 1.8$ & 298 & CGC & [2006PER/CON] \\
\hline $\mathrm{C}_{17} \mathrm{H}_{34} \mathrm{O}$ & $\begin{array}{c}\text { [540-08-9] } \\
\text { FUS } \\
\text { V }\end{array}$ & $\begin{array}{l}\text { 9-heptadecanone } \\
\text { (439-482) }\end{array}$ & $\begin{array}{c}66.68 \\
78.3\end{array}$ & $\begin{array}{c}323.9 \\
454\end{array}$ & $\begin{array}{c}\text { DSC } \\
\text { A, ME }\end{array}$ & $\begin{array}{l}\text { [1993VIL/HAM] } \\
\text { [1987STE/MAL, 1938UBB] }\end{array}$ \\
\hline $\mathrm{C}_{17} \mathrm{H}_{34} \mathrm{O}_{2}$ & $\begin{array}{l}\text { [112-39-0] } \\
\text { FUS } \\
\text { FUS } \\
\text { FUS } \\
\text { FUS } \\
\text { FUS } \\
\text { FUS }\end{array}$ & Methyl hexadecanoat & $\begin{array}{c}\text { te (methyl p } \\
56.17 \\
50.94 \\
56.0 \\
58.1 \\
53.8 \\
\text { U } 44.1\end{array}$ & $\begin{array}{l}\text { ate) } \\
305.1 \\
300.61 \\
305.2 \\
302.2 \\
302.2 \\
302.0\end{array}$ & $\begin{array}{l}\text { DSC } \\
\text { DSC } \\
\text { DSC } \\
\text { DSC } \\
\text { DSC } \\
\text { DSC }\end{array}$ & $\begin{array}{l}\text { [2016LIS/FAR] } \\
{[2013 \mathrm{BEN} / \mathrm{KHI}]} \\
{[2004 \mathrm{CHI} / \mathrm{ZHA}]} \\
{[2003 \mathrm{NIK} / \mathrm{MAR}]} \\
{[2003 \mathrm{SUP} / \mathrm{GOF}]} \\
{[1992 \mathrm{BAB} / \mathrm{HWA} 2]}\end{array}$ \\
\hline
\end{tabular}


TABLE 13. Phase change enthalpies of $\mathrm{C}_{17}$ to $\mathrm{C}_{18}$ organic compounds-Continued

\begin{tabular}{|c|c|c|c|c|c|c|}
\hline \multirow[b]{2}{*}{$\begin{array}{l}\text { Molecular } \\
\text { formula }\end{array}$} & \multirow{2}{*}{$\begin{array}{c}\text { CAS Registry } \\
\text { Number } \\
\text { Enthalpy }\end{array}$} & \multicolumn{5}{|l|}{ Compound } \\
\hline & & $\begin{array}{c}\text { Temperature } \\
\text { range }\end{array}$ & $\begin{array}{l}\Delta_{\text {trans }} H_{\mathrm{m}} \\
(\mathrm{kJ} / \mathrm{mol})\end{array}$ & $\begin{array}{l}T_{\mathrm{m}} \\
(\mathrm{K})\end{array}$ & Method & References \\
\hline & FUS & & 68.16 & 307.2 & DSC & [1993ACR, 1991CHI/BRA] \\
\hline & FUS & & 58.72 & 303.7 & $\mathrm{AC}$ & [1985ABE/KUR] \\
\hline & FUS & & 62.6 & 302.2 & & [1936KIN/GAR] \\
\hline & SUB & $(291-301)$ & $152.3 \pm 2$ & 296 & $\mathrm{ME}$ & [1965DAV/KYB, 1987STE/MAL] \\
\hline & $\mathrm{V}$ & & $101.8 \pm 3.1$ & 298 & CRT & [2015GOB/CHI] \\
\hline & $\mathrm{V}$ & & $95.3 \pm 4.3$ & 298 & $\mathrm{CGC}$ & [2015GOB/CHI] \\
\hline & $\mathrm{V}$ & & 93.4 & 350 & $\mathrm{CE}$ & [2002VAN/VAN] \\
\hline & $\mathrm{V}$ & & $83.3 \pm 0.4$ & 397 & $\mathrm{CE}$ & [2002VAN/VAN] \\
\hline & $\mathrm{V}$ & & $96.8 \pm 0.6$ & 298 & $\mathrm{CE}$ & [2002VAN/VAN] \\
\hline & $\mathrm{V}$ & $(463-523)$ & 96.4 & 298 & $\mathrm{GC}$ & [1997KRO/VEL] \\
\hline & $\mathrm{V}$ & $(433-473)$ & 93.2 & 298 & CGC & [1995CHI/HOS] \\
\hline & $\mathrm{V}$ & $(453-543)$ & 78.2 & 498 & $\mathrm{GC}$ & [1993HUS/SAR] \\
\hline & $\mathrm{V}$ & $(287-322)$ & U69.6 & 302 & A & [1987STE/MAL] \\
\hline & $\mathrm{V}$ & $(411-543)$ & 82.4 & 426 & A & [1987STE/MAL, 1963ROS/SCH] \\
\hline & $\mathrm{V}$ & $(378-445)$ & 82.6 & 393 & $\mathrm{MG}, \mathrm{OM}$ & {$[1952 \mathrm{SCO} / \mathrm{MAC}]$} \\
\hline & $\mathrm{V}$ & $(422-475)$ & 71.4 & 437 & & [1948BON/ATH] \\
\hline \multirow[t]{2}{*}{$\mathrm{C}_{17} \mathrm{H}_{34} \mathrm{O}_{2}$} & {$[110-27-0]$} & \multicolumn{5}{|c|}{ Isopropyl tetradecanoate } \\
\hline & V & $(413-466)$ & 70.2 & 428 & A & [1987STE/MAL, 1948BON/ATH, 1984BOU/FRI] \\
\hline \multirow[t]{2}{*}{$\mathrm{C}_{17} \mathrm{H}_{34} \mathrm{O}_{2}$} & {$[14303-70-9]$} & \multicolumn{5}{|c|}{ Propyl tetradecanoate } \\
\hline & $\mathrm{V}$ & $(420-474)$ & 71.3 & 435 & A & [1987STE/MAL, 1948BON/ATH, 1984BOU/FRI] \\
\hline \multirow[t]{10}{*}{$\mathrm{C}_{17} \mathrm{H}_{34} \mathrm{O}_{2}$} & {$[506-12-7]$} & \multicolumn{5}{|c|}{ Heptadecanoic acid (margaric acid) } \\
\hline & TRS & & 7.5 & 331.2 & & \\
\hline & FUS & & 46.5 & 333.5 & DSC & [2007GBA/NEG, 2008GBA/NEG] \\
\hline & TRS & $(90-345)$ & 7.44 & 329.2 & & \\
\hline & FUS & $(90-345)$ & 51.33 & 334.3 & $\mathrm{AC}$ & [1996DOM/HEA, 1982SCH/VAN2] \\
\hline & FUS & & 51.9 & 333.0 & DSC & [1976BER/BER, 1975BER/LEO] \\
\hline & TRS & & 7.3 & 329.6 & & \\
\hline & FUS & & 51.5 & 334.4 & & [1964ADR/DEK] \\
\hline & SUB & $(280-302)$ & 151 & & TPTD & [2005CHA/ZIE] \\
\hline & SUB & $(291-316)$ & 168 & & TPTD & [2001CHA/TOB] \\
\hline
\end{tabular}

[Note: Experimental values based on the TPTD method are often inconsistent values determined using other experimental methods.]

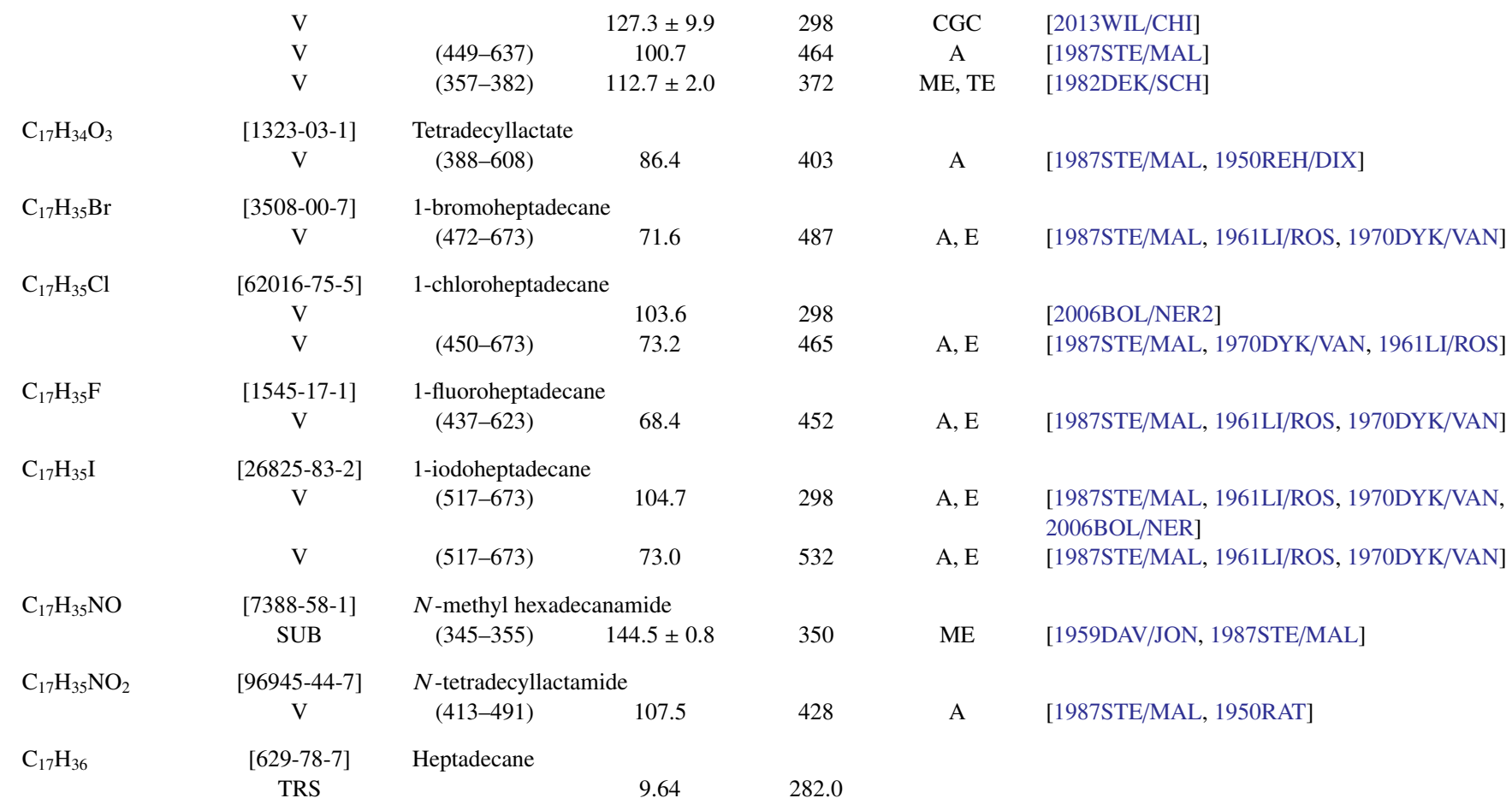


TABLE 13. Phase change enthalpies of $\mathrm{C}_{17}$ to $\mathrm{C}_{18}$ organic compounds-Continued

\begin{tabular}{|c|c|c|c|c|c|c|}
\hline \multirow[b]{2}{*}{$\begin{array}{l}\text { Molecular } \\
\text { formula }\end{array}$} & \multirow{2}{*}{$\begin{array}{l}\text { CAS Registry } \\
\text { Number } \\
\text { Enthalpy }\end{array}$} & \multicolumn{5}{|l|}{ Compound } \\
\hline & & $\begin{array}{l}\text { Temperature } \\
\text { range }\end{array}$ & $\begin{array}{l}\Delta_{\text {trans }} H_{\mathrm{m}} \\
(\mathrm{kJ} / \mathrm{mol})\end{array}$ & $\begin{array}{l}T_{\mathrm{m}} \\
(\mathrm{K})\end{array}$ & Method & References \\
\hline & FUS & & 39.68 & 294.5 & DSC & [2015VEL/ORT] \\
\hline & TRS & & 13.0 & 284.3 & & \\
\hline & FUS & & 41.5 & 294.7 & DSC & [2005HUA/SIM] \\
\hline & TRS & & 10.8 & & & \\
\hline & FUS & & 39.4 & & DSC & [2005ESP/WHI] \\
\hline & TRS & & 10.8 & 284.2 & & \\
\hline & FUS & & 39.4 & 294.7 & DSC & [2004MON/RAJ] \\
\hline & TRS & & 10.3 & 383.9 & & \\
\hline & FUS & & 39.4 & 294.8 & DSC & [1996ROB/ESP] \\
\hline & FUS & & 39.4 & 295.8 & DSC & [1992BAB/HWA, 1994BAB/BEN] \\
\hline & TRS & $(12-384)$ & 10.96 & 284.3 & & \\
\hline & FUS & $(12-384)$ & 40.17 & 295.1 & $\mathrm{AC}$ & [1996DOM/HEA, 1967MES/GUT] \\
\hline & SUB & & 125.1 & 298 & & [1972MOR3] \\
\hline & SUB & $(288-293)$ & $131.3 \pm 13$ & 290 & $\mathrm{ME}$ & [1949BRA/SHE, 1960JON] \\
\hline & $\mathrm{V}$ & & 86.5 & 298 & & [1994RUZ/MAJ] \\
\hline & $\mathrm{V}$ & $(289-320)$ & 91.1 & 304 & A & [1987STE/MAL] \\
\hline & $\mathrm{V}$ & $(488-577)$ & 62.9 & 503 & A & [1987STE/MAL] \\
\hline & $\mathrm{V}$ & & $86.0 \pm 0.8$ & 298 & $\mathrm{C}$ & [1972MOR] \\
\hline & $\mathrm{V}$ & & 86.2 & 298 & & [1971WIL/ZWO] \\
\hline & V & $(445-470)$ & 71.6 & 457 & $\mathrm{ME}$ & [1938UBB] \\
\hline \multirow[t]{2}{*}{$\mathrm{C}_{17} \mathrm{H}_{36}$} & {$[1560-92-5]$} & \multicolumn{2}{|l|}{ 2-methylhexadecane } & & & \\
\hline & V & $(428-569)$ & 63.5 & 443 & A & [1987STE/MAL, 1959TER/BRI] \\
\hline \multirow[t]{2}{*}{$\mathrm{C}_{17} \mathrm{H}_{36}$} & [6418-43-5] & \multicolumn{2}{|l|}{ 3-methylhexadecane } & & & \\
\hline & V & $(428-567)$ & 63.4 & 443 & A & [1987STE/MAL, 1959TER/BRI] \\
\hline \multirow[t]{2}{*}{$\mathrm{C}_{17} \mathrm{H}_{36}$} & [25117-26-4] & \multirow{2}{*}{$\begin{array}{l}\text { 4-methylhexadecane } \\
\text { (420-567) }\end{array}$} & & & & \\
\hline & V & & 58.7 & 435 & A & [1987STE/MAL, 1959TER/BRI] \\
\hline \multirow[t]{2}{*}{$\mathrm{C}_{17} \mathrm{H}_{36}$} & [25117-34-4] & \multicolumn{2}{|l|}{ 5-methylhexadecane } & & & \\
\hline & V & $(422-566)$ & 59.8 & 437 & A & [1987STE/MAL, 1959TER/BRI] \\
\hline \multirow[t]{2}{*}{$\mathrm{C}_{17} \mathrm{H}_{36}$} & [2882-97-5] & \multicolumn{2}{|c|}{ 2,3-dimethylpentadecane } & & & \\
\hline & V & $(424-569)$ & 60.6 & 439 & A & [1987STE/MAL, 1959TER/BRI] \\
\hline \multirow[t]{2}{*}{$\mathrm{C}_{17} \mathrm{H}_{36}$} & {$[61868-07-3]$} & \multicolumn{2}{|c|}{ 2,4-dimethylpentadecane } & & & \\
\hline & V & $(419-546)$ & 65.2 & 434 & A & [1987STE/MAL, 1959TER/BRI] \\
\hline \multirow[t]{2}{*}{$\mathrm{C}_{17} \mathrm{H}_{36}$} & {$[101791-53-1]$} & \multicolumn{2}{|c|}{ 2,4,6-trimethyltetradecane } & & & \\
\hline & $\mathrm{V}$ & $(411-534)$ & 64.5 & 426 & A & [1987STE/MAL] \\
\hline \multirow[t]{2}{*}{$\mathrm{C}_{17} \mathrm{H}_{36}$} & {$[93816-24-1]$} & \multicolumn{2}{|l|}{ 4,4-dipropylundecane } & & & \\
\hline & V & & $78.0 \pm 1.8$ & 298 & CGC & [1995CHI/HES] \\
\hline \multirow[t]{4}{*}{$\mathrm{C}_{17} \mathrm{H}_{36} \mathrm{O}$} & [1454-85-9] & \multicolumn{2}{|l|}{ 1-heptadecanol } & & & \\
\hline & TRS + FUS & & 63.4 & 325.3 & DSC & [2006NIC/KWE] \\
\hline & FUS & & 37.0 & 326.6 & DSC & [2004VEN/CAL] \\
\hline & FUS & $298-368)$ & 63.06 & 327.3 & & [2003VAN/VAN] \\
\hline
\end{tabular}

[Note: The enthalpy of fusion value includes the enthalpy of solid-to-solid transition that occurs at $323.2 \mathrm{~K}$.]

\begin{tabular}{|c|c|c|c|c|c|c|}
\hline & TRS & & 25.2 & 323.6 & & \\
\hline & FUS & & 37.0 & 326.6 & DSC & [2002VEN/RAM] \\
\hline & SUB & & $169.5 \pm 2.2$ & & & [1965DAV/KYB, 1970COX/PIL] \\
\hline & $\mathrm{V}$ & & $112.5 \pm 0.5$ & 298 & $\mathrm{CGC}$ & {$[2006 \mathrm{NIC} / \mathrm{KWE}]$} \\
\hline & $\mathrm{V}$ & $(460-620)$ & 78.3 & 475 & A & [1987STE/MAL] \\
\hline & $\mathrm{V}$ & $(473-623)$ & 75.9 & 488 & A & [1987STE/MAL] \\
\hline $\mathrm{C}_{17} \mathrm{H}_{36} \mathrm{O}$ & [103385-34-8] & 4-heptadecanol & & & & \\
\hline & FUS + TRS & & 35.7 & 311.5 & DSC & [2006NIC/KWE] \\
\hline $\mathrm{C}_{17} \mathrm{H}_{36} \mathrm{O}$ & [112283-13-3] & 6-heptadecanol & & & & \\
\hline & FUS + TRS & & 49.0 & 315.8 & DSC & [2006NIC/KWE] \\
\hline & $\mathrm{V}$ & & $108.6 \pm 1.0$ & 298 & $\mathrm{CGC}$ & [2006NIC/KWE] \\
\hline
\end{tabular}


TABLE 13. Phase change enthalpies of $\mathrm{C}_{17}$ to $\mathrm{C}_{18}$ organic compounds-Continued

\begin{tabular}{|c|c|c|c|c|c|c|}
\hline \multirow[b]{2}{*}{$\begin{array}{l}\text { Molecular } \\
\text { formula }\end{array}$} & \multirow{2}{*}{$\begin{array}{l}\text { CAS Registry } \\
\text { Number } \\
\text { Enthalpy }\end{array}$} & \multicolumn{5}{|l|}{ Compound } \\
\hline & & $\begin{array}{l}\text { Temperature } \\
\text { range }\end{array}$ & $\begin{array}{l}\Delta_{\text {trans }} H_{\mathrm{m}} \\
(\mathrm{kJ} / \mathrm{mol})\end{array}$ & $\begin{array}{l}T_{\mathrm{m}} \\
(\mathrm{K})\end{array}$ & Method & References \\
\hline \multirow[t]{2}{*}{$\mathrm{C}_{17} \mathrm{H}_{36} \mathrm{O}$} & $\begin{array}{c}{[93658-33-4]} \\
\text { FUS + TRS }\end{array}$ & 7-heptadecanol & 28.8 & 314.4 & DSC & [2006NIC/KWE] \\
\hline & $\mathrm{V}$ & & $108.2 \pm 0.8$ & 298 & CGC & [2006NIC/KWE] \\
\hline & $\mathrm{V}$ & & $108.5 \pm 0.4$ & 298 & CGC & [2006NIC/KWE] \\
\hline $\mathrm{C}_{17} \mathrm{H}_{36} \mathrm{O}_{2}$ & $\begin{array}{l}{[66577-59-1]} \\
\text { TRS } \\
\text { FUS }\end{array}$ & 1,17-heptadecane & $\begin{array}{l}34.9 \\
30.8\end{array}$ & $\begin{array}{c}98.4 \\
367.3\end{array}$ & DSC & [1999OGA/NAK] \\
\hline $\mathrm{C}_{17} \mathrm{H}_{36} \mathrm{O}_{3}$ & $\begin{array}{l}\text { [1561-06-4] } \\
\text { FUS }\end{array}$ & 3-(tetradecyloxy) & $\begin{array}{l}\text { 1,2-propanediol } \\
62.1\end{array}$ & 331.3 & DSC & [1993ACR, 1990VAN/VAN] \\
\hline $\mathrm{C}_{17} \mathrm{H}_{36} \mathrm{~S}$ & $\begin{array}{c}\text { [53193-22-9] } \\
\mathrm{V}\end{array}$ & $\begin{array}{l}\text { 1-heptadecanethi } \\
\text { (481-657) }\end{array}$ & 74.6 & 496 & & [1999DYK/SVO] \\
\hline $\mathrm{C}_{17} \mathrm{H}_{37} \mathrm{~N}$ & $\frac{[4200-95-7]}{\mathrm{V}}$ & $\begin{array}{l}\text { Heptadecylamine } \\
\quad(522-636)\end{array}$ & 68.2 & 537 & A & [1987STE/MAL, 1956MAN2] \\
\hline $\mathrm{C}_{17} \mathrm{H}_{37} \mathrm{NO}_{2}$ & $\begin{array}{c}\text { [111953-19-6] } \\
\text { FUS }\end{array}$ & 3-(tetradecylamin & $\begin{array}{l}\text { )-1,2-propanediol } \\
\quad 64.9\end{array}$ & 356.2 & DSC & [1993ACR, 1990VAN/VAN] \\
\hline $\mathrm{C}_{18} \mathrm{D}_{12}$ & $\begin{array}{c}{[1719-03-5]} \\
\mathrm{V}\end{array}$ & Chrysene- $\mathrm{d}_{12}$ & 106 & 298 & CGC & [2008ZHA/UNH] \\
\hline $\mathrm{C}_{18} \mathrm{H}_{10}$ & $\begin{array}{c}\text { [203-12-3] } \\
\text { TRS } \\
\text { TRS } \\
\text { TRS } \\
\text { FUS }\end{array}$ & Benzo[ghi]fluoral & $\begin{array}{l}\text { thene } \\
\begin{array}{r}5.35 \\
0.88 \\
0.44 \\
11.8\end{array}\end{array}$ & $\begin{array}{c}402.8 \\
402.1 \\
352.7 \\
424\end{array}$ & & [1980SMI] \\
\hline $\mathrm{C}_{18} \mathrm{H}_{10} \mathrm{BrNO}_{3}$ & $\begin{array}{c}\text { [10319-14-9] } \\
\text { SUB }\end{array}$ & $\begin{array}{l}\text { 2(4-bromo-3-hyd } \\
\quad(483-523)\end{array}$ & $\begin{array}{l}\text { oxy-2-quinolinyl) } \\
130.6\end{array}$ & $\begin{array}{l}H \text {-inden } \\
498\end{array}$ & $\begin{array}{c}2 H) \text {-dion } \\
\text { A }\end{array}$ & $\begin{array}{l}\text { C. I. disperse yellow 64) } \\
\text { [1987STE/MAL] }\end{array}$ \\
\hline $\mathrm{C}_{18} \mathrm{H}_{10} \mathrm{Br}_{2}$ & $\begin{array}{c}{[131222-99-6]} \\
\text { FUS }\end{array}$ & 6,12-dibromochry & 23.81 & 543.2 & DSC & [2012FU/SUU] \\
\hline & SUB & $(409-465)$ & $141.1 \pm 3.2$ & 437 & ME & [2012FU/SUU] \\
\hline $\mathrm{C}_{18} \mathrm{H}_{10} \mathrm{Cl}_{2} \mathrm{O}_{2} \mathrm{~S}_{2}$ & $\begin{array}{l}{[2379-74-0]} \\
\text { SUB }\end{array}$ & $\begin{array}{l}\text { 6-chloro-2-(6-chl } \\
\text { (C.I. Vat Red 1) } \\
\quad(519-634)\end{array}$ & $\begin{array}{l}\text { ro-4-methyl-3-ox } \\
148\end{array}$ & $\begin{array}{l}\text { enzo }[b] \mathrm{t} \\
577\end{array}$ & $\begin{array}{l}\text { 2(3H)-yli } \\
\text { GS }\end{array}$ & $\begin{array}{l}\text { e)-4-methyl-benzo }[b] \text { thiophen-3(2H)- one } \\
\text { [1986NIS/AND] }\end{array}$ \\
\hline $\mathrm{C}_{18} \mathrm{H}_{10} \mathrm{Cl}_{2} \mathrm{O}_{2} \mathrm{~S}_{2}$ & $\begin{array}{c}{[5462-29-3]} \\
\text { SUB }\end{array}$ & $\begin{array}{l}\text { 5-chloro-2-(5-chl } \\
\text { (C.I. Vat Violet 2) } \\
\quad(519-634)\end{array}$ & $\begin{array}{l}\text { ro-7-methyl-3-ox } \\
93.0\end{array}$ & $\begin{array}{l}\text { enzo }[b] \mathrm{t} \\
577\end{array}$ & $\begin{array}{l}\text { 2(3H)-yli } \\
\text { GS }\end{array}$ & $\begin{array}{l}\text { e)-7-methyl-benzo }[b] \text { thiophen-3(2H)- one } \\
\text { [1986NIS/AND] }\end{array}$ \\
\hline $\mathrm{C}_{18} \mathrm{H}_{10} \mathrm{~N}_{2}$ & $\begin{array}{c}\text { [22318-90-7] } \\
\text { FUS }\end{array}$ & $(5 \mathrm{E}, 11 \mathrm{E})$-dibenzo & $\begin{array}{l}a, e] \text { cycloctene- } 5 \\
31.1\end{array}$ & $\begin{array}{l}1-\text { dicarb } \\
463.7\end{array}$ & DSC & [2011PER/CON] \\
\hline $\mathrm{C}_{18} \mathrm{H}_{10} \mathrm{O}_{2}$ & $\begin{array}{l}\text { [2498-66-0] } \\
\text { SUB }\end{array}$ & 1,2-benzanthra-9, & $\begin{array}{l}\text { 0-quinone } \\
82.8 \pm 4.0\end{array}$ & & & [1956MAG, 1970COX/PIL] \\
\hline
\end{tabular}


TABLE 13. Phase change enthalpies of $\mathrm{C}_{17}$ to $\mathrm{C}_{18}$ organic compounds-Continued

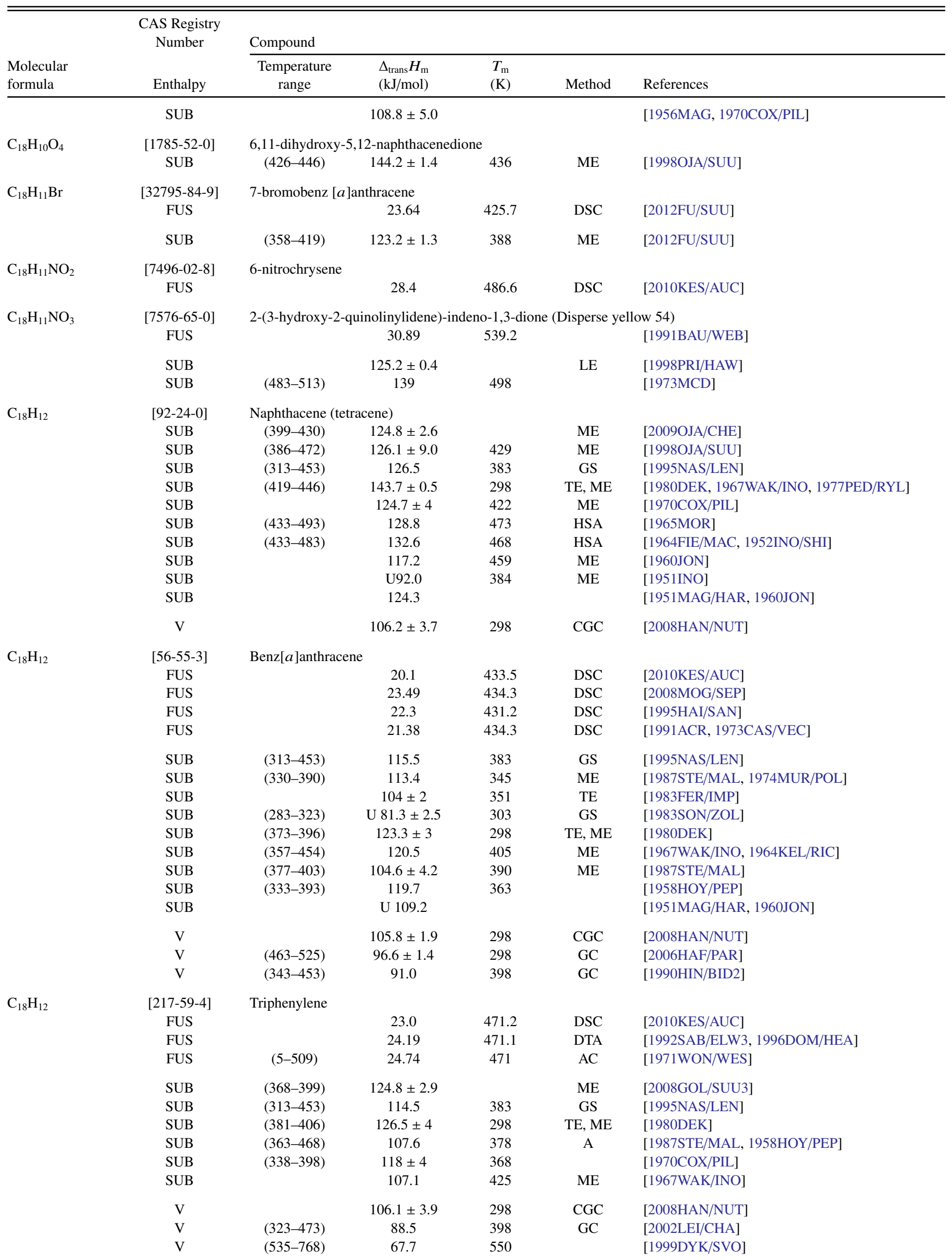


TABle 13. Phase change enthalpies of $\mathrm{C}_{17}$ to $\mathrm{C}_{18}$ organic compounds-Continued

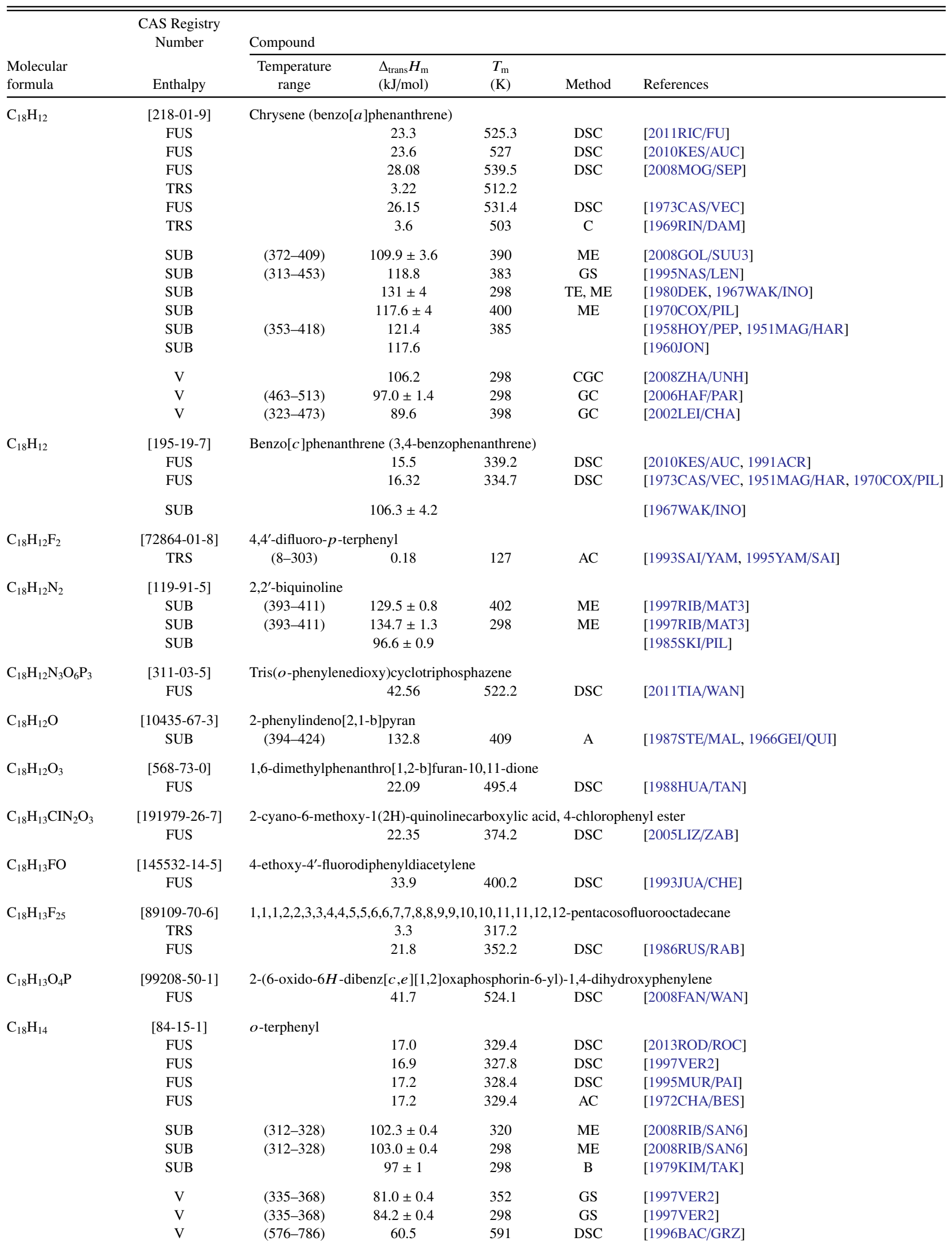


TABLE 13. Phase change enthalpies of $\mathrm{C}_{17}$ to $\mathrm{C}_{18}$ organic compounds-Continued

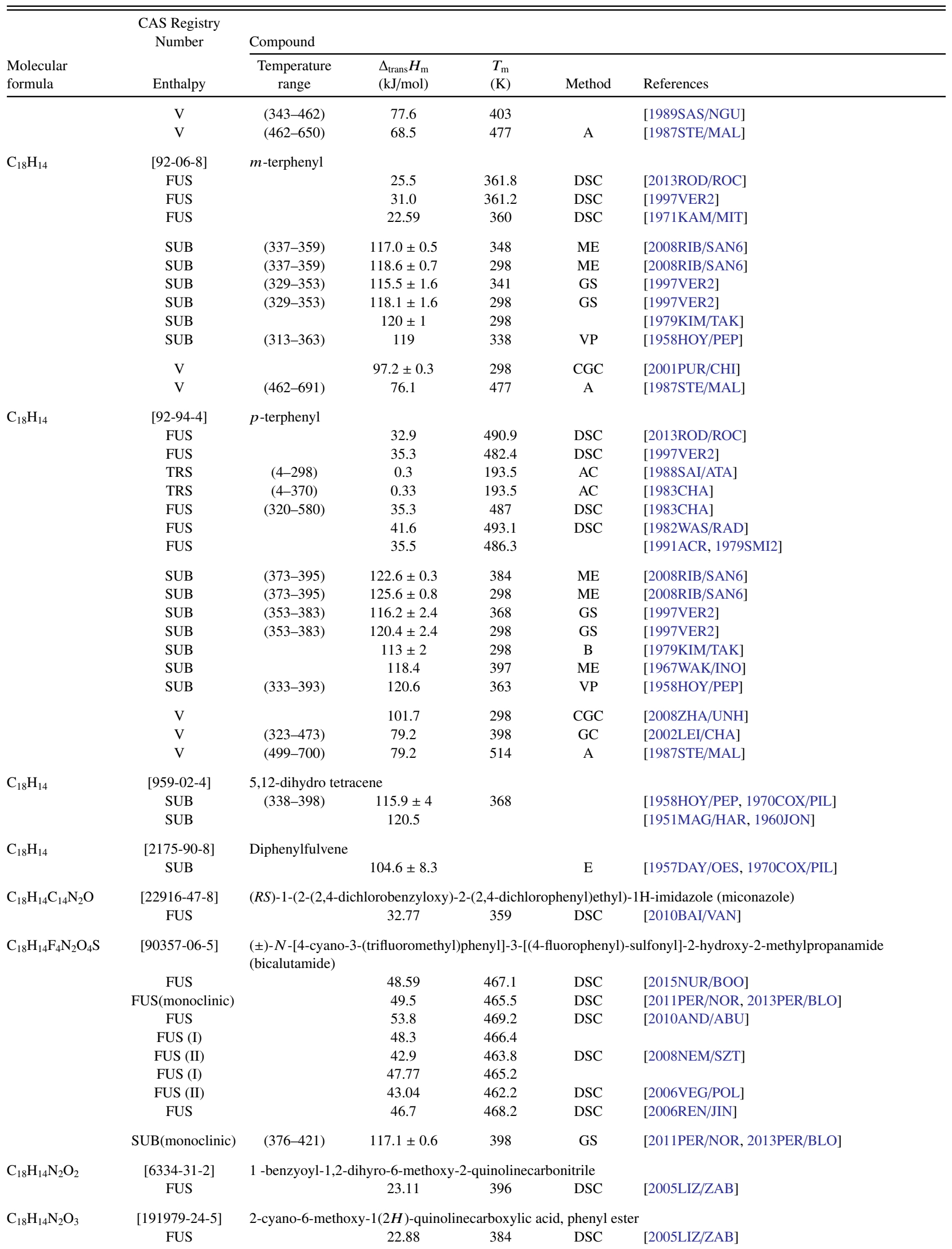


TABLE 13. Phase change enthalpies of $\mathrm{C}_{17}$ to $\mathrm{C}_{18}$ organic compounds-Continued

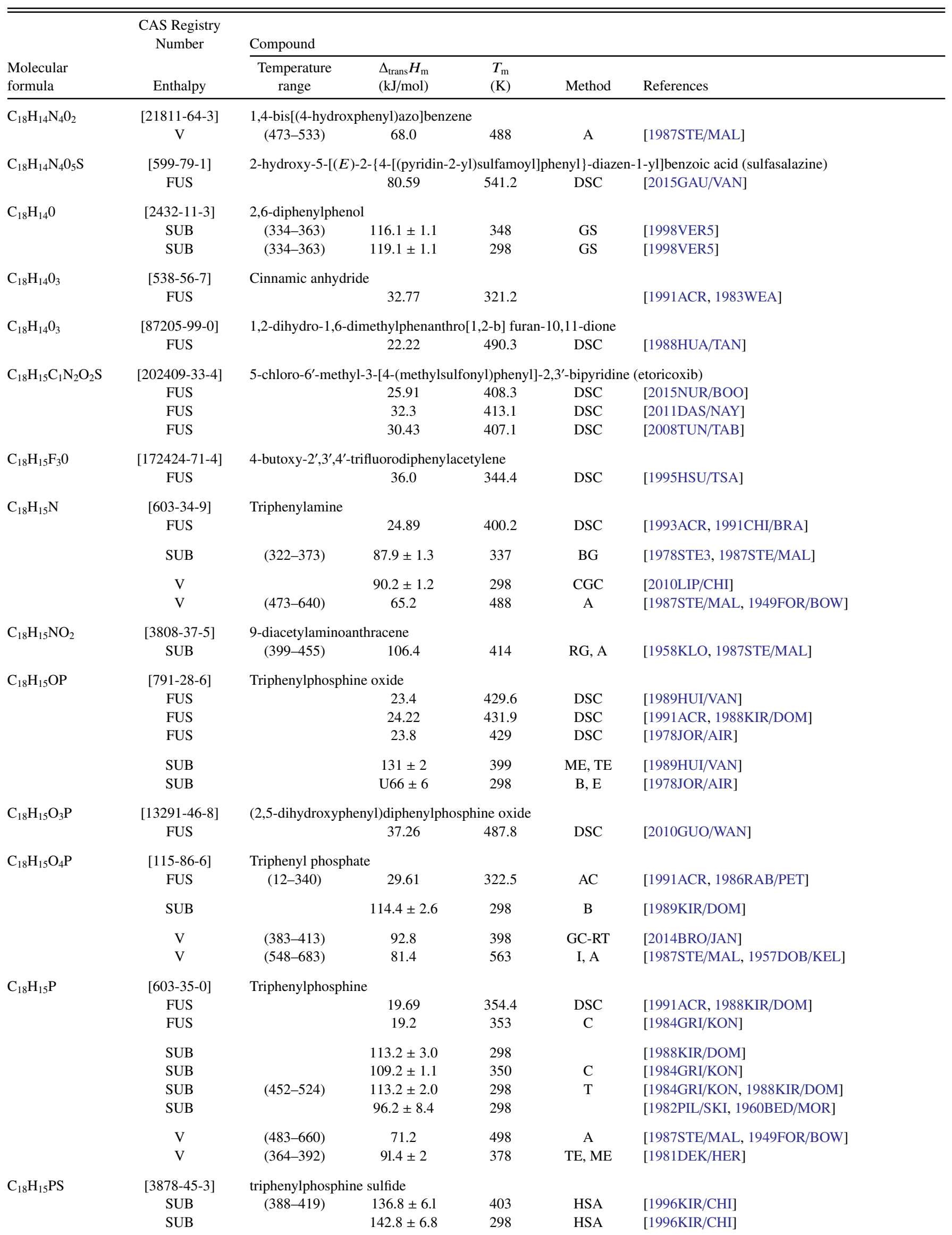


TABLE 13. Phase change enthalpies of $\mathrm{C}_{17}$ to $\mathrm{C}_{18}$ organic compounds-Continued

\begin{tabular}{|c|c|c|c|c|c|c|}
\hline \multirow[b]{2}{*}{$\begin{array}{l}\text { Molecular } \\
\text { formula }\end{array}$} & \multirow{2}{*}{$\begin{array}{l}\text { CAS Registry } \\
\text { Number } \\
\text { Enthalpy }\end{array}$} & \multicolumn{5}{|l|}{ Compound } \\
\hline & & $\begin{array}{c}\text { Temperature } \\
\text { range }\end{array}$ & $\begin{array}{l}\Delta_{\text {trans }} H_{\mathrm{m}} \\
(\mathrm{kJ} / \mathrm{mol})\end{array}$ & $\begin{array}{l}T_{\mathrm{m}} \\
(\mathrm{K})\end{array}$ & Method & References \\
\hline \multirow[t]{2}{*}{$\mathrm{C}_{18} \mathrm{H}_{16} \mathrm{FNO}_{2}$} & {$[86776-52-5]$} & \multicolumn{5}{|c|}{ 4-cyano-3-fluorophenyl 4-butylbenzoate } \\
\hline & FUS & $(5-360)$ & 22.22 & 288.4 & $\mathrm{AC}$ & [2012INA/SUZ] \\
\hline \multirow[t]{2}{*}{$\mathrm{C}_{18} \mathrm{H}_{16} \mathrm{~F}_{2}$} & {$[109970-65-2]$} & \multicolumn{5}{|c|}{ 4- $n$-butyl-3',4'-difluorodiphenylacetylene } \\
\hline & FUS & & 25.3 & 323.5 & DSC & [1995HSU/TSA] \\
\hline \multirow[t]{2}{*}{$\mathrm{C}_{18} \mathrm{H}_{16} \mathrm{NO}_{3} \mathrm{P}$} & [3848-51-9] & \multicolumn{5}{|c|}{ Diphenyl anilinophosphonate } \\
\hline & FUS & & 34.87 & 406.9 & DSC & [2015YU/WAN2] \\
\hline \multirow[t]{2}{*}{$\mathrm{C}_{18} \mathrm{H}_{16} \mathrm{~N}_{2} \mathrm{O}_{2}$} & & \multicolumn{5}{|c|}{ meso-2,3-dimethoxy-2,3-diphenylsuccinonitrile } \\
\hline & FUS & & 25.1 & 469.7 & & [1983ZAM/KAI] \\
\hline \multirow[t]{2}{*}{$\mathrm{C}_{18} \mathrm{H}_{16} \mathrm{~N}_{2} \mathrm{O}_{2}$} & {$[2479-46-1]$} & \multicolumn{5}{|c|}{ 1,3-bis(4-aminophenoxy )benzene } \\
\hline & FUS & & 0.463 & 389.2 & DSC & [2013ZHA/GAO] \\
\hline
\end{tabular}

[Note: The authors of [2013ZHA/GAO] reported the enthalpy of fusion in units of $\mathrm{J} \mathrm{mol}^{-1}$. We believe that the value is likely $46.3 \mathrm{~kJ} \mathrm{~mol}^{-1}$ ].

\begin{tabular}{|c|c|c|c|c|c|}
\hline \multirow[t]{2}{*}{$\mathrm{C}_{18} \mathrm{H}_{16} \mathrm{~N}_{4} \mathrm{O}$} & \multirow{2}{*}{$\begin{array}{c}\text { [1290504-01-6] } \\
\text { FUS }\end{array}$} & \multicolumn{4}{|c|}{ 5-benzyloxypyridine-2-aldehyde 2'-pyridinylhydrazone } \\
\hline & & 38 & 433.7 & DSC & [2013PER/KAZ] \\
\hline \multirow[t]{2}{*}{$\mathrm{C}_{18} \mathrm{H}_{16} \mathrm{~N}_{4} \mathrm{O}_{3}$} & {$[243445-13-8]$} & \multicolumn{4}{|c|}{ 2[4,5-dihydro-4-(4-methylphenyl)-5-oxo-3-(2-pyridinyl)-1,2,4-triazine-6(1H)-ylidene]acetic acid, methyl ester } \\
\hline & FUS & 42.8 & 468.3 & DSC & \\
\hline \multirow[t]{2}{*}{$\mathrm{C}_{18} \mathrm{H}_{16} \mathrm{~N}_{4}$} & [22119-35-3] & \multicolumn{4}{|l|}{ Dihydrodibenzotetra-aza-annulene } \\
\hline & SUB & $(443-583) \quad 81.5 \pm 6.4$ & 513 & $\mathrm{~T}$ & [1983ZVE/MOT] \\
\hline \multirow[t]{4}{*}{$\mathrm{C}_{18} \mathrm{H}_{16} \mathrm{O}_{2}$} & {$[84-47-9]$} & \multicolumn{4}{|l|}{ 2-tert-butyl-9,10-anthraquinone } \\
\hline & FUS & 19.12 & 376.2 & DSC & [2014JIA/YAN] \\
\hline & $\mathrm{V}$ & $(483-523)$ & 498 & A & [1987STE/MAL] \\
\hline & $\mathrm{V}$ & 97.7 & & & [1977SAS/FAL] \\
\hline \multirow[t]{2}{*}{$\mathrm{C}_{18} \mathrm{H}_{16} \mathrm{O}_{3}$} & {$[114390-57-7]$} & \multicolumn{4}{|c|}{ 1,8-diphenyl-2,3,5-trioxabicyclo[4.3.0]non-7-ene } \\
\hline & FUS & 21.7 & 371.2 & DSC & [1991JEF/JAB] \\
\hline \multirow[t]{2}{*}{$\mathrm{C}_{18} \mathrm{H}_{16} \mathrm{O}_{8}$} & {$[36063-07-7]$} & \multicolumn{4}{|l|}{ 1,2,3,4-tetracarbomethoxynaphthalene } \\
\hline & FUS & 35.9 & 423.7 & DSC & [1993ACR, 1978DOZ/FUJ] \\
\hline \multirow[t]{2}{*}{$\mathrm{C}_{18} \mathrm{H}_{16} \mathrm{O}_{8}$} & [68267-09-4] & \multicolumn{4}{|c|}{ 1,2,4,5-tetracarbomethoxynaphthalene } \\
\hline & FUS & 36.4 & 438.2 & DSC & [1993ACR, 1978DOZ/FUJ] \\
\hline \multirow[t]{2}{*}{$\mathrm{C}_{18} \mathrm{H}_{16} \mathrm{O}_{8}$} & {$[36063-08-8]$} & \multicolumn{4}{|c|}{ 1,2,5,6-tetracarbomethoxynaphthalene } \\
\hline & FUS & 42.1 & 470.2 & DSC & [1993ACR, 1978DOZ/FUJ] \\
\hline \multirow[t]{2}{*}{$\mathrm{C}_{18} \mathrm{H}_{16} \mathrm{O}_{8}$} & {$[68267-08-3]$} & \multicolumn{4}{|c|}{ 1,2,6,7-tetracarbomethoxynaphthalene } \\
\hline & FUS & 34.2 & 407.2 & DSC & [1993ACR, 1978DOZ/FUJ] \\
\hline \multirow[t]{2}{*}{$\mathrm{C}_{18} \mathrm{H}_{16} \mathrm{O}_{8}$} & {$[56110-97-5]$} & \multicolumn{4}{|l|}{ 2,3,6,7-tetracarbomethoxynaphthalene } \\
\hline & FUS & 42.2 & 458.2 & DSC & [1993ACR, 1978DOZ/FUJ] \\
\hline \multirow[t]{2}{*}{$\mathrm{C}_{18} \mathrm{H}_{16} \mathrm{O}_{8}$} & [31996-10-8] & \multicolumn{4}{|c|}{ 1,4,5,8-tetracarbomethoxynaphthalene } \\
\hline & FUS & 36.1 & 477.2 & DSC & [1993ACR, 1978DOZ/FUJ] \\
\hline \multirow[t]{2}{*}{$\mathrm{C}_{18} \mathrm{H}_{17} \mathrm{Cl}_{2} \mathrm{NO}_{3}$} & {$[22212-55-1]$} & \multicolumn{4}{|c|}{ Ethyl $N$-benzoyl- $N$-(3,4-dichlorophenyl)- $(d l)$-alaninate } \\
\hline & FUS & 27.06 & 341.7 & DSC & [1990DON/DRE] \\
\hline \multirow[t]{2}{*}{$\mathrm{C}_{18} \mathrm{H}_{17} \mathrm{~F}$} & [109970-63-0] & \multicolumn{4}{|l|}{ 4-n-butyl-4'-fluorodiphenylacetylene } \\
\hline & FUS & 18.5 & 329.9 & DSC & [1995HSU/TSA] \\
\hline \multirow[t]{2}{*}{$\mathrm{C}_{18} \mathrm{H}_{17} \mathrm{FO}$} & [130746-61-1] & 4-butoxy-4'-fluorodiphenylacetylene & & & \\
\hline & FUS & 25.4 & 346.7 & DSC & [1995HSU/TSA] \\
\hline $\mathrm{C}_{18} \mathrm{H}_{17} \mathrm{NO}_{3}$ & [483362-79-4] & 1-[(4-nitrophenyl)ethynyl]-4-butoxybe & nzene & & \\
\hline & FUS & 21.84 & 374.7 & DSC & [2002SPA/DZI] \\
\hline $\mathrm{C}_{18} \mathrm{H}_{17} \mathrm{NO}_{3}$ & [63610-08-2] & ( \pm )-2-(4-(1-oxoisoindolin-2-yl)phenyl & butanoic aci & ( \pm )-ind & fen) \\
\hline & FUS & 39.4 & 455.3 & DSC & [1995VIG/ZAM] \\
\hline $\mathrm{C}_{18} \mathrm{H}_{17} \mathrm{NO}_{3}$ & [118289-97-7] & $(S)-(+)-2-(4-(1-o x o i s o i n d o l i n-2-y l) p h$ & enyl)butanoi & $\operatorname{cid}((S)$ & lobufen) \\
\hline & FUS & 33.4 & 471.9 & DSC & [1995VIG/ZAM] \\
\hline
\end{tabular}


TABLE 13. Phase change enthalpies of $\mathrm{C}_{17}$ to $\mathrm{C}_{18}$ organic compounds-Continued

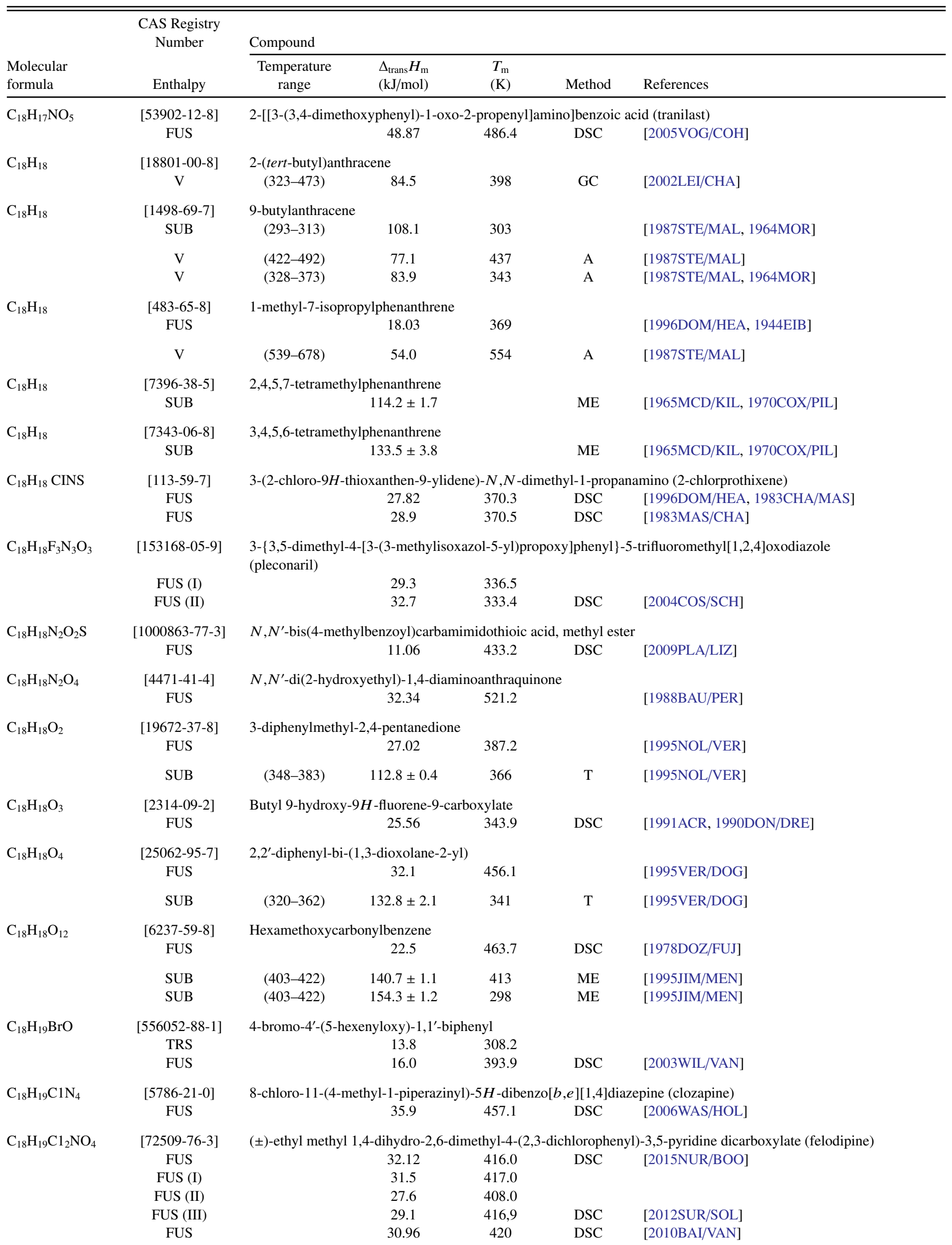


TABlE 13. Phase change enthalpies of $\mathrm{C}_{17}$ to $\mathrm{C}_{18}$ organic compounds-Continued

\begin{tabular}{|c|c|c|c|c|c|c|}
\hline \multirow[b]{2}{*}{$\begin{array}{l}\text { Molecular } \\
\text { formula }\end{array}$} & \multirow{2}{*}{$\begin{array}{l}\text { CAS Registry } \\
\text { Number } \\
\text { Enthalpy }\end{array}$} & \multicolumn{5}{|l|}{ Compound } \\
\hline & & $\begin{array}{c}\text { Temperature } \\
\text { range }\end{array}$ & $\begin{array}{l}\Delta_{\text {trans }} H_{\mathrm{m}} \\
(\mathrm{kJ} / \mathrm{mol})\end{array}$ & $\begin{array}{l}T_{\mathrm{m}} \\
(\mathrm{K})\end{array}$ & Method & References \\
\hline & FUS & & 34.8 & 412.3 & & [2007BER/WAS] \\
\hline & FUS & & 30.8 & 414.8 & DSC & [2006MAR/KON] \\
\hline & FUS & & 32.1 & 416.7 & DSC & [2004MAR/KOZ] \\
\hline & FUS (I) & & 31.5 & 417.4 & & \\
\hline & FUS (II) & & 26.7 & 405.7 & $\mathrm{DSC}$ & [2001ROL/BUR] \\
\hline & FUS & & 35.21 & 414.9 & $\mathrm{DSC}$ & {$[1992 \mathrm{SRC} / \mathrm{KER}]$} \\
\hline \multirow[t]{2}{*}{$\mathrm{C}_{18} \mathrm{H}_{19} \mathrm{Cl}_{2} \mathrm{NO}_{4}$} & [119945-59-4] & \multicolumn{5}{|c|}{ (+)-ethyl methyl 1,4-dihydro-2,6-dimethyl-4-(2,3-dichlorophenyl)-3,5-pyridine dicarboxylate(felodipine) } \\
\hline & FUS & & 25.4 & 415.7 & DSC & {$[2001 R O L / B U R]$} \\
\hline \multirow{2}{*}{$\mathrm{C}_{18} \mathrm{H}_{19} \mathrm{~N}$} & [63799-11-1] & \multicolumn{5}{|c|}{ (S)-4-(2-methylbutyl)-4'-cyanobiphenyl } \\
\hline & FUS & & 10.7 & 276 & DSC & [1999MAY/WIT] \\
\hline \multicolumn{7}{|c|}{ [Note: The compound may have phase transitions at lower temperatures.] } \\
\hline \multirow[t]{2}{*}{$\mathrm{C}_{18} \mathrm{H}_{19} \mathrm{NO}_{4}$} & {$[483362-68-1]$} & \multicolumn{5}{|c|}{ 2-(4-nitrophenyl)-1-(4-butoxyphenyl)ethanone } \\
\hline & FUS & & 24.94 & 336.1 & DSC & [2002SPA/DZI] \\
\hline \multirow[t]{2}{*}{$\mathrm{C}_{18} \mathrm{H}_{19} \mathrm{~N}_{3} \mathrm{O}$} & [99614-02-5] & \multicolumn{5}{|c|}{ 1,2,3,4-tetrahydro-9-methyl-3-[(2-methyl-1 $\boldsymbol{H}$-imidazol-1-yl)methyl]-9H-carbazol-4-one (ondanestron) } \\
\hline & FUS & & 45.05 & 493 & DSC & [2004DIM/DAL] \\
\hline \multirow[t]{6}{*}{$\mathrm{C}_{18} \mathrm{H}_{20}$} & [2913-24-8] & \multicolumn{5}{|c|}{ [3.3]para-cyclophane } \\
\hline & TRS & & 7.36 & 332 & & \\
\hline & TRS & & 0.46 & 351 & & \\
\hline & FUS & & 11.76 & 377 & DSC & [1969SHI/MCN] \\
\hline & SUB & $(322-343)$ & $103.3 \pm 1$ & 298 & $\mathrm{ME}$ & [1969SHI/MCN, 1977PED/RYL] \\
\hline & SUB & $(321-343)$ & 97.8 & 332 & A & [1987STE/MAL, 1969SHI/MCN] \\
\hline \multirow[t]{2}{*}{$\mathrm{C}_{18} \mathrm{H}_{20}$} & {$[115181-05-0]$} & \multicolumn{5}{|c|}{ 6-(4-biphenyl)-1-hexene } \\
\hline & FUS & & 15.1 & 274.5 & DSC & [1989MAL/KAN] \\
\hline \multirow[t]{2}{*}{$\mathrm{C}_{18} \mathrm{H}_{20} \mathrm{BrN}_{5} \mathrm{O}_{5}$} & [191355-38-1] & \multicolumn{5}{|c|}{ 8-bromo-( $R)$-7-[2-hydroxy-3-(4-acetylamino)-fenoxypropyl]-1,3-dimethylxanthine } \\
\hline & FUS & & 37.8 & 492.2 & DSC & [2000DAN/PRO] \\
\hline \multirow[t]{2}{*}{$\mathrm{C}_{18} \mathrm{H}_{20} \mathrm{Cl}_{2}$} & {$[72-56-0]$} & \multicolumn{5}{|c|}{ 1,1'-(2,2-dichloroethylidene)bis(4-ethylbenzene) } \\
\hline & FUS & & 23.34 & 331.6 & DSC & [1991ACR, 1990DON/DRE] \\
\hline \multirow[t]{3}{*}{$\mathrm{C}_{18} \mathrm{H}_{20} \mathrm{~N}_{2} \mathrm{O}_{2}$} & {$[3955-57-5]$} & \multicolumn{5}{|c|}{$N, N^{\prime}$-bis(salicylaldehydo)tetramethylenediimine } \\
\hline & FUS & & 35.54 & 362.8 & DSC & [2004RIB/GON] \\
\hline & SUB & $(349-361)$ & $165.1 \pm 3.1$ & 298 & ME & {$[2004 \mathrm{RIB} / \mathrm{GON}]$} \\
\hline $\mathrm{C}_{18} \mathrm{H}_{20} \mathrm{~N}_{2} \mathrm{O}_{4}$ & {$[332140-31-5]$} & Diethyl 2,4,6,8 & hydro-4,8-e & enzo $[1$, & 5-c']dipy & -1,7-dicarboxylate \\
\hline & FUS & & 18.2 & 439.3 & DSC & [2000UNO/ITO] \\
\hline $\mathrm{C}_{18} \mathrm{H}_{20} \mathrm{~N}_{2} \mathrm{O}_{4}$ & [54946-24-6] & $N$-pentylthalid & & & & \\
\hline & FUS & & 23.97 & 378.2 & DTA & [2002GOO/LAI] \\
\hline $\mathrm{C}_{18} \mathrm{H}_{20} \mathrm{~N}_{2} \mathrm{O}_{6}$ & [39562-70-4] & 3-ethyl-5-meth & 4-dihydro-2, & ethyl-4- & ophenyl)- & pyridinedicarboxylate $((R S)$ - nitrendipine) \\
\hline & FUS & & 39.6 & 432.6 & DSC & {$[2004 \mathrm{MAR} / \mathrm{KOZ}]$} \\
\hline & FUS & & 41.1 & 430.7 & DSC & [1997BUR/ROL] \\
\hline $\mathrm{C}_{18} \mathrm{H}_{20} \mathrm{~N}_{6} \mathrm{O}_{7}$ & [191355-39-2] & 8-nitro- $(R)-7-[$ & droxy-3-(4-a & $\operatorname{mino})-\mathrm{f}$ & ropyl]-1, & imethylxanthine \\
\hline & FUS & & 56.8 & 481.2 & DSC & [2000DAN/PRO] \\
\hline $\mathrm{C}_{18} \mathrm{H}_{20} \mathrm{OS}$ & {$[556052-90-5]$} & $4^{\prime}-(5$-hexenylox & 1,1'-bipheny & & & \\
\hline & TRS & & 11.6 & 358.4 & & \\
\hline & FUS & & 13.6 & 384.6 & DSC & [2003WIL/VAN] \\
\hline $\mathrm{C}_{18} \mathrm{H}_{20} \mathrm{O}_{2}$ & [56-53-1] & Diethylstilbestı & & & & \\
\hline & FUS & & 31.76 & 443.8 & DSC & [1990DON/DRE] \\
\hline $\mathrm{C}_{18} \mathrm{H}_{20} \mathrm{O}_{2}$ & [100923-74-8] & (2-hydroxyl-4, & nethylphenyl & 5-trimetl & nylmetha & \\
\hline & FUS & & 0.84 & 380.2 & DTA & [1989SAL/ABA] \\
\hline
\end{tabular}

[Note: Reported enthalpy of fusion is too small, and the published enthalpy and entropy of fusion data are internally inconsistent.]

$\mathrm{C}_{18} \mathrm{H}_{20} \mathrm{O}_{4}$

[39716-92-2] 2-hydroxy-4-butoxy-4'-methoxybenzophenone

FUS

33.7

345.6

DSC

[1999PRI/HAW] 
TABLE 13. Phase change enthalpies of $\mathrm{C}_{17}$ to $\mathrm{C}_{18}$ organic compounds-Continued

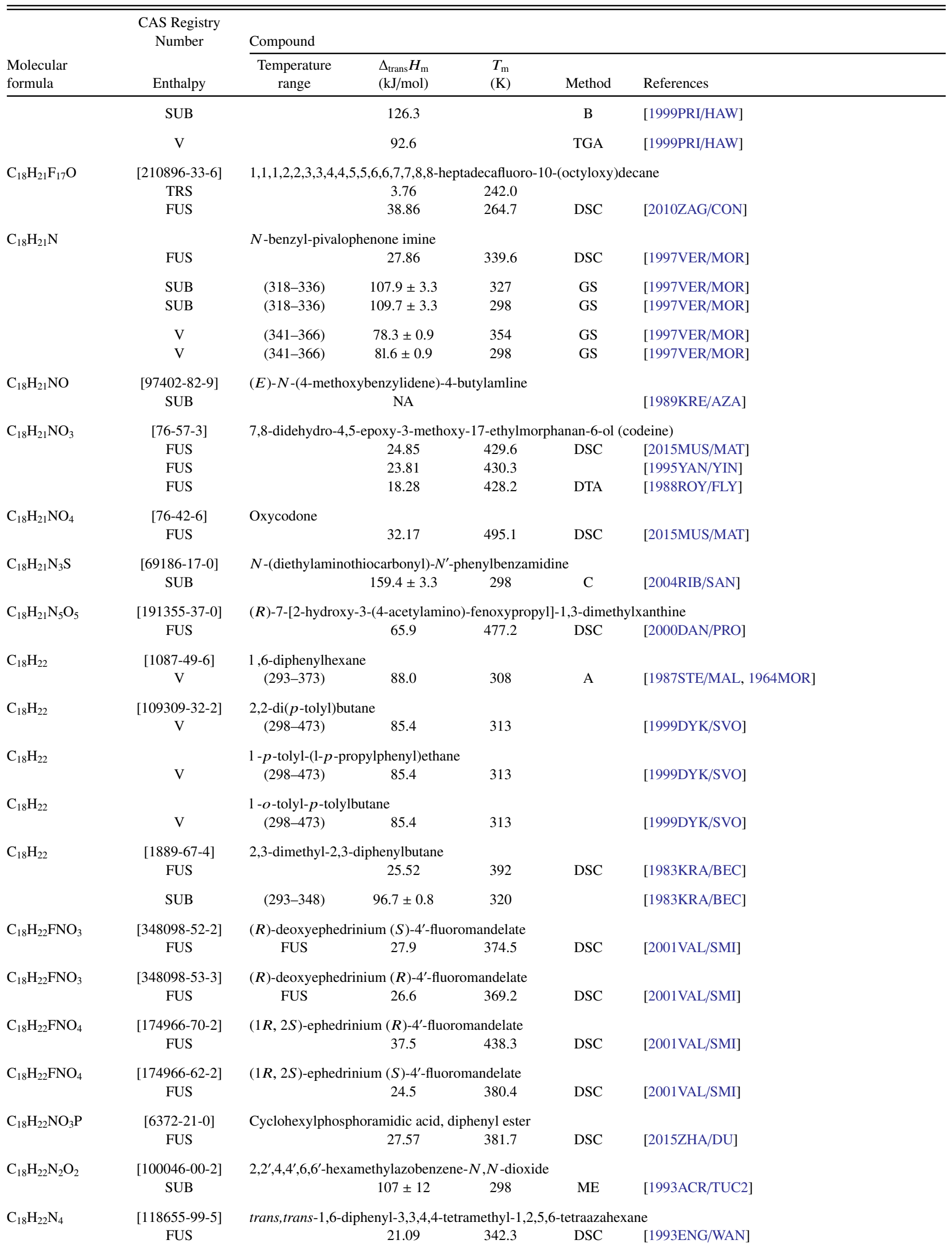


TABLE 13. Phase change enthalpies of $\mathrm{C}_{17}$ to $\mathrm{C}_{18}$ organic compounds-Continued

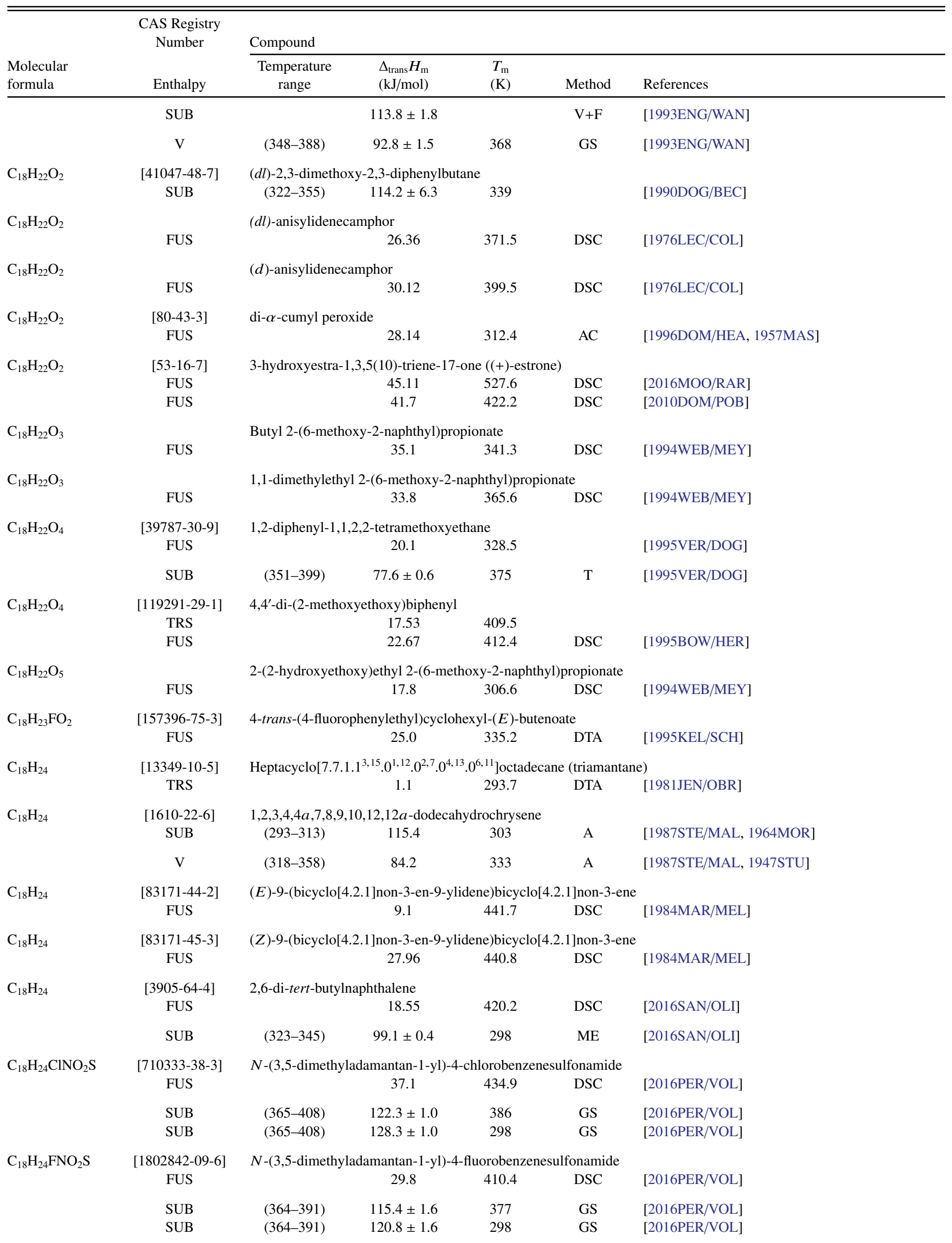


TABLE 13. Phase change enthalpies of $\mathrm{C}_{17}$ to $\mathrm{C}_{18}$ organic compounds-Continued

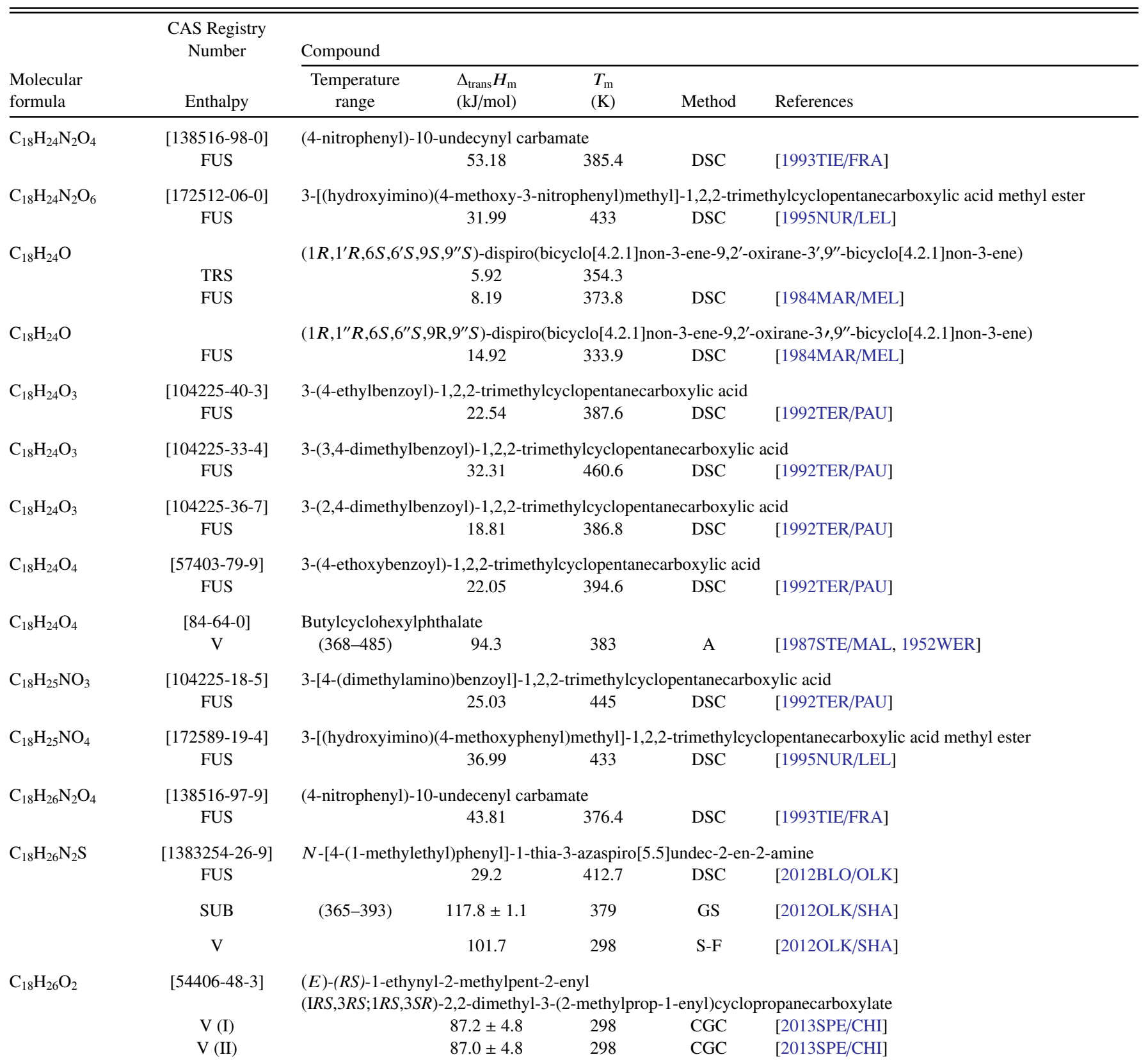

[Note: The authors of [2013SPE/CHI] reported the enthalpies of vaporization of the two diasteriomers in the commercial sample]

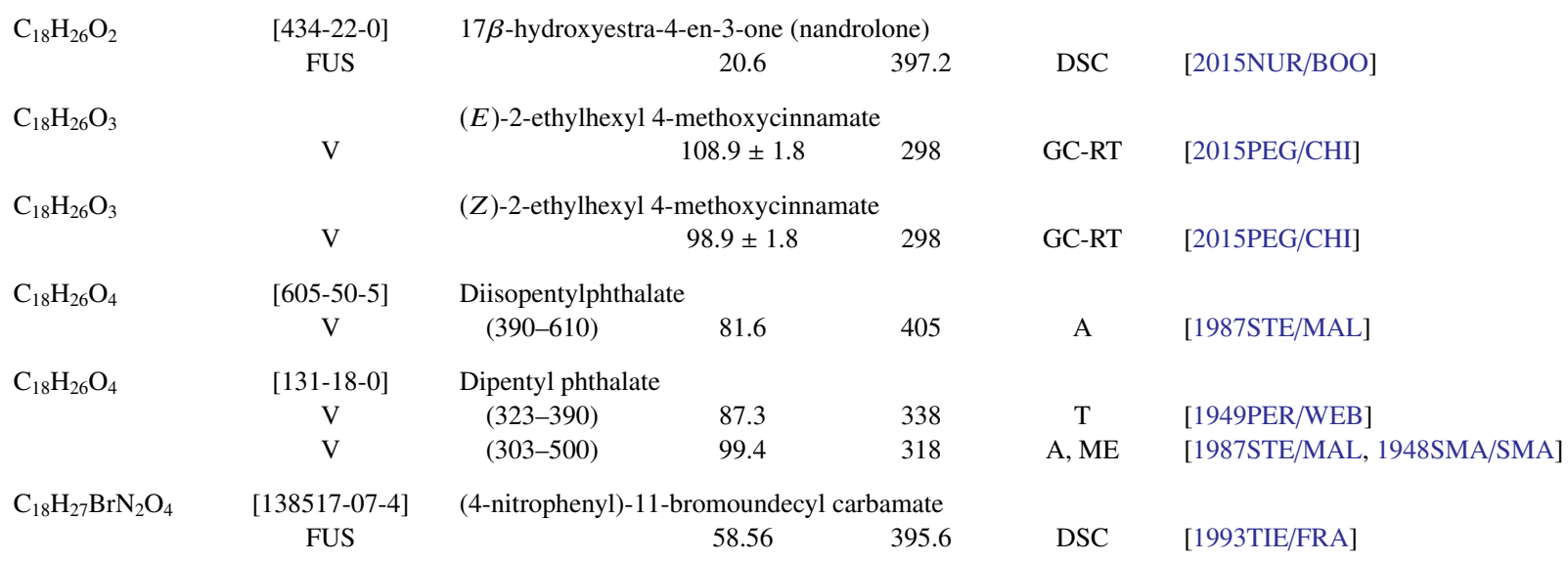


TABLE 13. Phase change enthalpies of $\mathrm{C}_{17}$ to $\mathrm{C}_{18}$ organic compounds-Continued

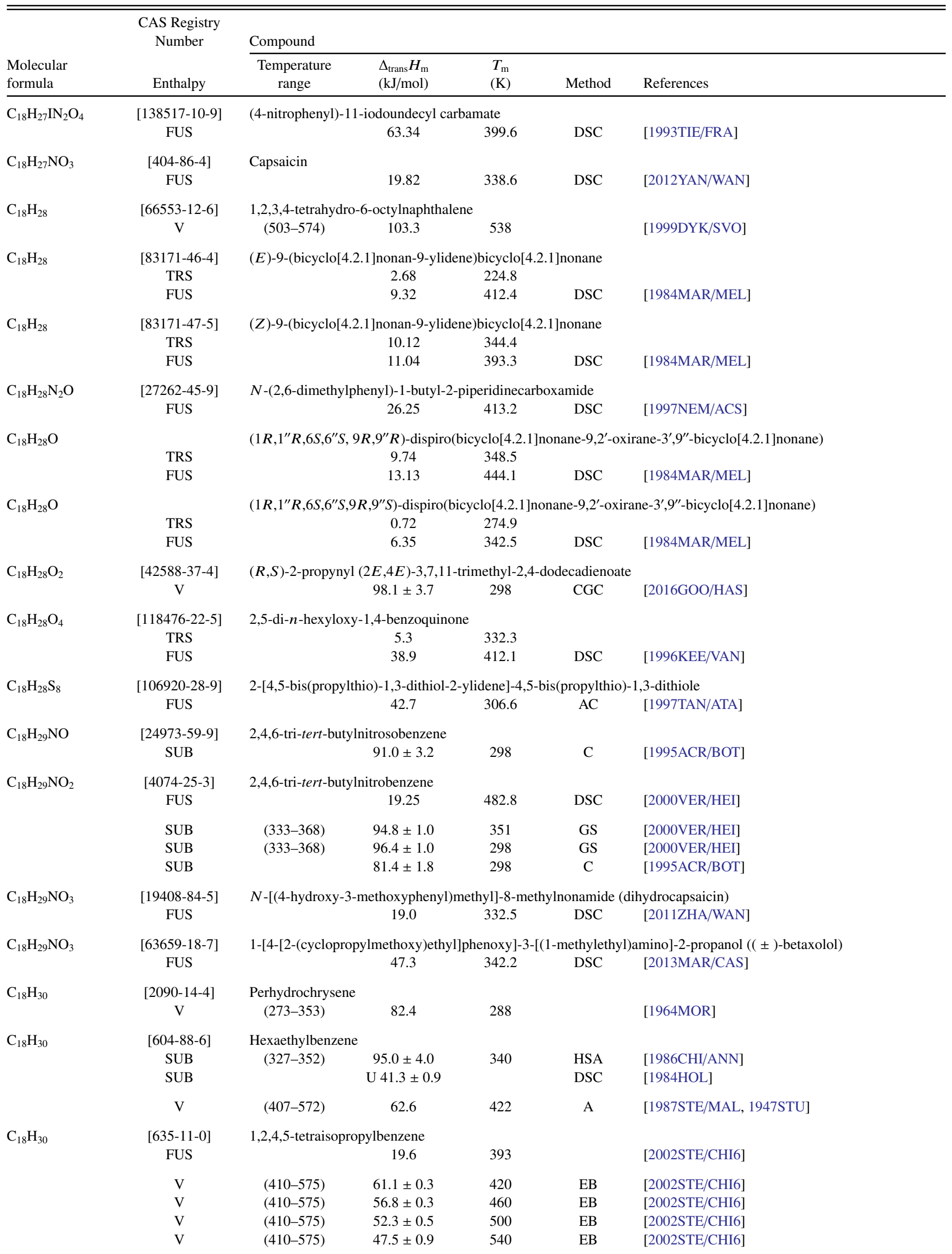


TABLE 13. Phase change enthalpies of $\mathrm{C}_{17}$ to $\mathrm{C}_{18}$ organic compounds-Continued

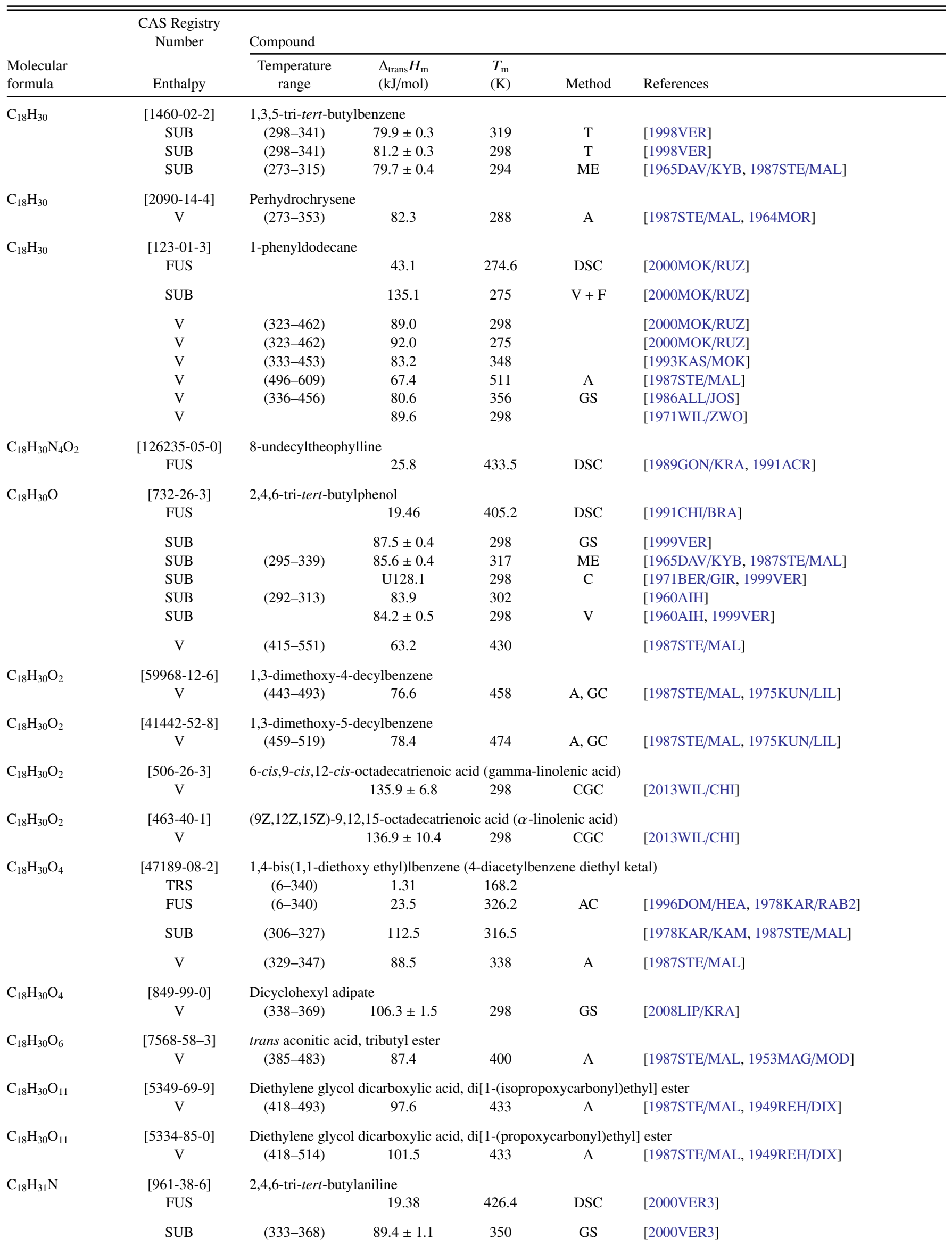


TABLE 13. Phase change enthalpies of $\mathrm{C}_{17}$ to $\mathrm{C}_{18}$ organic compounds-Continued

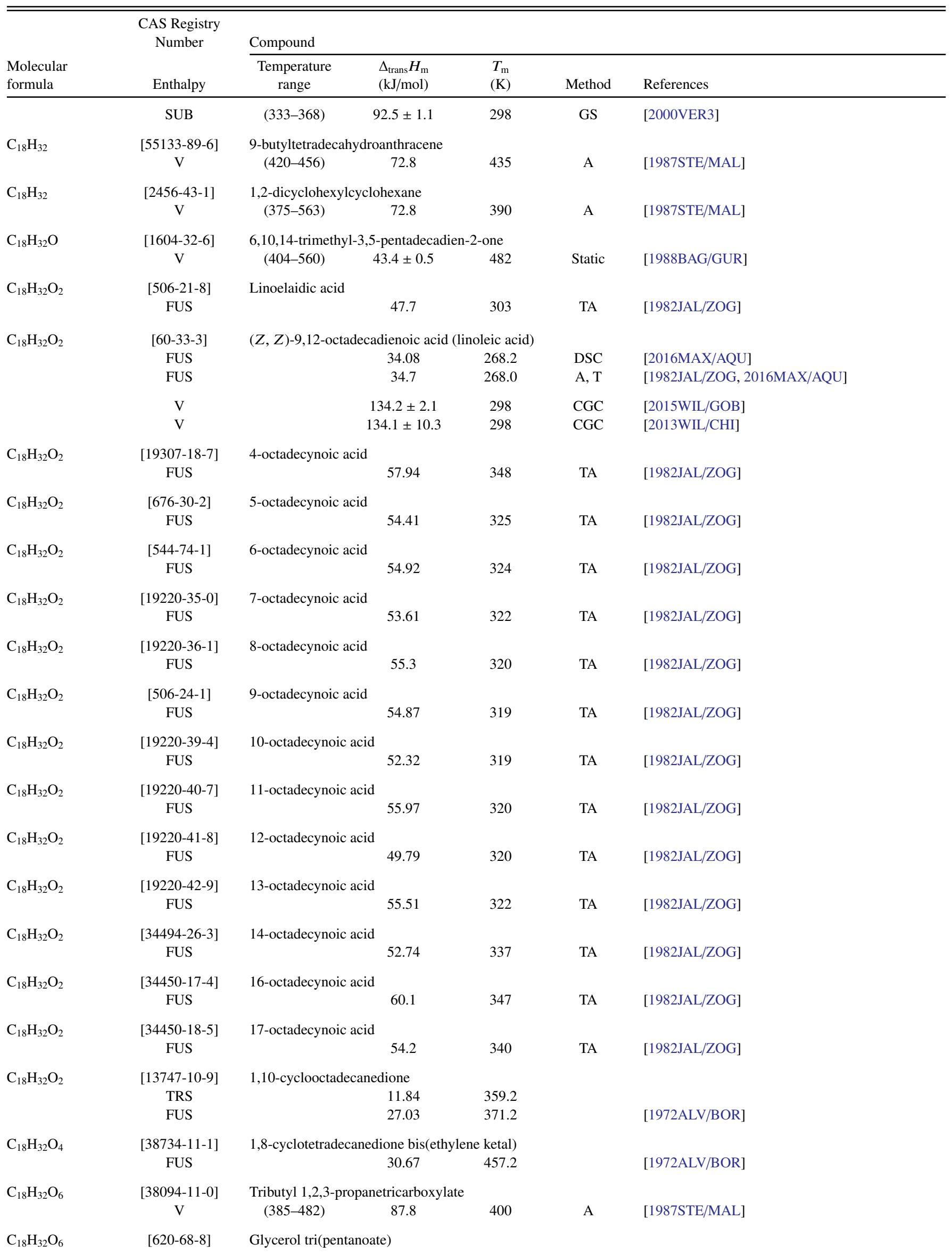


TABLE 13. Phase change enthalpies of $\mathrm{C}_{17}$ to $\mathrm{C}_{18}$ organic compounds-Continued

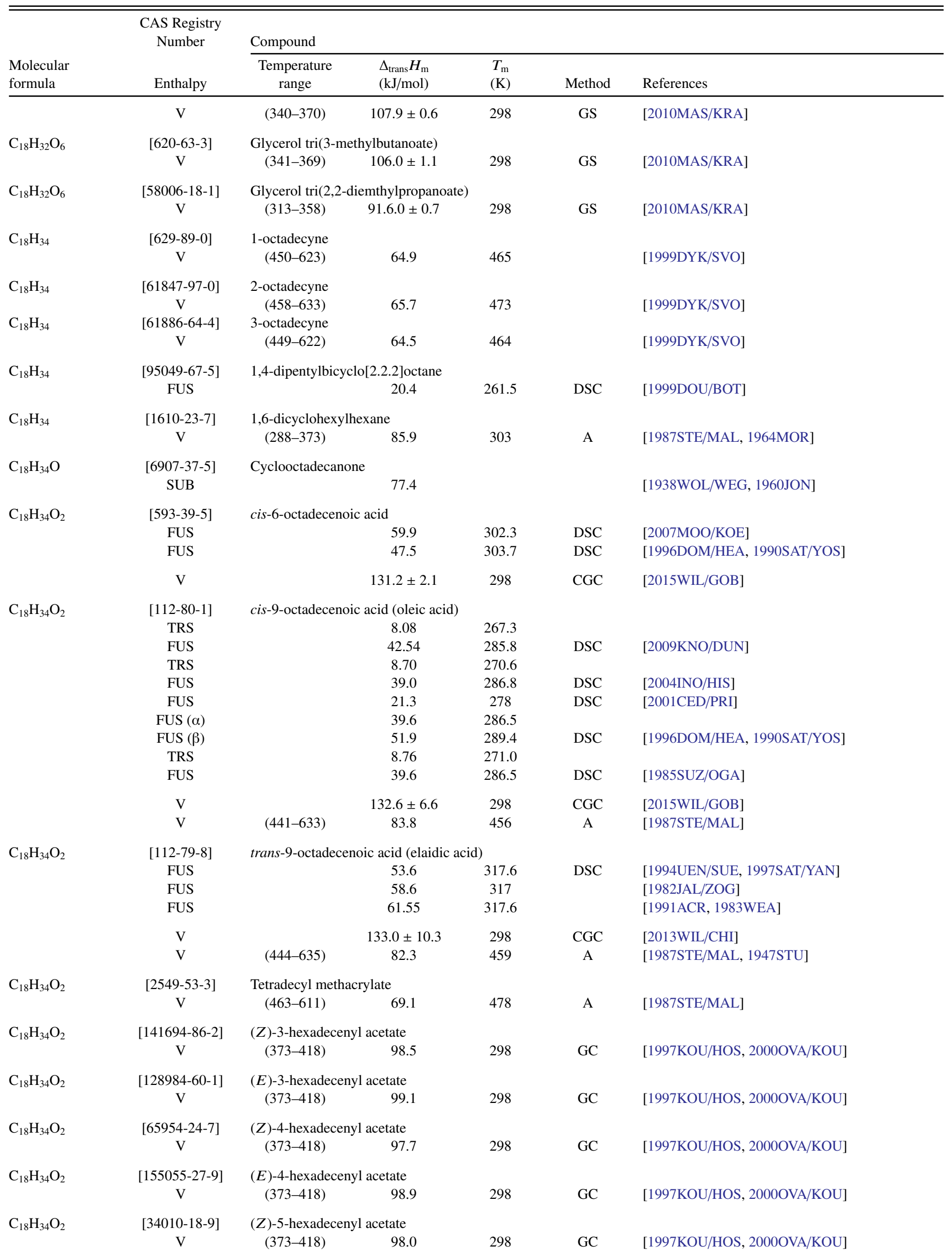


TABLE 13. Phase change enthalpies of $\mathrm{C}_{17}$ to $\mathrm{C}_{18}$ organic compounds-Continued

\begin{tabular}{|c|c|c|c|c|c|c|}
\hline \multirow[b]{2}{*}{$\begin{array}{l}\text { Molecular } \\
\text { formula }\end{array}$} & \multirow{2}{*}{$\begin{array}{c}\text { CAS Registry } \\
\text { Number } \\
\text { Enthalpy }\end{array}$} & \multicolumn{5}{|l|}{ Compound } \\
\hline & & $\begin{array}{c}\text { Temperature } \\
\text { range }\end{array}$ & $\begin{array}{l}\Delta_{\text {trans }} H_{\mathrm{m}} \\
(\mathrm{kJ} / \mathrm{mol})\end{array}$ & $\begin{array}{l}T_{\mathrm{m}} \\
(\mathrm{K})\end{array}$ & Method & References \\
\hline \multirow[t]{2}{*}{$\mathrm{C}_{18} \mathrm{H}_{34} \mathrm{O}_{2}$} & {$[56218-65-6]$} & \multicolumn{5}{|c|}{ (E)-5-hexadecenyl acetate } \\
\hline & $\mathrm{V}$ & $(373-418)$ & 98.8 & 298 & $\mathrm{GC}$ & [1997KOU/HOS, 2000OVA/KOU] \\
\hline \multirow{2}{*}{$\mathrm{C}_{18} \mathrm{H}_{34} \mathrm{O}_{2}$} & [34010-19-0] & \multicolumn{5}{|c|}{ (Z)-6-hexadecenyl acetate } \\
\hline & V & $(373-418)$ & 97.8 & 298 & GC & [1997KOU/HOS, 2000OVA/KOU] \\
\hline \multirow[t]{2}{*}{$\mathrm{C}_{18} \mathrm{H}_{34} \mathrm{O}_{2}$} & [56218-66-7] & \multicolumn{5}{|c|}{ (E)-6-hexadecenyl acetate } \\
\hline & $\mathrm{V}$ & $(373-418)$ & 98.6 & 298 & GC & [1997KOU/HOS, 2000OVA/KOU] \\
\hline \multirow[t]{2}{*}{$\mathrm{C}_{18} \mathrm{H}_{34} \mathrm{O}_{2}$} & {$[23192-42-9]$} & \multicolumn{5}{|c|}{ (Z)-7-hexadecenyl acetate } \\
\hline & V & $(373-418)$ & 97.8 & 298 & GC & [1997KOU/HOS, 2000OVA/KOU] \\
\hline \multirow{2}{*}{$\mathrm{C}_{18} \mathrm{H}_{34} \mathrm{O}_{2}$} & {$[23192-83-8]$} & \multicolumn{5}{|c|}{$(E)$-7-hexadecenyl acetate } \\
\hline & $\mathrm{V}$ & $(373-418)$ & 98.5 & 298 & GC & [1997KOU/HOS, 2000OVA/KOU] \\
\hline \multirow[t]{2}{*}{$\mathrm{C}_{18} \mathrm{H}_{34} \mathrm{O}_{2}$} & {$[56218-67-8]$} & \multicolumn{5}{|c|}{ (Z)-8-hexadecenyl acetate } \\
\hline & V & $(373-418)$ & 97.8 & 298 & GC & [1997KOU/HOS, 2000OVA/KOU] \\
\hline \multirow[t]{2}{*}{$\mathrm{C}_{18} \mathrm{H}_{34} \mathrm{O}_{2}$} & {$[56218-68-9]$} & \multicolumn{5}{|c|}{ (E)-8-hexadecenyl acetate } \\
\hline & V & $(373-418)$ & 98.6 & 298 & GC & [1997KOU/HOS, 2000OVA/KOU] \\
\hline \multirow{2}{*}{$\mathrm{C}_{18} \mathrm{H}_{34} \mathrm{O}_{2}$} & {$[34010-20-3]$} & \multicolumn{5}{|c|}{ (Z)-9-hexadecenyl acetate } \\
\hline & V & $(373-418)$ & 98.2 & 298 & GC & [1997KOU/HOS, 2000OVA/KOU] \\
\hline \multirow[t]{2}{*}{$\mathrm{C}_{18} \mathrm{H}_{34} \mathrm{O}_{2}$} & {$[56218-69-0]$} & (E)-9-hexadec & cetate & & & \\
\hline & V & $(373-418)$ & 98.9 & 298 & GC & [1997KOU/HOS, 2000OVA/KOU] \\
\hline $\mathrm{C}_{18} \mathrm{H}_{34} \mathrm{O}_{2}$ & {$[56218-70-3]$} & (Z)-10-hexade & acetate & & & \\
\hline & V & $(373-418)$ & 98.5 & 298 & GC & [1997KOU/HOS, 2000OVA/KOU] \\
\hline $\mathrm{C}_{18} \mathrm{H}_{34} \mathrm{O}_{2}$ & {$[56218-71-4]$} & (E)-10-hexade & acetate & & & \\
\hline & V & $(373-418)$ & 99.1 & 298 & GC & [1997KOU/HOS, 2000OVA/KOU] \\
\hline $\mathrm{C}_{18} \mathrm{H}_{34} \mathrm{O}_{2}$ & {$[34010-21-4]$} & (Z)-11-hexade & acetate & & & \\
\hline & V & $(373-418)$ & 98.9 & 298 & GC & [1997KOU/HOS, 2000OVA/KOU] \\
\hline $\mathrm{C}_{18} \mathrm{H}_{34} \mathrm{O}_{2}$ & {$[56218-72-5]$} & (E)-11-hexade & acetate & & & \\
\hline & V & $(373-418)$ & 99.5 & 298 & GC & [1997KOU/HOS, 2000OVA/KOU] \\
\hline $\mathrm{C}_{18} \mathrm{H}_{34} \mathrm{O}_{2}$ & {$[56218-73-6]$} & (Z)-12-hexade & acetate & & & \\
\hline & $\mathrm{V}$ & $(373-418)$ & 99.5 & 298 & GC & [1997KOU/HOS, 2000OVA/KOU] \\
\hline $\mathrm{C}_{18} \mathrm{H}_{34} \mathrm{O}_{2}$ & {$[64789-90-8]$} & (E)-12-hexade & acetate & & & \\
\hline & V & $(373-418)$ & 99.8 & 298 & GC & [1997KOU/HOS, 2000OVA/KOU] \\
\hline $\mathrm{C}_{18} \mathrm{H}_{34} \mathrm{O}_{2}$ & {$[56218-74-7]$} & (Z)-13-hexade & acetate & & & \\
\hline & V & $(373-418)$ & 100 & 298 & GC & [1997KOU/HOS, 2000OVA/KOU] \\
\hline $\mathrm{C}_{18} \mathrm{H}_{34} \mathrm{O}_{2}$ & {$[69282-67-3]$} & (E)-13-hexade & acetate & & & \\
\hline & V & $(373-418)$ & 100.3 & 298 & GC & [1997KOU/HOS, 2000OVA/KOU] \\
\hline $\mathrm{C}_{18} \mathrm{H}_{34} \mathrm{O}_{2}$ & {$[13161-77-8]$} & trans-3-octade & acid & & & \\
\hline & FUS & & 57.15 & 334 & TA & [1982JAL/ZOG] \\
\hline $\mathrm{C}_{18} \mathrm{H}_{34} \mathrm{O}_{2}$ & {$[34450-19-6]$} & trans-4-octade & acid & & & \\
\hline & FUS & & 55.88 & 333 & TA & [1982JAL/ZOG] \\
\hline $\mathrm{C}_{18} \mathrm{H}_{34} \mathrm{O}_{2}$ & {$[7056-85-1]$} & trans-5-octade & acid & & & \\
\hline & FUS & & 45.11 & 319 & TA & [1982JAL/ZOG] \\
\hline $\mathrm{C}_{18} \mathrm{H}_{34} \mathrm{O}_{2}$ & {$[593-40-8]$} & trans-6-octade & acid & & & \\
\hline & FUS & & 60.15 & 326 & TA & [1982JAL/ZOG] \\
\hline $\mathrm{C}_{18} \mathrm{H}_{34} \mathrm{O}_{2}$ & {$[5684-82-2]$} & trans-10-octad & ic acid & & & \\
\hline & FUS & & 58.52 & 326 & TA & [1982JAL/ZOG] \\
\hline $\mathrm{C}_{18} \mathrm{H}_{34} \mathrm{O}_{2}$ & {$[506-17-2]$} & cis-11-octadec & acid (ascle & & & \\
\hline & TRS & & 7.8 & 257.8 & & \\
\hline & FUS & & 39.8 & 287 & DSC & [1997SAT/YAN] \\
\hline $\mathrm{C}_{18} \mathrm{H}_{34} \mathrm{O}_{2}$ & [693-72-1] & trans-11-octad & ic acid & & & \\
\hline & FUS & & 58.49 & 317 & $\mathrm{TA}$ & [1982JAL/ZOG] \\
\hline
\end{tabular}


TABLE 13. Phase change enthalpies of $\mathrm{C}_{17}$ to $\mathrm{C}_{18}$ organic compounds-Continued

\begin{tabular}{|c|c|c|c|c|c|c|}
\hline \multirow[b]{2}{*}{$\begin{array}{l}\text { Molecular } \\
\text { formula }\end{array}$} & \multirow{2}{*}{$\begin{array}{c}\text { CAS Registry } \\
\text { Number } \\
\text { Enthalpy }\end{array}$} & \multicolumn{5}{|l|}{ Compound } \\
\hline & & $\begin{array}{c}\text { Temperature } \\
\text { range }\end{array}$ & $\begin{array}{l}\Delta_{\text {trans }} H_{\mathrm{m}} \\
(\mathrm{kJ} / \mathrm{mol})\end{array}$ & $\begin{array}{l}T_{\mathrm{m}} \\
(\mathrm{K})\end{array}$ & Method & References \\
\hline $\mathrm{C}_{18} \mathrm{H}_{34} \mathrm{O}_{2}$ & $\begin{array}{c}\text { [13126-38-0] } \\
\text { FUS }\end{array}$ & trans-12-octad & $\begin{array}{l}\text { ic acid } \\
56.71\end{array}$ & 325 & TA & [1982JAL/ZOG] \\
\hline $\mathrm{C}_{18} \mathrm{H}_{34} \mathrm{O}_{2}$ & $\begin{array}{c}{[693-71-0]} \\
\text { FUS }\end{array}$ & trans-13-octad & $\begin{array}{l}\text { ic acid } \\
55.62\end{array}$ & 318 & TA & [1982JAL/ZOG] \\
\hline $\mathrm{C}_{18} \mathrm{H}_{34} \mathrm{O}_{2}$ & $\begin{array}{c}\text { [13126-42-6] } \\
\text { FUS }\end{array}$ & trans-14-octad & $\begin{array}{l}57.06 \\
5 \text { acid }\end{array}$ & 327 & TA & [1982JAL/ZOG] \\
\hline $\mathrm{C}_{18} \mathrm{H}_{34} \mathrm{O}_{2}$ & $\begin{array}{c}\text { [13126-44-8] } \\
\text { FUS }\end{array}$ & trans-15-octad & $\begin{array}{l}\text { ic acid } \\
58.98\end{array}$ & 331 & TA & [1982JAL/ZOG] \\
\hline $\mathrm{C}_{18} \mathrm{H}_{34} \mathrm{O}_{4}$ & $\begin{array}{c}{[110-33-8]} \\
\mathrm{V}\end{array}$ & $\begin{array}{l}\text { Dihexyl adipat } \\
\quad(470-595)\end{array}$ & 80.4 & 485 & A & [1987STE/MAL] \\
\hline $\mathrm{C}_{18} \mathrm{H}_{34} \mathrm{O}_{4}$ & $\begin{array}{c}{[109-43-3]} \\
\mathrm{V} \\
\mathrm{V}\end{array}$ & $\begin{array}{l}\text { Dibutyl decane } \\
\quad(468-532) \\
(400-532)\end{array}$ & $\begin{array}{c}106.4 \\
87.6\end{array}$ & $\begin{array}{l}483 \\
465\end{array}$ & EB & $\begin{array}{l}\text { [2008ZHU/XU] } \\
\text { [2008ZHU/XU] }\end{array}$ \\
\hline
\end{tabular}

[Note: The first value is based on published Antoine constants determined from the authors' experimental data; the second value is what the authors reported from combining their experimental data with published literature data]

\begin{tabular}{|c|c|c|c|c|c|}
\hline V & & 88.1 & 327 & TGA & [1990KIS/SHO] \\
\hline V & & $91.8 \pm 3.2$ & 298 & TGA & [1990KIS/SHO] \\
\hline V & $(401-520)$ & 94.3 & 416 & A & [1987STE/MAL] \\
\hline
\end{tabular}

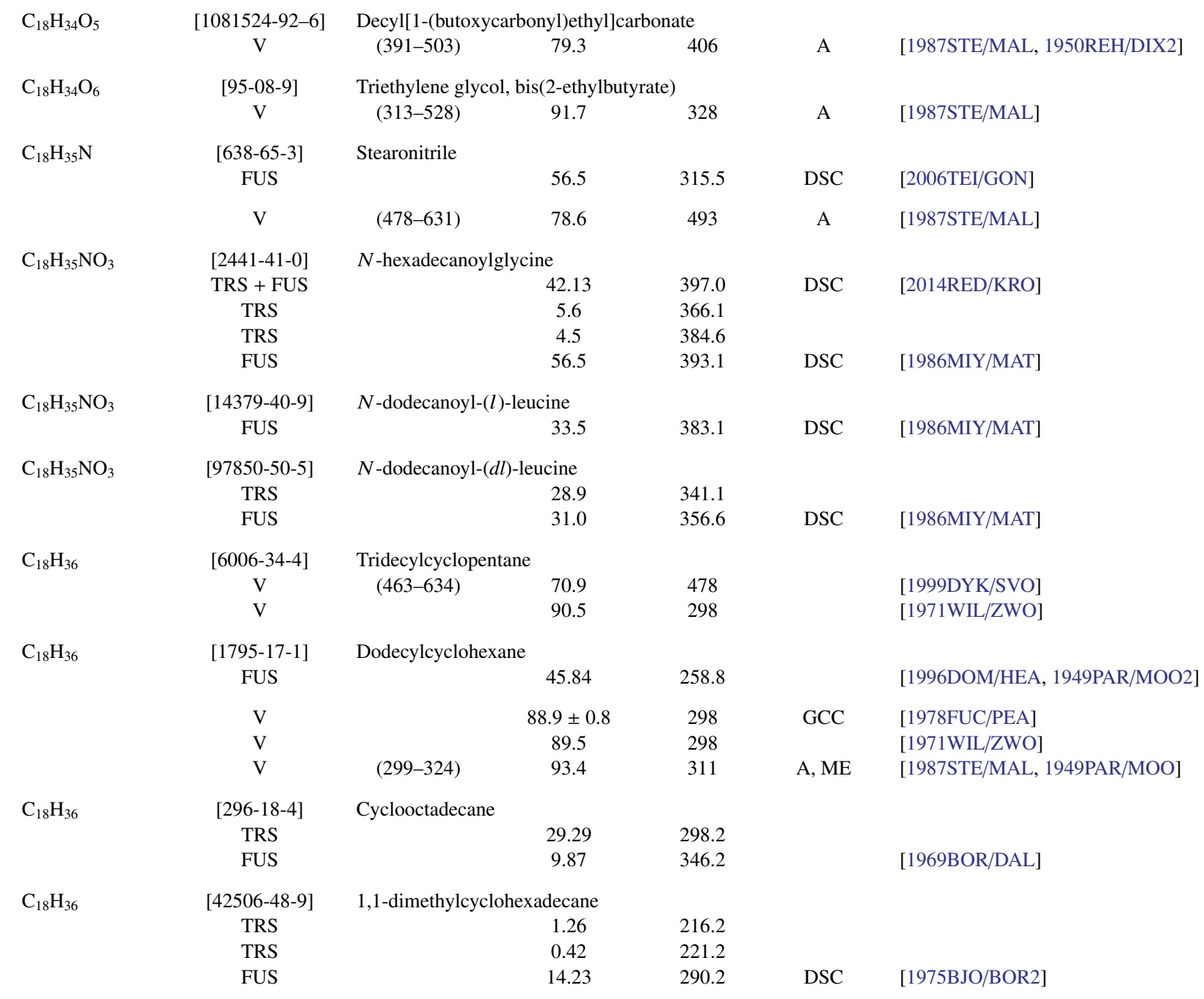

$\mathrm{C}_{18} \mathrm{H}_{36} \quad$ [112-88-9] 1-octadecene 
TABLE 13. Phase change enthalpies of $\mathrm{C}_{17}$ to $\mathrm{C}_{18}$ organic compounds-Continued

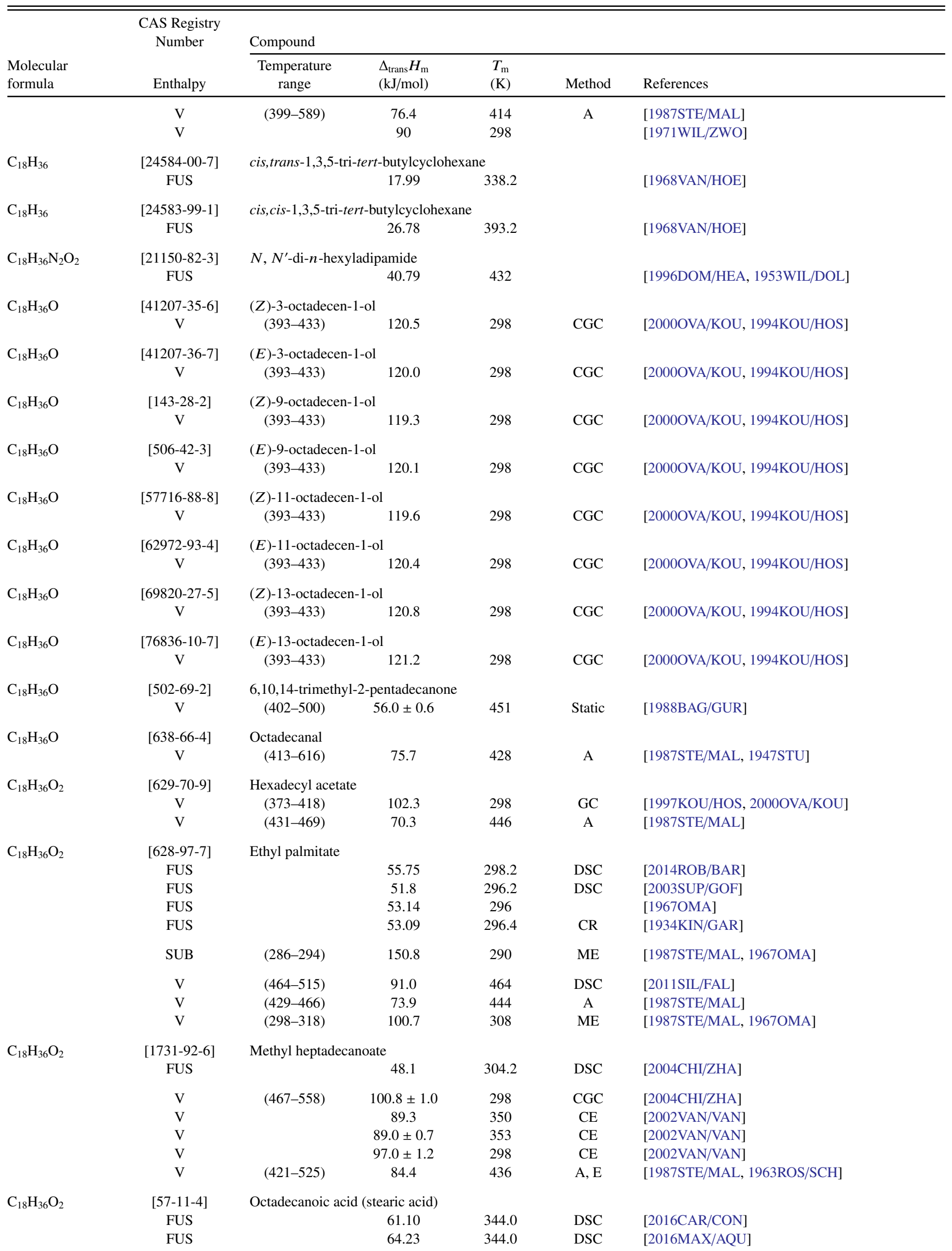


TABLE 13. Phase change enthalpies of $\mathrm{C}_{17}$ to $\mathrm{C}_{18}$ organic compounds-Continued

\begin{tabular}{|c|c|c|c|c|c|c|}
\hline \multirow[b]{2}{*}{$\begin{array}{l}\text { Molecular } \\
\text { formula }\end{array}$} & \multirow{2}{*}{$\begin{array}{c}\text { CAS Registry } \\
\text { Number } \\
\text { Enthalpy }\end{array}$} & \multicolumn{5}{|l|}{ Compound } \\
\hline & & $\begin{array}{c}\text { Temperature } \\
\text { range }\end{array}$ & $\begin{array}{l}\Delta_{\text {trans }} H_{\mathrm{m}} \\
(\mathrm{kJ} / \mathrm{mol})\end{array}$ & $\begin{array}{l}T_{\mathrm{m}} \\
(\mathrm{K})\end{array}$ & Method & References \\
\hline & FUS & & 63.02 & 342.8 & DSC & [2012BEN/KHI] \\
\hline & FUS & & 65.4 & 342.8 & DSC & [2011EGO/MAR] \\
\hline & FUS & & 67.56 & 344.0 & DSC & [2009COS/SAR] \\
\hline & FUS & & 72.5 & 342.1 & DSC & [2009SAR/BIC, 2010SAR/BIC] \\
\hline & TRS & & 5.4 & 324.4 & & \\
\hline & TRS & & 5.7 & 325.9 & & \\
\hline & FUS & & 63.2 & 342.8 & DSC & [2007MOR/COR] \\
\hline & FUS & & 60.4 & 338.3 & DSC & {$[2007 \mathrm{MOO} / \mathrm{KOE}]$} \\
\hline & FUS & & 57.8 & 344.1 & DSC & [2006TEI/GON] \\
\hline & FUS & & 61.0 & 340.2 & DSC & [2005GOF/SUP] \\
\hline & FUS & & 57.1 & 341.9 & DSC & [2004STU/WIT] \\
\hline & TRS & & 5.7 & 322.2 & & \\
\hline & FUS & & 68.3 & 342.2 & DSC & [2004MIR/ROH] \\
\hline & FUS & & 59.5 & 343.0 & DSC & [2004INO/HIS3] \\
\hline & FUS & & 60.0 & 343.9 & DSC & [2001CED/PRI] \\
\hline & FUS & & 50.93 & 340.2 & $\mathrm{AC}$ & [2000YU/MEN] \\
\hline & FUS & & 61.30 & 342.8 & & [1990SAT/YOS] \\
\hline & FUS & $(100-355)$ & 61.21 & 342.5 & $\mathrm{AC}$ & [1996DOM/HEA, 1982SCH/VAN] \\
\hline & FUS & & 61.50 & 344 & & [1982JAL/ZOG] \\
\hline & FUS & & 63.0 & 342.6 & & [1964ADR/DEK] \\
\hline & FUS & & 68.45 & 342.7 & & [1950SIN/WAR] \\
\hline & FUS & & 64.64 & 326.1 & & [1889EYK] \\
\hline & SUB & & $204.1 \pm 9$ & 298 & TPD & [2008CAP/LOV] \\
\hline & SUB & (291-309) & 158.5 & & TPTD & [2005CHA/ZIE] \\
\hline & SUB & $(296-319)$ & 158 & & TPTD & [2001CHA/TOB] \\
\hline
\end{tabular}

[Note: Experimental values based on the TPTD and TPD method are often inconsistent with values determined using other experimental methods.]

\begin{tabular}{|c|c|c|c|c|c|c|}
\hline & SUB & $(331-340)$ & $166.5 \pm 4.2$ & 336 & $\mathrm{ME}$ & [1961DAV/MAL, 1970COX/PIL] \\
\hline & $\mathrm{V}$ & & $132.6 \pm 8.6$ & 298 & CGC & [2013WIL/CHI] \\
\hline & $\mathrm{V}$ & $(349-415)$ & 124.3 & 364 & A & [1987STE/MAL] \\
\hline & $\mathrm{V}$ & $(457-649)$ & 100.6 & 472 & A & [1987STE/MAL] \\
\hline & $\mathrm{V}$ & $(366-389)$ & $118.9 \pm 2.0$ & 379 & ME, TE & [1982DEK/SCH] \\
\hline & $\mathrm{V}$ & & 79.8 & 515 & I & [1943CRA] \\
\hline $\mathrm{C}_{18} \mathrm{H}_{36} \mathrm{O}_{4}$ & {$[56444-62-3]$} & 2,2,11,11-tetramethy & yl-1,3,10,12-t & racyclo & cane & \\
\hline & FUS & & 35.1 & 373 & & [1975BOR] \\
\hline $\mathrm{C}_{18} \mathrm{H}_{37} \mathrm{Br}$ & {$[112-89-0]$} & 1-bromooctadecane & & & & \\
\hline & $\mathrm{V}$ & $(430-673)$ & 81.0 & 445 & $\mathrm{~A}, \mathrm{E}$ & [1987STE/MAL, 1961LI/ROS, 1970DYK/VAN] \\
\hline $\mathrm{C}_{18} \mathrm{H}_{37} \mathrm{Cl}$ & {$[3386-33-2]$} & 1-chlorooctadecane & & & & \\
\hline & $\mathrm{V}$ & & 108.8 & 298 & & [2006BOL/NER2] \\
\hline & $\mathrm{V}$ & $(333-393)$ & 96.9 & 333 & GC & [1980JON/MAT] \\
\hline & $\mathrm{V}$ & $(333-393)$ & 93.4 & 353 & GC & [1980JON/MAT] \\
\hline & $\mathrm{V}$ & $(333-393)$ & 88.4 & 373 & GC & [1980JON/MAT] \\
\hline & $\mathrm{V}$ & $(333-393)$ & 86.7 & 393 & GC & [1980JON/MAT] \\
\hline & $\mathrm{V}$ & $(472-673)$ & 74.2 & 487 & A & [1987STE/MAL, 1970DYK/VAN] \\
\hline $\mathrm{C}_{18} \mathrm{H}_{37} \mathrm{~F}$ & {$[1649-73-6]$} & 1-fluorooctadecane & & & & \\
\hline & $\mathrm{V}$ & $(477-633)$ & 68.2 & 492 & $\mathrm{~A}, \mathrm{E}$ & [1987STE/MAL, 1961LI/ROS, 1970DYK/VAN] \\
\hline $\mathrm{C}_{18} \mathrm{H}_{37} \mathrm{l}$ & {$[629-93-6]$} & 1-iodooctadecane & & & & \\
\hline & V & $(496-673)$ & 109.3 & 298 & $\mathrm{~A}, \mathrm{E}$ & $\begin{array}{l}\text { [1987STE/MAL, 1961LI/ROS, 1970DYK/VAN, } \\
\text { 2006BOL/NER] }\end{array}$ \\
\hline & $\mathrm{V}$ & $(496-673)$ & 77.2 & 511 & $\mathrm{~A}, \mathrm{E}$ & [1987STE/MAL, 1961LI/ROS, 1970DYK/VAN] \\
\hline $\mathrm{C}_{18} \mathrm{H}_{37} \mathrm{NO}$ & {$[124-26-5]$} & Octadecanamide & & & & \\
\hline & TRS & & 2.2 & 298.7 & & \\
\hline & FUS & & 54.8 & 379.7 & DSC & [2008ABA/BAD] \\
\hline & FUS & & 59.83 & 377.2 & DSC & [1993ACR, 1991CHI/BRA] \\
\hline & FUS & & 45.6 & 373.7 & DSC & [1975BER/CIN] \\
\hline & SUB & $(367-379)$ & $195.8 \pm 4.2$ & 373 & ME & [1959DAV/JON2, 1987STE/MAL] \\
\hline
\end{tabular}


TABlE 13. Phase change enthalpies of $\mathrm{C}_{17}$ to $\mathrm{C}_{18}$ organic compounds-Continued

\begin{tabular}{|c|c|c|c|c|c|c|}
\hline \multirow[b]{2}{*}{$\begin{array}{l}\text { Molecular } \\
\text { formula }\end{array}$} & \multirow{2}{*}{$\begin{array}{l}\text { CAS Registry } \\
\text { Number } \\
\text { Enthalpy }\end{array}$} & \multicolumn{5}{|l|}{ Compound } \\
\hline & & $\begin{array}{l}\text { Temperature } \\
\text { range }\end{array}$ & $\begin{array}{l}\Delta_{\text {trans }} H_{\mathrm{m}} \\
(\mathrm{kJ} / \mathrm{mol})\end{array}$ & $\begin{array}{l}T_{\mathrm{m}} \\
(\mathrm{K})\end{array}$ & Method & References \\
\hline \multirow[t]{2}{*}{$\mathrm{C}_{18} \mathrm{H}_{37} \mathrm{NO}$} & [41328-72-7] & \multicolumn{5}{|c|}{$N$-butyl tetradecanamide } \\
\hline & FUS & & 45.0 & 336.1 & DSC & [1993ACR, 1980CAR/BUS] \\
\hline $\mathrm{C}_{18} \mathrm{H}_{37} \mathrm{NO}$ & [146985-21-9] & \multicolumn{5}{|c|}{$N, N$-dihexyl hexanamide } \\
\hline \multirow[t]{29}{*}{$\mathrm{C}_{18} \mathrm{H}_{38}$} & [593-45-3] & \multicolumn{5}{|l|}{ Octadecane } \\
\hline & FUS & & 65.3 & 300.3 & DSC & [2016BOU/HAF] \\
\hline & FUS & & 56.8 & 301.6 & DSC & [2015QIU/LU] \\
\hline & FUS & & 62.0 & 300.2 & DSC & [2015VEL/KHA] \\
\hline & FUS & & 61.65 & 301.0 & DSC & [2014WEI/HAN] \\
\hline & FUS & & 61.34 & 301.5 & $\mathrm{CVC}$ & [2014FON/GUS] \\
\hline & FUS & & 59.8 & 301.1 & DSC & [1999MET/RAJ] \\
\hline & FUS & & U 50.9 & 301.6 & DSC & [1992BAB/HWA, 1994BAB/BEN] \\
\hline & FUS & & 61.5 & 301.5 & & {$[1991 \mathrm{BAR} / \mathrm{SCH}]$} \\
\hline & FUS & & 60.67 & 300.7 & & [1991CLA/LET] \\
\hline & FUS & & 60.76 & 301.0 & & [1985KOL/SYU] \\
\hline & FUS & \multirow{4}{*}{$(12-379)$} & 59.4 & 301.1 & DSC & [1983CHA/MAU] \\
\hline & FUS & & 61.71 & 301.3 & $\mathrm{AC}$ & [1967MES/GUT] \\
\hline & FUS & & 61.4 & 301.4 & $\mathrm{AC}$ & {$[1955 \mathrm{SCH} / \mathrm{BUS}]$} \\
\hline & FUS & & 61.5 & 301.3 & & [1996DOM/HEA, 1949PAR/MOO2] \\
\hline & SUB & & 142.3 & 298 & ME & [2014FON/GUS] \\
\hline & SUB & & 152.7 & 298 & $\mathrm{C}$ & [1972MOR3] \\
\hline & SUB & $(288-298)$ & $153.0 \pm 5$ & 293 & ME & [1949BRA/SHE, 1960JON, 1970COX/PIL] \\
\hline & $\mathrm{V}$ & $(501-548)$ & 64.8 & 516 & & [1987STE/MAL] \\
\hline & $\mathrm{V}$ & $(335-439)$ & 80.0 & 348 & GS & {$[1986 \mathrm{ALL} / \mathrm{JOS}]$} \\
\hline & $\mathrm{V}$ & \multirow[t]{7}{*}{$(318-361)$} & 84.3 & 333 & $\mathrm{~A}, \mathrm{GS}$ & [1987STE/MAL, 1979MAC/PRA] \\
\hline & $\mathrm{V}$ & & 72.5 & 343 & GC & {$[1977 \mathrm{NOV} / \mathrm{NOV}]$} \\
\hline & $\mathrm{V}$ & & 71.8 & 353 & GC & [1977NOV/NOV] \\
\hline & $\mathrm{V}$ & & 71.1 & 363 & $\mathrm{GC}$ & [1977NOV/NOV] \\
\hline & $\mathrm{V}$ & & 70.5 & 373 & $\mathrm{GC}$ & [1977NOV/NOV] \\
\hline & $\mathrm{V}$ & & 69.8 & 383 & $\mathrm{GC}$ & [1977NOV/NOV] \\
\hline & $\mathrm{V}$ & & 90.8 & 298 & & [1971WIL/ZWO] \\
\hline & $\mathrm{V}$ & $(447-474)$ & 78.1 & 460 & ME & [1938UBB] \\
\hline & $\mathrm{V}$ & $(447-590)$ & 69.4 & 462 & & [1882KRA, 1984BOU/FRI] \\
\hline \multirow[t]{2}{*}{$\mathrm{C}_{18} \mathrm{H}_{38}$} & {$[1560-89-0]$} & \multicolumn{2}{|l|}{ 2-methylheptadecane } & & & \\
\hline & $\mathrm{V}$ & $(442-581)$ & 67.8 & 457 & A & [1987STE/MAL, 1959TER/BRI] \\
\hline \multirow[t]{2}{*}{$\mathrm{C}_{18} \mathrm{H}_{38}$} & [6418-44-6] & \multicolumn{2}{|l|}{ 3-methylheptadecane } & & & \\
\hline & $\mathrm{V}$ & $(441-583)$ & 65.6 & 456 & A & [1987STE/MAL, 1959TER/BRI] \\
\hline $\mathrm{C}_{18} \mathrm{H}_{38}$ & [26429-11-8] & 4-methylheptadecane & & & & \\
\hline & $\mathrm{V}$ & $(429-580)$ & 58.9 & 444 & A & [1987STE/MAL, 1959TER/BRI] \\
\hline $\mathrm{C}_{18} \mathrm{H}_{38}$ & {$[26730-95-0]$} & 5-methylheptadecane & & & & \\
\hline & $\mathrm{V}$ & $(432-581)$ & 61.1 & 447 & A & [1987STE/MAL, 1959TER/BRI] \\
\hline $\mathrm{C}_{18} \mathrm{H}_{38}$ & [61868-02-8] & 2,3-dimethylhexadeca & ane & & & \\
\hline & V & $(466-583)$ & 64.9 & 481 & A & [1987STE/MAL, 1959TER/BRI] \\
\hline
\end{tabular}


TABLE 13. Phase change enthalpies of $\mathrm{C}_{17}$ to $\mathrm{C}_{18}$ organic compounds-Continued

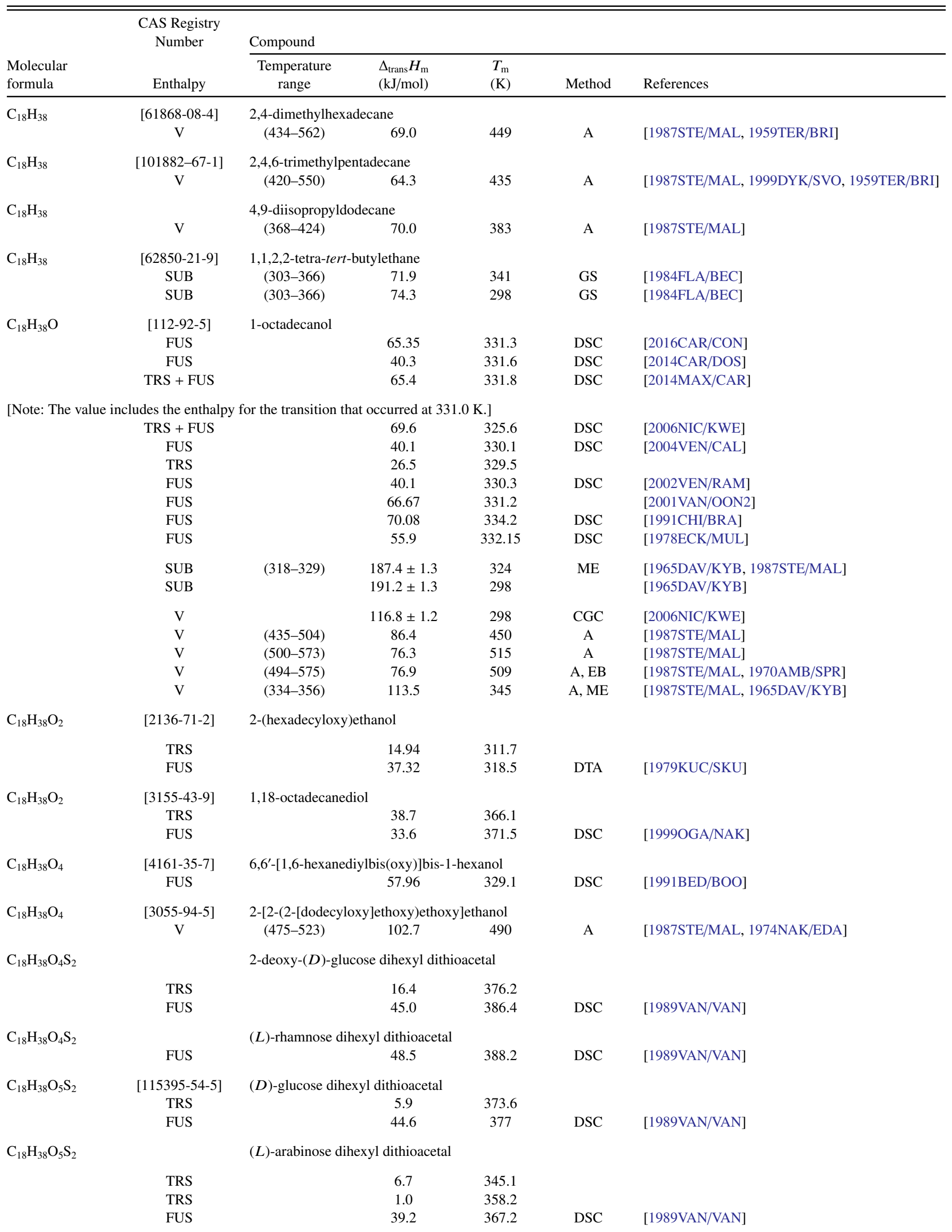


TABLE 13. Phase change enthalpies of $\mathrm{C}_{17}$ to $\mathrm{C}_{18}$ organic compounds-Continued

\begin{tabular}{|c|c|c|c|c|c|c|}
\hline \multirow[b]{2}{*}{$\begin{array}{l}\text { Molecular } \\
\text { formula }\end{array}$} & \multirow{2}{*}{$\begin{array}{l}\text { CAS Registry } \\
\text { Number } \\
\text { Enthalpy }\end{array}$} & \multicolumn{5}{|l|}{ Compound } \\
\hline & & $\begin{array}{l}\text { Temperature } \\
\text { range }\end{array}$ & $\begin{array}{l}\Delta_{\text {trans }} H_{\mathrm{m}} \\
(\mathrm{kJ} / \mathrm{mol})\end{array}$ & $\begin{array}{l}T_{\mathrm{m}} \\
(\mathrm{K})\end{array}$ & Method & References \\
\hline $\mathrm{C}_{18} \mathrm{H}_{38} \mathrm{O}_{9}$ & $\begin{array}{c}\text { [25990-94-7] } \\
\text { FUS }\end{array}$ & $1, \omega$-dimethoxy & $\begin{array}{l}\text { oxyethylene) } \\
60.1\end{array}$ & 276.2 & & [1997SCH/VER] \\
\hline $\mathrm{C}_{18} \mathrm{H}_{38} \mathrm{~S}$ & $\begin{array}{c}\text { [2885-00-9] } \\
\mathrm{V}\end{array}$ & $\begin{array}{l}\text { 1-octadecanethi } \\
(492-670)\end{array}$ & 77.1 & 507 & $\mathrm{E}$ & [1999DYK/SVO] \\
\hline $\mathrm{C}_{18} \mathrm{H}_{38} \mathrm{~S}_{2}$ & $\begin{array}{c}{[4485-77-2]} \\
\mathrm{V}\end{array}$ & $\begin{array}{l}\text { Dioctyl disulfid } \\
\quad(490-650)\end{array}$ & 78.3 & 514 & & [1999DYK/SVO] \\
\hline $\mathrm{C}_{18} \mathrm{H}_{39} \mathrm{~N}$ & $\begin{array}{c}{[112-69-6]} \\
\mathrm{V} \\
\mathrm{V}\end{array}$ & $\begin{array}{c}N, N \text {-dimethylh } \\
(483-671)\end{array}$ & $\begin{array}{c}\text { decylamine } \\
84.8 \pm 1.0 \\
67.3\end{array}$ & $\begin{array}{l}298 \\
498\end{array}$ & $\begin{array}{c}\text { CGC } \\
\text { A }\end{array}$ & $\begin{array}{l}\text { [2014GOB/VIK] } \\
\text { [1987STE/MAL] }\end{array}$ \\
\hline $\mathrm{C}_{18} \mathrm{H}_{39} \mathrm{~N}$ & $\begin{array}{c}{[124-30-1]} \\
\mathrm{V}\end{array}$ & $\begin{array}{l}\text { Octadecylamine } \\
\quad(450-635)\end{array}$ & 76.2 & 465 & A & [1987STE/MAL] \\
\hline $\mathrm{C}_{18} \mathrm{H}_{39} \mathrm{O}_{4} \mathrm{P}$ & $\begin{array}{c}\text { [2528-39-4] } \\
\text { V } \\
\text { V }\end{array}$ & $\begin{array}{l}\text { Trihexyl phosph } \\
\quad(483-513) \\
(493-523)\end{array}$ & $\begin{array}{l}104.8 \\
104.9\end{array}$ & $\begin{array}{l}298 \\
298\end{array}$ & CGC & $\begin{array}{l}\text { [2007PAN/ANT2] } \\
\text { [2007PAN/ANT2] }\end{array}$ \\
\hline $\mathrm{C}_{18} \mathrm{H}_{39} \mathrm{O}_{7} \mathrm{P}$ & $\begin{array}{c}{[78-51-3]} \\
\mathrm{V}\end{array}$ & $\begin{array}{l}\text { Tris(2-butoxyetl } \\
\quad(383-413)\end{array}$ & $\begin{array}{c}\text { ohosphate } \\
102.6\end{array}$ & 398 & GC-RT & [2014BRO/JAN] \\
\hline
\end{tabular}


TABle 14. Phase change enthalpies of $\mathrm{C}_{19}$ to $\mathrm{C}_{29}$ organic compounds

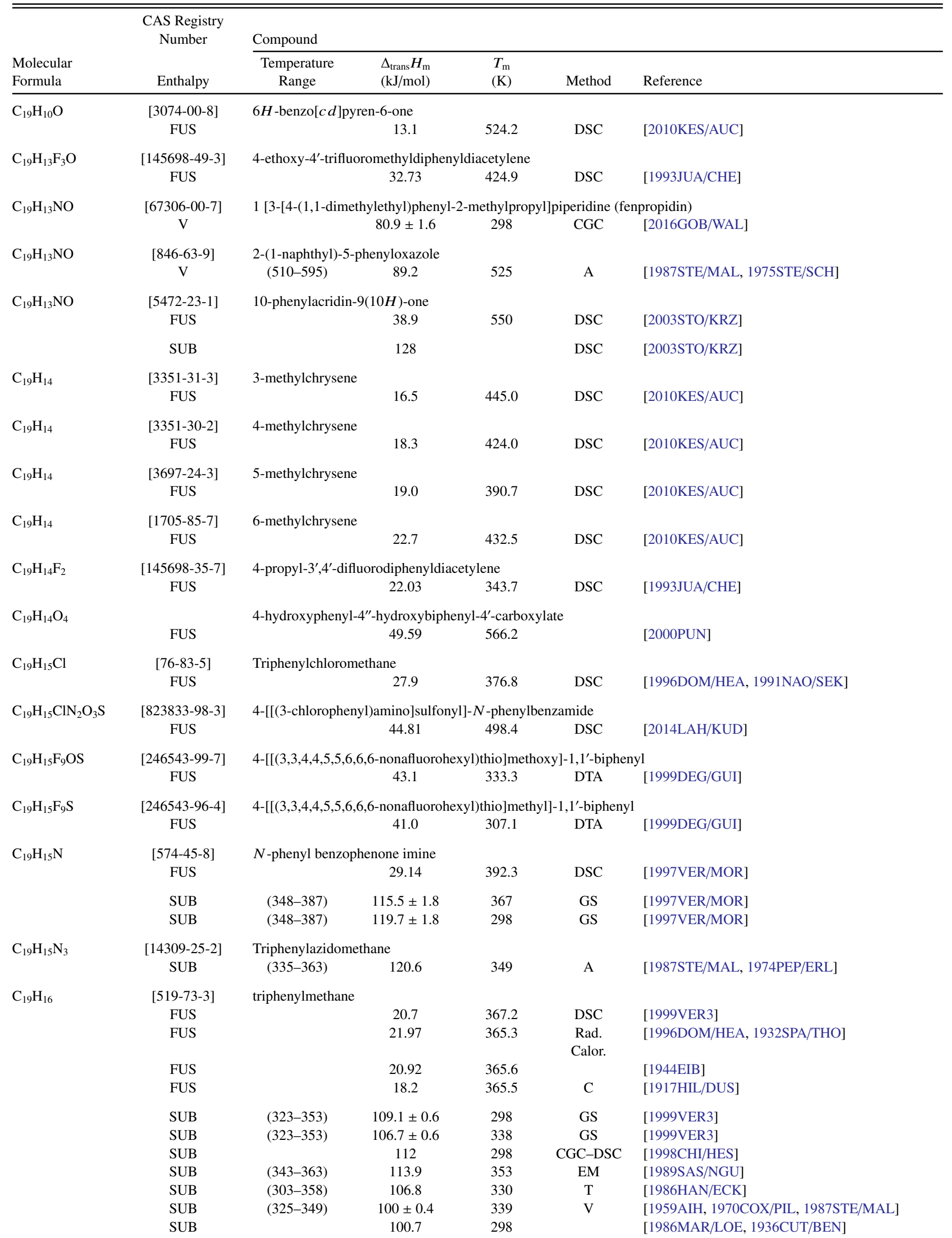


TABLE 14. Phase change enthalpies of $\mathrm{C}_{19}-\mathrm{C}_{29}$ organic compounds-Continued

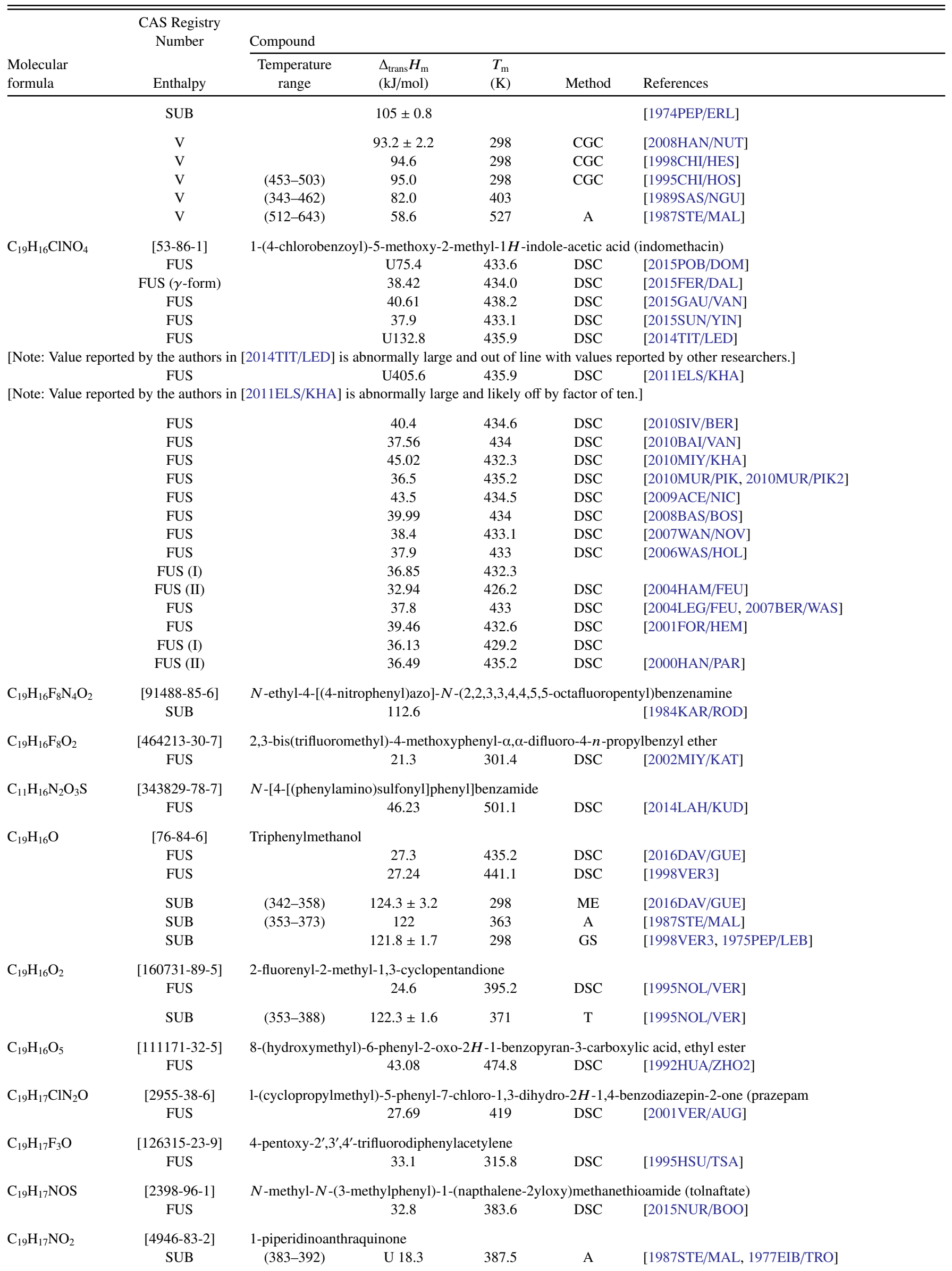


TABLE 14. Phase change enthalpies of $\mathrm{C}_{19}-\mathrm{C}_{29}$ organic compounds-Continued

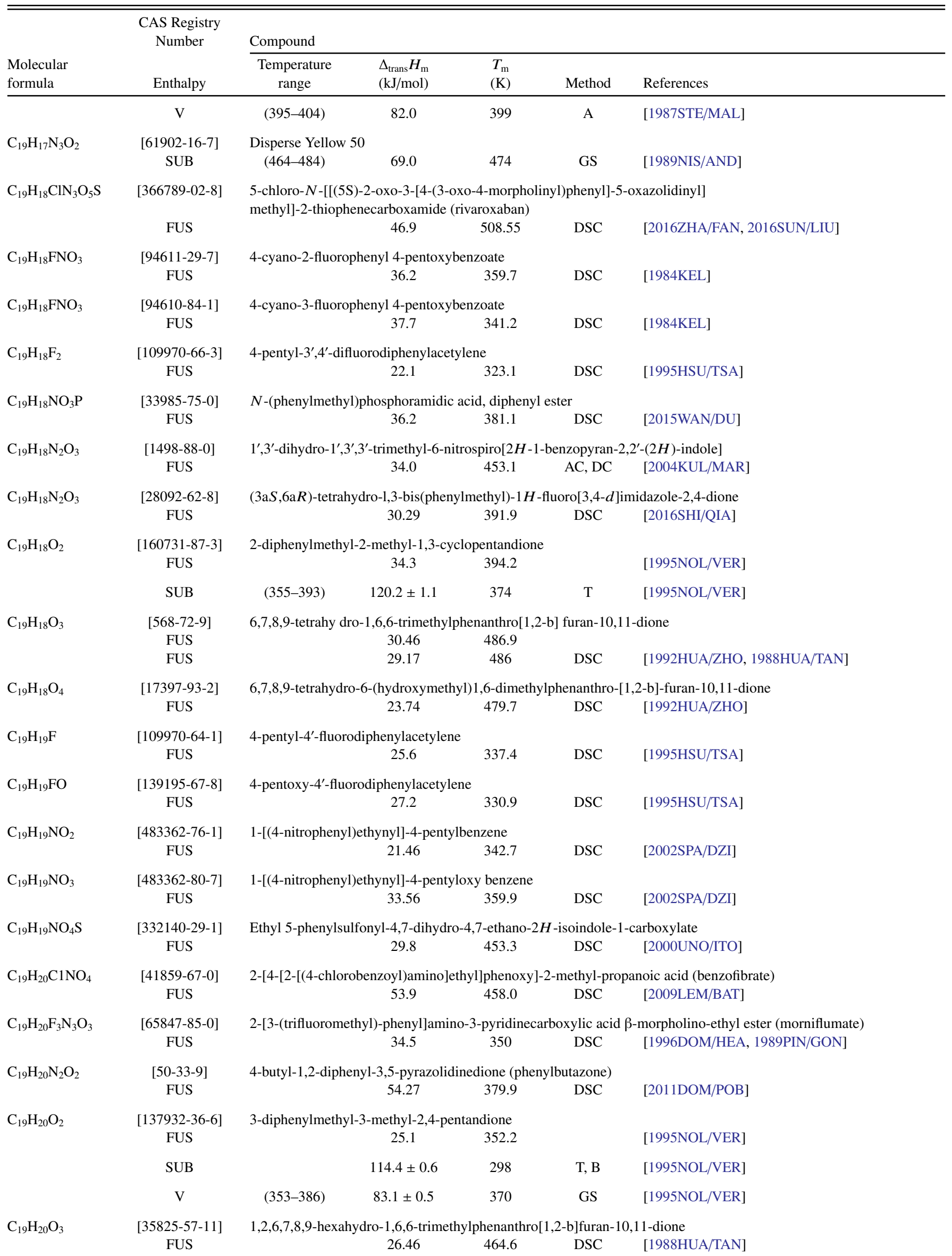


TABLE 14. Phase change enthalpies of $\mathrm{C}_{19}-\mathrm{C}_{29}$ organic compounds-Continued

\begin{tabular}{|c|c|c|c|c|c|c|}
\hline \multirow[b]{2}{*}{$\begin{array}{l}\text { Molecular } \\
\text { formula }\end{array}$} & \multirow{2}{*}{$\begin{array}{l}\text { CAS Registry } \\
\text { Number } \\
\text { Enthalpy }\end{array}$} & \multicolumn{5}{|l|}{ Compound } \\
\hline & & $\begin{array}{c}\text { Temperature } \\
\text { range }\end{array}$ & $\begin{array}{l}\Delta_{\text {trans }} H_{\mathrm{m}} \\
(\mathrm{kJ} / \mathrm{mol})\end{array}$ & $\begin{array}{l}T_{\mathrm{m}} \\
(\mathrm{K})\end{array}$ & Method & References \\
\hline \multirow[t]{2}{*}{$\mathrm{C}_{19} \mathrm{H}_{20} \mathrm{O}_{3}$} & & \multicolumn{5}{|c|}{ 1-(diphenylmethyl)-4-methyl-2,6,7-trioxabicyclo[2.2.2]octane } \\
\hline & FUS & & 32.6 & 443.2 & & [1995RAK/VER2] \\
\hline \multirow[t]{5}{*}{$\mathrm{C}_{19} \mathrm{H}_{20} \mathrm{O}_{4}$} & {$[85-68-7]$} & \multicolumn{5}{|c|}{ butyl benzyl phthalate } \\
\hline & V & & $109.8 \pm 2.6$ & 298 & CRT & [2015GOB/CHI] \\
\hline & $\mathrm{V}$ & & $107.9 \pm 0.9$ & 298 & CGC & [2015GOB/CHI] \\
\hline & $\mathrm{V}$ & & $106.2 \pm 2.4$ & 298 & CGC & [2014GOB/CHI] \\
\hline & $\mathrm{V}$ & $(416-516)$ & 89.0 & 431 & A & [1987STE/MAL] \\
\hline \multirow[t]{2}{*}{$\mathrm{C}_{19} \mathrm{H}_{20} \mathrm{O}_{4}$} & [74254-53-8] & \multicolumn{5}{|c|}{ dibenzyl ethyl malonate } \\
\hline & V & (403-483) & 94.1 & 418 & A & [1987STE/MAL] \\
\hline \multirow[t]{2}{*}{$\mathrm{C}_{19} \mathrm{H}_{21} \mathrm{ClN}_{2} \mathrm{O}_{3} \mathrm{~S}$} & [112529-15-4] & \multicolumn{5}{|c|}{ 5-[[5-[2-(5-ethyl-2-pyridinyl)ethoxy]phenyl]methyl]-2,4-thiazolidinedione monohydrochloride } \\
\hline & FUS & & 50.34 & 468.8 & DSC & [2013TAO/SUN] \\
\hline \multirow[t]{3}{*}{$\mathrm{C}_{19} \mathrm{H}_{21} \mathrm{~F}_{19}$} & {$[139277-01-3]$} & \multicolumn{5}{|c|}{ 1,1,1,2,3,3,4,4,5,5,6,6,7,7,8,8-hexadecafluoro-2-(trifluoromethyl)octadecane } \\
\hline & TRS & & 1.0 & 274 & & \\
\hline & FUS & & 25.0 & 298 & DSC & [1992HOP/MOL] \\
\hline \multirow[t]{2}{*}{$\mathrm{C}_{19} \mathrm{H}_{21} \mathrm{IO}_{3} \mathrm{~S}$} & [313057-07-7] & \multicolumn{5}{|c|}{ 4-(7-octenyloxy)phenyl 5-iodo-2-thiophene carboxylate } \\
\hline & FUS & & 80.33 & 324.9 & DSC & [2000WU/WAN] \\
\hline \multirow[t]{3}{*}{$\mathrm{C}_{19} \mathrm{H}_{21} \mathrm{NO}$} & {$[127529-16-2]$} & \multicolumn{5}{|c|}{$( \pm) 1,2$-diphenyl-2- $N$-piperidinyl-1-ethanone } \\
\hline & FUS & & 33.93 & 349.2 & & [1994WEL/VER] \\
\hline & SUB & & $147.1 \pm 1$ & & B & [1994WEL/VER] \\
\hline \multirow[t]{2}{*}{$\mathrm{C}_{19} \mathrm{H}_{21} \mathrm{NO}_{3}$} & {$[483362-65-8]$} & \multicolumn{5}{|c|}{ 2-(4-nitrophenyl)-1-(4-pentylphenyl)ethanone } \\
\hline & FUS & & 29.46 & 363.2 & DSC & [2002SPA/DZI] \\
\hline \multirow[t]{2}{*}{$\mathrm{C}_{19} \mathrm{H}_{21} \mathrm{NO}_{4}$} & {$[483362-69-2]$} & \multicolumn{5}{|c|}{ 2-(4-nitrophenyl)-1-(4-pentyloxyphenyl)ethanone } \\
\hline & FUS & & 27.07 & 353.4 & DSC & [2002SPA/DZI] \\
\hline $\mathrm{C}_{19} \mathrm{H}_{22} \mathrm{FN}_{3} \mathrm{O}$ & $\begin{array}{c}\text { [1649-18-9] } \\
\text { FUS }\end{array}$ & \multicolumn{5}{|c|}{ 1-(4-fluorophenyl)-4-[4-(2-pyridinyl)-1-piperazinyl]-1-butanone (azaperone) } \\
\hline \multirow[t]{2}{*}{$\mathrm{C}_{19} \mathrm{H}_{22} \mathrm{~F}_{8} \mathrm{O}_{2}$} & & \multicolumn{5}{|c|}{ 2,3-bis(trifluoromethyl)-4-methoxyphenyl-trans-4- $n$-propyl-cyclohexyl- $\alpha, \alpha$-difluoromethyl ether } \\
\hline & FUS & & 21.4 & 314.7 & & {$[2002 \mathrm{MIY} / \mathrm{KAT}]$} \\
\hline \multirow[t]{3}{*}{$\mathrm{C}_{19} \mathrm{H}_{23} \mathrm{NO}$} & {$[5219-49-8]$} & \multicolumn{5}{|c|}{$p$-hexyloxybenzylideneaniline } \\
\hline & TRS & $(16-385)$ & 0.19 & 73.41 & & \\
\hline & FUS & $(16-385)$ & 30.91 & 321.6 & $\mathrm{AC}$ & [1996DOM/HEA, 1982TSU/SOR] \\
\hline \multirow[t]{2}{*}{$\mathrm{C}_{19} \mathrm{H}_{23} \mathrm{NO}$} & {$[29743-08-6]$} & \multicolumn{5}{|c|}{ 4-butyl- $N$-[(4-ethoxyphenyl)methylene]benzenamine } \\
\hline & SUB (crys) & (291-309) & 30.0 & 309 & & [1981PIR/AZA] \\
\hline
\end{tabular}

[Note: The value is too small for an enthalpy of sublimation. The compound is likely not completely crystalline.]

\begin{tabular}{|c|c|c|c|c|c|}
\hline \multirow{4}{*}{$\mathrm{C}_{19} \mathrm{H}_{23} \mathrm{~N}_{3}$} & [33089-61-1] & \multicolumn{4}{|c|}{$N$-methyl- $N^{\prime}-2,4$-xylyl- $N$-(N-2,4-xylylformimodyl)formamidine (amitraz) } \\
\hline & FUS (I) & 26.77 & 355.3 & & \\
\hline & FUS (II) & 19.47 & 344.4 & & \\
\hline & FUS (III) & 53.14 & 388.6 & DSC & [2004DEV/VAN] \\
\hline \multirow[t]{4}{*}{$\mathrm{C}_{19} \mathrm{H}_{24}$} & {$[25566-92-1]$} & dicumenylmethane & & & \\
\hline & $\mathrm{V}$ & $(303-402)$ & 318 & & [1999DYK/SVO] \\
\hline & $\mathrm{V}$ & $(608-704)$ & 623 & & [1999DYK/SVO] \\
\hline & $\mathrm{V}$ & $(323-402)$ & 338 & A & [1987STE/MAL, 1958MAT/GEL] \\
\hline \multirow[t]{6}{*}{$\mathrm{C}_{19} \mathrm{H}_{24} \mathrm{~N}_{2} \mathrm{O}_{2}$} & {$[55268-74-1]$} & \multicolumn{4}{|c|}{ ( \pm ) 2-(cyclohexylcarbonyl)-1,2,3,6,7,11b-hexahydro- $4 H$-pyrazino-[2,1-a]isoqumolm-4-one $(( \pm)$ praziquantel $)$} \\
\hline & FUS & 28.3 & 414.4 & DSC & [2012RIB/AND] \\
\hline & FUS & 30.7 & 416.3 & DSC & [2006PAS/ALB] \\
\hline & FUS & 25.73 & 409.4 & DSC & [2004LIU/WAN] \\
\hline & FUS & 27.0 & 415.7 & DSC & [1999DEL/TOR] \\
\hline & FUS & 30.8 & 412.2 & DSC & [1998ELA/GIR] \\
\hline \multirow[t]{2}{*}{$\mathrm{C}_{19} \mathrm{H}_{24} \mathrm{~N}_{2} \mathrm{O}_{2}$} & {$[57452-97-8]$} & \multicolumn{4}{|c|}{ (+) 2-(cyclohexylcarbonyl)-1,2,3,6,7,11b-hexahydro- $4 H$-pyrazino-[2,1-a]isoqumolm-4-one $((+)$ praziquantel) } \\
\hline & FUS & 23.9 & 386 & DSC & [1998ELA/GIR] \\
\hline $\mathrm{C}_{19} \mathrm{H}_{24} \mathrm{~N}_{2} \mathrm{O}_{2}$ & [57452-98-9] & \multicolumn{4}{|c|}{ (-) 2-(cyclohexylcarbonyl)-1,2,3,6,7,11b-hexahydro-4H-pyrazino[2,1-a]-isoquinolin-4-one $((-)$ praziquantel) } \\
\hline
\end{tabular}


TABLE 14. Phase change enthalpies of $\mathrm{C}_{19}-\mathrm{C}_{29}$ organic compounds-Continued

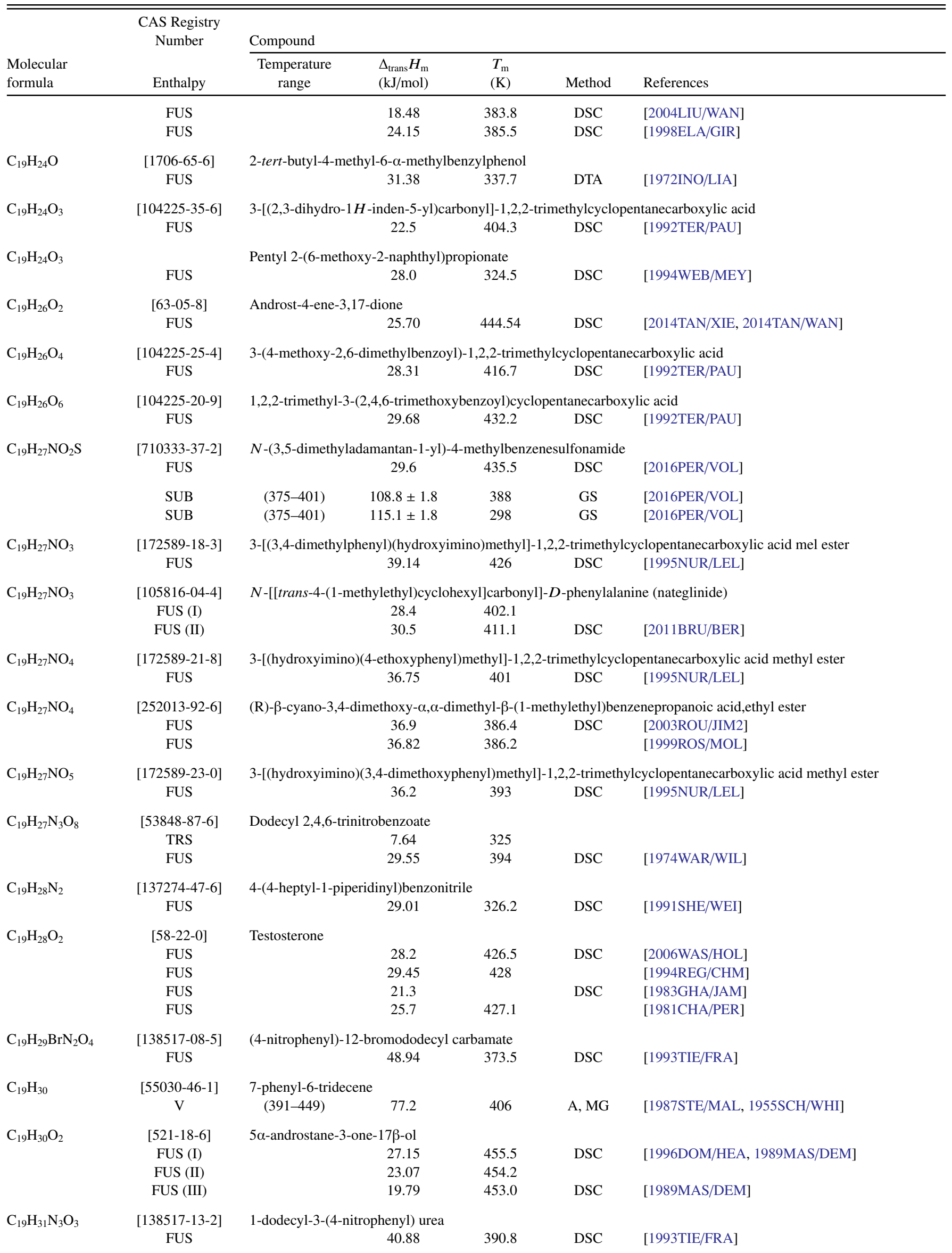


TABLE 14. Phase change enthalpies of $\mathrm{C}_{19}-\mathrm{C}_{29}$ organic compounds-Continued

\begin{tabular}{|c|c|c|c|c|c|c|}
\hline \multirow[b]{2}{*}{$\begin{array}{l}\text { Molecular } \\
\text { formula }\end{array}$} & \multirow{2}{*}{$\begin{array}{l}\text { CAS Registry } \\
\text { Number } \\
\text { Enthalpy }\end{array}$} & \multicolumn{5}{|l|}{ Compound } \\
\hline & & $\begin{array}{l}\text { Temperature } \\
\text { range }\end{array}$ & $\begin{array}{l}\Delta_{\text {trans }} H_{\mathrm{m}} \\
(\mathrm{kJ} / \mathrm{mol})\end{array}$ & $\begin{array}{l}T_{\mathrm{m}} \\
(\mathrm{K})\end{array}$ & Method & References \\
\hline $\mathrm{C}_{19} \mathrm{H}_{32}$ & $\begin{array}{c}{[123-02-4]} \\
\mathrm{V} \\
\mathrm{V} \\
\mathrm{V}\end{array}$ & $\begin{array}{l}\text { Tridecylbenzene } \\
\qquad(473-651) \\
(343-463)\end{array}$ & $\begin{array}{l}72.0 \\
90.0 \\
94.6\end{array}$ & $\begin{array}{l}488 \\
358 \\
298\end{array}$ & & $\begin{array}{l}\text { [1999DYK/SVO] } \\
{[1990 \mathrm{POM} / \mathrm{PIA}]} \\
{[1971 \mathrm{WIL} / \mathrm{ZWO}]}\end{array}$ \\
\hline $\mathrm{C}_{19} \mathrm{H}_{32}$ & $\begin{array}{c}{[2400-01-3]} \\
\mathrm{V}\end{array}$ & $\begin{array}{l}\text { 7-phenyltridecane } \\
\quad(413-470)\end{array}$ & 76.2 & 428 & $\mathrm{~A}, \mathrm{MG}$ & [1987STE/MAL, 1955SCH/WHI] \\
\hline $\mathrm{C}_{19} \mathrm{H}_{32} \mathrm{O}_{2}$ & $\begin{array}{c}{[301-00-8]} \\
\mathrm{V} \\
\mathrm{V} \\
\mathrm{V}\end{array}$ & $\begin{array}{l}\text { Methyl linolenate } \\
\qquad \begin{array}{l}(423-503) \\
(394-459)\end{array}\end{array}$ & $\begin{array}{c}110.5 \pm 0.5 \\
102.1 \\
87.7\end{array}$ & $\begin{array}{l}298 \\
298 \\
409\end{array}$ & $\begin{array}{c}\text { CGC } \\
\text { GC } \\
\mathrm{A}, \mathrm{MG} \\
\mathrm{OM}\end{array}$ & $\begin{array}{l}\text { [2007LIP/KAP] } \\
\text { [1997KRO/VEL] } \\
\text { [1987STE/MAL, 1952SCO/MAC] }\end{array}$ \\
\hline $\mathrm{C}_{19} \mathrm{H}_{32} \mathrm{O}_{3}$ & $\begin{array}{c}{[1422829-46-6]} \\
\text { TRS }\end{array}$ & Dipivaloyl(3-cyclo & $\begin{array}{l}\text { pentylpropano } \\
3.38\end{array}$ & $\begin{array}{l}\text { thane } \\
252.4\end{array}$ & $\mathrm{DSC}$ & {$[2013 \mathrm{STI} / \mathrm{KAI}]$} \\
\hline $\mathrm{C}_{19} \mathrm{H}_{34}$ & $\begin{array}{c}{[1610-24-8]} \\
\text { SUB } \\
\text { V } \\
\text { V }\end{array}$ & $\begin{array}{l}\text { Tricyclohexylmeth } \\
\qquad \begin{array}{l}(301-321) \\
(333-365) \\
(428-605)\end{array}\end{array}$ & $\begin{array}{r}117.4 \\
81.4 \\
73.3\end{array}$ & $\begin{array}{l}311 \\
348 \\
443\end{array}$ & $\begin{array}{l}\mathrm{A} \\
\mathrm{A} \\
\mathrm{A}\end{array}$ & $\begin{array}{l}\text { [1987STE/MAL, 1964MOR] } \\
\text { [1987STE/MAL, 1964MOR] } \\
\text { [1987STE/MAL] }\end{array}$ \\
\hline $\mathrm{C}_{19} \mathrm{H}_{34} \mathrm{O}$ & $\begin{array}{l}{[17687-74-0]} \\
\text { FUS }\end{array}$ & Tricyclohexylmeth & anol 23.8 & 367.2 & $\mathrm{AC}$ & [2007YAM/SAI] \\
\hline $\mathrm{C}_{19} \mathrm{H}_{34} \mathrm{O}_{2}$ & $\begin{array}{c}{[112-62-9]} \\
\mathrm{V} \\
\mathrm{V} \\
\mathrm{V} \\
\mathrm{V}\end{array}$ & $\begin{array}{l}\text { Linoleic acid, meth } \\
\qquad \begin{array}{l}(423-503) \\
(453-543) \\
(391-459)\end{array}\end{array}$ & $\begin{array}{c}\text { yl ester (meth } \\
107.8 \pm 0.6 \\
102.2 \\
77.2 \\
86.3\end{array}$ & $\begin{array}{c}\text { oleate) } \\
298 \\
298 \\
498 \\
406\end{array}$ & $\begin{array}{c}\text { CGC } \\
\text { GC } \\
\text { GC } \\
\mathrm{A}, \mathrm{MG}, \\
\mathrm{OM}\end{array}$ & $\begin{array}{l}\text { [2007LIP/KAP] } \\
{[1997 \mathrm{KRO} / \mathrm{VEL}]} \\
\text { [1993HUS/SAR] } \\
\text { [1987STE/MAL, 1952SCO/MAC] }\end{array}$ \\
\hline $\mathrm{C}_{19} \mathrm{H}_{36}$ & $\begin{array}{c}{[2090-15-5]} \\
\text { V } \\
\text { V }\end{array}$ & $\begin{array}{l}\text { 1,1-dicyclohexylhe } \\
\text { (293-368) } \\
(422-458)\end{array}$ & $\begin{array}{r}\text { ptane } \\
87.8 \\
73.8\end{array}$ & $\begin{array}{l}330 \\
437\end{array}$ & $\begin{array}{c}\text { A } \\
\mathrm{A}, \mathrm{MG}\end{array}$ & $\begin{array}{l}\text { [1987STE/MAL, 1999DYK/SVO] } \\
\text { [1987STE/MAL, 1955SCH/WHI] }\end{array}$ \\
\hline $\mathrm{C}_{19} \mathrm{H}_{36}$ & $\begin{array}{c}{[26186-01-6]} \\
V\end{array}$ & $\begin{array}{c}\text { 1-nonadecyne } \\
(462-637)\end{array}$ & 66.9 & 477 & & [1999DYK/SVO] \\
\hline $\mathrm{C}_{19} \mathrm{H}_{36}$ & $\begin{array}{c}{[61847-98-1]} \\
\mathrm{V}\end{array}$ & $\begin{array}{c}\text { 2-nonadecyne } \\
(469-648)\end{array}$ & 67.8 & 484 & & [1999DYK/SVO] \\
\hline $\mathrm{C}_{19} \mathrm{H}_{36}$ & $\begin{array}{c}{[61886-65-5]} \\
V\end{array}$ & $\begin{array}{l}\text { 3-nonadecyne } \\
(460-635)\end{array}$ & 66.5 & 475 & & [1999DYK/SVO] \\
\hline $\mathrm{C}_{19} \mathrm{H}_{36} \mathrm{O}$ & $\begin{array}{l}{[6907-38-6]} \\
\text { SUB }\end{array}$ & Cyclononadecanon & 82.4 & & & [1938WOL/WEG, 1960JON] \\
\hline $\mathrm{C}_{19} \mathrm{H}_{36} \mathrm{O}_{2}$ & $\begin{array}{c}\text { [112-62-9] } \\
\mathrm{V} \\
\mathrm{V} \\
\mathrm{V} \\
\mathrm{V} \\
\mathrm{V} \\
\mathrm{V} \\
\mathrm{V}\end{array}$ & $\begin{array}{l}\text { Methyl cis-9-octad } \\
\qquad \begin{array}{l}(423-503) \\
(433-473) \\
(453-543) \\
(428-486) \\
(401-458)\end{array}\end{array}$ & $\begin{array}{c}106.2 \pm 0.7 \\
103.3 \\
99.6 \\
77.2 \\
106.8 \pm 1.0 \\
83.0 \\
86.7\end{array}$ & $\begin{array}{l}\text { eate) } \\
298 \\
298 \\
298 \\
498 \\
298 \\
443 \\
416\end{array}$ & $\begin{array}{c}\text { CGC } \\
\text { GC } \\
\text { CGC } \\
\text { GC } \\
\text { GCC } \\
\text { A } \\
\text { MG, OM }\end{array}$ & $\begin{array}{l}\text { [2007LIP/KAP] } \\
{[1997 \mathrm{KRO} / \mathrm{VEL}]} \\
{[1995 \mathrm{CHI} / \mathrm{HOS}]} \\
{[1993 \mathrm{HUS} / \mathrm{SAR}]} \\
{[1980 \mathrm{FUC} / \mathrm{PEA}]} \\
{[1987 \mathrm{STE} / \mathrm{MAL}, 1964 \mathrm{ROS} / \mathrm{SCH}]} \\
{[1952 \mathrm{SCO} / \mathrm{MAC}]}\end{array}$ \\
\hline $\mathrm{C}_{19} \mathrm{H}_{36} \mathrm{O}_{2}$ & $\begin{array}{c}{[1937-62-8]} \\
V\end{array}$ & $\begin{array}{l}\text { Methyl elaidate } \\
\quad(453-543)\end{array}$ & 77.2 & 498 & GC & [1993HUS/SAR] \\
\hline $\mathrm{C}_{19} \mathrm{H}_{36} \mathrm{O}_{2}$ & $\begin{array}{l}{[43211-62-7]} \\
\text { FUS }\end{array}$ & Allyl hexadecanoat & 49.4 & 295.6 & $\mathrm{DSC}$ & [1992BAB/HWA2] \\
\hline $\mathrm{C}_{19} \mathrm{H}_{36} \mathrm{O}_{3}$ & $\begin{array}{c}{[141-24-2]} \\
\mathrm{V}\end{array}$ & $\begin{array}{l}\text { Methyl ricinoleate } \\
\quad(453-543)\end{array}$ & 89.3 & 498 & GC & [1993HUS/SAR] \\
\hline $\mathrm{C}_{19} \mathrm{H}_{36} \mathrm{O}_{4}$ & $\begin{array}{c}{[1429-66-9]} \\
\mathrm{V}\end{array}$ & $\begin{array}{l}\text { (2-hydroxy-3-octan } \\
\quad(512-567)\end{array}$ & $\begin{array}{c}\text { oyloxypropyl } \\
107\end{array}$ & $\begin{array}{r}\text { noate } \\
540\end{array}$ & DSC & [2014DAM/MAT] \\
\hline
\end{tabular}


TABLE 14. Phase change enthalpies of $\mathrm{C}_{19}-\mathrm{C}_{29}$ organic compounds-Continued

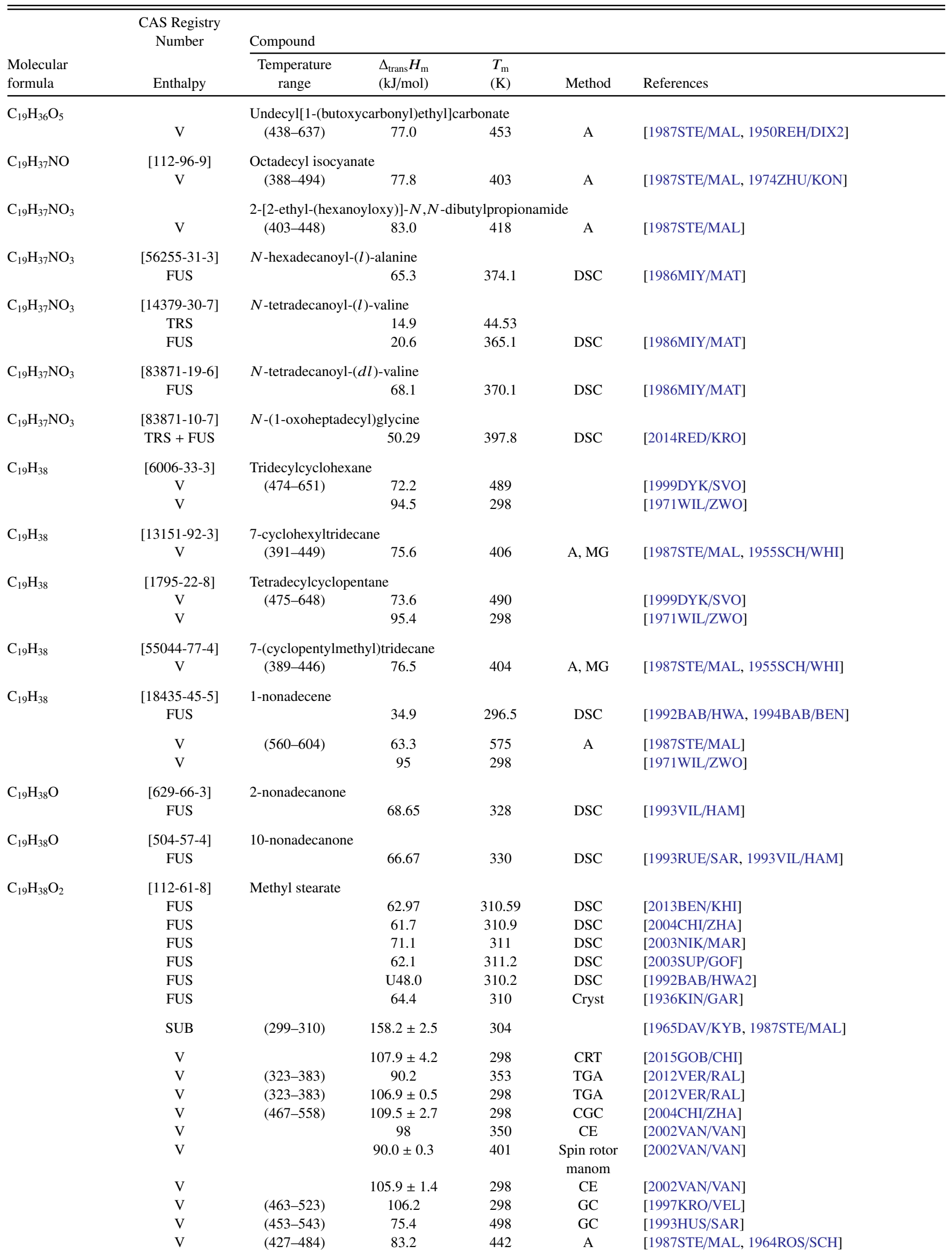


TABLE 14. Phase change enthalpies of $\mathrm{C}_{19}-\mathrm{C}_{29}$ organic compounds-Continued

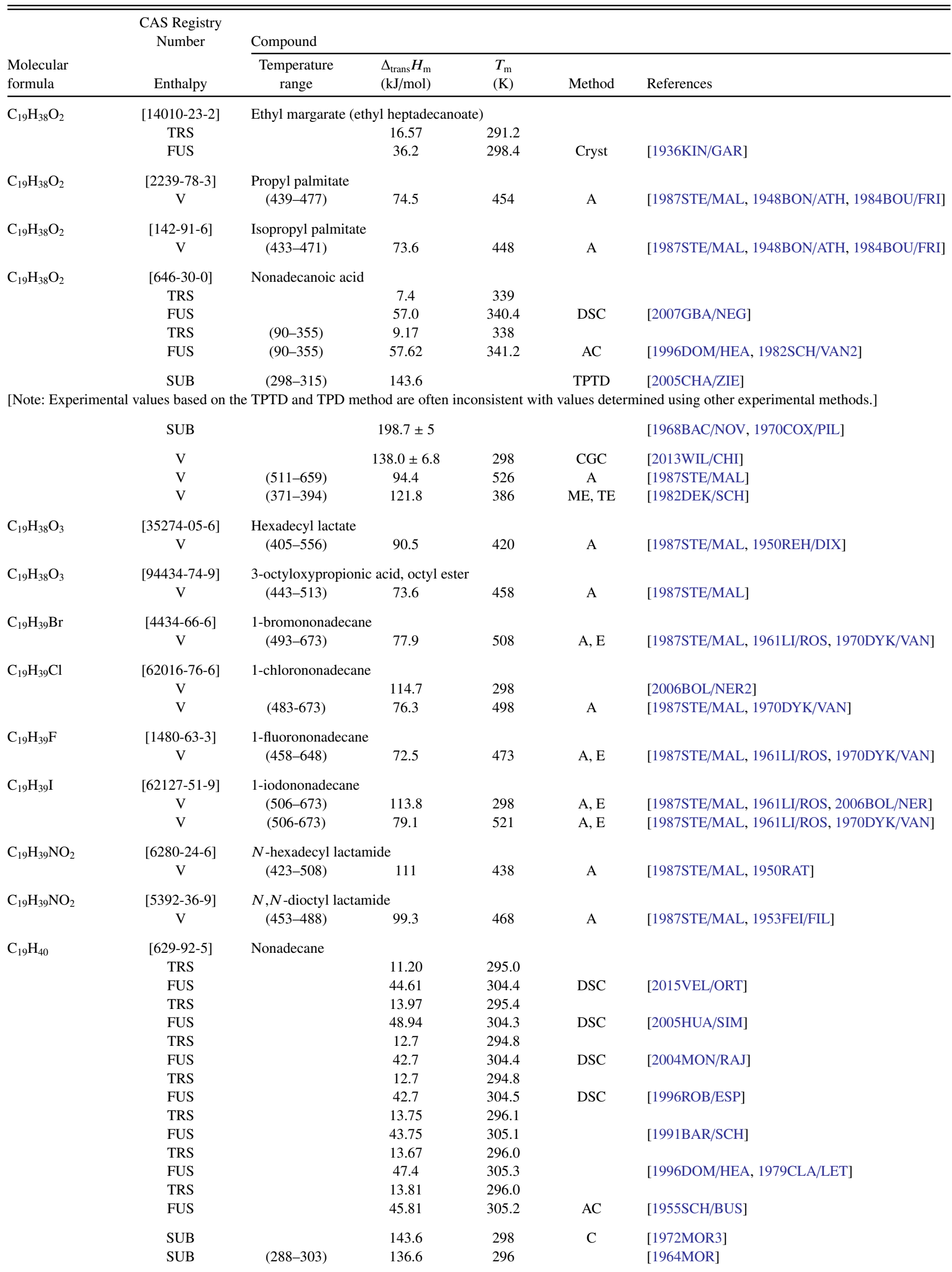


TABLE 14. Phase change enthalpies of $\mathrm{C}_{19}-\mathrm{C}_{29}$ organic compounds-Continued

\begin{tabular}{|c|c|c|c|c|c|c|}
\hline \multirow[b]{2}{*}{$\begin{array}{l}\text { Molecular } \\
\text { formula }\end{array}$} & \multirow{2}{*}{$\begin{array}{l}\text { CAS Registry } \\
\text { Number } \\
\text { Enthalpy }\end{array}$} & \multicolumn{5}{|l|}{ Compound } \\
\hline & & $\begin{array}{l}\text { Temperature } \\
\text { range }\end{array}$ & $\begin{array}{l}\Delta_{\text {trans }} H_{\mathrm{m}} \\
(\mathrm{kJ} / \mathrm{mol})\end{array}$ & $\begin{array}{l}T_{\mathrm{m}} \\
(\mathrm{K})\end{array}$ & Method & References \\
\hline & $\mathrm{V}$ & $(423-588)$ & 76.2 & 438 & & {$[1994 \mathrm{MOR} / \mathrm{KOB}]$} \\
\hline & $\mathrm{V}$ & & 96.4 & 298 & & [1994RUZ/MAJ] \\
\hline & $\mathrm{V}$ & $(456-606)$ & 73.0 & 471 & A & [1987STE/MAL] \\
\hline & V & & 95.8 & 298 & & {$[1971 \mathrm{WIL} / \mathrm{ZWO}]$} \\
\hline $\mathrm{C}_{19} \mathrm{H}_{40}$ & $\begin{array}{c}{[1560-88-9]} \\
\mathrm{V}\end{array}$ & $\begin{array}{l}\text { 2-methyloctadecane } \\
\quad(451-595)\end{array}$ & 67.5 & 466 & A & [1987STE/MAL, 1999DYK/SVO, 1959TER/BRI] \\
\hline $\mathrm{C}_{19} \mathrm{H}_{40}$ & $\begin{array}{c}{[6561-44-0]} \\
\mathrm{V}\end{array}$ & $\begin{array}{l}\text { 3-methyloctadecane } \\
\quad(455-597)\end{array}$ & 69.2 & 470 & A & [1987STE/MAL, 1999DYK/SVO, 1959TER/BRI] \\
\hline $\mathrm{C}_{19} \mathrm{H}_{40}$ & $\begin{array}{l}{[10544-95-3]} \\
\mathrm{V}\end{array}$ & $\begin{array}{l}\text { 4-methyloctadecane } \\
\quad(445-596)\end{array}$ & 63.3 & 460 & A & [1987STE/MAL, 1999DYK/SVO, 1959TER/BRI] \\
\hline $\mathrm{C}_{19} \mathrm{H}_{40}$ & $\begin{array}{l}{[25117-35-5]} \\
\mathrm{V}\end{array}$ & $\begin{array}{l}\text { 5-methyloctadecane } \\
\quad(445-595)\end{array}$ & 63.8 & 460 & A & [1987STE/MAL, 1999DYK/SVO, 1959TER/BRI] \\
\hline $\mathrm{C}_{19} \mathrm{H}_{40}$ & $\begin{array}{c}{[61868-03-9]} \\
\text { V } \\
\text { V }\end{array}$ & $\begin{array}{l}\text { 5-methyloctadecane } \\
\quad(447-598) \\
(493-598)\end{array}$ & $\begin{array}{l}64.1 \\
67.2\end{array}$ & $\begin{array}{l}462 \\
508\end{array}$ & A & $\begin{array}{l}\text { [1999DYK/SVO, 1959TER/BRI] } \\
\text { [1987STE/MAL] }\end{array}$ \\
\hline $\mathrm{C}_{19} \mathrm{H}_{40}$ & $\begin{array}{l}{[61868-09-5]} \\
\mathrm{V}\end{array}$ & $\begin{array}{l}\text { 2,4-dimethylheptadec } \\
\text { (444-574) }\end{array}$ & $\begin{array}{l}\text { cane } \\
70.6\end{array}$ & 459 & A & [1987STE/MAL, 1999DYK/SVO, 1959TER/BRI] \\
\hline $\mathrm{C}_{19} \mathrm{H}_{40}$ & $\begin{array}{c}\text { [102013-94-5] } \\
\text { V }\end{array}$ & $\begin{array}{l}\text { 2,4-dimethylheptadec } \\
\quad(435-568)\end{array}$ & $\begin{array}{l}\text { cane } \\
67.3\end{array}$ & 450 & A & [1987STE/MAL, 1999DYK/SVO, 1959TER/BRI] \\
\hline $\mathrm{C}_{19} \mathrm{H}_{40}$ & $\begin{array}{c}{[7225-66-3]} \\
\mathrm{V}\end{array}$ & $\begin{array}{l}\text { 7-hexyltridecane } \\
\text { (411-444) }\end{array}$ & 75.2 & 426 & A & [1987STE/MAL] \\
\hline $\mathrm{C}_{19} \mathrm{H}_{40} \mathrm{O}$ & $\begin{array}{c}\text { [1454-84-8] } \\
\text { FUS } \\
\text { TRS } \\
\text { FUS } \\
\text { FUS }\end{array}$ & 1-nonadecanol & $\begin{array}{c}43.3 \\
29.1 \\
43.3 \\
72.42\end{array}$ & $\begin{array}{l}333.9 \\
329.7 \\
333.9 \\
334.5\end{array}$ & $\begin{array}{l}\text { DSC } \\
\text { DSC }\end{array}$ & $\begin{array}{l}\text { [2004VEN/CAL] } \\
\text { [2002VEN/RAM] } \\
\text { [2001VAN/OON2] }\end{array}$ \\
\hline
\end{tabular}

[Note: The value includes the enthalpy of a solid/solid transition that occurs at about $331 \mathrm{~K}$.]

$\begin{array}{llllll}\mathrm{V} & (479-640) & 81.7 & 494 & \text { A } & \text { [1987STE/MAL] } \\ \mathrm{V} & (494-635) & 80.0 & 509 & \text { A } & \text { [1987STE/MAL] }\end{array}$

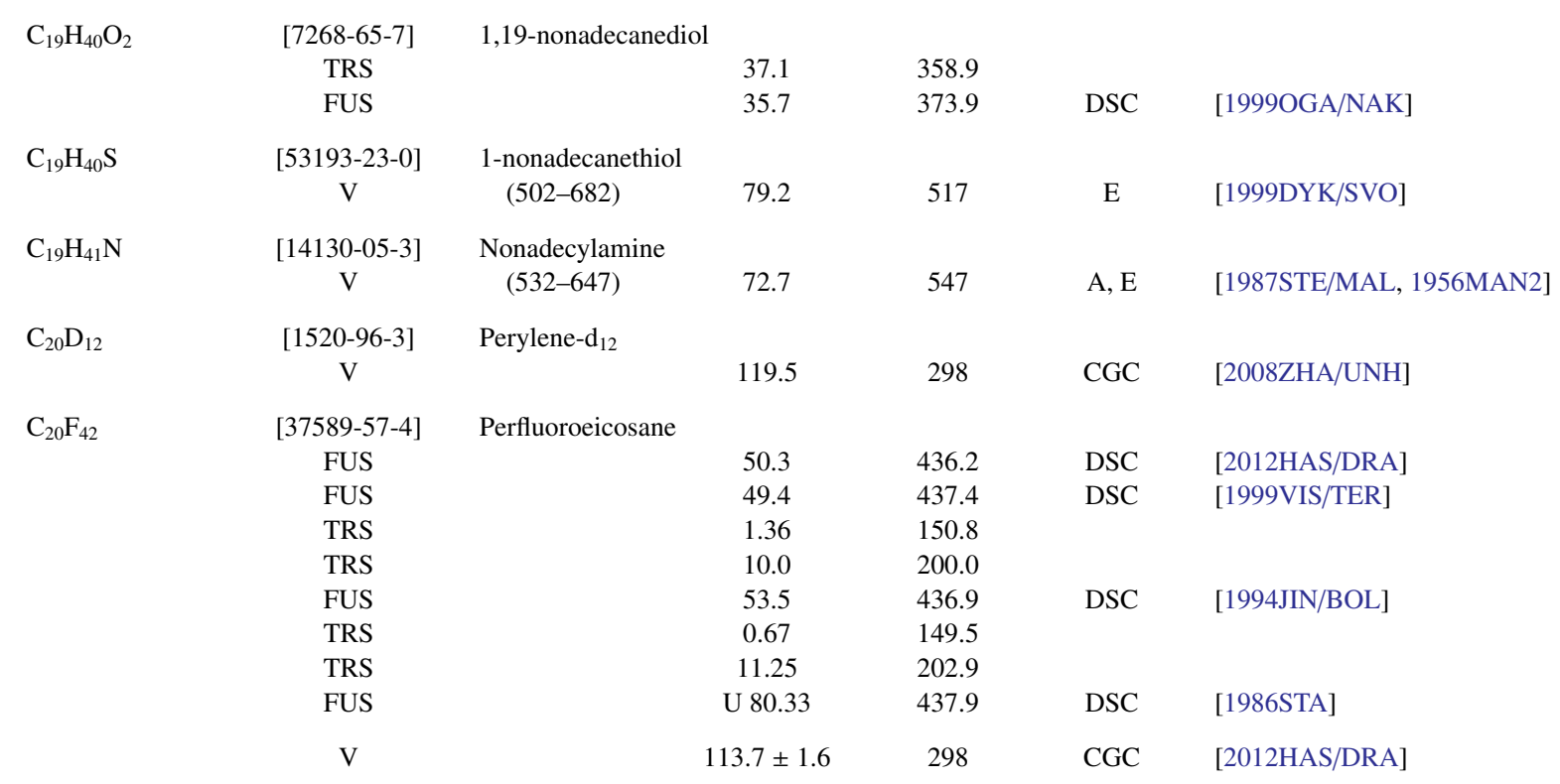

$\mathrm{C}_{20} \mathrm{H}_{4} \mathrm{Cl}_{4} \mathrm{~F}_{13} \mathrm{NO}_{2} \quad$ [433932-34-4] 4,5,6,7-dichloro-2-(4- $n$-tridecafluorohexylphenyl)isoindole-1,3-dione FUS $45.1 \quad 512.8 \quad$ DSC $\quad$ [2002JOW/DIN]

$\mathrm{C}_{20} \mathrm{H}_{6} \mathrm{Cl}_{2} \mathrm{~F}_{13} \mathrm{NO}_{2} \quad$ [433932-32-2] 5,6-dichloro-2-(4- $n$-tridecafluorohexylphenyl)isoindole-1,3-dione $\begin{array}{lllll}\text { FUS } & 40.7 & 459.6 & \text { DSC } & \text { [2002JOW/DIN] }\end{array}$ 
TABLE 14. Phase change enthalpies of $\mathrm{C}_{19}-\mathrm{C}_{29}$ organic compounds-Continued

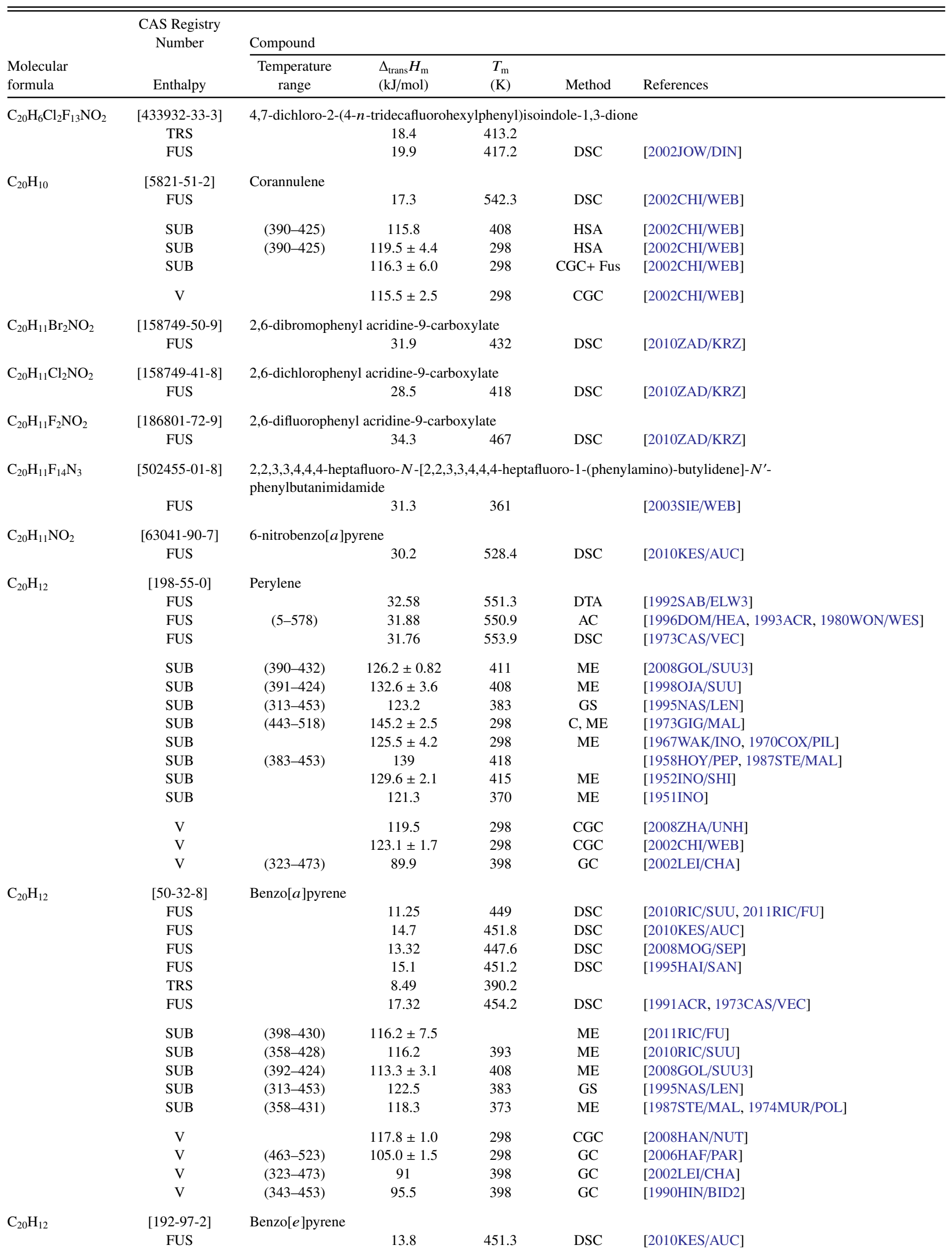


TABLE 14. Phase change enthalpies of $\mathrm{C}_{19}-\mathrm{C}_{29}$ organic compounds-Continued

\begin{tabular}{|c|c|c|c|c|c|c|}
\hline \multirow[b]{2}{*}{$\begin{array}{l}\text { Molecular } \\
\text { formula }\end{array}$} & \multirow{2}{*}{$\begin{array}{c}\text { CAS Registry } \\
\text { Number } \\
\text { Enthalpy }\end{array}$} & \multicolumn{5}{|l|}{ Compound } \\
\hline & & $\begin{array}{c}\text { Temperature } \\
\text { range }\end{array}$ & $\begin{array}{l}\Delta_{\text {trans }} H_{\mathrm{m}} \\
(\mathrm{kJ} / \mathrm{mol})\end{array}$ & $\begin{array}{l}T_{\mathrm{m}} \\
(\mathrm{K})\end{array}$ & Method & References \\
\hline & TRS & & 2.51 & 426.2 & & \\
\hline & FUS & & 16.57 & 454.4 & DSC & [1991ACR, 1973CAS/VEC] \\
\hline & SUB & $(313-453)$ & 117.9 & 383 & GS & [1995NAS/LEN] \\
\hline & SUB & $(359-423)$ & 119.1 & 373 & ME & [1987STE/MAL, 1974MUR/POL] \\
\hline & $\mathrm{V}$ & & $118.2 \pm 0.3$ & 298 & CGC & [2008HAN/NUT] \\
\hline & $\mathrm{V}$ & $(463-523)$ & $105.0 \pm 1.5$ & 298 & GC & [2006HAF/PAR] \\
\hline & V & $(343-453)$ & 92 & 398 & GC & [1990HIN/BID2] \\
\hline \multirow[t]{11}{*}{$\mathrm{C}_{20} \mathrm{H}_{12}$} & [207-08-9] & \multicolumn{5}{|c|}{ Benzo $[k]$ fluoranthene } \\
\hline & FUS & & 26.5 & & DSC & [2011FU/SUU] \\
\hline & FUS & & 32.4 & 489.7 & DSC & [2010KES/AUC] \\
\hline & FUS & & 27.5 & 490.6 & & [2002DIO/MIN] \\
\hline & SUB & $(384-424)$ & $121.5 \pm 3.3$ & & $\mathrm{ME}$ & [2011FU/SUU] \\
\hline & SUB & $(387-423)$ & $124.2 \pm 4.7$ & 298 & $\mathrm{ME}$ & [2002DIO/MIN] \\
\hline & SUB & $(363-430)$ & 130 & 378 & A & [1987STE/MAL] \\
\hline & SUB & & $120 \pm 10$ & & $\mathrm{TE}$ & [1983FER/QUA] \\
\hline & $\mathrm{V}$ & & $117.4 \pm 1.1$ & 298 & $\mathrm{CGC}$ & [2008HAN/NUT] \\
\hline & $\mathrm{V}$ & $(463-513)$ & $105.5 \pm 1.5$ & 298 & GC & [2006HAF/PAR] \\
\hline & $\mathrm{V}$ & $(323-473)$ & 88.5 & 398 & GC & [2002LEI/CHA] \\
\hline \multirow[t]{8}{*}{$\mathrm{C}_{20} \mathrm{H}_{12}$} & [205-99-2] & \multicolumn{5}{|c|}{ Benzo $[b]$ fluoranthene } \\
\hline & FUS & & 18.3 & & DSC & [2011FU/SUU] \\
\hline & FUS & & 19.6 & 441.5 & DSC & [2010KES/AUC] \\
\hline & SUB & $(364-414)$ & $118.8 \pm 0.8$ & & $\mathrm{ME}$ & [2011FU/SUU] \\
\hline & SUB & $(313-453)$ & 119.2 & 383 & GS & {$[1995 \mathrm{NAS} / \mathrm{LEN}]$} \\
\hline & $\mathrm{V}$ & & $116.8 \pm 1.6$ & 298 & $\mathrm{CGC}$ & [2008HAN/NUT] \\
\hline & $\mathrm{V}$ & $(463-513)$ & $104.0 \pm 1.5$ & 298 & GC & [2006HAF/PAR] \\
\hline & $\mathrm{V}$ & $(323-473)$ & 89.7 & 398 & $\mathrm{GC}$ & {$[2002 \mathrm{LEI} / \mathrm{CHA}]$} \\
\hline \multirow[t]{2}{*}{$\mathrm{C}_{20} \mathrm{H}_{12}$} & {$[205-82-3]$} & \multicolumn{5}{|c|}{ benzo $[j]$ fluoranthene } \\
\hline & FUS & & 17.9 & 438.3 & DSC & [2010KES/AUC] \\
\hline \multirow[t]{2}{*}{$\mathrm{C}_{20} \mathrm{H}_{12} \mathrm{BrNO}_{4}$} & {$[59722-76-8]$} & \multicolumn{5}{|c|}{ 1-amino-2-(4-bromophenoxy)-4-hydroxy-9,10-anthraquinone } \\
\hline & SUB & $(473-543)$ & 163.6 & 413 & & {$[1978 N I S / I S H]$} \\
\hline \multirow[t]{2}{*}{$\mathrm{C}_{20} \mathrm{H}_{12} \mathrm{FNO}_{2}$} & [1228096-41-0] & \multicolumn{5}{|c|}{ 2-fluorophenyl acridine-9-carboxylate } \\
\hline & FUS & & 28.1 & 437 & DSC & [2010ZAD/KRZ] \\
\hline \multirow[t]{2}{*}{$\mathrm{C}_{20} \mathrm{H}_{12} \mathrm{INO}_{2}$} & {$[1268261-57-9]$} & \multicolumn{5}{|c|}{ 2-iodophenyl acridine-9-carboxylate } \\
\hline & FUS & & 32.4 & 438 & DSC & [2010ZAD/KRZ] \\
\hline \multirow[t]{2}{*}{$\mathrm{C}_{20} \mathrm{H}_{12} \mathrm{~N}_{2} \mathrm{O}_{4}$} & {$[1093974-11-8]$} & \multicolumn{5}{|c|}{ 2-nitrophenyl acridine-9-carboxylate } \\
\hline & FUS & & 24.4 & 422 & DSC & [2010ZAD/KRZ] \\
\hline \multirow[t]{2}{*}{$\mathrm{C}_{20} \mathrm{H}_{12} \mathrm{O}$} & {$[13345-21-6]$} & \multicolumn{5}{|c|}{ 3-hydroxybenzo[ $a]$ pyrene } \\
\hline & FUS & & 24.1 & 469.6 & DSC & [2010KES/AUC] \\
\hline $\mathrm{C}_{2} \mathrm{OH}_{13} \mathrm{~N}$ & {$[194-59-2]$} & $7 H$-dibenzo $[c$, & arbazole & & & \\
\hline & FUS & & 20.1 & 429.8 & DSC & [2010KES/AUC] \\
\hline $\mathrm{C}_{20} \mathrm{H}_{13} \mathrm{NO}_{2}$ & {$[109392-90-7]$} & Phenyl acridine & arboxylate & & & \\
\hline & FUS & & 39.2 & 464 & DSC & [2010KRZ/MAL] \\
\hline $\mathrm{C}_{20} \mathrm{H}_{13} \mathrm{NO}_{4}$ & {$[17418-58-5]$} & 1-amino-4-hyd & -2-phenoxy-9 & thraqui & Disperse & 60) \\
\hline & FUS & & 30.79 & 458.2 & & [1991BAU/WEB] \\
\hline & SUB & $(423-448)$ & 104.9 & 436 & GC & {$[2002 \mathrm{SAW} / \mathrm{SHI}]$} \\
\hline & SUB & $(359-366)$ & 152.5 & 362.5 & A & [1987STE/MAL, 1977EIB/TRO] \\
\hline [Note: The tem & ure interval covere & in $[1987 \mathrm{STE} / \mathrm{M}$ & may be too $\mathrm{s}$ & or an ac & $\Delta_{S u b} H$ & rmination.] \\
\hline & SUB & & 141.8 & & & [1984KAR/KRU] \\
\hline & SUB & $(373-453)$ & 103.8 & 413 & & [1978NIS/ISH] \\
\hline $\mathrm{C}_{20} \mathrm{H}_{13} \mathrm{~N}_{5} \mathrm{O}_{3}$ & {$[194785-03-0]$} & 1-(2'-nitrobenz & ene)-2-phenaz & hydrazin & & \\
\hline & FUS & & 47.47 & 540.2 & DSC & [1997CIO/MEL] \\
\hline
\end{tabular}


TABLE 14. Phase change enthalpies of $\mathrm{C}_{19}-\mathrm{C}_{29}$ organic compounds-Continued

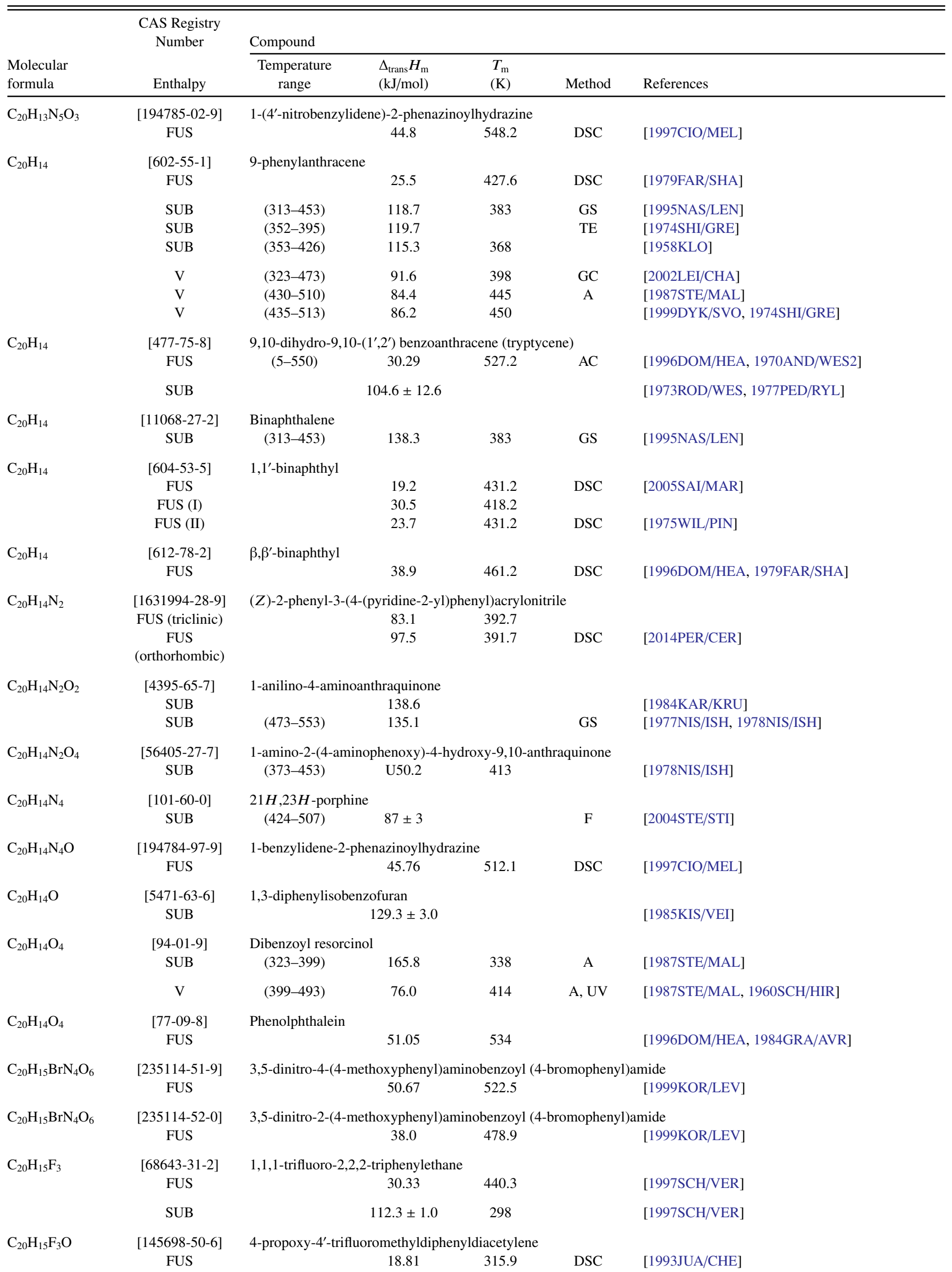


TABLE 14. Phase change enthalpies of $\mathrm{C}_{19}-\mathrm{C}_{29}$ organic compounds-Continued

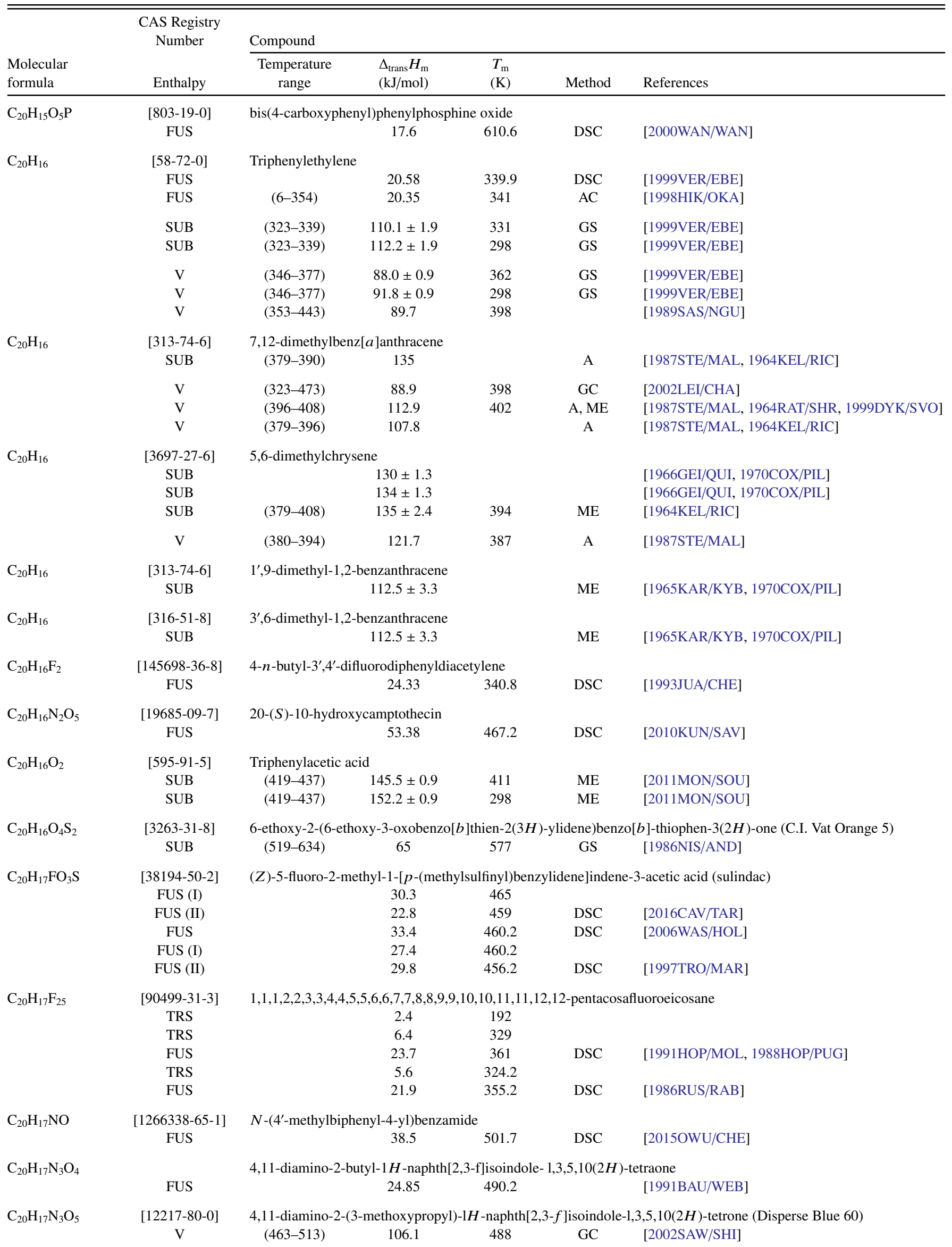


TABLE 14. Phase change enthalpies of $\mathrm{C}_{19}-\mathrm{C}_{29}$ organic compounds-Continued

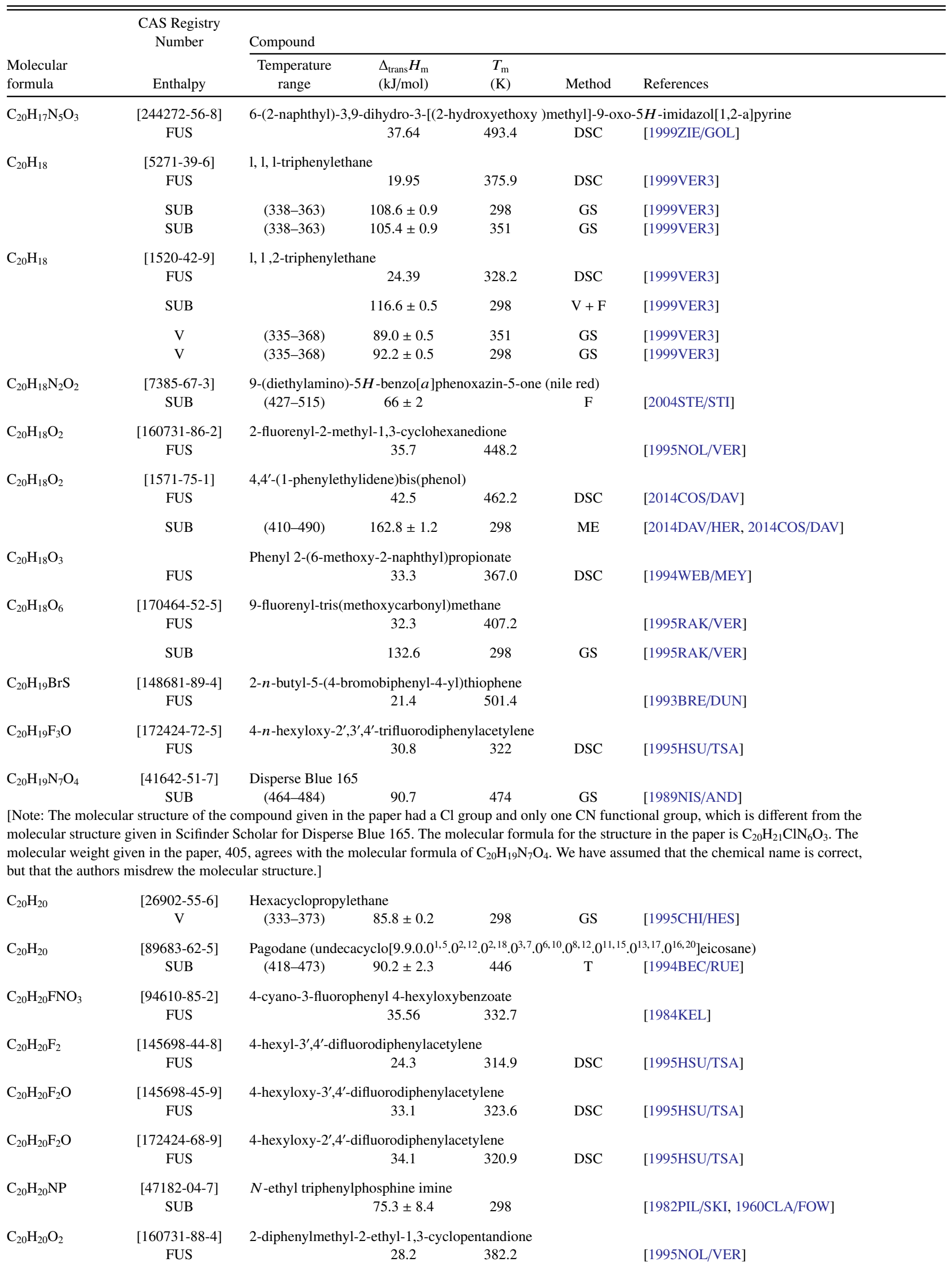


TABLE 14. Phase change enthalpies of $\mathrm{C}_{19}-\mathrm{C}_{29}$ organic compounds-Continued

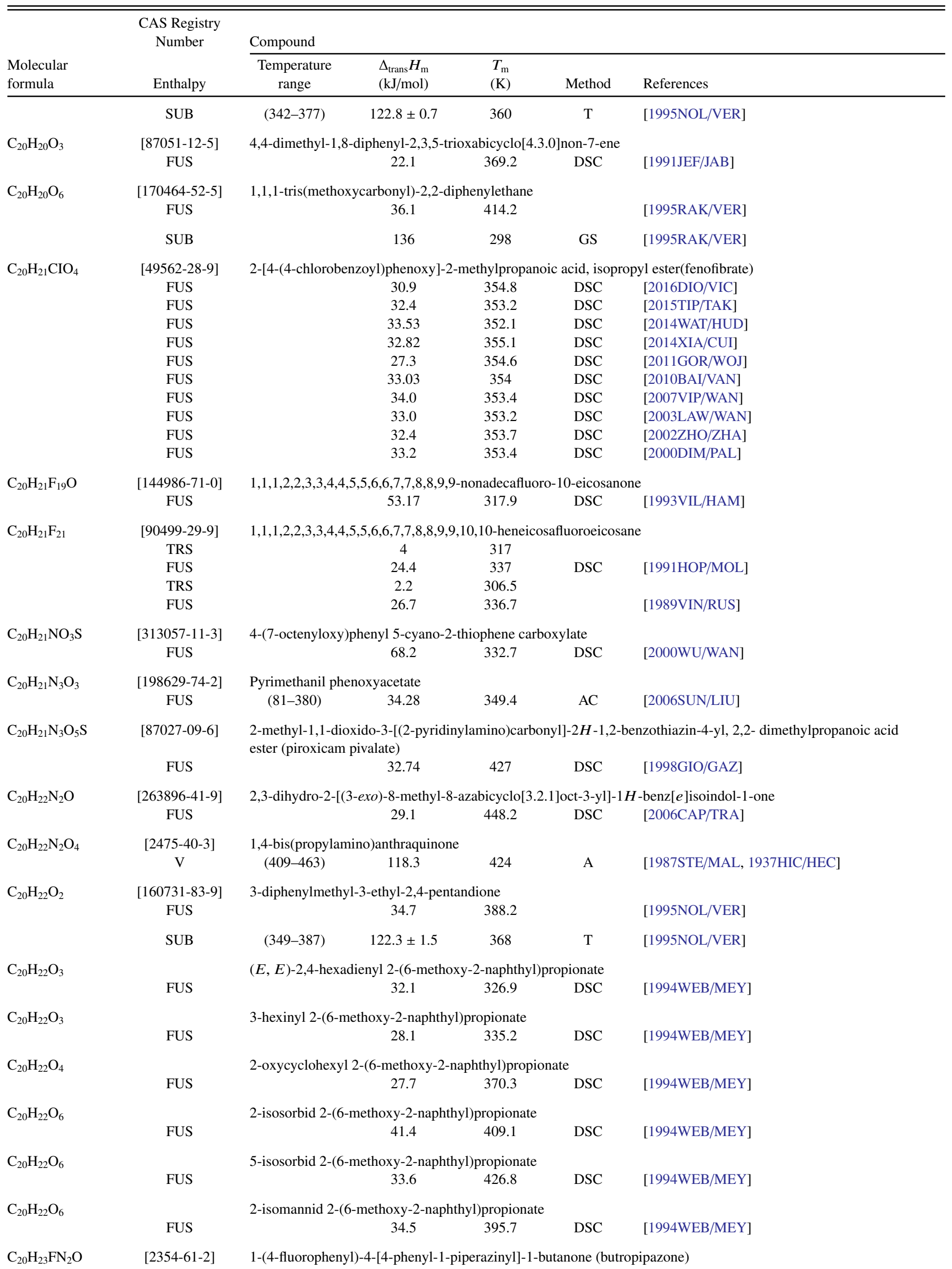


TABLE 14. Phase change enthalpies of $\mathrm{C}_{19}-\mathrm{C}_{29}$ organic compounds-Continued

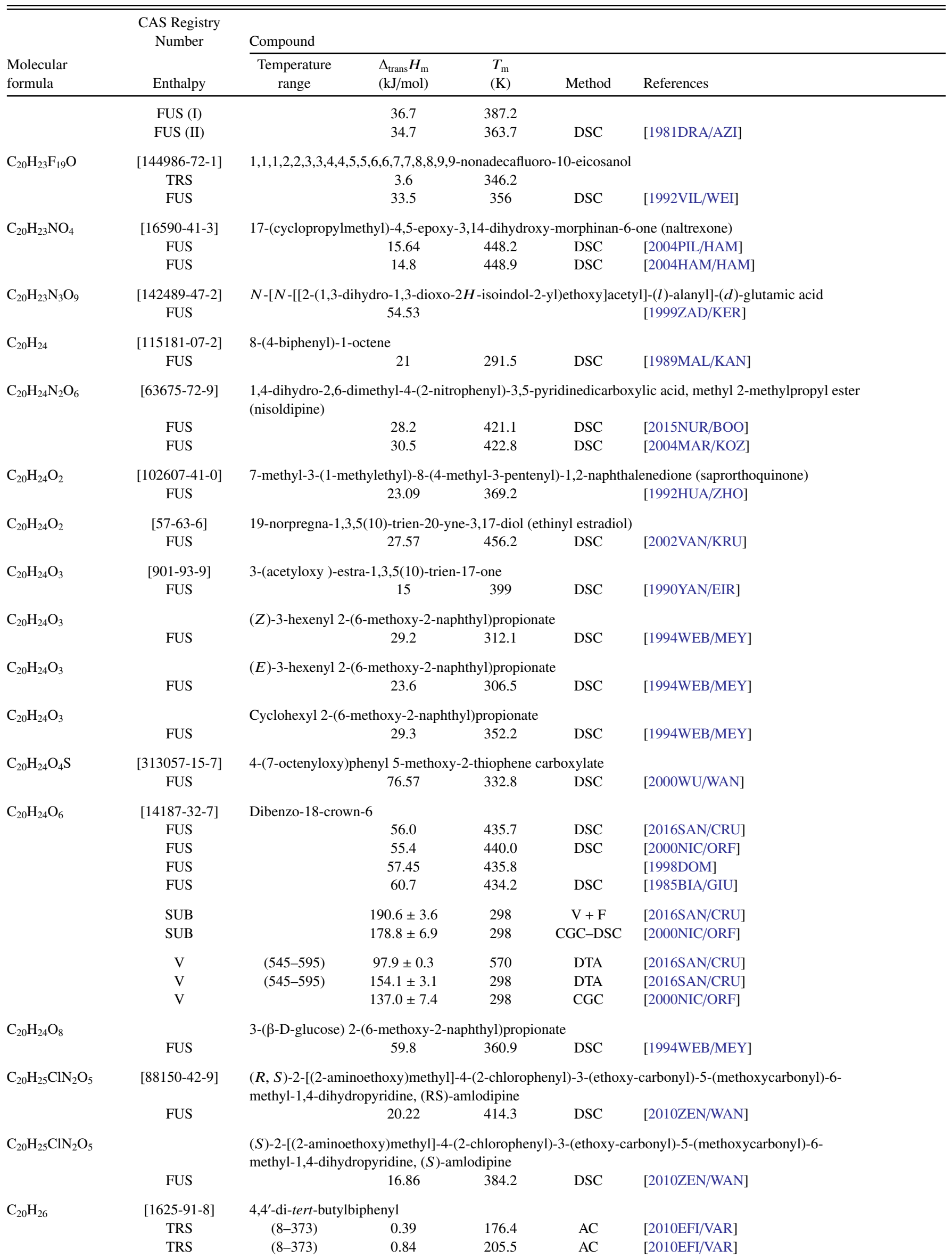


TABLE 14. Phase change enthalpies of $\mathrm{C}_{19}-\mathrm{C}_{29}$ organic compounds-Continued

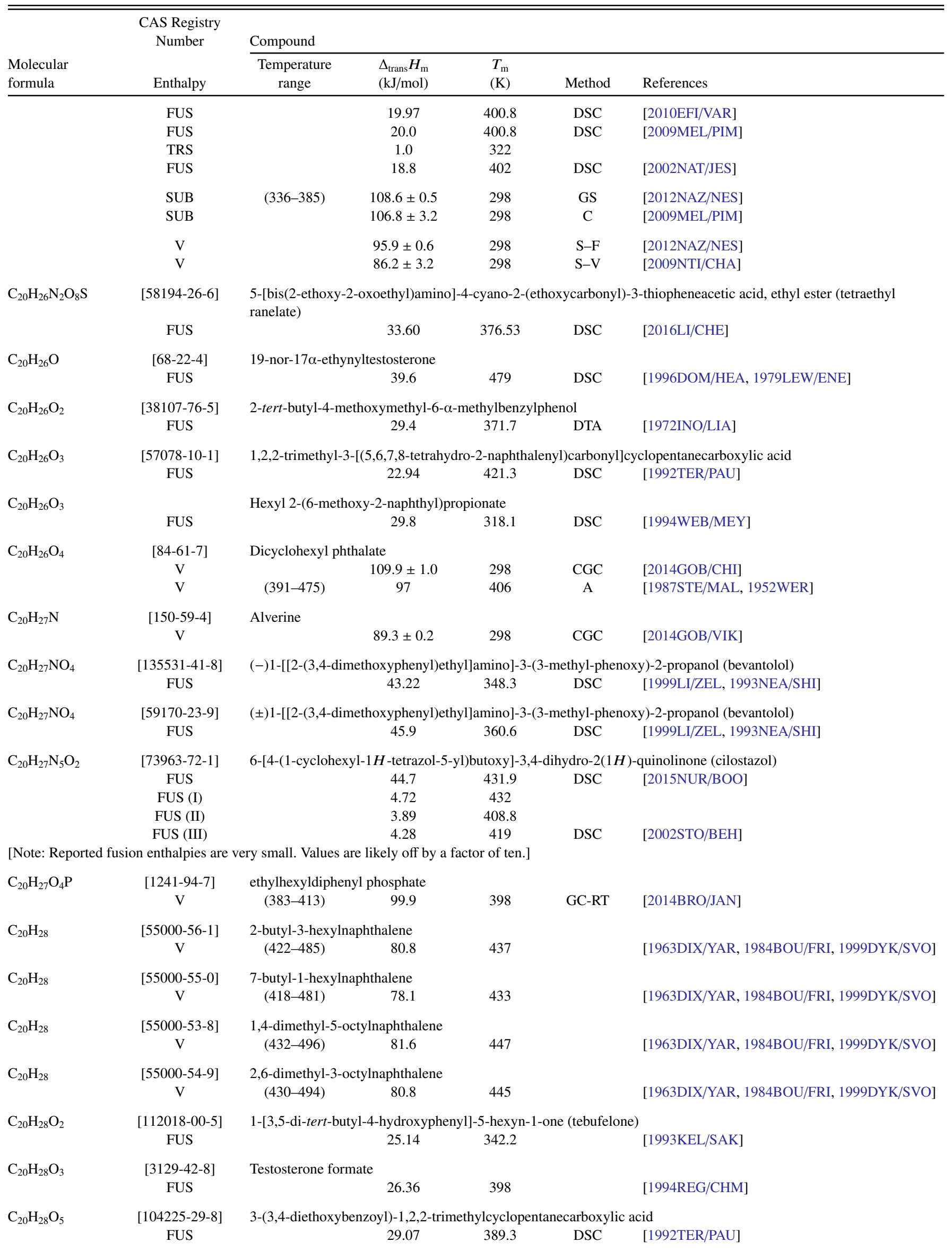


TABLE 14. Phase change enthalpies of $\mathrm{C}_{19}-\mathrm{C}_{29}$ organic compounds-Continued

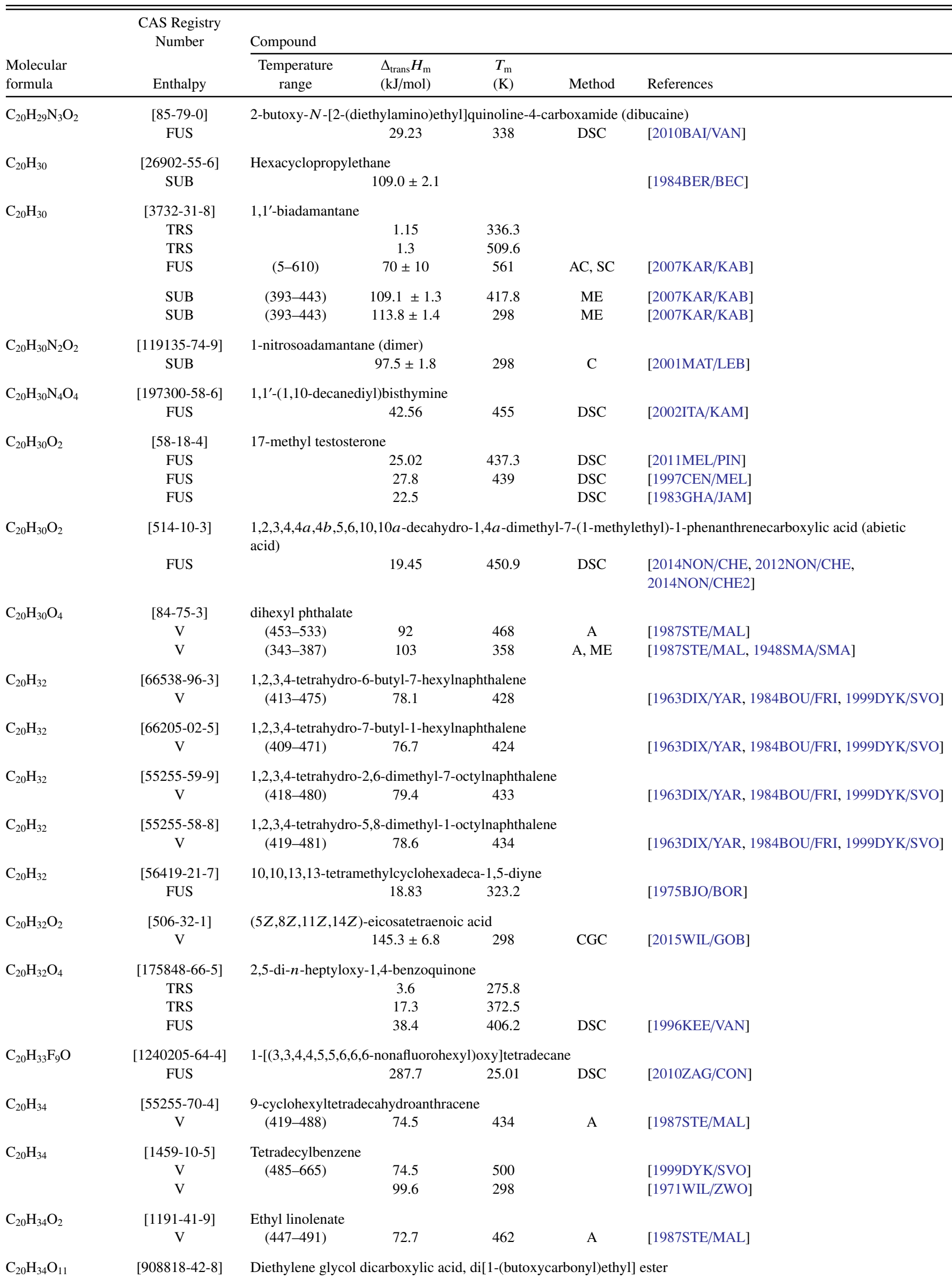


TABLE 14. Phase change enthalpies of $\mathrm{C}_{19}-\mathrm{C}_{29}$ organic compounds-Continued

\begin{tabular}{|c|c|c|c|c|c|c|}
\hline \multirow[b]{2}{*}{$\begin{array}{l}\text { Molecular } \\
\text { formula }\end{array}$} & \multirow{2}{*}{$\begin{array}{c}\text { CAS Registry } \\
\text { Number } \\
\text { Enthalpy }\end{array}$} & \multicolumn{5}{|l|}{ Compound } \\
\hline & & $\begin{array}{l}\text { Temperature } \\
\text { range }\end{array}$ & $\begin{array}{l}\Delta_{\text {trans }} H_{\mathrm{m}} \\
(\mathrm{kJ} / \mathrm{mol})\end{array}$ & $\begin{array}{l}T_{\mathrm{m}} \\
(\mathrm{K})\end{array}$ & Method & References \\
\hline & $\mathrm{V}$ & $(433-525)$ & 103.6 & 448 & A & [1987STE/MAL] \\
\hline $\mathrm{C}_{20} \mathrm{H}_{34} \mathrm{O}_{11}$ & $\mathrm{~V}$ & $\begin{array}{l}\text { Diethylene gly } \\
\quad(418-513)\end{array}$ & $\begin{array}{l}\text { carboxylic a } \\
103.1\end{array}$ & $\begin{array}{c}{[1-(\mathrm{sec}-} \\
433\end{array}$ & $\begin{array}{l}\text { ycarbony } \\
\text { A }\end{array}$ & $\begin{array}{l}\text { nyl] ester } \\
\text { [1987STE/MAL] }\end{array}$ \\
\hline $\mathrm{C}_{20} \mathrm{H}_{34} \mathrm{O}_{11}$ & $\begin{array}{c}{[5349-71-3]} \\
\mathrm{V}\end{array}$ & $\begin{array}{l}\text { Diethylene gly } \\
\quad(415-513)\end{array}$ & $\begin{array}{l}\text { carboxylic a } \\
103.1\end{array}$ & $\begin{array}{c}{[1-\text { (isol }} \\
430\end{array}$ & $\begin{array}{c}\text { carbonyl) } \\
\text { A }\end{array}$ & $\begin{array}{l}\text { l] ester } \\
\text { [1987STE/MAL, 1950REH/DIX3] }\end{array}$ \\
\hline $\mathrm{C}_{20} \mathrm{H}_{36} \mathrm{~N}_{2}$ & $\begin{array}{l}{[85688-86-4]} \\
\text { FUS }\end{array}$ & Tetraisobutylsu & nitrile & 360.2 & & [1983BAR/BEC] \\
\hline $\mathrm{C}_{20} \mathrm{H}_{36} \mathrm{O}_{2}$ & $\begin{array}{c}\text { [544-35-4] } \\
\mathrm{V} \\
\mathrm{V}\end{array}$ & $\begin{array}{c}\text { Ethyl linoleate } \\
\qquad \begin{array}{c}(487-537) \\
(448-497)\end{array}\end{array}$ & $\begin{array}{l}92.9 \\
72.6\end{array}$ & $\begin{array}{l}513 \\
463\end{array}$ & A & $\begin{array}{l}{[2011 \mathrm{SIL} / \mathrm{FAL}]} \\
{[1987 \mathrm{STE} / \mathrm{MAL}]}\end{array}$ \\
\hline $\mathrm{C}_{20} \mathrm{H}_{36} \mathrm{O}_{2}$ & $\begin{array}{l}{[14113-56-5]} \\
\text { FUS }\end{array}$ & 1,10-cycloeico & $\begin{array}{l}\text { lione } \\
55.06\end{array}$ & 327.2 & & [1972ALV/BOR] \\
\hline $\mathrm{C}_{20} \mathrm{H}_{36} \mathrm{O}_{2}$ & FUS & 1,9-cyclohexad & $\begin{array}{c}\text { edione bis et } \\
42.13\end{array}$ & $\begin{array}{l}\text { ketal } \\
404.2\end{array}$ & & [1972ALV/BOR] \\
\hline $\mathrm{C}_{20} \mathrm{H}_{36} \mathrm{O}_{4}$ & $\begin{array}{c}\text { [2424-62-6] } \\
\text { FUS }\end{array}$ & Hexadecyl mal & 71.4 & 345.0 & DSC & [2016RIC/DEL] \\
\hline $\mathrm{C}_{20} \mathrm{H}_{36} \mathrm{O}_{6}$ & $\mathrm{~V}$ & (syn-cis/anti-ci & $\begin{array}{l}\text { yclohexano- } \\
124.2 \pm 4.0\end{array}$ & $\begin{array}{r}\text { wn-6 } \\
298\end{array}$ & CGC & {$[2000 \mathrm{NIC} / \mathrm{ORF}]$} \\
\hline $\mathrm{C}_{20} \mathrm{H}_{38}$ & $\begin{array}{c}{[66455-55-8]} \\
\mathrm{V}\end{array}$ & $\begin{array}{l}\text { 2-butyl-3-hexy } \\
\text { (407-472) }\end{array}$ & $\begin{array}{c}\text { hydronaphth } \\
76.9\end{array}$ & 422 & & [1963DIX/YAR, 1984BOU/FRI, 1999DYK/SVO] \\
\hline $\mathrm{C}_{20} \mathrm{H}_{38}$ & $\begin{array}{c}{[66455-54-7]} \\
\mathrm{V}\end{array}$ & $\begin{array}{c}\text { 7-butyl-1-hexy } \\
\text { (407-467) }\end{array}$ & $\begin{array}{c}\text { hydronaphth } \\
80.0\end{array}$ & 422 & & [1963DIX/YAR, 1984BOU/FRI, 1999DYK/SVO] \\
\hline $\mathrm{C}_{20} \mathrm{H}_{38}$ & $\begin{array}{c}{[54964-83-9]} \\
\mathrm{V}\end{array}$ & $\begin{array}{c}\text { 1,4-dimethyl-5 } \\
\text { (404-466) }\end{array}$ & $\begin{array}{c}\text { ldecahydron } \\
73.9\end{array}$ & $\begin{array}{l}\text { lene } \\
419\end{array}$ & & [1963DIX/YAR, 1984BOU/FRI, 1999DYK/SVO] \\
\hline $\mathrm{C}_{20} \mathrm{H}_{38}$ & $\begin{array}{c}{[54964-85-1]} \\
\mathrm{V}\end{array}$ & $\begin{array}{l}\text { 2,6-dimethyl-3 } \\
\text { (406-469) }\end{array}$ & $\begin{array}{c}\text { ldecahydron } \\
76.4\end{array}$ & $\begin{array}{l}\text { lene } \\
421\end{array}$ & & [1963DIX/YAR, 1984BOU/FRI] \\
\hline $\mathrm{C}_{20} \mathrm{H}_{38}$ & $\begin{array}{c}{[26527-76-4]} \\
V\end{array}$ & $\begin{array}{l}\text { 3,4-dicyclohex } \\
\quad(343-365)\end{array}$ & $\begin{array}{l}\text { 4-dimethylhe } \\
78.4\end{array}$ & 359 & & [1999DYK/SVO, 1980BEC/KRA] \\
\hline $\mathrm{C}_{20} \mathrm{H}_{38}$ & $\begin{array}{c}{[765-27-5]} \\
V\end{array}$ & $\begin{array}{l}\text { 1-eicosyne } \\
\quad(473-651)\end{array}$ & 68.9 & 488 & & [1999DYK/SVO] \\
\hline $\mathrm{C}_{20} \mathrm{H}_{38}$ & $\begin{array}{c}{[61847-99-2]} \\
\mathrm{V}\end{array}$ & $\begin{array}{l}\text { 2-eicosyne } \\
\qquad(480-661)\end{array}$ & 69.8 & 495 & & {$[1999 \mathrm{DYK} / \mathrm{SVO}]$} \\
\hline $\mathrm{C}_{20} \mathrm{H}_{38}$ & $\begin{array}{c}{[61886-66-6]} \\
V\end{array}$ & $\begin{array}{l}\text { 3-eicosyne } \\
\quad(470-648)\end{array}$ & 68.4 & 485 & & [1999DYK/SVO] \\
\hline $\mathrm{C}_{20} \mathrm{H}_{38} \mathrm{O}$ & $\begin{array}{c}{[29171-23-1]} \\
\mathrm{V}\end{array}$ & $\begin{array}{c}\text { 3,7,11,15-tetra } \\
\quad(403-457)\end{array}$ & $\begin{array}{l}y \text {-1-hexadec } \\
43.8 \pm 1.9\end{array}$ & 430 & Static & [1988BAG/GUR] \\
\hline $\mathrm{C}_{20} \mathrm{H}_{38} \mathrm{O}_{2}$ & $\begin{array}{c}\text { [111-62-6] } \\
\mathrm{V} \\
\mathrm{V}\end{array}$ & $\begin{array}{l}\text { Ethyl oleate } \\
\qquad \begin{array}{r}(487-537) \\
(384-481)\end{array}\end{array}$ & $\begin{array}{l}87.6 \\
92.4\end{array}$ & $\begin{array}{l}513 \\
399\end{array}$ & $\begin{array}{c}\mathrm{DSC} \\
\mathrm{A}\end{array}$ & $\begin{array}{l}\text { [2011SIL/FAL] } \\
\text { [1987STE/MAL] }\end{array}$ \\
\hline $\mathrm{C}_{20} \mathrm{H}_{38} \mathrm{O}_{2}$ & $\begin{array}{c}{[2495-27-4]} \\
\mathrm{V}\end{array}$ & $\begin{array}{l}\text { Hexadecyl met } \\
\quad(431-541)\end{array}$ & $\begin{array}{l}\text { late } \\
73.1\end{array}$ & 446 & A & [1987STE/MAL] \\
\hline $\mathrm{C}_{20} \mathrm{H}_{38} \mathrm{O}_{2}$ & $\begin{array}{c}{[155055-33-7]} \\
\mathrm{V}\end{array}$ & $\begin{array}{l}(Z)-3 \text {-octadece } \\
\quad(393-438)\end{array}$ & $\begin{array}{l}\text { cetate } \\
108.7\end{array}$ & 298 & GC & [1997KOU/HOS, 2000OVA/KOU] \\
\hline $\mathrm{C}_{20} \mathrm{H}_{38} \mathrm{O}_{2}$ & $\begin{array}{c}{[155055-35-9]} \\
\mathrm{V}\end{array}$ & $\begin{array}{c}(E)-3 \text {-octadece } \\
(393-438)\end{array}$ & $\begin{array}{l}\text { cetate } \\
109.3\end{array}$ & 298 & GC & [1997KOU/HOS, 2000OVA/KOU] \\
\hline $\mathrm{C}_{20} \mathrm{H}_{38} \mathrm{O}_{2}$ & $\begin{array}{c}{[693-80-1]} \\
\mathrm{V}\end{array}$ & $\begin{array}{l}\text { (Z)-9-octadece } \\
\quad(393-438)\end{array}$ & $\begin{array}{l}\text { cetate } \\
107.8\end{array}$ & 298 & GC & [1997KOU/HOS, 2000OVA/KOU] \\
\hline $\mathrm{C}_{20} \mathrm{H}_{38} \mathrm{O}_{2}$ & $\begin{array}{c}{[22147-38-2]} \\
\mathrm{V}\end{array}$ & $\begin{array}{l}(E)-9 \text {-octadece } \\
\quad(393-438)\end{array}$ & $\begin{array}{l}\text { cetate } \\
108.7\end{array}$ & 298 & GC & [1997KOU/HOS, 2000OVA/KOU] \\
\hline
\end{tabular}


TABLE 14. Phase change enthalpies of $\mathrm{C}_{19}-\mathrm{C}_{29}$ organic compounds-Continued

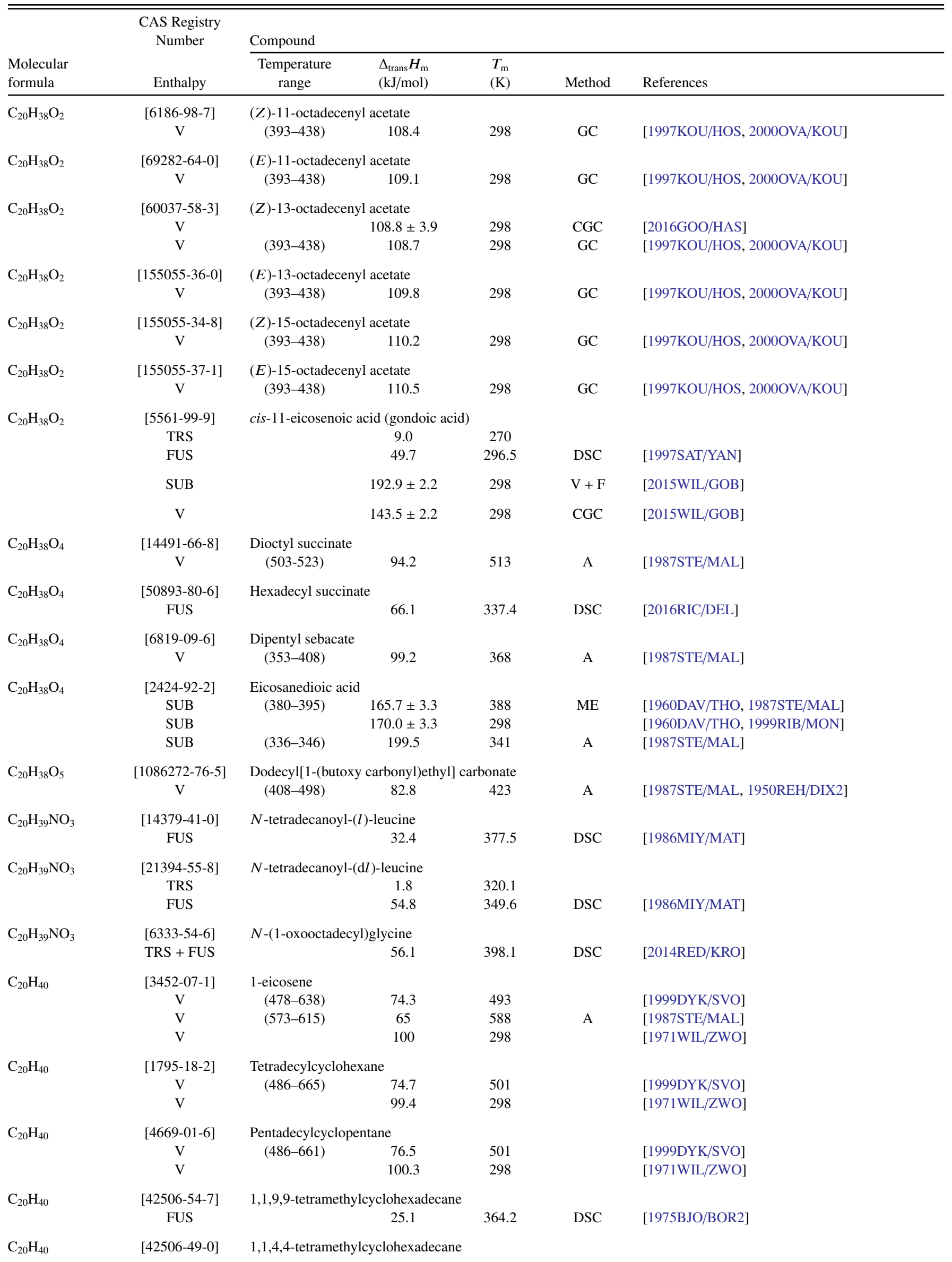


TABLE 14. Phase change enthalpies of $\mathrm{C}_{19}-\mathrm{C}_{29}$ organic compounds-Continued

\begin{tabular}{|c|c|c|c|c|c|c|}
\hline \multirow[b]{2}{*}{$\begin{array}{l}\text { Molecular } \\
\text { formula }\end{array}$} & \multirow{2}{*}{$\begin{array}{c}\text { CAS Registry } \\
\text { Number } \\
\text { Enthalpy }\end{array}$} & \multicolumn{5}{|l|}{ Compound } \\
\hline & & $\begin{array}{l}\text { Temperature } \\
\text { range }\end{array}$ & $\begin{array}{l}\Delta_{\text {trans }} H_{\mathrm{m}} \\
(\mathrm{kJ} / \mathrm{mol})\end{array}$ & $\begin{array}{l}T_{\mathrm{m}} \\
(\mathrm{K})\end{array}$ & Method & References \\
\hline & FUS & & 25.1 & 303.2 & $\mathrm{DSC}$ & [1975BJO/BOR2] \\
\hline $\mathrm{C}_{20} \mathrm{H}_{40}$ & $\begin{array}{l}{[54157-03-8]} \\
\text { FUS }\end{array}$ & 1,1-dimethylcycloo & $\begin{array}{c}\text { tadecane } \\
23.85\end{array}$ & 283.2 & & [1974BJO/BOR] \\
\hline $\mathrm{C}_{20} \mathrm{H}_{40} \mathrm{O}$ & $\begin{array}{c}{[60046-87-9]} \\
\mathrm{V}\end{array}$ & $\begin{array}{l}\text { 3,7,11,15-tetrameth } \\
\quad(439-468)\end{array}$ & $\begin{array}{l}y l-1 \text {-hexadec } \\
67.0 \pm 2.0\end{array}$ & 453 & Static & [1988BAG/GUR] \\
\hline $\mathrm{C}_{20} \mathrm{H}_{40} \mathrm{O}_{2}$ & $\begin{array}{c}{[822-23-1]} \\
\mathrm{V} \\
\mathrm{V}\end{array}$ & $\begin{array}{l}\text { Octadecyl acetate } \\
\quad(393-438) \\
(341-500)\end{array}$ & $\begin{array}{c}113.5 \\
94.3\end{array}$ & $\begin{array}{l}298 \\
356\end{array}$ & $\begin{array}{c}\mathrm{GC} \\
\mathrm{A}\end{array}$ & $\begin{array}{l}\text { [1997KOU/HOS, 2000OVA/KOU] } \\
\text { [1987STE/MAL] }\end{array}$ \\
\hline $\mathrm{C}_{20} \mathrm{H}_{40} \mathrm{O}_{2}$ & $\begin{array}{c}{[111-06-8]} \\
\mathrm{V}\end{array}$ & $\begin{array}{l}\text { Butyl palmitate } \\
\quad(353-383)\end{array}$ & 93.8 & 368 & A & [1987STE/MAL, 1958ROM/GOR] \\
\hline $\mathrm{C}_{20} \mathrm{H}_{40} \mathrm{O}_{2}$ & $\begin{array}{c}{[1654-86-0]} \\
\mathrm{V}\end{array}$ & $\begin{array}{l}\text { Decyl decanoate } \\
\quad(341-398)\end{array}$ & 97.8 & 356 & A & [1987STE/MAL] \\
\hline $\mathrm{C}_{20} \mathrm{H}_{40} \mathrm{O}_{2}$ & $\begin{array}{c}\text { [506-30-9] } \\
\text { TRS } \\
\text { TRS } \\
\text { FUS } \\
\text { FUS } \\
\text { FUS }\end{array}$ & Eicosanoic acid & $\begin{array}{c}4.1 \\
6.1 \\
71.6 \\
69.2 \\
72.0\end{array}$ & $\begin{array}{l}332.8 \\
333.3 \\
347.8 \\
348.2 \\
348.4\end{array}$ & $\begin{array}{l}\mathrm{DSC} \\
\mathrm{AC}\end{array}$ & $\begin{array}{l}\text { [2007MOR/COR] } \\
{[1996 \mathrm{DOM} / \mathrm{HEA}, 1982 \mathrm{SCH} / \mathrm{VAN}]} \\
{[1964 \mathrm{ADR} / \mathrm{DEK}]}\end{array}$ \\
\hline & SUB & $(305-323)$ & 148.4 & & TPTD & [2005CHA/ZIE] \\
\hline
\end{tabular}

[Note: Experimental values based on the TPTD method are often inconsistent with values determined using other experimental methods.]

\begin{tabular}{|c|c|c|c|c|c|c|}
\hline & SUB & $(337-346)$ & $199.6 \pm 7.5$ & 342 & ME & [1961DAV/MAL, 1970COX/PIL] \\
\hline & $\mathrm{V}$ & & $143.2 \pm 4.5$ & 298 & CGC & [2015WIL/GOB] \\
\hline & V & & $143.7 \pm 8.0$ & 298 & CGC & [2013WIL/CHI] \\
\hline & V & $(477-670)$ & 114.5 & 492 & A & [1987STE/MAL] \\
\hline & $\mathrm{V}$ & $(380-404)$ & 125.5 & 392 & ME, TE & [1982DEK/SCH] \\
\hline $\mathrm{C}_{20} \mathrm{H}_{40} \mathrm{O}_{2}$ & [111-61-5] & Ethyl stearate & & & & \\
\hline & FUS & & 59.81 & 307.4 & DSC & [2014ROB/BAR] \\
\hline & FUS & $(5-370)$ & 63.08 & 306.9 & $\mathrm{AC}$ & [2011AGA/VAR] \\
\hline & FUS & & 58.8 & 306.2 & DSC & [2003SUP/GOF] \\
\hline & FUS & & 59.83 & 307 & & [1967OMA] \\
\hline & FUS & & 59.87 & 304.3 & Cryst & [1934KIN/GAR] \\
\hline & SUB & $(297-306)$ & 161.4 & 301.5 & ME & [1987STE/MAL, 1967OMA] \\
\hline & $\mathrm{V}$ & & $109.3 \pm 3.2$ & 298 & CRT & [2015GOB/CHI] \\
\hline & V & & $109.7 \pm 0.3$ & 298 & CGC & [2015GOB/CHI] \\
\hline & $\mathrm{V}$ & $(355-412)$ & 93.5 & 383 & GS & [2012VER/RAL] \\
\hline & $\mathrm{V}$ & $(355-412)$ & $109.4 \pm 0.7$ & 298 & GS & [2012VER/RAL] \\
\hline & $\mathrm{V}$ & $(363-423)$ & 92.2 & 393 & TGA & [2012VER/RAL] \\
\hline & $\mathrm{V}$ & $(363-423)$ & $109.7 \pm 0.7$ & 298 & TGA & [2012VER/RAL] \\
\hline & V & $(491-534)$ & 101.7 & 513 & DSC & [2011SIL/FAL] \\
\hline & $\mathrm{V}$ & $(454-469)$ & 111.9 & 461 & A & [1987STE/MAL] \\
\hline & $\mathrm{V}$ & $(310-328)$ & 106.8 & 319 & A, ME & [1987STE/MAL, 1967OMA] \\
\hline $\mathrm{C}_{20} \mathrm{H}_{40} \mathrm{O}_{2}$ & [1731-94-8] & Methyl nonad & ate & & & \\
\hline & FUS & & 63.8 & 313.2 & DSC & [2004CHI/ZHA] \\
\hline & TRS & & 19.4 & 304.2 & & \\
\hline & FUS & & 42.8 & 313.2 & Cryst & [1936KIN/GAR] \\
\hline & $\mathrm{V}$ & $(467-558)$ & $109.5 \pm 5.4$ & 298 & CGC & [2004CHI/ZHA] \\
\hline & $\mathrm{V}$ & & 101.2 & 350 & $\mathrm{CE}$ & [2002VAN/VAN] \\
\hline & V & & $105.0 \pm 2.4$ & 326 & $\mathrm{CE}$ & [2002VAN/VAN] \\
\hline & $\mathrm{V}$ & & $109.5 \pm 2.7$ & 298 & $\mathrm{CE}$ & [2002VAN/VAN] \\
\hline & $\mathrm{V}$ & $(441-529)$ & 90.1 & 456 & $\mathrm{~A}, \mathrm{E}$ & [1987STE/MAL, 1963ROS/SCH] \\
\hline $\mathrm{C}_{20} \mathrm{H}_{40} \mathrm{O}_{2}$ & [20292-08-4] & 2-ethylhexyl li & & & & \\
\hline & $\mathrm{V}$ & $(371-452)$ & 91.4 & 386 & & [2001BUR/JOS] \\
\hline & V & $(443-503)$ & 104.5 & 298 & $\mathrm{GC}$ & [1997KRO/VEL] \\
\hline
\end{tabular}


TABLE 14. Phase change enthalpies of $\mathrm{C}_{19}-\mathrm{C}_{29}$ organic compounds-Continued

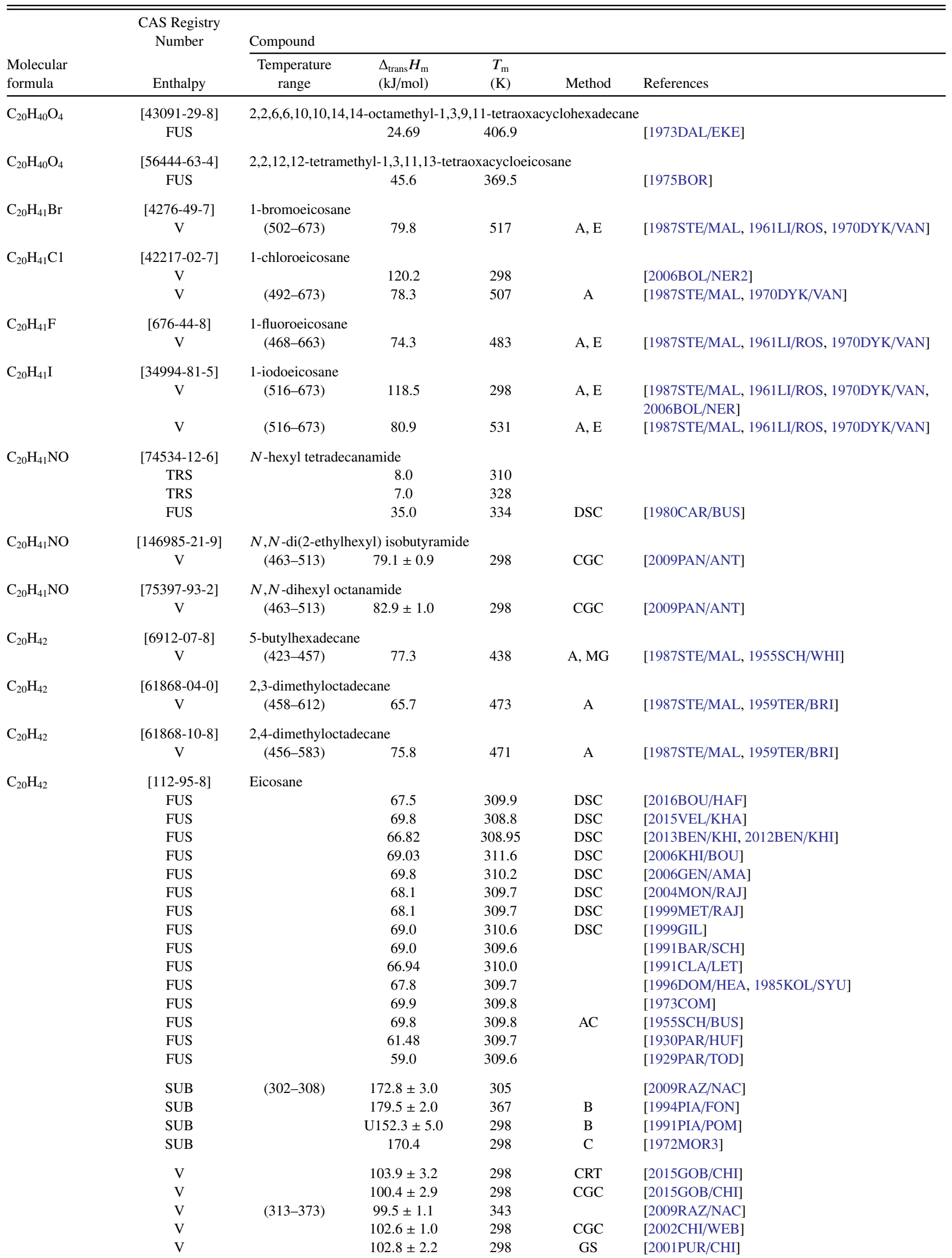


TABLE 14. Phase change enthalpies of $\mathrm{C}_{19}-\mathrm{C}_{29}$ organic compounds-Continued

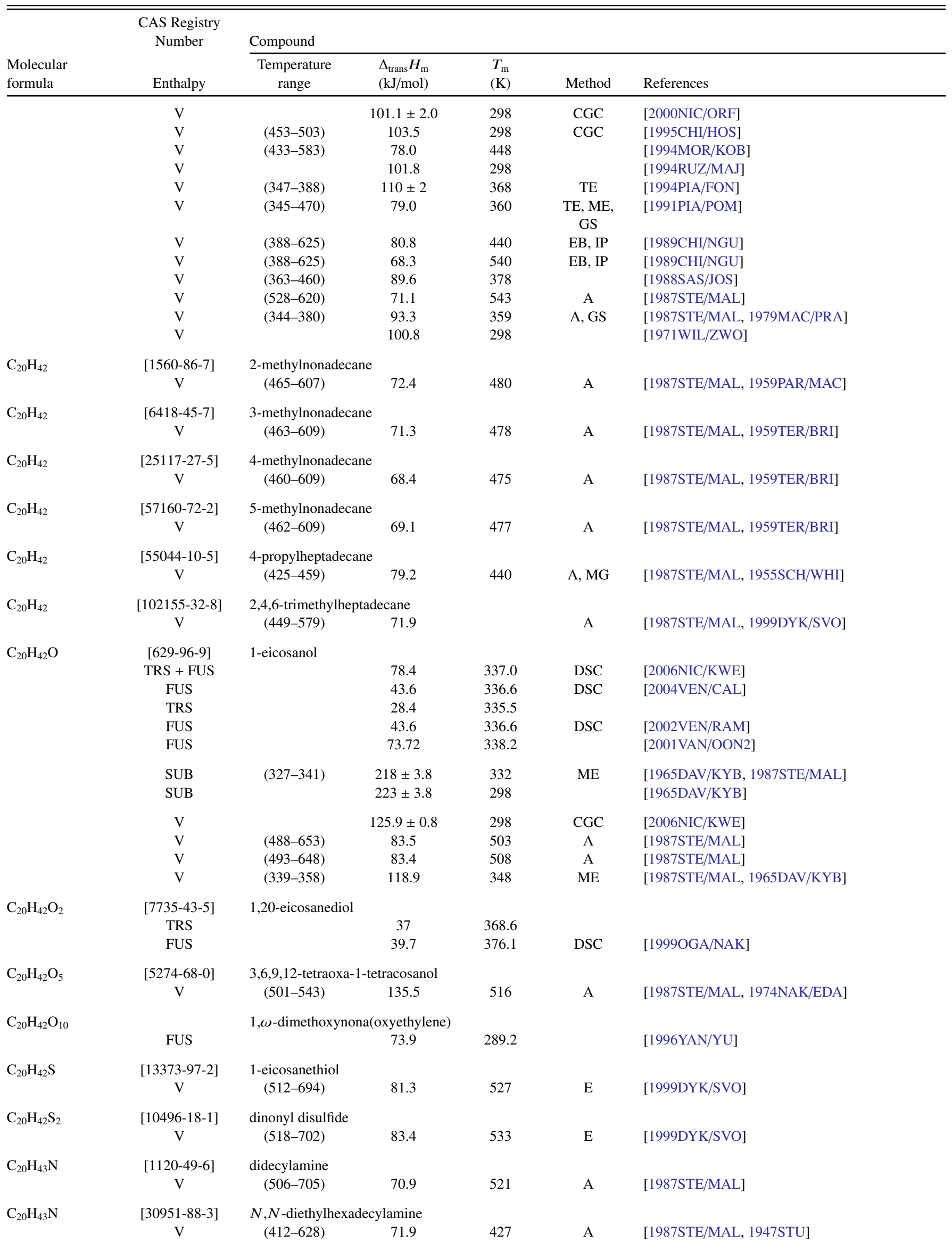


TABLE 14. Phase change enthalpies of $\mathrm{C}_{19}-\mathrm{C}_{29}$ organic compounds-Continued

\begin{tabular}{|c|c|c|c|c|c|c|}
\hline \multirow[b]{2}{*}{$\begin{array}{l}\text { Molecular } \\
\text { formula }\end{array}$} & \multirow{2}{*}{$\begin{array}{l}\text { CAS Registry } \\
\text { Number } \\
\text { Enthalpy }\end{array}$} & \multicolumn{5}{|l|}{ Compound } \\
\hline & & $\begin{array}{l}\text { Temperature } \\
\text { range }\end{array}$ & $\begin{array}{l}\Delta_{\text {trans }} H_{\mathrm{m}} \\
(\mathrm{kJ} / \mathrm{mol})\end{array}$ & $\begin{array}{l}T_{\mathrm{m}} \\
(\mathrm{K})\end{array}$ & Method & References \\
\hline $\mathrm{C}_{20} \mathrm{H}_{43} \mathrm{~N}$ & $\begin{array}{c}{[124-28-7]} \\
\mathrm{V}\end{array}$ & $\begin{array}{l}N, N \text {-dimethyl } \\
\quad(504-701)\end{array}$ & $\begin{array}{l}\text { lecylamine } \\
74.7\end{array}$ & 519 & A & [1987STE/MAL] \\
\hline $\mathrm{C}_{20} \mathrm{H}_{43} \mathrm{~N}$ & $\begin{array}{c}{[10525-37-8]} \\
\mathrm{V}\end{array}$ & $\begin{array}{l}\text { eicosylamine } \\
(543-659)\end{array}$ & 74.5 & 558 & A & [1987STE/MAL, 1956MAN2] \\
\hline $\mathrm{C}_{20} \mathrm{H}_{48} \mathrm{O}_{2}$ & $\begin{array}{c}\text { [302-79-4] } \\
\text { TRS } \\
\text { FUS (I) } \\
\text { FUS (II) }\end{array}$ & 3,7-dimethyl-9 & $\begin{array}{c}\text { 6-trimethyl- } \\
3.2 \\
37.1 \\
36.8\end{array}$ & $\begin{array}{r}\text { ohexen- } \\
419.8 \\
456.9 \\
456.3\end{array}$ & $2,4,6,8-n o$ & $\begin{array}{l}\text { etraenoic acid (retinoic acid) } \\
\text { [2006CAV/PAN] } \\
\text { [2006CAV/PAN] }\end{array}$ \\
\hline $\mathrm{C}_{21} \mathrm{H}_{6} \mathrm{~N}_{12} \mathrm{O}_{18}$ & $\begin{array}{c}\text { [49753-54-0] } \\
\text { SUB }\end{array}$ & $\begin{array}{l}2,4,6-\operatorname{tris}(2,4,6 \\
\quad(479-551)\end{array}$ & $\begin{array}{c}\text { trophenyl)-1 } \\
167.9\end{array}$ & $\begin{array}{r}\text { iazine } \\
494\end{array}$ & A & [1987STE/MAL, 1975COV] \\
\hline $\mathrm{C}_{21} \mathrm{H}_{8} \mathrm{~F}_{28} \mathrm{O}_{8}$ & $\begin{array}{c}\text { [464-40-4] } \\
\mathrm{V}\end{array}$ & $\begin{array}{c}\text { pentaerythritol, } \\
\quad(293-433)\end{array}$ & $\begin{array}{l}\text { perfluorobut } \\
35.5\end{array}$ & 308 & $\mathrm{I}, \mathrm{A}$ & [1987STE/MAL, 1957DOB/KEL] \\
\hline $\mathrm{C}_{21} \mathrm{H}_{13} \mathrm{~F}_{13} \mathrm{OS}$ & $\begin{array}{c}\text { [246543-94-2] } \\
\text { FUS } \\
\text { FUS }\end{array}$ & 2-(perfluoro- $n$ - & $\begin{array}{l}\text { 1)ethylthiom } \\
40.6 \\
40.4\end{array}$ & $\begin{array}{l}\text { oipheny } \\
344.8 \\
344.8\end{array}$ & $\begin{array}{l}\text { ether } \\
\text { DTA }\end{array}$ & $\begin{array}{l}\text { [1999DEG/GUI] } \\
\text { [1999TAF/GUI, 1999DEG/GUI] }\end{array}$ \\
\hline $\mathrm{C}_{21} \mathrm{H}_{13} \mathrm{~N}$ & $\begin{array}{c}\text { [215-62-3] } \\
\text { FUS }\end{array}$ & $\operatorname{Dibenz}[a, c] \mathrm{ac}$ & 27.8 & 477.4 & $\mathrm{DSC}$ & [2010KES/AUC] \\
\hline $\mathrm{C}_{21} \mathrm{H}_{13} \mathrm{~N}$ & $\begin{array}{c}\text { [226-36-8] } \\
\text { FUS }\end{array}$ & $\operatorname{Dibenz}[a, h] \mathrm{ac}$ & 30.6 & 499.7 & DSC & [2010KES/AUC] \\
\hline $\mathrm{C}_{21} \mathrm{H}_{13} \mathrm{~N}$ & $\begin{array}{c}\text { [226-92-6] } \\
\text { FUS }\end{array}$ & $\operatorname{Dibenz}[a, i] \mathrm{ac}$ & 29.6 & 483.5 & DSC & [2010KES/AUC] \\
\hline $\mathrm{C}_{21} \mathrm{H}_{13} \mathrm{~N}$ & $\begin{array}{c}\text { [224-42-0] } \\
\text { FUS }\end{array}$ & $\operatorname{Dibenz}[a, j] \mathrm{ac}$ & 25.5 & 492.7 & $\mathrm{DSC}$ & [2010KES/AUC] \\
\hline $\mathrm{C}_{21} \mathrm{H}_{14} \mathrm{~N}_{2} \mathrm{O}_{3}$ & $\begin{array}{c}\text { [13494-38-7] } \\
\text { SUB }\end{array}$ & 2-phenyl-3-ben & $\begin{array}{l}\text { quinoxaline- } \\
167.4 \pm 4.0\end{array}$ & $\begin{array}{l}\text { pxide } \\
298\end{array}$ & ME & [1997ACR/POW] \\
\hline $\mathrm{C}_{21} \mathrm{H}_{14} \mathrm{~N}_{2} \mathrm{O}_{3}$ & $\begin{array}{c}\text { [5166-47-2] } \\
\text { SUB }\end{array}$ & 1,4-diamino-2- & $\begin{array}{c}\text { pyl-9,10-antl } \\
168.5\end{array}$ & none & & [1984KAR/KRU] \\
\hline $\mathrm{C}_{21} \mathrm{H}_{15} \mathrm{BrN}_{2} \mathrm{O}_{2}$ & $\begin{array}{c}{[128-83-6]} \\
\text { SUB }\end{array}$ & $\begin{array}{c}\text { 1-amino-2-bror } \\
(418-438)\end{array}$ & $\begin{array}{l}-[(4-\text { methylp } \\
167.0 \pm 6.0\end{array}$ & $\begin{array}{c}\text { amino] } \\
428\end{array}$ & anthraquir & [1984KRI] \\
\hline $\mathrm{C}_{21} \mathrm{H}_{15} \mathrm{~F}_{13} \mathrm{~S}$ & $\begin{array}{c}{[246543-97-5]} \\
\text { FUS }\end{array}$ & 2-(perfluoro- $n$ & $\begin{array}{l}\text { 1)ethylthiom } \\
53.1\end{array}$ & $\begin{array}{l}\text { oipheny } \\
332.9\end{array}$ & DTA & [1999TAF/GUI, 1999DEG/GUI] \\
\hline $\mathrm{C}_{21} \mathrm{H}_{15} \mathrm{NO}_{2}$ & $\begin{array}{c}\text { [158749-37-2] } \\
\text { FUS }\end{array}$ & 2-methylpheny & $\begin{array}{c}\text { dine-9-carbo } \\
30.5\end{array}$ & 415 & $\mathrm{DSC}$ & [2010KRZ/MAL] \\
\hline $\mathrm{C}_{21} \mathrm{H}_{15} \mathrm{NO}_{2}$ & $\begin{array}{c}\text { [158749-58-7] } \\
\text { FUS }\end{array}$ & 3-methylpheny & $\begin{array}{c}\text { dine-9-carbo } \\
32\end{array}$ & 429 & $\mathrm{DSC}$ & [2010KRZ/MAL] \\
\hline $\mathrm{C}_{21} \mathrm{H}_{15} \mathrm{NO}_{2}$ & $\begin{array}{c}\text { [158749-59-8] } \\
\text { FUS }\end{array}$ & 4-methylpheny & $\begin{array}{c}\text { dine-9-carbo } \\
30.7\end{array}$ & 446 & DSC & [2010KRZ/MAL] \\
\hline $\mathrm{C}_{21} \mathrm{H}_{15} \mathrm{NO}_{3}$ & SUB & $\begin{array}{l}\text { 2-hydroxy-4-[( } \\
\text { (349-378) }\end{array}$ & $\begin{array}{l}\text { thylphenyl)a } \\
121.0 \pm 7.6\end{array}$ & $\begin{array}{c}9,10-\mathrm{a} \\
363\end{array}$ & uinone & [1984KRI] \\
\hline
\end{tabular}

[Note: Compound is listed as the 2-hydroxy-derivative in the paper; however, it is listed as the 1-hydroxy-derivative in Chem. Abstracts.]

$\mathrm{C}_{21} \mathrm{H}_{15} \mathrm{NO}_{3} \quad$ [1228096-42-1] 2-methoxyphenyl acridine-9-carboxylate

\begin{tabular}{|c|c|c|c|c|c|c|}
\hline & FUS & & 38.8 & 462 & DSC & [2010ZAD/KRZ] \\
\hline \multirow[t]{2}{*}{$\mathrm{C}_{21} \mathrm{H}_{15} \mathrm{~N}_{3}$} & [493-77-6] & 2,4,6-triphen & ine & & & \\
\hline & SUB & & $150.7 \pm 1.2$ & 298 & ME & [2011LIM/COS] \\
\hline \multirow[t]{3}{*}{$\mathrm{C}_{21} \mathrm{H}_{16}$} & {$[56-49-5]$} & 3-methylchol & & & & \\
\hline & SUB & $(401-425)$ & $127.2 \pm 2.4$ & 413 & A & [1987STE/MAL, 1964KEL/RIC] \\
\hline & V & $(323-473)$ & 93.8 & 398 & GC & [2002LEI/CHA] \\
\hline \multirow[t]{2}{*}{$\mathrm{C}_{21} \mathrm{H}_{16}$} & [611-48-3] & $1,2^{\prime}$-dinaphth & hane & & & \\
\hline & FUS & $(12-423)$ & 30.54 & 369.6 & & [1996DOM/HEA, 1977FIN/MES] \\
\hline
\end{tabular}


TABLE 14. Phase change enthalpies of $\mathrm{C}_{19}-\mathrm{C}_{29}$ organic compounds-Continued

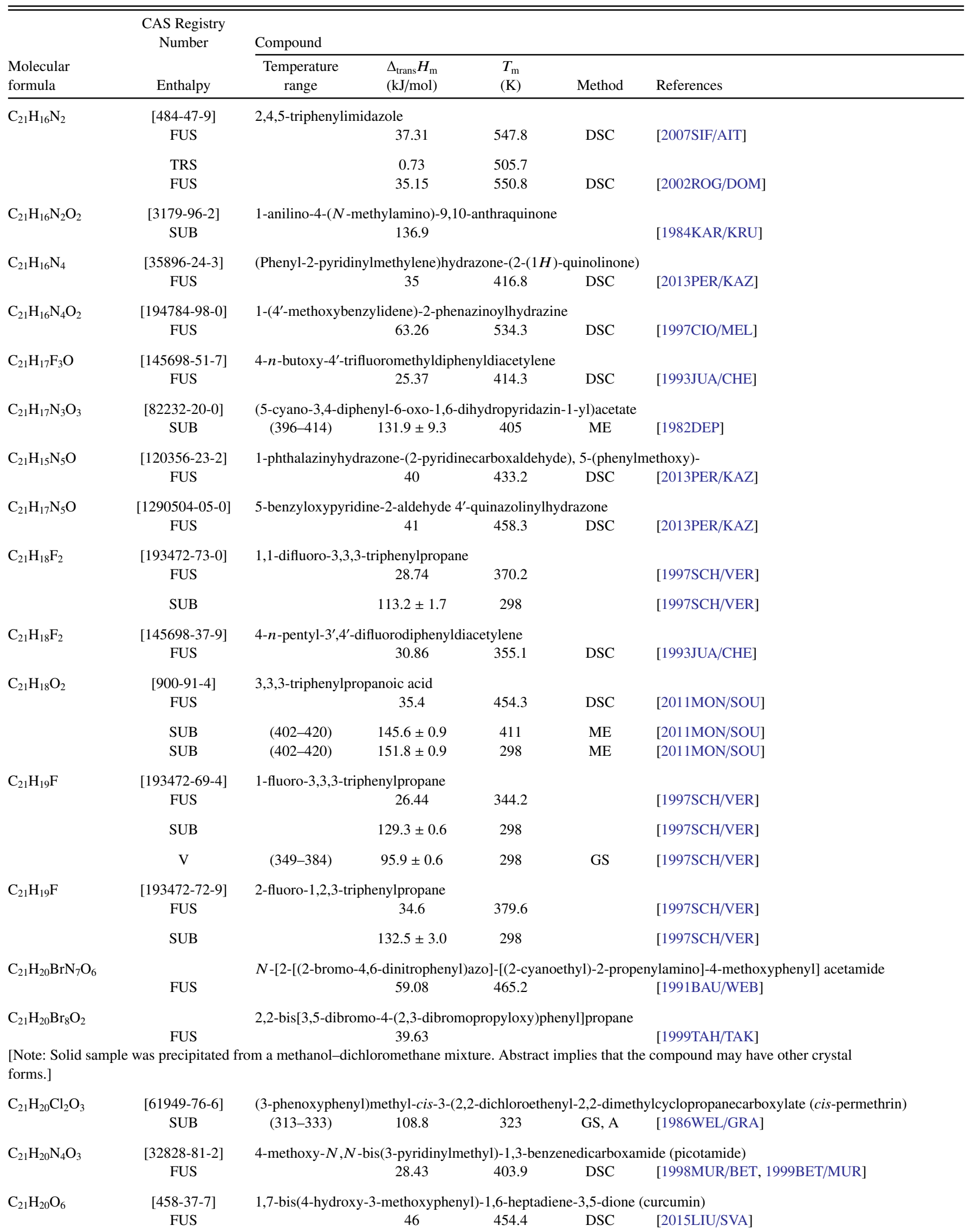


TABLE 14. Phase change enthalpies of $\mathrm{C}_{19}-\mathrm{C}_{29}$ organic compounds-Continued

\begin{tabular}{|c|c|c|c|c|c|c|}
\hline \multirow[b]{2}{*}{$\begin{array}{l}\text { Molecular } \\
\text { formula }\end{array}$} & \multirow{2}{*}{$\begin{array}{l}\text { CAS Registry } \\
\text { Number } \\
\text { Enthalpy }\end{array}$} & \multicolumn{5}{|l|}{ Compound } \\
\hline & & $\begin{array}{c}\text { Temperature } \\
\text { range }\end{array}$ & $\begin{array}{l}\Delta_{\text {trans }} H_{\mathrm{m}} \\
(\mathrm{kJ} / \mathrm{mol})\end{array}$ & $\begin{array}{l}T_{\mathrm{m}} \\
(\mathrm{K})\end{array}$ & Method & References \\
\hline \multirow[t]{2}{*}{$\mathrm{C}_{21} \mathrm{H}_{20} \mathrm{O}_{12}$} & [21637-25-2] & \multicolumn{5}{|c|}{ 2-(3,4-dihydroxyphenyl)-3-( $\beta$-D-glucofuranosyloxy)-5,7-dihydroxy-4H-1-benzopyran-4-one (isoquercitrin) } \\
\hline & FUS & & 49.8 & 471.2 & DSC & [2007CHE/HUM] \\
\hline \multirow{5}{*}{$\mathrm{C}_{21} \mathrm{H}_{21} \mathrm{~N}$} & [1159-53-1] & \multicolumn{5}{|c|}{ 4-methyl- $N, N$-bis(4-methylphenyl)benzenamine } \\
\hline & FUS & & 19.95 & 388.8 & DSC & [2006MAN/ROH] \\
\hline & FUS & & 22.96 & 388 & DSC & [2005MAN/ROH] \\
\hline & SUB & & 92.53 & & DSC & [2006MAN/ROH] \\
\hline & $\mathrm{V}$ & & 72.57 & & DSC & [2006MAN/ROH] \\
\hline \multirow[t]{4}{*}{$\mathrm{C}_{21} \mathrm{H}_{21} \mathrm{~N}$} & {$[117597-62-3]$} & \multicolumn{5}{|c|}{$N$-(3-methylphenyl)- $N, N$-bis(4-methylphenyl)amine } \\
\hline & FUS & & 21.71 & 329.9 & DSC & [2006MAN/ROH] \\
\hline & SUB & & 97.64 & & DSC & [2006MAN/ROH] \\
\hline & $\mathrm{V}$ & & 75.93 & & DSC & [2006MAN/ROH] \\
\hline \multirow[t]{4}{*}{$\mathrm{C}_{21} \mathrm{H}_{21} \mathrm{~N}$} & {$[97413-60-0]$} & \multicolumn{5}{|c|}{$N, N$-bis(3-methylphenyl)- $N$-(4-methylphenyl)amine } \\
\hline & FUS & & 26.39 & 362.7 & DSC & [2006MAN/ROH] \\
\hline & SUB & & 95.69 & & DSC & [2006MAN/ROH] \\
\hline & $\mathrm{V}$ & & 69.29 & & DSC & {$[2006 \mathrm{MAN} / \mathrm{ROH}]$} \\
\hline \multirow{4}{*}{$\mathrm{C}_{21} \mathrm{H}_{21} \mathrm{~N}$} & {$[20676-79-3]$} & \multicolumn{5}{|c|}{$N, N, N$-tris(3-methylphenyl)amine } \\
\hline & FUS & & 13.07 & 313 & DSC & [2006MAN/ROH] \\
\hline & SUB & & 50.66 & & DSC & [2006MAN/ROH] \\
\hline & $\mathrm{V}$ & & 37.59 & & DSC & [2006MAN/ROH] \\
\hline \multirow[t]{4}{*}{$\mathrm{C}_{21} \mathrm{H}_{21} \mathrm{~N}$} & [620-40-6] & \multicolumn{5}{|l|}{ Tribenzylamine } \\
\hline & TRS & & 1.1 & 342.5 & & \\
\hline & FUS & & 21.4 & 365.6 & DSC & [2014GOB/VIK] \\
\hline & $\mathrm{V}$ & & $92.4 \pm 1.4$ & 298 & CGC & [2014GOB/VIK] \\
\hline \multirow[t]{2}{*}{$\mathrm{C}_{21} \mathrm{H}_{21} \mathrm{NO}$} & [957-51-7] & \multicolumn{5}{|c|}{$N, N$-dimethyl-2,2-diphenylbenzeneacetamide } \\
\hline & FUS & & 25.43 & 402 & DSC & {$[1990 \mathrm{DON} / \mathrm{DRE}]$} \\
\hline \multirow[t]{2}{*}{$\mathrm{C}_{21} \mathrm{H}_{21} \mathrm{O}_{3} \mathrm{P}$} & {$[855-38-9]$} & \multicolumn{5}{|c|}{ Tris(4-methoxyphenyl)phosphine } \\
\hline & FUS & & 25.67 & 403.82 & DSC & [2010GUO/WAN2] \\
\hline \multirow[t]{3}{*}{$\mathrm{C}_{21} \mathrm{H}_{21} \mathrm{O}_{4} \mathrm{P}$} & {$[78-30-8]$} & \multicolumn{5}{|c|}{ Phosphoric acid, tris(2-tolyl) ester } \\
\hline & $\mathrm{V}$ & $(383-413)$ & 99.3 & 398 & GC-RT & [2014BRO/JAN] \\
\hline & V & $(293-700)$ & 86.8 & 308 & $\mathrm{~A}, \mathrm{I}$ & [1987STE/MAL, 1957DOB/KEL] \\
\hline $\mathrm{C}_{21} \mathrm{H}_{21} \mathrm{O}_{4} \mathrm{P}$ & {$[563-04-2]$} & Phosphoric acic & (3-tolyl) es & & & \\
\hline & V & $(383-413)$ & 103.7 & 398 & GC-RT & [2014BRO/JAN] \\
\hline & V & $(398-530)$ & 123.2 & 413 & A & {$[1987 \mathrm{STE} / \mathrm{MAL}]$} \\
\hline $\mathrm{C}_{21} \mathrm{H}_{21} \mathrm{O}_{4} \mathrm{P}$ & {$[78-32-0]$} & Phosphoric acic & (4-tolyl) es & & & \\
\hline & V & $(383-413)$ & 105.7 & 398 & GC-RT & [2014BRO/JAN] \\
\hline & $\mathrm{V}$ & $(388-530)$ & 104.9 & 408 & A & [1987STE/MAL] \\
\hline $\mathrm{C}_{21} \mathrm{H}_{21} \mathrm{P}$ & {$[1038-95-5]$} & Tris(4-tolyl)phc & & & & \\
\hline & FUS & & 28.52 & 417.84 & DSC & [2010GUO/WAN2] \\
\hline & $\mathrm{V}$ & $(372-394)$ & $126 \pm 5$ & 385 & $\mathrm{ME}, \mathrm{TE}$ & [1981DEK/HER] \\
\hline $\mathrm{C}_{21} \mathrm{H}_{23} \mathrm{BrFNO}_{2}$ & {$[10457-90-6]$} & 1-(4-fluorophen & -[4-hydrox & bromoph & -1-piperid & 1]-1-butanone (bromoperidol) \\
\hline & FUS & & 50.8 & 432.7 & DSC & [1981DRA/AZI] \\
\hline $\mathrm{C}_{21} \mathrm{H}_{23} \mathrm{ClFNO}_{2}$ & {$[52-86-8]$} & 1-(4-fluorophen & -[4-hydrox & chloroph & -1-piperid & 1]-1-butanone (haloperidol) \\
\hline & FUS & & 54.26 & 425 & DSC & [2010BAI/VAN] \\
\hline & FUS & & 48 & 422.7 & $\mathrm{DSC}$ & [1981DRA/AZI] \\
\hline $\mathrm{C}_{21} \mathrm{H}_{23} \mathrm{~F}_{2} \mathrm{NO}_{2}$ & {$[803-45-2]$} & 1-(4-fluorophen & -[4-hydrox & fluoroph & -1-piperidi & 1]-1-butanone \\
\hline & FUS & & 34 & 395.2 & DSC & [1981DRA/AZI] \\
\hline $\mathrm{C}_{21} \mathrm{H}_{23} \mathrm{NO}_{5}$ & {$[561-27-3]$} & Diacetylmorphi & leroin) & & & \\
\hline
\end{tabular}


TABLE 14. Phase change enthalpies of $\mathrm{C}_{19}-\mathrm{C}_{29}$ organic compounds-Continued

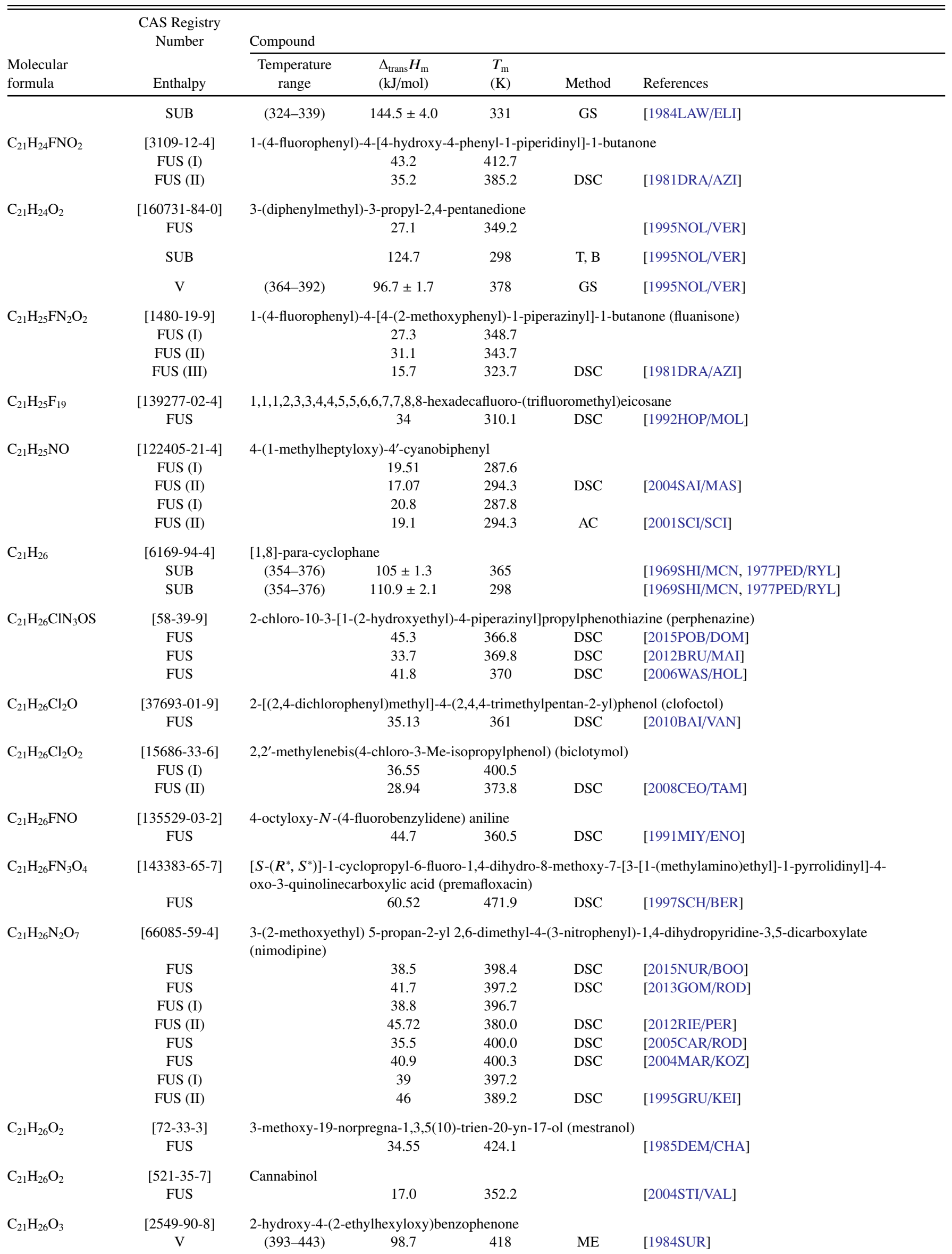


TABLE 14. Phase change enthalpies of $\mathrm{C}_{19}-\mathrm{C}_{29}$ organic compounds-Continued

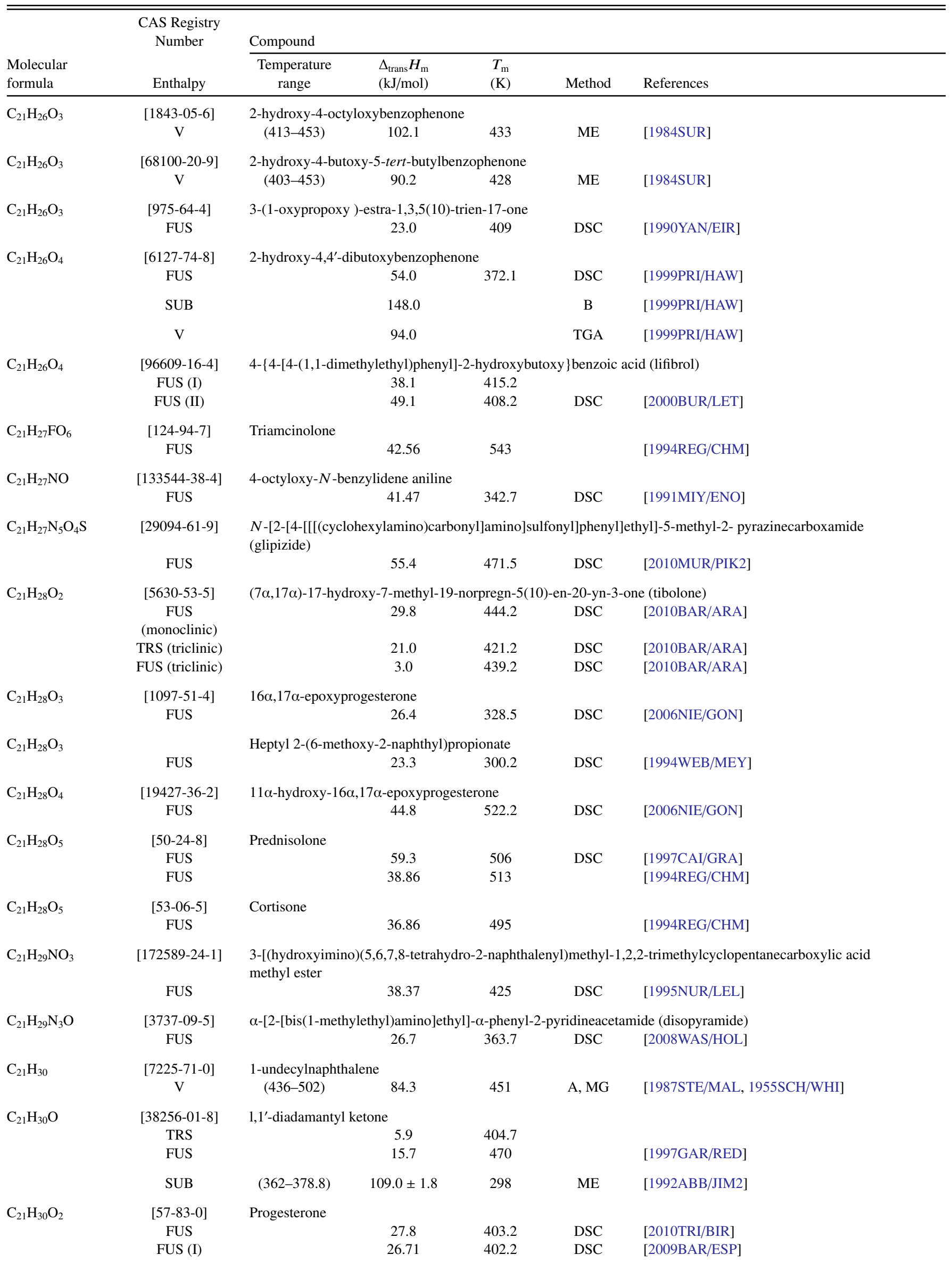


TABLE 14. Phase change enthalpies of $\mathrm{C}_{19}-\mathrm{C}_{29}$ organic compounds-Continued

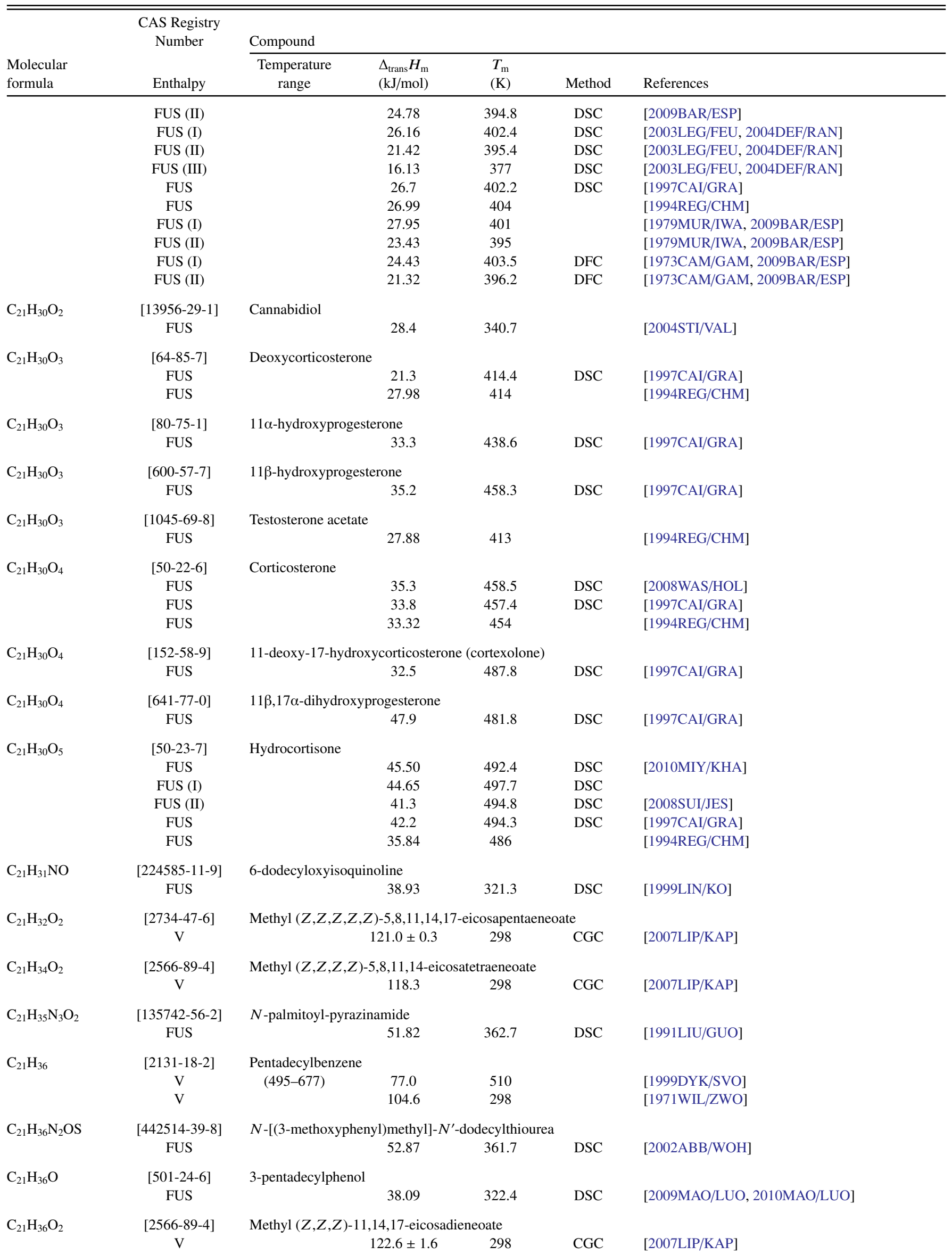


TABLE 14. Phase change enthalpies of $\mathrm{C}_{19}-\mathrm{C}_{29}$ organic compounds-Continued

\begin{tabular}{|c|c|c|c|c|c|c|}
\hline \multirow[b]{2}{*}{$\begin{array}{l}\text { Molecular } \\
\text { formula }\end{array}$} & \multirow{2}{*}{$\begin{array}{c}\text { CAS Registry } \\
\text { Number } \\
\text { Enthalpy }\end{array}$} & \multicolumn{5}{|l|}{ Compound } \\
\hline & & $\begin{array}{l}\text { Temperature } \\
\text { range }\end{array}$ & $\begin{array}{l}\Delta_{\text {trans }} H_{\mathrm{m}} \\
(\mathrm{kJ} / \mathrm{mol})\end{array}$ & $\begin{array}{l}T_{\mathrm{m}} \\
(\mathrm{K})\end{array}$ & Method & References \\
\hline \multirow[t]{2}{*}{$\mathrm{C}_{21} \mathrm{H}_{36} \mathrm{O}_{6}$} & & \multicolumn{5}{|c|}{ Triisopentyl-trans-aconitate } \\
\hline & $\mathrm{V}$ & $(396-499)$ & 88.3 & 411 & A & [1987STE/MAL, 1953MAG/MOD] \\
\hline \multirow[t]{2}{*}{$\mathrm{C}_{21} \mathrm{H}_{36} \mathrm{O}_{6}$} & [64617-29-4] & \multicolumn{5}{|c|}{ Tripentyl-trans-aconitate } \\
\hline & V & $(403-505)$ & 91.4 & 418 & A & [1987STE/MAL, 1953MAG/MOD] \\
\hline $\mathrm{C}_{21} \mathrm{H}_{38} \mathrm{O}_{2}$ & $\begin{array}{c}{[61012-46-2]} \\
\mathrm{V}\end{array}$ & \multicolumn{4}{|c|}{ Methyl $(Z, Z)$-11,14-eicosadieneoate } & [2007LIP/KAP] \\
\hline $\mathrm{C}_{21} \mathrm{H}_{38} \mathrm{O}_{4}$ & $\begin{array}{c}\text { [59223-30-2] } \\
\text { FUS }\end{array}$ & Hexadecyl itac & 74.6 & 358.8 & DSC & [2016RIC/DEL] \\
\hline $\mathrm{C}_{21} \mathrm{H}_{38} \mathrm{O}_{6}$ & $\begin{array}{c}{[621-70-5]} \\
\mathrm{V} \\
\mathrm{V} \\
\mathrm{V}\end{array}$ & $\begin{array}{c}\text { Glycerol tricap } \\
(356-410)\end{array}$ & $\begin{array}{c}99.9 \\
108.3 \pm 3.8 \\
94.2\end{array}$ & $\begin{array}{l}349 \\
298 \\
371\end{array}$ & $\begin{array}{l}\text { TGA } \\
\text { TGA } \\
\text { A, T }\end{array}$ & $\begin{array}{l}\text { [1990KIS/SHO] } \\
\text { [1990KIS/SHO] } \\
\text { [1987STE/MAL, 1949PER/WEB2] }\end{array}$ \\
\hline $\mathrm{C}_{21} \mathrm{H}_{38} \mathrm{O}_{6}$ & $\begin{array}{c}{[5333-56-2]} \\
\mathrm{V}\end{array}$ & $\begin{array}{l}\text { Triisopentyl 1,2 } \\
\quad(396-508)\end{array}$ & $\begin{array}{c}\text { propanetricarb } \\
88.2\end{array}$ & 411 & A & [1987STE/MAL, 1953MAG/MOD] \\
\hline $\mathrm{C}_{21} \mathrm{H}_{38} \mathrm{O}_{6}$ & $\begin{array}{c}{[5333-53-9]} \\
\mathrm{V}\end{array}$ & $\begin{array}{l}\text { Tripentyl 1,2,3 } \\
\quad(404-508)\end{array}$ & $\begin{array}{c}\text { panetricarbox } \\
90.2\end{array}$ & 419 & A & [1987STE/MAL, 1953MAG/MOD] \\
\hline $\mathrm{C}_{21} \mathrm{H}_{40}$ & $\begin{array}{c}{[66326-27-0]} \\
\mathrm{V}\end{array}$ & $\begin{array}{l}\text { 1-undecyldecal } \\
\text { (426-488) }\end{array}$ & $\begin{array}{c}\text { onaphthalene } \\
83.3\end{array}$ & 411 & A & [1987STE/MAL] \\
\hline $\mathrm{C}_{21} \mathrm{H}_{40}$ & $\begin{array}{c}\text { [95115-75-6] } \\
\text { FUS }\end{array}$ & trans-2-heptyl- & $\begin{array}{c}\text { ityldecalin } \\
31.8\end{array}$ & 295.3 & & [1985VAR/BRI] \\
\hline $\mathrm{C}_{21} \mathrm{H}_{40}$ & $\begin{array}{c}\text { [95115-78-9] } \\
\text { FUS }\end{array}$ & trans-2-heptyl- & $\begin{array}{c}\text { tyldecalin } \\
41\end{array}$ & 308.8 & & [1985VAR/BRI] \\
\hline $\mathrm{C}_{21} \mathrm{H}_{40} \mathrm{O}_{2}$ & $\begin{array}{c}\text { [2390-09-2] } \\
\mathrm{V}\end{array}$ & Methyl (Z)-11 & $115.8 \pm 0.7$ & 298 & CGC & [2007LIP/KAP] \\
\hline $\mathrm{C}_{21} \mathrm{H}_{40} \mathrm{O}_{2}$ & $\begin{array}{c}\text { [4813-57-4] } \\
\text { FUS }\end{array}$ & Octadecyl acry & 37.3 & 298.9 & DSC & [1992BAB/HWA] \\
\hline $\mathrm{C}_{21} \mathrm{H}_{41} \mathrm{NO}_{3}$ & $\begin{array}{l}{[45287-42-1]} \\
\text { TRS } \\
\text { FUS }\end{array}$ & $N$-hexadecano & $\begin{array}{r}\text {-valine } \\
29.1 \\
54.8\end{array}$ & $\begin{array}{l}349.1 \\
366.6\end{array}$ & DSC & [1986MIY/MAT] \\
\hline $\mathrm{C}_{21} \mathrm{H}_{41} \mathrm{NO}_{3}$ & $\begin{array}{c}\text { [83871-20-9] } \\
\text { FUS }\end{array}$ & $N$-hexadecano & $\begin{array}{r}l l) \text {-valine } \\
80.5\end{array}$ & 375.1 & $\mathrm{DSC}$ & [1986MIY/MAT] \\
\hline $\mathrm{C}_{21} \mathrm{H}_{41} \mathrm{NO}_{3}$ & $\begin{array}{c}\text { [914224-77-4] } \\
\text { TRS + FUS }\end{array}$ & $N$-(1-oxonona & $\begin{array}{r}\text { 1)glycine } \\
60.3\end{array}$ & 398.9 & $\mathrm{DSC}$ & [2014RED/KRO] \\
\hline $\mathrm{C}_{21} \mathrm{H}_{42}$ & $\begin{array}{c}\text { [1599-68-4] } \\
\mathrm{V}\end{array}$ & $\begin{array}{l}\text { 1-heneicosene } \\
\text { (392-628) }\end{array}$ & 92.8 & 407 & & [1999DYK/SVO] \\
\hline $\mathrm{C}_{21} \mathrm{H}_{42}$ & $\begin{array}{c}{[6812-39-1]} \\
\mathrm{V} \\
\mathrm{V}\end{array}$ & $\begin{array}{l}\text { Hexadecylcycl } \\
\quad(498-674)\end{array}$ & $\begin{array}{r}79.2 \\
105.3\end{array}$ & $\begin{array}{l}513 \\
298\end{array}$ & & $\begin{array}{l}\text { [1999DYK/SVO] } \\
\text { [1971WIL/ZWO] }\end{array}$ \\
\hline $\mathrm{C}_{21} \mathrm{H}_{42}$ & $\begin{array}{l}\text { [6006-95-7] } \\
\text { FUS } \\
\text { FUS }\end{array}$ & Pentadecylcycl & $\begin{array}{r}\text { xane } \\
58.7 \\
58.3\end{array}$ & 298.2 & $\begin{array}{l}\mathrm{DSC} \\
\mathrm{DSC}\end{array}$ & $\begin{array}{l}{[2001 \mathrm{YOU} / \mathrm{SCH}]} \\
{[2000 \mathrm{YOU} / \mathrm{DOL}]}\end{array}$ \\
\hline & $\begin{array}{l}\text { V } \\
\text { V }\end{array}$ & $(496-677)$ & $\begin{array}{c}77.2 \\
104.4\end{array}$ & $\begin{array}{l}511 \\
298\end{array}$ & & $\begin{array}{l}\text { [1999DYK/SVO] } \\
\text { [1971WIL/ZWO] }\end{array}$ \\
\hline $\mathrm{C}_{21} \mathrm{H}_{42} \mathrm{~N}_{3} \mathrm{PS}_{6}$ & $\begin{array}{c}{[100575-31-3]} \\
\text { SUB }\end{array}$ & Tris(dipropyldi & $\begin{array}{c}\text { carbamate)ph } \\
127.4 \pm 4.2\end{array}$ & rous & DSC, E & [1999NEV/GOU] \\
\hline $\mathrm{C}_{21} \mathrm{H}_{42} \mathrm{O}$ & $\begin{array}{c}\text { [22589-04-4] } \\
\text { FUS }\end{array}$ & 2-heneicosanor & 77.65 & 333.9 & $\mathrm{DSC}$ & [1993VIL/HAM] \\
\hline $\mathrm{C}_{21} \mathrm{H}_{42} \mathrm{O}$ & $\begin{array}{c}\text { [19781-72-7] } \\
\text { FUS }\end{array}$ & 11-heneicosanc & 76.2 & 336.7 & & [1993RUE/SAR] \\
\hline $\mathrm{C}_{21} \mathrm{H}_{42} \mathrm{O}_{2}$ & [1120-28-1] & Methyl eicosan & & & & \\
\hline
\end{tabular}


TABLE 14. Phase change enthalpies of $\mathrm{C}_{19}-\mathrm{C}_{29}$ organic compounds-Continued

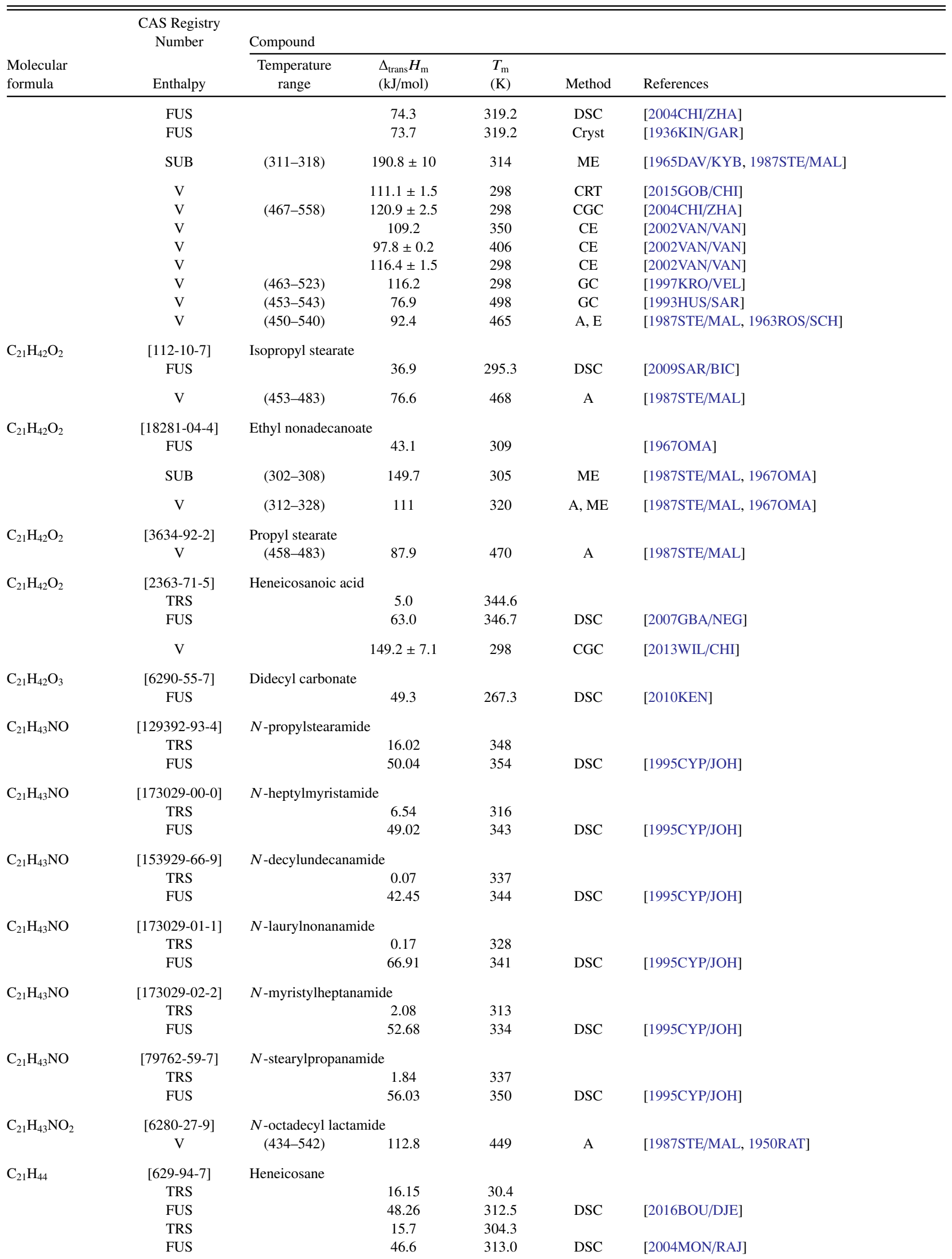


TABLE 14. Phase change enthalpies of $\mathrm{C}_{19}-\mathrm{C}_{29}$ organic compounds-Continued

\begin{tabular}{|c|c|c|c|c|c|c|}
\hline \multirow[b]{2}{*}{$\begin{array}{l}\text { Molecular } \\
\text { formula }\end{array}$} & \multirow{2}{*}{$\begin{array}{l}\text { CAS Registry } \\
\text { Number } \\
\text { Enthalpy }\end{array}$} & \multicolumn{5}{|l|}{ Compound } \\
\hline & & $\begin{array}{l}\text { Temperature } \\
\text { range }\end{array}$ & $\begin{array}{l}\Delta_{\text {trans }} H_{\mathrm{m}} \\
(\mathrm{kJ} / \mathrm{mol})\end{array}$ & $\begin{array}{l}T_{\mathrm{m}} \\
(\mathrm{K})\end{array}$ & Method & References \\
\hline & TRS & & 13.36 & 304.0 & & \\
\hline & FUS & & 45.21 & 313.2 & DSC & [2001CHE/BOU] \\
\hline & FUS & & 46.6 & 313.0 & DSC & [1999MET/RAJ] \\
\hline & TRS & & 15.48 & 305.7 & & \\
\hline & FUS & & 47.7 & 313.7 & $\mathrm{AC}$ & [1996DOM/HEA, 1955SCH/BUS] \\
\hline & SUB & & $141.8 \pm 10$ & 298 & B & [1991PIA/POM] \\
\hline & $\mathrm{V}$ & $(351-462)$ & 93.7 & 368 & & [2006SAW/MOK] \\
\hline & $\mathrm{V}$ & $(434-539)$ & 106.8 & 298 & CGC & [2004CHI/HAN] \\
\hline & $\mathrm{V}$ & & $109.4 \pm 2.6$ & 298 & CGC & [1997CHI/WIL] \\
\hline & $\mathrm{V}$ & $(365-400)$ & $110 \pm 2$ & 382 & $\mathrm{TE}$ & [1994PIA/FON] \\
\hline & $\mathrm{V}$ & $(352-478)$ & 84.7 & 367 & $\begin{array}{l}\text { TE, ME, } \\
\text { GS }\end{array}$ & [1991PIA/POM] \\
\hline & $\mathrm{V}$ & $(422-630)$ & 88.4 & 437 & $\mathrm{~A}, \mathrm{E}$ & [1987STE/MAL, 1966KUD/ZWO] \\
\hline $\mathrm{C}_{21} \mathrm{H}_{44}$ & $\begin{array}{c}{[1560-84-5]} \\
\mathrm{V}\end{array}$ & $\begin{array}{l}\text { 2-methyleicosane } \\
\quad(473-621)\end{array}$ & 70.3 & 488 & A & [1987STE/MAL] \\
\hline $\mathrm{C}_{21} \mathrm{H}_{44}$ & $\begin{array}{c}{[6418-46-8]} \\
\mathrm{V}\end{array}$ & $\begin{array}{l}\text { 3-methyleicosane } \\
\quad(477-620)\end{array}$ & 74.5 & 492 & A & [1987STE/MAL, 1999DYK/SVO, 1959TER/BRI] \\
\hline $\mathrm{C}_{21} \mathrm{H}_{44}$ & $\begin{array}{c}{[25117-28-6]} \\
\mathrm{V}\end{array}$ & $\begin{array}{l}\text { 4-methyleicosane } \\
\text { (471-621) }\end{array}$ & 70.2 & 486 & A & [1987STE/MAL, 1999DYK/SVO, 1959TER/BRI] \\
\hline $\mathrm{C}_{21} \mathrm{H}_{44}$ & $\begin{array}{c}{[25117-36-6]} \\
\mathrm{V}\end{array}$ & $\begin{array}{l}\text { 5-methyleicosane } \\
\quad(519-621)\end{array}$ & 73.2 & 534 & A & [1987STE/MAL, 1999DYK/SVO, 1959TER/BRI] \\
\hline $\mathrm{C}_{21} \mathrm{H}_{44}$ & $\begin{array}{c}{[75163-99-4]} \\
\mathrm{V}\end{array}$ & $\begin{array}{l}\text { 2,3-dimethylnonad } \\
\quad(493-635)\end{array}$ & $\begin{array}{l}\text { ecane } \\
68.8\end{array}$ & 508 & A & [1987STE/MAL, 1999DYK/SVO, 1959TER/BRI] \\
\hline $\mathrm{C}_{21} \mathrm{H}_{44}$ & $\begin{array}{c}\text { [115209-60-4] } \\
\mathrm{V}\end{array}$ & $\begin{array}{l}\text { 2,4-dimethylnonad } \\
\quad(465-594)\end{array}$ & $\begin{array}{l}\text { ecane } \\
77.0\end{array}$ & 480 & A & [1987STE/MAL, 1999DYK/SVO, 1959TER/BRI] \\
\hline $\mathrm{C}_{21} \mathrm{H}_{44}$ & $\begin{array}{c}\text { [114000-79-2] } \\
\mathrm{V}\end{array}$ & $\begin{array}{l}\text { 2,4,6-trimethylocta } \\
\quad(460-576)\end{array}$ & $\begin{array}{r}\text { decane } \\
74.9\end{array}$ & 475 & A & [1987STE/MAL, 1999DYK/SVO] \\
\hline $\mathrm{C}_{21} \mathrm{H}_{44}$ & $\begin{array}{c}{[13475-75-7]} \\
\mathrm{V}\end{array}$ & $\begin{array}{l}\text { 8-hexylpentadecan } \\
\quad(405-466)\end{array}$ & 78.5 & 420 & A & [1987STE/MAL] \\
\hline $\mathrm{C}_{21} \mathrm{H}_{44} \mathrm{O}_{2}$ & $\begin{array}{c}\text { [95008-70-1] } \\
\text { TRS } \\
\text { FUS }\end{array}$ & 1,21-heneicosaned & $\begin{array}{l}38.8 \\
41.7\end{array}$ & $\begin{array}{c}360 \\
377.5\end{array}$ & DSC & [1999OGA/NAK] \\
\hline $\mathrm{C}_{21} \mathrm{H}_{45} \mathrm{PO}$ & $\begin{array}{c}{[17262-51-0]} \\
\mathrm{V}\end{array}$ & $\begin{array}{l}\text { Triheptylphosphin } \\
\quad(507-638)\end{array}$ & $\begin{array}{l}\text { oxide } \\
70.8\end{array}$ & 573 & & [1971NAK/SMI] \\
\hline
\end{tabular}

The published abstract gives the formula for triheptylphosphine oxide; however, the title of the paper gives the name of trioctylphosphine oxide.

\begin{tabular}{|c|c|c|c|c|c|c|}
\hline \multirow[t]{2}{*}{$\mathrm{C}_{22} \mathrm{H}_{10} \mathrm{O}_{2}$} & \multirow{2}{*}{$\begin{array}{c}\text { [641-13-4] } \\
\text { SUB }\end{array}$} & \multicolumn{3}{|c|}{ Anthanthrone (dibenzochrysene-6,12-dione) } & \multirow[b]{2}{*}{ A } & \multirow[b]{2}{*}{ [1987STE/MAL] } \\
\hline & & $(450-550)$ & 152.2 & 465 & & \\
\hline \multirow[t]{10}{*}{$\mathrm{C}_{22} \mathrm{H}_{12}$} & [191-24-2] & Benzo[ghi]pe & & & & \\
\hline & FUS & & 16.3 & & DSC & [2011FU/SUU] \\
\hline & FUS & & 17.37 & 554.2 & DSC & [1991ACR, 1973CAS/VEC, 1980SMI] \\
\hline & SUB & $(399-454)$ & $128.0 \pm 2.0$ & & ME & [2011FU/SUU] \\
\hline & SUB & $(313-453)$ & 129.9 & 383 & GS & {$[1995 \mathrm{NAS} / \mathrm{LEN}]$} \\
\hline & SUB & $(389-468)$ & 127.8 & 404 & ME & [1987STE/MAL, 1974MUR/POL] \\
\hline & SUB & $(450-510)$ & 135.1 & 465 & A & [1987STE/MAL] \\
\hline & SUB & $(454-502)$ & 125.5 & 478 & ME & [1967WAK/INO] \\
\hline & $\mathrm{V}$ & & $128.9 \pm 1.5$ & 298 & CGC & [2008HAN/NUT] \\
\hline & $\mathrm{V}$ & $(323-473)$ & 96.1 & 398 & GC & [2002LEI/CHA] \\
\hline \multirow[t]{2}{*}{$\mathrm{C}_{22} \mathrm{H}_{12}$} & [191-26-4] & \multicolumn{3}{|c|}{ Anthranthrene (dibenzo[def,mno]chrysene) } & & \\
\hline & SUB & & $135 \pm 5$ & 479 & ME & [1952INO/SHI] \\
\hline \multirow[t]{2}{*}{$\mathrm{C}_{22} \mathrm{H}_{12}$} & [193-43-1] & \multicolumn{3}{|c|}{ Indeno[1,2,3-cd]fluoranthene } & & \\
\hline & FUS & & 23.2 & 542.3 & DSC & [2010KES/AUC] \\
\hline
\end{tabular}


TABLE 14. Phase change enthalpies of $\mathrm{C}_{19}-\mathrm{C}_{29}$ organic compounds-Continued

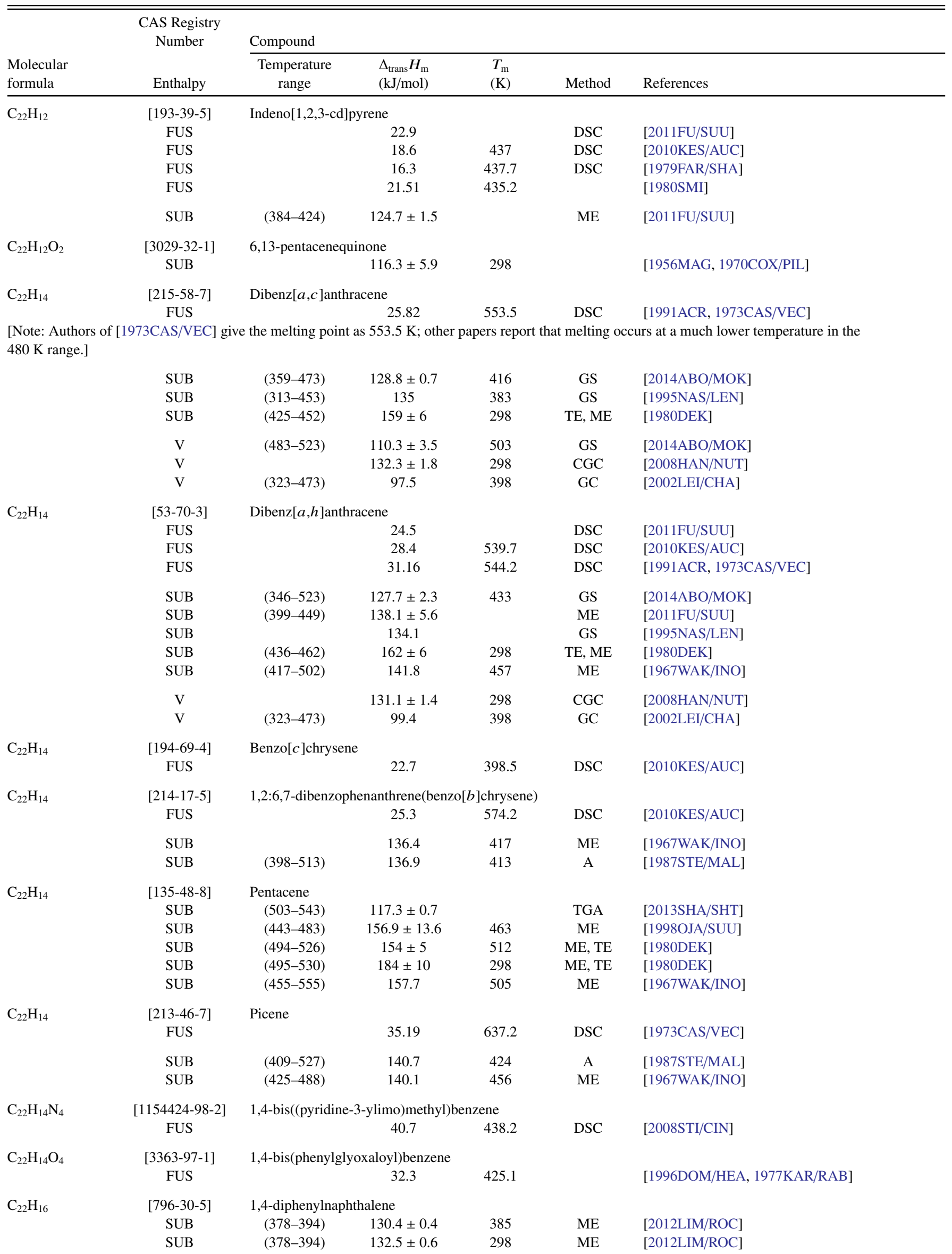


TABLE 14. Phase change enthalpies of $\mathrm{C}_{19}-\mathrm{C}_{29}$ organic compounds-Continued

\begin{tabular}{|c|c|c|c|c|c|c|}
\hline \multirow[b]{2}{*}{$\begin{array}{l}\text { Molecular } \\
\text { formula }\end{array}$} & \multirow{2}{*}{$\begin{array}{l}\text { CAS Registry } \\
\text { Number } \\
\text { Enthalpy }\end{array}$} & \multicolumn{5}{|l|}{ Compound } \\
\hline & & $\begin{array}{c}\text { Temperature } \\
\text { range }\end{array}$ & $\begin{array}{l}\Delta_{\text {trans }} H_{\mathrm{m}} \\
(\mathrm{kJ} / \mathrm{mol})\end{array}$ & $\begin{array}{l}T_{\mathrm{m}} \\
(\mathrm{K})\end{array}$ & Method & References \\
\hline \multirow[t]{3}{*}{$\mathrm{C}_{22} \mathrm{H}_{16}$} & {$[1038-67-1]$} & \multicolumn{5}{|c|}{ 1,8-diphenylnaphthalene } \\
\hline & SUB & (361-389) & $124.2 \pm 0.2$ & 376 & ME & [2012LIM/ROC] \\
\hline & SUB & (361-389) & $126.4 \pm 0.5$ & 298 & $\mathrm{ME}$ & [2012LIM/ROC] \\
\hline \multirow[t]{3}{*}{$\mathrm{C}_{22} \mathrm{H}_{16}$} & {$[82777-03-5]$} & \multicolumn{5}{|c|}{ 1-(4-biphenylyl)naphthalene } \\
\hline & SUB & $(390-414)$ & $136.1 \pm 0.5$ & 400 & $\mathrm{ME}$ & [2012LIM/ROC] \\
\hline & SUB & $(390-414)$ & $138.9 \pm 0.8$ & 298 & $\mathrm{ME}$ & [2012LIM/ROC] \\
\hline \multirow[t]{4}{*}{$\mathrm{C}_{22} \mathrm{H}_{16}$} & {$[68862-02-2]$} & \multicolumn{5}{|c|}{ 2-(biphen-4-yl)naphthalene } \\
\hline & FUS & & 25.1 & 489.5 & DSC & [2008ROU/LIM] \\
\hline & SUB & $(405-437)$ & $137.1 \pm 0.4$ & 421 & ME & [2008ROU/LIM] \\
\hline & SUB & $(405-437)$ & $140.2 \pm 1.3$ & 298 & $\mathrm{ME}$ & [2008ROU/LIM] \\
\hline \multirow[t]{4}{*}{$\mathrm{C}_{22} \mathrm{H}_{16}$} & [87294-80-2] & \multicolumn{5}{|c|}{ 2-(biphen-3-yl)naphthalene } \\
\hline & FUS & & 18.5 & 346.3 & $\mathrm{DSC}$ & [2008ROU/LIM] \\
\hline & $\mathrm{V}$ & $(381-413)$ & $104.4 \pm 1.2$ & 397 & ME & [2008ROU/LIM] \\
\hline & V & $(381-413)$ & $118.6 \pm 1.5$ & 298 & ME & [2008ROU/LIM] \\
\hline \multirow[t]{2}{*}{$\mathrm{C}_{22} \mathrm{H}_{16} \mathrm{Br}_{2} \mathrm{~N}_{2} \mathrm{~S}_{2}$} & {$[1448890-50-3]$} & \multicolumn{5}{|c|}{ 1,4-bis[[(4-bromophenyl)methyl]thio]phthalazine } \\
\hline & FUS & & 23.47 & 457.5 & DSC & [2013JIM/PLA] \\
\hline \multirow[t]{2}{*}{$\mathrm{C}_{22} \mathrm{H}_{16} \mathrm{C}_{12} \mathrm{~N}_{2} \mathrm{~S}_{2}$} & [1448890-48-9] & \multicolumn{5}{|c|}{ 1,4-bis[[(4-chlorophenyl)methyl]thio]phthalazine } \\
\hline & FUS & & 20.75 & 416.0 & DSC & [2013JIM/PLA] \\
\hline \multirow{2}{*}{$\mathrm{C}_{22} \mathrm{H}_{16} \mathrm{O}$} & {$[81-37-8]$} & \multicolumn{5}{|c|}{ 3,8-dimethylnaphtho[3,2,1-kl]xanthene (3,8-dimethylceroxene) } \\
\hline & SUB & $(373-433)$ & 138.2 & 388 & A & {$[1987 \mathrm{STE} / \mathrm{MAL}]$} \\
\hline \multirow[t]{3}{*}{$\mathrm{C}_{22} \mathrm{H}_{17} \mathrm{C} 1 \mathrm{~N}_{2}$} & {$[23593-75-1]$} & \multicolumn{5}{|c|}{ 1-[(2-chlorophenyl)(diphenyl)methyl]-1H-imidazole(clotrimazole) } \\
\hline & FUS & & 36.97 & 418.91 & DSC & [2015PAT/PAT] \\
\hline & FUS & & 33.34 & 418 & DSC & {$[2010 \mathrm{BAI} / \mathrm{VAN}]$} \\
\hline \multirow[t]{2}{*}{$\mathrm{C}_{22} \mathrm{H}_{17} \mathrm{NO}_{2}$} & {$[850804-64-7]$} & 2-ethylphenyl a & line-9-carboxy & & & \\
\hline & FUS & & 30.4 & 393 & DSC & [2010KRZ/MAL] \\
\hline $\mathrm{C}_{22} \mathrm{H}_{17} \mathrm{NO}_{2}$ & {$[128649-37-6]$} & 2,5-dimethylph & acridine-9-ca & ylate & & \\
\hline & FUS & & 37.9 & 457 & DSC & [2010KRZ/MAL] \\
\hline $\mathrm{C}_{22} \mathrm{H}_{17} \mathrm{NO}_{2}$ & [216668-66-5] & 2,6-dimethylph & 1 acridine-9-ca & ylate & & \\
\hline & FUS & & 29.1 & 435 & DSC & [2010KRZ/MAL] \\
\hline $\mathrm{C}_{22} \mathrm{H}_{17} \mathrm{NO}_{2}$ & [1262526-52-2] & 3,4-dimethylph & 1 acridine-9-c & ylate & & \\
\hline & FUS & & 29.2 & 442 & DSC & [2010KRZ/MAL] \\
\hline $\mathrm{C}_{22} \mathrm{H}_{17} \mathrm{NO}_{2}$ & [1262526-53-3] & 3,5-dimethylph & 1 acridine-9-ca & ylate & & \\
\hline & FUS & & 38.4 & 469 & DSC & [2010KRZ/MAL] \\
\hline $\mathrm{C}_{22} \mathrm{H}_{17} \mathrm{NO}_{3} \mathrm{~S}$ & [36245-88-2] & 2-(3-methyoxy & yl)-1 $H$-xanth & ,2,9-def & uinoline- & 2H)-dione \\
\hline & SUB & (605-647) & 111.8 & 620 & A & [1987STE/MAL] \\
\hline & SUB & $(647-685)$ & 150.8 & 662 & A & [1987STE/MAL] \\
\hline $\mathrm{C}_{22} \mathrm{H}_{17} \mathrm{NO}_{4}$ & [126430-58-8] & 2,6-dimethoxyl & yl acridine-9. & xylate & & \\
\hline & FUS & & 23.2 & 480 & DSC & [2010ZAD/KRZ] \\
\hline $\mathrm{C}_{22} \mathrm{H}_{18} \mathrm{Br}_{2} \mathrm{~N}_{4}$ & [1448890-41-2] & $N^{1}, N^{4}$-bis $[(4-\mathrm{b}$ & hophenyl)met & ,4-phtha & diamine & \\
\hline & FUS & & 21.87 & 555.2 & DSC & [2013JIM/PLA] \\
\hline $\mathrm{C}_{22} \mathrm{H}_{18} \mathrm{Cl}_{2} \mathrm{~N}_{4}$ & [1448890-39-8] & $N^{1}, N^{4}$-bis $[(4-c$ & rophenyl)metl & ,4-phtha & diamine & \\
\hline & FUS & & U1.63 & 444.6 & DSC & [2013JIM/PLA] \\
\hline $\mathrm{C}_{22} \mathrm{H}_{18} \mathrm{Cl}_{2} \mathrm{O}_{2}$ & [153977-22-1] & 2-[4-(4-chlorop & yl)cyclohexyl & loro- 1,4 & thoquinor & \\
\hline & FUS & & 35.0 & 457.2 & DSC & [2010MAL/FUG] \\
\hline $\mathrm{C}_{22} \mathrm{H}_{18} \mathrm{~F}_{2} \mathrm{O}$ & [145698-40-4] & 4-(6-hexenylox & ', $4^{\prime}$-difluorodi & ldiacety & & \\
\hline & FUS & & 37.45 & 370 & DSC & [1993JUA/CHE] \\
\hline $\mathrm{C}_{22} \mathrm{H}_{18} \mathrm{~F}_{2} \mathrm{O}$ & [153038-12-1] & 4-(cis-4-hexeny & y)-3', $4^{\prime}$-difluo & nenyldiac & & \\
\hline & FUS & & 35.32 & 364.4 & DSC & [1993JUA/CHE] \\
\hline $\mathrm{C}_{22} \mathrm{H}_{18} \mathrm{~F}_{2} \mathrm{O}$ & [153038-13-2] & 4-(cis-3-hexeny & y) $-3^{\prime}, 4^{\prime}$-difluo & henyldiac & & \\
\hline
\end{tabular}


TABLE 14. Phase change enthalpies of $\mathrm{C}_{19}-\mathrm{C}_{29}$ organic compounds-Continued

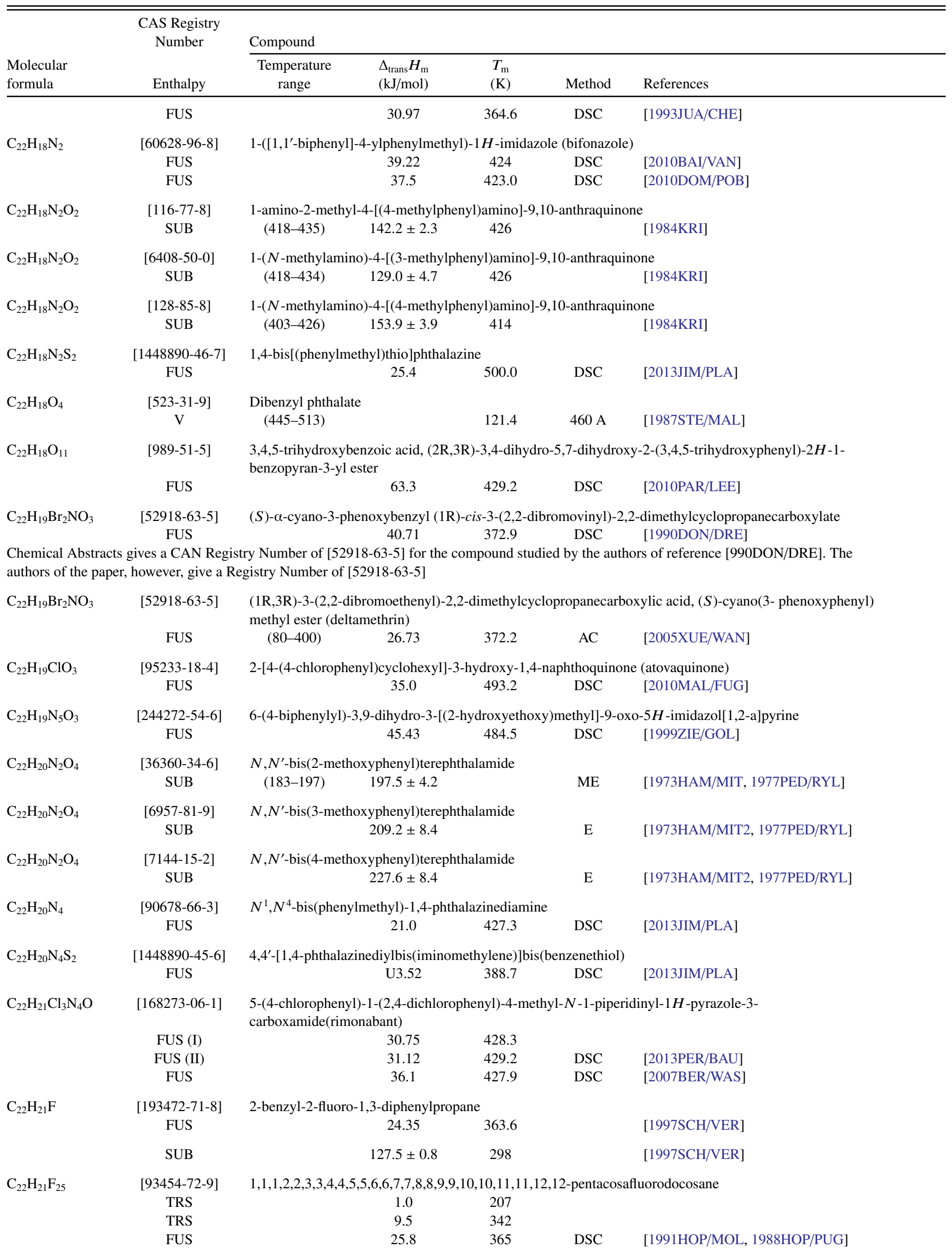


TABLE 14. Phase change enthalpies of $\mathrm{C}_{19}-\mathrm{C}_{29}$ organic compounds-Continued

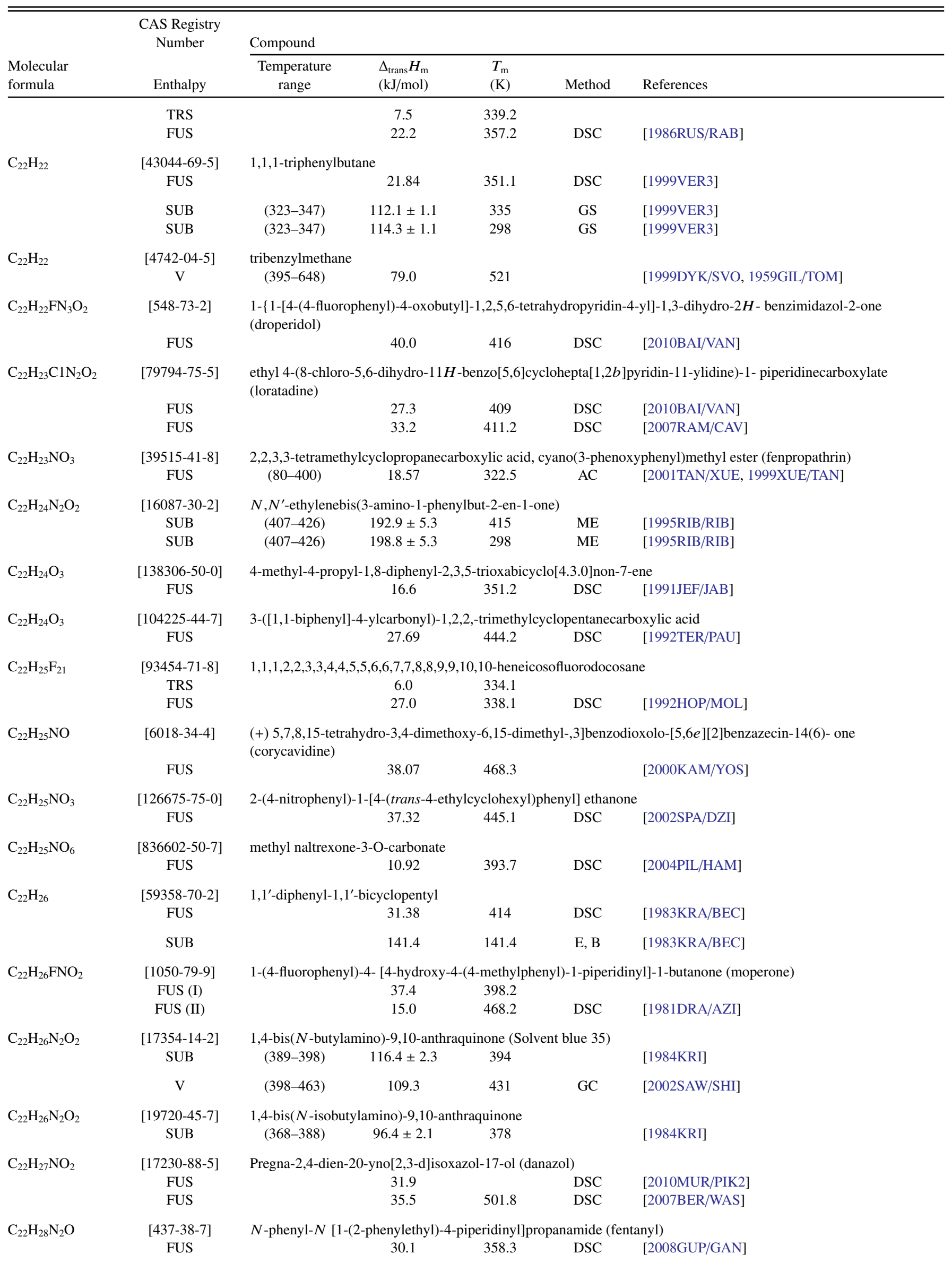


TABLE 14. Phase change enthalpies of $\mathrm{C}_{19}-\mathrm{C}_{29}$ organic compounds-Continued

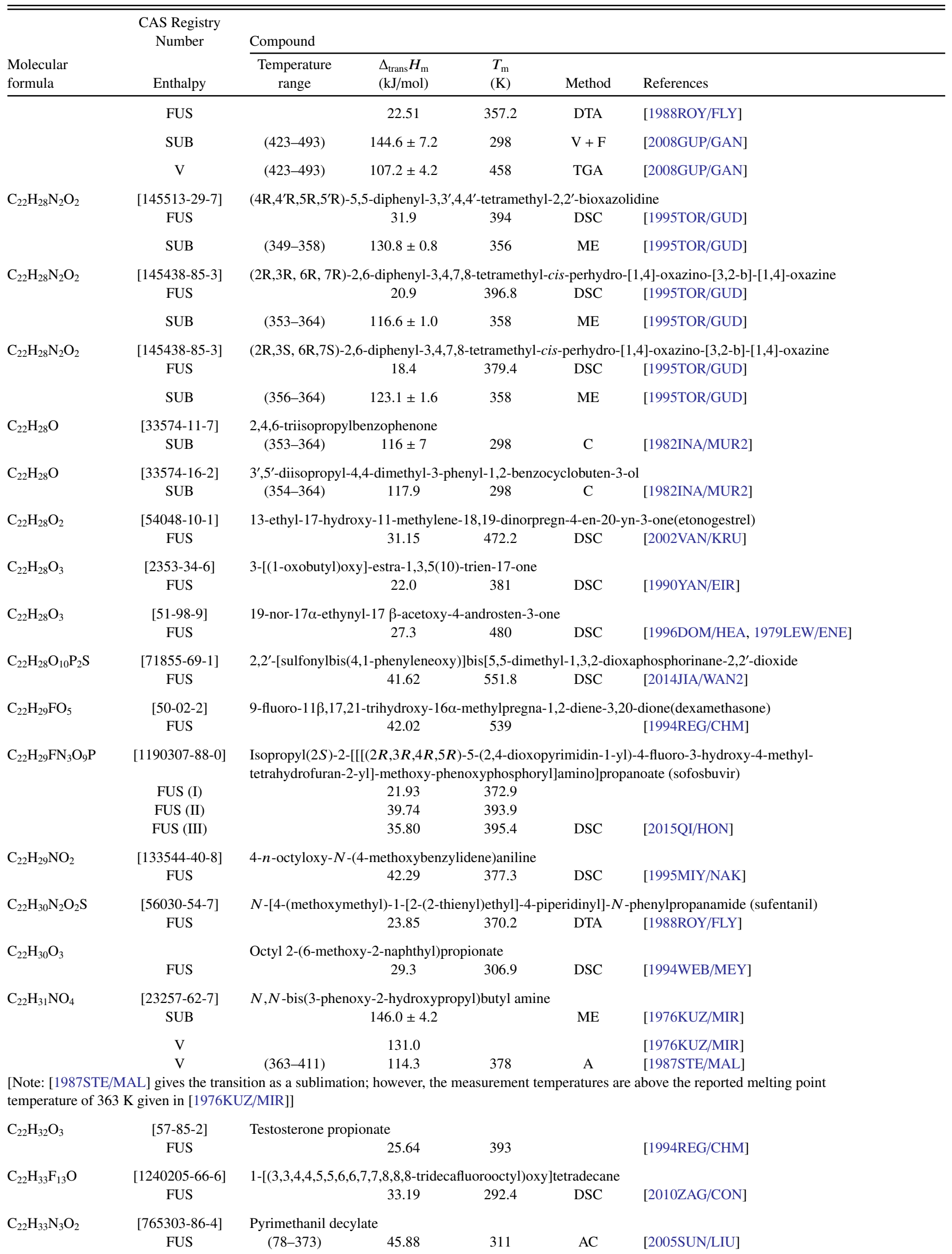


TABLE 14. Phase change enthalpies of $\mathrm{C}_{19}-\mathrm{C}_{29}$ organic compounds-Continued

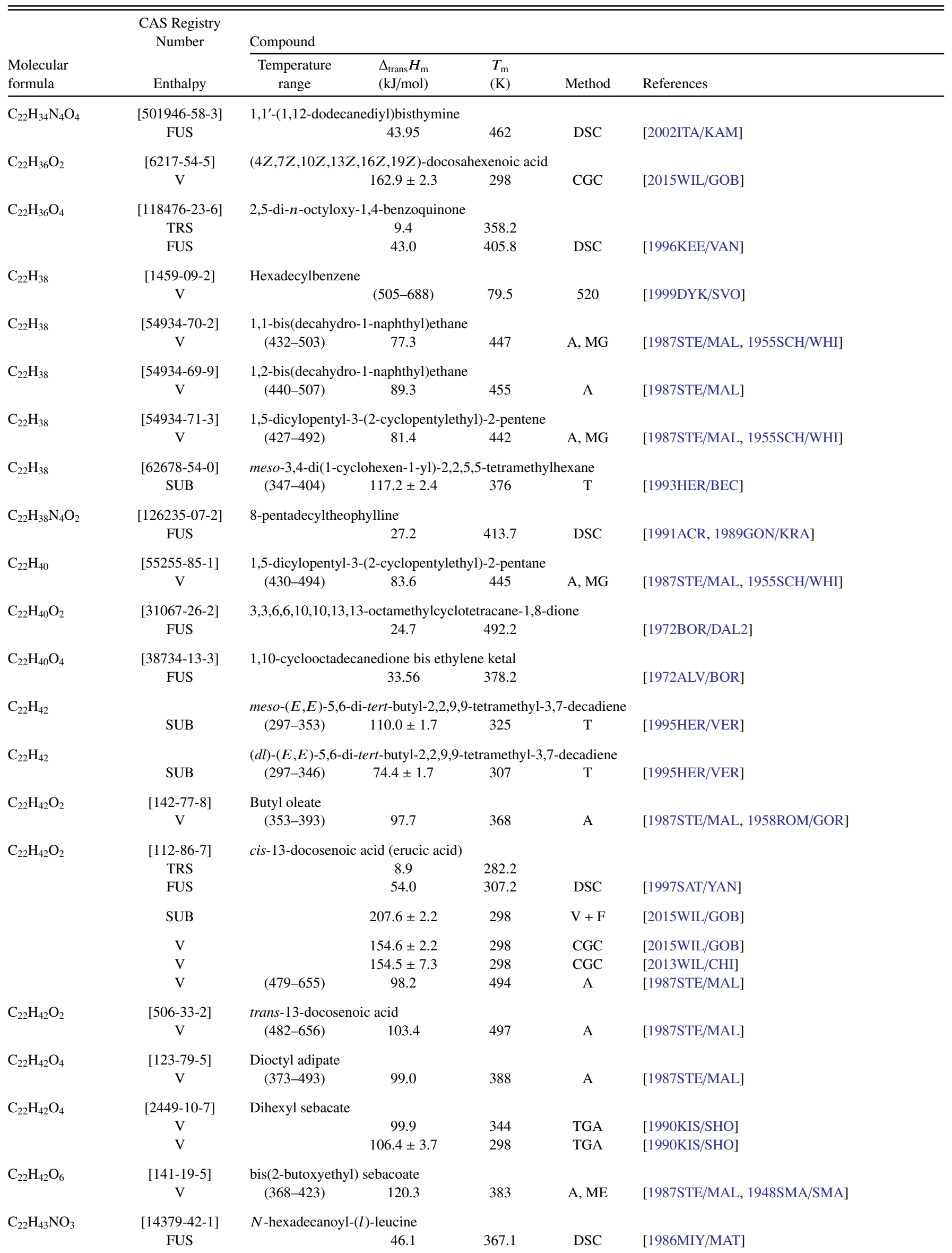


TABLE 14. Phase change enthalpies of $\mathrm{C}_{19}-\mathrm{C}_{29}$ organic compounds-Continued

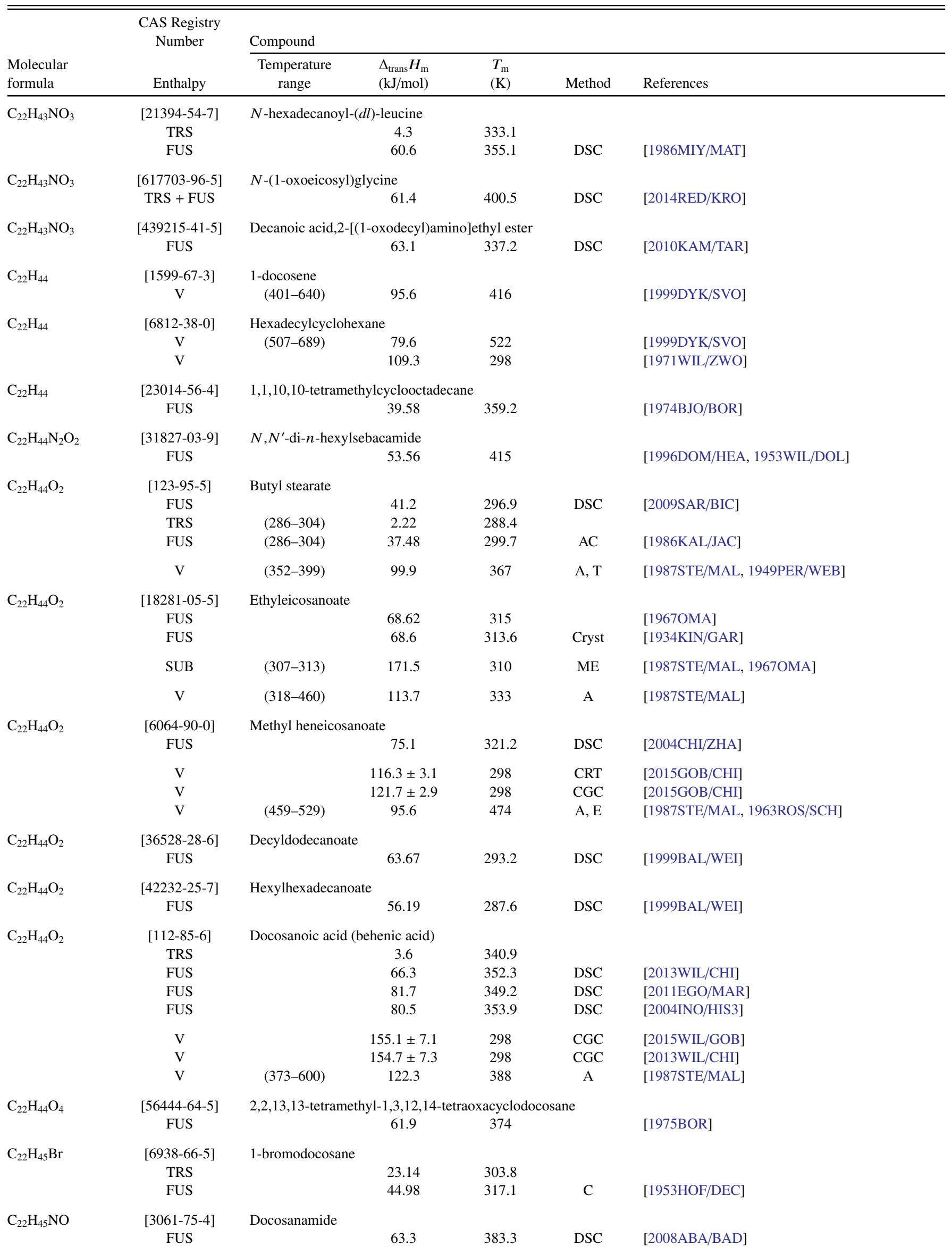


TABLE 14. Phase change enthalpies of $\mathrm{C}_{19}-\mathrm{C}_{29}$ organic compounds-Continued

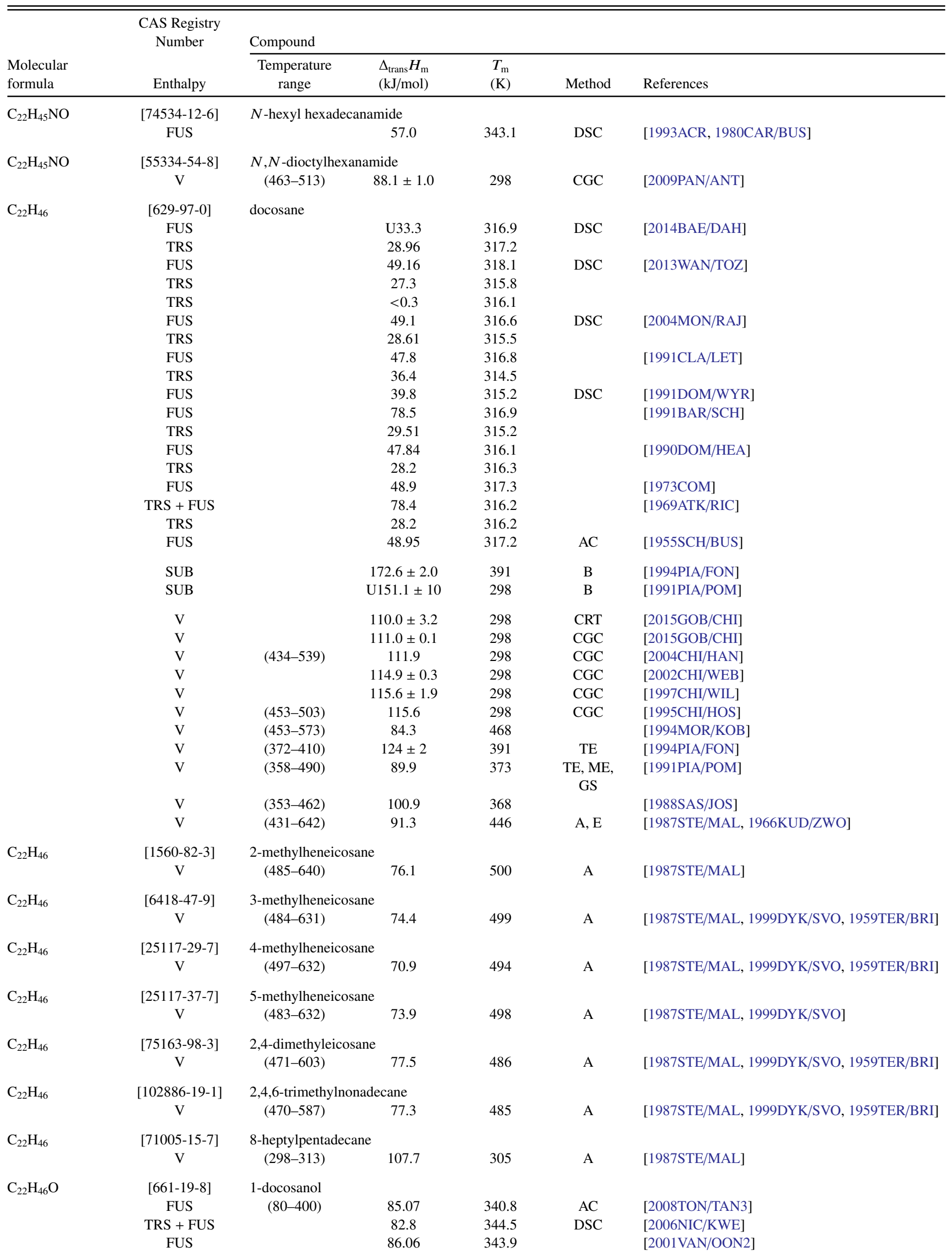


TABLE 14. Phase change enthalpies of $\mathrm{C}_{19}-\mathrm{C}_{29}$ organic compounds-Continued

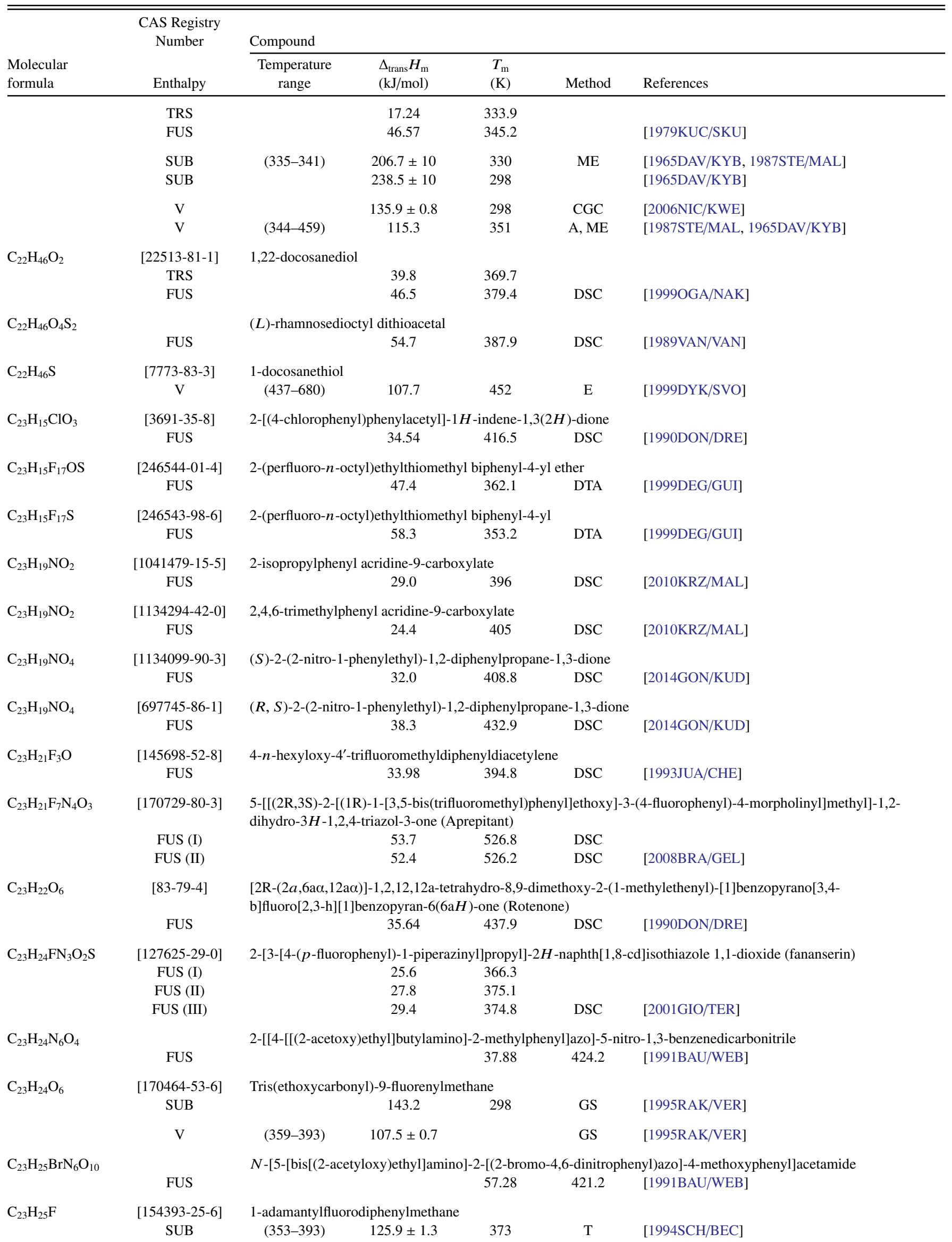


TABLE 14. Phase change enthalpies of $\mathrm{C}_{19}-\mathrm{C}_{29}$ organic compounds-Continued

\begin{tabular}{|c|c|c|c|c|c|c|}
\hline \multirow[b]{2}{*}{$\begin{array}{l}\text { Molecular } \\
\text { formula }\end{array}$} & \multirow{2}{*}{$\begin{array}{l}\text { CAS Registry } \\
\text { Number } \\
\text { Enthalpy }\end{array}$} & \multicolumn{5}{|l|}{ Compound } \\
\hline & & $\begin{array}{l}\text { Temperature } \\
\text { range }\end{array}$ & $\begin{array}{l}\Delta_{\text {trans }} H_{\mathrm{m}} \\
(\mathrm{kJ} / \mathrm{mol})\end{array}$ & $\begin{array}{l}T_{\mathrm{m}} \\
(\mathrm{K})\end{array}$ & Method & References \\
\hline \multirow[t]{4}{*}{$\mathrm{C}_{23} \mathrm{H}_{26} \mathrm{O}_{6}$} & [183212-67-1] & \multicolumn{5}{|c|}{ 1,1,1-tris(ethoxycarbonyl)-2,2-diphenylethane } \\
\hline & FUS & & 29.5 & 333.2 & & [1995RAK/VER] \\
\hline & SUB & & 140.1 & 298 & GS & [1995RAK/VER] \\
\hline & $\mathrm{V}$ & $(344-394)$ & $109.3 \pm 1.0$ & & GS & [1995RAK/VER] \\
\hline \multirow[t]{5}{*}{$\mathrm{C}_{23} \mathrm{H}_{27} \mathrm{C}_{12} \mathrm{~N}_{3} \mathrm{O}_{2}$} & [129722-12-9] & \multicolumn{5}{|c|}{ 7-[4-[4-(2,3-dichlorophenyl)-1-piperazinyl]butoxy]-3,4-dihydro-2(1H)-quinoline (aripiprazole) } \\
\hline & FUS (I) & & 38.36 & 422.1 & DSC & \\
\hline & FUS (II) & & 41.51 & 416.3 & DSC & \\
\hline & FUS (III) & & 39.97 & 412.4 & DSC & \\
\hline & FUS (IV) & & 40.76 & 408.1 & DSC & [2009BRA/GEL] \\
\hline \multirow[t]{2}{*}{$\mathrm{C}_{23} \mathrm{H}_{27} \mathrm{FN}_{4} \mathrm{O}_{2}$} & [106266-06-2] & \multicolumn{5}{|c|}{$\begin{array}{l}\text { 3-[2-[4-(6-fluoro-1,2-benzisoxazol-3-yl)-1-piperidinyl]ethyl]-6,7,8,9-tetrahydro-2-methyl-4H- } \\
\text { pyrido[1,2-a]pyrimidin-4-one }\end{array}$} \\
\hline & FUS & & 43.94 & 442.38 & DSC & [2014MEA/SVA] \\
\hline \multirow{2}{*}{$\mathrm{C}_{23} \mathrm{H}_{27} \mathrm{NO}_{3}$} & {$[126675-76-1]$} & \multicolumn{5}{|c|}{ 2-(4-nitrophenyl)-1-[4-(trans-4-propylcyclohexyl)phenyl]ethanone } \\
\hline & FUS & & 38.87 & 436.5 & DSC & [2002SPA/DZI] \\
\hline \multirow[t]{2}{*}{$\mathrm{C}_{23} \mathrm{H}_{27} \mathrm{NO}_{3} \mathrm{~S}$} & {$[313057-12-4]$} & \multicolumn{5}{|c|}{ 4-(7-undecenyloxy)phenyl 5-cyano-2-thiophenecarboxylate } \\
\hline & FUS & & 52.72 & 346.1 & DSC & [2000WU/WAN] \\
\hline \multirow[t]{2}{*}{$\mathrm{C}_{23} \mathrm{H}_{27} \mathrm{NO}_{6}$} & {$[836602-51-8]$} & \multicolumn{5}{|c|}{ Ethyl natrexone-3-O-carbonate } \\
\hline & FUS & & 18.99 & 404.2 & DSC & [2004PIL/HAM] \\
\hline \multirow[t]{5}{*}{$\mathrm{C}_{23} \mathrm{H}_{28} \mathrm{ClN}_{3} \mathrm{O}_{5} \mathrm{~S}$} & {$[10238-21-8]$} & \multicolumn{5}{|c|}{$\begin{array}{l}\text { 5-chloro- } N \text {-[2-[4-[[[(cyclohexylamino)carbonyl }] \text { amino }] \text { sulfonyl }] \text { phenyl }] \text { ethyl }]-2- \\
\text { methoxybenzamide(glyburide) }\end{array}$} \\
\hline & FUS & & 41.89 & 450.2 & DSC & [2015GAU/VAN] \\
\hline & FUS & & 55.4 & 446.9 & DSC & [2010MUR/PIK2] \\
\hline & FUS & & 46.3 & 446.8 & DSC & [2007BER/WAS, 2006WAS/HOL] \\
\hline & FUS & & 53.35 & 450.2 & DSC & {$[2000 \mathrm{HAN} / \mathrm{PAR}]$} \\
\hline \multirow[t]{2}{*}{$\mathrm{C}_{23} \mathrm{H}_{28} \mathrm{~N}_{2} \mathrm{O}_{5}$} & & \multicolumn{5}{|c|}{$N, N$-dimethyl naltrexone-3-O-carbamate } \\
\hline & FUS & & 22.01 & 480.2 & DSC & {$[2009 \mathrm{VAD} / \mathrm{BAN}]$} \\
\hline \multirow[t]{2}{*}{$\mathrm{C}_{23} \mathrm{H}_{30} \mathrm{O}_{3}$} & {$[128788-26-1]$} & \multicolumn{5}{|c|}{3 -[(1-oxopentyl)oxy]-estra-1,3,5(10)-trien-17-one } \\
\hline & FUS & & 25 & 398 & DSC & [1990YAN/EIR] \\
\hline $\mathrm{C}_{23} \mathrm{H}_{30} \mathrm{O}_{4} \mathrm{~S}$ & [313057-16-8] & 4-(7-undecenyl & phenyl 5-m & 2-thiopl & arboxylate & \\
\hline & FUS & & 61.92 & 334.1 & DSC & [2000WU/WAN] \\
\hline $\mathrm{C}_{23} \mathrm{H}_{30} \mathrm{O}_{6}$ & {$[52-21-1]$} & Prednisoloneac & & & & \\
\hline & FUS & & 42.3 & 515 & DSC & [1997CEN/MEL] \\
\hline & FUS & & 38.67 & 511 & & [1994REG/CHM] \\
\hline $\mathrm{C}_{23} \mathrm{H}_{30} \mathrm{O}_{6}$ & {$[50-04-4]$} & Cortisone acet: & & & & \\
\hline & FUS & & 38.43 & 509 & & [1994REG/CHM] \\
\hline $\mathrm{C}_{23} \mathrm{H}_{31} \mathrm{NO}$ & [164667-96-3] & 4-n-octyloxy- 1 & ,5-dimethyll & dene)an & & \\
\hline & FUS & & 37.73 & 324.7 & DSC & [1995MIY/NAK] \\
\hline $\mathrm{C}_{23} \mathrm{H}_{31} \mathrm{NO}_{3}$ & [164667-97-4] & 4-n-octyloxy-1 & ,5-dimethox & lidene)a & & \\
\hline & FUS & & 35.3 & 316.3 & DSC & [1995MIY/NAK] \\
\hline $\mathrm{C}_{23} \mathrm{H}_{32} \mathrm{~N}_{2} \mathrm{O}_{2} \mathrm{~S}$ & [910-86-1] & $N, N^{\prime}-[4-(3-\mathrm{m}$ & butoxy)pher & urea & & \\
\hline & FUS & & 38.1 & 415.3 & DSC & [2011LI/BOU] \\
\hline $\mathrm{C}_{23} \mathrm{H}_{32} \mathrm{O}_{2}$ & [119-47-1] & 3,3'-di-tert-but & 5'-dimethyl- & ydroxyc & ylmethane & \\
\hline & FUS & & 29.33 & 403.7 & DTA & [1972INO/LIA] \\
\hline $\mathrm{C}_{23} \mathrm{H}_{32} \mathrm{O}_{3}$ & [27811-56-9] & Estra-1,3,5(10) & ne-3-ol-17 $\beta$ & oate & & \\
\hline & FUS & & 29.45 & 420.7 & DSC & [1986DEM/MAS] \\
\hline $\mathrm{C}_{23} \mathrm{H}_{32} \mathrm{O}_{4}$ & {$[56-47-3]$} & Deoxycorticos & e acetate & & & \\
\hline & FUS & & 29.66 & 430 & & [1994REG/CHM] \\
\hline $\mathrm{C}_{23} \mathrm{H}_{32} \mathrm{O}_{6}$ & {$[50-03-3]$} & Hydrocortisone & tate & & & \\
\hline & FUS & & 53.64 & 480 & DSC & [1997CEN/MEL] \\
\hline & FUS & & 36.95 & 496 & & [1994REG/CHM] \\
\hline
\end{tabular}


TABLE 14. Phase change enthalpies of $\mathrm{C}_{19}-\mathrm{C}_{29}$ organic compounds-Continued

\begin{tabular}{|c|c|c|c|c|c|c|}
\hline \multirow[b]{2}{*}{$\begin{array}{l}\text { Molecular } \\
\text { formula }\end{array}$} & \multirow{2}{*}{$\begin{array}{c}\text { CAS Registry } \\
\text { Number } \\
\text { Enthalpy }\end{array}$} & \multicolumn{5}{|l|}{ Compound } \\
\hline & & $\begin{array}{l}\text { Temperature } \\
\text { range }\end{array}$ & $\begin{array}{l}\Delta_{\text {trans }} H_{\mathrm{m}} \\
(\mathrm{kJ} / \mathrm{mol})\end{array}$ & $\begin{array}{l}T_{\mathrm{m}} \\
(\mathrm{K})\end{array}$ & Method & References \\
\hline \multirow[t]{2}{*}{$\mathrm{C}_{23} \mathrm{H}_{34} \mathrm{O}_{2}$} & {$[2566-90-7]$} & \multicolumn{5}{|c|}{$(Z, Z, Z, Z, Z, Z)-4,7,10,13,16,19$-docosahexaeneoate } \\
\hline & $\mathrm{V}$ & & $131.8 \pm 0.2$ & 298 & CGC & [2007LIP/KAP] \\
\hline \multirow[t]{2}{*}{$\mathrm{C}_{23} \mathrm{H}_{34} \mathrm{O}_{3}$} & [3410-54-6] & \multicolumn{5}{|c|}{ Testosterone butyrate } \\
\hline & FUS & & 24.75 & 382 & & [1994REG/CHM] \\
\hline \multirow[t]{2}{*}{$\mathrm{C}_{23} \mathrm{H}_{35} \mathrm{~N}_{3} \mathrm{O}_{8}$} & {$[53848-86-5]$} & \multicolumn{5}{|c|}{ Hexadecyl 2,4,6-trinitrobenzoate } \\
\hline & TRS & & $\begin{array}{l}18.6 \\
29.54\end{array}$ & 349.3 & $\mathrm{DSC}$ & [1074WA R/WU ] \\
\hline \multirow[t]{5}{*}{$\mathrm{C}_{23} \mathrm{H}_{36} \mathrm{~N}_{2} \mathrm{O}_{2}$} & {$[98319-26-7]$} & \multicolumn{5}{|c|}{ (5 $\alpha, 17 \beta)-N$-(1,1-dimethylethyl)-3-oxo-4-azaandrost-1-ene-17-carboxamide(finasteride) } \\
\hline & TRS (I) & & 4.1 & 503.2 & & \\
\hline & FUS (I) & & 33.2 & 530.2 & & \\
\hline & FUS (II) & & 32.8 & 530.2 & DSC & [2000WEN/BAU] \\
\hline & SUB & $(463-488)$ & 143.7 & & TGA & [1997ELD] \\
\hline \multirow[t]{2}{*}{$\mathrm{C}_{23} \mathrm{H}_{37} \mathrm{BrN}_{2} \mathrm{O}_{4}$} & [138317-09-6] & \multicolumn{5}{|c|}{ (4-nitrophenyl)-16-bromohexadecyl carbamate } \\
\hline & FUS & & 62.62 & 382.9 & DSC & [1993TIE/FRA] \\
\hline \multirow[t]{2}{*}{$\mathrm{C}_{23} \mathrm{H}_{39} \mathrm{~N}_{3} \mathrm{O}_{3}$} & {$[6313-97-9]$} & \multicolumn{5}{|c|}{ 1-hexadecyl-3-(4-nitrophenyl) urea } \\
\hline & FUS & & 53.94 & 392.6 & DSC & [1993TIE/FRA] \\
\hline \multirow[t]{2}{*}{$\mathrm{C}_{23} \mathrm{H}_{40}$} & {$[14752-75-1]$} & \multicolumn{5}{|c|}{ Heptadecylbenzene } \\
\hline & $\mathrm{V}$ & (414-664) & 98.5 & 429 & & [1999DYK/SVO] \\
\hline \multirow[t]{2}{*}{$\mathrm{C}_{23} \mathrm{H}_{42} \mathrm{O}_{2}$} & {$[61012-47-3]$} & \multicolumn{5}{|c|}{ Methyl $(Z, Z)-13,16$-docosadienoate } \\
\hline & V & & 127.9 & 298 & CGC & [2007LIP/KAP] \\
\hline \multirow[t]{2}{*}{$\mathrm{C}_{23} \mathrm{H}_{42} \mathrm{O}_{3}$} & {$[5420-17-7]$} & \multicolumn{5}{|c|}{ Tetrahydrofurfuryl oleate } \\
\hline & V & $(353-398)$ & 98.7 & 368 & A & [1987STE/MAL] \\
\hline $\mathrm{C}_{23} \mathrm{H}_{44}$ & [95115-76-7] & trans-2-heptyl & xyldecalin & & & \\
\hline & FUS & & 38.91 & 312.2 & & [1985VAR/BRI] \\
\hline $\mathrm{C}_{23} \mathrm{H}_{44}$ & [95115-79-0] & trans-2-pentyl & tyldecalin & & & \\
\hline & FUS & & 43.51 & 314.2 & & [1985VAR/BRI] \\
\hline $\mathrm{C}_{23} \mathrm{H}_{44} \mathrm{O}_{2}$ & [1120-34-9] & methyl erucate & & & & \\
\hline & $\mathrm{V}$ & & $125.6 \pm 1.2$ & 298 & CGC & [2007LIP/KAP] \\
\hline & V & $(463-523)$ & 123.8 & 298 & GC & [1997KRO/VEL] \\
\hline & V & $(453-543)$ & 93.5 & 498 & GC & [1993HUS/SAR] \\
\hline $\mathrm{C}_{23} \mathrm{H}_{44} \mathrm{O}_{4}$ & {$[17598-93-5]$} & (3-decanoylox & ydroxypropy & anoate & & \\
\hline & V & $(536-565)$ & 124.1 & 551 & DSC & [2014DAM/MAT] \\
\hline $\begin{array}{l}\text { [Note: The CAS } \\
\text { the paper.] }\end{array}$ & istry Number of & $3988-07-1]$ that & thors of [20 & M/MAT & is not con & ent with the IUPAC chemical name i \\
\hline $\mathrm{C}_{23} \mathrm{H}_{44} \mathrm{O}_{5}$ & {$[820-17-7]$} & 1-aceto-3-stear & & & & \\
\hline & FUS & & 41.69 & 319.9 & & [1996DOM/HEA, 1955WAR/VIC] \\
\hline $\mathrm{C}_{23} \mathrm{H}_{45} \mathrm{NO}_{3}$ & & 2-lauryloxy- $N$ & libutylpropior & & & \\
\hline & V & $(443-458)$ & 90.6 & 450 & A & [1987STE/MAL] \\
\hline $\mathrm{C}_{23} \mathrm{H}_{45} \mathrm{NO}_{3}$ & {$[914224-79-6]$} & $N$-(1-oxohene & 1)glycine & & & \\
\hline & TRS + FUS & & 64.0 & 400.8 & DSC & [2014RED/KRO] \\
\hline $\mathrm{C}_{23} \mathrm{H}_{46}$ & {$[55124-77-1]$} & 9-cyclohexylh & ecane & & & \\
\hline & V & (456-492) & 83.9 & 471 & A & [1987STE/MAL] \\
\hline $\mathrm{C}_{23} \mathrm{H}_{46}$ & {$[19781-73-8]$} & Hexadecylcycl & ane & & & \\
\hline & V & (414-664) & 97.6 & 429 & & [1999DYK/SVO] \\
\hline $\mathrm{C}_{23} \mathrm{H}_{46}$ & {$[18835-32-0]$} & 1-tricosene & & & & \\
\hline & V & $(409-652)$ & 98.5 & 424 & & [1999DYK/SVO] \\
\hline $\mathrm{C}_{23} \mathrm{H}_{46}$ & [27519-02-4] & Z-9-tricosene & calure) & & & \\
\hline & V & & $114.4 \pm 1.0$ & 298 & CGC & [2013SPE/CHI] \\
\hline $\mathrm{C}_{23} \mathrm{H}_{46}$ & [35857-62-6] & $E$-9-tricosene & & & & \\
\hline
\end{tabular}


TABLE 14. Phase change enthalpies of $\mathrm{C}_{19}-\mathrm{C}_{29}$ organic compounds-Continued

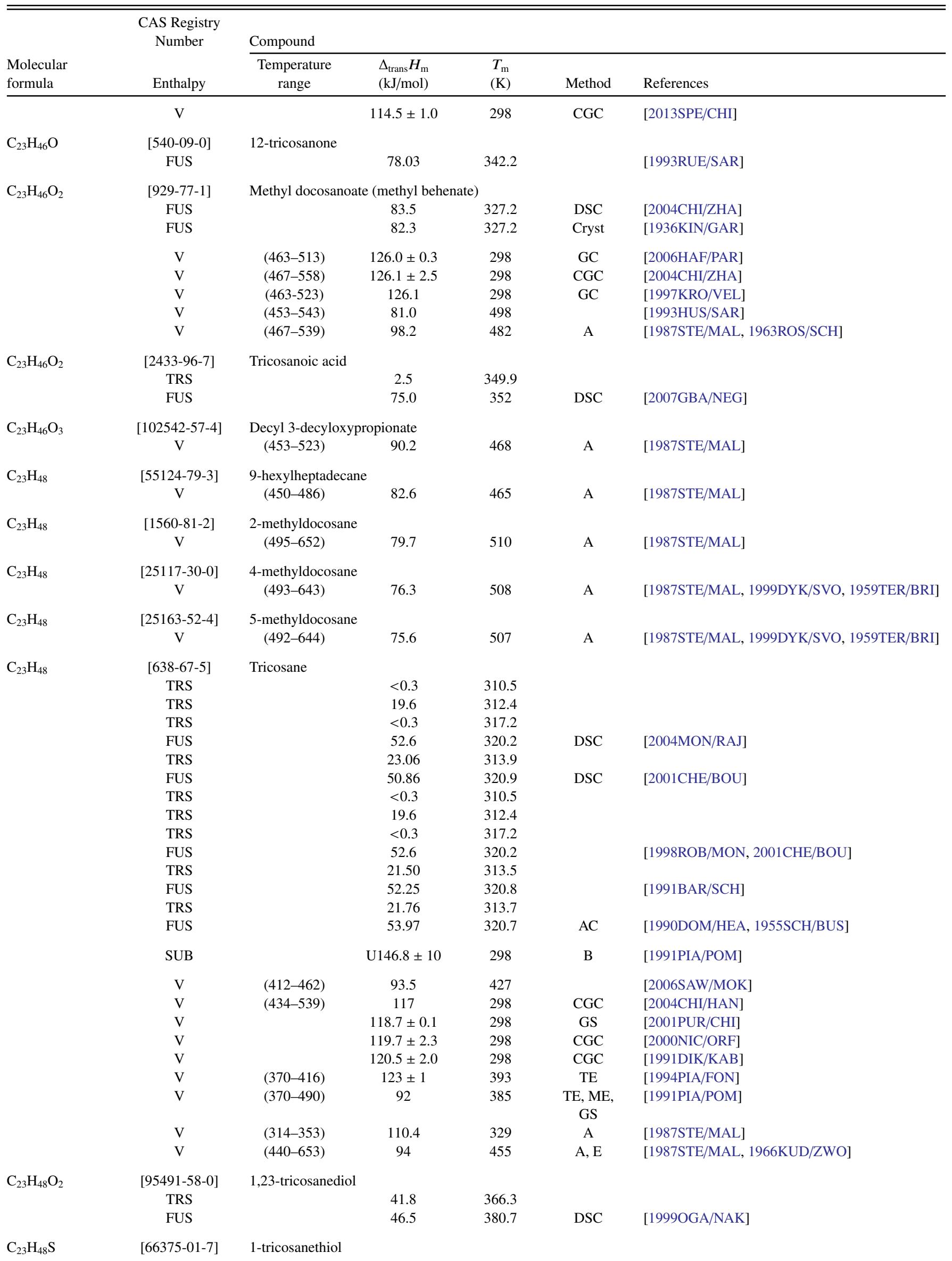


TABLE 14. Phase change enthalpies of $\mathrm{C}_{19}-\mathrm{C}_{29}$ organic compounds-Continued

\begin{tabular}{|c|c|c|c|c|c|c|}
\hline \multirow[b]{2}{*}{$\begin{array}{l}\text { Molecular } \\
\text { formula }\end{array}$} & \multirow{2}{*}{$\begin{array}{l}\text { CAS Registry } \\
\text { Number } \\
\text { Enthalpy }\end{array}$} & \multicolumn{5}{|l|}{ Compound } \\
\hline & & $\begin{array}{c}\text { Temperature } \\
\text { range }\end{array}$ & $\begin{array}{l}\Delta_{\text {trans }} H_{\mathrm{m}} \\
(\mathrm{kJ} / \mathrm{mol})\end{array}$ & $\begin{array}{l}T_{\mathrm{m}} \\
(\mathrm{K})\end{array}$ & Method & References \\
\hline & $\mathrm{V}$ & $(444-690)$ & 110.1 & 459 & $\mathrm{E}$ & [1999DYK/SVO] \\
\hline \multirow[t]{8}{*}{$\mathrm{C}_{24} \mathrm{~F}_{50}$} & {$[1766-41-2]$} & \multicolumn{5}{|c|}{ Perfluorotetracosane } \\
\hline & FUS & & 63.2 & 461.1 & DSC & [2012HAS/DRA] \\
\hline & FUS & & 56.0 & 461.7 & DSC & [1999VIS/TER] \\
\hline & TRS & & 14.0 & 217.5 & & \\
\hline & FUS & & 63.2 & 461.7 & $\mathrm{DSC}$ & [1994JIN/BOL] \\
\hline & TRS & & 3.89 & 202.7 & & \\
\hline & FUS & & U 100.8 & 465.2 & DSC & {$[1986 \mathrm{STA}]$} \\
\hline & $\mathrm{V}$ & & $141.0 \pm 1.7$ & 298 & CGC & [2012HAS/DRA] \\
\hline \multirow[t]{2}{*}{$\mathrm{C}_{24} \mathrm{H}_{8} \mathrm{O}_{6}$} & {$[128-69-8]$} & \multicolumn{5}{|c|}{ 3,4,9,10-perylenetetracarboxylic dianhydride } \\
\hline & SUB & $(653-793)$ & $185.6 \pm 0.5$ & & TGA & [2013SHA/SHT] \\
\hline \multirow[t]{14}{*}{$\mathrm{C}_{24} \mathrm{H}_{12}$} & [191-07-1] & coronene & & & & \\
\hline & FUS & & 21.2 & 709 & DSC & [2009TOR/CAM] \\
\hline & FUS & & 19.2 & 710.5 & & [1991ACR, 1980SMI] \\
\hline & SUB & $(473-483)$ & $126.6 \pm 1.7$ & 478 & $\mathrm{ME}$ & [2009TOR/CAM] \\
\hline & SUB & $(473-483)$ & $131.0 \pm 1.7$ & 298 & ME & [2009TOR/CAM] \\
\hline & SUB & $(421-504)$ & $133.1 \pm 5.1$ & 463 & $\mathrm{ME}$ & [1998OJA/SUU] \\
\hline & SUB & $(313-453)$ & 143.2 & 383 & GS & [1995NAS/LEN] \\
\hline & SUB & $(427-510)$ & 135.9 & 468 & $\mathrm{ME}$ & [1987STE/MAL, 1974MUR/POL] \\
\hline & SUB & & 128.4 & & $\mathrm{ME}$ & [1967WAK/INO] \\
\hline & SUB & $(433-513)$ & 147 & 473 & & [1958HOY/PEP] \\
\hline & SUB & $(476-555)$ & 143.2 & 407 & ME & [1952INO/SHI] \\
\hline & SUB & & 148.5 & 407 & ME & [1951INO] \\
\hline & $\mathrm{V}$ & & $148.0 \pm 0.5$ & 298 & $\mathrm{CGC}$ & [2002CHI/WEB] \\
\hline & $\mathrm{V}$ & $(323-473)$ & 104.2 & 398 & GC & [2002LEI/CHA] \\
\hline \multirow[t]{2}{*}{$\mathrm{C}_{24} \mathrm{H}_{12}$} & {$[102234-01-5]$} & \multicolumn{5}{|c|}{ bis-benzo[3,4]cyclobuta[1,2-a:1',2'-c]biphenylene ([4]phenylene) } \\
\hline & SUB & & $131.0 \pm 4.2$ & & & {$[2000 \mathrm{BEC} / \mathrm{FAU}]$} \\
\hline \multirow[t]{2}{*}{$\mathrm{C}_{24} \mathrm{H}_{12} \mathrm{O}_{2}$} & {$[3302-52-1]$} & \multicolumn{5}{|c|}{ 3,4:9,10-dibenzpyrene-5,8-quinone } \\
\hline & SUB & & $112.5 \pm 5.4$ & & & [1956MAG, 1970COX/PIL] \\
\hline \multirow[t]{5}{*}{$\mathrm{C}_{24} \mathrm{H}_{14}$} & {$[192-65-4]$} & \multicolumn{5}{|c|}{ Dibenzo $[a, e]$ pyrene } \\
\hline & FUS & & 32.1 & 517.9 & DSC & [2010KES/AUC] \\
\hline & FUS & & 30.5 & 520.2 & DSC & [1991ACR, 1973CAS/VEC] \\
\hline & SUB & $(414-506)$ & 146.4 & 429 & A & [1987STE/MAL] \\
\hline & SUB & $(434-526)$ & 137.6 & 480 & $\mathrm{ME}$ & [1967WAK/INO] \\
\hline \multirow[t]{3}{*}{$\mathrm{C}_{24} \mathrm{H}_{14}$} & [192-51-8] & \multicolumn{5}{|c|}{ Dibenzo[fg,op]naphthacene } \\
\hline & SUB & $(430-555)$ & 147.4 & 445 & A & [1987STE/MAL] \\
\hline & SUB & $(454-526)$ & 146.9 & 490 & ME & [1967WAK/INO] \\
\hline
\end{tabular}

(called 1,2,6,7-dibenzpyrene in paper, which we have taken to be dibenzo[fo,op]naphthacene based upon the melting point temperature reported in the paper)

\begin{tabular}{|c|c|c|c|c|c|}
\hline $\mathrm{C}_{24} \mathrm{H}_{14}$ & $\begin{array}{l}{[191-30-0]} \\
\text { FUS }\end{array}$ & Dibenzo $[a, l]$ pyrene & 501.2 & DSC & [1991ACR, 1973CAS/VEC] \\
\hline $\mathrm{C}_{24} \mathrm{H}_{14}$ & $\begin{array}{l}\text { [189-55-9] } \\
\text { FUS }\end{array}$ & $\begin{array}{r}\text { Benzo[rst }] \text { pentaphene } \\
27.87\end{array}$ & 556.8 & DSC & [1991ACR, 1973CAS/VEC] \\
\hline $\mathrm{C}_{24} \mathrm{H}_{14}$ & $\begin{array}{l}{[5385-75-1]} \\
\text { FUS }\end{array}$ & $\begin{array}{l}\text { Dibenzo }[a, e] \text { fluoranthene } \\
24.0\end{array}$ & 505.5 & DSC & [2010KES/AUC] \\
\hline $\mathrm{C}_{24} \mathrm{H}_{14} \mathrm{~S}_{6}$ & $\begin{array}{c}{[88493-55-4]} \\
\text { SUB } \\
\text { SUB }\end{array}$ & $\begin{array}{c}2,2^{\prime}, 5^{\prime}, 2^{\prime \prime}, 5^{\prime \prime}, 2^{\prime \prime \prime}, 5^{\prime \prime \prime}, 2^{\prime \prime \prime \prime}, 5^{\prime \prime \prime \prime}, 2^{\prime \prime \prime} \\
(503-563)\end{array}$ & ophene & $\begin{array}{l}\mathrm{ME} \\
\mathrm{ME}\end{array}$ & $\begin{array}{l}{[1998 \mathrm{KLO} / \mathrm{LAU}]} \\
{[1998 \mathrm{KLO} / \mathrm{LAU}]}\end{array}$ \\
\hline $\mathrm{C}_{24} \mathrm{H}_{15} \mathrm{Br}_{3}$ & $\begin{array}{l}{[7511-49-1]} \\
\text { SUB }\end{array}$ & $\begin{array}{r}\text { 1,3,5-tris(4-bromophenyl)ben } \\
177 \pm 3\end{array}$ & & ME & [2010GUT/HEC] \\
\hline $\mathrm{C}_{24} \mathrm{H}_{16}$ & $\begin{array}{c}\text { [14620-98-5] } \\
\text { SUB }\end{array}$ & $\begin{array}{l}\text { trans-heptacyclene } \\
\qquad 140.0 \pm 3\end{array}$ & 433 & $\mathrm{ME}$ & [2006SAN/BER] \\
\hline
\end{tabular}


TABLE 14. Phase change enthalpies of $\mathrm{C}_{19}-\mathrm{C}_{29}$ organic compounds-Continued

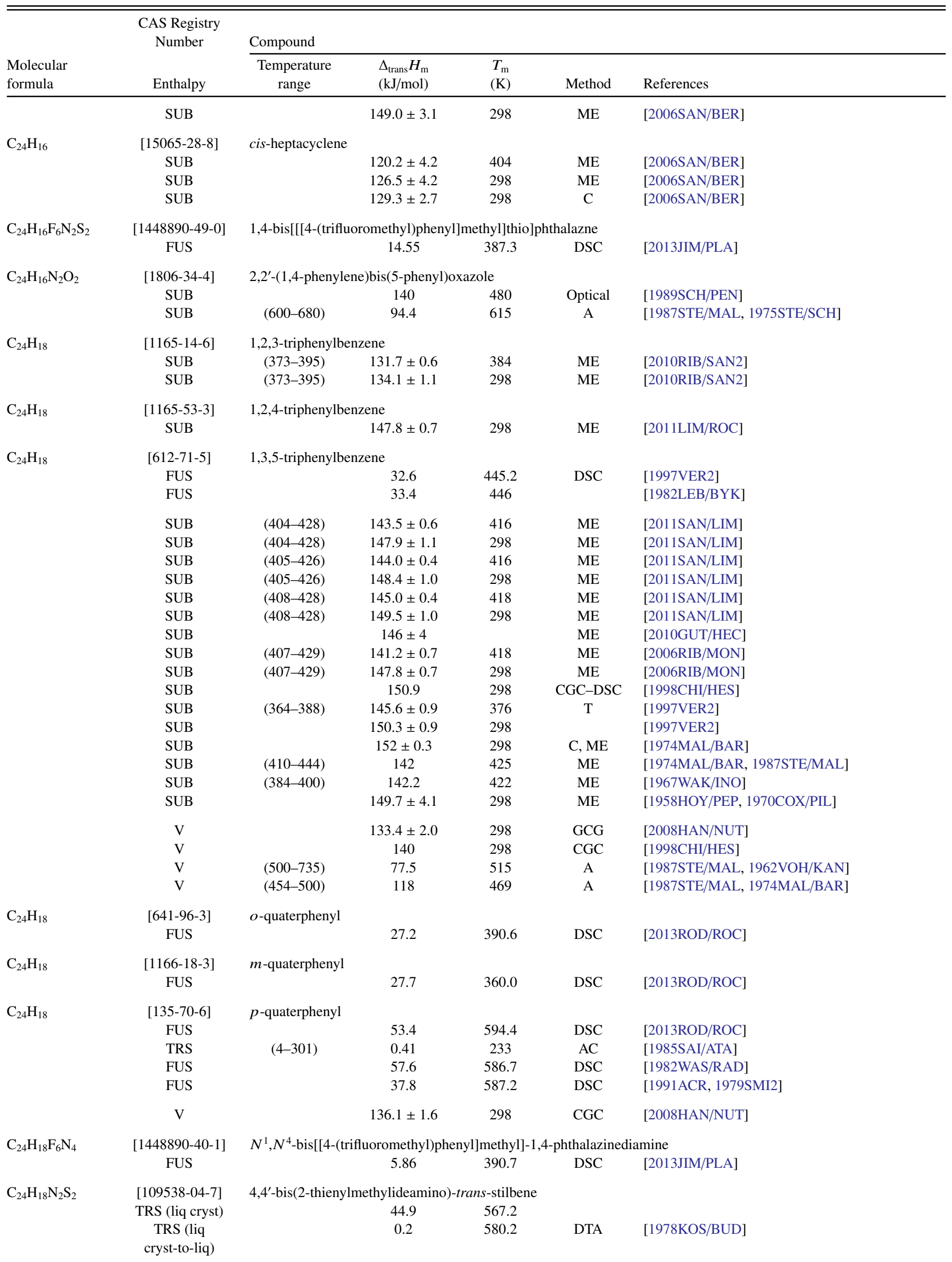


TABLE 14. Phase change enthalpies of $\mathrm{C}_{19}-\mathrm{C}_{29}$ organic compounds-Continued

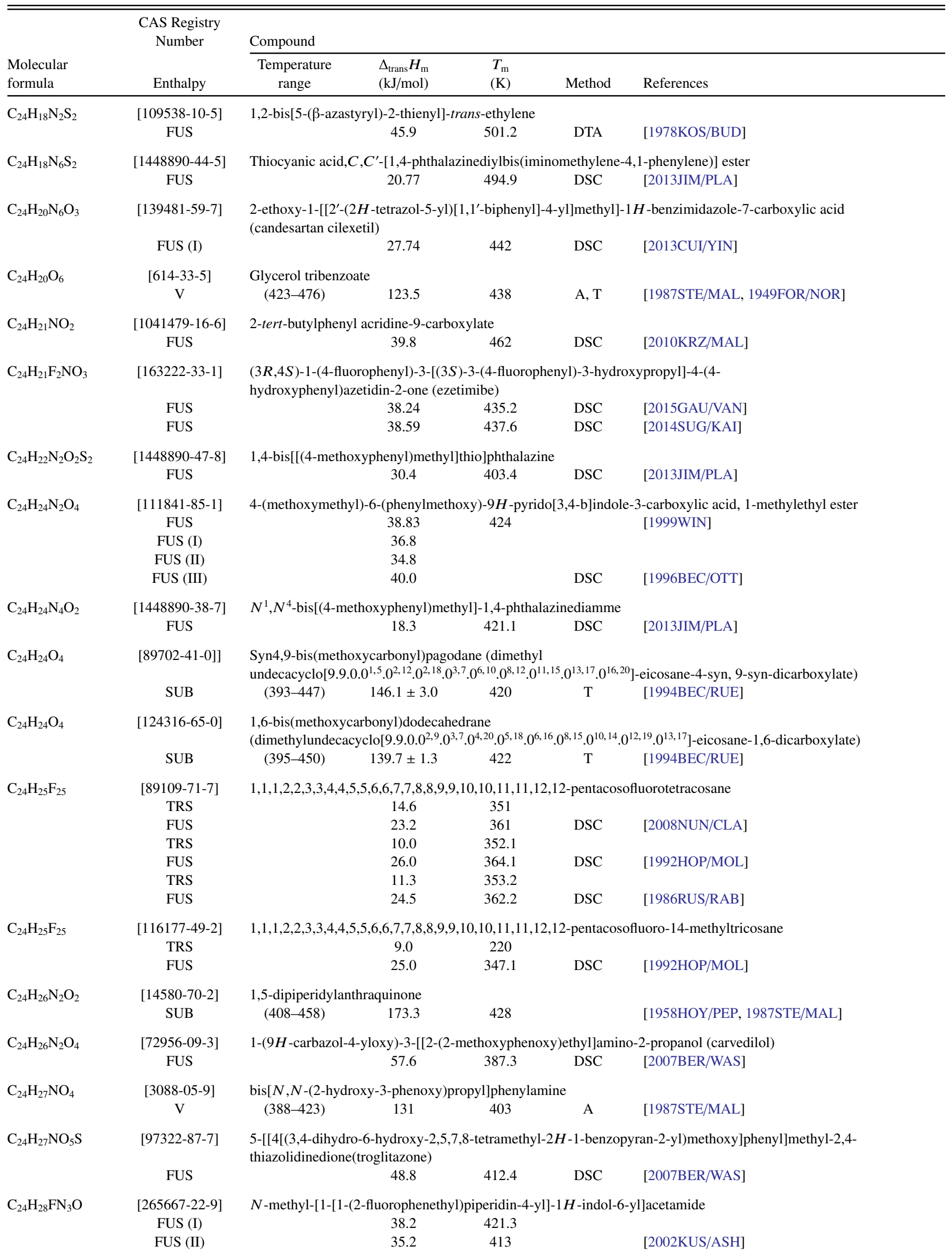


TABLE 14. Phase change enthalpies of $\mathrm{C}_{19}-\mathrm{C}_{29}$ organic compounds-Continued

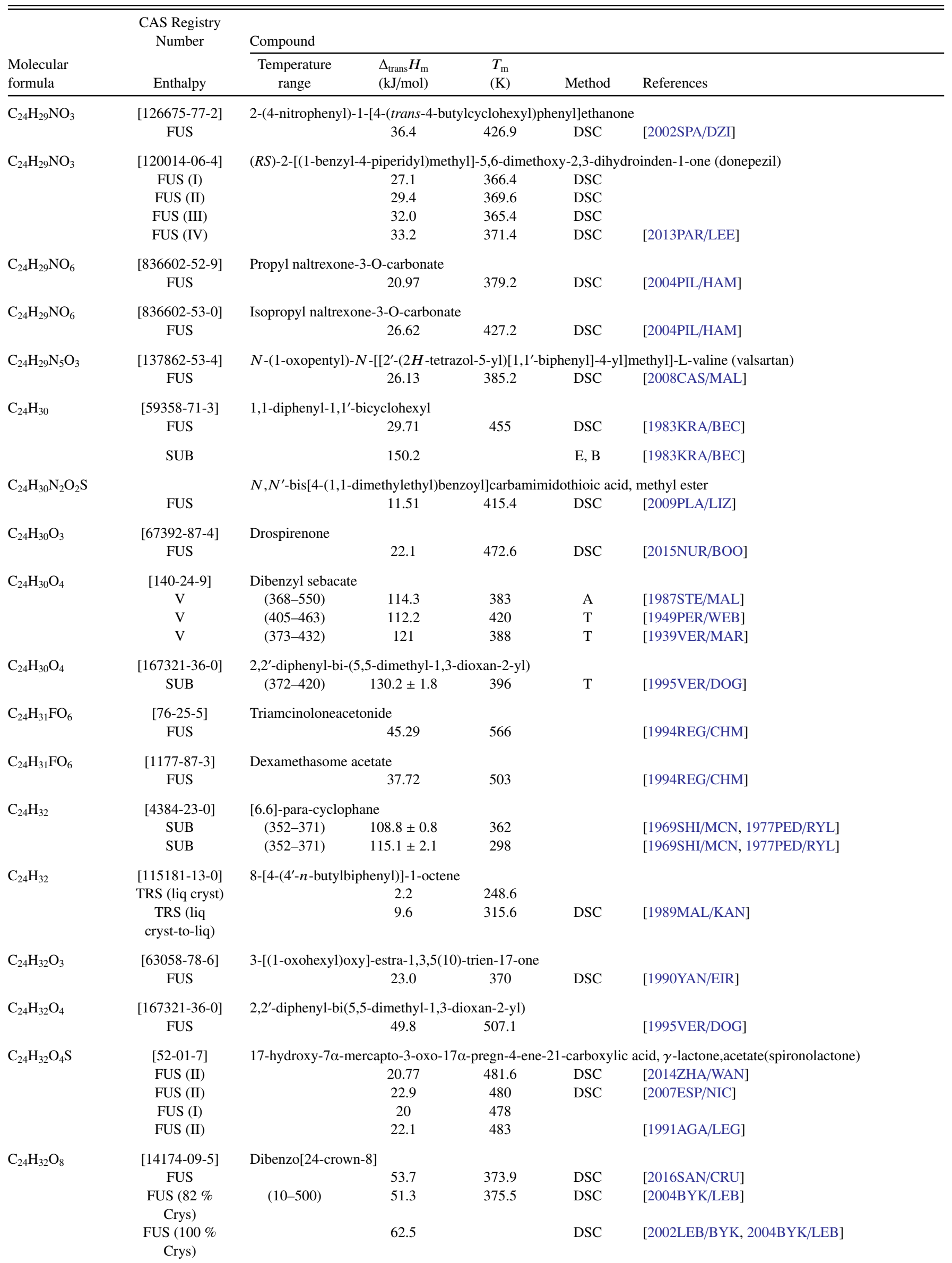


TABLE 14. Phase change enthalpies of $\mathrm{C}_{19}-\mathrm{C}_{29}$ organic compounds-Continued

\begin{tabular}{|c|c|c|c|c|c|c|}
\hline \multirow[b]{2}{*}{$\begin{array}{l}\text { Molecular } \\
\text { formula }\end{array}$} & \multirow{2}{*}{$\begin{array}{l}\text { CAS Registry } \\
\text { Number } \\
\text { Enthalpy }\end{array}$} & \multicolumn{5}{|l|}{ Compound } \\
\hline & & $\begin{array}{l}\text { Temperature } \\
\text { range }\end{array}$ & $\begin{array}{l}\Delta_{\text {trans }} H_{\mathrm{m}} \\
(\mathrm{kJ} / \mathrm{mol})\end{array}$ & $\begin{array}{l}T_{\mathrm{m}} \\
(\mathrm{K})\end{array}$ & Method & References \\
\hline & TRS & & 16.6 & 354.1 & & \\
\hline & FUS & & 52.25 & 375.4 & & [1998DOM, 1985RAE/SOL] \\
\hline & SUB & & $232.9 \pm 3.6$ & 298 & $\mathrm{~V}+\mathrm{F}$ & [2016SAN/CRU] \\
\hline & $\mathrm{V}$ & $(545-595)$ & $112.0 \pm 0.2$ & 570 & TGA & [2016SAN/CRU] \\
\hline & $\mathrm{V}$ & $(545-595)$ & $190.4 \pm 0.2$ & 298 & TGA & [2016SAN/CRU] \\
\hline \multirow[t]{2}{*}{$\mathrm{C}_{24} \mathrm{H}_{33} \mathrm{~F}_{17} \mathrm{O}$} & {$[699008-59-8]$} & \multicolumn{5}{|c|}{$1-[(3,3,4,4,5,5,6,6,7,7,8,8,9,9,10,10,10$-heptadecafluorodecyl)oxy]tetradecane } \\
\hline & FUS & & 45.12 & 300.3 & DSC & {$[2010 \mathrm{ZAG} / \mathrm{CON}]$} \\
\hline \multirow[t]{3}{*}{$\mathrm{C}_{24} \mathrm{H}_{34}$} & {$[1603-53-8]$} & \multicolumn{5}{|c|}{ 1,1-diphenyldodecane } \\
\hline & TRS & & 1.92 & 191 & & \\
\hline & FUS & & 38.83 & 281.4 & & [1996DOM/HEA, 1960KAR/STR] \\
\hline \multirow[t]{2}{*}{$\mathrm{C}_{24} \mathrm{H}_{34} \mathrm{~N}_{4} \mathrm{O}_{5} \mathrm{~S}$} & {$[93479-97-1]$} & \multicolumn{5}{|c|}{$\begin{array}{l}\text { 3-ethyl-2,5-dihydro-4-methyl- } N \text {-[2-[4-[[[[trans-4-methylcyclohexyl)amino] carbonyl] amino }] \\
\text { sulfonyl]phenyl] ethyl-2-oxo- } H \text {-pyrrole-1-carboxami(glimepiride) }\end{array}$} \\
\hline & FUS & & 53.3 & 485.7 & DSC & [2007BER/WAS] \\
\hline \multirow[t]{2}{*}{$\mathrm{C}_{24} \mathrm{H}_{36} \mathrm{O}_{3}$} & {$[3129-43-9]$} & \multicolumn{5}{|c|}{ Testosteronevalerate } \\
\hline & FUS & & 24.57 & 380 & & [1994REG/CHM] \\
\hline \multirow[t]{4}{*}{$\mathrm{C}_{24} \mathrm{H}_{36} \mathrm{O}_{5}$} & {$[75330-75-5]$} & \multicolumn{5}{|c|}{$\begin{array}{l}\text { 2-methylbutanoicacid, (1S,3R,7S,8S,8aR)-1,2,3,7,8,8a-hexahydro-3,7-dimethyl-8-[2-[(2R,4R)-tetrahydro-4- } \\
\text { hydroxy-6-oxo-2 } \mathrm{H} \text {-pyran-2-yl]ethyl]-1-naphthalenyl ester (lovastatin) }\end{array}$} \\
\hline & FUS & & 36.53 & 444.3 & DSC & {$[2008 \mathrm{TUN} / \mathrm{TAB}]$} \\
\hline & FUS & & 43.14 & 445.5 & DSC & [2008NTI/CHM] \\
\hline & FUS & & 43.1 & 445.2 & DSC & {$[2007 \mathrm{SOU} / \mathrm{CON}]$} \\
\hline $\mathrm{C}_{24} \mathrm{H}_{37} \mathrm{~N}_{3} \mathrm{O}$ & $\begin{array}{l}\text { [218765-43-6] } \\
\text { FUS }\end{array}$ & \multicolumn{5}{|c|}{ Pyrimethanil laurate } \\
\hline \multirow[t]{7}{*}{$\mathrm{C}_{24} \mathrm{H}_{38} \mathrm{O}_{4}$} & {$[117-81-7]$} & \multicolumn{5}{|c|}{ bis(2-ethylhexyl)phthalate } \\
\hline & V & & $116.9 \pm 3.8$ & 298 & CRT & {$[2015 \mathrm{GOB} / \mathrm{CHI}]$} \\
\hline & $\mathrm{V}$ & & $122.8 \pm 3.3$ & 298 & CGC & [2015GOB/CHI] \\
\hline & $\mathrm{V}$ & & $115.9 \pm 3.8$ & 298 & CGC & [2014GOB/CHI] \\
\hline & $\mathrm{V}$ & $(373-660)$ & 102.5 & 388 & A & [1987STE/MAL] \\
\hline & $\mathrm{V}$ & $(393-503)$ & 107.6 & 408 & & [1952WER] \\
\hline & V & $(385-440)$ & 110.7 & 390 & $\mathrm{~T}$ & [1949PER/WEB] \\
\hline \multirow[t]{2}{*}{$\mathrm{C}_{24} \mathrm{H}_{38} \mathrm{O}_{4}$} & {$[6422-86-2]$} & \multicolumn{5}{|c|}{ bis(2-ethylhexyl)terephthalate } \\
\hline & V & & $123.2 \pm 1.1$ & 298 & CGC & {$[2014 \mathrm{GOB} / \mathrm{CHI}]$} \\
\hline \multirow[t]{2}{*}{$\mathrm{C}_{24} \mathrm{H}_{38} \mathrm{O}_{4}$} & {$[131-15-7]$} & bis(1-methylhe & phthalate & & & \\
\hline & V & $(393-435)$ & 93.1 & 408 & A & [1987STE/MAL, 1952WER] \\
\hline $\mathrm{C}_{24} \mathrm{H}_{38} \mathrm{O}_{4}$ & {$[131-20-4]$} & bis(6-methylhe & phthalate & & & \\
\hline & V & $(383-490)$ & 92.4 & 398 & A & [1987STE/MAL, 1952WER] \\
\hline $\mathrm{C}_{24} \mathrm{H}_{38} \mathrm{O}_{4}$ & {$[117-84-0]$} & Dioctyl phthala & & & & \\
\hline & $\mathrm{V}$ & & $122.7 \pm 3.2$ & 298 & CRT & [2015GOB/CHI] \\
\hline & $\mathrm{V}$ & & $131.6 \pm 5.7$ & 298 & CGC & [2015GOB/CHI] \\
\hline & $\mathrm{V}$ & & $122.6 \pm 1.4$ & 298 & CGC & [2014GOB/CHI] \\
\hline & V & $(423-523)$ & 99.5 & 438 & A & [1987STE/MAL] \\
\hline & $\mathrm{V}$ & $(383-433)$ & 107.6 & 398 & $\mathrm{~T}$ & [1949PER/WEB] \\
\hline $\mathrm{C}_{24} \mathrm{H}_{40}$ & {$[62155-50-4]$} & 1-cyclohexyl-1 & nyldodecane & & & \\
\hline & FUS & & 35.19 & 275.8 & & [1996DOM/HEA, 1960KAR/STR] \\
\hline $\mathrm{C}_{24} \mathrm{H}_{40} \mathrm{~N}_{8} \mathrm{O}_{4}$ & {$[58-32-2]$} & $2,2^{\prime}, 2^{\prime \prime}, 2^{\prime \prime \prime}-[(4,8$ & 1-piperidinylp & $\operatorname{do}[5,4-\mathrm{c}$ & nidine-2, 6 & yl)-dinitrilo]-tetrakis-ethanol (dipyridamole) \\
\hline & FUS (I) & & 44.05 & 442.8 & & \\
\hline & FUS (II) & & 33.2 & 441.9 & DSC & [2006ADH/BAS] \\
\hline & FUS & & 28 & 438.9 & DSC & [2002BER/MAR] \\
\hline $\mathrm{C}_{24} \mathrm{H}_{40} \mathrm{O}_{3}$ & & 5-(1,1-dimethy & tyl)-2-[(1R,2R & 5-hydro & (3-hydrox & opyl)cyclohexyl]phenol \\
\hline & FUS & & 18.4 & 357 & & [2004VAL/KIP] \\
\hline $\mathrm{C}_{24} \mathrm{H}_{40} \mathrm{O}_{4}$ & [175848-64-3] & 2,5-di-n-nonyl & 1,4-benzoquir & & & \\
\hline & TRS & & 8.0 & 352.6 & & \\
\hline & TRS & & 24.2 & 383.8 & & \\
\hline
\end{tabular}


TABLE 14. Phase change enthalpies of $\mathrm{C}_{19}-\mathrm{C}_{29}$ organic compounds-Continued

\begin{tabular}{|c|c|c|c|c|c|c|}
\hline \multirow[b]{2}{*}{$\begin{array}{l}\text { Molecular } \\
\text { formula }\end{array}$} & \multirow{2}{*}{$\begin{array}{l}\text { CAS Registry } \\
\text { Number } \\
\text { Enthalpy }\end{array}$} & \multicolumn{5}{|l|}{ Compound } \\
\hline & & $\begin{array}{l}\text { Temperature } \\
\text { range }\end{array}$ & $\begin{array}{l}\Delta_{\text {trans }} H_{\mathrm{m}} \\
(\mathrm{kJ} / \mathrm{mol})\end{array}$ & $\begin{array}{l}T_{\mathrm{m}} \\
(\mathrm{K})\end{array}$ & Method & References \\
\hline & FUS & & 47.1 & 402.7 & DSC & [1996KEE/VAN] \\
\hline $\mathrm{C}_{24} \mathrm{H}_{41} \mathrm{~F}_{9} \mathrm{O}$ & $\begin{array}{c}\text { [1240205-69-9] } \\
\text { FUS }\end{array}$ & $1-[(3,3,4,4,5,5,6,6,6-1$ & $\begin{array}{c}\text { nonafluorol } \\
28.56\end{array}$ & $\begin{array}{l}\text { xy]octa } \\
291.7\end{array}$ & DSC & [2010ZAG/CON] \\
\hline $\mathrm{C}_{24} \mathrm{H}_{42}$ & $\begin{array}{c}{[2456-68-0]} \\
\mathrm{V}\end{array}$ & $\begin{array}{l}\text { Hexapropylbenzene } \\
\quad(458-606)\end{array}$ & 68.4 & 473 & A & [1987STE/MAL, 1937GRO/IPA] \\
\hline $\mathrm{C}_{24} \mathrm{H}_{42}$ & $\begin{array}{c}{[4445-07-2]} \\
\mathrm{V}\end{array}$ & $\begin{array}{l}\text { Octadecylbenzene } \\
\quad(423-675)\end{array}$ & 101 & 438 & & [1999DYK/SVO] \\
\hline $\mathrm{C}_{24} \mathrm{H}_{42} \mathrm{O}_{6}$ & $\begin{array}{c}{[64617-30-7]} \\
\mathrm{V}\end{array}$ & $\begin{array}{l}\text { trans trihexyl aconita } \\
\qquad(423-512)\end{array}$ & 98.2 & 438 & A & [1987STE/MAL, 1953MAG/MOD] \\
\hline $\mathrm{C}_{24} \mathrm{H}_{42} \mathrm{O}_{11}$ & $\mathrm{~V}$ & $\begin{array}{l}\text { Di[1-(2-ethylbutylox } \\
\quad(448-538)\end{array}$ & $\begin{array}{c}\text { ycarbonyl) } \\
110.1\end{array}$ & $\begin{array}{l}\text { diethyle } \\
463\end{array}$ & $\begin{array}{c}\text { col dicarb } \\
\mathrm{A}\end{array}$ & $\begin{array}{l}\text { late } \\
\text { [1987STE/MAL] }\end{array}$ \\
\hline $\mathrm{C}_{24} \mathrm{H}_{42} \mathrm{O}_{11}$ & $\mathrm{~V}$ & $\begin{array}{l}\text { Di[1-(2-hexyloxycart } \\
\quad(443-548)\end{array}$ & $\begin{array}{l}\text { bonyl)ethyl } \\
111\end{array}$ & $\begin{array}{l}\text { ylene gl } \\
458\end{array}$ & $\begin{array}{c}\text { icarboxyl } \\
\text { A }\end{array}$ & [1987STE/MAL] \\
\hline $\mathrm{C}_{24} \mathrm{H}_{44}$ & $\mathrm{~V}$ & $\begin{array}{l}\text { 9-decyltetradecahydr } \\
\quad(501-536)\end{array}$ & $\begin{array}{c}\text { oanthracen } \\
103.2\end{array}$ & 516 & A & [1987STE/MAL] \\
\hline $\mathrm{C}_{24} \mathrm{H}_{44}$ & $\mathrm{~V}$ & $\begin{array}{l}\text { 9-decyltetradecahydr } \\
\quad(502-542)\end{array}$ & $\begin{array}{l}\text { ophenanthr } \\
92.0\end{array}$ & 517 & A & [1987STE/MAL] \\
\hline $\mathrm{C}_{24} \mathrm{H}_{44} \mathrm{O}_{2}$ & $\begin{array}{c}\text { [31067-27-3] } \\
\text { FUS }\end{array}$ & $3,3,7,7,11,11,15,15-0$ & $\begin{array}{l}\text { ctamethylc } \\
34.3\end{array}$ & $\begin{array}{l}\text { xadecal } \\
423.2\end{array}$ & dione & [1972BOR/DAL2] \\
\hline $\mathrm{C}_{24} \mathrm{H}_{44} \mathrm{O}_{4}$ & $\begin{array}{c}\text { [38734-14-4] } \\
\text { FUS }\end{array}$ & 1,11-cycloeicosanedi & $\begin{array}{l}\text { Ione bis eth } \\
43.72\end{array}$ & $\begin{array}{l}\text { retal } \\
362.2\end{array}$ & & [1972ALV/BOR] \\
\hline $\mathrm{C}_{24} \mathrm{H}_{44} \mathrm{O}_{6}$ & $\begin{array}{c}{[140-04-5]} \\
\mathrm{V}\end{array}$ & $\begin{array}{l}\text { O-acetylricinoleic ac } \\
\quad(378-423)\end{array}$ & $\begin{array}{c}\text { id, butyl es } \\
105.2\end{array}$ & 393 & A & [1987STE/MAL] \\
\hline $\mathrm{C}_{24} \mathrm{H}_{44} \mathrm{O}_{6}$ & $\begin{array}{c}{[38094-13-2]} \\
\mathrm{V}\end{array}$ & $\begin{array}{l}\text { Trihexyl 1,2,3-propar } \\
\quad(422-526)\end{array}$ & $\begin{array}{l}\text { netricarbox } \\
\quad 98.1\end{array}$ & 437 & A & [1987STE/MAL] \\
\hline $\mathrm{C}_{24} \mathrm{H}_{44} \mathrm{O}_{6}$ & $\begin{array}{c}{[620-67-7]} \\
\mathrm{V}\end{array}$ & $\begin{array}{l}\text { Glyceroltriheptanoate } \\
\quad(401-452)\end{array}$ & 84.4 & 416 & & [2001BUR/JOS] \\
\hline $\mathrm{C}_{24} \mathrm{H}_{46}$ & $\begin{array}{c}{[18254-57-4]} \\
\text { FUS }\end{array}$ & 1,1-dicyclohexyldode & $\begin{array}{l}\text { ecane } \\
44.35\end{array}$ & 300.6 & & [1996DOM/HEA, 1960KAR/STR] \\
\hline $\mathrm{C}_{24} \mathrm{H}_{46}$ & $\begin{array}{c}\text { [95746-44-4] } \\
\text { FUS }\end{array}$ & 2,11-dicyclohexyldoc & $\begin{array}{l}\text { decane } \\
43.93\end{array}$ & 300.6 & & [1996DOM/HEA, 1960KAR/STR] \\
\hline $\mathrm{C}_{24} \mathrm{H}_{46}$ & $\begin{array}{c}{[95115-77-8]} \\
\text { FUS }\end{array}$ & trans-2,6-diheptyldec & $\begin{array}{l}\text { calin } \\
40.17\end{array}$ & 326.7 & & [1985VAR/BRI] \\
\hline $\mathrm{C}_{24} \mathrm{H}_{46} \mathrm{O}_{2}$ & $\begin{array}{c}\text { [506-37-6] } \\
\text { FUS }\end{array}$ & (15Z)-tetracosenoic a & acid 60.3 & 315 & DSC & [2015WIL/GOB] \\
\hline & SUB & & $228.9 \pm 2.4$ & 298 & $\mathrm{~V}+\mathrm{F}$ & [2015WIL/GOB] \\
\hline & $\mathrm{V}$ & & $170.6 \pm 2.3$ & 298 & CGC & [2015WIL/GOB] \\
\hline $\mathrm{C}_{24} \mathrm{H}_{46} \mathrm{O}_{4}$ & $\begin{array}{c}{[20270-50-2]} \\
\mathrm{V}\end{array}$ & $\begin{array}{l}\text { bis }(3,5,5 \text {-trimethylhe } \\
\quad(353-413)\end{array}$ & $\begin{array}{l}\text { xyl)adipate } \\
107.6\end{array}$ & 368 & $\mathrm{~A}, \mathrm{ME}$ & [1987STE/MAL, 1948SMA/SMA] \\
\hline $\mathrm{C}_{24} \mathrm{H}_{47} \mathrm{NO}_{3}$ & $\begin{array}{c}{[14246-59-4]} \\
\text { TRS + FUS }\end{array}$ & $N$-(1-oxododecyl)gly & $\begin{array}{l}\text { ycine } \\
71.0\end{array}$ & 402.2 & DSC & [2014RED/KRO] \\
\hline $\mathrm{C}_{24} \mathrm{H}_{47} \mathrm{NO}_{3}$ & $\begin{array}{c}\text { [1213779-59-9] } \\
\text { FUS }\end{array}$ & Undecanoic acid, 2-[ & $\begin{array}{l}\text { (1-oxounde } \\
53.6\end{array}$ & $\begin{array}{l}\text { ino]eth } \\
340.1\end{array}$ & DSC & [2010KAM/TAR] \\
\hline $\mathrm{C}_{24} \mathrm{H}_{48}$ & $\begin{array}{c}{[4445-06-1]} \\
\mathrm{V}\end{array}$ & $\begin{array}{l}\text { Octadecylcyclohexan } \\
\quad(422-675)\end{array}$ & 100.3 & 437 & & [1999DYK/SVO] \\
\hline $\mathrm{C}_{24} \mathrm{H}_{48}$ & $\begin{array}{c}{[10192-32-2]} \\
\mathrm{V}\end{array}$ & $\begin{array}{l}\text { 1-tetracosene } \\
\quad(418-663)\end{array}$ & 101 & 433 & & [1999DYK/SVO] \\
\hline
\end{tabular}


TABLE 14. Phase change enthalpies of $\mathrm{C}_{19}-\mathrm{C}_{29}$ organic compounds-Continued

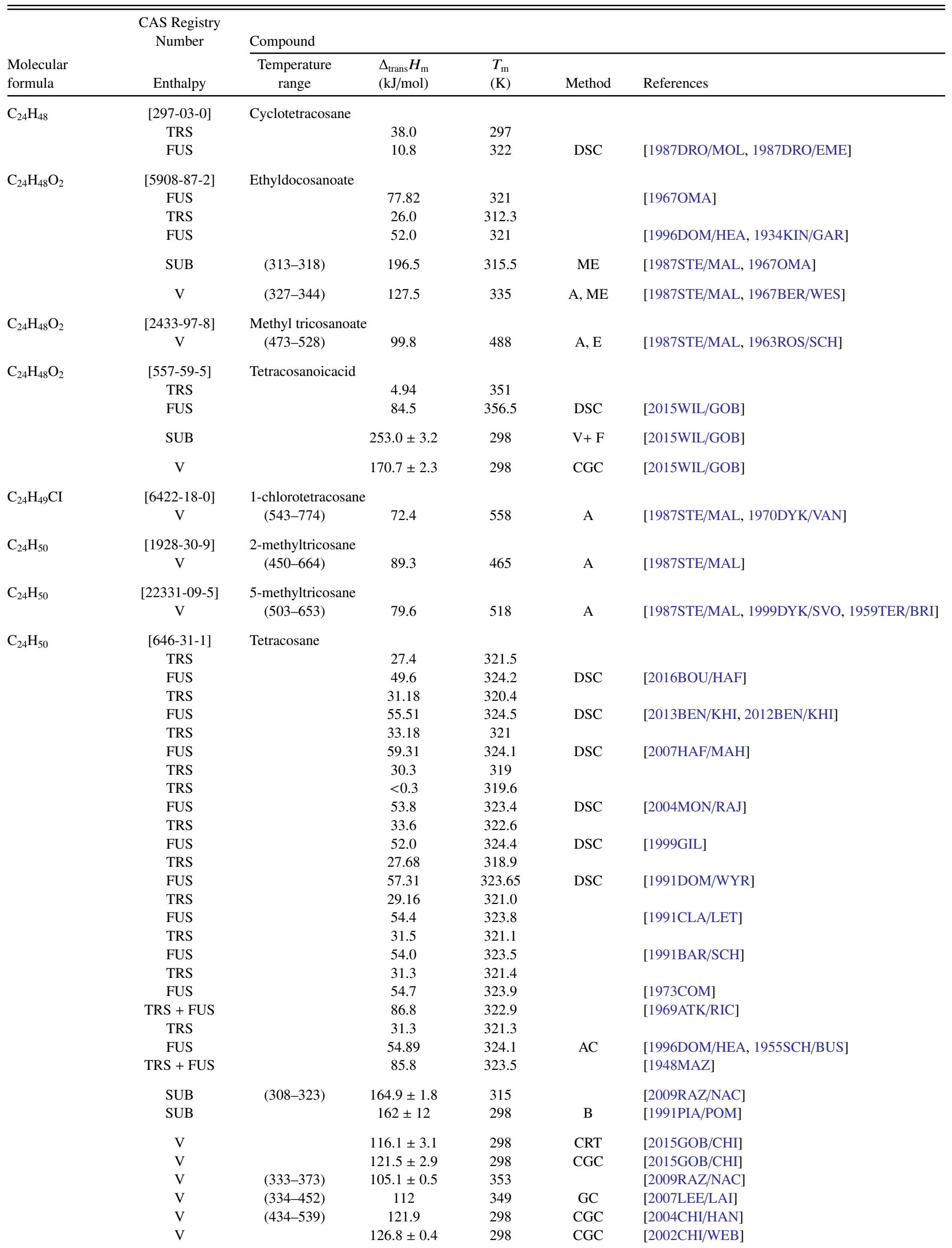


TABLE 14. Phase change enthalpies of $\mathrm{C}_{19}-\mathrm{C}_{29}$ organic compounds-Continued

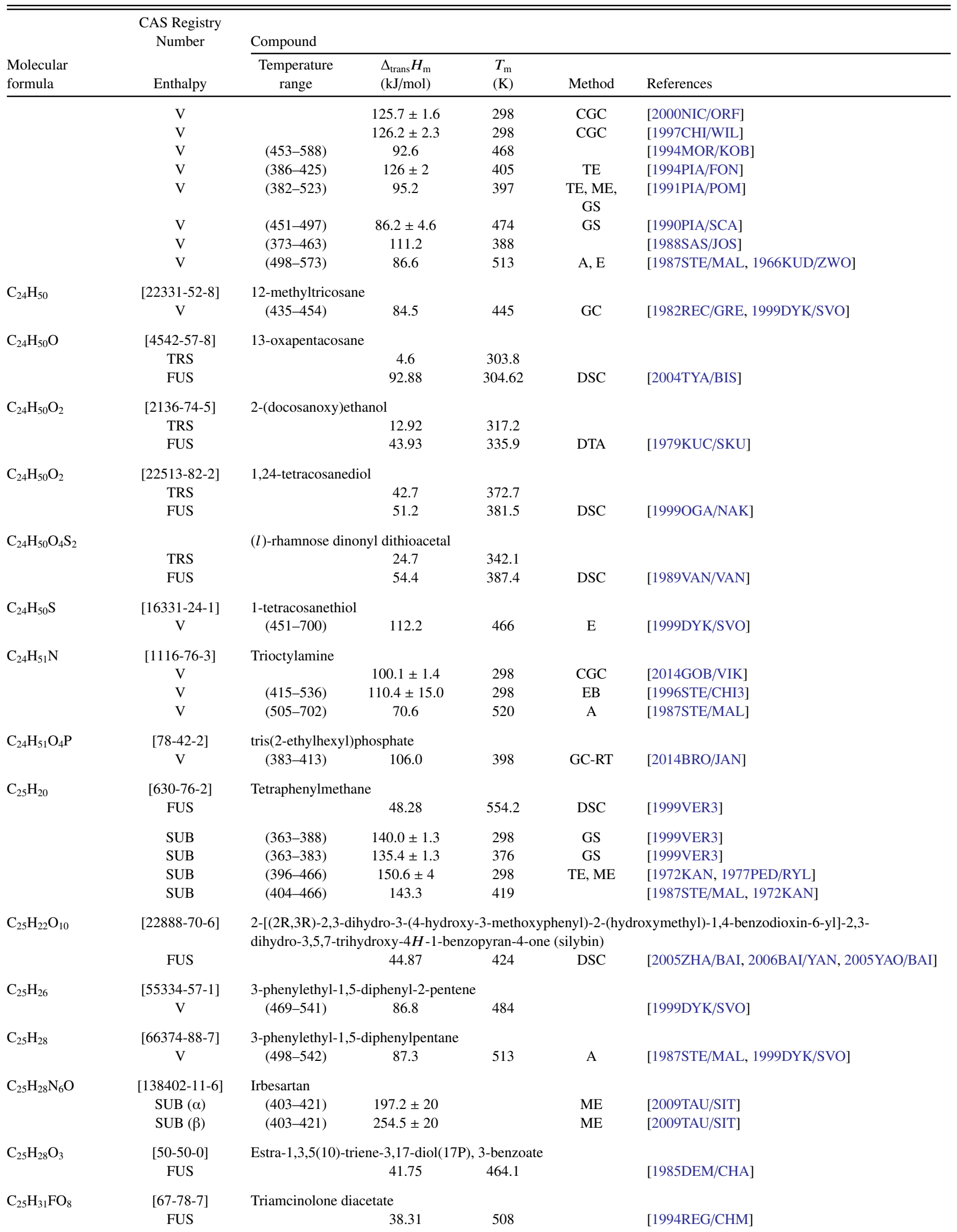


TABLE 14. Phase change enthalpies of $\mathrm{C}_{19}-\mathrm{C}_{29}$ organic compounds-Continued

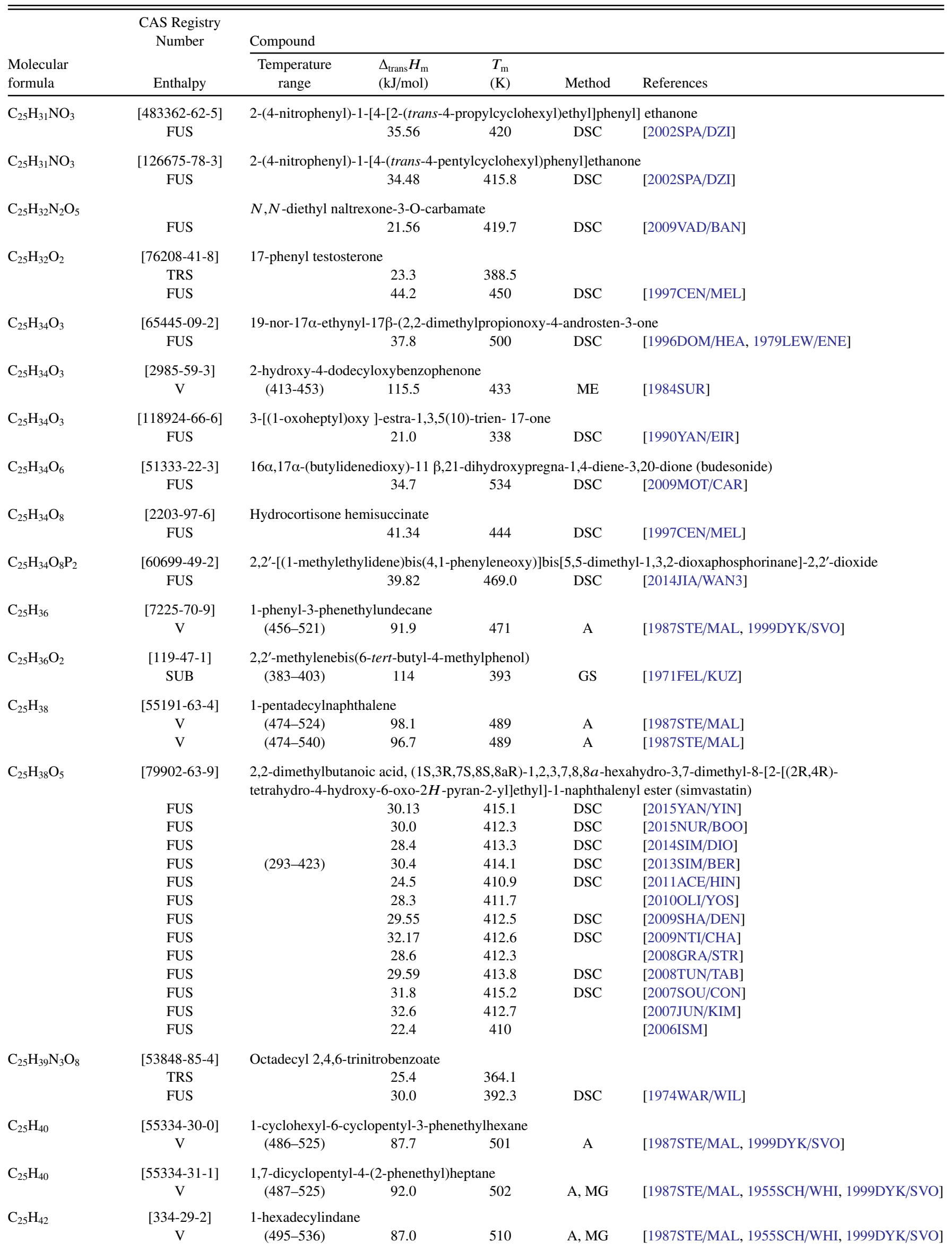


TABLE 14. Phase change enthalpies of $\mathrm{C}_{19}-\mathrm{C}_{29}$ organic compounds-Continued

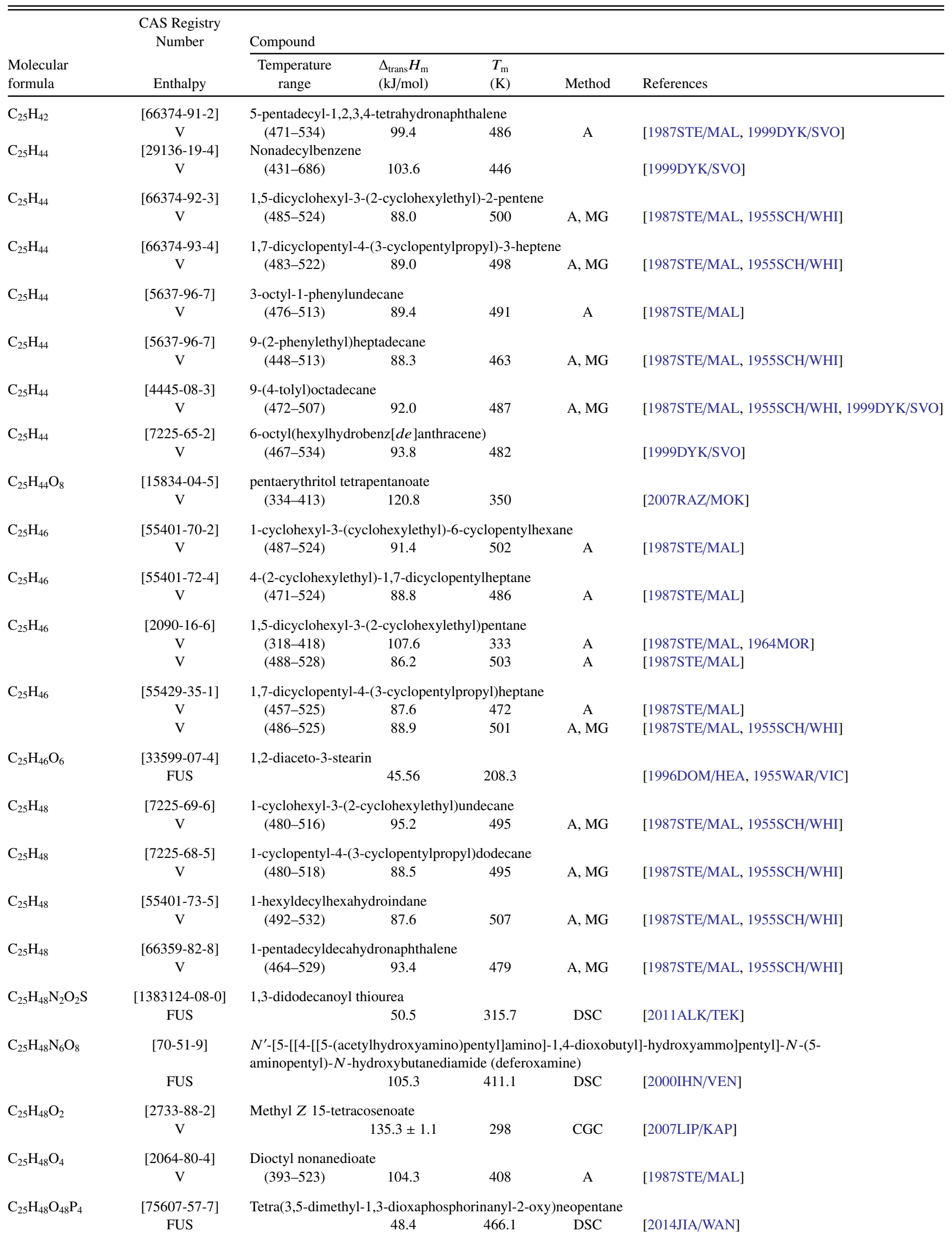


TABLE 14. Phase change enthalpies of $\mathrm{C}_{19}-\mathrm{C}_{29}$ organic compounds-Continued

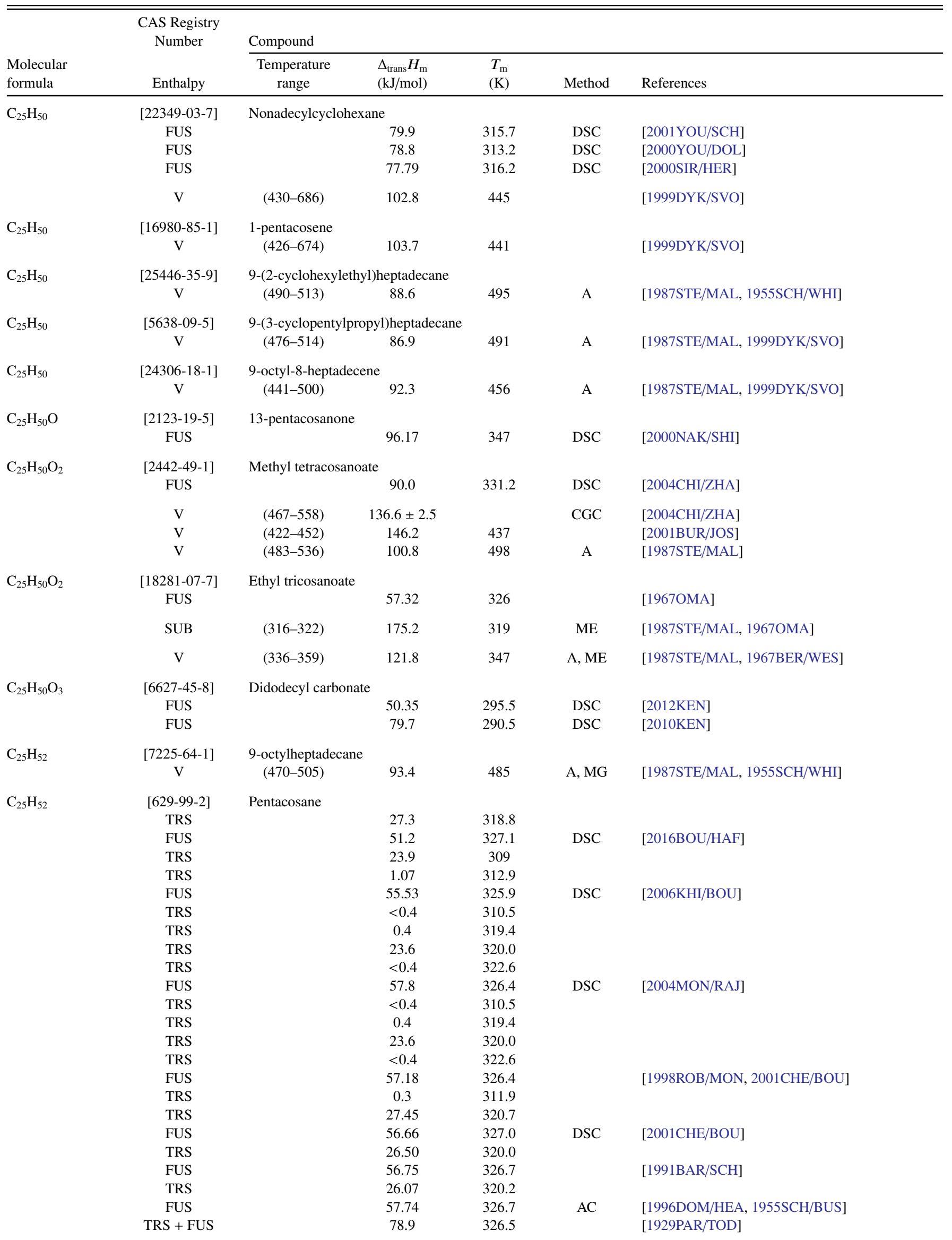


TABLE 14. Phase change enthalpies of $\mathrm{C}_{19}-\mathrm{C}_{29}$ organic compounds-Continued

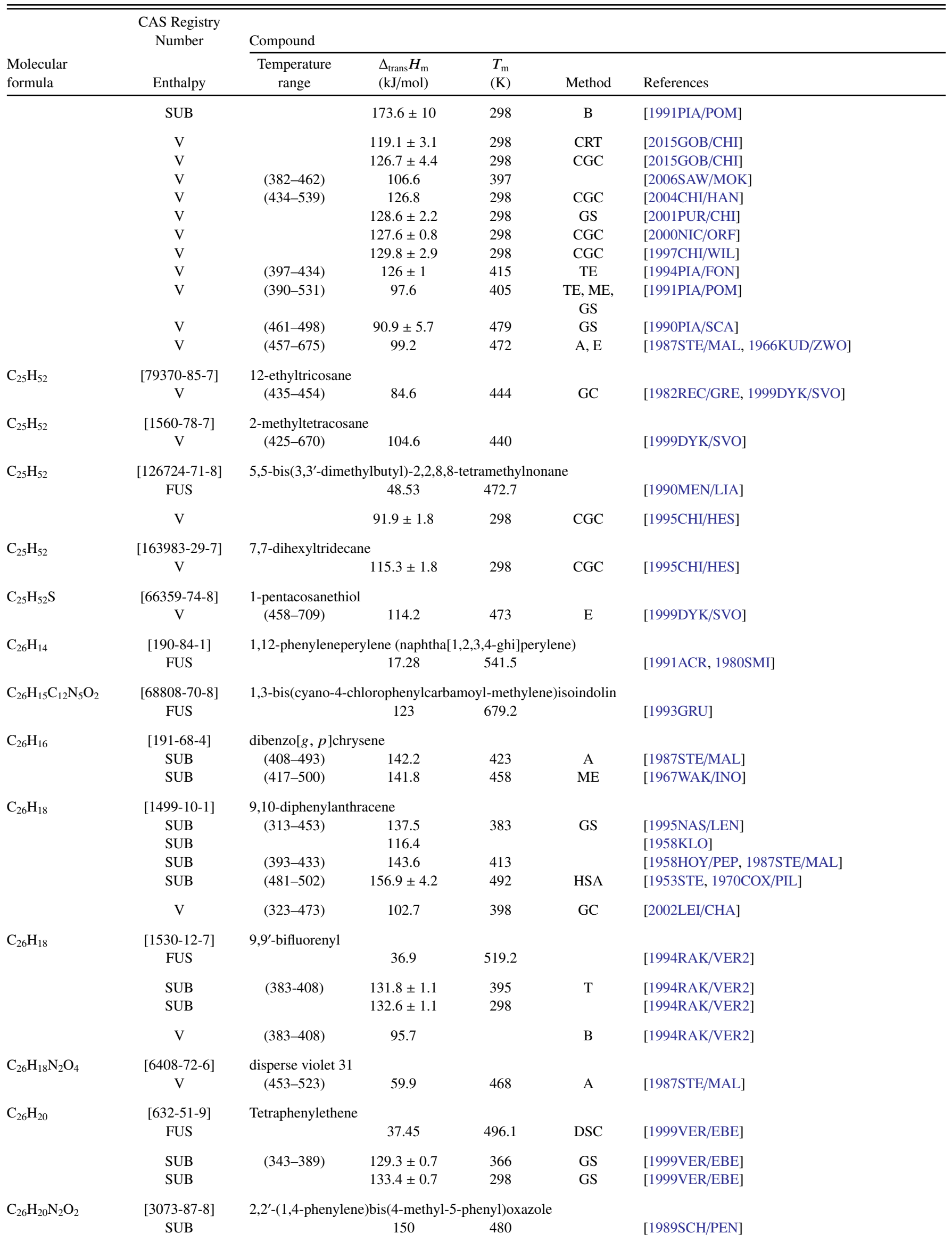


TABLE 14. Phase change enthalpies of $\mathrm{C}_{19}-\mathrm{C}_{29}$ organic compounds-Continued

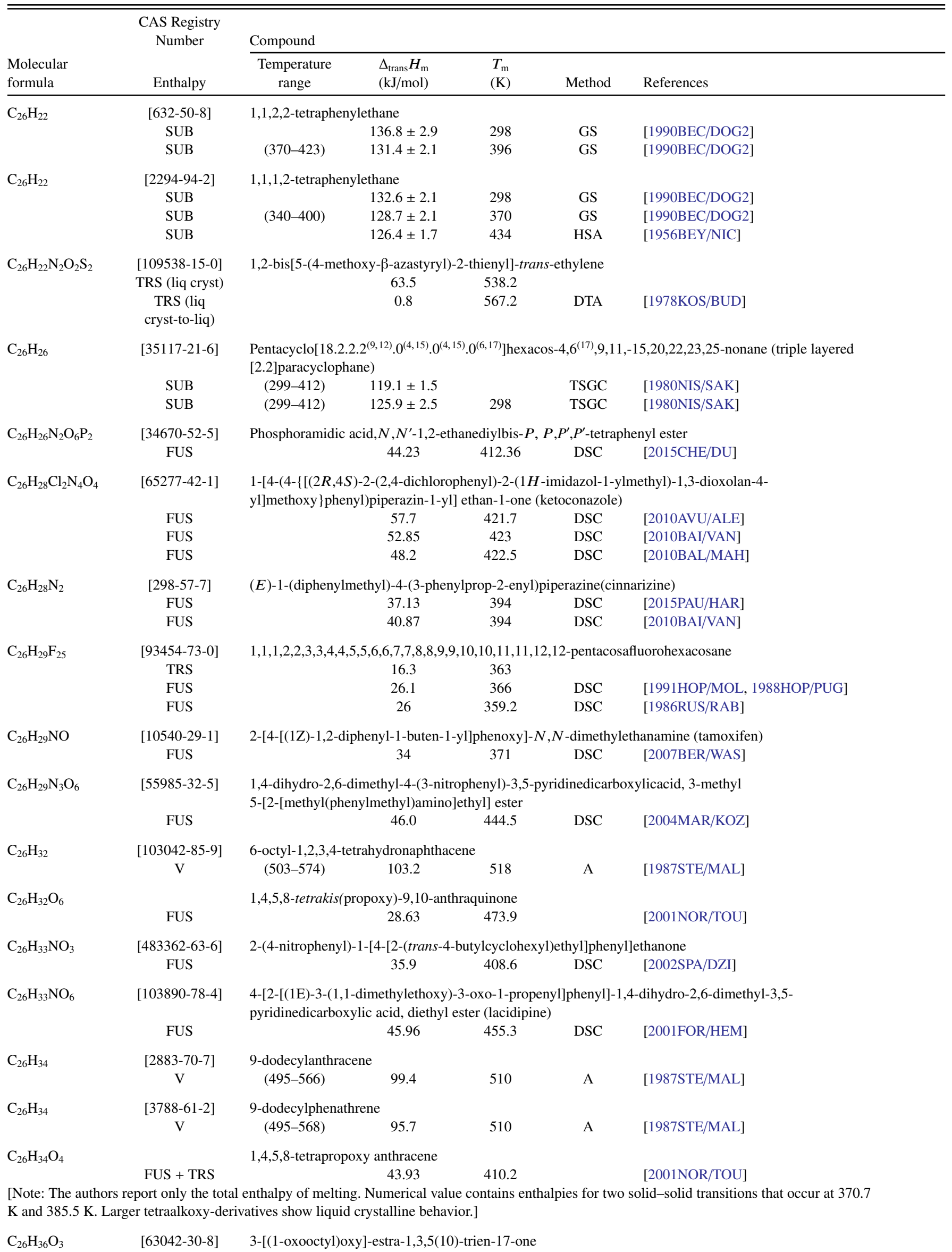


TABLE 14. Phase change enthalpies of $\mathrm{C}_{19}-\mathrm{C}_{29}$ organic compounds-Continued

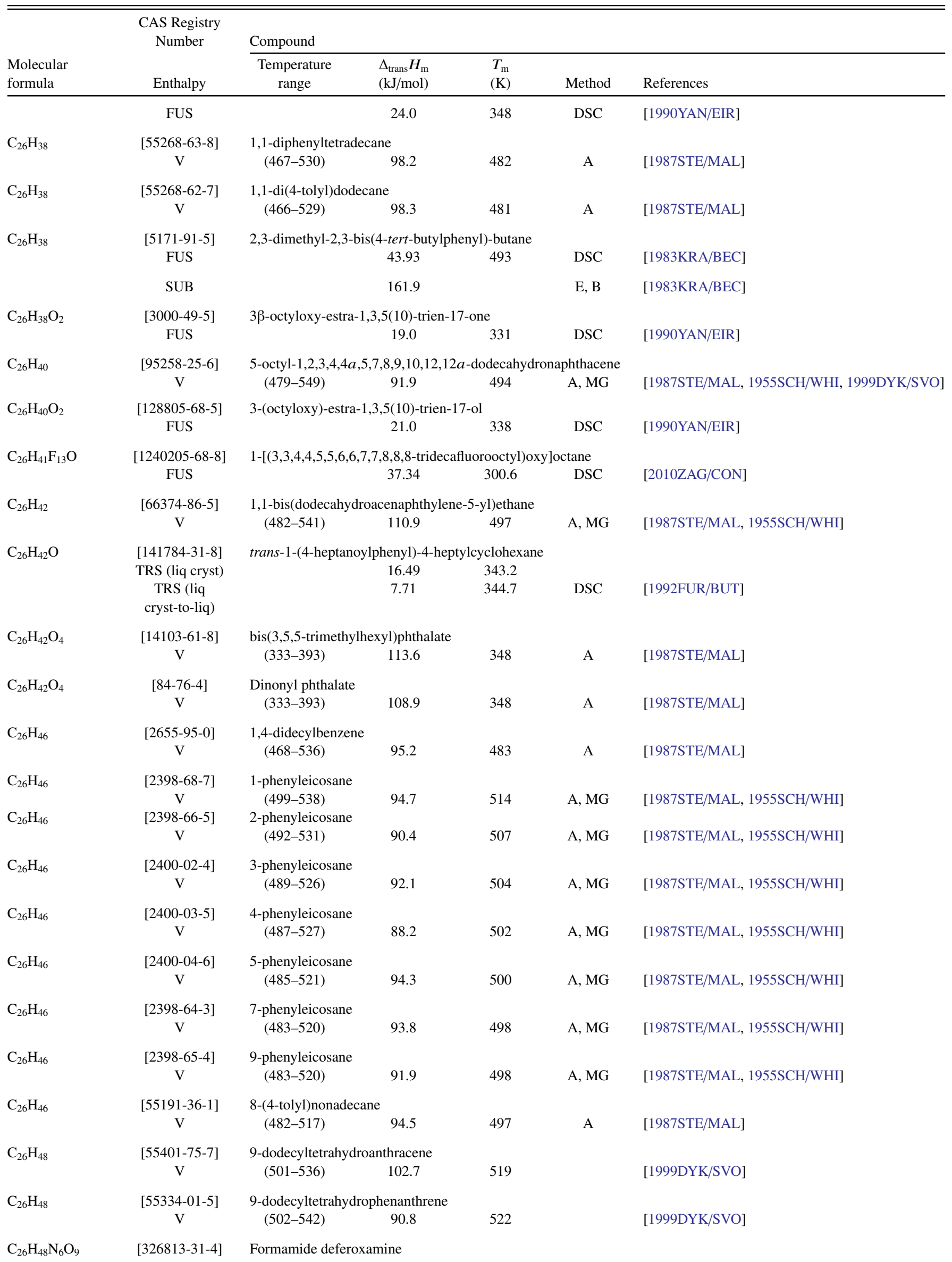


TABLE 14. Phase change enthalpies of $\mathrm{C}_{19}-\mathrm{C}_{29}$ organic compounds-Continued

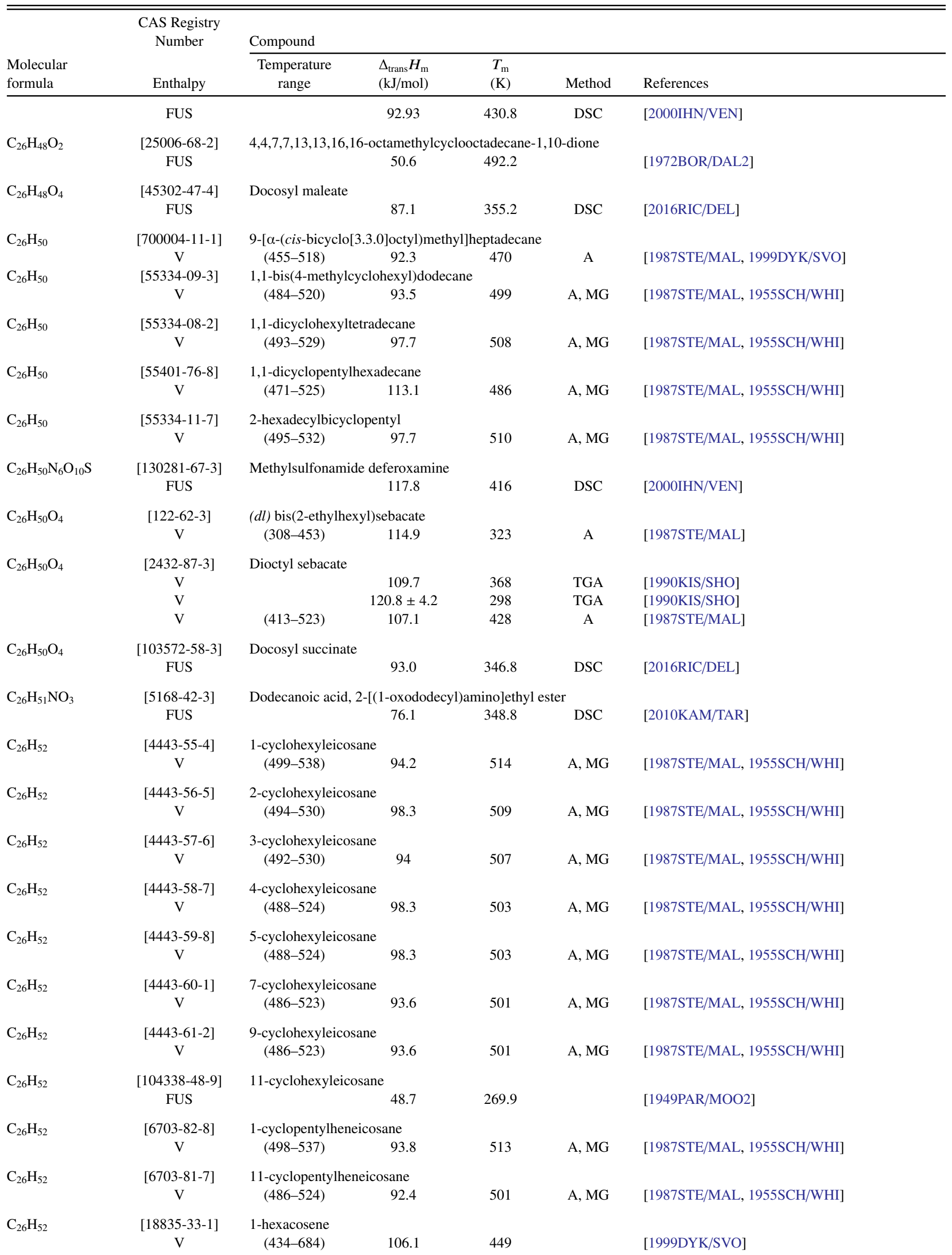


TABLE 14. Phase change enthalpies of $\mathrm{C}_{19}-\mathrm{C}_{29}$ organic compounds-Continued

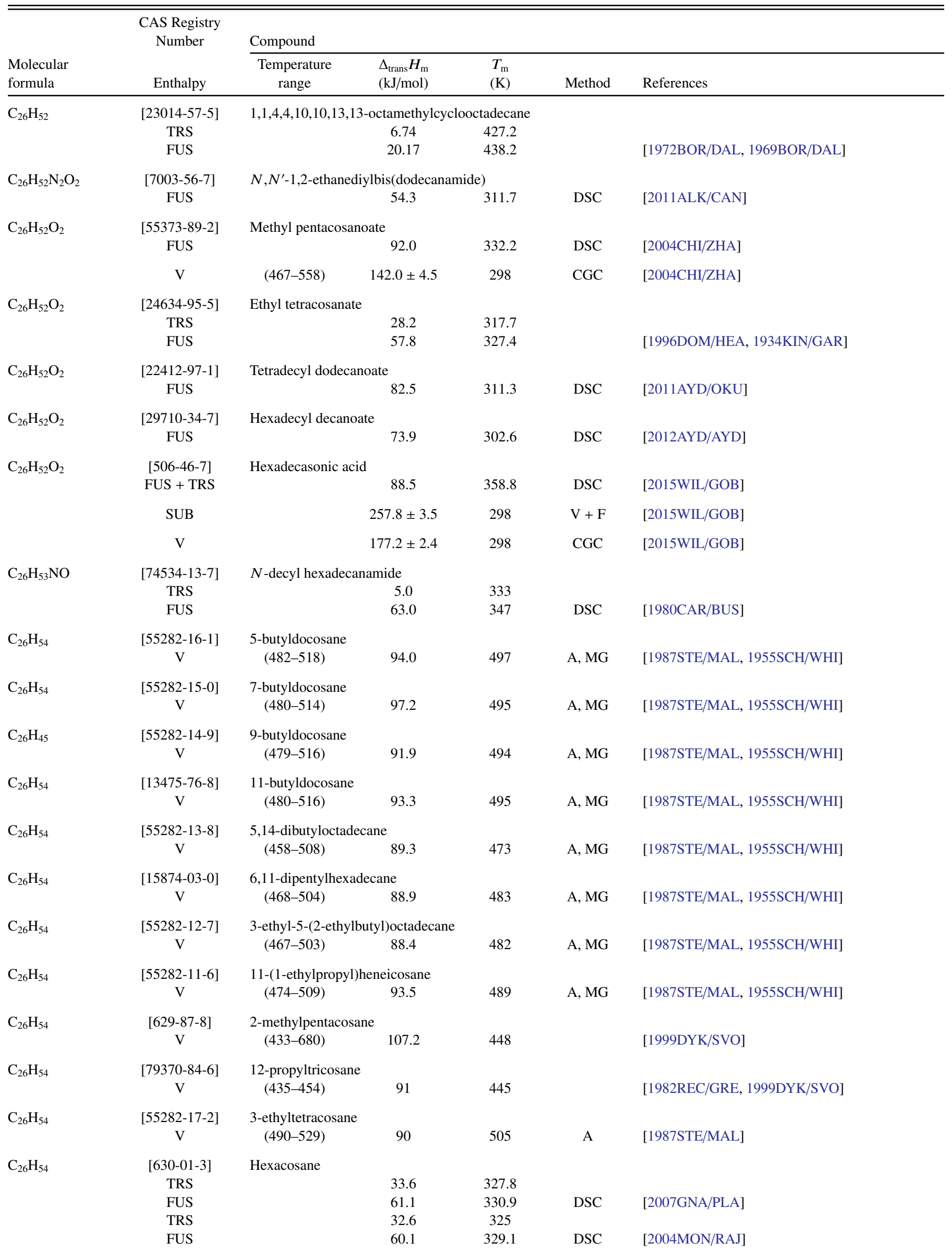


TABLE 14. Phase change enthalpies of $\mathrm{C}_{19}-\mathrm{C}_{29}$ organic compounds-Continued

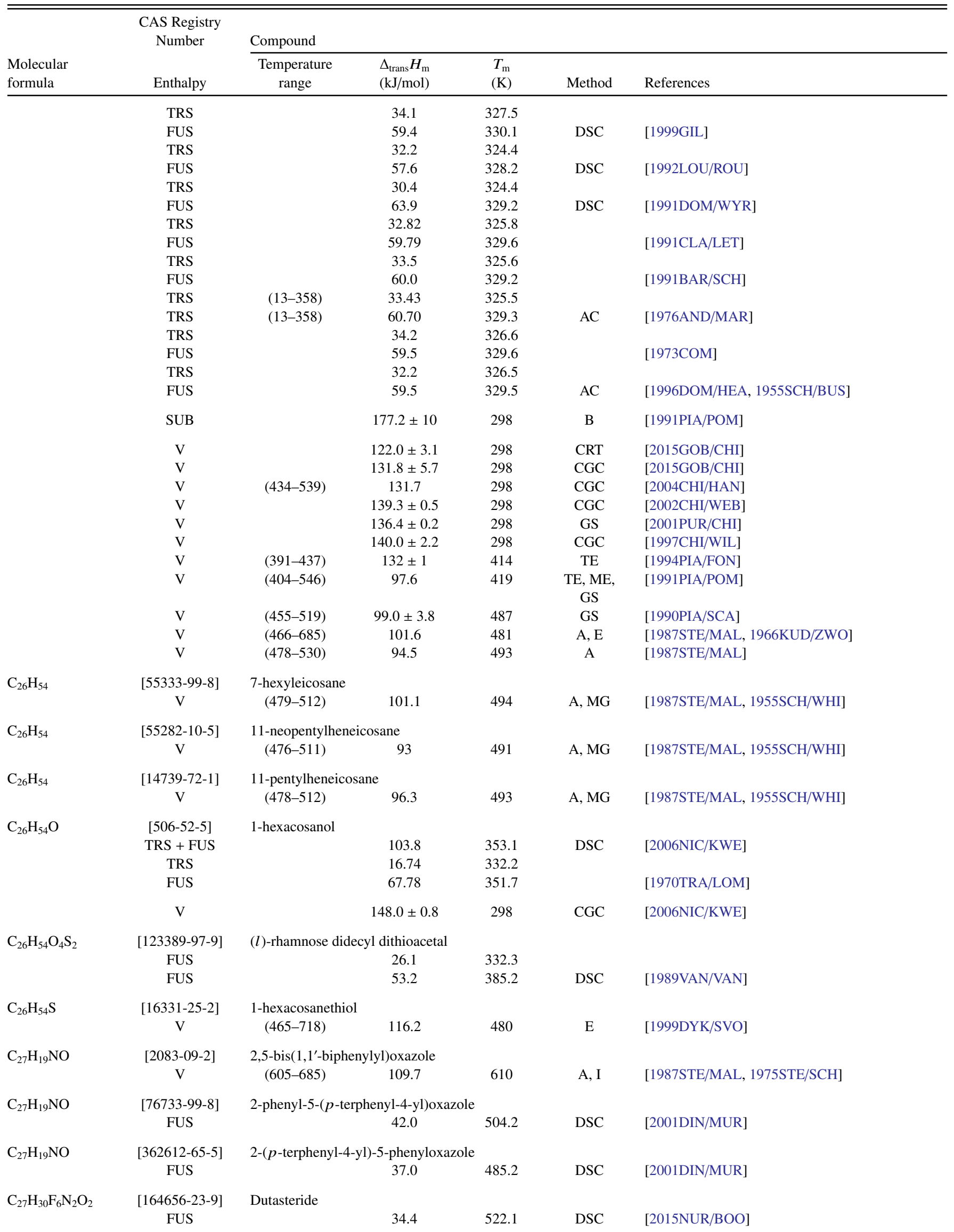


TABLE 14. Phase change enthalpies of $\mathrm{C}_{19}-\mathrm{C}_{29}$ organic compounds-Continued

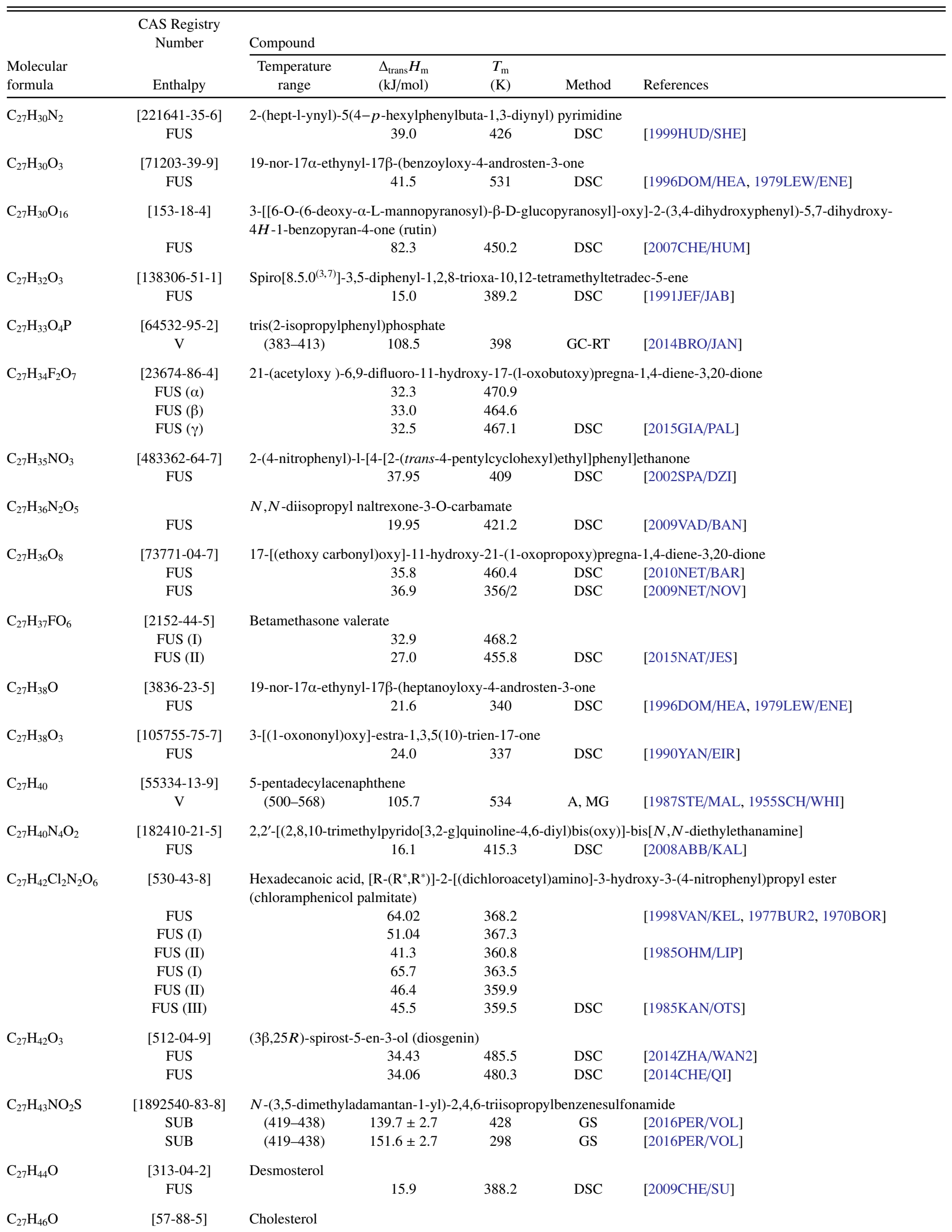


TABLE 14. Phase change enthalpies of $\mathrm{C}_{19}-\mathrm{C}_{29}$ organic compounds-Continued

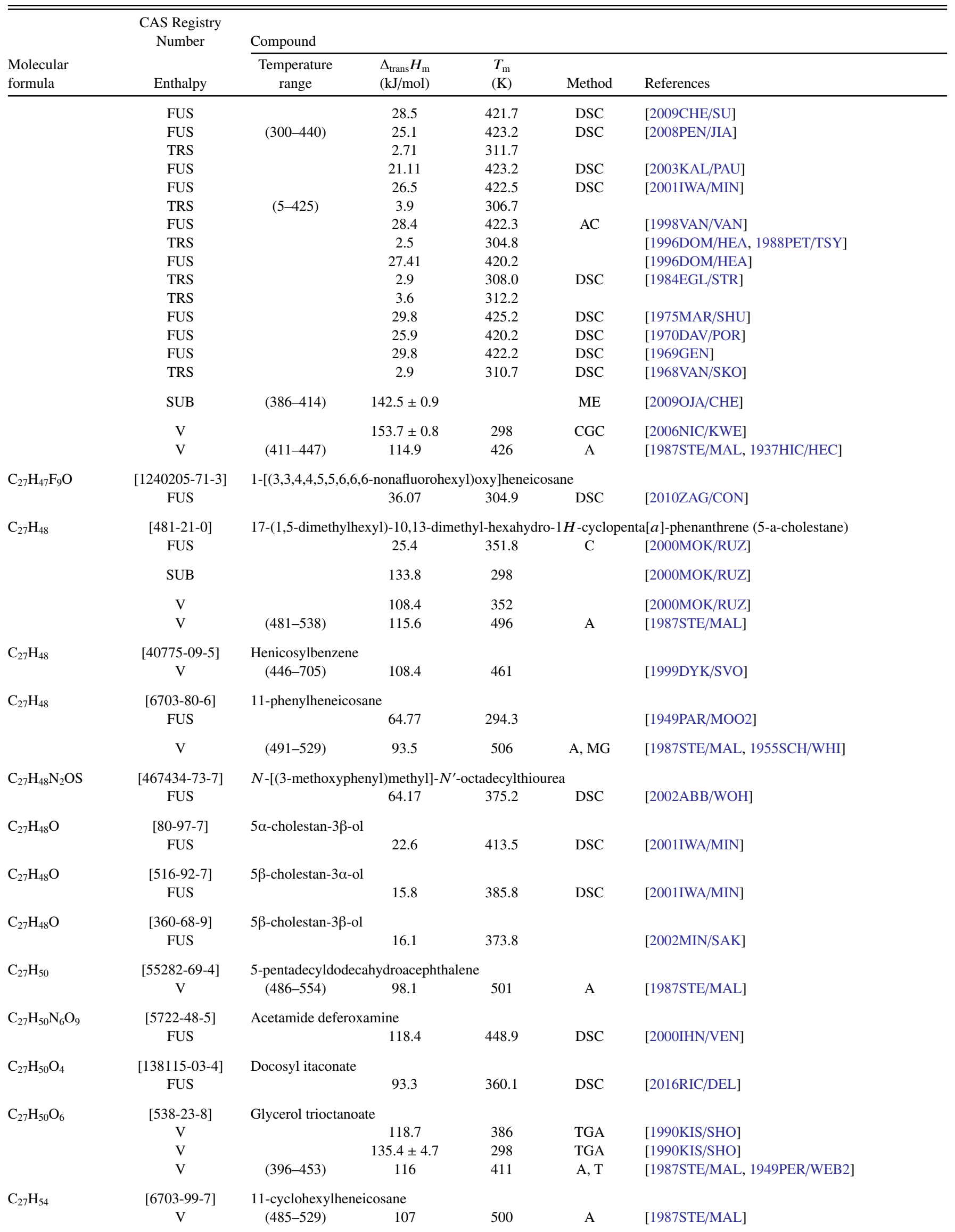


TABLE 14. Phase change enthalpies of $\mathrm{C}_{19}-\mathrm{C}_{29}$ organic compounds-Continued

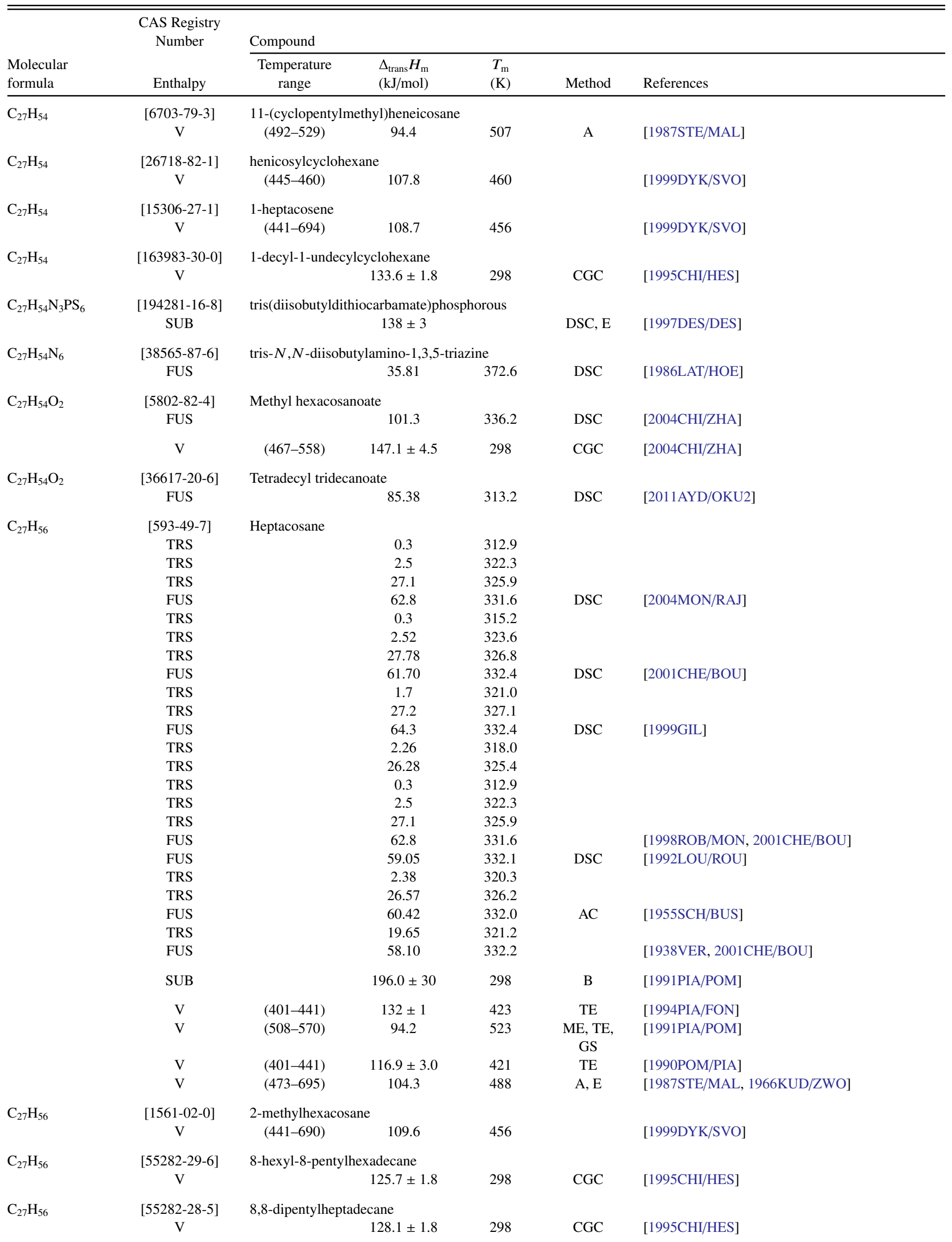


TABLE 14. Phase change enthalpies of $\mathrm{C}_{19}-\mathrm{C}_{29}$ organic compounds-Continued

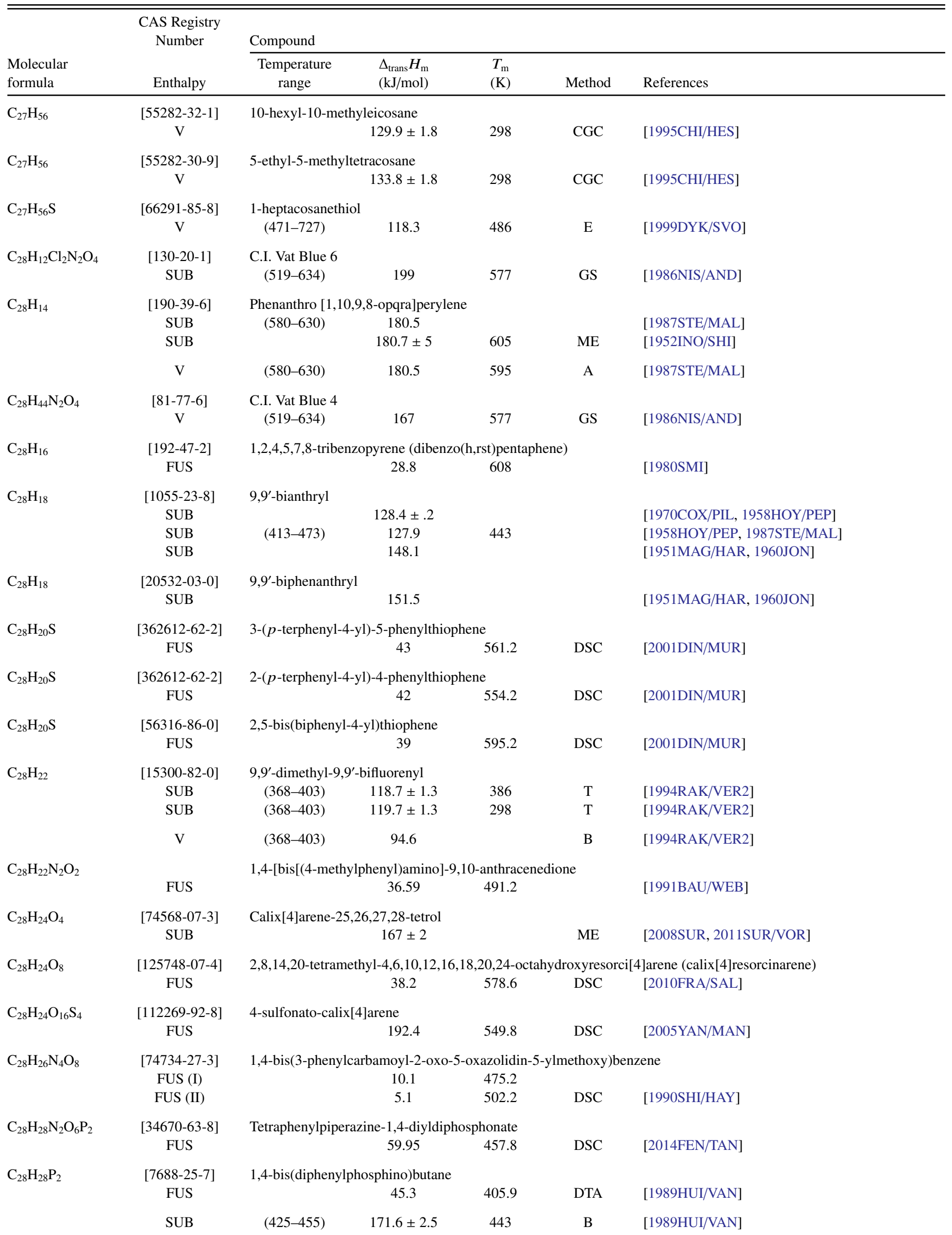


TABLE 14. Phase change enthalpies of $\mathrm{C}_{19}-\mathrm{C}_{29}$ organic compounds-Continued

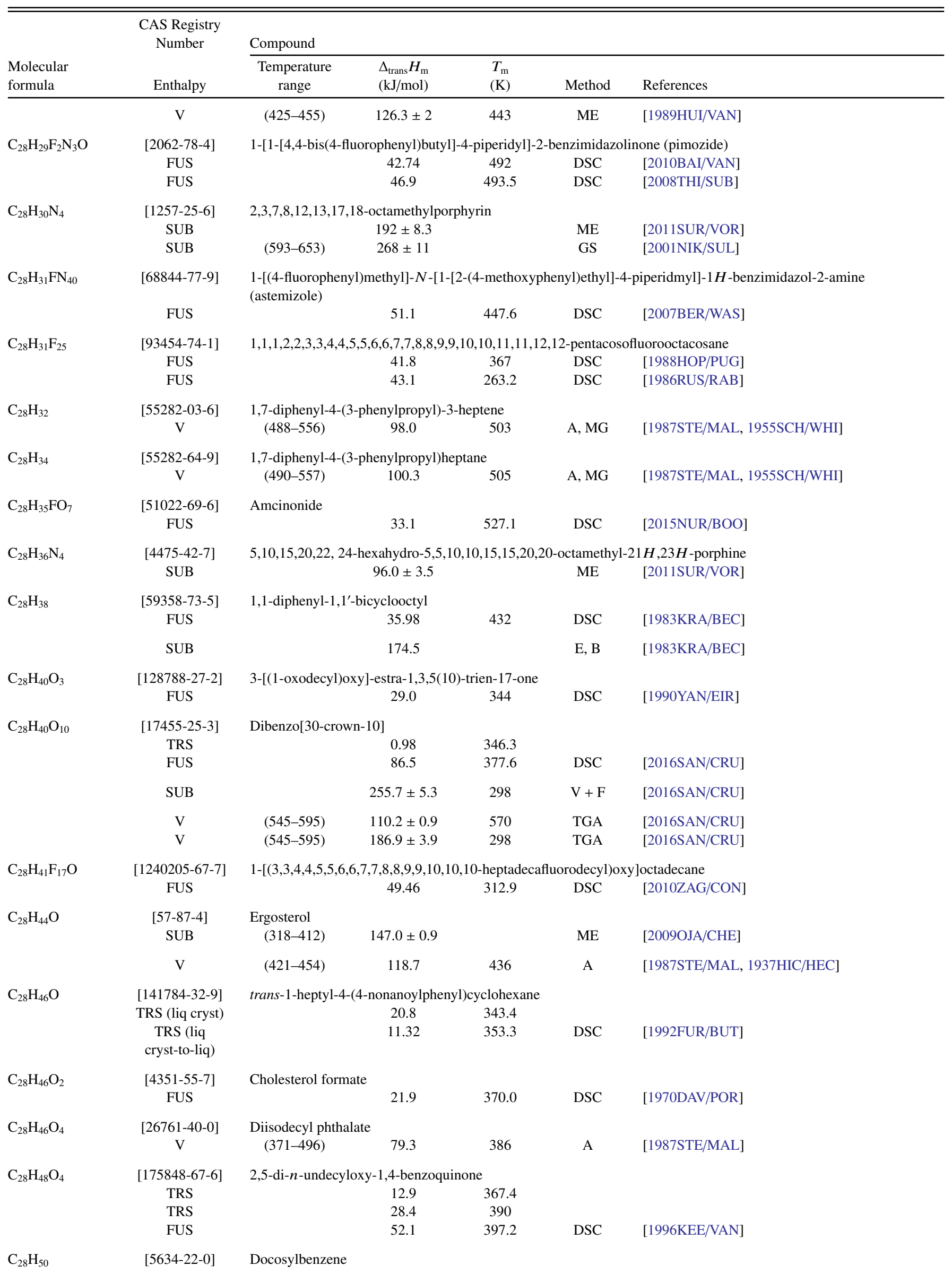


TABLE 14. Phase change enthalpies of $\mathrm{C}_{19}-\mathrm{C}_{29}$ organic compounds-Continued

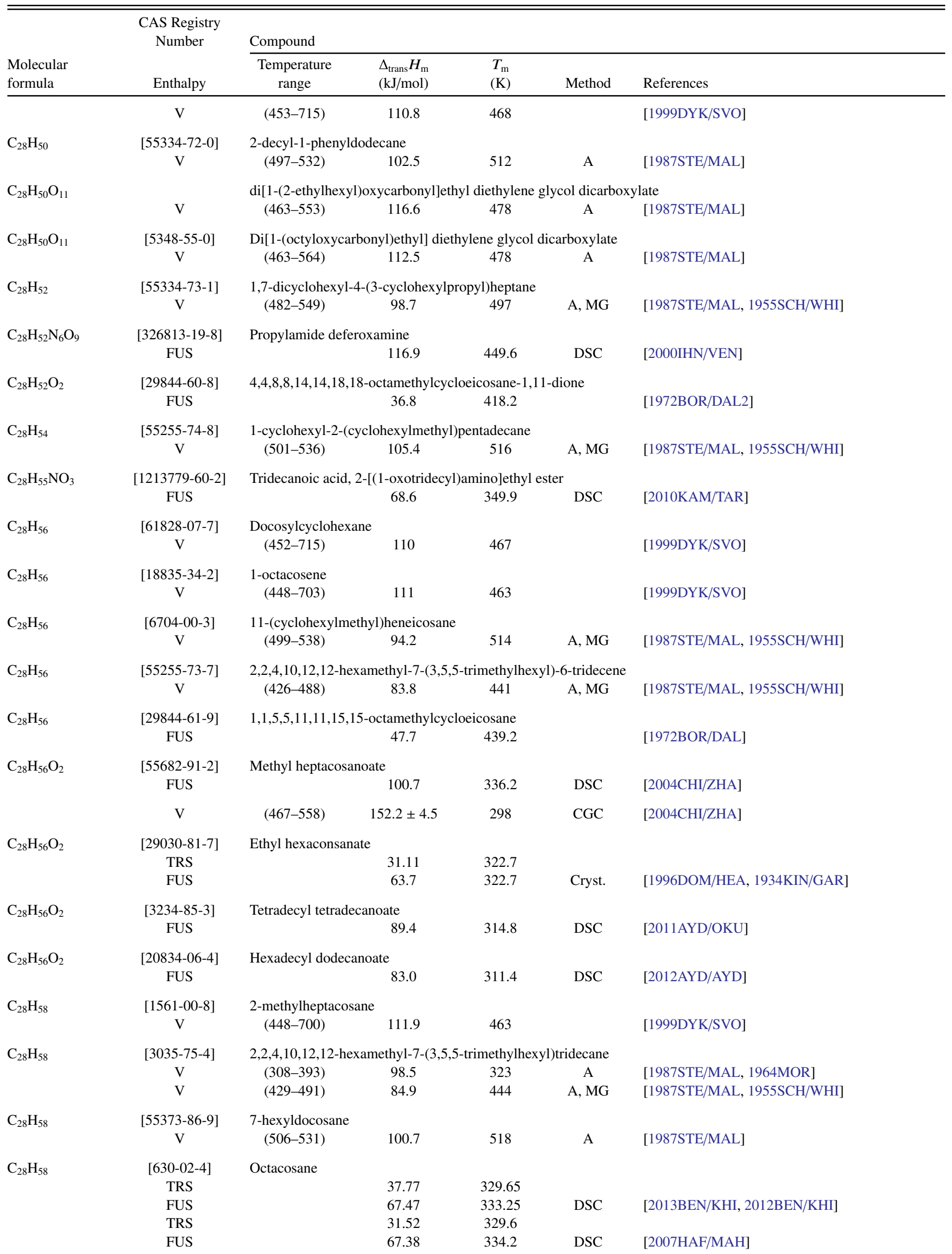


TABLE 14. Phase change enthalpies of $\mathrm{C}_{19}-\mathrm{C}_{29}$ organic compounds-Continued

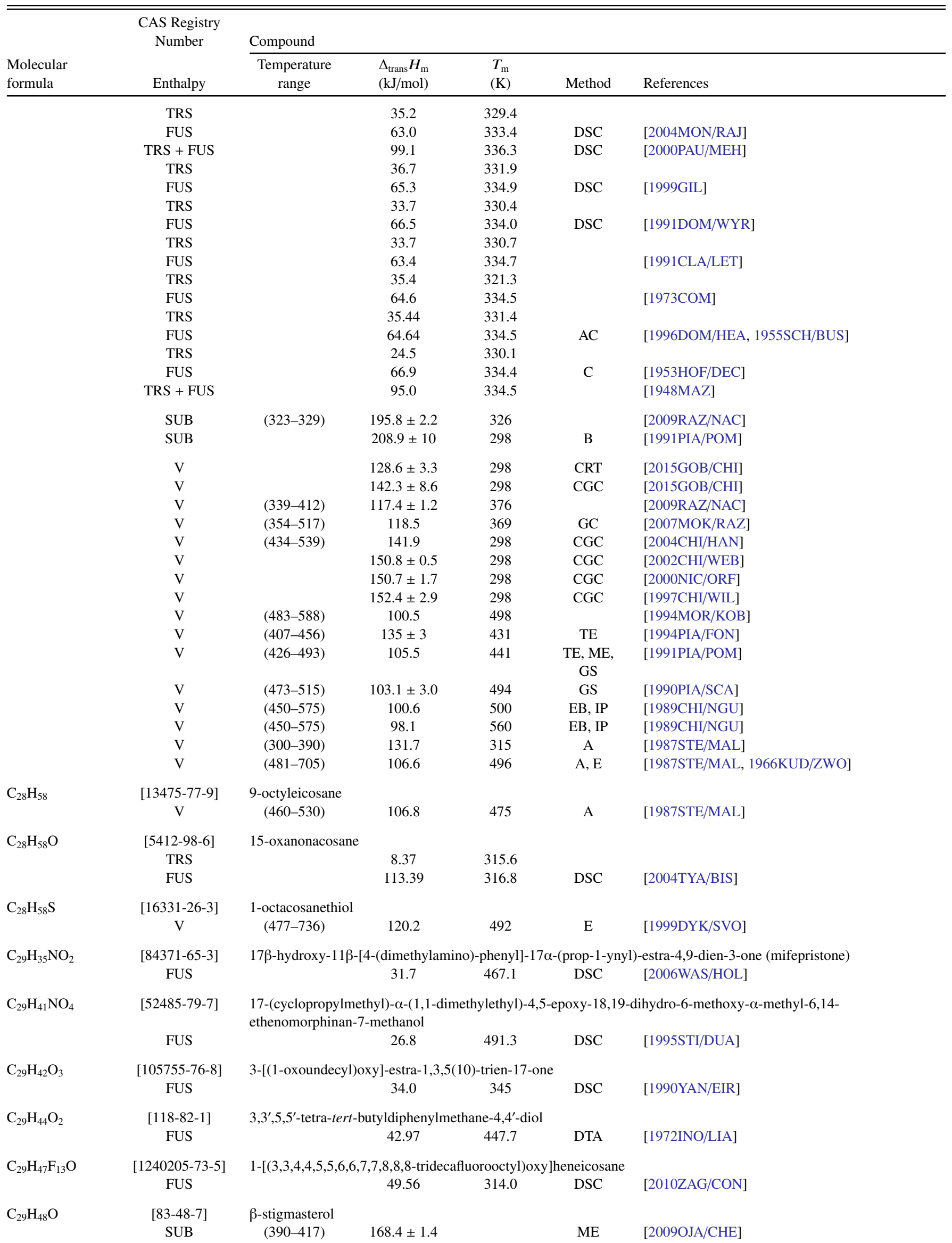


TABLE 14. Phase change enthalpies of $\mathrm{C}_{19}-\mathrm{C}_{29}$ organic compounds-Continued

\begin{tabular}{|c|c|c|c|c|c|c|}
\hline \multirow[b]{2}{*}{$\begin{array}{l}\text { Molecular } \\
\text { formula }\end{array}$} & \multirow{2}{*}{$\begin{array}{l}\text { CAS Registry } \\
\text { Number } \\
\text { Enthalpy }\end{array}$} & \multicolumn{5}{|l|}{ Compound } \\
\hline & & $\begin{array}{l}\text { Temperature } \\
\text { range }\end{array}$ & $\begin{array}{l}\Delta_{\text {trans }} H_{\mathrm{m}} \\
(\mathrm{kJ} / \mathrm{mol})\end{array}$ & $\begin{array}{l}T_{\mathrm{m}} \\
(\mathrm{K})\end{array}$ & Method & References \\
\hline $\mathrm{C}_{29} \mathrm{H}_{48} \mathrm{O}_{2}$ & $\begin{array}{c}\text { [604-35-3] } \\
\text { FUS }\end{array}$ & Cholesterol acetate & 20.4 & 387.8 & DSC & [1970DAV/POR] \\
\hline $\mathrm{C}_{29} \mathrm{H}_{50}$ & $\begin{array}{c}{[55373-90-5]} \\
\mathrm{V}\end{array}$ & $\begin{array}{l}\text { 11-(2,5-dimethylphe } \\
\quad(471-534)\end{array}$ & $\begin{array}{l}\text { enyl)-10-hene } \\
99.2\end{array}$ & ne 486 & A, MG & [1987STE/MAL, 1955SCH/WHI] \\
\hline $\mathrm{C}_{29} \mathrm{H}_{50} \mathrm{O}$ & $\begin{array}{l}{[83-46-5]} \\
\text { SUB }\end{array}$ & $\begin{array}{l}\beta \text {-sitosterol } \\
\quad(389-410)\end{array}$ & $143.8 \pm 0.5$ & & $\mathrm{ME}$ & [2009OJA/CHE] \\
\hline $\mathrm{C}_{29} \mathrm{H}_{52}$ & $\begin{array}{c}{[61828-04-4]} \\
\mathrm{V}\end{array}$ & $\begin{array}{l}\text { Tricosylbenzene } \\
\quad(459-724)\end{array}$ & 113.2 & 474 & & [1999DYK/SVO] \\
\hline $\mathrm{C}_{29} \mathrm{H}_{52}$ & $\begin{array}{c}{[18835-35-3]} \\
\mathrm{V}\end{array}$ & $\begin{array}{c}\text { 1-nonacosene } \\
(455-713)\end{array}$ & 113.3 & 470 & & [1999DYK/SVO] \\
\hline $\mathrm{C}_{29} \mathrm{H}_{52}$ & $\begin{array}{c}{[55373-91-6]} \\
\mathrm{V}\end{array}$ & $\begin{array}{l}\text { 11-(2,5-dimethylphe } \\
\quad(472-535)\end{array}$ & $\begin{array}{l}\text { enyl)heneicos } \\
100.8\end{array}$ & 487 & $\mathrm{~A}, \mathrm{MG}$ & [1987STE/MAL, 1955SCH/WHI] \\
\hline $\mathrm{C}_{29} \mathrm{H}_{52} \mathrm{~N}_{6} \mathrm{O}_{11}$ & $\begin{array}{c}\text { [84211-47-2] } \\
\text { FUS }\end{array}$ & Succinamide defero & $\begin{array}{r}\text { xamine } \\
101\end{array}$ & 436.2 & $\mathrm{DSC}$ & [2000IHN/VEN] \\
\hline $\mathrm{C}_{29} \mathrm{H}_{54} \mathrm{~N}_{6} \mathrm{O}_{9}$ & $\begin{array}{c}{[326813-21-2]} \\
\text { FUS }\end{array}$ & Butylamide deferox & $\begin{array}{l}\text { ramine } \\
111.4\end{array}$ & 451.1 & DSC & [2000IHN/VEN] \\
\hline $\mathrm{C}_{29} \mathrm{H}_{56} \mathrm{~N}_{2} \mathrm{O}_{2} \mathrm{~S}$ & $\begin{array}{c}\text { [1383124-22-8] } \\
\text { FUS }\end{array}$ & 1,3-ditetradecanoyl & $\begin{array}{r}\text { thiourea } \\
59.4\end{array}$ & 320.6 & DSC & [2011ALK/TEK] \\
\hline $\mathrm{C}_{29} \mathrm{H}_{56} \mathrm{O}_{4}$ & $\begin{array}{c}{[10525-39-0]} \\
\text { FUS }\end{array}$ & neopentyl glycol dil & $\begin{array}{r}\text { laurate } \\
45.8\end{array}$ & 283.9 & DSC & [2013SAR/ALK] \\
\hline $\mathrm{C}_{29} \mathrm{H}_{58}$ & $\begin{array}{c}{[61828-08-8]} \\
\mathrm{V}\end{array}$ & $\begin{array}{l}\text { tricosylcyclohexane } \\
\quad(459-724)\end{array}$ & 112.3 & 474 & & [1999DYK/SVO] \\
\hline $\mathrm{C}_{29} \mathrm{H}_{58} \mathrm{O}_{2}$ & $\begin{array}{c}{[55682-92-3]} \\
\text { FUS }\end{array}$ & methyl octacosanoa & 109.7 & 340.2 & $\mathrm{DSC}$ & [2004CHI/ZHA] \\
\hline & $\mathrm{V}$ & $(467-558)$ & $157.5 \pm 4.5$ & 298 & $\mathrm{CGC}$ & [2004CHI/ZHA] \\
\hline $\mathrm{C}_{29} \mathrm{H}_{58} \mathrm{O}_{2}$ & $\begin{array}{c}\text { [36617-31-9] } \\
\text { FUS }\end{array}$ & tetradecyl pentadeca & $\begin{array}{l}\text { anoate } \\
94.25\end{array}$ & 318.6 & DSC & [2011AYD/OKU2] \\
\hline $\mathrm{C}_{29} \mathrm{H}_{58} \mathrm{O}_{3}$ & $\begin{array}{c}\text { [153821-35-3] } \\
\text { FUS } \\
\text { FUS }\end{array}$ & ditetradecyl carbona & $\begin{array}{l}\text { ate } \\
\begin{array}{l}97.39 \\
103.2\end{array}\end{array}$ & $\begin{array}{l}309.5 \\
305.0\end{array}$ & $\begin{array}{l}\mathrm{DSC} \\
\mathrm{DSC}\end{array}$ & $\begin{array}{l}{[2012 \mathrm{KEN}]} \\
{[2010 \mathrm{KEN}]}\end{array}$ \\
\hline $\mathrm{C}_{29} \mathrm{H}_{60}$ & $\begin{array}{c}{[1560-98-1]} \\
\mathrm{V}\end{array}$ & $\begin{array}{l}\text { 2-methyloctacosane } \\
(455-709)\end{array}$ & 114.2 & 470 & & [1999DYK/SVO] \\
\hline $\mathrm{C}_{29} \mathrm{H}_{60}$ & $\begin{array}{c}\text { [630-03-5] } \\
\text { TRS } \\
\text { TRS } \\
\text { TRS } \\
\text { FUS } \\
\text { TRS } \\
\text { TRS } \\
\text { FUS } \\
\text { TRS } \\
\text { FUS }\end{array}$ & nonacosane & $\begin{array}{c}0.2 \\
2.47 \\
30.03 \\
66.94 \\
1.8 \\
30.5 \\
70.8 \\
29.71 \\
66.11\end{array}$ & $\begin{array}{l}314.2 \\
325.4 \\
331.8 \\
336.8 \\
325.1 \\
332.2 \\
337.1 \\
331.4 \\
336.6\end{array}$ & DSC & $\begin{array}{l}\text { [1999GIL] } \\
\text { [1996DOM/HEA, 1955SCH/BUS] }\end{array}$ \\
\hline & $\begin{array}{l}\mathrm{V} \\
\mathrm{V} \\
\mathrm{V} \\
\mathrm{V} \\
\mathrm{V}\end{array}$ & $\begin{array}{l}(422-452) \\
(434-539) \\
(423-457) \\
(423-456) \\
(488-714)\end{array}$ & $\begin{array}{c}112.5 \\
147.1 \\
137 \pm 3 \\
137.1 \pm 3.0 \\
109\end{array}$ & $\begin{array}{l}437 \\
298 \\
440 \\
439 \\
503\end{array}$ & $\begin{array}{l}\text { CGC } \\
\text { TE } \\
\text { TE } \\
\text { A, E }\end{array}$ & $\begin{array}{l}\text { [2006SAW/MOK] } \\
{[2004 \mathrm{CHI} / \mathrm{HAN}]} \\
{[1994 \mathrm{PIA} / \mathrm{FON}]} \\
{[1990 \mathrm{POM} / \mathrm{PIA}]} \\
\text { [1987STE/MAL, 1966KUD/ZWO] }\end{array}$ \\
\hline $\mathrm{C}_{29} \mathrm{H}_{60} \mathrm{~S}$ & $\begin{array}{c}{[66213-92-1]} \\
\mathrm{V}\end{array}$ & $\begin{array}{l}\text { 1-nonacosanethiol } \\
(483-744)\end{array}$ & 122 & 498 & $\mathrm{E}$ & [1999DYK/SVO] \\
\hline
\end{tabular}


TABLE 15. Phase change enthalpies of $\mathrm{C}_{30}$ to $\mathrm{C}_{49}$ organic compounds

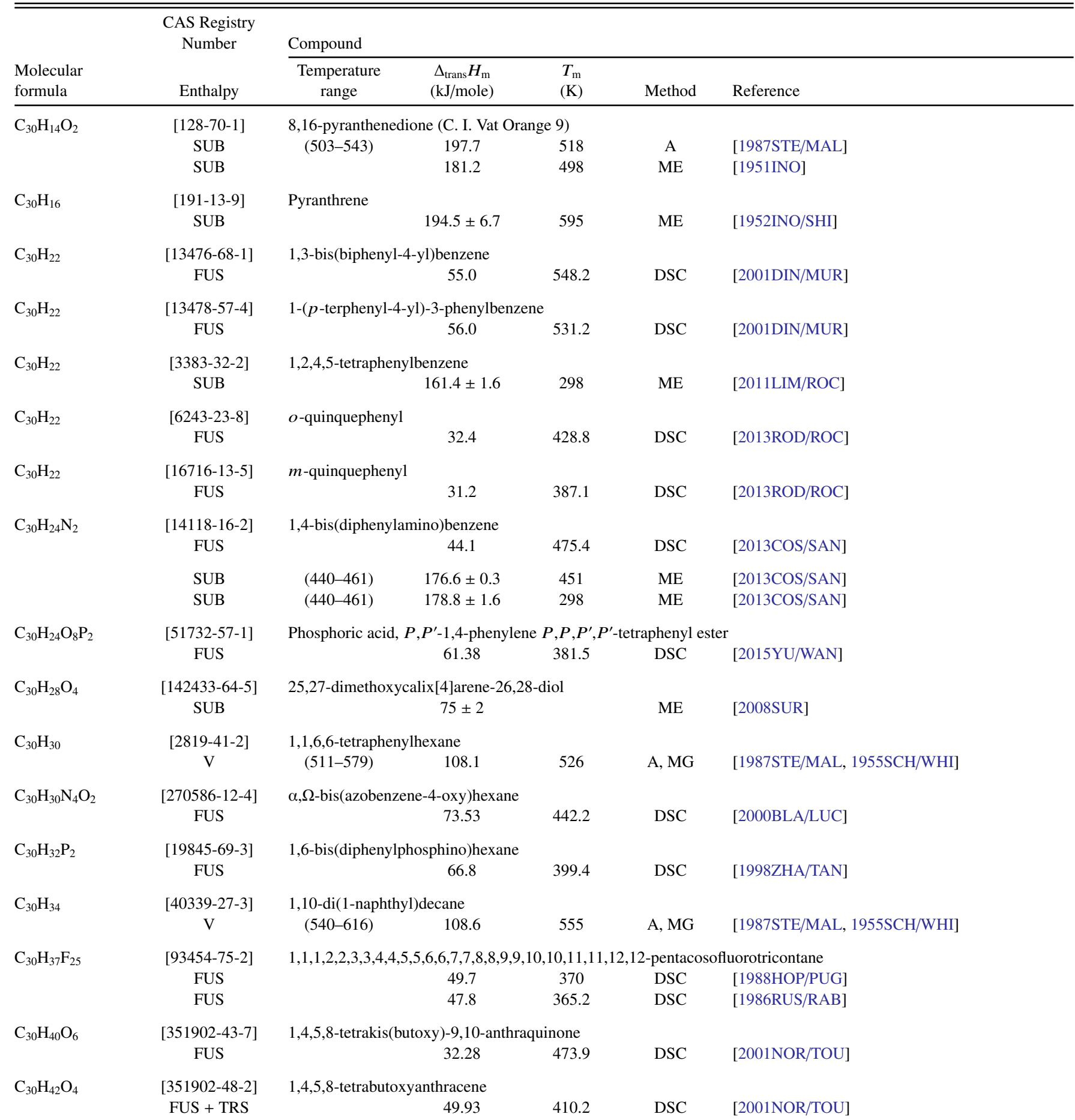

[Note: Authors report only the total enthalpy of melting. The numerical value contains enthalpies for two solid-solid transitions that occur at $370.7 \mathrm{~K}$ and $385.5 \mathrm{~K}$. Larger tetraalkoxy-derivatives show liquid crystalline behavior.]

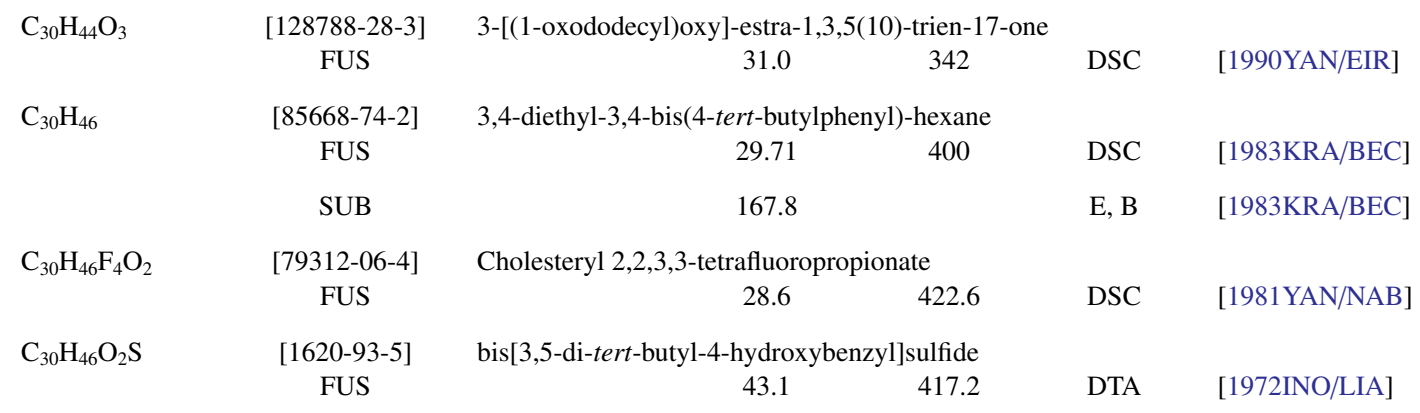


TABLE 15. Phase change enthalpies of $\mathrm{C}_{30}$ to $\mathrm{C}_{49}$ organic compounds-Continued

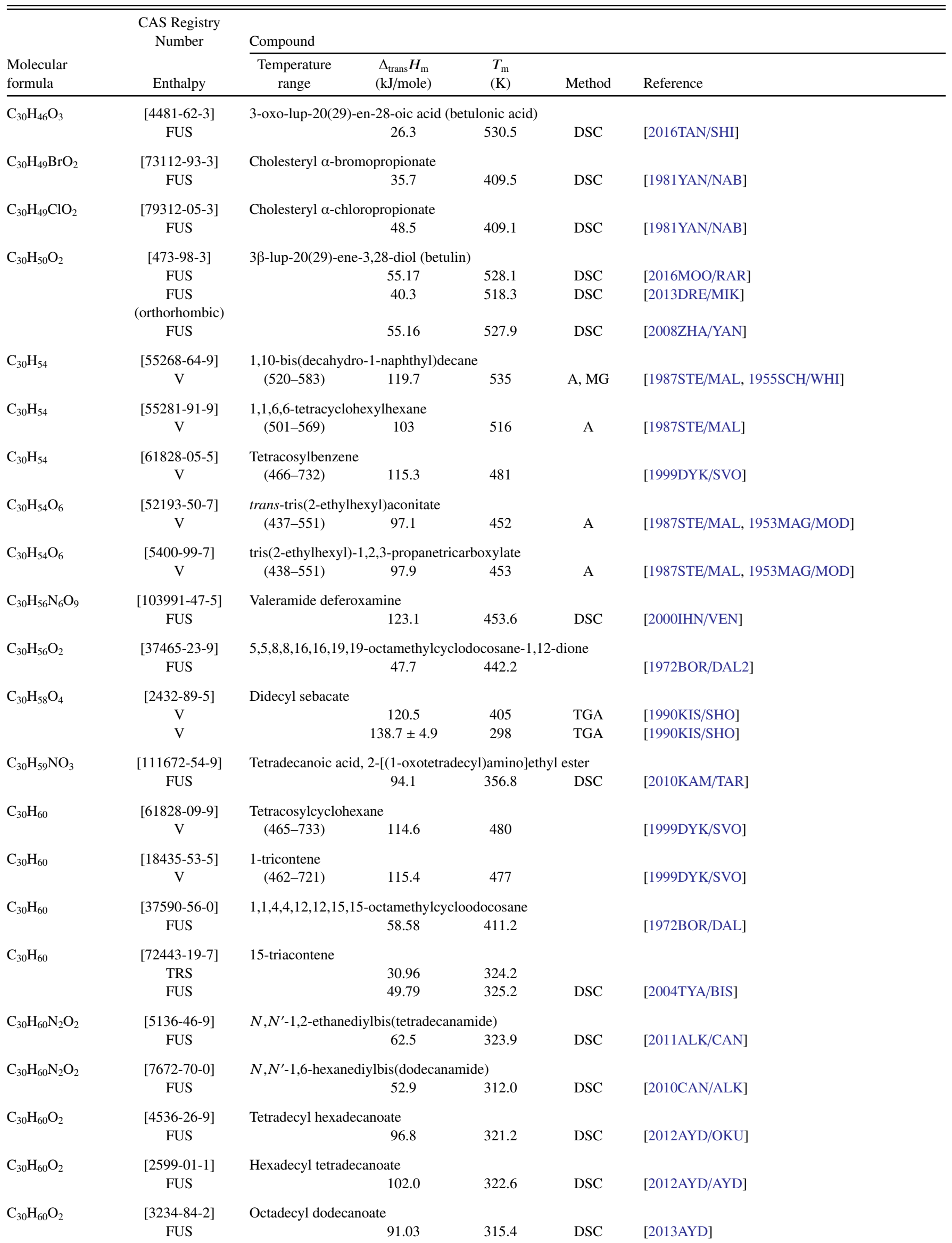


TABLE 15. Phase change enthalpies of $\mathrm{C}_{30}$ to $\mathrm{C}_{49}$ organic compounds-Continued

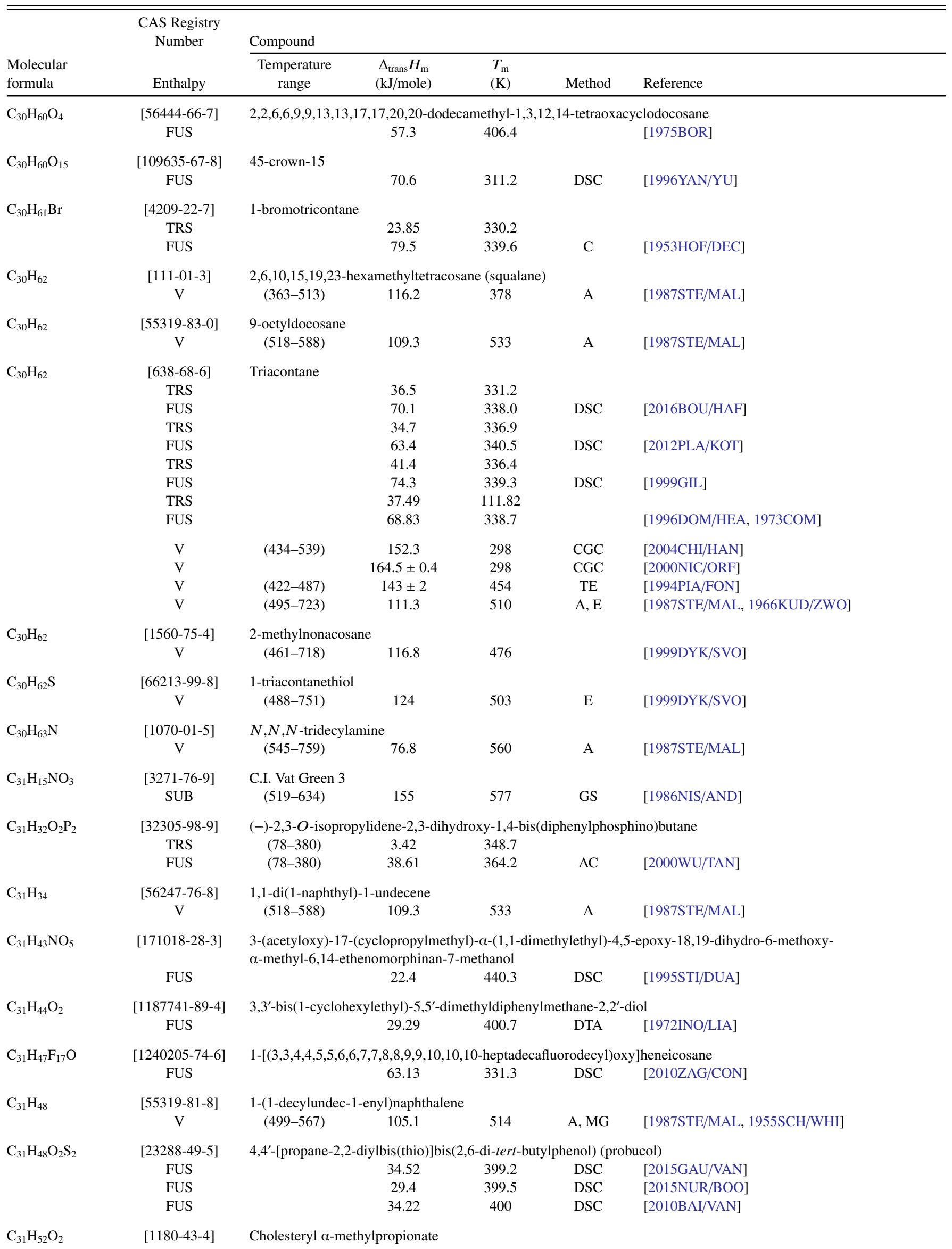


TABLE 15. Phase change enthalpies of $\mathrm{C}_{30}$ to $\mathrm{C}_{49}$ organic compounds-Continued

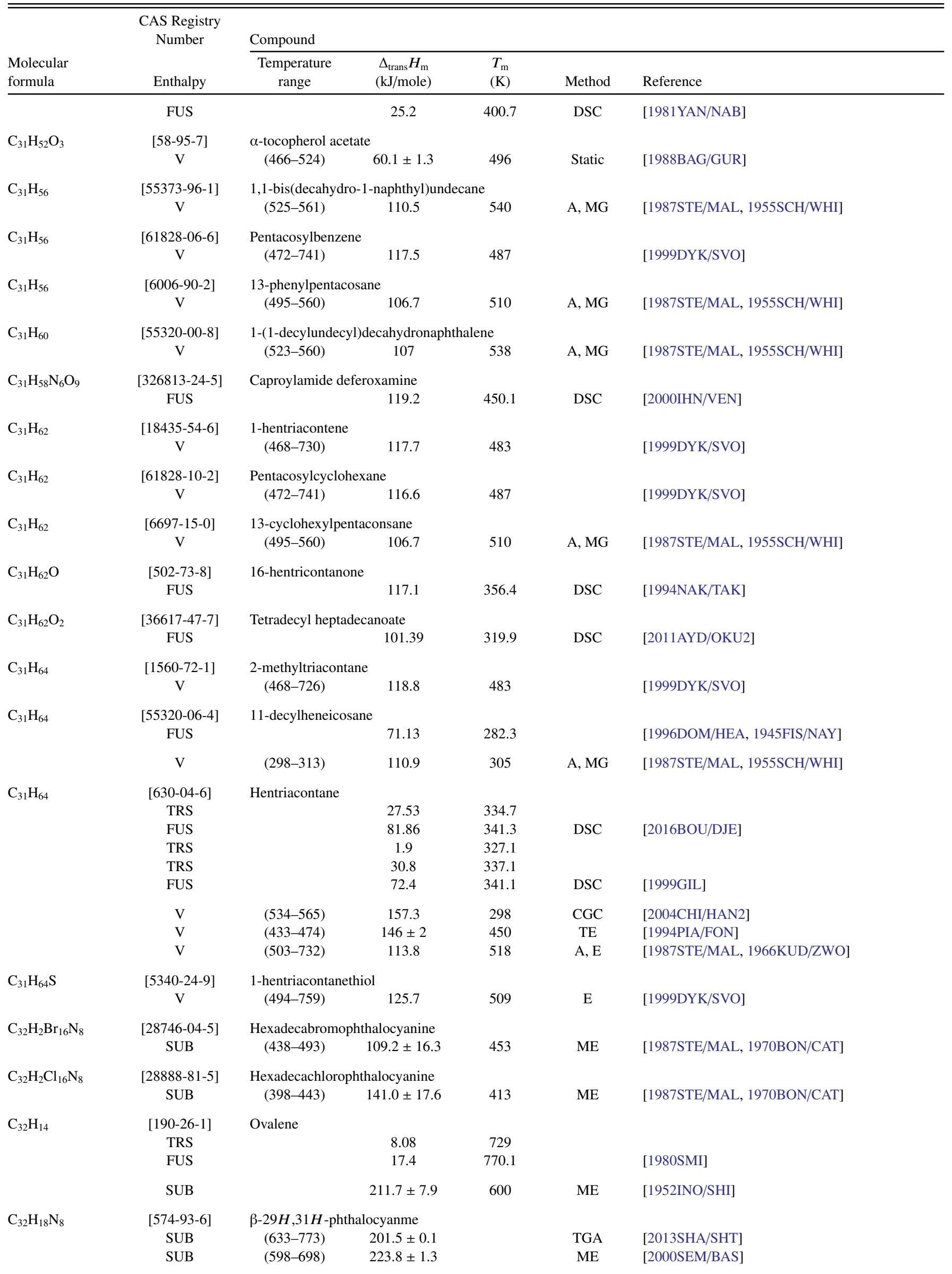


TABLE 15. Phase change enthalpies of $\mathrm{C}_{30}$ to $\mathrm{C}_{49}$ organic compounds-Continued

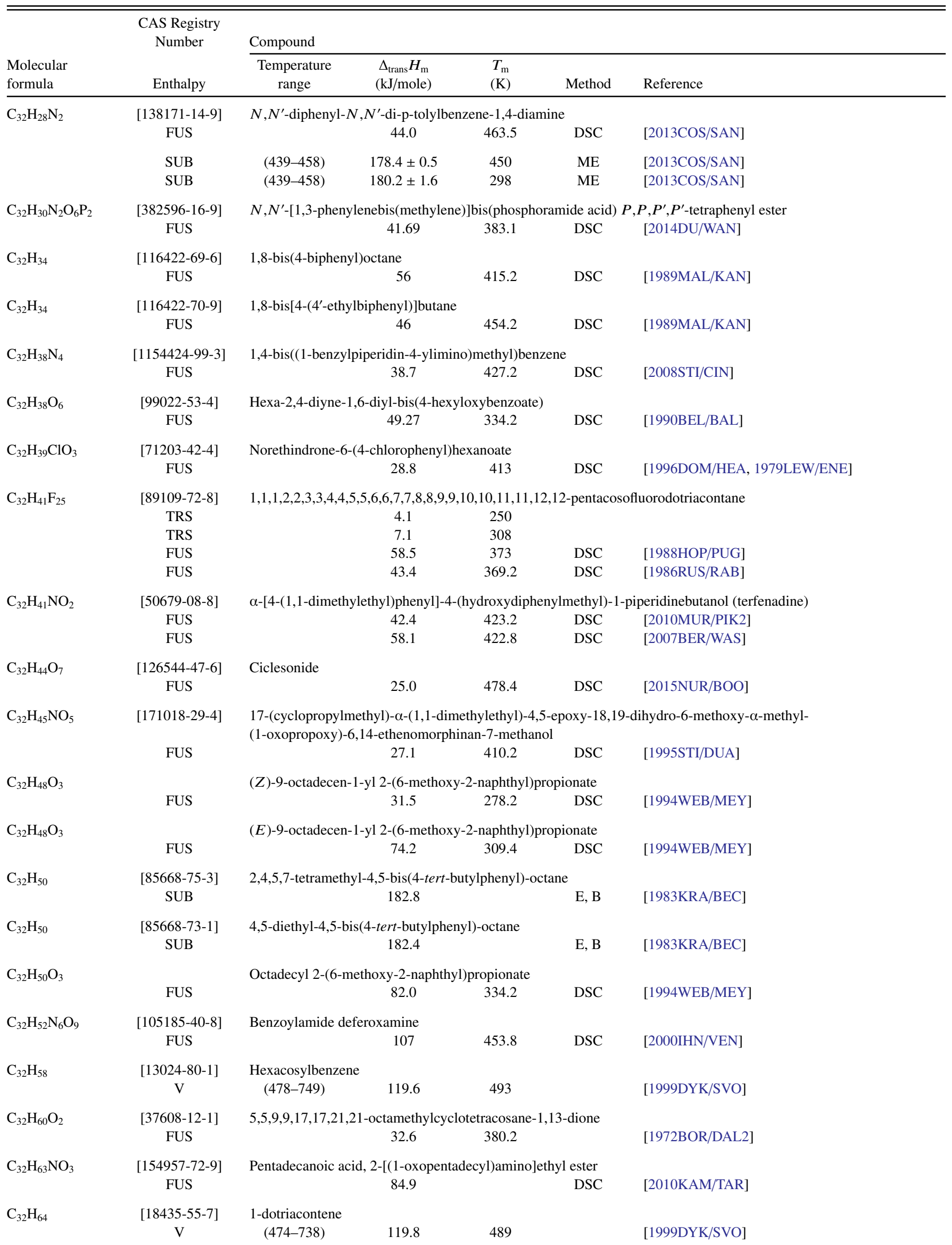


TABLE 15. Phase change enthalpies of $\mathrm{C}_{30}$ to $\mathrm{C}_{49}$ organic compounds-Continued

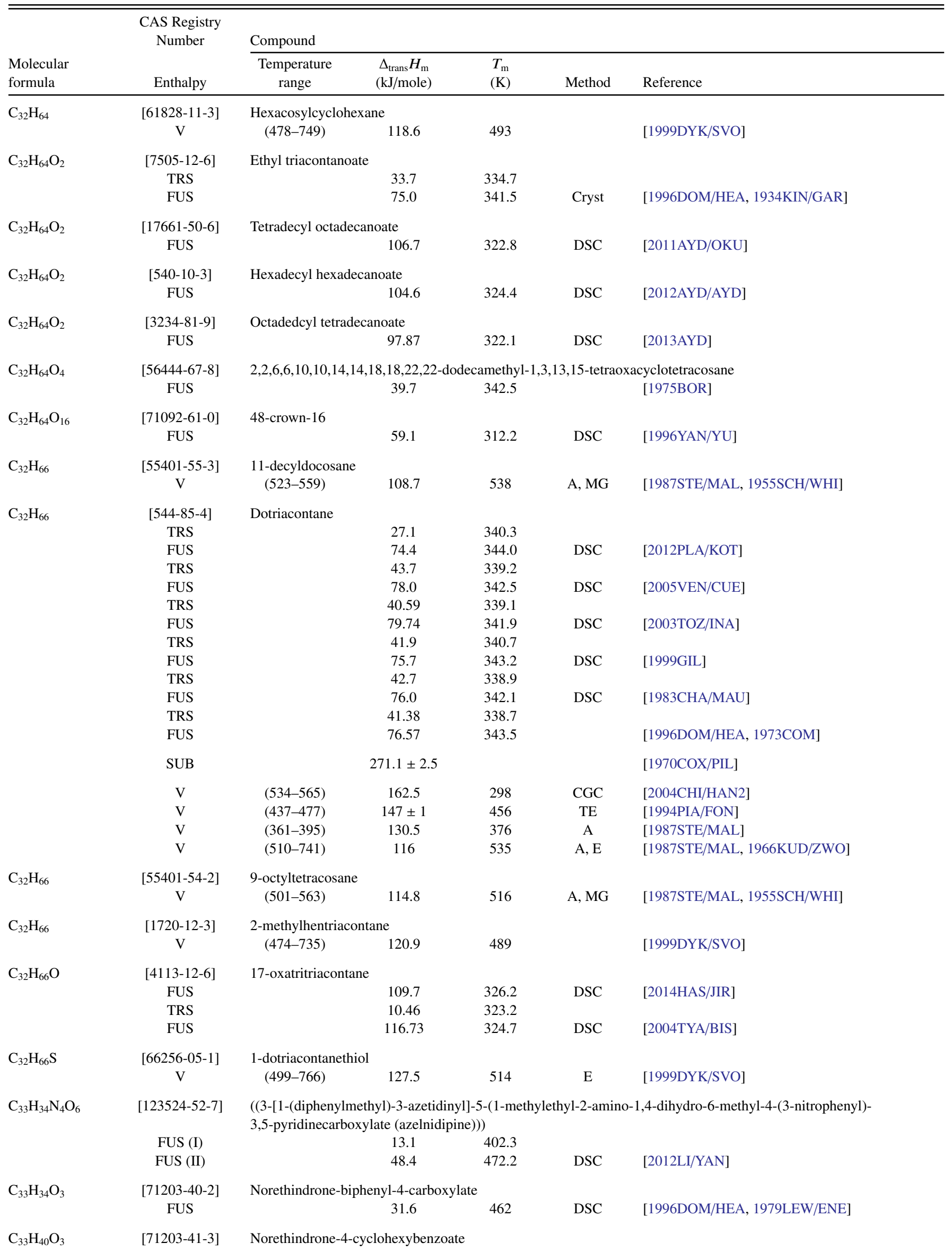


TABLE 15. Phase change enthalpies of $\mathrm{C}_{30}$ to $\mathrm{C}_{49}$ organic compounds-Continued

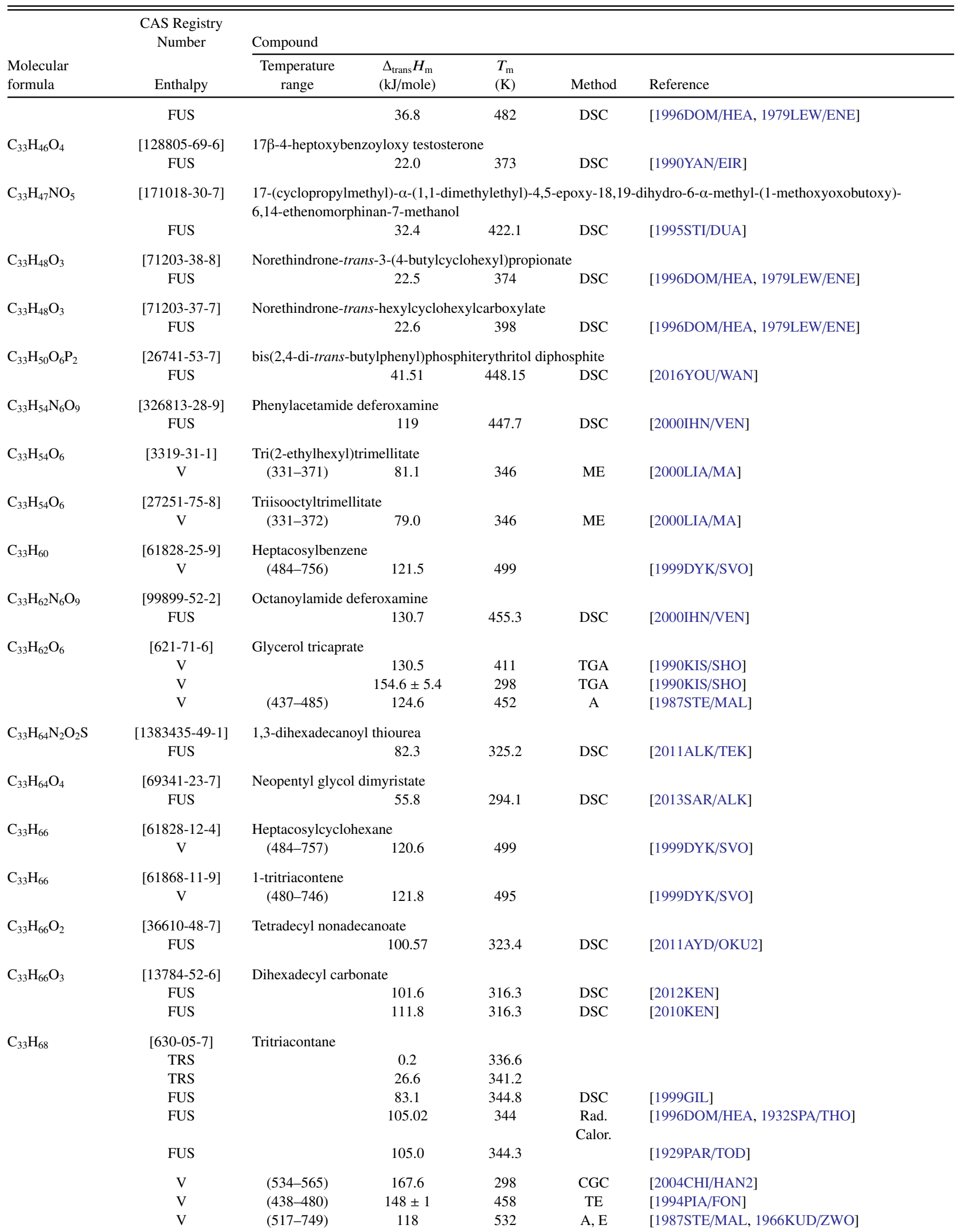


TABLE 15. Phase change enthalpies of $\mathrm{C}_{30}$ to $\mathrm{C}_{49}$ organic compounds-Continued

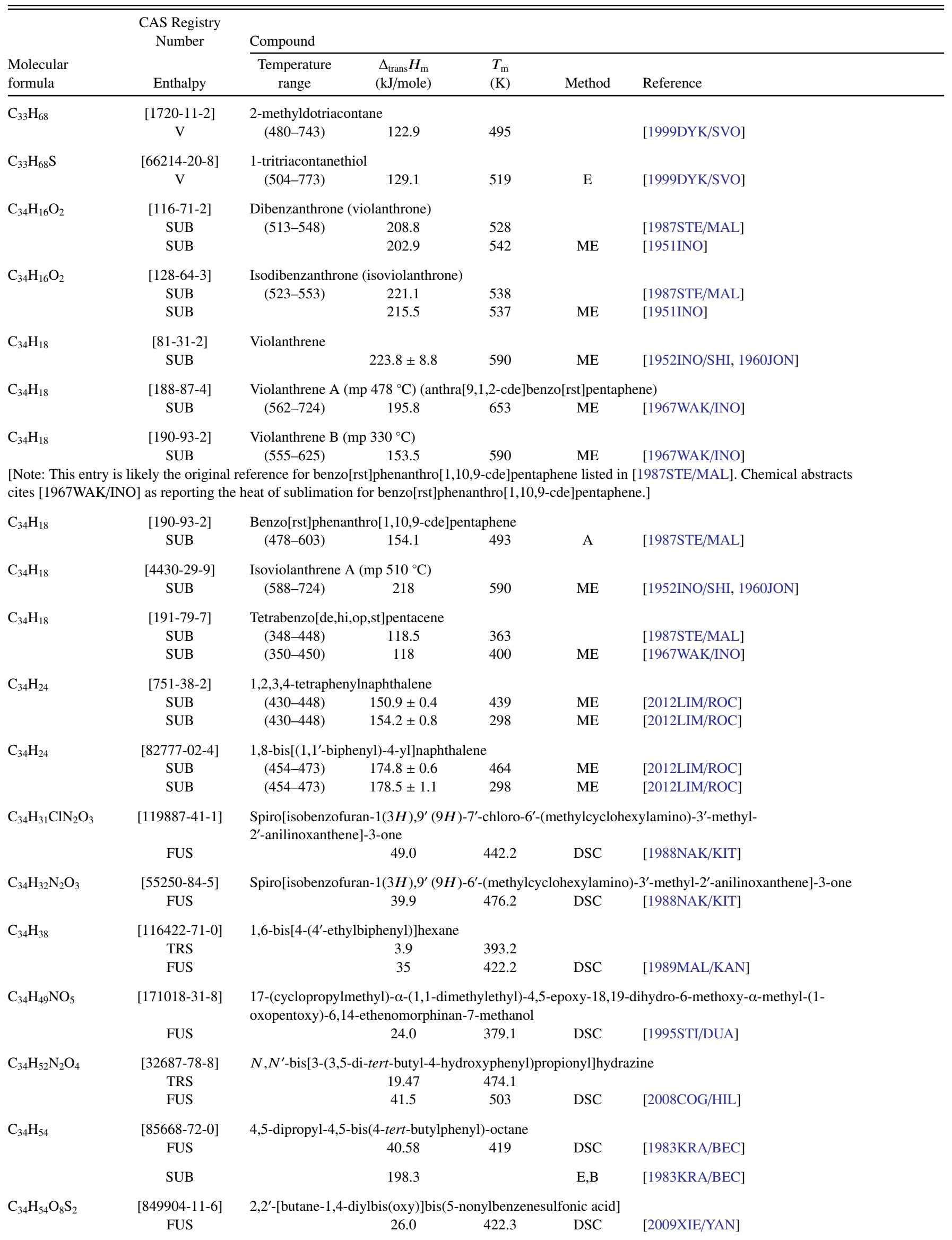


TABLE 15. Phase change enthalpies of $\mathrm{C}_{30}$ to $\mathrm{C}_{49}$ organic compounds-Continued

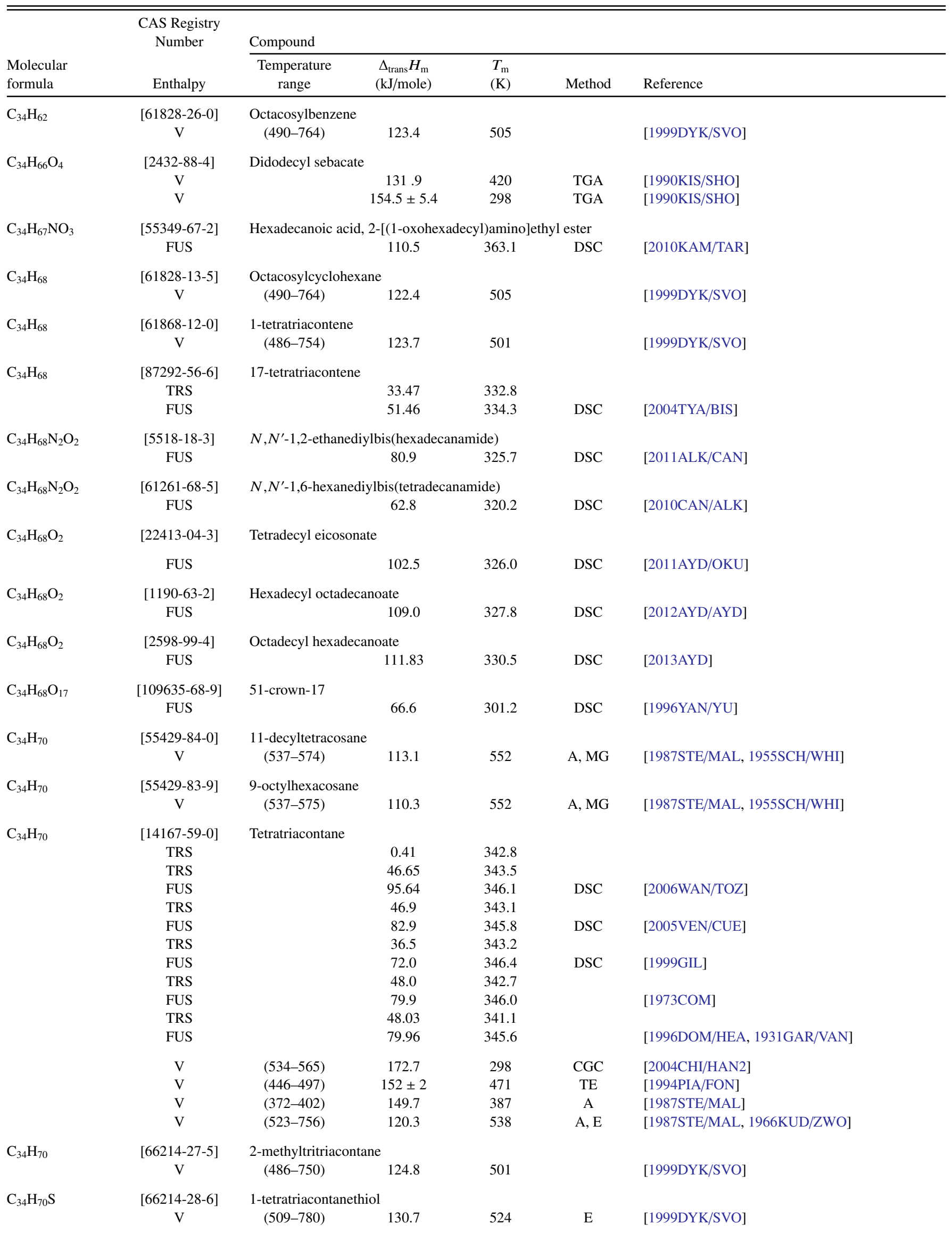


TABLE 15. Phase change enthalpies of $\mathrm{C}_{30}$ to $\mathrm{C}_{49}$ organic compounds-Continued

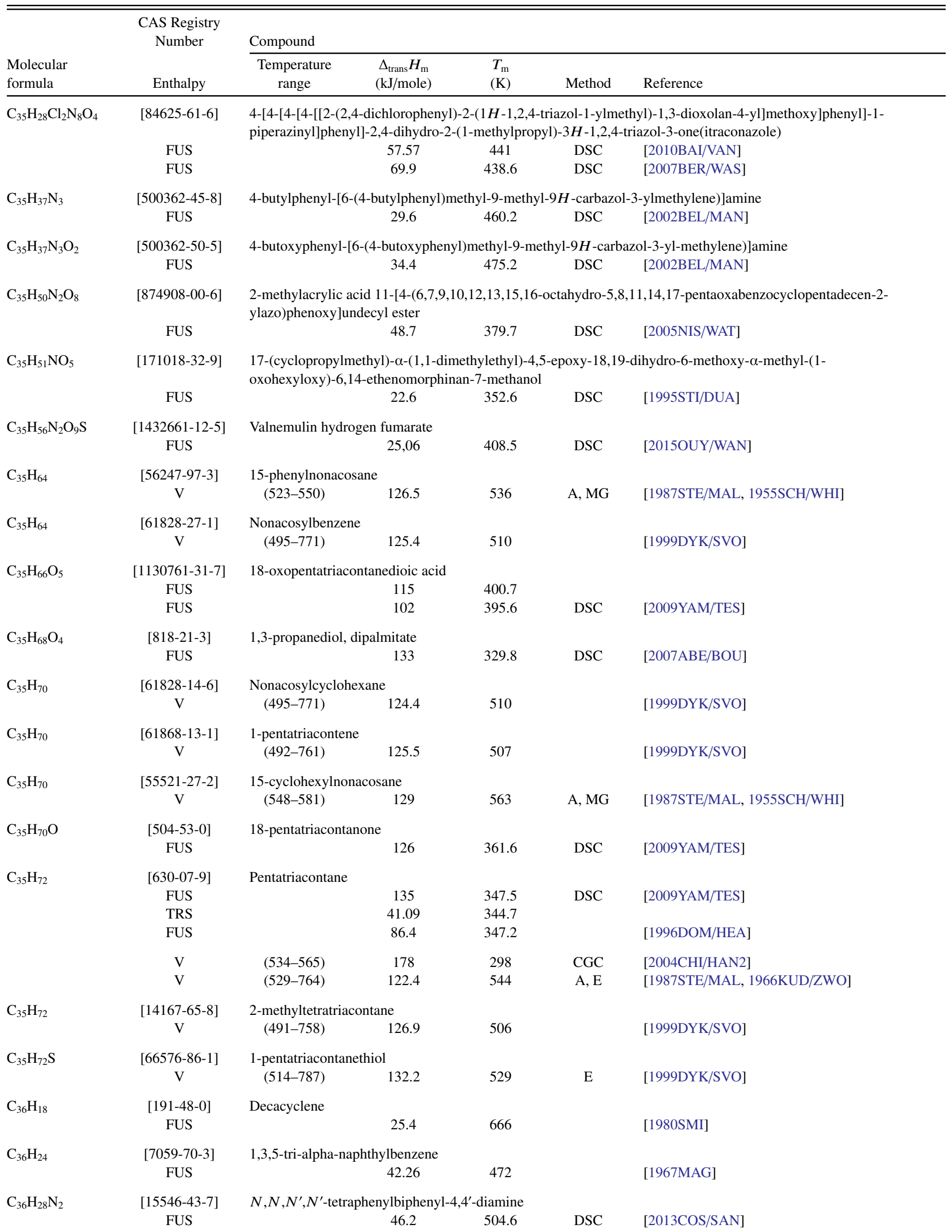


TABLE 15. Phase change enthalpies of $\mathrm{C}_{30}$ to $\mathrm{C}_{49}$ organic compounds-Continued

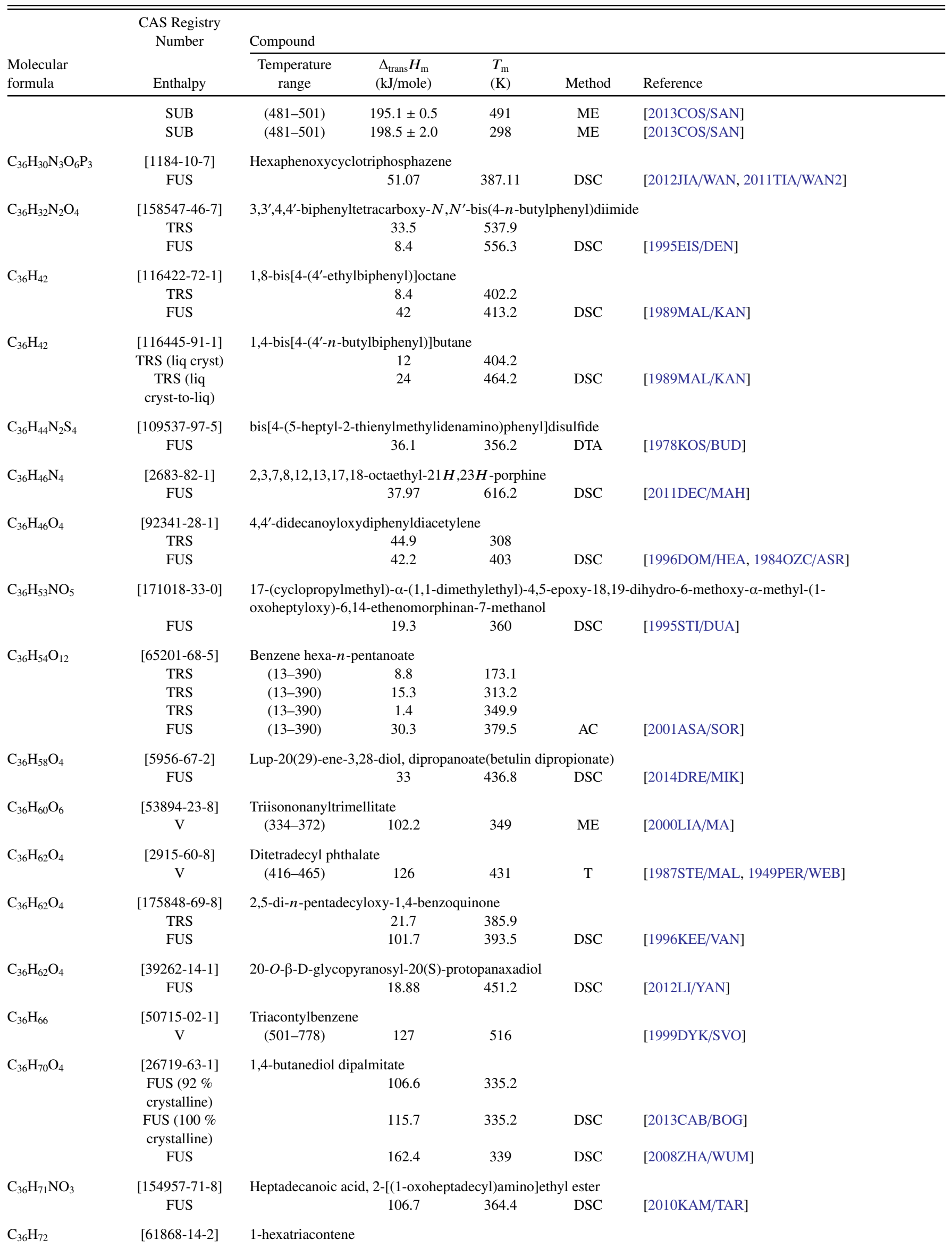


TABLE 15. Phase change enthalpies of $\mathrm{C}_{30}$ to $\mathrm{C}_{49}$ organic compounds-Continued

\begin{tabular}{|c|c|c|c|c|c|c|}
\hline \multirow[b]{2}{*}{$\begin{array}{l}\text { Molecular } \\
\text { formula }\end{array}$} & \multirow{2}{*}{$\begin{array}{l}\text { CAS Registry } \\
\text { Number } \\
\text { Enthalpy }\end{array}$} & \multicolumn{5}{|l|}{ Compound } \\
\hline & & $\begin{array}{c}\text { Temperature } \\
\text { range }\end{array}$ & $\begin{array}{c}\Delta_{\text {trans }} H_{\mathrm{m}} \\
(\mathrm{kJ} / \mathrm{mole})\end{array}$ & $\begin{array}{l}T_{\mathrm{m}} \\
(\mathrm{K})\end{array}$ & Method & Reference \\
\hline & $\mathrm{V}$ & $(497-768)$ & 127.4 & 512 & & [1999DYK/SVO] \\
\hline $\mathrm{C}_{36} \mathrm{H}_{72}$ & $\begin{array}{c}{[61828-15-7]} \\
\mathrm{V}\end{array}$ & $\begin{array}{l}\text { Triacontylcycl } \\
\quad(500-778)\end{array}$ & 126.3 & 515 & & [1999DYK/SVO] \\
\hline $\mathrm{C}_{36} \mathrm{H}_{72} \mathrm{O}_{2}$ & $\begin{array}{c}{[22413-05-4]} \\
\text { FUS }\end{array}$ & Hexadecyl eicc & 121.6 & 332.5 & $\mathrm{DSC}$ & [2012AYD/AYD] \\
\hline $\mathrm{C}_{36} \mathrm{H}_{72} \mathrm{O}_{2}$ & $\begin{array}{c}\text { [2778-96-3] } \\
\text { FUS }\end{array}$ & Octadecyl octa & $\begin{array}{l}\text { oate } \\
115.41\end{array}$ & 332.4 & $\mathrm{DSC}$ & [2013AYD] \\
\hline $\mathrm{C}_{36} \mathrm{H}_{72} \mathrm{O}_{18}$ & $\begin{array}{c}\text { [71092-62-1] } \\
\text { FUS }\end{array}$ & 54-crown-18 & 81.6 & 317.2 & DSC & [1996YAN/YU] \\
\hline $\mathrm{C}_{36} \mathrm{H}_{74}$ & $\begin{array}{c}\text { [630-06-8] } \\
\text { TRS } \\
\text { TRS } \\
\text { FUS } \\
\text { TRS } \\
\text { TRS } \\
\text { TRS } \\
\text { FUS } \\
\text { TRS } \\
\text { FUS } \\
\text { TRS } \\
\text { FUS } \\
\text { TRS } \\
\text { TRS } \\
\text { FUS } \\
\text { TRS } \\
\text { FUS } \\
\text { TRS } \\
\text { TRS } \\
\text { FUS }\end{array}$ & $\begin{array}{l}(80-370) \\
(80-370) \\
(80-370)\end{array}$ & $\begin{array}{c}11.73 \\
24.72 \\
81.6 \\
0.47 \\
15.02 \\
28.52 \\
102.51 \\
49.2 \\
173.5 \\
30.3 \\
87.6 \\
10.1 \\
32.1 \\
87.5 \\
43.4 \\
89.0 \\
15.3 \\
31.6 \\
86.0\end{array}$ & $\begin{array}{c}347.3 \\
348.3 \\
350.2 \\
343.6 \\
348.8 \\
347.1 \\
349.2 \\
346.0 \\
348.8 \\
347 \\
349 \\
345.4 \\
346.8 \\
348.9 \\
347.2 \\
349.4 \\
345.1 \\
346.9 \\
348.7\end{array}$ & $\begin{array}{l}\text { DSC } \\
\text { DSC }\end{array}$ & $\begin{array}{l}\text { [2006KHI/BOU] } \\
\text { [2006WAN/TOZ] } \\
\text { [2005VEN/CUE] } \\
\text { [2004MAR/KAI] }\end{array}$ \\
\hline
\end{tabular}

[Note: Authors of Ref. [1991JIN/WUN] reported that only the last two transition enthalpies and temperatures did not on crystallization change from the melt.]

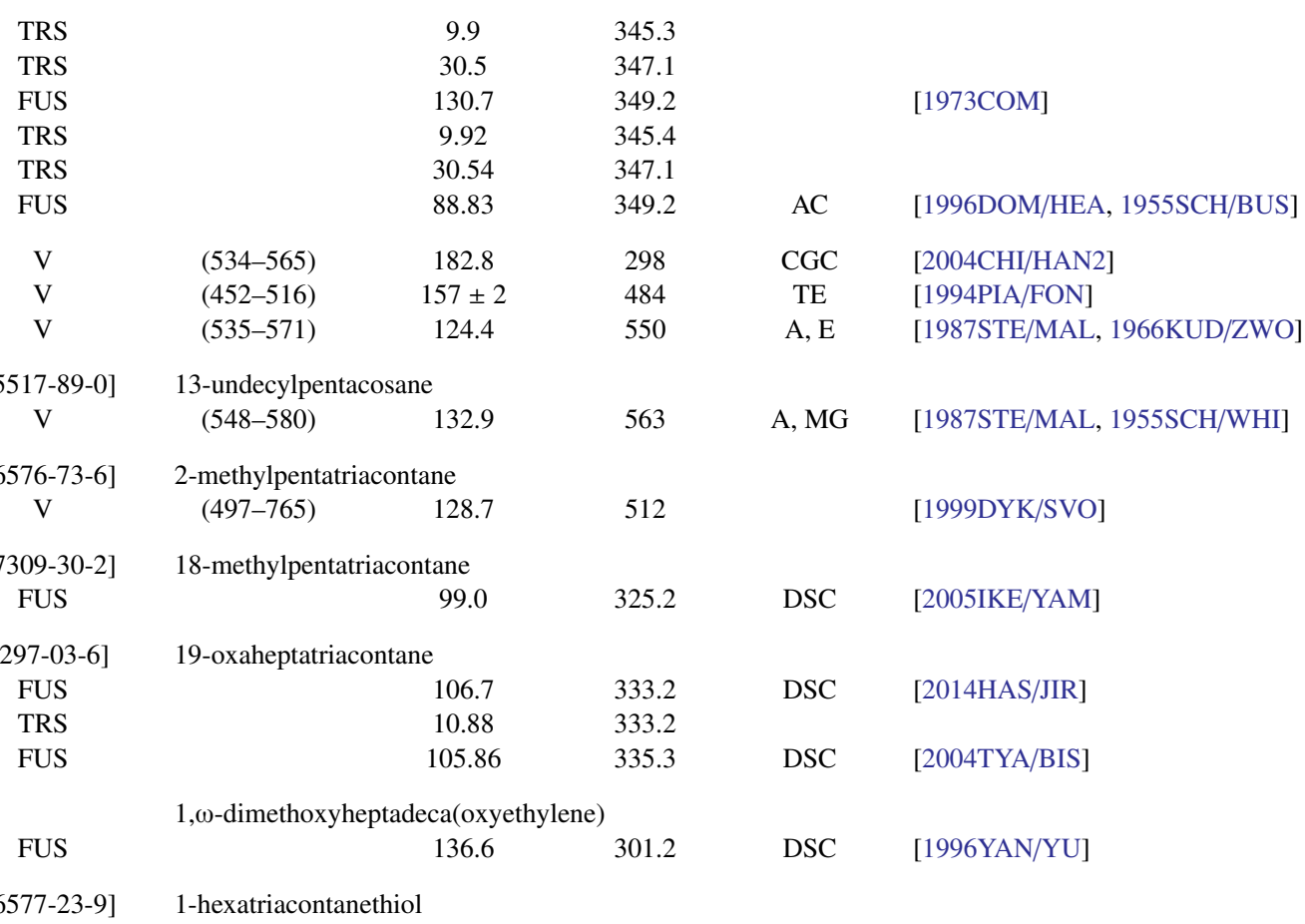


TABLE 15. Phase change enthalpies of $\mathrm{C}_{30}$ to $\mathrm{C}_{49}$ organic compounds-Continued

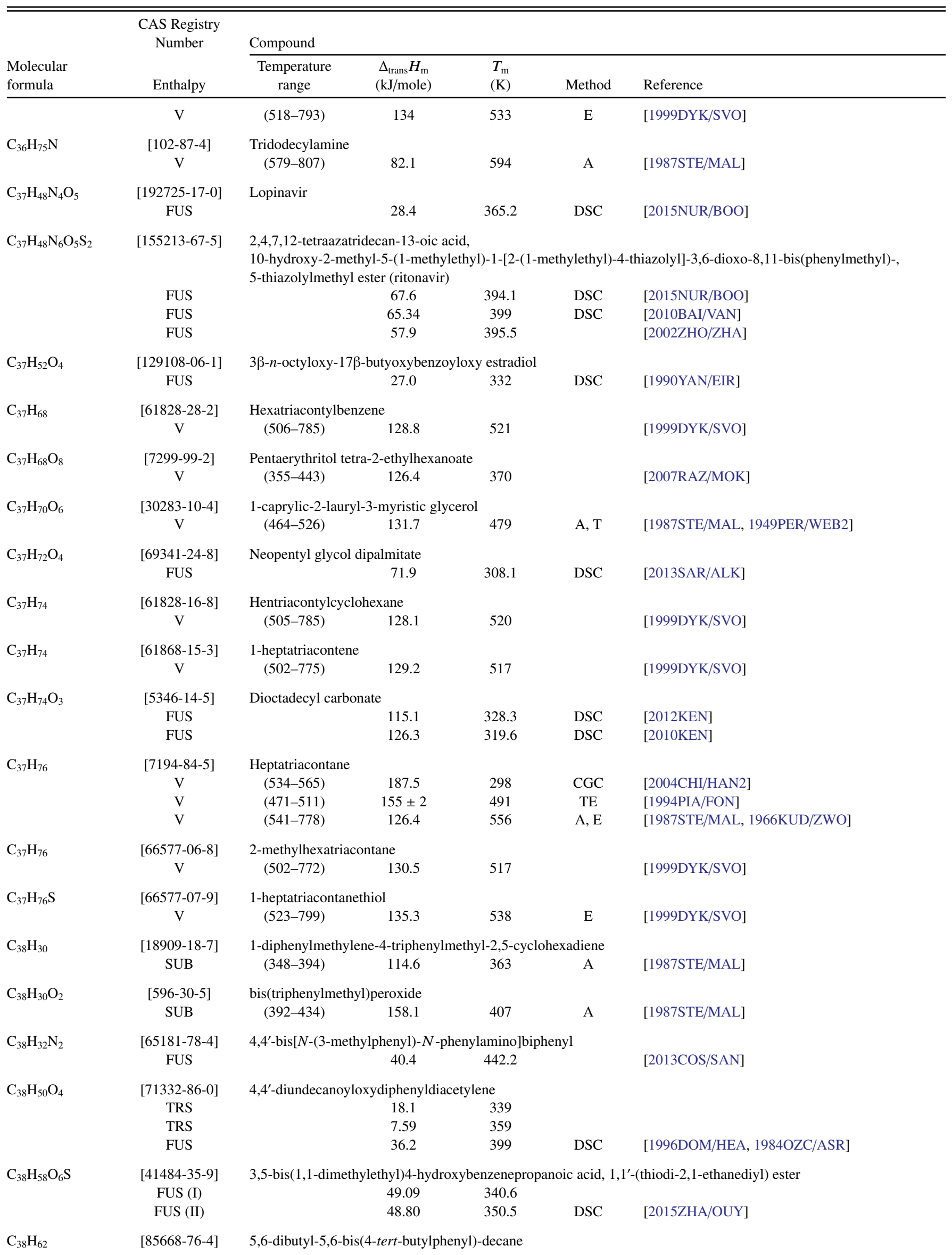


TABLE 15. Phase change enthalpies of $\mathrm{C}_{30}$ to $\mathrm{C}_{49}$ organic compounds-Continued

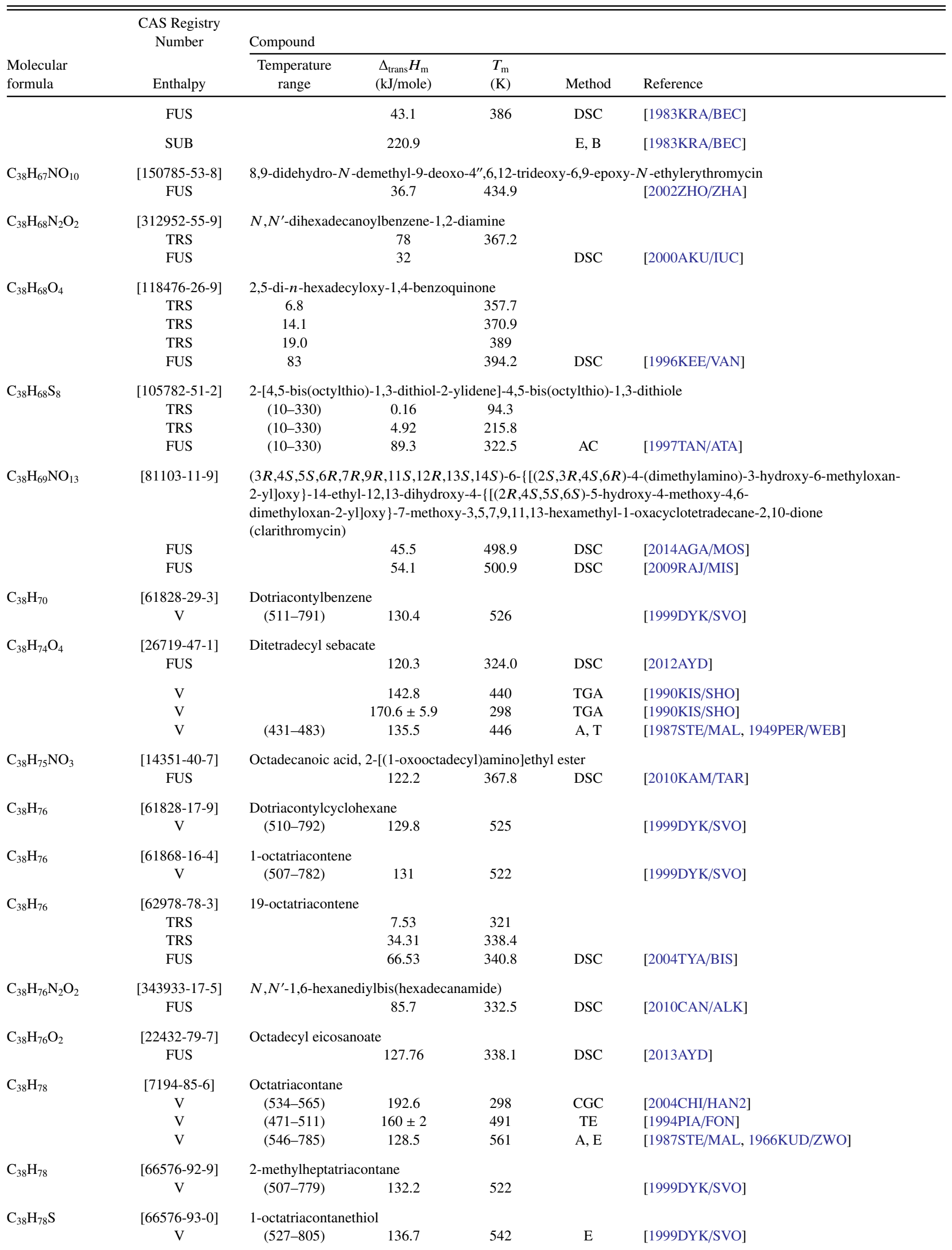


TABLE 15. Phase change enthalpies of $\mathrm{C}_{30}$ to $\mathrm{C}_{49}$ organic compounds-Continued

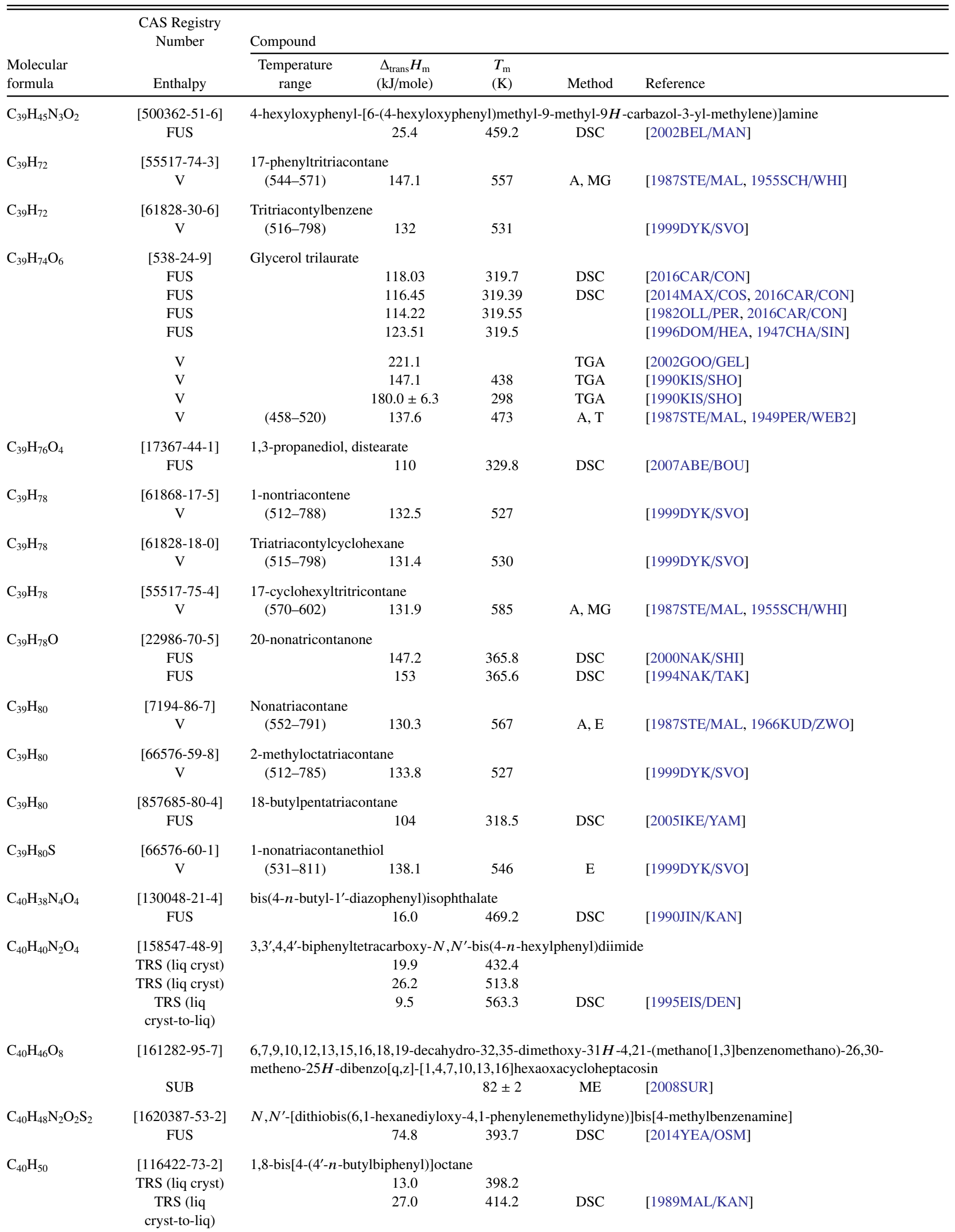


TABLE 15. Phase change enthalpies of $\mathrm{C}_{30}$ to $\mathrm{C}_{49}$ organic compounds-Continued

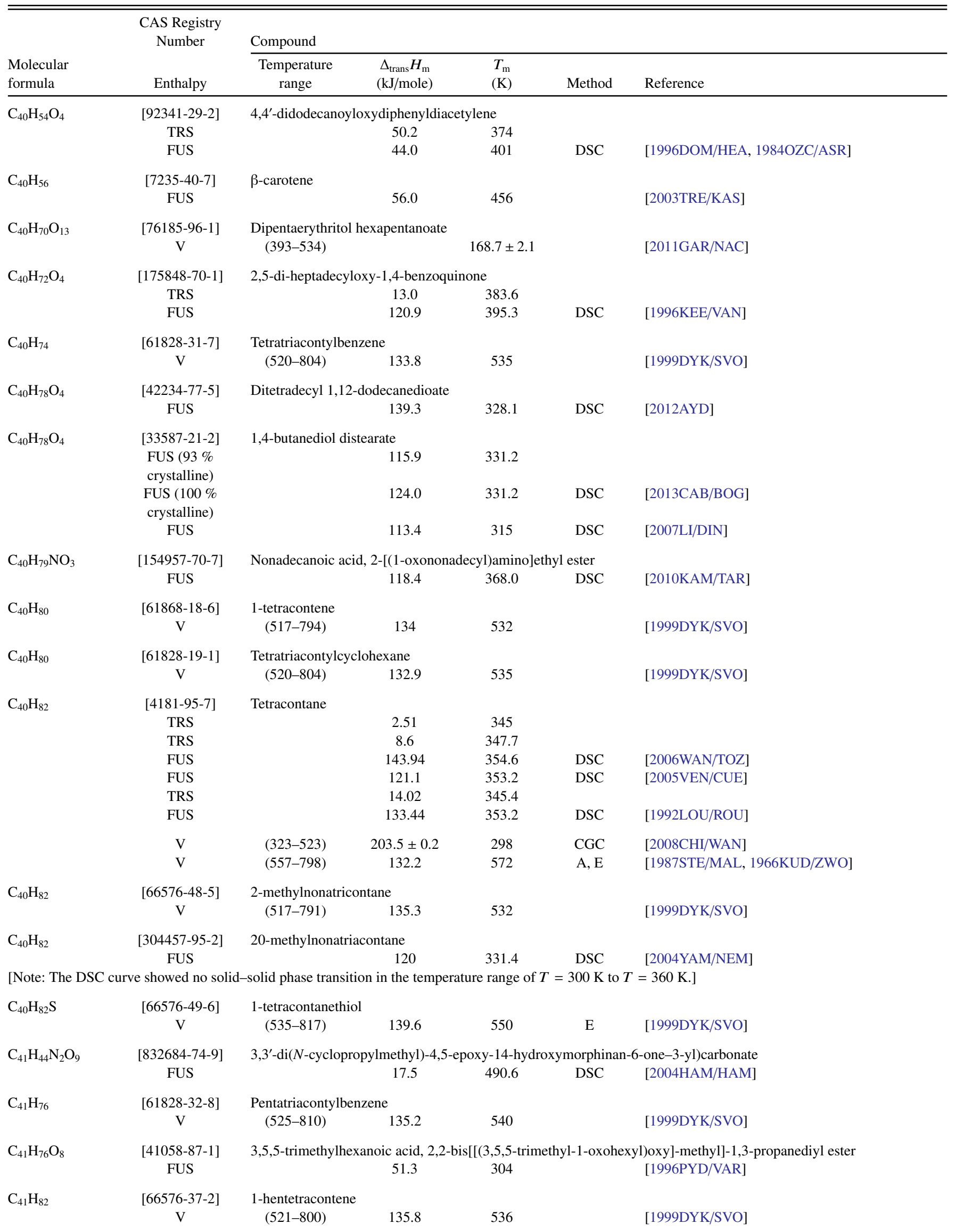


TABLE 15. Phase change enthalpies of $\mathrm{C}_{30}$ to $\mathrm{C}_{49}$ organic compounds-Continued

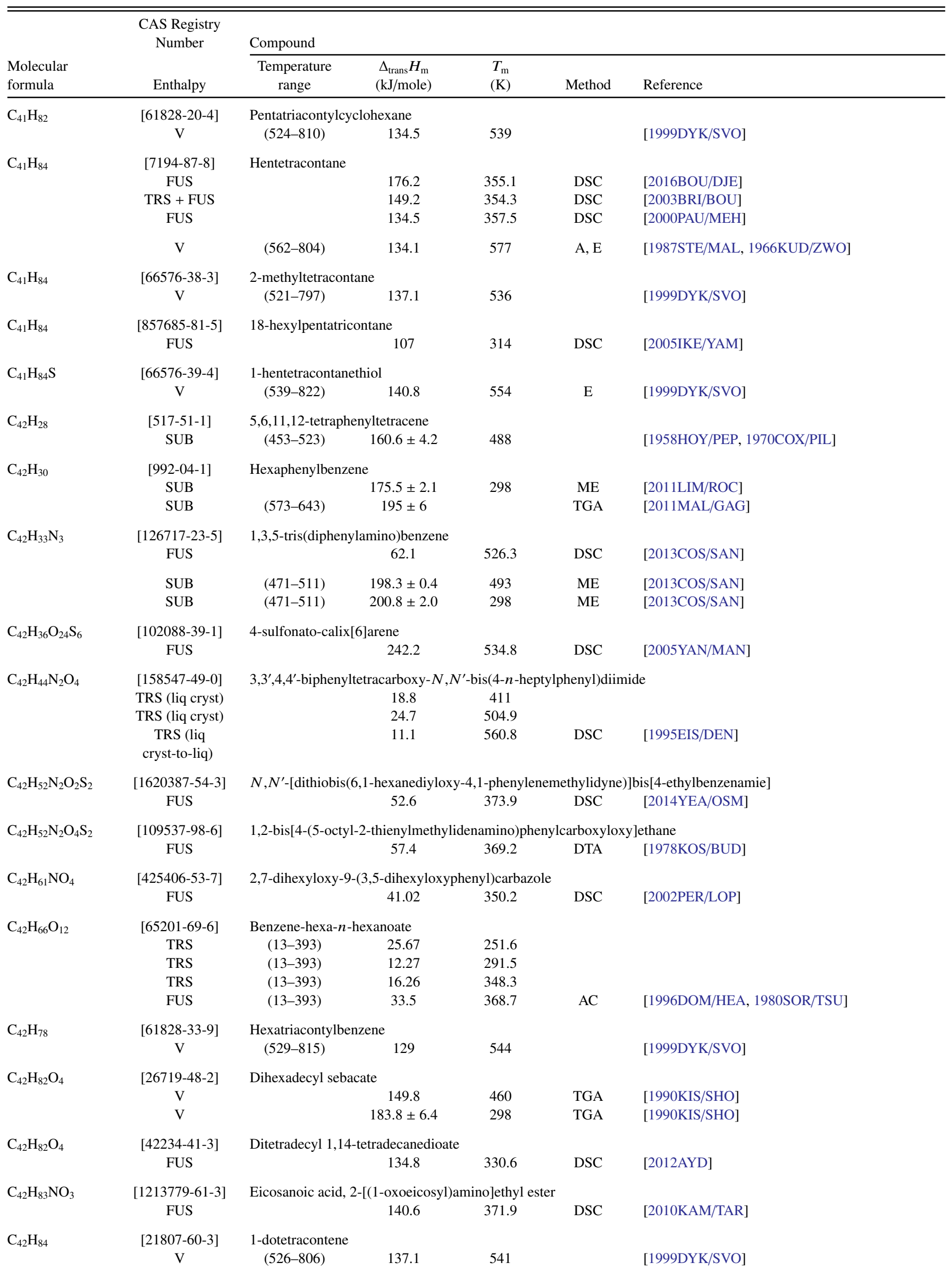


TABLE 15. Phase change enthalpies of $\mathrm{C}_{30}$ to $\mathrm{C}_{49}$ organic compounds-Continued

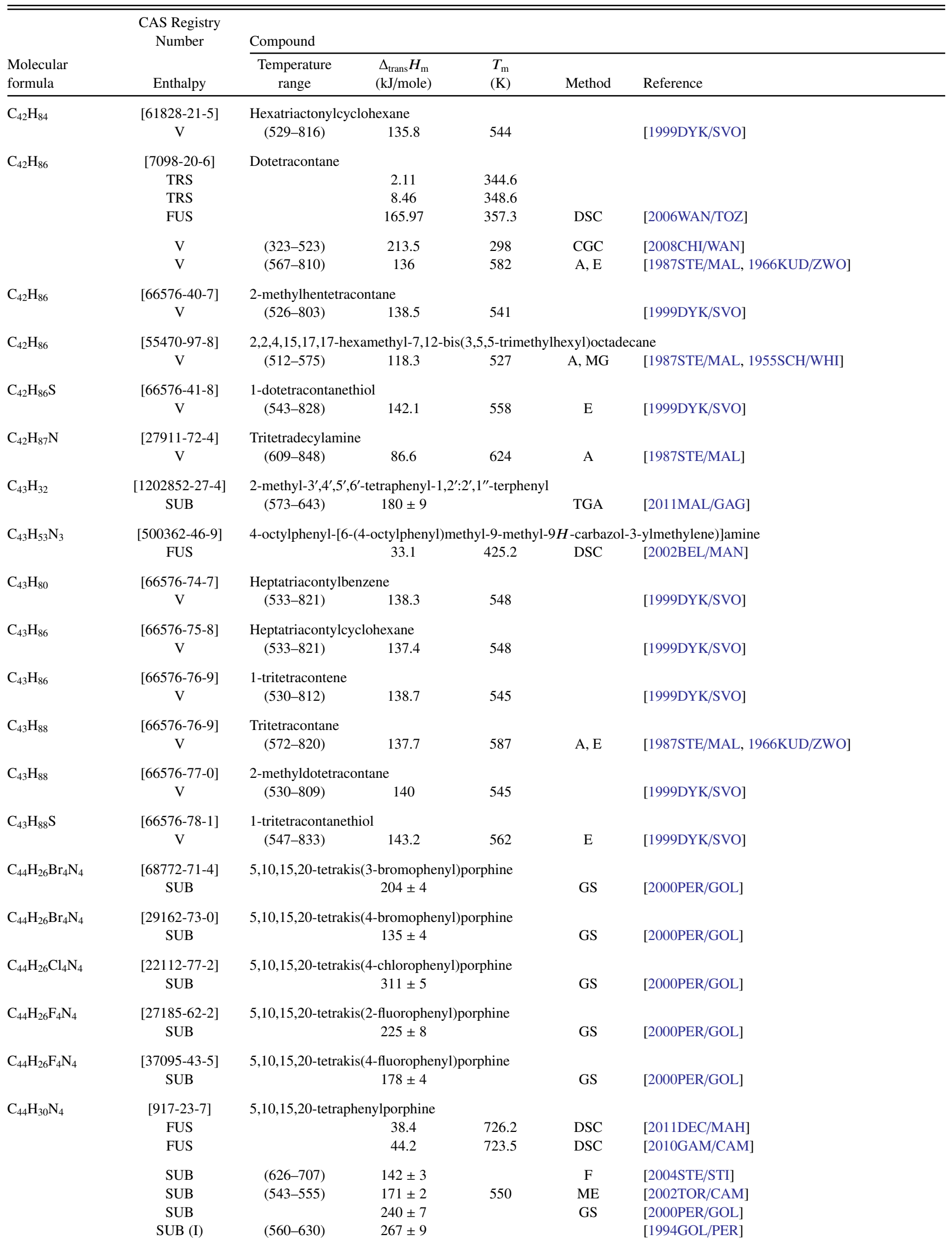


TABLE 15. Phase change enthalpies of $\mathrm{C}_{30}$ to $\mathrm{C}_{49}$ organic compounds-Continued

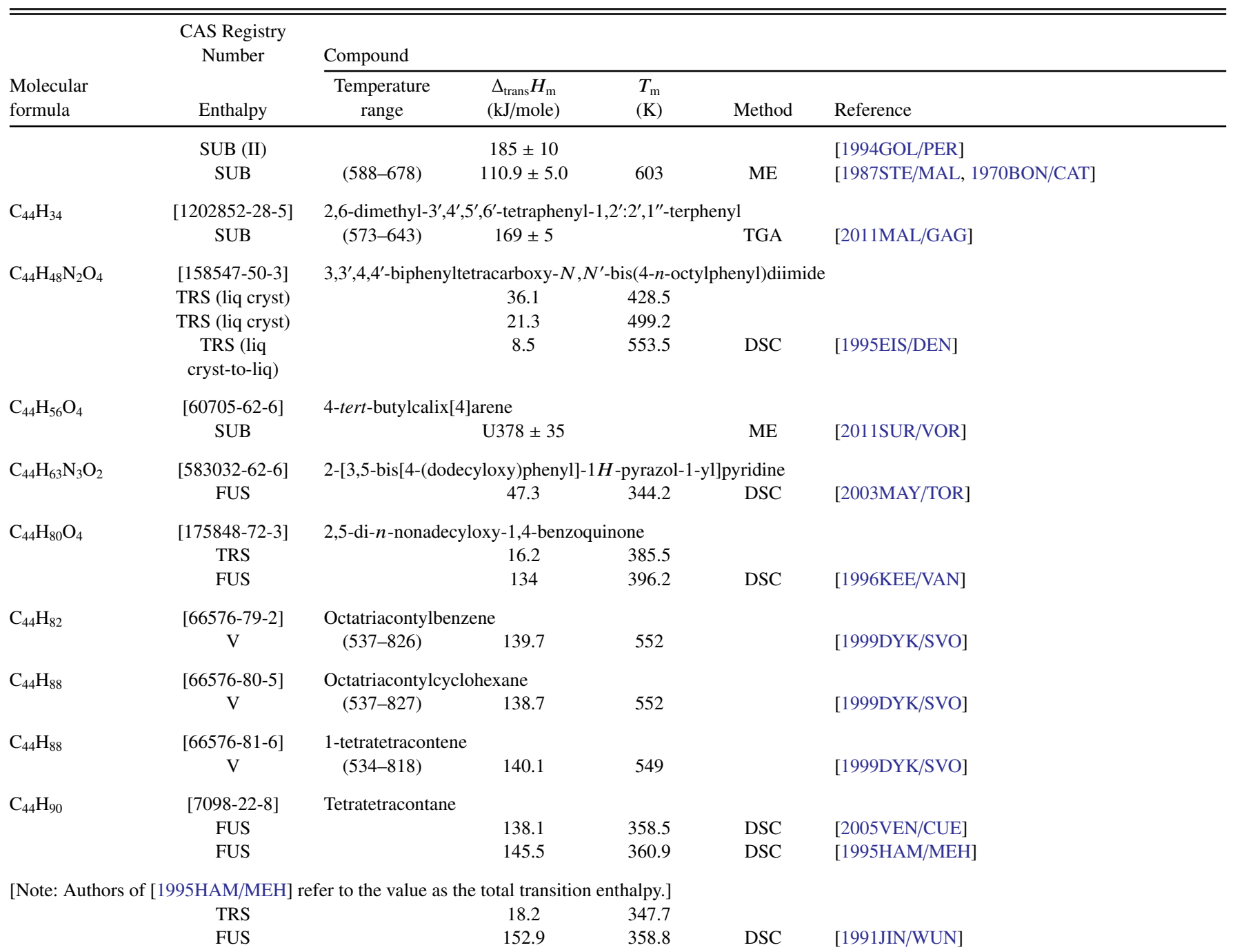

[Note: Authors of [1991JIN/WUN] reported that only the last transition enthalpy and temperature did not change on crystallization from the melt.]

$\begin{array}{lcccll}\mathrm{V} & (323-523) & 223.7 \pm 0.9 & 298 & \text { CGC } & {[\text { [2008CHI/WAN] }} \\ \mathrm{V} & & 146 & 387 & & {[1973 \mathrm{IVA} / \mathrm{GUJ}]} \\ \mathrm{V} & (577-821) & 139.3 & 592 & \text { A, E } & {[1987 \mathrm{STE} / \mathrm{MAL}, 1966 \mathrm{KUD} / \mathrm{ZWO}]}\end{array}$

\begin{tabular}{|c|c|c|c|c|c|}
\hline $\mathrm{C}_{44} \mathrm{H}_{90}$ & $\begin{array}{c}{[66576-82-7]} \\
V\end{array}$ & $\begin{array}{l}\text { 2-methyltritetracontane } \\
(534-815)\end{array}$ & 549 & & [1999DYK/SVO] \\
\hline $\mathrm{C}_{44} \mathrm{H}_{90} \mathrm{~S}$ & $\begin{array}{c}{[66576-83-8]} \\
\mathrm{V}\end{array}$ & $\begin{array}{l}\text { 1-tetratetracontanethiol } \\
\quad(551-838)\end{array}$ & 566 & $\mathrm{E}$ & [1999DYK/SVO] \\
\hline $\mathrm{C}_{45} \mathrm{H}_{39} \mathrm{~N}_{3}$ & $\begin{array}{c}{[138143-23-4]} \\
\text { FUS }\end{array}$ & $\begin{array}{r}\text { 1,3,5-tris[(3-methylphenyl)ph } \\
50.3\end{array}$ & $\begin{array}{l}\text { lo]benze } \\
455.8\end{array}$ & DSC & [2013COS/SAN] \\
\hline $\mathrm{C}_{45} \mathrm{H}_{84}$ & $\begin{array}{c}{[66576-61-2]} \\
\mathrm{V}\end{array}$ & $\begin{array}{l}\text { Nontriacontylbenzene } \\
\quad(541-832)\end{array}$ & 556 & & [1999DYK/SVO] \\
\hline $\mathrm{C}_{45} \mathrm{H}_{86} \mathrm{O}_{6}$ & $\begin{array}{c}{[60138-25-2]} \\
\mathrm{V}\end{array}$ & $\begin{array}{ll}(d l) \text {-1-lauric-2-myristic-3-palt } \\
(491-551) & 147.8\end{array}$ & $\begin{array}{c}\text { cerol } \\
506\end{array}$ & $\mathrm{~A}, \mathrm{~T}$ & [1987STE/MAL, 1949PER/WEB2] \\
\hline $\mathrm{C}_{45} \mathrm{H}_{86} \mathrm{O}_{6}$ & $\mathrm{~V}$ & $\begin{array}{l}(d l) \text {-1-myristic-2-capric-3-ste } \\
\quad(490-551)\end{array}$ & erol & A, $\mathrm{T}$ & [1987STE/MAL, 1949PER/WEB2] \\
\hline $\mathrm{C}_{45} \mathrm{H}_{86} \mathrm{O}_{6}$ & $\begin{array}{c}\text { [555-45-3] } \\
\text { FUS } \\
\text { FUS } \\
\text { FUS }\end{array}$ & Glycerol trimyristate & $\begin{array}{c}330.90 \\
305.2 \\
326.5\end{array}$ & $\begin{array}{l}\text { DSC } \\
\text { DSC } \\
\text { DSC }\end{array}$ & $\begin{array}{l}\text { [2014MAX/COS] } \\
{[2010 \mathrm{SAR} / \mathrm{BIC}]} \\
{[2007 \mathrm{HIM} / \mathrm{MAC}]}\end{array}$ \\
\hline
\end{tabular}

[Note: The value reported by [2007HIM/MAC] based on crystallization with a cooling scan rate of $0.1 \mathrm{~K} / \mathrm{min}$.]

$\begin{array}{ccccc}\text { FUS } & 152.2 & 330.2 & & {[1996 \mathrm{DOM} / \mathrm{HEA}, 1947 \mathrm{CHA} / \mathrm{SIN}]} \\ \mathrm{V} & 199.05 & & \text { TGA } \quad & {[2002 \mathrm{GOO} / \mathrm{GEL}]}\end{array}$


TABLE 15. Phase change enthalpies of $\mathrm{C}_{30}$ to $\mathrm{C}_{49}$ organic compounds-Continued

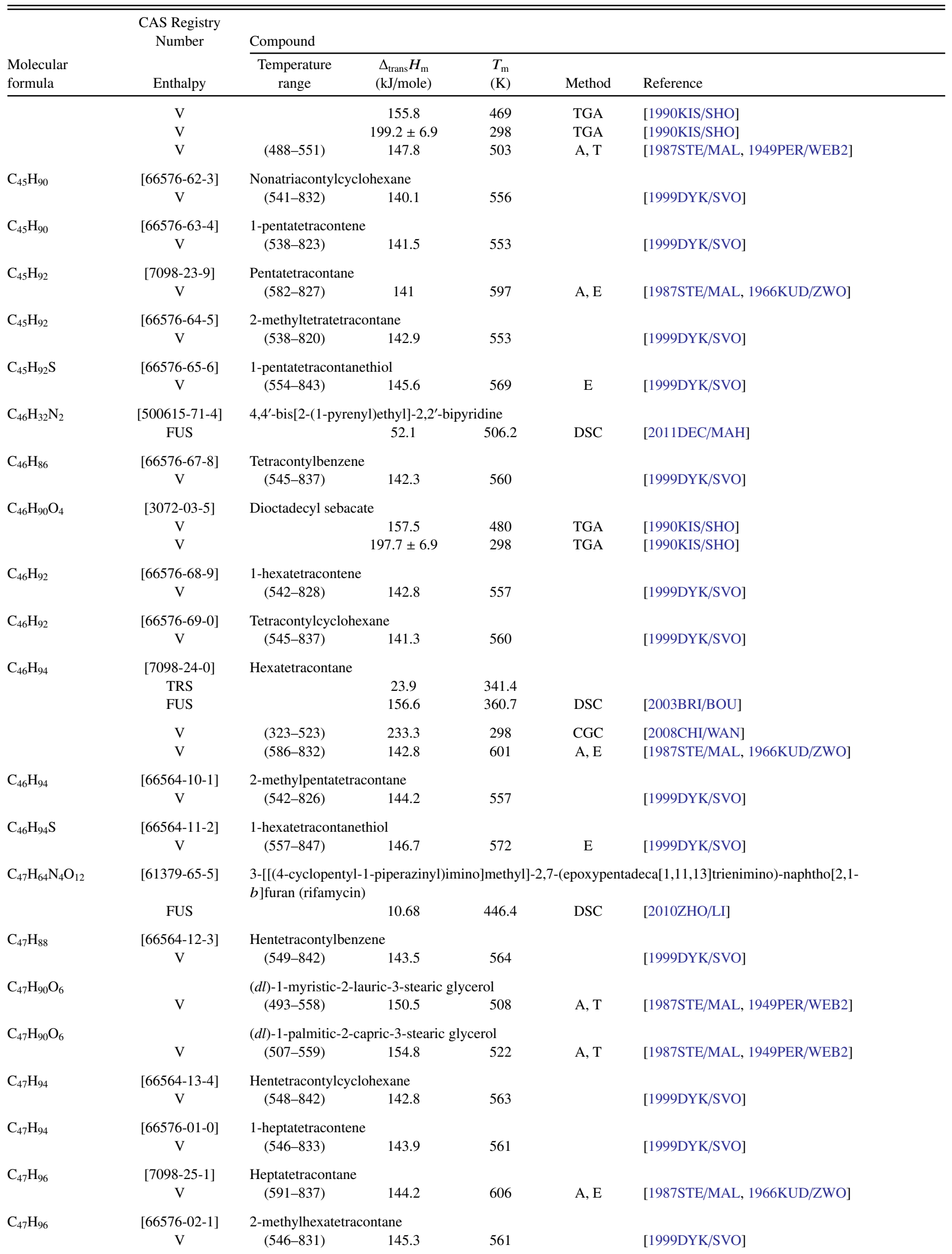


TABLE 15. Phase change enthalpies of $\mathrm{C}_{30}$ to $\mathrm{C}_{49}$ organic compounds-Continued

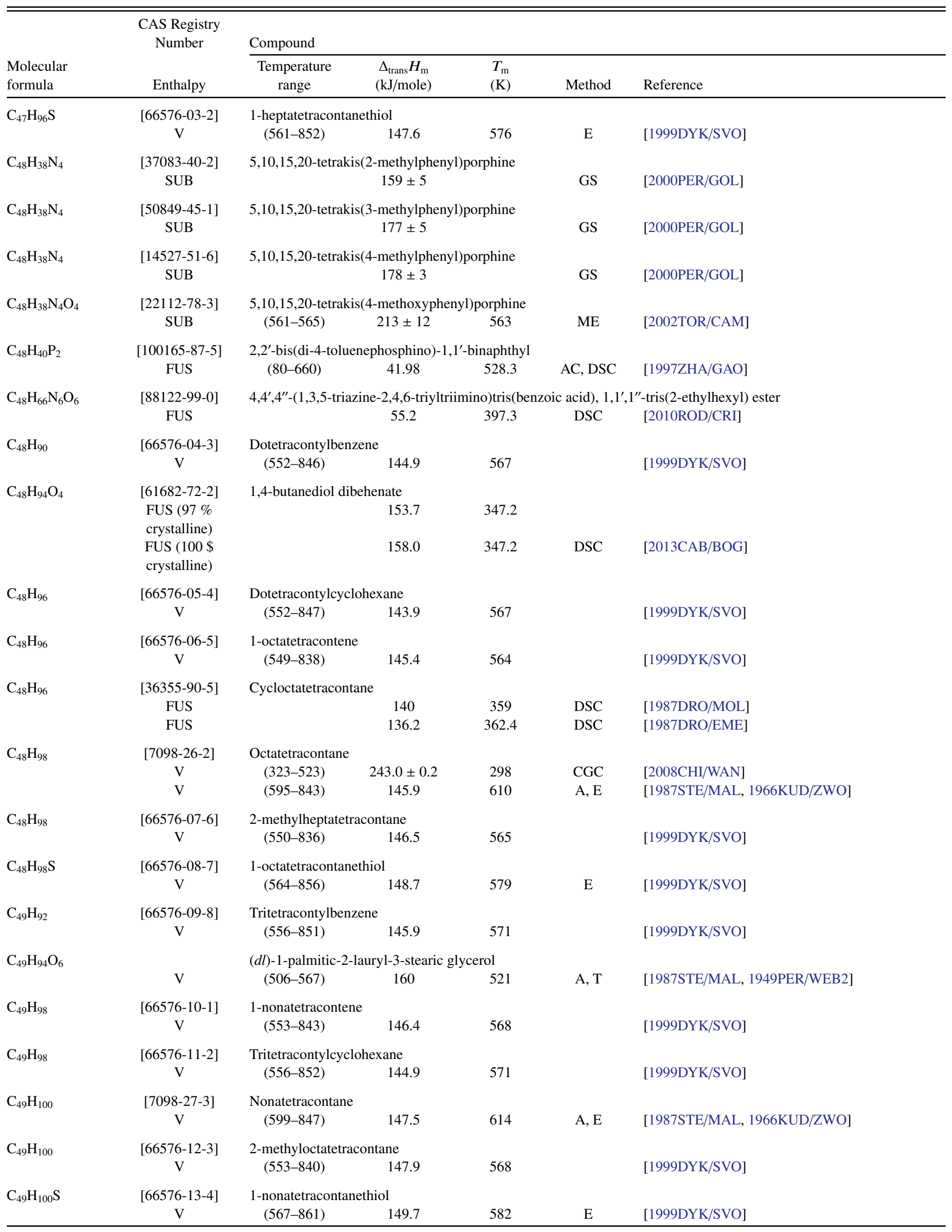


TABLE 16. Phase change enthalpies of $\mathrm{C}_{50}$ to $\mathrm{C}_{192}$ organic compounds

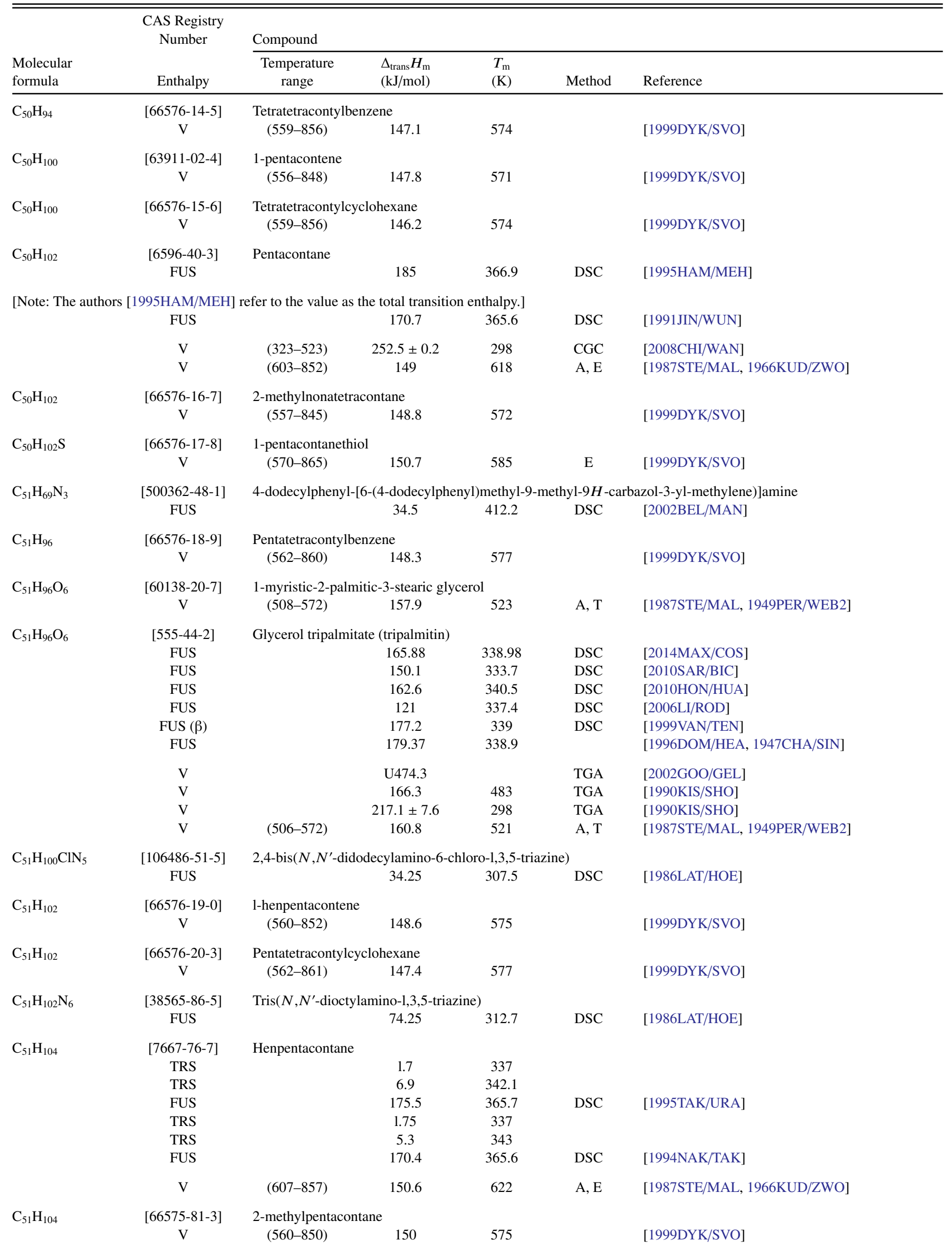


TABLE 16. Phase change enthalpies of $\mathrm{C}_{50}$ to $\mathrm{C}_{192}$ organic compounds-Continued

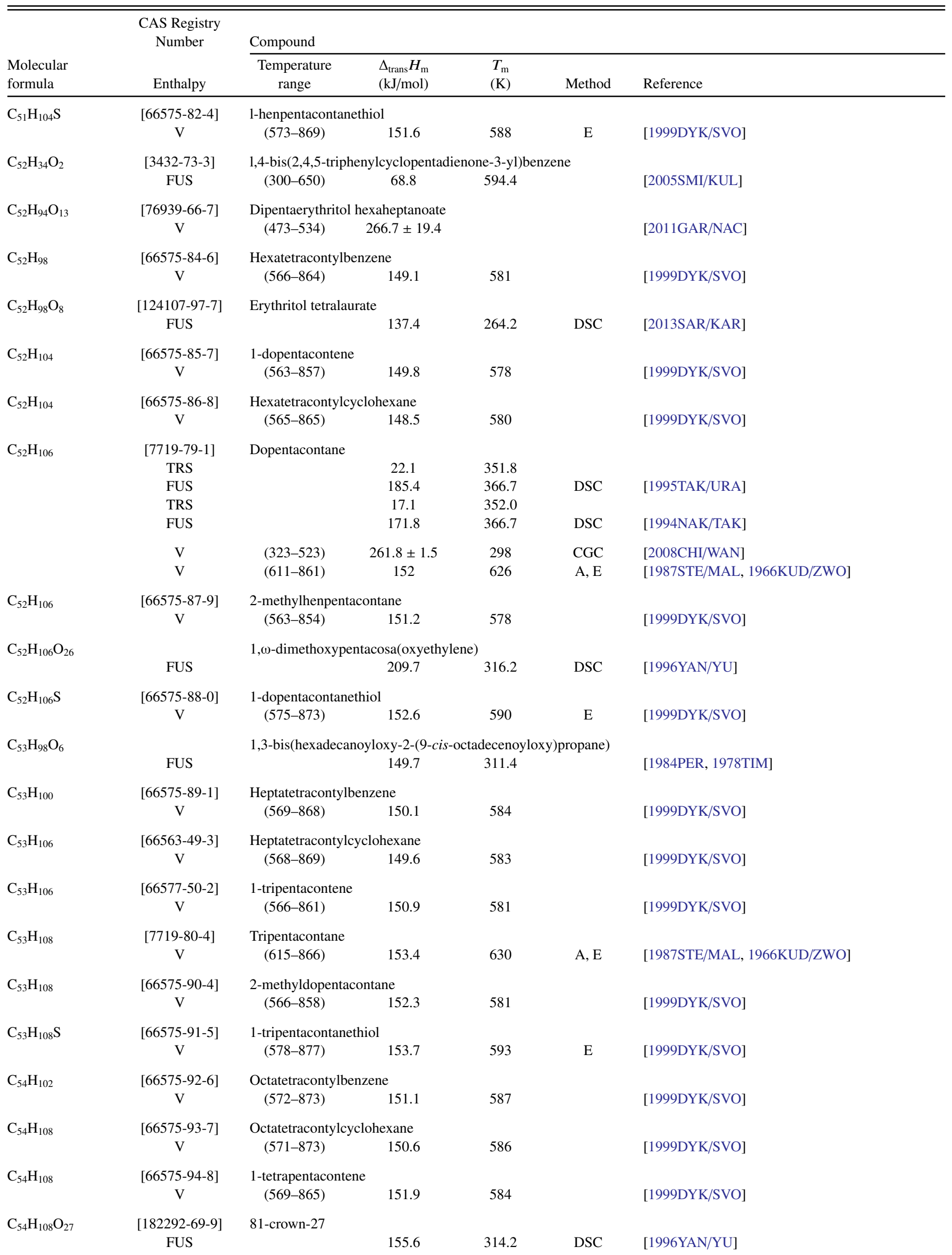


TABLE 16. Phase change enthalpies of $\mathrm{C}_{50}$ to $\mathrm{C}_{192}$ organic compounds-Continued

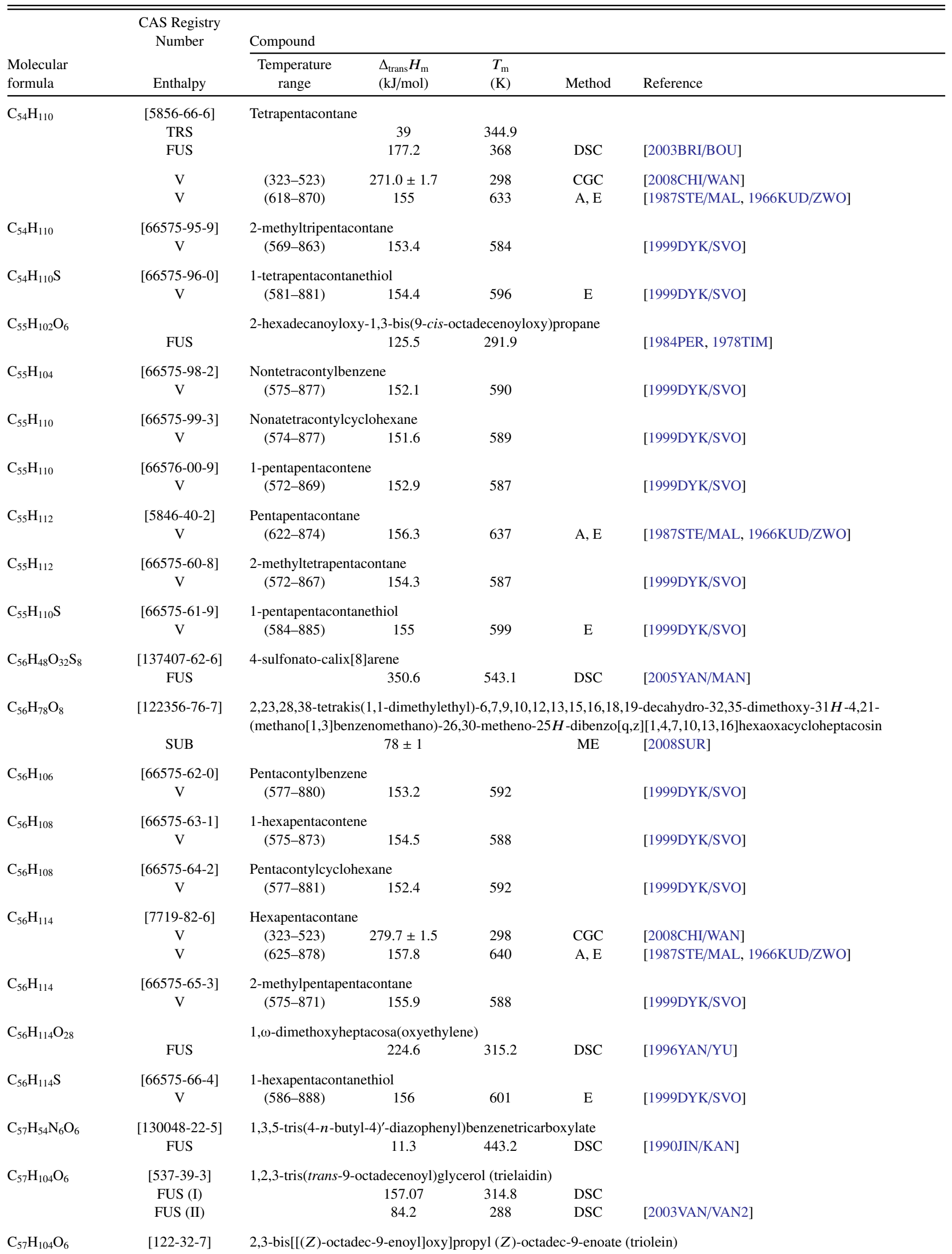


TABLE 16. Phase change enthalpies of $\mathrm{C}_{50}$ to $\mathrm{C}_{192}$ organic compounds-Continued

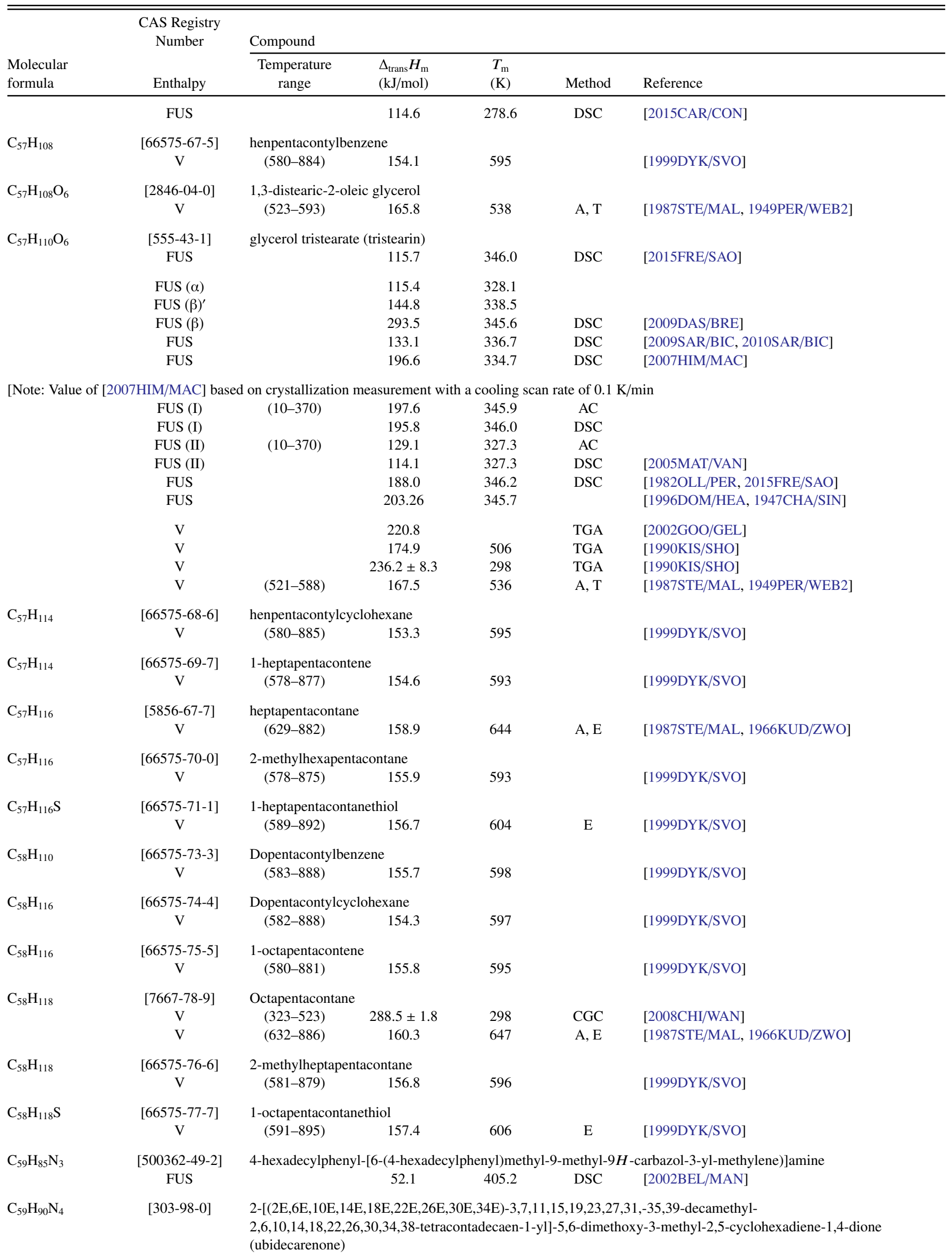


TABLE 16. Phase change enthalpies of $\mathrm{C}_{50}$ to $\mathrm{C}_{192}$ organic compounds-Continued

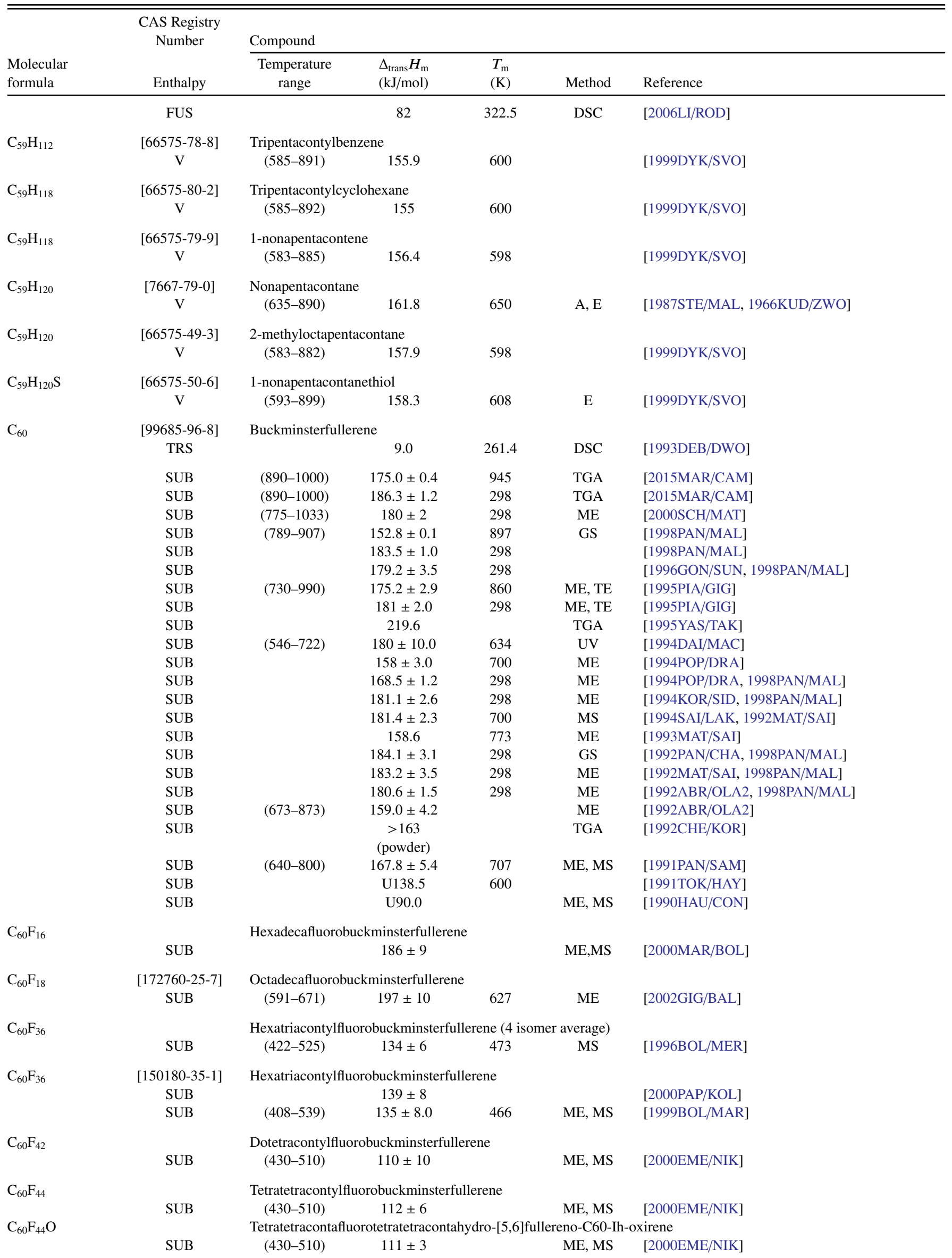


TABLE 16. Phase change enthalpies of $\mathrm{C}_{50}$ to $\mathrm{C}_{192}$ organic compounds-Continued

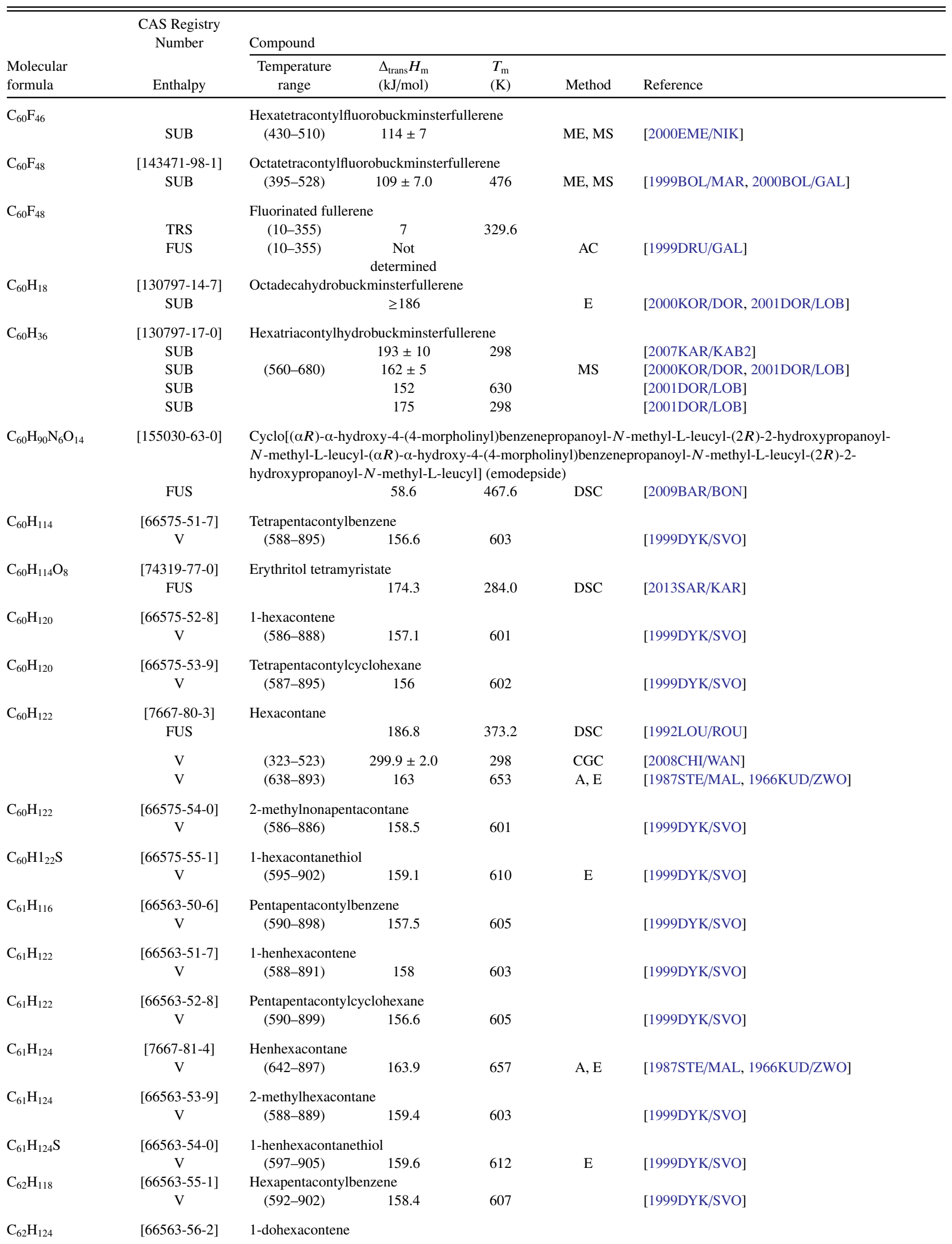


TABLE 16. Phase change enthalpies of $\mathrm{C}_{50}$ to $\mathrm{C}_{192}$ organic compounds-Continued

\begin{tabular}{|c|c|c|c|c|c|c|}
\hline \multirow[b]{2}{*}{$\begin{array}{l}\text { Molecular } \\
\text { formula }\end{array}$} & \multirow{2}{*}{$\begin{array}{l}\text { CAS Registry } \\
\text { Number } \\
\text { Enthalpy }\end{array}$} & \multicolumn{5}{|l|}{ Compound } \\
\hline & & $\begin{array}{l}\text { Temperature } \\
\text { range }\end{array}$ & $\begin{array}{l}\Delta_{\text {trans }} H_{\mathrm{m}} \\
(\mathrm{kJ} / \mathrm{mol})\end{array}$ & $\begin{array}{l}T_{\mathrm{m}} \\
(\mathrm{K})\end{array}$ & Method & Reference \\
\hline & $\mathrm{V}$ & $(590-895)$ & 158.6 & 605 & & [1999DYK/SVO] \\
\hline $\mathrm{C}_{62} \mathrm{H}_{124}$ & $\begin{array}{c}{[66563-57-3]} \\
\mathrm{V}\end{array}$ & $\begin{array}{l}\text { Hexapentacontylcycl } \\
\quad(592-902)\end{array}$ & $\begin{array}{c}\text { lohexane } \\
157.5\end{array}$ & 607 & & [1999DYK/SVO] \\
\hline $\mathrm{C}_{62} \mathrm{H}_{126}$ & $\begin{array}{c}{[7719-83-7]} \\
\mathrm{V} \\
\mathrm{V}\end{array}$ & $\begin{array}{l}\text { Dohexacontane } \\
\qquad(323-523) \\
(645-901)\end{array}$ & $\begin{array}{c}306.8 \pm 0.1 \\
165.2\end{array}$ & $\begin{array}{l}298 \\
660\end{array}$ & $\begin{array}{l}\text { CGC } \\
\mathrm{A}, \mathrm{E}\end{array}$ & $\begin{array}{l}\text { [2008CHI/WAN] } \\
\text { [1987STE/MAL, 1966KUD/ZWO] }\end{array}$ \\
\hline $\mathrm{C}_{62} \mathrm{H}_{126}$ & $\begin{array}{c}{[66563-58-4]} \\
\mathrm{V}\end{array}$ & $\begin{array}{l}\text { 2-methylhenhexacont } \\
\text { (590-892) }\end{array}$ & $\begin{array}{l}\text { ntane } \\
160.1\end{array}$ & 605 & & [1999DYK/SVO] \\
\hline $\mathrm{C}_{62} \mathrm{H}_{122} \mathrm{~S}$ & $\begin{array}{c}{[66563-59-5]} \\
\mathrm{V}\end{array}$ & $\begin{array}{l}\text { 1-dohexacontanethiol } \\
\text { (599-908) }\end{array}$ & 160.1 & 614 & $\mathrm{E}$ & [1999DYK/SVO] \\
\hline $\mathrm{C}_{63} \mathrm{H}_{120}$ & $\begin{array}{c}{[66563-60-8]} \\
V\end{array}$ & $\begin{array}{l}\text { Heptapentacontylben } \\
\quad(595-905)\end{array}$ & $\begin{array}{l}\text { nzene } \\
\qquad 158.9\end{array}$ & 610 & & [1999DYK/SVO] \\
\hline $\mathrm{C}_{63} \mathrm{H}_{126}$ & $\begin{array}{c}{[66563-61-9]} \\
\mathrm{V}\end{array}$ & $\begin{array}{l}\text { Heptapentacontylcyc } \\
\quad(594-905)\end{array}$ & $\begin{array}{c}\text { clohexane } \\
158.2\end{array}$ & 609 & & [1999DYK/SVO] \\
\hline $\mathrm{C}_{63} \mathrm{H}_{126}$ & $\begin{array}{c}{[66563-62-0]} \\
V\end{array}$ & $\begin{array}{l}\text { 1-trihexacontene } \\
\text { (593-899) }\end{array}$ & 159.8 & 608 & & [1999DYK/SVO] \\
\hline $\mathrm{C}_{63} \mathrm{H}_{126} \mathrm{~N}_{6}$ & $\begin{array}{c}\text { [106486-49-1] } \\
\text { FUS }\end{array}$ & $\operatorname{Tris}\left(N, N^{\prime}\right.$-didecylan & $\begin{array}{c}\operatorname{mino})-1,3,5- \\
87.68\end{array}$ & e 314.4 & $\mathrm{DSC}$ & {$[1986 \mathrm{LAT} / \mathrm{HOE}]$} \\
\hline $\mathrm{C}_{63} \mathrm{H}_{128}$ & $\begin{array}{c}{[7719-84-8]} \\
\mathrm{V}\end{array}$ & $\begin{array}{c}\text { Trihexacontane } \\
\text { (647-904) }\end{array}$ & 116.7 & 662 & $\mathrm{~A}, \mathrm{E}$ & [1987STE/MAL, 1966KUD/ZWO] \\
\hline $\mathrm{C}_{63} \mathrm{H}_{128}$ & $\begin{array}{c}{[66563-63-1]} \\
\mathrm{V}\end{array}$ & $\begin{array}{l}\text { 2-methyldohexaconta } \\
\text { (593-897) }\end{array}$ & $\begin{array}{l}\text { tane } \\
161.3\end{array}$ & 608 & & [1999DYK/SVO] \\
\hline $\mathrm{C}_{63} \mathrm{H}_{128} \mathrm{~S}$ & $\begin{array}{c}{[66563-64-2]} \\
\mathrm{V}\end{array}$ & $\begin{array}{l}\text { 1-trihexacontanethiol } \\
\quad(602-911)\end{array}$ & 161.1 & 617 & $\mathrm{E}$ & [1999DYK/SVO] \\
\hline $\mathrm{C}_{64} \mathrm{H}_{118} \mathrm{O}_{13}$ & $\begin{array}{c}\text { [127304-08-9] } \\
\mathrm{V}\end{array}$ & $\begin{array}{l}\text { Dipentaerythritol isor } \\
\quad(473-553)\end{array}$ & $\begin{array}{l}\text { ononanoate } \\
185.9 \pm 2.4\end{array}$ & & & [2011GAR/NAC] \\
\hline $\mathrm{C}_{64} \mathrm{H}_{122}$ & $\begin{array}{c}{[66563-65-3]} \\
V\end{array}$ & $\begin{array}{l}\text { Octapentacontylbenz } \\
\quad(597-908)\end{array}$ & $\begin{array}{l}\text { zene } \\
159.5\end{array}$ & 612 & & [1999DYK/SVO] \\
\hline $\mathrm{C}_{64} \mathrm{H}_{128}$ & $\begin{array}{c}{[66563-66-4]} \\
V\end{array}$ & $\begin{array}{l}\text { Octapentacontylcyclo } \\
\quad(596-908)\end{array}$ & $\begin{array}{r}\text { lohexane } \\
158.8\end{array}$ & 611 & & [1999DYK/SVO] \\
\hline $\mathrm{C}_{64} \mathrm{H}_{128}$ & $\begin{array}{c}{[66563-36-8]} \\
\mathrm{V}\end{array}$ & $\begin{array}{l}\text { 1-tetrahexacontene } \\
\quad(595-902)\end{array}$ & 160.5 & 610 & & [1999DYK/SVO] \\
\hline $\mathrm{C}_{64} \mathrm{H}_{130}$ & $\begin{array}{c}{[7719-87-1]} \\
\mathrm{V} \\
\mathrm{V}\end{array}$ & $\begin{array}{l}\text { Tetrahexacontane } \\
\qquad(323-523) \\
(650-907)\end{array}$ & $\begin{array}{c}315.4 \pm 0.4 \\
168.3\end{array}$ & $\begin{array}{l}298 \\
665\end{array}$ & $\begin{array}{l}\text { CGC } \\
\mathrm{A}, \mathrm{E}\end{array}$ & $\begin{array}{l}\text { [2008CHI/WAN] } \\
\text { [1987STE/MAL, 1966KUD/ZWO] }\end{array}$ \\
\hline $\mathrm{C}_{64} \mathrm{H}_{130}$ & $\begin{array}{c}{[66563-37-9]} \\
\mathrm{V}\end{array}$ & $\begin{array}{l}\text { 2-methyltrihexaconta } \\
\text { (595-900) }\end{array}$ & 161.9 & 610 & & [1999DYK/SVO] \\
\hline $\mathrm{C}_{64} \mathrm{H}_{130} \mathrm{~S}$ & $\begin{array}{c}{[66563-38-0]} \\
\mathrm{V}\end{array}$ & $\begin{array}{l}\text { 1-tetrahexacontanethi } \\
\quad(604-914)\end{array}$ & $\begin{array}{l}\text { hiol } \\
\qquad 161.6\end{array}$ & 619 & $\mathrm{E}$ & [1999DYK/SVO] \\
\hline $\mathrm{C}_{65} \mathrm{H}_{122} \mathrm{O}_{10}$ & FUS & Xylitol pentalaurate & 196.5 & 316.7 & DSC & [2015BIC/SAR] \\
\hline $\mathrm{C}_{65} \mathrm{H}_{124}$ & $\begin{array}{c}{[66563-39-1]} \\
\mathrm{V}\end{array}$ & $\begin{array}{l}\text { Nonapentacontylbenz } \\
\quad(599-911)\end{array}$ & $\begin{array}{l}\text { tzene } \\
160.1\end{array}$ & 614 & & [1999DYK/SVO] \\
\hline $\mathrm{C}_{65} \mathrm{H}_{130}$ & $\begin{array}{c}{[66563-40-4]} \\
\mathrm{V}\end{array}$ & $\begin{array}{l}\text { Nonapentacontylcycl } \\
\quad(599-912)\end{array}$ & $\begin{array}{c}\text { lohexane } \\
159.9\end{array}$ & 614 & & [1999DYK/SVO] \\
\hline $\mathrm{C}_{65} \mathrm{H}_{130}$ & $\begin{array}{c}{[66563-41-5]} \\
V\end{array}$ & $\begin{array}{l}\text { 1-pentahexacontene } \\
\text { (597-905) }\end{array}$ & 161.1 & 612 & & [1999DYK/SVO] \\
\hline $\mathrm{C}_{65} \mathrm{H}_{132}$ & $\begin{array}{c}{[7719-88-2]} \\
\mathrm{V}\end{array}$ & $\begin{array}{l}\text { Pentahexacontane } \\
\quad(653-910)\end{array}$ & 169 & 668 & $\mathrm{~A}, \mathrm{E}$ & [1987STE/MAL, 1966KUD/ZWO] \\
\hline
\end{tabular}


TABLE 16. Phase change enthalpies of $\mathrm{C}_{50}$ to $\mathrm{C}_{192}$ organic compounds-Continued

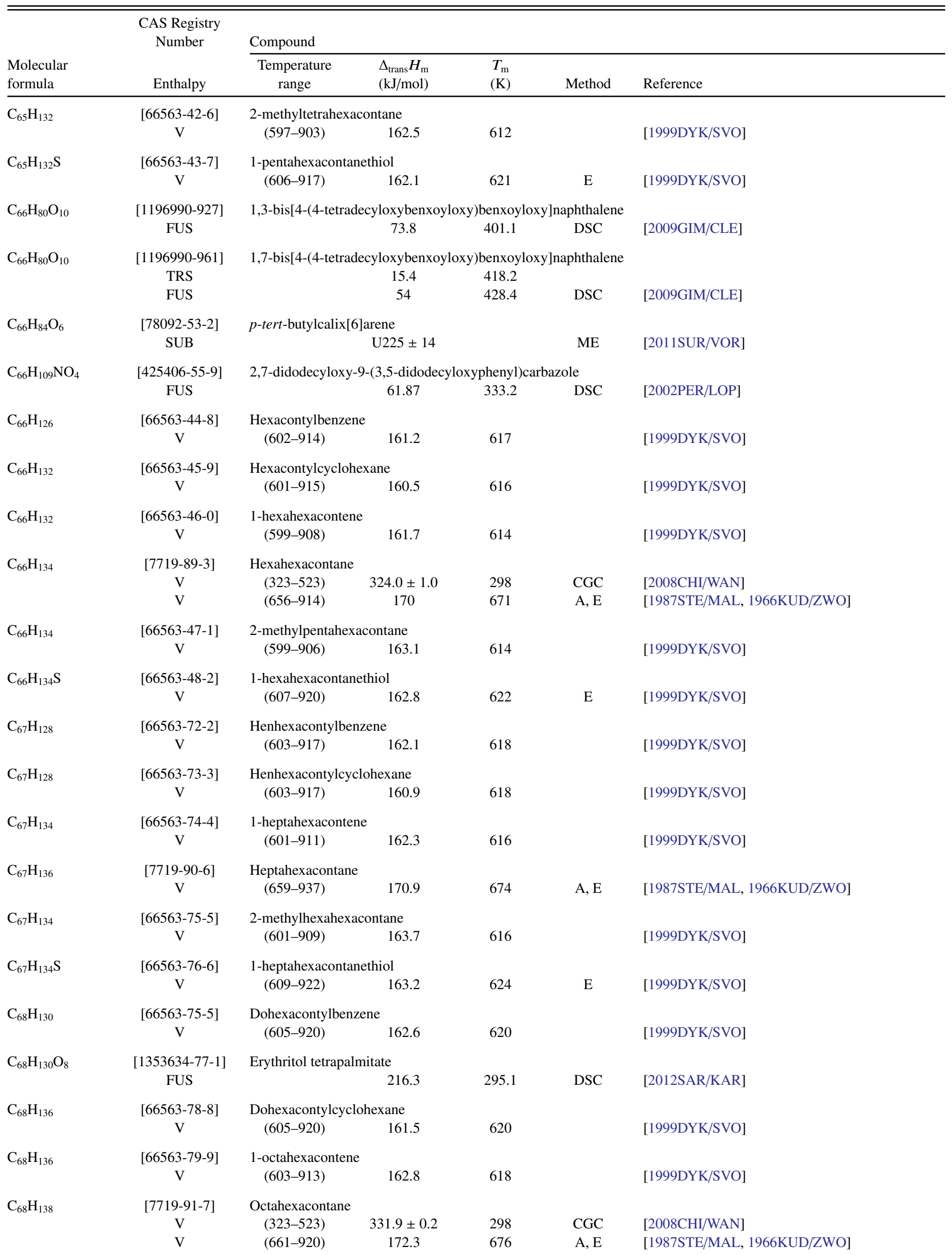


TABLE 16. Phase change enthalpies of $\mathrm{C}_{50}$ to $\mathrm{C}_{192}$ organic compounds-Continued

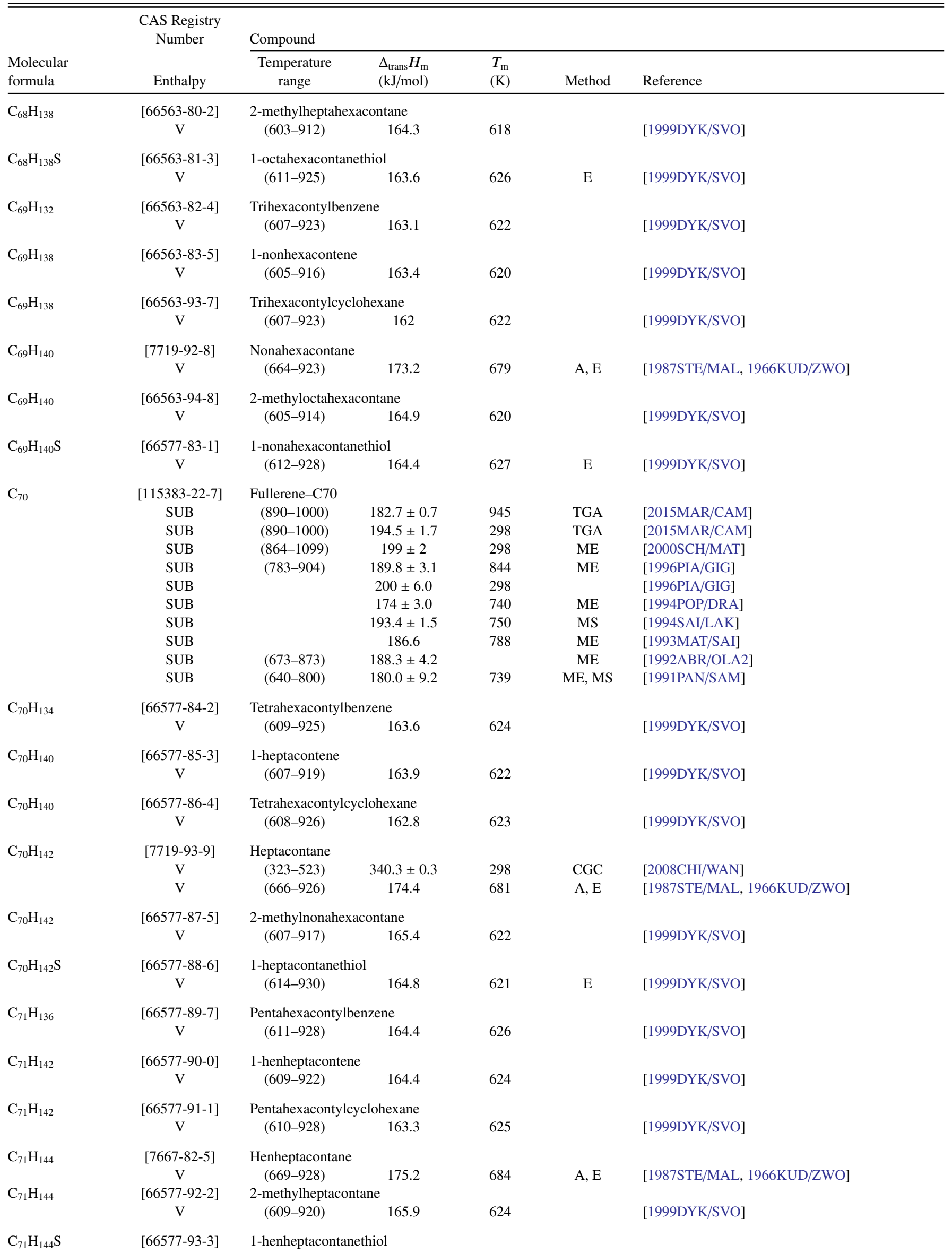


TABLE 16. Phase change enthalpies of $\mathrm{C}_{50}$ to $\mathrm{C}_{192}$ organic compounds-Continued

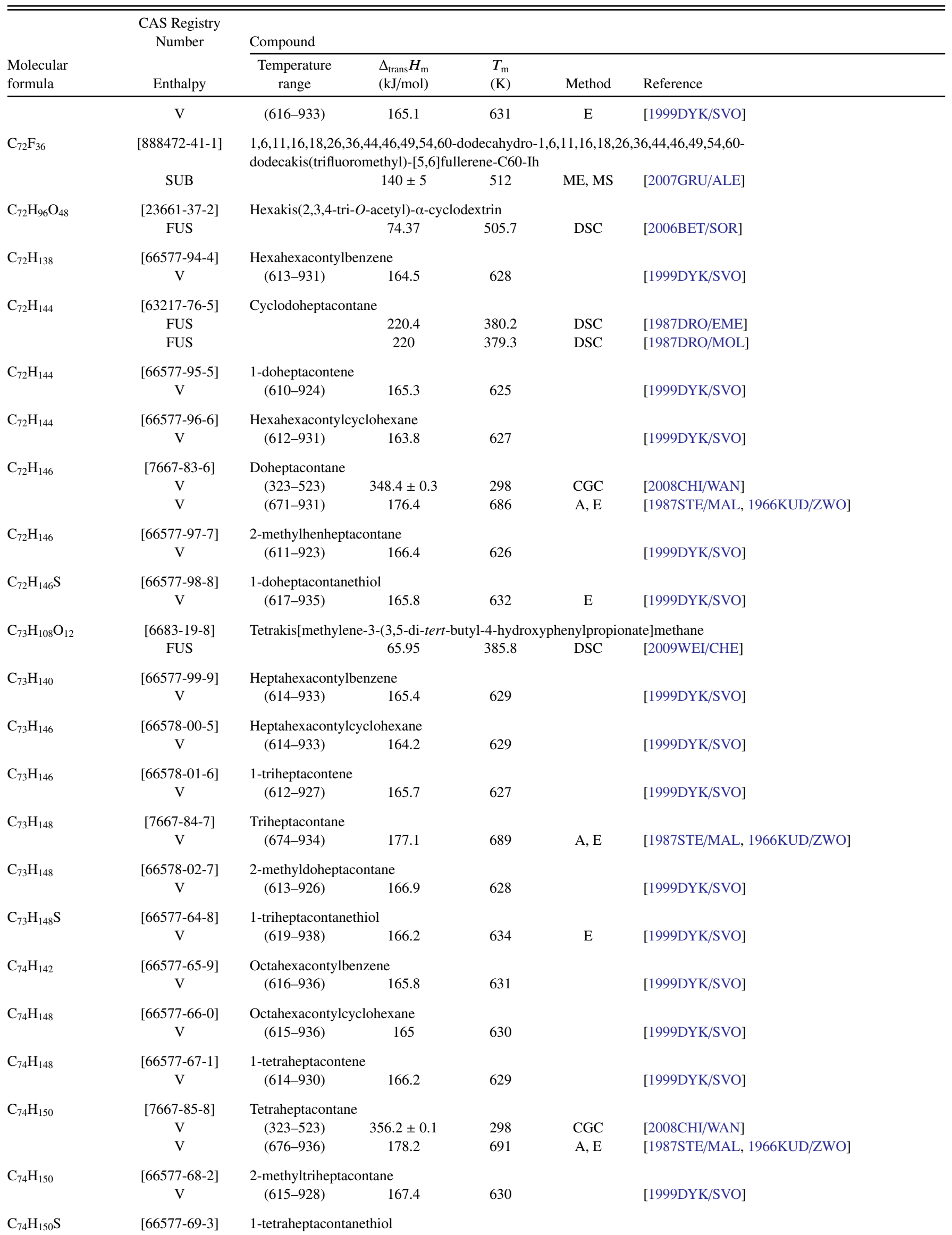


TABLE 16. Phase change enthalpies of $\mathrm{C}_{50}$ to $\mathrm{C}_{192}$ organic compounds-Continued

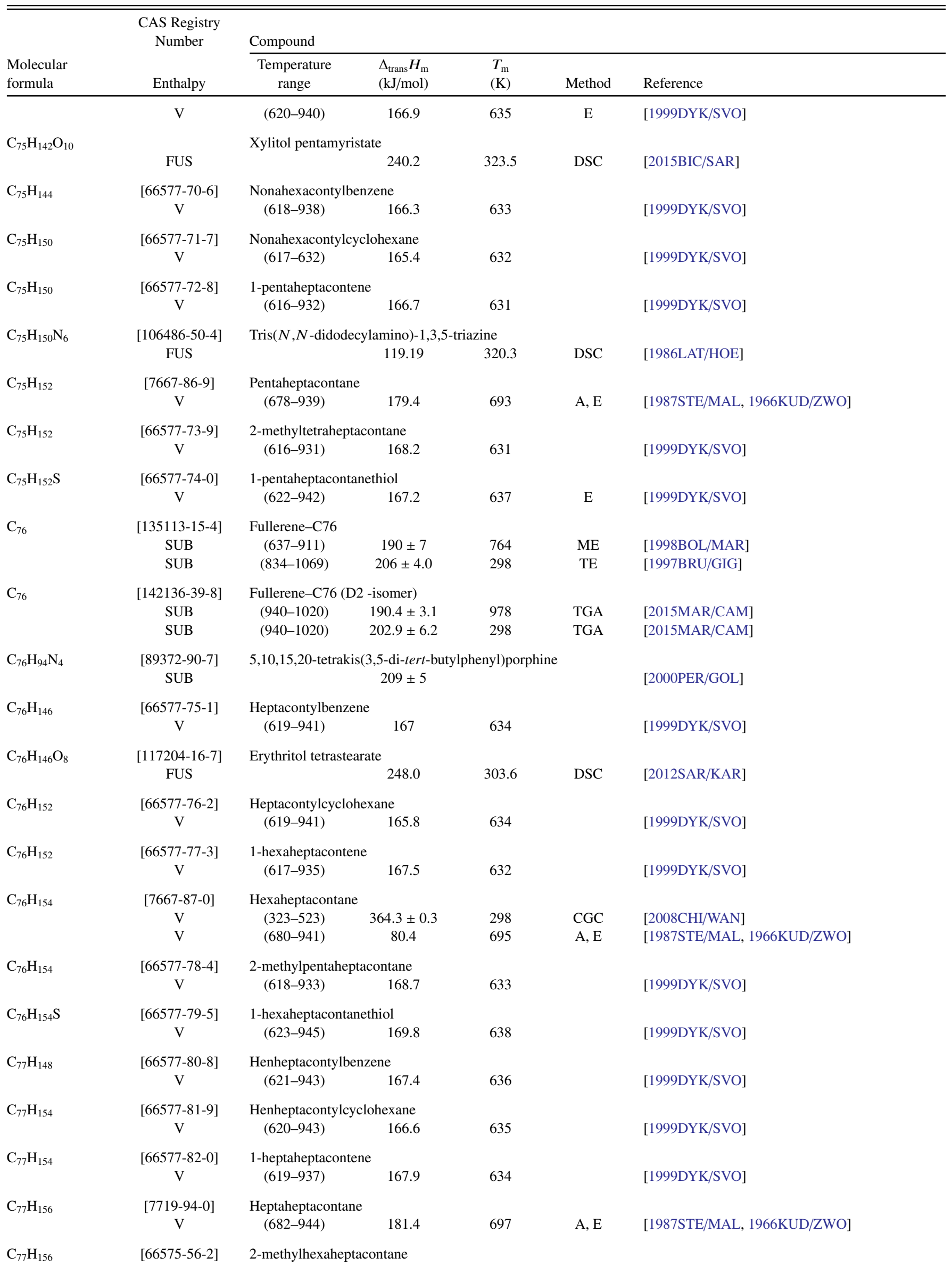


TABLE 16. Phase change enthalpies of $\mathrm{C}_{50}$ to $\mathrm{C}_{192}$ organic compounds-Continued

\begin{tabular}{|c|c|c|c|c|c|c|}
\hline \multirow[b]{2}{*}{$\begin{array}{l}\text { Molecular } \\
\text { formula }\end{array}$} & \multirow{2}{*}{$\begin{array}{l}\text { CAS Registry } \\
\text { Number } \\
\text { Enthalpy }\end{array}$} & \multicolumn{5}{|l|}{ Compound } \\
\hline & & $\begin{array}{l}\text { Temperature } \\
\text { range }\end{array}$ & $\begin{array}{l}\Delta_{\text {trans }} H_{\mathrm{m}} \\
(\mathrm{kJ} / \mathrm{mol})\end{array}$ & $\begin{array}{l}T_{\mathrm{m}} \\
(\mathrm{K})\end{array}$ & Method & Reference \\
\hline & $\mathrm{V}$ & $(620-936)$ & 169.1 & 635 & & [1999DYK/SVO] \\
\hline $\mathrm{C}_{77} \mathrm{H}_{156} \mathrm{~S}$ & $\begin{array}{c}{[66575-57-3]} \\
\mathrm{V}\end{array}$ & $\begin{array}{l}\text { 1-heptaheptaco } \\
\quad(625-947)\end{array}$ & $\begin{array}{l}\text { nethiol } \\
\qquad 168.2\end{array}$ & 640 & $\mathrm{E}$ & [1999DYK/SVO] \\
\hline $\mathrm{C}_{78} \mathrm{H}_{108}$ & $\begin{array}{c}{[125594-11-8]} \\
\text { FUS }\end{array}$ & $2,3,6,7,10,11-h$ & $\begin{array}{l}\text { kis(1-decynyl) } \\
63\end{array}$ & $\begin{array}{r}\text { nylene } \\
314.2\end{array}$ & DSC & [1996DOM/HEA, 1990PRA/KOH] \\
\hline $\mathrm{C}_{78} \mathrm{H}_{150}$ & $\begin{array}{c}{[66327-30-8]} \\
V\end{array}$ & $\begin{array}{l}\text { Doheptacontyl } \\
\quad(622-945)\end{array}$ & zene 167.3 & 637 & & [1999DYK/SVO] \\
\hline $\mathrm{C}_{78} \mathrm{H}_{156}$ & $\begin{array}{c}{[66327-32-0]} \\
\mathrm{V}\end{array}$ & $\begin{array}{l}\text { 1-octaheptacon } \\
\quad(621-940)\end{array}$ & 168.3 & 636 & & [1999DYK/SVO] \\
\hline $\mathrm{C}_{78} \mathrm{H}_{158}$ & $\begin{array}{c}\text { [7719-85-9] } \\
\mathrm{V} \\
\mathrm{V}\end{array}$ & $\begin{array}{l}\text { Octaheptacont } \\
\quad(638-691) \\
(685-946)\end{array}$ & $\begin{array}{c}372.1 \pm 3.7 \\
181.8\end{array}$ & $\begin{array}{l}298 \\
700\end{array}$ & $\begin{array}{l}\mathrm{CGC} \\
\mathrm{A}, \mathrm{E}\end{array}$ & $\begin{array}{l}\text { [2008CHI/LIP] } \\
\text { [1987STE/MAL, 1966KUD/ZWO] }\end{array}$ \\
\hline $\mathrm{C}_{78} \mathrm{H}_{158}$ & $\begin{array}{c}{[66327-33-1]} \\
\mathrm{V}\end{array}$ & $\begin{array}{l}\text { 2-methylheptal } \\
\text { (621-939) }\end{array}$ & $\begin{array}{l}\text { acontane } \\
169.9\end{array}$ & 636 & & [1999DYK/SVO] \\
\hline $\mathrm{C}_{78} \mathrm{H}_{158} \mathrm{~S}$ & $\begin{array}{c}{[66375-13-1]} \\
V\end{array}$ & $\begin{array}{l}\text { 1-octaheptacon } \\
\quad(626-949)\end{array}$ & $\begin{array}{l}\text { ethiol } \\
\qquad 168.8\end{array}$ & 641 & $\mathrm{E}$ & [1999DYK/SVO] \\
\hline $\mathrm{C}_{79} \mathrm{H}_{152}$ & $\begin{array}{c}{[66327-34-2]} \\
V\end{array}$ & $\begin{array}{l}\text { Triheptacontyl } \\
\text { (623-947) }\end{array}$ & 168 & 638 & & [1999DYK/SVO] \\
\hline $\mathrm{C}_{79} \mathrm{H}_{160}$ & $\begin{array}{c}{[66327-37-5]} \\
\mathrm{V}\end{array}$ & $\begin{array}{l}\text { 2-methyloctahe } \\
(622-940)\end{array}$ & $\begin{array}{l}\text { contane } \\
167.8\end{array}$ & 637 & & [1999DYK/SVO] \\
\hline $\mathrm{C}_{79} \mathrm{H}_{16} \mathrm{OS}$ & $\begin{array}{c}{[66327-38-6]} \\
V\end{array}$ & $\begin{array}{l}\text { 1-nonaheptaco } \\
\quad(628-952)\end{array}$ & $\begin{array}{l}\text { nethiol } \\
169.1\end{array}$ & 643 & $\mathrm{E}$ & [1999DYK/SVO] \\
\hline $\mathrm{C}_{80} \mathrm{H}_{154}$ & $\begin{array}{c}{[66327-39-7]} \\
V\end{array}$ & $\begin{array}{l}\text { Tetraheptacont } \\
\quad(625-949)\end{array}$ & $\begin{array}{l}\text { enzene } \\
\quad 168.4\end{array}$ & 640 & & [1999DYK/SVO] \\
\hline $\mathrm{C}_{80} \mathrm{H}_{160}$ & $\begin{array}{c}{[66327-40-0]} \\
\mathrm{V}\end{array}$ & $\begin{array}{l}\text { 1-octacontene } \\
\quad(624-945)\end{array}$ & 169.4 & 639 & & [1999DYK/SVO] \\
\hline $\mathrm{C}_{80} \mathrm{H}_{160}$ & $\begin{array}{c}{[66327-41-1]} \\
V\end{array}$ & $\begin{array}{l}\text { Tetraheptacont } \\
\quad(625-950)\end{array}$ & $\begin{array}{c}\text { clohexane } \\
168\end{array}$ & 640 & & [1999DYK/SVO] \\
\hline $\mathrm{C}_{80} \mathrm{H}_{162}$ & $\begin{array}{c}{[7667-88-1]} \\
\mathrm{V} \\
\mathrm{V}\end{array}$ & $\begin{array}{r}\text { Octacontane } \\
(638-691) \\
(689-951)\end{array}$ & $\begin{array}{l}379.6 \pm 3.8 \\
183.6\end{array}$ & $\begin{array}{l}298 \\
704\end{array}$ & $\begin{array}{l}\mathrm{CGC} \\
\mathrm{A}, \mathrm{E}\end{array}$ & $\begin{array}{l}\text { [2008CHI/LIP] } \\
\text { [1987STE/MAL, 1966KUD/ZWO] }\end{array}$ \\
\hline $\mathrm{C}_{80} \mathrm{H}_{162}$ & $\begin{array}{c}{[66327-42-2]} \\
V\end{array}$ & $\begin{array}{l}\text { 2-methylnonah } \\
\text { (624-943) }\end{array}$ & $\begin{array}{l}\text { acontane } \\
170.2\end{array}$ & 639 & & [1999DYK/SVO] \\
\hline $\mathrm{C}_{80} \mathrm{H}_{162} \mathrm{~S}$ & $\begin{array}{c}{[66327-43-3]} \\
\mathrm{V}\end{array}$ & $\begin{array}{l}\text { 1-octacontanet } \\
\quad(629-954)\end{array}$ & 169.6 & 644 & $\mathrm{E}$ & [1999DYK/SVO] \\
\hline $\mathrm{C}_{81} \mathrm{H}_{156}$ & $\begin{array}{c}{[66327-44-4]} \\
V\end{array}$ & $\begin{array}{l}\text { Pentaheptacont } \\
\quad(636-952)\end{array}$ & $\begin{array}{l}\text { enzene } \\
\quad 169.1\end{array}$ & 641 & & [1999DYK/SVO] \\
\hline
\end{tabular}


TABLE 16. Phase change enthalpies of $\mathrm{C}_{50}$ to $\mathrm{C}_{192}$ organic compounds-Continued

\begin{tabular}{|c|c|c|c|c|c|c|}
\hline \multirow[b]{2}{*}{$\begin{array}{l}\text { Molecular } \\
\text { formula }\end{array}$} & \multirow{2}{*}{$\begin{array}{l}\text { CAS Registry } \\
\text { Number } \\
\text { Enthalpy }\end{array}$} & \multicolumn{5}{|l|}{ Compound } \\
\hline & & $\begin{array}{l}\text { Temperature } \\
\text { range }\end{array}$ & $\begin{array}{l}\Delta_{\text {trans }} H_{\mathrm{m}} \\
(\mathrm{kJ} / \mathrm{mol})\end{array}$ & $\begin{array}{l}T_{\mathrm{m}} \\
(\mathrm{K})\end{array}$ & Method & Reference \\
\hline & $\mathrm{V}$ & $(625-946)$ & 169.3 & 640 & & [1999DYK/SVO] \\
\hline $\mathrm{C}_{81} \mathrm{H}_{162}$ & $\begin{array}{c}{[66327-46-6]} \\
V\end{array}$ & $\begin{array}{l}\text { Pentaheptacontylc } \\
\quad(627-52)\end{array}$ & $\begin{array}{c}\text { clohexane } \\
168.4\end{array}$ & 642 & & [1999DYK/SVO] \\
\hline $\mathrm{C}_{81} \mathrm{H}_{164}$ & $\begin{array}{c}{[66327-47-7]} \\
V\end{array}$ & $\begin{array}{l}\text { 2-methyloctancon } \\
\text { (625-945) }\end{array}$ & 17Q.9 & 640 & & [1999DYK/SVO] \\
\hline $\mathrm{C}_{81} \mathrm{H}_{164} \mathrm{~S}$ & $\begin{array}{c}{[66327-48-8]} \\
\mathrm{V}\end{array}$ & $\begin{array}{l}\text { 1-henoctacontanet } \\
\quad(630-955)\end{array}$ & iol 169.4 & 645 & $\mathrm{E}$ & [1999DYK/SVO] \\
\hline $\mathrm{C}_{82} \mathrm{H}_{164}$ & $\begin{array}{c}{[66327-09-1]} \\
\mathrm{V}\end{array}$ & $\begin{array}{l}\text { Hexaheptacontylc } \\
\quad(627-954)\end{array}$ & $\begin{array}{r}\text { clohexane } \\
168.5\end{array}$ & 642 & & [1999DYK/SVO] \\
\hline $\mathrm{C}_{82} \mathrm{H}_{166}$ & $\begin{array}{c}\text { [7719-95-1] } \\
\text { V } \\
\text { V }\end{array}$ & $\begin{array}{l}\text { Dooctacontane } \\
(638-691) \\
(693-955)\end{array}$ & $\begin{array}{c}387.2 \pm 3.8 \\
185.3\end{array}$ & $\begin{array}{l}298 \\
708\end{array}$ & $\begin{array}{l}\text { CGC } \\
\mathrm{A}, \mathrm{E}\end{array}$ & $\begin{array}{l}\text { [2008CHI/LIP] } \\
\text { [1987STE/MAL, 1966KUD/ZWO] }\end{array}$ \\
\hline $\mathrm{C}_{82} \mathrm{H}_{166}$ & $\begin{array}{c}{[66327-10-4]} \\
\mathrm{V}\end{array}$ & $\begin{array}{l}\text { 2-methylhenoctac } \\
\text { (627-947) }\end{array}$ & $\begin{array}{l}\text { ntane } \\
\quad 171.3\end{array}$ & 642 & & [1999DYK/SVO] \\
\hline $\mathrm{C}_{82} \mathrm{H}_{166} \mathrm{~S}$ & $\begin{array}{c}{[66327-11-5]} \\
\mathrm{V}\end{array}$ & $\begin{array}{l}\text { 1-dooctacontaneth } \\
\quad(631-57)\end{array}$ & 170 & 646 & $\mathrm{E}$ & [1999DYK/SVO] \\
\hline $\mathrm{C}_{83} \mathrm{H}_{168}$ & $\begin{array}{c}{[7667-90-5]} \\
V\end{array}$ & $\begin{array}{l}\text { Trioctacontane } \\
\quad(694-957)\end{array}$ & 186.5 & 709 & $\mathrm{~A}, \mathrm{E}$ & [1987STE/MAL, 1966KUD/ZWO] \\
\hline $\mathrm{C}_{83} \mathrm{H}_{168}$ & $\begin{array}{c}{[66327-15-9]} \\
\mathrm{V}\end{array}$ & $\begin{array}{l}\text { 2-methyldooctaco } \\
\text { (628-949) }\end{array}$ & $\stackrel{\operatorname{tane}}{171.1}$ & 643 & & [1999DYK/SVO] \\
\hline $\mathrm{C}_{83} \mathrm{H}_{168} \mathrm{~S}$ & $\begin{array}{c}{[66327-16-0]} \\
\mathrm{V}\end{array}$ & $\begin{array}{l}\text { 1-trioctacontaneth } \\
\quad(633-959)\end{array}$ & 170.2 & 648 & $\mathrm{E}$ & [1999DYK/SVO] \\
\hline $\mathrm{C}_{84}$ & $\begin{array}{c}\text { [135113-16-5] } \\
\text { SUB } \\
\text { SUB } \\
\text { SUB } \\
\text { SUB }\end{array}$ & $\begin{array}{c}\text { Fullerene-C84 } \\
(1053-1153) \\
(1053-1153) \\
(658-980) \\
(920-1190)\end{array}$ & $\begin{array}{c}205.4 \pm 1.4 \\
220.4 \pm 3.0 \\
202 \pm 4.0 \\
210 \pm 6\end{array}$ & $\begin{array}{l}1115 \\
298 \\
853 \\
950\end{array}$ & $\begin{array}{c}\text { TGA } \\
\text { TGA } \\
\text { ME } \\
\text { TE }\end{array}$ & $\begin{array}{l}\text { [2015MAR/CAM] } \\
{[2015 \mathrm{MAR} / \mathrm{CAM}]} \\
{[1998 \mathrm{BOL} / \mathrm{MAR} 2]} \\
{[1997 \mathrm{PIA} / \mathrm{PAL}]}\end{array}$ \\
\hline $\mathrm{C}_{84} \mathrm{H}_{112} \mathrm{O}_{56}$ & $\begin{array}{l}{[23739-88-0]} \\
\text { FUS }\end{array}$ & Heptakis(2,3,6-tri & $\begin{array}{l}\text {-acetyl)- } \beta \text {-cy } \\
\quad 82.73\end{array}$ & $\begin{array}{l}\text { xtrin } \\
491.7\end{array}$ & DSC & [2006BET/SOR] \\
\hline $\mathrm{C}_{84} \mathrm{H}_{162}$ & $\begin{array}{c}{[66327-17-1]} \\
\mathrm{V}\end{array}$ & $\begin{array}{l}\text { Octaheptacontylb } \\
\quad(630-957)\end{array}$ & $\begin{array}{l}\text { Izene } \\
\quad 169.9\end{array}$ & 645 & & [1999DYK/SVO] \\
\hline $\mathrm{C}_{84} \mathrm{H}_{168}$ & $\begin{array}{c}{[66327-18-2]} \\
V\end{array}$ & $\begin{array}{l}\text { Octaheptacontylc } \\
\quad(630-958)\end{array}$ & $\begin{array}{r}\text { lohexane } \\
169.5\end{array}$ & 645 & & [1999DYK/SVO] \\
\hline $\mathrm{C}_{84} \mathrm{H}_{168}$ & $\begin{array}{c}{[66327-19-3]} \\
\mathrm{V}\end{array}$ & $\begin{array}{l}\text { 1-tetraoctaconten } \\
\quad(629-953)\end{array}$ & 170.1 & 644 & & [1999DYK/SVO] \\
\hline $\mathrm{C}_{84} \mathrm{H}_{170}$ & $\begin{array}{c}{[7667-91-6]} \\
\mathrm{V} \\
\mathrm{V}\end{array}$ & $\begin{array}{l}\text { Tetraoctacontane } \\
(638-691) \\
(696-960)\end{array}$ & $\begin{array}{c}394.0 \pm 3.9 \\
187.3\end{array}$ & $\begin{array}{l}298 \\
711\end{array}$ & $\begin{array}{l}\mathrm{CGC} \\
\mathrm{A}, \mathrm{E}\end{array}$ & $\begin{array}{l}\text { [2008CHI/LIP] } \\
\text { [1987STE/MAL, 1966KUD/ZWO] }\end{array}$ \\
\hline
\end{tabular}


TABLE 16. Phase change enthalpies of $\mathrm{C}_{50}$ to $\mathrm{C}_{192}$ organic compounds-Continued

\begin{tabular}{|c|c|c|c|c|c|c|}
\hline \multirow[b]{2}{*}{$\begin{array}{l}\text { Molecular } \\
\text { formula }\end{array}$} & \multirow{2}{*}{$\begin{array}{l}\text { CAS Registry } \\
\text { Number } \\
\text { Enthalpy }\end{array}$} & \multicolumn{5}{|l|}{ Compound } \\
\hline & & $\begin{array}{c}\text { Temperature } \\
\text { range }\end{array}$ & $\begin{array}{l}\Delta_{\text {trans }} H_{\mathrm{m}} \\
(\mathrm{kJ} / \mathrm{mol})\end{array}$ & $\begin{array}{l}T_{\mathrm{m}} \\
(\mathrm{K})\end{array}$ & Method & Reference \\
\hline $\mathrm{C}_{84} \mathrm{H}_{170}$ & $\begin{array}{c}{[66327-20-6]} \\
\mathrm{V}\end{array}$ & $\begin{array}{l}\text { 2-methyltrioctaconta } \\
\text { (629-951) }\end{array}$ & $\begin{array}{l}\text { ane } \\
171.8\end{array}$ & 644 & & [1999DYK/SVO] \\
\hline $\mathrm{C}_{84} \mathrm{H}_{170} \mathrm{~S}$ & $\begin{array}{c}{[66327-21-7]} \\
\mathrm{V}\end{array}$ & $\begin{array}{l}\text { 1-tetraoctacontanethi } \\
\quad(634-962)\end{array}$ & iol 170.8 & 649 & $\mathrm{E}$ & [1999DYK/SVO] \\
\hline $\mathrm{C}_{85} \mathrm{H}_{162} \mathrm{O}_{10}$ & $\begin{array}{c}{[1426298-23-8]} \\
\text { FUS }\end{array}$ & Xylitol pentaplamitat & 228.6 & 291.9 & DSC & [2012BIC/SAR] \\
\hline $\mathrm{C}_{85} \mathrm{H}_{164}$ & $\begin{array}{c}{[66327-22-8]} \\
\mathrm{V}\end{array}$ & $\begin{array}{l}\text { Nonheptacontylbenz } \\
\quad(631-960)\end{array}$ & 170.6 & 646 & & [1999DYK/SVO] \\
\hline $\mathrm{C}_{85} \mathrm{H}_{170}$ & $\begin{array}{c}{[66327-23-9]} \\
\mathrm{V}\end{array}$ & $\begin{array}{l}\text { Nonheptacontylcyclo } \\
\quad(632-960)\end{array}$ & $\begin{array}{r}\text { ohexane } \\
169.8\end{array}$ & 647 & & [1999DYK/SVO] \\
\hline $\mathrm{C}_{85} \mathrm{H}_{172}$ & $\begin{array}{c}{[7719-96-2]} \\
\mathrm{V}\end{array}$ & $\begin{array}{l}\text { Pentaoctacontane } \\
\quad(698-962)\end{array}$ & 187.9 & 713 & $\mathrm{~A}, \mathrm{E}$ & [1987STE/MAL, 1966KUD/ZWO] \\
\hline $\mathrm{C}_{85} \mathrm{H}_{172}$ & $\begin{array}{c}{[66327-25-1]} \\
\mathrm{V}\end{array}$ & $\begin{array}{l}\text { 2-methyltetraoctacon } \\
\text { (631-953) }\end{array}$ & $\begin{array}{l}\text { ntane } \\
172.1\end{array}$ & 646 & & [1999DYK/SVO] \\
\hline $\mathrm{C}_{85} \mathrm{H}_{172} \mathrm{~S}$ & $\begin{array}{c}{[66327-26-2]} \\
\mathrm{V}\end{array}$ & $\begin{array}{l}\text { 1-pentaoctacontaneth } \\
\text { (634-963) }\end{array}$ & $\begin{array}{l}\text { hiol } \\
\qquad 170.8\end{array}$ & 649 & $\mathrm{E}$ & [1999DYK/SVO] \\
\hline $\mathrm{C}_{86} \mathrm{H}_{166}$ & $\begin{array}{c}{[66327-27-3]} \\
\mathrm{V}\end{array}$ & $\begin{array}{l}\text { Octacontylbenzene } \\
\quad(633-962)\end{array}$ & 170.9 & 648 & & [1999DYK/SVO] \\
\hline $\mathrm{C}_{86} \mathrm{H}_{172}$ & $\begin{array}{c}{[66327-28-4]} \\
\mathrm{V}\end{array}$ & $\begin{array}{l}\text { 1-hexaoctacontene } \\
\quad(631-957)\end{array}$ & 171.5 & 646 & & [1999DYK/SVO] \\
\hline $\mathrm{C}_{86} \mathrm{H}_{174} \mathrm{~S}$ & $\begin{array}{c}{[66326-89-4]} \\
\mathrm{V}\end{array}$ & $\begin{array}{l}\text { 1-hexaoctacontanethi } \\
\quad(636-965)\end{array}$ & iol 171.1 & 651 & $\mathrm{E}$ & [1999DYK/SVO] \\
\hline $\mathrm{C}_{87} \mathrm{H}_{168}$ & $\begin{array}{c}{[66326-90-7]} \\
V\end{array}$ & $\begin{array}{l}\text { Henoctacontylbenzer } \\
\quad(633-963)\end{array}$ & ne 171 & 648 & & [1999DYK/SVO] \\
\hline $\mathrm{C}_{87} \mathrm{H}_{174}$ & $\begin{array}{c}{[66326-91-8]} \\
\mathrm{V}\end{array}$ & $\begin{array}{l}\text { Henoctacontylcycloh } \\
\quad(633-964)\end{array}$ & $\begin{array}{l}\text { hexane } \\
\quad 170.6\end{array}$ & 648 & & [1999DYK/SVO] \\
\hline $\mathrm{C}_{87} \mathrm{H}_{174}$ & $\begin{array}{c}{[66326-92-9]} \\
V\end{array}$ & $\begin{array}{l}\text { 1-heptaoctacontene } \\
(633-959)\end{array}$ & 171.8 & 648 & & [1999DYK/SVO] \\
\hline $\mathrm{C}_{87} \mathrm{H}_{176}$ & $\begin{array}{c}{[7667-93-8]} \\
V\end{array}$ & $\begin{array}{l}\text { Heptaoctacontane } \\
\quad(702-966)\end{array}$ & 189.3 & 717 & $\mathrm{~A}, \mathrm{E}$ & [1987STE/MAL, 1966KUD/ZWO] \\
\hline $\mathrm{C}_{87} \mathrm{H}_{176}$ & $\begin{array}{c}{[66326-93-0]} \\
\mathrm{V}\end{array}$ & $\begin{array}{l}\text { 2-methylhexaoctacon } \\
\text { (633-957) }\end{array}$ & $\begin{array}{l}\text { ntane } \\
\quad 172.6\end{array}$ & 648 & & [1999DYK/SVO] \\
\hline $\mathrm{C}_{87} \mathrm{H}_{176} \mathrm{~S}$ & $\begin{array}{c}{[66326-94-1]} \\
\mathrm{V}\end{array}$ & $\begin{array}{l}\text { 1-heptaoctacontaneth } \\
\quad(637-967)\end{array}$ & $\begin{array}{l}\text { hiol } \\
\qquad 171.7\end{array}$ & 652 & E & [1999DYK/SVO] \\
\hline $\mathrm{C}_{84} \mathrm{H}_{144} \mathrm{O}_{6}$ & $\begin{array}{c}\text { [501447-88-7] } \\
\text { TRS } \\
\text { FUS }\end{array}$ & 2,3,6,7,10,11-hexakis & $\begin{array}{l}\mathrm{s}[[(2 \mathrm{R}, 4 \mathrm{R}, 6 \mathrm{P} \\
38 \\
5.2\end{array}$ & $\begin{array}{l}\text { 6-trime } \\
141.2 \\
237.2\end{array}$ & $\begin{array}{l}\text { ctyl]oxy]tr } \\
\text { DSC }\end{array}$ & $\begin{array}{l}\text { enylene } \\
\text { [2002SCH/LAS] }\end{array}$ \\
\hline $\mathrm{C}_{88} \mathrm{H}_{170}$ & $\begin{array}{c}{[66326-95-2]} \\
\mathrm{V}\end{array}$ & $\begin{array}{l}\text { Dooctacontylbenzen } \\
\quad(635-965)\end{array}$ & 171.3 & 650 & & [1999DYK/SVO] \\
\hline
\end{tabular}


TABLE 16. Phase change enthalpies of $\mathrm{C}_{50}$ to $\mathrm{C}_{192}$ organic compounds-Continued

\begin{tabular}{|c|c|c|c|c|c|c|}
\hline \multirow[b]{2}{*}{$\begin{array}{l}\text { Molecular } \\
\text { formula }\end{array}$} & \multirow{2}{*}{$\begin{array}{l}\text { CAS Registry } \\
\text { Number } \\
\text { Enthalpy }\end{array}$} & \multicolumn{5}{|l|}{ Compound } \\
\hline & & $\begin{array}{l}\text { Temperature } \\
\text { range }\end{array}$ & $\begin{array}{l}\Delta_{\text {trans }} H_{\mathrm{m}} \\
(\mathrm{kJ} / \mathrm{mol})\end{array}$ & $\begin{array}{l}T_{\mathrm{m}} \\
(\mathrm{K})\end{array}$ & Method & Reference \\
\hline & $\mathrm{V}$ & (635-966) & 170.8 & 650 & & [1999DYK/SVO] \\
\hline $\mathrm{C}_{88} \mathrm{H}_{176}$ & $\begin{array}{c}{[66326-97-4]} \\
\mathrm{V}\end{array}$ & $\begin{array}{l}\text { 1-octaoctacontene } \\
\quad(634-961)\end{array}$ & 172.5 & 649 & & [1999DYK/SVO] \\
\hline $\mathrm{C}_{88} \mathrm{H}_{178}$ & $\begin{array}{c}\text { [7667-94-9] } \\
\mathrm{V} \\
\mathrm{V}\end{array}$ & $\begin{array}{l}\text { Octaoctacontane } \\
\qquad(638-691) \\
(703-967)\end{array}$ & $\begin{array}{c}409.2 \pm 4.1 \\
190.4\end{array}$ & $\begin{array}{l}298 \\
718\end{array}$ & $\begin{array}{l}\mathrm{CGC} \\
\mathrm{A}, \mathrm{E}\end{array}$ & $\begin{array}{l}\text { [2008CHI/LIP] } \\
\text { [1987STE/MAL, 1966KUD/ZWO] }\end{array}$ \\
\hline $\mathrm{C}_{88} \mathrm{H}_{178}$ & $\begin{array}{c}{[66326-98-5]} \\
\mathrm{V}\end{array}$ & $\begin{array}{l}\text { 2-methylheptaocta } \\
\text { (634-959) }\end{array}$ & $\begin{array}{l}\text { ontane } \\
173.3\end{array}$ & 649 & & [1999DYK/SVO] \\
\hline $\mathrm{C}_{88} \mathrm{H}_{178} \mathrm{~S}$ & $\begin{array}{c}{[66326-99-6]} \\
V\end{array}$ & $\begin{array}{l}\text { 1-octacontanethiol } \\
\text { (639-969) }\end{array}$ & 171.9 & 654 & $\mathrm{E}$ & [1999DYK/SVO] \\
\hline $\mathrm{C}_{89} \mathrm{H}_{172}$ & $\begin{array}{c}{[66327-00-2]} \\
\mathrm{V}\end{array}$ & $\begin{array}{l}\text { Trioctacontylbenze } \\
\quad(636-967)\end{array}$ & 172 & 651 & & [1999DYK/SVO] \\
\hline $\mathrm{C}_{89} \mathrm{H}_{178}$ & $\begin{array}{c}{[66327-01-3]} \\
\mathrm{V}\end{array}$ & $\begin{array}{l}\text { 1-nonaoctacontene } \\
\quad(635-962)\end{array}$ & 172.3 & 650 & & [1999DYK/SVO] \\
\hline $\mathrm{C}_{89} \mathrm{H}_{178}$ & $\begin{array}{c}{[66327-02-4]} \\
\mathrm{V}\end{array}$ & $\begin{array}{l}\text { Trioctacontylcyclo } \\
\quad(636-968)\end{array}$ & $\begin{array}{l}\text { hexane } \\
171.5\end{array}$ & 651 & & [1999DYK/SVO] \\
\hline $\mathrm{C}_{89} \mathrm{H}_{180}$ & $\begin{array}{c}{[7719-76-8]} \\
\mathrm{V}\end{array}$ & $\begin{array}{l}\text { Nonaoctacontane } \\
\quad(705-969)\end{array}$ & 190.9 & 720 & $\mathrm{~A}, \mathrm{E}$ & [1987STE/MAL, 1966KUD/ZWO] \\
\hline $\mathrm{C}_{89} \mathrm{H}_{180}$ & $\begin{array}{c}{[66327-03-5]} \\
\mathrm{V}\end{array}$ & $\begin{array}{l}\text { 2-methyloctaoctace } \\
\quad(636-962)\end{array}$ & $\begin{array}{l}\text { ntane } \\
173.6\end{array}$ & 651 & & [1999DYK/SVO] \\
\hline $\mathrm{C}_{89} \mathrm{H}_{180} \mathrm{~S}$ & $\begin{array}{c}{[66327-04-6]} \\
\mathrm{V}\end{array}$ & $\begin{array}{l}\text { 1-nonaoctacontane } \\
\quad(639-970)\end{array}$ & $\begin{array}{l}\text { hiol } \\
\qquad 171.9\end{array}$ & 654 & $\mathrm{E}$ & [1999DYK/SVO] \\
\hline $\mathrm{C}_{90} \mathrm{H}_{174}$ & $\begin{array}{c}{[66327-05-7]} \\
\mathrm{V}\end{array}$ & $\begin{array}{l}\text { Tetraoctacontylben } \\
\quad(637-968)\end{array}$ & $\begin{array}{l}\text { zene } \\
171.7\end{array}$ & 652 & & [1999DYK/SVO] \\
\hline $\mathrm{C}_{90} \mathrm{H}_{182}$ & $\begin{array}{c}{[7667-51-8]} \\
\mathrm{V} \\
\mathrm{V}\end{array}$ & $\begin{array}{c}\text { Nonacontane } \\
\quad(638-691) \\
(707-971)\end{array}$ & $\begin{array}{c}416.4 \pm 4.3 \\
191.6\end{array}$ & $\begin{array}{l}298 \\
722\end{array}$ & $\begin{array}{l}\mathrm{CGC} \\
\mathrm{A}, \mathrm{E}\end{array}$ & $\begin{array}{l}\text { [2008CHI/LIP] } \\
\text { [1987STE/MAL, 1966KUD/ZWO] }\end{array}$ \\
\hline $\mathrm{C}_{91} \mathrm{H}_{184}$ & $\begin{array}{c}{[7719-97-3]} \\
\mathrm{V}\end{array}$ & $\begin{array}{l}\text { Hennonacontane } \\
\quad(708-973)\end{array}$ & 192.5 & 723 & $\mathrm{~A}, \mathrm{E}$ & [1987STE/MAL, 1966KUD/ZWO] \\
\hline $\mathrm{C}_{92} \mathrm{H}_{186}$ & $\begin{array}{c}{[7667-95-0]} \\
\mathrm{V} \\
\mathrm{V}\end{array}$ & $\begin{array}{l}\text { Dononacontane } \\
\quad(638-691) \\
(710-975)\end{array}$ & $\begin{array}{c}424.5 \pm 4.0 \\
193\end{array}$ & $\begin{array}{l}298 \\
725\end{array}$ & $\begin{array}{l}\mathrm{CGC} \\
\mathrm{A}, \mathrm{E}\end{array}$ & $\begin{array}{l}\text { [2008CHI/LIP] } \\
\text { [1987STE/MAL, 1966KUD/ZWO] }\end{array}$ \\
\hline $\mathrm{C}_{92} \mathrm{H}_{186} \mathrm{O}_{46}$ & FUS & 1, $\omega$-dimethoxypen & $\begin{array}{c}\operatorname{atetracosa}(\mathrm{ox} \\
374.8\end{array}$ & $\begin{array}{l}\text { ene) } \\
324.2\end{array}$ & DSC & [1996YAN/YU] \\
\hline $\mathrm{C}_{93} \mathrm{H}_{188}$ & $\begin{array}{c}{[7667-96-1]} \\
\mathrm{V}\end{array}$ & $\begin{array}{l}\text { Trinonacontane } \\
\quad(711-977)\end{array}$ & 194.1 & 726 & $\mathrm{~A}, \mathrm{E}$ & [1987STE/MAL, 1966KUD/ZWO] \\
\hline $\mathrm{C}_{94} \mathrm{H}_{190}$ & $\begin{array}{c}{[1574-32-9]} \\
\mathrm{V}\end{array}$ & $\begin{array}{l}\text { Tetranonacontane } \\
\quad(713-978)\end{array}$ & 194.5 & 728 & $\mathrm{~A}, \mathrm{E}$ & [1987STE/MAL, 1966KUD/ZWO] \\
\hline $\mathrm{C}_{95} \mathrm{H}_{182} \mathrm{O}_{10}$ & $\begin{array}{c}\text { [60869-87-6] } \\
\text { FUS }\end{array}$ & Xylitol pentasteara & 305.3 & 305.5 & $\mathrm{DSC}$ & {$[2012 \mathrm{BIC} / \mathrm{SAR}]$} \\
\hline $\mathrm{C}_{95} \mathrm{H}_{192}$ & $\begin{array}{c}{[7667-97-2]} \\
\mathrm{V}\end{array}$ & $\begin{array}{l}\text { Pentanonacontane } \\
\quad(714-980)\end{array}$ & 195.4 & 729 & $\mathrm{~A}, \mathrm{E}$ & [1987STE/MAL, 1966KUD/ZWO] \\
\hline $\mathrm{C}_{96} \mathrm{H}_{192}$ & $\begin{array}{c}\text { [63217-79-8] } \\
\text { FUS } \\
\text { FUS }\end{array}$ & Cyclohexanonacon & $\begin{array}{l}\text { tane } \\
302 \\
300\end{array}$ & $\begin{array}{c}389 \\
389.1\end{array}$ & $\begin{array}{l}\mathrm{DSC} \\
\mathrm{DSC}\end{array}$ & $\begin{array}{l}\text { [1987DRO/MOL] } \\
\text { [1987DRO/EME] }\end{array}$ \\
\hline $\mathrm{C}_{96} \mathrm{H}_{194}$ & $\begin{array}{c}{[7763-13-5]} \\
\mathrm{V}\end{array}$ & $\begin{array}{l}\text { Hexanonacontane } \\
\quad(716-982)\end{array}$ & 195.8 & 731 & $\mathrm{~A}, \mathrm{E}$ & [1987STE/MAL, 1966KUD/ZWO] \\
\hline $\mathrm{C}_{97} \mathrm{H}_{196}$ & $\begin{array}{c}{[7670-25-9]} \\
\mathrm{V}\end{array}$ & $\begin{array}{l}\text { Heptanonacontane } \\
\quad(717-983)\end{array}$ & 196.6 & 732 & $\mathrm{~A}, \mathrm{E}$ & [1987STE/MAL, 1966KUD/ZWO] \\
\hline
\end{tabular}


TABLE 16. Phase change enthalpies of $\mathrm{C}_{50}$ to $\mathrm{C}_{192}$ organic compounds-Continued

\begin{tabular}{|c|c|c|c|c|c|c|}
\hline \multirow[b]{2}{*}{$\begin{array}{l}\text { Molecular } \\
\text { formula }\end{array}$} & \multirow{2}{*}{$\begin{array}{c}\text { CAS Registry } \\
\text { Number } \\
\text { Enthalpy }\end{array}$} & \multicolumn{5}{|l|}{ Compound } \\
\hline & & $\begin{array}{l}\text { Temperature } \\
\text { range }\end{array}$ & $\begin{array}{l}\Delta_{\text {trans }} H_{\mathrm{m}} \\
(\mathrm{kJ} / \mathrm{mol}) \\
\end{array}$ & $\begin{array}{l}T_{\mathrm{m}} \\
(\mathrm{K}) \\
\end{array}$ & Method & Reference \\
\hline $\mathrm{C}_{98} \mathrm{H}_{198}$ & $\begin{array}{c}{[7670-26-0]} \\
\mathrm{V}\end{array}$ & $\begin{array}{l}\text { Octanonacontane } \\
\quad(719-985)\end{array}$ & 196.9 & 734 & $\mathrm{~A}, \mathrm{E}$ & [1987STE/MAL, 1966KUD/ZWO] \\
\hline $\mathrm{C}_{99} \mathrm{H}_{200}$ & $\begin{array}{c}{[7670-27-1]} \\
\mathrm{V}\end{array}$ & $\begin{array}{l}\text { Nonanonacontane } \\
\quad(720-986)\end{array}$ & 197.8 & 735 & $\mathrm{~A}, \mathrm{E}$ & [1987STE/MAL, 1966KUD/ZWO] \\
\hline $\mathrm{C}_{100} \mathrm{H}_{202}$ & $\begin{array}{c}\text { [6703-98-6] } \\
\text { TRS } \\
\text { FUS }\end{array}$ & Hectane & $\begin{array}{c}54.8 \\
331.8\end{array}$ & $\begin{array}{l}365.5 \\
338.5\end{array}$ & DSC & [1970HAY] \\
\hline & $\mathrm{V}$ & $(721-988)$ & 198.5 & 736 & $\mathrm{~A}, \mathrm{E}$ & [1987STE/MAL, 1966KUD/ZWO] \\
\hline $\mathrm{C}_{102} \mathrm{H}_{180} \mathrm{O}_{6}$ & $\begin{array}{c}\text { [501447-89-8] } \\
\text { TRS } \\
\text { FUS }\end{array}$ & 2,3,6,7,10,11-hexa & $\begin{array}{c}s[[(2 \mathrm{R}, 4 \mathrm{R}, \\
23.4 \\
6.6\end{array}$ & $\begin{array}{c}-2,4,6,8 \\
139.2 \\
236.2\end{array}$ & $\begin{array}{r}\text { nethyldec } \\
\text { DSC }\end{array}$ & $\begin{array}{l}\text { xy]triphenylene } \\
\text { [2002SCH/LAS] }\end{array}$ \\
\hline $\mathrm{C}_{114} \mathrm{H}_{218} \mathrm{O}_{12}$ & FUS & Galactitol hexastea & 447.1 & 321.0 & DSC & [2011SAR/BIC] \\
\hline $\mathrm{C}_{192} \mathrm{H}_{386}$ & $\begin{array}{l}\text { [96123-38-5] } \\
\text { FUS }\end{array}$ & $n$-dononacontahect & 661.1 & 399.1 & DSC & [1989STA/MAN] \\
\hline
\end{tabular}


TABle 17. Phase change enthalpies of organometallic and inorganic compounds

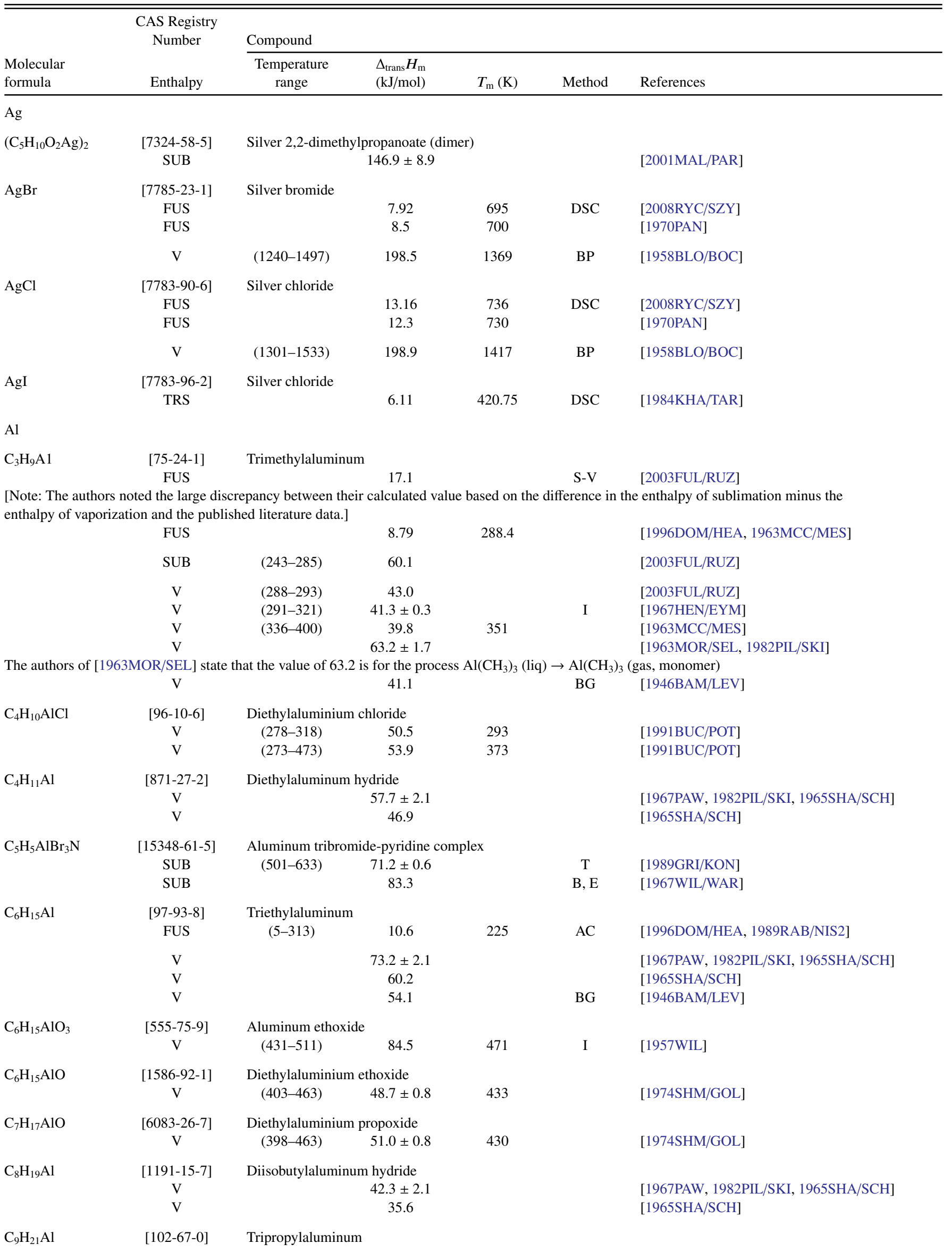


TABLE 17. Phase change enthalpies of organometallic and inorganic compounds-Continued

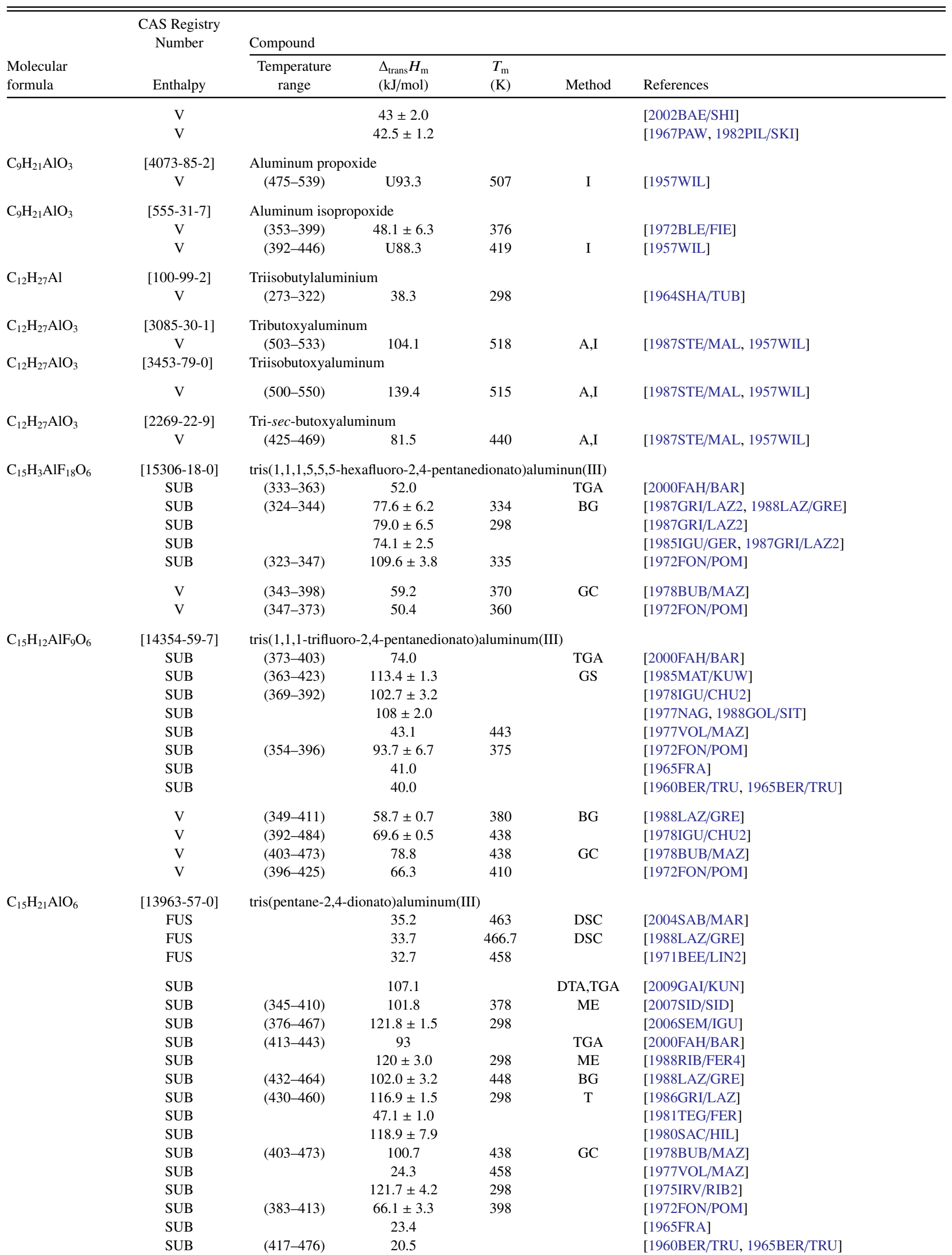


TABLE 17. Phase change enthalpies of organometallic and inorganic compounds-Continued

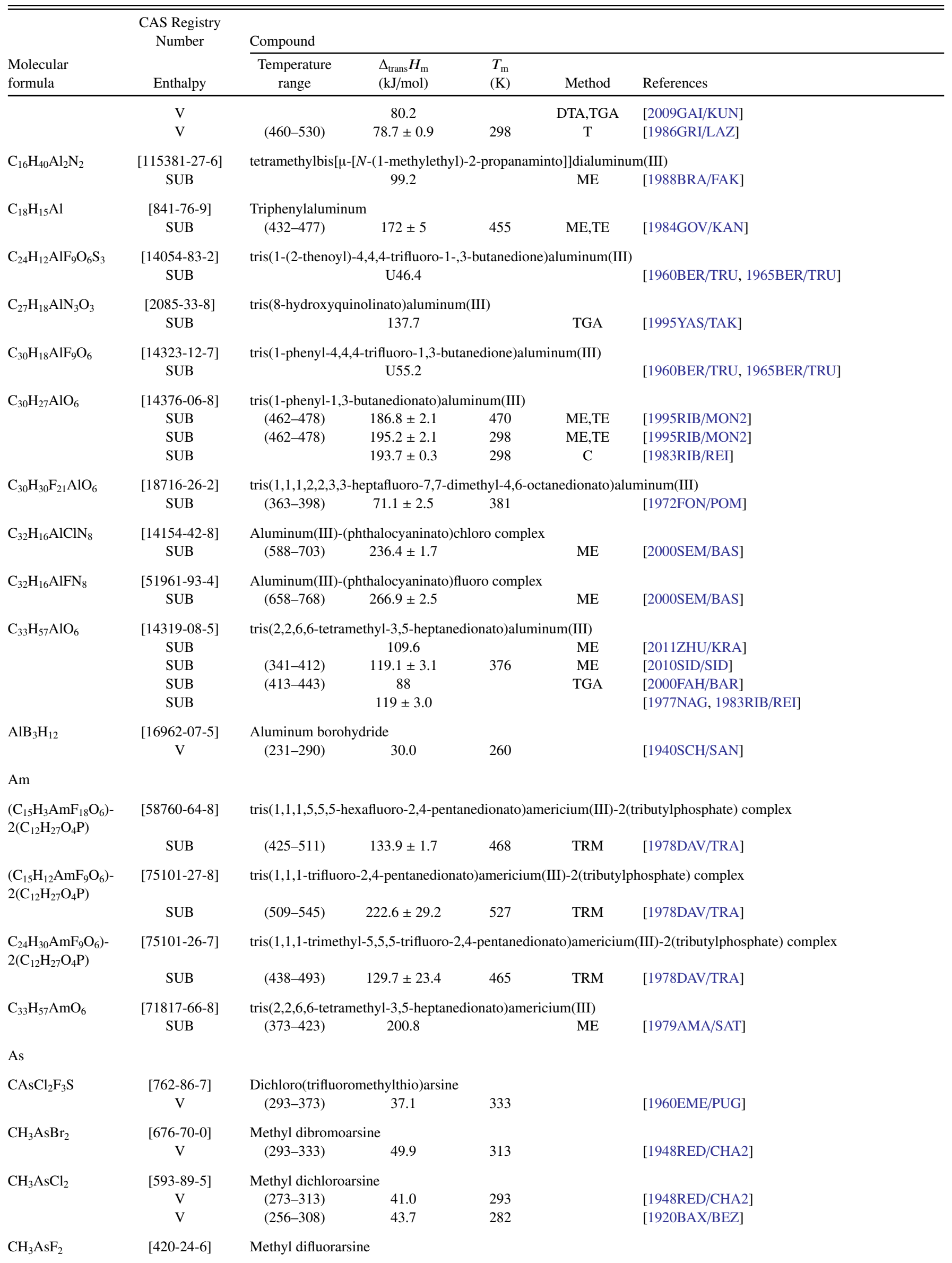


TABLE 17. Phase change enthalpies of organometallic and inorganic compounds-Continued

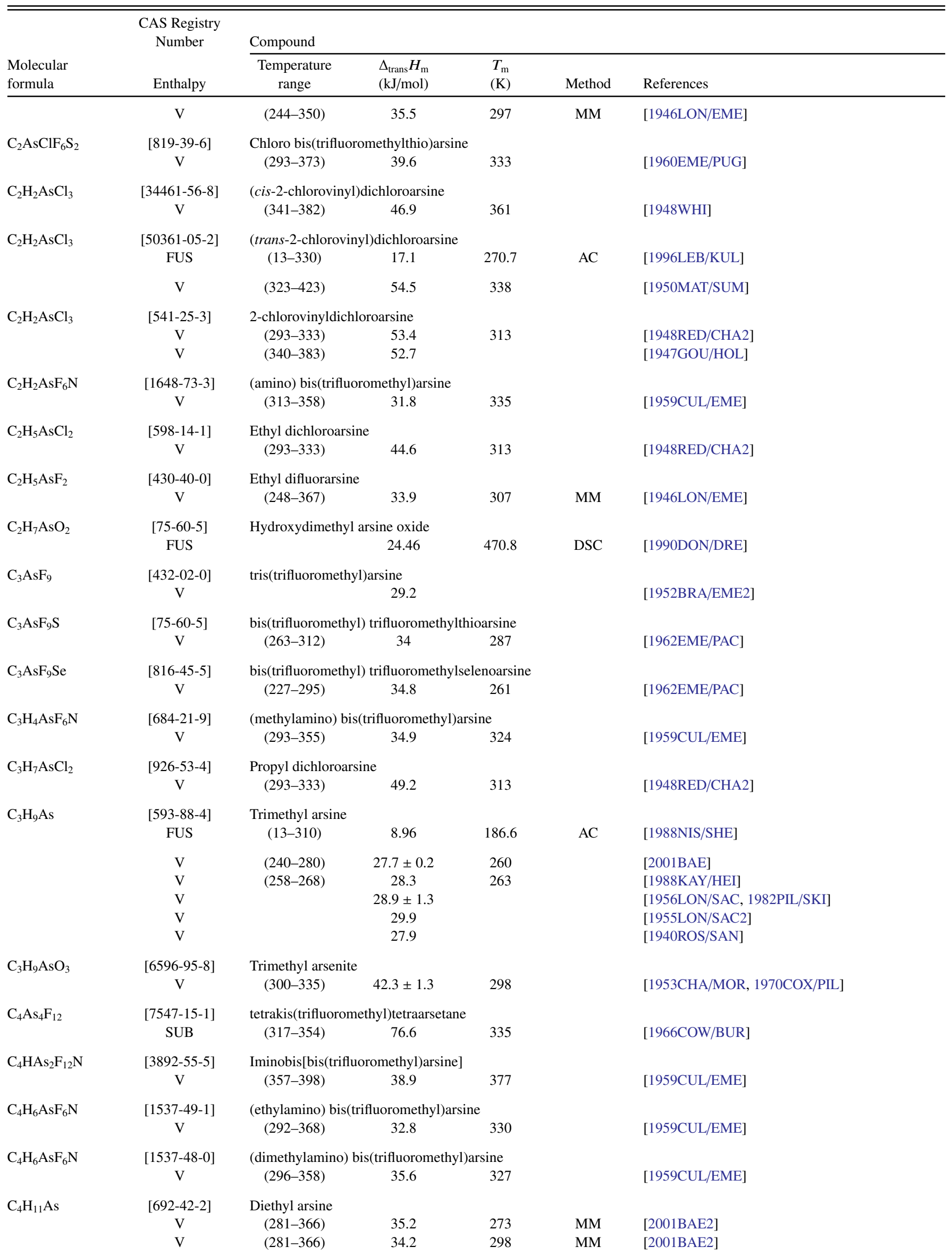


TABLE 17. Phase change enthalpies of organometallic and inorganic compounds-Continued

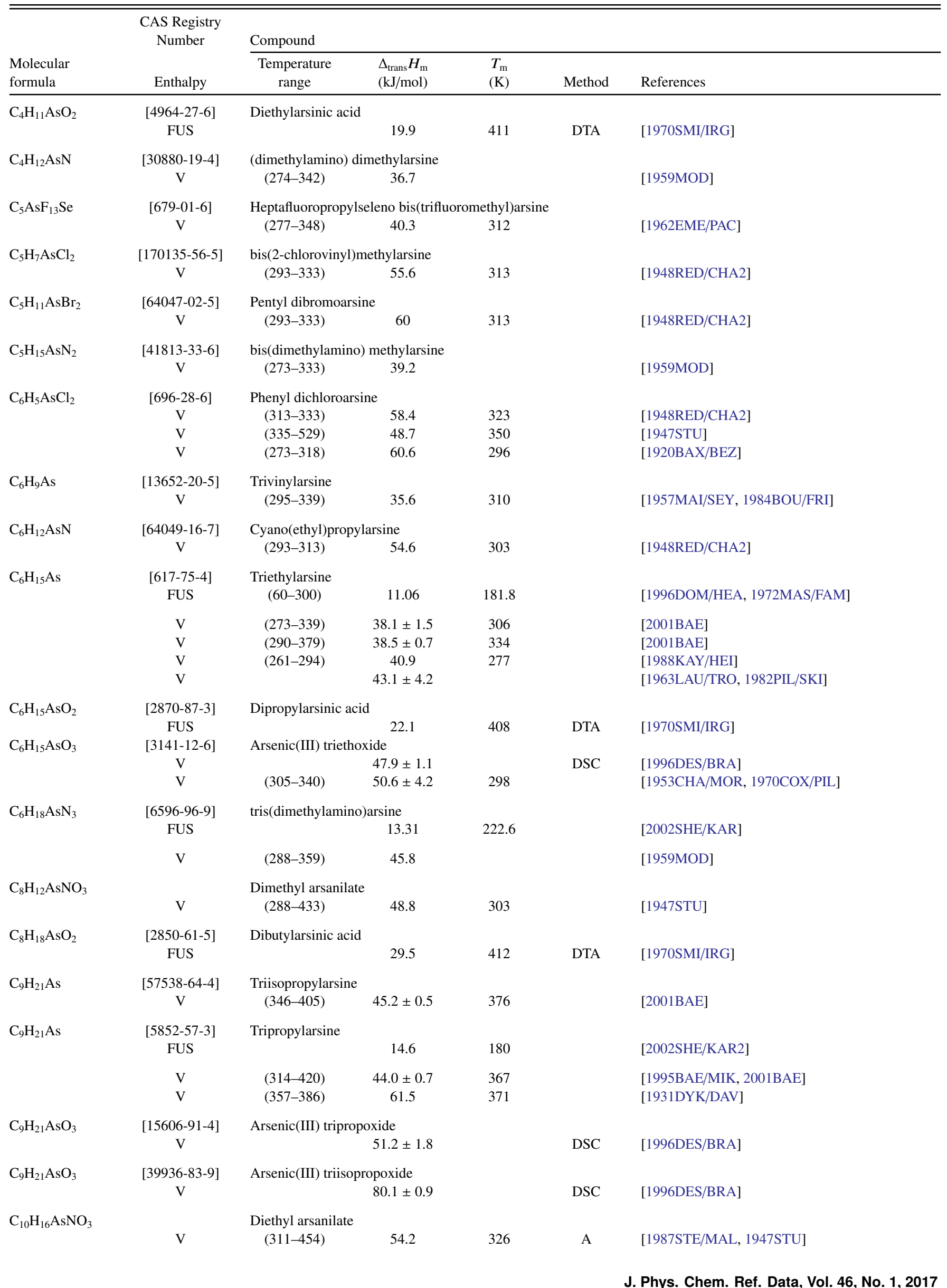


TABLE 17. Phase change enthalpies of organometallic and inorganic compounds-Continued

\begin{tabular}{|c|c|c|c|c|c|c|}
\hline \multirow[b]{2}{*}{$\begin{array}{l}\text { Molecular } \\
\text { formula }\end{array}$} & \multirow{2}{*}{$\begin{array}{l}\text { CAS Registry } \\
\text { Number } \\
\text { Enthalpy }\end{array}$} & \multicolumn{5}{|l|}{ Compound } \\
\hline & & $\begin{array}{c}\text { Temperature } \\
\text { range }\end{array}$ & $\begin{array}{l}\Delta_{\text {trans }} H_{\mathrm{m}} \\
(\mathrm{kJ} / \mathrm{mol})\end{array}$ & $\begin{array}{l}T_{\mathrm{m}} \\
(\mathrm{K})\end{array}$ & Method & References \\
\hline $\mathrm{C}_{12} \mathrm{H}_{10} \mathrm{AsCl}$ & $\begin{array}{c}{[712-48-1]} \\
\mathrm{V}\end{array}$ & $\begin{array}{l}\text { Diphenylchlor } \\
\quad(298-348)\end{array}$ & 63.0 & 323 & & [1920BAX/BEZ] \\
\hline $\mathrm{C}_{10} \mathrm{H}_{23} \mathrm{AsO}_{2}$ & $\begin{array}{c}\text { [4964-30-1] } \\
\text { FUS }\end{array}$ & Dipentyl arsen & 36 & 405 & DTA & [1970SMI/IRG] \\
\hline $\mathrm{C}_{12} \mathrm{H}_{27} \mathrm{AsO}_{2}$ & $\begin{array}{l}{[6727-92-0]} \\
\text { TRS } \\
\text { FUS }\end{array}$ & Dihexyl arseni & $\begin{array}{c}16.4 \\
24.35\end{array}$ & $\begin{array}{l}393 \\
405\end{array}$ & DTA & [1970SMI/IRG] \\
\hline $\mathrm{C}_{12} \mathrm{H}_{27} \mathrm{AsO}_{3}$ & $\begin{array}{c}{[3141-10-4]} \\
\mathrm{V}\end{array}$ & Arsenic(III) tri & $\begin{array}{l}\text { ide } \\
64.0 \pm 1.8\end{array}$ & & DSC & [1996DES/BRA] \\
\hline $\mathrm{C}_{12} \mathrm{H}_{27} \mathrm{AsO}_{3}$ & $\begin{array}{c}{[51587-28-1]} \\
\mathrm{V}\end{array}$ & Arsenic(III) tri & $\begin{array}{l}\text { toxide } \\
75.7 \pm 1.2\end{array}$ & & DSC & [1996DES/BRA] \\
\hline $\mathrm{C}_{13} \mathrm{H}_{10} \mathrm{AsN}$ & $\begin{array}{c}{[23525-22-6]} \\
\mathrm{V}\end{array}$ & $\begin{array}{l}\text { Diphenylarsine } \\
\quad(296-326)\end{array}$ & $\begin{array}{r}\text { onitrile } \\
84.6\end{array}$ & 311 & A & [1987STE/MAL] \\
\hline $\mathrm{C}_{14} \mathrm{H}_{31} \mathrm{AsO}_{2}$ & $\begin{array}{l}{[6757-53-5]} \\
\text { TRS } \\
\text { FUS }\end{array}$ & Diheptyl arsen & $\begin{array}{l}30.1 \\
20.3\end{array}$ & $\begin{array}{l}299 \\
389\end{array}$ & DTA & [1970SMI/IRG] \\
\hline $\mathrm{C}_{15} \mathrm{H}_{30} \mathrm{AsN}_{3} \mathrm{~S}_{6}$ & $\begin{array}{c}{[17767-20-3]} \\
\text { SUB }\end{array}$ & $\operatorname{tris}(N, N$-dieth & $\begin{array}{c}\text { hiocarbamate } \\
124 \pm 3\end{array}$ & $\begin{array}{l}\operatorname{iic}(\mathrm{III}) \\
298\end{array}$ & & [1987AIR/DES] \\
\hline $\mathrm{C}_{15} \mathrm{H}_{33} \mathrm{As}$ & $\begin{array}{c}{[5852-59-5]} \\
\mathrm{V}\end{array}$ & $\begin{array}{c}\text { Tripentylarsine } \\
\quad(408-466)\end{array}$ & 62.3 & 432 & & [1932JON/DYK] \\
\hline $\mathrm{C}_{18} \mathrm{AsF}_{15}$ & $\begin{array}{c}\text { [1259-34-3] } \\
\text { FUS }\end{array}$ & tris(pentafluorc & $\begin{array}{r}\text { y)arsine } \\
26.5\end{array}$ & 380.1 & DSC & [2008ZEL/CHU] \\
\hline $\mathrm{C}_{18} \mathrm{H}_{15} \mathrm{As}$ & $\begin{array}{c}{[603-32-7]} \\
\text { SUB }\end{array}$ & Triphenylarsin & $98.3 \pm 8.4$ & & & [1982PIL/SKI, 1964MOR/SEL] \\
\hline $\mathrm{C}_{18} \mathrm{H}_{15} \mathrm{AsO}$ & $\begin{array}{l}\mathrm{V} \\
{[1153-05-5]} \\
\text { SUB }\end{array}$ & $\begin{array}{l}(493-563) \\
\text { Triphenylarsin }\end{array}$ & $\begin{array}{l}75.7 \\
149.0 \pm 5.4\end{array}$ & 508 & A & $\begin{array}{l}\text { [1987STE/MAL, 1949FOR/BOW] } \\
\text { [1994LIE/MAR] }\end{array}$ \\
\hline $\mathrm{C}_{18} \mathrm{H}_{39} \mathrm{AsO}_{2}$ & $\begin{array}{l}\text { [6727-94-2] } \\
\text { TRS } \\
\text { FUS }\end{array}$ & Dinonyl arseni & $\begin{array}{l}24.3 \\
38.1\end{array}$ & $\begin{array}{l}383 \\
399\end{array}$ & DTA & [1970SMI/IRG] \\
\hline $\mathrm{C}_{19} \mathrm{H}_{37} \mathrm{AsO}_{7}$ & $\begin{array}{c}{[155325-38-5]} \\
\text { FUS }\end{array}$ & $(R)$-1,2-dicapry & $\begin{array}{l}\text { propyl-3-arss } \\
41.8\end{array}$ & $\begin{array}{l}\text { cid } \\
347.7\end{array}$ & DSC & [1993SER/SOT] \\
\hline $\mathrm{C}_{19} \mathrm{H}_{37} \mathrm{AsO}_{7}$ & $\begin{array}{c}\text { [155325-39-6] } \\
\text { FUS }\end{array}$ & $(S)$-1,2-dicapry & $\begin{array}{c}\text { propyl-3-ars } \\
37.66\end{array}$ & $\begin{array}{l}\text { cid } \\
346.7\end{array}$ & DSC & [1993SER/SOT] \\
\hline $\mathrm{C}_{2} \mathrm{H}_{43} \mathrm{AsO}_{2}$ & $\begin{array}{l}\text { [6727-95-3] } \\
\text { TRS } \\
\text { FUS }\end{array}$ & Di- $n$-decylarsi & $\begin{array}{r}24.5 \\
42.3\end{array}$ & $\begin{array}{l}380 \\
400\end{array}$ & DTA & [1970SMI/IRG] \\
\hline $\mathrm{C}_{21} \mathrm{H}_{42} \mathrm{AsN}_{3} \mathrm{~S}_{6}$ & $\begin{array}{c}{[86431-46-1]} \\
\text { SUB }\end{array}$ & tris(dipropyldit & $\begin{array}{l}\text { rbamate)ars } \\
145.1 \pm 5.3\end{array}$ & I) 298 & DSC,E & [1999NEV/GOU] \\
\hline $\mathrm{C}_{22} \mathrm{H}_{47} \mathrm{AsO}_{2}$ & $\begin{array}{l}\text { [6727-96-4] } \\
\text { TRS } \\
\text { FUS }\end{array}$ & Di- $n$-undecyl & $\begin{array}{l}\text { c acid } \\
30.1 \\
45.1\end{array}$ & $\begin{array}{l}384 \\
396\end{array}$ & DTA & [1970SMI/IRG] \\
\hline $\mathrm{C}_{23} \mathrm{H}_{45} \mathrm{AsO}_{7}$ & $\begin{array}{c}\text { [155325-40-9] } \\
\text { FUS }\end{array}$ & $(R)$-1,2-dicapr & $\begin{array}{l}\text { opyl-3-arsor } \\
68.2\end{array}$ & d 358.3 & DSC & [1993SER/SOT] \\
\hline $\mathrm{C}_{23} \mathrm{H}_{45} \mathrm{AsO}_{7}$ & $\begin{array}{c}{[155325-41-0]} \\
\text { FUS }\end{array}$ & $(S)$-1,2-dicapr & $\begin{array}{c}\text { opyl-3-arson } \\
54.39\end{array}$ & 358.5 & DSC & [1993SER/SOT] \\
\hline $\mathrm{C}_{24} \mathrm{H}_{51} \mathrm{AsO}_{2}$ & $\begin{array}{l}{[6727-97-5]} \\
\text { TRS } \\
\text { FUS }\end{array}$ & Di- $n$-dodecyl a & $\begin{array}{r}\text { c acid } \\
31.4 \\
49.4\end{array}$ & $\begin{array}{l}385 \\
398\end{array}$ & DTA & [1970SMI/IRG] \\
\hline $\mathrm{C}_{26} \mathrm{H}_{55} \mathrm{AsO}_{2}$ & [6727-98-6] & Di- $\boldsymbol{n}$-tridecyl a & c acid & & & \\
\hline
\end{tabular}


TABLE 17. Phase change enthalpies of organometallic and inorganic compounds-Continued

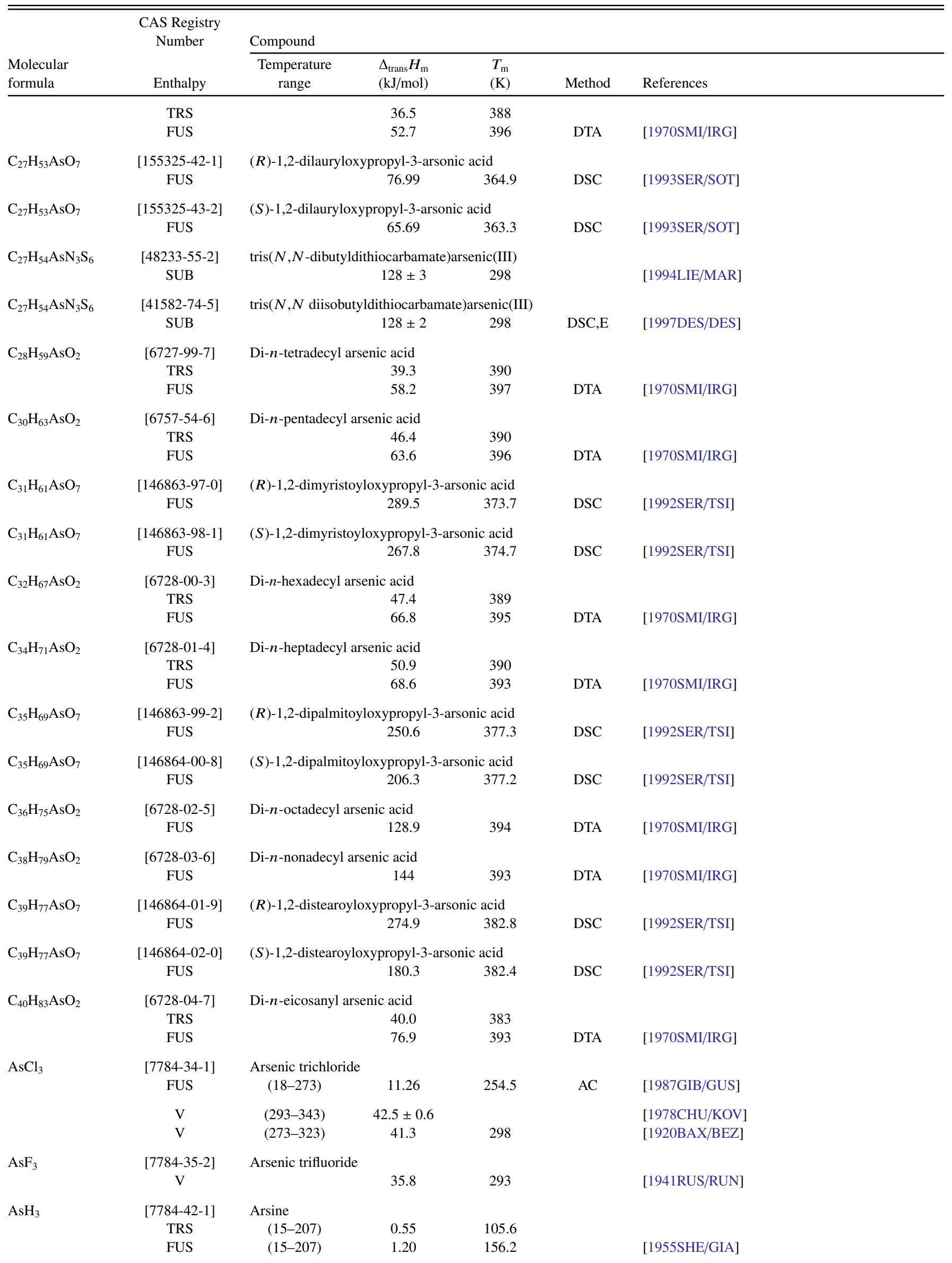


TABLE 17. Phase change enthalpies of organometallic and inorganic compounds-Continued

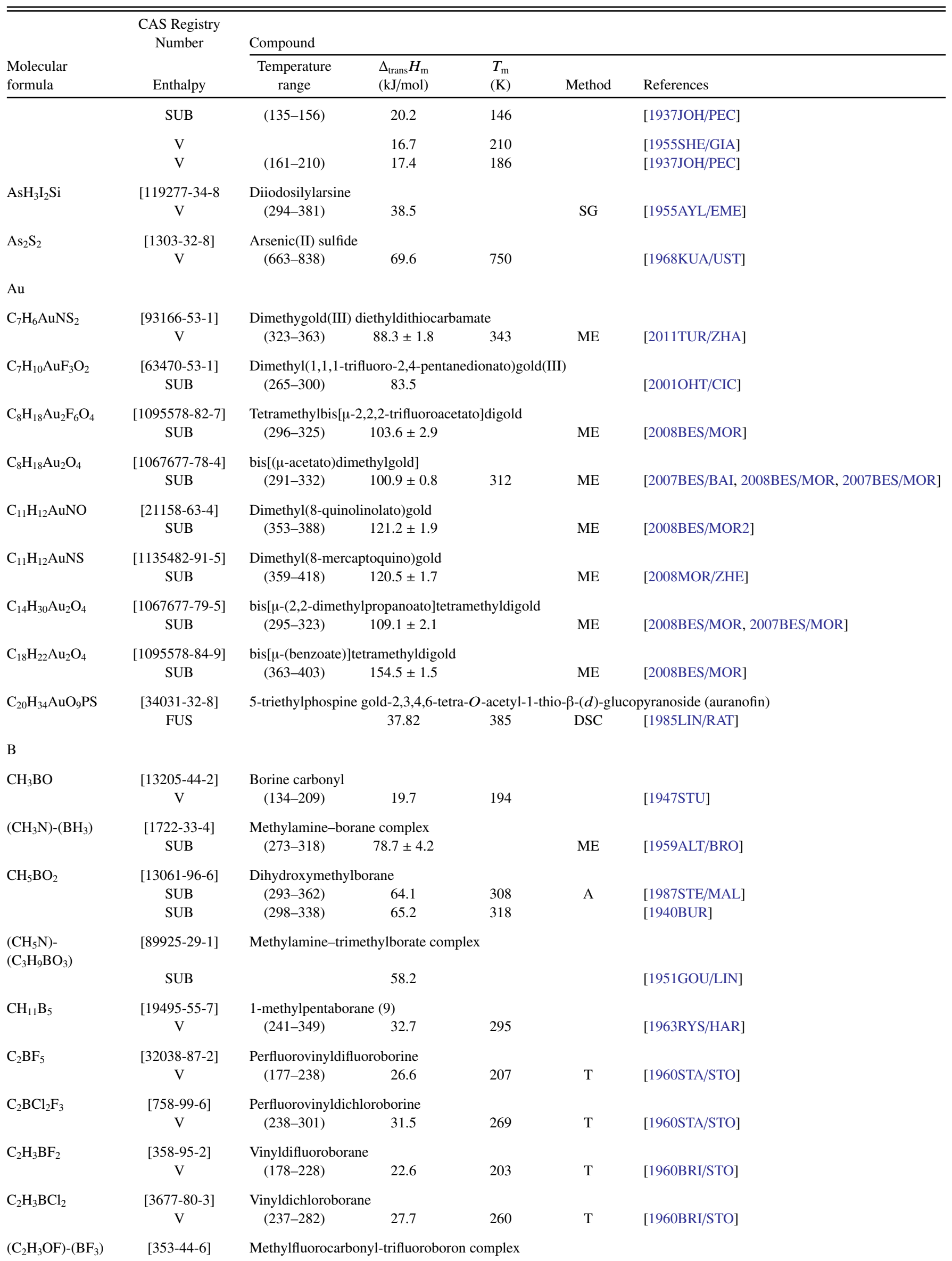


TABLE 17. Phase change enthalpies of organometallic and inorganic compounds-Continued

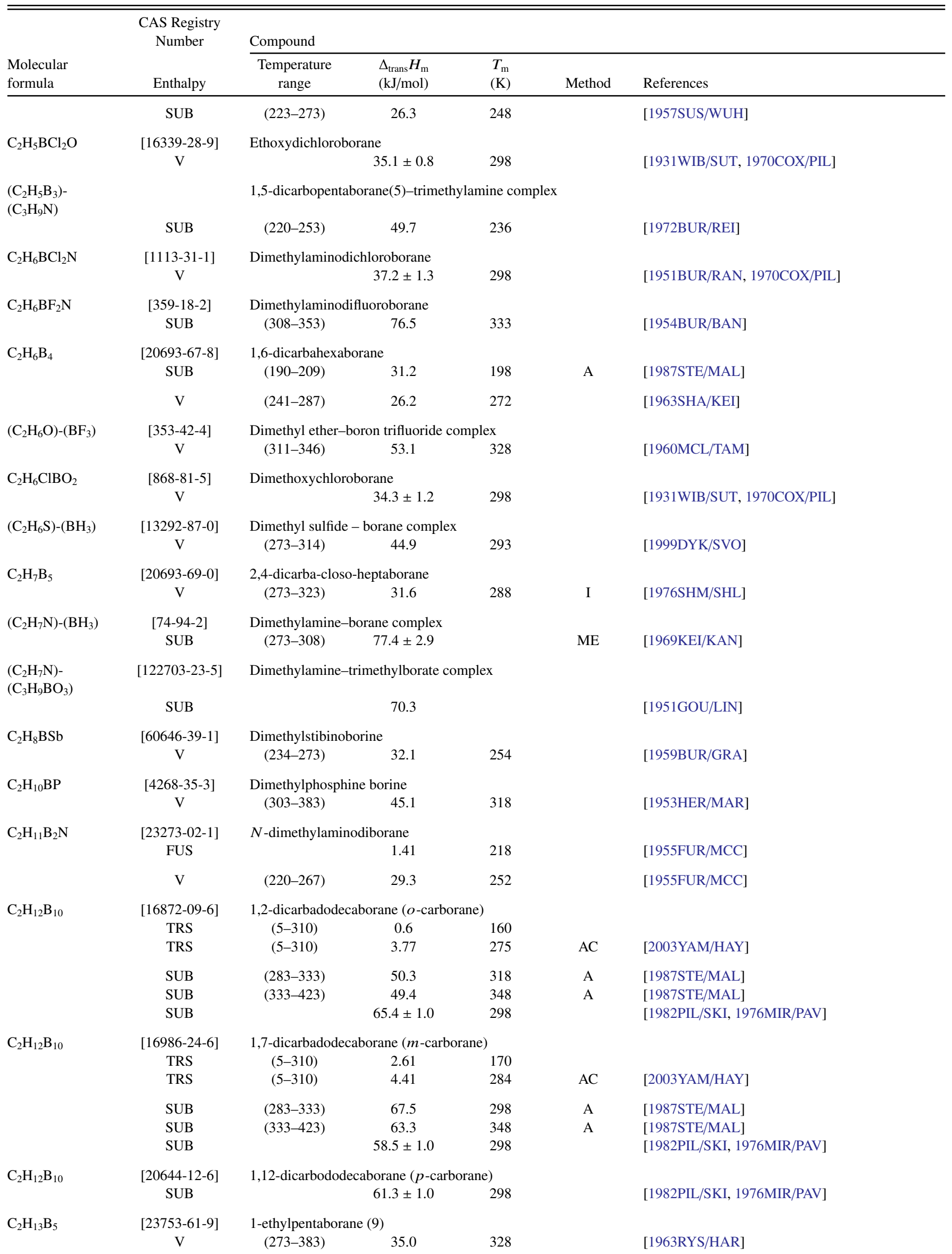


TABLE 17. Phase change enthalpies of organometallic and inorganic compounds-Continued

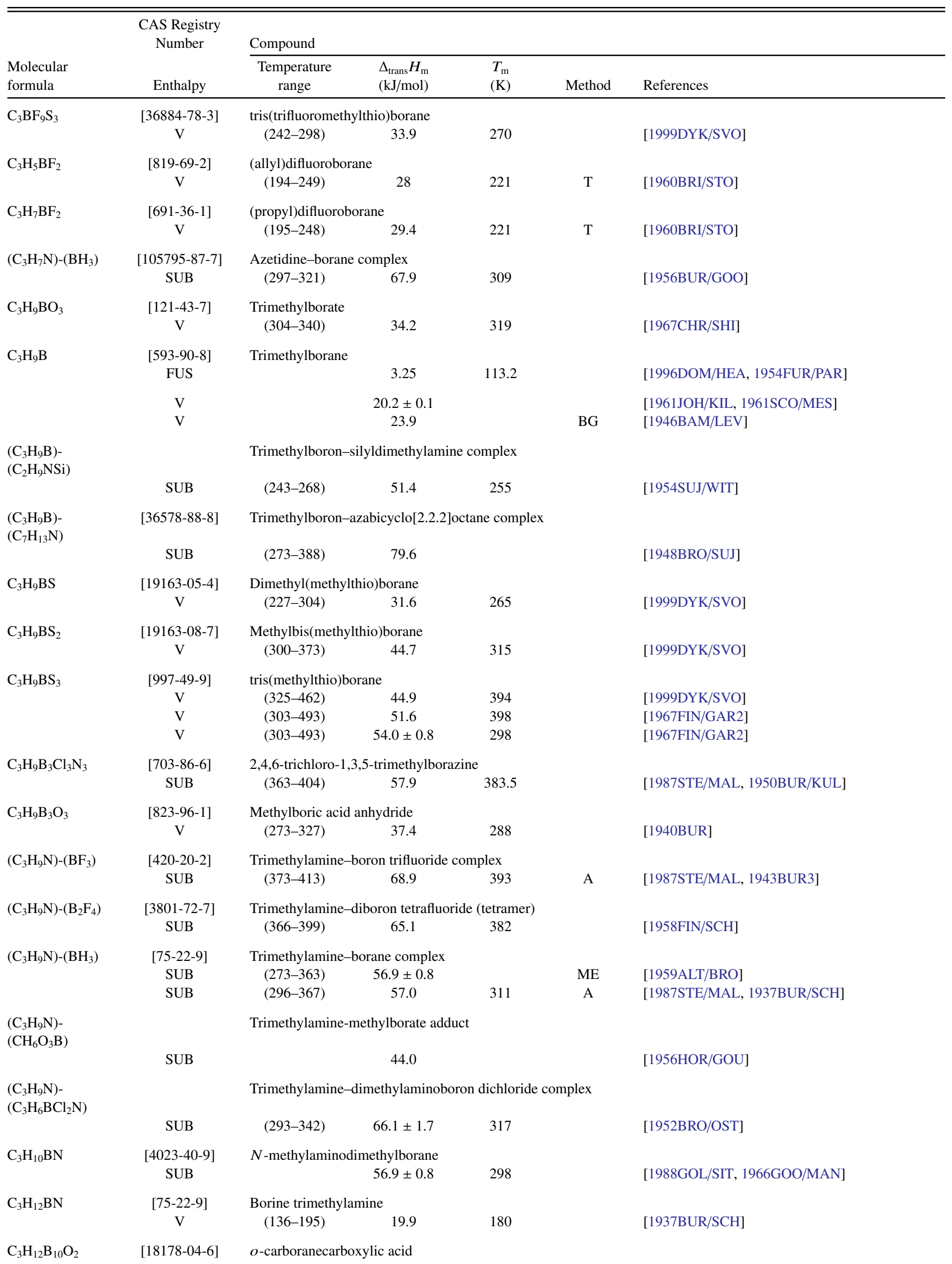


TABLE 17. Phase change enthalpies of organometallic and inorganic compounds-Continued

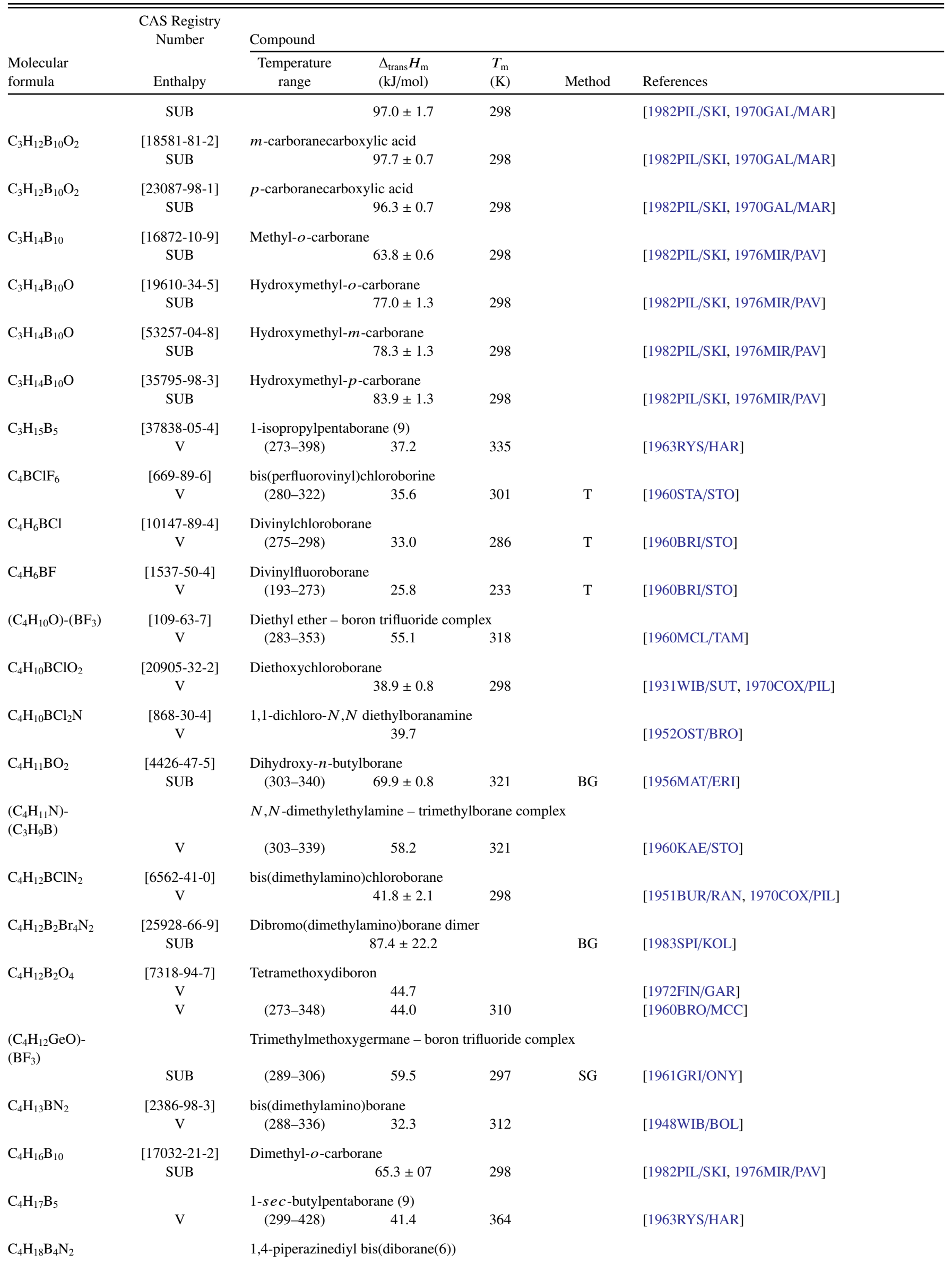


TABLE 17. Phase change enthalpies of organometallic and inorganic compounds-Continued

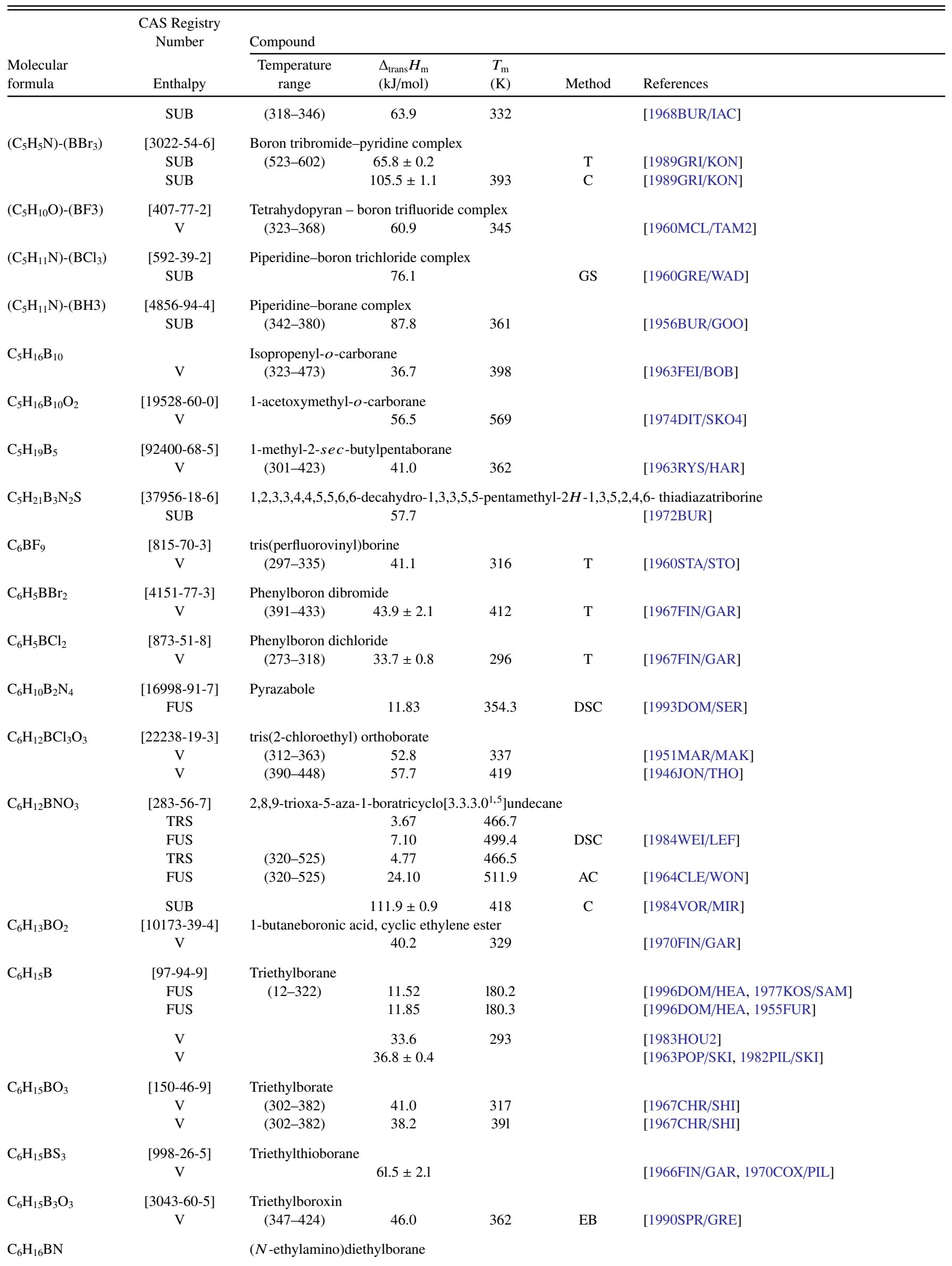


TABLE 17. Phase change enthalpies of organometallic and inorganic compounds-Continued

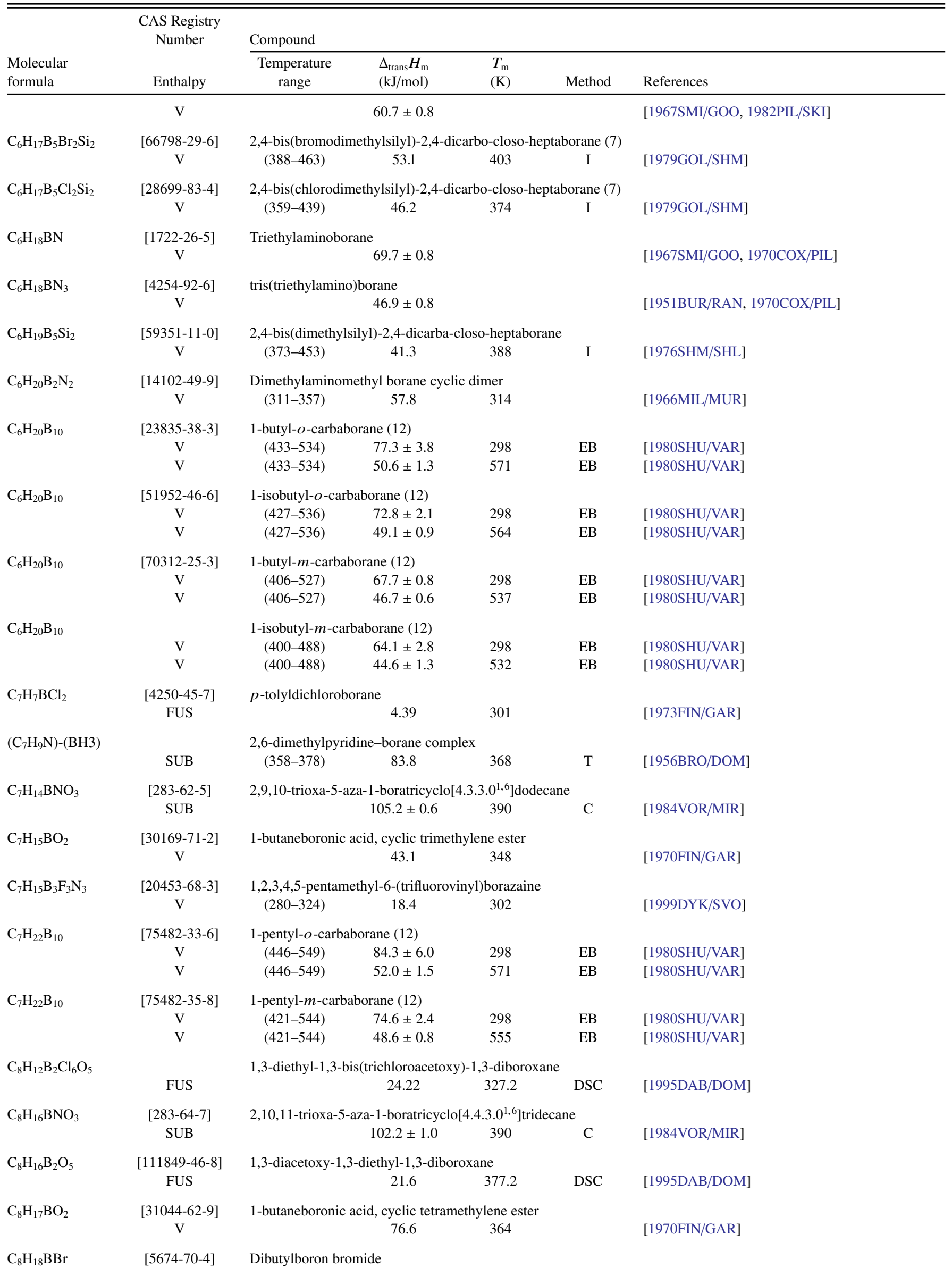


TABLE 17. Phase change enthalpies of organometallic and inorganic compounds-Continued

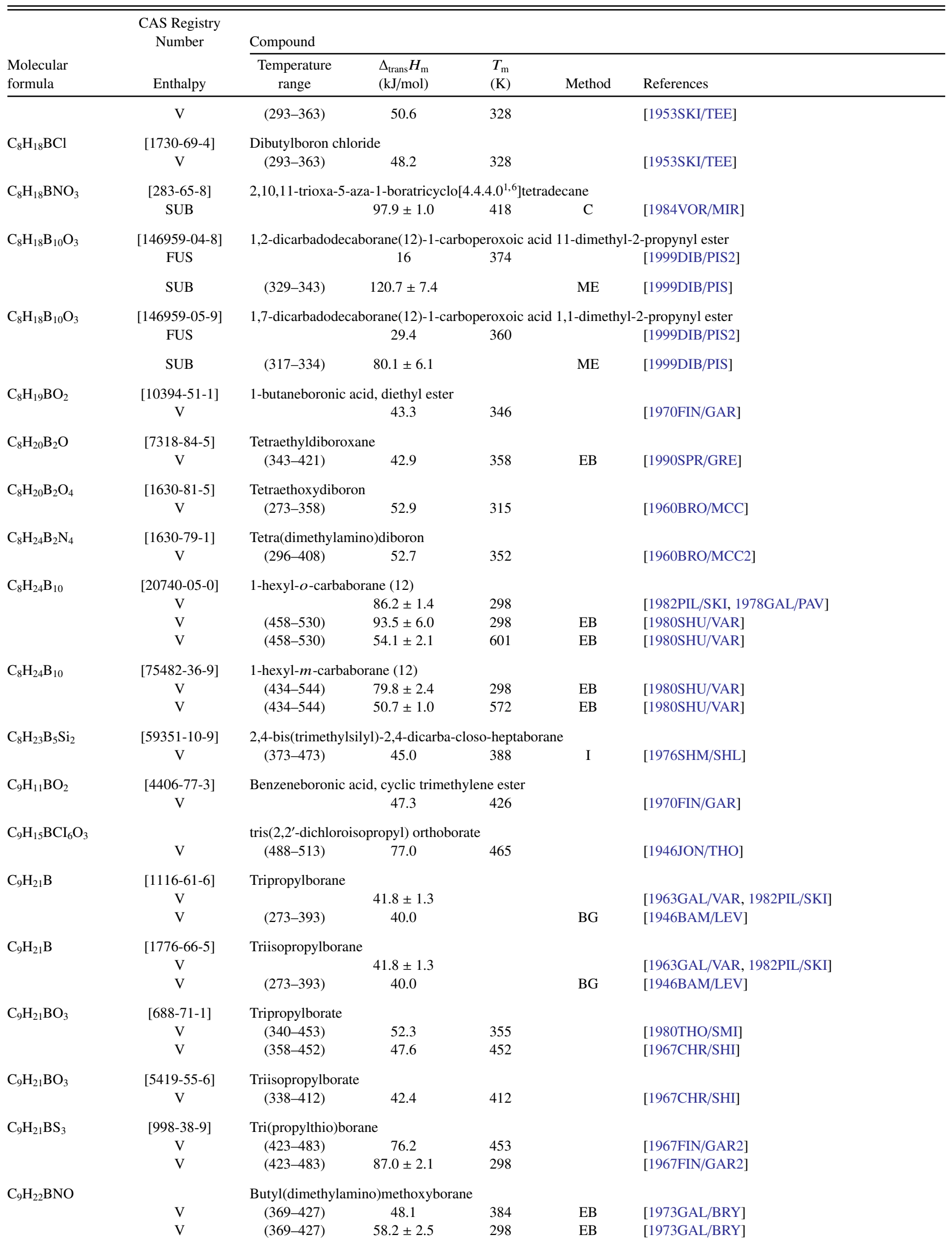


TABLE 17. Phase change enthalpies of organometallic and inorganic compounds-Continued

\begin{tabular}{|c|c|c|c|c|c|c|}
\hline \multirow[b]{2}{*}{$\begin{array}{l}\text { Molecular } \\
\text { formula }\end{array}$} & \multirow{2}{*}{$\begin{array}{l}\text { CAS Registry } \\
\text { Number } \\
\text { Enthalpy }\end{array}$} & \multicolumn{5}{|l|}{ Compound } \\
\hline & & $\begin{array}{l}\text { Temperature } \\
\text { range }\end{array}$ & $\begin{array}{l}\Delta_{\text {trans }} H_{\mathrm{m}} \\
(\mathrm{kJ} / \mathrm{mol})\end{array}$ & $\begin{array}{l}T_{\mathrm{m}} \\
(\mathrm{K})\end{array}$ & Method & References \\
\hline \multirow[t]{2}{*}{$\mathrm{C}_{10} \mathrm{H}_{13} \mathrm{BO}_{2}$} & [4406-76-2] & \multicolumn{5}{|c|}{ Benzeneboronic acid, cyclic tetramethylene ester } \\
\hline & $\mathrm{V}$ & & 57.3 & 441 & & [1970FIN/GAR] \\
\hline $\mathrm{C}_{10} \mathrm{H}_{15} \mathrm{BO}_{2}$ & $\begin{array}{c}{[31044-59-4]} \\
\mathrm{V}\end{array}$ & \multicolumn{5}{|c|}{ Benzeneboronic acid, diethyl ester } \\
\hline $\mathrm{C}_{11} \mathrm{H}_{24} \mathrm{~B}_{10} \mathrm{O}_{3}$ & $\mathrm{~V}$ & \multicolumn{5}{|c|}{ 3-methyl-3-(7-isopropyl-m-carboranoylperoxy)-1-butyne } \\
\hline $\mathrm{C}_{11} \mathrm{H}_{24} \mathrm{~B}_{10} \mathrm{O}_{3}$ & $\begin{array}{c}{[146959-06-0]} \\
\text { SUB }\end{array}$ & \multicolumn{5}{|c|}{ 1,2-dicarbadodecaborane(12)-1-carboperoxoic acid 2-(1-methylethyl)-1,1-dimethyl-2-propynyl ester } \\
\hline $\mathrm{C}_{11} \mathrm{H}_{24} \mathrm{~B}_{10} \mathrm{O}_{3}$ & FUS & \multicolumn{5}{|c|}{ 1,7-dicarbadodecaborane(12)-1-carboperoxoic acid, 7-(1-methylethyl)-1,1-dimethyl-2-propynyl ester } \\
\hline $\mathrm{C}_{12} \mathrm{H}_{10} \mathrm{BCl}$ & $\begin{array}{c}{[3677-81-4]} \\
V\end{array}$ & \multicolumn{4}{|c|}{ Diphenylboron chloride } & [1967FIN/GAR] \\
\hline $\mathrm{C}_{12} \mathrm{H}_{21} \mathrm{~B}$ & $\begin{array}{c}{[16664-33-8]} \\
\mathrm{V} \\
\mathrm{V}\end{array}$ & $\begin{array}{l}\text { Dodecahydro-9-b } \\
(304-404) \\
(294-404)\end{array}$ & $\begin{array}{l}\text { aphenalene } \\
53.1 \\
50.3\end{array}$ & $\begin{array}{l}319 \\
349\end{array}$ & A & $\begin{array}{l}\text { [1987STE/MAL] } \\
\text { [1960GRE/MOR] }\end{array}$ \\
\hline $\mathrm{C}_{12} \mathrm{H}_{27} \mathrm{~B}$ & $\begin{array}{c}{[122-56-5]} \\
V\end{array}$ & $\begin{array}{l}\text { Tributylboron } \\
\quad(293-363)\end{array}$ & 54.7 & 328 & & [1953SKI/TEE] \\
\hline $\mathrm{C}_{12} \mathrm{H}_{27} \mathrm{BO}_{3}$ & $\begin{array}{c}{[688-74-4]} \\
\mathrm{V} \\
\mathrm{V}\end{array}$ & $\begin{array}{l}\text { Tributylborate } \\
\quad(380-504) \\
(390-491)\end{array}$ & $\begin{array}{l}58.1 \\
55.9\end{array}$ & $\begin{array}{l}395 \\
405\end{array}$ & & $\begin{array}{l}{[1980 \mathrm{THO} / \mathrm{SMI}]} \\
{[1967 \mathrm{CHR} / \mathrm{SHI}]}\end{array}$ \\
\hline $\mathrm{C}_{14} \mathrm{H}_{26} \mathrm{~B}_{2} \mathrm{~N}_{4}$ & $\begin{array}{c}\text { [14695-69-3] } \\
\text { TRS } \\
\text { FUS }\end{array}$ & 4,4,8,8-tetraethyl & $\begin{array}{c}\text { razabole } \\
28.61 \\
3.22\end{array}$ & $\begin{array}{l}342.4 \\
379.2\end{array}$ & DSC & [1993DOM/SER] \\
\hline $\mathrm{C}_{15} \mathrm{H}_{32} \mathrm{~B}_{10} \mathrm{O}_{5}$ & $\begin{array}{c}{[141695-58-1]} \\
\mathrm{V}\end{array}$ & $\begin{array}{l}\text { 2,5-dimethyl-(2-t } \\
\quad(353-366)\end{array}$ & $\begin{array}{l}\text {-butylperoxy } \\
86.8 \pm 5.4\end{array}$ & $\begin{array}{c}\text { carbora } \\
360\end{array}$ & $\begin{array}{l}\text { eroxy)-3-h } \\
\text { ME }\end{array}$ & $\begin{array}{l}\text { yne } \\
\text { [1999DIB/PIS2] }\end{array}$ \\
\hline $\mathrm{C}_{15} \mathrm{H}_{33} \mathrm{BO}_{3}$ & $\begin{array}{c}{[621-78-3]} \\
V\end{array}$ & $\begin{array}{l}\text { Tripentylborate } \\
\quad(410-505)\end{array}$ & 67.7 & 425 & & [1980THO/SMI] \\
\hline $\mathrm{C}_{15} \mathrm{H}_{33} \mathrm{BS}_{3}$ & $\begin{array}{c}{[1116-74-1]} \\
\mathrm{V} \\
\mathrm{V}\end{array}$ & $\begin{array}{l}\text { Tri(pentylthio)bo } \\
\quad(446-503) \\
(446-503)\end{array}$ & $\begin{array}{l}92.3 \\
104.6 \pm 2.1\end{array}$ & $\begin{array}{l}474 \\
298\end{array}$ & & $\begin{array}{l}\text { [1967FIN/GAR2] } \\
\text { [1967FIN/GAR2] }\end{array}$ \\
\hline $\mathrm{C}_{18} \mathrm{H}_{12} \mathrm{BCl}_{3} \mathrm{O}_{3}$ & $\begin{array}{c}{[7359-58-2]} \\
\mathrm{V}\end{array}$ & $\begin{array}{l}\text { tris(4-chlorophen } \\
\quad(428-476)\end{array}$ & $\begin{array}{l}\text { y)borane } \\
30.6 \pm 0.9\end{array}$ & 452 & MM & [1973WIL/FEN] \\
\hline $\mathrm{C}_{18} \mathrm{H}_{12} \mathrm{BCl}_{3} \mathrm{O}_{3}$ & $\begin{array}{c}{[42080-72-8]} \\
\mathrm{V}\end{array}$ & $\begin{array}{l}\text { tris(3-chlorophen } \\
\quad(476-524)\end{array}$ & $\begin{array}{l}\text { y)borane } \\
49.6 \pm 1.6\end{array}$ & 500 & MM & [1973WIL/FEN] \\
\hline $\mathrm{C}_{18} \mathrm{H}_{15} \mathrm{~B}$ & $\begin{array}{l}{[960-71-4]} \\
\text { SUB } \\
\text { SUB } \\
\text { SUB }\end{array}$ & Triphenylborane & $\begin{array}{l}103.8 \pm 2.5 \\
92.1 \pm 2.5 \\
81.6 \pm 2.1\end{array}$ & $\begin{array}{l}360 \\
298\end{array}$ & TE,ME & $\begin{array}{l}\text { [1984GOV/KAN2] } \\
\text { [1978STE3] } \\
\text { [1982PIL/SKI, 1967FIN/GAR] }\end{array}$ \\
\hline & $\begin{array}{l}\mathrm{V} \\
\mathrm{V}\end{array}$ & $\begin{array}{l}(423-568) \\
(423-548)\end{array}$ & $\begin{array}{c}64.3 \\
64.4 \pm 2.1\end{array}$ & $\begin{array}{l}438 \\
486\end{array}$ & A & $\begin{array}{l}\text { [1987STE/MAL] } \\
{[1967 \mathrm{FIN} / \mathrm{GAR}]}\end{array}$ \\
\hline
\end{tabular}


TABLE 17. Phase change enthalpies of organometallic and inorganic compounds-Continued

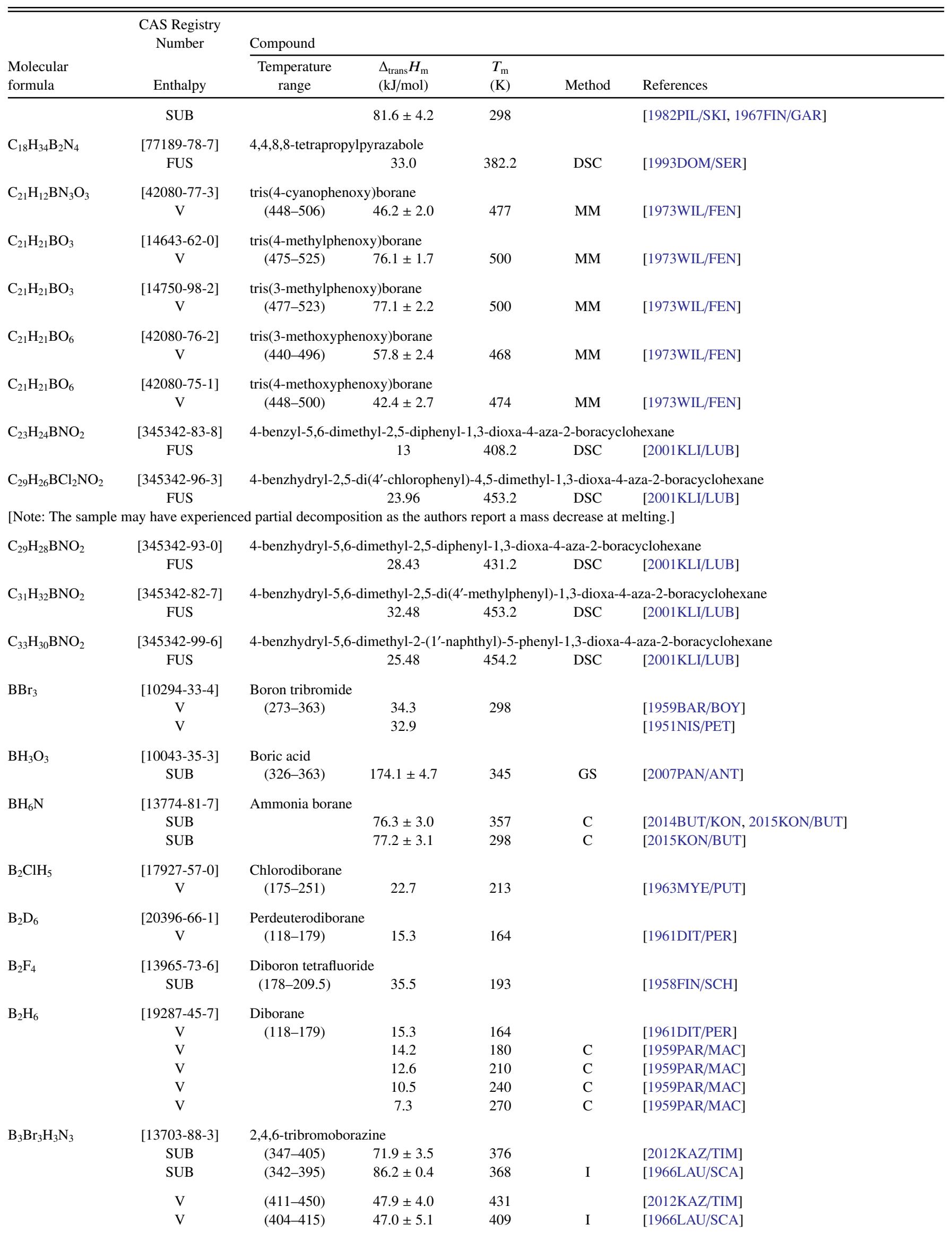

$\mathrm{B}_{3} \mathrm{Cl}_{3} \mathrm{H}_{3} \mathrm{~N}_{3} \quad$ [933-18-6] 2,4,6-trichloroborazine 
TABLE 17. Phase change enthalpies of organometallic and inorganic compounds-Continued

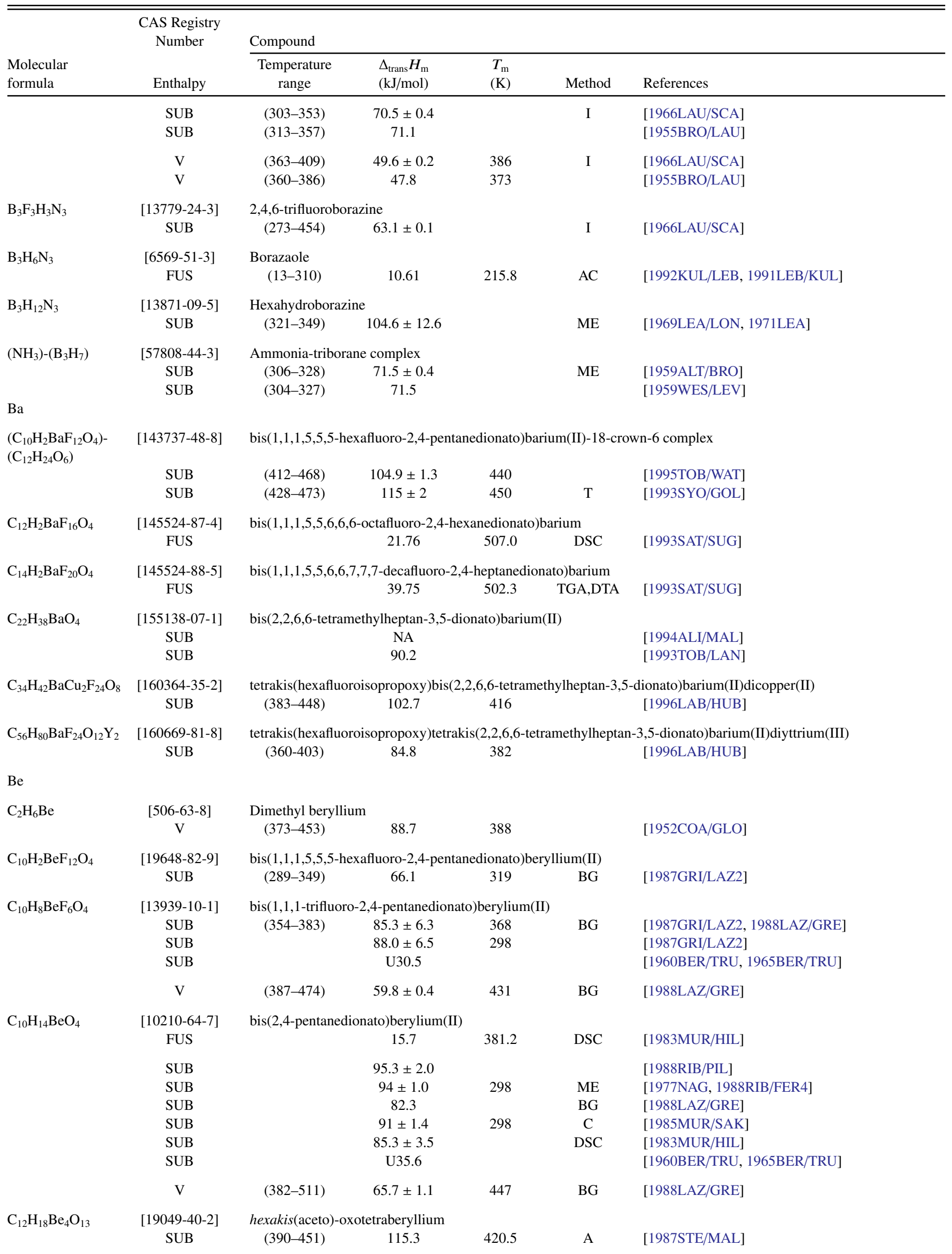


TABLE 17. Phase change enthalpies of organometallic and inorganic compounds-Continued

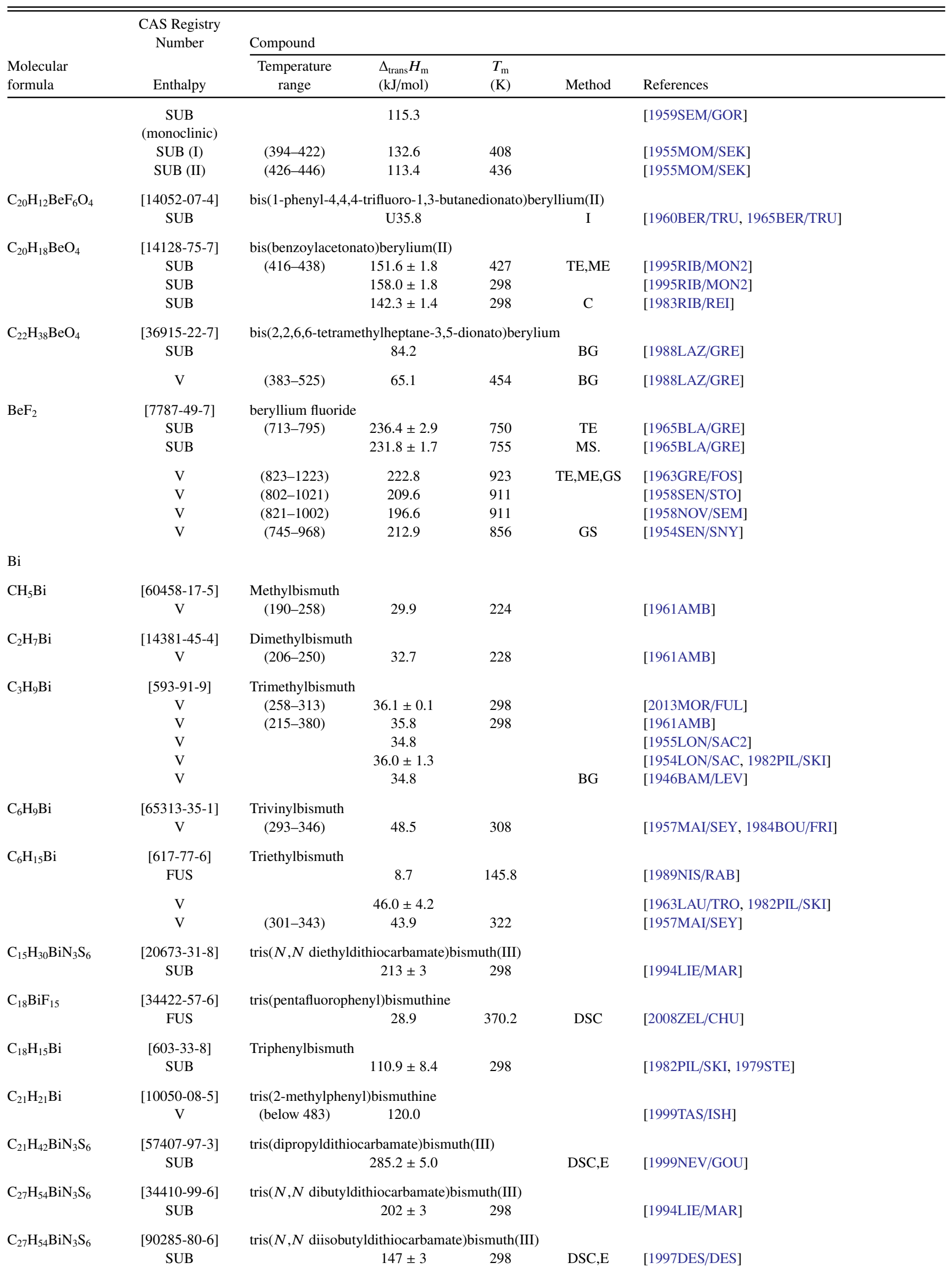


TABLE 17. Phase change enthalpies of organometallic and inorganic compounds-Continued

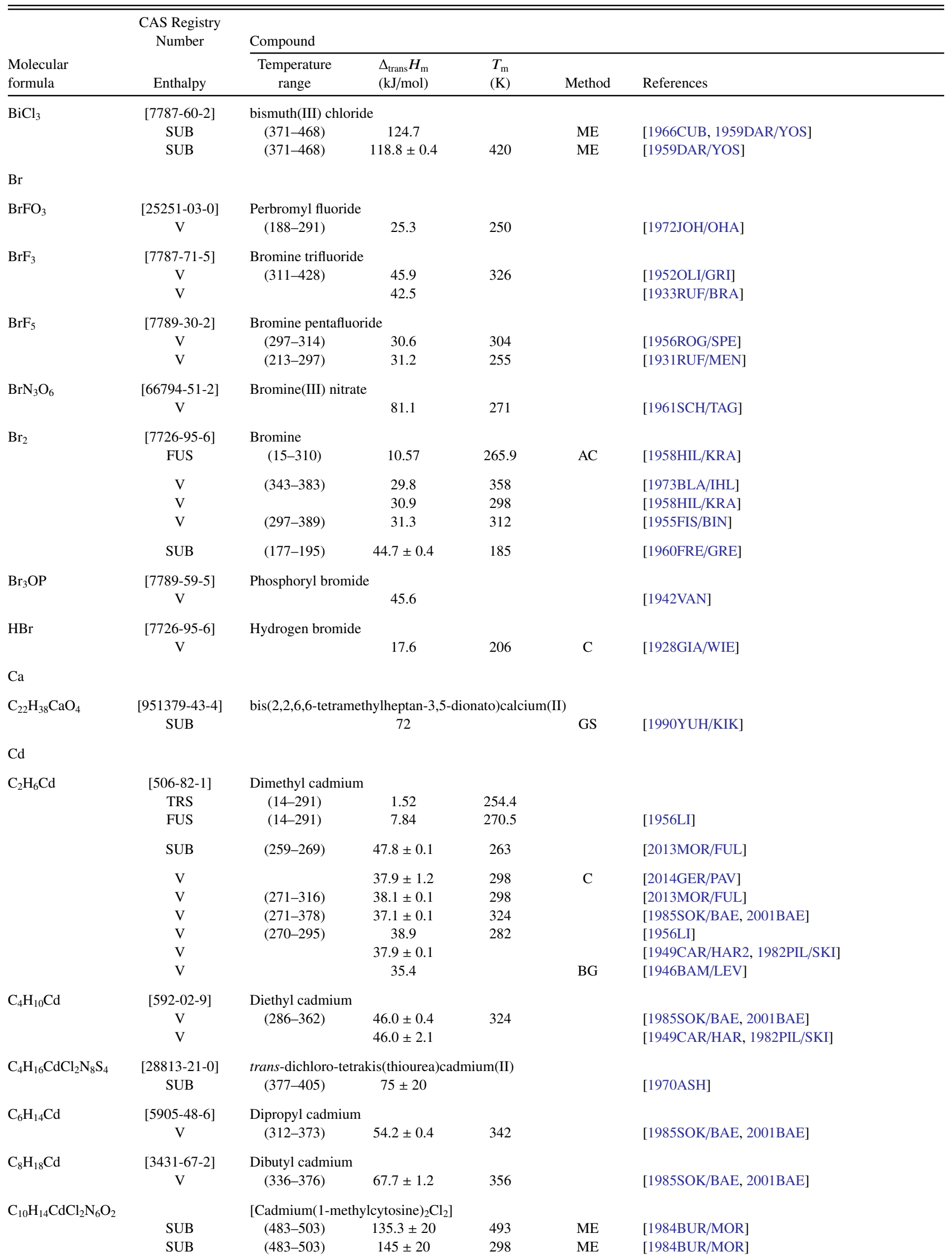


TABLE 17. Phase change enthalpies of organometallic and inorganic compounds-Continued

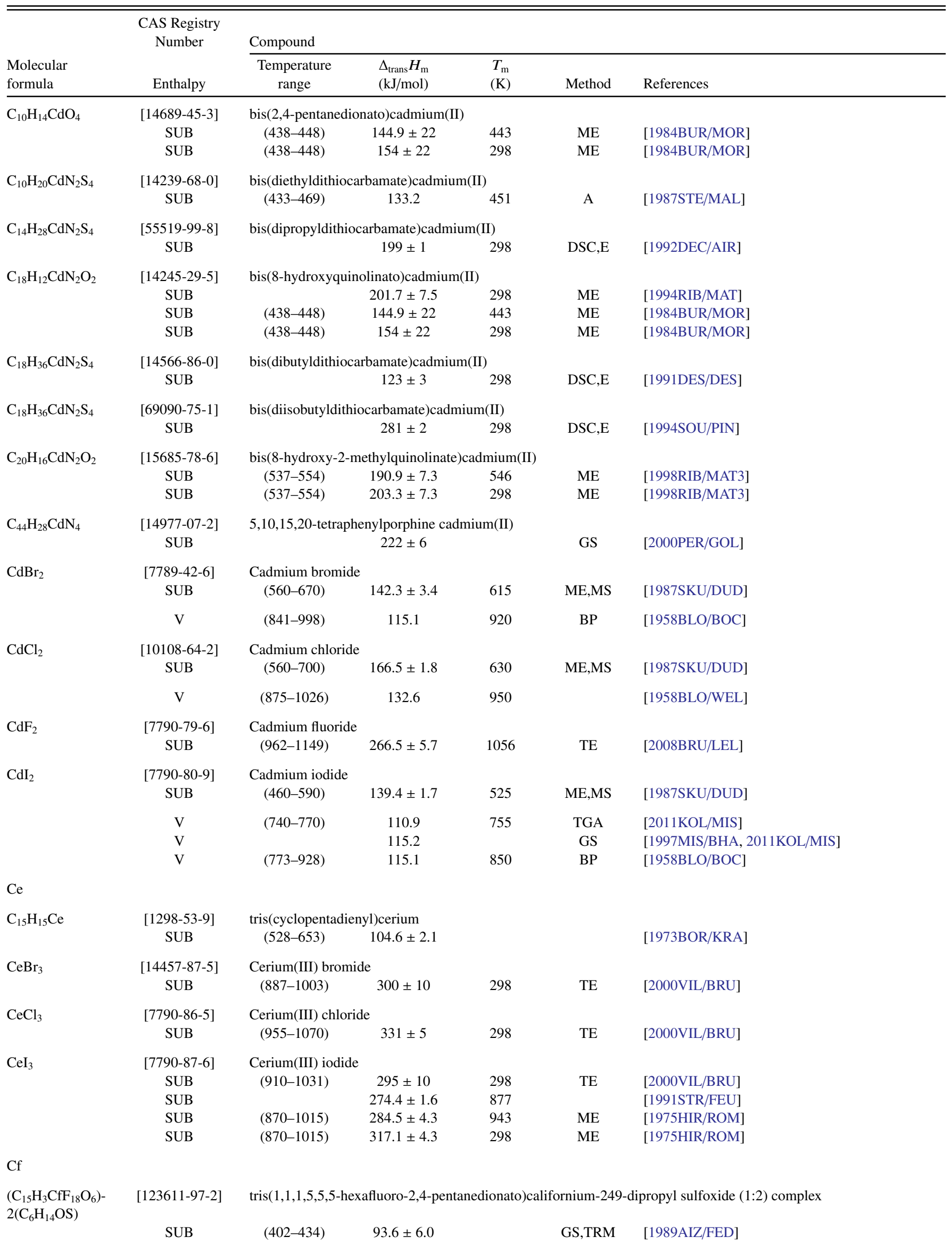


TABLE 17. Phase change enthalpies of organometallic and inorganic compounds-Continued

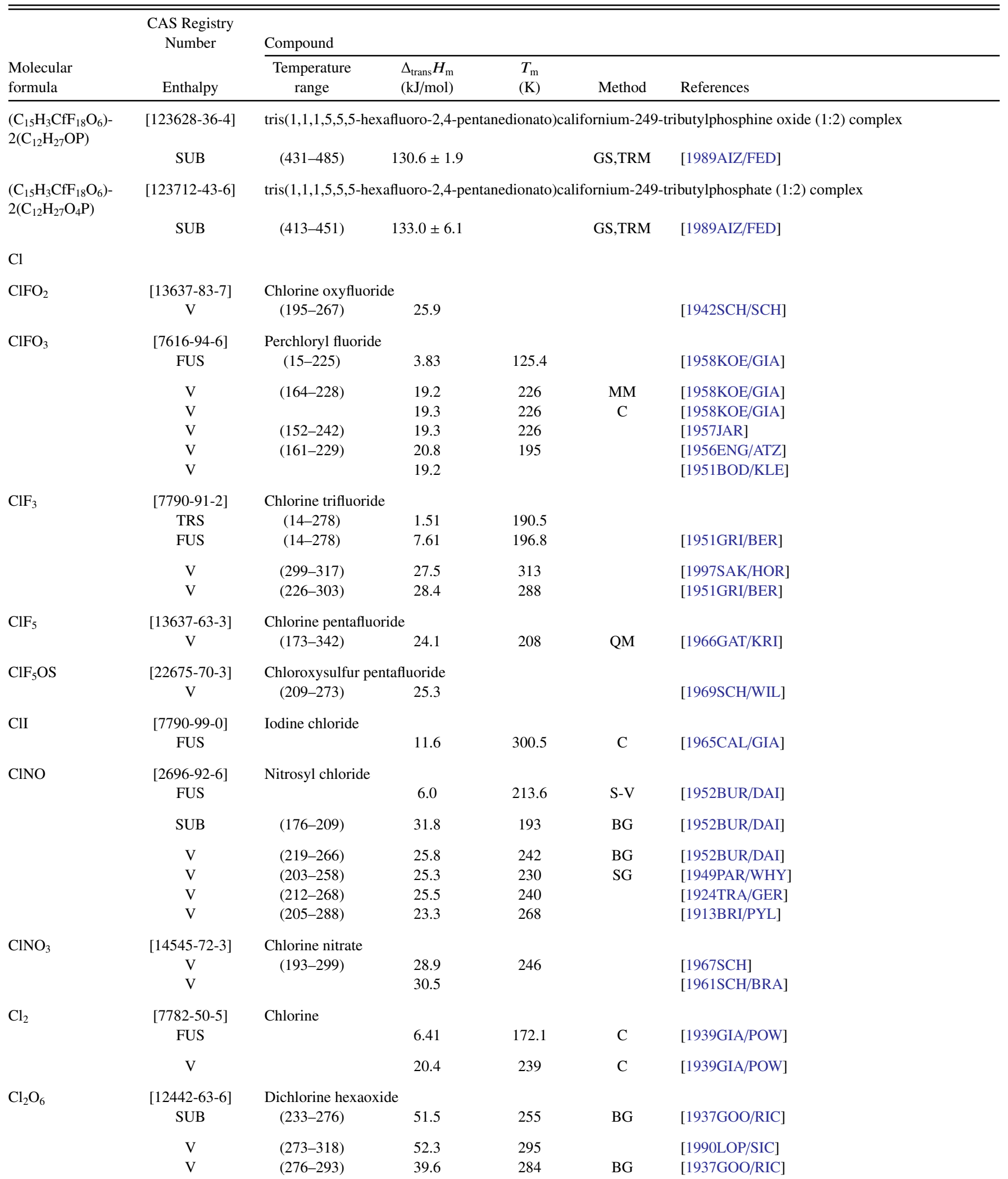

[Note: The authors of [1990LOP/SIC] noted the disagreement between their Trouton's constant and that reported by [1937GOO/RIC].

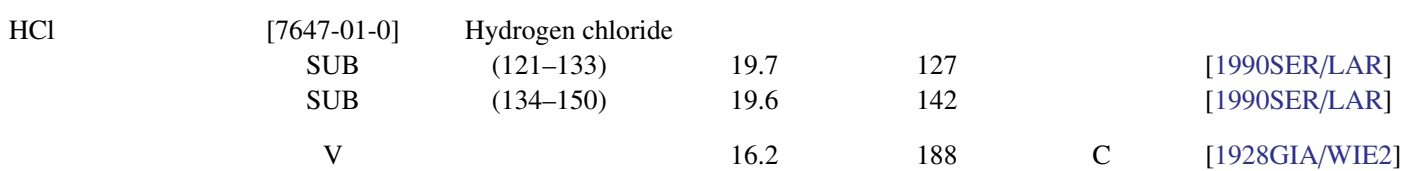

Co 
TABLE 17. Phase change enthalpies of organometallic and inorganic compounds-Continued

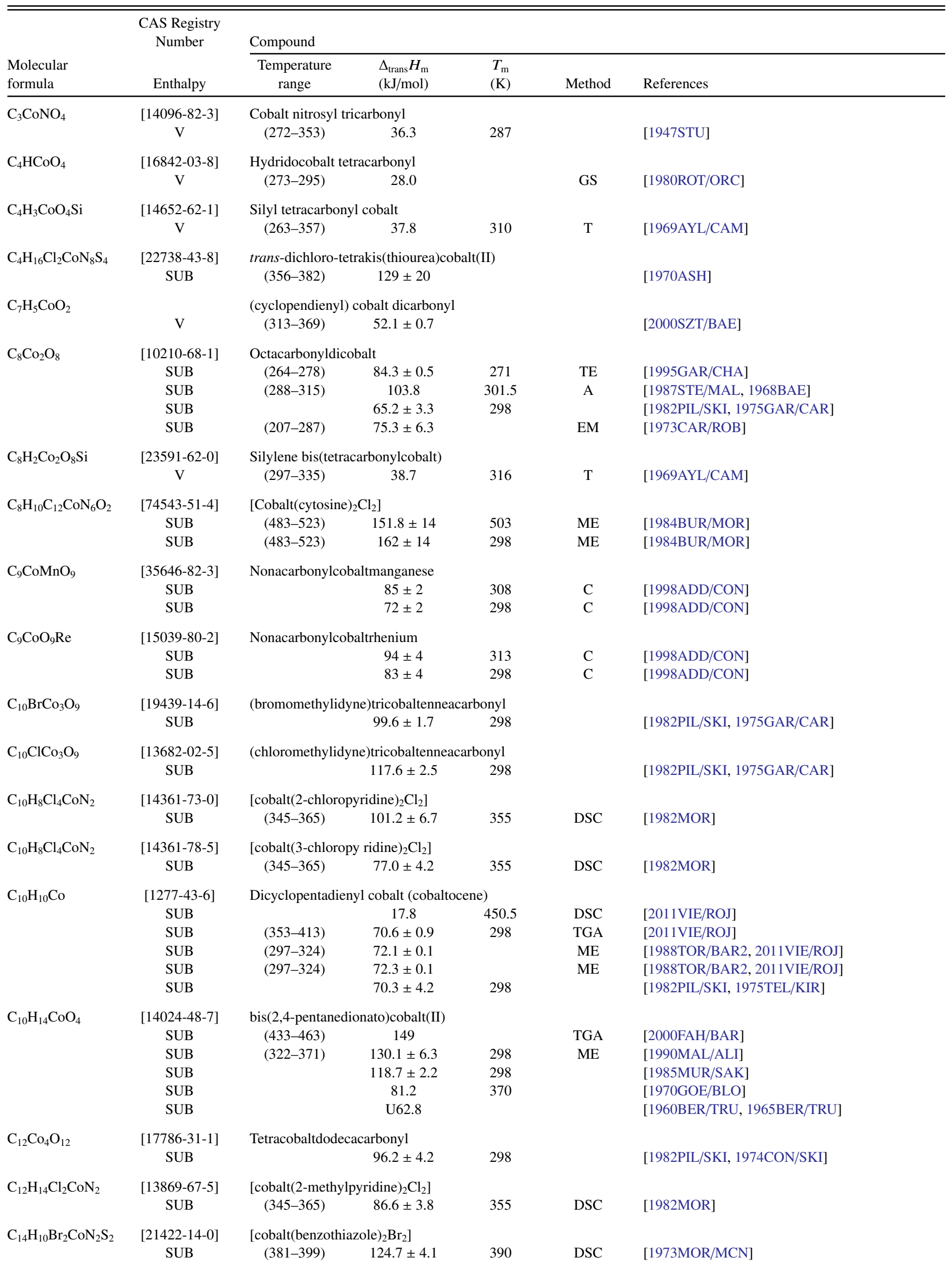


TABLE 17. Phase change enthalpies of organometallic and inorganic compounds-Continued

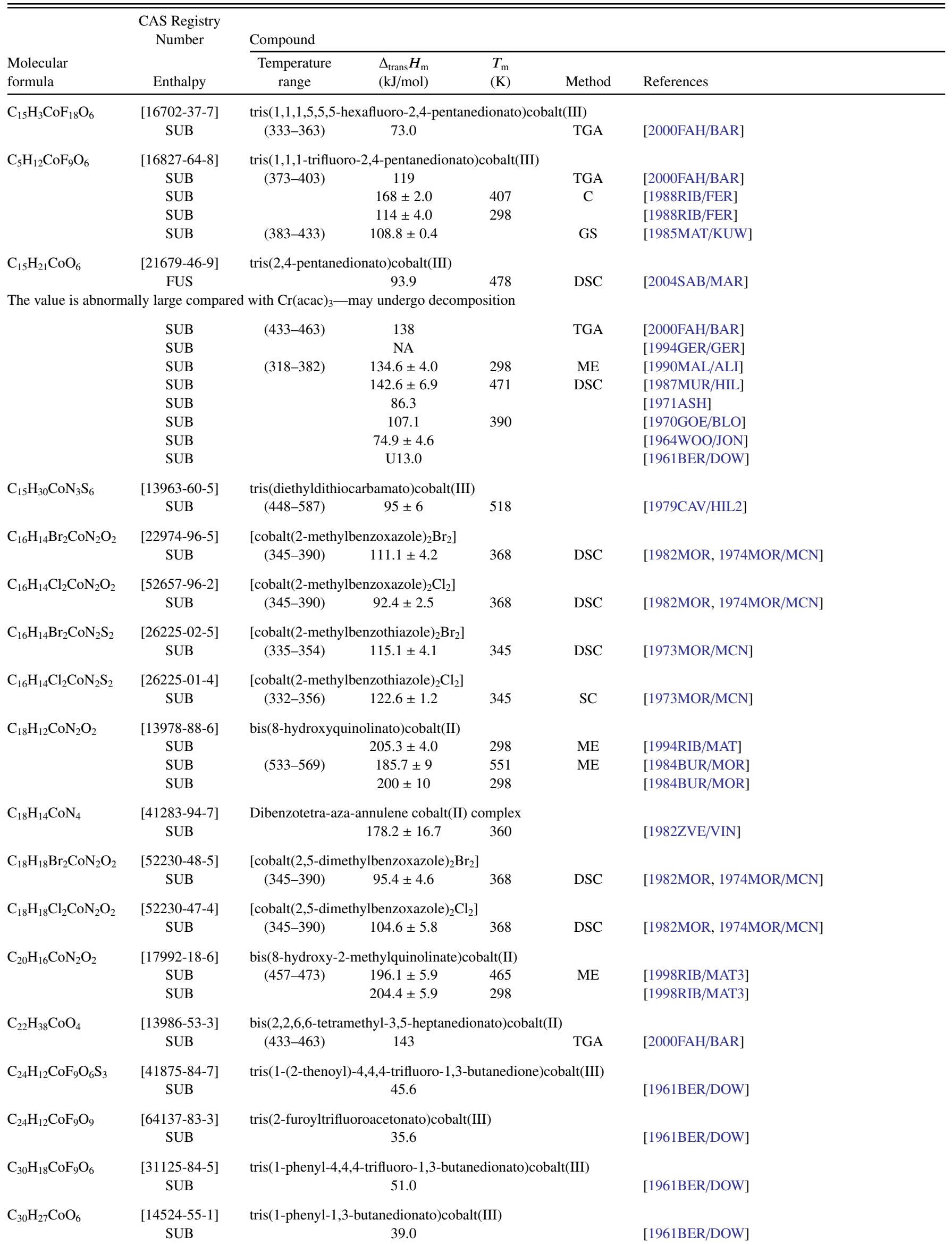


TABLE 17. Phase change enthalpies of organometallic and inorganic compounds-Continued

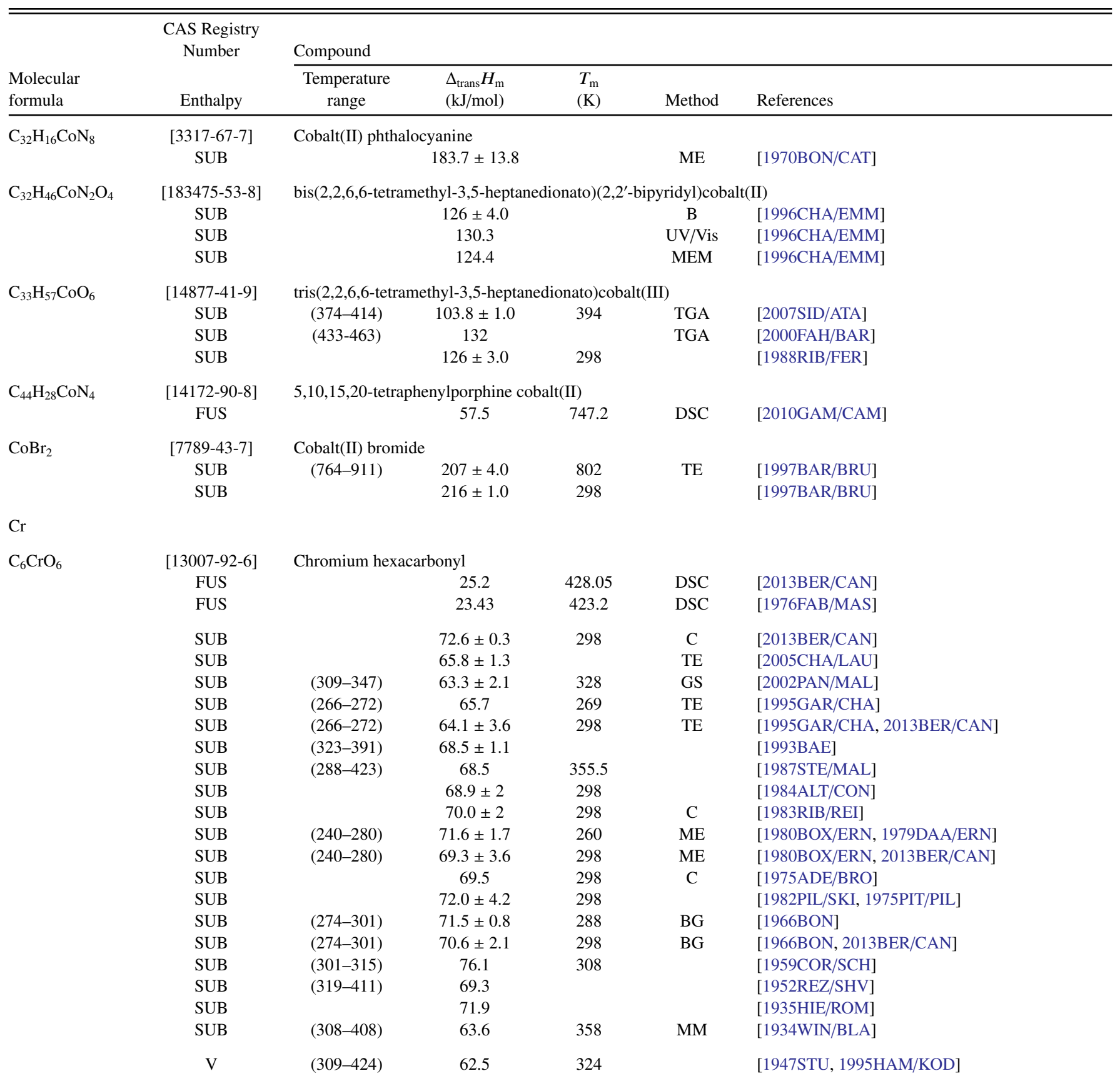

[Note: The authors of [2002PAN/MAL] question the value of $62.5 \mathrm{~kJ} / \mathrm{mol}$ for the enthalpy of vaporization. They suggest that the value is the sum of the enthalpy of vaporization plus the enthalpy of decomposition. The value is not consistent with the experimental enthalpies of fusion and sublimation. Given that the temperature range for the measurements falls below the melting point temperature, we believe that the value likely corresponds to the enthalpy of sublimation.]

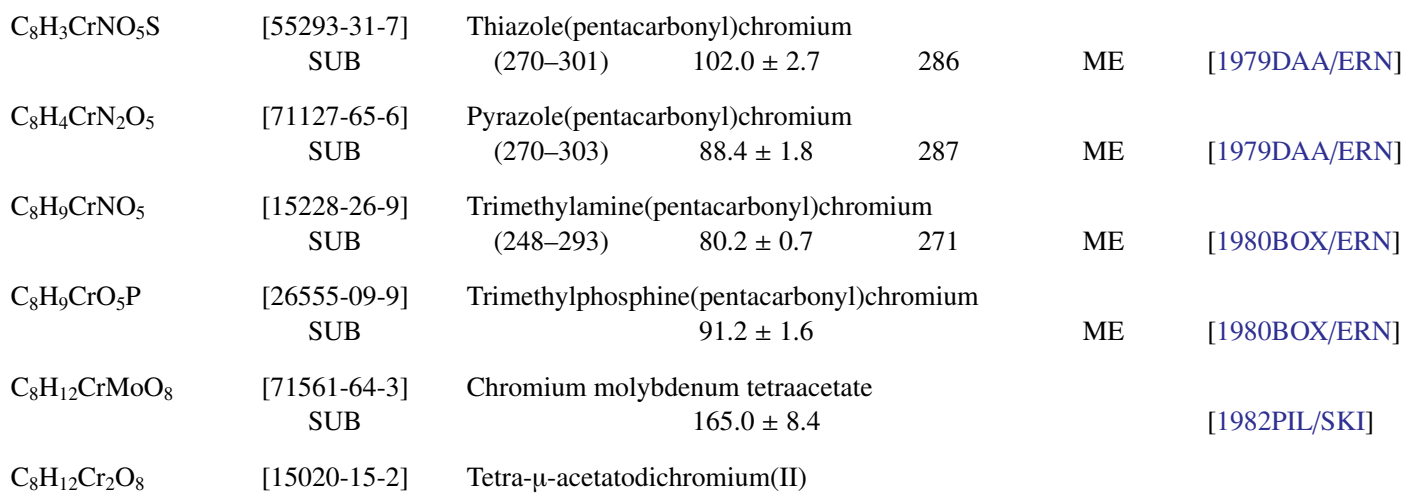


TABLE 17. Phase change enthalpies of organometallic and inorganic compounds-Continued

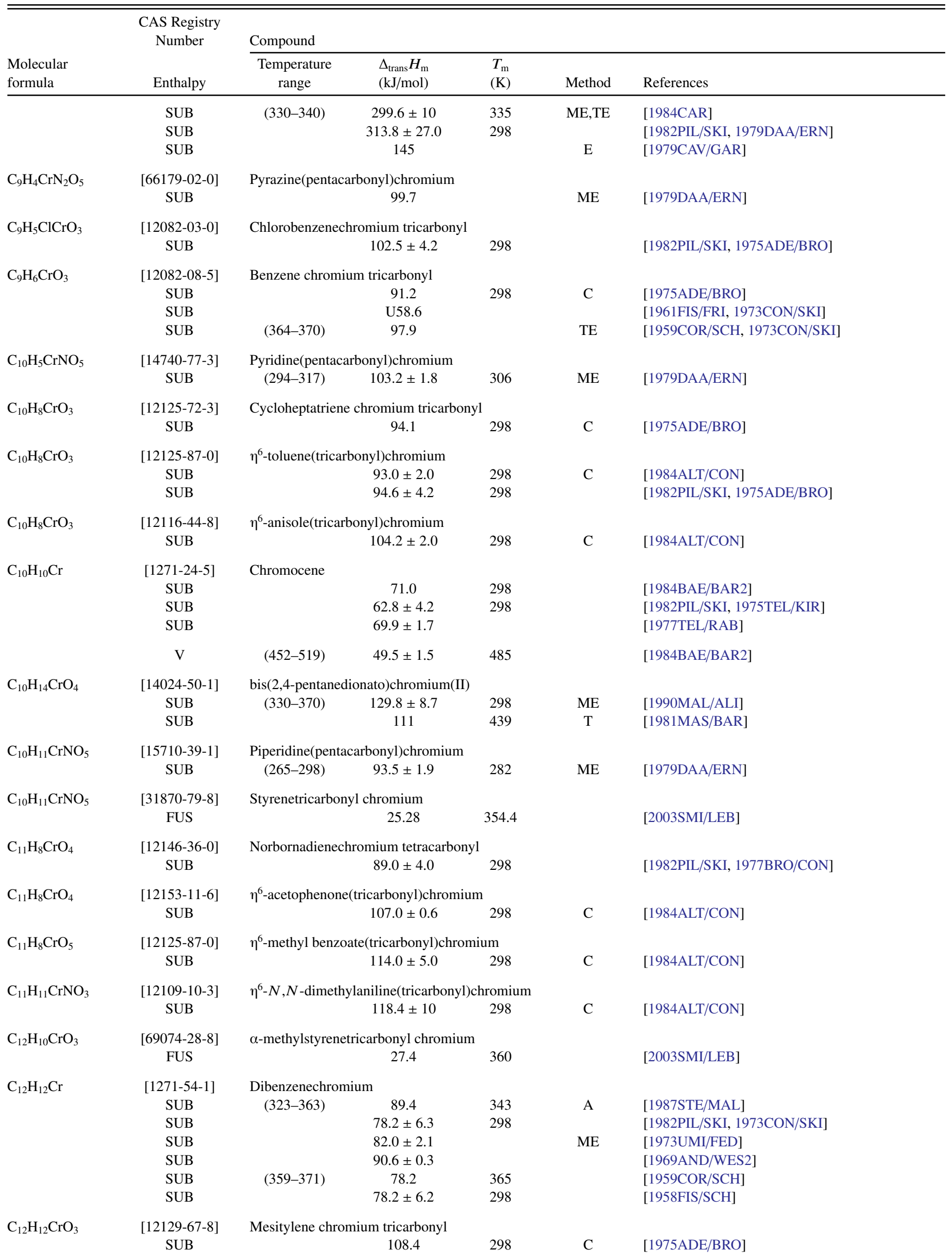


TABLE 17. Phase change enthalpies of organometallic and inorganic compounds-Continued

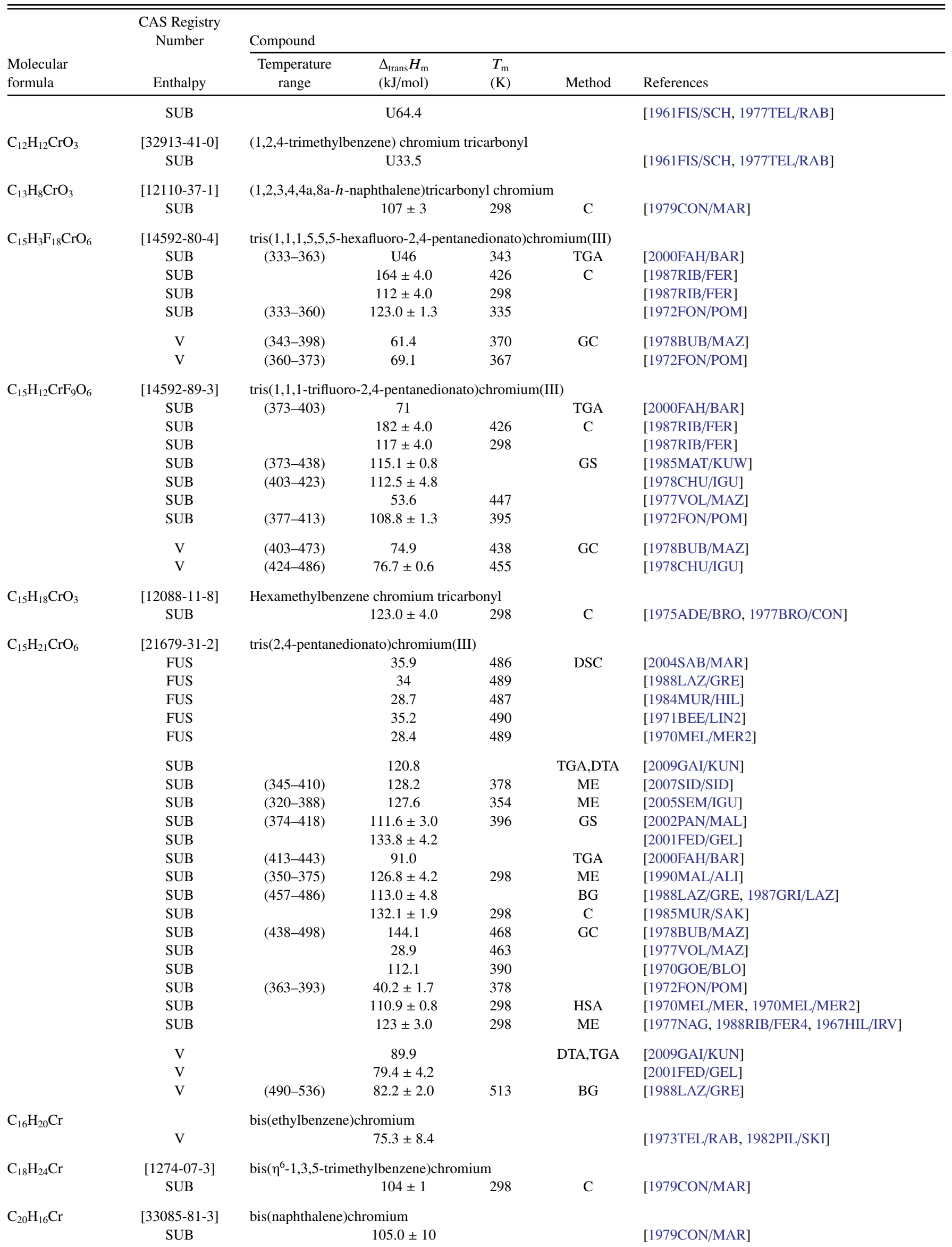


TABLE 17. Phase change enthalpies of organometallic and inorganic compounds-Continued

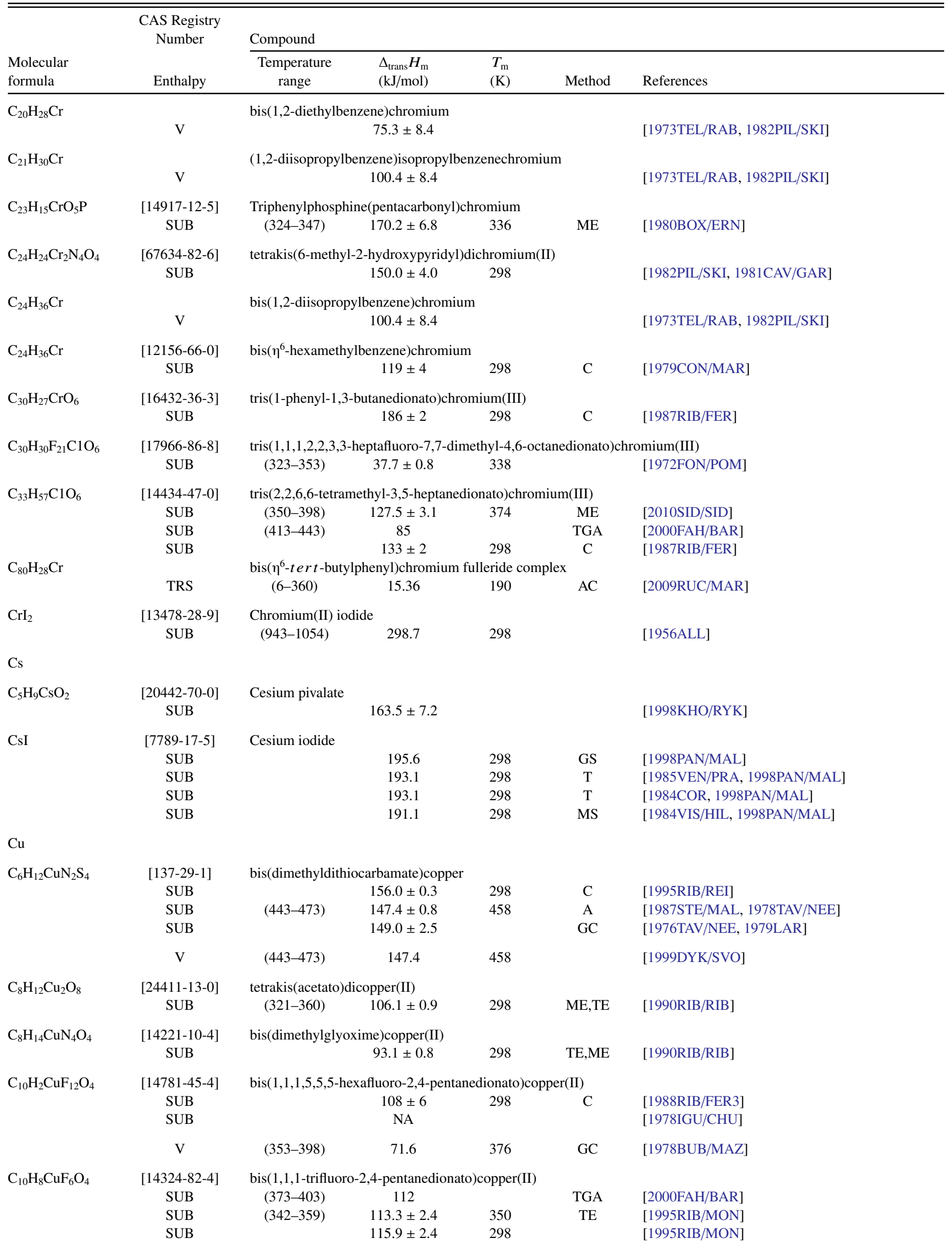


TABLE 17. Phase change enthalpies of organometallic and inorganic compounds-Continued

\begin{tabular}{|c|c|c|c|c|c|c|}
\hline \multirow[b]{2}{*}{$\begin{array}{l}\text { Molecular } \\
\text { formula }\end{array}$} & \multirow{2}{*}{$\begin{array}{c}\text { CAS Registry } \\
\text { Number } \\
\text { Enthalpy }\end{array}$} & \multicolumn{5}{|l|}{ Compound } \\
\hline & & $\begin{array}{c}\text { Temperature } \\
\text { range }\end{array}$ & $\begin{array}{l}\Delta_{\text {trans }} H_{\mathrm{m}} \\
(\mathrm{kJ} / \mathrm{mol})\end{array}$ & $\begin{array}{l}T_{\mathrm{m}} \\
(\mathrm{K})\end{array}$ & Method & References \\
\hline & SUB & $(342-359)$ & $114.4 \pm 1.6$ & 350 & $\mathrm{ME}$ & [1995RIB/MON] \\
\hline & SUB & & $117.0 \pm 1.6$ & 298 & & [1995RIB/MON] \\
\hline & SUB & & $112 \pm 3.0$ & 298 & $\mathrm{C}$ & [1988RIB/FER3] \\
\hline & SUB & $(383-463)$ & 110. \pm 0.8 & & GS & [1985MAT/KUW] \\
\hline & SUB & & NA & & & [1978IGU/CHU] \\
\hline & SUB & & U50.6 & & & [1960BER/TRU, 1965BER/TRU] \\
\hline & SUB & $(383-473)$ & 80.4 & 428 & GC & [1978BUB/MAZ] \\
\hline \multirow[t]{24}{*}{$\mathrm{C}_{10} \mathrm{H}_{14} \mathrm{CuO}_{4}$} & {$[13395-16-9]$} & \multicolumn{5}{|c|}{ bis(2,4-pentanedionato)copper(II) } \\
\hline & SUB & $(363-443)$ & $121.6 \pm 1.4$ & 403 & $\mathrm{ME}$ & [2009SID/SID] \\
\hline & SUB & $(413-443)$ & 120 & & TGA & [2000FAH/BAR] \\
\hline & SUB & $(377-398)$ & $122.5 \pm 1.2$ & 387 & TE & [1995RIB/MON] \\
\hline & SUB & & $127.0 \pm 1.2$ & 298 & & [1995RIB/MON] \\
\hline & SUB & $(377-398)$ & $122.6 \pm 0.7$ & 387 & $\mathrm{ME}$ & [1995RIB/MON] \\
\hline & SUB & & $127.1 \pm 1.2$ & 298 & & [1995RIB/MON] \\
\hline & SUB & & $122.3 \pm 1.1$ & 393 & $\mathrm{ME}$ & [1995RIB/MON] \\
\hline & SUB & & $127.0 \pm 1.1$ & 298 & & [1995RIB/MON] \\
\hline & SUB & & $116.6 \pm 2.0$ & 298 & $\mathrm{C}$ & [1994RIB/FER] \\
\hline & SUB & $(315-386)$ & $115.1 \pm 2.1$ & 298 & & [1991MAL/ALI] \\
\hline & SUB & & $142.6 \pm 6.9$ & 471 & DSC & [1987RIB/FER] \\
\hline & SUB & & $107.1 \pm 5.7$ & 492 & & [1987MUR/HIL] \\
\hline & SUB & & $127.5 \pm 3.2$ & 298 & & [1985MUR/SAK] \\
\hline & SUB & & $154 \pm 22$ & 298 & & [1984BUR/MOR] \\
\hline & SUB & & $109.9 \pm 3.4$ & 298 & $\mathrm{C}$ & [1984RIB/RIB] \\
\hline & SUB & & 57.1 & & $\mathrm{TE}$ & [1981TEG/FER] \\
\hline & SUB & $(423-473)$ & 106.1 & 448 & GC & [1978BUB/MAZ] \\
\hline & SUB & & NA & & & [1978IGU/CHU] \\
\hline & SUB & & 109.6 & & & [1972BOL, 2000DUN] \\
\hline & SUB & & 106.1 & & $\mathrm{TG}$ & [1971ASH] \\
\hline & SUB & & $109 \pm 6$ & 400 & & [1970GOE/BLO] \\
\hline & SUB & & 57.3 & & DSC & [1971BEE/LIN2] \\
\hline & SUB & & 62.8 & & & [1962JON/YOW] \\
\hline \multirow[t]{2}{*}{$\mathrm{C}_{10} \mathrm{H}_{16} \mathrm{CuN}_{2} \mathrm{O}_{2}$} & {$[14404-35-4]$} & \multicolumn{5}{|c|}{ bis(4-amino-3-penten-2-onato)copper } \\
\hline & SUB & $(393-463)$ & $114.2 \pm 1.3$ & & & [2003STA/BAI] \\
\hline \multirow[t]{6}{*}{$\mathrm{C}_{10} \mathrm{H}_{20} \mathrm{CuN}_{2} \mathrm{~S}_{4}$} & {$[13681-87-3]$} & \multicolumn{5}{|c|}{ bis(diethyldithiocarbamate)copper(II) } \\
\hline & SUB & $(420-465)$ & $149.1 \pm 0.4$ & 442.5 & A & [1987STE/MAL, 1978TAV/NEE] \\
\hline & SUB & & $103.8 \pm 2.4$ & & & [1979CAV/HIL2] \\
\hline & SUB & & $116.2 \pm 1.3$ & & & [1979CAV/HIL] \\
\hline & SUB & & $149.0 \pm 2.5$ & & & [1976TAV/NEE] \\
\hline & SUB & & $87 \pm 1.7$ & & I & [1969DAS/WEN] \\
\hline \multirow[t]{2}{*}{$\mathrm{C}_{12} \mathrm{H}_{12} \mathrm{CuF}_{6} \mathrm{O}_{4}$} & {$[13681-87-3]$} & \multicolumn{5}{|c|}{ bis(1,1,1-trifluorohexane-2,4-dione)copper(II) } \\
\hline & SUB & & $119.1 \pm 1.7$ & 298 & $\mathrm{ME}$ & [1998RIB/GON] \\
\hline \multirow[t]{4}{*}{$\mathrm{C}_{12} \mathrm{H}_{18} \mathrm{CuO}_{4}$} & {$[14781-49-8]$} & \multicolumn{5}{|c|}{ bis(3-methyl-2,4-pentanedionato)copper(II) } \\
\hline & SUB & & $130.7 \pm 1$ & 396.7 & $\mathrm{ME}$ & [1992RIB/FER3] \\
\hline & SUB & & $135.6 \pm 1$ & 298 & $\mathrm{ME}$ & [1992RIB/FER3] \\
\hline & SUB & & $132.7 \pm 2.5$ & 298 & $\mathrm{C}$ & [1992RIB/FER3] \\
\hline \multirow[t]{2}{*}{$\mathrm{C}_{14} \mathrm{H}_{16} \mathrm{CuF}_{6} \mathrm{O}_{4}$} & {$[33896-35-4]$} & \multicolumn{5}{|c|}{ bis(1,1,1-trifluoro-5-methylhexane-2,4-dione)copper(II) } \\
\hline & SUB & & $122.4 \pm 0.9$ & 298 & $\mathrm{ME}$ & [1998RIB/GON] \\
\hline \multirow[t]{3}{*}{$\mathrm{C}_{14} \mathrm{H}_{28} \mathrm{CuN}_{2} \mathrm{~S}_{4}$} & {$[14354-08-6]$} & \multicolumn{5}{|c|}{ bis(dipropyldithiocarbamate)copper } \\
\hline & SUB & & $118.4 \pm 3.3$ & & & [1978TAV/NEE] \\
\hline & $\mathrm{V}$ & $(422-453)$ & 118.4 & 437 & & [1999DYK/SVO] \\
\hline $\mathrm{C}_{14} \mathrm{H}_{28} \mathrm{CuN}_{2} \mathrm{~S}_{4}$ & {$[14354-07-5]$} & bis(dipropyldit & arbamate)cop! & & & \\
\hline & SUB & $(440-465)$ & $129.5 \pm 2.9$ & 452.5 & A & [1987STE/MAL, 1978TAV/NEE] \\
\hline $\mathrm{C}_{16} \mathrm{H}_{8} \mathrm{CuF}_{6} \mathrm{O}_{4} \mathrm{~S}_{2}$ & {$[13928-09-1]$} & bis(thenoyltrifl & acetonate)cop & & & \\
\hline & SUB & & $167.9 \pm 7.4$ & 298 & $\mathrm{C}$ & [2006RIB/SAN2] \\
\hline $\mathrm{C}_{16} \mathrm{H}_{8} \mathrm{CuF}_{6} \mathrm{O}_{6}$ & {$[13928-10-4]$} & bis(4,4,4-trifluc & -(2-furanyl)bu & 1,3-dior & pper(II) & \\
\hline & SUB & & $161.1 \pm 2.1$ & 298 & ME & [1998RIB/GON] \\
\hline
\end{tabular}


TABLE 17. Phase change enthalpies of organometallic and inorganic compounds-Continued

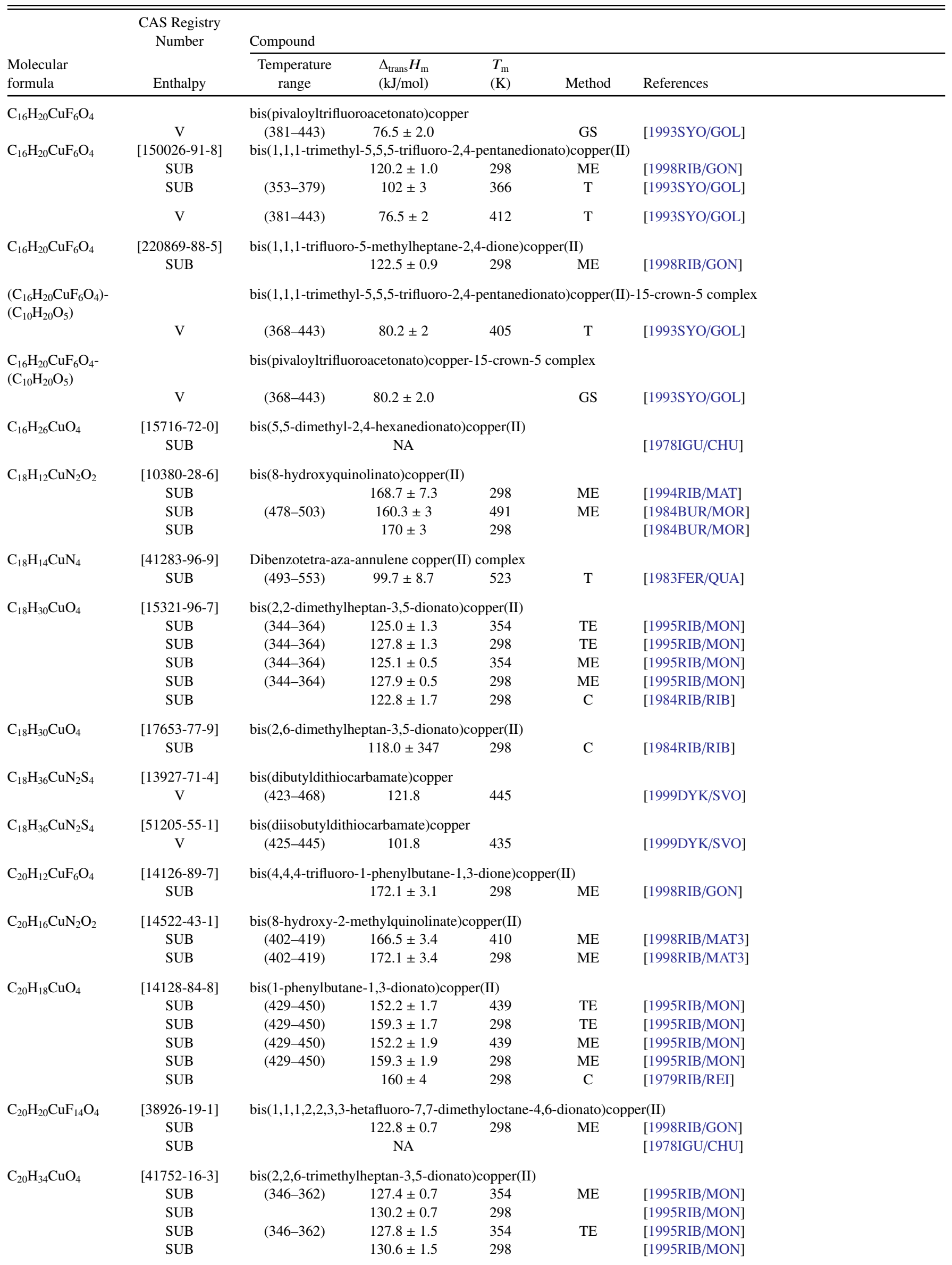


TABLE 17. Phase change enthalpies of organometallic and inorganic compounds-Continued

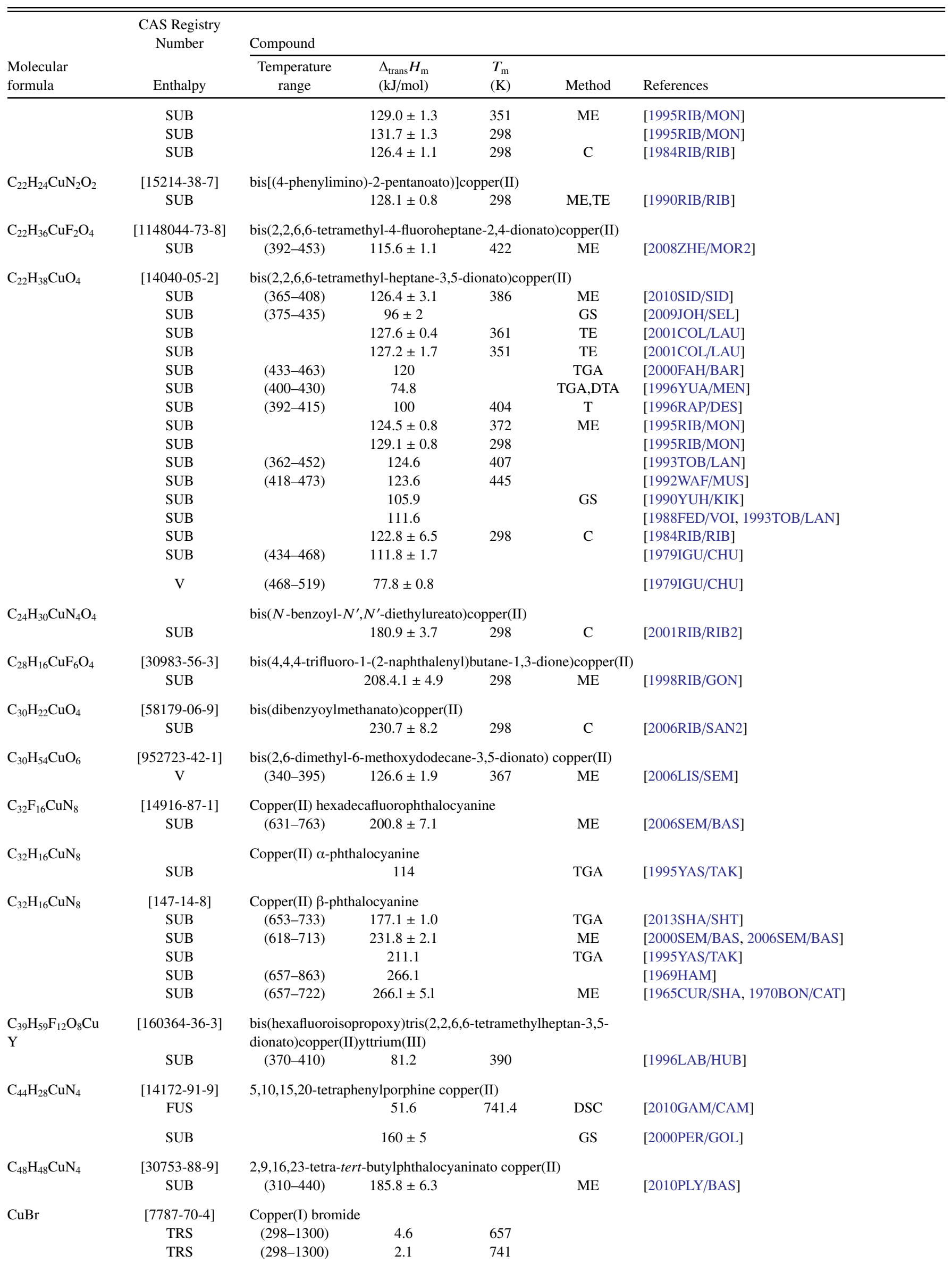


TABLE 17. Phase change enthalpies of organometallic and inorganic compounds-Continued

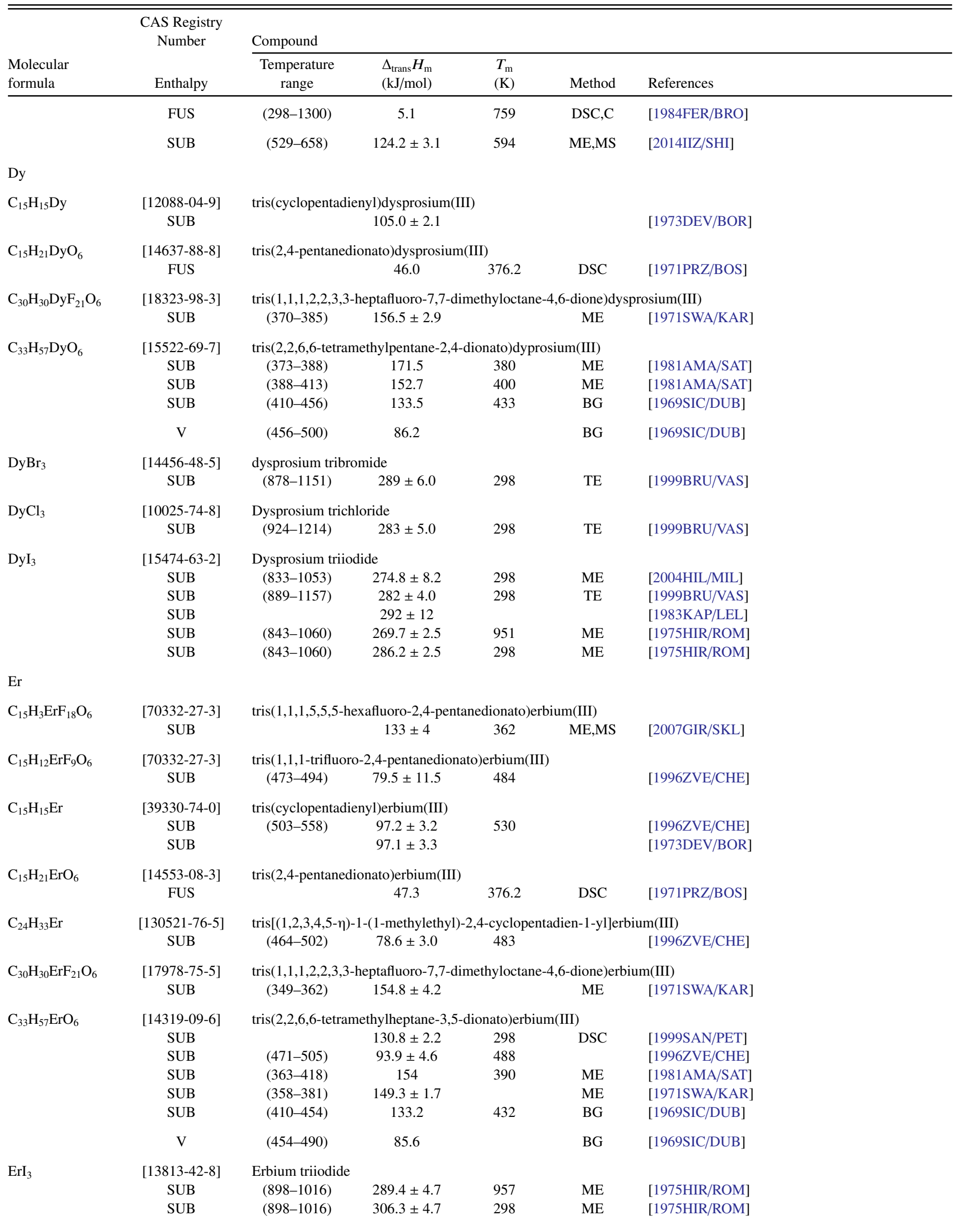

Eu 
TABLE 17. Phase change enthalpies of organometallic and inorganic compounds-Continued

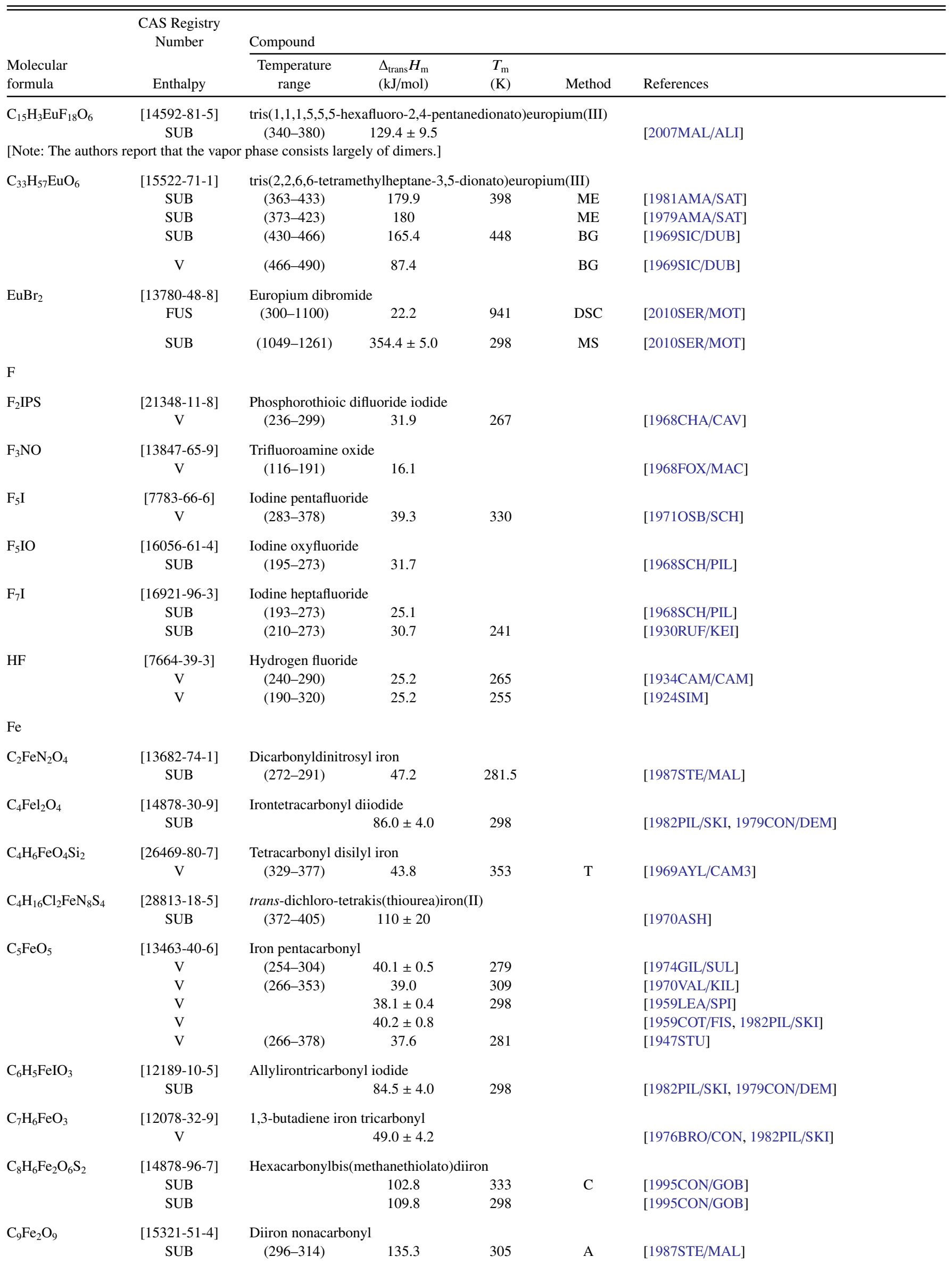


TABLE 17. Phase change enthalpies of organometallic and inorganic compounds-Continued

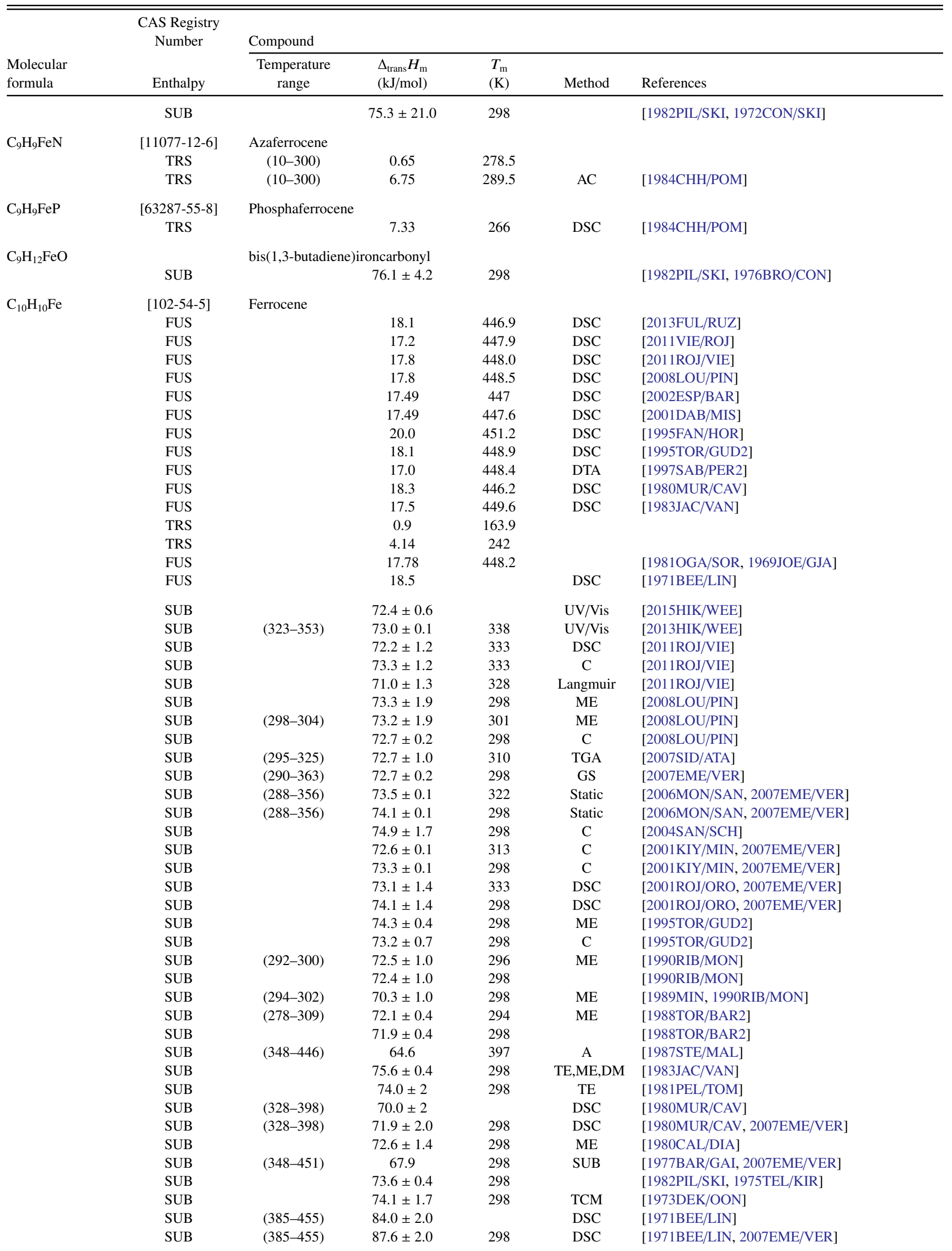


TABLE 17. Phase change enthalpies of organometallic and inorganic compounds-Continued

\begin{tabular}{|c|c|c|c|c|c|c|}
\hline \multirow[b]{2}{*}{$\begin{array}{l}\text { Molecular } \\
\text { formula }\end{array}$} & \multirow{2}{*}{$\begin{array}{l}\text { CAS Registry } \\
\text { Number } \\
\text { Enthalpy }\end{array}$} & \multicolumn{5}{|l|}{ Compound } \\
\hline & & $\begin{array}{l}\text { Temperature } \\
\text { range }\end{array}$ & $\begin{array}{l}\Delta_{\text {trans }} H_{\mathrm{m}} \\
(\mathrm{kJ} / \mathrm{mol})\end{array}$ & $\begin{array}{l}T_{\mathrm{m}} \\
(\mathrm{K})\end{array}$ & Method & References \\
\hline & SUB & & $72.7 \pm 2$ & 298 & ME & [1969AND/WES] \\
\hline & SUB & & $76.6 \pm 1$ & 298 & $\mathrm{ME}$ & [1962EDW/KIN] \\
\hline & SUB & $(295-303)$ & $76.8 \pm 0.9$ & 298 & ME & [1960EDW/KIN, 2007EME/VER] \\
\hline & SUB & $(323-367)$ & 83.3 & 345 & ME & {$[1959 \mathrm{COR} / \mathrm{SCH}]$} \\
\hline & SUB & $(323-367)$ & 84.7 & 298 & ME & [1959COR/SCH, 2007EME/VER] \\
\hline & SUB & $(357-455)$ & 70.5 & 406 & & {$[1952 \mathrm{KAP} / \mathrm{KES}]$} \\
\hline & SUB & $(357-455)$ & 73.7 & 298 & BG & [1952KAP/KES, 2007EME/VER] \\
\hline & $\mathrm{V}$ & $(456-523)$ & 47.3 & 471 & $\mathrm{~A}$ & [1987STE/MAL, 1999DYK/SVO] \\
\hline & $\mathrm{V}$ & $(451-523)$ & 49.8 & 466 & A & [1987STE/MAL, 1977BAR/GAI] \\
\hline & $\mathrm{V}$ & $(519-604)$ & 44.7 & 561 & $\mathrm{~EB}$ & {$[1972 \mathrm{NIS} / \mathrm{SOK}]$} \\
\hline & $\mathrm{V}$ & $(519-604)$ & $64.7 \pm 0.4$ & 298 & EB & [1972NIS/SOK, 2007EME/VER] \\
\hline & $\mathrm{V}$ & & 47.3 & 456 & & {$[1952 \mathrm{KAP} / \mathrm{KES}]$} \\
\hline \multirow[t]{3}{*}{$\mathrm{C}_{10} \mathrm{H}_{10} \mathrm{Fe}_{2} \mathrm{O}_{6} \mathrm{~S}_{2}$} & {$[28829-01-8]$} & \multicolumn{5}{|c|}{ Hexacarbonylbis(ethanethiolato)diiron } \\
\hline & SUB & & 105.4 & 340 & $\mathrm{C}$ & [1995CON/GOB] \\
\hline & SUB & & 112 & 298 & $\mathrm{C}$ & [1995CON/GOB] \\
\hline \multirow[t]{3}{*}{$\mathrm{C}_{10} \mathrm{H}_{14} \mathrm{FeO}_{4}$} & [14024-17-0] & \multicolumn{5}{|c|}{ bis(2,4-pentanedionato)iron(II) } \\
\hline & SUB & $(330-368)$ & $131.2 \pm 8.7$ & 298 & ME & [1990MAL/ALI] \\
\hline & SUB & & 117.6 & 385 & & {$[1970 \mathrm{GOE} / \mathrm{BLO}]$} \\
\hline \multirow[t]{2}{*}{$\mathrm{C}_{11} \mathrm{H}_{8} \mathrm{FeO}_{3}$} & {$[12093-05-9]$} & \multicolumn{5}{|c|}{ Cyclooctatetraeneirontricarbonyl } \\
\hline & SUB & & $87.0 \pm 4.0$ & 298 & & [1982PIL/SKI, 1979CON/DEM] \\
\hline \multirow[t]{12}{*}{$\mathrm{C}_{11} \mathrm{H}_{10} \mathrm{FeO}$} & {$[12093-10-6]$} & \multicolumn{5}{|c|}{ Ferrocenecarboxaldehdye } \\
\hline & TRS & & 11.6 & 317.4 & & \\
\hline & FUS & & 2.5 & 397.2 & DSC & [2008LOU/PIN] \\
\hline & TRS & & 0.89 & 293.5 & & \\
\hline & TRS & & 13.3 & 317 & & \\
\hline & FUS & $(13-405)$ & 2.76 & 397.6 & $\mathrm{AC}$ & [2007KAN/SOR] \\
\hline & TRS & & 11.7 & 316.4 & & \\
\hline & FUS & & 2.05 & 396.7 & & [1978DAN/LEA] \\
\hline & SUB & & $89.7 \pm 5.1$ & 298 & $\mathrm{ME}$ & [2008LOU/PIN] \\
\hline & SUB & $(302-312)$ & $89.9 \pm 5.1$ & 305 & $\mathrm{ME}$ & [2008LOU/PIN] \\
\hline & SUB & & $87.9 \pm 3.5$ & 298 & ME & [2008LOU/PIN] \\
\hline & SUB & $(302-312)$ & $87.3 \pm 3.5$ & 310 & $\mathrm{ME}$ & [2008LOU/PIN] \\
\hline \multirow[t]{6}{*}{$\mathrm{C}_{11} \mathrm{H}_{12} \mathrm{FeO}$} & {$[1273-86-5]$} & \multicolumn{5}{|c|}{ Ferrocenemethanol } \\
\hline & FUS & & 22.91 & 347.8 & $\mathrm{AC}$ & [2007KRO/DRU] \\
\hline & FUS & & 23.7 & 352.2 & DSC & [2007EME/VER] \\
\hline & FUS & & 23.82 & 351.4 & DSC & [2001DAB/MIS] \\
\hline & SUB & $(313-320)$ & $102.8 \pm 0.5$ & 298 & GS & [2007EME/VER] \\
\hline & $\mathrm{V}$ & $(353-393)$ & $87.0 \pm 0.8$ & 298 & GS & [2007EME/VER] \\
\hline \multirow[t]{2}{*}{$\mathrm{C}_{12} \mathrm{H}_{12} \mathrm{FeO}$} & {$[1271-55-2]$} & Acetylferrocen & & & & \\
\hline & SUB & (329-358) & $115.6 \pm 2.5$ & 298 & & [1981PEL/TOM] \\
\hline \multirow[t]{3}{*}{$\mathrm{C}_{12} \mathrm{H}_{14} \mathrm{Fe}$} & {$[1273-89-8]$} & Ethyl ferrocen & & & & \\
\hline & FUS & & 12.29 & 273.9 & & [2003KOZ/KAR, 2003KAR/KOZ] \\
\hline & $\mathrm{V}$ & $(297-320)$ & $65.1 \pm 2.7$ & 308 & $\mathrm{ME}$ & [2003KAR/KOZ] \\
\hline \multirow[t]{3}{*}{$\mathrm{C}_{12} \mathrm{H}_{14} \mathrm{Fe}$} & [1291-47-0] & $1,1^{\prime}$-dimethylfe & ene & & & \\
\hline & FUS & & 17.66 & 312.6 & DSC & [2008LOU/PIN] \\
\hline & SUB & & $84.5 \pm 1.9$ & 298 & $\mathrm{ME}$ & [2008LOU/PIN] \\
\hline \multirow[t]{4}{*}{$\mathrm{C}_{12} \mathrm{H}_{14} \mathrm{FeO}$} & [1277-49-2] & 1-ferrocenyl et & & & & \\
\hline & FUS & & 14.75 & 343.7 & DSC & [2008LOU/PIN] \\
\hline & FUS & & 26.65 & 366.5 & DSC & [2001DAB/MIS] \\
\hline & SUB & & $102.4 \pm 0.9$ & 298 & $\mathrm{C}$ & [2008LOU/PIN] \\
\hline $\mathrm{C}_{12} \mathrm{Fe}_{3} \mathrm{O}_{12}$ & $\begin{array}{l}{[17685-52-8]} \\
\text { SUB }\end{array}$ & \multicolumn{5}{|c|}{$\begin{array}{l}\text { Triiron dodecacarbonyl } \\
\qquad 96.0 \pm 21.0\end{array}$} \\
\hline
\end{tabular}


TABLE 17. Phase change enthalpies of organometallic and inorganic compounds-Continued

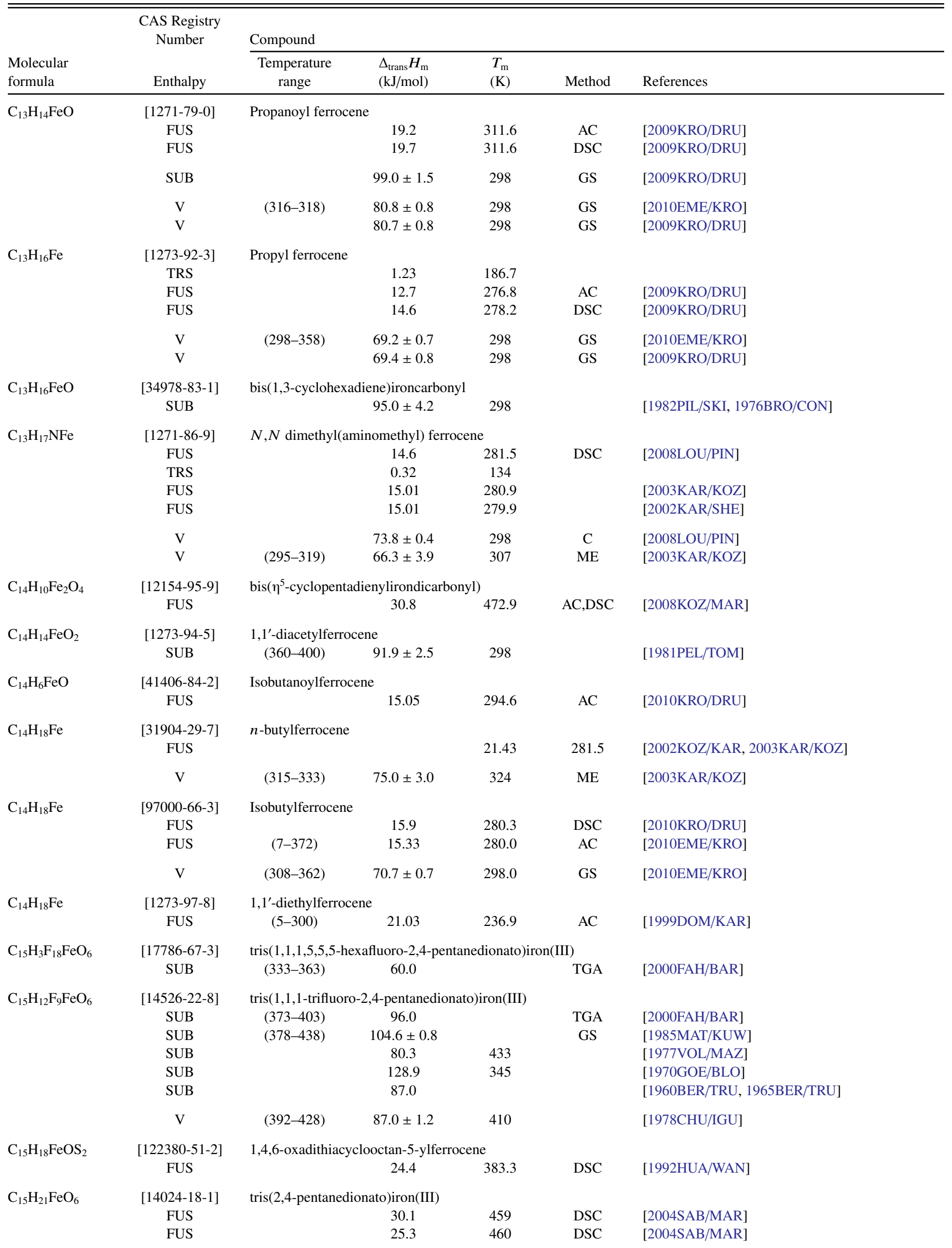


TABLE 17. Phase change enthalpies of organometallic and inorganic compounds-Continued

\begin{tabular}{|c|c|c|c|c|c|c|}
\hline \multirow[b]{2}{*}{$\begin{array}{l}\text { Molecular } \\
\text { formula }\end{array}$} & \multirow{2}{*}{$\begin{array}{l}\text { CAS Registry } \\
\text { Number } \\
\text { Enthalpy }\end{array}$} & \multicolumn{5}{|l|}{ Compound } \\
\hline & & $\begin{array}{l}\text { Temperature } \\
\text { range }\end{array}$ & $\begin{array}{l}\Delta_{\text {trans }} H_{\mathrm{m}} \\
(\mathrm{kJ} / \mathrm{mol})\end{array}$ & $\begin{array}{l}T_{\mathrm{m}} \\
(\mathrm{K})\end{array}$ & Method & References \\
\hline & FUS & & 22.6 & 462 & & [1984MUR/HIL] \\
\hline & FUS & & 25.9 & 454 & & [1970MEL/MER2] \\
\hline & SUB & $(413-443)$ & 112 & & TGA & [2000FAH/BAR] \\
\hline & SUB & & 118 & & TGA & [1997GIL/BOT] \\
\hline & SUB & $(369-388)$ & $124.6 \pm 0.9$ & 378 & TE,ME & [1996RIB/MON] \\
\hline & SUB & & $128.6 \pm 0.9$ & 298 & & [1996RIB/MON] \\
\hline & SUB & $(338-355)$ & $114.2 \pm 1.5$ & & & [1992GER/GER] \\
\hline & SUB & $(309-360)$ & $126.4 \pm 3.1$ & 298 & ME & [1990MAL/ALI] \\
\hline & SUB & & $138.4 \pm 5.2$ & 298 & $\mathrm{C}$ & [1985MUR/SAK] \\
\hline & SUB & & 100 & 395 & $\mathrm{~T}$ & [1981MAS/BAR] \\
\hline & SUB & & $113.6 \pm 3.8$ & & & [1980SAC/HIL] \\
\hline & SUB & & $99 \pm 0.8$ & & & $\begin{array}{l}\text { [1979RIB/REI, 1981MAS/BAR, } \\
\text { 1970MEL/MER2] }\end{array}$ \\
\hline & SUB & & 114.2 & 385 & & {$[1970 \mathrm{GOE} / \mathrm{BLO}]$} \\
\hline & SUB & & $65.3 \pm 3.3$ & 298 & & [1968HIL/IRV2] \\
\hline & SUB & & 97.9 & & I & [1982PIL/SKI, 1964FAR/JON] \\
\hline & SUB & & 81.6 & & & [1960BER/TRU, 1965BER/TRU] \\
\hline \multirow[t]{2}{*}{$\mathrm{C}_{15} \mathrm{H}_{3 \mathrm{O}} \mathrm{FeN}_{3} \mathrm{~S}_{6}$} & {$[34768-31-5]$} & tris(diethyldith & rbamoto)iron & & & \\
\hline & SUB & & $65.7 \pm 1.7$ & 246 & & [1970DAS/WEN] \\
\hline \multirow[t]{5}{*}{$\mathrm{C}_{17} \mathrm{H}_{14} \mathrm{FeO}$} & {$[1272-44-2]$} & Benzoylferroc & & & & \\
\hline & FUS & $(6-372)$ & 29.9 & 380.7 & $\mathrm{AC}$ & [2008KRO/DRU] \\
\hline & SUB & $(342-379)$ & $119.9 \pm 0.7$ & 298 & GS & [2007EME/VER] \\
\hline & SUB & $(358-382)$ & $116.3 \pm 6$ & 298 & TE,ME & [1983PEL/GIG] \\
\hline & $\mathrm{V}$ & $(384-429)$ & $98.2 \pm 0.3$ & 298 & GS & [2007EME/VER] \\
\hline \multirow[t]{4}{*}{$\mathrm{C}_{17} \mathrm{H}_{16} \mathrm{Fe}$} & [32994-54-0] & (phenylmethyl & ocene & & & \\
\hline & FUS & $(6-372)$ & 26.8 & 349.9 & $\mathrm{AC}$ & [2008KRO/DRU] \\
\hline & SUB & $(312-341)$ & $109.3 \pm 0.7$ & 298 & GS & [2007EME/VER] \\
\hline & $\mathrm{V}$ & $(351-377)$ & $90.6 \pm 0.6$ & 298 & GS & [2007EME/VER] \\
\hline \multirow[t]{2}{*}{$\mathrm{C}_{17} \mathrm{H}_{22} \mathrm{FeO}_{2} \mathrm{~S}_{2}$} & {$[122395-65-7]$} & 1,9-dioxa-4,6- & acyclundecan & errocene & & \\
\hline & FUS & & 29.4 & 371.7 & DSC & [1992HUA/WAN] \\
\hline \multirow[t]{2}{*}{$\mathrm{C}_{18} \mathrm{H}_{27} \mathrm{FeO}_{6}$} & {$[13978-46-6]$} & \multicolumn{5}{|c|}{ tris(3-methylpentane-2,4-dionato)iron(III) } \\
\hline & SUB & & 164.5 & 422 & & [1992RIB/FER] \\
\hline \multirow[t]{2}{*}{$\mathrm{C}_{19} \mathrm{H}_{26} \mathrm{FeO}_{2} \mathrm{~S}_{3}$} & {$[122395-70-4]$} & \multicolumn{5}{|c|}{ 1,9-dioxa-4,6,12-trithiacycltetracecan-5-ylferrocene } \\
\hline & FUS & & 40.0 & 367.1 & DSC & [1992HUA/WAN] \\
\hline \multirow[t]{2}{*}{$\mathrm{C}_{19} \mathrm{H}_{26} \mathrm{FeO}_{3} \mathrm{~S}_{2}$} & {$[122395-66-8]$} & \multicolumn{5}{|c|}{ 1,9,12-trioxa-4,6-dithiacyclotetradecan-5-ylferrocene } \\
\hline & FUS & & 32.1 & 349.7 & DSC & [1992HUA/WAN] \\
\hline \multirow[t]{7}{*}{$\mathrm{C}_{20} \mathrm{H}_{3 \mathrm{O}} \mathrm{Fe}$} & {$[12126-50-0]$} & \multicolumn{5}{|c|}{ bis $\left(\eta^{5}\right.$-pentamethylcyclopentadientyl)iron } \\
\hline & TRS & & 4.3 & 402.6 & & \\
\hline & TRS & & 4.87 & 503.7 & DSC & [2008LOU/PIN] \\
\hline & \multicolumn{6}{|c|}{ Decomposed upon melting } \\
\hline & SUB & & $99.0 \pm 2.4$ & 298 & ME & [2008LOU/PIN] \\
\hline & SUB & $(355-376)$ & $95.7 \pm 2.4$ & 365 & ME & {$[2008 \mathrm{LOU} / \mathrm{PIN}]$} \\
\hline & SUB & & $96.8 \pm 0.6$ & 298 & $\mathrm{C}$ & [2001KIY/MIN] \\
\hline \multirow[t]{2}{*}{$\mathrm{C}_{24} \mathrm{H}_{12} \mathrm{~F}_{9} \mathrm{FeO}_{6} \mathrm{~S}_{3}$} & {$[14319-78-9]$} & \multicolumn{5}{|c|}{ tris(1-(2-thenoyl)-4,4,4-trifluoro-1,3-butanedione)ironIII) } \\
\hline & SUB & & U46.4 & & & [1960BER/TRU, 1965BER/TRU] \\
\hline \multirow[t]{2}{*}{$\mathrm{C}_{24} \mathrm{H}_{18} \mathrm{FeO}_{2}$} & {$[12180-80-2]$} & $1,1^{\prime}$-dibenzoyl & cene & & & \\
\hline & SUB & $(358-381)$ & $109.3 \pm 6$ & 298 & TE,ME & [1983PEL/GIG] \\
\hline \multirow[t]{2}{*}{$\mathrm{C}_{3 \mathrm{O}} \mathrm{H}_{27} \mathrm{FeO}_{6}$} & {$[14323-17-2]$} & tris(benzoylace & to)iron(III) & & & \\
\hline & SUB & & $\mathrm{U} 45.6$ & & I & [1964FAR/JON] \\
\hline $\mathrm{C}_{32} \mathrm{H}_{16} \mathrm{FeN}_{8}$ & {$[132-16-1]$} & Iron(II) phthal & nine & & & \\
\hline & SUB & $(733-813)$ & $198.0 \pm 0.5$ & & TGA & [2013SHA/SHT] \\
\hline
\end{tabular}


TABLE 17. Phase change enthalpies of organometallic and inorganic compounds-Continued

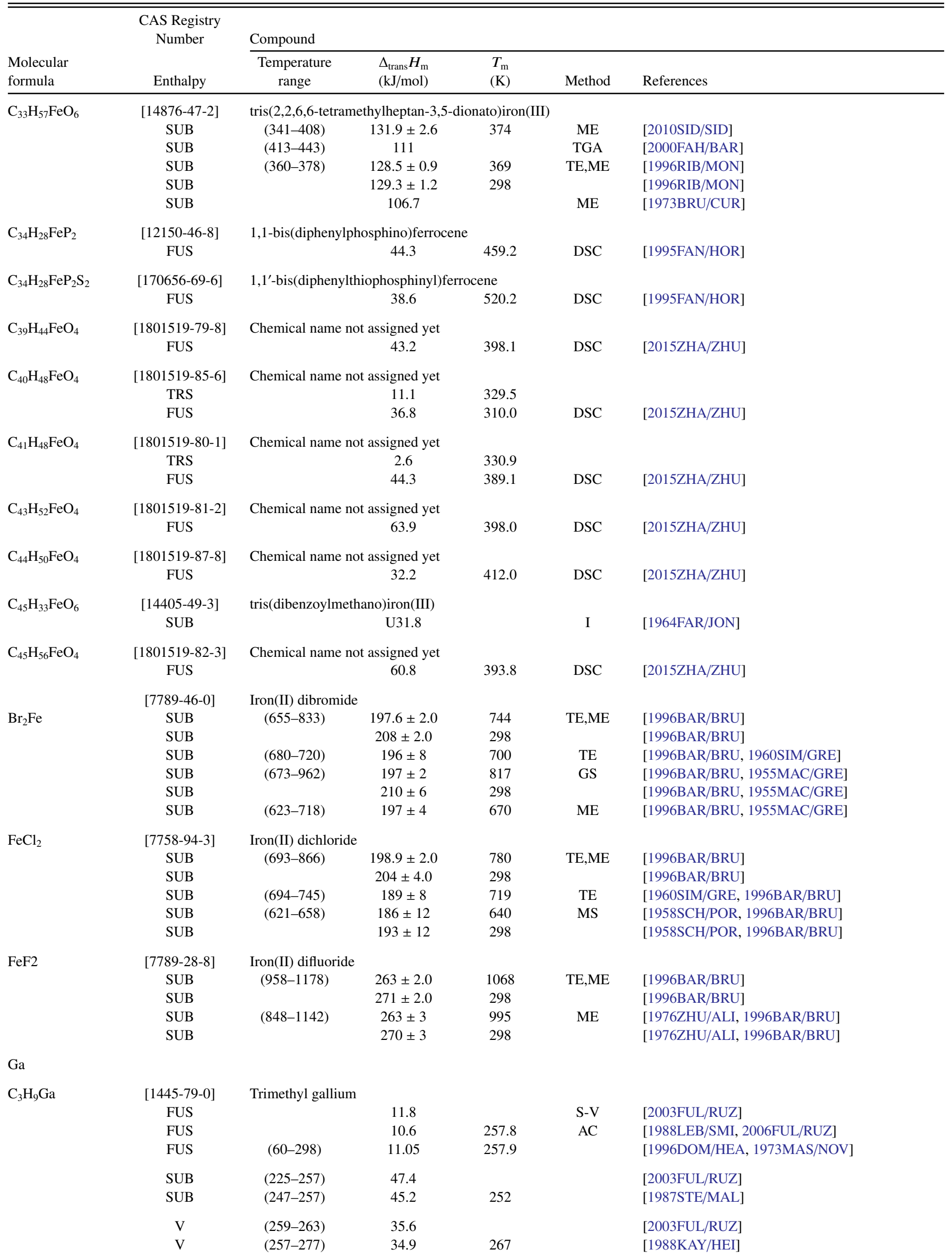


TABLE 17. Phase change enthalpies of organometallic and inorganic compounds-Continued

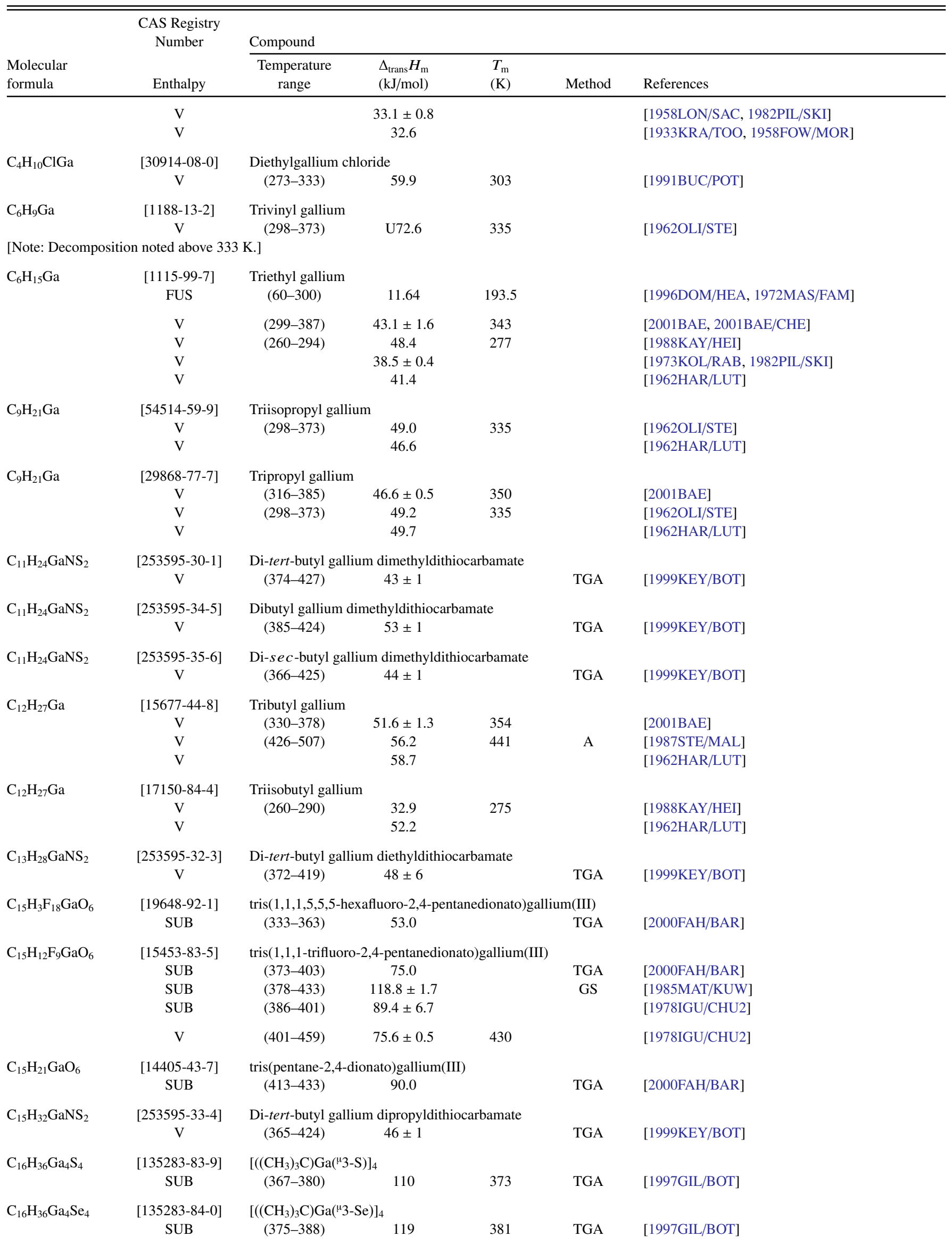


TABLE 17. Phase change enthalpies of organometallic and inorganic compounds-Continued

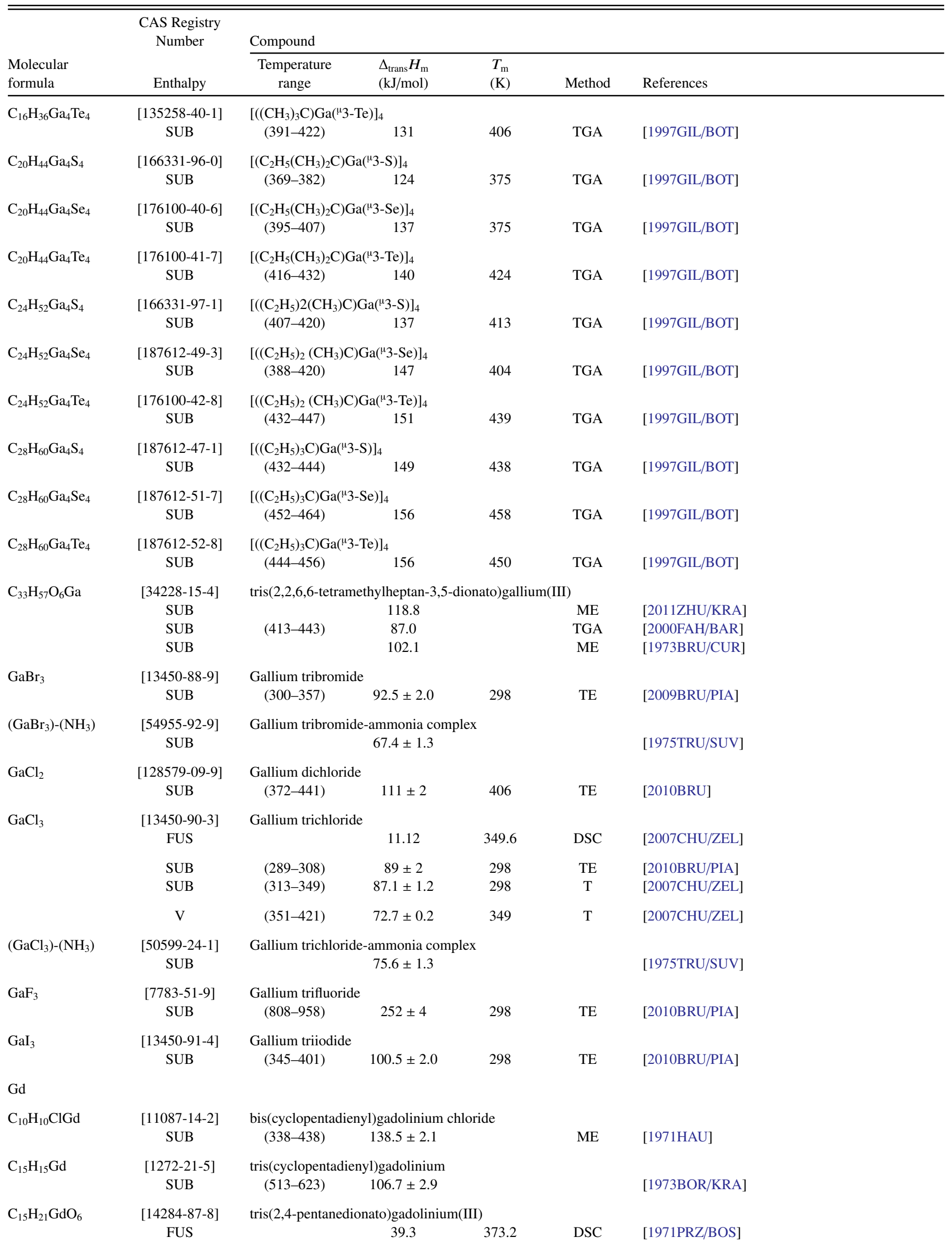


TABLE 17. Phase change enthalpies of organometallic and inorganic compounds-Continued

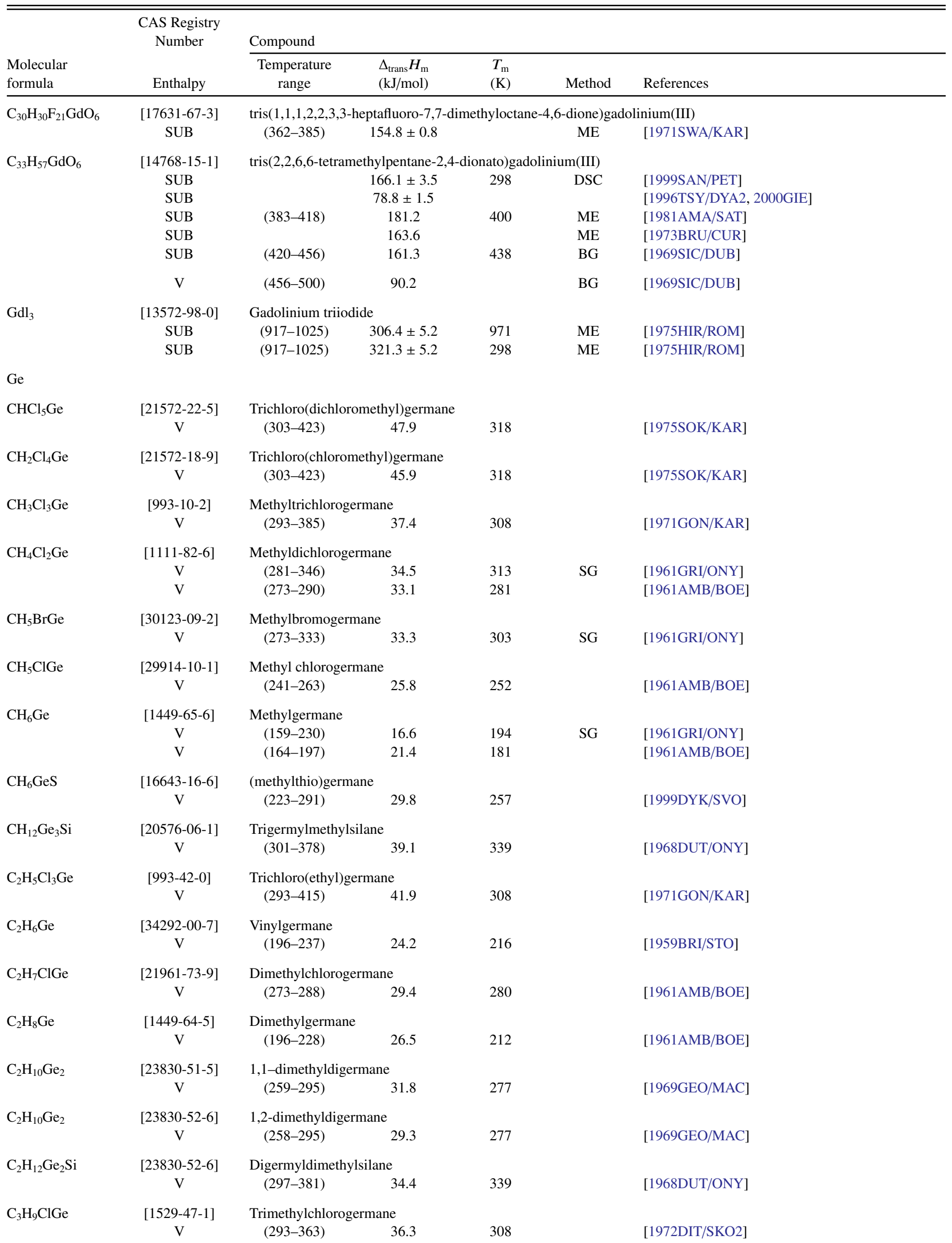


TABLE 17. Phase change enthalpies of organometallic and inorganic compounds-Continued

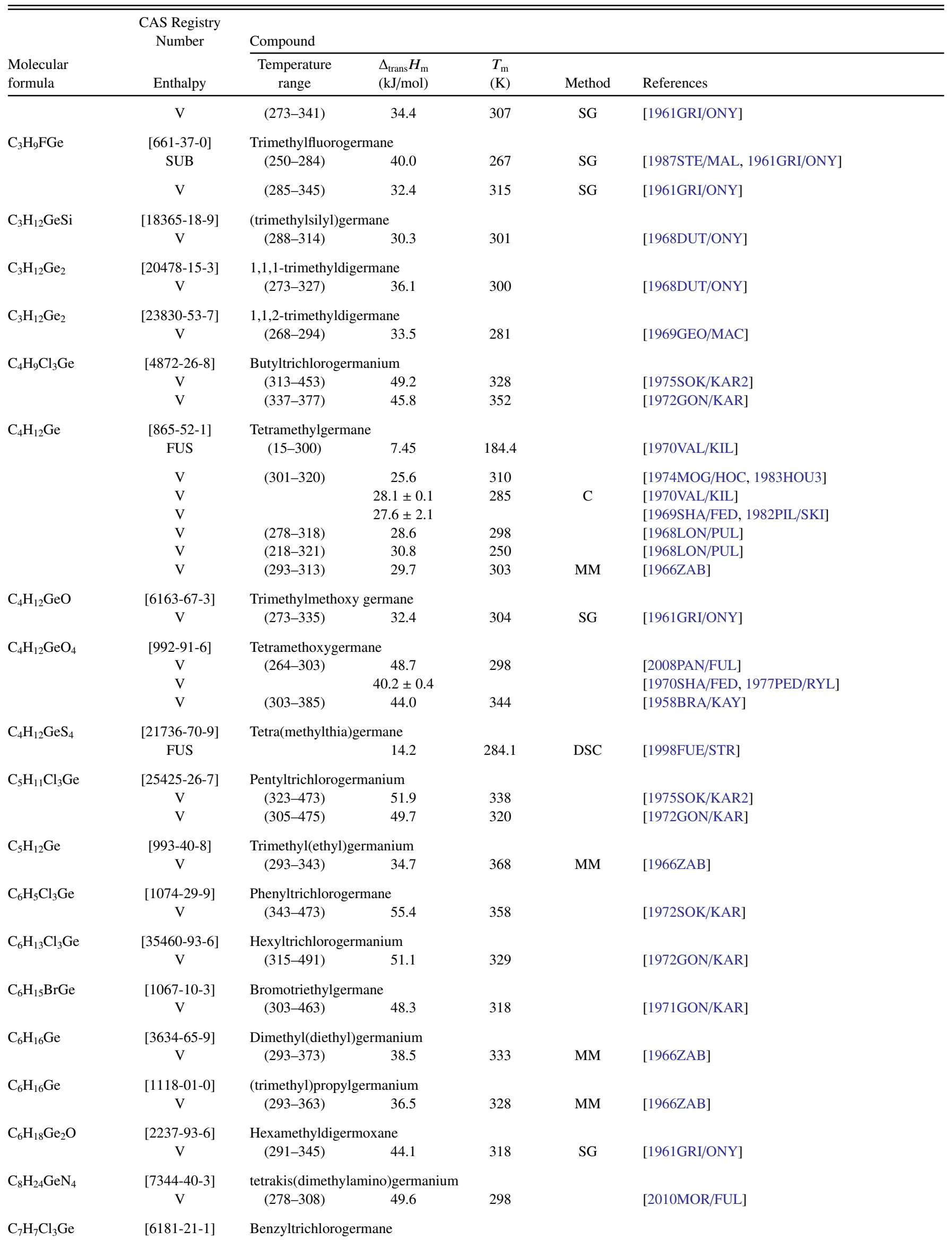


TABLE 17. Phase change enthalpies of organometallic and inorganic compounds-Continued

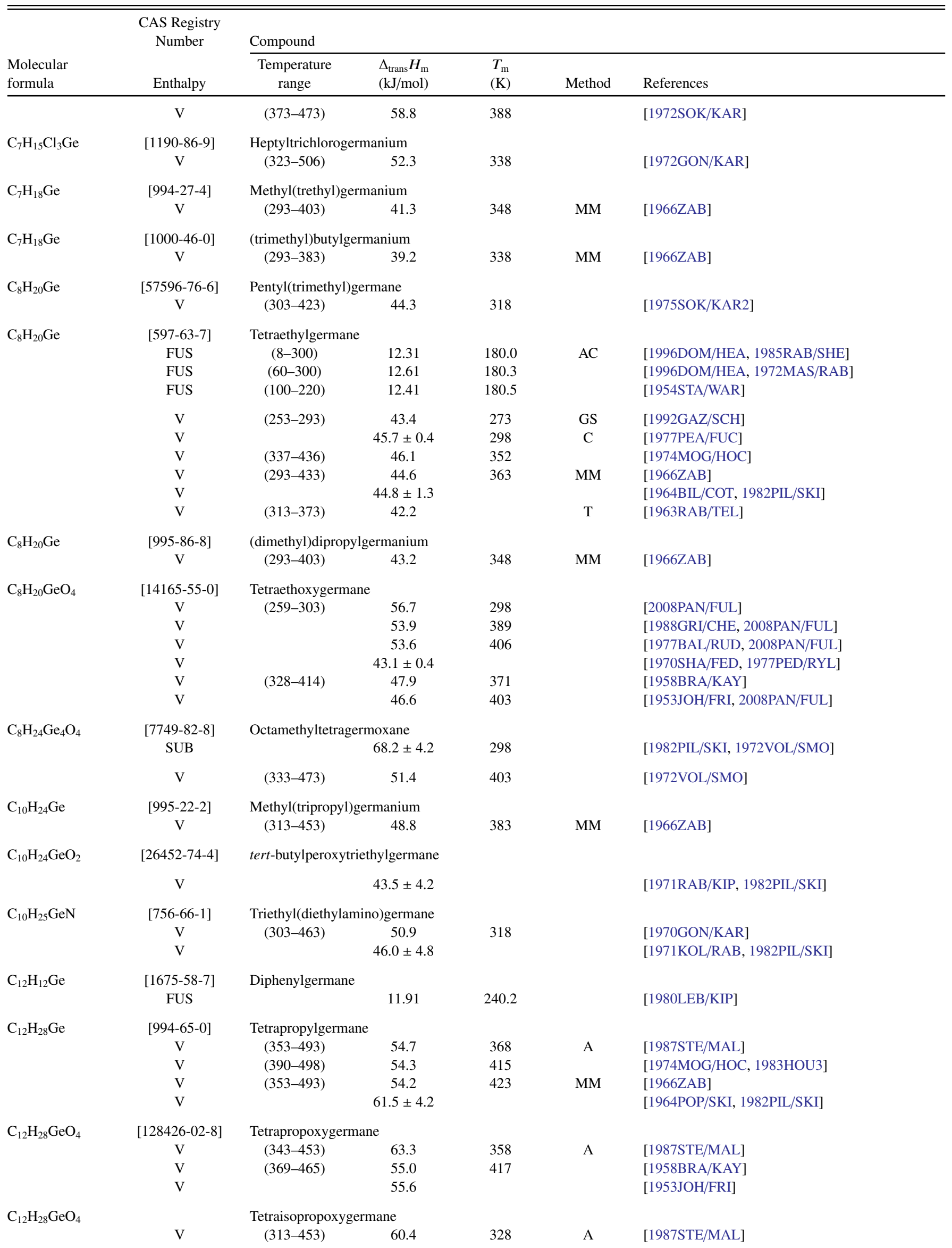


TABLE 17. Phase change enthalpies of organometallic and inorganic compounds-Continued

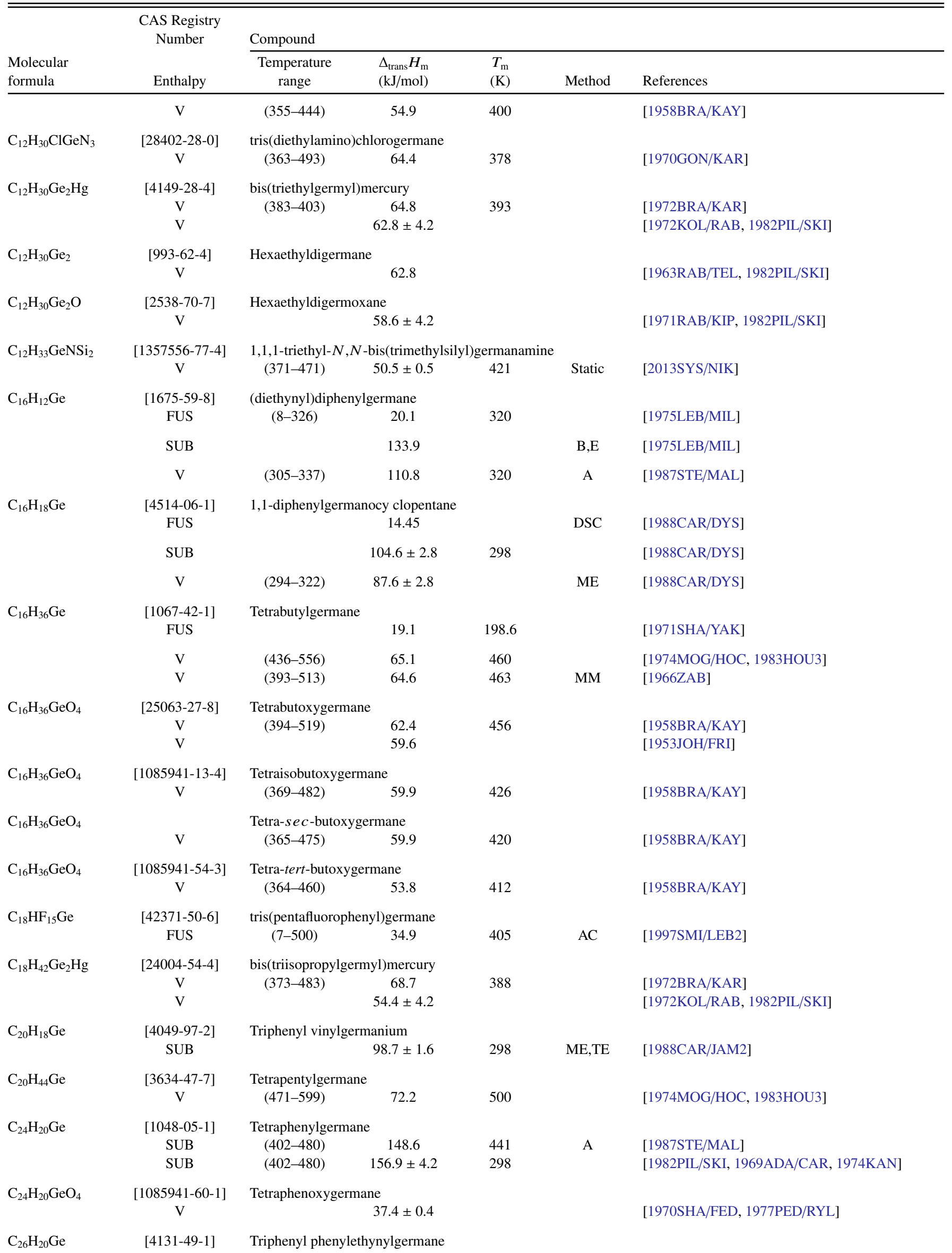


TABLE 17. Phase change enthalpies of organometallic and inorganic compounds-Continued

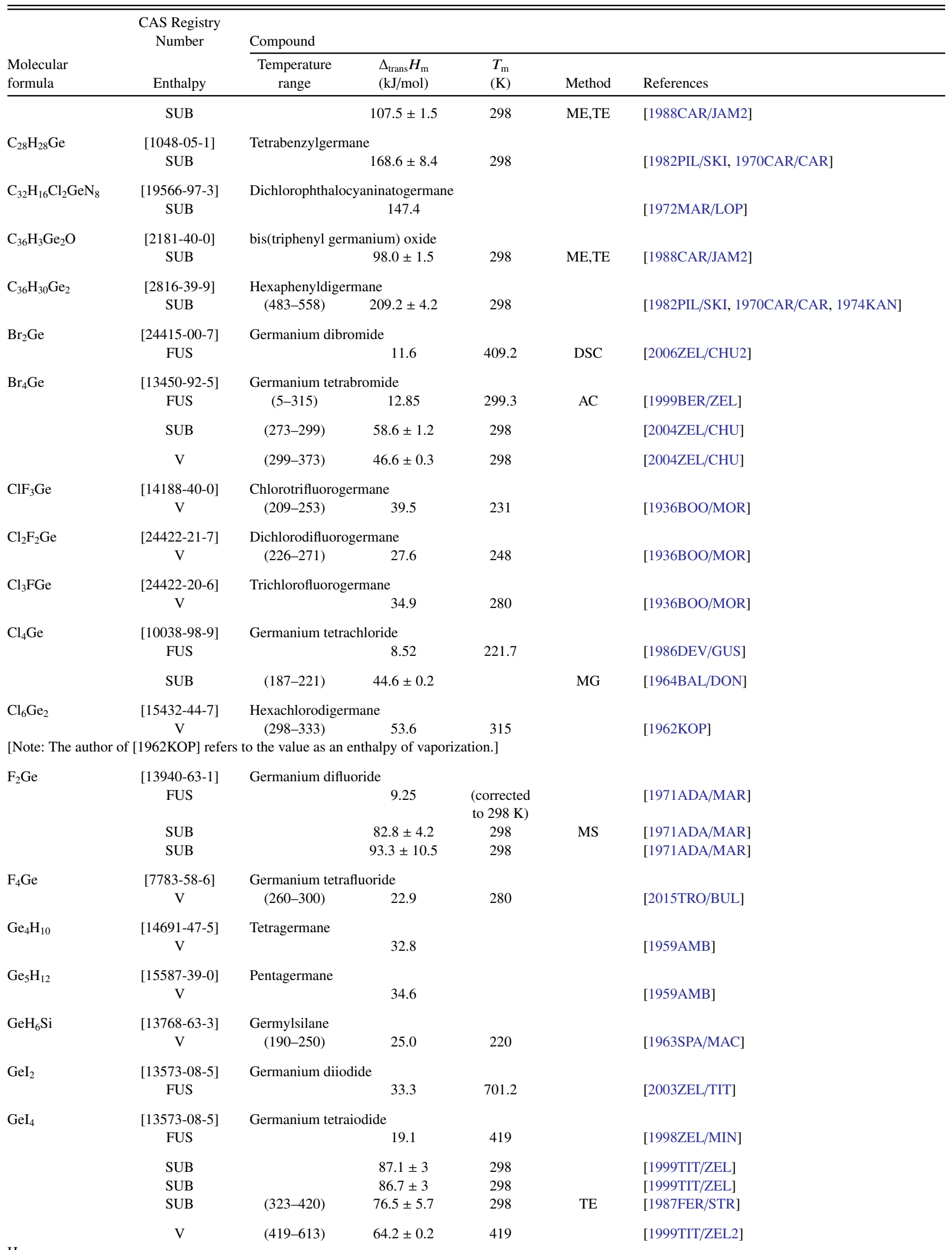

$\mathrm{Ha}$ 
TABLE 17. Phase change enthalpies of organometallic and inorganic compounds-Continued

\begin{tabular}{|c|c|c|c|c|c|c|}
\hline \multirow[b]{2}{*}{$\begin{array}{l}\text { Molecular } \\
\text { formula }\end{array}$} & \multirow{2}{*}{$\begin{array}{l}\text { CAS Registry } \\
\text { Number } \\
\text { Enthalpy }\end{array}$} & \multicolumn{5}{|l|}{ Compound } \\
\hline & & $\begin{array}{l}\text { Temperature } \\
\text { range }\end{array}$ & $\begin{array}{l}\Delta_{\text {trans }} H_{\mathrm{m}} \\
(\mathrm{kJ} / \mathrm{mol})\end{array}$ & $\begin{array}{l}T_{\mathrm{m}} \\
(\mathrm{K})\end{array}$ & Method & References \\
\hline $\mathrm{Cl}_{3} \mathrm{HaO}$ & $\begin{array}{c}\text { [143928-41-0] } \\
\text { SUB }\end{array}$ & $\begin{array}{l}\text { Hahnium(V) o } \\
\quad(298-607)\end{array}$ & $\begin{array}{l}\text { loride } \\
\qquad 152 \pm 18\end{array}$ & 298 & & [1996TUR/EIC] \\
\hline $\mathrm{Cl}_{5} \mathrm{Ha}$ & $\begin{array}{c}\text { [146837-09-4] } \\
\text { SUB }\end{array}$ & \multicolumn{4}{|c|}{ Hahnium(V) pentachloride } & [1996TUR/EIC] \\
\hline \multicolumn{7}{|l|}{ Hf } \\
\hline $\mathrm{C}_{10} \mathrm{H}_{10} \mathrm{Cl}_{2} \mathrm{Hf}$ & $\begin{array}{l}\text { [12116-66-4] } \\
\text { SUB } \\
\text { SUB } \\
\text { SUB } \\
\text { SUB } \\
\text { SUB }\end{array}$ & $\begin{array}{c}\text { bis(cyclopenta } \\
\text { (394-447) }\end{array}$ & $\begin{array}{c}110.2 \pm 2.9 \\
100.3 \\
106.7 \pm 2.1 \\
100.4 \pm 1.3 \\
107.3 \pm 2.4\end{array}$ & $\begin{array}{l}298 \\
420.5 \\
298 \\
298\end{array}$ & $\mathrm{ME}$ & $\begin{array}{l}\text { [2001DIO/PIE] } \\
\text { [1987STE/MAL] } \\
\text { [1982PIL/SKI, 1976KIR/TEL] } \\
\text { [1977BAL/BAR] } \\
\text { [1968KIS/DIL, 2001DIO/PIE] }\end{array}$ \\
\hline $\mathrm{C}_{12} \mathrm{H}_{16} \mathrm{Hf}$ & $\begin{array}{l}{[37260-88-1]} \\
\text { SUB }\end{array}$ & \multicolumn{5}{|c|}{ bis(cyclopentadienyl)dimethyl hafnium } \\
\hline \multirow[t]{2}{*}{$\mathrm{C}_{16} \mathrm{H}_{36} \mathrm{HfO}_{4}$} & $\begin{array}{c}\text { [2172-02-3] } \\
\text { FUS }\end{array}$ & tetrakis(tert-bu & $\begin{array}{c}\text { hafnium } \\
32.3\end{array}$ & \multicolumn{3}{|c|}{ tetrakis(tert-butoxy)hafnium } \\
\hline & $\mathrm{V}$ & $(280-308)$ & $64.8 \pm 0.4$ & 298 & & [2011FUL/RUZ2] \\
\hline $\mathrm{C}_{18} \mathrm{H}_{30} \mathrm{HfN}_{2}$ & $\begin{array}{c}\text { [159338-62-2] } \\
\text { SUB }\end{array}$ & \multicolumn{5}{|c|}{ bis(cyclopentadienyl)hafnium bis(diethylamide) } \\
\hline \multirow[t]{3}{*}{$\mathrm{C}_{20} \mathrm{H}_{16} \mathrm{~F}_{12} \mathrm{HfO}_{8}$} & $\begin{array}{c}\text { [17475-68-2] } \\
\text { FUS }\end{array}$ & tetrakis $(1,1,1-\mathrm{t}$ & \multicolumn{3}{|c|}{ tetrakis(1,1,1-trifluoro-2,4-pentanedionato)hafnium(IV) } & [2008ZHE/MOR] \\
\hline & $\begin{array}{l}\text { SUB } \\
\text { SUB } \\
\text { SUB } \\
\text { SUB }\end{array}$ & $\begin{array}{l}(358-393) \\
(358-398) \\
(383-438) \\
(383-438)\end{array}$ & $\begin{array}{l}133.0 \pm 1.8 \\
126.5 \pm 1.8 \\
129.7 \pm 3.8 \\
124.7 \pm 3.8\end{array}$ & 376 & $\begin{array}{l}\text { GS } \\
\text { GS }\end{array}$ & $\begin{array}{l}\text { [2008MOR/ZHE] } \\
{[2008 \mathrm{ZHE} / \mathrm{MOR}]} \\
{[1985 \mathrm{MAT} / \mathrm{KUW}]} \\
{[1985 \mathrm{MAT} / \mathrm{KUW}]}\end{array}$ \\
\hline & $\begin{array}{l}\mathrm{V} \\
\mathrm{V}\end{array}$ & $\begin{array}{l}(403-423) \\
(403-423)\end{array}$ & $\begin{array}{l}84.7 \pm 3.1 \\
83.2 \pm 2.0\end{array}$ & $\begin{array}{l}413 \\
413\end{array}$ & GS & $\begin{array}{l}\text { [2008MOR/ZHE] } \\
\text { [2008ZHE/MOR] }\end{array}$ \\
\hline $\mathrm{C}_{20} \mathrm{H}_{28} \mathrm{HfO}_{8}$ & $\begin{array}{c}{[17475-67-1]} \\
\text { SUB } \\
\text { SUB } \\
\text { SUB }\end{array}$ & $\begin{array}{l}\text { tetrakis(pentan } \\
\quad(408-433) \\
(408-443) \\
150.6 \pm 4.2\end{array}$ & $\begin{array}{l}138.7 \pm 7.4 \\
130.4 \pm 6.1\end{array}$ & $\begin{array}{l}\text { V) } \\
420 \\
425\end{array}$ & GS & $\begin{array}{l}\text { [2008MOR/ZHE] } \\
\text { [2008ZHE/MOR] } \\
{[1991 \mathrm{TEL} / \mathrm{LAR}]}\end{array}$ \\
\hline $\mathrm{C}_{32} \mathrm{H}_{40} \mathrm{~F}_{12} \mathrm{HfO}_{8}$ & $\begin{array}{c}\text { [916441-69-5] } \\
\text { SUB }\end{array}$ & \multicolumn{5}{|c|}{ tetrakis(1,1,1-trifluoro-5,5-dimethyl-2,4-hexanedionato)hafnium $N, N^{\prime}$} \\
\hline \multicolumn{7}{|c|}{$\begin{array}{l}\text { Note: The enthalpy of sublimation that was reported in the paper, } 97.5 \pm 1.7 \mathrm{~kJ} / \mathrm{mol} \text {, was not consistent with the reported Antoine constants. W } \\
\text { have recalculated the the enthalpy of sublimation assuming that the reported Antoine constants are correct. }\end{array}$} \\
\hline & $\begin{array}{l}\text { V } \\
\text { V }\end{array}$ & $\begin{array}{l}(426-493) \\
(424-472)\end{array}$ & $\begin{array}{l}92.1 \pm 1.3 \\
91.2 \pm 0.3\end{array}$ & 460 & GS & $\begin{array}{l}{[2008 \mathrm{MOR} / \mathrm{ZHE}]} \\
{[2008 \mathrm{ZHE} / \mathrm{MOR}]}\end{array}$ \\
\hline $\mathrm{C}_{44} \mathrm{H}_{76} \mathrm{HfO}_{8}$ & $\begin{array}{c}{[63370-90-1]} \\
\text { TRS }\end{array}$ & \multicolumn{5}{|c|}{ tetrakis(2,2,6,6-tetramethyl-3,5-heptanedionaot)hafnium(IV) } \\
\hline & FUS & & 5.4 & 630 & DSC & [2008ZHE/MOR] \\
\hline & $\begin{array}{l}\text { SUB } \\
\text { SUB }\end{array}$ & $\begin{array}{l}(453-553) \\
(368-428)\end{array}$ & $\begin{array}{l}103.5 \pm 0.6 \\
136.6 \pm 4.2\end{array}$ & $\begin{array}{l}503 \\
398\end{array}$ & $\begin{array}{l}\text { GS } \\
\mathrm{ME}\end{array}$ & $\begin{array}{l}\text { [2008MOR/ZHE] } \\
{[2008 \mathrm{MOR} / \mathrm{ZHE}]}\end{array}$ \\
\hline $\mathrm{HfCl}_{4}$ & $\begin{array}{c}\text { [13499-05-3] } \\
\text { SUB } \\
\text { SUB }\end{array}$ & $\begin{array}{l}\text { Hafnium tetrac } \\
\qquad(398-500) \\
(353-433)\end{array}$ & $\begin{array}{l}\text { ide } \\
\qquad 97.9 \pm 1.2 \\
107.9 \pm 0.8\end{array}$ & 499 & $\mathrm{~T}$ & $\begin{array}{l}\text { [1994TAN/BOS] } \\
{[1973 \mathrm{IZM} / \mathrm{KHO}]}\end{array}$ \\
\hline \multicolumn{7}{|l|}{$\mathrm{Hg}$} \\
\hline $\mathrm{CH}_{3} \mathrm{BrHg}$ & $\begin{array}{c}\text { [506-83-2] } \\
\text { SUB }\end{array}$ & \multicolumn{5}{|c|}{ Methylmercuric bromide } \\
\hline $\mathrm{CH}_{3} \mathrm{ClHg}$ & {$[115-09-3]$} & Methylmercuri & loride & & & \\
\hline
\end{tabular}


TABLE 17. Phase change enthalpies of organometallic and inorganic compounds-Continued

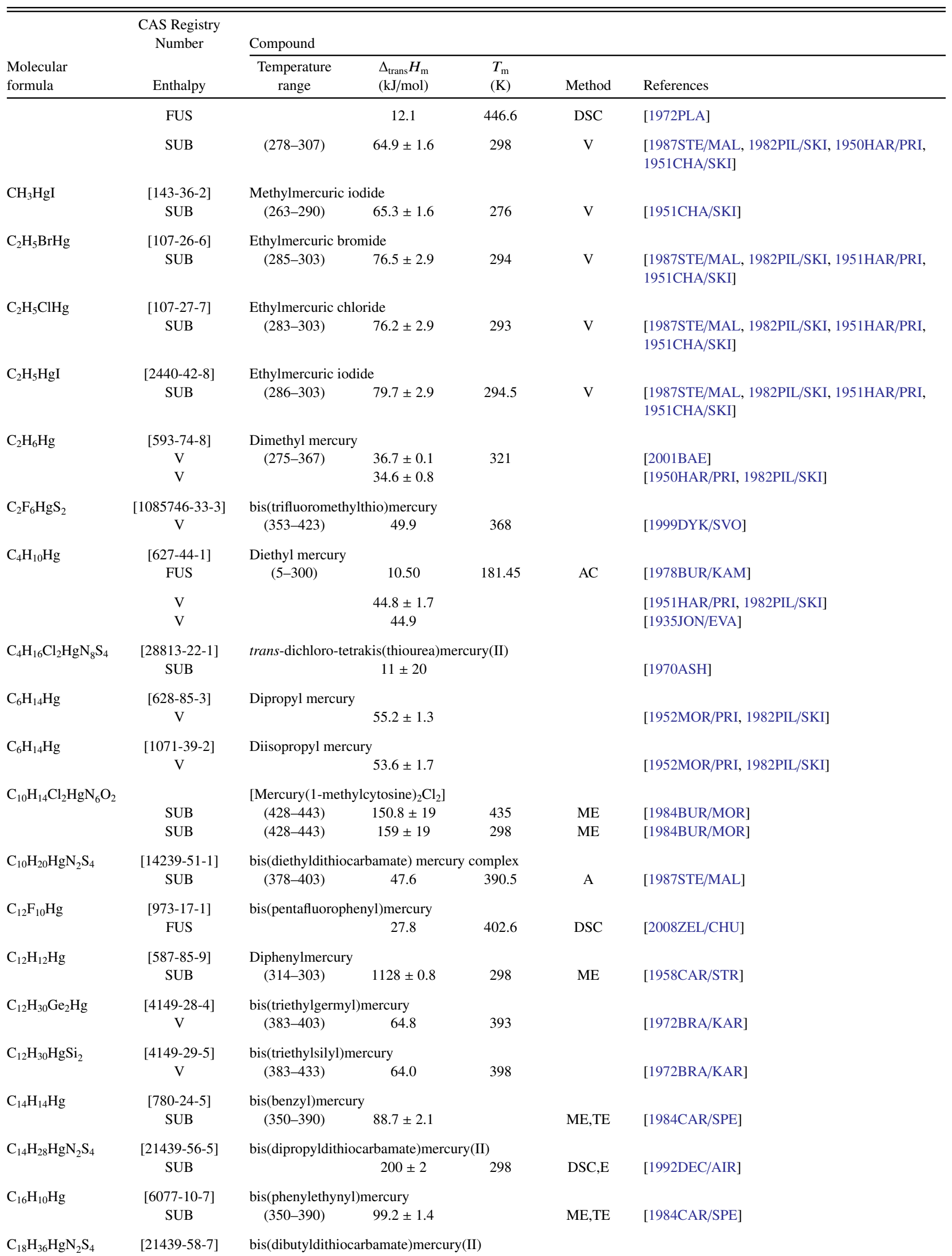


TABLE 17. Phase change enthalpies of organometallic and inorganic compounds-Continued

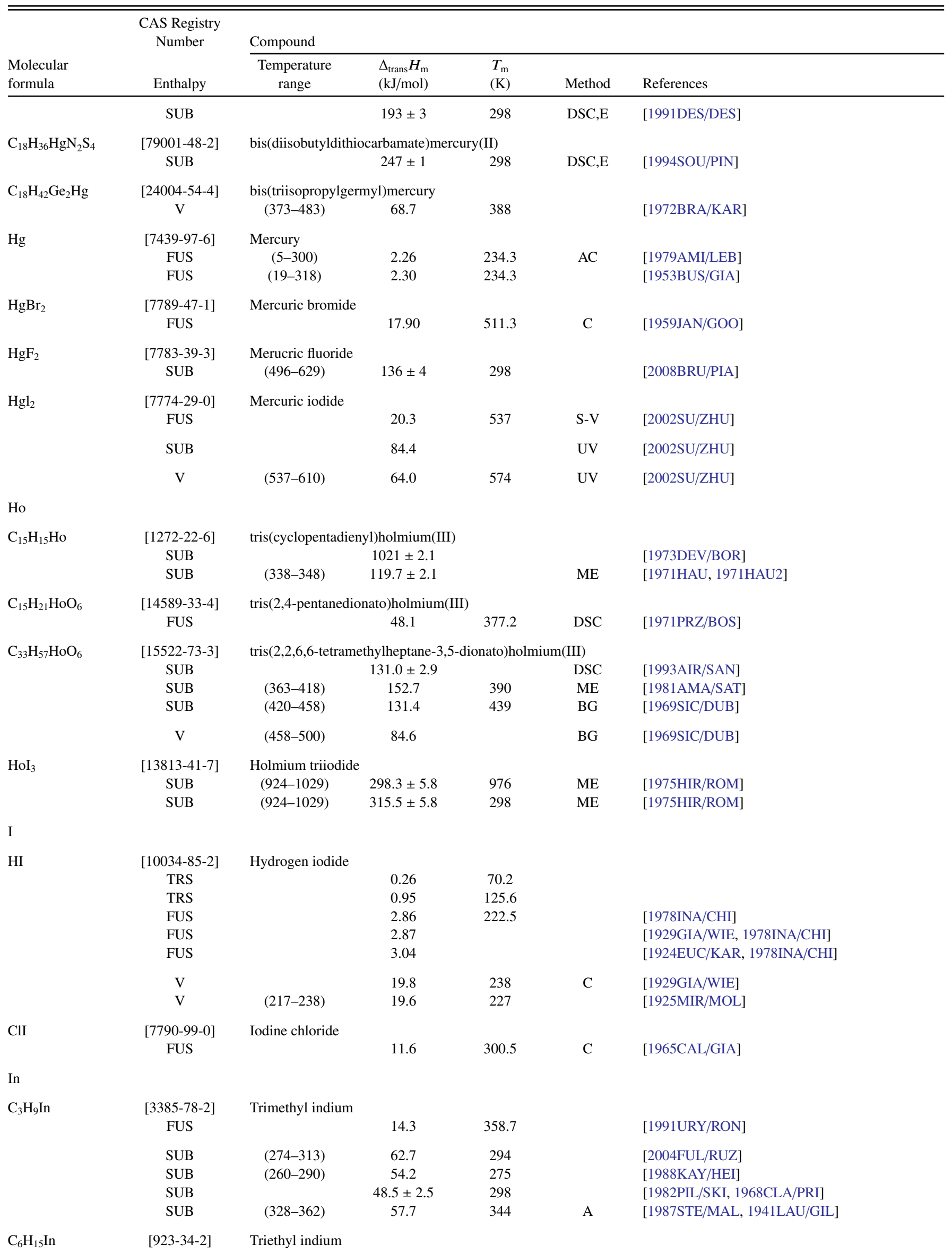


TABLE 17. Phase change enthalpies of organometallic and inorganic compounds-Continued

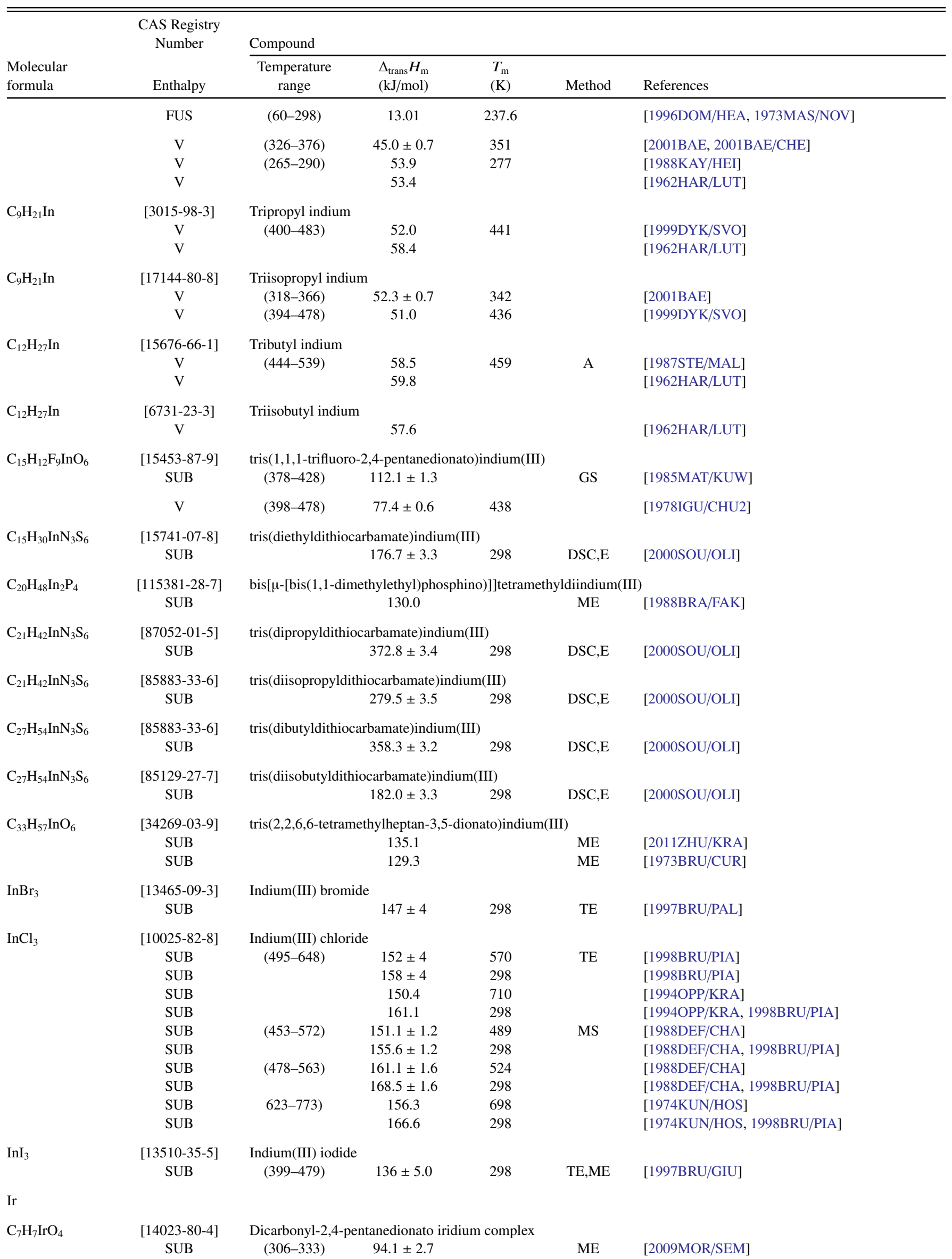


TABLE 17. Phase change enthalpies of organometallic and inorganic compounds-Continued

\begin{tabular}{|c|c|c|c|c|c|c|}
\hline \multirow[b]{2}{*}{$\begin{array}{l}\text { Molecular } \\
\text { formula }\end{array}$} & \multirow{2}{*}{$\begin{array}{l}\text { CAS Registry } \\
\text { Number } \\
\text { Enthalpy }\end{array}$} & \multicolumn{5}{|l|}{ Compound } \\
\hline & & $\begin{array}{l}\text { Temperature } \\
\text { range }\end{array}$ & $\begin{array}{l}\Delta_{\text {trans }} H_{\mathrm{m}} \\
(\mathrm{kJ} / \mathrm{mol})\end{array}$ & $\begin{array}{l}T_{\mathrm{m}} \\
(\mathrm{K})\end{array}$ & Method & References \\
\hline & SUB & $(286-325)$ & $92.0 \pm 1.3$ & 306 & ME & [1978JES/ERN, 1987STE/MAL] \\
\hline $\mathrm{C}_{7} \mathrm{H}_{13} \mathrm{Cl}_{2} \mathrm{IrO}_{2}$ & SUB & $\begin{array}{l}\text { bis(chloroethyl } \\
\quad(281-298)\end{array}$ & $\begin{array}{l}-2,4 \text {-pentaned } \\
89.5 \pm 4.2\end{array}$ & $\begin{array}{l}\text { iridiur } \\
290\end{array}$ & $\begin{array}{l}\text { plex } \\
\text { ME }\end{array}$ & [1978JES/ERN, 1987STE/MAL] \\
\hline $\mathrm{C}_{9} \mathrm{H}_{15} \mathrm{IrO}_{2}$ & $\begin{array}{l}{[52654-27-0]} \\
\text { SUB }\end{array}$ & \multicolumn{4}{|c|}{ bis(ethylene)-2,4-pentanedionato iridium complex } & [1978JES/ERN, 1987STE/MAL] \\
\hline $\mathrm{C}_{11} \mathrm{H}_{19} \mathrm{IrO}_{2}$ & $\begin{array}{l}{[66467-05-8]} \\
\text { SUB }\end{array}$ & $\begin{array}{l}\text { bis(propylene) } \\
\quad(269-304)\end{array}$ & $\begin{array}{l}\text { pentanedionat } \\
90 \pm 1.3\end{array}$ & $\begin{array}{l}\text { um cor } \\
287\end{array}$ & ME & {$[1978 J E S / E R N]$} \\
\hline $\mathrm{C}_{12} \mathrm{O}_{12} \mathrm{Ir}_{4}$ & $\begin{array}{l}{[11065-24-0]} \\
\text { SUB }\end{array}$ & \multirow{2}{*}{\multicolumn{5}{|c|}{$\begin{array}{l}\text { Tetrairidiumdodecacarbonyl } \\
\qquad 104.6 \pm 20 \\
\text { (pentamethylcyclopentadienyl)(dicarbonyl)iridium(I) }\end{array}$}} \\
\hline $\mathrm{C}_{12} \mathrm{H}_{15} \mathrm{IrO}_{2}$ & $\begin{array}{l}{[32660-96-1]} \\
\text { SUB }\end{array}$ & & & & & \\
\hline $\mathrm{C}_{13} \mathrm{H}_{19} \mathrm{IrO}_{2}$ & $\begin{array}{l}{[12154-84-6]} \\
\text { SUB }\end{array}$ & \multicolumn{5}{|c|}{ (acetylacetonato)(1,5-cyclooctadiene)iridium(I) } \\
\hline $\mathrm{C}_{13} \mathrm{H}_{19} \mathrm{IrO}_{6}$ & $\begin{array}{l}{[66467-07-0]} \\
\text { SUB }\end{array}$ & \multicolumn{5}{|c|}{ bis(vinyl acetate)-2,4-pentanedionato iridium complex } \\
\hline $\mathrm{C}_{13} \mathrm{H}_{19} \mathrm{IrO}_{6}$ & $\begin{array}{l}{[66467-08-1]} \\
\text { SUB }\end{array}$ & \multicolumn{4}{|c|}{ bis(methyl acrylate)-2,4-pentanedionato iridium complex } & {$[1978 J E S / E R N]$} \\
\hline $\mathrm{C}_{14} \mathrm{H}_{19} \mathrm{Ir}$ & $\begin{array}{c}{[132644-88-3]} \\
\text { SUB }\end{array}$ & \multicolumn{5}{|c|}{ (methylcyclopentadienyl)(1,5-cyclooctadiene)iridium(I) } \\
\hline & $\mathrm{V}$ & $(310-330)$ & $88.1 \pm 1.3$ & & ME & [2009MOR/SEM] \\
\hline $\mathrm{C}_{15} \mathrm{H}_{21} \mathrm{IrO}_{6}$ & $\begin{array}{c}{[15635-87-7]} \\
\text { SUB } \\
\text { SUB } \\
\text { SUB } \\
\text { SUB } \\
\text { SUB } \\
\text { SUB } \\
\text { SUB }\end{array}$ & $\begin{array}{l}\text { tris(2,4-pentan } \\
\qquad \begin{array}{l}(423-473) \\
(423-473) \\
(383-433) \\
(387-513) \\
(468-518)\end{array}\end{array}$ & $\begin{array}{c}\text { ato)iridium(I } \\
129.3 \pm 1.0 \\
128 \\
129.3 \pm 0.8 \\
130.5 \pm 3.4 \\
1016 \pm 1.8 \\
86.6 \pm 1.7 \\
\text { NA }\end{array}$ & & $\begin{array}{c}\text { GS } \\
\text { ME } \\
\text { MCV } \\
\text { SMZG }\end{array}$ & $\begin{array}{l}{[2010 \mathrm{SYS} / \mathrm{CHE}]} \\
{[2001 \mathrm{MOR} / \mathrm{ZHA}]} \\
{[2000 \mathrm{MOR} / \mathrm{SEM}]} \\
{[2000 \mathrm{MOR} / \mathrm{SEM}]} \\
{[2000 \mathrm{MOR} / \mathrm{SEM}]} \\
{[2000 \mathrm{MOR} / \mathrm{SEM}]} \\
{[1994 \mathrm{GER} / \mathrm{GER}]}\end{array}$ \\
\hline \multicolumn{7}{|l|}{$\mathrm{La}$} \\
\hline $\mathrm{C}_{15} \mathrm{H}_{15} \mathrm{La}$ & $\begin{array}{c}{[1272-23-7]} \\
\text { SUB } \\
\text { SUB }\end{array}$ & $\begin{array}{l}\text { tris(cyclopenta } \\
\qquad(548-663)\end{array}$ & $\begin{array}{l}\text { yl)lanthanum } \\
114.6 \pm 4.0 \\
102.1 \pm 2.9\end{array}$ & 298 & & $\begin{array}{l}\text { [1982PIL/SKI, 1974DEV/RAB] } \\
\text { [1973BOR/KRA] }\end{array}$ \\
\hline $\mathrm{C}_{30} \mathrm{H}_{30} \mathrm{~F}_{21} \mathrm{LaO}_{6}$ & $\begin{array}{l}{[19106-89-9]} \\
\text { SUB }\end{array}$ & \multicolumn{5}{|c|}{ tris(1,1,1,2,2,3,3-heptafluoro-7,7-dimethyloctane-4,6-dione)lanthanum(III) } \\
\hline $\mathrm{C}_{33} \mathrm{H}_{57} \mathrm{LaO}_{6}$ & $\begin{array}{c}{[14319-13-2]} \\
\text { SUB } \\
\text { SUB } \\
\text { SUB } \\
\text { SUB } \\
\text { SUB } \\
\text { SUB }\end{array}$ & $(388-423)$ & $\begin{array}{c}\text { thylheptan-3, } \\
156.0 \pm 4.6 \\
116.1 \pm 8.4 \\
107.9 \pm 4.6 \\
179.5 \\
164.4 \\
143.6\end{array}$ & 405 & $\begin{array}{l}\mathrm{ME} \\
\mathrm{ME} \\
\mathrm{BG}\end{array}$ & $\begin{array}{l}\text { [1997SAN/ROC, 2000GIE] } \\
\text { [1996TSY/DYA] } \\
\text { [1996TSY/DYA2, 2000GIE] } \\
\text { [1981AMA/SAT] } \\
\text { [1973BRU/CUR] } \\
\text { [1969SIC/DUB] }\end{array}$ \\
\hline $\mathrm{LaBr}_{3}$ & $\begin{array}{c}\text { [13536-79-3] } \\
\text { SUB } \\
\text { SUB }\end{array}$ & $\begin{array}{l}\text { Lanthanum tric } \\
\qquad \begin{array}{c}(955-1045) \\
(955-1045)\end{array}\end{array}$ & $\begin{array}{l}\text { ide } \\
\qquad \begin{array}{l}295 \pm 3 \\
308 \pm 5\end{array}\end{array}$ & $\begin{array}{c}1000 \\
298\end{array}$ & $\begin{array}{l}\mathrm{TE} \\
\mathrm{TE}\end{array}$ & $\begin{array}{l}{[2000 B R U / V I L]} \\
{[2000 B R U / V I L]}\end{array}$ \\
\hline $\mathrm{LaCl}_{3}$ & $\begin{array}{l}{[10099-58-8]} \\
\text { SUB } \\
\text { SUB } \\
\text { SUB }\end{array}$ & $\begin{array}{l}\text { Lanthanum tric } \\
(1006-1122) \\
(1137-1188) \\
(1006-1188)\end{array}$ & $\begin{array}{l}\text { ide } \\
\qquad \begin{array}{l}326 \pm 2 \\
268 \pm 5 \\
334 \pm 5\end{array}\end{array}$ & $\begin{array}{c}1064 \\
1162 \\
298\end{array}$ & $\begin{array}{l}\mathrm{TE} \\
\mathrm{TE} \\
\mathrm{TE}\end{array}$ & $\begin{array}{l}\text { [2000BRU/VIL] } \\
\text { [2000BRU/VIL] } \\
\text { [2000BRU/VIL] }\end{array}$ \\
\hline $\mathrm{LaI}_{3}$ & $\begin{array}{c}\text { [13813-22-4] } \\
\text { SUB } \\
\text { SUB }\end{array}$ & $\begin{array}{l}\text { Lanthanum trii } \\
\qquad \begin{array}{l}(827-978) \\
(827-978)\end{array}\end{array}$ & $\begin{array}{l}289 \pm 6 \\
304 \pm 6\end{array}$ & $\begin{array}{l}905 \\
298\end{array}$ & $\begin{array}{l}\text { ME,MS } \\
\text { ME,MS }\end{array}$ & $\begin{array}{l}\text { [2015DUN/KUD] } \\
\text { [2015DUN/KUD] }\end{array}$ \\
\hline
\end{tabular}


TABLE 17. Phase change enthalpies of organometallic and inorganic compounds-Continued

\begin{tabular}{|c|c|c|c|c|c|c|}
\hline \multirow[b]{2}{*}{$\begin{array}{l}\text { Molecular } \\
\text { formula }\end{array}$} & \multirow{2}{*}{$\begin{array}{l}\text { CAS Registry } \\
\text { Number } \\
\text { Enthalpy }\end{array}$} & \multicolumn{5}{|l|}{ Compound } \\
\hline & & $\begin{array}{c}\text { Temperature } \\
\text { range }\end{array}$ & $\begin{array}{l}\Delta_{\text {trans }} H_{\mathrm{m}} \\
(\mathrm{kJ} / \mathrm{mol})\end{array}$ & $\begin{array}{l}T_{\mathrm{m}} \\
(\mathrm{K})\end{array}$ & Method & References \\
\hline & SUB & $(932-1038)$ & $270 \pm 4$ & 985 & $\mathrm{TE}$ & [2000BRU/VIL, 2015DUN/KUD] \\
\hline & SUB & $(932-1038)$ & $288 \pm 4$ & 298 & TE & [2000BRU/VIL, 2015DUN/KUD] \\
\hline & SUB & $(1055-1123)$ & $216 \pm 6$ & 1098 & TE & [2000BRU/VIL, 2015DUN/KUD] \\
\hline & SUB & (1055-1123) & $287 \pm 6$ & 298 & $\mathrm{TE}$ & [2000BRU/VIL, 2015DUN/KUD] \\
\hline \multicolumn{7}{|l|}{$\mathrm{Li}$} \\
\hline \multirow[t]{2}{*}{$\mathrm{C}_{2} \mathrm{H}_{5} \mathrm{Li}$} & [811-49-4] & Ethyl lithium & & & & \\
\hline & SUB & (298-333) & 116.6 & 315.5 & A & [1987STE/MAL, 1962CHA] \\
\hline \multirow[t]{3}{*}{$\mathrm{C}_{4} \mathrm{H}_{9} \mathrm{Li}$} & [109-72-8] & Butyl lithium & & & & \\
\hline & SUB & (333-368) & 109.7 & 350.5 & A & [1987STE/MAL, 1962LEB/MIR] \\
\hline & $\mathrm{V}$ & & $107.1 \pm 2.9$ & & & [1961FOW/MOR, 1982PIL/SKI] \\
\hline
\end{tabular}

[Note: The authors of [1961FOW/MOR] noted that the experimental data were not very reproducible, and subject to considerable error.]

\begin{tabular}{|c|c|c|c|c|c|}
\hline \multirow[t]{2}{*}{$\mathrm{C}_{5} \mathrm{HF}_{6} \mathrm{LiO}_{2}$} & $\begin{array}{c}{[22466-51-9]} \\
\text { SUB }\end{array}$ & \multicolumn{3}{|c|}{ (1,1,1,5,5,5-hexafluoro-2,4-pentanedionato)lithium } & \multirow{2}{*}{ [2006FIL/SYS] } \\
\hline & \multicolumn{4}{|c|}{ The above value was calculated assuming the vapor phase is a dimer. } & \\
\hline \multirow[t]{3}{*}{$\mathrm{C}_{5} \mathrm{H}_{4} \mathrm{~F}_{3} \mathrm{LiO}_{2}$} & {$[127892-64-2]$} & \multicolumn{3}{|c|}{ (1,1,1-trifluoro-2,4-pentanedionato)lithium } & \\
\hline & SUB & $(453-523)$ & $145.6 \pm 3.0$ & & [2006FIL/SYS] \\
\hline & \multicolumn{4}{|c|}{ The above value was calculated assuming the vapor phase is a dimer. } & \\
\hline \multirow[t]{2}{*}{$\mathrm{C}_{8} \mathrm{H}_{10} \mathrm{~F}_{3} \mathrm{LiO}_{2}$} & [22441-09-4] & \multirow{2}{*}{\multicolumn{3}{|c|}{ (2-2-dimethyl-6,6,6-trifluoro-3,5-hexanedionato)lithium }} & \\
\hline & SUB & & & & [2006FIL/SYS] \\
\hline \multirow{6}{*}{$\mathrm{C}_{11} \mathrm{H}_{19} \mathrm{LiO}_{2}$} & \multicolumn{4}{|c|}{$\begin{array}{l}\text { The above value was calculated assuming the vapor phase is a dimer. } \\
{[22441-13-0] \quad(2,2,6,6 \text {-tetramethylheptane-3,5-dionato)lithium }}\end{array}$} & \\
\hline & SUB & $(400-450)$ & $191.2 \pm 7.1$ & ME,MS & [2006FIL/STA] \\
\hline & SUB & $(444-543)$ & $181.1 \pm 2.8$ & & [2006FIL/SYS] \\
\hline & SUB & $(537-545)$ & $198.0 \pm 15.1$ & & [2006FIL/SYS] \\
\hline & SUB & $(444-549)$ & $178.3 \pm 2.0$ & & [2006FIL/SYS] \\
\hline & SUB & & 174.5 & ME & [1973BRU/CUR] \\
\hline
\end{tabular}

The above four values were calculated assuming the vapor phase is a tetramer.
$\mathrm{V}$
$(550-581)$
$95.6 \pm 1.3$
[2006FIL/SYS]

The above value was calculated assuming the vapor phase is a tetramer.

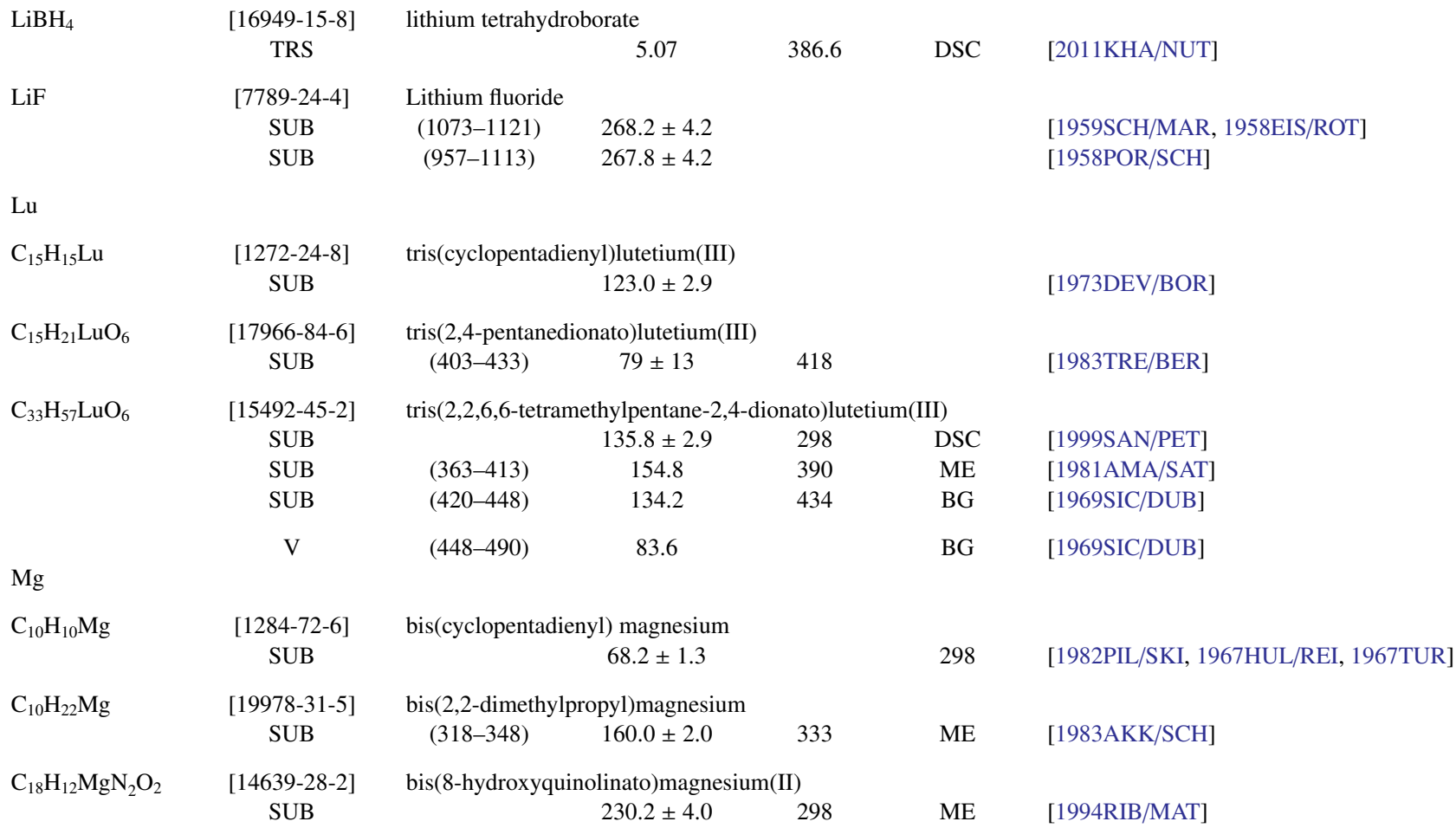


TABLE 17. Phase change enthalpies of organometallic and inorganic compounds-Continued

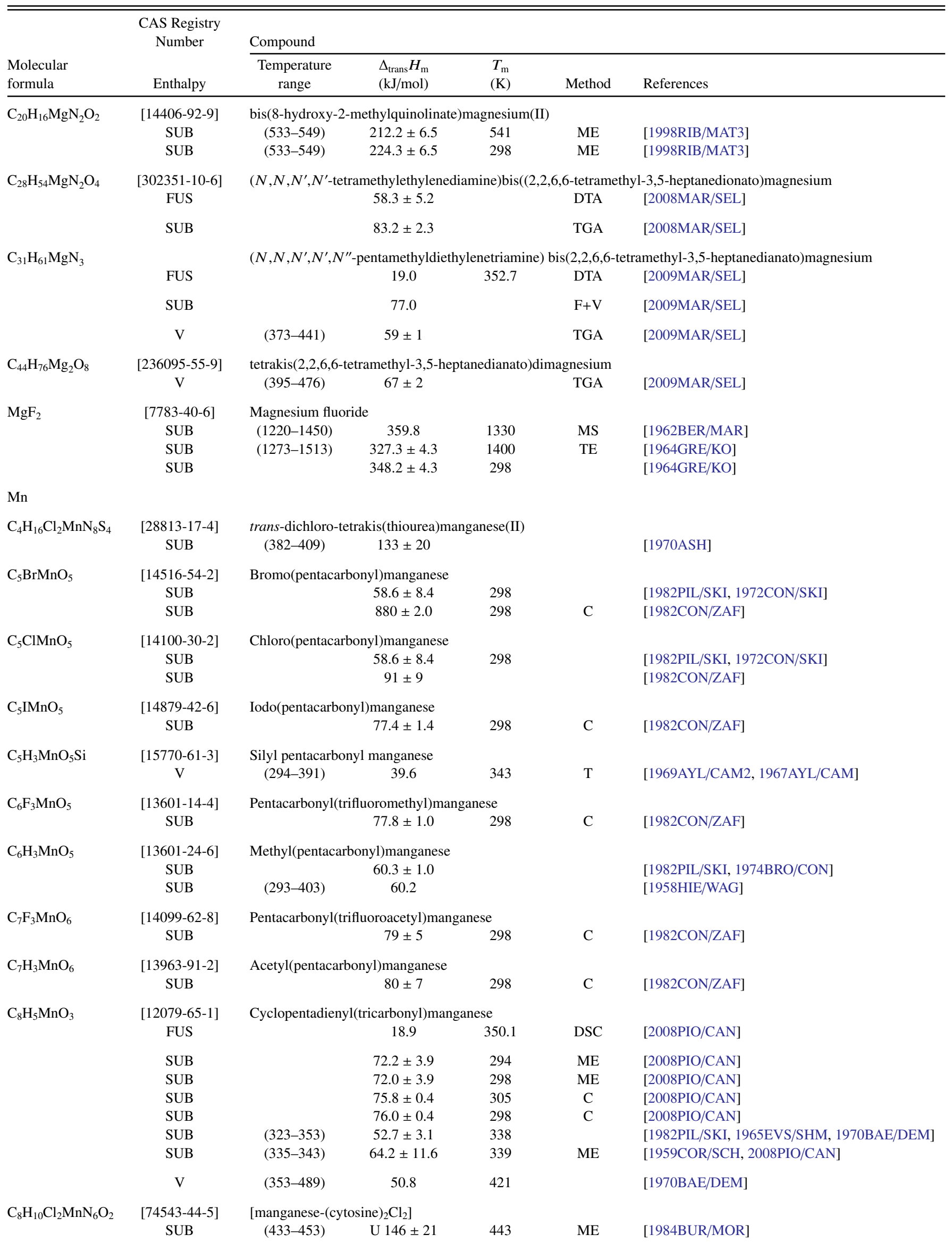


TABLE 17. Phase change enthalpies of organometallic and inorganic compounds-Continued

\begin{tabular}{|c|c|c|c|c|c|c|}
\hline \multirow[b]{2}{*}{$\begin{array}{l}\text { Molecular } \\
\text { formula }\end{array}$} & \multirow{2}{*}{$\begin{array}{c}\text { CAS Registry } \\
\text { Number } \\
\text { Enthalpy }\end{array}$} & \multicolumn{5}{|l|}{ Compound } \\
\hline & & $\begin{array}{c}\text { Temperature } \\
\text { range }\end{array}$ & $\begin{array}{l}\Delta_{\text {trans }} H_{\mathrm{m}} \\
(\mathrm{kJ} / \mathrm{mol})\end{array}$ & $\begin{array}{l}T_{\mathrm{m}} \\
(\mathrm{K})\end{array}$ & Method & References \\
\hline \multirow[t]{2}{*}{$\mathrm{C}_{8} \mathrm{H}_{5} \mathrm{MnO}_{3}$} & {$[12079-65-1]$} & \multicolumn{5}{|c|}{ Cyclopentadienylmanganese tricarbonyl } \\
\hline & SUB & $(335-343)$ & 43.1 & 339 & & {$[1959 \mathrm{COR} / \mathrm{SCH}]$} \\
\hline \multirow[t]{5}{*}{$\mathrm{C}_{10} \mathrm{MnO}_{10} \mathrm{Re}$} & {$[14693-30-2]$} & \multicolumn{5}{|c|}{ Decacarbonylmanganeserhenium } \\
\hline & SUB & & $109 \pm 4$ & 363 & $\mathrm{C}$ & [1998ADD/CON] \\
\hline & SUB & & $86 \pm 4$ & 298 & & [1998ADD/CON] \\
\hline & SUB & $(363-440)$ & 68.6 & 401 & MM & [1971BAE/DEM] \\
\hline & $\mathrm{V}$ & $(440-463)$ & 56.5 & 451 & & [1971BAE/DEM] \\
\hline \multirow[t]{5}{*}{$\mathrm{C}_{10} \mathrm{Mn}_{2} \mathrm{O}_{10}$} & {$[10170-69-1]$} & \multicolumn{5}{|c|}{ Decacarbonyldimanganese } \\
\hline & SUB & & $80.3 \pm 4.2$ & 298 & & [1982PIL/SKI, 1958GOO/FAI] \\
\hline & SUB & & $92.3 \pm 2.1$ & 298 & $\mathrm{C}$ & {$[1982 \mathrm{CON} / \mathrm{ZAF}]$} \\
\hline & SUB & $(351-428)$ & $80.3 \pm 2.1$ & 390 & MM & [1971BAE/DEM] \\
\hline & SUB & & $62.8 \pm 4.2$ & & & [1960COT/MON] \\
\hline \multicolumn{7}{|c|}{ [Note: The latter value is likely an enthalpy of vaporization.] } \\
\hline & $\mathrm{V}$ & $(428-463)$ & $60.7 \pm 1.3$ & 446 & & [1971BAE/DEM] \\
\hline \multirow[t]{2}{*}{$\mathrm{C}_{10} \mathrm{H}_{6} \mathrm{Mn}_{2} \mathrm{O}_{8} \mathrm{~S}_{2}$} & {$[21321-38-0]$} & \multicolumn{5}{|c|}{ bis( $\mu$-methanethiolato)octacarbonyldimanganese } \\
\hline & SUB & & $114.2 \pm 0.8$ & 340 & $\mathrm{C}$ & {$[1995 \mathrm{CON} / \mathrm{GOB} 2]$} \\
\hline \multirow[t]{5}{*}{$\mathrm{C}_{10} \mathrm{H}_{10} \mathrm{Mn}$} & {$[1271-27-8]$} & \multicolumn{5}{|c|}{ bis(cyclopentadienyl)manganese } \\
\hline & SUB & $(298-445)$ & 72.4 & 371.5 & A & [1987STE/MAL] \\
\hline & SUB & & $75.7 \pm 1.7$ & 298 & & [1982PIL/SKI, 1971TEL/RAB] \\
\hline & SUB & & 72.4 & & & {$[1956 \mathrm{WIL} / \mathrm{COT}]$} \\
\hline & $\mathrm{V}$ & $(378-435)$ & 58.0 & 393 & A & [1987STE/MAL] \\
\hline \multirow[t]{5}{*}{$\mathrm{C}_{10} \mathrm{H}_{14} \mathrm{MnO}_{4}$} & [14024-58-9] & \multicolumn{5}{|c|}{ bis(2,4-pentanedionato) manganese(II) } \\
\hline & SUB & $(390-440)$ & $139.3 \pm 2.5$ & 298 & ME & [1990MAL/ALI] \\
\hline & SUB & & 87.0 & 343 & & [1981MAS/BAR] \\
\hline & SUB & & 88.7 & & & [1972BOL, 2000DUN] \\
\hline & SUB & & 88.7 & 400 & & {$[1970 \mathrm{GOE} / \mathrm{BLO}]$} \\
\hline \multirow[t]{2}{*}{$\mathrm{C}_{11} \mathrm{H}_{5} \mathrm{MnO}_{5}$} & {$[13985-77-8]$} & \multicolumn{5}{|c|}{ Phenyl(pentacarbonyl)manganese } \\
\hline & SUB & & $84.9 \pm 4.4$ & 298 & $\mathrm{C}$ & {$[1982 \mathrm{CON} / \mathrm{ZAF}]$} \\
\hline \multirow[t]{2}{*}{$\mathrm{C}_{12} \mathrm{H}_{5} \mathrm{MnO}_{6}$} & {$[15612-92-7]$} & Benzoyl(pentac & nyl)manganes & & & \\
\hline & SUB & & $123 \pm 3$ & 298 & $\mathrm{C}$ & {$[1982 \mathrm{CON} / \mathrm{ZAF}]$} \\
\hline $\mathrm{C}_{12} \mathrm{H}_{7} \mathrm{MnO}_{5}$ & {$[14049-86-6]$} & Benzyl(pentaca & nyl)manganese & & & \\
\hline & SUB & & $84.5 \pm 0.7$ & 298 & $\mathrm{C}$ & {$[1982 \mathrm{CON} / \mathrm{ZAF}]$} \\
\hline $\mathrm{C}_{15} \mathrm{H}_{12} \mathrm{~F}_{9} \mathrm{MnO}_{6}$ & {$[14526-24-0]$} & $\operatorname{tris}(1,1,1-$ trifluc & ,4-pentanedio & manga & & \\
\hline & SUB & $(378-413)$ & $120.5 \pm 9.2$ & & GS & [1985MAT/KUW] \\
\hline & SUB & & 117.3 & & & [1971ASH] \\
\hline & SUB & & 77.8 & & & {$[1964 \mathrm{WOO} / \mathrm{JON}]$} \\
\hline $\mathrm{C}_{15} \mathrm{H}_{21} \mathrm{MnO}_{6}$ & {$[14284-89-0]$} & $\operatorname{tris}(2,4-$ pentane & lato) mangane & & & \\
\hline & FUS & & 27.7 & 421.9 & DSC & [2004SAB/MAR] \\
\hline & SUB & & $130.5 \pm 4.0$ & & MS & [2010KAM/YAR] \\
\hline & SUB & $(320-380)$ & $124.7 \pm 3.8$ & 298 & ME & [1990MAL/ALI] \\
\hline & SUB & & $120 \pm 10$ & 298 & $\mathrm{E}$ & [1988RIB/FER2] \\
\hline & SUB & & 99.0 & 392 & & [1981MAS/BAR] \\
\hline & SUB & & 113 & 370 & & [1970GOE/BLO] \\
\hline & SUB & & $77.8 \pm 0.8$ & 298 & & [1982PIL/SKI, 1968HIL/IRV] \\
\hline $\mathrm{C}_{18} \mathrm{H}_{12} \mathrm{MnN}_{2} \mathrm{O}_{2}$ & {$[14495-13-7]$} & bis(8-hydroxyq & linato) manga & & & \\
\hline & SUB & & $194.6 \pm 10.4$ & 298 & ME & [1994RIB/MAT] \\
\hline & SUB & $(615-650)$ & $208.4 \pm 14$ & 633 & ME & [1984BUR/MOR] \\
\hline & SUB & $(615-650)$ & $226 \pm 14$ & 298 & ME & [1984BUR/MOR] \\
\hline $\mathrm{C}_{20} \mathrm{H}_{16} \mathrm{MnN}_{2} \mathrm{O}_{2}$ & {$[14515-78-7]$} & bis(8-hydroxy- & thylquinolinat & anganes & & \\
\hline & SUB & $(521-541)$ & $199.6 \pm 7.2$ & 531 & $\mathrm{ME}$ & [1998RIB/MAT3] \\
\hline & SUB & $(521-541)$ & $211.2 \pm 7.2$ & 298 & ME & [1998RIB/MAT3] \\
\hline $\mathrm{C}_{30} \mathrm{H}_{27} \mathrm{MnO}_{6}$ & [14376-07-9] & tris(1-phenylbu & -1,3-dionato)n & anese(II & & \\
\hline
\end{tabular}


TABLE 17. Phase change enthalpies of organometallic and inorganic compounds-Continued

\begin{tabular}{|c|c|c|c|c|c|c|}
\hline \multirow[b]{2}{*}{$\begin{array}{l}\text { Molecular } \\
\text { formula }\end{array}$} & \multirow{2}{*}{$\begin{array}{l}\text { CAS Registry } \\
\text { Number } \\
\text { Enthalpy }\end{array}$} & \multicolumn{5}{|l|}{ Compound } \\
\hline & & $\begin{array}{l}\text { Temperature } \\
\text { range }\end{array}$ & $\begin{array}{l}\Delta_{\text {trans }} H_{\mathrm{m}} \\
(\mathrm{kJ} / \mathrm{mol})\end{array}$ & $\begin{array}{l}T_{\mathrm{m}} \\
(\mathrm{K})\end{array}$ & Method & References \\
\hline & SUB & & $195 \pm 10$ & 298 & $\mathrm{E}$ & [1988RIB/FER2] \\
\hline $\mathrm{C}_{33} \mathrm{H}_{57} \mathrm{MnO}_{6}$ & $\begin{array}{c}\text { [14324-99-3] } \\
\text { SUB } \\
\text { SUB } \\
\text { SUB }\end{array}$ & $\begin{array}{l}\operatorname{tris}(2,2,6,6-\text { tetr } \\
\qquad(350-408)\end{array}$ & $\begin{array}{c}\text { thylheptane- } 3 \\
89.0 \pm 7.0 \\
139.0 \pm 1.6 \\
140 \pm 10\end{array}$ & $\begin{array}{l}298 \\
379 \\
298\end{array}$ & $\begin{array}{c}\mathrm{MSe}(\mathrm{III}) \\
\mathrm{ME} \\
\mathrm{E}\end{array}$ & $\begin{array}{l}\text { [2011KAM/DEM] } \\
\text { [2010SID/SID] } \\
{[1988 \mathrm{RIB} / \mathrm{FER} 2]}\end{array}$ \\
\hline $\mathrm{C}_{44} \mathrm{H}_{28} \mathrm{MnN}_{4}$ & $\begin{array}{l}{[31004-82-7]} \\
\text { SUB }\end{array}$ & $5,10,15,20$-tetr & $\begin{array}{l}\text { nylporphine } \mathrm{n} \\
175 \pm 1\end{array}$ & anese(II) & UV & [1993SHE/KAR] \\
\hline $\mathrm{MnF}_{2}$ & $\begin{array}{c}\text { [7782-64-1] } \\
\text { SUB } \\
\text { SUB } \\
\text { SUB } \\
\text { V } \\
\text { V }\end{array}$ & $\begin{array}{c}\text { Manganese(II) } \\
(1054-1128) \\
(1054-1128) \\
(887-924) \\
(1130-1270) \\
(1132-1193)\end{array}$ & $\begin{array}{l}\text { ride } \\
305.4 \pm 6.7 \\
318.4 \pm 8.4 \\
319.7 \pm 2.1 \\
291.8 \pm 6.3 \\
300.8 \pm 6.7\end{array}$ & $\begin{array}{l}1103 \\
298 \\
298 \\
1165 \\
1159\end{array}$ & $\begin{array}{l}\text { ME } \\
\text { MS }\end{array}$ & $\begin{array}{l}{[1964 \mathrm{KEN} / \mathrm{EHL}]} \\
{[1964 \mathrm{KEN} / \mathrm{EHL}]} \\
{[1963 \mathrm{BAU} / \mathrm{MAR}]} \\
{[1969 \mathrm{HIT} / \mathrm{KAN}]} \\
{[1964 \mathrm{KEN} / \mathrm{EHL}]}\end{array}$ \\
\hline Mo & & & & & & \\
\hline $\mathrm{C}_{4} \mathrm{H}_{4} \mathrm{Mo}_{2} \mathrm{O}_{8}$ & $\begin{array}{l}{[51329-49-8]} \\
\text { SUB }\end{array}$ & Dimolybdenum & $\begin{array}{r}\text { tetraformate } \\
135.0 \pm 1.4\end{array}$ & 483 & & [2011SLY/KOZ] \\
\hline $\mathrm{C}_{6} \mathrm{MoO}_{6}$ & $\begin{array}{l}\text { [13939-06-5] } \\
\text { FUS } \\
\text { FUS } \\
\text { SUB } \\
\text { SUB } \\
\text { SUB } \\
\text { SUB } \\
\text { SUB } \\
\text { SUB } \\
\text { SUB } \\
\text { SUB } \\
\text { SUB }\end{array}$ & $\begin{array}{l}\text { Molybdenum h } \\
\begin{array}{l} \\
\\
(265-300) \\
(316-423) \\
(240-285) \\
(343-383) \\
(323-403) \\
(292-308)\end{array}\end{array}$ & $\begin{array}{l}\text { arbonyl } \\
25.1 \\
26.78 \\
73.4 \pm 0.3 \\
77.7 \\
69.1 \\
76.9 \pm 0.9 \\
73.8 \pm 1.0 \\
69.7 \\
72.5 \\
72.8 \\
68.2\end{array}$ & $\begin{array}{c}423.29 \\
419.2 \\
298 \\
\\
331 \\
263 \\
\\
363\end{array}$ & $\begin{array}{c}\text { DSC } \\
\text { DSC } \\
\text { C } \\
\text { A } \\
\text { ME }\end{array}$ & $\begin{array}{l}\text { [2013BER/CAN] } \\
\text { [1976FAB/MAS] } \\
\text { [2013BER/CAN] } \\
\text { [2001OHT/CIC] } \\
\text { [1987STE/MAL] } \\
\text { [1979DAA/ERN, 1980BOX/ERN] } \\
\text { [1975PIL/WAR, 1974BAR/PIL] } \\
\text { [1960MON/COT, 1947LAN/GER] } \\
\text { [1952REZ/SHV] } \\
\text { [1947LAN/GER] } \\
\text { [1935HIE/ROM] }\end{array}$ \\
\hline $\mathrm{C}_{7} \mathrm{H}_{3} \mathrm{MoNO}_{5}$ & $\begin{array}{l}{[17594-16-0]} \\
\text { SUB }\end{array}$ & $\begin{array}{l}\text { Acetonitrile mc } \\
\quad(260-279)\end{array}$ & $\begin{array}{l}\text { lenum pentac } \\
105.8 \pm 5.6\end{array}$ & 298 & & [1980CAV/ERN] \\
\hline $\mathrm{C}_{8} \mathrm{~F}_{12} \mathrm{Mo}_{2} \mathrm{O}_{8}$ & $\begin{array}{l}{[36608-07-8]} \\
\text { SUB } \\
\text { SUB }\end{array}$ & $\begin{array}{l}\text { Dimolybdenum } \\
\qquad(330-370)\end{array}$ & $\begin{array}{l}\text { atrifluoroacet } \\
115.3 \pm 1.2 \\
113.6 \pm 1.7\end{array}$ & $\begin{array}{l}420 \\
350\end{array}$ & $\mathrm{ME}, \mathrm{TE}$ & $\begin{array}{l}\text { [2011SLY/KOZ] } \\
{[1984 \mathrm{CAR}]}\end{array}$ \\
\hline $\mathrm{C}_{8} \mathrm{H}_{12} \mathrm{CrMoO}_{8}$ & $\begin{array}{l}{[71561-64-3]} \\
\text { SUB }\end{array}$ & Chromium mol & $\begin{array}{l}\text { num tetraace } \\
165.0 \pm 8.4\end{array}$ & & & [1982PIL/SKI] \\
\hline $\mathrm{C}_{8} \mathrm{H}_{12} \mathrm{Mo}_{2} \mathrm{O}_{8}$ & SUB & $\begin{array}{l}\text { Dimolybdenum } \\
\quad(400-420)\end{array}$ & $\begin{array}{l}\text { aacetate } \\
170.5 \pm 7\end{array}$ & 410 & $\mathrm{ME}, \mathrm{TE}$ & [1984CAR] \\
\hline $\mathrm{C}_{8} \mathrm{H}_{12} \mathrm{Mo}_{2} \mathrm{O}_{8}$ & $\begin{array}{l}{[14221-06-8]} \\
\text { SUB } \\
\text { SUB }\end{array}$ & Tetra- $\mu$-acetato & $\begin{array}{l}129 \pm 1 \\
165.0 \pm 8.4\end{array}$ & $\begin{array}{l}491 \\
298\end{array}$ & $\mathrm{C}$ & $\begin{array}{l}\text { [2008SLY/KON] } \\
\text { [1982PIL/SKI, 1979CAV/GAR] }\end{array}$ \\
\hline $\mathrm{C}_{8} \mathrm{H}_{24} \mathrm{MoN}_{4}$ & $\begin{array}{l}\text { [100207-68-9] } \\
\text { SUB } \\
\text { SUB }\end{array}$ & tetrakis(dimeth & $\begin{array}{l}\text { ino)molybde } \\
\begin{array}{l}88.4 \pm 3 \\
72.4 \pm 6\end{array}\end{array}$ & $\begin{array}{l}376 \\
298\end{array}$ & $\begin{array}{l}\mathrm{C} \\
\mathrm{C}\end{array}$ & $\begin{array}{l}\text { [1979ADE/CAV] } \\
{[1979 A D E / C A V, 1982 \mathrm{PIL} / \mathrm{SKI}]}\end{array}$ \\
\hline $\mathrm{C}_{9} \mathrm{H}_{9} \mathrm{MoN}_{3} \mathrm{O}_{3}$ & $\begin{array}{l}{[15038-48-9]} \\
\text { SUB } \\
\text { SUB }\end{array}$ & $\begin{array}{l}\text { tris(acetonitrile } \\
\qquad(283-308)\end{array}$ & $\begin{array}{l}\text { lybdenum tric } \\
111.3 \pm 3.0 \\
96.0 \pm 10.0\end{array}$ & $\begin{array}{l}\text { nyl } \\
298 \\
298\end{array}$ & & $\begin{array}{l}\text { [1980CAV/ERN] } \\
\text { [1982PIL/SKI, 1978ADE/CON] }\end{array}$ \\
\hline $\mathrm{C}_{10} \mathrm{H}_{5} \mathrm{MoNO}_{5}$ & $\begin{array}{l}{[14324-76-6]} \\
\text { SUB }\end{array}$ & $\begin{array}{l}\text { Pyridine(penta } \\
\quad(283-299)\end{array}$ & $\begin{array}{l}\text { nyl)molybde } \\
102.0 \pm 2.0\end{array}$ & 291 & $\mathrm{ME}$ & [1979DAA/ERN] \\
\hline $\mathrm{C}_{10} \mathrm{H}_{8} \mathrm{MoO}_{3}$ & $\begin{array}{l}{[12125-77-8]} \\
\text { SUB }\end{array}$ & Cycloheptatrie & $\begin{array}{l}\text { icarbonyl)mol } \\
88.0 \pm 4.0\end{array}$ & $\begin{array}{l}\text { num } \\
298\end{array}$ & & [1982PIL/SKI, 1977BRO/CON] \\
\hline $\mathrm{C}_{10} \mathrm{H}_{10} \mathrm{CI}_{2} \mathrm{Mo}$ & $\begin{array}{l}{[12184-22-4]} \\
\text { SUB }\end{array}$ & Dichlorobis $\left(\eta^{5}\right.$ & $\begin{array}{l}\text { cyclopentadie } \\
100.4 \pm 4.2\end{array}$ & $\begin{array}{l}\text { 1)molyb } \\
298\end{array}$ & $\mathrm{E}$ & [1976TEL/RAB] \\
\hline
\end{tabular}


TABLE 17. Phase change enthalpies of organometallic and inorganic compounds-Continued

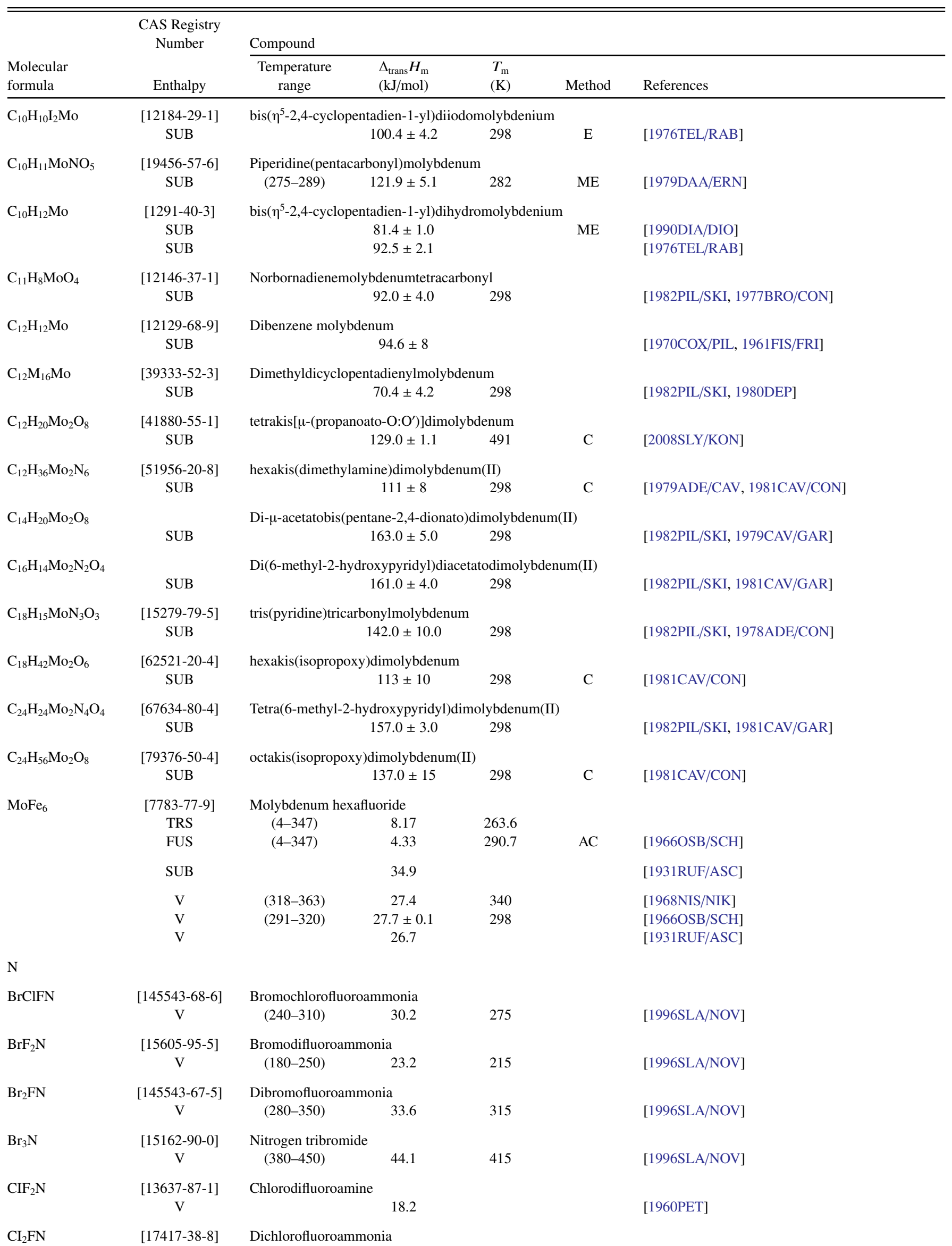


TABLE 17. Phase change enthalpies of organometallic and inorganic compounds-Continued

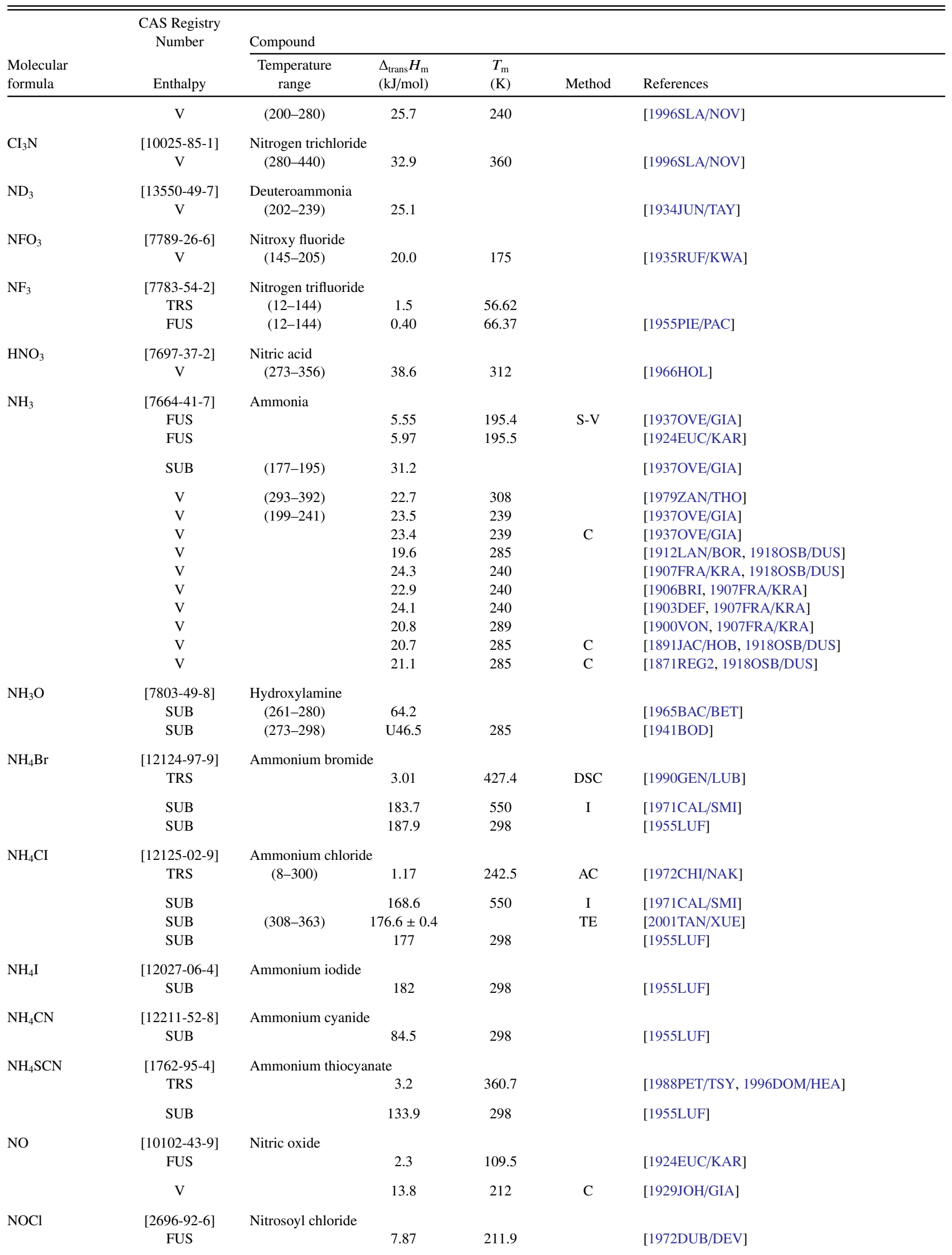

$\mathrm{N}_{2} \quad[7727-37-9] \quad$ Nitrogen


TABLE 17. Phase change enthalpies of organometallic and inorganic compounds-Continued

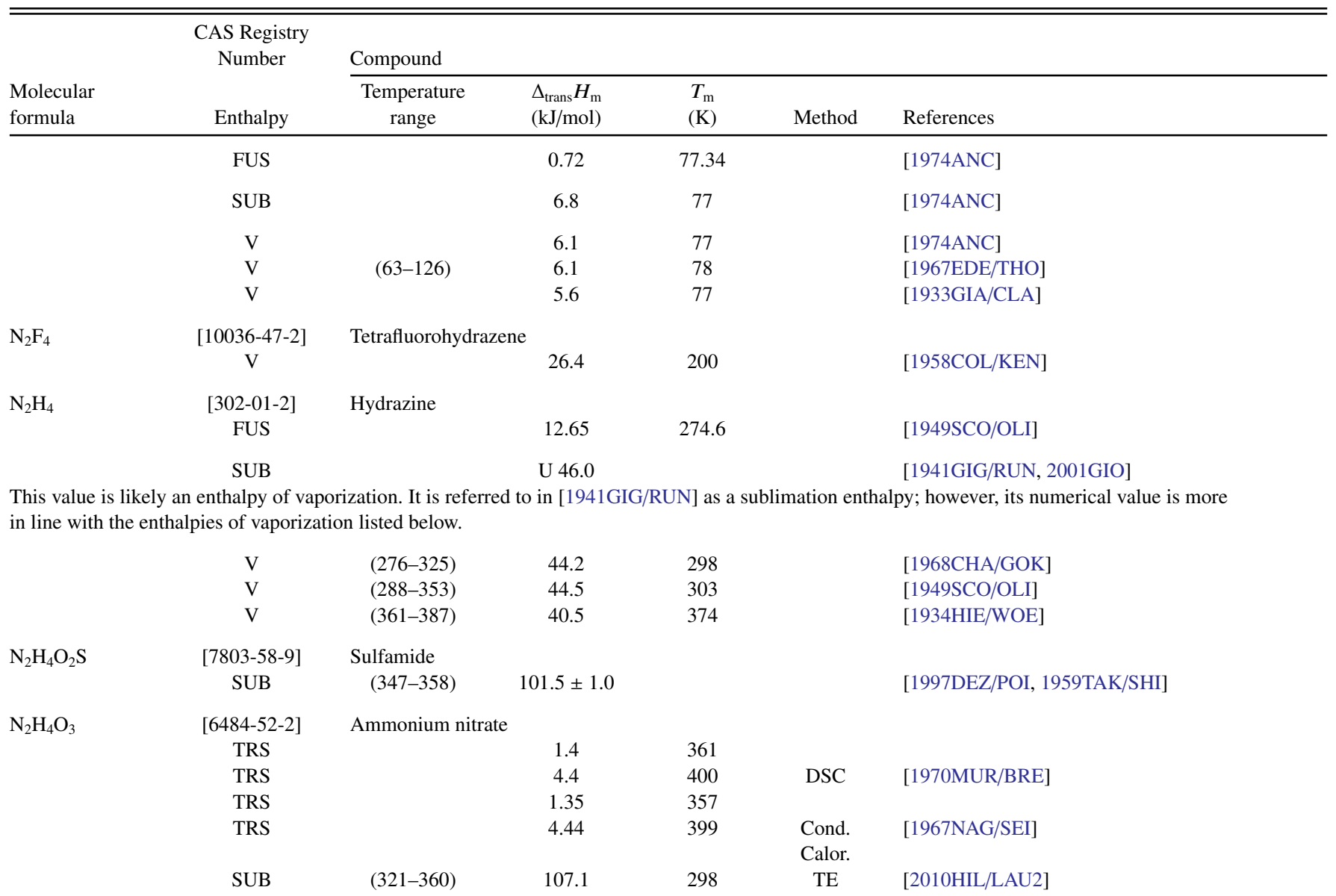

[Note: The authors reanalyzed the data in [2010HIL/LAU] using a different (presumably better) value for the equilibrium constant for the decomposition of ammonium nitrate to ammonia and nitric acid.]

$\begin{array}{lccccl}\text { SUB } & (321-360) & 87.4 \pm 8.4 & 298 & \text { TE } & {[2010 \mathrm{HIL} / \mathrm{LAU}]} \\ \text { SUB } & (349-438) & 178.7 & & & {[1962 \mathrm{BRA} / \mathrm{JUN}]} \\ \text { SUB } & & 174.9 & 298 & & {[1955 \mathrm{LUF}]}\end{array}$

\begin{tabular}{|c|c|c|c|c|c|c|}
\hline \multirow[t]{8}{*}{$\mathrm{N}_{2} \mathrm{O}$} & $\begin{array}{c}\text { [10024-97-2] } \\
\text { FUS }\end{array}$ & Nitrous oxide & 6.5 & 182.4 & & [1974ATA/CHI] \\
\hline & SUB & $(125-135)$ & 24.4 & 130 & & [1983TER] \\
\hline & SUB & $(135-147)$ & 24.2 & 141 & & [1983TER] \\
\hline & SUB & $(68-80)$ & $25.1 \pm 0.4$ & 74 & LE & [1974BRY/CAZ] \\
\hline & SUB & $(148-182)$ & 24.6 & 161 & & [1935BLU/GIA] \\
\hline & SUB & $(103-123)$ & 23.6 & 113 & MG & [1930BLA/VAN] \\
\hline & V & & 16.5 & 184.7 & & [1974ATA/CHI] \\
\hline & V & $(182-236)$ & 16.1 & 221 & & [1945HOG] \\
\hline \multirow[t]{2}{*}{$\mathrm{N}_{2} \mathrm{O}_{4}$} & [10544-72-6] & \multirow[t]{2}{*}{ Dinitrogen tetraoxide } & & & & \\
\hline & FUS & & 12.5 & 262 & & [1890RAM] \\
\hline \multirow[t]{2}{*}{$\mathrm{N}_{4} \mathrm{H}_{4}$} & [12164-94-2] & \multirow[t]{2}{*}{ Ammonium azide } & & & & \\
\hline & SUB & & 73.4 & & DSC & [1985NG/FIE] \\
\hline
\end{tabular}

$\mathrm{Na}$

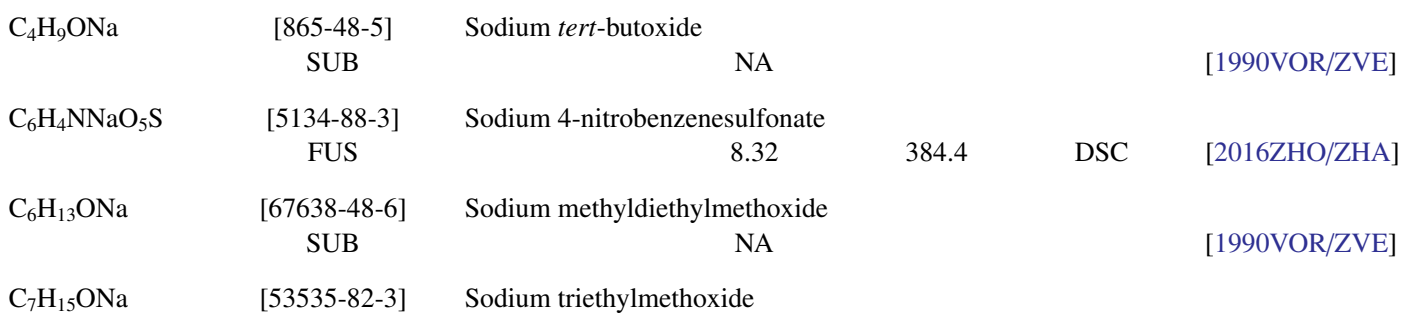


TABLE 17. Phase change enthalpies of organometallic and inorganic compounds-Continued

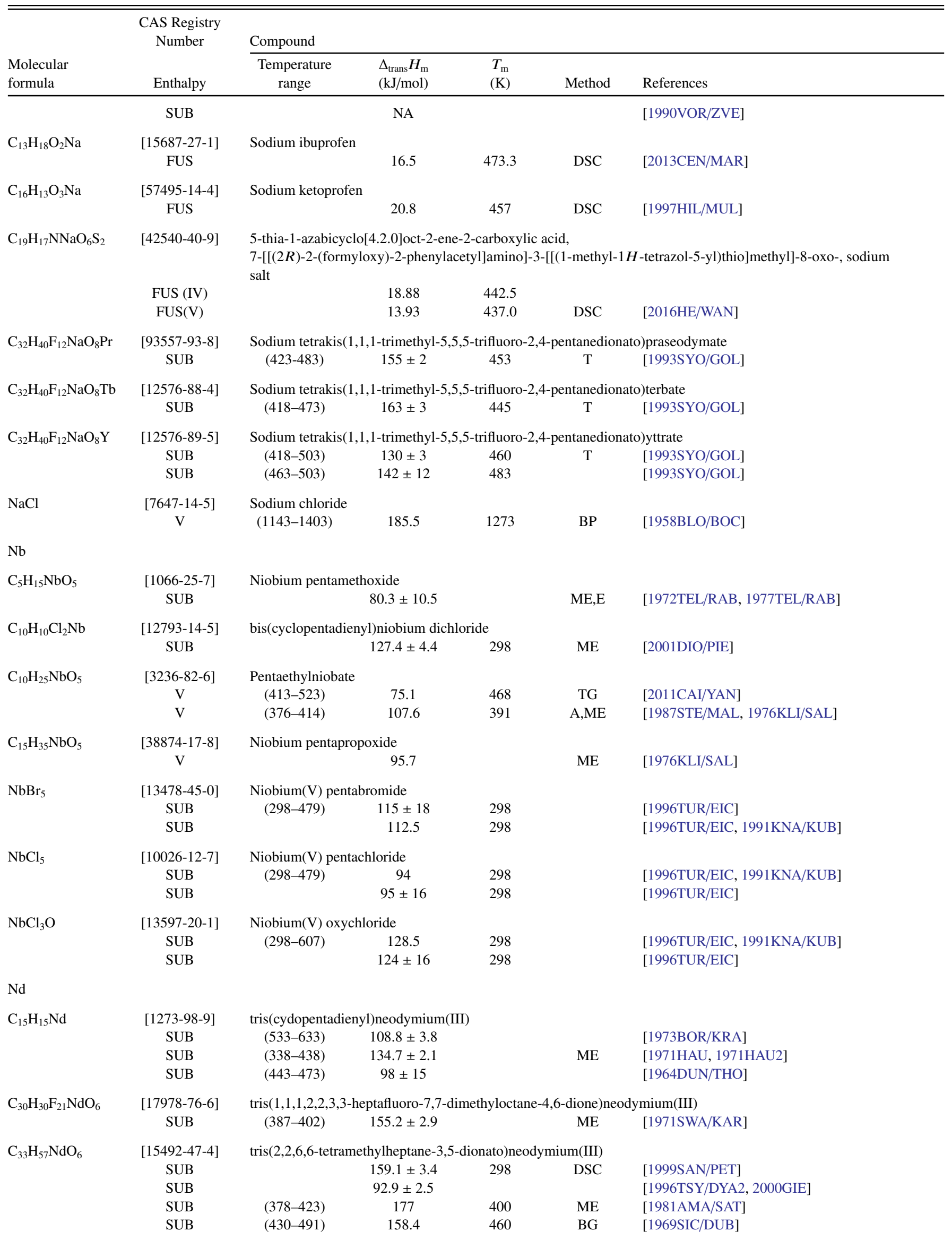


TABLE 17. Phase change enthalpies of organometallic and inorganic compounds-Continued

\begin{tabular}{|c|c|c|c|c|c|c|}
\hline \multirow[b]{2}{*}{$\begin{array}{l}\text { Molecular } \\
\text { formula }\end{array}$} & \multirow{2}{*}{$\begin{array}{l}\text { CAS Registry } \\
\text { Number } \\
\text { Enthalpy }\end{array}$} & \multicolumn{5}{|l|}{ Compound } \\
\hline & & $\begin{array}{l}\text { Temperature } \\
\text { range }\end{array}$ & $\begin{array}{l}\Delta_{\text {trans }} H_{\mathrm{m}} \\
(\mathrm{kJ} / \mathrm{mol})\end{array}$ & $\begin{array}{l}T_{\mathrm{m}} \\
(\mathrm{K})\end{array}$ & Method & References \\
\hline & V & $(491-510)$ & 99.1 & & BG & [1969SIC/DUB] \\
\hline $\mathrm{NdI}_{3}$ & $\begin{array}{c}\text { [13813-24-6] } \\
\text { SUB } \\
\text { SUB }\end{array}$ & $\begin{array}{c}\text { Neodymium tr } \\
(857-1037) \\
(857-1037)\end{array}$ & $\begin{array}{l}\text { de } \\
287.9 \pm 2.4 \\
320.5 \pm 2.4\end{array}$ & $\begin{array}{l}943 \\
298\end{array}$ & $\begin{array}{l}\mathrm{ME} \\
\mathrm{ME}\end{array}$ & $\begin{array}{l}{[1975 \mathrm{HIR} / \mathrm{ROM}]} \\
{[1975 \mathrm{HIR} / \mathrm{ROM}]}\end{array}$ \\
\hline $\begin{array}{l}\mathrm{Ni} \\
\left(\mathrm{C}_{3} \mathrm{MO}_{3}\right)- \\
\left(\mathrm{C}_{3} \mathrm{~F}_{9} \mathrm{P}\right)\end{array}$ & $\mathrm{V}$ & tris(trifluorom & phosphine - & tricarb & complex & [1958EME/SMI] \\
\hline $\mathrm{C}_{4} \mathrm{NiO}_{4}$ & $\begin{array}{c}\text { [13463-39-3] } \\
\text { SUB } \\
\text { V } \\
\text { V } \\
\text { V } \\
\text { V } \\
\text { V } \\
\text { V } \\
\text { V } \\
\text { V } \\
\text { V }\end{array}$ & $\begin{array}{l} \\
(250-315) \\
(273-298) \\
(253-316) \\
(263-316)\end{array}$ & $\begin{array}{l}1 \\
41.6 \pm 0.5 \\
29.8 \\
27.6 \pm 1.3 \\
28.0 \\
27.2 \\
30.2 \pm 0.1 \\
29.5 \\
30.1 \\
29.0 \\
27.2\end{array}$ & $\begin{array}{l}344 \\
298 \\
265 \\
286 \\
285\end{array}$ & & $\begin{array}{l}\text { [1953WAL] } \\
\text { [1970BAE] } \\
{[1957 \mathrm{FIS} / \mathrm{COT}, 1982 \mathrm{PIL} / \mathrm{SKI}]} \\
{[1955 \mathrm{SPI} / \mathrm{STA}]} \\
{[1955 \mathrm{SPI} / \mathrm{STA}]} \\
{[1953 \mathrm{WAL}]} \\
{[1947 \mathrm{STU}]} \\
{[1942 \mathrm{SUG} / \mathrm{SAT}, 1955 \mathrm{SPI} / \mathrm{STA}]} \\
{[1930 \mathrm{AND}]} \\
\text { [1903DEW/JON, 1955SPI/STA] }\end{array}$ \\
\hline $\mathrm{C}_{4} \mathrm{H}_{16} \mathrm{Cl}_{2} \mathrm{~N}_{8} \mathrm{NiS}_{8}$ & $\begin{array}{l}{[28813-19-6]} \\
\text { SUB }\end{array}$ & $\begin{array}{l}\text { trans-dichloro } \\
\quad(409-447)\end{array}$ & $\begin{array}{l}\text { kis(thiourea)r } \\
74 \pm 20\end{array}$ & & & {$[1970 \mathrm{ASH}]$} \\
\hline $\mathrm{C}_{6} \mathrm{H}_{12} \mathrm{~N}_{2} \mathrm{NiS}_{4}$ & $\begin{array}{c}{[15521-65-0]} \\
\mathrm{V}\end{array}$ & $\begin{array}{l}\text { bis(dimethyldi } \\
\quad(448-478)\end{array}$ & $\begin{array}{l}\text { arbamate)nic } \\
139.9 \pm 2.1\end{array}$ & 463 & & [1987STE/MAL, 1978TAV/NEE, 1999DYK/SVO] \\
\hline $\mathrm{C}_{8} \mathrm{~F}_{18} \mathrm{NiO}_{2} \mathrm{P}_{2}$ & $\begin{array}{l}{[15188-79-1]} \\
\text { SUB }\end{array}$ & $\begin{array}{l}\text { Dicarbonylbis } \\
\quad(293-302)\end{array}$ & $\begin{array}{l}\text { rifluoromethy } \\
47.2\end{array}$ & $\begin{array}{l}\text { phine] } \\
298\end{array}$ & & [1966BUR/STR] \\
\hline $\mathrm{C}_{8} \mathrm{~F}_{28} \mathrm{NiP}_{4}$ & $\begin{array}{l}{[14917-18-1]} \\
\text { SUB }\end{array}$ & $\begin{array}{l}\text { tetrakis }[\text { bis }(\text { tri } \\
\quad(305-331)\end{array}$ & $\begin{array}{l}\text { methyl)phor } \\
66.6\end{array}$ & $\begin{array}{l}\text { s fluori } \\
318\end{array}$ & ickel & [1966BUR/STR] \\
\hline $\mathrm{C}_{10} \mathrm{H}_{8} \mathrm{~F}_{6} \mathrm{NiO}_{4}$ & $\begin{array}{l}{[14324-83-5]} \\
\text { SUB }\end{array}$ & $\begin{array}{l}\text { bis }(1,1,1-\text { triflu } \\
\quad(416-473)\end{array}$ & $\begin{array}{l}\text { 4-pentanedic } \\
157.7 \pm 3.3\end{array}$ & hickel(1 & GS & [1985MAT/KUW] \\
\hline $\mathrm{C}_{10} \mathrm{H}_{10} \mathrm{Ni}$ & $\begin{array}{c}\text { [1271-28-9] } \\
\text { FUS } \\
\text { FUS }\end{array}$ & bis(cyclopenta & $\begin{array}{c}\text { 1) nickel (nic } \\
18.0 \\
19.0\end{array}$ & $\begin{array}{l}\text { ne) } \\
450.1 \\
450.8\end{array}$ & $\begin{array}{l}\text { DSC } \\
\text { DSC }\end{array}$ & $\begin{array}{l}\text { [2011 VIE/ROJ] } \\
\text { [2011ROJ/VIE] }\end{array}$ \\
\hline & $\begin{array}{l}\text { SUB } \\
\text { SUB } \\
\text { SUB } \\
\text { SUB } \\
\text { SUB } \\
\text { SUB } \\
\text { SUB } \\
\text { SUB }\end{array}$ & $\begin{array}{l}(348-413) \\
(284-306) \\
(284-306) \\
(353-419)\end{array}$ & $\begin{array}{l}72.6 \pm 0.7 \\
71.4 \pm 1.3 \\
72.0 \pm 2.2 \\
70.4 \pm 1.1 \\
71.5 \pm 0.6 \\
71.4 \pm 0.6 \\
70.2 \pm 1.5 \\
72.4 \pm 1.3\end{array}$ & $\begin{array}{l}298 \\
333 \\
333 \\
328 \\
\\
298 \\
298 \\
298\end{array}$ & $\begin{array}{c}\text { TGA } \\
\text { DSC } \\
\text { C } \\
\text { Langmuir } \\
\text { ME } \\
\text { ME } \\
\text { MM }\end{array}$ & $\begin{array}{l}\text { [2011VIE/ROJ] } \\
\text { [2011ROJ/VIE] } \\
\text { [2011ROJ/VIE] } \\
\text { [2011ROJ/VIE] } \\
{[1988 \mathrm{TOR} / \mathrm{BAR} 2,2011 \mathrm{VIE} / \mathrm{ROJ}]} \\
{[1988 \mathrm{TOR} / \mathrm{BAR} 2,2011 \mathrm{VIE} / \mathrm{ROJ}]} \\
\text { [1984BAE/BAR2] } \\
\text { [1982PIL/SKI, 1975TEL/KIR, 1967TUR] }\end{array}$ \\
\hline $\mathrm{C}_{10} \mathrm{H}_{14} \mathrm{NiO}_{4}$ & $\begin{array}{l}\text { [3264-82-2] } \\
\text { SUB } \\
\text { SUB } \\
\text { SUB } \\
\text { SUB } \\
\text { SUB } \\
\text { SUB } \\
\text { SUB } \\
\text { SUB } \\
\text { SUB }\end{array}$ & $\begin{array}{l}\text { bis(2,4-pentan } \\
\quad(357-420)\end{array}$ & $\begin{array}{c}\text { ato)nickel(II) } \\
126.4 \pm 4.4 \\
108.2 \pm 5 \\
108.2 \pm 4.9 \\
155 \pm 80 \\
127.7 \pm 10 \\
132 \pm 10 \\
69.0 \\
95.4 \\
69.0\end{array}$ & $\begin{array}{l}298 \\
207 \\
480 \\
298 \\
381 \\
298 \\
400\end{array}$ & $\begin{array}{c}\text { ME } \\
\text { DSC } \\
\text { DSC } \\
\text { C } \\
\text { ME } \\
\text { ME } \\
\text { I }\end{array}$ & $\begin{array}{l}\text { [1990MAL/ALI] } \\
{[1987 \mathrm{MUR} / \mathrm{HIL}]} \\
{[1987 \mathrm{RIB} / \mathrm{FER}]} \\
{[1985 \mathrm{MUR} / \mathrm{SAK}]} \\
{[1984 \mathrm{BUR} / \mathrm{MOR}]} \\
{[1984 \mathrm{BUR} / \mathrm{MOR}]} \\
{[1971 \mathrm{ASH}]} \\
{[1970 \mathrm{GOE} / \mathrm{BLO}]} \\
\text { [1960BER/TRU, 1965BER/TRU] }\end{array}$ \\
\hline $\mathrm{C}_{10} \mathrm{H}_{20} \mathrm{~N}_{2} \mathrm{NiS}_{4}$ & $\begin{array}{l}{[14267-17-5]} \\
\text { SUB } \\
\text { SUB } \\
\text { SUB } \\
\text { SUB }\end{array}$ & $\begin{array}{l}\text { bis(diethyldith } \\
\quad(448-478) \\
\quad(440-478) \\
(507-650) \\
(443-543)\end{array}$ & $\begin{array}{c}\text { bamato)nicke } \\
157.3 \pm 6.0 \\
152 \pm 0.8 \\
98.8 \pm 6 \\
91.9 \pm 6\end{array}$ & $\begin{array}{l}459 \\
579 \\
493\end{array}$ & $\begin{array}{c}\mathrm{C} \\
\mathrm{DSC} \\
\mathrm{DSC}\end{array}$ & $\begin{array}{l}\text { [1989RIB/REI] } \\
\text { [1987STE/MAL, 1978TAV/NEE] } \\
\text { [1979CAV/HIL2] } \\
\text { [1979CAV/HIL2] }\end{array}$ \\
\hline
\end{tabular}


TABLE 17. Phase change enthalpies of organometallic and inorganic compounds-Continued

\begin{tabular}{|c|c|c|c|c|c|c|}
\hline \multirow[b]{2}{*}{$\begin{array}{l}\text { Molecular } \\
\text { formula }\end{array}$} & \multirow{2}{*}{$\begin{array}{l}\text { CAS Registry } \\
\text { Number } \\
\text { Enthalpy }\end{array}$} & \multicolumn{5}{|l|}{ Compound } \\
\hline & & $\begin{array}{c}\text { Temperature } \\
\text { range }\end{array}$ & $\begin{array}{l}\Delta_{\text {trans }} H_{\mathrm{m}} \\
(\mathrm{kJ} / \mathrm{mol})\end{array}$ & $\begin{array}{l}T_{\mathrm{m}} \\
(\mathrm{K})\end{array}$ & Method & References \\
\hline & SUB & & $151.9 \pm 2.1$ & & & [1976TAV/NEE] \\
\hline & SUB & & $61.1 \pm 1.7$ & & $\mathrm{I}$ & [1969DAS/WEN] \\
\hline \multirow[t]{2}{*}{$\mathrm{C}_{12} \mathrm{H}_{8} \mathrm{~N}_{2} \mathrm{NiO}_{4}$} & {$[17653-01-9]$} & bis(picolinato)r & l(II) & & & \\
\hline & SUB & & 76.6 & & I & [1963WOO/JON] \\
\hline \multirow{2}{*}{$\mathrm{C}_{13} \mathrm{H}_{6} \mathrm{~F}_{24} \mathrm{~N}_{2} \mathrm{Ni}_{2} \mathrm{O}_{3} \mathrm{P}_{4}$} & [14402-98-3] & \multicolumn{5}{|c|}{$\mu$-carbonyldicarbonylbis $[\mu-[($ methylimino)bis[bis(trifluoromethyl)phophine $]]]$ dinickel } \\
\hline & SUB & $(370-390)$ & 92.3 & 380 & & [1968SIN/BUR] \\
\hline \multirow[t]{2}{*}{$\mathrm{C}_{14} \mathrm{H}_{10} \mathrm{NiO}_{4}$} & {$[14263-01-5]$} & \multicolumn{5}{|c|}{ bis(salicyladehydato)nickel(II) } \\
\hline & SUB & & 85.4 & & I & [1963WOO/JON] \\
\hline \multirow[t]{2}{*}{$\mathrm{C}_{14} \mathrm{H}_{12} \mathrm{~N}_{2} \mathrm{NiO}_{2}$} & [14283-99-9] & \multicolumn{5}{|c|}{ bis(salicyliminato)nickel(II) } \\
\hline & SUB & & 158.2 & & I & {$[1963 \mathrm{WOO} / \mathrm{JON}]$} \\
\hline \multirow[t]{3}{*}{$\mathrm{C}_{14} \mathrm{H}_{12} \mathrm{~N}_{2} \mathrm{NiO}_{4}$} & {$[14363-30-5]$} & \multicolumn{5}{|c|}{ bis(salicylaldoximato)nickel(II) } \\
\hline & SUB & $(403-423)$ & $106.6 \pm 29$ & 413 & & [1984BUR/MOR] \\
\hline & SUB & & $112 \pm 29$ & 298 & & [1984BUR/MOR] \\
\hline \multirow[t]{4}{*}{$\mathrm{C}_{14} \mathrm{H}_{28} \mathrm{~N}_{2} \mathrm{NiS}_{4}$} & {$[14516-30-4]$} & \multicolumn{5}{|c|}{ bis(dipropyldithiocarbamate)nickel } \\
\hline & SUB & & $147.2 \pm 5.0$ & & $\mathrm{C}$ & [1989RIB/REI] \\
\hline & SUB & & $126.1 \pm 0.8$ & & & [1978TAV/NEE] \\
\hline & $\mathrm{V}$ & $(433-462)$ & 126.1 & 448 & & [1999DYK/SVO] \\
\hline \multirow[t]{3}{*}{$\mathrm{C}_{14} \mathrm{H}_{28} \mathrm{~N}_{2} \mathrm{NiS}_{4}$} & {$[15694-55-0]$} & \multicolumn{5}{|c|}{ bis(diisopropyldithiocarbamate) nickel complex } \\
\hline & SUB & & $148.0 \pm 5.0$ & & $\mathrm{C}$ & [1989RIB/REI] \\
\hline & SUB & $(442-477)$ & $143.4 \pm 2.1$ & 459.5 & & [1987STE/MAL, 1978TAV/NEE] \\
\hline \multirow[t]{2}{*}{$\mathrm{C}_{16} \mathrm{H}_{8} \mathrm{~F}_{6} \mathrm{NiO}_{2} \mathrm{~S}_{4}$} & {$[14239-90-8]$} & \multicolumn{5}{|c|}{ bis(monothiothenoyltrifluoroacetonate)nickel(II) } \\
\hline & SUB & & $161.0 \pm 5.1$ & 298 & $\mathrm{C}$ & [2007RIB/SAN2] \\
\hline \multirow[t]{2}{*}{$\mathrm{C}_{16} \mathrm{H}_{14} \mathrm{~N}_{2} \mathrm{MO}_{2}$} & {$[14167-20-5]$} & \multicolumn{5}{|c|}{$N, N$-bis(salicylidene)ethylenediaminonickel(II) } \\
\hline & SUB & $(459-545)$ & $149.8 \pm 7.0$ & & ME & [1999ALI/MAL] \\
\hline $\mathrm{C}_{16} \mathrm{H}_{16} \mathrm{~N}_{2} \mathrm{NiO}_{2}$ & {$[57377-56-7]$} & bis(2-hydroxya & phenamine)ni & & & \\
\hline & SUB & $(486-582)$ & $130.2 \pm 7.2$ & & GS & [2009ARO/MAL] \\
\hline $\mathrm{C}_{16} \mathrm{H}_{34} \mathrm{NiP}_{2} \mathrm{~S}_{4}$ & {$[83053-11-6]$} & $\operatorname{bis}[P, P$-bis $(2-$ & lylpropyl)pho & dithiat & l(II) & \\
\hline & SUB & $(433-513)$ & $104.2 \pm 2.0$ & 473 & GS & [2010KOK/SEM] \\
\hline $\mathrm{C}_{18} \mathrm{H}_{12} \mathrm{~N}_{2} \mathrm{NiO}_{2}$ & {$[14100-15-3]$} & bis(8-hydroxyq & linato)nickel( & & & \\
\hline & SUB & & $175.4 \pm 6.7$ & 298 & $\mathrm{ME}$ & [1994RIB/MAT] \\
\hline & SUB & $(468-503)$ & $129.9 \pm 6$ & 486 & $\mathrm{ME}$ & [1984BUR/MOR] \\
\hline & SUB & & $139 \pm 6$ & 298 & & [1984BUR/MOR] \\
\hline $\mathrm{C}_{18} \mathrm{H}_{14} \mathrm{~N}_{4} \mathrm{Ni}$ & {$[39251-81-5]$} & Dibenzotetra-a & anulene nicke & omplex & & \\
\hline & SUB & $(463-553)$ & $116.6 \pm 5.5$ & 508 & $\mathrm{~T}$ & [1983FER/QUA] \\
\hline $\mathrm{C}_{18} \mathrm{H}_{20} \mathrm{~N}_{2} \mathrm{NiO}_{2}$ & [1161880-17-6] & bis(2-hydroxyp & ophenamine) $\mathrm{r}$ & & & \\
\hline & SUB & $(443-552)$ & $113.0 \pm 7.5$ & & GS & [2009ARO/MAL] \\
\hline $\mathrm{C}_{18} \mathrm{H}_{36} \mathrm{~N}_{2} \mathrm{NiS}_{4}$ & {$[13927-77-0]$} & bis(dibutyldithi & bamate)nicke & & & \\
\hline & SUB & & $132.6 \pm 5.0$ & & $\mathrm{C}$ & [1989RIB/REI] \\
\hline & $\mathrm{V}$ & $(438-562)$ & 136.6 & 500 & & [1999DYK/SVO] \\
\hline $\mathrm{C}_{18} \mathrm{H}_{36} \mathrm{~N}_{2} \mathrm{NiO}_{4}$ & {$[28371-07-5]$} & bis(diisobutyld & carbamate)nic & & & \\
\hline & SUB & & $133.6 \pm 5.0$ & & $\mathrm{C}$ & [1989RIB/REI] \\
\hline & SUB & $(423-443)$ & $152.1 \pm 1.3$ & 433 & A & [1987STE/MAL, 1978TAV/NEE] \\
\hline & $\mathrm{V}$ & $(453-473)$ & 124 & 463 & & [1999DYK/SVO] \\
\hline $\mathrm{C}_{2} \mathrm{H}_{16} \mathrm{~N}_{2} \mathrm{NiO}_{2}$ & [15200-70-1] & bis(8-hydroxy- & thylquinolina & $\operatorname{kel}(\mathrm{II})$ & & \\
\hline & SUB & $(489-505)$ & $170.9 \pm 3.7$ & 496 & $\mathrm{ME}$ & [1998RIB/MAT3] \\
\hline & SUB & & $180.9 \pm 3.7$ & 298 & & [1998RIB/MAT3] \\
\hline $\mathrm{C}_{22} \mathrm{H}_{38} \mathrm{NiO}_{4}$ & [14481-08-4] & $\operatorname{bis}(2,2,6,6$-tetr & hylheptane-3, & hato)nic & & \\
\hline & SUB & $(360-408)$ & $137.8 \pm 2.3$ & 384 & ME & [2010SID/SID] \\
\hline & SUB & $(453-493)$ & 111 & & MEM & [1999EMM/PIC] \\
\hline & SUB & & $145.2 \pm 10$ & & $\mathrm{ME}$ & [1978IRV/SCH] \\
\hline
\end{tabular}


TABLE 17. Phase change enthalpies of organometallic and inorganic compounds-Continued

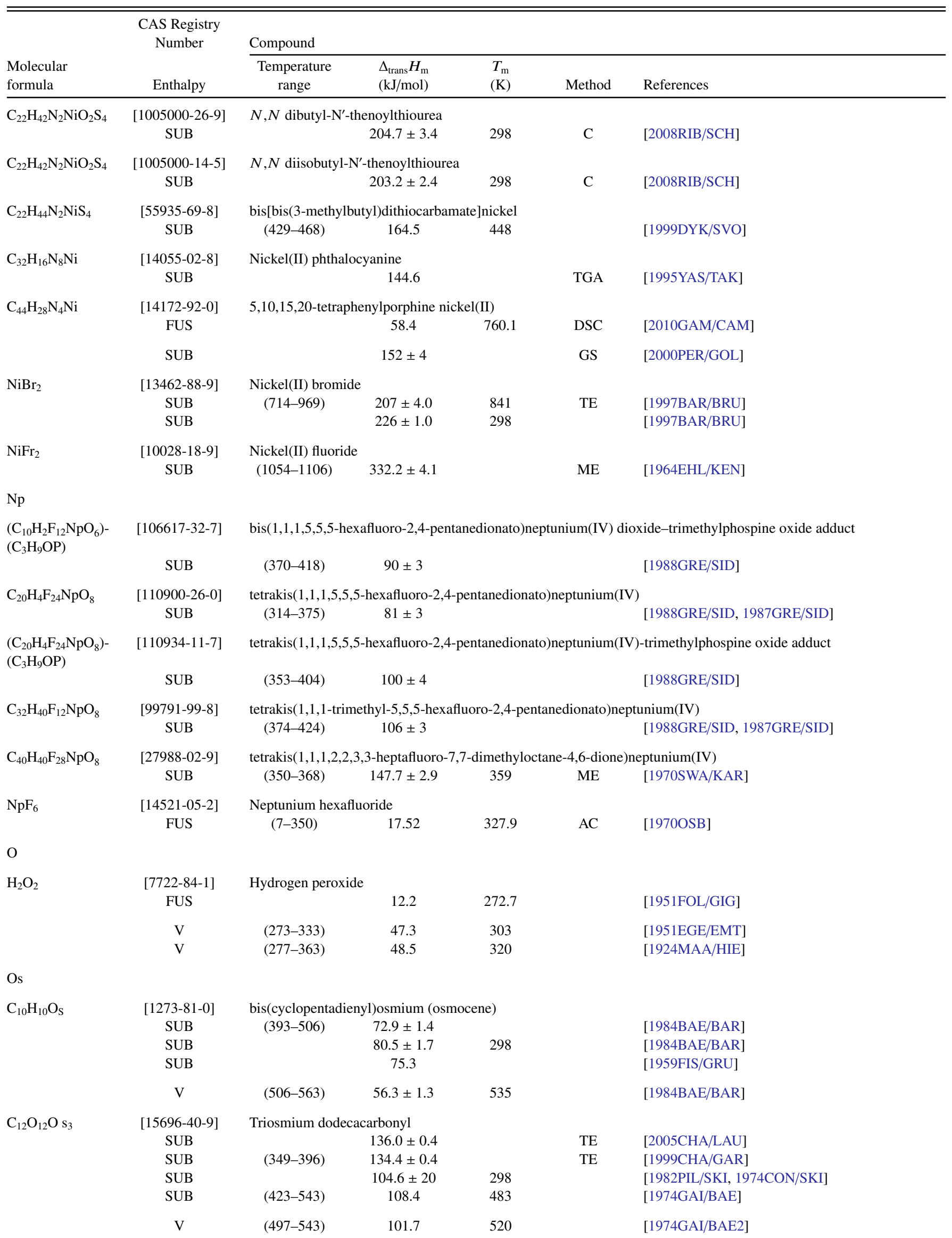


TABLE 17. Phase change enthalpies of organometallic and inorganic compounds-Continued

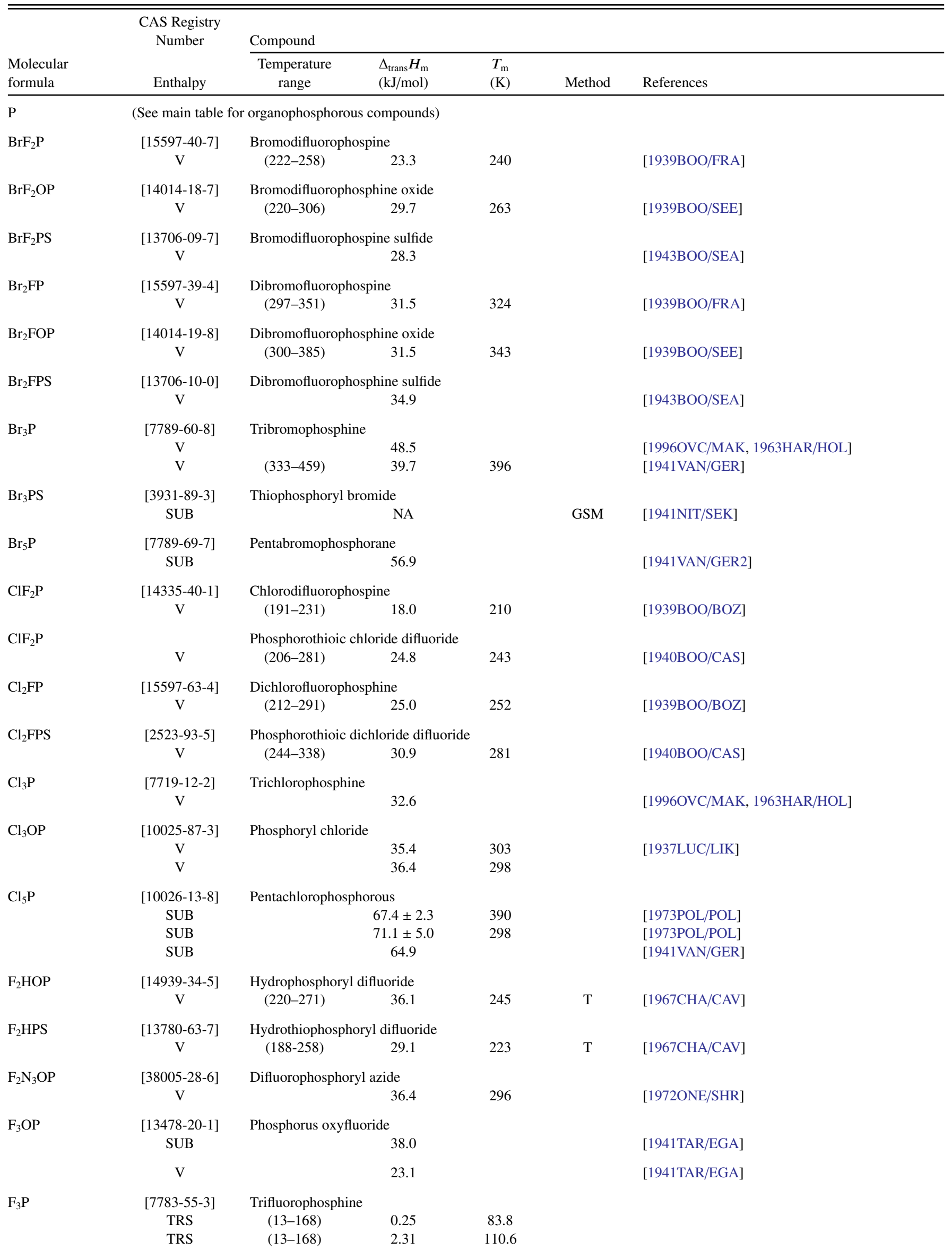


TABLE 17. Phase change enthalpies of organometallic and inorganic compounds-Continued

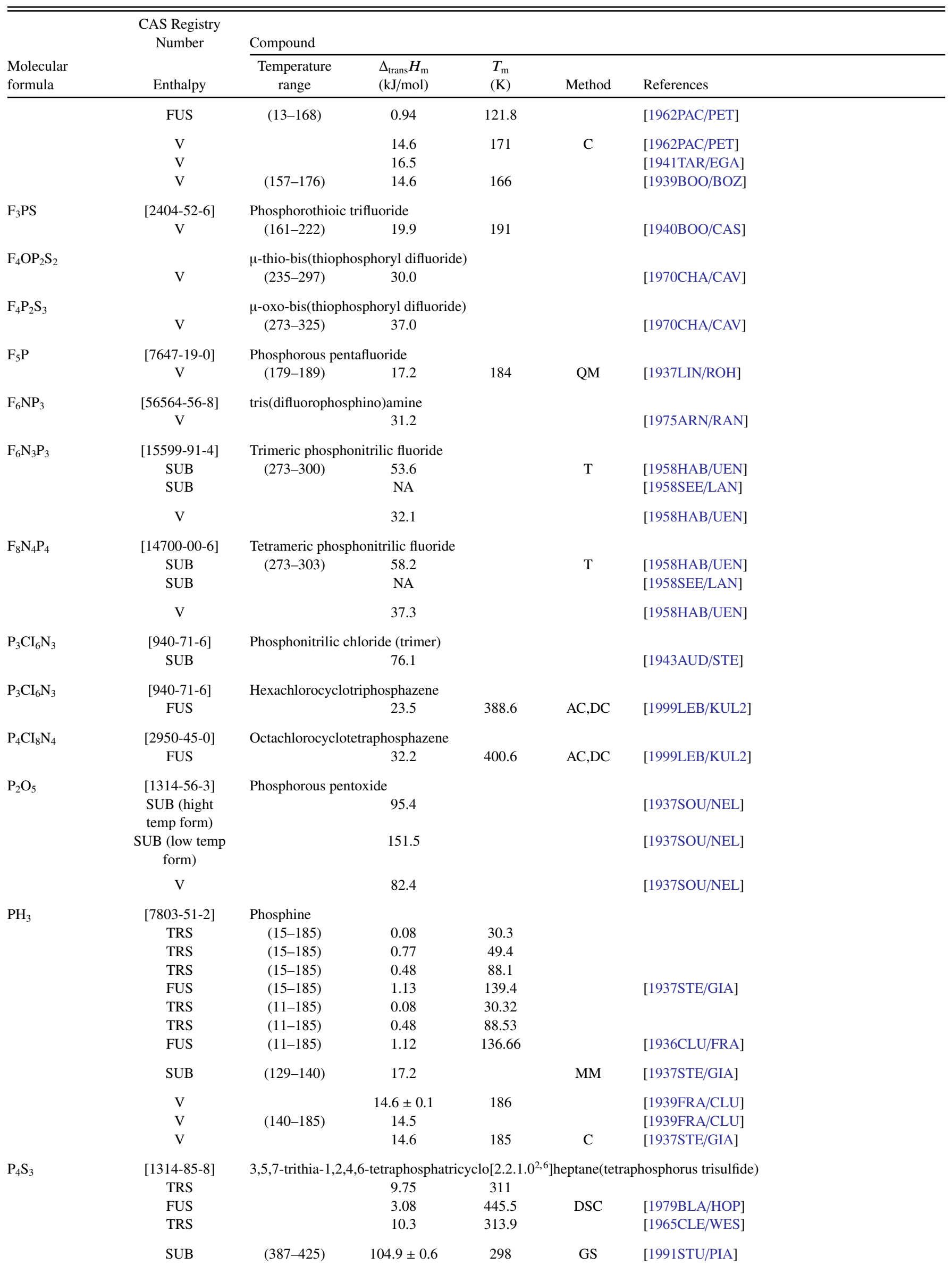


TABLE 17. Phase change enthalpies of organometallic and inorganic compounds-Continued

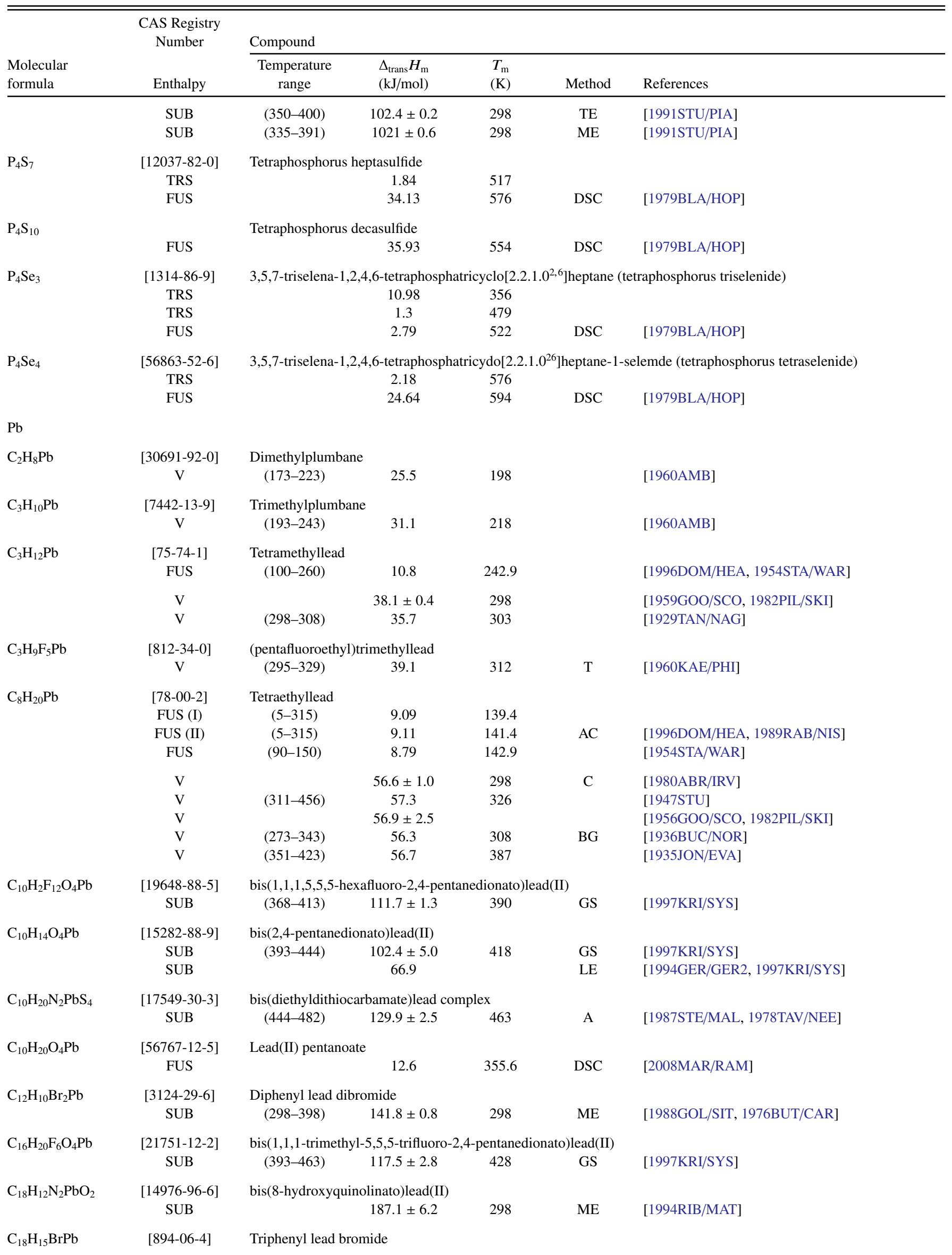


TABLE 17. Phase change enthalpies of organometallic and inorganic compounds-Continued

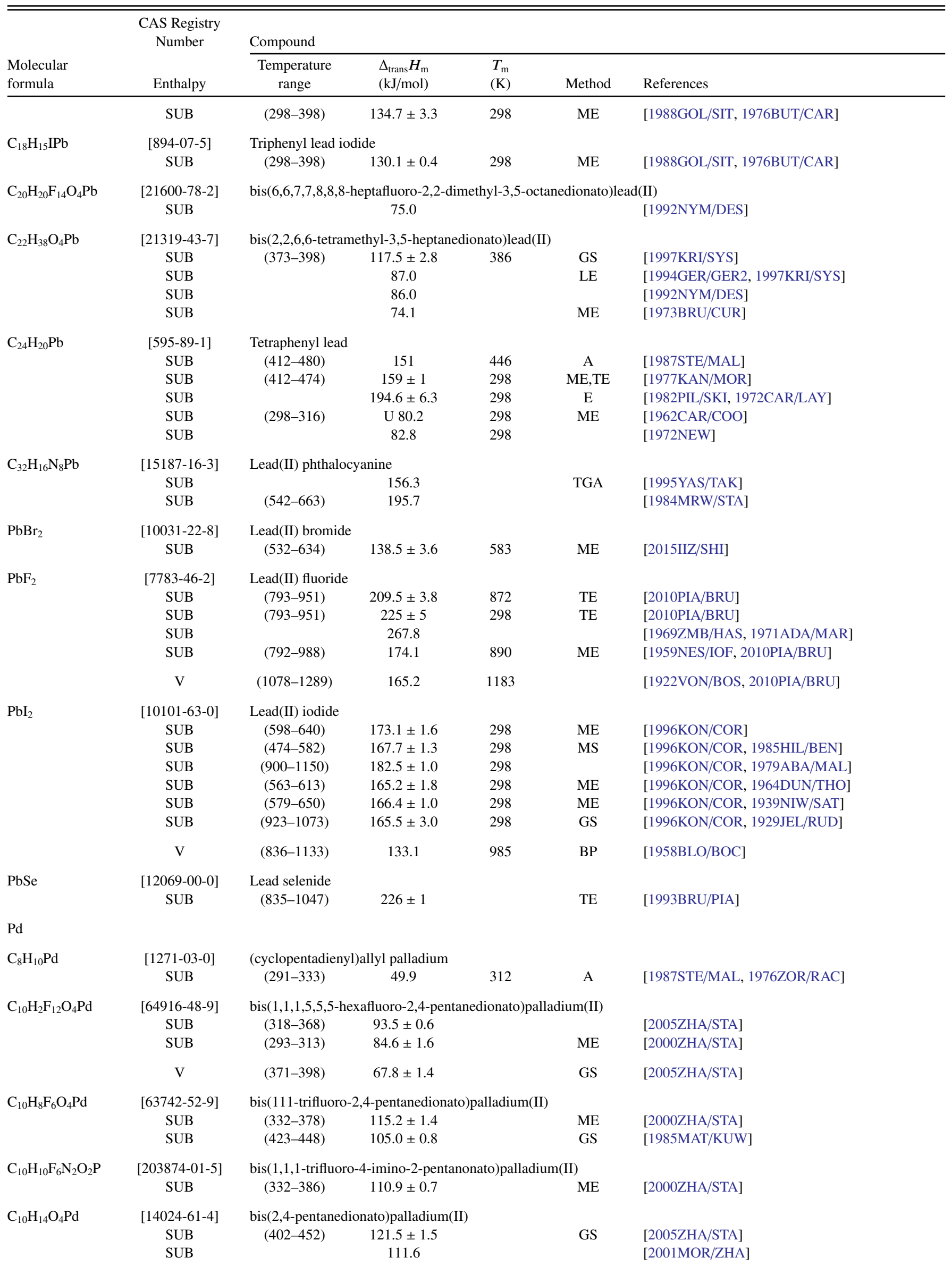


TABLE 17. Phase change enthalpies of organometallic and inorganic compounds-Continued

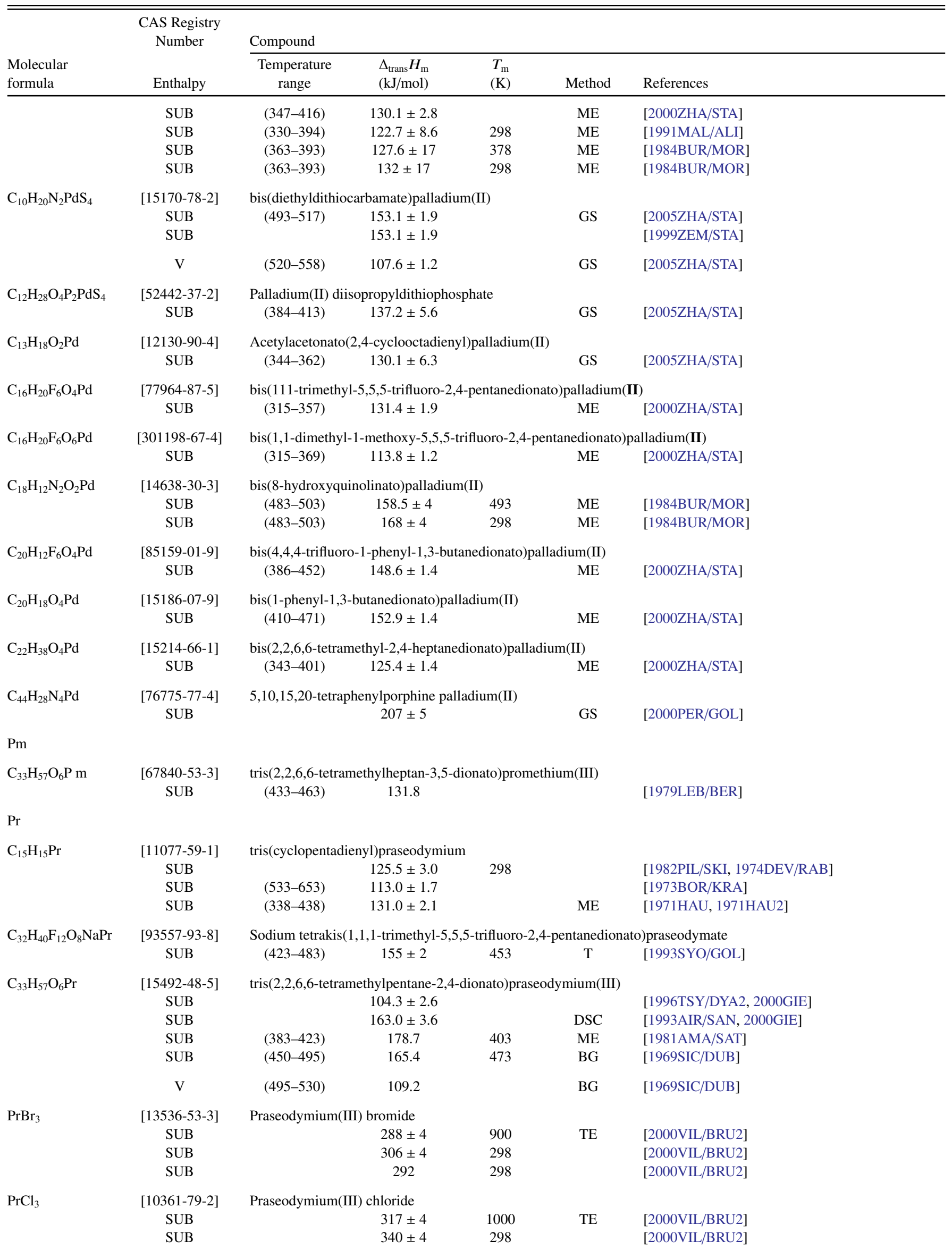


TABLE 17. Phase change enthalpies of organometallic and inorganic compounds-Continued

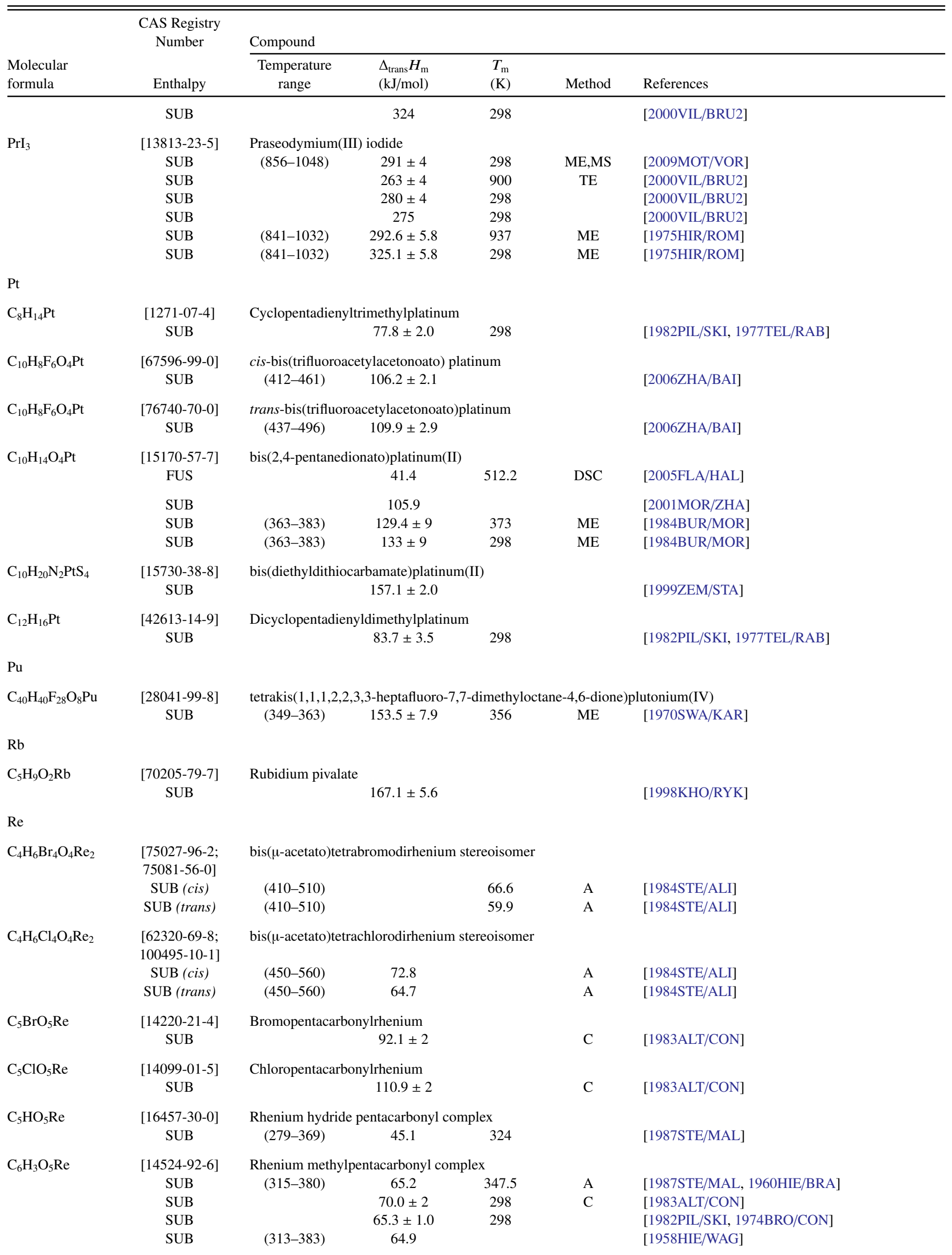


TABLE 17. Phase change enthalpies of organometallic and inorganic compounds-Continued

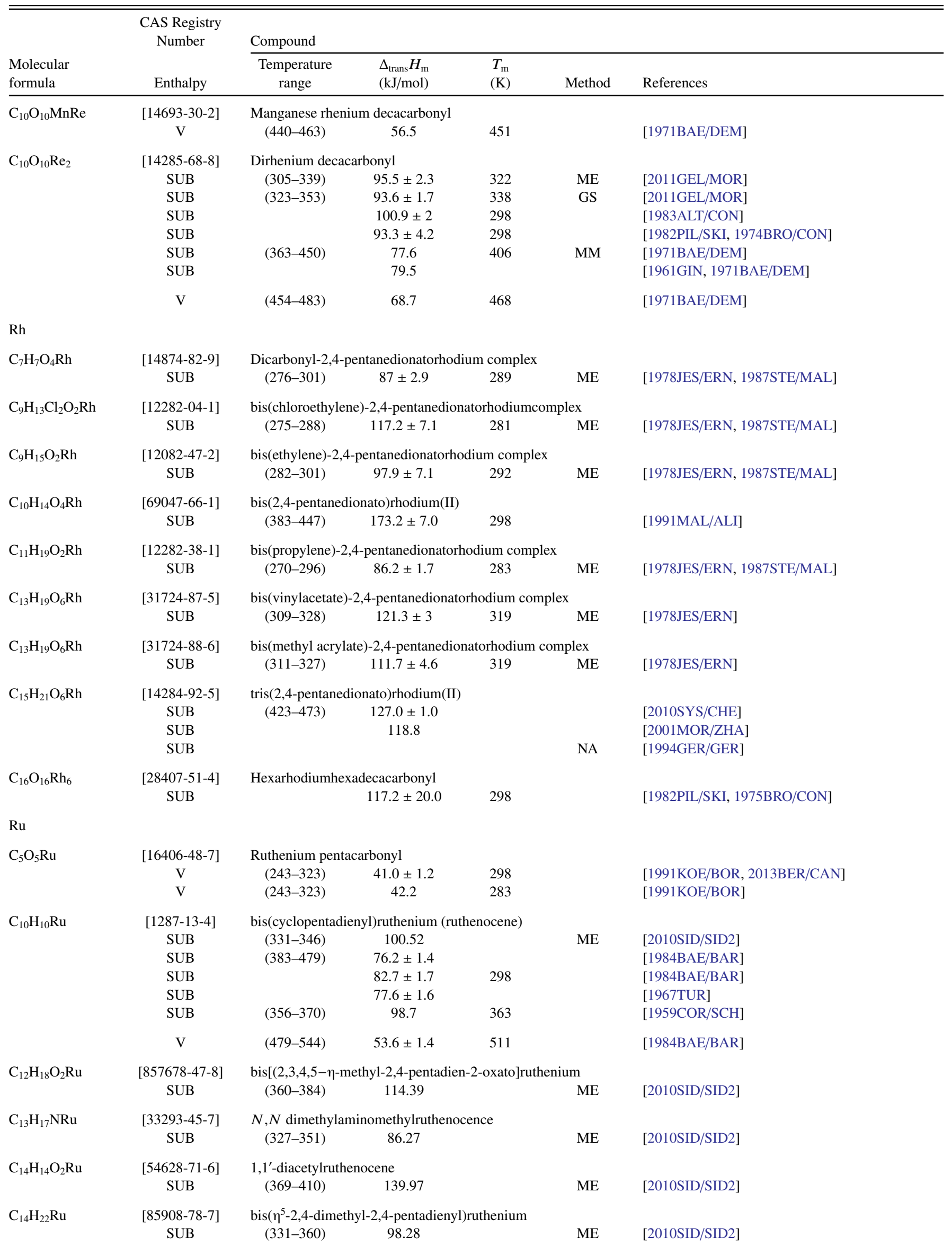


TABLE 17. Phase change enthalpies of organometallic and inorganic compounds-Continued

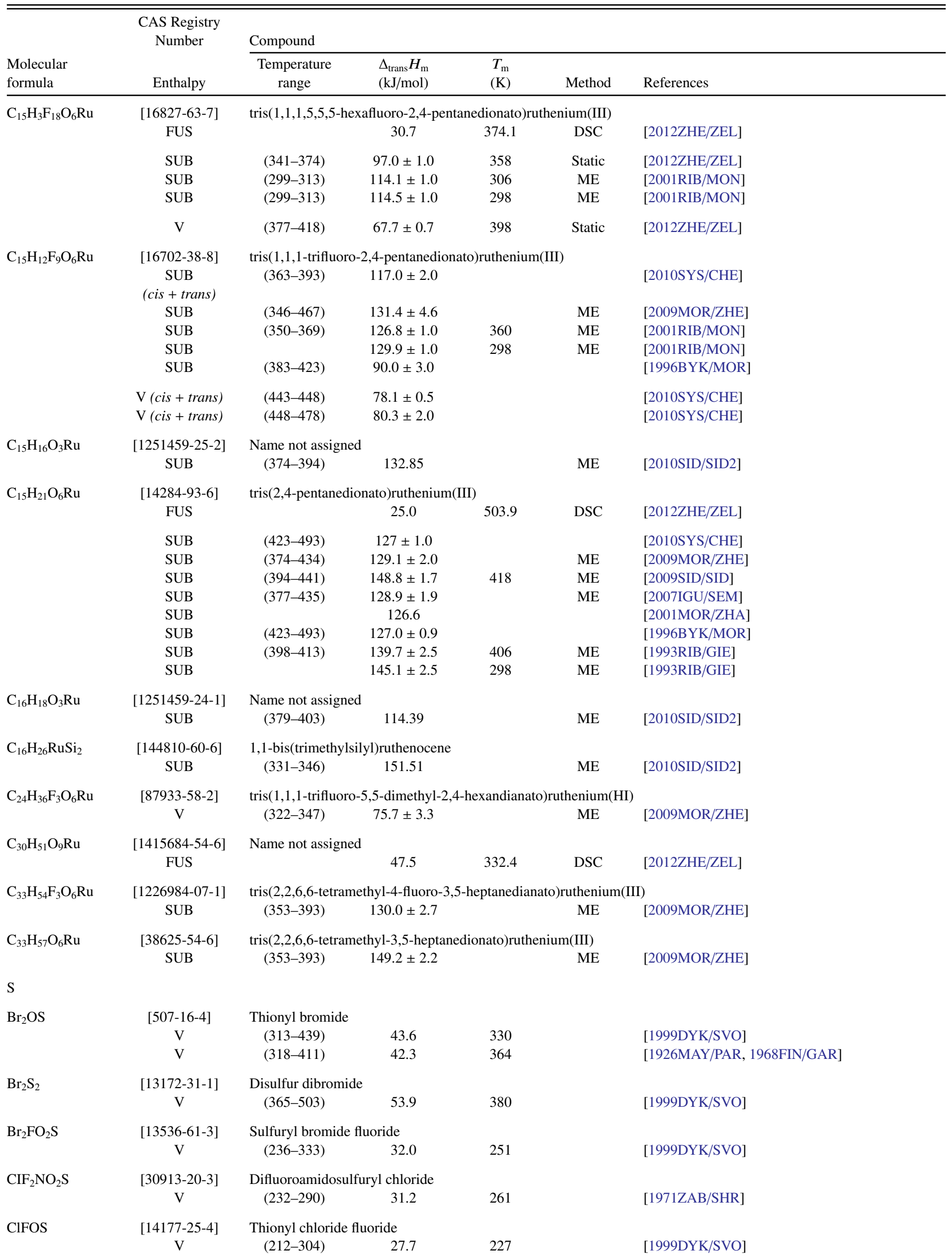


TABLE 17. Phase change enthalpies of organometallic and inorganic compounds-Continued

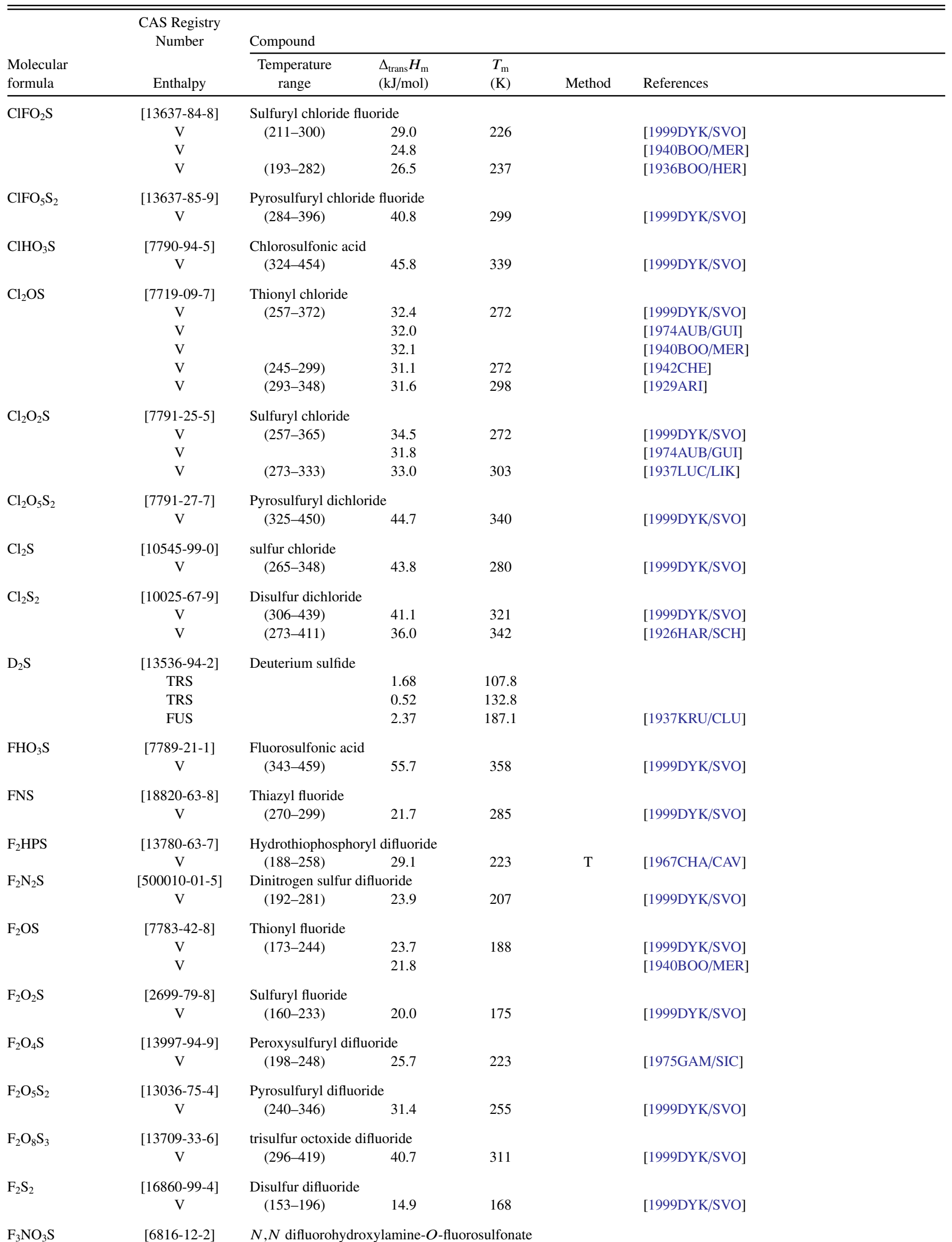


TABLE 17. Phase change enthalpies of organometallic and inorganic compounds-Continued

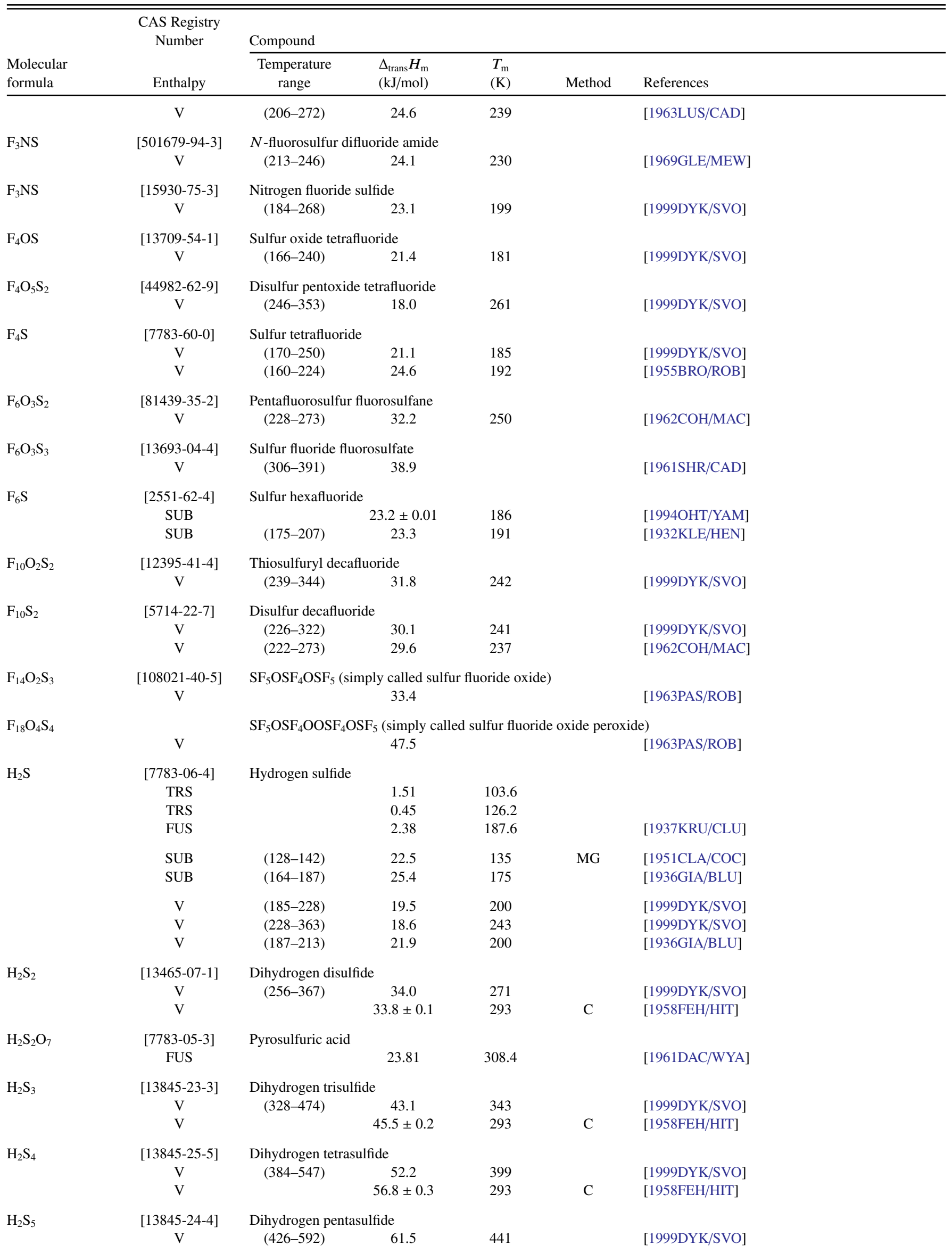


TABLE 17. Phase change enthalpies of organometallic and inorganic compounds-Continued

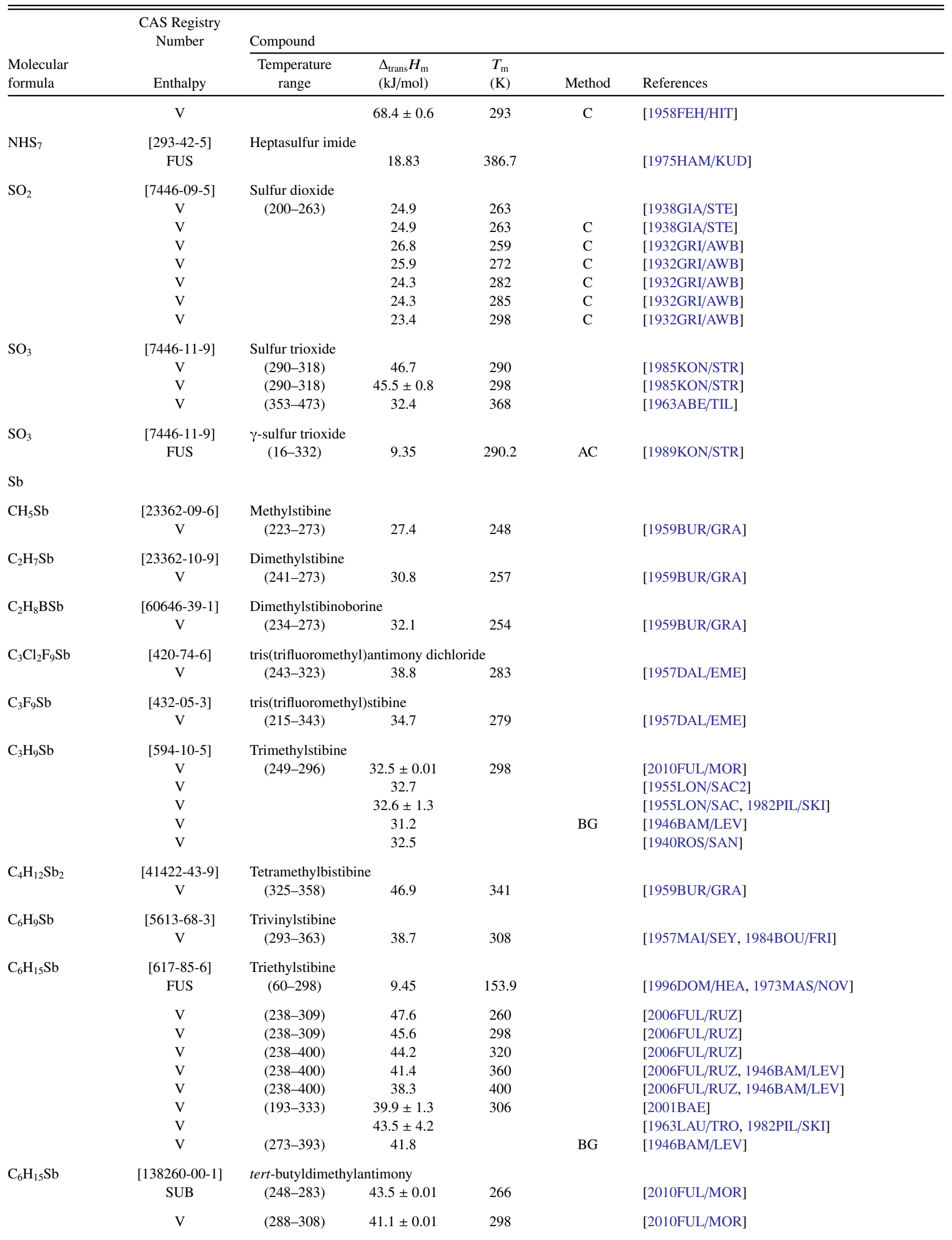


TABLE 17. Phase change enthalpies of organometallic and inorganic compounds-Continued

\begin{tabular}{|c|c|c|c|c|c|c|}
\hline \multirow[b]{2}{*}{$\begin{array}{l}\text { Molecular } \\
\text { formula }\end{array}$} & \multirow{2}{*}{$\begin{array}{c}\text { CAS Registry } \\
\text { Number } \\
\text { Enthalpy }\end{array}$} & \multicolumn{5}{|l|}{ Compound } \\
\hline & & $\begin{array}{l}\text { Temperature } \\
\text { range }\end{array}$ & $\begin{array}{l}\Delta_{\text {trans }} H_{\mathrm{m}} \\
(\mathrm{kJ} / \mathrm{mol})\end{array}$ & $\begin{array}{l}T_{\mathrm{m}} \\
(\mathrm{K})\end{array}$ & Method & References \\
\hline $\mathrm{C}_{6} \mathrm{H}_{18} \mathrm{NSb}$ & $\begin{array}{c}{[7289-92-1]} \\
\mathrm{V}\end{array}$ & \multicolumn{4}{|c|}{ tris(dimethylamino)antimony } & [2013MOR/FUL] \\
\hline $\mathrm{C}_{15} \mathrm{H}_{30} \mathrm{~N}_{3} \mathrm{~S}_{6} \mathrm{Sb}$ & $\begin{array}{l}{[22914-48-3]} \\
\text { SUB }\end{array}$ & \multicolumn{4}{|c|}{$\operatorname{tris}(N, N$-diethyldithiocarbamate)antimony(III) } & [1994LIE/MAR] \\
\hline $\mathrm{C}_{18} \mathrm{~F}_{15} \mathrm{Sb}$ & $\begin{array}{l}\text { [3910-39-2] } \\
\text { FUS }\end{array}$ & \multicolumn{3}{|c|}{ tris(pentafluorophenyl)antimony } & DSC & [2008ZEL/CHU] \\
\hline \multirow[t]{2}{*}{$\mathrm{C}_{18} \mathrm{H}_{15} \mathrm{Sb}$} & $\begin{array}{l}{[603-36-1]} \\
\text { SUB }\end{array}$ & \multicolumn{4}{|c|}{$\begin{array}{l}\text { Triphenylantimony } \\
\qquad 106.3 \pm 8.4\end{array}$} & [1982PIL/SKI, 1960BIR] \\
\hline & $\mathrm{V}$ & $(503-553)$ & 83.3 & 518 & A & [1987STE/MAL, 1949FOR/BOW] \\
\hline $\mathrm{C}_{21} \mathrm{H}_{42} \mathrm{~N}_{3} \mathrm{~S}_{6} \mathrm{Sb}$ & $\begin{array}{c}\text { [226980-30-9] } \\
\text { SUB }\end{array}$ & \multicolumn{3}{|c|}{ tris(dipropyldithiocarbamate)antimony(III) } & DSC,E & [1999NEV/GOU] \\
\hline $\mathrm{C}_{26} \mathrm{H}_{25} \mathrm{O}_{4} \mathrm{Sb}$ & $\begin{array}{c}{[1305343-65-0]} \\
\text { TRS }\end{array}$ & \multicolumn{3}{|c|}{ Triphenylantimony dimethacrylate } & $\mathrm{AC}$ & [2011MAR/LET2] \\
\hline $\mathrm{C}_{27} \mathrm{H}_{54} \mathrm{~N}_{3} \mathrm{~S}_{6} \mathrm{Sb}$ & $\begin{array}{l}{[14907-93-8]} \\
\text { SUB }\end{array}$ & \multicolumn{3}{|c|}{$\operatorname{tris}(N, N$-dibutyldithiocarbamate)antimony(III) } & & [1994LIE/MAR] \\
\hline $\mathrm{C}_{27} \mathrm{H}_{54} \mathrm{~N}_{3} \mathrm{~S}_{6} \mathrm{Sb}$ & $\begin{array}{l}\text { [41594-79-0] } \\
\text { SUB }\end{array}$ & \multicolumn{3}{|c|}{$\operatorname{tris}(N, N$-diisobutyldithiocarbamate)antimony(III) } & DSC,E & [1997DES/DES] \\
\hline $\mathrm{C}_{34} \mathrm{H}_{31} \mathrm{~N}_{2} \mathrm{O}_{2} \mathrm{Sb}$ & $\begin{array}{c}\text { [474647-34-2] } \\
\text { FUS }\end{array}$ & \multicolumn{4}{|c|}{ Triphenylantimony bis(acetophenoneoximate) } & [2011MAR/LET] \\
\hline $\mathrm{C}_{40} \mathrm{H}_{45} \mathrm{O}_{4} \mathrm{Sb}$ & $\begin{array}{l}\text { [1168154-05-9] } \\
\text { FUS }\end{array}$ & \multicolumn{3}{|c|}{ Triphenylantimony bis(1-adamantanecarboxylate) } & DSC & [2013LET/MAR] \\
\hline $\mathrm{SbBr}_{3}$ & $\begin{array}{c}\text { [7789-61-9] } \\
\text { V } \\
\text { V } \\
\text { V }\end{array}$ & \multicolumn{4}{|c|}{$\begin{array}{l}\text { Tribromostibine } \\
\qquad \begin{array}{l}(399-588) \\
(399-588) \\
(435-561)\end{array}\end{array}$} & $\begin{array}{l}{[1973 \mathrm{MAE}]} \\
{[1973 \mathrm{MAE}]} \\
{[1963 \mathrm{SIM}]}\end{array}$ \\
\hline $\mathrm{SbCl}_{3}$ & $\begin{array}{c}\text { [10025-91-9] } \\
\text { V } \\
\text { V } \\
\text { V } \\
\text { V }\end{array}$ & $\begin{array}{l}\text { Trichlorostibin } \\
\qquad \begin{array}{l}(351-492) \\
(381-491) \\
(381-491) \\
(363-463)\end{array}\end{array}$ & $\begin{array}{c}49.0 \pm 1.3 \\
47.2 \\
60.4 \\
46.7\end{array}$ & $\begin{array}{l}422 \\
495 \\
298 \\
496\end{array}$ & $\begin{array}{l}\mathrm{BG} \\
\mathrm{BG}\end{array}$ & $\begin{array}{l}\text { [1974UST/PET] } \\
{[1973 \mathrm{MAE}]} \\
{[1973 \mathrm{MAE}]} \\
{[1967 \mathrm{OPP}]}\end{array}$ \\
\hline $\mathrm{SbCl}_{5}$ & $\begin{array}{c}{[7647-18-9]} \\
\mathrm{V} \\
\mathrm{V}\end{array}$ & $\begin{array}{l}\text { Pentachloroant } \\
\quad(329-360) \\
(323-393)\end{array}$ & $\begin{array}{c}46.4 \pm 0.8 \\
43.4\end{array}$ & $\begin{array}{l}344 \\
449\end{array}$ & & $\begin{array}{l}{[1974 \mathrm{UST} / \mathrm{PET}]} \\
{[1967 \mathrm{OPP}]}\end{array}$ \\
\hline $\mathrm{Sbl}_{3}$ & $\begin{array}{c}{[7790-44-5]} \\
\mathrm{V}\end{array}$ & \multirow{2}{*}{\multicolumn{2}{|c|}{$\begin{array}{l}\text { Triiodostibine } \\
\quad(510-629)\end{array}$}} & 570 & & [1963SIM] \\
\hline \multicolumn{5}{|l|}{ Sc } & & \\
\hline $\mathrm{C}_{15} \mathrm{H}_{3} \mathrm{~F}_{18} \mathrm{O}_{6} \mathrm{Sc}$ & $\begin{array}{c}\text { [18990-42-6] } \\
\text { SUB } \\
\text { SUB } \\
\text { SUB }\end{array}$ & $\begin{array}{l}\operatorname{tris}(1,1,1,5,5,5 \\
\qquad \begin{array}{l}(333-363) \\
(313-348)\end{array}\end{array}$ & $\begin{array}{l}\text { Afluoro-2,4-pe } \\
113.3 \\
55.0 \\
60.2 \pm 1.2\end{array}$ & onato)sc & $\begin{array}{l}\text { m(III) } \\
\text { ME,MS } \\
\text { TGA } \\
\text { I }\end{array}$ & $\begin{array}{l}\text { [2012BEL/GIR] } \\
\text { [2000FAH/BAR] } \\
\text { [1978KOM/GUR] }\end{array}$ \\
\hline $\mathrm{C}_{15} \mathrm{H}_{12} \mathrm{~F}_{9} \mathrm{O}_{6} \mathrm{Sc}$ & $\begin{array}{c}\text { [14634-68-5] } \\
\text { SUB } \\
\text { SUB } \\
\text { SUB }\end{array}$ & $\begin{array}{l}\operatorname{tris}(1,1,1-\text { triflu } \\
\quad(373-403) \\
(363-433) \\
(366-413)\end{array}$ & $\begin{array}{c}\text { 2,4-pentanedi } \\
78.0 \\
117.6 \pm 1.7 \\
53.2 \pm 1.0\end{array}$ & scandiu & $\begin{array}{c}\text { TGA } \\
\text { I }\end{array}$ & $\begin{array}{l}\text { [2000FAH/BAR] } \\
{[1985 \mathrm{MAT} / \mathrm{KUW}]} \\
{[1978 \mathrm{KOM} / \mathrm{GUR}]}\end{array}$ \\
\hline & $\mathrm{V}$ & $(397-457)$ & $82.2 \pm 0.8$ & 427 & & [1978CHU/IGU] \\
\hline $\mathrm{C}_{15} \mathrm{H}_{15} \mathrm{Sc}$ & $\begin{array}{l}\text { [1298-54-0] } \\
\text { SUB }\end{array}$ & tris(cyclopenta & $\begin{array}{l}\text { yl)scandium } \\
97.1 \pm 3.5\end{array}$ & 298 & & [1982PIL/SKI, 1974DEV/RAB] \\
\hline $\mathrm{C}_{15} \mathrm{H}_{21} \mathrm{O}_{6} \mathrm{Sc}$ & $\begin{array}{l}{[14284-94-7]} \\
\text { FUS }\end{array}$ & tris(2,4-pentan & $\begin{array}{l}\text { tato) scandiun } \\
28.8\end{array}$ & 460 & & [1970MEL/MER2] \\
\hline & SUB & & 119 & & ME,MS & [2012BEL/GIR] \\
\hline
\end{tabular}


TABLE 17. Phase change enthalpies of organometallic and inorganic compounds-Continued

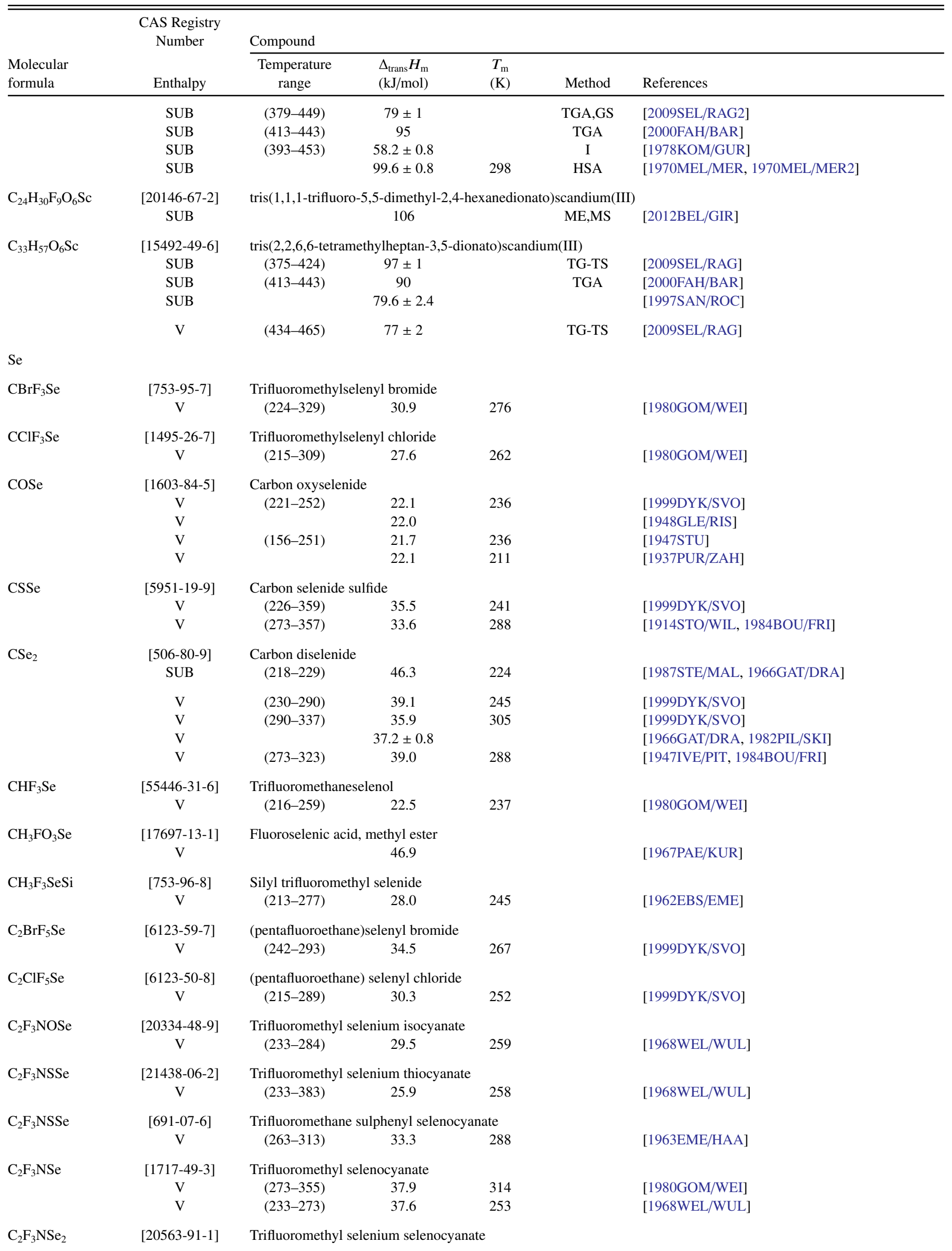


TABLE 17. Phase change enthalpies of organometallic and inorganic compounds-Continued

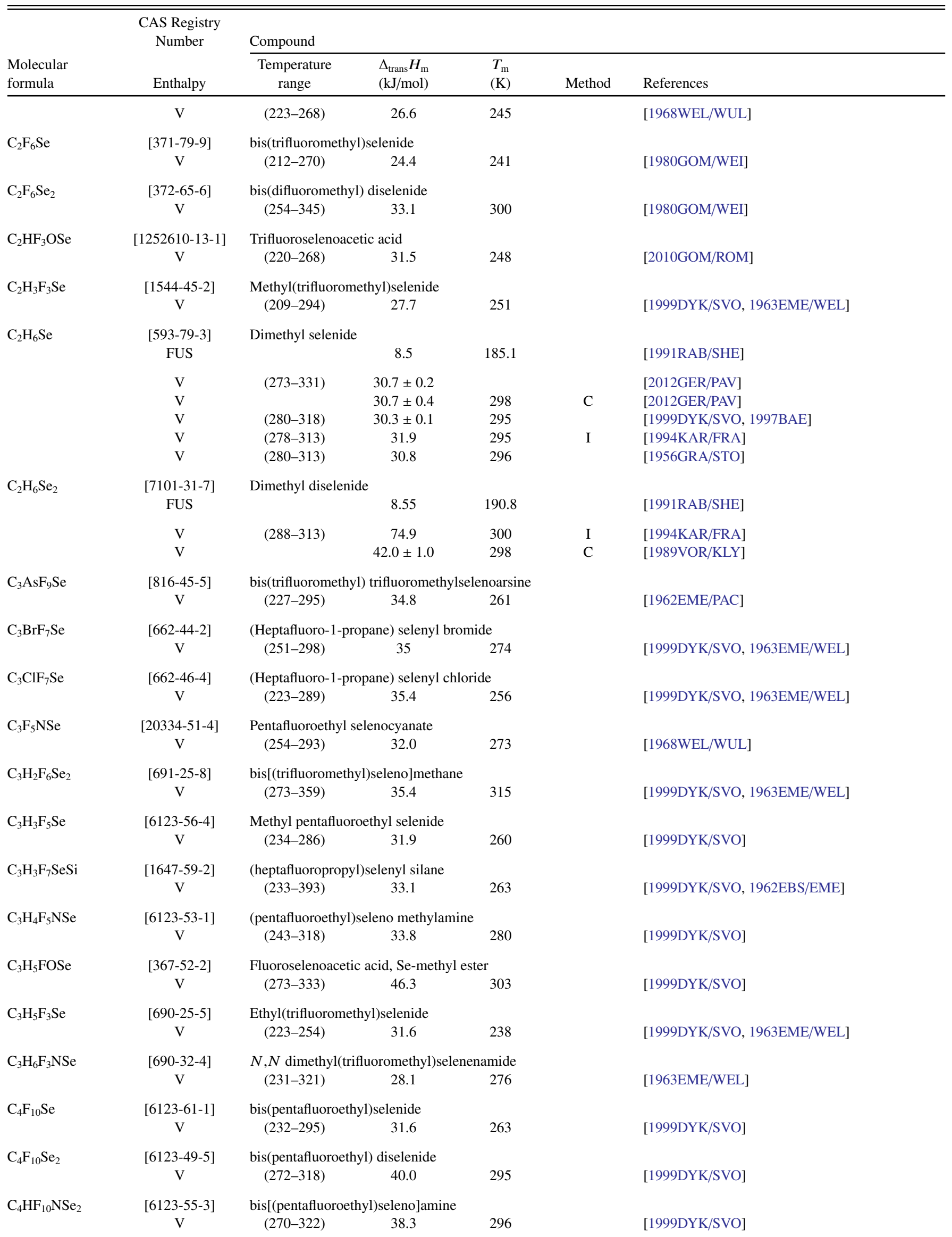


TABLE 17. Phase change enthalpies of organometallic and inorganic compounds-Continued

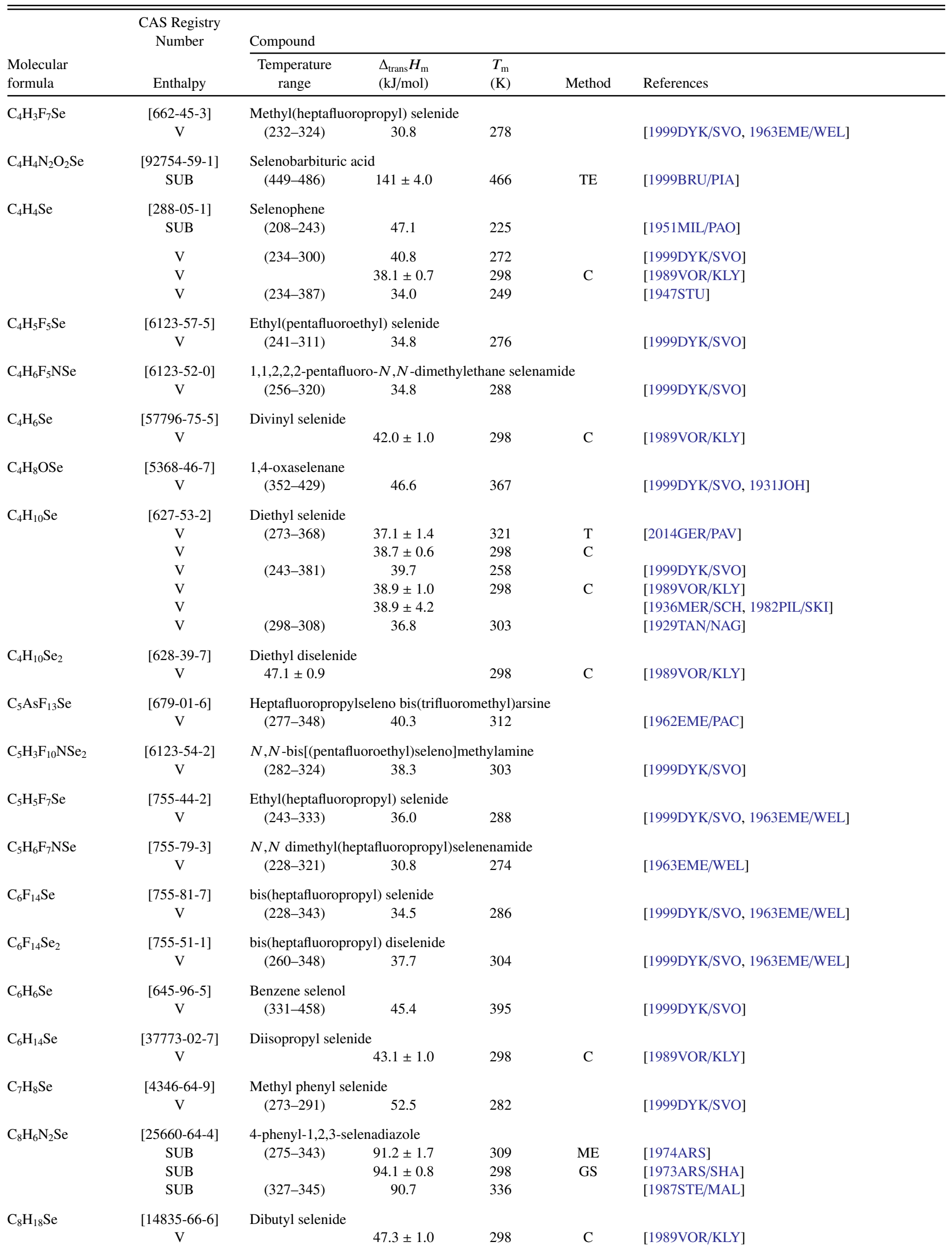


TABLE 17. Phase change enthalpies of organometallic and inorganic compounds-Continued

\begin{tabular}{|c|c|c|c|c|c|c|}
\hline \multirow[b]{2}{*}{$\begin{array}{l}\text { Molecular } \\
\text { formula }\end{array}$} & \multirow{2}{*}{$\begin{array}{l}\text { CAS Registry } \\
\text { Number } \\
\text { Enthalpy }\end{array}$} & \multicolumn{5}{|l|}{ Compound } \\
\hline & & $\begin{array}{l}\text { Temperature } \\
\text { range }\end{array}$ & $\begin{array}{l}\Delta_{\text {trans }} H_{\mathrm{m}} \\
(\mathrm{kJ} / \mathrm{mol})\end{array}$ & $\begin{array}{l}T_{\mathrm{m}} \\
(\mathrm{K})\end{array}$ & Method & References \\
\hline $\mathrm{C}_{10} \mathrm{H}_{22} \mathrm{Se}$ & $\begin{array}{c}{[14835-67-7]} \\
V\end{array}$ & Dipentyl selenide & $51.9 \pm 1.0$ & 298 & $\mathrm{C}$ & [1989VOR/KLY] \\
\hline $\mathrm{C}_{12} \mathrm{H}_{10} \mathrm{Se}$ & $\begin{array}{c}\text { [1132-39-4] } \\
\text { SUB } \\
\text { V } \\
\text { V } \\
\text { V }\end{array}$ & $\begin{array}{l}\text { Diphenyl selenide } \\
\qquad(302-324) \\
(379-575) \\
(378-575)\end{array}$ & $\begin{array}{c}116.7 \pm 2.5 \\
63.4 \\
61.9 \\
63.6 \pm 2.5\end{array}$ & $\begin{array}{l}313 \\
394 \\
393\end{array}$ & ME & $\begin{array}{l}\text { [1980MOR/WAT] } \\
\text { [1999DYK/SVO] } \\
\text { [1987STE/MAL] } \\
\text { [1973BAR/MOR, 1982PIL/SKI] }\end{array}$ \\
\hline $\mathrm{C}_{14} \mathrm{H}_{10} \mathrm{Cl}_{2} \mathrm{~N}_{4} \mathrm{O}_{2} \mathrm{Se}$ & $\begin{array}{c}\text { [1000863-66-0] } \\
\text { FUS }\end{array}$ & $\mathrm{N}, N^{\prime}$-bis $[(2$-chloro & $\begin{array}{c}\text { 3-pyridinyl)c } \\
17.64\end{array}$ & $\begin{array}{l}\text { yl]carbc } \\
460\end{array}$ & $\begin{array}{l}\text { doselenoi } \\
\text { DSC }\end{array}$ & $\begin{array}{l}\text { cid, methyl ester } \\
\text { [2009PLA/LIZ] }\end{array}$ \\
\hline $\mathrm{C}_{14} \mathrm{H}_{12} \mathrm{~N}_{4} \mathrm{O}_{2} \mathrm{Se}$ & FUS & $N, N^{\prime}$-bis $[(3-$ pyridi & $\begin{array}{c}\text { nyl)carbonyl] } \\
12.85\end{array}$ & $\begin{array}{l}\operatorname{mimido} \\
431.5\end{array}$ & $\begin{array}{l}\text { ic acid, m } \\
\text { DSC }\end{array}$ & $\begin{array}{l}\text { yl ester } \\
\text { [2009PLA/LIZ] }\end{array}$ \\
\hline $\mathrm{C}_{14} \mathrm{H}_{14} \mathrm{Se}_{2}$ & $\begin{array}{c}{[1482-82-2]} \\
\text { SUB }\end{array}$ & $\begin{array}{l}\text { Dibenzyl diselenide } \\
\quad(291-330)\end{array}$ & 130.5 & & ME & [1974ARS, 1973ARS/SHA] \\
\hline $\mathrm{C}_{16} \mathrm{H}_{12} \mathrm{Cl}_{2} \mathrm{~N}_{2} \mathrm{O}_{2} \mathrm{Se}$ & $\begin{array}{c}{[1000863-72-8]} \\
\text { FUS }\end{array}$ & $N, N^{\prime}$-bis(4-chlorol & $\begin{array}{c}\text { enzoyl)carba } \\
16.23\end{array}$ & $\begin{array}{c}\text { doselen } \\
467.6\end{array}$ & $\begin{array}{l}\text { d, methyl } \\
\text { DSC }\end{array}$ & 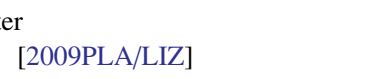 \\
\hline $\mathrm{C}_{16} \mathrm{H}_{14} \mathrm{~N}_{2} \mathrm{O}_{2} \mathrm{Se}$ & $\begin{array}{c}\text { [1000863-67-1] } \\
\text { FUS }\end{array}$ & $N, N^{\prime}$-dibenzoylca & $\begin{array}{c}\text { rbamimidosel } \\
10.56\end{array}$ & $\begin{array}{l}\text { acid, m } \\
412.7\end{array}$ & DSC & [2009PLA/LIZ] \\
\hline $\mathrm{C}_{17} \mathrm{H}_{16} \mathrm{~N}_{2} \mathrm{O}_{2} \mathrm{Se}$ & $\begin{array}{c}{[1000863-68-2]} \\
\text { FUS }\end{array}$ & $N, N^{\prime}$-dibenzoylca & $\begin{array}{c}\text { rbamimidosel } \\
10.38\end{array}$ & $\begin{array}{l}\text { acid,etl } \\
379.1\end{array}$ & DSC & [2009PLA/LIZ] \\
\hline $\mathrm{C}_{18} \mathrm{H}_{12} \mathrm{~F}_{6} \mathrm{~N}_{4} \mathrm{O}_{2} \mathrm{Se}$ & $\begin{array}{c}{[1000863-75-1]} \\
\text { FUS }\end{array}$ & $N, N^{\prime}$-bis $[4-($ trifluc & $\begin{array}{l}\text { romethyl)ben } \\
14.77\end{array}$ & $\begin{array}{c}\text { carbami } \\
446.1\end{array}$ & $\begin{array}{l}\text { elenoic ac } \\
\text { DSC }\end{array}$ & $\begin{array}{l}\text { methyl ester } \\
\text { [2009PLA/LIZ] }\end{array}$ \\
\hline $\mathrm{C}_{18} \mathrm{H}_{12} \mathrm{~N}_{4} \mathrm{O}_{2} \mathrm{Se}$ & $\begin{array}{c}{[1000863-76-2]} \\
\text { FUS }\end{array}$ & $N, N^{\prime}$-bis(4-cyanol & $\begin{array}{l}\text { enzoyl)carba } \\
17.55\end{array}$ & $\begin{array}{c}\text { doselen } \\
492.1\end{array}$ & $\begin{array}{l}\text { d, methyl } \\
\text { DSC }\end{array}$ & [2009PLA/LIZ] \\
\hline $\mathrm{C}_{18} \mathrm{H}_{16} \mathrm{Cl}_{2} \mathrm{~N}_{2} \mathrm{O}_{2} \mathrm{Se}$ & $\begin{array}{c}\text { [1000863-73-9] } \\
\text { FUS }\end{array}$ & $N, N^{\prime}$-bis(4-chloro & $\begin{array}{c}\text { benzoyl)carba } \\
13.97\end{array}$ & $\begin{array}{l}\text { doselen } \\
437.8\end{array}$ & $\begin{array}{l}\text { id, 1-metl } \\
\text { DSC }\end{array}$ & $\begin{array}{l}\text { thyl ester } \\
\text { [2009PLA/LIZ] }\end{array}$ \\
\hline $\mathrm{C}_{18} \mathrm{H}_{18} \mathrm{~N}_{2} \mathrm{O}_{2} \mathrm{Se}$ & $\begin{array}{c}\text { [1000863-69-3] } \\
\text { FUS }\end{array}$ & $N, N^{\prime}$-dibenzoylca & $\begin{array}{l}\text { rbamimidosel } \\
8.04\end{array}$ & $\begin{array}{c}\text { acid, } 1- \\
380.2\end{array}$ & $\begin{array}{l}\text { lethyl est } \\
\text { DSC }\end{array}$ & [2009PLA/LIZ] \\
\hline $\mathrm{C}_{18} \mathrm{H}_{18} \mathrm{~N}_{2} \mathrm{O}_{2} \mathrm{Se}$ & $\begin{array}{c}{[1000863-78-4]} \\
\text { FUS }\end{array}$ & $N, N^{\prime}$-bis(4-methy & $\begin{array}{c}\text { lbenzoyl)carb } \\
12.21\end{array}$ & $\begin{array}{l}\text { idoseler } \\
421.7\end{array}$ & $\begin{array}{l}\text { id, methy } \\
\text { DSC }\end{array}$ & $\begin{array}{l}\text { ster } \\
\text { [2009PLA/LIZ] }\end{array}$ \\
\hline $\mathrm{C}_{20} \mathrm{H}_{22} \mathrm{~N}_{2} \mathrm{O}_{6} \mathrm{Se}$ & $\begin{array}{c}{[1000863-70-6]} \\
\text { FUS }\end{array}$ & $N, N^{\prime}$-bis $(3,5$-dime & $\begin{array}{c}\text { thoxybenzoyl } \\
18.43\end{array}$ & $\begin{array}{c}\text { amimid } \\
436.8\end{array}$ & $\begin{array}{l}\text { oic acid, } \\
\text { DSC }\end{array}$ & $\begin{array}{l}\text { thyl ester } \\
\text { [2009PLA/LIZ] }\end{array}$ \\
\hline $\mathrm{C}_{22} \mathrm{H}_{16} \mathrm{Br}_{2} \mathrm{~N}_{2} \mathrm{Se}_{2}$ & $\begin{array}{c}{[1448890-32-1]} \\
\text { FUS }\end{array}$ & 1,4-bis[[(4-bromop & $\begin{array}{c}\text { nenyl)methyl] } \\
16.9\end{array}$ & $\begin{array}{l}\text { o]phtha } \\
379.0\end{array}$ & $\mathrm{DSC}$ & [2013JIM/PLA] \\
\hline $\mathrm{C}_{22} \mathrm{H}_{16} \mathrm{~N}_{4} \mathrm{O}_{4} \mathrm{Se}_{2}$ & $\begin{array}{c}\text { [1448890-33-2] } \\
\text { FUS }\end{array}$ & 1,4-bis[[(4-nitrophe & $\begin{array}{l}\text { nyl)methyl]se } \\
\text { U3.07 }\end{array}$ & $\begin{array}{l}\text { phthala } \\
420.0\end{array}$ & DSC & [2013JIM/PLA] \\
\hline $\mathrm{C}_{22} \mathrm{H}_{18} \mathrm{~N}_{2} \mathrm{~S}_{2} \mathrm{Se}_{2}$ & $\begin{array}{c}{[1448890-51-4]} \\
\text { FUS }\end{array}$ & 4,4'-[1,4-phthalazin & $\begin{array}{l}\text { ediylbis(thion } \\
\text { U7.56 }\end{array}$ & $\begin{array}{l}\text { lene)]bi } \\
395.6\end{array}$ & $\begin{array}{l}\text { eneselenc } \\
\text { DSC }\end{array}$ & [2013JIM/PLA] \\
\hline $\mathrm{C}_{22} \mathrm{H}_{20} \mathrm{~N}_{4} \mathrm{Se}_{2}$ & $\begin{array}{c}{[1448890-43-4]} \\
\text { FUS }\end{array}$ & 4,4'-[1,4-phthalazin & $\begin{array}{l}\text { ediylbis(imin } \\
\text { U4.83 }\end{array}$ & $\begin{array}{l}\text { nylene)] } \\
392.3\end{array}$ & $\begin{array}{l}\text { DSenesele } \\
\text { DSC }\end{array}$ & [2013JIM/PLA] \\
\hline $\mathrm{C}_{22} \mathrm{H}_{26} \mathrm{~N}_{2} \mathrm{O}_{6} \mathrm{Se}$ & $\begin{array}{c}{[1000863-71-7]} \\
\text { FUS }\end{array}$ & $N, N^{\prime}$-bis $(3,5$-dime & $\begin{array}{c}\text { thoxybenzoy } \\
19.34\end{array}$ & $\begin{array}{l}\text { amimid } \\
426.9\end{array}$ & $\begin{array}{l}\text { oic acid, } \\
\text { DSC }\end{array}$ & $\begin{array}{l}\text { hethyl ethyl ester } \\
\text { [2009PLA/LIZ] }\end{array}$ \\
\hline $\mathrm{C}_{24} \mathrm{H}_{16} \mathrm{~F}_{6} \mathrm{~N}_{2} \mathrm{Se}_{2}$ & $\begin{array}{c}\text { [1448890-30-9] } \\
\text { FUS }\end{array}$ & 1,4-bis[[[]4-(trifluor & $\begin{array}{l}\text { methyl)phen } \\
28.52\end{array}$ & $\begin{array}{c}\text { thyl]sel } \\
391.9\end{array}$ & $\begin{array}{l}\text { thalazine } \\
\text { DSC }\end{array}$ & [2013JIM/PLA] \\
\hline $\mathrm{C}_{24} \mathrm{H}_{16} \mathrm{~N}_{4} \mathrm{~S}_{2} \mathrm{Se}_{2}$ & $\begin{array}{c}\text { [1448890-53-6] } \\
\text { FUS }\end{array}$ & Selenocyanic acid, & $\begin{array}{c}C C^{\prime}-[1,4-\text { phth } \\
13.11\end{array}$ & $\begin{array}{l}\text { ediylbi } \\
408.1\end{array}$ & $\begin{array}{c}\text { nethylene } \\
\text { DSC }\end{array}$ & $\begin{array}{l}\text { I-phenylene)] ester } \\
\text { [2013JIM/PLA] }\end{array}$ \\
\hline $\mathrm{C}_{24} \mathrm{H}_{16} \mathrm{~N}_{4} \mathrm{Se}_{2}$ & $\begin{array}{c}\text { [1448890-34-3] } \\
\text { FUS }\end{array}$ & 4,4'-[1,4-phthalazin & $\begin{array}{c}\text { ediylbis(selen } \\
18.97\end{array}$ & $\begin{array}{l}\text { hylene) } \\
461.1\end{array}$ & $\begin{array}{l}\text { nzonitrile } \\
\text { DSC }\end{array}$ & [2013JIM/PLA] \\
\hline
\end{tabular}


TABLE 17. Phase change enthalpies of organometallic and inorganic compounds-Continued

\begin{tabular}{|c|c|c|c|c|c|c|}
\hline \multirow[b]{2}{*}{$\begin{array}{l}\text { Molecular } \\
\text { formula }\end{array}$} & \multirow{2}{*}{$\begin{array}{l}\text { CAS Registry } \\
\text { Number } \\
\text { Enthalpy }\end{array}$} & \multicolumn{5}{|l|}{ Compound } \\
\hline & & $\begin{array}{c}\text { Temperature } \\
\text { range }\end{array}$ & $\begin{array}{l}\Delta_{\text {trans }} H_{\mathrm{m}} \\
(\mathrm{kJ} / \mathrm{mol})\end{array}$ & $\begin{array}{l}T_{\mathrm{m}} \\
(\mathrm{K})\end{array}$ & Method & References \\
\hline & FUS & & 10.31 & 427.8 & DSC & [2013JIM/PLA] \\
\hline $\mathrm{C}_{24} \mathrm{H}_{22} \mathrm{~N}_{2} \mathrm{O}_{2} \mathrm{Se}_{2}$ & $\begin{array}{c}{[1448890-28-5]} \\
\text { FUS }\end{array}$ & 1,4-bis[[(4-me & $\begin{array}{l}\text { phenyl)methy } \\
26.58\end{array}$ & $\begin{array}{l}\text { eno]phtl } \\
387.0\end{array}$ & DSC & [2013JIM/PLA] \\
\hline $\mathrm{C}_{24} \mathrm{H}_{22} \mathrm{~N}_{2} \mathrm{~S}_{2} \mathrm{Se}_{2}$ & $\begin{array}{c}{[1448890-52-5]} \\
\text { FUS }\end{array}$ & 1,4-bis[[[4-(me & $\begin{array}{c}\text { seleno)phenyl } \\
10.03\end{array}$ & $\begin{array}{l}\text { yl]thio] } \\
382.2\end{array}$ & $\begin{array}{l}\text { lazine } \\
\text { DSC }\end{array}$ & [2013JIM/PLA] \\
\hline $\mathrm{C}_{24} \mathrm{H}_{22} \mathrm{~N}_{2} \mathrm{Se}_{2}$ & $\begin{array}{c}{[1448890-31-0]} \\
\text { FUS }\end{array}$ & 1,4-bis[[(4-me & $\begin{array}{c}\text { henyl)methyl } \\
28.14\end{array}$ & $\begin{array}{l}\text { o]phtha } \\
397.7\end{array}$ & DSC & [2013JIM/PLA] \\
\hline $\mathrm{C}_{24} \mathrm{H}_{30} \mathrm{~N}_{2} \mathrm{O}_{2} \mathrm{Se}$ & $\begin{array}{c}{[1000863-79-5]} \\
\text { FUS }\end{array}$ & $N, N^{\prime}$-bis $[4-(1$ & $\begin{array}{c}\text { methylethyl)b } \\
13.04\end{array}$ & $\begin{array}{c}\text { yl]carba } \\
437.5\end{array}$ & $\begin{array}{l}\text { doselenoic } \\
\text { DSC }\end{array}$ & $\begin{array}{l}\text { id, methyl ester } \\
\text { [2009PLA/LIZ] }\end{array}$ \\
\hline $\mathrm{C}_{28} \mathrm{H}_{38} \mathrm{~N}_{2} \mathrm{O}_{2} \mathrm{Se}$ & $\begin{array}{c}\text { [1396294-64-6] } \\
\text { FUS }\end{array}$ & 2-[4-(butylsele & $\begin{array}{l}\text { henyl }]-5-[4-(d \\
37.6\end{array}$ & $\begin{array}{l}\text { xy)pher } \\
356.9\end{array}$ & $\begin{array}{l}\text { 3,4-oxadi } \\
\text { DSC }\end{array}$ & $\begin{array}{l}\text { le } \\
\text { [2012FRI/RAM] }\end{array}$ \\
\hline $\mathrm{C}_{30} \mathrm{H}_{22} \mathrm{~N}_{4} \mathrm{~S}_{4} \mathrm{Se}_{2}$ & $\begin{array}{c}{[1448890-35-4]} \\
\text { FUS }\end{array}$ & $4,4^{\prime}-[$ dithiobis ( & $\begin{array}{c}\text { hthalazinediy } \\
40.51\end{array}$ & $\begin{array}{c}\text { methyle } \\
471.0\end{array}$ & $\begin{array}{l}\text { S(benzene } \\
\text { DSC }\end{array}$ & $\begin{array}{l}\text { nol) } \\
\text { [2013JIM/PLA] }\end{array}$ \\
\hline $\mathrm{C}_{32} \mathrm{H}_{26} \mathrm{~N}_{4} \mathrm{~S}_{4} \mathrm{Se}_{2}$ & $\begin{array}{c}{[1448890-36-5]} \\
\text { FUS }\end{array}$ & 1,1'-dithiobis $[4$ & $\begin{array}{c}\text {-(methylselen } \\
25.07\end{array}$ & $\begin{array}{l}\text { enyl]me } \\
399.2\end{array}$ & $\begin{array}{l}\text { io]phthal } \\
\text { DSC }\end{array}$ & [2013JIM/PLA] \\
\hline $\mathrm{C}_{35} \mathrm{H}_{52} \mathrm{O}_{2} \mathrm{Se}$ & $\begin{array}{c}\text { [1714083-30-3] } \\
\text { FUS }\end{array}$ & cholest-5-en-3 & $\begin{array}{c}\beta), 3-[2-(\text { phen } \\
26.4\end{array}$ & $\begin{array}{l}371.2 \\
37 \text { acet }\end{array}$ & DSC & [2015FRI/RAF] \\
\hline $\mathrm{C}_{36} \mathrm{H}_{54} \mathrm{~N}_{2} \mathrm{O}_{2} \mathrm{Se}$ & $\begin{array}{c}\text { [1396294-61-3] } \\
\text { FUS }\end{array}$ & 3-[4-(decyloxy & $\begin{array}{c}\text { nyl }]-5-[4-(\mathrm{doc} \\
30.6\end{array}$ & $\begin{array}{l}\text { eleno)p } \\
346.6\end{array}$ & $\begin{array}{l}-1,2,4-\mathrm{ox} \\
\text { DSC }\end{array}$ & $\begin{array}{l}\text { Izole } \\
\text { [2012FRI/RAM] }\end{array}$ \\
\hline $\mathrm{C}_{36} \mathrm{H}_{54} \mathrm{O}_{2} \mathrm{Se}$ & $\begin{array}{c}\text { [1714083-31-4] } \\
\text { FUS }\end{array}$ & Cholest-5-en-3 & $\begin{array}{c}3 \beta), 3-[3-(\text { phe } \\
16.5\end{array}$ & $\begin{array}{c}\text { leno)pro } \\
320.3\end{array}$ & DSC & [2015FRI/RAF] \\
\hline $\mathrm{C}_{58} \mathrm{H}_{94} \mathrm{O}_{4} \mathrm{Se}_{2}$ & $\begin{array}{c}\text { [1714083-33-6] } \\
\text { FUS }\end{array}$ & Cholest-5-en-3 & $\begin{array}{c}3 \beta), 3,3^{\prime}-\left(2,2^{\prime}\right. \\
18.0\end{array}$ & $\begin{array}{c}\text { enodiace } \\
433.9\end{array}$ & DSC & [2015FRI/RAF] \\
\hline $\mathrm{Cl}_{2} \mathrm{OSe}$ & $\begin{array}{c}{[7791-23-3]} \\
\mathrm{V} \\
\mathrm{V}\end{array}$ & $\begin{array}{l}\text { Selenium oxyc } \\
\qquad(352-476) \\
(353-453)\end{array}$ & $\begin{array}{l}59.1 \\
46.9\end{array}$ & $\begin{array}{l}367 \\
403\end{array}$ & & $\begin{array}{l}\text { [1999DYK/SVO] } \\
\text { [1971NIS/TRE] }\end{array}$ \\
\hline $\mathrm{D}_{2} \mathrm{Se}$ & $\begin{array}{c}\text { [13536-95-3] } \\
\text { TRS } \\
\text { TRS } \\
\text { FUS }\end{array}$ & Hydrogen sele & $\begin{array}{r}-\mathrm{d}_{2} \\
\\
1.94 \\
1.18 \\
2.49\end{array}$ & $\begin{array}{c}90.5 \\
176.0 \\
206.6\end{array}$ & & [1937KRU/CLU] \\
\hline & $\mathrm{V}$ & $(202-256)$ & 22.2 & 217 & & [1999DYK/SVO] \\
\hline $\mathrm{F}_{2} \mathrm{OSe}$ & $\begin{array}{c}\text { [7783-43-9] } \\
\text { FUS }\end{array}$ & Seleninyl diflu & 8.08 & 288 & & [1979CAR/CLA, 1977BOU/CAR] \\
\hline & $\begin{array}{l}\mathrm{V} \\
\mathrm{V} \\
\mathrm{V}\end{array}$ & $(316-420)$ & $\begin{array}{c}52.1 \\
46.9 \pm 0.8 \\
46.7\end{array}$ & $\begin{array}{l}331 \\
298\end{array}$ & $\mathrm{C}$ & $\begin{array}{l}\text { [1999DYK/SVO] } \\
{[1979 \mathrm{CAR} / \mathrm{CLA}]} \\
{[1977 \mathrm{BOU} / \mathrm{CAR}]}\end{array}$ \\
\hline $\mathrm{F}_{4} \mathrm{Se}$ & $\begin{array}{c}{[13465-66-2]} \\
\mathrm{V}\end{array}$ & $\begin{array}{l}\text { Selenium tetra } \\
\quad(297-398)\end{array}$ & de 46.4 & 312 & & [1999DYK/SVO] \\
\hline $\mathrm{F}_{6} \mathrm{Se}$ & $\begin{array}{c}\text { [7783-79-1] } \\
\text { SUB } \\
\text { SUB }\end{array}$ & $\begin{array}{r}\text { Selenium hexa } \\
\text { (194-226) }\end{array}$ & $\begin{array}{l}\text { ide } \\
24.96 \pm 0.04 \\
\quad 23.5\end{array}$ & $\begin{array}{l}205 \\
210\end{array}$ & $\mathrm{C}$ & $\begin{array}{l}{[1996 \mathrm{OHT} / \mathrm{OSA}]} \\
{[1932 \mathrm{KLE} / \mathrm{HEN}]}\end{array}$ \\
\hline $\mathrm{F}_{6} \mathrm{O}_{2} \mathrm{Se}$ & $\begin{array}{c}{[27069-91-6]} \\
\mathrm{V}\end{array}$ & $\begin{array}{l}\text { trans bis(fluor } \\
\quad(241-286)\end{array}$ & $\begin{array}{l}\text { tetrafluorosele } \\
26.5\end{array}$ & 263 & & [1970SMI/CAD] \\
\hline $\mathrm{H}_{2} \mathrm{Se}$ & $\begin{array}{c}\text { [7783-07-5] } \\
\text { TRS } \\
\text { TRS } \\
\text { FUS }\end{array}$ & Hydrogen sele & $\begin{array}{l}1.57 \\
1.12 \\
2.52\end{array}$ & $\begin{array}{c}82.3 \\
172.5 \\
207.4\end{array}$ & & [1937KRU/CLU] \\
\hline & V & $(231-304)$ & 19.8 & & & [1902DEF/FON] \\
\hline
\end{tabular}


TABLE 17. Phase change enthalpies of organometallic and inorganic compounds-Continued

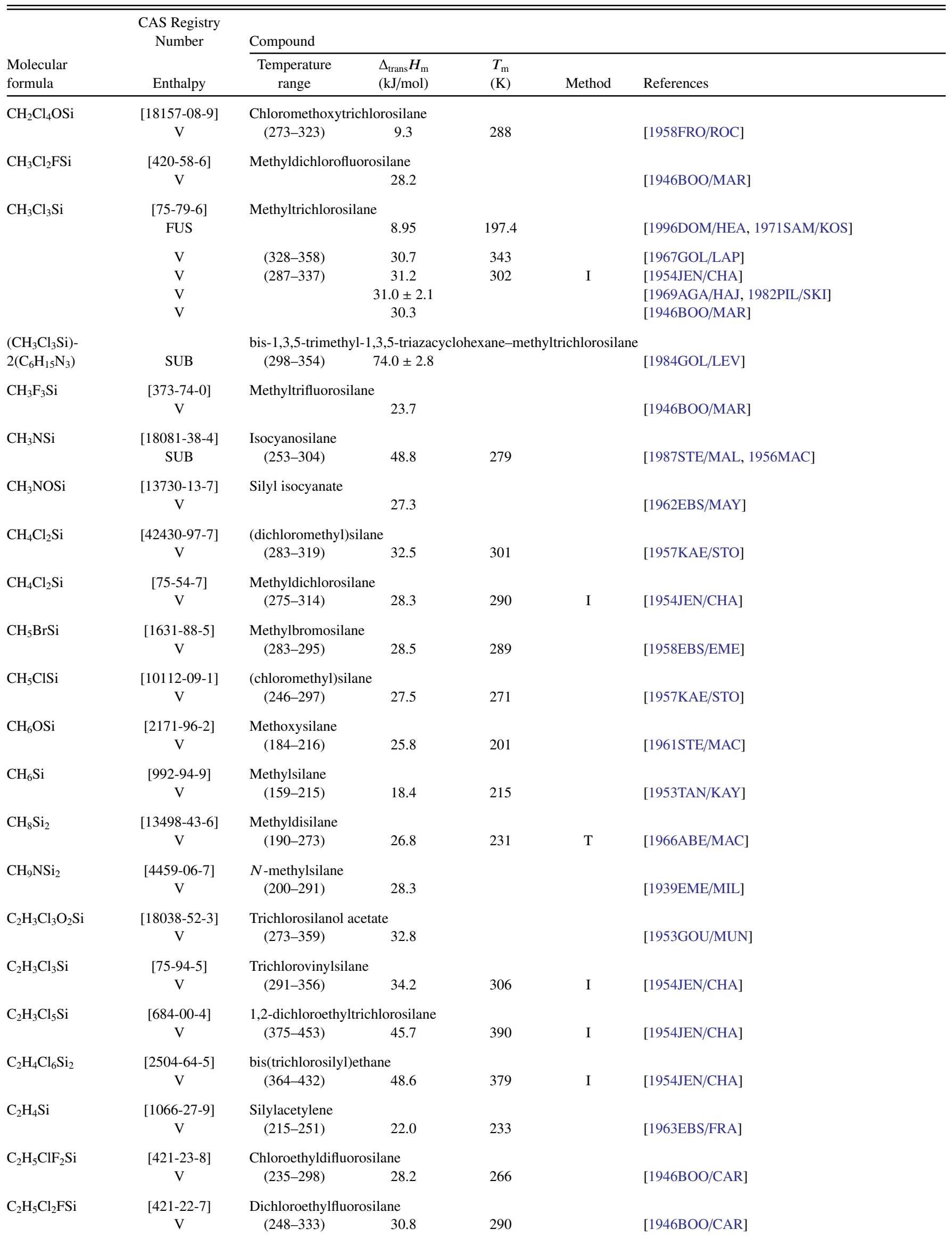


TABLE 17. Phase change enthalpies of organometallic and inorganic compounds-Continued

\begin{tabular}{|c|c|c|c|c|c|c|}
\hline \multirow[b]{2}{*}{$\begin{array}{l}\text { Molecular } \\
\text { formula }\end{array}$} & \multirow{2}{*}{$\begin{array}{l}\text { CAS Registry } \\
\text { Number } \\
\text { Enthalpy }\end{array}$} & \multicolumn{5}{|l|}{ Compound } \\
\hline & & $\begin{array}{l}\text { Temperature } \\
\text { range }\end{array}$ & $\begin{array}{l}\Delta_{\text {trans }} H_{\mathrm{m}} \\
(\mathrm{kJ} / \mathrm{mol})\end{array}$ & $\begin{array}{l}T_{\mathrm{m}} \\
(\mathrm{K})\end{array}$ & Method & References \\
\hline $\mathrm{C}_{2} \mathrm{H}_{5} \mathrm{Cl}_{3} \mathrm{Si}$ & $\begin{array}{l}{[115-21-9]} \\
\text { FUS }\end{array}$ & $\begin{array}{l}\text { Ethyltrichlorosilane } \\
\qquad(13-300)\end{array}$ & 6.96 & 165.3 & & [1996DOM/HEA, 1969NAG/DZH] \\
\hline & $\begin{array}{l}\mathrm{V} \\
\mathrm{V} \\
\mathrm{V}\end{array}$ & $\begin{array}{l}(303-363) \\
(301-368) \\
(263-373)\end{array}$ & $\begin{array}{l}35.1 \\
35.9 \\
32.2\end{array}$ & $\begin{array}{l}318 \\
316 \\
318\end{array}$ & I & $\begin{array}{l}{[1970 \mathrm{SOK} / \mathrm{KAR}]} \\
{[1954 \mathrm{JEN} / \mathrm{CHA}]} \\
{[1946 \mathrm{BOO} / \mathrm{CAR}]}\end{array}$ \\
\hline $\mathrm{C}_{2} \mathrm{H}_{5} \mathrm{~F}_{3} \mathrm{Si}$ & $\begin{array}{c}{[353-89-9]} \\
\mathrm{V} \\
\mathrm{V}\end{array}$ & $\begin{array}{l}\text { Ethyltrifluorosilane } \\
\qquad \begin{array}{l}(193-268) \\
(201-268)\end{array}\end{array}$ & $\begin{array}{l}25.6 \\
25.9\end{array}$ & 230 & & $\begin{array}{l}\text { [1946BOO/CAR] } \\
{[1944 \mathrm{EME} / \mathrm{WIL}]}\end{array}$ \\
\hline $\mathrm{C}_{2} \mathrm{H}_{5} \mathrm{~F}_{3} \mathrm{OSi}$ & $\begin{array}{c}{[460-55-9]} \\
\mathrm{V}\end{array}$ & $\begin{array}{l}\text { Ethoxy trifluorosilane } \\
\quad(206-248)\end{array}$ & 26.8 & 227 & & [1949EME/HEA] \\
\hline $\mathrm{C}_{2} \mathrm{H}_{3} \mathrm{~F}_{3} \mathrm{O}_{2} \mathrm{Si}$ & $\begin{array}{c}{[6876-44-4]} \\
V\end{array}$ & $\begin{array}{l}\text { Silyl trifluoroacetate } \\
\quad(273-293)\end{array}$ & 30.7 & 283 & & [1987STE/MAL, 1967EBS/THO] \\
\hline $\mathrm{C}_{2} \mathrm{H}_{6} \mathrm{ClFSi}$ & $\begin{array}{c}{[420-57-5]} \\
V\end{array}$ & Chlorofluorodimethyls & $\begin{array}{l}\text { lsilane } \\
28.4\end{array}$ & & & {$[1946 \mathrm{BOO} / \mathrm{SUT}]$} \\
\hline $\mathrm{C}_{2} \mathrm{H}_{6} \mathrm{Cl}_{2} \mathrm{Si}$ & $\begin{array}{l}{[75-78-5]} \\
\text { FUS }\end{array}$ & Dichlorodimethylsilan & ne 8.83 & 199 & & [1996DOM/HEA, 1971SAM/KOS] \\
\hline & $\begin{array}{l}\mathrm{V} \\
\mathrm{V}\end{array}$ & $(301-345)$ & $\begin{array}{l}31.5 \\
31.5\end{array}$ & 316 & I & $\begin{array}{l}{[1954 J E N / C H A]} \\
{[1946 \mathrm{BOO} / \mathrm{SUT}]}\end{array}$ \\
\hline $\mathrm{C}_{2} \mathrm{H}_{6} \mathrm{Cl}_{2} \mathrm{Si}$ & $\begin{array}{c}{[1789-58-8]} \\
\mathrm{V} \\
\mathrm{V}\end{array}$ & $\begin{array}{l}\text { Dichloroethylsilane } \\
\qquad \begin{array}{l}(279-346) \\
(301-345)\end{array}\end{array}$ & $\begin{array}{l}31.5 \\
31.6\end{array}$ & $\begin{array}{l}294 \\
316\end{array}$ & I & $\begin{array}{l}\text { [1954JEN/CHA] } \\
\text { [1954JEN/CHA, 1987STE/MAL] }\end{array}$ \\
\hline $\mathrm{C}_{2} \mathrm{H}_{6} \mathrm{Cl}_{4} \mathrm{Si}_{2}$ & $\begin{array}{c}{[4518-98-3]} \\
\mathrm{V}\end{array}$ & $\begin{array}{l}\text { 1,1,2,2-tetrachloro- } 1,2 \\
\quad(300-375)\end{array}$ & $\begin{array}{l}\text { 2-dimethy } \\
42.4\end{array}$ & 337 & & [1967REE/URR] \\
\hline $\mathrm{C}_{2} \mathrm{H}_{6} \mathrm{~F}_{2} \mathrm{Si}$ & $\begin{array}{c}{[353-66-2]} \\
\mathrm{V}\end{array}$ & Difluorodimethylsilan & 25.8 & & & {$[1946 \mathrm{BOO} / \mathrm{SUT}]$} \\
\hline $\mathrm{C}_{2} \mathrm{H}_{6} \mathrm{~F}_{3} \mathrm{NSi}$ & $\begin{array}{c}{[812-14-6]} \\
\mathrm{V}\end{array}$ & $\begin{array}{l}\text { 1,1,1-trifluoro- } N, N-\mathrm{c} \\
\quad(225-288)\end{array}$ & $\begin{array}{l}\text {-dimethyla } \\
28.5\end{array}$ & $\begin{array}{l}\text { lane } \\
273\end{array}$ & & [1961GRO/KLE] \\
\hline $\mathrm{C}_{2} \mathrm{H}_{6} \mathrm{Si}$ & $\begin{array}{c}{[7291-09-0]} \\
\mathrm{V}\end{array}$ & $\begin{array}{l}\text { Vinylsilane } \\
\quad(186-250)\end{array}$ & 21.4 & 250 & & [1953TAN/KAY] \\
\hline $\mathrm{C}_{2} \mathrm{H}_{7} \mathrm{ISi}$ & $\begin{array}{c}{[2441-21-6]} \\
\mathrm{V}\end{array}$ & $\begin{array}{l}\text { Dimethyliodosilane } \\
\quad(273-323)\end{array}$ & 28.3 & 298 & & [1958EME/SMY] \\
\hline $\mathrm{C}_{2} \mathrm{H}_{8} \mathrm{Si}$ & $\begin{array}{c}{[1111-74-6]} \\
\mathrm{V}\end{array}$ & $\begin{array}{l}\text { Dimethylsilane } \\
(187-251)\end{array}$ & 21.3 & 253 & & [1953TAN/KAY] \\
\hline $\mathrm{C}_{2} \mathrm{H}_{8} \mathrm{Si}$ & $\begin{array}{c}{[2814-79-1]} \\
\mathrm{V}\end{array}$ & $\begin{array}{l}\text { Ethylsilane } \\
\qquad(198-257)\end{array}$ & 22.3 & 260 & & [1953TAN/KAY] \\
\hline $\mathrm{C}_{2} \mathrm{H}_{9} \mathrm{NSi}$ & $\begin{array}{c}{[2875-98-1]} \\
\text { SUB }\end{array}$ & $\begin{array}{l}\text { Dimethylaminosilane } \\
\quad(228-264)\end{array}$ & 58.8 & 246 & A & [1987STE/MAL, 1954SUJ/WIT] \\
\hline $\mathrm{C}_{2} \mathrm{H}_{10} \mathrm{Si}_{2}$ & $\begin{array}{c}{[870-26-8]} \\
\mathrm{V}\end{array}$ & $\begin{array}{l}\text { 1,2-dimethyldisilane } \\
\quad(227-273)\end{array}$ & 25.4 & 258 & & [1962CRA/MAC] \\
\hline $\mathrm{C}_{2} \mathrm{H}_{11} \mathrm{NSi}_{2}$ & $\begin{array}{c}{[14396-26-0]} \\
\mathrm{V}\end{array}$ & $\begin{array}{l}N, N \text { dimethyldisilany } \\
\quad(207-273)\end{array}$ & $\begin{array}{c}\text { ylamine } \\
35.4\end{array}$ & 240 & $\mathrm{~T}$ & [1963ABE/MAC] \\
\hline $\mathrm{C}_{3} \mathrm{H}_{4} \mathrm{Cl}_{3} \mathrm{NSi}$ & $\begin{array}{c}{[2621-01-4]} \\
\mathrm{V}\end{array}$ & $\begin{array}{l}\text { Trichloro- } \beta \text {-cyanoethy } \\
\quad(343-443)\end{array}$ & $\begin{array}{c}\text { ylsilane } \\
53.5\end{array}$ & 358 & & [1978SHM/SHL] \\
\hline $\mathrm{C}_{3} \mathrm{H}_{4} \mathrm{Cl}_{3} \mathrm{NSi}$ & $\begin{array}{c}{[1071-22-3]} \\
\text { FUS }\end{array}$ & $\beta$-trichlorosilylpropior & $\begin{array}{l}\text { nitrile } \\
21.24\end{array}$ & 307.9 & & {$[1975 \mathrm{KOS} / \mathrm{SAM}]$} \\
\hline $\mathrm{C}_{3} \mathrm{H}_{5} \mathrm{Cl}_{3} \mathrm{Si}$ & $\begin{array}{c}{[107-37-9]} \\
\mathrm{V}\end{array}$ & $\begin{array}{l}\text { Allyltrichlorosilane } \\
\quad(319-388)\end{array}$ & 40.1 & 333 & I & [1954JEN/CHA] \\
\hline $\mathrm{C}_{3} \mathrm{H}_{6} \mathrm{Cl}_{4} \mathrm{Si}$ & $\begin{array}{c}{[2550-06-3]} \\
\mathrm{V}\end{array}$ & $\begin{array}{l}\gamma \text {-chloropropyltrichlor } \\
\quad(313-443)\end{array}$ & $\begin{array}{r}\text { rsilane } \\
49.7\end{array}$ & 328 & & [1972SOK/BRA] \\
\hline
\end{tabular}


TABLE 17. Phase change enthalpies of organometallic and inorganic compounds-Continued

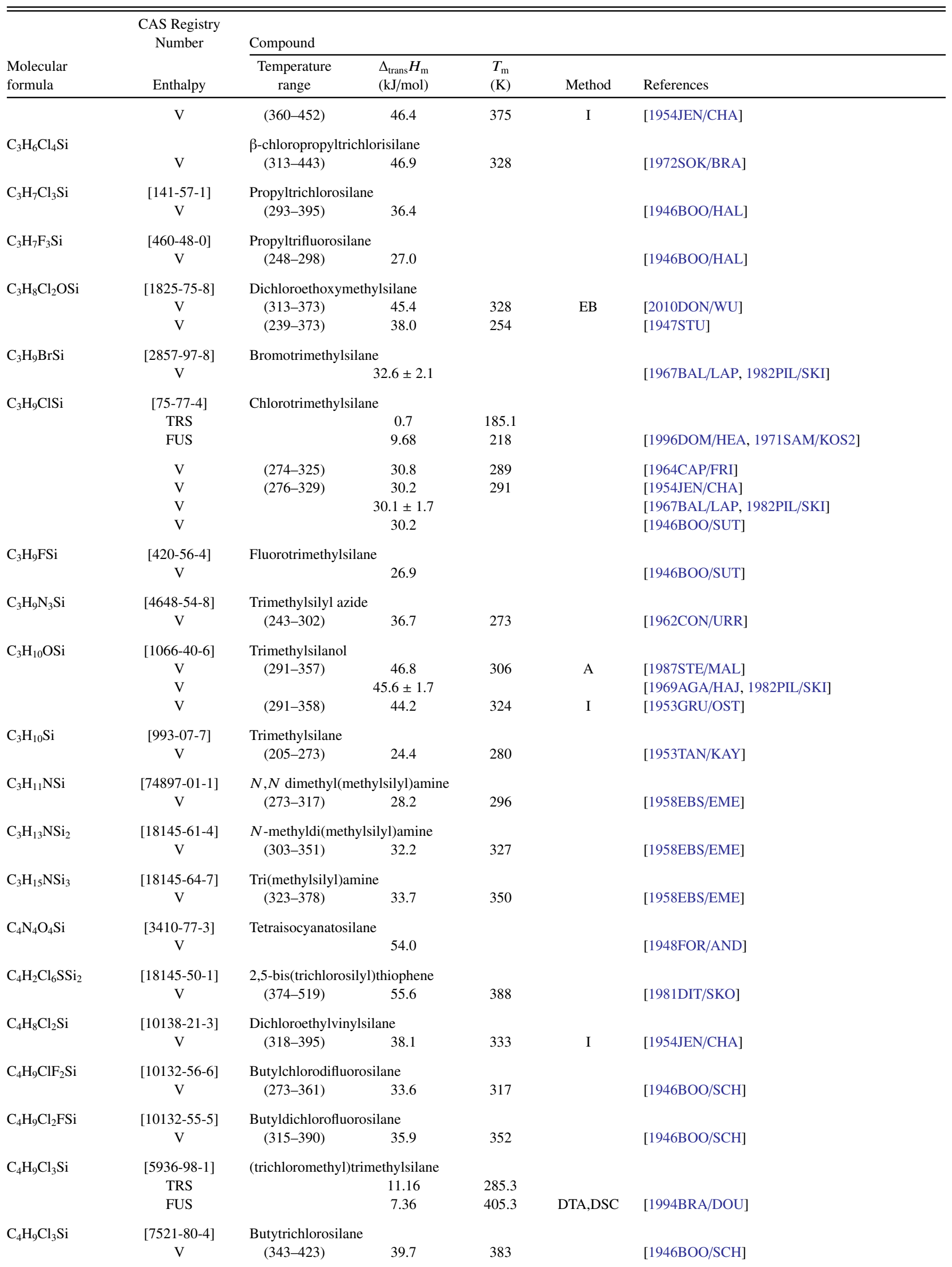


TABLE 17. Phase change enthalpies of organometallic and inorganic compounds-Continued

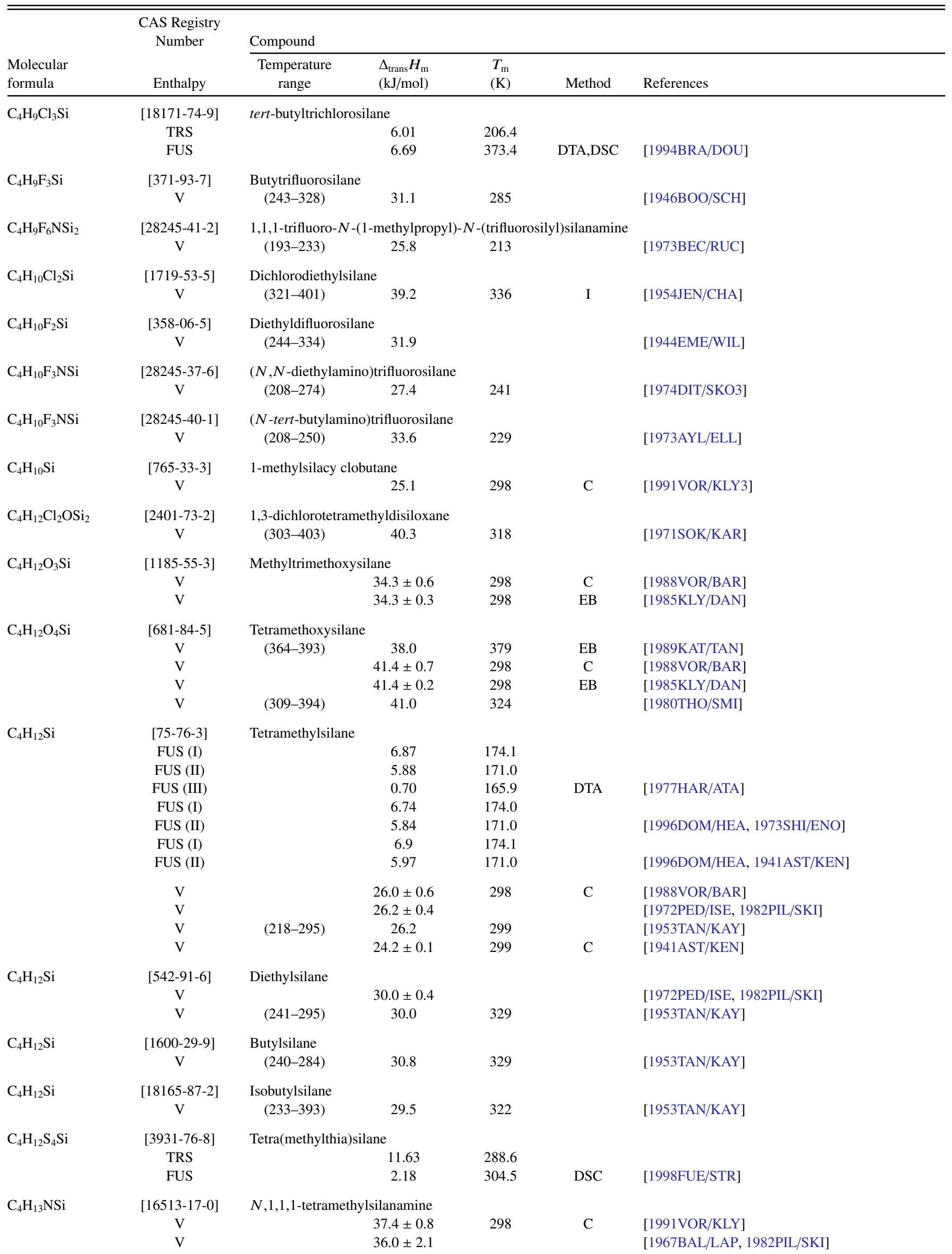


TABLE 17. Phase change enthalpies of organometallic and inorganic compounds-Continued

\begin{tabular}{|c|c|c|c|c|c|c|}
\hline \multirow[b]{2}{*}{$\begin{array}{l}\text { Molecular } \\
\text { formula }\end{array}$} & \multirow{2}{*}{$\begin{array}{c}\text { CAS Registry } \\
\text { Number } \\
\text { Enthalpy }\end{array}$} & \multicolumn{5}{|l|}{ Compound } \\
\hline & & $\begin{array}{l}\text { Temperature } \\
\text { range }\end{array}$ & $\begin{array}{l}\Delta_{\text {trans }} H_{\mathrm{m}} \\
(\mathrm{kJ} / \mathrm{mol})\end{array}$ & $\begin{array}{l}T_{\mathrm{m}} \\
(\mathrm{K})\end{array}$ & Method & References \\
\hline \multirow[t]{2}{*}{$\mathrm{C}_{4} \mathrm{H}_{14} \mathrm{~N}_{2} \mathrm{Si}$} & [4693-04-3] & \multicolumn{5}{|c|}{ bis(dimethylamino)silane } \\
\hline & $\mathrm{V}$ & $(288-344)$ & 32.4 & 316 & $\mathrm{~T}$ & [1964AYL/PET] \\
\hline \multirow[t]{2}{*}{$\mathrm{C}_{4} \mathrm{H}_{14} \mathrm{~N}_{2} \mathrm{Si}$} & {$[18148-26-0]$} & \multicolumn{5}{|c|}{ bis(aminomethyl)dimethylsilane } \\
\hline & $\mathrm{V}$ & $(293-413)$ & 39.3 & & & [1962GOU/FRO] \\
\hline \multirow{2}{*}{$\mathrm{C}_{4} \mathrm{H}_{14} \mathrm{OSi}$} & {$[3277-26-7]$} & \multicolumn{5}{|c|}{ 1,1,3,3-tetramethyldisiloxane } \\
\hline & V & $(273-323)$ & 30.3 & 298 & & [1958EME/SMY] \\
\hline \multirow[t]{2}{*}{$\mathrm{C}_{4} \mathrm{H}_{14} \mathrm{SSi}_{2}$} & {$[16642-70-9]$} & \multicolumn{5}{|c|}{ 1,1,3,3-tetramethyldisilthiane } \\
\hline & $\mathrm{V}$ & $(273-323)$ & 28.7 & 298 & & [1958EME/SMY] \\
\hline \multirow[t]{2}{*}{$\mathrm{C}_{4} \mathrm{H}_{16} \mathrm{~N}_{2} \mathrm{Si}_{2}$} & {$[18148-05-5]$} & \multicolumn{5}{|c|}{$N, N, N^{\prime}, N^{\prime}$-tetramethyldisilanyldiamine } \\
\hline & $\mathrm{V}$ & $(311-354)$ & 39.3 & 332 & $\mathrm{~T}$ & {$[1963 \mathrm{ABE} / \mathrm{MAC}]$} \\
\hline \multirow[t]{2}{*}{$\mathrm{C}_{4} \mathrm{H}_{16} \mathrm{O}_{4} \mathrm{Si}_{4}$} & {$[2370-88-9]$} & \multicolumn{5}{|c|}{ 2,4,6,8-tetramethylcyclotetrasiloxane } \\
\hline & $\mathrm{V}$ & & 41.2 & & $\mathrm{~EB}$ & {$[2012 Y U E / D O N]$} \\
\hline \multirow[t]{2}{*}{$\mathrm{C}_{5} \mathrm{H}_{5} \mathrm{~N}_{3} \mathrm{O}_{4} \mathrm{Si}$} & {$[18243-45-3]$} & \multicolumn{5}{|c|}{ Ethoxytriisocyanatosilane } \\
\hline & $\mathrm{V}$ & & 48.1 & & & [1948FOR/AND] \\
\hline \multirow[t]{2}{*}{$\mathrm{C}_{5} \mathrm{H}_{6} \mathrm{Cl}_{2} \mathrm{SSi}$} & {$[18243-72-6]$} & \multicolumn{5}{|c|}{ 2-(methyldichlorosilyl)thiophene } \\
\hline & $\mathrm{V}$ & $(341-467)$ & 46.4 & 356 & & {$[1981 \mathrm{DIT} / \mathrm{SKO}]$} \\
\hline \multirow[t]{2}{*}{$\mathrm{C}_{5} \mathrm{H}_{9} \mathrm{~F}_{6} \mathrm{NOSSi}$} & {$[34556-30-4]$} & \multicolumn{5}{|c|}{$S, S$-bis(trifluoromethyl)- $N$-(trimethylsilyl)sulfoximine } \\
\hline & $\mathrm{V}$ & & 33.5 & 378 & I & [1972SAU/SHR] \\
\hline \multirow[t]{2}{*}{$\mathrm{C}_{5} \mathrm{H}_{9} \mathrm{~F}_{6} \mathrm{PSSi}$} & [38680-96-5] & \multicolumn{5}{|c|}{ bis(trifluoromethyl)(trimethylsilylthio)phosphine } \\
\hline & $\mathrm{V}$ & (273-328) & 46.6 & 301 & & [1973GOS/MIL] \\
\hline \multirow[t]{2}{*}{$\mathrm{C}_{5} \mathrm{H}_{10} \mathrm{~F}_{3} \mathrm{NSi}$} & [33552-49-7] & \multicolumn{5}{|c|}{ 1-(trifluorosilyl)piperidine } \\
\hline & $\mathrm{V}$ & $(250-282)$ & 33.9 & 266 & & [1973AYL/ELL] \\
\hline \multirow[t]{2}{*}{$\mathrm{C}_{5} \mathrm{H}_{12} \mathrm{Si}$} & & \multicolumn{5}{|c|}{ 1,2-dimethylsilacyclobutane } \\
\hline & $\mathrm{V}$ & & 33.1 & 298 & $\mathrm{C}$ & [1991VOR/KLY3] \\
\hline
\end{tabular}

The authors list the compound as 1,2-dimethylsilacyclobutane in Table 1 and as 1,1-dimethylsilacyclobutane in Table 2 of their paper.

\begin{tabular}{|c|c|c|c|c|c|}
\hline \multirow[t]{5}{*}{$\mathrm{C}_{5} \mathrm{H}_{12} \mathrm{Si}$} & [2295-12-7] & \multicolumn{4}{|l|}{ 1,1-dimethylsilacyclobutane } \\
\hline & FUS & 6.76 & 155.5 & & [1975GUS/KAR] \\
\hline & $\mathrm{V}$ & 32.1 & 356 & & [1975GUS/KAR] \\
\hline & $\mathrm{V}$ & $33.0 \pm 0.8$ & 298 & I & [1974BES/MAR] \\
\hline & $\mathrm{V}$ & $34.7 \pm 2.1$ & & & [1972PED/ISE, 1982PIL/SKI] \\
\hline \multirow[t]{3}{*}{$\mathrm{C}_{5} \mathrm{H}_{12} \mathrm{Si}$} & {$[754-05-2]$} & Vinyltrimethylsilane & & & \\
\hline & FUS & 7.66 & 141.7 & & [1996DOM/HEA, 1975RAB/LEB] \\
\hline & $\mathrm{V}$ & $33.1 \pm 0.6$ & 298 & $\mathrm{C}$ & [1988VOR/BAR] \\
\hline \multirow[t]{2}{*}{$\mathrm{C}_{5} \mathrm{H}_{13} \mathrm{NSi}$} & [2116-90-7] & Trimethylsilylethyleneimine & & & \\
\hline & FUS & 10.5 & 192.5 & & [1999KUL/LEB] \\
\hline \multirow[t]{4}{*}{$\mathrm{C}_{5} \mathrm{H}_{14} \mathrm{OSi}$} & {$[1825-62-3]$} & Ethoxytrimethylsilane & & & \\
\hline & $\mathrm{V}$ & $38.4 \pm 0.6$ & 298 & $\mathrm{C}$ & [1988VOR/BAR] \\
\hline & $\mathrm{V}$ & $38.4 \pm 0.3$ & 298 & $\mathrm{~EB}$ & [1985KLY/DAN] \\
\hline & $\mathrm{V}$ & $(223-349)$ & 238 & & [1947STU] \\
\hline \multirow[t]{2}{*}{$\mathrm{C}_{5} \mathrm{H}_{14} \mathrm{O}_{3} \mathrm{SSi}$} & {$[57557-66-1]$} & Trimethoxy[(methylthio)methyl] & & & \\
\hline & $\mathrm{V}$ & $40.2 \pm 0.6$ & 298 & $\mathrm{C}$ & [1989VOR/SOR] \\
\hline \multirow[t]{7}{*}{$\mathrm{C}_{5} \mathrm{H}_{14} \mathrm{O}_{3} \mathrm{Si}$} & {$[5314-55-6]$} & Ethyl(trimethoxy)silane & & & \\
\hline & $\mathrm{V}$ & $(306-397) \quad 43.6$ & 316 & $\mathrm{~EB}$ & [2010WU/LIU] \\
\hline & $\mathrm{V}$ & $(306-397)$ & 339 & $\mathrm{~EB}$ & [2010WU/LIU] \\
\hline & $\mathrm{V}$ & $(306-397)$ & 360 & EB & [2010WU/LIU] \\
\hline & $\mathrm{V}$ & $(306-397)$ & 371 & EB & [2010WU/LIU] \\
\hline & $\mathrm{V}$ & $(306-397)$ & 380 & $\mathrm{~EB}$ & [2010WU/LIU] \\
\hline & $\mathrm{V}$ & $(306-397)$ & 390 & $\mathrm{~EB}$ & [2010WU/LIU] \\
\hline \multirow[t]{2}{*}{$\mathrm{C}_{5} \mathrm{H}_{14} \mathrm{Si}$} & {$[760-32-7]$} & Methyldiethylsilane & & & \\
\hline & $\mathrm{V}$ & $34.6 \pm 0.7$ & 298 & $\mathrm{C}$ & [1988VOR/BAR] \\
\hline
\end{tabular}


TABLE 17. Phase change enthalpies of organometallic and inorganic compounds-Continued

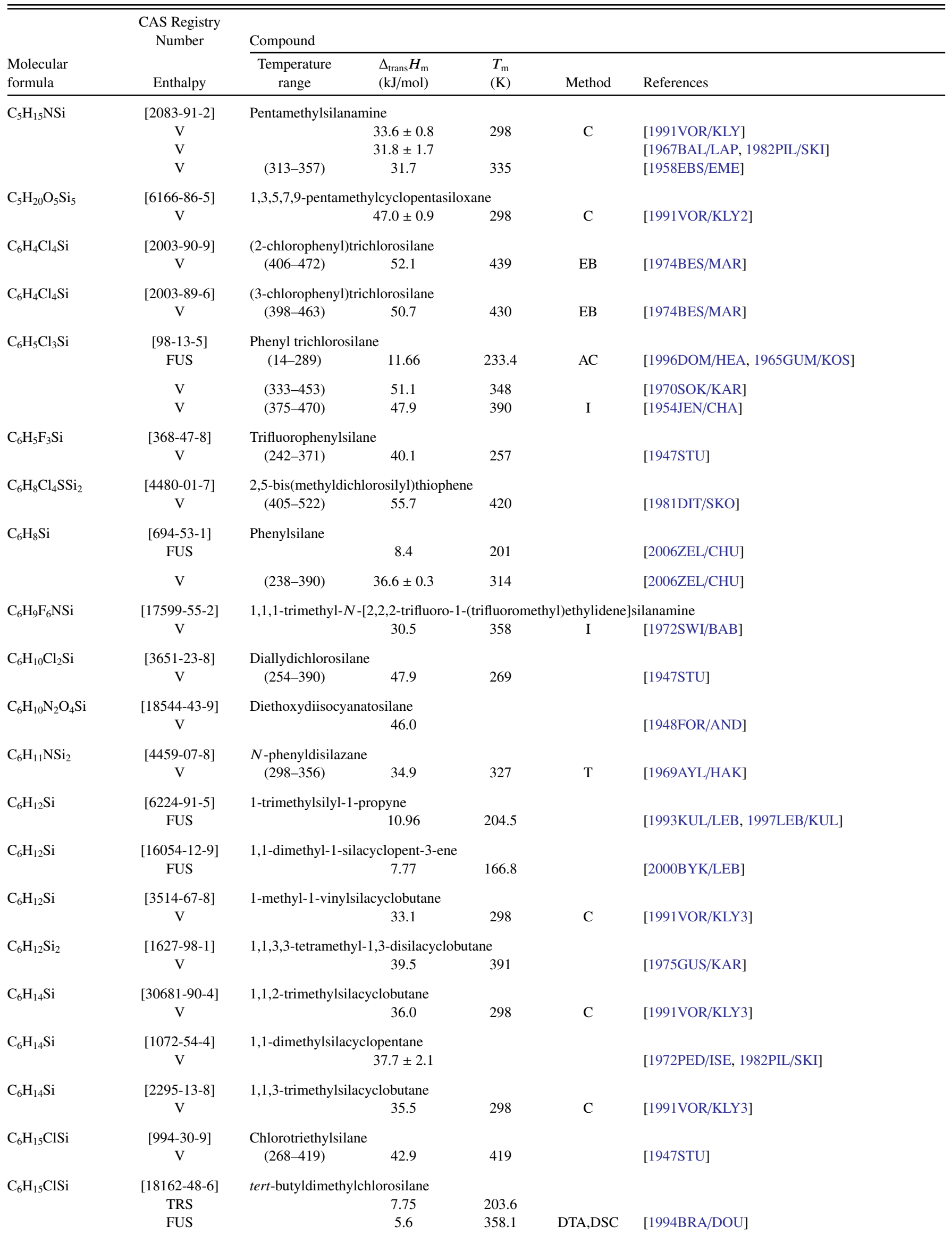


TABLE 17. Phase change enthalpies of organometallic and inorganic compounds-Continued

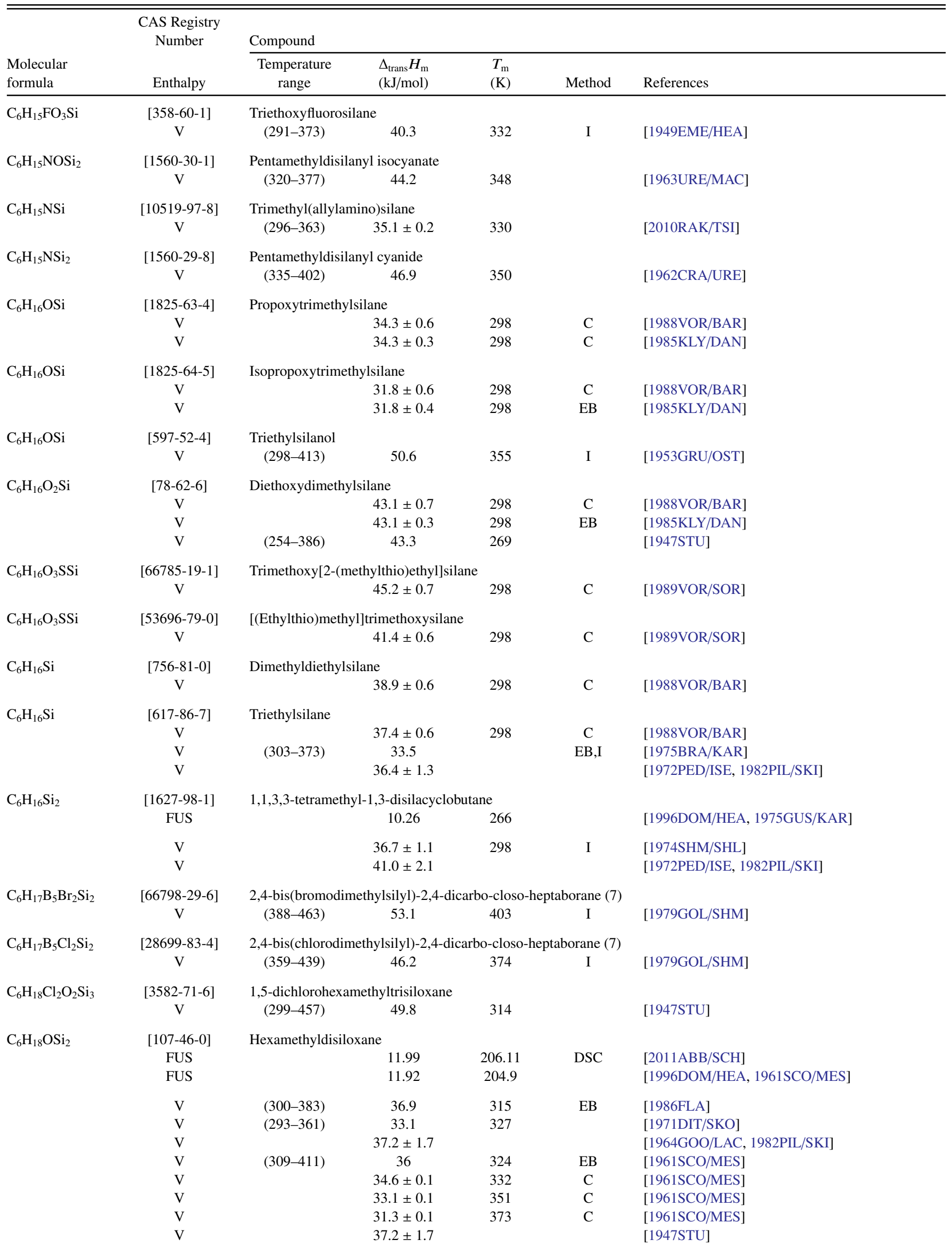


TABLE 17. Phase change enthalpies of organometallic and inorganic compounds-Continued

\begin{tabular}{|c|c|c|c|c|c|c|}
\hline \multirow[b]{2}{*}{$\begin{array}{l}\text { Molecular } \\
\text { formula }\end{array}$} & \multirow{2}{*}{$\begin{array}{l}\text { CAS Registry } \\
\text { Number } \\
\text { Enthalpy }\end{array}$} & \multicolumn{5}{|l|}{ Compound } \\
\hline & & $\begin{array}{c}\text { Temperature } \\
\text { range }\end{array}$ & $\begin{array}{l}\Delta_{\text {trans }} H_{\mathrm{m}} \\
(\mathrm{kJ} / \mathrm{mol})\end{array}$ & $\begin{array}{l}T_{\mathrm{m}} \\
(\mathrm{K})\end{array}$ & Method & References \\
\hline \multirow[t]{10}{*}{$\mathrm{C}_{6} \mathrm{H}_{18} \mathrm{O}_{3} \mathrm{Si}_{3}$} & [541-05-9] & \multicolumn{5}{|c|}{ Hexamethylcyclotrisiloxane } \\
\hline & FUS & & 19.4 & 344 & DSC & [1995OUT/KLO] \\
\hline & FUS & & 16.61 & 335.2 & & [1996DOM/HEA, 1982KUL/DZH] \\
\hline & FUS & & 11.8 & 335.2 & & {$[1971 \mathrm{ALV} / \mathrm{DAL}]$} \\
\hline & FUS & & 15.5 & 341.2 & $\mathrm{~S}-\mathrm{V}$ & [1953OST/GRU] \\
\hline & SUB & $(297-335)$ & $55.2 \pm 0.4$ & 316 & & [1953OST/GRU] \\
\hline & $\mathrm{V}$ & $(342-419)$ & 40.8 & 357 & $\mathrm{~EB}$ & [1986FLA] \\
\hline & $\mathrm{V}$ & $(353-403)$ & 39.7 & 368 & & [1974BRA/KAR] \\
\hline & $\mathrm{V}$ & $(339-407)$ & 39.0 & 373 & & [1971DIT/SKO] \\
\hline & $\mathrm{V}$ & $(343-388)$ & 39.7 & 365 & & [1953OST/GRU] \\
\hline \multirow[t]{7}{*}{$\mathrm{C}_{6} \mathrm{H}_{18} \mathrm{Si}_{2}$} & {$[1450-14-2]$} & \multicolumn{5}{|c|}{ Hexamethyldisilane } \\
\hline & TRS & & 9.75 & 221.8 & & \\
\hline & FUS & & 3.02 & 287.7 & & [1996DOM/HEA, 1959SUG/SEK] \\
\hline & $\mathrm{V}$ & $(305-387)$ & 36.3 & 320 & EB & [1986TAK/ISH] \\
\hline & $\mathrm{V}$ & & $37.4 \pm 0.4$ & & & [1972PED/ISE, 1982PIL/SKI] \\
\hline & $\mathrm{V}$ & $(288-310)$ & 37.2 & & & [1959SUG/SEK, 1986TAK/ISH] \\
\hline & $\mathrm{V}$ & $(294-334)$ & 36.8 & & & [1941BRO/DAV, 1986TAK/ISH] \\
\hline \multirow{2}{*}{$\mathrm{C}_{6} \mathrm{H}_{19} \mathrm{~B}_{5} \mathrm{Si}_{2}$} & {$[59351-11-0]$} & \multicolumn{5}{|c|}{ 2,4-bis(dimethylsilyl)-2,4-dicarba-closo-heptaborane } \\
\hline & $\mathrm{V}$ & $(373-453)$ & 41.3 & 388 & I & [1976SHM/SHL] \\
\hline \multirow[t]{5}{*}{$\mathrm{C}_{6} \mathrm{H}_{19} \mathrm{NSi}_{2}$} & [999-97-3] & \multicolumn{5}{|c|}{ Hexamethyldisilazane } \\
\hline & V & $(293-382)$ & $37.4 \pm 0.4$ & 338 & Static & [2015ERM/SYS] \\
\hline & $\mathrm{V}$ & & $42.2 \pm 0.9$ & 298 & $\mathrm{C}$ & [1991VOR/KLY] \\
\hline & $\mathrm{V}$ & $(294-395)$ & 36.0 & 344 & & [1972DIT/SKO2] \\
\hline & $\mathrm{V}$ & & $41.4 \pm 2.1$ & & & [1966BEE/MOR, 1982PIL/SKI] \\
\hline \multirow[t]{2}{*}{$\mathrm{C}_{6} \mathrm{H}_{19} \mathrm{~N}_{3} \mathrm{Si}$} & [15112-89-7] & \multicolumn{5}{|c|}{ tris(dimethylamino)silane } \\
\hline & $\mathrm{V}$ & (309-387) & 41.1 & 348 & $\mathrm{~T}$ & [1964AYL/PET] \\
\hline \multirow[t]{3}{*}{$\mathrm{C}_{6} \mathrm{H}_{21} \mathrm{~N}_{3} \mathrm{Si}_{3}$} & {$[1009-93-4]$} & \multicolumn{5}{|c|}{ Hexamethylcyclotrisilazane } \\
\hline & FUS & & 15.17 & 254.4 & & [1996DOM/HEA, 1981MEK/KAR] \\
\hline & $\mathrm{V}$ & $(342-456)$ & 45.6 & 399 & & [1972DIT/SKO2] \\
\hline \multirow[t]{2}{*}{$\mathrm{C}_{7} \mathrm{H}_{8} \mathrm{Cl}_{2} \mathrm{Si}$} & [18173-99-4] & \multicolumn{5}{|c|}{ Benzyl dichlorosilane } \\
\hline & $\mathrm{V}$ & $(318-467)$ & 58.5 & 333 & & [1947STU, 1999DYK/SVO] \\
\hline \multirow[t]{2}{*}{$\mathrm{C}_{7} \mathrm{H}_{8} \mathrm{Cl}_{2} \mathrm{Si}$} & {$[149-74-6]$} & \multicolumn{5}{|c|}{ Phenyldichloromethylsilane } \\
\hline & V & $(309-479)$ & 51.2 & 323 & & [1947STU, 1999DYK/SVO] \\
\hline \multirow[t]{2}{*}{$\mathrm{C}_{7} \mathrm{H}_{8} \mathrm{Cl}_{2} \mathrm{Si}$} & {$[13272-80-5]$} & Dichloro-4-toly & & & & \\
\hline & V & $(319-469)$ & 58 & 334 & & [1947STU, 1999DYK/SVO] \\
\hline $\mathrm{C}_{7} \mathrm{H}_{8} \mathrm{~F}_{2} \mathrm{Si}$ & {$[13272-80-5]$} & Difluoromethy & ylsilane & & & \\
\hline & $\mathrm{V}$ & $(303-413)$ & 44.6 & 318 & & [1999DYK/SVO] \\
\hline $\mathrm{C}_{7} \mathrm{H}_{9} \mathrm{~F}_{8} \mathrm{NOSSi}$ & {$[77589-40-3]$} & $2,2,3,3,4,4,5,5$ & luoro-1,1,2,3 & exahydr & trifluorom & yl)silyl]imino]thiophene-1-oxide \\
\hline & V & & 31.4 & 383 & & [1981ABE/SHR2] \\
\hline $\mathrm{C}_{7} \mathrm{H}_{9} \mathrm{~F}_{2} \mathrm{~N}_{2} \mathrm{OSSi}$ & {$[62609-67-0]$} & 1,1,1-trifluoro- & 2,2-trifluoro & fluorom & ethyliden & $\mathrm{V}^{\prime}$-(trimethylsilyl)methanesulfonimidamide \\
\hline & $\mathrm{V}$ & & 39.3 & 429 & I & [1977KIT/SHR, 1999DYK/SVO] \\
\hline $\mathrm{C}_{7} \mathrm{H}_{15} \mathrm{NO}_{3} \mathrm{Si}$ & {$[2288-13-3]$} & 1-methyl-2,8,9 & a-5-aza-1-si & $\operatorname{clo}[3.3 .3$ & cane & \\
\hline & SUB & & $82 \pm 0.8$ & & & [1989VOR/BAR] \\
\hline $\mathrm{C}_{7} \mathrm{H}_{15} \mathrm{NO}_{4} \mathrm{Si}$ & {$[18340-01-7]$} & Triethoxyisocy & silane & & & \\
\hline & V & & 45.6 & & & [1948FOR/AND] \\
\hline $\mathrm{C}_{7} \mathrm{H}_{16} \mathrm{O}_{3} \mathrm{SSi}$ & [57877-58-4] & Trimethoxy[(2 & enylthio)met & lane & & \\
\hline & $\mathrm{V}$ & & $38.6 \pm 0.5$ & 298 & $\mathrm{C}$ & [1989VOR/SOR] \\
\hline $\mathrm{C}_{7} \mathrm{H}_{17} \mathrm{ClSi}$ & [18817-17-9] & (1-chloroethyl) & ylmethylsila & & & \\
\hline & $\mathrm{V}$ & $(353-445)$ & 41.8 & 400 & & [1999DYK/SVO] \\
\hline $\mathrm{C}_{7} \mathrm{H}_{17} \mathrm{NSi}$ & {$[18387-12-7]$} & $N$-( $\beta$-trimethy & thyl)ethyleni & & & \\
\hline
\end{tabular}


TABLE 17. Phase change enthalpies of organometallic and inorganic compounds-Continued

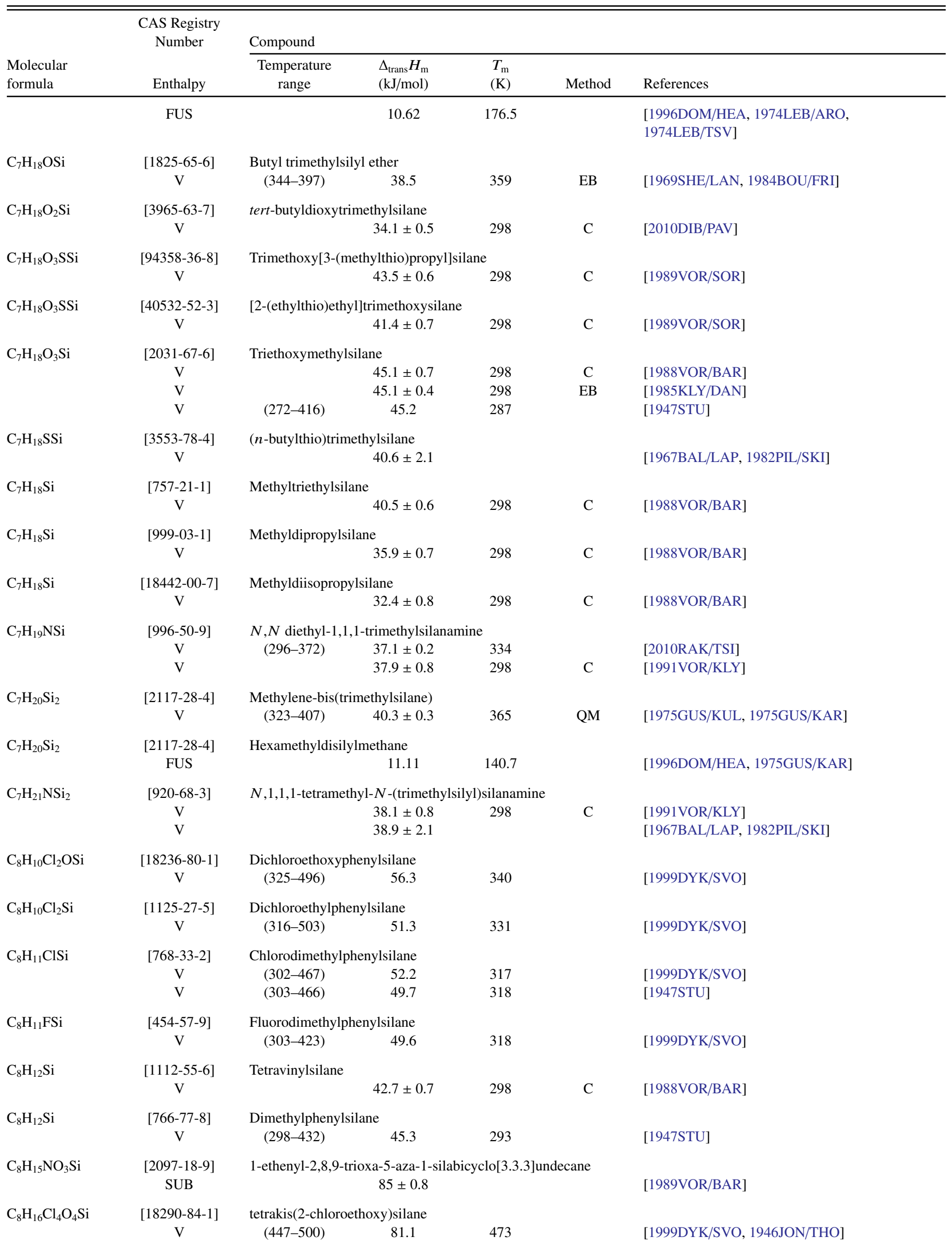


TABLE 17. Phase change enthalpies of organometallic and inorganic compounds-Continued

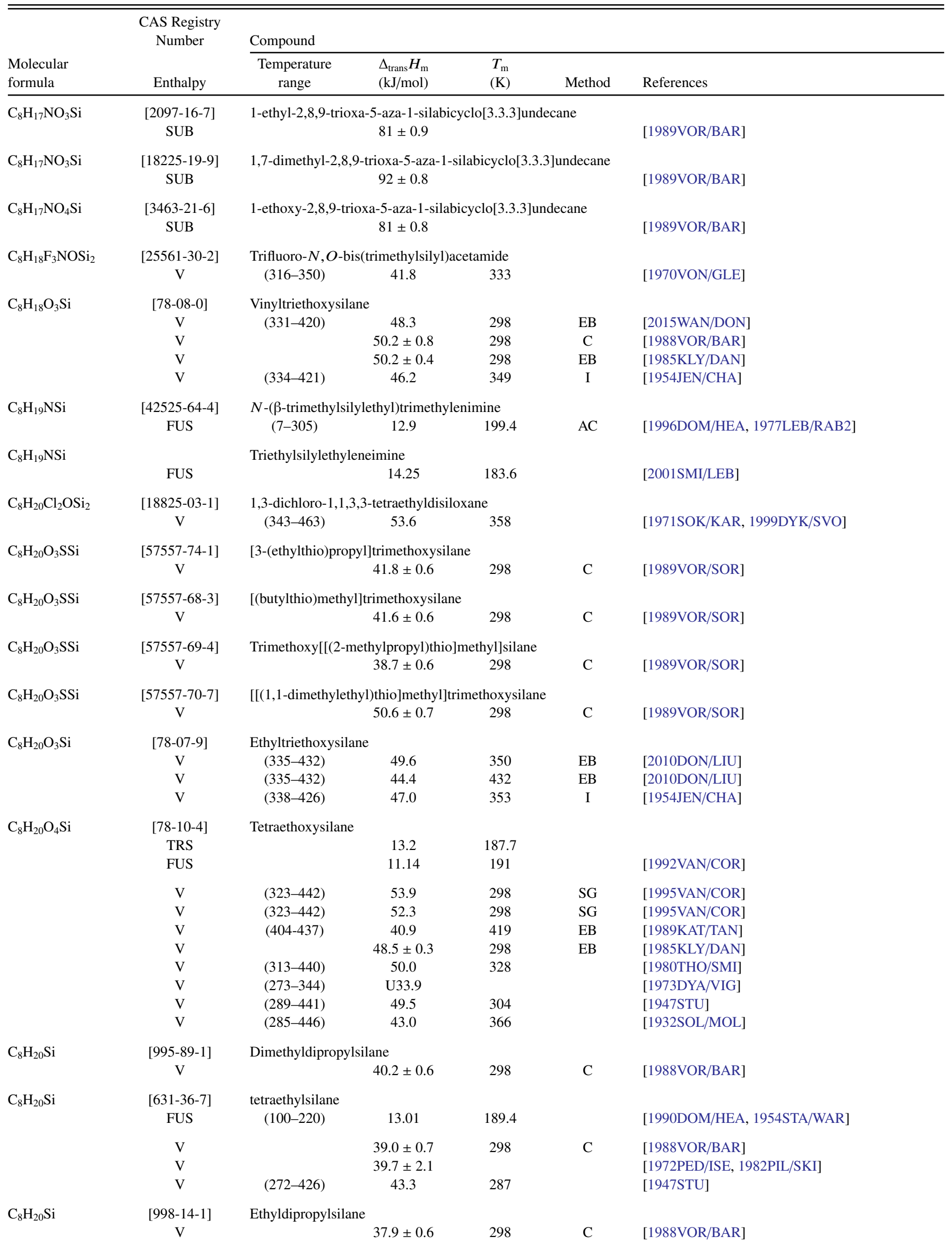


TABLE 17. Phase change enthalpies of organometallic and inorganic compounds-Continued

\begin{tabular}{|c|c|c|c|c|c|c|}
\hline \multirow[b]{2}{*}{$\begin{array}{l}\text { Molecular } \\
\text { formula }\end{array}$} & \multirow{2}{*}{$\begin{array}{l}\text { CAS Registry } \\
\text { Number } \\
\text { Enthalpy }\end{array}$} & \multicolumn{5}{|l|}{ Compound } \\
\hline & & $\begin{array}{c}\text { Temperature } \\
\text { range }\end{array}$ & $\begin{array}{l}\Delta_{\text {trans }} H_{\mathrm{m}} \\
(\mathrm{kJ} / \mathrm{mol})\end{array}$ & $\begin{array}{l}T_{\mathrm{m}} \\
(\mathrm{K})\end{array}$ & Method & References \\
\hline \multirow[t]{2}{*}{$\mathrm{C}_{8} \mathrm{H}_{20} \mathrm{Si}$} & {$[17591-40-1]$} & \multicolumn{5}{|c|}{ Ethyldiisopropylsilane } \\
\hline & $\mathrm{V}$ & & $38.1 \pm 0.7$ & 298 & $\mathrm{C}$ & [1988VOR/BAR] \\
\hline \multirow[t]{2}{*}{$\mathrm{C}_{8} \mathrm{H}_{20} \mathrm{Si}$} & {$[30736-07-3]$} & \multicolumn{5}{|c|}{ di-tert-butylsilane } \\
\hline & $\mathrm{V}$ & $(242-288)$ & 41.4 & 257 & & [2005FUL/RUZ] \\
\hline \multirow[t]{2}{*}{$\mathrm{C}_{8} \mathrm{H}_{23} \mathrm{~B}_{5} \mathrm{Si}_{2}$} & [59351-10-9] & \multicolumn{5}{|c|}{ 2,4-bis(trimethylsilyl)-2,4-dicarba-closo-heptaborane } \\
\hline & $\mathrm{V}$ & $(373-473)$ & 45.0 & 388 & $\mathrm{I}$ & [1976SHM/SHL] \\
\hline \multirow[t]{2}{*}{$\mathrm{C}_{8} \mathrm{H}_{24} \mathrm{Cl}_{2} \mathrm{O}_{3} \mathrm{Si}_{4}$} & {$[2474-02-4]$} & \multicolumn{5}{|c|}{ 1,7-dichloro-1,1,3,3,5,5,7,7-octamethyltetrasiloxane } \\
\hline & V & $(326-495)$ & 53.8 & 341 & & [1999DYK/SVO] \\
\hline \multirow[t]{2}{*}{$\mathrm{C}_{8} \mathrm{H}_{24} \mathrm{~N}_{4} \mathrm{Si}$} & [1624-01-7] & \multicolumn{5}{|c|}{ tetrakis(dimethylamino)silane } \\
\hline & $\mathrm{V}$ & $(361-415)$ & 40.0 & 388 & $\mathrm{~T}$ & [1964AYL/PET] \\
\hline \multirow[t]{5}{*}{$\mathrm{C}_{8} \mathrm{H}_{24} \mathrm{O}_{2} \mathrm{Si}_{3}$} & {$[107-51-7]$} & \multicolumn{5}{|c|}{ Octamethyltrisiloxane } \\
\hline & FUS & & 13.6 & 186.7 & DSC & {$[2011 \mathrm{ABB} / \mathrm{SCH}]$} \\
\hline & $\mathrm{V}$ & $(346-446)$ & 43.2 & 361 & $\mathrm{~EB}$ & [1986FLA] \\
\hline & $\mathrm{V}$ & & $39.7 \pm 2.1$ & & & [1972PED/ISE, 1982PIL/SKI] \\
\hline & $\mathrm{V}$ & $(345-417)$ & 40.2 & 381 & & {$[1971 \mathrm{SKO} / \mathrm{DIT}]$} \\
\hline \multirow[t]{13}{*}{$\mathrm{C}_{8} \mathrm{H}_{24} \mathrm{O}_{4} \mathrm{Si}_{4}$} & {$[556-67-2]$} & \multicolumn{5}{|c|}{ Octamethylcyclotetrasiloxane } \\
\hline & FUS & & 19.06 & 291.12 & DSC & [2011ABB/SCH] \\
\hline & TRS & & 4.87 & 258 & & \\
\hline & FUS & & 23.77 & 290.5 & $\mathrm{C}$ & [1996DOM/HEA, 1971ALV/DAL] \\
\hline & FUS & & 18.4 & 290.7 & & {$[1953 \mathrm{OST} / \mathrm{GRU}]$} \\
\hline & SUB & & $64 \pm 2$ & & B & [1953OST/GRU, 1960JON] \\
\hline & $\mathrm{V}$ & $(308-368)$ & 48 & & GCRT & [2010LEI/WAN] \\
\hline & $\mathrm{V}$ & & $57.0 \pm 0.8$ & 298 & $\mathrm{C}$ & [1991VOR/KLY2] \\
\hline & $\mathrm{V}$ & $(361-469)$ & 47.6 & 376 & $\mathrm{~EB}$ & [1986FLA] \\
\hline & $\mathrm{V}$ & $(334-423)$ & 44.1 & 378 & & [1971DIT/SKO] \\
\hline & $\mathrm{V}$ & $(303-428)$ & 56.1 & 298 & $\mathrm{I}$ & [1954OST/GRU] \\
\hline & $\mathrm{V}$ & $(303-428)$ & 48.5 & 373 & $\mathrm{I}$ & [1954OST/GRU] \\
\hline & V & $(303-428)$ & 45.6 & 398 & $\mathrm{I}$ & [1954OST/GRU] \\
\hline \multirow[t]{2}{*}{$\mathrm{C}_{8} \mathrm{H}_{24} \mathrm{O}_{12} \mathrm{Si}_{8}$} & {$[57348-79-5]$} & \multicolumn{5}{|c|}{ Octamethyldodecaoxooctasilicon } \\
\hline & SUB & $(463-563)$ & 110.5 & 513 & A & [1987STE/MAL, 1975TIT/CHU] \\
\hline \multirow[t]{2}{*}{$\mathrm{C}_{8} \mathrm{H}_{24} \mathrm{Si}_{3}$} & {$[3704-44-7]$} & Octamethyltris & & & & \\
\hline & V & & $46.0 \pm 0.8$ & & & [1972PED/ISE, 1982PIL/SKI] \\
\hline $\mathrm{C}_{8} \mathrm{H}_{28} \mathrm{~N}_{4} \mathrm{Si}_{4}$ & {$[1020-84-4]$} & Octamethylcyc & asilazane & & & \\
\hline & FUS & & 25.05 & 367.7 & & [1996DOM/HEA, 1981MEK/KAR] \\
\hline & $\mathrm{V}$ & $(388-513)$ & 52.3 & 450 & & [1972DIT/SKO2] \\
\hline $\mathrm{C}_{9} \mathrm{H}_{5} \mathrm{~N}_{3} \mathrm{O}_{3} \mathrm{Si}$ & {$[17883-47-5]$} & Triisocyanatop & silane & & & \\
\hline & $\mathrm{V}$ & & 58.2 & & & [1948FOR/AND] \\
\hline $\mathrm{C}_{9} \mathrm{H}_{9} \mathrm{~F}_{5} \mathrm{Si}$ & {$[1206-46-8]$} & Pentafluorophe & rimethyl)sila & & & \\
\hline & FUS & & 8.4 & 223 & DSC & [2006ZEL/CHU, 2008ZEL/CHU] \\
\hline & $\mathrm{V}$ & $(273-440)$ & $40.6 \pm 0.3$ & 357 & & [2006ZEL/CHU] \\
\hline $\mathrm{C}_{9} \mathrm{H}_{14} \mathrm{OSi}$ & {$[1529-17-5]$} & Phenoxytrimet & lane & & & \\
\hline & V & & $56.9 \pm 0.8$ & 298 & $\mathrm{C}$ & [1988VOR/BAR] \\
\hline $\mathrm{C}_{9} \mathrm{H}_{14} \mathrm{Si}$ & [768-32-1] & Trimethylphen & & & & \\
\hline & V & (296-404) & $43.8 \pm 0.7$ & 350 & ST & [2015ERM/SYS] \\
\hline $\mathrm{C}_{9} \mathrm{H}_{15} \mathrm{NSi}$ & {$[3768-55-6]$} & Trimethyl(pher & ino)silane & & & \\
\hline & $\mathrm{V}$ & $(311-455)$ & $45.7 \pm 0.7$ & 383 & Static & [2015ERM/SYS] \\
\hline & $\mathrm{V}$ & $(312-452)$ & $46 \pm 1$ & 383 & & [2010RAK/TSI] \\
\hline $\mathrm{C}_{9} \mathrm{H}_{19} \mathrm{NO}_{3} \mathrm{Si}$ & [26053-77-0] & 1-propyl-2,8,9 & a-5-aza-1-sil & clo[3.3.3 & cane & \\
\hline & SUB & & $84 \pm 0.8$ & & & [1989VOR/BAR] \\
\hline $\mathrm{C}_{9} \mathrm{H}_{19} \mathrm{NO}_{3} \mathrm{Si}$ & [2097-17-8] & 1-(1-methyleth & ,8,9-trioxa-5 & -silabicy & .3.3]undec & \\
\hline
\end{tabular}


TABLE 17. Phase change enthalpies of organometallic and inorganic compounds-Continued

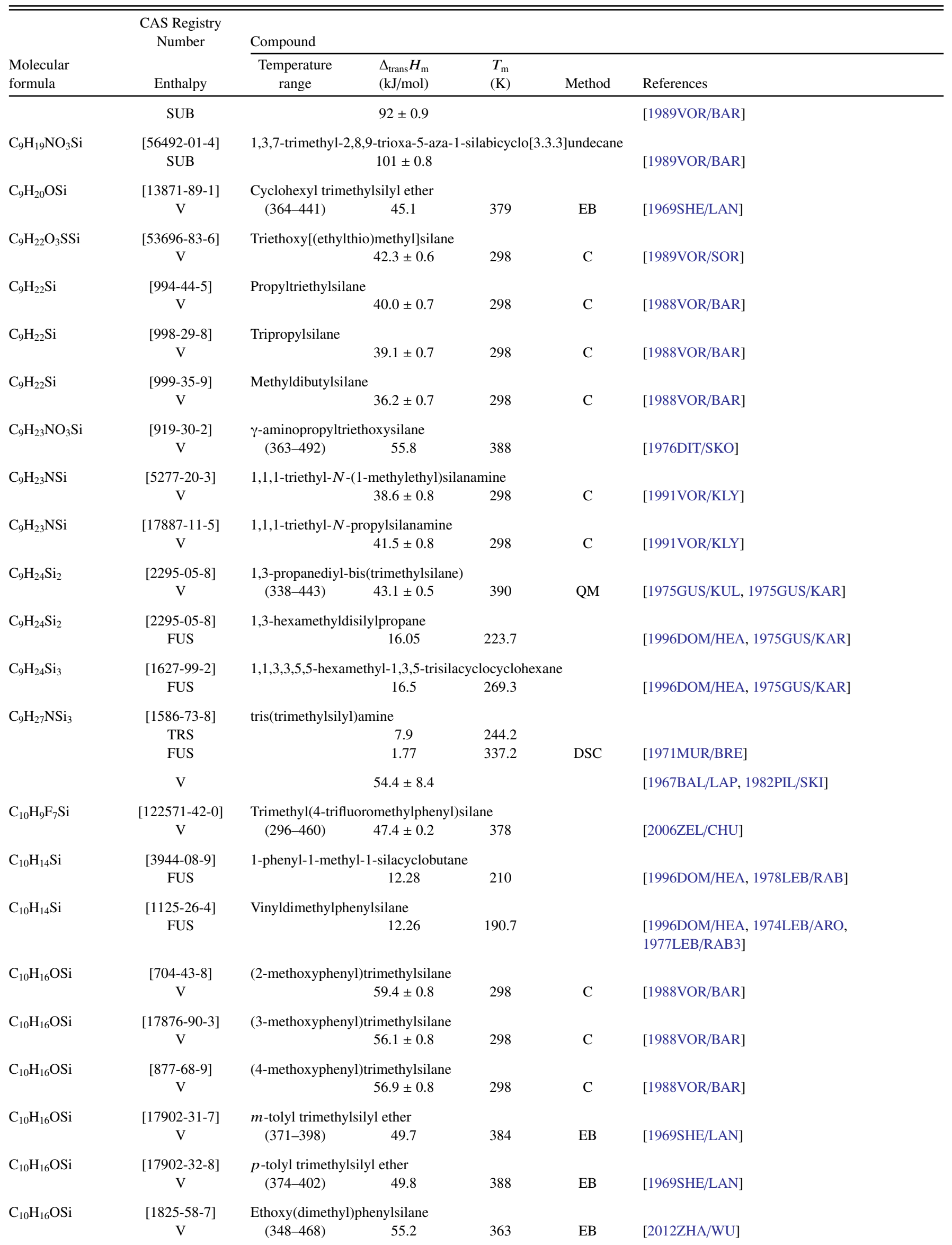


TABLE 17. Phase change enthalpies of organometallic and inorganic compounds-Continued

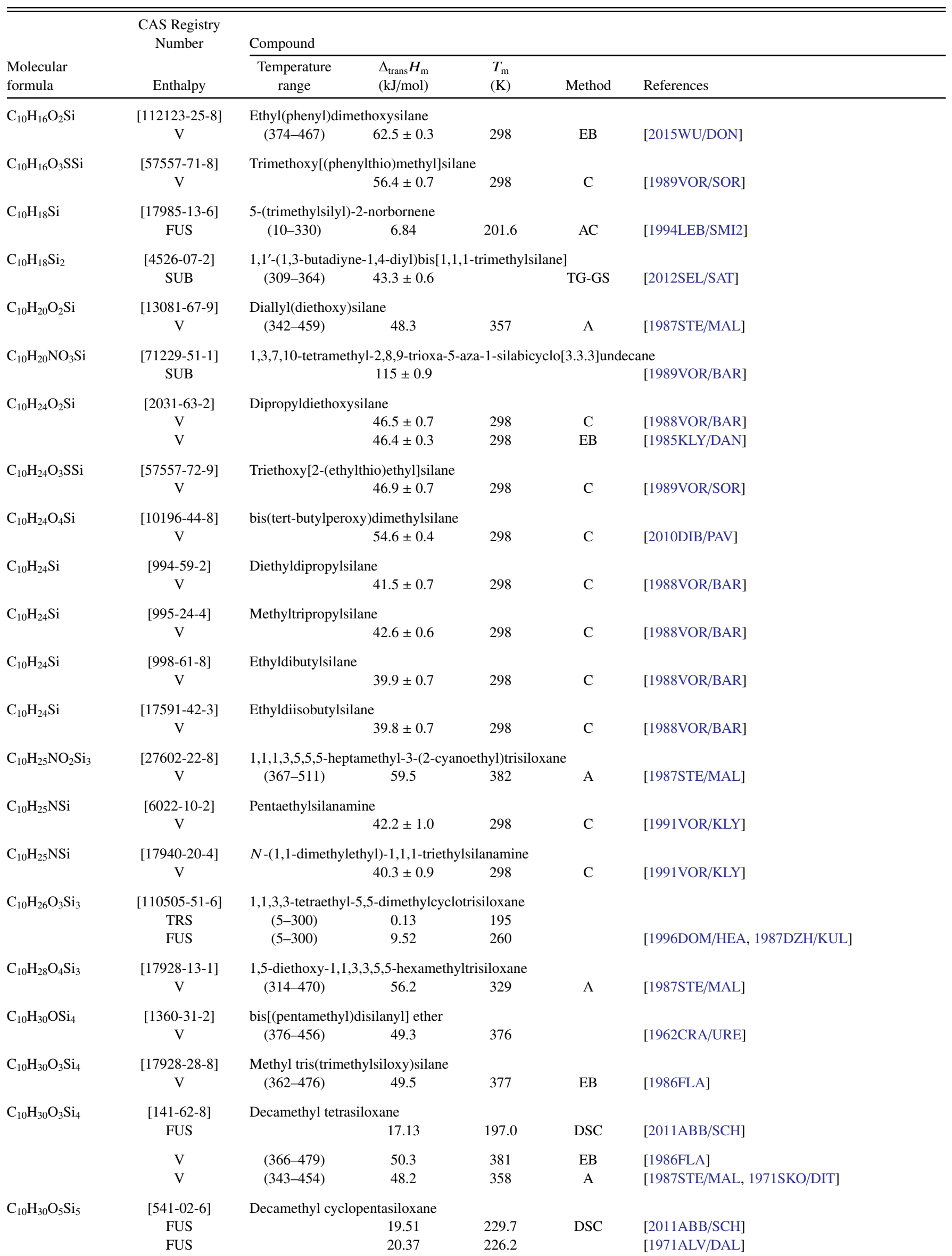


TABLE 17. Phase change enthalpies of organometallic and inorganic compounds-Continued

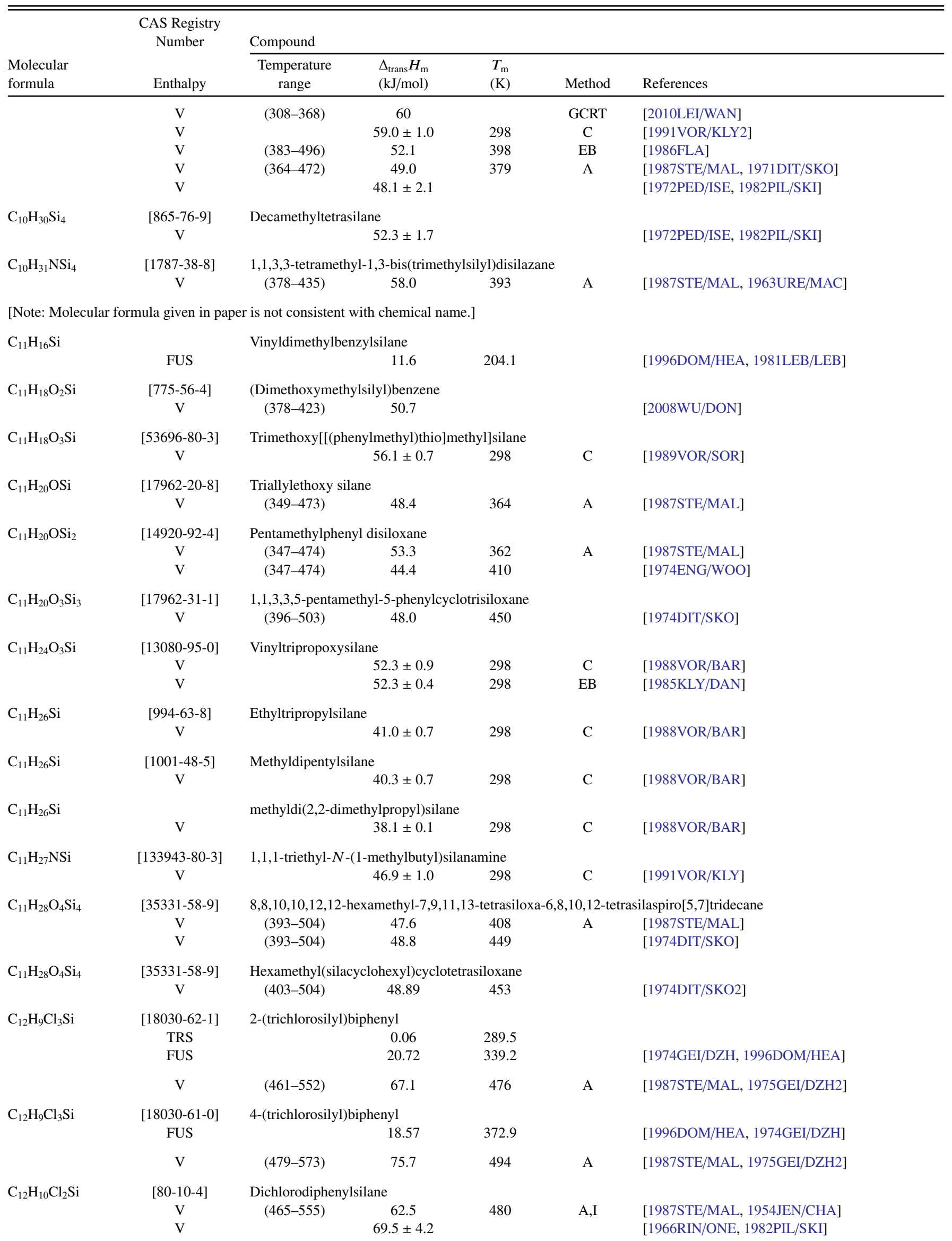


TABLE 17. Phase change enthalpies of organometallic and inorganic compounds-Continued

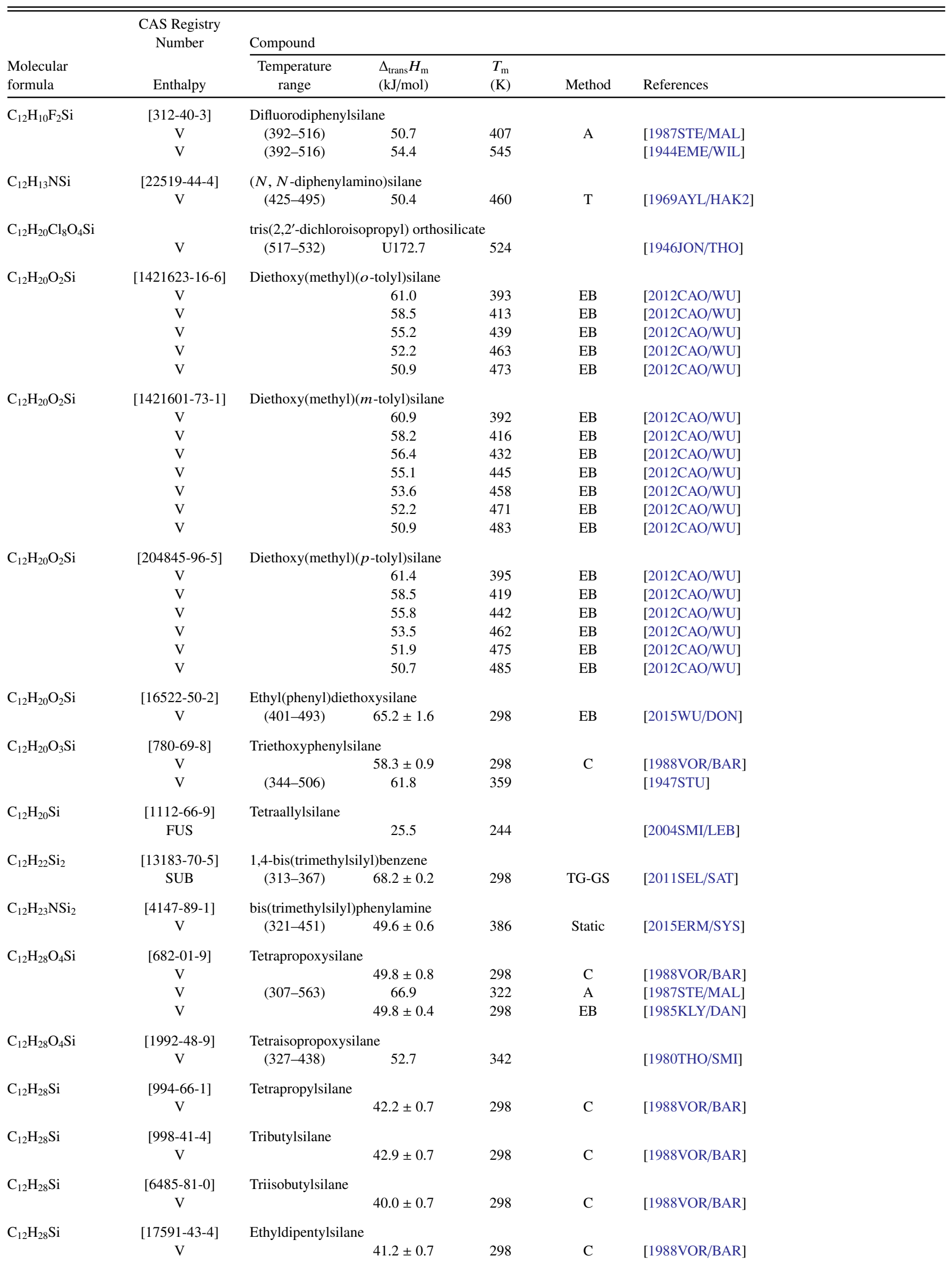


TABLE 17. Phase change enthalpies of organometallic and inorganic compounds-Continued

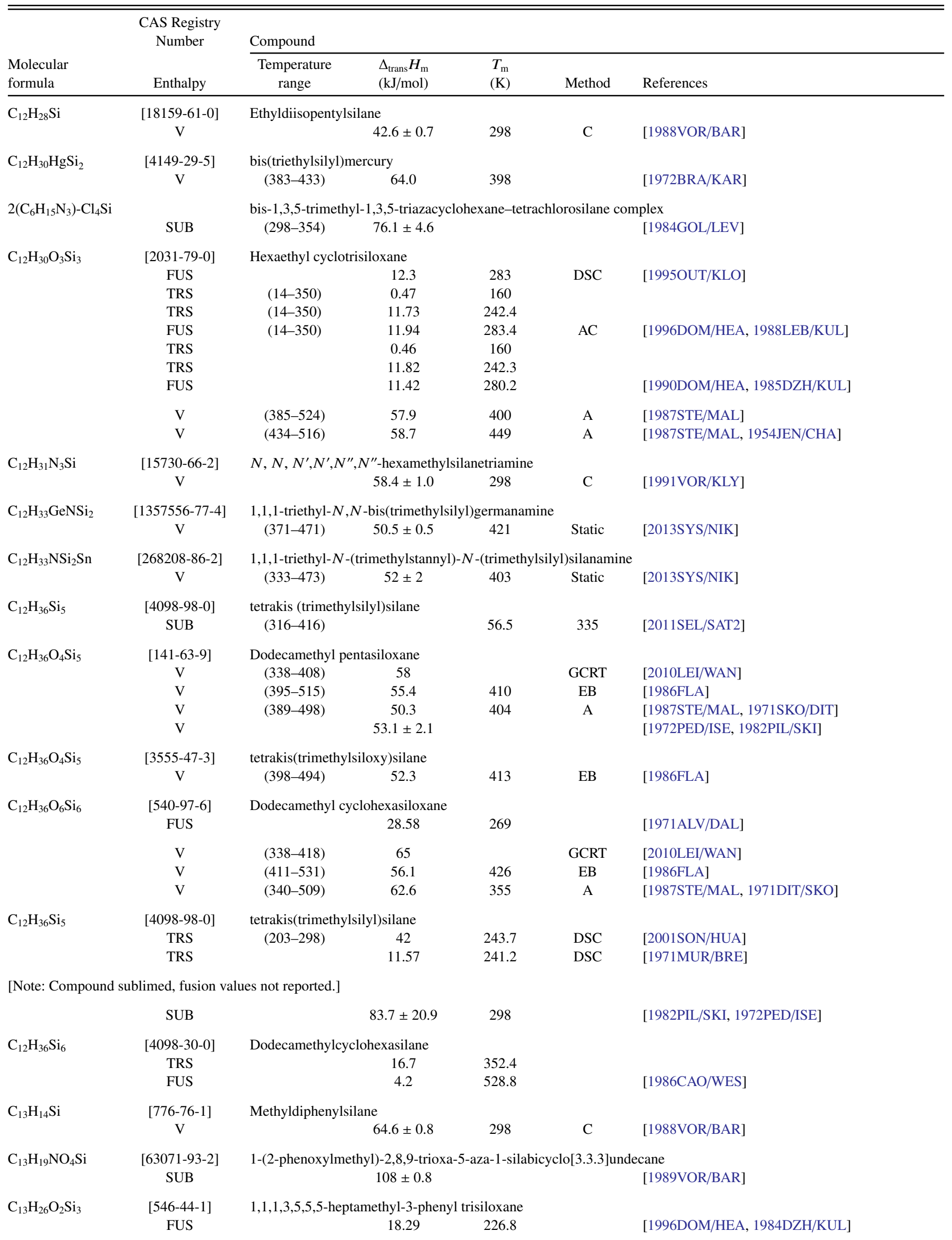


TABLE 17. Phase change enthalpies of organometallic and inorganic compounds-Continued

\begin{tabular}{|c|c|c|c|c|c|c|}
\hline \multirow[b]{2}{*}{$\begin{array}{l}\text { Molecular } \\
\text { formula }\end{array}$} & \multirow{2}{*}{$\begin{array}{c}\text { CAS Registry } \\
\text { Number } \\
\text { Enthalpy }\end{array}$} & \multicolumn{5}{|l|}{ Compound } \\
\hline & & $\begin{array}{l}\text { Temperature } \\
\text { range }\end{array}$ & $\begin{array}{l}\Delta_{\text {trans }} H_{\mathrm{m}} \\
(\mathrm{kJ} / \mathrm{mol})\end{array}$ & $\begin{array}{l}T_{\mathrm{m}} \\
(\mathrm{K})\end{array}$ & Method & References \\
\hline & $\mathrm{V}$ & $(357-492)$ & 61.5 & 372 & A & [1987STE/MAL] \\
\hline $\mathrm{C}_{13} \mathrm{H}_{26} \mathrm{O}_{4} \mathrm{Si}_{4}$ & $\begin{array}{c}{[10448-09-6]} \\
\mathrm{V}\end{array}$ & $\begin{array}{l}2,4,4,6,6,8,8-\mathrm{h} \\
\quad(397-514)\end{array}$ & $\begin{array}{c}\text { lethyl-2-pher } \\
65.6\end{array}$ & $\begin{array}{c}\text { otetras } \\
412\end{array}$ & A & [1987STE/MAL] \\
\hline $\mathrm{C}_{13} \mathrm{H}_{30} \mathrm{O}_{3} \mathrm{SSi}$ & $\begin{array}{c}{[57557-75-2]} \\
\mathrm{V}\end{array}$ & [3-(butylthio)p & $\begin{array}{l}\text { ]triethoxysil } \\
47.1 \pm 0.6\end{array}$ & 298 & $\mathrm{C}$ & [1989VOR/SOR] \\
\hline $\mathrm{C}_{13} \mathrm{H}_{30} \mathrm{O}_{6} \mathrm{Si}$ & $\begin{array}{c}{[10196-45-9]} \\
\mathrm{V}\end{array}$ & tris(tert-butylp & $\begin{array}{l}\text { methylsilan } \\
70.4 \pm 0.7\end{array}$ & 298 & $\mathrm{C}$ & [2010DIB/PAV] \\
\hline $\mathrm{C}_{13} \mathrm{H}_{30} \mathrm{Si}$ & $\begin{array}{c}{[18414-75-0]} \\
\mathrm{V}\end{array}$ & $\begin{array}{c}\text { Decyltrimethyl } \\
\quad(340-513)\end{array}$ & 57.8 & 355 & & [1947STU] \\
\hline $\mathrm{C}_{13} \mathrm{H}_{30} \mathrm{Si}$ & $\begin{array}{c}{[1001-46-3]} \\
\mathrm{V}\end{array}$ & Methyldihexyl & $42.6 \pm 0.7$ & 298 & $\mathrm{C}$ & [1988VOR/BAR] \\
\hline $\mathrm{C}_{14} \mathrm{H}_{6} \mathrm{~F}_{10} \mathrm{Si}$ & $\begin{array}{c}{[10536-62-6]} \\
\mathrm{V}\end{array}$ & $\begin{array}{l}\text { Di(pentafluoro } \\
\quad(366-463)\end{array}$ & $\begin{array}{l}\text { 1)dimethylsi } \\
55.3 \pm 0.6\end{array}$ & 414 & & [2006ZEL/CHU] \\
\hline $\mathrm{C}_{14} \mathrm{H}_{10} \mathrm{~N}_{2} \mathrm{O}_{2} \mathrm{Si}$ & $\begin{array}{c}{[4756-54-1]} \\
\mathrm{V}\end{array}$ & Diisocyanatodi & $\begin{array}{r}\text { ylsilane } \\
65.7\end{array}$ & & & [1948FOR/AND] \\
\hline $\mathrm{C}_{14} \mathrm{H}_{16} \mathrm{O}_{2} \mathrm{Si}$ & $\begin{array}{c}{[6843-66-9]} \\
\mathrm{V}\end{array}$ & Diphenoxydim & $\begin{array}{l}\text { silane } \\
64.4 \pm 0.9\end{array}$ & 298 & $\mathrm{C}$ & [1988VOR/BAR] \\
\hline $\mathrm{C}_{14} \mathrm{H}_{16} \mathrm{Si}$ & $\begin{array}{c}{[7535-07-1]} \\
\mathrm{V}\end{array}$ & Ethyldiphenyls & $66.1 \pm 0.8$ & 298 & $\mathrm{C}$ & [1988VOR/BAR] \\
\hline $\mathrm{C}_{14} \mathrm{H}_{16} \mathrm{Si}$ & $\begin{array}{c}{[15458-91-0]} \\
\mathrm{V}\end{array}$ & $\begin{array}{l}\text { Dibenzylsilane } \\
\quad(357-577)\end{array}$ & 56.0 & 467 & & [1959GIL/TOM] \\
\hline $\mathrm{C}_{14} \mathrm{H}_{19} \mathrm{NO}_{5} \mathrm{Si}$ & $\begin{array}{c}{[79791-55-2]} \\
\text { SUB }\end{array}$ & 2,8,9-trioxa-5- & $\begin{array}{c}\text {-silabicyclo[ } \\
109 \pm 0.9\end{array}$ & Indecan & lethanol b & $\begin{array}{l}\text { oate ester } \\
\text { [1989VOR/BAR] }\end{array}$ \\
\hline $\mathrm{C}_{14} \mathrm{H}_{21} \mathrm{NO}_{3} \mathrm{Si}$ & $\begin{array}{c}{[63330-92-7]} \\
\text { SUB }\end{array}$ & 1-(2-phenyleth & $\begin{array}{c}8,9-\text { trioxa-5 } \\
108 \pm 0.9\end{array}$ & silabicy & .3.3] undec & [1989VOR/BAR] \\
\hline $\mathrm{C}_{14} \mathrm{H}_{30} \mathrm{O}_{6} \mathrm{Si}$ & $\begin{array}{c}{[15188-09-7]} \\
\mathrm{V}\end{array}$ & Vinyltris(tert-b & $\begin{array}{c}\text { eroxy)silane } \\
68.8 \pm 0.5\end{array}$ & 298 & $\mathrm{C}$ & [2010DIB/PAV] \\
\hline $\mathrm{C}_{14} \mathrm{H}_{32} \mathrm{Si}$ & $\begin{array}{c}{[10175-53-8]} \\
\mathrm{V}\end{array}$ & $\begin{array}{c}\text { Triethyloctylsi } \\
\quad(347-535)\end{array}$ & 56.1 & 361 & & [1947STU] \\
\hline $\mathrm{C}_{14} \mathrm{H}_{32} \mathrm{Si}$ & $\begin{array}{c}{[17591-45-6]} \\
V\end{array}$ & Ethyldihexylsil & $44.8 \pm 0.7$ & 298 & $\mathrm{C}$ & [1988VOR/BAR] \\
\hline $\mathrm{C}_{14} \mathrm{H}_{32} \mathrm{Si}$ & $\begin{array}{c}{[994-76-3]} \\
\mathrm{V}\end{array}$ & Dipropyldibuty & $44.0 \pm 0.8$ & 298 & $\mathrm{C}$ & [1988VOR/BAR] \\
\hline $\mathrm{C}_{14} \mathrm{H}_{33} \mathrm{NSi}$ & $\begin{array}{c}{[17995-32-3]} \\
V\end{array}$ & $N, N$ dibutyl-1 & $\begin{array}{l}\text { riethylsilana } \\
56.3 \pm 1.0\end{array}$ & 298 & $\mathrm{C}$ & [1991VOR/KLY] \\
\hline $\mathrm{C}_{14} \mathrm{H}_{33} \mathrm{NSi}$ & $\begin{array}{c}{[133943-79-0]} \\
\mathrm{V}\end{array}$ & 1,1,1-triethyl- $\Lambda$ & $\begin{array}{l}\text { bis }(1-\text { methyl } \\
51.4 \pm 0.9\end{array}$ & $\begin{array}{l}\text { silana } \\
298\end{array}$ & $\mathrm{C}$ & [1991VOR/KLY] \\
\hline $\mathrm{C}_{14} \mathrm{H}_{33} \mathrm{NSi}$ & $\begin{array}{c}{[133943-81-4]} \\
V\end{array}$ & 1,1,1-triethyl- $\perp$ & $\begin{array}{l}\text { ylsilanamine } \\
59.1 \pm 1.0\end{array}$ & 298 & $\mathrm{C}$ & [1991VOR/KLY] \\
\hline $\mathrm{C}_{14} \mathrm{H}_{42} \mathrm{O}_{2} \mathrm{Si}_{6}$ & $\begin{array}{c}{[1787-37-7]} \\
\mathrm{V}\end{array}$ & $\begin{array}{l}\text { bis }[(\text { pentameth } \\
\quad(442-474)\end{array}$ & $\begin{array}{l}\text { ilanoxy]disi } \\
62.2\end{array}$ & 457 & & [1962CRA/URE] \\
\hline $\mathrm{C}_{14} \mathrm{H}_{42} \mathrm{O}_{5} \mathrm{Si}_{6}$ & $\begin{array}{c}{[107-52-8]} \\
\mathrm{V} \\
\mathrm{V} \\
\mathrm{V}\end{array}$ & $\begin{array}{c}\text { Tetradecameth } \\
\quad(338-438) \\
(449-545) \\
(397-522)\end{array}$ & $\begin{array}{c}\text { xasiloxane } \\
68 \\
56.9 \\
56.6\end{array}$ & $\begin{array}{l}464 \\
412\end{array}$ & $\begin{array}{c}\text { GCRT } \\
\text { EB } \\
\text { A }\end{array}$ & $\begin{array}{l}\text { [2010LEI/WAN] } \\
{[1986 \mathrm{FLA}]} \\
{[1987 \mathrm{STE} / \mathrm{MAL}, 1971 \mathrm{SKO} / \mathrm{DIT}]}\end{array}$ \\
\hline $\mathrm{C}_{14} \mathrm{H}_{42} \mathrm{O}_{7} \mathrm{Si}_{7}$ & $\begin{array}{c}{[107-50-6]} \\
\text { FUS }\end{array}$ & Tetradecameth & $\begin{array}{l}\text { loheptasilox } \\
20.88\end{array}$ & 237.7 & & [1971ALV/DAL] \\
\hline & $\begin{array}{l}\mathrm{V} \\
\mathrm{V}\end{array}$ & $\begin{array}{l}(338-418) \\
(359-537)\end{array}$ & $\begin{array}{c}74 \\
58.6\end{array}$ & 374 & $\begin{array}{c}\text { GCRT } \\
\text { A }\end{array}$ & $\begin{array}{l}\text { [2010LEI/WAN] } \\
\text { [1987STE/MAL, 1974BRA/KAR] }\end{array}$ \\
\hline
\end{tabular}


TABLE 17. Phase change enthalpies of organometallic and inorganic compounds-Continued

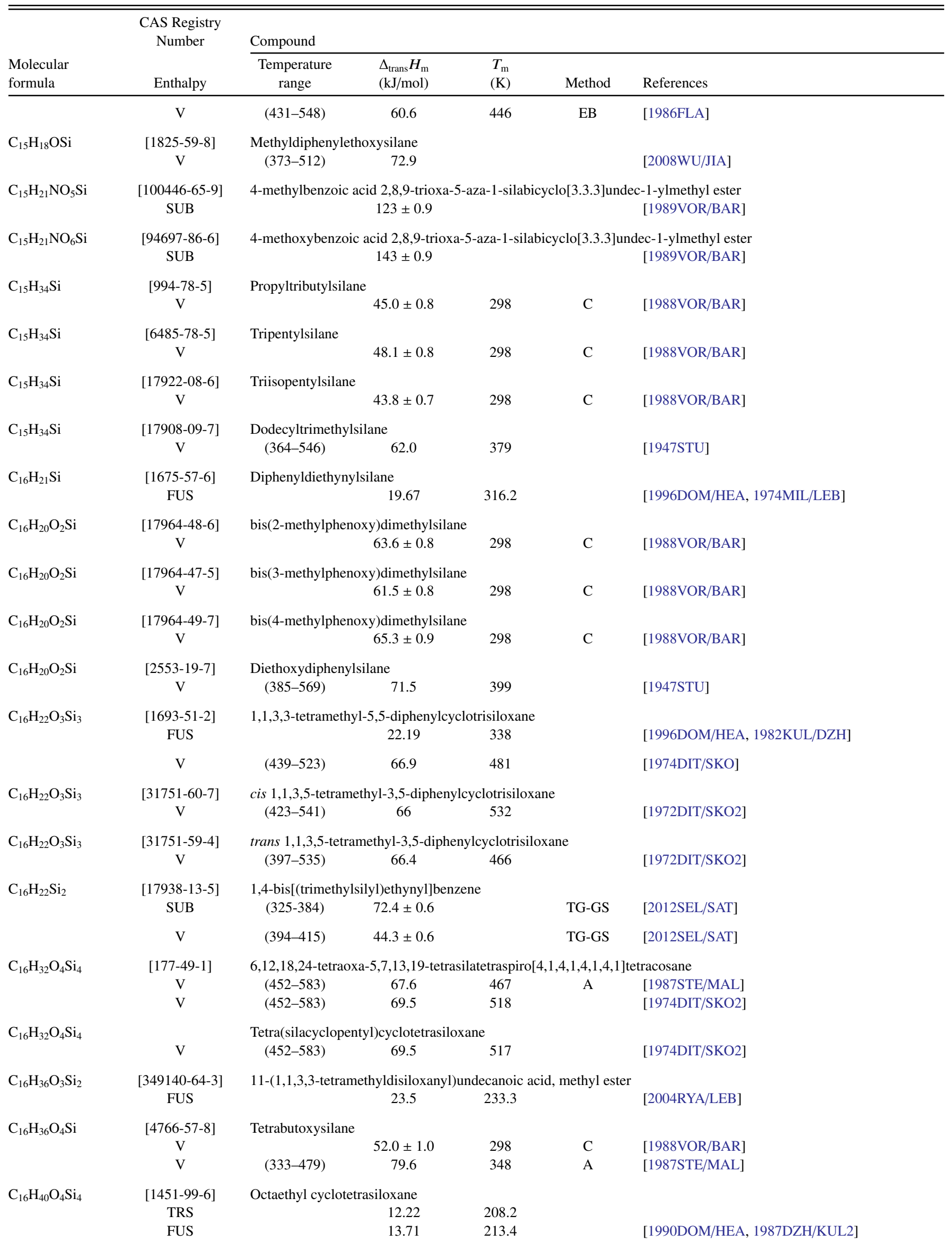


TABLE 17. Phase change enthalpies of organometallic and inorganic compounds-Continued

\begin{tabular}{|c|c|c|c|c|c|c|}
\hline \multirow[b]{2}{*}{$\begin{array}{l}\text { Molecular } \\
\text { formula }\end{array}$} & \multirow{2}{*}{$\begin{array}{c}\text { CAS Registry } \\
\text { Number } \\
\text { Enthalpy }\end{array}$} & \multicolumn{5}{|l|}{ Compound } \\
\hline & & $\begin{array}{l}\text { Temperature } \\
\text { range }\end{array}$ & $\begin{array}{l}\Delta_{\text {trans }} H_{\mathrm{m}} \\
(\mathrm{kJ} / \mathrm{mol})\end{array}$ & $\begin{array}{l}T_{\mathrm{m}} \\
(\mathrm{K})\end{array}$ & Method & References \\
\hline & $\mathrm{V}$ & $(420-574)$ & 69.2 & 435 & A & [1987STE/MAL] \\
\hline $\mathrm{C}_{16} \mathrm{H}_{46} \mathrm{O}_{7} \mathrm{Si}_{6}$ & $\begin{array}{c}{[18143-15-2]} \\
\mathrm{V}\end{array}$ & $\begin{array}{l}\text { 1,11-diethoxy- } \\
\quad(376-547)\end{array}$ & $\begin{array}{c}3,5,7,7,9,9, \\
66.9\end{array}$ & $\begin{array}{l}\text { odecan } \\
391\end{array}$ & $\begin{array}{c}\text { hexasilox } \\
\text { A }\end{array}$ & [1987STE/MAL] \\
\hline $\mathrm{C}_{16} \mathrm{H}_{48} \mathrm{O}_{6} \mathrm{Si}_{7}$ & $\begin{array}{c}{[541-01-5]} \\
\mathrm{V} \\
\mathrm{V} \\
\mathrm{V}\end{array}$ & $\begin{array}{l}\text { Hexadecameth } \\
\quad(338-438) \\
(443-468) \\
(443-551)\end{array}$ & $\begin{array}{c}\text { tasiloxane } \\
89 \\
63.8 \\
60.8\end{array}$ & $\begin{array}{l}459 \\
458\end{array}$ & $\begin{array}{l}\text { GCRT } \\
\text { EB } \\
\text { A }\end{array}$ & $\begin{array}{l}\text { [2010LEI/WAN] } \\
\text { [1986FLA] } \\
\text { [1987STE/MAL, 1971SKO/DIT] }\end{array}$ \\
\hline $\mathrm{C}_{16} \mathrm{H}_{48} \mathrm{O}_{8} \mathrm{Si}_{8}$ & $\begin{array}{c}{[556-68-3]} \\
\mathrm{V} \\
\mathrm{V} \\
\mathrm{V}\end{array}$ & $\begin{array}{l}\text { Hexadecameth } \\
\quad(338-418) \\
(378-563) \\
(454-576)\end{array}$ & $\begin{array}{c}\text { looctasilox } \\
83 \\
66.6 \\
64.5\end{array}$ & $\begin{array}{l}391 \\
469\end{array}$ & $\begin{array}{c}\text { GCRT } \\
\text { A } \\
\text { EB }\end{array}$ & $\begin{array}{l}\text { [2010LEI/WAN] } \\
\text { [1987STE/MAL, 1974BRA/KAR] } \\
\text { [1986FLA] }\end{array}$ \\
\hline $\mathrm{C}_{17} \mathrm{H}_{26} \mathrm{O}_{4} \mathrm{Si}_{4}$ & $\begin{array}{c}{[13093-12-4]} \\
\mathrm{V}\end{array}$ & $\begin{array}{l}\text { Hexamethyl(si } \\
\quad(466-548)\end{array}$ & $\begin{array}{l}\text { naphthenyl) } \\
68.6\end{array}$ & $\begin{array}{c}\text { trasilox } \\
507\end{array}$ & & [1974DIT/SKO2] \\
\hline $\mathrm{C}_{17} \mathrm{H}_{32} \mathrm{O}_{2} \mathrm{Si}$ & $\begin{array}{c}{[261766-69-2]} \\
\mathrm{V}\end{array}$ & $\begin{array}{c}\text { 3-methyl-3-[2- } \\
\text { (307-318) }\end{array}$ & $\begin{array}{l}\text { hexylpropyl } \\
74.2 \pm 2.0\end{array}$ & ]-1-trin & $\begin{array}{l}\text { silyl-1-but } \\
\text { ME }\end{array}$ & [1999DIB/LUS] \\
\hline $\mathrm{C}_{17} \mathrm{H}_{38} \mathrm{Si}$ & $\begin{array}{c}{[18558-18-4]} \\
\mathrm{V}\end{array}$ & $\begin{array}{c}\text { Tetradecyltrim } \\
\quad(393-573)\end{array}$ & $\begin{array}{l}\text { ilane } \\
70.9\end{array}$ & 408 & & {$[1947 \mathrm{STU}]$} \\
\hline $\mathrm{C}_{18} \mathrm{H}_{15} \mathrm{ClSi}$ & $\begin{array}{c}{[76-86-8]} \\
\text { FUS }\end{array}$ & Triphenylchlor & 26.88 & 370.6 & & [1996DOM/HEA, 1968KOS/MOS] \\
\hline $\mathrm{C}_{18} \mathrm{H}_{16} \mathrm{Si}$ & $\begin{array}{c}{[789-25-3]} \\
\mathrm{V}\end{array}$ & $\begin{array}{c}\text { Triphenylsilan } \\
\text { (395-457) }\end{array}$ & $33.9 \pm 0.2$ & & TG-GS & [2012SEL/SAT] \\
\hline $\mathrm{C}_{18} \mathrm{H}_{28} \mathrm{O}_{2} \mathrm{Si}_{3}$ & $\begin{array}{c}{[797-77-3]} \\
\text { FUS }\end{array}$ & 1,1,1,5,5,5-hex & $\begin{array}{l}\text { hyl-3,3-diph } \\
\text { 22.75 }\end{array}$ & $\begin{array}{l}\text { siloxan } \\
270.5\end{array}$ & & [1996DOM/HEA, 1981SHA/DZH] \\
\hline $\mathrm{C}_{18} \mathrm{H}_{28} \mathrm{O}_{4} \mathrm{Si}_{4}$ & $\begin{array}{c}{[18604-02-9]} \\
\mathrm{V}\end{array}$ & $\begin{array}{l}2,2,4,4,6,8-h e x \\
\quad(459-576)\end{array}$ & $\begin{array}{l}\text { hyl-6,8-diph } \\
\quad 70.5\end{array}$ & $\begin{array}{l}\text { clotetra } \\
474\end{array}$ & A & [1987STE/MAL] \\
\hline $\mathrm{C}_{18} \mathrm{H}_{28} \mathrm{O}_{4} \mathrm{Si}_{4}$ & $\begin{array}{c}\text { [1693-44-3] } \\
\text { FUS }\end{array}$ & $1,1,3,3,5,5$-hex & $\begin{array}{l}\text { hyl-7,7-diph } \\
\quad 42.73\end{array}$ & $\begin{array}{l}\text { clotetra } \\
305\end{array}$ & & $\begin{array}{l}\text { [1996DOM/HEA, 1975MEK/KAR, } \\
\text { 1981MEK/KAR] }\end{array}$ \\
\hline $\mathrm{C}_{18} \mathrm{H}_{40} \mathrm{Si}$ & $\begin{array}{c}{[2929-52-4]} \\
V\end{array}$ & Trihexylsilane & $51.0 \pm 0.7$ & 298 & $\mathrm{C}$ & [1988VOR/BAR] \\
\hline $\mathrm{C}_{18} \mathrm{H}_{40} \mathrm{Si}$ & $\begin{array}{c}{[51502-64-8]} \\
\mathrm{V}\end{array}$ & Ethyldioctylsil & $47.3 \pm 0.7$ & 298 & $\mathrm{C}$ & [1988VOR/BAR] \\
\hline $\mathrm{C}_{18} \mathrm{H}_{42} \mathrm{O}_{3} \mathrm{Si}_{3}$ & $\begin{array}{l}\text { [92411-29-5] } \\
\text { FUS }\end{array}$ & 2,2,4,4,6,6-hex & $\begin{array}{l}\text { ylcyclotrisi } \\
30.6\end{array}$ & 297 & DSC & [1995OUT/KLO] \\
\hline $\mathrm{C}_{18} \mathrm{H}_{48} \mathrm{Si}_{6}$ & $\begin{array}{l}\text { [76750-22-6] } \\
\text { TRS } \\
\text { FUS }\end{array}$ & $1,2,3,4,5,6-$ hex & $\begin{array}{l}\text { hyl- } 1,2,3,4,5 \\
\quad 3.8 \\
1.8\end{array}$ & $\begin{array}{l}\text { aethylc } \\
226.3 \\
439.2\end{array}$ & xasilane & [1986CAO/WES] \\
\hline $\mathrm{C}_{18} \mathrm{H}_{54} \mathrm{O}_{7} \mathrm{Si}_{8}$ & $\begin{array}{c}{[556-69-4]} \\
\mathrm{V} \\
\mathrm{V} \\
\mathrm{V}\end{array}$ & $\begin{array}{c}\text { Octadecamethy } \\
\quad(338-438) \\
(378-563) \\
(464-586)\end{array}$ & $\begin{array}{c}\text { asiloxane } \\
98 \\
67.7 \\
68.4\end{array}$ & $\begin{array}{l}393 \\
479\end{array}$ & $\begin{array}{c}\text { GCRT } \\
\text { A } \\
\text { EB }\end{array}$ & $\begin{array}{l}\text { [2010LEI/WAN] } \\
\text { [1987STE/MAL, 1974BRA/KAR2] } \\
\text { [1986FLA] }\end{array}$ \\
\hline $\mathrm{C}_{18} \mathrm{H}_{54} \mathrm{O}_{9} \mathrm{Si}_{9}$ & $\begin{array}{l}{[556-71-8]} \\
\text { FUS }\end{array}$ & Octadecamethy & $\begin{array}{l}\text { lononasilox } \\
25.64\end{array}$ & 246.2 & & [1971ALV/DAL] \\
\hline & $\begin{array}{l}\mathrm{V} \\
\mathrm{V} \\
\mathrm{V}\end{array}$ & $\begin{array}{l}(338-418) \\
(463-584) \\
(473-578)\end{array}$ & $\begin{array}{c}91 \\
67.9 \\
68\end{array}$ & $\begin{array}{l}478 \\
488\end{array}$ & $\begin{array}{c}\text { GCRT } \\
\text { A } \\
\text { EB }\end{array}$ & $\begin{array}{l}\text { [2010LEI/WAN] } \\
{[1987 \mathrm{STE} / \mathrm{MAL}]} \\
{[1986 \mathrm{FLA}]}\end{array}$ \\
\hline $\mathrm{C}_{19} \mathrm{H}_{15} \mathrm{NOSi}$ & $\begin{array}{c}{[18678-65-4]} \\
\text { V }\end{array}$ & Isocyanatotripl & $\begin{array}{l}\text { ilane } \\
67.4\end{array}$ & & & [1948FOR/AND] \\
\hline $\mathrm{C}_{19} \mathrm{H}_{18} \mathrm{O}_{3} \mathrm{Si}$ & $\begin{array}{c}{[3439-97-2]} \\
\mathrm{V}\end{array}$ & Methyltriphen & $\begin{array}{l}\text { ane } \\
71.5 \pm 0.9\end{array}$ & 298 & $\mathrm{C}$ & [1988VOR/BAR] \\
\hline
\end{tabular}


TABLE 17. Phase change enthalpies of organometallic and inorganic compounds-Continued

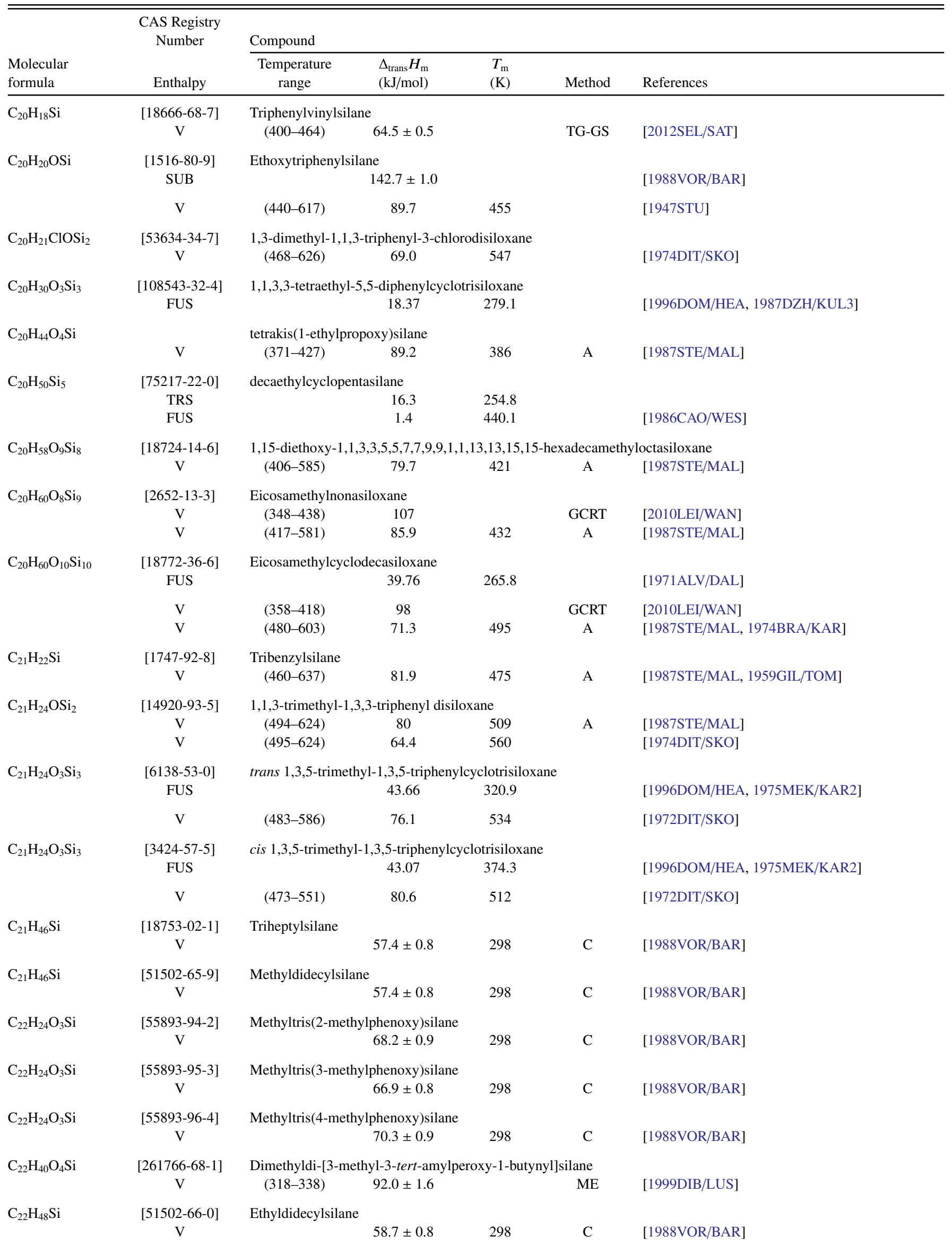


TABLE 17. Phase change enthalpies of organometallic and inorganic compounds-Continued

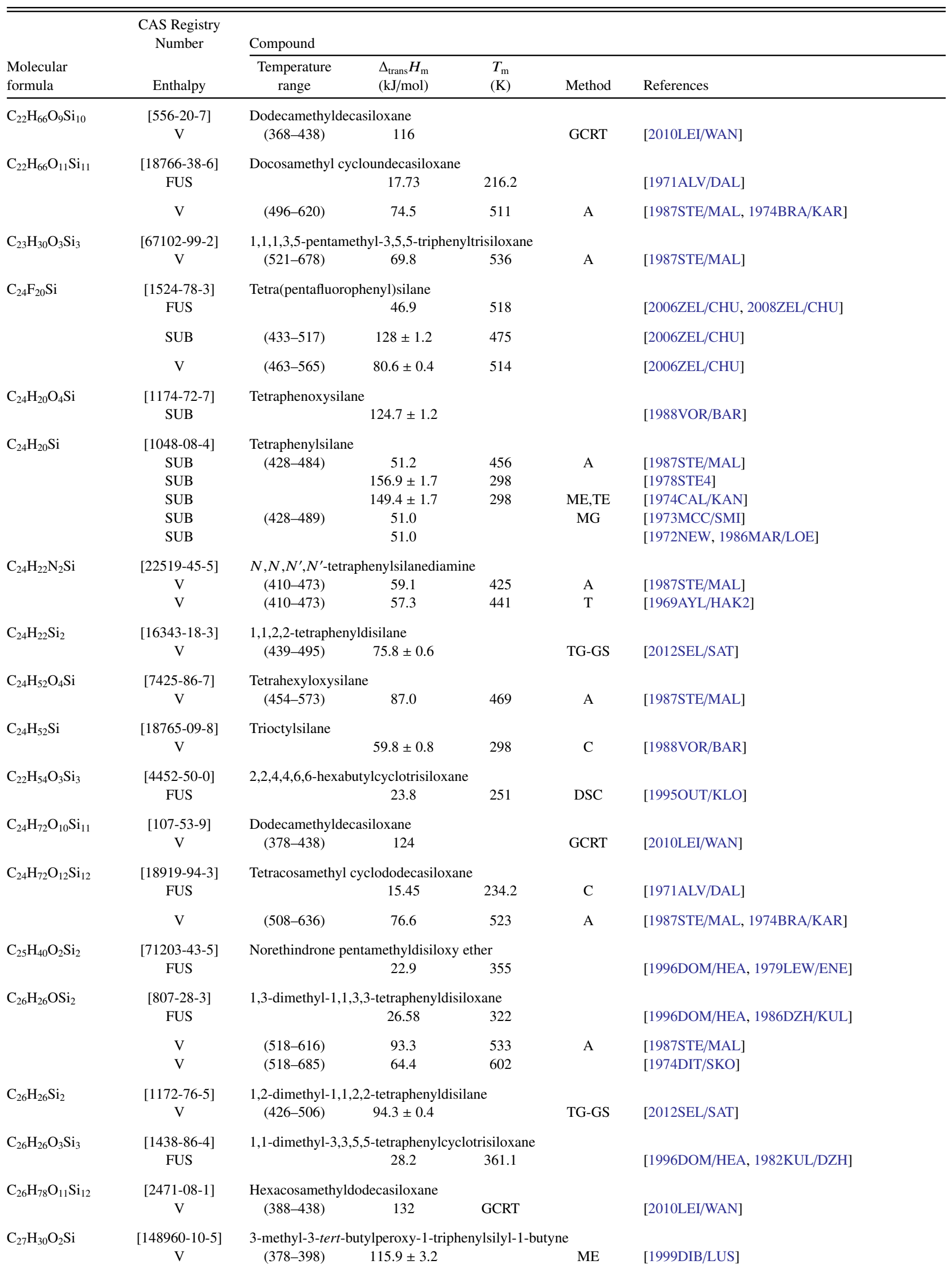


TABLE 17. Phase change enthalpies of organometallic and inorganic compounds-Continued

\begin{tabular}{|c|c|c|c|c|c|c|}
\hline \multirow[b]{2}{*}{$\begin{array}{l}\text { Molecular } \\
\text { formula }\end{array}$} & \multirow{2}{*}{$\begin{array}{l}\text { CAS Registry } \\
\text { Number } \\
\text { Enthalpy }\end{array}$} & \multicolumn{5}{|l|}{ Compound } \\
\hline & & $\begin{array}{l}\text { Temperature } \\
\text { range }\end{array}$ & $\begin{array}{l}\Delta_{\text {trans }} H_{\mathrm{m}} \\
(\mathrm{kJ} / \mathrm{mol})\end{array}$ & $\begin{array}{l}T_{\mathrm{m}} \\
(\mathrm{K})\end{array}$ & Method & References \\
\hline $\mathrm{C}_{27} \mathrm{H}_{58} \mathrm{Si}$ & $\begin{array}{c}{[51502-67-1]} \\
\mathrm{V}\end{array}$ & Trinonylsilane & $61.8 \pm 0.8$ & 298 & $\mathrm{C}$ & [1988VOR/BAR] \\
\hline $\mathrm{C}_{28} \mathrm{H}_{28} \mathrm{O}_{4} \mathrm{Si}$ & $\begin{array}{c}{[16714-40-2]} \\
\mathrm{V}\end{array}$ & tetrakis(2-meth & $\begin{array}{c}\text { enoxy)silane } \\
76.2 \pm 1.0\end{array}$ & 298 & $\mathrm{C}$ & [1988VOR/BAR] \\
\hline $\mathrm{C}_{28} \mathrm{H}_{28} \mathrm{O}_{4} \mathrm{Si}$ & $\begin{array}{c}{[16714-54-8]} \\
\mathrm{V}\end{array}$ & tetrakis(3-meth & $\begin{array}{c}\text { enoxy)silane } \\
73.6 \pm 0.9\end{array}$ & 298 & $\mathrm{C}$ & [1988VOR/BAR] \\
\hline $\mathrm{C}_{28} \mathrm{H}_{28} \mathrm{O}_{4} \mathrm{Si}$ & $\begin{array}{c}{[16714-41-3]} \\
\mathrm{V}\end{array}$ & tetrakis(4-meth & $\begin{array}{c}\text { enoxy)silane } \\
97.1 \pm 1.0\end{array}$ & 298 & $\mathrm{C}$ & [1988VOR/BAR] \\
\hline $\mathrm{C}_{28} \mathrm{H}_{32} \mathrm{O}_{2} \mathrm{Si}$ & $\begin{array}{c}\text { [261766-66-9] } \\
\text { V }\end{array}$ & $\begin{array}{l}\text { 3-methyl-3-tert } \\
\text { (378-393) }\end{array}$ & $\begin{array}{l}\text { ylperoxy-1-tri } \\
120.3 \pm 5.8\end{array}$ & lsilyl-1 & $\mathrm{ME}$ & [1999DIB/LUS] \\
\hline $\mathrm{C}_{28} \mathrm{H}_{32} \mathrm{O}_{2} \mathrm{Si}_{3}$ & $\begin{array}{c}{[67103-00-8]} \\
\mathrm{V}\end{array}$ & $\begin{array}{c}\text { 1,1,1,3-tetramet } \\
\quad(549-678)\end{array}$ & $\begin{array}{c}3,5,5,5 \text {-tetrap } \\
82.6\end{array}$ & $\begin{array}{l}\text { risilox } \\
564\end{array}$ & A & [1987STE/MAL] \\
\hline $\mathrm{C}_{28} \mathrm{H}_{32} \mathrm{O}_{2} \mathrm{Si}_{3}$ & $\begin{array}{c}{[67142-05-6]} \\
\mathrm{V}\end{array}$ & $\begin{array}{c}\text { 1,1,3,5-tetrame } \\
(566-666)\end{array}$ & $\begin{array}{c}1,3,5,5 \text {-tetrap } \\
90.9\end{array}$ & $\begin{array}{l}\text { risilox } \\
581\end{array}$ & A & [1987STE/MAL] \\
\hline $\mathrm{C}_{28} \mathrm{H}_{32} \mathrm{O}_{2} \mathrm{Si}_{3}$ & $\begin{array}{c}{[3982-82-9]} \\
\mathrm{V}\end{array}$ & $\begin{array}{c}\text { 1,3,3,5-tetramet } \\
\quad(544-686)\end{array}$ & $\begin{array}{c}1,1,5,5 \text {-tetrap } \\
88.3\end{array}$ & $\begin{array}{l}\text { risilox } \\
559\end{array}$ & A & [1987STE/MAL] \\
\hline $\mathrm{C}_{28} \mathrm{H}_{32} \mathrm{O}_{3} \mathrm{Si}$ & $\begin{array}{c}{[167283-26-3]} \\
\mathrm{V}\end{array}$ & $\begin{array}{c}{[[4-[(1,1-\text { dimetl }} \\
\quad(354-386)\end{array}$ & $\begin{array}{l}\text { thyl)dioxy]-4 } \\
87.6 \pm 3.7\end{array}$ & $\begin{array}{l}\text { 1-2-pe } \\
370\end{array}$ & loxy]triph & $\begin{array}{l}\text { lsilane } \\
\text { [2006DIB/MEL] }\end{array}$ \\
\hline $\mathrm{C}_{28} \mathrm{H}_{32} \mathrm{O}_{4} \mathrm{Si}_{4}$ & $\begin{array}{c}\text { [1693-47-6] } \\
\text { TRS } \\
\text { TRS } \\
\text { FUS }\end{array}$ & 1,1,3,3-tetrame & $\begin{array}{c}5,5,7,7 \text {-tetrap } \\
0.24 \\
1.05 \\
27.05\end{array}$ & $\begin{array}{l}\text { cyclote } \\
186.5 \\
271.5 \\
346.2\end{array}$ & $\begin{array}{l}\text { xane } \\
\text { AC }\end{array}$ & [1996DOM/HEA, 1976KUL/DZH] \\
\hline $\mathrm{C}_{28} \mathrm{H}_{32} \mathrm{O}_{4} \mathrm{Si}_{4}$ & $\begin{array}{l}{[77-63-4]} \\
\text { FUS }\end{array}$ & $1,1,5^{\prime}, 7^{\prime}$-tetram & $\begin{array}{l}1-1^{\prime}, 3^{\prime}, 5,7-\text { tetr } \\
24.62\end{array}$ & $\begin{array}{l}\text { ylcyclo } \\
373.4\end{array}$ & $\begin{array}{r}\text { loxane } \\
\mathrm{AC}\end{array}$ & [1996DOM/HEA, 1975MEK/KAR3] \\
\hline $\mathrm{C}_{28} \mathrm{H}_{84} \mathrm{O}_{12} \mathrm{Si}_{13}$ & $\begin{array}{c}{[2471-09-2]} \\
\mathrm{V}\end{array}$ & $\begin{array}{l}\text { octacosamethyl } \\
(398-438)\end{array}$ & $\begin{array}{l}\text { easiloxane } \\
121\end{array}$ & & GCRT & [2010LEI/WAN] \\
\hline $\mathrm{C}_{29} \mathrm{H}_{34} \mathrm{O}_{2} \mathrm{Si}$ & $\begin{array}{c}{[261766-67-0]} \\
\mathrm{V}\end{array}$ & $\begin{array}{l}\text { 3-methyl-3-tert } \\
\text { (383-398) }\end{array}$ & $\begin{array}{l}\text { ylperoxy-1-tr } \\
126.3 \pm 3.0\end{array}$ & ylsilyl- & ne $\mathrm{ME}$ & [1999DIB/LUS] \\
\hline $\mathrm{C}_{29} \mathrm{H}_{44} \mathrm{O}_{6} \mathrm{Si}_{2}$ & $\begin{array}{c}\text { [179108-75-9] } \\
\text { TRS } \\
\text { FUS }\end{array}$ & 4-[[1-oxo-11-(1 & $\begin{array}{c}\text { 3-tetramethy } \\
20.1 \\
5.4\end{array}$ & $\begin{array}{c}\text { xanyl)t } \\
322 \\
331\end{array}$ & l]oxy]ben & $\begin{array}{l}\text { c acid, 4-methoxyphenyl ester } \\
\text { [2004RYA/LEB] }\end{array}$ \\
\hline $\mathrm{C}_{29} \mathrm{H}_{44} \mathrm{O}_{6} \mathrm{Si}_{2}$ & $\begin{array}{c}\text { [349149-95-7] } \\
\text { TRS } \\
\text { FUS }\end{array}$ & 4-methoxybenz & $\begin{array}{c}\text { acid, } 4-[[1-\mathrm{ox} \\
24.3 \\
4.7\end{array}$ & $\begin{array}{c}1,1,3,3 \\
324 \\
334\end{array}$ & nethyldisic & $\begin{array}{l}\text { nyl)undecyl]oxy]phenyl ester } \\
\text { [2004RYA/LEB] }\end{array}$ \\
\hline $\mathrm{C}_{30} \mathrm{H}_{40} \mathrm{~F}_{30} \mathrm{O}_{3} \mathrm{Si}_{4}$ & $\mathrm{~V}$ & $\begin{array}{l}1,1,1,3,5,5,5-\text { he } \\
\quad(502-652)\end{array}$ & $\begin{array}{c}\gamma \text {-trifluoropro } \\
64.3\end{array}$ & $\begin{array}{c}\operatorname{tris}(\gamma- \\
671\end{array}$ & ropropyl)s & $\begin{array}{l}\text { xytrisiloxane } \\
\text { [1974DIT/SKO3] }\end{array}$ \\
\hline $\mathrm{C}_{30} \mathrm{H}_{64} \mathrm{Si}$ & $\begin{array}{c}{[18765-73-6]} \\
V\end{array}$ & tris(decyl)silane & $65.3 \pm 0.8$ & 298 & $\mathrm{C}$ & [1988VOR/BAR] \\
\hline $\mathrm{C}_{30} \mathrm{H}_{66} \mathrm{O}_{3} \mathrm{Si}_{3}$ & $\begin{array}{l}{[152656-68-3]} \\
\text { FUS }\end{array}$ & 2,2,4,4,6,6-hex & $\begin{array}{l}\text { tylcyclotrisilo } \\
38.6\end{array}$ & 266 & $\mathrm{DSC}$ & [1995OUT/KLO] \\
\hline $\mathrm{C}_{32} \mathrm{H}_{16} \mathrm{Cl}_{2} \mathrm{~N}_{8} \mathrm{Si}$ & $\begin{array}{l}{[19333-10-9]} \\
\text { SUB }\end{array}$ & silicon phthaloc & $\begin{array}{c}\text { ine dichloride } \\
151.3\end{array}$ & & & [1972MAR/LOP] \\
\hline $\mathrm{C}_{32} \mathrm{H}_{70} \mathrm{Si}_{10}$ & $\begin{array}{c}\text { [206868-23-7] } \\
\text { FUS }\end{array}$ & $1,1,2,2,3,3,4,4$, & $\begin{array}{c}, 6,7,7,8,8,9,9 \\
56.1\end{array}$ & $\begin{array}{l}- \text {-eicosa } \\
391.2\end{array}$ & 1-1,10-dip & $\begin{array}{l}\text { ryldecasilane } \\
\text { [2001YAT/MIN] }\end{array}$ \\
\hline $\mathrm{C}_{33} \mathrm{H}_{34} \mathrm{O}_{2} \mathrm{Si}_{3}$ & $\begin{array}{c}{[67103-01-9]} \\
\mathrm{V}\end{array}$ & $\begin{array}{l}\text { 1,1,3-trimethyl- } \\
\quad(603-711)\end{array}$ & $\begin{array}{c}\text { 5,5,5-pentaph } \\
91.3\end{array}$ & $\begin{array}{l}\text { isiloxa } \\
618\end{array}$ & A & [1987STE/MAL] \\
\hline $\mathrm{C}_{33} \mathrm{H}_{34} \mathrm{O}_{2} \mathrm{Si}_{3}$ & $\begin{array}{c}{[3390-61-2]} \\
\mathrm{V}\end{array}$ & $\begin{array}{c}\text { 1,3,5-trimethyl- } \\
\quad(575-625)\end{array}$ & $\begin{array}{c}\text { 3,5,5-pentaph } \\
87.3\end{array}$ & $\begin{array}{l}\text { siloxa } \\
590\end{array}$ & A & [1987STE/MAL] \\
\hline $\mathrm{C}_{33} \mathrm{H}_{34} \mathrm{O}_{4} \mathrm{Si}_{4}$ & [32395-60-1] & 1,3,5-trimethyl- & 5,7,7-pentaph & yclotetr & ane & \\
\hline
\end{tabular}


TABLE 17. Phase change enthalpies of organometallic and inorganic compounds-Continued

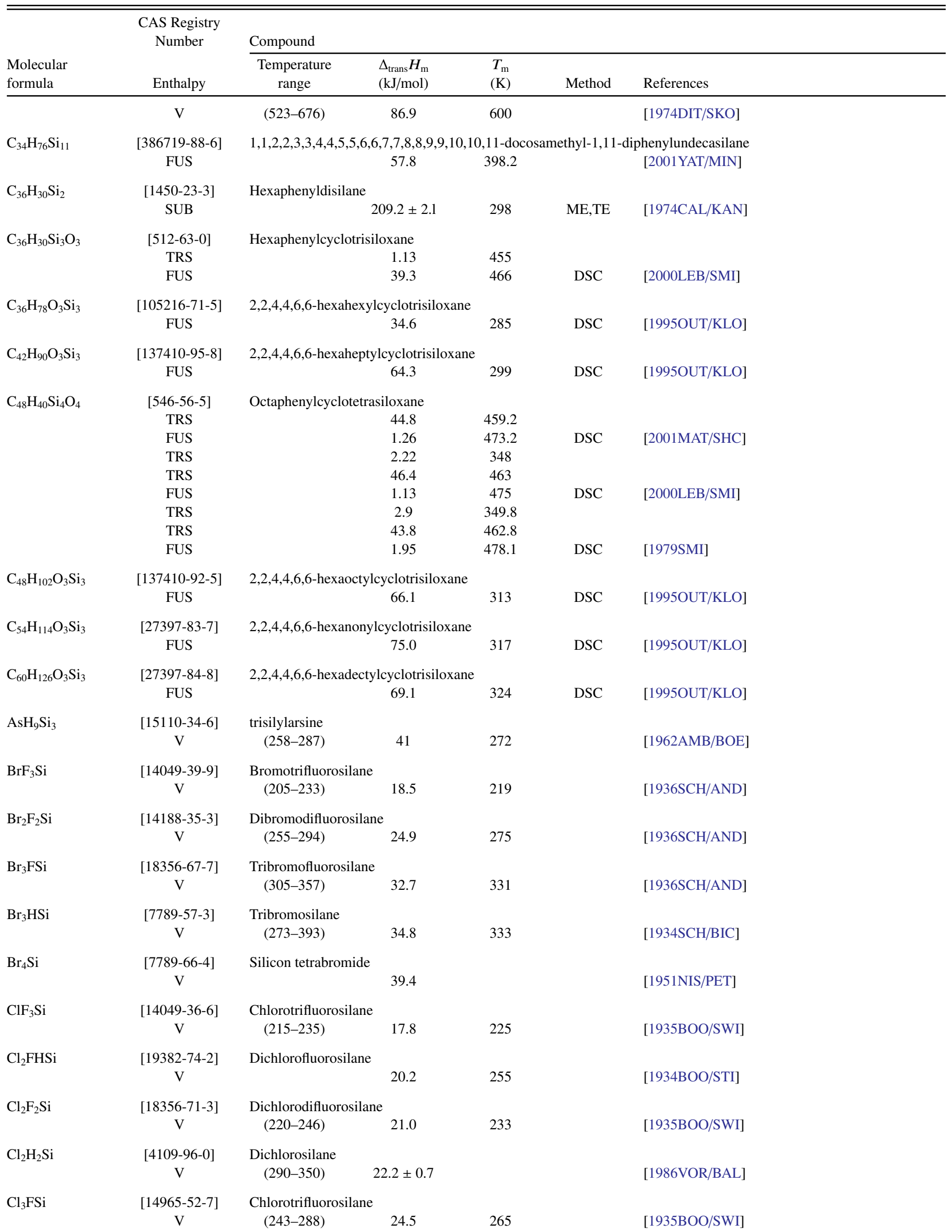


TABLE 17. Phase change enthalpies of organometallic and inorganic compounds-Continued

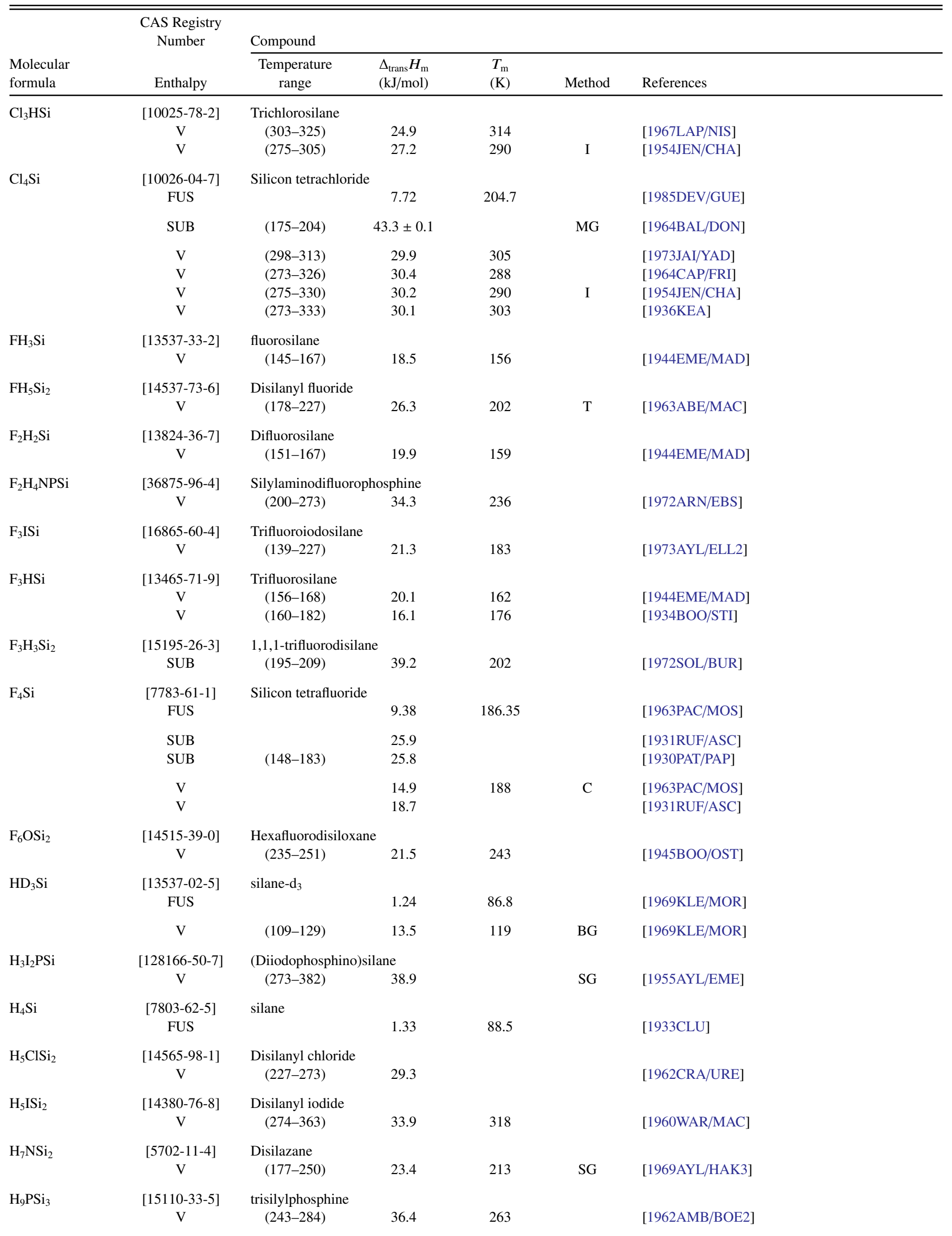


TABLE 17. Phase change enthalpies of organometallic and inorganic compounds-Continued

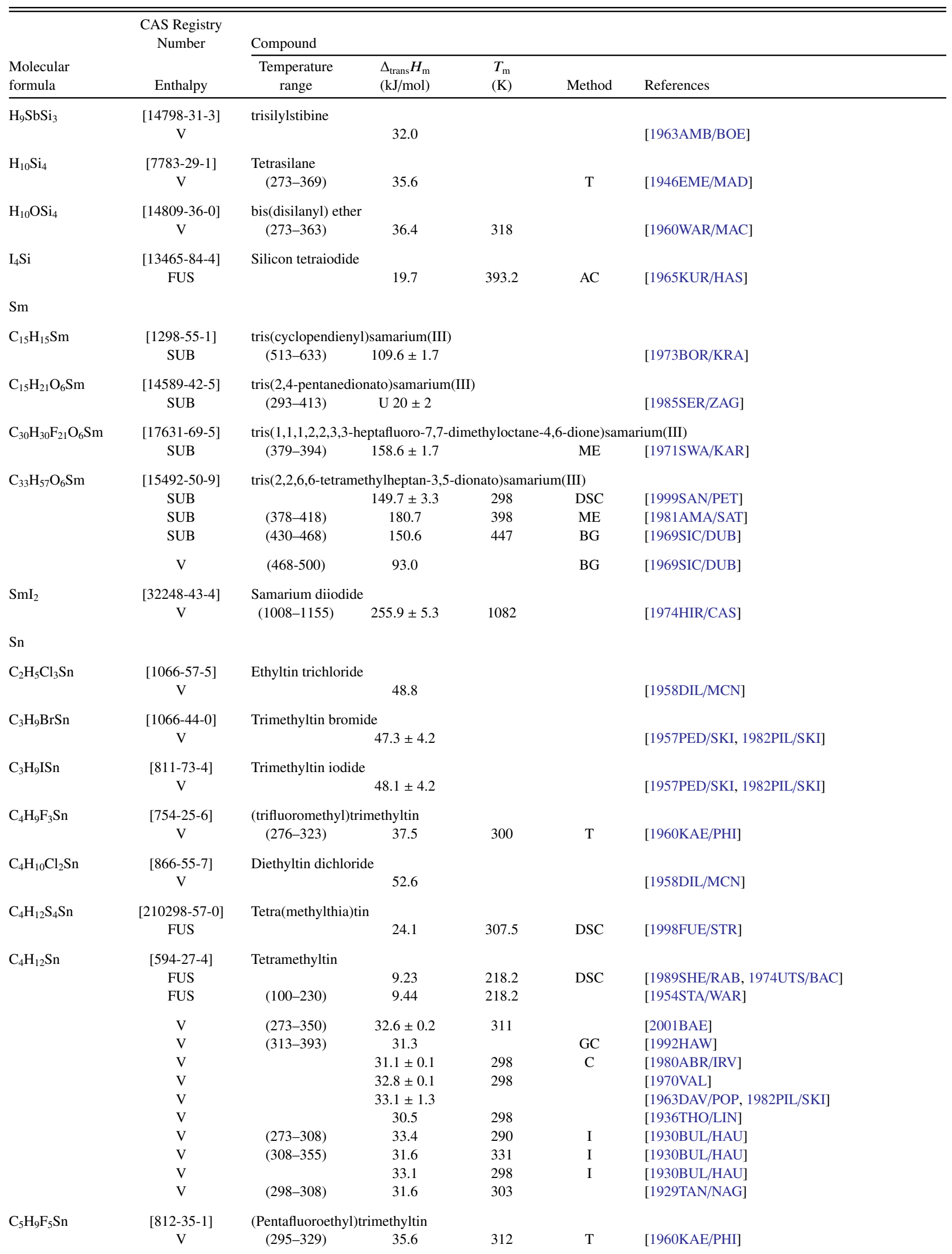


TABLE 17. Phase change enthalpies of organometallic and inorganic compounds-Continued

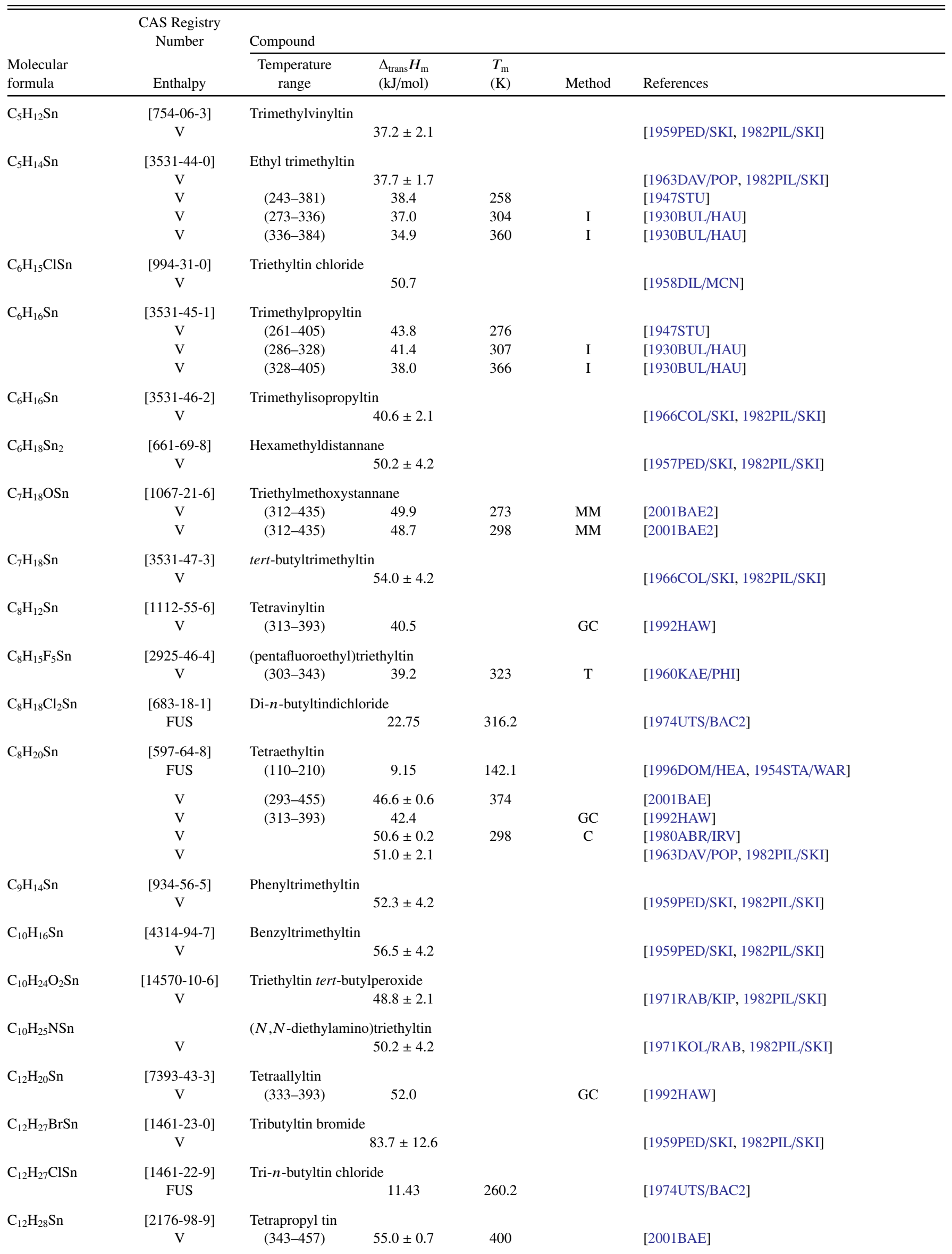


TABLE 17. Phase change enthalpies of organometallic and inorganic compounds-Continued

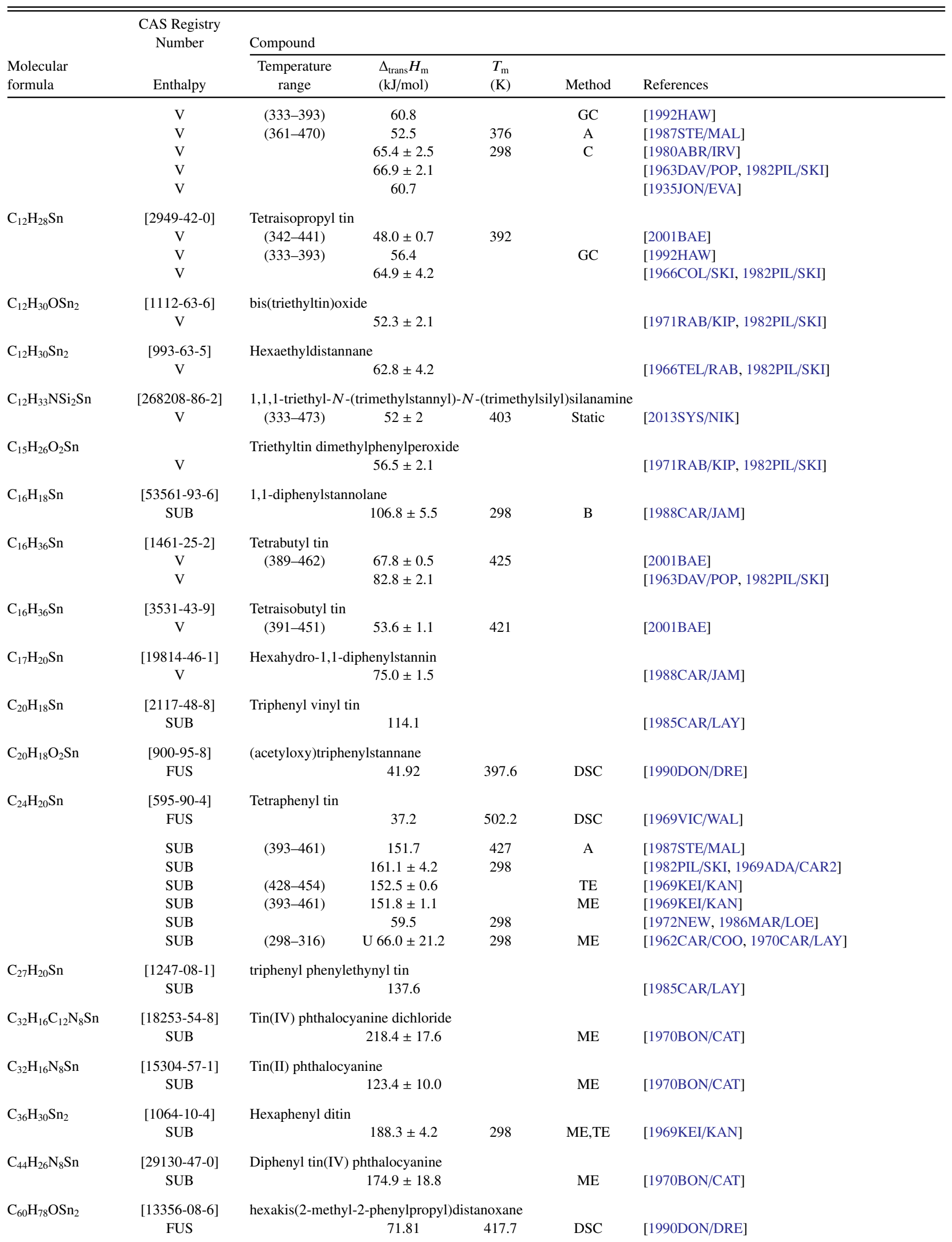


TABLE 17. Phase change enthalpies of organometallic and inorganic compounds-Continued

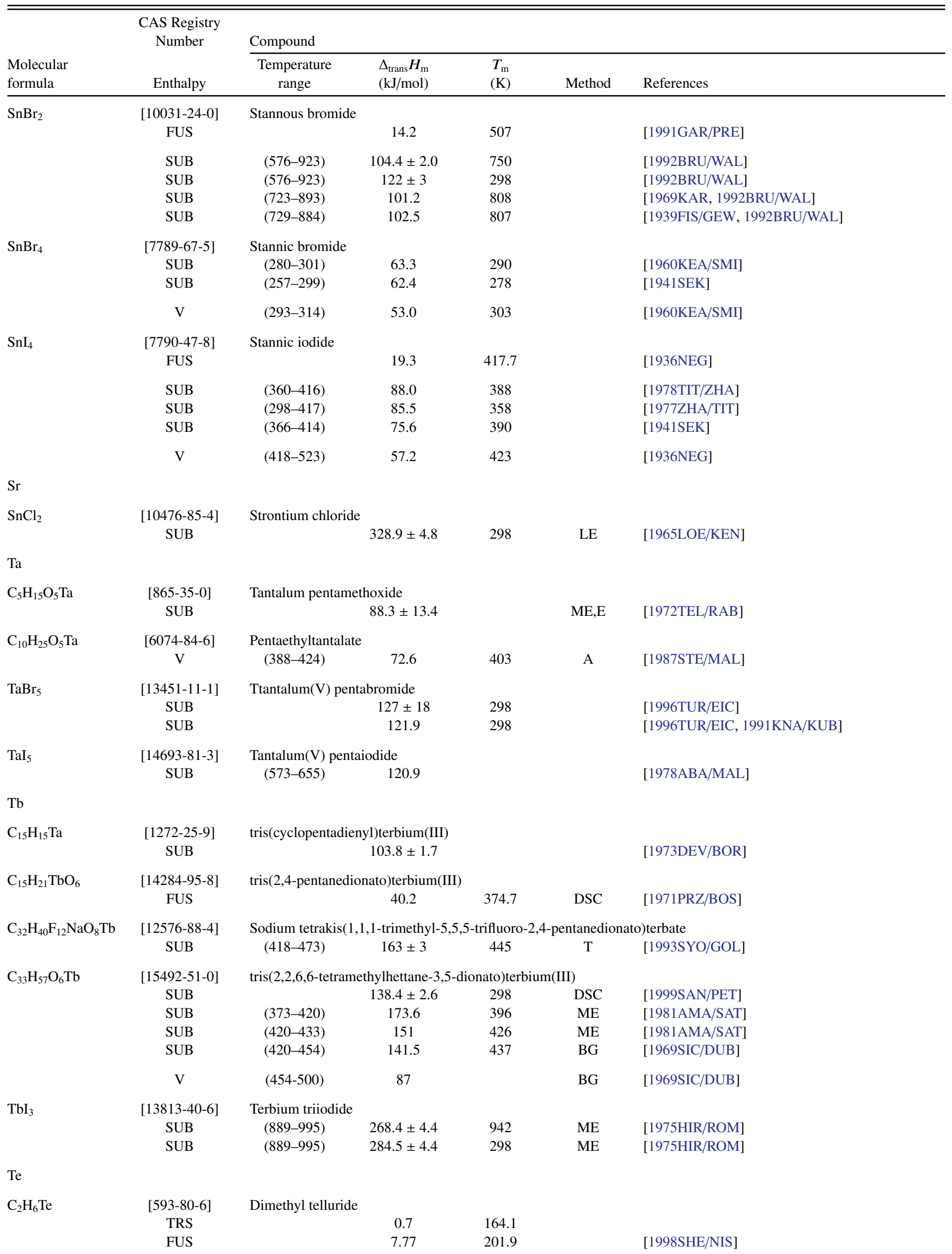


TABLE 17. Phase change enthalpies of organometallic and inorganic compounds-Continued

\begin{tabular}{|c|c|c|c|c|c|c|}
\hline \multirow[b]{2}{*}{$\begin{array}{l}\text { Molecular } \\
\text { formula }\end{array}$} & \multirow{2}{*}{$\begin{array}{l}\text { CAS Registry } \\
\text { Number } \\
\text { Enthalpy }\end{array}$} & \multicolumn{5}{|l|}{ Compound } \\
\hline & & $\begin{array}{l}\text { Temperature } \\
\text { range }\end{array}$ & $\begin{array}{l}\Delta_{\text {trans }} H_{\mathrm{m}} \\
(\mathrm{kJ} / \mathrm{mol})\end{array}$ & $\begin{array}{l}T_{\mathrm{m}} \\
(\mathrm{K})\end{array}$ & Method & References \\
\hline & $\mathrm{V}$ & & $36.1 \pm 0.6$ & 298 & $\mathrm{C}$ & [2014GER/PAV] \\
\hline & $\mathrm{V}$ & $(298-367)$ & 34.4 & 313 & & [1999DYK/SVO] \\
\hline & $\mathrm{V}$ & $(273-372)$ & $35.6 \pm 0.1$ & 323 & & [1997BAE, 1996BAE/POD] \\
\hline & $\mathrm{V}$ & $(267-369)$ & 36.9 & 282 & BG & [1996VAN/COR] \\
\hline & $\mathrm{V}$ & $(267-369)$ & $36.1 \pm 1.0$ & 298 & BG & [1996VAN/COR] \\
\hline & $\mathrm{V}$ & & $37.4 \pm 0.7$ & 298 & $\mathrm{C}$ & [1989VOR/KLY] \\
\hline & $\mathrm{V}$ & & $36.0 \pm 2.1$ & & & [1988TEL/LAR] \\
\hline \multirow[t]{4}{*}{$\mathrm{C}_{4} \mathrm{H}_{6} \mathrm{Te}$} & {$[63000-06-6]$} & Divinyl telluride & & & & \\
\hline & FUS & & 9.59 & 182.6 & & [1999NIS/SHE] \\
\hline & $\mathrm{V}$ & & $44.8 \pm 0.8$ & 298 & $\mathrm{C}$ & [1989VOR/KLY] \\
\hline & $\mathrm{V}$ & & $38.1 \pm 2.1$ & & & [1988TEL/LAR] \\
\hline \multirow[t]{5}{*}{$\mathrm{C}_{4} \mathrm{H}_{10} \mathrm{Te}$} & {$[627-54-3]$} & Diethyl telluride & & & & \\
\hline & FUS & & 7.62 & 161.5 & & [1996SHE/KAM] \\
\hline & $\mathrm{V}$ & $(295-411)$ & 41.8 & 310 & & [1999DYK/SVO] \\
\hline & $\mathrm{V}$ & $(273-415)$ & $41.6 \pm 0.2$ & 344 & & [1996BAE/POD] \\
\hline & $\mathrm{V}$ & & $41.6 \pm 0.8$ & 298 & $\mathrm{C}$ & [1989VOR/KLY] \\
\hline \multirow[t]{3}{*}{$\mathrm{C}_{4} \mathrm{H}_{14} \mathrm{Te}$} & {$[64501-17-3]$} & Dipropyl telluride & & & & \\
\hline & $\mathrm{V}$ & $(298-434)$ & $45.5 \pm 0.3$ & 366 & & [1996BAE/POD] \\
\hline & $\mathrm{V}$ & & $46.5 \pm 0.7$ & 298 & $\mathrm{C}$ & [1989VOR/KLY] \\
\hline \multirow[t]{2}{*}{$\mathrm{C}_{6} \mathrm{H}_{14} \mathrm{Te}$} & [51112-72-2] & Diisopropyl telluride & & & & \\
\hline & $\mathrm{V}$ & (298-399) & $40.4 \pm 0.1$ & 349 & & [1996BAE/POD] \\
\hline \multirow[t]{2}{*}{$\mathrm{C}_{6} \mathrm{H}_{14} \mathrm{Te}_{2}$} & [79971-42-9] & Dipropyl ditelluride & & & & \\
\hline & V & & $52.7 \pm 1.0$ & 298 & $\mathrm{C}$ & [1989VOR/KLY] \\
\hline \multirow[t]{3}{*}{$\mathrm{C}_{8} \mathrm{H}_{18} \mathrm{Te}$} & {$[38788-38-4]$} & Dibutyl telluride & & & & \\
\hline & $\mathrm{V}$ & $(303-423)$ & $53.4 \pm 0.1$ & 358 & & [1996BAE/POD] \\
\hline & V & & $51.0 \pm 1.0$ & 298 & $\mathrm{C}$ & [1989VOR/KLY] \\
\hline \multirow[t]{2}{*}{$\mathrm{C}_{8} \mathrm{H}_{18} \mathrm{Te}$} & [83817-01-0] & Diisobutyl telluride & & & & \\
\hline & $\mathrm{V}$ & $(303-410)$ & $47.6 \pm 0.1$ & 356 & & [1996BAE/POD] \\
\hline \multirow[t]{2}{*}{$\mathrm{C}_{8} \mathrm{H}_{18} \mathrm{Te}$} & {$[83817-20-3]$} & Di-sec-butyl tellurid & & & & \\
\hline & $\mathrm{V}$ & $(303-372)$ & $49.6 \pm 0.9$ & 338 & & [1996BAE/POD] \\
\hline \multirow[t]{2}{*}{$\mathrm{C}_{8} \mathrm{H}_{18} \mathrm{Te}_{2}$} & [77129-69-2] & Dibutyl ditelluride & & & & \\
\hline & $\mathrm{V}$ & & $57.3 \pm 1.0$ & 298 & $\mathrm{C}$ & [1989VOR/KLY] \\
\hline \multirow[t]{3}{*}{$\mathrm{C}_{10} \mathrm{H}_{22} \mathrm{Te}$} & {$[71475-88-2]$} & Dipentyl telluride & & & & \\
\hline & FUS & & 23.1 & 215.4 & & [1994TEL/SHE] \\
\hline & $\mathrm{V}$ & $(343-403)$ & $59.5 \pm 0.8$ & 373 & & [1996BAE/POD] \\
\hline \multirow[t]{2}{*}{$\mathrm{C}_{10} \mathrm{H}_{22} \mathrm{Te}$} & [110346-75-3] & Diisopentyl telluride & & & & \\
\hline & $\mathrm{V}$ & $(343-403)$ & $51.9 \pm 0.7$ & 373 & & [1996BAE/POD] \\
\hline \multirow[t]{2}{*}{$\mathrm{C}_{12} \mathrm{~F}_{10} \mathrm{Te}$} & [18064-76-1] & bis(pentafluoropheny & yl) telluride & & & \\
\hline & FUS & & 16.3 & 322.3 & DSC & [2008ZEL/CHU] \\
\hline \multirow[t]{2}{*}{$\mathrm{C}_{12} \mathrm{H}_{10} \mathrm{Te}$} & [1202-36-4] & Diphenyl telluride & & & & \\
\hline & FUS & & 15.35 & 268.4 & & [1996TEL/SHE] \\
\hline \multirow[t]{2}{*}{$\mathrm{TeBr}_{4}$} & [10031-27-3] & Tellurium tetrabromi & ide & & & \\
\hline & SUB & & $129.7 \pm 2.1$ & 298 & & [1974OPP/STO] \\
\hline \multirow[t]{4}{*}{$\mathrm{TeCl}_{4}$} & [10026-07-0] & Tellurium tetrachlori & ide & & & \\
\hline & SUB & & $105 \pm 2$ & 298 & $\mathrm{TE}$ & [1994DAL/FER] \\
\hline & $\mathrm{V}$ & & $87.9 \pm 2.1$ & 298 & & [1974OPP/STO] \\
\hline & $\mathrm{V}$ & $(506-660)$ & 77 & 583 & GS & [1930SIM] \\
\hline $\mathrm{TeF}_{6}$ & $\begin{array}{c}{[7783-80-4]} \\
\text { SUB }\end{array}$ & $\begin{array}{l}\text { Tellurium hexafluoric } \\
\quad(194-233)\end{array}$ & ide 25.6 & 214 & & [1932KLE/HEN] \\
\hline
\end{tabular}


TABLE 17. Phase change enthalpies of organometallic and inorganic compounds-Continued

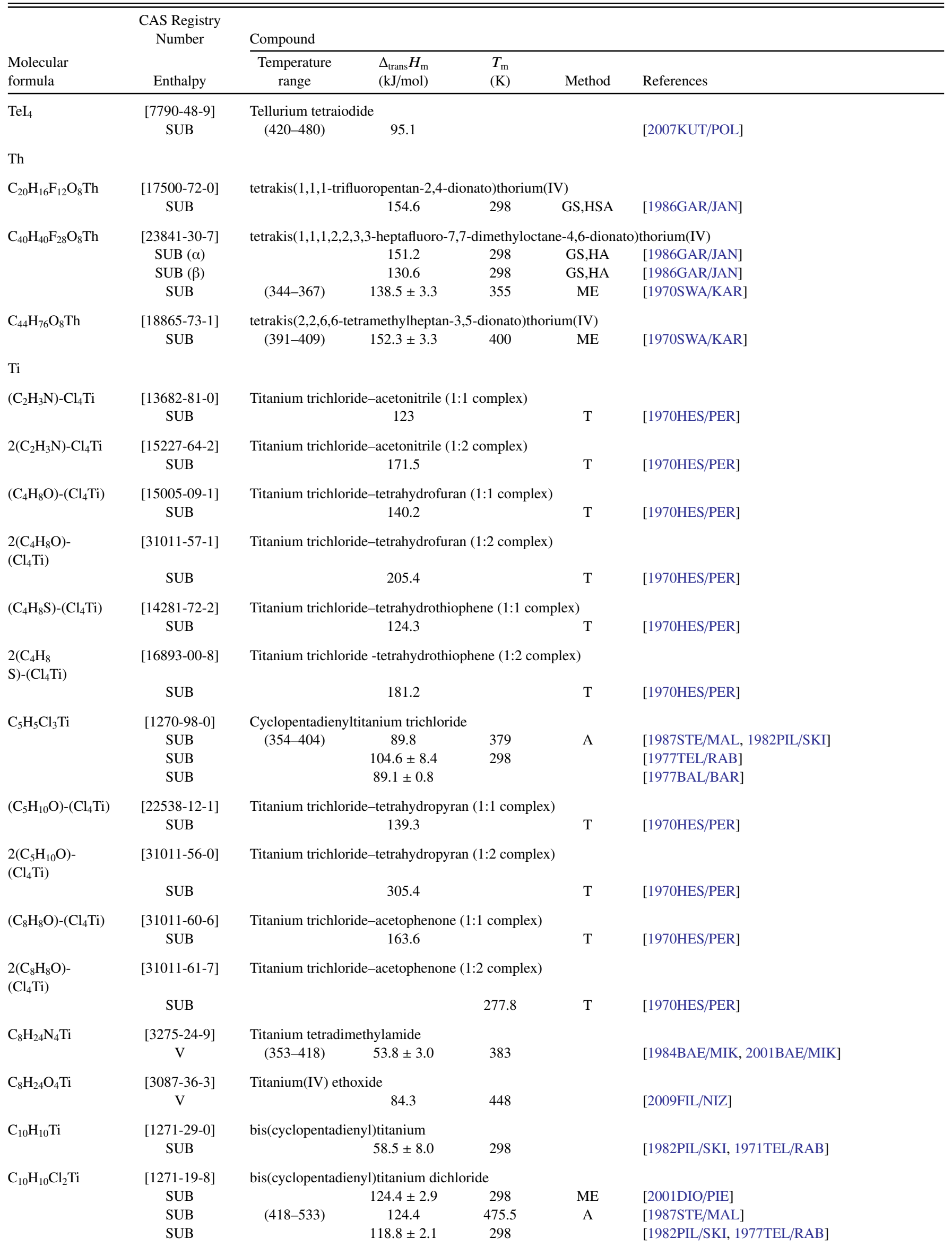


TABLE 17. Phase change enthalpies of organometallic and inorganic compounds-Continued

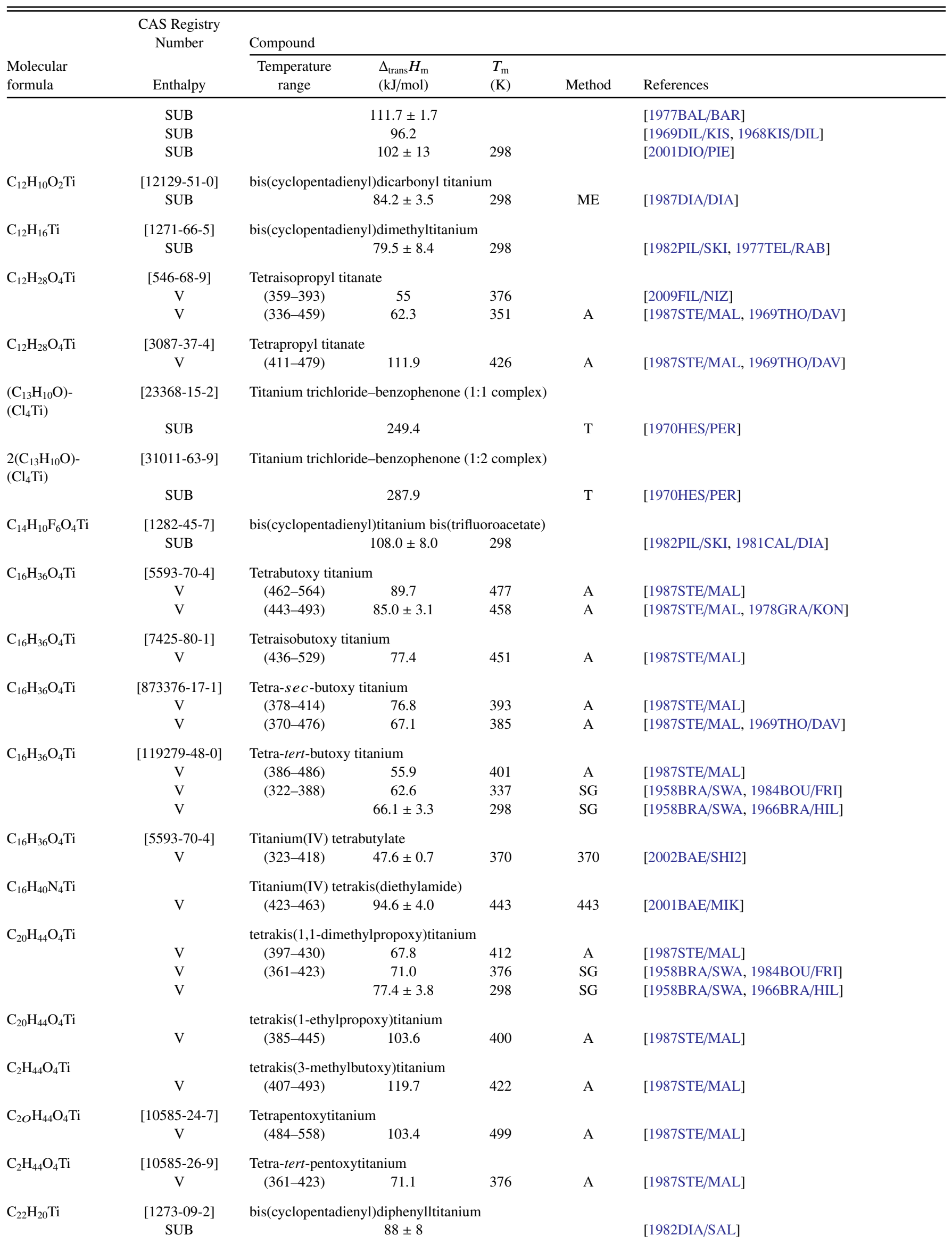


TABLE 17. Phase change enthalpies of organometallic and inorganic compounds-Continued

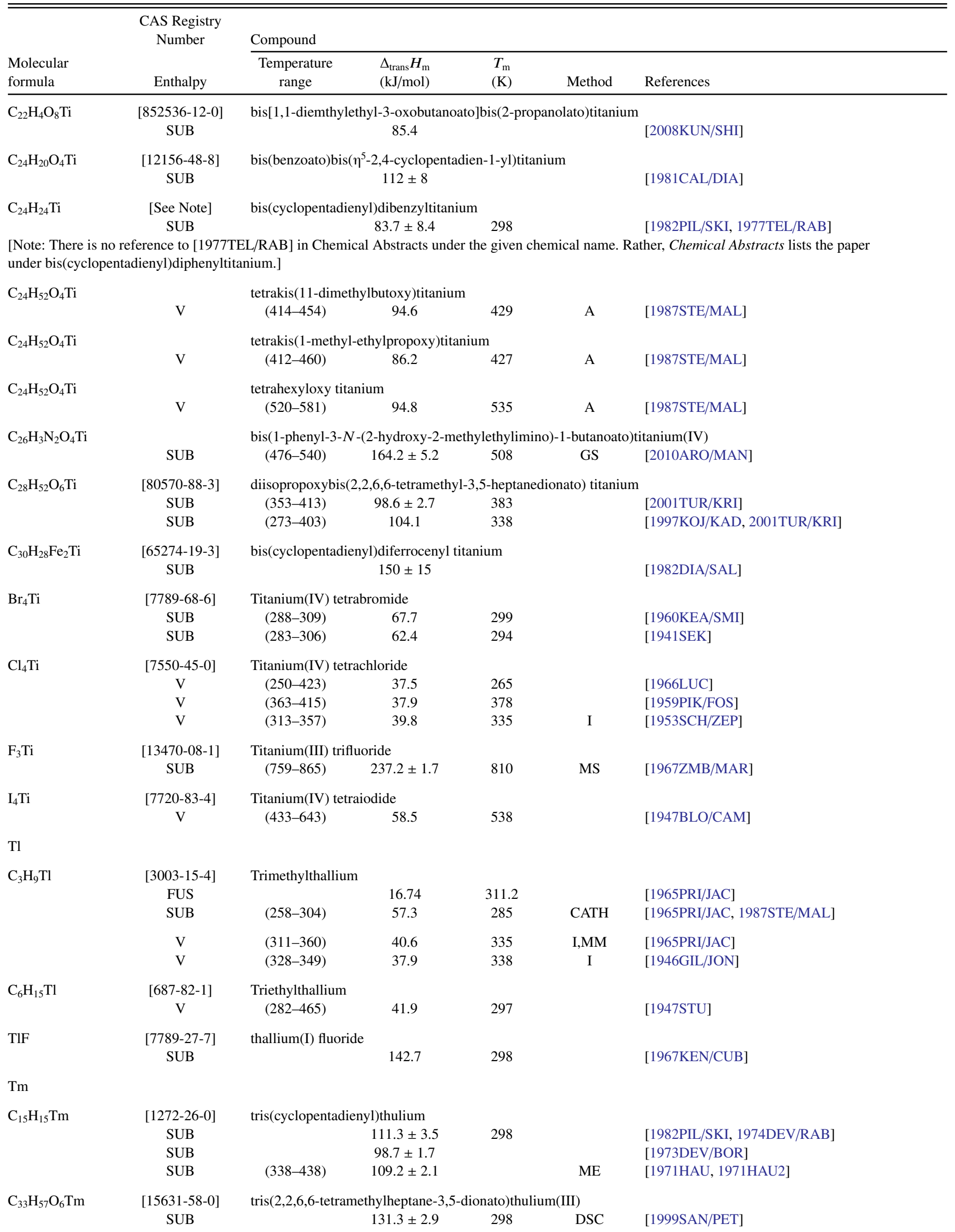


TABLE 17. Phase change enthalpies of organometallic and inorganic compounds-Continued

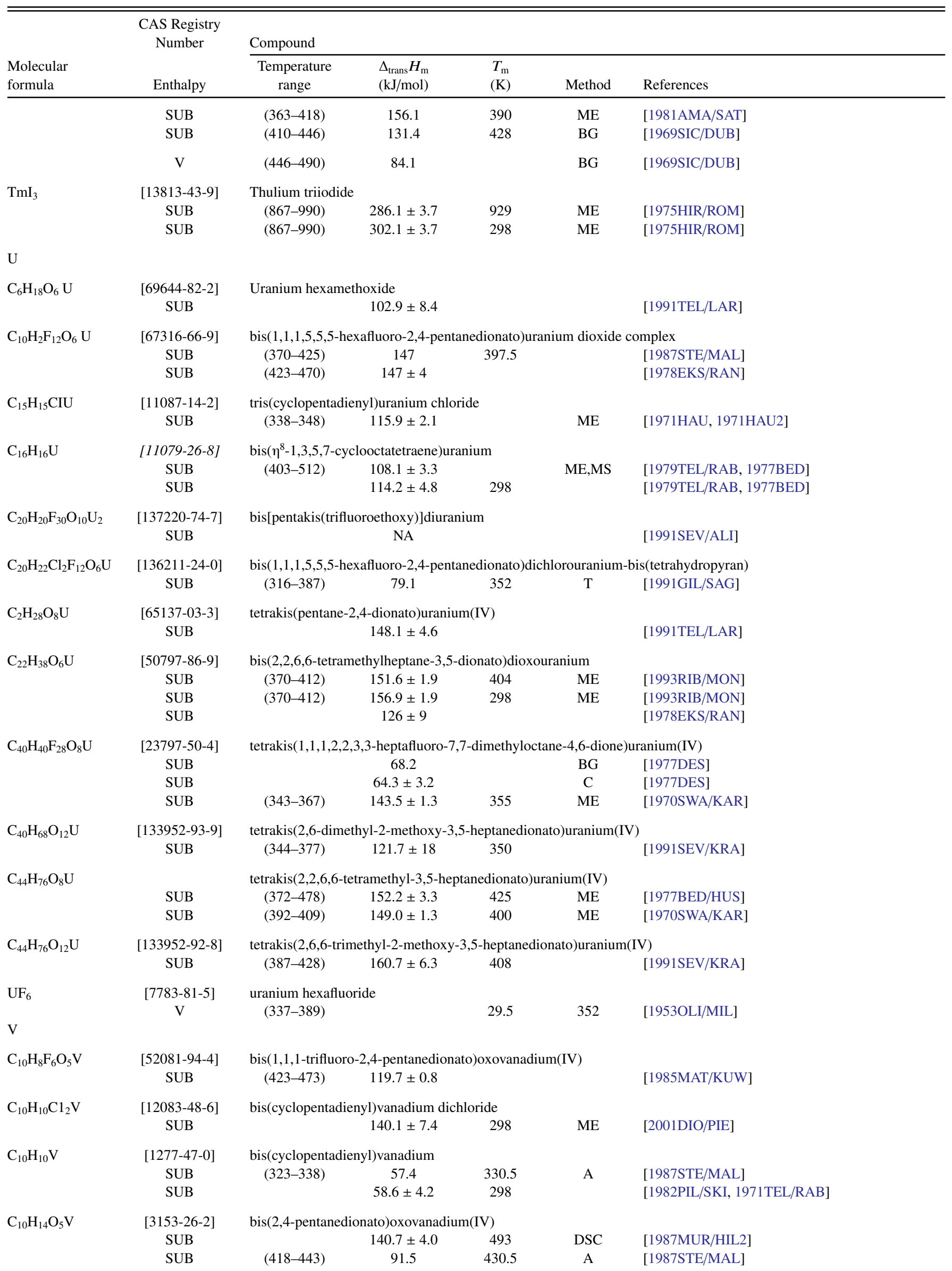


TABLE 17. Phase change enthalpies of organometallic and inorganic compounds-Continued

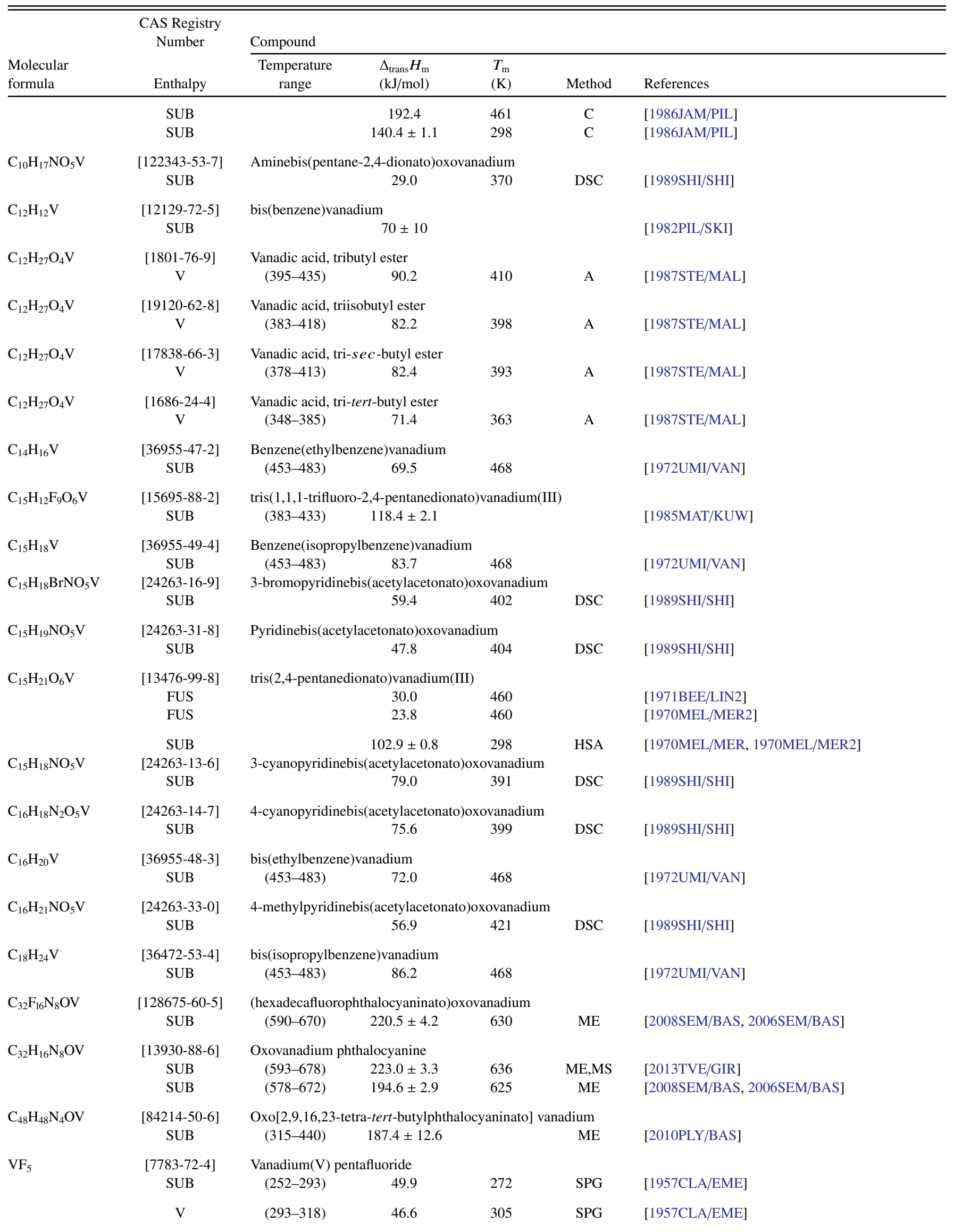


TABLE 17. Phase change enthalpies of organometallic and inorganic compounds-Continued

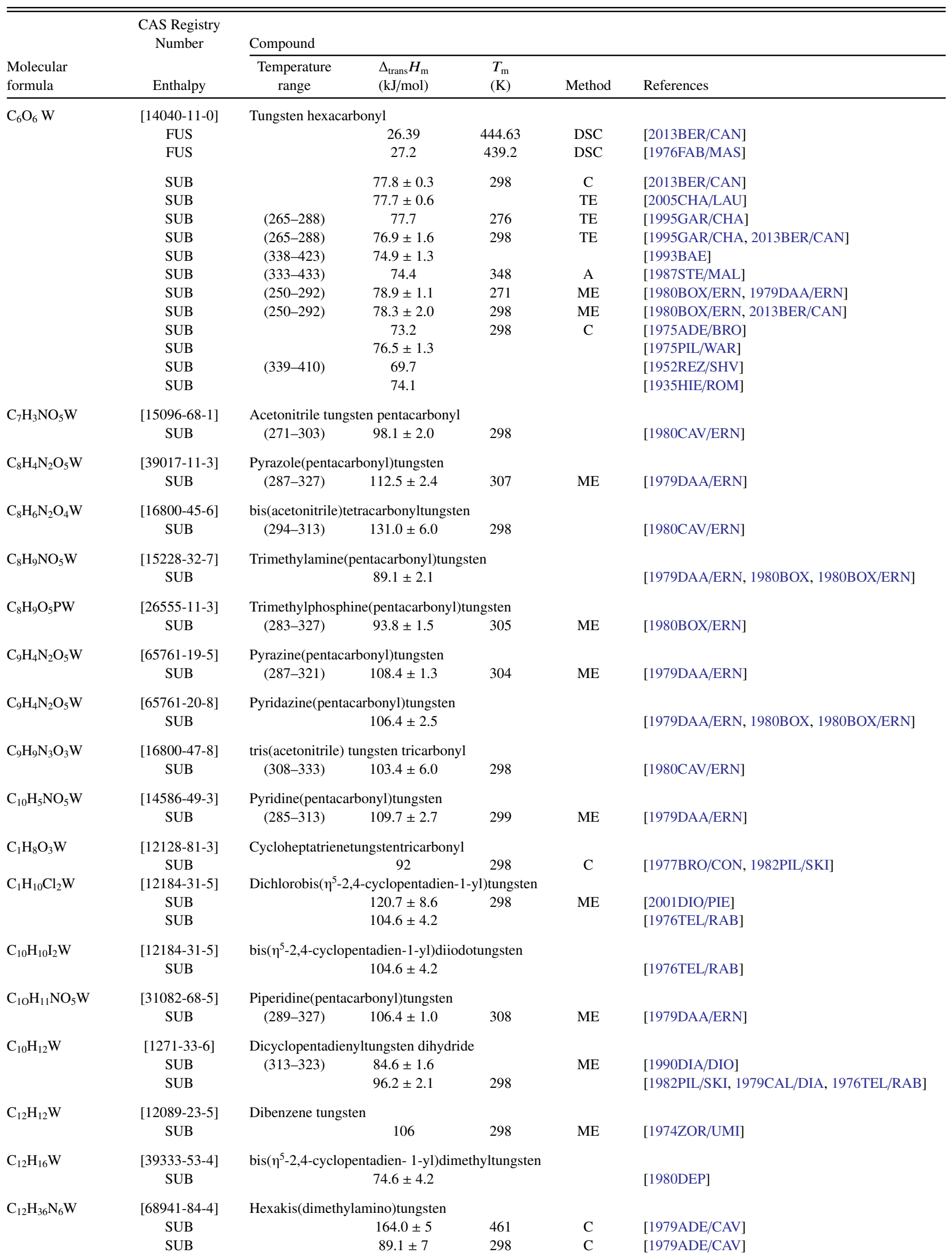


TABLE 17. Phase change enthalpies of organometallic and inorganic compounds-Continued

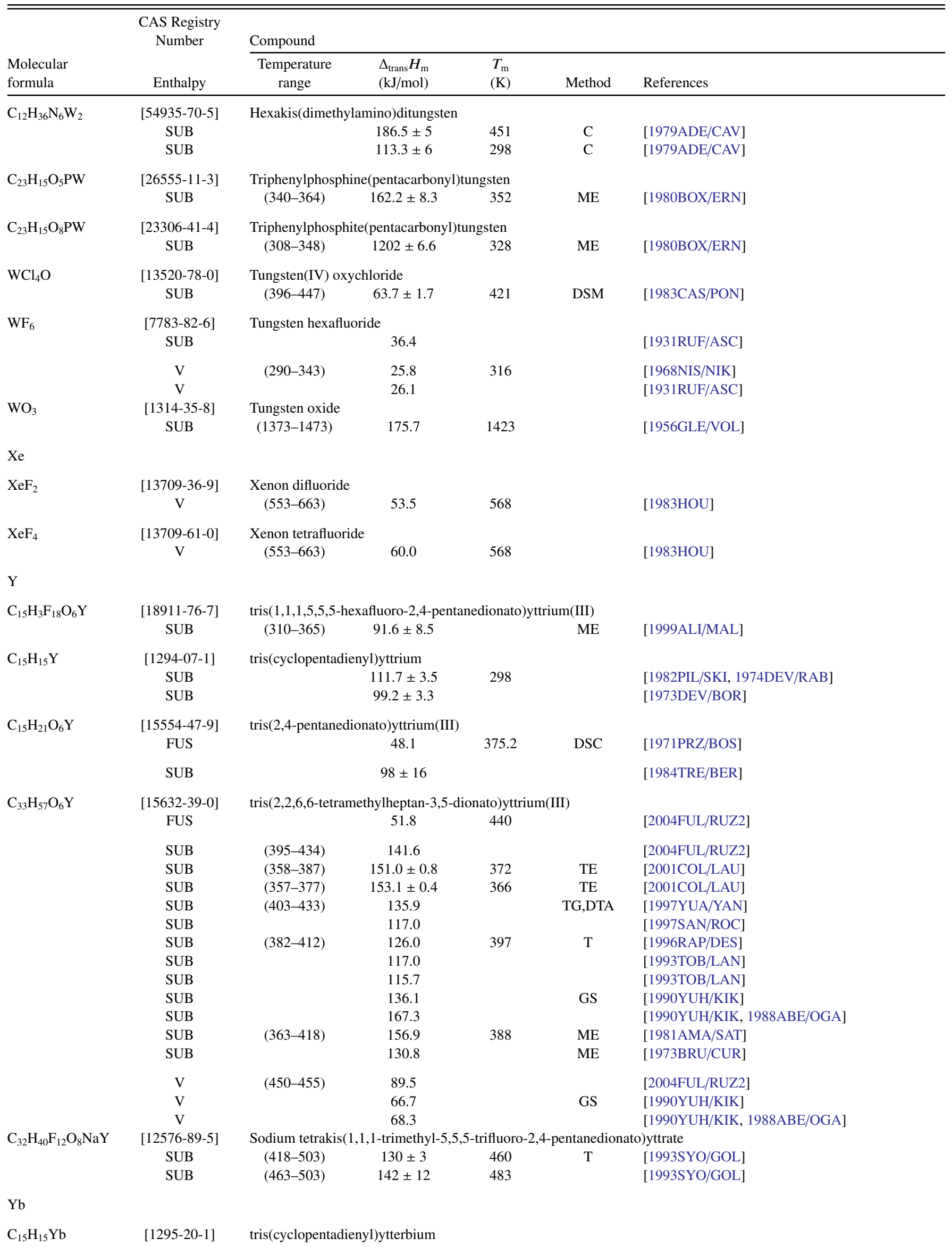


TABLE 17. Phase change enthalpies of organometallic and inorganic compounds-Continued

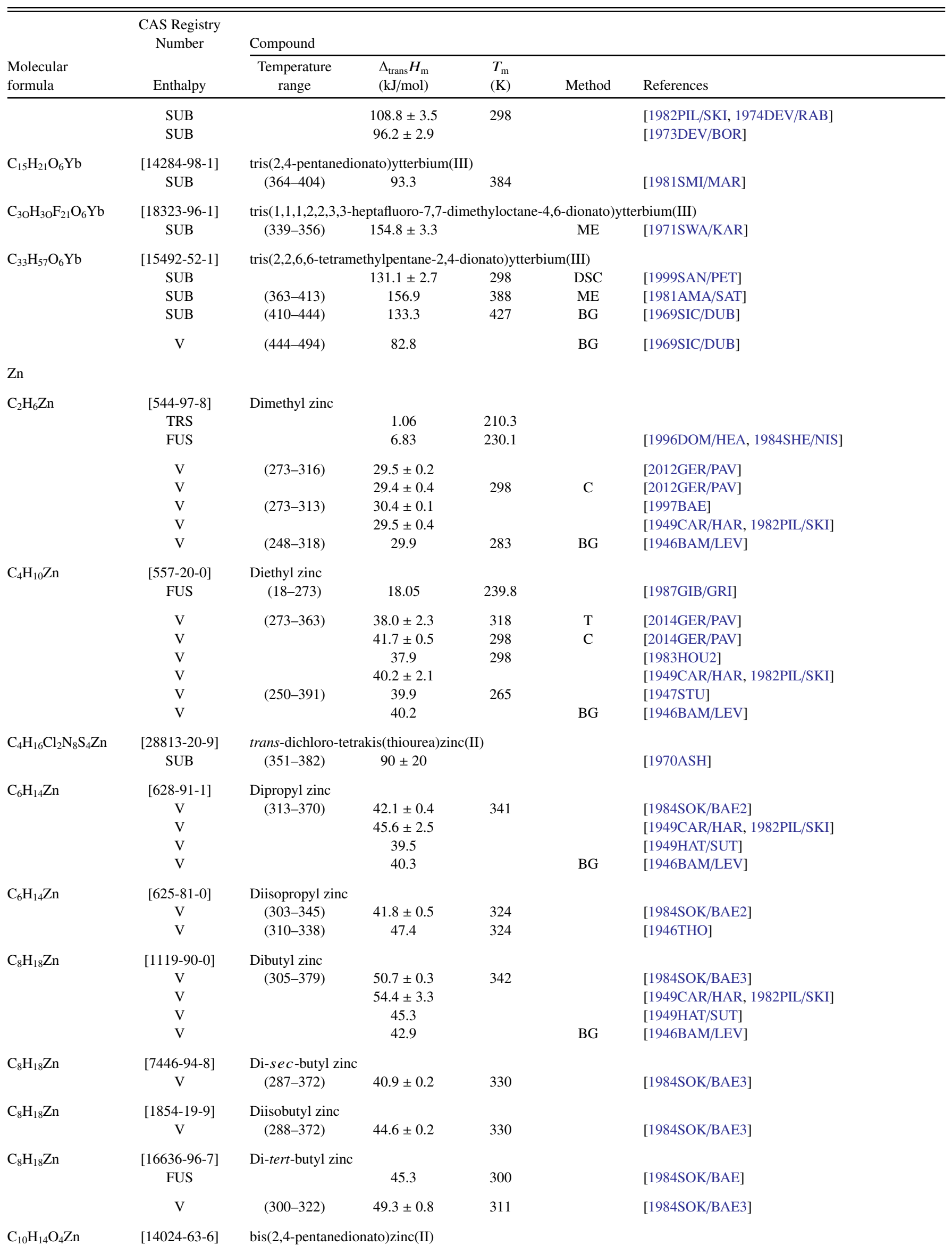


TABLE 17. Phase change enthalpies of organometallic and inorganic compounds-Continued

\begin{tabular}{|c|c|c|c|c|c|c|}
\hline \multirow[b]{2}{*}{$\begin{array}{l}\text { Molecular } \\
\text { formula }\end{array}$} & \multirow{2}{*}{$\begin{array}{c}\text { CAS Registry } \\
\text { Number } \\
\text { Enthalpy }\end{array}$} & \multicolumn{5}{|l|}{ Compound } \\
\hline & & $\begin{array}{l}\text { Temperature } \\
\text { range }\end{array}$ & $\begin{array}{l}\Delta_{\text {trans }} H_{\mathrm{m}} \\
(\mathrm{kJ} / \mathrm{mol})\end{array}$ & $\begin{array}{l}T_{\mathrm{m}} \\
(\mathrm{K})\end{array}$ & Method & References \\
\hline & $\begin{array}{l}\text { SUB } \\
\text { SUB }\end{array}$ & & $\begin{array}{c}132.6 \pm 8 \\
117 \pm 3\end{array}$ & 298 & $\mathrm{C}$ & $\begin{array}{l}\text { [1985MUR/SAK, 1988RIB/PIL] } \\
\text { [1980SAC/HIL] }\end{array}$ \\
\hline $\mathrm{C}_{10} \mathrm{H}_{20} \mathrm{~N}_{2} \mathrm{~S}_{4} \mathrm{Zn}$ & $\begin{array}{c}\text { [14324-55-1] } \\
\text { SUB } \\
\text { SUB } \\
\text { SUB }\end{array}$ & $\begin{array}{l}\text { bis(diethyldith } \\
\text { (401-444) }\end{array}$ & $\begin{array}{c}\text { bamate)zinc( } \\
115 \pm 15 \\
143.1 \\
142.7 \pm 2.5\end{array}$ & $\begin{array}{c}298 \\
422.5\end{array}$ & $\begin{array}{c}\text { DSC,E } \\
\text { A } \\
\text { GC }\end{array}$ & $\begin{array}{l}\text { [2000DEA/SOU] } \\
{[1987 \mathrm{STE} / \mathrm{MAL}]} \\
{[1976 \mathrm{TAV} / \mathrm{NEE}]}\end{array}$ \\
\hline $\mathrm{C}_{10} \mathrm{H}_{22} \mathrm{Zn}$ & $\begin{array}{c}{[14402-93-8]} \\
\mathrm{V}\end{array}$ & Dipentyl zinc & 48.6 & & & [1949HAT/SUT] \\
\hline $\mathrm{C}_{12} \mathrm{H}_{26} \mathrm{Zn}$ & $\begin{array}{c}{[13822-55-4]} \\
\mathrm{V}\end{array}$ & Dihexyl zinc & 56.2 & & & [1949HAT/SUT] \\
\hline $\mathrm{C}_{14} \mathrm{H}_{28} \mathrm{~N}_{2} \mathrm{~S}_{4} \mathrm{Zn}$ & $\begin{array}{l}{[15694-56-1]} \\
\text { SUB }\end{array}$ & bis(dipropyldit & $\begin{array}{l}\text { arbamate)zinc } \\
147 \pm 2\end{array}$ & 298 & $\mathrm{DSC}, \mathrm{E}$ & [1992DEC/AIR] \\
\hline $\mathrm{C}_{14} \mathrm{H}_{30} \mathrm{Zn}$ & $\begin{array}{c}{[14402-95-0]} \\
\mathrm{V}\end{array}$ & Diheptyl zinc & 62.3 & & & [1949HAT/SUT] \\
\hline $\mathrm{C}_{18} \mathrm{H}_{12} \mathrm{~N}_{2} \mathrm{O}_{2} \mathrm{Zn}$ & $\begin{array}{c}\text { [13978-85-3] } \\
\text { SUB } \\
\text { SUB } \\
\text { SUB }\end{array}$ & $\begin{array}{l}\text { bis(8-hydroxyc } \\
(473-513)\end{array}$ & $\begin{array}{c}\text { linato)zinc(II } \\
183.2 \pm 6.3 \\
167.9 \pm 6 \\
178 \pm 6\end{array}$ & $\begin{array}{l}298 \\
493 \\
298\end{array}$ & $\begin{array}{l}\text { ME } \\
\text { ME }\end{array}$ & $\begin{array}{l}\text { [1994RIB/MAT] } \\
\text { [1984BUR/MOR] } \\
\text { [1984BUR/MOR] }\end{array}$ \\
\hline $\mathrm{C}_{18} \mathrm{H}_{36} \mathrm{~N}_{2} \mathrm{~S}_{4} \mathrm{Zn}$ & $\begin{array}{l}{[136-23-2]} \\
\text { SUB }\end{array}$ & bis(dibutyldith & $\begin{array}{l}\text { bamate }) \text { zinc( } \\
107 \pm 3\end{array}$ & 298 & $\mathrm{DSC}, \mathrm{E}$ & [1991DES/DES] \\
\hline $\mathrm{C}_{18} \mathrm{H}_{36} \mathrm{~N}_{2} \mathrm{~S}_{4} \mathrm{Zn}$ & $\begin{array}{l}{[36190-62-2]} \\
\text { SUB }\end{array}$ & bis(diisobutyld & $\begin{array}{l}\text { carbamate)zil } \\
283 \pm 2\end{array}$ & 298 & DSC,E & [1994SOU/PIN] \\
\hline $\mathrm{C}_{20} \mathrm{H}_{16} \mathrm{~N}_{2} \mathrm{O}_{2} \mathrm{Zn}$ & $\begin{array}{c}\text { [14128-73-5] } \\
\text { SUB } \\
\text { SUB }\end{array}$ & $\begin{array}{c}\text { bis(8-hydroxy- } \\
\quad(437-556) \\
(437-556)\end{array}$ & $\begin{array}{l}\text { thylquinolin } \\
172.0 \pm 5.0 \\
179.4 \pm 5.0\end{array}$ & $\begin{array}{l}\mathrm{c}(\mathrm{II}) \\
541 \\
298\end{array}$ & $\begin{array}{l}\text { ME } \\
\text { ME }\end{array}$ & $\begin{array}{l}\text { [1998RIB/MAT3] } \\
\text { [1998RIB/MAT3] }\end{array}$ \\
\hline $\mathrm{C}_{22} \mathrm{H}_{38} \mathrm{O}_{4} \mathrm{Zn}$ & $\begin{array}{l}{[14363-14-5]} \\
\text { SUB }\end{array}$ & bis(2,2,6,6-tetr & $\begin{array}{l}\text { hylheptan-3,5 } \\
136\end{array}$ & ato)zin & $\mathrm{ME}$ & [1973BRU/CUR] \\
\hline $\mathrm{C}_{22} \mathrm{H}_{44} \mathrm{~N}_{2} \mathrm{~S}_{4} \mathrm{Zn}$ & $\begin{array}{l}{[15337-18-5]} \\
\text { SUB }\end{array}$ & bis(dipentyldit & $\begin{array}{l}\text { rbamate)zinc } \\
127 \pm 3\end{array}$ & 298 & $\mathrm{DSC}, \mathrm{E}$ & [2000DEA/SOU] \\
\hline $\mathrm{C}_{32} \mathrm{~F}_{16} \mathrm{~N}_{8} \mathrm{Zn}$ & $\begin{array}{l}{[14320-04-8]} \\
\text { SUB }\end{array}$ & $\begin{array}{l}1,2,3,4,8,9,10, \\
\quad(658-711)\end{array}$ & $\begin{array}{l}16,17,18,22 \\
236.4 \pm 1.7\end{array}$ & $\begin{array}{c}\text { 25-hex } \\
685\end{array}$ & $\begin{array}{l}\text { luorophth } \\
\text { ME }\end{array}$ & $\begin{array}{l}\text { cyanine zinc(II) } \\
\text { [2008SEM/BAS, 2006SEM/BAS] }\end{array}$ \\
\hline $\mathrm{C}_{32} \mathrm{H}_{16} \mathrm{~N}_{8} \mathrm{Zn}$ & $\begin{array}{l}{[14320-04-8]} \\
\text { SUB }\end{array}$ & $\begin{array}{l}\text { Zinc(II)phthal } \\
\quad(693-813)\end{array}$ & $\begin{array}{l}\text { line } \\
207.5 \pm 0.5\end{array}$ & & TGA & [2013SHA/SHT] \\
\hline & $\mathrm{V}$ & $(664-709)$ & $202.3 \pm 7.5$ & 686 & $\mathrm{ME}$ & [2008SEM/BAS, 2006SEM/BAS] \\
\hline
\end{tabular}

[Note: The authors of [2013SHA/SHT] refer to the value as the enthalpy of sublimation; while the authors of [2006SEM/BAS] refer to the value as the vaporization enthalpy.]

\begin{tabular}{|c|c|c|c|c|c|c|}
\hline \multirow[t]{7}{*}{$\mathrm{C}_{44} \mathrm{H}_{28} \mathrm{~N}_{4} \mathrm{Zn}$} & [14074-80-7] & \multicolumn{5}{|c|}{ 5,10,15,20-tetraphenylphorphine zinc(II) } \\
\hline & FUS & & 54.9 & 770.4 & DSC & [2010GAM/CAM] \\
\hline & SUB & $(555-567)$ & $183 \pm 3$ & 559 & ME & [2002PAT/CAM] \\
\hline & SUB & $(555-567)$ & $196 \pm 3$ & 298 & ME & [2002PAT/CAM] \\
\hline & SUB & & $208 \pm 4$ & & GS & [2000PER/GOL] \\
\hline & SUB & $(563-663)$ & $213 \pm 3$ & & & [1994PER/NAN, 2002PAT/CAM] \\
\hline & SUB & & 109 & 666 & $\mathrm{UV} / \mathrm{Vis}$ & [1971EDW/DOL, 2002PAT/CAM] \\
\hline \multirow[t]{4}{*}{$\mathrm{C}_{48} \mathrm{H}_{36} \mathrm{~N}_{4} \mathrm{O}_{4} \mathrm{Zn}$} & [57715-42-1] & \multicolumn{5}{|c|}{ (5,10,15,20-tetrakis(4-methoxyphenyl)porphyrinato)zinc } \\
\hline & TRS & & 2.36 & 313 & & [2007PAT/CAM] \\
\hline & SUB & $(583-593)$ & $223.7 \pm 4.4$ & 588 & ME & [2007PAT/CAM] \\
\hline & SUB & (583-593) & $238.2 \pm 4.4$ & 298 & ME & [2007PAT/CAM] \\
\hline \multirow[t]{3}{*}{$\mathrm{Br}_{2} \mathrm{Zn}$} & [7699-45-8] & Zinc bromide & & & & \\
\hline & FUS & & 15.5 & 675 & $\mathrm{C}$ & [1964CUB/ELD] \\
\hline & SUB & $(453-574)$ & $115.3 \pm 2.1$ & 514 & ME,MS & [2014IIZ/SHI] \\
\hline
\end{tabular}


TABLE 17. Phase change enthalpies of organometallic and inorganic compounds-Continued

\begin{tabular}{|c|c|c|c|c|c|c|}
\hline \multirow[b]{2}{*}{$\begin{array}{l}\text { Molecular } \\
\text { formula }\end{array}$} & \multirow{2}{*}{$\begin{array}{l}\text { CAS Registry } \\
\text { Number } \\
\text { Enthalpy }\end{array}$} & \multicolumn{4}{|l|}{ Compound } & \multirow[b]{2}{*}{ References } \\
\hline & & $\begin{array}{c}\text { Temperature } \\
\text { range }\end{array}$ & $\begin{array}{l}\Delta_{\text {trans }} H_{\mathrm{m}} \\
(\mathrm{kJ} / \mathrm{mol})\end{array}$ & $\begin{array}{l}T_{\mathrm{m}} \\
(\mathrm{K})\end{array}$ & Method & \\
\hline & $\mathrm{V}$ & $(701-923)$ & 118.6 & 812 & $\mathrm{BP}$ & [1958BLO/BOC] \\
\hline \multirow[t]{2}{*}{$\mathrm{Cl}_{2} \mathrm{Zn}$} & $\begin{array}{c}\text { [7646-85-7] } \\
\text { FUS }\end{array}$ & Zinc chloride & 10.3 & 591 & $\mathrm{AC}$ & [1964CUB/ELD] \\
\hline & $\begin{array}{l}\mathrm{V} \\
\mathrm{V}\end{array}$ & $\begin{array}{l}(695-826) \\
(743-963)\end{array}$ & $\begin{array}{l}134.5 \\
126.6\end{array}$ & $\begin{array}{l}760 \\
851\end{array}$ & $\mathrm{BP}$ & $\begin{array}{l}\text { [1958BLO/WEL] } \\
{[1958 \mathrm{BLO} / \mathrm{BOC}]}\end{array}$ \\
\hline $\mathrm{F}_{2} \mathrm{Zn}$ & $\begin{array}{l}{[7783-49-5]} \\
\text { SUB } \\
\text { SUB }\end{array}$ & $\begin{array}{r}\text { Zinc fluoride } \\
(846-1047) \\
(901-1125)\end{array}$ & $\begin{array}{c}239.5 \pm 7.7 \\
252.4\end{array}$ & $\begin{array}{c}946 \\
1015\end{array}$ & $\begin{array}{l}\mathrm{TE} \\
\mathrm{ME}\end{array}$ & $\begin{array}{l}\text { [2008BRU/LEL] } \\
{[1973 \mathrm{BIE} / \mathrm{EIC}]}\end{array}$ \\
\hline \multicolumn{7}{|l|}{$\mathrm{Zr}$} \\
\hline \multirow[t]{2}{*}{$\mathrm{C}_{10} \mathrm{H}_{10} \mathrm{Cl}_{2} \mathrm{Zr}$} & $\begin{array}{c}\text { [1291-32-3] } \\
\text { SUB } \\
\text { SUB } \\
\text { SUB } \\
\text { SUB } \\
\text { SUB } \\
\text { SUB }\end{array}$ & $\begin{array}{l}\text { bis(cyclopenta } \\
\text { (393-457) }\end{array}$ & $\begin{array}{c}\text { yl)zirconium } \\
108.5 \pm 4.6 \\
100.3 \\
105.0 \pm 2.1 \\
100.4 \pm 1.7 \\
96.7 \\
103 \pm 13\end{array}$ & $\begin{array}{l}\text { ide } \\
298 \\
425 \\
298 \\
\\
298\end{array}$ & $\begin{array}{c}\mathrm{ME} \\
\mathrm{A}\end{array}$ & $\begin{array}{l}\text { [2001DIO/PIE] } \\
\text { [1987STE/MAL] } \\
\text { [1982PIL/SKI, 1976KIR/TEL] } \\
\text { [1977BAL/BAR] } \\
\text { [1969DIL/KIS] } \\
\text { [1968KIS/DIL, 2001DIO/PIE] }\end{array}$ \\
\hline & $\mathrm{V}$ & $(408-449)$ & $87 \pm 5$ & & & [2008ARU/MAT] \\
\hline $\mathrm{C}_{12} \mathrm{H}_{16} \mathrm{Zr}$ & $\begin{array}{c}\text { [1291-32-3] } \\
\text { SUB }\end{array}$ & bis(cyclopenta & $\begin{array}{l}\text { yl)dimethylzir } \\
81.2 \pm 2.1\end{array}$ & 298 & & [1982PIL/SKI, 1976KIR/TEL] \\
\hline $\mathrm{C}_{12} \mathrm{H}_{36} \mathrm{~N}_{4} \mathrm{Zr}$ & $\begin{array}{c}\text { [175923-04-3] } \\
\text { SUB }\end{array}$ & $\begin{array}{l}\text { tetrakis(methy } \\
\quad(278-333)\end{array}$ & $\begin{array}{c}\text { lamino)zircon } \\
79.4 \pm 2.4\end{array}$ & & $\mathrm{ME}$ & [2009MON/NUT] \\
\hline $\begin{array}{l}\text { [Note: The autho } \\
\text { is linear over the } \\
\text { enthalpy of vapo }\end{array}$ & $\begin{array}{l}\text { tate in the paper } t \\
\text { re temperature ra } \\
\text { tion given the aut }\end{array}$ & $\begin{array}{l}\text { the compound } \\
\text { re. The authors } \\
\text { rs' statement th }\end{array}$ & $\begin{array}{l}\text { liquid at room } \\
\text { to the enthalp } \\
\text { e compound is }\end{array}$ & $\begin{array}{l}\text { erature } \\
\text { le enth } \\
\text { iid. Th }\end{array}$ & $\begin{array}{l}\text { e } 3 \text { in the } \\
\text { sublimat } \\
\text { ound's } m\end{array}$ & $\begin{array}{l}\text { er shows that the plot of } \ln \mathrm{P} \text { ver } \\
\text { We have taken the value to be th } \\
\text { ag point temperature is unknown }\end{array}$ \\
\hline $\mathrm{C}_{16} \mathrm{H}_{36} \mathrm{O}_{4} \mathrm{Zr}$ & & Tetra-tert-butc & rconium & & & \\
\hline & $\mathrm{V}$ & (374-587) & 56.6 & 389 & A & [1987STE/MAL] \\
\hline $\mathrm{C}_{20} \mathrm{H}_{4} \mathrm{~F}_{24} \mathrm{O}_{8} \mathrm{Zr}$ & $\begin{array}{l}\text { [19530-02-0] } \\
\text { SUB }\end{array}$ & $\begin{array}{l}\text { tetrakis }(1,1,1, \\
(333-363)\end{array}$ & $\begin{array}{l}\text { hexafluoro-2, } \\
59.0\end{array}$ & anedio & $\begin{array}{l}\text { rconium( } \\
\text { TGA }\end{array}$ & [2000FAH/BAR] \\
\hline & $\mathrm{V}$ & $(366-456)$ & $48.6 \pm 0.6$ & 411 & $\mathrm{~T}$ & [1996MOR/SYS] \\
\hline $\mathrm{C}_{20} \mathrm{H}_{16} \mathrm{~F}_{12} \mathrm{O}_{8} \mathrm{Zr}$ & $\begin{array}{c}{[17499-68-2]} \\
\text { SUB } \\
\text { SUB } \\
\text { SUB } \\
\text { SUB } \\
\text { SUB }\end{array}$ & $\begin{array}{l}\text { tetrakis }(1,1,1- \\
\qquad \begin{array}{l}(373-403) \\
(368-398) \\
(383-438) \\
(383-438)\end{array}\end{array}$ & $\begin{array}{l}\text { oro-2,4-pentan } \\
94 \\
133.6 \pm 2.0 \\
118.7 \pm 3.1 \\
126.4 \pm 1.7 \\
119.2 \pm 1.7\end{array}$ & $\begin{array}{l}\text { ato)zir } \\
383 \\
298\end{array}$ & $\begin{array}{l}\text { (IV) } \\
\text { TGA } \\
\text { SMZG } \\
\text { C } \\
\text { GS } \\
\text { GS }\end{array}$ & $\begin{array}{l}\text { [2000FAH/BAR] } \\
{[1996 \mathrm{MOR} / \mathrm{SYS}]} \\
{[1992 \mathrm{RIB} / \mathrm{FER} 2]} \\
{[1985 \mathrm{MAT} / \mathrm{KUW}]} \\
{[1985 \mathrm{MAT} / \mathrm{KUW}]}\end{array}$ \\
\hline $\mathrm{C}_{20} \mathrm{H}_{28} \mathrm{O}_{8} \mathrm{Zr}$ & $\begin{array}{l}{[17501-44-9]} \\
\text { SUB } \\
\text { SUB } \\
\text { SUB } \\
\text { SUB } \\
\text { SUB }\end{array}$ & $\begin{array}{l}\text { tetrakis }(2,4-\mathrm{pe} \\
\qquad(413-443) \\
\quad(403-433)\end{array}$ & $\begin{array}{c}\text { dionato)zirco } \\
126 \\
138.8 \pm 2 \\
125.8 \pm 2.9 \\
132.0 \pm 6.8 \\
116 \pm 34\end{array}$ & $\begin{array}{l}\text { IV) } \\
418 \\
298 \\
463\end{array}$ & $\begin{array}{l}\text { TGA } \\
\text { SMZG } \\
\text { C }\end{array}$ & $\begin{array}{l}\text { [2000FAH/BAR] } \\
{[1996 \mathrm{MOR} / \mathrm{SYS}]} \\
{[1992 \mathrm{RIB} / \mathrm{FER} 2]} \\
{[1987 \mathrm{MUR} / \mathrm{HIL} 2]} \\
{[1984 \mathrm{TRE} / \mathrm{BER}]}\end{array}$ \\
\hline $\mathrm{C}_{20} \mathrm{H}_{40} \mathrm{O}_{8} \mathrm{Zr}$ & $\begin{array}{c}{[228997-54-4]} \\
\text { SUB }\end{array}$ & $\begin{array}{l}\text { Zirconium(IV } \\
\quad(403-482)\end{array}$ & $168.1 \pm 8.2$ & 443 & & [2006KUZ/ALT] \\
\hline $\mathrm{C}_{20} \mathrm{H}_{44} \mathrm{O}_{4} \mathrm{Zr}$ & $\mathrm{V}$ & $\begin{array}{l}\text { tetrakis(1,1-di } \\
\quad(392-426)\end{array}$ & $\begin{array}{c}\text { ylpropoxy)zir } \\
68\end{array}$ & 407 & A & [1987STE/MAL] \\
\hline $\mathrm{C}_{20} \mathrm{H}_{44} \mathrm{O}_{4} \mathrm{Zr}$ & $\mathrm{V}$ & $\begin{array}{c}\text { Tetra-tert-pent } \\
\quad(361-435)\end{array}$ & $\begin{array}{r}\text { irconium } \\
74.1\end{array}$ & 361 & A & [1987STE/MAL] \\
\hline $\mathrm{C}_{22} \mathrm{H}_{20} \mathrm{Zr}$ & $\begin{array}{c}\text { [51177-89-0] } \\
\text { SUB }\end{array}$ & bis(cyclopenta & $\begin{array}{l}\text { 1)diphenylzir } \\
92.0 \pm 4.2\end{array}$ & & & [1976KIR/TEL] \\
\hline $\mathrm{C}_{24} \mathrm{H}_{52} \mathrm{O}_{4} \mathrm{Zr}$ & $\mathrm{V}$ & $\begin{array}{l}\text { tetrakis(1,1-di } \\
\qquad(406-449)\end{array}$ & $\begin{array}{c}\text { ylbutoxy)zirco } \\
93.3\end{array}$ & 421 & A & [1987STE/MAL] \\
\hline
\end{tabular}


TABLE 17. Phase change enthalpies of organometallic and inorganic compounds-Continued

\begin{tabular}{|c|c|c|c|c|c|c|}
\hline \multirow[b]{2}{*}{$\begin{array}{l}\text { Molecular } \\
\text { formula }\end{array}$} & \multirow{2}{*}{$\begin{array}{l}\text { CAS Registry } \\
\text { Number } \\
\text { Enthalpy }\end{array}$} & \multicolumn{5}{|l|}{ Compound } \\
\hline & & $\begin{array}{l}\text { Temperature } \\
\text { range }\end{array}$ & $\begin{array}{l}\Delta_{\text {trans }} H_{\mathrm{m}} \\
(\mathrm{kJ} / \mathrm{mol})\end{array}$ & $\begin{array}{l}T_{\mathrm{m}} \\
(\mathrm{K})\end{array}$ & Method & References \\
\hline \multirow[t]{2}{*}{$\mathrm{C}_{24} \mathrm{H}_{52} \mathrm{O}_{4} \mathrm{Zr}$} & & \multicolumn{5}{|c|}{ tetrakis(1-methyl-1-ethylpropoxy)zirconium } \\
\hline & $\mathrm{V}$ & $(423-460)$ & 91.4 & 438 & A & [1987STE/MAL] \\
\hline \multirow[t]{2}{*}{$\mathrm{C}_{32} \mathrm{H}_{40} \mathrm{~F}_{12} \mathrm{O}_{8} \mathrm{Zr}$} & {$[56044-44-1]$} & \multicolumn{5}{|c|}{ tetrakis(1,1,1-trimethyl-5,5,5-trifluoro-2,4-pentanedionato)zirconium(IV) } \\
\hline & $\mathrm{V}$ & $(394-425)$ & \multicolumn{2}{|l|}{$71 \pm 1$} & & [2008ARU/MAT] \\
\hline \multirow{2}{*}{$\mathrm{C}_{44} \mathrm{H}_{76} \mathrm{O}_{8} \mathrm{Zr}$} & {$[18865-74-2]$} & \multicolumn{5}{|c|}{ tetrakis(2,2,6,6-tetramethylheptan-3,5-dionato)zirconium(IV) } \\
\hline & TRS & \multicolumn{3}{|c|}{$11.6 \quad 446$} & & \\
\hline \multicolumn{7}{|c|}{ Note: The value also includes the enthalpy of the solid/solid transition at $438 \mathrm{~K}$. } \\
\hline \multicolumn{3}{|c|}{ FUS } & 5.7 & 616 & DSC & [2008ZHE/MOR] \\
\hline & SUB & $(413-443)$ & 120 & & TGA & [2000FAH/BAR] \\
\hline \multirow[t]{2}{*}{$\mathrm{ZrCl}_{4}$} & [10026-11-6] & \multicolumn{2}{|c|}{ Zirconium tetrachloride } & & & \\
\hline & SUB & $(405-518)$ & $98.9 \pm 0.5$ & 512 & $\mathrm{~T}$ & [1994TAN/BOS] \\
\hline \multirow[t]{8}{*}{$\mathrm{ZrF}_{4}$} & [7783-64-4] & \multicolumn{2}{|c|}{ Zirconium tetrafluoride } & & & \\
\hline & SUB & $(685-828)$ & 228 & 756 & $\mathrm{TE}$ & [2011BRU/PIA] \\
\hline & SUB & $(685-828)$ & $239 \pm 2$ & 298 & TE & [2011BRU/PIA] \\
\hline & SUB & $(696-856)$ & $240.0 \pm 0.1$ & 298 & $\mathrm{TE}$ & [1994KON/HIL] \\
\hline & SUB & 796 & 243 & 298 & MS & [1965SID/AKI, 1994KON/HIL] \\
\hline & SUB & (983-1177) & $241.1 \pm 0.1$ & 298 & & [1964FIS/PET, 1994KON/HIL] \\
\hline & SUB & $(681-913)$ & $242.6 \pm 1.7$ & 298 & MS & [1963AKA/BEL, 1994KON/HIL] \\
\hline & SUB & $(713-873)$ & $232.3 \pm 1.2$ & 298 & & [1963GAL/TUM, 1994KON/HIL] \\
\hline
\end{tabular}


TABLE 18. Phase change enthalpies of organic salts and ionic liquids

\begin{tabular}{|c|c|c|c|c|c|c|}
\hline \multirow[b]{2}{*}{$\begin{array}{l}\text { Molecular } \\
\text { formula }\end{array}$} & \multirow{2}{*}{$\begin{array}{l}\text { CAS Registry } \\
\text { Number } \\
\text { Enthalpy }\end{array}$} & \multicolumn{5}{|l|}{ Compound } \\
\hline & & $\begin{array}{l}\text { Temperature } \\
\text { range }\end{array}$ & $\begin{array}{l}\Delta_{\text {trans }} H_{\mathrm{m}} \\
(\mathrm{kJ} / \mathrm{mol})\end{array}$ & $\begin{array}{l}T_{\mathrm{m}} \\
(\mathrm{K})\end{array}$ & Method & Reference \\
\hline $\mathrm{CH}_{6} \mathrm{BrN}$ & $\begin{array}{c}\text { [6876-37-5] } \\
\text { TRS } \\
\text { TRS } \\
\text { FUS }\end{array}$ & Methylammon & $\begin{array}{r}\text { omide } \\
1.60 \\
3.51 \\
8.34\end{array}$ & $\begin{array}{l}394.3 \\
485.0 \\
522.7\end{array}$ & DSC & [1990GEN/LUB] \\
\hline $\mathrm{CH}_{6} \mathrm{BrN}$ & $\begin{array}{c}\text { [6876-37-5] } \\
\text { TRS } \\
\text { TRS } \\
\text { FUS } \\
\text { TRS }\end{array}$ & Methylammon & $\begin{array}{r}\text { omide } \\
1.60 \\
3.51 \\
8.34 \\
1.28\end{array}$ & $\begin{array}{l}397.7 \\
488.4 \\
531.9 \\
389.0\end{array}$ & DSC & $\begin{array}{l}\text { [1996DOM/HEA, 1990GEN/LUB] } \\
\text { [1967TSA/GIL] }\end{array}$ \\
\hline $\mathrm{CH}_{6} \mathrm{C} 1 \mathrm{~N}$ & $\begin{array}{c}{[593-51-1]} \\
\text { TRS } \\
\text { TRS }\end{array}$ & Methylammon & $\begin{array}{r}\text { loride } \\
1.78 \\
2.82\end{array}$ & $\begin{array}{l}220.4 \\
264.5\end{array}$ & & [1996DOM/HEA, 1946AST/ZIE] \\
\hline $\mathrm{C}_{2} \mathrm{H}_{8} \mathrm{BF}_{4} \mathrm{~N}$ & $\begin{array}{l}\text { [16970-97-1] } \\
\text { TRS } \\
\text { FUS }\end{array}$ & Dimethylamm & $\begin{array}{l}\text { tetrafluoro } \\
7.50 \\
3.50\end{array}$ & $\begin{array}{c}283.5 \\
375\end{array}$ & & [1996DOM/HEA, 1992ISH/IWA] \\
\hline $\mathrm{C}_{2} \mathrm{H}_{8} \mathrm{BrN}$ & $\begin{array}{c}\text { [593-55-5] } \\
\text { TRS } \\
\text { FUS } \\
\text { TRS } \\
\text { FUS }\end{array}$ & Ethylammoniu & $\begin{array}{l}\text { mide } \\
\qquad \begin{array}{c}12.07 \\
8.52 \\
11.30 \\
8.37\end{array}\end{array}$ & $\begin{array}{c}369.9 \\
439.5 \\
363 \\
434\end{array}$ & DSC & $\begin{array}{l}\text { [1996DOM/HEA, 1990GEN/LUB] } \\
\text { [1967TSA/GIL] }\end{array}$ \\
\hline $\mathrm{C}_{2} \mathrm{H}_{8} \mathrm{C} 1 \mathrm{~N}$ & $\begin{array}{c}{[506-59-2]} \\
\mathrm{V} \\
\mathrm{V}\end{array}$ & $\begin{array}{l}\text { Dimethylamm } \\
\quad(429-533) \\
(533-569)\end{array}$ & $\begin{array}{c}\text { chloride } \\
95.6 \\
143.9\end{array}$ & $\begin{array}{l}444 \\
548\end{array}$ & $\begin{array}{l}\mathrm{A}, \mathrm{I} \\
\mathrm{A}, \mathrm{I}\end{array}$ & $\begin{array}{l}\text { [1987STE/MAL, 1967KIS] } \\
\text { [1987STE/MAL, 1967KIS] }\end{array}$ \\
\hline $\mathrm{C}_{2} \mathrm{H}_{8} \mathrm{C} 1 \mathrm{~N}$ & $\begin{array}{c}{[557-66-4]} \\
\mathrm{V}\end{array}$ & $\begin{array}{l}\text { Ethylammoniu } \\
\quad(382-480)\end{array}$ & $\begin{array}{l}\text { oride } \\
\qquad 34.3\end{array}$ & 397 & $\mathrm{~A}, \mathrm{I}$ & [1987STE/MAL, 1967KIS] \\
\hline $\mathrm{C}_{3} \mathrm{H}_{8} \mathrm{~N}_{2}$ & $\begin{array}{c}{[51283-80-8]} \\
\mathrm{V}\end{array}$ & $\begin{array}{l}\text { Dimethyl amm } \\
(251-295)\end{array}$ & $\begin{array}{l}\text { cyanide } \\
49.0\end{array}$ & 280 & A & [1987STE/MAL, 1973DIE/MAR] \\
\hline $\mathrm{C}_{3} \mathrm{H}_{10} \mathrm{BrN}$ & $\begin{array}{l}\text { [4905-83-3] } \\
\text { FUS } \\
\text { FUS }\end{array}$ & Propylammoni & $\begin{array}{l}\text { omide } \\
13.3 \\
12.51\end{array}$ & $\begin{array}{c}464.6 \\
456\end{array}$ & $\mathrm{DSC}$ & $\begin{array}{l}\text { [1996DOM/HEA, 1990GEN/LUB] } \\
\text { [1967TSA/GIL] }\end{array}$ \\
\hline $\mathrm{C}_{4} \mathrm{H}_{5} \mathrm{~N}_{3} \mathrm{O}_{3}$ & $\begin{array}{l}\text { [156204-43-2] } \\
\text { FUS }\end{array}$ & 1-methylimida & $\begin{array}{r}\text { nitrate } \\
19.2\end{array}$ & 342.6 & DSC & [2009EME/VER3] \\
\hline $\mathrm{C}_{4} \mathrm{H}_{10} \mathrm{~N}_{2}$ & $\begin{array}{l}{[28871-28-5]} \\
\text { SUB }\end{array}$ & $\begin{array}{l}\text { Trimethylamm } \\
\quad(219-236)\end{array}$ & $\begin{array}{c}\text { cyanide } \\
45.0\end{array}$ & 227.5 & & [1987STE/MAL] \\
\hline $\mathrm{C}_{4} \mathrm{H}_{11} \mathrm{NO}_{3}$ & $\begin{array}{c}\text { [54300-24-2] } \\
\text { FUS }\end{array}$ & 2-(hydroxyethy & $\begin{array}{c}\text { nonium ac } \\
15.7\end{array}$ & 336 & DSC & [2014PEN/UUS] \\
\hline $\mathrm{C}_{4} \mathrm{H}_{12} \mathrm{BF}_{4} \mathrm{~N}$ & $\begin{array}{c}\text { [661-36-9] } \\
\text { TRS } \\
\text { TRS }\end{array}$ & Tetramethylam & $\begin{array}{l}\text { Im tetrafluc } \\
0.5 \\
1.7\end{array}$ & $\begin{array}{l}154 \\
601\end{array}$ & $\mathrm{DSC}$ & [1987ZAB/FER] \\
\hline $\mathrm{C}_{4} \mathrm{H}_{12} \mathrm{BrN}$ & $\begin{array}{c}\text { [6274-12-0] } \\
\text { TRS } \\
\text { TRS } \\
\text { TRS } \\
\text { FUS }\end{array}$ & Diethylammon & $\begin{array}{r}\text { romide } \\
2.2 \\
1.6 \\
2.3 \\
10.4\end{array}$ & $\begin{array}{l}283 \\
329 \\
342 \\
481\end{array}$ & DSC & [1997SHI/TAN] \\
\hline $\mathrm{C}_{4} \mathrm{H}_{12} \mathrm{CIN}$ & $\begin{array}{c}{[3858-78-4]} \\
\mathrm{V}\end{array}$ & $\begin{array}{c}\text { Butylammoniu } \\
\quad(489-508)\end{array}$ & $\begin{array}{l}\text { oride } \\
\qquad 62.1\end{array}$ & 498 & $\mathrm{~A}, \mathrm{I}$ & [1987STE/MAL, 1967KIS] \\
\hline $\mathrm{C}_{4} \mathrm{H}_{12} \mathrm{CIN}$ & $\begin{array}{c}\text { [75-57-0] } \\
\text { TRS } \\
\text { TRS } \\
\text { TRS }\end{array}$ & $\begin{array}{l}\text { Tetramethylam } \\
\qquad \begin{array}{c}(5-350) \\
(5-350)\end{array}\end{array}$ & $\begin{array}{c}\text { chlorid } \\
9.91 \\
0.18 \\
0.28\end{array}$ & $\begin{array}{c}536 \\
75.76 \\
184.45\end{array}$ & $\begin{array}{l}\mathrm{DSC} \\
\mathrm{AC}\end{array}$ & $\begin{array}{l}\text { [1996DOM/HEA, 1970MUR/BRE] } \\
\text { [1996DOM/HEA, 1962CHA/WES] }\end{array}$ \\
\hline $\mathrm{C}_{4} \mathrm{H}_{12} \mathrm{ClN}$ & $\begin{array}{c}{[660-68-4]} \\
\mathrm{V}\end{array}$ & $\begin{array}{c}\text { Diethylamine } 1 \\
\quad(513-558)\end{array}$ & $\begin{array}{r}\text { hloride } \\
177.6\end{array}$ & 528 & $\mathrm{~A}, \mathrm{I}$ & [1987STE/MAL, 1967KIS] \\
\hline
\end{tabular}


TABLE 18. Phase change enthalpies of organic salts and ionic liquids-Continued

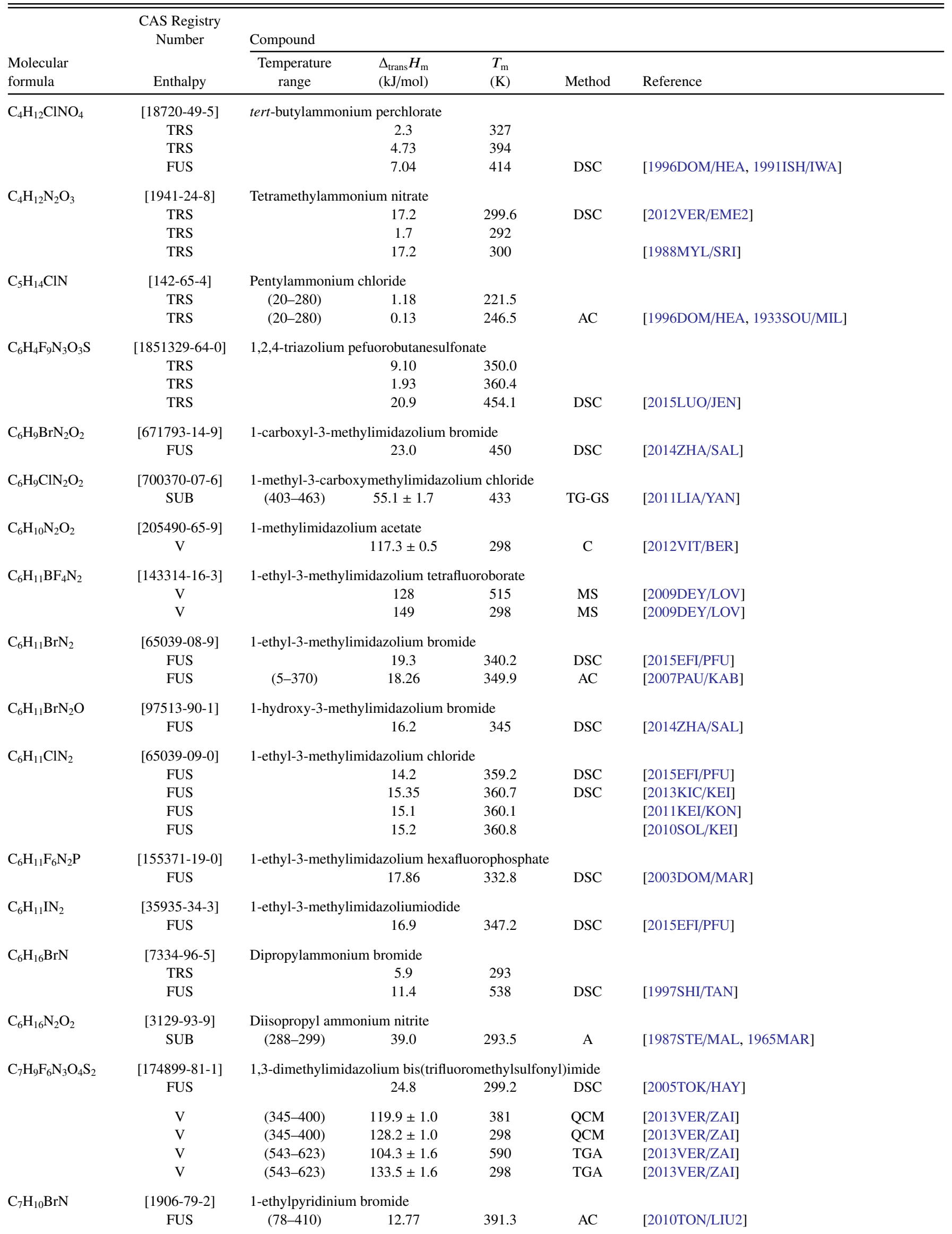


TABLE 18. Phase change enthalpies of organic salts and ionic liquids-Continued

\begin{tabular}{|c|c|c|c|c|c|c|}
\hline \multirow[b]{2}{*}{$\begin{array}{l}\text { Molecular } \\
\text { formula }\end{array}$} & \multirow{2}{*}{$\begin{array}{l}\text { CAS Registry } \\
\text { Number } \\
\text { Enthalpy }\end{array}$} & \multicolumn{5}{|l|}{ Compound } \\
\hline & & $\begin{array}{l}\text { Temperature } \\
\text { range }\end{array}$ & $\begin{array}{l}\Delta_{\text {trans }} H_{\mathrm{m}} \\
(\mathrm{kJ} / \mathrm{mol})\end{array}$ & $\begin{array}{l}T_{\mathrm{m}} \\
(\mathrm{K})\end{array}$ & Method & Reference \\
\hline $\mathrm{C}_{7} \mathrm{H}_{10} \mathrm{CIN}$ & $\begin{array}{l}\text { [3287-99-8] } \\
\text { TRS }\end{array}$ & \multicolumn{5}{|c|}{ Phenylmethylammonium chloride } \\
\hline $\mathrm{C}_{7} \mathrm{H}_{10} \mathrm{~F}_{6} \mathrm{NP}$ & $\begin{array}{l}{[103173-73-5]} \\
\text { FUS }\end{array}$ & \multicolumn{4}{|c|}{$N$-ethylpyridinium hexafluorophosphate } & [2014LIU/TAN] \\
\hline $\mathrm{C}_{7} \mathrm{H}_{10} \mathrm{~N}_{2} \mathrm{O}_{3}$ & $\begin{array}{l}{[49580-44-1]} \\
\text { TRS }\end{array}$ & \multicolumn{4}{|c|}{ Phenylmethylammonium nitrate } & [1996DOM/HEA, 1989VAN/WHI] \\
\hline $\mathrm{C}_{7} \mathrm{H}_{11} \mathrm{BrN}_{2} \mathrm{O}_{2}$ & $\begin{array}{c}{[637743-77-2]} \\
\text { FUS }\end{array}$ & & \multicolumn{3}{|c|}{ 1,2-dimethyl-3-carboyxlimidazolium bromide } & [2014ZHA/SAL] \\
\hline \multirow[t]{2}{*}{$\mathrm{C}_{7} \mathrm{H}_{11} \mathrm{C} 1 \mathrm{~N}_{2}$} & $\begin{array}{l}{[65039-10-3]} \\
\text { TRS }\end{array}$ & \multicolumn{5}{|c|}{ 1-allyl-3-methylimidazolium chloride } \\
\hline & FUS & & 0.52 & 307.5 & DSC & [2012MEL/ROD] \\
\hline $\mathrm{C}_{7} \mathrm{H}_{11} \mathrm{~N}_{3} \mathrm{~S}$ & $\begin{array}{c}\text { [331717-63-6] } \\
\text { V } \\
\text { V }\end{array}$ & 1-ethyl-3-methy & $\begin{array}{c}\text { idazolium thi } \\
133 \\
151\end{array}$ & $\begin{array}{l}\text { late } \\
490 \\
298\end{array}$ & $\begin{array}{l}\text { MS } \\
\text { MS }\end{array}$ & $\begin{array}{l}{[2009 \mathrm{DEY} / \mathrm{LOV}]} \\
{[2009 \mathrm{DEY} / \mathrm{LOV}]}\end{array}$ \\
\hline $\mathrm{C}_{7} \mathrm{H}_{13} \mathrm{BrN}_{2}$ & $\begin{array}{c}\text { [85100-76-1] } \\
\text { FUS } \\
\text { FUS (I) } \\
\text { FUS (II) }\end{array}$ & $\begin{array}{l}\text { 1-propyl-3-met } \\
\quad(5-370)\end{array}$ & $\begin{array}{c}\text { midazolium b } \\
19.11 \\
13.5 \\
21.4\end{array}$ & $\begin{array}{l}\text { le } \\
309.5 \\
314.7 \\
310.4\end{array}$ & $\mathrm{AC}$ & [2007PAU/KAB] \\
\hline $\mathrm{C}_{7} \mathrm{H}_{14} \mathrm{~F}_{6} \mathrm{~N}_{2} \mathrm{O}_{4} \mathrm{~S}_{2}$ & $\begin{array}{c}\text { [173274-74-3] } \\
\text { FUS }\end{array}$ & \multicolumn{5}{|c|}{ trimethyl(ethyl)ammonium bis(trifluoromethylsulfonyl)imide } \\
\hline $\mathrm{C}_{8} \mathrm{H}_{13} \mathrm{~F}_{6} \mathrm{~N}_{2} \mathrm{P}$ & $\begin{array}{c}{[216300-12-8]} \\
\text { FUS }\end{array}$ & \multicolumn{5}{|c|}{ 1-propyl-3-methylimidazolium hexafluorophosphate } \\
\hline \multirow[t]{23}{*}{$\mathrm{C}_{8} \mathrm{H}_{11} \mathrm{~F}_{6} \mathrm{~N}_{3} \mathrm{O}_{4} \mathrm{~S}_{2}$} & $\begin{array}{c}\text { [174899-82-2] } \\
\text { FUS } \\
\text { FUS }\end{array}$ & $\begin{array}{l}\text { 1-ethyl-3-meth } \\
\quad(50-300)\end{array}$ & $\begin{array}{c}\text { idazolium bis } \\
\begin{array}{c}21.3 \\
24.8\end{array}\end{array}$ & $\begin{array}{l}271.4 \\
255.2\end{array}$ & $\begin{array}{c}\text { lfonyl)imide } \\
\text { AC } \\
\text { DSC }\end{array}$ & $\begin{array}{l}{[2009 \mathrm{SHI} / \mathrm{OHT}]} \\
{[2005 \mathrm{TOK} / \mathrm{HAY}]}\end{array}$ \\
\hline & $\mathrm{V}$ & & $117 \pm 2$ & 458 & ME-MS & [2016DUN/MOT] \\
\hline & V & $(423-498)$ & $121.7 \pm 0.7$ & 459 & ME-MS & [2015CHI/MED] \\
\hline & $\begin{array}{l}\mathrm{V} \\
\mathrm{V}\end{array}$ & $\begin{array}{l}(423-498) \\
(500-750)\end{array}$ & $\begin{array}{c}130 \pm 3 \\
108.2 \pm 1.9\end{array}$ & $\begin{array}{l}298 \\
625\end{array}$ & $\begin{array}{l}\text { ME-MS } \\
\text { DFSC }\end{array}$ & $\begin{array}{l}\text { [2015CHI/MED] } \\
\text { [2014AHR/BRI] }\end{array}$ \\
\hline & $\mathrm{V}$ & $(500-750)$ & 126 & 298 & DFSC & [2014AHR/BRI] \\
\hline & $\mathrm{V}$ & $(500-750)$ & $110.0 \pm 1.2$ & 625 & DFSC & [2014AHR/BRI] \\
\hline & $\mathrm{V}$ & $(500-750)$ & 128.3 & 298 & DFSC & [2014AHR/BRI] \\
\hline & $\mathrm{V}$ & $(362-395)$ & $118.6 \pm 1.0$ & 378 & QCM & [2013VER/ZAI] \\
\hline & $\mathrm{V}$ & $(362-395)$ & $129.6 \pm 1.0$ & 298 & QCM & [2013VER/ZAI] \\
\hline & $\mathrm{V}$ & $(480-570)$ & $110.5 \pm 1.5$ & 520 & TGA & [2012VER/RAL] \\
\hline & $\mathrm{V}$ & $(480-570)$ & $119.4 \pm 1.5$ & 298 & TGA & [2012VER/RAL] \\
\hline & $\mathrm{V}$ & $(445-483)$ & $114.6 \pm 0.4$ & 464 & QCM-ME & [2011ROC/LIM, 2012VER/RAL] \\
\hline & $\mathrm{V}$ & $(445-483)$ & $121.2 \pm 0.4$ & 298 & QCM-ME & [2011ROC/LIM, 2012VER/RAL] \\
\hline & $\mathrm{V}$ & $(362-395)$ & $118.6 \pm 1.0$ & 378 & QCM-LE & [2011VER/ZAI2, 2012VER/RAL] \\
\hline & $\mathrm{V}$ & $(362-395)$ & $126.6 \pm 1.0$ & 298 & QCM-LE & [2011VER/ZAI2, 2012VER/RAL] \\
\hline & $\mathrm{V}$ & $(359-436)$ & $122.0 \pm 3.0$ & 398 & TPD-MS & $\begin{array}{l}\text { [2010LOV/DEY, 2011VER/ZAI2, } \\
\text { 2012VER/RAL] }\end{array}$ \\
\hline & $\mathrm{V}$ & $(359-436)$ & $123.8 \pm 3.0$ & 298 & TPD-MS & $\begin{array}{l}\text { [2010LOV/DEY, 2011 VER/ZAI2, } \\
\text { 2012VER/RAL] }\end{array}$ \\
\hline & $\mathrm{V}$ & $(545-600)$ & $109.3 \pm 1.7$ & 573 & TPD-UV & $\begin{array}{l}\text { [2010WAN/LUO, 2011VER/ZAI2, } \\
\text { 2012VER/RAL] }\end{array}$ \\
\hline & $\mathrm{V}$ & $(545-600)$ & $122.7 \pm 1.7$ & 298 & TPD-UV & $\begin{array}{l}\text { [2010WAN/LUO, 2011 VER/ZAI2, } \\
\text { 2012VER/RAL] }\end{array}$ \\
\hline & $\mathrm{V}$ & $(473-523)$ & $120.6 \pm 2.1$ & 496 & TGA & $\begin{array}{l}\text { [2008LUO/BAK, 2011VER/ZAI2, } \\
\text { 2012VER/RAL] }\end{array}$ \\
\hline & $\mathrm{V}$ & $(473-523)$ & $128.5 \pm 2.1$ & 298 & TGA & $\begin{array}{l}\text { [2008LUO/BAK, } 2011 \mathrm{VER} / \mathrm{ZAI} 2, \\
\text { 2012VER/RAL] }\end{array}$ \\
\hline & $\mathrm{V}$ & $(499-538)$ & $114.7 \pm 6.8$ & 517 & GS & $\begin{array}{l}\text { [2007EME/VER2, } 2011 \mathrm{VER} / \mathrm{ZAI} 2 \text {, } \\
\text { 2012VER/RAL] }\end{array}$ \\
\hline & $\mathrm{V}$ & $(499-538)$ & $123.4 \pm 6.8$ & 298 & GS & $\begin{array}{l}\text { [2007EME/VER2, } 2011 \mathrm{VER} / \mathrm{ZAI} 2 \text {, } \\
\text { 2012VER/RAL] }\end{array}$ \\
\hline
\end{tabular}


TABLE 18. Phase change enthalpies of organic salts and ionic liquids-Continued

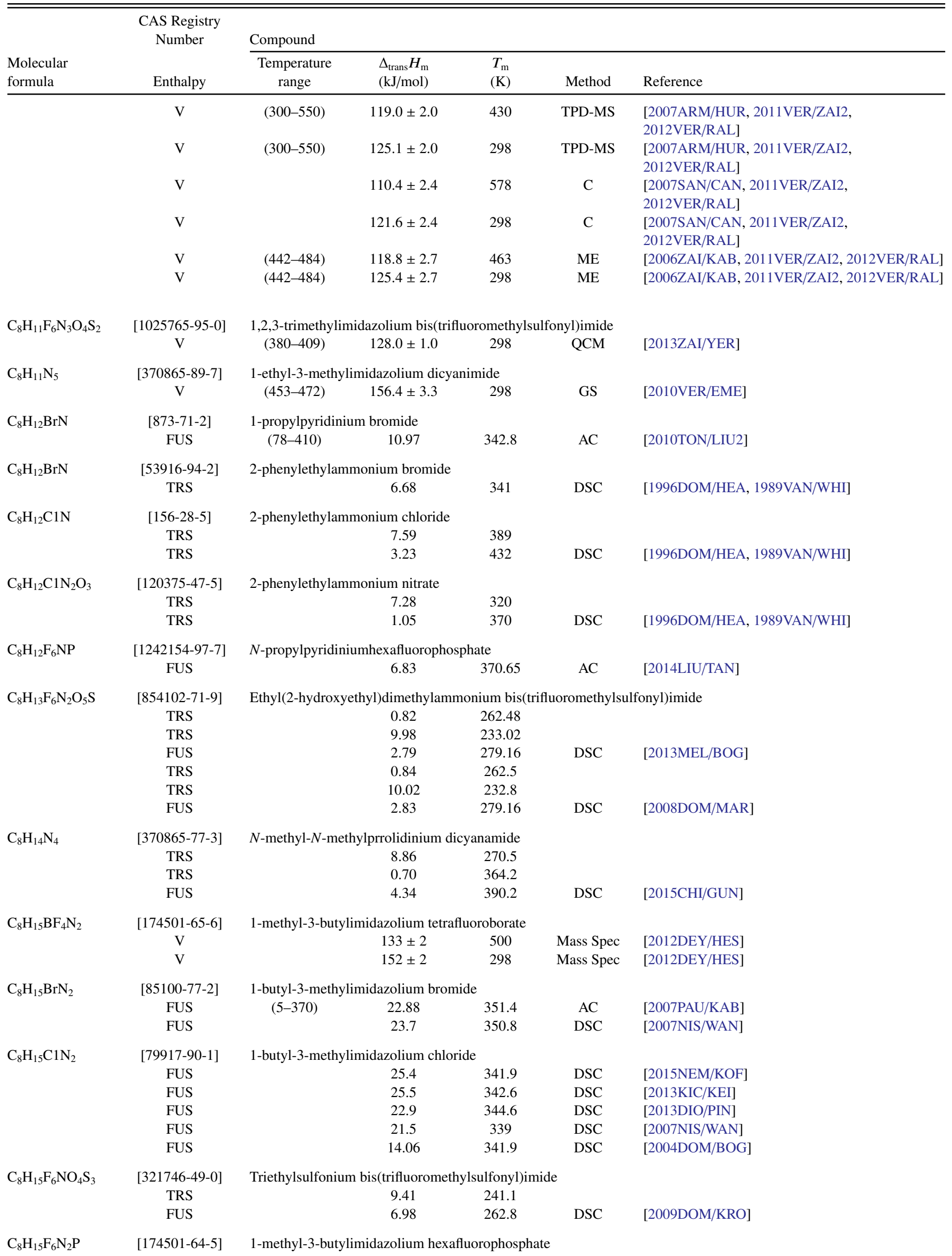


TABLE 18. Phase change enthalpies of organic salts and ionic liquids-Continued

\begin{tabular}{|c|c|c|c|c|c|c|}
\hline \multirow[b]{2}{*}{$\begin{array}{l}\text { Molecular } \\
\text { formula }\end{array}$} & \multirow{2}{*}{$\begin{array}{l}\text { CAS Registry } \\
\text { Number } \\
\text { Enthalpy }\end{array}$} & \multicolumn{5}{|l|}{ Compound } \\
\hline & & $\begin{array}{c}\text { Temperature } \\
\text { range }\end{array}$ & $\begin{array}{l}\Delta_{\text {trans }} H_{\mathrm{m}} \\
(\mathrm{kJ} / \mathrm{mol})\end{array}$ & $\begin{array}{l}T_{\mathrm{m}} \\
(\mathrm{K})\end{array}$ & Method & Reference \\
\hline & FUS & & 20.9 & 282.3 & DSC & [2015NEM/KOF] \\
\hline & FUS (I) & & 13.1 & 285.8 & & \\
\hline & FUS (II) & & 22.6 & 285.3 & DSC & [2013END/NIS] \\
\hline & FUS & $(80-390)$ & 20.67 & 281.8 & $\mathrm{AC}$ & [2010ZHA/CUI] \\
\hline & FUS & & 13.3 & 284.3 & $\mathrm{C}$ & [2010END/KAT] \\
\hline & FUS & & 12 & 282 & DSC & [2008JIN/OHA] \\
\hline & FUS & & 19.9 & 280.0 & DSC & [2006TRO/CER] \\
\hline & FUS & $(5-310)$ & 19.6 & 283.5 & $\mathrm{AC}$ & [2004KAB/BLO] \\
\hline & TRS & & 10.67 & 258.0 & & \\
\hline & FUS & & 9.21 & 276.4 & DSC & [2003DOM/MAR] \\
\hline & FUS & & 13.2 & 281 & $\mathrm{DSC}$ & [2003FOX/AWA] \\
\hline \multirow[t]{3}{*}{$\mathrm{C}_{8} \mathrm{H}_{15} \mathrm{IN}_{2}$} & [65039-05-6] & 1-butyl-3-meth & lazolium io & & & \\
\hline & FUS & $(5-370)$ & 18.99 & 291.9 & $\mathrm{AC}$ & [2014PAU/BLO] \\
\hline & FUS & & 19.0 & 291.9 & $\mathrm{AC}$ & [2010KAB/PAU] \\
\hline \multirow[t]{5}{*}{$\mathrm{C}_{8} \mathrm{H}_{15} \mathrm{~N}_{3} \mathrm{O}_{3}$} & [179075-88-8] & 1-butyl-3-meth & lazolium ni & & & \\
\hline & TRS & $(5-370)$ & 2.08 & 278.8 & & \\
\hline & TRS & $(5-370)$ & 0.36 & 288.1 & & \\
\hline & TRS & $(5-370)$ & 0.15 & 292.2 & & \\
\hline & FUS & $(5-370)$ & 17.99 & 309.2 & $\mathrm{AC}$ & [2008STR/KAB] \\
\hline \multirow[t]{3}{*}{$\mathrm{C}_{8} \mathrm{H}_{17} \mathrm{BrN}_{2}$} & [202256-55-1] & 1-ethyl-4-aza-1 & abicyclo[2. & ane bror & & \\
\hline & TRS & & 1.1 & 348.6 & & \\
\hline & FUS & & 13.4 & 467.3 & $\mathrm{DSC}$ & [2012LAU/RUT] \\
\hline \multirow[t]{3}{*}{$\mathrm{C}_{8} \mathrm{H}_{17} \mathrm{~F}_{3} \mathrm{~N}_{2} \mathrm{O}_{4} \mathrm{~S}$} & [805247-89-6] & $O$-ethyl- $N, N, \Lambda$ & tetramethyl & hium trif & methanesulf & nate \\
\hline & $\mathrm{V}$ & & $110 \pm 1$ & 425 & Mass Spec & [2012DEY/HES] \\
\hline & V & & $122 \pm 1$ & 298 & Mass Spec & [2012DEY/HES] \\
\hline \multirow[t]{3}{*}{$\mathrm{C}_{8} \mathrm{H}_{18} \mathrm{BrN}$} & [608140-09-6] & 1-propyl-1-met & rrolidinium & & & \\
\hline & TRS & & 2.92 & 465.1 & & \\
\hline & FUS & & 10.85 & 476.7 & $\mathrm{DSC}$ & [2014ZAW/KRO] \\
\hline \multirow[t]{4}{*}{$\mathrm{C}_{8} \mathrm{H}_{18} \mathrm{~F}_{6} \mathrm{NP}$} & [327022-58-2] & 1-propyl-1-met & rrolidinium & uoropho & & \\
\hline & TRS & & 2.81 & 346.6 & & \\
\hline & TRS & & 2.31 & 359.5 & & \\
\hline & FUS & & 3.41 & 382.5 & $\mathrm{DSC}$ & {$[2014 \mathrm{MAX} / \mathrm{SAN}]$} \\
\hline \multirow[t]{3}{*}{$\mathrm{C}_{8} \mathrm{H}_{20} \mathrm{BF}_{4} \mathrm{~N}$} & {$[429-06-1]$} & Tetraethylamm & n tetrafluor & & & \\
\hline & FUS & & 29.2 & 364.2 & DSC & [2013BHA/GOH] \\
\hline & TRS & & 11.7 & 336 & DSC & [1987ZAB/FER] \\
\hline \multirow[t]{3}{*}{$\mathrm{C}_{8} \mathrm{H}_{20} \mathrm{BrN}$} & {$[71-91-0]$} & Tetraethylamm & a bromide & & & \\
\hline & TRS & & 20.0 & 448.3 & & \\
\hline & TRS & & 1.48 & 462.6 & DSC & [1992XEN/CHE] \\
\hline & FUS & & 20.3 & 447 & DSC & [1996DOM/HEA, 1974BUR/VER] \\
\hline \multirow[t]{4}{*}{$\mathrm{C}_{8} \mathrm{H}_{20} \mathrm{BrN}$} & {$[10435-44-6]$} & Dibutylammor & romide & & & \\
\hline & TRS & & 3.1 & 228 & & \\
\hline & TRS & & 2.1 & 254 & & \\
\hline & FUS & & 11.3 & 563 & $\mathrm{DSC}$ & [1997SHI/TAN] \\
\hline \multirow[t]{2}{*}{$\mathrm{C}_{8} \mathrm{H}_{20} \mathrm{BrNO}_{3}$} & {$[82150-35-4]$} & Tetraethylamm & a bromate & & & \\
\hline & FUS & & 30.5 & 319.2 & $\mathrm{DSC}$ & {$[2013 \mathrm{BHA} / \mathrm{GOH}]$} \\
\hline \multirow[t]{2}{*}{$\mathrm{C}_{8} \mathrm{H}_{20} \mathrm{ClN}$} & {$[6287-40-7]$} & Dibutylammor & hloride & & & \\
\hline & $\mathrm{V}$ & $(553-563)$ & 116.7 & 558 & A & [1987STE/MAL, 1999DYK/SVO, 1967KIS] \\
\hline \multirow[t]{2}{*}{$\mathrm{C}_{8} \mathrm{H}_{20} \mathrm{~F}_{6} \mathrm{NP}$} & & Tetraethylamm & n hexafluor & hate & & \\
\hline & FUS & & 36.3 & 355.2 & DSC & {$[2013 \mathrm{BHA} / \mathrm{GOH}]$} \\
\hline \multirow[t]{2}{*}{$\mathrm{C}_{8} \mathrm{H}_{20} \mathrm{IN}$} & {$[68-05-3]$} & Tetraethylamm & iodide & & & \\
\hline & TRS & & 20.87 & 471.3 & DSC & [1992XEN/CHE] \\
\hline
\end{tabular}


TABLE 18. Phase change enthalpies of organic salts and ionic liquids-Continued

\begin{tabular}{|c|c|c|c|c|c|c|}
\hline \multirow[b]{2}{*}{$\begin{array}{l}\text { Molecular } \\
\text { formula }\end{array}$} & \multirow{2}{*}{$\begin{array}{l}\text { CAS Registry } \\
\text { Number } \\
\text { Enthalpy }\end{array}$} & \multicolumn{5}{|l|}{ Compound } \\
\hline & & $\begin{array}{l}\text { Temperature } \\
\text { range }\end{array}$ & $\begin{array}{l}\Delta_{\text {trans }} H_{\mathrm{m}} \\
(\mathrm{kJ} / \mathrm{mol})\end{array}$ & $\begin{array}{l}T_{\mathrm{m}} \\
(\mathrm{K})\end{array}$ & Method & Reference \\
\hline & FUS & & 21.3 & 465 & & [1971LEV/KOH] \\
\hline $\mathrm{C}_{8} \mathrm{H}_{20} \mathrm{INO}_{3}$ & FUS & Tetraethylamm & $\begin{array}{r}\text { um iodate } \\
44.9\end{array}$ & 326.2 & DSC & [2013BHA/GOH] \\
\hline $\mathrm{C}_{8} \mathrm{H}_{20} \mathrm{~N}_{2} \mathrm{O}_{3}$ & $\begin{array}{l}\text { [1941-26-0] } \\
\text { FUS } \\
\text { TRS }\end{array}$ & Tetraethylamm & $\begin{array}{r}\text { um nitrate } \\
26.4 \\
10.8\end{array}$ & $\begin{array}{l}388.2 \\
371.5\end{array}$ & $\begin{array}{l}\text { DSC } \\
\text { DSC }\end{array}$ & $\begin{array}{l}\text { [2013BHA/GOH] } \\
\text { [2012VER/EME2] }\end{array}$ \\
\hline $\mathrm{C}_{9} \mathrm{H}_{10} \mathrm{~F}_{6} \mathrm{~N}_{2} \mathrm{O}_{4} \mathrm{~S}_{2}$ & $\begin{array}{c}\text { [712354-97-7] } \\
\text { V } \\
\text { V } \\
\text { V }\end{array}$ & $\begin{array}{l}\text { 1-ethylpyridini } \\
(493-504)\end{array}$ & $\begin{array}{c}\text { bis(trifluorom } \\
120.1 \pm 0.6 \\
125.3 \pm 1.0 \\
131.4\end{array}$ & $\begin{array}{l}\text { sulfonyl) } \\
498 \\
400 \\
298\end{array}$ & $\begin{array}{l}\text { e } \\
\text { QCM, ME } \\
\text { QCM } \\
\text { QCM }\end{array}$ & $\begin{array}{l}{[2013 \mathrm{ROC} / \mathrm{SAN}]} \\
{[2012 \mathrm{ZAI} / \mathrm{YER}]} \\
{[2012 \mathrm{ZAI} / \mathrm{YER}]}\end{array}$ \\
\hline $\mathrm{C}_{9} \mathrm{H}_{13} \mathrm{~F}_{6} \mathrm{~N}_{3} \mathrm{O}_{4} \mathrm{~S}_{2}$ & $\begin{array}{l}\mathrm{V} \\
\mathrm{V}\end{array}$ & $\begin{array}{c}\text { 1-methyl-3-iso } \\
\begin{array}{c}(343-390) \\
(343-390)\end{array}\end{array}$ & $\begin{array}{c}\text { ylimidazoliur } \\
123.3 \pm 1.0 \\
128.0 \pm 1.4\end{array}$ & $\begin{array}{l}\text { trifluoro } \\
363 \\
298\end{array}$ & $\begin{array}{l}\text { ylsulfonyl)i } \\
\text { QCM } \\
\text { QCM }\end{array}$ & $\begin{array}{l}\text { ide } \\
\text { [2016ZAI/VAR] } \\
\text { [2016ZAI/VAR] }\end{array}$ \\
\hline $\mathrm{C}_{9} \mathrm{H}_{13} \mathrm{~F}_{6} \mathrm{~N}_{3} \mathrm{O}_{4} \mathrm{~S}_{2}$ & $\begin{array}{c}\text { [174899-88-8] } \\
\text { FUS } \\
\text { V } \\
\text { V } \\
\text { V }\end{array}$ & $\begin{array}{l}\text { 1,3-diethylimic } \\
\begin{array}{r}(455-480) \\
(455-480) \\
(455-480)\end{array}\end{array}$ & $\begin{array}{l}\text { lium bis(triflu } \\
20.4 \\
\\
109.2 \pm 0.5 \\
110.1 \pm 0.5 \\
129.0 \pm 1.0\end{array}$ & $\begin{array}{c}\text { ethylsul } \\
262.6 \\
468 \\
460 \\
298\end{array}$ & $\begin{array}{l}\text { imide } \\
\text { DSC } \\
\text { ME } \\
\text { ME } \\
\text { ME }\end{array}$ & $\begin{array}{l}{[2008 \mathrm{DOM} / \mathrm{REK}]} \\
{[2012 \mathrm{ROC} / \mathrm{COU}]} \\
{[2012 \mathrm{ROC} / \mathrm{COU}]} \\
{[2012 \mathrm{ROC} / \mathrm{COU}]}\end{array}$ \\
\hline $\mathrm{C}_{9} \mathrm{H}_{14} \mathrm{BrN}$ & $\begin{array}{c}\text { [120375-53-3] } \\
\text { TRS } \\
\text { TRS }\end{array}$ & 3-phenylpropy & $\begin{array}{l}\text { nonium brom } \\
10.8 \\
0.11\end{array}$ & $\begin{array}{l}357 \\
402\end{array}$ & DSC & [1996DOM/HEA, 1989VAN/WHI] \\
\hline $\mathrm{C}_{9} \mathrm{H}_{14} \mathrm{CIN}$ & $\begin{array}{c}\text { [30684-05-0] } \\
\text { TRS } \\
\text { TRS }\end{array}$ & 3-phenylpropy & $\begin{array}{l}\text { nonium chlori } \\
6.22 \\
4.24\end{array}$ & $\begin{array}{l}343 \\
368\end{array}$ & DSC & [1996DOM/HEA, 1989VAN/WHI] \\
\hline $\mathrm{C}_{9} \mathrm{H}_{14} \mathrm{CIN}$ & $\begin{array}{l}\text { [1124-64-7] } \\
\text { FUS }\end{array}$ & 1-butylpyridini & $\begin{array}{l}\text { chloride } \\
20.7\end{array}$ & 393.3 & DSC & [2012VER/ZAI] \\
\hline $\mathrm{C}_{9} \mathrm{H}_{15} \mathrm{BrN}_{2} \mathrm{O}_{2}$ & $\begin{array}{c}\text { [1456904-69-0] } \\
\text { FUS }\end{array}$ & 1,2-dimethyl-3 & $\begin{array}{l}\text { ylacetateimida } \\
\quad 24.5\end{array}$ & $\begin{array}{c}\text { Im brom } \\
423\end{array}$ & DSC & [2014ZHA/SAL] \\
\hline $\mathrm{C}_{9} \mathrm{H}_{15} \mathrm{BrN}_{2} \mathrm{O}_{2}$ & $\begin{array}{c}\text { [1309877-52-8] } \\
\text { FUS }\end{array}$ & 1-carboxyl-3-b & $\begin{array}{l}\text { Imidazolium b } \\
\quad 12.2\end{array}$ & de 430 & DSC & [2014ZHA/SAL] \\
\hline $\mathrm{C}_{9} \mathrm{H}_{15} \mathrm{ClN}_{2} \mathrm{O}_{2}$ & $\begin{array}{c}{[1584745-11-8]} \\
\text { FUS }\end{array}$ & 1,2-dimethyl-3 & $\begin{array}{c}\text { ylacetateimida } \\
23.0\end{array}$ & $\begin{array}{c}\text { Im chlor } \\
434\end{array}$ & DSC & [2014ZHA/SAL] \\
\hline $\mathrm{C}_{9} \mathrm{H}_{15} \mathrm{~F}_{3} \mathrm{~N}_{2} \mathrm{O}_{3} \mathrm{~S}$ & $\begin{array}{c}\text { [174899-66-2] } \\
\text { TRS } \\
\text { FUS }\end{array}$ & $\begin{array}{l}\text { 3-butyl-1-meth } \\
\quad(5-370) \\
(5-370)\end{array}$ & $\begin{array}{l}\text { idazolium, } 1,1 \\
0.02 \\
19.43\end{array}$ & $\begin{array}{l}\text { fluorom } \\
120 \\
291.0\end{array}$ & $\begin{array}{l}\text { hesulfonate } \\
\text { AC }\end{array}$ & [2010PAU/KOH] \\
\hline $\mathrm{C}_{9} \mathrm{H}_{16} \mathrm{~N}_{2} \mathrm{O}_{2}$ & $\begin{array}{c}{[865627-64-1]} \\
\mathrm{V}\end{array}$ & 1-methyl-3-eth & $\begin{array}{c}\text { idazolium pro } \\
111.1\end{array}$ & 438 & TGA & [2014HON/LIU] \\
\hline
\end{tabular}


TABLE 18. Phase change enthalpies of organic salts and ionic liquids-Continued

\begin{tabular}{|c|c|c|c|c|c|c|}
\hline \multirow[b]{2}{*}{$\begin{array}{l}\text { Molecular } \\
\text { formula }\end{array}$} & \multirow{2}{*}{$\begin{array}{c}\text { CAS Registry } \\
\text { Number } \\
\text { Enthalpy }\end{array}$} & \multicolumn{5}{|l|}{ Compound } \\
\hline & & $\begin{array}{c}\text { Temperature } \\
\text { range }\end{array}$ & $\begin{array}{l}\Delta_{\text {trans }} H_{\mathrm{m}} \\
(\mathrm{kJ} / \mathrm{mol})\end{array}$ & $\begin{array}{l}T_{\mathrm{m}} \\
(\mathrm{K})\end{array}$ & Method & Reference \\
\hline \multirow[t]{4}{*}{$\mathrm{C}_{9} \mathrm{H}_{16} \mathrm{~N}_{2} \mathrm{O}_{2}$} & {$[1005328-08-4]$} & \multicolumn{5}{|c|}{ 1-propyl-3-methylimidazolium acetate } \\
\hline & $\mathrm{V}$ & $(418-438)$ & 104.4 & 438 & ITGA & [2014ZHA/WEI2] \\
\hline & $\mathrm{V}$ & $(418-438)$ & 106.1 & 418 & ITGA & [2014ZHA/WEI2] \\
\hline & $\mathrm{V}$ & $(418-438)$ & 116.8 & 298 & ITGA & [2014ZHA/WEI2] \\
\hline \multirow[t]{4}{*}{$\mathrm{C}_{9} \mathrm{H}_{20} \mathrm{~F}_{6} \mathrm{NP}$} & {$[327022-58-2]$} & \multicolumn{5}{|c|}{ 1-propyl-1-methylpiperidinium hexafluorophosphate } \\
\hline & TRS & & 8.08 & 311.7 & & \\
\hline & TRS & & 2.74 & 352.4 & & \\
\hline & FUS & & 5.11 & 368.5 & DSC & {$[2014 \mathrm{MAX} / \mathrm{SAN}]$} \\
\hline \multirow[t]{2}{*}{$\mathrm{C}_{9} \mathrm{H}_{20} \mathrm{BrN}$} & {$[93457-69-3]$} & \multicolumn{5}{|c|}{ 1-butyl-1-methylpyrrolidinium bromide } \\
\hline & FUS & & 13.12 & 487.7 & DSC & {$[2014 \mathrm{ZAW} / \mathrm{KRO}]$} \\
\hline \multirow[t]{2}{*}{$\mathrm{C}_{9} \mathrm{H}_{20} \mathrm{~N}_{2} \mathrm{~S}$} & {$[4587-19-3]$} & \multicolumn{5}{|c|}{ Tetraethylammonium thiocyanate } \\
\hline & FUS & & 24.0 & 335.2 & DSC & [2013BHA/GOH] \\
\hline \multirow[t]{2}{*}{$\mathrm{C}_{9} \mathrm{H}_{21} \mathrm{NO}_{3}$} & {$[17351-61-0]$} & \multicolumn{5}{|c|}{ Tetraethylammonium carbonate } \\
\hline & FUS & & 27.2 & 347.2 & DSC & [2013BHA/GOH] \\
\hline \multirow[t]{5}{*}{$\mathrm{C}_{10} \mathrm{H}_{11} \mathrm{~N}_{5}$} & {$[666823-18-3]$} & \multicolumn{5}{|c|}{ 1-ethyl-3-methylimidazolium tricyanomethanide } \\
\hline & TRS & & 1.62 & 237 & & \\
\hline & FUS & & 6.48 & 257.2 & DSC & [2013DOM/KRO] \\
\hline & $\mathrm{V}$ & $(440-448)$ & $123.0 \pm 1.0$ & 423 & QCM-LE & [2011EME/ZAI] \\
\hline & V & $(440-448)$ & $138.8 \pm 5.0$ & 298 & QCM-LE & [2011EME/ZAI] \\
\hline \multirow[t]{3}{*}{$\mathrm{C}_{10} \mathrm{H}_{11} \mathrm{~F}_{10} \mathrm{~N}_{2} \mathrm{O}_{2} \mathrm{P}$} & {$[852616-00-3]$} & \multicolumn{5}{|c|}{ 1-ethyl-3-methylimidazolium bis(pentafluoroethyl)phosphate } \\
\hline & $\mathrm{V}$ & & $120 \pm 2$ & 400 & Mass Spec & [2012DEY/HES] \\
\hline & $\mathrm{V}$ & & $130 \pm 2$ & 298 & Mass Spec & [2012DEY/HES] \\
\hline \multirow[t]{3}{*}{$\mathrm{C}_{10} \mathrm{H}_{12} \mathrm{~F}_{6} \mathrm{~N}_{2} \mathrm{O}_{4} \mathrm{~S}_{2}$} & $\begin{array}{c}\text { [1104525-90-7] } \\
\mathrm{V}\end{array}$ & 1-propylpyridi & bis(trifluoron & $\begin{array}{l}\text { sulfony } \\
504\end{array}$ & de & \\
\hline & V & $(498-511)$ & $\begin{array}{l}124.1 \pm 0.5 \\
128.0 \pm 1.0\end{array}$ & $\begin{array}{l}504 \\
398\end{array}$ & $\begin{array}{l}\text { QCM, ME } \\
\text { OCM }\end{array}$ & [2012ZAI/YER] \\
\hline & $\mathrm{V}$ & & 134.5 & 298 & QCM & [2012ZAI/YER] \\
\hline $\mathrm{C}_{10} \mathrm{H}_{12} \mathrm{~F}_{6} \mathrm{~N}_{2} \mathrm{O}_{4} \mathrm{~S}_{2}$ & {$[841251-37-4]$} & $N$-ethyl-3-metl & ridinium bis( & rometh & onyl)imide & \\
\hline & $\mathrm{V}$ & $(400-430)$ & $162 \pm 35$ & 419 & $\mathrm{UV} / \mathrm{Vis}$ & [20130GU/AKA] \\
\hline $\mathrm{C}_{10} \mathrm{H}_{13} \mathrm{~F}_{6} \mathrm{~N}_{3} \mathrm{O}_{4} \mathrm{~S}_{2}$ & & 1-cyclopropyln & 1-3-methylim & ium bis & Ioromethylsu & onyl)imide \\
\hline & $\mathrm{V}$ & $(350-398)$ & $126.5 \pm 1.0$ & 374 & QCM & [2016ZAI/VAR] \\
\hline & V & (350-398) & $131.9 \pm 1.6$ & 298 & QCM & [2016ZAI/VAR] \\
\hline $\mathrm{C}_{10} \mathrm{H}_{15} \mathrm{~F}_{3} \mathrm{~N}_{2} \mathrm{O}_{2}$ & [174899-94-6] & 1-butyl-3-meth & idazolium trif & acetate & & \\
\hline & TRS & & 0.29 & 274 & & \\
\hline & FUS & & 19.14 & 296.4 & $\mathrm{AC}$ & [2008STR/PAU] \\
\hline $\mathrm{C}_{10} \mathrm{H}_{15} \mathrm{~F}_{6} \mathrm{~N}_{3} \mathrm{O}_{4} \mathrm{~S}_{2}$ & [174899-83-3] & 1-butyl-3-meth & idazolium bis & oromet & Ifonyl)imide & \\
\hline & FUS & & 25.0 & 269.6 & DSC & [2016XUE/GUR] \\
\hline & FUS & $(15-300)$ & 23.8 & 270.4 & $\mathrm{AC}$ & [2012SHI/OHT, 2007SHI/OHT] \\
\hline & FUS & $(5-370)$ & 23.8 & 270.2 & $\mathrm{AC}$ & {$[2008 \mathrm{BLO} / \mathrm{PAU}]$} \\
\hline & FUS & & 21.0 & 271 & DSC & [2008JIN/OHA] \\
\hline & FUS & & 22.43 & 367.7 & DSC & [2006TRO/CER] \\
\hline & FUS & & 20.9 & 270 & DSC & [2005TOK/HAY] \\
\hline & $\mathrm{V}$ & $(502-567)$ & $111.3 \pm 1.6$ & 534 & ME,MS & [2014BRU/CIC] \\
\hline & $\mathrm{V}$ & $(453-507)$ & $114.9 \pm 1.2$ & 489 & ME,MS & [2014BRU/CIC] \\
\hline & $\mathrm{V}$ & $(398-458)$ & $118.4 \pm 1.5$ & 427 & ME,MS & [2014BRU/CIC] \\
\hline & $\mathrm{V}$ & $(400-430)$ & $137 \pm 11$ & 418 & $\mathrm{UV} / \mathrm{Vis}$ & [2013OGU/AKA] \\
\hline & $\mathrm{V}$ & $(362-395)$ & $124.4 \pm 1.0$ & 378 & QCM & [2013VER/ZAI] \\
\hline & $\mathrm{V}$ & $(362-395)$ & $132.4 \pm 1.0$ & 298 & QCM & [2013VER/ZAI] \\
\hline & $\mathrm{V}$ & $(513-572)$ & $113.5 \pm 1.5$ & 542 & TGA & [2013VER/ZAI] \\
\hline & $\mathrm{V}$ & $(513-572)$ & $137.8 \pm 1.5$ & 298 & TGA & [2013VER/ZAI] \\
\hline & $\mathrm{V}$ & $(362-395)$ & $124.4 \pm 2.5$ & 378 & QCM & [2011ZAI/VER] \\
\hline & V & $(262-395)$ & 132.4 & 298 & QCM & [2011ZAI/VER] \\
\hline & $\mathrm{V}$ & $(458-517)$ & $120 \pm 5$ & 487 & ME & [2005PAU/ZAI] \\
\hline $\mathrm{C}_{10} \mathrm{H}_{15} \mathrm{~F}_{6} \mathrm{~N}_{3} \mathrm{O}_{4} \mathrm{~S}_{2}$ & & 1-isobutyl-3-m & limidazolium & fluoron & sulfonyl)imi & \\
\hline & $\mathrm{V}$ & (345-393) & $126.0 \pm 1.0$ & 369 & QCM & [2016ZAI/VAR] \\
\hline
\end{tabular}


TABLE 18. Phase change enthalpies of organic salts and ionic liquids-Continued

\begin{tabular}{|c|c|c|c|c|c|c|}
\hline \multirow[b]{2}{*}{$\begin{array}{l}\text { Molecular } \\
\text { formula }\end{array}$} & \multirow{2}{*}{$\begin{array}{l}\text { CAS Registry } \\
\text { Number } \\
\text { Enthalpy }\end{array}$} & \multicolumn{5}{|l|}{ Compound } \\
\hline & & $\begin{array}{l}\text { Temperature } \\
\text { range }\end{array}$ & $\begin{array}{l}\Delta_{\text {trans }} H_{\mathrm{m}} \\
(\mathrm{kJ} / \mathrm{mol})\end{array}$ & $\begin{array}{l}T_{\mathrm{m}} \\
(\mathrm{K})\end{array}$ & Method & Reference \\
\hline & $\mathrm{V}$ & $(345-393)$ & $131.4 \pm 1.6$ & 298 & QCM & [2016ZAI/VAR] \\
\hline $\mathrm{C}_{1} \mathrm{H}_{15} \mathrm{~F}_{6} \mathrm{~N}_{3} \mathrm{O}_{4} \mathrm{~S}_{2}$ & $\begin{array}{l}\mathrm{V} \\
\mathrm{V}\end{array}$ & $\begin{array}{c}\text { 1-sec-butyl-3-m } \\
\quad(346-393) \\
(346-393)\end{array}$ & $\begin{array}{c}\text { ylimidazoliun } \\
123.0 \pm 1.0 \\
128.4 \pm 1.6\end{array}$ & $\begin{array}{l}\text { rifluoror } \\
369 \\
298\end{array}$ & $\begin{array}{l}\text { ylsulfonyl)in } \\
\text { QCM } \\
\text { QCM }\end{array}$ & $\begin{array}{l}\text { de } \\
\text { [2016ZAI/VAR] } \\
\text { [2016ZAI/VAR] }\end{array}$ \\
\hline $\mathrm{C}_{10} \mathrm{H}_{15} \mathrm{~F}_{6} \mathrm{~N}_{3} \mathrm{O}_{4} \mathrm{~S}_{2}$ & $\begin{array}{c}{[169051-76-7]} \\
\mathrm{V} \\
\mathrm{V}\end{array}$ & $\begin{array}{c}\text { 1-propyl-2,3-di } \\
(423-513) \\
(423-513)\end{array}$ & $\begin{array}{l}\text { hylimidazoliu } \\
123.6 \pm 1.8 \\
135 \pm 4\end{array}$ & $\begin{array}{l}\text { (trifluorc } \\
468 \\
298\end{array}$ & $\begin{array}{l}\text { hylsulfonyl)i } \\
\text { ME-MS } \\
\text { ME-MS }\end{array}$ & $\begin{array}{l}\text { ide } \\
\text { [2015CHI/MED] } \\
\text { [2015CHI/MED] }\end{array}$ \\
\hline $\mathrm{C}_{10} \mathrm{H}_{15} \mathrm{~F}_{6} \mathrm{~N}_{3} \mathrm{O}_{5} \mathrm{~S}_{2}$ & $\begin{array}{c}{[1103335-97-2]} \\
\text { V } \\
\text { V } \\
\text { V }\end{array}$ & $\begin{array}{c}\text { 3-(2-methoxyet } \\
\quad(358-406) \\
(512-575)\end{array}$ & $\begin{array}{r}-1,2 \text {-dimethy } \\
122.5 \pm 1.0 \\
102.5 \pm 1.2 \\
132.4 \pm 1.0\end{array}$ & $\begin{array}{l}\text { zolium } \\
381 \\
543 \\
298\end{array}$ & $\begin{array}{l}\text { rifluoromethy } \\
\text { QCM } \\
\text { TGA }\end{array}$ & $\begin{array}{l}\text { sulfonyl)imide } \\
\text { [2013ZAI/YER2] } \\
\text { [2013ZAI/YER2] } \\
\text { [2013ZAI/YER2] }\end{array}$ \\
\hline $\mathrm{C}_{10} \mathrm{H}_{16} \mathrm{~F}_{6} \mathrm{NP}$ & $\begin{array}{c}\text { [955127-11-4] } \\
\text { FUS }\end{array}$ & $N$-pentylpyridir & $\begin{array}{c}\text { n hexafluorop } \\
5.9\end{array}$ & $\begin{array}{l}\text { ate } \\
328.35\end{array}$ & $\mathrm{AC}$ & [2014LIU/TAN] \\
\hline $\mathrm{C}_{10} \mathrm{H}_{16} \mathrm{BrN}$ & $\begin{array}{c}\text { [120375-52-2] } \\
\text { TRS } \\
\text { TRS }\end{array}$ & 4-phenylbutyla & $\begin{array}{c}\text { onium bromic } \\
9.60 \\
1.40\end{array}$ & $\begin{array}{l}353 \\
393\end{array}$ & DSC & [1996DOM/HEA, 1989VAN/WHI] \\
\hline $\mathrm{C}_{10} \mathrm{H}_{16} \mathrm{ClN}$ & $\begin{array}{c}\text { [30684-06-1] } \\
\text { TRS } \\
\text { TRS } \\
\text { TRS }\end{array}$ & 4-phenylbutyla & $\begin{array}{c}\text { onium chlorid } \\
4.03 \\
0.65 \\
0.27\end{array}$ & $\begin{array}{l}243 \\
274 \\
295\end{array}$ & DSC & [1996DOM/HEA, 1989VAN/WHI] \\
\hline $\mathrm{C}_{10} \mathrm{H}_{16} \mathrm{ClN}$ & $\begin{array}{c}\text { [125652-55-3] } \\
\text { FUS }\end{array}$ & 1-butyl-3-meth & $\begin{array}{c}\text { ridinium chlo } \\
28\end{array}$ & 384.5 & DSC & [2011PER/ROD] \\
\hline $\mathrm{C}_{10} \mathrm{H}_{17} \mathrm{NO}_{4} \mathrm{~S}$ & $\begin{array}{c}{[885456-20-2]} \\
\mathrm{V} \\
\mathrm{V}\end{array}$ & $N$-butylpyridini & $\begin{array}{c}\text { methanesulfa } \\
\begin{array}{c}144 \pm 2 \\
164 \pm 2\end{array}\end{array}$ & $\begin{array}{l}510 \\
298\end{array}$ & $\begin{array}{l}\text { Mass Spec } \\
\text { Mass Spec }\end{array}$ & $\begin{array}{l}{[2012 \mathrm{DEY} / \mathrm{HES}]} \\
{[2012 \mathrm{DEY} / \mathrm{HES}]}\end{array}$ \\
\hline $\mathrm{C}_{10} \mathrm{H}_{18} \mathrm{~N}_{2} \mathrm{O}_{2}$ & $\begin{array}{c}{[1374313-75-3]} \\
\mathrm{V}\end{array}$ & 1-methyl-3-pro & $\begin{array}{c}\text { midazolium } \mathrm{p} \\
115.8\end{array}$ & $\begin{array}{l}\text { oate } \\
\qquad 443\end{array}$ & TGA & [2014HON/LIU] \\
\hline $\mathrm{C}_{10} \mathrm{H}_{18} \mathrm{~N}_{2} \mathrm{O}_{2}$ & $\begin{array}{c}{[284049-75-8]} \\
\text { V } \\
\text { V }\end{array}$ & $\begin{array}{l}\text { 1-butyl-3-meth } \\
\quad(408-448) \\
(408-448)\end{array}$ & $\begin{array}{c}\text { hidazolium ac } \\
127.8 \pm 4.2 \\
134.8\end{array}$ & $\begin{array}{l}428 \\
298\end{array}$ & $\begin{array}{l}\text { ITGA } \\
\text { ITGA }\end{array}$ & $\begin{array}{l}{[2015 \mathrm{WEI} / \mathrm{BU}]} \\
{[2015 \mathrm{WEI} / \mathrm{BU}]}\end{array}$ \\
\hline $\mathrm{C}_{10} \mathrm{H}_{20} \mathrm{~N}_{2} \mathrm{~S}$ & $\begin{array}{c}\text { [507477-22-7] } \\
\text { TRS } \\
\text { FUS }\end{array}$ & 1-butyl-1-meth & $\begin{array}{l}\text { rroldinium th } \\
8.68 \\
2.06\end{array}$ & $\begin{array}{l}\text { nate } \\
245.3 \\
295.4\end{array}$ & DSC & [2011DOM/KRO] \\
\hline $\mathrm{C}_{10} \mathrm{H}_{22} \mathrm{BrN}$ & $\begin{array}{c}\text { [833446-30-3] } \\
\text { TRS } \\
\text { FUS }\end{array}$ & 1-pentyl-1-metl & $\begin{array}{c}\text { yrrolidinium } \\
1.95 \\
8.97\end{array}$ & $\begin{array}{l}\text { de } \\
326.9 \\
431.6\end{array}$ & DSC & [2014ZAW/KRO] \\
\hline $\mathrm{C}_{10} \mathrm{H}_{24} \mathrm{ClN}$ & $\begin{array}{c}\text { [23307-02-0] } \\
\text { TRS }\end{array}$ & $\begin{array}{l}\text { Dipentylammor } \\
\quad(25-350)\end{array}$ & $\begin{array}{c}\text { n chloride } \\
13.1\end{array}$ & 243.8 & $\mathrm{AC}$ & [1996DOM/HEA, 1988VAN/WHI] \\
\hline $\mathrm{C}_{10} \mathrm{H}_{24} \mathrm{ClN}$ & $\begin{array}{c}\text { [143-09-9] } \\
\text { TRS } \\
\text { TRS } \\
\text { TRS }\end{array}$ & $\begin{array}{c}\text { Decylammoniu } \\
\quad(80-380) \\
(80-380) \\
(80-380)\end{array}$ & $\begin{array}{l}\text { hloride } \\
\qquad \begin{array}{r}6.39 \\
5.85 \\
2.04\end{array}\end{array}$ & $\begin{array}{l}307.5 \\
325.0 \\
327.3\end{array}$ & $\mathrm{AC}$ & [2011DAN/DI] \\
\hline $\mathrm{C}_{10} \mathrm{H}_{24} \mathrm{~F}_{6} \mathrm{NP}$ & $\begin{array}{c}{[1020810-59-6]} \\
\text { FUS }\end{array}$ & Dibutyl(dimeth & $\begin{array}{l}\text { Immonium he } \\
12.9\end{array}$ & $\begin{array}{l}\text { rophosp } \\
436.7\end{array}$ & DSC & [2008BUS/LAH] \\
\hline
\end{tabular}


TABLE 18. Phase change enthalpies of organic salts and ionic liquids-Continued

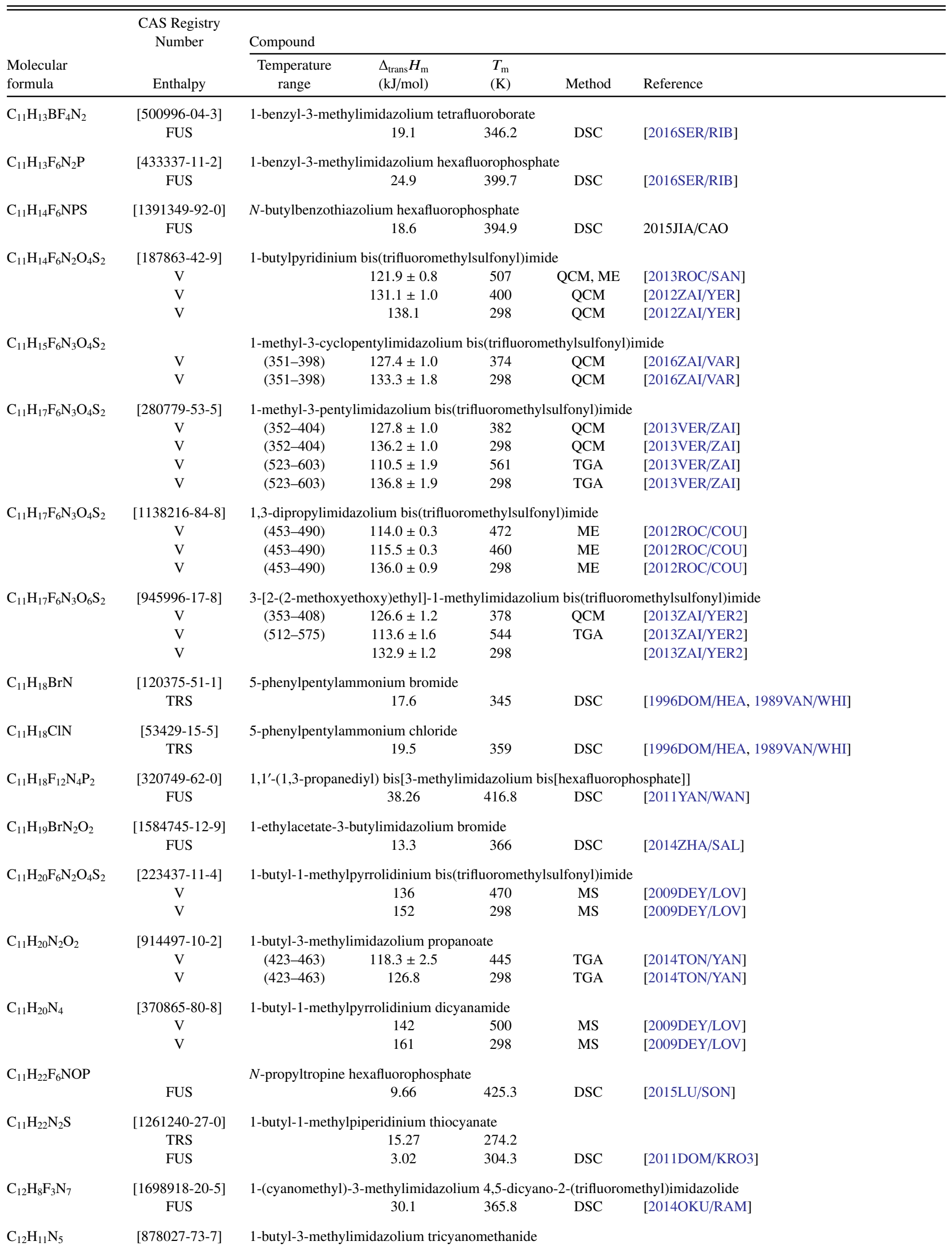


TABLE 18. Phase change enthalpies of organic salts and ionic liquids-Continued

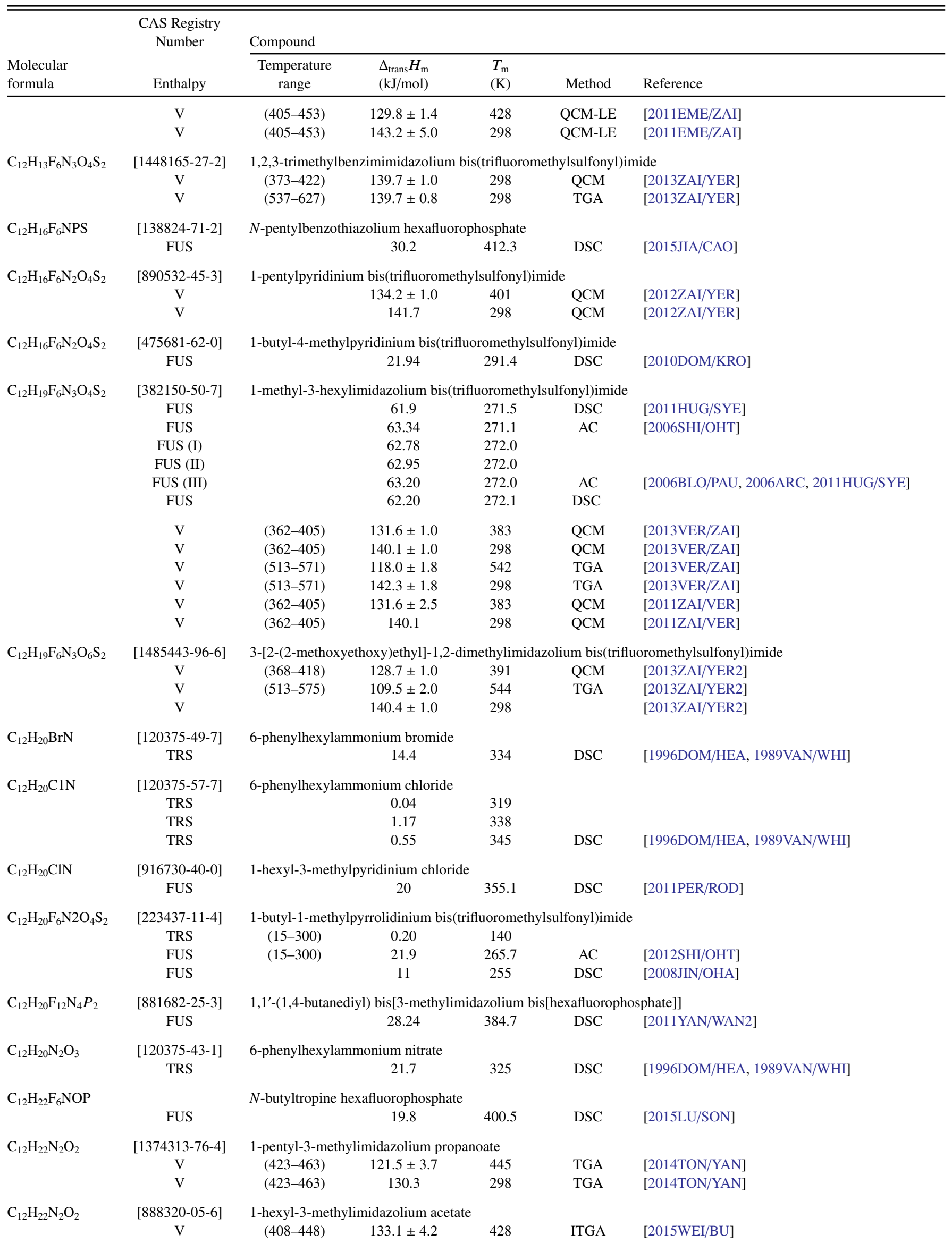


TABLE 18. Phase change enthalpies of organic salts and ionic liquids-Continued

\begin{tabular}{|c|c|c|c|c|c|c|}
\hline \multirow[b]{2}{*}{$\begin{array}{l}\text { Molecular } \\
\text { formula }\end{array}$} & \multirow{2}{*}{$\begin{array}{l}\text { CAS Registry } \\
\text { Number } \\
\text { Enthalpy }\end{array}$} & \multicolumn{5}{|l|}{ Compound } \\
\hline & & $\begin{array}{c}\text { Temperature } \\
\text { range }\end{array}$ & $\begin{array}{l}\Delta_{\text {trans }} H_{\mathrm{m}} \\
(\mathrm{kJ} / \mathrm{mol})\end{array}$ & $\begin{array}{l}T_{\mathrm{m}} \\
(\mathrm{K})\end{array}$ & Method & Reference \\
\hline & $\mathrm{V}$ & $(408-448)$ & 140.7 & 298 & ITGA & {$[2015 \mathrm{WEI} / \mathrm{BU}]$} \\
\hline $\mathrm{C}_{12} \mathrm{H}_{23} \mathrm{ClN}$ & $\begin{array}{c}{[64697-40-1]} \\
\mathrm{V} \\
\mathrm{V}\end{array}$ & 1-octyl-3-meth & $\begin{array}{l}\text { lazolium ch } \\
151 \pm 10 \\
166 \pm 10\end{array}$ & $\begin{array}{l}455 \\
298\end{array}$ & $\begin{array}{l}\text { TPD,MS } \\
\text { TPD,MS }\end{array}$ & $\begin{array}{l}\text { [2014LOV/ARM] } \\
\text { [2014LOV/ARM] }\end{array}$ \\
\hline $\mathrm{C}_{12} \mathrm{H}_{23} \mathrm{~F}_{6} \mathrm{~N}_{2} \mathrm{P}$ & $\begin{array}{c}\text { [304680-36-2] } \\
\text { FUS }\end{array}$ & 1-octyl-3-meth & $\begin{array}{l}\text { dazolium he } \\
12.9\end{array}$ & $\begin{array}{l}\text { rophosp } \\
272.3\end{array}$ & DSC & [2015NEM/KOF] \\
\hline $\mathrm{C}_{12} \mathrm{H}_{23} \mathrm{IN}$ & $\begin{array}{c}{[188589-28-8]} \\
\mathrm{V} \\
\mathrm{V}\end{array}$ & 1-octyl-3-meth & $\begin{array}{l}\text { dazolium io } \\
149 \pm 8 \\
167 \pm 8\end{array}$ & $\begin{array}{l}480 \\
298\end{array}$ & $\begin{array}{l}\text { TPD,MS } \\
\text { TPD,MS }\end{array}$ & $\begin{array}{l}\text { [2014LOV/ARM] } \\
\text { [2014LOV/ARM] }\end{array}$ \\
\hline $\mathrm{C}_{12} \mathrm{H}_{28} \mathrm{BF} 4 \mathrm{~N}$ & $\begin{array}{c}\text { [338-38-5] } \\
\text { TRS } \\
\text { TRS } \\
\text { FUS } \\
\text { TRS } \\
\text { FUS }\end{array}$ & Tetrapropylam & $\begin{array}{c}\text { Im tetrafluo } \\
1.5 \\
16.3 \\
19.2 \\
14.6 \\
14.2\end{array}$ & $\begin{array}{l}289 \\
392 \\
511 \\
397 \\
512\end{array}$ & $\begin{array}{l}\text { DSC } \\
\text { DSC }\end{array}$ & $\begin{array}{l}\text { [1987ZAB/FER] } \\
\text { [1970COK/AMB] }\end{array}$ \\
\hline $\mathrm{C}_{12} \mathrm{H}_{28} \mathrm{BrN}$ & $\begin{array}{c}\text { [1941-30-6] } \\
\text { TRS } \\
\text { TRS }\end{array}$ & Tetrapropylam & $\begin{array}{c}\text { Im bromide } \\
17.0 \\
0.35\end{array}$ & $\begin{array}{l}382.2 \\
395.8\end{array}$ & DSC & [1992XEN/CHE] \\
\hline $\mathrm{C}_{12} \mathrm{H}_{28} \mathrm{BrN}$ & $\begin{array}{c}\text { [26204-55-7] } \\
\text { TRS } \\
\text { TRS } \\
\text { TRS }\end{array}$ & $\begin{array}{c}\text { Dodecylammo } \\
\quad(78-400) \\
(78-400) \\
(78-400)\end{array}$ & $\begin{array}{c}\text { bromide } \\
8.58 \\
7.60 \\
9.65\end{array}$ & $\begin{array}{l}329.3 \\
337.8 \\
347.4\end{array}$ & $\mathrm{AC}$ & [2010LIU/DI] \\
\hline $\mathrm{C}_{12} \mathrm{H}_{28} \mathrm{ClN}$ & $\begin{array}{c}\text { [929-73-7] } \\
\text { TRS } \\
\text { TRS } \\
\text { FUS }\end{array}$ & $\begin{array}{c}\text { Dodecylammo } \\
\quad(79-396) \\
(79-396)\end{array}$ & $\begin{array}{c}\text { chloride } \\
25.72 \\
5.05 \\
7.83\end{array}$ & $\begin{array}{l}330.8 \\
345.1 \\
456.7\end{array}$ & $\begin{array}{c}\mathrm{AC} \\
\mathrm{DSC}\end{array}$ & [2009KON/DI] \\
\hline $\mathrm{C}_{12} \mathrm{H}_{28} \mathrm{ClN}$ & $\begin{array}{c}\text { [2296-13-1] } \\
\text { TRS } \\
\text { TRS }\end{array}$ & $\begin{array}{l}\text { Dihexylammo } \\
\qquad \begin{array}{l}(25-350) \\
(25-350)\end{array}\end{array}$ & $\begin{array}{r}\text { hloride } \\
0.91 \\
15.95\end{array}$ & $\begin{array}{l}115.3 \\
279.4\end{array}$ & $\mathrm{AC}$ & [1996DOM/HEA, 1988VAN/WHI] \\
\hline $\mathrm{C}_{12} \mathrm{H}_{28} \mathrm{ClNO} 4$ & $\begin{array}{c}\text { [15780-02-6] } \\
\text { TRS } \\
\text { TRS } \\
\text { FUS }\end{array}$ & Tetrapropylam & $\begin{array}{c}\text { Im perchlor } \\
11.2 \\
4.47 \\
14.2\end{array}$ & $\begin{array}{l}444.2 \\
446.7 \\
514.2\end{array}$ & DSC & [1989NAK/KUW] \\
\hline $\mathrm{C}_{12} \mathrm{H}_{28} \mathrm{IN}$ & $\begin{array}{c}\text { [631-40-3] } \\
\text { TRS } \\
\text { TRS }\end{array}$ & Tetrapropylam & $\begin{array}{c}\text { Im iodide } \\
1.44 \\
15.0\end{array}$ & $\begin{array}{l}225.4 \\
418.9\end{array}$ & DSC & [1992XEN/CHE] \\
\hline [Note: The author & $\begin{array}{c}\text { f Ref. [1992XEN } \\
\text { TRS } \\
\text { TRS } \\
\text { FUS } \\
\text { TRS }\end{array}$ & HE] state that $\mathrm{t}$ & $\begin{array}{c}\text { sition at } 41 \\
1.35 \\
2.93 \\
13.81 \\
32.6\end{array}$ & $\begin{array}{l}\text { s a tran } \\
218 \\
225 \\
418 \\
419\end{array}$ & to a plastic & $\begin{array}{l}\text { ystal.] } \\
\text { [1996DOM/HEA, 1973JOH/MAR] } \\
\text { [1971LEV/KOH] } \\
\text { [1970COK/AMB] }\end{array}$ \\
\hline $\mathrm{C}_{12} \mathrm{H}_{28} \mathrm{~N}_{2} \mathrm{O}_{3}$ & $\begin{array}{c}\text { [1941-28-2] } \\
\text { TRS } \\
\text { FUS }\end{array}$ & Tetrapropylam & $\begin{array}{l}\text { Im nitrate } \\
8.97 \\
\text { Not given }\end{array}$ & 330.8 & DSC & [1989NAK/KUW] \\
\hline $\mathrm{C}_{13} \mathrm{H}_{14} \mathrm{~F}_{4} \mathrm{~N}_{2} \mathrm{O}_{3} \mathrm{~S}$ & FUS & 1-benzyl-3-me & $\begin{array}{l}\text { idazoium } \\
23.6\end{array}$ & $\begin{array}{c}\text { tetraflu } \\
315.4\end{array}$ & $\begin{array}{l}\text { Ianesulfona } \\
\text { DSC }\end{array}$ & [2016SER/RIB] \\
\hline $\mathrm{C}_{13} \mathrm{H}_{18} \mathrm{~N}_{2} \mathrm{O}_{3} \mathrm{~S}$ & $\begin{array}{c}{[328090-25-1]} \\
\text { FUS }\end{array}$ & 3-ethyl-1-meth & $\begin{array}{c}\text { dazolium to } \\
20.49\end{array}$ & 322.3 & DSC & [2010DOM/KRO2] \\
\hline $\mathrm{C}_{13} \mathrm{H}_{18} \mathrm{~F}_{6} \mathrm{NPS}$ & $\begin{array}{c}{[1330158-81-0]} \\
\text { FUS }\end{array}$ & $N$-hexylbenzot & $\begin{array}{c}\text { lium hexafl } \\
23.5\end{array}$ & $\begin{array}{l}\text { osphate } \\
358.8\end{array}$ & DSC & [2015JIA/CAO] \\
\hline $\mathrm{C}_{13} \mathrm{H}_{18} \mathrm{~F}_{6} \mathrm{~N}_{2} \mathrm{O}_{4} \mathrm{~S}_{2}$ & $\begin{array}{c}{[460983-97-5]} \\
\mathrm{V} \\
\mathrm{V}\end{array}$ & 1-hexylpyridin & $\begin{array}{l}\text { is(trifluoror } \\
137.3 \pm 1.0 \\
145.9\end{array}$ & $\begin{array}{l}\text { ulfonyl } \\
406 \\
298\end{array}$ & $\begin{array}{l}\text { QCM } \\
\text { QCM }\end{array}$ & $\begin{array}{l}\text { [2012ZAI/YER] } \\
\text { [2012ZAI/YER] }\end{array}$ \\
\hline
\end{tabular}


TABLE 18. Phase change enthalpies of organic salts and ionic liquids-Continued

\begin{tabular}{|c|c|c|c|c|c|c|}
\hline \multirow[b]{2}{*}{$\begin{array}{l}\text { Molecular } \\
\text { formula }\end{array}$} & \multirow{2}{*}{$\begin{array}{l}\text { CAS Registry } \\
\text { Number } \\
\text { Enthalpy }\end{array}$} & \multicolumn{5}{|l|}{ Compound } \\
\hline & & $\begin{array}{l}\text { Temperature } \\
\text { range }\end{array}$ & $\begin{array}{l}\Delta_{\text {trans }} H_{\mathrm{m}} \\
(\mathrm{kJ} / \mathrm{mol})\end{array}$ & $\begin{array}{l}T_{\mathrm{m}} \\
(\mathrm{K})\end{array}$ & Method & Reference \\
\hline & $\mathrm{V}$ & & 139 & 440 & MS & [2009DEY/LOV] \\
\hline & $\mathrm{V}$ & & 152 & 298 & MS & [2009DEY/LOV] \\
\hline $\mathrm{C}_{13} \mathrm{H}_{19} \mathrm{~F}_{6} \mathrm{~N}_{3} \mathrm{O}_{4} \mathrm{~S}_{2}$ & & \multicolumn{5}{|c|}{ 1-methyl-3-cyclohexylmethylimidazolium bis(trifluoromethylsulfonyl)imide } \\
\hline \multirow[t]{4}{*}{$\mathrm{C}_{13} \mathrm{H}_{21} \mathrm{~F}_{6} \mathrm{~N}_{3} \mathrm{O}_{4} \mathrm{~S}_{2}$} & [749921-07-1] & \multicolumn{5}{|c|}{ 1,3-dibutylimidazolium bis(trifluoromethylsulfonyl)imide } \\
\hline & $\mathrm{V}$ & $(455-488)$ & $116.8 \pm 0.4$ & 472 & ME & [2012ROC/COU] \\
\hline & $\mathrm{V}$ & $(455-488)$ & $118.4 \pm 0.4$ & 460 & ME & [2012ROC/COU] \\
\hline & $\mathrm{V}$ & $(455-488)$ & $140.8 \pm 0.9$ & 298 & ME & [2012ROC/COU] \\
\hline $\mathrm{C}_{13} \mathrm{H}_{21} \mathrm{~F}_{6} \mathrm{~N}_{3} \mathrm{O}_{4} \mathrm{~S}_{2}$ & [425382-14-5] & \multicolumn{5}{|c|}{ 1-methyl-3-heptylimidazolium bis(trifluoromethylsulfonyl)imide } \\
\hline \multirow{4}{*}{$\mathrm{C}_{13} \mathrm{H}_{21} \mathrm{~F}_{6} \mathrm{~N}_{3} \mathrm{O}_{7} \mathrm{~S}_{2}$} & [947407-82-1] & \multicolumn{5}{|c|}{ 3-[2-[2-(2-methoxyethoxy)ethoxy]ethyl]-1-methylimidazolium bis(trifluoromethylsulfonyl)imide } \\
\hline & $\mathrm{V}$ & $(363-418)$ & $133.6 \pm 1.0$ & 388 & QCM & [2013ZAI/YER2] \\
\hline & $\mathrm{V}$ & $(519-582)$ & $117.8 \pm 1.8$ & 551 & TGA & [2013ZAI/YER2] \\
\hline & $\mathrm{V}$ & & $142.4 \pm 1.0$ & 298 & & [2013ZAI/YER2] \\
\hline \multirow[t]{2}{*}{$\mathrm{C}_{13} \mathrm{H}_{22} \mathrm{BrN}$} & {$[120375-50-0]$} & \multicolumn{5}{|c|}{ 7-phenylheptylammonium bromide } \\
\hline & TRS & & 9.3 & 332 & DSC & [1996DOM/HEA, 1989VAN/WHI] \\
\hline \multirow[t]{2}{*}{$\mathrm{C}_{13} \mathrm{H}_{22} \mathrm{ClN}$} & {$[120375-56-6]$} & \multicolumn{5}{|c|}{ 7-phenylheptylammonium chloride } \\
\hline & TRS & & 10.1 & 310 & DSC & [1996DOM/HEA, 1989VAN/WHI] \\
\hline \multirow[t]{2}{*}{$\mathrm{C}_{13} \mathrm{H}_{24} \mathrm{~F}_{6} \mathrm{~N}_{2} \mathrm{O}_{4} \mathrm{~S}_{2}$} & [380497-19-8] & \multicolumn{5}{|c|}{ 1-hexyl-1-methylpyrroldinium bis(trifluoromethylsulfonyl)imide } \\
\hline & $\mathrm{V}$ & & 141 & 460 & MS & {$[2009 \mathrm{DEY} / \mathrm{LOV}]$} \\
\hline $\mathrm{C}_{13} \mathrm{H}_{30} \mathrm{BrN}$ & [2082-84-0] & Decyl(trimethy & monium brom & & & \\
\hline & TRS & & 32.4 & 369.5 & DSC & [1981IWA/OHN] \\
\hline $\mathrm{C}_{14} \mathrm{H}_{17} \mathrm{~F}_{6} \mathrm{~N}_{3} \mathrm{O}_{4} \mathrm{~S}_{2}$ & [956592-35-1] & 1-hexyl-4-cyan & ridinium bis(tr & omethyl & nyl)imide & \\
\hline & FUS & & 18.83 & 280.2 & DSC & [2013DOM/SKI] \\
\hline $\mathrm{C}_{14} \mathrm{H}_{17} \mathrm{NO}_{3} \mathrm{~S}$ & [78105-28-9] & 1,4-dimethylpy & nium tosylate & & & \\
\hline & FUS & & 30.89 & 424.8 & DSC & [2009DOM/KRO] \\
\hline $\mathrm{C}_{14} \mathrm{H}_{20} \mathrm{~F}_{6} \mathrm{NPS}$ & [1667725-22-5] & $N$-heptylbenzo & zolium hexaflu & nosphate & & \\
\hline & FUS & & 20.1 & 359.3 & DSC & [2015JIA/CAO] \\
\hline $\mathrm{C}_{14} \mathrm{H}_{23} \mathrm{~F}_{6} \mathrm{~N}_{3} \mathrm{O}_{4} \mathrm{~S}_{2}$ & [178631-04-4] & 1-methyl-3-oct & idazolium bis & orometh & fonyl)imic & \\
\hline & FUS & & 57.7 & 249.8 & DSC & [2011HUG/SYE] \\
\hline & FUS & & 59.9 & 251.4 & $\mathrm{AC}$ & [2007PAU/BLO] \\
\hline & $\mathrm{V}$ & $(372-402)$ & $136.8 \pm 1.0$ & 387 & QCM & [2013VER/ZAI] \\
\hline & V & $(372-402)$ & $145.7 \pm 1.0$ & 298 & QCM & [2013VER/ZAI] \\
\hline & $\mathrm{V}$ & $(513-571)$ & $122.6 \pm 1.0$ & 542 & TGA & [2013VER/ZAI] \\
\hline & $\mathrm{V}$ & $(513-571)$ & $147.0 \pm 1.0$ & 298 & TGA & [2013VER/ZAI] \\
\hline & V & $(372-402)$ & $136.8 \pm 2.5$ & 387 & QCM & [2011ZAI/VER] \\
\hline & $\mathrm{V}$ & $(372-402)$ & 145.7 & 298 & QCM & [2011ZAI/VER] \\
\hline $\mathrm{C}_{14} \mathrm{H}_{23} \mathrm{~F}_{6} \mathrm{~N}_{3} \mathrm{O}_{7} \mathrm{~S}_{2}$ & [1485443-99-9] & $3-[2-[2-(2-m e t]$ & rethoxy)ethoxy & 1]-1,2-d & ylimidazo & m bis(trifluoromethylsulfonyl)imide \\
\hline & $\mathrm{V}$ & $(378-428)$ & $134.4 \pm 1.0$ & 401 & $\mathrm{QCM}$ & [2013ZAI/YER2] \\
\hline & $\mathrm{V}$ & & $147.8 \pm 1.0$ & 298 & & [2013ZAI/YER2] \\
\hline $\mathrm{C}_{14} \mathrm{H}_{23} \mathrm{~N}_{5}$ & [905972-84-1] & 1-octyl-3-meth & idazolium dic & mide & & \\
\hline
\end{tabular}


TABLE 18. Phase change enthalpies of organic salts and ionic liquids-Continued

\begin{tabular}{|c|c|c|c|c|c|c|}
\hline \multirow[b]{2}{*}{$\begin{array}{l}\text { Molecular } \\
\text { formula }\end{array}$} & \multirow{2}{*}{$\begin{array}{l}\text { CAS Registry } \\
\text { Number } \\
\text { Enthalpy }\end{array}$} & \multicolumn{5}{|l|}{ Compound } \\
\hline & & $\begin{array}{c}\text { Temperature } \\
\text { range }\end{array}$ & $\begin{array}{l}\Delta_{\text {trans }} H_{\mathrm{m}} \\
(\mathrm{kJ} / \mathrm{mol})\end{array}$ & $\begin{array}{l}T_{\mathrm{m}} \\
(\mathrm{K})\end{array}$ & Method & Reference \\
\hline & $\mathrm{V}$ & & 162 & 298 & MS & [2009DEY/LOV] \\
\hline $\mathrm{C}_{14} \mathrm{H}_{24} \mathrm{BrN}$ & $\begin{array}{c}\text { [120396-93-2] } \\
\text { TRS } \\
\text { TRS }\end{array}$ & 8-phenyloctyla & $\begin{array}{c}\text { nium bromi } \\
0.69 \\
12.1\end{array}$ & $\begin{array}{l}356 \\
379\end{array}$ & DSC & [1996DOM/HEA, 1989VAN/WHI] \\
\hline $\mathrm{C}_{14} \mathrm{H}_{24} \mathrm{ClN}$ & $\begin{array}{c}\text { [17734-26-8] } \\
\text { TRS } \\
\text { TRS }\end{array}$ & 8-phenyloctyla & $\begin{array}{c}\text { nium chlori } \\
7.02 \\
2.85\end{array}$ & $\begin{array}{l}332 \\
347\end{array}$ & DSC & [1996DOM/HEA, 1989VAN/WHI] \\
\hline $\mathrm{C}_{14} \mathrm{H}_{24} \mathrm{ClN}$ & $\begin{array}{c}\text { [864461-36-9] } \\
\text { FUS }\end{array}$ & 1-octyl-3-meth & $\begin{array}{l}\text { ridinium chl } \\
15\end{array}$ & 353.2 & DSC & [2011PER/ROD] \\
\hline $\mathrm{C}_{14} \mathrm{H}_{24} \mathrm{~N}_{2} \mathrm{O}_{3}$ & $\begin{array}{c}\text { [120375-41-9] } \\
\text { TRS }\end{array}$ & 8-phenyloctyla & $\begin{array}{c}\text { nium nitrate } \\
23.0\end{array}$ & 334 & DSC & [1996DOM/HEA, 1989VAN/WHI] \\
\hline $\mathrm{C}_{14} \mathrm{H}_{26} \mathrm{~F}_{6} \mathrm{NOP}$ & FUS & $N$-hexyltropine & $\begin{array}{l}\text { afluorophos! } \\
15.2\end{array}$ & 362.3 & DSC & [2015LU/SON] \\
\hline $\mathrm{C}_{15} \mathrm{H}_{16} \mathrm{~F}_{6} \mathrm{~N}_{2} \mathrm{O}_{4} \mathrm{~S}_{2}$ & $\begin{array}{c}\text { [1289382-58-6] } \\
\text { FUS }\end{array}$ & 1-butylquinolin & $\begin{array}{l}\text { bis(trifluoro } \\
\quad 44.14\end{array}$ & $\begin{array}{l}\text { sulfony } \\
329.1\end{array}$ & DSC & [2010DOM/ZAW] \\
\hline $\mathrm{C}_{15} \mathrm{H}_{16} \mathrm{~F}_{6} \mathrm{~N}_{2} \mathrm{O}_{4} \mathrm{~S}_{2}$ & $\begin{array}{c}\text { [957763-47-2] } \\
\text { FUS }\end{array}$ & 2-butylisoquinc & $\begin{array}{c}\text { Im bis(triflu } \\
46.1\end{array}$ & $\begin{array}{c}\text { hylsulfo } \\
321.0\end{array}$ & $\begin{array}{l}\text { nide } \\
\text { DSC }\end{array}$ & [2011DOM/ZAW3] \\
\hline $\mathrm{C}_{15} \mathrm{H}_{18} \mathrm{~F}_{12} \mathrm{~N}_{6} \mathrm{O}_{8} \mathrm{~S}_{4}$ & $\begin{array}{c}{[844468-61-7]} \\
\text { V } \\
\text { V }\end{array}$ & 3,3'-(1,3-propa & $\begin{array}{c}\text { yl)bis[1-met } \\
158 \\
190\end{array}$ & $\begin{array}{l}\text { dazoliun } \\
545 \\
298\end{array}$ & $\begin{array}{l}\text { (trifluoro } \\
\text { MS } \\
\text { MS }\end{array}$ & $\begin{array}{l}\text { hylsulfonyl)imide] } \\
\text { [2009DEY/LOV] } \\
\text { [2009DEY/LOV] }\end{array}$ \\
\hline $\mathrm{C}_{15} \mathrm{H}_{19} \mathrm{NO}_{3} \mathrm{~S}$ & $\begin{array}{c}\text { [59229-09-3] } \\
\text { FUS }\end{array}$ & 2,4,6-trimethyl & $\begin{array}{l}\text { linium tosyl } \\
22.88\end{array}$ & 403.7 & DSC & [2009DOM/KRO] \\
\hline $\mathrm{C}_{15} \mathrm{H}_{20} \mathrm{~F}_{18} \mathrm{NP}$ & $\begin{array}{c}{[851856-47-8]} \\
\mathrm{V} \\
\mathrm{V}\end{array}$ & 1-butyl-1-meth & $\begin{array}{c}\text { rrolidinium } \\
138 \\
152\end{array}$ & $\begin{array}{l}\text { tafluoro } \\
450 \\
298\end{array}$ & $\begin{array}{l}\text { trifluorop } \\
\text { MS } \\
\text { MS }\end{array}$ & $\begin{array}{l}\text { phate } \\
\text { [2009DEY/LOV] } \\
\text { [2009DEY/LOV] }\end{array}$ \\
\hline $\mathrm{C}_{15} \mathrm{H}_{21} \mathrm{~F}_{6} \mathrm{~N}_{2} \mathrm{O}_{3} \mathrm{P}$ & FUS & $N$-(4-nitrobenz & $\begin{array}{c}\text { opine hexafl } \\
25.8\end{array}$ & $\begin{array}{c}\text { osphate } \\
518.3\end{array}$ & DSC & [2015LU/SON] \\
\hline $\mathrm{C}_{15} \mathrm{H}_{22} \mathrm{~F}_{6} \mathrm{NOP}$ & FUS & $N$-benzyltropin & $\begin{array}{l}\text { xafluoropho } \\
22.5\end{array}$ & 427.0 & DSC & [2015LU/SON] \\
\hline $\mathrm{C}_{15} \mathrm{H}_{22} \mathrm{~F}_{6} \mathrm{NPS}$ & $\begin{array}{c}{[1667725-23-6]} \\
\text { FUS }\end{array}$ & $N$-octylbenzoth & $\begin{array}{c}\text { lium hexaflu } \\
15.0\end{array}$ & $\begin{array}{l}\text { osphate } \\
334.2\end{array}$ & DSC & [2015JIA/CAO] \\
\hline $\mathrm{C}_{15} \mathrm{H}_{22} \mathrm{~N}_{2} \mathrm{O}_{3} \mathrm{~S}$ & $\begin{array}{c}\text { [410522-18-8] } \\
\text { FUS } \\
\text { TRS } \\
\text { FUS } \\
\text { FUS } \\
\text { FUS }\end{array}$ & $\begin{array}{l}\text { 1-butyl-3-meth } \\
\begin{array}{c}(5-370) \\
(5-370) \\
(5-370) \\
(315-470)\end{array}\end{array}$ & $\begin{array}{c}\text { idazolium to } \\
16.35 \\
0.19 \\
19.9 \\
21.6 \\
22.8\end{array}$ & $\begin{array}{c}330.2 \\
152 \\
342.5 \\
343.9\end{array}$ & $\begin{array}{c}\text { AC } \\
\text { DSC }\end{array}$ & $\begin{array}{l}\text { [2007STR/PAU] } \\
\text { [2007STR/PAU] }\end{array}$ \\
\hline $\mathrm{C}_{15} \mathrm{H}_{25} \mathrm{~F}_{6} \mathrm{~N}_{2} \mathrm{O}_{4} \mathrm{~S}_{2}$ & $\begin{array}{c}{[1138216-85-9]} \\
\text { V } \\
\text { V } \\
\text { V }\end{array}$ & $\begin{array}{c}\text { 1,3-dipentylim } \\
(463-496) \\
(463-496) \\
(463-496)\end{array}$ & $\begin{array}{l}\text { lium bis(tri } \\
123.6 \pm 0.2 \\
126.5 \pm 0.2 \\
150.6 \pm 0.9\end{array}$ & $\begin{array}{l}\text { nethylsu } \\
480 \\
460 \\
298\end{array}$ & $\begin{array}{r}\text { imide } \\
\mathrm{ME} \\
\mathrm{ME} \\
\mathrm{ME}\end{array}$ & $\begin{array}{l}{[2012 \mathrm{ROC} / \mathrm{COU}]} \\
{[2012 \mathrm{ROC} / \mathrm{COU}]} \\
{[2012 \mathrm{ROC} / \mathrm{COU}]}\end{array}$ \\
\hline $\mathrm{C}_{15} \mathrm{H}_{26} \mathrm{BrN}$ & $\begin{array}{c}{[120375-48-6]} \\
\text { TRS }\end{array}$ & 9-phenylnonyla & $\begin{array}{c}\text { onium brom } \\
8.93\end{array}$ & 309 & DSC & [1996DOM/HEA, 1989VAN/WHI] \\
\hline $\mathrm{C}_{15} \mathrm{H}_{26} \mathrm{ClN}$ & $\begin{array}{c}{[120375-55-5]} \\
\text { TRS } \\
\text { TRS }\end{array}$ & 9-phenylnonyla & $\begin{array}{c}\text { onium chlor } \\
10.0 \\
7.59\end{array}$ & $\begin{array}{l}320 \\
331\end{array}$ & DSC & [1996DOM/HEA, 1989VAN/WHI] \\
\hline $\mathrm{C}_{15} \mathrm{H}_{28} \mathrm{~F}_{6} \mathrm{~N}_{2} \mathrm{O}_{4} \mathrm{~S}_{2}$ & $\begin{array}{c}{[927021-43-0]} \\
\mathrm{V} \\
\mathrm{V}\end{array}$ & 1-octyl-1-meth & $\begin{array}{c}\text { rrolidinium } \\
145 \\
161\end{array}$ & $\begin{array}{l}\text { uorome } \\
\qquad \begin{array}{l}470 \\
298\end{array}\end{array}$ & $\begin{array}{l}\text { lfonyl)im } \\
\text { MS } \\
\text { MS }\end{array}$ & $\begin{array}{l}\text { [2009DEY/LOV] } \\
\text { [2009DEY/LOV] }\end{array}$ \\
\hline $\mathrm{C}_{15} \mathrm{H}_{32} \mathrm{~F}_{12} \mathrm{~N}_{2} \mathrm{P}_{2}$ & $\begin{array}{c}\text { [1669342-29-3] } \\
\text { FUS }\end{array}$ & 1,1'-(1,3-propa & $\begin{array}{l}\text { 1)-bis(1-me } \\
8.58\end{array}$ & $\begin{array}{l}\text { erdiniur } \\
410.2\end{array}$ & $\begin{array}{l}\text { exafluoro } \\
\text { DSC }\end{array}$ & $\begin{array}{l}\text { sphate } \\
\text { [2014HAD/VIL] }\end{array}$ \\
\hline
\end{tabular}


TABle 18. Phase change enthalpies of organic salts and ionic liquids-Continued

\begin{tabular}{|c|c|c|c|c|c|c|}
\hline \multirow[b]{2}{*}{$\begin{array}{l}\text { Molecular } \\
\text { formula }\end{array}$} & \multirow{2}{*}{$\begin{array}{l}\text { CAS Registry } \\
\text { Number } \\
\text { Enthalpy }\end{array}$} & \multicolumn{5}{|l|}{ Compound } \\
\hline & & $\begin{array}{c}\text { Temperature } \\
\text { range }\end{array}$ & $\begin{array}{l}\Delta_{\text {trans }} H_{\mathrm{m}} \\
(\mathrm{kJ} / \mathrm{mol})\end{array}$ & $\begin{array}{l}T_{\mathrm{m}} \\
(\mathrm{K})\end{array}$ & Method & Reference \\
\hline \multirow{2}{*}{$\mathrm{C}_{15} \mathrm{H}_{34} \mathrm{BrN}$} & [1119-94-4] & \multicolumn{5}{|c|}{ Dodecyl(trimethyl)ammonium bromide } \\
\hline & TRS & & 42.3 & 370.4 & DSC & [1981IWA/OHN] \\
\hline \multirow[t]{2}{*}{$\mathrm{C}_{15} \mathrm{H}_{34} \mathrm{C} 1 \mathrm{~N}$} & {$[112-00-5]$} & \multicolumn{5}{|c|}{ Dodecyl(trimethyl)ammonium chloride } \\
\hline & TRS & & 29.6 & 357.2 & DSC & [1981IWA/OHN] \\
\hline \multirow[t]{3}{*}{$\mathrm{C}_{16} \mathrm{H}_{19} \mathrm{~F}_{18} \mathrm{~N}_{2} \mathrm{P}$} & [713512-19-7] & \multicolumn{5}{|c|}{ 1-methyl-3-hexylimidazolium tris(pentafluoroethyl)trifluorophospate } \\
\hline & $\mathrm{V}$ & & $131 \pm 2$ & 430 & Mass Spec & [2012DEY/HES] \\
\hline & V & & $143 \pm 2$ & 298 & Mass Spec & [2012DEY/HES] \\
\hline \multirow[t]{2}{*}{$\mathrm{C}_{16} \mathrm{H}_{20} \mathrm{~F}_{6} \mathrm{NP}$} & {$[12260-70-7]$} & \multicolumn{5}{|c|}{ Dibenzyl(dimethyl)ammonium hexafluorophosphate } \\
\hline & FUS & & 37.6 & 491.0 & DSC & [2008BUS/LAH] \\
\hline \multirow[t]{2}{*}{$\mathrm{C}_{16} \mathrm{H}_{21} \mathrm{~F}_{6} \mathrm{~N}_{3} \mathrm{O}_{4} \mathrm{~S}_{2}$} & {$[1447958-52-2]$} & \multicolumn{5}{|c|}{ 1-octyl-3-cyanopyridinium bis(trifluoromethylsulfonyl)imide } \\
\hline & FUS & & 17.71 & 287.9 & DSC & [2013DOM/SKI] \\
\hline \multirow[t]{2}{*}{$\mathrm{C}_{16} \mathrm{H}_{24} \mathrm{~F}_{6} \mathrm{NO}_{2} \mathrm{P}$} & & \multicolumn{5}{|c|}{$N$-(3-methoxybenzyl)tropine hexafluorophosphate } \\
\hline & FUS & & 29.8 & 439.3 & DSC & [2015LU/SON] \\
\hline \multirow[t]{9}{*}{$\mathrm{C}_{16} \mathrm{H}_{27} \mathrm{~F}_{6} \mathrm{~N}_{3} \mathrm{O}_{4} \mathrm{~S}_{2}$} & {$[433337-23-6]$} & \multicolumn{5}{|c|}{ 1-methyl-3-decylimidazolium bis(trifluoromethylsulfonyl)imide } \\
\hline & TRS & $(5-370)$ & 2.51 & 249 & & \\
\hline & FUS & $(5-370)$ & 28.67 & 277.3 & $\mathrm{AC}$ & [2016PAU/BLO] \\
\hline & $\mathrm{V}$ & $(380-410)$ & $142.5 \pm 1.0$ & 395 & QCM & [2013VER/ZAI] \\
\hline & $\mathrm{V}$ & $(380-410)$ & $152.1 \pm 1.0$ & 298 & QCM & [2013VER/ZAI] \\
\hline & $\mathrm{V}$ & $(493-552)$ & $125.2 \pm 1.0$ & 522 & TGA & [2013VER/ZAI] \\
\hline & $\mathrm{V}$ & $(493-552)$ & $147.5 \pm 1.0$ & 298 & TGA & [2013VER/ZAI] \\
\hline & $\mathrm{V}$ & $(380-410)$ & $142.5 \pm 2.5$ & 394 & QCM & [2011ZAI/VER] \\
\hline & $\mathrm{V}$ & $(380-410)$ & 152.1 & 298 & QCM & [2011ZAI/VER] \\
\hline \multirow{3}{*}{$\mathrm{C}_{16} \mathrm{H}_{28} \mathrm{BrN}$} & [120396-92-1] & \multicolumn{5}{|c|}{ 10-phenyldecylammonium bromide } \\
\hline & TRS & & 14.2 & 323 & & \\
\hline & TRS & & 16.0 & 369 & DSC & [1996DOM/HEA, 1989VAN/WHI] \\
\hline $\mathrm{C}_{16} \mathrm{H}_{28} \mathrm{ClN}$ & {$[120375-54-4]$} & 10-phenyldecy & nonium chlor & & & \\
\hline & TRS & & 17.7 & 357 & & \\
\hline & TRS & & 7.2 & 368 & DSC & [1996DOM/HEA, 1989VAN/WHI] \\
\hline $\mathrm{C}_{16} \mathrm{H}_{28} \mathrm{ClN}$ & {$[29529-26-8]$} & 1-decyl-3-metl & ridinium chlc & & & \\
\hline & FUS & & 14 & 352.3 & DSC & [2011PER/ROD] \\
\hline $\mathrm{C}_{16} \mathrm{H}_{30} \mathrm{Br}_{2} \mathrm{~N}_{2}$ & {$[51523-43-4]$} & 1,2-bis(methyl & ylammonium & e dibron & & \\
\hline & TRS & & 3.01 & 371 & DSC & [1996DOM/HEA, 1974BUR/VER] \\
\hline $\mathrm{C}_{16} \mathrm{H}_{31} \mathrm{~F}_{6} \mathrm{~N}_{2} \mathrm{P}$ & {$[219947-93-0]$} & 1-dodecyl-3-m & limidazolium & uoroph & & \\
\hline & FUS & & 27.2 & 326.2 & DSC & {$[2015 \mathrm{NEM} / \mathrm{KOF}]$} \\
\hline & FUS & & 25.88 & 326.3 & DSC & [2014MAX/SAN] \\
\hline $\mathrm{C}_{16} \mathrm{H}_{32} \mathrm{~N}_{2} \mathrm{O}_{4} \mathrm{~S}$ & {$[445473-58-5]$} & 1-methyl-3-me & midazolium & lfate & & \\
\hline & $\mathrm{V}$ & & $161 \pm 2$ & 510 & Mass Spec & [2012DEY/HES] \\
\hline & $\mathrm{V}$ & & $181 \pm 2$ & 298 & Mass Spec & [2012DEY/HES] \\
\hline $\mathrm{C}_{16} \mathrm{H}_{34} \mathrm{~F}_{12} \mathrm{~N}_{2} \mathrm{P}_{2}$ & {$[741188-72-7]$} & $1,1^{\prime}-(1,4-$ butan & 1)-bis(1-methy & cidinium & exafluorophc & hate \\
\hline & FUS & & 6.10 & 376.5 & DSC & [2014HAD/VIL] \\
\hline $\mathrm{C}_{16} \mathrm{H}_{36} \mathrm{BF}_{4} \mathrm{~N}$ & [429-42-5] & Tetrabutylamm & Im tetrafluoro & & & \\
\hline & TRS & & 7.9 & 335 & & \\
\hline & FUS & & 12.1 & 429 & DSC & [1987ZAB/FER] \\
\hline & TRS & & 6.7 & 341 & & \\
\hline & FUS & & 10.5 & 439 & DSC & [1970COK/AMB] \\
\hline $\mathrm{C}_{16} \mathrm{H}_{36} \mathrm{BrN}$ & [24447-58-3] & Dioctylammon & bromide & & & \\
\hline & TRS & & 25 & 303 & DSC & [2005STE/VOI] \\
\hline $\mathrm{C}_{16} \mathrm{H}_{36} \mathrm{BrN}$ & {$[1643-19-2]$} & Tetrabutylamm & Im bromide & & & \\
\hline & TRS & & 15.1 & 367.0 & & \\
\hline & TRS & & 0.38 & 379.2 & & \\
\hline & FUS & & 14.8 & 393.9 & DSC & [1992XEN/CHE] \\
\hline & TRS & & 0.07 & 293 & & \\
\hline
\end{tabular}


TABLE 18. Phase change enthalpies of organic salts and ionic liquids-Continued

\begin{tabular}{|c|c|c|c|c|c|c|}
\hline \multirow[b]{2}{*}{$\begin{array}{l}\text { Molecular } \\
\text { formula }\end{array}$} & \multirow{2}{*}{$\begin{array}{l}\text { CAS Registry } \\
\text { Number } \\
\text { Enthalpy }\end{array}$} & \multicolumn{5}{|l|}{ Compound } \\
\hline & & $\begin{array}{c}\text { Temperature } \\
\text { range }\end{array}$ & $\begin{array}{l}\Delta_{\text {trans }} H_{\mathrm{m}} \\
(\mathrm{kJ} / \mathrm{mol})\end{array}$ & $\begin{array}{l}T_{\mathrm{m}} \\
(\mathrm{K})\end{array}$ & Method & Reference \\
\hline & TRS & & 1.63 & 367 & & \\
\hline & TRS & & 0.34 & 383 & & [1996DOM/HEA, 1974BUR/VER] \\
\hline & FUS & & 16.15 & 395 & DSC & \\
\hline & TRS & & 14.2 & 372 & & \\
\hline & FUS & & 15.5 & 395 & DSC & {$[1970 \mathrm{COK} / \mathrm{AMB}]$} \\
\hline \multirow[t]{2}{*}{$\mathrm{C}_{16} \mathrm{H}_{36} \mathrm{C} 1 \mathrm{~N}$} & [1112-67-0] & Tetrabutylamm & chloride & & & \\
\hline & FUS & & 20.5 & 314 & DSC & {$[1970 \mathrm{COK} / \mathrm{AMB}]$} \\
\hline \multirow[t]{3}{*}{$\mathrm{C}_{16} \mathrm{H}_{36} \mathrm{C} 1 \mathrm{~N}$} & [2296-14-2] & Dioctylammon & hloride & & & \\
\hline & TRS & & 37 & 294 & DSC & [2005STE/VOI] \\
\hline & TRS & $(25-350)$ & 33.61 & 297.7 & $\mathrm{AC}$ & [1996DOM/HEA, 1988VAN/WHI] \\
\hline \multirow[t]{2}{*}{$\mathrm{C}_{16} \mathrm{H}_{36} \mathrm{C} 1 \mathrm{NO}_{4}$} & {$[10081-31-9]$} & Dioctylammon & erchlorate & & & \\
\hline & TRS & & 37 & 296 & DSC & [2005STE/VOI] \\
\hline \multirow[t]{3}{*}{$\mathrm{C}_{16} \mathrm{H}_{36} \mathrm{C} 1 \mathrm{NO}_{4}$} & {$[1923-70-2]$} & Tetrabutylamm & perchlor & & & \\
\hline & TRS & & 3.10 & 329.2 & & \\
\hline & FUS & & 12.3 & 487.7 & DSC & [1989NAK/KUW] \\
\hline \multirow[t]{6}{*}{$\mathrm{C}_{16} \mathrm{H}_{36} \mathrm{~F}_{6} \mathrm{NP}$} & [3109-63-5] & Tetrabutylamm & hexafluo & hate & & \\
\hline & TRS & & 1.78 & 300.4 & & \\
\hline & TRS & & 5.80 & 356.1 & & \\
\hline & FUS & & 13.02 & 520.9 & DSC & [2014MAX/SAN] \\
\hline & TRS & & 10.5 & 366 & & \\
\hline & FUS & & 17.2 & 517 & DSC & {$[1970 \mathrm{COK} / \mathrm{AMB}]$} \\
\hline \multirow[t]{3}{*}{$\mathrm{C}_{16} \mathrm{H}_{36} \mathrm{~F}_{6} \mathrm{P}_{2}$} & [111928-21-3] & Tetrabutylphos & um hexaflı & sphate & & \\
\hline & TRS & & 1.84 & 265.0 & & \\
\hline & FUS & & 13.18 & 497.5 & DSC & [2014MAX/SAN] \\
\hline \multirow[t]{2}{*}{$\mathrm{C}_{16} \mathrm{H}_{36} \mathrm{IN}$} & [24447-59-4] & Dioctylammon & dide & & & \\
\hline & TRS & & 21 & 271 & DSC & [2005STE/VOI] \\
\hline \multirow[t]{9}{*}{$\mathrm{C}_{16} \mathrm{H}_{36} \mathrm{IN}$} & [311-28-4] & Tetrabutylamm & iodide & & & \\
\hline & TRS & & 29.4 & 394.0 & & \\
\hline & FUS & & 8.96 & 420.6 & DSC & [1992XEN/CHE] \\
\hline & TRS & & 27.1 & 300.3 & & \\
\hline & FUS & & 9.18 & 419.2 & DSC & [1989NAK/KUW] \\
\hline & TRS & & 28.0 & 392 & & \\
\hline & FUS & & 9.2 & 418 & & [1971LEV/KOH] \\
\hline & TRS & & 28.0 & 392 & & \\
\hline & FUS & & 9.6 & 419 & DSC & [1970COK/AMB] \\
\hline \multirow[t]{7}{*}{$\mathrm{C}_{16} \mathrm{H}_{36} \mathrm{~N}_{2} \mathrm{O}_{3}$} & [1941-27-1] & Tetrabutylamm & nitrate & & & \\
\hline & TRS & & 0.2 & 361.4 & & \\
\hline & FUS & & 14.3 & 391.1 & DSC & [2012VER/EME2] \\
\hline & TRS & & 0.21 & 362.3 & & \\
\hline & FUS & & 15.6 & 389.3 & DSC & [1989NAK/KUW] \\
\hline & TRS & & 0.2 & 366 & & \\
\hline & FUS & & 14.6 & 392 & DSC & [1970COK/AMB] \\
\hline \multirow[t]{2}{*}{$\mathrm{C}_{16} \mathrm{H}_{36} \mathrm{~N}_{2} \mathrm{O}_{3}$} & [10081-30-8] & Dioctylammon & itrate & & & \\
\hline & TRS & & 53.5 & 318 & DSC & [2005STE/VOI] \\
\hline \multirow[t]{2}{*}{$\mathrm{C}_{16} \mathrm{H}_{38} \mathrm{Br}_{2} \mathrm{~N}_{2}$} & [1067-62-5] & 1,4-bis(triethyl & nium)buta & omide & & \\
\hline & TRS & & 50.2 & 518 & DSC & [1996DOM/HEA, 1974BUR/VER] \\
\hline \multirow[t]{2}{*}{$\mathrm{C}_{17} \mathrm{H}_{20} \mathrm{~F}_{6} \mathrm{~N}_{2} \mathrm{O}_{4} \mathrm{~S}_{2}$} & [1263302-30-2] & 1-hexylquinoli & ois(trifluor & lsulfony & & \\
\hline & FUS & & 63.54 & 317.2 & DSC & [2011DOM/ZAW2] \\
\hline \multirow[t]{3}{*}{$\mathrm{C}_{17} \mathrm{H}_{20} \mathrm{~F}_{6} \mathrm{~N}_{2} \mathrm{O}_{4} \mathrm{~S}_{2}$} & {$[957763-49-4]$} & 2-hexylisoquin & $m$ bis(trifl & hylsulf & mide & \\
\hline & TRS & & 2.45 & 193.8 & & \\
\hline & FUS & & 58.64 & 327.2 & DSC & [2011DOM/ZAW] \\
\hline \multirow[t]{2}{*}{$\mathrm{C}_{17} \mathrm{H}_{23} \mathrm{NO}_{3} \mathrm{~S}$} & {$[1256080-48-4]$} & 1-butyl-3-meth & dinium to & & & \\
\hline & FUS & & 11.34 & 315.8 & DSC & [2010LET/RAM] \\
\hline
\end{tabular}


TABLE 18. Phase change enthalpies of organic salts and ionic liquids-Continued

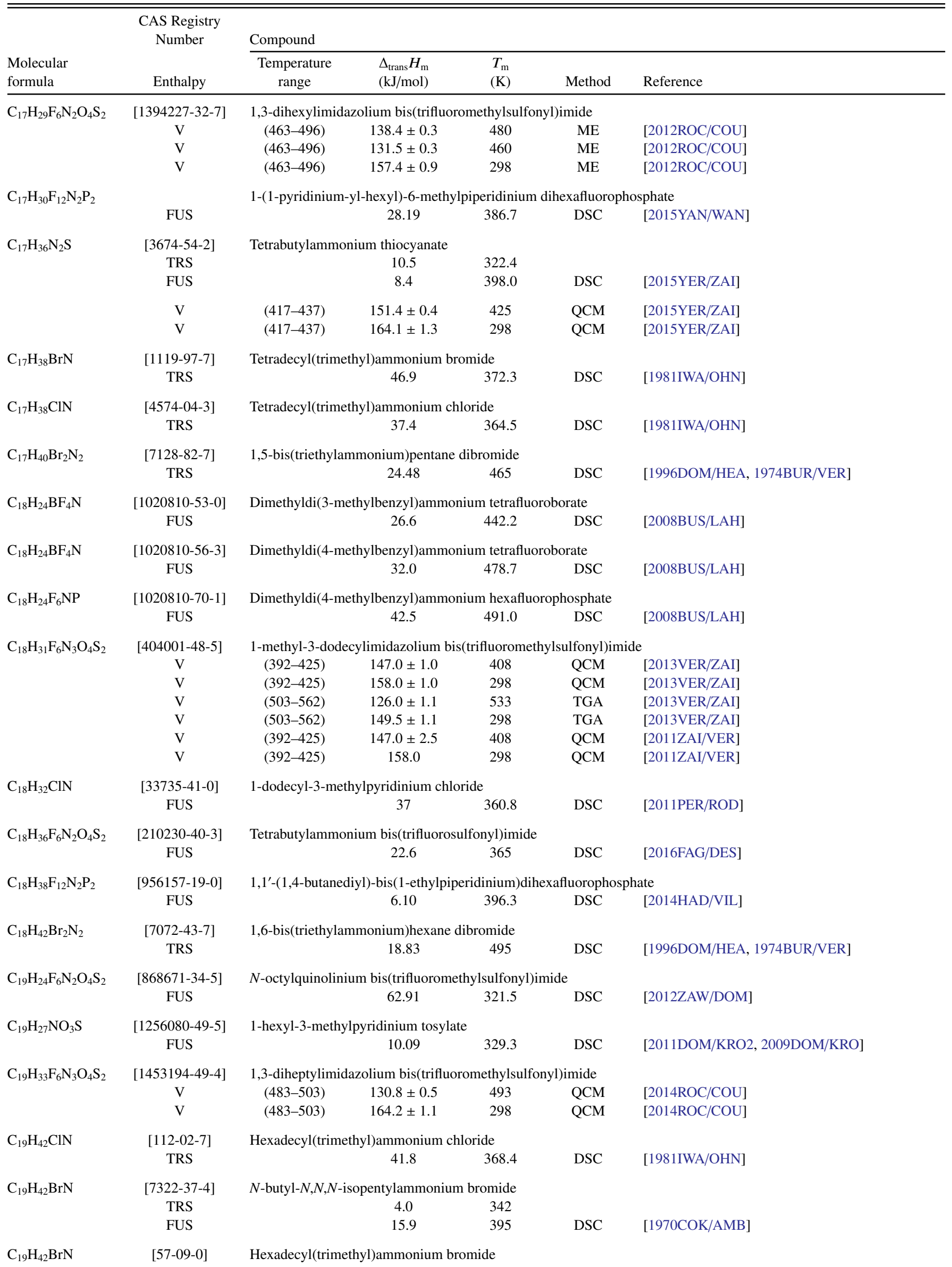


TABLE 18. Phase change enthalpies of organic salts and ionic liquids-Continued

\begin{tabular}{|c|c|c|c|c|c|c|}
\hline \multirow[b]{2}{*}{$\begin{array}{l}\text { Molecular } \\
\text { formula }\end{array}$} & \multirow{2}{*}{$\begin{array}{l}\text { CAS Registry } \\
\text { Number } \\
\text { Enthalpy }\end{array}$} & \multicolumn{5}{|l|}{ Compound } \\
\hline & & $\begin{array}{l}\text { Temperature } \\
\text { range }\end{array}$ & $\begin{array}{l}\Delta_{\text {trans }} H_{\mathrm{m}} \\
(\mathrm{kJ} / \mathrm{mol})\end{array}$ & $\begin{array}{l}T_{\mathrm{m}} \\
(\mathrm{K})\end{array}$ & Method & Reference \\
\hline & TRS & & 51.5 & 376.1 & DSC & [1981IWA/OHN] \\
\hline $\mathrm{C}_{20} \mathrm{H}_{35} \mathrm{~F}_{6} \mathrm{~N}_{3} \mathrm{O}_{4} \mathrm{~S}_{2}$ & $\begin{array}{c}\text { [404001-49-6] } \\
\text { TRS } \\
\text { FUS } \\
\text { V } \\
\text { V } \\
\text { V } \\
\text { V } \\
\text { V } \\
\text { V } \\
\text { V }\end{array}$ & $\begin{array}{c}\text { 1-methyl-3-tet } \\
(5-370) \\
(5-370) \\
(498-528) \\
(400-432) \\
(400-432) \\
(513-571) \\
(513-571) \\
(400-432) \\
(400-432)\end{array}$ & $\begin{array}{c}\text { cylimidazoliuı } \\
4.25 \\
45.8 \\
140.6 \\
152.5 \pm 1.0 \\
164.3 \pm 1.0 \\
132.7 \pm 1.0 \\
157.1 \pm 1.0 \\
152.5 \pm 2.5 \\
164.3\end{array}$ & $\begin{array}{c}\text { trifluorc } \\
295.5 \\
308.8 \\
513 \\
416 \\
298 \\
542 \\
298 \\
416 \\
298\end{array}$ & $\begin{array}{c}\text { ylsulfonyl } \\
\text { AC } \\
\text { ME } \\
\text { QCM } \\
\text { QCM } \\
\text { TGA } \\
\text { TGA } \\
\text { QCM } \\
\text { QCM }\end{array}$ & $\begin{array}{l}\text { ide } \\
\text { [2016PAU/BLO] } \\
\text { [2016PAU/BLO] } \\
\text { [2013VER/ZAI] } \\
\text { [2013VER/ZAI] } \\
\text { [2013VER/ZAI] } \\
\text { [2013VER/ZAI] } \\
\text { [2011ZAI/VER] } \\
\text { [2011ZAI/VER] }\end{array}$ \\
\hline $\mathrm{C}_{20} \mathrm{H}_{36} \mathrm{ClN}$ & $\begin{array}{l}{[102-43-2]} \\
\text { FUS }\end{array}$ & \multicolumn{5}{|c|}{ 1-tetradecyl-3-methylpyridinium chloride } \\
\hline $\mathrm{C}_{20} \mathrm{H}_{44} \mathrm{BrN}$ & $\begin{array}{l}{[24447-61-8]} \\
\text { TRS }\end{array}$ & Didecylammo & $\begin{array}{l}\text { bromide } \\
38\end{array}$ & 330 & DSC & [2005STE/VOI] \\
\hline $\mathrm{C}_{20} \mathrm{H}_{44} \mathrm{BrN}$ & $\begin{array}{c}\text { [866-97-7] } \\
\text { FUS } \\
\text { FUS }\end{array}$ & Tetrapentylam & $\begin{array}{c}\text { ium bromide } \\
36.6 \\
41.4\end{array}$ & $\begin{array}{c}374.0 \\
376\end{array}$ & $\begin{array}{l}\text { DSC } \\
\text { DSC }\end{array}$ & $\begin{array}{l}{[1992 \mathrm{XEN} / \mathrm{CHE}]} \\
{[1970 \mathrm{COK} / \mathrm{AMB}]}\end{array}$ \\
\hline $\mathrm{C}_{20} \mathrm{H}_{44} \mathrm{ClN}$ & $\begin{array}{c}\text { [4965-17-7] } \\
\text { TRS } \\
\text { FUS }\end{array}$ & Tetrapentylam & $\begin{array}{c}\text { ium chloride } \\
2.8 \\
1.3\end{array}$ & $\begin{array}{l}281 \\
295\end{array}$ & $\mathrm{DSC}$ & {$[1970 \mathrm{COK} / \mathrm{AMB}]$} \\
\hline $\mathrm{C}_{20} \mathrm{H}_{44} \mathrm{ClN}$ & $\begin{array}{c}\text { [2486-84-2] } \\
\text { TRS } \\
\text { TRS }\end{array}$ & $\begin{array}{l}\text { Didecylammo } \\
\text { (25-350) }\end{array}$ & $\begin{array}{c}\text { chloride } \\
40 \\
50.59\end{array}$ & $\begin{array}{c}321 \\
321.5\end{array}$ & $\begin{array}{c}\mathrm{DSC} \\
\mathrm{AC}\end{array}$ & $\begin{array}{l}\text { [2005STE/VOI] } \\
\text { [1996DOM/HEA, 1988VAN/WHI] }\end{array}$ \\
\hline [Note: Numerical & lue contains the $\mathrm{e}$ & halpy for the tra & on that occurr & $320 \mathrm{~K}]$. & & \\
\hline $\mathrm{C}_{20} \mathrm{H}_{44} \mathrm{ClNO}_{3}$ & $\begin{array}{l}{[619671-14-6]} \\
\text { TRS }\end{array}$ & Didecylammo & $\begin{array}{c}\text { chlorate } \\
59\end{array}$ & 328.7 & DSC & [2005STE/VOI] \\
\hline $\mathrm{C}_{20} \mathrm{H}_{44} \mathrm{ClNO}_{4}$ & $\begin{array}{c}\text { [4328-09-0] } \\
\text { TRS } \\
\text { FUS } \\
\text { TRS } \\
\text { FUS }\end{array}$ & Tetrapentylam & $\begin{array}{c}\text { ium perchlora } \\
16.3 \\
36.1 \\
36.8 \\
18.0\end{array}$ & $\begin{array}{l}362.1 \\
389.2 \\
364.2 \\
391.2\end{array}$ & $\begin{array}{l}\text { DSC } \\
\text { DSC }\end{array}$ & $\begin{array}{l}\text { [1989NAK/KUW] } \\
\text { [1978GOR/RAO] }\end{array}$ \\
\hline $\mathrm{C}_{20} \mathrm{H}_{44} \mathrm{ClNO}_{4}$ & $\begin{array}{l}{[68963-42-8]} \\
\text { FUS }\end{array}$ & $N$-butyl- $N, N-\mathrm{c}$ & $\begin{array}{c}\text { tyl- } N \text {-hexylan } \\
26.4\end{array}$ & $\begin{array}{l}\text { um perc } \\
355.7\end{array}$ & DSC & [1978GOR/RAO] \\
\hline $\mathrm{C}_{20} \mathrm{H}_{44} \mathrm{ClNO}_{4}$ & $\begin{array}{c}\text { [68963-44-0] } \\
\text { FUS }\end{array}$ & $N, N$-dibutyl- $N$ & $\begin{array}{c}\text { ihexylammon } \\
34.3\end{array}$ & $\begin{array}{l}\text { erchlora } \\
355.2\end{array}$ & DSC & {$[1978 \mathrm{GOR} / \mathrm{RAO}]$} \\
\hline $\mathrm{C}_{20} \mathrm{H}_{44} \mathrm{ClNO}_{4}$ & $\begin{array}{c}\text { [68963-45-1] } \\
\text { FUS }\end{array}$ & $N, N, N$-trihexy & $\begin{array}{l}\text { thylammoniu } \\
18.8\end{array}$ & $\begin{array}{l}\text { chlorate } \\
320.2\end{array}$ & $\mathrm{DSC}$ & {$[1978 \mathrm{GOR} / \mathrm{RAO}]$} \\
\hline $\mathrm{C}_{20} \mathrm{H}_{44} \mathrm{ClNO}_{4}$ & $\begin{array}{c}\text { [68963-47-3] } \\
\text { FUS }\end{array}$ & $N, N, N$-tripropy & $\begin{array}{c}\text { undecylammo } \\
25.1\end{array}$ & $\begin{array}{l}\text { perchlo } \\
335.2\end{array}$ & DSC & {$[1978 \mathrm{GOR} / \mathrm{RAO}]$} \\
\hline $\mathrm{C}_{20} \mathrm{H}_{44} \mathrm{ClNO}_{4}$ & $\begin{array}{c}\text { [68963-48-4] } \\
\text { FUS }\end{array}$ & $N, N$-diethyl- $N$ & $\begin{array}{l}\text { ioctylammoni } \\
50.2\end{array}$ & $\begin{array}{l}\text { rchlorat } \\
333.2\end{array}$ & $\mathrm{DSC}$ & {$[1978 \mathrm{GOR} / \mathrm{RAO}]$} \\
\hline $\mathrm{C}_{20} \mathrm{H}_{44} \mathrm{ClNO}_{4}$ & $\begin{array}{c}\text { [68963-49-5] } \\
\text { TRS } \\
\text { FUS }\end{array}$ & $N, N N$-triethyl $/$ & $\begin{array}{c}\text { radecylammor } \\
12.6 \\
50.2\end{array}$ & $\begin{array}{c}\text { perchlor } \\
338 \\
425.2\end{array}$ & DSC & [1978GOR/RAO] \\
\hline $\mathrm{C}_{20} \mathrm{H}_{44} \mathrm{ClNO}_{4}$ & $\begin{array}{c}\text { [68963-50-8] } \\
\text { TRS } \\
\text { TRS } \\
\text { FUS }\end{array}$ & $N, N$-dimethyl- & $\begin{array}{c}\text { hyl- } N \text {-hexade } \\
5.4 \\
18.4 \\
19.2\end{array}$ & $\begin{array}{l}\text { moniun } \\
352.7 \\
359.2 \\
429.2\end{array}$ & $\begin{array}{l}\text { hlorate } \\
\text { DSC }\end{array}$ & [1978GOR/RAO] \\
\hline $\mathrm{C}_{20} \mathrm{H}_{44} \mathrm{ClNO}_{4}$ & $\begin{array}{c}\text { [2452-81-5] } \\
\text { TRS } \\
\text { FUS }\end{array}$ & Tetraisopentyl & $\begin{array}{l}\text { onium perchlc } \\
6.60 \\
22.4\end{array}$ & $\begin{array}{l}371.2 \\
386.2\end{array}$ & DSC & [1989NAK/KUW] \\
\hline
\end{tabular}


TABLE 18. Phase change enthalpies of organic salts and ionic liquids-Continued

\begin{tabular}{|c|c|c|c|c|c|c|}
\hline \multirow[b]{2}{*}{$\begin{array}{l}\text { Molecular } \\
\text { formula }\end{array}$} & \multirow{2}{*}{$\begin{array}{l}\text { CAS Registry } \\
\text { Number } \\
\text { Enthalpy }\end{array}$} & \multicolumn{5}{|l|}{ Compound } \\
\hline & & $\begin{array}{l}\text { Temperature } \\
\text { range }\end{array}$ & $\begin{array}{l}\Delta_{\text {trans }} H_{\mathrm{m}} \\
(\mathrm{kJ} / \mathrm{mol})\end{array}$ & $\begin{array}{l}T_{\mathrm{m}} \\
(\mathrm{K})\end{array}$ & Method & Reference \\
\hline $\mathrm{C}_{20} \mathrm{H}_{44} \mathrm{ClNO}_{4}$ & $\begin{array}{c}\text { [59785-31-8] } \\
\text { FUS }\end{array}$ & Tributyloctylar & $\begin{array}{l}\text { hium perchlor } \\
28.9\end{array}$ & 338.2 & DSC & [1978GOR/RAO] \\
\hline $\mathrm{C}_{20} \mathrm{H}_{44} \mathrm{IN}$ & $\begin{array}{c}\text { [2498-20-6] } \\
\text { TRS } \\
\text { FUS } \\
\text { TRS } \\
\text { FUS } \\
\text { TRS } \\
\text { FUS } \\
\text { TRS } \\
\text { FUS }\end{array}$ & Tetrapentylamı & $\begin{array}{c}\text { um iodide } \\
16.7 \\
38.7 \\
15.2 \\
38.2 \\
13.8 \\
37.7 \\
17.2 \\
39.3\end{array}$ & $\begin{array}{c}404.6 \\
412.1 \\
394.2 \\
407.2 \\
403 \\
410 \\
405 \\
412\end{array}$ & $\begin{array}{l}\text { DSC } \\
\text { DSC }\end{array}$ & $\begin{array}{l}\text { [1992XEN/CHE] } \\
\text { [1989NAK/KUW] } \\
\text { [1971LEV/KOH] } \\
\text { [1970COK/AMB] }\end{array}$ \\
\hline $\mathrm{C}_{20} \mathrm{H}_{44} \mathrm{IN}$ & $\begin{array}{c}\text { [68963-51-9] } \\
\text { FUS }\end{array}$ & $N$-methyl- $N, N$ - & $\begin{array}{c}\text { opyl- } N \text {-tridec } \\
37.7\end{array}$ & $\begin{array}{c}\text { monium } \\
345.2\end{array}$ & DSC & [1978GOR/RAO] \\
\hline $\mathrm{C}_{20} \mathrm{H}_{44} \mathrm{~N}_{2} \mathrm{O}_{3}$ & $\begin{array}{c}\text { [682-02-0] } \\
\text { TRS } \\
\text { FUS } \\
\text { TRS } \\
\text { FUS }\end{array}$ & Tetrapentylamı & $\begin{array}{c}\text { um nitrate } \\
11.5 \\
29.4 \\
12.6 \\
26.5\end{array}$ & $\begin{array}{c}355.7 \\
383.7 \\
366 \\
387\end{array}$ & $\begin{array}{l}\text { DSC } \\
\text { DSC }\end{array}$ & $\begin{array}{l}\text { [1989NAK/KUW] } \\
\text { [1970COK/AMB] }\end{array}$ \\
\hline $\mathrm{C}_{20} \mathrm{H}_{44} \mathrm{~N}_{2} \mathrm{O}_{3}$ & $\begin{array}{c}\text { [22377-07-7] } \\
\text { TRS }\end{array}$ & Didecylammon & $\begin{array}{l}\text { nitrate } \\
\quad 64\end{array}$ & 333 & DSC & [2005STE/VOI] \\
\hline $\mathrm{C}_{21} \mathrm{H}_{44} \mathrm{~N}_{2} \mathrm{~S}$ & $\begin{array}{c}\text { [3475-60-3] } \\
\text { TRS } \\
\text { FUS } \\
\text { V }\end{array}$ & $\begin{array}{c}\text { Tetrapentylam } \\
22.6\end{array}$ & $\begin{array}{c}\text { um thiocyana } \\
315 \\
19.7 \\
\\
159.0\end{array}$ & $\begin{array}{c}322.7 \\
437\end{array}$ & $\begin{array}{l}\text { DSC } \\
\text { QCM }\end{array}$ & $\begin{array}{l}\text { [1970COK/AMB] } \\
\text { [2015YER/ZAI] }\end{array}$ \\
\hline $\mathrm{C}_{21} \mathrm{H}_{42} \mathrm{~F}_{6} \mathrm{~N}_{3} \mathrm{O}_{4} \mathrm{~S}_{2}$ & $\begin{array}{c}\text { [1031250-01-7] } \\
\text { TRS } \\
\text { FUS }\end{array}$ & Hexadecyl(trim & $\begin{array}{l}\text { 1)ammonium } \\
29.4 \\
25.6\end{array}$ & $\begin{array}{l}\text { rifluorost } \\
319 \\
335\end{array}$ & $\begin{array}{l}\text { 1)imide } \\
\text { DSC }\end{array}$ & [2016FAG/DES] \\
\hline $\mathrm{C}_{22} \mathrm{H}_{46} \mathrm{BrN}$ & $\begin{array}{c}{[1120-02-1]} \\
\text { TRS }\end{array}$ & Octadecyl(trim & $\begin{array}{l}\text { ammonium } \\
64.9\end{array}$ & $\begin{array}{l}\text { ide } \\
378.3\end{array}$ & DSC & [1981IWA/OHN] \\
\hline $\mathrm{C}_{22} \mathrm{H}_{46} \mathrm{ClN}$ & $\begin{array}{c}{[112-03-8]} \\
\text { TRS }\end{array}$ & Octadecyl(trim & $\begin{array}{l}\text { ammonium } \\
42.7\end{array}$ & $\begin{array}{l}\text { ide } \\
374.7\end{array}$ & DSC & [1981IWA/OHN] \\
\hline $\mathrm{C}_{22} \mathrm{H}_{46} \mathrm{IN}$ & $\begin{array}{c}\text { [4292-25-5] } \\
\text { TRS }\end{array}$ & Octadecyl(trim & $\begin{array}{l}\text { )ammonium } \\
46.9\end{array}$ & 393.9 & DSC & [1981IWA/OHN] \\
\hline $\mathrm{C}_{22} \mathrm{H}_{39} \mathrm{~F}_{6} \mathrm{~N}_{3} \mathrm{O}_{4} \mathrm{~S}_{2}$ & $\begin{array}{c}\text { [404001-50-9] } \\
\text { TRS } \\
\text { FUS } \\
\text { V } \\
\text { V } \\
\text { V } \\
\text { V } \\
\text { V } \\
\text { V }\end{array}$ & $\begin{array}{c}\text { 1-methyl-3-hex } \\
(5-370) \\
(5-370) \\
(508-533) \\
(405-445) \\
(405-445) \\
(513-571) \\
(513-571) \\
(405-445)\end{array}$ & $\begin{array}{c}\text { ylimidazoliu } \\
5.34 \\
51.28 \\
147.1 \\
156.3 \pm 1.0 \\
169.0 \pm 1.0 \\
136.4 \pm 1.4 \\
160.8 \pm 1.4 \\
156.3 \pm 2.5\end{array}$ & $\begin{array}{c}\text { (trifluoro } \\
305.5 \\
319.25 \\
520 \\
425 \\
298 \\
542 \\
298 \\
425\end{array}$ & $\begin{array}{c}\text { lsulfonyl } \\
\text { AC } \\
\text { ME } \\
\text { QCM } \\
\text { QCM } \\
\text { TGA } \\
\text { TGA } \\
\text { QCM }\end{array}$ & $\begin{array}{l}\text { ide } \\
\text { [2016PAU/BLO] } \\
\text { [2016PAU/BLO] } \\
\text { [2013VER/ZAI] } \\
\text { [2013VER/ZAI] } \\
\text { [2013VER/ZAI] } \\
\text { [2013VER/ZAI] } \\
\text { [2011ZAI/VER] }\end{array}$ \\
\hline
\end{tabular}


TABLE 18. Phase change enthalpies of organic salts and ionic liquids-Continued

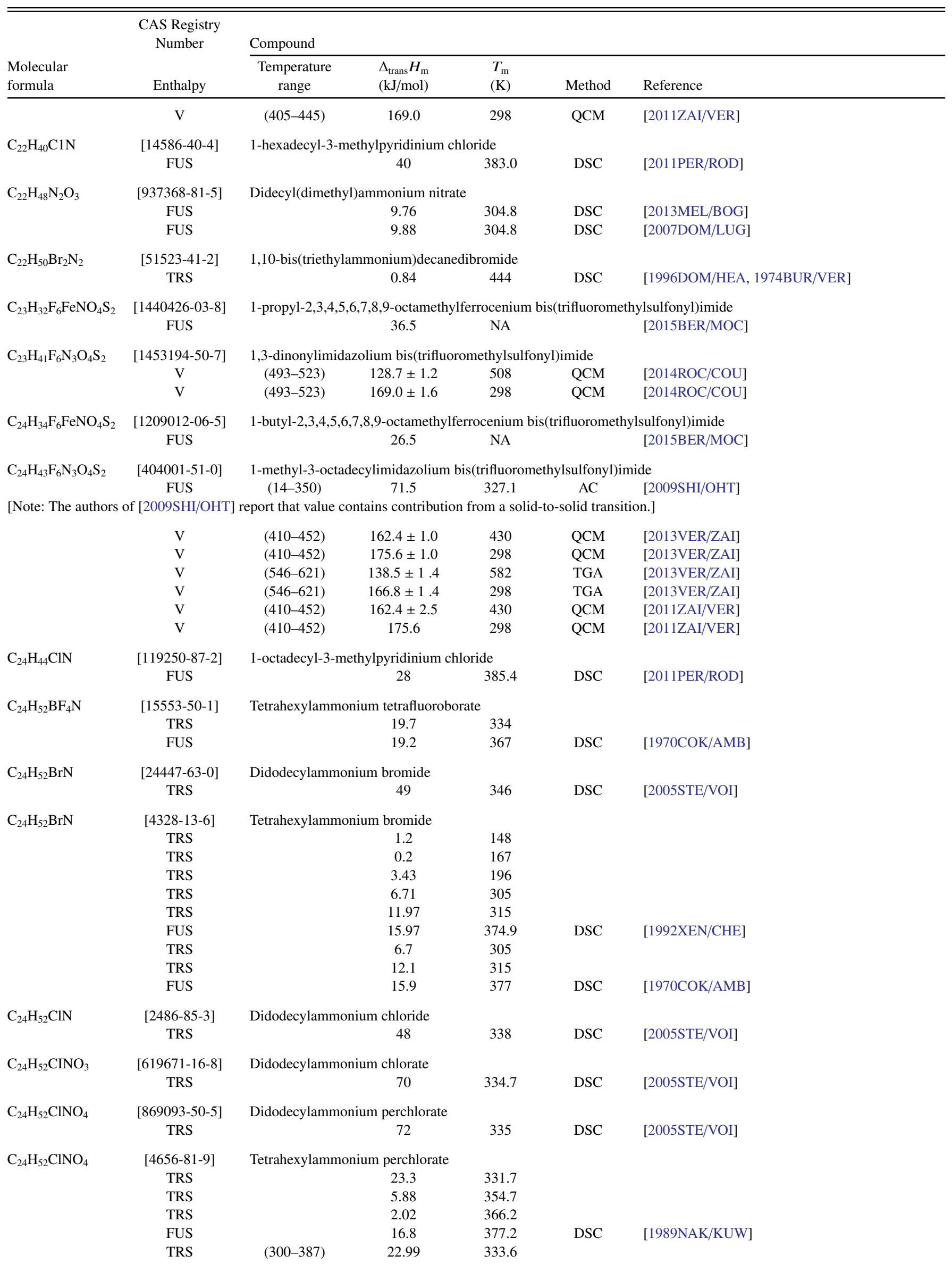


TABLE 18. Phase change enthalpies of organic salts and ionic liquids-Continued

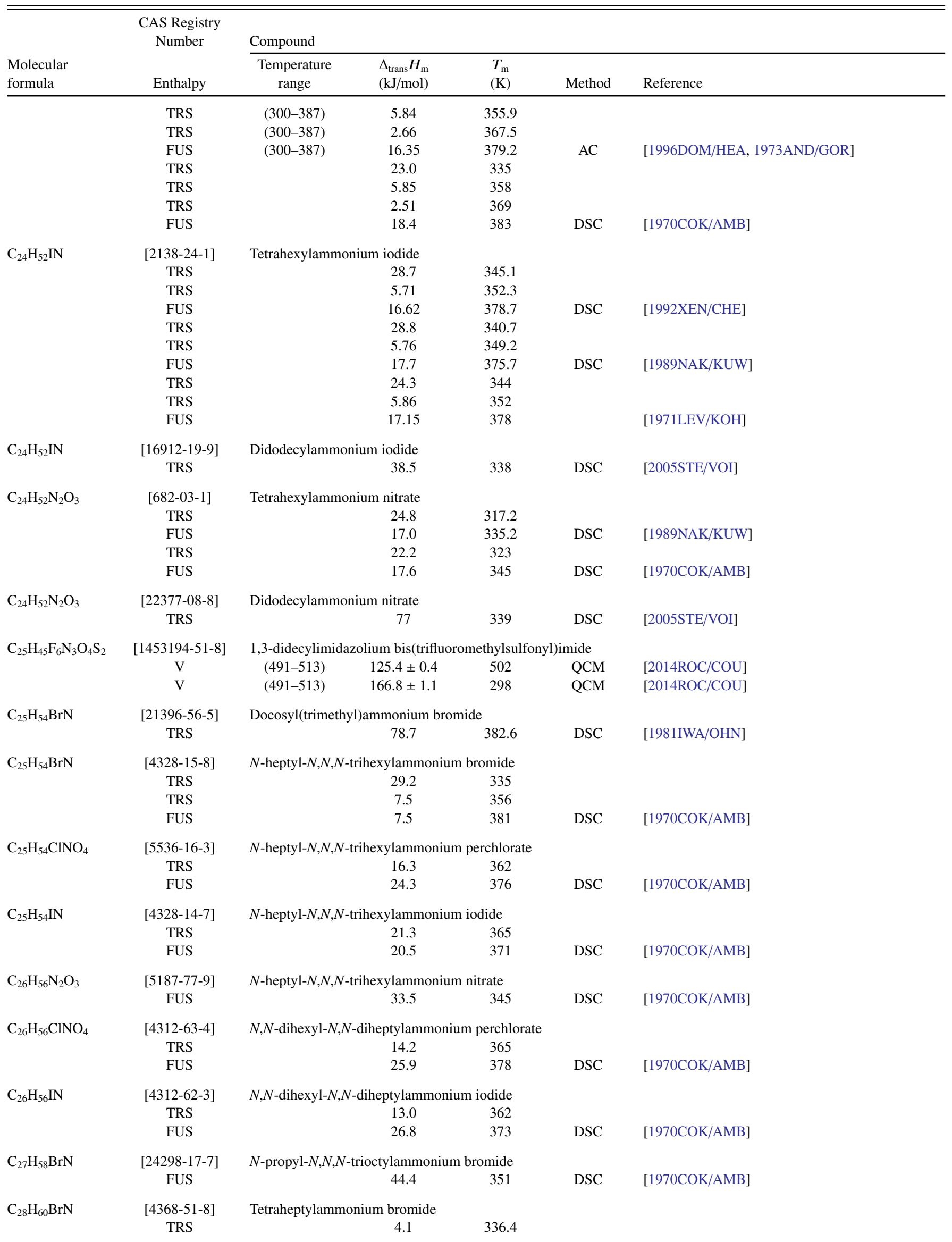


TABLE 18. Phase change enthalpies of organic salts and ionic liquids-Continued

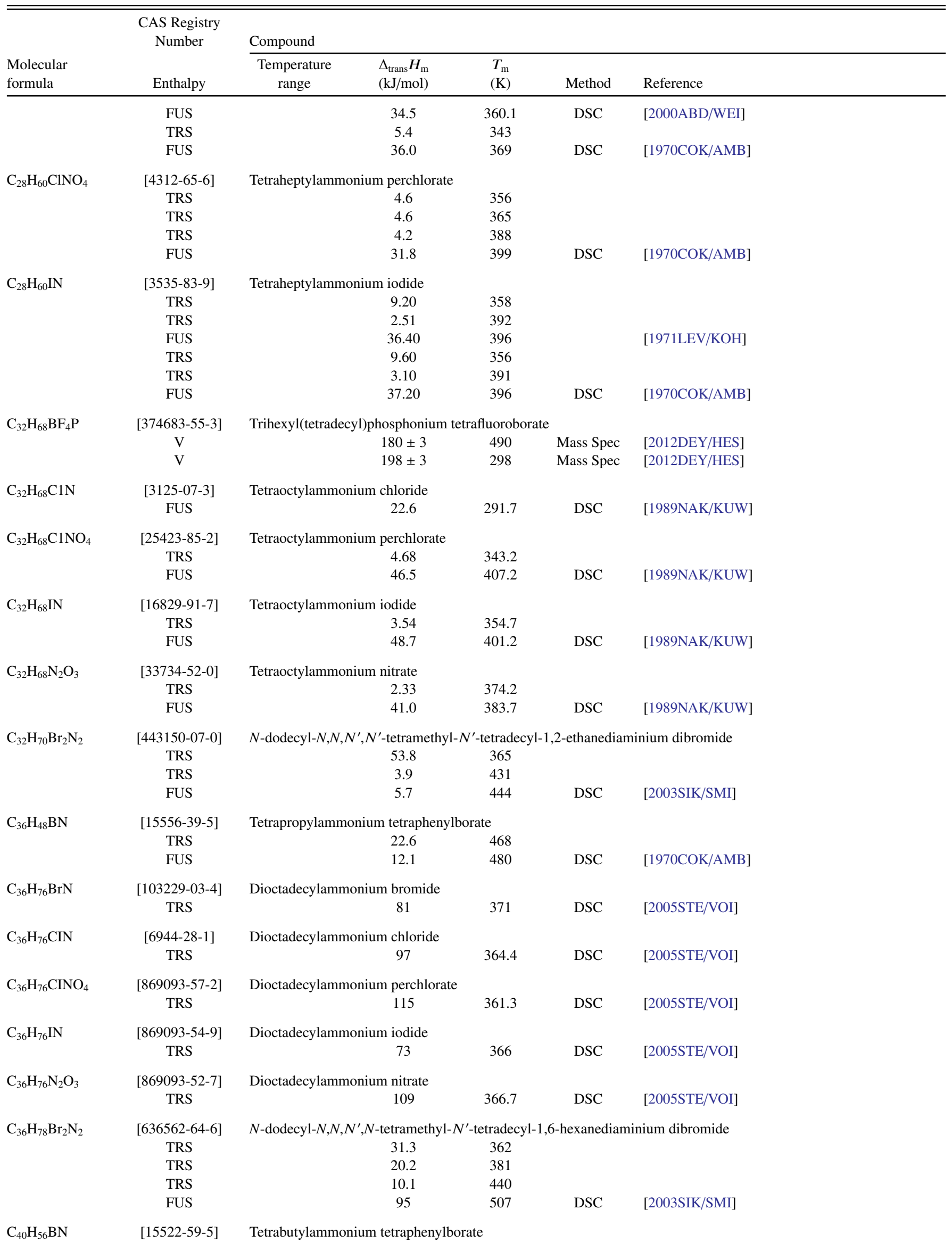


TABLE 18. Phase change enthalpies of organic salts and ionic liquids-Continued

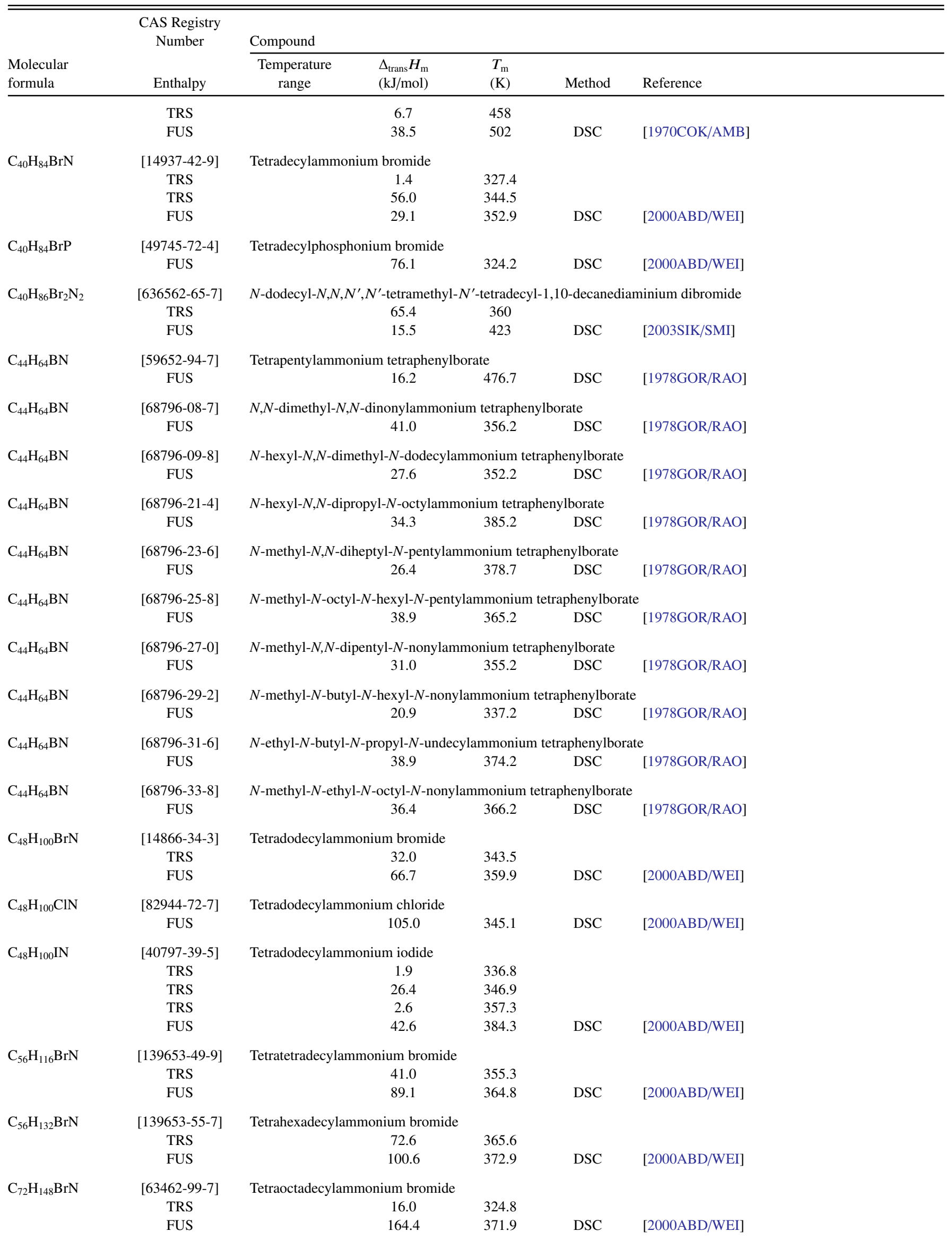


TABLE 18. Phase change enthalpies of organic salts and ionic liquids-Continued

\begin{tabular}{|c|c|c|c|c|c|c|}
\hline \multirow[b]{2}{*}{$\begin{array}{l}\text { Molecular } \\
\text { formula }\end{array}$} & \multirow{2}{*}{$\begin{array}{l}\text { CAS Registry } \\
\text { Number } \\
\text { Enthalpy }\end{array}$} & \multicolumn{5}{|l|}{ Compound } \\
\hline & & $\begin{array}{l}\text { Temperature } \\
\text { range }\end{array}$ & $\begin{array}{l}\Delta_{\text {trans }} H_{\mathrm{m}} \\
(\mathrm{kJ} / \mathrm{mol})\end{array}$ & $\begin{array}{l}T_{\mathrm{m}} \\
(\mathrm{K})\end{array}$ & Method & Reference \\
\hline $\mathrm{C}_{72} \mathrm{H}_{148} \mathrm{BrP}$ & $\begin{array}{c}\text { [58237-06-2] } \\
\text { FUS }\end{array}$ & Tetraoctadecyl & $\begin{array}{c}\text { honium b } \\
201.6\end{array}$ & 368.0 & DSC & [2000ABD/WEI] \\
\hline $\mathrm{C}_{72} \mathrm{H}_{148} \mathrm{ClNO}_{4}$ & $\begin{array}{c}\text { [139653-59-1] } \\
\text { TRS } \\
\text { TRS } \\
\text { TRS } \\
\text { FUS }\end{array}$ & Tetraoctadecyla & $\begin{array}{c}\text { nium perc } \\
0.4 \\
1.7 \\
84.8 \\
8.2\end{array}$ & $\begin{array}{l}340.6 \\
349.7 \\
378.4 \\
379.2\end{array}$ & DSC & [2000ABD/WEI] \\
\hline $\mathrm{C}_{72} \mathrm{H}_{148} \mathrm{ClO}_{4} \mathrm{P}$ & $\begin{array}{c}\text { [258888-10-7] } \\
\text { TRS } \\
\text { TRS } \\
\text { FUS }\end{array}$ & Tetraoctadecylp & $\begin{array}{c}\text { honium pe } \\
1.3 \\
1.6 \\
119.6\end{array}$ & $\begin{array}{l}330.3 \\
337.7 \\
364.6\end{array}$ & DSC & [2000ABD/WEI] \\
\hline $\mathrm{C}_{72} \mathrm{H}_{148} \mathrm{IN}$ & $\begin{array}{c}\text { [183552-31-0] } \\
\text { TRS } \\
\text { FUS }\end{array}$ & Tetraoctadecyla & $\begin{array}{c}\text { nium iodi } \\
11.7 \\
115.6\end{array}$ & $\begin{array}{l}355.3 \\
387.0\end{array}$ & DSC & [2000ABD/WEI] \\
\hline $\mathrm{C}_{72} \mathrm{H}_{148} \mathrm{IP}$ & $\begin{array}{c}\text { [245071-84-5] } \\
\text { FUS }\end{array}$ & Tetraoctadecyl & $\begin{array}{c}\text { honium io } \\
143.2\end{array}$ & 97.8 & DSC & [2000ABD/WEI] \\
\hline
\end{tabular}

\section{Acknowledgment}

The authors would like to acknowledge the assistance of Don Burgess in preparing the manuscript for publication.

\section{References}

\begin{tabular}{|c|c|}
\hline 871REG2 & M. Regnailt, Ann. Chim. Phys. (4th Series) 23, 73 (1871). \\
\hline 1882KRA & Krafft, Ber. Bunsen-Ges. Phys. Chem. 15, 1687 (1882). \\
\hline 85STO/WIL & $\begin{array}{l}\text { F. Stohmann and H. Wilsing, J. Prakt. Chem. 140, } 80 \\
\text { (1885). }\end{array}$ \\
\hline 1889EYK & J. F. Eykman, Z. Phys. Chem. 4, 497-519 (1889). \\
\hline 90RAM & W. Ramsay, J. Chem. Soc., Trans. 57, 590 (1890). \\
\hline 91JAC/HOB & $\begin{array}{l}\text { D. S. Jacobus and N. J. Hoboken, Trans. Am. Inst. Mech. } \\
\text { Eng. 12, } 307 \text { (1891). }\end{array}$ \\
\hline 94BRU & L. Bruner, Ber. Dtsch. Chem. Ges. 27, 2102 (1894). \\
\hline & G. Ta \\
\hline $\mathrm{ON}$ & H. von Strombeck, J. Franklin Inst. 13 \\
\hline 02DEF/FON & $\begin{array}{l}\text { d H. Fonzeo-Diacon, C. R. Hebd. } \\
\text { 34, } 229 \text { (1902). }\end{array}$ \\
\hline & d, Ann. Chim. Phys. 28, 539 (1903). \\
\hline 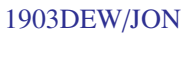 & $\begin{array}{l}\text { J. Dewar and H. O. Jones, Proc. R. Soc. London 71, } 427 \\
\text { (1902). }\end{array}$ \\
\hline 904JAQ/WAS & $\begin{array}{l}\text { A. Jaquerod and E. Wassmer, Ber. Dtsch. Chem. Ges. 37, } \\
\text { 2531-2534 (1904). }\end{array}$ \\
\hline 06BRI & O. Brill, Drude's Ann. 21, 179 (1906). \\
\hline 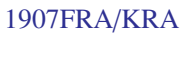 & $\begin{array}{l}\text { E. C. Franklin and C. A. Kraus, J. Phys. Chem. 11, } 553 \\
\text { (1907). }\end{array}$ \\
\hline 1908BOG/WIN & $\begin{array}{l}\text { A. Bogojawlenski and N. Windogradow, Z. Phys. Chem. } \\
\text { 64, } 251 \text { (1908). }\end{array}$ \\
\hline 1912LAN/BOR & $\begin{array}{l}\text { H. Landolt and R. Bornstein, Physikalsch-Chemische } \\
\text { Tabellen (Springer, Berlin, 1912). }\end{array}$ \\
\hline 1913BRI/PYL & $\begin{array}{l}\text { E. Briner and M. Z. Pylkoff, J. Chim. Phys. Phys.-Chim. } \\
\text { Biol. 10, } 640 \text { (1913); J. Chem. Soc., Abstr. 104(II), } 317 \\
\text { (1913). }\end{array}$ \\
\hline 914STO/WIL & $\begin{array}{l}\text { A. Stock and E. Willfroth, Ber. Dtsch. Chem. Ges. 47, } \\
\text { 144-154 (1914). }\end{array}$ \\
\hline
\end{tabular}

1915BRI P. W. Bridgman, Phys. Rev. 6, 94 (1915).

1915CRA J. M. Crafts, J. Chim. Phys. Phys.-Chim. Biol. 13, 105-161 (1915).

1917HIL/DUS J. H. Hildebrand, A. D. Duschak, A. H. Foster, and C. W. Beebe, J. Am. Chem. Soc. 39, 2293 (1917).

1918 OSB/DUS N. S. Osborne and M. S. Van Dusen, J. Am. Chem. Soc. 40, 14-25 (1918).

1919PAD M. Padoa, Atti Linc. 28, 239 (1919).

1920ATK/HEY R. H. Atkinson, C. T. Heycock, and W. J. Pope, J. Chem. Soc., Trans. 117, 1410 (1920)

1920BAX/BEZ G. P. Baxter, F. K. Bezzenberger, and C. H. Wilson, J. Am. Chem. Soc. 42, 1386-1392 (1920).

1922NEL/SEN O. A. Nelson and C. E. Senseman, Ind. Eng. Chem. 14, 58-62 (1922).

1922STR/PAR K. Stratton and J. R. Partington, Philos. Mag. 43, 436 (1922).

1922 VON/BOS H. Von Wartenberg and O. Bosse, Z. Elektrochem. Angew. Phys. Chem. 28, 354 (1922).

1923MOR/MUR F. S. Mortimer and R. V. Murphy, Ind. Eng. Chem. 15, 1140-1142 (1923).

1923SEN/NEL C. E. Senseman and O. A. Nelson, Ind. Eng. Chem. 15, 382-383 (1923).

1924EUC/KAR A. Eucken and E. Karwat, Z. Phys. Chem. 112, 467 (1924).

1924GAR/RAN W. E. Garner and F. C. Randall, J. Chem. Soc., Trans. 125, 881 (1924).

1924MAA/HIE O. Maas and P. G. Hiebert, J. Am. Chem. Soc. 46, 2693-2700 (1924).

1924SIM J. Simons, J. Am. Chem. Soc. 46, 2179-2183 (1924).

1924STR/PAR K. Stratton and J. R. Partington, Philos. Mag. 48, 1085 (1924).

1924TRA/GER M. Trautz and W. Gerwig, Z. Anorg. Allg. Chem. 134, 409 (1924).

1925MIR/MOL E. Miravelles and E. Moles, An. R. Soc. Esp. Fis. Quim. 23, 509 (1925).

1925VOL/KIR M. Volmer and P. Kirchhoff, Z. Phys. Chem. 115, 223-228 (1925).

1926HAR/SCH E. H. Harvey and H. A. Schuette, J. Am. Chem. Soc. 48, 2065 (1926).

1926MAY/PAR H. A. Mayes and J. R. Partingon, J. Chem. Soc. 129, 2594 (1926). 
1927STE/JOH L. E. Steiner and J. J. Johnston, J. Phys. Chem. 32, 912-939 (1927).

1928GIA/WIE W. F. Giauque and R. Wiebe, J. Am. Chem. Soc. 50, 2193-2202 (1928).

1928GIA/WIE2 W. F. Giauque and R. Wiebe, J. Am. Chem. Soc. 50, 101-122 (1928).

1929ARI

1929GIA/WIE K. Arii, Rikagaku Kenkyusho Iho 8, 719 (1929); Chem. Abstr. 24, 2304 (1930).

1929JEL/RUD K. Jellinek and A. Rudat, Z. Phys. Chem. 143, 55-61 (1929).

1929JOH/GIA H. L. Johnston and W. F. Giauque, J. Am. Chem. Soc. 51, 3194-3214 (1929)

1929PAR/TOD G. S. Parks and S. S. Todd, J. Ind. Eng. Chem. 21, 1235 (1929).

1929TAN/NAG Y. Tanaka and Y. Nagai, Proc. Imp. Acad. 5, 78-79 (1929); Chem. Abstr. 23, 2622 (1929).

1930AND

1930BLA/VAN

J. S. Anderson, J. Chem. Soc. 1930, 1653.

H. K. Black, G. van Praagh, and B. Topley, Trans. Faraday Soc. 26, 196-197 (1930)

1930BUL/HAU R. H. Bullard and A. C. Haussman, J. Phys. Chem. 34, 743-747 (1930).

1930CUN G. V. Cunningham, Power 72, 374-377 (1930).

1930EVA/DAV D. P. Evans, W. C. Davies, and W. J. Jones, J. Chem. Soc. 1930, 1310.

1930HUF/PAR2 H. M. Huffman, G. S. Parks, and A. C. Daniels, J. Am. Chem. Soc. 52, 1547 (1930).

1930MAC/SMI F. H. MacDougall and L. I. Smith, J. Am. Chem. Soc. 52, 1998 (1930).

1930NEL O. A. Nelson, Ind. Eng. Chem. 22, 971-972 (1930).

1930PAR/HUF G. S. Parks, H. M. Huffman, and S. B. Thomas, J. Am. Chem. Soc. 52, 1032 (1930).

1930PAT/PAP W. I. Patnode and J. Papish, J. Phys. Chem. 34, 1494-1496 (1930).

1930RUF/KEI O. Ruff and R. Keim, Z. Anorg. Allg. Chem. 193, 176 (1930).

1930SIM

1931DYK/DAV

J. H. Simons, J. Am. Chem. Soc. 52, 3488-3493 (1930).

W. J. C. Dyke, G. Davies, and W. J. Jones, J. Chem. Soc. 1931, 185.

1931GAR/VAN W. E. Garner, K. Van Bibber, and A. M. King, J. Chem. Soc. 1931, 1533.

1931HUf/PAR H. M. Huffman, G. S. Parks, and M. Barmore, J. Am. Chem. Soc. 53, 3876 (1931).

$1931 \mathrm{JOH}$

1931RUF/ASC

J. D. A. Johnson, J. Chem. Soc. 1931, 1913.

O. Ruff and E. Ascher, Z. Anorg. Allg. Chem. 196, 413 (1931).

1931RUF/MEN O. Ruff and W. Menzel, Z. Anorg. Allg. Chem. 202, 49-61 (1931).

1931SCH/COW H. A. Schuette and M. A. Cowley, J. Am. Chem. Soc. 53, 3485-3489 (1931).

1931WIB/SUT $\quad$ E. Wiberg and W. Sütterlin, Z. Anorg. Allg. Chem. 202, 1-21 (1931).

1932GRI/AWB E. Griffiths and J. H. Awbery, Proc. Phys. Soc. 44, 121 (1932).

1932JON/DYK W. J. Jones, W. J. C. Dyke, G. Davies, D. C. Griffiths, and J. H. Webb, J. Chem. Soc. 1932, 2285-2293.

1932KLE/HEN W. Klemm and P. Henkel, Z. Anorg. Allg. Chem. 207, 73-86 (1932).

1932NEU/VOL K. Neumann and E. Volker, Z. Phys. Chem. A161, 33-45 (1932).

1932SOL/MOL E. Solana and E. Moles, An. R. Soc. Esp. Fis. Quim. 30, 886 (1932).

1932SPA/THO M. E. Spaght, S. B. Thomas, and G. S. Parks, J. Phys. Chem. 36, 882-888 (1932).

1933CLU K. Clusius, Z. Phys. Chem. B. 23, 213 (1933).

$1933 \mathrm{COW} / \mathrm{SCH}$ M. A. Cowley and H. A. Schuette, J. Am. Chem. Soc. 55, 387-391 (1933).

1933FER/THO J. D. Ferry and S. B. Thomas, J. Phys. Chem. 37, 253 (1933).

1933GIA/CLA W. F. Giauque and J. O. Clayton, J. Am. Chem. Soc. 55, 4875-4889 (1933).
1933HEN/MUR

H. R. Henze and T. J. Murchison, J. Am. Chem. Soc. 55, 4255-4259 (1933).

1933 KRA/TOO C. A. Kraus and F. E. Toonder, Proc. Natl. Acad. Sci. U. S. A. 19, 292-298 (1933); Chem. Abstr. 27, 2646 (1933).

1933RUF/BRA O. Ruff and A. Braida, Z. Anorg. Allg. Chem. 214, 91 (1933).

1933SOU/MIL J. C. Southard, R. T. Milner, and S. B. Hendricks, J. Chem. Phys. 1, 95 (1933).

1934BOO/STI H. S. Booth and W. D. Stillwell, J. Am. Chem. Soc. 56, 1531 (1934).

1934CAM/CAM A. N. Campbell and A. J. Campbell, Trans. Faraday Soc. 30, 1109-1114 (1934).

1934HIE/WOE W. Hieber and A. Woerner, Z. Elektrochem. Angew. Phys. Chem. 40, 252-256 (1934).

1934JUN/TAY J. C. Jungers and H. S. Taylor, J. Chem. Phys. 2, 373 (1934).

1934KIN/GAR A. M. King and W. E. Garner, J. Chem. Soc. 1934, 1449.

1934PAR/LIG G. S. Parks and D. W. Light, J. Am. Chem. Soc. 56, 1511 (1934).

1934SCH/BIC W. C. Schumb and F. A. Bickford, J. Am. Chem. Soc. 56, 852-858 (1934).

1934WIN/BLA M. M. Windsor and A. A. Blanchard, J. Am. Chem. Soc. 56, 823-825 (1934).

1934WOL/TRI K. L Wolf and H. G. Trieschmann, Z. Phys. Chem. B 27, 376-380 (1934).

1935BLU/GIA R. W. Blue and W. F. Giauque, J. Am. Chem. Soc. 57, 991-997 (1935).

1935BOO/SWI H. S. Booth and C. F. Swinehart, J. Am. Chem. Soc. 57, 1333 (1935).

1935HIE/ROM W. Hieber and E. Romberg, Z. Anorg. Allg. Chem. 221, 332-336 (1935); Chem. Abstr. 29, 2467 (1935).

1935JON/EVA W. J. Jones, D. P. Evans, T. Gulwell, and D. C. Griffiths, J. Chem. Soc. 1935, 39-47.

1935RUF/KWA O. Ruff and W. Kwasnik, Angew. Chem. 48, 238 (1935).

1935SKA

1936BOO/HER

1936BOO/MOR E. L. Skau, J. Phys. Chem. 39, 761 (1935).

H. S. Booth and C. V. Herrmann, J. Am. Chem. Soc. 58, 63 (1936).

1936BUC/NOR E. J. Buckler and R. G. W. Norrish, J. Chem. Soc. 1936, $1567-1569$.

1936CLU/FRA K. Clusius and A. Frank, Z. Phys. Chem. B 34, 405 (1936).

1936CUT/BEN G. R. Cuthbertson and H. E. Bent, J. Am. Chem. Soc. 58 2000-2003 (1936).

1936GIA/BLU W. F. Giauque and R. W. Blue, J. Am. Chem. Soc. 58, 831-837 (1936).

1936KEA

1936KIN/GAR

K. Kearby, J. Am. Chem. Soc. 58, 374-375 (1936).

1936MER/SCH H. Merten and H. Schlüter, Ber. Dtsch. Chem. Ges. 69, H. Merten and H. Sch
1364-1366 (1936).

1936NEG G. R. Negishi, J. Am. Chem. Soc. 58, 2293-2296 (1936).

1936SCH/AND W. C. Schumb and H. H. Anderson, J. Am. Chem. Soc. 58, 994 (1936).

1936THO/LIN H. W. Thompson and J. W. Linnett, Trans. Faraday Soc. 32, 681-685 (1936).

1937BUR/SCH A. B. Burg and H. I. Schlesinger, J. Am. Chem. Soc. 59, 780-787 (1937).

1937GOO/RIC C. F. Goodeve and F. D. Richardson, J. Chem. Soc. 1937, 294.

1937GRO/IPA A. V. Grosse and V. N. Ipatieff, J. Org. Chem. 2, 447-458 (1937).

1937HIC/HEC K. C. D. Hickman, J. C. Hecker, and N. D. Embree, Ind. Eng. Chem., Anal. Ed. 9, 264 (1937).

1937JOH/PEC W. C. Johnson and A. Pechukas, J. Am. Chem. Soc. 59, 2065 (1937).

1937KRU/CLU A. Kruis and K. Clusius, Z. Phys. Chem. B. 38, 156 (1937).

1937LIN/ROH R. Linke and W. Rohrmann, Z. Phys. Chem. B 35, 256-260 (1937).

1937LUC/LIK G. P. Luchinskii and A. I. Likacheva, Zh. Fiz. Khim. 9, 65 (1937). 
1937OVE/GIA R. Overstreet and W. F. Giauge, J. Am. Chem. Soc. 59, 254-259 (1937).

1937PUR/ZAH R. H. Purcell and F. D. Zahoorbux, J. Chem. Soc. 1937, 1029-1035.

1937RUD/KOR G. A. Rudakov and S. Y. Korotov, Zh. Prikl. Khim. 10, 312 (1937); Chem. Abstr. 31, 32399 (1937).

1937 SOU/NEL J. C. Southard and R. A. Nelson, J. Am. Chem. Soc. 59, 911 (1937).

1937STE/GIA C. C. Stephenson and W. F. Giauque, J. Chem. Phys. 5, 149-158 (1937).

1938GIA/STE W. F. Giauque and C. C. Stephenson, J. Am. Chem. Soc. 60, 1389-1394 (1938).

1938UBB A. R. Ubbelohde, Trans. Faraday Soc. 34, 282-292 (1938).

1938VER G. Verdonk, Chem. Weekbl. 35, 741 (1938).

1938WEG H. Weghofer, Ph.D. dissertation, Phys. Chem. Institut der Universital Halle, Germany, 1938.

1938WOL/WEG K. L. Wolf and H. Z. Weghofer, Z. Phys. Chem. B 39, 194-208 (1938).

1939BOO/BOZ H. S. Booth and A. R. Bozarth, J. Am. Chem. Soc. 61, 2927 (1939).

1939BOO/FRA H. S. Booth and S. G. Frary, J. Am. Chem. Soc. 61, 2934 (1939).

1939BOO/SEE H. S. Booth and C. G. Seegmiller, J. Am. Chem. Soc. 61, 3120 (1939).

1939EME/MIL H. J. Emeleus and N. Miller, J. Chem. Soc. 1939, 819.

1939FIS/GEW W. Fischer and R. Gewehr, Z. Anorg. Allg. Chem. 242, 188 (1939).

1939FRA/CLU A. Frank and K. Clusius, Z. Phys. Chem. 42B, 395-421 (1939).

1939GIA/POW W. F. Giauque and T. M. Powell, J. Am. Chem. Soc. 61, 1970 (1939).

1939NIW/SAT K. Niwa, M. Sato, and M. Yosiyama, J. Chem. Soc. Jpn. 60, 918-928 (1939).

1939VER/MAR F. H. Verhoek and A. L. Marshall, J. Am. Chem. Soc. 61, 2736-2742 (1939).

1940BOO/CAS H. S. Booth and M. C. Cassidy, J. Am. Chem. Soc. 62, 2369 (1940).

1940BOO/MER H. S. Booth and F. C. Mericola, J. Am. Chem. Soc. 62, 640 (1940).

1940BUR

1940ROS/SAN E. J. Rosenbaum and C. R. Sandberg, J. Am. Chem. Soc. 62, 1622 (1940).

1940SCH/SAN H. I. Schlesinger, R. T. Sanderson, and A. B. Burg, J. Am. Chem. Soc. 62, 3421-3425 (1940).

1940ZIL A. A. Zil'berman-Granovskaya, J. Phys. Chem. USSR 14, 759-767 (1940); 14, 768-773 (1940); 1004-1006 (1940); Chem. Abstr. 35, 3867 (1941).

1941AST/KEN J. G. Aston, R. M. Kennedy, and G. H. Messerly, J. Am. Chem. Soc. 63, 2343-2348 (1941).

1941BOD M. Z. Bodenstein, Z. Elktrochem. Angew. P. 47, 501-518 (1941); Chem. Abstr. 36, 4014 (1942).

1941BRO/DAV L. O. Brockway and N. R. Davidson, J. Am. Chem. Soc. 63, 3287 (1941).

$1941 \mathrm{GIG} / \mathrm{RUN} \quad$ P. A. Giguere and R. E. Rundle, J. Am. Chem. Soc. 63, 1135-1137 (1941).

1941LAU/GIL A. W. Laubengayer and W. F. Gilliam, J. Am. Chem. Soc. 63, 477-479 (1941).

1941MAI/STR $\quad$ B. J. Mair and A. J. Streiff, J. Res. Natl. Bur. Stand. 27, 343 (1941)

1941 NIT/SEK I. Nitta and S. Seki, J. Chem. Soc. Jpn. 62, 581-586 (1941); Chem. Abstr. 36, 15 (1942).

1941RUS/RUN H. Russell, Jr., R. E. Rundle, and D. M. Yost, J. Am. Chem. Soc. 63, 2825-2828 (1941).

1941SCH W. R. Schmidt, M.S. thesis, Washington University, St. Louis, 1941.

$\begin{array}{ll}\text { 1941SEK } & \text { S. Seki, J. Chem. Soc. Jpn. 62, 789-795 (1941). } \\ \text { 1941TAR/EGA } & \text { G. Tarbutton, E. P. Egan, Jr., and S. G. Frary, J. Am. Chem. }\end{array}$ Soc. 63, 1782 (1941).

1941VAN/GER M. van Driel and H. Gerding, Recl. Trav. Chim. Pays-Bas 60, 943 (1941).

1941 VAN/GER2 M. van Driel and H. Gerding, Recl. Trav. Chim. Pays-Bas 60, 869 (1941).
1942CHE

1942NEL/SMI

1942SCH/AST

$1942 \mathrm{SCH} / \mathrm{SCH}$

1942SUG/SAT

1942VAN

1943AUD/STE

1943BAC/PER

1943BOO/SEA

1943BUR3

1943CRA

1943HAL/REI

1943STE

1943WES/EUC

1944ALT/TRI

1944AST/SAG

1944EIB

1944EME/MAD

1944EME/WIL 1944MCL/EDW

1945BOO/OST

1945FIS/NAY

1945HOG

1945WIL/TAY

1946ALB/WIL

1946AST/ZIE

1946BAM/LEV

1946BOO/CAR

1946BOO/HAL

1946BOO/MAR

1946BOO/SCH

1946BOO/SUT

1946EME/MAD

1946GIL/JON

1946JON/THO

1946LON/EME

1946THO

1947BAL

1947BLO/CAM
R. Cherton, Bull. Soc. R. Sci. Liege 11, 54 (1942).

O. A. Nelson and L. E. Smith, J. Am. Chem. Soc. 64 3035-3037 (1942).

S. C. Schumann, J. G. Aston, and M. Sagenkahn, J. Am. Chem. Soc. 64, 1039-1043 (1942); Erratum, J. Am. Chem. Soc. 64, 30690 (1942).

H. Schmitz and H. J. Schumacher, Z. Anorg. Allg. Chem. 249, 238 (1942).

B. Suginuma and K. Satozaki, Rikagaku Kenkyusho Iho 41, 432 (1942).

M. van Driel, Recl. Trav. Chim. Pays-Bas 61, 748 (1942).

L. F. Audrieth, R. Steinman, and A. D. F. Toy, Chem. Rev. 32, 109-133 (1943).

H. J. Backer and W. G. Perdok, Recl. Trav. Chim. PaysBas 62, 533 (1943).

H. S. Booth and C. A. Seabright, J. Am. Chem. Soc. 65, 1834 (1943).

A. B. Burg, J. Am. Chem. Soc. 65, 1838-1841 (1943)

J. S. N. Cramer, Recl. Trav. Chim. Pays-Bas 62, 606-610 (1943); Chem. Zentr. II 2234 (1943); Chem. Abstr. 38, 6148 (1944).

W. P. Hall and E. E. Reid, J. Am. Chem. Soc. 65, 1466-1468 (1943).

D. R. Stevens, Ind. Eng. Chem. 35, 655-660 (1943).

O. Westphal and M. Eucken, Ber. Dtsch. Chem. Ges. 76, 1137 (1943).

P. M. Althouse and H. O. Triebold, Ind. Eng. Chem., Anal. Ed. 16, 605-606 (1944).

J. G. Aston, M. L. Sagenkahn, G. J. Szasz, G. W. Moessen, and H. F. Zuhr, J. Am. Chem. Soc. 66, 1171-1177 (1944).

J. Eibert, M.S. thesis, Washington University, St. Louis, 1944.

H. L. Emeleus and A. G. Maddock, J. Chem. Soc. 1944 293-296.

H. J. Emeleus and C. J. Wilkins, J. Chem. Soc. 1944, 454.

C. R. McLellan and W. R. Edwards, Jr., J. Am. Chem. Soc. 66, 409 (1944).

H. S. Booth and R. A. Osten, J. Am. Chem. Soc. 67, 1092 (1945).

F. B. Fischl, B. F. Naylor, C. W. Naylor, C. W. Ziemer, G. S. Parks, and J. G. Aston, J. Am. Chem. Soc. 67, 2075 (1945).

H. J. Hoge, J. Res. Natl. Bur. Stand. 34, 281-293 (1945). C. B. Willingham, W. J. Taylor, J. M. Pignocco, and F. D. Rossini, J. Res. Natl. Bur. Stand. 35, 219-224 (1945).

A. Albert and J. B. Willis, Nature 157, 341 (1946); J. B. Willis, Trans. Faraday Soc. 43, 97-102 (1946).

J. G. Aston and C. W. Ziemer, J. Am. Chem. Soc. 68, 1405 (1946).

H. Bamford, D. L. Levi, and D. M. Newitt, J. Chem. Soc. 1946, 468-471.

H. S. Booth and P. H. Carnell, J. Am. Chem. Soc. 68, 2650 (1946).

H. S. Booth and H. S. Halbedel, J. Am. Chem. Soc. 68, 2652 (1946).

H. S. Booth and W. F. Martin, J. Am. Chem. Soc. 68, 2655 (1946).

H. S. Booth and A. A. Schwartz, J. Am. Chem. Soc. 68 2662 (1946)

H. S. Booth and J. F. Suttle, J. Am. Chem. Soc. 68, 2658 (1946).

H. J. Emeleus and A. G. Maddock, J. Chem. Soc. 1946, 1131-1134.

H. Gilman and R. G. Jones, J. Am. Chem. Soc. 68, 517-520 (1946).

W. J. Jones, L. H. Thomas, E. H. Pritchard, and S. T. Bowden, J. Chem. Soc. 1946, 824-827.

L. H. Long, H. J. Emeleus, and H. V. A. Briscoe, J. Chem. Soc. 1946, 1123-1126.

G. W. Thompson, Chem. Rev. 38, 1-39 (1946).

E. W. Balson, Trans. Faraday Soc. 43, 54-60 (1947).

J. M. Blocher, Jr. and I. E. Campbell, J. Am. Chem. Soc. 69, 2100-2101 (1947). 
1947BOL/COL J. M. Bolling, A. R. Collett, and C. L. Lazzell, Proc. W. Va. Acad. Sci. 19, 61 (1947).

1947CHA/SIN G. H. Charbonnet and W. S. Singleton, J. Am. Oil Chem. Soc. 24, 140 (1947).

1947GOL/MAR K. B. Goldblum, R. W. Martin, and R. B. Young, Ind. Eng. Chem. 39, 1474-1476 (1947).

1947GOU/HOL C. Gould, Jr., G. Holzman, and C. Niemann, Anal. Chem. 19, 204-206 (1947).

1947IVE/PIT D. J. G. Ives, R. W. Pittman, and W. Wardlaw, J. Chem. Soc. 1947, 1080-1083.

1947LAN/GER J. J. Lander and L. H. Germer, Am. Inst. Mining Met. Engrs., Inst. Metals Div., Metals Technol., 14(6), Tech. Pub. No. 2259, 1947; as quoted in Ref. [1960MON/COT].

1947REH/DIX C. E. Rehberg, M. B. Dixon, and C. H. Fisher, J. Am. Chem. Soc. 69, 2966 (1947).

1947STU D. R. Stull, Ind. Eng. Chem. 39, 517-540 (1947).

1947WIL/EDW H. B. Williams and W. R. Edwards, J. Am. Chem. Soc. 69, 336 (1947).

1948BEN/FRA H. E. Bent and R. J. Francel, J. Am. Chem. Soc. 70, 634-637 (1948).

1948BON/ATH C. E. Bonhorst and P. M. Athouse, Ind. Eng. Chem. 40, 2379-2384 (1948).

1948BRO/SUJ H. C. Brown and S. Sujishi, J. Am. Chem. Soc. 70, 2878-2881 (1948).

1948COA/SUT G. E. Coates and L. E. Sutton, J. Chem. Soc. 1948, 1187-1196.

1948DIX/REH $\quad$ M. B. Dixon, C. E. Rehberg, and C. E. Fisher, J. Am. Chem. Soc. 70, 3733 (1948).

1948FEI/FIS M. L. Fein and C. M. Fisher, J. Org. Chem. 13, 749 (1948).

1948FOR/AND G. S. Forbes and H. H. Anderson, J. Am. Chem. Soc. 70, 1043 (1948).

1948GLE/RIS O. Glemser and T. Risler, Z. Naturforsch., B 3, 1 (1948).

1948MAZ

1948RED/CHA2 C. E. Rede, Recl. Trav. Chim. Pays-Bas 67, 197 (1948).

Benedict, J. Am. Chem. Soc. 70, 637-639 (1948).

1948SMA/SMA P. A. Small, K. W. Small, and P. Cowley, Trans. Faraday Soc. 44, 810-816 (1948).

1948TSC

1948WHI

1948WIB/BOL

H. Tschamler, Monatsh. Chem. 79, 162 (1948).

G. H. Whiting, J. Chem. Soc. 1948, 1209-1210.

1949BIR/BRA J. Birks and R. S. Bradley, Proc. R. Soc. A 198, 226-239 (1949).

1949BRA/SHE R. S. Bradley and A. D. Shellard, Proc. R. Soc. A 198, 239-251 (1949).

1949BRA/SHE2 R. S. Bradley and A. D. Shellard, Trans. Faraday Soc. 45, 501-507 (1949).

1949BUC/COL F. R. Buck, K. F. Coles, G. T. Kennedy, and F. Morton, J. Chem. Soc. 1949, 2377-2383.

1949CAR/HAR A. S. Carson, K. Hartley, and H. A. Skinner, Trans. Faraday Soc. 45, 1159-1167 (1949).

1949CAR/HAR2 A. S. Carson, K. Hartley, and H. A. Skinner, Proc. R. Soc. A 195, 500-512 (1949).

1949DIC/RAL F. H. Dickey, J. H. Raley, F. F. Rust, R. S. Treseder, and W. E. Vaughn, Ind. Eng. Chem. 41, 1673 (1949).

1949DRE/MAR R. R. Dreisbach and R. A. Martin, Ind. Eng. Chem. 41, 2875-2878 (1949).

1949DRE/SHR R. R. Dreisbach and S. A. Shrader, Ind. Eng. Chem. 41, 2879-2880 (1949).

1949EME/HEA H. J. Emeleus and H. G. Heal, J. Chem. Soc. 1949, 1696-1699.

1949FOR/BOW M. V. Forward, S. T. Bowden, and J. Jones, J. Chem. Soc. 1949, S121-S126.

1949FOR/NOR A. F. Forziati, W. R. Norris, and F. D. Rossini, J. Res. Natl. Bur. Stand. 43, 555-563 (1949).

1949HAT/SUT L. F. Hatch, G. Sutherland, and W. J. Ross, J. Org. Chem. 14, 1130-1136 (1949), Note: vapor pressure data presented graphically as $\log \mathrm{P}$ versus $1 / \mathrm{T}$ in paper.

1949KLA F. Klages, Chem. Ber. 82, 358-375 (1949).

1949KRE/WIE C. B. Kretschmer and R. Wiebe, J. Am. Chem. Soc. 71, 1793-1797 (1949).

1949KUH/MAS W. Kuhn and P. Massini, Helv. Chim. Acta 32, 1530-1542 (1949).
1949NIT/SEK

I. Nitta, S. Seki, and H. Chihara, Nippon Kagaku Zasshi 70, 387-390 (1949); Chem. Abstr. 45, 2737d (1951).

1949PAR/MOO G. S. Parks and G. E. Moore, J. Chem. Phys. 17, 1151-1153 (1949).

1949PAR/MOO2 G. S. Parks, G. E. Moore, M. L. Renquist, B. F. Naylor, L. A. McClaine, P. S. Fujii, and J. A. Hatton, J. Am. Chem. Soc. 71, 3386-3389 (1949).

1949PAR/WHY J. R. Partington and A. L. Whynes, J. Phys. Chem. 53, 500-503 (1949).

1949PER/WEB E. S. Perry and W. H. Weber, J. Am. Chem. Soc. 71, 3726-3730 (1949).

1949PER/WEB2 E. S. Perry, W. H. Weber, and B. F. Baubert, J. Am. Chem. Soc. 71, 3720-3726 (1949).

1949REH/DIX C. E. Rehberg, M. B. Dixon, and C. H. Fisher, J. Org. Chem. 14, 593 (1949).

1949SCO/OLI D. W. Scott, G. D. Oliver, M. E. Gross, W. N. Hubbard, and H. M. Huffman, J. Am. Chem. Soc. 71, 2293-2297 (1949).

1949SEA/HOP2 G. W. Sears and E. R. Hopke, J. Am. Chem. Soc. 71, 1632-1634 (1949).

1950BRI/CAR N. F. H. Bright, T. Carson, and T. A. Dyson, Research (London) 3, 185-186 (1950); Chem. Abstr. 44, 9367c (1950).

1950BUR/KUL A. B. Burg and E. S. Kuljian, J. Am. Chem. Soc. 72, 3103-3107 (1950).

1950CRO/SMY R. W. Crowe and C. P. Smyth, J. Am. Chem. Soc. 72, 1098-1106 (1950).

1950FOR/CAM A. F. Forziati, D. L. Camin, and F. D. Rossini, J. Res. Natl. Bur. Stand. 45, 406-410 (1950).

1950HAR/PRI K. Hartley, H. O. Pritchard, and H. A. Skinner, Trans. Faraday Soc. 46, 1019-1025 (1950).

1950KAK/SAK Y. Kakiuchi, T. Sakurai, and T. Suzuki, J. Phys. Soc. Jpn. 5, 369 (1950).

1950MAT/SUM J. B. Matthews, J. F. Sumner, and E. A. Moellyn-Hughes, Trans. Faraday Soc. 46, 797-803 (1950).

1950NIT/SEK I. Nitta, S. Seki, M. Momotani, and K. Sato, Nippon Kagaku Zasshi 71, 378-382 (1950); Chem. Abstr. 45, 6448g (1951).

1950NIT/SEK3 I. Nitta, S. Seki, and M. Momotani, Nippon Kagaku Zasshi 71, 430-433 (1950); Chem. Abstr. 45, 6448i (1951).

1950RAT

1950REH/DIX W. R. Ratchford, J. Org. Chem. 15, 326 (1950).

1950REH/DIX2 C. E. Rehberg and M. B. Dixon, J. Org. Chem. 15, 565 (1950).

1950REH/DIX3 C. E. Rehberg, M. B. Dixon, and C. H. Fisher, J. Org. Chem. 15, 560 (1950).

1950SIN/WAR W. S. Singleton, T. L. Ward, and F. G. Dollear, J. Am. Oil Chem. Soc. 27, 143 (1950).

1950UEB/ORT K. Ueberreiter and H.-J. Orthmann, Z. Naturforsch. 5A 101 (1950).

1951BOD/KLE H. Bode and E. Klesper, Z. Anorg. Allg. Chem. 266, 275 (1951).

1951BRA/WAG R. S. Bradley and G. S. C. Waghorn, Proc. R. Soc. A 206, 65-72 (1951).

1951BRI

1951BUR/RAN A. B. Burg and C. L. Randolph, J. Am. Chem. Soc. 73, 953-957 (1951).

1951CHA/SKI T. Charnley and H. A. Skinner, J. Chem. Soc. 1951, 1921-1924.

1951CLA/COC A. M. Clark, A. H. Cockett, and H. S. Eisner, Proc. R. Soc. A 209, 408-415 (1951).

1951EGE/EMT A. C. Egerton, W. Emte, and G. J. Minkoff, Discuss. Faraday Soc. 278-282 (1951).

1951FOL/GIG W. T. Foley and P. A. Giguere, Can. J. Chem. 29, 895 (1951).

1951FUR/GIN G. T. Furukawa, D. C. Ginnings, R. E. Mcgoskey, and R. A. Nelson, J. Res. Natl. Bur. Stand. 46, 195 (1951).

1951FUR/MCC G. T. Furukawa, R. E. McCoskey, and G. T. King, J. Res. Natl. Bur. Stand. 47, 256 (1951).

1951GOU/LIN J. Goubeau and R. Link, Z. Anorg. Allg. Chem. 267, 27-37 (1951). 
1951GRI/BER J. W. Grisard, H. A. Bernhardt, and G. D. Oliver, J. Am. Chem. Soc. 73, 5725-2727 (1951).

1951HAR/PRI K. Hartley, H. O. Pritchard, and H. A. Skinner, Trans. Faraday Soc. 47, 254-263 (1951).

1951HOP/SEA E. R. Hopke and G. W. Sears, J. Chem. Phys. 19, 1345-1351 (1951).

1951INO H. Inokuchi, Nippon Kagaku Zasshi 72, 552-555 (1951).

1951MAG/HAR A. Magnus, H. Hartman, and F. Becker, Erdoel Kohle 4, 115-118 (1951); Z. Phys. Chem. 197, 75-91 (1951).

1951MAR/MAK D. R. Martin and L. S. Mako, J. Am. Chem. Soc. 73, 2674 (1951).

1951MCE/RIG W. S. McErvan and M. W. Rigg, J. Am. Chem. Soc. 73, 4725-4727 (1951).

1951MIL/PAO G. Milazzo and L. Paoloni, Ann. Chim. (Rome) 41, 673-684 (1951); Rend. Ist. Super. Sanita (Rome) 14(9), 673-685 (1951); Chem. Abstr. 46, 3355f (1952).

1951 NIS/PET L. A. Nisel'son and I. V. Petrusevich, Zh. Neorg. Khim. 6, 748 (1951).

1951NIT/SEK I. Nitta, S. Seki, H. Chihara, and K. Suzuki, Science Papers Osaka Univ. No 29, 1951; Chem. Abstr. 46, 3820 (1952).

1952BRA/EME G. A. R. Brandt, H. J. Emeleus, and R. N. Haszeldine, J. Chem. Soc. 1952, 2198-2205.

1952BRA/EME2 G. A. R. Brandt, H. J. Emeleus, and R. N. Haszeldine, J. Chem. Soc. 1952, 2552.

1952BRA/PLE D. S. Brackman and P. H. Plesch, J. Chem. Soc. 1952, 2188-2190.

1952BRO/OST C. A. Brown and R. C. Osthoff, J. Am. Chem. Soc. 74, 2340-2345 (1952).

1952BUR/DAI W. G. Burns and F. S. Dainton, Trans. Faraday Soc. 48, 21 (1952).

1952COA/GLO G. E. Coates, F. Glocling, and N. D. Huck, J. Chem. Soc. 1952, 4496-4501.

1952INO/SHI H. Inokuchi, S. Shiba, T. Handa, and H. Akamatu, Bull. Chem. Soc. Jpn. 25, 299-302 (1952).

1952KAP/KES L. Kaplan, W. L. Kester, and J. J. Katz, J. Am. Chem. Soc. 74, 5531-5532 (1952).

1952MOR/PRI C. T. Mortimer, H. O. Pritchard, and H. A. Skinner, Trans. Faraday Soc. 48, 220-228 (1952).

1952OLI/GRI G. D. Oliver and J. W. Grisard, J. Am. Chem. Soc. 74, 2705-2707 (1952).

1952OST/BRO R. C. Osthoff and C. A. Brown, J. Am. Chem. Soc. 74, 2378 (1952).

1952REZ/SHV T. N. Rezukhina and V. V. Shvyrev, Vestn. Mosk. Univ. 7, 41-46 (1952); Chem. Abstr. 47, 4676h (1953).

1952SCO/MAC T. A. Scott, Jr., D. MacMillan, and E. H. Melvin, Ind. Eng. Chem. 44, 172-175 (1952).

1952WAR/SIN T. L. Ward and W. S. Singleton, J. Phys. Chem. 56, 696 (1952).

1952WER A. C. Werner, Ind. Eng. Chem. 44, 2736 (1952).

1953AIH A. Aihara, Nippon Kagaku Zasshi 74, 437-441 (1953); 74, 631-635 (1953); Chem. Abstr. 48, 1088h (1954).

1953BRA/CLE R. S. Bradley and T. G. Cleasby, J. Chem. Soc. 1953, 1690-1692.

1953BRA/CLE2 R. S. Bradley and T. G. Cleasby, J. Chem. Soc. 1953, $1681-1684$

1953BUS/GIA R. H. Busey and W. F. Giauque, J. Am. Chem. Soc. 75, 806 (1953).

1953CHA/MOR T. Charnley, C. T. Mortimer, and H. A. Skinner, J. Chem. Soc. 1953, 1181-1184.

1953FEI/FIL M. L. Fein and E. M. Filachione, J. Am. Chem. Soc. 75, 2097 (1953).

1953 GIN/FUR D. C. Ginnings and G. T. Furukawa, J. Am. Chem. Soc. 75, 522 (1953).

1953GOU/MUN J. Goubeau and R. Mundiel, Z. Anorg. Allg. Chem. 272, $313(1953)$

1953GRU/OST W. T. Grubb and R. C. Osthoff, J. Am. Chem. Soc. 75, 2230-2232 (1953).

1953HER/MAR E. F. G. Herington and J. F. Martin, Trans. Faraday Soc. 49, 154-162 (1953).

1953HOF/DEC J. D. Hoffman and B. F. Decker, J. Phys. Chem. 57, 520-529 (1953).
1953JOH/FRI

O. H. Johnson and H. E. Fritz, J. Am. Chem. Soc. 75, 718-720 (1953).

1953MAG/MOD F. C. Magne and R. R. Mod, Ind. Eng. Chem. 45, 1546 (1953).

1953MEL/VIO E. F. Mellon, S. J. Viola, and S. R. Hooper, J. Phys. Chem. 57, 607-608 (1953).

1953OLI/MIL G. D. Oliver, H. T. Milton, and J. W. Grisard, J. Am. Chem. Soc. 75, 2827-2829 (1953).

1953OST/GRU R. C. Osthoff, W. T. Grubb, and C. A. Burkhard, J. Am. Chem. Soc. 75, 2227-2230 (1953).

1953SCH/ZEP H. Schaefer and F. Z. Zeppernick, Z. Anorg. Allg. Chem. 272, 274-278 (1953).

1953SEK/SUZ S. Seki and K. Suzuki, Bull. Chem. Soc. Jpn. 26, 209-213 (1953); S. Seki and K. Suzuki, Bull. Chem. Soc. Jpn. 26, 372-380 (1953).

1953SER/VOI V. V. Serpinskii, S. A. Voitkevich, and N. Y. Lyuboshits, Zh. Fiz. Khim. 27, 1032-1038 (1953); Chem. Abstr. 49, 3594e (1955).

1953SKI/TEE H. A. Skinner and T. F. S. Tees, J. Chem. Soc. 1953 3378-3383.

1953STA/MUL H. Stage, E. Müller, and P. Faldix, Erdoel Kohle 6, 375-380 (1953); Note: Enthalpies of vaporization were calculated by fitting published vapor pressure data, taken from various sources to the Antoine equation. Some of the references are to unpublished data measured by chemical companies.

1953STE $\quad$ B. Stevens, J. Chem. Soc. 1953, 2973-2974.

1953TAN/KAY S. Tannenbaum, S. Kaye, and G. Fihewenz, J. Am. Chem. Soc. 75, 3753 (1953).

1953WAG/BUR R. I. Wagner and A. B. Burg, J. Am. Chem. Soc. 75, 3869-3871 (1953).

1953WAL

K. A. Walsh, U. S. Atomic Energy Comm. LA-1649 (1953); Chem. Abstr. 55, 19319c (1961); 55, 19319d (1961).

1953WIL/DOL R. C. Wilhoit and M. Dole, J. Phys. Chem. 57, 14 (1953).

1954BUR/BAN A. B. Burg and J. Banus, J. Am. Chem. Soc. 76, 3903-3905 (1954).

1954 CAM/FOR D. L. Camin, A. F. Forziati, and F. D. Rossini, J. Phys. Chem. 58, 440-442 (1954).

1954DAV/JEN M. Davies and D. G. Jenkin, J. Chem. Soc. 1954, 2374-2377.

1954DUN $\quad$ S. A. Dunn, J. Am. Chem. Soc. 76, 6191-6192 (1954).

1954FIN/GRO2 H. L. Finke, M. E. Gross, G. Waddington, and H. M. Huffman, J. Am. Chem. Soc. 76, 333 (1954).

1954FUR/PAR G. T. Furukawa and R. P. Park, "Heat capacity, heats of fusion, transition, and vaporization, and vapor pressure of trimethylborane $\mathrm{B}\left(\mathrm{CH}_{3}\right)_{3}$," National Bureau of Standards Report, 1954.

1954JEN/CHA A. C. Jenkins and G. F. Chambers, Ind. Eng. Chem. 46, 2367-2369 (1954).

1954JOR

T. E. Jordan, Vapour Pressure of Organic Compounds (Wiley, New York, 1954).

1954LON/SAC L. H. Long and J. F. Sackman, Trans. Faraday Soc. 50, 1177-1182 (1954).

1954OST/GRU R. C. Osthoff and W. T. Grubb, J. Am. Chem. Soc. 76, 399-401 (1954)

1954SEN/SNY K. A. Sense, M. J. Snyder, and J. W. Clegg, J. Phys. Chem. 58, 223-224 (1954).

1954SEN/SNY2 K. A. Sense, M. J. Snyder, and R. B. Filbert, Jr., J. Phys. Chem. 58, 995-996 (1954).

1954SER/VOI V. V. Serpinskii, S. A. Voitkevich, and N. Y. Lyuboshits, Zh. Fiz. Khim. 28, 810-813 (1954); 28, 1969-1974 (1954); 49, 6677C (1955); 50, 4573h-4573i (1956).

1954SPR/WHI H. D. Springall and T. R. White, J. Chem. Soc. 1954, 2764-2766.

1954STA/WAR L. A. K. Staveley, J. B. Warren, H. P. Paget, and D. J. Dowrick, J. Chem. Soc. 1954, 1992.

1954SUJ/WIT S. Sujishi and S. Witz, J. Am. Chem. Soc. 76, 4631-4636 (1954).

1955AIH3 A. Aihara, Nippon Kagaku Zasshi 76, 492-494 (1955); Chem. Abstr. 49, 15323c (1955).

1955AYL/EME $\quad$ B. J. Aylett, H. J. Emeleus, and A. G. Maddock, J. Inorg. Nucl. Chem. 1, 187 (1955). 
1955BRO/LAU C. A. Brown and A. W. Laubengayer, J. Am. Chem. Soc. 77, 3699-3700 (1955).

1955BRO/ROB F. Brown and P. L. Robinson, J. Chem. Soc. 1955, 3147-3151.

1955CAM/ROS D. L. Camin and F. D. Rossini, J. Phys. Chem. 59, 1173-1179 (1955).

1955CAS/SPR R. C. Cass, H. D. Springall, and P. G. Quincey, J. Chem. Soc. 1955, 1188-1190.

1955ENG J. J. Engelsman, Ph.D. thesis, Vrije Universiteit te Amsterdam, 1955.

1955FIS/BIN J. Fischer and J. Bingle, J. Am. Chem. Soc. 77, 6511-6512 (1955).

1955FUR

G. T. Furukawa, "Heat capacity, heats of fusion and vaporization, and vapor pressure of triethylborane, $\left(\mathrm{C}_{2} \mathrm{H}_{5}\right)_{3}$ B," NBS Report No. 3712, 1-16, 1955.

1955FUR/MCC G. T. Furukawa, R. E. McCoskey, M. L. Reilly, and A. W. Herman, J. Res. Natl. Bur. Stand. 55, 201-207 (1955).

1955LON/SAC L. H. Long and J. F. Sackman, Trans. Faraday Soc. 51, 1062-1064 (1955).

1955LON/SAC2 L. H. Long and J. F. Sackman, Res. Corresp. 5, S23 (1955); Chem. Abstr. 50, 47201 (1956).

1955LUF N. W. Luft, Ind. Chem. 31, 502-504 (1955); Chem. Abstr. 50, 5388c (1956).

1955MAC/GRE R. O. MacLaren and N. W. Gregory, J. Phys. Chem. 59, 184-186 (1955).

1955MOM/SEK M. Momotani, S. Seki, H. Chihara, and H. Suga, Bull. Chem. Soc. Jpn. 28, 325-330 (1955).

1955PIE/PAC L. Pierce and E. L. Pace, J. Chem. Phys. 23, 551 (1955).

1955 SCH/BUS $\quad$ A. A. Schaerer, C. J. Busso, A. E. Smith, and L. B. Skinner, J. Am. Chem. Soc. 77, 2017 (1955).

1955SCH/WHI R. W. Schiessler and F. C. Whitmore, Ind. Eng. Chem. 47, 1660-1665 (1955); Note: Data is deposited as supplementary material, Document No. 4597.

1955SER/VOI V. V. Serpinskii, S. A. Voitkevich, and N. Y. Lyuboshits, Zh. Fiz. Khim. 29, 653-657 (1955); Chem. Abstr. 50, 16231e (1956).

1955SHE/GIA R. H. Sherman and W. F. Giauque, J. Am. Chem. Soc. 77, 2154-2160 (1955).

1955SPI/STA J. E. Spice, L. A. Staveley, and G. A. Harrow, J. Chem. Soc. 1955, 100-104.

1955TSY/YA O. Tsypikina and J. Ya, J. Appl. Chem. (U.S.S.R.) 28, 167-172 (1955); Chem. Abstr. 49, 9967h (1955).

$1955 T U N / S T O \quad$ D. D. Tunnicliff and H. Stone, Anal. Chem. 27, 73 (1955).

1955WAR/VIC T. L. Ward, E. J. Vicknair, W. S. Singleton, and R. O. Feuge, J. Phys. Chem. 59, 4 (1955).

1956ALL T. L. Allen, J. Am. Chem. Soc. 78, 5476-5478 (1956).

1956BEY/NIC J. H. Beynon and G. R. Nicholson, J. Sci. Instrum. 33, 376-380 (1956).

1956BRO/DOM H. C. Brown and L. Domash, J. Am. Chem. Soc. 78, 5384-5386 (1956).

1956BUR/GOO A. B. Burg and C. D. Good, J. Inorg. Nucl. Chem. 2, 237-245 (1956).

1956DIC W. Dickinson, Trans. Faraday Soc. 52, 31-35 (1956).

1956ENG/ATZ A. Engelbrecht and H. Atzwanger, J. Inorg. Nucl. Chem. 2, 348 (1956).

1956GLE/VOL O. Glemser and H. G. Volz, Naturwissenschaften 43, 33 (1956).

1956GOO/SCO W. D. Good, D. W. Scott, and G. Waddington, J. Phys. Chem. 60, 1090-1095 (1956).

1956GRA/STO W. A. G. Graham and F. G. A. Stone, J. Inorg. Nucl. Chem. 3, 164 (1956).

1956HOR/GOU H. Horn and E. S. Gould, J. Am. Chem. Soc. 78, 5772 (1956).

1956LI J. C. M. Li, J. Am. Chem. Soc. 78, 1081-1083 (1956).

1956LI/PIT J. C. M. Li and K. S. Pitzer, J. Am. Chem. Soc. 78, 1077-1080 (1956).

1956LON/SAC L. H. Long and J. F. Sackman, Trans. Faraday Soc. 52, 1201-1206 (1956).

1956MAC A. G. MacDiarmid, J. Inorg. Nucl. Chem. 2, 88-94 (1956).

1956MAG

1956MAJ
1956MAJ2

1956MAN2

T. G. Majury, J. Soc. Dyers Colour. 72, 41-49 (1956).

Manufacturing Chemists Association Research Project 1956, Tables 23-18-2(1.01111)-k and 23-18-2(1.01112)k; Note: The authors suspect that the tabulated Antoine Constants for the various compounds were generated in the manner discussed in [1961LI/ROS].

1956MAT/ERI H. C. Mattraw, C. E. Erickson, and A. W. Laubengayer, J. Am. Chem. Soc. 78, 4901-4904 (1956).

1956MOM/SUG M. Momotani, H. Suga, S. Seki, and I. Nitta, Proc. Natl. Acad. Sci., India, Sect. A 25, 74 (1956).

1956ROG/SPE M. T. Rogers and J. L. Speirs, J. Phys. Chem. 60, 1462-1463 (1956).

1956SER/VOI V. V. Serpinskii, S. A. Voitkevich, and N. Y. Lyuboshits, Zh. Fiz. Khim. 30, 177-183 (1956).

1956SMI/DOL C. W. Smith and M. Dole, J. Polym. Sci. 20, 37 (1956).

1956WIL/COT G. Wilkinson, F. A. Cotton, and J. Birmingham, J. Inorg. Nucl. Chem. 2, 95-113 (1956).

1957CAR/DAV E. L. Carpenter and H. S. Davis, J. Appl. Chem. 7, 671-676 (1957)

1957CLA/EME H. C. Clark and H. J. Emeleus, J. Chem. Soc. 1957, 2119.

1957DAL/EME J. W. Dale, H. J. Emeleus, R. N. Haszeldine, and J. H. Moss, J. Chem. Soc. 1957, 3708-3713.

1957DAY/OES J. H. Day and C. Oestruch, J. Org. Chem. 22, 214-216 (1957).

1957DOB/KEL A. Dobry and R. Keller, J. Phys. Chem. 61, 1448-1449 (1957).

1957FIS/COT A. K. Fischer, F. A. Cotton, and J. Wilkinson, J. Am. Chem. Soc. 79, 2044-2046 (1957).

1957GLA/RUL F. Glaser and H. Ruland, Chem. Ing. Tech. 29, 772-775 (1957).

1957JAR R. L. Jarry, J. Phys. Chem. 61, 498 (1957).

1957KAE/STO H. D. Kaesz and F. G. A. Stone, J. Chem. Soc. 1957, 1433-1435.

1957LIT

1957MAI/SEY

1957MAS

R. Littlewood, J. Chem. Soc. 1957, 2419-2420.

1957MCC/FIN Am. Chem. Soc. 79, 5884-5889 (1957).

S. V. R. Mastrangelo, Anal. Chem. 29, 841 (1957).

$\begin{array}{ll}\text { 1957MCC/FIN2 } & \text { J. P. McCullough, H. L. Finke, J. F. Messerly, S. S. Todd, }\end{array}$ T. C. Kincheloe, and G. Waddington, J. Phys. Chem. 61, 1105 (1957).

1957PED/SKI J. B. Pedley, H. A. Skinner, and C. L. Chernick, Trans. Faraday Soc. 53, 1612-1617 (1957).

1957SER/VOI V. V. Serpinskii, S. A. Voitkevich, and N. Y. Lyuboshits, Zh. Fiz. Khim. 31, 1278-1284 (1957); Chem. Abstr. 52, 3270f (1958).

1957SPI L. H. Spinar, Ph.D. dissertation, University of Wisconsin, 1957; as mentioned in W. S. Johnson, J. L. Margrave, V. J. Bauer, M. A. Frisch, L. H. Dreger, and W. N. Hubbard, J. Am. Chem. Soc. 82, 1255-1256 (1960).

1957SUS/WUH $\quad$ B. P. Susz and J.-J. Wuhrmann, Helv. Chim. Acta 722-730 (1957).

1957VAN A. VanKamp, Ph.D. dissertation, Free University of Amsterdam, 1957.

1957WIL

1958BLO/BOC

R. C. Wilhoit, J. Phys. Chem. 61, 114-116 (1957).

1958BLO/WEL H. Bloom and B. J. Welch, J. Phys. Chem. 62, 1594-1595 (1958).

1958BRA/KAY D. C. Bradley, L. J. Kay, J. D. Swanick, and W. Wardlaw, J. Chem. Soc. 1958, 3656-3659.

1958BRA/SWA $\quad$ D. C. Bradley and J. D. Swanwick, J. Chem. Soc. 1958 3207-3214.

1958CAN/NEW S. Cantor, R. F. Newton, W. R. Grimes, and F. F. Blankenship, J. Phys. Chem. 62, 96-99 (1958).

1958CAR/STR A. S. Carson, D. R. Stranks, and B. R. Wihmshurst, Proc. R. Soc. A 244, 72-84 (1958).

1958CAS/FLE3 R. C. Cass, S. E. Fletcher, C. T. Mortimer, H. D. Springall, and T. R. White, J. Chem. Soc. 1958, 1406-1410.

1958COL/KEN C. B. Colburn and A. Kennedy, J. Am. Chem. Soc. 80, 5004 (1958). 
1958DIL/MCN

C. R. Dillard, E. H. McNeill, D. E. Simmons, and J. B. Yeldell, J. Am. Chem. Soc. 80, 3607 (1958).

1958DUN/HAN A. S. Dunn and A. Hanrahan, J. Chem. Soc. 1958, 534.

1958DUN/MUR R. D. Dunlap, C. J. Murphy, Jr., and R. C. Bedford, J. Am. Chem. Soc. 80, 83-85 (1958).

1958EBS/EME E. A. V. Ebsworth and H. J. Emeleus, J. Chem. Soc. 1958, 2150-2156.

1958EIS/ROT M. Eisenstadt, G. M. Rothberg, and P. Kusch, J. Chem. Phys. 29, 797-804 (1958).

1958EME/SMI H. J. Emeleus and J. D. Smith, J. Chem. Soc. 1958, $527-528$.

1958EME/SMY H. J. Emeleus and L. E. Smythe, J. Chem. Soc. 1958, 609.

1958FEH/HIT F. Feher and G. Hitzemann, Z. Anorg. Allg. Chem. 294, 50-62 (1958).

1958FIN/SCH A. Finch and H. I. Schlesinger, J. Am. Chem. Soc. 80, 3573-3574 (1958).

1958FIS/SCH $\quad$ E. O. Fischer and S. Schreiner, Chem. Ber. 91, 2213-2215 (1958).

1958FOW/MOR P. A. Fowell and C. T. Mortimer, J. Chem. Soc. 1958, 3734-3736.

1958FRO/ROC R. E. Frost and E. G. Rochow, J. Inorg. Nucl. Chem. 52, 201-206 (1958); Chem. Abstr. 52, 6999c (1958).

1958GOO/FAI W. D. Good, D. M Fairbrother, and G. Wadddington, J. Phys. Chem. 62, 853-856 (1958).

1958 HAB/UEN $\quad$ C. P. Haber and R. K. Uenishi, Ind. Eng. Chem. Chem. Eng. Data Ser. 3, 323-324 (1958); Chem. Abstr. 53, 16781b (1959)

1958HIE/WAG W. Hieber and G. Wagner, Justus Liebigs Ann. Chem. 618, 24-30 (1958).

1958 HIL/KRA $\quad$ D. L. Hildenbrand, W. R. Kramer, R. A. McDonald, and D. R. Stull, J. Am. Chem. Soc. 80, 4129 (1958).

1958HOY/PEP H. Hoyer and W. Peperle, Z. Electrochem. 62, 61-66 (1958).

1958KLO

V. P. Klochkov, Zh. Fiz. Khim. 32, 1177 (1958); Chem. Abstr. 52, 19394f (1958).

1958KOE/GIA J. K. Koehler and W. F. Giauque, J. Am. Chem. Soc. 80, 2659-2662 (1958).

1958LON/SAC L. H. Long and J. F. Sackman, Trans. Faraday Soc. 54, 1797-1803 (1958).

1958MAT/GEL I. G. Matveev, N. I. Gel'perin, K. V. Vil'shau, and R. L. Globus, Zh. Prikl. Khim. 31, 868 (1958)

1958 NOV/SEM A. V. Novoselova, K. N. Semenenko, and O. Ya Turova, Vestn. Mosk. Univ., Ser. Mat., Mekh., Astron., Fiz., Khim. 13, 139-147 (1958).

1958POR/SCH R. F. Porter and R. C. Schoonmaker, J. Chem. Phys. 29, 1070-1074 (1958).

1958ROM/GOR U. D. Roman and A. M. Gorriz, An. R. Soc. Esp. Fis. Quim. 54B, 559 (1958); Chem. Abstr. 53, 92394 (1959).

1958ROS/PAP A. Rose, B. T. Paphronis, and E. T. Williams, J. Chem. Eng. Data 3, 216-219 (1958).

1958SCH/POR R. C. Schoonmaker and R. F. Porter, J. Chem. Phys. 29, 116-120 (1958).

1958SCH/WAG K. Schafer and U. Wagner, Z. Elektrochem. 62, 328 (1958).

1958SEE/LAN F. Seel and J. Langer, Z. Anorg. Allg. Chem. 295, 315-326 (1958).

1958SEN/STO K. A. Sense and R. W. Stone, J. Phys. Chem. 62, 453-457 (1958).

1958SER/VOI V. V. Serpinskii, S. A. Voitkevich, and N. Y. Lyuboshits, Trudy Vsesoyuz. Nauch.-Issledovatel. Inst. Sintet. I. Natural. Dushistykh Veshchestv 4, 125-130 (1958).

1958VAR

1959AIH M. C. P. Varma, J. Appl. Chem. 8, 117 (1958)

A. Aihara, Bull. Chem. Soc. Jpn. 32, 1242-1248 (1959).

1959ALT/BRO E. R. Alton, R. D. Brown, J. C. Carter, and R. C. Taylor, J. Am. Chem. Soc. 81, 3550-3551 (1959).

1959AMB E. Amberger, Angew. Chem. 71, 372-373 (1959); Chem. Abstr. 53, 16780c (1959).

1959BAR/BOY W. F. Barber, C. F. Boynton, and P. E. Gallagher, U. S. Depart. Commerce, Tech. Services PB Report 148374, 1959 ; Chem. Abstr. 56, 71437 (1962).

1959BRI/STO F. E. Brinckman and F. G. A. Stone, J. Inorg. Nucl. Chem. 11, 24 (1959).
1959BUR/GRA

A. B. Burg and L. R. Grant, J. Am. Chem. Soc. 81, 1-5 (1959).

$1959 \mathrm{COR} / \mathrm{SCH} \quad$ J. F. Cordesand and S. Schreiner, Z. Anorg. Allg. Chem. 299, 87-91 (1959).

1959COT/FIS F. A. Cotton, A. K. Fischer, and J. Wilkinson, J. Am. Chem. Soc. 81, 800-803 (1959).

1959CUL/EME W. R. Cullen and H. J. Emeleus, J. Chem. Soc. 1959, 372-375.

1959DAR/YOS A. J. Darnell and S. J. Yosim, J. Phys. Chem. 63 1813-1815 (1959).

1959DAV/JON M. Davies and A. H. Jones, Trans. Faraday Soc. 55, 1329-1332 (1959).

1959DAV/JON2 M. Davies, A. H. Jones, and G. H. Thomas, Trans. Faraday Soc. 55, 1100-1108 (1959).

1959FIS/GRU E. O. Fischer and H. Grubert, Chem. Ber. 92, 2302-2309 (1959).

1959FOL/MOR P. A. Folwell and C. T. Mortimer, J. Chem. Soc. 1959, 2913-2918.

1959GIL/TOM H. Gilman, R. A. Tomasi, and D. Wittenberg, J. Org. Chem. 24, 821 (1959)

1959GOO/SCO W. D. Good, D. W. Scott, J. L. Lacina, and J. P. McCullough, J. Phys. Chem. 63, 1139-1142 (1959).

1959JAN/GOO G. J. Janz and J. Goodkin, J. Phys. Chem. 63, 1975 (1959).

1959LEA/SPI A. J. Leadbetter and J. E. Spice, Can. J. Chem. 37, 1923-1929 (1959).

1959MCD/SHR R. A. McDonald, S. A. Shrader, and D. R. Stull, J. Chem. Eng. Data 4, 311-313 (1959).

1959MOD K. Mödritzer, Chem. Ber. 92, 2637-2640 (1959); Chem. Abstr. 54, 3180e (1959)

1959NES/IOF A. N. Nesmeyanov and B. Z. Iofa, Zh. Neorg. Khim. 4 486 (1959).

1959PAR/MAC L. J. Paridon, G. E. MacWood, and J. -H. Hu, J. Phys. Chem. 63, 1998-1999 (1959).

1959PED/SKI J. B. Pedley and H. A. Skinner, Trans. Faraday Soc. 55, 544-547 (1959).

1959PIK/FOS F. P Pike and C. F. Foster, Jr., J. Chem. Eng. Data 4 305-306 (1959).

1959SCH/MAR R. S. Scheffee and J. L. Margrave, J. Chem. Phys. 31, 1682-1683 (1959).

1959SEM/GOR K. N. Semenenko and I. V. Gordeev, Zh. Neorg. Khim. 4, 952-954 (1959); Chem. Abstr. 54, 8206d (1960).

1959SUG/SEK H. Suga and S. Seki, Bull. Chem. Soc. Jpn. 32, 1088 (1959).

1959TAK/SHI S. Takagi, R. Shintani, H. Chihara, and S. Seki, Bull. Chem. Soc. Jpn. 32, 137-142 (1959); Chem. Abstr. 54, $1958 \mathrm{~g}(1960)$.

1959TER/BRI E. Terres, L. Brinkmann, D. Fischer, D. Hüllstrung, W. Loz, and G. Weisbrod, Brennst.-Chem. 40, 279-280 (1959).

1959WES/LEV E. F. Westrum, Jr. and N. W. Levitin, J. Am. Chem. Soc. 81, 3544-3547 (1959).

1960AIH A. Aihara, Bull. Chem. Soc. Jpn. 33, 194-200 (1960).

1960AIH2

1960AMB

1960BED/MOR

A. Aihara, Bull. Chem. Soc. Jpn. 33, 1188-1194 (1960). E. Amberger, Angew. Chem. 72, 494 (1960); Chem. Abstr. 55, 22103f (1961); 60, 4171d (1964).

1960BER/TRU E. W. Berg and J. T. Truemper, J. Phys. Chem. 64, 487-490 (1960); Note: In the manuscript the authors imply that some of the experimental values may be enthalpies of vaporization; however, in the tabulation of experimental data all numerical values are labeled as enthalpies of sublimation.

1960BIR K.-H. Birr, Z. Anorg. Allg. Chem. 306, 21-24 (1960).

1960BRA/BIR R. S. Bradley, C. L. Bird, and F. Jones, Trans. Faraday Soc. 56, 23-28 (1960).

1960BRI/STO F. E. Brinckman and F. G. A. Stone, J. Am. Chem. Soc. 82, 6218-6223 (1960).

1960BRO/MCC R. J. Brotherton, A. L. McCloskey, J. L. Boone, and H. M. Manasevit, J. Am. Chem. Soc. 82, 6245-6248 (1960).

1960BRO/MCC2 R. J. Brotherton, A. L. McCloskey, L. L. Petterson, and H. Steinberg, J. Am. Chem. Soc. 82, 6242-6245 (1960). 
1960BUD

S. Budurov, Izv. Khim. Inst., Bulg. Akad. Nauk. 7, 281-301 (1960).

1960CLA/FOW A. P. Claydon, P. A. Fowell, and C. T. Mortimer, J. Chem. Soc. 1960, 3284-3287.

1960COT/MON F. A. Cotton and R. R. Monchamp, J. Chem. Soc. 1960, 533-536.

1960DAV/THO M. Davies and G. H. Thomas, Trans. Faraday Soc. 56, 185-192 (1960).

1960EDW/KIN J. W. Edwards, G. L. Kington, and R. Mason, Trans. Farday Soc. 56, 660-669 (1960).

1960EME/PUG H. J. Emeleus and H. Pugh, J. Chem. Soc. 1960, 1108-1112.

1960FRE/GRE M. B. Frey and N. W. Gregory, J. Am. Chem. Soc. 82, 1068 (1960).

1960GRE/MOR N. N. Greenwood and J. N. Morris, J. Chem. Soc. 1960, 2922.

1960GRE/WAD N. N. Greenwood and K. Wade, J. Chem. Soc. 1960, $1130-1141$.

1960HAL/LAN S. Hala and S. Landa, Collect. Czech. Chem. Commun. 25, 2692 (1960).

1960HIE/BRA W. Hieber, G. Braun, and W. Beck, Chem. Ber. 93, 901-908 (1960).

1960JON A. H. Jones, J. Chem. Eng. Data 5, 196-200 (1960).

1960KAE/PHI H. D. Kaesz, J. P. Phillips, and F. G. A. Stone, J. Am. Chem. Soc. 82, 6228-6232 (1960).

1960KAE/STO H. D. Kaesz and F. G. A. Stone, J. Am. Chem. Soc. 82, 6213-6218 (1960).

1960KAR/STR K. A. Karasharli and P. G. Strelkov, Dokl. Akad. Nauk SSSR 131, 568 (1960).

1960KEA/SMI J. J. Keavney and N. O. Smith, J. Phys. Chem. 64, 737 (1960).

1960MAL/MAL N. A. Malafeev, V. A. Malyusov, N. N. Umnik, I. V. Podgornaya, and V. M. Zhavoronkov, Dokl. Akad. Nauk SSSR 135, 659 (1960); Chem. Abstr. 56, 5115 (1962).

$1960 \mathrm{MCL} / \mathrm{TAM} \quad$ D. E. McLaughlin and M. Tamres, J. Am. Chem. Soc. 82, 5618-5621 (1960).

1960MCL/TAM2 D. E. McLaughlin, M. J. Tamres, and S. Searless, Jr., J. Am. Chem. Soc. 82, 5621-5625 (1960).

$1960 \mathrm{MON} / \mathrm{COT} \quad$ R. R. Monchamp and F. A. Cotton, J. Chem. Soc. 1960, 1438-1440.

1960PET R. C. Petry, J. Am. Chem. Soc. 82, 2400 (1960).

$1960 \mathrm{SCH} / \mathrm{HIR} \quad$ R. G. Schmitt and R. C. Hirt, J. Polym. Sci. 45, 35-47 (1960).

1960SIM/GRE R. G. Sime and N. W. Gregory, J. Phys. Chem. 64, 86-89 (1960).

1960STA/STO S. L. Stafford and F. G. A. Stone, J. Am. Chem. Soc. 82, 6238-6240 (1960).

1960WAR/MAC L. G. L. Ward and A. G. MacDiarmid, J. Am. Chem. Soc. 82, 2151-2153 (1960).

1960WEY/KLI F. Weygand, G. Klipping, and D. Palm, Chem. Ber. 93, 2619-2625 (1960).

1961AMB E. Amberger, Chem. Ber. 94, 1447-1452 (1961); Chem. Abstr. 56, 8279e (1962).

$1961 \mathrm{AMB} / \mathrm{BOE} \quad$ E. Amberger and H. Boeters, Angew. Chem. 73, 114 (1961); Chem. Abstr. 55, 16403h (1961).

1961BER/DOW E. W. Berg and H. W. Dowling, J. Chem. Eng. Data 6, 556-557 (1961).

1961DAC/WYA B. Dacre and P. A. H. Wyatt, Trans. Faraday Soc. 57, 1281-1285 (1961).

1961DAV/MAL M. Davies and V. E. Malpass, J. Chem. Soc. 1961, 1048-1055.

1961DIT/PER J. F. Ditter, J. C. Perrine, and I. Shapiro, J. Chem. Eng. Data 6, 271 (1961).

1961FIS/FRI $\quad$ E. O. Fischer and H. P. Fritz, Angew. Chem. 73, 353-364 (1961).

1961FIS/SCH E. O. Fischer, S. Schreiner, and A. Reckziegel, Chem. Ber. 94, 258-262 (1961).

1961FOW/MOR P. A. Fowell and C. T. Mortimer, J. Chem. Soc. 1961, 3793-3796.

1961GIN A. A. Ginzburg, Zh. Prikl. Khim. 34, 2569 (1961).

$1961 \mathrm{GRI} / \mathrm{ONY}$ J. E. Griffiths and M. Onyszchuk, Can. J. Chem. 39, 339-347 (1961).
1961GRO/KLE

H. Grosse-Ruyken and R. Kleesaat, Z. Anorg. Allg. Chem. 308, 122-132 (1961).

1961JOH/KIL W. H. Johnson, M. V. Kilday, and E. J. Prosen, J. Res. Natl. Bur. Stand., Sect. A 65A, 215-219 (1961).

1961LI/ROS

J. C. M. Li and F. D. Rossini, J. Chem. Eng. Data 6, 268-270 (1961).

1961ROZ/POL I. L. Rozenfel'd, M. N. Polteva, and V. P. Persiantseva, Zh. Fiz. Khim. 35, 1474-1477 (1961).

1961SCH/BRA M. Schmeisser and K. Braendle, Angew. Chem. 73, 388-393 (1961).

1961SCH/TAG M. Schmeisser and L. Taglinger, Chem. Ber. 94, 1533-1539 (1961).

1961SCO/MES ～D. W. Scott, J. F. Messerly, S. S. Todd, G. B. Gutherie, I. A. Hosenlopp, R. T. Moore, A. Osborn, W. T. Berg, and J. P. McCullough, J. Phys. Chem. 65, 1320-1326 (1961).

1961SHR/CAD J. M. Shreeve and G. H. Cady, J. Am. Chem. Soc. 83, 4521 (1961).

1961STE/MAC B. Sternback and A. G. MacDiarmid, J. Am. Chem. Soc. 83, 3384-3388 (1961).

1961WAG/NEU H. Wagner and K. Neumann, Z. Phys. Chem. 28, 51-70 (1961).

1961ZIM/GEI H. Zimmermann and H. Geisenfelder, Z. Electrochem. 65 368-371 (1961).

1962AMB/BOE E. Amberger and H. Boeters, Angew. Chem. 74, 293 (1962); Chem. Abstr. 57, 4276f (1962).

1962AMB/BOE2 E. Amberger and H. Boeters, Angew. Chem. 74, 32-33 (1962); Chem. Abstr. 57, 12522e (1962).

1962BER/MAR J. Berkowitz and J. R. Marquart, J. Chem. Phys. 37, 1853-1865 (1962).

1962BRA/JUN J. D. Brandner, N. N. Junk, J. W. Lawrence, and J. Robins, J. Chem. Eng. Data 7, 227-228 (1962).

1962CAR/COO A. Carson, R. Cooper, and D. R. Stranks, Trans. Faraday Soc. 58, 2125-2133 (1962).

1962CHA

A. M. Chaikin, Zh. Fiz. Khim. 36, 130-131 (1962); Chem. Abstr. 58, 7387e (1962).

1962CHA/WES S.-S. Chang and E. F. Westrum, Jr., J. Chem. Phys. 36, 2420 (1962).

1962COH/MAC B. Cohen and A. G. MacDiarmid, Inorg. Chem. 1, 754-756 (1962).

1962CON/URR J. W. Connolly and G. Urry, Inorg. Chem. 1, 718 (1962).

1962CRA/MAC A. D. Craig and A. G. MacDiarmid, J. Inorg. Nucl. Chem. 24, 161-164 (1962).

1962CRA/URE A. D. Craig, J. V. Urenovitch, and A. G. MacDiarmid, J. Chem. Soc. 1962, 548-553.

1962DOW

1962EBS/EME

A. J. Downs, J. Chem. Soc. 1962, 4361-4366.

1962EBS/MAY E. A. V. Ebsworth and M. J. Mays, J. Chem. Soc. 1962,

Chem. Soc. 1962, 2290-2293. 4844.

1962EDW/KIN J. W. Edwards and G. L. Kingston, Trans. Faraday Soc. 58, 1323-1333 (1962).

1962EME/PAC H. J. Emeleus, K. J. Packer, and N. Welcman, J. Chem. Soc. 1962, 2529-2531.

1962GEI/QUI

G. Geiseler, K. Quitzsch, J. Hoffmann, and W. Moeschler, Z. Phys. Chem. (Leipzig) 200, 391-396 (1962).

1962GEI/QUI2 G. Geiseler, K. Quitzsch, J. Hesselbach, and R. Huettig, Z. Phys. Chem. (Leipzig) 200, 79-88 (1962).

1962GOU/FRO J. Goubeau and H. D. From, Z. Anorg. Allg. Chem. 317, 41 (1962).

1962HAR/LUT H. Hartmann and H. Lutsche, Naturwissenschaften 49, 182 (1962); Chem. Abstr. 57, 62860 (1962).

1962JOH/MCE I. B. Johns, E. A. McElhill, and J. O. Smith, J. Chem. Eng. Data 7, 277-281 (1962)

1962JON/YOW M. M. Jones, B. W. Yow, and W. R. May, Inorg. Chem. 1 , 166 (1962).

1962KOP R. W. Kopp, United States Atomic Energy Commission [Unclassified and Declassified Reports Published by the Atomic Energy Commission and Its Contractors], UCRL10437, 53 (1962).

1962KRA/BER M. Kraus, L. Beranek, K. Kochloeff, and V. Bazant, Chem. Prumsyl 12, 649-652 (1962); Chem. Abstr. 58, $10746 \mathrm{c}$ (1963). 
1962LEB/MIR Y. A. Lebedev, E. A. Miroshnichenko, and A. M. Chaikin, Dokl. Akad. Nauk SSSR 145, 1288-1289 (1962); Chem. Abstr. 58, 2906g (1963).

1962MAC/MAY3 H. Mackle and R. G. Mayrick, Trans. Faraday Soc. 58, 238-243 (1962).

1962OLI/STE J. P. Oliver and L. G. Stevens, J. Inorg. Nucl. Chem. 24, 953-960 (1962).

1962PAC/PET $\quad$ E. L. Pace and R. V. Petrella, J. Chem. Phys. 36, 2991 (1962).

1962RAB/TEL I. B. Rabinovich, V. I. Tel'noi, L. M. Terman, A. S. Kirillova, and G. A. Razuvaev, Dokl. Akad. Nauk SSSR 143, 133-136 (1962); Eng. Edit. 143, 171 (1962); Chem. Abstr. 57, 4557e (1962).

1962VOH/KAN S. P. Vohra, T. L. Kang, K. A. Kobe, and J. J. McKetta, J. Chem. Eng. Data 7, 150-155 (1962).

1963ABE/MAC M. Abedini and A. G. MacDiarmid, Inorg. Chem. 2, 608-613 (1963).

1963ABE/TIL D. C. Abercromby and P. F. Tiley, J. Chem. Soc. 1963, 4902-4903.

1963AKA/BEL P. A. Akashin, V. I. Belousov, and L. N. Sidorov, Russ. J. Inorg. Chem. 8, 789-790 (1963).

1963AMB/BOE E. Amberger and H. Boeters, Z. Naturforsch., B 18, 157 (1963); Chem. Abstr. 59, 5194g (1963).

1963BAU/MAR R. G. Bautista and J. L. Margrave, J. Phys. Chem. 67, 1564 (1963).

1963BES

1963BON

R. J. Best, J. Chem. Eng. Data 8, 267-270 (1963).

1963BOY

1963DAV/POP

A. Bondi, J. Chem. Eng. Data 8, 371-381 (1963).

R. H. Boyd, J. Chem. Phys. 38, 2529-2535 (1963).

J. V. Davies, A. E. Pope, and H. A. Skinner, Trans. Faraday Soc. 59, 2233-2242 (1963).

1963DIX/YAR J. A. Dixon, G. R. Yarnell, and J. A. Mountain, J. Chem. Eng. Data 8, 572-573 (1963).

1963EBS/FRA E. A. V. Ebsworth and S. G. Frankiss, J. Chem. Soc. 1963, 661-665.

1963EME/HAA H. J. Emeleus and A. Haas, J. Chem. Soc. 1963, $1272-1275$

1963EME/WEL H. J. Emeleus and N. Welcman, J. Chem. Soc. 1963, $1268-1271$.

1963FEI/BOB M. M. Fein, J. Bobinski, N. Mayes, N. Schwartz, and M. S. Cohen, Inorg. Chem. 2, 1111-1115 (1963).

1963GAL/TUM N. P. Galkin, Y. N. Tumanov, V. I. Tarasov, and Y. D. Shishov, Russ. J. Inorg. Chem. 8, 1054-1055 (1963).

1963GAL/VAR G. L. Gal'chenko and R. M. Varushchenko, Zh. Fiz. Khim. 37, 2513-2519 (1963).

1963GRE/FOS M. A. Greenbaum, J. N. Foster, M. L. Arin, and M. Farber, J. Phys. Chem. 67, 36-40 (1963).

1963HAR/HOL S. B. Hartley, W. S. Holmes, J. K. Jaques, M. F. Mole, and J. C. McCoubrey, Q. Rev., Chem. Soc. 17, 204 (1963).

1963LAU/TRO W. F. Lautsch, A. Tröber, W. Zimmer, L. Mehner, W. Linck, H. M. Lehman, H. Brandenberger, H. Korner, H.J. Metschker, K. Wagner, and R. Kaden, Z. Chem. 3, 415-421 (1963).

1963LUS/CAD M. Lustig and G. H. Cady, Inorg. Chem. 2, 388-390 (1963).

1963MAR/FRI J. L. Margrave, M. A. Frisch, R. G. Bautista, R. L. Clarke, and W. S. Johnson, J. Am. Chem. Soc. 85, 546-548 (1963).

1963MCC/MES J. P. McCullough, J. F. Messerly, R. T. Moore, and S. S. Todd, J. Phys. Chem. 67, 677-679 (1963).

1963 MOR/SEL C. T. Mortimer and P. Sellers, J. Chem. Soc. 1963, 1978-1981.

1963MOR/SUN $\quad$ E. Morawetzer and S. Sunner, Acta Chem. Scand. 17, 473 (1963).

1963MYE/PUT H. W. Myers and R. F. Putnam, Inorg. Chem. 2, 655 (1963).

1963PAC/MOS E. L. Pace and J. S. Mosser, J. Chem. Phys. 39, 154 (1963).

1963PAS/ROB G. Pass and H. L. Roberts, Inorg. Chem. 2, 1016-1019 (1963).

1963POP/SKI A. E. Pope and H. A. Skinner, J. Chem. Soc. 1963, 3704-3712.

1963RAB/TEL I. B. Rabinovich, V. I. Tel'noi, N. V. Karyakin, and G. A. Razuvaev, Dokl. Akad. Nauk SSSR 149, 324-326(1963).
1963RAS/NIG

R. P. Rastogi, R. P. Nigam, R. N. Sharma, and H. L. Girdhar, J. Chem. Phys. 39, 3042-3045 (1963).

1963ROS/SCH A. Rose and V. N. Schrodt, J. Chem. Eng. Data 8, 9-13 (1963).

1963RYS/HAR G. E. Ryschkewitsch, S. W. Harris, E. J. Mezley, H. H. Sisler, E. A. Weilmuenster, and A. B. Garrett, Inorg. Chem. 2, 890 (1963).

1963SHA/KEI I. Shapiro, B. Keilin, R. E. Williams, and C. D. Good, J. Am. Chem. Soc. 85, 3167-3171 (1963).

1963SIM

1963SPA/MAC R. J. Sime, J. Phys. Chem. 67, 501 (1963).

1963URE/MAC E. J. Spanier and A. G. MacDiarmid, Inorg. Chem. 2, 214-215 (1963)

1963WOO/JON

J. V. Urenovitch and A. G. MacDiarmid, J. Chem. Soc. 1963, 1091-1095.

1964ADR/DEK N. Adriaanse, H. Dekker, and J. Coops, Recl. Trav. Chim. Pays-Bas 83, 557 (1964).

1964ARN

1964AYL/PET H. Arnold, Z. Phys. Chem. (Leipzig) 225, 45 (1964).

1964BAL/DON B. J. Aylett and L. K. Peterson, J. Chem. Soc. 1964, 3429-3436.

1964BIL/COT

P. Balk and D. Dong, J. Phys. Chem. 68, 960-962 (1964).

J. A. Bills and F. A. Cotton, J. Phys. Chem. 68, 806-810 (1964).

1964CAP/FRI

A. Capkova and V. Fried, Collect. Czech. Chem. Commun. 29, 336-340 (1964).

1964CLE/WON H. L. Clever, W.-K. Wong, C. A. Wulff, and E. F. Westrum, Jr., J. Phys. Chem. 68, 1967 (1964).

1964CUB/ELD D. Cubicciotti and H. Elding, J. Chem. Phys. 40, 978 (1964).

1964DUN/THO J. F. Duncan and F. G. Thomas, J. Chem. Soc. 1964, 360-364.

1964EHL/KEN R. C. Ehlert, R. A. Kent, and J. L. Margrave, J. Am. Chem. Soc. 86, 5093-5095 (1964).

1964FAR/JON $\quad$ D. T. Farrar and M. M. Jones, J. Phys. Chem. 68, 1717-1721 (1964).

1964FIE/MAC P. E. Fielding and A. G. Mackay, Aust. J. Chem. 17, 1288-1290 (1964).

1964FIS/PET W. Fischer, T. Petzel, and S. Lauter, Z. Anorg. Allg. Chem. 333, 226-234 (1964).

1964FRI/BAU M. A. Frisch, R. G. Bautista, J. L. Margrave, C. G. Parsons, and J. H. Wotez, J. Am. Chem. Soc. 86, 335-336 (1964).

1964GOO/LAC W. D. Good, J. L. Lacina, B. L. De Prater, and J. P. McCullough, J. Phys. Chem. 68, 579-586 (1964).

1964GRE/KO

M. A. Greenbaum, H. C. Ko, M. Wong, and M. Farber, J. Phys. Chem. 68, 965-968 (1964).

1964JON/SED

1964KEL/RIC

F. Jones and R. Seddon, Text. Res. J. 34, 373-380 (1964).

J. D. Kelley and F. O. Rice, J. Phys. Chem. 68, 3794-3796 (1964).

1964KEN/EHL R. A. Kent, T. C. Ehlert, and J. L. Margrave, J. Am. Chem. Soc. 86, 5090-5093 (1964)

1964MAC/OHA H. Mackle and P. A. G. O'Hare, Trans. Faraday Soc. 60, 506-509 (1964).

1964MAL/WEC B. Malanowska and J. Wecsile, Bull. Acad. Pol. Sci. 12 , 239 (1964).

1964MAN E. G. Mangalin, Khim. Prom. 4, 304-306 (1964).

1964MOR D. W. Morecroft, J. Chem. Eng. Data 9, 488-490 (1964).

1964MOR/SEL C. T. Mortimer and P. Sellers, J. Chem. Soc. 1964, 1965-1970.

1964POP/SKI A. E. Pope and H. A. Skinner, Trans. Faraday Soc. 60, 1404-1407 (1964).

1964RAS/BAS R. P. Rastogi and P. S. Bassi, J. Phys. Chem. 68, 2398 (1964).

1964RAT/SHR C. T. Ratcliffe and J. M. Shreeve, Inorg. Chem. 3, 631-633 (1964).

1964ROB

$1964 \mathrm{ROS} / \mathrm{SCH}$

H. L. Roberts, J. Chem. Soc. 1964, 4538.

1964SHA/TUB Y. K. Shaulov, V. S. Tubyanskaya, E. V. Evstegneeva, and G. O. Shmyreva, Russ. J. Phys. Chem. 38, 967-970 (1964). 
1964SMI/GOR N. K. Smith, G. Gorin, W. D. Good, and J. P. McCullough, J. Phys. Chem. 68, 940-946 (1964).

1964WOO/JON J. L. Wood and M. M. Jones, Inorg. Chem. 3, 1553-1556 (1964).

1965BAC/BET R. A. Back and J. Betts, Can. J. Chem. 43, 2157-2161 (1965).

1965BER/TRU E. W. Berg and J. T. Truemper, Anal. Chim. Acta 32, 245-252 (1965)

1965BLA/GRE J. A. Blauer, M. A. Greenbaum, and M. Farber, J. Phys. Chem. 69, 1069-1070 (1965).

1965BOY/CHR R. H. Boyd, R. L. Christensen, and R. Pua, J. Am. Chem. Soc. 87, 3554-3559 (1965).

1965 CAL/GIA G. V. Calder and W. F. Giauque, J. Phys. Chem. 69, 2443 (1965).

1965CLE/WES H. L. Clever, E. F. Westrum, Jr., and A. W. Cordes, J. Phys. Chem. 69, 1214-1219 (1965).

1965COL/COU R. R. Collerson, R. Handley, J. F. Martin, and C. H. S. Sprake, J. Chem. Soc. 1965, 3697-3700.

1965CUR/SHA J. Curry and R. W. Shaw, J. Phys. Chem. 69, 344-346 (1965).

1965DAV/KYB M. Davies and B. Kybett, Trans. Faraday. Soc. 61, 1608-1617 (1965); 61, 1893-1895 (1965).

1965EVS/SHM E. V. Evstigneeva and G. O. Shmyreva, Russ. J. Phys. Chem. 39, 529-530 (1965).

1965FIN/MES H. L. Finke, J. F. Messerly, and S. S. Todd, J. Phys. Chem. 69, 2094-2100 (1965).

1965FRA

W. A. Frankhauser, M.S. dissertation, Air Force Institute of Technology, Wright Patterson Air Force Base, Ohio, 1965; as cited in Ref. [1972FON/POM].

1965FRA/AST M. Frankosky and J. G. Aston, J. Phys. Chem. 69, 3126-3131 (1965).

1965GAK/BAB I. J. Gakh and E. P. Babin, Russ. J. Phys. Chem. 39, 924 (1965).

1965GUM/KOS D. O. Gumbatov and V. N. Kostryukov, Zh. Fiz. Khim. 39, 116 (1965).

1965KAL/ROZ L. A. Kalashnikova, E. G. Rozantsev, and A. M. Chaikin, Izv. Akad. Nauk SSSR 1965, 800-805; Chem. Abstr. 63, 4972c (1965).

1965KAR/KYB H. A. Karnes, B. Kybett, M. H. Wilson, J. L. Margrave, and M. S. Newman, J. Am. Chem. Soc. 87, 5554-5558 (1965).

1965KUR/HAS T. Kurosawa, R. Hasegawa, and T. Yagihasi, Trans. Natl. Res. Inst. Met. 7, 222 (1965).

1965LOE/KEN R. E. Loehman, R. A. Kent, and J. L. Margrave, J. Chem. Eng. Data 10, 296-297 (1965).

1965MAR P. Martin, J. Chem. Eng. Data 10, 292-294 (1965).

$1965 \mathrm{MCD} / \mathrm{KIL} \quad$ L. A. McDougall and J. E. Kilpatrick, J. Chem. Phys. 42, 2311-2321 (1965).

1965MES/TOD J. F. Messerly, S. S. Todd, and H. L. Finke, J. Phys. Chem. 69, 353 (1965).

1965MOR G. C. Morris, J. Mol. Spectrosc. 18, 42-50 (1965).

1965PRI/JAC S. J. Price and M. G. Jacko, Can. J. Chem. 43, 3481-3482 (1965).

1965SHA/SCH Y. K. Shaulov, G. O. Schmyreva, and V. S. Tubyanskaya, Zh. Fiz. Khim. 39, 105-109 (1965).

1965 SID/AKI L. N. Sidorov, P. A. Akishin, V. B. Shol'ts, and Y. M. Kotenev, Russ. J. Phys. Chem. 39, 1146-1150 (1965).

1966ABE/MAC M. Abedini and A. G. MacDiarmid, Inorg. Chem. 5, 2040-2042 (1966).

1966ARN/DAM R. A. Arndt and A. C. Damask, J. Chem. Phys. 45, 755 (1966).

1966BEE/MOR A. E. Beezer and C. T. Mortimer, J. Chem. Soc. A 1966, 514-516 (1966).

1966BON A. A. Boni, J. Electrochem. Soc. 113, 1089-1090 (1966); Chem. Abstr. 65, 17721h (1966).

1966BOY R. H. Boyd, Tetrahedron 22, 119-122 (1966).

1966BRA/HIL D. C. Bradley and M. J. Hillyer, Trans. Faraday Soc. 62, 2374-2381 (1966).

1966BUR/STR A. B. Burg and G. B. Street, Inorg. Chem. 5, 1532-1537 (1966).

1966COL/SKI D. J. Coleman and H. A. Skinner, Trans. Faraday Soc. 62, 1721-1755 (1966).
1966COW/BUR A. H. Cowley, A. B. Burg, and W. R. Cullen, J. Am. Chem. Soc. 88, 3178-3179 (1966).

1966CUB

1966FIN/GAR

D. Cubicciotti, J. Phys. Chem. 70, 2410-2413 (1966).

1966GAT/DRA

Soc. 62, 1072-1081 (1966)

1966GAT/KRI

G. Gattrow and M. Draeger, Z. Anorg. Allg. Chem. 343, 232-243 (1966); Chem. Abstr. 64, 18513f (1966).

1966GEI/QUI G. Geiseler, K. Quitzsch, H.-J. Rauh, H. Schaffernicht,

R. Gatti, R. L. Krieger, J. E. Sicre, and H. J. Schumacker, J. Inorg. Nucl. Chem. 28, 655 (1966). and H. Walther, Ber. Bunsen-Ges. Phys. Chem. 70, 551-556 (1966).

1966GOO/MAN W. D. Good and M. Mansson, J. Phys. Chem. 70, 97-104 (1966).

1966GRA/BUR D. N. Gray and G. Burton, J. Chem. Eng. Data 11, 59-60 (1966).

$1966 \mathrm{HOL}$ I. Holeci, Chem. Prumysl 16, 267-270 (1966).

1966JEN/SCH

D. J. Jensen and E. D. Schall, J. Agric. Food Chem. 14, 123-126 (1966).

1966JON/KRA F. Jones and J. Kraska, J. Soc. Dyers Colour. 82, 333-338 (1966).

1966KUD/ZWO A. P. Kudchadker and B. J. Zwolinski, J. Chem. Eng. Data 11, 253-255 (1966).

1966LAU/SCA

A. W. Laubengayer and C. W. Scaife, J. Chem. Eng. Data 11, 172-174 (1966).

1966LUC

1966MAT

1966MEY/MET

G. P. Luchinskii, Zh. Fiz. Khim. 40, 593-598 (1966).

S. Matsumoto, Bull. Chem. Soc. Jpn. 39, 1811 (1966).

1966MEY/WAG

R. Meyer and J. C. R. Metzger, Acad. Sci., Paris, Ser. C 263, 1333-1335 (1966).

1966MIL/MUR E. F. Meyer and
3162-3168 (1966).

$\begin{array}{ll} & \text { Chem. 5, 1832-1834 (1966). } \\ 19660 S B / S C H & \text { D. W. Osborne, F. Schreiner, J. G. Malm, H. Selig, and L. }\end{array}$ Rochester, J. Chem. Phys. 44, 2802 (1966).

1966RIN/ONE M. A. Ring, H. E. O'Neal, A. H. Kadhim, and F. J. Jappe, Organomet. Chem. 5, 124-129 (1966).

1966ROS J. M. Rosen (private communication), as quoted in Ref. [1968MAR/ARM].

1966TEL/RAB V. I. Tel'noi and I. B. Rabinovich, Russ. Zh. Fiz Khim. 40, 1556-1563 (1966).

1966ZAB R. Zablotina, Bull. Acad. Pol. Sci., Ser. Sci. Chim. 14, 835 (1966).

1966ZAL/STR A. A. Zalykin and Y. A. Strepikheev, J. Appl Chem. USSR 39, 2448 (1966).

1967AYL/CAM B. J. Aylett and J. M. Campbell, Inorg. Nucl. Chem. Lett. 3, 137-139 (1967).

1967BAL/LAP J. C. Baldwin, M. F. Lappert, J. B. Pedley, and J. A. Treverton, J. Chem. Soc. A 1980-1984 (1967).

1967BAR/POR E. M. Barrall II, R. S. Porter, and J. F. Johnson, J. Phys. Chem. 71, 895 (1967).

1967BER/WES H. A. Berman and E. D. West, J. Chem. Eng. Data 12, 197-199 (1967).

1967BRA/SMI M. X. Brady and N. O. Smith, Can. J. Chem. 45, 1125 (1967).

1967CHA/CAV T. L. Charlton and R. G. Cavell, Inorg. Chem. 6, 2204-2208 (1967).

1967CHA/HOR S. S. Chang, J. A. Horman, and A. B. Bestul, J. Res. Natl. Bur. Stand., Sect. A 71A, 293 (1967).

1967 CHI/SIM $\quad$ Y. T. Chia and H. E. Simmons, J. Am. Chem. Soc. 89, 2638-2643 (1967).

1967CHR/SHI P. M. Christopher and A. Shilman, J. Chem. Eng. Data 12, 333-335 (1967).

1967DAT/KAN K. V. Datye, P. J. Kangle, and B. Milicevic, Textilveredlung 2, 263 (1967).

1967EBS/THO E. A. V. Ebsworth and J. C. Thompson, J. Chem. Soc. A 1967, 69-72.

1967EDE/THO M. P. Edejer and G. Thodos, J. Chem. Eng. Data 12, 206-209 (1967).

1967FIN/GAR A. Finch, P. J. Gardner, E. J. Pearn, and G. B. Watts, Trans. Faraday Soc. 63, 1880-1888 (1967).

1967FIN/GAR2 A. Finch, P. J. Gardner, and G. B. Watts, Trans. Faraday Soc. 63, 1603-1607 (1967). 
1967FIN/GRA D. H. Fine and P. Gray, Combust. Flame 11, 71-78 (1967); Chem. Abstr. 66, 117537j (1967).

1967GOL/LAP Y. V. Golubkov, I. I. Lapidus, and L. A. Nisel'son, Russ. J. Phys. Chem. 41, 1122-1123 (1967).

1967GRE/JON H. S. Green and F. Jones, Trans. Faraday Soc. 63, 1612-1619 (1967)

1967HEN/EYM C. H. Henrickson and D. P. Eyman, Inorg. Chem. 6, 1461 (1967).

1967HER A. J. Herbert, Trans. Faraday Soc. 63, 555-560 (1967).

1967HIL/IRV J. O. Hill and R. J. Irving, J. Chem. Soc. A 1967, 1413-1416.

1967HUL/REI H. S. Hull, A. F. Reid, and A. G. Turnbull, Inorg. Chem. 6, 805-807 (1967).

1967KEN/CUB F. J. Keneshea and D. Cubicciotti, J. Phys. Chem. 71, 1958-1960 (1967).

1967KIS

1967LAP/NIS

A. Kisza, Roczniki Chim. 41, 351 (1967).

1967MAG

1967MES/GUT

L. L. Lapidus, L. A. Nisel'son, and A. A. Karateeva, Russ. J. Phys. Chem. 41, 241-243 (1967).

J. H. Magill, J. Chem. Phys. 47, 2802-2807 (1967).

J. F. Messerly, G. B. Guthrie, S. S. Todd, and H. L. Finke, J. Chem. Eng. Data 12, 338 (1967).

1967NAG/SEI M. Nagatani, T. Seiyama, M. Sakiyama, H. Suga, and S. Seki, Bull. Chem. Soc. Jpn. 40, 1833 (1967).

1967OMA M. M. Omar, J. Chem. Soc. C 2038-2040 (1967).

1967OPP

1967PAC

1967PAE/KUR

H. Oppermann, Z. Anorg. Allg. Chem. 356, 1 (1967).

P. Pacor, Anal. Chim. Acta 37, 200 (1967).

1967PAW S. Pawlenko, Chem. Ber. 100, 3591-35981 (1967).

1967REE/URR D. Reedy and G. Urry, Inorg. Chem. 6, 2117-2118 (1967).

1967RIN/DAM H. Ringel, A. C. Damask, and R. A. Arndt, Mol. Cryst. 3, 145 (1967).

$1967 \mathrm{SCH}$

1967SMI/GOO

C. J. Schack, Inorg. Chem. 6, 1938 (1967).

N. K. Smith and W. D. Good, J. Chem. Eng. Data 12, 570-572 (1967).

1967TSA/GIL J. Tsau and D. F. R. Gilson, J. Phys. Chem. 72, 4082 (1967).

1967TUR

1967TUR2

A. G. Turnbull, Aust. J. Chem. 20, 2757-2760 (1967).

C. Turrión, Presiones de vapor y calores de sublimación de los ácidos bencenecarboxilicos y sus ésteres metilicos. Beca "Juan March" de estudios en España, grupo B., Madrid, 1967.

1967WAK/INO N. Wakayama and H. Inokuchi, Bull. Chem. Soc. Jpn. 40, 2267-2271 (1967); Chem. Abstr. 68, 38935a (1968).

1967WIL/WAR J. M. Wilson and I. J. Worrall, J. Chem. Soc. A 1967, 392-394.

1967ZMB/MAR K. F. Zmbov and J. L. Margrave, J. Phys. Chem. 71, 2893-2895 (1967).

1968BAC/NOV D. P. Baccanari, J. A. Novinski, Y.-C. Pan, M. M. Yevitz, and H. A. Swain, Trans. Faraday Soc. 64, 1201-1205 (1968).

1968BAE A. K. Baev, Vestsi Akad. Navuk Belarusi, Ser. Khim. Navuk 1968, 76-80; Chem. Abstr. 70, 61310t (1969).

1968BUR/IAC A. B. Burg and B. Iachia, Inorg. Chem. 7, 1670-1672 (1968).

1968CHA/CAV T. L. Charlton and R. G. Cavell, Inorg. Chem. 7, 2195 (1968).

1968CHA/GOK E. T. Chang and N. A. Gokcen, J. Phys. Chem. 72, 2556 (1968).

1968CLA/PRI W. D. Clarke and S. J. W. Price, Can. J. Chem. 46, 1633-1634 (1968).

1968DAV/BAT Z. V. Davydova, P. S. Bataev, I. A. Makolkin, and M. E. Yanitskaya, Trudy Moskov. Inst. Narodnog Khozyaistva 46, 32 (1968).

1968DUT/ONY W. A. Dutton and M. Onyszchuk, Inorg. Chem. 7, 1735-1739 (1968).

1968FIN/GAR A. Finch, P. J. Gardner, and K. Radcliffe, J. Chem. Eng. Data 13, 176 (1968).

1968FOX/MAC W. B. Fox, J. S. MacKenzie, E. R. McCarthy, J. R. Holmes, R. F. Stahl, and R. Juurik, Inorg. Chem. 7, 2064-2067 (1968).
1968HIL/IRV

J. O. Hill and R. J. Irving, J. Chem. Soc. A $\mathbf{1 9 6 8 ,}$ 3116-3118.

1968HIL/IRV2 J. O. Hill and R. J. Irving, J. Chem. Soc. A 1968 , $1052-1054$.

1968KAR/RAB N. V. Karyakin, I. B. Rabinovich, and L. G. Pakhomov, Russ. J. Phys. Chem. 42, 954-955 (1968).

1968KIS/DIL R. W. Kiser, J. G. Dillard, and D. L. Dugger, Adv. Chem. Ser. 72, 153-180 (1968).

1968KOS/MOS V. N. Kostryukov, A. M. Mosin, O. P. Samorukov, and V. G. Shlyakova, Russ. J. Phys. Chem. 42, 968 (1968).

1968 KUA/UST $\quad$ B. M. Kuadzhe, G. P. Ustyugov, and A. A. Kudryavtsev, Tr. Mosk. Khim. Tekhnol. Inst. 58, 27-28 (1968); Chem. Abstr. 71, 6728 (1969).

1968LON/PUL L. H. Long and C. I. Pulford, J. Inorg. Nucl. Chem. 30, 2071 (1968).

1968MAR/ARM S. Marantz and G. T. Armstrong, J. Chem. Eng. Data 13, 118-121 (1968).

1968NIS/NIK L. A. Nisel'son, R. K. Nikolaev, T. D. Sokolova, V. I. Stolyarov, and Y. M. Korolev, Izv.Sib. Otd. Akad. Nauk. SSSR, Ser. Khim. Nauk 1968, 109-114; Chem. Abstr. 69, 69834s (1969).

1968SCH/PIL C. J. Schack, D. Pilipovich, S. N. Cohz, and D. F. Sheehan, J. Phys. Chem. 72, 4697 (1968).

1968SIN/BUR R. A. Sinclair and A. B. Burg, Inorg. Chem. 7, 2160-2162 (1968).

1968TSU/KOJ S. Tsuruoka and H. Kojima, Sen'i Gakkaishi 24, 27-34 (1968).

1968VAN/HOE H. Van Bekkum, M. A. Hoefnagel, L. de Lavieter, A. Van Veen, P. E. Verkade, A. Wemmers, B. M. Wepster, J. H. Palm, L. Schafer, H. Dekker, C. Mosselman, and G. Somsen, Recl. Trav. Chim. Pays-Bas 86, 1363-1371 (1968).

1968VAN/SKO K. Van Putte, W. Skoda, and M. Petroni, Chem. Phys. Lipids 2, 361-371 (1968).

1968WEL/WUL N. Welcman and M. Wulf, Isr. J. Chem. 6, 37-41 (1968).

1969ADA/CAR G. P. Adams, A. S. Carson, and P. G. Laye, Trans. Faraday Soc. 65, 113-120 (1969).

1969ADA/CAR2 G. P. Adams, A. S. Carson, and P. G. Laye, J. Chem. Thermodyn. 1, 393-396 (1969).

1969AGA/HAJ M. Y. Agarunov and S. N. Hajiev, Dokl. Akad. Nauk SSSR 185, 221 (1969).

1969AND/WES J. T. S. Andrews and E. F. Westrum, Jr., J. Organomet. Chem. 17, 349-352 (1969).

1969AND/WES2 J. T. S. Andrews, E. F. Westrum, Jr., and N. Bjerrum, J. Organomet. Chem. 17, 293-302 (1969).

1969ATK/RIC C. M. L. Atkinson and M. J. Richardson, Trans. Faraday Soc. 65, 1749 (1969).

1969AYL/CAM B. J. Aylett and J. M. Campbell, J. Chem. Soc. A 1969, 1910-1916.

1969AYL/CAM2 B. J. Aylett and J. M. Campbell, J. Chem. Soc. A 1969, 1916-1920.

1969AYL/CAM3 B. J. Aylett, J. M. Campbell, and A. Walton, J. Chem. Soc. A 1969, 2110-2113.

1969AYL/HAK B. J. Aylett and M. J. Hakim, J. Chem. Soc. A 1969, 800-803.

1969AYL/HAK2 B. J. Aylett and M. J. Hakim, J. Chem. Soc. A 1969, 636-638.

1969AYL/HAK3 B. J. Aylett and M. J. Hakim, J. Chem. Soc. A 1969, 639-642.

1969BOR/DAL G. Borgen and J. Dale, J. Chem. Soc., Chem. Commun. 1969, 447-448.

1969COR/GOO J. M. Corkill, J. F. Goodman, and J. R. Tate, Trans. Faraday Soc. 65, 1742 (1969).

1969DAS/WEN G. D’Ascenzo and W. W. Wendlandt, J. Therm. Anal. 1, 423-434 (1969).

1969DAV/MAK Z. V. Davydova, I. A. Makolkin, and P. S. Bataev, Zh. Obshch. Khim. 39, 1668-1669 (1969).

1969DAV/SMI R. R. Davidson and W. H. Smith, Chem. Eng. Sci. 24, 1589 (1969).

1969DIL/KIS J. G. Dillard and R. W. Kiser, J. Organomet. Chem. 16, 265-273 (1969).

1969GEN W. L. G. Gent, J. Sci. Instrum. 2, 69 (1969). 
1969GEO/MAC R. D. George and K. M. Mackay, J. Chem. Soc. A 1969, 2122-2126.

1969GLE/MEW O. Glemser, R. Mews, and H. W. Roesky, J. Chem. Soc., Chem. Commun. 1969, 914.

1969HAM C. Z. Hamann, Z. Chem. 9, 195-196(1969); Chem. Abstr. 71, 24938d (1969).

1969HIT/KAN W. C. Hitchingham and A. S. Kana'an, High Temp. Sci. 1, 216 (1969).

1969JOE/GJA O. Joens and J. Gjaldbaek, Chr. Dan. Tidsskr. Farm. 43, 151-155 (1969); Chem. Abstr. 72, 6776r (1969).

1969KAR N. V. Karpenko, Vestn. Keningrad. Univ. Fiz. Khim. 1969, 77.

1969KEI/KAN D. Keiser and A. S. Kana'an, J. Phys. Chem. 73, 4264-4269 (1969).

1969KEM/KRE R. H. Kemme and S. I. Kreps, J. Chem. Eng. Data 14, 98-102 (1969)

1969KLE/MOR M. L. Klein, J. A. Morrison, and R. D. Weir, Discuss. Faraday Soc. 48, 93 (1969).

1969KRO N. G. Krokhin, Russ. J. Phys. Chem. 43, 1342-1343 (1969).

1969LEA/LON D. R. Leavers, J. R. Long, S. G. Shore, and W. J. Taylor, J. Chem. Soc. 1969, 1580-1581.

1969MAC/MCN H. Mackle, D. V. McNally, and W. V. Steele, Trans. Faraday Soc. 65, 2060-2068 (1969).

1969MAC/MCN2 H. Mackle and D. V. McNally, Trans. Faraday Soc. 65, 1738-1741 (1969).

1969MAC/STE H. Mackle and W. V. Steele, Trans. Faraday. Soc 65, 2069-2072 (1969); 65, 2073-2077 (1969).

1969NAG/DZH M. E. Nagiev, O. I. Dzhafarov, K. A. Karasharli, and V. N. Kostryukov, Dokl. Akad. Nauk SSSR 184, 648 (1969).

$19690 V E / S T E \quad$ J. E. Overberger, W. A. Steele, and J. G. Aston, J. Chem. Thermodyn. 1, 535-542 (1969).

1969PLA/GLA C. Plato and A. R. Glasgow, Jr., Anal. Chem. 41, 330-336 (1969).

1969RIN/DAM H. Ringel, A. C. Damask, and R. A. Arndt, Mol. Cryst. 5, 295 (1969).

1969ROB/SCO P. M. Robinson and H. G. Scott, Mol. Cryst. Liq. Cryst. 5, 387-404 (1969).

1969ROS/DIC J. M. Rosen and C. Dickinson, J. Chem. Eng. Data 14, 120-124 (1969).

1969SAD/STE H. W. Sadowska, G. B. Stepniewska, and R. M. Recko, Przem. Chem. 48, 282 (1969).

1969SCH/WIL C. J. Schack, R. D. Wilson, J. S. Muirhead, and S. N. Cohz, J. Am. Chem. Soc. 91, 2907 (1969).

1969SHA/FED Y. K. Shaulov, A. K. Federov, and V. G. Genchel, Zh. Fiz Khim. 185, 1336-1337 (1969).

1969SHE/LAN R. J. Sheehan and S. H. Langer, J. Chem. Eng. Data 14, 248-250 (1969).

1969SHI/MCN C.-F. Shieh, D. McNally, and R. H. Boyd, Tetrahedron 25, 3653-3665 (1969).

1969SIC/DUB J. E. Sicre, J. T. Dubois, K. J. Eisentraut, and R. E. Sievers, J. Am. Chem. Soc. 91, 3476-3481 (1969).

1969SPE/CLI W. F. Spencer and M. M. Cliath, Environ. Sci. Technol. 3, 670-674 (1969).

1969THO/DAV L. H. Thomas and G. H. Davies, J. Chem. Soc. A 1969, 1271.

1969VIC/WAL M. Victoria and J. Walkley, Trans. Faraday Soc. 65, 57-61 (1969).

1969ZMB/HAS K. F. Zmbov, J. W. Hastie, R. Hauge, and J. L. Margrave, Proceedings of the 3rd IUPAC Conference on High Temperature Technology (Butterworths, London, 1969), p. 345; as quoted in Ref. [1971ADA/MAR].

1970AMB/SPR D. Ambrose and C. H. S. Sprake, J. Chem. Thermodyn. 2 , 631-645 (1970).

1970AND/WES J. T. S. Andrews and E. F. Westrum, Jr., J. Phys. Chem. 74, 2170-2174 (1970).

1970AND/WES2 J. T. S. Andrews and E. F. Westrum, Jr., J. Chem. Thermodyn. 2, 245 (1970).

1970ASH S. J. Ashcroft, J. Chem. Soc. A 1970, 1020-1024.

1970BAE A. K. Baev, Obshch. Prikl. Khim. 1970, 146-160; Chem. Abstr. 74, 91934z (1971).

1970BAE/DEM A. K. Baev and V. V. Demyanchuck, Obshch. Prikl. Khim. 6, 65-72 (1970); Chem. Abstr. 74, 103799 (1971).
1970BAR/HAS

1970BON/CAT

1970BOR

1970BOR/DAL

1970CAR/CAR

1970CAR/LAY

1970CHA/CAV

1970COK/AMB

1970COX/PIL

1970DAS/WEN

1970DAV/POR

1970DYK

1970DYK/VAN

1970FIN/GAR

1970GAL/MAR

1970GOE/BLO

1970GON/KAR

1970GOU/GIR

1970GUA/SAR

1970HAY

1970HES/PER

1970KOJ

1970MEL/MER

1970MEL/MER

1970MUR/BRE

1970OSB

1970PAN

1970POL/PER

1970SHA/FED

1970SMI/CAD

1970SMI/IRG

1970SOK/KAR

1970SWA/KAR

1970TRA/LOM

1970VAL

1970VAL/KIL
M. G. Barlow, R. N. Haszeldine, and R. Hubbard, J. Chem. Soc. C 1970, 1232-1237.

D. Bonderman, E. D. Cater, and W. Bennett, J. Chem. Eng. Data 15, 396-400 (1970).

L. Borka, Acta Pharm. Suec. 7, 1-6 (1970).

G. Borgen and J. Dale, J. Chem. Soc., Chem. Commun 1970, 1340-1342.

A. S. Carson, E. M. Carson, P. G. Laye, J. A. Spencer, and W. Steele, Trans. Faraday Soc. 66, 2459-2463 (1970).

A. S. Carson, P. G. Laye, J. A. Spencer, and W. Steele, J. Chem. Thermodyn. 2, 659-664 (1970).

T. L. Charlton and R. G. Cavell, Inorg. Chem. 9, 379 (1970).

T. G. Coker, J. Ambrose, and G. J. Janz, J. Am. Chem. Soc. 92, 5293 (1970).

J. D. Cox and G. Pilcher, Thermochemistry of Organic and Organometallic Compounds (Academic Press, London, 1970).

G. D'Ascenzo and W. W. Wendlandt, J. Inorg. Nucl. Chem. 32, 2431-2433 (1970).

G. J. Davis, R. S. Porter, and E. M. Barrall II, Mol. Cryst. Liq. Cryst. 10, 1 (1970).

J. Dykyj, J. Petrochem. 10, 51-59 (1970); 10, 123-146 (1970); 10, 187-206 (1970).

J. Dykyj and A. Vanko, Petrochemia 10, 3-22 (1970).

A. Finch, P. J. Gardner, P. McNamara, and G. R. Wellum, J. Chem. Soc. A 1970, 3339-3345.

G. L. Gal'chenko, L. N. Martynovskaya, V. I. Stanko, and A. I. Klimova, Dokl. Akad. Nauk SSSR 193, 483 (1970). H. J. Goetze, K. Bloss, and H. Molketin, Z. Phys. Chem. 73, 314-320 (1970).

A. K. Goncharov, M. K. Karapet'yants, and G. M. Kol'yakova, Russ. J. Phys. Chem. 44, 466-467 (1970).

P. Goursot, H. L. Girdhar, and E. F. Westrum, Jr., J. Phys. Chem. 74, 2538 (1970).

G. Guarini and P. Sarti-Fantoni, Mol. Cryst. Liq. Cryst. 6, 423-426 (1970).

J. N. Hay, J. Polym. Sci., Part B 8, 395-399 (1970).

B. Hessett and P. G. Perkins, J. Chem. Soc. A 1970 , 3229-3234.

H. Kojima, Sen'i Gakkaishi 26, 530-532 (1970).

1489-1493 (1970).

T. P. Melia and R. Merrifield, J. Inorg. Nucl. Chem. 32, 2573-2579 (1970).

E. Murrill and L. Breed, Thermochim. Acta 1, 239-246 (1970).

D. W. Osborne, J. Chem. Phys. 52, 1803 (1970).

L. B. Pankratz, "Thermodynamic data for silver chloride and silver bromide," U.S. Bureau of Mines Report of Investigation 7430, 1970.

M. N. Polteva, V. P. Persiantseva, and I. L. Rozenfel'd, Corrosion and Protection of Metals (Nauka, 1970), p. 140; as quoted in Ref. [1972ROZ/POL].

Y. K. Shaulov, A. K. Federov, G. Y. Zueva, G. V. Borisyuk, and V. G. Genchen, Russ. J. Phys. Chem. 44, 1181-1182 (1970); Zh. Fiz. Khim. 44, 2081-2083 (1970); Chem. Abstr. 74, 7230m (1971).

J. E. Smith and G. H. Cady, Inorg. Chem. 9, 1293-1294 (1970).

M. R. Smith, K. J. Irgolic, E. A. Meyers, and R. A. Zingaro, Thermochim. Acta 1, 51-60 (1970).

V. B. Sokolov, M. K. Karapet'yants, and A. K. Goncharov, Tr. Mosk. Khim.-Tekhnol. Inst. 67, 37-39 (1970); Chem. Abstr. 75, 122149z (1971).

H. A. Swain, Jr. and D. G. Karraker, Inorg. Chem. 9 1766-1769 (1970).

A. A. Trapeznikov and T. A. Lomonosova, Zh. Fiz. Khim. 44, 2121-2128 (1970).

A. J. Valerga, Diss. Abstr. 31, 3316-B (1970-71).

4545-4549 (1970). 
1970VON/GLE S. P. Von Halasz and O. Glemser, Chem. Ber. 103, 553-560 (1970).

1971ADA/MAR G. P. Adams, J. L. Margrave, R. P. Steiger, and P. W. Wilson, J. Chem. Thermodyn. 3, 297-305 (1971).

1971ALV/DAL T. Alvik and J. Dale, Acta Chem. Scand. 25, 2131-2141 (1971).

1971ASH

1971BAE/DEM

S. J. Ashcroft, Thermochim. Acta 2, 512-514 (1971).

A. K. Baev, V. V. Dem'yanchuk, G. Mirzoev, G. I. Noikov, and N. E. Kolobova, Russ. J. Phys. Chem. 45, 777-779 (1971); Chem. Abstr. 75, 80991k (1971); as cited by D. Lalage, S. Brown, J. A. Connor, and H. A. Skinner, J. Organomet. Chem. 81, 403-409 (1974).

1971BEE/LIN G. Beech and R. M. Lintonbon, Thermochim. Acta 2, 86-88 (1971).

1971BEE/LIN2 G. Beech and R. M. Lintonbon, Thermochim. Acta 3, 97-105 (1971).

1971BER/GIR G. Bertholon, M. Giray, R. Perrin, and M. F. VincentFalquet-Berny, Bull. Soc. Chim. Fr. 1971, 3180-3187.

1971BOY/SAN R. H. Boyd, S. N. Sanwal, S. Shary-Tehrany, and D. McNally, J. Phys. Chem. 75, 1264-1271 (1971).

1971BUT/CAR R. S. Butler, A. S. Carson, P. G. Laye, and W. V. Steele, J. Chem. Thermodyn. 3, 277-280 (1971).

1971CAL/SMI J. E. Callanan and N. O. Smith, J. Chem. Thermodyn. 3, 531-538 (1971).

1971CAR/FIN A. S. Carson, D. H. Fine, P. Gray, and P. G Laye, J. Chem. Soc. B 1971, 1611-1615.

1971CAR/LAY A. S. Carson, P. G. Laye, W. V. Steele, D. E. Johnston, and M. A. McKervey, J. Chem. Thermodyn. 3, 915-918 (1971).

1971CAR/WES H. G. Carlson and E. F. Westrum, Jr., J. Phys. Chem. 54, 1464 (1971).

1971DIT/SKO V. E. Ditsent, I. I. Skorokhodov, N. A. Terent'eva, and M. N. Zolotareva, Russ. J. Phys. Chem. 45, 901-902 (1971).

1971EDW/DOL L. Edwards, D. H. Dolphin, M. Gouterman, and A. D. Adler, J. Mol. Spectrosc. 38, 16-32 (1971).

1971 FEL/KUZ L. S. Fel'dshtein and A. S. Kuz'minskii, Kauch. Rezina 30, 15-18 (1971).

1971 GET/WAR F. W. Getzen and T. M. Ward, Ind. Eng. Chem. Prod. Res. Dev. 10, 122 (1971).

1971 GON/KAR A. K. Goncharov, M. K. Karapet'yants, and T. K. Gar, Russ. J. Phys. Chem. 45, 104-105 (1971).

1971HAU H. O. Haug, Ges. Kernforsch m.b.H. [Rep.] KFK-1283, 23, 1971 ; Chem. Abstr. 75, 122126q (1971).

1971HAU2 H. O. Haug, J. Organomet. Chem. 30, 53-57 (1971); Chem. Abstr. 75, 122161x (1971).

1971KAM/MIT H. Kambe, I. Mita, and R. Yokata, in Proceedings of the Third International Conference on Thermal Analysis and Calorimetry, Volume 3, Davos, Switz, 1971, pp. 387-395, 1971; Chem. Abstr. 79, 5824n (1973).

1971 KIP/RAB $\quad$ E. G. Kiparisova and I. B. Rabinovitch, Dokl. Akad. Nauk SSSR 199, 1075 (1971); Eng. Edit. 675-677.

1971 KOL/RAB G. M. Kol'yakova, I. B. Rabinovich, and N. S. Vyazankin, Dokl. Akad. Nauk. SSSR 200, 111-113 (1971).

1971LEA D. R. Leavers, Ph.D. dissertation, The Ohio State University, 1971.

1971LEB/GUT N. D. Lebedeva, N. M. Gutner, and V. L. Ryadnenko, Russ. J. Phys. Chem. 45, 561 (1971).

1971LEV/KOH J. Levkov, W. Kohr, and R. A. Mackay, J. Phys. Chem. 75, 2066 (1971).

1971MAS/CHE W. L. Masterton, C. H. Chen, C. E. Waring, R. Oksala, 1. Beatty, and A. Krause, J. Chem. Thermodyn. 3, 243 (1971).

1971MES/FIN J. F. Messerly and H. L. Finke, J. Chem. Thermodyn. 3, 675 (1971)

1971 MEY/REN E. F. Meyer, T. A. Renner, and K. S. Stec, J. Phys. Chem. 75, 642-648 (1971).

1971MUR/BRE E. Murrill and L. W. Breed, Inorg. Chem. 10, 641-643 (1971).

1971 NAK/SMI I. E. Nakhutin, N. M. Smirnova, V. I. Krivenko, and G. A. Loshakov, Zh. Obshch. Khim. 41, 940-943 (1971); Chem. Abstr. 75, 122154x (1971).

1971 NIS/TRE $\quad$ L. A. Nisel'son, K. V. Tret'yakova, E. P. Paremuzov, and E. N. Torbina, Izv. Akad. Nauk SSSR, Neorg. Mater. 7, 792-794 (1971); Chem. Abstr. 75, 67746q (1971).
1971OSB/SCH

1971PRI

1971PRZ/BOS

1971RAB/KIP

1971ROG

1971ROS/HOL

1971SAM/KOS

1971SAM/KOS2

1971SEL

1971SHA/YAK

1971SKO/DIT

1971SOK/KAR

1971SUN/EIS

1971SWA/KAR

1971TEL/RAB

1971VOI/SHC

1971WIL/ZWO

1971WON/WES

1971ZAB/SHR

1972ALV/BOR

1972ARN/EBS

1972BLE/FIE

1972BOL

1972BOO/HAU

1972BOR/DAL

1972BOR/DAL2

1972BRA/KAR

1972BUR

1972BUR/REI

1972CAR/LAY

1972CHA/BES

1972CHA/HAG

1972CHI/NAK

1972CON/SKI

1972DAL/KRI
D. W. Osborne, F. Schreiner, and H. Selig, J. Chem. Phys. 54, 3790-3798 (1971).

V. P. Privalko, Russ. J. Phys. Chem. 45, 900 (1971).

J. K. Przystal, W. G. Bos, and I. B. Liss, J. Inorg. Nucl. Chem. 33, 679 (1971).

I. B. Rabinovich, E. G. Kiparisov, and Y. A. Aleksandrov, Dokl. Akad. Nauk SSSR 200, 1116-1118 (1971).

F. E. Rogers, J. Phys. Chem. 75, 1734-1737 (1971).

J. M. Rosen, J. R. Holden, and H. T. Simmons Sr., Microscope 19, 151-156 (1971).

O. P. Samorukov and V. N. Kostryukov, Russ. J. Phys. Chem. 45, 747 (1971).

O. P. Samorukov, V. N. Kostryukov, and N. K. Samorukova, Russ. J. Phys. Chem. 45, 562 (1971).

P. Sellers, Acta Chem. Scand. 25, 2099-2102 (1971).

Y. K. Shaulov and E. S. Yakubov, Zh. Fiz. Khim. 45, 188 (1971).

I. I. Skorokhodov, V. E. Ditsent, N. A. Teret'eva, and M. N. Zolotarev, Russ. J. Phys. Chem. 45, 902 (1971).

V. B. Sokolov, M. K. Karapet'yants, N. D. Rumyantseva, and V. A. Drozdov, Russ. J. Phys. Chem. 45, 1332-1333 (1971).

C. Sunwoo and H. Eisen, J. Pharm. Sci. 60, 238-244 (1971).

H. A. Swain, Jr. and D. G. Karraker, J. Inorg. Nucl. Chem. 33, 2851-2856 (1971).

V. I. Tel'noi, I. B. Rabinovitch, V. N. Latyaeva, and A. N. Lineva, Dokl. Akad. Nauk SSSR 197, 353 (1971).

S. A. Voitkevich, M. M. Shchedrina, N. P. Solov'eva, and T. A. Rudol'fi, Maslozhir. Prom-st. 37, 27-30 (1971).

R. C. Wilhoit and B. J. Zwolinski, Handbook of Vapor Pressures and Heats of Vaporization of Hydrocarbons and Related Compounds, API44-TRC Publications in Science and Engineering (Thermodynamics Research Center, College Station, TX, 1971).

W.-K. Wong and E. F. Westrum, Jr., J. Chem. Thermodyn. 3, 105-124 (1971).

407-409 (1971).

T. Alvik, G. Borgen, and J. Dale, Acta Chem. Scand. 26, 1805-1816 (1972).

D. E. J. Arnold, E. A. V. Ebsworth, H. F. Jessep, and D. W. H. Rankin, J. Chem. Soc., Dalton Trans. 1972, 1681-1687.

R. H. T. Bleyerveld and W. Fieggen, Recl. Trav. Chim. Pays-Bas 91, 477-482 (1972); Chem. Abstr. 76, 145167p (1972).

M. W. G. Bolster, Ph.D. thesis, University of Leiden, 1972, as quoted in Ref. [2000DUN].

H. J. Booss and K. R. Hauschildt, Fresenius' Z. Anal. Chem. 261, 32 (1972).

G. Borgen and J. Dale, Acta Chem. Scand. 26, 1149-1154 (1972).

G. Borgen and J. Dale, Acta Chem. Scand. 26, 1799-1804 (1972).

G. P. Brajin, M. K. Karapet'yants, A. K. Goncharov, and G. M. Kol'yakova, Tr. Mosk. Khim.-Tekhnol. Inst. 71, 10-12 (1972); Chem. Abstr. 80, 100458a (1974).

A. B. Burg, Inorg. Chem. 11, 2283-2285 (1972).

A. B. Burg and T. J. Reilly, Inorg. Chem. 11, 1962-1964 (1972).

A. S. Carson, P. J. Laye, J. A. Spencer, and W. J. Steele, J. Chem. Thermodyn. 4, 783-787 (1972).

S. S. Chang and A. B. Bestul, J. Chem. Phys. 56, 503-516 (1972).

N. B. Chanh, Y. Haget, and J. Bedouin, J. Bull. Soc. Fr. Mineral Cristallogr. 95, 281 (1972).

H. Chihara and M. Nakamura, Bull. Chem. Soc. Jpn. 45, 133 (1972).

J. A. Connor, H. A. Skinner, and Y. Virmani, J. Chem. Soc., Faraday Trans. 1 168, 1754-1763 (1972).

J. Dale and P. O. Kristiansen, Acta Chem. Scand. 26, 1471-1478 (1972). 
1972DIT/SKO V. E. Ditsent, I. I. Skorokhodov, N. A. Terent'eva, and M. N. Zolotareva, Russ. J. Phys. Chem. 46, 317-318 (1972); Zh. Fiz. Khim. 46, 544 (1972); Chem. Abstr. 77, 9812p (1972).

1972DIT/SKO2 V. E. Ditsent, I. I. Skorokhodov, N. A. Terent'eva, and M. N. Zolotareva, Russ. J. Phys. Chem. 46, 1088 (1972); Zh. Fiz. Khim. 46, 1887-1888 (1972); Chem. Abstr. 77, 106168r (1972); 77, 106166p (1972).

1972DUB/DEV C. Dubois and C. Devin, C. R. Seances Acad. Sci., Ser. C 274, 1289 (1972).

1972FIN/GAR A. Finch, P. J. Gardner, and A. F. Webb, J. Chem. Thermodyn. 4, 495-498 (1972).

1972FON/POM R. Fontaine, C. Pommier, and Guiochon, Bull. Soc. Chim. Fr. 1972, 3011-3015.

1972GEI/SAW G. Geiseler and J. Sawistowsky, Z. Phys. Chem. (Leipzig) 250, 43-48 (1972).

1972 GON/KAR A. K. Goncharov and M. H. Karapetiyaits, Zh. Fiz. Khim. 46, 228-230 (1972).

1972INO/LIA P. P. Inozemtsev, A. G. Liakumovich, and Z. D. Gracheva, Russ. J. Phys. Chem. 46, 914 (1972).

1972JOH/OHA G. K. Johnson, P. A. G. O’Hare, and E. H. Appelman, Inorg. Chem. 11, 800-802 (1972).

1972KAN

A. S. Kana' an, J. Chem. Thermodyn. 4, 893-901 (1972).
G. M. Kol'yakova, I. B. Rabinovich, E. N. Gladyshev, and N. S. Vyazankin, Dokl. Akad. Nauk SSSR 204, 356-358 (1972).

1972MAR/LOP I. Y. Markova, I. L. Lopatkina, Y. K. Shaulov, and Y. A. Priselkov, Tr. Khim. Khim. Tecknol. 1972, 61-64; Chem. Abstr. 79, 832724u (1973).

1972MAS/FAM V. A. Maslova and L. A. Faminskaya, Tr. Khim. Khim. Teknol. 1972, 51.

1972MAS/RAB V. A. Maslova, I. B. Rabinovich, V. P. Nistoalov, and L. A. Faminskaya, Tr. Khim. Khim. Teknol. 1972, 44.

1972MIL G. D. Mills, Diss. Abstr. Int., B 33, 1485 (1972); Chem. Abstr. 78, 42653f (1973).

1972MOR

1972MOR2

1972MOR3

1972NEW

1972NIS/SOK

1972ONE/SHR S. R. O'Neill and J. M. Shreeve, Inorg. Chem. 11, 1629-1631 (1972)

1972PED/ISE J. B. Pedley and B. S. Iseard, CATCH Tables, Silicon Compounds (University of Sussex, Brighton, UK, 1972).

1972PIN/CAV A. A. Pinkerton and R. G. Cavell, J. Am. Chem. Soc. 94, 1870 (1972).

1972PLA C. Plato, Anal. Chem. 44, 1531-1534 (1972).

1972ROG/ORT R. N. Rogers and L. W. Ortiz, Thermochim. Acta 3, 379 (1972).

1972ROG2 F. E. Rogers, J. Phys. Chem. 76, 106-109 (1972).

1972ROZ/POL I. L. Rozenfel'd, M. N. Polteva, and V. P. Persiantesva, Izv. Akad. Nauk SSSR, Ser. Khim. 1972, 720-722; Chem. Abstr. 77, 66496n (1972); Note: Compound is incorrectly named in the abstract.

1972SAU/SHR D. T. Sauer and J. M. Shreeve, Inorg. Chem. 11, 238-242 (1972).

1972SOK/BRA V. B. Sokolov, G. P. Bragin, and M. K. Karapet'yants, Tr. Mosk. Khim.-Tekhnol. Inst. 71, 8-9 (1972); Chem. Abstr. 80, 100457z (1974).

1972SOK/KAR V. B. Sokolov, M. K. Karapet'yants, S. P. Kolesnikov, B. L. Perl'mutter, and O. M. Nefedov, Tr. Mosk. Khim.Tekhnol. Inst. 71, 6-7 (1972); Chem. Abstr. 80, 100456y (1974).

1972SOL/BUR D. Solan and A. B. Burg, Inorg. Chem. 11, 1253-1258 (1972).

1972SPE/CLI W. F. Spencer and M. M. Cliath, J. Agric. Food Chem. 20, 645-649 (1972).

1972SWI/BAB $\quad$ R. F. Swindell, D. B. Babb, T. J. Ouellette, and J. M. Shreeve, Inorg. Chem. 11, 242-245 (1972).
1972TEL/RAB

V. I. Tel'noi, I. B. Rabinovich, B. I. Kozyrkin, B. A. Salamantin, and K. V. Kir'yanov, Dokl. Akad. Nauk SSSR 205, 364-366 (1972); Chem. Abstr. 77, 131500a (1972).

1972UMI/VAN V. A. Umilin and V. K. Vanchagova, Izv. Akad. Nauk SSSR, Ser. Khim. 1972, 651-653.

1972VOL/SMO E. A. Volchkova, D. D. Smol'yanimova, V. G. Genchel, K. Lapatkina, and Y. K. Shaulov, Russ. J. Phys. Chem. 46, 1053-1054 (1972).

1972WAU/GET R. D. Wauchope and F. W. Getzen, J. Chem. Eng. Data 17, 38 (1972).

1972WIE H. G. Wiedemann, Thermochim. Acta 3, 355-366 (1972).

1972WOL

1973AND/GOR

G. Wolf, Helv. Chim. Acta 55, 1446-1458 (1972).

1973ARS/SHA

J. T. S. Andrews and J. E. Gordon, J. Chem. Soc., Faraday Trans. 1 69, 546 (1973).

1973AYL/ELL

M. R. Arshadi and M. Shabrang, J. Chem. Soc., Perkin Trans. 2 1973, 1732-1734.

1973AYL/ELL2 B. J. Aylett, I. A. Ellis, and J. R. Richmond, J. Chem. Soc., Dalton Trans. 1973, 981-987.

1973BAR/MAL G. Bardi, L. Malaspina, and V. Piacenti, J. Chem. Eng. Data 18, 126-130 (1973).

1973BAR/MOR D. S. Barnes and C. T. Mortimer, J. Chem. Thermodyn. 5, 371-377 (1973).

1973BEC/RUC H.-D. Beckhaus and C. Rüchardt, Tetrahedron Lett. 1971-1974 (1973).

1973BIE/EIC R. M. Biefeld and H. A. Eick, J. Chem. Thermodyn. 5, 353-360 (1973).

1973BLA/IHL A. Blair and H. Ihle, J. Inorg. Nucl. Chem. 35, 3795-3803 (1973).

1973BOR/KRA G. K. Borisov, S. G. Krasnova, and G. G. Devyatykh, Russ. J. Inorg. Chem. 18, 346-348 (1973).

1973BRU/CUR H. R. Brunner and B. J. Curtis, J. Therm. Anal. 5, 111-119 (1973).

1973CAM/GAM R. Cameroni, G. Gamberini, M. T. Bernabei, and M. Facchini, Farmaco 28, 621-635 (1973).

1973CAR/ROB A. Cartner, B. Robinson, and P. J. Gardner, J. Chem. Soc., Chem. Commun. 1973, 317-318.

1973CAS/VEC F. Casellato, C. Vecchi, A. Girell, and B. Casu, Thermochim. Acta 6, 361-368 (1973).

$1973 \mathrm{COM}$

1973CON/SKI

J. C. Company, Chem. Eng. Sci. 28, 318 (1973).

1973DAL/EKE

J. A. Connor, H. A. Skinner, and Y. Virmani, J. Chem. Soc., Faraday Trans. 1 69, 1218-1225 (1973).

1973DEK/OON J. Dale and T. Ekeland, Acta Chem. Scand. 27, 1519-1525 (1973).

1973DEV/BOR

C. G. DeKruif and H. A. Oonk, Chem. Ing. Tech. 45, 455-461 (1973); Chem. Abstr. 78, 152198u (1973).

1973DIE/MAR G. G. Devyatykh, G. K. Borisov, L. F. Zyuzina, and S. G. Krasnov, Dokl. Phys. Chem. 212, 703-705 (1973).

1973DYA/VIG E. A. Dietz and D. R. Martin, J. Inorg. Nucl. Chem. 35, 3681-3684 (1973).

1973FEL/SAV N. N. D'yachkova, E. N. Vigdorovich, and L. A Ivanyutin, Russ. J. Phys. Chem. 47, 258-259 (1973).

1973FIN/GAR A. Finch, P. J. Gardner, N. Hill, and K. S. Hussian, J I. N. Fel'dman, V. V. Savko, U. I. Mamai, and M. Finkel'shtein, Russ. J. Phys. Chem. 47, 1531 (1973). Chem. Soc., Dalton Trans. 1973, 2543-2545.

1973GAL/BRY G. L. Gal'chenko, E. P. Brykina, R. M. Varushchenko, L. S. Vasil'ev, and B. N. Mikhailov, Russ. J. Phys. Chem. 47, 914 (1973).

1973GEI/DZH K. I. Geidarov, O. I. Dzhafarov, K. A. Karasharli, and V. N. Kostryukov, Zh. Fiz. Khim. 47, 275 (1973); Chem. Abstr. 78, 129067s (1973).

1973GEI/SAW G. Geiseler and J. Sawistowsky, Z. Phys. Chem. (Leipzig) 253, 333-336 (1973).

1973GIG/MAL R. Gigli, L. Malaspina, and G. Bardi, Ann. Chim. (Rome) 63, 627-633 (1973); Chem. Abstr. 82, 77775g (1975).

1973GOS/MIL K. Gosling and J. L. Miller, Inorg. Nucl. Chem. Lett. 9, 355-358 (1973).

1973HAM/MIT J. V. Hamilton and G. M. Mitchell, J. Chem. Eng. Data 18, 38-39 (1973). 
1973HAM/MIT2 J. V. Hamilton, G. M. Mitchell, and D. A. Ayers, J. Chem. Eng. Data 18, 364-366 (1973).

1973IVA/GUJ G. A. Ivanov, V. G. Gujlya, and A. A. Zhukhovitskii, Zavadskaya Lab. 39, 15-17 (1973); Chem. Abstr. 78, 129058 (1973).

1973IZM/KHO A. S. Izmailovich, Y. S. Khodeev, and V. I. Tsirel'nikov, Zh. Fiz. Khim. 47, 1565-1566 (1973); Chem. Abstr. 79, 108298y (1973).

1973JAI/YAD D. V. S. Jain and O. P. Yadav, Indian J. Chem. 11, 28-30 (1973).

1973JOH/MAR D. A. Johnson and J. T. Martin, J. Chem. Soc., Dalton Trans. 1973, 1585

1973KOL/RAB G. M. Kol'yakova, I. B. Rabinovich, and E. N. Zorina, Dokl. Akad. Nauk SSSR 209, 616-617 (1973).

1973MAE K. Maeda, Sci. Pap. Inst. Phys. Chem. Res. 67, 143 (1973).

1973MAL/BAR L. Malaspina, R. Gigli, and G. Bardi, J. Chem. Thermodyn. 5, 845-850 (1973).

1973MAL/GIG L. Malaspina, R. Gigli, and G. Bardi, J. Chem. Phys. 59, 387-394 (1973).

1973MAS/NOV V. A. Maslova, N. V. Novoselova, E. M. Moseeva, N. D. Berezhnaya, and I. B. Rabinovich, Tr. Khim. Khim. Technol. 1973, 51 .

1973MCC/SMI J. A. McCauley and N. O. Smith, J. Chem. Thermodyn. 5, 31-40 (1973).

1973MCD W. McDowell, J. Soc. Dyers Colour. 89, 177 (1973).

1973MCE/SAN D. M. McEachern and O. Sandoval, J. Phys. E 6, 155-161 (1973).

1973MEY/HOT E. F. Meyer and R. D. Hotz, J. Chem. Eng. Data 18, 359-362 (1973).

1973 MOR/MCN C. T. Mortimer and J. L. McNaughton, Thermochim. Acta 6, 269-274 (1973).

1973POL/POL L. D. Polyachenok and O. G. Polyachenok, Zh. Fiz. Khim. 47, 498 (1973).

1973RAU/GEY H.-J. Rauh, W. Geyer, H. Schmidt, and G. Geiseler, Z. Phys. Chem. (Leipzig) 253, 43-48 (1973).

1973ROD/WES D. L. Rodgers, E. F. Westrum, Jr., and J. T. S. Andrews, J. Chem. Thermodyn. 5, 733-739 (1973).

1973SHI/ENO T. Shinoda, H. Enokida, Y. Maeda, H. Tomita, and Y. Mashiko, Bull. Chem. Soc. Jpn. 46, 48 (1973).

1973TEL/RAB V. I. Tel'noi, I. B. Rabinovich, and V. A. Umilin, Dokl. Akad. Nauk SSSR 209, 127-129 (1973).

1973 UMI/FED V. A. Umilin, I. E. Fedorov, Y. B. Zverev, and A. Gorbunova, Tr. Khim. Khim. Tekhnol. 1973, 63-64; Chem. Abstr. 81, 140914y (1974).

1973WIL/FEN J. W. Wilson and J. T. F. Fenurck, J. Chem. Thermodyn. 5, 341-345 (1973).

1973WIL/ZWO R. C. Wilhoit and B. J. Zwolinski, J. Phys. Chem. Ref. Data 2(Suppl. 1), 1-420 (1973); Note: The authors report Antoine constants for numerous alcohols that were calculated from vapor pressure data taken from the published literature. For several of the alcohols, the authors had only a very limited number of experimental data points that were pooled from several sources.

1974AMB/ELL D. Ambrose and J. H. Ellender, J. Chem. Thermodyn. 6, 909-914 (1974).

1974ANC J. Ancsin, Can. J. Phys. 52, 1521 (1974).

1974ARS M. R. Arshadi, J. Chem. Soc., Faraday Trans. 1 70, 1569-1571 (1974).

1974ATA/CHI T. Atake and H. Chihara, Bull. Chem. Soc. Jpn. 49, 2126-2136 (1974).

1974AUB/GUI M. Aubry and R. Guiraud, Bull. Soc. Chim. Fr. 1, 1857 (1974).

1974AUG/BOR E. Augdahl, G. Borgen, J. Dale, and J. Krane, Acta Chem. Scand. 28B, 125-129 (1974).

1974BAR/PIL D. S. Barnes, G. Pilcher, D. A. Pittam, H. A. Skinner, D. Todd, and Y. Virmani, J. Less-Common Met. 36, 177-186 (1974); ibid. 38, 53 (1974).

1974BES/MAR N. A. Bessareb and Y. M. Martynov, Russ. J. Phys. Chem. 48, 1547-1548 (1974).

1974BJO/BOR S. L. Björnstad, G. Borgen, and G. Gaupset, Acta Chem. Scand. 28B, 821-822 (1974).

1974BOR
1974BRA/KAR

G. P. Bragin and M. K. Karapet'yants, Russ. J. Phys. Chem. 48, 612 (1974).

1974BRA/KAR2 G. P. Bragin and M. K. Karapet'yants, Zh. Fiz Khim. 48 , 1049 (1974).

1974BRO/CON D. L. S. Brown, J. A. Connor, and H. A. Skinner, J. Organomet. Chem. 81, 403-409 (1974).

1974BRY/CAZ C. E. Bryson III, V. Cazcarra, and L. L. Levenson, J. Chem. Eng. Data 19, 107-110 (1974).

1974BUR/VER I. A. Burns and R. E. Verrall, Thermochim. Acta 9, 277 (1974).

1974CAL/KAN L. M. Calle and A. S. Kana'an, J. Chem. Thermodyn. 6, 935-942 (1974)

1974CAN/JAC J. Canceill, J. Jacques, and M. C. Perucaud-Brianso, Bull. Soc. Chim. Fr. 1974, 2833-2838.

1974CIN/BER A. Cingolani and G. Berchiesi, J. Therm. Anal. 6, 87 (1974).

1974CLA/MCK T. Clark, M. A. McKervey, H. Mackle, and J. Rooney, J. Chem. Soc., Faraday Trans. 1 70, 1279-1291 (1974).

1974COL/ROU M. Colomina, M. V. Roux, and C. Turrion, J. Chem. Thermodyn. 6, 149-155 (1974).

1974 CON/SKI J. A. Connor, H. A. Skinner, and Y. Virmani, Faraday Symp. High Temp. Chem. 8, 18 (1974).

1974DEV/RAB C. G. Devyatykh, I. B. Rabinovitch, V. I. Tel'noi, G. K. Borisov, and L. F. Zyazina, Dokl. Akad. Nauk SSSR 217, 673 (1974).

1974DIT/SKO V. E. Ditsent, I. I. Skorokhodov, N. A. Terent'eva, and M. N. Zolotareva, Russ. J. Phys. Chem. 48, 447 (1974); Zh. Fiz. Khim. 48, 2152-2153 (1974); Chem. Abstr. 81, $158939 \mathrm{v}(1974)$.

1974DIT/SKO2 V. E. Ditsent, I. I. Skorokhodov, N. A. Terent'eva, M. N. Zolotareva, and M. B. Lotarev, Russ. J. Phys. Chem. 48, 1279 (1974); Zh. Fiz. Khim. 48, 2152 (1974); Chem. Abstr. 81, 158938u (1974).

1974DIT/SKO3 V. E. Ditsent, I. I. Skorokhodov, N. A. Terent'eva, M. N. Zolotareva, N. E. Rodzecich, and O. I. Minosyan, Zh. Fiz. Khim. 48, 2153 (1974); Chem. Abstr. 81, 158940p (1974).

1974DIT/SKO4 V. E. Ditsent, I. I. Skorokhodov, N. A. Terent'eva, M. N. Zolotareva, B. N. Parfenov, and O. I. Minosyan, Zh. Fiz. Khim. 48, 2151 (1974); Chem. Abstr. 81, 158937t (1974).

1974ENG/WOO P. S. Engel, J. L. Wood, J. A. Sweet, and J. L. Margrave, J. Am. Chem. Soc. 96, 2381-2387 (1974).

1974FRO H. Frostling, Acta Chem. Scand. 28A, 83-85 (1974).

1974GAI/BAE I. L. Gaidym, A. K. Baev, V. G. Syrkin, A. A. Uel'skii, and A. V. Medvedeva, Zh. Fiz. Khim. 48, 1871 (1974); Chem. Abstr. 81, 160019p (1974).

1974GAI/BAE2 I. L. Gaidym, A. K. Baev, V. G. Syrkin, A. A. Uel'skii, and A. V. Medvedeva, Russ. J. Phys. Chem. 48, 1111 (1974); Chem. Abstr. 81, 160019p (1974).

1974GEI/DZH K. I. Geidarov, O. I. Dzhafarov, K. A. Karasharli, and V. N. Kostryukov, Zh. Fiz. Khim. 48, 1058 (1974).

1974GEI/DZH2 K. I. Geidarov, O. I. Dzharfarov, K. A. Karasharli, and V. N. Kostryukov, Zh. Fiz. Khim. 48, 1147 (1974).

1974GIL/SUL A. G. Gilbert and K. G. P. Sulzmann, J. Electrochem. Soc. 121, 832-834 (1974).

1974HIR/CAS C. Hirayama, P. M. Castle, R. W. Liebermann, R. J. Zollweg, and F. E. Camp, Inorg. Chem. 13, 2804-2807 (1974).

1974KAN

1974KUN/HOS

A. S. Kana'an, J. Chem. Thermodyn. 6, 191-199 (1974).

1974LEB/ARO

20-25 (1974); see [1998BRU/PIA]

B. V. Lebedev and B. M. Aron, Tr. Khim. Khim. Tekhnol. 1974, 75.

1974LEB/TSV $\quad$ B. V. Lebedev and L. Y. Tsvetkova, Tr. Khim. Khim. Tekhnol. 1974, 143.

1974MAL/BAR L. Malaspina, G. Bardi, and R. Gigli, J. Chem. Thermodyn. 6, 1053-1064 (1974).

1974MAN/LOG G. D. Mantyukov, M. A. Loginova, B. N. Bychkov, and S. S. Rozhkov, Zh. Prikl. Khim. 47, 2585 (1974).

1974MAN3 M. Mansson, Acta Chem. Scand., Ser. B 28, 677-680 (1974).

1974MAN4 M. Mansson, J. Chem. Thermodyn. 6, 1153-1159(1974). 
1974MIL/LEB V. I. Milov, B. V. Lebedev, L. Y. Tsvetkova, A. M. Sladkov, L. K. Luneva, N. K. Lebedev, and A. A. Evstropov, Tr. Khim. Khim. Tekhnol. Gor'kii 1, 40 (1974).

1974MOG/HOC P. H. Mogul, M. C. Hochberg, R. Michiel, G. K. Nestel, B. L. Wamsley, and S. D. Coren, J. Chem. Eng. Data 19, 4-6 (1974)

1974 MOR/MCN C. T. Mortimer and J. L. McNaughton, Thermochim. Acta 8, 265-269 (1974).

1974MOS/MOU C. Mosselman and J. Mouric, J. Chem. Thermodyn. 6, 477-489 (1974).

1974MUR/POL J. J. Murray, R. F. Pollie, and C. Pupp, Can. J. Chem. 52, 557-563 (1974).

1974NAK/EDA M. Nakamura, K. Eda, and K. Kosiyama, Bull. Chem. Soc. Jpn. 47, 2877 (1974).

1974OPP/STO H. Opperman, G. Stoever, and E. Wolf, Z. Anorg. Allg. Chem. 410, 179 (1974).

1974OSB/DOU A. G. Osborn and D. R. Douslin, J. Chem. Eng. Data 19, 114-117 (1974).

1974PEP/ERL V. I. Pepekin, R. D. Erlikh, Y. N. Matyushin, and Y. A. Lebedev, Dokl. Phys. Chem. 214, 123-125 (1974).

1974PRI/POU J. Pribilova and J. Pouchly, Collect. Czech. Chem. Commun. 39, 1118-1124 (1974).

1974RAD/KAT L. G. Radchenko and A. I. Kataigorodskii, Zh. Fiz. Khim. 48, 2702-2074 (1974); Eng. Edit. 48, 1595-1597 (1974).

1974ROU/TUR M. V. Roux, C. Turrion, M. Colomina, and R. PerezOssario, An. Quim. 70, 201-207 (1974); Chem. Abstr. 81, 119718c (1974).

1974RYB/MAR J. Rybicky and J. P. Marton, Thermochim. Acta 9, 450 (1974).

1974SAB R. Sabbah, Energetique de Liaison Inter-et Intramoleculaires, C.N.R.S., Marseille, 1974.

1974SAB/CHA2 R. Sabbah, R. Chastel, and M. Laffite, Thermochim. Acta 10, 353-358 (1974).

1974SAB/GIL R. Sabbah and E. M. Gilbert, Thermochim. Acta 10, 345-351 (1974).

1974SHI/GRE C. F. Shieh and N. W. Gregory, J. Chem. Eng. Data 19, 11-14 (1974).

1974SHM/GOL G. O. Shmyreva, R. M. Golosova, G. B. Sakharovskaya, A. F. Popov, N. N. Korneev, and A. A. Smolyaninova, Russ. J. Phys. Chem. 48, 447 (1974).

1974SHM/SHL G. O. Shmyreva, G. B. Sakharovskaya, R. M. Golosova, G. G. Filippov, E. A. Volnina, E. N. Burdasov, and L. E. Gusel'nikov, Russ. J. Phys. Chem. 48, 1699-1700 (1974).

1974SOL/GRU J. F. Solsky and E. Grushka, J. Phys. Chem. 78, 275 (1974).

1974UST/PET A. I. Ustinov and E. S. Petrov, Izv. Sibirskogo Otdeleniya Akad. Nauk SSSR, Ser. Khim. Nauk 1974, 20.

1974UTS/BAC H. Utschick, G. Bachmann, and H. Kapitza, Chem. Tech. 26, 423-424 (1974).

1974UTS/BAC2 H. Utschick, G. Bachmann, and H. Kapitza, Chem. Tech. 26, 422 (1974).

1974VOI/SHC S. A. Voitkevich, M. M. Shchedrina, K. K. Prilepskaya, and T. A. Rudol'fi, Masiozhirovanya Prom. 1974, 29.

1974WAR/WIL M. D. Warren and W. S. Wilson, Thermochim. Acta 10, 33-38 (1974).

1974ZHU/KON E. Z. Zhuravlev and I. I. Konstantinov, Zh. Prikl. Khim. 47, 931 (1974).

1974ZOR/UMI A. D. Zorin, V. A. Umilin, and V. K. Vanchagova, Zh. Obschchei Khim. 44, 592 (1974); as cited by J. A. Connor, M. I. El Saied, J. A. Martinho-Simoes, and H. A. Skinner, J. Organomet. Chem. 212, 405-410 (1981).

1975ADE/BRO F. A. Adedeji, D. L. S. Brown, J. A. Connor, M. L. Leung, I. M. Paz-Andrade, and H. A. Skinner, J. Organomet. Chem. 97, 221-228 (1975).

1975AMB/ELL D. Ambrose, J. H. Ellender, E. B. Lees, C. H. S. Sprake, and R. J. Townsend, J. Chem. Thermodyn. 7, 453-472 (1975).

1975AND/MAR R. J. L. Andon and J. F. Martin, J. Chem. Thermodyn. 7, 593 (1975)

1975ARN/RAN D. E. Arnold and D. W. H. Rankin, J. Chem. Soc., Dalton Trans. 1975, 889-894.
1975AUB/MAY

M. Aubry, M. N. Mayoral, and P. Villardry, Bull. Soc. Chim. Fr. 112, 500-502 (1975).

1975BAG/AND G. V. Bagrov, S. N. Andreev, M. F. Smirnova, and L. A. Vol'f, Deposited Doc. VINITI, 1196, 1975 ; Chem. Abstr. 87, 59208y (1977).

1975BER/CIN G. Berchiesi, A. Cingolani, and D. Leonesi, J. Therm. Anal. 8, 427 (1975).

1975BER/LEO G. Berchiesi, D. Leonesi, and A. Cingolani, J. Therm. Anal. 7, 659 (1975).

1975BER/PER G. Bertholon and R. Perrin, Bull. Soc. Chim. Fr. 7/8 1537-1544 (1975).

1975BJO/BOR S. L. Björnstad and G. Borgen, Acta Chem. Scand. 29B, 13-16 (1975).

1975BJO/BOR2 S. L. Björnstad, G. Borgen, J. Dale, and G. Gaupset, Acta Chem. Scand. 29B, 320-324 (1975).

1975BOR

1975BRA/KAR

G. Borgen, Acta Chem. Scand. 29B, 265-272 (1975).

1975BRO/CON D. L. S. Brown, J. A. Connor, and H. A. Skinner, J. Chem.

. Karapet'yants, Tr. Khim. Khim Tekhnol. 1975, 78-79; Chem. Abstr. 85, 83381d (1976). Soc., Faraday Trans. 1 71, 699-700 (1975).

1975CAR/LAY A. S. Carson, P. G. Laye, and H. Morris, J. Chem. Thermodyn. 7, 993-996 (1975).

1975CAS/VEC F. Casellato, C. Vecchi, A. Girelli, and P. G. Farrell, Thermochim. Acta 13, 37 (1975).

1975CHI

1975CLA/KNO

J. S. Chickos, J. Chem. Educ. 52, 134-136 (1975).

T. Clark, T. Knox, H. Mackle, M. A. McKervey, and J. J. Rooney, J. Chem. Soc., Faraday Trans. 1 71, 2107-2110 (1975).

$1975 \mathrm{COV} \quad$ D. F. Covell, U.S. NTIS, AD-A Report, Issue No. 008141, 40, 1975.

1975CUC A. Cuccuru, Thermochim. Acta 13, 96-99 (1975).

1975CUC2

1975DEK/VAN

1975FEN/HAR

A. Cuccuru, Thermochim. Acta 11, 247-251 (1975).

C. G. DeKruif, C. H. D. van Ginkel, and J. Voogd, 4th International Conference on Chemical Thermodynamics, Montpellier, France (1975), Vol. 8/2, p. 11.

1975GAM/SIC M. Gambaruto, J. E. Sicre, and H. J. Schumacher, J. Fluorine Chem. 5, 175-179 (1975).

1975GAR/CAR P. J. Gardner, A. Cartner, R. G. Cunninghame, and B. H. Robinson, J. Chem. Soc., Dalton Trans. 1975, 2582-2586.

1975GEI/DZH K. I. Geidarov, O. I. Dzhafarov, and K. A. Karasharli, Russ. J. Phys. Chem. 49, 197-198 (1975).

1975GEI/DZH2 K. I. Geidarov, O. I. Dzhafarov, and K. A. Karasharli, Russ. J. Phys. Chem. 49, 1278 (1975).

1975GUS/KAR A. Z. Guseinov, K. A. Karasharli, O. I. Dzhafarov, G. G. Nurullaev, N. S. Nametkin, L. E. Gusel'nikov, E. A. Volnina, E. N. Burdasov, and V. N. Vdovin, Doklad. Acad. Nauk SSSR 222, 1369-1372 (1975).

1975 GUS/KUL A. Z. Guseinov, A. A. Kuliev, K. A. Karasharli, and O. I. Dzhafarov, Azerb. Khim. Zh. 1975, 72-74; Chem. Abstr. 84, 65359c (1976).

1975HAM/KUD S. Hamada, Y. Kudo, and M. Kawano, Bull. Chem. Soc. Jpn. 48, 2963-2964 (1975).

1975HIR/ROM C. Hirayama, J. F. Rome, and F. E. Camp, J. Chem. Eng. Data 20, 1 (1975).

1975IRV/RIB R. J. Irving and M. A. V. Ribeiro da Silva, J. Chem. Soc., Dalton Trans. 1975, 798-800.

1975IRV/RIB2 R. J. Irving and M. A. V. Ribeiro da Silva, J. Chem. Soc., Dalton Trans. 1975, 1257-1260.

1975KOS/SAM V. N. Kostryukov, O. P. Samorukov, N. K. Samorukova, and E. K. Chaplygina, Russ. J. Phys. Chem. 49, 944 (1975).

1975KUN/LIL H. Kundel, U. Lille, and N. Kaidas, Tr. Tallin. Politekh. Inst. 390, 107-115 (1975).

1975KUS/SAI K. Kusano and Y. Saito, Preprints 33rd Ann. Meeting Chem. Soc. Japan 1, 123 (1975); as quoted in [1985MAJ/SVO].

1975LEB/MIL B. V. Lebedev, V. I. Milov, L. Y. Tsvetkova, N. K. Lebedev, Y. G. Kirparisova, and A. A. Evstropov, Zh. Fiz. Khim. 49, 1591 (1975); Eng. Edit. 49, 944 (1975). 
1975MAL/GIG L. Malaspina, R. Gigli, G. Bardi, and G. DeMaria, Conf. Int. Thermodyn. Chim., [C. R.], 4th 1, 54-60 (1975); in Cent. Rech. Microcalorimetrie Thermochim. C.N.R.S., Marseille, France, edited by J. Rouquerol and R. Sabbah (1975); Chem. Abstr. 84, 179387r (1976).

1975MAR/SHU S. P. Marfey and A. R. Schultz, Artery 1, 299 (1975).

1975MCE/INI D. M. McEachern, J. C. Iniquez, and H. C. Ornelas, J. Chem. Eng. Data 20, 226-228 (1975).

1975 MCE/SAN D. M. McEachern, O. Sandoval, and J. C. Iniguez, J. Chem. Thermodyn. 7, 299-306 (1975).

1975MEK/KAR S. A. Mekhtiev, K. A. Karasharli, and D. I. Dzhafarov, Russ. J. Phys. Chem. 49, 259 (1975).

1975MEK/KAR2 S. A. Mekhtiev, K. A. Karasharli, D. I. Dzhafarov, and A. G. Kuznetsova, Russ. J. Phys. Chem. 49, 1914-1916 (1975).

1975MEK/KAR3 S. A. Mekhtiev, K. A. Karasharli, and D. I. Dzhafarov, Zh. Fiz. Khim. 49, 2578 (1978).

1975OBS/DOU A. G. Osborn and D. R. Douslin, J. Chem. Eng. Data 20, 229-232 (1975).

1975OSB/DOU A. G. Osborn and D. R. Douslin, J. Chem. Eng. Data 20, 229-231 (1975).

1975PAR/STE W. Parker, W. V. Steele, W. Stirling, and I. Watt, J. Chem. Thermodyn. 7, 795-802 (1975).

1975PEP/LEB V. I. Pepekin, V. P. Lebedev, and A. A. Balepin, USSR Acad. Sci. Bull Chem. Ser. 10, 2204 (1975).

1975PET/SHR3 K. E. Peterman and J. M. Shreeve, J. Fluorine Chem. 6, 83-92 (1975).

1975PIL/WAR G. Pilcher, M. J. Ware, and D. A. Pittam, J. Less-Common Met. 42, 223-228 (1975).

1975PIT/PIL D. A. Pittam, G. Pilcher, D. S. Barnes, H. A. Skinner, and D. Todd, J. Less-Common Met. 42, 217-222 (1975).

1975RAB/LEB I. B. Rabinovich, B. V. Lebedev, L. Y. Tsvetkova, N. S. Nametkin, S. G. Durgar'yan, V. S. Khotimskii, and Y. G. Kiparisova, Vysokomol. Soedin. A17, 1421 (1975).

1975 SOK/KAR V. B. Sokolov, M. K. Karapet'yants, T. N. Sergeeva, S. P. Kolesnikov, and B. L. Perl'mutter, Izv. Vyssh. Uchebn. Zaved., Khim. Khim. Teknol. 18, 1319-1321 (1975); Chem. Abstr. 84, 22433e (1976).

1975 SOK/KAR2 V. B. Sokolov, M. K. Karapet'yants, T. N. Sergeeva, S. P. Kolesnikov, and B. L. Perl'mutter, Izv. Vyssh. Uchebn. Zaved., Khim. Khim. Teknol. 18, 1234-1237 (1975); Chem. Abstr. 84, 9167b (1976).

1975STE/SCH $\quad$ B. Steyer and F. P. Schafer, Appl. Phys. 7, 113-122 (1975).

1975STR/SUN G. Stridh and S. Sunner, J. Chem. Thermodyn. 7, 161-168 (1975).

1975SUB/ZWO D. J. Subach and B. J. Zwolinski, J. Chem. Eng. Data 20, 232-234 (1975).

1975TEL/KIR V. I. Tel'noi, K. V. Kirynov, V. I. Ermolaev, and I. B. Rabinovich, Dokl. Akad. Nauk SSSR 220, 1088 (1975).

1975 TIT/CHU V. A. Titov, T. P. Chusova, and G. A. Kokovin, Izv. Sib. Otd. Akad. Nauk SSSR, Ser. Khim. Nauk 1975, 3-6; Chem. Abstr. 83, 183512z (1975).

1975TRU/SUV V. I. Trusov, A. V. Suvorov, and R. N. Abakumova, Zh. Neorg. Khim. 20, 501-503 (1975); Chem. Abstr. 82, 161007r (1975).

1975VAN/DEK C. H. D. van Ginkel, G. C. Dekruif, and F. E. B. DeWaal, J. Phys. E: Sci. Instrum. 8, 490-492 (1975).

1975VIL/PER R. Vilcu, S. Perisanu, and I. Ciocazanu, Conf. Int. Thermodyn. Chim., [C. R.], 4th 1, 105-112 (1975).

1975WIL/PIN K. R. Wilson and R. E. Pincock, J. Am. Chem. Soc. 97, 1474-1478 (1975).

1976AMB/ELL D. Ambrose, J. H. Ellender, C. H. S. Sprake, and R. Townsend, J. Chem. Thermodyn. 8, 165-178 (1976).

1976AMB/LAW D. Ambrose, I. J. Lawrenson, and C. H. S. Sprake, J. Chem. Thermodyn. 8, 503-504 (1976).

1976AND/MAR R. J. L. Andon and J. F. Martin, J. Chem. Thermodyn. 8, 1159 (1976).

1976BER/BER G. Berchiesi, M. A. Bercuiesi, G. G. Lobbia, and D. Leonesi, Gazz. Chim. Ital. 106, 549-555 (1976).

1976BOR/DAL G. Borgen and J. Dale, Acta Chem. Scand. 30B, 711-715 (1976).
1976BRO/CON

D. L. S. Brown, J. A. Connor, M. L. Leung, M. I. PazAndrade, and H. A. Skinner, J. Organomet. Chem. 110, 79-89 (1976)

1976BUT/CAR R. S. Butler, A. S. Carson, P. G. Laye, and W. V. Steele, J. Chem. Thermodyn. 8, 1153-1158 (1976).

1976DIT/SKO V. E. Ditsent, I. I. Skorokhodov, N. A. Terent'eva, M. N. Zolotareva, Z. V. Belyakova, and Z. V. Belikova, Zh. Fiz. Khim. 50, 1905-1906 (1976); Chem. Abstr. 85, 166806x (1976).

1976ENG/MEL P. S. Engel, R. A. Melaugh, M. Mansson, J. W. Timberlake, A. Garner, and F. D. Rossini, J. Chem. Thermodyn. 8, 607-621 (1976).

1976FAB/MAS F. Fabbrizzi, R. Masherini, and P. Paoletti, J. Chem. Soc., Faraday Trans. 1 72, 896 (1976).

1976HON/SIN H. C. Hon, R. P. Singh, and A. P. Kudchadker, J. Chem. Eng. Data 21, 430-431 (1976).

1976HOP/BOS H. P. Hopkins, Jr., D. Bostwick, and C. J. Alexander, J. Am. Chem. Soc. 98, 1355-1357 (1976).

1976KIP/TSV E. G. Kiparisova, L. Y. Tsvetkova, and B. V. Lebedev, Russ. J. Phys. Chem. 50, 638 (1976).

1976KIR/TEL K. V. Kir'yanov, V. I. Tel'noi, G. A. Vasil'eva, and I. B. Rabinovich, Dokl. Akad. Nauk SSSR 231, 1021 (1976).

1976KLI/SAL S. A. Klinchikova, B. A. Salamatin, A. S. Pashinkin, B. I. Kozyrkin, and B. G. Gibov, Izvest. Vyssh. Uchebn. Zaved. Khim. Khim. Technol. 19, 585 (1976).

1976KUL/DZH A. M. Kuliev, O. I. Dzhafarov, K. A. Karasharli, and A. G. Kuznetsova, Zh. Fiz. Khim. 50, 1903 (1976); Chem. Abstr. 85, 183105 (1976).

1976KUZ/MIR V. P. Kuznetsova, E. A. Miroshinichenko, A. Z. Zelenetskii, G. V. Rokova, Y. A. Lebedev, and N. S. Enikolopyan, Dokl. Phys. Chem. 226, 147-150 (1976).

1976LEC/COL M. Leclercq, A. Collet, and J. Jacques, Tetrahedron 32, 821-828 (1976)

1976MEL/MAN R. A. Melaugh, M. Mansson, and F. D. Rossini, J. Chem. Thermodyn. 8, 623-626 (1976).

1976MEY/HOT E. F. Meyer and C. A. Hotz, J. Chem. Eng. Data 21, 274-279 (1976)

1976MIR/PAV E. A. Miroschnichenko, V. K. Pavloich, A. Yu, Y. A. Lebedev, V. I. Stanko, and G. L. Gal'chenko, Termodin. Org. Soedin. 5, 3-8 (1976).

1976SHM/SHL G. O. Shmyreva, V. G. Shlyakova, R. M. Golosova, A. B. Petrunin, D. B. Bekker, and A. F. Zhigach, Russ. J. Phys. Chem. 50, 478 (1976).

1976STR2 G. Stridh, J. Chem. Thermodyn. 8, 895-899 (1976).

1976STR3

1976TAV/NEE

G. Stridh, J. Chem. Thermodyn. 8, 901-906 (1976).

A. Tavlaridis and R. Neeb, Naturwissenschaften 63, 146-147 (1976), as quoted in [1979LAR].

1976TAY/CRO J. W. Taylor and R. J. Crookes, J. Chem. Soc., Faraday Trans. 1 72, 723-729 (1976).

1976TEL/RAB V. I. Tel'noi, I. B. Rabinovich, K. V. Kir'yanov, and A. S. Smirnov, Dokl. Akad. Nauk SSSR 231, 733-736 (1976).

1976ZHU/ALI L. V. Zhuravleva, A. S. Alikhanyan, and L. N. Sidorov, Russ. J. Phys. Chem. 50, 818 (1976).

1976ZOR/RAC A. D. Zorin, O. F. Rachkova, and N. S. Belousova, Termodin. Org. Soedin. 1976, 40-41.

1977BAL/BAR G. Balducci, G. Bardi, G. de Maria, and R. Gigli, in VII All-Union Calorimetry Conference, Moscow, 1977.

1977BAL/RUD T. S. Balanevskaya, V. V. Rudnev, N. G. Klishina, and A. A. Efremov, Tr. VNII Khim. Reakt. Osobo Chist. Khim. Veshehestv 1977, 98-101.

1977BAR/GAI A. A. Barkatin, I. L. Gaidym, and A. K. Baev, Khim. Khim. Technol. USSR 12, 44-48 (1977).

1977BED

1977BED/HUS R. G. Bedford, J. Phys. Chem. 81, 1284-1289 (1977).

1977BOU/CAR R. G. Bedford and E. B. Huss, J. Chem. Eng. Data 22, 239-241 (1977).

J. Bousquet, J. Carre, P. Claudy, M. Kollmanns-Berger, J. Thourey, and P. Barberi, Journ. Calorim. Anal. Therm. 8, 83-88 (1977).

1977BRO/CON D. L. S. Brown, J. A. Connor, C. P. Demain, M. L. Lueng, J. A. Martinho-Simoes, H. A. Skinner, and M. T. Zarfarani-Moattar, J. Organomet. Chem. 142, 321-335 (1977).

1977BUR2 A. Burger, Sci. Pharm. 45, 269 (1977). 
1977CLA/KNO T. Clark, T. M. O. Knox, H. Mackle, and M. A. McKervey, J. Chem. Soc., Faraday Trans. 1 73, 1224-1231 (1977).

1977DEK/VAN C. G. Dekruif and C. H. D. van Ginkel, J. Chem. Thermodyn. 9, 725-730 (1977).

1977DES M. L. Desauge, Journ. Calorim. Anal. Therm. 8, 89-99 (1977); Chem. Abstr. 87, 206628r (1977).

1977DWO/FUC A. Dworkin and A. H. Fuchs, J. Chem. Phys. 67, 1789-1790 (1977).

1977DYG/STE R. S. Dygdala, K. Stefanski, and J. Wonikowski, Bull. Acad. Pol. Sci., Ser. Sci., Math., Astron. Phys. 25, 439-446 (1977).

1977EIB/TRO J. Eibl, W. Tröger, and K.-H. Sender, Melliand Textilber. Int. 58, 844-850 (1977).

1977FIN/MES H. L. Finke, J. F. Messerly, S. H. Lee, A. G. Osborn, and D. R. Douslin, J. Chem. Thermodyn. 9, 937-956 (1977).

1977 GAF/PIE J. S. Gaffney, R. C. Pierce, and L. Friedman, J. Am. Chem. Soc. 99, 4293-4298 (1977).

1977HAR/ATA M. Harada, T. Atake, and H. Chihara, J. Chem. Thermodyn. 9,523 (1977).

1977KAN/MOR A. S. Kana'an and T. I. Morrison, J. Chem. Thermodyn. 9, 423-429 (1977).

1977KAR/RAB N. V. Karyakin, I. B. Rabinovich, V. N. Sapozhnikov, G. P. Kamslova, E. S. Krongauz, V. V. Korshak, and A. P. Travnikova, Vysokomol. Soedin., Ser. B 19, 457 (1977).

1977KIT/SHR T. Kitazume and J. M. Shreeve, Inorg. Chem. 16, 1818-1819 (1977).

1977KOS/SAM V. N. Kostryukov, O. P. Samorukov, N. K. Samorukova, A. M. Krasavin, and A. B. Petrunin, Vses. Konf. Kalorim., 7th 2, 387 (1977).

1977LEB/EVS $\quad$ B. V. Lebedev, A. A. Evstropov, G. B. Sadikov, and L. F. Larina, Russ. J. Phys. Chem. 51, 762 (1977).

1977LEB/RAB $\quad$ B. V. Lebedev, I. B. Rabinovich, E. G. Kiparisova, A. A. Evstropov, V. V. Korshak, and V. A. Pankratov, Dokl. Akad. Nauk SSSR 237, 383 (1977).

1977LEB/RAB2 B. V. Lebedev, I. B. Rabinovich, and A. A. Evstropov, J. Chem. Thermodyn. 9, 101 (1977).

1977LEB/RAB3 B. V. Lebedev, I. B. Rabinovich, N. K. Lebedev, B. G. Tsvetkov, S. G. Durhar'yan, and V. S. Khotimskii, Vysokomol. Soedin., Ser. A 19, 513 (1977).

1977MAN/SEL M. Mansson, P. Sellers, G. Stridh, and S. Sunner, J. Chem. Thermodyn. 9, 91-97 (1977).

1977NAG H. Naghibi-Bidokhti, Ph.D. thesis, The University of Surrey, 1977.

1977NIS/ISH K. Nishida, E. Ishihara, T. Osaka, and M. Koukitu, J. Soc. Dyers Colour. 93, 52-54 (1977).

1977 NOV/NOV N. Novakova and J. Novak, J. Chromatogr. 135, 13-24 (1977).

1977PEA/FUC L. A. Peacock and R. Fuchs, J. Am. Chem. Soc. 99, 5524-5525 (1977).

1977PED/RYL $\quad$ J. B. Pedley and J. Rylance, N. P. L. Computer Analysed Thermochemical Data: Organic and Organometallic Compounds (School of Molecular Sciences, University of Sussex, Brighton, UK, 1977).

1977SAS/FAL T. M. Sas, V. A. Falin, N. E. Mazepova, I. A. Krasavin, and I. I. Bogomolov, Zh. Fiz. Khim. 51, 1273 (1977).

1977SCH/PET F.-W. Schulze, H.-J. Petrick, H. K. Canmenga, and H. Klinge, Z. Phys. Chem. 107, 1-19 (1977).

1977SEL P. Sellers, J. Chem. Thermodyn. 9, 139-142 (1977).

1977STE/WAT W. V. Steele and I. Watt, J. Chem. Thermodyn. 9, 843-849 (1977).

1977STRI/SUN $\quad$ G. Stridh, S. Sunner, and C. Svenson, J. Chem. Thermodyn. 9, 1005-1010 (1977).

1977TEL/RAB V. I. Tel'noi and I. B. Rabinovich, Usp. Khim. 46, 1337-1367 (1977); Eng. Edit. 46, 689-705 (1977).

1977VAR/AMM R. M. Varushchenko, M. M. Ammar, and L. L. Bulgakova, Zh. Fiz. Khim. 51, 278-279 (1977).

1977VAR/AMM2 R. M. Varushchenko, M. M. Ammar, and L. L. Bulgakova, Russ. J. Phys. Chem. 51, 167-168 (1977).

1977VOI/SHC S. A. Voitkevich, M. M. Shchedrina, K. K. Prilepskaya, N. P. Solov'eva, T. M. Tsirkel, and T. A. Rudol'fi, Maslozhir. Prom-st. 1977, 23.

1977VOI/SHC2 S. A. Voitkevich, M. M. Shchedrina, K. K. Prilepskaya, N. P. Solov'eva, T. M. Tsirkel, and T. A. Rudol'fi, Maslozhir. Prom-st. 1977, 35.
1977VOL/MAZ

S. V. Volkov, E. A. Mazurenko, and Z. N. Bublik, Str. Svoistva Primen. B[Beta]-Diketonatov Met., [Mater. Vses. Semin.] 3rd 1977, 119-122; Chem. Abstr. 91, 79727c (1977).

1977ZHA/TIT N. N. Zhamskaya, V. A. Titov, A. A. Titov, and G. A. Kokovia, Zh. Fiz. Khim. 51, 1277 (1977).

1978ABA/MAL A. S. Abakumov and M. L. Malyshev, Russ. J. Inorg. Chem. 23, 1442-1444 (1978).

1978ADE/CON F. A. Adedeji, J. A. Connor, C. P. Demain, J. A. MartinhoSimoes, H. A. Skinner, and M. T. Zafarani-Moattar, J. Organomet. Chem. 149, 333-343 (1978).

1978ARO/STE M. Arora and W. V. Steele, J. Chem. Thermodyn. 10, 403-407 (1978).

1978BUB/MAZ Z. N. Bublik, E. A. Mazurenko, and S. V. Volkov, Ukr. Biokhim. Zh. 44, 1214 (1978).

1978BUR/KAM G. B. Burchalova, G. P. Kamelova, V. P. Nistratov, M. S. Sheiman, and I. B. Rabinovich, Termodin. Org. Soedin. 7, 21 (1978).

1978CHU/IGU Y. V. Chumachenko, I. K. Igumenov, and S. V. Zemskov, Russ. J. Phys. Chem. 52, 1393-1394 (1978).

1978CHU/KOV T. P. Chusova, N. L. Kovalenko, L. E. Gorsh, V. A. Titov, L. I. Chernyavskii, and G. A. Kokovin, Izvest. Sibirskogo Otdeleniya Akad. Nauk SSSR, Ser. Khim. Nauk 1978, 35 .

1978DAN/LEA D. M. Daniel, A. L. Leadbetter, R. E. Meads, and W. G. Parker, J. Chem. Soc., Faraday Trans. 2 74, 456-465 (1978).

1978DAV/TRA A. V. Davydov, S. S. Travnikov, E. V. Fedoseev, L. A. Ivanova, and B. F. Myasoedov, Probl. Khim. Primen. B [Beta]-Diketonatov Met., [Mater. Veses. Semin.] 1978, 02-100 (Publ. 1982).

1978DOZ/FUJ Y. Dozen, H. Fujishima, and H. Shingu, Thermochim. Acta 25, 209-216 (1978).

1978ECK/MUL T. Eckert and J. Muller, Arch. Pharm. 311, 31 (1978).

1978EKS/RAN A. Ekstrom and C. H. Randall, J. Phys. Chem. 82, 2180-2183 (1978).

1978FUC/PEA R. Fuchs and L. A. Peacock, Can. J. Chem. 56, 2493-2498 (1978).

1978GAL/PAV G. L. Gal'chenko, V. K. Pavlovich, V. N. Siryatskaya, A. F. Zhigach, and A. F. Termodinamika, Org. Soedin. 7, 38-43 (1978).

1978GOR/RAO J. E. Gordon and G. N. S. Rao, J. Am. Chem. Soc. 100, 7445 (1978).

1978GRA/KON T. D. Grabik, S. G. Konstantinov, G. P. Dudchik, and O. G. Polyachenok, Russ. J. Phys. Chem. 52, 894-895 (1978).

1978GUZ/LAR J. Guzman and J. J. Largo-Cabrerizo, Heterocycl. Chem. 15, 1531-1533 (1978).

1978HAM/BEN W. S. Hamilton, S. Benton, J. French, D. McCormick, S. Pustejovsky, and P. J. Thomspon, J. Chem. Eng. Data 23, 201-203 (1978).

1978IGU/CHU I. K. Igumenov, Y. V. Chumachenko, and S. V. Zemskov, Koordinocjonnaja Chim. 4, 164 (1978); ibid. 5, 34-38 (1979).

1978IGU/CHU2 I. K. Igumenov, Y. U. Chumanchenko, and S. V. Zemskov, Zh. Fiz. Khim. 52, 2664-2666 (1978); Russ. J. Phys. Chem. 52, 1531-1532 (1978)

1978INA/CHI A. Inaba and H. Chihara, J. Chem. Thermodyn. 10, 45 (1978).

1978IRV/SCH R. J. Irving, R. A. Schulz, and H. Naghihi, unpublished work cited in R. irving and M. A. V. Riberio Da Silva, J. Chem. Soc., Dalton Trans. 1978, 399-402.

1978JES/ERN A. C. Jesse, J. M. Ernsting, D. J. Stufkens, and K. Vrieze, Thermochim. Acta 25, 69-75 (1978).

1978JOR/AIR R. A. Jorge, C. Airolde, and A. P. Chagas, J. Chem. Soc., Dalton Trans. 1978, 1102-1104.

1978KAR/KAM N. V. Karyakin, G. P. Kamelova, and V. N. Sapozhnikov, Termodin. Org. Soedin. 7, 52-54 (1978); Chem. Abstr. 92, 75658d (1980).

1978KAR/RAB2 N. V. Karyakin, I. B. Rabinovich, and V. P. Nistratov, Termodin. Org. Soedin. 1978, 12.

1978KOM/GUR L. N. Komissarova, M. Z. Gurevich, T. S. Sas, and B. D Stepin, Zh. Neorg. Khim. 23, 3145-3147 (1978); Chem. Abstr. 90, 61378j (1979). 
1978KOS/BUD G. Koßmehl and D. Z. Budwell, Z. Naturforsch 42B, 478-488 (1978); Note: Names of the compounds were in German, and were translated.

1978LEB/RAB $\quad$ B. V. Lebedev, I. B. Rabinovich, N. K. Lebedev, and N. V. Ushakov, Dokl. Akad. Nauk SSSR 239, 1140 (1978).

1978MAN M. Mansson, 1978, unpublished work as referenced in Ref. [1978MON/ROS].

1978MAR/CIO2 D. I. Marchidan and M. Ciopec, J. Therm. Anal. 14, 131 (1978).

1978MON/ROS R. L. Montgomery, F. D. Rossini, and M. Mansson, J. Chem. Eng. Data 23, 125-129 (1978).

1978NIS/ISH K. Nishida, E. Ishihara, M. Osaka, and M. Koukitu, Galaxia 72 10, 13-14 (1978).

1978 OSB/SCO A. G. Osborn and D. W. Scott, J. Chem. Thermodyn. 10, 619-628 (1978).

1978RIB/IRV M. A. V. Ribeiro da Silva and R. G. Irving, Rev. Port. Quim. 20, 36-46 (1978); Chem. Abstr. 90, 211054s (1979).

1978SAB/LAF2 R. Sabbah and M. Laffitte, Thermochim. Acta 23, 196-198 (1978).

1978SEK/TSU K. Sekiguchi, Y. Tsuda, M. Kanke, E. Suzuki, and M. Iwatsuru, Chem. Pharm. Bull. 26, 1279 (1978).

1978SHM/SHL G. O. Shmyreva, V. G. Shlyakova, and R. M. Golosova, Russ. J. Phys. Chem. 52, 135-136 (1978); 52, 894-895 (1978).

1978SMI/ZEL G. E. Smirnova, Y. D. Zel'venski, V. A. Shalygin, G. A. Sukhorukova, and T. X. Dzhekseneva, Mosk. Khim.Teknol. Inst. Mockow, USSR Avali. Deposited Doc. VINITI 3298-78, 9, 1978.

1978SPI/AND G. M. Spinella, J. T. S. Andrews, W. E. Bacon, R. C. Fort, Jr., and J. Sabo, J. Chem. Thermodyn. 10, 1023 (1978).

1978STE3

1978STE4

1978STR/ROG

W. V. Steele, J. Chem. Thermodyn. 10, 441-444 (1978) W. V. Steele, J. Chem. Thermodyn. 10, 445-452 (1978). R. Stryjek, M. Rogalski, T. Treszczanowicz, and M. Luszczyk, Bull. Acad. Pol. Sci., Ser. Sci. Chim. 26, 327 (1978).

1978SUN/VIS S. Sundaram and D. S. Viswanath, J. Chem. Eng. Data 23, 62-64 (1978).

1978TAV/NEE A. Tavlaridis and R. Z. Neeb, Anal. Chem. 293, 211 (1978), as quoted in Ref. [1999ZEM/STA].

1978TIM R. E. Timms, Chem. Phys. Lipids 21, 113-129 (1978).

1978TIT/ZHA V. A. Titov, N. N. Zhamskaya, A. A. Titov, and G. A. Kokonin, Izv. Sibirskogo Otdeleniya Akad. Nauk SSSR, Ser. Khim. Nauk 1978, 10.

1978WES/MCK E. F. Westrum, Jr., M. A. McKervey, J. T. S. Andrews, R. C. Fort, Jr., and T. Clark, J. Chem. Thermodyn. 10, 959 (1978).

1979ABA/MAL A. S. Abakumov and M. L. Malyhev, Zh. Neorg. Khim. 24, 642-645 (1979); Eng. Edit. 24, 357-359.

1979ADE/CAV F. A. Adedeji, J. K. Cavell, S. Cavell, J. A. Connor, G. Pilcher, H. A. Skinner, and M. T. Zafarami-Moattar, J. Chem. Soc., Faraday Trans. 1 75, 603-613 (1979).

1979AMA/SAT R. Amano, A. Sato, and S. Suzuki, Radiochem. Radioanal. Lett. 39, 441-450 (1979); Chem. Abstr. 91, 181625e (1979).

1979AMI/LEB $\quad$ E. B. Amitin, E. G. Lebedeva, and I. E. Paukov, Zh. Fiz. Khim. 53, 2666 (1979).

1979ARM/JAM N. A. Armstrong, K. C. James, and C. K. Wong, J. Pharm. Pharmacol. 31, 627-631 (1979).

1979ARN/CHA E. M. Arnett and B. Chawla, J. Am. Chem. Soc. 101, 7141 (1979).

1979BAL/FRI M. Balish and V. Fried, J. Chem. Eng. Data 24, 91-92 (1979).

1979BER/ANG G. Berthon, V. Angot, V. Beden, and O. Enea, J. Chem. Thermodyn. 11, 539-546 (1979).

1979BLA/HOP R. Blachnik and A. Hoppe, Z. Anorg. Allg. Chem. 457, 91 (1979).

1979BLU/BAE V. I. Bludilina, A. K. Baev, V. K. Matveev, I. L. Gaidym, and E. I. Shcherbina, Zh. Fiz. Khim. 53, 1052-1053 (1979); Chem. Abstr. 91, 9585f (1979).

1979BOT/CAM H. Bothe and H. K. Cammenga, J. Therm. Anal. 16, 267-275 (1979).
1979BRI/VAN

1979CAL/DIA

1979CAR/CLA

1979CAV/GAR

1979CAV/HIL

1979CAV/HIL2

1979CLA/KNO

1979CLA/LET

1979COL/JIM

1979COL/JIM2

1979CON/DEM

1979CON/MAR

979DAA/ERN

1979FAR/SHA

1979FUC/PEA

1979GOL/SHM

1979IGU/CHU

1979KHE/LAL

1979KIM/TAK

1979KUC/SKU

1979KUD/KUD2

1979LEB/BER

1979LAR

1979LEE/HOS

1979LEW/ENE

1979MAC/PRA

1979MAS/MAL

1979MIZ/DAN

1979MUR/IWA

1979RIB/REI

1979SAB

1979SAB2

1979SAL/PEA
W. J. Briels and J. C. van Miltenburg, J. Chem. Phys. 70, 1064 (1979).

J. C. G. Calado, A. R. Dias, A. R. Martinho-Simoes, and M. A. V. Riberio da Silva, J. Organomet. Chem. 174, 77-80 (1979)

J. Carre, P. Claudy, J. M. Letoffe, M. Kollmannsberger, and J. Bousquet, J. Fluorine Chem. 14, 139-152 (1979).

K. J. Cavell, C. D. Garner, G. Pilcher, and S. Parks, J. Chem. Soc., Dalton Trans. 1714-1717 (1979).

K. J. Cavell, J. O. Hill, and R. J. Magee, Thermochim. Acta 33, 155-160 (1979).

K. J. Cavell, J. O. Hill, and R. J. Magee, Thermochim. Acta 33, 383-386 (1979).

T. Clark, T. M. Knox, M. A. McKervey, H. Mackle, and J. Rooney, J. Am. Chem. Soc. 101, 2404-2410 (1979).

P. Claudy, J. M. Letoffe, and J. Bousquet, Journ. Calorim. Anal. Therm. 10, AD.6.6-AD.6.9 (1979).

M. Colomina, P. Jimenez, R. Perez-Ossorio, and C. Turrion, J. Chem. Thermodyn. 11, 1179-1184 (1979).

M. Colomina, P. Jimenez, M. V. Roux, and C. Turrion, An. Quim. 75, 620-624 (1979); Chem. Abstr. 92, 75685k (1980).

J. A. Connor, C. P. Demain, H. A. Skinner, and M. T. Zafarani-Moattar, J. Organomet. Chem. 170, 117-130 (1979).

J. A. Connor, J. A. Martinho-Simoes, H. A. Skinner, and M. T. Zafarani-Moattar, J. Organomet. Chem. 179, 331-356 (1979).

H. Daamen, J. M. Ernsting, and A. Oskam, Thermochim. Acta 33, 217-223 (1979).

R. G. Farrell, F. Shahidi, F. Casellato, C. Vecchi, and A. Girelli, Thermochim. Acta 33, 275 (1979).

R. Fuchs and L. A. Peacock, Can. J. Chem. 57, 2302-2304 (1979).

R. M. Golosova, G. O. Shmyreva, V. G. Shlyakova, A. B Petrunin, D. B. Bekker, and A. F. Zhigach, Zh. Fiz. Khim. 53, 766-767 (1979).

I. K. Igumenov, Y. V. Chumachenko, and S. V. Zemskov, Khim. Termodin. Termokhim. 1979, 65-66; Chem. Abstr. 91, 217803c (1979).

S. C. Khetarpal, K. Lal, and H. L. Bhainagar, Aust. J. Chem. 32, 49 (1979).

T. Kimura and S. Takagi, J. Chem. Thermodyn. 11, 47-55 (1979).

Y. K. Kuchhal, R. N. Shukla, and A. B. Biswas, Thermochim. Acta 31, 61-70 (1979).

S. A. Kudchadker, A. P. Kudchadker, and B. J. Zwolinski, J Chem. Thermodyn. 11, 1051-1059 (1979).

S. Y. Lebedev, S. S. Berdonosov, I. V. Melikhov, N. B. Mekheev, and I. A. Rumer, Radiokhimiya 21, 470-472 (1979); Chem. Abstr. 91, 146146c (1979).

S. V. Larionov, Russ. J. Inorg. Chem. 24, 802 (1979).

S. H. Lee-Bechtold, I. A. Hossenlop, D. W. Scott, A. G. Osborn, and W. D. Good, J. Chem. Thermodyn. 11, 469-482 (1979).

G. A. Lews and R. P. Enever, Int. J. Pharm. 22, 203 (1979).

A. B. Macknick and J. M. Prausnitz, J. Chem. Eng. Data 24, 175-178 (1979).

R. Masse, R. Malaviolle, and A. Chauvet, J. Therm. Anal. 16, 341-345 (1979).

E. N. Mizinov, S. M. Danov, G. L. Afraimovich, and M. A. Chugunov, Zh. Prikl. Khim. 52, 1431 (1979).

M. Muramatsu, M. Iwahashi, and U. Takeuchi, J. Pharm. Sci. 68, 175-177 (1979).

M. A. V. Riberio da Silva and A. M. M. V. Reis, Bull. Chem. Soc. Jpn. 52, 3080-3083 (1979).

R. Sabbah, Bull. Soc. Chem. Fr. 9-10(1), 434-437 (1979).

R. Sabbah, C. R. Seances Acad. Sci., Ser. C 289, 153-156 (1979); Chem. Abstr. 92, 58049p (1980).

P. P. S. Saluja, L. A. Peacock, and R. Fuchs, J. Am. Chem. Soc. 101, 1958-1962 (1979). 
1979SAN/EPS D. J. Sandman, A. J. Epstein, J. S. Chickos, J. Ketchum, J. S. Fu, and H. A. Scheraga, J. Chem. Phys. 70, 305-313 (1979).

1979SEV C. Sevensson, J. Chem. Thermodyn. 11, 593-596(1979).

1979SMI

1979SMI2

1979SPE/SHO

G. W. Smith, Phase Transitions 1, 107-129 (1979).

G. W. Smith, Mol. Cryst. Liq. Cryst. 49, 207 (1979).

W. F. Spencer, T. D. Shoup, M. M. Cliath, W. J. Farmer, and R. J. Hague, J. Agric. Food Chem. 27, 273-278 (1979).

1979STE W. V. Steele, J. Chem. Thermodyn. 11, 187-192 (1979).

1979SUN/SVE S. Sunner and C. Svensson, J. Chem. Soc., Faraday Trans. 1 75, 2359-2365 (1979).

1979SUN/SVE2 S. Sunner, C. Svensson, and A. S. Zelepuga, J. Chem. Thermodyn. 11, 491-495 (1979).

1979TEL/RAB V. I. Tel'noi, I. B. Rabinovich, M. R. Leonov, G. V. Solov'eva, and N. I. Gramoteeva, Dokl. Phys. Chem. 245, 363-365 (1979).

1979YAN/TEP I. K. Yanson, A. B. Teplitsky, and L. F. Sukhodub, Biopolymers 18, 1149-1170 (1979).

1979ZAN/THO M. Zander and W. Thomas, J. Chem. Eng. Data 24, 1-2 (1979).

1980ABR/IRV M. H. Abraham and R. J. Irving, J. Chem. Thermodyn. 12, 539-544 (1980).

1980AIH

1980AND/CON

A. Aihara, Denki Tsushin Daigaku Gakuho 31, 65-68 (1980); Chem. Abstr. 94, 174045z (1981).

1980ARN/SCH $\quad$ H. Arntz and G. M. Schneider, Faraday Discuss. Chem. Soc. 69, 139-145 (1980).

1980ARS M. R. Arshadi, J. Chem. Thermodyn. 12, 903-906 (1980).

1980BEC/KRA H.-D. Beckhaus, G. Kratt, K. Lay, J. Greiselmann, C. Rüchardt, B. Kitschke, and H. J. Lindner, Chem. Ber. 113, 3441-3455 (1980).

1980BOX G. Boxhoorn, Ph.D. dissertation, University of Amsterdam, 1980, Appendix p. 149 as cited by [1980CAV/ERN].

1980BOX/ERN G. Boxhoorn, J. M. Ernsting, D. J. Stufkens, and A. Oskam, Thermochim. Acta 42, 315-321 (1980).

1980CAL/DIA J. C. R. Calado, A. R. Dias, M. E. Minas da Piedade, and J. A. Martinho Simoes, Rev. Port. Quim. 22, 53-58 (1980), see [1983JAC/VAN].; Chem. Abstr. 96, 52441w (1981).

1980CAR/BUS C. Carfagna, V. Busico, V. Salerno, and M. Vacatello, Thermochim. Acta 37, 31-36 (1980).

1980CAV/ERN K. J. Cavell, J. M. Ernsting, and D. J. Stufkens, Thermochim. Acta 42, 343-348 (1980).

1980CLA/KNO T. Clark, T. M. Knox, M. A. McKervey, and H. Mackle, J. Chem. Soc., Perkin Trans. 2 1980, 1686-1689.

1980COL/JIM2 M. Colomina, P. Jimenez, C. Turrion, J. A. Fernandez, and C. Monzon, An. Quim., Ser. A 76, 245-248 (1980).

1980DEK

1980DEK/GOV C. G. DeKruif and H. A. J. Govers, J. Chem. Phys. 73, 553-555 (1980).

1980DEP $\quad$ R. S. DePablo, J. Phys. D: Appl. Phys. 13, 313-319 (1980).

1980DYG/STE R. S. Dygdala and K. Stefanski, Chem. Phys. 53, 51-62 (1980).

1980FUC/PEA R. Fuchs and L. A. Peacock, Can. J. Chem. 58, 2796-2799 (1980).

1980GOM/WEI W. Gombler and H.-U. Weiler, J. Fluorine Chem. 15, 279-288 (1980).

1980JON/MAT J. A. Jonsson, L. Mathiasson, and A.-M. Olsson, Acta Chem. Scand. 34A, 147-150 (1980).

1980KRA/PIG A. Krajewska and K. Pigon, Thermochim. Acta 41, 187-197 (1980).

1980LEB/KIP N. K. Lebedev, E. G. Kiparisova, B. V. Lebedev, A. M. Sladkov, and N. A. Vasmeva, Bull. Acad. Sci. USSR, Div. Chem. Sci. 29, 374 (1980).

1980MEY/AWE E. F. Meyer, M. J. Awe, and R. E. Wagner, J. Chem. Eng. Data 25, 371-374 (1980).

1980MOR/WAT C. T. Mortimer and J. Waterhouse, J. Chem. Thermodyn. 12, 961-965 (1980).
1980MUR/CAV

J. P. Murray, K. J. Cavell, and J. O. Hill, Thermochim. Acta 36, 97-101 (1980).

1980NAS/HWA P. Nasir, S. C. Hwang, and R. Kobayashi, J. Chem. Eng. Data 25, 298-301 (1980).

1980NIG/DEP G. D. Nigam and B. Deppisch, Z. Kristallogr. - Cryst. Mater. 151, 185-191 (1980).

1980NIS/SAK K. Nishiyama, N. Sakiyama, S. Seki, H. Horita, T. Otsubo, and S. Misumi, Bull. Chem. Soc. Jpn. 53, 869-877 (1980).

1980 OSB/SCO A. G. Osborn and D. W. Scott, J. Chem. Thermodyn. 12, 429-438 (1980).

1980PIT/KIS $\quad$ V. S. Pityugin, T. L. Kislitsyna, R. F. Shakirov, L. N. Sharif'yanova, and E. N. Maslennikov, Khim. Prom-st., Ser.: Khlornaya Prom-st. 1980, 11-14.

1980RAD/RAD M. Radomska and R. Radomski, Thermochim. Acta 40, 405 (1980).

1980ROD/KRU G. N. Rodionova, I. V. Krutovskaya, A. N. Rodionov, Y. G. Tuchin, and V. V. Karpov, Zh. Prikl. Spektrosk. 32, 623-628 (1980)

1980ROT A. M. Rothman, J. Agric. Food Chem. 28, 1225-1228 (1980).

1980ROT/ORC J. A. Roth and M. Orchin, J. Organomet. Chem. 187, 103-105 (1980).

1980SAC/HIL J. Sachinides and J. O. Hill, Thermochim. Acta 35, 59-66 (1980).

1980SHA/SAD L. N. Sharif'yanova, F. M. Sadykova, and S. G. Akhmerova, Khim. Prom-st., Ser.: Khlornaya Prom-st. 1980, 16-18.

1980SHU/VAR P. Shul'tse, R. M. Varushchenko, G. L. Gal'chenko, T V. Klimova, and V. I. Stanko, J. Gen. Chem. USSR 50, 1482-1488 (1980); Zh. Obshch. Khim. 50, 1818-1825 (1980).

1980SMI G. W. Smith, Mol. Cryst. Liq. Cryst. 64, 15 (1980).

1980SMI/STE N. K. Smith, R. C. Stewart, Jr., A. G. Osborn, and D. W. Scott, J. Chem. Thermodyn. 12, 919-926 (1980).

1980SOR/TSU M. Sorai, K. Tsuji, H. Suga, and S. Seki, Mol. Cryst. Liq. Cryst. 59, 33 (1980).

1980STE W. V. Steele, J. Chem. Thermodyn. 12, 187-192 (1980).

1980SWA/KWA H. A. Swain, Jr., Y. C.-Y. Kwan, and H.-N. Sung, J. Phys. Chem. 84, 1347-1349 (1980).

1980TEP/YAN A. B. Teplitskii, I. K. Yanson, O. T. Glukhova, A. Zielenkiewicz, W. Zielenkiewicz, and K. L. Wierzchowski, Biophys. Chem. 11, 17-21 (1980).

1980THO/SMI L. H. Thomas, H. Smith, and G. H. Davis, J. Chem. Technol. Biotechnol. 30, 476-480 (1980).

1980URB/GIG M. Urbani, R. Gigli, and V. Picente, J. Chem. Eng. Data 25, 97-100 (1980).

1980VAN/PRA C. Van de Rostyne and J. M. Prausnitz, J. Chem. Eng. Data 25, 1-3 (1980).

1980WIE/KOB S. A. Wieczorek and R. Kobayashi, J. Chem. Eng. Data 25, 302 (1980).

1980WON/WES W.-K. Wong and E. F. Westrum, Jr., Mol. Cryst. Liq. Cryst. 61, 207 (1980).

1981ABE/SHR2 T. Abe and J. M. Shreeve, Inorg. Chem. 20, 2432-2434 (1981).

1981AMA/SAT R. Amano, A. Sato, and S. Suzuki, Bull. Chem. Soc. Jpn. 54, 1368-1374 (1981).

1981BRO/MCE W. Brostow, D. M. McEachern, and J. A. Valdez, Mater. Chem. 6, 187-195 (1981).

1981BYS

1981CAL/DIA

K. Bystrom, J. Chem. Thermodyn. 13, 139-145 (1981). J. C. G. Calado, A. R. Dias, M. S. Salema, and J. A. Martinho-Simoes, J. Chem. Soc., Dalton Trans. 1981, $1174-1177$.

1981CAV/CON K. J. Cavell, J. A. Connor, G. Pilcher, M. A. V. Riberio da Silva, M. D. M. C. Riberio Da Silva, H. A. Skinner, Y. Virmani, and M. T. Zafarami-Moattar, J. Chem. Soc., Faraday Trans. 1 77, 1585-1594 (1981).

1981CAV/GAR K. J. Cavell, C. D. Garner, J. A. Martinho-Simoes, G. Pilcher, H. Al-Samman, H. A. Skinner, G. Al-Takhin, I. B. Walton, and M. T. J. Zafarami-Moattar, J. Chem. Soc., Faraday Trans. 1 77, 2927-2938 (1981).

1981CHA/PER A. Chauvet, R. Perrier, and J. Masse, Thermochim. Acta 43, 161 (1981). 
1981CHI/GAR J. S. Chickos, D. L. Garin, M. Hitt, and G. Schilling, Tetrahedron 37, 2255-2259 (1981).

1981DEK/HER K. G. De Kruif, J. M. Herman, and P. J. Van den Berg, J. Chem. Eng. Data 26, 359-361 (1981).

1981DIT/SKO V. E. Ditsent, I. I. Skorokhodov, M. N. Zolotareva, V. I. Savuskina, and B. N. Tabenko, Zh. Prikl. Khim. (Leningrad) 54, 1617-1619 (1981).

1981DRA/AZI M. Draguet-Brughmans, M. Azibi, and R. Bouche, Proc. Eur. Symp. Therm. Anal. 2, 615-617 (1981).

1981EDW/PRA D. R. Edwards and J. M. Prausnitz, J. Chem. Eng. Data 26, 121-124 (1981).

1981 GUS/KAS S. N. Guseinova, V. V. Kas'yanov, and Y. V. Politanskii, Khim. Prom-st. Ser., Khlornaya Prom-st. 1981, 28-30; Chem. Abstr. 95, 225804b (1981).

1981HAL/COG J. L. Hales, R. C. Cogman, and W. J. Frith, J. Chem. Thermodyn. 13, 591-601 (1981).

1981IWA/OHN K. Iwamoto, Y. Ohnuki, K. Sawada, and M. Seno, Mol. Cryst. Liq. Cryst. 73, 95 (1981).

1981JEN/OBR T. E. Jenkins and P. O’Brien, Phys. Status Solidi A 67, K161 (1981).

1981LEB/LEB $\quad$ B. V. Lebedev, N. K. Lebedev, V. S. Khotimskii, S. G. Durgar'yan, and N. S. Nametkin, Dokl. Akad. Nauk SSSR 259, 629 (1981).

1981LEB/YEV $\quad$ B. V. Lebedev, A. A. Yevstropov, and Y. G. Kiparisova, J. Chem. Thermodyn. 13, 1185-1204 (1981).

1981LEE/FIN S. H. Lee-Bechtold, H. L. Finke, J. F. Meserly, and D. W. Scott, J. Chem. Thermodyn. 13, 213 (1981).

1981MAS/BAR J. Maslowska and J. B. Baranowski, Chem. Anal. 26, 1017-1027 (1981).

1981MEK/KAR S. A. Mekhtiev and K. A. Karasharli, Azerb. Khim. Zh. 1981, 85 .

1981MRA/ORO S. C. Mraw and D. F. O'Rourke, J. Chem. Thermodyn. 13, 199 (1981).

1981NAV/HAU P. Navard and J. M. Haudin, J. Therm. Anal. 22, 107-118 (1981).

1981OGA/SOR K. Ogasahara, M. Sorai, and H. Suga, Mol. Cryst. Liq. Cryst. 71, 189-211 (1981).

1981PEL/TOM M. Pelino, M. Tomassetti, V. Piacente, and G. D’Ascenzo, Thermochim. Acta 44, 89-99 (1981).

1981PIR/AZA A. I. Pirogov, G. I. Azarova, and V. I. Klopov, Izv. Vyssh Uchebn. Zaved., Khim. Khim. Tekhnol. 24, 827-829 (1981); Chem. Abstr. 95, 138901n (1981).

1981SHA/DZH T. N. Shakhtaktinskii, O. I. Dzhafarov, K. A. Karasharli, and A. M. Kuliev, Dokl. Akad. Nauk. Azerb. SSR 37, 54 (1981).

1981SMI/MAR E. V. Smirnov, I. A. Marav'eva, L. I. Martynenko, and V. I. Spitsyn, Zh. Neorg. Khim. 26, 1709 (1981); Chem. Abstr. 95, 86430m (1981).

1981TEG/FER R. Teghil, D. Ferro, L. Bencivenni, and M. Pelino, Thermochim. Acta 44, 213-222 (1981).

1981TEK/MAJ V. Tekac, V. Majer, V. Svoboda, and V. Hynek, J. Chem. Thermodyn. 13, 659-662 (1981).

1981TOD/BEL D. T. Todorov, M. N. Belikov, V. A. Shalygin, and G. E. Smirnova, Zh. Priklad. Khim. 54, 1175 (1981).

1981VAR/BUL R. M. Varushchenko, L. L. Bulgakova, P. S. Minzabekyants, and K. N. Makarov, Russ. J. Phys. Chem. 55, 1480-1482 (1981).

1981 WES/SIM J. W. Westcott, C. G. Simon, and J. F. Bidleman, Environ. Sci. Technol. 15, 1375-1378 (1981).

1981WIE/KOB S. A. Wieczorek and P. Kobayashi, J. Chem. Eng. Data 26, 8-11 (1981); 26, 11-13 (1981).

1981YAN/NAB S. Yano, Y. Nabata, and K. Aoki, Mol. Cryst. Liq. Cryst. 70, 163-168 (1981).

1982ATA/GYO T. Atake, H. Gyoten, and H. Chihara, J. Chem. Phys. 76, 5535 (1982).

1982CON/ZAF J. A. Connor, M. T. Zafarani-Moattar, J. Bickerton, N. I. El-Saied, S. Suradi, R. Carson, G. Al Takkhin, and H. A. Skinner, Organometallics 1, 1166-1174 (1982).

1982DEK/SCH G. C. DeKruif, R. C. F. Schaake, J. C. Van Meltenburg, K. Van der Klauw, and J. G. Blok, J. Chem. Thermodyn. 14, 791-798 (1982).

1982DEP R. S. DePablo, J. Chem. Eng. Data 27, 374-375 (1982).
1982DIA/SAL A. R. Dias, M. S. Salema, and J. A. Martinho Simoes, Organometallics 1, 971-973 (1982).

1982FUC/HAL R. Fuchs, J. H. Hallman, and M. O. Perlman, Can. J. Chem. 60, 1832-1835 (1982).

1982GAM/CAL $\quad$ B. E. Gammon, J. E. Callanan, I. A. Hossenlopp, A. G. Osborn, and W. D. Good, Proc. Symp. Thermophys. Prop. 8, 402-408 (1982).

1982GRA/FOS B. T. Grayson and L. A. Fosbraey, Pestic. Sci. 13, 269-278 (1982).

1982INA/MUR2 S. Inagaki, S. Murata, M. Sakiyama, Y. Ito, Y. Umehara, T. Hijiya, and T. Matsuura, Bull. Chem. Soc. Jpn. 55, 2803-2807 (1982).

1982INI/LOP J. C. Iniguez, M. E. Lopez, and D. M. McEachern, Rev. Soc. Quim. Mex. 26, 122-124 (1982); Chem. Abstr. 97, $144252 \mathrm{j}(1982)$.

1982INV

1982JAL/ZOG C. Invernizzi, Termotecnica 4, 78-85 (1982).

1982KUL/DZH Chem. Phys. Lipids 31, 395-404 (1982). N. Kostryukov, Zh. Fiz. Khim. 56, 310 (1982).

1982LEB/BYK B. V. Lebedev, T. A. Bykova, N. N. Smirnova, and T. G. Kulagina, Zh. Obshch. Khim. 52, 2630-2636 (1982).

1982MAR/MIR A. Martin and M. J. Miralles, J. Pharm. Sci. 71, 439-442 (1982).

1982MOR C. T. Mortimer, in Thermochemistry and Its Applications to Chemical and Biochemical Systems, edited by Riberio Da Silva, Series C: Mathematical and Physical Sciences Vol. 119 (D. Reidel Publishing Company, Boston MA, 1982), pp. 47-60.

1982MUR/SAK S. Murata, M. Sakiyama, and S. Seki, J. Chem. Thermodyn. 14, 707-721 (1982); 14, 723-731 (1982).

1982 NUZ M. Nuzzi, Rivista Comb. 36, 41 (1982).

1982OLL/PER M. Ollivon and R. Perron, Thermochim. Acta 53, 183 (1982).

1982PIL/SKI G. Pilcher and H. A. Skinner, in The Chemistry of the Metal-Carbon Bond, edited by F. R. Hartley and S. Patai (John Wiley \& Sons, New York, 1982), Chap. 2.

1982REC/GRE P. Reche and M.-F. Grenier-Loustalot, J. Chromatogr. 238 317-334 (1982)

1982SCH/VAN R. C. F. Schaake, J. C. van Miltenberg, and C. G. De Kruif, J. Chem. Thermodyn. 14, 771 (1982).

1982SCH/VAN2 R. C. F. Schaake, J. C. van Miltenberg, and C. G. De Kruif, J. Chem. Thermodyn. 14, 763 (1982).

1982SIV/KOB $\quad$ A. Sivaraman and R. Kobayashi, J. Chem. Eng. Data 27, 264 (1982).

1982TRE/VAU C. Treiner, C. Vaution, and G. N. Cave, J. Pharm. Pharmacol. 34, 539 (1982).

1982TSU/SOR K. Tsuji, M. Sorai, H. Suga, and S. Seki, Mol. Cryst. Liq. Cryst. 87, 305 (1982).

1982WAS/RAD J. Wasicki, M. Radomska, and R. Radomski, J. Therm. Anal. 25, 509 (1982).

1982ZVE/VIN V. V. Zvezdina, E. A. Vinogradova, V. P. Bochin, B. D. Berezin, and O. A. Golubchikov, Zh. Neorg. Khim. 27, 2818-2822 (1982).

1983 AKK/SCH $\quad$ O. S. Akkerman, G. Schat, E. A. I. M. Evers, and F. Bickelhaupt, Recl.: J. R. Neth. Chem. Soc. 102, 109 (1983).

1983ALT/CON G. Al-Takhin, J. A. Connor, and H. A. Skinner, J. Organomet. Chem. 259, 313 (1983).

1983BAR/BEC W. Barbe, H.-D. Beckhaus, H. J. Lindner, and C. Rüchardt, Chem. Ber. 116, 1017 (1983).

1983BEN/BIE R. Bender, V. Bieling, and G. Mauer, J. Chem. Thermodyn. 15, 585 (1983).

1983CAS/PON A. E. Castro Luna, M. I. Ponzi, and J. B. Rivarola, J. Chem. Eng. Data 28, 349 (1983).

1983CHA $\quad$ S. S. Chang, J. Chem. Phys. 79, 6229 (1983).

1983CHA/MAS A. Chauvet and J. Masse, Thermochim. Acta 64, 267 (1983).

1983CHA/MAU S. S. Chang, J. R. Maurey, and W. J. Plummer, J. Chem. Eng. Data 28, 187 (1983).

1983COL/JIM M. Colomina, P. Jimenez, C. Turrion, M. Kaminski, and W. Zielenkiewicz, Thermochim. Acta 68, 371 (1983).

1983DEK/VAN C. G. DeKruif, J. C. Van Miltenburg, and J. G. Blok, J. Chem. Thermodyn. 15, 129 (1983). 
1983ELV/KUU A. Elvelt, M. Kuus, and L. S. Kudryavtseva, Eesti NSV Tead. Akad. Toim. Keem. 32, 172 (1983).

1983FER/IMP $\quad$ D. Ferro, P. Imperatori, and C. Quangliata, J. Chem. Eng. Data 28, 242 (1983).

1983FER/PIA D. Ferro, V. Piacente, and P. Scardala, Thermochim. Acta 68, 329 (1983).

1983FER/QUA D. Ferro, C. Quagliata, and M. R. Conte, Thermochim. Acta 60, 211 (1983).

1983GHA/JAM M. Gharavi and K. C. James, Int. J. Pharm. 14, 325 (1983).

1983GRA/ABO D. J. Grant and I. K. A. Abougela, Int. J. Pharm. 17, 77 (1983).

1983HAA/PAU W. Haase, H. Paulus, and R. Pendzialek, Mol. Cryst. Liq. Cryst. 100, 211 (1983).

$1983 \mathrm{HOL}$

$1983 \mathrm{HOU}$

1983HOU2

1983HOU3

1983JAC/VAN

1983KAP/LEL

1983KHA

1983KRA/BEC

1983MAJ/AZZ

M. R. Holdiness, Thermochim. Acta 68, 375 (1983).

J. E. House, Jr., J. Fluorine Chem. 22, 299 (1983).

J. E. House, Jr., Thermochim. Acta 71, 215 (1983).

J. E. House, Jr., Thermochim. Acta 69, 361 (1983).

M. H. G. Jacobs, P. J. van Ekeren, and C. G. Dekruif, J.

Chem. Thermodyn. 15, 619 (1983).

O. Kaposi, L. Lelik, and K. Balthazar, High Temp. Sci. 16, 299 (1983).

F. I. Khattab, Thermochim. Acta 61, 253 (1983).

G. Kratt, H.-D. Bechhaus, W. Bernloehr, and C. Rüchardt, Thermochim. Acta 62, 279 (1983).

$\begin{array}{ll}\text { 1983MAS/CHA } & \text { Trans. 1 79, } 675 \text { (1983). } \\ \text { J. Masse and A. Chauvet, Ann. Pharm. Fr. 41, } 579 \text { (1983). }\end{array}$

1983MAS/STE H. Maskill and W. V. Steele, J. Chem. Thermodyn. 15, 481 (1983).

1983MUR/HIL J. P. Murray and J. O. Hill, Thermochim. Acta 63, 211 (1983).

1983OLS/JON A.-M. Olsson, J. A. Jonsson, B. Thelin, and T. Liljefors, J. Chem. Ecol. 9, 375 (1983).

1983ORO/MRA D. R. O'Rourke and S. C. Mraw, J. Chem. Thermodyn. 15, 489 (1983).

1983PEL/GIG M. Pelino, R. Gigli, and M. Tomassette, Thermochim. Acta 61, 301 (1983).

1983RAE/SCH A. Raemy and T. F. Schweizer, J. Therm. Anal. 28, 95 (1983).

1983RIB/REI M. A. V. Ribeiro Da Silva and A. M. M. V. Reis, J. Chem. Thermodyn. 15, 957 (1983).

1983SIV/KOB A. Sivaraman and R. Kobayashi, J. Chem. Thermodyn. 15, 1127 (1983).

1983SIV/MAR A. Sivaraman, R. J. Martin, and R. Kobayashi, Fluid Phase Equilib. 12, 175 (1983).

1983SON/ZOL W. J. Sonnefeld, W. H. Zoller, and W. E. May, Anal. Chem. 55, 275 (1983).

1983SPI/KOL V. I. Spitsyn, I. D. Kolli, E. A. Balabanova, E. V. Smirnov, and I. A. Murav'eva, Zh. Neorg. Khim. 28, 2697 (1983); Eng. Edit. 28, 1530 (1983).

1983TER A. Terlain, J. Chim. Phys. Phys.-Chim. Biol. 1983, 805.

1983 TRE/BER G. V. Trembovetskii, S. S. Berdonosov, I. Murav'eva, and L. I. Martynenko, Zh. Neorg. Khim. 28, 3032 (1983); Chem. Abstr. 100, 39999r (1984).

1983VAN/JAC P. J. Van Ekeren, M. H. G. Jacobs, J. C. A. Offringa, and C. G. J. Dekruif, J. Chem. Thermodyn. 15, 409 (1983).

1983WEA by R. C. Weast (CRC Press, Boca Raton, FL, 1983).
M. Zamkanei, J. H. Kaiser, H. Birkhofer, H.-D. Beckhaus, and C. Rüchardt, Chem. Ber. 116, 3216 (1983).

1983ZVE/MOT V. V.Zvezdina, O. R. Motorina, G. Y. Guara, V. P. Bochin, O. A. Golubchikov, and B. D. Berezin, Russ. J. Phys. Chem. 57, 1422 (1983).

1984ALT/CON G. Al-Takhin, J. A. Connor, H. A. Skinner, and M. T. Zafarani-Moattan, J. Organomet. Chem. 260, 189 (1984).

1984BAE/BAR A. K. Baev and A. A. Barkatin, Russ. J. Phys. Chem. 58, 195 (1984).

1984BAE/BAR2 A. K. Baev, A. A. Barkatin, and L. M. Dyagileva, Vestsi Akad. Navuk BSSR, Ser. Khim. Navuk 1984, 60.

1984BAE/MIK A. K. Baev, V. E. Mikhailov, and A. A. Baev, Vestsi Akad. Navuk BSSR, Ser. Khim. Navuk 1984, 75.
1984BEC/RUC

1984BER/BEC

H.-D. Beckhaus, C. Rüchardt, and M. Smisek, Thermochim. Acta 79, 149 (1984).

W. Bernloehr, H.-D. Beckhaus, K. Peters, H. G. Von Schnering, and C. Ruechardt, Chem. Ber. 117, 1013 (1984).

1984BER/BEC2 W. Bernloehr, H.-D. Beckhaus, and C. Rüchardt, Chem. Ber. 117, 1026 (1984).

1984BOU/FRI T. Boublik, V. Fried, and E. Hala, The Vapour Pressures of Pure Substances: Selected Values of the Temperature Dependence of the Vapour Pressures of Some Pure Substances in the Normal and Low Pressure Region, 2nd Revised ed. (Elsevier, Amsterdam, 1984); Note: The vaporization enthalpies were calculated from the vapor pressures obtained from the Antoine constants reported in this compendium. In cases where the Antoine constant $\mathrm{C}=0$, the Antoine Equation $(\log 10 \mathrm{P}=A-B /(\mathrm{C}+\mathrm{T}))$ reduces to the integrated form of the Clausius Clapeyron equation directly. This was the case for many vaporization enthalpies. In those cases where $\mathrm{C}^{1} 0$, the vaporization enthalpy was calculated as DvapHm $(\mathrm{T})=2.303 \mathrm{R} \mathrm{B}[\mathrm{T} /(\mathrm{T}$ $+\mathrm{C})] 2$.

1984BOU/OON J. A. Bouwstra, H. A. J. Oonk, J. G. Blok, and C. G. De Kruif, J. Chem. Thermodyn. 16, 403 (1984).

1984BUR/ARM L. P. Burkhard, D. E. Armstrong, and A. Andren, J. Chem. Eng. Data 29, 248 (1984).

1984BUR/MOR P. M. Burkinshaw and C. T. Mortimer, J. Chem. Soc., Dalton Trans. 1984, 75.

1984CAR A. S. Carson, J. Chem Thermodyn. 16, 427 (1984).

1984CAR/SPE A. S. Carson and J. A. Spencer, J. Chem Thermodyn. 16, 423 (1984).

1984CHH/POM K. Chhor, C. Pommier, and M. Diot, J. Chem. Thermodyn. 16, 503 (1984).

1984COR E. H. P. Cordfunke, Thermochim. Acta 108, 45 (1984).

1984DOM/EVA E. S. Domalski, W. H. Evans, and E. D. Hearing, J. Phys. Chem. Ref. Data 13(Suppl. 1) (1984).

1984DZH/KUL O. I. Dzhafarov, A. M. Kuliev, and A. G. Kuznestsova, Zh Fiz. Khim. 58, 1630 (1984).

1984EGL/STR U. H. Egli, R. A. Streuli, and E. Dubler, Biochemistry 23, 148 (1984).

1984FER/BRO M. J. Ferrante and R. R. Brown, Rep. Invest.-U.S. Bur. Mines R1, 8917 (1984).

1984FLA/BEC M. A. Flamm-Ter Meer, H.-D. Beckhaus, and C. Rüchardt, Thermochim. Acta 80, 81 (1984).

1984FOU/AMO M. Foulon, J. P. Amoureux, J. L. Sauvajol, J. P. Cavrot, and M. Muller, J. Phys. C 17, 4213 (1984).

1984GOL/LEV G. S. Gol'din, E. I. Levites, A. I. L'vov, and N. L. Sin'ko, Koord. Khim. 20, 563 (1984).

1984GOV/KAN S. W. Govorchin and A. S. Kana'an, J. Chem. Thermodyn. 16, 437 (1984).

1984GOV/KAN2 S. W. Govorchin, A. S. Kana'an, and J. M. Kanameuller, J. Chem. Thermodyn. 16, 703 (1984).

1984GRA/AVR E. Grantscharova, I. Avramov, and I. Gutzow, Dokl. Bolg. Akad. Nauk 37, 1521 (1984).

1984GRI/KON A. A. Grigor'ev, Y. V. Kontrat'ev, and A. V. Suvorov, Zh. Obshch. Khim. 54, 1935 (1984); as cited by D. R. Kirklin E. S. Domalski, J. Chem. Thermodyn. 20, 743 (1988).

1984HOL M. R. Holdiness, Thermochim. Acta 78, 435 (1984).

1984KAN/YAM N. Kaneniwa, T. Yamaaguchi, N. Watari, and M. Otsuka, Yakugashi Zasshi 104, 184 (1984).

1984KAR/KRU V. V. Karpov, I. V. Krutovskaya, and G. N. Rodionova, Zh. Prikl. Khim. (Leningrad) 57, 1348 (1984).

1984KAR/ROD V. V. Karpov, G. N. Rodionova, I. V. Krutovskaya, L. Z. Gandel'sman, L. A. Khomenko, and L. M. Yagupol'skii, Dyes Pigm. 5, 285 (1984).

1984KEL S. M. Kelly, Helv. Chim. Acta 67, 1572 (1984).

1984KER/OPP von F. Kersten and H. Oppermann, Z. Phys. Chem. (Leipzig) 265, 1207 (1984).

1984KHA/TAR A. Khandkar, V. B. Tare, A. Navrotsky, and J. B. Wagner, Jr., J. Electrochem. Soc. 131, 2683 (1984).

1984KRI G. Krien, Thermochim. Acta 81, 29 (1984)

1984LAW/ELI A. H. Lawrence, L. Elias, and M. Authier-Martin, Can. J. Chem. 62, 1886 (1984). 
1984LEB/BYK $\quad$ B. V. Lebedev, T. A. Bhykova, V. G. Kiparisova, V. A. Pankratov, V. V. Korshak, and V. M. Latkinov, Zh. Obshch. Khim. 54, 417 (1984).

1984LEB/GUT2 N. V. Lebedeva, N. M. Gutner, Y. A. Katin, N. M. Kozlova, N. N. Kiseleva, E. F. Makhina, and S. L. Dobychin, J. Appl. Chem. (USSR) 57, 2118 (1984).

1984LEB/LEB $\quad$ B. V. Lebedev, N. K. Lebedev, Y. G. Kiparisova, K. A. Yesayan, L. K. Golova, and Y. B. Amerik, Vysokomol. Soedin. A26, 909 (1984).

1984MAJ/SVO3 V. Majer, V. Svoboda, J. Pechacek, and S. Hala, J. Chem. Thermodyn. 16, 567 (1984).

1984MAR/MEL M. Marcos, E. Melendez, J. L. Serrano, P. Camps, M. Figueredo, and C. Jaime, J. Chem. Soc., Perkin Trans. 2 1984, 7.

1984MIL/GHO M. M. Miller, S. Ghodbane, S. P. Wasik, Y. B. Tewari, and D. E. Martire, J. Chem. Eng. Data 29, 184 (1984).

1984MRA/KEW S. C. Mraw and C. F. Keweshan, J. Chem. Thermodyn. 16, 873 (1984).

1984MRW/STA A. Mrwa, M. Starke, and C. Harmann, Z. Phys. Chem. (Leipzig) 265, 577 (1984).

1984MUR/HIL J. P. Murray and J. O. Hill, Thermochim. Acta 72, 341 (1984).

1984OZC/ASR Y. Ozcayir, J. Asrar, and A. Blumstein, Mol. Cryst. Liq. Cryst. 110, 263 (1984).

1984PER

1984RIB/RIB

R. R. Perron, Rev. Fr. Corps Gras 31, 171 (1984).

M. D. M. C. Ribeiro Da Silva, M. A. V. Ribeiro Da Silva, A. P. S. M. C. Carvalho, M. J. Akello, and G. Pilcher, J. Chem. Thermodyn. 16, 137 (1984).

1984SHE/NIS M. S. Sheiman, V. P. Nistratov, G. P. Kamelova, and I. B. Rabinovich, Prob. Kalorim. Khim. Termodin. Dokl. Vses. Konf. 10th 2, 457 (1984).

1984SIV/KOB A. Sivaraman and R. Kobayashi, J. Chem. Eng. Data 29, 375 (1984).

1984SOK/BAE A. E. Sokolovskii and A. K. Baev, Russ. J. Phys. Chem. 58, 1635 (1984).

1984SOK/BAE2 A. E. Sokolovskii and A. K. Baev, Vestsi Akad. Navuk BSSR Ser. Khim. Navuk 1984, 115 (1984).

1984SOK/BAE3 A. Sokolovskii and A. K. Baev, Russ. J. Phys. Chem. 58, 195 (1984).

1984STA/WAD P. Starzewski, I. Wadso, and W. Zielenkiewicz, J. Chem. Thermodyn. 16, 331 (1984).

1984STE/ALI A. V. Steblevskii, A. S. Alikhanyan, L. G. Vedenkina, A. V. Shtemenko, A. S. Kotel'nikova, and V. I. Gorgoraki, Koord. Khim. 10, 72 (1984).

1984SUR $\quad$ E. Surova, Chem. Zvesti 38, 93 (1984).

1984TRE/BER G. V. Trembovetskii, S. S. Berdonosov, I. A. Murav'eva, and L. I. Martynenko, Zh. Neorg. Khim. 29, 2159 (1984); Chem. Abstr. 101, 137451w (1984).

1984VAN/BOU J. C. Van Miltenburg and J. A. Bouwstra, J. Chem. Thermodyn. 16, 61 (1984).

1984VIS/HIL R. Viswanathan and K. Hilpert, Ber. Bunsen-Ges. Phys. Chem. 88, 125 (1984).

1984 VOR/MIR V. P. Vorob'eva, E. A. Miroshnichenko, I. I. Solomennikova, F. I. Zelchan, E. Lukevits, and Y. A. Lebedev, Bull. Acad. Sci. USSR, Div. Chem. Sci. 33, 1372 (1984).

1984WEI/LEF D. I. Weinstein, A. J. Leffler, and J. A. Currie, Mol. Cryst. Liq. Cryst. 104, 95 (1984).

1985 ABE/KUR I. Abe, T. Kuroya, and K. Kusano, J. Jpn. Oil Chem. Soc. 34, 681 (1985).

1985BIA/GIU A. Bianchi, J. Giusti, and P. Paoletti, Thermochim. Acta 90, 109 (1985).

1985BOU/DEL J. A. Bouwstra, V. V. De Leeuw, and J. C. van Miltenburg, J. Chem. Thermodyn. 17, 685 (1985).

1985CAM/FER A. R. Campanelli, D. Ferro, and N. V. Pavel, Thermochim. Acta 87, 231 (1985); Chem. Abstr. 103, 93861q (1985).

1985CAR/LAY A. S. Carson, P. G. Laye, and J. A. Spencer, J. Chem.

1985CHA S. M. Chatham, Ph.D. thesis, University of London, 1985; Thermodyn. 17, 277 (1985). as quoted in Ref. [1990TIM/CRA].

1985DEM/CHA G. De Maury, A. Chauvet, and J. Masse, Thermochim. Acta 87, 189 (1985).

1985DEV/GUE $\quad$ G. G. Devyatykh, A. V. Guesev, A. M. Gibin, N. V. Zhernenkov, L. M. Zakharov, M. Y. Antipin, and Y. M. T. Struchov, Russ. J. Inorg. Chem. 30, 780 (1985).
1985DZH/KUL O. I. Dzhafarov and A. M. Kuliev, Zh. Fiz. Khim. 59, 308 (1985).

1985FRA/COM R. Francesconi and P. Comelli, J. Chem. Eng. Data 30, 352 (1985).

1985HIL/BEN K. Hilpert, L. Bencivenni, and B. Saha, Ber. Bunsen-Ges. Phys. Chem. 89, 1292 (1985).

1985IGU/GER I. K. Igumenov, T. Y. Gerasimenko, and V. G. Isakova, Izv. Sib. Otdel Akad. Nauk SSSR, Ser. Khim. Nauki 1985, 42 (1985).

1985KAN/OTS N. Kaneniwa and M. Otsuka, Chem. Pharm. Bull. 33, 1660 (1985).

1985KAR/ABD M. K. Karabaev, T. P. Abduzhaminov, M. M. Kenisarin, and A. A. Saidov, Izv. Akad. Nauk Uzb. SSR, Ser. FizMat. Nauk 1985, 74 (1985).

1985KIS/VEI V. D. Kiselev, E. A. Veisman, B. N. Solomonov, and A. I. Konovalov, Zh. Obshch. Khim. 55, 1965 (1985).

1985KLY/DAN V. A. Klyuchnikov, T. F. Danilova, M. G. Voronkov, and V. M. D’yakov, Dokl. Phys. Chem. 281, 373 (1985).

1985 KOL/SYU S. I. Kolesnikov and Z. I. Syunyaev, Zh. Prikl. Khim. 58, 2267 (1985).

1985 KON/STR $\quad$ S. N. Kondrat'ev and B. V. Strizhov, Zh. Fiz. Khim. 59, 2933 (1985)

1985KUS

1985LIN/RAT

1985 MAJ/SVO V. Majer and V. Svoboda, Enthalpies of Vaporization of

K. Kusano, Thermochim. Acta 88, 109 (1985).

S. Lindenbaum, E. S. Rattie, G. E. Zuber, M. E. Miller, and L. J. Ravin, Int. J. Pharm. 26, 123 (1985). Organic Compounds: A Critical Review and Data Compilation (Blackwell Scientific Publishers, Oxford, U.K., 1985).

1985MAT/KUW N. Matsubara and T. Kuwamoto, Inorg. Chem. 24, 2697 (1985).

1985MAU/RAM L. Maury, J. Rambaud, B. Pauvert, G. Berge, M. Audran, and Y. Lasserre, Pharm. Acta Helv. 60, 22 (1985).

1985MUR/SAK S. Murata, M. Sakiyama, and S. Seki, Thermochim. Acta 88, 121 (1985).

1985 NG/FIE $\quad$ W. L. Ng and J. E. Field, Thermochim. Acta 84, 133 (1985).

1985NOV/TSV N. V. Novoselova, L. Y. Tsvetkova, I. B. Rabinovich, E. M. Moseeva, and L. A. Faminskaya, Zh. Fiz. Khim. 59, 604 (1985).

$19850 C H \quad$ A. B. Ochsner, Ph.D. dissertation, The Ohio State University, 1985.

1985OHM/LIP A. Ohm and B. C. Lippold, Int. J. Pharm. Technol. Prod. Manuf. 6, 1 (1985).

1985PRI/PUC V. P. Privalko, G. A. Puchkovskaya, E. N. Shermatov, and A. A. Yakubov, Mol. Cryst. Liq. Cryst. 126, 289 (1985).

1985RAB/SHE I. B. Rabinovich, M. S. Sheiman, V. P. Nistratov, G. P. Kamelova, and A. D. Zorin, Zh. Fiz. Khim. 59, 2414 (1985).

1985RAE/SOL O. A. Raewskii, B. P. Solov'ev, and L. W. Govorkova, Zh. Obshch. Khim. 55, 1381 (1985).

1985SAI/ATA K. Saito, T. Atake, and H. Chihara, J. Chem. Thermodyn. 17, 539 (1985).

1985SCH/HIL J. M. Schroy, F. D. Hileman, and S. C. Cheng, Chemosphere 14, 877 (1985).

1985SER/ZAG G. B. Sergeev, V. V. Zagorskii, A. M. Kosolapov, and S. E. Kondakov, Zh. Neog. Khim. 30, 3212 (1985); Chem. Abstr. 104, 121894u (1986).

1985SKI/PIL H. A. Skinner, G. Pilcher, and G. Al-Takhin, J. Chem. Soc., Perkin Trans. 2 1985, 811, personal comment to M. Faour and T. S. Akasheh.

1985SOK/BAE A. E. Sokolovski and A. K. Baev, Vestsi Akad. Navuk BSSR, Ser. Khim. Navuk 1985, 112.

1985STE/GAN W. V. Steele, B. E. Gannon, N. K. Smith, A. Greenburg, J. S. Chickos, and J. F. Liebman, J. Chem. Thermodyn. 17, 505 (1985).

1985SUZ/OGA M. Suzuki, T. Ogaki, and K. Sato, J. Am. Oil Chem. Soc. 62, 1600 (1985).

1985TRU/KRA V. I. Trusov, I. M. Krasikova, A. A. Grigor'ev, A. I. Altsybeeva, A. V. Suvorov, A. A. Khonikevich, and P. A. Vinogradov, Zashch. Met. 21, 299 (1985); Chem. Abstr. 102, 173623h (1985). 
1985VAR/BRI D. Varech, M. J. Brienne, and J. Jacques, Tetrahedron Lett. 26, 61 (1985).

1985VEN/PRA V. Venugopal, R. Prasad, and D. D. Sood, J. Nucl. Mater. 130, 115 (1985).

1985YOS/FUJ Y. Yoshimoto, T. Fujiwara, T. Atake, and H. Chihara, Chem. Lett. 14, 1347 (1985).

1986ACH/HAS P. J. Achorn, W. G. Haseltine, and J. K. Miller, J. Chem. Eng. Data 31, 385 (1986).

1986AIR/SIL C. Airoldi, M. L. C. P. Silva, and A. P. Chagas, J. Chem. Soc., Dalton Trans. 1986, 1913.

1986ALL/JOS N. Allemand, J. Jose, and J. C. Merlin, Thermochim. Acta 105, 79 (1986).

1986BER/COL M. Bertaut, M. Collet, and R. Nowak, Solid State Commun. 58, 613 (1986).

1986CAO/WES M. Cao, J. Wesson, K. Loufakis, B. Wunderlich, and M. Moller, Mol. Cryst. Liq. Cryst. 140, 231 (1986).

1986CHI/ANN J. S. Chickos, R. S. Annunziata, L. H. Ladon, A. S. Hyman, and J. F. Liebman, J. Org. Chem. 51, 4311 (1986).

1986CIH/VOJ M. Cihova, J. Vojtko, and J. Ilavsky, Petrochemia 26, 69 (1986).

1986DEM/MAS G. De Maury and J. Masse, J. Therm. Anal. 31, 1263 (1986).

1986DEV/GUS G. G. Devyatykh, A. V. Gusev, A. M. Gibin, N. V. Zhernenkov, and A. V. Kabanov, Russ. J. Inorg. Chem. 31, 1281 (1986).

1986DZH/KUL A. Deyko, K. R. J. Lovelock, J.-A. Corfield, A. W. Taylor, P. N. Gooden, I. J. Villar-Garcia, P. Licence, R. G. Jones, V. G. Grasovskiy, E. A. Chernikova, and L. M. Kustov, Phys. Chem. Chem. Phys. 11, 8544 (2009).

1986ELV/KUD A. Elvelt, L. Kudryantseva, H. Kirss, and O. Eisen, Eesti NSV Tead. Akadem. Toimetised Keem. 35, 280 (1986).

1986FLA

1986FLA/BEC

1986GAR/JAN G. Gärtner, P. Janiel, H. Rau, H. A. M. van Hal, and H. J. P. O. L. Flaningan, J. Chem. Eng. Data 31, 266 (1986).

Nabben, Ber. Bunsen-Ges. Phys. Chem. 90, 459 (1986).

Y. K. Grinberg, V. B. Lazarev, A. Y. Zavernyaev, V. A. Shreider, and S. D. Chepik, Zh. Fiz. Khim. 60, 1386 (1986); Chem. Abstr. 105, 86041k (1986).

1986HAN/ECK P. C. Hansen and C. A. Eckert, J. Chem. Eng. Data 31, 1 (1986).

1986HAR/GIL $\quad$ P. D. Harvey, D. F. R. Gilson, and I. S. Butler, J. Phys. Chem. 90, 136 (1986).

1986JAM/PIL E. H. Jamea and G. Pilcher, Thermochim. Acta 97, 77 (1986).

1986KAL/JAC B. Kalinowska and A. W. Jackowski, J. Chem. Thermodyn. 18, 715 (1986).

1986KIR/ACR J. J. Kirchner, W. E. Acree, Jr., G. Pilcher, and S. Li, J. Chem. Thermodyn. 18, 793 (1986).

1986KUN/YUK H. Kunisada and Y. Yuki, Kobunshi Ronbunshu 43, 895 (1986); Chem. Abstr. 106, 85109 (1987).

1986LAT/HOE G. Latterman and H. Hoecker, Mol. Cryst. Liq. Cryst. 133, 245 (1986).

1986MAR/LOE Y. Marcus and A. Loewenschuss, J. Chem. Soc., Faraday Trans. 1 82, 993 (1986).

1986MAU/RAM L. Maury, J. Rambaud, B. Pauvert, G. Berge, Y. Lasserre, and M. Audran, Farmaco 41, 26 (1986).

1986MIY/MAT S. Miyagishi, S. Matumura, T. Asakawa, and M. Nishida, Bull. Chem. Soc. Jpn. 59, 557 (1986).

1986NIL/WAD S.-O. Nilsson and I. Wadso, J. Chem. Thermodyn. 18, 673 (1986).

1986NIS/AND K. Nishida, Y. Ando, S. Sunagawa, A. Ogihara, I. Tanaka, and A. Koukitsu, J. Soc. Dyers Colour. 102, 18 (1986).

1986RAB/PET I. B. Rabinovich, V. I. Pet'kov, S. S. Zarudaeva, and E. Y. Ovchinnikov, Russ. J. Phys. Chem. 60, 767 (1986).

1986ROR B. F. Rordorf, Chemosphere 15, 1325 (1986).

1986ROR2 B. F. Rordorf, in Proceedings of the 5th International Symposium on Chlorinated Dioxins and Related Compounds, Chemosphere, Bayreuth, FRG, 16-19 September 1986.

1986RUS/RAB T. P. Russell, J. F. Rabolt, R. J. Twieg, R. L. Siemens, and B. L. Farmer, Macromolecules 19, 1135 (1986).

1986SAT/INO N. Sato, H. Inomata, K. Arai, and S. Saito, J. Chem. Eng. Jpn. 19, 145 (1986).
1986SHA/BRI

1986STA

1986TAK/ISH

1986VOR/BAL

A. C. Shah and N. J. Britten, J. Pharm. Pharmacol. 39, 736 (1986).

H. W. Starkweather, Jr., Macromolecules 19, 1131 (1986).

S. Takagi, M. Ishikawa, M. Kumada, T. Kimura, and R. Fujishiro, Thermochim. Acta 109, 55 (1986).

Z. M. Vorotyntsev, V. V. Balabanov, and D. A. Shamrakov,
(196. Ko, 234 (1986); Chem. Abstr. 104, 96047k (1986).

1986WEL/GRA ～D. Wells, B. T. Grayson, and E. Langner, Pestic. Sci. 17, 473 (1986).

1986WHI

1987AIR/DES

1987AZA

C. M. White, J. Chem. Eng. Data 31, 198 (1986).

$1987 \mathrm{CHI} / \mathrm{HOS}$

Trans. 1987, 2955.

E. C. Azandegbe, Analusis 15, 370 (1987).

$\begin{array}{ll} & \text { Steele, NIPER Report 247, 1987, p. } 110 . \\ 1987 \text { CHI/HOS2 } & \text { R. D. Chirico, I. A. Hossenlopp, A. Nguyen, and W. V. }\end{array}$ R. D. Chirico, I. A. Hossenlopp, A. N
Steele, NIPER Report 239, 1987, p. 67.

1987DIA/DIA A. R. Dias, P. B. Dias, H. P. Diogo, A. M. Galvao, M. E. Minas da Piedade, and J. A. Martinho Simoes, Organometallics 6, 1427 (1987).

1987DRO/EME H. Drotloff, D. Emeis, R. F. Waldron, and M. Möller, Polymer 28, 1200 (1987).

1987DRO/MOL H. Drotloff and M. Moller, Thermochim. Acta 112, 57 (1987).

1987DRO/ROT H. Drotloff, H. Rotter, D. Emeis, and M. Móller, J. Am. Chem. Soc. 109, 7797 (1987).

1987DZH/KUL O. L. Dzhafarov and A. M. Kuliev, Zh. Fiz. Khim. 61, 1504 (1987).

1987DZH/KUL2 O. L. Dzhafarov and A. M. Kuliev, Zh. Fiz. Khim. 61, 602 (1987).

1987DZH/KUL3 O. L. Dzhafarov and A. M. Kuliev, Zh. Fiz. Khim. 61, 606 (1987).

1987ECO/BER C. Ecolivet, A. Bertaut, A. Miezeyewski, and A. Collet, in Dynamics of Molecular Crystals, edited by $\mathrm{J}$. Lascombe (Elsevier, Amsterdam, 1987), p. 187.

1987FER/DEL D. Ferro and G. Della Gatta, Thermochim. Acta 122, 189 (1987).

1987FER/STR D. Ferro and S. Stranges, Thermochim. Acta 119, 373 (1987).

1987GIB/GRI A. M. Gibin, N. D. Grishnova, A. V. Gusev, A. N. Moiseev, I. I. Remeshkova, and Y. M. Salganskii, Vysokochist. Veshchestva 1987, 28; Chem. Abstr. 109, 136102 (1988).

$1987 \mathrm{GIB} / \mathrm{GUS}$ A. M. Gibin, A. V. Gusev, and I. I. Remeshkova, Vysokochist. Veshchestva 1987, 35.

1987GRE/SID N. R. Grebenshchikov, G. V. Sidorenko, and D. N. Suglobov, Radiokhim. 29, 296 (1987).

1987GRI/LAZ Y. K. Grinberg and V. B. Lazarev, Zh. Neorg. Khim. 32, 3110 (1987).

1987GRI/LAZ2 Y. K. Grinberg, V. B. Lazarev, V. V. Petukhov, G. Y. Novikova, G. A. Sharpataya, and Z. P. Ozerova, Dokl. Akad. Nauk SSSR 297, 633 (1987).

1987MIL/FEN2 P. L. Mills, R. L. Fenton, and F. G. Schaefer, J. Chem. Eng. Data 32, 251 (1987).

1987MUR/HIL J. P. Murray and J. O. Hill, Thermochim. Acta 109, 383 (1987).

1987MUR/HIL2 J. P. Murray and J. O. Hill, Thermochim. Acta 109, 391 (1987).

1987PIT/VAL D. Pitre and E. Valoti, Arch. Pharm. 320, 859 (1987).

1987RIB/FER M. A. V. Ribeiro da Silva and M. L. C. C. H. Ferrao, J. Chem. Thermodyn. 19, 645 (1987).

1987SAB/ANT R. Sabbah and I. Antipine, Bull. Soc. Chim. Fr. 1987, 392.

1987SHI/OHK T. Shimizu, S. Ohkubo, M. Kimura, I. Tabata, and T. Hori, J. Soc. Dyers Colour. 103, 132 (1987).

1987SKU/DUD K. Skudlarski, J. Dudek, and J. Kapala, J. Chem. Thermodyn. 19, 857 (1987).

1987STE/MAL R. M. Stephenson and S. Malanowski, Handbook of the Thermodynamics of Organic Compounds (Elsevier, New York, 1987). The data in this listing was obtained directly from this compendium which does not contain literature references. The sublimation enthalpies were calculated 
from the vapor pressures obtained from the Antoine constants reported in this compendium. In cases where the Antoine constant $\mathrm{C}=0$, the Antoine Equation $(\log 10 \mathrm{P}$ $=\mathrm{A}-\mathrm{B} /(\mathrm{C}+\mathrm{T}))$ reduces to the integrated form of the Clausius Clapeyron equation directly. This was the case for most sublimation enthalpies. In those cases where this condition was not met, vapor pressures were calculated over a $30 \mathrm{~K}$ temperature range closest to $\mathrm{T}=298 \mathrm{~K}$ and the temperature dependence was reformulated in terms of the integrated form of the Clausius Clapeyron equation.

1987VAR/LOS2 R. M. Varushchenko, O. L. Loseva, and A. I. Druzhinina, Zh. Fiz. Khim. 61, 638 (1987)

1987ZAB/FER G. Zabinska, P. Ferloni, and M. Sanesi, Thermochim. Acta 122, 87 (1987).

1988ABE/OGA T. Abe, R. Ogawa, and Y. Kuniya, in Abstracts of 49th Autumn Meeting of the Japan Society of Applied Physics, Toyama, Japan, October (1988), p. 116; as quoted in Ref. [1990YUH/KIK].

1988AMB/GHI D. Ambrose and N. B. Ghiassee, J. Chem. Thermodyn. 20, 1231-1232 (1988).

1988ASH/UCH K. Ashizawa, K. Uchikawa, T. Hattori, T. Sato, and Y. Miyake, J. Pharm. Sci. 77, 635 (1988).

1988BAG/GUR A. K. Baglay, L. L. Gurarly, and G. G. Kuleshov, J. Chem. Eng. Data 33, 512-518 (1988).

1988BAU/PER G. L. Baughman and T. A. Perenich, Environ. Toxicol. Chem. 7, 183-199 (1988).

1988BRA/FAK D. C. Bradley, M. M. Faktor, D. M. Frigo, and K. V. Young, Chemtronics 3, 50-53 (1988); Chem. Abstr. 109, 43735e (1988).

1988CAR/DYS $\quad$ A. S. Carson, J. Dyson, P. G. Laye, and J. A. Spencer, J. Chem. Thermodyn. 20, 1423-1426 (1988).

1988CAR/JAM A. S. Carson, E. H. Jamea, P. G. Laye, and J. A. Spencer, J. Chem. Thermodyn. 20, 923-926 (1988).

1988CAR/JAM2 A. S. Carson, E. H. Jamea, P. G. Laye, and J. A. Spencer, J. Chem. Thermodyn. 20, 1223-1229 (1988).

1988COL/JIM M. Colomina, P. Jimenez, R. Perez-Ossorio, M. V. Roux, and C. Turrion, J. Chem. Thermodyn. 20, 575-580 (1988).

1988COO/SED J. E. Coon, W. B. Sediawan, J. E. Auwaerter, and E. McLaughlin, J. Solution Chem. 17, 519 (1988).

1988DEF/CHA F. Defoort, C. Chatillon, and C. Bernard, J. Chem. Thermodyn. 20, 1443-1456 (1988).

1988FED/VOI N. E. Fedotova, A. A. Voityuk, A. A. Blitznyuk, and I. K. Igumenov, Koord. Khim. 14, 1493-1496 (1988).

1988GOL/SIT O. F. Golovanova, G. V. Sitonina, V. I. Pepekin, and F. I. Korsunskii, Izv. Akad. Nauk. SSSR, Ser. Khim. 1988, 1012-1016; Chem. Abstr. 110, 38466j (1989).

1988GRE/SID N. R. Grebenshchikov, G. V. Sidorenko, D. N. Suglobov, L. L. Shcherbakova, and V. M. Adamov, Inorg. Chim. Acta 145, 315-325 (1988).

1988GRI/CHE E. E. Grinberg, N. G. Chernaya, and A. A. Efremov, Vysokochist. Veshchestva 1988, 180-184.

1988HOP/PUG J.Höpken, C. Pugh, W. Richtering, and M. Móller, Makromol. Chem. 189, 911-925 (1988).

1988HUA/TAN W. Huang, F. Tan, and H. Luo, Zhongguo Yaoke Daxue Xuebao 19, 90-92 (1988).

1988KAT H. Katayama, Bull. Chem. Soc. Jpn. 61, 3326-3328 (1988).

1988KAT2 H. Katayama, J. Chem. Eng. Data 33, 75-77 (1988).

1988KAY/HEI O. Kayser, H. Heinecke, A. Brauers, H. Luth, and P. Balk, Chemtronics 3, 90 (1988).

1988KIR/DOM D. R. Kirklin and E. S. Domalski, J. Chem. Thermodyn. 20, 743-754 (1988).

1988LAZ/GRE V. B. Lazarev, Y. K. Greenberg, Z. P. Ozerova, and G. A. Sharpataya, J. Therm. Anal. 33, 797-799 (1988).

1988LEB/KUL $\quad$ B. V. Lebedev, T. G. Kulagina, and N. N. Smirnova, J. Chem. Thermodyn. 20, 1383-1396 (1988).

1988LEB/SMI $\quad$ B. V. Lebedev, N. N. Smirnova, V. G. Vasil'ev, E. N. Karataev, E. N. Dregigh, and I. A. Fischenko, Vysokochist. Veshehestva 1988, 56.

1988MES/FIN J. F. Messerly, H. L. Finke, W. D. Good, and B. Gammon, J. Chem. Thermodyn. 20, 485-501 (1988).
1988MES/TOD

J. F. Messerly, S. S. Todd, H. L. Finke, W. D. Good, and B. E. Gammon, J. Chem. Thermodyn. 20, 209 (1988).

1988MYL/SRI M. Mylrajan and T. K. K. Srinivasan, J. Phys. C: Solid State Phys. 21, 1673 (1988).

1988NAK/KIT H. Nakazumi, T. Kitao, and K. Ohnishi, J. Heterocycl. Chem. 25, 1422-1426 (1988).

1988NIS/SHE V. P. Nistratov, M. S. Sheiman, I. B. Rabinovich, V. G. Vasil'ev, E. N. Karataev, and I. A. Feshchenko, Zh. Fiz. Khim. 62, 2219-2221 (1988).

1988PET/TSY N. N. Petropavlov, I. G. Tsygankova, and L. A. Teslenko, Sov. Phys. Crystallogr. 33, 853 (1988).

1988RIB/FER M. A. V. Ribeiro da Silva and M. L. C. C. H. Ferrao, Bull Chem. Soc. Jpn. 61, 1755-1759 (1988).

1988RIB/FER2 M. A. V. Ribeiro da Silva and M. L. C. C. H. Ferrao, Can J. Chem. 66, 651-654 (1988).

1988RIB/FER3 M. A. V. Ribeiro da Silva, M. L. C. C. H. Ferrao, and C. C. H. Luisa, J. Chem. Thermodyn. 20, 359-361 (1988).

1988RIB/FER4 M. A. V. Ribeiro da Silva and M. L. C. C. H. Ferrao, Pure Appl. Chem. 60, 1225-1234 (1988).

1988RIB/PIL M. A. V. Ribeiro da Silva, G. Pilcher, and R. J. Irving, J. Chem. Thermodyn. 20, 95-102 (1988).

1988ROY/FLY S. D. Roy and G. L. Flynn, Pharm. Res. 5, 580-586 (1988).

1988SAB/ELW R. Sabbah and L. El Watik, Bull. Soc. Chim. Fr. 1988, 626-630.

1988SAB/ELW2 R. Sabbah, L. El Watik, and C. C. R. Minadakis, Acad. Sci., Paris 307((Series II)), 239-244 (1988).

1988SAI/ATA

K. Saito, T. Atake, and H. Chihara, Bull. Chem. Soc. Jpn. 61, 2327-2336 (1988).

1988SAS/JOS K. Sasse, J. Jose, and J.-C. Merlin, Fluid Phase Equilib. 42, 287-304 (1988).

1988SOP/KEA P. A. Sopade, M. W. Kearsley, and G. A. Le Grys, Int. Sugar J. 90, 38 (1988).

1988TEL/LAR V. I. Tel'noi, V. N. Larina, E. N. Karataev, and E. N. Deryagina, Zh. Fiz. Khim. 62, 3108-3109 (1988).

1988TOR/BAR L. A. Torres-Gomez, G. Barreiro-Rodriguez, and A. Galarza-Mondragon, Thermochim. Acta 124, 229-233 (1988).

1988TOR/BAR2 L. A. Torres-Gomez, G. Barreiro-Rodriguez, and F. Mendez-Ruiz, Thermochim. Acta 124, 179-183 (1988).

1988VAN/WHI M. J. M. Van Oort and M. A. White, Ber. Bunsen-Ges. Phys. Chem. 92, 168 (1988).

1988VOR/BAR M. G. Voronkov, V. P. Baryshok, V. A. Klyuchnikov, T. F. Danilova, V. I. Pepekin, A. N. Korchagina, and Y. I. Khudobin, J. Organomet. Chem. 345, 27-38 (1988).

1989ABB/JIM J.-L. Abboud, P. Jimenez, M. V. Roux, C. Turrion, and C. Lopez-Mardomingo, J. Chem. Thermodyn. 21, 859-865 (1989).

1989ACR/KIR W. E. Acree, Jr., J. J. Kirchner, S. A. Tucker, G. Pilcher, and D. M. C. Riberio da Silva, J. Chem. Thermodyn. 21, 443-448 (1989).

1989AIZ/FED M. I. Aizenberg, E. V. Fedoseev, S. S. Travnikov, A. V. Davydov, and B. F. Myasoedov, J. Radioanal. Nucl. Chem. Lett. 136, 405-409 (1989).

1989BRA/RYT B. K. Braxton and J. H. Rytting, Thermochim. Acta 154, 27-47 (1989).

1989CHI/HOS R. D. Chirico, I. A. Hossenlopp, A. Nguyen, W. V. Steele, and B. E. Gammon, J. Chem. Thermodyn. 21, 179-201 (1989).

1989CHI/KNI R. D. Chirico, S. E. Knipmeyer, A. Nguyen, and W. V. Steele, J. Chem. Thermodyn. 21, 1307-1331 (1989).

1989CHI/NGU R. D. Chirico, A. Nguyen, W. V. Steele, M. M. Strube, and C. Tsonopoulos, J. Chem. Eng. Data 34, 149-156 (1989).

1989COL/JIM M. Colomina, P. Jimenez, M. V. Roux, and C. Turrion, J. Chem. Thermodyn. 21, 275-281 (1989).

1989GON/KRA T. Gondova, P. Kralik, and J. Gonda, Thermochim. Acta 156, 147-155 (1989).

1989GRI/KON A. A. Grigor'ev, Y. V. Kondrat'ev, A. V. Suvorov, and V. I. Nikolaeva, Zh. Obshch. Khim. 59, 1834-1841 (1989).

1989HUI/VAN J. Huinink, K. Van Miltenburg, H. A. Oonk, and A. Schuijff, J. Chem. Eng. Data 34, 99-100 (1989).

1989KAT/TAN M. Kato and M. Tanaka, J. Chem. Eng. Data 34, 206-209 (1989). 
1989KHO/PUL M. G. Khodzhayeva, M. A. Pulatova, D. A. Nadirova, N. R. Gabzalilova, and T. S. Ismailov, Khim.-Pharm. Zh. 23, 378 (1989).

1989 KIR/CHU $\quad$ D. R. Kirklin, K. L. Churney, and E. S. Domalski, J. Chem. Thermodyn. 21, 1105 (1989).

1989KIR/DOM D. R. Kirklin and E. S. Domalski, J. Chem. Thermodyn. 21, 449-456 (1989).

1989KON/STR S. N. Kondrat'ev and B. V. Strizhov, Russ. J. Phys. Chem. 63, 656 (1989).

1989KRE/AZA A. G. Krestov and G. I. Azarova, Izv. Vyssh. Uchebn Zaved., Khim. Khim. Teckhnol. 32, 61-63 (1989); Chem. Abstr. 111, 219794f (1989).

1989LUB/JAN J. Lubkowski T. Janiak, J. Czerminski, and J. Blazejowski, Thermochim. Acta 155, 7-28 (1989).

1989MAL/KAN J. J. Mallon and S. W. Kanto, Macromolecules 22, 2070-2077 (1989).

1989MAS/DEM J. Masse, G. de Maury, A. Chauvet, and A. Terol, Thermochim. Acta 149, 1 (1989).

1989MIN M. E. Minas da Piedade, Ph.D. thesis, Technical University of Lisbon, 1989.

1989NAK/KUW H. Nakayama, H. Kuwata, N. Yamamoto, Y. Akagi, and H. Matsui, Bull. Chem. Soc. Jpn. 62, 985 (1989).

1989NEA/FLY S. H. Neau, G. L. Flynn, and S. H. Yalkowsky, Int. J. Pharm. 49, 223 (1989).

1989NIS/AND K. Nishida, Y. Ando, K. Ohwada, T. Mori, M. Koide, and A. Koukitsu, J. Soc. Dyers Colour. 105, 112-113 (1989).

1989NIS/RAB V. P. Nistratov, I. B. Rabinovich, M. S. Sheiman, N. N. Smirnova, I. A. Zelyaev, and I. A. Feshchenko, Zh. Fiz. Khim. 63, 1779-1783 (1989).

1989PIN/GON J. J. Pinvidic, A. Gonthier-Vassal, H. Szwarc, R. Ceolin, P. Toffoli, J. M. Teulon, and C. Guechot, Thermochim. Acta 153, 37-45 (1989).

1989RAB/NIS I. B. Rabinovich, V. P. Nistratov, M. S. Sheiman, E. N. Karataev, G. P. Kamelova, and I. A. Feschchenko, Zh. Fiz. Khim. 63, 2520 (1989).

1989RAB/NIS2 I. B. Rabinovich, V. P. Nistratov, M. S. Sheiman, K. N. Klimov, G. P. Kamelov, and A. D. Zorin, Zh. Fiz. Khim. 63, 522 (1989).

1989RIB/REI M. A. V. Ribeiro Da Silva and A. M. M. V. Reis, J. Chem. Thermodyn. 21, 423-429 (1989).

1989RIB/RIB M. A. V. Ribeiro Da Silva, M. D. M. C. Ribeiro Da Silva, J. A. S. Taixeira, J. M. Bruce, P. M. Guyan, and G. Pilcher, J. Chem. Thermodyn. 21, 265-274 (1989).

1989RIB/SOU M. D. M. C. Ribeiro Da Silva, P. Souza, and G. Pilcher, J. Chem. Thermodyn. 21, 173-178 (1989).

1989ROR $\quad$ B. F. Rordorf, Chemosphere 18, 783-788 (1989).

1989ROR/RUT ～B. F. Rordorf, S. Rutschmann, and P. Schiess, Int. J. Mass Spectrom. Ion Processes 95, 211-222 (1989).

1989SAB/ELW R. Sabbah and E. El Watik, Thermochim. Acta 138, 241-247 (1989).

1989SAK/IWA A. Sakoguichi, Y. Iwai, and J. Takenaka, Kagaku Kogaku Ronbunshu 15, 166 (1989).

1989SAL/ABA S. R. Salman and K. F. Abas, Thermochim. Acta 152, 381-386 (1989).

1989SAS/NGU K. Sasse, J. N'Guimbi, J. Jose, and J. C. Merlin, Thermochim. Acta 146, 53-61 (1989).

1989SCH/PEN J. Schmidt and A. Penzkofer, J. Chem. Phys. 91, 1403-1409 (1989).

1989SHE/RAB M. S. Sheiman, I. B. Rabinovich, V. P. Nistratov, G. P. Kamelova, E. N. Karataev, and A. I. Feshchenko, Zh. Fiz. Khim. 63, 836-838 (1989).

1989SHI/SHI Y. Shibutani and K. Shinra, Bull. Chem. Soc. Jpn. 62, 1477-1481 (1989).

1989STA/MAN G. M. Stack, L. Mandelkern, C. Krohnke, and G. Wegner, Macromolecules 22, 4351-4361 (1989).

1989STE/CHI W. V. Steele, R. D. Chirico, I. A. Hossenlopp, A. Nguyen, N. K. Smith, and B. E. Gammon, J. Chem. Thermodyn. 21, 81-107 (1989).

1989VAN/VAN H. A. Van Doren, R. Van der Geest, C. A. Keuning, R. M. Kellogg, and H. Wynberg, Liq. Cryst. 5, 265-283 (1989).

1989VAN/WHI M. J. M. Van Oort and M. A. White, Thermochim. Acta 139, 205 (1989).
1989VIN/RUS

C. Viney, T. P. Russell, L. E. Depero, and R. J. Twieg, Mol. Cryst. Liq. Cryst. 168, 63-82 (1989).

1989VOR/BAR M. G. Voronkov, V. P. Baryshok, V. A. Klyuchnikov, A. N Korchagina, and V. I. Pepekin, J. Organomet. Chem. 359, 169-177 (1989).

1989VOR/KLY M. G. Voronkov, V. A. Klyuchnikov, S. N. Kolabin, G. N. Shvets, P. I. Varushin, E. N. Deryagina, N. A. Korchevin, and S. I. Tsvetnitskaya, Dokl. Phys. Chem. 307, 650-653 (1989).

1989VOR/SOR M. G. Voronkov, M. S. Sorokin, V. A. Klyuchnikov, G. N. Shvetz, and V. I. Pepekin, J. Organomet. Chem. 359, 301-313 (1989).

1989WAN/YIN K. Wang, X.-G. Ying, M.-S. Xia, J.-Z. Xia, and Y. Hu, J. Chem. Eng. Data 34, 126-130 (1989).

1989ZHA/HUA X. Zhang, W. Huang, F. Tan, G. Xu, and Z. Wu, Chin. Sci. Bull. 34, 1844 (1989).

1990BEC/DOG2 H. D. Beckhaus, B. Dogan, J. Schaetzer, S. Hellman, and C. Rüchardt, Chem. Ber. 123, 137 (1990).

1990BEL/BAL K. Belina, G. Balazs, G. Varga, G. Hardy, J. Horvath, K. Nyitrai, A. R. Werninck, A. J. Shand, and G. H. W. Milburn, Liq. Cryst. 7, 399 (1990).

1990BRI/WAD L. E. Briggner and I. Wadso, J. Chem. Thermodyn. 22, 143 (1990).

1990CHI/GAM R. D. Chirico, B. E. Gammon, S. E. Kripmeyer, A. Nguyen, M. M. Strube, C. Tsonopoulos, and W. V. Steele, J. Chem. Thermodyn. 22, 1075 (1990).

1990CHI/HES J. S. Chickos, D. G. Hesse, and J. F. Liebman, J. Org. Chem. 55, 383 (1990).

1990DIA/DIO A. R. Dias, H. P. Diogo, M. E. Minas da Piedade, J. A. Martinho-Simoes, A. S. Carson, and E. H. Jamea, J. Organomet. Chem. 391, 361 (1990).

1990DOG/BEC B. Dogan, H. D. Beckhaus, H. Birkhofer, and C. Rúchardt, Chem. Ber. 123, 1365 (1990).

1990DOG/BEC2 H. Dogan, H.-D. Beckhaus, J. Schaetzer, S. Hellmann, and C. Rüchardt, Chem. Ber. 123, 137-144 (1990).

1990DOM U. Domanska, Fluid Phase Equilib. 55, 125 (1990).

1990DOM/HEA E. S. Domalski and E. D. Hearing, J. Phys. Chem. Ref. Data 19, 881 (1990).

1990DOM2 U. Domanska, Ind. Eng. Chem. Res. 29, 470 (1990).

1990DON/DRE J. R. Donnelly, L. A. Drewes, R. L. Johnson, W. D. Munslow, K. K. Knapp, and G. W. Sovocool, Thermochim. Acta 167, 155 (1990)

1990ERT/HEA K. D. Ertel, R. A. Heasley, C. Koegel, A. Chakrabaathi, and J. T. Carstensen, J. Pharm. Sci. 79, 552 (1990).

1990FIN/PAY A. Finch and J. Payne, Thermochim. Acta 164, 55 (1990).

1990FRI/DOG K. Fritzsche, B. Dogan, H.-D. Beckhaus, and C. Rüchardt, Thermochim. Acta 160, 147 (1990).

1990GEN/LUB O. Generowicz, L. Lubkowski, and J. Blazejowski, J. Therm. Anal. 36, 2211 (1990).

1990HAU/CON R. E. Haufler, J. Conceicao, L. P. F. Chibante, Y. Chai, N E. Byrne, S. Flanagan, M. M. Haley, S. C. O'Brien, C. Pan, Z. Xiao, W. E. Billups, M. A. Cinfolini, R. H. Hauge, J. L. Margrave, L. J. Wilson, R. F. Curl, and R. E. Smalley, J. Phys. Chem. 94, 8634 (1990).

1990HIN/BID2 H. D. Hinckley, T. F. Bidleman, W. T. Foreman, and J. R. Tuschall, J. Chem. Eng. Data 35, 232 (1990).

1990JIM/ROU P. Jimenez, M. V. Roux, and C. Turrion, J. Chem. Thermodyn. 22, 721 (1990).

1990JIN/KAN J.-I. Jin, C.-S. Kang, and B. Y. Chung, Bull. Korean Chem. Soc. 11, 245 (1990).

1990KAL/DRE J. Kalinowski, Z. Dreger, J. Godlewski, and P. Mondalski, Chem. Phys. Lett. 172, 522 (1990).

1990KIS/SHO K. Kishore, H. K. Shobha, and G. J. Mattamal, J. Phys. Chem. 94, 1642 (1990).

1990LEI/PIL M. M. P. Leitao, G. Pilcher, W. E. Acree, Jr., A. I. Zvaigzne, S. A. Tucker, and M. D. M. C. Ribeiro da Silva, J. Chem. Thermodyn. 22, 923 (1990).

1990LOP/SIC M. I. Lopez and J. E. Sicre, J. Phys. Chem. 94, 3860 (1990).

1990MAL/ALI I. P. Malkerova, A. S. Alikhanyan, V. G. Sevvast'yanov, Y. K. Grinberg, and V. I. Gorgoraki, Zh. Neorg. Khim. 35, 413 (1990); Chem. Abstr. 112, 186852p (1990). 
1990MEN/LIA G. F. Mendenhall, S. X. Liang, and E. H. T. Chen, J. Org. Chem. 55, 3697 (1990).

1990MES/TOD J. F. Messerly, S. S. Todd, H. L. Finke, S. H. Lee-Bechtold, G. B. Guthrie, W. V. Steele, and R. D. R. D. Chirico, J. Chem. Thermodyn. 22, 1107 (1990).

1990NEA/FLY S. H. Neau and G. L. Flynn, Pharm. Res. 7, 1157 (1990). 1990NES/NAZ T. N. Nesterova, A. G. Nazmutdinov, V. S. Tsvetkov, A. M. Rozhnov, and I. Y. Roshchupkina, J. Chem. Thermodyn. 22, 365 (1990).

1990PIA/SCA V. Piacente and P. Scardala, Thermochim. Acta 159, 193 (1990).

1990POM/PIA T. Pompili and V. Piacente, Thermochim. Acta 170, 289 (1990).

1990PRA/KOH K. Praefcke, B. Kohne, and D. Singer, Angew. Chem. 102, 200 (1990).

1990RIB/MON M. A. V. Ribeiro Da Silva and M. J. S. Monte, Thermochim. Acta 171, 169 (1990).

1990RIB/RIB M. A. V. Ribeiro Da Silva, M. D. M. C. Ribeiro Da Silva, M. C. S. S. Rangel, G. Pilcher, M. J. Akello, A. S. Carson, and E. H. Jamea, Thermochim. Acta 160, 267 (1990).

1990SAT/YOS K. Sato, N. Yoshimoto, M. Suzuki, M. Kobayashi, and F. Kaneko, J. Phys. Chem. 94, 3180 (1990).

1990SER/LAR F. Ser and Y. Larher, J. Chem. Thermodyn. 22, 407 (1990).

1990SHI/HAY ～C. Shimasaki, S. Hayase, A. Murai, J. Takai, E. Tsukurimichi, and T. Yoshimura, Bull. Chem. Soc. Jpn. 63, 1074 (1990).

1990SOH/OKA M. Sohda, M. Okazaki, Y. Iwai, Y. Arai, A. Sakoguchi, R. Ueoka, and Y. Kato, J. Chem. Thermodyn. 22, 607 (1990).

1990SPR/GRE A. Sprozynski and J. Gregorowicz, Main Group Met. Chem. 13, 1 (1990).

1990TIM/CRA M. G. Timoney and D. Q. M. Craig, J. Pharm. Pharmacol. 42(S1), 122P (1990).

1990VAN/VAN H. A. Van Doren, R. van der Geest, R. M. Kellog, and H. Wynberg, Recl. Trav. Chim. Pays-Bas 109, 197 (1990).

1990 VOR/ZVE V. M. Vorotyntsev, Y. B. Zverev, and D. A. Shamrakov, Vysokochist Veshchestra 1990, 123.

1990WUN B. Wunderlich, in Thermal Analysis (Academic, New York, 1990), Chap. 5, pp. 417-431, ATHAS Appendix.

1990YAN/EIR S. Yano, M. Eiraku, M. Tomiyama, and T. Sugihara, Liq. Cryst. 7, 537 (1990).

1990YUH/KIK S. Yuhya, K. Kikuchi, M. Yoshida, Y. Sugawara, and Y. Shiohara, Mol. Cryst. Liq. Cryst. 184, 231 (1990).

1991ACR

1991AGA/LEG

W. E. Acree, Jr., Thermochim. Acta 189, 37 (1991), and references therein.

1991ASA/SOR $\quad$ S. Asahina, M. Sorai, and R. Eidenschink, Liq. Cryst. 10, 675 (1991).

1991BAR/SCH P. Barbillon, 1. Schuffenecker, J. Dellacherie, D. Balesdent, and M. Dirand, J. Chim. Phys. Phys.-Chim. Biol. 88, 91 (1991).

1991BAS/SVO P. Basarova and V. Svoboda, Fluid Phase Equilib. 68, 13 (1991).

1991BAU/WEB G. L. Baughman and E. J. Weber, Dyes Pigm. 16, 261 (1991).

1991BED/BOO A. D. Bedells, C. Booth, and K. Viras, Makromol. Chem. 192, 2099 (1991).

1991BUC/POT N. I. Buchan, R. M. Potemski, and T. F. Kuech, J. Chem. Eng. Data 36, 372 (1991).

1991CEO/DUG R. Ceolin, J. Dugue, J. C. Rouland, C. Ralambosoa, and F. Lepage, Int. J. Pharm. 74, 77 (1991).

1991CHI/BRA J. S. Chickos, C. M. Braton, D. G. Hesse, and J. F. Liebman, J. Org. Chem. 56, 927 (1991); and references and supplemental material therein.

1991CHI/KNI R. D. Chirico, S. E. Knipmeyer, A. Nguyen, and W. V. Steele, J. Chem. Thermodyn. 23, 431 (1991).

1991CLA/LET P. Claudy and J. M. letoffe, Calorim. Anal. Therm. 22, 281 (1991).

1991DES/DES A. G. De Souza, J. H. De Souza, and C. Airoldi, J. Chem. Soc., Dalton Trans. 1991, 1751.
1991DIK/KAB

V. V. Dikii and G. Y. Kabo, Dokl. Akad. Nauk BSSR 35, 158 (1991); Chem. Abstr. 114, 206233 j (1991).

1991DOM/WYR U. Domanska and D. Wyrzykowska-Starkiewicz, Thermochim. Acta 179, 265 (1991).

1991ELW/SAB ～L. El Watik and R. Sabbah, Bull. Soc. Chim. Fr. 128, 344 (1991).

1991GAR/PRE P. J. Gardner and S. R. Preston, Thermochim. Acta 185, 219 (1991).

1991GIL/SAG M. S. Gill and V. S. Sagoria, Indian J. Chem., Sect. A: Inorg., Bio-inorg., Phys., Theor. Anal. Chem. 30A, 727 (1991); Chem. Abstr. 115, 149065a (1991).

1991HOP/MOL J. Hopken, M. Moller, and S. Boileau, New Polym. Mater. 2, 339 (1991).

1991HOR

1991ISH/IWA

1991JEF/JAB

T. Hori, Shikizai Kyokaishi 64, 83 (1991).

H. Ishida, T. Iwachido, N. Hayama, D. Nakamura, and R. Ikedaa, Bull. Chem. Soc. Jpn. 64, 3613 (1991).

1991JIN/WUN

C. W. Jefford, A. Jaber, J. Boukouvalas, and P. Tissot, Thermochim. Acta 188, 337 (1991).

1991KNA/KUB Thermochemical Properties of Inorganic Substances

Y. Jin and B. Wunderlich, J. Phys. Chem. 95, 9000 (1991). II, edited by O. Knacke, O. Kubaaschewski, and $\mathrm{K}$. Hesselmann (Springer-Verlag, Berlin, 1991).

1991 KOE/BOR R. Koelliker and G. Bor, Inorg. Chem. 30, 2236 (1991).

1991LEB/KUL B. V. Lebedev and T. G. Kulagina, J. Chem. Thermodyn. 23, 1097 (1991).

1991LIU/GUO Z. Z. Liu, X. D. Guo, L. E. Straub, G. Erdos, R. J. Prankerd, R. J. Gonzalez-Rothi, and H. Schreier, Drug Des. Discovery 8, 57 (1991).

1991MAL/ALI I. P. Malkerova, A. S. Alikhanyan, I. Y. Filatov, I. L. Kazanskaya, and V. G. Sevast'yanov, Zh. Neorg. Khim. 36, 3112 (1991); Chem. Abstr. 116, 114007b (1992).

1991MIY/ENO S. Miyajima, T. Enomoto, T. Kusanagi, and T. Chiba, Bull. Chem. Soc. Jpn. 64, 679 (1991).

1991NAO/SEK M. Naoki, M. Seki, H. Kugo, F. Saito, and T. Taioka, J. Phys. Chem. 95, 5628 (1991).

1991PAN/SAM C. Pan, M. P. Sampson, Y. Chai, R. H. Hauge, and J. L. Margrave, J. Phys. Chem. 95, 2944 (1991).

1991PIA/POM V. Piacente, T. Pompili, P. Scardala, and D. Ferro, J. Chem. Thermodyn. 23, 379 (1991)

1991RAB/SHE I. B. Rabinovich, M. S. Sheiman, G. P. Kamelova, V. P. Nistratov, and E. N. Karataev, Zh. Fiz. Khim. 65, 2071 (1991).

1991SAB/WAT R. Sabbah and L. E. Watik, Thermochim. Acta 176, 163 (1991).

1991SEV/ALI V. G. Sevast'yanov, A. S. Alikhanyan, T. I. Krasovskaya, I. Y. Filatov, S. V. Krasnodub-skaya, and N. T. Kuznetsov, Vysokochist. Veshchestva 1991, 137; Chem. Abstr. 115, 246646y (1991).

1991SEV/KRA V. G. Sevast'yanov, S. V. Krasnodubskaya, O. V. Kurtasov, A. S. Alikhanyan, N. T. Kuznetsov, and A. S. Zanina, Vysokochist. Veshchestva 1991, 190; Chem. Abstr. 114, 239291e (1991).

1991SHE/WEI $\quad$ B. M. Sheikh-Ali and R. G. Weiss, Liq. Cryst. 10, 575 (1991).

1991STE/CHI W. V. Steele, R. D. Chirico, S. E. Knipmeyer, and A. Nguyen, J. Chem. Thermodyn. 23, 957 (1991).

1991STR/FEU C. W. Struck and A. E. Feuersanger, High Temp. Sci. 31, 127 (1991).

1991STU/PIA G. Stubos and V. Piacenti, Thermochim. Acta 188, 287-292 (1991)

1991TEL/LAR V. I. Tel'noi, V. N. Larina, N. T. Kuznetsov, V. G. Sevast'yanov, S. V. Krasnodubskaya, and I. Y. Felatov, Vysokochist. Veshchestva 1991, 194.

1991TOK/HAY A. Tokmakoff, D. R. Haynes, and S. M. George, Chem. Phys. Lett. 186, 450 (1991).

1991URY/RON V. F. Ur'yash, O. V. Ronina, E. N. Karataev, I. V. Zimina, T. V. Kuznetsova, T. V. Petrun, I. A. Feshchenko, and A. D. Zorin, Metalloogranicheskaya Khimiya 4, 1050 (1991); Chem. Abstr. 116, 6605 (1992).

1991 VOR/KLY M. G. Voronkov, V. A. Klyuchnikov, L. I. Marenkova, T. F. Danilova, G. N. Shvets, S. I. Tsvetnitskaya, and Y. I. Khudobin, J. Organomet. Chem. 406, 99 (1991). 
1991VOR/KLY2 M. G. Voronkov, V. A. Klyuchnikov, E. V. Mironenko, G. N. Shvets, T. F. Danilova, and Y. I. Khudobin, J. Organomet. Chem. 406, 91 (1991).

1991VOR/KLY3 M. G. Voronkov, V. A. Klyuchnikov, E. V. Sokolova, T. F. Danilova, G. N. Shvets, A. N. Korchagina, L. E. Gussel'nikov, and V. V. Volkova, J. Organomet. Chem. 401, 245 (1991).

1991WIB/WAL K. B. Wiberg and R. F. Waldron, J. Am. Chem. Soc. 113, 7697 (1991).

1991WU/XIO S. Wu and W. Xiong, Xibei Daxue Xuebao, Ziran Kexueban 21, 39 (1991).

1991ZHA/HUA X. Zhang, W. Huang, F. Tan, G. Xu, and Z. Wu, Acta Phys.-Chim. Sin. 7, 106 (1991).

1992ABB/JIM J. L. M. Abboud, P. Jimenez, M. V. Roux, C. Turrion, and C. Lopez-Mardomingo, J. Chem. Thermodyn. 24, 1299 (1992).

1992ABB/JIM2 J.-L. M. Abboud, P. Jimenez, M. V. Roux, C. Turrion, C. Lopez-Mardoningo, and G. Sanz, J. Chem. Thermodyn. 24, 217 (1992).

1992ABR/OLA2 J. Abrefah, D. R. Olander, M. Balooch, and W. J. Siekhaus, Appl. Phys. Lett. 60, 1313 (1992).

1992BAB/HWA M. W. Babich, S. W. Hwang, and R. D. Mounts, Thermochim. Acta 210, 83 (1992).

1992BAB/HWA2 M. W. Babich, S. W. Hwang, and R. D. Mounts, Thermochim. Acta 210, 77 (1992).

1992BRU/WAL T. R. Brumleve, S. R. Walston, S. A. Mucklejohn, and N. W. O'Brien, J. Chem. Thermodyn. 24, 773 (1992).

1992CHA/MOU A. Chauvet, A. El Moussaoui, and J. Masse, Thermochim. Acta 210, 133 (1992).

1992CHE/KOR H. S. Chen, A. R. Kortan, R. C. Haddon, and D. A. Fleming, J. Phys. Chem. 96, 1016 (1992).

1992CHI/HES J. S. Chickos, D. G. Hesse, S. Y. Panshin, D. W. Rogers, M. Saunders, P. M. Uffer, and J. F. Liebman, J. Org. Chem. 57, 1897 (1992).

1992DEC/AIR M. A. R. P. de Carvalho, C. Airoldi, and A. G. de Souza, J. Chem. Soc., Dalton Trans. 1992, 1235.

1992DIA/MIN A. R. Dias, M. E. Minas da Piedade, J. A. Martinho Simoes, J. A. Simoni, C. Teixeira, H. P. Diogo, Y. MengYan, and G. Pilcher, J. Chem. Thermodyn. 24, 439 (1992).

1992DWI/SAT S. K. Dwivedi, S. Sattari, F. Jamali, and A. G. Mitchell, Int. J. Pharm. 87, 95 (1992).

1992FUR/BUT I. Furman, R. J. Butcher, R. M. Catchings, and R. G. Weiss, J. Am. Chem. Soc. 114, 6023 (1992).

1992GAZ/SCH M. Gazicki, H. Schalko, P. Svasek, F. Olcaytug, and F. Kohl, J. Vac. Sci. Technol., A 10, 51 (1992).

1992GER/GER P. A. Gerasimova, A. I. Gerasimova, N. E. Fedotova, N. B. Morozova, and I. K. Igumenov, Izv. Vyssh. Uchebn. Zaved., Khim. Khim. Tekhnol. 35, 38 (1992).

1992HAW D. C. Hawker, Chemosphere 25, 427 (1992)

1992HOP/FAU J. Hopken, S. Faulstich, and M. Moller, Mol. Cryst. Liq. Cryst. Sci. Technol., Sect. A 210, 59 (1992).

1992HOP/MOL J. Hopken and M. Moller, Macromolecules 25, 2482 (1992).

1992HUA/WAN Z. Huang, Y. Wang, W. Huang, Y. Zhou, and F. Tan, Huaxue Wuli Xuebao 5, 445 (1992).

1992HUA/ZHO W. Huang, Y. Zhou, F. Tan, X. Huang, and B. Yang, Acta Phys.-Chim. Sin. 8, 795 (1992).

1992HUA/ZHO2 W. Huang, Y. Zhou, F. Tan, G. Xu, Y. Hu, and H. Hu, Huaxue Wuli Xuebao 5, 178 (1992).

1992ISH/IWA H. Ishida, T. Iwachido, and R. Ikeda, Ber. Bunsen-Ges. Phys. Chem. 96, 1468 (1992).

1992KUL/LEB T. G. Kulagina, B. V. Lebedev, E. N. Karataev, and Y. I. Amosov, Zh. Fiz. Khim. 66, 1694 (1992).

1992LEE/DEM C.-H. Lee, D. M. Dempsey, R. S. Mohamed, and G. D. Holder, J. Chem. Eng. Data 37, 183 (1992).

1992LOU/ROU D. Lourdin, A. H. Roux, J.-P. E. Grolier, and J.-M. Buisine, Thermochim. Acta 204, 99 (1992).

1992MAR/KOZ L. I. Marachuk, A. A. Kozyro, and V. V. Simirsky, Zh. Prikl. Khim. 64, 875 (1992).

1992MAT/SAI C. K. Mathews, M. Sai Baba, T. S. L. Narasimhan, R. Balasubramanian, N. Sivaraman, T. G. Srinivasan, and P. R. V. Rao, J. Phys. Chem. 96, 3566 (1992).
1992NGU/KAS

J. N'Guimbi, H. Kasehgari, I. Mokbel, and J. Jose, Thermochim. Acta 196, 367 (1992).

1992NYM/DES

M. Nyman and S. B. Desu, Mater. Res. Soc. Symp. Proc. 243, 381 (1992); Chem. Abstr. 119, 172856a (1993).

1992PAN/CHA C. Pan, M. S. Chandasekharaiah, D. Agan, R. H. Hauge, and J. L. Margrave, J. Phys. Chem. 96, 6752 (1992).

1992RAI/GEO U. S. Rai and S. George, Can. J. Chem. 70, 2869 (1992).

1992REI/HAN H. Reinke, M. Hans, and H. Dehne, Mikrochim. Acta 108, 143 (1992).

1992RIB/FER M. A. V. Ribeiro da Silva, M. L. C. C. H. Ferrao, and R M. G. Esteves da Silva, J. Chem. Thermodyn. 24, 1293 (1992).

1992RIB/FER2 M. A. V. Ribeiro Da Silva, M. L. C. C. H. Ferrao, R. M. C. Marques, and J. M. T. Lima, J. Chem. Thermodyn. 24, 595 (1992).

1992RIB/FER3 M. A. V. Ribeiro Da Silva, M. L. C. C. H. Ferrao, M. J. S. Monte, R. M. G. Esteves Da Silva, and J. C. Ribeiro, J. Chem. Thermodyn. 24, 585 (1992).

1992RIB/MON M. A. V. Ribeiro da Silva and M. J. S. Monte, J. Chem. Thermodyn. 24, 1219 (1992).

1992RIC/MCC P. J. Richardson, D. F. McCafferty, and A. D. Woolfson, Int. J. Pharm. 78, 189 (1992).

1992SAB/ELW R. Sabbah and L. El Watik, Can. J. Chem. 70, 24 (1992).

1992SAB/ELW2 R. Sabbah and L. El Watik, Thermochim. Acta 197, 381 (1992).

1992SAB/ELW3 R. Sabbah and L. El Watik, J. Therm. Anal. 38, 855 (1992).

1992SAB/WAT R. Sabbah and L. E. Watik, J. Therm. Anal. 38, 803 (1992).

1992SER/TSI S. V. Serves, G. M. Tsivoulis, D. N. Sotiropoulos, P. V. Ioannou, and M. K. Jain, Phosphorus, Sulfur Silicon Relat. Elem. 71, 99 (1992).

1992 SHA/SHA $\quad$ B. L. Sharma, N. K. Sharma, and M. Rambal, Thermochim. Acta 206, 71 (1992).

1992SRC/KER S. Srcic, J. Kerc, U. Urleb, I. Zupancic, G. Lahajnar, B. Kofler, and J. Smid-Korbar, Int. J. Pharm. 87, 1 (1992).

1992STE/CHI W. V. Steele, R. D. Chirico, S. E. Knipmeyer, and A. Nguyen, J. Chem. Thermodyn. 24, 245 (1992).

1992TER/PAU A. Terol, B. Pauvert, A. Bouassab, P. Chevallet, and G. Cassanas, Thermochim. Acta 211, 279 (1992).

1992VAN D. W. Van Krevelen, "Part I: Group contribution methods," in Computational Modeling of Polymers, edited by J. Bicerano (Marcel Dekker, New York, 1992).

1992VAN/COR M. G. M. Van der Vis, E. H. P. Cordfunke, R. J. M. Konings, G. J. K. Van der Berg, and J. C. Van Miltenburg, J. Chem. Thermodyn. 24, 1103 (1992).

1992VER/BEC S. P. Verevkin, H.-D. Beckhaus, and C. Rüchardt, Thermochim. Acta 197, 27 (1992).

1992VIG/ZAM A. Vigevani, M. Zampieri, and S. Pellizzato, J. Pharm. Biomed. Anal. 10, 31 (1992).

1992VIL/WEI P. M. Vilalta and R. G. Weiss, Liq. Cryst. 12, 531 (1992).

1992WAF/MUS E. Waffenschmidt, J. Musolf, M. Heuken, and K. Heime, J. Supercond. 5, 119 (1992).

1992XEN/CHE A. Xenopoulous, J. Cheng, M. Yasuniwa, and B. Wunderlich, Mol. Cryst. Liq. Cryst. 214, 63 (1992).

1993ABB/JIM J.-L. M. Abboud, P. Jimenez, M. V. Roux, C. Turrion, and C. Lopez-Mardomingo, Calorim. Anal. Therm. 24, 181 (1993).

1993ACR W. E. Acree, Jr., Thermochim. Acta 219, 97 (1993); and references therein.

1993ACR/TUC W. E. Acree, Jr., S. A. Tucker, G. Pilcher, M. I. P Andrade, and M. D. C. Ribeiro da Silva, J. Chem. Thermodyn. 25, 653 (1993).

1993ACR/TUC2 W. E. Acree, Jr., S. A. Tucker, G. Pilcher, A. Chowdhary, M. D. C. Ribeiro da Silva, and M. J. S. Monte, J. Chem. Thermodyn. 25, 1253 (1993).

1993AIR/SAN C. Airoldi and L. S. Santos, Struct. Chem. 4, 323 (1993).

1993BAE

1993BRE/DUN A. K. Baev, Russ. J. Phys. Chem. 67, 2161 (1993).

1993BRU/PIA $\quad$ B. Brunetti and V. Piacente, J. Mater. Sci. Lett. 12, 1738 (1993). 
1993BYK/KIP T. A. Bykova, E. G. Kiparisova, B. V. Lebedev, N. G. Senchenya, K. A. Mager, and Y. G. Gololobov, Polym. Sci. U.S.S.R. 35, 8 (1993).

1993CHI/HOS J. S. Chickos, S. Hosseini, D. G. Hesse, and J. F. Liebman, Struct. Chem. 4, 261 (1993); Note: Heat capacity corrections to $298 \mathrm{~K}$ were estimated by the procedure outlined in the article.

1993CHI/KNI R. D. Chirico, S. E. Knipmeyer, A. Nguyen, and W. V. Steele, J. Chem. Thermodyn. 25, 1461 (1993).

1993 CHI/KNI2 R. D. Chirico, S. E. knipmeyer, A. Nguyen, N. K. Smith, and W. V. Steele, J. Chem. Thermodyn. 25, 729 (1993); Note: Calculated from the vapor pressuretemperature data.

1993CON/VIA M. D. Contreras Claramonte, A. P. Vialard, and F. G. Vilchez, Int. J. Pharm. 94, 23 (1993).

1993DEB/DWO J. De Brulin, A. Dworkin, H. Szwarc, J. Godard, R. Ceolin, C. Fabre, and A. Rassat, Europhys. Lett. 24, 551 (1993).

1993DIO/MIN H. P. Diogo, M. E. Minas da Piedade, A. C. Fernandes, J. A. Martinho Simoes, M. A. V. Riberio da Silva, and M. J. S. Monte, Thermochim. Acta 228, 15 (1993).

1993DOM/SER U. Domanska, J. Serwatowski, A. Sporzynski, and M. Dabrowski, Thermochim. Acta 222, 279 (1993).

1993ENG/WAN P. S. Engel, C. Wang, Y. Chen, C. Rüchardt, and H.-D. Beckhaus, J. Am. Chem. Soc. 115, 65 (1993).

1993GRU A. Grubenmann, Dyes Pigm. 21, 273 (1993).

1993HER/BEC C. Herberg, H.-D. Beckhaus, T. Koertvelysi, and C. Rüchardt, Chem. Ber. 126, 117 (1993).

1993HUS/SAR S. Husain, P. N. Sarma, G. Y. S. K. Swamy, and K. S. Devi, J. Am. Oil Chem. Soc. 70, 149 (1993).

1993JUA/CHE T. M. Juang, Y. N. Chen, S. H. Lung, Y. H. Lu, C. S. Hsu, and S. T. Wu, Liq. Cryst. 15, 529 (1993).

1993KAS/MOK H. Kasehgari, I. Mokbel, C. Viton, and J. Jose, Fluid Phase Equilib. 87, 133 (1993).

1993KEL/SAK G. R. Kelm and A. A. Sakr, Drug Dev. Ind. Pharm. 19, 809-826 (1993).

1993KUL/LEB T. T. Kulagina and B. V. Lebedev, Journ. Mediterr. Calorim. Anal. Therm. 24, 253 (1993); Chem. Abstr. 122, 57054n (1995)

1993LEB/BYK B. V. Lebedev, T. A. Bykova, V. G. Vasil'ev, and B. Wunderlich, Khim. Termodin. Termokhim. 2, 62 (1993).

1993LEE/HOL C.-H. Lee and G. D. Holder, J. Chem. Eng. Data 38, 320 (1993).

1993MAT/SAI C. K. Mathews, M. Sai Baba, T. S. Lakshmi Narasimhan, R. Balasubramanian, N. Sivaraman, T. G. Srinivasan, and P. R. Vasudeva Rao, Fullerene Sci. Technol. 1, 101 (1993); see also J. Phys. Chem. 96, 3566 (1992).

1993NEA/SHI S. H. Neau, M. K. Shinwari, and E. W. Hellmuth, Int. J. Pharm. 99, 303 (1993).

1993PIA/FER V. Piacente, D. Ferro, and G. Della Gatta, Thermochim. Acta 223, 65 (1993).

1993RAM/BOU J. Rambaud, A. Bouassab, B. Pauvert, P. Chevallet, J.-P. Declercq, and A. Terol, J. Pharm. Sci. 82, 1262 (1993).

1993RIB/GIE M. A. V. Ribeiro da Silva, E. Giera, and M. J. S. Monte, J. Alloys Compd. 197, 105 (1993).

1993RIB/MON M. A. V. Ribeiro Da Silva, M. J. S. Monte, E. Giera, and W. Kakolowicz, J. Chem. Thermodyn. 25, 1263 (1993).

1993RIB/RIB M. A. V. Ribeiro da Silva, M. D. M. C. Ribeiro da Silva, J. P. A. Paiva, I. M. C. S. Nogueira, A. M. Damas, J. V. Barley, M. M. Harding, M. J. Akello, and G. Pilcher, J. Chem. Soc., Perkin Trans. 2 1993, 1765.

1993ROM/RHO A. J. Romero and C. T. Rhodes, J. Pharm. Pharmacol. 45, 258 (1993)

1993RUE/SAR P. Ruelle, E. Sarraf, L. van den Berge, K. Seghers, M. Buchmann, and U. W. Kesselring, Pharm. Acta Helv. 68, 49 (1993).

1993SAI/YAM K. Saito, Y. Yamamura, H. Saitoh, H. Matsuyama, K. Kikuchi, and I. Ikemoto, Solid State Commun. 87, 903 (1993).

1993SAT/SUG H. Sato and H. Sugawara, Inorg. Chem. 32, 1941 (1993).

1993SCH/KVA B. Schneider, J. Kvarda, P. Schmidt, M. Suchoparek, and I. Prokopova, Collect. Czech. Chem. Commun. 58, 2403 (1993).
1993SER/SOT

1993SHE/KAR

1993STE/CHI

1993SYO/GOL

1993TER/BOU

1993TIE/FRA

1993TOB/LAN

1993VIL/HAM

1994ALI/MAL

1994BAB/BEN

1994BEC/RUE

1994BLU/PRA

1994BRA/DOU

1994CHE/WES

1994CHI/GAM

1994DAI/MAC

1994DAL/FER

1994ESC/BUS

1994FAL/BID

1994GER/GER

1994GER/GER2

1994GOL/PER

1994JIN/BOL

1994KAB/KOZ

1994KAR/FRA

1994KON/HIL

1994KOR/SID

1994KOU/HOS

1994LEB/BYK

1994LEB/SMI2

1994LIE/MAR
S. V. Serves, D. N. Sotiropoulos, P. V. Ioannou, and M. K. Jain, Phosphorus, Sulfur Silicon Relat. Elem. 81, 181 (1993).

V. B. Sheinin, V. V. Karasyov, S. A. Klyuyev, and B. D. Berezin, Zh. Khim. Termodin. Termokhim. 2, 186-189 (1993); Chem. Abstr. 122, 116291n (1995).

W. E. Steele, R. D. Chirico, S. E. Knipmeyer, and A. Nguyen, J. Chem. Thermodyn. 25, 965 (1993).

S. V. Syoev, A. N. Golubenko, L. D. Nikulina, and T. N. Martynova, Thermochim. Acta 225, 137 (1993).

A. Tero, A. Bouassab, B. Pauvert, P. Chevallet, and G. Cassanas, Thermochim. Acta 225, 97 (1993).

G. V. D. Tiers and C. V. Francis, Thermochim. Acta 226, 311 (1993).

P. Tobaly and G. Lanchec, J. Chem. Thermodyn. 25, 503 (1993).

P. M. Vilalta, G. S. Hammond, and R. G. Weiss, Langmuir 9, 1910 (1993).

A. S. Alikhanyan, I. P. Malkerova, N. P. Kuz'mina, V. K. Ivanov, and A. R. Kaul, Zh. Neorg. Khim. 39, 1534 (1994).

M. W. Babich, R. Benrashid, and R. D. Mounts, Thermochim. Acta 243, 193 (1994).

H.-D. Beckhaus, C. Rüchardt, D. R. Lagerwall, L. A Pauette, F. Wahl, and H. Prinzbach, J. Am. Chem. Soc. 116, 11775 (1994).

D. Blunk, K. Praefcke, and G. Legler, Liq. Cryst. 17, 841 (1994).

S. Braeuninger, S.-Q. Dou, H. Fuess, W. Schmahl, R. Strauss, and A. Weiss, Ber. Bunsen-Ges. Phys. Chem. 98, 1096 (1994).

J. A. R. Cheda and E. F. Westrum, Jr., J. Phys. Chem. 98, 2482 (1994).

R. D. Chirico, B. E. Gammon, I. A. Hossenlopp, and W. V. Steele, J. Chem. Thermodyn. 26, 469 (1994).

S. Dai, L. Mac Toth, G. D. Del Cul, and D. H. Metcalf, J. Chem. Phys. 101, 4470 (1994).

L. D'Alessio, D. Ferro, and V. Piacente, J. Alloys Compd. 209, 207 (1994).

J. B. Escalera, P. Bustamante,

R. L. Falconer and T. F. Bidleman, Atmos. Environ. 28, 547 (1994).

P. A. Gerasimov, A. I. Gerasimova, V. G. Isakova, and I. K. Igumenov, Izv. Vyssh. Uchebn. Zaved., Khim. Khim. Technol. 37, 48 (1994); Chem. Abstr. 120, $228675 f$ (1994).

P. A. Gerasimov, A. I. Gerasimova, V. V. Krisyuk, and I. K. Igumenov, Russ. J. Phys. Chem. 68, 199 (1994).

O. A. Golubchikov, G. L. Perlovich, and B. K. Naneishvili, Zh. Fiz. Khim. 68, 2120 (1994).

Y. Jin, A. Boller, B. Wunderlich, and B. V. Lebedev, Thermochim. Acta 234, 103 (1994).

G. J. Kabo, A. A. Kozyro, A. P. Marchand, V. V. Diky, V. V. Simirsky, L. S. Ivashkevich, A. P. Krasulin, V. M. Sevruk, and M. L. Frenkel, J. Chem. Thermodyn. 26, 129 (1994).

U. Karlson, W. T. Frankenberger, Jr., and W. F. Spencer, J. Chem. Eng. Data 39, 608 (1994).

R. J. M. Konings and D. L. Hildenbrand, J. Chem. Thermodyn. 26, 155 (1994).

M. V. Korobov and L. N. Sidorov, J. Chem. Thermodyn. 26, 61 (1994).

B. Koutek, M. Hoskovec, P. Vrkova, K. Konecny, and L. Feltl, J. Chromatogr. A 679, 307 (1994).

B. V. Lebedev, T. A. Bukova, V. G. Vasil'ev, and B. Wunderlich, Zh. Fiz. Khim. 68, 15 (1994).

B. Lebedev, N. Smirnova, N. Novosyolova, K. Makovetskii, and I. Ostrovskaya, Macromol. Chem. Phys. 195, 1807 (1994)

J. F. Liebman, J. A. Martinho-Simoes, and S. W. Slayden, in The Chemistry of Organic Arsenic, Antimony and Bismuth Compounds, edited by S. Patai (John Wiley and Sons, 1994), Chap. 4. 
1994MAR/MAR M. C. Martinez-Oharriz, C. Martin, M. M. Goni, C. Rodriguez-Espinosa, M. C. Tros de Ilarduya-Apaolaza, and M. Sanchez, J. Pharm. Sci. 83, 174 (1994).

1994MEL/LIT A. P. Melissaris and M. H. Litt, Macromolecules 27, 888 (1994).

1994MOR/KOB D. L. Morgan and R. Kobayashi, Fluid Phase Equilib. 97, 211 (1994).

1994NAK/TAK K. Nakasone, K. Takamizawa, K. Shiokawa, and Y. Urabe, Thermochim. Acta 233, 175 (1994).

1994OHT/YAM T. Ohta, O. Yamamuro, and H. Suga, J. Chem. Thermodyn. 26, 319 (1994).

1994OPP/KRA H. Opperman, R. Krausze, U. Bruhn, and M. Balarin, Z. Anorg. Allg. Chem. 620, 1110 (1994).

1994PER/NAN G. L. Perlovich, B. K. Naneishvili, and O. A. Golubchikov, Zh. Fiz. Khim. 68, 1932 (1994).

1994PIA/FER V. Piacente, D. Ferro, and G. Della Gatta, Thermochim. Acta 232, 317 (1994); Erratum, 235, 291-292 (1994).

1994PIA/FON V. Piacente, D. Fontana, and P. Scardala, J. Chem. Eng. Data 39, 231 (1994).

1994POP/DRA A. Popovic, G. Drazic, and J. Marsel, Rapid Commun. Mass Spectrom. 8, 985 (1994).

1994RAK/VER K. Rakus, S. P. Verevkin, H.-D. Beckhaus, and C. Rüchardt, Chem. Ber. 127, 2225 (1994).

1994RAK/VER2 K. Rakus, S. P. Verevkin, J. Schaetzer, H.-D. Beckhaus, and C. Rüchardt, Chem. Ber. 127, 1095 (1994).

1994REG/CHM A. Regosz, A. Chmielewska, T. Pelplinska, and P. Kowalski, Pharmazie 49, 371 (1994).

1994RIB/FER M. A. V. Ribeiro da Silva and M. L. C. C. H. Ferrao, J. Chem. Thermodyn. 26, 315 (1994).

1994RIB/MAT M. A. V. Ribeiro da Silva, M. A. R. Matos, J. M. Goncalves, P. M. Johnson, G. Pilcher, P. M. Burkinshaw, and C. T. Mortimer, Thermochim. Acta 247, 245 (1994).

1994RUZ/MAJ K. Ruzicka and V. Majer, J. Phys. Chem. Ref. Data 23, 1 (1994).

1994RUZ/ZAB V. Ruzicka, Jr., M. Zabransky, K. Ruzicka, and V. Majer, Thermochim. Acta 245, 121 (1994).

1994SAB/TAB R. Sabbah, D. Tabet, and S. T. Belaadi, Thermochim. Acta 247, 193 (1994).

1994SAB/TAB2 R. Sabbah, D. Tabet, and S. T. Belaadi, Thermochim. Acta 247, 201 (1994).

1994SAI/LAK M. Sai Baba, T. S. Lakshmi Narasimhan, R. Balasubramanian, N. Sivaraman, and C. K. Mathews, J. Phys. Chem. 98, 1333 (1994).

1994SAN/DEF N. Santilli, P. de Filippio, and A. Chianese, J. Chem. Eng. Data 39, 179 (1994).

1994SCH/BEC F. Schaffer, H.-D. Beckhaus, H.-J. Rieger, and C. Rüchardt, Chem. Ber. 127, 557 (1994).

1994SOU/PIN $\quad$ A. G. Souza, C. D. Pinheiro, L. C. Santos, and M. L. M. Melo, Thermochim. Acta 231, 31 (1994).

1994TAN/BOS R. P. Tangri and D. K. Bose, Thermochim. Acta 244, 249 (1994).

1994TEL/SHE V. I. Tel'noi, M. S. Sheiman, G. P. Kamelova, and V. N. Larina, Russ. J. Gen. Chem. 64, 977 (1994).

1994TER/CAS A. Terol, G. Cassanas, J. Nurit, B. Pauvert, A. Bouassab, J. Rambaud, and P. Chevallet, J. Pharm. Sci. 83, 1437 (1994).

1994UEN/SUE S. Ueno, T. Suetake, Y. Yano, M. Suzuki, and K. Sato, Chem. Phys. Lipids 72, 27 (1994).

1994WAN/KUO C.-C. Wang, J.-F. Kuo, and C.-Y. Chen, Macromol. Chem. Phys. 195, 1493 (1994).

1994WAN/SHU F. Wania, W.-Y. Shui, and D. Mackay, J. Chem. Eng. Data 39, 572 (1994).

1994WEB/MEY H. Weber and K. Meyer-Trumpener, Arch. Pharm. (Weinheim) 327, 337 (1994).

1994WEL/VER F. Welle, S. P. Verevkin, M. Keller, H.-D. Beckhaus, and C. Rüchardt, Chem. Ber. 127, 697 (1994).

1994YU/ZIP X. Yu, G. L. Zipp, and G. W. R. Davidson III, Pharm. Res. 11, 522 (1994).

1994ZIE/ZIE W. Zielenkiewicz, A. Zielenkiewicz, and K. L. Wierzchowski, J. Solution Chem. 23, 1125 (1994).

1995ABB/JIM J.-L. M. Abboud, P. Jimenez, M. V. Roux, C. Turrion, C. Lopez-Mardomingo, A. Podosenin, D. W. Rogers, and J. F. Liebman, J. Phys. Org. Chem. 8, 15 (1995).
1995ACR/BOT

W. E. Acree, Jr., S. G. Bott, S. A. Tucker, M. D. M. C. Ribeiro da Silva, M. A. R. Matos, and G. Pilcher, J. Chem. Thermodyn. 27, 1433 (1995).

1995BAE/MIK A. K. Baev, V. E. Mikhailov, and I. N. Chernyak, Russ. J. Gen. Chem. 65, 977 (1995).

1995BEC/RUC H.-D. Beckhaus, C. Rüchardt, S. I. Kozhushkov, V. N. Belov, S. P. Verevkin, and A. de Meijere, J. Am. Chem. Soc. 117, 11854 (1995).

1995BOW/HER C. J. Bowden, T. M. Herrington, A. M. Moseley, and R. Richardson, Liq. Cryst. 18, 825 (1995).

1995BUS/ESC P. Bustamante and B. Escalera, J. Pharm. Pharmacol. 47, 550 (1995).

1995CHI/HES J. S. Chickos, D. G. Hesse, S. Hosseini, J. F. Liebman, G. D. Mendenhall, S. P. Verevkin, K. Rakus, H.-D. Beckhaus, and C. Rüchardt, J. Chem. Thermodyn. 27, 693 (1995).

1995CHI/HOS J. S. Chickos, S. Hosseini, and D. G. Hesse, Thermochim. Acta 249, 41 (1995).

1995CIA/DU X. Cia, D. Du, Y. Jin, and Y. Qian, J. Therm. Anal. 45, 193 (1995).

1995CON/GOB J. A. Connor and A. Göbel, J. Chem. Thermodyn. 27, 605 (1995).

1995CON/GOB2 J. A. Connor and A. Göbel, Polyhedron 14, 3107 (1995).

1995CYP/JOH C. C. Cypcar, G. Johnson, and L. Mathias, Polym. Prepr. (Am. Chem. Soc., Div. Polym. Chem.) 36, 402 (1995).

1995DAB/DOM M. Dabrowski, U. Domanska, J. Serwatowski, and A. Sporzynski, Thermochim. Acta 250, 19 (1995).

1995EIS/DEN P. Eiselt, S. Denzinger, and H.-W. Schmidt, Liq. Cryst. 18, 257 (1995).

1995ESP/BIS F. Espitalier, B. Biscans, P. S. Peyrigain, and C. Laguerie, Fluid Phase Equilib. 113, 151 (1995).

1995FAN/HOR Z.-G. Fang, T. S. A. Hor, Y.-S. Wen, L.-K. Liu, and T. C. W. Mak, Polyhedron 14, 2403 (1995).

1995FUJ/FUJ H. Fujita, H. Fujimori, and M. Oguni, J. Chem. Thermodyn. 27, 927 (1995)

1995GAR/CHA M. L. Garner, D. Chandra, and K. H. Lau, J. Phase Equilib 16, 24 (1995).

1995GRU/KEI A. Grunenberg, B. Keil, and J.-O. Henck, Int. J. Pharm. 118, 11 (1995).

1995HAI/SAN R. L. S. Haines and S. I. Sandler, J. Chem. Eng. Data 40 833 (1995).

1995HAM/KOD M. J. Hampden Smith and T. T. Kodas, Chem. Vap. Deposition $\mathbf{1}, 8$ (1995).

1995HAM/MEH A. Hammami and A. K. Mehrotra, Fluid Phase Equilib. 111, 253 (1995).

1995HER/VER C. Herberg, S. P. Verevkin, M. Nölke, H.-D. Beckhaus, and C. Rüchardt, Liebigs Ann. 1995, 515.

1995HSU/TSA C. S. Hsu, K. T. Tsay, A. C. Chang, S. R. Wang, and S. T. Wu, Liq. Cryst. 19, 409 (1995).

1995JIM/MEN P. Jiménez, V. Menéndez, M. V. Roux, and C. Turrión, J. Chem. Thermodyn. 27, 679 (1995).

1995KAB/KOZ G. J. Kabo, A. A. Kozyro, V. V. Diky, V. V. Simirsky, L. S. Ivashkevich, A. P. Krasulin, V. M. Sevruk, A. P. Marchand, and M. L. Frenkel, J. Chem. Thermodyn. 27, 707 (1995).

1995KEL/SCH S. M. Kelly, M. Schadt, and H. Seiberle, Liq. Cryst. 18 581 (1995).

1995KRI/VES A. Kristl and G. Vesnaver, J. Chem. Soc., Faraday Trans. 91, 995 (1995).

1995KSI/NAG A. Ksiazczak and I. Nagata, Thermochim. Acta 254, 31 (1995).

1995MIY/NAK S. Miyajima, A. Nakazato, N. Sakoda, and T. Chiba, Liq. Cryst. 18, 651 (1995)

1995MOK/GUE I. Mokbel, T. Guetachew, and J. Jose, ELDATA: Int. Electron. J. Phys.-Chem. Data 2, 167 (1995).

1995MUR/PAI S. S. N. Murthy, A. Paikaray, and N. Arya, J. Chem. Phys. 102, 8213 (1995).

1995NAS/LEN K. Nass, D. Lenoir, and A. Kettrup, Angew. Chem., Int. Ed. Engl. 34, 1735 (1995).

1995NOL/VER M. Nolke, S. P. Verevkin, H.-D. Beckhaus, and C. Rüchardt, Liebigs Ann. 1995, 41.

1995NUR/LEL J. Nurit, J. M. Leloup, B. Pauvert, O. Doumbia, P. Chevallet, and A. Terol, J. Therm. Anal. 45, 535 (1995). 
1995OUT/KLO G. J. J. Out, H.-A. Klok, M. Moller, and D. Oelfin, Macromol. Chem. Phys. 196, 195 (1995).

1995PIA/GIG V. Piacente, G. Gigli, P. Scradala, A. Giustini, and D. Ferro, J. Phys. Chem. 99, 14052 (1995).

1995RAK/VER K. Rakus, S. P. Verevkin, M. Keller, H.-D. Beckhaus, and C. Rüchardt, Liebigs Ann. 1995, 1483.

1995RAK/VER2 K. Rakus, S. P. Verevkin, W.-H. Peng, H.-D. Beckhaus, and C. Rüchardt, Liebigs Ann. 1995, 2059.

1995RIB/MAT3 M. A. V. Ribeiro da Silva, M. A. R. Matos, and L. M. P. F. Amaral, J. Chem. Thermodyn. 27, 1141 (1995).

1995RIB/MON M. A. V. Ribeiro da Silva, M. J. S. Monte, and J. Huinink, J. Chem. Thermodyn. 27, 175 (1995).

1995RIB/MON2 M. A. V. Ribeiro da Silva, M. J. S. Monte, and J. Huinink, J. Alloys Compd. 224, 181 (1995).

1995RIB/REI M. A. V. Ribeiro da Silva, A. M. M. V. Reis, and R. I. M. C. P. Faria, J. Chem. Thermodyn. 27, 1365 (1995).

1995RIB/RIB M. A. V. Ribeiro da Silva, M. D. M. C. Ribeiro da Silva, M. J. S. Monte, J. M. Goncalves, and E. M. R. Fernandes, J. Chem. Thermodyn. 27, 613 (1995).

1995RUL/RAK J. Rulewski, J. Rak, P. Dorurno, P. Skurski, and J. Blazejowski, J. Therm. Anal. 45, 839 (1995).

1995STE/CHI W. V. Steele, R. D. Chirico, A. B. Cowell, A. Nguyen, and S. E. Knipmeyer, J. Chem. Thermodyn. 27, 1407 (1995).

1995STI/DUA A. L. Stinchcomb, R. Dua, A. Paliwal, R. W. Woodard, and G. L. Flynn, Pharm. Res. 12, 1526 (1995).

1995STR/ARG L. Stradella and M. Argentero, Thermochim. Acta 268, 1 (1995).

1995TAK/URA K. Takamizawa, Y. Urabe, J. Fujimoto, H. Ogata, and Y. Ogawa, Thermochim. Acta 267, 297 (1995).

1995TOB/WAT P. Tobaly and I. M. Watson, J. Chem. Thermodyn. 27, 1211 (1995).

1995TOR/GUD L. A. Torres, G. Gudiño, I. Hernandez-Contreras, M. E. Ochoa, N. Farfan, and R. L. Santillan, J. Chem. Thermodyn. 27, 779 (1995).

1995TOR/GUD2 L. A. Torres, R. Gudino, R. Sabbah, and J. A. Guardado, J. Chem. Thermodyn. 27, 1261 (1995).

1995VAN/COR M. G. M. Van der Vis and E. H. P. Cordfunke, Thermochim. Acta 265, 129 (1995).

1995VAR/DRO R. M. Varushchenko and A. I. Droujinina, J. Chem. Thermodyn. 27, 355 (1995).

1995VER/BEC S. P. Verevkin, H.-D. Beckhaus, and C. Rüchardt, Thermochim. Acta 249, 1 (1995).

1995VER/DOG S. P. Verevkin, B. Dogan, J. Hadrich, H.-D. Beckhaus, and C. Rüchardt, J. Prakt. Chem. 337, 93 (1995).

1995 VIE/CDE $\quad$ E. F. S. Vieira, J. C. de Queiroz, and F. S. Dias, Thermochim. Acta 256, 249 (1995).

1995VIG/ZAM A. Vigevani and M. Zampieri, Chirality 7, 575 (1995).

1995WUL/ALD M. Wulff and M. Alden, Thermochim. Acta 256, 151 (1995).

1995YAM/SAI Y. Yamamura, K. Saito, H. Saitoh, H. Matsuyama, K. Kikucki, and I. Ikemoto, J. Phys. Chem. Solids 56, 107 (1995).

1995YAN/YIN L. Yang, H. Yin, W. Zhu, and S. Niu, J. Therm. Anal. 45, 207 (1995).

1995YAS/TAK K. Yase, Y. Takahashi, N. Ara-kato, and A. Kawazu, Jpn. J. Appl. Phys., Part 1 34, 636 (1995).

1996BAC/GRZ D. D. Back, L. R. Grzyll, and M. Corrigan, Thermochim. Acta 272, 53 (1996).

1996BAE/POD A. K. Baev, A. I. Podkovirov, and V. I. Kosirkin, Izv. Vyssh. Uchebn. Zaved., Khim. Khim. Tekhnol. 39, 10 (1996).

1996BAR/BRU G. Bardi, B. Brunetti, and V. Piacente, J. Chem. Eng. Data 41, 14 (1996).

1996BEC/OTT W. Beckmann and W. H. Otto, Chem. Eng. Res. Des. 74, 750 (1996).

1996BOL/MER O. V. Boltalina, V. Y. Merkov, A. Y. Borschevskiy, and A. Popovitch, Mendeleev Commun. 6, 253 (1996).

1996BUR/DAG H. D. Burrows, M. da Graca, M. Miguel, A. P. Varela, and R. S. Becker, Thermochim. Acta 279, 77 (1996).

1996BUR/KOL A. Burger, K. T. Koller, and W. M. Schiermeir, Eur. J. Pharm. Biopharm. 42, 142 (1996).

1996BYK/MOR A. F. Bykov, N. B. Morozova, I. K. Igumenov, and S. V. Sysoev, J. Therm. Anal. 46, 1551 (1996).
1996CAM/FIG

H. K. Cammenga, L. O. Figura, and B. Zielasko, J. Therm. Anal. 47, 427 (1996).

1996CHA/EMM P. Chassot and F. Emmenegger, Inorg. Chem. 35, 5931 (1996).

1996CIO/MEL I. Ciocazanu and V. Meltzer, J. Therm. Anal. 47, 1755 (1996).

1996DES/BRA A. G. de Souza, M. G. A. Brasilino, and C. Airoldi, J. Chem. Thermodyn. 28, 1359 (1996).

1996DOM/HEA E. S. Domalski and E. D. Hearing, J. Phys. Chem. Ref. Data 25, 1 (1996), and references therein.

1996FON/ROS L. Fontana, L. Rosati, A. Sala, and L. Dobetti, Pharm. Acta Helv. 71, 341 (1996).

1996GON/SUN Q. Gong, Y. Sun, Z. Huang, X. Zhou, Z. Gu, and D. Qiang, J. Phys. B: At., Mol. Opt. Phys. 29, 4981 (1996).

1996GOV/RUT

H. A. J. Govers, E. Ruts, F. W. M. van der Wielen, and A. G. van Haelst, Polycyclic Aromat. Compd. 9, 75 (1996).

1996KEE/VAN E. M. D. Keegstra, V. van der Mieden, J. W. Zwikker, L. W. Jenneskens, A. Schouten, H. Kooijman, N. Veldman, and A. L. Spek, Chem. Mater. 8, 1092 (1996).

1996 KIR/CHI $\quad$ D. R. Kirklin, J. S. Chickos, and J. F. Liebman, Struct. Chem. 7, 355 (1996).

1996KON/COR R. J. M. Konings, E. H. P. Cordfunke, and R. R. van der Laan, Thermochim. Acta 273, 231 (1996).

1996KOU/HOS B. Koutek, M. Hoskovec, P. Vrkocova, K. Konecny, L. Feltl, and J. Vrkoc, J. Chromatogr. A 719, 391 (1996).

1996LAB/HUB F. Labrize, L. G. Hubert-Pfalzgraf, L.-C. Daran, S. Halut, and P. Tobaly, Polyhedron 15, 2707 (1996).

1996LEB/BYK B. V. Lebedev, T. A. Bykova, E. G. Kiparisova, and V. G. Vasil'yev, Macromol. Chem. Phys. 197, 1553 (1996).

1996LEB/KUL B. V. Lebedev, T. G. Kulagina, A. A. Cheremukhina, and E. N. Karataev, Russ. J. Gen. Chem. 66, 880 (1996).

1996 MOR/SYS N. B. Morozova, S. V. Sysoev, I. K. Igumenov, and A. N. Golubenko, J. Therm. Anal. 46, 1367 (1996).

1996OHT/OSA T. Ohta, Y. Osamu, T. Matsuo, and H. Suga, J. Phys. Chem. 100, 2353 (1996).

19960VC/MAK V. V. Ovchinnikov, T. B. Makeeva, L. I. Lapteva, and A. I. Konovalov, Thermochim. Acta 277, 145 (1996).

1996PAP/KOL T. S. Papina, V. P. Kolesov, V. P. Vorobieva, and V. F. Golovkov, J. Chem. Thermodyn. 28, 307 (1996).

1996PIA/GIG V. Piacente, G. Gigli, P. Scardala, A. Giustini, and G. Bardi, J. Phys. Chem. 100, 9815 (1996).

1996PYD/VAR M. Pyda, M. Varma-Nair, W. Chen, H. S. Aldrich, R. H. Schlosberg, and B. Wunderlich, J. Therm. Anal. 46, 1093 (1996).

1996RAP/DES ～B. J. Rappoli and W. J. Desisto, Appl. Phys. Lett. 68, 2726 (1996).

1996RIB/MON M. A. V. Ribeiro da Silva, M. J. S. Monte, and J. Huinink, J. Chem. Thermodyn. 28, 413 (1996).

1996ROB/ESP L. Robles, P. Espeau, D. Mondieg, Y. Haget, and H. A. J. Oonk, Thermochim. Acta 274, 61 (1996).

1996ROU/JIM M. V. Roux, P. Jimenez, J. Z. DaValos, J. L. M. Abboud, and M. T. Molina, J. Chem. Thermodyn. 28, 1029 (1996).

$1996 \mathrm{SCH}$

1996SHE/KAM F. P. Schwarz, J. Solution Chem. 25, 471 (1996).

1996SLA/NOV I. B. Sladkov and N. Y. Novikova, Zh. Prikl. Khim. (S.-

M. S. Sheiman, G. P. Kamelova, K. G. Shvetsova, and V. P. Nistratov, Russ. J. Phys. Chem. 70, 1105 (1996). Peterburg) 96, 1840 (1996).

1996STE/CHI W. V. Steele, R. D. Chirico, S. E. Knipmeyer, and A. Nguyen, J. Chem. Eng. Data 41, 1255 (1996).

1996STE/CHI2 W. V. Steele, R. D. Chirico, S. E. Knipmeyer, A. Nguyen, and N. K. Smith, J. Chem. Eng. Data 41, 1285 (1996).

1996STE/CHI3 W. V. Steele, R. D. Chirico, S. E. Knipmeyer, A. Nguyen, N. K. Smith, and I. R. Tasker, J. Chem. Eng. Data 41, 1269 (1996).

1996TEL/SHE V. I. Tel'noi, M. S. Sheiman, V. N. Larina, and G. P. Kamelova, Russ. J. Phys. Chem. 70, 1261 (1996).

1996TSY/DYA E. I. Tsyganova and L. M. Dyagileva, Russ. Chem. Rev. 65, 315 (1996).

1996TSY/DYA2 E. I. Tsyganova and L. M. Dyagileva, Usp. Khim. 65, 334 (1996), as quoted in Ref. [2000GIE].

1996TUR/EIC A. Türler, B. Eichler, D. T. Jost, D. Piqquet, H. W. Gäggeler, K. E. Gregorich, B. Kadkhodayan, S. A. Kreek, D. M. Lee, M. Mohar, E. Sylwester, D. C. Hoffman, and S. Hübener, Radiochim. Acta 73, 55 (1996). 
1996VAN/COR M. G. M. Van der Vis, E. H. P. Cordfunke, R. J. M. Konings, and A. Oskam, J. Chem. Soc., Faraday Trans. 92, 973 (1996).

1996VAN/VAN A. G. van Haelst, F. W. M. van der Wielen, and H. A. J. Govers, J. Chromatogr. A 727, 265 (1996).

1996VAR/DRU R. M. Varushchenko, A. I. Druzhinina, and I. L. Pashchenko, Fluid Phase Equilib. 126, 93 (1996).

1996VAR/PAS R. M. Varushchenko, L. L. Pashchenko, and A. I. Druzhinina, Russ. J. Phys. Chem. 70, 208 (1996).

1996VER/PEN S. P. Verevkin, W.-H. Peng, H.-D. Beckhaus, and C. Rüchardt, Struct. Chem. 7, 397 (1996).

1996VIT/CHA C. Viton, M. Chavret, and J. Jose, ELDATA: Int. Electron. J. Phys.-Chem. Data 2, 103 (1996).

1996WEB/DEF2 L. A. Weber and D. R. Defibaugh, J. Chem. Eng. Data 41, 382 (1996).

1996YAN/YU Z. Yang, C.-E. Yu, J. Cooke, Z. Ali-Adib, K. Viras, H. Matsuura, A. J. Ryan, and C. Booth, J. Chem. Soc., Faraday Trans. 92, 3173 (1996).

1996YUA/MEN Z. Yuan and G. Meng, Huaxue Shiji 18, 76 (1996); Chem. Abstr. 125, 47592j (1996).

1996ZIE/EIK T. Ziegler, O. Eikenberg, U. Bilitewski, and M. Grol, Analyst 121, 119 (1996).

1996ZVE/CHE Y. B. Zverev and S. G. Chesnokova, Vysokochist. Veshchestva 1996, 75.

1997ACR/POW W. E. Acree, Jr., J. R. Powell, S. A. Tucker, M. D. M. C. Ribeiro da Silva, M. A. R. Matos, J. M. Goncalves, L. M. N. B. F. Santos, V. M. F. Morais, and G. Pilcher, J. Org. Chem. 62, 3722 (1997).

1997BAE

1997BAR/BRU G. Bardi, B. Brunetti, E. Ciccariello, and V. Piacente, J. Alloys Compd. 247, 202 (1997).

1997BRU/GIG B. Brunetti, G. Gigli, E. Giglio, V. Piacente, and P. Scardala, J. Phys. Chem. B 101, 10715 (1997).

1997BRU/GIU B. Brunetti, A. Giustini, and V. Piacente, J. Chem. Thermodyn. 29, 239 (1997).

1997BRU/PAL B. Brunetti, C. Palchetti, and V. Piacente, J. Mater. Sci. Lett. 16, 1395 (1997).

1997BUR/ROL A. Burger, J. M. Rollinger, and R. Brueggellar, J. Pharm. Sci. 86, 674 (1997).

1997CAI/GRA X. Cai, D. J. W. Grant, and T. S. Wiedmann, J. Pharm. Sci. 86, 372 (1997).

1997CEN/MEL Z. Cenuse and V. Meltzer, Analele Universit F. Atii Bucure W. Sti. Chimie 6, 101 (1997).

1997CHA/YUE K.-T. Chan, K.-H. Yuen, H. Takayangi, S. Janadasa, and K.-K. Peh, Phytochemistry 46, 1209 (1997).

1997CHI/WIL J. S. Chickos and J. A. Wilson, J. Chem. Eng. Data 42, 190 (1997).

1997CIO/MEL I. Ciocazanu, V. Meltzer, A. Nicolae, and R. Vilcu, Calorim. Anal. Therm. 28, 267 (1997).

1997DAS/DAS J. P. Dasilva and A. M. Dasilva, J. Chem. Eng. Data 42, 538 (1997).

1997DEF/CAR D. R. Defibaugh, E. Carrillo-Nava, J. J. Hurly, M. R. Moldover, J. W. Schmidt, and L. A. Weber, J. Chem. Eng. Data 42, 488 (1997).

1997DES/DES A. G. de Souza, F. de Souza Neto, J. H. de Souza, R. O. Macedo, J. B. L. de Oliveira, and C. D. Pinheiro, J. Therm. Anal. 49, 679 (1997).

1997DEZ/POI V. de Zea Bermudez, C. Poinsignon, and M. B. Armand, J. Mater. Chem. 7, 1677 (1997).

1997ELD

1997GAR/RED

J. P. Elder, J. Therm. Anal. 49, 897 (1997)

Roux, J. Chem. Soc., Faraday Trans. 93, 2509 (1997).

1997GIL/BOT E. G. Gillan, S. G. Bott, and A. R. Barron, Chem. Mater. 9, 796 (1997).

1997 GOO M. A. Goodman, J. Chem. Eng. Data 42, 1227 (1997).

1997GUD/TOR R. Gudino, L. A. Torres, M. Campos, R. L. Santillan, and N. Farfan, J. Chem. Thermodyn. 29, 565 (1997).

1997HIL/MUL G. E. Hildebrand and C. C. Muller-Goymann, J. Pharm. Sci. 86, 854 (1997).

1997IBR/FRA W. G. A. Ibrom and A. W. Frahm, Arzneim.-Forsch./Drug Res. 47, 662 (1997).

1997JIM/ROU P. Jimenez, M. V. Roux, J. Z. Davalos, M. A. MarrtinLuengo, and J. L. M. Abboud, J. Chem. Thermodyn. 29, 1281 (1997).
1997KER/LOC

P. Kerkoc, S. Lochran, R. T. Bailey, F. R. Cruickshank, D. Pugh, and J. Sherwood, J. Appl. Phys. 81, 624 (1997); Chem. Abstr. 126, 136332n (1997).

1997KOJ/KAD Y. Kojima, H. Kadokura, Y. Okuhara, M. Matsumoto, and T. Mogi, Integr. Ferroelectr. 18, 183 (1997).

1997 KOU/HOS $\quad$ B. Koutek, M. Hoskovec, P. Vrkocova, and L. Feltl, J. Chromatogr. A 759, 93 (1997).

1997 KRI/SYS V. V. Krisyuk, S. V. Sysoev, N. E. Fedotova, I. K. Igumenov, and N. V. Grigorieva, Thermochim. Acta 307, 107 (1997).

1997KRO/VEL H. B. Krop, M. J. M. Velzen, J. R. Parsons, and H. A. J. Govers, J. Am. Oil Chem. Soc. 74, 309 (1997).

1997LEB/KUL B. V. Lebedev and T. G. Kulagina, J. Chem. Thermodyn. 29, 595 (1997).

1997LUK/KOL V. A. Luk'yanova, V. P. Kolesov, N. V. Avramenko, and V. F. Vorob'eva, Russ. J. Phys. Chem. 71, 338 (1997).

1997MAK/KAB Y. V. Maksimuk, G. J. Kabo, A. A. Kozyro, V. V. Simirsky, A. P. Krasulin, and V. M. Sevruk, J. Chem. Thermodyn. 29, 687 (1997).

1997MIS/BHA R. Mishra, S. R. Bharadwaj, A. S. Kerkar, and S. R. Dharwadkar, J. Nucl. Mater. 240, 236 (1997).

1997NEA/BHA S. H. Neau, S. V. Bhandarkar, and E. W. Hellmuth, Pharm. Res. 14, 601 (1997).

1997NEM/ACS K. Nemak, M. Acs, D. Kozma, and E. Fogassy, J. Therm. Anal. 48, 691 (1997).

1997PAN/BOS F. Pan, C. Bosshard, M. S. Wong, C. Serbutoviez, K. Schenk, V. Gramlich, and P. Günter, Chem. Mater. 9, 1328 (1997).

1997PIA/PAL V. Piacente, C. Palchetti, G. Gigli, and P. Scardala, J. Phys. Chem. 101, 4303 (1997).

1997RIB/GON M. A. V. Ribeiro da Silva, J. M. Goncalves, and G. Pilcher, J. Chem. Thermodyn. 29, 253 (1997).

1997RIB/MAT3 M. A. V. Ribeiro da Silva, M. A. R. Matos, and L. M. P. F. Amaral, J. Chem. Thermodyn. 29, 1129 (1997).

1997RIB/MAT4 M. A. V. Ribeiro da Silva, M. A. R. Matos, C. M. A. do Rio, and V. M. F. Morais, J. Chem. Soc., Faraday Trans. 93, 3061 (1997).

1997ROU/JIM M. V. Roux, P. Jimenez, M. A. Martin-Luengo, J. Z. Davalos, Z. Sun, R. S. Hosmane, and J. F. Liebman, J. Org. Chem. 62, 2732 (1997).

1997SAB/PER2 R. Sabbah and J. A. G. Perez, Thermochim. Acta 297, 17 (1997).

1997SAK/HOR T. Sako, S. Horiguchi, H. Ichimaru, and S. Nakagawa, J. Chem. Eng. Data 42, 169 (1997).

1997SAN/ROC L. S. Santos, Jr., S. Roca, and C. Airoldi, J. Chem. Thermodyn. 29, 661 (1997).

1997SAT/YAN K. Sato, J. Yano, I. Kawada, M. Kawano, F. Kaneko, and M. Suzuki, J. Am. Oil Chem. Soc. 74, 1153 (1997).

1997SCH/BER W. C. Schinzer, M. S. Bergren, D. S. Aldrich, F. S. Chao, M. J. Dunn, A. Jeganathan, and L. M. Madden, J. Pharm. Sci. 86, 1426 (1997).

1997SCH/VER F. Schaffer, S. P. Verevkin, H.-J. Rieger, H.-D. Beckhaus, and C. Rüchardt, Liebigs Ann./Recl. 1997, 1333.

1997SHI/TAN T. Shimizu, S. Tanaka, N. Onoda-Yamamuro, S. Ishimaru, and R. Ikeda, J. Chem. Soc., Faraday Trans. 93, 321 (1997).

1997SMI/LEB2 N. N. Smirnova, B. V. Lebedev, and V. G. Vasil'ev, Zh. Obshch. Khim. 66, 199 (1997).

1997SQU/NEE E. Squillante, T. Needham, and H. Zia, Int. J. Pharm. 159, 171 (1997).

1997STE/CHI W. V. Steele, R. D. Chirico, A. B. Cowell, S. E. Knipmeyer, and A. Nguyen, J. Chem. Eng. Data 42, 1053 (1997).

1997STE/CHI2 W. V. Steele, R. D. Chirico, S. E. Knipmeyer, and A. Nguyen, J. Chem. Eng. Data 42, 1008 (1997).

1997STE/CHI3 W. V. Steele, R. D. Chirico, S. E. Knipmeyer, and A. Nguyen, J. Chem. Eng. Data 42, 1021 (1997).

1997STE/CHI4 W. V. Steele, R. D. Chirico, S. E. Knipmeyer, A. Nguyen, and N. K. Smith, J. Chem. Eng. Data 42, 1037 (1997).

1997TAN/ATA T. Tanaka, T. Atake, Z. Shi, C. Nakano, T. Enoki, G. Saito, and H. Inokuchi, J. Phys. Chem. Solids 58, 295 (1997).

1997TES/PIK M. Tesconi, M. J. Pikal, and S. H. Yalkowsky, J. Pharm. Sci. 86, 1299 (1997). 
1997TRO/MAR M. C. Tros de Ilardaya, C. Martin, M. M. Coni, and M. C. Martinez-Oharriz, J. Pharm. Sci. 86, 248 (1997).

1997VER

1997VER/MOR S. P. Verevkin, J. Morgenthaler, and C. Rüchardt, J. Chem. Thermodyn. 29, 1175 (1997).

1997VER/NOL S. P. Verevkin, M. Nölke, H.-D. Beckhaus, and C. Rüchardt, J. Org. Chem. 62, 4683 (1997).

1997VER2

1997VER3

1997WEL/VER

S. P. Verevkin, J. Chem. Thermodyn. 29, 1495 (1997).

S. P. Verevkin, Thermochim. Acta 307, 17 (1997).

F. M. Welle, S. P. Verevkin, H.-D. Beckhaus, and C. Rüchardt, Liebigs Ann./Recl. 1977, 155.

1997YUA/YAN Z. Yuan, P. Yang, G. Meng, and D. Peng, Wuji Huaxue Xuebao 13, 168 (1997); Chem. Abstr. 127, 144299t (1997).

1997ZHA/GAO J.-B. Zhang, X.-G. Gao, Z.-C. Tan, S.-H. Meng, L.-M. Zhang, and S.-H. Li, Thermochim. Acta 297, 1 (1997).

1998ADD/CON S. J. Addison, J. A. Connor, and J. A. Kinkaid, J. Organomet. Chem. 554, 123 (1998).

1998ASA/SOR S. Asahina, M. Sorai, and R. Eidenshink, Liq. Cryst. 24, 201 (1998).

1998BOL/MAR O. V. Boltalina, V. Y. Markov, A. Y. Borschevskii, L. N. Sidorov, V. N. Bezmelnitsin, A. V. Eletskii, and R. Taylor, Rapid Commun. Mass Spectrom. 12, 1028 (1998).

1998BOL/MAR2 O. V. Boltalina, V. Y. Markov, A. Y. Borschevskii, V. Y. Davydov, L. N. Sidorov, V. N. Bezmelnitsin, A. N. Eletskii, and R. Taylor, Mendeleev Commun. 8, 141 (1998).

1998BOT/ELL M. Botoshansky, A. Ellern, N. Gasper, J.-O. Henck, and F. H. Herbstein, Acta Crystallogr., Sect. B: Struct. Sci. 54, 277 (1998).

1998BRU/PIA B. Brunetti, V. Piacenti, and P. Scardala, J. Chem. Eng. Data 43, 101 (1998).

1998BUS/PEN P. Bustamante, M. A. Pena, and J. Barra, Int. J. Pharm. 174, 141 (1998).

1998BUS/PEN2 P. Bustamante, M. A. Pena, and J. Barra, J. Pharm. Pharmacol. 50, 975 (1998).

1998BUS/ROM P. Bustamante, S. Romero, A. Pena, B. Escalera, and A. Reillo, J. Pharm. Sci. 87, 1590 (1998).

1998CHI/COW R. D. Chirico, A. B. Cowell, W. D. Good, T. D. Klots, S. E. Knipmeyer, A. A. Nguyen, R. Rau, J. W. Reynolds, N. K. Smith, and W. V. Steele, J. Chem. Thermodyn. 30, 1423 (1998).

1998CHI/HES J. S. Chickos, D. Hesse, S. Hosseini, G. Nichols, and P. Webb, Thermochim. Acta 313, 101 (1998).

1998DOM U. Domanska, Pol. J. Chem. 72, 925 (1998).

1998DOM/VEN U. Domanska and P. Venkatesu, J. Chem. Eng. Data 43, 919 (1998).

1998DRO/TOM K. G. Drouillard, G. T. Tomy, D. C. G. Muir, and K. J. Friesen, Environ. Toxicol. Chem. 17, 1252 (1998).

1998ELA/GIR S. K. El-Arini, D. Giron, and H. Leuenberger, Pharm. Dev. Technol. 3, 557 (1998).

1998FUE/STR H. Fuess and R. Strauss, J. Phys. Chem. B 102, 5329 (1998).

1998GIO/GAZ F. Giordano, A. Gazzaniga, J. R. Moyano, P. Ventura, M. Zanol, T. Peveri, and L. Carima, J. Pharm. Sci. 87, 333 (1998).

1998GON/SZW A. Gonthier-Vassal and H. Szwarc, Thermochim. Acta 320, 141 (1998).

1998GUD/TOR R. Gudino, L. A. Torres, R. L. Santillan, and N. Farfan, J. Chem. Thermodyn. 30, 671 (1998).

1998HIK/OKA T. Hikima, N. Okamoto, M. Hanaya, and M. Oguni, J. Chem. Thermodyn. 30, 509 (1998).

1998ISS/ELA Y. M. Issa, A. L. El-Ansary, and W. Selim, Anal. Lett. 31, 131 (1998)

1998JAM/PAL M. E. Jamroz, M. Palczewska-Tulinska, D. Wyrzykowska-Stankiewicz, A. M. Szafranski, J. Polaczek, J. C. Dobrowolski, M. H. Jamroz, and A. P. Mazurek, Fluid Phase Equilib. 152, 307 (1998).

1998KHO/RYK N. M. Khoretonenko, A. N. Rykov, and Y. M. Korenev, Zh. Neorg. Khim. 43, 584 (1998); Chem. Abstr. 129, 45952k (1998).

1998KIS/KAS V. D. Kiselev, E. A. Kashaeva, and A. I. Konovalov, Russ. J. Gen. Chem. 68, 1246 (1998).
1998KLO/LAU

C. Kloc and R. A. Laudise, J. Cryst. Growth 193, 563 (1998).

1998KOL/PIM V. P. Kolesov, S. M. Pimenova, V. A. Lukyanova, T. S Kuznetsova, and M. P. Kozina, J. Chem. Thermodyn. 30, 1455 (1998).

1998LEB/CHI V. P. Lebedev, V. V. Chirnonov, V. P. Vorob'eva, and Y. N. Matyushin, Khim. Fiz. 17, 54 (1998).

1998MAK/KAB Y. V. Maksimuk, G. J. Kabo, V. V. Simirsky, A. A. Kozyro, and V. M. Sevruck, J. Chem. Eng. Data 43, 293 (1998).

1998MOK/RAU I. Mokbel, E. Rauzy, J. P. Meille, and J. Jose, Fluid Phase Equilib. 147, 271 (1998).

1998MUR/BET P. Mura, G. P. Bettinetti, M. T. Faucci, and P. L. Parrini, Thermochim. Acta 321, 59 (1998).

1998MUR/BET2 P. Mura, G. P. Bettinetti, A. Manderioli, M. T. Faucci, G. Bramanti, and M. Sorrenti, Int. J. Pharm. 166, 189 (1998).

1998 NIL/SOE F. Nilsson, O. Söederman, and I. Johansson, J. Colloid Interface Sci. 203, 131 (1998).

19980JA/SUU V. Oja and E. M. Suuberg, J. Chem. Eng. Data 43, 486 (1998).

1998PAL/WEH G. R. Palmieri, P. Wehrle, and S. Martelli, Drug Dev. Ind. Pharm. 24, 653 (1998).

1998PAN/MAL R. Pankajavalli, C. Mallika, O. M. Sreedharan, M. Premila, and P. Gopalan, Thermochim. Acta 316, 101 (1998).

1998PAP/KOL T. S. Papina, V. P. Kolesov, V. A. Lukyanova, V. F. Golovkov, C. A. Chernov, and V. P. Vorobieva, J. Chem. Thermodyn. 30, 431 (1998).

1998PAP/LUK T. S. Papina, V. A. Luk'yanova, V. P. Golovkov, S. A. Chernov, and V. P. Vorob'eva, Zh. Fiz. Khim. 72, 7 (1998).

1998PRI/HAW ～D. M. Price and M. Hawkins, Thermochim. Acta 315, 19 (1998).

1998RAI/RAI U. S. Rai and R. N. Rai, J. Therm. Anal. 53, 883 (1998).

1998REI/ZIM G. Reinwald and I. Zimmermann, J. Pharm. Sci. 87, 745 (1998).

1998RIB/GON M. A. V. Ribeiro da Silva and J. M. Goncalves, J. Chem. Thermodyn. 30, 1465 (1998).

1998RIB/MAT3 M. A. V. Ribeiro da Silva, M. A. R. Matos, and J. M. Goncalves, J. Chem. Thermodyn. 30, 299 (1998).

1998ROB/MON L. Robles, D. Mondieig, Y. Haget, and M. A. CuevasDiarte, J. Chim. Phys. Phys.-Chim. Biol. 95, 92-111 (1998).

1998RUU/MOK K. Ruuzicka, I. Mokbel, V. Majer, V. Ruuzicka, J. Jose, and M. Zabransky, Fluid Phase Equilib. 148, 107 (1998).

1998SHE/NIS M. S. Sheiman, V. P. Nistratov, and G. P. Kamelova, Russ. J. Phys. Chem. 72, 1620 (1998).

1998SOR/NEG M. Sorrenti, A. Negri, and G. P. Bettinetti, J. Therm. Anal. 51, 993 (1998).

1998STE/ZAW Z. Stec and J. Zawadiak, Pol. J. Appl. Chem. XLII, 237 (1998).

1998STO/KRZ P. Storoniak, K. Krzyminski, and J. Blazejowski, J. Therm. Anal. 54, 183 (1998).

1998VAN/AUG G. Van den Mooter, P. Augustijns, and R. Kinget, Int. J. Pharm. 164, 81 (1998).

1998VAN/KEL P. J. C. M. van Hoof and E. Kellenbach, J. Therm. Anal. Calorim. 53, 957 (1998).

1998VAN/VAN J. C. van Miltenburg, A. C. G. van Genderen, and G. J. K. van den Berg, Thermochim. Acta 319, 151 (1998).

1998VER

1998VER/BEC S. P. Verevkin, J. Chem. Thermodyn. 30, 1029 (1998).

1998VER/KUM S. P. Verevkin, H.-D. Beckhaus, U. Schüle, and C. Rüchardt, Struct. Chem. 9, 1 (1998).

$\begin{array}{ll} & \text { and C. Rüchardt, Eur. J. Org. Chem. 1998, } 579 . \\ \text { S. P. Verevkin, W.-H. Peng, H. D. Beckhaus, and C. }\end{array}$ S. P. Verevkin, W.-H. Peng, H. D. Beckh
Rücuardt, Eur. J. Org. Chem. 1998, 2323.

1998VER/WEL S. P. Verevkin and F. M. Welle, Struct. Chem. 9, 215 (1998).

1998VER3 S. P. Verevkin, Struct. Chem. 9, 375 (1998).

1998VER4 S. P. Verevkin, Thermochim. Acta 310, 229 (1998).

1998VER5

1998VER6

1998VER7
S. P. Verevkin, J. Chem. Thermodyn. 30, 389 (1998).

S. P. Verevkin, Thermochim. Acta 316, 131 (1998).
S. P. Verevkin, Struct. Chem. 9, 113 (1998). 
1998ZEL/MIN L. N. Zelenina, Y. F. Minenkov, Y. G. Stenin, V. A. Titov, and T. P. Chusova, Zh. Fiz. Khim. 72, 803 (1998).

1998ZHA/TAN J.-B. Zhang, Z.-C. Tan, X.-G. Gao, S.-H. Meng, and L. Li, Thermochim. Acta 322, 89 (1998).

1999ALI/MAL A. S. Alikhanyan, I. P. Malkerova, N. P. Kuz'mina, A. Gleizes, M. Julve-Olsina, J. L. Sanz, and I. L. Eremenko, Zh. Neorg. Khim. 44, 969 (1999); Chem. Abstr. 131, 234185q (1999).

1999BAL/WEI J. E. Baldvins and R. G. Weiss, Liq. Cryst. 26, 897 (1999). 1999BAR/BER M. Bartolomei, P. Bertocchi, M. C. Ramusino, N. Santucci, and L. Valvo, J. Pharm. Biomed. Anal. 21, 299 (1999).

1999BER/ZEL G. A. Berezovskii, L. M. Zelenina, T. P. Chusova, and I. E. Paukov, Russ. J. Phys. Chem. 73, 1347 (1999).

1999BET/MUR G. Bettinetti, P. Mura, M. Sorrenti, M. T. Faucci, and A. Negri, J. Pharm. Sci. 88, 1133 (1999).

1999BET/SOR G. Bettinetti, M. Sorrenti, A. Negri, M. Setti, P. Mura, and F. Melani, Pharm. Res. 16, 689 (1999).

1999BOL/MAR O. V. Boltalina, V. Y. Markov, A. Y. Borschevskii, N. A. Galeva, L. N. Sidorov, G. Gigli, and G. Balducci, J. Phys. Chem. B. 103, 3828 (1999).

1999BRU/PIA B. Brunetti and V. Piacenti, J. Chem. Eng. Data 44, 809 (1999).

1999BRU/VAS B. Brunetti, P. Vassalo, V. Piacenti, and P. Scardala, J. Chem. Eng. Data 44, 509 (1999).

1999CHA/GAR D. Chandra, M. L. Garner, and K. H. Lau, J. Phase Equilib. 20, 565 (1999).

1999DEG/GUI E. T. De Givenchy, F. Guittard, F. Bracon, and A. Cambon, Liq. Cryst. 26, 1371 (1999).

1999DEL/TOR P. De La Torre, S. Torrado, and S. Torrado, Chem. Pharm. Bull. 47, 1629 (1999).

1999DIB/LUS V. N. Dibrivnyi, G. V. Lustiv, Y. Y. Van-Chin-Syan, A. P. Yuvchenko, and E. A. Dikusar, Russ. J. Phys. Chem. 73, 2040 (1999).

1999DIB/PIS $\quad$ V. N. Dibrivnyi, Z. E. Pistun, Y. Y. Van-Chin-Syan, A. P. Yuvchenko, and T. D. Zvereva, Zh. Fiz. Khim. 73, 2257 (1999).

1999DIB/PIS2 V. N. Dibrivnyi, Z. E. Pistun, Y. Y. Van-Chin-Syan, A. Yuvchenko, and T. D. Zvereva, Russ. J. Phys. Chem. 73, 2043 (1999).

1999DOM/KAR L. G. Domracheva, N. V. Karyakin, M. S. Sheiman, G. V. Kamelova, V. N. Larina, O. N. Suvorova, and G. A. Domrachev, Russ. Chem. Bull. 48, 1647 (1999).

1999DOU/BOT A. G. Douglass, B. Both, and P. Kaszynski, J. Mater. Chem. 9, 683 (1999).

1999DRU/GAL A. I. Druzhinina, N. A. Galeva, R. M. Varushchenko, and O. V. Boltalina, J. Chem. Thermodyn. 31, 1469 (1999).

1999DYK/SVO J. Dykyj, J. Svoboda, R. C. Wilhoit, M. L. Frenkel, and K. R. Hall, Vapor Pressure of Chemicals: Part A. Vapor Pressure and Antoine Constants for Hydrocarbons and Sulfur, Selenium, Tellurium and Hydrogen Containing Organic Compounds (Springer, Berlin, 1999); Note: The vaporization enthalpies were calculated from the vapor pressures obtained from the Antoine constants reported in this compendium. In cases where the Antoine constant $\mathrm{C}=0$, the Antoine Equation $(\log 10 \mathrm{P}=\mathrm{A}-\mathrm{B} /(\mathrm{C}+\mathrm{T}))$ reduces to the integrated form of the Clausius Clapeyron equation directly. This was the case for many vaporization enthalpies. In those cases where this condition was not met the vaporization enthalpy was calculated as $\operatorname{DvapHm}(\mathrm{T})$ $=2.303 \mathrm{R} \mathrm{B}[\mathrm{T} /(\mathrm{T}+\mathrm{C})] 2$.

1999EMM/PIC F. Emmenegger and M. Piccand, J. Therm. Anal. Calorim. 57, 235 (1999).

1999GAL/COL Z. Galewski and H. J. Coles, J. Mol. Liq. 79, 77 (1999). 1999GIL 1999GIO/BET

1999HEN/KUH J.-O. Henck and M. Kuhnert-Brandstaetter, J. Pharm. Sci. 88, 103 (1999).

1999HUd/SHE $\quad$ C. M. Hudson, R. A. Shenoy, M. E. Neubert, and R. G. Petschek, Liq. Cryst. 26, 241 (1999).

E. P. Gilbert, Phys. Chem. Chem. Phys. 1, 1517 (1999).

F. Giordano, R. Bettini, C. Donini, A. Gazzaniga, M. R. Caira, G. G. Z. Zhang, and D. J. W. Grant, J. Pharm. Sci.
1999HUZ/SAI

1999JIM/ROU

1999KEY/BOT

1999KIM/HIR

1999KOL/DOR

1999KUL/LEB

1999LEB/KUL2

1999LI/ZEL

1999LIN/KO

1999MAY/WIT

1999MET/RAJ

1999MON/HIL

1999MUR/FAU

1999NEV/GOU

1999NGU/BER

1999NIS/SHE

1999OGA/NAK

1999OJA/SUU

1999PRI/HAW

1999RAI/SHE

1999RIB/FER

1999RIB/MAT2

1999RIB/MON

1999ROM/ESC

1999ROS/MOL

1999ROU/DAV

1999SAN/PET

1999TAF/GUI

1999TAH/TAK

1999TAS/ISH

1999TIT/ZEL

1999TIT/ZEL2

1999VAN/TEN

1999VER
S. Huzisawa, K. Saito, and I. Ikemoto, J. Phys. Chem. Solids 60, 723 (1999).

P. Jimenez, M. V. Roux, J. Z. Davalos, J. L. M. Abboud, and M. T. Molina, J. Chem. Thermodyn. 31, 263 (1999).

A. Keys, S. G. Bott, and A. R. Barron, Chem. Mater. 11, 3578 (1999).

K. Kimura, F. Hirayama, and K. Uekama, J. Pharm. Sci. 88, 385 (1999).

V. P. Kolesov, O. V. Dorofeeva, V. S. Iorish, T. S. Papina, V. A. Lukyanova, and S. V. Melkhanova, Mendeleev Commun. 9, 143 (1999).

A. P. Korobko, I. V. Levakova, S. V. Krasheninnikov, A. I. Stash, N. A. Kon'kova, V. V. Kuz'min, N. V. Kozlova, and T. A. Korobko, Kristallografiya 44, 262 (1999).

T. G. Kulagina and B. V. Lebedev, Russ. J. Phys. Chem. 73, 1909 (1999)

B. V. Lebedev, T. G. Kulagina, and D. R. Tur, J. Chem. Thermodyn. 31, 697 (1999).

Z. J. Li, M. T. Zell, E. J. Munson, and D. J. W. Grant, J. Pharm. Sci. 88, 337 (1999).

H. C. Lin, C.-W. Ko, K. Guo, and T. W. Cheng, Liq. Cryst. 26, 613 (1999).

J. Mayer, W. Witko, M. Massalska-Arodz, G. Williams, and R. Dabrowski, Phase Transitions 69, 199 (1999).

V. Metivaud, F. Rajabalee, H. A. J. Oonk, D. Mondieig, and Y. Haget, Can. J. Chem. 77, 332 (1999).

M. J. S. Monte and D. M. Hillesheim, J. Chem. Thermodyn. 31, 1443 (1999).

P. Mura, M. T. Faucci, P. L. Parrini, S. Furlanetto, and S. Penzauti, Int. J. Pharm. 179, 117 (1999).

L. P. M. Neves, A. Gouveia de Souza, J. B. L. de Oliveira, and C. Airoldi, Thermochim. Acta 328, 217 (1999).

J. N'Guimbi, C. Berro, I. Mokbel, E. Rauzy, and J. Jose, Fluid Phase Equilib. 162, 143 (1999).

V. P. Nistratov, M. S. Sheiman, and G. P. Kamelova, Russ. J. Phys. Chem. 73, 842 (1999).

Y. Ogawa and N. Nakamura, Bull. Chem. Soc. Jpn. 72, 943 (1999).

V. Oja and E. M. Suuberg, J. Chem. Eng. Data 44, 26 (1999).

D. M. Price and M. Hawkins, Thermochim. Acta 329, 73 (1999).

U. S. Rai and H. Shekhar, Asian J. Chem. 11, 453 (1999). M. D. M. C. Ribeiro da Silva, M. L. C. C. H. Ferrao, M. J. S. Monte, J. M. Goncalves, and F. Jiye, J. Chem. Thermodyn. 31, 1067 (1999).

M. A. V. Ribeiro da Silva, M. A. R. Matos, V. M. F. Morais, and M. S. Miranda, J. Org. Chem. 64, 8816 (1999).

M. A. V. Ribeiro da Silva, M. J. S. Monte, and J. R. Ribeiro, J. Chem. Thermodyn. 31, 1093 (1999).

S. Romero, B. Escalera, and P. Bustamante, Int. J. Pharm. 178, 193 (1999).

F. Ros and M. T. Molina, Eur. J. Org. Chem. 1999, 3179. M. V. Roux, J. Z. Davalos, P. Jimenez, H. Flores, J. L. Saiz, J. L. M. Abboud, and E. Juaristi, J. Chem. Thermodyn. 31, 635 (1999).

L. S. Santos, Jr., G. P. Petrucelli, and C. Airoldi, Polyhedron 18, 969 (1999); Chem. Abstr. 131, 50237 (1999).

E. Taffin de Givenchy, F. Guittard, F. Bracon, and A. Cambon, Liq. Cryst. 26, 1163 (1999).

Y. Tahira and Y. Taketani, Patent, Chem. Abstr. 130, 124888 (1999).

Y. Tasaki, J. Ishiai, S. Yoshizawa, and Y. Kuniya, Nippon Kagaku Kaishi 1, 45 (1999); Chem. Abstr. 130, 190408 (1999).

V. A. Titov, L. N. Zelenina, and T. P. Chusova, Zh. Fiz. Khim. 73, 951 (1999).

V. A. Titov, L. N. Zelenina, and T. P. Chusova, Russ. J. Phys. Chem. 73, 835 (1999).

J. C. van Miltenburg and E. ten Grotenhuis, J. Chem. Eng. Data 44, 721 (1999).

S. P. Verevkin, J. Chem. Thermodyn. 31, 1397 (1999). 
1999VER/EBE S. P. Verevkin and J. Ebenhoch, Struct. Chem. 10, 401 (1999).

1999VER/HEI S. P. Verevkin and A. Heintz, J. Chem. Eng. Data 44, 1240 (1999).

1999VER2

1999VER3

1999VER5

1999VER6

1999VER7

1999VER8

1999VIS/TER

1999WAN/TAN

1999WIN

1999XUE/TAN

S. P. Verevkin, J. Chem. Thermodyn. 31, 559 (1999).

S. P. Verevkin, J. Chem. Eng. Data 44, 557 (1999).

S. P. Verevkin, J. Chem. Eng. Data 44, 175 (1999).

S. P. Verevkin, Thermochim. Acta 326, 17 (1999).

S. P. Verevkin, Phys. Chem. Chem. Phys. 1, 127 (1999).

S. P. Verevkin, Thermochim. Acta 332, 27 (1999).

J. Visjager, T. A. Tervoort, and P. Smith, Polymer 40, 4533 (1999).

L. Wang, Z.-C. Tan, S.-H. Meng, and D.-B. Liang, Thermochim. Acta 342, 59 (1999).

G. Winter, "Polymorphs and solvates of molecular solids in the pharmaceutical industry," in Reactivity of Molecular Solids, edited by E. Boldyreva and V. Boldyrev (John Wiley and Sons, New York, NY, 1999), Chap. 7.

B. Xue, Z.-C. Tan, S.-W. Lu, S.-H. Meng, and X.-H. Yuan, Huaxue Xuebao 57, 881 (1999); Chem. Abstr. 131, 224854 (1999).

1999ZAD/KER D. Zadravec, J. Kerc, and J. Kristl, Farm. Vest. (Ljubljana) 50, 350 (1999); Chem. Abstr. 132, 313492 (2000).

1999ZEM/STA S. M. Zemskova, P. A. Stabnikov, S. V. Susoev, and I. K. Igumenov, Electrochem. Soc., Proc. 98-23, 286 (1999).

1999ZIE/GOL W. Zielenkiewicz, B. Golankiewicz, G. L. Pelovich, and M. Kozbial, J. Solution Chem. 28, 731 (1999).

2000ABD/WEI D. J. Abdallah and R. G. Weiss, Chem. Mater. 12, 406 (2000).

2000AKU/IUC T. Akutagawa, K. Iuchi, and Y. Matsunaga, Liq. Cryst. 27, 1399 (2000)

2000BEC/FAU H.-D. Beckhaus, R. Faust, D. L. Matzger, D. L. Mohler, D. W. Rogers, C. Rüchardt, A. K. Sawhney, S. P. Verevkin, K. P. C. Volhardt, and S. Wolff, J. Am. Chem. Soc. 122, 7819 (2000).

2000BLA/LUC A. E. Blatch and G. R. Luckhurst, Liq. Cryst. 27, 775 (2000).

2000BOL/GAL O. V. Boltalina and N. A. Galeva, Russ. Chem. Rev. 69, 609 (2000)

2000BRU/VIL B. Brunetti, A. R. Villani, V. Piacente, and P. Cardala, J. Chem. Eng. Data 45, 231 (2000).

2000BUR/LET A. Burger and A. Lettenbichler, Eur. J. Pharm. Biopharm. 49, 65 (2000).

2000BUR/VAN A. Burger and C. Van den Boom, Mikrochim. Acta 135, 63 (2000).

2000BYK/LEB T. A. Bykova, B. V. Lebedev, N. V. Ushakov, and E. S. Finkel'shtein, Polym. Sci., Ser. A 42, 863 (2000); Note: Compound used was only $83 \%$ crystalline. Authors reported a corrected value of $7.77 \mathrm{~kJ} \mathrm{~mol}^{-} 1$ for the enthalpy of fusion for a sample of $100 \%$ degree of crystallinity.

2000DAN/PRO G. H. Danila, L. Profire, G. G. Bumbu, and C. Vasile, Thermochim. Acta 343, 69 (2000).

2000DEA/SOU J. A. de A. Imeida Sales, A. G. Souza, G. F. Goncalves de Freitas, S. Prasad, M. F. S. Trindade, L. H. Carvalho, and P. O. Dunstan, Thermochim. Acta 356, 9 (2000).

2000DEF/VAN K. M. De Fina, T. T. Van, K. A. Fletcher, and W. E. Acree, Jr., Can. J. Chem. 78, 449 (2000).

2000DI/TAN2 Y.-Y. Di, Z.-C. Tan, Z.-N. Li, Y.-J. Song, Y. Zheng, S.-H. Meng, and S.-S. Qu, Thermochim. Acta 362, 7 (2000).

2000DIM/PAL P. Di Martino, G. F. Palmieri, and S. Martelli, Pharmazie 55, 625 (2000).

2000DRU/VAR A. I. Druzhinina, R. M. Varushchenko, V. S. Sarisova, and A. A. Pimerzin, Zh. Fiz. Khim. 74, 404 (2000).

2000DRU/VAR2 A. I. Druzhinina, R. M. Varushchenko, V. S. Sarkisova, and A. A. Pimerzin, Russ. J. Phys. Chem. 74, 333 (2000).

2000DUN

2000EME/NIK P. O. Dunstan, Thermochim. Acta 356, 19 (2000).

Sukhoverkhov, Russ. J. Inorg. Chem. 45, 1565 (2000).

2000FAH/BAR $\quad$ B. D. Fahlman and A. R. Barron, Adv. Mater. Opt. Electron. 10, 223 (2000).

2000GIE E. Giera, J. Chem. Thermodyn. 32, 821 (2000).

2000HAN/PAR $\quad$ B. C. Hancock and M. Parks, Pharm. Res. 17, 397 (2000).
2000IHN/VEN

2000KAM/YOS

P. M. Ihnat, J. L. Vennerstrom, and D. H. Robinson, J. Pharm. Sci. 89, 1525 (2000).

M. Kamigauchi, M. Yoshida, K. Saiki, M. Sugiura, J. Nishijo, Y. In, and T. Ishida, Bull. Chem. Soc. Jpn. 73, 1233 (2000).

2000KOR/DOR M. V. Korobov, P. A. Dorozhko, A. S. Lobach, A. A. Popov, and V. M. Senyavin, Schr. Forschungszent. Juelich, Reihe Energietech./Energy Technol. 15, 371 (2000).

2000LEB/SMI B. V. Lebedev, N. N. Smirnova, V. S. Papkov, M. I. Buzin, and I. I. Dubovik, Polym. Sci., Ser. A 42, 1111 (2000).

2000LIA/MA Y.-H. Liang and P.-S. Ma, Shiyou Huagong 29, 939 (2000).

2000LIS/JAM Z. Lisicki and M. E. Jamroz, J. Chem. Thermodyn. 32, 1335 (2000)

2000MAC/COU J. J. B. Machado, J. A. Coutinho, and E. A. Macedo, Fluid Phase Equilib. 173, 121 (2000).

2000MAH/SOL R. Mahmoud, R. Solimando, M. Bouroukba, and M. Rogalski, J. Chem. Eng. Data 45, 433 (2000).

2000MAR/BOL V. Y. Markov, O. V. Boltalina, A. A. Gorjunkov, A. Y. Lukonin, L. N. Sidorov, G. Gigli, G. Balducci, and R. Taylor, Proc. - Electrochem. Soc. 2000-12, 109 (2000); Chem. Abstr. 134, 121607x (2001).

2000MAR/MIK A. Marbeuf, D. Mikailitchenko, P. Negrier, M.-A. CuevasDiarte, and T. Calvet-Pallas, Chem. Mater. 12, 3280 (2000).

2000MEL/PIM S. V. Melkhanova, S. M. Pimenova, V. P. Kolesov, A. A. Pimerzin, and V. S. Sarkisova, J. Chem. Thermodyn. 32, 1311 (2000).

2000MOK/RUZ I. Mokbel, K. Ruzicka, V. Majer, V. Ruzicka, M. Ribeiro, J. Jose, and M. Zabransky, Fluid Phase Equilib. 169, 191 (2000).

2000MOR/HAR K. Moriya, F. Harada, S. Yano, and S. Kagabu, Liq. Cryst. 27, 1647 (2000)

2000MOR/SEM N. B. Morozova, P. P. Semyannikov, S. V. Sysoev, V. M. Grankin, and I. K. Igumenov, J. Therm. Anal. Calorim. 60, 489 (2000).

2000NAK/SHI N. Nakasone, K. Shiokawa, Y. Urabe, and N. Nemoto, J. Phys. Chem. B 104, 7483 (2000).

2000NIC/ORF G. Nichols, J. Orf, S. M. Reiter, J. Chickos, and G. W. Gokel, Thermochim. Acta 346, 15 (2000).

2000OVA/KOU P. V. Ova, B. Koultek, and M. Hoskovec, in Practice Orietned Results on Use and Production of Neem Ingredients and Pheromones VI, edited by H. Kleeberg and C. P. W. Zebit (Druck and Graphic, Giessen, 2000), pp. 211-218.

2000PAP/KOL T. S. Papaina, V. P. Kolesova, V. A. Lukyanova, O. V. Boltalina, A. Y. Lukonin, and L. N. Sidorov, J. Phys. Chem. B 104, 5403 (2000).

2000PAS/KOR L. L. Pashenko, M. V. Korpunova, M. V. Varushchenko, and A. I. Druzhinina, Zh. Fiz. Khim. 74, 1007 (2000).

2000PAU/MEH I. Paunovic and A. K. Mehrotra, Thermochim. Acta 356, 27 (2000)

2000PER/GOL G. L. Perlovich, O. A. Golubchikov, and M. E. Klueva, J. Porphyrins Phthalocyanines 4, 699 (2000).

2000PUN P. Punchaipetch, Ph.D. dissertation, University of North Texas, Denton, TX, 2000.

2000RIB/MAT2 M. A. V. Ribeiro da Silva, M. A. R. Matos, C. A. Rio, V. M. F. Morais, J. Wang, G. Nichols, and J. S. Chickos, J. Phys. Chem. A 104, 1774 (2000).

2000RIB/RIB M. A. V. Ribeiro da Silva, M. D. M. C. Ribeiro da Silva, L. C. M. Silva, F. Dietz, and E. Hoyer, J. Chem. Thermodyn. 32, 1113 (2000).

2000ROH/CEN V. Rohac, M. Censky, D. Zala, V. Ruzicka, K. Ruzicka, K. Sporka, and K. Aim, J. Chem. Eng. Data 45, 1205 (2000).

2000ROU/JIM M. A. Roux, P. Jimenez, J. Z. Davalos, M. A. MartinLuengo, V. M. Rotello, A. O. Cuello, and J. F. Liebman, Struct. Chem. 11, 1 (2000).

2000SCH/MAT E. Schonherr, K. Matsumoto, and K. Murakami, Proc. Electrochem. Soc. 2000-12, 89 (2000); Chem. Abstr. 134, 121606w (2001).

2000SEM/BAS P. P. Semyannikov, T. V. Basova, V. M. Grankin, and I. K. Igumenov, J. Porphyrins Phthalocyanines 4, 271 (2000). 
2000SIR/HER E. B. Sirota, A. B. Herhold, and M. Varma-Nair, J. Chem. Phys. 113, 8225 (2000).

2000SOU/OLI A. G. Souza, M. M. Oliveira, A. D. Gondim, P. O. Dunstan, and D. M. de A. Melo, Thermochim. Acta 344, 29 (2000).

2000SZT/BAE $\quad$ B. Sztaray and T. Baer, J. Am. Chem. Soc. 122, 9219 (2000).

2000UNO/ITO H. Uno, S. Ito, M. Wada, H. Watanabe, M. Nagai, A. Hayashi, T. Murashima, and N. Uno, J. Chem. Soc., Perkin Trans. 1 2000, 4347.

2000VER/HEI S. P. Verevkin and A. Heintz, J. Chem. Thermodyn. 32, 1169 (2000).

2000VER/SCH S. P. Verevkin and C. Schick, J. Chem. Eng. Data 45, 946 (2000).

2000VER/WAN S. P. Verevkin, D. Wandschneder, and A. Heintz, J. Chem. Eng. Data 45, 618 (2000).

2000VER3 S. P. Verevkin, J. Chem. Thermodyn. 32, 247 (2000).

2000VIL/BRU A. R. Villani, B. Brunetti, and V. Piacente, J. Chem. Eng. Data 45, 823 (2000).

2000 VIL/BRU2 A. R. Villani, B. Brunetti, and V. Piacente, J. Chem. Eng. Data 45, 1167 (2000).

2000WAN/WAN L.-S. Wang and X.-L. Wang, J. Chem. Eng. Data 45, 743 (2000).

2000WEN/BAU R. M. Wenslow, M. W. Baum, R. G. Ball, J. M. McCauley, and R. J. Varsolona, J. Pharm. Sci. 89, 1271 (2000).

2000WU/TAN X.-M. Wu, Z.-C. Tan, Y. Ji-Song, S.-H. Meng, and S.-S. $\mathrm{Qu}$, Thermochim. Acta 346, 57 (2000).

2000WU/WAN L.-H. Wu, Y.-C. Wang, and C.-S. Hsu, Liq. Cryst. 27, 1503 (2000).

2000YOU/DOL P. H. Young, D. Dollimore, and C. A. Schall, J. Therm. Anal. Calorim. 62, 163 (2000).

2000YU/MEN S. Yu, S. Meng, Z. Tan, L. Li, and J. Zhang, Taiyangneng Xuebao 21, 171 (2000); Chem. Abstr. 133, 166195 (2001).

2000YU/STE L. Yu, G. A. Stephenson, C. A. Mitchell, C. A. Bunnell, S. V. Snorek, J. J. Bowyer, T. B. Borchardt, J. G. Stowell, and S. R. Byrn, J. Am. Chem. Soc. 122, 585 (2000).

$2000 Z$ ZHA/STA G. I. Zharkova, P. A. Stabnikov, V. M. Grankin, P. P. Semyannikov, and I. K. Igumenov, Russ. J. Coord. Chem. 26, 576 (2000).

2001ASA/SOR S. Asahina and M. Sorai, Liq. Cryst. 28, 1085 (2001).

2001BAE A. K. Baev, Izv. Vyssh. Uchebn. Zaved., Khim. Khim. Tekhnol. 44, 3 (2001).

2001BAE/CHE A. K. Baev and I. N. Chernyak, Russ. J. Inorg. Chem. 46, 310 (2001); Zh. Neorg. Khim. 46, 361 (2001); Note: enthalpies of vaporization are presented graphically in paper for binary mixtures.

2001BAE/MIK A. K. Baev and V. E. Mikhailov, Russ. J. Appl. Chem. 74, 1435 (2001).

2001BAE2 A. K. Baev, Russ. J. Appl. Chem. 74, 162 (2001).

2001BUR/JOS N. Bureau, J. Jose, I. Mokbel, and J.-C. de Hemptinne, J. Chem. Thermodyn. 33, 1485 (2001).

$2001 \mathrm{CED} / \mathrm{PRI} \quad$ F. O. Cedeno, M. M. Prieto, A. Espina, and J. R. Garcia, Thermochim. Acta 369, 39 (2001).

2001CHA/DOL K. Chatterjee, D. Dollimore, and K. Alexander, Instrum. Sci. Technol. 29, 133 (2001); Chem. Abstr. 135, 16629t (2001); Note: reported values are referred to as enthalpies of vaporization.

2001CHA/TOB S. Chattopadhyay, H. J. Tobias, and P. J. Ziemann, Anal. Chem. 73, 3797 (2001).

2001CHE/BOU V. Chevallier, M. Bouroukba, D. Petitjean, D. Barth, P. Dupuis, and M. Dirand, J. Chem. Eng. Data 46, 1114 (2001).

2001COL/LAU C. Colominas, K. H. Lau, D. L. Hildenbrand, S. CrouchBaker, and A. Sanjurjo, J. Chem. Eng. Data 46, 446 (2001).

2001DAB/MIS M. Dabrowski, B. Misterkiewicz, and A. Sporzynski, J. Chem. Eng. Data 46, 1627 (2001).

2001DAM/BLA F. Damian, N. Blaton, P. Auguestijns, L. Naesens, J. Balzarini, R. Kinget, and G. Van den Mooter, Thermochim. Acta 366, 61 (2001).

2001DI/SUN Y.-Y. Di, X.-H. Sun, Y.-F. Liu, Z.-C. Tan, S.-H. Meng, S.-L. Gao, and S.-S. Qu, Huaxue Xuebao 59, 496 (2001); Chem. Abstr. 134, 372309w (2002).
2001DIN/MUR

T. J. Dingemans, N. S. Murthy, and E. T. Samulski, J. Phys. Chem. B 105, 8845 (2001).

2001DIO/PIE

H. P. Diogo, M. E. Minas da Piedade, J. M. Goncalves, M. J. S. Monte, and M. A. V. Ribeiro da Silva, Eur. J. Inorg. Chem. 2001, 257.

2001DOR/LOB P. A. Dorozhko, A. S. Lobach, A. A. Popov, V. M. Senyavin, and M. V. Korobov, Chem. Phys. Lett. 336, 39 (2001).

2001EDW/SKE A. D. Edwards, B. Y. Skekunov, R. T. Forbes, J. G. Grossmann, and P. York, J. Pharm. Sci. 90, 106 (2001).

2001FED/GEL N. E. Fedotova, N. V. Gelfond, I. K. Igumenov, A. N. Mikheev, N. B. Morozova, and R. H. Tuffias, Int. J. Therm. Sci. 40, 469 (2001).

2001FOR/HEM A. Forster, J. Hempenstall, I. Tucker, and T. Rades, Int. J. Pharm. 226, 147 (2001).

$2001 \mathrm{GIO}$

2001GIO/TER

D. Giordano, J. Chem. Eng. Data 46, 486 (2001).

2001GIO/TER

J. Giovanni, L. Ter Minassian, R. Ceolin, S. Toscani, M.-A. Perrin, D. Louer, and F. Leveiller, J. Phys. IV France 11, Pr10-123 (2001).

2001HE/GRI

X. He, U. J. Griesser, J. G. Stowell, T. B. Borchardt, and S. B. Byrn, J. Pharm. Sci. 90, 371 (2001).

2001IWA/MIN M. Iwahashi, H. Minami, T. Suzuki, M. Koyanagi, H. Hao, K. Ema, and K. Ashizawa, J. Oleo Sci. 50, 693 (2001).

2001JAM/DOB M. E. Jamroz, J. C. Dobrowolski, J. Placzek, A. M. Szafranski, J. K. Kazimirski, and Z. Lisicki, J. Chem. Thermodyn. 33, 565 (2001).

2001KIY/MIN T. Kiyobayashi and M. E. Minas da Piedade, J. Chem. Thermodyn. 33, 11 (2001).

2001KLI/LUB Ｗ. Kliegel, G. Lubkowitz, J. O. Pokriefke, S. J. Rettig, and J. Trotter, Can. J. Chem. 79, 226 (2001).

$2001 \mathrm{KOZ} / \mathrm{BLO}$ A. A. Kozyro, A. V. Blokhin, G. J. Kabo, and Y. U. Paulechka, J. Chem. Thermodyn. 33, 305 (2001).

2001 KUL/VER2 D. Kulikov, S. P. Verevkin, and A. Heintz, Fluid Phase Equilib. 192, 187 (2001).

2001LAI/LEE ～L.-L. Lai, L.-J. Lee, E. Wang, and F.-Y. Su, Liq. Cryst. 28 , 381 (2001).

2001LAL/SCH J. O. Lalah, K.-W. Schramm, D. Lenoir, B. Henkelmann, N. Hertkorn, G. Matuschek, A. Kettrup, and K. Günther, Chem. - Eur. J. 7, 4790 (2001).

2001LEB/VAN N. Lebrun and J. C. van Miltenburg, J. Alloys Compd. 320, 320 (2001).

2001LEG/BAZ B. Legendre, G. Baziard-Mouysser, M. Anastassiadou, J. M. Leger, and M. Payard, J. Therm. Anal. Calorim. 66, 659 (2001).

2001LI/GRA Z. J. Li, W. H. Ojala, and D. J. W. Grant, J. Pharm. Sci. 90, 1523 (2001).

2001LI/HE J. Li, Y. He, K. Ishida, T. Yamane, and Y. Inoue, Polym. J. (Tokyo, Jpn.) 33, 773 (2001).

2001MAL/PAR I. P. Malkerova, S. E. Paramonov, A. S. Alikhanyan, and N. P. Kuz'mina, Zh. Neorg. Khim. 46, 1700 (2001).

2001MAR/GOM F. Martinez and A. Gomez, J. Solution Chem. 30, 909 (2001).

2001MAT/LEB Y. N. Matyushin, V. P. Lebedev, E. A. Miroshnichenko, L. M. Kostikova, and Y. O. Inosemzev, in International Annual Conference of ICT (2001), pp. 102/1-102/12.

2001MAT/SHC E. V. Matukhina, O. I. Shchegolikhina, N. N. Makarova, Y. A. Pozdniakova, and D. E. Katsoulis, Liq. Cryst. 28, 869 (2001).

2001MON/HIL M. J. S. Monte and D. M. Hillesheim, J. Chem. Eng. Data 46, 1601 (2001).

2001MON/HIL4 M. J. S. Monte and D. M. Hillesheim, J. Chem. Thermodyn. 33, 837 (2001).

2001MON/HIL5 M. J. S. Monte and D. M. Hillesheim, J. Chem. Thermodyn. 33, 849 (2001).

2001MOR/ZHAＮ. B. Morozova, S. I. Zharkova, P. P. Semyannikov, I. K. Igumenov, N. E. Fedotova, and N. V. Gelford, J. Phys. IV 11, Pr3-609 (2001); Chem. Abstr. 136, 43187 (2001).

2001NAI/SIR Z. Naima, T. Siro, G.-D. Juan-Manuel, C. Chantal, C. Rene, and D. Jerome, Eur. J. Pharm. Sci. 12, 395 (2001).

2001 NIK/SUL G. E. Nikitina, B. Suleiman, A. S. Semeikin, and O. A. Golubchikov, Russ. J. Phys. Chem. 75, 675 (2001).

2001NOR/TOU S. Norvez, F.-G. Tournilhac, P. Bassoul, and P. Herson, Chem. Mater. 13, 2552 (2001). 
2001OHT/CIC T. Ohta, F. Cicoira, P. Doppelt, L. Beitone, and P. Hoffmann, Chem. Vap. Deposition 7, 33 (2001); Chem. Abstr. 134, 153247w (2001).

2001PUR/CHI S. Puri, J. S. Chickos, and W. Welsh, J. Anal. Chem. 73, 1480 (2001)

2001RAI/VAR R. N. Rai and K. B. R. Varma, Mater. Lett. 48, 356 (2001).

2001RIB/MAT M. A. V. Ribeiro da Silva, M. A. R. Matos, M. S. Miranda, M. H. F. A. Sousa, R. M. Borges dos Santos, M. M. Bizarro, and J. A. Martinho Simoes, Struct. Chem. 12, 171 (2001).

2001RIB/MON M. A. V. Ribeiro da Silva, M. J. S. Monte, and E. Giera, J. Chem. Thermodyn. 33, 369 (2001).

2001RIB/RIB2 M. A. V. Ribeiro da Silva, M. D. M. C. Ribeiro da Silva, L. C. M. da Silva, F. Dietz, and E. Hoyer, Thermochim. Acta 378, 45 (2001).

2001RIT L. Rittfeldt, Anal. Chem. 73, 2405 (2001).

2001ROJ/ORO A. Rojas-Aguilar, E. Orozco-Guareno, and M. MartinezHerra, J. Chem. Thermodyn. 33, 1405 (2001).

2001ROL/BUR J. M. Rollinger and A. Burger, J. Pharm. Sci. 90, 949 (2001).

2001ROL/KRA M. P. Rolemberg and M. A. Krahnbuhl, J. Chem. Eng. Data 46, 256 (2001).

2001SCI/SCI J. Sciesinski, E. Sciesinski, M. Massalska-Arodz, T. Wasiutynski, P. M. Zielinski, and W. Witko, IEEE Trans. Dielectr. Electr. Insul. 8, 522 (2001).

2001SHI/YAM E. Shibata, S. Yamamoto, H. Koyo, T. Ikeda, E. Kasai, M. Maeda, and T. Nakamura, Mater. Trans. 42, 2531 (2001).

2001SMI/LEB N. N. Smirnova and B. V. Lebedev, Russ. J. Phys. Chem. (Suppl. 1) 75, S118 (2001).

2001SON/HUA Y. Song, Y. Huang, E. A. Havenga, and I. S. Butler, Vib. Spectrosc. 27, 127 (2001).

2001TAN/XUE Z.-C. Tan, B. Xue, S.-W. Lu, S. H. Meng, X.-H. Yuan, and Y. J. Song, J. Therm. Anal. Calorim. 63, 297 (2001).

2001TIT/TOM S. A. Tittlemier and G. T. Tomy, Environ. Toxicol. Chem. 20, 146 (2001).

$2001 T U R / K R I \quad$ A. E. Turgambaeva, V. V. Krisyuk, S. V. Sysoev, and I. K. Igumenov, Chem. Vap. Deposition 7, 121 (2001).

2001VAL/SMI E. J. Valente, T. N. Smith, and M. E. Harris, Chirality 13, 244 (2001)

2001VAN/OON2 J. C. van Miltenburg, H. A. J. Oonk, and L. Ventola, J. Chem. Eng. Data 46, 90 (2001).

2001VAR/PAS R. M. Varushchenko, L. L. Paschenko, A. I. Druzhinina, A. V. Abramenkov, and A. A. Pimersin, J. Chem. Thermodyn. 33, 733 (2001).

2001VER/AUG S. Verheyen, P. Augustijns, R. Kinget, and G. Van den Mooter, Int. J. Pharm. 228, 199 (2001).

2001VER/HEI S. P. Verevkin and A. Heintz, J. Chem. Eng. Data 46, 984 (2001).

2001VER/HEI2 S. P. Verevkin and A. Heintz, J. Chem. Eng. Data 46, 41 (2001).

2001WON/LEI A. Wong, Y. D. Lei, M. Alaee, and F. Wania, J. Chem. Eng. Data 46, 239 (2001).

2001YAT/MIN T. Yatabe, N. Minami, H. Okumoto, A. Kaito, K. Ueno, and Y. Tanabe, Mol. Cryst. Liq. Cryst. 365, 7 (2001).

$2001 \mathrm{YOU} / \mathrm{SCH} \quad$ P. H. Young and C. A. Schall, Thermochim. Acta 367-368, 387 (2001)

2002ABB/WOH T. P. Abbott, A. Wohlman, T. Isbell, F. A. Momany, C. Cantrell, D. Garlotta, and D. Weisleder, Ind. Crops Prod. 16, 43 (2002).

2002BAE/SHI A. K. Baev and M. A. Shishko, Russ. J. Appl. Chem. 75, 25 (2002).

2002BAE/SHI2 A. K. Baev and M. A. Shiskho, Russ. J. Appl. Chem. 75, 156 (2002).

2002BEL/MAN M. Belloni, M. Manickam, and J. A. Preece, Ferroelectrics 276, 103 (2002).

2002BER/MAR V. Berbenni, A. Marini, G. Bruni, A. Maggioni, and P. Cogliati, J. Therm. Anal. Calorim. 68, 413 (2002).

2002BOR/CES B. Borde and A. Cesaro, J. Therm. Anal. Calorim. 69, 267 (2002).

2002CAI/BET M. R. Caira, G. Bettinetti, and M. Sorrenti, J. Pharm. Sci. 91, 467 (2002).

2002CHA/DOL K. Chatterjee, D. Dollimore, and K. S. Alexander, Thermochim. Acta 392-393, 107 (2002).
2002CHI/KNI2

R. D. Chirico, S. E. Knipmeyer, and W. V. Steele, J. Chem. Thermodyn. 34, 1885 (2002).

2002CHI/WEB J. S. Chickos, P. Webb, G. Nichols, T. Kiyobayashi, P.C. Cheng, and L. Scott, J. Chem. Thermodyn. 34, 1195 (2002).

2002DAL/DEL L. Dall'acqua, G. Della Gatta, B. Nowicka, and P. Ferloni, J. Chem. Thermodyn. 34, 1 (2002).

2002DAS/ZAL Z. Daszkiewicz, J. Zaleski, E. M. Nowakowska, and J. B. Kyziol, Pol. J. Chem. 76, 1113 (2002).

2002DIA/LOP J. Diaz-Hernandez, A. Lopez-Echarri, I. Ruiz-Laurren, A Fraile-Rodriquez, and T. Breczewski, Ferroelectrics 268, 753 (2002).

2002DIO/KIY H. P. Diogo, T. Kiyobayashi, M. E. Minas da Piedade, N. Burlak, D. W. Rogers, D. McMasters, G. Persy, J. Wirz, and J. F. Liebman, J. Am. Chem. Soc. 124, 2065 (2002).

2002DIO/MIN H. P. Diogo and M. E. Minas da Piedade, J. Chem. Thermodyn. 34, 173 (2002).

2002ESP/BAR P. Espeau, M. Barrio, D. O. Lopez, J. L. Tamarit, R. Ceolin, H. Allouchi, V. Agatfonov, F. Masin, and H. Szwarc, Chem. Mater. 14, 321 (2002).

2002GIG/BAL G. Gigli, G. Balducci, V. Y. Markov, O. V. Boltalina, A. A. Goryunkov, N. Sidorov, and R. Taylor, J. Chem. Thermodyn. 34, 57 (2002).

2002GOO/GEL J. W. Goodrum and D. P. Geller, Bioresour. Technol. 84, 75 (2002); Note: The vaporization enthalpies that were calculated with the Clausius-Clapeyron equation coefficients given in the paper were less than $1 \mathrm{~kJ} / \mathrm{mole}$. Through personal correspondence with the authors we learned that the coefficients are to be multiplied by 1000 .

2002GOO/LAI C. Goosen, T. J. Laing, J. du Plessis, T. C. Goosen, and J. L. Flynn, Pharm. Res. 19, 13 (2002).

2002GRA/RAS S. Gracin and A. C. Rasmuson, J. Chem. Eng. Data 47, 1379 (2002).

2002HAN/HIK M. Hanaya, T. Hikirna, M. Hatase, and M. Oguni, J. Chem. Thermodyn. 34, 1173 (2002).

2002HER/ACK A. K. Hermetet, L. J. Ackerman, J. K. Swearingen, C. A. Presto, D. R. Kelman, J. M. Giesen, K. I. Goldberg, W. Kaminsky, and D. M. West, J. Chem. Crystallogr. 32, 17 (2002).

2002ITA/KAM T. Itahara, J. Kamada, S. Ikeda, and T. Ueda, Chem. Lett. 31, 1122 (2002).

2002JIM/ROU P. Jimenez, M. V. Roux, J. Z. Davalos, and M. T. Molina, J. Chem. Thermodyn. 34, 1117 (2002).

2002JOW/DIN K. G. Jow and T. J. Dingemans, Liq. Cryst. 29, 573 (2002).

2002KAR/SHE N. V. Karyakin, M. S. Sheiman, M. S. Kozlova, G. P. Kamelova, V. N. Larina, and A. S. Smirnov, Russ. J. Phys. Chem. 76, 1061 (2002).

2002KEL/SZC ～D. R. Kelman, L. F. Szczepura, K. I. Goldberg, W. Kaminsky, A. K. Hermetet, L. G. Ackerman, J. K. Swearingen, and D. X. West, J. Mol. Struct. 610, 143 (2002).

2002KOZ/KAR M. S. Kozlova, N. V. Karyakin, M. S. Sheiman, G. P. Kamelova, and V. N. Larina, Russ. J. Phys. Chem. 76, 1068 (2002).

2002KRA/VAS E. L. Krasnykh, T. V. Vasiltsova, S. P. Verevkin, and A. Heintz, J. Chem. Eng. Data 47, 1372 (2002).

2002KUS/ASH I. Kushida and K. Ashizawa, J. Pharm. Sci. 91, 2193 (2002).

2002LEB/BYK B. V. Lebedev, T. A. Bykova, A. V. Markin, A. R. Korigodskii, V. V. Kireev, and N. N. Sanina, Polym. Sci., Ser. A 44, 1327 (2002).

2002LEI/CHA Y. D. Lei, R. Chankalal, A. Chan, and F. Wania, J. Chem. Eng. Data 47, 801 (2002).

2002LI/SHI X.-W. Li, E. Shibata, E. Kasai, and T. Nakamura, Mater. Trans. 43, 2903 (2002).

2002LIP/SCH M. Lipovska, H.-G. Schmidt, V. Rohac, V. Ruicka, G. Wolf, and M. Zabranska, J. Therm. Anal. Calorim. 68, 753 (2002).

2002LU/SON Q.-Y. Lu, Y.-J. Song, S.-H. Meng, J. Xing, and Z.-C. Tan, Wuli Huaxue Xuebao 18, 166 (2002); Chem. Abstr. 136, 134441e (2002).

2002MAR/GOM F. Martinez and A. Gomez, Phys. Chem. Liq. 40, 411 (2002). 
2002MIN/SAK H. Minami, Y. Sakamoto, and M. Iwahashi, J. Oleo Sci. 51, 11 (2002).

2002MIY/KAT K. Miyazawa, T. Kato, M. Itoh, and M. Ushioda, Liq. Cryst. 29, 1483 (2002).

2002MUR/GRA P. Mura, P. Gratteri, and M. T. Faucci, J. Therm. Anal. Calorim. 68, 541 (2002).

2002NAT/JES C. Nather, I. Jess, Z. Havlas, M. Bolte, N. Nagel, and S. Nick, Solid State Sci. 4, 859 (2002).

2002PAN/MAL R. Pankajavalli, C. Mallika, O. M. Sreedharan, V. S. Raghunathan, P. A. Premkumar, and K. S. Negaraja, Chem. Eng. Sci. 57, 3603 (2002).

2002PAT/CAM R. Patino, M. Campos, and L. A. Torres, J. Chem. Thermodyn. 34, 193 (2002).

2002PER/HAN G. L. Perlovich, L. K. Hansen, and A. Bauer-Brandl, J. Pharm. Sci. 91, 1036 (2002).

2002PER/LOP E. Perea, F. Lopez-Calahorra, and D. Velasco, Liq. Cryst. 29, 421 (2002).

2002PIM/MEL S. M. Pimehova, S. V. Melkhanova, V. P. Kolesov, P. I. Demyanov, A. N. Fedotov, and V. P. Vorbieva, J. Chem. Thermodyn. 34, 385 (2002).

2002RIB/RIB M. A. V. Ribeiro da Silva, M. D. M. C. Ribeiro da Silva, L. C. M. da Silva, and F. Dietze, J. Chem. Thermodyn. 34, 155 (2002).

2002ROC/GRI G. D. Rockwell, T. B. Grindley, K. C. Smith, and M. A. White, J. Phys. Chem. B 106, 12311 (2002).

2002ROG/DOM M. Rogalski, U. Domanska, D. Czyrny, and D. Dyczko, Chem. Phys. 285, 355 (2002).

2002ROL/GST J. M. Rollinger, E. M. Gstrein, and A. Burger, Eur. J. Pharm. Biopharm. 53, 75 (2002).

2002SAW/SHI Y. Sawanoi, Y. Shimbo, I. Tabata, K. Hisada, and T. Hori, Dyes Pigm. 52, 29 (2002).

2002SCH/LAS A. Schulz, S. Laschat, M. Morr, S. Diele, M. Dreyer, and G. Bringmann, Helv. Chim. Acta 85, 3909 (2002).

2002 SCH/LEN E. Schnitzler, K. Lencone, and M. Kobelnik, Exact Soil Sci., Agrarian S. Eng. 8, 91 (2002).

2002 SHE/KAR V. M. Sheiman, N. V. Karyakin, B. I. Kozyrkin, and I. A. Zelyaev, Russ. J. Phys. Chem. 76, 885 (2002).

2002 SHE/KAR2 V. M. Sheiman, N. V. Karyakin, M. S. Sheiman, and I. A. Zelyaev, Russ. J. Phys. Chem. 76, 881 (2002).

2002SPA/DZI A. Spadlo, J. Dziaduszek, R. Dabrowski, K. Cyuprynski, T. Stolarz, and S. T. Wu, Proc. SPIE 4759, 79 (2002).

2002 STE/CHI3 W. V. Steele, R. D. Chirico, S. E. Knipmeyer, and A. Nguyen, J. Chem. Eng. Data 47, 689 (2002).

2002STE/CHI6 W. V. Steele, R. D. Chirico, A. B. Cowell, S. E. Knipmeyer, and A. Nguyen, J. Chem. Eng. Data 47, 725 (2002).

2002STO/BEH G. W. Stowell, R. J. Behme, S. M. Denton, I. Pfeiffer, F. D. Sancilio, L. B. Whittall, and R. R. Whittle, J. Pharm. Sci. 91, 2481 (2002).

2002SU/ZHU C.-H. Su, S. Zhu, N. Ramachandran, and A. Burger, J. Cryst. Growth 235, 313 (2002).

2002SZC/KEL L. F. Szczepura, D. R. Kelman, A. K. Hermetet, L. J. Ackerman, K. I. Goldberg, K. A. Claborn, W. Kaminsky, and D. X. West, J. Mol. Struct. 608, 245 (2002).

2002 TOR/CAM L. A. Torres, M. Campos, E. Enriquez, and R. Patino, J. Chem. Thermodyn. 34, 293 (2002).

2002VAL/HER J. Vales-Martinez, S. Hernandez-Ortega, G. EspinosaPerez, C. A. Presto, A. K. Hermetet, K. D. Haslow, L. J. Ackerman, L. F. Szczepura, K. I. Goldberg, W. Kaminsky, and D. X. West, J. Mol. Struct. 608, 77 (2002).

2002 VAN/KRU J. A. H. van Laarhoven, M. A. B. Kruft, and H. Vromans, Int. J. Pharm. 232, 163 (2002).

2002 VAN/PAR A. van Roon, J. R. Parsons, and H. A. J. Govers, J. Chromatogr. A 955, 105 (2002).

2002VAN/VAN A. C. G. van Genderen, J. C. van Miltenburg, J. G. Blok, M. J. van Bommel, P. J. van Ekeren, G. J. K. van den Berg, and H. A. J. Oonk, Fluid Phase Equilib. 202, 109 (2002).

2002VEN/RAM L. Ventola, M. Ramirez, T. Calvet, X. Solans, M. A. Cuevas-Diarte, N. Negrier, D. Mondieig, J. C. van Miltenburg, and H. A. J. Oonk, Chem. Mater. 14, 508 (2002). S. P. Verevkin, J. Chem. Eng. Data 47, 1071 (2002).

2002VER

2002VER/HEI S. P. Verevkin and A. Heintz, J. Chem. Soc., Perkin Trans. 2 2002, 728.
2002VER2

2002ZHO/ZHA

2003ASA/SOR

2003BRI/BOU

2003CHA/BLO2

S. P. Verevkin, J. Chem. Thermodyn. 34, 263 (2002).

D. Zhou, G. G. Z. Zhang, D. Law, D. J. W. Grant, and E. A. Schmitt, J. Pharm. Sci. 91, 1863 (2002).

S. Asahina and M. Sorai, J. Chem. Thermodyn. 35, 649-666 (2003).

A.-J. Briard, M. Bouroukba, D. Petitjean, N. Hubert, and M. Dirand, J. Chem. Eng. Data 48, 497 (2003).

2003 CHA/BLO2 M. B. Charapennikau, A. V. Blokhin, G. J. Kabo, V. M. Sevruk, and A. P. Krasulin, Thermochim. Acta 405, 85 (2003).

2003CHA/GUP C. Chawla, P. Gupta, R. Thilagavathi, A. K. Chakraborti, and A. K. Bansal, Eur. J. Pharm. Sci. 20, 305 (2003).

2003 CHI/ACR J. S. Chickos and W. E. Acree, Thermochim. Acta 395, 59-113 (2003).

2003CHI/HIL J. S. Chickos, D. M. Hillesheim, S. P. Verevkin, M. V. Roux, M. Temprado, M. Segura, R. Notario, D. E. Demasters, and J. F. Liebman, Mol. Phys. 101, 1311 (2003).

2003CLO/JAN K. Clou, J. F. Janssens, N. Blaton, A. T. H. Lenstra, and H. O. Desseyn, Thermochim. Acta 298, 47 (2003).

2003DEM/KOZ A. de Meijere, S. I. Kozhushkov, K. Rauch, H. Schill, S. P. Verevkin, M. Kuemmerlin, H.-D. Beckhaus, C. Rüchardt, and D. S. Yufit, J. Am. Chem. Soc. 125, 15110 (2003).

2003DOM/MAR U. Domanska and A. Marciniak, J. Chem. Eng. Data 48, 451 (2003).

2003FOX/AWA ～D. M. Fox, W. H. Awad, J. W. Gilman, P. H. Maupin, H. C. De Long, and P. Trulov, Green Chem. 5, 724 (2003).

2003FUL/RUZ M. Fulem, K. Ruzicka, E. Hulicius, T. Simecek, K. Melichar, J. Pangrac, S. A. Rushworth, and L. M. Smith, J. Cryst. Growth 248, 99 (2003).

2003GIO/ROS F. Giordano, A. Rossi, I. Pasquali, R. Bettini, E. Frigo, A. Gazzaniga, M. E. Sangalli, V. Mileo, and S. Catinella, J. Therm. Anal. Calorim. 73, 509 (2003).

2003GRZ/LAN A. L. Grzesiak, M. Lang, K. Kim, and A. J. Matzger, J. Pharm. Sci. 92, 2260 (2003).

2003 HIN/RAF $\quad$ D. Hinks, M. I. Rafiq, D. M. Price, G. A. Montero, and B. Smith, Color. Technol. 119, 84 (2003).

2003KAL/PAU J. Kaloustian, A.-M. Pauli, P. Lechene de la Porte, H. Lafont, and H. Portugal, J. Therm. Anal. Calorim. 71, 341 (2003).

2003KAR/KOZ N. V. Karyakin, M. S. Kozlova, M. S. Sheiman, G. P. Kamelova, and V. N. Larina, Zh. Fiz. Khim. 77, 1375 (2003).

2003KOZ/KAR M. S. Kozlova, N. V. Karyakin, M. S. Sheiman, G. P. Kamelova, V. N. Larina, and A. S. Smirnov, Russ. J. Phys. Chem. 77, 26 (2003).

2003LAW/WAN D. Law, W. Wang, E. A. Schmitt, Y. Qiu, S. L. Krill, and J. J. Fort, J. Pharm. Sci. 92, 505 (2003).

2003LEB/VAN N. Lebrun, J. C. Van Miltenburg, D. Bustin, and M. Descamps, Phase Transitions 76, 841 (2003).

2003LEE/CHO M.-J. Lee, Y.-H. Chou, and H.-M. Lin, Ind. Eng. Chem. Res. 42, 3434 (2003).

2003LEG/FEU B. Legendre, Y. Feutelais, and G. Defossemont, Thermochim. Acta 400, 213 (2003).

2003MAR/AVI F. Martinez, C. M. Avila, and A. Gomez, J. Braz. Chem. Soc. 4, 803 (2003).

2003MAY/TOR M. J. Mayoral, M. C. Torralba, M. Cano, J. A. Campo, and J. V. Heras, Inorg. Chem. Commun. 6, 626 (2003).

2003NIK/MAR R. Nikolic, M. Marinovic-Cincovic, S. Gadzuric, and I. Zsigrai, Sol. Energy Mater. Sol. Cells 79, 285 (2003).

2003NOK/BOL A. Nokhodchi, N. Bolourtchian, and R. Dinarvand, Int. J. Pharm. 250, 85 (2003).

2003PAJ/ROS J. Pajak, M. Rospenk, Z. Galewski, and L. Sobczyk, J. Mol. Liq. 105, 53 (2003).

2003PER/CON S. Perisanu, I. Contineanu, M. D. Banciu, J. F. Liebman, B. S. Farivar, M. A. Mullan, J. S. Chickos, N. Rath, and D. M. Hillesheim, Thermochim. Acta 400, 109 (2003).

2003PER/KUR G. L. Perlovich, S. V. Kurkov, and A. Bauer-Brandl, Eur. J. Pharm. Sci. 19, 423 (2003).

2003PER/KUR2 G. L. Perlovich, S. V. Kurkov, A. N. Kinchin, and A. Bauer-Brandl, J. Pharm. Sci. 92, 2502 (2003).

2003ROJ/ORO A. Rojas and E. Orozco, Thermochim. Acta 405, 93 (2003). 
2003ROU/JIM2 M. V. Roux, P. Jimenez, A. Vacas, F. H. Cano, M. del Carmen Apreda-Rojas, and F. Ros, Eur. J. Org. Chem. 2003, 2084.

2003SHA/KAN $\quad$ B. L. Sharma, R. Kant, R. Sharma, and S. Tandon, Mater. Chem. Phys. 82, 216 (2003).

2003SIE/WEB A. R. Siedle, R. J. Webb, F. E. Behr, R. A. Newmark, D. A. Weil, K. Erikson, R. Naujok, M. Bronstrom, M. Mueller, S.-H. Chou, and V. G. Young, Jr., Inorg. Chem. 42, 932 (2003).

2003SIK/SMI M. Sikiric, I. Smit, L. Tusek-Bozic, V. Tomasic, I. Pucic, I. Primozic, and N. Filipovic-Vincekovic, Langmuir 19, 10044 (2003).

2003SMI/LEB N. N. Smirnova and B. V. Lebedev, Russ. J. Gen. Chem. 73, 247 (2003)

2003STA/BAI P. A. Stabnikov, I. A. Baidina, S. V. Sysoev, N. S. Vanina, N. B. Morozova, and I. K. Igumenov, J. Struct. Chem. 44, 1054 (2003).

2003STO/KRZ P. Storoniak, K. Krzyminski, A. Bouzyk, E. P. Koval'chuk, and J. Blazejowski, J. Therm. Anal. Calorim. 74, 443 (2003).

2003SUP/GOF G. J. Suppes, M. J. Goff, and S. Lopes, Chem. Eng. Sci. 58, 1751 (2003).

2003TOZ/INA K.-I. Tozaki, H. Inaba, H. Hayashi, C. Quan, N. Nemoto, and T. Kimura, Thermochim. Acta 397, 155 (2003).

2003TRE/KAS T. Treszczanowicz, T. Kaspryzycka-Guttman, and A. Treszcyanowicz, J. Chem. Eng. Data 48, 1517 (2003).

2003VAN/VAN J. C. Van Miltenburg, G. J. K. van den Berg, and M. Ramirez, J. Chem. Eng. Data 48, 36 (2003).

2003VAN/VAN2 J. C. van Miltenburg, P. J. van Ekeren, F. G. Gandolfo, and E. Flöter, J. Chem. Eng. Data 48, 1245 (2003).

2003VER S. P. Verevkin, J. Chem. Thermodyn. 35, 1237 (2003).

2003VER/KRA S. P. Verevkin, E. L. Krasnykh, T. V. Vasiltsova, and A. Heintz, J. Chem. Eng. Data 48, 591 (2003).

2003 VER/KRA2 S.P. Verevkin, E. L. Krasnykh, T. V. Vasiltsova, B. Koutek, J. Doubsky, and A. Heintz, Fluid Phase Equilib. 206, 331 (2003).

2003VER/ROU S. P. Verevkin, M. V. Roux, R. Notario, D. E. Demasters, and J. F. Liebman, Mol. Phys. 21, 3231 (2003).

2003VER2

2003WIL/VAN

S. P. Verevkin, Phys. Chem. Chem. Phys. 5, 710 (2003).

H. T. A. Wilderbeek, M. G. M. Van der Meer, M. A. G. Jansen, L. Nelissen, H. R. Fisher, J. J. G. S. Van Es, C. W. M. Bastiaansen, J. Lub, and D. J. Broer, Liq. Cryst. 30, 93 (2003).

2003YAM/HAY O. Yamamura, M. Hayashi, T. Matsuo, and P. Lunkenheimer, J. Chem. Phys. 119, 4775 (2003).

2003YU/TAN H.-G. Yu, Z.-C. Tan, Y. Liu, X.-Z. Lan, F. Xu, X.M. Huang, and L.-X. Sun, Thermochim. Acta 404, 89 (2003).

2003 YU/TAN2 P. Yu, Z. C. Tan, S. H. Meng, S. W. Lu, X. Z. Lan, L. X. Sun, F. Xu, T. Zhang, and S. X. Hu, J. Therm. Anal. Calorim. 74, 867 (2003).

2003ZAI/VER D. H. Zaitsau, S. P. Verevkin, Y. U. Paulechka, G. J. Kabo, and V. M. Sevuk, J. Chem. Eng. Data 48, 1393 (2003).

2003ZEL/TIT L. N. Zelenina, V. A. Titov, T. P. Chusov, Y. G. Stenin, and A. A. Titov, J. Chem. Thermodyn. 35, 1601 (2003).

2004AMB/MAH A. A. Ambike, K. R. Mahadik, and A. Paradkar, Int. J. Pharm. 282, 151 (2004).

2004BEN/KHI M. Benkhennouf, K. Khimeche, and A. Dahmani, J. Phys. IV (France) 113, 7 (2004).

2004B YK/LEB T. A. Bykova and B. V. Lebedev, Russ. J. Gen. Chem. 74, 250 (2004).

2004CAI/BOU M. R. Caira, S. A. Bourne, and C. L. Oliver, J. Therm. Anal. Calorim. 77, 597 (2004).

2004CAI/FOP M. R. Caira, A. Foppoli, M. E. Sangalli, L. Zema, and F. Giordano, J. Therm. Anal. Calorim. 77, 653 (2004).

2004CHI/HAN J. S. Chickos and W. J. Hanshaw, Chem. Eng. Data 49, 77 (2004).

2004CHI/HAN2 J. S. Chickos and W. J. Hanshaw, J. Chem. Eng. Data 49, 620 (2004).

2004CHI/ZHA J. S. Chickos, H. Zhao, and G. Nichols, Thermochim. Acta 424, 111 (2004).

2004COS/SCH S. Coste, J.-M. Schneider, M.-N. Petit, and G. Coquerel, Cryst. Growth Des. 4, 1237 (2004).
2004DEF/RAN

2004DEV/VAN

G. Defossemont, S. L. Randzio, and B. Legendre, Cryst. Growth Des. 4, 1169 (2004).

M. M. de Villiers, C. M. Van Eeden, W. Liebenberg, M. Song, W. M. Kolling, and M. R. Caira, J. Agric. Food Chem. 52, 7362 (2004)

2004DI/TAN Y.-Y. Di, Z.-C. Tan, X.-H. Sun, M.-H. Wang, F. Xu, Y.-F. Liu, L.-X. Sun, and H.-T. Zhang, J. Chem. Thermodyn. 36, 79 (2004).

2004DIM/DAL ～D. A. Dimas, P. P. Dallas, and D. M. Rekkas, Pharm. Dev. Technol. 9, 311 (2004).

2004DOM/BOG U. Domanska and E. Bogel-Lukasik, Fluid Phase Equilib. 218, 123 (2004).

2004FOP/SAN A. Foppoli, M. E. Sangalli, A. Maroni, A. Gazzaniga, M. R. Caira, and F. Giordano, J. Pharm. Sci. 93, 521 (2004).

2004FUL/RUZ M. Fulem, K. Ruzicka, V. Ruzicka, E. Hulicius, T. Simecek, J. Pangrac, S. A. Rushworth, and L. M. Smith, J. Cryst. Growth 272, 42 (2004).

2004FUL/RUZ2

M. Fulem, K. Ruzicka, V. Ruzicka, T. Simecek, E. Hulicius, and J. Pangrac, J. Cryst. Growth 264, 192 (2004).

2004GON/KOS

T. Gondova and D. Koscakova, J. Therm. Anal. Calorim. 76, 133 (2004).

2004GRI/WEI

U. J. Griesser, D. Weigand, J. M. Rollinger, M. Haddow, and E. Gstrein, J. Therm. Anal. Calorim. 77, 511 (2004).

2004GUP/SIN

R. K. Gupta and R. A. Singh, J. Cryst. Growth 267, 340 (2004).

2004HAM/FEU N. Hamdi, Y. Feutelais, N. Yagoubi, D. de Girolamo, and B. Legendre, J. Therm. Anal. Calorim. 76, 985 (2004).

2004HAM/HAM D. C. Hammell, M. Hamad, H. K. Vaddi, P. A. Crooks, and A. L. Stinchcomb, J. Controlled Release 97, 283 (2004).

2004HIL/MIL K. Hilpert, M. Miller, and F. Ramondo, Thermochim. Acta 417, 163 (2004).

2004INO/HIS T. Inoue, Y. Hisatsugu, R. Ishikawa, and M. Suzuki, Chem. Phys. Lipids 127, 161 (2004).

2004INO/HIS3 T. Inoue, Y. Hisatsuga, R. Yamamoto, and M. Suzuki, Chem. Phys. Lipids 127, 143 (2004).

2004KAB/BLO G. J. Kabo, A. V. Blokhin, Y. U. Paulechka, A. G. Kabo, M. P. Shymanovich, and J. W. Magee, J. Chem. Eng. Data 49, 453 (2004).

2004KUL/MAR T. G. Kulagina, A. V. Markin, T. A. Bykova, N. N. Smirnova, L. A. Smirnova, and V. A. Barachevskii, Russ. J. Phys. Chem. 78, 139 (2004).

2004LEG/FEU B. Legendre and Y. Feutelais, J. Therm. Anal. Calorim. 76, 255 (2004).

2004LI/SHI X.-W. Li, E. Shibata, E. Kasai, and T. Nakamura, Environ. Toxicol. Chem. 23, 348 (2004).

2004LIU/WAN Y. Liu, X. Wang, J.-K. Wang, and C. B. Ching, J. Pharm. Sci. 93, 3039 (2004).

2004LU/CHI

2004MAR/KAI

Y. H. Lu and C. B. Ching, Chirality 16, 541 (2004).

2004MAR/KOZ B. Marciniec, M. Kozak, and M. Ogrodowczyk, J. Therm. Anal. Calorim. 77, 581 (2004).

2004MAT/MIR2 M. A. R. Matos, M. S. Miranda, D. V. S. S. Martins, N. A. B. Pinto, V. M. F. Morais, and J. F. Liebman, Org. Biomol. Chem. 2, 1353 (2004).

2004MCG/SAU C. McGregor, M. H. Saunders, G. Buckton, and R. D. Saklatvala, Thermochim. Acta 417, 231 (2004).

2004MIR/ROH M. Mirmehrabi and S. Rohani, Can. J. Chem. Eng. 82, 335 (2004).

2004MON/ALM M. J. S. Monte, A. R. R. P. Almeida, and M. A. V. Ribeiro da Silva, J. Chem. Thermodyn. 36, 385 (2004).

2004MON/RAJ D. Mondieig, F. Rajabalee, V. Metivaud, H. A. J. Oonk, and M. A. Cuevas-Diarte, Chem. Mater. 16, 786 (2004).

2004NUN/EUS S. C. C. Nunes, M. E. Eusebio, M. L. P. Leitão, and J. S. Redinha, Int. J. Pharm. 285, 13 (2004).

2004PAU/ZAI Y. U. Paulechka, D. H. Zaitsau, and G. J. Kabo, J. Mol. Liq. 115, 105 (2004).

2004PER/KUR G. L. Perlovich, S. V. Kurkov, A. N. Kinchin, and A. Bauer-Brandl, Eur. J. Pharm. Biopharm. 57, 411 (2004).

2004PIA/SUG X. Piao, Y. Sugihara, and J. Nakayama, Heteroat. Chem. 15, 424 (2004).

2004PIL/HAM O. Pillai, M. O. Hamad, P. A. Crooks, and A. L. Stinchcomb, Pharm. Res. 21, 1146 (2004); Note: Enthalpies of 
fusion were calculated from the tabulated melting point temperature and thermodynamic activity data given in paper.

2004QU/BAI H. Qu, P. Bai, Z. Yang, and G. Yu, Chin. J. Chem. Eng. 12, 294 (2004)

2004RAM/COR J. J. M. Ramos, N. T. Correia, and H. P. Diogo, Phys. Chem. Chem. Phys. 6, 793 (2004).

2004RAM/DIO K. J. M. Ramos, H. P. Diogo, M. H. Godinho, C. Cryz, and K. Merkel, J. Phys. Chem. B 108, 7955 (2004).

2004RIB/GOM2 M. D. M. C. Ribeiro da Silva, J. R. B. Gomes, J. M. Goncalves, E. A. Sousa, S. Pandey, and W. E. Acree, Jr., J. Org. Chem. 69, 2785 (2004).

2004RIB/GON M. D. M. C. Ribeiro da Silva, J. M. Goncalves, A. R. L. Silva, A. M. R. O. A. Silva, P. C. F. C. Oliveira, and M. A. V. M. A. V. Ribeiro da Silva, Thermochim. Acta 420, 67 (2004).

2004RIB/SAN M. A. V. Ribeiro da Silva, L. M. N. B. F. Santos, B. Schroder, and L. Beyer, J. Chem. Thermodyn. 36, 555 (2004).

2004RIB/SAN2 M. A. V. Ribeiro da Silva, L. M. N. B. F. Santos, B. Schroder, F. Dietze, and L. Beyer, J. Chem. Thermodyn. 36, 491 (2004).

2004RIB/SAN3 M. A. V. Ribiero da Silva and L. M. N. B. F. Santos, J. Chem. Thermodyn. 36, 447 (2004).

2004ROH/RUZ V. Rohac, K. Ruzicka, V. Ruzicka, D. H. Zaitsau, G. J. Kabo, V. Dicky, and K. Aim, J. Chem. Thermodyn. 36, 929 (2004).

2004ROM/BUS S. Romero, P. Bustamante, B. Escalera, M. Cirri, and P. Mura, J. Therm. Anal. Calorim. 77, 541 (2004).

2004ROM/BUS2 S. Romero, P. Bustamante, B. Escalera, P. Mura, and M. Cirri, J. Pharm. Biomed. Anal. 35, 715 (2004).

2004ROM/ROC J. J. M. Romos, S. Rocha, and H. P. Diogo, J. Non-Cryst. Solids 344, 119 (2004).

2004ROU/TEM M. V. Roux, M. Temprado, R. Notario, S. P. Verevkin, V. N. Emel'Yanenko, D. Demasters, and J. F. Liebman, Mol. Phys. 102, 1909 (2004).

2004RYA/LEB M. V. Ryabkov, B. V. Lebedev, N. N. Smirnova, E. V. Agina, S. Ponomarenko, E. A. Makeev, N. I. Boiko, and V. P. Shibaev, Russ. J. Phys. Chem. 78, 1718 (2004).

2004SAB/MAR J. Sabolovi, E. Marak, S. Kotrun, and A. Janekovi, Inorg. Chem. 43, 8479 (2004).

2004SAI/MAS K. Saito, M. Massalska-Arodz, S. Ikeuchi, M. Maekawa, J. Sciesinski, E. Sciesinska, J. Mayer, T. Wasiutynski, and M. Sorai, J. Phys. Chem. B 108, 5785 (2004).

2004 SAN/SCH $\quad$ L. M. N. B. F. Santos, B. Schroder, O. O. P. Fernandex, and M. A. V. Ribeiro da Silva, Thermochim. Acta 415, 15 (2004).

2004SAW/MOK T. Sawaya, I. Mokbel, E. Rauzy, J. Saab, C. Berro, and J. Jose, Fluid Phase Equilib. 226, 283 (2004).

2004 SHA/TAN $\quad$ B. L. Sharma, S. Tandon, R. Kant, and R. Sharma, Thermochim. Acta 421, 161 (2004).

2004SMI/LEB N. N. Smirnova, B. V. Lebedev, O. V. Stepanova, A. M. Muzafarov, and E. A. Tatarinova, Russ. J. Phys. Chem. 78, 12 (2004).

2004SOH/SEO Y. T. Sohn and H. O. Seo, Arch. Pharm. Res. 27, 357 (2004).

2004SON/TAN Y.-J. Song, Z. C. Tan, S. W. Lu, and Y. Xue, J. Therm. Anal. Calorim. 77, 873 (2004).

2004STE/CHI W. V. Steele, R. D. Chirico, S. E. Knipmeyer, and A. Nguyen, J. Chem. Thermodyn. 36, 845 (2004).

2004 STE/CHI2 W. V. Steele, R. D. Chirico, A. B. Cowell, A. Nguyen, and S. E. Knipmeyer, J. Chem. Thermodyn. 36, 497 (2004).

2004STE/STI A. Stefanov, A. Stibor, A. Dominguez-Clarimon, and M. Arndt, J. Chem. Phys. 121, 6935 (2004).

2004STI/VAL A. L. Stinchcomb, S. Valiveti, D. C. Hammell, and D. R. Ramsey, J. Pharm. Pharmacol. 56, 291 (2004).

2004STU/WIT L. Sturz, V. T. Witusiewicz, U. Hecht, and S. Rex, J. Cryst. Growth 270, 273 (2004).

2004SUN/LIU X.-H. Sun, Y.-F. Liu, Z.-C. Tan, Y.-Y. Di, H.-F. Wang, and M.-H. Wang, J. Chem. Thermodyn. 36, 895 (2004).

2004SUN/SON X.-H. Sun, J.-R. Song, Z.-C. Tan, Y.-Y. Di, M.-H. Wang, M.-X. Ma, H.-F. Wang, and L.-X. Sun, Youji Huazue 24, 409 (2004).
2004SUN/SON2 X-H. Sun, J.-R. Song, Z.-C. Tan, Y.-Y. Di, H.-X. Ma, M.H. Wang, and L.-X. Sun, Thermochim. Acta 413, 261 (2004).

2004TYA/BIS O. S. Tyagi, H. S. Bisht, and A. K. Chatterjee, J. Phys. Chem. B 108, 3010 (2004).

2004UUS/POK P. Uusi-Kyyny, J.-P. Pokki, Y. Khim, and J. Aittamaa, J. Chem. Eng. Data 49, 251 (2004).

2004VAL/KIP S. Valiveti, P. K. Kiptoo, D. C. Hammell, and A. L. Stinchcomb, Int. J. Pharm. 278, 173 (2004).

2004VAN/MAL E. V. Van Tonder, T. S. P. Maleka, W. Liebenberg, M. Song, D. E. Wurster, and M. M. de Villiers, Int. J. Pharm. 269, 417 (2004).

2004VEN/CAL L. Ventola, T. Calvet, M. A. Cuevas-Diarte, M. Ramirez, H. A. J. Oonk, D. Mondieig, and P. Negrier, Phys. Chem. Chem. Phys. 6, 1786 (2004).

2004VER S. P. Verevkin, Fluid Phase Equilib. 225, 145 (2004).

2004VER/VAS S. P. Verevkin and T. V. Vasiltsova, J. Chem. Eng. Data 49, 1717 (2004).

2004WAN/TAN M.-H. Wang, Z.-C. Tan, X.-H. Sun, F. Xu, L.-B. Kong, L.-X. Sun, and T. Zhang, Thermochim. Acta 411, 203 (2004).

2004XU/SUN $\quad$ F. Xu, L.-X. Sun, Z.-C. Tan, J.-G. Liang, and R.-L. Li, Thermochim. Acta 412, 33 (2004).

2004YAM/NEM H. Yamamoto, N. Nemoto, and K. Tashiro, J. Phys. Chem. B 108, 5827 (2004).

2004ZEL/CHU L. N. Zelenina, T. P. Chusova, and Y. G. Stenin, Zh. Fiz. Khim. 78, 598 (2004).

2005ALT/COP M. Altamura, G. Coppini, P. Cuda, P. Dapporto, A. Guerri, A. Guidi, C. Nativi, P. Paoli, and P. Rossi, J. Mol. Struct. 749, 20 (2005).

2005CAR/ROD T. M. Cardoso, P. O. Rodrigues, H. K. Stulzer, M. Antonio, S. Silva, and J. do Rosario Matos, Drug Dev. Ind. Pharm. 31, 631 (2005).

2005CHA/LAU D. Chandra, K. H. Lau, W.-M. Chien, and M. Garner, J. Phys. Chem. Solids 66, 241 (2005).

2005CHA/ZIE S. Chattopadhyay and P. Ziemann, J. Aerosol Sci. Technol. 39, 1085 (2005).

$2005 \mathrm{CHI} / \mathrm{STE} 2$ R. D. Chirico and W. V. Steele, J. Chem. Eng. Data 50, 1052 (2005).

2005DOM/MAR U. Domanska and M. Marciniak, Fluid Phase Equilib. 235, 30 (2005).

2005EME/STR V. N. Emel'yanenko, A. Strutynska, and S. P. Verevkin, J. Phys. Chem. A 109, 4375 (2005).

2005EME/VER V. N. Emel'yanenko, S. P. Verevkin, B. Koutek, and J. Doubsky, J. Chem. Thermodyn. 37, 73 (2005).

2005ESP/WHI

2005FAT/KAS P. Espeau and J. W. White, Carbon 43, 1885 (2005).

A. Fattahi, S. R. Kass, J. F. Liebman, M. A. R. Matos, M. S. Miranda, and V. M. F. Morais, J. Am. Chem. Soc. 127, 6116 (2005).

2005FLA/HAL S. Flanagan, E. Hall, W. Bowie, J. W. Fuhs, R. Logan, F. Maniel, and A. Hunt, Green Chem. 7, 333 (2005).

2005FUL/RUZ M. Fulem, K. Ruzicka, V. Ruzicka, T. Sinecsk, E. Hulicius, J. Pangrac, J. Becker, J. Koch, and A. Salzmann, J. Chem. Eng. Data 50, 1613 (2005).

2005GOF/SUP M. J. Goff, G. J. Suppes, and M. A. Dasari, Fluid Phase Equilib. 238, 149 (2005).

2005HAS/TAJ M. Hashimoto, T. Tajima, K. Eda, K. Yamamura, and T. Okazaki, J. Mol. Struct. 734, 23 (2005).

2005HOS/GRY M. Hoskovec, D. Grygarova, J. Cvacka, L. Strinz, J. Zima, S. P. Verevkin, and B. Koutek, J. Chromatogr. A 1083, 161 (2005).

2005HUA/SIM D. Huang, S. L. Simon, and G. B. McKenna, J. Chem. Phys. 122, 084907 (2005).

2005IKE/YAM K. Ikedou, H. Yamamoto, H. Nagashima, N. Nemoto, and K. Tashiro, J. Phys. Chem. B 109, 10668 (2005).

2005LEE/SU M.-J. Lee, C.-C. Su, and H.-M. Lin, J. Chem. Eng. Data 50, 1535 (2005).

2005LIZ/ZAB E. Lizarraga, C. Zabaleta, and J. A. Palop, Thermochim. Acta 427, 171 (2005).

2005MAN/ROH T. Manifar, S. Rohani, and M. Saban, Ind. Eng. Chem. Res. 44, 970 (2005).

2005MAT/MIR2 M. A. R. Matos, M. S. Miranda, N. A. B. Pinto, V. M. F. Morais, N. Dhananjaya, and J. F. Liebman, Mol. Phys. 103, 1885 (2005). 
2005MAT/VAN M. Matovic, J. C. van Miltenburg, J. Los, F. G. Gandolfo, and E. Flöter, J. Chem. Eng. Data 50, 1624 (2005).

2005MET/LEF V. Metivaud, A. Lefevre, L. Ventola, P. Negrier, E. Moreno, T. Calvet, D. Mondieig, and M. A. CuevasDiarte, Chem. Mater. 17, 3302 (2005).

2005MUR/BET P. Mura, G. P. Bettinetti, M. Cirri, F. Maestrelli, M. Sorrenti, and L. Catenacci, Eur. J. Pharm. Biopharm. 59, 99 (2005).

2005NAK/SHI K. Nakajoh, E. Shibata, T. Todoroki, A. Ohara, K. Nishizawa, and T. Nakamura, Environ. Toxicol. Chem. 24, 1602 (2005).

2005NAZ/NES A. G. Nazmutdinov, I. A. Nesterov, T. N. Nesterova, and T. A. Nazmutdinov, Izvest. Vyssh. Uchebn. Zaved. Khim. Khim. Technol. 48, 18 (2005).

2005NIS/WAT Y. Nishikawa, T. Watanabe, H. Yoshida, and M. Ikeda, Thermochim. Acta 431, 81 (2005).

2005PAU/ZAI Y. U. Paulechka, D. H. Zaitsau, G. J. Kabo, and A. A. Strechan, Thermochim. Acta 439, 158 (2005).

2005PER/BAN S. Perisanu, M. D. Bancia, I. Contineau, A. Neascu, and L. Chivu, Rev. Chim. (Bucuresti) 56, 813 (2005).

2005PER/ROD G. L. Perlovich, S. V. Rodionov, and A. Bauer-Brandl, Eur. J. Pharm. Sci. 24, 25 (2005).

2005RES/GON J. M. Resa, C. Gonzalez, S. Ortiz de Landaluce, and J. M. Goenaga, J. Chem. Eng. Data 50, 319 (2005).

2005RIB/GOM M. A. V. Ribeiro da Silva, J. R. B. Gomes, and A. I. M. C. L. Ferreira, J. Phys. Chem. B 109, 13356 (2005).

2005RIB/RIB M. A. V. Ribeiro da Silva, M. D. M. C. Ribeiro da Silva, L. M. P. F. Amaral, J. Elguero, P. Jimenez, M. V. Roux, J. Z. Davalos, M. Temprado, P. Cabildo, R. M. Claramunt, O. Mo, and M. Yanez, J. Chem. Thermodyn. 37, 1168 (2005).

2005ROU/DAV M. V. Roux, J. Z. Davalos, P. Jimenez, R. Notorio, O. Castano, J. S. Chickos, W. Hanshaw, H. Zhao, N. Rath, J. F. Liebman, B. S. Farivar, and A. Bashir-Hashemi, J. Org. Chem. 70, 5461 (2005).

2005ROU/TEM M. V. Roux, M. Temprado, and J. S. Chickos, J. Chem. Thermodyn. 37, 941 (2005).

2005RYA/GUL S. P. Ryan, B. K. Gullett, D. Tabor, L. Oudejans, and A. Touati, Chem. Eng. Sci. 60, 787 (2005).

2005SAI/MAR C. I. Sainz-Diaz, A. P. Martin-Islan, and J. H. E. Cartwright, J. Phys. Chem. B 109, 18758 (2005).

2005SBI/VEC N. Sbirrazzuoli, S. Vecchio, and A. Catalani, Int. J. Chem. Kinet. 37, 74 (2005).

2005SCH A. C. Schmidt, Int. J. Pharm. 298, 186 (2005).

2005SEM/IGU P. P. Semyannikov, I. K. Igumenov, S. V. Trubin, T. P. Chusova, and Z. I. Semenova, Thermochim. Acta 432, 91 (2005).

2005SIK/MOD M. Sikorska-Iwan and B. Modzelewska-Banachrewicz, J. Therm. Anal. Calorim. 81, 119 (2005).

2005SMI/KUL N. N. Smirnova, T. G. Kulagina, A. V. Markin, Z. B. Shifrina, and A. L. Rusanov, Thermochim. Acta 425, 39 (2005).

2005STE/VOI S. Steinert, W. Voigt, R. Glausch, and M. Neuschutz, Thermochim. Acta 435, 28 (2005).

2005STR/SPO A. Strutynska, A. Sporzynski, J. Serwatowski, and S. P. Verevkin, Fluid Phase Equilib. 227, 283 (2005).

2005SUN/LIU X.-H. Sun, Y.-F. Liu, Z.-C. Tan, M.-H. Wang, and J.-Q. Jia, Chem. Res. Chin. Univ. 21, 697 (2005).

2005SUN/LIU2 X.-H. Sun, Y.-F. Liu, Z.-C. Tan, Y.-Q. Jia, M.-H. Wang, and Y.-Y. Di, Chin. J. Chem. 23, 23 (2005).

2005SUN/LIU4 X.-H. Sun, Y.-F. Liu, Z.-C. Tan, Y.-Q. Jia, J.-W. Yang, and M.-H. Wang, Chin. J. Chem. 23, 501 (2005).

2005TOK/HAY H. Tokuda, K. Hayamizu, K. Ishii, M. A. B. H. Susan, and M. Watanabe, J. Phys. Chem. B 109, 6103 (2005)

2005TOM/MIZ S. Tomitaka, M. Mizukami, F. Paladi, and M. Oguni, J. Therm. Anal. Calorim. 81, 637 (2005).

2005VAN/OON J. C. van Miltenburg and H. A. J. Oonk, J. Chem. Eng. Data 50, 1348 (2005).

2005VAR/DRU R. M. Varushchenko, A. I. Druzhinina, V. M. Senyavin, and V. S. Sarkisova, J. Chem. Thermodyn. 37, 141 (2005).

2005 VEC/BRU S. Vecchio and B. Brunetti, J. Chem. Eng. Data 50, 666 (2005); Note: Complete chemical numbers for several of the compounds were obtained through personal corresponence with S. Vecchio.
2005VEN/CUE

L. Ventola, M. A. Cuevas-Diarte, T. Calvet, I. Angulo, M. Vivanco, M. Bernar, G. Bernar, M. Melero, and D. Mondieig, J. Phys. Chem. Solids 66, 1668 (2005).

2005VOG/COH F. G. Vogt, D. E. Cohen, J. D. Bowman, G. P. Spoors, G. E. Zuber, G. A. Trescher, P. C. Dell'orco, L. M. Katrincic, G. W. Debrosse, and R. C. Haltiwanger, J. Pharm. Sci. 94, 651 (2005).

2005XU/SUN F. Xu, L.-X. Sun, Z.-C. Tan, R.-L. Li, Q.-F. Tian, and T. Zhang, Wuli Huaxue Xuebao 21, 1 (2005).

2005XUE/WAN B. Xue, J. Wang, Z.-C. Tan, T. Wu, Z. Zhang, and H. Chen, Huagong Xuebao 56, 570 (2005).

2005YAN/DEV W. Yang and M. M. de Villiers, AAPS J. 7, E241 (2005).

2005YAN/MAN W. Yang, R. Manek, W. M. Kolling, M. Brits, W. Liebenberg, and M. M. De Villiers, Supramol. Chem. 17, 485 (2005).

2005YAO/BAI W.-W. Yao, T.-C. Bai, J.-P. Sun, C.-W. Zhu, J. Hu, and H.-L. Zhang, Thermochim. Acta 437, 17 (2005).

2005YI/WAN W. Yi, C. Wang, H. Li, and S. Han, J. Chem. Eng. Data 50, 1837 (2005).

2005ZHA/BAI H.-L. Zhang, T.-C. Bai, G.-B. Yan, and J. Hu, Fluid Phase Equilib. 238, 186 (2005).

2005ZHA/STA G. I. Zharkova, P. A. Stabnikov, S. A. Sysoev, and I. K. Igumenov, J. Struct. Chem. 46, 320 (2005).

2006ACR/CHI W. E. Acree, Jr. and J. S. Chickos, J. Phys. Chem. Ref. Data 35, 1051-1330 (2006).

2006ADH/BAS R. Adhiyaman and S. K. Basu, Int. J. Pharm. 321, 27 (2006).

2006ARC D. G. Archer, NIST Internal Report 6645 (National Institute of Standards, Gaithersburg, 2006), as quoted in Ref. [2011HUG/SYE].

2006BAD/DEL E. Badea, G. Della Gata, D. D. Angelo, B. Brunetti, and Z. Reckova, J. Chem. Thermodyn. 38, 1546 (2006).

2006BAI/YAN T.-C. Bai, G.-B. Yan, J. Hu, H.-L. Zhang, and C.-G. Huang, Int. J. Pharm. 308, 100 (2006).

2006BAR/DAV J. Baran, N. A. Davydova, M. Drozd, and A. Pietraszko, J. Phys.: Condens. Matter 18, 5695 (2006).

2006BET/SOR G. Bettinetti, M. Sorrenti, L. Catenacci, F. Ferrari, and S Rossi, J. Pharm. Biomed. Anal. 41, 1205 (2006).

2006BLO/PAU A. V. Blokhin, Y. U. Paulechka, and G. J. Kabo, J. Chem. Eng. Data 51, 1377 (2006).

2006BOL/NER M. F. Bolotnikov and Y. A. Neruchev, Fluid Phase Equilib. 243, 121 (2006).

2006BOL/NER2 M. F. Bolotnikov and Y. A. Neruchev, Russ. J. Phys. Chem. 80, 1191 (2006).

2006CAP/TRA A. Cappelli, V. Travagli, I. Zanardi, M. Anzini, G. Giorgi, A. Donati, M. Aggravi, M. Casolaro, M. Fresta, E. Paccagnini, F. Makovec, and S. Vomero, J. Pharm. Sci. 95, 2706 (2006)

2006CAV/PAN G. Caviglioli, M. Pani, P. Gatti, B. Parodi, S. Cafaggi, and G. Bignardi, J. Pharm. Sci. 95, 2207 (2006).

2006CHE/OJA X. Chen, V. Oja, W. G. Chan, and M. R. Hajaligol, J. Chem. Eng. Data 51, 386 (2006).

2006COR/LOP P. Correia, C. Lopez, M. E. M. Piedade, J. A. A. Lourenco, and M. L. Serrano, J. Chem. Eng. Data 51, 1306 (2006).

2006DAB/SPO A. Dabrowska, A. Sporzynski, and S. P. Verevkin, Fluid Phase Equilib. 249, 115 (2006).

2006DIB/MEL V. N. Dibrivnyi, G. V. Mel'nik, Y. Y. Van-Chin-Syan, and A. P. Yuvchenko, Russ. J. Phys. Chem. 80, 330 (2006).

2006DiO/PIN H. P. Diogo, S. S. Pinto, and J. J. M. Ramos, J. Therm. Anal. Calorim. 83, 361 (2006).

2006DRE/SHA V. A. Drebushchak, T. P. Shakhtshneider, S. A. Apenina, T. N. Dresushchak, A. S. Medvedeva, L. P. Safronova, and V. V. Boldyrev, J. Therm. Anal. Calorim. 84, 643 (2006).

2006DRU/KRO A. I. Druzhinina, O. V. Krol, A. A. Efimova, R. M. Varushchenko, and L. L. Gervits, Russ. J. Phys. Chem. 80, 1742 (2006).

2006FIL/STA E. S. Filatov, P. A. Stabnikov, P. P. Semyannikov, S. V. Trubin, and I. K. Igumenov, Russ. J. Coord. Chem. 32, 126 (2006).

2006FIL/SYS E. S. Filatov, S. V. Sysoev, L. N. Zelenina, T. P. Chusova, V. A. Logvinenko, P. P. Semyannikov, and I. K. Igumenov, J. Therm. Anal. Calorim. 86, 537 (2006). 
2006FUL/RUZ M. Fulem, K. Ruzicka, V. Ruzicka, T. Simecek, E. Hulicius, and J. Pangrac, J. Chem. Thermodyn. 38, 312 (2006).

2006GEN/AMA A. Genovese, G. Amarasinghe, M. Glewis, D. Mainwaring, and R. A. Shanks, Thermochim. Acta 443, 235 (2006).

2006HAF/PAR J. J. H. Haftka, J. R. Parsons, and H. A. J. Govers, J. Chromatogr. A 1135, 91 (2006).

2006ISM F. A. Ismail, Drug Dev. Ind. Pharm. 32, 1199 (2006).

2006KHI/BOU K. Khimeche, Y. Boumrah, M. Benziane, and A. Dahmani, Thermochim. Acta 444, 166 (2006).

2006KHI/DAH K. Khimeche and A. Dahmani, J. Therm. Anal. Calorim. 84, 47 (2006).

2006KHI/DAH2 K. Khimeche and A. Dahmani, J. Chem. Eng. Data 51, 382 (2006).

2006KRA/VER E. L. Krasnykh, S. P. Verevkin, B. Koutek, and J. Doubsky, J. Chem. Thermodyn. 38, 717 (2006).

2006KUR/PER S. V. Kurkov, G. L. Perlovich, and W. Zielenkiewicz, J. Therm. Anal. Calorim. 83, 549 (2006).

2006KUZ/ALT N. P. Kuz'mina, A. E. Altsybeev, I. P. Malkerova, A. S. Alikhanyan, and I. E. Korsakov, Russ. J. Inorg. Chem. 51, 1750 (2006).

2006LI/ROD J. Li, M. Rodriques, A. Paiva, H. A. Matos, and E. G. de Azevedo, Fluid Phase Equilib. 241, 196 (2006).

2006LI/STO H. Li, J. G. Stowell, T. B. Borchardt, and S. R. Bryn, Cryst. Growth Des. 11, 2469 (2006).

2006LIS/SEM T. I. Liskovskaya, P. P. Semyannikov, I. A. Baidina, P. A. Stabnikov, N. V. Pervukhina, V. A. Logvinenko, I. K. Igumenov, and I. E. Sokolov, J. Struct. Chem. 47, 726 (2006).

2006MAN/ROH T. Manifar, S. Rohani, M. Jennings, D. Hairsine, and I. Dance, CrystEngComm 8, 59 (2006).

2006MAR/KON P. J. Marsac, H. Konno, and L. S. Taylor, Pharm. Res. 23, 2306 (2006)

2006MAR/STA B. Marchiniec, M. Stawny, M. Kozak, and M. Naskrent, J. Therm. Anal. Calorim. 84, 741 (2006).

2006MAT/MIR2 M. A. R. Matos, M. S. Miranda, V. M. F. Morais, and J. F. Liebman, Mol. Phys. 104, 2855 (2006).

2006MON/SAN M. J. S. Monte, L. M. N. B. F. Santos, M. Fulem, J. M. S. Fonseca, and C. A. D. Sousa, J. Chem. Eng. Data 51, 757 (2006).

2006MUL/MOZ P. Mulder, O. Mozenson, S. Lin, C. E. S. Bernardes, M. E. Minas da Piedade, A. F. L. O. Santos, M. A. V. Ribeiro da Silva, G. A. DiLabio, H.-G. Korth, and K. U. Ingold, J. Phys. Chem. A 110, 9949 (2006).

2006NAK/SHI K. Nakajoh, E. Shibota, T. Todoroki, A. Ohara, K. Nishizawa, and T. Nakamura, Environ. Toxicol. Chem. 25, 327 (2006).

2006NAS/NEU K. Nasurzadeh, R. Neueder, and W. Kunz, J. Chem. Eng. Data 51, 7 (2006).

2006NIC/KWE G. Nichols, S. Kweskin, M. Frericks, S. Reiter, G. Wang, J. Orf, B. Carvallo, D. Hillesheim, and J. S. Chickos, J. Chem. Eng. Data 51, 475 (2006).

2006NIE/GON Q. Nie, J. B. Gong, J. K. Wang, and S. Wang, Ind. Eng. Chem. Res. 45, 432 (2006).

2006PAS/ALB N. Passerini, B. Albertini, B. Perissutti, and L. Rodriguez, Int. J. Pharm. 318, 92 (2006)

2006PER/CON S. Perisanu, I. Continanu, M. D. Banciu, H. Zhao, N. Ruth, and J. S. Chickos, Struct. Chem. 17, 639 (2006).

2006REN/JIN F. Ren, Q. Jing, Y. Tang, Y. Shen, J. Chen, F. Gao, and J. Cui, Drug Dev. Ind. Pharm. 32, 967 (2006).

2006RIB/AMA2 M. A. V. Ribeiro da Silva, L. M. P. F. Amaral, A. F. L. O. M. Santos, and J. R. B. Gomes, J. Chem. Thermodyn. 38, 367 (2006)

2006RIB/AMA3 M. A. V. Ribeiro da Silva, L. M. P. F. Amaral, A. F. L. O. M. Santos, and J. R. B. Gomes, Chem. Thermodyn. 38, 748 (2006).

2006RIB/AMA4 M. A. V. Ribeiro da Silva, L. M. P. F. Amaral, F. C. R. Guedes, and J. R. B. Gomes, J. Phys. Org. Chem. 19, 689 (2006).

2006RIB/CAB M. A. V. Ribeiro da Silva and J. I. T. A. Cabral, J. Chem. Eng. Data 51, 1556 (2006).
2006RIB/CAB2

M. A. V. Ribeiro da Silva and J. I. T. A. Cabral, J. Chem. Eng. Data 51, 767 (2006).

2006RIB/MON M. A. V. Ribeiro da Silva, M. J. S. Monte, and L. M. N B. F. Santos, J. Chem. Thermodyn. 38, 778 (2006).

2006RIB/SAN M. A. V. Ribeiro da Silva, C. P. F. Santos, M. J. S. Monte, and C. A. D. Sousa, J. Therm. Anal. Calorim. 83, 533 (2006).

2006RIB/SAN2 M. A. V. Ribeiro da Silva and L. M. N. F. F. Santos, J. Chem. Thermodyn. 38, 817 (2006).

2006RIB/SAN3 M. A. V. Ribero da Silva, L. M. N. B. F. Santos, and B. Schoder, J. Chem. Thermodyn. 38, 1455 (2006).

2006SAN/BER R. C. Santos, C. E. S. Bernardes, H. P. Diogo, M. F. M. Piedade, J. N. C. Lopes, and M. E. Minas da Piedade, J. Phys. Chem. A 110, 2299 (2006).

2006SAW/MOK T. Sawaya, I. Mokbel, N. Ainous, E. Rauzy, C. Berro, and J. Jose, J. Chem. Eng. Data 51, 854 (2006).

$2006 \mathrm{SCH} / \mathrm{SCH} \quad$ A. C. Schmidt, I. Schwarz, and K. Mereiter, J. Pharm. Sci. 95, 1097 (2006).

2006SEM/BAS P. Semyannikov, T. Basova, S. Trubin, E. Kol'tsov, and I. Igumenov, J. Porphyrins Phthalocyanines 10, 1034 (2006).

2006SEM/IGUＰ. P. Semyannikov, I. K. Igumenov, S. V. Trubin, and Z. I. Semenova, Thermochim. Acta 451, 80 (2006).

2006SHE/KAB M. P. Shevelyova, G. J. Kabo, A. V. Blokhin, A. G. Kabo, J. A. Jursha, and A. A. Rajko, J. Chem. Eng. Data 51, 40 (2006).

2006SHI/OHT Y. Shimizu, Y. Ohte, Y. Yamamura, K. Saito, and T. Atake, J. Phys. Chem. B 110, 13970 (2006).

2006SUN/LIU X.-H. Sun, Y.-F. Liu, Z.-C. Tan, Y.-Q. Jia, and M.H. Wang, Gaodeng Xuexia Huaxue Xuebao 27, 1109 (2006); Chem. Abstr. 145, 426927 (2006).

2006 TEI/GON $\quad$ A. C. T. Teixeira, A. M. P. S. Goncalves da Silva, and A. C. Fernandes, Chem. Phys. Lipids 144, 160 (2006).

2006TEO/BAR M. Teodorescu, A. Barhala, and D. Dragoescu, J. Chem. Thermodyn. 38, 1432 (2006).

2006TRO/CER J. Troncoso, C. A. Cerdeirina, Y. A. Sammamed, L. Romani, and L. P. N. Rebelo, J. Chem. Eng. Data 51, 1856 (2006).

2006UMN/KWE P. Umnahanant, S. Kweskin, G. Nichols, M. J. Dunn, H. Smart-Ebinne, and J. S. Chickos, J. Chem. Eng. Data 51, 2246 (2006)

2006VEG/PET D. Vega, A. Petragalli, D. Fernandez, and J. A. Ellena, J. Pharm. Sci. 95, 1075 (2006).

2006VEG/POL D. R. Vega, G. Polla, A. Martinez, E. Mendioroz, and M. Reinoso, Int. J. Pharm. 328, 112 (2006).

2006VEN/MET L. Ventola, V. Metivaud, L. Bayes, R. Benzges, M. A. Cuevas-Diarte, T. Calvet, and D. Mondieig, Helv. Chim. Acta 89, 2027 (2006).

2006VER

2006VER/KOZ S. P. Verevkin, J. Chem. Thermodyn. 38, 1111 (2006).

S. P. Verevkin, S. A. Kozlova, V. N. Emel'yanenko, E. D. Nikitin, A. P. Popov, and E. L. Krasnykh, J. Chem. Eng. Data 51, 1896 (2006).

2006WAN/TAN M.-H. Wang, Z.-C. Tan, Q. Shi, L.-X. Sun, and T. Zhang, J. Therm. Anal. Calorim. 84, 413 (2006).

2006WAN/TOZ S. Wang, K. Tozaki, H. Hayashi, H. Inaba, and H. Yamamoto, Thermochim. Acta 448, 73 (2006).

2006WAS/HOL C. M. Wassvik, A. G. Holmen, C. A. S. Bergstrom, I Zamora, and P. Artursson, Eur. J. Pharm. Sci. 29, 294 (2006).

2006ZAI/KAB I. H. Zaitsau, G. J. Kabo, A. A. Strechan, Y. U. Paulechka, A. Tschersich, S. P. Verevkin, and A. Heintz, J. Phys. Chem. A 110, 7303 (2006).

2006ZEL/CHU L. N. Zelenina, T. P. Chusova, Y. G. Stenin, and V. V. Bakovets, Russ. J. Phys. Chem. 80, 139 (2006).

$2006 Z E L / C H U 2$ L. N. Zelenina, T. P. Chusova, Y. G. Stenin, and G. A. Berezovskii, Russ. J. Phys. Chem. A 80, 1911 (2006).

2006ZHA/BAI G. I. Zharkova, I. A. Baidina, P. A. Stabnikov, and I. K. Igumenov, J. Struct. Chem. 47, 716 (2006).

2007ABE/BOU M. Abes, L. Bouzidi, and S. S. Narine, Chem. Phys. Lipids 150, 89 (2007).

2007ARM/HUR J. P. Armstrong, C. Hurst, R. G. Jones, P. Licence, K. R. J. Lovelock, C. J. Satterley, and I. J. Villar-Garcia, Phys. Chem. Chem. Phys. 9, 982 (2007). 
2007BAB/SUB P. R. S. Babu, C. V. S. Subrahmanyam, J. Thimmasetty, R. Manavalan, and K. Valliappan, Pak. J. Pharm. Sci. 20, 311 (2007)

2007BAD/BLA E. Badea, I. Blanco, and G. Della Gatta, J. Chem. Thermodyn. 39, 1392 (2007).

2007BAR/PRO R. Barbas, R. Prohens, and G. Puigjaner, J. Therm. Anal. Calorim. 89, 687 (2007).

2007BAS/AND A. Bashkirava, P. C. Andrews, P. C. Junk, E. G. Robertson, L. Spiccia, and N. Vanderhoek, Chem. - Asian J. 2, 530 (2007).

2007BER/WAS C. A. S. Bergstrom, C. A. M. Wassvik, K. Johansson, and I. Hubatsch, J. Med. Chem. 50, 5858 (2007).

2007BES/BAI A. A. Bessonov, I. A. Baidina, N. B. Morozova, P. P. Semyannikov, S. V. Trubin, N. V. Gelfond, and I. K. Igumenov, J. Struct. Chem. 48, 282 (2007).

2007BES/MOR A. A. Bessonov, N. B. Morozova, N. V. Gelfond, P. P. Semyannikov, S. V. Trubin, Y. V. Shevtsov, Y. V. Shubin, and I. K. Igumenov, Surf. Coat. Technol. 201, 9099 (2007).

2007BLA/SCH P. Blasi, A. Schoubben, S. Giovagnoli, L. Perioli, M. Ricci, and C. Rossi, AAPS PharmSciTech 8, 37 (2007).

2007BON/CAT M. G. Bonicelli, A. Catalani, G. Mariano, and S. Vecchio, Thermochim. Acta 466, 69 (2007).

2007CAI/BET M. R. Caira, G. Bettinetti, M. Sorrenti, and L. Catenacci, J. Pharm. Sci. 96, 996 (2007).

2007CAN/EYL A. I. Canizo, G. N. Eyler, and G. P. Barreto, Chromatographia 65, 21 (2007).

2007CAP/LOV C. D. Cappa, E. R. Lovejoy, and A. R. Ravishankara, J. Phys. Chem. A 111, 3099 (2007).

2007CHE/HUM L. Chebil, C. Humeau, J. Anthoni, F. Dehez, J.-M. Engasser, and M. Ghoul, J. Chem. Eng. Data 52, 1552 (2007).

2007CHE/KIM Y.-H. Cheon and K.-J. Kim, J. Chem. Eng. Data 52, 1390 (2007).

2007CHI/JOH R. D. Chirico, R. D. Johnson III, and W. V. Steele, J. Chem. Thermodyn. 39, 698 (2007).

2007CHU/ZEL T. P. Chusova, L. N. Zelenina, Y. G. Stenin, Z. I. Semenova, and V. A. Titov, Russ. Chem. Bull. 56, 1313 (2007).

2007DEF/RAN G. Defossemont, S. Randzio, and B. Legendre, J. Therm. Anal. Calorim. 89, 751 (2007).

2007DOM/LUG U. Domanska, K. Lugowska, and J. Pernak, J. Chem. Thermodyn. 39, 729 (2007).

2007DRA/JAN L. C. Draucker, M. Janakat, M. J. Lazzaroni, D. Bush, C. A. Eckert, T. C. Frank, and J. D. Olson, Ind. Eng. Chem. Res. 46, 2198 (2007).

2007DRU/EFI A. I. Druzhinina, A. A. Efimova, R. M. Varushchenko, and N. V. Chelovskaya, Russ. J. Phys. Chem. A 81, 2090 (2007).

2007EME/VER V. N. Emel'yanenko, S. P. Verevkin, O. V. Krol, R. M. Varushchenko, and N. V. Chelovskaya, J. Chem. Thermodyn. 39, 594 (2007).

2007EME/VER2 V. N. Emel'yanenko, S. P. Verevkin, and A. Heintz, J. Am. Chem. Soc. 129, 3930 (2007).

2007ESP/NIC P. Espeau, B. Nicolai, R. Ceolin, M.-A. Perrin, L. Zaske, J. Giovannini, and F. Leveiller, J. Therm. Anal. Calorim. 90, 341 (2007).

2007GBA/NEG G. Gbabode, P. Negrier, D. Mondieig, E. M. Calvo, T. Calvet, and M. A. Cuevas-Diarte, Chem. - Eur. J. 13, 3150 (2007).

2007GIR/SKL G. V. Girichev, S. A. Sklykov, N. I. Giricheva, A. V. Krasnov, I. O. Zyabko, N. P. Kuz'mina, and I. G. Zaitseva, Russ. J. Phys. Chem. A 81, 524 (2007).

2007GNA/PLA I. I. Gnatyuk, N. V. Platovova, G. A. Puchkovskaya, E. N. Kotelnikova, S. K. Filatov, J. Baran, and M. Drozd, J. Struct. Chem. 48, 654 (2007).

2007GOE/MCC A. Goel, L. L. McConnell, and A. Torrents, J. Environ. Sci. Health, Part B 42, 343 (2007).

$2007 \mathrm{GOM} / \mathrm{SOU}$ J. R. B. Gomes, E. A. Sousa, J. M. Goncalves, L. Gales, A. M. Damas, P. Gomes, S. Pandey, W. E. Acree, Jr., and M. D. M. C. Ribeiro da Silva, J. Phys. Org. Chem. 20, 491-498 (2007).

2007GOM/SOU2 J. R. B. Gomes, E. A. Sousa, P. Gomes, N. Vale, J. M. Goncalves, S. Pandey, W. E. Acree, Jr., and M. D. M. C. Ribeiro da Silva, J. Phys. Chem. B 111, 2075 (2007).
2007GRO/DEV

D. Grooff, M. M. De Villiers, and W. Liebenberg, Thermochim. Acta 454, 33 (2007).

2007GRU/ALE N. I. Gruzinskaya, V. E. Aleshina, A. Y. Borshchevskii, S. I. Troyanov, and L. N. Sidorov, Russ. J. Phys. Chem. A 81, 312 (2007).

2007GUP/SIN R. K. Gupta, S. K. Singh, and R. A. Singh, J. Cryst. Growth 300, 415 (2007).

2007HAF/MAH S. L. Hafsaoui and R. Mahmoud, J. Therm. Anal. Calorim. 88, 565 (2007).

2007HEI/KAP A. Heintz, S. Kapteina, and S. P. Verevkin, J. Phys. Chem. A 111, 6552 (2007).

2007HIM/MAC C. Himanwan, W. MacNaughtan, I. A. Farhat, and A. G. F. Stapley, Eur. J. Lipid Sci. Technol. 109, 49 (2007).

2007IGU/SEM I. K. Igumenov, P. P. Semyannikov, S. V. Trubin, N. B. Morozova, N. V. Gelfond, A. V. Mischenko, and J. A. Norman, Surf. Coat. Technol. 201, 9003 (2007).

2007JUN/KIM S. W. Jun, M. S. Kim, J. S. Kim, H. J. Park, S. Lee, J. S. Woo, and S. J. Hwang, Eur. J. Pharm. Biopharm. 66, 413 (2007).

2007KAN/SOR Y. Kaneko and M. Sorai, Phase Transitions 80, 517 (2007).

2007KAR/KAB L. S. Karpushenkava, G. J. Kabo, A. B. Bazyleva, A. V. Blokhin, A. G. Kabo, D. H. Zaitsau, A. A. Pinerzin, and V. S. Sarkisova, Thermochim. Acta 459, 104 (2007).

$2007 \mathrm{KAR} / \mathrm{KAB} 2$ L. S. Karpushenkava, G. J. Kabo, and V. V. Diky, Fullerenes, Nanotubes, Carbon Nanostruct. 15, 227 (2007).

2007KRO/DRU O. V. Krol, A. I. Druzhinina, R. M. Varushchenko, O. V. Dorofeyeva, M. D. Rechetova, and N. Y. Borisova, Vest. Moskov. Univer., Ser. 2: Khim. 48, 372 (2007).

2007KUR/MAE H. Kuramochi, K. Maeda, and K. Kawamoto, Chemosphere 67, 1858 (2007).

2007 KUT/POL A. M. Kut'in, V. S. Polyakov, M. F. Churbanov, and G. E. Snopatin, Inorg. Mater. 43, 1018 (2007).

2007LAR/PER F. Lara-Ochoa, G. E. Perez, and F. Miyangos-Santiago, J. Mol. Struct. 840, 97 (2007).

2007LEE/LAI M.-J. Lee, C.-H. Lai, T.-B. Wang, and H.-M. Lin, J. Chem. Eng. Data 52, 1291 (2007).

2007LI/DIN

2007LIP/KAP

2007LIU/LIA

W.-D. Li and E.-Y. Ding, Mater. Lett. 61, 1526 (2007). Chickos, Thermochim. Acta 456, 94 (2007).

2007LUK/PAP V. A. Luk'yanova, T. S. Papina, V. P. Kolesov, N. V. Yu Jishu 14, 25 (2007). Chelovskaya, A. N. Fedotov, P. I. Dem'yanov, N. V. Avramenko, and V. P. Korob'eva, Russ. J. Phys. Chem. 81, 1 (2007).

2007MAL/ALI I. P. Malkerova, A. S. Alikhanyan, S. V. Eliseeva, V. A. Ketsko, and N. P. Kuz'mina, Russ. J. Inorg. Chem. 52, 918 (2007).

2007MAT/MOR M. A. R. Matos, V. M. F. Morais, C. C. S. Sousa, M. V. Roux, R. Notario, and J. F. Liebman, Mol. Phys. 105, 1789 (2007).

2007MIS/MIS A. K. Misra, M. Misra, G. M. Panpalia, and A. K. Dorle, J. Macromol. Sci., Part A 44, 685 (2007).

2007MOK/RAZ M. Mokbel, A. Razzouk, A. Hajjaji, N. Misakni, and J. Jose, J. Chem. Eng. Data 52, 1720 (2007).

2007MON/PER M. Moneghini, B. Perissutti, F. Vecchione, I. Kikic, P. Alessi, and A. Cortesi, Curr. Drug Delivery 4, 241 (2007).

2007MOO/KOE D. J. Moore, D. Koelmel, D. Laura, and E. Bedford, Chem. Phys. Lipids 150, 109 (2007).

2007MOR/COR E. Moreno, R. Cordobilla, T. Calvet, M. A. Cuevas-Diarte, G. Gbabode, P. Negrier, D. Mondieig, and H. A. J. Oonk, New J. Chem. 31, 947 (2007).

2007NIS/WAN K. Nishikawa, S. Wang, H. Katayanagi, S. Hayashi, H. Hamaguchi, Y. Koga, and K.-I. Tozaki, J. Phys. Chem. B 111, 4894 (2007)

2007NOV/PEE H. Novoa de Armas, O. M. Peeters, G. Van den Mooter, and N. Blaton, J. Pharm. Sci. 96, 1114 (2007).

2007PAN/ANT R. Pankajavalli, S. Anthonysamy, K. Ananthasivan, and P. P. V. Rao, J. Nucl. Mater. 362, 128 (2007).

2007PAN/ANT2 K. Panneerselvam, M. P. Antony, T. G. Srinivasan, and P. R. Vasudeva Rao, Thermochim. Acta 466, 49 (2007). 
2007PAS/BET I. Pasquali, R. Bettini, and F. Giordano, J. Therm. Anal. Calorim. 90, 903 (2007).

2007PAS/KUZ L. L. Pashchenko and T. S. Kuznetsova, Russ. J. Phys. Chem. A 81, 1238 (2007).

2007PAT/CAM R. Patino, M. Campos, and L. A. Torres, Inorg. Chem. 46, 9332 (2007).

2007PAU/BLO Y. U. Paulechka, A. V. Blokhin, G. J. Kabo, and A. A. Strechan, J. Chem. Thermodyn. 39, 866 (2007).

2007PAU/KAB Y. U. Paulechka, G. J. Kabo, A. V. Blokhin, A. S. Shaplov, E. I. Lozinskaya, and Y. S. Vygodskii, J. Chem. Thermodyn. 39, 158 (2007).

2007PER/STR G. L. Perlovich, N. N. Strakhova, V. P. Kazachenko, T. V. Volkova, V. V. Tkachev, K.-J. Schaper, and O. A. Raevsky, Int. J. Pharm. 334, 115 (2007).

2007PER/SUR G. L. Perlovich, A. O. Surov, L. K. Hansen, and A. BauerBrandl, J. Pharm. Sci. 96, 1031 (2007).

2007PER/SUR2 G. L. Perlovich, A. O. Surov, and A. Bauer-Brandl, J. Pharm. Biomed. Anal. 45, 679 (2007).

2007PER/VOL G. L. Perlovich, T. V. Volkova, and A. Bauer-Brandl, Mol. Pharm. 4, 929 (2007).

2007PIN/BER S. S. Pinto, C. E. S. Bernandes, H. P. Diogo, and M. E. Minas da Piedade, J. Chem. Thermodyn. 39, 1384 (2007).

2007RAM C. H. Ramirez, Ph.D. dissertation, Ohio State University, 2007.

2007RAM/CAV L. A. Ramos and E. T. G. Cavalheiro, J. Therm. Anal. Calorim. 87, 831 (2007).

2007RAM/ROJ L. G. Ramierez-Verduzco, A. Rojas-Aguilar, J. A. De los Reyes, J. A. Munoz-Arroyo, and F. Murrieta-Guevara, J. Chem. Eng. Data 52, 2212 (2007).

2007RAZ/MOK A. Razzouk, I. Mokbel, J. Garcia, J. Fernandez, N. Msakni, and J. Jose, Fluid Phase Equilib. 260, 248 (2007).

2007RIB/AMA2 M. A. V. Ribeiro da Silva, L. M. P. F. Amaral, and J. R. B. Gomes, J. Phys. Chem. B 111, 13033 (2007).

2007RIB/CAB M. A. V. Ribeiro da Silva and J. I. T. A. Cabral, J. Therm. Anal. Calorim. 90, 865 (2007).

2007RIB/MON M. A. V. Ribeiro da Silva, I. M. M. Monteiro, L. M. N. B. F. Santos, and B. Schroder, J. Chem. Thermodyn. 39, 767 (2007).

2007RIB/SAN M. A. V. Ribeiro da Silva and A. F. L. O. M. Santos, J. Therm. Anal. Calorim. 88, 7 (2007).

2007RIB/SAN2 M. A. V. Ribeiro da Silva, L. M. N. B. F. Santos, and E. Giera, J. Chem. Thermodyn. 39, 361 (2007).

2007RIB/SAN3 M. A. V. Ribeiro da Silva, C. P. F. Santos, M. J. S. Monte, and C. A. D. Sousa, J. Chem. Thermodyn. 39, 1363 (2007).

2007SAN/CAN L. M. N. B. F. Santos, J. N. Canongia Lopes, J. A. P. Coutinho, J. M. S. S. Esperanca, L. R. Gomes, I. M. Marrucho, and L. P. N. Rebelo, J. Am. Chem. Soc. 129, 284 (2007).

2007SHI/OHT Y. Shimizu, Y. Ohte, Y. Yamamura, and K. Saito, Chem. Lett. 36, 1484 (2007).

2007SID/ATA M. A. Siddiqi and B. Atakan, Thermochim. Acta 452, 128 (2007).

2007SID/SID M. A. Siddiqu, R. A. Siddiqui, and B. Atakan, Surf. Coat. Technol. 201, 9055 (2007).

2007SIF/AIT H. Sifaoui, A. Ait-Kaci, A. Mondarressi, and M. Rogalski, Thermochim. Acta 456, 114 (2007).

2007SOU/CON M. A. F. Souza, M. M. Conceicao, M. C. D. Silva, L. E. B. Soledade, and A. G. Sousa, J. Therm. Anal. Calorim. 87, 859 (2007).

2007STR/PAU A. A. Strechan, Y. U. Paulechka, A. G. Kabo, A. V. Blokhin, and G. J. Kabo, J. Chem. Eng. Data 52, 1791 (2007).

2007TUR/UPP M. Turk, G. Upper, M. Steurenthaler, K. Hussein, and M. A. Wahl, J. Supercrit. Fluids 39, 435 (2007).

2007VEC S. Vecchio, J. Therm. Anal. Calorim. 87, 79 (2007).

2007VER/GEO S. P. Verevkin, M. Georgieva, and S. V. Melkhanova, J. Chem. Eng. Data 52, 286 (2007).

2007VIP/WAN S. R. Vippagunta, Z. Wang, S. Hornung, and S. L. Krill, J. Pharm. Sci. 96, 294 (2007).

2007WAN/NOV X. Wang, H. Novoa de Armas, N. Blaton, A. Michoel, and G. Van den Mooter, Int. J. Pharm. 345, 95 (2007).
2007WAN/TAN

2007YAM/SAI

S.-X. Wang, Z.-C. Tan, Y.-S. Li, Y. Li, Q. Shi, and B. Tong, Thermochim. Acta 463, 21 (2007).

2008ABA/BAD

Y. Yamamura, H. Saitoh, M. Sumaito, and K. Saito, J. Phys.: Condens. Matter 19, 176219 (2007).

2008ABB/KAL

L. Abate, E. Badea, I. Blando, and G. Della Gatta, J. Chem. Eng. Data 53, 959 (2008).

D. Abbas, J. Kaloustian, C. Orneto, P. Piccerelle, H. Portugal, and A. Nicolay, J. Therm. Anal. Calorim. 93, 353 (2008).

2008ARU/MAT T. S. Arul Jeevan, T. Mathews, V. S. Raghunathan, and K. S. Nagaraja, Thermochim. Acta 478, 41 (2008).

2008BAS/BOS

S. Basavoju, D. Bostrom, and S. Velaga, Pharm. Res. 25, 530 (2008).

2008BES/MOR A. A. Bessonov, N. B. Morozova, P. P. Semyannikov, S. V. Trubin, N. V. Gel'fond, and I. K. Igumenov, J. Therm. Anal. Calorim. 92, 751 (2008).

2008BES/MOR2 A. A. Bessonov, N. B. Morozova, P. P. Semynannikov, S. V. Trubin, N. V. Gel'fond, and I. K. Igumenov, Russ. J. Coord. Chem. 34, 186 (2008).

2008BLO/PAU A. V. Blokhin, Y. U. Paulechka, A. A. Strechan, and G. J. Kabo, J. Phys. Chem. B 112, 4357 (2008).

2008BRA/GEL D. E. Braun, T. Gelbrich, V. Kahlenberg, G. Laus, J. Wieser, and U. J. Griesser, New J. Chem. 32, 1677 (2008).

2008BRE/BRE A. A. Bredikhin, Z. A. Bredikhina, V. G. Novikova, A. V. Pashagin, D. V. Zakharychev, and A. T. Gubaidullin, Chirality 20, 1092 (2008).

2008BRU/LEL B. Brunetti, D. Lelo, V. Piacente, and P. Scardala, J. Chem. Eng. Data 53, 2101 (2008).

2008BRU/PIA B. Brunetti, V. Piacente, A. Latini, and P. Scardala, J. Chem. Eng. Data 53, 2493 (2008).

2008BUS/LAH S. Busi, M. Lahtinen, J. Valkonen, and K. Rissanen, J. Mol. Struct. 875, 549 (2008).

2008CAP/LOV C. D. Cappa, E. R. Lovejoy, and A. R. Ravishankara, J. Phys. Chem. A 112, 3959 (2008).

2008CAS/MAL G. Castaldi, L. Malpezzi, G. Razzetti, and G. Ventimiglia, Ital. patent IT 1349272 B1 20081120, 2008. [Chemical Abstracts Number 152:509949].

2008CEO/TAM R. Ceolin, J.-L. Tamarit, M. Barrio, D. O. Lopez, B. Nicolai, N. Veglio, M.-A. Perrin, and P. Espeau, J. Pharm. Sci. 97, 3927 (2008).

2008CHI/LIP J. S. Chickos and D. Lipkind, J. Chem. Eng. Data 53, 2432 (2008).

2008CHI/STE R. D. Chirico and W. V. Steele, J. Chem. Thermodyn. 40, 806 (2008).

2008CHI/WAN J. S. Chickos, T. Wang, and E. Sharma, J. Chem. Eng. Data 53, 481 (2008).

2008COG/HIL J. M. Cogen and A. J. Hilmer, Polym. Degrad. Stab. 93, $2193(2008)$

2008DI/WAN Y.-Y. Di, D.-Q. Wang, S. Quan, and Z.-C. Tan, Chin. Phys. B 17, 2859 (2008).

2008DIO/PIN H. P. Diogo, S. S. Pinto, and J. J. M. Ramos, Int. J. Pharm. 358, 192 (2008).

2008DOM/MAR U. Domanska, A. Marciniak, and M. Krlikowski, J. Phys. Chem. B 112, 1218 (2008).

2008DOM/REK U. Domanska, A. Rekawek, and A. Marciniak, J. Chem. Eng. Data 53, 1126 (2008).

2008DUD/DAN E. Dudognon, F. Danede, M. Descamps, and N. T. Correia, Pharm. Res. 25, 2853 (2008).

2008EGO/MAR V. M. Egorov, V. A. Marikhin, and L. P. Myasnikova, Phys. Solid State 50, 126 (2008).

2008FAN/WAN R. L. Fan, L.-S. Wang, and M.-Y. Li, J. Chem. Eng. Data 53, 228 (2008).

2008FRI/ACR V. L. S. Frietas, W. E. Acree, Jr., and M. D. M. C. Ribeiro da Silva, J. Chem. Eng. Data 53, 1820 (2008).

2008GBA/NEG G. Gbabode, P. Negrier, D. Mondieig, E. Moreno, T. Calvet, and M. A. Cuevas-Diarte, Chem. Phys. Lipids 154, 68 (2008).

2008GOL/SUU J. L. Goldfarb and E. M. Suuberg, Environ. Toxicol. Chem. 27, 1244 (2008).

2008GOL/SUU2 J. L. Goldfarb and E. M. Suuberg, J. Chem. Thermodyn. 40, 460 (2008).

2008GOL/SUU3 J. L. Goldfarb and E. M. Suuberg, J. Chem. Eng. Data 53, 670 (2008). 
2008GOM/AMA J. R. B. Gomes, L. M. P. F. Amaral, and M. A. V. Ribeiro da Silva, J. Phys. Org. Chem. 21, 365 (2008).

2008GRA/STR K. A. Graeser, C. J. Strachan, J. E. Patterson, K. C. Gordon, and T. Rades, Cryst. Growth Des. 8, 128 (2008).

2008GUA/YAN Y. Gua, F. Yang, Y. Xing, D. Li, W. Fang, and R. Lin, Energy Fuels 22, 510 (2008).

2008GUP/GAN P. K. Gupta, K. Ganesan, P. K. Gutch, L. Manral, and D. K. Dubey, J. Chem. Eng. Data 53, 841 (2008).

2008HAN/NUT W. Hanshaw, M. Nutt, and J. S. Chickos, J. Chem. Eng. Data 53, 1903 (2008); Erratum, ibid. 53, 2721 (2008).

2008JEE/ARO T. S. A. Jeevan, S. Arockiasamy, T. Mathews, V. S. Rahunathan, and K. S. Nagaraja, Mater. Lett. 62, 4170 (2008).

2008JIN/OHA H. Jin, B. O'Hare, J. Dong, S. Azhantsev, G. A. Baker, J. F. Wishart, A. J. Benesi, and M. Maroncelli, J. Phys. Chem. B 112, 81 (2008).

2008KOZ/EME S. A. Kozlova, V. N. Emel'yanenko, M. Georgieva, S. P. Verevkin, Y. Chernyak, B. Schaffner, and A. Borner, J. Chem. Thermodyn. 40, 1136 (2008).

2008KOZ/MAR M. S. Kozlova, A. V. Markin, V. N. Larina, and N. V. Karyzkin, Russ. J. Phys. Chem. A 82, 1993 (2008).

2008KRO/DRU O. V. Krol, A. I. Druzhinina, R. M. Varushchenko, O. V. Dorofeeva, M. D. Reshetova, and N. E. Borisova, J. Chem. Thermodyn. 40, 549 (2008).

2008 KUN/SHI G. V. Kunte, S. A. Shivashankar, and A. M. Umarji, Meas. Sci. Technol. 19, 025704 (2008).

2008KUR/KAW H. Kuramochi, K. Kawamoto, K. Miyazaki, K. Nagahama, K. Maeda, X.-W. Li, E. Shibata, T. Nakamura, and S.-I. Sakai, Environ. Toxicol. Chem. 27, 2413 (2008).

2008KUR/PER S. V. Kurkov and G. L. Perlovich, Int. J. Pharm. 357, 100 (2008).

2008LAG/JIM A. F. Lago, P. Jimenez, R. Herrero, J. Z. Davalos, and J.-L. M. Abboud, J. Phys. Chem. A 112, 3201 (2008).

2008LIP/KRA S. V. Lipp, E. L. Krasnykh, and S. V. Levanova, Russ. J. Phys. Chem. A 82, 2025 (2008).

2008LOU/PIN $\quad$ C. M. Lousada, S. S. Pinto, J. N. Canongia Lopes, M. F. Minas da Piedade, H. P. Diogo, and M. E. Minas da Piedade, J. Phys. Chem. A 112, 2977 (2008).

2008LUO/BAK H. Luo, G. A. Baker, and S. Dai, J. Phys. Chem. B 112, 10077 (2008).

2008MAR/RAM F. J. Martinez Casado, M. Ramos Riesco, A. Sanchez Arenas, M. V. Garci Perez, M. I. Redondo, S. LopezAndras, L. Garrido, and J. A. R. Cheda, J. Phys. Chem. B 112, 16601 (2008).

2008MAR/SEL M. Maria, J. Selvakumar, V. S. Raghunathan, T. Mathews, and K. S. Nagaraja, Thermochim. Acta 474, 87 (2008).

2008MAR/STA B. Marciniec, M. Stawny, M. Hofman, and M. Naskrent, J. Therm. Anal. Calorim. 93, 733 (2008).

2008MOG/SEP E. Mogalian, K. Sepassi, and P. B. Myrdal, Drug Dev. Ind. Pharm. 34, 930 (2008).

2008MON/SAN M. J. S. Monte, L. M. N. B. F. Santos, C. A. D. Sousa, and M. Fulem, J. Chem. Eng. Data 53, 1922 (2008).

2008MOR/ZHE N. B. Morozova, K. V. Zherikova, I. A. Baidina, S. V. Sysoev, P. P. Semyannikov, L. V. Yakovkina, T. P. Smirnova, N. V. Gelfond, I. K. Igumenov, G. Carta, and G. Rossetto, J. Phys. Chem. Solids 69, 673 (2008).

2008NEM/SZT Z. Nemet, J. Sztatisz, and A. Demeter, J. Pharm. Sci. 97, 3222 (2008).

2008NTI/CHM J. Nti-Gyabaah, R. Chmielowski, V. Chan, and Y. C. Chiew, Int. J. Pharm. 359, 111 (2008).

2008NUN/CLA E. Nunez, C. G. Clark, Jr., W. Cheng, A. Best, G. Floudas, A. N. Semenov, G. Fytas, and K. Mullen, J. Phys. Chem. B 112, 6542 (2008).

2008PAN/FUL J. Pangrac, M. Fulem, E. Hulicius, K. Melichar, T. Simecek, K. Ruzicka, P. Moravek, V. Ruzicka, and S. A. Rushworth, J. Cryst. Growth 310, 4720 (2008).

2008PEN/JIA L. Peng, X. Jianjun, M. Fangquan, L. Xi, and Z. Chaocan, J. Therm. Anal. Calorim. 93, 485 (2008).

2008PER/STR G. L. Perlovich, N. N. Strakhova, V. P. Kazachenko, T. V. Volkova, V. V. Tkachev, K.-J. Shaper, and O. A. Raevsky, Int. J. Pharm. 349, 300 (2008).

2008PIN/DIO S. S. Pinto and H. P. Diogo, J. Pharm. Sci. 97, 5354 (2008).
2008PIO/CAN

2008RIB/SAN6

2008RIB/SCH

2008ROU/LIM

2008ROU/TEM

2008RYC/SZY

2008SEM/BAS

2008SHA/GUP

2008SIN/DAS

2008SIN/MUR2

2008SLY/KON

2008STI/CIN

2008STR/KAB

2008STR/PAU

2008SUI/JES

2008SUR

2008SUR/SUR

2008THI/SUB

2008TON/TAN3

2008TUN/TAB

2008VEN/BAY

2008VER/EME2

2008VER/KOZ2

2008WAS/HOL

2008WEI

2008WEI/PEI

2008WU/DON

2008WU/JIA

2008XIN/TAN

2008ZEL/CHU

2008ZHA/UNH
R. Piociochi, J. N. Cananaja Lopes, H. P. Diogo, and M. E. Minas da Piedade, J. Phys. Chem. A 112, 10429 (2008). M. A. V. Ribeiro da Silva, L. M. N. B. F. Santos, and L. M. S. S. Lima, J. Chem. Thermodyn. 40, 375 (2008).

M. A. V. Ribeiro da Silva, B. Schroder, V. B. M. Castro, and L. M. N. B. F. Santos, J. Chem. Thermodyn. 40, 599 (2008).

A. A. Roucha, C. F. R. A. C. Lima, and L. M. N. B. F. Santos, J. Chem. Thermodyn. 40, 1458 (2008).

M. V. Roux, M. Temprado, J. S. Chickos, and Y. Nagano, J. Phys. Chem. Ref. Data 37, 1855 (2008).

L. Rycerz, M. Szymanska-Kolodziej, P. Kolodziej, and M. Gaune-Escard, J. Chem. Eng. Data 53, 1116 (2008).

P. P. Semyannikov, T. V. Basova, S. V. Trubin, E. K. Kol'tsov, V. A. Plyashkevich, and I. K. Igumenov, Russ. J. Phys. Chem. A 82, 159 (2008).

B. L. Sharma, S. Gupta, S. Tandon, and R. Kant, Mater. Chem. Phys. 111, 423 (2008).

N. B. Singh, S. S. Das, P. Gupta, and M. K. Dwivedi, J. Cryst. Growth 311, 118 (2008).

L. P. Singh and S. S. N. Murthy, J. Chem. Phys. 129, 094501 (2008).

I. V. Slyusarev, Y. V. Kondrat'ev, A. O. Kozin, and L. P. Belorukova, Vestnik. St.-Peterburg. Universit., Ser. 4 Fiz. Khim. 64 (2008).

V. Stilinovic, D. Cincic, and B. Kaitner, Acta Chim. Slov. 55, 874 (2008).

A. A. Strechan, A. G. Kabo, Y. U. Paulechka, A. V. Blokhin, G. J. Kabo, A. S. Shaplov, and E. I. Lozinskay, Thermochim. Acta 474, 25 (2008).

A. A. Strechan, Y. U. Paulechka, A. V. Blokhin, and G. J. Kabo, J. Chem. Thermodyn. 40, 632 (2008).

V. Suitchmezian, I. Jess, and C. Naether, J. Pharm. Sci. 97, 4516 (2008).

O. V. Surov, Russ. J. Gen. Chem. 78, 602 (2008).

A. O. Surov and O. V. Surov, Russ. J. Gen. Chem. 78, 1481 (2008).

J. Thimmasetty, C. V. S. Subrahmanyam, P. R. S. Babu, M. A. Maulik, and B. A. Viswanath, J. Solution Chem. 37, 1365 (2008).

B. Tong, Z.-C. Tan, and S.-X. Wang, Wuli Huaxue Xuebao 24, 1699 (2008); Chem. Abstr. 150, 11821 (2009).

H.-H. Tung, J. Tabora, N. Variankaval, D. Bakken, and C.C. Chen, J. Pharm. Sci. 97, 1813 (2008).

L. Ventola, L. Bayes, R. Benzges, F. J. Novegil-Anleo, M. A. Cuevas-Diarte, T. Calvet, and D. Mondieig, Helv. Chim. Acta 91, 1286 (2008).

S. P. Verevkin, V. N. Emel'yanenko, and S. A. Kozlova, J. Phys. Chem. A 112, 10667 (2008).

S. P. Verevkin, S. A. Kozlova, V. N. Emel'yanenko, P. Goodrich, and C. Hardacre, J. Phys. Chem. A 112, 11273 (2008).

C. M. Wassvik, A. G. Holmen, R. Draheim, P. Artursson, and C. A. S. Bergstrom, J. Med. Chem. 51, 3035 (2008). D. Wei, Thermochim. Acta 479, 32 (2008).

D. Wei and Y. Pei, Ind. Eng. Chem. Res. 47, 8953 (2008). C. Wu, H. Dong, X. Yang, J. Jiang, G. Lai, and H. Qui, Huaxue Gongcheng 36, 47 (2008); Chem. Abstr. 150, 549100 (2009); Note: In Chemical Abstracts the same journal reference is given for both [2008WU/JIA] and [2008WU/DON], the abstracts are quite different however.

C. Wu, J. Jiang, G. Lia, and H. Qiu, Huaxue Gongcheng 36, 47 (2008); Chem. Abstr. 150, 475170 (2009); Note: In Chemical Abstracts the same journal reference is given for both [2008WU/JIA] and [2008WU/DON], the abstracts are quite different however.

J. Xing, Z.-C. Tan, Q. Shi, B. Tong, S.-X. Wang, and Y.-S. Li, J. Therm. Anal. Calorim. 92, 375 (2008).

L. N. Zelenina, T. P. Chusova, Y. G. Stenin, and V. V. Kakovets, J. Therm. Anal. Calorim. 92, 723 (2008).

H. Zhao, P. Unhannanant, W. Hanshaw, and J. S. Chickos, J. Chem. Eng. Data 53, 1545 (2008). 
2008ZHA/WAN G. Q. Zhang, L. S. Wang, R.-L. Fan, X.-Z. Shao, and X.-F. Wang, J. Chem. Eng. Data 53, 1192 (2008).

2008ZHA/WUM L. Zhang, E. Wumanjian, Q. Meng, and G. Cai, Yingyong Huagnong 37, 802 (2008).

2008ZHA/YAN G. Zhao and W. Yan, Fluid Phase Equilib. 267, 79 (2008).

2008ZHE/MOR K. V. Zherikova, N. B. Morozova, L. N. Zelenina, S. V. Sysoev, T. P. Chusova, and I. K. Igumenov, J. Therm. Anal. Calorim. 92, 729 (2008)

2008ZHE/MOR2 K. V. Zherikova, N. B. Morozova, I. A. Baidina, S. V. Sysoev, and I. K. Igumenov, J. Struct. Chem. 49, 58 (2008).

2008ZHO/ZHA D. Zhou, G. G. Z. Zhang, D. Law, D. J. W. Grant, and E. A. Schmitt, Mol. Pharmaceutics 5, 927 (2008).

2008ZHU/XU R. Zhu, X. Xu, Z. Dong, and Y. Tian, Fluid Phase Equilib. 272, 60 (2008).

2009CHI/ACR W. E. Acree, Jr. and J. S. Chickos, Thermochim. Acta 495, 5-13 (2009).

2009ACE/NIC J. M. Aceves-Hernandez, I. Nicolas-Vazquez, F. J. Aceves, J. Hinojosa-Torres, M. Paz, and V. M. Castano, J. Pharm. Sci. 98, 2448 (2009).

2009ARA/SOS D. M. Aragon, A. Sosnik, and F. Martinez, J. Solution Chem. 38, 1493 (2009).

2009ARO/MAL S. Arockiasamy, C. Mallika, O. M. Sreetharan, V. S. Raghunathan, and K. S. Nagaraja, Inorg. Chim. Acta 362, 1977 (2009).

2009BAR/BON J. Baronsky, S. Bongaerts, M. Traeubel, H.-C. Weiss, and N. Urbanetz, Eur. J. Pharm. Biopharm. 71, 88 (2009).

2009BAR/ESP M. Barrio, P. Espeau, J. L. Tamarit, M.-A. Perrin, N. Veglio, and R. Ceolin, J. Pharm. Sci. 98, 1657 (2009).

2009BRA/GEL D. E. Braun, T. Gelbrich, V. Kahlenberg, R. Tessadri, J. Wieser, and U. J. Griesser, J. Pharm. Sci. 98, 2010 (2009).

2009BRU/PIA B. Brunetti, V. Piacente, and P. Scardala, J. Chem. Eng. Data 54, 2273 (2009).

2009CAM/EME H. Cammenga, V. N. Emel'yanenko, and S. P. Verevkin, Ind. Eng. Chem. Res. 48, 10120 (2009).

2009CHE/SU W. Chen, B. Su, H. Xing, Y. Yang, and Q. Ren, Fluid Phase Equilib. 287, 1 (2009).

2009CHI/STE R. D. Chirico and W. V. W. V. Steele, J. Chem. Thermodyn. 41, 392 (2009).

2009CIR/MAE M. Cirri, F. Maestrelli, N. Mennini, and P. Mura, J. Pharm. Biomed. Anal. 50, 690 (2009).

2009CLA/GOM R. A. Clara, A. C. Gomez Marigliano, and H. N. Solimo, J. Chem. Eng. Data 54, 1087 (2009).

2009COS/SAR M. C. Costa, M. Sardo, M. P. Rolemberg, J. A. P. Coutinho, A. J. R. Meirelles, P. Ribeiro-Claro, and M. A. Krahenbuhl, Chem. Phys. Lipids 160, 85 (2009).

2009DAS/BRE E. da Silva, S. Bresson, and D. Rousseau, Chem. Phys. Lipids 157, 113 (2009).

2009DEY/LOV A. Deyko, K. R. J. Lovelock, J.-A. Corfield, A. W. Taylor, P. N. Gooden, I. J. Villar-Garcia, P. Licence, R. G. Jones, V. G. Grasovskiy, E. A. Chernikova, and L. M. Kustov, Phys. Chem. Chem. Phys. 11, 8544 (2009).

2009DOM/KRO U. Domanska, M. Krolkowski, and K. Slesinska, J. Chem. Thermodyn. 41, 1303 (2009).

2009DOM/POB U. Domanska, A. Pobudkowska, A. Pelczarska, and P. Gierycz, J. Phys. Chem. B 113, 8941 (2009).

2009EGO/MAR V. M. Egorov, V. A. Marikhin, L. P. Myasnikova, and N. Nakamura, Phys. Solid State 51, 2129 (2009).

2009EME/VER3 V. N. Emel'yanenko, S. P. Verevkin, A. Heintz, K. Voss, and A. Schulz, J. Phys. Chem. B 113, 9871 (2009).

2009FIL/NIZ E. S. Filatov, H. Nizard, P. P. Semyannikov, S. V. Sysoev, S. V. Trubin, N. B. Morozova, K. V. Zherikova, and N. V. Gelfond, ECS Trans. 25, 55 (2009).

2009FRE/GOM V. L. S. Freitas, J. R. B. Gomes, and M. D. M. C. Ribeiro da Silva, J. Chem. Thermodyn. 41, 1199 (2009).

2009FRE/GOM2 V. L. S. Freitas, J. R. B. Gomes, and M. D. M. C. Ribeiro da Silva, J. Therm. Anal. Calorim. 97, 827 (2009).

2009FRE/MON V. L. S. Freitas, M. J. S. Monte, L. M. N. B. F. Santos, J. R. B. Gomes, and M. D. C. M. Ribeiro da Silva, J. Phys. Chem. A 113, 12988 (2009).

2009GAI/KUN A. Gairola, G. V. Kunte, A. M. Umarji, and S. A. Shivashankar, Thermochim. Acta 488, 17 (2009).
2009GAS/CEN

Z. Gashi, R. Censi, L. Malaj, R. Gobetto, M. Mozzicafreddo, M. Angeletti, A. Masic, and P. Di Martino, J. Pharm. Sci. 98, 4216 (2009).

2009GBA/NEG G. Gbabode, P. Negrier, D. Mondieig, E. Moreno, T. Calvet, and M. A. Cuevas-Diarte, J. Alloys Compd. 469, 539 (2009).

2009GIM/CLE N. Gimeno, M. J. Clemente, P. Forcen, J. L. Serrano, and M. B. Ros, New J. Chem. 33, 2007 (2009).

2009GOM/MON J. R. B. Gomes, A. R. Monteiro, B. B. Campos, P. Gomes, and M. D. M. C. Riberio da Silva, J. Phys. Org. Chem. 22, 17 (2009).

2009GOO/ROD D. J. Good and N. Rodriguez-Hornedo, Cryst. Growth Des. 9, 2252 (2009).

2009JOH/SEL M. G. Johnson, J. Selvakumar, and K. S. Nagaraja, Thermochim. Acta 495, 38 (2009).

2009KNO/DUN G. Knothe and R. O. Dunn, J. Am. Oil Chem. Soc. 86, 843 (2009).

2009KON/DI

Y.-X. Kong, Y.-Y. Di, Y.-Q. Zhang, W.-W. Yang, and Z.-C. Tan, Int. J. Thermophys. 30, 1960 (2009).

2009KRO/DRU O. V. Krol, A. I. Druzhinina, R. M. Varuxhchenko, M D. Reshetova, N. E. Borisova, O. V. Dorofeeva, and S. I. Troyanov, J. Chem. Eng. Data 54, 263 (2009).

2009LEG/BAC B. Legendre, P. Bac, M. German, and Y. Feutelais, J. Therm. Anal. Calorim. 98, 91 (2009).

2009LEM/BAT A. Lemmerer, N. B. Bathori, C. Esterhuysen, S. A Bourne, and M. R. Caira, Cryst. Growth Des. 9, 2646 (2009).

2009LIP/CHI D. Lipkind and J. S. Chickos, in Abstract from 38th Great Lakes Regional Meeting of the American Chemical Society, Chicago, IL, 13-16 May 2009. Abstract available online through Scifinder Scholar.

2009LIP/HAN D. Lipkind, W. Hanshaw, and J. S. Chickos, J. Chem. Eng. Data 54, 2930 (2009).

2009MAO/LUO Z.-B. Mao, T.-L. Luo, T.-B. Cui, Y. Wang, and G.-J. Liu, J. Zhengzhou Univ. (Nat. Sci. Ed.) 41, 84 (2009).

2009MAR/SEL M. Maria, J. Selvakumar, V. S. Raghunathan, and K. S. Nagaraja, Surf. Coat. Technol. 204, 222 (2009).

2009MAT/SOU2 M. A. R. Matos, C. C. S. Sousa, and V. M. F. Morais, J. Chem. Thermodyn. 41, 69 (2009).

2009MEL/PIM S. V. Melkhanova, S. M. Pimenova, N. V. Chelovskaya, E. A. Miroshichenko, L. L. Pashehenko, I. A. Nesterov, and P. V. Naumkin, J. Chem. Thermodyn. 41, 651 (2009).

2009MIR/PAS E. A. Miroshnichenko and L. L. Paschenko, in XVII International Conference on Chemical Thermodynamics, Russia (2009), p. 73.

2009MON/NUT D. Monnier, I. Nuta, C. Chatillon, M. Gross-Jean, F. Volpi, and E. Blanquet, J. Electrochem. Soc. 156, H71 (2009).

2009MOR/SEM N. B. Morozova, P. P. Semyannikov, S. V. Trubin, P. P. Stabnikov, A. A. Bessonov, K. V. Zherikova, and I. K. Igumenov, J. Therm. Anal. Calorim. 96, 261 (2009).

2009MOR/ZHE N. B. Morozova, K. V. Zherikova, P. P. Semyannikov, S. V. Trubin, and I. K. Igumenov, J. Therm. Anal. Calorim. 98, 395 (2009).

2009MOT/CAR F. L. Mota, A. P. Carneiro, A. J. Queimada, S. P. Pinho, and E. A. Macedo, Eur. J. Pharm. Sci. 37, 499 (2009).

2009MOT/VOR V. B. Motalov, D. E. Vorobiev, L. S. Kudin, and T. Markus, J. Alloys Compd. 473, 36 (2009).

2009NET/NOV H. S. Neto, C. Novak, and J. R. Matos, J. Therm. Anal Calorim. 97, 367 (2009).

2009NTI/CHA J. Nti-Gyabaah, V. Chan, and Y. C. Chiew, Fluid Phase Equilib. 280, 35 (2009).

2009OJA/CHE V. Oja, X. Chen, M. R. Hajaligol, and W. G. Chan, J. Chem. Eng. Data 54, 730 (2009).

2009OLI/BER P. R. Olieira, L. S. Bernadi, F. S. Murakami, C. Mendes, and M. A. S. Silva, J. Therm. Anal. Calorim. 97, 741 (2009).

2009PAN/ANT K. Panneerselvam, M. P. Antony, T. G. Srinivasan, and P. R. V. Rao, Thermochim. Acta 495, 1 (2009).

2009PEN/ESC M. A. Pena, A. Escalera, A. Reillo, A. B. Sanchez, and P. Bustamante, J. Pharm. Sci. 98, 1129 (2009).

2009PER/TKA G. L. Perlovich, V. V. Tkachev, N. N. Strakhova, V. P. Kazachenko, T. V. Volkova, O. V. Surov, K.-J. Schaper, and O. A. Raevsky, J. Pharm. Sci. 98, 4738 (2009). 
2009PLA/LIZ D. Plano, E. Lizarraga, M. Font, J. A. Palop, and C. Sanmartin, J. Therm. Anal. Calorim. 98, 559 (2009).

2009RAI/RED R. N. Rai and R. S. B. Reddi, Thermochim. Acta 496, 13 (2009).

2009RAJ/MIS P. S. Rajinikanth and B. Mishra, Chem. Pharm. Bull. 57, 1068 (2009).

2009RAZ/HAJ A. Razzouk, A. Hajjaji, I. Mokbel, P. Mougin, and J. Jose, Fluid Phase Equilib. 282, 11 (2009).

2009RAZ/NAC A. Razzouk, R. A. Naccoul, I. Mokbel, J. Saab, and J. Jose, J. Chem. Eng. Data 54, 1214 (2009).

2009ROY/MAT S. Roy and A. J. Matzger, Angew. Chem., Int. Ed. 48, 8505 (2009).

2009RUC/MAR V. A. Ruchenin, A. V. Markin, N. N. Smirnova, G. V. Markin, Y. A. Shevelev, V. K. Cherkasov, V. A. Kuropatov, S. Y. Ketkov, M. A. Lopatin, and G. A. Domrachev, Bull. Chem. Soc. Jpn. 82, 65 (2009).

2009SAR/BIC A. Sari, A. Bicer, and A. Karaipekli, Mater. Lett. 63, 1213 (2009).

2009SEL/RAG J. Selvakumar, V. S. Raghunathan, and K. S. Nagaraja, J. Phys. Chem. C 113, 19011 (2009).

2009SEL/RAG2 J. Selvakumar, V. S. Raghunathan, and K. S. Nagaraja, Chem. Vap. Deposition 15, 262-268 (2009).

2009SHA/DEN P. Sharma, W. A. Denny, and S. Garg, Int. J. Pharm. 380, 40 (2009).

2009SHI/OHT Y. Shimizu, Y. Hote, Y. Yamamura, and K. Saito, Chem. Phys. Lett. 470, 295 (2009).

2009SID/SID M. A. Siddiqi, R. A. Siddiqui, and B. Atakan, J. Chem. Eng. Data 54, 2795 (2009).

2009SOU/MAT C. C. S. Sousa, M. A. R. Matos, and V. M. F. Morais, J. Chem. Thermodyn. 41, 1408 (2009).

2009SUR/PER A. O. Surov and G. L. Perlovich, Russ. J. Phys. Chem. A 83, 1823 (2009).

2009SUR/SZT A. O. Surov, P. Szterner, W. Zielenkiewicz, and G. L. Perlovich, J. Pharm. Biomed. Anal. 50, 831 (2009).

2009SUR/TER A. O. Surov, I. V. Terekhova, A. Bauer-Brandl, and G. L. Perlovich, Cryst. Growth Des. 9, 3265 (2009).

2009TAM/MIR R. E. Tamagawa, E. A. Miranda, C. C. Santana, and M. Giulietti, J. Chem. Eng. Data 54, 16 (2009).

2009TAU/SIT P. Taulelle, G. Sitja, G. Pepe, E. Garcia, C. Hoff, and S. Veesler, Cryst. Growth Des. 9, 4706 (2009).

2009THI/SUB J. Thimmasetty, C. V. S. Subrahmanyam, B. A. Vishwanath, and P. R. Sathesh Babu, Asian J. Res. Chem. 2, 188-195 (2009).

2009TOR/CAM L. A. Torres, M. Campos, M. Martinez, and A. Rojas, J. Chem. Thermodyn. 41, 957 (2009).

2009VAD/BAN H. K. Vaddi, S. L. Banks, J. Chen, D. C. Hammell, P. A. Crooks, and A. L. Stinchcomb, J. Pharm. Sci. 98, 2611 (2009).

2009VAN/WES J. T. H. van Eupen, R. Westheim, M. A. Deij, A. Meekes, P. Bennema, and E. Vlieg, Int. J. Pharm. 368, 146 (2009).

2009VER/EME3 S. P. Verevkin, V. N. Emel'yanenko, A. V. Toktonov, P. Goodrich, and C. Hardacre, J. Phys. Chem. B 113, 12704 (2009).

2009WEI/CHE D. Wei and L. Chen, Fluid Phase Equilib. 277, 9 (2009).

2009XIE/YAN J. Xie, J. Yang, and G. Chen, J. Dispersion Sci. Technol. 30, 1350 (2009)

2009YAM/TES H. Yamamoto, S. Teshima, N. Nemoto, and K. Tashiro, J. Phys. Chem. A 113, 2632 (2009).

2009ZAI/PAU D. H. Zaitsau, Y. U. Paulenchka, A. V. Blokhin, A. V. Yermalayeu, A. G. Kabo, and M. R. Ivanets, J. Chem. Eng. Data 54, 3026 (2009).

2009ZEN/CAO J. L. Zeng, Z. Cao, D. W. Yang, F. Xu, L. X. Sun, L. Zhang, and X. F. Zhang, J. Therm. Anal. Calorim. 95, 501 (2009).

2010AND/ABU G. P. Andrews, O. A. Abudiak, and D. S. Jones, J. Pharm. Sci. 99, 1322 (2010).

2010ARO/MAN S. Arockiasamy, K. Manoj, M. M. Ghadbhade, C. Mallika, and K. S. Kagaraja, Inorg. Chim. Acta 363, 2243 (2010).

2010AVU/ALE S. G. Avula, K. Alexander, and A. Riga, J. Therm. Anal. Calorim. 99, 655 (2010).

2010BAI/VAN J. A. Baird, B. van Eerdenbrugh, and L. S. Taylor, J. Pharm. Sci. 99, 3787 (2010).

2010BAL/MAH G. Balata, M. Mahdi, and R. A. Bakera, Asian J. Pharm. Sci. 5, 1 (2010).
2010BAN/ARC

G. Bannach, R. Arcaro, D. C. Ferroni, A. B. Siqueira, O. Treu-Filho, M. Ionashiro, and E. Schnitzler, J. Therm. Anal. Calorim. 102, 163 (2010).

2010BAR/ARA G. L. Barros de Araju, D. L. Araujo de Faria, M. H. Zaim, F. M. de Souza Carvalho, F. R. Dias de Andrade, and J. do Rosario Matos, J. Therm. Anal. Calorim. 102, 233 (2010).

2010BOG/GON R. Bogel-Lukasik, L. M. N. Goncalves, and E. BogelLukasik, Green Chem. 12, 1947 (2010).

2010BRA/GRE

D. Braga, F. Grepioni, L. Maini, P. P. Mazzeo, and K. Rubini, Thermochim. Acta 507-508, 1 (2010).

2010BRU

2010BRU/PIA

B. Brunetti, J. Chem. Eng. Data 55, 2455 (2010).

2010CAN/ALK Data 55, 98 (2010).

2010CAN/CAS

G. Canik and C. Alkan, Sol. Energy 84, 666 (2010).

J. Canotilho, R. A. E. Castro, M. J. S. Rosado, S. C. C. Nunes, M. S. C. Cruz, and J. S. Redinha, J. Therm. Anal. Calorim. 100, 423 (2010).

2010CEN/ROH M. Censky, V. Rohac, K. Ruzicka, M. Fulem, and K. Aim, Fluid Phase Equilib. 298, 192 (2010).

2010CHA/LAY E. L. Charsley, P. G. Laye, and T. Le Goff, Thermochim. Acta 497, 72 (2010).

2010CHI/DEG ～D. A. Chiappetta, J. Degrossi, R. A. Liazrazo, D. L. Salinas, F. Martinez, and A. Sosnik, in Polymer, Aging, Stabilizers and Amphiphilic Block Copolymers, edited by L. Segewicz and M. Petrowski (Nova Publishers, New York, 2010), preproduction as quoted in Ref. [2009ARA/SOS].

$2010 \mathrm{CHI} / \mathrm{KAZ} \quad$ R. D. Chirico, A. F. Kazakov, and W. V. Steele, J. Chem. Thermodyn. 42, 581 (2010).

$2010 \mathrm{CHI} / \mathrm{KAZ} 2$ R. D. Chirico, A. F. Kazakov, and W. V. Steele, J. Chem. Thermodyn. 42, 571 (2010).

2010CIL/ALB

F. Cilurzo, E. Alberti, P. Minghetti, C. G. M. Gennari, A. Casiraghi, and L. Montanari, Int. J. Pharm. 386, 71 (2010).

2010COR/NEG Y.Corvis, P. Negrier, M. Lazerges, S. Massip, J.-M. Leger, and P. Espeau, J. Phys. Chem. B 114, 5420 (2010).

2010DAV/GUE J. Z. Davalos, A. Guerrero, R. Herrero, P. Jimenez, A. Chana, J. L. M. Abboud, C. F. R. A. C. Lima, L. M. N. B. F. Santos, and A. F. Lago, J. Org. Chem. 75, 2564 (2010).

2010DIB/PAV V. N. Dibrivnyi, Y. P. Pavlovskii, and Y. Y. Van-ChinSyan, Russ. J. Phys. Chem. A 84, 778 (2010).

2010DOM/KRO U. Domanska, M. Krolikowski, D. Ramjugernath, T. M. Letcher, and K. Tumba, J. Phys. Chem. B 114, 15011 (2010).

2010DOM/KRO2 U. Domanska, M. Krolikowska, and M. Krolikowski, Fluid Phase Equilib. 294, 72 (2010).

2010DOM/KRO3 U. Domanska and M. Krolikowski, J. Chem. Thermodyn. 42, 355 (2010).

2010DOM/POB U. Domanska, A. Pobudkowska, P. Pelczarska, M. Winiarska-Tusznio, and P. Gierycz, J. Chem. Thermodyn. 42, 1465 (2010).

2010DOM/ZAW U. Domanska, M. Zawadzki, M. M. Tshibangu, D. Ramjugernath, and T. M. Letcher, J. Chem. Thermodyn. 42, 1180 (2010).

2010DON/LIU H. Dong, L. Liu, C. Wu, and G. Lai, J. Chem. Eng. Data 55, 5347 (2010).

2010DON/WU H. Dong, C. Wu, X. Yang, and G. Lai, J. Chem. Eng. Data $\mathbf{5 5}, 889$ (2010).

2010EFI/VAR A. A. Efimova, R. M. Varushchenko, A. I. Druzhinina, N V. Chelovskaya, E. S. Tkachenko, I. A. Nesterov, and T. N. Nesterova, Russ. J. Phys. Chem. A 84, 343 (2010).

2010ELK/ESS A. A. Elkordy and E. A. Essay, Pak. J. Pharm. Sci. 23, 284 (2010).

2010EME/KRO V. N. Emel'yankenko, O. V. Krol, R. M. Varushechenko, A. I. Druzhinina, and S. P. Verevkin, Russ. J. Phys. Chem. A 84, 1089 (2010).

2010END/KAT T. Endo, T. Kato, K.-I. Tozaki, and K. Nishikawa, J. Phys. Chem. B 114, 407 (2010).

2010FAN/WAN C. L. Fan and L. S. Wang, J. Chem. Eng. Data 55, 479 (2010).

2010FON/SAN J. M. S. Fonseca, L. M. N. B. F. Santos, and M. J. S. Monte, J. Chem. Eng. Data 55, 2238 (2010). 
2010FRA/SAL L. S. Franco, Y. P. Salamanca, M. Maldonado, and E. F. Vargas, J. Chem. Eng. Data 55, 1042 (2010).

2010FRE/GOM V. L. S. Freitas, J. R. B. Gomes, and M. D. M. C. Ribeiro da Silva, J. Chem. Thermodyn. 42, 251 (2010).

2010FRE/GOM2 V. L. S. Freitas, J. R. B. Gomes, and M. D. M. C. Ribeiro da Silva, J. Chem. Thermodyn. 42, 1248 (2010).

2010FRE/GOM3 V. L. S. Freitas, J. R. B. Gomes, L. Gales, A. M. Damas, and M. D. M. C. Ribeiro da Silva, J. Chem. Eng. Data 55, 5009 (2010).

2010FU/RIC J. Fu, J. W. Rice, and E. M. Suuberg, Fluid Phase Equilib. 298, 219 (2010).

2010FUC/QUR K. Fucke, N. Qureshi, D. S. Yufit, A. K. Howard, and J. W. Steed, Cryst. Growth Des. 10, 880 (2010).

2010FUL/MOR M. Fulem, P. Moravek, J. Pangrac, E. Hulicius, T. Simecek, K. Ruzicka, V. Ruzicka, B. Kozyrkin, and V. Shatunov, J. Chem. Eng. Data 55, 362 (2010).

2010GAM/CAM M. Gamboa, M. Campos, and L. A. Torres, J. Thermodyn. Chem. 42, 666 (2010).

2010GHU/DON P. Ghugare, V. Dongre, P. Karmuse, R. Rana, D. Singh, A. Kumar, and Z. Filmwala, J. Pharm. Biomed. Anal. 51, 532 (2010).

2010GOL/KUL J. L. Goldfarb and I. Kulaots, J. Therm. Anal. Calorim. 102, 1063 (2010).

2010GOL/SUU J. L. Goldfarb and E. M. Suuberg, J. Chem. Thermodyn. 42, 781 (2010)

2010GOM/ROM J. A. Gomez Castano, R. M. Romano, H. Beckers, H. Willner, and C. O. Della Vedova, Inorg. Chem. 49, 9972 (2010).

2010GUO/WAN X.-Z. Guo, L.-S. Wang, and N.-N. Tian, J. Chem. Eng. Data 55, 1745 (2010).

2010GUO/WAN2 X.-Z. Guo and L.-S. Wang, J. Chem. Eng. Data 55, 4709 (2011).

2010GUT/HEC R. Gutzler, W. M. Heckl, and M. Lackinger, Rev. Sci. Instrum. 81, 015108 (2010).

2010HAH/GRA I. Hahnenkamp, G. Graubner, and J. Gmehling, Int. J. Pharm. 388, 73 (2010).

2010HIL/LAU D. L. Hildenbrand, K. H. Lau, and D. Chandra, J. Phys. Chem. B 114, 330 (2010).

2010HIL/LAU2 $\quad$ D. L. Hildenbrand, K. H. Lau, and D. Chandra, J. Phys. Chem. A 114, 11654 (2010).

2010HON/HUA J. Hong, D. Hua, X. Wang, H. Wang, and J. Li, J. Chem. Eng. Data 55, 297 (2010).

2010HU/WAN F.-H. Hu, L.-S. Wang, and S.-F. Cai, J. Chem. Eng. Data 55, $492(2010)$.

2010KAB/PAU G. N. Kabo, Y. U. Paulechka, A. G. Kabo, and A. V. Blokhin, J. Chem. Thermodyn. 42, 1292 (2010).

2010KAM/TAR R. K. Kamlekar, P. K. Tarafdar, and M. J. Swamy, J. Lipid Res. 51, 42 (2010).

2010KAM/YAR N. N. Kamkin, N. G. Yaryshev, and A. S. Alikhanyan, Russ. J. Inorg. Chem. 55, 1443-1447 (2010).

2010KEN J. A. Kenar, Sol. Energy Mater. Sol. Cells 94, 1697 (2010).

2010KES/AUC V. Kestens, G. Auclair, K. Drozdzewska, A. Held, G. Roebben, and T. Linsinger, J. Therm. Anal. Calorim. 99, 245 (2010).

2010KOK/SEM T. E. Kokina, P. P. Semyannikov, S. V. Trubin, S. V. Sysoev, and S. V. Larinov, Russ. J. Inorg. Chem. 55, 1438 (2010).

2010KRO/DRU O. V. Krol, A. I. Druzhinina, R. M. Varushchenko, P. A. Zhizhko, M. D. Reshetova, N. E. Borisova, and N. V. Chetovskaya, Russ. J. Phys. Chem. 84, 771 (2010).

2010KRU/MAJ A. Krupa, D. Majda, R. Jachowicz, and W. Mozgawa, Thermochim. Acta 509, 12 (2010).

2010KRZ/MAL K. Krzyminski, P. Malecha, P. Storoniak, B. Zadykowicz, and J. Blazejowski, J. Therm. Anal. Calorim. 100, 217 (2010).

2010KUN/SAV S. Kunadharaju and M. Savva, J. Chem. Eng. Data 55, 103 (2010).

2010LAV/PIR Z. Lavric, J. Pirnat, J. Luznik, J. Seliger, V. Zagar, Z. Trontelj, and S. Srcic, J. Pharm. Sci. 99, 4857 (2010).

2010LAZ/RIE M. Lazerges, I. V. Rietveld, Y. Corvis, R. Ceolin, and P. Espeau, Thermochim. Acta 497, 124 (2010).
2010LEI/WAN

2010LET/RAM

Y. D. Lei, F. Wania, and D. Mathers, J. Chem. Eng. Data $\mathbf{5 5}, 5868$ (2010).

T. M. Letcher, D. Ramjugernath, K. Tumba, M. Krolikowski, and U. Domanska, Fluid Phase Equilib. 294, 89 (2010).

2010 LIM/SOU $\quad$ C. F. R. A. C. Lima, C. A. D. Sousa, J. E. RodriguezBorges, A. Melo, L. R. Gomes, J. N. Low, and L. M. N. B. F. Santos, Phys. Chem. Chem. Phys. 12, 11228 (2010).

2010LIP/CHI D. Lipkind, J. S. Chickos, and J. F. Liebman, J. Chem. Eng. Data 55, 1628 (2010).

2010LIP/PLI

D. Lipkind, G. Plienrasri, and J. S. Chickos, J. Phys. Chem. B 114, 16959 (2010).

2010LIU/DI

Y. Liu, Y. Di, W. Dan, and D. He, Z. Phys. Chem. 224, 1371 (2010).

2010LOV/DEY

K. R. J. Lovelock, A. Deyko, P. Licence, and R. G. Jones, Phys. Chem. Chem. Phys. 12, 8893 (2010).

2010LUT/MAT K. M. Lutker and A. J. Matzger, J. Pharm. Sci. 99, 794 (2010).

2010MAL/FUG L. Malpezzi, C. Fuganti, E. Maccaroni, N. Masciocchi, and A. Nardi, J. Therm. Anal. Calorim. 102, 203 (2010).

2010MAO/LUO Z.-B. Mao, T.-L. Luo, T.-B. Cui, Y. Wang, and G.-J. Liu, J. Chem. Eng. Data 55, 543 (2010).

2010MAS/KRA A. S. Maslakova, E. L. Krasnykh, and S. V. Levanova, Russ. J. Phys. Chem. 84, 163 (2010).

2010MEK/KHI A. Mekki, K. Khimeche, and A. Dahmani, J. Chem. Thermodyn. 42, 1050 (2010).

2010MIR/MOR M. S. Miranda, V. M. F. Morais, M. A. R. Matos, and J. F. Liebman, J. Chem. Thermodyn. 42, 1094 (2010).

2010MIY/KHA Y. Miyako, N. Khalef, K. Matsuzaki, and R. Pinal, Int. J. Pharm. 393, 48 (2010).

$2010 \mathrm{MON} / \mathrm{SOU}$ M. J. S. Monte, C. A. D. Sousa, J. M. S. Fonseca, and L. M. N. B. F. Santos, J. Chem. Eng. Data 55, 5264 (2010).

2010MOR/FUL P. Moravek, M. Fulem, K. Ruzicka, J. Pangrac, E. Hulicius, T. Simecek, V. Ruzicka, and S. A. Rushworth, J. Chem. Eng. Data 55, 4095 (2010).

2010MUR/PIK $\quad$ S. B. Murdande, M. J. Pikal, R. M. Shanker, and R. H. Bogner, J. Pharm. Sci. 99, 1254 (2010).

2010MUR/PIK2 $\quad$ S. B. Murdande, M. J. Pikal, R. M. Shanker, and R. H. Bogner, Pharm. Sci. 27, 2704 (2010).

2010NET/BAR H. S. Neto, F. A. P. Barros, F. M. de Sousa Carvalho, and J. R. Matos, J. Therm. Anal. Calorim. 102, 277 (2010).

2010NOK/AMI A. Nokhodchi, O. Amire, and M. Jelvehgari, Daru 18, 74 (2010).

2010OLI/YOS M. A. Oliveira, M. I. Yoshida, E. C. L. Gomes, W. N. Mussel, C. D. Vianna-Soares, and G. A. Pianetti, Quim. Nova 8, 653 (2010).

2010PAN/SAR E. Panteli, P. Saratsioti, H. Stamatis, and E. Voutsas, J. Chem. Eng. Data 55, 745 (2010).

2010PAR/LEE K. A. Park, H. J. Lee, and I. K. Hong, J. Ind. Eng. Chem. 16, 490 (2010).

2010PAU/KOH Y. U. Paulechka, S. V. Kohut, V. Sviataslau, A. V. Blokhin, and G. J. Kabo, Thermochim. Acta 511, 119 (2010).

2010PER/VOL G. L. Perlovich, T. V. Volkova, A. N. Proshin, D. Y. Sergeev, C. T. Bui, L. N. Petrova, and S. O. Bachurin, J. Pharm. Sci. 99, 3754 (2010).

2010PIA/BRU V. Piacente, B. Brunetti, and P. Scardala, J. Chem. Eng. Data 55, 3731 (2010).

2010PLY/BAS V. Plyashkevich, T. Basova, P. Semyannikov, and A. Hassan, Thermochim. Acta 501, 108 (2010).

2010RAK/TSI V. I. Rakhlin, I. P. Tsirendorzhieva, M. G. Voronkov, L. D. Nikulina, S. V. Sysoev, and M. L. Kosinova, Glass Phys. Chem. 36, 376 (2010).

2010RIB/FER3 M. A. V. Ribeiro da Silva, A. I. M. C. L. Ferreira, and F. M. Maciel, J. Chem. Thermodyn. 42, 220 (2010).

2010RIB/GON M. D. M. C. Ribeiro da Silva, M. V. Goncalves, and M. J. S. Monte, J. Chem. Thermodyn. 42, 472 (2010).

2010RIB/RIB2 M. A. V. Ribeiro da Silva, M. D. M. C. Ribeiro da Silva, A. F. L. O. M. Santos, M. V. Roux, C. Foces-Foces, R. Notario, R. Guzman-Mejia, and E. Juaristi, J. Phys. Chem. B 114, 16471 (2010).

2010RIB/RIB2a M. D. M. C. Ribeiro da Silva, M. A. V. Riberio da Silva, V. L. S. Freitas, M. V. Roux, P. Jimenez, J. Z. Davalos, P. Cabildo, R. M. Claramunt, E. Pinilla, M. R. Torres, and J. Elguero, J. Chem. Thermodyn. 42, 536 (2010). 
2010RIB/SAN M. A. V. Ribeiro da Silva and A. F. L. O. M. Santos, J. Chem. Thermodyn. 42, 128 (2010).

2010RIB/SAN2 M. A. V. Ribeiro da Silva, L. M. N. B. F. Santos, and L. M. S. S. Lima, J. Chem. Thermodyn. 42, 134 (2010).

2010RIB/SAN5 M. A. V. Ribeiro da Silva and A. F. L. O. M. Santos, J. Phys. Chem. B 114, 16214 (2010).

2010RIC/FU J. W. Rice, J. Fu, and E. M. Suuberg, J. Chem. Eng. Data 55, 3598 (2010).

2010RIC/SUU J. W. Rice and E. M. Suuberg, J. Chem. Thermodyn. 42, $1356(2010)$

2010ROD/CRI S. J. Rodriguez, D. M. Cristancho, P. C. Neita, E. F. Vargas, and F. Martinez, J. Solution Chem. 39, 1122 (2010).

2010SAN/SAR P. Sanphui, B. Sarma, and A. Nangia, Cryst. Growth Des. 10, 4550 (2010)

2010SAR/BIC A. Sari, A. Bicer, A. Karaipekli, C. Alkan, and A. Karadag, Sol. Energy Mater. Sol. Cells 94, 1711 (2010).

2010SER/MOT D. N. Sergeev, V. B. Motatov, M. F. Butman, L. S. Ludin, K. W. Kraemer, L. Rycerz, and M. Gaune-Escard, Russ. J. Phys. Chem. A 84, 554 (2010).

2010SID/SID M. A. Siddiqi, R. A. Siddiqui, and B. Atakan, J. Chem. Eng. Data 55, 2149 (2010)

2010SID/SID2 M. A. Siddiqi, R. A. Siddiqui, B. Atakan, N. Roth, and H. Lang, Materials 3, 1172 (2010).

2010SIV/BER A. Sivert, V. Berard, and C. Andres, J. Pharm. Sci. 99, 1399 (2010).

2010SOL/KEI J. L. Sola-Cervera, P. Keil, and A. Konig, Chem. Eng. Technol. 33, 821 (2010).

2010SUR/PER A. O. Surov and G. L. Perlovich, J. Struct. Chem. 51, 308 (2010).

2010SYS/CHE $\quad$ S. V. Sysoev, T. N. Cheremisina, L. N. Zelemina, S. V. Tkachev, K. V. Zherikova, N. B. Morozova, and N. V. Kuratieva, J. Therm. Anal. Calorim. 101, 41 (2010).

2010THI/AIT S. Thirunahari, S. Aitipamula, P. S. Chow, and R. B. H. Tan, J. Pharm. Sci. 99, 2975 (2010).

2010TON/LIU2 B. Tong, Q.-S. Liu, Z.-C. Tan, and U. Welz-Biermann, J. Phys. Chem. A 114, 3782 (2010).

2010TRI/BIR R. Tripathi, S. V. Biradar, B. Mishra, and A. R. Paradkar, AAPS PharmSciTech 11, 1493 (2010).

2010VAR/EFI R. M. Varushchenko, A. A. Efimova, A. I. Druzhinina, E. S. Tkachenko, I. A. Nesterov, T. N. Nesterova, and S. P. Verevkin, J. Chem. Thermodyn. 42, 1265 (2010).

2010VER/EME S. P. Verevkin, V. N. Emel'yanenko, D. H. Ziitsau, A. Heintz, C. D. Muzny, and M. Frenkel, Phys. Chem. Chem. Phys. 12, 14994 (2010).

2010WAN/LUO C. Wang, H. Luo, H. Li, and S. Dai, Phys. Chem. Chem. Phys. 12, 7246 (2010).

2010WEI/WAN D. Wei, L. Wang, F. Yan, and C. Zhang, Fluid Phase Equilib. 291, 66 (2010).

2010WU/LIU C. Wu, L. Liu, H. Dong, X. Yang, and G. Lai, J. Chem. Eng. Data 55, 3952 (2010).

2010YUA/CAP X. Yuan and A. C. Capomacchia, Drug Dev. Ind. Pharm. 36, 1168 (2010).

2010ZAD/KRZ B. Zadykowicz, K. Krzyminski, P. Storoniak, and J. Blazejowski, J. Therm. Anal. Calorim. 101, 429 (2010).

$2010 Z A G / C O N \quad$ A. Zaggia, L. Conte, G. Pardoan, and F. Ceretta, J. Fluorine Chem. 131, 844 (2010).

2010ZEN/GEL N. Zencirci, T. Gelbrich, D. C. Apperley, R. K. Harris, V. Kalenberg, and U. J. Griesser, Cryst. Growth Des. 10, 302 (2010).

2010ZEN/WAN A. Zeng, C. Wang, B. Yuan, G. Yang, and Q. Fu, Drug Dev. Ind. Pharm. 36, 724 (2010).

2010ZHA/CUI Z. H. Zhang, T. Cui, J. L. Zhang, h. Xiong, G. P. Li, L. X. Sun, F. Xu, Z. Cao, F. Li, and J. J. Zhao, J. Therm. Anal. Calorim. 101, 1143 (2010).

2010ZHA/SUN J.-N. Zhao, L.-X. Sun, and Z. C. Tan, J. Chem. Eng. Data 55, 4267 (2010).

2010ZHO/LI K. Zhou, J. Li, J. Luo, and D. Zheng, Front. Chem. Eng. China 4, 65 (2010).

$2011 \mathrm{ABB} / \mathrm{SCH} \quad$ R. Abbas, A. Schedemann, C. Ihmels, S. Enders, and J. Gmehling, Ind. Eng. Chem. Res. 50, 9748 (2011).

2011 ACE/HIN J. M. Aceves-Hernandez, J. Hinojosa-Torres, I. NicolasVazquez, R. M. Ruvalcaba, and R. M. L. Garcia, J. Mol. Struct. 995, 41 (2011).
2011AGA/VAR

L. E. Agafonova, R. M. Varushchenko, A. I. Druzhinina, O. V. Polyakova, and Y. S. Kolesov, Russ. J. Phys. Chem. 85, 1516 (2011).

2011AIT/CHO S. Aitipamula, P. S. Chow, and R. B. H. Tan, Cryst. Growth Des. 11, 4101 (2011).

2011ALK/CAN C. Alkan, G. Canik, H. Dunya, and A. Sari, Sol. Energy Mater. Sol. Cells 95, 1203 (2011).

2011 ALK/TEK C. Alkan, Y. Tek, and D. Kahraman, Turk. J. Chem. 35, 769 (2011).

2011AMA/PIN P. Amador, B. Pineda, A. Lopez, and H. Flores, J. Chem. Thermodyn. 43, 1414 (2011).

2011AYD/OKU A. A. Aydin and H. Okutan, Sol. Energy Mater. Sol. Cells 95, 2752 (2011).

2011AYD/OKU2 A. A. Aydin and H. Okutan, Sol. Energy Mater. Sol. Cells 95, 2417 (2011).

2011BEN/KHI M. Benziane, K. Khimeche, I. Mokbel, T. Sawaya, A. Dahmani, and J. Jose, J. Chem. Eng. Data 56, 4736 (2011).

2011BRA/ARD D. E. Braun, M. Ardid-Candel, E. D'Oria, P. G Karamertzanis, J.-B. Arlin, A. J. Florence, A. G. Jones, and S. L. Price, Cryst. Growth Des. 11, 5659 (2011).

2011BRU/BER G. Bruni, V. Berbenni, C. Milanese, A. Girella, A. Cardini, S. Lanfranconi, and A. Marini, J. Pharm. Biomed. Anal. 54, 1196 (2011).

2011BRU/PIA B. Brunetti, V. Piacente, and P. Scardala, J. Chem. Eng. Data 56, 1970 (2011).

2011CAI/XIA Z. Cairong, S. Xiaohua, C. Lingling, and W. Haifeng, Fluid Phase Equilib. 302, 123 (2011).

2011CAI/YAN Y.-N. Cai, S.-H. Yang, S.-M. Jin, H.-P. Yang, G.-F. Hou, and J.-Y. Xia, J. Cent. South Univ. Technol. 18,73 (2011).

2011CAS/RIB R. A. E. Castro, J. D. B. Ribeiro, T. M. R. Maria, M. R. Silva, C. Yueste-Vivas, J. Canotilho, and M. E. S. Eusebio, Cryst. Growth Des. 11, 5396 (2011).

2011COS/LIM J. C. S. Costa, C. F. R. A. C. Lima, M. A. A. Rocha, L. R. Gomes, and L. M. N. B. F. Santos, J. Chem. Thermodyn. 43, 133 (2011).

2011DAN/DI W.-Y. Dan, Y.-Y. Di, D.-H. He, and Y.-P. Liu, Phase Transitions 84, 135 (2011).

2011DAN/JIN H. Dan, H. Jingdui, and L. Jun, Fluid Phase Equilib. 302, 190 (2011).

2011DAS/NAY M. Das, A. K. Nayak, B. Mohanty, and S. Panda, ISRN Pharm., 819765/1, 2011.

2011DEC/MAH G. P. Dechaine, Y. Maham, X. Tan, and M. R. Gray, Energy Fuels 25, 737 (2011).

2011DEL/HOL D. R. Delgado, A. R. Holguin, O. A. Almanza, F. Martinez, and Y. Marcus, Fluid Phase Equilib. 305, 88 (2011).

2011DOM/KRO U. Domanska and M. Krolikowska, Fluid Phase Equilib. 308, 55 (2011).

2011DOM/KRO2 U. Domanska and M. Krolikowska, J. Chem. Thermodyn. 43, 1488 (2011).

2011DOM/KRO3 U. Domanska, M. Krolikowska, and K. Paduszynski, Fluid Phase Equilib. 303, 1 (2011).

2011DOM/PAD U. Domanska, K. Pauduszynski, and J. Dabska, J. Chem. Eng. Data 56, 881 (2011).

2011DOM/POB U. Domanska, A. Pobudkowska, P. Pelczarska, and L. Zukowski, Int. J. Pharm. 403, 115 (2011).

2011DOM/ZAW U. Domanska, M. Zawadzki, M. Tshibangu, D. Ramjugernath, and T. M. Letcher, J. Phys. Chem. B 115, 4003 (2011).

2011DOM/ZAW2 U. Domanska, M. Zawadzki, and M. Zowlinska, J. Chem. Thermodyn. 43, 775 (2011).

2011DOM/ZAW3 U. Domanska and M. Zawadzki, J. Chem. Thermodyn. 43, 989 (2011).

2011EGO/MAR V. M. Egorov, V. A. Marikhin, and L. P. Myasnikova, Polym. Sci., Ser. A 53, 906 (2011).

2011ELS/KHA A. Elshaer, S. Kan, D. Perumal, P. Hanson, and A. R. Mohammed, Curr. Drug Delivery 8, 363 (2011).

2011EME/POR V. N. Emel'yanenko, S. V. Portnova, S. P. Verevkin, A. Skrzypczak, and T. Schubert, J. Chem. Thermodyn. 43, 1500 (2011).

2011 EME/POR2 V. N. Emel'yanenko, S. V. Portnova, S. P. Verevkin, and A. Skrzypczak, J. Chem. Eng. Data 56, 3532 (2011). 
2011EME/VER V. N. Emel'yanenko, S. P. Verevkin, E. N. Stepurko, and G. N. Roganov, Russ. J. Phys. Chem. A 85, 348 (2011).

2011EME/VER3 V. N. Emel'yanenko, S. P. Verevin, E. N. Stepurko, and G. N. Roganov, Russ. J. Phys. Chem. 85, 2093 (2011).

2011EME/ZAI V. N. Emel'yanenko, D. H. Zaitsau, S. P. Verevkin, A. Heintz, K. Voß, and A. Schulz, J. Phys. Chem. B 115, 11712 (2011).

2011FEL/RAM H. Felix-Rivera, M. L. Ramirez-Cedeno, R. A. SanchezCuprill, and S. P. Hernandez-Rivera, Thermochim. Acta 514, 37 (2011).

2011FON/PFO J. M. S. Fonseca, D. Pfohl, and R. Dohrn, J. Chem. Thermodyn. 43, 1942 (2011).

2011FU/SUU J. Fu and E. M. Suuberg, J. Chem. Thermodyn. 43, 1660 (2011).

2011FU/SUU2 J. Fu and E. M. Suuberg, Environ. Toxicol. Chem. 30, 2216 (2011).

2011FUL/RUZ M. Fulem, K. Ruzicka, and M. Ruzicka, Fluid Phase Equilib. 303, 205 (2011).

2011FUL/RUZ2 M. Fulem and K. Ruzicka, Fluid Phase Equilib. 311, 25 (2011).

2011GAR/NAC J. Garcia, R. A. Naccoul, J. Fernandez, A. Razzouk, and I. Mokbel, Ind. Eng. Chem. Res. 50, 4231 (2011).

$2011 \mathrm{GEL} / \mathrm{MOR} \quad$ N. V. Gelfond, N. B. Morozova, K. V. Zkerikova, P. P. Semyannikov, S. V. Trubin, S. V. Sysoev, and I. K. Igumenov, J. Chem. Thermodyn. 43, 1646 (2011).

2011GOR/WOJ A. Gorniak, A. Wojakowska, B. Karolewicz, and J. Pluta, J. Therm. Anal. Calorim. 104, 1195 (2011).

2011GRO/LIE D. Grooff, W. Liebenberg, and M. M. De Villiers, J. Pharm. Sci. 100, 1944 (2011).

2011HUG/SYE T. J. Hughes, T. Syed, B. F. Graham, K. N. Marsh, and E. F. May, J. Chem. Eng. Data 56, 2153 (2011).

2011JET/BHO R. K. R. Jetti, B. R. Bhogala, A. R. Gorantla, N. R. Kurusula, and D. Datta, Cryst. Growth Des. 11, 2039 (2011).

2011KAM/DEM N. N. Kamkin, A. I. Dement'ev, N. G. Yaryshev, A. S. Alikhanian, and A. V. Kharchenko, Inorg. Mater. 47, 324 (2011).

$2011 \mathrm{KEI} / \mathrm{KON} \quad$ P. Keil and A. Konig, Thermochim. Acta 524, 202 (2011).

2011KHA/NUT A. El Kharbachi, I. Nuta, F. Hodaj, and M. Baricco, Thermochim. Acta 520, 75 (2011).

2011 KOL/MIS $\quad$ S. Kolay, R. Mishra, D. Das, and S. R. Dharwadkar, Thermochim. Acta 512, 110 (2011).

$2011 \mathrm{KOV} / \mathrm{GOL} \quad$ N. A. Kovalenko, N. B. Golvina, A. G. Bogachev, and I. A. Uspenskaya, Russ. J. Phys. Chem. 85, 1570 (2011).

2011LEE/THO J. W. Lee, L. C. Thomas, and S. J. Schmidt, J. Agric. Food Chem. 59, 684 (2011).

2011LI/BOU J. Li, S. A. Bourne, M. M. de Villiers, A. M. Crider, and M. R. Caira, Cryst. Growth Des. 11, 4950 (2011).

2011LIA/YAN R. Liang, M.-R. Yang, G.-X. Xi, and X.-P. Xuan, Henan Shifan Daxue Xuebao, Ziran Kexueban 39, 95 (2011).

2011 LIM/COS $\quad$ C. F. R. A. C. Lima, J. C. S. Costa, and L. M. N. B. F. Santos, J. Phys. Chem. A 115, 9249 (2011).

2011 LIM/ROC $\quad$ C. F. R. A. C. Lima, M. A. A. Rocha, A. Melo, R. R. Gomes, J. N. Low, and L. M. N. B. F. Santos, J. Phys. Chem. A 115, 11876 (2011).

2011LIP/KRA S. V. Lipp, E. L. Krasnykh, and S. P. Verevkin, J. Chem. Eng. Data 56, 800 (2011).

2011LOR/VON H. Lorenz, J. von Langermann, G. Sadiq, C. C. Seaton, R. J. Davey, and A. Seidel-Morgenstern, Cryst. Growth Des. 11, 1549 (2011).

2011MAL/GAG K. E. Maly, E. Gagnon, and J. D. Wuest, Chem. Commun. 47, 5163-5165 (2011).

2011MAR/LET A. V. Markin, I. A. Letyanina, N. N. Smirnova, V. V. Sharutin, and O. V. Molokova, Russ. J. Phys. Chem. A 85, 1315 (2011).

2011MAR/LET2 A. V. Markin, I. A. Letyanina, V. A. Ruchenin, N. V. Smirnova, A. V. Gushchin, and D. V. Shashkin, J. Chem. Eng. Data 56, 3657 (2011).

2011MAS/KRA A. S. Maslakova, E. L. Krasnykh, and S. V. Levanova, Russ. J. Phys. Chem. 85, 1695-1700 (2011).

2011MEL/PIN V. Meltzer and E. Pincu, J. Chem. Thermodyn. 33, 1011 (2011).

2011MIR/MAT M. S. Miranda, M. A. R. Matos, V. M. F. Morais, and J. F. Liebman, J. Chem. Thermodyn. 43, 364 (2011).
$2011 \mathrm{MON} / \mathrm{SOU}$

2011PAS/MIR

2011PER/CON

2011PER/NOR

2011PER/ROD

2011PER/RYZ

2011POR/KRA

2011POZ/VER

2011RIB/AMA3

2011RIB/FER

2011RIB/FER2

2011RIB/FER3

2011RIB/SAN

2011RIC/FU

2011ROC/LIM

2011ROJ/VIE

2011ROU/MAR

2011SAN/LIM

2011SAN/SAR

2011SAN/SAR2

2011SAP/POK

2011SAR/BIC

2011SEL/SAT

2011SEL/SAT2

2011SIL/FAL

2011SLY/KOZ

2011SUR/PER

2011SUR/VOR

2011TIA/WAN

2011TIA/WAN2
M. J. S. Monte and C. A. D. Sousa, J. Therm. Anal. Calorim. 106, 913 (2011).

L. L. Paschenko, E. A. Miroshnichenko, S. M. Pimenova, and I. A. Nesterov, Butler. Soobshch. 26, 66 (2011).

S. Perisanu, I. Contineanu, A. Neascu, R. Notario, M. V. Roux, J. F. Liebman, and B. J. Dodson, Struct. Chem. 22, 89-94 (2011).

R. Persson, S. Nordholm, G. Perlovich, and L. Lindfors, J. Phys. Chem. B 115, 3062 (2011).

A. B. Pereiro, A. Rodriguez, M. Blesic, K. Shimizu, J. N. Canongia Lopes, and L. P. N. Rebelo, J. Chem. Eng. Data 56, 4356 (2011).

G. L. Perlovich, A. M. Ryzhakov, N. N. Strakhova, V. P. Kazachenko, K.-J. Schaper, and O. A. Raevsky, J. Chem. Thermodyn. 43, 683 (2011).

S. V. Portnova, E. L. Krasnykh, and S. P. Verevkin, Fluid Phase Equilib. 309, 114 (2011).

V. A. Pozdeev and S. P. Verevkin, J. Chem. Thermodyn. 43, 1791 (2011).

M. A. V. Ribeiro da Silva, L. M. P. F. Amaral, and R. V. Ortiz, J. Chem. Thermodyn. 43, 546 (2011).

M. A. V. Ribeiro da Silva, A. I. M. C. L. Ferreira, A. L. M. Barros, A. R. C. Bessa, B. C. S. A. Brito, J. A. S. Vieira, and S. A. P. Martins, J. Chem. Thermodyn. 43, 1306 (2011).

M. A. V. Ribeiro da Silva, A. I. M. C. L. Ferreira, and A. Cimas, J. Org. Chem. 76, 3754 (2011).

M. A. V. Ribeiro da Silva, A. I. M. C. L. Ferreira, and A. Cimas, J. Chem. Thermodyn. 43, 1857 (2011).

M. A. V. Ribeiro da Silva, A. F. L. O. M. Santos, L. P. T. Carneiro, R. F. Mendes, A. S. M. C. Rodrigues, P. J. O. Ferreira, and R. M. C. Ramos, J. Chem. Thermodyn. 43, 172 (2011).

J. W. Rice, J. Fu, and E. M. Suuberg, Ind. Eng. Chem. Res. 50, 3613 (2011).

M. A. A. Rocha, C. F. R. A. C. Lima, L. M. S. R. Gomes, B. Schoder, J. A. P. Coutinho, I. M. Marrucho, J. M. S. S. Esperanca, L. P. N. Rebelo, K. Shimizu, J. N. A. C. Lopez, and L. M. N. F. G. Santos, J. Phys. Chem. B 115, 10919 (2011).

A. Rojas and M. T. Vieyra-Eusebio, J. Chem. Thermodyn. 43, 1738 (2011).

M. V. Roux, G. Martin-Valcarcel, R. Notario, S. Kini, J. S. Chickos, and J. F. Liebman, J. Chem. Eng. Data 56, 1220 (2011).

L. M. B. F. Santos, L. M. S. S. Lima, C. F. R. A. C. Lima, F. D. Magalhaes, M. C. Torres, B. Schroder, and M. A. V. Ribeiro da Silva, J. Chem. Thermodyn. 43, 834 (2011).

P. Sanphui, B. Sarma, and A. Nangia, J. Pharm. Sci. 100, 2287 (2011).

P. Sanphui, B. Sarma, and A. Nangia, Cryst. Growth Des. 10, 4550 (2010).

E. Sapei, J.-P. Pokki, P. Uusi-Kyyny, K. I. Keskinen, and V. Alopaeus, Fluid Phase Equilib. 301, 200 (2011).

A. Sari, A. Bicer, O. Lafci, and M. Ceylan, Sol. Energy 85, 2061 (2011).

J. Selvakumar, D. Sathiyamoorthy, and K. S. Nagaraja, Surf. Coat. Technol. 205, 3493 (2011).

J. Selvakumar, D. Sathiyamoorthy, and K. S. Nagaraja, Mater. Chem. Phys. 129, 62 (2011).

L. Y. A. Silva, R. M. M. Falleiro, A. J. A. Meirelles, and M. A. Krahenbuhl, J. Chem. Thermodyn. 43, 943 (2011). I. V. Slyusareva, A. O. Kozin, I. A. Dement'ev, and Y. V. Kondrat'ev, Vestnik Sankt-Peterburgskogo Universiteta Ser. 4 (2011); Fiz. Khim. 2011, 129.

A. O. Surov, G. L. Perlovich, V. N. Emel'yanenko, and S. P. Verevkin, J. Chem. Eng. Data 56, 4325 (2011).

O. V. Surov, M. I. Voronova, N. Z. Mamardashvili, and A. G. Zakharov, Tetrahedron Lett. 52, 705 (2011).

N.-N. Tian, L.-S. Wang, and R.-Y. Jiang, J. Chem. Eng. Data 56, 3208 (2011).

N.-N. Tian, L.-S. Wang, M.-Y. Li, and R.-Y. Jiang, J. Chem. Eng. Data 56, 661 (2011). 
2011TIT/FUL $\quad$ B. Tita, A. Fulias, G. Bandur, E. Marian, and D. Tita, J. Pharm. Biomed. Anal. 56, 211 (2011).

2011TKA/DRU Y. S. Tkachenko, A. I. Druzhinina, N. V. Avramenko, R. M. Varushchenko, A. I. Emelina, I. A. Nesterov, and T. N. Nesterova, Vestnik. Mosk. Univer., Ser. 2; Khim. 52, 341 (2011).

2011TKA/VAR E. S. Tkachenko, R. M. Varuschenko, A. I. Druzhinina, M. D. Reshetova, and N. E. Borisova, J. Chem. Eng. Data 56, 4700 (2011).

2011TUR/ZHA A. E. Turgambaeva, G. Zharkova, P. Semyannikov, V. V. krisyuk, T. Koretskaya, S. Trubin, B. Kuchumov, and I. Igumenov, Gold Bull. 44, 177 (2011).

2011UMN/CHI P. Umnahanant and J. S. Chickos, J. Pharm. Sci. 100, 1847 (2011).

2011VER/EME S. P. Verevkin, V. N. Emel'yanenko, A. A. Pimerzin, and E. E. Vishnevskay, J. Phys. Chem. A 115, 1992 (2011).

2011VER/ZAI2 S. P. Verevkin, D. H. Zaitsau, V. N. Emel'yanenko, and A. Heintz, J. Phys. Chem. B 115, 12889 (2011).

2011 VIE/ROJ M. T. Vieyra-Eusebio and A. Rojas, J. Chem. Eng. Data 56, 5008 (2011).

2011WEI/LI D. Wei, H. Li, C. Liu, and B. Wang, Ind. Eng. Chem. Res. 50, 2473 (2011).

2011YAN/WAN X. Yang, J. Wang, and X. Sun, Huaxue Gongcheng 39, 61 (2011).

2011YAN/WAN2 X. Z. Yang, J. Wang, and X. K. Sun, Beijing Huagong Daxue Xuebao, Ziran Kexueban 38, 27 (2011).

2011ZAI/VER D. H. Zaitsau, S. P. Verevkin, V. N. Emel'yanenko, and A. Heintz, ChemPhysChem 12, 3609 (2011).

2011ZEN/YU J.-L. Zeng, S.-B. Yu, B. Tong, L.-X. Sun, Z.-C. Tan, Z. Cao, D.-W. Yang, and J.-N. Zhang, J. Therm. Anal. Calorim. 103, 1087 (2011).

2011ZHA/WAN Y. Zhang, L. Wang, R. Zhou, and X. Liu, J. Chem. Eng. Data 56, 2090 (2011).

2011ZHO/SHI C. R. Zhou, X.-H. Shi, H.-F. Wang, and D.-G. Jiang, Gaoxiao Huaxue Gongcheng Xuebao 25, 442 (2011).

2011ZHU/KRA T. A. Zhukova, A. V. Krasnov, N. V. Belova, G. V. Firichev, Y. A. Zhabanov, and N. P. Kuz'mina, Izvest. Vyssh. Uchebn. Zaved. Khim. Khim. Tecknol. 54, 31 (2011).

2011ZUO/LI J. Zuo, W. Li, and L. Weng, Appl. Therm. Eng. 31, 1352 (2011).

2012AYD A. A. Aydin, Sol. Energy Mater. Sol. Cells 104, 102 (2012).

2012AYD/AYD A. A. Aydin and A. Aydin, Sol. Energy Mater. Sol. Cells 96, 93 (2012).

2012AYD/AYDa A. A. Aydin, Sol. Energy Mater. Sol. Cells 104, 102 (2012).

2012AYD/OKU A. A. Aydin and H. Okutan, Sol. Energ. Mat. Sol. Cells 95, 2752-2762 (2011).

2012BAG/RED P. P. Bag and C. M. Reddy, Cryst. Growth Des. 12, 2740 (2012).

2012BAN/MAH P. V. Bankar and O. P. Mahatma, Int. J. Drug Delivery 4, 498 (2012).

2012BEI/RUE T. Beierling and F. Ruether, Chem. Eng. Sci. 77, 71 (2012).

2012BEL/GIR N. V. Belova, G. V. Girichev, N. I. Giricheva, I. G. Zaitseva, I. O. Zyabko, A. V. Krasnov, N. P. Kuz'mina, and S. A. Shlykov, Izvest. Vysshikh Uchebnykh Zavedenii, Khim. Khimich. Tekhnol. 55, 50 (2012).

2012BEN/KHI M. Benziane, K. Khimeche, A. Dahmani, S. Nezar, and D. Trache, Mol. Phys. 110, 1383 (2012).

2012BIC/SAR A. Bicer and A. Sari, Sol. Energy Mater. Sol. Cells 102, 125 (2012).

2012BLO/OLK S. V. Blokhina, M. V. Ol'khovich, A. V. Sharapova, A. N. Proshin, and G. L. Perlovich, J. Chem. Eng. Data 57, 1996 (2012).

2012BRU/MAI G. Bruni, M. Maielta, L. Maggi, M. Bini, D. Capsoni, S. Ferrari, M. Boiocchi, V. Berbenni, C. Milanesi, and A. Marini, CrystEngComm 14, 6035 (2012).

2012CAO/WU J. Cao, C. Wu, H. Dong, D. Cheng, Y. Zhang, and K. Wu, Fluid Phase Equilib. 334, 137 (2012).

2012CHA/ARO R. Chadha, P. Arora, A. Saini, and D. S. Jain, J. Pharm. Pharm. Sci. 15, 234 (2012).
2012CHA/LAY

2012CHI/KAZ

2012DEY/HES

2012ELK/ASH

2012ELS/HAN

2012EME/VER

2012FRE/GOM

2012FRI/RAM

2012FU/SUU

2012GAR/VER

2012GER/PAV

2012HAS/DRA

2012INA/SUZ

2012JEG/PRA

2012JIA/WAN

2012KAC/RUS

2012KAN/RAI

2012KAZ/TIM

2012KEN

2012KHA/ACH

2012LAU/RUT

2012LI/YAN

2012LIM/JAN

2012LIM/ROC

2012MAX/CHI

2012MEL/ROD

2012MER/ROD

2012MON/NOT

2012MON/NOT2

2012MON/PIN

2012NAZ/NES

E. L. Charsley, P. G. Laye, and H. M. Markham, Thermochim. Acta 539, 115 (2012).

R. D. Chirico, A. F. Kazakov, and W. V. Steele, J. Chem. Thermodyn. 54, 278 (2012).

A. Deyko, S. G. Hessey, P. Licence, E. A. Chernikova, V. G. Krasovskiy, L. M. Kustov, and R. G. Jones, Phys. Chem. Chem. Phys. 14, 3181 (2012).

A. A. Elkordy, A. Ashoore, and E. A. Essa, J. Appl. Pharm. 4, 614 (2012).

A. ElShaer, P. Hanson, T. Worthington, P. Lambert, and A. R. Mohammed, Pharmaceutics 4, 179 (2012).

V. N. Emel'yanenko, S. P. Verevkin, R. V. Ralys, V. V. Turovtsev, and V. Y. Orlov, Russ. J. Phys. Chem. A 86, 1493 (2012).

V. L. S. Freitas, J. R. B. Gomes, and M. D. M. C. Ribeiro da Silva, J. Chem. Thermodyn. 54, 108 (2012).

T. E. Frizon, D. S. Rampon, H. Gallardo, A. A. Merlo, P. H. Schneider, O. E. D. Rodrigues, and A. L. Braga, Liq. Cryst. 39, 769 (2012).

J. Fu and E. M. Suuberg, Environ. Toxicol. Chem. 31, 486 (2012).

I. V. Garist, S. P. Verevkin, J. E. Bara, M. S. Hindman, and S. P. O. Danielson, J. Chem. Eng. Data 57, 1803 (2012).

S. I. Gerasimchuk, Y. P. Pavlovskii, and Y. Y. Van-ChinSyan, Russ. J. Phys. Chem. A 86, 1500 (2012).

D. Hasty, J. Drapekin, T. Subramanian, T. C. Winter, J. S. Chickos, A. A. Samarov, A. V. Yermalayeu, and S. P. Verevkin, J. Chem. Eng. Data 57, 2350 (2012).

A. Inaba, H. Suzuki, M. Massalska, and T. Rozwadowski, J. Chem. Thermodyn. 54, 204 (2012).

B. Jeganathan and V. Prakya, Iran. J. Pharm. Sci. 8, 83 (2012).

R.-Y. Jiang and L.-S. Wang, J. Solution Chem. 41, 2107 (2012).

I. Kacso, L. Rus, M. Pop, G. Borodi, and I. Brata, Spectroscopy 27, 49 (2012).

S. Kant, U. S. Rai, and R. N. Rai, J. Therm. Anal. Calorim. 110, 551 (2012).

I. V. Kazakov and A. Y. Timoshkin, Russ. J. Inorg. Chem. 57, 557 (2012).

J. A. Kenar, Eur. J. Lipid Sci. Technol. 114, 63 (2012).

M. Kharwadi, G. Achyuta, C. V. S. Subrhmanyan, and P. P. S. Babu, J. Solution Chem. 41, 1364 (2012).

Y. Lauw, T. Ruther, M. D. Horne, K. S. Wallwork, B. W. Skelton, I. C. Madsen, and T. Rodopoulos, Cryst. Growth Des. 12, 2802 (2012).

R. Li, H. Yan, Z. Wang, and J. Gong, Ind. Eng. Chem. Res. 51, 8141 (2012).

J. Lim, S. Jang, M. S. Shin, and H. Kim, Fluid Phase Equilib. 332, 144 (2012).

C. F. R. A. C. Lima, M. A. A. Rocha, B. Shröder, L. R. Gomes, J. N. Low, and L. M. N. B. F. Santos, J. Phys. Chem. B 116, 3557 (2012).

R. Maxwell and J. S. Chickos, J. Pharm. Sci. 101, 805 (2012).

C. I. Melo, A. I. Rodrigues, R. Bogel-Lukasik, and E. Bogel-Lukasik, J. Phys. Chem. A 116, 1765 (2012).

K. C. Mercado, G. A. Rodriguez, D. R. Delgado, F. Martinez, and A. Romdhani, Quim. Nova 35, 1967 (2012).

M. J. S. Monte, R. Notario, S. P. Pinto, A. I. M. C. L. Ferreira, and M. D. M. C. Ribeiro da Silva, J. Chem. Thermodyn. 49, 159 (2012).

M. J. S. Monte, R. Notario, M. M. G. Calvinho, A. R. R. P. Almeida, L. M. P. F. Amaral, A. I. M. C. Lobo Ferreira, and M. D. M. C. Ribeiro da Silva, J. Chem. Eng. Data 57, 2486 (2012).

M. J. S. Monte, S. P. Pinto, A. I. M. L. Ferreira, L. M. P. Amaral, V. L. S. Freitas, and M. D. M. C. Ribeiro da Silva, J. Chem. Thermodyn. 45, 53 (2012).

A. G. Nazmutdinov, I. A. Nesterov, T. A. Nazmutdinov, T. N. Nesterova, S. V. Tarazanov, S. V. Vostrikov, L. L. Pashchenko, E. A. Miroshnchenko, and S. P. Verevkin, Fluid Phase Equilib. 335, 88 (2012). 
2012NON/CHE W. Nong, X. Chen, L. Wang, J. Liang, and Z. Tong, Gaoxiao Huaxue Gongcheng Xuebao 26, 552 (2012).

2012OLK/SHA M. V. Ol'khovich, A. V. Sharapova, S. V. Blokhina, G. L. Perlovich, and A. N. Proshin, J. Chem. Eng. Data 57, 3452 (2012).

2012PAL/ORA M. Palczewska-Tulinska and P. Oracz, J. Chem. Eng. Data 57, 3176 (2012)

2012PLA/KOT N. V. Platonova, E. N. Kotel'nikova, S. K. Filatov, G. A. Puchkovskaya, I. I. Gnatyuk, J. Baran, and M. Drozd, J. Struct. Chem. 53, 973 (2012).

2012RIB/AND A. L. Ribeiro Souza, T. Andreani, F. M. Nunes, D. L. Cassimiro, A. E. Almeida, C. A. Ribeiro, V. H. V. Sarmento, M. P. D. Gremiao, A. M. Silva, and E. B. Souto, J. Therm. Anal. Calorim. 108, 353 (2012).

2012RIE/PER M. K. Riekes, R. N. Pereira, G. S. Rauber, S. L. Cuffini, C. E. Maduro de Campos, M. A. S. Silva, and H. K. Stulzer, J. Pharm. Biomed. Anal. 70, 188 (2012).

2012ROC/COU M. A. A. Rocha, J. A. P. Coutinho, and L. M. N. B. F. Santos, J. Phys. Chem. B 116, 10922 (2012).

2012ROS/GEL D. Rossi, T. Gelbrich, V. Kahlenberg, and U. J. Griesser, CrystEngComm 14, 2494 (2012).

2012ROZ/BRU J. Rozmus, I. Brunella, P. Mougin, and J. C. de Hemptinne, J. Chem. Eng. Data 57, 2915 (2012).

2012SAM/NAZ A. A. Samarov, A. G. Nazmutdinov, and S. P. Verevkin, Fluid Phase Equilib. 334, 70 (2012).

2012SAR/KAR A. Sari and A. Karaipekli, Appl. Therm. Eng. 37, 208 (2012).

2012SEL/SAT J. Selvakumar and D. Sathiyamoorthy, J. Mater. Chem. 22, 7551 (2012).

2012SHA/LAL $\quad$ B. L. Sharma, P. Lal, M. Sharma, and A. K. Sharma, J. Therm. Anal. Calorim. 109, 447 (2012).

2012SHI/OHT Y. Shimizu, Y. Ohte, Y. Yamamura, S. Tsuzuki, and K. Saito, J. Phys. Chem. B 116, 5406 (2012).

2012 SIL/PER V. M. T. M. Silva, C. S. M. Pereira, A. E. Rodrigues, S. P. Verevkin, V. N. Emel'yanenko, I. V. Garist, and J. Gmehling, Ind. Eng. Chem. Res. 39, 12723 (2012).

2012SMI/LET N. N. Smirnova, L. A. Letyanina, Y. A. Zakhorova, A. A. Pimerzin, and E. E. Vishnevskaya, J. Chem. Thermodyn. 48, 118 (2012).

2012SUR/SOL A. O. Surov, K. A. Solanko, A. D. Bond, G. L. Perlovich, and A. Bauer-Brandl, Cryst. Growth Des. 12, 4022 (2012).

2012 TEV/BRO D. E. Tevault, A. Brozena, J. H. Buchanan, P. L. Abercrombie-Thomas, and L. C. Beuttner, J. Chem. Eng. Data 57, 1970 (2012).

2012TOT/BHO S. Tothadi, B. R. Bhogala, A. R. Gorantla, T. S. Thakur, R. K. R. Jetti, and G. R. Desiraju, Chem. - Asian J. 7, 330 (2012).

2012UMN/CHI P. Umnahanant and J. Chickos, J. Chem. Eng. Data 57, 1331 (2012).

2012UMN/HAS P. Umnahanant, D. Hasty, and J. S. Chickos, J. Pharm. Sci. 101, 2045 (2012).

2012VER/EME2 S. P. Verevkin, V. N. Emel'yanenko, I. Krossing, and A. Kalb, J. Chem. Thermodyn. 51, 107 (2012).

2012VER/EME3 S. P. Verevkin, V. N. Emel'yanenko, A. Heintz, K. Stark, and W. Arlt, Ind. Eng. Chem. Res. 51, 12150 (2012).

2012 VER/RAL S. P. Verevkin, R. V. Ralys, D. H. Zaitsau, V. N. Emel'yanenko, and C. Schick, Thermochim. Acta 538, 55 (2012).

2012VER/ZAI S. P. Verevkin, D. H. Zaitsau, V. N. Emel'yanenko, R. V. Ralys, C. Schick, M. Geppert-Rybezynska, S. Jayaraman, and E. J. Maginn, Aust. J. Chem. 65, 1487 (2012).

2012VIT/BER J. Vitorino, C. E. S. Bernardes, and M. E. Minas da Piedade, Phys. Chem. Chem. Phys. 14, 4440 (2012).

2012VIV/FRE M. L. F. Viveiros, V. L. S. Freitas, N. Vale, J. R. B. Gomes, P. Gomes, and M. D. M. C. Ribeiro da Silva, J. Phys. Org. Chem. 25, 420 (2012).

2012YAM/SUZ Y. Yamamura, Y.-T. Suzuki, M. Sumita, and K. Saito, J. Phys. Chem. 116, 3938 (2012).

2012YAN/THA H. Yang, J. Thati, and A. C. Rasmuson, J. Chem. Thermodyn. 48, 150 (2012).

2012YAN/WAN H. Yan, Z. Wang, and J. Wang, Ind. Eng. Chem. Res. 51, 2808 (2012).
2012YUE/DON

2012ZAI/YER

2012ZAW/DOM

2012ZHA/WU

2012ZHE/ZEL

2013ACE/VAZ

2013AGA/MOS

2013ALM/MON

Y. Yue, H. Dong, C. Wu, G. Lai, and J. Jiang, Nanjing Gongye Daxue Xuebao, Ziran Kexueban 34, 111 (2012). D. H. Zaitsau, A. V. Yermalayeu, V. N. Emel'yanenko, S. P. Verevkin, U. Welz-Biermann, and T. Schubert, Sci. China: Chem. 55, 1525 (2012).

M. Zawadzski and U. Domanska, J. Chem. Thermodyn. 48, 276 (2012).

B. Zhang, C. Wu, H. Dong, and J. Jiang, Nanjing Gongye Daxue Xuebao, Ziran Kexueban 34, 106 (2012).

K. V. Zherikova, L. N. Zelenina, N. B. Morozova, and T. P. Chusova, J. Therm. Anal. Calorim. 108, 1325 (2012).

J. M. Aceves-Hernandez, I. N. Vazquez, J. HinojosaTorres, G. P. Carrillo, G. A. Razo, and R. M. Rubalcaba, J. Mol. Struct. 1038, 163 (2013).

E. V. Agafonova, Y. V. Moshchenskii, and M. L. Tkachenko, Russ. J. Phys. Chem. A 87, 1291 (2013).

2013ALM/MON2 A. R. R. P. Almeida, M. J. S. Monte, M. A. R. Matos, and V. M. F. Morais, J. Chem. Thermodyn. 59, 222 (2013).

2013ALM/MON3 A. R. R. P. Almeida and M. J. S. Monte, J. Chem. Thermodyn. 65, 150 (2013).

2013AMA/RIB L. M. P. F. Amaral and M. A. V. Ribeiro da Silva, J. Chem. Thermodyn. 57, 301 (2013).

2013AMA/SAN L. M. P. F. Amaral, A. F. L. O. M. Santos, M. D. M. C. Ribeiro da Silva, and R. Notario, J. Chem. Thermodyn. 58, 315 (2013).

2013AMA/SZT L. M. P. F. Amaral, P. Szterner, and M. A. V. Ribeiro da Silva, J. Chem. Thermodyn. 64, 187 (2013).

2013AYD

2013BAR/GIL A. A. Aydin, Sol. Energy Mater. Sol. Cells 113, 44 (2013).

2013BEN/KHI Cabeza, Sol. Energy 94, 344 (2013).

2013BEN/KHI2

M. Benziane, K. Khimeche, A. Dahmani, S. Nezar, and D. Trache, J. Therm. Anal. Calorim. 112, 229 (2013).

2013BER/CAN J. Jose, J. Chem. Eng. Data 58, 492 (2013).

2013BHA/GOH

C. E. S. Bernardes, J. N. Canongia Lopes, and M. E. Minas da Piedade, J. Phys. Chem. A 117, 11107 (2013).

2013BLO/KOH (2013).

2013BOU/TEY

A. V. Blokhin, S. V. Kohut, G. J. Kabo, E. N. Stepurko, Y. U. Paulechka, and O. V. Voitkevich, Thermochim. Acta 565, 221 (2013)

B. Bouillot, S. Teychene, and B. Biscans, Ind. Eng. Chem. Res. 52, 9276 (2013).

2013BRA/AMH C. Brandel, Y. Amharar, J. M. Rollinger, U. J. Griesser, Y. Cartigny, S. Petit, and G. Coquerel, Mol. Pharm. 10, 3850 (2013).

2013BRE/GUB A. A. Bredikhin, A. T. Gubaidullin, Z. A. Bredikhina, R. R. Fayzullin, A. I. Samigullina, and D. V. Zakharychev, J. Mol. Struct. 1045, 104 (2013).

2013BUR/STR A. U. Burman and K. H. U. Strom, J. Chem. Eng. Data 58, 257 (2013).

2013CAB/BOG

S. Cabus, K. Bogaerts, J. Van Mechelen, M. Smet, and B. Goderis, Cryst. Growth Des. 13, 3438 (2013).

2013CAR/LUC S. P. Carvalho, E. F. Lucas, G. Gonzalez, and L. S. Spinelli, J. Braz. Chem. Soc. 24, 1998 (2013).

2013CAR/RHA L. Carpentier, K. F. Rharrassi, P. Derollez, and Y. Guinet, Thermochim. Acta 556, 63 (2013).

2013CEN/MAR R. Censi, V. Martena, E. Hoti, L. Malag, and P. di Martino, J. Therm. Anal. Calorim. 111, 2009 (2013).

2013CHA/ARO R. Chadha, P. Arora, M. Garg, S. Bhandari, and D. S. Jain, J. Therm. Anal. Calorim. 111, 2133 (2013).

2013CHE/XIE Z. Chen, C. Xie, Z. Xu, Y. Wang, H. Zhao, and H. Hao, J. Chem. Eng. Data 58, 143 (2013).

2013CHI/DER N. Chiali-Baba-Ahmed, F. Dergal, L. Negadi, and I. Mokbel, J. Chem. Thermodyn. 63, 44 (2013).

2013COS/SAN J. C. S. Costa and L. M. N. B. F. Santos, J. Phys. Chem. C 117, 10919 (2013).

2013CUI/YIN P. Cui, Q. Yin, J. Gong, Y. Wang, H. Hao, C. Xie, Y. Bao, M. Zhang, B. Hou, and J. Wang, Fluid Phase Equilib. 337, 354 (2013). 
2013DAV/JIM J. Z. Davalos, P. Jimenez, M. V. Roux, M. T. Molina, T. Filipova, E. Lewars, and J. F. Liebman, Struct. Chem. 24, 2027 (2013).

2013DAV/KIL P. S. Davis and J. E. Kilpatrick, J. Chem. Thermodyn. 58, 134 (2013).

2013DIO/PIN H. P. Diogo, S. S. Pinto, and J. J. M. Ramos, J. Mol. Liq. 178, 142 (2013).

2013DIX/KUL M. Dixit, P. K. Kulkarni, and R. S. Vaghela, Trop. J. Pharm. Res. 12, 317 (2013).

2013DOM/KRO U. Domanska, M. Krolikowska, and K. and walczak, Colloids Surf., A 436, 504 (2013).

2013DOM/SKI U. Domanska, K. Skiba, M. Zawadzki, K. Paduszynski, and M. Krolikowski, J. Chem. Thermodyn. 56, 153 (2013).

2013DOS/MOR A. D. dos Santos, A. R. C. Morais, C. Melo, R. BogelLukasik, and E. Bogel-Lukasik, Fluid Phase Equilib. 356, 18 (2013).

2013DRE/MIK V. A. Drebushchak, M. A. Mikhailenko, T. P. Shakhtshneider, and S. A. Kuznetsova, J. Therm. Anal. Calorim. 111, 2005 (2013).

2013DUD/COR E. Dudognon, N. T. Correia, F. Danede, and M. Descamps, Pharm. Res. 30, 81 (2013).

2013EME/VER V. N. Emel'yanenko and S. P. Verevkin, Vest. St. Peter. Ser. Fiz. Khim. 2013, 113.

2013END/NIS T. Endo and K. Nishikawa, Chem. Phys. Lett. 584, 79 (2013).

2013FER/RIB $\quad$ A. I. M. C. L. Ferreira and M. A. V. Ribeiro da Silva, J. Chem. Thermodyn. 59, 94 (2013).

2013FLO/MEN H. Flores, J. Mentado, E. A. Camarillo, A. Ximello, and M. A. Amador, Struct. Chem. 24, 2083 (2013).

2013FRE/GOM V. L. S. Freitas, J. R. B. Gomes, and M. D. M. C. Ribeiro da Silva, Struct. Chem. 24, 1923 (2013).

2013FRI/VIL C. P. Frizzo, M. A. Villetti, A. Z. Tier, I. M. Gindri, L. Buriol, F. A. Rosa, R. M. Claramunt, D. Sanz, and M. A. P. Martins, Thermochim. Acta 574, 63 (2013).

2013FUL/RUZ M. Fulem, K. Ruzicka, C. Cervinka, M. A. A. Rocha, L. M. N. B. F. Santos, and R. F. Berg, J. Chem. Thermodyn. 57, 530 (2013).

2013FUL/VLA A. Fulias, G. Vlase, C. Grigorie, I. Ledeti, P. Albu, M. Bilanin, and T. Vlase, J. Therm. Anal. Calorim. 113, 265 (2013).

2013GAL/RIB T. L. P. Galvao, M. D. M. C. Ribeiro da Silva, and M. A. V. Ribeiro da Silva, J. Chem. Thermodyn. 62, 186 (2013).

2013GAL/ROC T. L. P. Galvao, I. M. Rocha, M. D. M. C. Ribeiro da Silva, and M. A. V. Ribeiro da Silva, J. Phys. Chem. C 117, 5826 (2013).

2013GAN/BAR I. Gana, M. Barrio, B. Do, J.-L. Tamarit, R. Ceolin, and I. B. Rietveld, Int. J. Pharm. 456, 480 (2013).

2013GAU/FOR A. Gaujac, J. L. Ford, N. M. Dempster, J. Bittencourt de Andrade, and S. D. Brandt, Microchem. J. 110, 146 (2013).

2013GOB/GUT C. Gobble, A. Gutterman, and J. S. Chickos, Struct. Chem. 24, 1903 (2013).

2013GOB/RAT C. Gobble, N. Rath, and J. Chickos, J. Chem. Eng. Data 58, 2600 (2013).

2013GOM/ROD J. L. Gomez, G. A. Rodriguez, D. M. Cristancho, D. R. Delgado, and F. Martinez, Phys. Chem. Liq. 51, 651 (2013).

2013GRI/MAR G. S. Grigoryan and S. L. A. Markaryan, Russ. J. Phys. Chem. 87, 191 (2013).

2013HIK/WEE W. M. Hikal and B. L. Weeks, Chem. Phys. 415, 228 (2013).

2013HUA/LU J. Huang, S. Lu, X. Kong, S. Liu, and Y. Li, Materials 6, 4758 (2013).

2013HUA/LV G. Huang and C. Lv, J. Chem. Eng. Data 58, 279 (2013).

2013JEO/JEO S.-G. Jeong, J. Jeon, O. Chung, S. Kim, and S. Kim, J. Therm. Anal. Calorim. 114, 689 (2013).

2013JIA/HU X.-M. Jiang, Y.-H. Hu, W.-G. Yang, Z.-Y. Lei, R.-R. Tang, and F. Shen, J. Solution Chem. 42, 272 (2013).

2013JIA/LI D. Jia, Y. Li, Y. Li, and C. Li, J. Chem. Eng. Data 58, 3183 (2013).

2013JIM/PLA I. Jimenez, D. Plano, J. A. Palop, C. Sanmartin, and E. Lizarraga, J. Therm. Anal. Calorim. 111, 605 (2013).
2013JUS/ZIE

2013KAN

2013KHA/SUB

2013KIC/KEI

2013KOV/PUS

2013KUH/SVA

2013KUM/NAN

2013LET/MAR

2013LI/LU

2013LI/TAN

2013LI/ZHA

2013LIM/JAN

2013LIM/JAN2

2013LIU/YIN

2013LIU/ZHA

2013LOM/GIN

2013LOM/LAF

2013MAH/MAN

2013MAN/VOR

2013MAN/WIL

2013MAR/CAS

2013MEJ/OLI

2013MEK/BEN

2013MEL/BOG

2013MIR/MAT

2013MOD/DAN

2013MOR/ELL

2013MOR/FUL

2013NAT/JES

2013NEG/BAR

2013NOT/EME

2013OGU/AKA
E. Juszynska-Galazka, P. M. Zielinski, M. MassalskaArodz, and J. Krawczyk, Acta Phys. Pol., A 124, 917 (2013).

V. Kant, Bull. Pharm. Med. Sci. 1, 117 (2013).

M. Kharwade, C. V. S. Subrahmanyam, and P. R. S. Babu, J. Pharm. Res. 7, 409 (2013).

M. Kick, P. Keil, and A. Konig, Fluid Phase Equilib. 338, 172 (2013).

N. A. Kovalenko, E. A. Pustovgar, and I. A. Uspenskay, J. Chem. Eng. Data 58, 159 (2013).

M. Kuhs, M. Svard, and A. C. Rasmuson, J. Chem. Thermodyn. 66, 50 (2013).

(2013)

I. A. Letyanina, A. V. Marken, N. N. Smirnova, S. S. Sologubov, and V. V. Shartutin, J. Chem. Eng. Data 58, 3087 (2013).

Q Li, F. Lu, Y. Tian, S. Feng, Y. Shen, and B. Wang, J. Chem. Eng. Data 58, 1020 (2013).

R.-C. Li and Z.-C. Tan, J. Chem. Eng. Data 58, 2137 (2013).

Y. Li, H. Zhao, M. Mao, and Z. Yang, J. Chem. Eng. Data 58, 1629 (2013).

J. Lim, S. Jang, H. K. Cho, M. S. Shin, and H. Kim, J. Chem. Thermodyn. 57, 295 (2013).

J. Lim, S. Jang, H. Kim, H. K. Cho, and M. S. Shin, Korean J. Chem. Eng. 30, 181 (2013).

Z. Liu, Q. Yin, X. Zhang, H. Zhang, J. Gong, and J. Wang, Fluid Phase Equilib. 356, 38 (2013).

R. Liu, T. Zhang, Y. Liu, L. Yang, and Z. Zhou, J. Therm. Anal. Calorim. 112, 1523 (2013).

L. Lomba, B. Giner, C. Lafuente, S. Martin, and H. Artigas, J. Chem. Eng. Data 58, 1193 (2013).

L. Lomba, C. Lafuente, M. Garcia-Mardones, I. Gascon, and B. Giner, J. Chem. Thermodyn. 65, 34 (2013).

M. P. Maheswaran, D. Mantheni, I. Perera, H. Venumuddala, A. Riga, and K. Alexander, J. Therm. Anal. Calorim. 111, 1987 (2013).

A. N. Manin, A. P. Voronin, and G. L. Perlovich, Thermochim. Acta 551, 57 (2013).

A. Manhieu, J.-F. Willart, E. Dudognon, M. D. Eddleston, W. Jones, F. Danede, and M. Descamps, J. Pharm. Sci. 102, 462 (2013).

T. M. R. Maria, R. A. E. Castro, M. R. Silva, M. L. Ramos, L. L. G. Justino, H. D. Burrows, J. Canotilho, and M. E. R. Eusebio, J. Therm. Anal. Calorim. 111, 2171 (2013). A. Mejia, M. B. Oliveira, H. Segura, M. Cartes, and J. A. P. Coutinho, Fluid Phase Equilib. 345, 60 (2013).

A. Mekki-Berrada, S. Bennici, J.-L. Dubois, and A. Auroux, J. Am. Oil Chem. Soc. 90, 1621 (2013).

C. I. Melo, R. Bogel-Lukasik, M. N. Ponte, and E. BogelLukasik, Fluid Phase Equilib. 338, 209 (2013).

M. S. Miranda, M. A. R. Matos, and V. M. F. Morais, J. Chem. Thermodyn. 62, 170 (2013).

S. R. Modi, A. K. Dantuluri, V. Puri, Y. B. Pawar, P. Nadekar, A. T. Sangamwar, S. R. Perumalia, C. C. Sun, and A. K. Bansal, Cryst. Growth Des. 13, 2824 (2013).

K. M. Morgan, J. A. Ellis, J. Lee, A. Fulton, S. L. Wilson, P. S. Dupart, and R. Dastorri, J. Org. Chem. 78, 4303 (2013).

P. Moravek, M. Fulem, J. Pangrac, E. Hulicius, and K. Ruzicka, Fluid Phase Equilib. 360, 106 (2013).

C. Nather, I. Jess, P. G. Jones, C. Taouss, and N. Teschmit, Cryst. Growth Des. 13, 1676 (2013).

P. Negrier, M. Barrio, J. L. Tamarit, D. Mondieig, M. J. Zuriaga, and S. C. Perez, Cryst. Growth Des. 13, 2143 (2013).

R. Notario, V. N. Emel'yanenko, M. V. Roux, F. Ros, S. P. Verevkin, J. S. Chickos, and J. F. Liebman, J. Phys. Chem. A 117, 244 (2013).

T. Ogura, N. Akai, A. Kawai, and K. Shibuya, Chem. Phys. Lett. 555, 110 (2013). 
2013OLI/CAL J. A. S. A. Oliveira, M. M. Calvinho, R. Notario, M. J. S. Monte, and M. D. M. C. Ribeiro da Silva, J. Chem. Thermodyn. 62, 222 (2013).

2013OLK/BLO M. V. Ol'khovich, S. V. Blokhina, A. V. Sharapova, G. L. Perlovich, and A. N. Proshin, Thermochim. Acta 569, 61 (2013).

2013PAL/MCC K. J. Paluch, T. McCabe, H. Muller-Bunz, O. I. Corrigan, A. M. Healy, and L. Tajber, Mol. Pharm. 10, 3640 (2013).

2013PAR/LEE Y. Park, J. Lee, S. H. Lee, H. G. Choi, C. Mao, S. K. Kang, S.-E. Choi, and E. H. Lee, Cryst. Growth Des. 13, 5450 (2013).

2013PER/BAU M.-A. Perrin, M. Bauer, M. Barrio, J.-L. Tamarit, R. Ceolin, and I. B. Rietveld, J. Pharm. Sci. 102, 2311 (2013).

2013PER/BLO G. L. Perlovich, S. V. Blokhina, N. G. Manin, T. V. Volkova, and V. V. Tkachev, J. Therm. Anal. Calorim. 111, 655 (2013).

2013PER/BLO2 G. L. Perlovich, S. V. Blokhina, M. V. Ol'khovich, A. V. Sharapova, and A. N. Proshin, J. Solution Chem. 42, 2057 (2013).

2013PER/CON S. Perisanu, I. Contineanu, A. Neascu, N. P. Rath, J. S. Chickos, R. Notario, and J. F. Liebman, Struct. Chem. 24, 1975 (2013).

2013PER/KAZ G. L. Perlovich, V. P. Kazachenko, N. N. Strakhova, K.-J. Schaper, and O. A. Raevsky, J. Chem. Eng. Data 58, 2659 (2013).

2013PER/RYZ G. L. Perlovich, A. M. Ryzhakov, V. V. Tkachev, L. K. Hansen, and O. A. Raevsky, Cryst. Growth Des. 13, 4002 (2013).

2013QI/WAN ～C.-M. Qi, L.-S. Wang, J.-C. Du, G.-M. Yu, and G.-Q. Sun, Fluid Phase Equilib. 360, 343 (2013).

2013RED/BEN P. Reddy, T. P. Benecke, and D. Ramjugernath, J. Chem. Thermodyn. 58, 330 (2013).

2013RIB/AMA M. A. V. Ribeiro da Silva, L. M. P. F. Amaral, and P. Szterner, J. Chem. Thermodyn. 57, 380 (2013).

2013ROC/GAL I. M. Rocha, T. L. P. Galvao, M. D. M. C. Ribeiro da Silva, and M. A. V. Ribeiro da Silva, Struct. Chem. 24, 1935 (2013).

2013ROC/GAL2 I. M. Rocha, T. L. P. Galvao, E. Sapei, M. D. M. C. Ribeiro da Silva, and M. A. V. Ribeiro da Silva, J. Chem. Eng. Data 58, 1813 (2013).

2013ROC/SAN M. A. A. Rocha and L. M. N. B. F. Santos, Chem. Phys. Lett. 585, 59 (2013).

2013ROD/ROC A. S. M. C. Rodriques, M. A. A. Rocha, and L. M. N. B. F. Santos, J. Chem. Thermodyn. 63, 78 (2013).

2013SAN/AMA A. F. L. O. M. Santos, L. M. P. F. Amaral, M. D. M. C. Ribeiro da Silva, M. V. Roux, and R. Notario, J. Chem. Thermodyn. 58, 29 (2013).

2013SAN/RIB A. F. L. M. Santos and M. A. V. Ribeiro da Silva, J. Chem. Thermodyn. 57, 454 (2013).

2013SAN/RIB2 A. F. L. M. Santos and M. A. V. Ribeiro da Silva, J. Chem. Thermodyn. 58, 476 (2013).

2013SAN/RIB3 A. F. L. O. M. Santos and M. A. V. Ribeiro da Silva, Struct. Chem. 24, 1981 (2013).

2013SAN/RIB4 A. F. L. O. M. Santos and M. A. V. Ribeiro da Silva, J. Phys. Chem. A 117, 5195 (2013).

2013 SAN/RIB5 A. F. L. O. M. Santos and M. A. V. Ribeiro da Silva, J. Chem. Thermodyn. 67, 190 (2013).

2013SAR/ALK A. Sari, C. Alkan, and A. Bicer, Ind. Eng. Chem. Res. 52, 18269 (2013).

2013SAR/KAR A. Sari, A. Karaipekli, R. Eroglu, and A. Bicer, Energy Sources, Part A 35, 1285 (2013)

2013SCH/BUC $\quad$ B. J. Schindler, J. H. Buchanan, J. H. Mahle, G. W. Peterson, and T. G. Glover, J. Chem. Eng. Data 58, 1806 (2013).

2013SHA/SHT O. Shalev and M. Shtein, Org. Electron. 14, 94 (2013).

2013SIL/CIM A. L. R. Silva, A. Cimas, and M. D. M. C. Ribeiro da Silva, J. Chem. Thermodyn. 57, 212 (2013).

2013SIL/CIM2 A. L. R. Silva, A. Cimas, N. Vale, P. Gomes, M. J. S. Monte, and M. D. M. C. Ribeiro da Silva, J. Chem. Thermodyn. 58, 158 (2013).

2013SIL/CIM3 A. L. R. Silva, A. Cimas, and M. D. M. C. Ribeiro da Silva, Struct. Chem. 24, 1863 (2013).
2013SIM/BER

R. G. Simoes, C. E. S. Bernardes, H. P. Diogo, F. Agapito, and M. E. Minas da Piedade, Mol. Pharm. 10, 2713 (2013).

2013SIM/BER2 R. G. Simoes, C. E. S. Bernardes, and M. E. Minas da Piedade, Cryst. Growth Des. 13, 2803 (2013).

2013SIN/PAN M. Singh, P. Pandey, R. N. Rai, and U. S. Rai, J. Therm. Anal. Calorim. 113, 977 (2013).

2013SOS/MOR C. C. S. Sosa, V. M. F. Morais, and M. A. R. Matos, J. Chem. Thermodyn. 56, 83 (2013).

2013SOT/HOL R. Sotomayor, A. R. Holguin, D. M. Cristancho, D. R Delgado, and F. Martinez, J. Mol. Liq. 180, 34 (2013).

2013SOU/MAT C. C. S. Sousa, M. A. R. Matos, L. M. N. B. F. Santos, and V. M. F. Morais, J. Chem. Thermodyn. 67, 210 (2013).

2013SPE/CHI J. Spencer and J. Chickos, J. Chem. Eng. Data 58, 3513 (2013).

2013STE/FUL V. Stejfa, M. Fulem, K. Ruzicka, C. Cervinka, M. A. A. Rocha, L. M. N. B. F. Santos, and B. Schroder, J. Chem. Thermodyn. 60, 117 (2013).

2013STI/KAI V. Stilinovic and B. Kaitner, Cryst. Growth Des. 13, 1703 (2013).

2013SUN/LI Y. Sun, Z. Li, C. Xie, W. Chen, and C. Zhang, Front. Chem. Sci. Eng. 7, 10 (2013).

2013SUN/WAN G.-Q. Sun, L.-S. Wang, J.-C. Du, and C.-M. Qi, Fluid Phase Equilib. 360, 97 (2013).

2013SUR/BUI

A. O. Surov, C. T. Bui, A. N. Proshin, P. Roussel, A. Idrissi, and G. L. Perlovich, J. Phys. Chem. B 117, 10414 (2013).

2013SYS/NIK S. V. Sysoev, L. D. Nikulina, E. N. Ermakova, M. L. Kosinova, V. I. Rakhlin, I. P. Tsyrendorzhieva, A. V. Lis, and M. G. Voronkov, Inorg. Mater. 49, 363 (2013).

2013TAO/SUN M. Tao, H. Sun, Z. Wang, P. Cui, and J. Wang, Fluid Phase Equilib. 352, 14 (2013).

2013THO/CHI M. Thornton, J. Chickos, I. V. Garist, M. A. Varfolomeev, A. A. Svetlov, and S. P. Verevkin, J. Chem. Eng. Data 58, 2018 (2013).

2013TKA/DRU E. S. Tkachenko, A. I. Druzhinina, R. M. Varushchenko, S. V. Tarazanov, T. N. Nesterova, M. D. Rechetova, and O. D. Poyakova, Russ. J. Phys. Chem. A 87, 705 (2013).

2013TRA/KHI D. Trache, K. Khimeche, R. Benelmir, and A. Dahmani, Thermochim. Acta 565, 8 (2013).

2013TRA/KHI2 D. Trache, K. Khimeche, and A. Dahmani, Int. J. Thermophys. 34, 226 (2013).

2013TVE/GIR

N. V. Tverdova, G. V. Girichev, A. V. Krasnov, O. A. Pimenov, and O. I. Koifman, Struct. Chem. 24, 883 (2013).

2013TYU/BAD E. Y. Tyunina and V. G. Badelin, Russ. J. Gen. Chem. 83, 708 (2013).

2013UUS/DEL P. Uusi-Kyyny, C. Dell'Era, A. Penttila, M. Pakkanen, and V. Alopaeus, Fluid Phase Equilib. 338, 164 (2013).

2013VEC S. Vecchio, Struct. Chem. 24, 1821 (2013).

2013VEC/BRU S. Vecchio and B. Brunetti, Fluid Phase Equilib. 338, 148 (2013).

2013VEN/BEN J. G. Veneral, T. Benazzi, M. A. Mazutti, F. A. P. Voll, L. Cardozo-Filho, M. I. Corazza, R. Guirardello, and J. V. Oliveira, J. Chem. Thermodyn. 58, 398 (2013).

2013VER/ZAI S. P. Verevkin, D. H. Zaitsau, V. N. Emel'yanenko, A. V. Yermalayeu, C. Schick, H. Liu, E. J. Maginn, S. Bulut, I. Krossing, and R. Kalb, J. Phys. Chem. B 117, 6473 (2013).

2013WAN L. Wang, J. Mol. Liq. 188, 55 (2013).

2013WAN/GUO Y. Wang, C. Guo, X. Liu, S. Huang, B. Wang, and H. Zheng, Fluid Phase Equilib. 342, 47 (2013).

2013WAN/LI H. Wang, L. Li, and C. Ye, Fluid Phase Equilib. 342, 42 (2013).

2013WAN/LV L. Wang and T.-T. Lv, J. Mol. Liq. 181, 29 (2013).

2013WAN/TOZ S. Wang, K. Tozaki, H. Hayashi, and H. Inaba, Thermochim. Acta 571, 8 (2013).

2013WEI/ZHA $\quad$ D. Wei, X. Zhang, and H. Li, J. Chem. Thermodyn. 60, 94 (2013).

2013WIL/CHI J. A. Wilson and J. S. Chickos, J. Chem. Eng. Data 58, 322 (2013).

2013WIL/HUL ～B. R. Williams, M. S. Hulet, A. Brozena, R. W. Miles, Jr., and D. E. Tavault, J. Chem. Eng. Data 58, 1679 (2013). 
2013XU/LI D. Xu, H. Li, and Z. Li, J. Chem. Eng. Data 58, 3110 (2013).

2013YAD/KUM P. S. Yadav, V. Kumar, U. P. Singh, H. R. Bhat, and B Mazumder, Saudi Pharm. J. 21, 77 (2013).

2013 YAN/YIN $\quad$ L. Yang, Q. Yin, B. Hou, Y. Wang, Y. Bao, J. Wang, and H. Hao, Ind. Eng. Chem. Res. 52, 2477 (2013).

2013YE/DON C. S. Ye, J. Dong, and L. Li, J. Chem. Eng. Chin. Univ. 27, 21 (2013).

2013 YOU/GAO Y. You, T. Gao, F. Qiu, Y. Wang, X. Chen, W. Jia, and R. Li, J. Chem. Eng. Data 58, 1845 (2013).

2013YUA/CAP X. Yuan and A. C. Capomacchia, J. Pharm. Sci. 102, 1957 (2013).

2013ZAI/YER D. H. Zaitsau, A. V. Yermalayeu, V. N. Emel'yanenko, C. Schick, S. P. Verevkin, A. A. Samarov, S. Schlenk, and P. Wasserscheid, Z. Phys. Chem. 227, 205 (2013).

2013ZAI/YER2 $\quad$ D. H. Zaitsau, A. V. Yermalayeu, S. P. Verevkin, J. E. Bara, and A. D. Stanton, Ind. Eng. Chem. Res. 52, 16615 (2013).

2013ZHA/GAO J.-Q. Zhang, H.-X. Gao, T.-Z. Ji, M. Chao, H.-X. Ma, K. Z. Xu, and R.-Z. Hu, J. Therm. Anal. Calorim. 114, 441 (2013).

2013ZHA/LU Q. Zhang, L. Lu, W. Dai, and X. Mei, Cryst. Growth Des. 13, 2198 (2013).

2013ZHA/LV Z. Zhang, M. Lv, D. Huang, P. Jia, D. Sun, and W. Li, J. Chem. Eng. Data 58, 3054 (2013).

2013ZHA/XIA H. Zhang, Q. Xia, Y. Yang, F.-B. Zhang, and G. L. Zhang, Ind. Eng. Chem. Res. 52, 5230 (2013).

2013ZHA/YAN W. Zhang, Z. Q. Yang, J. Lu, and J. Lu, J. Chem. Eng. Data 58, 2307 (2013).

2014ABO/MOK R. Abou-Naccoul, I. Mokbel, G. Bassil, J. Saab, K. Stephan, and J. Jose, Chemosphere 95, 41 (2014).

2014ACH/EME $\quad$ F. Achrainer, V. N. Emel'yanenko, W. Tantawy, S. P. Verevkin, and H. Zipse, J. Phys. Chem. B 118, 10426 (2014).

2014AGA/MOS E. V. Agafonova, Y. V. Moshchenskiy, and M. L. Tkachenko, Thermochim. Acta 580, 1 (2014).

2014AHR/BRI M. Ahrenberg, M. Brinckmann, J. W. P. Schelzer, M. Beck, C. Schmidt, O. Kessler, U. Kragl, S. P. Verevkin, and C. Schick, Phys. Chem. Chem. Phys. 16, 2971 (2014).

2014ALM/CUN A. R. R. P. Almeida, A. F. G. Cunha, M. A. R. Matos, V. M. F. Morais, and M. J. S. Monte, J. Chem. Thermodyn. 78, 43 (2014).

2014ALM/MON A. R. R. P. Almeida, M. J. S. Monte, M. A. R. Matos, and V. M. F. Morais, J. Chem. Thermodyn. 73, 12 (2014).

2014ALM/MON2 A. R. R. P. Almeida and M. J. S. Monte, J. Chem. Thermodyn. 77, 46 (2014).

2014AMA/DEC L. M. P. F. Amaral, T. M. T. de Carvalho, J. I. T. A. Cabral, M. D. M. C. Ribeiro da Silva, and M. A. V. Ribeiro da Silva, J. Therm. Anal. Calorim. 151, 803 (2014).

2014AMA/MOR L. M. P. F. Amaral, V. M. F. Morais, and M. A. V. Ribeiro da Silva, J. Chem. Thermodyn. 74, 22 (2014).

2014AMA/RIB I. M. P. F. Amaral and M. A. V. Ribeiro da Silva, J. Chem. Thermodyn. 73, 44 (2014).

2014AMA/RIB2 L. M. P. F. Amaral and M. A. V. Ribeiro da Silva, J. Chem. Thermodyn. 78, 254 (2014).

2014AMA/SZT L. M. P. F. Amaral, P. Szterner, M. S. Miranda, and M. A. V. Ribeiro da Silva, J. Chem. Thermodyn. 75, 106 (2014).

2014ARD/ELN A. Ard-Elbary, M. A. El Nabarawi, D. H. Hassen, and A. A. Taha, Int. J. Pharm. Pharm. Sci. 6, 183 (2014).

2014BAD/DEL E. Badea, G. Della Gatta, and B. Palecz, J. Chem. Thermodyn. 73, 178 (2014).

2014BAD/NOW E. Badea, B. Nowicka, and G. Della Gatta, J. Chem. Thermodyn. 68, 90 (2014).

2014BAE/DAH A. C. Baelhadj, O. Dahmani, R. Mahmoud, and N. F. Cherif, J. Mol. Liq. 198, 134 (2014).

2014BEB/ROS S. V. S. Bebiano, M. T. S. Rosado, R. A. E. Castro, M. Ramos Cilva, J. Cantilho, T. M. R. Maria, and M. E. S. Eusebio, J. Mol. Struct. 1078, 10 (2014).

2014BEN/AIT H. Ben-Makhlouf-Hakem, A. Ait-Kaci, and J. Jose, Fluid Phase Equilib. 373, 34 (2014).

2014BEN/NEG L. Bendiaf, A. Negadi, I. Mokbel, and L. Negadi, Fuel 122, 247 (2014).
2014BER/SIM

2014BLO/OLK

C. E. S. Bernardes, R. G. Simoes, H. P. Diogo, and M. E. Minas da Piedade, J. Chem. Thermodyn. 73, 140 (2014). S. V. Blokhina, M. V. Ol'khovich, A. V. Sharapova, G. L. Perlovich, and A. N. Proshin, J. Chem. Thermodyn. 69, 107 (2014).

2014BOU/NEG Z. Bouzina, A. Negadi, I. Mokbel, J. Jose, and L. Negadi, J. Chem. Thermodyn. 84, 81 (2015).

2014BRE/ZAK $\quad$ A. A. Bredikhin, D. V. Zakharychev, A. T. Gubaidullin, R. R. Fayzullin, A. V. Pashagin, and Z. A. Bredikhina, Cryst. Growth Des. 14, 1676 (2014).

2014BRO/BUC A. Brozena, J. H. Buchanan, R. W. Miles, Jr., B. R. Williams, and M. S. Hulet, J. Chem. Eng. Data 59, 2649 (2014).

2014BRO/JAN S. Brommer, L. M. Jantunen, T. F. Bidleman, S. Harrad, and M. L. Diamond, J. Chem. Eng. Data 59, 1441 (2014).

2014BRO/TEV A. Brozena, D. E. Tevault, and K. Irwin, J. Chem. Eng. Data 59, 307 (2014).

2014BRU/CIC B. Brunetti, A. Ciccioli, G. Gigli, A. Lapi, N. Misceo, L. Tanzi, and S. V. Ciprioti, Phys. Chem. Chem. Phys. 16, 15653 (2014).

2014BUT/KON A. V. Butlak, Y. V. Kondrat'ev, and A. Y. Timoshkin, Russ. J. Gen. Chem. 84, 2455 (2014).

2014CAB/GRA $\quad$ D. Cabaleiro, C. Gracia-Fernandez, and L. Lugo, J. Chem. Thermodyn. 74, 43 (2014).

2014CAM/MEN E. A. Camarillo, J. Mentado, H. Flores, and J. M. Hernandez-Perez, J. Chem. Thermodyn. 73, 269 (2014).

2014CAR/CAS N. D. D. Carareto, T. Castagnaro, M. C. Costa, and A. J. A. Meirelles, J. Chem. Thermodyn. 78, 99 (2014).

2014CAR/DOS N. D. D. Carareto, A. O. dos Santos, M. P. Rolemberg, L. P. Cardoso, M. C. Costa, and A. J. A. Meirelles, Thermochim. Acta 589, 137 (2014).

2014CHE/LI ～L. Chen, H.-R. Li, R.-J. Xu, Y. Xiong, T. Xu, B.-Y. Huang, and Y.-J. Shu, Propellants, Explos., Pyrotech. 39, 217 (2013).

2014CHE/QI F.-X. Chen, Z.-L. Qi, L. Feng, J.-Y. Miao, and B.-Z. Ren, J. Chem. Thermodyn. 71, 231 (2014).

2014CHI/STE R. D. Chirico, W. V. Steele, and A. F. Kazakov, J. Chem. Thermodyn. 73, 241 (2014).

2014COR/GUI Y. Corvis, N. Guiblin, and P. Espeau, J. Phys. Chem. B 118, 1925 (2014).

2014COS/DAV J. C. S. Costa, J. Z. Davalos, and L. M. N. B. F. Santos, J. Phys. Chem. A 118, 9712 (2014).

2014CUD/POD M. F. Cuddy, A. R. Poda, and M. A. Chappell, Propellants, Explos., Pyrotech. 39, 236 (2013).

2014DAM/MAT D. S. Damaceno, R. M. Matricarde Falleiro, M. A. Krahenbuhl, A. J. A. Meirelles, and R. Ceriani, J. Chem. Eng. Data 59, 1515 (2014).

2014DAV/HER J. Z. Davalos, R. Herrero, J. C. S. Costa, L. M. N. B. F Santos, and J. F. Liebman, J. Phys. Chem. A 118, 3705 (2014).

2014DIB/RAE V. N. Dibrivnyl, Y. A. Raevskii, and V. V. Sergeev, Russ. J. Gen. Chem. 84, 1069 (2014).

2014DIC/LEG E. Dichi, B. Legendre, and M. Sghaier, J. Therm. Anal. Calorim. 115, 1551 (2014).

2014DRE/MIK V. A. Drebushchak, M. A. Mikhailenko, T. P. Shakhtshneider, T. V. Drebushchak, S. A. Kuznetsova, and J. N. Malyar, J. Therm. Anal. Calorim. 115, 2521 (2014).

2014DU/WAN C.-J. Du, L.-S. Wang, and G.-M. Yu, J. Chem. Eng. Data 59, 1533 (2014).

2014EME/ALG V. N. Emel'yaneko, M. Algarra, J. C. G. Esteves da Silva, J. Hierrezuelo, J. M. Lopez-Romero, and S. P. Verevkin, Thermochim. Acta 597, 78 (2014).

2014FEN/TAN Y. Feng, W. Tang, Y. Huang, Y. Xiong, L. Chen, Y. Liu, and Y. Li, J. Chem. Thermodyn. 78, 143 (2014).

2014FLO/XIM H. Flores, A. Ximello, E. A. Camarillo, and P. Amador, J. Therm. Anal. Calorim. 117, 433 (2014).

2014FON/GUS J. M. S. Fonseca, N. Gushterov, and R. Dohrn, J. Chem. Thermodyn. 73, 148 (2014).

2014FRE/GOM V. L. S. Freitas, J. R. B. Gomes, and M. D. M. C. Ribeiro da Silva, J. Chem. Thermodyn. 73, 110 (2014).

2014FRE/GOM2 V. L. S. Freitas, J. R. B. Gomes, and M. D. M. C. Ribeiro da Silva, J. Chem. Eng Data 59, 312 (2014). 
2014FRE/LEI V. L. S. Freitas, S. Leirosa, R. Notario, and M. D. M. C. Ribeiro da Silva, J. Org. Chem. 79, 11583 (2014).

2014FUL/RUZ M. Fulem, K. Ruzicka, C. Cervinka, A. Bazyleva, and G. Della Gatta, Fluid Phase Equilib. 371, 93 (2014).

2014GAL/RIB T. L. P. Galvao, M. D. M. C. Ribeiro da Silva, and M. A. V. Ribeiro da Silva, J. Phys. Chem. A 118, 4816 (2014).

2014GAL/ROC T. L. P. Galvao, I. M. Rocha, M. D. M. C. Ribeiro da Silva, and M. A. V. Ribeiro da Silva, J. Phys. Chem. A 118, 3360 (2014).

2014GAR/AMA M. A. Garcia-Castro, P. Amador, J. M. Hernandez-Perez, A. E. Medina-Vavela, and H. Flores, J. Phys. Chem. A 118, 3820 (2014).

2014GER/PAV S. I. Gerasimchuk, Y. P. Pavlovskii, I. B. Sobechko, and Y. Y. Van-Chin-Syan, Russ. J. Phys. Chem. 88, 365 (2014).

2014GOB/CHI C. Gobble, J. Chickos, and S. P. Verevkin, J. Chem. Eng. Data 59, 1353 (2014).

2014GOB/VIK C. Gobble, J. Vikman, and J. S. Chickos, J. Chem. Eng. Data 59, 2551 (2014).

2014GON/AGA E. M. Goncalves, F. Agapito, T. S. Almeida, and J. A. M. Simoes, J. Chem. Thermodyn. 73, 90 (2014).

2014GON/KUD K. Gonczi, V. Kudar, Z. Jaszay, P. Bombicz, F. Faigl, and J. Madarasz, Thermochim. Acta 580, 46 (2014).

2014GUO/YIN Y. Guo, Q. Yin, H. Hao, M. Zhang, Y. Bao, B. Hou, W. Chen, H. Zhang, and W. Cong, J. Chem. Eng. Data 59, 1326 (2014).

2014HAD/VIL B. Haddad, D. Villemin, E.-H. Belarbi, N. Bar, and M. Rahmouni, Arabian J. Chem. 7, 781 (2014).

2014HAS/JIR T. Hasl and I. Jiricek, Energy Procedia 46, 301 (2014).

2014HON/LIU M. Hong, R.-J. Liu, H.-X. Yang, W. Guan, J. Tong, and J.-Z. Yang, J. Chem. Thermodyn. 70, 214 (2014).

2014HOR/SEI E. Horosanskaia, A. Seidel-Morgenstern, and H. Lorenz, Thermochim. Acta 578, 74 (2014).

2014HU/ZHA P. Hu, P.-P. Zhao, Y. Jin, and Z.-S. Chen, Sol. Energy 102, 91 (2014).

2014IIZ/SHI A. Iizuka, E. Shibata, M. Sato, N. Onodera, and T. Nakamura, Thermochim. Acta 593, 1 (2014).

2014JIA/WAN $\quad$ L.-K. Jiang, L.-S. Wang, C.-J. Du, G.-Q. Sun, and C.-M. Qi, Fluid Phase Equilib. 367, 117 (2014).

2014JIA/WAN2 L.-K. Jiang, L.-S. Wang, and G.-M. Yu, Fluid Phase Equilib. 373, 55 (2014).

2014JIA/WAN3 L.-K. Jiang and L.-S. Wang, J. Chem. Pharm. Res. 6, 47 (2014).

2014JIA/YAN X. Jia, Y. Yang, C. Liu, and Z. Pan, Fluid Phase Equilib. 376, 165 (2014).

2014KHE/MUT A. Khelassi-Sefaoui, F. Mutelet, L. Negadi, and I. Mokbel, J. Chem. Thermodyn. 72, 134 (2014).

2014KIM/LIM H. Kim, J. Lim, J. H. Hong, A. R. Kim, M. S. Shin, and H. Kim, J. Chem. Thermodyn. 77, 1 (2014).

2014KOZ/GOB M. Kozlovskiy, C. Gobble, and J. S. Chickos, J. Chem. Thermodyn. 73, 262 (2014).

2014KRI/RAJ V. Krishnakumar and M. Ragaboopathi, J. Therm. Anal. Calorim. 115, 723 (2014).

2014KUR/TAK H. Kuramochi, H. Takigami, M. Scheringer, and S.-I. Sakai, J. Chem. Eng. Data 59, 8 (2014).

2014LAH/KUD M. Lahtinen, J. Kudva, P. Hegde, K. Bhat, E. Kolehmainen, Nonappa, Venkatesh, and D. Naral, J. Mol. Struct. 1060, 280 (2014).

2014LEY/LOS J. Leys, P. Losada-Perez, E. Slenders, C. Glorieux, and J. Thoen, Thermochim. Acta 582, 68 (2014).

2014LI/SUN $\quad$ D. Li, X. Sun, L. Li, S. Wang, L. Wang, and X. Liu, J. Taiwan Inst. Chem. Eng. 45, 823 (2014).

2014LIM/CRE C. E. Lima de Oliveira and M. A. Cremasco, Thermochim. Acta 577, 1 (2014).

2014LIU/TAN Q.-S. Liu, Z.-C. Tan, U. Welz-Biermann, and X.-X. Liu, J. Chem. Thermodyn. 68, 82 (2014).

2014LIU/ZOU T. Liu, W. Zou, W. Zhao, F.-B. Zhang, G.-L. Zhang, and Q. Xia, Fluid Phase Equilib. 374, 102 (2014).

2014LOM/GIN L. Lomba, B. Giner, E. Zuriaga, I. Gascon, and C. Lafuente, Thermochim. Acta 575, 305 (2014).

2014LOM/GIN2 L. Lomba, B. Giner, M. C. Lopez, L. Aldea, and C. Lafuente, J. Chem. Eng. Data 59, 329 (2014).

2014LOV/ARM K. R. J. Lovelock, J. P. Armstrong, P. Licence, and R. G. Jones, Phys. Chem. Chem. Phys. 16, 1339-1353 (2014).
2014MAG/WUR A. Magon, A. Wurm, C. Schick, P. Pangloli, S. Zivanovic, M. Skotnicki, and M. Pyda, Thermochim. Acta 589, 183 (2014).

2014MAN/MAH D. R. Mantheni, M. P. K. Maheswaram, R. Munigeti, I. Perera, A. Riga, and K. S. Alexander, J. Therm. Anal. Calorim. 115, 2253 (2014).

2014MAN/VOR A. N. Manin, A. P. Voronin, and G. L. Perlovich, Thermochim. Acta 583, 72 (2014).

2014MAN/VOR2 A. N. Manin, A. P. Voronin, N. G. Manin, M. V. Vener, A. V. Shishkina, A. S. Lermontov, and G. L. Perlovich, J. Phys. Chem. B 118, 6803 (2014).

2014MAX/CAR G. J. Maximo, N. D. D. Carereto, M. C. Costa, A. O. dos Santos, L. P. Cardoso, M. A. Krahenbuhl, and A. J. A. Meirelles, Fluid Phase Equilib. 366, 88 (2014).

2014MAX/COS G. J. Maximo, M. C. Costa, and A. J. A. Meirelles, Phys. Chem. Chem. Phys. 16, 16740 (2014).

2014MAX/SAN G. J. Maximo, R. J. B. N. Santos, P. Brandao, J. M. S S. Esperanca, M. C. Costa, A. J. A. Meirelles, M. G. Freire, and J. A. P. Coutinho, Cryst. Growth Des. 14, 4270 (2014).

2014MBA/KNO J. Mbah, D. Knot, and S. Steward, Talanta 129, 586 (2014).

2014MEA/SVA D. Mealey, M. Svard, and A. C. Rasmuson, Fluid Phase Equilib. 375, 73 (2014).

2014MEN/BEJ D. A. Meneses, A. Bejarano, and J. C. de la Fuente, J. Chem. Thermodyn. 74, 16 (2014).

2014MEN/ZHU Q. Meng, D. Zhu, Z.-C. Tan, Y. Dong, and W. Li, Appl. Mech. Mater. 525, 133 (2014).

2014MIR/CHI M. S. Miranda, J. S. Chickos, J. C. G. Esteves da Silva, and J. F. Liebman, J. Chem. Thermodyn. 73, 69 (2014).

2014NEG/BAR P. Negrier, M. Barrio, J. L. Tamarit, and D. Mondieig, J. Phys. Chem. B 118, 9595 (2014).

2014NEG/MOK L. Negadi, I. Mokbel, N. Chiali-Baba-Ahmed, and L. Kara-Zaitri, J. Chem. Thermodyn. 70, 233 (2014).

2014NOG/ILD B. A. Nogueira, G. O. Ildiz, J. Canotilho, E. M. S. Eusebio, and R. Fausto, J. Phys. Chem. A 118, 5994 (2014).

2014NON/CHE W. Nong, X. Chen, L. Wang, J. Liang, H. Wang, Y. Huang, and Z. Tong, J. Chem. Thermodyn. 68, 199 (2014).

2014NON/CHE2 W. Nong, X. Chen, L. Wang, J. Liang, H. Wang, L. Long, Y. Huang, and Z. Tong, Fluid Phase Equilib. 367, 74 (2014).

2014NOT/ROU R. Notario, M. V. Roux, F. Ros, V. N. Emel'yanenko, and S. P. Verevkin, J. Chem. Thermodyn. 74, 144 (2014).

2014NOT/ROU2 R. Notario, M. V. Roux, F. Ros, V. N. Emel'yanenko, D. H. Zaitsau, and S. P. Verevkin, J. Chem. Thermodyn. 77, 151 (2014).

2014OKU/RAM P. Okuniewska, D. Ramjugernath, P. Naidoo, and U. Domanska, Fluid Phase Equilib. 376, 55 (2014).

2014 OLI/MON J. A. S. A. Oliveira, M. J. S. Monte, R. Notario, and M. D. M. C. Ribeiro da Silva, J. Chem. Thermodyn. 76, 56 (2014).

2014OZA/NAK S. Ozaki, Y. Nakagawa, O. Shirai, and K. Kano, J. Pharm. Sci. 103, 3524 (2014).

2014PAU/BLO Y. U. Paulechka and A. V. Blokhin, J. Chem. Thermodyn. 79, 94 (2014).

2014PEN/UUS A. Penttila, P. Uusi-Kyyny, A. Salminen, J. Seppala, and V. Alopaeus, Int. J. Greenhouse Gas Control 22, 313 (2014).

2014PER/CER M. J. Percino, M. Ceron, P. Ceballos, G. Soriano-Moro, M. E. Castro, V. M. Chapela, J. Bonilla-Cruz, M. ReyesReyes, P. Lopez-Sandoval, and M. A. Siegler, J. Mol. Struct. 1078, 74 (2014).

2014PER/KAZ G. L. Perlovich, V. P. Kazachenko, N. N. Strakhova, and O. A. Raevsky, J. Chem. Eng. Data 59, 4217 (2014).

2014PIM/PAS S. M.Pimenova, L. L. Pashchenko, E. A. Miroshnichenko, and I. A. Nesterov, Russ. J. Phys. Chem. A 88, 557 (2014).

2014POB/DOM A. Pobudkowski, U. Domanska, and J. A. Kryska, J. Chem. Thermodyn. 79, 41 (2014).

2014RAG T. Rager, J. Pharm. Sci. 103, 1673 (2014).

2014RAM/OGI S. L. L. M. Ramos, M. Ogino, and M. Oguni, J. Therm. Anal. Calorim. 115, 1353 (2014). 
2014RAT/DES P. B. Rathi and K. V. Deshpande, J. Solution Chem. 43, 1886 (2014).

2014RED/KRO S. T. Reddy, K. P. Krovi, and M. J. Swamy, Cryst. Growth Des. 14, 4944 (2014).

2014RIO/ORT R. Rios, J. Ortega, L. Fernandez, I. de Nuez, and J. Wisniak, J. Chem. Eng. Data 59, 125 (2014).

2014ROB/BAR M. D. Robustillo, D. F. Barbosa, A. J. de Almeida Meirelles, and P. de Alcantara Pessoa Filho, Fluid Phase Equilib. 361, 188 (2014).

2014ROC/COU M. A. A. Rocha, J. A. P. Coutinho, and L. M. N. B. F. Santos, J. Phys. Chem. 141, 134501 (2014).

2014ROC/GAL I. M. Rocha, T. L. P. Galvao, M. D. M. C. Ribeiro da Silva, and M. A. V. Ribeiro da Silva, J. Phys. Chem. A 118, 1502 (2014).

2014RUZ/FUL K. Ruzicka, M. Fulem, P. B. P. Serra, O. Vlk, and I. Krakovsky, Thermochim. Acta 596, 98 (2014).

2014SAI/MUR M. K. Saini and S. S. N. Murthy, Thermochim. Acta 575, 195 (2014).

2014SAN/RIB $\quad$ A. F. L. O. M. Santos and M. A. V. Ribeiro da Silva, J. Chem. Thermodyn. 75, 1 (2014).

2014SAN/SIL A. F. L. O. M. Santos, A. L. R. Silva, O. D. F. Santiago, J. M. Goncalves, S. Pandey, W. E. Acree, Jr., and M. D. M. C. Ribeiro da Silva, J. Chem. Thermodyn. 73, 62 (2014).

2014SHA/SRE S. Shantikumar, G. Sreekanth, K. V. S. Nath, S. J. Valli, and N. Satheeshkumar, J. Therm. Anal. Calorim. 115, 2423 (2014).

2014SII/KAM C. Siitsman, I. Kamenev, and V. Oja, Thermochim. Acta 595, 35 (2014).

2014SIL/CIM A. L. R. Silva, A. Cimas, and M. D. M. C. Ribeiro da Silva, J. Chem. Thermodyn. 73, 3 (2014).

2014SIL/FRE $\quad$ A. L. R. Silva, V. L. S. Freitas, and M. D. M. C. Ribeiro da Silva, Chemosphere 107, 203 (2014).

2014SIL/MON $\quad$ A. L. R. Silva, M. J. S. Monte, V. M. F. Morais, and M. D. M. C. Ribeiro da Silva, J. Chem. Thermodyn. 74, 67 (2014).

2014SIL/MOR A. L. R. Silva, V. M. F. Morais, and M. D. M. C. Ribeiro da Silva, J. Mol. Struct. 1078, 197 (2014).

2014SIM/AGA R. G. Simoes, F. Agapito, H. P. Diogo, and M. E. Minas da Piedade, J. Phys. Chem. A 118, 11026 (2014).

2014SIM/DIO R. G. Simoes, H. P. Diogo, A. Dias, M. C. Oliveira, C. Cordeiro, C. E. S. Bernardes, and M. E. S. Minas da Piedade, J. Pharm. Sci. 103, 241 (2014).

2014SOB/VAN I. B. Sobechko, Y. Y. Van-Chin-Syan, V. V. Kochubei, R. T. Prokop, N. I. Velychkivska, Y. I. Gorak, V. N. Dibrivnyi, and M. D. Obushak, Russ. J. Phys. Chem. 88, 2046 (2014).

2014SOU/MAT C. C. S. Sousa, M. A. R. Matos, and V. M. F. Morais, J. Chem. Thermodyn. 73, 101 (2014).

2014STE/FUL V. C. Stejfa, M. Fulem, K. Ruzicka, and C. Cervinka, J. Chem. Thermodyn. 79, 272 (2014).

2014STE/FUL2 V. C. Stejfa, M. Fulem, K. Ruzicka, and C. Cervinka, J. Chem. Thermodyn. 79, 280 (2014).

2014STE/PAU $\quad$ E. N. Stepurko, Y. U. Paulechka, A. V. Blokhin, G. J. Kabo, S. V. Voitelhovich, A. S. Lyakhov, S. V. Kohut, and T. E. Kazarovets, Thermochim. Acta 592, 10 (2014).

2014SUG/KAI K. Sugandha, S. Kaity, S. Mukherjee, J. Isaac, and A. Ghosh, Cryst. Growth Des. 14, 4475 (2014).

2014SUN/HAO Z. Sun, H. Hao, C. Xie, Z. Xu, Q. Yin, Y. Bao, B. Hou, and Y. Wang, Ind. Eng. Chem. Res. 53, 13506 (2014).

2014SUR/PRO A. O. Surov, A. N. Proshin, and G. L. Perlovich, Acta Crystallogr., Sect. B: Struct. Sci., Cryst. Eng. Mater. 70, 47 (2014).

2014SZT/GAL P. Szterner, T. L. P. Galvao, L. M. P. F. Amaral, M. D. M. C. Ribeiro da Silva, and M. A. V. Ribeiro da Silva, Thermochim. Acta 588, 68 (2014)

2014TAN/WAN W. Tang, Z. Wang, Y. Feng, C. Xie, J. Wang, C. Yang, and J. Gong, Ind. Eng. Chem. Res. 53, 11538 (2014).

2014TAN/XIE W. Tang, C. Xie, Z. Wang, S. Wu, Y. Feng, X. Wang, J. Wang, and J. Gong, Fluid Phase Equilib. 363, 86 (2014).

2014TEM/NOT M. Temprado, R. Notario, M. V. Roux, and S. P. Verevkin, J. Chem. Thermodyn. 73, 97 (2014).

2014THO/GOB M. Thornton, C. Gobble, and J. Chickos, J. Chem. Thermodyn. 73, 51 (2014).
2014TIT/LED

2014TON/YAN

B. Tita, I. Ledeti, G. Bandur, and D. Tita, J. Therm. Anal. Calorim. 118, 1293 (2014).

J. Tong, H.-X. Yang, R.-J. Liu, C. Li, L.-X. Xia, and J.-Z. Yang, J. Phys. Chem. B 118, 12972 (2014).

2014TU/CHE W. Tu, Z. Chen, Y. Gao, Z. Li, Y. Zhang, R. Liu, Y. Tian, and L.-M. Wang, Phys. Chem. Chem. Phys. 16, 3586 (2014).

2014TYU/KRA V. V. Tyunina, A. V. Krasnov, E. Y. Tyunina, V. G. Baldelin, and G. V. Girichev, J. Chem. Thermodyn. 74, 221 (2014).

2014VER/EME S. P. Verevkin, V. N. Emel'yanenko, M. A. Varfolomeev, B. N. Solomonov, and K. V. Zherikova, Fluid Phase Equilib. 380, 67 (2014).

2014VER/EME2 S. P. Verevkin, V. N. Emel'yanenko, M. A. Varfolomeev, B. N. Solomonov, K. V. Zherikova, and S. V. Melkhanova, J. Phys. Chem. A 118, 14479 (2014).

2014VIT/AGA J. Vitorino, F. Agapito, M. F. M. Piedade, C. E. S. Bernardes, H. P. Diogo, J. P. Leal, and M. E. Minas da Piedade, J. Chem. Thermodyn. 77, 179 (2014).

2014WAN/HUA Y.-S. Wang, N.-R. Huan, B.-H. Xu, B.-Y. Wang, and Z. S. Bai, J. Chem. Eng. Data 59, 494 (2014).

2014WAT/HUD S. Watterson, S. Hudson, M. Svard, and A. C. Rasmuson, Fluid Phase Equilib. 367, 143 (2014).

2014WEI/HAN D. Wei, S. Han, and B. Wang, Fluid Phase Equilib. 373, 84 (2014).

2014WIS/PER J. Wisniak, J. R. Perez-Correa, A. Mejia, and H. Segura, J. Chem. Eng. Data 59, 942 (2014).

2014WU/DAN H. Wu, L. Dang, and H. Wei, Ind. Eng. Chem. Res. 53, 1707 (2014).

2014XIA/CUI I. Xia, F. Cui, Y. Gan, H. Mu, and M. Yang, J. Pharm. Sci. 103, 697 (2014)

2014XIM/FLO A. Ximello, H. Flores, A. Rojas, E. A. Carmarillo, and M. P. Amador, J. Chem. Thermodyn. 79, 33 (2014).

2014XU/ZHA H. Xu, B. Zhang, Z. Yang, G. Yao, and H. Zhao, J. Chem. Eng. Data 59, 1281 (2014).

2014YAN/KOU Z.-Q. Yang, L.-G. Kou, W. Mao, J. Lu, W. Zhang, and J. Lu, J. Chem. Eng. Data 59, 157 (2014).

2014YAN/RAS H. Yang and A. C. Rasmuson, Fluid Phase Equilib. 376, 69 (2014).

2014YAN/WAN W. Yang, C. Wang, Y. Hu, Y. Li, and K. Wang, Fluid Phase Equilib. 363, 276 (2014).

2014YAO/YAN G. Yao, Z. Yang, B. Zhang, H. Xu, and H. Zhao, Fluid Phase Equilib. 367, 103 (2014).

2014YEA/OSM C.-Y. Yeap, F. Osman, W. A. K. Mahmood, and D. Takeuchi, J. Mol. Struct. 1074, 666 (2014).

2014ZAW/KRO M. Zawadzki, M. Krolikowska, and P. Lipinski, Thermochim. Acta 589, 148 (2014).

2014ZEN/GRI N. Zencirci, U. J. Griesser, T. Gelbrich, D. C. Apperley, and R. K. Harris, Mol. Pharm. 11, 338 (2014).

2014ZEN/PAN A.-G. Zeng, X.-L. Pang, N. Wu, D. Wang, G.-J. Nan, G.D. Yang, and X.-L. Bian, Fluid Phase Equilib. 366, 127 (2014).

2014ZEN/YAN Z. Zeng, Z. Yang, W. Xue, and X. Li, Chin. J. Chem. Eng. 22, 1145 (2014).

2014ZHA/QIA X. Zhang, G. Qian, X. Yang, C. Hu, and X. Zhou, Fluid Phase Equilib. 363, 228 (2014).

2014ZHA/SAL Z. Zhang, A. A. M. Salih, M. Li, and B. Yang, Energy Fuels 28, 2802 (2014).

2014ZHA/WAN J. Zhang, Y. Wang, G. Wang, H. Hao, H. Wang, Q. Luan, and C. Jiang, J. Chem. Thermodyn. 79, 61 (2014).

2014ZHA/WAN2 M.-R. Zhao, H.-J. Wang, S.-Y. Wang, and X.-X. Yue, Russ. J. Phys. Chem. A 88, 2280 (2014).

2014ZHA/WEI1 W. Zhang, Z.-Q. Wei, J. Lu, and J. Lu, Gaoxiao Xauxue Gongcheng Xuebao 28, 449 (2014).

2014ZHA/WEI W. Zhang, Z.-Q. Wei, J. Lu, and J. Lu, Gaoxiao Xauxue Gongcheng Xuebao 28, 449 (2014).

2014ZHA/WEI2 Z. H. Zhang, J. Wei, X. X. Ma, W. G. Xu, J. Tong, W. Guan, and J. J. Yang, Zhongguo Kexue Huaxue 44, 1005 (2014).

2014ZHA/ZHO L. Zhang, C.-R. Zhou, Z.-P. Liu, and M.-X. Huang, J. Therm. Anal. Calorim. 117, 355 (2014).

2015 ABR/KRA V. K. Abrosimov, A. V. Krasnov, Y. A. Zhabanov, and E. V. Ivanov, Izv. Vyssh. Uchebn. Zaved., Khim. Khim. Tekhnol. 58, 3 (2015). 
2015ALM/OLI A. R. R. P. Almeida, J. A. S. A. Oliveira, and M. J. S. Monte, J. Chem. Thermodyn. 82, 108 (2015).

2015ALM/SOU A. R. R. P. Almeida, C. A. D. Sousa, L. M. N. B. F. Santos, and M. J. S. Monte, J. Chem. Thermodyn. 86, 6 (2015).

2015AMA/FRE I. M. P. F. Amaral, V. L. S. Freitas, J. F. R. Goncalves, M. Barbosa, J. S. Chickos, and M. D. M. C. Ribeiro da Silva, Struct. Chem. 26, 137 (2015).

2015AMA/SZT L. M. P. F. Amaral, P. Szterner, V. M. F. Morais, and M. A. V. Ribeiro da Silva, J. Chem. Thermodyn. 91, 452 (2015).

2015BER/MOC C.E. S. Bernardes, T. Mochida, and J. N. Canongia Lopes, Phys. Chem. Chem. Phys. 17, 10200 (2015).

2015BIC/SAR A. Bicer, A. Sari, and O. Lafci, Energy Sources, Part A 37, 2702 (2015).

2015BLO/SHA S. Blokhina, A. Sharapova, M. Ol'khovich, T. Volkova, and G. Perlovich, J. Therm. Anal. Calorim. 120, 1053 (2015).

2015BLO/SHA2 S. Blokhina, A. Sharapova, M. Ol'khovich, T. Volkova, and G. Perlovich, Thermochim. Acta 622, 97 (2015).

2015BOL/ARK E. V. Boldyreva, S. G. Arkhipov, T. N. Drebushchak, V. A. Drebushchak, E. A. Losev, A. A. Matvienko, V. S. Minkov, D. A. Rychkov, Y. V. Seryotkin, J. Stare, and B. A. Zakharov, Chem. - Eur. J. 21, 15395 (2015).

2015BOU/BOU Y. Boumrah, S. Bouanani, K. Khimeche, and A. Dahmani, J. Therm. Anal. Calorim. 120, 583 (2015).

2015BOU/NEG2 Z. Bouzina, A. Negadi, F. Dergal, I. Mokbel, J. Jose, and L. Negadi, J. Mol. Liq. 201, 83 (2015).

2015BRU/IRR B. Brunetti, S. Irrera, and G. Portalone, J. Chem. Eng. Data 60, 74 (2015).

2015CAR/AMA T. M. T. Carvalho, L. M. P. F. Amaral, V. M. F. Morais, and M. D. M. C. Ribeiro da Silva, J. Chem. Thermodyn. 85, 129 (2015)

2015CAR/CON F. Cardoso de Matos, M. Conceicao da Costa, A. J. A. Meirelles, and E. A. C. Batista, Fluid Phase Equilib. 404, 1 (2015).

2015CAR/DUB A. Carletta, J. Dubois, A. Tilborg, and J. Wouters, CrystEngComm 17, 3509 (2015).

2015CHE/DU B. Chen, C. Du, H. Zhou, J. Wang, and L. Wang, J. Chem. Eng. Data 60, 1693 (2015).

2015CHE/KHA D. Cheuk, D. Khamer, P. McArdle, and A. C. Rasmuson, J. Chem. Eng. Data 60, 2110 (2015).

2015CHE/SVA D. Cheuk, M. Svard, C. Seaton, P. McArdle, and A. C. Rasmuson, CrystEngComm 17, 3985 (2015).

2015CHI/GUN T. Chimdi, D. Gunzelmann, J. Vongsvivut, and M. Forsyth, Solid State Ionics 272, 74 (2015).

2015CHI/KAZ R. D. Chirico and A. F. Kazakov, J. Chem. Thermodyn. 86, 90 (2015).

2015CHI/MED N. S. Chilingarov, A. A. Medvedev, G. S. Dekyo, L. M. Kustov, E. A. Chernikova, L. M. Glukhov, V. Yu Markov, I. N. Ioffe, V. M. Senyavin, M. V. Polyakova, and L. N. Sidorov, Rapid Commun. Mass Spectrom. 29, 1227 (2015).

2015 CHI/STE R. D. Chirico, W. V. Steele, and A. F. Kazakov, J. Chem. Thermodyn. 86, 106 (2015).

2015DIB/SOB V. Dibrivnyl, I. Sobechko, M. Puniak, Y. Horak, M. Obushak, Y. Van-Chin-Syan, M. Andriy, and N. Velychkivska, Chem. Cent. J. 9, 67/1 (2015).

2015DIN/YIN S. Ding, Q. Yin, W. Du, X. Sun, B. Hou, M. Zhang, and Z. Wang, J. Chem. Eng. Data 60, 1401 (2015).

2015DRU/PIM A. I. Druzhinina, S. M. Pimenova, S. V. Tarazanova, T. N. Nesterova, and R. M. Varushchenko, J. Chem. Thermodyn. 87, 69 (2015).

2015DUN/KUD A. M. Dunaev, L. S. Kudin, V. B. Motalov, D. A. Ivanov, M. F. Butman, and K. W. Kramer, Thermochim. Acta 622, 82 (2015).

2015EFI/PFU A. Efimova, L. Pfutzner, and P. Schmidt, Thermochim. Acta 604, 129 (2015).

2015EME/PIM V. N. Emel'yanenko, A. A. Pimerzin, V. V. Turovtsev, and S. P. Verevkin, J. Phys. Chem. A 119, 2142 (2015).

2015EME/TUR V. N. Emel'yanenko, V. V. Turovtsev, and Y. D. Orlov, Russ. J. Phys. Chem. A 89, 1146 (2015).

2015EME/VAR V. N. Emel'yanenko, M. A. Varfolomeev, S. P. Verevkin, K. Stark, K. Muller, M. Muller, A. Bosmann, P. Waserscheid, and W. Ailt, J. Phys. Chem. C 119, 26381 (2015).
2015EME/VER

V. N. Emel'yanenko and S. P. Verevkin, J. Chem. Thermodyn. 85, 111 (2015).

2015EME/VER2 V. N. Emel'yanenko, S. P. Verevkin, and R. Notario, J. Chem. Thermodyn. 87, 129 (2015).

2015EME/VER3 V. N. Emel'yanenko and S. P. Verevkin, Russ. J. Phys. Chem. A 89, 1740 (2015).

2015EME/ZAI V. N. Emel'yanenko, K. V. Zaitseva, F. Agapito, J. A Martinho, and S. P. Verevkin, J. Chem. Thermodyn. 85, 155 (2015).

2015EME/ZAI2 V. N. Emel'yanenko, D. H. Zaitsau, E. Shoifet, F. Meurer, S. P. Verevkin, C. Schick, and C. Held, J. Phys. Chem. A 119, 9680 (2015).

2015ERM/SYS E. N. Ermakova, S. V. Sysoev, L. D. Nikulina, I. P. Tsyrendorzhieva, V. I. Rakhlin, and M. L. Kosinova, Thermochim. Acta 622, 2 (2015).

2015FAN/ZHA J. Fang, M. Zhang, P. Zhu, J. Ouyang, J. Gong, W. Chen, and F. Xu, J. Chem. Thermodyn. 85, 202 (2015).

2015FER/DAL V. Ferretti, A. Dalpiaz, V. Bertolassi, L. Ferraro, S. Beggiato, F. Spizzo, E. Spisni, and B. Pavan, Mol. Pharm. 12, 1501 (2015).

2015FRE/SAO T. P. P. Freire, A. Sao Pedro, R. Fialho, E. C. Albuquerque, A. Bertucco, and G. M. N. Costa, J. Chem. Thermodyn. 88, 144 (2015).

2015FRI/RAF T. E. Frizon, J. Rafique, S. Saba, I. H. Bechtold, H. Gallardo, and A. L. Braga, Eur. J. Org. Chem. 2015, 3470.

2015GAO/XUE X. Gao, W.-L. Xue, Z.-X. Zeng, and X.-R. Fan, J. Chem. Eng. Data 60, 2273 (2015).

2015GAU/VAN N. Gautschi, P. Van Hoogevest, and M. Kuentz, Int. J. Pharm. 491, 218 (2015).

2015GIA/PAL C. Giacobbe, G. Palmisano, G. B. Giovenzana, L. Giovannelli, R. Negri, and N. Masciocchi, J. Pharm. Biomed. Anal. 102, 305 (2015).

2015GOB/CHI C. Gobble and J. S. Chickos, J. Chem. Eng. Data 60, 2739 (2015).

2015HAS/NEG $\quad$ B. B. Hassine, P. Negrier, M. Barrio, D. Mondieig, S. Massip, and J. L. Tamarit, Cryst. Growth Des. 15, 4149 (2015).

2015HIK/WEE W. M. Kikal and B. L. Weeks, J. Therm. Anal. Calorim. 122, 1055 (2015).

2015HUA/JIA Z. Huang, H. Jiang, L. Li, H. Wang, and T. Qui, J. Chem. Thermodyn. 86, 75 (2015).

2015HUA/WAN X. Huang, J. Wang, H. Hao, J. Ouyang, Y. Gao, Y. Bao, Y. Wang, and Q. Yin, Fluid Phase Equilib. 394, 148 (2015).

2015IIZ/SHI A. Iizuka, E. Shibata, M. Sato, and T. Nakamura, Thermochim. Acta 622, 103 (2015).

2015JAK/SPO M. Jakubczk, A. Sporznski, V. N. Emel'yanenko, M. A. Varfolomeev, and S. P. Verevkin, Thermochim. Acta 615, 88 (2015).

2015JIA/CAO C. Jia, Y. Cao, T.Zuo, R. Hu, T. Yao, and H. Song, J. Chem. Eng. Data 60, 999 (2015).

2015KAB/PAU G. J. Kabo, Y. U. Paulechka, O. V. Voitkevich, A. V. Blokhin, E. N. Stepurko, S. V. Kohut, and Y. V. Voznyi, J. Chem. Thermodyn. 85, 101 (2015).

$2015 \mathrm{KON} / \mathrm{BUT} \quad$ Y. V. Kondrat'ev, A. V. Butlak, I. V. Kazakov, and A. Y. Timoshkin, Thermochim. Acta 622, 64 (2015).

2015KOZ/GOB M. Kozlovskiy, C. Gobble, and J. S. Chickos, J. Chem. Thermodyn. 86, 65 (2015).

2015KUM/THI $\quad$ D. Kumar, R. Thipparaboina, S. R. Modi, A. K. Bansal, and N. R. Shastri, J. Cryst. Growth 422, 44 (2015).

2015LI/DU R.-R. Li, C.-B. Du, S. Han, J. Wang, G.-B. Yao, and H.-K. Zhao, Fluid Phase Equilib. 396, 35 (2015).

2015LI/WAN W. Li, J. Wang, G. Yao, and H. Zhao, J. Solution Chem. 44, 2042 (2015).

2015LI/YAN H.-Y. Li, B. Yan, K.-Q. Bai, H. Liu, H.-X. Ma, J.-R. Song, and L. Yan, J. Chem. Thermodyn. 91, 240 (2015).

2015LIU/SVA J. Liu, M. Svard, P. Hippen, and A. C. Rasmuson, J. Pharm. Sci. 104, 2183 (2015).

2015LU/SON J. Lu, H. Song, Y. Yang, G. Qian, L. Nie, and S. Yao, J. Mol. Liq. 209, 648 (2015).

2015LUO/JEN J. Luo, A. H. Jensen, N. R. Brooks, J. Sniekers, M. Knipper, D. Ailli, Q. Li, B. Vanroy, M. Wubbenhorst, F. Yan, L. Van Meervelt, Z. Shao, J. Fang, Z.-H. Luo, D. E. De Vos, K. Binnemans, and J. Fransaer, Energy Environ. Sci. 8, $1276(2015)$. 
2015MAD/SWA D. Maddileti, D. Swapna, and A. Nangia, Cryst. Growth Des. 15, 1745 (2015).

2015MAR/CAM M. Martinez-Herrera, M. Campos, L. A. Torres, and A. Rojas, Thermochim. Acta 622, 72 (2015).

2015MAR/PIN D.-C. Marinescu, E. Pincu, P. Oancea, G. Bruni, A. Marini, and V. Meltzer, J. Therm. Anal. Calorim. 120, 829 (2015).

2015MAT/MOR H. Matsuda, K. Mori, M. Tomioka, K. Kariyasu, T. Fukami, K. Kurihara, K. Tochigi, and K. Tomono, Fluid Phase Equilib. 406, 116 (2015).

2015MBA/KNO J. Mbah, D. Knott, S. Steward, and D. Cornett, Int. J. Energ. Mater. Chem. Propul. 14, 321 (2015).

2015MEN/XU Q. Y. Meng, D. Xu, Y. Y. Di, Z. F. Gao, and J. M. Dou, J. Therm. Anal. Calorim. 119, 1923 (2015).

2015MOR/DAS P. Morgado, G. Das, C. McCabe, and E. J. M. Filipe, J. Phys. Chem. B 119, 1623 (2015).

2015MUS/MAT U. M. Musazzi, C. Matera, C. Dallanoce, F. Vacondio, M. De Amici, G. Vistoli, F. Cilurzo, and P. Minghetti, Int. J. Pharm. 489, 177 (2015).

2015NAT/JES C. Nather, I. Jess, L. Seyfarth, K. Barwinkel, and J. Senker, Cryst. Growth Des. 15, 366 (2015).

2015NAT/USH V. Natarajan, S. Usharani, M. Arivanandhan, P. Anandan, and Y. Hayakawa, Spectrochim. Acta, Part A 145, 329 (2015).

2015NEM/KOF F. Nemoto, M. Kofu, and O. Yamanuro, J. Phys. Chem. B 119, 5028 (2015).

2015NOK/HOM A. Nokhodchi, A. Homayouni, R. Araya, W. Kaialy, W. Obeidat, and K. Asare-Addo, RSC Adv. 5, 46119 (2015).

2015NUR/BOO K. Nurzynska, J. Booth, C. J. Roberts, J. McCabe, I. Dryden, and P. M. Fisher, Mol. Pharm. 12, 3389 (2015).

2015 OLI/SAN J. A. S. A. Oliveira, A. F. L. O. M. Santos, M. D. M. C. Ribeiro da Silva, and M. J. S. Monte, J. Chem. Thermodyn. 89, 134 (2015).

2015OUY/WAN J. Ouyang, J. Wang, X. Huang, Y. Bao, Y. Wang, Q. Yin, A. Liu, X. Li, and H. Hao, J. Chem. Thermodyn. 91, 73 (2015).

2015OWU/CHE S. K. Owusu-Ware, A. J. Cherry, C. B. Baltus, J. Spencer, and M. D. Antonijevic, J. Therm. Anal. Calorim. 121, 437 (2015).

2015PAN/MEH R. B. Pandya, T. A. Mehta, and M. C. Gohel, Int. J. Pharm. Sci. Res. 6, 4236 (2015).

2015PAS/MIR L. L. Pashchenko and E. A. Miroshnichenko, J. Therm. Anal. Calorim. 120, 1375 (2015).

2015PAT/JAG J. Patel, M. Jagia, A. K. Bansal, and S. Patel, J. Pharm. Sci. 104, 3722 (2015).

2015PAT/PAT S. V. Patel and S. Patel, Eur. J. Pharm. Sci. 77, 161 (2015).

2015PAU/HAR R. Paus, E. Hert, Y. Ji, and G. Sadowski, J. Chem. Eng. Data 60, 2256 (2015).

2015PEG/CHI C. N. Pegoraro, M. S. Chiappero, and H. A. Montejano, Chemosphere 138, 546 (2015).

2015PER/CAS P. S. Pereira Silva, R. A. E. Castro, E. Melro, M. R. Silva, T. M. R. Maria, J. Canotilho, and M. E. S. Eusebio, J. Therm. Anal. Calorim. 120, 667 (2015).

2015PFU/CHA L. Y. Pfund, B. L. Chamberlin, and A. J. Matzger, Cryst. Growth Des. 15, 2047 (2015).

2015POB/DOM A. Pobudkowska, U. Domanska, B. A. Jurkowska, and K. Dymczuk, Fluid Phase Equilib. 392, 56 (2015).

2015POB/JUR A. Pobudkowska, B. A. Jurkowska, and M. A. Wiatrowski, Fluid Phase Equilib. 406, 209 (2015).

2015PRI D. M. Price, Thermochim. Acta 622, 44 (2015)

2015QI/HON M.-H. Qi, M.-H. Hong, Y. Liu, E.-F. Wang, F. Ren, and G. Ren, Cryst. Growth Des. 15, 5062 (2015).

2015QIU/LU X. Qiu, L. Lu, J. Wang, G. Tang, and G. Song, Thermochim. Acta 620, 10 (2015).

2015RAM/CHI $\quad$ S. L. L. M. Ramos, A. K. Chigira, and M. Oguni, J. Phys. Chem. B 119, 4076 (2015).

2015RAM/MAR M. Ramos-Riesco, F. J. Martinez-Casado, J. A. R. Cheda, M. I. R. Yelamos, A. Fernandez-Mertinez, and S. LopezAndres, Cryst. Growth Des. 15, 497 (2015).

2015RIC/FU J. W. Rice, J. Fu, E. Sandstrom, J. C. Ditto, and E. M. Suuberg, J. Chem. Thermodyn. 90, 79 (2015).

2015ROJ/VAL A. Rojas, A. Valdes-Ordonez, M. Martinez-Herra, L. A. Torres, M. Campos, J. Hernandez-Obregon, R. Herrera, and J. Tamariz, J. Phys. Chem. A 119, 4953 (2015).
2015ROT/VRB

J. Rotrekl, P. Vrbka, Z. Sedlakova, Z. Wagner, J. Jacquemin, and M. Bendova, Pure Appl. Chem. 87, 453 (2015).

2015RUZ/GON V. Ruz, M. M. Gonzalez, D. Winant, Z. Rodriguez, and G. Van den Mooter, Molecules 20, 15175 (2015).

2015SAN/OLI A. F. L. O. M. Santos, J. A. S. A. Oliveira, and M. J. S. Monte, J. Chem. Thermodyn. 90, 282 (2015).

$2015 \mathrm{SCH} / \mathrm{HAR} \quad$ S. M. Schultz, H. H. Harris, and J. S. Chickos, J. Chem. Thermodyn. 89, 306 (2015).

2015SHA/GE X. Shao, H. Ge, Z. Li, C. Ren, and J. Wang, Fluid Phase Equilib. 390, 7 (2015).

2015SIN/RAI M. Singh, R. N. Rai, and U. S. Rai, J. Cryst. Growth 419, 114 (2015).

2015SIN/SIN $\quad$ J. Singh and N. B. Singh, Fluid Phase Equilib. 386, 168 (2015).

2015SIN/SIN2 J. Singh and N. B. Singh, J. Chem. Thermodyn. 89, 197 (2015).

2015SOB/VAN I. B. Sobechko, Y. Y. Van-Chin-Syan, Y. I. Gorak, V. V. Kochubei, R. T. Prokop, N. I. Velychkivska, Y. I. Gorak, V. N. Dibrivnyi, and M. D. Obushak, Russ. J. Phys. Chem. 89, 919 (2015).

2015SOL/ROD I. A. Solomina, M. N. Rodnikova, M. P. Kiselev, and A. V. Khoroshilov, Russ. J. Phys. Chem. 89, 910 (2015).

2015SOL/VAR B. N. Solomonov, M. A. Varfolomeev, R. N. Nagrimanov, V. B. Norikov, M. A. Ziganshin, A. V. Gerasimov, and S. P. Verevkin, J. Chem. Eng. Data 60, 748 (2015).

2015SON/LI Y. Song, J. Q. Li, X. Sun, Z. Feng, Y. Lin, J. Wang, J. Ding, H. Zhang, and W. Li, Fluid Phase Equilib. 388, 16 (2015).

2015STA/EME K. Stark, V. N. Emel'yanenko, A. A. Zhabina, M. A Varfolomeev, S. P. Verevkin, K. Muller, and W. Arlt, Ind. Eng. Chem. Res. 54, 7953 (2015).

2015STE/DER V. Stejfa, F. Dergal, I. Mokbel, M. Fulem, J. Jose, and K. Ruzicka, Fluid Phase Equilib. 406, 124 (2015).

2015STE/FUL V. Stejfa, M. Fulem, K. Ruzicka, and P. Matejka, Fluid Phase Equilib. 402, 18 (2015)

2015SUN/YIN X. Sun, Q. Yin, S. Ding, Z. Shen, Y. Bao, J. Gong, B. Hou, H. Hao, Y. Wang, J. Wang, and C. Xie, J. Chem. Thermodyn. 85, 171 (2015).

2015SUR/SIM A. O. Surov, A. A. Simagina, N. G. Manin, L. G. Kuzmina, A. V. Churakov, and G. L. Perlovich, Cryst. Growth Des. 15, 228 (2015).

2015TAN/DAI W. Tang, H. Dai, Y. Feng, S. Wu, Y. Bao, J. Wang, and J. Gong, J. Chem. Thermodyn. 90, 28 (2015).

2015TIP/TAK P. Tipduangta, K. Takieddin, L. Fabian, P. Belton, and S. Qi, Cryst. Growth Des. 15, 5011 (2015).

2015TRO/BUL O. Y. Troshin, A. D. Bulanov, T. G. Sorochkina, and A. N. Kolesnikov, Inorg. Mater. 51, 718 (2015).

2015VEL/KHA C. Velez, M. Khayet, and J. M. Ortiz de Zarate, Appl. Energy 143, 383 (2015).

2015VEL/ORT C. Velez, J. M. Ortiz de Zarate, and M. Khayet, Int. J. Therm. Sci. 94, 139 (2015).

2015VER/EME S. P. Verevkin, V. N. Emel'yanenko, M. A. Varfolomeev, B. N. Solomonov, K. V. Zherikova, and S. V. Melkhanova, Fluid Phase Equilib. 387, 160 (2015).

2015VER/EME2 S. P. Verevkin, V. N. Emel'yanenko, and I. V. Garist, J. Chem. Thermodyn. 87, 34 (2015).

2015VER/EME3 S. P. Verevkin and V. N. Emel'yanenko, Thermochim. Acta 608, 40 (2015).

2015VER/SAZ S. P. Verevkin, A. Y. Sazonova, V. E. Emel'yanenko, D. H. Zaitsau, M. A. Varfolomeev, B. N. Solomonov, and K. V. Zerkikova, J. Chem. Eng. Data 60, 89 (2015).

2015VER/ZAI S. P. Verevkin, D. H. Zaitsau, V. E. Emel'uanenko, E. N. Stepurko, and K. S. Zherikova, Thermochim. Acta 622, 18 (2015).

2015VER/ZAI2 S. P. Verevkin, D. H. Zaitsau, V. N. Emel'yanenko, and A A. Zhabina, Fluid Phase Equilib. 397, 87 (2015).

2015VER/ZAI3 S. P. Verevkin, K. V. Zaitseva, A. D. Stanton, M. S. Hindman, A. C. Irvin, and J. E. Bara, Ind. Eng. Chem. Res. 54, 9850 (2015).

2015VIT/AGA J. Vitorino, F. Agapita, C. E. S. Bernardes, and M. E. Minas da Piedade, J. Chem. Thermodyn. 80, 59 (2015).

2015WAN/DON X. Wang, H. Dong, Z. Zeng, and C. Wu, J. Solution Chem. 44, 67 (2015). 
2015WAN/DU L. Wang, C. Du, X. Wang, H. Zeng, J. Yao, and B. Chen, J. Chem. Eng. Data 60, 1814 (2015).

2015WAN/LI ～L. Y. Wang, X.-C. Li, L. Zhu, Z.-L. Sha, Y.-F. Wang, and L.-B. Yang, J. Mol. Liq. 208, 211 (2015).

2015WAN/WAN C. Wang, Y. Wang, Q. Yin, Z. Xu, Y. Bao, B. Hou, W. Liu, and H. Hao, J. Chem. Eng. Data 60, 3053 (2015).

2015WEI/BU J. Wei, X. Bu, W. Guan, N. Xing, D. Fang, and Y. Wu, RSC Adv. 5, 70333 (2015).

2015WIL/GOB J. Wilson, C. Gobble, and J. Chickos, J. Chem. Eng. Data 60, 202 (2015).

2015WU/DON X. Wu, H. Dong, X. Wang, Z. Zeng, and C. Wu, J. Chem. Eng. Data 60, 3106 (2015).

2015YAN/WAN X. Yang, J. Wang, W. Zou, and J. Wu, Korean J. Chem. Eng. 32, 2369 (2015).

2015YAN/WU J. Yang, H. Wu, Y. Wang, Q. Luan, J. Zhang, G. Wang, and H. Hao, J. Chem. Thermodyn. 83, 77 (2015).

2015YAN/YIN J. Yan, Q. Yin, C. Jiang, J. Gong, M. Zhang, Y. Wang, B. Hou, and H. Hao, Fluid Phase Equilib. 406, 77 (2015).

2015YER/ZAI A. V. Yermalayeu, D. H. Zaitsau, V. N. Emel'yanenko, and S. P. Verevkin, J. Solution Chem. 44, 754 (2015).

2015YU/WAN G.-M. Yu, L.-S. Wang, C.-J. Du, and J. Sun, Fluid Phase Equilib. 388, 6 (2015).

2015 YU/WAN2 G.-M. Yu, L.-S. Wang, C.-J. Du, and J. Sun, Fluid Phase Equilib. 396, 50 (2015).

2015ZAI/EME K. V. Zaitseva, V. N. Emel'yanenko, F. Agapito, A. A. Pimerzin, M. A. Varfolomeev, and S. P. Verevkin, J. Chem. Thermodyn. 91, 186 (2015).

2015ZAI/PAU D. Zaitsau, E. Paulechka, D. S. Firaha, A. V. Blokhin, F. J. Kabo, A. Bazyleva, A. G. Kabo, M. A. Varfolomeev, and V. M. Sevruk, J. Chem. Thermodyn. 91, 459 (2015).

$2015 Z A I / V E R \quad$ D. H. Zaitsau, S. P. Verevkin, and A. Y. Sazonova, Fluid Phase Equilib. 386, 140 (2015).

2015ZHA/CAI P. Zhang, S. Cai, L. Song, L. Zhang, H. Fan, L. Zhou, R. Lin, G. Yang, X. Bian, W. Wang, and J. Zhang, J. Mol. Liq. 211, 197 (2015).

2015ZHA/DU Q.-A. Zhang and C.-J. Du, J. Mol. Liq. 211, 527 (2015).

2015ZHA/JIN L. Zhang, H. Jing, W. Zhu, F.-B. Zhang, G.-L. Zhang, and Q. Xia, J. Chem. Thermodyn. 80, 142 (2015).

2015ZHA/LIU Y. Zhang, J. J. Liu, L. Zhang, and X. Z. Wang, J. Chem. Eng. Data 60, 1968 (2015).

2015ZHA/OUY H. Zhang, J. Ouyang, J. Zhang, Y. Xiao, X. Huang, H. Hao, Y. Wang, and Q. Yin, J. Chem. Eng. Data 60, 740 (2015).

2015ZHA/QIA X. Zhang, G. Qian, R. Wang, X. Yang, L. Hao, H. Wei, and X. Zhou, Fluid Phase Equilib. 388, 59 (2015).

2015ZHA/ZHU H. Zhao, X. Zhu, D. Wang, S. Chen, and Z. Bian, Aust. J. Chem. 68, 1035 (2015).

2015ZHU/WAN L. Zhu, L.-Y. Wang, X.-C. Li, Z. L. Sha, Y.-F. Wang, and L.-B. Yang, J. Chem. Thermodyn. 91, 369 (2015).

2016ABI/ARM A. O. Abioye, R. Armitage, and A. T. Kola-Mustapha, Pharm. Res. 33, 337 (2016); Erratum, 33, 804 (2016).

2016ACR/CHI $\quad$ W. E. Acree, Jr. and J. S. Chickos, J. Phys. Chem. Ref. Data 45, 033101 (2016).

2016ALM/MON A. R. R. P. Almeida and M. J. S. Monte, J. Chem. Thermodyn. 92, 118 (2016).

2016AMA/DEG F. Aman-Pommier, G. Degobert, and C. Jallut, Fluid Phase Equilib. 408, 284 (2016).

2016AMB/CER B. Ambrozini, P. Cervini, and E. T. G. Cavalheiro, J. Therm. Anal. Calorim. 123, 1013 (2016).

2016BAR/SZT A. Bartyzel, M. Sztanke, and K. Sztanke, J. Therm. Anal. Calorim. 123, 2053 (2016).

2016BOU/DJE I. Boudouh, I. Djemai, J. A. Gonzalez, and D. Barkat, J. Mol. Liq. 216, 764 (2016).

2016BOU/HAF I. Boudouh, S. L. Hafsaoui, R. Mahmoud, and D. Barkat, J. Therm. Anal. Calorim. 125, 793 (2016).

2016BRU/LAP B. Brunetti, A. Lapri, and S. V. Ciprioti, Thermochim. Acta 636, 71 (2016).

2016CAR/AMA T. M. T. Carvalho, L. M. P. F. Amaral, V. M. F. Morais, and M. D. M. C. Ribeiro da Silva, J. Chem. Thermodyn. 97, 70 (2016).

2016CAR/CON F. Cardoso de Matos, M. C. Conceicao da Costa, A. J. D. Meirelles, and E. A. C. Bastista, Fluid Phase Equilib. 423, 74 (2016).
2016CAV/TAR

C. Cavallari, F. Tarterini, and A. Fini, Thermochim. Acta 633, 129 (2016).

2016DAV/GUE J. Z. Davalos, A. Guerrero, A. C. Valderrama-Negron, V. Romero, and A. F. Lago, J. Chem. Thermodyn. 97, 315 (2016).

2016DEL/ALM D. R. Delgado, O. A. Almanza, F. Martinez, M. A. Pena, A. Jouyban, and W. E. Acree, Jr., J. Chem. Thermodyn. 97, 264 (2016).

2016DIO/VIC H. P. Diogo, M. T. Viciosa, and J. J. Moura Ramos, Thermochim. Acta 623, 29 (2016)

2016DU/XU C. Du, R. Xu, S. Han, J. Xu, L. Meng, J. Wang, and H. Zhao, J. Chem. Thermodyn. 96, 187 (2016).

2016DUN/MOT A. M. Dunaev, V. B. Motalov, L. S. Kudin, and M. F. Butman, J. Mol. Liq. 219, 599 (2016).

2016EME/YER V. N. Emel'yanenko, A. V. Yermalayeu, M. Voges, C. Held, G. Sadowski, and S. P. Verevkin, Fluid Phase Equilib. 422, 99 (2016).

2016FAG/DES $\quad$ D. P. Fagnant, Jr., M. A. Desilva, and J. F. Brenneck, J. Chem. Eng. Data 61, 958 (2016).

2016GOB/WAL C. Gobble, B. Walker, and J. S. Chickos, J. Chem. Eng. Data 61, 896 (2016).

2016GON/ZHA N. Gong, G. Zhang, G. Jin, G. Du, and Y. Lu, J. Pharm. Sci. 105, 1387 (2016).

2016GOO/HAS S. Goodrich, J. Hasanovic, C. Gobble, and J. S. Chickos, J. Chem. Eng. Data 61, 1524 (2016).

2016HAN/MEN S. Han, L. Meng, C. Du, J. Xu, C. Cheng, J. Wang, and H. Zhao, J. Chem. Thermodyn. 97, 17 (2016).

2016HE/WAN F. He, Y. Wang, Q. Yin, L. Tao, J. Lv, Z. Xu, J. Wang, and H. Hao, Fluid Phase Equilib. 422, 56 (2016).

2016KOL/TAR K. Kolodziejczyk, M. Tarnacka, E. Kaminska, M. Dulski, K. Kamisnski, and M. Paluch, Cryst. Growth Des. 16, 1218 (2016).

2016LED/CAM J. M. Ledo, E. A. Camarillo, H. Flores, F. Ramos, and A. Rojas, J. Therm. Anal. Calorim. 123, 2391 (2016).

2016LI/CHE W. Li, J. Chen, S. Han, C. Du, and H. Zhao, J. Mol. Liq. 216, 771 (2016).

2016LIS/FAR $\quad$ L. C. Liston, Y. Farnam, M. Krafcik, J. Weiss, K. Erk, and B. Y. Tao, Appl. Therm. Eng. 96, 501 (2016).

2016MAX/AQU G. J. Maximo, R. T. Aquino, A. J. A. Meirelles, M. A. Krahenbuhl, and M. C. Costa, Fluid Phase Equilib. 426, 119 (2016).

2016MOO/RAR K. Moodley, J. Rarey, and D. Ramjugernath, J. Chem. Thermodyn. 98, 42 (2016).

2016OLI/FRE T. S. M. Oliveira, V. L. S. Freitas, and M. D. M. C. Ribeiro da Silva, J. Chem. Thermodyn. 94, 7 (2016).

20160ST/UUS A. Ostonen, P. Uusi-Kyyny, M. Pakkanen, and V. Olopaeus, Fluid Phase Equilib. 408, 79 (2016).

2016PAS/ABA K. I. Pashanova, G. A. Abakumov, A. V. Markin, A. V. Piskunov, and N. N. Smirnova, J. Chem. Thermodyn. 92, 76 (2016).

2016PAU/BLO E. Paulechka, A. V. Blokhin, A. S. M. C. Rodrigues, M. A. A. Rocha, and L. M. N. B. F. Santos, J. Chem. Thermodyn. 97, 331 (2016).

2016PER/VOL G. L. Perlovich, T. V. Volkova, A. V. Sharapova, V. P. Kazachenko, N. V. Strakhova, and A. N. Proshin, Phys. Chem. Chem. Phys. 18, 9281 (2016).

2016RIC/DEL J.-V. Richard, C. Delaite, G. Riess, and A.-S. Schuller, Thermochim. Acta 623, 136 (2016).

2016SAN/CRU T. Sanchez-Bulas, O. Cruz-Vasquez, J. HernandezObregon, and A. Rojas, "Enthalpies of fusion, vaporisation and sublimation of crown ethers determined by thermogravimetry and differential scanning calorimetry," Thermochim. Acta (published online).

2016SAN/OLI A. F. L. O. M. Santos, J. A. S. A. Oliveira, M. D. M. C. Ribeiro da Silva, and M. J. S. Monte, Chemosphere 146, 173 (2016).

2016SER/RIB $\quad$ P. B. P. Serra, F. M. S. Ribeiro, M. A. A. Rocha, M. Fulem, K. Ruzicka, and L. M. N. B. F. Santos, J. Chem. Thermodyn. 100, 124 (2016).

2016SHA/GAN P. Sharma, D. Gangopadhyay, S. Umrao, S. Kumar, A. K Gosh, P. C. Mishra, and R. K. Singh, J. Raman Spectrosc. 47, 468 (2016). 
2016SHI/QIA Y.-L. Shi, C. Qian, and X.-Z. Chen, J. Chem. Eng. Data 61, 1509 (2016).

2016SIM/GOB $\quad$ D. Simmons, C. Gobble, and J. Chickos, J. Chem. Thermodyn. 92, 126 (2016).

2016STA/KEI K. Stark, P. Keil, S. Schug, K. Muller, P. Wasserscheid, and W. Arlt, J. Chem. Eng. Data 61, 1441 (2016).

2016SUN/LIU J. Sun, X. Liu, Z. Fang, S. Mao, L. Zhang, S. Rohani, and J. Lu, J. Chem. Eng. Data 61, 495 (2016).

2016TAN/SHI N. Tang, W. Shi, and W. Yan, J. Chem. Eng. Data 61, 35 (2016).

2016VOL/BLO T. V. Volkova, S. V. Blokhina, A. M. Ryzhakov, A. V. Sharapova, M. V. Ol'khovich, and G. L. Perlovich, J. Therm. Anal. Calorim. 123, 841 (2016).

2016WAN/YAO D.-C. Wang, S. Yao, Y. Cao, T. Yao, and H. Song, J. Chem. Thermodyn. 92, 55 (2016).

2016XU/WAN J. Xu, Y. Wang, G. Wang, C. Huang, H. Hao, and Q. Yin, J. Chem. Thermodyn. 92, 12 (2016).

2016 XU/WAN2 R. Xu, J. Wang, C. Du, S. Han, L. Meng, and H. Zhao, J. Chem. Thermodyn. 99, 86 (2016).

2016XUE/GUR L. Xue, E. Gurung, G. Tamas, Y. P. Koh, M. Shadeck, S. L. Simon, M. Maroncelli, and E. L. Quintevis, J. Chem. Eng. Data 61, 1078 (2016).
2016YOU/WAN

2016ZAI/VAR

2016ZHA/FAN

2016ZHA/WAN

2016ZHE/SVE

2016ZHO/ZHA

2016ZHU/CHE

$\mathrm{UR} / \mathrm{CHI}$

UR/DEK

$\mathrm{UR} / \mathrm{MAC}$

$\mathrm{UR} / \mathrm{STU}$

UR/VER
K. You, B. Wang, S. Chen, and D. Wei, J. Chem. Eng. Data 61, 1205 (2016).

D. M. Zaitsau, M. A. Varafolomeev, S. P. Verevkin, A. D. Stanton, M. S. Hindman, and J. E. Baro, J. Chem. Thermodyn. 93, 151 (2016).

M. Zhang, Z. Fang, J. Zhai, S. Mao, L. Zhang, S. Rohani, and J. Lu, J. Chem. Thermodyn. 94, 1 (2016).

F. Zhang, L. Wang, Y. Tang, X. Gao, L. Xu, and G. Liu, Fluid Phase Equilib. 409, 98 (2016).

K. V. Zherikova, A. A. Svetlov, M. A. Varfolomeev, S. P. Verevkin, and C. Held, Fluid Phase Equilib. 409, 399 (2016).

G. Zhou, Y. Zhang, Y. You, H. Chen, Q. Zhou, A. Zhong, and R. Li, J. Chem. Eng. Data 61, 636 (2016).

P. Zhu, Y. Chen, J. Fang, Z. Wang, C. Xie, B. Hou, W. Chen, and F. Xu, J. Chem. Thermodyn. 92, 198 (2016).

J. S. Chickos (unpublished).

C. G. de Kruif (unpublished).

H. Mackle, W. V. Steele, and D. V. McNally (unpublished).

D. Stull (unpublished).

S. P. Verevkin, E. Krasnykh, T. Vasiltsova, and A. Heintz, unpublished data as quoted in Ref. [2002VER] 